\title{
International Workshop on the Physics of Compressible Turbulent Mixing
}

Edited By: O. Schilling

November 28, 2001

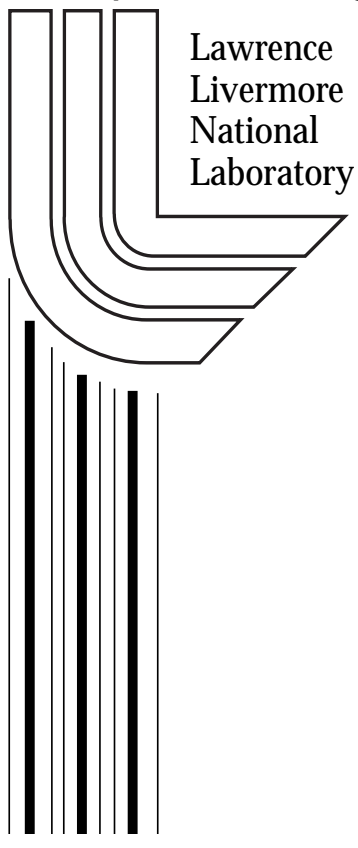


$8^{\text {th }}$ International Workshop on the Physics of Compressible Turbulent Mixing,

Pasadena, CA (2001)

\section{Disclaimer and Auspices}

This document was prepared as an account of work sponsored by an agency of the United States Government. Neither the United States Government nor the University of California nor any of their employees, makes any warranty, express or implied, or assumes any legal liability or responsibility for the accuracy, completeness, or usefulness of any information, apparatus, product, or process disclosed, or represents that its use would not infringe privately owned rights. Reference herein to any specific commercial product, process, or service by trade name, trademark, manufacturer, or otherwise, does not necessarily constitute or imply its endorsement, recommendation, or favoring by the United States Government or the University of California. The views and opinions of authors expressed herein do not necessarily state or reflect those of the United States Government or the University of California, and shall not be used for advertising or product endorsement purposes.

This work was performed under the auspices of the U.S. Department of Energy by University of California, Lawrence Livermore National Laboratory under Contract W-7405-Eng-48. 
$8^{\text {th }}$ International Workshop on the Physics of Compressible Turbulent Mixing,

Pasadena, CA (2001)

\section{Instructions for use of the CD-ROM}

\section{To View Presentations}

To view the contributed, unedited presentations from the $8^{\text {th }}$ International Workshop on the Physics of Compressible Turbulent Mixing, open the PDF document CONTENTS.PDF and click with the cursor on the presentation number, animation, or title (for the Summary and Review Talks): this will open the corresponding presentation in Adobe ${ }^{\circledR}$ Portable Document Format (PDF), graphic (gif format), or animation ( $\mathrm{avi}$ format). See below to obtain freely-downloadable software to view these files.

The summary presentation "Summary of the $8^{\text {th }}$ International Workshop on the Physics of Compressible Turbulent Mixing (9-14 December 2001, Pasadena, CA)" (Schilling) can be viewed, and has links to all of the contributed presentations on the CD-ROM.

\section{To View the Abstract Book}

To view the Abstract Book in PDF, navigate to the bottom of CONTENTS.PDF and click on ABSTRACT BOOK.

\section{To View the Program Summary}

To view the Program Summary in PDF, navigate to the bottom of CONTENTS.PDF and click on PROGRAM SUMMARY.

\section{To View the Author Contact Information}

To view the Author Contact Information in PDF, navigate to the bottom of CONTENTS.PDF and click on AUTHOR CONTACT INFORMATION.

\section{To View the Photographs from the Banquet and Workshop}

To view the Photographs from the Banquet and Workshop in PDF, navigate to the bottom of CONTENTS.PDF and click on PHOTOGRAPHS FROM THE BANQUET AND WORKSHOP.

\section{To Obtain Adobe ${ }^{\circledR}$ Acrobat ${ }^{\circledR}$ Reader ${ }^{\circledR}$}

To obtain the freely available Adobe ${ }^{\circledR}$ Acrobat ${ }^{\circledR}$ Reader $®$, click on http://www.adobe.com/products/acrobat/readstep.html

\section{To Obtain RealOne ${ }^{\mathrm{TM}}$ Player}

To obtain the freely available RealOne ${ }^{\mathrm{TM}}$ Player for avi files, click on http://www.real.com/realoneplayer.html 
$8^{\text {th }}$ International Workshop on the Physics of Compressible Turbulent Mixing,

Pasadena, CA (2001)

\section{OVERVIEW}

\section{CD-ROM Table of Contents}

O. Schilling

Summary of the $8^{\text {th }}$ International Workshop on the Physics of Compressible Turbulent Mixing

\section{CONTRIBUTED REVIEW PRESENTATIONS}

J.-F. Haas and S. G. Zaytsev

Review on RTI, RMI and TM Experiments

\section{L. Youngs}

Review of Numerical Simulation of Mixing due to Rayleigh-Taylor and Richtmyer-Meshkov Instabilities

D. Kartoon, D. Oron, L. Arazi, A. Rikanati, O. Sadot, A. Yosef-Hai, U. Alon, G. Ben-Dor, and D. Shvarts

Three Dimensional Multi-Mode Rayleigh-Taylor and Richtmyer-Meshkov Instabilities at All Density Ratios (T14)

\section{CONTRIBUTED EXPERIMENTAL PRESENTATIONS}

Yu. A. Kucherenko, S. I. Balabin, R. I. Ardashova, O. E. Kozelkov, A. V. Dulov, and I. A. Romanov Experimental Study of the Influence of the Stabilizing Properties of Transitional Layers on the Turbulent Mixing Evolution (E3)

C. W. Barnes, S. H. Batha, A. M. Dunne, N. E. Lanier, G. R. Magelssen, T. J. Murphy, K. W. Parker, S. Rothman, J. M. Scott, and D. Youngs

Improvements to Convergent Cylindrical Plasma Mix Experiments Using Laser Direct Drive (E4)

S. H. Batha, K. W. Parker, C. W. Barnes, A. M. Dunne, N. E. Lanier, G. R. Magelssen, T. J. Murphy, S. Rothman, J. M. Scott, and D. L. Youngs

Mixing Between Two Compressing Cylinders (E5)

M. Bliznetsov, V. Dudin, S. Gerasimov, L. Houas, G. Jourdan, A. Logvinov, E. Meshkov, and Yu. Vlasov

Development of the Method for Interaction Between Shock Wave and Flame (E6)

M. V. Bliznetsov, E. E. Meshkov, N. V. Nevmerzhitzky, A. A. Nikulin, E. D. Sen'kovsky, and E. A. Sotskov

Influence of Scales of Initial Perturbations on Rayleigh-Taylor Instability Growth on Gas-Liquid Interface (E7)

M. Brouillette and C. H. Hebért

Formation and Propagation of Shock-Generated Vortex Rings (E8) 
R. P. Drake

Design of Flyer-Plate-Driven Compressible-Turbulent-Mix Experiments (E9)

R. P. Drake, P. Keiter, K. E. Korreck, K. Dannenberg, H. A. Robey, T. S. Perry, J. O. Kane, O. A. Hurricane, B. A. Remington, D. D. Ryutov, R. J. Wallace, J. Knauer, R. Teyssier, A. Calder, B. Fryxell, R. Rosner, Y. Zhang, J. Glimm, J. Grove, D. Arnett, R. McCray, N. Turner, and J. Stone,

Compressible Hydrodynamics on the Omega Laser: Motivated by Astrophysics (E10)

S. G. Glendinning, D. G. Braun, M. J. Edwards, W. W. Hsing, B. F. Lasinski, H. Louis, A. Miles, J. Moreno, T. A. Peyser, B. A. Remington, H. F. Robey, E. J. Turano, C. P. Verdon, and Y. Zhou An Experimental Study of the Effect of Shock Proximity on the Richtmyer-Meshkov Instability at High Mach Number (E12)

D. A. Holder, C. J. Barton, A. V. Smith, and D. L. Youngs

Mix Experiments Using a Two-Dimensional Convergent Shock Tube (E13)

J. M. Holford, S. B. Dalziel, and D. Youngs

Rayleigh-Taylor Instability at a Tilted Interface in Incompressible Laboratory Experiments and Compressible Numerical Simulations (E14)

S. H. R. Hosseini and K. Takayama

Production of Diverging and Converging Spherical Shock Waves and Eccentric Interaction of Converging Shock Waves with Cylindrical Interfaces (E15)

L. Houas, G. Jourdan, L. Schwaederle, and E. E. Meshkov

Linear and Non-Linear Stages of the Richtmyer-Meshkov Instability Development in a Large Cross Section Shock-Tube (E17)

J. W. Jacobs and V. V. Krivets

Experimental Investigation of Richtmyer-Meshkov Instability After a Second Interaction with a Reflected Shock Wave (E18)

D. H. Kalantar, J. Belak, J. D. Colvin, M. Kumar, K. T. Lorenz, K. O. Mikaelian, S. Pollaine, B. A. Remington, S. V. Weber, L. G. Wiley, J. S. Wark, A. Loveridge, A. M. Allen, M. A. Meyers, and M. Schneider Laser-Based High Pressure, High-Strain-Rate Materials Experiments (E19)

Yu. A. Kucherenko, O. E. Shestachenko, S. I. Balabin, and A. P. Pylaev

RFNC-VNIITF Multifunctional Shock Tube for Investigating the Evolution of Instabilities in Unstationary Gasdynamic Flows (E20)

G. Layes, G. Jourdan, P. Roualdes, and L. Houas

Hydrodynamic Instabilities at a Shock Accelerated Bubble Gas-Gas Interface (E22)

K. Levy, Sadot, A. Rikanati, D. Kartoon, Y. Srebro, A. Yosef-Hai, G. Ben-Dor, and D. Shvarts

Scaling in the Shock-Bubble Interaction (E23) 
$8^{\text {th }}$ International Workshop on the Physics of Compressible Turbulent Mixing,

Pasadena, CA (2001)

C. E. Niederhaus and J. W. Jacobs

Single-Mode Incompressible Richtmyer-Meshkov Instability Experiments (E26)

M. H. Anderson, J. G. Oakley, B. Puranik, and R. Bonazza

Experimental Study of a Strongly Shocked Gas Interface with Visualized Initial Conditions (E27 and animation)

Yu. A. Kucherenko, O. E. Shestachenko, Yu. A. Piskunov, E. V. Sviridov, V. M. Medvedev, and A. I. Baishev

Experimental Investigation into the Self-Similar Mode of Mixing of Different Density Gases in the Earth's Gravitational Field (E28)

S. Pollaine, D. Petersen, D. Kalantar, B. Remington, J. Belak, J. Colvin, M. Kumar, T. Lorenz, S. Weber, J. Wark, A. Loveridge, A. Allen, and M. Meyers

Modeling Laser Material Strength Experiments (E29)

K. Prestridge, C. Tomkins, C. Zoldi, M. Marr-Lyon, P. Vorobieff, P. Rightley, and R. Benjamin Experiments and Simulations of Instabilities in Shock-Accelerated Gas Cylinders (E30)

Yu. A. Kucherenko, A. P. Pylaev, V. D. Murzakov, A. V. Belomestnih, V. N. Popov, and A. A. Tyaktev

Experimental Study into Rayleigh-Taylor Turbulent Mixing Zone Heterogeneous Structure (E31)

\section{J. Andrews and P. Ramaprabhu}

Measurements of Turbulence Correlations in Low Atwood Number Rayleigh-Taylor Mixing (E32, animation1, animation2, and animation3)

H. F. Robey, T. S. Perry, R. I. Klein, J. A. Greenough, H. Louis, P. Davis, J. O. Kane, and T. R. Boehly

Experimental Study of the Interaction of a Strong Shock with a Spherical Density Inhomogeneity (E34)

H. F. Robey, Y. K. Zhou, A. C. Buckingham, P. Keiter, B. A. Remington, and R. P. Drake

Turbulent Transition in a High Reynolds Number, Rayleigh-Taylor Unstable Plasma Flow (E35)

O. Sadot, A. Rikanati, D. Oron, A. Yosef-Hai, G. Ben-Dor, and D. Shvarts

High Mach Number and High Initial Amplitude Effects on the Evolution of the Single-Mode RichtmyerMeshkov Instability-An Experimental Study (E36)

L. Schwaederlé, G. Jourdan, L. Houas, and J.-F. Haas

Measurements Within a Richtmyer-Meshkov Mixing Zone Using a Triple Hot Wire Probe Technique (E37)

Yu. A. Kucherenko, O. E. Shestachenko, Yu. A. Piskunov, E. V. Sviridov, V. M. Medvedev, and A. I. Baishev

Experimental Investigation into the Evolution of Turbulent Mixing of Gases by Using the Multifunctional Shock Tube (E38) 
A. V. Smith, D. A. Holder, C. J. Barton, A. P. Morris, and D. L. Youngs

Shock Tube Experiments on Richtmyer-Meshkov Instability Across a Chevron Profiled Interface (E39, animation1, and animation2)

C. Tomkins, K. Prestridge, P. Rightley, M. Marr-Lyon, R. Benjamin, J. Doyle, M. Schneider, P. Vorobieff, and C. Zoldi

The Evolution and Interaction of Two Shock-Accelerated, Unstable Gas Cylinders (E40)

R. I. Klein, H. Robey, T. Perry, J. Kane, J. Greenough, and M. Marinak

The Interaction of Supernova Blast Waves with Interstellar Clouds: Experiments on the Omega Laser (E42)

\section{H. Azechi et al.}

Ablative Rayleigh-Taylor Instability at Short Wavelengths (E45)

H. F. Robey, S. G. Glendinning, J. A. Greenough, and S. V. Weber

A Vortex Model for Studying the Effect of Shock Proximity on Richtmyer-Meshkov Instability at High Mach Number (E46)

\section{CONTRIBUTED COMPUTATIONAL PRESENTATIONS}

\section{G. Anuchin, V. E. Neuvazhayev, and I. E. Parshukov}

Application of Ke-Model for the Description of an Atmospheric Surface Layer (C2)

W. Ashurst and A. Kerstein

Computational Modeling of Low-Mach-Number High-Atwood-Number Turbulent Mixing (C4)

A. W. Cook, W. Cabot, J. A. Greenough, and S. V. Weber

Spectral and High-Order Methods for Shock-Induced Mixing (C8)

R. M. Darlington and K. S. Budil

Numerical Simulation of Mode Coupling in Laser-Driven Rayleigh-Taylor Instability Experiments (C9)

S. Dutta, E. George, J. Grove, J. Glimm, X. Li, A. Marchese, D. Sharp, Z. Xu, and

Y. Zhang

Numerical Methods for Determination of RT and RM Mixing (C11 and animation)

R. Epstein, J. A. Delettrez, V. Yu. Glebov, V. N. Goncharov, P. W. Mckenty, P. B. Radha, S. Skupsky, V. A. Smalyuk, C. Stoeckl, J. Frenje, C. K. Li, R. D. Petrasso, and F. H. Séguin One-Dimensional Simulation of the Effects of Unstable Mix on Neutron and Charged-Particle Yield from Laser-Driven Implosions (C13)

E. S. Gavrilova, E. V. Gubkov, V. A. Zhmailo, and Yu. V. Yanilkin

3D Computation for Surface Perturbations Evolution in Plasma Cloud During its Expansion in Magnetic Field (C14) 
$8^{\text {th }}$ International Workshop on the Physics of Compressible Turbulent Mixing,

\section{J. A. Greenough, W. J. Rider, C. Zoldi, and J. R. Kamm}

Code-to-Code Comparisons for the Problem of Shock Acceleration of a Diffuse Dense Gaseous Cylinder (C16)

\section{B. Grieves}

Implementation of a Turbulent Mix Model in a 2D ALE Code (C17)

E. V. Gubkov, V. A. Zhmailo, and Yu. V. Yanilkin

Semiempirical Model of Turbulent Magnetic Field Diffusion to Driven Plasma (C19)

\section{N. J. Zabusky, S. Gupta, Y. Gulak, G. Peng, and R. Samtaney}

Localization and Spreading of Interfaces (Contact Discontinuities) in PPM and WENO Simulations of the Compressible Euler Equations (C20)

S. W. Haan, T. Dittrich, G. Strobel, M. Marinak, D. Munro, G. Glendinning, P. Amendt, and R. Turner

Instability Modeling for NIF Ignition Targets and Omega Experiments (C22)

\section{G. Lebo and V. D. Zvorykin}

Laser Shock Tube for the Study of Supersonic Gas Flows and the Development of Hydrodynamic Instabilities in Layered Media (C25)

K. Nishihara, V. Zhakhovskii, and M. Abe

Molecular Dynamics Simulation of Shocks and Richtmyer-Meshkov Instability in Cylindrical Geometry (C26, animation 1, and animation 2)

\section{G. Peng, S. Gupta, S. Zhang, and N. J. Zabusky}

Dispersal of Mass and Circulation Following Shock-Sphere and Shock-Cylinder Interactions: Effects Arising from Shock-Cavity Collapse, Vortex Bilayers, Density-Gradient Intensification and Vortex Projectiles (C29)

W. J. Rider, J. R. Kamm, and C. Zoldi

A Numerical Study of Shocked Gas Cylinders (C31)

R. Samtaney, D. I. Pullin, T. Voelkl, and D. J. Hill

Large Eddy Simulation of Strong-Shock Richtmyer-Meshkov Instability (C33)

P. Seytor and M. Legrand

Numerical Investigation of a Laser Induced Turbulent Mixing Zone (C35)

D. Souffland and F. Renaud

A Mix-Model For One-Dimensional Simulations of Laser-Driven Implosion Experiments (C36)

Y. Srebro, D. Kushnir, Y. Elbaz, and D. Shvarts

Modeling Turbulent Mixing in Inertial Confinement Fusion Implosions (C37) 
H. Takabe, S. Yamada, K. Kobayashi, A. Mizuta, and K. Nomoto

Turbulent Mixing Nuclear Burning in Type Ia Supernova Explosion Based on Bubble Statistical Mechanics (C38)

N. Toqué

Turbulent Diffusion in Solar Type Star (C39)

E. Vold

Recent Computational Simulations of Rayleigh-Taylor Mix Layer Growth With a Multi-Fluid Model (C40)

S. P. Wang, M. H. Anderson, J. G. Oakley, and R. Bonazza

An Efficient and High Resolution Solver for the Two-Dimensional Numerical Simulation of the Richtmyer-Meshkov Instability (C41)

S. V. Weber, G. Dimonte, and M. M. Marinak

ALE Simulations of Turbulent Rayleigh-Taylor Instability in 2-D and 3-D (C42)

Yu. V. Yanilkin, V. P. Statsenko, S. V. Rebrov, O. G. Sin'kova, and A. L. Stadnik

Study of Gravitational Turbulent Mixing at Large Density Differences Using Direct 3D Numerical

Simulation (C44)

\section{L. Youngs}

Development and Validation of a 2D Turbulent Mix Model (C46)

D. L. Youngs and A. Llor

Preliminary Results of LES Simulations of Self-Similar Variable Acceleration RT Mixing Flows (C47)

\section{S. Zhang and N. J. Zabusky}

Shock-Planar Curtain Interactions: Strong Secondary Baroclinic Deposition and Emergence of Coherent and Random Vortex Projectiles (VPs), and Decaying Stratified Turbulence (C48)

\section{S. Zhang, N. J. Zabusky, K. Nishihara, and Y.-G. Kang}

Rapid Turbulization Arising from Vortex Double Layers in Interactions of "Complex" Blast Waves and Cylindrical/Spherical Bubbles (C49)

C. A. Zoldi

Simulations of a Shock-Accelerated Gas Cylinder and Comparison with Experimental Images and Velocity Fields (C50)

A. N. Razin, V. I. Kozlov, and I. V. Sapozhnikov

Simulations of Turbulent Mixing of Two Fluids With Variable Acceleration Laws (C51)

V. I. Kozlov and A. N. Razin

The Behaviour of Axial Variance Resulting from Turbulent Mixing Zone Interaction with Shocks (C52)

D. E. Eliason, W. H. Cabot, and Y. Zhou

A New Two-Scale Mix Model: Towards a Multi-Component Model of Turbulent Mixing (C53) 
$8^{\text {th }}$ International Workshop on the Physics of Compressible Turbulent Mixing,

Pasadena, CA (2001)

W. S. Don, D. Gottlieb, C.-W. Shu, and L. Jameson

High Order Methods for 2D Richtmyer-Meshkov Instability (C54)

\section{A. M. Dimits}

Examination of Rayleigh-Taylor $\alpha$ in Three Cases: PPM Simulations, Vasilenko et al. Shock Tube

Experiment, and Meshkov-Nevmerzhitsky Accelerated Tank Experiment (C55)

A. Cook, P. Dimotakis, and T. Mattner

Transition Stages of Rayleigh-Taylor Instability Between Miscible Fluids (C56)

A. Miles, J. Edwards, and G. Glendinning

CALE Simulation of Richtmyer-Meshkov Instability Experiments at High Mach Number (C57)

S. M. Bakrakh, N. A. Volodina, P. N. Nizotsev, V. F. Spiridonov, and E. V. Shuvalova

Numerical Simulation of Initial Perturbation Growth in Oblique Impact of Metal Plates

\section{CONTRIBUTED THEORETICAL PRESENTATIONS}

\section{S. I. Abarzhi}

Nonlinear Evolution of Unstable Fluid Interface (T1)

\section{S. I. Abarzhi, J. Glimm, and A. der Lin}

Nonlinear Asymptotic Solutions to Rayleigh-Taylor and Richtmyer-Meshkov Problems for Fluids With a Finite Density Contrast (T2)

\section{S. I. Abarzhi}

Turbulent Mixing in RTI as Order-Disorder Process (T3)

P. Bailly and A. Llor

A New Turbulent Two-Fluid RANS Model for KH, RT and RM Mixing Layers (T4)

\section{R. E. Breidenthal}

How to Inhibit Rayleigh-Taylor Mixing (T5)

\section{B. Cheng, J. Glimm, and D. Sharp}

Theoretical Methods for the Determination of Mix (T7)

\section{T. Clark and F. Harlow}

Modeling Radiation Effects in Mixing Layers (T8)

\section{S. B. Dalziel}

Toy Models for Rayleigh-Taylor Instability (T10) 
Y. Elbaz, Y. Srebro, O. Sadot, and D. Shvarts

A General Buoyancy-Drag Model for the Evolution of the Rayleigh-Taylor and Richtmyer-Meshkov

Instabilities (T11)

N. Inogamov, M. Tricottet, A. Oparin, and S. Bouquet

Nonlinear RT and RM Single Modes (Analytic) (T13)

V. M. Ktitorov

Stability of Diverging Shock Waves (T15)

V. M. Ktitorov

Stability of Reflected from the Center Self-Similar Converging Shock Wave (T16)

A. Llor

Response of Turbulent RANS Models to Self-Similar Variable Acceleration RT-Mixing: An Analytical “0D” Analysis (T18)

K. Nishihara, C. Matsuoka, and Y. Fukuda

Nonlinear Evolution of an Interface in the Richtmyer-Meshkov Instability (T19)

A. Rikanati, U. Alon, and D. Shvarts

Vortex-Merger Statistical Model for the Late Time Self-Similar Evolution of the Kelvin Helmholtz

Instability (T22)

\section{A. Rikanati, D. Oron, O. Sadot, and D. Shvarts}

High Mach Number and High Initial Amplitude Effects on the Evolution of the Single-Mode RichtmyerMeshkov Instability-Theoretical Study (T23)

\section{D. Ryutov}

Compressible MHD Turbulence in Strongly Radiating Molecular Clouds in Astrophysics (T25)

O. Schilling

Single-Velocity, Multi-Component Turbulent Transport Models for Interfacial Instability-Driven Flows (T26)

\section{O. Schilling and A. W. Cook}

Large- and Small-Scale Dynamics of Variable-Density Rayleigh-Taylor Instability-Induced Turbulent Mixing (T28)

M. Tricottet and S. Bouquet

Analytical Study of the RTI in Compressible Fluids (T30)

M. Vandenboomgaerde

Analytical Growth Rate of a Non-Linear Single-Mode R-M Instability (T31) 
$8^{\text {th }}$ International Workshop on the Physics of Compressible Turbulent Mixing,

Pasadena, CA (2001)

M. Vandenboomgaerde, S. Gauthier, D. Galmiche, C. Cherfils, and P. A. Raviart

Efficient Perturbation Methods for Richtmyer-Meshkov and Rayleigh-Taylor Instabilities: Weakly

Nonlinear Stage and Beyond (T32)

P. Wilson, M. Andrews, and F. Harlow

Combined Shear and Buoyancy Instabilities (T33)

J. G. Wouchuk

Rate of Growth of the Linear Richtmyer-Meshkov Instability (T34)

A. Yosef-Hai, O. Sadot, D. Kartoon, D. Oron, L. A. Levin, E. Sarid, Y. Elbaz, G. Ben-Dor, and D. Shvarts

The Dependence of the Shock Induced Richtmyer-Meshkov Instability on Dimensionality and Density Ratio (T35)

Y. Zhou, H. F. Robey, and A. C. Buckingham, B. A. Remington, A. Dimits, W. Cabot, J. Greenough, S. Weber, O. Schilling, T. A. Peyser, D. Eliason, P. Keiter, and R. P. Drake

Mixing Transition in Time-Dependent Flows (T36)

A. L. Kuhl and R. E. Ferguson

Spherical Combustion Layer in a TNT Explosion (T37)

B. Afeyan, P. Ramaprabhu, and M. J. Andrews

Discrete Wavelet Transform Techniques for Denoising, Pattern Detection and Compression of Turbulent Rayleigh-Taylor Mix Data (T39)

\section{ABSTRACT BOOK \\ PROGRAM SUMMARY}

AUTHOR CONTACT INFORMATION

PHOTOGRAPHS FROM THE BANQUET AND WORKSHOP 

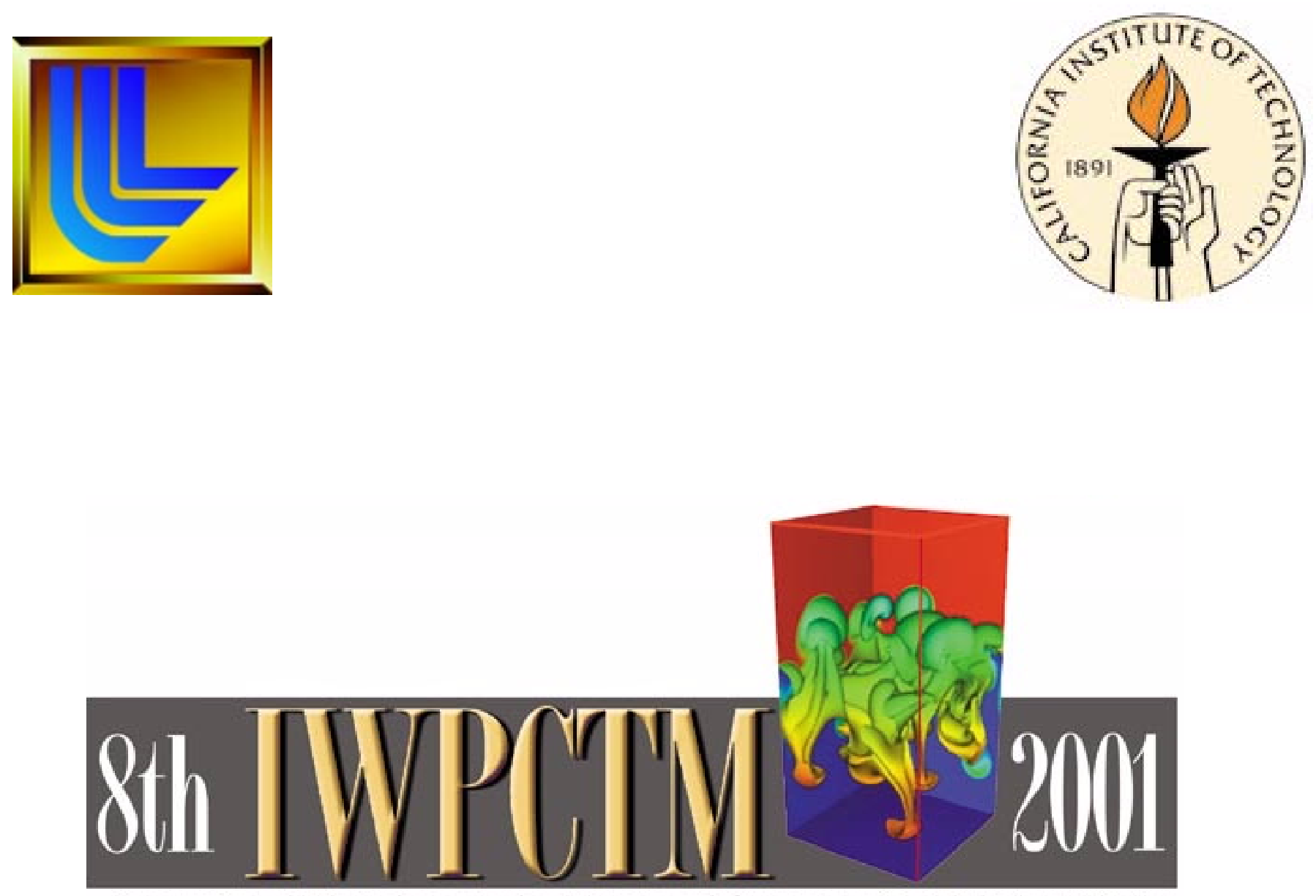

International Workshop on the Physics of Compressible Turbulent Mixing

\author{
December 9-14, 2001 \\ Pasadena, California, USA
}

\title{
ABSTRACTS
}

Oleg Schilling, Chairman 


\section{DISCLAIMER}

This document was prepared as an account of work sponsored by an agency of the United States Government. Neither the United States Government nor the University of California nor any of their employees, makes any warranty, express or implied, or assumes any legal liability or responsibility for the accuracy, completeness, or usefulness of any information, apparatus, product, or process disclosed, or represents that its use would not infringe privately owned rights. Reference herein to any specific commercial product, process, or service by trade name, trademark, manufacturer, or otherwise, does not necessarily constitute or imply its endorsement, recommendation, or favoring by the United States Government or the University of California. The views and opinions of authors expressed herein do not necessarily state or reflect those of the United States Government or the University of California, and shall not be used for advertising or product endorsement purposes.

This work was performed under the auspices of the U. S. Department of Energy by the University of California, Lawrence Livermore National Laboratory under Contract No. W-7405-Eng-48.

This report has been reproduced directly from the best available copy.

Available to DOE and DOE contractors from the Office of Scientific and Technical Information

P.O. Box 62, Oak Ridge, TN 37831

Prices available from (423) 576-8401 http://apollo.osti.gov/bridge/

Available to the public from the National Technical Information Service

U.S. Department of Commerce 5285 Port Royal Rd., Springfield, VA 22161 http://www.ntis.gov/

OR

Lawrence Livermore National Laboratory Technical Information Department's Digital Library http://www.llnl.gov/tid/Library.html 
$8^{\text {th }}$ International Workshop on the Physics of Compressible Turbulent Mixing,

Pasadena, CA (2001)

\section{Local Organizing Committee}

G. Dimonte, Lawrence Livermore National Laboratory, Livermore, CA, USA

B. Goodwin, Lawrence Livermore National Laboratory, Livermore, CA, USA

D. Meiron, California Institute of Technology, Pasadena, CA, USA

T. McAbee, Lawrence Livermore National Laboratory, Livermore, CA, USA

T. Peyser, Lawrence Livermore National Laboratory, Livermore, CA, USA

B. Remington, Lawrence Livermore National Laboratory, Livermore, CA, USA

O. Schilling (Chairman), Lawrence Livermore National Laboratory, Livermore, CA, USA

C. Verdon, Lawrence Livermore National Laboratory, Livermore, CA, USA

\section{International Scientific Committee}

G. Ben-Dor, Ben-Gurion University of the Negev, Beer-Sheeva, IL

D. Besnard, Commissariat à l'Energie Atomique, FR

A. Buckingham, Lawrence Livermore National Laboratory, Livermore, CA, USA

T. Clark, Los Alamos National Laboratory, Los Alamos, NM, USA

S. Dalziel, University of Cambridge, Cambridge, UK

D. Galmiche, Commissariat à l'Energie Atomique, FR

S. Gauthier, Commissariat à l'Energie Atomique, FR

J. Glimm, State University of New York at Stony Brook, NY, USA

B. Goodwin, Lawrence Livermore National Laboratory, Livermore, CA, USA

N. Hoffman, Los Alamos National Laboratory, Los Alamos, NM, USA

L. Houas, IUSTI Universite de Provence, Marseille, FR

J. Jacobs, University of Arizona, Tuscon, AZ, USA

Yu. Kucherenko, Russian Federal Nuclear Center-VNIITF, Snezhinsk, RU

P. Linden, University of California at San Diego, San Diego, CA, USA

D. Meiron, California Institute of Technology, Pasadena, CA, USA

E. Meshkov, Russian Federal Nuclear Center-VNIIEF, Sarov, RU

V. Neuvazhaev, Russian Federal Nuclear Center-VNIITF, Snezhinsk, RU

T. Peyser, Lawrence Livermore National Laboratory, Livermore, CA, USA

J. Redondo, Universitat Politecnica de Catalunya, Barcelona, SP

B. Remington, Lawrence Livermore National Laboratory, Livermore, CA, USA

V. Rozanov, P. N. Lebedev Physical Institute, Moscow, RU

O. Schilling, Lawrence Livermore National Laboratory, Livermore, CA, USA

D. Sharp, Los Alamos National Laboratory, Los Alamos, NM, USA

D. Shvarts, Nuclear Research Center, Beer-Sheeva, IL

E. Son, Moscow Physical and Technical Institute, Moscow, RU

H. Takabe, ILE, Osaka University, Osaka, JP

K. Takayama, Tohoku University, Sendai, JP

D. Youngs, Atomic Weapons Establishment, Aldermaston, UK

S. Zaytsev, ENIN, Moscow, RU 


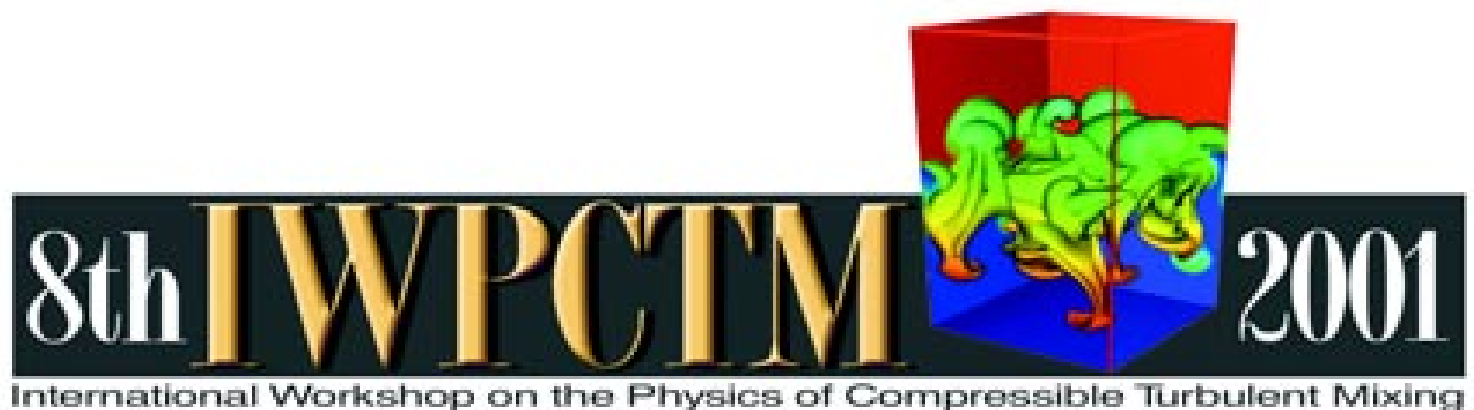

\section{General Information}

The workshop is hosted by the Lawrence Livermore National Laboratory and is held at the California Institute of Technology.

Schedule (Oral and poster presentations will be at the California Institute of Technology):

\begin{tabular}{|c|c|c|c|}
\hline Day & Date & Time & Activity \\
\hline Sunday & December 9, 2001 & 5:00 PM - 9:00 PM & Registration - Pasadena Hilton \\
\hline Sunday & December 9, 2001 & 6:00 PM - 9:00 PM & Reception - Pasadena Hilton \\
\hline Monday & December 10, 2001 & $8: 15 \mathrm{AM}-4: 15 \mathrm{PM}$ & $\begin{array}{c}\text { Opening Remarks/Oral } \\
\text { Presentations }\end{array}$ \\
\hline Monday & December 10, 2001 & 4:15 PM - 6:00 PM & Poster Presentations \\
\hline Tuesday & December 11,2001 & $8: 15 \mathrm{AM}-4: 15 \mathrm{PM}$ & $\begin{array}{c}\text { Announcements/Oral } \\
\text { Presentations }\end{array}$ \\
\hline Tuesday & December 11, 2001 & 4:15 PM - 6:00 PM & $\begin{array}{c}\text { Experimental Discussion/ } \\
\text { Computational and Theoretical } \\
\text { Poster Presentations }\end{array}$ \\
\hline Wednesday & December 12, 2001 & $8: 15$ AM - 4:15 PM & $\begin{array}{c}\text { Announcements /Oral } \\
\text { Presentations } \\
\end{array}$ \\
\hline Wednesday & December 12, 2001 & 4:15 PM - 6:00 PM & $\begin{array}{c}\text { Computational } \\
\text { Discussion/Experimental and } \\
\text { Theoretical Poster Presentations }\end{array}$ \\
\hline Wednesday & December 12,2001 & 6:00 PM - 9:00 PM & Banquet - Pasadena Hilton \\
\hline Thursday & December 13, 2001 & $8: 15$ AM - 4:15 PM & $\begin{array}{c}\text { Announcements /Oral } \\
\text { Presentations } \\
\end{array}$ \\
\hline Thursday & December 13, 2001 & 4:15 PM - 6:00 PM & $\begin{array}{c}\text { Theoretical } \\
\text { Discussion/Computational and } \\
\text { Experimental Poster Presentations }\end{array}$ \\
\hline Friday & December 14, 2001 & $8: 15$ AM - 12:00 PM & $\begin{array}{c}\text { Announcements/Oral } \\
\text { Presentations/Summary } \\
\text { Remarks/Closing Remarks }\end{array}$ \\
\hline
\end{tabular}




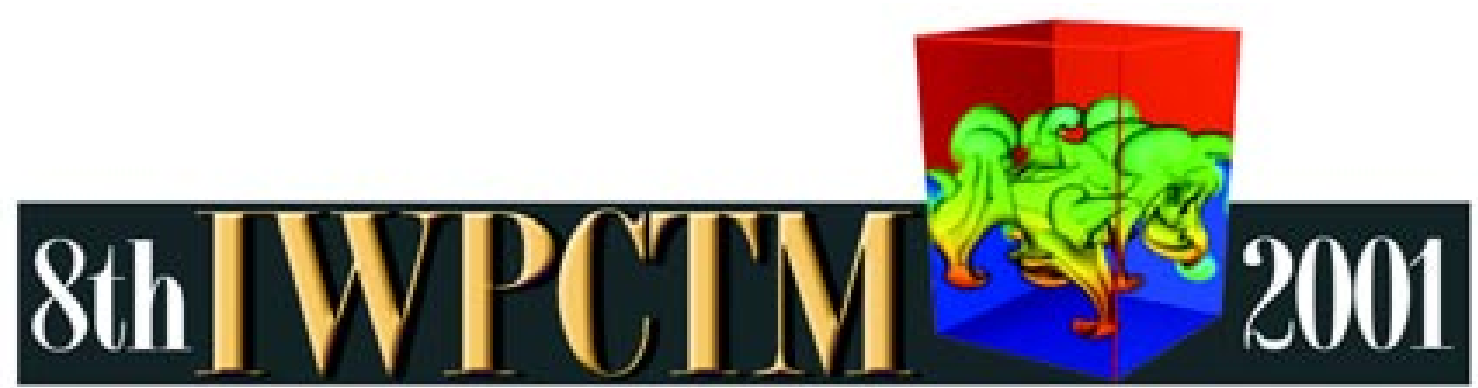

International Workshop on the Physics of Compressible Turbulent Mixing

\section{Invited Guest Speakers:}

Dr. Edward I. Moses, National Ignition Facility (NIF) Project Manager at the Lawrence Livermore National Laboratory, will be the guest speaker at the Reception on Sunday evening, December 9. Z. Nagin Cox, Mission Operations Engineer for the Mars Sample Return Mission at the NASA Jet Propulsion Laboratory, will be the guest speaker at the Banquet on Wednesday evening, December 12.

\section{Message Line:}

A phone will be located in the lobby of the Beckman Institute Auditorium for messages: the telephone number is (626) 395-5035.

\section{Shuttles:}

The Pasadena Hilton will provide shuttles to and from the hotel and Caltech in the morning and afternoon (parking on or near campus is extremely limited).

\section{Pasadena Convention Center:}

The Pasadena Convention Center will have representatives available for scheduling tourist activities. Representatives will be available on Sunday during the Registration and Tuesday through Thursday from 4:00 PM - 8:00 PM in the Pasadena Hilton Hotel lobby.

\section{Administrative Staff:}

C. Cantlin, Lawrence Livermore National Laboratory, Livermore, CA, USA

S. Davis, Lawrence Livermore National Laboratory, Livermore, CA, USA

K. Evans, Lawrence Livermore National Laboratory, Livermore, CA, USA 
$8^{\text {th }}$ International Workshop on the Physics of Compressible Turbulent Mixing,

Pasadena, CA (2001)

\section{Table of Contents}

REVIEW TALKS

J.-F. Haas and S. G. Zaytsev

A Review on RT and RM Instability and TM Experiments

S. G. Zaytsev

The Experimental Study of Excitation and Development of the Hydrodynamic Instability in the Mixing Zone Separating Gases of Different Densities at their Accelerated Motion

D. L. Youngs

Review of Numerical Simulation of Mixing due to Rayleigh-Taylor and Richtmyer-Meshkov Instabilities

D. Shvarts

Modeling Late-Time Nonlinear Evolution of Hydrodynamic Instabilities and their Role in Inertial Confinement Fusion

EXPERIMENTAL ABSTRACTS

Yu. A. Kucherenko, S. I. Balabin, R. I. Ardashova, A. P. Pylaev, O. E. Kozelkov, and V. D. Murzakov

Experimental Investigations of the Heavy and Light Media Separation in the Rayleigh-Taylor Turbulence Zone at Different Atwood Numbers (E2)

Yu. A. Kucherenko, S. I. Balabin, R. I. Ardashova, O. E. Kozelkov, A. V. Dulov, and I. A. Romanov

Experimental Investigations into Influence of Stablizing Properties of Transitional Layers Upon the Turbulent Mixing Evolution (E3)

C. W. Barnes, S. H. Batha, A. M. Dunne, N. E. Lanier, G. R. Magelssen, T. J. Murphy, K. W. Parker, S. Rothman, J. M. Scott, and D. Youngs

Improvements to Convergent Cylindrical Plasma Mix Experiments Using Laser Direct Drive (E4)

S. H. Batha, K. W. Parker, C. W. Barnes, A. M. Dunne, N. E. Lanier, G. R. Magelssen, T. J. Murphy, S. Rothman, J. M. Scott, and D. Youngs

Mixing Between Two Compressing Cylinders (E5)

M. Bliznetsov, V. Dudin, S. Gerasimov, L. Houas, G. Jourdan, A. Logvinov, E. Meshkov, and Yu. Vlasov

Development of a Method for Studying the Interaction Between Shock Wave and a Flame Front (E6)

M. Bliznetsov, E. Meshkov, N. Nevmerzhitzky, A. Nikulin, E. Sen'kovsky, and E. Sotskov

The Influence of Scaling for Periodical Perturbations on Development of Turbulent Mixing on a Gas-Liquid Interface (E7) 
M. Brouillette and C. H. Hebért

Pasadena, CA (2001)

Compressible Vortex Rings (E8)

R. P. Drake

Design of Flyer-Plate-Driven Compressible Turbulent Mix Experiments (E9)

R. P. Drake, P. Keiter, K. E. Korreck, K. Dannenberg, H. A. Robey, T. Perry, J. O. Kane,

B. A. Remington, R. J. Wallace, O. A. Hurricane, D. D. Ryutov, J. Knauer, R. Teyssier,

A. Calder, R. Rosner, B. Fryxell, D. Arnett, Y. Zhang, J. Glimm, N. Turner, J. Stone,

R. McCray, and J. Grove

Compressible Hydrodynamics on the Omega Laser, Motivated by Astrophysics (E10)

O. B. Drennov, A. L. Mikhaylov, P. N. Nizovtsev, and V. A. Raevskii

Growth of Perturbation on Metals Interface at Oblique Collission with Supersonic Velocity of Contact Point Motion (E11)

S. G. Glendinning, D. G. Braun , M. J. Edwards, W. W. Hsing, B. F. Lasinski, H. Louis, J. Moreno, T. A. Peyser, B. A. Remington, H. F. Robey, E. J. Turano, C. P. Verdon, and Y. Zhou

An Experimental Study of the Effect of Shock Proximity on the Richtmyer-Meshkov

Instability at High Mach Number (E12)

D. A. Holder, C. Barton, and A. V. Smith

Mix Experiments using a Two Dimensional Convergent Shock Tube (E13)

J. M. Holford, S. B. Dalziel, and D. Youngs

Rayleigh-Taylor Instability at a Tilted Interface in Incompressible Laboratory Experiments and Compressible Numerical Simulations (E14)

S. H. R. Hosseini and K. Takayama

Study of Diverging and Converging Spherical Shock Waves Induced by Micro Explosives and their Interaction with Product Gases (E15)

S. H. R. Hosseini and K. Takayama

Interaction of Converging Shock Waves with Cylindrical Heavy Gas Interfaces in an Eccentric Arrangement (E16)

L. Houas, G. Jourdan, L. Schwaederle, and E. E. Meshkov

From Linear to Turbulent Stages of the Richtmyer-Meshkov Instability Development in a Large Cross Section Shock Tube (E17)

J. W. Jacobs and V. V. Krivets

PLIF Flow Visualization of a Shock-Accelerated Air/SF ${ }_{6}$ Interface (E18)

D. H. Kalantar, J. Belak, J. D. Colvin, M. Kumar, K. T. Lorenz, K. O. Mikaelian, S. Pollaine, B. A. Remington, S. V. Weber, L. G. Wiley, A. M. Allen, A. LoveridgeSmith, J. S. Wark, and M. A. Meyers

Laser-Based High Pressure, High Strain-Rate Solid-State Experiments (E19) 
$8^{\text {th }}$ International Workshop on the Physics of Compressible Turbulent Mixing,

Pasadena, CA (2001)

Yu. A. Kucherenko, O. E. Shestachenko, S. I. Balabin, and A. P. Pylaev

RFNC-VNIITF Multifunctional Shock Tube to Investigate the Evolution of Instabilities in Nonstationary Gas Dynamic Flows (E20)

A. Lassis, P. Montlaurent, C. Rayer and J.-F. Haas

Planar Laser Sheet Visualization and Laser Doppler Velocity Measurements in Shock-Induced

Turbulent Mixing Zones (E21)

G. Layes, G. Jourdan, P. Roualdes, and L. Houas

Hydrodynamic Instabilities at a Shock Accelerated Bubble Gas-Gas Interface (E22)

K. Levy, O. Sadot, D. Oron, Y. Srebro, Y. Elbaz, A. Josef-Hai, G. Ben-Dor, and

D. Shvarts

Experimental and Numerical Study of Shock Wave-Bubble Interaction (E23)

K. T. Lorenz, D. Kalantar, J. Edwards, J. D. Colvin, and B. Remington

Laser-Driven Near Isentropic Compression of an Aluminum Flyer Plate (E24)

C. E. Niederhaus and J. W. Jacobs

Single-Mode Incompressible Richtmyer-Meshkov Instability Experiments (E26)

J. G. Oakley, M. H. Anderson, and R. Bonazza

Experimental Study of a Strongly-Shocked Gas Interface with Visualized Initial Conditions (E27)

Yu. A. Kucherenko, O. E. Shestachenko, Yu. A. Piskunov, E. V. Sviridov, V. M. Medvedev, and A. I. Baishev

Experimental Investigations of the Self-Similar Mixing Mode of Different Density Gases in the Earth's Gravitational Field (E28)

S. Pollaine, D. Kalantar, B. Remington, J. Belak, J. D. Colvin, J. Edwards, R. Minich, K. O. Mikaelian, K. T. Lorenz, S. V. Weber, L. G. Wiley, D. Paisley, A. Hauer, J. S. Wark, A. Loveridge, A. M. Allen, T. R. Boehly, and M. A. Meyers Modeling Laser Material Strength Experiments (E29)

K. Prestridge, C. A. Zoldi, P. Vorobieff , P. M. Rightley, and R. F. Benjamin

Experiments and Simulations of Instabilities in a Shock-Accelerated Gas Cylinder (E30)

Yu. A. Kucherenko, A. P. Pylaev, V. D. Murzakov, A. V. Belomestnih, V. N. Popov, and A. A. Tyaktev

Experimental Study into Rayleigh-Taylor Turbulent Mixing Zone Heterogeneous Structure (E31)

P. Ramaprabhu and M. J. Andrews

Measurements of Turbulence Correlations in Low Atwood Number Rayleigh-Taylor

Mixing (E32) 
H. F. Robey, T. S. Perry, R. I. Klein, J. A. Greenough, J. O. Kane, and T. R. Boehly Experimental Study of the Interaction of a Strong Shock with a Spherical Density

Inhomogeneity (E34)

H. F. Robey, Y. K. Zhou, A. C. Buckingham, P. Keiter, B. A. Remington, and R. P. Drake

Turbulent Transition in a High Reynolds Number, Rayleigh-Taylor Unstable Plasma Flow (E35)

O. Sadot, A. Yosef-Hai, A. Rikanati, D. Oron, G. Ben-Dor, and D. Shvarts

Effects of High Initial Amplitudes and High Mach Numbers on the Evolution of the RM

Instability II. Experimental Study (E36)

L. Schwaederlé, G. Jourdan, L. Houas, and J.-F. Haas

Measurements within a Richtmyer-Meshkov Mixing Zone using a Triple Hot Wire Probe

Technique (E37)

Yu. A. Kucherenko, O. E. Shestachenko, Yu. A. Piskunov, E. V. Sviridov, V. M. Medvedev, and A. I. Baishev

Experimental Study into Evolution of Gravitational Turbulent Mixing of Gases at the Multifunctional Shock Tube (E38)

A. V. Smith, D. A. Holder, C. J. Barton, A. P. Morris, and D. L. Youngs

Shock Tube Experiments on Richtmyer-Meshkov Instability Across a Chevron Profiled

Interface (E39)

C. Tomkins, K. Prestridge, P. Rightley, C. Zoldi, and R. Benjamin

The Evolution and Interaction of Two Shock-Accelerated Unstable Gas Cylinders (E40)

D. J. Ward, K. S. Budil, T. A. Peyser, B. A. Remington, P. L. Miller, R. J. Wallace, H. Louis, and A. Demiris

Doubly-Shocked Richtmyer-Meshkov Instability Experiments at Nova (E41)

R. I. Klein, H. Robey, T. Perry, and J. Greenough

The Interaction of Supernova Blast Waves with Interstellar Clouds: Experiments on the OMEGA Laser (E42)

S. G. Zaytsev, V. V. Krivets, I. M. Mazilin, S. N. Titov, E. I. Chebotareva, V. V. Nikishin, V. F. Tishkin, S. Bouquet, and J.-F. Haas

Evolution of the Mixing Zone of Different Densities Gases Being Interaction to Compression Waves (E43)

A. Lebedev, P. Nizovtcev, and V. Raevsky

Studies of Rayleigh-Taylor Instability in Aluminum Under Shock-Wave and Shock Less Loading (E44)

H. Azechi, T. Sakaiya, M. Nakai, H. Shiraga, K. Shigemori, N. Miyanaga, M. Nishikino, S. Fujioka, Y. Tamari, H. Nagatomo, H. Takabe, and A. Sunahara

Ablative Rayleigh-Taylor Instability at Short Wavelengths (E45) 
$8^{\text {th }}$ International Workshop on the Physics of Compressible Turbulent Mixing,

Pasadena, CA (2001)

H. F. Robey and S. G. Glendinning

A Vortex Model for Studying the Effect of Shock Proximity on Richtmyer-Meshkov Instability at High Mach Number (E46)

COMPUTATIONAL ABSTRACTS

V. I. Anisimov and A. V. Polionov

Modes' Interaction on Nonlinear Stage of Richtmyer-Meshkov Instability Evolution (C1)

M. G. Anuchin, V. E. Neuvazhayev, and I. E. Parshukov

Application of Ke-Model for the Description of an Atmospheric Surface Layer (C2)

Wm. T. Ashurst and A. R. Kerstein

Computational Modeling of Low-Mach-Number High-Atwood-Number Turbulent Mixing (C4) 56

A. W. Cook, W. H. Cabot, and J. A. Greenough

Spectral and High-Order Compact Methods for Shock-Induced Mixing (C8)

R. M. Darlington and K. S. Budil

Numerical Simulation of Mode Coupling in Laser-Driven Rayleigh-Taylor Instability

Experiments (C9)

G. Dimonte, A. Dimits, S. Weber, D. L. Youngs, A. C. Calder, B. Fryxell, J. Biello, L. Dursi, P. MacNeice, K. Olson, P. Ricker, R. Rosner, F. Timmes, H. Tufo, Y.-N. Young, M. Zingale, M. J. Andrews, P. Ramaprabhu, S. Wunsch, C. Garasi, and A. Robinson A Comparison of High-Resolution 3D Numerical Simulations Of Turbulent Rayleigh-Taylor (RT) Instability: Alpha-Group Collaboration (C10)

S. Dutta, E. George, J. Glimm, J. Grove, X. Li, A. Marchese, D. H. Sharp, Z. Xu, and Y. Zhang

Numerical Methods for Determination of Mix (C11)

Y. Elbaz, A. Rikanati, D. Oron, and D. Shvarts

Rayleigh-Taylor Instability in Compressible Fluids (C12)

R. Epstein, J. A. Delettrez, V. Yu. Glebov, V. N. Goncharov, P. W. Mckenty, P. B. Radha, S. Skupsky, V. A. Smalyuk, and C. Stoeckl

One-Dimensional Simulation of the Effects of Unstable Mix on Neutron and Charged-Particle Yield from Laser-Driven Implosion Experiments (C13)

E. S. Gavrilova, E. V. Gubkov, V. A. Zhmailo, and Yu. V. Yanilkin

3D Computation for Surface Perturbation Evolution of Plasma Cloud During its Expansion in Magnetic Field (C14)

M. J. Graham, K. S. Budil, J. Grove, and B. A. Remington

The Richtmyer-Meshkov Instability in Cylindrical Geometry: Experiments and Simulation (C15) 
J. A. Greenough, W. J. Rider, C. A. Zoldi, and J. R. Kamm

Pasadena, CA (2001)

Code to Code Comparisons for the Problem of Shock Acceleration of a Diffuse Dense Gaseous

Cylinder (C16)

B. Grieves

Implementation of a Turbulent Mix Model in a 2D ALE Code (C17)

J. W. Grove

Error Estimation for Strong Shock Hydrodynamics (C18)

E. V. Gubkov, V. A. Zhmailo, and Yu. V. Yanilkin

A Semi-Empirical Model for Turbulent Diffusion of Magnetic Field to Accelerated Plasma (C19)

S. Gupta, N. J. Zabusky, R. Samtaney, and Y. Gulak

Localization and Spreading of Interfaces (Contact Discontinuities) in Direct Numerical

Simulations of the Compressible Euler Equations (C20)

S. W. Haan, T. Dittrich, S. Hatchett, D. Hinkel, M. Marinak, D. Munro, O. Jones, S. Pollaine, and L. Suter

Update on Instability Modeling for the NIF Ignition Targets (C22)

J. O. Kane, D. D. Ryutov, B. A. Remington, S. G. Glendinning, J. Nash, M. Pound, and D. Arnett

Pillars of Creation $(\mathrm{C} 23)$

I. G. Lebo and V. D. Zvorykin

Application of a Laser Shock Tube for the Study of Supersonic Gas Flows and the Development of Hydrodynamic Instabilities in Layered Media (C25)

K. Nishihara, V. Zhakhovskii, and M. Abe

Molecular Dynamic Simulation of Shock and Richtmyer-Meshkov Instability in Cylindrical Geometry (C26)

C. Pantano and S. Sarkar

Compressibility Effects in a High-Speed, Reacting Shear Layer: An Investigation Using DNS (C27)

K. W. Parker, A. M. Dunne, S. Rothman, D. Youngs, C. W. Barnes, S. H. Batha, N. E. Lanier, G. R. Magelssen, T. J. Murphy, and J. M. Scott

Computational Modeling of Two-Shell Cylindrical Implosions with Mix (C28)

\section{G. Peng and N. J. Zabusky}

Dispersal of Mass and Circulation Following $M=2.5-\mathrm{M}=10$ Shock-Bubble Interactions: Effects Arising from Shock-Cavity Collapse-\& Re-Expansion, Vortex Double Layers (Vdls) and Vortex Projectiles (Vps) (C29)

V. A. Raevski, S. N. Sinitsina, and Yu. V. Yanilkin

Influence of Turbulent Mixing Zone on Growth of Local Perturbation in Environments of Rayleigh-Taylor Instability (Numerical Simulation) (C30) 
$8^{\text {th }}$ International Workshop on the Physics of Compressible Turbulent Mixing,

Pasadena, CA (2001)

W. J. Rider, J. R. Kamm, and C. A. Zoldi

A Statistical Comparison of Gas Cylinder Experiments with their Simulation (C31)

R. Samtaney, T. Voelkl, and D. I. Pullin

Large Eddy Simulation of Strong Shock Richtmyer-Meshkov Instability (C33)

P. Seytor and M. Legrand

Numerical Investigation of a Laser Induced Turbulent Mixing Zone (C35)

D. Souffland and F. Renaud

A Mix-Model For One-Dimensional Simulations of Laser-Driven Implosion Experiments (C36)

Y. Srebro, D. Kushnir, Y. Elbaz, and D. Shvarts

Modeling Turbulent Mixing in Inertial Confinement Fusion Implosions (C37)

H. Takabe, S. Yamada, K. Kobayashi, A. Mizuta, and K. Nomoto

Turbulent Mixing Nuclear Burning in Type Ia Supernova Explosion Based on Bubble Statistical Mechanics (C38)

N. Toqué

Turbulent Diffusion in Solar Type Star (C39)

E. Vold

Recent Computational Simulations of Rayleigh-Taylor Mix Layer Growth With a Multi-Fluid Model (C40)

S. P. Wang, M. H. Anderson, J. G. Oakley, and R. Bonazza

An Efficient and High Resolution Solver for the Two-Dimensional Numerical Simulation of the

Richtmyer-Meshkov Instability (C41)

S. V. Weber, G. Dimonte, and M. M. Marinak

ALE Simulations of Turbulent Rayleigh-Taylor Instability in 2-D and 3-D (C42)

Yu. V. Yanilkin, V. P. Statsenko, S. V. Rebrov, N. I. Selchenkova, O. G. Sin'kova, A. L. Stadnik, and A. Ya. Uchayev

Study of Turbulent Gravitational Mixing at Large Density Differences Using Direct 3D

Numerical Simulation (C44)

D. L. Youngs

Development and Validation of a 2D Turbulent Mix Model (C46)

D. L. Youngs, X. Silvani, J. Magnaudet, and A. Llor

Preliminary Results of DNS and LES Simulations of Self-Similar Variable Acceleration

RT-Mixing Flows (C47)

\section{S. Zhang and N. J. Zabusky}

Shock-Planar Curtain Interactions: Strong Secondary Baroclinic Deposition and the Emergence of Coherent and Random Vortex Projectiles (VPs) and Decaying Stratified Turbulence (C48) 92 
S. Zhang, Y.-G. Kang, K. Nishihara, N. J. Zabusky, and H. Kim

Rapid Turbulization Arising from Vortex Double Layers in Interactions of "Complex" Blast

Waves and Cylindrical and Spherical Bubbles (C49)

C. A. Zoldi, K. Prestridge, P. M. Rightley, and R. F. Benjamin

Simulations of a Shock-Accelerated Gas Cylinder and Comparison with Experimental Images and Velocity Fields (C50)

V. I. Kozlov, A. N. Razin, and I. V. Sapozhnikov

Turbulent Flow Simulations of Two Fluids Moving with Different Laws of Acceleration (C51)

V. I. Kozlov and A. N. Razin

The Behaviour of Velocity Variance Resulting from Turbulent Mixing Zone-Shock Interaction (C52)

D. E. Eliason, W. H. Cabot, and Y. Zhou

An Assessment of Multi-Velocity Versus Single Velocity in a Multi-Component Model of Turbulent

Mixing (C53)

W.-S. Don, D. Gottlieb, L. Jameson, and C.-W. Shu

High Order Numerical Methods for the 2D Richtmyer-Meshkov Instability (C54)

A. M. Dimits

Mixing Due to the Rayleigh-Taylor Instability (C55)

A. W. Cook and P. E. Dimotakis

Transition Stages of Rayleigh-Taylor Instability Between Miscible Fluids (C56)

100

A. Miles, J. Edwards, and G. Glendinning

CALE Simulation of Richtmyer-Meshkov Experiments at High Mach Number (C57)

S. I. Abarzhi

Nonlinear Evolution of Unstable Fluid Interface (T1)

S. I. Abarzhi

Nonlinear Asymptotic Solutions to RT and RM Problems for Fluids With Close Densities (T2)

S. I. Abarzhi

Turbulent Mixing in RTI as Order-Disorder Process (T3)

P. Bailly and A. Llor

A New Turbulent Two-Fluid RANS Model for KH, RT and RM Mixing Layers (T4)

R. E. Breidenthal

Super-Exponential Rayleigh-Taylor Flow (T5)

B. Cheng, J. Glimm, and D. H. Sharp

Theoretical Methods for Determination of Mix (T7) 
$8^{\text {th }}$ International Workshop on the Physics of Compressible Turbulent Mixing,

Pasadena, CA (2001)

T. Clark and F. Harlow

Modeling Radiation Effects in Mixing Layers (T8)

109

J. D. Colvin, M. Legrand, B. A. Remington, G. Schurtz, and S. V. Weber

A Model for Instability Growth in Accelerated Solid Metals (T9)

110

S. B. Dalziel

Toy Models for the Growth Rate of Rayleigh-Taylor Instability (T10)

Y. Elbaz, Y. Srebro, O. Sadot, and D. Shvarts

A General Buoyancy-Drag Model for the Evolution of the Rayleigh-Taylor and RichtmyerMeshkov Instabilities (T11)

N. A. Inogamov, A. M. Oparin, M. Tricottet, and S. Bouquet

3D Rayleigh-Taylor and Richtmyer-Meshkov Single-Modes (T12)

113

N. A. Inogamov, M. Tricottet, A. M. Oparin, and S. Bouquet

Rayleigh-Taylor Instability for Compressible and Incompressible Media (T13)

114

D. Kartoon, D. Oron, L. Arazi, A. Rikanati, U. Alon, and D. Shvarts

Three Dimensional Multi-Mode Rayleigh-Taylor and Richtmyer-Meshkov Instabilities at All Density Ratios (T14)

V. M. Ktitorov

Stability of Diverging Shock Waves (T15)

V. M. Ktitorov

Stability of Reflected from the Center Self-Similar Converging Shock Wave (T16)

R. R. Linn and F. H. Harlow

Using a Turbulence Transport Approach to Study Shocks Through Polycrystalline Metal (T17)

A. Llor

Response of Turbulent RANS Models to Self-Similar Variable Acceleration RT-Mixing: An Analytical 0D Analysis (T18)

C. Matsuoka, K. Nishihara, and Y. Fukuda

Nonlinear Evolution of an Interface in the Richtmyer-Meshkov Instability (T19)

K. O. Mikaelian

Evolution of Arbitrary Perturbations in the Richtmyer-Meshkov Instability (T20)

A. M. Oparin, N. A. Inogamov, and A. Yu. Dem'yanov

RT Turbulence: Dramatic Dynamics of Interpenetration (Fast Jets, Sharp Decelerations and Accelerations) (T21) 


\section{A. Rikanati, U. Alon, and D. Shvarts}

Statistical Mechanics Large Scale Model for the Evolution of the Multi-Mode Kelvin Helmholtz Instability (T22)

A. Rikanati, D. Oron, O. Sadot, and D. Shvarts

Effects of High Initial Amplitudes and High Mach Numbers on the Evolution of the RM Instability: I. Theoretical Study (T23)

D. D. Ryutov and B. A. Remington

Compressible MHD Turbulence in Strongly Radiating Molecular Clouds in Astrophysics (T25) 125

O. Schilling

Single-Velocity, Multi-Component Turbulent Transport Models for Interfacial Instability-Driven Flows (T26)

O. Schilling and A. W. Cook

Large- and Small-Scale Dynamics of Variable-Density Rayleigh-Taylor Instability-Induced Turbulent Mixing (T28)

M. Tricottet and S. Bouquet

Analytical Study of the Rayleigh-Taylor Instability in Compressible Fluids (T30)

M. Vandenboomgaerde

Analytic Nonlinear Growth of A Single-Mode Richtmyer-Meshkov Instability (T31)

M. Vandenboomgaerde, C. Cherfils, D. Galmiche, S. Gauthier, and P. A. Raviard Efficient Perturbation Methods for Richtmyer-Meshkov and Rayleigh-Taylor Instabilities: Weakly Nonlinear Stage and Beyond (T32)

P. N. Wilson, M. J. Andrews, and F. H. Harlow

Combined Shear and Buoyancy Instabilities (T33)

J. G. Wouchuk

Rate of Growth of the Linear Richtmyer-Meshkov Instability (T34)

A. Yosef-Hai, O. Sadot, D. Kartoon, D. Oron, E. Sarid, G. Ben-Dor, and D. Shvarts The Dependence of the Shock Induced Richtmyer-Meshkov Instability on Dimensionality and Density Ratio (T35)

Y. Zhou, H. F. Robey, and A. C. Buckingham

A New Framework for Transitional and Turbulent Mixing (T36)

A. L. Kuhl and R. E. Ferguson

Spherical Combustion Layer in a TNT Explosion (T37)

V. F. Tishkin and N. V. Zmitrenko

Spectral Analysis of Turbulent Flows Induced by RT and RM Instabilities (T38) 
Pasadena, CA (2001)

B. B. Afeyan, P. Ramaprabhu, and M. J. Andrews

Pattern Detection, Compression and Denoising of Rayleigh-Taylor Mix Data Using Discrete

Wavelet Transform Techniques (T39)

Author Contact Information

Author Index 
$8^{\text {th }}$ International Workshop on the Physics of Compressible Turbulent Mixing,

Pasadena, CA (2001)

REVIEW TALKS 


\title{
A Review on RT and RM Instability and TM Experiments
}

\author{
J.-F. Haas ${ }^{1}$ and S. G. Zaytsev ${ }^{2}$ \\ ${ }^{1}$ Commissariat à l'Energie Atomique, Bruyères-le-Châtel, France \\ ${ }^{2}$ Krzhizhanovsky Power Engineering Institute, Moscow, Russia
}

We analyse the state of the art for experiments on Rayleigh-Taylor, Richtmyer-Meshkov instabilities (RTI, RMI) and the resulting (possibly compressible) turbulent mixing (TM). There is a very wide spectrum of state of matter (in addition to acceleration history and magnitude) ranging from « ordinary » gas or liquid dynamics experiments, through intermediate complexity detonation-driven setups often involving materials with strength, all the way to large laser driven experiments for the study of high energy density plasmas. Experiments are needed for a better understanding of fundamental mechanisms (e.g. nonlinear regime of the RTI and RMI, complex natural phenomena (supernovae explosions) and future applications (inertial confinement fusion). We consider first the field of « simple» gas dynamics RMI and TM experiments performed in ordinary shock tubes. It was suggested at the experimental roundtable of the $7^{\text {th }}$ IWPCTM that the experiment type could be presented on a map with an horizontal axis for a compressibility parameter (such as the incident shock Mach number) and a vertical axis for the instability strength (e.g., according to the linear RMI formula, the product of Atwood number, perturbation wave number and initial amplitude). A second map was proposed, with a horizontal axis tentatively called "usefulness to theory " and a vertical one labelled " complexity of diagnostics ». The classical visualization by refractive effects (shadowgraph, schlieren, Mach-Zehnder or differential interferometry) is useful for geometrical observations of the mixing zones and instability patterns, but of limited quantitative value in TM. The modern laser sheet method provides a 2D map of the density or concentration field (via Mie or Rayleigh scattering or fluorescence). Flash X-rays absorption by Xe, infrared emission or infrared $\mathrm{CO}_{2}$ laser absorption by shock heated $\mathrm{CO}_{2}$ allow density measurements within binary mixing zones containing these gases. Laser Doppler Velocimetry (giving the velocity history at a given position) and Particle Image Velocimetry (giving a velocity map at a given time) have recently been introduced. The Hot Wire Anemometer provides the time evolution at a fixed position of the Nusselt number, which depends on velocity, concentration and temperature. The initial gas separation is best membrane-less for RMI and with microfilm for fine scale TM. Often, a good experiment for theory benefits from an imaginative conception while advanced diagnostics are useful for a quantitative comparison with numerical simulation and TM modelling. Among the cold hydrodynamics RTI experiments, some based on gravitational mixing of liquids (molecularly miscible or not, with or without surface tension) increasingly benefit from modern diagnostics. The experiments on gases in modified shock- or combustion tubes allow the investigation of compressibility and acceleration nonsteadiness, but with usually less precise diagnostics. There is a variety of gas detonation or combustion experiments in which the effect of cylindrical geometries are tested. Effects of initial interfacial perturbations and material strength can be investigated with jellies. Solid explosive drivers are needed for the measurement of such RT/RM processes in metals. Among recent high energy laser driven plasma experiments, the high quality visualizations in some very high Mach number experiments are fascinating. Our challenge is to insure that simpler and cheaper gas or liquid dynamics experiments will remain useful with the advent of the next generation of ICF lasers.

PACS Nos.: 42.79, 47.20, 47.27, 47.40, 52.57 
$8^{\text {th }}$ International Workshop on the Physics of Compressible Turbulent Mixing,

\title{
The Experimental Study of Excitation and Development of the Hydrodynamic Instability in the Mixing Zone Separating Gases of Different Densities at their Accelerated Motion
}

\author{
S. G. Zaytsev \\ Krzhizhanovsky Power Engineering Institute, Moscow, Russia
}

The properties of the mixing zone between gases of different densities during accelerated and decelerated motion caused by compression waves have been analyzed. The design of the experimental set-up for study of the mentioned processes has been described.

The wave diagrams of possible regimes of flows and basic parameters such as velocity $\mathrm{u}$, acceleration $\mathrm{g}$, density $\rho$, pressure $\mathrm{p}$, and temperature $\mathrm{T}$ have been under consideration.

The properties of excitation and development of the Rayleigh-Taylor instability (RTI) at the stages of accelerated and decelerated motion of the mixing zone have been described.

The characteristics of the mixing zone at the stage of "stratification" caused by interaction with a reflected compression wave - non-shocked deceleration- have been defined. The mixing zone volume decreases in this case.

The generation of shocks during evolution of compression waves and their interaction with the mixing zone results in growth of the mixing volume caused by excitation of the Richtmyer-Meshkov instability (RMI).

The amount of the substance involved into mixing during accelerated motion of the mixing zone separating gases of different densities has been defined. The problem of influence of the working media compressibility on process of the mixing has been discussed. 


\title{
Review of Numerical Simulation of Mixing due to Rayleigh-Taylor and Richtmyer-Meshkov Instabilities
}

\author{
D. L. Youngs \\ Atomic Weapons Establishment, Aldermaston, United Kingdom
}

Since the late 1960s numerical simulation has been very successfully used to gain insight into the non-linear growth of Rayleigh-Taylor (RT) and Richtmyer-Meshkov(RM) instabilities. The first calculations were for single-mode growth in 2D. Then in the 1980s, 2D multimode simulations became feasible. 3D simulations of fully-developed turbulent mixing in simple situations have been performed during the past few years. There are many examples where numerical simulation has been used to study the affect of additional physical processes, such as ablation stabilisation or material strength, on instability growth. The progress made is reviewed and instances where numerical simulation has enhanced our understanding are highlighted. The main emphasis of this review will be on RT and RM instability. However, some reference will be made to simulations of turbulent shear flow and homogeneous turbulence, especially where ideas from these areas are relevant to RT/RM studies.

The numericalmethods used will be discussed. RT and RM problems involve discontinuities, contact surfaces or shocks. TVD schemes have proved popular and number of researchers have used interface tracking techniques. For 3D LES (Large Eddy Simulation)dissipation at high-wavenumbers is needed. Many researchers, especially those who work on turbulent shear flow, favour the use of an explicit sub-grid model to represent this effect. The TVD methods have high-wavenumber dissipation inherent in the numerical scheme and when applied to turbulence simulations are referred to as MILES schemes (Monotone Implicit LES). There have been a number of examples of the application of MILES to RT and RM turbulent mixing and also some examples of 3D DNS (Direct Numerical Simulation) in which the effects of viscosity and diffusivity are resolved.

The future role of numerical simulation will be discussed. 2D simulation will continue to be useful for understanding complex experiments or the effect of additional physics, where 3D simulation is impractical. However, with the advent of very powerful supercomputers, 3D simulation (LES or DNS) will become increasingly useful and will give a detailed understanding of turbulent mixing in simplified situations. It is likely that RANS (Reynolds-AveragedNavier-Stokes)models will contine to be essential for modelling the most complex real applications. However, 3D simulation can make an important contribution here as well. In addition to experimentaldata, the results of 3D simulations should be used to validate the RANS models in simplified situations. 
$8^{\text {th }}$ International Workshop on the Physics of Compressible Turbulent Mixing,

Pasadena, CA (2001)

\title{
Modeling Late-Time Nonlinear Evolution of Hydrodynamic Instabilities and their Role in Inertial Confinement Fusion
}

\author{
D. Shvarts \\ Ben-Gurion University, Beer Sheeva, Israel and Nuclear Research Center, Negev, Israel
}

Abstract not available at time of printing. 


\section{EXPERIMENTAL ABSTRACTS}


$8^{\text {th }}$ International Workshop on the Physics of Compressible Turbulent Mixing,

\title{
Abstract No. E2 \\ Experimental Investigations of the Heavy and Light Media Separation in the Rayleigh-Taylor Turbulence Zone at Different Atwood Numbers
}

\author{
Yu. A. Kucherenko, S. I. Balabin, R. I. Ardashova, A. P. Pylaev, O. E. Kozelkov, and \\ V. D. Murzakov \\ Russian Federal Nuclear Center - VNIITF, Snezhinsk, Russia
}

In the paper the experimental results with respect to the nondimensional rate of separation in the Rayleigh-Taylor turbulence zone are presented. In the experiments two different density liquids separated by a plane contact boundary were accelerated so that in the first phase of acceleration the Rayleigh-Taylor instability evolved and the definite zone of the turbulent mixing formed. At the second phase the sign of acceleration was jumpwise changed into the opposite one. As a result, the system of two different density liquids became stable. At these instants of time, in the turbulent mixing zone the separation processes of the heavy and light liquids evolved.

For three values of Atwood numbers the experiments were performed. for each of Atwood numbers the nondimensional rate of separation was determined. 


\title{
Abstract No. E3 \\ Experimental Investigation into Influence of Stabilizing Properties of Transitional Layers Upon the Turbulent Mixing Evolution
}

\author{
Yu. A. Kucherenko, S. I. Balabin, R. I. Ardashova, O. E. Kozelkov, A. V. Dulov, and \\ I. A. Romanov \\ Russian Federal Nuclear Center - VNIITF, Snezhinsk, Russia
}

It is presented the results of the experimental investigation of the transitionallayers width influence upon the evolution of turbulent mixing caused by the Rayleigh-Taylor instability. In experiments, mutual soluble liquids with density relation been equal to two were used. A transitional layer having continuous distribution of density arises in the region of liquids contact because of molecular diffusion. In experiments, it has been determined the dependence of the turbulent mixing evolution delay on both the initial perturbation region size and the characteristic width of the transitional layer. 
$8^{\text {th }}$ International Workshop on the Physics of Compressible Turbulent Mixing,

\title{
Abstract No. E4 \\ Improvements to Convergent Cylindrical Plasma Mix Experiments Using Laser Direct Drive
}

\author{
C. W. Barnes ${ }^{1}$, S. H. Batha ${ }^{1}$, A. M. Dunne ${ }^{2}$, N. E. Lanier ${ }^{1}$, G. R. Magelssen ${ }^{1}$, \\ T. J. Murphy ${ }^{1}$, K. W. Parker ${ }^{2}$, S. Rothman ${ }^{2}$, J. M. Scott ${ }^{1}$, and D. Youngs ${ }^{2}$ \\ ${ }^{1}$ Los Alamos National Laboratory, Los Alamos, NM \\ ${ }^{2}$ Atomic Weapons Establishment, Aldermaston, United Kingdom
}

Experiments studying mix in a compressible, convergent, miscible, plasma system are being conducted on the OMEGA Laser at the Laboratory for Laser Energetics at the University of Rochester.1.2 Thin-walled polystyrene cylinders $2.25-\mathrm{mm}$ long and $0.86 \mathrm{~mm}$ inner diameter with foam inside are directly illuminated with $351-\mathrm{nm}$ wavelength light from 50 laser beams in a 1-ns square laser pulse. The turbulence driven by the Richtmyer-Meshkov instability by shock passage across a density discontinuity mixes marker material that is radiographically opaque. Initial work using a high-density, high-opacity marker layer of gold between the plastic ablator and foam clearly demonstrated significant measurable mix width ${ }^{2}$. However, the high opacity of the gold prevented determination of a density profile in the mix region, and it was also overly sensitive to hydrodynamic effects at the end of the marker layer. Use of lower opacity marker material will be described and its impact on end effects and the measurements of mix density profile described.

1 C. W. Barnes et al., Rev . Sci. Instrum. 70 (1999) 471.

2 C. W. Barnes et al., submitted to Physical Review Letters (2001). 
Abstract No. E5

\title{
Mixing Between Two Compressing Cylinders
}

\author{
S. H. Batha ${ }^{1}$, K. W. Parker ${ }^{2}$, C. W. Barnes ${ }^{1}$, A. M. Dunne ${ }^{2}$, \\ N. E. Lanier ${ }^{1}$, G. R. Magelssen ${ }^{1}$, T. J. Murphy ${ }^{1}$, S. Rothman ${ }^{2}$, \\ J. M. Scott ${ }^{1}$, and D. Youngs ${ }^{2}$ \\ ${ }^{1}$ Los Alamos National Laboratory, Los Alamos, NM \\ ${ }^{2}$ Atomic Weapons Establishment, Aldermaston, United Kingdom
}

Foam-filled cylinders have been imploded by the OMEGA laser at the University of Rochester. A marker layer of heavier material is placed between the foam and the outside ablator. The marker layer is hydrodynamically unstable when a strong shock passes through both these interfaces and the marker layer material mixes into the foam and the ablator. These experiments thus measure mix in the compressible, convergent, miscible, strong-shock regime.

These experiments are being extended by placing a solid cylinder at the center of the foam, forming a set of concentric cylinders separated by foam. The initial shock converges on the central cylinder and then rebounds and expands. The shock is predicted to create even more mixing of the marker layer as it traverses the previously mixed region. We present experimental measurements of this configuration. 
$8^{\text {th }}$ International Workshop on the Physics of Compressible Turbulent Mixing,

\title{
Development of a Method for Studying the Interaction Between Shock Wave and a Flame Front
}

\author{
M. Bliznetsov ${ }^{1}$, V. Dudin ${ }^{1}$, S. Gerasimov ${ }^{1}$, L. Houas ${ }^{3}$, G. Jourdan ${ }^{3}$, A. Logvinov ${ }^{1,2}$, \\ E. Meshkov ${ }^{1,2}$, and Yu. Vlasov ${ }^{1,2}$ \\ ${ }^{1}$ Russian Federal Nuclear Center - VNIIEF, Sarov, Russia \\ ${ }^{2}$ SarPTI, Sarov, Russia \\ ${ }^{3}$ IUSTI/CNRS, Université de Provence, Marseille, France
}

Tomsk University has carried out research into explosive method for extinguishing the wild fires (A.M.Grishin, Kovalev Yu.M., 1994, Grishin, 1994, Grishin et al., 2000). This method has been experimentally checked but it is not currently used in practice. According to Grishin's hypothesis (A.M.Grishin, Kovalev Yu.M., 1994, Grishin, 1994), the explosivemethod for extinguishingthe wild fires is based on "blowing out" the flame by a shock. There exists another idea (Meshkov, 1999) according to which this extinguishing is due to development of hydrodynamic instabilities. Experimental study of this method in natural conditions is rather complex, dangerous, and expensive. Hence, modeling this method in laboratory conditions is of interest. We report on results of an experimental study of developing such a method for interaction between shock and flame.

\section{References}

Grishin A.M., Babaev V.M., Gruzin A.D., Zverev V.G., Abaltusov V.E., Mamontov G.Ya. (1985). Meaning of extinguishing wild fires A.D. 1136811 USSR. Published 30.01.85. Bul.4

Grishin A.M., Kovalev Yu.M. (1989). Experimentaland theoretical study of the interaction between explosion and crown fire. FGV, 6, pp. $72-79$

Grishin A.M., Zima V.P., Mashovich A.Ya., Samoilov V.I. (2000). Experimental study of the interaction between a shock induced by point charges, and crowns. Proceedings of international conference "Common problems on mechanics and ecology", Tomsk University, pp.83-85

Meshkov E.E. (2000). Turbulent mixing associated with hydrodynamic instabilities in modern practical problems. Proceedings of international conference "Common problems on mechanics and ecology", Tomsk University, pp.156-158. 


\title{
Abstract No. E7 \\ The Influence of Scaling for Periodical Perturbations on Development of Turbulent Mixing on a Gas-Liquid Interface
}

\author{
M. Bliznetsov, E. Meshkov, N. Nevmerzhitsky, A. Nikulin, E. Sen'kovsky, and E. Sotskov \\ Russian Federal Nuclear Center - VNIIEF, Sarov, Russia
}

We report on results of experimentalstudy of the influence of scaling for 3D periodical perturbations on dynamics of turbulent mixing on a gas-liquid interface, associated with R-T instability. The liquid was modeled with layers of a low-strength $(=0.01 \mathrm{MPa})$ water-solved gelatin jellies driven in a squared (40x40-mm) channel with helium compressed to $13 \mathrm{~atm}$. The perturbations imposed on unstable surface had quadrilateral pyramidal structures with a height equal to the perturbation wavelength $(=(0=0.25 ; 1 ; 2,2.86 \mathrm{~mm}$. The experimental results obtained in tests without imposed perturbations of a given shape are also presented. The acceleration of layers was of $3 \times 10^{4} \mathrm{~m} / \mathrm{s}^{2}$. The obtained results show:

- When the perturbations are not originally given or when they have $(=(0=0.25$ on unstable surface, the turbulent mixing develops with an initial delay, and then it grows linearly as $(\mathrm{l}=\mathrm{dhl} / \mathrm{d}(2 \mathrm{~S})=0.12(0.025$, where (l- intensity for penetration of light substance into heavy, hl- depth of the gas- into- jelly penetration, $\mathrm{S}$ - layer displacement,

- For $(=(0=0.25 ; 1 ; 2,2.86$, the R-T instability grows simultaneouslywith the layer movement, and turbulent mixing has linear regime for light-into-heavy penetration $(1=0.1(0.14$. $(1$ increases from 0.13 to 0.42 with growing the perturbation amplitude; hence, for the given range of initial periodical perturbations their amplitudes influence weakly on the rate of the light-into-heavy penetration, but sufficiently on the heavy-into-light. 
$8^{\text {th }}$ International Workshop on the Physics of Compressible Turbulent Mixing,

\title{
Abstract No. E8 \\ Compressible Vortex Rings
}

\author{
M. Brouillette and C. Hébert \\ Université de Sherbrooke, Quebec, Canada
}

We present the results of an experimental study aimed at characterising compressible and viscous effects on the generation and propagation of vortex rings. The overall aim of this study is to characterise basic vortical structures in the context of compressibleturbulence, but these findingsalso have applications in the study of shock-vortex interaction, for example. In the context of the Richtmyer-Meshkov instability, these rings can be viewed as rapidly mushrooming spikes at moderate Atwood numbers.

The vortices are produced by the diffraction of a shock wave from the open end of the driven section of a specially-built shock tube. By varying the pressure ratio across the driver and driven sections we could control the strength of the incident shock wave. Also, by altering the length of the driver, we could modify the ejection velocity history, also known as the ejection velocity program, at the orifice end of the tube. Finally, by changing the diameter of the end orifice, we could change the size, i.e., Reynolds number, of the vortex ring. Our instrumentation comprised fast-response piezoelectric pressure transducers and flow visualisation was achieved with shadow and schlieren photography along with holographic interferometry.

A major difference between incompressible and compressible vortex ring formation is in the maximum circulation attained in the ring. Previous studies have found that the vorticity saturation threshold of incompressible vortex rings was not a strong function of the ejection velocity program; we found that this was not the case for compressible vortex rings. In the present study, we found that a higher normalised circulation was possible, for a given incident shock wave, with a continuous jet at the exit of the tube than with a rapidly attenuated jet.

The appearance of a shock wave within the recirculating region of the vortex ring is also strongly dependant on the amount of vorticity deposited within the ring. In fact, the onset of appearance of this shock wave and other shock and vortical features around the main vortex ring can now be related to vorticity deposition through the ejection velocity program.

Although the vortex formation mechanism of the present study is inherently compressible and nonlinear, the propagation of these vortex rings is similar to that of incompressible rings reported in numerous previous studies. The principal compressibility effect is in the structure of the vortex core, which appears to exhibit a Reynolds number dependence.

Because of the wide range of viscous and convectivescales present in this problem, experiments such as these can also pose an interesting challenge to direct numerical simulation in the context of compressible turbulence. 


\title{
Abstract No. E9 \\ Design of Flyer-Plate-Driven Compressible Turbulent Mix Experiments
}

\author{
R. P. Drake \\ University of Michigan, Ann Arbor, MI
}

In this work we consider the optimization of experiments that use flyer plates to study compressible turbulent mixing. There are now at least two types of flyer plates that can be used for such purposes. The recent advent of high-velocity $(>20 \mathrm{~km} / \mathrm{s})$, solid state flyers on the $\mathrm{Z}$ machine at Sandia National Laboratories, along with the pending activation of $x$-ray backlighting, will make possible very clean experiments. In addition, any large laser can shock and accelerate a slab of material, producing a "plasma flyer" that can deliver energy and momentum to a desired target. If the laser is large enough $(\sim 1 \mathrm{~kJ})$, then the plasma flyer can have sufficient lateral size to permit studies of mixing. Here we consider the problem of designing of an optimized experiment.

This poster will discuss the optimization of a flyer-driven experiment for either Rayleigh Taylor (RT) or Richtmyer Meshkov (RM) experiments. In RT experiments, one wants to decelerate an interface immediately after it is shocked (with minimum coasting time), and to move the interface as far as possible. In RM experiments, one wants to cause the interface to coast steadily after it is shocked, for the longest possible time. This poster will present an analysis and analytic relations that can guide the achievement of these goals, and hydrodynamic simulations showing what one can do using flyer plates on $\mathrm{Z}$. 
$8^{\text {th }}$ International Workshop on the Physics of Compressible Turbulent Mixing,

Abstract No. E10

\title{
Compressible Hydrodynamics on the Omega Laser, Motivated by Astrophysics
}

\author{
R. P. Drake ${ }^{1}$, P. Keiter ${ }^{1}$, K. E. Korreck ${ }^{1}$, K. Dannenberg ${ }^{1}$, H. A. Robey ${ }^{2}$, T. Perry ${ }^{2}$, \\ J. O. Kane ${ }^{2}$, B. A. Remington ${ }^{2}$, R. J. Wallace ${ }^{2}$, O. A. Hurricane ${ }^{2}$, D. D. Ryutov ${ }^{2}$, \\ J. Knauer ${ }^{3}$, R. Teyssier ${ }^{4}$, A. Calder ${ }^{5}$, R. Rosner ${ }^{5}$, B. Fryxell ${ }^{5}$, D. Arnett ${ }^{6}$, Y. Zhang ${ }^{7}$, \\ J. Glimm ${ }^{7}$, N. Turner ${ }^{8}$, J. Stone ${ }^{8}$, R. McCray ${ }^{9}$, J. Grove $^{10}$ \\ ${ }^{1}$ University of Michigan, Ann Arbor, MI \\ ${ }^{2}$ Lawrence Livermore National Laboratory, Livermore, $C A$ \\ ${ }^{3}$ Laboratory for Laser Energetics, University of Rochester, Rochester, NY \\ ${ }^{4}$ Commissariat à l'Energie Atomique, Saclay, France \\ ${ }^{5}$ University of Chicago, Chicago, $I L$ \\ ${ }^{6}$ University of Arizona, Tuscon, AZ \\ ${ }^{7}$ State University New York Stony Brook, Stony Brook, NY \\ ${ }^{8}$ University of Maryland, College Park, $M D$ \\ ${ }^{9}$ University of Colorado, Boulder, $\mathrm{CO}$ \\ ${ }^{10}$ Los Alamos National Laboratory, Los Alamos, NM
}

Compressible turbulent mixing is an inherent feature in supernovae, supernova remnants, and related systems. Our scientific team collaborates to produce, in the laboratory, hydrodynamic mechanisms that are important for the evolution of such systems. These experiments are designed to be well scaled from astrophysical systems to the laboratory. This talk will provide an overview of this work and will highlight our most recent results. Our work is motivated by the specific fact that highresolution 2D and 3D numerical simulations have proven unable to reproduce certain aspects of observations of supernova SN 1987A, and by the general need to provide experimental tests of modeling of hydrodynamic systems. The experiments take place on the Omega Laser at the Laboratory for Laser Energetics, University of Rochester. We have explored the coupling between unstable interfaces, instability growth in a diverging system, the comparison of 2D and 3D systems, the comparison of single mode and multimode systems, and the production and diagnosis of a radiative-precursor shock. In each of these cases, the experiment begins by using the laser to drive a strong shock into a target material. This produces a hydrodynamic initial state that can be modeled by any astrophysical or laboratory hydrodynamics code. The shock subsequently interacts with other structures in the target, which we design in order to explore a specific physical issue. In each case, we then compare the results of the experiments with those of computer simulations. The US DOE and NASA supported this work.

This work was performed under the auspices of the U.S. Department of Energy by the University of California Lawrence Livermore National Laboratory under contract No. W-7405-Eng-48. 
Abstract No. E11

\title{
Growth of Perturbations on Metals Interface at Oblique Collission with Supersonic Velocity of Contact Point Motion
}

\author{
O. B. Drennov, A. L. Mikhaylov, P. N. Nizovtsev, and V. A. Raevskii \\ Russian Federal Nuclear Center - VNIIEF, Sarov, Russia
}

By now the subsonic mode of collision is studied in detail. In the supersonic mode, when shock waves arrive to the contact point, jet formation is impossible. It is assumed that perturbations growth at metals interface is also impossible. It is obtained in our experiments that perturbations are formed in the mode of supersonic jetless oblique collision at the metals interface. Analytical consideration of the problem determined existence of the critical value of Mach number characterizing transition from the stability area to the instable area. Numerical calculations with use of the twodimensional Lagrange technique showed presence of an area with large gradient of velocity and high intensity of strains near the contact point. It results in fulfillment of the conditions for growth of Kelvin-Helmholtz instability. Comparison of calculated and experimental values of amplitude of occurred perturbations showed a rather good agreement between them. 
$8^{\text {th }}$ International Workshop on the Physics of Compressible Turbulent Mixing,

Abstract No. E12

\title{
An Experimental Study of the Effect of Shock Proximity on the Richtmyer-Meshkov Instability at High Mach Number
}

\author{
S. G. Glendinning, D. G. Braun, M. J. Edwards, W. W. Hsing, B. F. Lasinski, \\ H. Louis, J. Moreno, T. A. Peyser, B. A. Remington, H. F. Robey, E. J. Turano, \\ C. P. Verdon, and Y. Zhou \\ Lawrence Livermore National Laboratory, Livermore, CA
}

The effect of shock proximity on the non-linear evolution of Richtmyer-Meshkov instability of a sinusoidal perturbation at high Mach number was examined on experiments at the Omega laser at the Laboratory for Laser Energetics, University of Rochester. We will present results from experiments using a laser drive of about $3 \times 10^{13} \mathrm{~W} / \mathrm{cm}^{2}$ and targets made with polycarbonate as a pusher and carbon foam $(\rho=0.1 \mathrm{~g} / \mathrm{cc})$ as a payload. This provided an incident shock of Mach number $\sim 10$, a nearly constant interface velocity for $10 \mathrm{~ns}$, and a transmitted shock to interface velocity ratio of about 1.22. Wavelengths studied varied between $50 \mu \mathrm{m}$ and $150 \mu \mathrm{m}$. Different amplitudes were chosen to allow linear growth, nonlinear growth, or proximate-shock growth to dominate.

This work was performed under the auspices of the U.S. Department of Energy by the University of California Lawrence Livermore National Laboratory under contract No. W-7405-Eng-48. 
Abstract No. E13

\title{
Mix Experiments using a Two Dimensional Convergent Shock Tube
}

\author{
D. A. Holder, C. Barton, and A. V. Smith \\ Atomic Weapons Establishment, Aldermaston, United Kingdom
}

This paper reports on the first Richtmyer Meshkov instability mix experiments using the improved version of the AWE detonation driven Convergent Shock Tube (CST). Results from an early prototype presented at the $7^{\text {th }}$ IWPCTM demonstrated concept feasibility, but also the need for refinement of the multi-point ignition process that critically controlled cylindricity of the generated shock. This paper includesa brief description of the modifications undertaken to achieve the required performance; also images recording the origin of the detonation wave formation and combination process. The CST facility has been created to allow an extension of earlier RMI studies using a conventional linear shock tube at low Mach number to two-dimensional studies at Mach number 2 -3 .

The current configuration is as shown, with height (internal) $1.02 \mathrm{~m}$ : depth $50 \mathrm{~mm}$ : apex angle $30^{\circ}$. Detonation of an oxyacetylene gas mixture by 30 sparkplugs drives a cylindrically converging shock of order 10 bar into a dense gas region, bounded by cylindrically curved microfilm membranes, supported by fine wire meshes. Maximum compression of the dense gas during its motion into the apex region is $\sim 40$ for sulphur hexafluoride, or optionally, $\sim 20$ for xenon.

Visualisation is currently by shadowgraphy using a pulsed copper vapour laser and drum camera. This provides a timed sequence of images of the mixing development over the dense gas region. Results from basic experiments with two unperturbed interfaces will be presented, with comparisons to TURMOIL3D code calculations.

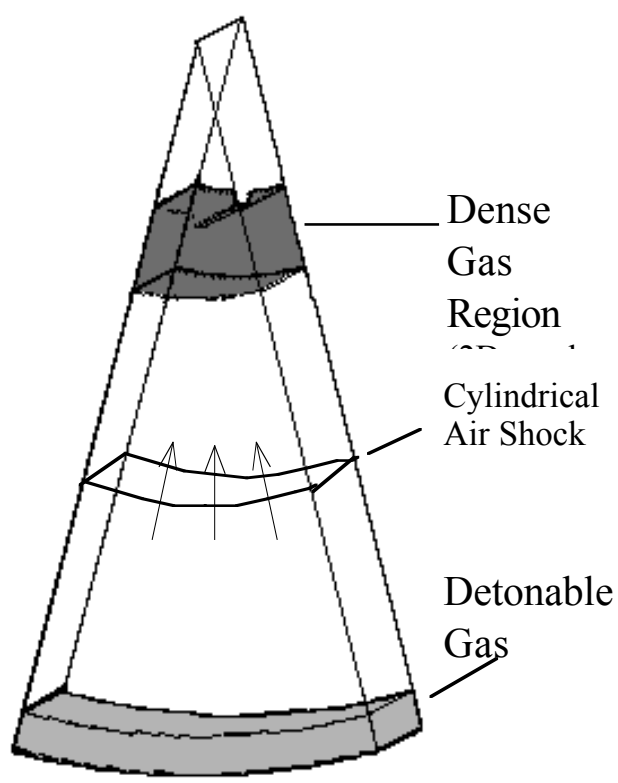

Additionally results will be shown from the first experiments to feature a perturbation superimposed on one interface. These will serve as a forerunner to the proposed investigations in 2D geometry of a series of perturbation profiles previously investigated using the AWE linear shock tube [1]. The results will be used to validate 2D turbulent mix models. Proposals will also be included for incorporating improved diagnostic techniques including the laser sheet technique with ICCD camera recording to facilitate image analysis and derivation of quantitative data.

PACS No.: 47.20.Ma 
$8^{\text {th }}$ International Workshop on the Physics of Compressible Turbulent Mixing,

Abstract No. E14

\title{
Rayleigh-Taylor Instability at a Tilted Interface in Incompressible Laboratory Experiments and Compressible Numerical Simulations
}

\author{
J. M. Holford ${ }^{1}$, S. B. Dalziel ${ }^{1}$, and D. Youngs ${ }^{2}$ \\ ${ }^{1}$ Cambridge University, Cambridge, United Kingdom \\ ${ }^{2}$ Atomic Weapons Establishment, Aldermaston, United Kingdom
}

An important feature of Rayleigh-Taylor (RT) instability is the significant amount of molecular mixing that occurs, due to the small scales that are created. Molecular mixing is important both as a control on the rate of reaction between chemically active species, and because it creates a sink for available energy by allowing the background potential energy of the flow to increase. RT instability is the most efficient known mixing process, and laboratory measurements show that, over the whole flow evolution, up to $40 \%$ of the initial available energy increases the potential energy of the background stratification, while the balance is lost to viscous dissipation.

Regions of locally unstable stratification are frequently created in perturbed stratified flows, for example in breaking gravity waves and shear-driven billows. However, in these naturally occurring statically unstable regions, the initial conditions are far from the idealised classical RT instability. In this study, we investigate the mixing that occurs in RT instability at an interface that is initially tilted at an angle to the horizontal, introducing a competition between the local overturning of RT instability and a large-scale overturning within the whole domain.

RT instability is initialised in a water tank in the laboratory by withdrawing a barrier separating dense salt water above from fresh water below. Measurements of both the density distribution and in-plane velocity field are made in a vertical slice through the centre of the tank. For the first time, measurements of the instantaneous efficiency of mixing are made. The instantaneous efficiency at early times can be higher than the cumulative efficiency, with the rate of increase of potential energy of the background stratification reaching $50 \%$ of the rate of decrease of available energy. The reduction in the cumulative mixing efficiency as the angle of the initial interface increases is quantified.

The experiments have been modelled numerically using the compressible code TURMOIL3D. The initial conditions are carefully chosen to model the incompressibleexperiments as closelyas possible. Analyses of energy and concentration fluctuation spectra are used to understand the mixing and dissipation processes. The combination of experimental and computational modelling is shown to be useful both as a validation of the numerical methods and as a tool for understanding the basic dynamics of the flow. 


\title{
Abstract No. E15 \\ Study of Diverging and Converging Spherical Shock Waves Induced by Micro Explosives and their Interaction with Product Gases
}

\author{
S. H. R. Hosseini and K. Takayama \\ Tohoku University, Sendai, Japan
}

The paper reports an experimental study of production and propagation of spherical shock waves. In order to quantitatively observe spherical shock waves and the flow field behind them, an aspheric spherical transparent test section was designed and constructed. This $150 \mathrm{~mm}$ inner-diameter aspheric lens shaped test section permits the collimated visualization laser beam to traverse the test section parallel and emerge parallel. Spherical diverging shock waves were produced at the center of the spherical test section. In order to generate shock waves, irradiation of a pulsed Nd:YAG laser beam on micro silver azide pellets were used. The weight of silver azide pellets ranged from 5 to 20 $\mathrm{mg}$, with their corresponding energy of 9 to $36 \mathrm{~J}$. Pressure histories at different points over the test section were measured to validate production of uniform shock waves. After reflection of spherical shock wave from the test section, a converging spherical shock wave was produced and its interaction with the interface of explosive product gas was studied. Double exposure holographic interferometry and time resolved high speed photography were used for flow visualization. The whole sequence of diverging and converging spherical shock waves propagation and their interaction with product gases were observed.

PACS No.: 47.40.Nm 
$8^{\text {th }}$ International Workshop on the Physics of Compressible Turbulent Mixing,

\title{
Abstract No. E16 \\ Interaction of Converging Shock Waves With Cylindrical Heavy Gas Interfaces in an Eccentric Arrangement
}

\author{
S. H. R. Hosseini and K. Takayama \\ Tohoku University, Sendai, Japan
}

Paper reports a study on interaction of converging and diverging cylindrical shock waves in air with non-uniform gaseous media and resulting Richtmyer-Meshkov instability. An annular vertical coaxial diaphragmless shock tube was used to produce converging cylindrical shock waves. Cylindrical soap bubbles filled with $\mathrm{SF}_{6}$ heavy gas were placed out of the geometricalcenter of shock tube's test section. As a result of asymmetry between converging cylindrical shock waves and cylindrical interfaces, a complex wave motion and interaction was produced. Pressure histories at different radii were measured during the converging and diverging shock wave propagation in the test section after interaction. A strong secondary shock wave focusing in the $\mathrm{SF}_{6}$ test gas with a high peak overpressure was observed. Double exposure holographic interferometry was used for flow visualization. The time evolution of turbulent mixing zone between the air/ $\mathrm{SF}_{6}$ light/heavy gases and interfacial deformations were quantitatively studied. After the secondary shock wave focusing in the $\mathrm{SF}_{6}$ a relatively strong jet, which was penetrating to the air in the direction of geometricalcenter, was produced.

PACS No.: 47.40.Nm 
Abstract No. E17

\title{
From Linear to Turbulent Stages of the Richtmyer-Meshkov Instability Development in a Large Cross Section Shock Tube
}

\author{
L. Houas ${ }^{1}$, G. Jourdan ${ }^{1}$, L. Schwaederlé ${ }^{1}$, and E. E. Meshkov² \\ ${ }^{1}$ IUSTI, CNRS, Université de Provence, Technopôle de Château-Gombert, \\ Marseille, France \\ ${ }^{2}$ Russian Federal Nuclear Center - VNIIEF, Sarov, Russia
}

The aim of the present investigation is to contribute to the understanding of the turbulence transition phases by the help of the development of the Richtmyer-Meshkov instability (RMI). In this way, we have built a new shock tube which allows to follow a Richtmyer-Meshkov instability induced mixing from its beginning to the fully turbulent developed stage with both control and knowledge of the initial conditions. Up today, all experiments developed to study the RMI have been focussed on the observation of the growth of the initial perturbations in the linear and the non-linear regimes and not far, or the investigation of the turbulent phase without knowing the real initial conditions, i.e. when the shock wave interacts with the thin membrane which initially separates the two fluids of different densities. Thus, the new shock tube has first a large square cross section $(20 \mathrm{~cm}$ by $20 \mathrm{~cm})$ in order to prevent from wall effects. Furthermore a suitable experimental chamber permits to observe and control the initial perturbations we impose to the thin material interface which initially separates the two gases expected to mix together after the incident shock wave accelerates their common interface. The total length of the shock tube is of $7 \mathrm{~m}$, the experimental chamber is $50 \mathrm{~cm}$ total length and its field of view starts from $4 \mathrm{~cm}$ before the initial position of the interface to $46 \mathrm{~cm}$ after. To follow the development of the initial perturbationsand the mixing of the two gases, we have carried out a Mie scattering laser sheet technique. A $50 \mathrm{Khz}$ Oxford copper vapor laser beam is transformed as a laser sheet before crossing the experimental chamber in its length direction. This ultra rapid laser is coupled with a 321 Cordin model high speed camera, which together allow to record, during the same run, about 100 plane frames of the experiment spaced by 100 to $20 \mu \mathrm{s}$ depending on the laser frequency (from 10 to $50 \mathrm{Khz}$ ). The maximum recorded image rates is of about 50,000 pictures per second. Moreover, a suitablerotating mirror device accurately coupled and synchronized with the laser-camera system, permits to translate, during the same run, the laser sheet from the center axis of the experimental chamber to its walls in order to obtain a $3 \mathrm{D}$ visualization of the phenomenon. The gas initially present in the experimental chamber is seeded with water vapor particles. The test gases are air/He, air/Ar and air/Kr in order to investigate the cases where the shock wave passes from a heavy to a light gas and vice-versa. The initial pressure is $1 \mathrm{~atm}$. and the shock wave Mach number in air is of 1.3. The principle of the experiment and a view of the experimental set up are shown on Figure 1. We are now running the first experiments and we hope to present in the full paper the first results illustrating, during the same run, the development of the different 
Pasadena, CA (2001)

stages of the instability, the transient phases as well as some information on the fully turbulent regime.

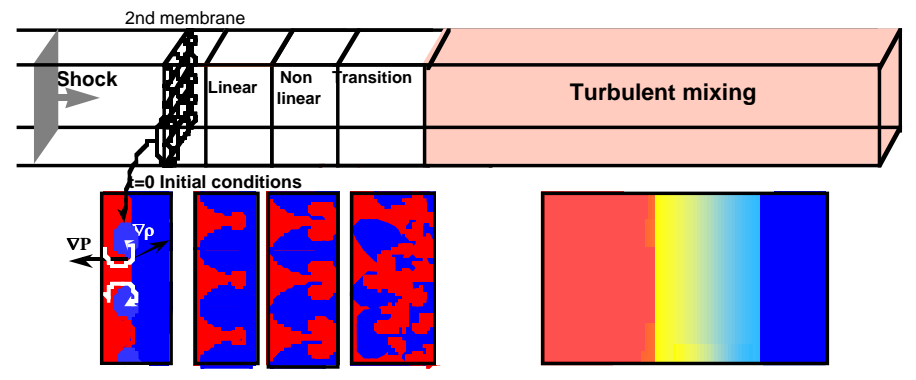

(a)

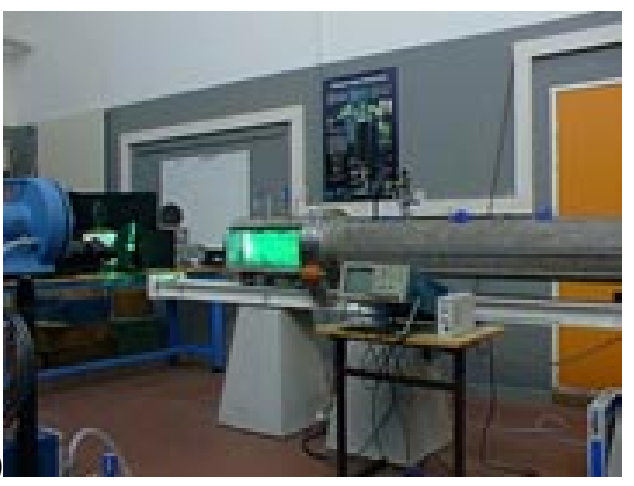

Fig. 1: Principle of the experiment (a) and view of the new large cross section shock tube of IUSTI coupled with a Copper vapor Mie scattering laser sheet technique (b). 


\title{
Abstract No. E18 \\ PLIF Flow Visualization of a Shock-Accelerated Air/SF 6 Interface
}

\author{
J. W. Jacobs and V. V. Krivets \\ University of Arizona, Tucson, AZ
}

A vertical shock tube is used to study the Richtmyer-Meshkovinstability of a membraneless Air/ $/ \mathrm{SF}_{6}$ interface. The two gases enter the shock tube at opposite ends of the driven section and are allowed to exit through slots in the shock tube wall to produce a flat, slightly diffuse interface in the test section. A sinusoidal perturbation is then given to the interface by oscillating the shock tube in the lateral direction to produce standing waves. Planar laser induced fluorescence (PLIF) is used to visualize the flow by seeding the air with acetone vapor and illuminating it with a sheet of light produced by a pulsed Nd:YAG laser. The resulting fluorescentsignal is then recorded using a cooled CCD camera. Images obtained from these experiments show very clearly the development of the instability far into the nonlinear regime in which the interface is contorted into pronounced mushroom structures. New results using $\mathrm{M}=1.3$ shock waves will be presented which clearly show the transition to turbulence in this flow at late times. The transition process begins with the development of Kelvin-Helmholtz instability on the vortex spirals. After formation, the initially coherent Kelvin-Helmholtzpattern very quickly decays into turbulence. Eventually the turbulence, which is initially confined to the vortex cores, begins to erode the remainder of the mushroom structures. Experiments will also be presented that study the effects of reshock on different stages of the instability. In these experiments a false wall is used to vary the distance between the initial interface location and the end wall in order to control the arrival time of the reflected shock wave. 
$8^{\text {th }}$ International Workshop on the Physics of Compressible Turbulent Mixing,

Abstract No. E19

\title{
Laser-Based High Pressure, High Strain-Rate Solid-State Experiments
}

\author{
D. H. Kalantar ${ }^{1}$, J. Belak ${ }^{1}$, J. D. Colvin ${ }^{1}$, M. Kumar ${ }^{1}$, K. T. Lorenz ${ }^{1}$, K. O. Mikaelian ${ }^{1}$, \\ S. Pollaine ${ }^{1}$, B. A. Remington ${ }^{1}$, S. V. Weber ${ }^{1}$, L. G. Wiley ${ }^{1}$, A. M. Allen ${ }^{2}$, \\ A. Loveridge-Smith ${ }^{2}$, J. S. Wark ${ }^{2}$, and M. A. Meyers ${ }^{3}$ \\ ${ }^{1}$ Lawrence Livermore National Laboratory, Livermore, $C A$ \\ ${ }^{2}$ Oxford University, Oxford, United Kingdom \\ ${ }^{3}$ University of California at San Diego, San Diego, CA
}

We have performed a high pressure solid state instability growth experiment using an x-ray ablative drive on the Nova laser [1]. In this experiment, an Al foil is shock compressed to a peak pressure of 1.8 Mbar with a sequence of shocks. A preimposed sinusoidal modulation grows by the Rayleigh-Taylorinstability. At early time, the growth is nearly fluid-like, but it is suppressed at late time. The growth of the instability provides information about the strength of the metal at high pressure $[2,3]$. In order to model this experiment, we invoke softening by shear bands and recovery of strength following dissipation of the heat associated with the localized shear bands.

In order to develop a lattice level understanding of response of these samples at high pressure, we perform dynamic x-ray diffraction of shocked materials to verify the state of the material under compression. In these experiments, we record x-rays diffracted from orthogonal lattice planes of shock compressed single crystal $\mathrm{Cu}$. The shift of the Bragg diffraction from these orthogonal planes confirms that the lattice undergoes a 3D compression. By comparison, $\mathrm{Si}$ is observed to respond with uniaxial compression. [4]

We are also developing shocked sample recovery techniques to characterize the residual deformation microstructure. This residual structure is studied by optical and electron microscopy techniques.

Results of the RT, diffraction, and recovery experiments will be discussed.

[1] D. H. Kalantar, B. A. Remington, J. D. Colvin, et al, Phys. Plasmas 7, 1999 (2000).

[2] J. F. Barnes, P. J. Blewett, R. G> McQueen, et al, J. Appl. Phys. 45, 727 (1974).

[3] A. I. Lebedev, P. N. Nizovtsev, V. A. Raevskii, V. P. Solov'ev, Phys. Dokl. 41, 328 (1996).

[4] A. Loveridge-Smith, A. Allen, J. Belak, et al, Phys. Rev. Letters 86, 2349 (2001).

This work was performed under the auspices of the U.S. Department of Energy by the University of California Lawrence Livermore National Laboratory under contract No. W-7405-Eng-48. 


\title{
Abstract No. E20 \\ RFNC-VNIITF Multifunctional Shock Tube to Investigate the Evolution of Instabilities in Nonstationary Gas Dynamic Flows
}

\author{
Yu. A. Kucherenko, O. E. Shestachenko, S. I. Balabin, and A. P. Pylaev \\ Russian Federal Nuclear Center - VNIITF, Snezhinsk, Russia
}

In the paper, at the shock tube operation in three modes, the parameters of the flows in the RFNCVNIITF were given.

In the first mode, in the shock tube the stationary shock waves are formed. This makes it possible to investigate the evolution of the Richtmyer-Meshkov instability and turbulence.

In the second mode, in the shock tube a nonstationary shock wave is formed that makes it possible to carry out the investigation of the behaviour of the contact boundaries between different density gases when there are conditions for the evolution of the Rayleigh-Taylor and Richtmyer-Meshkov instabilities.

In the third mode, in the shock tube a compression wave is formed. This makes it possible to investigate the evolution of the Rayleigh-Taylor instability and turbulence. 
$8^{\text {th }}$ International Workshop on the Physics of Compressible Turbulent Mixing,

Abstract No. E21

\title{
Planar Laser Sheet Visualization and Laser Doppler Velocity Measurements in Shock-Induced Turbulent Mixing Zones
}

\author{
A. Lassis, P. Montlaurent, C. Rayer, and J.-F. Haas \\ Commissariat à l'Energie Atomique, Bruyères-le-Châtel, France
}

We plan to present measurements on gaseous turbulent mixing arising from the Richtmyer-Meshkov instability (RMI) in a vertical shock tube of driver (below) and driven (above) sections lengths $1 \mathrm{~m}$ and 4-5 m respectively and of square internal cross section throughout $(13 \mathrm{~cm}$ by $13 \mathrm{~cm})$. In its present configuration, the maximum driver pressure is 8 bar and the driven section is initially at local atmospheric pressure ( 1 bar), thus the shock tube is limited to Mach numbers of about 1.5 maximum. The main diaphragm is made of two plastic layers (kapton) with conducting wires between them. In a typical low Mach number experiment, the initial pressure of air in the driver is set at 3 bar, and a Mach 1.2 shock is driven in the test section air at a controlled time by Joule heating the wires and locally melting (and weakening) the kapton.

We will characterize the mixing arising when the shock wave (propagating upwards in the $\mathrm{z}$ direction) accelerates a planar horizontal contact surface made of a thin $(0.5 \mu \mathrm{m})$ nitrocellulose membrane laid against a thin stainless steel wire mesh (wire diameter and spacing 1010 and $80 \mu \mathrm{m}$ ). The purpose of the film-mesh combinationis to force the small scales ( 1 by $1 \mathrm{~mm}$ in the $\mathrm{x}$ and $\mathrm{y}$ directions) of the RMI, thus insuring an early transition to turbulence of a thin planar mixing zone after shock passage. The gas pairs of initial interest are SF6-air and air-SF6. As it was several years ago, our primary goal is to provide an experimental data base (density structure and turbulent kinetic energy) for verification and validation of turbulent mixing models (1) imbedded in one- or twodimensional hydrodynamic codes. The same laser-doppler velocimeter will be used (Dantec two component system) for the measurement of the kinematic parameters of the mixing zone. Compared to the earlier effort performed in a shorter $(3.8 \mathrm{~m})$ and narrower shock tube (cross section 8 by $8 \mathrm{~cm}$ ), we expect to improve the quality of the flow because the wall

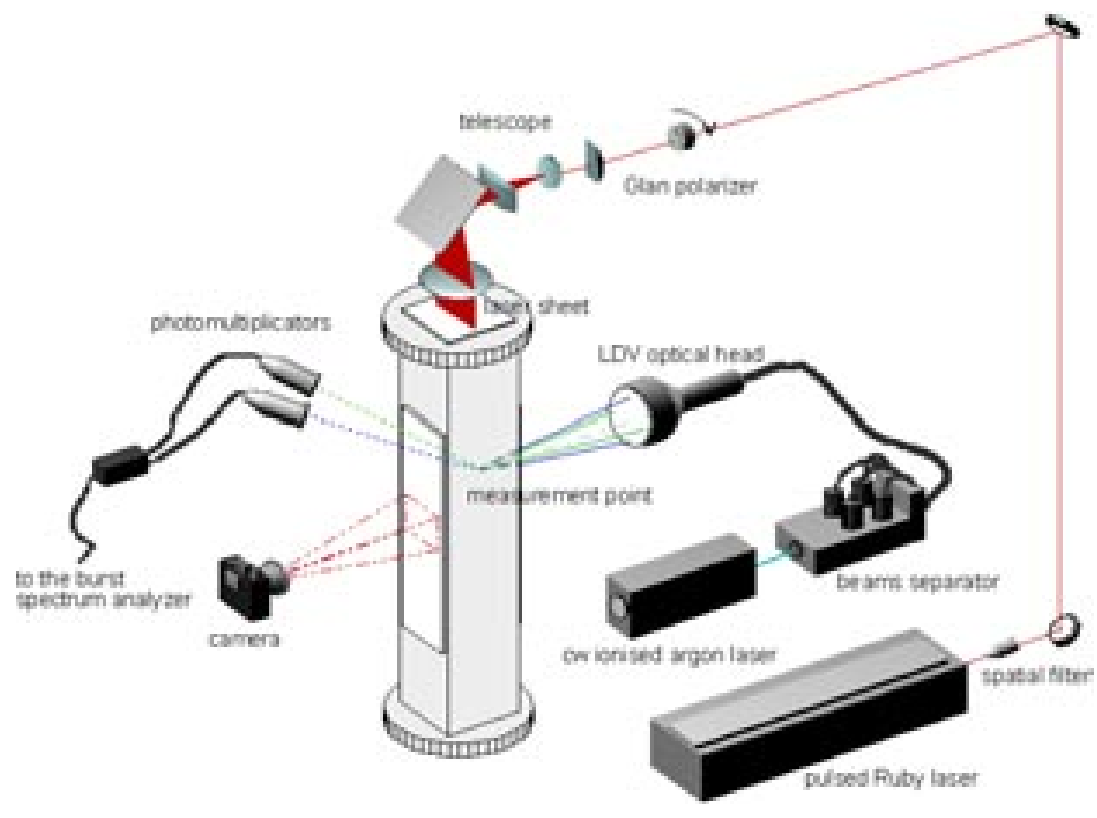


effects will be much less disruptive. We are also preparing a planar visualization system using a short pulse ruby laser to produce a light sheet (thickness 0.5 to $1 \mathrm{~mm}$ ) entering the shock tube from the top end-plate. Thus we hope to measure the local structure of the mixing zone without the optical signature from wall-located mixing which perturbed our earlier visualizations in the smaller tube (2).

\section{References :}

1. D. Souffland et al., Measurements and Simulations of the Turbulent Energy Levels in Mixing Zones Generated in Shock Tubes. pp. 486-491 in the proceedings of the $6^{\text {th }}$ IWPCTM, Marseilles, June 1997, Jourdan and Houas eds.

2. I. Galametz at al., Visualization of shocked mixing zones using differential interferometry and X-rays. pp. 178-184 in the proceedings of the $5^{\text {th }}$ IWPCTM, Stony Brook, July 1995, Young, Glimm and Boston eds., World Scientific. 
$8^{\text {th }}$ International Workshop on the Physics of Compressible Turbulent Mixing,

Abstract No. E22

\title{
Hydrodynamic Instabilities at a Shock Accelerated Bubble Gas-Gas Interface
}

\author{
G. Layes ${ }^{1}$, G. Jourdan ${ }^{1}$, P. Roualdes ${ }^{2}$, and L. Houas ${ }^{1}$ \\ ${ }^{1}$ IUSTI, CNRS, Université de Provence, Technopôle de Château-Gombert, \\ Marseille, France \\ ${ }^{2}$ Centre d'Etudes de Gramat, Gramat, France
}

The aim of the present work is to investigate the interaction of a plane shock wave with one gas bubble within another gas of different density in order to better understand the Richtmyer-Meshkov instability process in spherical geometry. These experiments are performed in the new $500 \mathrm{~mm}$ circular cross section shock tube installation of IUSTI (donation of DGA Gramat). It is a $12 \mathrm{~m}$ total length shock-tube with a $2 \mathrm{~m}$ high pressure chamber long and an experimental chamber total field of view of $475 \mathrm{~mm}$ long by $320 \mathrm{~mm}$ high. The shock tube is coupled with a Schlieren high speed camera system and PCB piezoelectric transducers are flush mounted on the shock tube side walls for both recording the pressure evolutions and triggering the acquisition device.

Spherical volumes of gas (He, Ar or Kr) with density, and sound speed, differing from that of the surrounding atmosphere (air) are accelerated by a relatively weak shock wave. The incident shock wave Mach number in air is around about 1.2. From successive Schlieren pictures (up to 30000 frames per second), we hope to investigate the hydrodynamic interface instability and the bubble distortion. Finally, we plan to generate several neighboring bubbles in the experimental chamber in order to study the bubble coupling during their acceleration. 
Abstract No. E23

\title{
Experimental and Numerical Study of Shock Wave-Bubble Interaction
}

\author{
K. Levy ${ }^{1,2}$, O. Sadot ${ }^{1,2}$, D. Oron ${ }^{1,2}$, Y. Srebro ${ }^{2}$, Y. Elbaz ${ }^{2}$, A. Yosef-Hai ${ }^{1,2}$, \\ G. Ben-Dor ${ }^{3}$, and D. Shvarts ${ }^{1,2}$ \\ ${ }^{1}$ Ben-Gurion University, Beer-Sheva, Israel \\ ${ }^{2}$ Nuclear Research Center - Negev, Israel
}

This work presents a study of the interaction of a shock wave with a sphericalbubble, which results in the formation of vortex rings and a jet. Similar studies which presents various stages of the interaction evolution were published in [1-5].

In the present work two configurations were studied in which a spherical bubble of SF6 (Heavy bubble) or He (light bubble) was imbedded in the shock tube at ambient conditions. The evolution of the flow due to the interaction of the shock wave with the bubble was followed experimentally and numerical. The results reveal that in the first case a jet is formed due to a converge shock wave towards the bubble center, which formed high-pressure region on the bubble axis that forced the heavy fluid forward. In the second case a vortex ring is formed around the bubble creating a region of high pressure in the heavy gas forcing the heavy fluid forward and forming a jet. A good agreement was found comparing the results of the experiments to those of the simulations.

\section{References}

1. Quirk et al., J. Fluid Mechanics, 318, pp. 129 (1996).

2. Picone et al., J. Fluid Mechanics, 189, pp. 23 (1988).

3. Yang, et al., J. Fluid Mechanics, 258, pp.217 (1994).

4. Haas et al., J. Fluid Mechanics, 181, pp. 41 (1987).

5. Smith et al. in the proceeding of the MIX 91 workshop. 
$8^{\text {th }}$ International Workshop on the Physics of Compressible Turbulent Mixing,

Pasadena, CA (2001)

Abstract No. E24

\title{
Laser-Driven Near Isentropic Compression of an Aluminum Flyer Plate
}

\author{
K. T. Lorenz, D. Kalantar, J. Edwards, J. D. Colvin, and B. Remington \\ Lawrence Livermore National Laboratory, Livermore, CA
}

A new design for producing a ramped pressure wave for the study of material response in solid media under nearly isentropic compression conditions will be discussed. A plasma source, initiated from laser heating of a low-density carbon foam, unloads across a vacuum gap onto an $\mathrm{Al}$ target to provide a ramped, shockless, pressure load. Experiments using HE to create shockless drives have previously been demonstrated by Barnes, et al. [1] and Levedev, et al. [2,3]. This type pressure drive is coupled to targets having modulated surfaces for the study of material response and strength. The current design configuration of our near isentropic drive will provide peak pressures and strain rates on order of $0.4 \mathrm{Mbar}$ and $10^{6}-10^{7} \mathrm{sec}^{-1}$, respectively. Initial experiments using VISAR, x-ray radiography and thin $\mathrm{Al}$ foils will examine both the planarity and the time-dependent nature of the pressure loading in the target. Recent experimental results and as well as experimental simulations scaled to the laser drive conditions will be presented.

[1] J.F. Barnes, P.J. Blewett, R.G. McQueen, K.A. Meyer and D. Venable, J. Appl. Phys. 45, 727 (1974).

[2] A.I. Lebedev, P.N. Nizovtsev, V.A. Rayevsky, in the Proceedings of the $4^{\text {th }}$ International Workshop on the Physics of Compressible Turbulent Mixing, 29 March - 1 April, Cambridge, England (Cambridge University Press, Cambridge, 1993), p. 81.

[3] A.I. Lebedev, P.N. Nizovtsev, V.A. Raevskiiand V.P. Solov'ev, Dokl. Akad. Nauk. 349 (MAIK Nauka / Interperiodica Publishing, Moscow July 1996), pp. 332-4. Translation: Phys. Dokl. 41, 328 (1996).

This work was performed under the auspices of the U.S. Department of Energy by the University of California Lawrence Livermore National Laboratory under contract No. W-7405-Eng-48. 


\title{
Abstract No. E26 \\ Single-Mode Incompressible Richtmyer-Meshkov Instability Experiments
}

\author{
C. E. Niederhaus ${ }^{1}$ and J. W. Jacobs ${ }^{2}$ \\ ${ }^{1} \mathrm{NASA}$ Glenn, Cleveland, $\mathrm{OH}$ \\ ${ }^{2}$ University of Arizona, Tucson, $A Z$
}

The Richtmyer-Meshkov instability of a moderate Atwood number, miscible, two-liquid system is experimentally investigated. The instability is generated by dropping a fluid container onto a coil spring, producing a nearly impulsive acceleration followed by a period of freefall The initialdensity interface has a well-defined, 2-D, single-mode sinusoidal perturbation generated by laterally oscillating the fluid container. The perturbation quickly inverts and then grows in amplitude after undergoing the impulsive acceleration. Planar laser-induced fluorescence is used for flow visualization, providing clear views of the fluids far into the nonlinear regime. Disturbance amplitudes are measured and compared to theoretical predictions in the linear, weakly nonlinear, and nonlinear regimes. The effects of Reynolds number (based on circulation) on the vortex core evolution and overall growth rate of the interface are also investigated. In addition, an instability in the vortex cores is observed and criteria established for its occurrence. 
$8^{\text {th }}$ International Workshop on the Physics of Compressible Turbulent Mixing,

Abstract No. E27

\title{
Experimental Study of a Strongly-Shocked Gas Interface with Visualized Initial Conditions
}

\author{
J. G. Oakley, M. H. Anderson, and R. Bonazza \\ University of Wisconsin at Madison, Madison, WI
}

The Richtmyer-Meshkov (RM) instability is studied for a strongly shocked gas-gas interface in the nonlinear regime. The impulsive acceleration of the interface by a shock wave imparts a velocity to the interface and baroclinic vorticity $(\nabla \rho \times \nabla p)$ causes the amplitude of a single mode perturbation to grow. Experiments for studying the compressible, turbulent mixing of a gas-gas interface are conducted in a shock tube. The shock tube is oriented vertically $(9.3 \mathrm{~m}$ high), has a large square cross-section $(25.4 \mathrm{~cm})$, is modular (for studying interfaces of different ages) and has a structural capacity of $20 \mathrm{MPa}$ [1]. The driven and test section gases are initially separated with a thin copper plate that has been formed with a sinusoidal perturbation along its length. The sine wave plate has three wavelengths of $\lambda=38.1 \mathrm{~mm}$ and an amplitude, $a_{0}=3.2 \mathrm{~mm}$ which forms an initial condition in the linear to nonlinear transition with a wavelength amplitude product of $k a_{0}=0.52$. The sine wave plate is retracted from the shock tube forming a membraneless, single-mode perturbation between the driven and test gases. Using a heavy-above-light gas configuration, the Rayleigh-Taylor (RT) instability develops and the perturbation amplitudes grow in time forming the initial condition for the RM experiment. A continuous wave laser is used in the interface section to illuminate the interface, and the RT instability is imaged using a 256x256 pixel array, 8-bit CCD camera framing at $100 \mathrm{fps}$. The test gas is seeded with smoke particles $(\sim 0.5 \mu \mathrm{m})$ and Mie scattering is used to visualize the interface the two interface gases. After acceleration by the planar shock wave, the interface travels down the shock tube and is imaged in the test section using a pulsed YAG laser and 1024x1024 pixel array, 16-bit CCD camera. One post-shock image is obtained per experiment. The experimental images are processed to determine the initial and post-shocked perturbation amplitudes. The experimental results are compared with linear and nonlinear RM theories. The gas pair combination $\mathrm{CO}_{2}$-air is studied in the strongly shocked regime, $M=2.90$.

[1] Anderson, M.H., B.P. Puranik, J.G. Oakley, P.W. Brooks and R. Bonazza, "Shock tube Investigation of Hydrodynamic Issues Related to Inertial Confinement Fusion," Shock Waves, 10(5), pp. 377-387, 2000. 


\title{
Abstract No. E28 \\ Experimental Investigations of the Self-Similar Mixing Mode of Different Density Gases in the Earth's Gravitational Field
}

\author{
Yu. A. Kucherenko, O. E. Shestachenko, Yu. A. Piskunov, \\ E. V. Sviridov, V. M. Medvedev, and A. I. Baishev \\ Russian Federal Nuclear Center - VNIITF, Snezhinsk, Russia
}

At the installation OSA the experiments on the investigation of the self-similar mixing mode of different density gases in the Earth's gravitational field were performed. When so doing the heavy gas was placed over the light one. By means of the specter-diaphragm the gases were separated. At some instant of time the specter-diaphragm was quickly ruptured into small-scale fragments under the action of the external force. At the formed contact boundary of two different density gases the Rayleigh-Taylor instability and the unstationary zone of turbulent mixing were evolved.

For three values of Atwood numbers the experiments were carried out. In the experiments the trajectories of the mixing fronts in the light and heavy gases were registered. The mixing asymmetry coefficient and the constant alpha specifying the nondimensional mixing rate were determined. 
$8^{\text {th }}$ International Workshop on the Physics of Compressible Turbulent Mixing,

Abstract No. E29

\title{
Modeling Laser Material Strength Experiments
}

\author{
S. Pollaine ${ }^{1}$, D. Kalantar ${ }^{1}$, B. Remington ${ }^{1}$, J. Belak ${ }^{1}$, J. D. Colvin ${ }^{1}$, \\ J. Edwards ${ }^{1}$, R. Minich ${ }^{1}$, K. O. Mikaelian ${ }^{1}$, K. T. Lorenz ${ }^{1}$, S. V. Weber ${ }^{1}$, L. G. Wiley ${ }^{1}$, \\ D. Paisley ${ }^{2}$, A. Hauer ${ }^{2}$, J. S. Wark ${ }^{3}$, A. Loveridge ${ }^{3}$, A. M. Allen ${ }^{3}$, T. R. Boehly ${ }^{4}$, and \\ M. A. Meyers ${ }^{5}$ \\ ${ }^{1}$ Lawrence Livermore National Laboratory, Livermore, $C A$ \\ ${ }^{2}$ Los Alamos National Laboratory, Los Alamos, NM \\ ${ }^{3}$ Oxford University, Oxford, United Kingdom \\ ${ }^{4}$ Laboratory for Laser Energetics, University of Rochester, Rochester, NY \\ ${ }^{5}$ University of California at San Diego, San Diego, CA
}

We have done many experiments on the Omega and Janus lasers to measure material strength and other properties of $\mathrm{Al}, \mathrm{Si}$ and $\mathrm{Cu}$ at high pressures $(100 \mathrm{~kb}-1 \mathrm{Mb})$ and strain rates (1.e5 - 1.e8). These experiments are diagnosed by VISAR (velocity measurement), $\mathrm{x}$-ray diffraction and material recovery. We simulate these experiments with the Steinberg-Guinanconstitutive model that includes shear strength, yield and melting temperature as a function of pressure and temperature.

This work was performed under the auspices of the U.S. Department of Energy by the University of California Lawrence Livermore National Laboratory under contract No. W-7405-Eng-48. 
Abstract No. E30

\title{
Experiments and Simulations of Instabilities in a Shock-Accelerated Gas Cylinder
}

\author{
K. Prestridge ${ }^{1}$, C. A. Zoldi ${ }^{1,2}$, P. Vorobieff ${ }^{3}$, P. M. Rightley, and R. F. Benjamin ${ }^{1}$ \\ ${ }^{1}$ Los Alamos National Laboratory, Los Alamos, NM \\ ${ }^{2}$ State University New York, Stony Brook, Stony Brook, NY \\ ${ }^{3}$ University of New Mexico, Albuquerque, NM
}

The interaction of a planar $(\mathrm{M}=1.2)$ shock with a heavy-gas $\mathrm{SF}$, round cylinder surrounded by air produces strong vorticity, driven by the shock wave's pressure gradient interacting with density gradients at the air/SF6 interface. The growth of the cylinder is measured using six images of the density profiles of each experimentalevent, unlike earlier studies, which captured only one image per event. The velocity field is measured at one time using Particle Image Velocimetry (PIV). We also present two-dimensional computational simulations, using the RAGE code, which utilize the actual initial conditions measured in the experiment. The simulation has the same spatial resolution as the experimental diagnostics, and for the first time, the width of the computational domain has been matched to that of the experiment, allowing us to consider sidewall effects. Experimental images show an instability growth rate somewhat higher than the results of the RAGE simulation. Velocity fields measured experimentally qualitatively agree with simulations, but the quantitative difference in velocity magnitudes is substantial. 
$8^{\text {th }}$ International Workshop on the Physics of Compressible Turbulent Mixing,

\section{Abstract No. E31 \\ Experimental Study into Rayleigh-Taylor Turbulent Mixing Zone Heterogeneous Structure}

Yu. A. Kucherenko, A. P. Pylaev, V. D. Murzakov, A. V. Belomestnih, V. N. Popov, and

A. A. Tyaktev

Russian Federal Nuclear Center - VNIITF, Snezhinsk, Russia

The heterogeneous structure study has been performed by means of a "light-sheet" technique at the SOM gas-dynamic accelerator. The investigated system consisted of three layers of differentdensity liquids. For leading out the information from the mixing zone inner region illuminated by the "lightsheet", visualizing particles were seeded into one of the liquids. The visualizing particles, which got into the "light-sheet", diffused light, and at the same time photo images of the liquid fragments, contained the visualizing particles, were formed by a light-sensitive receiver. For the error reduction refractive indexes of all the three liquids were equalized. A special test has been conducted for determining of measurements inaccuracy. Experiments have been performed for two values of acceleration of artificial field of gravity. Distributions of liquid fragments sizes are showed in the form of bar charts for different moments of time. 
Abstract No. E32

\title{
Measurements of Turbulence Correlations in Low Atwood Number Rayleigh-Taylor Mixing
}

\author{
P. Ramaprabhu and M. J. Andrews \\ Texas A \& M University, College Station, TX
}

Simultaneous measurement of velocity and density fields in a statistically-steady, low Atwood number $\left(\sim 10^{-3}\right)$, Rayleigh-Taylor experiment have been made. The experiment allows long data collection times and thus extensive spectral characterization. The method used is referred to as Particle Image Velocimetry-Scalar(PIV-S), and is a variant of the PIV technique. The PIV-S method uses different concentrations of particles to mark fluids of different densities. Tracking the motion of individual particles yields velocity measurements, while local particle concentrationsgives density measurements. Two-dimensional fields of $\left\langle\rho^{\prime 2}\right\rangle,\left\langle u^{\prime 2}\right\rangle,\left\langle v^{\prime 2}\right\rangle,\left\langle u^{\prime} v^{\prime}\right\rangle,\left\langle\rho^{\prime} u^{\prime}\right\rangle$, and $\left\langle\rho^{\prime} v^{\prime}\right\rangle$ correlations, with associated power spectra will be presented. The density measurements compare well with corresponding temperature data from thermocouple experiments. 
$8^{\text {th }}$ International Workshop on the Physics of Compressible Turbulent Mixing,

Pasadena, CA (2001)

Abstract No. E34

\title{
Experimental Study of the Interaction of a Strong Shock with a Spherical Density Inhomogeneity
}

\author{
H. F. Robey ${ }^{1}$, T. S. Perry ${ }^{1}$, R. I. Klein ${ }^{1,2}$, J. A. Greenough ${ }^{1}$, J. O. Kane ${ }^{1}$, and \\ T. R. Boehly ${ }^{3}$ \\ ${ }^{1}$ Lawrence Livermore National Laboratory, Livermore, $C A$ \\ ${ }^{2}$ University California at Berkeley, Department of Astronomy, Berkeley, CA \\ ${ }^{3}$ Laboratory for Laser Energetics, University of Rochester, Rochester, NY
}

Laser-driven experiments conducted on the Omega Laser are described which probe the interaction of a very strong shock with a spherical density inhomogeneity. The interaction is viewed simultaneously from two orthogonal directions. This enables visualization of both the initial distortion of the sphere into a double vortex ring structure as well as the onset of an azimuthal instability that ultimately results in the three-dimensional breakup of the ring. The experimental results are compared with three-dimensional numerical simulations using an adaptive mesh refinement technique. The agreement between experiment and simulationis shown to be quite good. The experimental results completely define the three-dimensional topology of the flow, and the three-dimensional breakup is shown to be in remarkable agreement with the incompressible theory of Widnall et al.

This work was performed under the auspices of the U.S. Department of Energy by the University of California Lawrence Livermore National Laboratory under contract No. W-7405-Eng-48. 


\title{
Abstract No. E35 \\ Turbulent Transition in a High Reynolds Number, Rayleigh-Taylor Unstable Plasma Flow
}

\author{
H. F. Robey ${ }^{1}$, Y. K. Zhou ${ }^{1}$, A. C. Buckingham ${ }^{1}$, \\ P. Keiter ${ }^{2}$, B. A. Remington ${ }^{1}$, and R. P. Drake ${ }^{2}$ \\ ${ }^{1}$ Lawrence Livermore National Laboratory, Livermore, $C A$ \\ ${ }^{2}$ University of Michigan, Ann Arbor, MI
}

A high Reynolds number, Rayleigh-Taylor unstable plasma flow driven by laser radiation is described. Given enough time at these experimental conditions, the interfacial mixing layer will eventually transition to turbulence. The experiments are limited, however, in the very short time duration of the available flow. The Reynolds number characterizing the mixing layer is determined from the experimentally measured length and velocity scales together with the plasma kinematic viscosity determined from a corresponding 1D numerical simulation. From these, the Reynolds number is determined to be sufficiently large $\left(\operatorname{Re}>10^{5}\right)$ to support a turbulent flow. An estimate of the developing Taylor and Kolmogorov dissipation scales, however, shows that the temporal duration of the flow is insufficient to allow for the appearance of a turbulent inertial subrange. A methodology is described for estimating the time required for the development of a fully turbulent flow at these conditions.

This work was performed under the auspices of the U.S. Department of Energy by the University of California Lawrence Livermore National Laboratory under contract No. W-7405-Eng-48. 
$8^{\text {th }}$ International Workshop on the Physics of Compressible Turbulent Mixing,

Abstract No. E36

\title{
Effects of High Initial Amplitudes and High Mach numbers on the Evolution of the RM instability: II. Experimental Study
}

\author{
O. Sadot ${ }^{1,2}$, A. Yosef-Hai ${ }^{2}$, A. Rikanati ${ }^{1,2}$, D. Oron ${ }^{1}$, G. Ben-Dor ${ }^{2}$, and D. Shvarts ${ }^{1,2}$ \\ ${ }^{1}$ Nuclear Research Center Negev, Israel \\ ${ }^{2}$ Ben-Gurion University, Beer-Sheva, Israel
}

Recent theoretical work [Rikanati et. al. this conference] suggested that the reduction in the RM instability initial growth rate observed in recent experiments $[1,2]$ is mainly a result of high initial amplitudes used in those experiments, rather then high Mach number effects.

In the present work, effects of high initial amplitudes and high Mach number are studied experimentally. Results from a shock tube apparatus at low Mach number $(\mathrm{M}=1.2)$ with high initial amplitudes shows velocity reduction similar to the theoretical predictions. Preliminary experiments studying the RM instability at high Mach numbers were done, using a newly constructedshuck tube, to confirm the velocity reduction due to effects of high Mach numbers.

\section{References}

1) Aleshin et. al., in Proceedings of the Sixth International Workshop on the Physics of Compressible Turbulent Mixing edited by G. Jourdan \& L. Houas, Marsielle France 1997. Page 1.

2) Dimonte G., Frerking C.E., Schnider M. and Remington B., Phys. of Plasmas 12, 304 (1996). 
Abstract No. E37

\title{
Measurements within a Richtmyer-Meshkov Mixing Zone using a Triple Hot Wire Probe Technique
}

\author{
L. Schwaederlé ${ }^{1}$, G. Jourdan ${ }^{1}$, L. Houas ${ }^{1}$, and J.-F. Haas ${ }^{2}$ \\ ${ }^{1}$ IUSTI, CNRS, Université de Provence, Technopôle de Château-Gombert, Marseille, France \\ ${ }^{2}$ Commissariat à l'Energie Atomique, Bruyères-le-Châtel, France
}

A triple probe constant temperature hot wire anemometer(CTHWA) investigation is undertaken in a shock tube to characterize the turbulent mixing zone induced by the Richtmyer-Meshkov instability (RMI) when the shock wave propagates through the interface between two gases of different densities. The first gas is air and the second is He (lighter), $\mathrm{Ar}$ (moderately heavier) and $\mathrm{Kr}$ (much heavier). The experiments are conducted in a $8.5 \mathrm{~cm}$ square cross section shock tube of which test section is represented on Fig. 1 . The two gases are separated by a thin $(0.4 \mu \mathrm{m})$ nitrocellulose film resting on an orthogonal grid made of 9_9 wires (180 $\mu \mathrm{m}$ diameter, $8.5 \mathrm{~mm}$ spacing) which is accelerated by a Mach number 1.25 shock wave in air at atmospheric pressure. Fig 2. summarizes the principle and the aim of the present investigation.

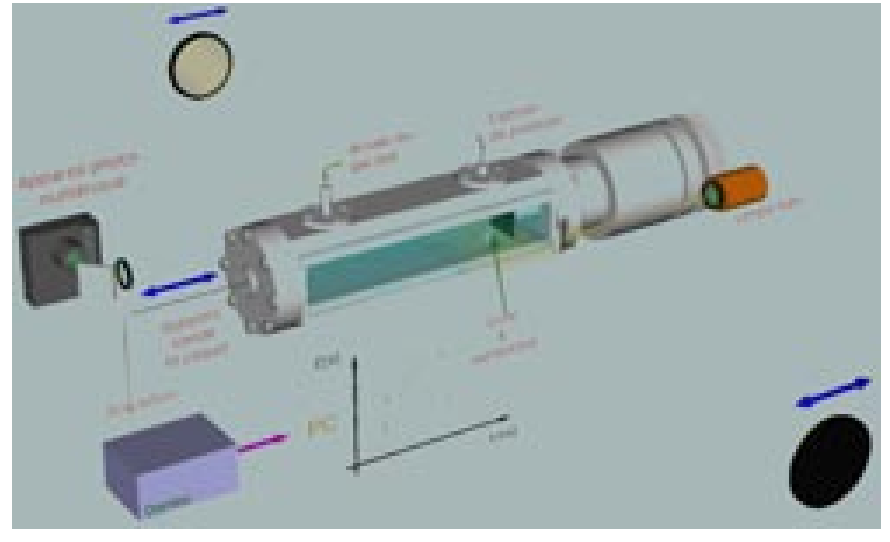

Fig 1. Experimental set-up

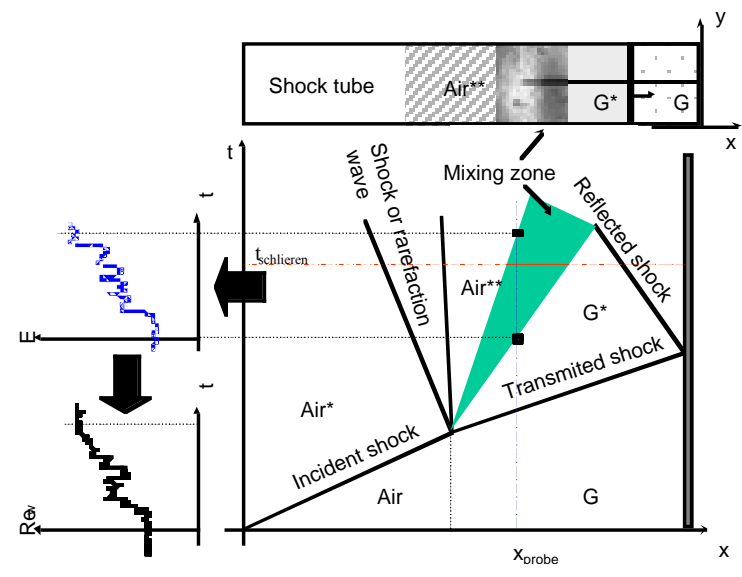

Fig 2. Principle of the investigation

The CTHWA output voltage is a function of local Reynolds number, heat conductivity and temperature with empirical constants. With the simplifying assumption of linear profiles for both temperature (with a jump less than $30 \mathrm{~K}$ in all cases) and heat conductivity across the mixing zone, and using the Rankine-Hugoniot calculations in pure and premixed gases, the determination of the constants, given by a suitable calibration procedure with varying concentrations (by steps of 10\%), provides the evolution of the Reynolds number within the mixing zone. An example of both (a) raw hot-wire signal and (b) deduced Reynolds number evolution in air/Ar mixing zone are represented in Fig 3. 
In order to obtain separately the mixing density, temperature and velocity we positioned three HWA probes $(5 \mu \mathrm{m}$ in diameter and $1.25 \mathrm{~mm}$ in length), inserted from the end plate along the shock tube axis and working at different temperatures. We intend to present the local Reynolds number evolution across

(a)

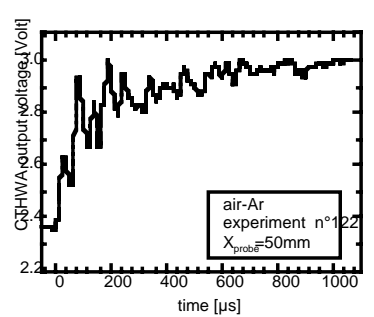

Fig. 3. Typical hot-wire signal and local Reynolds number evolution the mixing zone, the estimates of density, velocity and temperatures with statistical results based on identical shock tube experiments, and carry this study at different positions both axially and laterally. 
Abstract No. E38

\title{
Experimental Study into Evolution of Gravitational Turbulent Mixing of Gases at the Multifunctional Shock Tube
}

\author{
Yu. A. Kucherenko, O. E. Shestachenko, Yu. A. Piskunov, E. V. Sviridov, \\ V. M. Medvedev, and A. I. Baishev \\ Russian Federal Nuclear Center - VNIITF, Snezhinsk, Russia
}

\begin{abstract}
At initial moment of time investigated different density gases are placed inside the multifunctional shock tube and separated with the "Spectre-diaphragm". Next the "Spectre-diaphragm"is destroyed into small-scale fragments by an external force. The gaseous interfaceis accelerated by a compression wave formed in the shock tube. At that, the Rayleigh-Taylor instability arises at the contact boundary of different density gases, and a non-stationary zone of gravitational turbulent mixing forms. According to the experimental results, the dependence of the turbulent mixing zone width on the interface displacement has been built, and the non-dimensional rate of mixing alpha has been obtained.
\end{abstract}


$8^{\text {th }}$ International Workshop on the Physics of Compressible Turbulent Mixing,

Pasadena, CA (2001)

Abstract No. E39

\title{
Shock Tube Experiments on Richtmyer-Meshkov Instability Across a Chevron Profiled Interface
}

\author{
A. V. Smith, D. A. Holder, C. J. Barton, A. P. Morris, and D. L. Youngs \\ Atomic Weapons Establishment, Aldermaston, United Kingdom
}

This paper reports on the latest experiment in the series of Richtmyer-Meshkov instability (RMI) shock tube experiments. They feature a dense gas / air interface in the form of a chevron of central obtuse angle $157^{\circ}$ and full test cell height. The interest in an inclined interface of this angle was initiated at the $5^{\text {th }}$ IWPCTM $^{[1]}$.

The experiments were conducted at shock Mach number 1.26 (70kPa overpressure), using the 200 $\mathrm{x} 100 \mathrm{~mm}$ shock tube with a three zone test cell arrangement of air / dense gas / air. The dense gas is optionally sulphur hexafluoride $\left(\mathrm{SF}_{6}\right)$ or xenon $(\mathrm{Xe})$ which provide Atwood numbers of 0.67 and 0.64 respectively. Gas separation was by means of microfilm membranes, supported by fine wire meshes. Visualisation of the gas mixing was by laser sheet illumination of the seeded dense gas using a copper vapour laser pulsing at $12.5 \mathrm{kHz}$. Mie scattered light was recorded using a $35 \mathrm{~mm}$ rotating drum camera to capture a sequence of 50 images per experiment; or alternatively a single image from an ICCD camera.

Sample laser sheet images are compared to those from corresponding 3-D hydrocode calculations. Quantitative analysis will be of the form of derived relative intensity data from line-outs through experimental images and their code equivalents. Comparisons will reveal substantial agreement on major features.

A video will also be available showing a full sequence of images from one experiment with corresponding computed code images.

1. Bashurov et al. Experimental and Numerical Evolution Studies for 2-D Perturbations of the Interface Accelerated by Shock Waves. $5^{\text {th }}$ IWPCTM

PACS No.: 47.20.Ma 
Abstract No. E40

\title{
The Evolution and Interaction of Two Shock-Accelerated Unstable Gas Cylinders
}

\author{
C. Tomkins, K. Prestridge, P. Rightley, C. Zoldi, and R. Benjamin \\ Los Alamos National Laboratory, Los Alamos, NM
}

The interaction of two Richtmyer-Meshkov-unstable gas cylinders is investigated experimentally. The dense-gas cylinders are initially configured with separation $S$ in the spanwise direction $(S=1.1$ to 2.0 times the diameter, center-to-center), and subject to acceleration by a planar shockwave. The evolution of the resulting flow structures is captured downstream by flow visualization and PIV.

In the single-cylinder case (Prestridge et al.), the flow structure is dominated by two spanwiseseparated vortices. In the double-cylinderconfiguration, the innermost vortices interact (e.g., Figure 1). The nature and degree of the interaction - and hence the morphology of the resulting flow structures - is observed to be highly sensitive to the initial cylinder spacing. The effects of the interaction on both the initial baroclinic vorticity production, and the subsequent evolution of this deposited vorticity, are investigated.

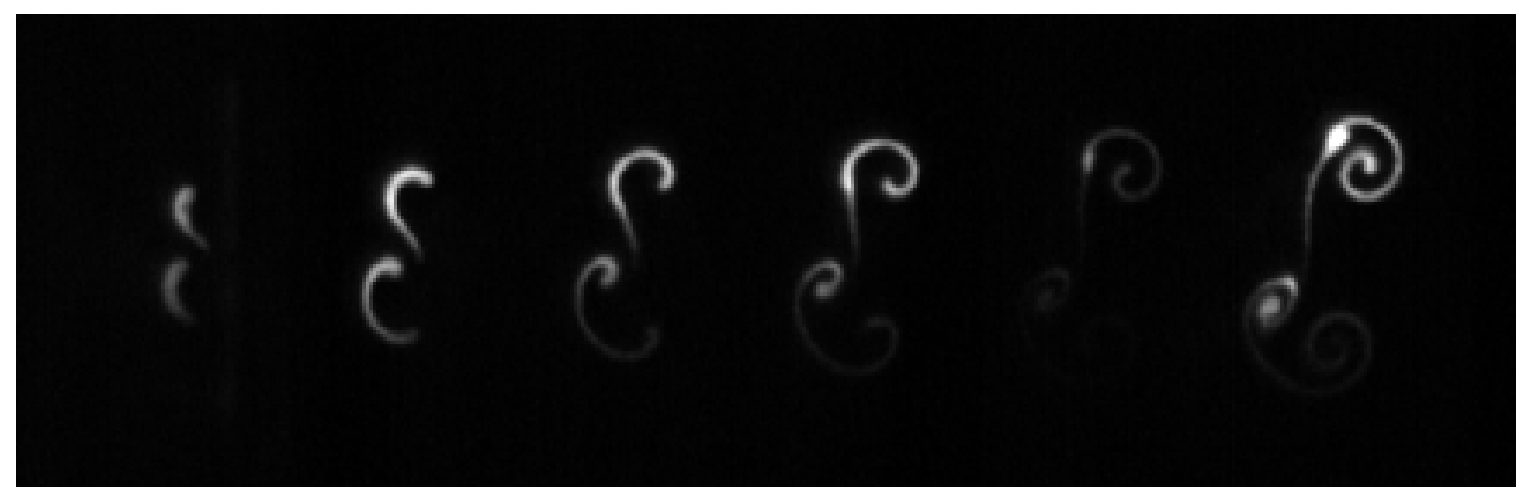

Figure 1. Flow visualization example of interaction between adjacent shock-acceleratedgas cylinders.

\section{References}

K. Prestridge, et al., "Experiments and Simulations of Instabilities in a Shock-Accelerated Gas Cylinder", submitted to Phys. Fluids.

PACS No.: 47.20Ma 
$8^{\text {th }}$ International Workshop on the Physics of Compressible Turbulent Mixing,

Pasadena, CA (2001)

Abstract No. E41

\title{
Doubly-Shocked Richtmyer-Meshkov Instability Experiments at Nova
}

\author{
D. J. Ward, K. S. Budil, T. A. Peyser, B. A. Remington, \\ P. L. Miller, R. J. Wallace, H. Louis, and A. Demiris \\ Lawrence Livermore National Laboratory, Livermore, $C A$
}

Hydrodynamic instabilities are present in many physical systems, ranging from very small inertial confinement fusion capsules to supernovae. A great deal of effort, computational, theoretical and experimental, has been focused on the evolution of buoyancy-driven (Rayleigh-Taylor),shear-driven (Kelvin-Helmholz) and shock-driven (Richtmyer-Meshkov) instabilities. For astrophysics the interaction of shock waves with molecular clouds in the interstellar medium is a common occurrence and a problem that has been studied extensively. A slightly more complex problem is the interaction of multiple shock waves with such a cloud, in either a co- or counter-propagating geometry. This is the system that we chose to address with these experiments.

We will present the results of a series of experiments that investigated hydrodynamic instabilities in doubly shocked systems. A half-hohlraum driver was used to launch a shock into a miniature shock tube that then crossed a rippled interface, causing the ripples at the interface to grow via the Richtmyer-Meshkov instability. A second, counterpropagating shock was launched from the opposite end of the shock tube by a second half-hohlraum driver that impacted the developing mix region at some later time. This unique geometry allowed independent control of the relative timing of the two shocks and their relative strength. However, for ease of experimentalimplementation we have chosen to begin with the case of two roughly equal strength, counter-propagating shock waves. The evolution of the mixing region was observed via radiography.

The quality of the data obtained in this experiment was greatly improved over prior experiments by the use of a layered ablator, constructed by using two density matched plastic materials, only one of which was radiographically opaque to the backlighter $\mathrm{X}$ rays. The opaque material was confined to the central 100 microns along the line-of-sight, thus virtually eliminating the complications due to shock curvature in that direction. The initial perturbation was a $100 \mu \mathrm{m}$ wavelength ripple with an initial amplitude of $1 \mu \mathrm{m}$.

The experimentalresults show good agreement with two-dimensional radiation-hydrodynamicscode simulations. We will also discuss comparisons to existing analytic models for the evolution of the RM instability.

This work was performed under the auspices of the U.S. Department of Energy by the University of California Lawrence Livermore National Laboratory under contract No. W-7405-Eng-48. 
Abstract No. E42

\title{
The Interaction of Supernova Blast Waves with Interstellar Clouds: Experiments on the OMEGA Laser
}

\author{
R. I. Klein ${ }^{1,2}$, H. Robey ${ }^{1}$, T. Perry ${ }^{1}$, and J. Greenough ${ }^{1}$ \\ ${ }^{1}$ Lawrence Livermore National Laboratory, Livermore, $C A$ \\ ${ }^{2}$ University of California at Berkeley, Berkeley, $C A$
}

The interaction of strong shock waves, such as those generated by the explosion of supernovae with interstellar clouds, is a problem of fundamental importance in understanding the evolution and the dynamics of the interstellar medium (ISM) as it is disrupted by shockwaves.. The physics of this essential interaction is critical to understanding the evolution $\mathrm{f}$ the ISM, the mixing of interstellar clouds with the ISM and the viability of this mechanism for triggered star formation. We present the results of a series of new OMEGA laser experiments investigating the evolution of a high density sphere embedded in a low density medium after the interaction of a strong shock wave, emulating the supernova shock-cloud interaction. The interaction is viewed from two orthogonal directions using face-on and side-on x-ray radiography enabling visualization of the both the initial distortion of the sphere into a vortex ring as well as the onset of a powerful azimuthal 3D instability that ultimately results in the three-dimensional breakup of the ring. These studies augment the previous studies of Klein et al. $(2000,2001)$ on the NOVA laser by enabling the full three-dimensional topology of the interaction to be understood. We compare the experimental results for the vortex ring with the incompressible theory of Widnall et al. 1974 and we discuss high resolution 3D numerical simulations that recover all of the essential features of the interactionincluding RichtmyerMeshkov, Rayleigh-Taylor and Kelvin-Helmholtz instabilities. We discuss implications for mixing in the ISM.

This work was performed under the auspices of the U.S. Department of Energy by the University of California Lawrence Livermore National Laboratory under contract No. W-7405Eng-48. 
$8^{\text {th }}$ International Workshop on the Physics of Compressible Turbulent Mixing,

\title{
Abstract No. E43 \\ Evolution of the Mixing Zone of Different Densities Gases Being Interaction to Compression Waves
}

\author{
S. G. Zaytsev ${ }^{1}$, V. V. Krivets ${ }^{1}$, I. M. Mazilin ${ }^{1}$, S. N. Titov ${ }^{1}$, E. I. Chebotareva ${ }^{1}$, \\ V. V. Nikishin' ${ }^{2}$, V. F. Tishkin ${ }^{2}$, S. Bouquet ${ }^{3}$, and J.-F. Haas ${ }^{3}$ \\ ${ }^{1}$ Krzhizhanovsky Power Engineering Institute, Moscow, Russia \\ ${ }^{2}$ Institute of Mathematical Modeling, Moscow, Russia \\ ${ }^{3}$ Commissariat à l'Energie Atomique, Bruyères-le-Châtel, France
}

The experimental and numerical study of the mixing zone evolution between a combustible mixture (hydrogen-oxygen, molecular weight is 18.5) and argon was carried out during accelerated, and then decelerated motion. The acceleratedmotion was formed by compression waves generated by a flame front in a combustible mixture. The magnitude of acceleration was about $10^{4}$ acceleration of gravity. In experiments the density distribution and shape of the mixing zone in the test-section were observed.

One-dimensional (1D) and two-dimensional (2D) models of process were used in numerical calculations.

The analysis of numerical and experimental results has shown:

1. The one-dimensional model satisfactorily describes a trajectory of the mixing zone motion and density distribution outside the mixing zone during accelerated, and then decelerated motion.

2. The shape of perturbations in the mixing zone generated as a result of the Rayleigh-Taylor instability evolution at the stage of accelerated motion is satisfactorily described by twodimensional model.

3. At the stage of deceleration two qualitatively different ways of the mixing zone evolution are observed:

4. At deceleration caused by the reflected shock, the decrease of the perturbation amplitude is observed - non-shocked deceleration.

5. In the given design of experiments, there are waves, which observed sometimes inside the incident compression wave. They form reflected shock waves with the Mach number just slightly exceeding $M=1$. The interaction of these extremely weak shocks with the mixing zone resulted in the perturbation amplitude growth inside the mixing zone - shock-induced deceleration. 
Abstract No. E44

\title{
Studies of Rayleigh-Taylor Instability in Aluminum Under Shock-Wave and Shock Less Loading
}

\author{
A. Lebedev, P. Nizovtcev, and V. Raevsky \\ Russian Federal Nuclear Center - VNIIEF, Sarov, Russia
}

The paper present results of experimentalstudies of Rayleigh-Taylorinstability growth in aluminum alloys AMg-6 and 6061-T6 subjected to shock-wave and shock less loading up pressures of 45Gpa. Fast growth of perturbations was recorded at the initial stage of acceleration in experiments with shock-wave loading. This testifies to short-time reduction of strength of tested material. To explain this phenomenon, the authors suggest a relaxation model of aluminum strength, taking into account heterogeneous character of deformation at shock wave front. Results of micro structural analysis of samples subjected to shock less and shock-wave loading are presented.

The study was performed at financial support under Agreement 512964 between Lawrence Livermore National Laboratory University of California and All-Russia Research Institute of Experimental Physics 
$8^{\text {th }}$ International Workshop on the Physics of Compressible Turbulent Mixing,

Pasadena, CA (2001)

\author{
Abstract No. E45
}

\title{
Ablative Rayleigh-Taylor Instability at Short Wavelengths
}

\author{
H. Azechi ${ }^{1}$, T. Sakaiya ${ }^{1}$, M. Nakai ${ }^{1}$, H. Shiraga ${ }^{1}$, K. Shigemori ${ }^{1}$, N. Miyanaga ${ }^{1}$, \\ M. Nishikino ${ }^{1}$, S. Fujioka ${ }^{1}$, Y. Tamari ${ }^{1}$, H. Nagatomo ${ }^{1}$, H. Takabe ${ }^{1}$, and A. Sunahara ${ }^{2}$ \\ ${ }^{1}$ Osaka University, Osaka, Japan \\ ${ }^{2}$ University of Rochester, Rochester, NY
}

The Rayleigh-Taylor (RT) instability in inertial confinement fusion (ICF) targets and in some astrophysical objects has an essential difference from the classical RT instability: material ablation. Since the ablation removes the RT perturbation away from the unstable surface, the RT growth is expected to be substantially reduced from its classical growth. Accordingly the RT instability at short wavelengths provides a critical test of various theories. To date, few experiment has addressed the short wavelength RT instability because of the wavelength of interest is around or even below the diagnostic spatial resolution. We will report in this Workshop the short wavelength RT instability growth rates which are measured for the first time by utilizing the newly innovated moire interferometry. The measured growth rates are reasonably well reproduced by the simulation that solves the Fokker-Plank equation for non-local heat transport. 
Abstract No. E46

\title{
A Vortex Model for Studying the Effect of Shock Proximity on Richtmyer-Meshkov Instability at High Mach Number
}

\author{
H. F. Robey and S. G. Glendinning \\ Lawrence Livermore National Laboratory, Livermore, CA
}

The effect of shock proximity on the non-linear evolution of Richtmyer-Meshkov instability of a sinusoidal perturbation at high Mach number is investigated analytically using a vortex model. The presence of the time-dependent shock boundary condition is incorporated using a system of image vortices of opposite sign located at the shock-to-interface distance ahead of the shock. For certain conditions, the perturbation growth rate is predicted by the linear theory to exceed the velocity of the transmitted shock relative to the mean interface. The effect of the image vortices is to initially suppress the growth of the perturbation while the shock remains close to the unstable interface. Later in time as the shock separates from the interface, the growth rate rebounds to a value slightly greater than would have occurred in the absence of the proximity effect. The model is compared with data from recent high Mach number RM experiments conducted on the Omega Laser and is shown to provide very reasonable agreement.

This work was performed under the auspices of the U.S. Department of Energy by the University of California Lawrence Livermore National Laboratory under contract No. W-7405-Eng-48. 
$8^{\text {th }}$ International Workshop on the Physics of Compressible Turbulent Mixing,

Pasadena, CA (2001)

\section{COMPUTATIONAL ABSTRACTS}




\title{
Abstract No. C1 \\ Modes' Interaction on Nonlinear Stage of Richtmyer-Meshkov Instability Evolution
}

\author{
V. I. Anisimov and A. V. Polionov \\ Russian Federal Nuclear Center - VNIITF, Snezhinsk, Russia
}

Universal dependence, permitting to describe linear and non-linear stages of Richtmyer-Meshkov instability evolution for single mode for the wide range of Mach and Atwood numbers was obtained earlier. In the present paper we are making an attempt to describemodes' interaction. For each single mode its own turbulent viscosity is determined. During modes interaction it is supposed that evolution of each mode damps because of total viscosity all modes being in the presence. The obtained results are compared with direct numerical simulation by $\mathrm{MACH}$ code. 
$8^{\text {th }}$ International Workshop on the Physics of Compressible Turbulent Mixing,

Pasadena, CA (2001)

\title{
Abstract No. C2 \\ Application of Ke-Model for the Description of an Atmospheric Surface Layer
}

\author{
M. G. Anuchin, V. E. Neuvazhayev, and I. E. Parshukov \\ Russian Federal Nuclear Center - VNIITF, Snezhinsk, Russia
}

The problem on determination of non-dimensional characteristics of turbulent flow in atmospheric surface layer is considered within $\mathrm{k} \varepsilon$-model. $\mathrm{K} \varepsilon$-equations and their singular points are investigated. The mathematical program for calculations of characteristicsof turbulent flow in surface atmospheric layer is developed. From the set of integral curves those curves are chosen which correspond to the solution of formulated task and ensure the satisfactory experiments description. Here the basic model constants are chosen according to the conventional criteria. At the same it is shown that the parameter responding to convection source term of an $\varepsilon$-equation should be chosen depending on stability conditions. The best agreement with experimental results is reached if for steady stratification and for unstable stratification. By a numerical choice of value and factor of turbulent diffusion the quite satisfactory description of experimental observations known as analytical interpolar dependencies is received. 


\title{
Abstract No. C4 \\ Computational Modeling of Low-Mach-Number High-Atwood-Number Turbulent Mixing
}

\author{
Wm. T. Ashurst and A. R. Kerstein \\ Sandia National Laboratories, Livermore, CA
}

A prerequisite for physical understanding of compressibleturbulent mixing is clarification of low-Ma high-At turbulent mixing mechanisms. Remarkably, uncertainty persists concerning the interpretation of the fundamental experiments in this regime, such as the seminal mixing-layer study by Brown and Roshko [1] and subsequentGALCIT experiments. It is difficultto perform numerical simulations directly comparable to the pertinent experiments, and theoretical progress has been limited. The present study provides an integrated picture of low-Ma high-At turbulent mixing using a new computational model for stochastic simulation of variable-density turbulent mixing. Comparison of model results to various published and unpublished experimental and numerical results clarifies the physical mechanisms underlying the diverse results and demonstrates novel predictive capabilities. A planned extension of the model to compressible flow is outlined.

G. L. Brown and A. Roshko, J. Fluid Mech. 64, 775 (1974).

PACS Nos.: 47.27.Eq, 47.27.Nz, 47.27.Jv 
$8^{\text {th }}$ International Workshop on the Physics of Compressible Turbulent Mixing,

Abstract No. C8

\title{
Spectral and High-Order Compact Methods for Shock-Induced Mixing
}

\author{
A. W. Cook, W. H. Cabot, and J. A. Greenough \\ Lawrence Livermore National Laboratory, Livermore, CA
}

A methodology, based on high-order compact and spectral schemes, is described for computing multicomponent turbulent flows at any Mach number. Filters are employed to stabilize the numerical integration and high-order artificial transport coefficients are introduced to control Gibbs oscillations. The equations and numerical scheme are formulated such that, under grid refinement, the method approaches a DNS. The method is evaluated for flows in 1, 2, and 3 dimensions, including comparisons with lower-order schemes. The dissipative character of the filter and artificial terms appears to be of little consequence for strongly forced flows which evolve over short periods of time; however, the dissipation is more noticeable for unforced flows which evolve over long periods of time.

PACS Nos.: 02.70.Hm, 47.20.Ma

This work was performed under the auspices of the U.S. Department of Energy by the University of California Lawrence Livermore National Laboratory under contract No. W-7405-Eng-48. 


\title{
Abstract No. C9 \\ Numerical Simulation of Mode Coupling in Laser-Driven Rayleigh-Taylor Instability Experiments
}

\author{
R. M. Darlington and K. S. Budil \\ Lawrence Livermore National Laboratory, Livermore, $C A$
}

This study addresses the simulation of multimode laser-driven Rayleigh-Taylor instability experiments. The linear and transition stages of the instability will be examined, with particularstudy of the mode coupling between short and long wavelengths. The experiments, conducted at the Nova laser facility at LLNL, consisted of ablatively-accelerated planar composite foils mounted onto the side of a gold hohlraum. A modulation was machined at the interface between a brominated plastic ablator layer (40 um thick) and a titanium payload (15 um thick) and its growth was diagnosed by measuring the changing optical depth modulation via face-on radiography. In this work we will focus on the evolution of a superposition of a 20 and a 4 um mode and contrast this to the evolution of a 20 um single mode perturbation. The shape and growth rate of

the resulting instability will be examined, as well as the effect of numerical methods on the simulation. Similar simulations will also be used to examine the evolution of this perturbation in a more idealized situation where the target layers will be much thicker in order to mitigate thin foil effects, and the laser drive can be sustained for much longer durations. This will allow us to investigate the experimental conditions required to follow the instability further toward the turbulent regime.

This work was performed under the auspices of the U.S. Department of Energy by the University of California Lawrence Livermore National Laboratory under contract No. W-7405-Eng-48. 
$8^{\text {th }}$ International Workshop on the Physics of Compressible Turbulent Mixing,

Abstract No. C10

\title{
A Comparison of High-Resolution 3D Numerical Simulations Of Turbulent Rayleigh-Taylor (RT) Instability: Alpha-Group Collaboration
}

\author{
G. Dimonte ${ }^{1}$, A. Dimits ${ }^{1}$, S. Weber ${ }^{1}$, D. L. Youngs ${ }^{2}$, A. C. Calder ${ }^{3}$, B. Fryxell ${ }^{3}$, J. Biello ${ }^{3}$,

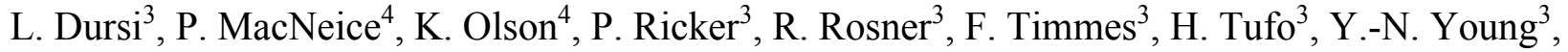 \\ M. Zingale ${ }^{3}$, M. J. Andrews ${ }^{5}$, P. Ramaprabhu ${ }^{5}$, S. Wunsch ${ }^{6}$, C. Garasi ${ }^{6}$, and A. Robinson ${ }^{6}$ \\ ${ }^{1}$ Lawrence Livermore National Laboratory, Livermore, $C A$ \\ ${ }^{2}$ Atomic Weapons Establishment, Aldermaston, United Kingdom \\ ${ }^{3}$ University of Chicago, Chicago, IL \\ ${ }^{4}$ NASA Goddard Space Flight Center, Greenbelt, MD \\ ${ }^{5}$ Texas A \& M University, College Station, TX \\ ${ }^{6}$ Sandia National Laboratories, Livermore, $C A$
}

The RT instability is investigated by comparing high resolution( $256 \times 256 \times 512$ zones $)$ simulations using various (5-7) numerical techniques with identical initial conditions. The fluids have a density ratio $\rho_{2} / \rho_{1}=3$ and an ideal gas specific heat ratio of $\gamma=5 / 3$. The hydrostatic equilibrium is adiabatic with a pressure $\sim 2 \pi\left(\rho_{1}+\rho_{2}\right) \mathrm{gL}$ ( $\mathrm{g}=$ acceleration, $\mathrm{L}=$ box width) at the interface to keep the velocities sub-sonic $($ Mach $<0.2)$. The initial perturbations have an $\mathrm{RMS}$ amplitude $\mathrm{h}_{\mathrm{o}} / \mathrm{L} \sim 3 \times 10^{-4}$ with mode numbers randomly distributed in a cylindrical shell $32 \leq \mathrm{n} \leq 64$. This paper compares the self-similar growth $\sim \mathrm{gt}^{2}$ of the mixing zone and internal scales, the atomic mixing, and the energy budget from the different codes and with available experiments.

This work was performed under the auspices of the U.S. Department of Energy by the University of California Lawrence Livermore National Laboratory under contract No. W-7405-Eng-48. 
Abstract No. C11

\title{
Numerical Methods for Determination of Mix
}

\author{
S. Dutta ${ }^{1}$, E. George ${ }^{1}$, J. Glimm ${ }^{1,3}$, J. Grove $^{2}$, X. $\mathrm{Li}^{1}$, \\ A. Marchese ${ }^{1}$, D. H. Sharp ${ }^{2}$, Z. Xu ${ }^{1}$, and Y. Zhang ${ }^{1}$ \\ ${ }^{1}$ State University of New York at Stony Brook, Stony Brook, NY \\ ${ }^{2}$ Los Alamos National Laboratory, Los Alamos, NM \\ ${ }^{3}$ Brookhaven National Laboratory, Brookhaven, $N Y$
}

We present numerical studies of the growth of a 3D mixing layer due to Rayleigh-Taylor (RT) or Richtmyer-Meshkov(RM) instabilities. Simulations based on the Front Tracking code FronTier give a mixing rate alpha for the bubble growth in planar RT mixing within the range determined by experiments of Youngs-Reed, Smeeton-Youngs and Dimonte et. al. Identical simulation problems, solved with a TVD capturing code, give an alpha below this range of experiment. We present an analysis (based on theory and on diagnostics from the two simulations) to indicate that the difference between simulations is primarily due to diffusion of mass across the fluid interface in the TVD (capturing) simulation.

Axisymmetric 3D spherical RM mixing studies show dependence of the mixing rate on the azimuthal angle, especially after reshock. Statistical mix quantities (volume fraction, etc.) are recorded and compared with mix model equations of the authors and co-workers.

PACS Nos.: 47.20Bp, $47.52+\mathrm{j}$ 
$8^{\text {th }}$ International Workshop on the Physics of Compressible Turbulent Mixing,

Pasadena, CA (2001)

Abstract No. C12

\title{
Rayleigh-Taylor Instability in Compressible Fluids
}

\author{
Y. Elbaz ${ }^{1,2}$, A. Rikanati ${ }^{1,2}$, D. Oron ${ }^{3}$, and D. Shvarts ${ }^{1,2}$ \\ ${ }^{1}$ Nuclear Research Center Negev, Israel \\ ${ }^{2}$ Ben Gurion University, Beer-Sheva, Israel \\ ${ }^{3}$ Weizmann Institue of Science, Rehovot, Israel
}

The behavior of the single mode and multimode Rayleigh-Taylor (RT) instability in compressible fluids is studied using full two-dimensional numerical simulations and analytical theoretical models. Due to the finite mass of the heavier fluid above the bubble, resulting from the initial density distribution, the perturbation growth causes a state of pressure non-equilibrium on the heavier fluid, leading to a bulk acceleration of the heavier fluid as in the case of RT instability in a finite layer of an incompressible fluid. Also, the finite sound speed of the heavier fluid causes an effective mass accumulation in time, therefore changing the pressure gradient on the interface with time.

Analyzing the instability dynamics in a frame of reference moving with the accelerated physical system shows a small effect of compressibility on the instability dynamics. In this frame of reference the simulation results coincide with the known incompressible results - a constant velocity in the single mode case and $\alpha \sim 0.04-0.06$ in the multimode case. However, due to the bulk acceleration of the heavier fluid, in the laboratory frame of reference the bubble velocity continuously increases in the single mode case, and in the multimode case an $\alpha \mathrm{gt}^{2}$ growth rate is obtained, with $\alpha$ continuously increasing as well. 
Abstract No. C13

\title{
One-Dimensional Simulation of the Effects of Unstable Mix on Neutron and Charged-Particle Yield from Laser-Driven Implosion Experiments
}

\author{
R. Epstein, J. A. Delettrez, V. Yu. Glebov, V. N. Goncharov, P. W. McKenty, \\ P. B. Radha, S. Skupsky, V. A. Smalyuk, and C. Stoeck1 \\ Laboratory for Laser Energetics, University of Rochester, Rochester, NY
}

The effects of Rayleigh-Taylor flow in laser-driven implosion experiments are simulated in one dimension by the hydrodynamics code LILAC. Mix is modeled as a diffusive transport process affecting material constituents, thermal energy, and turbulent mix-motion energy within a mix region whose boundaries are derived from a saturable, linear, multimode model of the Rayleigh-Taylor instability. The growth rates and the coupling between perturbations of different unstable interfaces are obtained analytically in terms of the one-dimensional fluid profiles. The initial perturbations are due to beam-energy imbalance, hydrodynamic imprint of short-scale laser nonuniformity, and target surface roughness. The effects of fuel-pusher mix on neutron production and secondary particle yields are characterized and compared with data from implosion experiments. The limitations of one-dimensional mix as an approximation to the multidimensional distortion of the fuel-pusher interface will be considered. 
$8^{\text {th }}$ International Workshop on the Physics of Compressible Turbulent Mixing,

Abstract No. C14

\title{
3D Computation for Surface Perturbation Evolution of Plasma Cloud During its Expansion in Magnetic Field
}

\author{
E. S. Gavrilova, E. V. Gubkov, V. A. Zhmailo, and Yu. V. Yanilkin \\ Russian Federal Nuclear Center - VNIIEF, Sarov, Russia
}

Previously, ref. [1] considered the 2D problem of initially spherical plasma cloud expansion in the axial magnetic field. In particular, it was noted that the plasma surface was unstable to evolution of perturbations (of "chute" type).

This paper solves the above problem with taking into account the growth of the perturbations. The computation is performed with 3D code TREK [2]. Two methods to solve the problem are discussed:

- it is assumed that outside the cloud there is plasma of quite low density as well which magnetic field is "frozen into", in this case appropriate MHD equations are used to compute magnetic field variations;

- it is assumed that outside the cloud there is vacuum, in this case quasi-stationary approximation [1] is used to compute the magnetic field.

Two initial perturbation types are given: one mode and random. The computed data for the linear stage of the one mode perturbation growth is compared to the analytical data.

The results of the problem computation with random perturbations are averaged (over azimuth). Thus obtained plasma density and magnetic field profiles, in particular, dependence of the transition zone width in the profiles on the problem parameters are considered. Applicability of this plasma model is discussed.

1. Bakhrakh S.M., Gubkov E.V., Zhmailo V.A., Terekhin V.A. "Plasma cloud expansion in homogeneous magnetic field”. PMTF, 1974, No. 4, pp. 146-150.

2. Yanilkin Yu.V., Tarasov V.I., Stadnik A.L., Bazhenov S.V., Bashurov V.V., Belyaev S.P., Bondarenko Yu.A., Bykova E.A., Gavrilova E.S., Gorev V.V., Dibirov O.A., Ivanova G.G., Kovalev N.P., Korol'kova T.V., Pevnaya P.I., Sofronov V.N., Toropova T.A., Shanin A.A. Program System TREK for Numerical Simulation of 3D Multi-component Medium Flows. Proceedings of workshop "New Models and Numerical Codes for Shock Wave Processes in Condensed Media”, Oxford, 1997, pp 413-422, 1997. 
Abstract No. C15

\title{
The Richtmyer-Meshkov Instability in Cylindrical Geometry: Experiments and Simulation
}

\author{
M. J. Graham ${ }^{1}$, K. S. Budil ${ }^{1}$, J. Grove ${ }^{2}$, and B. A. Remington ${ }^{1}$ \\ ${ }^{1}$ Lawrence Livermore National Laboratory, Livermore, $C A$ \\ ${ }^{2}$ Los Alamos National Laboratory, Los Alamos, NM
}

Hydrodynamic instabilities are fundamentally important to a wide range of fields, including astrophysics, inertial confinement fusion (ICF), and inertial fusion energy (IFE). The most common of these instabilities is the Rayleigh-Taylor (RT), or buoyancy-driven instability, is caused when a material of higher density is accelerated by a material of lower density. The Richtmyer-Meshkov $(\mathrm{RM})$, or shock-driven instability is produced when an incident shock wave impulsively accelerates a material interface causing small disturbances to grow.

The RT interface is unstable only when the external force acts from the heavy material to the lighter material, whereas the RM instability is present whether the incident shock travels from light to heavy or vice versa. The majority of the theoretical, computational and experimental work has been successfully performed for the RM instability in planar geometry. In most physical applications the RM instability occurs in a curved geometry, eithercylindrical or spherical. This curved geometry complicates the system considerably. For example, the unperturbed system does not have an analytical solution, while the unperturbed system in plane geometry does. The occurrence of reacceleration or re-shock of the material interface caused by the waves reflecting back from the origin is unavoidable in curved geometry.

The Nova Laser was used to test critical ingredients of our understanding of the fundamental properties of the RM instability in the strong-shock, high-compression regime. A shock was launched into a copper hemicylinder with a thin plastic ablator layer by focusing 6 KPP-smoothed, $1 \mathrm{~ns}$ square laser beams at $3 \omega$ onto the interior of the target. A single-mode sinusoidal perturbation was machined onto the outer surface of the copper, which was embedded in a thick layer of plastic. The expanding interface was diagnosed by side-on radiography and radiographs were recorded at several times.

We will show numerical simulations of this experiment using two difference codes: FronTier and CALE. In the FronTier method a lower dimensional grid is fitted to and moves dynamically with discontinuities in the flow. CALE is a continuous adaptive Lagrangian Eulerian method.

PACS Nos.: 52.35.Tc, $47.11+\mathrm{j}$ 
$8^{\text {th }}$ International Workshop on the Physics of Compressible Turbulent Mixing,

Abstract No. C16

\title{
Code to Code Comparisons for the Problem of Shock Acceleration of a Diffuse Dense Gaseous Cylinder
}

\author{
J. A. Greenough ${ }^{1}$, W. J. Rider ${ }^{2}$, C. A. Zoldi ${ }^{2}$, and J. R. Kamm ${ }^{2}$ \\ ${ }^{1}$ Lawrence Livermore National Laboratory, Livermore, $C A$ \\ ${ }^{2}$ Los Alamos National Laboratory, Los Alamos, NM
}

The current computational study is motivated by large-scale (and small-scale)discrepancies between ongoing calculations and experiments of a shock wave accelerating a diffuse cylinder of $\mathrm{SF}_{6}$ ("Experiments and simulations of instabilities in a shock accelerated cylinder," K. Prestridge, C. A. Zoldi, P. Vorobieff, P.M. Rightley, and R. F. Benjamin, Los Alamos Report LAUR -00-3973). Three different Eulerian based codes, Rage (LANL), Cuervo (LANL) and Raptor (LLNL), are applied to an idealized two-dimensional version of the experiment. The model problem consists of a Gaussian shaped $\mathrm{SF}_{6}$ inhomogeneity in air that is accelerated by a $\mathrm{M}=1.2$ shock wave. The initial diffuse cylinder evolves into a quasi-vortex dipole at intermediate times until finally becoming unstable at late times. The integral (large) scale features, which include the length and width of the evolving structure, will be measured from the calculations and compared. The sub-integral scale, small-scale vortical features in the central roll-up, will also be examined quantitatively and compared at intermediate times. An assessment of the degree of convergence of the simulations as well as factors accounting for computed differences will be discussed.

This work was performed under the auspices of the U.S. Department of Energy by the University of California Lawrence Livermore National Laboratory under contract No. W-7405-Eng-48. 


\title{
Abstract No. C17 \\ Implementation of a Turbulent Mix Model in a 2D ALE Code
}

\author{
B. Grieves \\ Atomic Weapons Establishment, Aldermaston, United Kingdom
}

The addition of a turbulent mix model to a two dimensional finite element ALE hydrocode, CORVUS, is discussed. Use is made of the existing mixed-cell data structure to facilitate the inclusion of the model.

This first stage of the model is based on the multiphase flow equations, and is a simplified form of the model implemented by Youngs (See paper at this workshop) in a 2D Eulerian Code. This is applicable to simple Rayleigh-Taylor and Richtmyer-Meshkov instabilities, and some results are presented.

A simple buoyancy-drag model is used to calculate the early stages of the instability growth at interface nodes, and this is used to initialise the turbulent mix model calculation. 
$8^{\text {th }}$ International Workshop on the Physics of Compressible Turbulent Mixing,

\title{
Abstract No. C18 \\ Error Estimation for Strong Shock Hydrodynamics
}

\author{
J. W. Grove \\ Los Alamos National Laboratory, Los Alamos, NM
}

The quantification of uncertainty is a fundamental problem in mathematical modeling. Sources of uncertainty include incomplete physical models, poorly defined initial conditions, and the effect of numerical methods. Traditional numerical analysis is extremely limited in accessing the accuracy of a computation, especially in highly nonlinear regimes. Predictive calculations require a more detailed assessment of solution error, including a quantitative model for the probability distribution of the error in a simulation.

This talk will describe a prototype methodology, developed in collaboration with researchers at the University at Stony Brook, for studying uncertainty in a computational model. We apply this methodology to a simple strong shock refraction test problem. Assuming known probability distributions for a set of initialization and flow parameters, we perform a statistical study of the generation and propagation of solution error. Error is computed by comparing fine and coarse grid computations for different mesh sizes, and numerical methods. We obtain a space-time field of probability distributions for a variety of state variables, and seek stochastic models for the generation and propagation of solution error as a function of flow state and numerical method.

PACS Nos.: 07.05.Tp, 47.11.+j, 47.40.-x 
Abstract No. C19

\title{
A Semi-Empirical Model for Turbulent Diffusion of Magnetic Field to Accelerated Plasma
}

\author{
E. V. Gubkov, V. A. Zhmailo, and Yu. V. Yanilkin \\ Russian Federal Nuclear Center - VNIIEF, Sarov, Russia
}

A nonlinear phase of instability development at the accelerated plasma/magnetic field interface is studied. The paper considers the case with short wavelength and incidental initial perturbations. A semi-empirical model which structure is similar to that of the hydrodynamic model from $/ 1 /$ is proposed to describe perturbations of such a kind.

Two problems are solved using the proposed model: a one-dimensional problem of a converging cylindrical liner with axial magnetic field in cavity (ultra-high magnetic field generator "MK-1" /2/); a two-dimensional problem of a plasma cloud expansion in external magnetic field /3-5/.

By comparing computation results with the corresponding experimental data, some constants introduced to the model are determined, as well as frames of its applicability are specified.

1. Yanilkin Yu.V., Nikiforov V.V., Zharova G.V. A Two-Equation Model and a Method for Turbulent Mixing Computations in 2D CompressibleFlows. - VANT, Ser.:MMPhP, 1994, Iss.4.

2. Sakharov A.D., LudaevR.Z., Smirnov E.N., Plyushcheyev Yu.I., Pavlovskii A.I., et al. DAN SSSR, 1965, V.196, No.1, pp.65-68.

3. Bakhrakh S.M., Gubkov E.V., Zhmailo V.A., Terekhin V.A. Expansion of a Plasma Cloud in Uniform Magnetic Field. - PMTPh, 1974, No.4, pp.146-150.

4. Zakharov Yu.P., Orishich A.T., Ponomarenko A.G. "Plasma Physics" Journal, 1986, V.12, p.674.

5. Pisarczuk T., Kasprczuk A., Karpinski L., et al. Application of Interferometric Methods to Investigation of Laser-Produced Plasma in Strong External Magnetic Field. -In "Advances in laser interaction with matter and inertial fusion", Madrid, 1996. 
$8^{\text {th }}$ International Workshop on the Physics of Compressible Turbulent Mixing,

Abstract No. C20

\title{
Localization and Spreading of Interfaces (Contact Discontinuities) in PPM and WENO Simulations of the Inviscid Compressible Euler Equations
}

\author{
S. Gupta, N. J. Zabusky, R. Samtaney, and Y. Gulak \\ Rutgers, StateUniversity of New Jersey, Piscataway, NJ
}

The physical or numerically- "motion" of interfaces or contact discontinuities (CD) between two fluids of different density or temperature governs the mixing of species, particularly during the late time ("asymptotic ") epochs.

Using the methods of Vorozhtzov and Yanenko, [1] we show that for the equation $\frac{f \rho}{f t}+u_{0} \frac{f \rho}{f x}=0$, the continuum $\quad$ limit $\quad \operatorname{sields} \frac{f \rho}{f t}+u_{0} \frac{f \rho}{f x}=(-1)^{r+1} \mu\left(h, \tau, u_{0}, p_{0}\right) \frac{f^{r+1} \rho}{f x^{\prime+1}}(\underline{\mathrm{A}})$ where $r$ is the order of the scheme ( $r=1$ for 1 st order schemes, such as Lax, etc.), $\mathrm{h}$ is the grid size and $\tau$ is the time-step of the integration. For $\mathrm{r}=1$ and 2 , the exact solution of (A) with a discontinuous initial density function, $\left(\rho=\rho_{1}, x<x_{0} ; \rho=\rho_{2}, x>x_{0}\right)$, is $\rho(x, t)=\left(\rho_{1}+\mathrm{r} \rho_{2}\right) /(\mathrm{r}+1)+\left(\rho_{2}-\rho_{1}\right) \mathrm{F}(\xi(x, \mathrm{t})),(\underline{\mathrm{B}})$, where $\xi(x, t)=\left(x-x_{0}-u_{0} t\right) /\left(c_{r} \mu t\right)^{1 /(r+1)} \quad$ and $\mathbf{F}$ is the solution of an ODE arising in a self-similarstudy. In our numerical PPM [3] solutions, we find accurate agreement with the constant term in (B), i.e. the center of a spreading interface depends on $\mathrm{r}$. This explains the lack of convergence in attempting to localize the CD previously [2]. However, the numerical spreading, $\xi_{P P M}(x, t)$, produced by PPM artificially steepens the density over two grid intervals if $\rho_{2}>\rho_{1}$ and spreads it according to a power law if $\rho_{2}<\rho_{1}$. This asymmetry will prove troublesome for reshock and reacceleration problems at late time epochs. We also comment on higher-order algorithms [4] and the effects of vorticity on the interface in 2D.

[1] E. V. Vorozhtzov and N. N. Yanenko, 1990. Methods for the Localization of Singularities in Numerical Solutions of Gas Dynamics Problems, Springer.

[2] R. Samtaney and N. J. Zabusky, 2001. High gradient compressible flows: Visualization, feature extraction and quantification, In Flow Visualization: Techniques and Examples, Editors T. T. Lim and A. Smits, Imperial College Press.

[3] J.M Blondin et al at NCSU.VH-1. A Lagrangian remap code based on PPM.

[4] Ravi Samtaney, Caltech . Higher order WENO code 


\title{
Abstract No. C22 \\ Update on Instability Modeling for the NIF Ignition Targets
}

\author{
S. W. Haan, T. Dittrich, S. Hatchett, D. Hinkel, M. Marinak, D. Munro, O. Jones, \\ S. Pollaine, and L. Suter \\ Lawrence Livermore National Laboratory, Livermore, $C A$
}

This talk is a general update on the hydrodynamic instability modeling that we do for ignitiontargets for the National Ignition Facility. Recent results include design of a polystyrene-ablator target, analysis of Rayleigh-Taylor growth on beryllium targets driven at $250 \mathrm{eV}$ at various scales, simulations of the effect of fill tubes on the implosion,, and simulations of 3D asymmetry and its impact. Hydrodynamic instability modeling is done with direct numerical simulations, since the targets are designed to avoid short wavelength instability growth.

PACS Nos.: $52.57 . \mathrm{Bc}, 52.57 . \mathrm{Fg}$ 
$8^{\text {th }}$ International Workshop on the Physics of Compressible Turbulent Mixing,

Pasadena, CA (2001)

Abstract No. C23

\title{
Pillars of Creation
}

\author{
J. O. Kane ${ }^{1}$, D. D. Ryutov ${ }^{1}$, B. A. Remington ${ }^{1}$, S. G. Glendinning ${ }^{1}$, J. Nash ${ }^{1}$, \\ M. Pound ${ }^{2}$, and D. Arnett ${ }^{3}$ \\ ${ }^{1}$ Lawrence Livermore National Laboratory, Livermore, $C A$ \\ ${ }^{2}$ University of Maryland, College Park, $M D$ \\ ${ }^{3}$ University of Arizona, Tuscon, $A Z$
}

The towering 'Pillars of Creation' of the Eagle Nebula are a long-standing astrophysical mystery. A new initiative is underway to develop a model for the formation of the Pillars, employing threedimensional numerical modeling and scaled verification experiments using intense lasers. In the Rayleigh-Taylorinstability (RT) model of the Pillars advanced almost fifty years ago by Spitzer and Frieman (Spitzer, L. 1954, ApJ 120, 1, Frieman, E. A. 1954, ApJ 120, 18), radiation from nearby stars photo-evaporates and accelerates the cloud surface, and the Pillars are falling 'spikes' of dense gas. Recently, fluid velocities and column densities in the Pillars have been measured (Pound, M. W. 1998, ApJ 493, L113). Preliminary two-dimensional numerical simulations of the RT model have been performed which produce results consistent these observations, assuming compressible fluids and a thin initial cloud. Since the radiation may impact the surface at an angle, a 'Tilted Radiation' instability (LLNL report UCRL-JC-138744, May 2000; .D. Ryutov, B.A. Remington, H.F. Robey, R.P. Drake. Phys. Plasmas, 8, 1804 (2001)) can cause the spikes to translate as waves whose tips may 'break', producing the small gas 'bullets' visible near the Pillars in images taken by the Hubble Space Telescope. In an alternate model for the Pillars, the cometary model, the Pillars consist of gas swept behind dense preexisting nuclei, but it appears difficult to reproduce the observed velocities and densities in numerical models with dense preexisting nuclei as the initial condition. . However, the effect of radiative cooling and magnetic fields remains to be explored. The maturing field of laser astrophysics presents an opportunity for testing models for the Pillars in the laboratory. Theoretical and numerical evaluations of variousmodels, implications for observations, and plans for verification experiments are presented.

This work was performed under the auspices of the U.S. Department of Energy by the University of California Lawrence Livermore National Laboratory under contract No. W-7405-Eng-48. 
Abstract No. C25

\title{
Application of a Laser Shock Tube for the Study of Supersonic Gas Flows and the Development of Hydrodynamic Instabilities in Layered Media
}

\author{
I. G. Lebo ${ }^{1}$ and V. D. Zvorykin ${ }^{2}$ \\ ${ }^{1}$ Tekhnikal University-MIREA, Moscow, Russia \\ ${ }^{2}$ P. N. Lebedev Physical Institute of the Russian Academy of Sciences, Moscow, Russia
}

The study of the evolution of hydrodynamic instabilities of the interface between two media found in the field of acceleration is a problem of great importance in inertial confinement fusion (ICF), physics of high energy densities, cosmology, and astrophysics. The passage of strong shock waves (with Mach number $\mathrm{M}>>1$ ) through contact surfaces of two gases or plasma with different densities causes the formation and development of complex vortex structures, which are of interest for present-day nonlinear hydrodynamics and for studying the problem of a change from an ordered state to chaos. Another problem, which is important for the development of modern aerospace engineering and protecting the Earth from collisions with space objects, is the study of supersonic flow past bodies of complex shape at large Mach numbers. Usually, such experiments in gases are carried out at relatively small Mach numbers $M=1-4$ with help of shock tubes. The pressure amplitudes in shock wave are about 2-10 bar. The design of a miniature laser shock tube for the study of a wide range of hydrodynamic phenomena in liquids at pressures greater than $10 \mathrm{kbar}$ and supersonic flows in gases with large Mach numbers (greater than 10) is discussed in this paper. In the system considered here, the confinement of a laser-producedplasma and the excitation of plane shock waves take place inside a miniature tube, which restricts lateral unloading. The design of such a laser shock tube (LST) is based on the use of the following basic components: a shock tube chamber; a powerful $\mathrm{KrF}$ laser [1]; an original laser focusing system; and 2D numerical codes. The technique proposed here for exciting shock waves in gases and compression waves in liquids by $\mathrm{KrF}$ laser radiation has some advantages in comparison with the conventional technique used in experiments with shock tubes: 1) large Mach numbers in gas flows $(M>20)$ and pressure pulses greater than $10 \mathrm{kbar}$ in liquids [2]; 2) economy of noble gases and other supplies (laser driven shock tube volume is less in $\sim 10^{3}-10^{4}$ times). This study is supported by RFBR, grant N0101-00023

References.

1. Zvorykin V.D., Lebo I.G. Laser and Particle Beams, 17, 69, 1999

2. Zvorykin V.D., Lebo I.G. Quantum Electronics, 30, 540, 2000 
$8^{\text {th }}$ International Workshop on the Physics of Compressible Turbulent Mixing,

Abstract No. C26

\title{
Molecular Dynamic Simulation of Shock and Richtmyer-Meshkov Instability in Cylindrical Geometry
}

\author{
K. Nishihara, V. Zhakhovskii, and M. Abe \\ Osaka University, Osaka, Japan
}

Molecular dynamic (MD) approach has been applied to study the converging cylindrical shock waves and the Richtmyer-Meshkov instability in a dense Lennard-Jones fluid. MD method is based on tracking of the atom motion and hence it has fundamental advantages over hydrodynamic methods that assume a shock as a structureless discontinuity and require an equation of state. In addition, hydrodynamic simulation has a limitation in grid resolution, especially, in the cylindrical geometry. It is found that the one million particles is enough to simulate propagation of a cylindrical shock in close detail due to small thickness of shock fronts (a few Angstroms for Argon) in liquid.

We investigate the stability of converging shocks with different perturbation modes and its mixture for different Mach numbers. The converging shock is unstable for low mode number perturbation in large Mach number. It was shown that the amplitude of a shock front ripple increases and the Mach stems are formed. Supersonic jets generated by interaction of reflected shocks in downstream flow are observed. We also study the Richtmyer-Meshkov instability of an interface between two L-J liquids of different densities in the cylindrical geometry. The turbulent mixing is observed when the reflected shock near the center passes again through the unstable interface. 
Abstract No. C27

\title{
Compressibility Effects in a High-Speed, Reacting Shear Layer: An Investigation Using DNS
}

\author{
C. Pantano and S. Sarkar \\ University of California at San Diego, La Jolla, CA
}

In technological applications such as combustion, high-speed propulsion and energetic devices, the variation in thermodynamic variables associated with large heat release interacts with and modifies the underlying turbulent flow. Direct numerical simulations of the reacting shear layer using up to 20 million grid points are performed over a wide range of heat release rates and convective Mach numbers to quantify and understand some of these modifications to the turbulence evolution and structure. Large heat release rates typical of hydrocarbon combustion are considered, and not only the overall growth characteristics but also the turbulence structure is investigated. The single-step irreversible combustion of a diluted methane stream mixing with an air stream is considered. An infinitely fast reaction rate is assumed, that is, the heat release is confined to an infinitely thin region in mixture fraction space located at the stoichiometric value.

A longitudinal snapshot of the density field is shown in Fig. 1. The upper air stream moves to the left while the lower fuel stream moves to the right. The mean location of the flame sheet is displaced to the upper air side. However, the convective stirring of the flame sheet by the turbulent motion spans the entire width of the shear layer so that an instantaneous snapshot such as Fig.1 shows a wide central core of hot, low-density fluid separated from the cold, high-density fluid on either side by thin regions with large values of the density gradient. The thickness growth rate of the shear layer is the overall quantity of primary interest. With increasing values of convective Mach number, the growth rate of the nonreacting cases shows the well-known large reduction. What is perhaps less expected is the effect of Mach number on the growth rate of the reacting cases. The growth rate is already quite low at the low-Mach number reacting case and a further increase in the Mach number causes only a relatively small additional reduction. The Reynolds shear stress profiles show that, in the low-speed case, there is a significant reduction of its peak value in the case with the highest heat release. However, at the largest convective Mach number, any additional change in the Reynolds shear stress is relatively small. The width of the profiles of Reynolds shear stress (not shown) as well as other Reynolds stresses scale well with the vorticity thickness but not the momentum thickness. The full paper will present results regarding all Reynolds stress components as well as thermodynamic correlations and cross-correlations 
Pasadena, CA (2001)

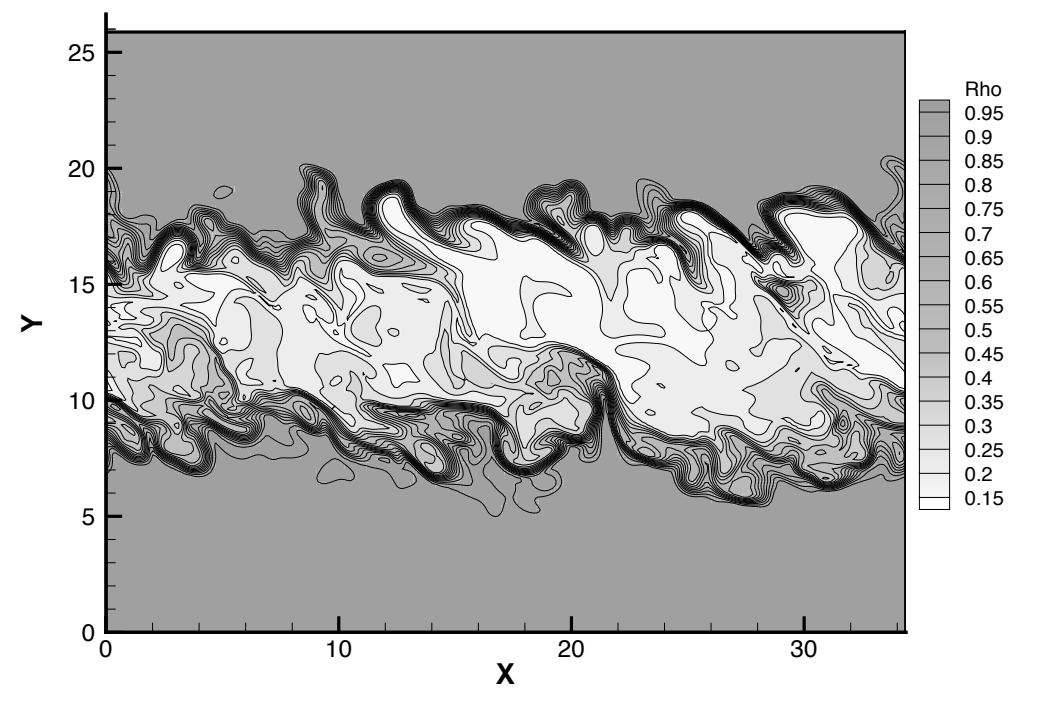


Abstract No. C28

\title{
Computational Modelling of Two-Shell Cylindrical Implosions with Mix
}

\author{
K. W. Parker ${ }^{1}$, A. M. Dunne ${ }^{1}$, S. Rothman ${ }^{1}$, D. Youngs ${ }^{1}$, C. W. Barnes ${ }^{2}$, \\ S. H. Batha ${ }^{2}$, N. E. Lanier ${ }^{2}$, G. R. Magelssen ${ }^{2}$, T. J. Murphy', and J. M. Scott ${ }^{2}$ \\ ${ }^{1}$ Atomic Weapons Establishment, Aldermaston, United Kingdom \\ ${ }^{2}$ Los Alamos National Laboratory, Los Alamos, NM
}

Experiments to look at mix in a compressible, convergent geometry have been carried out on the Omega Laser Facility. These employ a radio-graphically opaque marker layer, which is sandwiched between the polystyrene ablator and low-density foam. As the implosion proceeds, a strong shock is launched which causes the marker to become mixed into both the foam and the ablator. More recently, these experiments have introduced a high-density core to the targets, such that a shock is reflected from the core back through the mix layer at late time. Presented here are calculations for these '2-shell' targets. Where possible, comparisons are made to the experimental results. 
$8^{\text {th }}$ International Workshop on the Physics of Compressible Turbulent Mixing,

Abstract No. C29

\title{
Dispersal of Mass and Circulation Following Shock-Sphere (axisymmetric) and Shock Cylinder Interactions: Effects Arsing From Shock Cavity Collapse, Vortex Double Layers; Density-Gradient Intensification and Vortex Projectiles
}

\author{
G. Peng, S. Gupta, S. Zhang, and N. J. Zabusky \\ Rutgers, StateUniversity of New Jersey, Piscataway, NJ
}

We quantify and scale the dispersal and mixing (transport) of mass and vorticity following a spherical (axisymmetric) shock-bubble interaction. We use planar shocks of Mach =2.5, 5.0 and 10 and a density ratio - bubble/ambient- of 10.0, a parameter domain beyond that explored earlier [1], where new effects arise. We correlate and scale the transports with: the primary circulation layer deposited by the passing incident shock in epoch 1; the collapsing transmitted shock cavity circulation layer (TSCCL); and a vortex "double layer" (VDL) on the downstream boundary. The TSCSL is generated at the sharp kink of the collapsing transmitted shock (where numerous shocklets arise, e.g. a "penta-point" shock for M= 2.5) and is responsible for an epoch 1 appearing and upstream-moving Vortex Projectile (VP) (with an associated density enhancement). The VDL arises from two shock wave sources incident on the downstream side of the bubble: from inside, the re-expanding cavity and from outside the incident shock as it passes the rear side of the bubble. These phenomena evolve into a chaotic downstream array of vortex projectiles (VPs) which in axisymmetry are complex-shaped stratified rings of opposite polarity. We observe strong circulation generating baroclinic effects during this epoch [2]. In 3D, these VPs will be rapidly unstable and lead to domains of reconnecting vortices and stratified turbulence. The collapse of the shock cavity produces: large short-time enhancements of pressure, density and temperature, which we scale; and subsequent reverberationeffects in the bubble interior and exterior, which we quantify. We simulated the 2d axisymmetric Euler equations with the Colella \& Woodward (1984) PPM in a Galilean frame translating uniformly with the velocity equal to 20 percent of the post-shock velocity. Our study was made at three resolutions, (z, r): (1) $\{803,123\}$; (2) $\{1606,246\}$ and $\{3212,492\}$. At our high Mach numbers and resolutions fast instabilities arise which yield coherent structures (e.g.[3]) and we comment on their relevance to the new observed phenomena.

[1] N.J. Zabusky and S-M. Zeng, J. Fluid Mechanics 362, pp. 327-346, 1998.

Shock cavity implosion morphologies and vortical projectile generation in axisymmetric shockspherical F/S bubble interactions. Also,: N.J. Zabusky, Annual Review Fluid Mechanics 31, pp. 495536,1999

[2] S. Zhang and N. J. Zabusky Shock - planar curtain interactions: Strong secondary baroclinic deposition and the emergence of coherent and random vortex projectiles (VPs) and decaying stratified turbulence. 8th IWPCTM: International Workshop on the Physics of Compressible Turbulent Mixing (this volume) [3] R. Samtaney and D.I. Pullin, Physics of Fluids 8, pp. 2650-2655, 1996

*This work was supported mainly by DOE (Grant No. DE-FG0293ER25179.A000) and monitored by Dr. Daniel Hitchcock. Additional support was provided by Rutgers University SROA program \# This paper is dedicated to Brad Sturtevant whose experiments inspired important configurations for accelerated flows.

PACS Nos.: 47.40.-x, 47.40.Nm, 52.57.Fg, 47.20 Ma, 47.27.-I, 47.27.Eq, 47.11.+j 


\title{
Abstract No. C30 \\ Influence of Turbulent Mixing Zone on Growth of Local Perturbation in Environments of Rayleigh-Taylor Instability (Numerical Simulation)
}

\author{
V. A. Raevski, S. N. Sinitsina, and Yu. V. Yanilkin \\ Russian Federal Nuclear Center - VNIIEF, Sarov, Russia
}

It is common knowledgethat self-similargrowth of local perturbation occurs following the law in the case of absence of turbulent mixing zone. The growth constant is about 3 times higher than the constant of growth of self-similar turbulent mixing zone. Basing on two-dimensional numerical computations by Euler technique EGAK, it is revealed that continuous continuum of self-similar solutions occurs, where is function of the relation and, if at the initial time there are local perturbation and the perturbations forming further the turbulent mixing zone. 
$8^{\text {th }}$ International Workshop on the Physics of Compressible Turbulent Mixing,

\title{
A Statistical Comparison of Gas Cylinder Experiments with Their Simulation
}

\author{
W. J. Rider, J. R. Kamm, and C. A. Zoldi \\ Los Alamos National Laboratory, Los Alamos, NM
}

We present the statistical analysis of the evolution of a diffuse cylinder of $\mathrm{SF}_{6}$ shocked by a Mach 1.2 shock. The cylinder baroclinically develops a vortical structure and subsequently mixes with the surrounding air. The experimental diagnostics are images of tracer particles in the $\mathrm{SF}_{6}$ and particle image velocimetry. We examine the nature of the mixing using a variety of tools includingimage analysis using correlations, wavelets, and fractal dimension. Our efforts follow the path of earlier investigations of a gas curtain geometry. There we found significant departures in behavior between the details of the experimentally measured mixing and that computed with the hydrodynamic codes. These statistics from the experiment are then compared with complementary simulations using several computer codes. In each case, we examine the sensitivity of the results to variations in mesh resolution and numerical algorithms. Figure 1 contains plots showing that both the integral size of the evolving cylinder and the magnitude of the velocity field computed in hydrodynamic codes do not match the experimentally
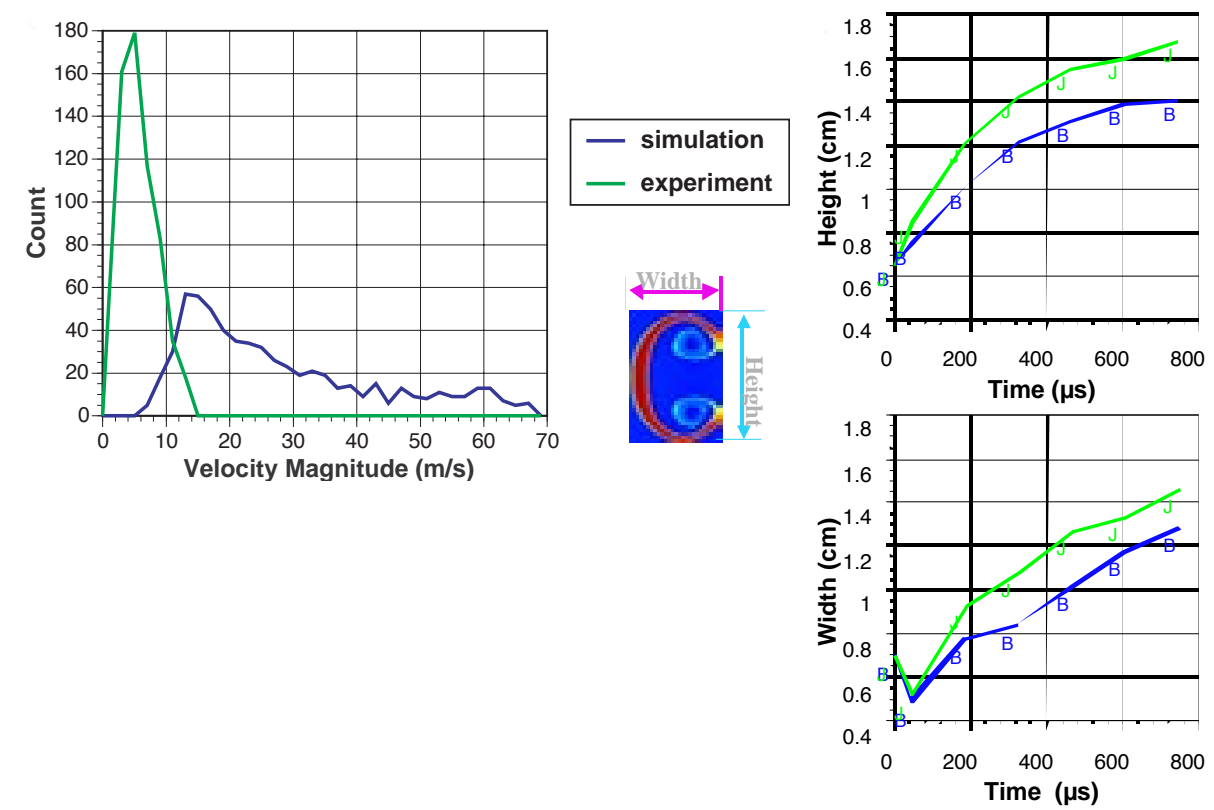

measured results.

Fig. 1. The plot on the left shows a distribution of the velocity magnitude for the experiment (green) and simulation (blue). The plots on the right show the time evolution of the height and width of the evolving shocked cylinder structure. 
Abstract No. C33

\title{
Large Eddy Simulation of Strong Shock Richtmyer-Meshkov Instability
}

\author{
R. Samtaney, T. Voelkl, and D. I. Pullin \\ Graduate Aeronautical Laboratories, California Institute of Technology, Pasadena, CA
}

In previous studies of isotropic compressible turbulence [1], it was demonstrated that low-order difference schemes are unsuitable for large eddy simulations(LES) of compressible turbulence. In this paper, we present results from formally high-order accurate LES of the Richtmyer-Meshkov (RM) instability. We chose fifth and seventh-order accurate Weighted Essentially Non-Oscillatory (WENO) schemes as the numerical method. These were suitably modified to suppress the so-called carbuncle numerical instability of the shock front. The physical details of the simulations are as follows. The physical domain is a shock-tube of square cross-section. A smooth flat interface with a hyperbolic tangent profile between two gases is initially deformed with a prescribed spectrum giving it multiple harmonic perturbations. This interface is accelerated with a strong (Mach number $=10$ ) shock. The boundary conditions are periodic in the transverse directions, and inflow and reflecting along the length of the shock-tube.

The sub-grid-scale (SGS) model employed in the LES is the stretched vortex (SV) SGS model [2]. This model assumes sub-grid motion to be generated by nearly axisymmetric vortices. The sub-grid heat flux is modeled by advection of a passive scalar taken as the temperature. This model was successfully demonstrated in a posteriori comparisons between LES and direct numerical simulations of moderate turbulent Mach number decaying isotropic compressible turbulent simulations in the presence of shocklets [1]. It requires the velocity gradient tensor and the temperature gradient, both of which are calculated with an explicit fourth-order finite difference method.

We will present the evolution of the mixing width as a function of time computed using a level-set approach and a variety of diagnostic procedures, the transverse spectra and evolution of the turbulent kinetic energy (both sub-grid and resolved). In particular, we focus on the effects on these variables due to reshock. Finally, we will endeavor to shed light on the modified wavenumber characteristics of the WENO method and it's suitability for the LES of RM flows.

Acknowledgement: We gratefully acknowledge support of this work by the Academic Strategic Alliances Program of the Accelerated Strategic Computing Initiative(ASCI/ASAP) under subcontract no. B341492 of DOE contract W-7405-ENG-48. Useful discussions with Paul Dimotakis, Tony Leonard, Dan Meiron, and Branko Kosovic are gratefully acknowledged.

References:

[1] Branko Kosovic, Dale I. Pullin, and Ravi Samtaney. Subgrid-scale modeling for large-eddy simulation of compressible turbulence. Physics of fluids, sub-judice.

[2] Tobias Voelkl and D.I Pullin. A physical-space version of the stretched-vortex subgrid-stress model for large-eddy simulation. Physics of Fluids, Vol. 12, pp1810-1825 
$8^{\text {th }}$ International Workshop on the Physics of Compressible Turbulent Mixing,

Abstract No. C35

\title{
Numerical Investigation of a Laser Induced Turbulent Mixing Zone
}

\author{
P. Seytor and M. Legrand \\ Commissariat à l'Energie Atomique, Bruyères-le-Châtel, France
}

We have used high Mach number (M 30) mix instability experiments ${ }^{1}$ which have been conducted using Nova laser system to investigate the growth of the Richtmyer-Meshkov instability resulting from a strong shock wave. The initial nonlinear single-mode two dimensionnal pertubation was machined into a brominated plastic ablator $\left(1.22 \mathrm{~g} / \mathrm{cm}^{3}\right)$ adjacent to a low density carbon foam $(0.10$ $\mathrm{g} / \mathrm{cm}^{3}$ ). We compared the experimental measurements with LLNL simulations (CALE 1D/2D) and our own numerical simulations(FCI1/FCI2). We found both experiment and simulation to be in good agreement with a k-_model and also with recent theories for the non linear evolution of instability relevant to an other work presented at this meeting ${ }^{2}$.

${ }^{1}$ D.R. Farley, L. M. Logory, S.D. Murray and E. W. Burke PHYS; Plasmas 6, 4304, (1999).

${ }^{2} \mathrm{M}$. Vandenboomgaerde this meeting $8^{\text {th }}$ IWPCTM 2001. 


\title{
Abstract No. C36 \\ A Mix-Model For One-Dimensional Simulations of Laser-Driven Implosion Experiments
}

\author{
D. Souffland and F. Renaud \\ Commissariat à l'Energie Atomique, Bruyères-le-Châtel, France
}

In laser-driven implosion experiments, hydrodynamic instabilities can growth at interfaces between components as well as at ablation fronts. These processes have various origins and evolve through different ways, but they have in common to favor interpenetration of different fluid components or of fluid regions differing only by their thermodynamic states. The complete study of these intrinsically three-dimensional phenomena, involving a large range of length scales, is still unworkable. We thus need simplified models to assess the impact of variations in the definition of the target, the hohlraum or the laser drive.

The main hypothesis for the present mix-model is that, at the scale of the mesh size, an intimate mixing can simulate the interpenetration region. The description of the model, called hereafter $\mathrm{MeDiC}$, specifies the treatment for the two main cases: density interface instabilities and nonmaterial front instabilities. Diffusive terms are added to model heat and momentum transfers. In the first case, an additional equation for the mass concentration of one component of the mixing is calculate, when, for the second one, the boundaries of the mixing region are imposed. The thickening of the interpenetration zone is, indeed, supposed to be known from experimentaldata or from postprocessing of two-dimensional computations results. This information is used to set the boundary locations, in the non-material front instabilities case, and, in both cases, to calculate the evolution of the diffusion coefficients.

We will discuss examples of mixings due to the Richtmyer-Meshkov instability occurring at the interface between the plastic shell and the fuel, on one hand, and to the ablation front instability occurring at the edge of the hot spot during its formation, on the other hand.

PACS Nos.: 52.57.Fg, 51.20, 42.27.Qb 
$8^{\text {th }}$ International Workshop on the Physics of Compressible Turbulent Mixing,

\title{
Modeling Turbulent Mixing in Inertial Confinement Fusion Implosions
}

\author{
Y. Srebro ${ }^{1,2}$, D. Kushnir ${ }^{2,3}$, Y. Elbaz ${ }^{1,2}$, and D. Shvarts ${ }^{1,2}$ \\ ${ }^{1}$ Ben-Gurion University, Beer-Sheva, Israel \\ ${ }^{2}$ Nuclear Research Center, Negev, Israel \\ ${ }^{3}$ Hebrew University, Jerusalem, Israel
}

A series of high uniformity spherical implosion experiments has recently been conducted on the OMEGA laser system in the University of Rochester. In these experiments 3-15atm gas-filled plastic shells of diameter $\sim 1 \mathrm{~mm}$ were irradiated with $1 \mathrm{~ns}$ square laser pulses of total energy $\sim 20 \mathrm{~kJ}$. Fusion yields were measured experimentally to be $10-40 \%$ of one-dimensionalnumerical simulations' prediction, probably because of core-shell mixing.

Perturbations to these implosions include inner and outer surface roughness, beam-to-beam power imbalance and single-beam laser nonuniformity, which has been reduced to a minimal level using $1 \mathrm{THz} 2 \mathrm{D}-\mathrm{SSD}$.

Two-dimensional numerical simulations, describing the Rayleigh-Taylor growth of multimode perturbations during the deceleration-stage, were performed to determine mix region width. Reductions in the temporal neutron production rate, attained from the simulations assuming various levels of atomic mixing in the mixed region, were compared to experimental results for implosions with different convergence ratios. 
Abstract No. C38

\title{
Turbulent Mixing Nuclear Burning in Type Ia Supernova Explosion Based on Bubble Statistical Mechanics
}

\author{
H. Takabe ${ }^{1}$, S. Yamada ${ }^{1}$, K. Kobayashi ${ }^{1}$, A. Mizuta ${ }^{1}$, and K. Nomoto ${ }^{2}$ \\ ${ }^{1}$ Osaka University, Osaka, Japan \\ ${ }^{2}$ University of Tokyo, Tokyo, Japan
}

It is well known that Type Ia supernovae explode when the masses of white dwarfs become close to the Chandrasekhar limiting mass. This is the reason why the Type Ia explosion is used as a standard candle in the universe to determine, for example, the Hubble constant and dark energy. The scale of explosion has been well studied with one-dimensional code with some mixing model; however, the physical mechanism has not determined from the first principle, yet. There are many works to understand the physics with large scale computing based on hydrodynamics in twodimension or mostly three-dimension in these days [1]. It seems, however, that the smaller scale fluctuation appears, the smaller the grid size, and it is still open question how the instability grows and evolves into nonlinear stage and enhance the energy release by nuclear reactions.

In the present report, we would like to model the growth of the Rayleigh-Taylorinstability coupled with the Landau-Darrieusinstability. In the nonlinear stage, we consider the statistical mechanics of the bubbles following the way developed by Don Shvarts[2] and estimate the increase in the nuclear burning rate due to the increase in the surface area of the burning wave in the form of fractal structure. This model is coupled with the multi-dimensional explosion code to predict the scale of explosion. Such work is expected to be used to identify the physical mechanismof the time evolution of the burning wave, which may change from deflagration wave to detonation wave.

Reference:

[1]W. Hillebrandt and J. Niemeyer, Ann. Rev. Astron. Astrophys. 38, 191-230

(2000)

[2]D. Shvarts et al, Physics of Plasmas 2. 2465 (1995). 
$8^{\text {th }}$ International Workshop on the Physics of Compressible Turbulent Mixing,

Pasadena, CA (2001)

\author{
Abstract No. C39
}

\title{
Turbulent Diffusion in Solar Type Star
}

\author{
N. Toqué \\ University of Montreal, Quebec, Canada
}

The shear layers with the instability of Kelvin-Helmoltzare common topics of the fluids mechanics. They are less common when they are assumed to be in a solar type star to partly explain the anomalies of abundances at the photosphere. Thanks to the rolling-up of the convective zone, the ionized species, which are produced in the radiative core of the star, such as the Lithium, have to migrate to the top of it. However, they are not enough detected at the photophere to validate the standard stellar model. So, it is assumed that at the vicinity of the tachoclyne, the goin-up of the light abundances is blocked by horizontal turbulence in shear layers.

This poster introduces the content of the numerical 2D code and the assumptions made to simplify the modelisation of the physical problem. It shows results which enforce the influence of the turbulence and quantify its effect on the going-up of the ionized species to the photophere of the star. 


\title{
Abstract No. C40 \\ Recent Computational Simulations of Rayleigh-Taylor Mix Layer Growth With a Multi-Fluid Model
}

\author{
E. Vold \\ Los Alamos National Laboratory, Los Alamos, NM
}

Recent results of computational simulations of the Rayleigh-Taylor mix layer are presented and discussed. Our previous work is summarized briefly comparing mix layer growth characteristics observed in different simulation modes including single fluid with initial density discontinuity, twofluids with interface reconstruction and in a full multi-fluid dynamic approach. Recent comparisons under varying compressibility are presented showing negligible influence of compressibility on the mix layer growth rate. Using spectral analyses, perturbations intentionally introduced in the initial conditions are compared to long wave length perturbations introduced inadvertently in these initial conditions. The influence of these initialconditions on late time growth and growthrate are explored. The compressible multi-fluid model allows each fluid to have its own 'drift velocity' relative to the mass averaged fluid velocity. This can be applied in several ways within the mix layer to represent a real molecular mixing, a turbulent enhanced diffusive mixing, or an individual species 'sub-grid' convective drift flux. Examples of these in the Rayleigh-Taylormix layer are discussed. Finally, we consider the combination of these factors which best matches the experimental results for mixing layer growth rates in incompressible experiments, and how these results may apply to compressible fluids. 
$8^{\text {th }}$ International Workshop on the Physics of Compressible Turbulent Mixing,

Abstract No. C41

\title{
An Efficient and High Resolution Solver for the Two-Dimensional Numerical Simulation of the Richtmyer-Meshkov Instability
}

\author{
S. P. Wang, M. H. Anderson, J. G. Oakley, and R. Bonazza \\ University of Wisconsin at Madison, Madison, WI
}

The development of a consistent and fully conservative model and a corresponding efficient and high resolution solver for the numerical simulation of multicomponent or multifluid flows is presented. This theoretical and numerical work was developed to support the Wisconsin shock tube investigation of hydrodynamic issues related to the Richtmyer-Meshkov and Rayleigh-Taylor instabilities.

A consistent and fully conservative treatment of contact discontinuities is proposed for the simulation of compressible multifluid flows. The model is capable of capturing contact discontinuities with significantly reduced numerical uncertainties compared to conventional conservative models. Starting from the concept of total enthalpy conservation for the mixture, a new formulation is defined for the determination of the ratio of the specific heats of the mixture, and a governing equation in conservative form for pressure is obtained subsequently. With continuity equations for the individual components, a governing equation in conservative form for the ratio of specific heats of the mixture is easily derived. These two derived equations, combined with mass balance and momentum balance equations form the full system for the description of multifluid flows.

The conservative governing equations are then solved with an efficientand high resolution Godunovtype solver which is based upon the exact Pike(1993) Riemann solver. To improve the accuracy of the scheme, by preserving monotonicity of the variables at shock waves and contact surfaces, a Monotonic Upstream-Centred Scheme for Conservation Laws (MUSCL) technique for the data reconstruction of fluxes is used. Second order accuracy is achieved by using a piece-wise linear method and a piece-wise spline method is introduced to achieve higher-order accuracy especially useful for capturing contact discontinuities such as the Richtmyer-Meshkov instability (fourth order accuracy has been achieved even for non-uniform mesh sizes).

Several 1-D multifluid flows with both strong and weak shocks are simulated using the model. Comparisons of numerical results obtained by the proposed model, conventional models and exact solutions are made. They show that the proposed model and the methods are accurate, robust and generate oscillation-freesolutions near material interfaces. Finally, the proposed model and method are extended to 2-D multifluid flow problems and compared to experimental Richtmyer-Meshkov instability growth measurements conducted in the University of Wisconsin shock tube. 
Abstract No. C42

\title{
ALE Simulations of Turbulent Rayleigh-Taylor Instability in 2-D and 3-D
}

\author{
S. V. Weber, G. Dimonte, and M. M. Marinak \\ Lawrence Livermore National Laboratory, Livermore, CA
}

We have performed simulations of the evolution of the turbulent Rayleigh-Taylor instability with the ALE code HYDRA3, including interface reconstruction. The test problem is that of the "alpha group", discussed in the presentation of Dimonte et al. at this conference. Perfect $\gamma=5 / 3$ gases of densities 1 and 3 are accelerated by constant gravity. The initial interface perturbation is a random spectrum of modes in the range $32 \leq \mathrm{n} \leq 64$. We employed meshs of $256 \times 512,512 \times 1028$, and 1028 x 2048 in two dimensions (2-D) and $128^{2}$ x 512 and $256^{2}$ x 512 in 3-D. The shortest seed modes have only 4 zones/wavelength at the nominal (coarsest) resolution. Consequently, linear growth is suppressed by under-resolution, and is not fully converged even at the highest resolution. However, as the growth transitions toward turbulence, turn-over of the growth rate in the 2-D simulations occurs earlier and at smaller amplitude with higher resolution. Results for mixing layer growth in the self-similar $\sim \mathrm{gt}^{2}$ regime and sub-structure of the mixing layer will be discussed.

1 M. Marinak et al., Phys. Plasmas 3, 2070 (1996).

This work was performed under the auspices of the U.S. Department of Energy by the University of California Lawrence Livermore National Laboratory under contract No. W-7405-Eng-48. 
$8^{\text {th }}$ International Workshop on the Physics of Compressible Turbulent Mixing,

Abstract No. C44

\title{
Study of Turbulent Gravitational Mixing at Large Density Differences Using Direct 3D Numerical Simulation
}

\author{
Yu. V. Yanilkin, V. P. Statsenko, S. V. Rebrov, N. I. Selchenkova, O. G. Sin'kova, \\ A. L. Stadnik and A. Ya. Uchayev \\ Russian Federal Nuclear Center - VNIIEF, Sarov, Russia
}

3D hydrocode TREK is used for numerical study of turbulence evolution in the field of gravity at a plane interface between two incompressible fluids (gases) with a large density difference, $3 \leq \rho_{2} / \rho_{1}$ $\leq 40$.

The computations were conducted on a fine computational grid with parallelization on several tens of processors.

The computed data was processed (averaged) in order to obtain moments of hydrodynamic values: diagonal components of Reynolds tensor (turbulent energy), turbulent flows, density profiles and mean-square pulsation. The resultant values are compared to predictions with phenomenological turbulence models and known experimental data.

The dissipation problems in these computations are disscussed.

A one-point function of concentration probability density is constructed using processed results of the direct numerical simulation. The results are compared to computed data obtained elsewhere. A fractal analysis of turbulent vortex scales is also conducted, which demonstrates that in the turbulent mixing zone the fractal size does not essentially change and is close to the measured value and the value from 3D computations by other investigators. 


\title{
Abstract No. C46 \\ Development and Validation of a 2D Turbulent Mix Model
}

\author{
D. L. Youngs \\ Atomic Weapons Establishment, Aldermaston, United Kingdom
}

A 2D turbulence model based on the equations of multiphase flow with turbulent diffusion effects added, is used to model mixing by Rayleigh-Taylor and Richtmyer-Meshkov instabilities in situations where the mean flow is two dimensional. For simple 1D flows it is relativelyeasy to check that the turbulence model gives satisfactory mix distributions. However, this is much more difficult to do for the case when the mean flow is two dimensional. In order to validate the $2 \mathrm{D}$ turbulence model, results are compared to the tilted-interface Rayleigh-Taylormixing experiments presented by J.M.Holford at this meeting and the 'chevron' shock tube experiments presented by A.V.Smith at this meeting. Experimental measurements of mix distributions are difficult to make in 2D. Hence 3D Large Eddy Simulation is able to make a very useful contribution. The TURMOIL3D code is used to perform 3D simulations which give a satisfactory match to the experimental results. The mix distributions obtained by averaging the calculational results in the third dimension may then be compared directly with the 2D turbulence model results.

At present 3D LES is often not practical for complex real applications. However, 3D LES for simplified problems does have a very useful role in helping to validate the turbulence models which can be applied to complex problems. 
$8^{\text {th }}$ International Workshop on the Physics of Compressible Turbulent Mixing,

\title{
Abstract No. C47 \\ Preliminary Results of DNS and LES Simulations of Self-Similar Variable Acceleration RT-Mixing Flows
}

\author{
D. L. Youngs ${ }^{1}$, X. Silvani ${ }^{2}$, J. Magnaudet ${ }^{2}$, and A. Llor $^{3}$ \\ ${ }^{1}$ Atomic Weapons Establishment, Aldermaston, United Kingdom \\ ${ }^{2}$ Institut de Mécanique des Fluides de Toulouse, Toulouse, France \\ ${ }^{3}$ Commissariat à l'Energie Atomique, Bruyères-le-Châtel, France
}

The importance of self-similar variable acceleration RT flows (SSVARTs) for the design an calibration of turbulent mixing models has been shown in an other presentation to the present workshop.

Because experimental results on SSVARTs are not, and will probably not be available in any close future, we are currently investigating such flows by means of DNS and LES.

This first presentation of our preliminary results will be devoted to discussing the technical issues (compressibility effects, subgrid models, initial conditions, mesh size, Atwood number...) whose influence must be carefully controled due to the lack of experimental data. The behaviour of the observed growth rates and large scale turbulent structures will also be analysed. 
Abstract No. C48

\title{
Shock-Planar Curtain Interactions: Strong Secondary Baroclinic Deposition and the Emergence of Coherent and Random Vortex Projectiles (VPs) and Decaying Stratified Turbulence
}

\author{
S. Zhang and N. J. Zabusky \\ Rutgers, State University of New Jersey, Piscataway, NJ
}

We continue our previous investigation [1] of the interaction of a shock with a planar, inclined curtain ( $\mathrm{s} / \mathrm{f} / \mathrm{s})$ to higher Mach numbers, $(\mathrm{M}=1.5,2.5$ \& 5.0), longer times (epochs) and alternate configurations in $2 \mathrm{D}$ and $3 \mathrm{D}$ (e.g. a fast/slow/fast or (f/s/f)). In all cases, the qualitative features may be explained in terms of opposite-signed vortex layers (deposited by shock waves in epoch 1) that move in opposite directions and collide at one boundary to form a complex vortex double layer $(\boldsymbol{V D L})$ that traverses the shock tube. (This causes early-time "breakthrough" [1]). We focus on longer evolution times where, eventually, the transversely moving VDLs collide with the opposite horizontal boundary and evolve into upstream \& downstream moving stratified vortex projectiles (VPs) [2]. In 2D, we compare these near-stationary, inhomogeneous coherent structures to the Lamb-Chaplygin vortex of 2D homogeneous flow. We also display and quantify: (1) strong nonacoustic circulationgenerationvia baroclinic processes during traversal of the VDL across the shock tube (epoch 2); and (2) evolution and decay ( epochs $>2$ ). of a stratified turbulent domain that arises between the two dominant VPs. We compare with images from Sturtevant's 1985 experiments and comment on the unusual advantages of this configuration as well as the convergence of results under mesh refinement.

[1] Yang, X., N.J. Zabusky, and I-L. Chern. Phys. Fluids A 2(6),892-895, 1990. "Breakthrough" via Dipolar-Vortex/Jet Formation in Shock-Accelerated Stratified Layers. [2] N.J. Zabusky and S-M. Zeng, J. Fluid Mechanics 362, pp. 327-346, 1998. Shock cavity implosion morphologies and vortical projectile generation in axisymmetric shockspherical F/S bubble interactions.

*This work was supported mainly by DOE (Grant No. DE-FG0293ER25179.A000) and monitored by Dr. Daniel Hitchcock. Additional support was provided by SROA program and the Jacobs Chair of Applied Physics at Rutgers University 
$8^{\text {th }}$ International Workshop on the Physics of Compressible Turbulent Mixing,

Abstract No. C49

\title{
Rapid Turbulization Arising from Vortex Double Layers in Interactions of "Complex" Blast Waves and Cylindrical and Spherical Bubbles
}

\author{
S. Zhang ${ }^{1}$, Y.-G. Kang ${ }^{2}$, K. Nishihara, N. J. Zabusky ${ }^{1}$, and H. Kim² \\ ${ }^{1}$ Institute of Laser Engineering, Osaka, Japan \\ ${ }^{2}$ Rutgers, State University of New Jersey, Piscataway, $N J$
}

\begin{abstract}
${ }^{1}$ We examinethe interaction of both cylindrical and spherical bubbles and a complex blast wave which consists of an approaching shock/contact discontinuity/shock. Such configurations arise following a supernova explosion, e.g. SN 1987A where a complex blast wave is presently approaching a high density ring ("inner circumstellar"), and may lead to rapid onset of turbulence on the upstream part of the bubble ${ }^{2}$, not an occurrence at low Mach numbers and density ratios ${ }^{3}$. The mixing in this turbulent domain will affect the electromagnetic radiation processes. Using $\mathrm{PPM}^{4}$, we examine a parameter domain containing SN 1987A parameters to validate the occurrence of this process which is related to shock reverberations and vortex double layers and their rapid instabilities.
\end{abstract}

*At Rutgers, this work was supported mainly by DOE (Grant No. DE-FG0293ER25179.A000) and monitored by Dr. Daniel Hitchcock. Additional support was provided by the SROA program and the Jacobs Chair of Applied Physics at Rutgers University. ${ }^{2}$ Laser Plasma Laboratory, Dept. of Materials Sci. \& Eng., Kwangju Institute of Science and Technology, 1 Oryong-dong, Puk-gu, Kwangju, Korea

${ }^{\mathrm{T}} \mathrm{Y}-\mathrm{G}$ Kang, et al, "A novel experiment on the blast wave-sphere interaction using a laser produced plasma” Phys. Rev E, submitted May 2001.

${ }^{2}$ K.J.Borkowski, J.M. Blondin and R. McCray. Astrophys J. 477, 281-293, 1997

${ }^{3}$ N.J. Zabusky and S-M. Zeng, J. Fluid Mechanics 362, 327-346, 1998.

${ }^{4} \mathrm{M}$. Blondin et al, Code VH-1, NCSU. A lagrangian remap code based on PPM.

$8^{\text {th }}$ International Workshop on the Physics of Compressible Turbulent Mixing, 
Abstract No. C50

\title{
Simulations of a Shock-Accelerated Gas Cylinder and Comparison with Experimental Images and Velocity Fields
}

\author{
C. A. Zoldi ${ }^{1,2}$, K. Prestridge ${ }^{1}$, P. M. Rightley ${ }^{1}$, and R. F. Benjamin ${ }^{1}$ \\ ${ }^{1}$ Los Alamos National Laboratory, Los Alamos, NM \\ ${ }^{2}$ State University New York at Stony Brook, Stony Brook, NY
}

The evolution of a cylinder of $\mathrm{SF}_{6}$ gas accelerated by a Mach 1.2 shock wave is studied both experimentally and computationally. Images of the initial conditions and the time evolution of the cylinder are obtained from the experiment. Velocity measurements are determined at one time using Particle Image Velocimetry. Using an image of the experimental initial conditions, 2D simulations are performed with the adaptive mesh Eulerian code, RAGE. Although qualitative agreement is achieved, significant differences exist in quantitative measurements. The linear dimensions of the cylinder measured over time are approximately $15 \%$ smaller in the simulation than in the experiment. In addition, although the directions of the velocity vectors are similar, the peak magnitude of the velocity is a factor of three larger in the simulation. The effect of turbulent mixing, which has not been considered in previous analyses, is examined using the BHR K-S-a-b mix model recently added to the RAGE code.

PACS No.: 47.20.Ma 
$8^{\text {th }}$ International Workshop on the Physics of Compressible Turbulent Mixing,

\title{
Abstract No. C51 \\ Turbulent Flow Simulations of Two Fluids Moving with Different Laws of Acceleration
}

\author{
V. I. Kozlov, A. N. Razin, and I. V. Sapozhnikov \\ Russian Federal Nuclear Center - VNIIEF, Sarov, Russia
}

With Dimonte tests as an example, turbulent mixing (TM) evolution is discussed that results from Rayleigh-Taylor instability at the interface between two fluids. In these tests, an ampoule with two molecularly immiscible fluids (freon and water) was accelerated while the acceleration law being varied in these tests.

The VIKHR code was used for the numerical simulations of the Dimonte's tests. This VIKHR technique includes V.V. Nikiforov's semiempirical model of turbulent mixing. This model treats various characteristics of a turbulent field, like the kinetic energy of turbulence, the turbulence energy dissipation rate, the average square density fluctuations and the turbulent mass transfer velocity.

The calculations were performed with a sequence of refined grids with different initial TM zone widths for increasing, decreasing, pulse and constant accelerations of the ampoule (the acceleration laws were the same as those given in the paper by Dimonte). TM zone growth laws versus the ampoule's path and the initial TM zone width have been obtained. The numerical results have been shown to be in good agreement with the measured data provided additional constrains have been included in the V.V. Nikiforov's model. These constraints can be interpreted as effective treatment of turbulent motion energy being transferred to the interface energy of the boundary between the fluids that are molecularly immiscible.

References

Dimonte Guy, Schneider Marilyn. Turbulent Rayleigh-Taylor instability experiments with variable acceleration // Physical Review E, 1996, v. 54, p.3740-3743. 
Abstract No. C52

\title{
The Behaviour of Velocity Variance Resulting from Turbulent Mixing Zone-Shock Interaction
}

\author{
V. I. Kozlov and A. N. Razin \\ Russian Federal Nuclear Center - VNIIEF, Sarov, Russia
}

Turbulent mixing (TM) caused by Richtmyer-Meshkovinstability at an interface between two gases with differentdensities is discussed. VIKHR code simulations of shock tube tests by Poggi et al have been performed. The feature of these tests is that the fluid's instantaneous mass velocities were measured by Doppler's laser anemometer.

The VIKHR code includes the 1D version of the semiempirical TM model suggested by V.V. Nikiforov that treats eddies' anisotropy. The VIKHR technique permits to precisely calculate various quantities of a turbulent field, like for instance, the average square variance of different velocity components and the average square density fluctuations, along with the flow's gasdynamical parameter distributions.

The calculations gave TM zone width and location versus time. The temporal evolution of the average square axial velocity variance was also obtained for several Eulerian points. No special calculation algorithm for turbulence quantities was used at the shock front in these calculations.

On the whole, there is satisfactory agreement with the test data by Poggi et al. Meanwhile, behind the front of the first wave (whose intensity is the highest) reflected from the tube's dead end is approximately two time higher than that measured in the tests. (Note that the same quantity calculated by Souffland et. al. exceeds the observed value by an order of magnitude). These results have shown that the correlations used in the Nikiforov's model to treat shock wave-turbulent field interactions, need to be improved.

\section{References}

1. F. Poggi, M.-H. Thorembey, G. Rodriguez. Physics of Fluids, v. 10, _ 11, 1998, pp. 2698-2700.

2. D Souffland, O. Gregoire, S. Gauthier, F. Poggi, J.M. Koenig. $6^{\text {th }}$ International Workshop on the physics of compressible turbulent mixing (Marseille, France), 1997, pp. 486-491. 
$8^{\text {th }}$ International Workshop on the Physics of Compressible Turbulent Mixing,

Pasadena, CA (2001)

Abstract No. C53

\title{
An Assessment of Multi-Velocity Versus Single Velocity in a Multi- Component Model of Turbulent Mixing
}

\author{
D. E. Eliason, W. H. Cabot, and Y. Zhou \\ Lawrence Livermore National Laboratory, Livermore, CA
}

Turbulent mixing of the fluids in a multi-componentsystem is of interest in situations such as inertial confinement fusion (ICF) and core-collapse supernovae[1]. We report results of a project to include a model of turbulent mixing in a multi-component hydrodynamics and physics model called KULL, which is used for ICF. Because KULL is a complex, multi-dimensional model, we have developed a simplified, one-dimensional version called sKULL to speed-up the development of the turbulent mixing model.

Of primary interest in the development of a turbulent mixing model for a multi-component fluid is the question of whether it is necessary to allow each component of the fluid to retain its own velocity. A recently developed model of turbulent mixing, consisting of an extended buoyancy-drag model and two-equation turbulent transport model [2], treats all components of the fluid as if they had the same velocity. In contrast, multi-velocity turbulent mixing models allow separate velocities for each component of the fluid [3]. However, the necessity to carry separate velocities for each component of the fluid greatly increases the memory requirements and complexity of the computer implementation. We will report results of a comparison between single velocity and multi-velocity turbulent mixing models in sKULL with the intention of answering the question of whether the full multi-velocity treatment is really necessary.

This work was performed under the auspices of the U.S. Department of Energy by the University of California Lawrence Livermore National Laboratory under contract No. W-7405-Eng-48.

[1] Remington, B.A., D. Arnett, R.P. Drake, and H. Takabe, Modeling Astrophysical Phenomena in the Laboratory with Intense Lasers, Science, 284, 1488 (1999).

[2] Zhou, Y., G. Zimmerman, and E.B. Burke, submitted to Phys. Rev. E., (2001).

[3] Youngs, D.L., Laser \& Particle Beams 12, 725 (1994). 
Abstract No. C54

\title{
High Order Numerical Methods for the 2D Richtmyer-Meshkov Instability
}

\author{
W.-S. Don ${ }^{1}$, D. Gottlieb ${ }^{1}$, L. Jameson ${ }^{2}$, and C.-W. Shu ${ }^{1}$ \\ ${ }^{1}$ Brown University, Providence, $R I$ \\ ${ }^{2}$ Lawrence Livermore National Laboratory, Livermore, $C A$
}

The primary goal of this presentation is to examine several numerical methodologies with high order accuracy for the investigation of the two dimensional (and eventually three dimensional) RichtmyerMeshkov instability. The high order schemes employed are the Spectral methods and the high order Weighted Essentially Non-Oscillatory (WENO) finite difference scheme. We will briefly discuss several important aspects of the numerical schemes when applied to the Euler equations. Multispecies full Navier-Stokes equations will be implemented in the near future.

A series of numerical simulations are carried out to investigate the convergence properties of the schemes and long time behavior of the interface evolution. Numerical results from the simulation of shock interaction with a single mode perturbation of interface separating the heavy (Xenon) to light (Argon) gases will be presented with various interface thickness and different Mach numbers. It can be observed that the large and median scale structures such as the spike and bubble, transmitted shock, shocked-interface velocity and shock triple point obtained by the different schemes are basically in excellent agreement with each other and with available experimental data. Also convergence studies had been made. Some minor discrepancies of the finest scale structures along the gaseous interface, as can be expected for numerical simulations of the Euler equations with this sensitive nature, are observed.

This work was performed under the auspices of the U.S. Department of Energy by the University of California Lawrence Livermore National Laboratory under contract No. W-7405-Eng-48. 
$8^{\text {th }}$ International Workshop on the Physics of Compressible Turbulent Mixing,

Abstract No. C55

\title{
Mixing Due to the Rayleigh-Taylor Instability
}

\author{
A. M. Dimits \\ Lawrence Livermore National Laboratory, Livermore, $C A$
}

Several aspects of mixing due to the Rayleigh-Taylor (RT) instability are investigated.

Analysis of 3D multimode simulations using the PPM code [D.H. Porter and P.R. Woodward, Astrophys. J. Suppl. 93, 309 (1994), and references therein.] show that there are regions of the parameter space of the initial conditions in which the growth rate is independent of variations in the initial conditions. The simulated growth rates are found to increase as the Navier-Stokes viscosity is increased. It is investigated whether this couterintuitiveresult is due to the suppression of material mixing at the molecular level for larger viscosities.

Analyses of two RT experiments, one in which water is accelerated by a compressed gas (E.E. Meshkov and N.V. Nevmerzhitsky, Proc. ${ }^{\text {rd }}$ Int. Workshop. on the Physics of Compressible Turbulent Mixing, 1991) and one in which an interface between gases of different density is decellerated in the post-shock region of a shock in an electromagnetic shock tube (A.M. Vasilenko et al., ibid.), are presented. Direct compressibility effects on the RT growth are shown to be negligible in the former. Various effects of the expansion of the gases in the region of the interface on the RT growth rates are investigated for the latter experiment, both analytically and with 1D simulations. These effects are found to be insufficient to reconcile the growth rates observed in the Vasilenko et al. experiments with some other experimental and simulation results.

This work was performed under the auspices of the U.S. Department of Energy by the University of California Lawrence Livermore National Laboratory under contract No. W-7405-Eng-48. 
Abstract No. C56

\title{
Transition Stages of Rayleigh-Taylor Instability Between Miscible Fluids
}

\author{
A. W. Cook and P. E. Dimotakis \\ Lawrence Livermore National Laboratory, Livermore, $C A$ \\ California Institute of Technology, Pasadena, CA
}

\begin{abstract}
Direct Numerical Simulations are presented of three-dimensional, Rayleigh-Taylor instability between two incompressible, miscible fluids, with a 3:1 density ratio. Periodic boundary conditions are imposed in the horizontal directions of a rectangular domain, with no-slip top and bottom walls. Solutions are obtained for the Navier-Stokes equations, augmented by a species transport-diffusion equation, with various initial perturbations. Three of the simulations (Cases A, B and C) were performed at a resolution of $256 \times 256 \times 1024$ grid points, and the fourth simulation (Case D) was performed at a resolution of $512 \times 512 \times 2040$ grid points. The A, B and C cases achieved outer-scale Reynolds numbers, based on height and rate of growth of mixing-zone, in excess of 3000; Case D achieved an outer-scale Reynolds number of 5500. Initial diffusive growth is captured in the simulations. The onset of nonlinear growth is as predicted by linear stability theory. Following the diffusive stage, growth rates are found to depend on the initial perturbations through the end of the simulations. Mixing is found to be even more sensitive to initial conditions than growth rates. Taylor microscales and Reynolds numbers are anisotropic throughout the simulations. Improved collapse of many statistics is achieved if the height of the mixing zone, rather than time, is used as the progress variable. Mixing has dynamical consequences for this flow, since it is driven by the action of the imposed acceleration field on local density differences.
\end{abstract}

This work was performed under the auspices of the U.S. Department of Energy by the University of California Lawrence Livermore National Laboratory under contract No. W-7405-Eng-48. 
$8^{\text {th }}$ International Workshop on the Physics of Compressible Turbulent Mixing,

Abstract No. C57

\title{
CALE Simulation of Richtmyer-Meshkov Experiments at High Mach Number
}

\author{
A. Miles ${ }^{1,2}$, J. Edwards $^{2}$, G. Glendinning ${ }^{2}$ \\ ${ }^{1}$ Lawrence Livermore National Laboratory, Livermore, $C A$ \\ ${ }^{2}$ University of Maryland, College Park, MD
}

Richtmyer-Meshkov instability experiments, recently conducted on the Omega laser, are simulated via the C-based Arbitrary Lagrangian-Eulerian (CALE) hydrodynamics code in $2 \mathrm{D}$. In the experiments, a high Mach number shock $(M \approx 10)$ is incident on a corrugated plastic-foam interface $(\mathrm{ka}=0.92)$. The ratio of plastic to foam density is $12: 1$. After passage of the incident shock, the perturbation amplitude grows in time. Computational grids initially rectangular and conforming to the initial amplitude perturbation are both considered, as are zoning effects. Discrepancies between the experimentand simulation are considered, including the growth rate at early times, the post-shock amplitude, and the shock-interface proximity as the transmitted shock propagates through the foam. A modified input velocity source is presented which results in a time-dependent growth rate that agrees with the experimental observations much better than does the original source, which is produced by a 1D Lasnex simulation. Various EOS models are used, and their predictions compared. 


\section{THEORETICAL ABSTRACTS}


$8^{\text {th }}$ International Workshop on the Physics of Compressible Turbulent Mixing,

\author{
Abstract No. T1
}

\title{
Nonlinear Evolution of Unstable Fluid Interface
}

\author{
S. I. Abarzhi \\ State University of New York at Stony Brook, Stony Brook, NY
}

We study the nonlinear evolution of the fluid interface generated by the Richtmyer-Meshkov instability. For the first time we find the theoretical solutions, which capture the interplay of harmonics in the nonlinear dynamics of 3D and 2D flows. A new type of the evolution of the bubble front in RMI is discovered [1]. It is shown that the nonlinear RM bubbles flatten in time and the shapes of Rayleigh-Taylor and Richtmyer-Meshkov bubbles differ significantly.

To perform the multi-mode analysis for the RM flow, we generalized the method developed in [2] for RTI, and based our approach on symmetry theory. From the conservation aws we derived a dynamical system governing the local dynamics of the nonlinear bubble. To capture the interplay of harmonics in the local dynamics, we extended the functional space, involved all bubbles allowed by symmetry of the flow, and found a family of regular asymptotic solutions. The physically dominant solution in this family, i.e. the fastest stable one, corresponds to a flattened bubble, not to a bubble with finite curvature as in [3].

The theory reveals deficiency of previous theoretical approaches in [3], explains existing experiments, and establishes control parameters to be monitored in experiments.

1.S.I.Abarzhi, Nonlinear evolution of unstable fluid interface, Phys.Rev.Lett. submitted 2.S.I.Abarzhi, PRL89, 1332 (1998)

3.J.Hecht, U.Alon, D.Shvarts, Phys.Fluids 6, 4019 (1994); N.A.Inogamov, Sov.Phys.JETP 80, 890 (1995); K.O.Mikaelian, Phys.Rev.Lett.80, 508 (1998); Q.Zhang, Phys.Rev.Lett. 81,3391 (1998); S.Abarzhi, Phys.Fluids 12, N12 (2000). 
Abstract No. T2

\title{
Nonlinear Asymptotic Solutions to RT and RM Problems for Fluids With Close Densities
}

\author{
S. I. Abarzhi \\ State University of New York at Stony Brook, Stony Brook, NY
}

We consider the interface dynamics in the Rayleigh-Taylorand Richtmyer-Meshkov instabilities for fluids with close densities, the Atwood number $\mathrm{A}<1$. We find the analytical solutions to the equations governing the interface dynamics in $3 \mathrm{D}$ and $2 \mathrm{D}$ (conservation laws, potential approximation), and analyze their regular and singular asymptotic behavior. First we derive a nonlinear solution of the Layzer-type, i.e. single-mode solution [1]. For this solution the normal component of velocity is discontinuous, and the flux of mass through the interface is significant. We resolve this paradox and find a multi-mode nonlinear solution with NO FLUX of MASS through the interface. This solution is the fastest one in the family of asymptotic solutions to the conservation laws [1].

The theory [1] determines parameters to distinguish between the Layzer-type solution and the nonlinear solution with NO FLUX. In RTI the bubble with NO FLUX is in few times narrower than the Layzer-type bubble, while in RMI the bubble with NO FLUX is flattened. The singular asymptotes (spikes) are also analyzed, and the influence of vorticity on the spike motion is evaluated. We conclude that there is a non-trivial dependence on the Atwood number for the parameters of the nonlinear motion (such as velocity of the bubble or spike) in either RTI or RMI cases. The RT/RM mixing process is discussed.

1. S.I.Abarzhi, The dependence of the nonlinear RT/RM motion on the Atwood number, in preparation, 2001. 
$8^{\text {th }}$ International Workshop on the Physics of Compressible Turbulent Mixing,

Abstract No. T3

\title{
Turbulent Mixing in RTI as Order-Disorder Process
}

\author{
S. I. Abarzhi \\ State University of New York at Stony Brook, Stony Brook, NY
}

The cascades of energy and the dynamics of large-scale coherent structure are fundamental issues in the problem of Rayleigh-Taylor turbulent mixing. The large-scale structure is a periodic array of bubbles and spikes in the plane normal to the direction of gravity. We study dynamics of this structure based on group theory, and analyze transitions associated with the growth of length scale of the flow. In the limiting case of 2D flow, the scale growth occurs as a doubling of the spatial period, in agreement with Sharp and Wheeler model, and a stable observable coherent structure appears under this transition. In contrast, for a 3D flow the growth of length scale leads eventually to anisotropy of the flow in the plane normal to the direction of gravity and no isotropic structure occurs. We see that in RT turbulent mixing a balance between the inverse and direct cascades is required to keep isotropy of the flow. These two processes may lead in a generation of an internal structure with hexagonal symmetry and with close packing in the plane normal to the direction of gravity. The concept of self-similarity in the RT mixing is discussed. 
Abstract No. T4

\title{
A New Turbulent Two-Fluid RANS Model for KH, RT and RM Mixing Layers
}

\author{
P. Bailly and A. Llor \\ Commissariat à l'Energie Atomique, Bruyères-le-Châtel, France
}

Our aim is to develop an accurate turbulent mixing model for combined RT, RM and KH type of instabilities, with arbitrarily variable accelerations.

Following the recent analysis of the RT and RM cases by G. Dimonte, and of the self-similar variable acceleration RT flows (SSVARTs) in an other presentation to the present workshop, we have considered as crucial to capture the following physical aspects by the corresponding model features:

- the directed transport by a two-fluid approach,

- the correct buoyancy force by including mass transfer between the fluids,

- the turbulence diffusion by including most of the standard $k-\varepsilon$ features,

- the geometrical aspects by consistent closures of the length scales.

This yielded a two-fluid two-turbulence model whose specific and original features will be discussed. 1D numerical results of this model applied to self-similar flows will be presented. 
$8^{\text {th }}$ International Workshop on the Physics of Compressible Turbulent Mixing,

Pasadena, CA (2001)

\title{
Abstract No. T5 \\ Super-Exponential Rayleigh-Taylor Flow
}

\author{
R. E. Breidenthal \\ University of Washington, Seattle, WA
}

A new class of forced, self-similarturbulence is proposed. In it, the rotation period of the large-scale vortices is forced to decrease by a constant factor at each rotation. This is achieved by imposing an e-folding time scale on the flow that decreases linearly with time. Based on experimental results in analogous flows, super-exponential turbulence may exhibit extraordinarily low entrainment and mixing rates. One application is in inertial-confinementfusion, where super-exponentialacceleration may play a useful role in achieving ignition. It is shown that super-exponential flows are the mirror image of unforced turbulence, and both are members of a closely related family of self-similar turbulence. 
Abstract No. T7

\title{
Theoretical Methods for Determination of Mix
}

\author{
B. Cheng ${ }^{1}$, J. Glimm ${ }^{2,3}$, and D. H. Sharp ${ }^{1}$ \\ ${ }^{1}$ Los Alamos National Laboratory, Los Alamos, NM \\ ${ }^{2}$ State University of New York at Stony Brook, Stony Brook, NY \\ ${ }^{3}$ Brookhaven National Laboratory, Brookhaven, $N Y$
}

We present a theoretical description of the growth of a planar 3D mixing layer due to RayleighTaylor (RT) or Richtmyer-Meshkov (RM) instabilities. The methods yield agreement with all known experiments. They also possess advantageous theoretical features, such as real characteristic speeds and improved mixed cell EOS.

The first method is a bubble merger model, validated by comparison to experiments of Smeeton and Youngs and of Dimonte et al. The model is based on a renormalization group fixed point calculation, incorporating the self similar behavior of RT instability. The second method is based on the dynamic motion of the RT center of mass; it couples the bubble and spike mixing zone edges, and predicts the RT spike growth as a function of the RT bubble growth. The third method is a drag buoyancy model, with a phenomenological drag coefficient, set to agree with the RT edge motion models above.

This model predicts RM edge motion. The final method is a mix model, i.e. a set of averaged equations, with prediction of the behavior of mixed average flow quantities such as volume fraction, as a function of the mixing zone edges.

PACS Nos.: 47.20Bp, $47.52+\mathrm{j}$ 
$8^{\text {th }}$ International Workshop on the Physics of Compressible Turbulent Mixing,

Abstract No. T8

\title{
Modeling Radiation Effects in Mixing Layers
}

\author{
T. Clark and F. Harlow \\ Los Alamos National Laboratory, Los Alamos, NM
}

Radiative heat transport and resulting material phase changes can have a pronounced effect on the evolution of Rayleigh-Taylor and Richtmyer-Meshkov mixing layers. For sufficiently high temperature differences across the materials in these layers, the radiation effects may significantly alter the rate of growth of these mixing layers. Direct numerical simulation of these processes is generally not possible for practical circumstances due to the rapid growth of fine-scale structures as well as the inherentstochasticity of the mixingprocess. Consequently,we are developing a turbulent mix model that incorporates the effects of radiative heat transfer and ablation in a computationally tractable fashion. The model under development describes the ablation of the cold material as a surface phenomenon in which a thin skin of the cold material is ablated by the radiation through a thin skin of the hot material. As a first approximation we have assumed that only two materials are present. Thus after the cold material is ablated it is indistinguishable from the hot material. The goal of the model is to derive a model with sufficient predictive power to determine the mixing layer growth rates, and to distinguish the circumstances under which the ablation process (i.e., "firepolishing") will overwhelm the tendency towards hydrodynamic mixing intrinsic to these mixing layers. We will discuss both model development and computed results.

PACS Nos.: 47.27.Eq, 47.27.Te, 47.55.Kf, 47.70.Mc 
Abstract No. T9

\title{
A Model for Instability Growth in Accelerated Solid Metals
}

\author{
J. D. Colvin ${ }^{1}$, M. Legrand ${ }^{2}$, B. A. Remington ${ }^{1}$, G. Schurtz ${ }^{2}$, and S. V. Weber ${ }^{1}$ \\ ${ }^{1}$ Lawrence Livermore National Laboratory, Livermore, $C A$ \\ ${ }^{2}$ Commissariat à l'Energie Atomique, Bruyères-le-Châtel, France
}

We present the derivation of an approximate analytical dispersion relationship for elastic-plastic acceleration-driveninstability growth. We have applied this model, where applicable, to perturbation growth measurements made in three separate types of experiments: HE-drivenplanar Al plates, HEdriven implosions of steel cylinders, and planar Al foils driven indirectly by LLNL's Nova laser. We have also compared the analytical modeling with 2-D simulations. We find that for the moderate strain rates of the HE experiments the simulations and analytical modeling agree with each other and with the data, with an equivalent plastic viscosity consistent with the von Mises criterion. For the high strain rates of the Nova experiments, on the other hand, the viscosity needed in the analytical model to match the data is about one-tenth of what the simulations predict. This initial material weakening, followed by a relaxation to a strengthened state, is consistent with a "relaxation model" in which plastic flow at high strain rate is confined to discrete shear bands. We also derive a characteristic scale for the plastic viscosity and find under what conditions the growth is independent of initial amplitude.

This work was performed under the auspices of the U.S. Department of Energy by the University of California Lawrence Livermore National Laboratory under contract No. W-7405-Eng-48. 
$8^{\text {th }}$ International Workshop on the Physics of Compressible Turbulent Mixing,

\title{
Abstract No. T10 \\ Toy Models for the Growth Rate of Rayleigh-Taylor Instability
}

\author{
S. B. Dalziel \\ Cambridge University, Cambridge, United Kingdom
}

There remains an on-going debate about one of the most basic characteristics of the instability: the growth-rate coefficient $\alpha$. In many respects this is surprising, but at the same time it is perhaps inevitable. After undergoing a period of convergence between experiments and numerics for values of this important quantity, some recent studies show a continued decline in the growth rate for some numerical models, at the same time as the models offer overall improvement in the resolution and quality of results. This paper makes use of a range of toy models for Boussinesq Rayleigh-Taylor instability in an effort to understand and reconcile the issues.

The paper begins by returning to the classical Layzer model and reconciles it with the behaviour of other buoyancy-driven flows, before exploring the possible growth rates it predicts for the developing instability. Attention is then turned to a shell model for the instability. Shell models are normally used to help explain the behaviour of turbulence in homogeneous fluids subject to forcing at low wavenumbers. In this study, a very simple model is adapted to take account of buoyancydriven forcing over the entire range of available scales. The results offer an interesting comparison with Rayleigh-Taylorinstability, and offer insight into the behaviour that determines the growth rate $\alpha$. 
Abstract No. T11

\title{
A General Buoyancy-Drag Model for the Evolution of the Rayleigh-Taylor and Richtmyer-Meshkov Instabilities
}

\author{
Y. Elbaz ${ }^{1,2}$, Y. Srebro ${ }^{1,2}$, O. Sadot ${ }^{2}$, and D. Shvarts ${ }^{1,2}$ \\ ${ }^{1}$ Ben-Gurion University, Beer-Sheva, Israel \\ ${ }^{2}$ Nuclear Research Center - Negev, Israel
}

The growth of a single-modeperturbation is described by a buoyancy-drag equation, which describes all instability stages (linear, non-linear and asymptotic) at time-dependant Atwood number and acceleration profile. The evolution of a multi-mode spectrum of perturbations from a short wavelength random noise is described using a single characteristic wavelength. The temporal evolution of this wavelength allows the description of both the linear stage and the late time selfsimilar behavior.

The model includes additional effects, such as shock compression and spherical convergence. In addition to the mixing zone fronts, the internal density profile of the mixing region has been investigated using a simple diffusion-like model.

Model results are compared to full 2D and 3D numerical simulations and shock-tube experiments of random perturbations, studying the various stages of the evolution. 
$8^{\text {th }}$ International Workshop on the Physics of Compressible Turbulent Mixing,

Abstract No. T12

\title{
3D Rayleigh-Taylor and Richtmyer-Meshkov Single-Modes
}

\author{
N. A. Inogamov ${ }^{1}$, A. M. Oparin ${ }^{2}$, M. Tricottet, and S. Bouquet ${ }^{3}$ \\ ${ }^{1}$ Landau Institute for Theoretical Physics, Moscow, Russia \\ ${ }^{2}$ Institute for Computer Aided Design, Moscow, Russia, \\ ${ }^{3}$ Commissariat à l'Energie Atomique, Bruyères-le-Châtel, France
}

We study 3D topology of Rayleigh-Taylor (RT) and Richtmyer-Meshkov (RM) single-modes. 3D case is much richer than 2D case. For example, in addition to the well-known bubbles and jets, 3D saddles appear. Saddles are points of stagnation - as are the tips of bubbles and jets - and, therefore, they play the same important role. We present a 3D analytic description of the interface as a whole,from bubbles to jets. Hexagonal, square and triangular lattices of bubbles are investigated both analytically and numerically. 
Abstract No. T13

\title{
Rayleigh-Taylor Instability for Compressible and Incompressible Media
}

\author{
N. A. Inogamov ${ }^{1,2}$, M. Tricottet ${ }^{2}$, A. M. Oparin ${ }^{3}$, and S. Bouquet ${ }^{2}$ \\ ${ }^{1}$ Landau Institute for Theoretical Physics, Moscow, Russia \\ ${ }^{2}$ Commissariat à l'Energie Atomique, Bruyères-le-Châtel, France \\ ${ }^{3}$ Institute of Computer-Aided Design, Moscow, Russia
}

After a brief Reminding of the role played by Rayleigh-Taylor Instabilities in astrophysics (Type II supernovae, supernovae remnants, insterstellar clouds driven by ablation), some developments concerning instability criteria, linear growth for compressible media will be presented.

Then, the 3D structure of non-linear single-mode instability interfaces is investigated, and proves to be much richer than in 2D. Accumulation points are analytically studied, up to high-order development, for arbitrary long times - including asymptotic behaviour.

Finally, some comparisons will be effected to numerical simulations with the predictions concerning this points and some shape factor.

PACS Nos.: 47.20.Ma, 47.40.Nm 
$8^{\text {th }}$ International Workshop on the Physics of Compressible Turbulent Mixing,

Abstract No. T14

\title{
Three Dimensional Multi-Mode Rayleigh-Taylor and Richtmyer-Meshkov Instabilities at All Density Ratios
}

\author{
D. Kartoon ${ }^{1,2}$, D. Oron ${ }^{1}$, L. Arazi ${ }^{3}$, A. Rikanati ${ }^{1,2}$, U. Alon ${ }^{4}$, and D. Shvarts ${ }^{1,2}$ \\ ${ }^{1}$ Nuclear Research Center Negev, Israel \\ ${ }^{2}$ Ben-Gurion University, Beer-Sheva, Israel \\ ${ }^{3}$ Tel-Aviv University, Tel-Aviv, Israel \\ ${ }^{4}$ Weizmann Institute, Rehovot, Israel
}

The three dimensional turbulent mixing zone (TMZ) evolution was studied using two approaches. First, an extensivenumerical study was made, investigating the growth of a random three dimensional (3D) perturbation under Rayleigh-Taylor and Richtmyer-Meshkov unstable conditions in a wide range of density ratios. Following that, a new 3D statistical model was developed based on the same logic as the $2 \mathrm{D}$ statistical model - binary interactions between bubbles growing at a $3 \mathrm{D}$ asymptotic rate.

The results for the growth rate of the 3D bubble front attained from the theoretical model show good agreement with both the experimental [1] and the 3D simulation results. The simulation results also agree well with the experimental spike front growth rate. Further approval for the theoretical model was gained by detailed comparison of the bubble size distribution to the numerical simulations, and by comparison to a 3D multi-mode drag-buoyancy model [2].

The good agreement between the theoretical models, the 3D numerical simulations and the experimental results, together with the clear differences between the $2 \mathrm{D}$ and the $3 \mathrm{D}$ results, suggest that the discrepancies between the experiments and the previously developed models are due to geometrical effects.

[1] G. Dimonte, Phys. Plasmas 6, 2009 (1999).

[2] D. Oron, L. Arazi, D. Kartoon, A. Rikanati, U. Alon, D. Shvarts, Phys. Plasmas 8 (June 2001) 
Abstract No. T15

\title{
Stability of Diverging Shock Waves
}

\author{
V. M. Ktitorov \\ Russian Federal Nuclear Center - VNIIEF, Sarov, Russia
}

For the first time the self-similar approach was successfully applied to the stability problem of diverging shock waves when calculating evolution of small perturbations of self-similar point blast wave. The new sort of Rayleigh-Taylorinstability was found in this case when $\gamma<1.2$ (See instability region on Fig.1 /1/). The results of calculations were later proved in laboratory experiment $/ 2 /$ as well as in computer simulation $/ 3 /$.

In this paper we summarize the solutions of stability problems of various types of diverging shock waves (both spherical and cylindrical) which were obtained with the help of the self-similar approach. The following cases are considered:

Point blast wave in non-ideal gas and in the gas which density depends on radius, Reflected from the center converging shock wave.

We calculate the values of complex exponents of power time dependence of front perturbations in a wide region of values of harmonic number and of gas adiabatic exponent. We also determine the region of instability.

V.M.Ktitorov, Khim.Fizika(Chem.Phys.Issues),V.14,No2-3,p.169,1995

J.Grun et al, Phys.Rev.Let.,V.66,No21,p.2738,1991

V.Ktitorov,V.Meltsas, Proceedings of the $6^{\text {th }}$ IWPCTM,Marseille, 1997

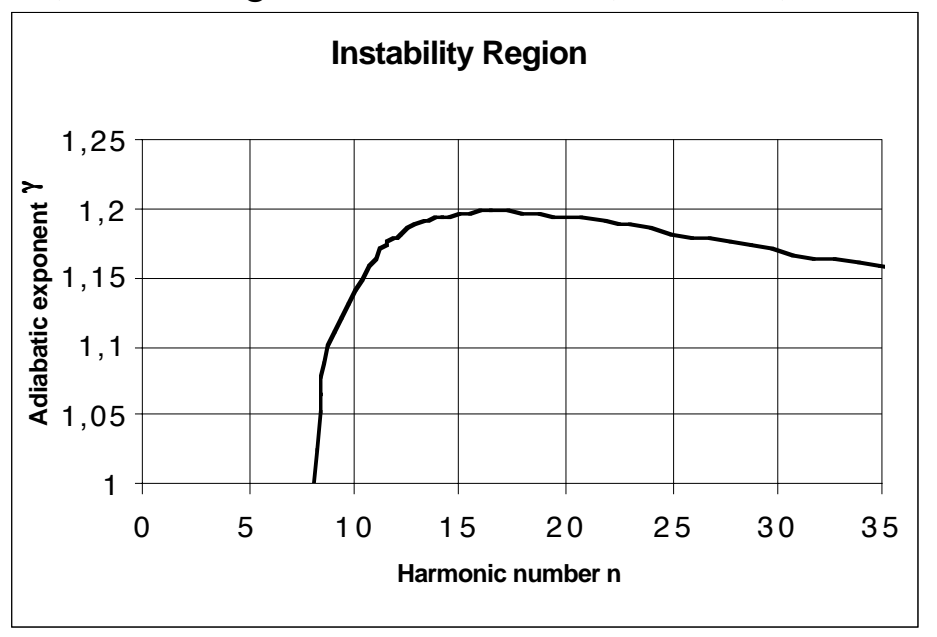


$8^{\text {th }}$ International Workshop on the Physics of Compressible Turbulent Mixing,

Abstract No. T16

\title{
Stability of Reflected from the Center Self-Similar Converging Shock Wave
}

\author{
V. M. Ktitorov \\ Russian Federal Nuclear Center-VNIIEF, Sarov, Russia
}

The technique first used in /1-3/ for solving the point blast wave stability problem is applied for the one of the self-similar converging shock wave after focusing.

Small (linear) perturbations of the shock wave are expanded into spherical harmonics the components of expansion being presented in the self-similar form. The stability problem is reduced to the solving of the system of the ordinary differential equations, which are to be solved simultaneously with the main spherically symmetric equations of the shock wave.

The eigenvalue problem is formulated. This problem is solved, complex values of power exponent (they describe a time dependence of front perturbations) are calculated as eigenvalues. The eigenvalues are calculated numerically in the general case of arbitrary values of harmonic number $n$ and gas adiabatic exponent $\gamma$. The region of instability is defined on the plane $n-\gamma$.

V.M.Ktitorov (Russian Atomic Science and Technique Issues, Ser. Theoretical and Applied Physics), No2, p.28, (1984);

D.Ryu and E.T.Vishniac, Astrophys.J. 313, 820 (1987);

V.M.Ktitorov, Khimicheskaya Fizika (Chemical Physics Issues) V.14, No 2-3, p.169, (1995); 
Abstract No. T17

\title{
Using a Turbulence Transport Approach to Study Shocks Through Polycrystalline Metal
}

\author{
R. R. Linn and F. H. Harlow \\ Los Alamos National Laboratory, Los Alamos, NM
}

\begin{abstract}
A polycrystalline metal is composed of close packed crystals in each of which the elastic modulus is non-isotropic to a degree that ranges from slight to extreme (as in delta-phase plutonium where there is a seven to one variation). We have made considerable progress in the development of a stochastic model by which to describe the collective behavior as a strong shock or rarefaction passes through the material. The basic idea is to start with the laws of mass, momentum, and energy conservation, decompose each variable into mean and fluctuating parts, ensemble average the equations, and then derive transport equations and closures for the higher-order moments. The first version has been obtained and tested numerically for a self-similar traveling wave, and results show deficiencies, together with strong clues for their remediation. We have made much progress in developing a second, improved version for which results will be presented during the talk.
\end{abstract}


$8^{\text {th }}$ International Workshop on the Physics of Compressible Turbulent Mixing,

Abstract No. T18

\title{
Response of Turbulent RANS Models to Self-Similar Variable Acceleration RT-Mixing: An Analytical 0D Analysis
}

\author{
A. Llor \\ Commissariat à l'Energie Atomique, Bruyères-le-Châtel, France
}

So far, the validation of RANS models applicable to variable acceleration RT mixing flows (as found in ICF) has mostly been carried out by fitting experimental or numerical data obtained for constant (RT) and impulsive (RM) accelerations. Further checks are also possible on the few available data for variable acceleration, such as in mixing-demixing flows induced by reverting the gravity field. Although this approach is widely applied and accepted, it is unsatisfactory because of the complex relationship between the model features and coefficients and the experimental measurements.

It is shown here that self-similar variable acceleration RT (SSVART) provides an appealing alternative since it extends the usual calibration techniques of turbulent RANS models based on simple self-similar flows. The general model equations in 1D (PDEs) are still too complex for full analytical calculations of SSVART flows, but using reasonable assumptions, simple OD (ODEs) approximations can be derived and solved analytically.

This approach is applied to an extended k- $\varepsilon$ model derived from Andronov's and to Young's twofluid model. The behaviour of the mixing layer growth rate and integral turbulent scales provides important informations on the accuracy of these models.

Finally, general qualitative arguments will be discussed showing the importance and the difficulty of capturing accurately a broad range of SSVARTs with a single simple model. 
Abstract No. T19

\title{
Nonlinear Evolution of an Interface in the Richtmyer-Meshkov Instability
}

\author{
C. Matsuoka ${ }^{1}$, K. Nishihara ${ }^{2}$, and Y. Fukuda ${ }^{2}$ \\ ${ }^{1}$ Ehime University, Ehime, Japan \\ ${ }^{2}$ Institute of Laser Engineering, Osaka University, Osaka, Japan
}

We have developed an analytical model that describes a fully nonlinear evolution of an interface in the Richtmyer-Meshkov instability. Proper boundary conditions at the interface are derived and the temporal evolution of the interface is investigated as a vortex sheet using them. It is shown that the created vorticity on the interface has a strong inhomogeneity, which causes the stretching and compression of the sheet. We discuss the inhomogeneity in detail, from which we show the interface has a double spiral structure. We also show a good agreement in the analytical solutions of the interface shape with two-dimensional hydrodynamic simulations.

We present the proper kinematic boundary condition, the modified Birkhoff-Rott equation, in order to describe the nonlinear evolution of the interface with the temporal evolution of the circulation, corresponding to the Bernoulli equation, on the interface for an arbitrary Atwood number. The analytical solutions show that the interface is stretched to the tangential direction proportional to time. In the nonlinear stage, the modes in the normal and tangential directions mutually interact to yield to the large deformation of the spike. By introducing the self-similar form of the velocity potential first found by Rott, we can construct a fully nonlinear evolution of the double spiral structure in the spike. 
$8^{\text {th }}$ International Workshop on the Physics of Compressible Turbulent Mixing,

Pasadena, CA (2001)

Abstract No. T20

\title{
Evolution of Arbitrary Perturbations in the Richtmyer-Meshkov Instability
}

\author{
K. O. Mikaelian \\ Lawrence Livermore National Laboratory, Livermore, $C A$
}

\begin{abstract}
We present analytical and numerical calculations on the evolution of arbitrarily shaped initial perturbations undergoing the Richtmyer-Meshkov instability. In many cases a simple, explicit, analytic formula can be written down for the linear regime. These formulas serve as nontrivial tests of hydrocodes, and we present simulations with the Arbitrary Lagrangian-EulerianhydrocodeCALE that cover the linear as well as the deeply nonlinear regime of the instability. A brief outline and code calculations for possible experiments will also be presented.
\end{abstract}

PACS No.: 52.35.Py

This work was performed under the auspices of the U.S. Department of Energy by the University of California Lawrence Livermore National Laboratory under contract No. W-7405-Eng-48. 
Abstract No. T21

\title{
RT Turbulence: Dramatic Dynamics of Interpenetration (Fast Jets, Sharp Decelerations and Accelerations)
}

\author{
A. M. Oparin ${ }^{1}$, N. A. Inogamov ${ }^{2}$, and A. Yu. Dem'yanov ${ }^{3}$ \\ ${ }^{1}$ Institute for Computer Aided Design, Moscow, Russia \\ ${ }^{2}$ Landau Institute for Theoretical Physics, Moscow, Russia \\ ${ }^{3}$ Moscow Institute for Physics and Technology, Moscow, Russia
}

Dynamics of turbulent mixing due to the Rayleigh-Taylor instability is considered. The mixing layer consists of a single horizontal array of large scale structures. The characteristics of these structures are studied by the spectral and statistical methods. Stimulation of mixing by long-wavelength noise is studied. It is demonstrated that, for a typical homogeneous unscaled noise,time-squared selfsimilarity is retained. The threshold amplitude of random broadband noise is determined, below which these noise can be ignored. The mixing deceleration by the side boundaries is studied. The stimulation and deceleration effects sizably influence the mixing coefficient, increasing and decreasing it respectively. 
Abstract No. T22

\title{
Statistical Mechanics Large Scale Model for the Evolution of the Multi-Mode Kelvin Helmholtz Instability
}

\author{
A. Rikanati ${ }^{1,2}$, U. Alon ${ }^{3}$, and D. Shvarts ${ }^{1,2}$ \\ ${ }^{1}$ Nuclear Research Center Negev, Israel \\ ${ }^{2}$ Ben-Gurion University, Negev, Israel \\ ${ }^{3}$ Weizmann Institute of Science, Israel
}

The nonlinear growth of the multi-mode incompressible Kelvin-Helmholtz (KH) shear flow instability at all density ratios is treated by a large scale statistical-mechanics eddy-pairing model, based on a vortex model for the single eddy behavior and the process of two eddy-pairing. Using an adaptation of the statistical merger model by Alon et. al [1994], a linear time growth of the mixing zone is obtained, resulting in the linear time growth coefficient for several density ratios as well as an asymptotic lognormal eddy size distribution and the average eddy life time probability. Very good agreement with full numerical simulations and experiments is achieved.

References:

U. Alon, J. Hecht, D. Mukamel and D. Shvarts, Phys. Rev. Lett. 72, 2867 (1994). 
Abstract No. T23

\title{
Effects of High Initial Amplitudes and High Mach Numbers on the Evolution of the RM Instability: I. Theoretical Study
}

\author{
A. Rikanati ${ }^{1,2}$, D. Oron ${ }^{1}$, O. Sadot ${ }^{1,2}$, and D. Shvarts ${ }^{1,2}$ \\ ${ }^{1}$ Nuclear Research Center Negev, Israel \\ ${ }^{2}$ Ben-Gurion University, Beer-Sheva, Israel
}

Recent shock tube experiments [Aleshin et al. 1997] and laser driven experiments [Dimonte et al.1998] resulted in an initial bubble growth velocity smaller then that predicted by the matching impulsive models [Richtmyer et. al. 196? and Meyer-Blewett 1972]. It was suggested that the reduction can be attributed to effects of both High Mach number and high initial amplitudes [Holems 2000].

In the present work two models are formulated describing the velocity reduction caused by the two effects. A vorticity deposition model is formulated for effects of high initial amplitudes and a "wall" model is formulated for describing effects of high Mach number caused by the proximity of the shock wave with the two fluid interface. Both in good agreement with the matching experimental results.

Implementing the above models for a range of initial conditions (low to high initial amplitudes and Mach numbers) and with the aid of full numerical simulations, previous experiments and new low and high Mach number shock tube experiments [Sadot et. al., present conference], the range of initial conditions is divided into regions of high Mach dominance, regions of high initial amplitudes dominance and "classical " regions were the two effects are negligible. Using the above mapping, it was found that effects of high initial amplitudes dominates most of the previous experiments.

References:

Aleshin et. al., in Proceedings of the Sixth International Workshop on the Physics of Compressible Turbulent Mixing edited by G. Jourdan \& L. Houas, Marsielle France 1997. Page 1 .

Dimonte G., Frerking C.E., Schnider M. and Remington B., Phys. of Plasmas 12, 304 (1996).

Holmes et. al., J. Fluid Mech 187, 329 (1999).

Meyer K.M. and Blewett P.J., Phys. of. Fluids 15, 753 (1972).

Richtmyer R.D., Commun. Pure Appl. Math. 13, 297 (1960). 
$8^{\text {th }}$ International Workshop on the Physics of Compressible Turbulent Mixing,

Abstract No. T25

\title{
Compressible MHD Turbulence in Strongly Radiating Molecular Clouds in Astrophysics
}

\author{
D. D. Ryutov and B. A. Remington \\ Lawrence Livermore National Laboratory, Livermore, CA
}

Molecular clouds in astrophysics are often subjected to intense irradiation by nearby young stars. The ablation process ensues and a strong shock is driven into the cloud. In a number of cases, the radiative cooling time of the shocked matter is much shorter than the dynamical time of the cloud evolution. In such situations, possible pre-existing turbulent motions and turbulent magnetic fields can potentially contribute to the "stiffness" of the shocked material. We suggest simple models allowing quick evaluation of these effects. We conclude that the presence of a turbulent magnetic field can play a significantrole, provided its amplitude is beyond some critical level, whereas the turbulent ram pressure of the unmagnetized medium can play only a relatively minor role. Implications for the dynamics of astrophysical molecular clouds are discussed.

PACS Nos.: 47.27.Jv, 47.65.+a, 95.30.Qd. 98.58.Db

This work was performed under the auspices of the U.S. Department of Energy by the University of California Lawrence Livermore National Laboratory under contract No. W-7405-Eng-48. 
Abstract No. T26

\title{
Single-Velocity, Multi-Component Turbulent Transport Models for Interfacial Instability-Driven Flows
}

\author{
O. Schilling \\ Lawrence Livermore National Laboratory, Livermore, $C A$
}

\begin{abstract}
A family of two-equation turbulent transport models is proposed for three-dimensional, singlevelocity, multi-component turbulent flows driven by Rayleigh-Taylor, Richtmyer-Meshkov, and Kelvin-Helmholtzinstabilities. The models are compressibleversions of $K$ - $Z$ models, where $K$ is the turbulent kinetic energy and $Z$ is an auxiliary variable such as the turbulent kinetic energy dissipation rate, turbulent frequency, or turbulent lengthscale. Terms are proposed in these equations that account for buoyancy and compressibility effects. The relative merits of different $K-Z$ models will be discussed, and preliminary a priori and a posteriori tests of the models using direct numerical simulation data for Rayleigh-Taylor instability-induced turbulent mixing will be presented. Future plans for model tests and applications to Richtmyer-Meshkov instability-induced turbulent mixing will also be discussed.
\end{abstract}

PACS Nos.: 47.20.Ma, 47.27.Eq, 47.40.Nm

This work was performed under the auspices of the U.S. Department of Energy by the University of California Lawrence Livermore National Laboratory under contract No. W-7405-Eng-48. 
$8^{\text {th }}$ International Workshop on the Physics of Compressible Turbulent Mixing,

Pasadena, CA (2001)

Abstract No. T28

\title{
Large- and Small-Scale Dynamics of Variable-Density Rayleigh-Taylor Instability-Induced Turbulent Mixing
}

\author{
O. Schilling and A. W. Cook \\ Lawrence Livermore National Laboratory, Livermore, CA
}

The statistical dynamics of the large- and small-scales in a three-dimensional turbulent mixing layer induced by Rayleigh-Taylorinstability is studied using $512^{2}$ x 2040 direct numerical simulation data. The terms in the evolution equations for the density-weighted kinetic energy, density-weighted enstrophy, and squared density are evaluated to study their relative contributions during the timeevolution. Particular consideration is given to the role of the baroclinic production and turbulent diffusion terms, as well as to the coupling between the density and velocity fields. The traditional method used to study the flow of energy between resolved (supergrid) and unresolved (subgrid) scales by introducing a cutoff wavenumber in Fourier space is generalized using a multi-resolution wavelet analysis, and used to quantify the forward cascade of kinetic energy, enstrophy, and the squared density from large to smaller scales, as well as the backward cascade from small to larger scales. The implications of this study for developing and assessing subgrid-scale and backscatter models for large-eddy simulation of Rayleigh-Taylor mixing are discussed. Wavelet analysis is ideally suited to studying evolving, anisotropic turbulent mixing, as wavelet-transformed spectra yield information regarding both the scale of structures and their location within the flow. The use of wavelet methods provides additional insight into the coupling between the large-scale, coherent flow (the bubbles and spikes formed during the merger process) and the small-scale, incoherent background flow (the smaller scale turbulence induced by Kelvin-Helmholtz instability and the turbulent energy transfer process).

PACS Nos.: 47.20.Ma, 47.27.Eq

This work was performed under the auspices of the U.S. Department of Energy by the University of California Lawrence Livermore National Laboratory under contract No. W-7405-Eng-48. 
Abstract No. T30

\title{
Analytical Study of the Rayleigh-Taylor Instability in Compressible Fluids
}

\author{
M. Tricottet and S. Bouquet \\ Commissariat à l'Energie Atomique, Bruyères-le-Châtel, France
}

Since the observation of the explosion of the type II supernova, SN87a, Rayleigh-Taylorinstabilities (RTI) are suspected to play a key-role during the process of expansion of the envelope (due to the passage of the shock) $[1,2,3,4]$.

In this paper we perform an analytical study of the RTI. In contrast to most of all previous studies $[5,6,7]$, we examine the case of compressible fluids. In addition, both the static (time-independent acceleration) and the dynamical (time-dependent acceleration) cases are presented.

For these two cases we are able to derive a non-trivial analytical dispersion relationship. Comparisons are made, first, with the models developped for incompressiblefluids and, as expected, for wave number, k, going to infinity (wavelengths going to zero) we recover the well-known result valid for incompressibility.

On the other hand, the influence of the time-dependence in the acceleration is shown and the differences with the static case are emphasized.

[1] Clayton, Principles of Stellar Evolution and Nucleosynthesis, Univ. of Chicago Press (1983)

[2] Fryxell et al., ApJ. 367, 619 (1991)

[3] Glanz, Science 276, 351 (1997)

[4] Kane et al., ApJ. Lett. 478, 75 (1997)

[5] Abarzhi, Phys. Rev. Lett. 81(2), 337 (1998)

[6] Velasquez et al., Astron. Astrophys. 334, 1060 (1998)

[7] Mikaelian, Phys. Rev. Lett. 80(3), 508 (1998) 
$8^{\text {th }}$ International Workshop on the Physics of Compressible Turbulent Mixing,

Pasadena, CA (2001)

Abstract No. T31

\title{
Analytic Nonlinear Growth of A Single-Mode Richtmyer-Meshkov Instability
}

\author{
M. Vandenboomgaerde \\ Commissariat à l'Energie Atomique, Bruyères-le-Châtel, France
}

Perturbation method where only the most secular terms are retained gives a simple result for the weakly nonlinear growth of a single-mode shock-accelerated interface [M.Vandenboomgaerde, C.Mugler, and S.Gauthier, Proceedings, 7th IWPCTM, St. Petersbourg, 1999]. This result writes as a series in integer powers of time. It can be considered as the Taylor expansion of an analytic function. We believe that such a function has been identified; it describes the evolution of the instability from the linear to intermediate nonlinear regime. Whereas the series has a finite radius of convergence, the function has no singularity. The accuracy of this analytic formula is checkedagainst various 2D simulations. Comparisons with previous theoretical models are also presented. 
Abstract No. T32

\title{
Efficient Perturbation Methods for Richtmyer-Meshkov and Rayleigh-Taylor Instabilities: Weakly Nonlinear Stage and Beyond
}

\author{
M. Vandenboomgaerde, C. Cherfils, D. Galmiche, S. Gauthier, and P. A. Raviard \\ Commissariat à l'Energie Atomique, Bruyères-le-Châtel, France
}

A perturbation method has been derived by Q.Zhang and S-I.Sohn [Phys.Fluids 9, 1106 (1997)] in order to predict the weakly nonlinear stage of the Richtmyer-Meshkov instability. Retaining the most secular terms has allowed us to drastically simplify this theory [M.Vandenboomgaerde, C.Mugler, and S.Gauthier, Proceedings, 7th IWPCTM, St Petersbourg, 1999].

We use this simplified but accurate approach to show the importance of the sign of the amplitude of the modes in the selection mode process. Such process is also studied beyond the weakly nonlinear stage. A class of homothetic interfaces is deduced fromthe theory. Its validity is checked against 2D simulations, even in the intermediate nonlinear regime.

Finally, this approach is used in order to solve the equations of the nonlinear stage of the RayleighTaylor instability. We present comparisons between theory and various published test cases. 
$8^{\text {th }}$ International Workshop on the Physics of Compressible Turbulent Mixing,

Pasadena, CA (2001)

Abstract No. T33

\title{
Combined Shear and Buoyancy Instabilities
}

\author{
P. N. Wilson ${ }^{1}$, M. J. Andrews ${ }^{1}$, and F. H. Harlow ${ }^{2}$ \\ ${ }^{1}$ Texas A \& M University, College Station, $T X$ \\ ${ }^{2}$ Los Alamos National Laboratory, Los Alamos, NM
}

Mixing layer experiments were performed at Texas A\&M University with flows in shear, with an unstable buoyancy configuration and combined shear and (stabilizing, de-stabilizing) buoyancy. Two-time density correlations were measured, as well as single-point, second-order velocity correlations for the various flow configurations. A turbulence spectral transport model and a singlepoint turbulence transport model were investigated with their local formulation and later with nonlocal formulation in both physical and wave number space. Numerical simulations of the mixing layer were compared with experimental data and gave good agreement with the addition of terms to model non-local processes, such as, pressure fluctuations propagating into the surrounding fluid from the mix region, advection of small-scale eddies by large-scale structures, and vortex pairing resulting from Kelvin-Helmholtz instabilities. Results of the comparisons between theoretical models and numerical simulations and experimental data are presented. 
Abstract No. T34

\title{
Rate of Growth of the Linear Richtmyer-Meshkov Instability
}

\author{
J. G. Wouchuk \\ E. T. S. I. Industriales, University of Castilla, Salamanca, Spain
}

\begin{abstract}
A theoretical model is presented that calculates the exact asymptotic rate of growth of the perturbations present at a shocked corrugated contact surface. The model covers both situations: whether a shock or a rarefaction are reflected back in the first fluid. The asymptotic growth rate can be calculated with the desired accuracy for any value of the incident shock Mach number, fluids compressibilities or initial density contrast. The growth rate is obtained as the solution, either of a system of two coupled functional equations in the shock reflected situation, or of only one functional equation in the rarefaction reflected case. The model includes the compressible history of the sound wave reverberations between the corrugated fronts and the material interface. It is seen a quite high speed of convergence for the intermediatecalculations. Good agreement with previous numerical and experimental works is shown.
\end{abstract}

PACS Nos.: 47.20.-k, 52.35.Py-, 52.35-T 
$8^{\text {th }}$ International Workshop on the Physics of Compressible Turbulent Mixing,

Abstract No. T35

\title{
The Dependence of the Shock Induced Richtmyer-Meshkov Instability on Dimensionality and Density Ratio
}

\author{
A. Yosef-Hai ${ }^{1}$, O. Sadot ${ }^{1,2}$, D. Kartoon ${ }^{1,2}$, D. Oron ${ }^{2}$, E. Sarid ${ }^{2}$, G. Ben-Dor ${ }^{1}$, and D. Shvarts ${ }^{1,2}$ \\ ${ }^{1}$ Ben-Gurion University, Beer-Sheva, Israel \\ ${ }^{2}$ Nuclear Research Center, Negev, Israel
}

The RM instability occurs when a shock wave passes through a perturbed interface between two fluids. As a result of the instability, small perturbations on the initial interface develop into an array of bubbles and spikes. The bubble front was found to be dominated by bubbles rising and competing $^{1}$. It was previously shown that this evolution of a multi-mode random initial perturbations is strongly related to the evolution of the single-mode case.

For a single-mode perturbation the instability can be described by a linear stage, during which the growth is characterized by a constant velocity, followed by a nonlinear stage, during which the growth velocity reaches an asymptotic $1 /$ behavior $^{2}$. Simple drag-buoyancy considerations can be used to derive the acceleration of a single bubble. Assuming two fluids with different densities $\rho_{\mathrm{H}}$ and $\rho_{L}$ and bubble of wave length $\lambda$ the equation of motion is:

(1) $\left(\rho_{\mathrm{L}}+\mathrm{C}_{\mathrm{a}} \rho_{\mathrm{H}}\right)_{\mathrm{dt}}^{\mathrm{dU}}=\left(\rho_{\mathrm{H}}-\rho_{\mathrm{L}}\right) \mathrm{g}-\frac{\mathrm{C}_{\mathrm{d}}}{\lambda} \rho_{\mathrm{H}} \mathrm{U}^{2}$

The two constants $C_{a}$ and $C_{d}$, which are the added mass coefficient and the drag coefficient respectively, are determined by equating Eq. 1 to the prediction of a potential flow model ${ }^{2}$. For the $2 D$ case $C_{a}=2$ and $C_{d}=6 \pi$, for the $3 D$ case $C_{a}=1$ and $C_{d}=2 \pi$. The asymptotic solution of Eq. 1 for $\mathrm{RM}$ is achieved by neglecting the effect of the shock $(\mathrm{g}=0)$. By doing so the bubble asymptotic acceleration is derived:

(2)

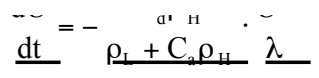

The growth rate is the solution of Eq.2. By introducing the coefficients as described above, the dependence of the late time growth rate on the dimensionality and density ratio is found. The results are summarized in the following Table:

\begin{tabular}{|c|c|c|}
\hline & ${ }_{0} / l_{0_{1} \rightarrow 1}$ & $\rho / \rho_{\mathrm{L}} \rightarrow$ \\
\hline $2 \mathrm{D}$ & $\frac{-}{\lambda}-\bar{\lambda}$ & $-3 \pi-1$ \\
\hline $3 \mathrm{D}$ & $\overline{1}_{\bar{\lambda}}$ & $7-\bar{\lambda}$ \\
\hline
\end{tabular}


In the present work a set of shock tube experiments were made to verify the model's prediction. A thin membrane, on which the 2D and 3D initial perturbations were imposed, separated the two gases. Different pairs of gases were used to achieve different density ratios. The evolution of the shockwave induced mixing zone was measured by high speed laser schlieren photography. The linear and asymptotic stages were observed. The results were found to be in good agreement with both model and simulations. See the following Figure for the case of $\rho_{\mathrm{H}} \rho_{\mathrm{L}}=5$.

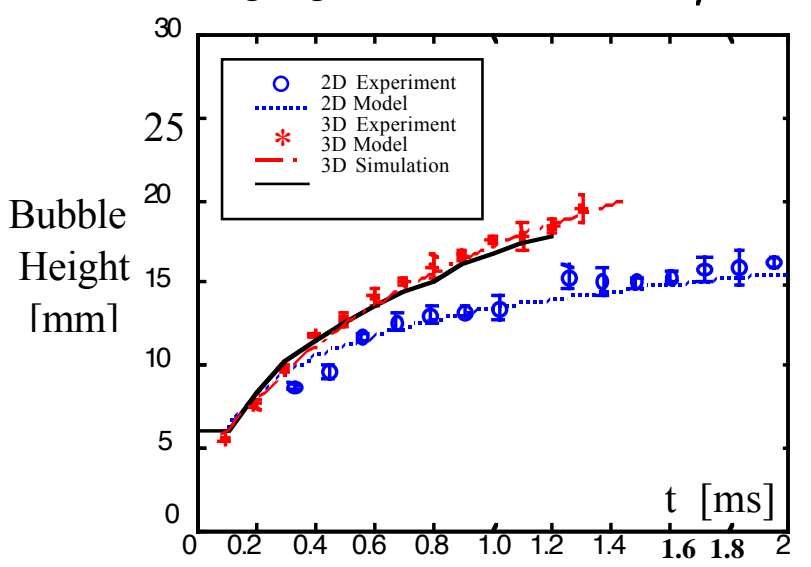

\section{References:}

[1] Alon U., Hecht J., Ofer D. and Shvarts D. 1995 Phys. Rev. Lett. 74, 534.

[2] Hecht J., Alon U. and Shvarts D., 1994 Phys. Fluids 6, 4019. 
$8^{\text {th }}$ International Workshop on the Physics of Compressible Turbulent Mixing,

Pasadena, CA (2001)

Abstract No. T36

\title{
A New Framework for Transitional and Turbulent Mixing
}

\author{
Y. Zhou, H. F. Robey, and A. C. Buckingham \\ Lawrence Livermore National Laboratory, Livermore, CA
}

We develop a framework, based on the current knowledge of turbulence theory and using phenomenological treatment, for the characterization of turbulent mixing evolving from shock and gravity driven instabilities. The procedure is designed to supplement and connect the history of the flow development from the early stages of the Rayleigh-Taylorand Richtmyer-Meshkovinstabilities into the fully developed turbulent flow regime under the conditions of high pressure, high temperature, elevated Reynolds number flow and material mixing. We first demonstrate an analogy between the buoyancy drag model and the one equation turbulence transport closure model. We show that in simplifying the latter transport model to form the buoyancy drag model, the multiple length scales of physical turbulence are drastically reduced to essentially a single, dominant length scale. Furthermore, we show that in the buoyancy drag model several other terms representing specific additional contributions of physical turbulence in the one equation transport model are omitted in the simplification. Next, we compile the key parameters that are needed to characterize both initial transitional flow and its subsequent evolution into fully developed turbulent flow. Although all important length scales are well known and well described in turbulent fluid dynamics literature, we pay special attention here to their time dependent features because of our special focus on description of the transient states and their evolution in transitional and turbulent material mixing. As a result, we have formed a generalization of the transition criteria proposed by Dimotakis $(J$. Fluid Mechanics, 409, 69 (2000)). We illustrate the utility of our framework for transitional and turbulent mixing by applying it to a classical fluid dynamics RTI experimentconducted at Cambridge University and, a laser experiment carried out in the Omega laser facility at the University of Rochester.

This work was performed under the auspices of the U.S. Department of Energy by the University of California Lawrence Livermore National Laboratory under contract No. W-7405-Eng-48. 
Abstract No. T37

\title{
Spherical Combustion Layer in a TNT Explosion
}

\author{
A. L. Kuhl ${ }^{1}$ and R. E. Ferguson ${ }^{2}$ \\ ${ }^{1}$ Lawrence Livermore National Laboratory, Livermore, $C A$ \\ ${ }^{2}$ Krispin Technologies Inc, Rockville, MD
}

A theoretical model of combustion in explosions at large Reynolds, Peclet and Damkohler numbers is described. A key feature of the model is that combustion is treated as material transformations in the Le Chatelier plane, rather than "heat release". In the limit considered here, combustion becomes concentrated in thin exothermic sheets (boundaries between fuel and oxidizer). The products expand along the sheet, thereby inducing vorticity on either side of the sheet that continues to feed the process. The results illustrate the linking between turbulence (vorticity) and exothermicity (dilatation) in the limit of fast chemistry, thereby demonstrating the controlling role that fluid dynamics plays in such flows.
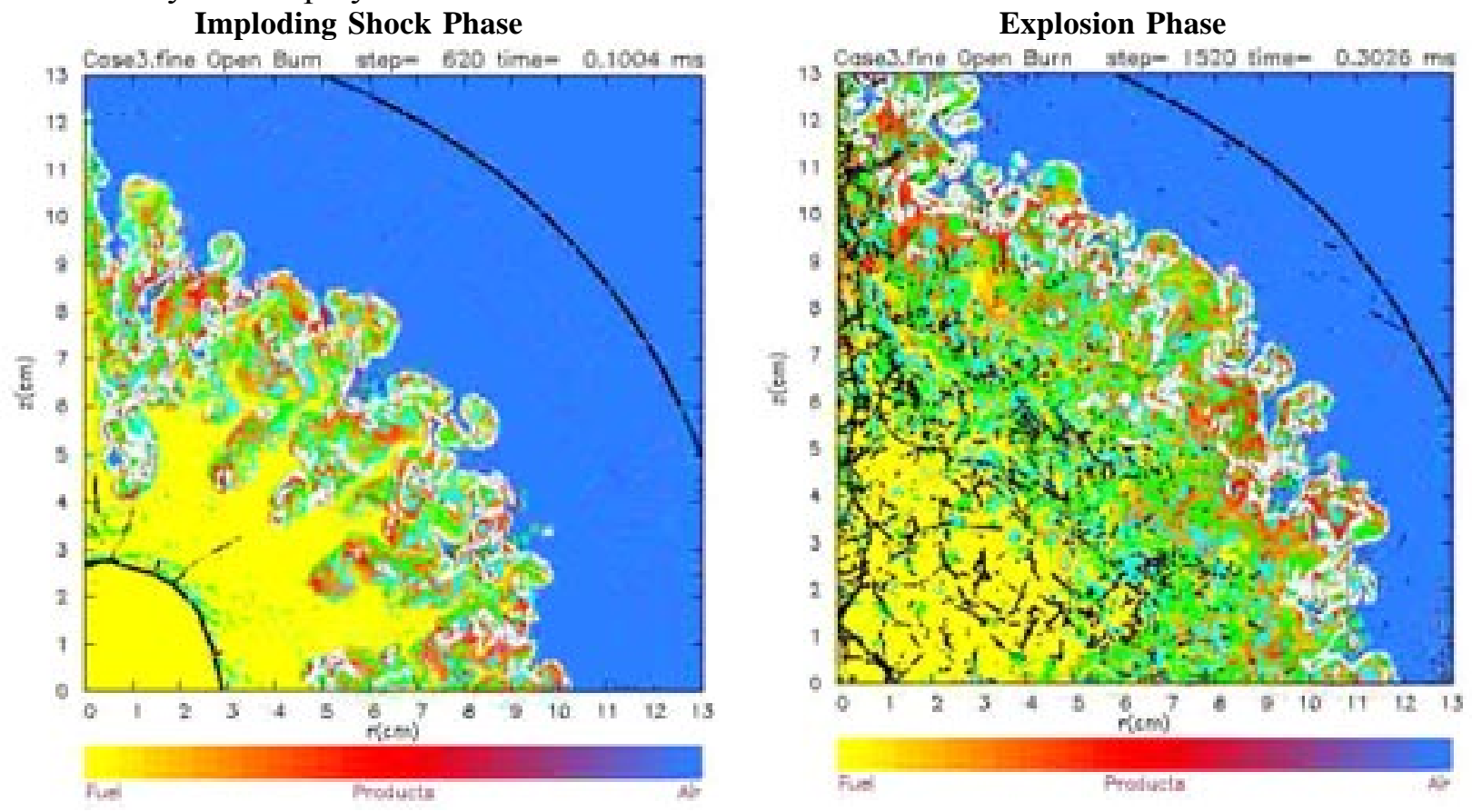

Figure 1. Cross-sectionalview of the combustion field generated by the detonation of a 1-g spherical TNT charge. TNT explosion products (shown as yellow) mix with air (depicted in blue) to form combustion products (represented as red). Exothermic cells are marked with white dots. Vorticity contours are turquoise (positive) and chartreuse (negative), while compressional dilatation contours are black.

This work was performed under the auspices of the U.S. Department of Energy by the University of California Lawrence Livermore National Laboratory under contract No. W-7405Eng-48. 
$8^{\text {th }}$ International Workshop on the Physics of Compressible Turbulent Mixing,

Pasadena, CA (2001)

\title{
Spectral Analysis of Turbulent Flows Induced by RT and RM Instabilities
}

\author{
V. F. Tishkin and N. V. Zmitrenko \\ Institute for Mathematical Modeling of the Russian Academy of Sciences, Moscow, Russia
}

The results of analysis of spectral characteristics of a velocity field for two unstable hydrodynamic problems are examined in this report. One of this problem involves direct simulation of turbulent mixing experiment, which has done for the case of Richtmyer-Meshkov instability. Results of simulation describe main characteristics of developing mixing zone, i.e. shape and sizes of turbulent layer. The spectral analysis has revealed a presence of an interval connected with an enstrophy transfer to small-scale oscillations.

Another problem relates to classic case or Rayleigh-Taylor instability. The analysis of this case has shown that an inertial interval is observed.

Both problems were treated with the help of 3D hydrodynamic code NUT. The spectral analysis was fulfilled by means of specially alaborated for this problems code SPAN.

Supported by ISTC project \# 1495 . 
Abstract No. T39

\title{
Pattern Detection, Compression and Denoising of Rayleigh-Taylor Mix Data Using Discrete Wavelet Transform Techniques
}

\author{
B. B. Afeyan ${ }^{1}$, P. Ramaprabhu ${ }^{2}$, and M. J. Andrews ${ }^{2}$ \\ ${ }^{1}$ Polymath Research Incorporated, Pleasanton, $C A$ \\ ${ }^{2}$ Texas A \& M University, College Station, TX
}

Sequential single point density measurements have been collected from a statistically steady Rayleigh-Taylor mix. The experiment allows long collection times, and thus highly detailed statistical analyses are possible. We have studied this data using a number of discrete wavelet transform techniques in order to denoise, compress and detect patterns and correlations in these ideal representations of intermittent data. By comparing the statistical properties of the evolution of RT at various points downstream, we can establish the minimum number of wavelet coefficients whose evolution can capture the most significant aspects of the turbulent flow. To this end, scale based as well as largest coefficient thresholding are compared and contrasted and the choice of optimum wavelets for the tasks as hand identified. instability-induced turbulent mixing will also be discussed. 

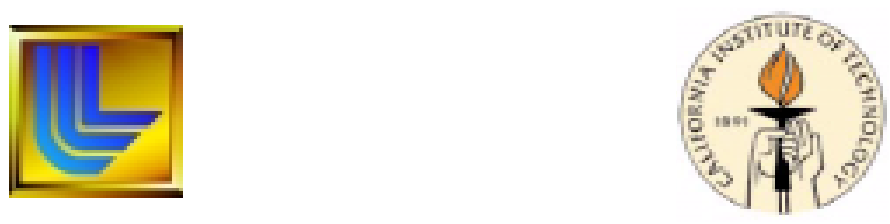

\section{TH INTERNATIONAL WORKSHOP ON THE}

Physics of Compressible

\section{Turbulent MiXing}

December 9 - 14, 2001

California Institute of Technology

Pasadena, California, USA

\section{Program Summary}

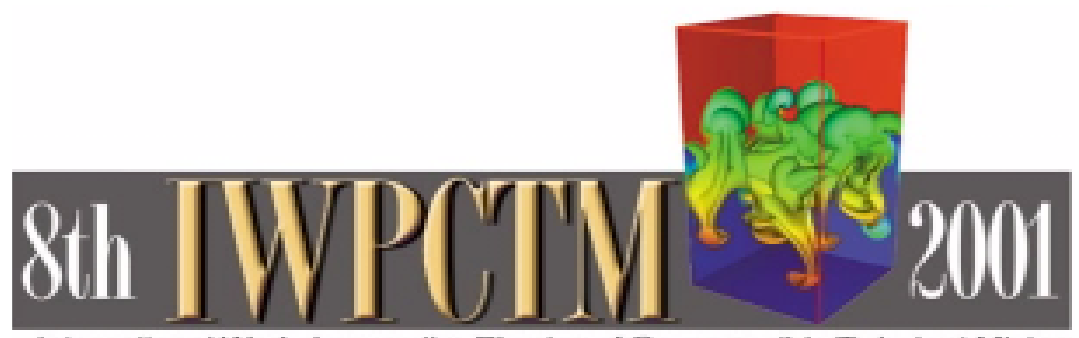

Intemational Workshop on the Physics of Compressible Turbulent Mixing 


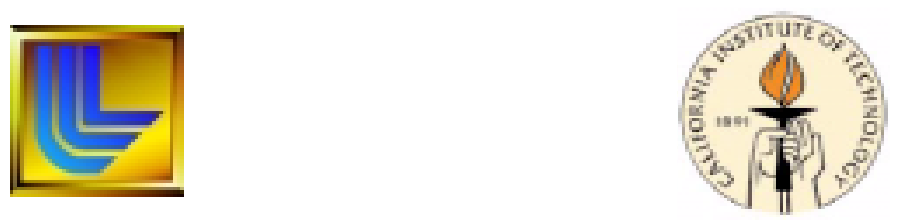

The 8th IWPCTM is hosted by the

Lawrence Livermore National Laboratory and is co-hosted by the California Institute of Technology ${ }^{\dagger}$

All oral sessions and discussions will be held in the Beckman Institute Auditorium

Poster sessions will be held in the Winnett Lounge and Club Room

"Partial financial support was generously provided by the California Institute of Technology 


\section{Transportation}

Buses depart from the Pasadena Hilton to campus at 7:45 Monday - Friday, December 10 - 14 .

Buses depart from campus to the Pasadena Hilton at 17:50 Monday - Thursday, December 10 - 13, and at 12:15 on Friday, December 14.

\section{REFRESHMENTS}

Refreshments will be available at 8:00 and at breaks Monday - Friday, December 10 - 14 . 


\section{SundaY, DeCEMber 9, 2001}

17:00 - 21:00 Registration

Pasadena Hilton

18:00 - 21:00 Reception

Pasadena Hilton

Guest Speaker: E. I. Moses

NIF Project Manager

(Lawrence Livermore National Laboratory) 


\section{Monday, DeCember 10, 2001}

8:15 - 8:30 Welcome and Opening Remarks:

S. Koonin / O. Schilling

\section{Experimental Session I}

Chair: H. F. Robey

(Lawrence Livermore National Laboratory)

8:30 - 9:30 Review Talk:

A Review on RT and RM Instability and TM Experiments J.-F. Haas and S. G. Zaytsev (Comissariat à l'Energie

Atomique and Krzhizhanovsky Power Engineering Institute)

9:30 - 9:50 The Influence of Scaling for Periodical Perturbations on Development of Turbulent Mixing on a Gas-Liquid Interface (E7)

M. Bliznetsov, E. Meshkov, N. Nevmerzhitsky,

A. Nikulin, E. Sen'kovsky, and E. Sotskov (Russian Federal Nuclear Center-VNIIEF)

9:50 - 10:10 Experimental Study Into Rayleigh-Taylor Turbulent Mixing Zone Heterogeneous Structure (E31)

Yu. A. Kucherenko, A. P. Pylaev, V. D. Murzakov,

A. V. Belomestnih, V. N. Popov, and A. A. Tyaktev (Russian Federal Nuclear Center-VNIITF)

10:10 - 10:30 Rayleigh-Taylor Instability at a Tilted Interface in Incompressible Laboratory Experiments and Compressible Numerical Simulations (E14)

J. M. Holford, S. B. Dalziel, and D. L. Youngs (Cambridge University and Atomic Weapons Establishment)

10:30 - 10:50 Break: Beckman Institute (courtyard)

Experimental Session II

Chair: J.-F. Haas

(Commissariat à l'Energie Atomique)

10:50 - 11:10 Measurements of Turbulence Correlations in Low Atwood Number Rayleigh-Taylor Mixing (E32)

P. Ramaprabhu and M. J. Andrews (Texas A \& M University) 
11:10 - 11:30 Experimental Investigations of the Self-Similar Mixing Mode of Different Density Gases in the Earth's Gravitational Field (E28)

Yu. A. Kucherenko, O. E. Shestachenko, Yu. A. Puskunov, E. V. Sviridov, V. M. Medvedev, and A. I. Baishev (Russian Federal Nuclear Center - VNIITF)

11:30 - 11:50 Mix Experiments Using a Two Dimensional Convergent Shock Tube (E13)

D. A. Holder, C. Barton, and A. V. Smith (Atomic Weapons Establishment)

12:00 - 13:15 Lunch: Winnett Lounge

\section{Experimental Session III \\ Chair: J. W. Jacobs \\ (University of Arizona)}

13:15 - 13:35 The Evolution and Interaction of Two Shock-Accelerated Unstable Gas Cylinders (E40)

C. Tomkins, K. Prestridge, P. Rightley, C. A. Zoldi, and R. Benjamin, (Los Alamos National Laboratory)

13:35 - 13:55 PLIF Flow Visualization of a Shock-Accelerated Air/SF6 Interface (E18)

$\underline{\text { J. W. Jacobs and V. V. Krivets (University of Arizona, Tuscon) }}$

13:55 - 14:15 Shock Tube Experiments on Richtmyer-Meshkov Instability Across a Chevron Profiled Interface (E39)

A. V. Smith, D. A. Holder, C. J. Barton, A. P. Morris, and D. L. Youngs (Atomic Weapons Laboratory)

14:15 - 14:35 Study of Diverging and Converging Spherical Shock Waves Induced by Micro Explosives and Their Interaction with Product Gases (E15)

S.H. R. Hosseini and K. Takayama (Tohoku University)

\section{4:35 - 14:55 Break: Beckman Institute (courtyard)}




\section{Experimental Session IV}

Chair: K. Budil

(Lawrence Livermore National Laboratory)

14:55 - 15:15 The Dependence of the Shock Induced Richtmyer-Meshkov Instability on Dimensionality and Density Ratio (T35)

A. Yosef-Hai, O. Sadot, D. Kartoon, D. Oron, E. Sarid, G. Ben-Dor, and D. Shvarts (Ben-Gurion University, Nuclear Research Center, Negev)

15:15 - 15:35 Effects of High Initial Amplitudes and High Mach Numbers on the Evolution of the RM Instability: II. Experimental Study (E36)

O. Sadot, A. Yosef-Hai, A. Rikanati, D. Oron, G. Ben-Dor, and D. Shvarts (Nuclear Research Center, Negev and Ben-Gurion University)

15:35 - 15:55 Experimental Study of a Strongly-Shocked Gas Interface With Visualized Initial Conditions (E27)

J. G. Oakley, M. H. Anderson, and $\underline{R}$. Bonazza (University of Wisconsin, Madison)

15:55 - 16:15 Compressible Vortex Rings (E8)

M. Brouillette and C. Hébert (Université de Sherbrooke)

16:15 - 17:45 General Poster Session: Winnett Lounge and Club Room 


\section{Tuesday, December 11, 2001}

$8: 15-8: 30$

$8: 30-9: 30$

$9: 30-9: 50$

$9: 50-10: 10$

$10: 10-10: 30$

Announcements: O. Schilling

Experimental Session V

Chair: T. A. Peyser

(Lawrence Livermore National Laboratory)

Review Talk: The Experimental Study of Excitation and Development of the Hydrodynamic Instability in the Mixing Zone Separating Gases of Different Densities at their Accelerated Motion

S. G. Zaytsev (Krzhizhanovsky Power Engineering Institute)

Compressible Hydrodynamics on the Omega Laser, Motivated by Astrophysics (E10)

R. P. Drake, P. Keiter, K. E. Korreck, K. Dannenberg,

H. F. Robey, T. Perry, J. O. Kane, B. A. Remington,

R. J. Wallace, O. A. Hurricane, D. D. Ryutov, J. Knauer,

R. Teyssier, A. Calder, R. Rosner, B. Fryxell, D. Arnett, Y. Zhang, J. Glimm, N. Turner, J. Stone, R. McCray, and

$J$. Grove (University of Michigan, Lawrence Livermore National Laboratory, University of Rochester, Laboratory for Laser Energetics, Comissariat à l'Energie Atomique, University of Chicago, University of Arizona, State University of New York, Stony Brook, University of Maryland, University of Colorado, and Los Alamos National Laboratory)

Improvements to Convergent Cylindrical Plasma Mix Experiments Using Laser Direct Drive (E4)

C. W. Barnes, S. H. Batha, A. M. Dunne, N. E. Lanier, G. R. Magelssen, T. J. Murphy, K W. Parker, S. Rothman, J. M. Scott, and D. Youngs (Los Alamos National Laboratory and Atomic Weapons Establishment)

The Interaction of Supernova Blast Waves with Interstellar Clouds: Experiments on the OMEGA Laser (E42) R. I. Klein, H. Robey, T. Perry, and J. Greenough (Lawrence Livermore National Laboratory and University of California, Berkeley)

10:30 - 10:50 Break: Beckman Institute (courtyard) 


\section{Experimental Session VI \\ Chair: G. Dimonte \\ (Lawrence Livermore National Laboratory)}

10:50 - 11:10 An Experimental Study of the Effect of Shock Proximity on the Richtmyer-Meshkov Instability at High Mach Number (E12)

S. G. Glendinning, D. G. Braun, M. J. Edwards,

W. W. Hsing, B. F. Lasinski, H. Louis, J. Moreno, T. A. Peyser, B. A. Remington, H. F. Robey, E. J. Turano, C. P. Verdon, and Y. Zhou (Lawrence Livermore National Laboratory)

11:10 - 11:30 A Vortex Model for Studying the Effect of Shock Proximity on Richtmyer-Meshkov Instability at High Mach Number (E46) H. F. Robey and S. G. Glendinning (Lawrence Livermore National Laboratory)

11:30 - 11:50 Laser-Based High Pressure, High Strain-Rate Solid-State Experiments (E19)

D. H. Kalantar, J. Belak, J. D. Colvin, M. Kumar, K. T. Lorenz,

K. O. Mikaelian, S. Pollaine, B. A. Remington, S. V. Weber,

L. G. Wiley, A. M. Wiley, A. Loveridge-Smith, J. S. Wark, and

M. A. Myers (Lawrence Livermore National Laboratory, Oxford University, and University of California, San Diego)

\section{2:00 - 13:15 Lunch: Winnett Lounge}

\section{Computational Session I}

Chair: T. L. McAbee

(Lawrence Livermore National Laboratory)

13:15 - 13:35 A Comparison of High-Resolution 3D Numerical Simulations of Turbulent Rayleigh-Taylor (RT) Instability: Alpha-Group Collaboration (C10)

G. Dimonte, A. Dimits, S. Weber, D. L. Youngs, A. C. Calder,

B. Fryxell, J. Biello, L. Dursi, P. MacNiece, K. Olson,

P. Ricker, R. Rosner, F. Timmes, H. Tufo, Y.-N. Young,

M. Zingale, M. J. Andrews, P. Ramaprabhu, S. Wunsch,

C. Garasi, and A. Robinson (Lawrence Livermore National

Laboratory, Atomic Weapons Establishment, University of

Chicago, NASA Goddard Space Flight Center, Texas A \& M University, and Sandia National Laboratories) 
13:35 - 13:55 Study of Turbulent Gravitational Mixing at Large

DensityDifferences Using Direct 3D Numerical Simulation (C44)

Yu. V. Yanilkin, V. P. Statsenko, S. V. Rebrov,

N. I. Selchenkova, O. G. Sin'kova, A. L. Stadnik, and

A. Ya. Uchayev (Russian Federal Nuclear Center - VNIIEF)

13:55 - 14:15 Numerical Methods for Determination of Mix (C11)

S. Dutta, E. George, J. Glimm, J. Grove, X. Li, A. Marchese,

D. H. Sharp, Z. Xu, and Y. Zhang (State University of

New York, Stony Brook, Los Alamos National Laboratory, and

Brookhaven National Laboratory)

14:15 - 14:35 Effects of High Initial Amplitudes and High Mach Numbers on the Evolution of the RM Instability: II. Experimental Study (E36)

O. Sadot, A. Yosef-Hai, A. Rikanati, D. Oron, G. Ben-Dor, and D. Shvarts (Nuclear Research Center, Negev and Ben-Gurion University)

\section{4:35 - 14:55 Break: Beckman Institute (courtyard)}

\section{Computational Session II}

\section{Chair: H. Takabe}

(Osaka University)

14:55 - 15:15 Numerical Investigation of a Laser Induced Turbulent Mixing Zone (C35)

P. Seytor and M. Legrand (Commissariat à l'Energie

Atomique)

15:15 - 15:35 Development and Validation of a 2D Turbulent Mix Model (C46)

D. L. Youngs (Atomic Weapons Establishment)

15:35 - 15:55 Computational Modeling of Low-Mach-Number HighAtwood-Number Turbulent Mixing (C4)

Wm. T. Ashurst and A. R. Kerstein (Sandia National Laboratories)

15:55 - 16:15 Simulation of a Shock-Accelerated Gas Cylinder and Comparison with Experimental Images and Velocity Fields (C50) C. A. Zoldi, K. Prestridge, P. M. Rightley, and R. F. Benjamin (Los Alamos National Laboratory and State University of New York, Stony Brook) 
16:15 - 17:45 Experimental Discussion: Beckman Institute Auditorium

Computational and Theoretical Poster Session: Winnett Lounge 


\section{WedNeSDay, DeCeMber 12, 2001}

8:15 - 8:30 Announcements: O. Schilling

Computational Session III

Chair: J. Glimm

(State University of New York, Stony Brook)

8:30 - 9:30 Review Talk:

Review of Numerical Simulation of Mixing due to RayleighTaylor and Richtmyer-Meshkov Instabilities

D. L. Youngs (Atomic Weapons Establishment)

9:30 - 9:50 Transition Stages of Rayleigh-Taylor Instability Between Miscible Fluids (C56)

A. W. Cook and P. E. Dimotakis (Lawrence Livermore National Laboratory and California Institute of Technology)

9:50 - 10:10 Application of a Laser Shock Tube for the Study of Supersonic Gas Flows and the Development of Hydrodynamic Instabilities in Layered Media (C25)

I. G. Lebo and V. D. Zvorykin (Technical University MIREA and P. N. Lebedev Physical Institute)

10:10 - 10:30 Shock-Planar Curtain Interactions: Strong Secondary Baroclinic Deposition and the Emergence of Coherent and Random Vortex Projectiles (VPs) and Decaying Stratified Turbulence (C48)

S. Zhang and N. J. Zabusky (Rutgers University)

10:30 - 10:50 Break: Beckman Institute (courtyard)

Computational Session IV

Chair: J. Grove

(Los Alamos National Laboratory)

10:50 - 11:10 One-Dimensional Simulation of the Effects of Unstable Mix on Neutron and Charged-Particle Yield from Laser-Driven Implosion Experiments (C13)

R. Epstein, J. A. Delettrez, V. Yu. Glebov, V. N. Goncharov, P. W. McKenty, P. B. Radha, S. Skupsky, V. A. Smalyuk, and C. Stoeckl (University of Rochester, Laboratory for Laser Engineering) 
11:10 - 11:30 Modeling Turbulent Mixing in Inertial Confinement Fusion Implosions (C37)

Y. Srebro, D. Kushnir, Y. Elbaz, and D. Shvarts (Ben-Gurion University, Nuclear Research Center, Negev, and Hebrew University)

11:30 - 12:00 Dispersal of Mass and Circulation Following Shock-Sphere (Axisymmetric) and Shock Cylinder Interactions: Effects Arising from Shock Cavity Collapse, Vortex Double Layers; Density-Gradient Intensification and Vortex Projectiles (C29)

G. Peng, S. Gupta, S. Zhang, and N. J. Zabusky (Rutgers University)

\section{2:00 - 13:15 Lunch: Winnett Lounge}

\section{Computational Session V}

Chair: N. J. Zabusky

(Rutgers University)

13:15 - 13:35 Code to Code Comparisons for the Problem of Shock Acceleration of Diffuse Dense Gaseous Cylinder (C16) J. A. Greenough, W. J. Rider, C. A. Zoldi, and J. R. Kamm (Lawrence Livermore National Laboratory and Los Alamos National Laboratory)

13:35 - 13:55 Molecular Dynamic Simulation of Shock and RichtmyerMeshkov Instability in Cylindrical Geometry (C26) K. Nishihara, V. Zhakhovskii, and M. Abe (Osaka University, Institute of Laser Engineering)

13:55 - 14:15 Large Eddy Simulation of Strong Shock Richtmyer-Meshkov Instability (C33)

R. Samtaney, T. Voelkl, and D. I. Pullin (California Institute of Technology)

14:15 - 14:35 Spectral and High-Order Compact Methods for ShockInduced Mixing (C8)

A. W. Cook, W. Cabot, and J. A. Greenough (Lawrence Livermore National Laboratory)

\section{4:35 - 14:55 Break: Beckman Institute (courtyard)}




\section{Computational Session VI \\ Chair: B. T. Goodwin \\ (Lawrence Livermore National Laboratory)}

14:55 - 15:15 Turbulent Mixing Nuclear Burning in Type Ia Supernova Explosion Based on Bubble Statistical Mechanics (C38) H. Takabe, S. Yamada, K. Kobayashi, A. Mizuta, and K. Nomoto (Osaka University, Institute of Laser Engineering and University of Tokyo)

15:15 - 15:35 High Order Numerical Methods for the 2D RichtmyerMeshkov Instability (C54)

W.-S. Don, D. Gottlieb, L. Jameson, and C.-W. Shu (Brown

University and Lawrence Livermore National Laboratory)

15:35 - 15:55 Compressibility Effects in a High-Speed, Reacting Shear Layer: An Investigation Using DNS (C27)

C. Pantano and $\underline{S}$. Sarkar (University of California, San Diego)

15:55 - 16:15 A Semi-Empirical Model for Turbulent Diffusion of Magnetic Field to Accelerated Plasma (C19)

E. V. Gubkov, V. A. Zhmailo, and Yu. V. Yanilkin (Russian Federal Nuclear Center-VNIIEF)

16:15 - 17:45 Computational Discussion: Beckman Institute Auditorium

Experimental and Theoretical Poster Session:

Winnett Lounge

18:00 - 21:00 Banquet: Pasadena Hilton

Guest Speaker: Z. Nagin Cox

(NASA, Jet Propulsion Laboratory) 


\section{Thursday, DeCember 13, 2001}

8:15 - 8:30 Announcements: O. Schilling

Theoretical Session I

Chair: D. I. Meiron

(California Institute of Technology)

8:30 - 9:30 Review Talk:

Three Dimensional Multi-Mode Rayleigh-Taylor and Richtmyer-Meshkov Instabilities at All Density Ratios (T14) D. Kartoon, D. Oron, L. Arazi, A. Rikanati, U. Alon, and D. Shvarts (Nuclear Research Center, Negev, Ben-Gurion University, Tel-Aviv University, and Weizmann Institute)

9:30 - 9:50 Theoretical Methods for Determination of Mix (T7) B. Cheng, J. Glimm, and D. H. Sharp (Los Alamos National Laboratory, State University of New York, Stony Brook, and Brookhaven National Laboratory)

9:50 - 10:10 Effects of High Initial Amplitudes and High Mach Numbers on the Evolution of the RM Instability: I. Theoretical Study (T23)

A. Rikanati, D. Oron, O. Sadot, and D. Shvarts (Nuclear Research Center, Negev and Ben-Gurion University)

10:10 - 10:30 Evolution of Arbitrary Perturbations in the RichtmyerMeshkov Instability (T20)

K. O. Mikaelian (Lawrence Livermore National Laboratory)

10:30 - 10:50 Break: Beckman Institute (courtyard)

Theoretical Session II

Chair: S. B. Dalziel

(Cambridge University)

10:50 - 11:10 Spectral Analysis of Turbulent Flows Induced by RT and RM Instabilities (T38)

V. F. Tishkin and N. V. Zmitrenko (Institute for Mathematical Modeling, Russian Academy of Sciences) 
11:10 - 11:30 A New Framework for Transitional and Turbulent Mixing (T36) Y. Zhou, H. F. Robey, and A. C. Buckingham (Lawrence Livermore National Laboratory)

11:30 - 11:50 Raleigh-Taylor and Richtmyer-Meshkov Instabilities for Fluids with a Finite Density Contrast (T2)

S. I. Abarzhi, J. Glimm, and A der Lin (State University of New York, Stony Brook)

12:00 - 13:15 Lunch: Winnett Lounge

Theoretical Session III

Chair: T. T. Clark

(Los Alamos National Laboratory)

13:15 - 13:35 Nonlinear Evolution of an Interface in the RichtmyerMeshkov Instability (T19)

K. Nishihara, C. Matsuoka, and Y. Fukuda (Ehime University and Osaka University Institute of Laser Engineering)

13:35 - 13:55 Nonlinear Evolution of Unstable Fluid Interface (T1) S. I. Abarzhi (State University of New York, Stony Brook)

13:55 - 14:15 Analytical Study of the Rayleigh-Taylor Instability in Compressible Fluids (T30)

M. Tricottet and $\underline{\text { S. Bouquet (Commissariat à l'Energie }}$ Atomique)

14:15 - 14:35 Non-Linear Stages for the RT and RM Instabilities (T13)) N. A. Inogamov, M. Tricottet, A. M. Oparin, and S. Bouquet (Landau Institute for Theoretical Physics, Institute of Computer-Aided Design, and Commissariat à l'Energie Atomique)

\section{4:35 - 14:55 Break: Beckman Institute (courtyard)}




\section{Theoretical Session IV}

Chair: D. L. Youngs

(Atomic Weapons Establishment)

14:55 - 15:15 Rate of Growth of the Linear Richtmyer-Meshkov Instability (T34)

J. G. Wouchuk (University of Castilla)

15:15 - 15:35 Efficient Perturbation Methods for Richtmyer-Meshkov and Rayleigh-Taylor Instabilities: Weakly Nonlinear Stage and Beyond (T32)

M. Vandenboomegaerde, C. Cherfils, D. Galmiche,

S. Gauthier, and P. A. Raviard (Commissariat à l'Energie Atomique)

15:35 - 15:55 Response of Turbulent RANS Models to Self-Similar Variable Acceleration RT-Mixing: An Analytical 0D Analysis (T18) A. Llor (Commissariat à l'Energie Atomique)

15:55 - 16:15 Combined Shear and Buoyancy Instabilities (T33) P. N. Wilson, M. J. Andrews, and F. H. Harlow (Texas A \& M University and Los Alamos National Laboratory)

16:15 - 17:45 Theoretical Discussion: Beckman Institute Auditorium

Experimental and Computational Poster Session: Winnett Lounge 


\section{Friday, December 14, 2001}

$8: 15-8: 30$

$8: 30-8: 50$

$8: 50-9: 10$

$9: 10-9: 30$

$9: 30-9: 50$

$9: 50-10: 10$

$10: 10-10: 30$

10:30 - 10:50

$10: 50-11: 10$
Announcements: O. Schilling

\section{Theoretical Session V}

Chair: O. Schilling

(Lawrence Livermore National Laboratory)

Rayleigh-Taylor Instability in Compressible Fluids (C12) Y. Elbaz, A. Rikanati, D. Oron, and D. Shvarts (Nuclear Research Center Negev, Ben Gurion University, and Weizmann Institute of Science)

A Model for Instability Growth in Accelerated Solid Metals (T9) J. D. Colvin, M. Legrand, B. A. Remington, G. Schurtz, and S. V. Weber (Lawrence Livermore National Laboratory and Comissariat à l'Energie Atomique)

Toy Models for the Growth Rate of Rayleigh-Taylor Instability (T10)

S. B. Dalziel (Cambridge University)

Spherical Combustion Layer in a TNT Explosion (T37)

A. L. Kuhl and R. E. Ferguson (Lawrence Livermore National Laboratory and Krispin Technologies)

3D Rayleigh-Taylor and Richtmyer-Meshkov Single -Modes (T12)

N. A. Inogamov, A. M. Oparin, M. Tricottet, and S. Bouquet (Landau Institute for Theoretical Physics, Institute for Computer Aided Design, and Commissariat à l'Energie Atomique)

Modeling Radiation Effects in Mixing Layers (T8)

T. Clark and F. H. Harlow (Los Alamos National Laboratory)

\section{Break: Beckman Institute (courtyard)}

Large- and Small-Scale Dynamics of Variable-Density Rayleigh-Taylor Instability-Induced Turbulent Mixing (T28) O. Schilling and A. W. Cook (Lawrence Livermore National Laboratory)

\section{1:10 - 11:55 Summary Remarks}

11:55 - 12:00 Closing Remarks: O. Schilling / D. Meiron 


\section{Experimental Posters}

Experimental Investigation of the Heavy and Light Media Separation in the Rayleigh-Taylor Turbulence Zone at Different Atwood Numbers (E2)

Yu. A. Kucherenko, S. I. Balabin, R. I. Ardashova, A. P. Pylaev,

O. E. Kozelkov, and V. D. Murzakov (Russian Federal Nuclear CenterVNIITF)

Experimental Investigation into Influence of Stabilizing Properties of Transitional Layers Upon the Turbulent Mixing Evolution (E3) Yu. A. Kucherenko, S. I. Balabin, R. I. Ardashova, O. E. Kozelkov,

A. V. Dulov, and I. A. Romanov (Russian Federal Nuclear Center-VNIITF)

Mixing Between Two Compressing Cylinders (E5)

S. H. Batha, K. W. Parker, C. W. Barnes, A. M. Dunne, N. E. Lanier, G. R. Magelssen, T. J. Murphy, S. Rothman J. M. Scott, and D. Youngs (Los Alamos National Laboratory and Atomic Weapons Establishment)

Development of a Method for Studying the Interaction between Shock Wave and a Flame Front (E6)

M. Bliznetsov, V. Dudin, S. Gerasimov, L. Houas, G. Jourdan, and

A. Logvinov (Russian Federal Nuclear Center - VNIIEF, SarPTI, IUSTI/CNRS)

Design of Flyer-Plate-Driven Compressible Turbulent Mix Experiments (E9)

R. P. Drake (University of Michigan)

Growth of Perturbations on Metals Interface at Oblique Collision with Supersonic Velocity of Contact Point Motion (E11)

O. B. Drennov, A. L. Mikhaylov, P. N. Nizovtsev, and V. A. Raevskii (Russian Federal Nuclear Center - VNIIEF)

From Linear to Turbulent Stages of the Richtmyer-Meshkov Instability Development in a Large Cross Section Shock Tube (E17)

L. Houas, G. Jourdan, E. E. Meshkov, and L. Schwaederlé (Université de Provence and Russian Federal Nuclear Center - VNIIEF)

RFNC-VNIITF Multifunctional Shock Tube to Investigate the Evolution of Instabilities in Nonstationary Gas Dynamic Flows (E20)

Yu. A. Kucherenko, O. E. Shestachenko, S. I. Balabin, and A. P. Pylaev (Russian Federal Nuclear Center - VNIITF) 
Hydrodynamic Instabilities at a Shock Accelerated Bubble Gas-Gas Interface (E22)

G. Layes, G. Jourdan, P. Roualdes, and L. Houas (IUSTI and Centre d'Etudes de Gramat)

Experimental and Numerical Study of Shock Wave-Bubble Interaction (E23)

K. Levy, O. Sadot, D. Oron, Y. Srebro, Y. Elbaz, A. Josef-Hai, G. Ben-Dor, and D. Shvarts (Ben-Gurion University and Nuclear Research Center, Negev)

Laser-Driven Near Isentropic Compression of an Aluminum Flyer Plate (E24) K. T. Lorenz, D. Kalantar, J. Edwards, J. D. Colvin, and B. Remington (Lawrence Livermore National Laboratory)

Single-Mode Incompressible Richtmyer-Meshkov Instability Experiments (E26) C. E. Niederhaus and J. W. Jacobs (NASA Glenn and University of Arizona)

Modeling Laser Material Strength Experiments (E29)

S. Pollaine, D. Kalantar, B. Remington, J. Belak, J. D. Colvin, J. Edwards, R. Minich, K. O. Mikaelian, K. T. Lorenz, S. V. Weber, L. G. Wiley, D. Paisley, A. Hauer, J. S. Wark, A. Loveridge, A. M. Allen, T. R. Boehly, and M. A. Meyers (Lawrence Livermore National Laboratory, Los Alamos National Laboratory, Oxford University, University of Rochester, and University of California, San Diego)

Experiments and Simulations of Instabilities in a Shock-Accelerated Gas Cylinder (E30)

K. Prestridge, C. A. Zoldi, P. Vorobieff, P. M. Rightley, and R. F. Benjamin (Los Alamos National Laboratory, State University New York, Stony Brook, and University of New Mexico)

Experimental Study of the Interaction of a Strong Shock with a Spherical Density Inhomogeneity (E34)

H. F. Robey, T. S. Perry, R. I. Klein, J. A. Greenough, J. O. Kane, and

T. R. Boehly (Lawrence Livermore National Laboratory, University of California, Berkeley, University of Rochester)

Turbulent Transition in a High Reynolds Number, Rayleigh-Taylor Unstable Plasma Flow (E35)

H. F. Robey, Y. K. Zhou, A. C. Buckingham, P. Keiter, B. A. Remington, and R. P. Drake (Lawrence Livermore National Laboratory and University of Michigan) 
Measurements Within a Richtmyer-Meshkov Mixing Zone Using a Triple Hot Wire Probe Technique (E37)

L. Schwaederlé, G. Jourdan, L. Houas, and J.-F. Haas (IUSTI and Commissariat à l'Energie Atomique)

Experimental Study into Evolution of Gravitational Turbulent Mixing of Gases at the Multifunctional Shock Tube (E38)

Yu. A. Kucherenko, O. E. Shestachenko, Yu. A. Piskunov, E. V. Sviridov,

V. M. Medvedev, and A. I. Baishev (Russian Federal Nuclear Center-VNITF)

Doubly-Shocked Richtmyer-Meshkov Instability Experiments at Nova (E41)

D. J. Ward, K. S. Budil, T. A. Peyser, B. A. Remington, P. L. Miller, R. J. Wallace,

H. Louis, and A. Demiris (Lawrence Livermore National Laboratory)

Evolution of the Mixing Zone of Different Densities Gases Being Interaction to Compression Waves (E43)

S. G. Zaytsev, V. V. Krivets, I. M. Mazlin, S. N. Titov, E. I. Chebotareva, V. V. Nikishin, V. F. Tishkin, S. Bouquet, and J.-F. Haas (Krzhizhanovsky Power Engineering Institute, Institute of Mathematical Modeling, and Commissariat à l'Energie Atomique)

Studies of Rayleigh-Taylor Instability in Aluminum Under Shock-Wave and Shock Less Loading (E44)

A. Lebedev, P. Nizovtcev, and V. Raevsky (Russian Federal Nuclear Center - VNIIEF)

Ablative Rayleigh-Taylor Instability at Short Wavelengths (E45)

H. Azechi, T. Sakaiya, M. Nakai, H. Shiraga, K. Shigemori, N. Miyanaga,

M. Nishikino, S. Fujioka, Y. Tamari, H. Nagatomo, A. Sunahara, and

H. Takabe (Osaka University and Rutgers University) 


\section{Computational Posters}

Modes' Interaction on Nonlinear Stage of Richtmyer-Meshkov Instability Evolution (C1)

V. I. Anisimov and A. V. Polionov (Russian Federal Nuclear Center-VNIITF)

Application of ke-Model for the Description of an Atmospheric Surface Layer (C2) M. G. Anuchin, V. E. Neuvazhayev, and I. E. Parshukov (Russian Federal Nuclear Center-VNIITF)

Numerical Simulation of Mode Coupling in Laser-Driven Rayleigh-Taylor Instability Experiments (C9)

R. Darlington and K. Budil (Lawrence Livermore National Laboratory)

3D Computation for Surface Perturbation Evolution of Plasma Cloud During its Expansion in Magnetic Field (C14)

E. S. Gavrilova, E. V. Gubkov, V. A. Zhmailo, and Yu. V. Yanilkin (Russian Federal Nuclear Center - VNIIEF)

The Richtmyer-Meshkov Instability in Cylindrical Geometry: Experiments and Simulation (C15)

M. J. Graham, K. S. Budil, J. Grove, and B. A. Remington (Lawrence Livermore National Laboratory and Los Alamos National Laboratory)

Implementation of a Turbulent Mix Model in a 2D ALE Code (C17)

B. Grieves (Atomic Weapons Establishment)

Error Estimation for Strong Shock Hydrodynamics (C18)

J. W. Grove (Los Alamos National Laboratory)

Localization and Spreading of Interfaces (Contact Discontinuities) in PPM and WENO Simulations of the Inviscid Compressible Euler Equations (C20) Y. Gupta, N. J. Zabusky, R. Samtaney, and Y. Gulak (Rutgers University)

Update on Instability Modeling for the NIF Ignition Targets (C22) S. W. Haan, T. Dittrich, S. Hatchett, D. Hinkel, M. Marinak, D. Munro, O. Jones, S. Pollaine, and L. Suter (Lawrence Livermore National Laboratory)

Pillars of Creation (C23)

J. O. Kane, D. D. Ryutov, B. A. Remington, S. G. Glendinning, J. Nash, M. Pound, and D. Arnett (Lawrence Livermore National Laboratory, University of Maryland, and University of Arizona) 
Computational Modeling of Two-Shell Cylindrical Implosions with Mix (C28)

K. W. Parker, A. M. Dunne, S. Rothman, D. Youngs, C. Barnes, S. H. Batha,

N. E. Lanier, G. R. Magelssen, T. J. Murphy, and J. M. Scott (Atomic

Weapons Establishment and Los Alamos National Laboratory)

Influence of Turbulent Mixing Zone on Growth of Local Perturbation in

Environments of Rayleigh-Taylor Instability (Numerical Simulation) (C30)

V. A. Raevski, A. N. Sinitsina, and Yu. V. Yanilkin (Russian Federal Nuclear Center-VNIIEF)

A Statistical Comparison of Gas Cylinder Experiments with Their Simulation (C31)

W. J. Rider, J. R. Kamm, and C. A. Zoldi (Los Alamos National Laboratory)

A Mix-Model for One-Dimensional Simulations of Laser-Driven Implosion Experiments (C36)

D. Souffland and F. Renaud (Commissariat à l'Energie Atomique)

Turbulent Diffusion in Solar Type Star (C39)

N. Toqué (University of Montreal)

Recent Computational Simulations of Rayleigh-Taylor Mix Layer Growth with a Multi-Fluid Model (C40)

E. Vold (Los Alamos National Laboratory)

An Efficient and High Resolution Solver for the Two-Dimensional Numerical Simulation of the Richtmyer-Meshkov Instability (C41) S. P. Wang, M. H. Anderson, J. G. Oakley, and R. Bonazza (University of Wisconsin, Madison)

ALE Simulations of Turbulent Rayleigh-Taylor Instability in 2-D and 3-D (C42) S. V. Weber, G. Dimonte, and M. M. Marinak (Lawrence Livermore National Laboratory)

Preliminary Results of DNS and LES Simulations of Self-Similar Variable Acceleration RT-Mixing Flows (C47)

D. L. Youngs, X. Silvani, J. Magnaudet, and A. Llor (Atomic Weapons Establishment, Institute de Mécanique des Fluides de Toulouse, and Commissariat à l'Energie Atomique)

Rapid Turbulization Arising from Vortex Double Layers in Interactions of "Complex" Blast Waves and Cylindrical and Spherical Bubbles (C49)

S. Zhang, Y.-G. Kang, K. Nishihara, N. J. Zabusky, and H. Kim (Rutgers

University and Kwangju Institute of Science and Technology) 
Turbulent Flow Simulations of Two Fluids Moving with Different Laws of Acceleration (C51)

V. I. Kozlov, A. N. Razin, and I. V. Saphzhnikov (Russian Federal Nuclear Center - VNIIEF)

The Behavior of Velocity Variance Resulting From Turbulent Mixing Zone-Shock Interaction (C52)

V. I. Kozlov and A. N. Razin (Russian Federal Nuclear Center-VNIIEF)

An Assessment of Multi-Velocity Versus Single Velocity in a Multi-Component Model of Turbulent Mixing (C53)

D. Eliason, W. Cabot, and Y. Zhou (Lawrence Livermore National Laboratory)

Mixing Due to the Rayleigh-Taylor Instability (C55)

A. M. Dimits (Lawrence Livermore National Laboratory)

CALE Simulation of Richtmyer-Meshkov Experiments at High Mach Number (C57) A. Miles, J. Edwards, and S. G. Glendinning (Lawrence Livermore National Laboratory and University of Michigan) 


\section{Theoretical Posters}

Nonlinear Asymptotic Solutions to RT and RM Problems for Fluids with Close Densities (T2)

S. I. Abarzhi (State University of New York at Stony Brook)

Turbulent Mixing in RTI as Order-Disorder Process (T3)

S. I. Abarzhi (State University of New York at Stony Brook)

ANew Turbulent Two-Fluid RANS Model for KH, RT, and RM Mixing Layers (T4) P. Bailly and A. Llor (Commissariat à l'Energie Atomique)

Super-Exponential Rayleigh-Taylor Flow (T5)

R. E. Breidenthal (University of Washington)

A General Buoyancy-Drag Model for the Evolution of the Rayleigh-Taylor and Richtmyer-Meshkov Instabilities (T11)

Y. Elbaz, Y. Srebro, O. Sadot, and D. Shvarts (Ben Gurion University and Nuclear Research Center)

Stability of Diverging Shock Waves (T15)

V. M. Ktitorov (Russian Federal Nuclear Center - VNIIEF)

Stability of Reflected From the Center Self-Similar Converging Shock Wave (T16) V. M. Ktitorov (Russian Federal Nuclear Center - VNIIEF)

Using a Turbulence Transport Approach to Study Shocks Through Polycrystalline Metal (T17)

R. R. Linn and F. H. Harlow (Los Alamos National Laboratory)

Statistical Mechanics Large Scale Model for the Evolution of the Multi-Mode Kelvin Helmholtz Instability (T22)

A. Rikanati, U. Alon, and D. Shvarts (Nuclear Research Center, Ben-Gurion University, and Weizmann Institute of Science)

Compressible MHD Turbulence in Strongly Radiating Molecular Clouds in Astrophysics (T25)

D. D. Ryutov and B. A. Remington (Lawrence Livermore National Laboratory)

Single-Velocity, Multi-Component Turbulent Transport Models for Interfacial Instability-Driven Flows (T26)

O. Schilling (Lawrence Livermore National Laboratory) 
Analytic Nonlinear Growth of a Single-Mode Richtmyer-Meshkov Instability (T31)

M. Vandenboomgaerde (Commissariat à l'Energie Atomique)

Pattern Detection, Compression and Denoising of RT Mix Data Using Discrete Wavelet Transform (T39)

B. B. Afeyan, P. Ramaprabhu, and M. J. Andrews (PolyMath Research Incorporated and Texas $A \& M$ University) 


\section{Numerical simulation of initial perturbation growth in oblique impact of metal plates \\ Bakhrakh S.M., Volodina N.A., Nizovtsev P.N., Spiridonov V.F., Shuvalova E.V. VNIIEF-RUSSIA}

\section{Introduction}

An oblique impact of metal layers results in development of intensive shear strains at their interface, the near-boundary metal layers become severely heated and produce shaped jets. These effects lead to the interface profile distortion. Both regular and asymmetric distorted waves appear. In this case a hydrodynamic instability, the Kelvin-Helmholtz instability, appears [1].

The subsonic regime of the oblique impact has been comprehensively studied recently, where $U_{c}<C_{s}\left(U_{c}\right.$ - contact point velocity, $C_{s}$ - sound speed in the material). Under these loading conditions, a shaped jet forms at the contact point, if the pressure about the impact point is higher than the metal strength.

The initial perturbation growth is known to occur in the subsonic impact conditions in the gas dynamics approximation. The situation is more complex in the supersonic impact of strong plates.

When the impact angle is constant, there is critical velocity $\mathrm{U}_{\mathrm{cr}}$. If $\mathrm{C}_{\mathrm{s}}<\mathrm{U}_{\mathrm{c}}<\mathrm{U}_{\mathrm{cr}}$, detached oblique shock waves form in the flow. During the transition across the wave front the flow transforms from supersonic to subsonic. In the impact zone the shaped jet forms and the perturbations grow like in the case of the subsonic impact.

When $U_{c}>U_{c r}$, attached oblique shock waves are established at the contact point and no jet formation is observed. The perturbation evolution is considered as impossible in this case, as there is no principal perturbation generator, i.e. the shaped jet.

The material interface state in such supersonic loading conditions has been inadequately studied. It was anticipated that essentially prompt cessation of the perturbation evolution process would be obtained in the transition to the jet-free conditions of the plate impact. That is, as soon as $\mathrm{U}_{\mathrm{c}}=\mathrm{U}_{\mathrm{cr}}$, no peturbations should appear. However, the perturbation growth was observed in the experiments. Ref. [2] analyzes the experimental data and explains this phenomenon theoretically.

The direct numerical simulation of the experiments is of interest.

\section{Numerical codes. Setting up problems}

The numerical simulations were performed with the program complex that implements code LEGAK [3].

The strength properties are included using code [4].

The material state was described in the elastic-plastic approximation. Pressure was estimated by Mie-Grueneisen equation of state [5] with the following parameters:

$$
\rho_{0}=2.64 \frac{\mathrm{g}}{\mathrm{cm}^{3}}, c_{0}=5.55 \frac{\mathrm{km}}{\mathrm{s}}, \boldsymbol{n}=\mathbf{3 . 2}, \boldsymbol{\Gamma}=\mathbf{2 . 1 4} \text {. }
$$


Yield strength was taken as a function of pressure and temperature:

$$
\boldsymbol{Y}=\boldsymbol{Y}_{\mathbf{0}}(\mathbf{1}+\alpha \cdot \boldsymbol{P})\left(\mathbf{1}-\beta \cdot \frac{\boldsymbol{E}_{\boldsymbol{T}}}{\boldsymbol{E}_{\boldsymbol{m}}}\right),
$$

where $\boldsymbol{Y}_{\mathbf{0}}=\mathbf{0 . 3}, \alpha=\frac{\mathbf{1}}{\mathbf{3}}, \beta=\mathbf{1}, \boldsymbol{E}_{T}$ is heat energy, $E_{m}=1.1 \frac{\mathrm{kJ}}{\mathrm{g}}$ is melting energy, $\vartheta=\mathbf{0 . 3 3}$ is Poisson ratio.

The problem geometry is presented in Fig.1. Impact of two aluminum plates at an angle of 14 degrees was simulated. The perturbation of $\lambda_{0}=0.5 \mathrm{~mm}$ wavelength and $a_{0}=0.01 \mathrm{~mm}$ amplitude was given on the surface of the lower rod (Fig.2).

The computational grid is depicted in Fig.3.

Domain 1: impacting plate of $4 \mathrm{~mm}$ thickness.

Domain 2: immovable plate of $15 \mathrm{~mm}$ thickness, on the external boundary of which initial perturbation $a=a_{0} \cdot \sin \frac{2 \pi}{\lambda_{0}} x$ is given.

Domain 3: vacuum.

During the computation the grid remains immovable, fixed. The material moves relative to the immovable computational grid. This allows computations with severe interface strains.

To follow the evolution of the interface between the upper and lower aluminum rods, they were marked with different marks, to be more exact, as "different materials" with identical physical properties. Hence, if material from the upper and lower plates gets into the same computational cell, it is possible to determine the plate interface position by the material concentrations and follow the perturbation evolution.

The computational grid was taken such, that there were on the order of 20 computational cells per wavelength. The total number of the points is $1120 \times 740=828800$. The computations in the scalar (one-processor) regime are problematic. So the computations were performed on the shared memory multi-processor computer with complex LEGAK-MP [6]. 


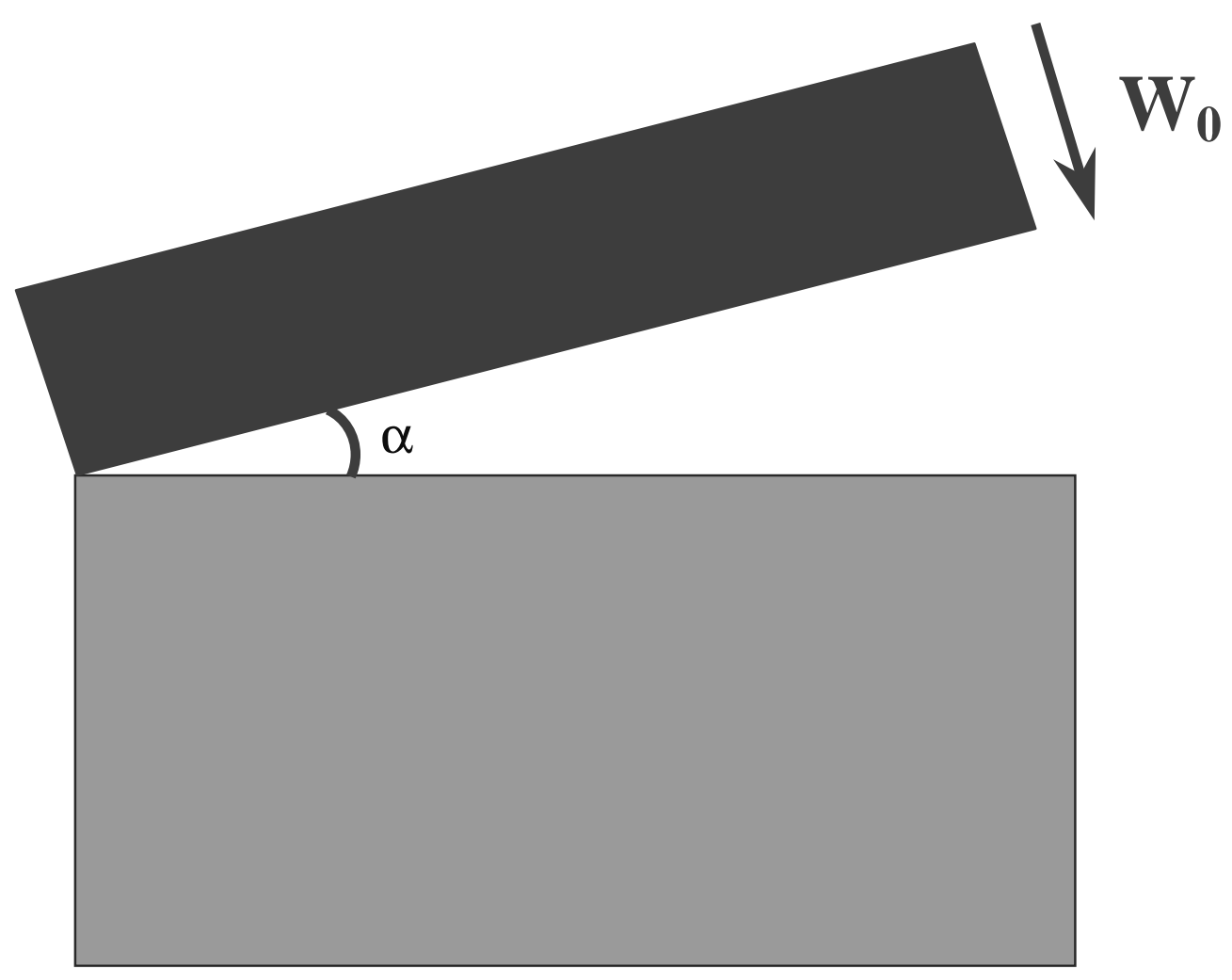

Fig.1 Problem geometry

Time $=0.000000 \mathrm{e}+000$

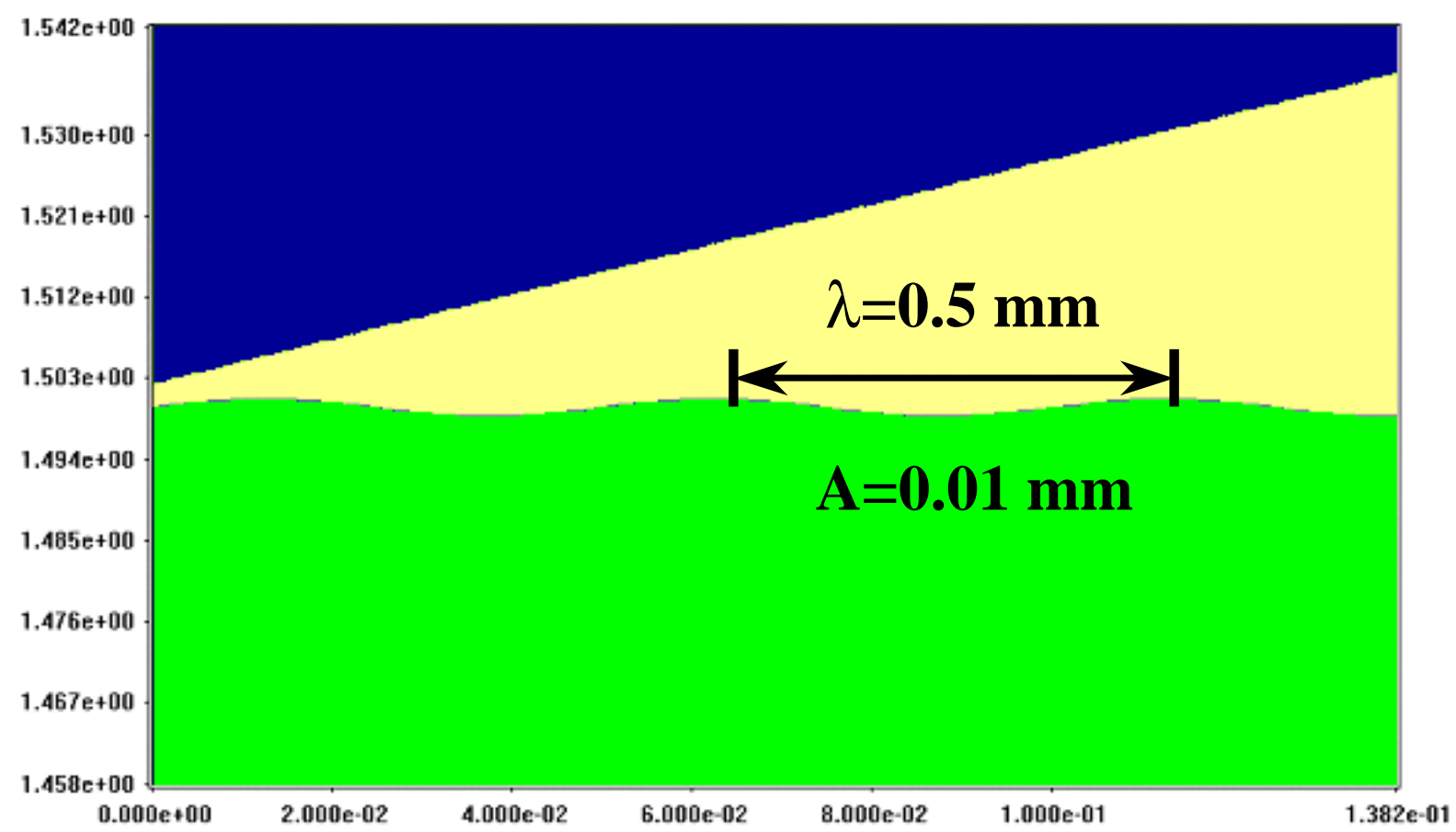

Fig.2 Initial perturbation 
Time $=0.000000 \mathrm{e}+000$

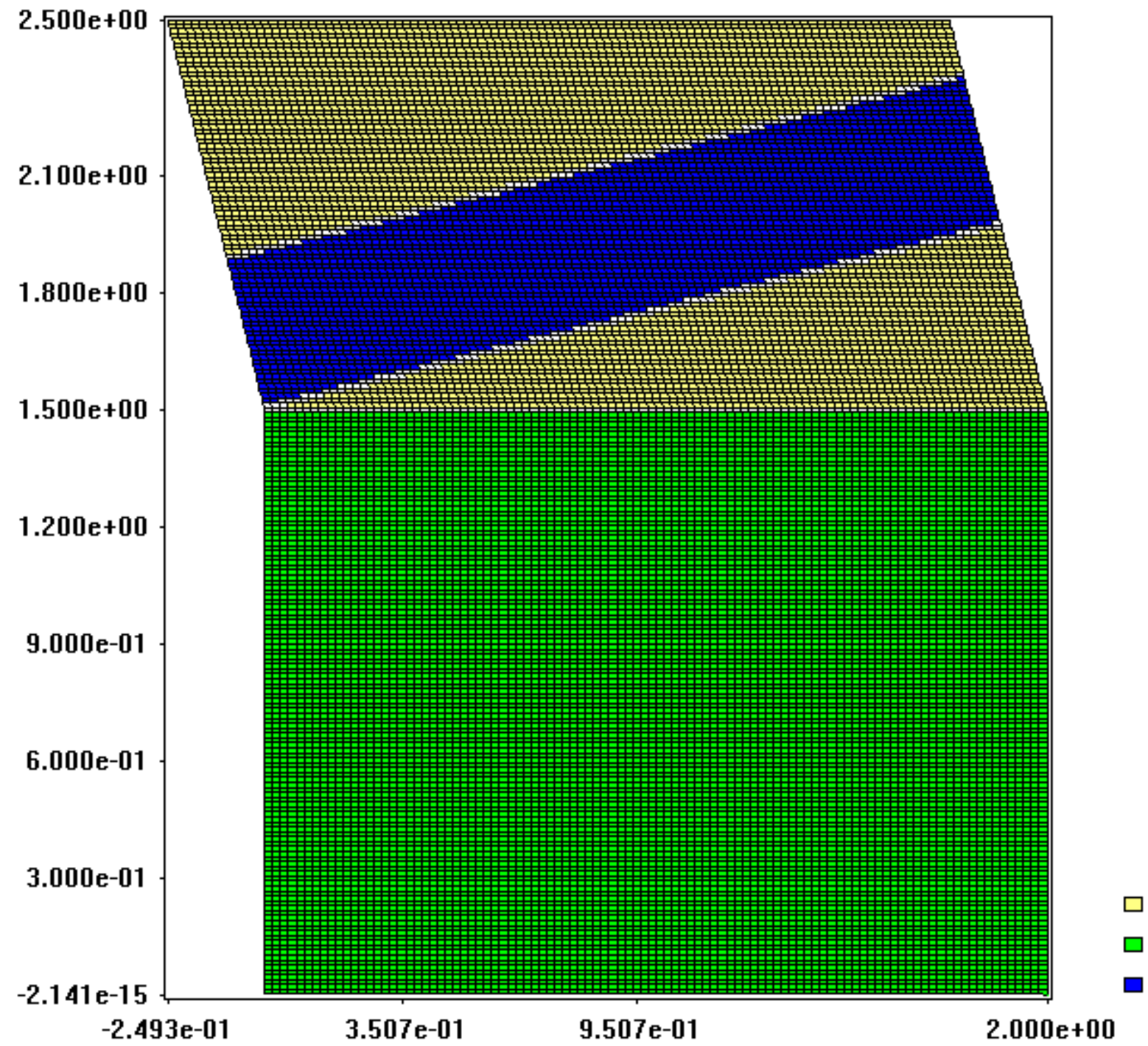

Fig.3 Computational grid (Eulerian computation)

At the initial study phase, the computations were performed in the quasiLagrangian formulation. In these computations, both the upper (launched) and lower plates were considered as separate regions, whose boundaries were Lagrangian lines, between which there was no interaction. In the areas of the plates, the distances between which became less than $\mathbf{0 . 1 h}$ (where $\boldsymbol{h}$ is the computational grid size) during the motion, their boundary segments were integrated, thereby the inter-plate interaction was given. A number of computations were conducted in this formulation. The computed data for early times are in a good agreement with the computations on the Eulerian grid. However, because of the grid distortion (due to the complex flow) and other computational "phenomena" it was impossible to perform the computations up to the times of our concern. For this reason our choice was in favor of the computations on the immovable Eulerian computational grid. 


\section{Results of the computations}

Several computational series were conducted. The following was varied in the computations:

- plate impact velocity $\boldsymbol{W}_{\boldsymbol{0}}$;

- perturbation wavelength $\lambda_{0}$;

- material properties:

○ gas-dynamic approximation;

○ elastic-plastic approximation with different medium parameters:

- variations of strengthening $\alpha$,

- variations of yield strength $\boldsymbol{Y}_{\boldsymbol{0}}$,

- variations of melting heat $\boldsymbol{E}_{\boldsymbol{m}}$,

- determination of $\boldsymbol{E}_{\boldsymbol{m}}$ according to the Lindemann model.

It is of interest to perform the computations including the elastic stress relaxation. This model is being introduced to the program complex LEGAK, and we are planning the studies at the next phase of the work. We are inspired by the fact that the relaxation models most adequately describe material shear strain that is responsible for the perturbation growth in the oblique impact of plates.

\subsection{Impact velocity effect}

The impact velocity was varied in the computations.

The results for all the computations are presented for the time, when the contact point has traveled a distance of $\boldsymbol{L}=\mathbf{2 0} \mathbf{m m}$.

The computations were conducted with strengthening factor $\alpha=\frac{1}{3}$, melting heat $E_{m}=1.1 \frac{\mathrm{kJ}}{\mathrm{g}}$, and perturbation wavelength $\lambda_{0}=0.5 \mathrm{~mm}$.

The computed amplitude increase factors are summarized in Table 1.

Table 1

\begin{tabular}{|c|c|}
\hline Initial velocity $W_{0}, \mathrm{~km} / \mathrm{s}$ & $\begin{array}{l}\text { Amplitude increase } \\
\text { factor } \\
\boldsymbol{a} / \boldsymbol{a}_{\mathbf{0}}\end{array}$ \\
\hline 1.5 & 6 \\
\hline 1.75 & 2.5 \\
\hline 2 & 1 \\
\hline
\end{tabular}

The perturbation evolution pattern for $\mathrm{W}_{0}=1.5$ and $1.75 \mathrm{~km} / \mathrm{s}$ is presented in Figs. 8 and 4.

Thus, the direct numerical simulation reproduces the experimentally detected effect of the perturbation increase. 
Time $=2.600300 \mathrm{e}-001$

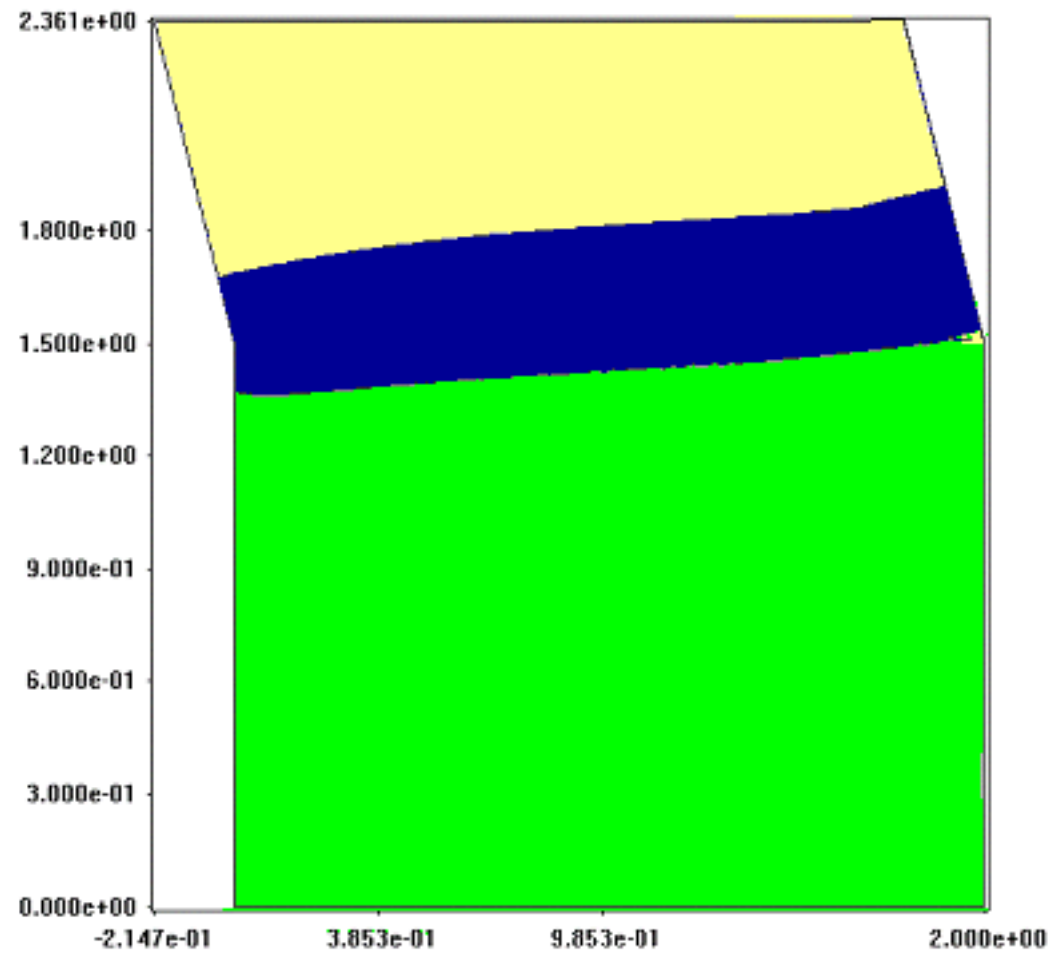

Time $=2.800212 \mathrm{e}-001$

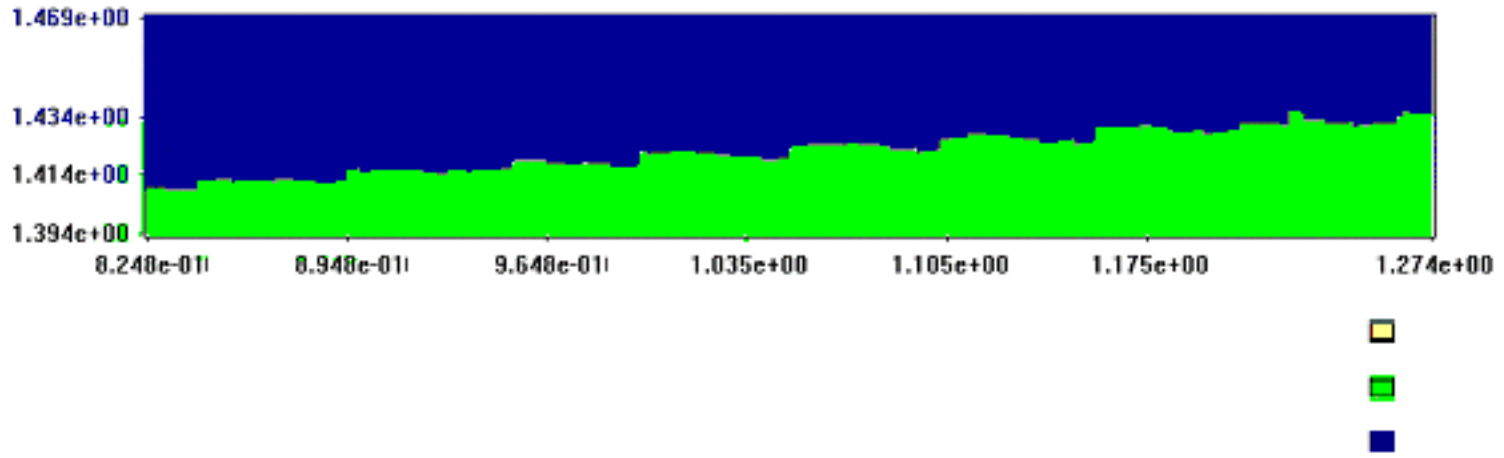

Time $=2.800212 \mathrm{e}-001$

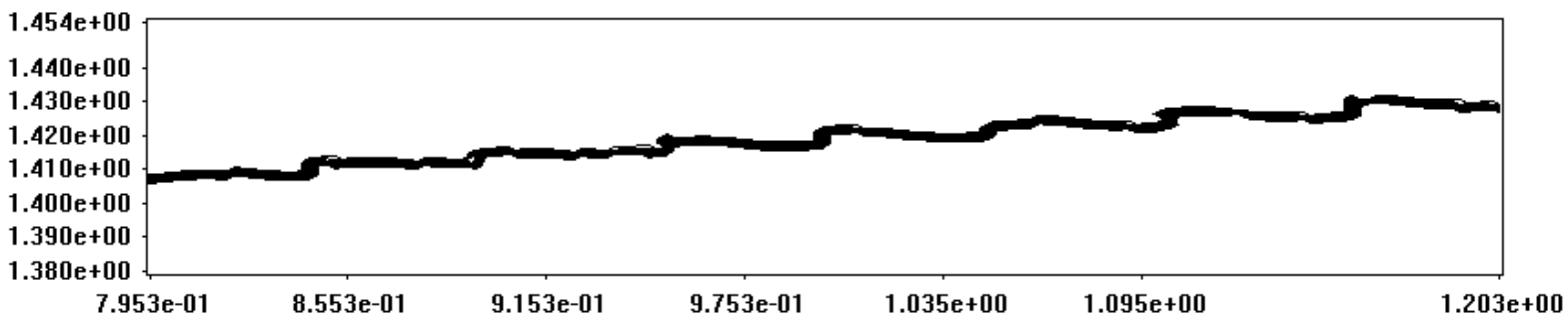

Fig.4 Geometry and isolines (UP $\alpha=1 / 3, U_{0}=1.75, \lambda_{0}=0.5 \mathrm{~mm}$ ) 


\subsection{Simulation of metal interface instability with change in strength properties}

The material strength properties were varied in the computations.

The computations were conducted with initial velocity $\mathrm{W}_{0}=1.5 \mathrm{~km} / \mathrm{s}$, melting heat $E_{m}=1.1 \frac{\mathrm{kJ}}{\mathrm{g}}$, and perturbation wavelength $\lambda_{0}=0.5 \mathrm{~mm}$.

The perturbation growth pattern is depicted in Figs. 5-8. The material strength effect on the perturbation growth is summarized in Table 2.

\section{Table 2}

\begin{tabular}{|c|c|}
\hline Material model & $\begin{array}{c}\text { Amplitude increase } \\
\text { factor } \\
\boldsymbol{a} / \boldsymbol{a}_{\mathbf{0}}\end{array}$ \\
\hline Gas dynamics & 93 \\
\hline $\begin{array}{l}\text { Elasticity-plasticity } \\
\qquad \alpha=\mathbf{0}\end{array}$ & 65 \\
\hline $\begin{array}{c}\text { Elasticity-plasticity } \\
\alpha=\mathbf{0 . 1}\end{array}$ & 53 \\
\hline $\begin{array}{l}\text { Elasticity-plasticity } \\
\qquad \alpha=\mathbf{1} / \mathbf{3}\end{array}$ & 6 \\
\hline $\begin{array}{l}\text { Elasticity-plasticity } \\
\qquad \alpha=\mathbf{2}\end{array}$ & 2 \\
\hline $\begin{array}{c}\text { Elasticity-plasticity } \\
\qquad \alpha=1\end{array}$ & 1 \\
\hline
\end{tabular}

\subsection{Simulation of metal interface instability with different values of melting heat}

The value of melting heat was varied in the computations.

The computations were conducted with initial velocity $\mathrm{W}_{0}=1.5 \mathrm{~km} / \mathrm{s}$, strengthening factor $\alpha=\frac{\mathbf{1}}{\mathbf{3}}$, and perturbation wavelength $\lambda_{0}=0.5 \mathrm{~mm}$.

The effect of different melting heat values on the perturbaton growth is summarized in Table 3. 
Table 3

\begin{tabular}{|c|c|}
\hline $\begin{array}{c}\text { Melting heat } \\
\boldsymbol{E}_{\boldsymbol{m}}\left(\frac{\boldsymbol{\kappa} \text { Дж}}{2}\right)\end{array}$ & $\begin{array}{c}\text { Amplitude increase } \\
\text { factor } \\
\boldsymbol{a} / \boldsymbol{a}_{\mathbf{0}}\end{array}$ \\
\hline 0.4 & 29 \\
\hline 1.1 & 6 \\
\hline 1.5 & 2 \\
\hline 2.0 & 1 \\
\hline
\end{tabular}

\subsection{Simulation of metal interface instability with different initial perturbation wavelengths}

The computations were conducted in the elastic-plastic approximation $\left(\boldsymbol{Y}_{\mathbf{0}}=\mathbf{0 . 3}\right.$, $\left.\alpha=\frac{\mathbf{1}}{\mathbf{3}}, \beta=\mathbf{1}\right)$ for initial velocity $\mathrm{W}_{0}=1.5 \mathrm{~km} / \mathrm{s}$ with different initial perturbation wavelengths.

The results are summarized in Table 4.

Table 4

\begin{tabular}{|c|c|}
\hline \multirow{2}{*}{ Initial wavelength $(\mathbf{m m})$} & $\begin{array}{l}\text { Amplitude increase } \\
\text { factor }\end{array}$ \\
\hline & $a / a_{0}$ \\
\hline 0.25 & 1 \\
\hline 0.5 & 6 \\
\hline 1.0 & 1 \\
\hline
\end{tabular}

Thus, the $\sim 0.5$-mm-wavelength perturbations grow most intensively under the discussed conditions. This can explain the fact that among the whole spectrum of the perturbations that take place on "smooth" plates by virtue of their machining it is $0.5 \div 0.6-\mathrm{mm}$-wavelength perturbations that grow and are observed in the experiments.

\subsection{Change in perturbation wavelength}

The computations revealed an interesting fact of increase in the initial perturbation wavelength. The computed data for initial velocity $\mathrm{W}_{0}=1.5 \mathrm{~km} / \mathrm{s}$ and wavelength $\lambda_{0}=0.05 \mathrm{~cm}$ are presented in Table 5 . 
Time $=2.600246 \mathrm{e}-001$

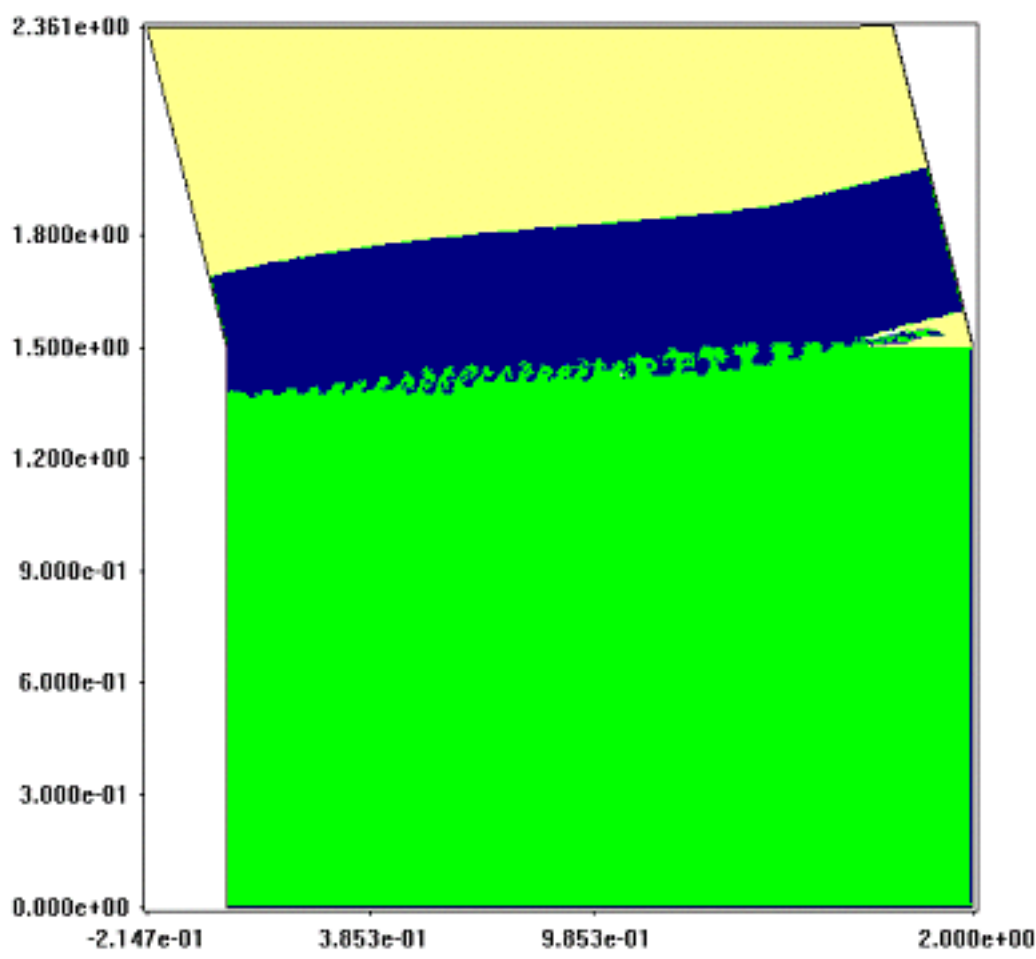

Time * z.BbnzTse-no1

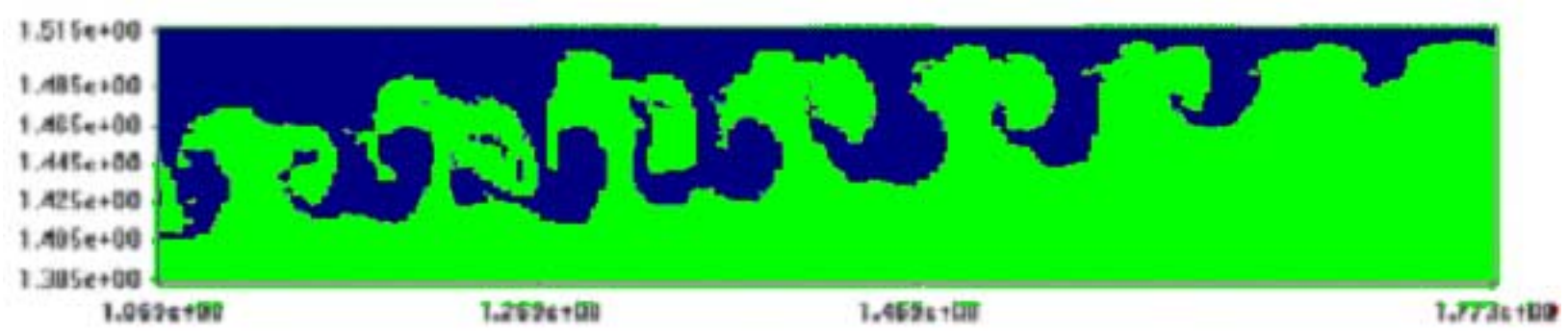

Time $=2.800275 \mathrm{e}-001$

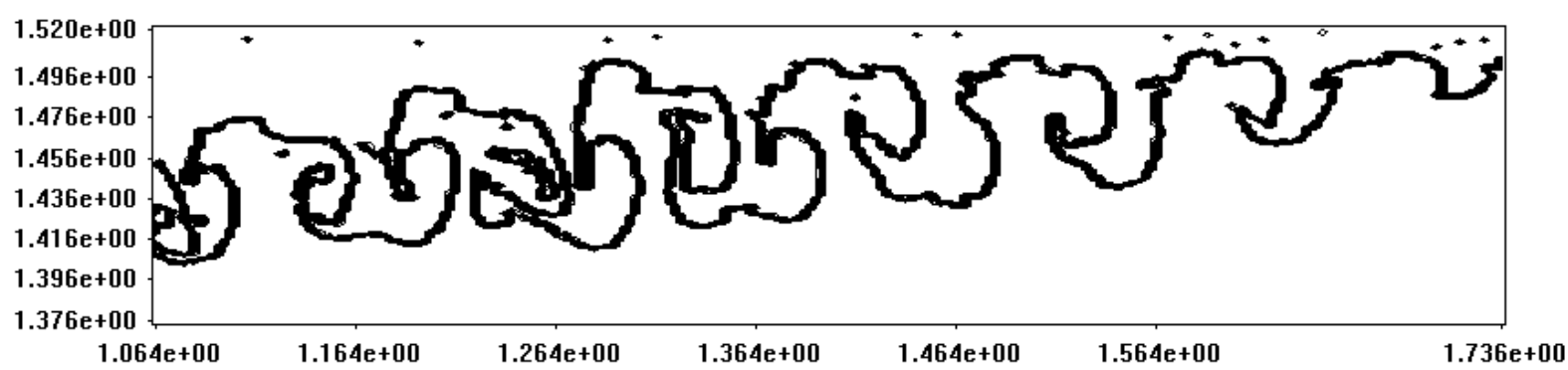

Fig.5 Geometry and isolines $\left(G D, U_{0}=1.5, \lambda_{0}=0.5 \mathrm{~mm}\right)$ 
Time $=2.793961 \mathrm{e}-001$

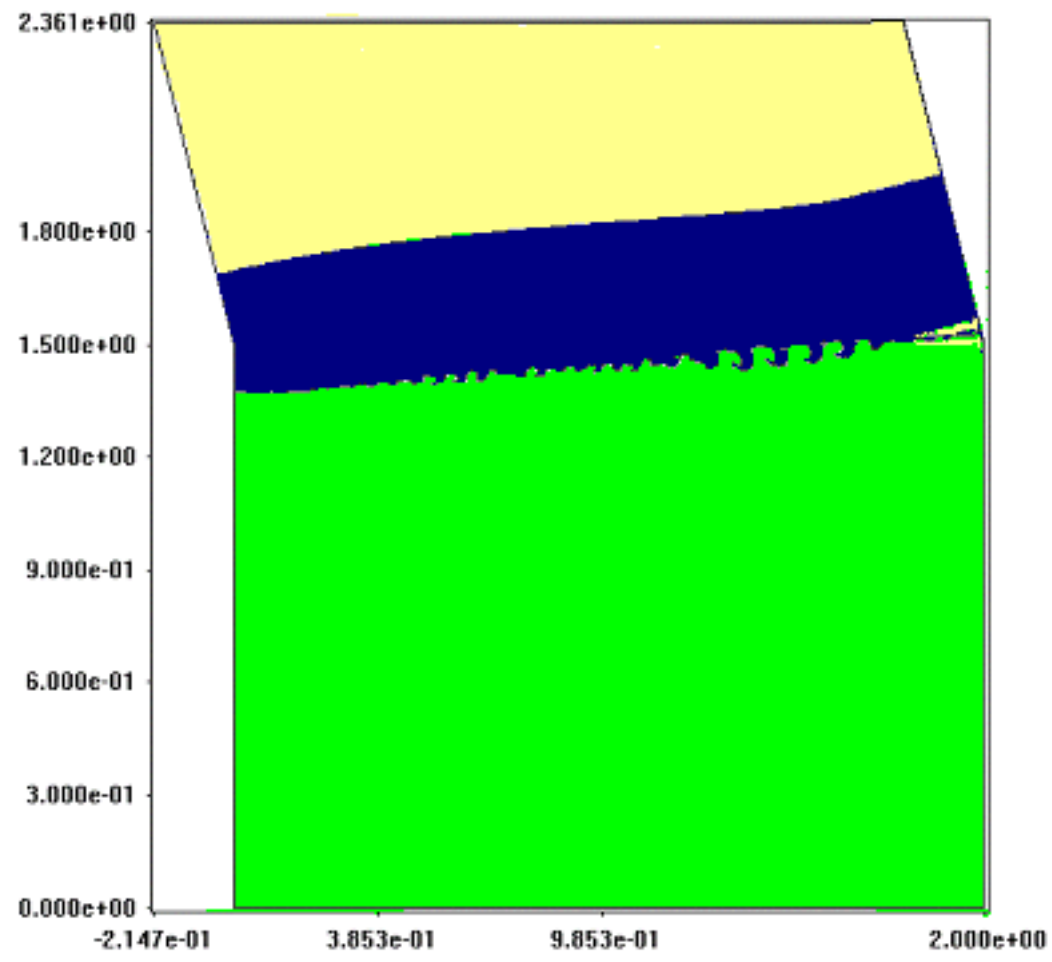

Time $=2.793961 \mathrm{e}-001$
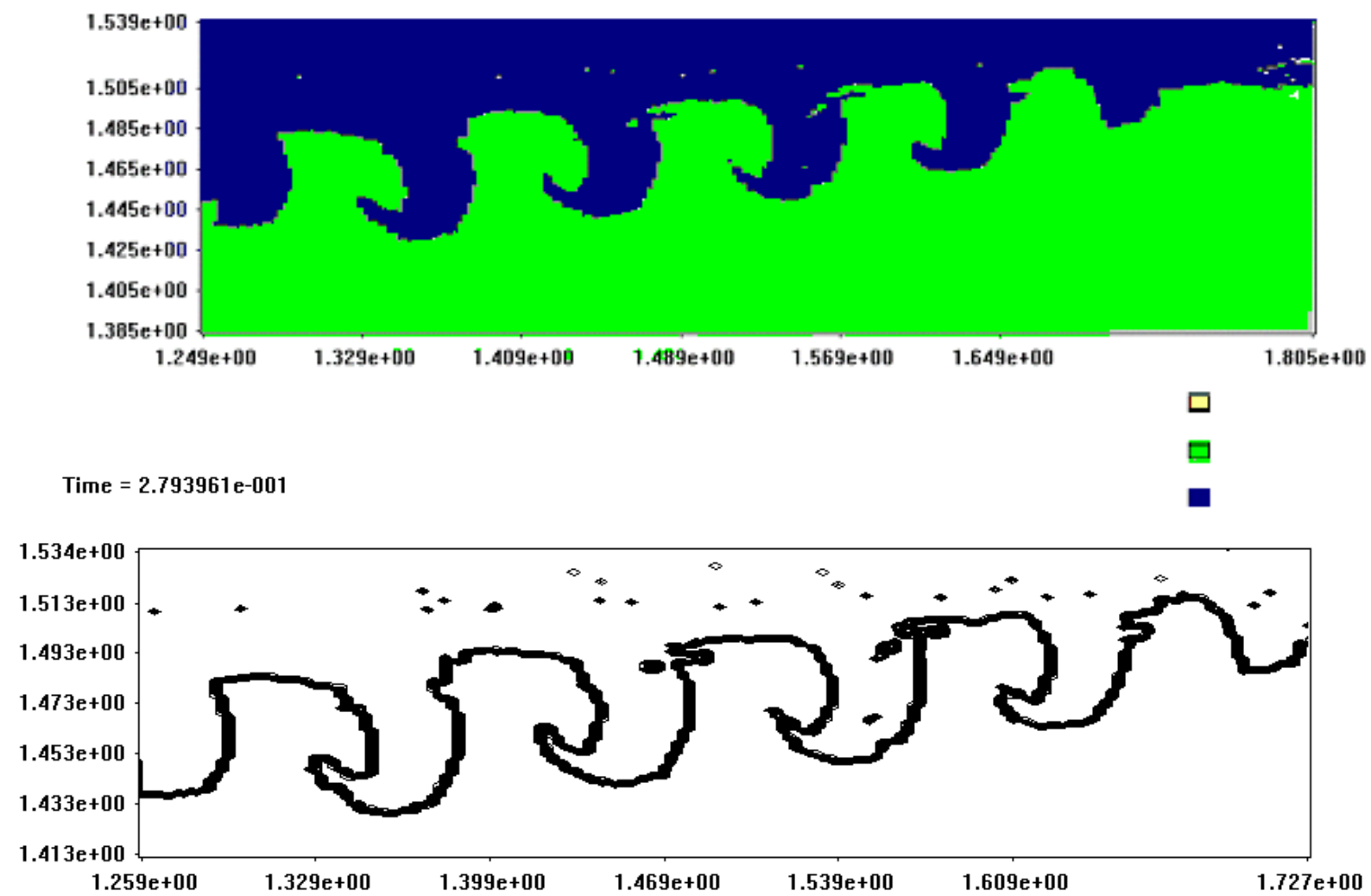

Fig.6 Geometry and isolines $\left(U P \alpha=0, U_{0}=1.5, \lambda_{0}=0.5 \mathrm{~mm}\right)$ 
Time $=2.800099 \mathrm{e}-001$

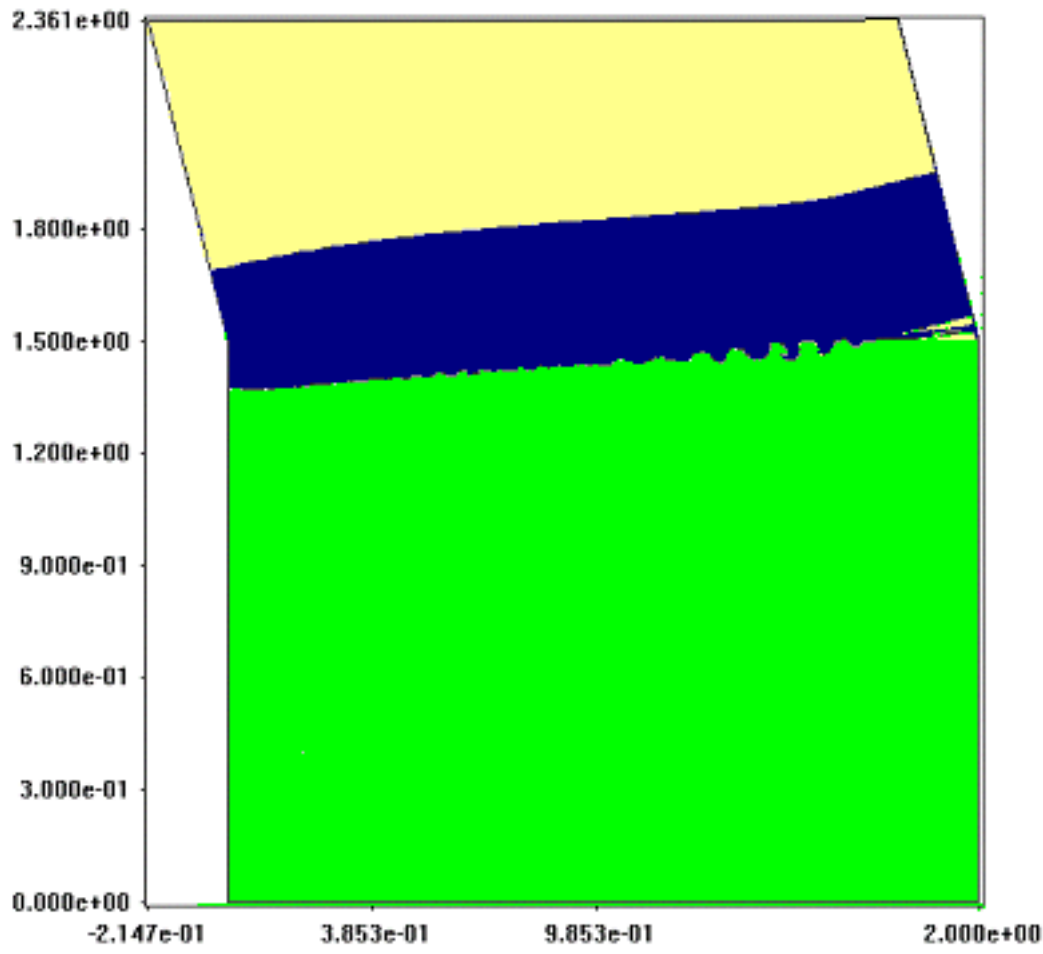

Time $=3.000110 \mathrm{e}-001$

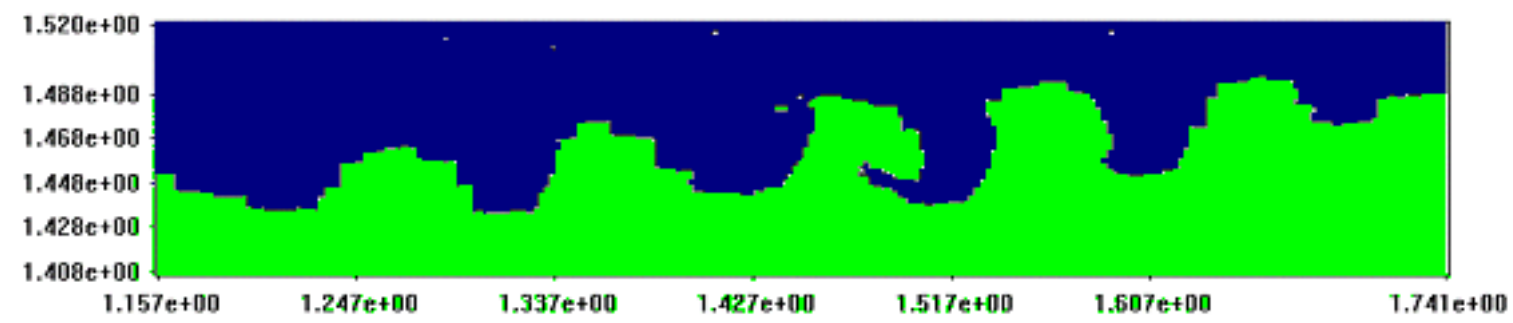

Time $=3.000110 \mathrm{e}-001$

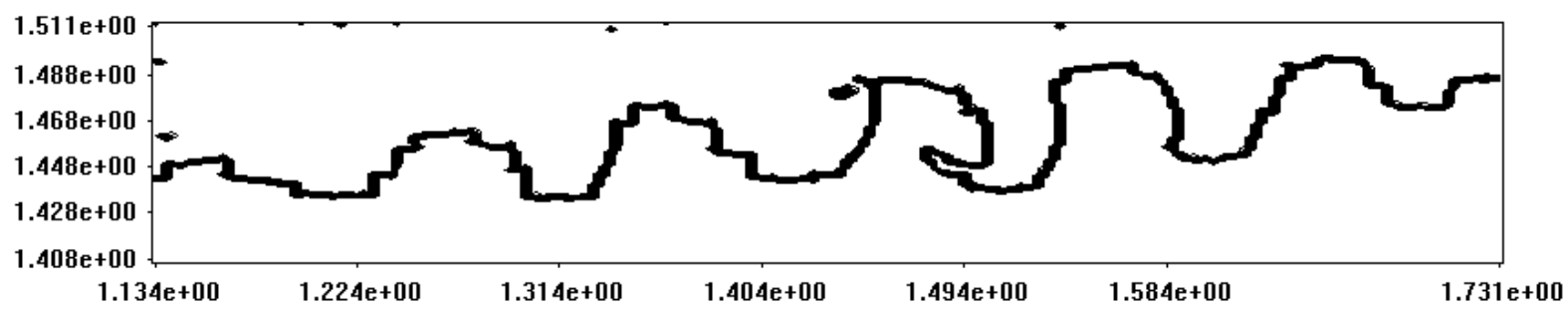

Fig.7 Geometry and isolines ( $\left.U P \alpha=0.1, U_{0}=1.5, \lambda_{0}=0.5 \mathrm{~mm}\right)$ 
Time $=2.800099 \mathrm{e}-00$

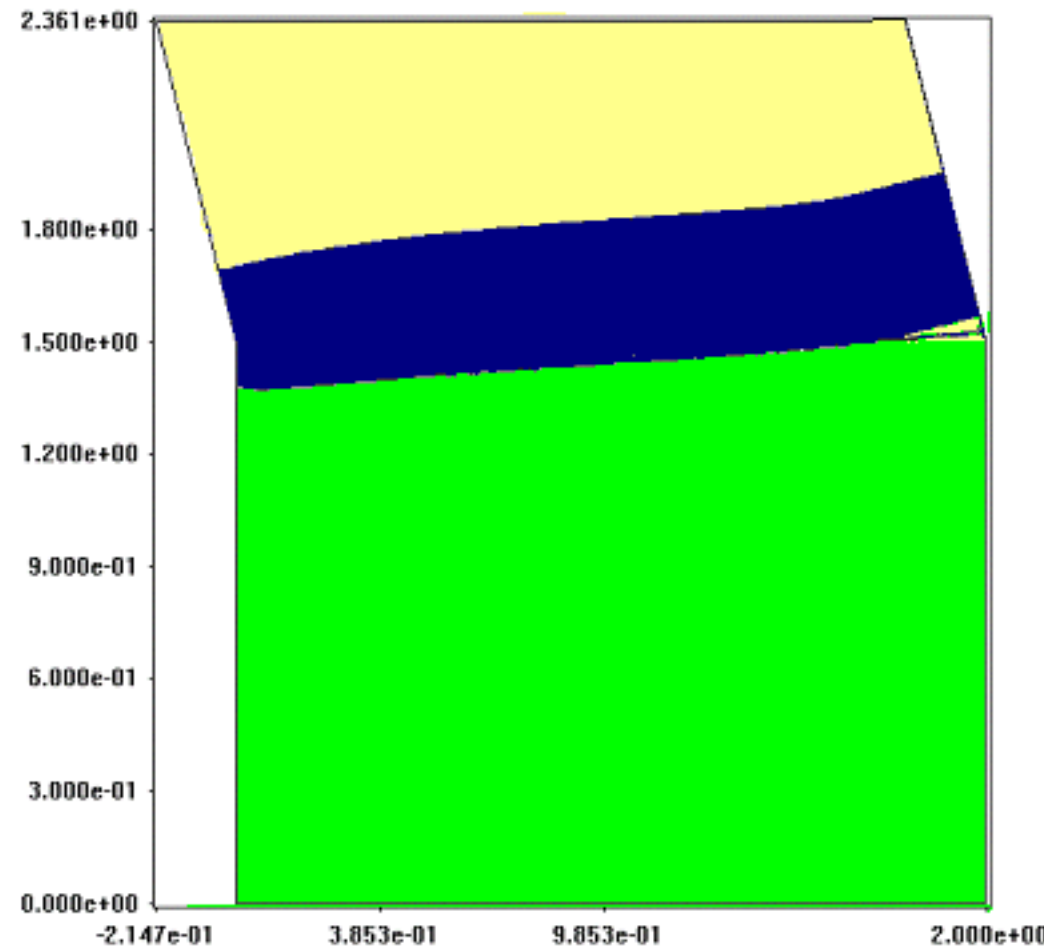

Time $=3.000177 \mathrm{e}-001$

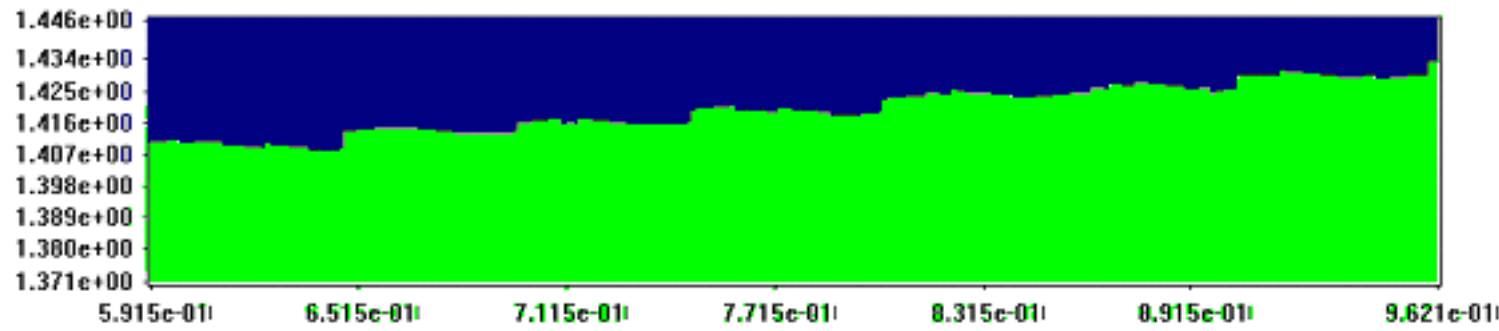

Time $=3.000177 \mathrm{e}-001$

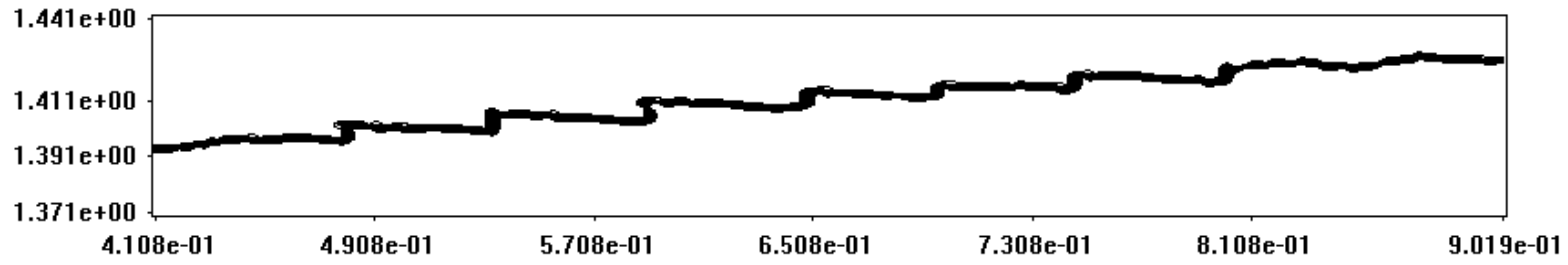

Fig.8 Geometry and isolines $\left(U P \alpha=1 / 3, U_{0}=1.5, \lambda_{0}=0.5 \mathrm{~mm}\right)$ 
Table 5

\begin{tabular}{|c|c|}
\hline Material model & $\begin{array}{c}\text { Final wavelength } \\
\lambda(\mathbf{m m})\end{array}$ \\
\hline Gas dynamics & 1 \\
\hline $\begin{array}{c}\text { Elasticity-plasticity } \\
\alpha=\mathbf{0}\end{array}$ & 0.9 \\
\hline $\begin{array}{c}\text { Elasticity-plasticity } \\
\boldsymbol{\alpha}=\mathbf{0 . 1}\end{array}$ & 0.8 \\
\hline $\begin{array}{c}\text { Elasticity-plasticity } \\
\boldsymbol{\alpha}=\mathbf{1} / \mathbf{3}\end{array}$ & 0.5 \\
\hline
\end{tabular}

Whereas the wavelength increases by a factor of about 2 in the gas-dynamic approximation, it remains essentially unchanged in strong medium. This phenomenon requires more comprehensive study, both numerical and experimental.

\section{Conclusion}

The computed data for the oblique plate impact agrees with the experimental data. In particular, it is numerically corroborated that the perturbations of wavelength $\lambda=0.5 \mathrm{~mm}$ grow most intensively. The numerical simulation revealed the effect of the wavelength growth in low-strength material, which needs more comprehensive study, both experimental and computational.

The work was carried out under the support by Russia Fundamental Research Foundation, Grant 99-01-00812 and 02-01-00796, and LNLN/VNIIEF Agreement B512135. 


\section{References}

1. Deribas A.A. Strengthening and explosive welding physics. Novosibirsk, Nauka Publishers. 1980.

2. Drennov O.B., Mikhailov A.L., Nizovtsev P.N., Rayevsky V.A. Perturbation evolution at metal interface in oblique impact with contact point moving at supersonic velocity. VANT. Ser. Teoreticheskaya i Prikladnaya Fizika (to be published).

3. Bakhrakh S.M., Spiridonov V.F., Shanin A.A. A method for heterogeneous medium gas-dynamic flow computations in Lagrangian-Eulerian coordinates// DAN SSSR, 1984, V. 278, No. 4, pp. 829-833.

4. Bakhrakh S.M., Kovalev N.P., Pavlusha I.N. A method for elastic-plastic flow computations// Proceedings of All-Union Conference on Numerical Methods of Elasticity and Plasticity Theory. Part I. Novosibirsk, Nauka Publishers. 1974, pp.22-36.

5. Zeldovich Ya.B., Raizer Yu.P. Physics of shock waves and high-temperature hydrodynamic phenomena. Moscow, Fizmatgiz Publishers, 1963, 632p.

6. Avdeyev P.A., Artamonov M.V., Bakhrakh S.M., Velichko S.V., Volodina N.A., Vorobyeva N.M., Yegorshin S.P., Yesayeva E.N., Kovaleva A.D., Luchinin M.V., Pronevich S.N., Spiridonov V.F., Taradai I.Yu., Tarasova A.N., Shuvalova E.V. Program complex LEGAK and principles of its parallelization on multiprocessor computers// VANT. Ser. Matematicheskoye Modelirovaniye Fizicheskikh Protsessov (to be published). 


\title{
APPLICATION OF KE-MODEL FOR THE DESCRIPTION OF AN ATMOSPHERIC SURFACE LAYER
}

\author{
Anuchin M.G., Neuvazhayev V.E., Parshukov I.E. \\ (RFNC-VNIITF, Snezhinsk, Chelyabinsk region)
}

The problem on determination of non-dimensional characteristics of turbulent flow in atmospheric

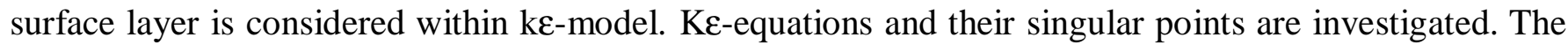
mathematical program for calculations of characteristics of turbulent flow in surface atmospheric layer is developed. From the set of integral curves those curves are chosen which correspond to the solution of formulated task and ensure the satisfactory experiments description. Here the basic model constants are chosen according to the conventional criteria. At the same it is shown that the parameter $C_{\theta}$ responding to convection source term of an $\varepsilon$-equation should be chosen depending on stability conditions. The best agreement with experimental results is reached if $C_{\theta}=0$ for steady stratification and $C_{\theta} \neq 0$ for unstable stratification. By a numerical choice of value $C_{\theta}$ and factor of turbulent diffusion $\hat{\alpha}$ the quite satisfactory description of experimental observations known as analytical interpolar dependencies is received.

\section{Introduction}

Semiempirical $K \varepsilon$ models of turbulence are widely used for the description of different classes of turbulent flows [1-3] (jet streams and channels streams, gravitational turbulent mixing of fluid layers with different densities and others).

$K \varepsilon$ model was used for solving of the micrometeorology problems as well. In [4,5] the calculations of universal profile functions of atmosphere surface layer were conducted. On the basis of $K \varepsilon$ model the technique of calculation reconstruction of vertical profiles of meteorological values in the atmosphere boundary layer was developed according to the data of standard surface meteorological measurements [6,7].

In the current paper we are also analyzing the problem about atmosphere surface layer (ASL) within the frameworks of $K \varepsilon$ model. ASL is an example of the stratified flow, which is interested by the fact that it includes simultaneously both shear and convective (gravitational) mechanisms of turbulence generation. Moreover, the profile characteristics of stationary ASL have been studied experimentally quite well up to present. These conditions make the ASL problem by a good test for any semiempirical turbulence model.

The conducted theoretical and numerical investigations of the problem under consideration allowed to perform the procedure of selection of the empirical model constants more accurately and to obtain more accurate description of the experimental profiles as compared with the previous papers [4,5]. As the analysis showed, the best agreement with the observation data is obtained in case we assume the empirical parameter $C_{\theta}$ (which regulates the convective source of turbulence in $\varepsilon$ equation) depends on the ASL stability status: $C_{\theta}=0$ in the stable stratification area and $C_{\theta} \neq 0$ in the unstable stratification area. 


\section{Atmosphere surface layer. Theory of similarity, dimensionless form of equations of $K \varepsilon$ model.}

To describe turbulent flow in stationary and horizontally homogeneous ASL the following system of equations is applicable

$$
\begin{aligned}
& k \frac{\partial U}{\partial z}=u_{*}^{2} ; \\
& -\alpha_{\theta} k \frac{\partial \theta}{\partial z}=q ; \\
& k\left(\frac{\partial U}{\partial z}\right)^{2}-\frac{g}{T} \alpha_{\theta} k \frac{\partial \theta}{\partial z}-\varepsilon+\alpha_{\mathrm{b}} \frac{\partial}{\partial z} k \frac{\partial b}{\partial z}=0 ; \\
& C_{\varepsilon 1} k\left(\frac{\partial U}{\partial z}\right)^{2}-C_{\theta} \alpha_{\theta} \frac{g}{T} k \frac{\partial \theta}{\partial z}-C_{\varepsilon 2} \varepsilon+C_{\varepsilon} \frac{b}{\varepsilon} \frac{\partial}{\partial z} \frac{b^{2}}{\varepsilon} \frac{\partial \varepsilon}{\partial z}=0 ; \\
& k=C \frac{b^{2}}{\varepsilon} .
\end{aligned}
$$

Here the axis $O z$ is up-directed, $T$ is temperature of ground surface, $\theta$ is potential temperature, $U$ is velocity, $g$ is gravitational acceleration, $u_{*}$ is dynamic friction velocity, which determines turbulent frictional force between horizontal layers, $q$ is turbulent temperature flow on the surface, $b$ is turbulent energy pulsation (turbulent kinetic energy), $\mathcal{E}$ is turbulent energy dissipation, $k$ is turbulent viscosity (diffusion) coefficient. $\alpha_{b}, \alpha_{\theta}, C_{\varepsilon}, C_{\varepsilon 1}, C_{\varepsilon 2}, C_{\theta}, C$ are empirical constants.

At the height much above the significant dimensions of surface roughness the dimensional parameters of the problem are: $u_{*}, q, \frac{g}{T}$. With them it is possible to construct the single length parameter $L=-\frac{u_{*}^{3}}{\kappa \frac{g}{T} q}=\frac{u_{*}^{2}}{\kappa \frac{g}{T} \theta_{*}}$ presenting the characteristic of stratification (relational influence of dynamic and heat factors). Here $\kappa$ is Karman constant, $\theta_{*}=-\frac{q}{u_{*}}$ is temperature scale.

If $L>0$, turbulent heat flow is down-directed (air substratum is cooled with a colder ground surface), stratification is stable. If $L<0$, turbulent heat flow is up-directed (air is heated with a hotter surface), stratification is unstable. The limit $L \rightarrow \infty$ corresponds to neutral stratification (heat flow equals to zero, thermal convection is absent).

All ASL characteristics are the functions of dimensionless height $\xi=\frac{z}{L}$ and may be presented in the form

$$
U(z)=\frac{u_{*}}{\kappa} u_{n}(\xi), \quad k(z)=\kappa u_{*} L k_{n}(\xi), \quad b(z)=\frac{u_{*}^{2}}{\sqrt{C}} b_{n}(\xi), \quad \varepsilon(z)=\frac{u_{*}^{3}}{\kappa L} \varepsilon_{n}(\xi), \theta(z)=\theta_{0}+\frac{\theta_{*}}{\kappa \alpha_{\theta}} \theta_{n}(\xi) .
$$

Here the dimensionless functions are indicated with the index " $n$ ", the empirical constants are traditionally introduced for convenience.

In the dimensionless variables instead of (1.1)-(1.4) after some transformations we finally have the following system of equations: 


$$
\begin{aligned}
& \frac{1}{k_{n}}-1-\varepsilon_{n}+\hat{\alpha} \frac{d}{d \xi} k_{n} \frac{d b_{n}}{d \xi}=0, \\
& \frac{C_{\varepsilon 1}}{k_{n}}-C_{\theta}-C_{\varepsilon 2} \varepsilon_{n}+\hat{\sigma} \frac{b_{n}}{\varepsilon_{n}} \frac{d}{d \xi} k_{n} \frac{d \varepsilon_{n}}{d \xi}=0, \\
& k_{n}=\frac{b_{n}^{2}}{\varepsilon_{n}} .
\end{aligned}
$$

Here $\hat{\alpha}=\alpha_{b} \frac{\mathrm{K}^{2}}{\sqrt{C}} ; \hat{\sigma}=C_{\varepsilon} \frac{\mathrm{\kappa}^{2}}{\sqrt{C}}$.

To interpret the experimental observations we use the dimensionless function $\varphi_{u}(\xi)=\frac{k z}{u_{*}} \frac{d U}{d z}=\xi \frac{\partial u_{n}}{\partial \xi}=\frac{\xi}{k_{n}}$ and Richardson flow number $R f(\xi)=-\frac{\frac{g}{T} q}{u_{*}^{2} \frac{\partial U}{\partial z}}=\frac{\xi}{\varphi_{u}}=k_{n}$, which is monotonous and single-valued function of $\xi$.

For limiting cases of neutral stratification $(z / L \rightarrow 0)$, strong instability $(z / L \rightarrow-\infty)$ and strong stability $(z / L \rightarrow+\infty)$ the similarity considerations allow to make a number of specific conclusions without solving the system (1.5)-(1.6).

If $\xi=z / L \rightarrow 0(L \rightarrow \infty$ or $q \rightarrow 0$ or $z \rightarrow 0)$, the parameter $q$ ceases to be the parameter of the problem, and dependence of the functions $\frac{\partial U}{\partial z}, \frac{\partial \theta}{\partial z}, \varepsilon$ upon it should fall out. The length scale also disappears, i.e. that regime is self-similar. It is possible only if at $\xi \rightarrow 0$ $\frac{\partial u_{n}}{\partial \xi}, \frac{\partial \theta_{n}}{\partial \xi}, \varepsilon_{n} \rightarrow \frac{1}{\xi}$, hence it follows that the values of universal functions approach the constants: $\varphi_{u}(0)=1, \quad \varphi_{\varepsilon}(0)=1$. Such constant values are provided by Karman constant $\kappa$ previously introduced into the definition of universal functions.

Thus, near to zero the following decompositions are true:

$$
\left.\begin{array}{rl}
u_{n} & =\ln \xi+\beta_{1} \xi+\text { const } \\
\theta_{n} & =\ln \xi+\beta_{1} \xi+\text { const } \\
b_{n} & =1+\gamma_{1} \xi+\cdots \\
\varepsilon_{n} & =\frac{1}{\xi}+\delta_{1}+\cdots \\
k_{n} & =\xi\left(1-\beta_{1} \xi\right)+\cdots \\
\varphi_{u} & =1+\beta_{1} \xi+\cdots
\end{array}\right\},
$$

It is verified by multiple atmosphere observations and laboratory experiments by studying surface layers in unstratified fluid.

According to the necessity of asymptotic (1.7) existence if $\xi \rightarrow 0$, the additional limitation for the values of $K \varepsilon$ model empirical constants follows: $\hat{\sigma}=C_{\varepsilon 2}-C_{\varepsilon 1}$. At this for the decomposition coefficients it is possible to obtain:

$$
\gamma_{1}=\frac{1}{\hat{\alpha}-2} ; \quad \delta_{1}=\frac{3 C_{\varepsilon 1}-C_{\varepsilon 2}-C_{\theta}(2-\hat{\alpha})}{2(2-\hat{\alpha}) \hat{\sigma}} ; \quad \beta_{1}=\delta_{1}-2 \gamma_{1}=\frac{3 C_{\varepsilon 2}-C_{\varepsilon 1}-C_{\theta}(2-\hat{\alpha})}{2(2-\hat{\alpha}) \hat{\sigma}}
$$

The limiting case $z / L \rightarrow-\infty$ is the regime of purely convective turbulence, which may be obtained at $u_{*} \rightarrow 0$, i.e. $u_{*}$ falls out from the determining parameters of the problem, hence it 
follows: $\varphi_{u} \approx C_{u}(-\xi)^{-1 / 3}, \varphi_{\theta} \approx C_{\theta}(-\xi)^{-1 / 3}, \varphi_{\varepsilon} \approx-C_{\varepsilon} \xi$. Then $R f=k_{n} \approx-\frac{1}{C_{u}}(-\xi)^{4 / 3}, b_{n} \approx C_{b}(-\xi)^{2 / 3}$ $\left(C_{u}, C_{\theta}, C_{\varepsilon}, C_{b}\right.$ are constants.)

The obtained asymptotic presents just qualitative information about behavior of universal functions and does not allow uniquely formulating the boundary conditions for $b$ and $\varepsilon$ for numerical solution of the system of equations (1.5), (1.6) at finite integrating interval.

The universal functions are considered rather well known, though different authors propose different formulas for experimental data interpolating. However, for the stratification, which is not very far from indifferent $(|\xi|<1)$, different sources give close results especially for convective conditions. In [10] by processing of multiple experiments the formula adjusted for convective and stable conditions was obtained:

$$
\varphi_{u}=\frac{\xi}{k_{n}}=\left\{\begin{array}{c}
1+\xi\{1+0.667 \exp (-0.35 \xi)[1+0.35(14.3-\xi)]\}, \quad \xi>0 \\
(1-19 \xi)^{-1 / 4}, \quad \xi<0 .
\end{array}\right.
$$

Below we present the results of calculations of ASL characteristics within the frameworks of $K \varepsilon$ model.

\section{Statement of boundary problem. Selection of values of $\mathbf{k \varepsilon}$ model empirical constants.}

As the basic sought functions we will use $b_{n}$ and $k_{n} . b_{n}$ corresponds to turbulent kinetic energy and is a positive and monotone decreasing function. Function $k_{n}$ is also monotone and, according to the experimental observations, increase from $-\infty$ up to some finite value $k_{n}(\infty) \leq 1$. Thus, the system of equations (1.5)-(1.6) is to be solved, which, if $\hat{\alpha} \neq 0$, with the variables $b_{n}$ and $k_{n}$ assumes the following form:

$$
\begin{aligned}
& k_{n} b_{n}^{\prime \prime}+k_{n}^{\prime} b_{n}^{\prime}=\frac{1}{\hat{\alpha} k_{n}}\left(b_{n}^{2}+k_{n}-1\right) \\
& b_{n} k_{n}^{\prime \prime}+4 k_{n}^{\prime} b_{n}^{\prime}-2 \frac{k_{n}}{b_{n}} b_{n}^{22}-\frac{b_{n} k_{n}^{\prime 2}}{k_{n}}==\frac{1}{\hat{\alpha} \hat{\sigma} k_{n}}\left[\left(2 \hat{\sigma}-\hat{\alpha} C_{\varepsilon 2}\right) b_{n}^{2}+\left(2 \hat{\sigma}-\hat{\alpha} C_{\theta}\right) k_{n}+\hat{\alpha} C_{\varepsilon 1}-2 \hat{\sigma}\right]
\end{aligned}
$$

Here the stroke sign denotes $\xi$ differentiation.

If we head for the dependencies (1.7) and (1.9) and attempt to approach them along the whole interval $-\infty<\xi<+\infty$, the boundary conditions for the system (2.1)-(2.2) will be as follows, taking into account the form of the functions $k_{n}, b_{n}$ :

$$
\begin{aligned}
& b_{n}=+\infty, k_{n}=-\infty \text { at } \xi=-\infty \\
& b_{n}=0, k_{n}=1 \text { at } \xi=+\infty . \\
& b_{n}=1, k_{n}=0 \text { at } \xi=0
\end{aligned}
$$

Formally the model constants $C_{\varepsilon 2}, C_{\varepsilon 1}, C_{\theta}, \hat{\alpha}$ remain undefined. The constants $C_{\varepsilon 2}, C_{\varepsilon 1}, \hat{\sigma}=C_{\varepsilon 2}-C_{\varepsilon 1}, \hat{\alpha}$ are supposed to be uniform for the whole area $-\infty<\xi<+\infty$, where the solution is sought. If we consider the decomposition of the function (1.9), obtained from the experimental data, in series in the neighborhood $\xi=0$, we will obtain different decompositions to the right and to the left. Taking into account (1.7) the coefficient $\beta_{1}$ should be discontinuous that is $C_{\theta}$ should be taken as piecewise with discontinuity at $\xi=0$. 
In meteorology the following constant values are considered to be conventional $C_{\varepsilon 2}=2 ; C_{\varepsilon 1}=1.45 ; \hat{\sigma}=C_{\varepsilon 2}-C_{\varepsilon 1}=0.55 ; \hat{\alpha}=0.54 ; C_{\theta}=1$.

However such selection of the constants is not unique [2,14]. For example, in [2] the description of self-similar profiles in experiments [13] is the basis for constant selection, and it is obtained that $C_{\varepsilon 2}=1.92 ; C_{\varepsilon 1}=1.43 ; \hat{\alpha}=1.7 \cdot \hat{\sigma}=0.83$.

Values $C_{\varepsilon 2}, C_{\varepsilon 1}$ in these sets differ insignificantly. One can notice the difference in $\hat{\alpha}$ and the value $C_{\theta}$ remains virtually indefinite.

Below we describe the algorithms for selecting the values $\hat{\alpha}, C_{\theta}$ for best description of the experimental observations at the finite interval $\xi \in[-a ;+a]$ for the both nominal sets of constants $C_{\varepsilon 2}$ and $C_{\varepsilon 1}$ (which are assumed already known).

\section{Behavior of solutions of the systems of equations of surface layer.}

Let us investigate the behavior of the integral curves of the system (2.1), (2.2). We equate the right-hand sides to zero and obtain:

$$
b_{n 1}^{2}=\frac{C_{\varepsilon 1}-C_{\theta}}{C_{\varepsilon 2}-C_{\theta}} ; \quad k_{n 1}=\frac{C_{\varepsilon 2}-C_{\varepsilon 1}}{C_{\varepsilon 2}-C_{\theta}} .
$$

The obtained flock of points is the solution of the system. Decomposition of the sought solution in the neighborhood $\xi=0$ is presented with the formulas (1.7), and the behavior of the integral curves near this point will be considered below.

It is known that $C_{\varepsilon 2}>C_{\varepsilon 1}$. We consider the behavior of the solution of the equations (2.1)(2.2) at $\xi \in(0 ;+\infty)$. It would be natural to suppose that the solution should pass through the critical points $\left.b_{n}\right|_{\xi=0}=1,\left.k_{n}\right|_{\xi=0}=0$ и $\left.b_{n}\right|_{\xi=+\infty}=b_{n 1},\left.k_{n}\right|_{\xi=+\infty}=k_{n 1}$. Since $0<k_{n 1} \leq 1$ and $b_{n 1}^{2} \geq 0$ should be satisfied, then one should assume $0 \leq C_{\theta} \leq C_{\varepsilon 1}$ at $\xi>0$.

When $\xi=-\infty$, the empirical formula (1.9) gives $\left.k_{n}\right|_{\xi=-\infty} \approx c_{k}(-\xi)^{5 / 4}$. Let us find the decomposition of the solution of the equations (2.1)-(2.2) in the neighborhood $\xi=-\infty$ in the form $\left.k_{n}\right|_{\xi=-\infty} \approx c_{k}(-\xi)^{\alpha},\left.b_{n}\right|_{\xi=-\infty} \approx c_{b}(-\xi)^{\beta}$. We obtain that the exponents $\alpha$ and $\beta$ depend on the used values of the parameters $C_{\varepsilon 1}, C_{\varepsilon 2}, C_{\theta}, \hat{\alpha}$. From the allowed decompositions at $\xi=-\infty$ we select those, which correspond to the physical meaning of the problem, i.e. along the whole interval $\xi \in(-\infty ; 0)$ the conditions of monotone increase of the function $k_{n}$ from $-\infty$ to 0 and monotone decrease of $b_{n}$ from $+\infty$ to 1 should be satisfied. We obtain $3 / 2 \leq \alpha<2$ and $\beta=2-\alpha$ (that is $0<\beta \leq 1 / 2$ ). Thus, at no values of the used parameters it is possible to obtain the solution of the equations (2.1)-(2.2), which would satisfy asymptotic of the empirical formula (1.9) at $\xi=-\infty$.

We consider the behavior of the solution at $\xi \approx 0$. Taking into account (1.7), at the fixed values $C_{\varepsilon 1}, C_{\varepsilon 2}$ it is always possible to select values $C_{\theta}, \hat{\alpha}$, which allow to obtain coincidences for solution of the problem (2.1)-(2.2) with the values of the experimental curve slope at the right and at the left of $\xi=0$.

Thus, while solving the equations (2.1)-(2.2) for the selected fixed values $C_{\varepsilon 1}, C_{\varepsilon 2}$, at the expense of selecting $\hat{\alpha}$ and value $C_{\theta}$ discontinuous at $\xi=0$, one may accurately describe individually or the behavior of the experimental curve in the neighborhood $\xi=0$ or $\xi=+\infty$.

Though decompositions by $\xi= \pm \infty$, following from $K \varepsilon$ model, do not agree with the experimental formulas, which have different asymptotic at infinity with different authors, there 
may be no sense to try to satisfy them accurately. The experimental observations relate to the limited interval of change of dimensionless height, therefore the numerical integration of the equations (2.1)-(2.2) should be performed at the limited interval of height change. If we do not try to satisfy the empirical asymptotic at $\xi= \pm \infty$, but consider just the mean square deviation of the obtained solution from the experimental data at the limited interval, for example $-2 \leq \xi \leq 2$, then one may obtain good agreement with the experiment at the expense of parameter selection $C_{\theta}, \hat{\alpha}$.

\section{Numerical integration of surface layer system of equations}

We solve the system of equation (2.1)-(2.2) for the functions $k_{n}, b_{n}$. Since the coefficients included into the equations may be discontinuous at $\xi=0$, integrating is performed by the two intervals: $[0.01 ; 2]$ and $[-2 ;-0.01]$. In the points $\xi_{0}= \pm 0.01$ the values $b_{n}$ and $k_{n}$ are determined from the decomposition (1.7).

Let us determine the right boundary condition at the interval [0.01;2]. The performed studying of the problem shows that at the right the values $b_{n}$ and $k_{n}$ quickly approach some constant positive values, which are formally dependent on the constants $C_{\varepsilon 1}, C_{\varepsilon 2}, C_{\theta}, \hat{\alpha}$. Therefore it is natural to take $\left.\frac{d b_{n}}{d \xi}\right|_{\xi_{R}}=\left.\frac{d k_{n}}{d \xi}\right|_{\xi_{R}}=0$ as a boundary condition.

We consider the interval $[-2 ;-0.01] . k_{n} \rightarrow-\infty, b_{n} \rightarrow+\infty$ is to be satisfied within the limit $\xi \rightarrow-\infty$. If we take into account the kind of the solution decomposition at $\xi=-\infty,\left.\quad \frac{d b_{n}}{d \xi}\right|_{\xi=-\infty}=0$ is to be satisfied as well. This condition is satisfied with adequate accuracy already for values $\xi$ at small module, therefore one may take the derivative value close to zero: $\left.\frac{d b_{n}}{d \xi}\right|_{\xi_{L}}=-0.001$ as the left boundary condition for $b_{n}$. The value of $k_{n}$ in the point $\xi_{L}=-2$ we determine from the condition $k_{n}\left(\xi_{L}=-2\right)=\frac{\xi_{L}}{\varphi_{u}\left(\xi_{L}\right)} \approx-\frac{2}{0.4}$, where $\varphi_{u}\left(\xi_{L}=-2\right)=0.4$ is the experimental value determined from (1.9).

The system of equations (2.1)-(2.2) with specified boundary conditions was solved numerically by chaser method at the intervals $[0.01 ; 2]$ and $[-2 ;-0.01]$. The program of numerical solution of the given system was created. Calculations were made with various number of points $N$. Convergence of the results was obtained at $N>50$ in positive and negative areas.

It was assumed that the values $C_{\varepsilon 1}, C_{\varepsilon 2}$ have been determined earlier and are constant for both intervals. Value $\hat{\alpha}$ is assumed to be constant and value $C_{\theta}$ is assumed to be discontinuous: $C_{\theta}=\left\{\begin{array}{cc}C_{\theta_{+}}: & \xi>0 \\ C_{\theta_{-}}: & \xi<0\end{array}\right.$. A great number of calculations with different values of $\hat{\alpha}, C_{\theta}$ was performed with the purpose to determine such their values, which allow to obtain numerical solution describing in the best way (that is mean-square deviation) the empirical functional dependence (1.9) at the interval $\xi \in[-2 ; 2]$. 


\section{Comparison of numerical solution with experimental observations}

As we have noted above, the following set of constant values is typically used in meteorology: $C_{\varepsilon 2}=2 ; C_{\varepsilon 1}=1.45 ; \hat{\sigma}=C_{\varepsilon 2}-C_{\varepsilon 1}=0.55 ; \hat{\alpha}=0.54 ; C_{\theta}=1$.

It was obtained that the resulting solution at $\xi>0$ very weekly depends on $\hat{\alpha}$ and strongly depends on $C_{\theta}$. At this the calculated values $k_{n}, b_{n}$ approach very quickly (just at $\xi \approx 1$ ) the constant values, which coincide with the values in the critical points (3.1) (that verifies the correctness of the numerical solution of the problem).

Actually, having numerical solution at the finite right interval of height change and knowing the solution behavior beyond the interval at large values of $\xi$, we can construct the solution along the whole interval $\xi \in(0 ;+\infty)$. For $k_{n}$ the best agreement with the empirical dependence is obtained at $C_{\theta}=0$.

Thus, it is necessary to assume $C_{\theta}=0$ at $\xi \in(0 ;+\infty)$ and it is required to match $\hat{\alpha}$ and $C_{\theta}$ at the left integrating interval.

Let us take the value $C_{\theta}=1$ at $\xi<0$. According to calculation results the optimal value of the left parameter $\hat{\alpha}=1$ was selected, which provides the best agreement between calculation and experimental data. The results of calculation of the case $\hat{\alpha}=1, C_{\theta}=\left\{\begin{array}{cc}0: & \xi>0 \\ 1: & \xi<0\end{array}\right.$ are presented in Figure 1 as compared with the empirical data.

Let $\hat{\alpha}=0.54$. According to the calculation results the optimal value $C_{\theta}=1.6$ at $\xi<0$. The results of calculation of the case $\hat{\alpha}=0.54, C_{\theta}=\left\{\begin{array}{c}0: \quad \xi>0 \\ 1.6: \quad \xi<0\end{array}\right.$ are presented in Figure 1 as well and almost coincide with the previous case.

Above we presented another used set of constants of ke model $C_{\varepsilon 2}=1.92 ; C_{\varepsilon 1}=1.43$; $\hat{\alpha}=1.7 \cdot \hat{\sigma}=0.83$. The results of calculation of this case provide the following optimal values of parameters $C_{\theta}=\left\{\begin{array}{cc}0: & \xi>0 \\ 1.2: & \xi<0\end{array}\right.$. The calculating results are presented in Figure 1 as well.

As is seen, the solutions for all the obtained sets of constants almost coincide between each other and are close to the experimental data. The calculations at various values of parameters $\hat{\alpha}$ and $C_{\theta}$ were conducted, however, the available results would not be improved.

If we know the values of the used parameters, we may determine the character of the solution behavior at $\xi \approx 0$ and $\xi= \pm \infty$.

For example, the set of parameters $C_{\varepsilon 2}=2 ; C_{\varepsilon 1}=1.45 ; \hat{\sigma}=0.55 ; \hat{\alpha}=1, C_{\theta}=\left\{\begin{array}{cc}0: & \xi>0 \\ 1: & \xi<0\end{array}\right.$ provides the following behavior of the functions: $\left.k_{n}\right|_{\xi=-\infty} \sim(-\xi)^{1.62},\left.\quad b_{n}\right|_{\xi=-\infty} \sim(-\xi)^{0.38}$, and the decomposition coefficient for $k_{n}$ in the neighborhood $\xi \approx-0$ (see (1.7)) $\beta_{1}=3.23$. At $\xi>0$ the values $k_{n}, b_{n}$ quickly approach the constant values coincident with the values in the critical points (3.1), at this in the neighborhood $\xi \approx+0$ the value $\beta_{1}=4.17$.

The parameter set $C_{\varepsilon 2}=2 ; C_{\varepsilon 1}=1.45 ; \hat{\sigma}=0.55 ; \hat{\alpha}=0.54, C_{\theta}=\left\{\begin{array}{c}0: \quad \xi>0 \\ 1.6: \xi<0\end{array}\right.$ provides the following results: $\left.k_{n}\right|_{\xi=-\infty} \sim(-\xi)^{1.54},\left.\quad b_{n}\right|_{\xi=-\infty} \sim(-\xi)^{0.46}, \quad \beta_{1}=1.38$ at $\xi \approx-0$ and $\beta_{1}=2.83$ at $\xi \approx+0$. 
If $C_{\varepsilon 2}=1.92 ; C_{\varepsilon 1}=1.43 ; ; \hat{\alpha}=0.83, C_{\theta}=\left\{\begin{array}{cc}0: & \xi>0 \\ 1.2: & \xi<0\end{array}\right.$, then $\left.k_{n}\right|_{\xi=-\infty} \sim(-\xi)^{1.59},\left.b_{n}\right|_{\xi=-\infty} \sim(-\xi)^{0.41}$, $\beta_{1}=2.55$ at $\xi \approx-0$ and $\beta_{1}=3.78$ at $\xi \approx+0$.

Thus, the values of the model parameters are obtained, at which the numerical solutions of the system (2.1)-(2.2) describe well the experimental dependence (1.9) along the interval $\xi \in[-2 ; 2]$.

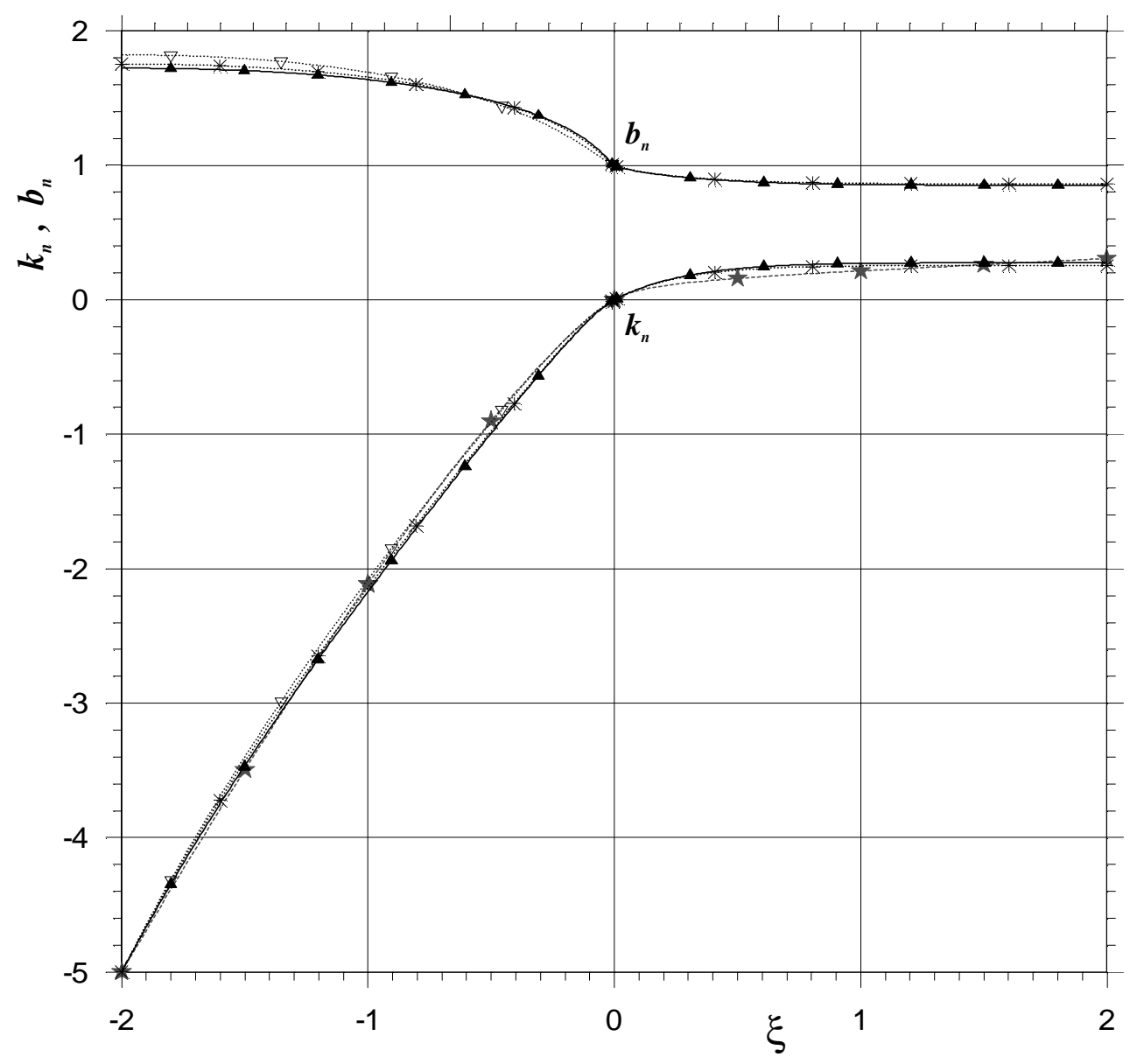

Figure 1. Dependence of functions $k_{n}, b_{n}$ on dimensionless height $\xi$ :

-- $\Delta$-- calculation with $C_{\varepsilon 2}=2 ; C_{\varepsilon 1}=1.45 ; \hat{\sigma}=0.55 ; \hat{\alpha}=1, C_{\theta}=\left\{\begin{array}{cc}0: & \xi>0 \\ 1: & \xi<0\end{array}\right.$;

- - calculation with $C_{\varepsilon 2}=2 ; C_{\varepsilon 1}=1.45 ; \hat{\sigma}=0.55 ; \hat{\alpha}=0.54, C_{\theta}=\left\{\begin{array}{ll}0: & \xi>0 \\ 1.6: & \xi<0\end{array}\right.$;

- - * - calculation with $C_{\varepsilon 2}=1.92 ; C_{\varepsilon 1}=1.43 ; ; \hat{\alpha}=0.83, C_{\theta}=\left\{\begin{array}{l}0: \quad \xi>0 \\ 1.2: \quad \xi<0\end{array} ;\right.$

- - $\star$ - - the empirical curve [10], 


\section{Conclusions}

Within the frameworks of $K \varepsilon$-model the problem about determination of dimensionless characteristics of turbulent flow in atmosphere surface layer is considered.

The complete investigation of $K \varepsilon$-equations and their critical points are conducted. From the variety of the integral curves the ones are selected, which correspond to the solution of the stated problem and describe quite satisfactorily all experiments. At this the basic empirical constants of the model $\left(C_{\varepsilon 1}, C_{\varepsilon 2}\right)$ are selected according to the conventional criteria. At the same time it is shown that parameter $C_{\theta}$ responsible for the convective source term in the equation for $\varepsilon$ should be selected different depending on stability status of the surface layer. At this the best agreement with experimental observations is obtained, if $C_{\theta}=0$ in the area of stable stratification and $C_{\theta} \neq 0$ in the area of unstable stratification.

The program, which allows solving the obtained model equations, is created. The calculations for various values $C_{\theta}$ and $\hat{\alpha}$ are conducted.

By the numerical matching of values $C_{\theta}$ and turbulent diffusion coefficient $\hat{\alpha}$ the quite satisfactory description of the experimental observations is obtained at the finite interval of dimensionless height change for any state of atmosphere stability.

Taking into account the obtained asymptotic of the model equation solutions for the values of dimensionless height $\xi= \pm \infty$, the solution is virtually constructed along the whole interval $\xi \in(-\infty ;+\infty)$.

\section{References}

1. Kharsha P. Turbulent kinetic energy transfer models. // Turbulence. Application principles. M., Mir, 1980, pp. 207-261.

2. Neuvazhayev V.E., Yakovlev V.G. Description of gravitational turbulent mixing by ke-model // VANT, Theoretical and Applied Physics, 1986, V1, p. 28.

3. Neuvazhayev V.E. Characteristics of models of gravitational turbulent mixing in non-self-similar problems // VANT, Mathematical Modeling of Physical Processes, 1994, V3, pp.3-10.

4. Nadezhina E.D. On using differential equations for second moments in models of atmosphere boundary layer // GTO Proceedings, 1975, V362, pp. 3-14.

5. Vager B.G., Nadezhina E.D. Using differential equation of dissipation transfer at modeling of atmospheric surface layer // News of AS USSR. Physics of atmosphere and ocean, 1976, V12, № 4, pp. 345-355.

6. Shneidman V.A., Bondarenko V.N. Quantitative description of vertical structure of surface sublayer in model of planet boundary atmosphere layer // IEM Proceedings, 1981, V 27 (100), pp. 22-28.

7. Stepanenko S.N., Shneidman V.A. Comparison of results of generalization of experimental data with calculations by three-parameter model of atmosphere boundary layer // GTO Proceedings, 1983, V 481, pp. 144-151.

8. Matveev L.T. Course of general meteorology. Aerophysics. - Leningrad: Gidrometeoizdat, 1976.

9. Atmosphere. Reference Book. - Leningrad: Gidrometeoizdat, 1991.

10. Boughton B.A. and DeLaurentis A.M. Description and validation of ERAD: an atmospheric dispersion model for high explosive detonations, Sandia National Laboratories Report №SAND922069, 1992.

11. Leichtman D.L. Physics of atmosphere boundary layer.- Leningrad: Gidrometeoizdat, 1970.

12. Simonov V.V. On using of equation for dissipation velocity while modeling turbulent flow above wavy surface // GTO Proceedings, 1986, V504, pp. 119-132.

13. Anuchina N.N., Kucherenko Yu.A., Neuvazhayev V.E. et al. Turbulent mixing at accelerating interface of fluids of different densities // MZhG, 1978, №6, pp. 157-160.

14. Sonin A.A. Calibration of the ke turbulence model for the diffusion of turbulence // Phys. Fluids, 1983, 26, 2769. 


\author{
Computational Modeling of Low-Mach-Number \\ High-Atwood-Number Turbulent Mixing (C4) \\ William Ashurst and Alan Kerstein \\ ashurs@ca.sandia.gov and kerstein@ca.sandia.gov \\ Sandia National Laboratories, Livermore
}

\title{
Introduction
}

One Dimensional Turbulence (ODT) is a stochastic model which tries to capture the essence of turbulent combustion: fully resolved molecular fluxes within a turbulent flow. To do this task a three dimensional velocity vector is described along a line (assumed to be the inhomogeneous direction in a temporal or spatially developing flow). Molecular processes are solved numerically and the turbulent flow effects of compressive strain rate and entrainment are accomplished with rearrangement events which are called the eddies. Upon random selection of an eddy length and location, guided by an assumed probability distribution, the proposed eddy energy is used to determine the eddy time scale, or frequency. This time scale is compared with an event rate distribution using a Bernoulli trial process; the eddy acceptance probability is kept low by the choice of the eddy time step so that the probability of more than one eddy occuring at one time vanishes. The action of the eddy is to rearrange all quantities within the eddy length - this is done by compressing the spatial scale to one-third of the eddy length, making two copies of the compressed result and placing them to fill out the eddy domain, the middle copy is then reversed. This triplet map will make a sawtooth curve out of a region with a linear gradient. Repeated application of the triplet map, at random locations, will generate a lognormal distribution of gradients.

In addition to the triplet mapping the eddy exchanges energy (energy flux) in the temporal (spatial) flow to mimic the pressure-strain-rate effects in turbulence. Using available direct numerical simulations (DNS) of Navier-Stokes turbulence in two shear flows, planar shear layer and planar wake, we have calibrated the ODT model by setting one rate term in order to match the temporal growth rate found in the DNS simulations. Comparison of first, second and third order turbulence quantities reveals nice agreement considering the simplicity of the ODT assumptions (Kerstein et al., 2001). 
New ODT work considers the temporal and spatial planar mixing layers in which the density of one stream may be a thousand times larger than the density of the other stream. This density contrast is achieved within incompressible flow by using two species with different molecular weights.

In this presentation we compare the variable density temporal shear with recent DNS compressible simulations by Pantano and Sarkar (2002). The spatially evolving ODT results are compared with the experiments performed by Brown and Roshko (1974). These experiments approximate incompressible flow while the DNS temporal simulations with density ratios up to eight were done with a convective Mach number of 0.7 .

\section{ODT Flow Equations}

We achieve variable-density, incompressible flow by using two species which have different molecular weights. The number density is constant and pressure gradients are also neglected (boundary layer approximation). We apply ODT to a shear layer which evolves in time (temporal) or in space (spatial) and conservation of streamwise momentum yields different governing equations between these two cases. The streamwise momentum in two dimensions is

$$
\frac{\partial \rho u}{\partial t}+\frac{\partial \rho u^{2}}{\partial x}+\frac{\partial \rho u v}{\partial y}=v i s
$$

where vis is the viscous term. In the temporal case this reduces to $\partial \rho u / \partial t=v i s$ and there is no convective velocity, that feature is accomplished by the eddies. In steady spatial flow the momentum relation reduces to

$$
\frac{\partial \rho u U}{\partial x}+\frac{\partial \rho u V}{\partial y}=v i s
$$

where $U, V$ are the convection velocities with $U=u$, but $V \neq v$ because convection is accomplished by the eddies. Thus, in the spatial case it is the flux of momentum which is conserved while in the temporal it is the momentum itself which is conserved. To mimic pressure scrambling of turbulence we exchange kinetic energy among the vector components in the temporal flow, but we exchange kinetic energy flux in the spatial flow, that is, exchange $\rho u^{2} U$ with $\rho v^{2} U$ and $\rho w^{2} U$. 


\section{Time Developing Flow Equations}

The ODT velocity components on the $1 \mathrm{D}$ domain are denoted $v_{i}(y, t), i=1,2,3$, where $y$ (corresponding to $i=2$ ) is the domain coordinate. These velocities do no convection, convection is done by the eddies. The number density is constant (incompressible flow), but in a binary mixture with unequal molecular weights, the mass and momentum fluxes are not zero when the species gradient is not zero.

Density diffusion:

$$
\frac{\partial \rho}{\partial t}=\kappa \frac{\partial^{2} \rho}{\partial y^{2}}
$$

\section{Momentum:}

$$
\frac{\partial \rho v_{i}}{\partial t}=\mu \frac{\partial^{2} v_{i}}{\partial y^{2}}+\kappa \frac{\partial}{\partial y} v_{i} \frac{\partial \rho}{\partial y}
$$

\section{Spatially Developing Flow Equations}

Each ODT realization is a steady, planar flow. The streamwise advecting velocity $U$ is the same as $v_{1}$, while the lateral advecting velocity $V$ is different from $v_{2}$. This distinction is made because the $v_{i}$ velocities are regarded as being storage of kinetic energy, and this energy is displaced by the eddy mappings. Therefore, the $v_{i}$ velocities are not convective but evolve formally as passive scalars advected by $U$ and $V$ and diffused by viscosity.

\section{Continuity:}

$$
\frac{\partial \rho U}{\partial x}+\frac{\partial \rho V}{\partial y}=0
$$

\section{Density diffusion:}

$$
\frac{\partial U}{\partial x}+\frac{\partial V}{\partial y}=-\frac{\partial}{\partial y} \frac{\kappa}{\rho} \frac{\partial \rho}{\partial y}
$$

note: incompressible flow has zero volume flux, and the mass average velocity is the sum of volume flux $V^{\prime}$ plus the diffusion flux, $V=V^{\prime}-(\kappa / \rho)(\partial \rho / \partial y)$.

Momentum:

$$
\frac{\partial \rho v_{i} U}{\partial x}+\frac{\partial \rho v_{i} V}{\partial y}=\mu \frac{\partial^{2} v_{i}}{\partial y^{2}}
$$




\section{Temporal Shear Layer}

In our ODT temporal shear layer the one-dimensional line is in the lateral direction and we impose streamwise boundary velocities of $U_{1}=0.5$ for $y>0$ and $U_{2}=-0.5$ for $y<0$. We normalize velocities with $\Delta U=U_{1}-U_{2}$ and set $\rho_{2}=s \rho_{1}$ with $\rho_{1}=1$. With incompressible flow there is no equation of state.

Pantano and Sarkar (2002) have performed compressible DNS of the temporal shear layer using an ideal gas with constant specific heats, dynamic viscosity, thermal conductivity and diffusion. The energy equation is expressed as a dynamical equation for the pressure. Reference values are: $L_{o}, \rho_{o}, p_{o}, U_{o}$ with the condition that $\gamma M a_{o}^{2}=1$ leading to $p_{o}=\rho_{o} U_{o}^{2}$. The convective Mach number is $M_{c}=\Delta U /\left(c_{1}+c_{2}\right)$. The average density is fixed to unity $\rho_{o}=\left(\rho_{1}+\rho_{2}\right) / 2=1$ with $s=\rho_{2} / \rho_{1}$ and $s \geq 1$ (by symmetry the flow with $s=1 / s$ is the same flow). Their flow is periodic in the spanwise $(z)$ and streamwise $(x)$ directions while a free boundary is used in the lateral direction $(y)$. The initial conditions include broadband fluctuations, isotropic turbulence spectrum, in the shear region with an initial turbulence intensity of ten percent. They computed with convective Mach numbers of $0.3,0.7$ and 1.1 with a density ratio different from unity only in the 0.7 case.

The ODT parameters are those developed in the constant density work (Kerstein et al., 2001), no new parameters were needed to include variable density, only the specification of a diffusivity was required. With constant dynamic viscosity $\mu$, the diffusivity is $D_{12}=0.6 \mu\left(\left(\rho_{1}+\rho_{2}\right) /\left(\rho_{1} \rho_{2}\right)\right)$. The ODT mean streamwise velocity and density profiles agree with the DNS results $(s=1,2,4,8)$ and the ODT results indicate a reduced growth rate with increasing density ratio, similar to the behavior of the compressible layer. The ODT turbulent kinetic energy production, dissipation and transport have a peak amplitude which is about twice that of the compressible results. Future ODT work which includes a compressible velocity component may show that this amplitude difference is a compressibility effect. 


\section{Temporal Shear Layer}

Comparison with Compressible DNS, $M_{c}=0.7$

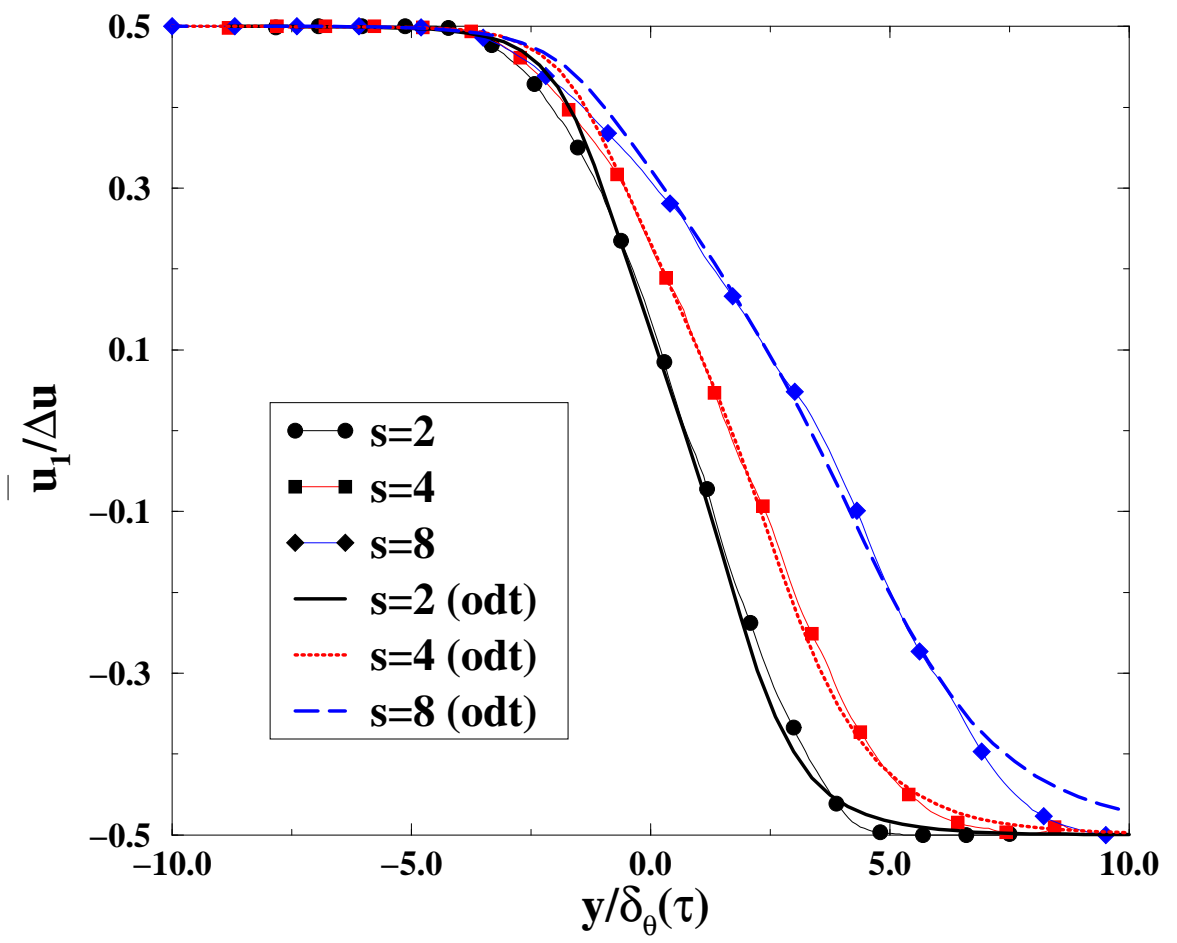

Mean streamwise velocity $u$ from Pantano \& Sarkar (JFM).

Momentum Thickness (Favre velocity: $\tilde{u}=\overline{\rho u} / \bar{\rho}$ )

$$
\delta_{\theta}=\frac{1}{\rho_{o} \Delta U^{2}} \int \bar{\rho}\left(u_{1}-\tilde{u}\right)\left(\tilde{u}-u_{2}\right) d y
$$




\section{Temporal Shear Layer}

Comparison with Compressible DNS, $M_{c}=0.7$

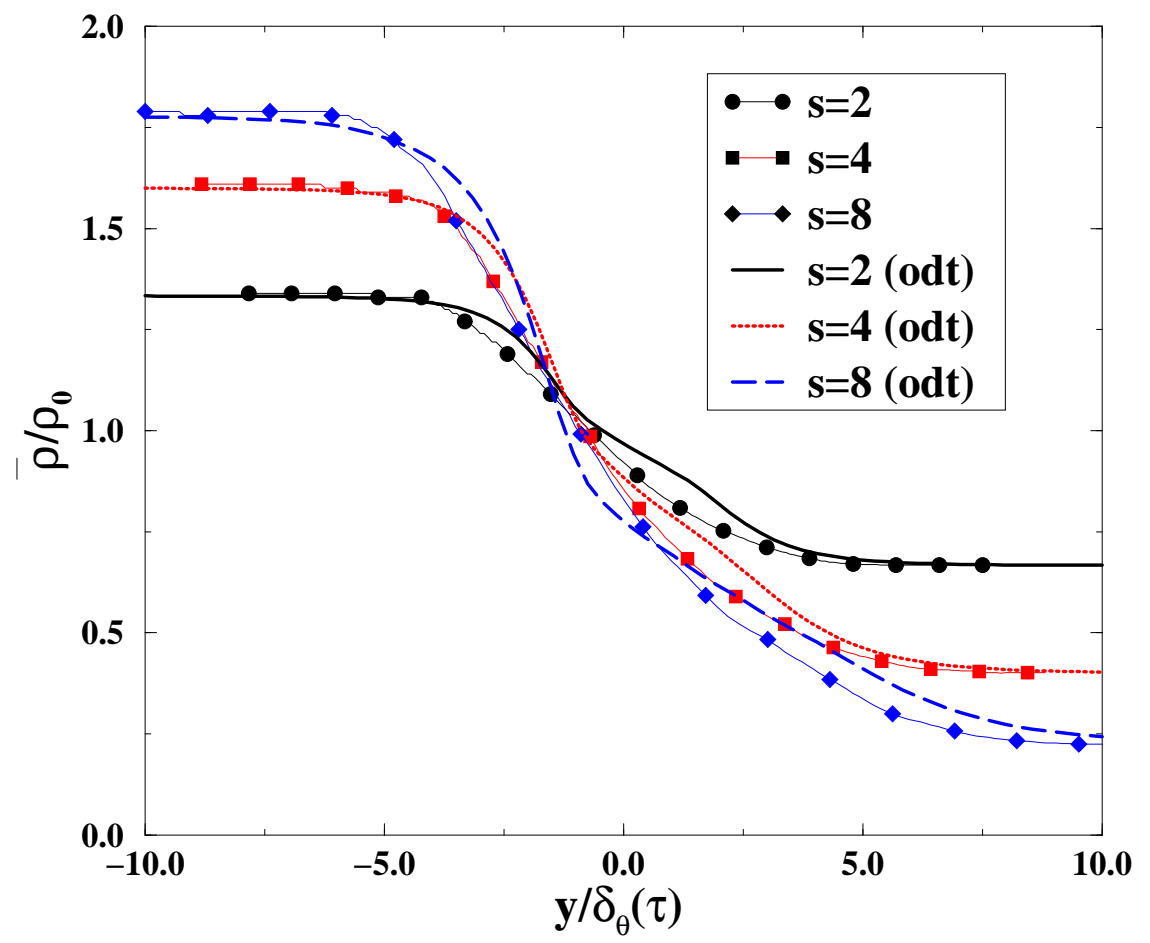

Mean density $\rho$ from Pantano \& Sarkar (JFM). 


\section{Temporal Shear Layer}

Comparison with Compressible DNS, $M_{c}=0.7$

\section{Pantano \& Sarkar, Mc = 0.7}

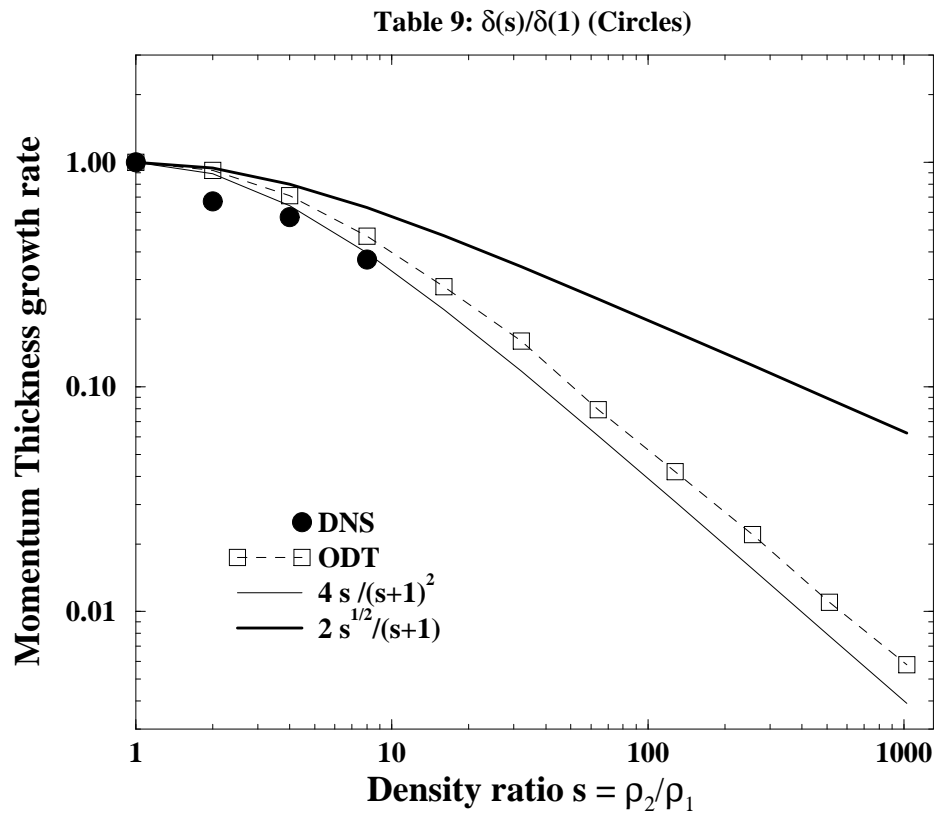

Momentum Thickness $\delta_{\theta}$ growth rate from Pantano \& Sarkar (JFM).

$$
\delta_{\theta}=\frac{2}{\left(\rho_{1}+\rho_{2}\right)(\Delta U)^{2}} \int \bar{\rho}\left(u_{1}-\tilde{u}\right)\left(\tilde{u}-u_{2}\right) d y
$$

Favre velocity: $\tilde{u}=\overline{\rho u} / \bar{\rho}$

Ramshaw (2000) has presented a model of shear layer growth, which when only the Kelvin-Helmholtz instability is active becomes

$$
h \approx \Delta U t \frac{\sqrt{\rho_{1} \rho_{2}}}{\left(\rho_{1}+\rho_{2}\right)}
$$

however, the ODT results indicate that the square of the density term is a better approximation

$$
h \approx \Delta U t \frac{\rho_{1} \rho_{2}}{\left(\rho_{1}+\rho_{2}\right)^{2}}
$$

which, with density ratio $s=\rho_{2} / \rho_{1}$ gives the $s /(s+1)^{2}$ effect shown in the figure above. Of course, being unit-less, there is no way to determine the exponent of this term by scaling laws. 


\section{Temporal Shear Layer}

Comparison with Compressible DNS, $M_{c}=0.7$

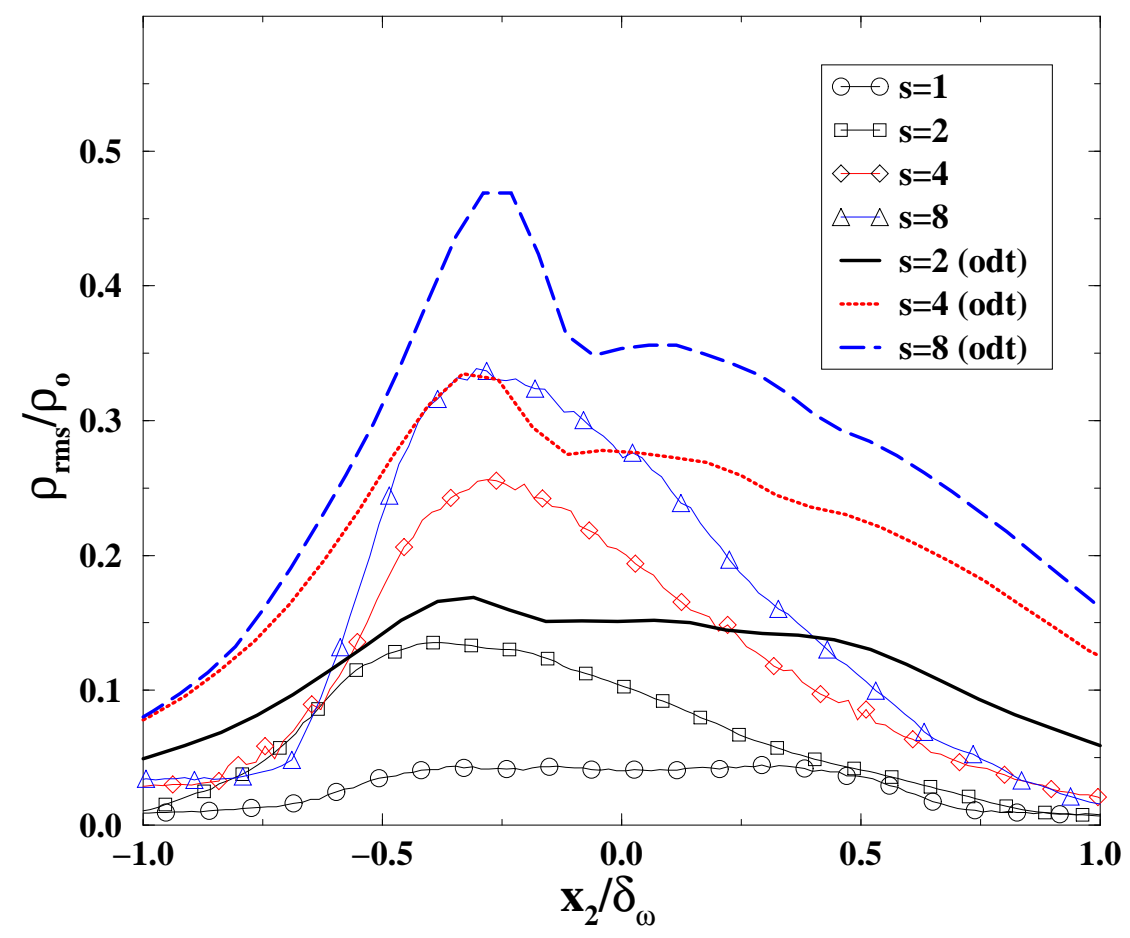

Density rms versus vorticity thickness $\delta_{\omega}$ (from Pantano \& Sarkar, JFM)

Vorticity Thickness $\delta_{\omega}=\Delta U /(\partial u / \partial y)_{\max }$ 


\section{Temporal Shear Layer}

Comparison with Compressible DNS, $M_{c}=0.7$

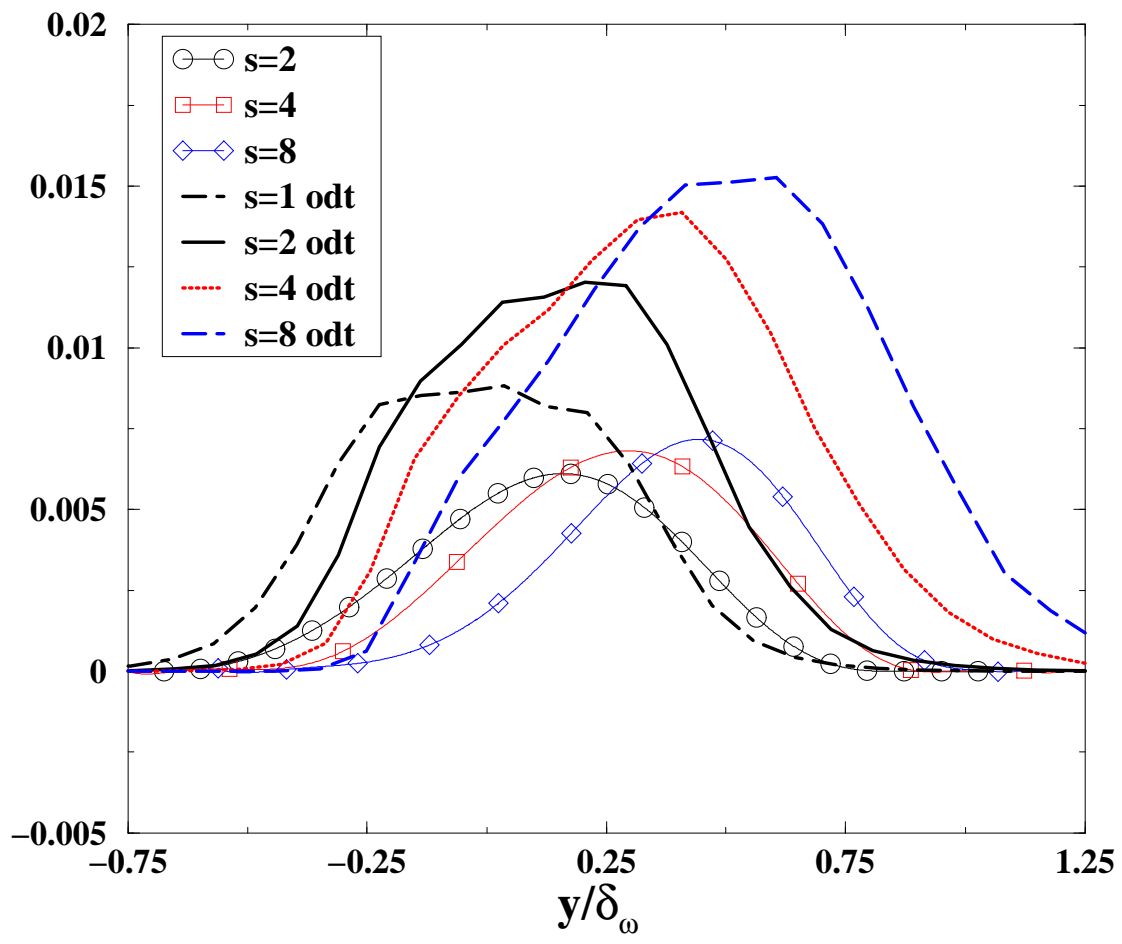

Turbulent kinetic energy production versus vorticity thickness $\delta_{\omega}$ (from Pantano \& Sarkar, JFM)

Vorticity Thickness $\delta_{\omega}=\Delta U /(\partial u / \partial y)_{\max }$ 


\section{Temporal Shear Layer}

Comparison with Compressible DNS, $M_{c}=0.7$

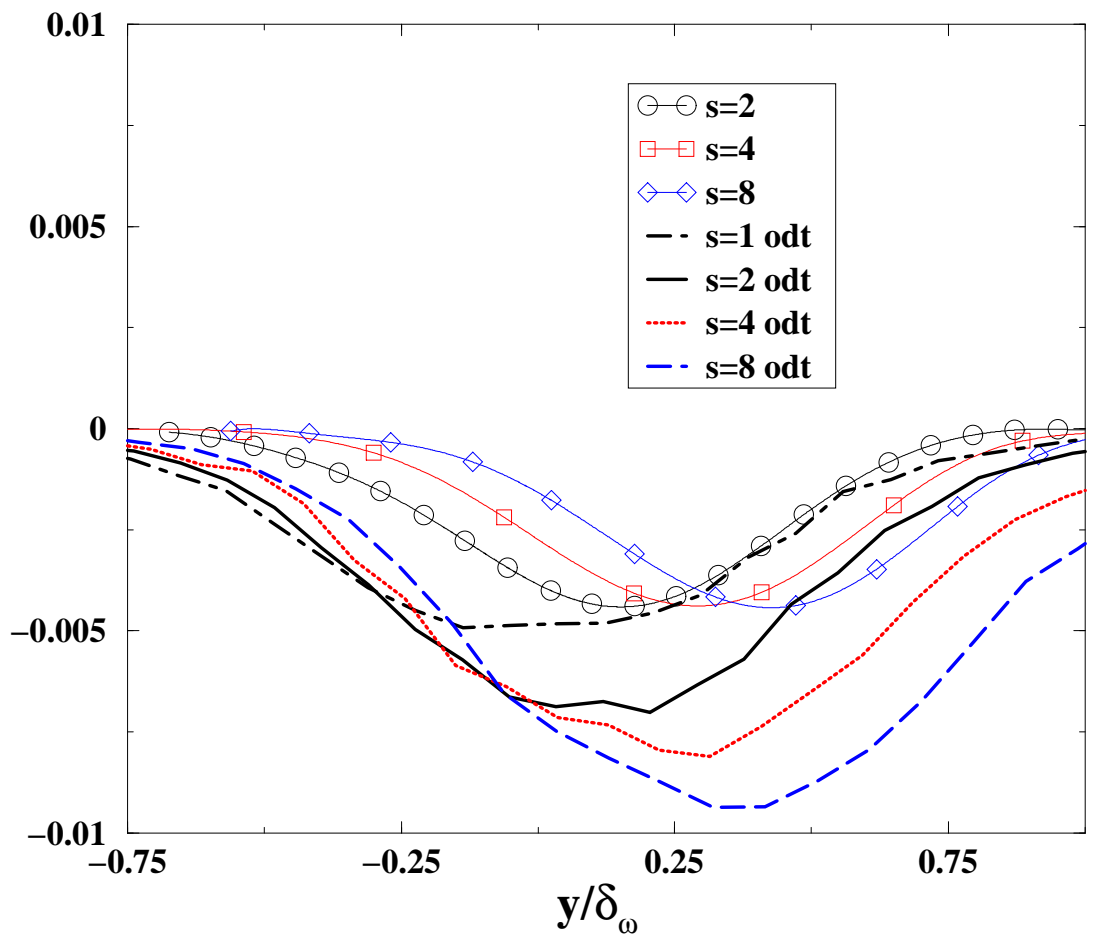

Turbulent kinetic energy dissipation versus vorticity thickness $\delta_{\omega}$ (from Pantano \& Sarkar, JFM) 


\section{Temporal Shear Layer}

Comparison with Compressible DNS, $M_{c}=0.7$

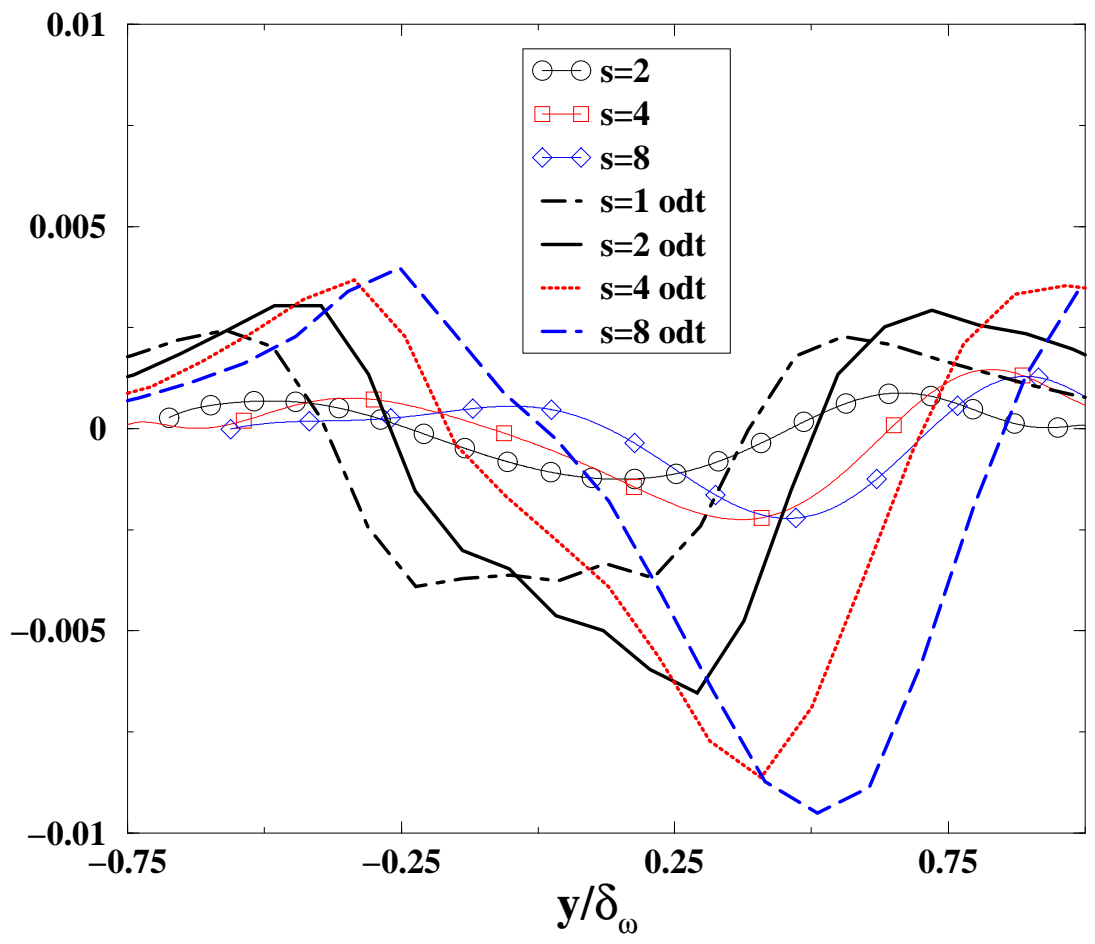

Turbulent kinetic energy transport versus vorticity thickness $\delta_{\omega}$ (from Pantano \& Sarkar, JFM) 


\section{SPATIALLY DEVELOPING SHEAR LAYER}

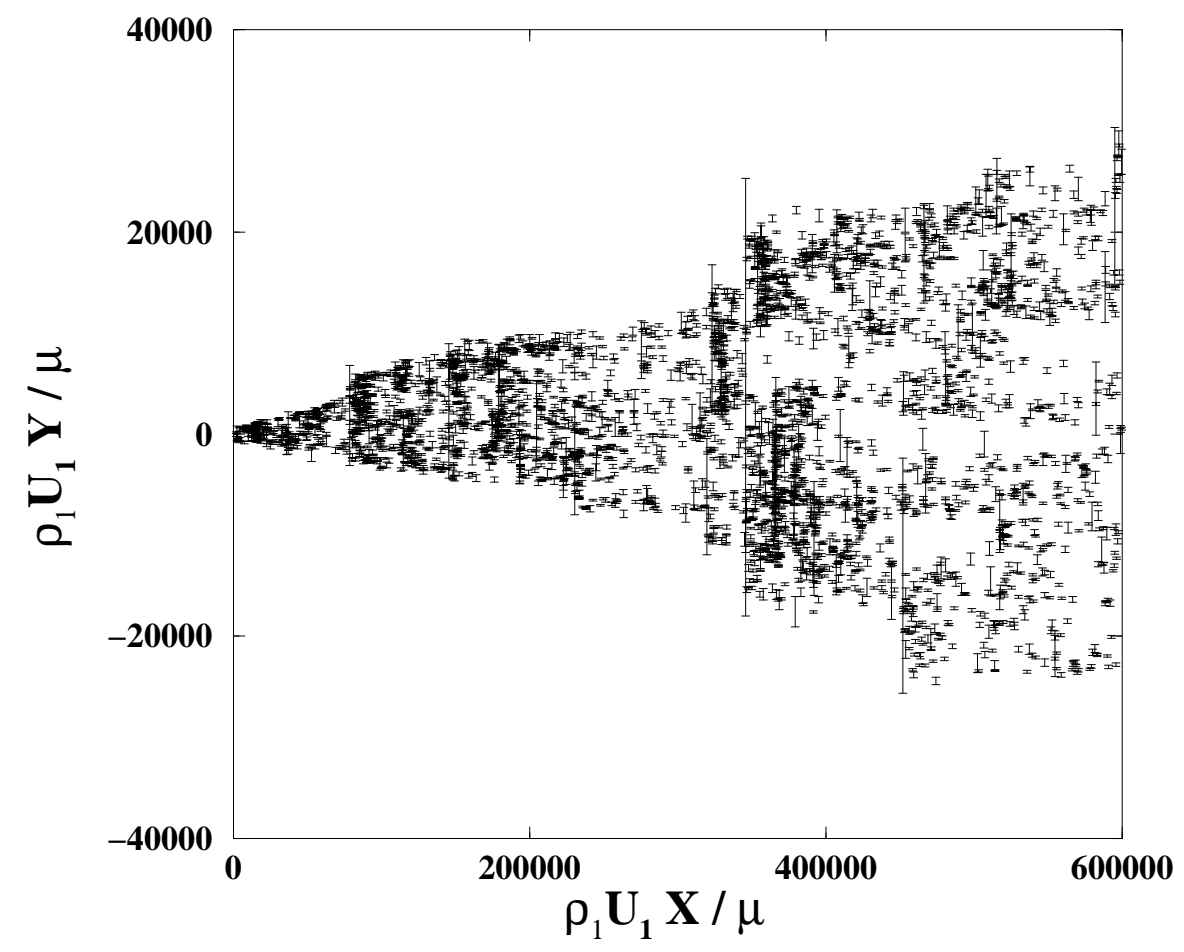

The density ratio is unity $s=1$, the upper flow has $U_{2}=0.38$ and the lower flow has $U_{1}=1$, the Reynolds number is comparable to the experimental value.

One ODT realization is shown by drawing vertical lines corresponding to the eddy length and location. To be accepted the eddy energy, given by velocity component in the direction of the ODT line, combined with the eddy length must give a time scale which is smaller than the viscous time scale for that eddy length. An additional barrier to accepting an eddy is examination of eddy segments, each segment is one-third of the eddy, if any segment fails to be acceptable then the eddy is rejected. In this manner an eddy may protrude into the quiet freestream and thus cause an abrupt growth in the layer thickness. Notice the increased eddy activity downstream of the larger eddies, the larger eddy has enhanced the local shear which favors smaller eddies - thus forming a cascade of shear to small length scales. 


\section{SPATIALLY DEVELOPING SHEAR LAYER}

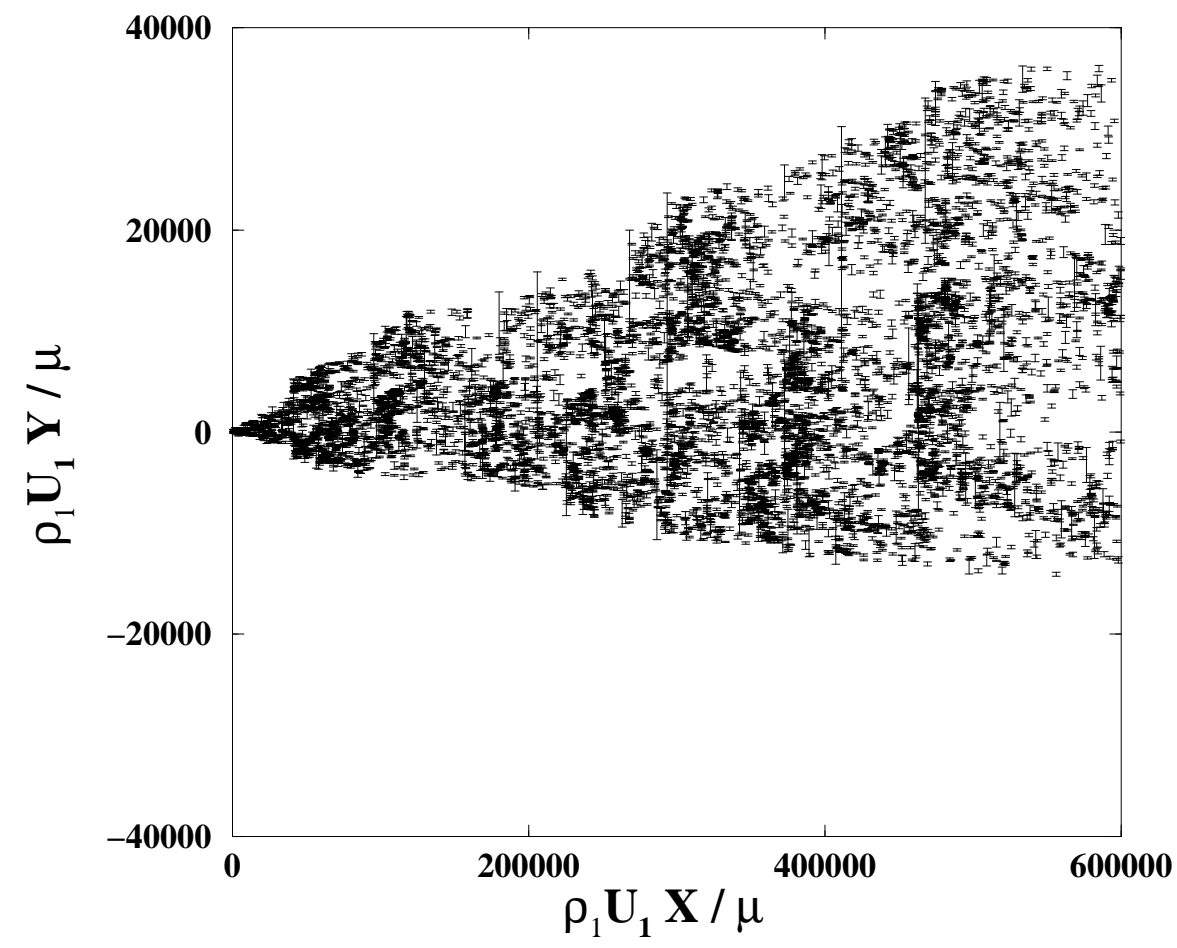

The density ratio is $s=7=\rho_{2} / \rho_{1}$, the upper flow has $U_{1}=1$ and the lower flow has $U_{2}=0.38$.

Notice that the spreading of the eddies into the lower flow, which has the larger inertia, is reduced by comparison with the upper flow. 


\section{SPATIALLY DEVELOPING SHEAR LAYER}

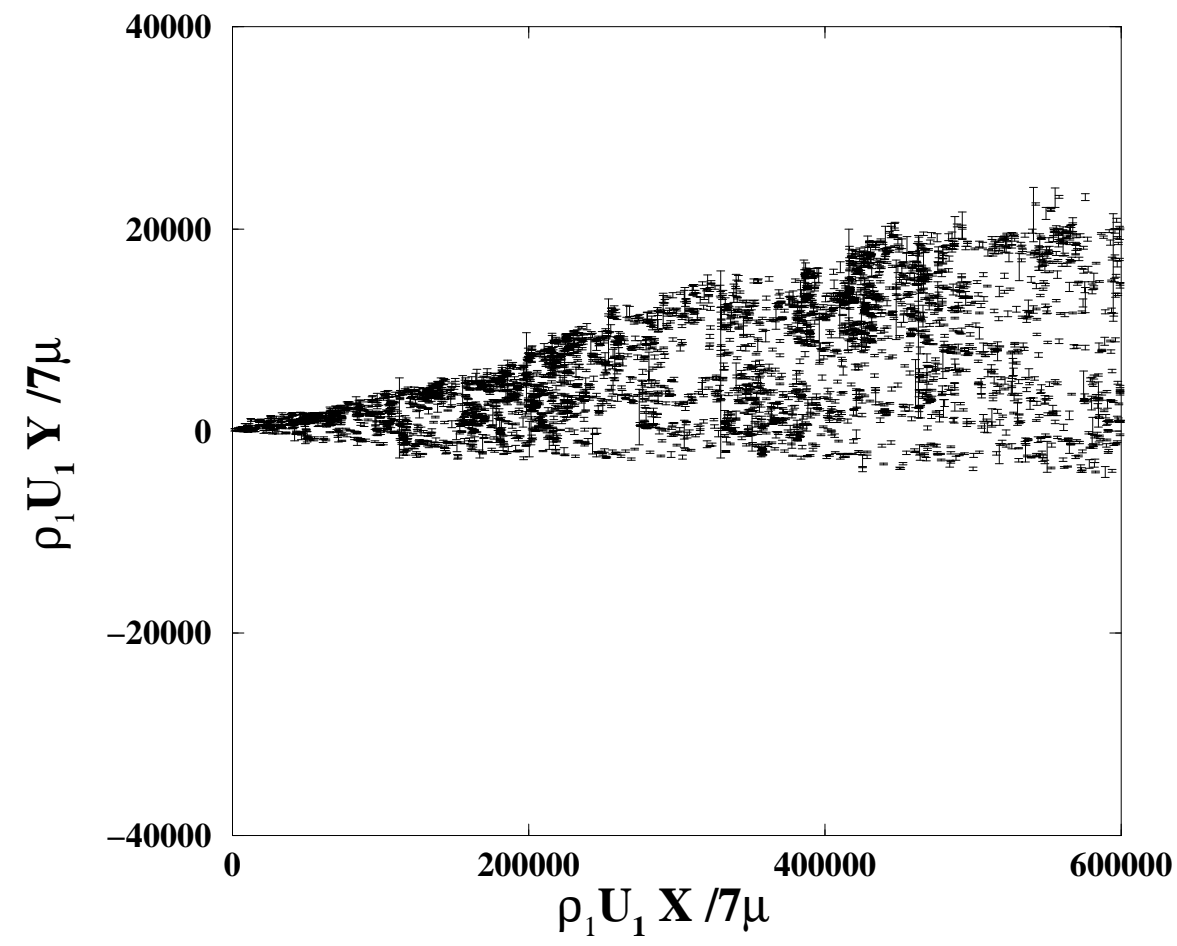

The density ratio is $s=1 / 7=\rho_{2} / \rho_{1}$, the upper flow has $U_{2}=0.38$ and the lower flow has $U_{1}=1$.

Notice that the faster flow which now has the larger inertia has very few eddies, and the overall growth rate is much less than when the denser flow is the slower stream (previous case with $s=7$ ). 


\section{SPATIALLY DEVELOPING SHEAR LAYER}

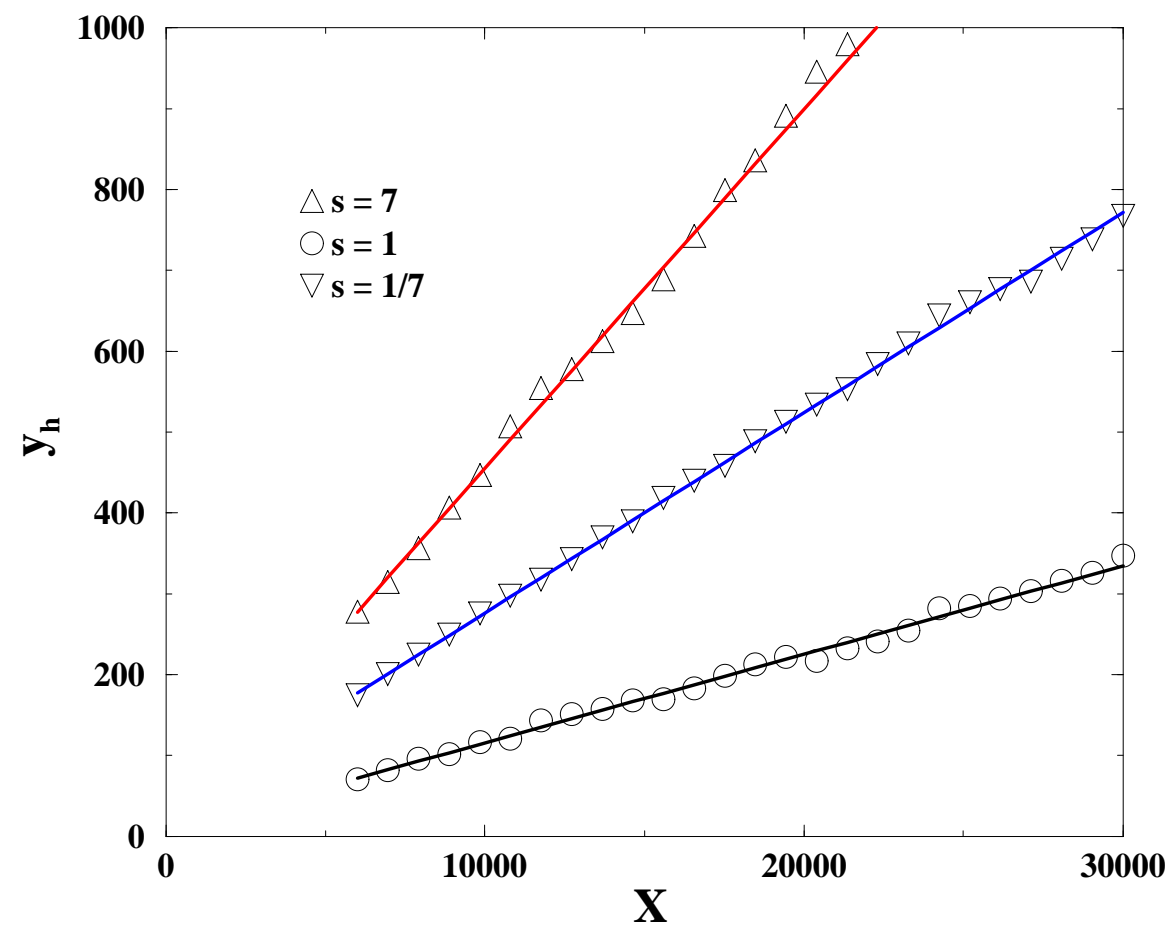

With each density ratio $s=7,1,1 / 7$, one thousand realizations are used to determine mean profiles. An origin in the lateral direction is determined by the location where the Favre velocity equals the mean of the freestream velocities, $\tilde{u}=\left(U_{1}+U_{2}\right) / 2$, this defines $y_{h}$. The symbols denote $y_{h}$ locations and the lines are least square fits which determine the virtual origin $x_{o}$ and the growth rate. The lateral profiles will be shown as a function of the similarity coordinate $\left(y-y_{h}\right) /\left(x-x_{o}\right)$. In the spatial units shown here the dynamic viscosity has the value of $1 / 20$. 


\section{SPATIALLY DEVELOPING SHEAR LAYER}

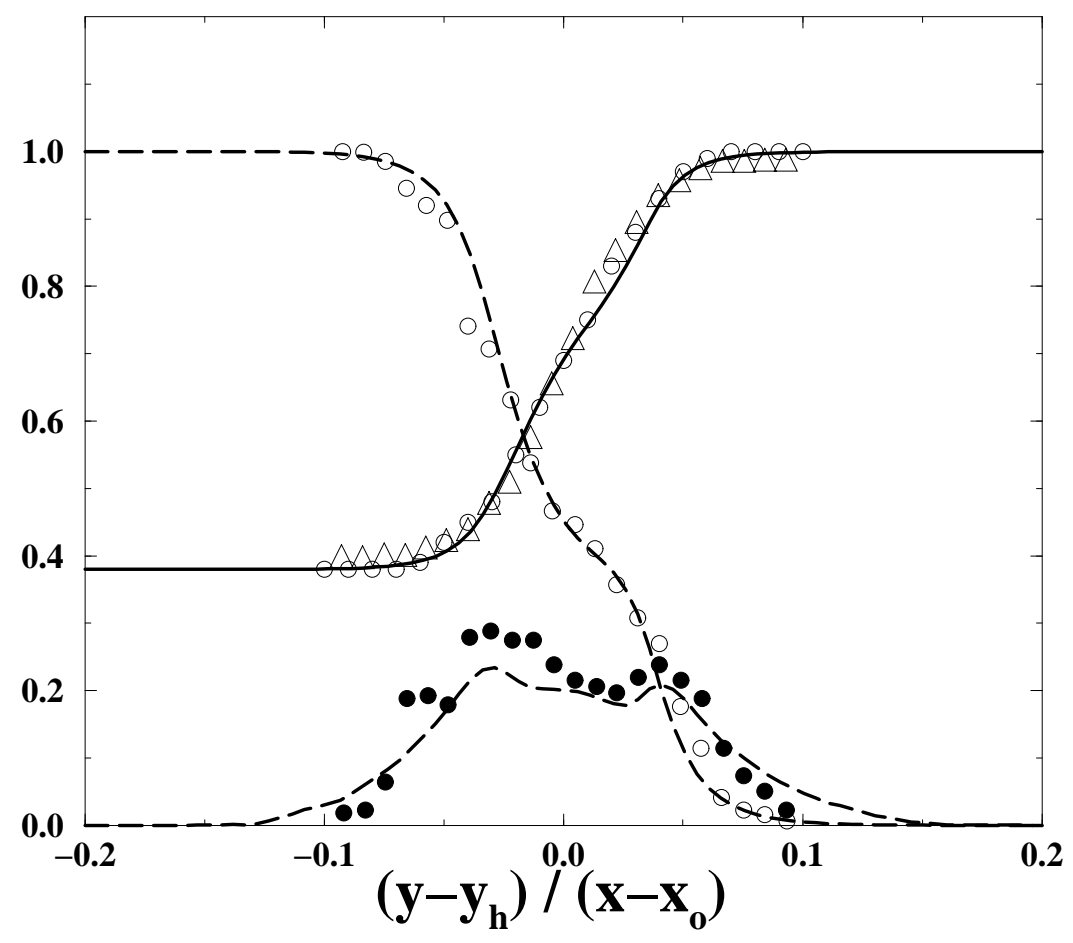

Lateral profiles of velocity (ODT, solid line) and concentration and concentration rms values (ODT, dashed lines) with constant density $s=1$; the open triangles are from Brown \& Roshko (velocity only) and the circles are from Konrad (velocity and concentration, open circle and concentration rms, filled circle). The ODT and Konrad's results have been scaled to match the velocity integral thickness of Brown \& Roshko; this sets the eddy rate constant in the ODT simulations (which is used in the other density cases) and requires a factor of 0.7 times Konrad's lateral coordinate values. The velocity is reduced by the maximum velocity. 


\section{SPATIALLY DEVELOPING SHEAR LAYER}

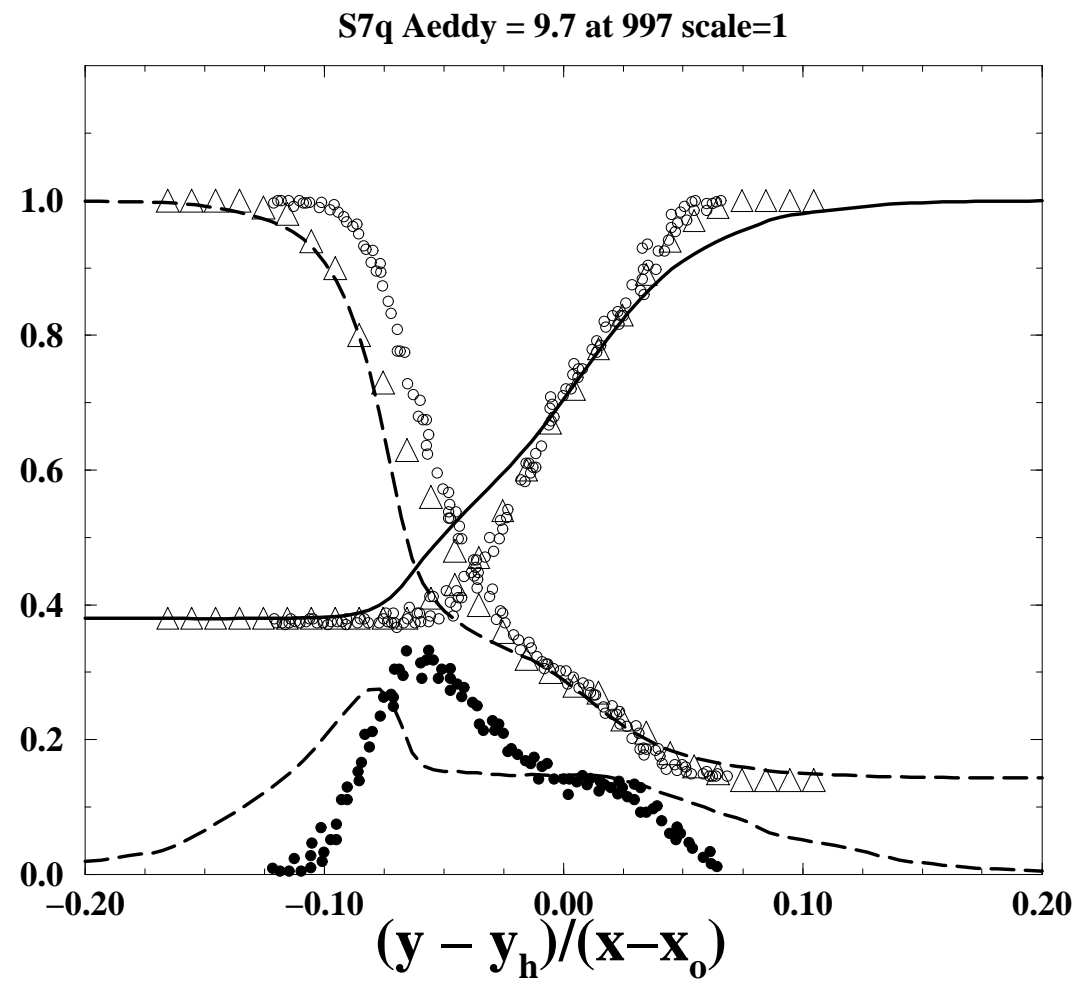

Lateral profiles of velocity (ODT, solid line) and density and rms density values (ODT, dashed lines) with density ratio $s=7$; the open triangles are from Brown \& Roshko (velocity and density only) and the circles are from Konrad (velocity and density, open circle and rms density, filled circle). The lateral scale factors are the same as given in $s=1$ results; the density, and its rms, are normalized by the maximum density. 


\section{SPATIALLY DEVELOPING SHEAR LAYER}

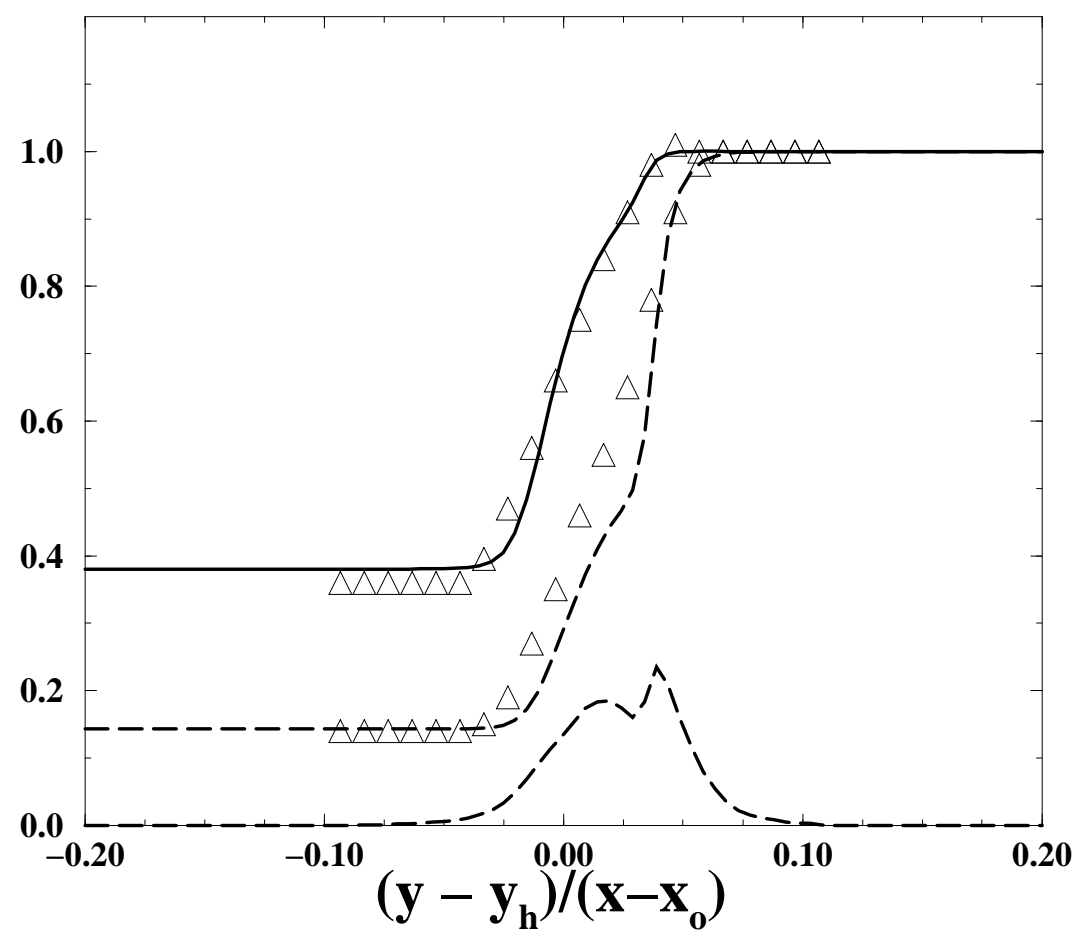

Lateral profiles of velocity (ODT, solid line) and density and rms density values (ODT, dashed lines) with density ratio $s=1 / 7$; the open triangles are from Brown \& Roshko (velocity and density only). The lateral scale factors and normalizations are the same as given in $s=7$ results. 


\section{SPATIALLY DEVELOPING SHEAR LAYER}

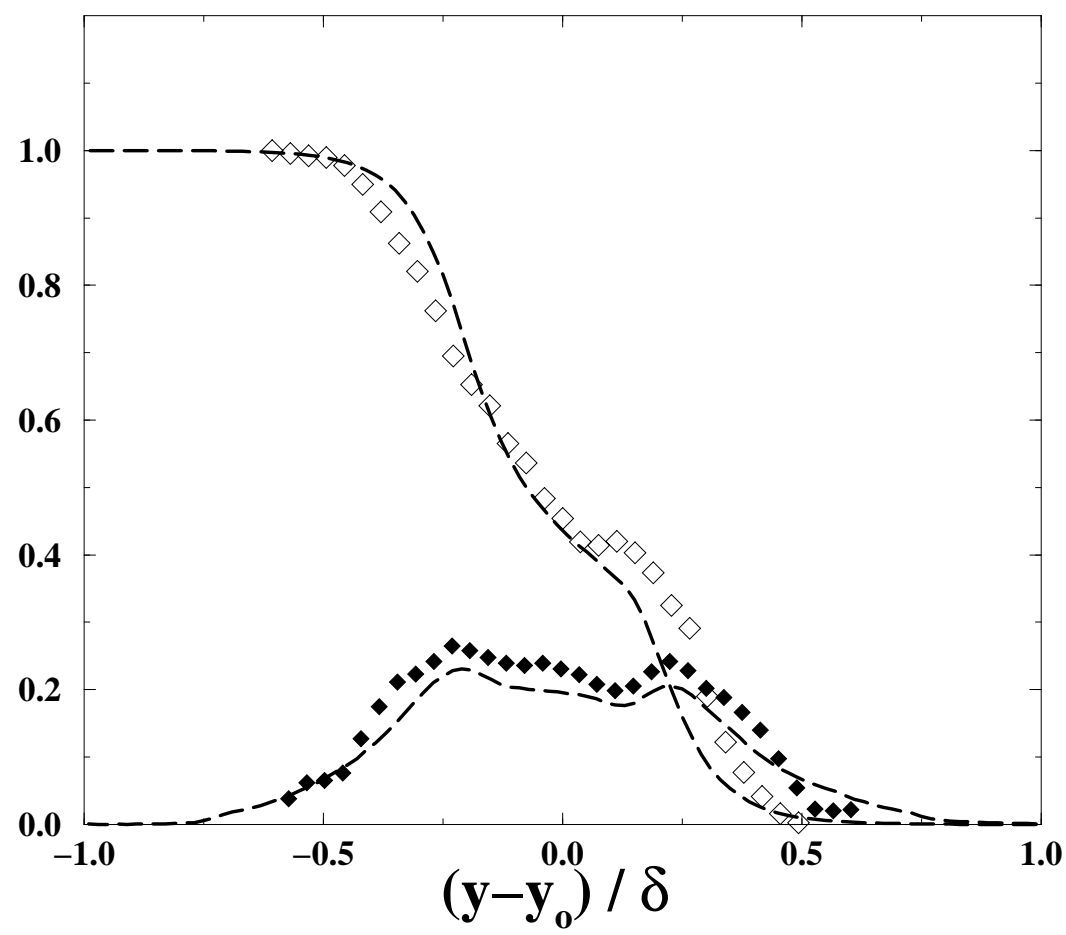

Concentration, and its rms, in constant density flow, velocity ratio of 0.4 (Pickett and Ghandhi, Phys. Fluids, 2002, symbols) and ODT (dashed lines). Lateral scaling is from the concentration one-percent distance $\delta$, the lateral origin is the mean of those locations. The high speed stream is at positive $y$ values. 


\section{SPATIALLY DEVELOPING SHEAR LAYER}

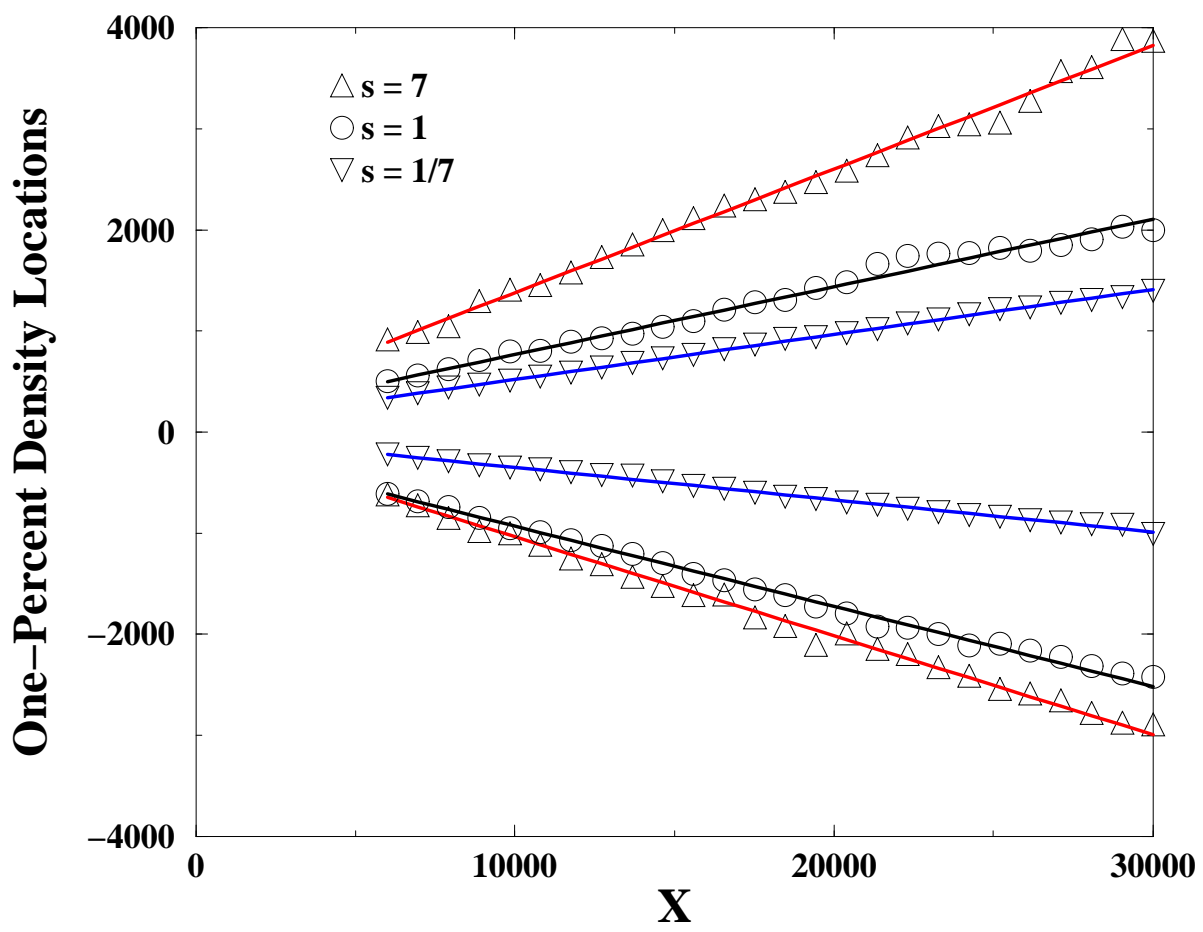

With each density ratio $s=7,1,1 / 7$, one thousand realizations are used to determine mean profiles. The symbols denote the locations where the concentration is at a onepercent difference from its freestream value. The lines are least-square fits and the slope differences give the density growth rate. 


\section{SPATIALLY DEVELOPING SHEAR LAYER}

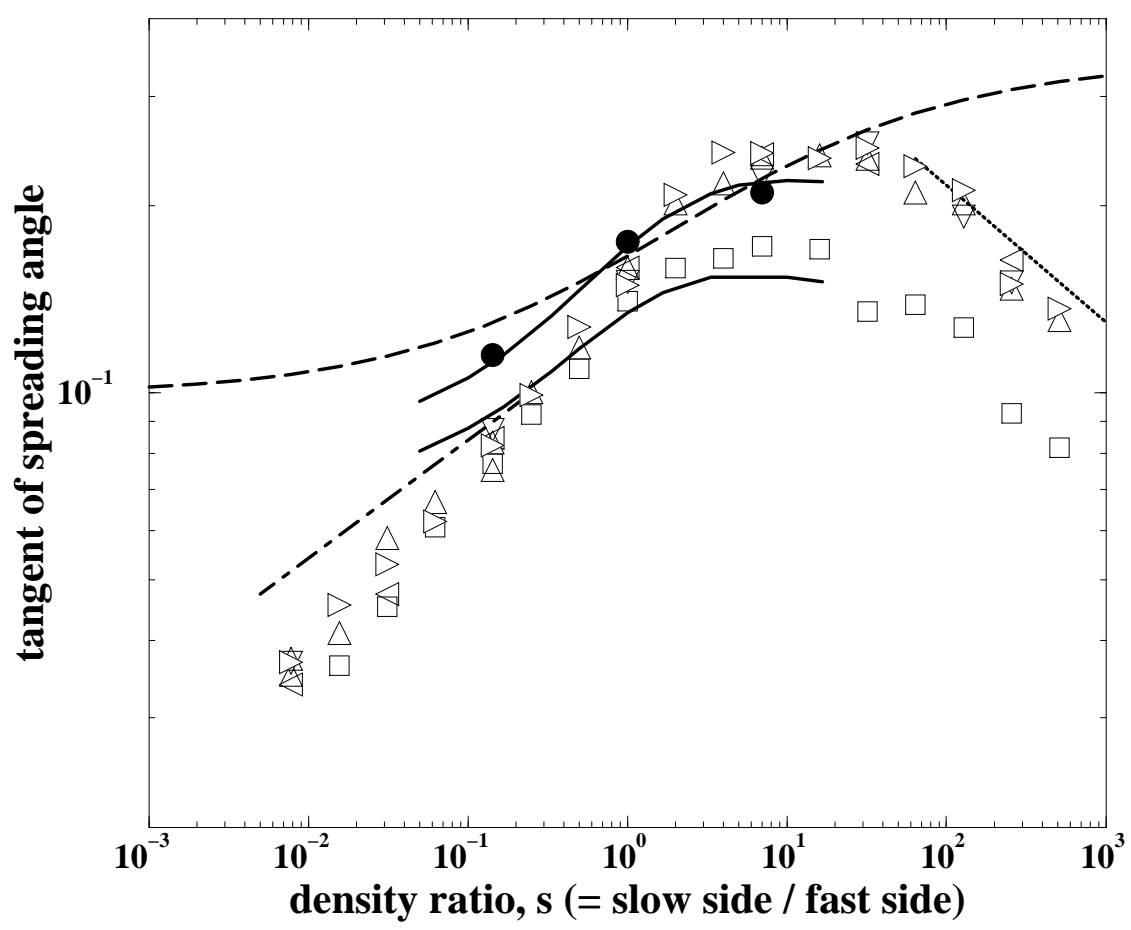

Density growth rate as a function of density ratio $s$ and velocity ratio $r=U_{2} / U_{1}$. The ODT simulations have been matched to the experimental velocity integral thickness at $s=1, r=0.38$, no other adjustments have been made for other density ratios or the other velocity ratio. The visual growth rate from Brown \& Roshko (their figure 7) are given by the filled circles. The model of Dimotakis is the dashed line, while the two solid lines are given by the laminar stability of a compressible boundary layer, recently computed by Sandham at velocity ratios of 0.38 (upper) and 0.48 (lower). The ODT results $(r=0.38$, triangles and $r=0.48$, squares) indicate a power-law behavior when $s$ is very small and when $s$ is large. Similar behavior has been found in round jets: Siebers has found an exponent of 0.19 over the range of $0.005<s<0.24$ in spray jets and Witze has found an exponent of -0.22 over the range of $1<s<7$ in gaseous jets. These exponents of 0.19 (dot-dash) and -0.22 (dotted) are shown above with amplitudes to allow comparison with the ODT behavior. 


\section{Acknowledgments}

We thank Professor Sarkar and Dr. Pantano for sharing their DNS results and thank Professor Sandham for providing the stability results. Discussions with Professor Roshko have been helpful. This work supported by the Division of Chemical Sciences, Office of Basic Energy Sciences, U. S. Department of Energy.

\section{References}

Brown, G. L. and Roshko, A., "On density effects and large structure in turbulent mixing layers", J. Fluid Mech. 64, 775 (1974).

Dimotakis, P. E., "Two-Dimensional Shear-Layer Entrainment", AIAA Jour. 24, 1791 (1986).

Kerstein, A. R., Ashurst, W. T., Wunsch, S. and Nilsen, V., "One-dimensional turbulence: vector formulation and application to free shear flows", J. Fluid Mech. 447, 85 (2001).

Konrad, J. H., "An Experimental Investigation of Mixing in Two-Dimensional Turbulent Shear Flows with Application to Diffusion-Limited Chemical Reactions", Project SQUID Tech. Rept. CIT-8-PU, Dec. 1976.

Pantano, C. and Sarkar, S., "A study of compressibility effects in the high-speed turbulent shear layer using direct simulation", J. Fluid Mech. 451, 329 (2002).

Ramshaw, J. D., "Simple model for mixing at accelerated fluid interfaces with shear and compression", Phys. Rev. E 61, 5339 (2000).

Sandham, N. D. and Reynolds, W. C., "Compressible mixing layer: Linear theory and direct simulation", AIAA J. 28, 618 (1990) and private communication (2001).

Siebers, D. L., "Scaling Liquid-Phase Fuel Penetration in Diesel Sprays Based on MixingLimited Vaporization", SAE Paper 1999-01-0528.

Witze, P. O., "Centerline Velocity Decay of Compressible Free Jets", AIAA Jour. 12, 417 (1974). 


\section{Numerical Methods for Determination of RT and RM Mixing}

S. Dutta, E. George, J. Grove, J. Glimm, X. L. Li,

A. Marchese, D. Sharp, Z. Xu and Y. Zhang

Department of Applied Math and Statistics

SUNY at Stony Brook

Stony Brook, NY 11794 


\section{Outline of the Talk}

- Tracking the contact interface

- Simulations of Random RT Mixing

- Comparison with untracked code

- Analysis of buoyancy acceleration

- Simulation of axisymmetric RM mixing

- Conclusion 


\section{The Front Tracking Method}

- Interface divides space into subdomains

- Riemann solution to propagate interface

- Finite difference with ghost cells

- Coupling interface-interior solutions

- Dynamic resolution of interface topology

- Clear separation of discontinuous states

- Clear separation of material (EOS) 


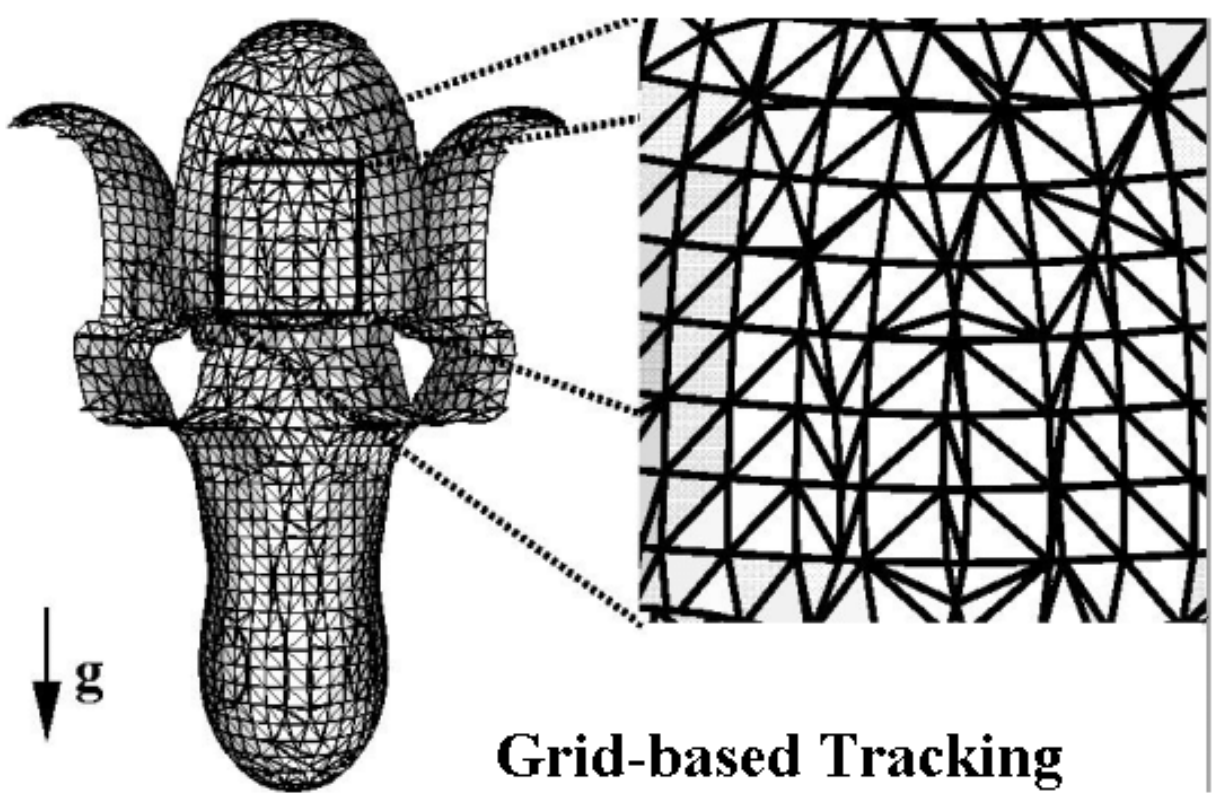


Basic FronTier Test Simulations

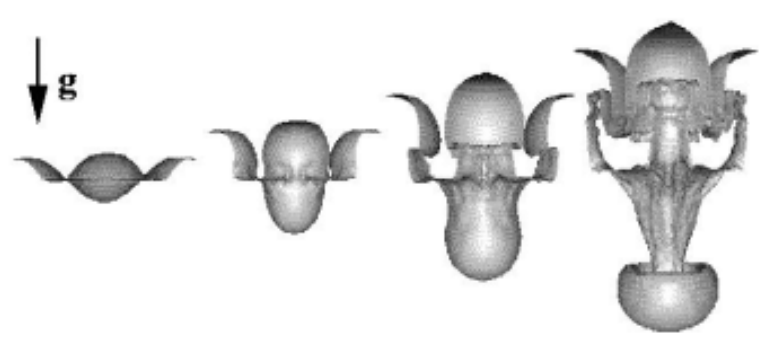

Case Single-2

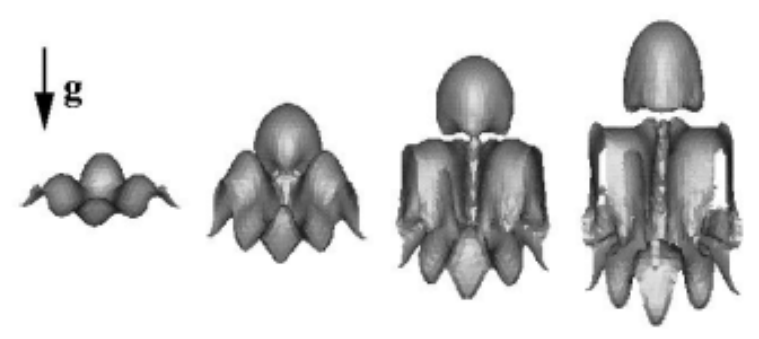

Case Bifure-1 


\section{Separating fluids via ghost-cell}

1. Ghost-cell method

Front Tracking (Glimm, McBryan, etc 1980)

2. Ghost cell has been the key design component of front tracking 


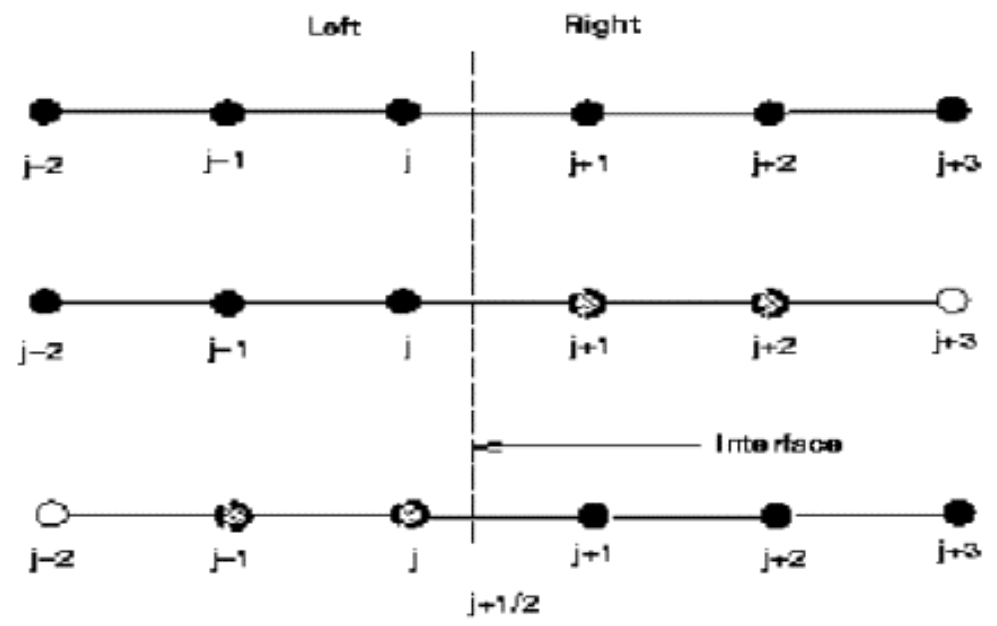

The ghost-cell method 
Numerical diffusion in 1st order upwinding

$$
\begin{gathered}
D u=u_{t}-a u_{x}=0 \\
D_{h} u=\frac{u_{j}^{n+1}-u_{j}^{n}}{\Delta t}-a \frac{u_{j}^{n}-u_{j-1}^{n}}{\Delta x}=0 \\
D_{h} u-D u=-\frac{a \Delta x}{2}\left(1-a \frac{\Delta t}{\Delta x}\right) u_{x x} \\
a \frac{\Delta t_{C F L}}{\Delta x}=1
\end{gathered}
$$

Numerical diffusion is large if $\Delta t<<\Delta t_{C F L}$ 


\section{Numerical dissipation at contact discontinuity}

1. Most higher order schemes switch to 1 st order at discontinuity to avoid oscillation, using either artificial viscosity or limiter.

2. For three characteristic wave speed $u \pm c, u$, the motion of contact is the inertial motion with speed $u$.

3. For first order scheme, the smaller is $\Delta t$ away from the CFL condition, the more dissipative it becomes.

4. It is particularly dissipative at contact for low compressibility

$$
c>u \quad \Delta t=C F L \times \frac{\Delta x}{|u|+c} \ll C F L \times \frac{\Delta x}{|u|}
$$




\section{The $\alpha$ Paradox}

$$
h_{b}=\alpha A g t^{2}
$$

David Youngs and K.Read (1984) 


\section{Read's Experiment (1984)}

3D alcohol/air $\quad$ Exp \#29

39

58

3D NaI soln./Pentane Exp \#33

35

3D NaI soln./Hexane Exp \#62

60
Alpha $=0.073$

0.076

0.077

Alpha $=0.066$

0.071

Alpha $=0.063$

0.073 


\section{Summary of Experiments}

\begin{tabular}{|lc|ll|}
\hline \hline \multicolumn{4}{|c|}{ Experiments } \\
\hline Read/Youngs & $' 84$ & $\alpha_{b} \sim 0.58-0.65$ & $2 \mathrm{D}$ \\
& & $\alpha_{b} \sim 0.063-0.077$ & $3 \mathrm{D}$ \\
\hline Kucherenko & 91 & $\alpha_{b} \sim 0.07$ & 3D \\
\hline Snider/Andrews & 94 & $\alpha_{b} \sim 0.07 \pm 0.007$ & $3 \mathrm{D}$ \\
\hline Schneider/Dimonte/Remington & 99 & $\alpha_{b} \geq 0.054$ & 3D \\
\hline Dimonte/Schneider & 99 & $\alpha_{b} \sim 0.05 \pm 0.01$ & 3D \\
\hline \hline
\end{tabular}

Bliznetsov $\quad 0.1$

Shestachchenko $\quad 0.04$ 


\section{FronTier Simulation of Random RT Mixing}

\section{Simulation Random-3}

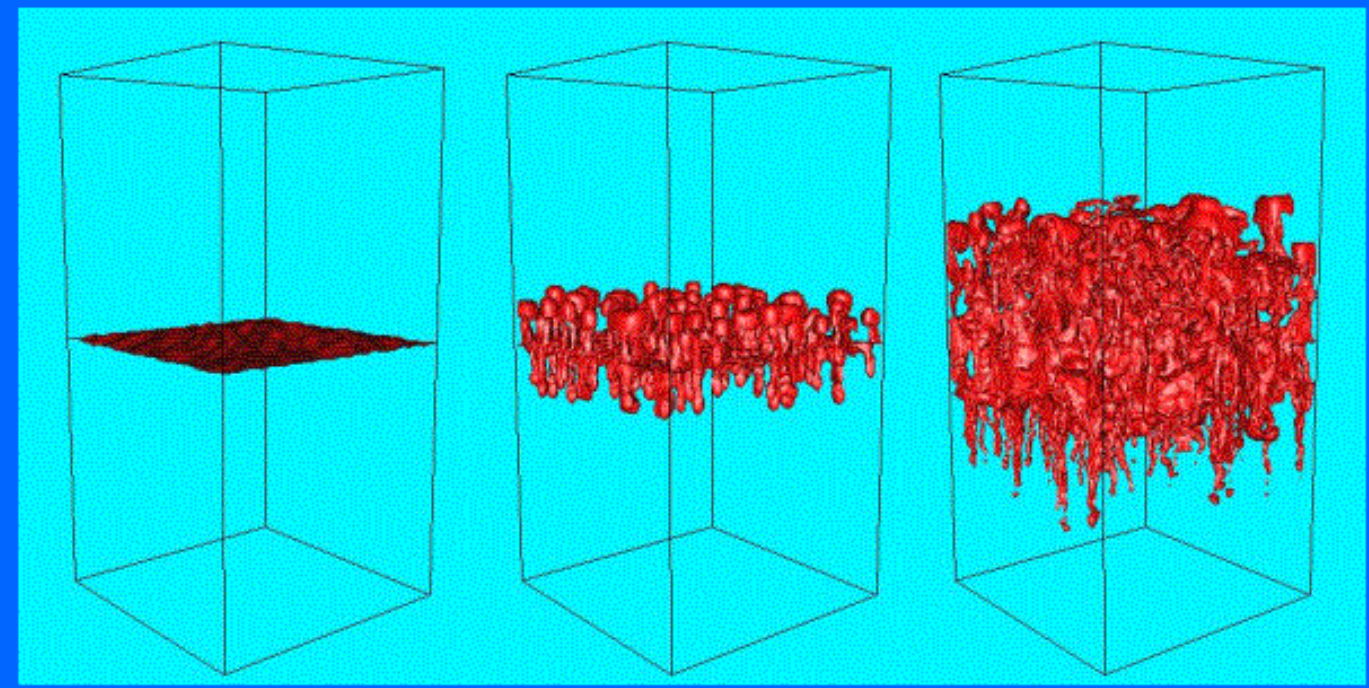

$$
\text { Compressibility }=0.06 \quad \text { Alpha } \sim 0.08
$$




\section{Movie Random-4}

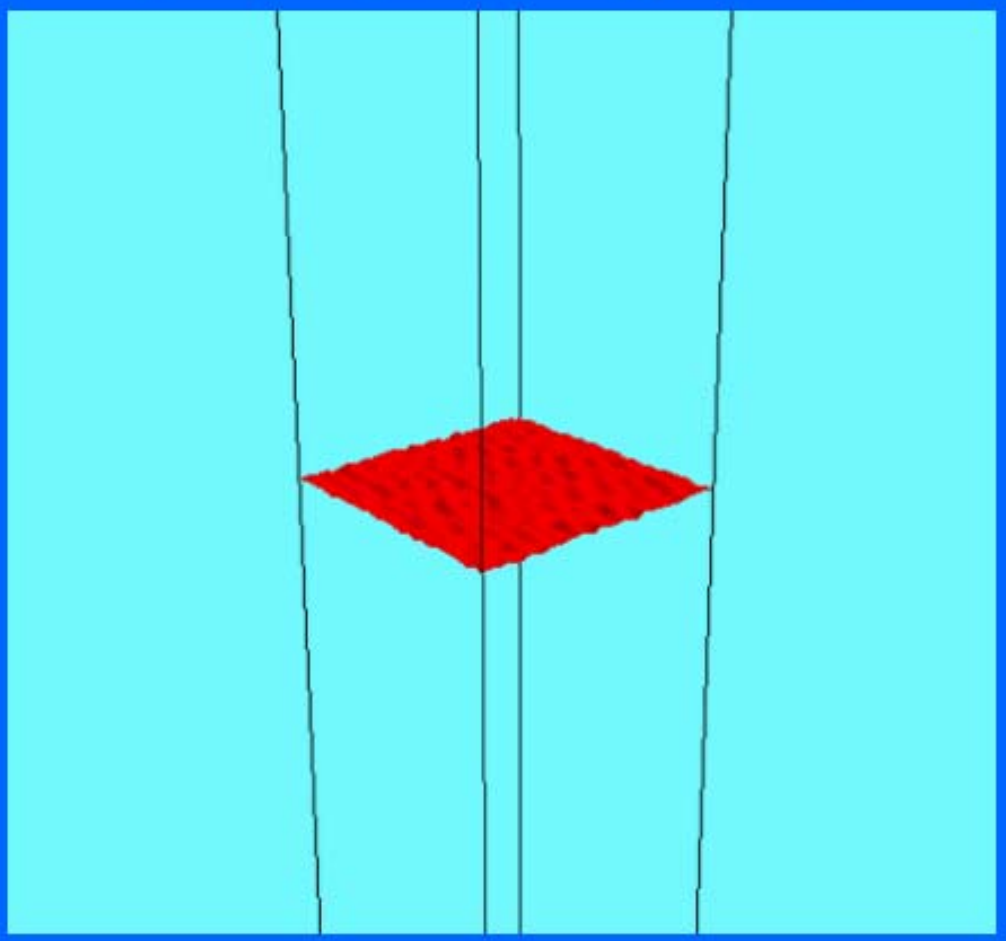



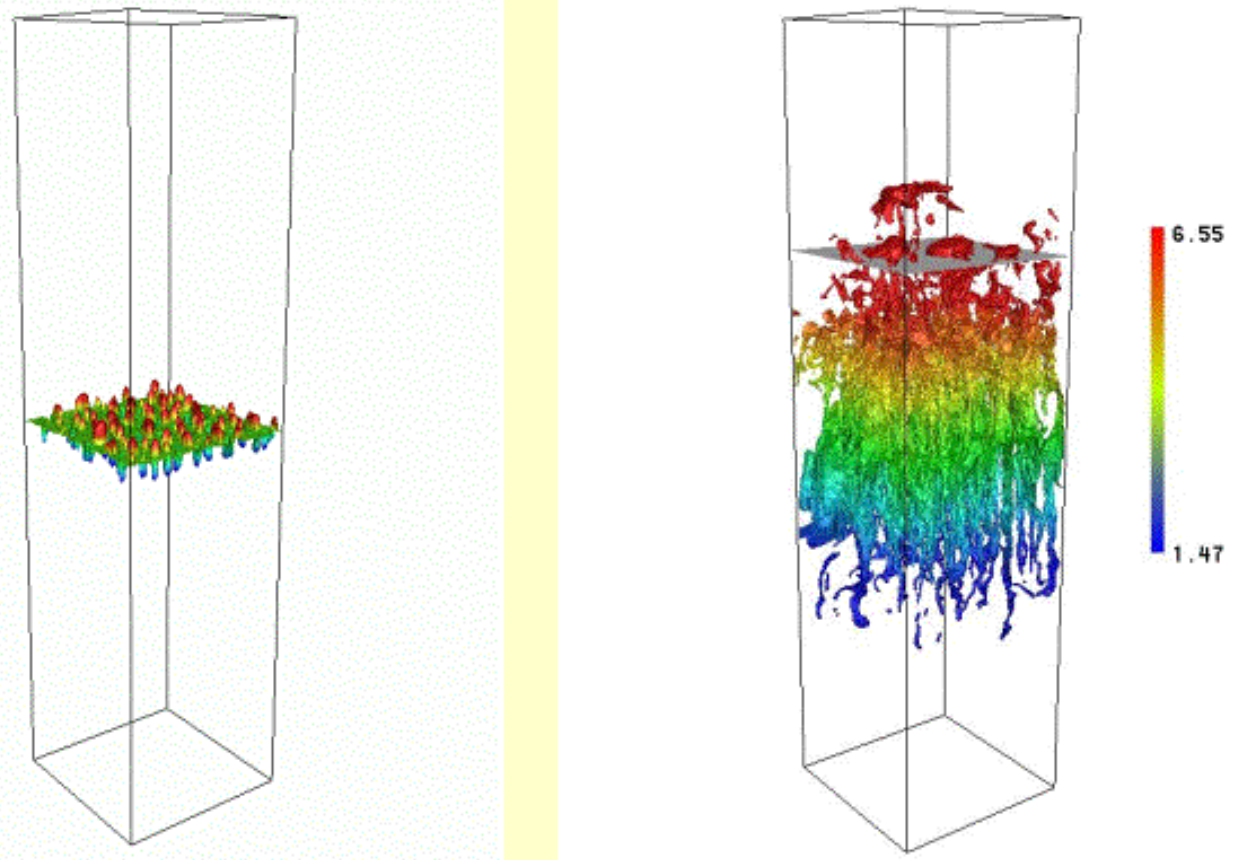

FronTier simulation of RT mixing, with $\mathrm{A}=0.5$.

Left: early time. Right: late time. On $128 * 128 * 512$ grid 


\section{The Alpha of Bubbles}

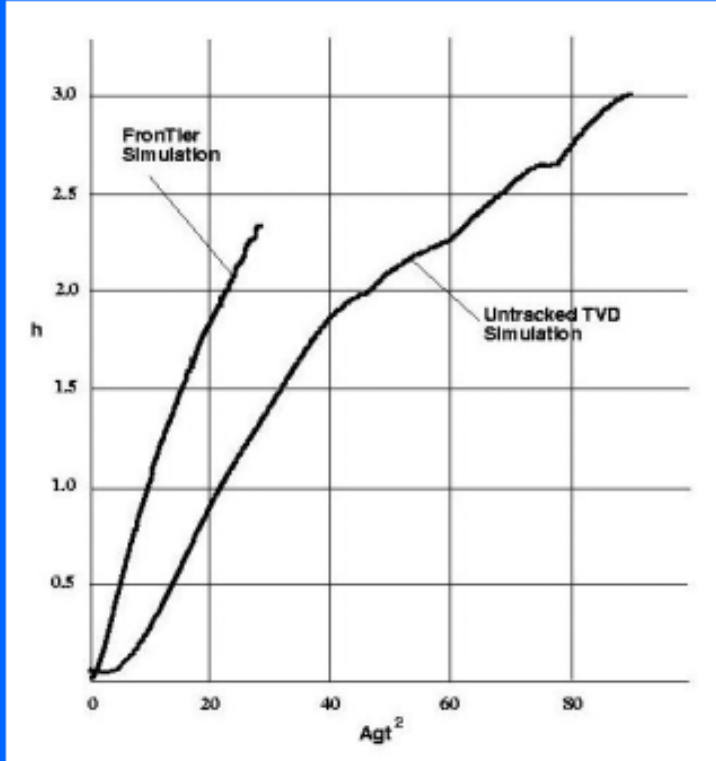

FronTier:

Alpha $=0.08$

TVD:

Alpha $=0.025-0.045$ 


\section{FronTier}
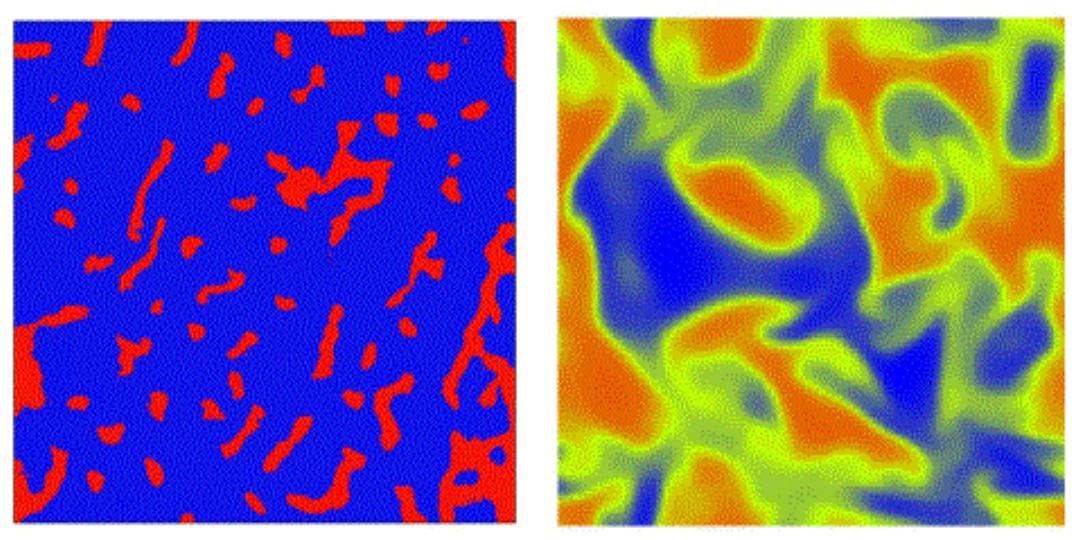

Agt $\stackrel{2}{=} 25.3 \quad h=4.16$ Density plot 


\section{The Archimedes Principle}

- The buoyancy force on an immersed body equals to the weight of the fluid the immersed body has repelled.

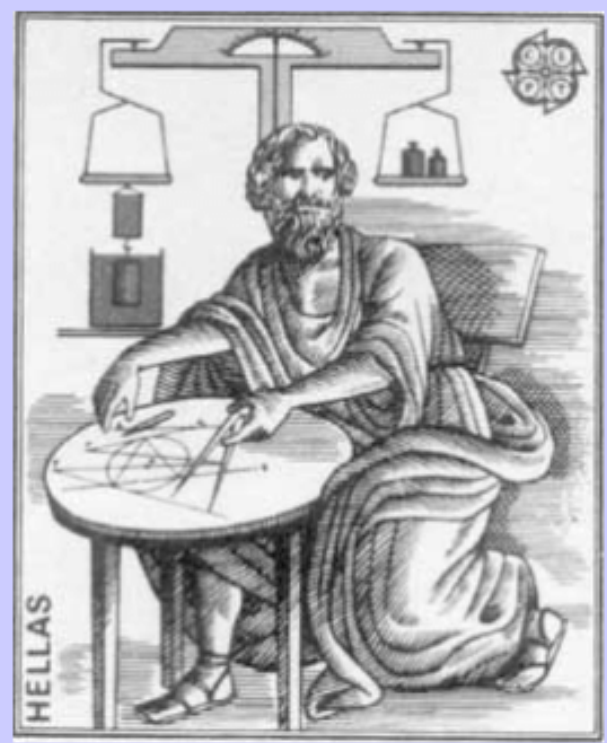



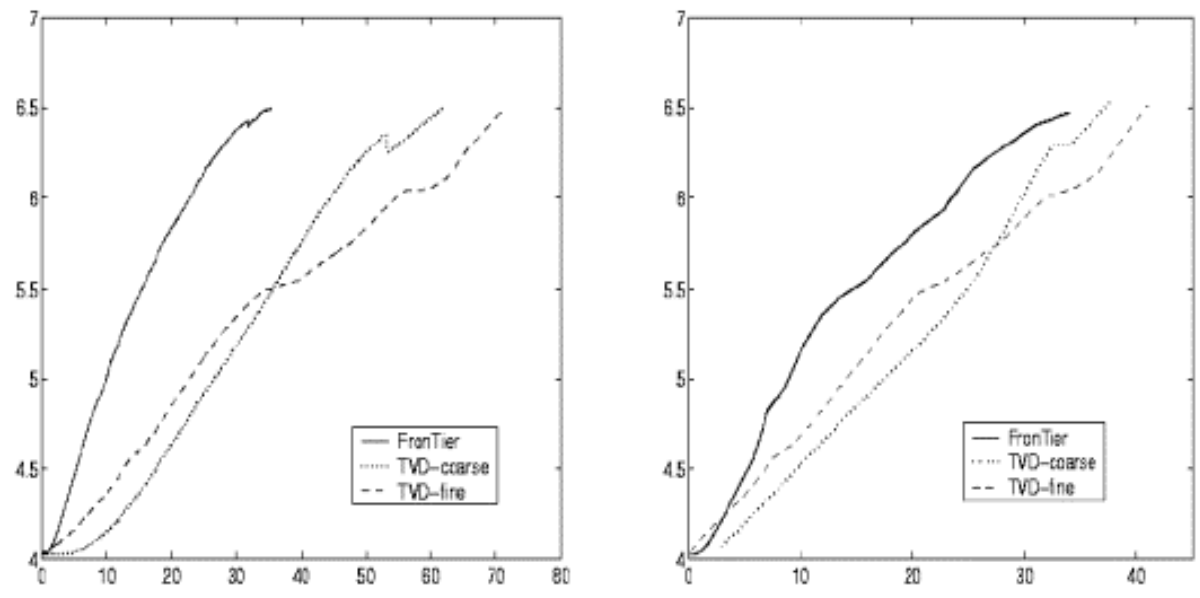

Normalization of alpha with effective Atwood number 

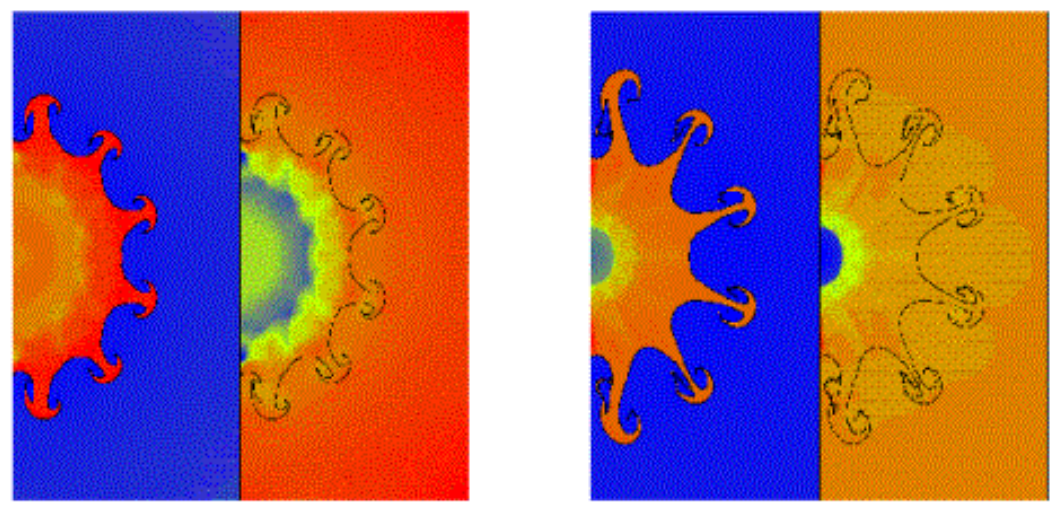

A 2D simulation of spherical RM instability with axisymmetry The Mach number of the imploding shock is 1.2 and the Atwood number is $2 / 3$. The inner gas is $S F_{6}$ and the outer gas is air. 


\section{FronTier Simulation of NLUF 2 Experiment}

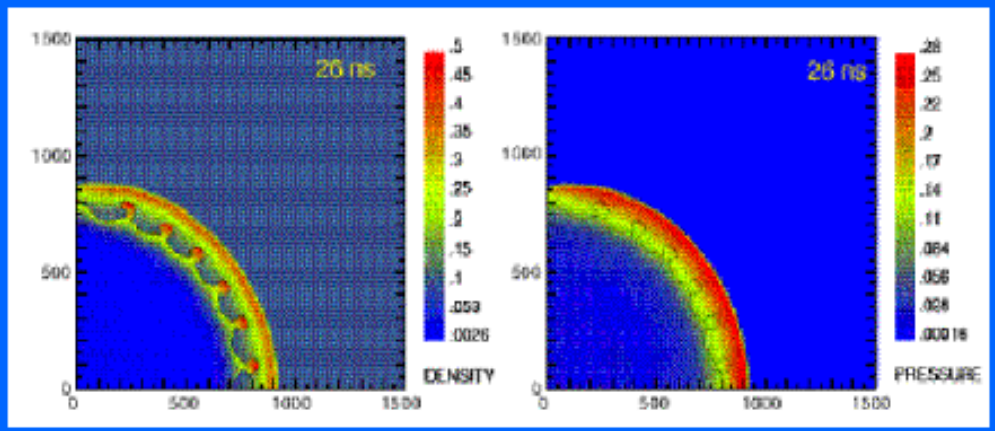

CHGe capsule surrounded by CRF foam. The RM instability is driven by strong shock of Mach number 300 by the Omega laser 


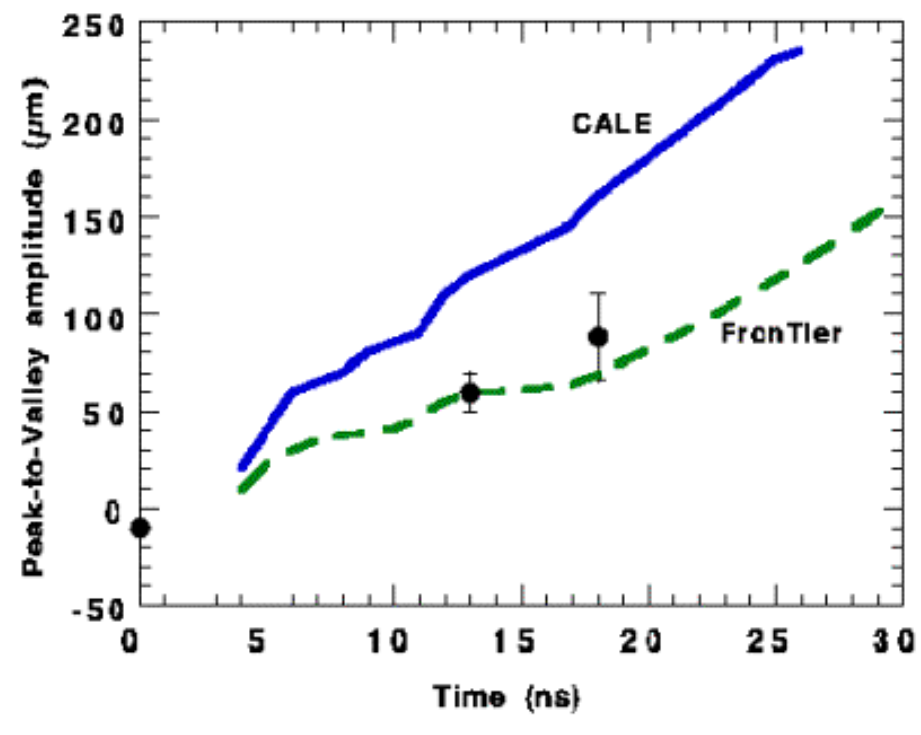

Comparison of FronTier amplitude and the CALE Simulation with the experiment 


\section{Conservative Interface-Interior Coupling}

The conservation law:

$$
u_{t}+f(u)_{x}=0
$$

The Rankine-Hugoniot condition:

$$
f\left(u_{L}\right)-s u_{L}=f\left(u_{R}\right)-s u_{R}
$$


Neglect higher order term and note that

$$
\int_{\Delta V} u d V=\int_{S_{M}} u v_{n} d S \Delta t
$$

We have the integral form of conservation

$$
\frac{\partial}{\partial t} \int_{V} u d V-\int_{s_{M}} u v_{n} d S+\int_{S} F_{n}(u) d S=0
$$

This can also be written as

$$
\frac{\partial}{\partial t} \int_{V} u d V+\int_{S_{F}} F_{n}(u)+\int_{S_{M}}\left(F_{n}(u)-v_{n} u\right) d S=0
$$

Or simply

$$
\frac{\partial}{\partial t} \int_{V} u d V+\oint_{S}\left(F_{n}(u)-v_{n} u\right) d S=0
$$




\section{Conclusion}

1. Numerical diffusion is a serious problem in simulation of contact surface in gas dynamics, especially when $c>u$.

2. Front tracking prevents such diffusion through ghost-cell method, retains correct buoyancy force in RT simulation.

3. The introduction of local effective Atwood number bridges the gap between tracked and untracked simulations.

4. Front tracking shows agreement in axisymmetric spherical RM instability.

5. Front tracking is will replace ghost-cell by conservative tracking using dynamic flux at a cell with moving boundary. 


\section{One-Dimensional Simulation of the Effects of Unstable Mix on Neutron and Charged-Particle Yield from Laser-Driven Implosions}

UR 粼 MIT

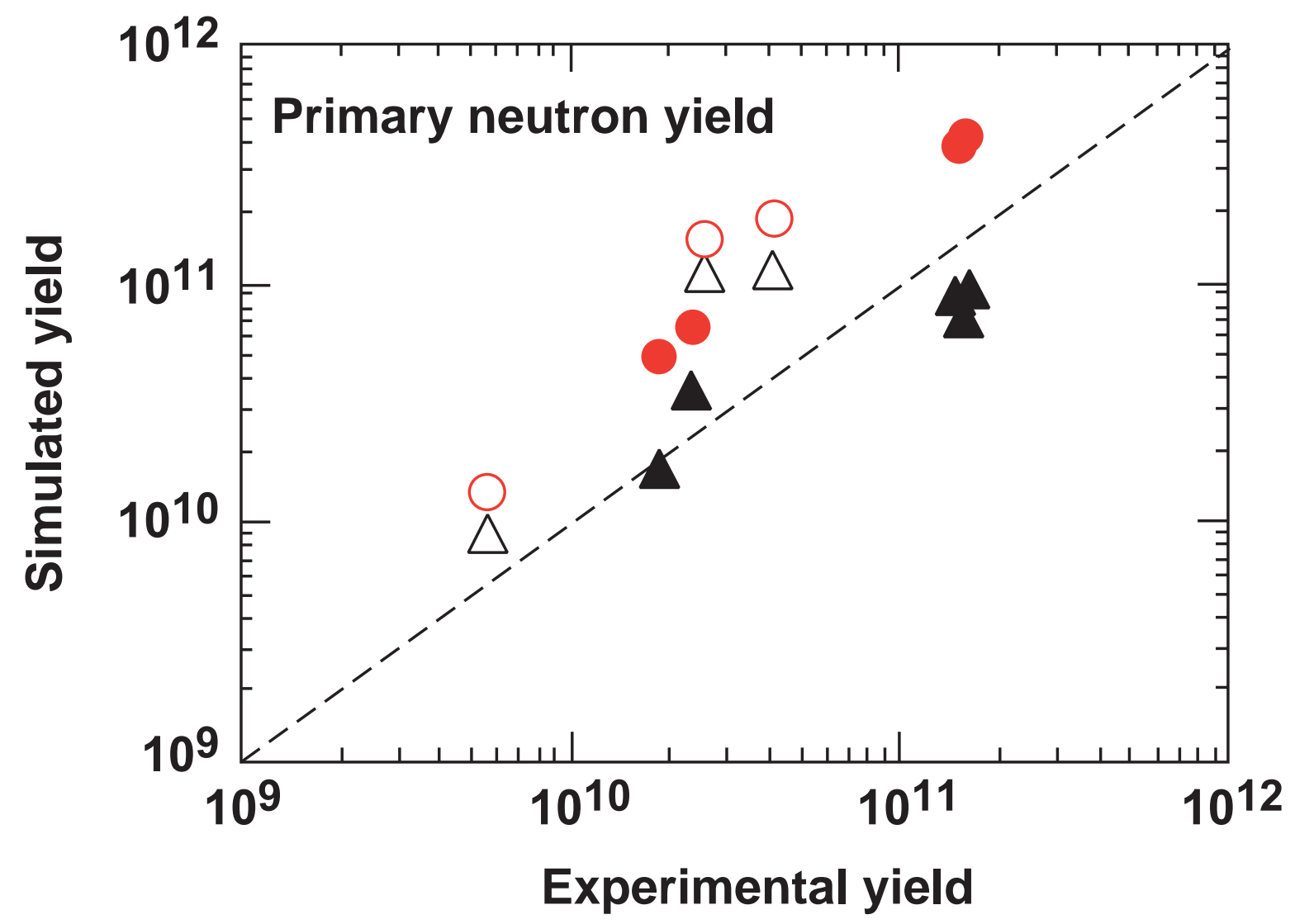

R. Epstein

University of Rochester

Laboratory for Laser Energetics 8th International Workshop on the Physics of Compressible Turbulent Mixing

Pasadena, CA 10-14 December 2001 


\section{Collaborators}

J. A. Delettrez, V. Yu. Glebov, V. N. Goncharov, P. W. McKenty,

P. B. Radha, S. Skupsky, V. A. Smalyuk, and C. Stoeckl

Laboratory for Laser Energetics

University of Rochester

J. Frenje, C. K. Li, R. D. Petrasso, and F. H. Séguin

Massachusetts Institute of Technology 


\section{Summary}

Mix effects on particle yields can be described effectively by mix modeling in the 1-D hydrocode LILAC

- The mix model includes the transport of target constituents, thermal energy, and turbulent energy due to both the acceleration and deceleration instabilities.

- Including mix in 1-D simulations of experiments provides improved predictions of primary and secondary particle yields over a broad range of target performance. 


\section{Outline}

- Modeling of mix in 1-D

- Comparison of simulated and experimental yields

- Secondary neutron and proton production

- Conclusions 


\section{"Bubble and spike" mixing thickness is obtained from a multimode Rayleigh-Taylor perturbation model ${ }^{*}$

- - Mixing boundaries - Ablation surface and fuel-pusher interface

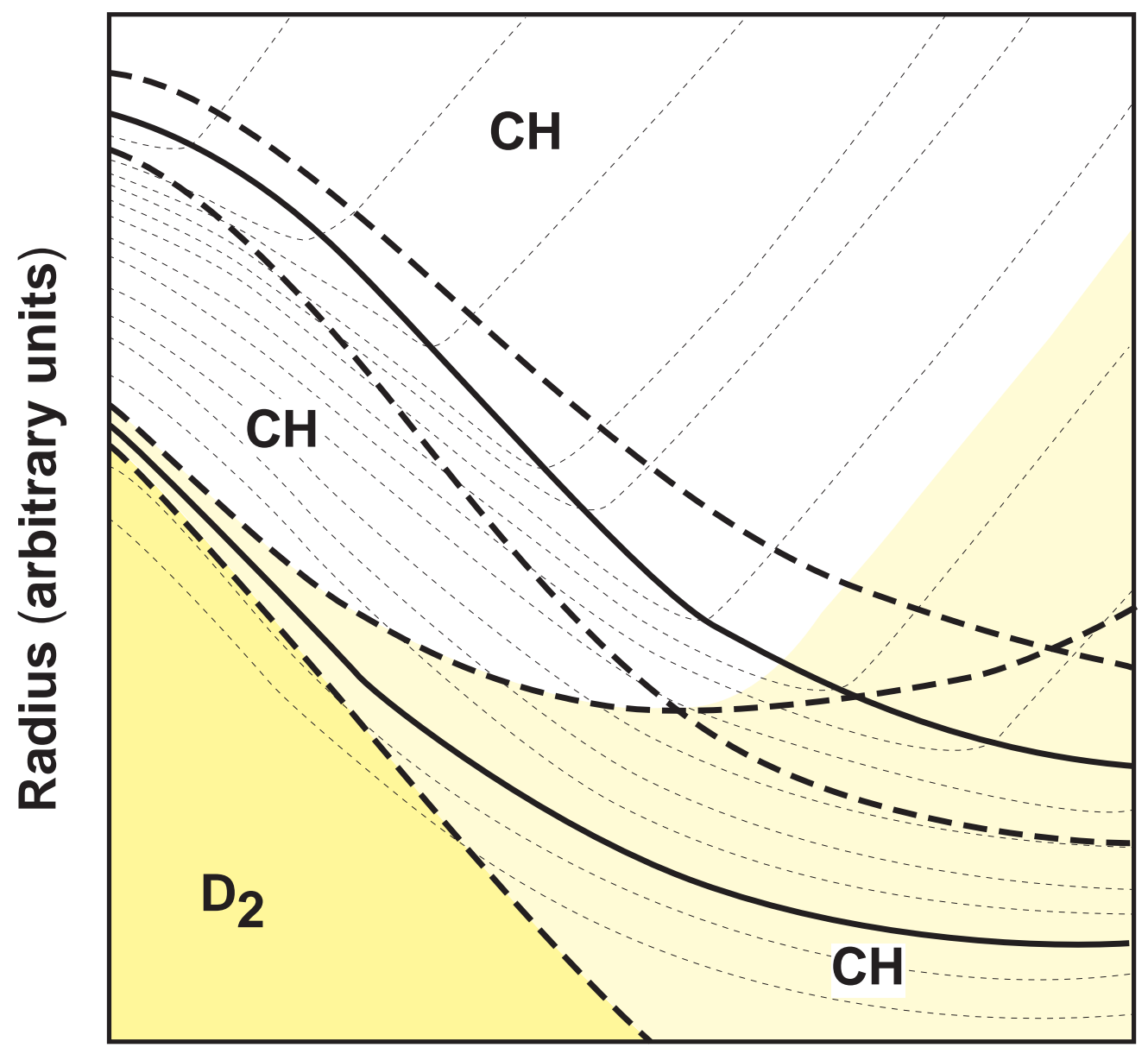

Time (arbitrary units)
- $\frac{\mathbf{d}^{2}}{\mathbf{d t}^{2}} \mathbf{A}_{\ell}=\gamma^{2}(\mathbf{t}) \mathbf{A}_{\ell}$ including Bell-Plesset effects

- Takabe/Betti form for $\gamma^{2}(t)$

- Haan saturation procedure for

$$
\mathbf{A}_{\ell}(\mathrm{t})>\frac{2 \mathrm{R}(\mathrm{t})^{*}}{\ell^{2}}
$$

- Initial perturbation spectrum $A_{\ell}\left(t=t_{0}\right)$ specified at ablation surface and fed through to fuel-pusher interface over time.

- Mix is modeled as a diffusive transport process.

*S. W. Haan, Phys. Rev. A 39, 5812 (1989). 


\section{The mix model is based on carefully formulated phenomenology}

- Perturbations due to single-beam imprint were obtained from $O R C H I D$ calculations based on measured single-beam nonuniformity.

- Beam-imbalance effects are based on power-imbalance measurements from each shot and the geometrical superposition of the acceleration distributions of 60 beams.

- The formulation of the perturbation growth using fully time-dependent perturbation equations allows secular nonuniform irradiation effects and "feedthrough" from the outer to the inner instabilities to be treated as driving terms, rather than as instantaneous effects.

- Plausible flux limitation of the diffusive mix transport is obtained by allowing that the mixed constituent profiles can remain self-similar under expansion. 


\section{Perturbation equations are best written in terms of a mass amplitude}

Incompressible planar approximation

$$
\begin{array}{ll}
\frac{\mathbf{d}^{2}}{\mathbf{d t}^{2}} \mathbf{A}_{\ell}=\gamma_{0}^{2} \mathbf{A}_{\ell} & \gamma_{ \pm}= \pm \gamma_{0} \\
\mathbf{A}_{\ell \pm}=\mathbf{A}_{\ell \mathbf{0}} \mathbf{e}^{\gamma_{ \pm} \mathbf{t}} & \gamma_{0}^{2}=\frac{\ell}{\mathbf{R}}\left(\frac{\rho_{2}-\rho_{1}}{\rho_{2}+\rho_{1}}\right) \ddot{\mathbf{R}}
\end{array}
$$

Compressible spherical solution (i.e., Bell-Plesset ${ }^{\star}$ )

$$
\begin{aligned}
& \left(-\gamma_{\rho}-\gamma_{\mathbf{R}}+\frac{\mathbf{d}}{\mathbf{d t}}\right) \frac{\mathbf{d}}{\mathbf{d t}}\left(\mathbf{A}_{\ell} \rho \mathbf{R}^{2}\right)=\gamma_{\mathbf{0}}^{2}\left(\mathbf{A}_{\ell} \rho \mathbf{R}^{2}\right) \quad \gamma_{\mathbf{R}}=\dot{\mathbf{R}} / \mathbf{R}, \gamma_{\rho}=\dot{\rho} / \rho \\
& \gamma_{0}^{2}=\frac{\ell(\ell+\mathbf{1})}{\mathbf{R}} \frac{\left(\rho_{\mathbf{2}}-\rho_{\mathbf{1}}\right) \ddot{\mathbf{R}}}{\left[\ell \rho_{\mathbf{2}}+(\ell+\mathbf{1}) \rho_{\mathbf{1}}\right]} \quad \gamma_{ \pm}=\frac{1}{\mathbf{2}}\left(\gamma_{\rho}+\gamma_{\mathbf{R}}\right) \pm \sqrt{\gamma_{0}^{2}+\frac{1}{\mathbf{4}}\left(\gamma_{\rho}+\gamma_{\mathbf{R}}\right)^{2}}
\end{aligned}
$$




\section{Mix is modeled in 1-D as a diffusive transport process}

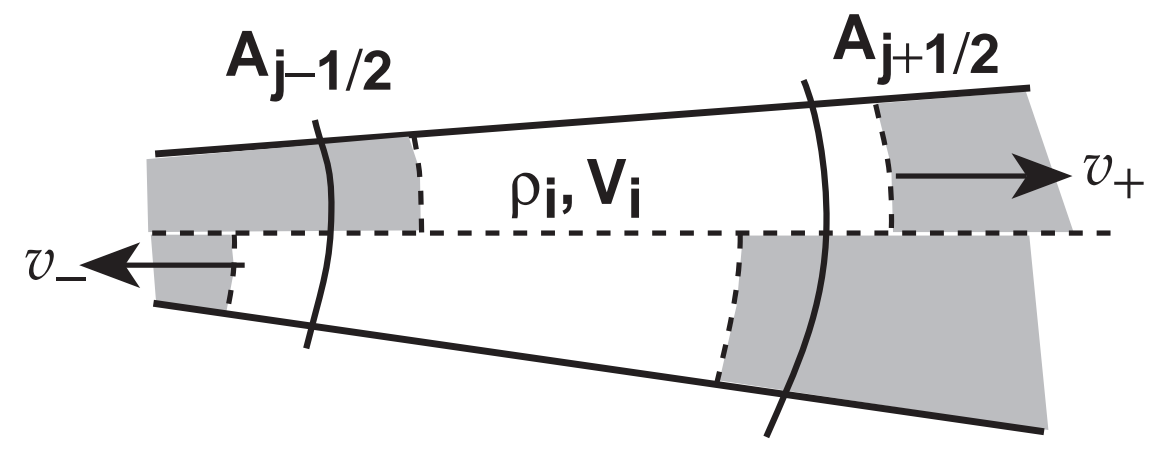

Advection to and from nearest-neighbor zones is expressed as diffusion in 1-D.

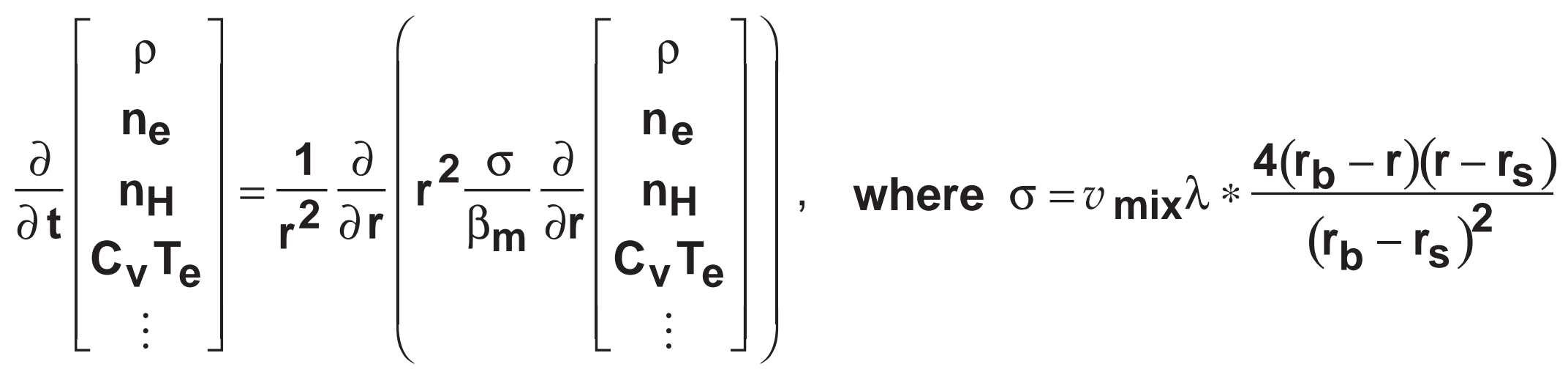

$v_{\text {mix }}$ : obtained from trajectories of mix-region boundaries

$\lambda$ : scale length of turbulence structure from rms perturbation wavelength

f: "flux limit" parameter $\frac{\sigma}{\beta_{\mathbf{m}}}\left|\frac{\partial \rho}{\partial \mathbf{r}}\right| \Rightarrow \operatorname{Min}\left[\frac{\sigma}{\beta_{\mathbf{m}}}\left|\frac{\partial \rho}{\partial \mathbf{r}}\right|, \mathbf{f} \rho v_{\text {mix }}\right]$ 


\section{The mix computation is done as a separate step within the 1-D hydrocode}

- Diffusive transport of constituent densities $\{\phi\}$ keeps zone masses constant:

$$
\begin{aligned}
& \frac{\mathbf{d}}{\mathbf{d t}}\left(\mathbf{V}_{\phi}\right)_{\mathbf{j}}=\left[\mathbf{A}\left(\mathbf{u}+\frac{\sigma}{\beta_{\mathbf{m}}} \frac{\partial}{\partial \mathbf{r}}\right)_{\hat{\mathbf{j}+\mathbf{1} / \mathbf{2}}}-\left[\mathbf{A}\left(\mathbf{u}+\frac{\sigma}{\beta_{\mathbf{m}}} \frac{\partial}{\partial \mathbf{r}}\right) \phi_{\mathbf{j}-\mathbf{1} / \mathbf{2}}\right.\right. \\
& (\rho \mathbf{u})_{\mathbf{j}+\mathbf{1} / \mathbf{2}}=-\left[\frac{\sigma}{\beta_{\mathbf{m}}} \frac{\partial \rho}{\partial \mathbf{r}}\right]_{\mathbf{j}+\mathbf{1} / \mathbf{2}} \\
& \frac{\mathbf{d}}{\mathbf{d t}} \mathbf{M}_{\mathbf{j}}=\frac{\mathbf{d}}{\mathbf{d t}}\left(\mathbf{V}_{\rho}\right)_{\mathbf{j}}=\mathbf{0}
\end{aligned}
$$

- Hydrodynamics in terms of total mass velocity ${ }^{*} v_{\mathbf{j}+\mathbf{1} / \mathbf{2}}=\left\langle v_{\mathbf{j}+\mathbf{1} / \mathbf{2}}\right\rangle+\mathbf{u}_{\mathbf{j}+\mathbf{1} / \mathbf{2}}$

$$
\frac{\mathbf{d} \rho}{\mathbf{d t}}=\frac{-\rho}{\mathbf{r}^{\mathbf{2}}} \frac{\partial}{\partial \mathbf{r}}\left(\mathbf{r}^{\mathbf{2} v}\right), \quad \rho \frac{\mathbf{d} v}{\mathbf{d t}}=-\frac{\partial}{\partial \mathbf{r}}\left(\mathbf{P}+\mathbf{Q}+\mathbf{P}_{\mathbf{T}}+\mathbf{Q}_{\mathbf{T}}\right)
$$

*Leith, UCRL-96036 (1986). 


\section{Mix-motion energy is computed as turbulent energy in a " $k-\lambda$ " model}

- Turbulent energy density k:

$$
\mathbf{P}_{\mathbf{T}}=\frac{2}{3} \mathbf{k}, \quad \mathbf{Q}_{\mathbf{T}}=-\frac{\mathbf{4}}{\mathbf{3}} \frac{\sigma}{\beta_{\mathbf{q}}} \frac{\partial v}{\partial \mathbf{r}}, \quad \sigma=v_{\text {mix }} \lambda
$$

- Buoyant force as source of $k$ :

$$
\mathbf{S}=\max \left(\rho \mathbf{u} \frac{\mathbf{d} \mathbf{v}}{\mathbf{d t}}, \mathbf{0}\right), \quad \rho \mathbf{u}=-\frac{\sigma}{\beta_{\mathbf{m}}} \frac{\partial \rho}{\partial \mathbf{r}}
$$

- Dissipation rate:

$$
\varepsilon=C_{\varepsilon} \frac{k^{3 / 2}}{\rho^{1 / 2} \lambda}
$$

- Evolution:

$$
\begin{aligned}
& \frac{\mathbf{d k}}{\mathbf{d t}}=\frac{\mathbf{1}}{\mathbf{r}^{2}} \frac{\partial}{\partial \mathbf{r}}\left(\mathbf{r}^{2} \frac{\sigma}{\beta_{\mathbf{k}}} \frac{\partial \mathbf{k}}{\partial \mathbf{r}}\right)-\left(\mathbf{P}_{\mathbf{T}}+\mathbf{Q}_{\mathbf{T}}\right) \frac{\mathbf{1}}{\mathbf{r}^{2}} \frac{\partial}{\partial \mathbf{r}}\left(\mathbf{r}^{2} v\right)+\mathbf{S}-\varepsilon \\
& \frac{d E_{i}}{d t}=\frac{1}{r^{2}} \frac{\partial}{\partial r}\left(r^{2} \frac{\sigma}{\beta_{m}} \frac{\partial E_{i}}{\partial r}\right)+\varepsilon-S+\ldots \text { etc. }
\end{aligned}
$$




\section{Growth rates of perturbations of arbitrary density profiles are estimated using Sturm-Liouville theory}

- The Rayleigh perturbation equation for an arbitrary density profile is a Sturm-Liouville eigenvalue equation:

$$
\frac{1}{\mathbf{r}^{2}} \frac{\mathbf{d}}{\mathbf{d r}}\left[\mathbf{r}^{2} \rho \frac{\mathbf{d} \mathbf{w}_{\ell}}{\mathbf{d r}}\right]-\rho \frac{\ell(\ell+1)}{\mathbf{r}^{2}} \mathbf{w}_{\ell}+\frac{1}{\gamma_{\ell}^{2}} \frac{\ell(\ell+1)}{\mathbf{r}^{2}} \mathbf{g} \frac{\mathbf{d} \rho}{\mathbf{d r}} \mathbf{w}_{\ell}=0
$$

- The perturbation growth rate is given by a variational expression:

$$
\frac{\gamma_{\ell}^{2}}{\mathbf{g}}=\operatorname{Max}\left\{\int_{0}^{\infty} \frac{\ell(\ell+1)}{\mathbf{r}^{2}} \frac{\mathbf{d} \rho}{\mathbf{d r}} \mathbf{w}_{\ell}^{2} \mathbf{r}^{2} \mathbf{d r} / \int_{0}^{\infty} \rho\left[\left(\frac{\mathbf{d} \mathbf{w}_{\ell}}{\mathbf{d r}}\right)^{2}+\frac{\ell(\ell+1)}{\mathbf{r}^{2}} \mathbf{w}_{\ell}^{2}\right] \mathbf{r}^{2} \mathbf{d r}\right\}
$$

- Estimates obtained using only moderately accurate eigenfunctions are accurate to second order in variations of the postulated eigenfunction:

$$
\mathbf{w}_{\ell} \approx\left[(\mathbf{r} / \mathbf{L})^{\ell}, \mathbf{r}<\mathbf{L} / \mathbf{2} ; \mathbf{a}+\mathbf{b}\left(\mathbf{r}-\mathbf{r}_{\mathbf{0}}\right)+\mathbf{c}\left(\mathbf{r}-\mathbf{r}_{\mathbf{0}}\right)^{2},-\mathbf{L} / \mathbf{2}<\mathbf{r}<\mathbf{L} / \mathbf{2} ;(\mathbf{r} / \mathbf{L})^{-(\ell+1)}, \mathbf{r}>\mathbf{L} / \mathbf{2}\right]
$$




\section{A constrained set of static model core properties reproduces most experimental observables}

- 1-ns square, $23 \mathrm{~kJ}, 20-\mu \mathrm{m}-\mathrm{CH}$ shells, 15 atm fill, CR 15
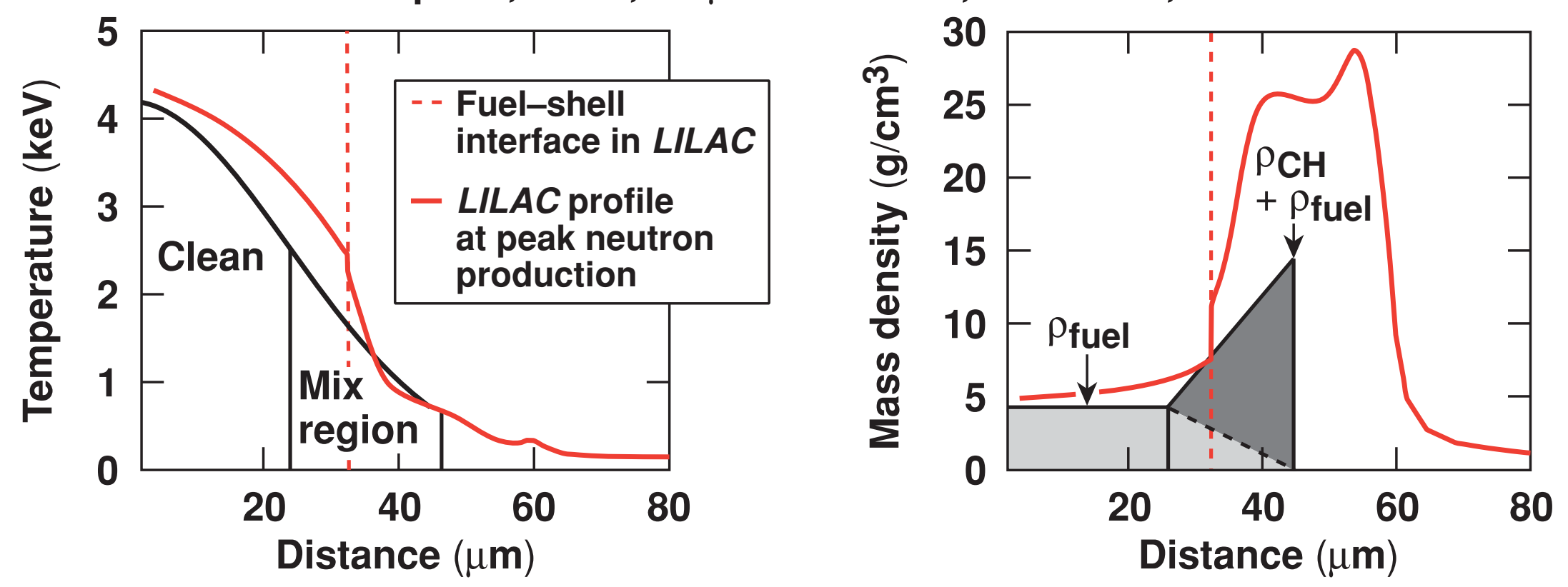

\begin{tabular}{|l|l|c|c|}
\cline { 3 - 4 } \multicolumn{2}{c|}{} & Measured & $\%$ of model \\
\hline \multirow{2}{*}{$\mathrm{D}_{2}$} & Max: neutron rate & $(9 \pm 2) \times 10^{20}$ & 120 \\
\cline { 3 - 4 } & Burn width $(\mathrm{ps})$ & $170 \pm 20$ & 94 \\
\cline { 3 - 4 } & $\mathrm{T}_{\text {ion }}\left(\mathrm{D}_{2}\right)(\mathrm{keV})$ & $3.7 \pm 0.5$ & 90 \\
\cline { 2 - 4 } & Secondary neutron ratio & $(2.1 \pm 0.4) \times 10^{-3}$ & 90 \\
\cline { 2 - 4 } & Secondary proton ratio & $(1.8 \pm 0.3) \times 10^{-3}$ & 100 \\
\hline
\end{tabular}




\section{Mix modeling improves the agreement of simulated primary neutron yield with implosion data}

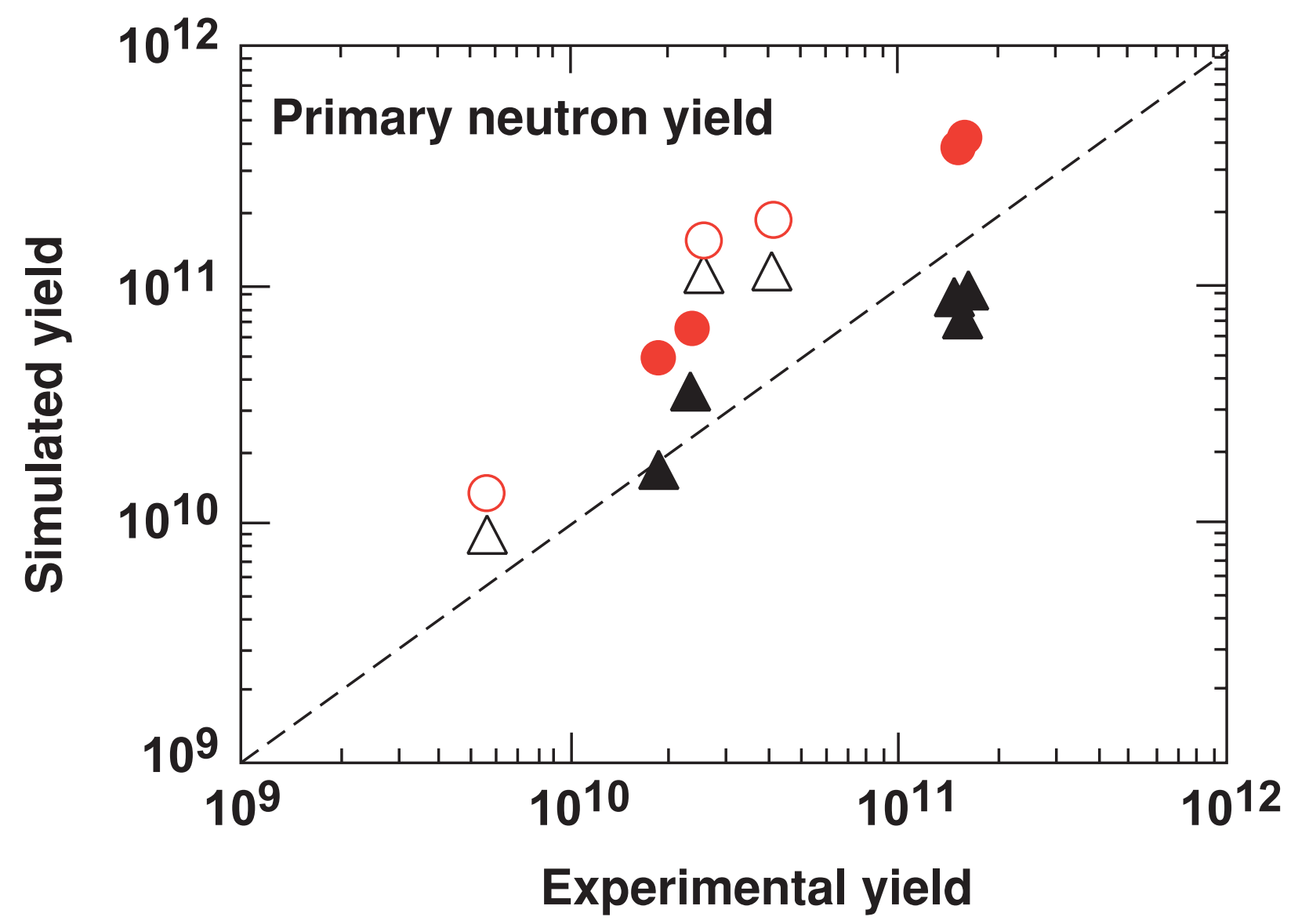

MIT

\begin{tabular}{l}
\hline Clean \\
$\Delta$ Mix \\
Clean, 3 atm \\
$\triangle$ Mix, 3 atm
\end{tabular}

- Data from eight shots (August 2000)

- Pure-CH shells, 20-27 $\mu \mathrm{m}, 900-\mu \mathrm{m}$ diameter, $\mathrm{D}_{2}$ fill, 3-25 atm 
Primary yield ratios indicate that implosion degradation is comparable to the predictions of mix modeling

LLE

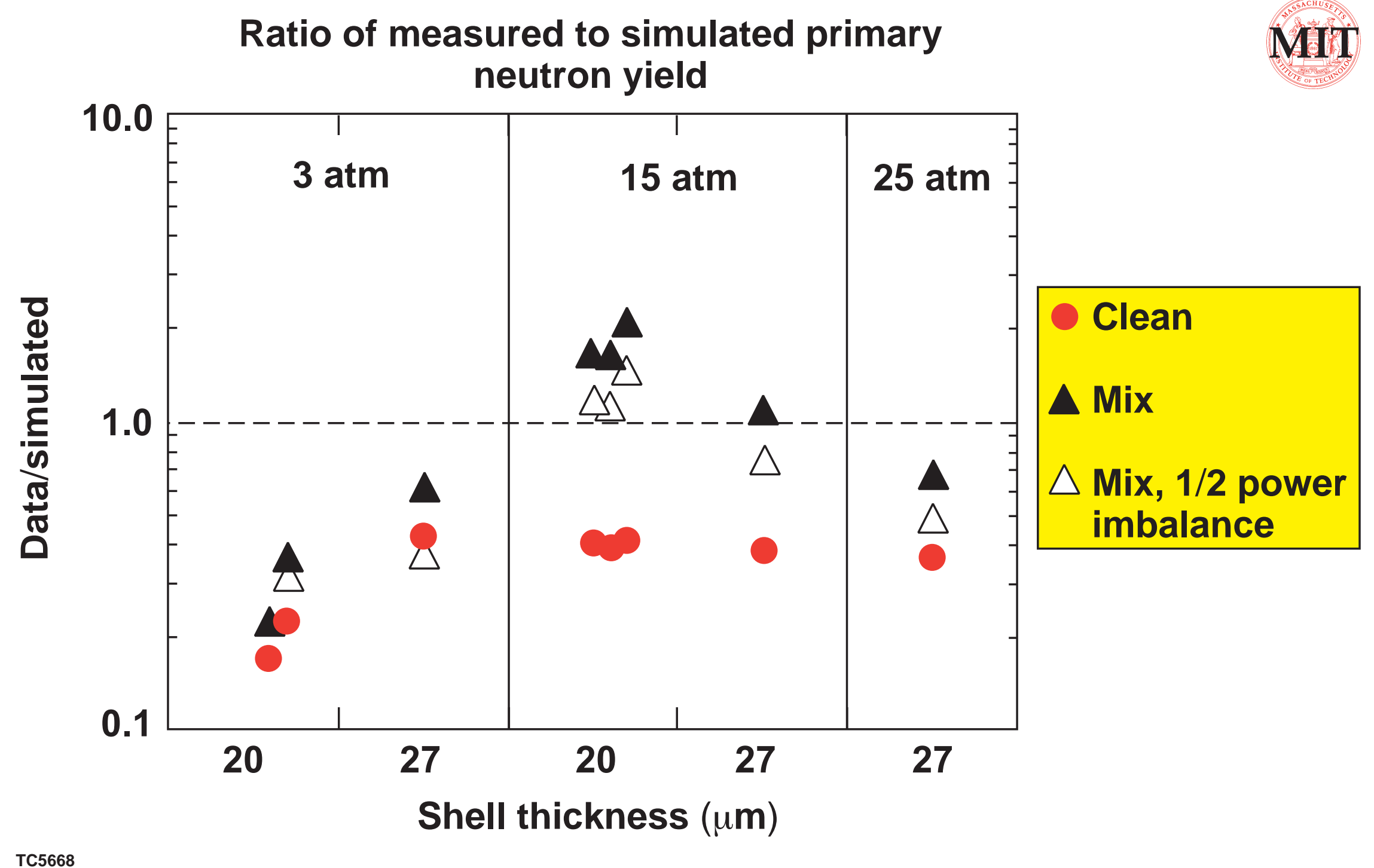


Simulated and measured neutron-averaged temperatures show some improved agreement with mix modeling

UR 䊁

MIT

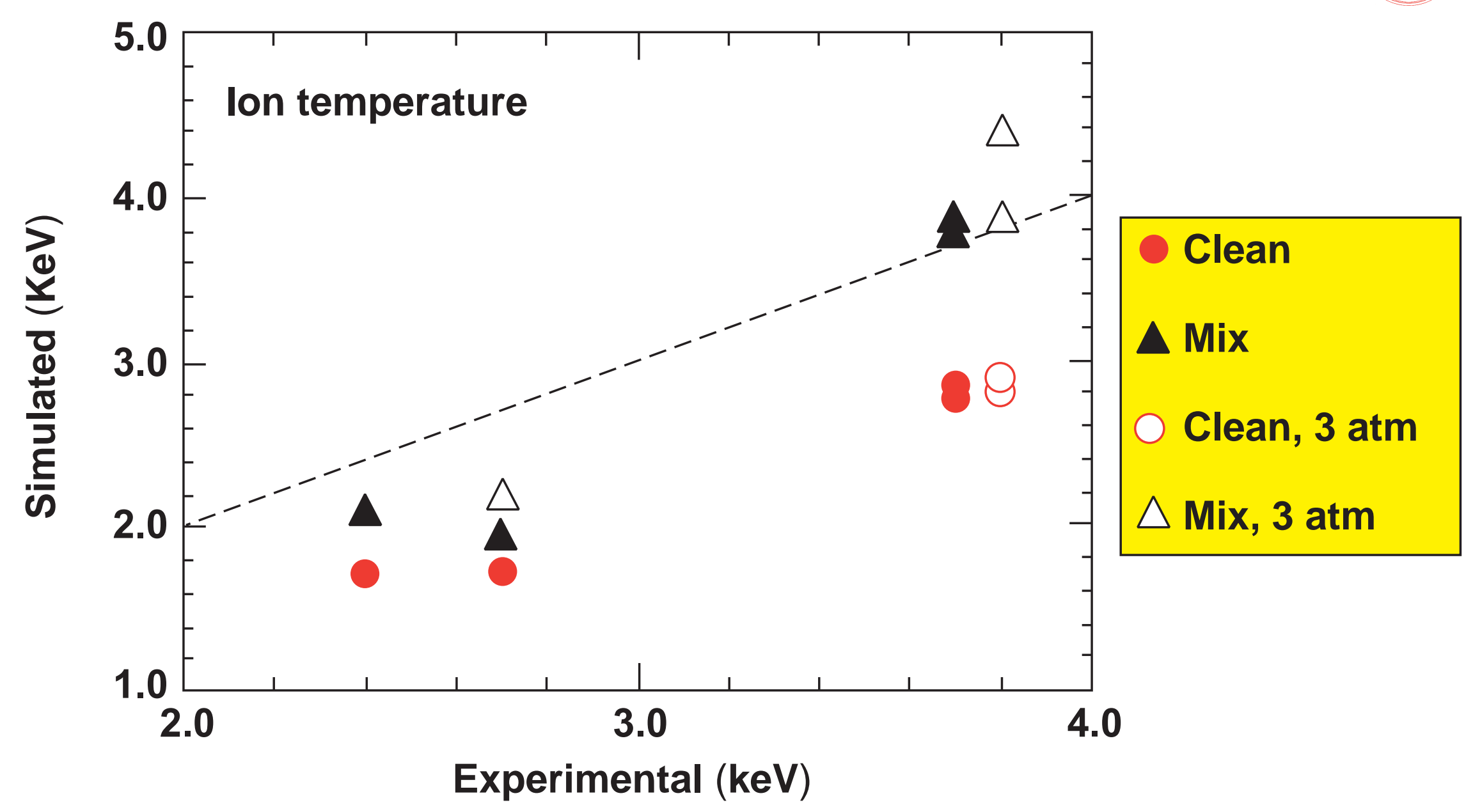


Secondary particle yields reflect different slowing rates and cross sections with contrasting energy dependence

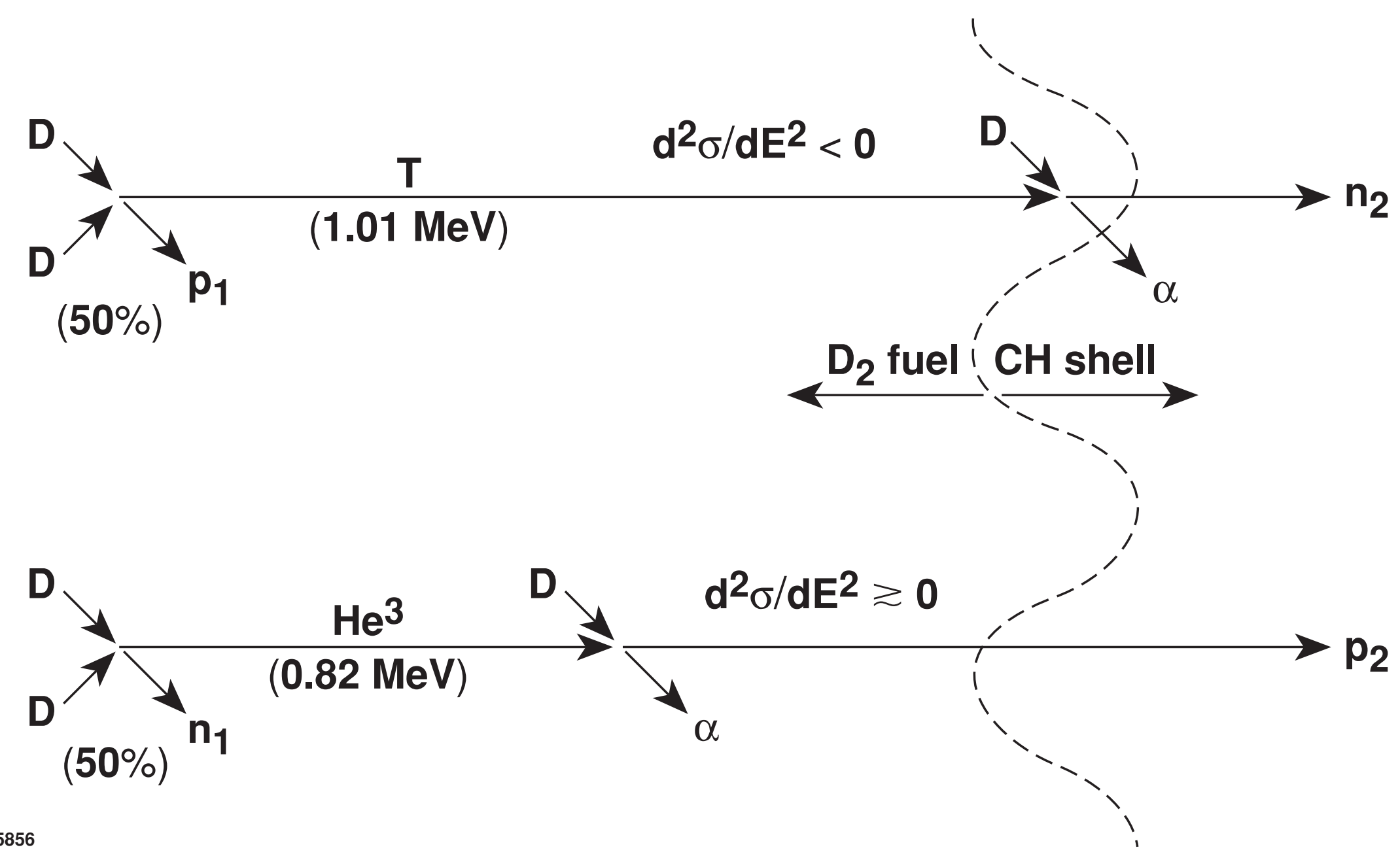




\section{Comparison of simulated with measured secondary particle yield ratios suggests sensitivity to dynamics}

Secondary production efficiencies

MIT
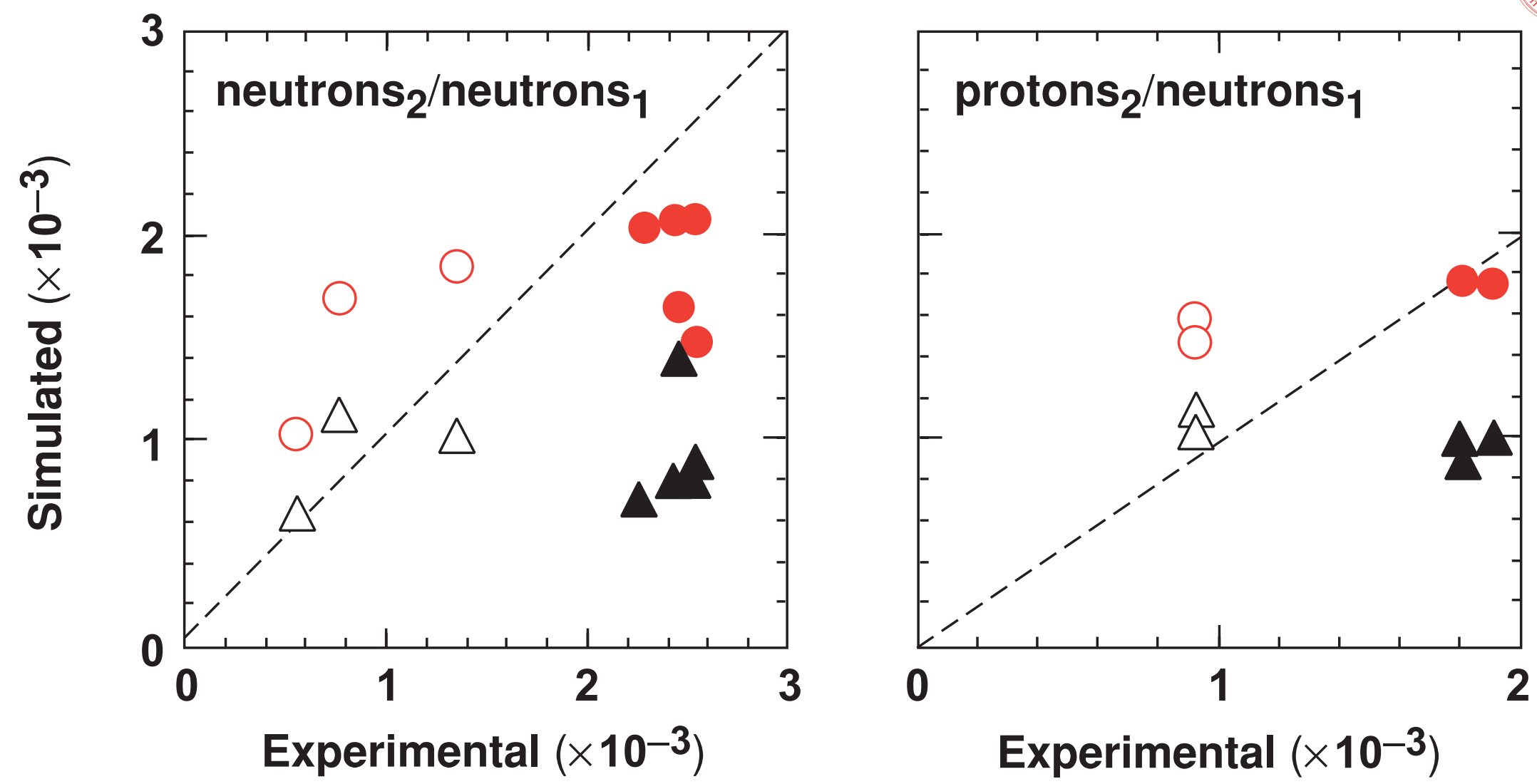

Clean $\bigcirc$ Clean, 3 atm

$\triangle$ Mix $\triangle$ Mix, 3 atm 


\section{The spatial distribution of secondary particle production depends on the extent of mix}

Total particle yields

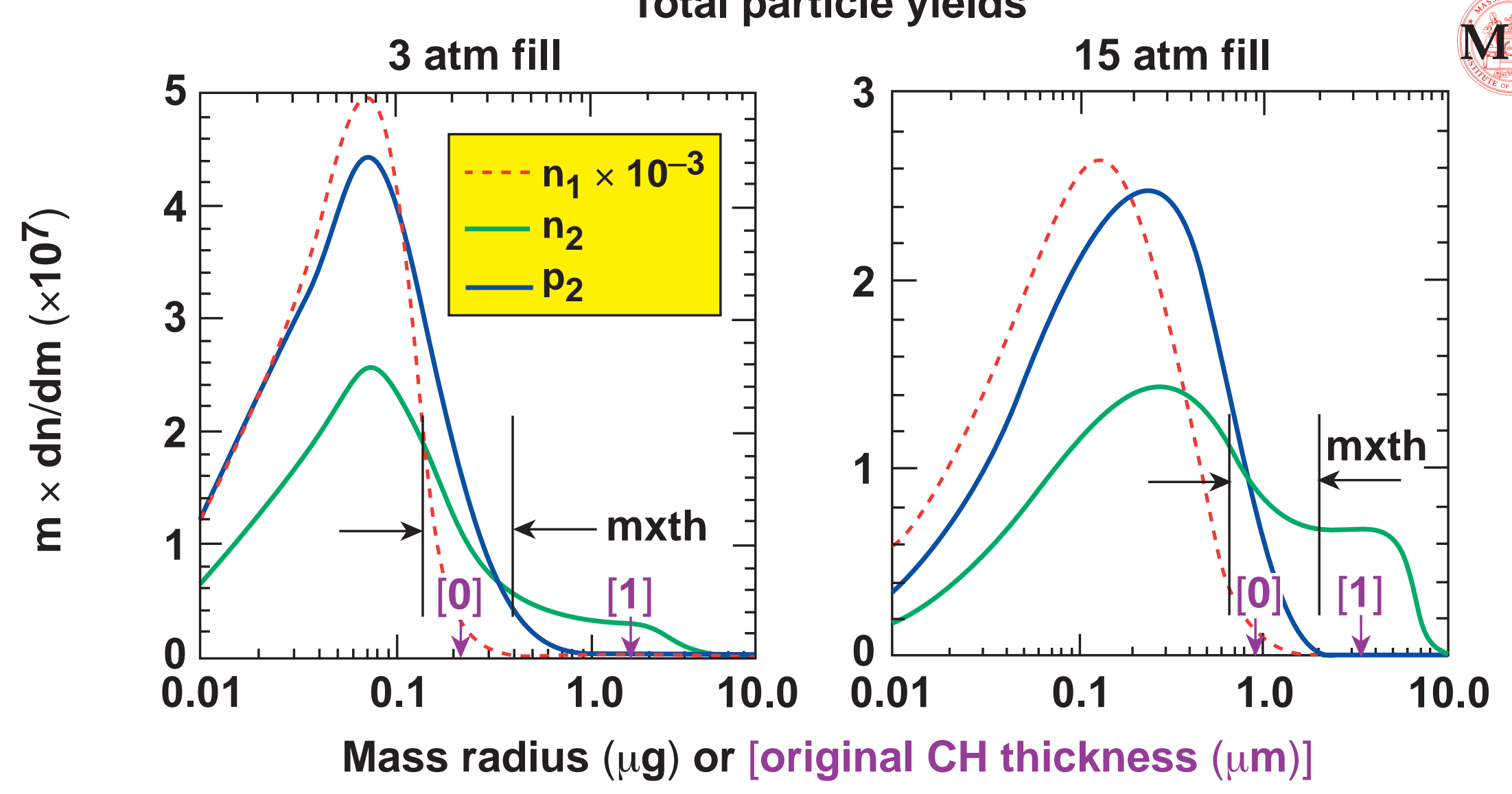

- Mix thickness (mxth) is from the 1:3 to $3: 1 \mathrm{mix}$ points at the time of peak $n_{1}$ production rate.

- With the mass-spatial distribution as plotted here, the area under the curve is preserved. 
The relative timing of peak neutron production and peak compression does not affect the coincidence of primary and secondary production times
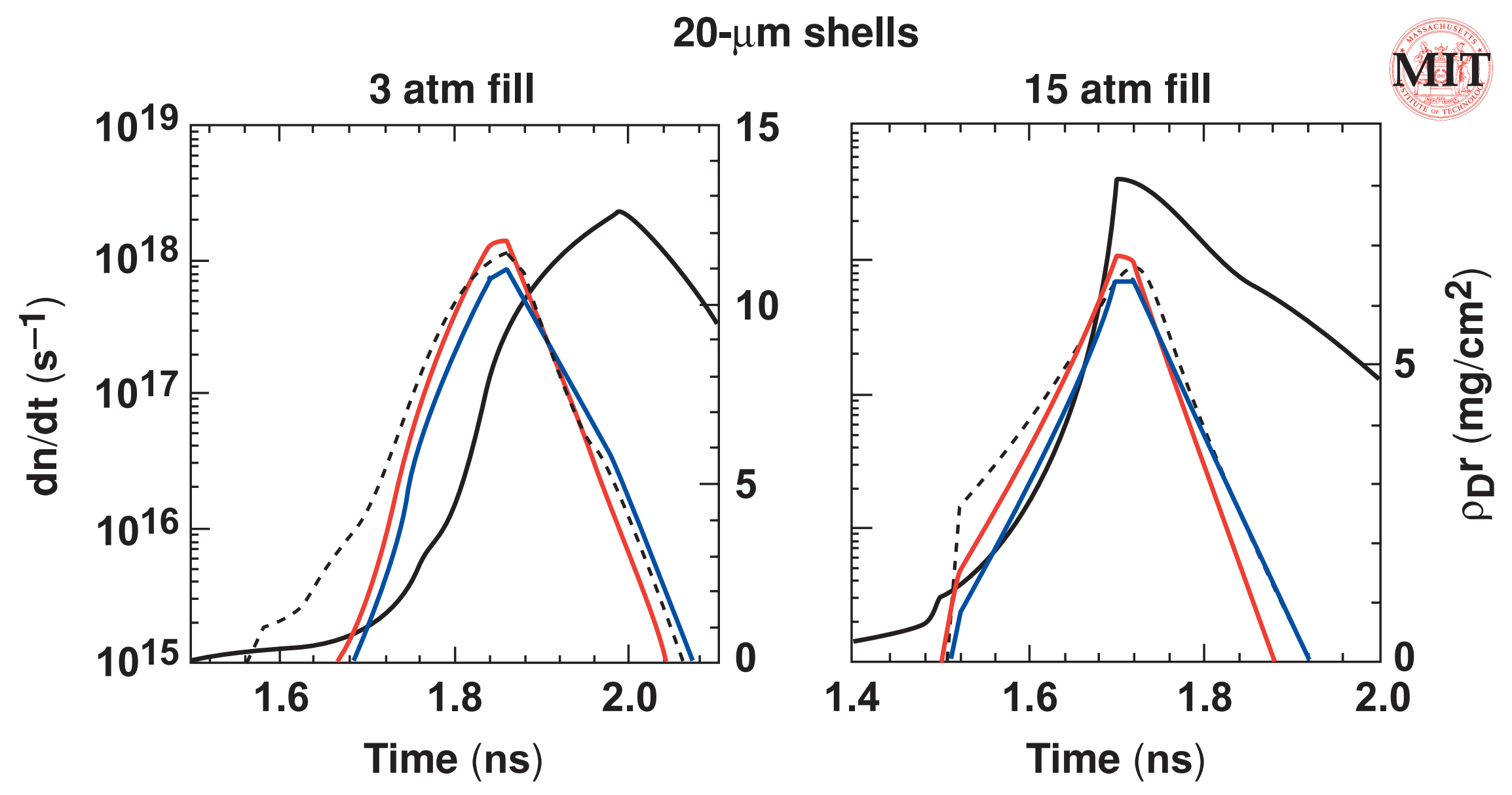

$$
\begin{aligned}
& -\rho_{D^{r}}-p_{2} \\
& -n_{2} \cdots n_{1} \times 10^{-3}
\end{aligned}
$$




\section{Summary/Conclusion}

\section{Mix effects on particle yields can be described effectively by mix modeling in the 1-D hydrocode LILAC}

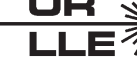

MIT

- The mix model includes the transport of target constituents, thermal energy, and turbulent energy due to both the acceleration and deceleration instabilities.

- Including mix in 1-D simulations of experiments provides improved predictions of primary and secondary particle yields over a broad range of target performance.

- The validity of approximating multidimensional hydrodynamics with a spherically symmetric model remains an issue. 


\title{
3D computation of surface perturbations evolution in plasma cloud during its expansion in magnetic field.
}

\author{
E.S. Gavrilova, E.V. Gubkov, V.A. Zhmailo, Yu.V. Yanilkin \\ RFNC-VNIIEF, 607190, Sarov, Nizhni Novgorod region \\ E-mail: vaz@,vniief.ru
}

\section{Paper presented at the $8^{\text {th }}$ International Workshop on the Physics of Compressible Turbulent Mixing $\left(8^{\text {th }}\right.$ IWPCTM, Pasadena, 2001)}

Previously, refs. [1-3] considered a 2D problem of initially spherical plasma cloud expansion in axial magnetic field. The papers indicated, in particular, that the cloud surface was nonresistant to "chute" type instability evolution and estimated the instability growth increments.

The objective of the paper is tracking the evolution of the above instabilities with account for their actual, i.e. three-dimensional, nature.

Two approaches are used for this purpose:

- $\quad$ the initial stage of the perturbation growth is considered analytically under the assumption of the perturbation smallness,

- $\quad$ the nonlinear stage is computed with 3D numerical code TREK [4].

\section{Unperturbed plasma cloud dynamics in magnetic field.}

Recall the features of solving the problem of dynamics of a plasma cloud expanding in the external magnetic field.

Consider a cloud of energy $\mathrm{E}$, mass $\mathrm{M}$ and assume the magnetic field to be axial, homogeneous, of strength $\vec{H}=\left(0,0, H_{0}\right)$ with $\mathrm{r} \rightarrow \infty$. Also, assume that the initial shape cloud is spherical of radius $r_{0}$.

A detailed pattern of the cloud expansion and deformation is obtained by numerical computations and discussed in ref. [3]. To find out the qualitative pattern, an approximate model can be used, which implies that the motion of each "sector" of the cloud depends on magnetic pressure on its surface. If the pressure is given (by relations presented in ref. [1]), then we can obtain the equation for the cloud surface radius:

$$
R(\Theta, t)=\left[r_{0}^{3}+R_{0}^{3} \cdot \frac{\sin ^{2} \omega t}{\sin ^{2} \Theta}\right]^{\frac{1}{3}}
$$

where $u_{0}=\left(\frac{E}{M}\right)^{\frac{1}{2}}$ is initial expansion rate, $\omega(\Theta)=\frac{\omega_{0}}{\sin \Theta}, \omega_{0} \cong \frac{u_{0}}{R_{0}}, R_{0}=\left(\frac{E}{\frac{4}{3} \pi \cdot p_{m}^{0}}\right)^{\frac{1}{3}}$, $p_{m}^{0}=\frac{H_{0}^{2}}{8 \pi}, \Theta$ is an angle with respect to the axis of symmetry.

Thus, from the solution it follows that the expanding cloud is decelerated by the magnetic field, its radius periodically changes depending on time $t$, with the deceleration being most noticeable at the "equator" $\left(\Theta=\frac{\pi}{2}\right)$ and missing at the poles $(\Theta=0)$.

As the comparison to the data of ref. [3] suggests, this simple cloud dynamics model is valid at $\omega_{0} t \leq \pi$.

The plasma cloud deceleration by the magnetic field leads to the perturbation evolution on the cloud surface (by analogy with the R-T instability in hydrodynamics: the role of the "heavy" fluid is played by the cloud plasma, that of the "light" by the magnetic field). 


\section{Linear stage of instability growth}

When considering this stage of the perturbation growth on the plasma cloud surface, of interest to us will be most hazardous of them, that is such, for which increment $\gamma>>\omega_{0}$. In this case the unperturbed surface can be considered as spherically symmetric and the unperturbed magnetic field near the surface as having only one, tangential, component $H_{\Theta}=\frac{3}{2} H_{0} \sin \Theta[1]$.

If the surface perturbation form is given as

$$
R(\Theta, \varphi, t)=R_{0}(t)+\sum_{l m} \xi_{l m}(t) \cdot Y_{l m}(\Theta, \varphi)
$$

where $Y_{l m}(\Theta, \varphi)$ are spherical harmonics, then, by extending the well-known conclusion [5] for plane plasma-magnetic field interface to the spherical case under consideration, the following equation system can be obtained for harmonics $\xi_{l m}(t)$ :

$$
\frac{\partial}{\partial t}\left(R^{2} \frac{\partial \sigma_{l m}}{\partial t}\right)=\sum_{k=l, l \pm 2} a_{k m}^{l m} \cdot \sigma_{k m}
$$

where $a_{k m}^{l m}=-\left[\ddot{R}_{0} \cdot R_{0} \cdot \lambda_{k m}^{l m}+c_{A}^{2} \cdot \mu_{k m}^{l m}\right] \cdot l$

where $c_{A}^{2}=\frac{\left(\frac{3}{2} H_{0}\right)^{2}}{4 \cdot \pi \cdot \rho_{0}(t)}, \lambda_{k m}^{l m}$ and $\mu_{k m}^{l m}$ are algebraic functions 1 and $\mathrm{m}$. The expressions for the functions prove quite cumbersome, here it is sufficient to note that for $l>>1, \lambda_{k m}^{l m} \sim \delta_{k l}+\delta \lambda_{k m}^{l m}$, where $\delta \lambda_{k m}^{l m} \sim \frac{1}{m+1}, \mu_{k m}^{l m} \sim(l-m)$.

Note that the "engagement" of harmonics 1 and $1 \pm 2$ in equation system (2.2) is a consequence of the fact that the pressure of the unperturbed magnetic field on the surface depends on $\Theta\left(p_{m} \sim \sin ^{2} \Theta\right)$. This "engagement" disappears, when the initial perturbation is localized at $\Theta=\Theta_{0} \pm \Delta \Theta$, where $\Delta \Theta<<1$. Assuming $\mathrm{m}=0$ and 1 such that $l \Delta \Theta>>1$, equation (2.2) can be reduced to a simpler form:

$$
\frac{\partial}{\partial t}\left(R^{2} \frac{\partial \sigma_{l m}}{\partial t}\right)=-\sigma_{l m}\left[l \cdot \ddot{R}_{0}\left(\Theta_{0}\right) \cdot R_{0}+c_{A}^{2}\left(\Theta_{0}\right) \cdot l^{2}\right]
$$

where $\ddot{R}_{0}\left(\Theta_{0}\right) \sim c_{A}^{2}\left(\Theta_{0}\right) \sim \sin ^{2} \Theta_{0}$ are surface acceleration and Alfven velocity characteristic of a given angle.

In the quasi-static case $\left(\gamma>>\omega_{0}\right)$ from (2.4) it follows that

$\gamma_{l}^{2}=-\left[k \cdot \ddot{R}\left(\Theta_{0}\right)+c_{A}^{2}\left(\Theta_{0}\right) \cdot k^{2}\right]$

where $k=\frac{l}{R_{0}}$. This expression coincides with that obtained previously [5] for the plane interface with parameters corresponding to angle $\Theta_{0}$.

If we continue to consider $1>>1$, but $\mathrm{m}=1-\Delta$, where $\Delta \sim 1$, then from (2.2) it readily follows that

$$
\frac{\partial}{\partial t}\left(R^{2} \frac{\partial \sigma_{l m}}{\partial t}\right)=-\sigma_{l m}\left[l \cdot \ddot{R}_{0} \cdot R_{0}+c_{A}^{2} \cdot l \cdot \Delta\right]
$$

As seen from the comparison between (2.3) and (2.5), for such perturbations the stabilizing role of the magnetic field (addend $\sim \mathrm{c}^{2}{ }_{\mathrm{A}}$ in 2.5 ) is noticeably less than that for $\mathrm{m}=0$. In 
particular, for $c_{A}^{2} \ll<\left(\ddot{R}_{0} \cdot R_{0}\right)(2.5)$ yields an expression for increment $\gamma_{l m}$ similar to that known [6] for a cylindrical problem with longitudinal magnetic field $\left(\gamma \sim \sqrt{-m \frac{\ddot{R}_{0}}{R_{0}}}\right)$.

\section{Numerical calculations}

The computations were with code TREK [3]. The scaling was as follows: $p_{1}=\frac{H_{0}^{2}}{8 \pi}$ for pressure, $r_{1}=\left(\frac{E}{\frac{4}{3} \pi \cdot p_{1}}\right)^{\frac{1}{3}}$ for length, $u_{1}=\left(\frac{E}{M}\right)^{\frac{1}{2}}$ for velocity, $\frac{r_{1}}{u_{1}}$ for time, $\rho_{1}=\frac{M}{\frac{4}{3} \pi \cdot r_{1}^{3}}=\frac{p_{1}}{u_{1}^{2}}$ for density.

The magnetic pressure on the cloud surface was calculated using two apporoaches:

- $\quad$ Vacuum was assumed outside the cloud; the magnetic field in it was calculated using quasi-stationary approximation [2]. In this case the problem is characterized with an only dimensionless parameter, $r_{0}{ }^{\prime}=\frac{r_{0}}{r_{1}}$, with the dependence on the parameter disappearing at $r_{0}^{\prime}<<1, r^{\prime} \sim 1$.

"Background" plasma of quite a small density with a magnetic field frozen in it was assumed outside the cloud. In this case the magnetic field changes are calculated using MHD approximation; besides the parameter $r_{0}$, the problem is also characterized with parameter $M_{A}^{2}=\frac{u_{1}^{2}}{c_{A}^{2}}$, where $c_{A}^{2}=\frac{H_{0}^{2}}{4 \pi \cdot \rho_{1}} ;$ options with $M_{A}^{2}<<1$ were considered, in which the dependence on $M_{A}^{2}$ disappears.

At $\mathrm{t}=0$, sphere surface radial velocity perturbations were given, i.e.

$u(0, \mu, \varphi)=u_{0}+u_{l m} \cdot Y_{l m}(\mu, \varphi)$

was assumed. Here $u_{0}=\sqrt{\frac{10}{3}} u_{1}$ is free expansion velocity of "cold" spherical cloud having a linear velocity profile and constant density. $u_{l m}=\alpha \cdot u_{0}$ was assumed.

Four problems were calculated.

In the first of them, $\alpha=0$ (unperturbed surface) was assumed. The results of the calculation are plotted in Figs. 1 through $3\left(M_{A}^{2}=0.05, r_{0}{ }^{\prime}=1, \mathrm{t}=0.25 ; 2.0\right.$ and 3 , respectively). The expansion pattern qualitatively agrees with relation (1.1): the plasma spreading in the longitudinal direction and oscillating motion (with period $\sim \pi$ ) in the transversal direction occur.

In the second problem, "meridional" velocity perturbations were given at the initial time: $\alpha=0.1,1=12, \mathrm{~m}=0$, the other parameters are the same as in problem 1 . The calculation results are illustrated in Figs. 4 and 5.

In this case the ratio of the first addend to the second in (2.3) is $\sim \frac{u_{0}^{2}}{c_{A}^{2} \cdot l}<<1$ (here $c_{A}^{2}$ is estimated by plasma cloud density $\rho_{0} \sim 1$ at $\mathrm{t} \leq 1$ ), hence, a significant perturbation growth stabilization can be expected thanks to the magnetic field.

This assumption is confirmed by comparison between the results of the calculation and similar calculation 3, which differs from the former in the fact that instead of "magnetic" pressure, "thermal" pressure of the same magnitude is given in the "external" plasma. In this case the cloud dynamics will remain about the same as in problem 2, however, the perturbation growth depends only on the first addend in (2.3), i.e. is significantly faster than in problem 2 . 
Problem 4 differs from problem 2 in given $m=1=12$, i.e. the initial perturbation was localized near "equator" and its amplitude depended on azimuthal angle $\varphi$. Its solution results appear in Figs. 8 and 9. In this case, according to (2.6), we can expect that increment $\gamma^{2}>0$ (unsteady conditions) and that the cloud surface will be perturbed, in the main, across azimuth. These assumptions agree with the calculated data presented in Figs. 8 and 9.

\section{Conclusion}

More extensive computational series with varying parameters $r_{0}, M_{A}^{2}, 1, \mathrm{~m}$, as well as setting "random" surface perturbations are being planned.

The authors are thankful to T. Yu. Odintsova for the performed numerical computations and assistance in the paper execution.

\section{References}

1. Raizer Yu.P. "On plasma cloud expansion into a vacuum magnetic field”. RMTF, 1967, No.5

2. J.W.Poukey "Expansion of a Plasma Shell into a Vacuum Magnetic Field", Phys FI, 1969, vol12, No.7, pp 1452

3. Bakhrakh S.M., Gubkov E.V., Zhmailo V.A., Terekhin V.A. "Plasma cloud expansion in homogeneous magnetic field". RMTF, 1974, No.4, pp 146-150

4. Yanilkin Yu.V., Tarasov V.I., Stadnik A.L. "Program System "TREK" for Numerical Simulation of 3D Multicomponent Medium Flows". Proceedings of Workshop "New models and Numerical Codes for Shock Processes in Condensed Media", Oxford, 1997, pp 413-422

5. Kruskal M., Schwarzschild M., "Some instabilities of completely ionized plasma", Proc. of the Roy. Soc., vol223, p 348-361, 1954

6. Harris E.G. "Rayleigh-Taylor Instabilities of a Collapsing Cylindrical Shell in a Magnetic Field”, Phys FI, 1962, vol5, No.9, pp 1057-1062 


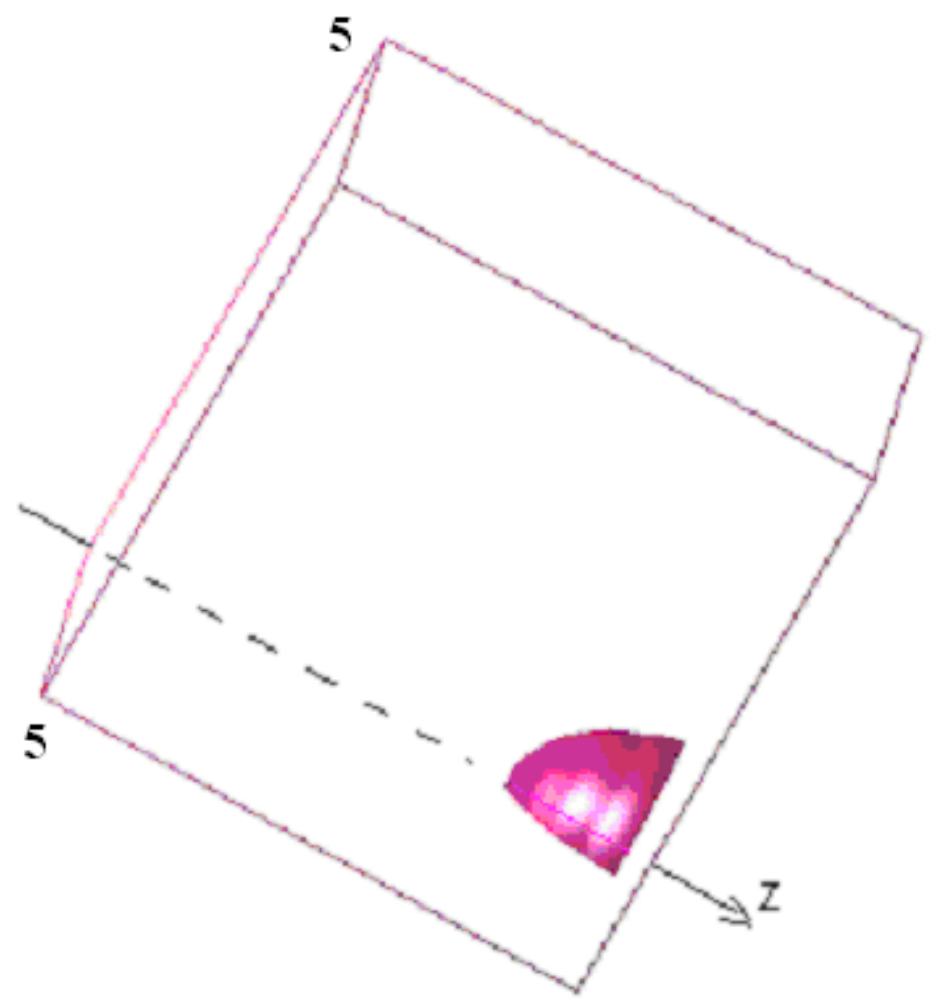

fig1. Plasma cloud expansion in the magnetic field (unperturbed surface), $\mathrm{t}=0.25, M_{A}^{2}=0.05$

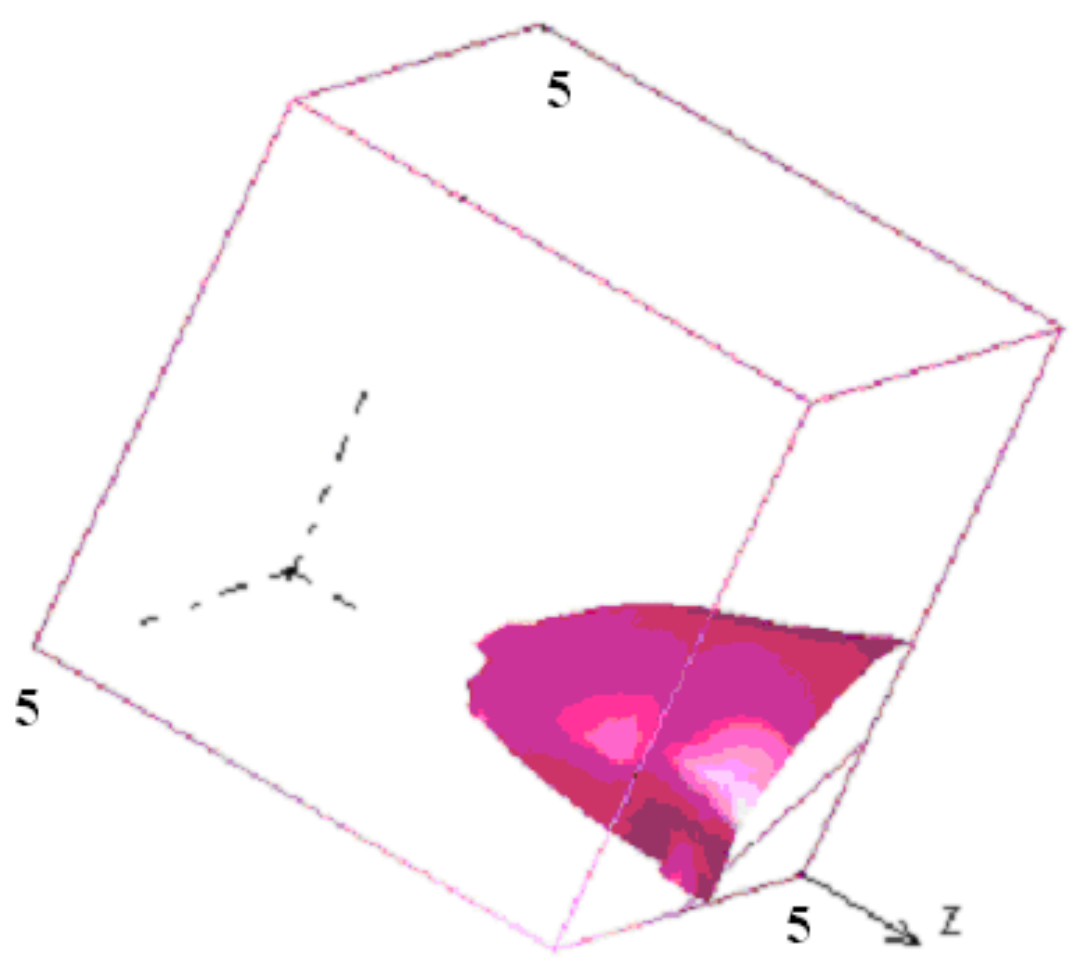

fig2. Plasma cloud expansion in the magnetic field (unperturbed surface), $\mathrm{t}=2.0, M_{A}^{2}=0.05$ 


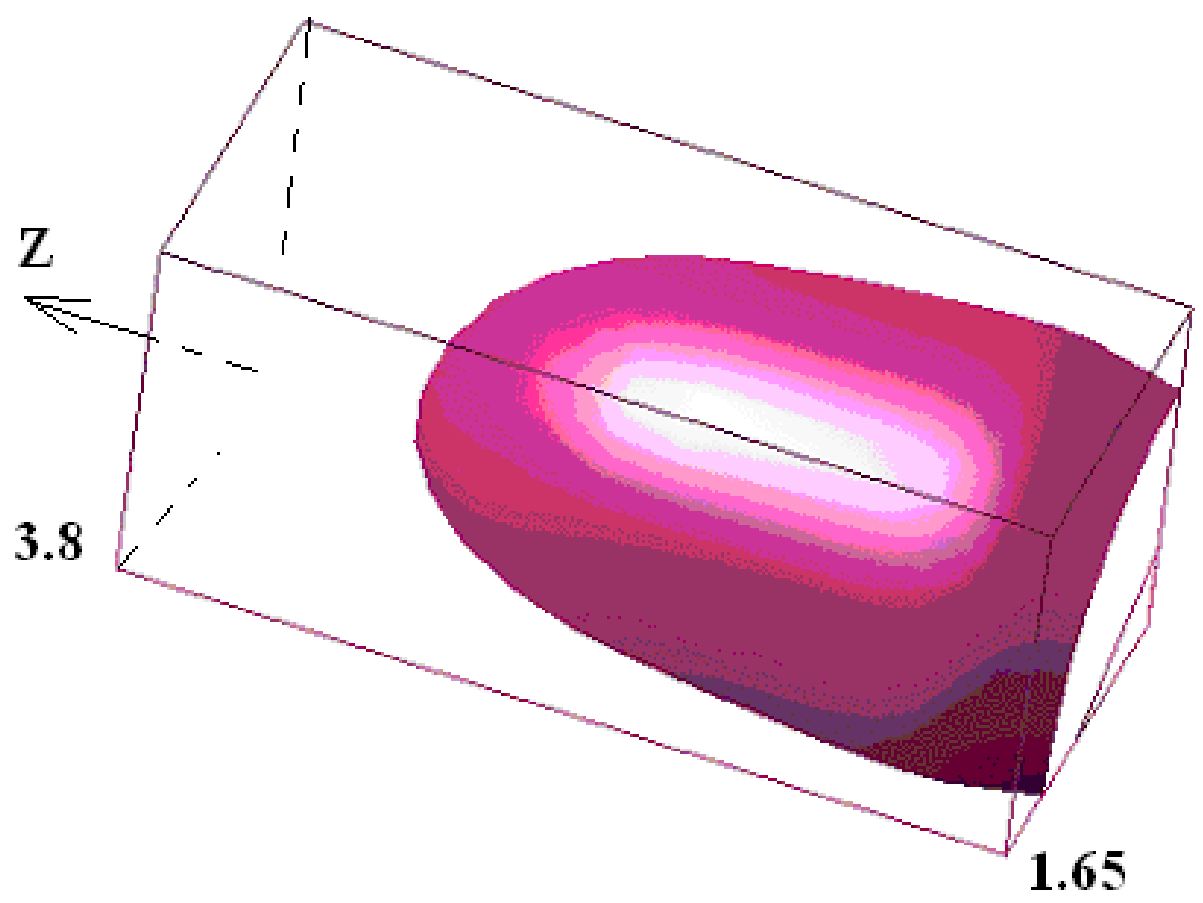

fig3. Plasma cloud expansion in the magnetic field (unperturbed surface), $\mathrm{t}=3.0, M_{A}^{2}=0.05$

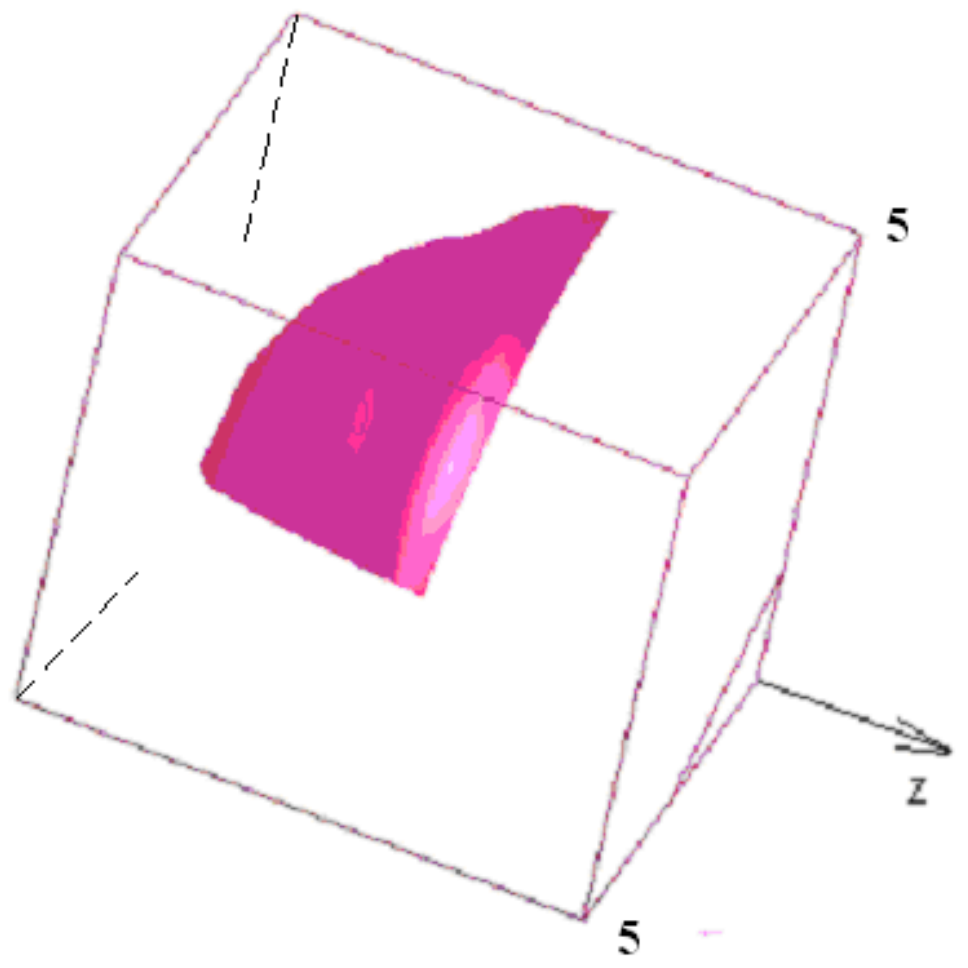

fig4. Plasma cloud expansion in the magnetic field (meridianal perturbations, $\left.\mathrm{l}=12, \mathrm{~m}=0, M_{A}^{2}=0.05\right), \mathrm{t}=0.25$ 


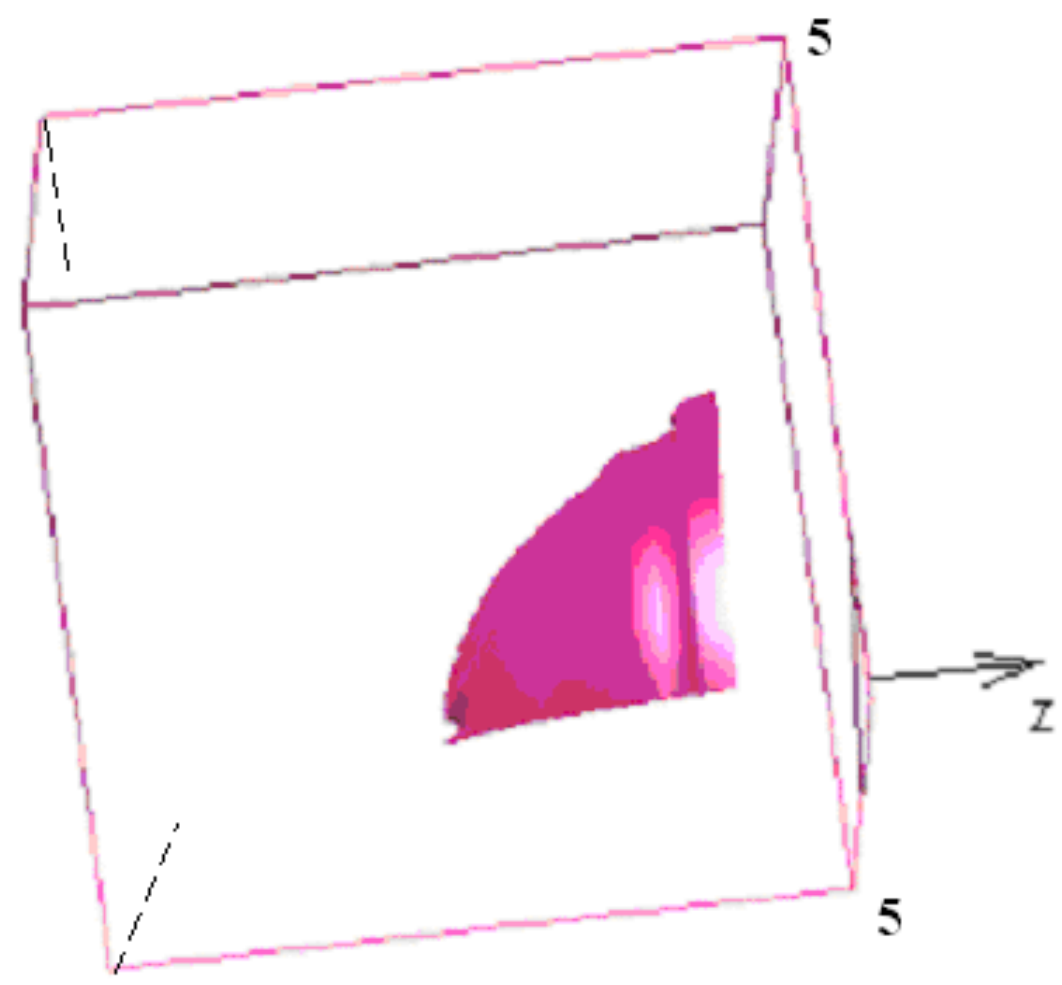

fig5. Plasma cloud expansion in the magnetic field (meridianal perturbations, $\left.\mathrm{l}=12, \mathrm{~m}=0, M_{A}^{2}=0.05\right), \mathrm{t}=0.5$

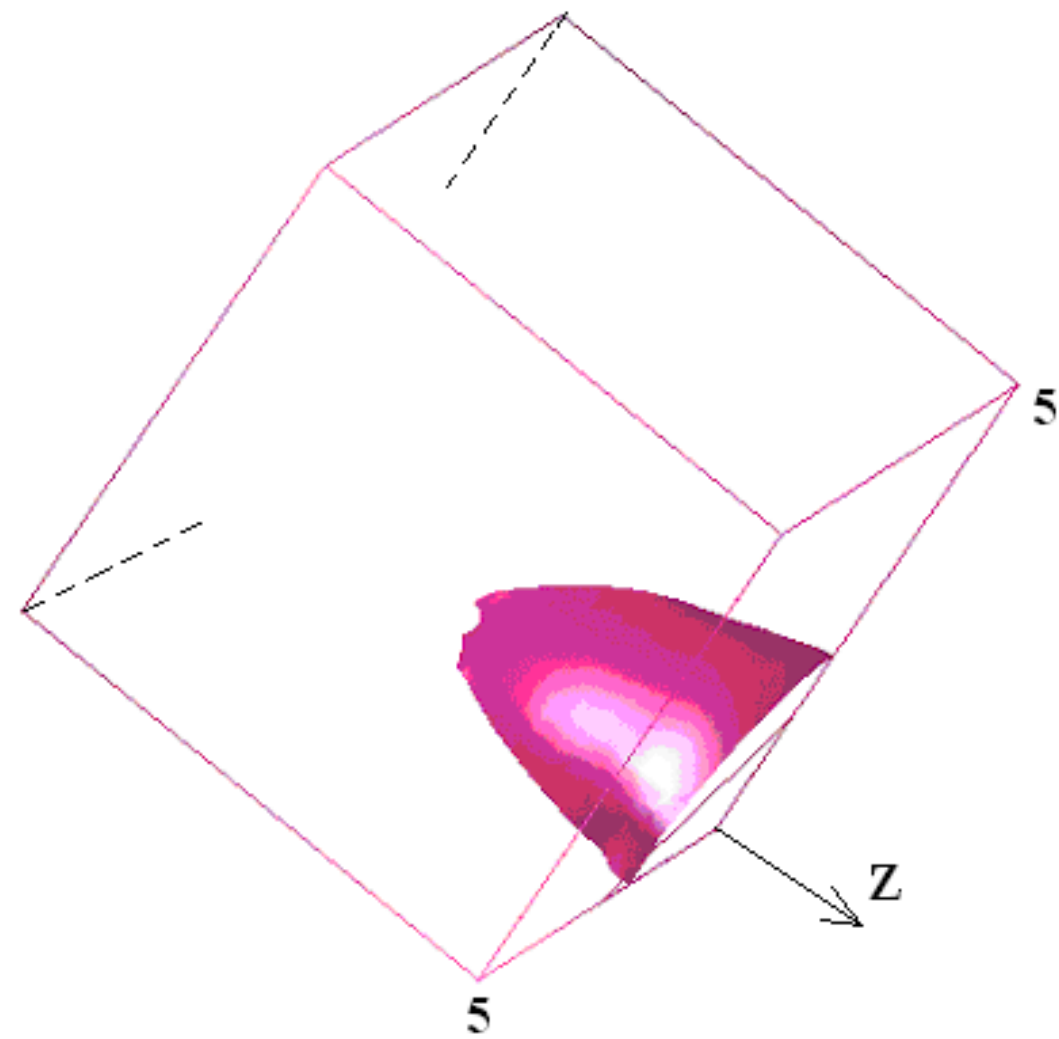

fig6. Plasma cloud expansion against termal pressure (meridianal perturbations), $M_{\text {th }}^{2}=0.05, \mathrm{P}_{\mathrm{m}}=0, \mathrm{l}=12, \mathrm{~m}=0, \mathrm{t}=0.5$ 


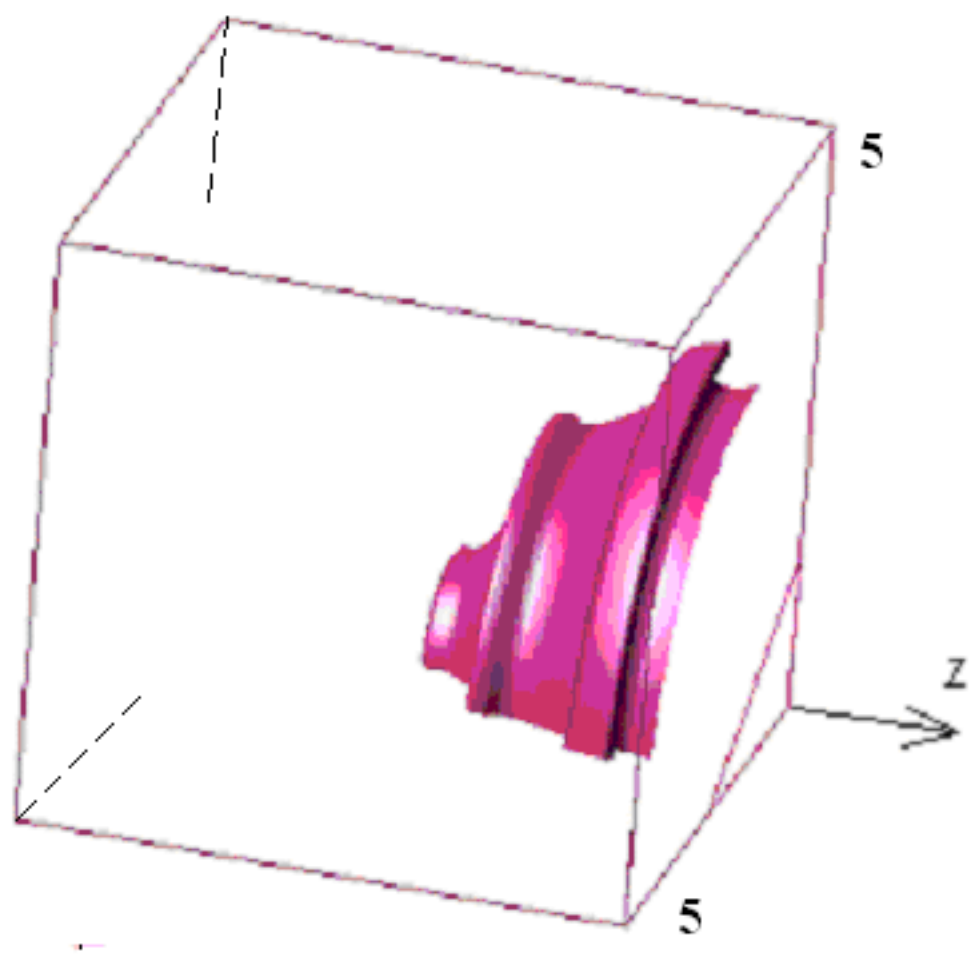

fig7. Plasma cloud expansion against termal pressure (meridianal perturbations), $M_{t h}^{2}=0.05, \mathrm{P}_{\mathrm{m}}=0, \mathrm{l}=12, \mathrm{~m}=0, \mathrm{t}=3.0$

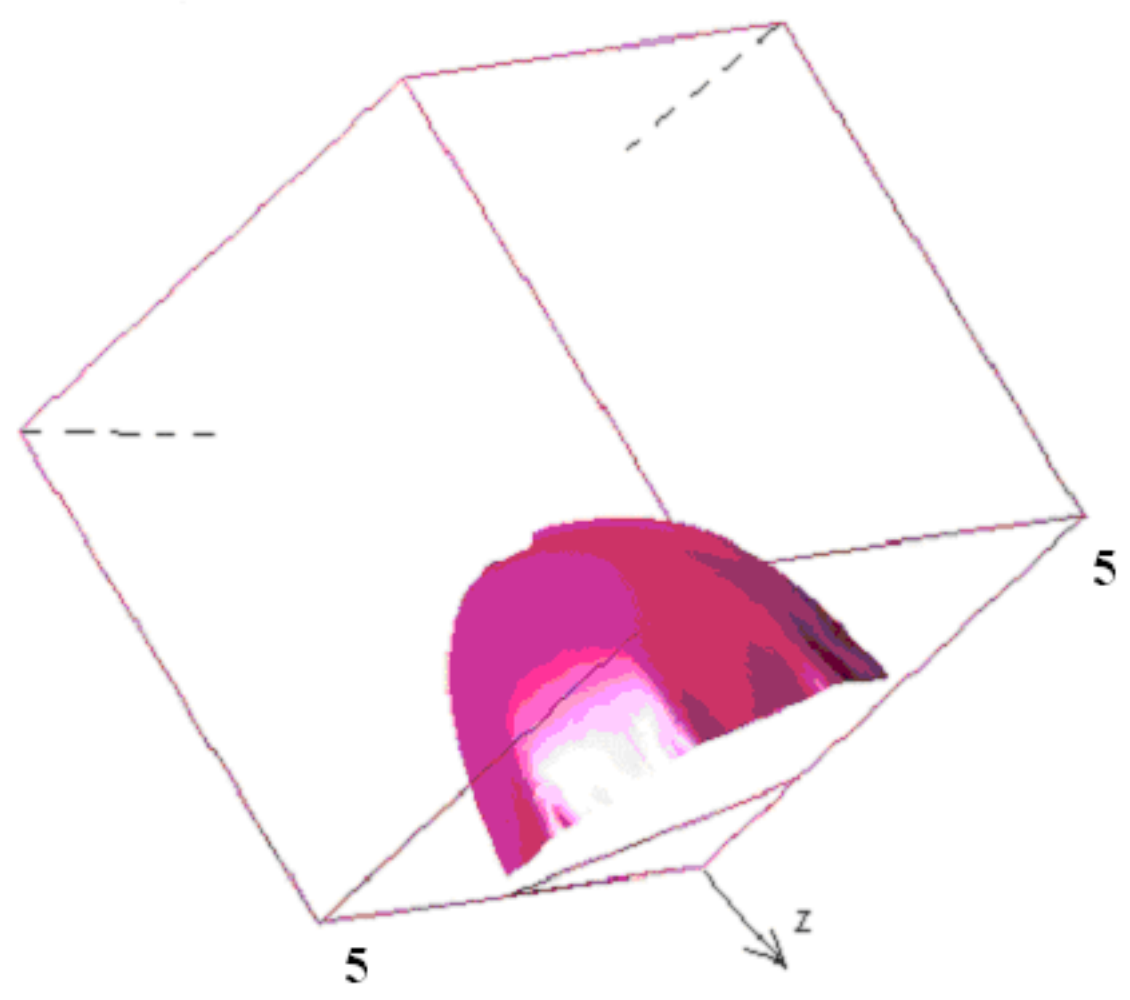

fig8. Plasma cloud expansion in the magnetic field (perturbation $1=12$, $\left.\mathrm{m}=12, M_{A}^{2}=0.05\right), \mathrm{t}=0.4$ 


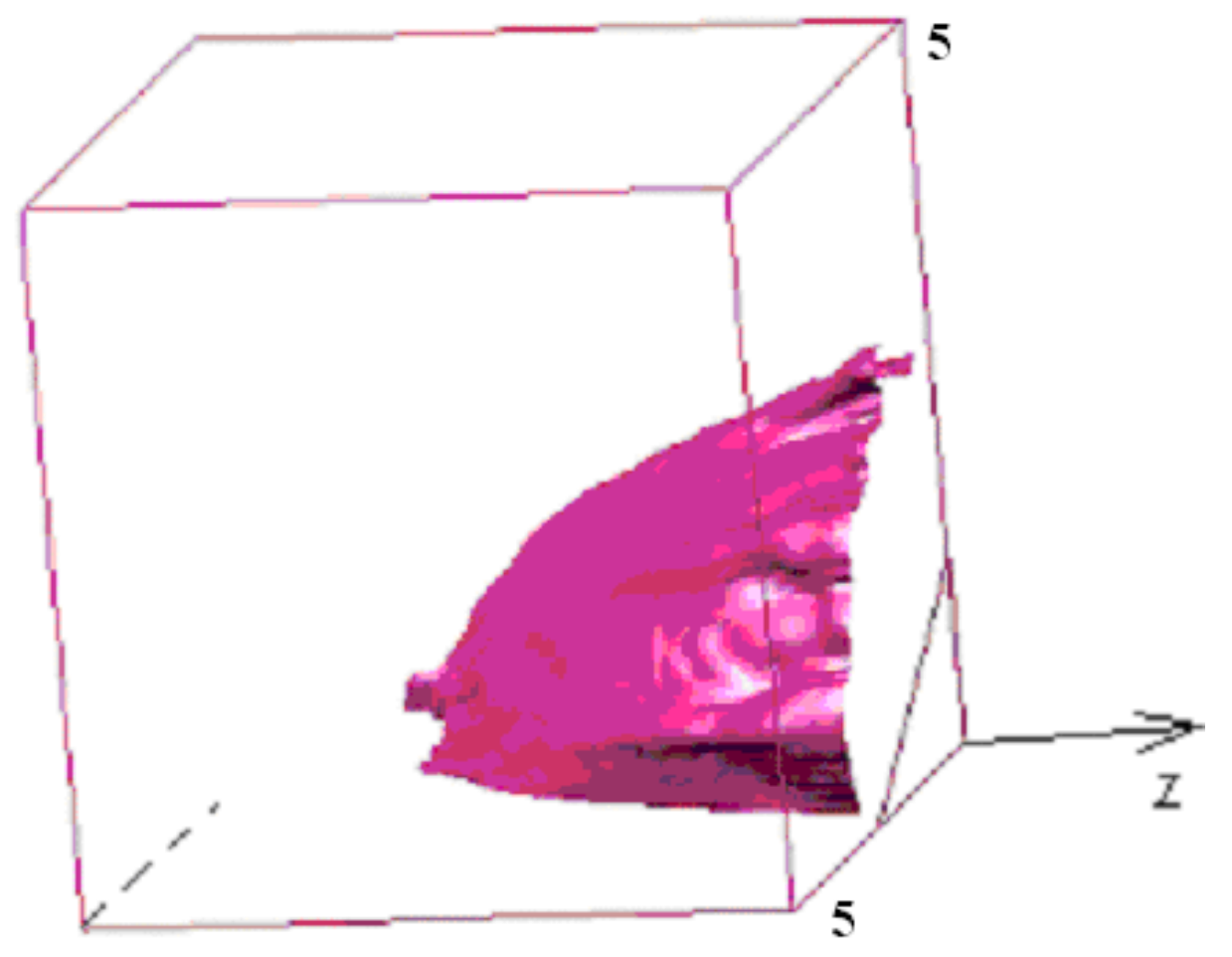

fig9. Plasma cloud expansion in the magnetic field (perturbation $1=12$,

$$
\left.\mathrm{m}=12, M_{A}^{2}=0.05\right), \mathrm{t}=1 \text {. }
$$




\title{
Code-to-Code Comparisons for the Problem of Shock Acceleration of a Diffuse Dense Gaseous Cylinder
}

J.A. Greenough ${ }^{1}$, W.J. Rider ${ }^{2}$, C. Zoldi ${ }^{2}$, J.R. Kamm²

\author{
$8^{\text {th }}$ IWPCTM \\ December 9-14, 2001 \\ Pasadena, CA
}

${ }^{1}$ Lawrence Livermore National Laboratory, ${ }^{2}$ Los Alamos National Laboratory 


\section{Motivation}

- Focus on computational issues as cause for disagreement between Rage and ongoing LANL shock/cylinder experiments:

- Large scale (dipole aspect ratio) differences

- Quantitative velocity measurements (PIV)

- Remove experimental uncertainities/unknowns:

- Use well-defined initial conditions

- Analysis and comparisons based on computational data

- Use different codes for comparison 


\section{Motivation}

- Use this research to also examine:

- What does convergence mean for evolving flows \& instabilities?

- What are the resolution requirements for "fully-resolved" calculations of this class of flow?

- What quality of results can we obtain from low-order codes (secondorder) in this regime?

- Our guide will be existing \& on-going experiments 


\section{Experimental Configuration}

- "Pour" $\mathrm{SF}_{6}$ in the shocktube as a laminar stream

- LANL experiments seed gas with glycol/water droplets (original CalTech experiments used biacetyl)

- Laser sheet illumination with multiple frames per experiment

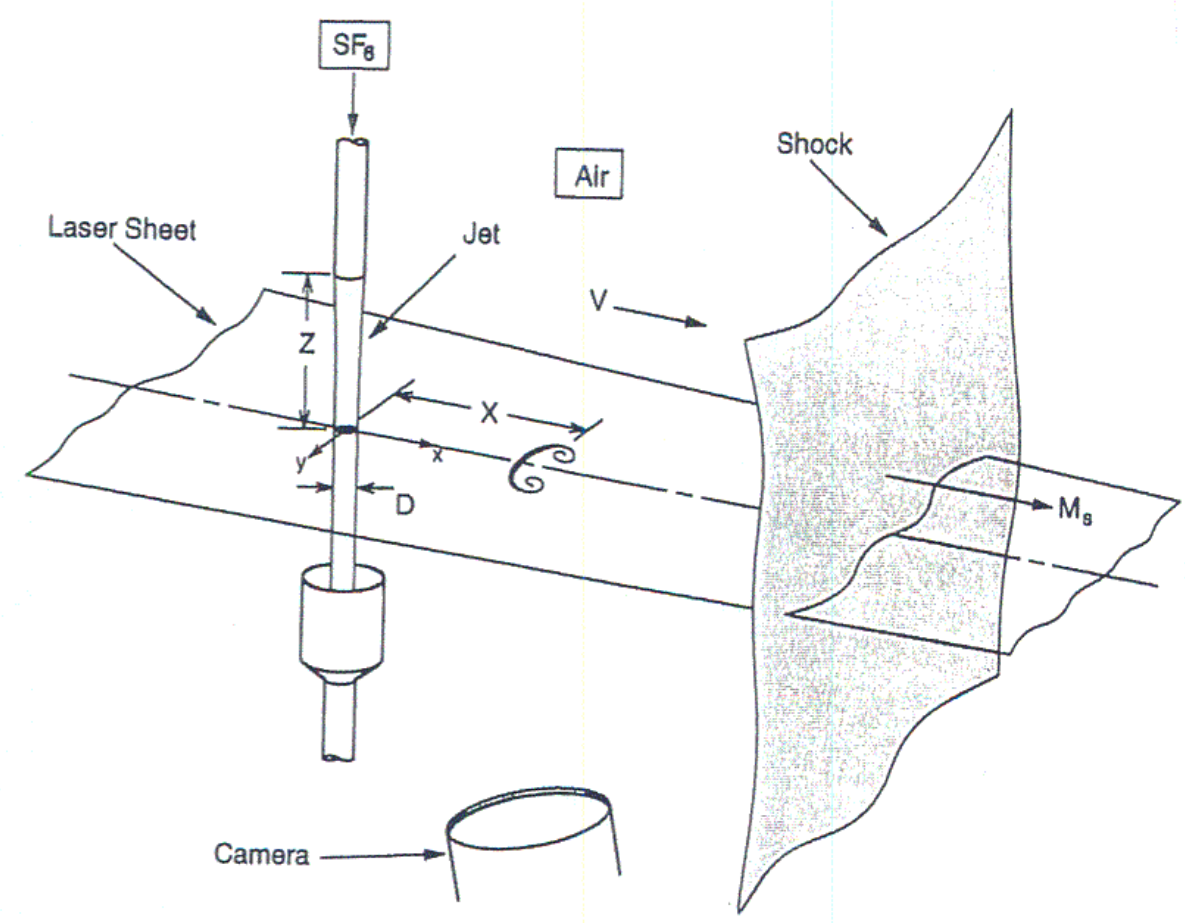

FIG. 1. Drawing of the jet/PLIF configuration. 
Comparison Between Experiment and Simulation
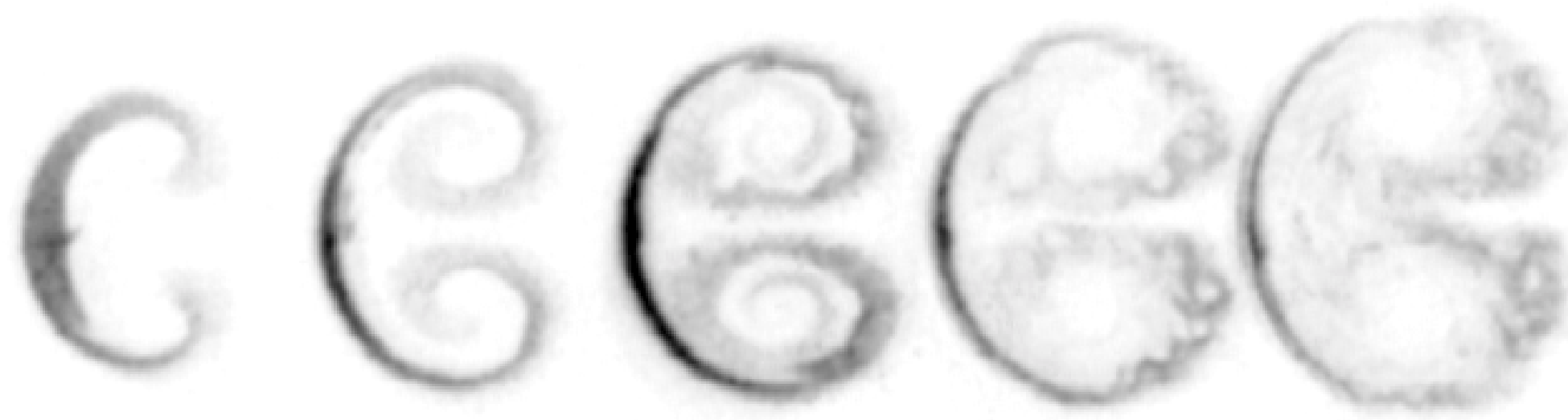

$50 \mu \mathrm{s}$

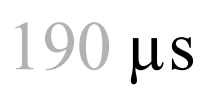

$330 \mu \mathrm{s}$

$470 \mu \mathrm{s}$

$610 \mu \mathrm{s}$

$750 \mu \mathrm{s}$
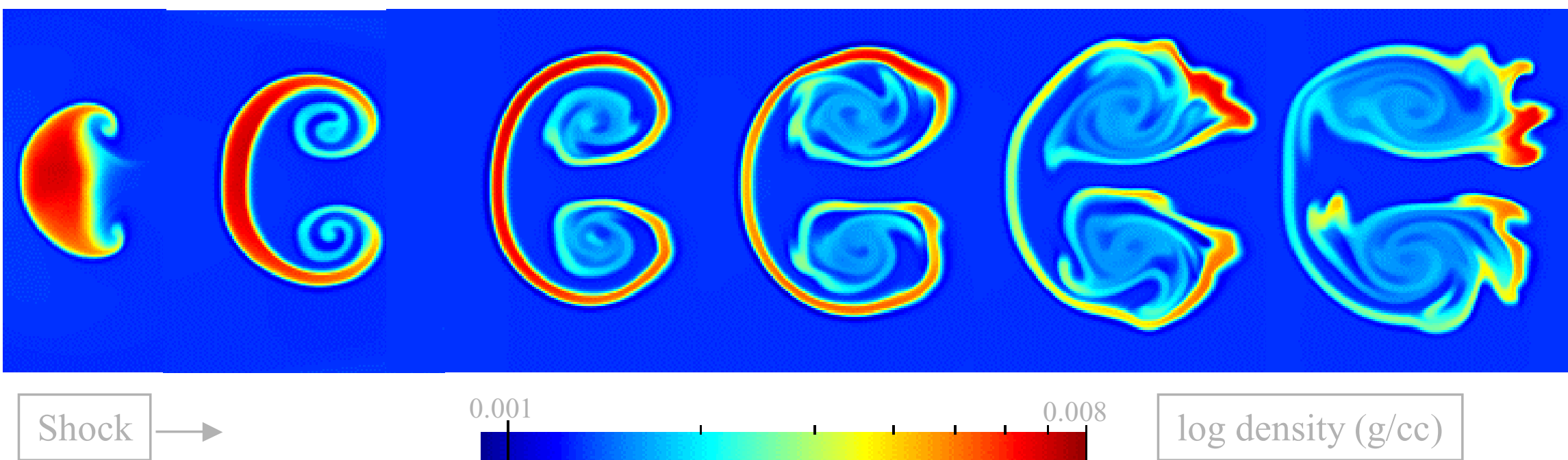

0.001

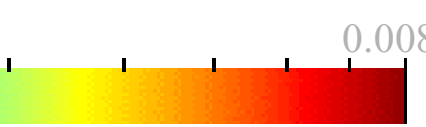

$\log$ density (g/cc) 


\section{Quantitative Measurements}
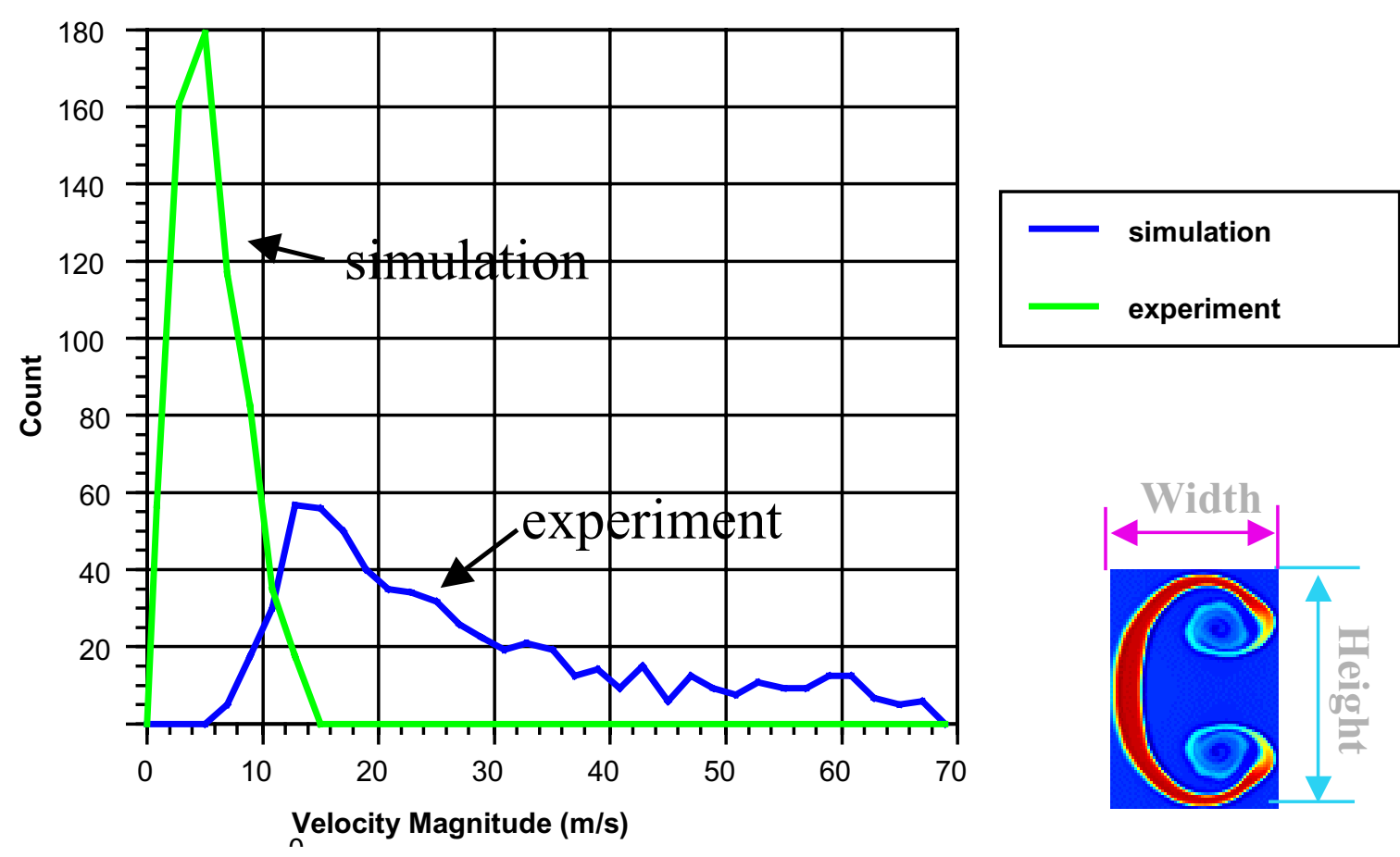

Simulation has larger velocities and smaller lengths compared to the experimental data.
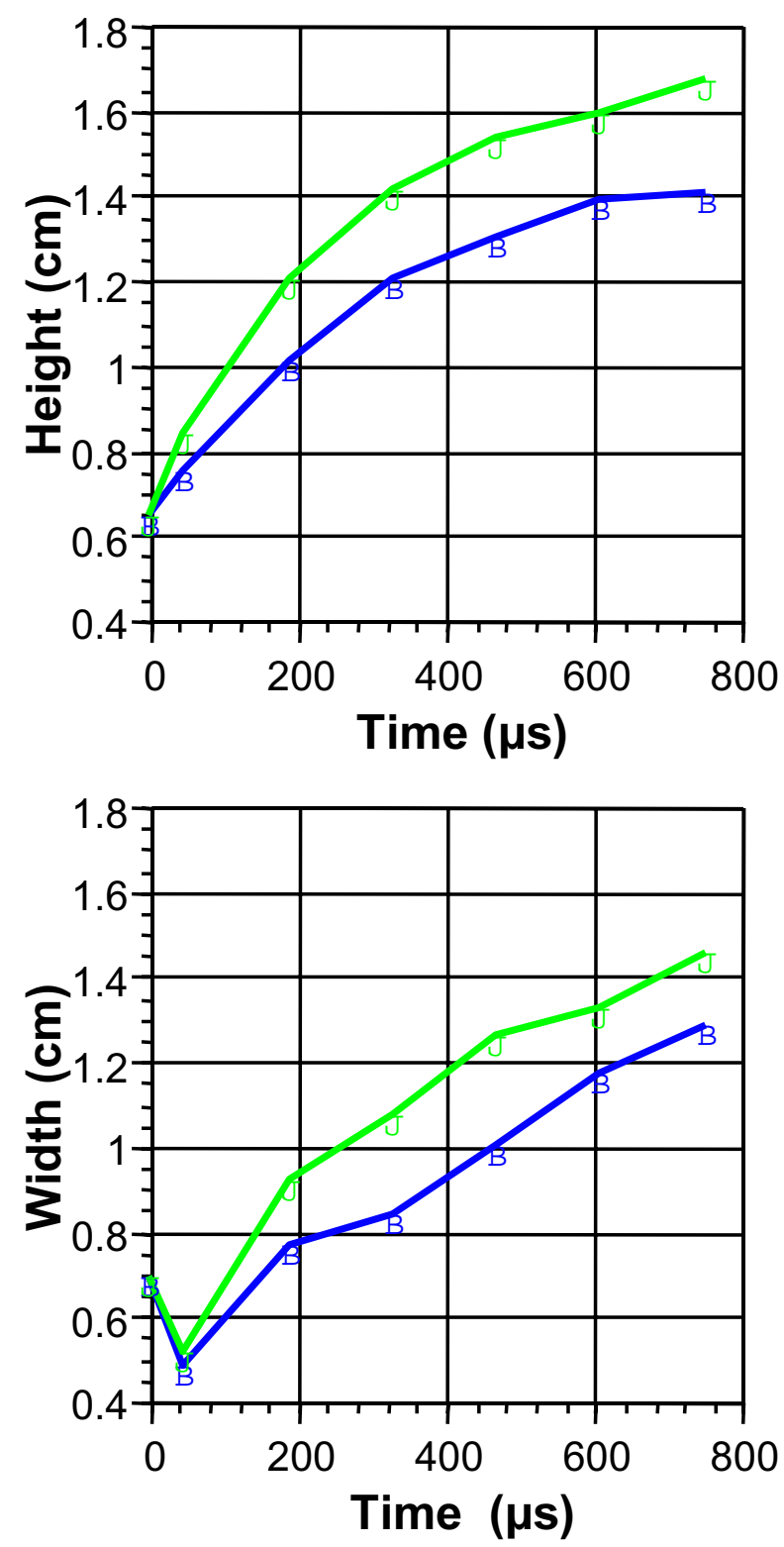


\section{Codes}

- Rage (LANL; Gittings et al.)

- Eulerian (Lagrange + Remap); directionally split

- Unstructured AMR (point-wise adaptivity)

- Multi-component formulation (mass fraction); one energy equation

- Euler equations (inviscid)

- Cuervo (LANL; Rider \& Kamm)

- Eulerian (direct); directionally and temporally unsplit

- Rectangular uniform grids

- single-component formulation (gamma blending); one energy equation

- Navier-Stokes equations (constant properties)

- Raptor (LLNL; Greenough et al.)

- Eulerian (direct); directionally split

- Block-structured AMR (patch-based adaptivity)

- VOF formulation (volume fraction); $\mathrm{N}$ energy equations

- Navier-Stokes equations (Chapman-Enskog, Sutherland's formula) 


\section{Model Problem}

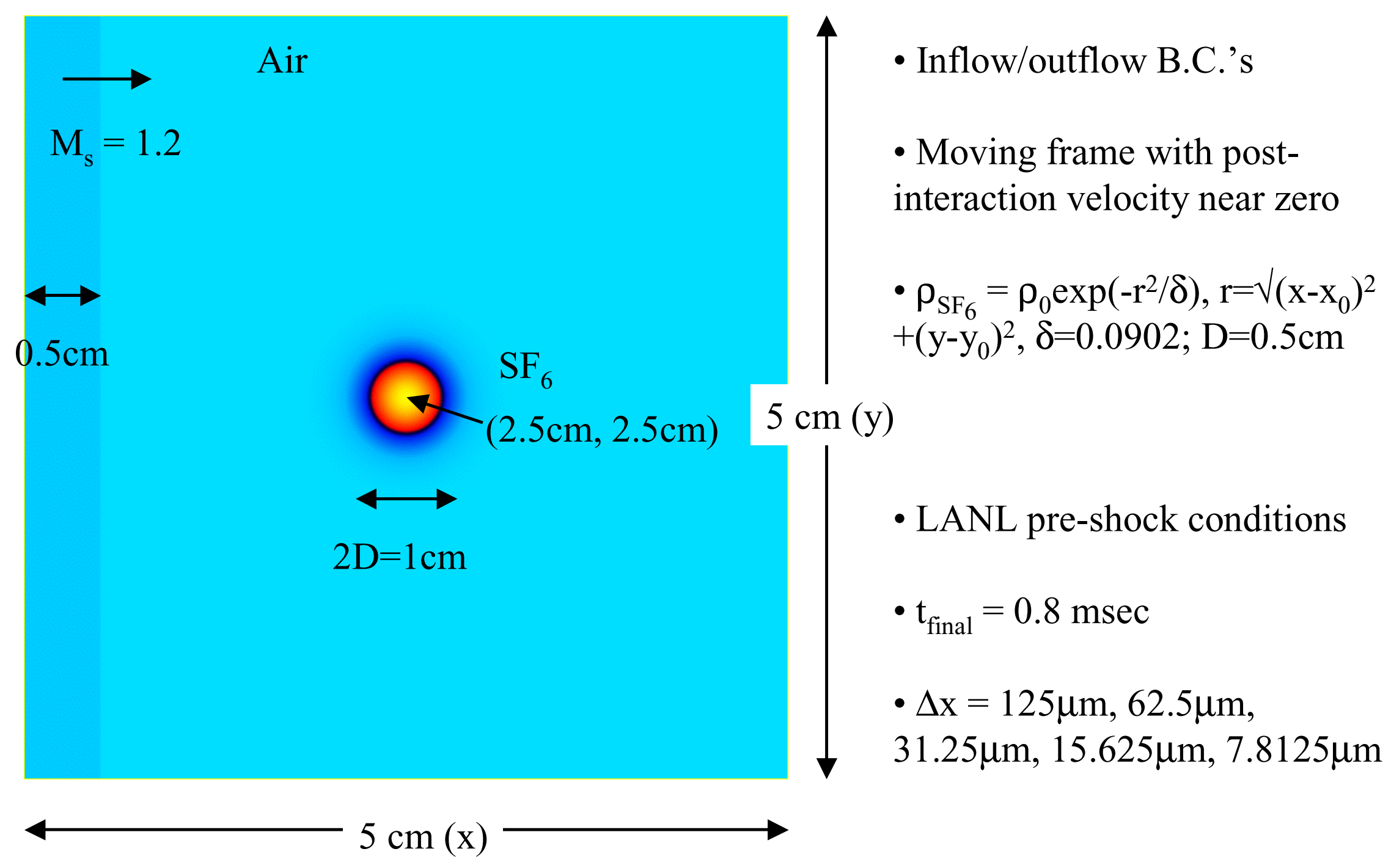




\section{Integral Lengths/Flow}

125 micron zoning, $\mathrm{t}=0.8 \mathrm{msec}$
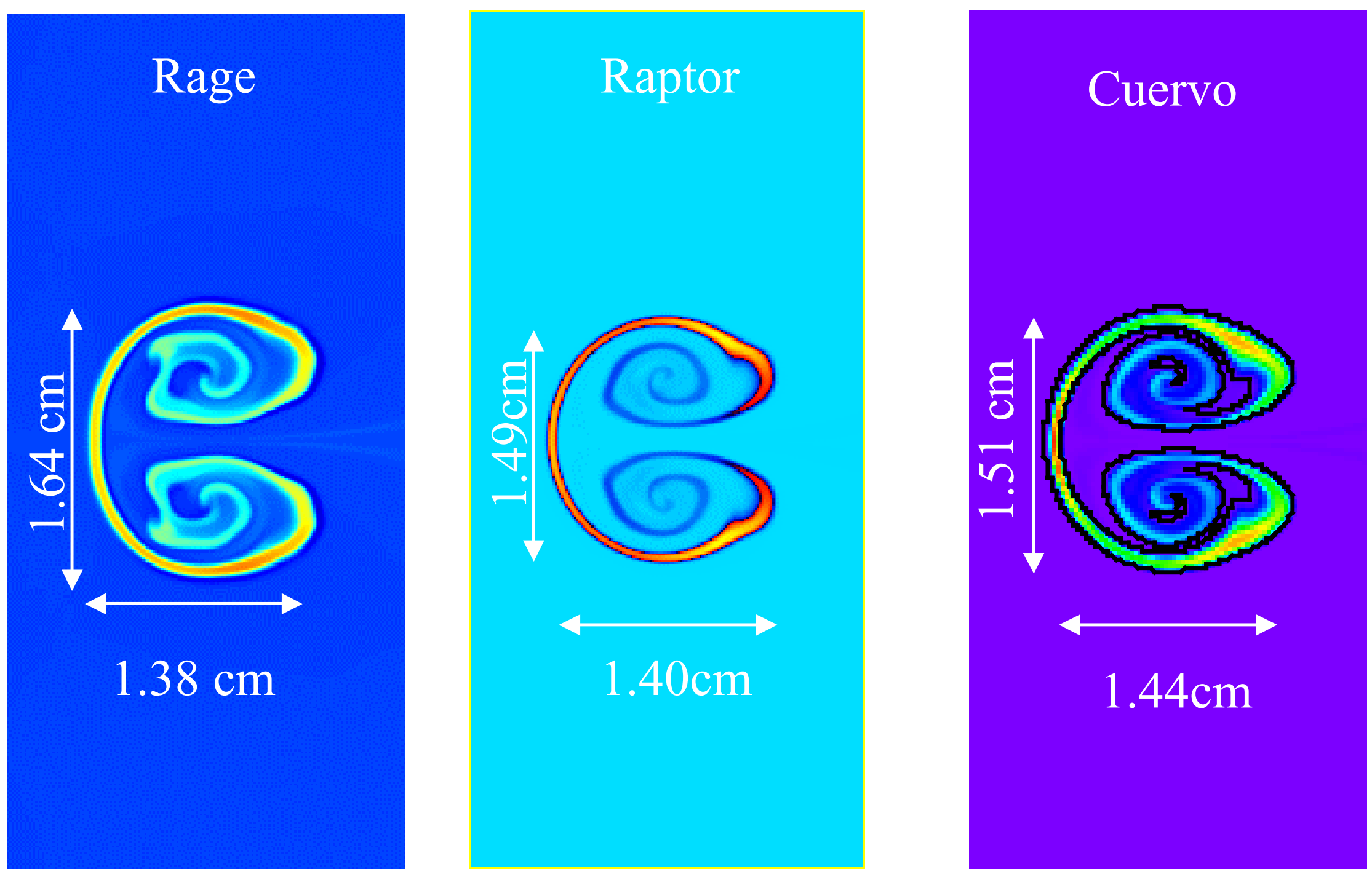


\section{Integral Lengths/Flow}

62.5 micron zoning, $\mathrm{t}=0.8 \mathrm{msec}$
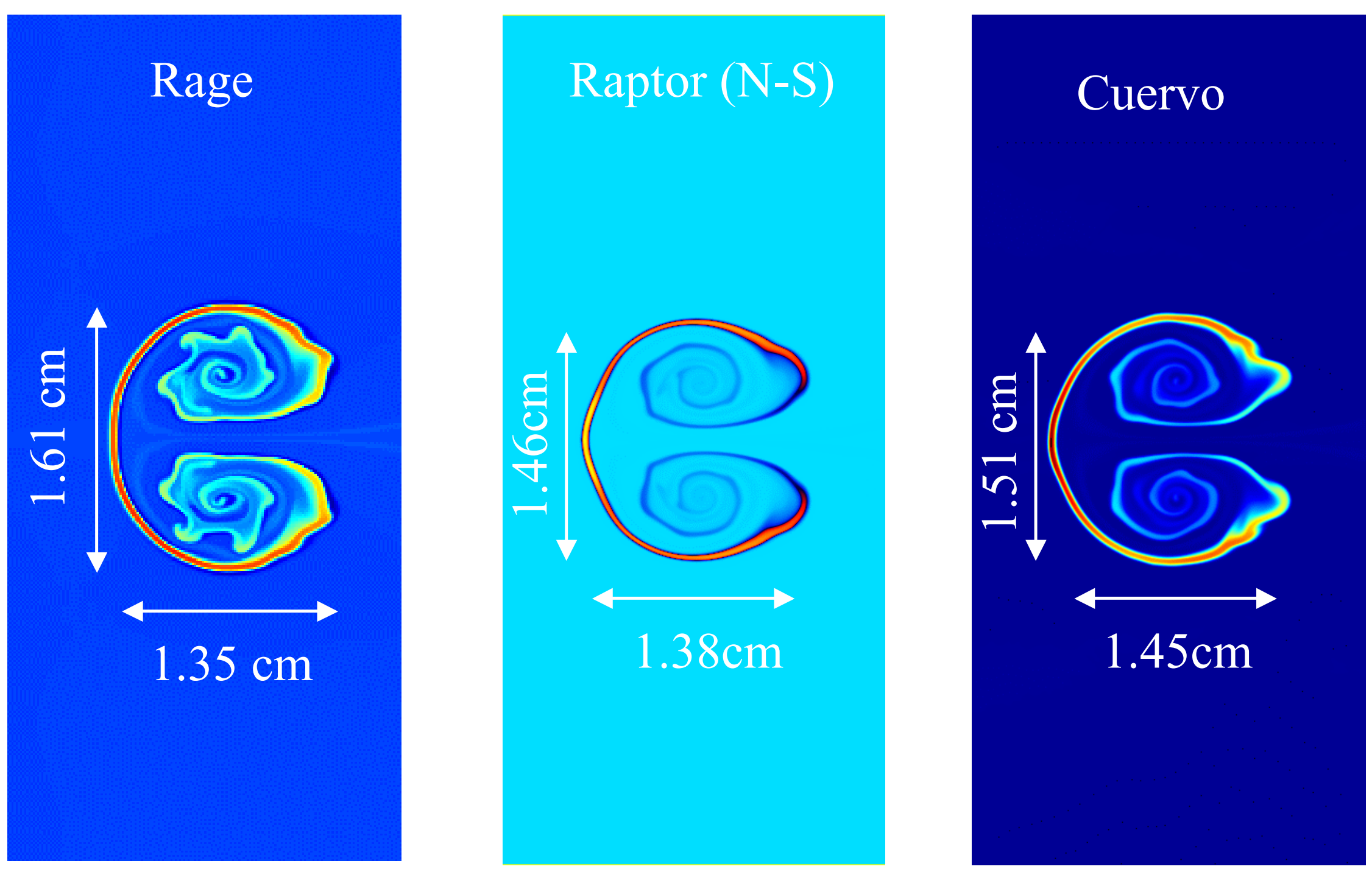


\section{Integral Lengths/Flow}

31.25 micron zoning, $\mathrm{t}=0.8 \mathrm{msec}$
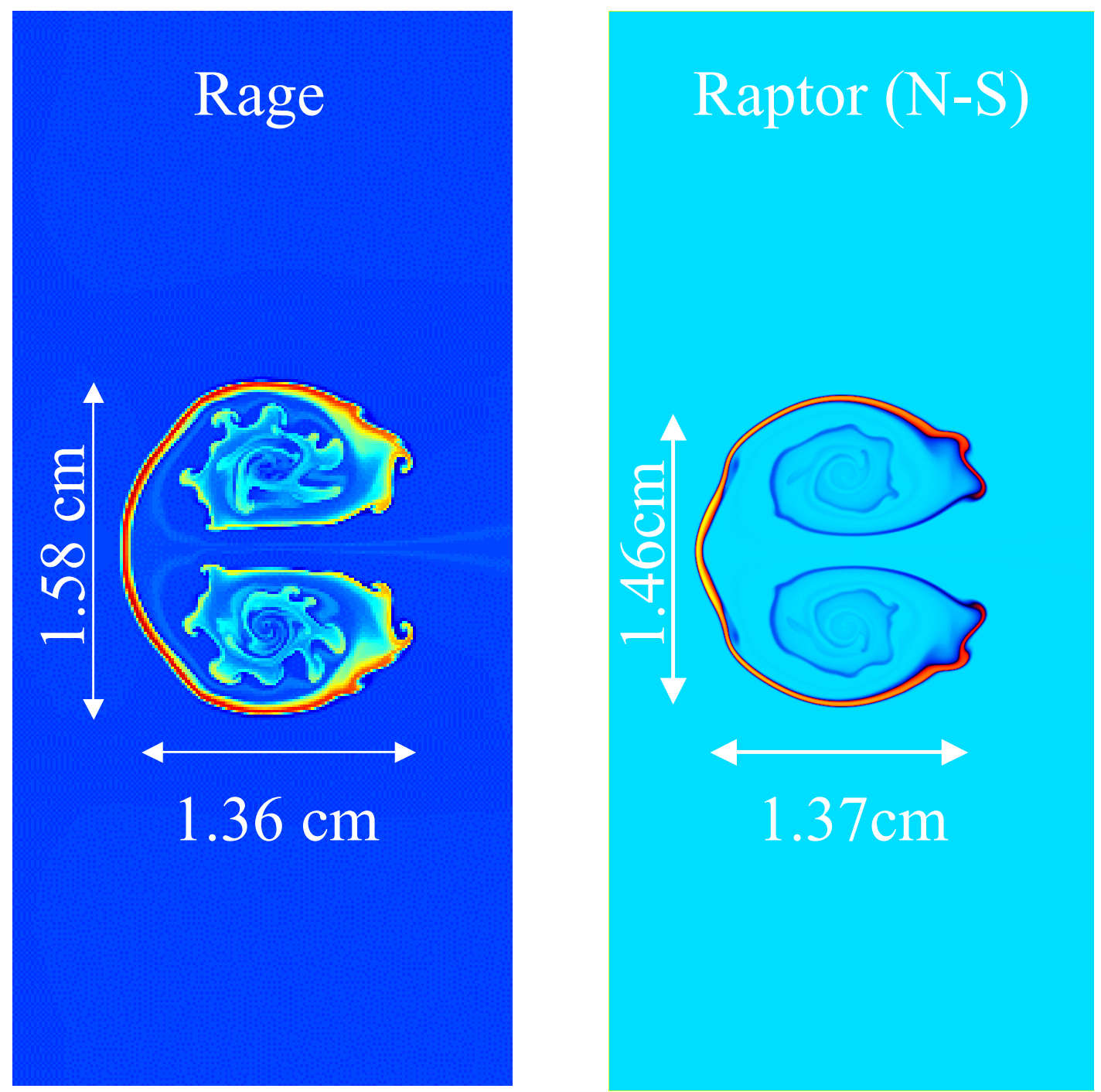


\section{Integral Lengths/Flow}

15.125 micron zoning

\section{Raptor (N-S)}

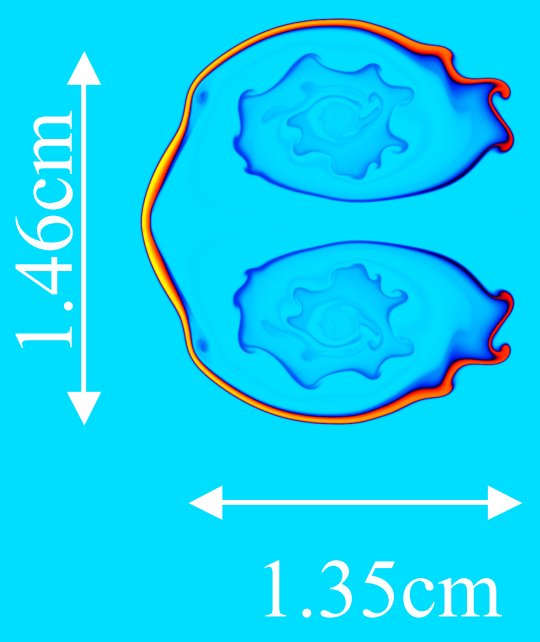

7.8125 micron zoning

3.90625 micron zoning

Raptor (N-S)
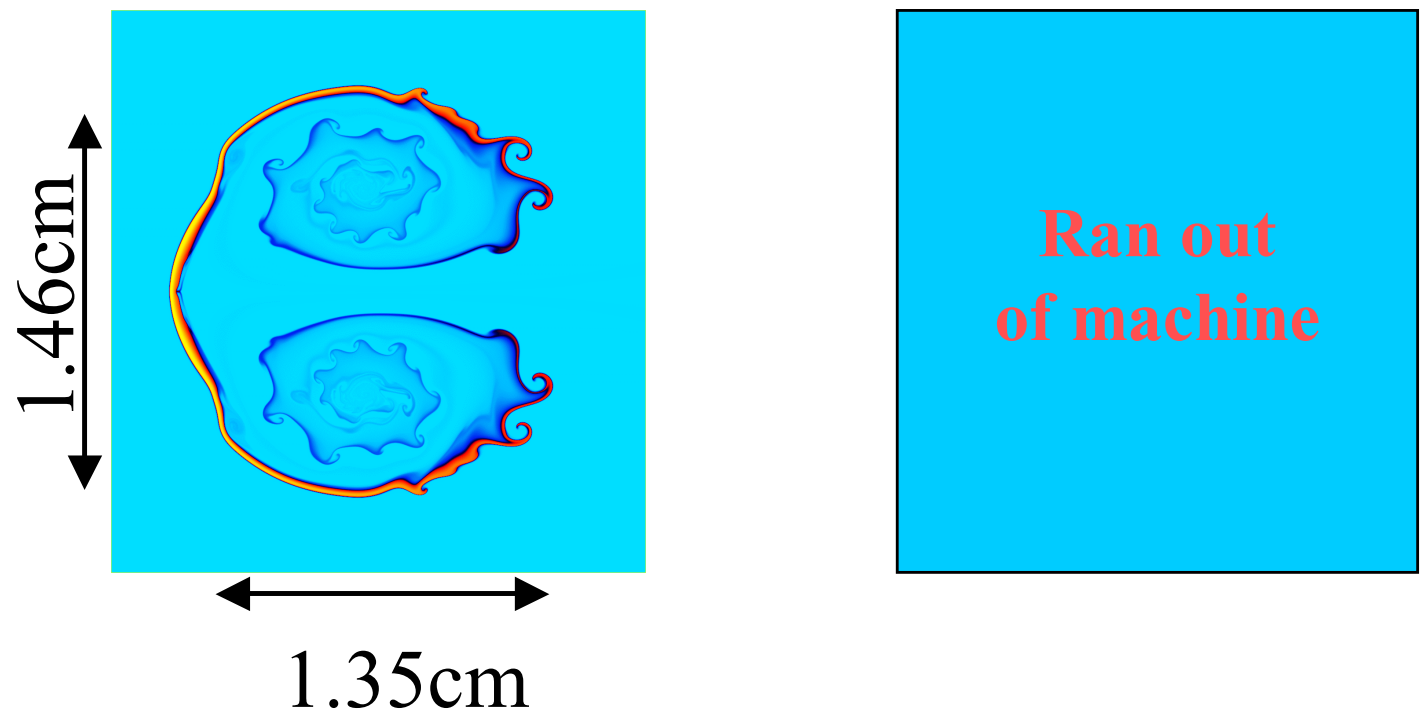


\section{Integral Lengths - Summary}
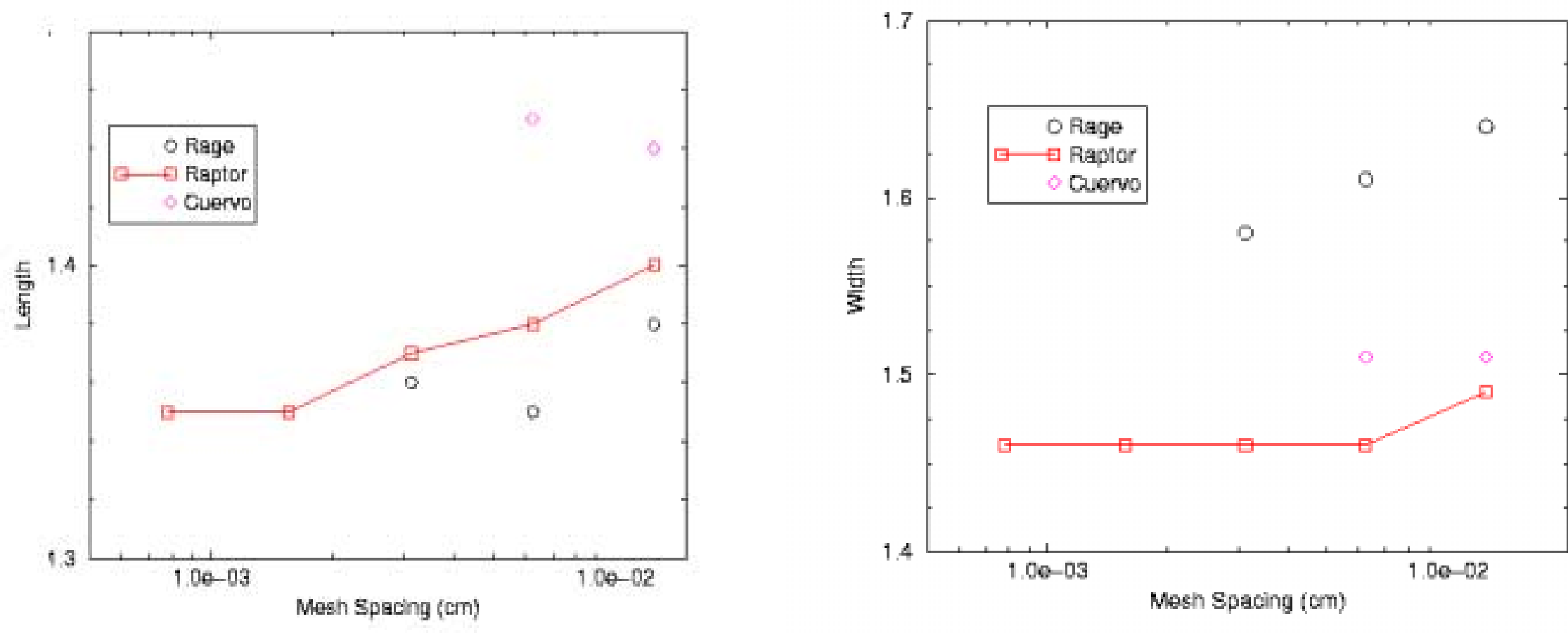

\section{Convergence Rates}

Cuervo $\sim \Delta \mathrm{x}^{1.28}$

Raptor $\sim \Delta \mathrm{x}^{1.58}$

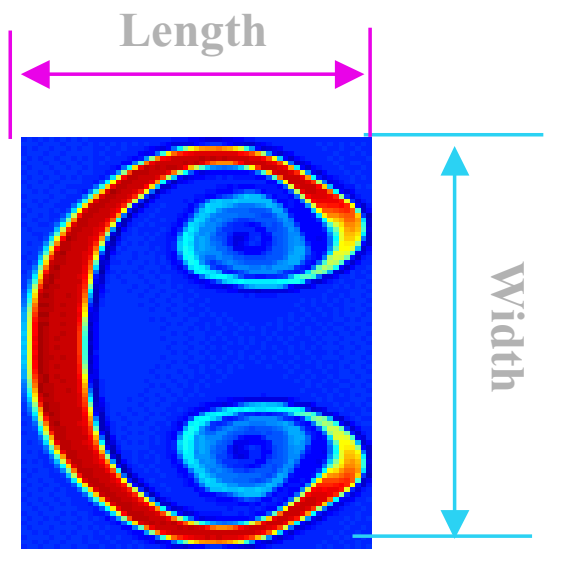

\section{Convergence Rates}

Cuervo $\sim \Delta \mathrm{x}^{0.74}$

Raptor $\sim \Delta \mathrm{x}^{0.28}$ 


\section{Mixing Fraction}

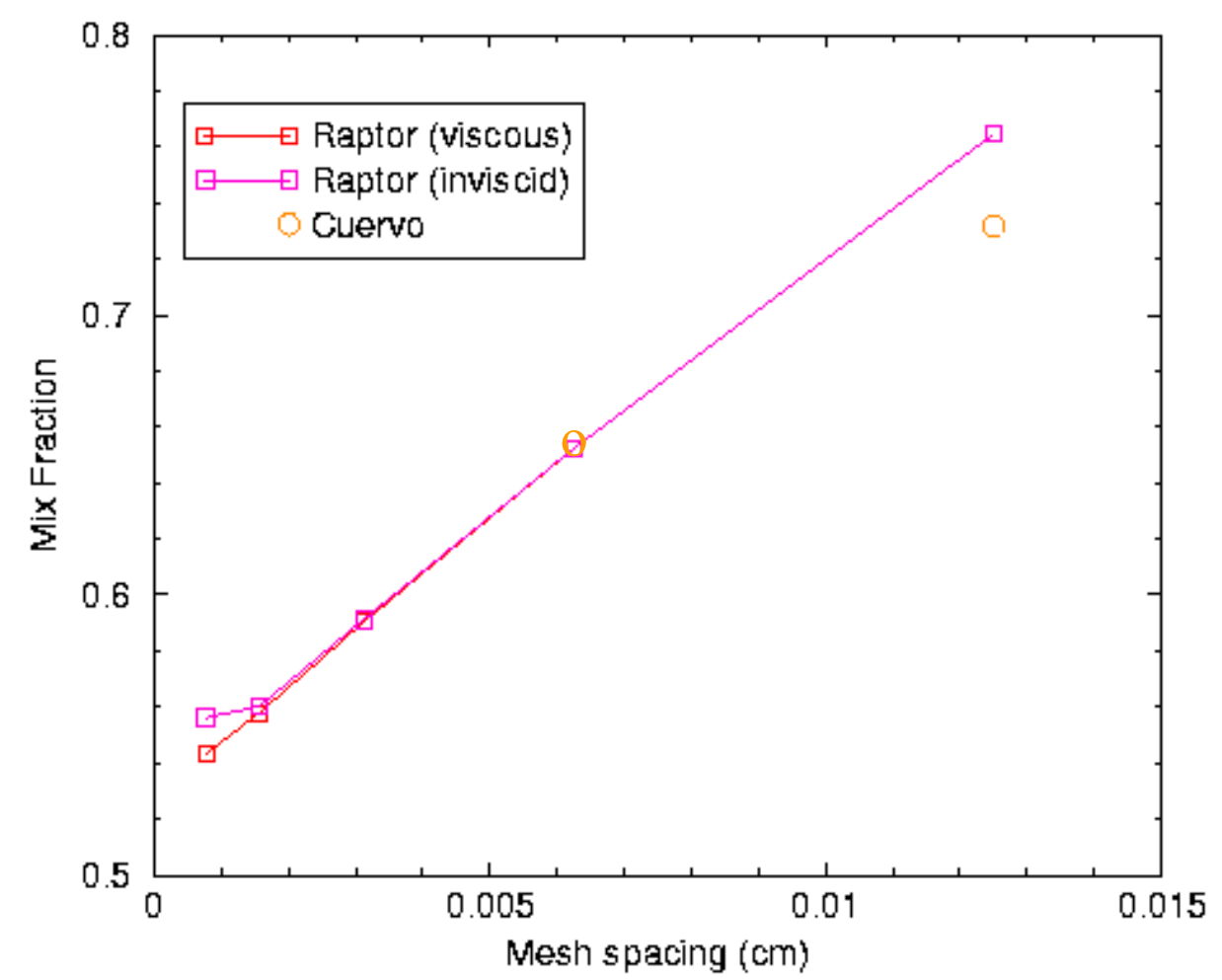

$$
\theta=\frac{\sum \mathrm{f}_{\mathrm{SF}_{6}}\left(1-\mathrm{f}_{\mathrm{SF}_{6}}\right) \Delta \mathrm{x} \Delta \mathrm{y}}{\left(\sum \mathrm{f}_{\mathrm{SF}_{6}} \Delta \mathrm{x} \Delta \mathrm{y}\right)\left(\sum\left(1-\mathrm{f}_{\mathrm{SF}_{6}}\right) \Delta \mathrm{x} \Delta \mathrm{y}\right)}
$$

\section{Convergence Rates}

Cuervo $\sim \Delta \mathrm{x}^{0.28}$

Raptor $\sim \Delta \mathrm{x}^{1.02}$ 


\section{Vortex Spacing}

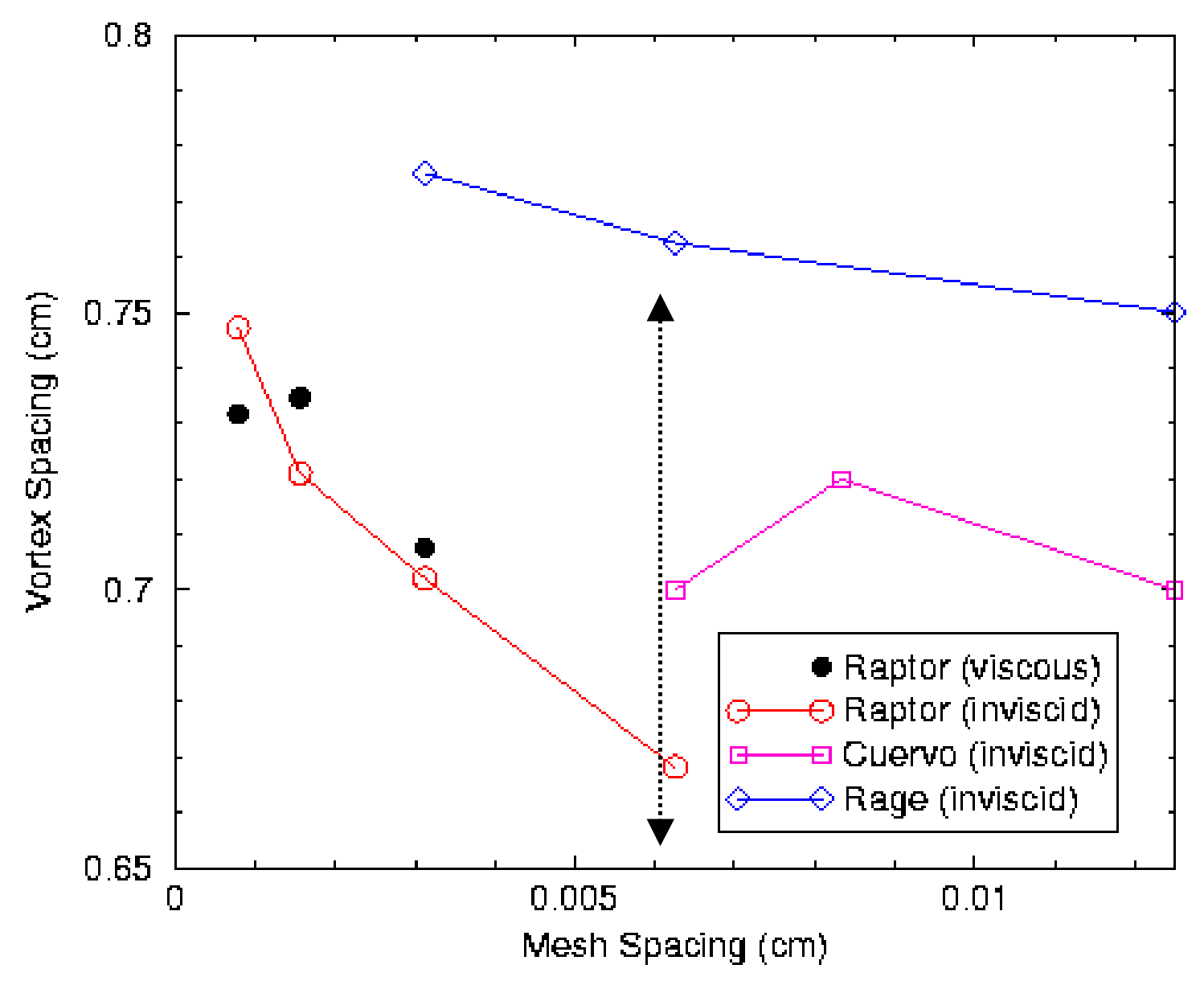

- Experimental data range shown for comparison

- cf. J.W. Jacobs, Phys. Fluids 1993; $\mathrm{M}=1.095$, $\mathrm{D}=0.43$

\section{Convergence Rates}

Raptor $\sim \Delta \mathrm{x}^{0.87}$ 


\section{Circulation Budget}
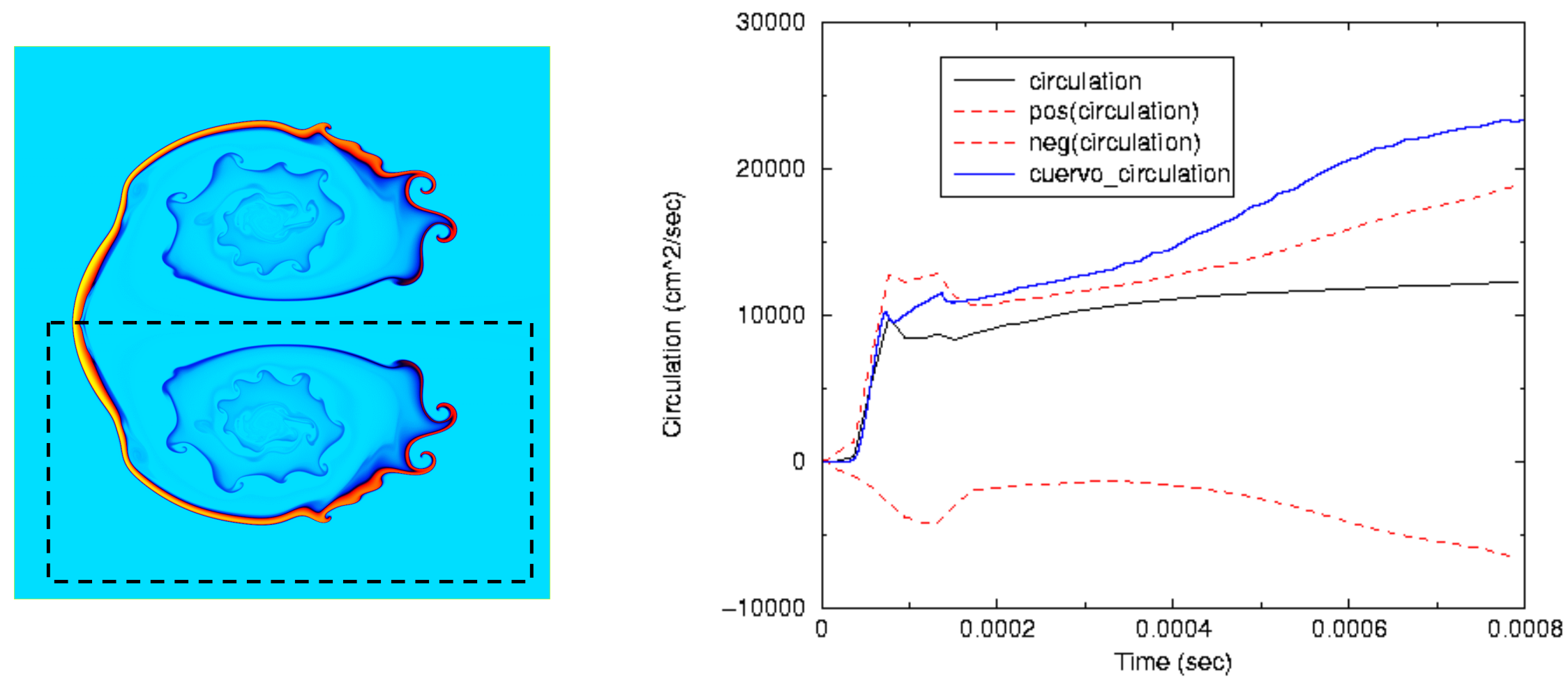

- Deposition by shock (positive)

- Counter-sign production (baroclinic)

- Late-time equilibration 


\section{Flow Dynamics}

- Early time

- Vortex blob deposition (shock-passage time $\sim 30 \mu \mathrm{sec}$ )

- Intermediate time

- Blob dipole transformation

- Counter-sign production

- Later time

- Dipole configuration established

- Balanced net vorticity (i.e. $\Gamma \sim$ constant) 


\section{Flow Dynamics - Density}
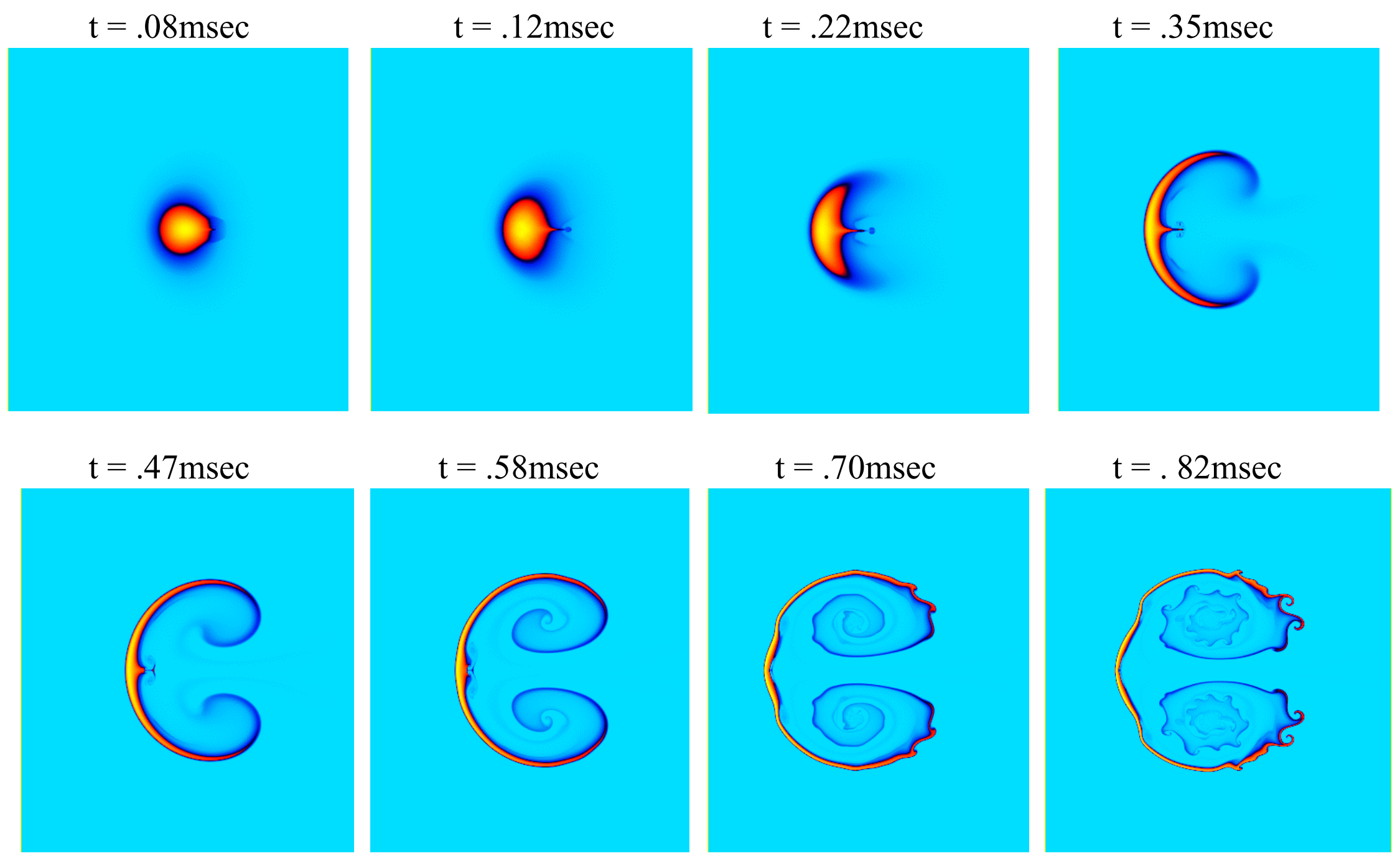


\section{Flow Dynamics - Vorticity}

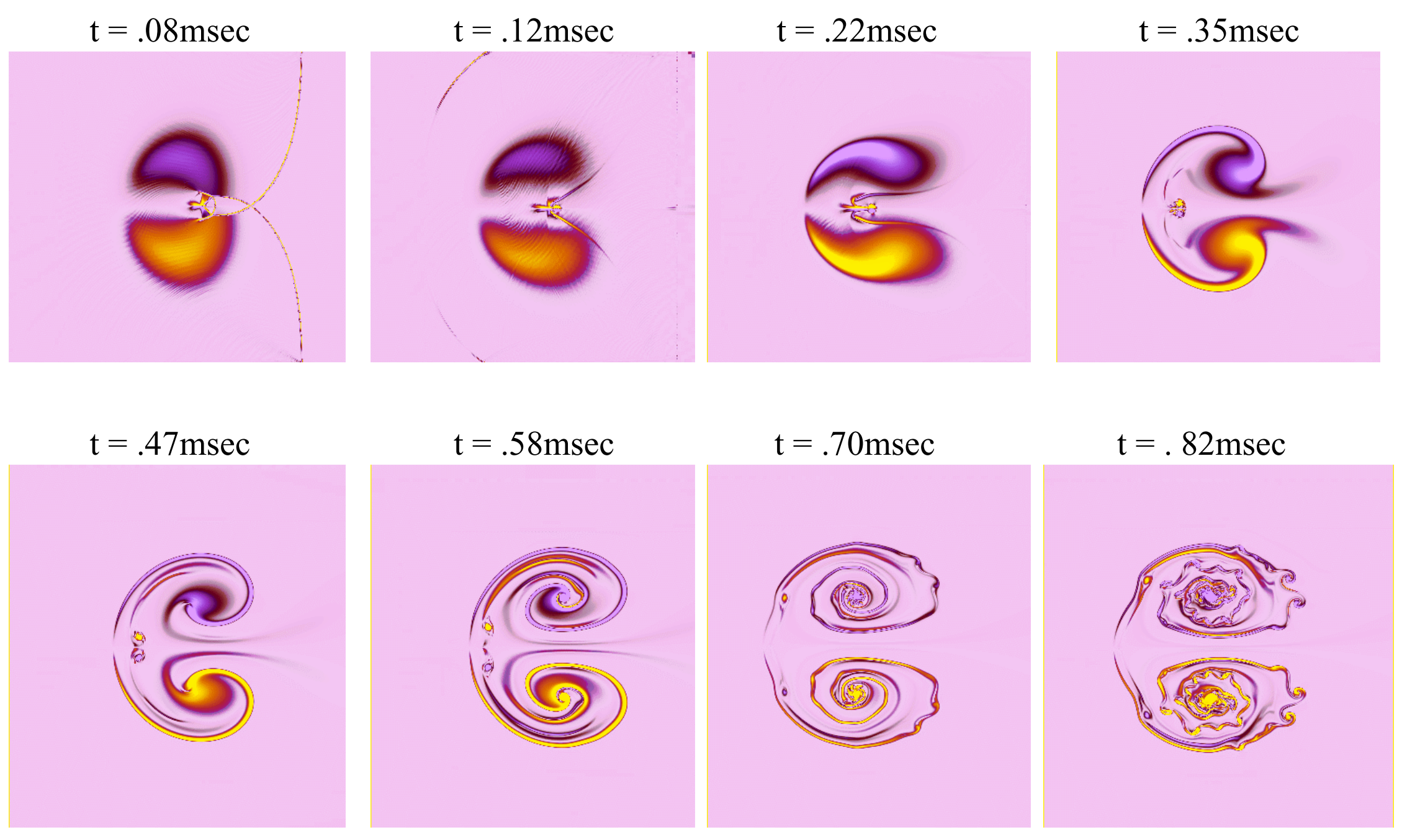




\section{Flow Dynamics - Baroclinic Generation}

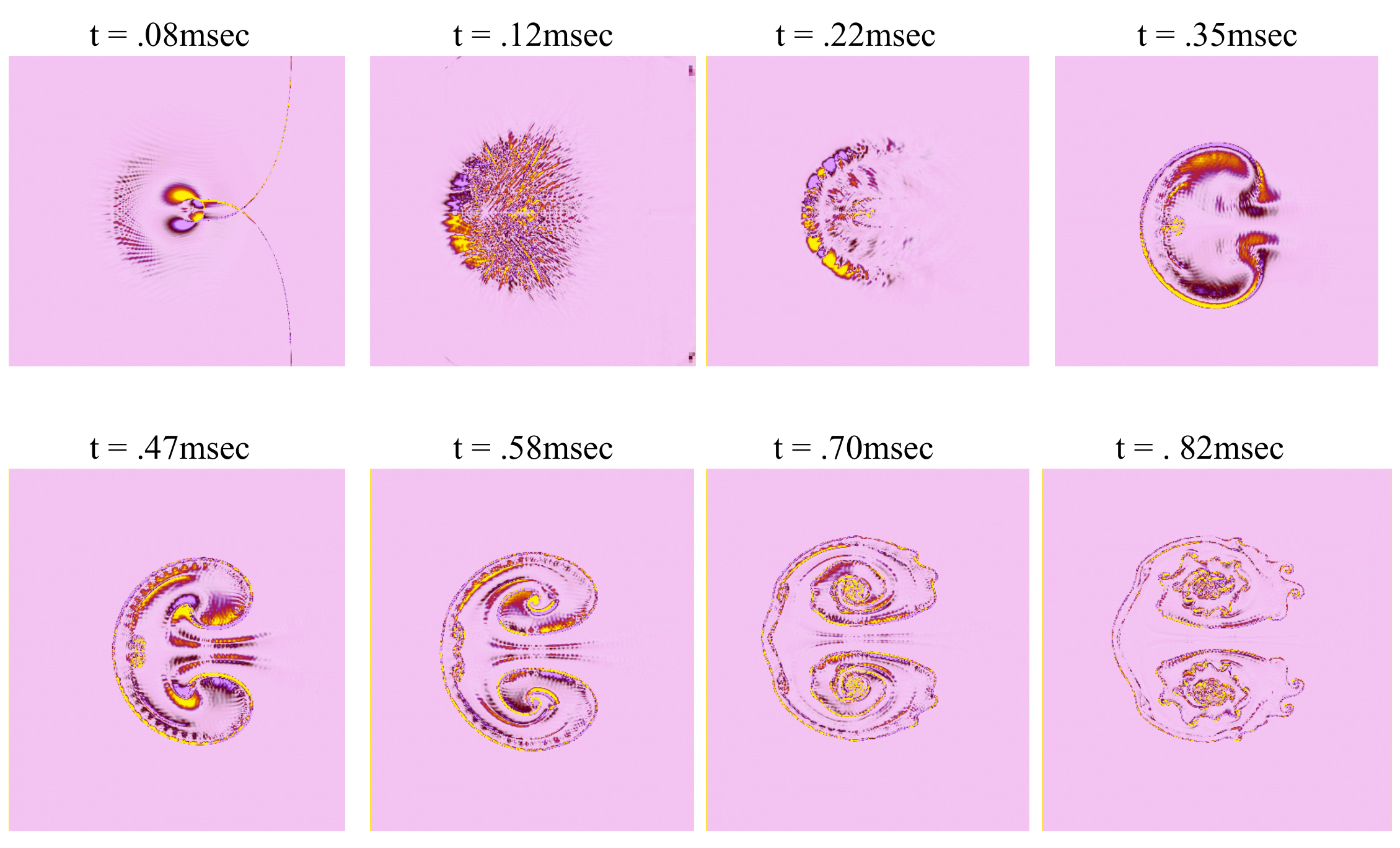




\section{Raptor Summary}

$31.25 \mu \mathrm{m}, 15.625 \mu \mathrm{m}, 7.8125 \mu \mathrm{m}$
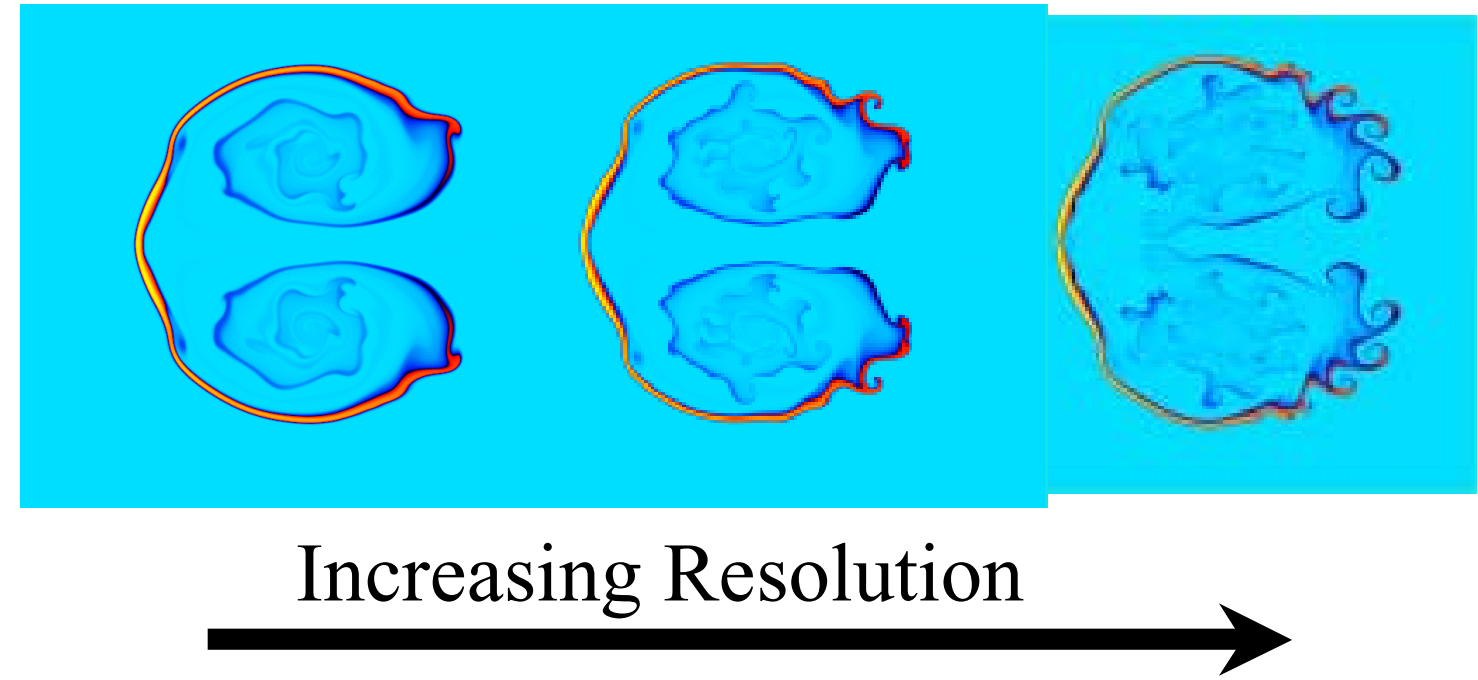

Inviscid
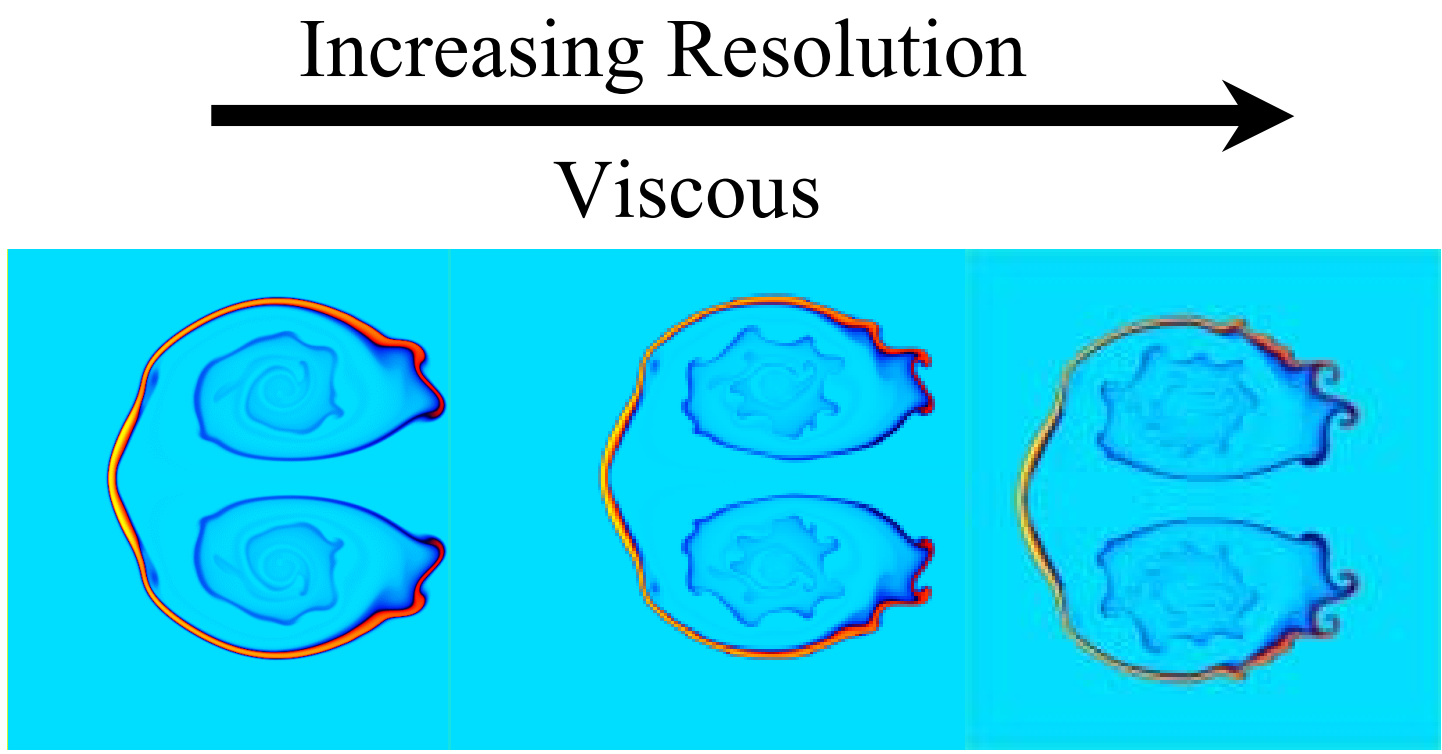
NEW Raptor Summary No prelax, viscosity fix

$31.25 \mu \mathrm{m}, 15.625 \mu \mathrm{m}, 7.8125 \mu \mathrm{m}$

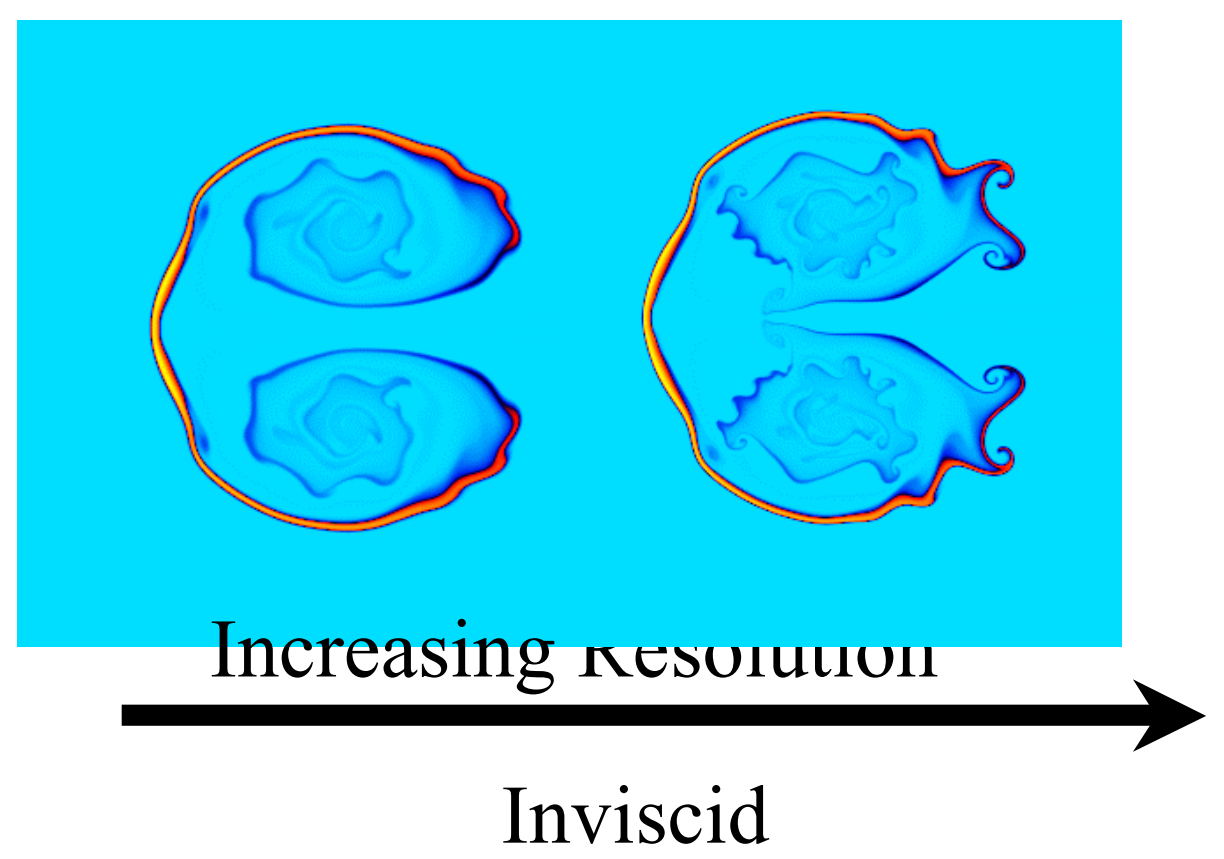

Increasing Resolution

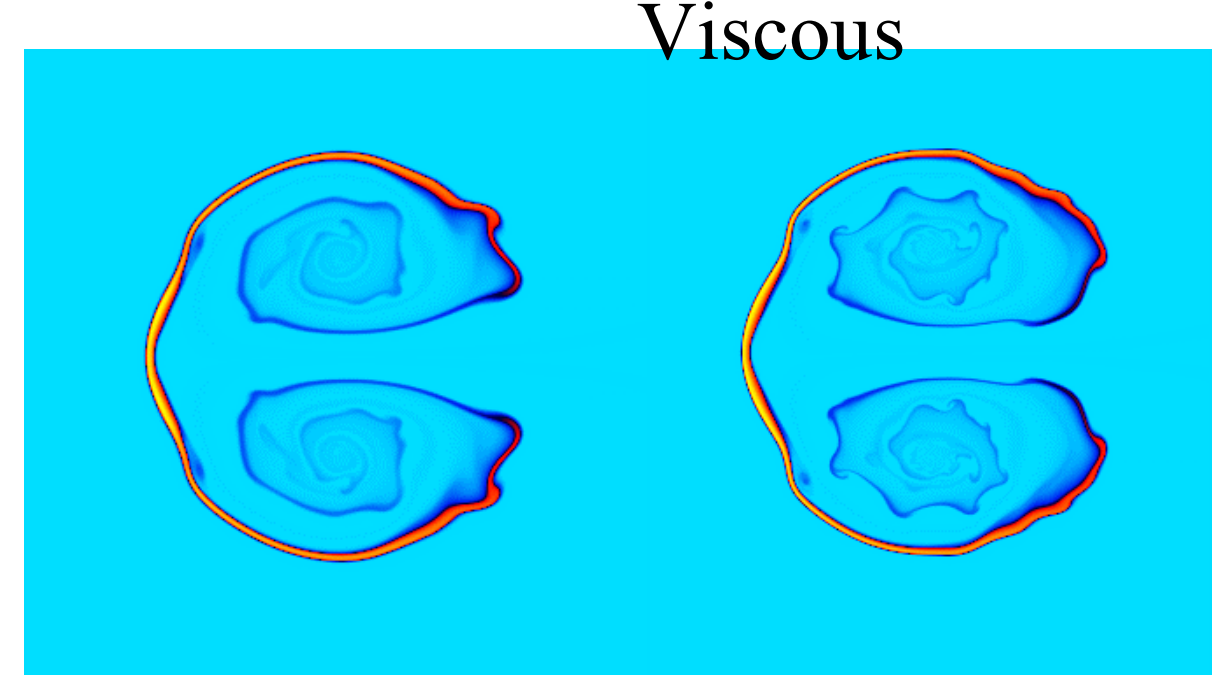




\section{Lengthscale estimates}

- Using order of magnitude considerations (Tennekes and Lumley)

- $\mathrm{U} \approx 2,000 \mathrm{~cm} / \mathrm{sec}, v \approx 0.1 \mathrm{~cm}^{2} / \mathrm{sec}, \mathrm{L}=0.1 \mathrm{~cm} \quad \mathrm{Re}=2,000$

$\cdot \eta / \mathrm{L} \sim \mathrm{Re}^{-0.75} \quad \eta \sim 3 \mu \mathrm{m}$ (Kolmgorov scale)

- $\lambda / \mathrm{L} \sim \mathrm{Re}^{-0.5} \quad \lambda \sim 90 \mu \mathrm{m}$ (Taylor scale)

- At $7.8125 \mu \mathrm{m}$ resolution, we have about 12 points/ $\lambda$ resolvable

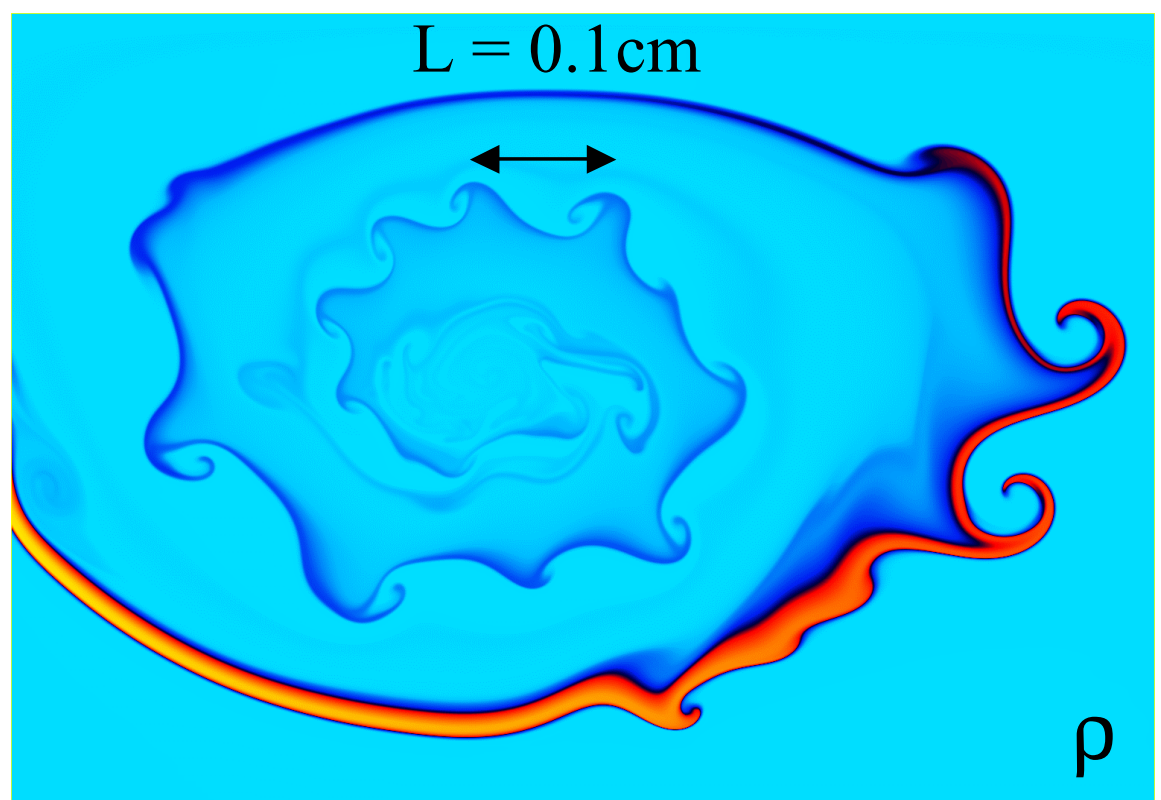




\section{Conclusions}

- Have we demonstrated convergence?

- Maybe. Some diagnostics show convergence while others do not.

- Include addition diagnostics (statistical, wavelet analysis).

- Have demonstrated what resolutions and physics are required for resolved calculations.

- Directly compute mm wavelength vortices. This is a robust feature present in analogous flow (Jacobs' Diffuse Interface R-M).

- Rage calculations appear to be the outlier; much more structure and different integral measurements. Vorticity?

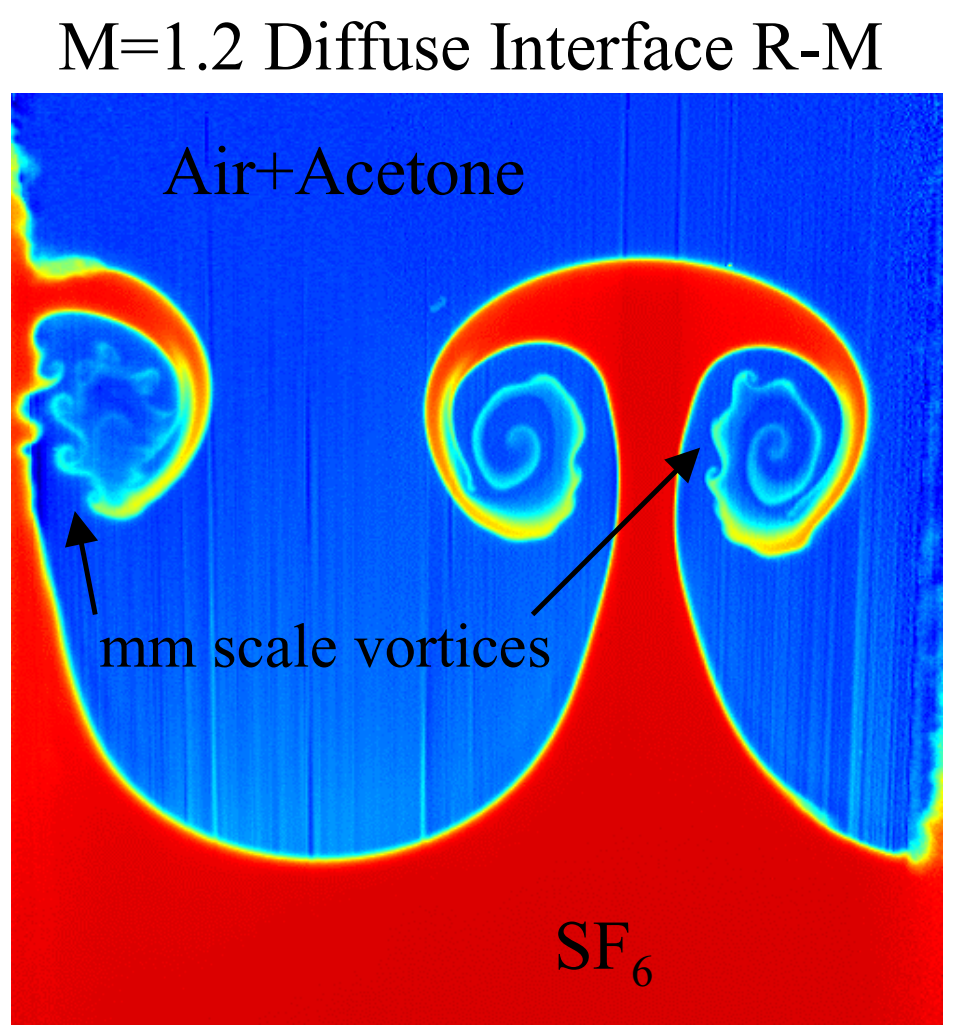

Courtesy of Prof. J.W. Jacobs 


\section{NEEDS}

- High(er) resolution experimental imaging

- PLIF visualization. LANL facility appears to generate a "more stable" evolving flow better pictures. Isolate mm-scale vortices

- More direct measurements

- Mixing measurements (Rayleigh scattering). Complementary to Helium jet work by J. Budzinski.

- More computing resources (never have enough) would allow definitive simulations.

- e.g. highest resolution run took $\sim 70$ hrs wall clock on 128 CPU's of an SP-3; AMR required 4.7 Mzones compared to 43 Mzones single grid. 


\section{LANL Experimental Activity}

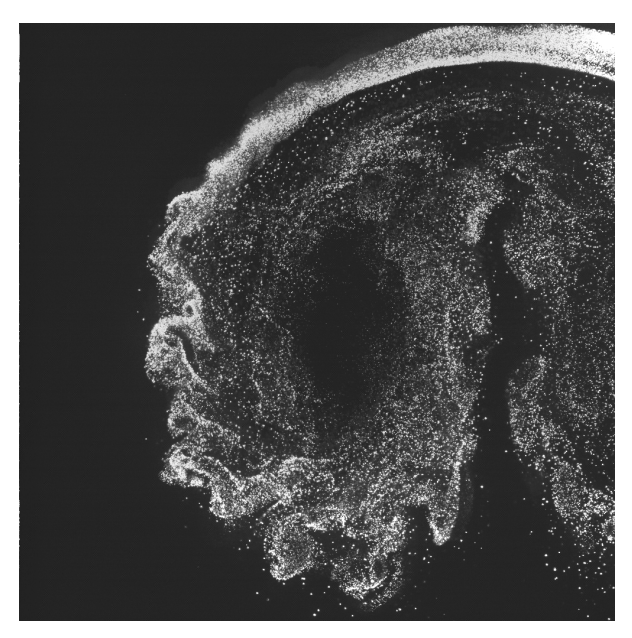

- No outer flow seeding

Varying the seeding densities \& light intensity
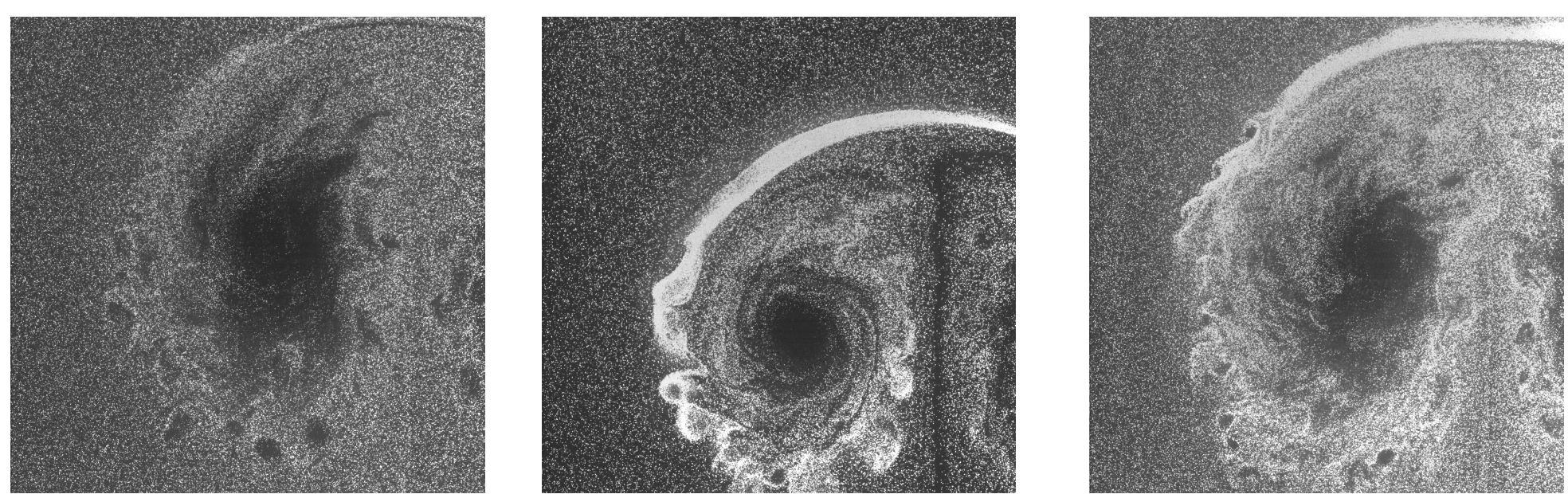

Images courtesy C. Tomkins, LANL, DX-3 


\section{Implementation of a}

Turbulent Mix Model in a 2D ALE Code

B Grieves

AWE 


\section{Abstract}

The addition of a turbulent mix model to a two dimensional finite element ALE hydrocode, CORVUS, is discussed. Use is made of the existing mixed-cell data structure package to facilitate the inclusion of the model.

This first stage of the model is based on the multiphase flow equations, and is a simplified form of the model implemented by Youngs (see paper at this workshop) in a 2D eulerian code. This is applicable to simple Rayleigh-Taylor and Richtmyer-Meshkov instabilities and some results are presented.

A simple buoyancy-drag model is used to calculate the early stages of the instability growth at internal nodes, and this is used to initialise the turbulent mix model calculation. 


\section{D ALE Code, CORVUS}

Corvus is a 2D finite element arbitrary lagrangian eulerian (ALE) hydrocode. It uses a staggered quadrilateral grid which is explicitly integrated using bilinear finite elements; pressure and internal energy are cell centred and the velocity is node centred. Each region is logically quadrilateral and ALE works across adjacent regions. The time step is split into a lagrangian and rezone phase.

Corvus uses a predictor corrector scheme for time discretisation and the rezone is accomplished using a second order van leer scheme. This gives a nominal second order accuracy both spatially and temporally. Corvus also incorporates strength, slide, friction, void opening and void closure.

The purpose of this work is to add a mixing phase to the time step.

A. J. Barlow, "ALE in Corvus", proceedings of new models and numerical codes for shock wave processes in condensed media, oxford, 1997, 581-596 


\section{The Model}

Equations for the transport of mass, momentum and internal energy.

$\frac{\partial}{\partial t}\left(f_{r} \rho_{r}\right)+\frac{\partial}{\partial x_{j}}\left(f_{r} \rho_{r} u_{r j}\right)=0$

$\frac{\partial}{\partial t}\left(f_{r} \rho_{r} u_{r i}\right)+\frac{\partial}{\partial x_{j}}\left(f_{r} \rho_{r} u_{r i} u_{r j}\right)=-f_{r} \frac{\partial p}{\partial x_{i}}$

$$
+\sum_{s} A_{r s i}+\sum_{s} D_{r s i}+f_{r} \rho_{r} g_{i}
$$

$\frac{\partial}{\partial t}\left(f_{r} \rho_{r} e_{r}\right)+\frac{\partial}{\partial x_{j}}\left(f_{r} \rho_{r} e_{r} u_{r j}\right)=-h_{r} f_{r} \rho_{r} \frac{\partial \bar{u}_{j}}{\partial x_{j}}+f_{r} \varepsilon$

$\mathrm{f}_{\mathrm{r}}=$ volume fraction of material $\mathrm{r} \mathrm{h}_{\mathrm{r}}=$ compressibility factor of material $\mathrm{r}$ $\rho_{\mathrm{r}}=$ density of material $\mathrm{r} \quad \mathrm{u}_{\mathrm{rj}}=$ velocity of material $\mathrm{r}$ in direction $\mathrm{j}$

$\mathrm{e}_{\mathrm{r}}=$ internal energy of material $\mathrm{r} \quad \mathrm{A}_{\mathrm{rsi}}=$ Added mass of fluid $\mathrm{r}$ due to $\mathrm{s}$

$\mathrm{g}_{\mathrm{i}}=$ gravity $\quad \mathrm{D}_{\mathrm{rsi}}=$ Drag on fluid $\mathrm{r}$ due to $\mathrm{s}$ 


\section{The Model - Mixing phase}

Volume fluxes calculated

$$
\begin{aligned}
& f_{r j}^{1} V_{j}^{1}=f_{r j}^{0} V_{j}+\delta V_{r j}-\delta V_{r j+1} \\
& \delta V_{r j}=A_{j j} x_{r j}\left(u_{r j}^{0}-\bar{u}_{j}^{0}+\delta u\right) \Delta t \\
& A_{j}=\text { area } \\
& f_{r j}^{x}=\text { van leer limited advection value } \\
& u_{r j}^{0}=\text { fluid r velocity } \\
& \delta u=\text { correction used to ensure } \Sigma \delta V=0
\end{aligned}
$$

Density Fluxes

$$
\rho_{r j}^{1} f_{r j}^{1} V^{1}{ }_{j}=\rho_{r j}^{0} f^{0}{ }_{r j} V_{j}^{0}+\delta M_{r j}-\delta M_{r j+1}
$$

Internal Energy

$$
e^{1}{ }_{r} \rho_{r j}^{1} f_{r j}^{1} V_{j}^{1}=e^{1}{ }_{r} \rho_{r j}^{0} f_{r j} V_{j}{ }_{j}+\delta E_{r j}-\delta E_{r j+1}
$$




\section{The model - Van Leer}

Van Leer limited advection flux calculated as follows if $\left(u_{r j}^{0}-\bar{u}_{j}^{0}+\delta u\right)>0$ then $a=j-2, b=j-1, c=j$

otherwise $a=j+1, b=j, c=j-1$

define $\varepsilon_{r j}=\left(\left|u_{r j}^{0}-\bar{u}_{j}^{0}+\delta u\right| A_{j} \Delta t\right) / V_{b}$ then

$f_{r j}=f_{r b}+\left(1-\varepsilon_{r j}\right) D_{r j} / 2$

$D_{r j}$ which represents the change in $f$ across the upwind cell is obtained by Van Leer limiting. This is the minimum of:

$$
\begin{aligned}
& \Delta_{1}=\mathrm{fo}_{\mathrm{rb}}-\mathrm{ff}_{\mathrm{ra}} \\
& \Delta_{2}=\mathrm{f}_{\mathrm{rc}}-\mathrm{f}_{\mathrm{rb}}
\end{aligned}
$$$$
D_{r j}^{0}=V_{b}\left(p \frac{\Delta_{1}}{\frac{1}{2}\left(V_{a}+V_{b}\right)}+(1-p) \frac{\Delta_{2}}{\frac{1}{2}\left(V_{b}+V_{v}\right)}\right)
$$$$
\text { where } p=\frac{V_{a}+\varepsilon_{r j} V_{b}}{V_{a}+V_{b}+V_{c}}
$$

Similarly $\delta M_{r j}=\rho_{r j}{ }_{r j} \delta V_{r j}$ and $\delta E_{r j}=e_{r j}{ }_{r j} \delta M_{r j}$ where $\rho_{r j}^{x}$ and $e^{x}{ }_{r j}$ are also Van Leer limited values 


\section{Implementation in Corvus}

The mix model is initialised by specifying the interfaces across which the mixing is calculated. A list of cells is generated on each side of this interface.

The mixing routines are then called after the lagrangian and rezone phases of each time step. The equations are solved along "rows" and "columns" of cells. The cell numbers of the edges are stored and the line of cells between these edges is generated and passed to the mixing routines one at time. The volume fractions are stored using the ALE package which calculates the effect of mesh movement. Other variables are stored on a "mixed cell" basis, in the same way as the ALE package. This minimises storage and utilises the pre-existing code. 


\section{Results - buoyancy-drag model}

A simple problem is used to illustrate the model. This is two regions of density 1 and 3 . The Equation of State of both fluids is $-\frac{1}{\rho} \frac{\partial p}{\partial x}=0.5$ with boundary conditions $\mathrm{p}=400$ and $\mathrm{p}=200$. This gives mixing limits of 54.0 and 70.0 at $\mathrm{t}=60$.

The table below gives the results.

\begin{tabular}{|l|l|l|}
\hline Time & $\begin{array}{l}\text { Mix } \\
\text { penetration }\end{array}$ & $\begin{array}{l}\text { Mix } \\
\text { penetration }\end{array}$ \\
\hline $0.000 \mathrm{E}+00$ & $1.000 \mathrm{E}-05$ & $1.000 \mathrm{E}-05$ \\
\hline $2.500 \mathrm{E}+00$ & $1.243 \mathrm{E}-01$ & $9.594 \mathrm{E}-02$ \\
\hline $5.000 \mathrm{E}+00$ & $4.918 \mathrm{E}-01$ & $3.794 \mathrm{E}-01$ \\
\hline $1.000 \mathrm{E}+01$ & $1.955 \mathrm{E}+00$ & $1.508 \mathrm{E}+00$ \\
\hline $1.500 \mathrm{E}+01$ & $4.391 \mathrm{E}+00$ & $3.387 \mathrm{E}+00$ \\
\hline $2.000 \mathrm{E}+01$ & $7.798 \mathrm{E}+00$ & $6.015 \mathrm{E}+00$ \\
\hline $2.500 \mathrm{E}+01$ & $1.217 \mathrm{E}+01$ & $9.394 \mathrm{E}+00$ \\
\hline $3.000 \mathrm{E}+01$ & $1.753 \mathrm{E}+01$ & $1.352 \mathrm{E}+01$ \\
\hline $3.500 \mathrm{E}+01$ & $2.385 \mathrm{E}+01$ & $1.840 \mathrm{E}+01$ \\
\hline $4.000 \mathrm{E}+01$ & $3.115 \mathrm{E}+01$ & $2.403 \mathrm{E}+01$ \\
\hline $4.250 \mathrm{E}+01$ & $3.516 \mathrm{E}+01$ & $2.712 \mathrm{E}+01$ \\
\hline $4.500 \mathrm{E}+01$ & $3.942 \mathrm{E}+01$ & $3.041 \mathrm{E}+01$ \\
\hline $4.750 \mathrm{E}+01$ & $4.392 \mathrm{E}+01$ & $3.388 \mathrm{E}+01$ \\
\hline $5.000 \mathrm{E}+01$ & $4.866 \mathrm{E}+01$ & $3.753 \mathrm{E}+01$ \\
\hline $5.250 \mathrm{E}+01$ & $5.364 \mathrm{E}+01$ & $4.138 \mathrm{E}+01$ \\
\hline $5.500 \mathrm{E}+01$ & $5.887 \mathrm{E}+01$ & $4.541 \mathrm{E}+01$ \\
\hline $5.750 \mathrm{E}+01$ & $6.434 \mathrm{E}+01$ & $4.963 \mathrm{E}+01$ \\
\hline $6.000 \mathrm{E}+01$ & $7.006 \mathrm{E}+01$ & $5.404 \mathrm{E}+01$ \\
\hline & & \\
\hline
\end{tabular}




\section{Results - buoyancy-drag model}

This is a mesh plot, the stars show the mixed cells.

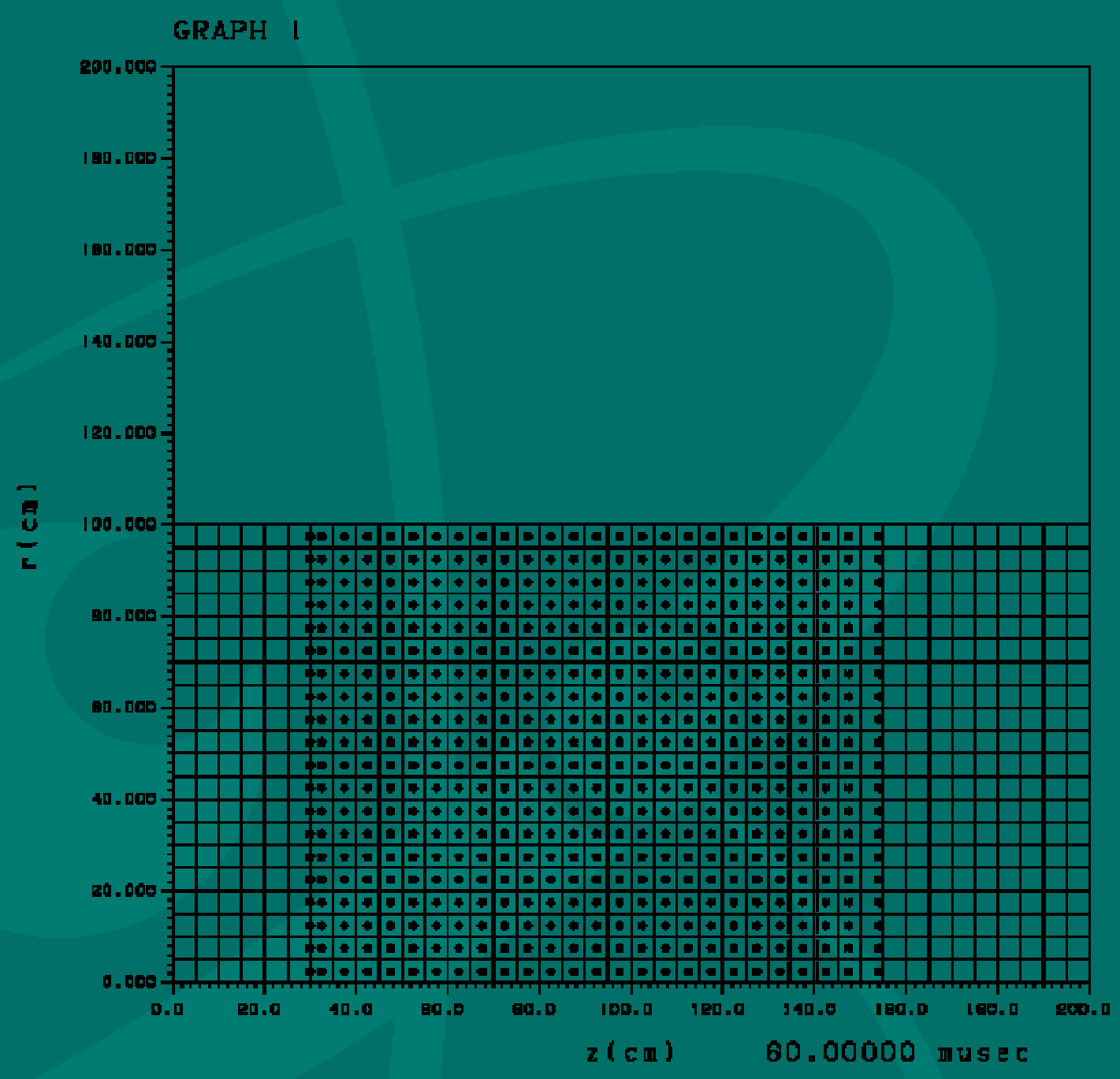




\section{Results}

These are the volume fractions along a line.
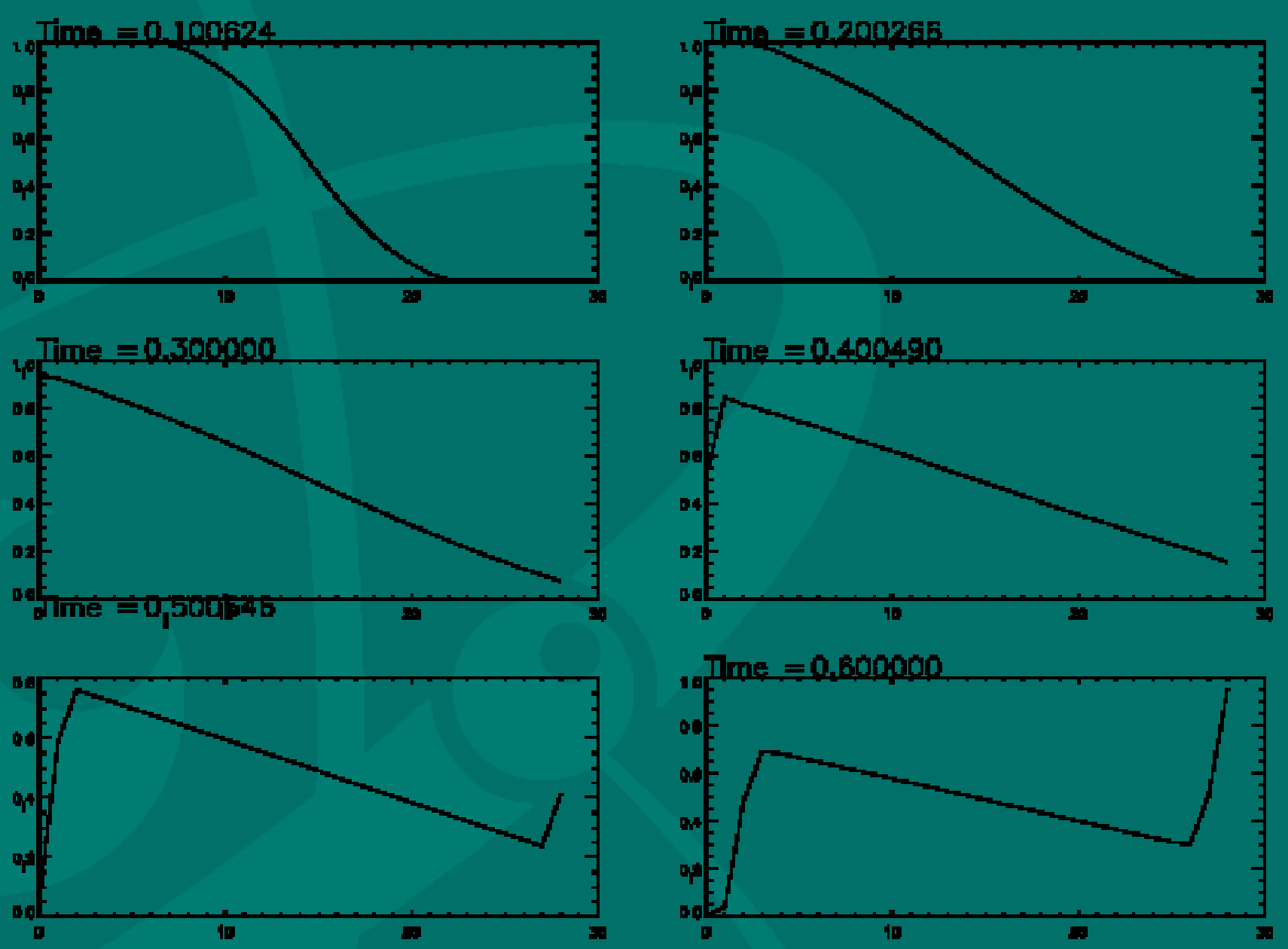


\section{Results}

This is a meshplot showing volume fractions. GRAPH ALE

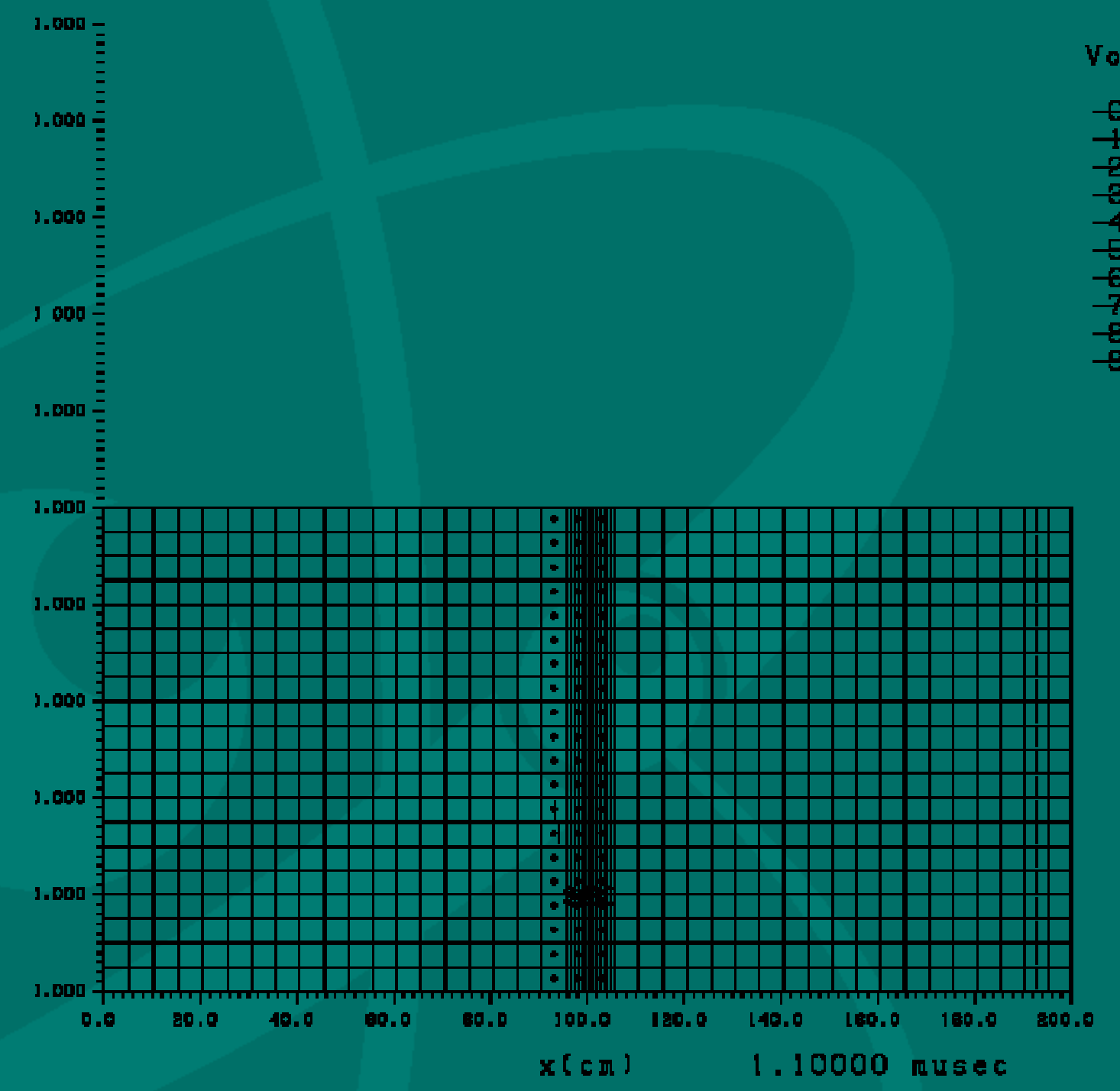

Volume Fraction

- 0.9090909E-

$+0.1818182 E 1$

2. d.2727273E

I- 0.3636364E

$40.4545456 E$

$50.5454545 E$

0.6969636E

$0.7272727 E_{1}$

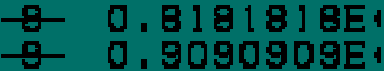




\section{Further work}

Initialisation using a simple buoyancy-drag model to start the turbulent model, this should increase the accuracy of the early time behaviour on a course mesh.

Mass exchange in the cells. Currently each mix cell consists of separate fluids.

\section{Conclusions}

A turbulent mix model has been implemented in a 2D ALE code, Corvus. It has been used on a simple problem and the results shown. 


\section{Semiempirical model of turbulent magnetic field diffusion to driven plasma}

E.V.Gubkov, V.A.Zhmailo, Yu.V.Yanilkin

RFNC-VNIIEF, 607190, Sarov, Nizhni Novgorod region

E-mail: vaz@vniief.ru

\section{Paper to be presented at the $8^{\text {th }}$ IWPCTM, Pasadena, 2001.}

There are quite many astrophysical and geophysical problems [1,2] as well as CTF related problems [3], in which a significant role is played by plasma-magnetic field interface instability. Among the numerous types of the instability, the magnetohydrodynamic instabilities were studied first. A most well-known example of this instability type is a so-called "chute" instability arising at the plasma-magnetic field interface during the interface acceleration.

This instability type is studied in a large number of papers, (see ref. [3]). The papers, as a rule, consider the linear stage of its evolution. The studies of the later, nonlinear stage became possible only recently thanks to availability of numerical methods. The approaches can be exemplified by refs. $[4,5,8]$. The first of them treats collisionless plasma and uses a "hybrid" model, the others consider plasma as "collisional" and perform the computation in the MHD approximation.

It was possible to obtain many useful results for the RT instability evolution at the plasma-magnetic field interface using this kind of approaches, however, all examples known to us of using these approaches are limited to problems, where unperturbed flow is one-dimensional. (A typical example is plasma cylinder expansion.) This limitation relates to the fact that such approaches prove quite complex, cumbersome, and do not allow us to follow the computed flow behavior in quite small scales.

A similar difficulty is encountered in unstable flow computations in hydrodynamics. A method to avoid this is using "semiempirical" turbulent mixing (TM) models. The model for "gravitational" TM problems was formulated for the first time in ref. [6], later the "semiempirical" models were developed quite extensively, became more complex, and found wide use for computation of a different kinds of turbulent flows. The models can be exemplified with those of refs. [7,12,13]. 
The studies of the RT instabilities in hydrodynamics and MHD flows revealed quite a close analogy between them at the linear stage [3]. This fact allows us to expect that this analogy may be also valid for a later, "turbulent" stage of the problem.

This paper discusses a semiempirical model for computing characteristics of a transitional layer at the accelerated plasma cloud - magnetic field interface. The model was developed on the basis of the hydrodynamic models [6,7] with using the abovementioned analogy. Results of some 1D and 2D computations by the model are presented.

\section{Derivation of governing equations}

Assume that the flow can be described by MHD equations [9].

Thus, we have:

$$
\begin{aligned}
& \frac{\partial \rho}{\partial \mathrm{t}}+\operatorname{div} \rho \overrightarrow{\mathrm{u}}=0 \\
& \frac{\partial(\rho \overrightarrow{\mathrm{u}})}{\partial \mathrm{t}}+\operatorname{Div}(\overrightarrow{\mathrm{S}})=\overrightarrow{\mathrm{f}} \\
& \frac{\partial \mathrm{Q}}{\partial \mathrm{t}}+\operatorname{div}\left[\rho \overrightarrow{\mathrm{u}}\left(\frac{\mathrm{u}^{2}}{2}+\omega\right)-(\overrightarrow{\mathrm{u}} \cdot \vec{\sigma})+\frac{1}{4 \pi}[\overrightarrow{\mathrm{H}} \overrightarrow{\mathrm{E}}]\right]=0 \\
& \overrightarrow{\mathrm{f}}=\frac{1}{4 \pi}[\operatorname{rot} \overrightarrow{\mathrm{H}} \cdot \overrightarrow{\mathrm{H}}] \\
& \text { where } \mathrm{S}_{\mathrm{ik}}=\mathrm{P} \delta_{\mathrm{ik}}+\rho \mathrm{u}_{\mathrm{i}} \mathrm{u}_{\mathrm{k}}-\sigma_{\mathrm{ik}} \\
& \mathrm{Q}=\frac{\rho \mathrm{u}^{2}}{2}+\rho \mathrm{E}+\frac{\mathrm{H}^{2}}{8 \pi} \\
& \overrightarrow{\mathrm{E}}=\frac{1}{\mathrm{c}}[\overrightarrow{\mathrm{u}} \overrightarrow{\mathrm{H}}]-\frac{\mathrm{c}}{4 \pi \Sigma} \operatorname{rot}_{\mathrm{H}} \\
& \omega=\mathrm{E}+\frac{\mathrm{P}}{\rho} \\
& \vec{\sigma} \quad \text { is } \quad \operatorname{viscous} \text { stress tensor, } \Sigma \quad \text { - conductivity, other notations are }
\end{aligned}
$$
conventional.

This system is complemented with equations for magnetic field:

$$
\begin{aligned}
& \frac{\partial \overrightarrow{\mathrm{H}}}{\partial \mathrm{t}}+(\overrightarrow{\mathrm{u}} \nabla) \overrightarrow{\mathrm{H}}=(\overrightarrow{\mathrm{H}} \nabla) \overrightarrow{\mathrm{u}}+v_{\mathrm{m}} \Delta \mathrm{H} \\
& \operatorname{div} \overrightarrow{\mathrm{H}}=0
\end{aligned}
$$


where $v_{m}=\frac{C^{2}}{4 \pi \sigma}$ is "magnetic" viscosity factor, as well as with the equation for different concentrations $\alpha_{i}$ :

$$
\frac{\partial \rho_{\mathrm{i}}}{\partial \mathrm{t}}+\operatorname{div} \rho_{\mathrm{i}} \overrightarrow{\mathrm{u}}=\operatorname{div}\left(\rho \mathrm{i} \nabla \alpha_{\mathrm{i}}\right)
$$

where $\rho_{i}=\rho \alpha_{i}, D-$ coefficient of molecular diffusion, and equation of state:

$$
\mathrm{P}=\mathrm{P}\left(\rho, \mathrm{E}, \alpha_{\mathrm{i}}\right)
$$

As usual, divide the quantities to be calculated into low- and high-frequency addends, then make appropriate averaging [10,11], and use the commonly accepted expressions for the addends containing the third or higher order moments to obtain the following from set 1.1 through 1.12 for averaged values:

For density: Eq. 1.1

For velocity: Eq. 1.2

For concentrations : Eq. 1.11

For "magnetic" force: Eq. 1.4.

The equations for velocity and density therewith involve the "turbulent" tensor of viscous pressures, $\sigma_{t}$, and diffusion coefficient, $\mathrm{D}_{\mathrm{t}}$, instead of relevant "molecular" values.

For internal energy:

$$
\begin{aligned}
& \frac{\partial}{\partial t}(\rho \cdot E)+\operatorname{div}(\rho E \vec{u})=c_{\alpha} \operatorname{div}(\rho D \nabla(\alpha E))-P \operatorname{div} \vec{u}+\rho \varepsilon+\frac{(\vec{j})^{2}}{\Sigma_{T}} \\
& \vec{j}=\frac{c}{4 \pi} \operatorname{rot} \vec{H}
\end{aligned}
$$

Here $\varepsilon$ is dissipation rate of turbulent energy $\mathrm{k}, \Sigma_{T}$ is effective conductivity. The following relations are used for functions $\sigma_{t}$ and $\mathrm{D}_{\mathrm{t}}[7]$ :

$$
\begin{gathered}
D_{t}=C_{D} \frac{k^{2}}{\varepsilon} \\
\sigma_{t}^{i j}=\rho D_{t}\left[\frac{\partial u_{i}}{\partial x_{j}}+\frac{\partial u_{j}}{\partial x_{i}}-\frac{2}{3} \delta_{i j} \frac{\partial u_{r}}{\partial x_{r}}\right]-\frac{2}{3} \rho k \delta_{i j}
\end{gathered}
$$


Also, note that relations 1.11 to 1.16 were derived assuming Reynolds numbers $\mathrm{Re}$ and $\mathrm{Re}_{\text {mag }} \rightarrow \infty$ and addends, such as "turbulent pressure" $\mathrm{P}_{\mathrm{T}}$ and "magnetic turbulent pressure, were neglected.

A number of additional assumptions were used in this paper to calculate averaged magnetic field:

- cylindrical symmetry of the problem was assumed,

- the geometry of the initial magnetic field was assumed "poloidal",

- "magnetic viscosity" can be neglected.

Under these assumptions, the magnetic field components $H_{\rho}$ and $H_{z}$ (where $z$ is axis of symmetry) are related with $\mathrm{A}_{\phi}$, azimuthal potential vector component, as

$$
\begin{aligned}
& \mathrm{H}_{\rho}=-\frac{\partial \mathrm{A}_{\varphi}}{\partial \mathrm{z}} \\
& \mathrm{H}_{\mathrm{z}}=\frac{1}{\rho} \frac{\partial}{\partial \rho}\left(\mathrm{A}_{\varphi} \rho\right)
\end{aligned}
$$

$$
\mathrm{A}_{\varphi} \rho=\mathrm{m}
$$

where

$$
\begin{aligned}
& \frac{\partial \mathrm{m}}{\partial \mathrm{t}}+\overrightarrow{\mathrm{u}} \nabla \mathrm{m}=\operatorname{div} \overrightarrow{\mathrm{R}}_{\mathrm{m}} \\
& \overrightarrow{\mathrm{R}}_{\mathrm{m}}=-\left\langle\overrightarrow{\mathrm{u}}^{\prime} \mathrm{m}^{\prime}\right\rangle=\widetilde{\mathrm{D}} \nabla \mathrm{m}
\end{aligned}
$$

Assume that $\widetilde{D}=C_{m} D$, where $C_{m}$ is an empirical constant.

When considering the equations for turbulent values $\mathrm{k}$ and $\varepsilon$, turbulence anisotropization by magnetic field must be included. To do this, a simplest method is using the equation system for $k_{i j}=\left\langle u_{i}^{\prime} u_{j}^{\prime}\right\rangle$ instead of a single equation for $\mathrm{k}$ [7]. By analogy with this equation [7], the following can be written for diagonal components $\mathrm{k}_{\mathrm{j}}$ :

$$
\begin{array}{cc}
\frac{\partial}{\partial t}\left(\rho k_{j}\right)=\rho\left(G_{j}-\varepsilon_{j}\right)+C_{k} d i v \rho D_{j} \nabla k_{j}+\Phi_{j}-\operatorname{Re} l\left(\rho k_{j}\right)-d i v \rho k_{j} \vec{u}-\frac{2}{3} \rho k_{j} d i v \vec{u} & 1.22 \\
G_{j}=G_{j}^{(1)}+G_{j}^{(2)} & 1.23 \\
G_{j}^{(1)}=\sigma_{j l} \frac{\partial u_{j}}{\partial x_{l}} \text { - shear generation } \\
G_{j}^{(2)}=\frac{w_{j} \gamma_{j}}{\rho} \text { - gravitational generation }
\end{array}
$$


$\vec{\gamma}=\nabla\left(P+P_{m}\right)-\frac{1}{4 \pi}(\vec{H} \nabla \vec{H})$

$\vec{w}=\frac{\nabla P}{\gamma P}-\frac{\nabla \rho}{\rho}, \vec{w}-$ turbulent flow

$\operatorname{Re} l\left(\rho k_{j}\right)=\frac{\varepsilon}{k} \rho\left(k_{j}-\frac{k}{3}\right)$

$\Phi_{j}$ - exchange term (between velocity and magnetic field fluctuation)

$\left.\Phi_{j}=\frac{1}{4 \pi} \cdot 2 \cdot\left[H_{l}<u_{j} \frac{\partial h_{j}}{\partial x_{l}}\right\rangle+\frac{\partial H_{j}}{\partial x_{l}} r_{j l}-\left\langle u_{j} \frac{\partial}{\partial x_{j}} P_{m}^{\prime}\right\rangle\right]$

here $\quad r_{j l}=\left\langle u_{j} h_{l}\right\rangle, P_{m}^{\prime}=\left(H_{k}+\frac{h_{k}}{2}\right) h_{k}, h_{k}$ - fluctuating part of magnetic field, $\varepsilon_{j}$ dissipation of the $k_{j}$.

To "close" system 1.21-1.29, $\Phi_{j}$ and $\varepsilon_{j}$ should be expressed in terms of the previously introduced functions.

The last addend in 1.29 can be written (by analogy with Rotta's hypothesis [10]) as a sum of the diffusion and relaxation terms for tensor components $\pi_{i k}=\left\langle h_{i} h_{k}\right\rangle$. If then the first addend in 1.29 is neglected, it is possible to express function $\Phi_{j}$ in terms of $r_{j l}$ and $\pi_{i k}$.

The equation system for these functions can be derived from 1.2 and 1.9. By solving this system, they can be expressed in terms of $k_{i j}$ and average velocities and fields.

The equation for $\mathcal{E}_{j}$ can be written, following [11], as

$$
\varepsilon_{j}=\frac{k_{j}^{\frac{3}{2}}}{l_{j}}
$$

where $1_{\mathrm{j}}$ is the mixing path length that depends on the orientation of unit vector $\vec{e}_{j}$ relative to the magnetic field. When $\vec{e}_{j} \| \vec{H}$ the $l_{j} \rightarrow \infty$ if $\vec{H} \rightarrow \infty$ [11].It is suggested that the methods for determination of $\Phi_{j}$ and $\varepsilon_{j}$ should be discussed separately. 


\section{Setting up the computations and discussion}

The computations discussed below assumed that the magnetic field was not very strong and its effect on the anisotropy and turbulence dissipation could be neglected. This allowed us to neglect addend $\Phi_{j}$ in 1.22, i.e. to use one equation for $\mathrm{k}$ and one equation for $\varepsilon$ presented in [7].

In so doing the magnetic field action on plasma is expressed in terms of force $\vec{f}$ in equation 1.2 and by relevant addends $\vec{\gamma}$ (1.26) in "generation" term $\mathrm{G}_{2}$ for turbulent energy.

The plasma action on magnetic field leads to its displacement, however turbulence causes field diffusion into the plasma, with the diffusion coefficient itself depending on the field.

Two problems of plasma cloud expansion to surrounding, "background", lowdensity plasma ("vacuum") with magnetic field were calculated.

Problem 1. One-dimensional problem of cylindrical plasma cloud expansion in magnetic field.

It is agreed that at $\mathrm{t}=0$ there is a cylindrical symmetric plasma cloud of radius $r_{0}$, energy E, and mass M (per unit length) surrounded by cold "background" plasma of density $\rho_{0}$ and by magnetic field oriented along axis of symmetry z, i.e. $\vec{H}=\left(0,0, H_{1}\right)$.

It is convenient to consider this problem in dimensionless variables. Introduce scaling: $u_{1}=\left(\frac{E}{M}\right)^{1 / 2}$ for velocity, $r_{1}=\left(\frac{E}{P_{1}}\right)^{1 / 2}$ for distance, $\frac{r_{1}}{u_{1}}$ for time, $\frac{M}{r_{1}{ }^{2}}$ for density, $H_{1}$ for magnetic field (here $P_{1}$ is unperturbed magnetic field pressure).

In this scaling the problem is characterized with two dimensionless parameters, $\mathrm{r}_{0}$ and $\rho_{0}^{\prime}=\frac{\rho_{0}}{\rho_{1}}=\left(\frac{\mathrm{u}_{1}}{\mathrm{C}_{\mathrm{A}}}\right)^{2}$, where $\mathrm{C}_{\mathrm{A}}^{2}=\frac{\mathrm{H}_{1}^{2}}{4 \pi \rho_{0}}$.

Next, restrict our consideration to $\rho_{0}^{\prime} \rightarrow 0$, i.e. "sub-Alfven" plasma expansion. The dimensionless initial data are summarized in Table 2.1 
Table 2.1

\begin{tabular}{|l|l|l|l|l|}
\hline & $\rho$ & $\varepsilon$ & $\mathrm{u}$ & $\mathrm{H}$ \\
\hline $\mathrm{r}<\mathrm{r}_{0}$ & $\frac{1}{\pi \mathrm{r}_{0}^{\prime 2}}$ & 1 & 0 & 0 \\
\hline $\mathrm{r}>\mathrm{r}_{0}$ & $\rho_{0}^{\prime}$ & 0 & 0 & 1 \\
\hline
\end{tabular}

To calculate the turbulent mixing with the code of ref. [7], the data of initial turbulent energy profiles $\mathrm{k}$ and turbulent energy dissipation $\varepsilon$ should be added to these initial data. It was assumed that at $\mathrm{t}=0$ these functions are nonzero only in the layer of thickness $\delta \mathrm{r}_{0}<<\mathrm{r}_{0}$ near boundary $\mathrm{r}_{0}$. In the computation, $\mathrm{C}_{\mathrm{m}}=1$.

The computed data for $\rho_{0}^{\prime}=\frac{10^{-2}}{\pi}$ and $r_{0}=0.1$ is plotted in Figs. 1.1-1.3.

The figures depict turbulent mixing zone (TMZ) boundaries with tine (here $\mathrm{R}_{1}(\mathrm{t})$ is radius determined by level $H\left(R_{1}\right)=0.05, \mathrm{R}_{2}(\mathrm{t})$ is that by level $\left.H\left(R_{2}\right)=0.95\right)$ (Fig. 1); magnetic field profiles at times $t=0.5 \tau$, and $\tau$, where $\tau$ is period of one cylinder radius oscillation (Fig. 2), plasma density profiles at the same times (Fig. 3).

As seen in these figures, a noticeable magnetic field and plasma interpenetration ("turbulent diffusion") is observed. At $\rho_{0}^{\prime}<<1$ and $\mathrm{r}_{0}{ }^{\prime}<<1$ the result weakly depends on specific magnitudes of the parameters and on initial profiles $\mathrm{k}$ and $\varepsilon$.

Problem 2. Two-dimensional problem of spherical cloud expansion.

Assume that at $\mathrm{t}=0$ there is a spherical plasma cloud of energy $\mathrm{E}$, mass $\mathrm{M}$, radius $\mathrm{r}_{0}$ surrounded with "background" plasma of density $\rho_{0}$, thermal pressure $\mathrm{P}_{0}$ and by magnetic field, whose strength tends to $\vec{H}=\left(0,0, H_{0}\right)$ with $\mathrm{r} \rightarrow \infty$.

Like previously, introduce scaling $u_{1}^{2}=\frac{E}{M}$ for velocity, $r_{1}=\left(\frac{E}{P_{1}}\right)^{1 / 3}$ for distance, $\mathrm{P}_{1}=\frac{\left(3 / 2 \mathrm{H}_{0}\right)^{2}}{8 \pi}+P_{0}$ for pressure, $H_{1}=H_{0}$ for magnetic field.

It was solved two spherical tasks №2.1 and 2.2. We assumed $\mathrm{H}_{0}=0, P_{0} \neq 0$ in the task 2.1 (so, this task become one-dimensional) and $H_{0} \neq 0, P_{0}=0$ in the task 2.2.

The dimensionless initial data for the problem are summarized in Table 2.2. Table 2.2 


\begin{tabular}{|l|l|l|l|l|}
\hline & $\rho$ & $\varepsilon$ & $\mathrm{u}$ & $\mathrm{H}$ \\
\hline $\mathrm{r}<\mathrm{r}_{0}$ & $\frac{3}{4 \pi \mathrm{r}_{0}{ }^{3}}$ & 1 & 0 & 0 \\
\hline $\mathrm{r}>\mathrm{r}_{0}$ & 0,1 & $\varepsilon_{0}$ & 0 & $\mathrm{H}_{0}$ \\
\hline
\end{tabular}

Here $\varepsilon_{0}=10, \mathrm{H}_{0}=0$ in the task 2.1 and $\varepsilon_{0}=0, \mathrm{H}_{0}=1$ in task 2.2, $\mathrm{r}_{0}=0,5, \gamma=2$,

In contrast to Problem 1, here magnetic field in region $r>r_{0}$ is not constant and corresponds to the dipole magnetic field of radius $r_{0}$ in the external field of strength $(0,0,1)$.

The initial data for the "turbulent" functions $\mathrm{k}$ and $\varepsilon$ were given like in Problem 1: in a thin layer near interface $r_{0}$.

It was assumed that $\mathrm{C}_{\mathrm{m}}=1$.

The results of the computation are plotted in the figures 4-9.

The first of them depicts the TMZ time dependence for 1D task 2.1. We can see TMZ width $\mathrm{L}_{\mathrm{t}}$ is comparable with the cloud radious.

The fig. 5 depict the TMZ time dependence for 2D task $2.2\left(\theta=\frac{\pi}{2}\right)$.

Comparing fig. 4 and 5 we can note although the plasma clouds dynamics are similar, but the magnetic field diminish value of $\mathrm{L}_{\mathrm{t}}$.

Figs. 6,7 plot the plasma density isolines at times $\mathrm{t}=0,4,0,6$, Figs. 8,9 depict the magnetic pressure isolines at the same times .

As seen, a noticeable field and plasma inter-diffusion is observed in this problem as well at the selected value of $\mathrm{C}_{\mathrm{m}}$, with this being significantly stronger at the $\theta=\frac{\pi}{4}$. 


\section{Conclusion}

This paper discusses first our attempt to develop a semi-empirical model of turbulent mixing in inhomogeneous plasma with magnetic field.

To describe the MHD effects, the simplest form of the model is shown to require setting two additional constants determining the turbulent magnetic field diffusion factor and dissipative addends in the equation of energy.

To determine the constants, it is reasonable to involve experimental data on pinches and plasma liners.

It is being planned to make more accurate $2 \mathrm{D}$ computation and include turbulent energy anisotropy in it.

The authors would like to thank A.S.Pavlunin for his assistance in the computations and L.A. Rogacheva for help in execution of this paper. 


\section{References}

1. Shklovsky I.S. Supernovae. Moscow, Nauka Publishers, 1976.

2. Dahlburg J.P., Fyfe D.E., Gardner J.H. et al., Phys. Plasmas 2. 2453(1995).

3. Kadomtsev B.B. Collective phenomena in plasma. Moscow, Nauka Publishers, 1988.

4. Winske Dan, "Regimes of magnetized RT instability" LA-UR-96-818, 1996

5. Hassam A.B., Huba J.D., Phys Fluids 31, 318(1988).

6. Belen'ky S.Z., Fradkin E.S. Theory of turbulent mixing. Trudy FIAN. V. XXIX, 1965.

7. Yanilkin Yu.V., Nikiforov V.V., Zharova G.V. A two-equation model and a code for turbulent mixing in 2D flows. VANT, Ser. MMFP, No.4, 1994.

8. Zhmailo V.A., Yanilkin Yu.V. "Direct numerical simulation of magnetic field turbulent diffusion into accelerated plasma", $7^{\text {th }}$ IWPCTM, St. Petersburg, 1999

9. Landau L.D., Lifshits E.M. Continuum electrodynamics. Moscow. 1962.

10. Monin A.S., Yaglom A.M. Statistical hydromechanics. V.1. Moscow, Nauka Publishers, 1965.

11. Ievlev V.M. Turbulent high-temperature media flow. Moscow, Nauka Publishers, 1975.

12. Levellen V. A method for invariant modeling. In: Turbulence: Principles and Applications. Moscow, Mir Publishers, 1980.

13. Bakhrakh S.M., Darova N.S., Statsenko V.P., Zhmailo V.A. "The effects of turbulence and viscosity on structure of thin vortex rings" $4^{\text {th }}$ JWPCTM, Cambridge, 1993. 
Cylindrical cloud.

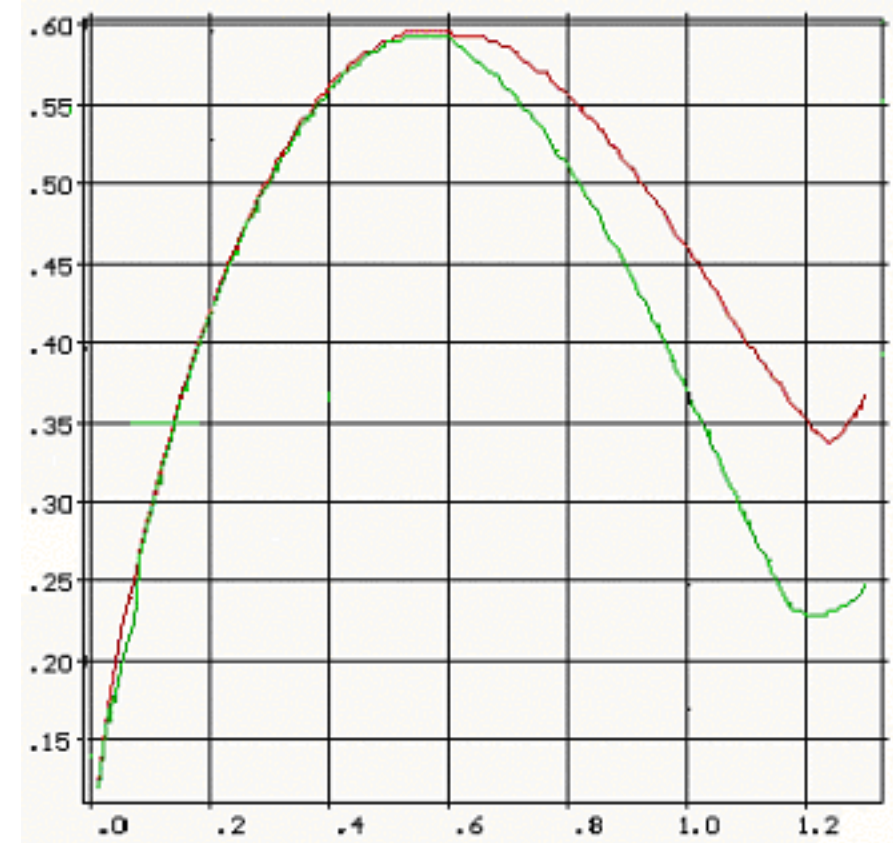

fig1. TMZ time dependence

(concentration levels 0.05 and 0.95 ), cylindr. task, $\mathrm{P}_{\mathrm{m}}=1.0$

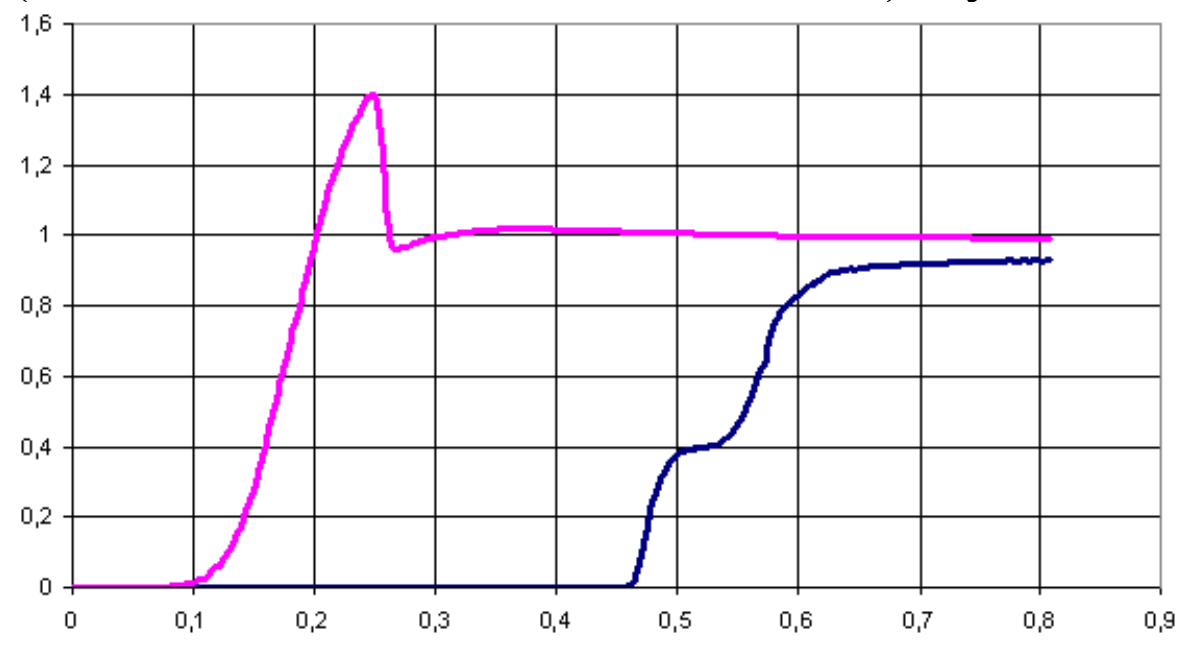

fig2. Magnetic field profiles, cylindr. task, $\mathrm{P}_{\mathrm{m}}=1.0, \mathrm{t}=0.6, \mathrm{t}=1.2$

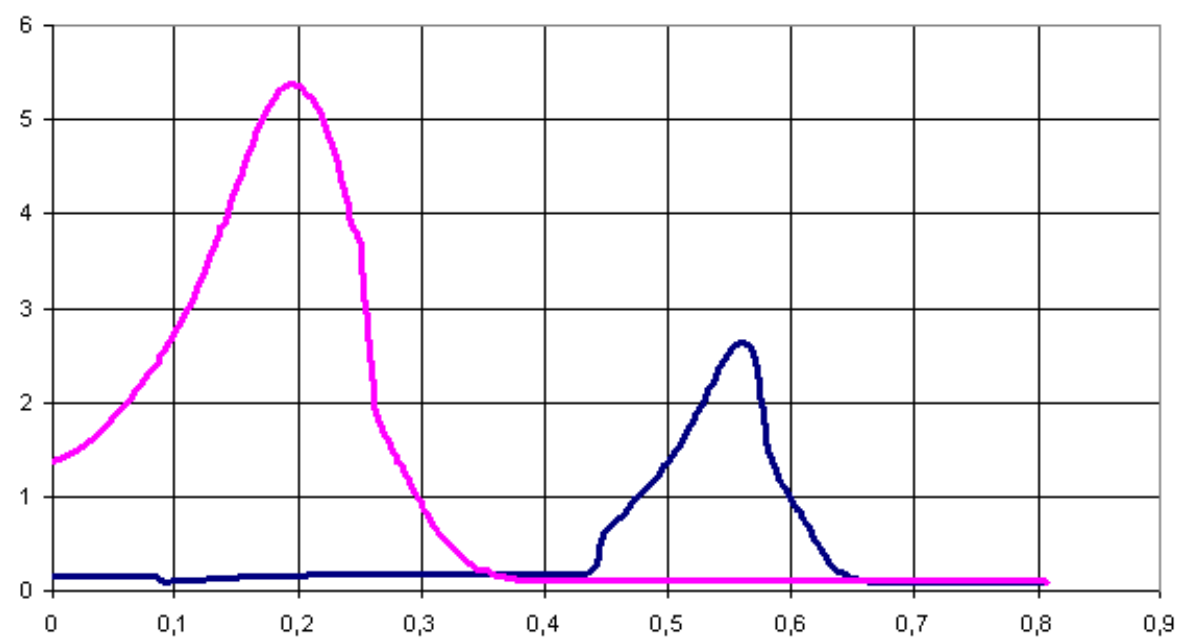

fig3. Plasma density profiles, cylindr. task, $\mathrm{P}_{\mathrm{m}}=1.0, \mathrm{t}=0.6, \mathrm{t}=1.2$ Spherical cloud. 


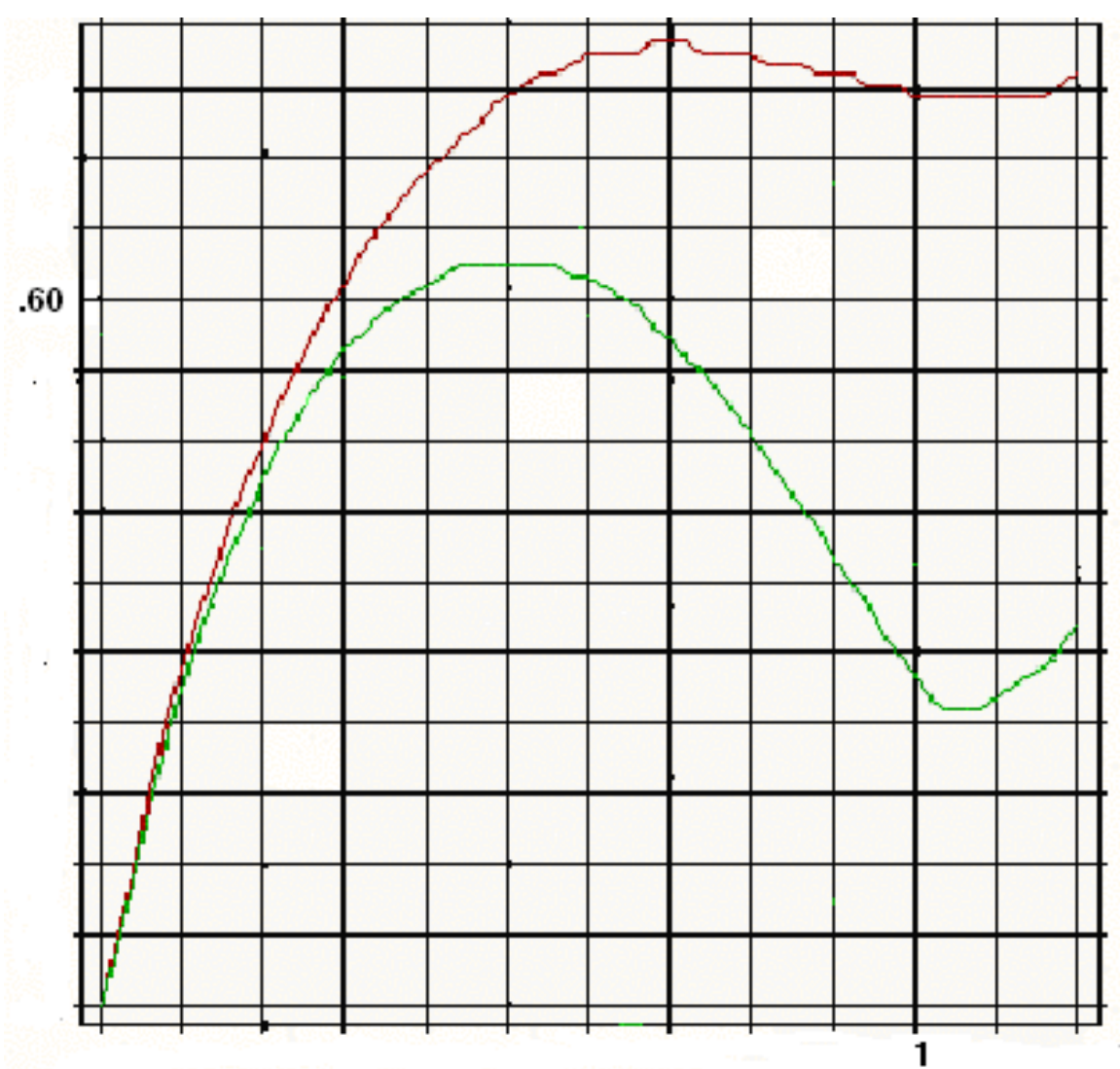

fig4. TMZ time dependence, spher. task, $\mathrm{P}_{\mathrm{th}}=0.1, \mathrm{P}_{\mathrm{m}}=0$

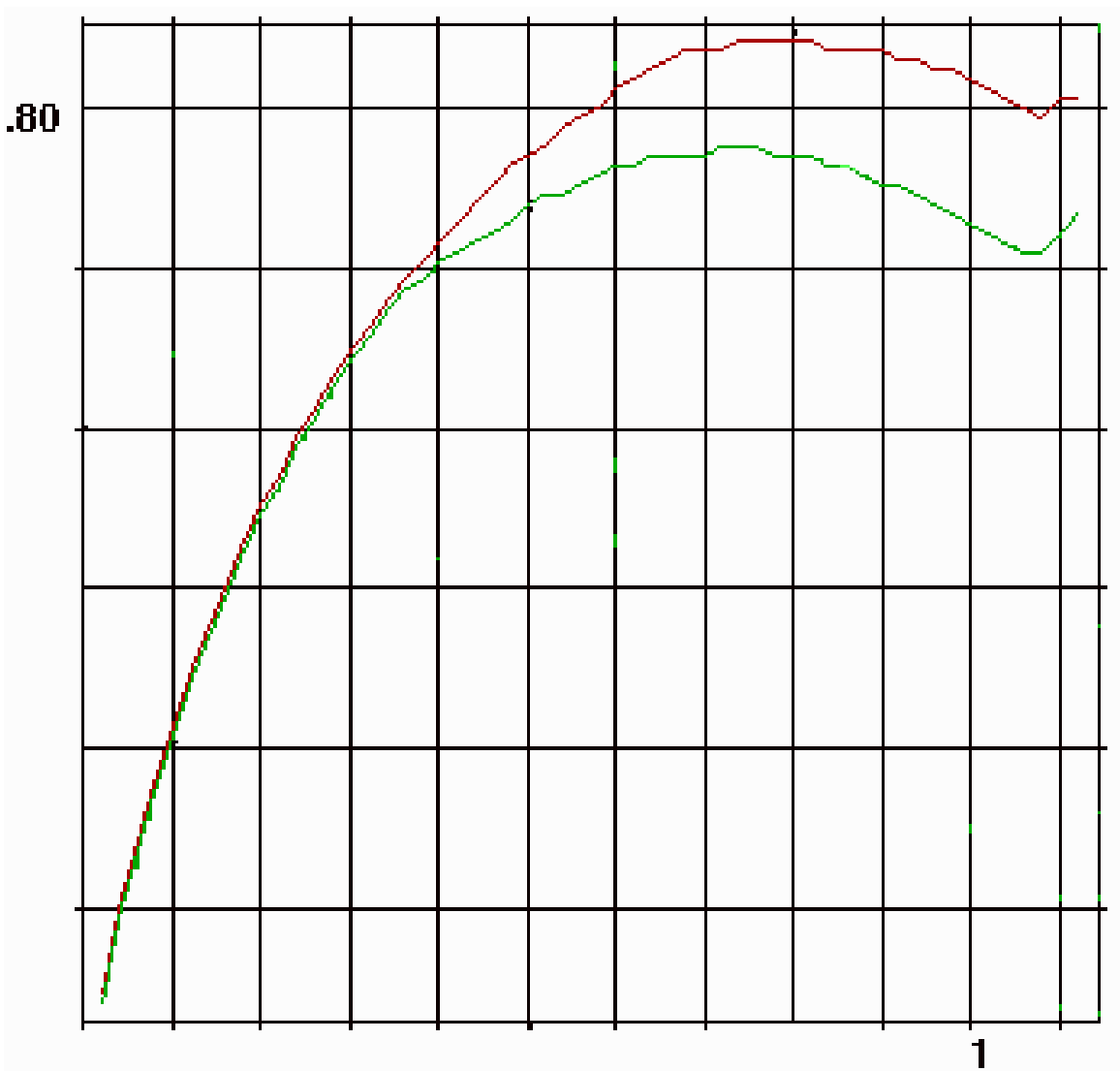

fig5. TMZ time dependence, $2 \mathrm{D}$ task, $\mathrm{P}_{\mathrm{m}}=0.44, \Theta=\pi / 2$ (equator) 


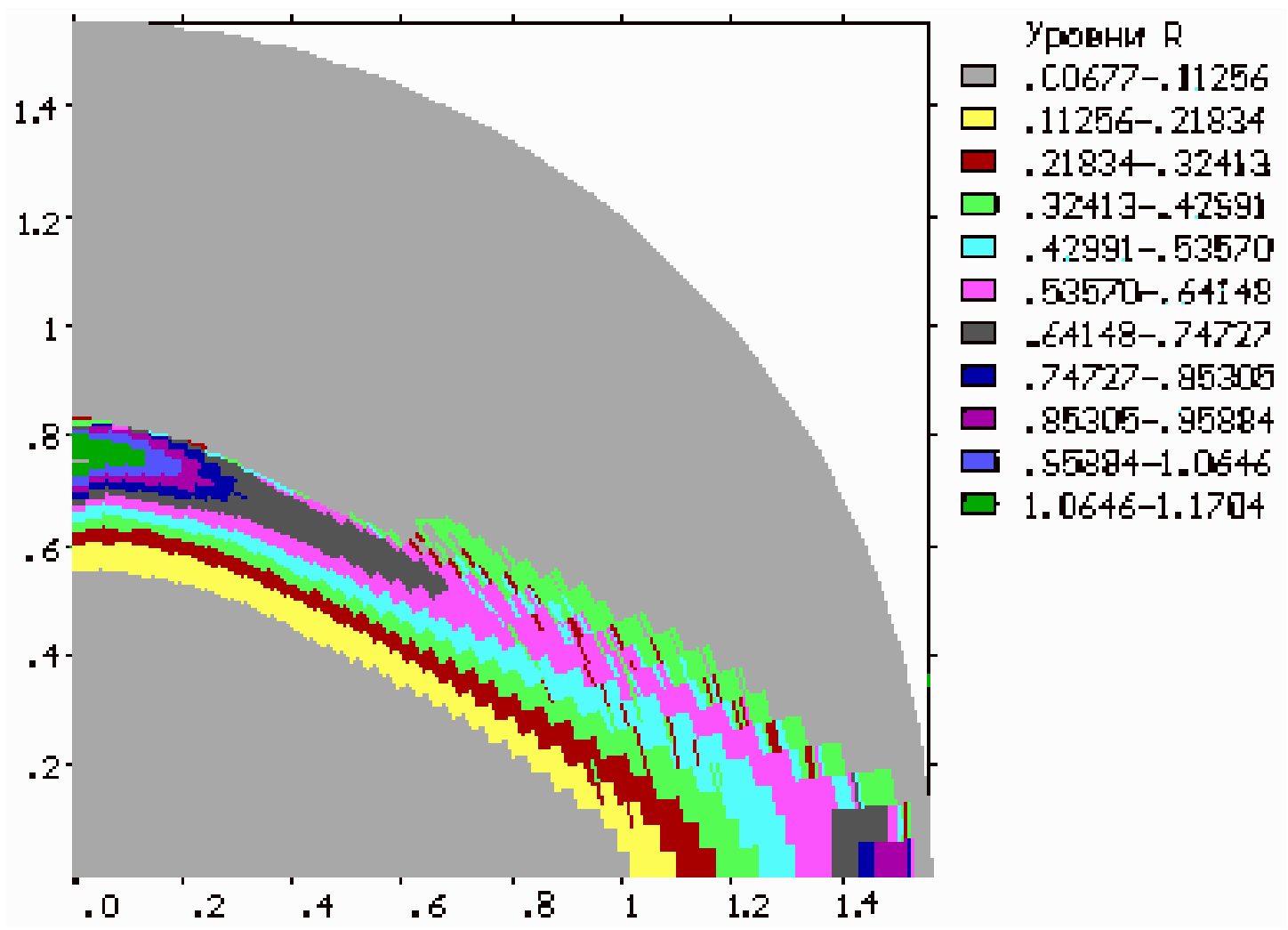

fig6. Plot of the plasma density, 2D task, $\mathrm{P}_{\mathrm{m}}=0.44, \mathrm{t}=0.8$

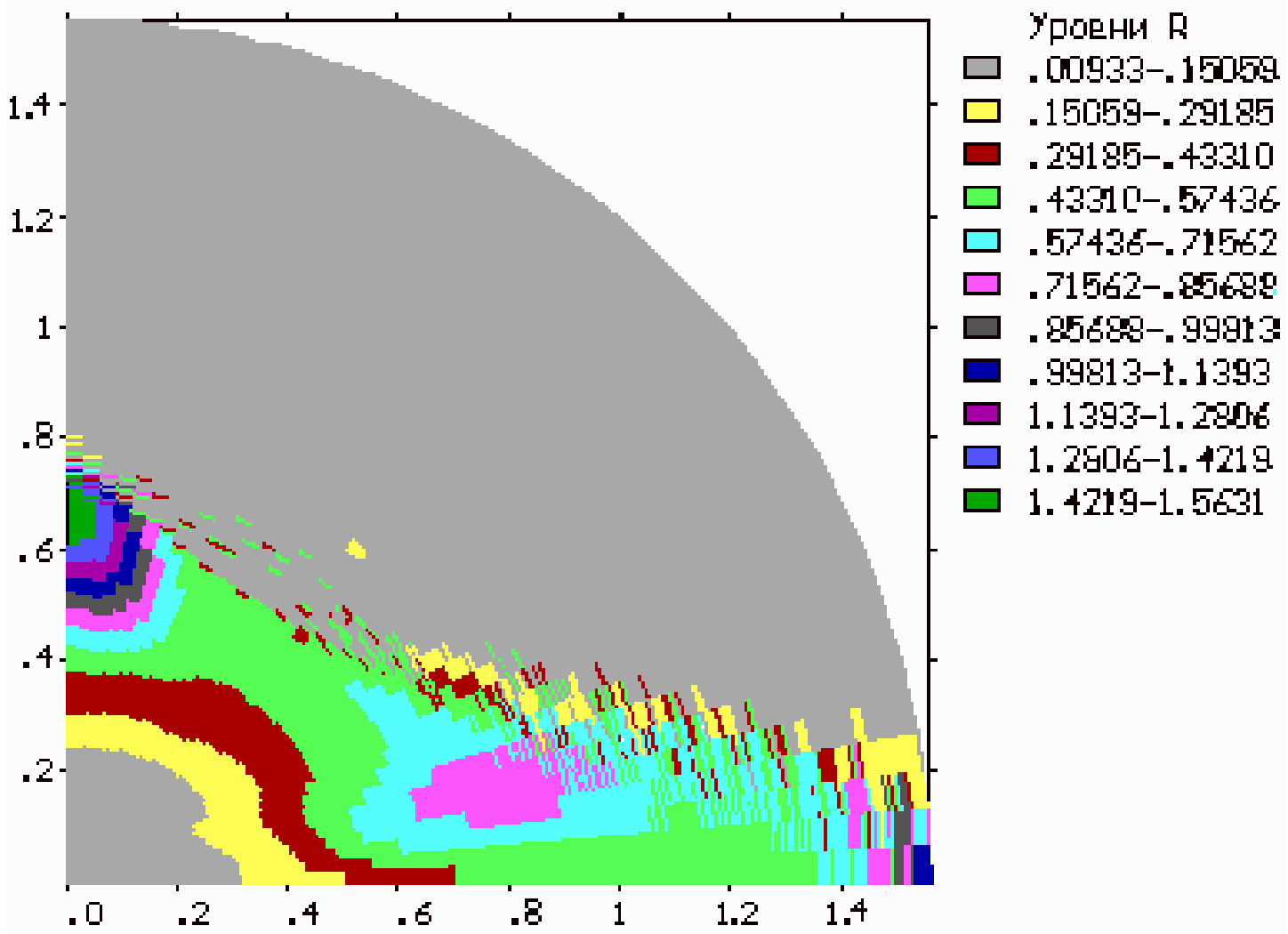

fig7. Plot of the plasma density, $2 \mathrm{D}$ task, $\mathrm{P}_{\mathrm{m}}=0.44, \mathrm{t}=1.2$ 


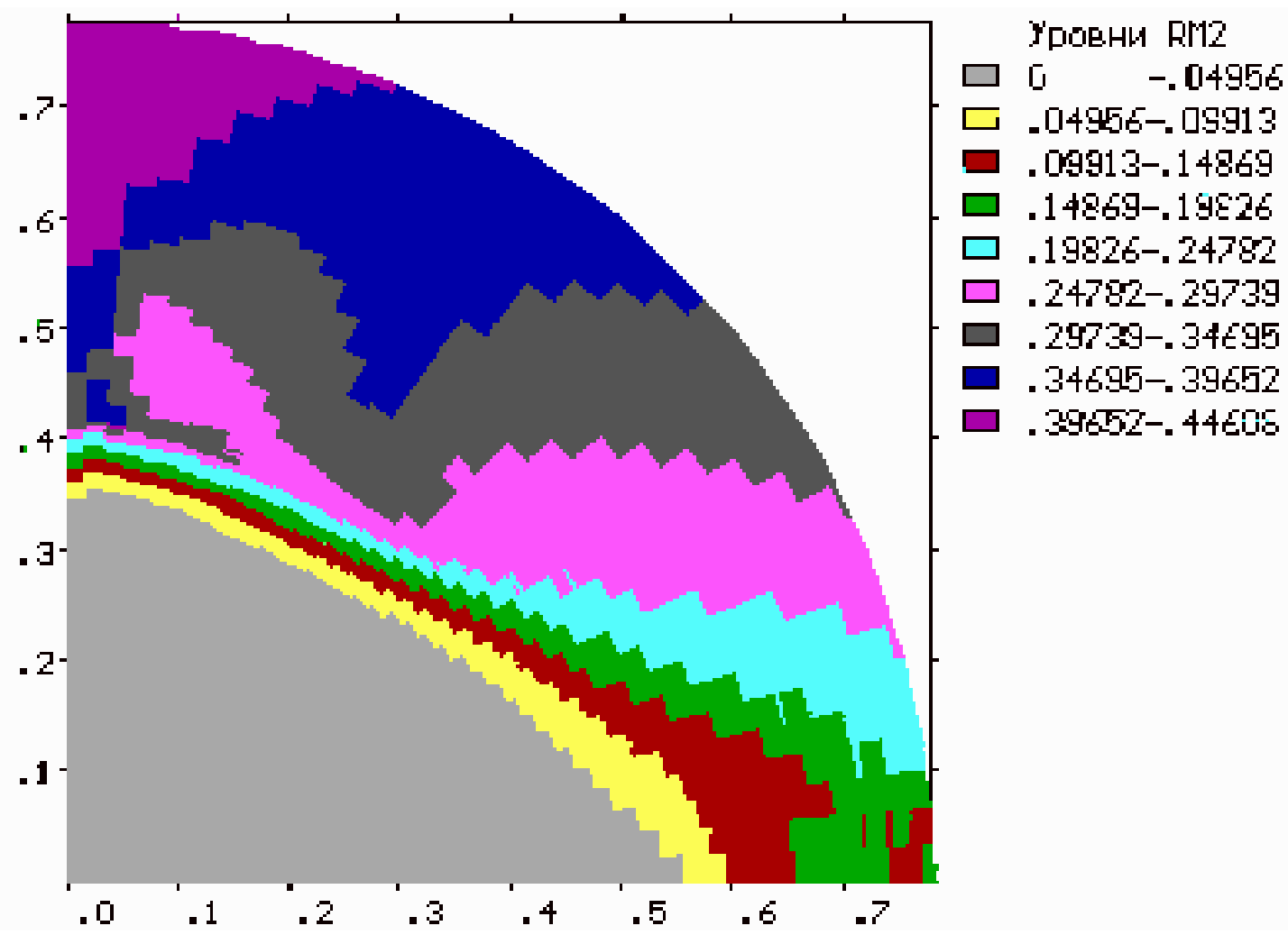

fig8. Plot of the "magnetic" pressure, 2D task, $\mathrm{P}_{\mathrm{m}}=0.44, \mathrm{t}=0.4$

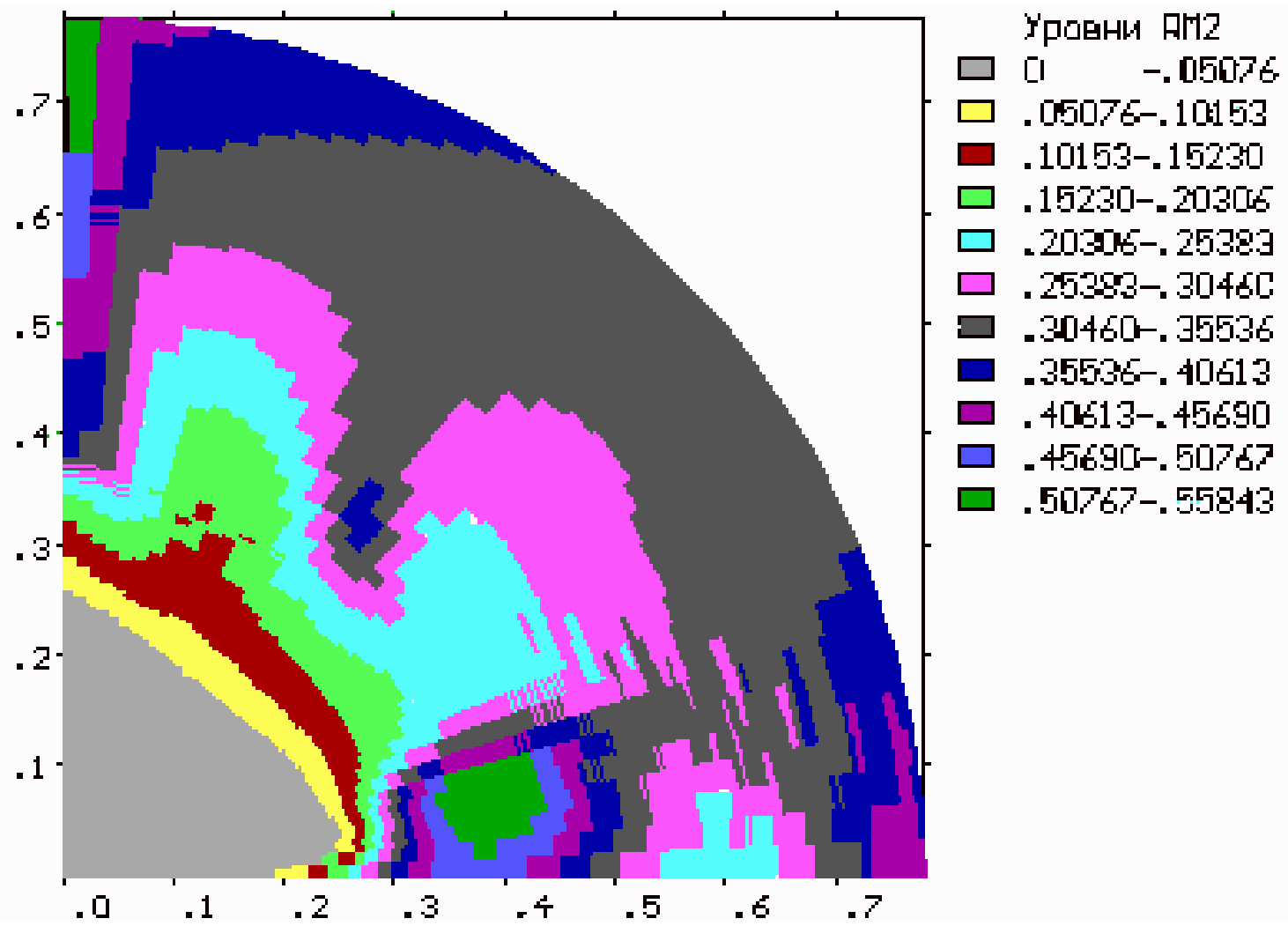

fig9. Plot of the "magnetic" pressure, $2 \mathrm{D}$ task, $\mathrm{P}_{\mathrm{m}}=0.44, \mathrm{t}=0.6$ 


\section{LOCALIZATION AND SPREADING OF INTERFACES (CONTACT DISCONTINUITIES) IN PPM AND WENO SIMULATIONS OF THE INVISCID COMPRESSIBLE EULER EQUATIONS}

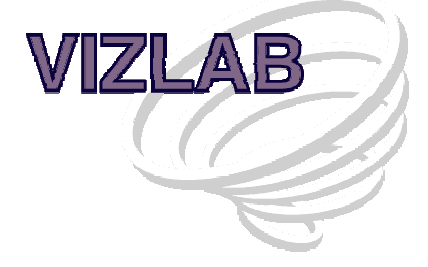

N.J Zabusky ${ }^{1}$, S. Gupta ${ }^{1}$, Y. Gulak ${ }^{1}$, G. Peng ${ }^{1}$, R. Samtaney ${ }^{2}$, ${ }^{1}$ Rutgers University,NJ ${ }^{2}$ Princeton Plasma Physics Lab.,NJ 


\section{OBJECTIVE}

$>$ Systematic approach to examine localization and temporal spreading of contact discontinuities(CDs) in 1D and 2D.

$>\quad$ Validity of near contact simulations of accelerated flows of high-gradient compressible media (RT and RM).

$>$ Evolution of sinusoidal RM interface at late time and interfacial growth rate.

Reflecting Boundary

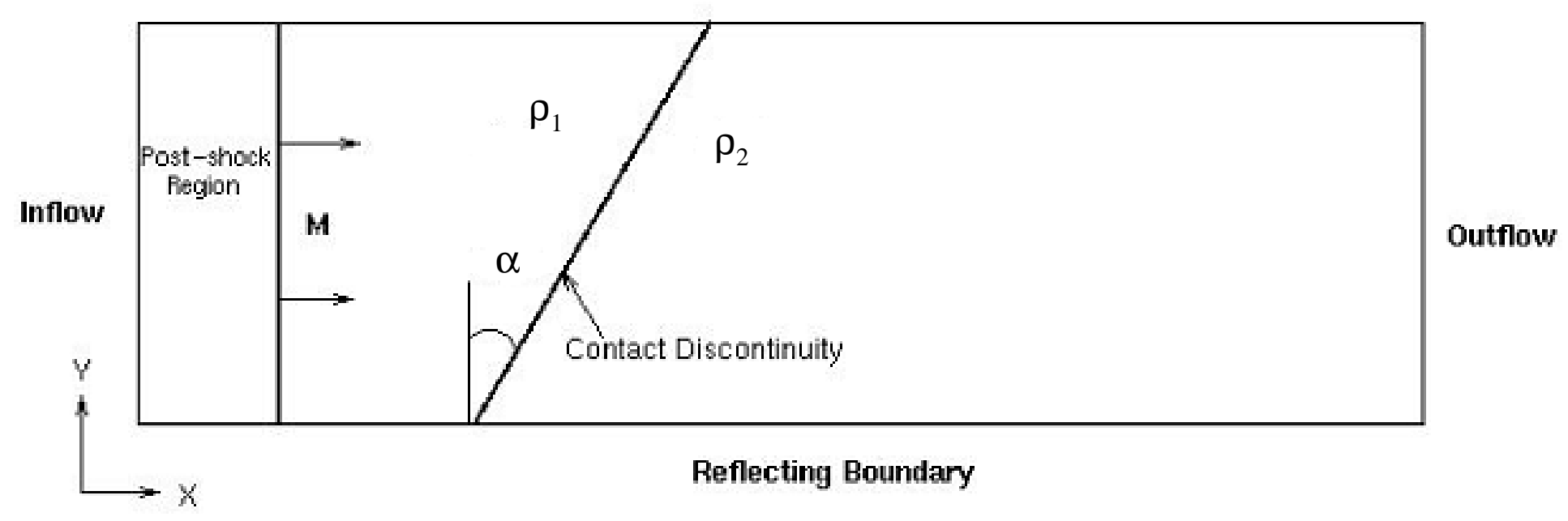

Schematic of Shock Interaction with an Inclined Discontinuity. $M$ is the Mach number, $\alpha$ is the angle between shock and contact discontinuity, $\rho_{1}$ and $\rho_{2}$ are the densities of two gases. 


\section{MOTIVATION}

$>$ Study by Samtaney \& Zabusky: Visualization and quantification of compressible flows in Flow Visualization(1999).

$>$ Non-convergence of position of contact discontinuity $\left(\mathrm{x}_{\text {num }}-\mathrm{x}_{\text {anal }}\right) / \mathrm{h}$ to exact analytical solution for 1D.

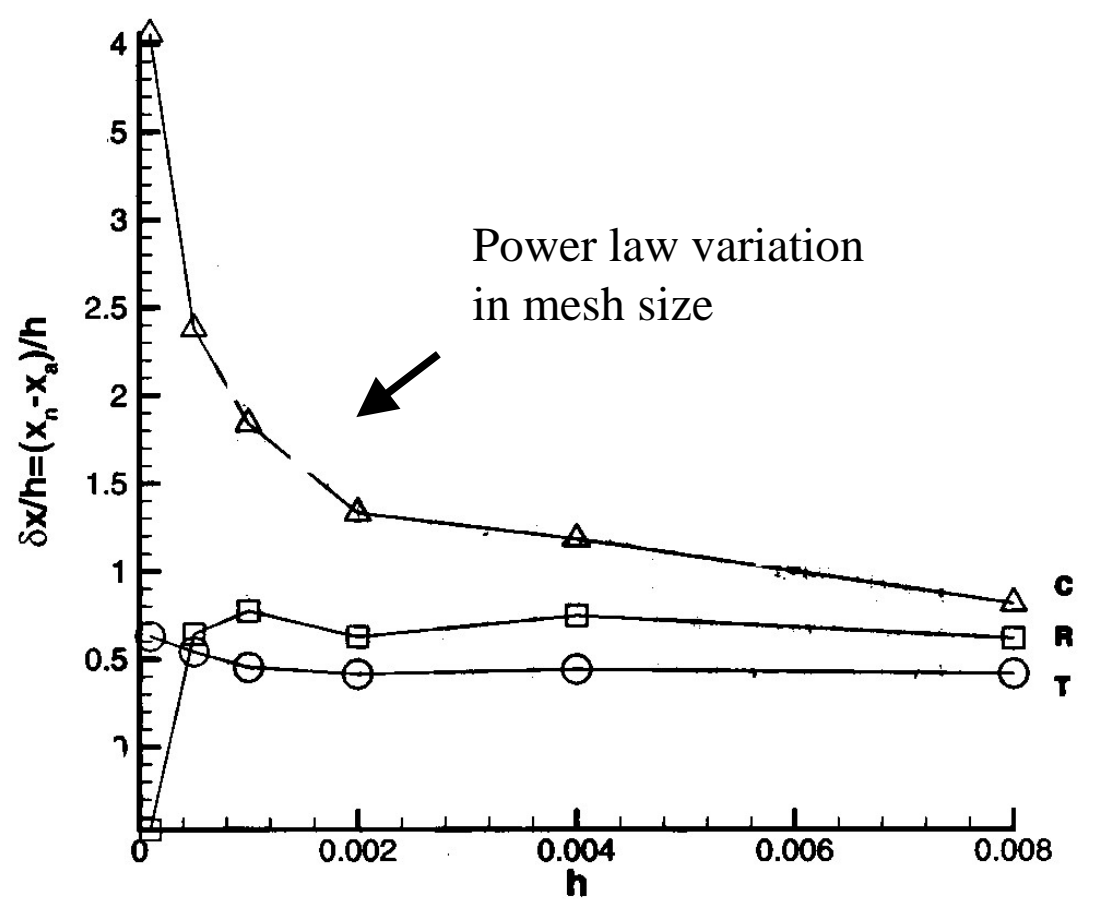

Convergence study using difference in the numerical and analytical locations of high gradient regions (shocks and CDs) vs mesh size $h . M=3.0$ shock interacts with a density discontinuity (CD, $\rho_{2} / \rho_{1}=3.0$ ) and yields a moving $C D(C)$, upstream reflected shock $(R)$, and downstream transmitted shock $(\mathrm{T})$. 


\section{CONTINUUM LIMITS \& DIFFERENTIAL APPROXIMATION}

Consider a 1D Riemann problem for Euler System

$$
\frac{\partial \mathrm{U}}{\partial \mathrm{t}}+\frac{\partial F(\mathrm{U})}{\partial \mathrm{x}}=0 \text {. }
$$

with initial conditions,

$$
\mathrm{u}(\mathrm{x}, 0)=\mathrm{u}_{0}, \mathrm{p}(\mathrm{x}, 0)=\mathrm{p}_{0}, \rho(\mathrm{x}, 0)=\left\{\begin{array}{l}
\rho_{1}, \mathrm{x}<\mathrm{x}_{0} \\
\rho_{2}, \mathrm{x}>\mathrm{x}_{0}
\end{array}\right.
$$

Using Differential Approximation (Vorozhtsov and Yanenko, Springer1990) for a numerical method of $\mathbf{r}$-th order spatial accuracy, system reduces to,

$$
\frac{\partial \rho}{\partial \mathrm{t}}+\mathrm{u}_{0} \frac{\partial \rho}{\partial \mathrm{x}}=(-1)^{\mathrm{r}+1} \mu_{\mathrm{r}+1} \frac{\partial^{\mathrm{r}+1} \rho}{\partial \mathrm{x}^{\mathrm{r}+1}}
$$

For $r=1$,

$$
\rho(\mathrm{x}, \mathrm{t})=0.5\left(\rho_{1}+\rho_{2}\right)[1+\operatorname{erf}(\chi)]
$$

For $r=2$,

$$
\rho(\mathrm{x}, \mathrm{t})=\left(2 \rho_{2}+\rho_{1}\right) / 3+\left(\rho_{2}-\rho_{1}\right) \int_{0}^{\chi} \operatorname{Ai}\left(\chi^{\prime}\right) \mathrm{d} \chi^{\prime}
$$

where

$$
\chi(\mathrm{x}, \mathrm{t})=\left(\mathrm{x}-\mathrm{x}_{0}-\mathrm{u}_{0} \mathrm{t}\right) /\left((\mathrm{r}+1) \mu_{\mathrm{r}+1} \mathrm{t}\right)^{1 / \mathrm{r}+1}
$$




\section{EXTRACTION OF CONTACT DISCONTINUITY}

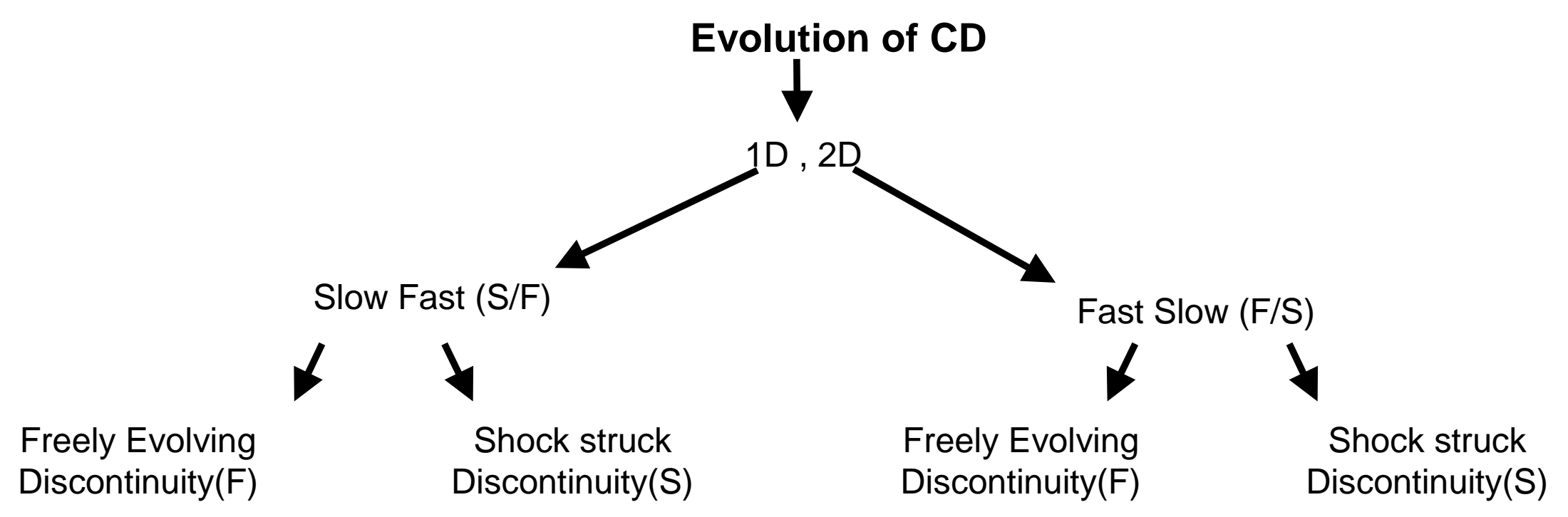

For $2 \mathrm{D}$ case, we examine a slice at $\mathrm{y}=\mathrm{YMAX} / 2$

\section{NUMERICAL METHODS}

$>$ Piecewise Parabolic Method (PPM)

> Weighted Essentially Non- Oscillatory (WENO, $r=5$ ) 


\section{EXTRACTION PROCEDURE FOR CD}

$>$ Point-wise Algorithm ( $A$ variation of edge detection technique)

$>\quad$ Width of $C D=X\left(d^{2} \rho_{\max }\right)-X\left(d^{2} \rho_{\min }\right)$ where $d^{2} \rho$ is the second central difference

$>$ Shock - Elimination using cost functions

- $\quad$ Divergence of velocity $|\nabla . U|<|\nabla . U|_{\text {thresh }}$

- $\quad$ Normalized pressure jump $\mathrm{dP}<\mathrm{dP}$ thresh 


\section{LOCALIZATION OF CD UNDER MESH REFINEMENT}
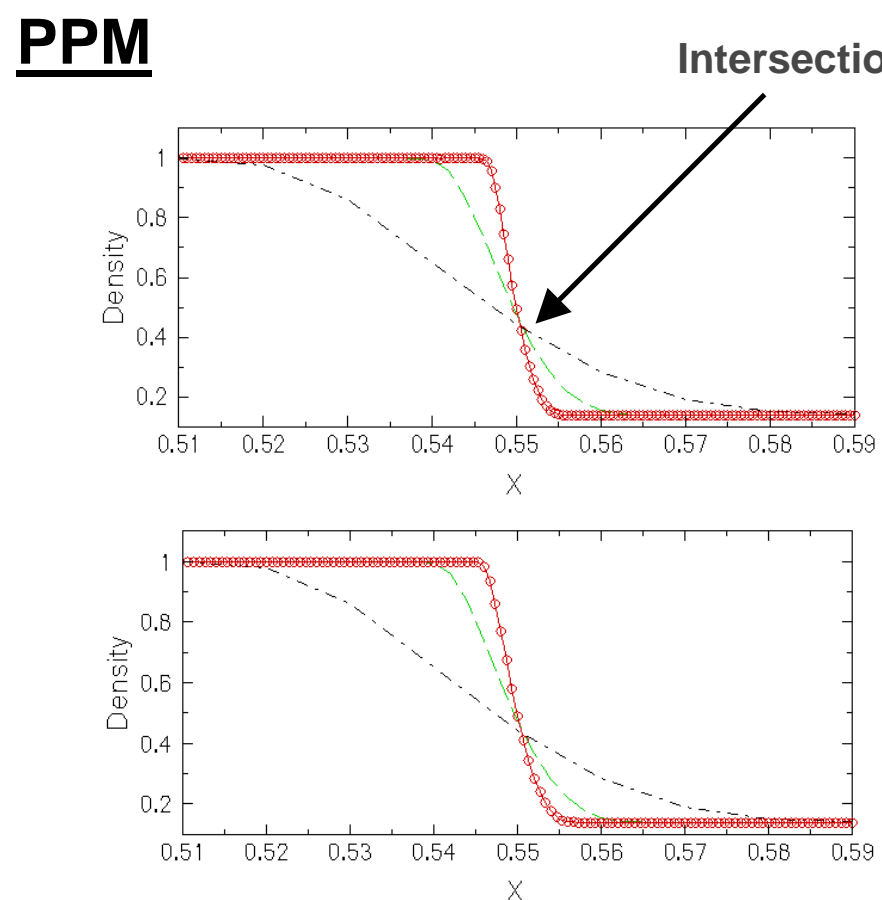

(a)
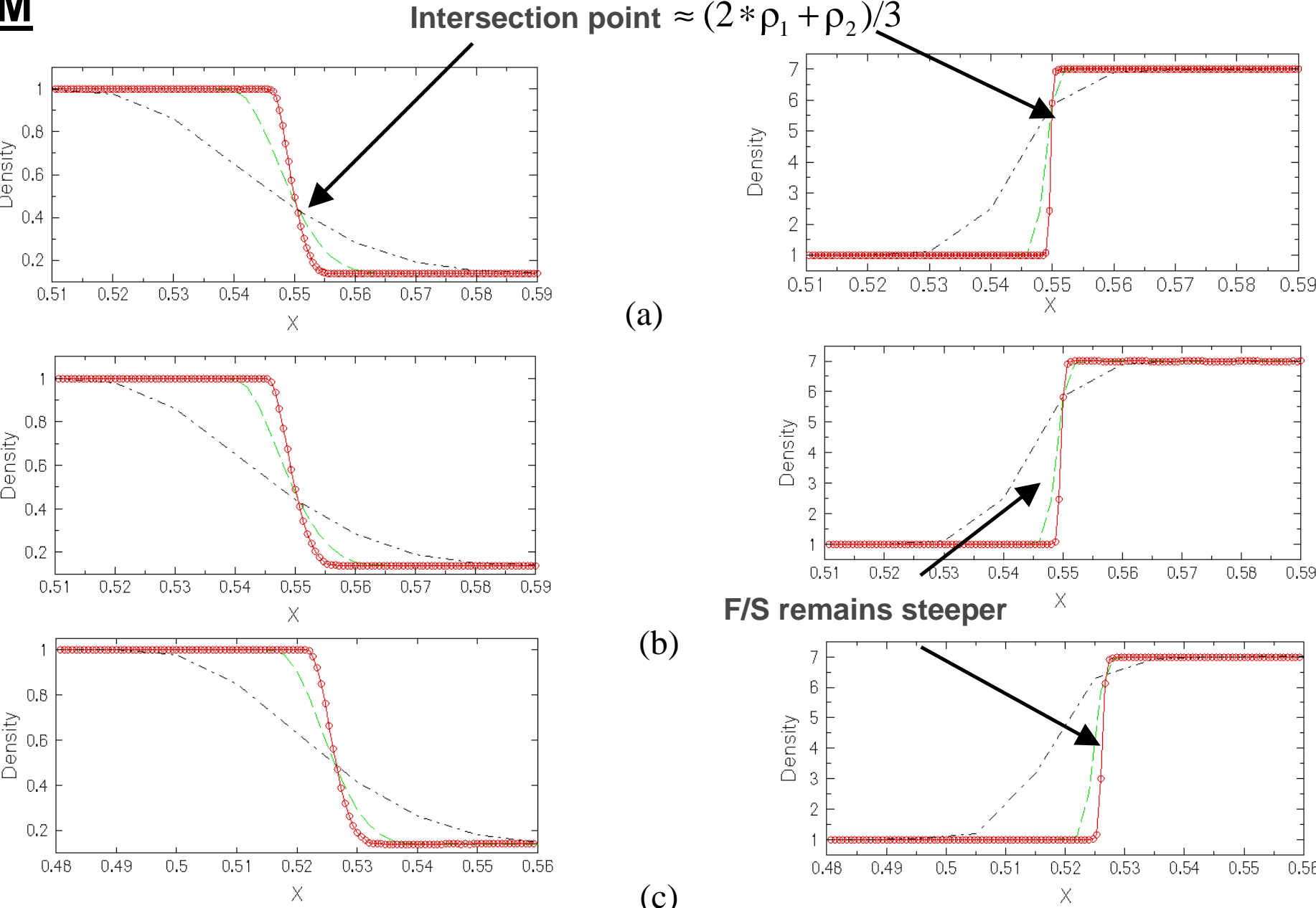

(b)

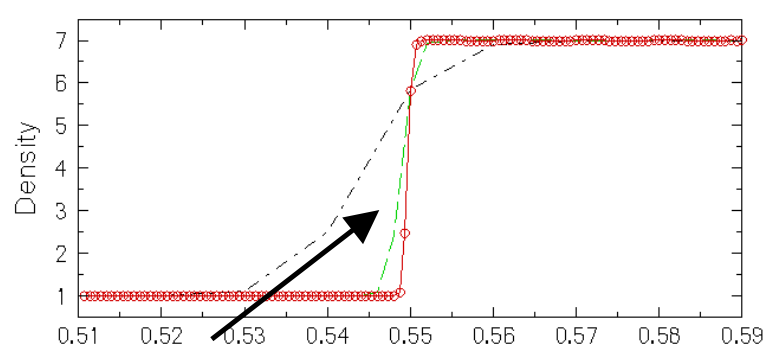

F/S remains steeper

$\mathrm{S} / \mathrm{F}($ density ratio $=0.14)$

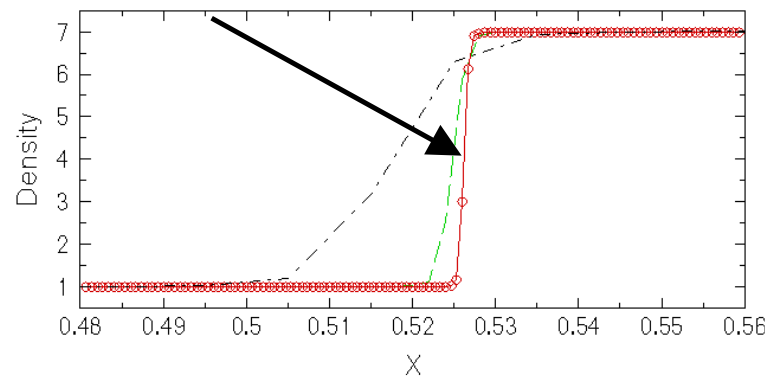

$\mathrm{F} / \mathrm{S}($ density ratio=7.0)

Density profiles for Diffusing Contact Discontinuity $(u 0=1.5)$ at $t=0.3$. Top to Down (a) $1 \mathrm{D}$ (b) $2 \mathrm{D}, \alpha$ $=0$ (c) 2D, $\alpha=30$. The solid line with open circles is the highest resolution 0.0005 and $-\cdots$ and $-\cdots$ are 0.002 and 0.01 respectively. 

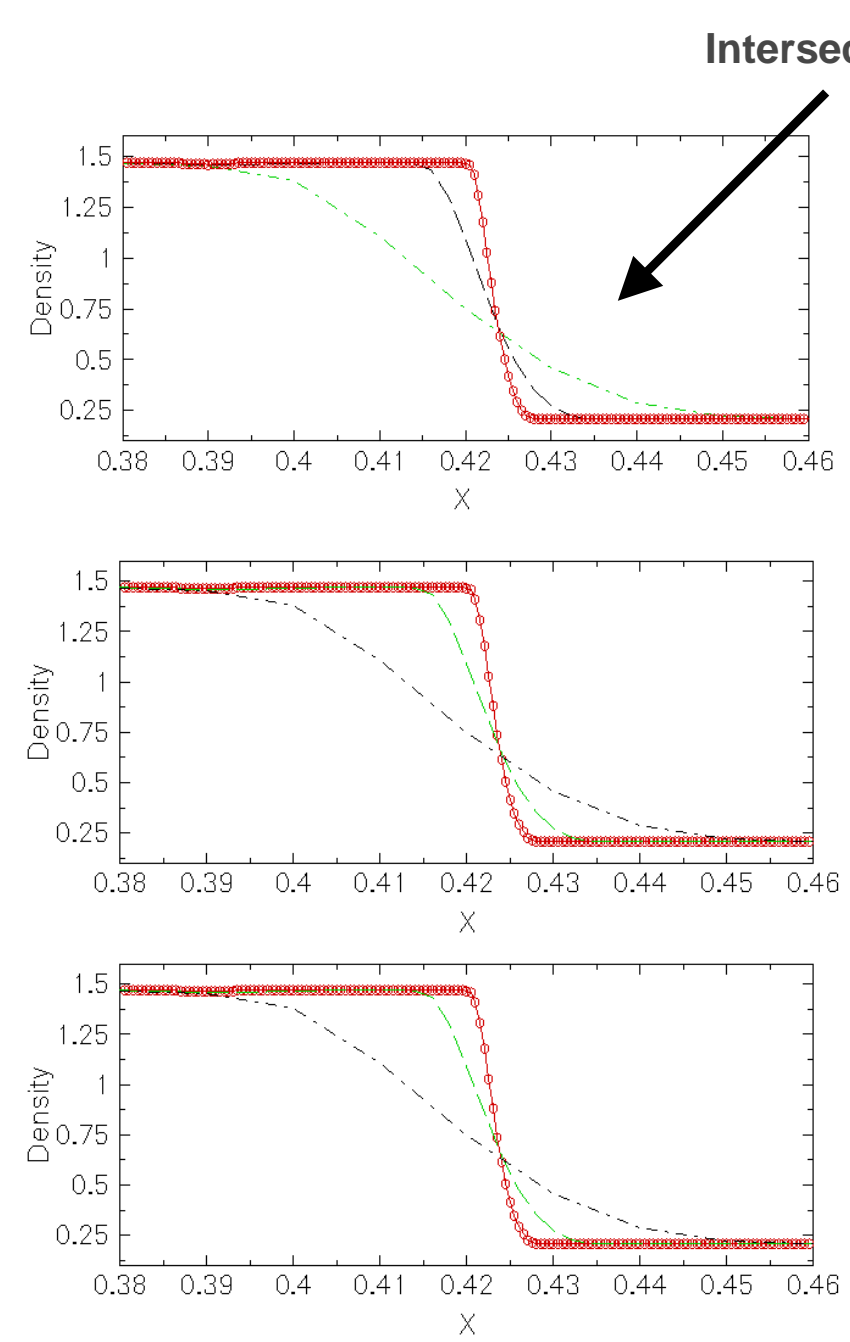

$\mathrm{S} / \mathrm{F}($ density ratio $=0.14)$ (a)
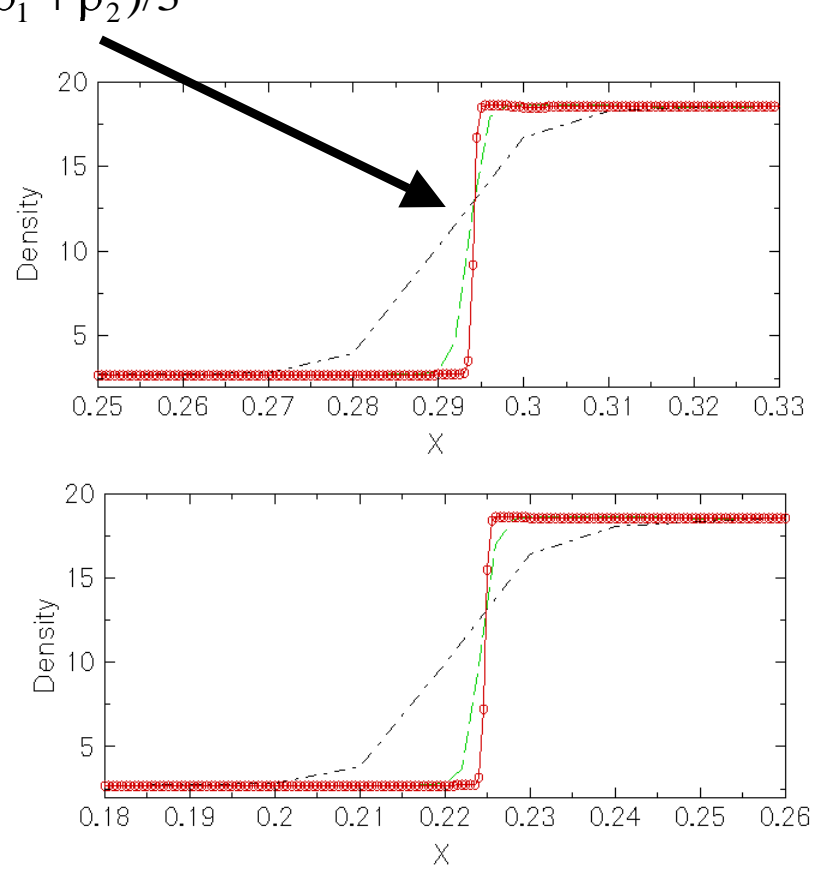

(b)

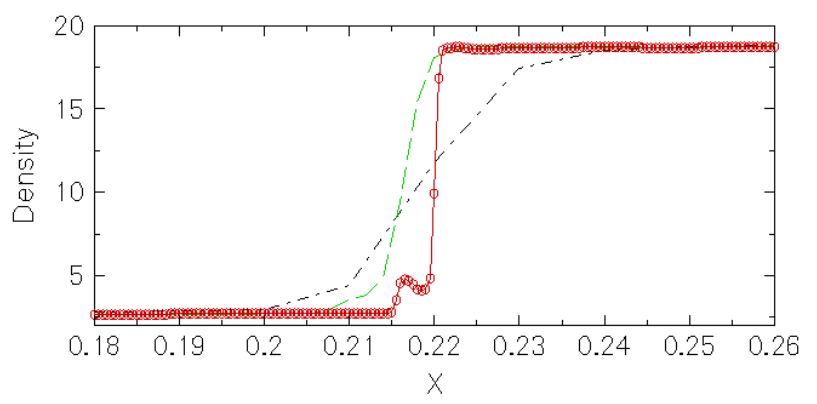

F/S (density ratio=7.0)

Density profiles for Shock Contact Discontinuity Interaction $(M=1.5)$ at $t=0.3$. Top to Down (a) $1 \mathrm{D}$ (b) $2 \mathrm{D}, \alpha=0$ (c) $2 \mathrm{D}, \alpha=10$. The solid line with open circles is the highest resolution 0.0005 and - - - and - . - - are 0.002 and 0.01 respectively. $\rho_{1}{ }^{*}, \rho_{2}{ }^{*}$ are the post shock densities. 


\section{LOCALIZATION OF CD UNDER MESH REFINEMENT(CONT.)}

\section{WENO}
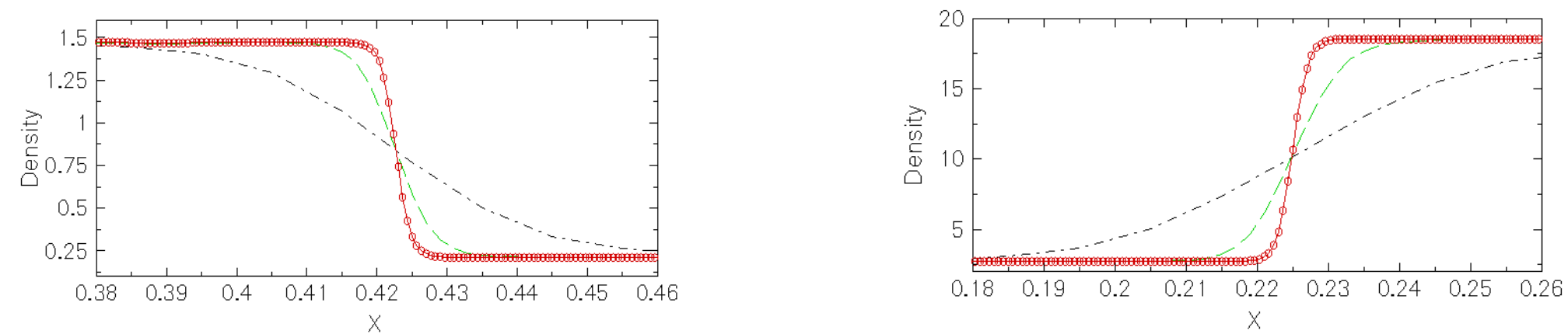

Density profiles for 1D Shock Contact Discontinuity Interaction $(M=1.5)$ at $\mathrm{t}=0.3$. The solid line with open circles is the highest resolution 0.000667 and - - and $-\cdots$ - are 0.002 and 0.01 respectively. 


\section{SPREADING OF CD UNDER MESH REFINEMENT}

PPM (width $\left.\propto t^{1 / 3}\right)$

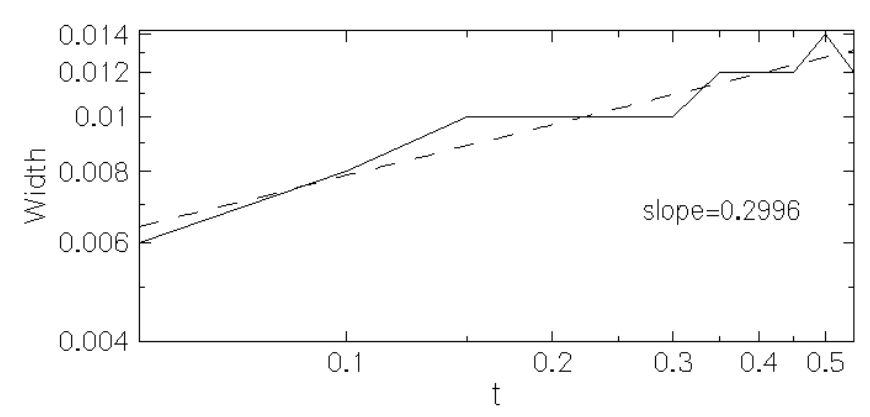

(a)

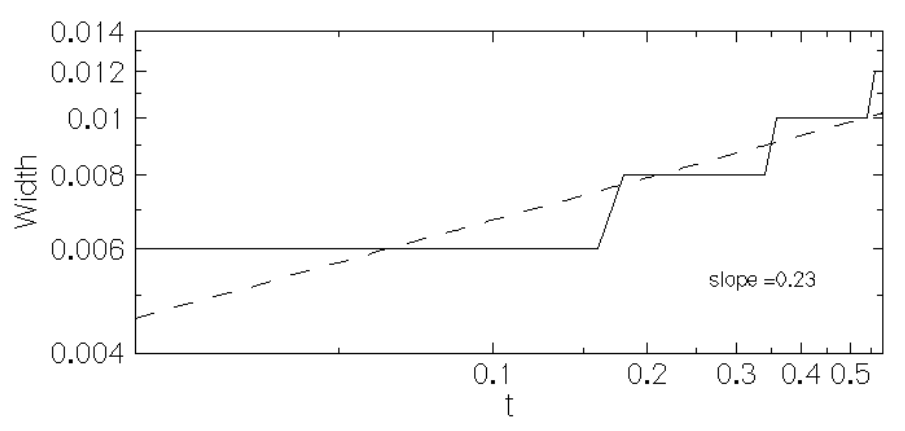

(c)

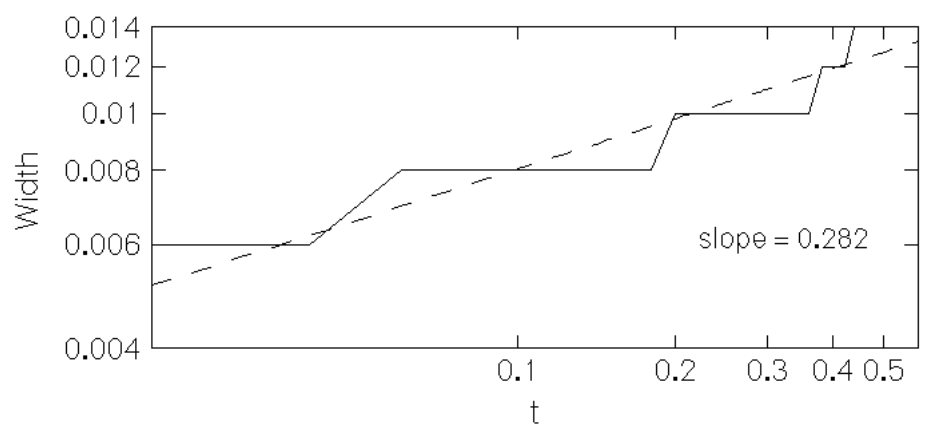

(b)

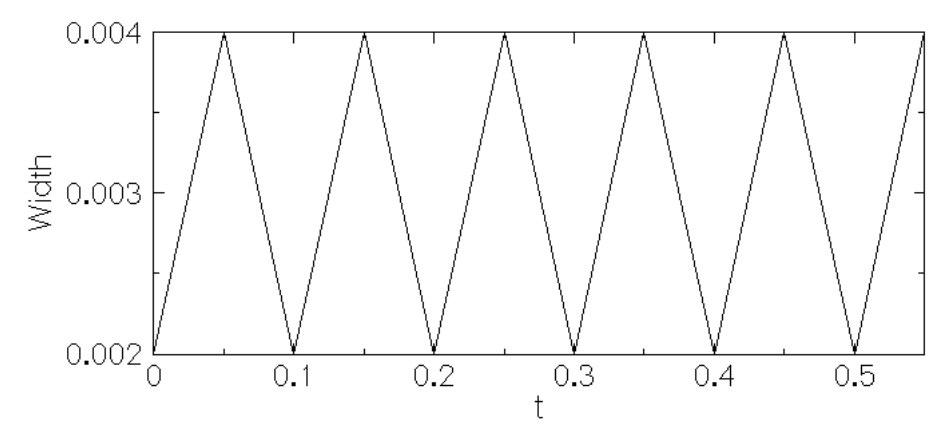

(d)

Growth of width of $C D$ with time in Diffusing Contact for a resolution of 0.002 (a) $S / F$ (1D) (b) $S / F(2 D, \alpha=0)(c) S / F(2 D, \alpha=30)$. For (d)F/S, 1D Width oscillates between two values. Dashed line is the power law fit. 


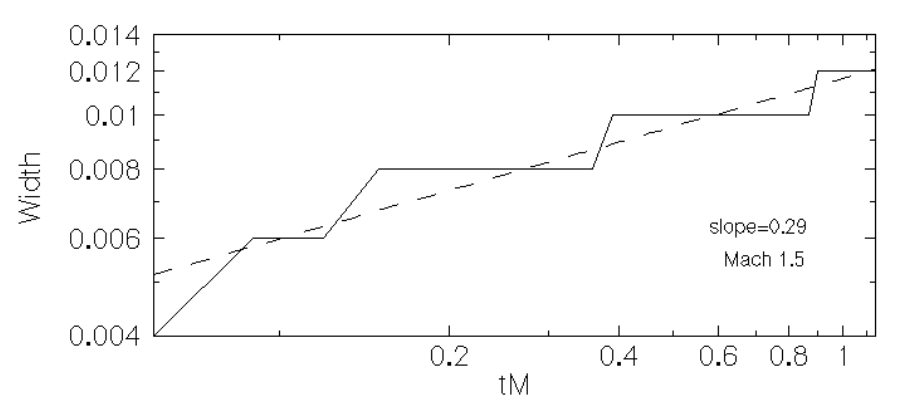

(a)

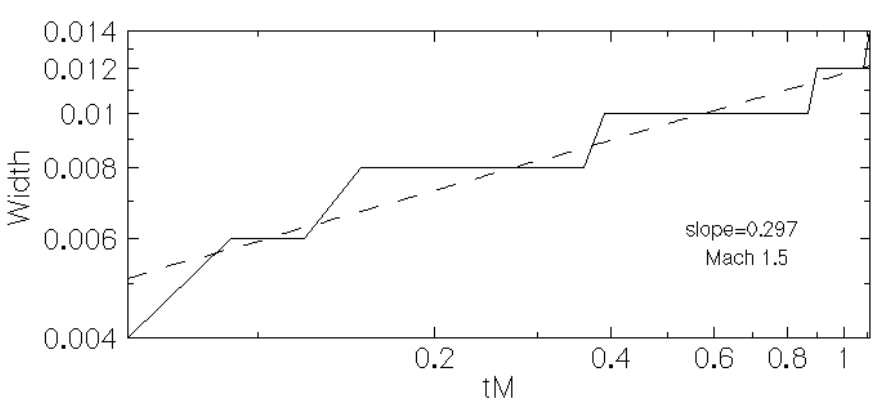

(b)

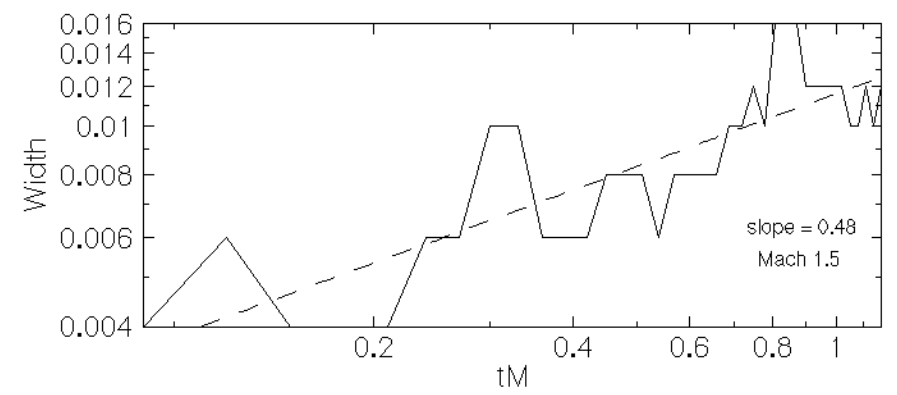

(c)

Growth of width of CD with time in Shock Contact interaction (Mach 1.5) for a resolution of 0.002 (a) $S / F(1 D)(b) S / F(2 D, \alpha=0)$ (c) $S / F(2 D, \alpha=10)$. Dashed line is the power law fit. WENO (width $\propto t^{1 / 4}$ )

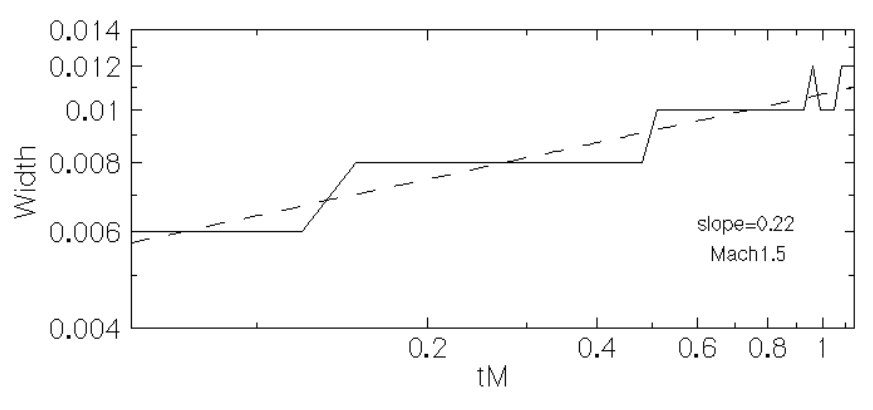

(a)

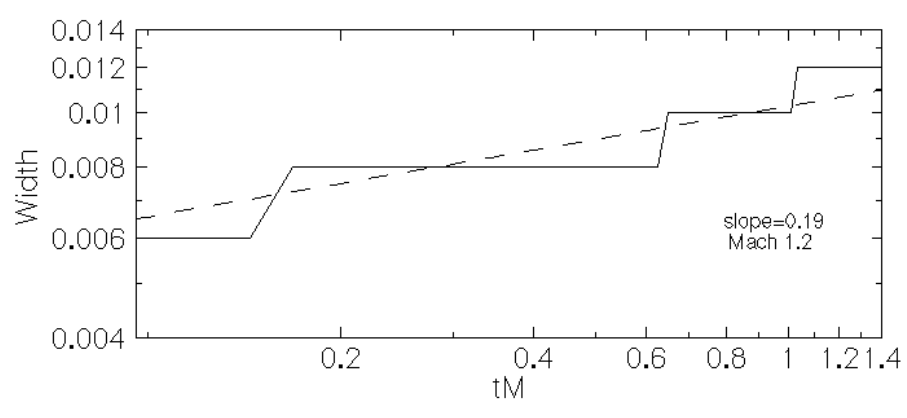

(b)

Growth of width of CD with time in Shock Contact interaction for a resolution of 0.002 (a)S/F (b) F/S Dashed line is the power law fit. 


\section{SPREADING RATES}

\begin{tabular}{|c|c|c|c|c|c|c|}
\hline Evolution & $\eta$ or $\eta^{*}$ & Vel $\left(\mathrm{U}_{0}\right.$ or $\left.\mathrm{M}\right)$ & $\mathrm{nD}$ & $\alpha$ & $\mathrm{C} / \mathrm{r}$ & Exponent(p) \\
\hline $\mathrm{F}$ & 0.14 & 1.5 & 1 & $\mathrm{~N} / \mathrm{A}$ & $\mathrm{PPM} / 2$ & 0.2996 \\
\hline $\mathrm{F}$ & 0.14 & 1.5 & 2 & 0 & $\mathrm{PPM} / 2$ & 0.282 \\
\hline $\mathrm{F}$ & 7.0 & 1.5 & 1 & $\mathrm{~N} / \mathrm{A}$ & $\mathrm{PPM} / 2$ & Oscillating \\
\hline $\mathrm{S}$ & 0.142 & 1.2 & 1 & $\mathrm{~N} / \mathrm{A}$ & $\mathrm{PPM} / 2$ & 0.245 \\
\hline $\mathrm{S}$ & 0.142 & 1.5 & 1 & $\mathrm{~N} / \mathrm{A}$ & $\mathrm{PPM} / 2$ & 0.31 \\
\hline $\mathrm{S}$ & 0.142 & 2.0 & 1 & N/A & $\mathrm{PPM} / 2$ & 0.337 \\
\hline $\mathrm{S}$ & 0.142 & 2.5 & 1 & N/A & PPM/2 & 0.327 \\
\hline $\mathrm{S}$ & 0.142 & 1.5 & 2 & 0 & $\mathrm{PPM} / 2$ & 0.297 \\
\hline $\mathrm{S}$ & 0.142 & 1.2 & 2 & 10 & $\mathrm{PPM} / 2$ & 0.26 \\
\hline $\mathrm{S}$ & 0.142 & 1.5 & 2 & 30 & $\mathrm{PPM} / 2$ & 0.16 \\
\hline $\mathrm{S}$ & 0.142 & 1.2 & 1 & N/A & WENO/3 & 0.18 \\
\hline $\mathrm{S}$ & 0.142 & 1.5 & 1 & N/A & WENO/3 & 0.22 \\
\hline $\mathrm{S}$ & 0.142 & 2.0 & 1 & N/A & WENO/3 & 0.25 \\
\hline $\mathrm{S}$ & 6.83 & 1.2 & 1 & N/A & WENO/3 & 0.19 \\
\hline $\mathrm{S}$ & 6.83 & 2.0 & 1 & N/A & WENO/3 & 0.25 \\
\hline
\end{tabular}

F or S - Freely evolving or Shock struck $\eta$ or $\eta^{*}$ - Density ratio before and after shock passage

$\mathrm{U}_{0}$ or $\mathrm{M}-$ Constant initial velocity or Mach number

$\mathrm{nD}$ - Number of dimensions $\quad \mathrm{C} / \mathrm{r}$ - Code/Order of code 


\section{VORTEX LOCALIZATION \& NONLINEAR EVOLUTION SINGLE-MODE RM INTERFACE}

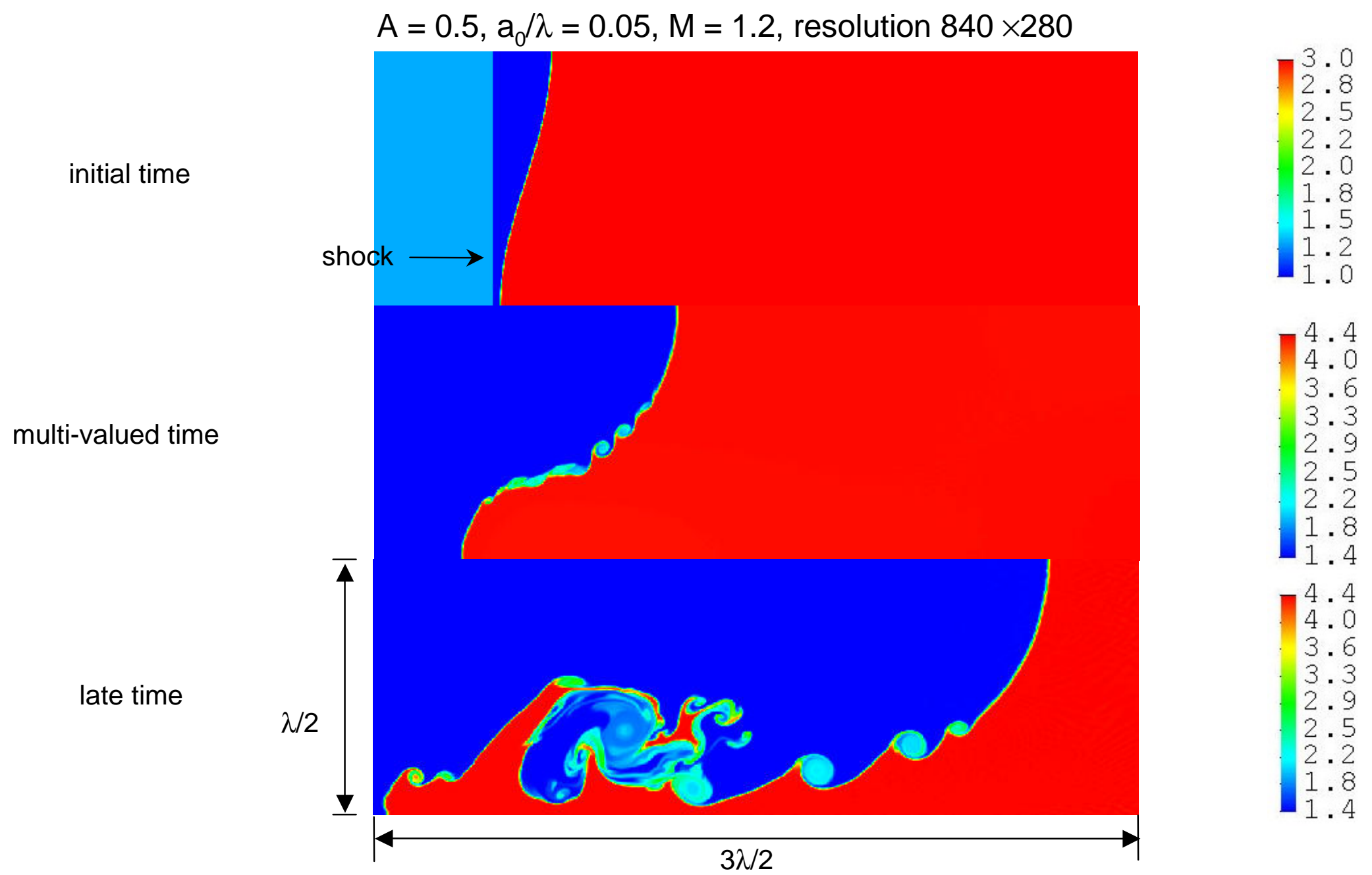

Three stages(density) of RM instability in shock-sinusoidal interaction : "initial time", "multi-valued" and "late time." The actual times are 0, 6.0 and 36; Atwood number is 0.5 ; initial density ratio is 3.0; Incident shock is $M=1.2$; the perturbation is $a_{0} / \lambda=0.05$; resolution $840 \times 280(P P M)$ 


\section{INTERFACIAL GROWTH RATE AND GLOBAL CIRCULATION}

$$
A=0.5, a_{0} / \lambda=0.05, M=1.2
$$

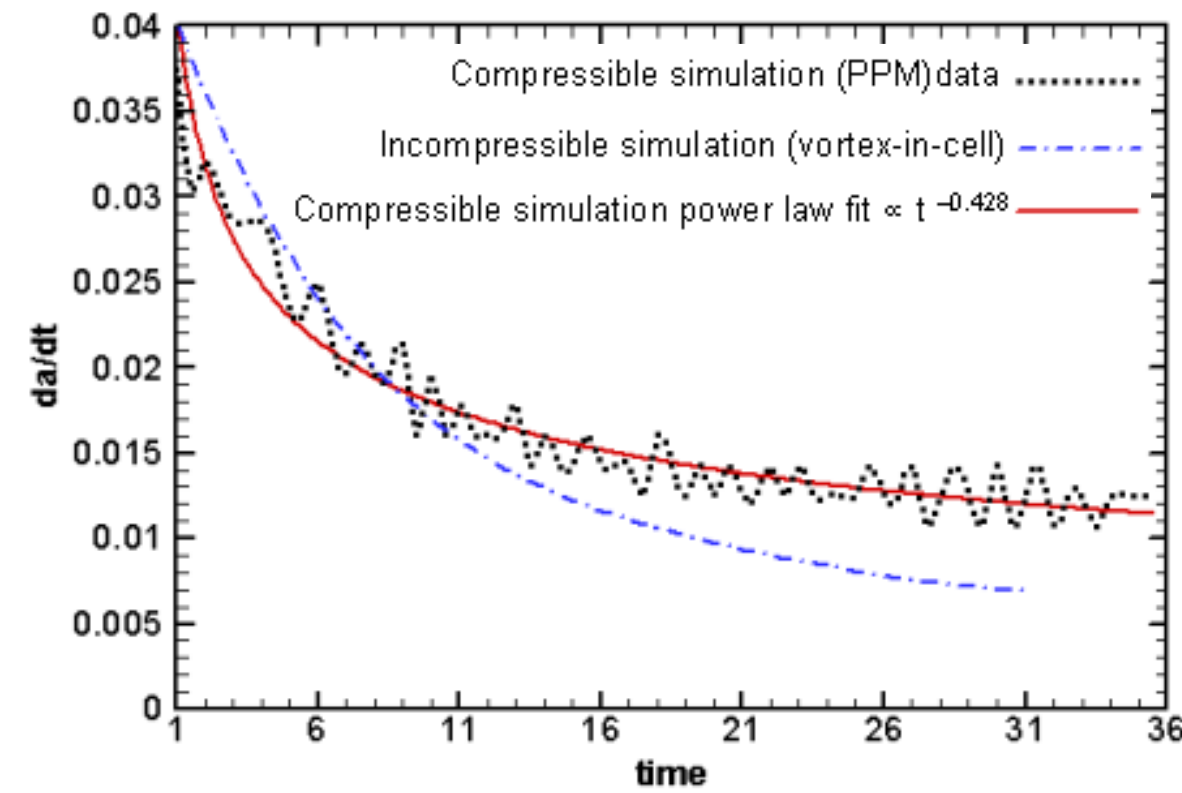

Interfacial growth rate obtained from compressible simulation(PPM), incompressible simulation (vortexin-cell) and power law fitting for compressible simulation (PPM) data. For the power fitting: total RMS error $\sim 8.7 \%$, but for $16<\mathrm{t}<36, \mathrm{da} / \mathrm{dt}=0.013$ $0.15 / \mathrm{t}$ and $\mathrm{RMS}$ error $\sim 7 \%$.

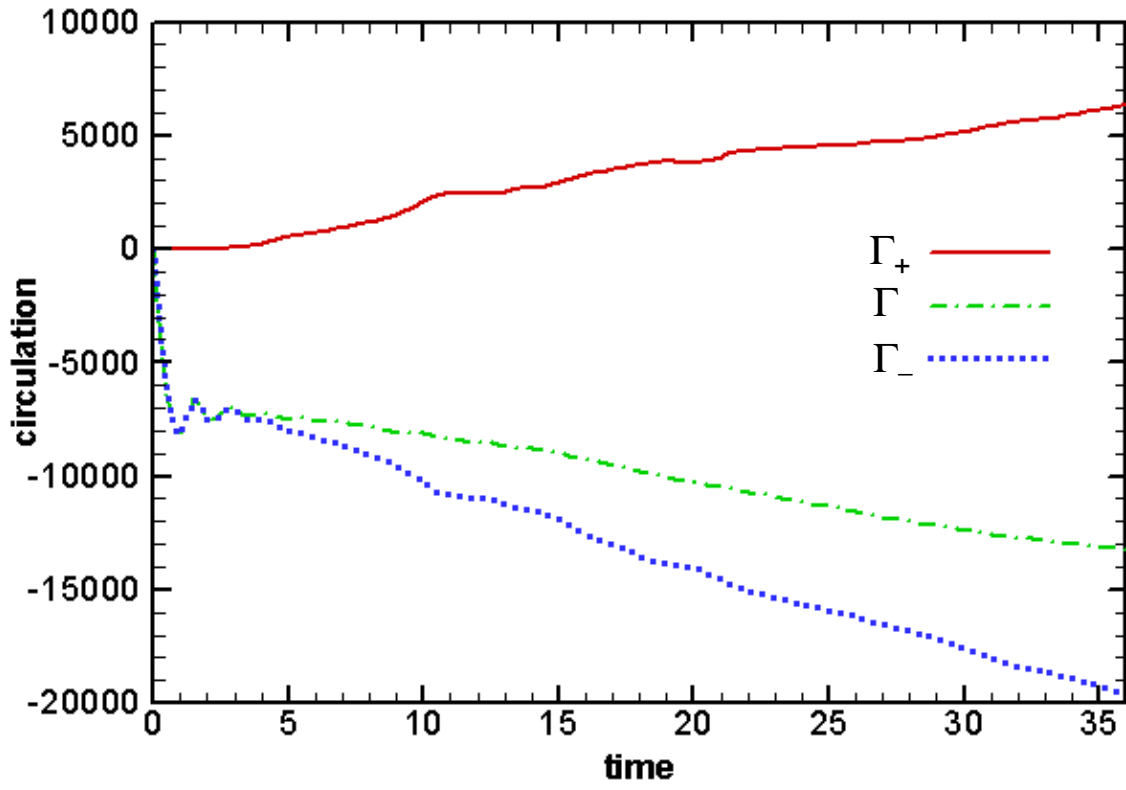

Positive $\left(\Gamma_{+}\right.$, negative $\left(\Gamma_{-}\right)$and net $(\Gamma)$ circulations obtained from compressible simulation (PPM). Secondary vorticity generation and associated instability contribute to interfacial growth rate. 


\section{CONCLUSIONS}

$>$ We present a systematic approach to quantify interfacial localization and temporal spreading in one dimension.

$>$ We observe asymmetry in interfacial spreading rates for the one and two dimensional PPM simulations for F/S and S/F configurations. These are not present in a WENO simulations.

$>$ Evolution of sinusoidal RM interface at late time exhibits large growth of positive and negative "secondary" baroclinic circulation. Interfacial growth rate is not $(1 / t)$ and depends on Atwood number. 


\section{Instability modeling for NIF ignition targets and Omega experiments}

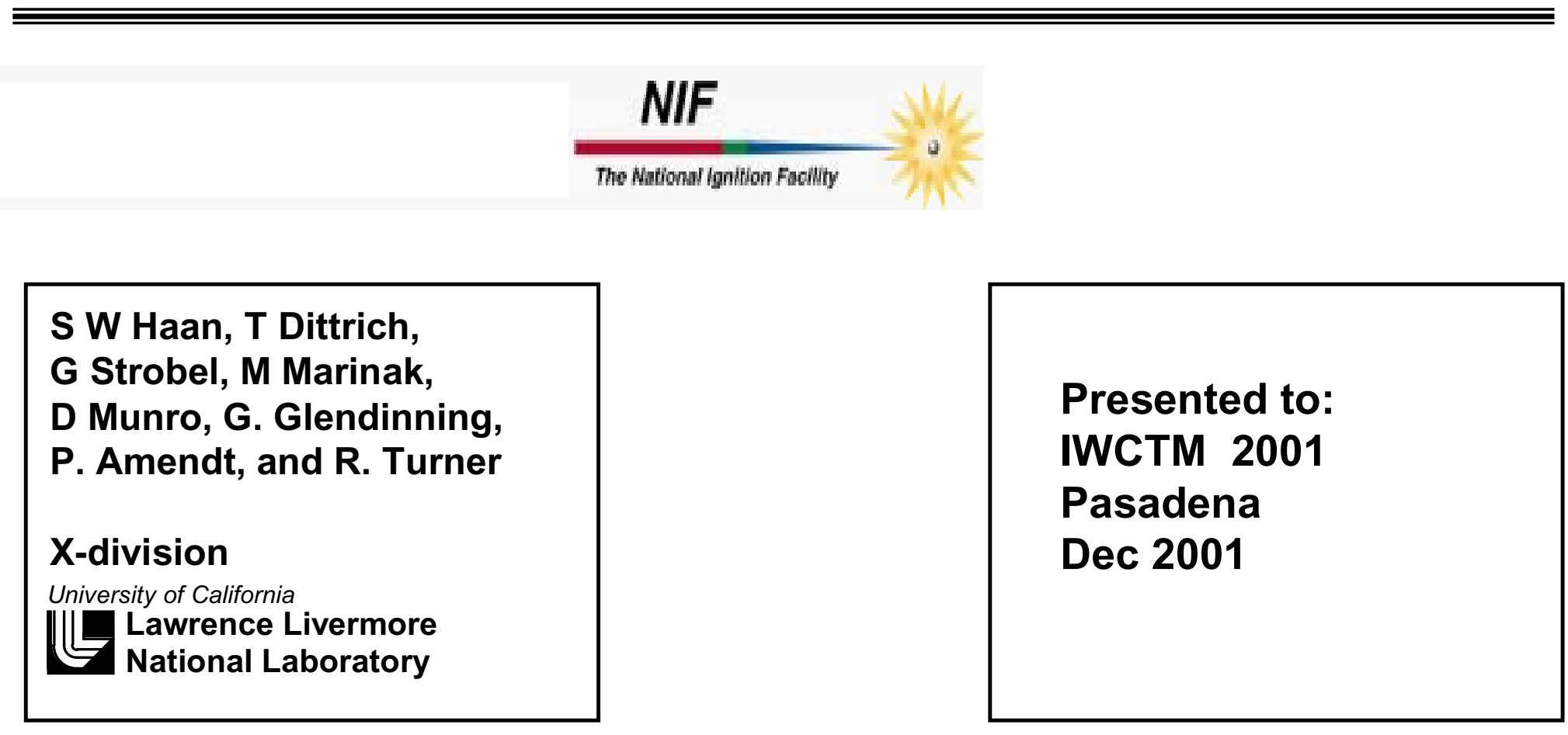

${ }^{*}$ This work was performed under the auspices of the U.S. Department of Energy by the University of California, Lawrence Livermore National Laboratory under contract No. W-7405-Eng-48. 
Summary: We are continuing to explore hydro instability issues on NIF targets, and verifying modeling with Omega experiments

Specifications are being completed for a variety of indirect drive targets:

Beryllium, polyimide, $\mathrm{CH}(\mathrm{Ge})$ ablators

Drive temperatures $250-\mathbf{3 5 0} \mathrm{eV}$, spectra for gold or cocktail hohlraum Scales from $100 \mathrm{~kJ}$ to $600 \mathrm{~kJ}$ into capsule (NIF energy $\sim 1.8 \mathrm{MJ}$ )

Details such as ${ }^{3} \mathrm{He}$ buildup in the core are being analyzed

Modeling of Omega planar polyimide Rayleigh-Taylor foils is close to experiments

A new design for convergent Rayleigh-Taylor experiments on Omega will test other aspects of the modeling 


\section{Generically the ignition targets all look the same as for the last 10 years or so}

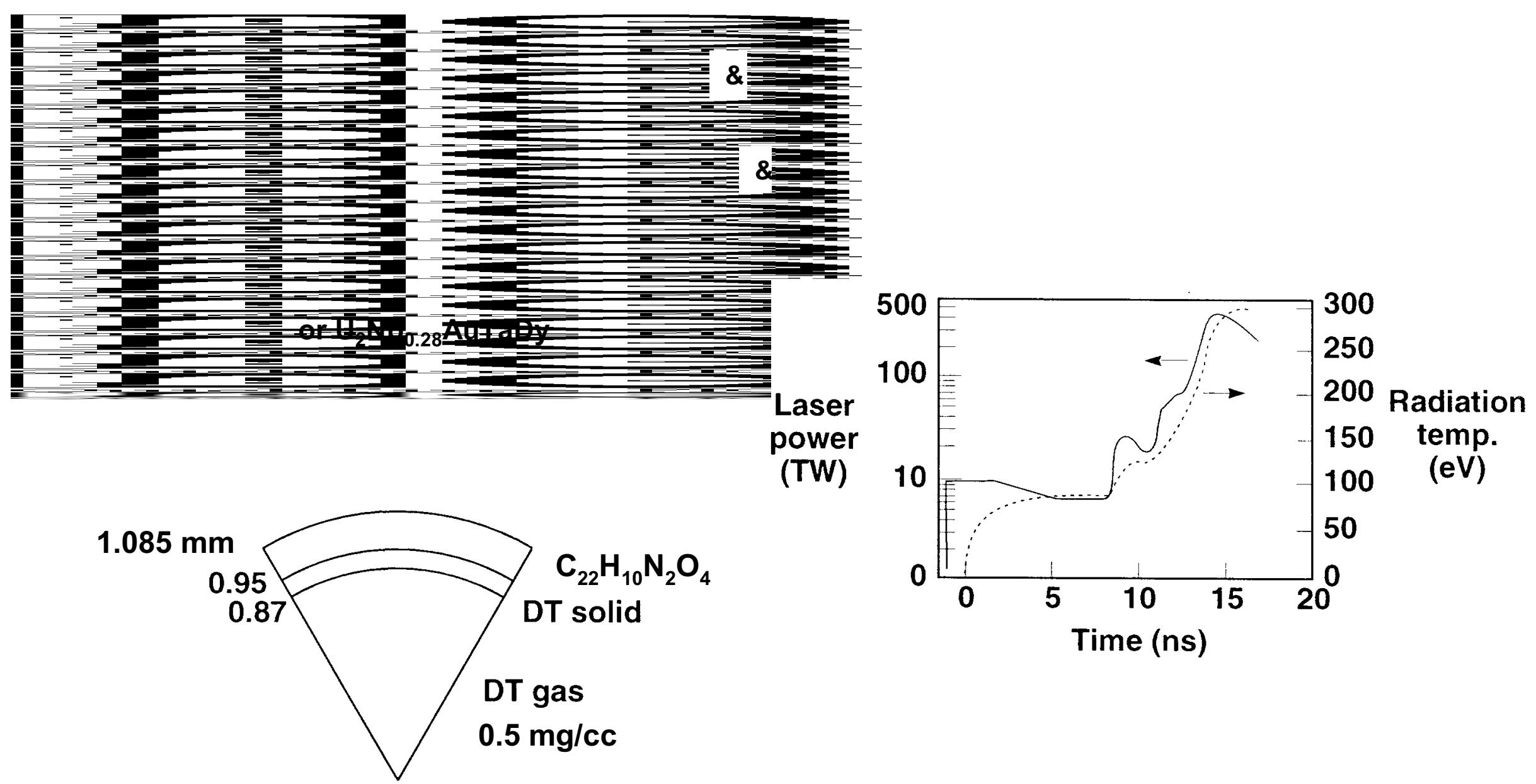


Our current instability modeling is based entirely on explicit full simulations of perturbation growth and its impact on ignition and burn

- Single shell cryogenic capsules are ablatively stabilized on outside during acceleration, and on inside during deceleration

- Simulations indicate that modes beyond about 120 do have any appreciable amplitudes at any times of interest

-Experiments have generally been compatible with simulations giving us confidence in them -Modeling is done in 2D (LASNEX and Hydra) and 3D (HYDRA) for single modes, and for multiple modes over various solid angles

-Biggest uncertainties are considered to be in the input: spectrum of drive radiation, opacities, characterization of initial perturbations 


\section{There are three failure modes we see in our simulations}

-Acceleration: Modes $l \sim 100$ grow and disrupt the shell Especially a problem if shell is too thin

-Deceleration: Modes $l \sim 15$ create spikes that cool the hotspot Especially a problem if shell is too thick

- Low modes: If there is much solid angle with $\rho r<1 \mathrm{~g} / \mathrm{cm}^{2}$, bubbles blow out and yield is reduced

A successful target is optimized to trade off the first two issues, and has enough 1D pr to minimize the third. Requires power and energy to have room to trade them off! 


\section{This plot summarizes ablator-seeded Rayleigh- Taylor results for the different capsules}

\section{Rms roughness}

for $50 \%$ YOC, $\mathrm{nm}$

All with gold hohlraum spectrum

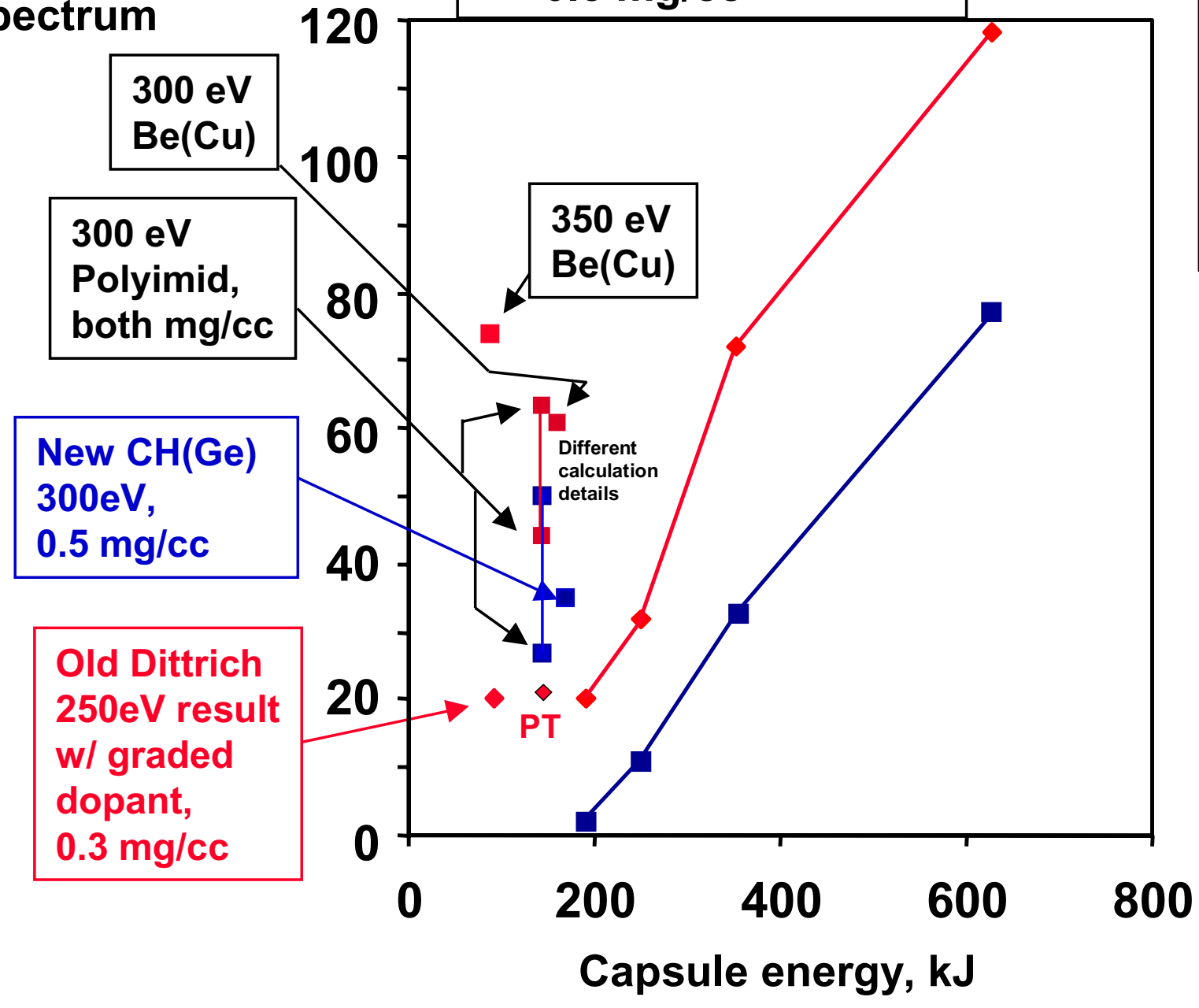

$\mathrm{Be}(\mathrm{Cu})$ is better, and higher $T_{R}$ helps a lot 


\section{$600 \mathrm{~kJ}$ capsules might be constrained in foot length, at a significant energy price}

Largest scale might have foot increased in order to keep total pulse length close to 20 ns

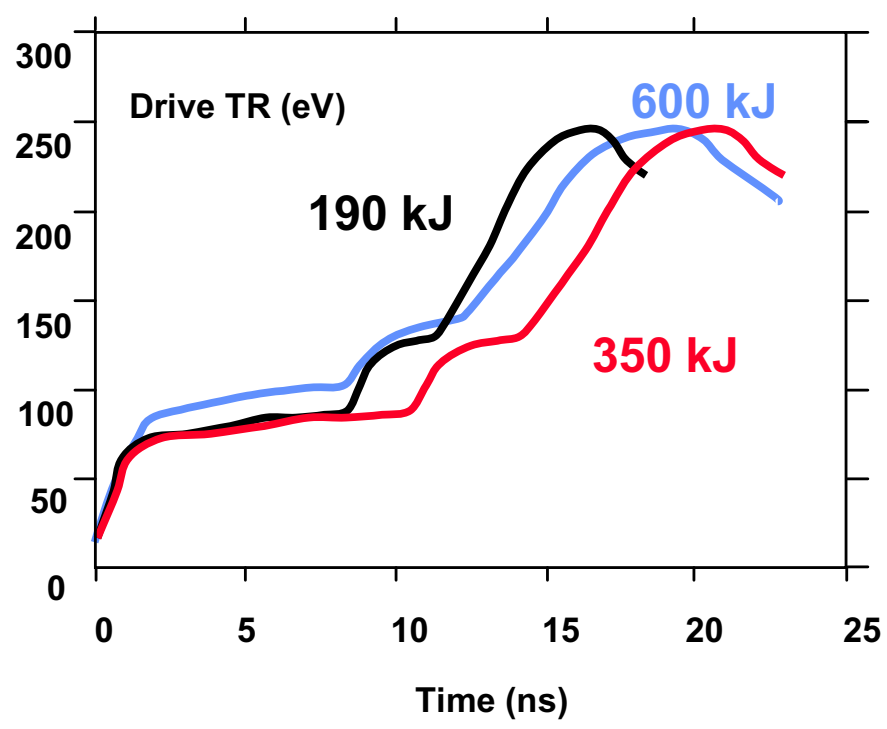

If shock-crossing time is fixed, velocity $\sim \mathrm{S}^{1}$

foot level flux $F \sim S^{2}$

Adiabat $\beta \sim \mathbf{S}^{1.2}$

Margin $\sim S^{3} \beta^{-1.5} \sim S^{1.2} \sim E^{0.4}$ instead of $E^{1}$

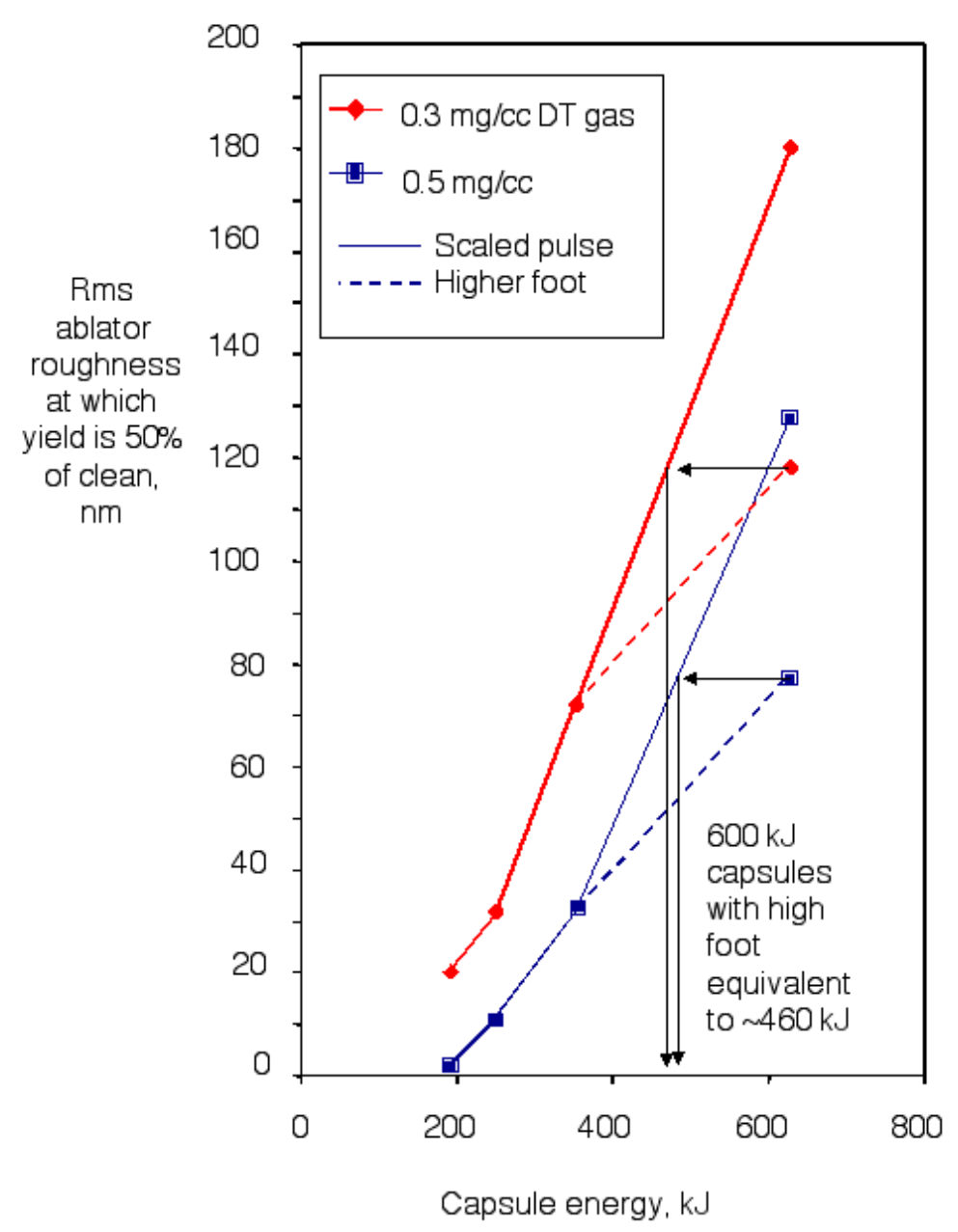




\section{Surface roughness specifications are tighter if there}

\section{is ${ }^{1} \mathrm{H}$ or ${ }^{3} \mathrm{He}$ in the central gas}

-Both are "dead weight" w/ respect to hydro, ignition \& burn

-Atom-for-atom, ${ }^{3} \mathrm{He}$ is worse-more electrons and ion

charge, increases radiative and conductive losses

-But gram-for-gram, ${ }^{1} \mathrm{H}$ is slightly worse-3x more atoms/g

Relative

ablator

roughness

requirement

(ablator

roughness

for $50 \%$ YOC,

normalized)

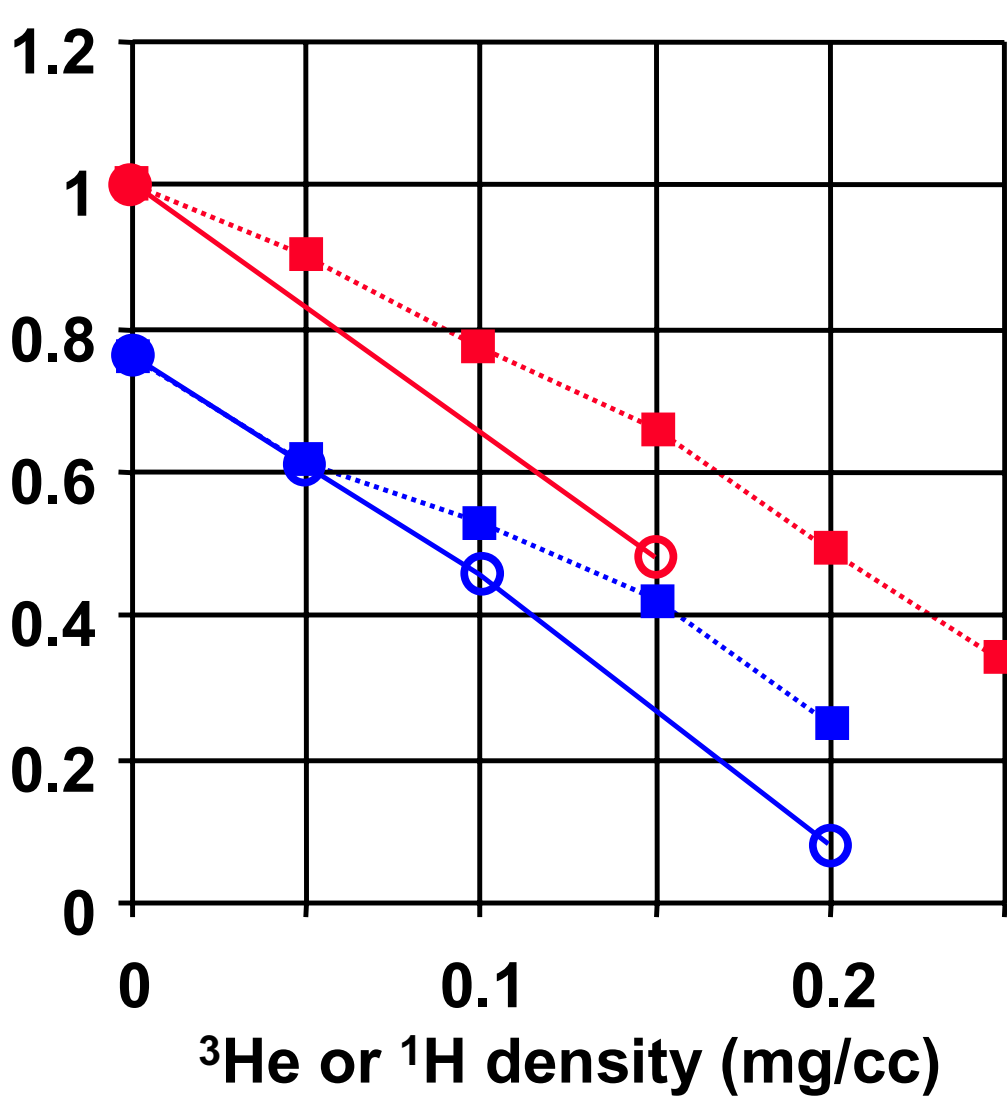

${ }^{1} \mathrm{H}$,

$0.3 \mathrm{mg} / \mathrm{cc}$ DT $+{ }^{1} \mathrm{H}$

- $0.5 \mathrm{mg} / \mathrm{cc}$ DT $+{ }^{1} \mathrm{H}$ ${ }^{3} \mathrm{He}:$

$0.3 \mathrm{mg} / \mathrm{cc} \mathrm{DT}+{ }^{3} \mathrm{He}$

$0.5 \mathrm{mg} / \mathrm{cc} \mathrm{DT}+{ }^{3} \mathrm{He}$

2D simulations (ablator roughness for $50 \%$ yield, normalized to $65 \mathrm{~nm}$, include $0.93 \mu \mathrm{m}$ DT $\mathrm{rms}$ ) 


\section{The calculated NIF cocktail spectrum is intermediate between Planckian and gold}

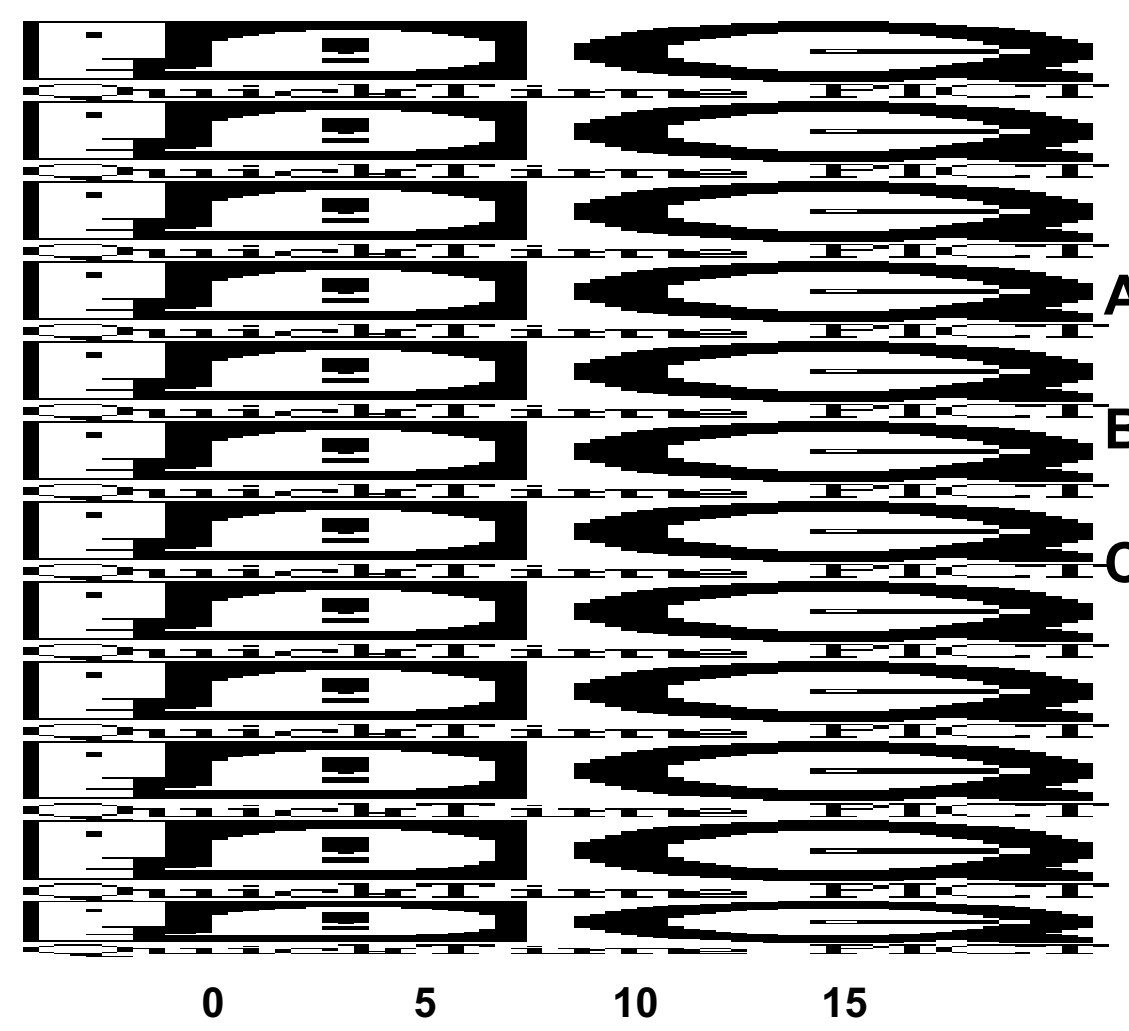

A (black) typical gold spectrum

B (red) cocktail calculation (Pollaine)

C (blue) Planckian w/ same flux

Need to do simulations of effect on Rayleigh-

Taylor of actual

cocktail spectrum

Time (ns) 


\section{With a Planckian drive, baseline polyimide NIF capsule shows $\mathbf{8 5 \%}$ more Rayleigh-Taylor growth}

Growth in 2D simulations, very small multi-mode pert on ablator initially

- Black Au

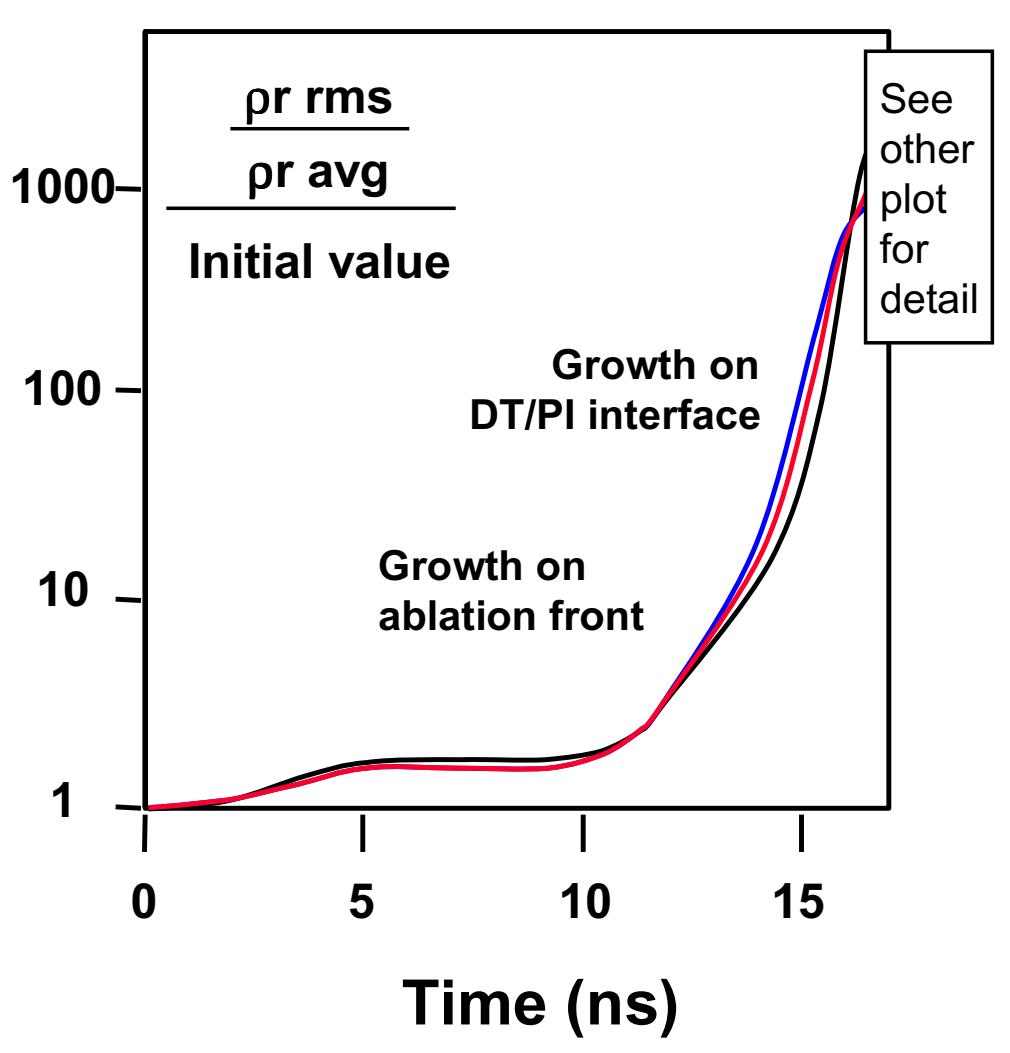

Red cocktail hohIraum wall

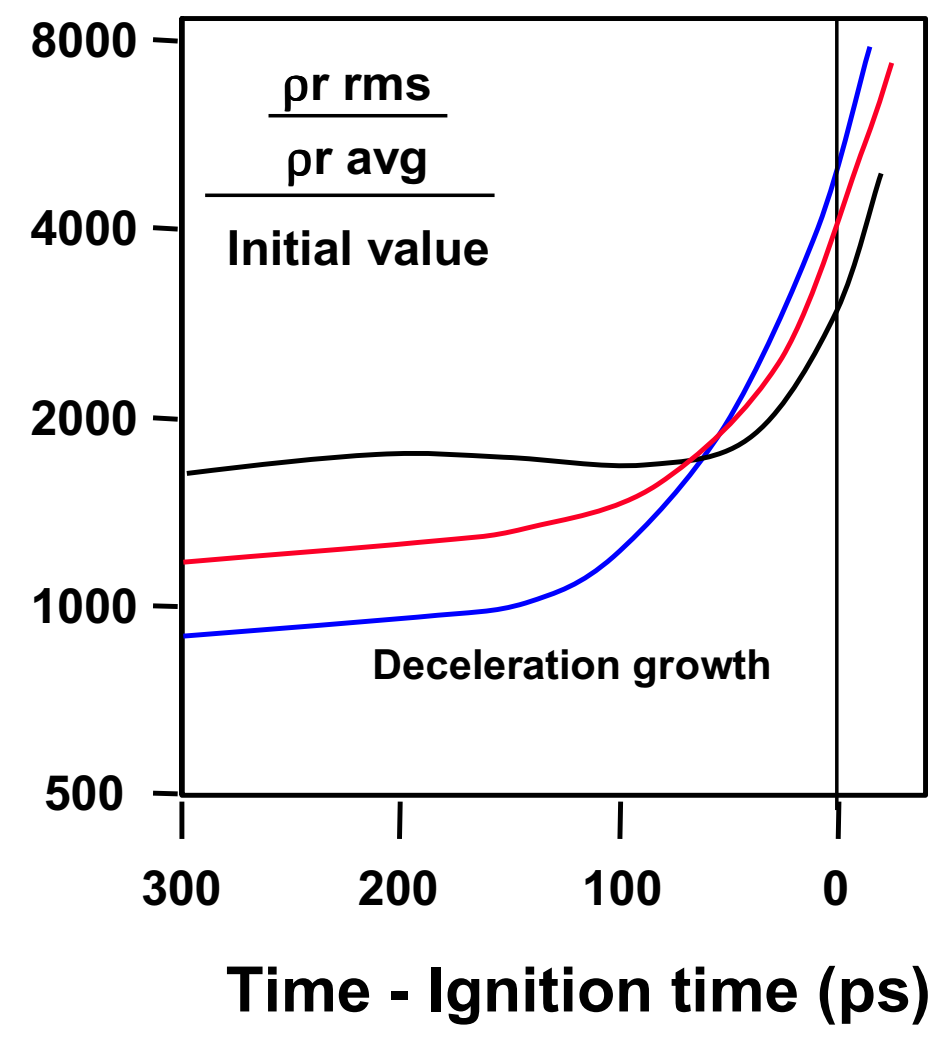

Complicated interplay of growth on the various interfaces With doped ablators, may be able to reoptimize w/ cocktail wall 
We are doing Rayleigh-Taylor experiments on Omega to verify modeling of polyimide

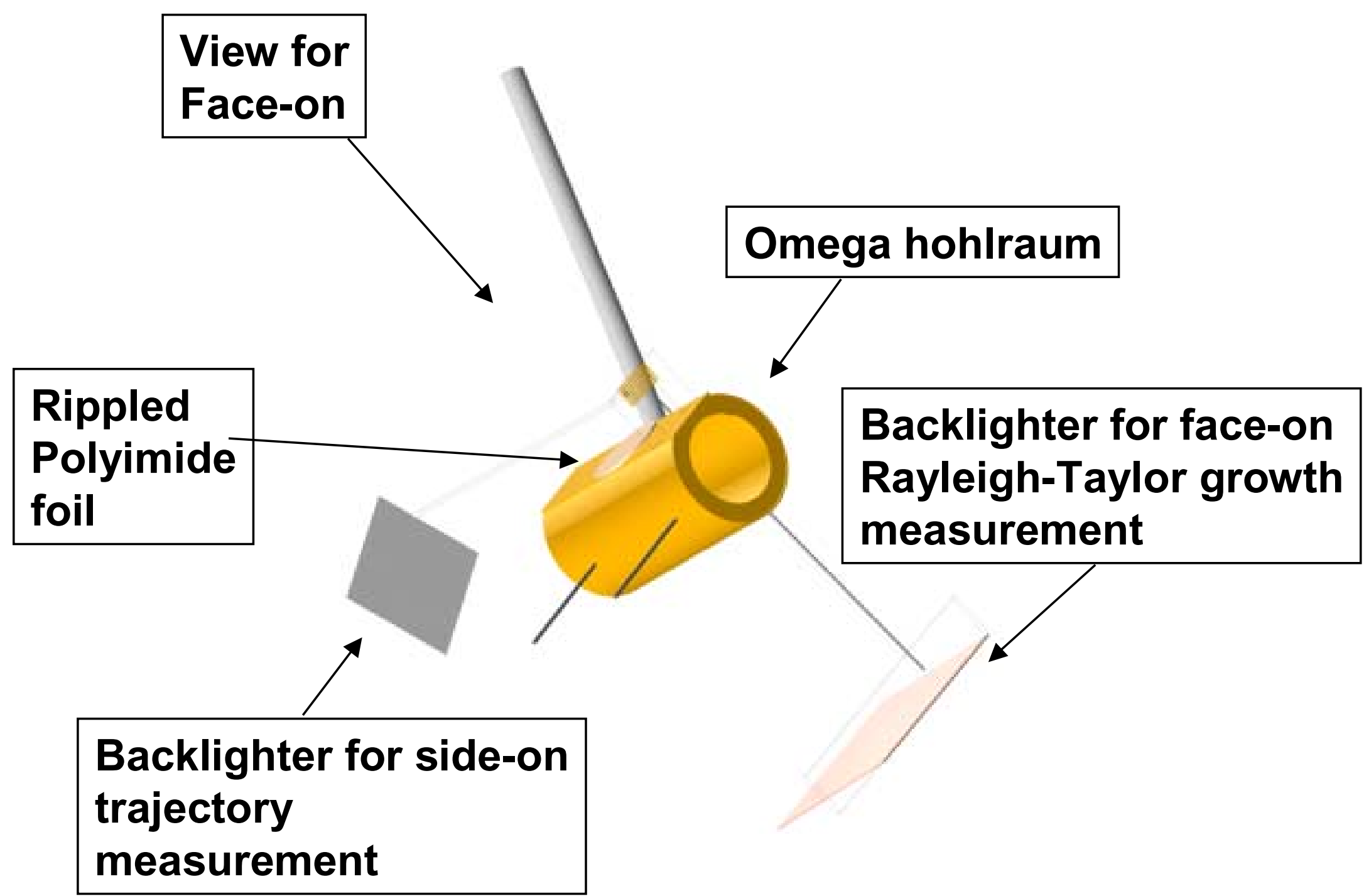




\section{Peter Amendt has done hohlraum simulations that fit the Dante flux measurement}

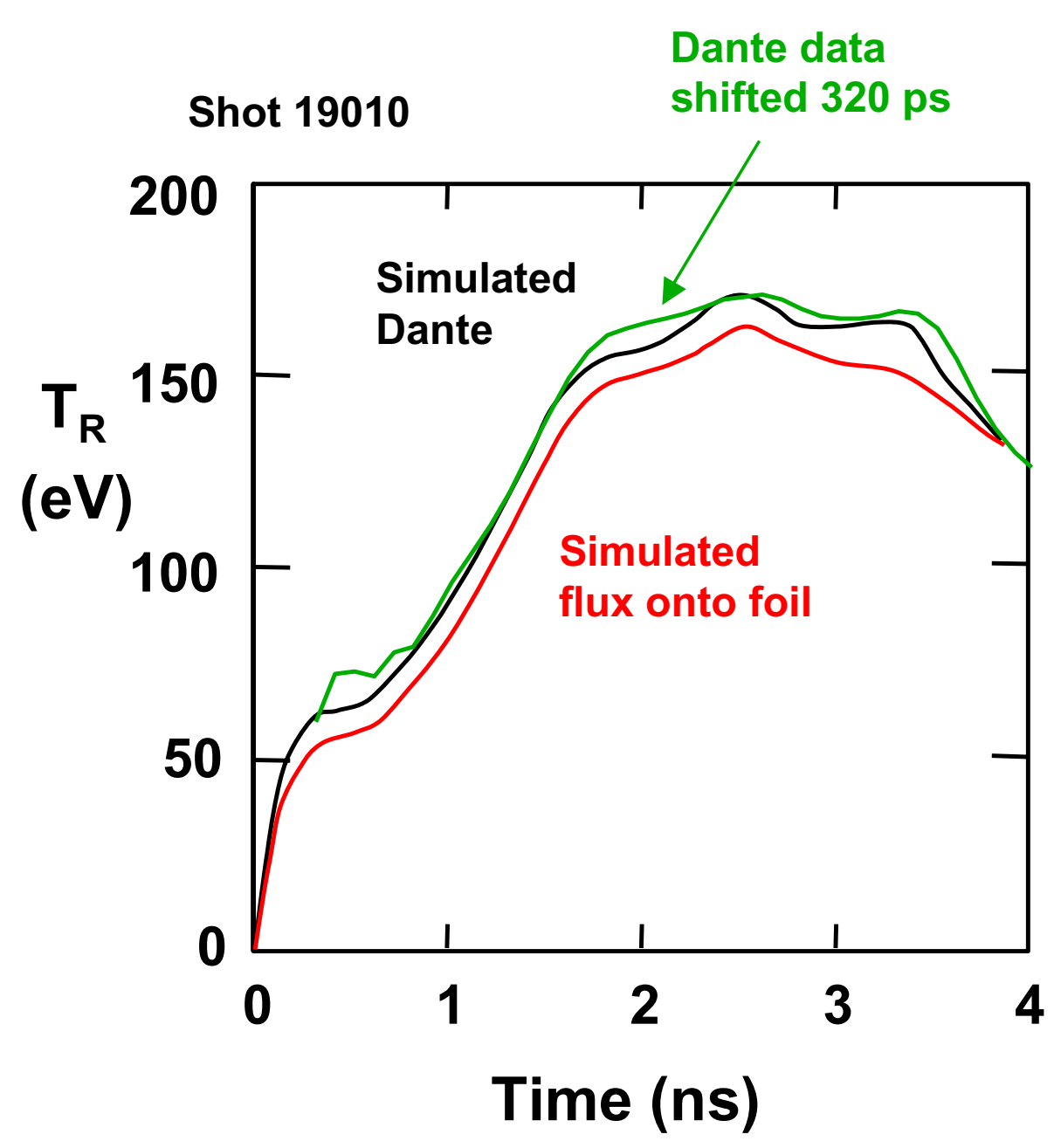

Post-process to simulate Dante: almost high enough to fit data (black curve compared to green).

Simulated drive for package is red curve, about $10 \mathrm{eV}$ lower

There's a significant geometrical correction (like the old albedo correction, but now in the other direction) that we need to incorporate 


\section{Simulated drive extracted from Peter's hohlraum calculations makes sideons very close to data}

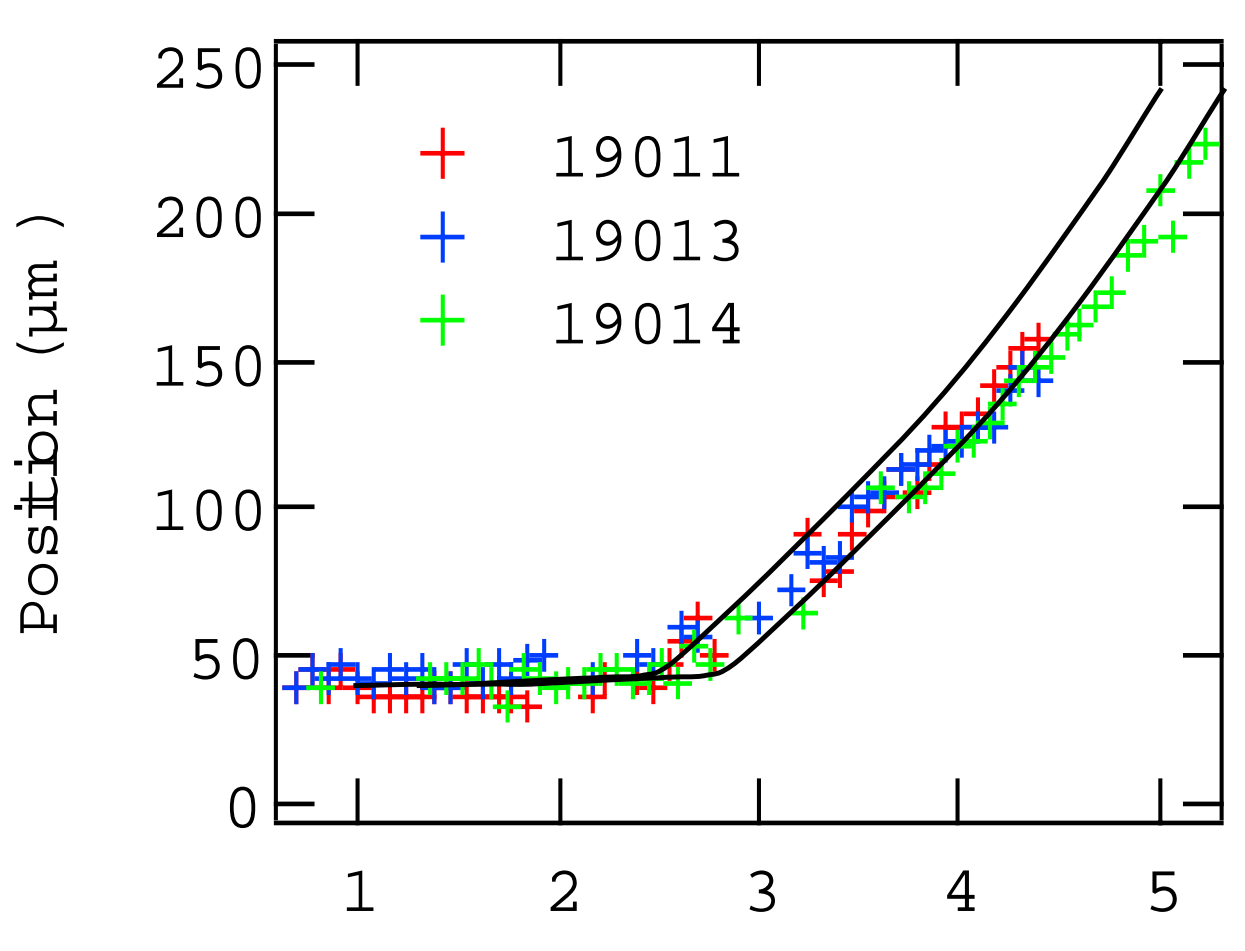

Time (ns
- Simulation using Peter's simulated drive

Also shown shifted in time, improves fit

Peter's hohlraum simulations include a foil, its side-on motion agrees with my foil-only simulation 


\section{I have finished one case faceon and sideon from June 00 shots with the new source info}

Source was Dante-25eV, with M-band adjusted (by factor of several) to match Dante M-band fraction

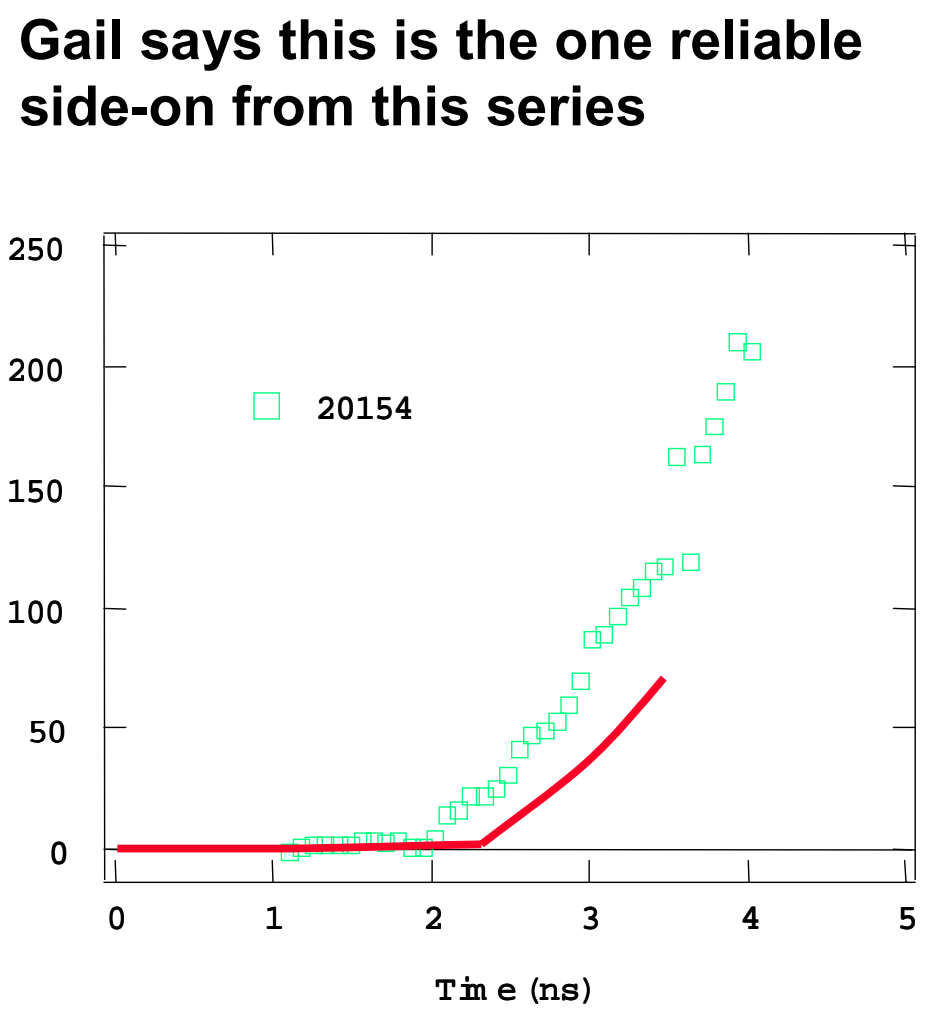

- Old drive New drive
Face-on

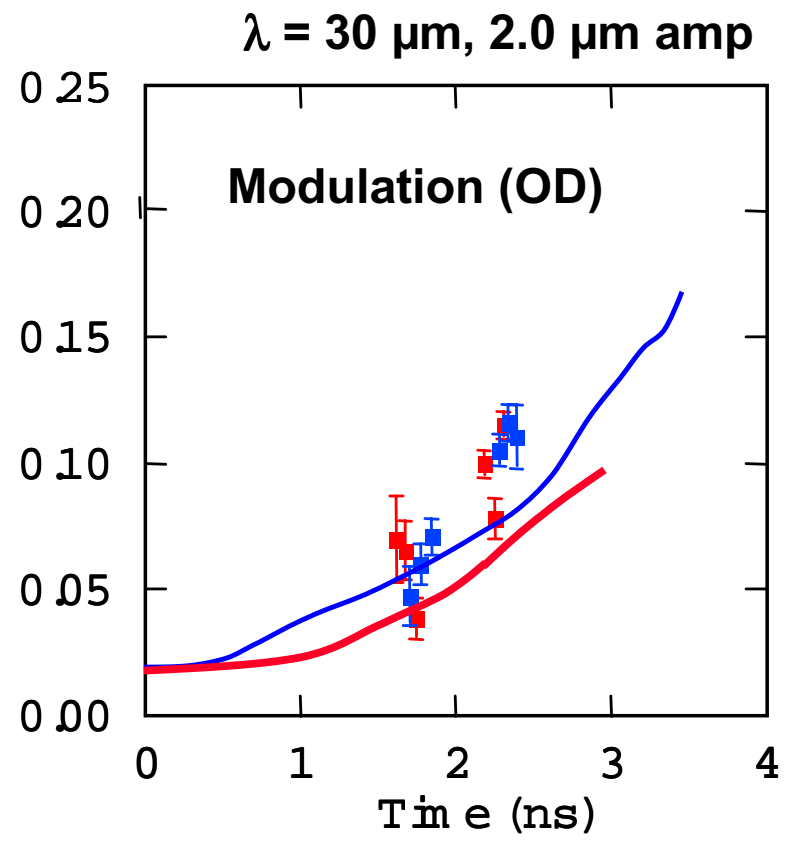

This is late and slow, meaning we've overcorrected the drive, which is very good news 


\section{The simulations l've shown previously for the June 00 faceons used this drive profile}

— Profile I used for old face-on work

. - . - 19010 simulated source from Peter (aruguably fits sideons)

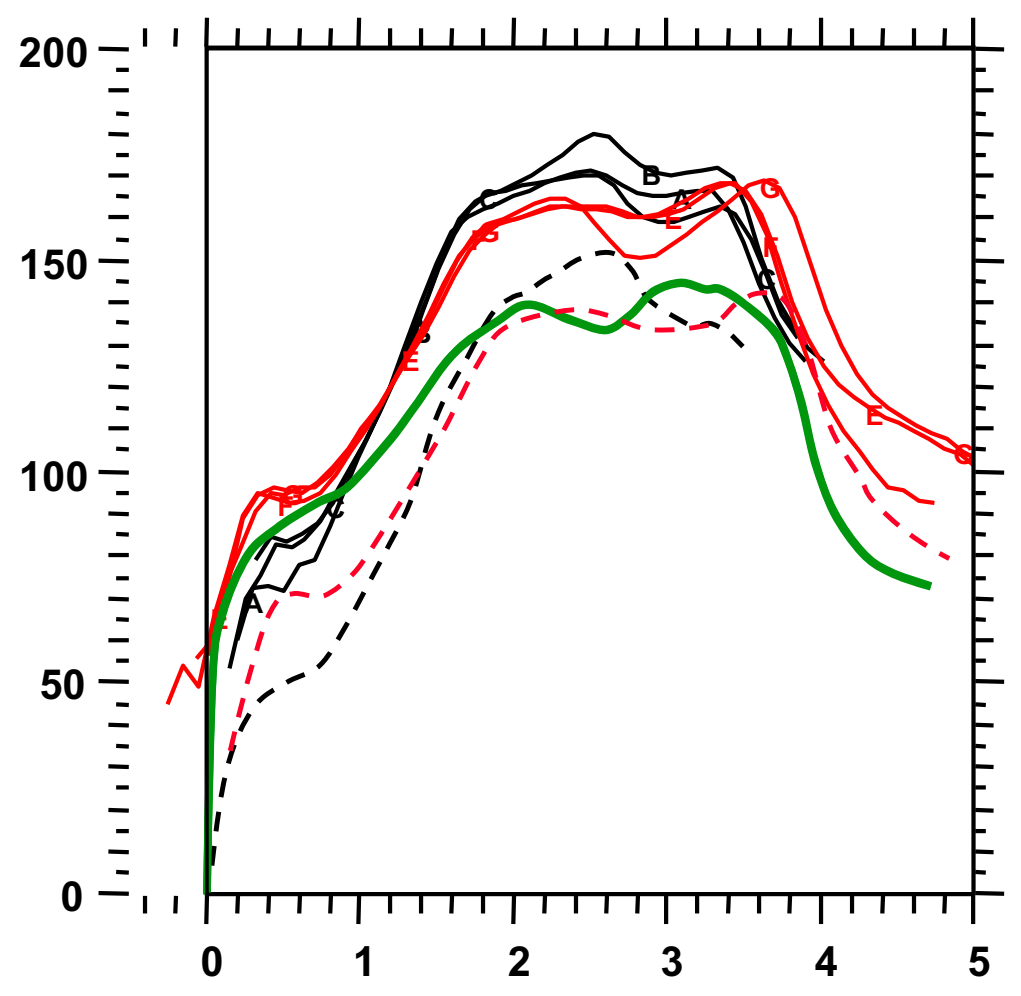

Dante:

Black 19010, 1, 3 (Feb 00)

(sideons we've been trying to fit) Red 2015456 (June 00)

(faceon shots)

All Dante retimed to go through

$(1.2 \mathrm{~ns}, 120 \mathrm{eV})$

All plots are with CEA calibration

Black solid to black dashed is geometry correction $+\sim 10 \mathrm{eV}$ that Dante is still high compared to simulations. (Arguably fits sideons) Same correction to red curves would be "right" profile, compare to green curve.

Red dash is face-on Dante $-25 e V$, shifted 0.1 ns to get good time 0 -best guess at drive for faceons 20154-6. On old green profile, foot was too high, peak not bad 


\section{With that profile I had a decent fit, need to revisit now that sideons are more or less sorted out}

- Better simulations use opacity tables generated from OPAL code

_ Increases growth slightly, improves agreement at $\mathbf{3 0}$ microns

Simulations using XSN opacities, Dante drive, calculated spectrum (same as above)
- OPAL opacities, drive shown above and calculated spectrum
---- OPAL opacities, Planckian spectrum
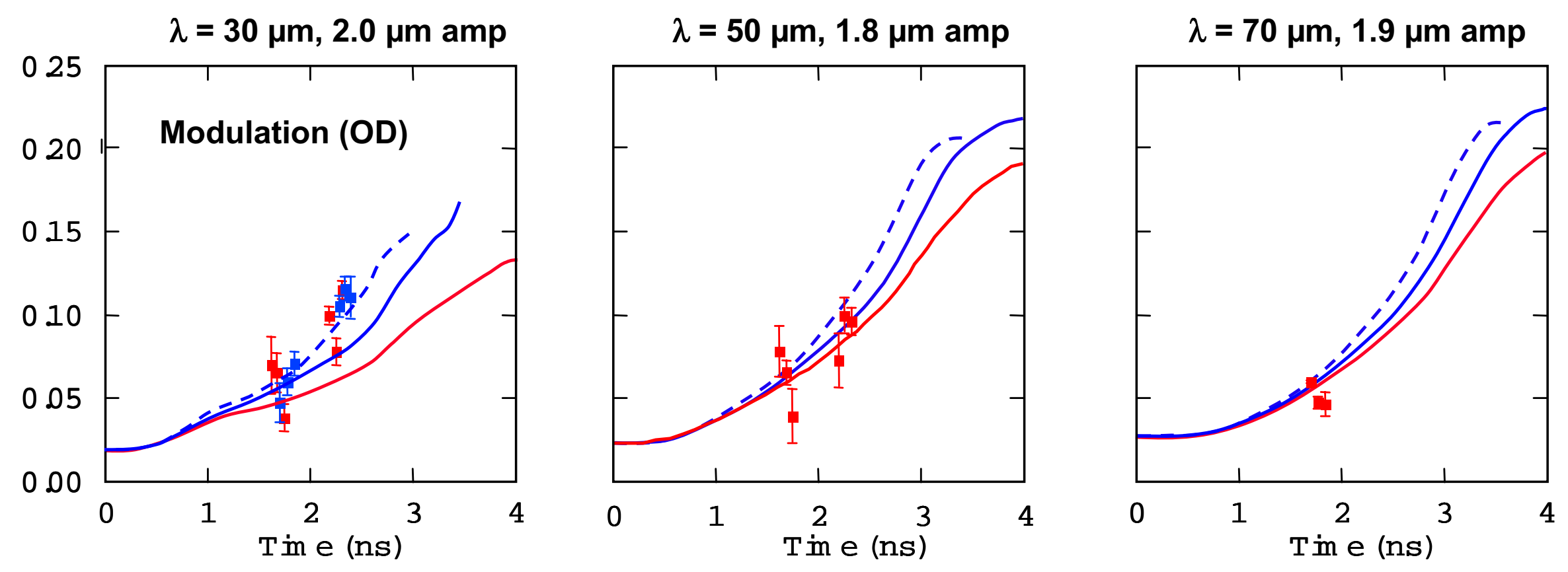


\section{Recent shots in cocktail hohIraum had this drive}

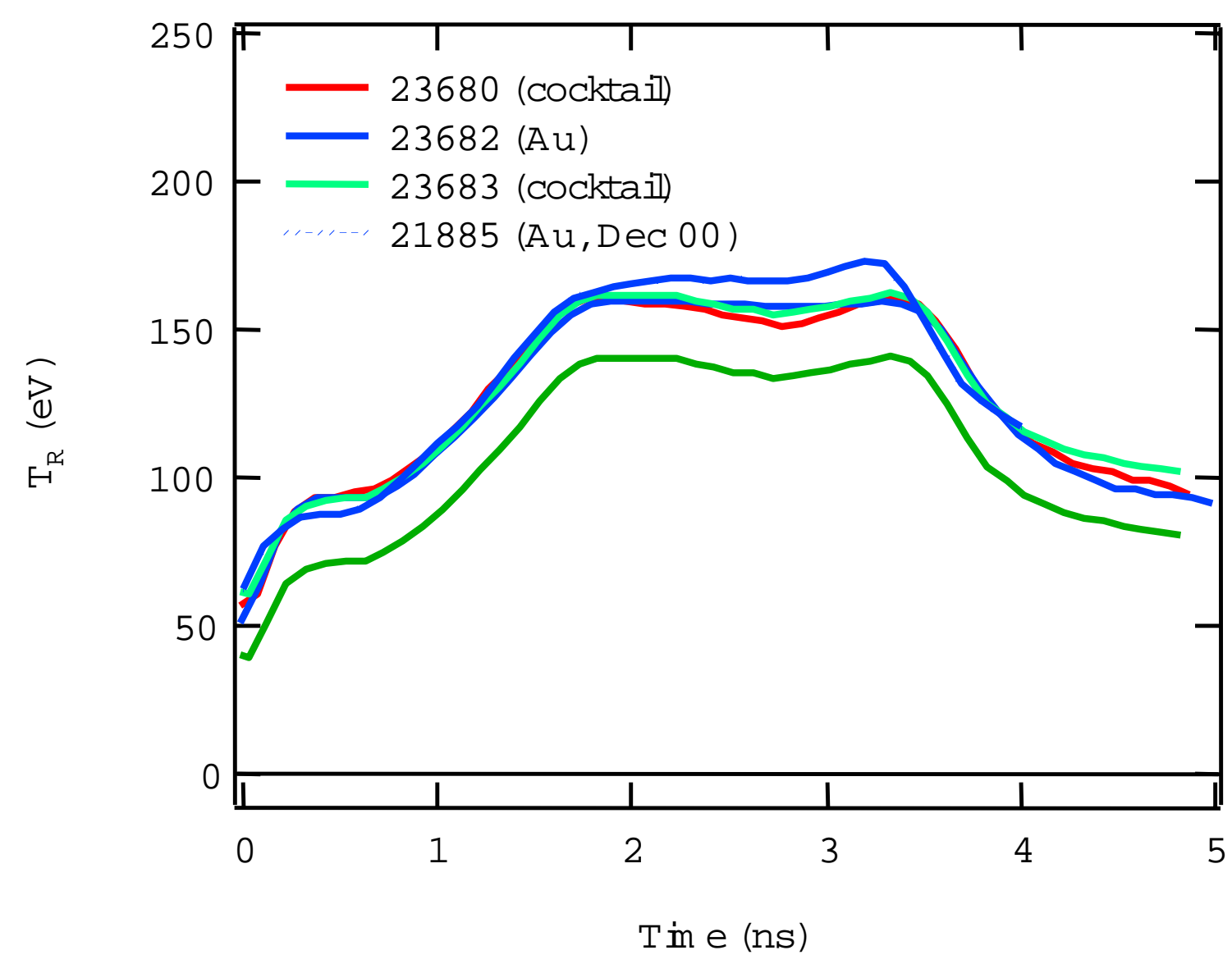

Dante curve 23683, minus $25 \mathrm{eV}$ from "typical" temperature for this series, shifted +200ps for Dante timing -- used as source for foil simulations 


\section{At 70 micron wavelength, we see good agreement between simulated and observed perturbation}

\section{growth}

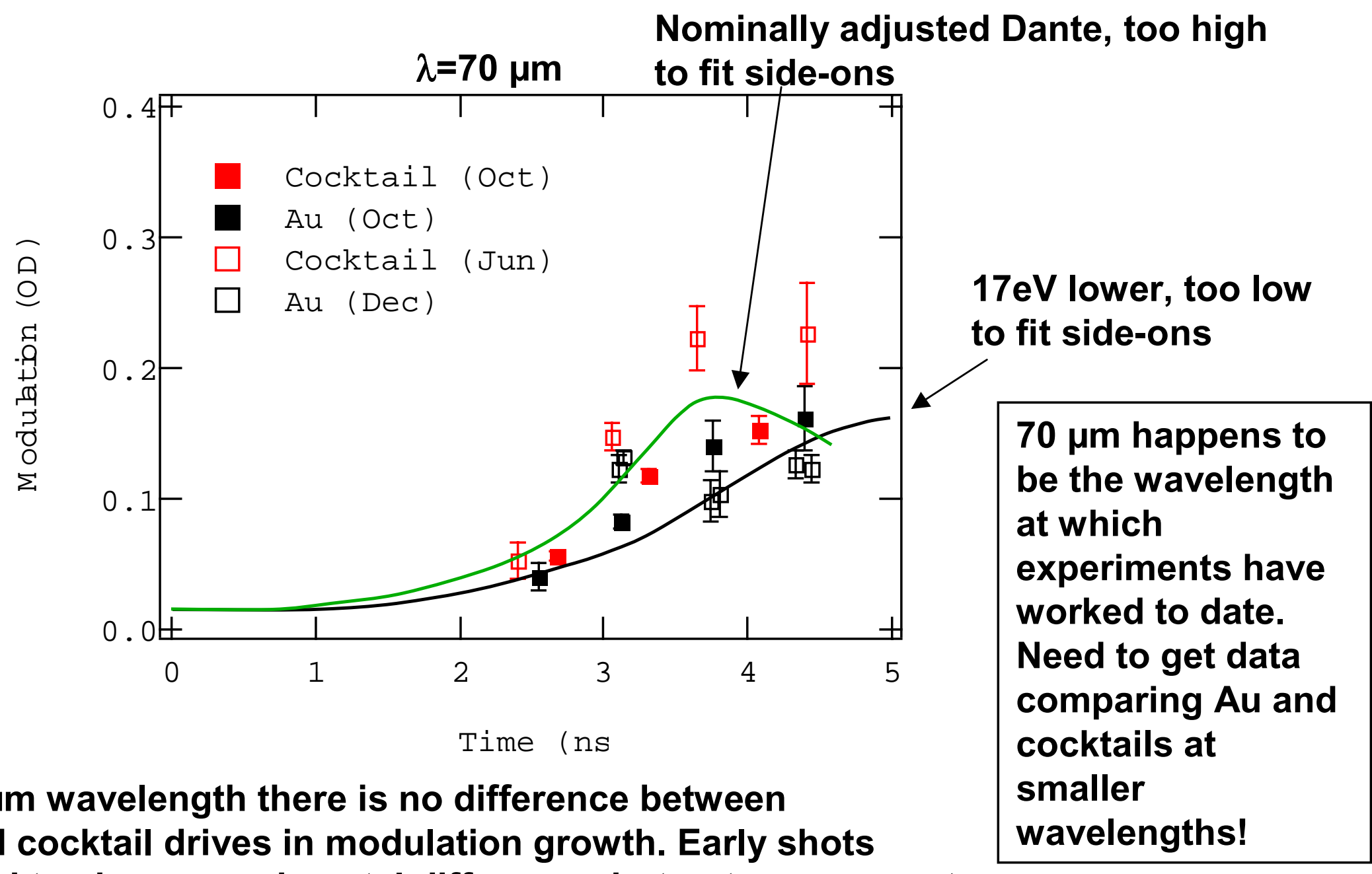

At $70 \mu \mathrm{m}$ wavelength there is no difference between Au and cocktail drives in modulation growth. Early shots seemed to show experimental difference, but not more recent data 


\section{We are also planning convergent Rayleigh-Taylor experiments with a mock fuel layer}

- On NIF capsules, perturbation ends up growing on interface between ablator and fuel, which becomes increasingly unstable as shells implode

-Converging geometry is a big part of the physics determining densities, plus something we haven't done enough with yet

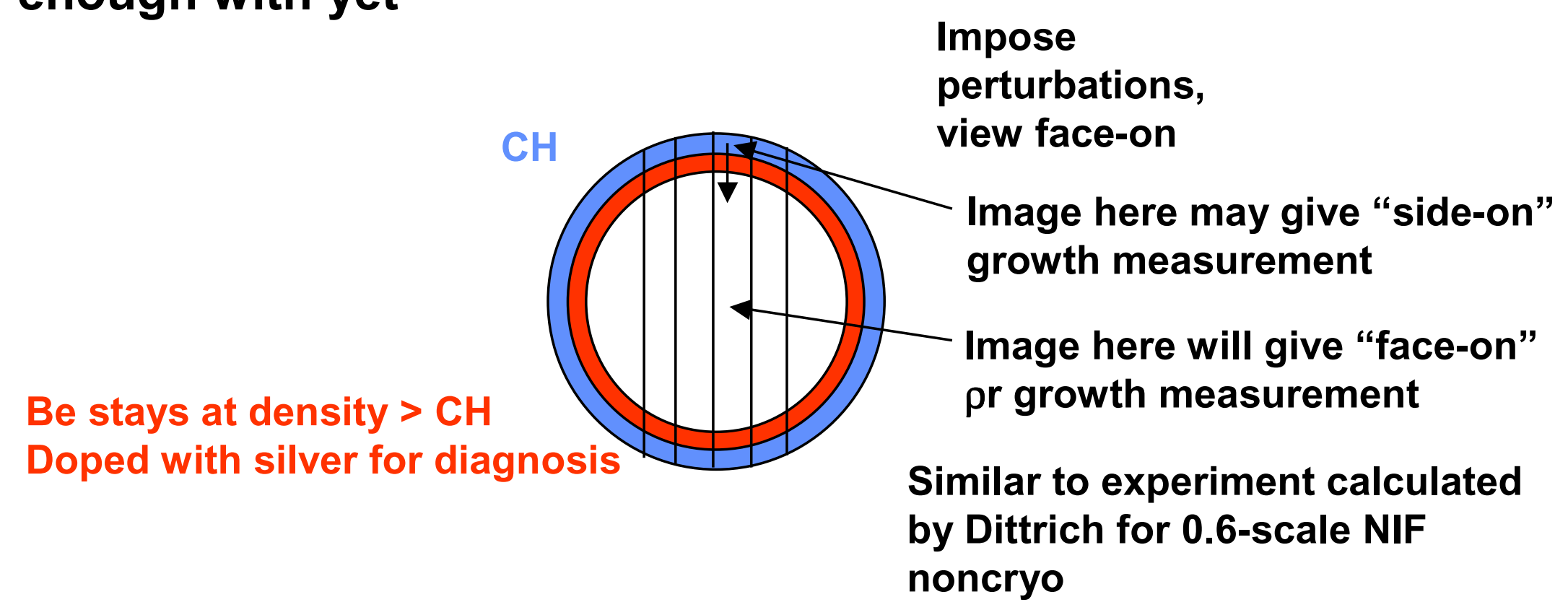




\section{With a beryllium mock fuel layer we do a decent job of mocking up the interface instability}

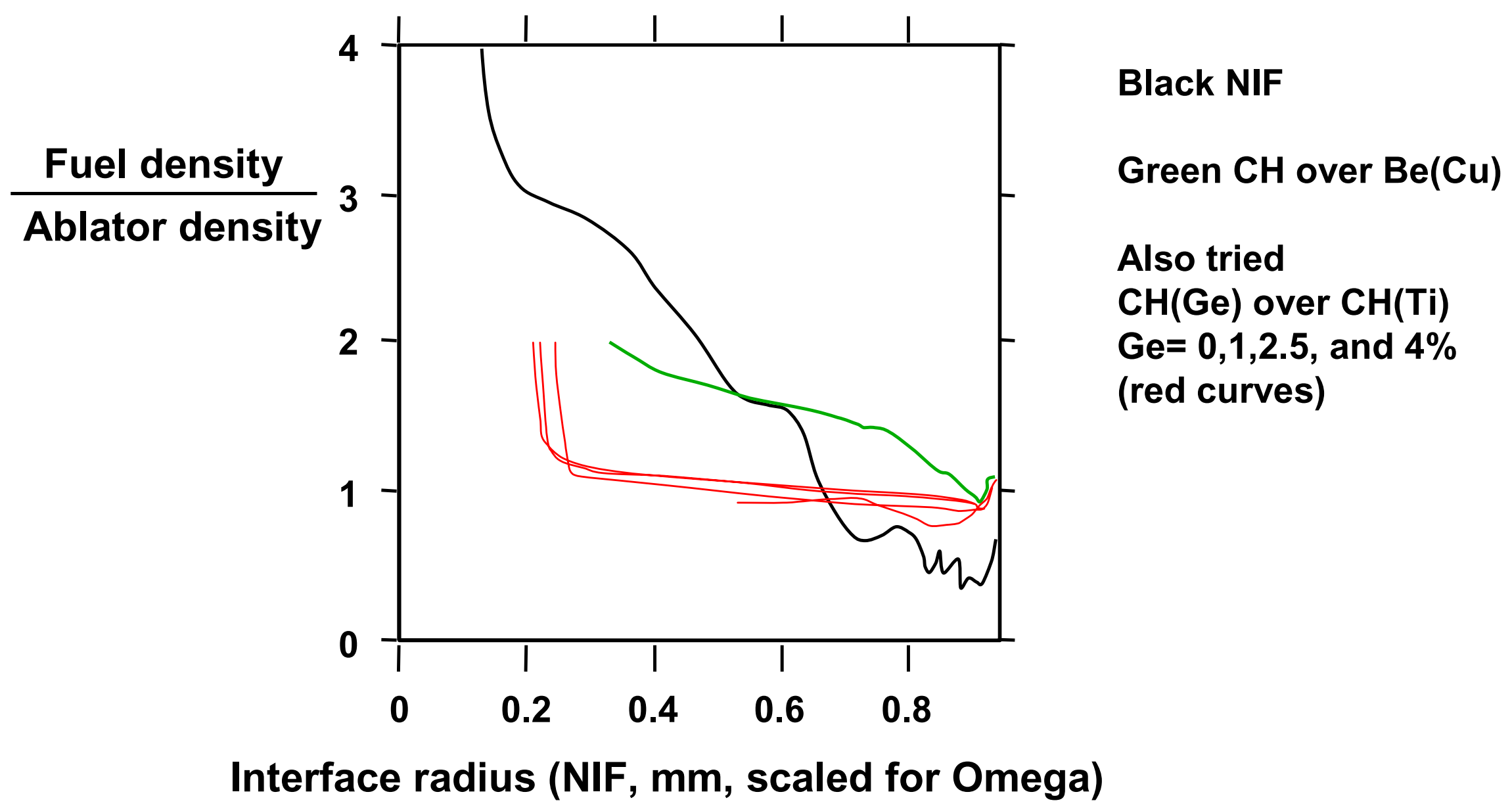




\section{We are working on optimizing this experiment}

Current thinking: ramp pulse that pushered single shell program developed, they are verifying symmetry

Capsule $210 \mu \mathrm{m}$ outer radius,

$23 \mu \mathrm{m} \mathrm{CH} / 4 \mu \mathrm{m} \mathrm{Be}+0.5 \% \mathrm{Ag} / 3 \mu \mathrm{m}$ mandrel

Gives good density profiles, and good images
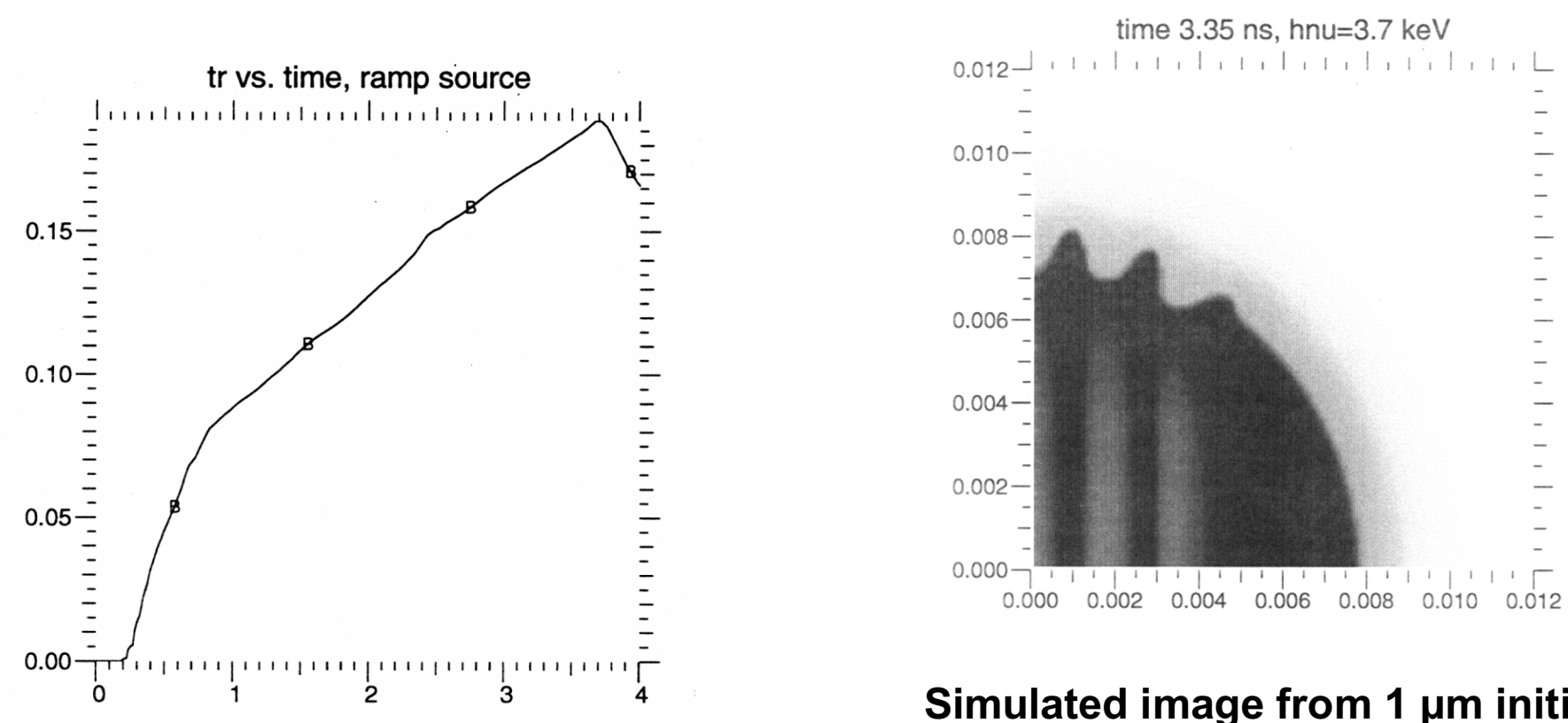

Simulated image from $1 \mu \mathrm{m}$ initial amp, $50 \mu \mathrm{m}$ initial wavelength, 2 1/2 waves at waist cut into ablator 


\section{Molecular Dynamics Simulation of Shocks and Richtmyer-Meshkov Instability in Cylindrical Geometry}

angular velocity at $t=30$

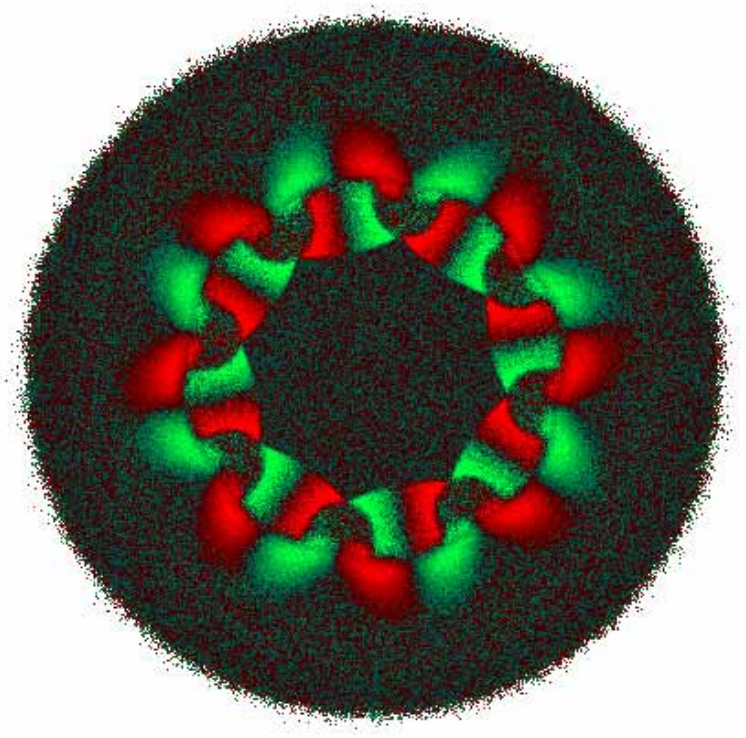

density at $t=100$

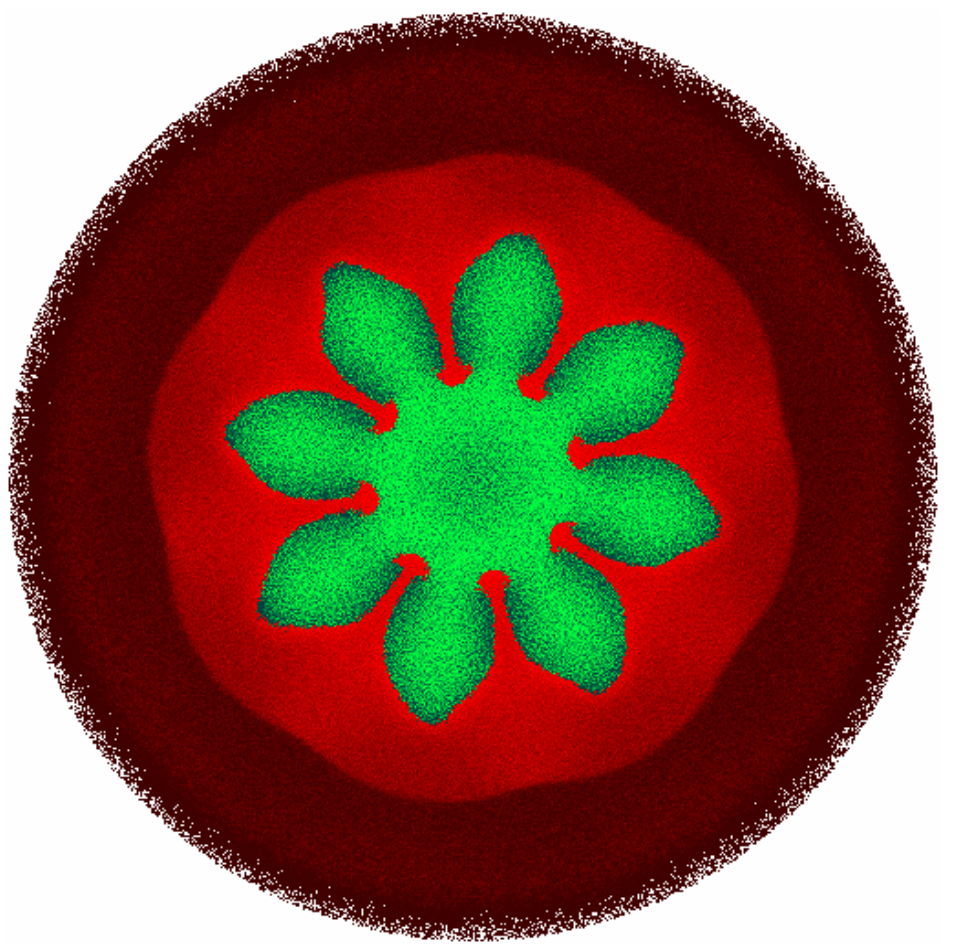

18 IWPCTM,

December 10-14, 2001 Pasadena, USA 
Introduction

Difficulty in hydrodynamic simulation in converging geometry

-Singularity at the center

- Numerical instability and viscosity depend on the grid structure

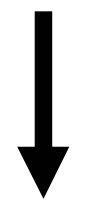

Molecular Dynamic Simulation

Advantage

spatial mesh and EOS are not required non LTE system can be treated viscosity, heat conduction are automatically taken into account conservation laws are automatically satisfied

Disadvantage

system size limited by computer power limited temperature 
Today's hydrodynamic codes looks well but they are still inaccurate

RM gas curtain experiments and simulations

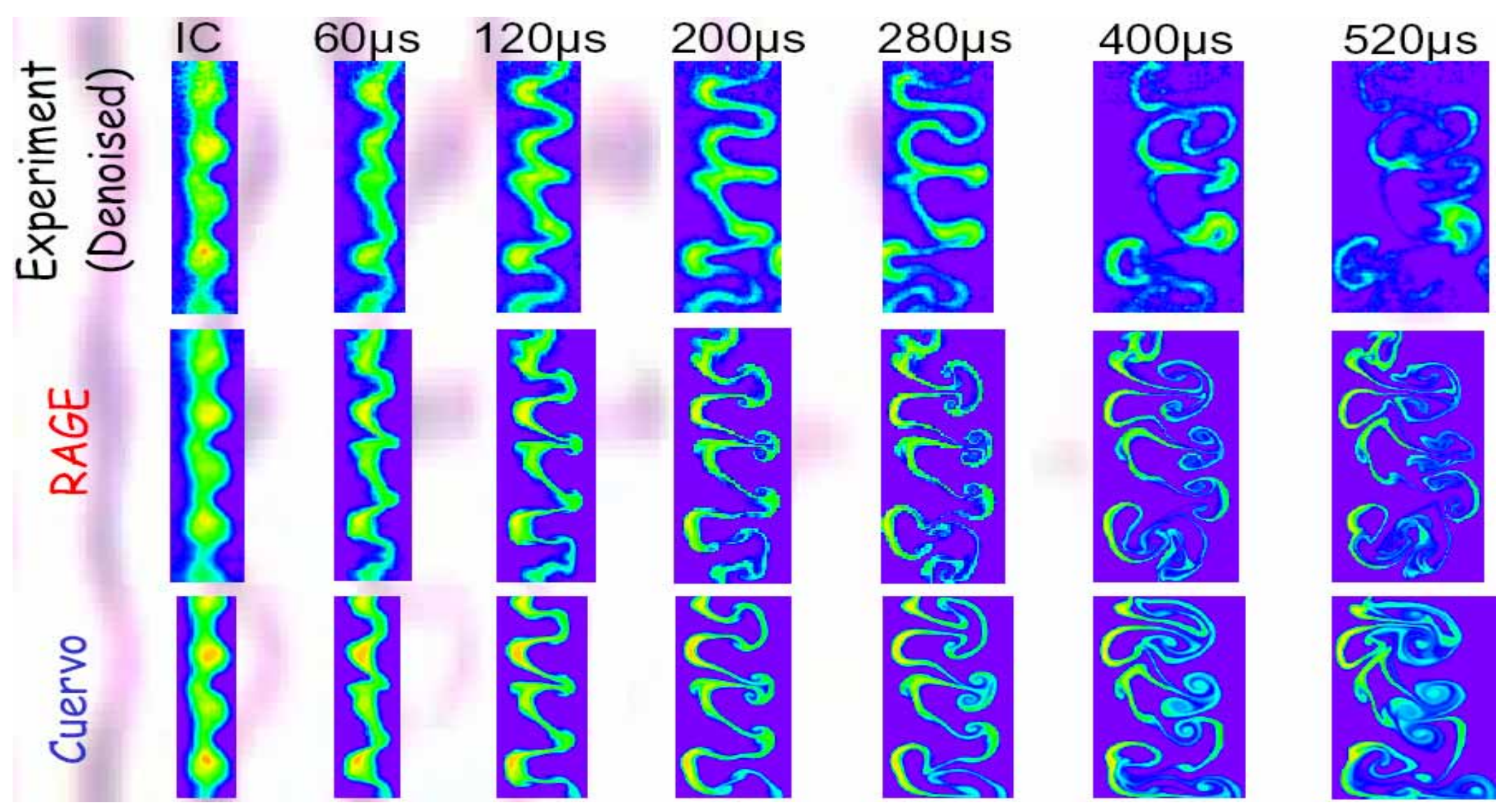

Pictures from W.Rider and J.Kamm, LANL 
executive summary

- MD simulation provides a new tool for a study of hydrodynamic instabilities, when CFD fails.

- Mode dependence of nonlinear growth is investigated for cylindrical RMI. Higher mode decays slower.

- Growth of mixing zone in cylindrical RMI is much larger than that in planar RMT (shocks hit interface repeatedly).

- In a shell case (two interfaces), many higher modes appear on its inner surface, and thickness of mixing zone is thinner than that on single interface. 


\section{Outline of Talk}

- Introduction of Molecular Dynamic simulation

- Shock wave in cylindrical geometry structure and stability of rippled front

- Richtmyer-Meshkov instability in cylindrical geometry nonlinear dynamics of RMI dependence of nonlinear growth on mode number comparison with planar geometry shell structure (animation) 


\section{Molecular dynamics simulation technique}

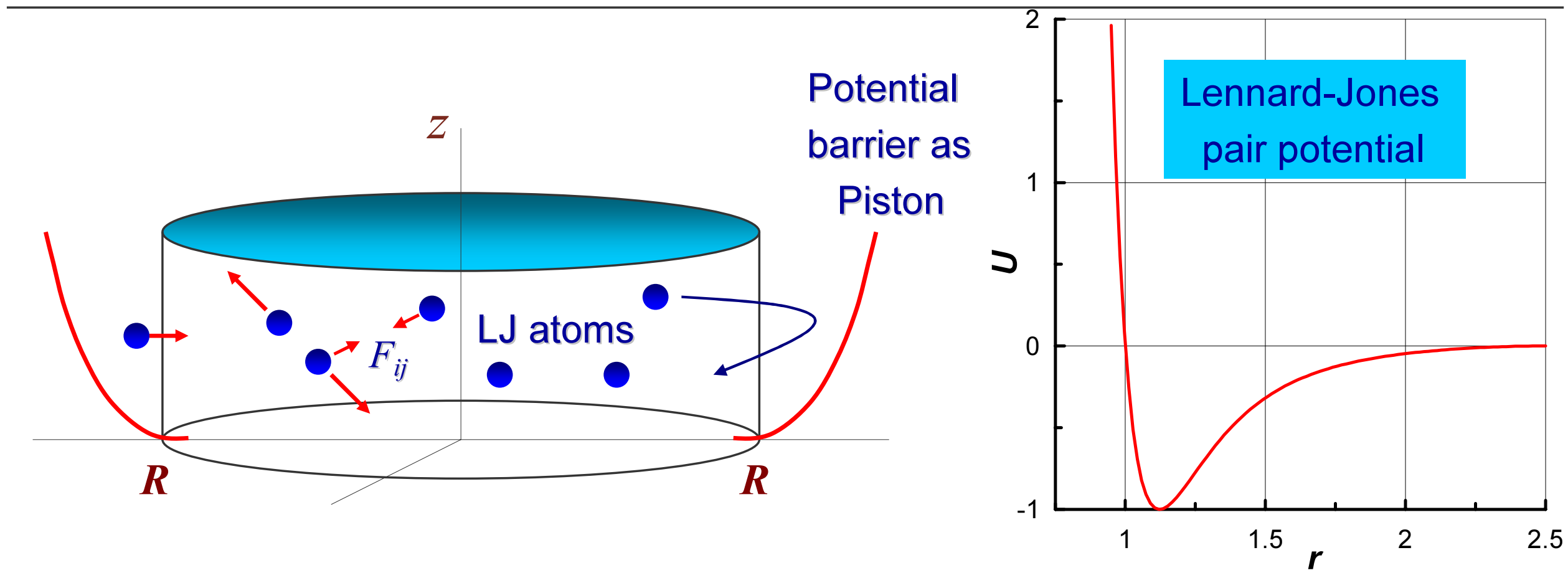

- Periodical boundary conditions are imposed on the system along z-axis

- The atoms interact via Lennard-Jones (LJ) pair potential with cutoff at $r_{c}$

$$
\phi_{L J}(r)=4 \varepsilon\left[(\sigma / r)^{12}-(\sigma / r)^{6}\right] \quad \text { if } \quad 0<r<r_{c}=2.5 \sigma
$$

- The piston is simulated by an external potential $\sim\left[\boldsymbol{r}_{i}-\boldsymbol{R}\left(\phi_{i}, t\right)\right]^{2}$ and its position may depend on angles in case of perturbed boundary as well as on time to generate shock waves. 


\section{Shock $\mathrm{R}-\mathrm{H}$ relations are satisfied within accuracy of $10^{-5}$}

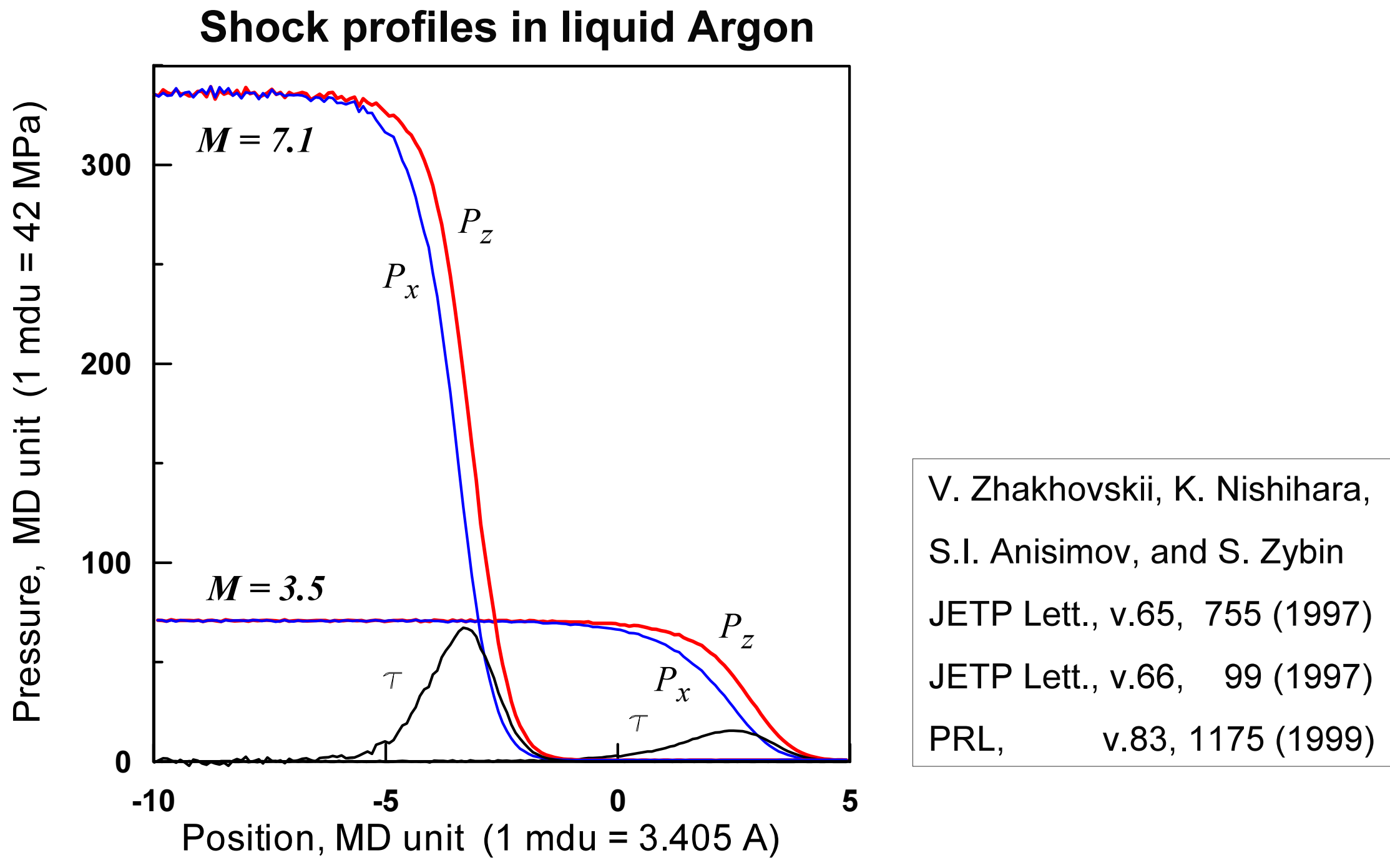

Shock front thickness is enough thin compared with perturbation amplitude 


\section{Molecular dynamics simulation technique}

Typical snapshots of MD systems at the beginning of compression

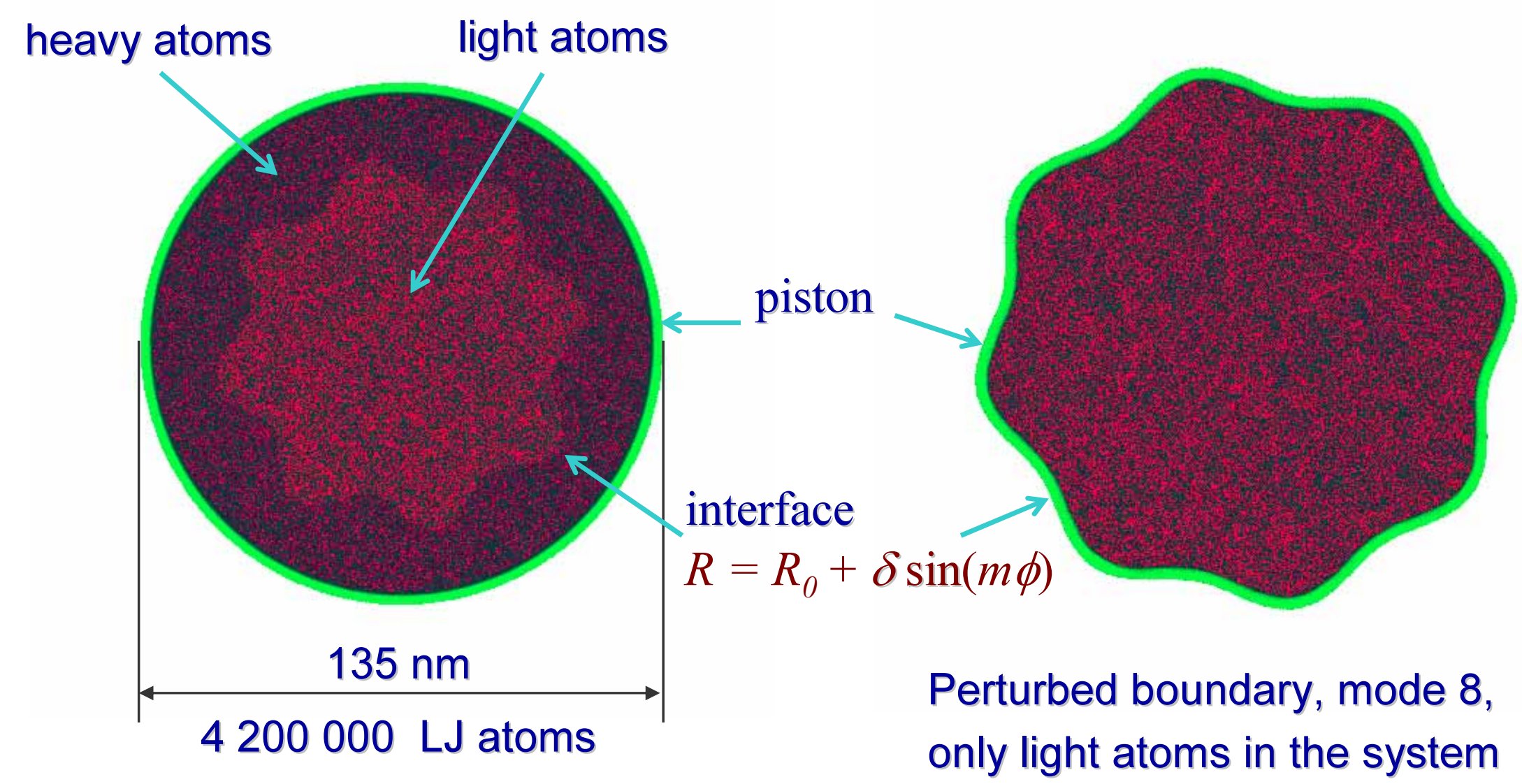

- Each pixel represents a small domain, which is occupied approximately 30 atoms

- Green colors correspond negative radial velocity of a domain, red colors mean positive velocity. Brightness is proportional to absolute values and balances according maximal amplitude. 


\section{MD simulation of perturbed shock front, of which ripples oscillate in time.}

First generation of Mach stems appears at very early stage of SW converging Brightness is proportional to density.

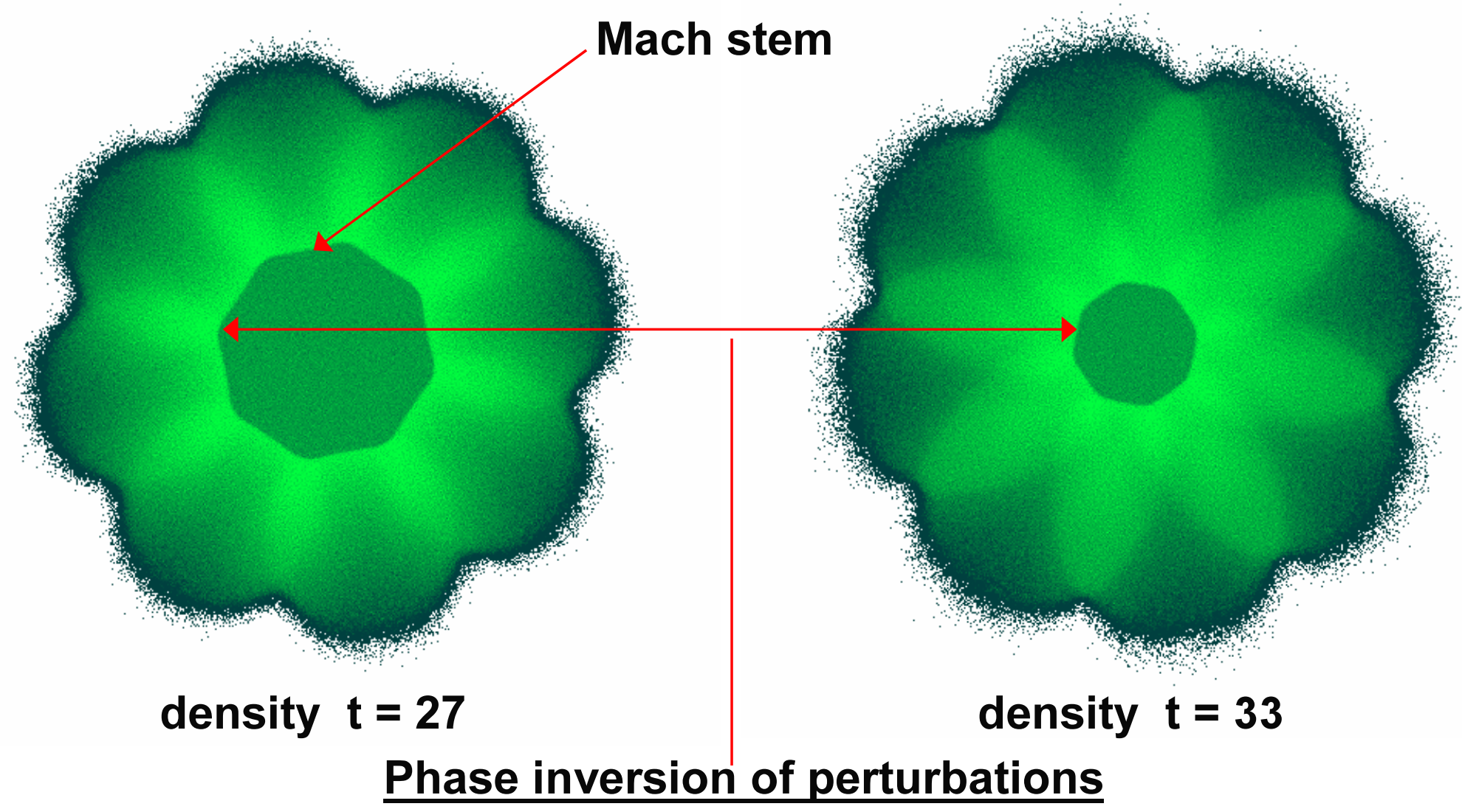

instead of circle the polygons ( 8 or 16 sides for mode 8 ) converge to center 
Converging shock wave is unstable for low mode number
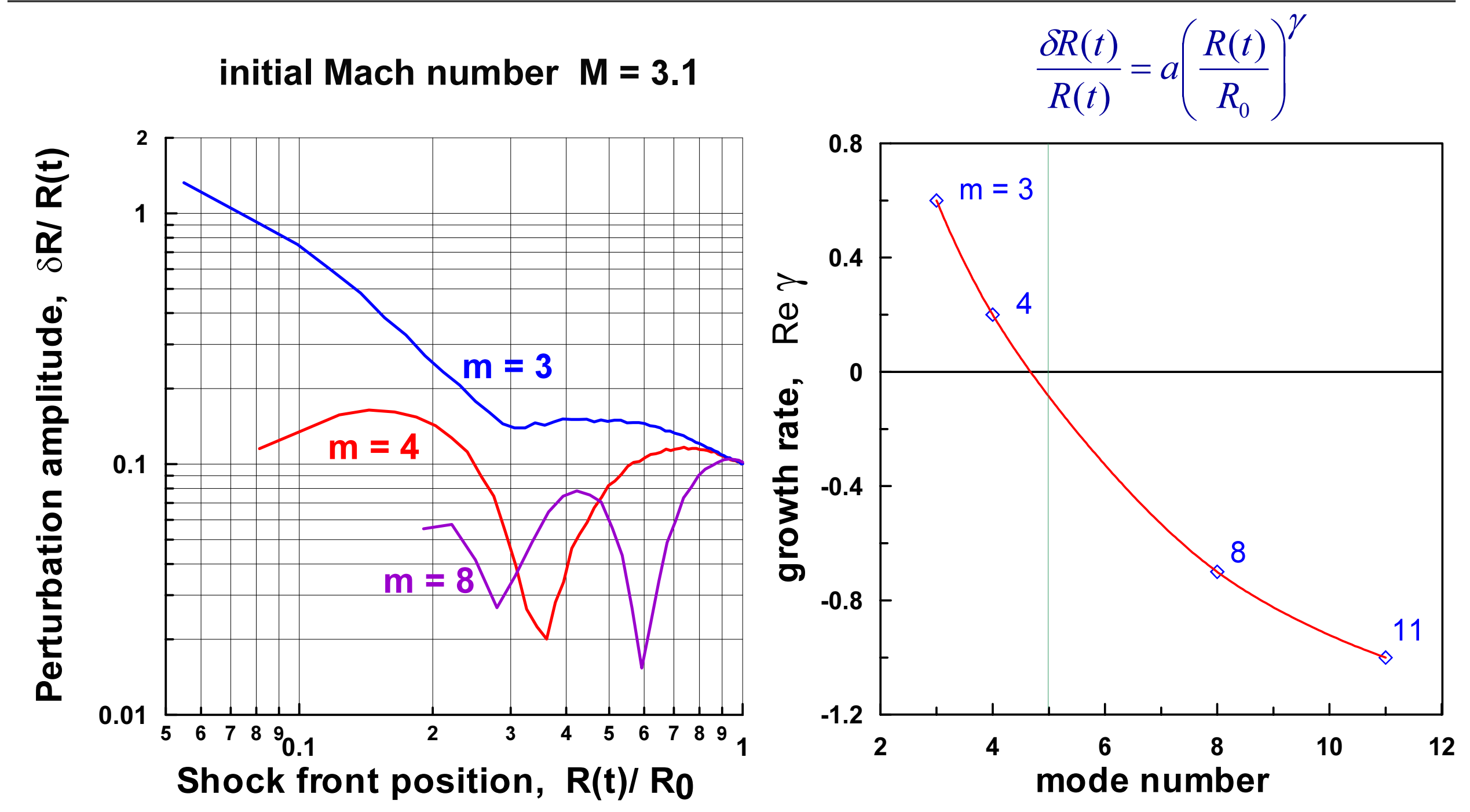

Perturbations grows if the mode number $m<5$ 


\section{MD simulation of RM instability}

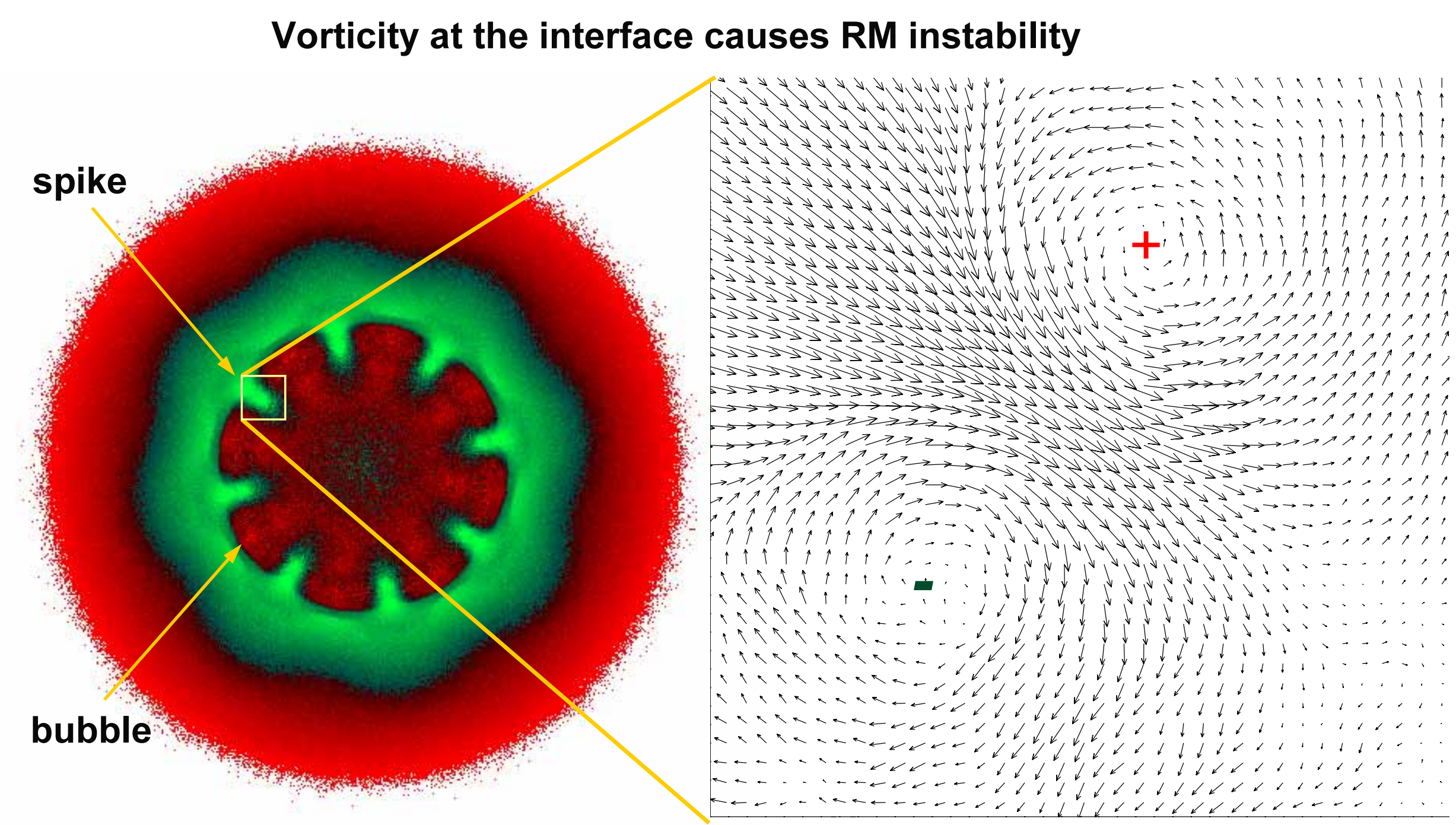

Radial velocity map,

Velocity field, each arrow indicates green means negative value (to origin) average velocity of about 100 atoms 


\section{Richtmyer-Meshkov instability in cylindrical geometry}

shock passing interface at $\underline{\mathrm{t}=20}$

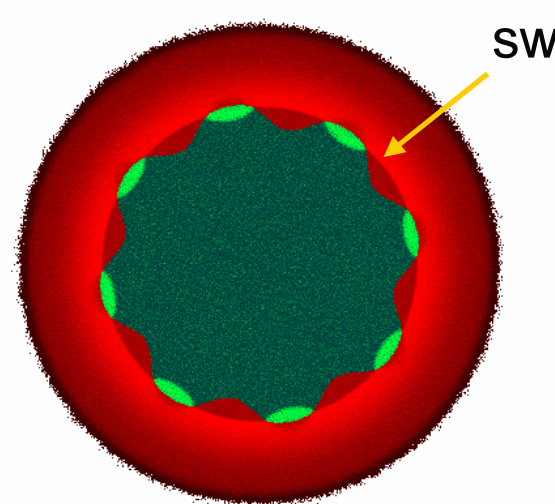

number density
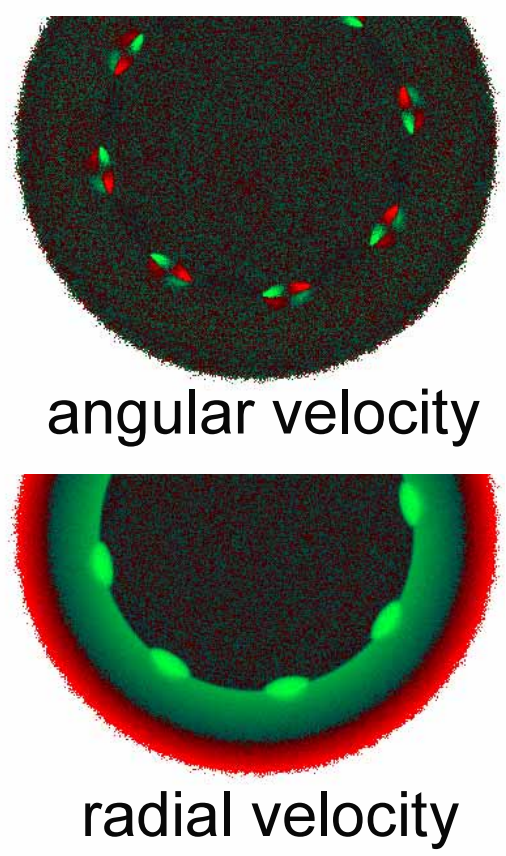

converging stage

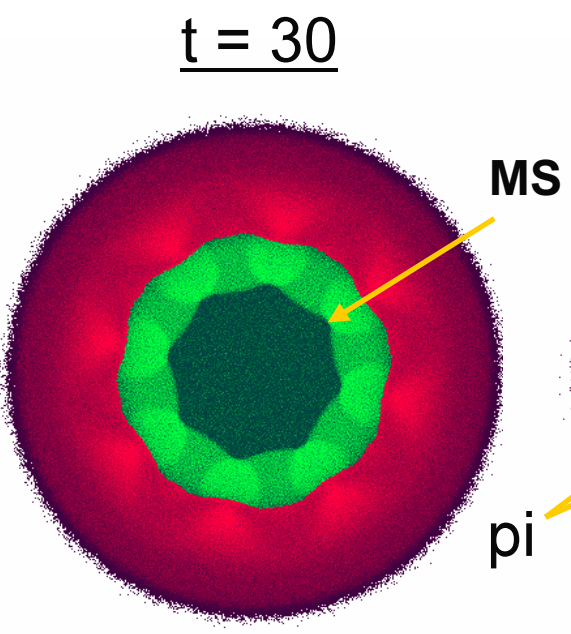

MS

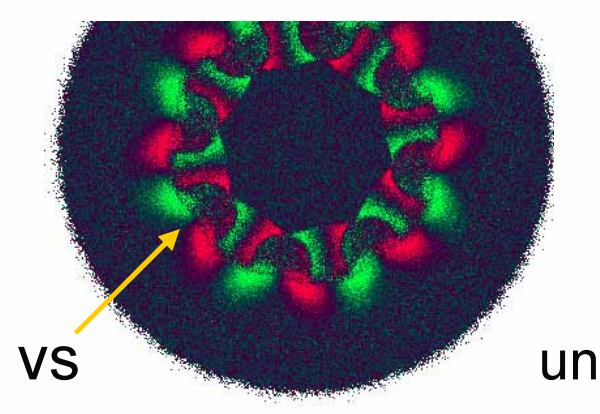

un

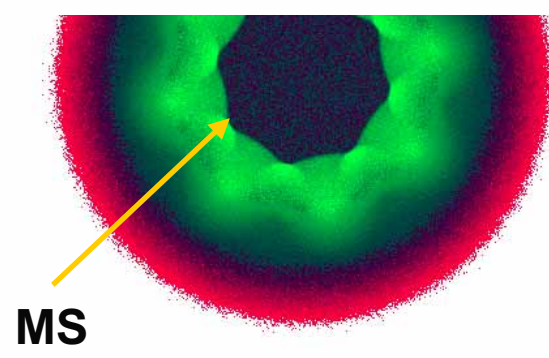

reflected stage

$\underline{t}=40$

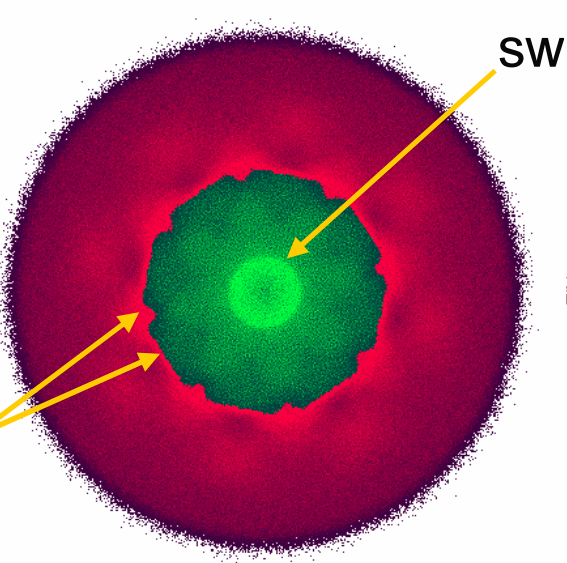

bubbles/spikes $\underline{t=100}$
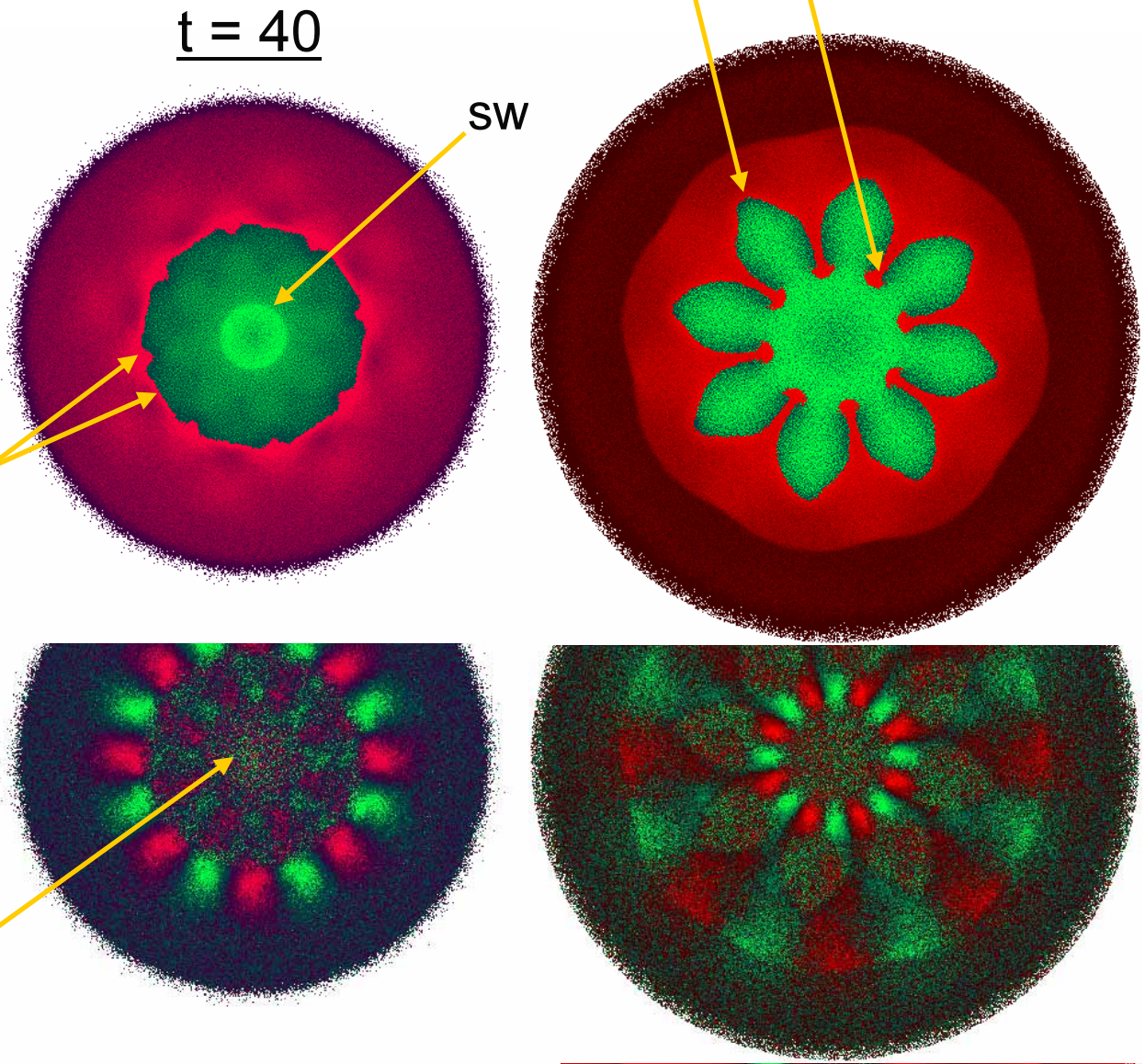

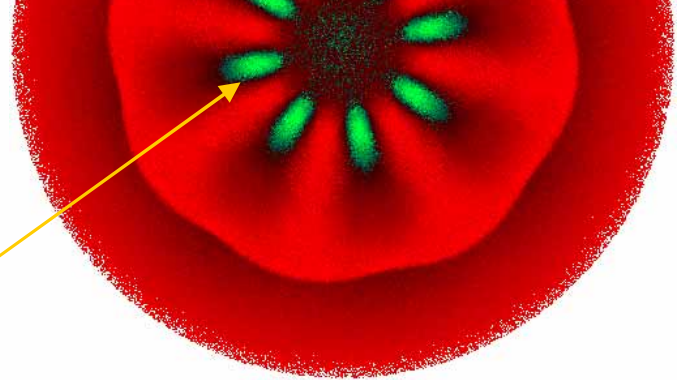




\section{Molecular dynamics simulations show RM growth driven by multiple shocks for different mode numbers.}

Decay of nonlinear growth is mode dependent and higher mode decays slower
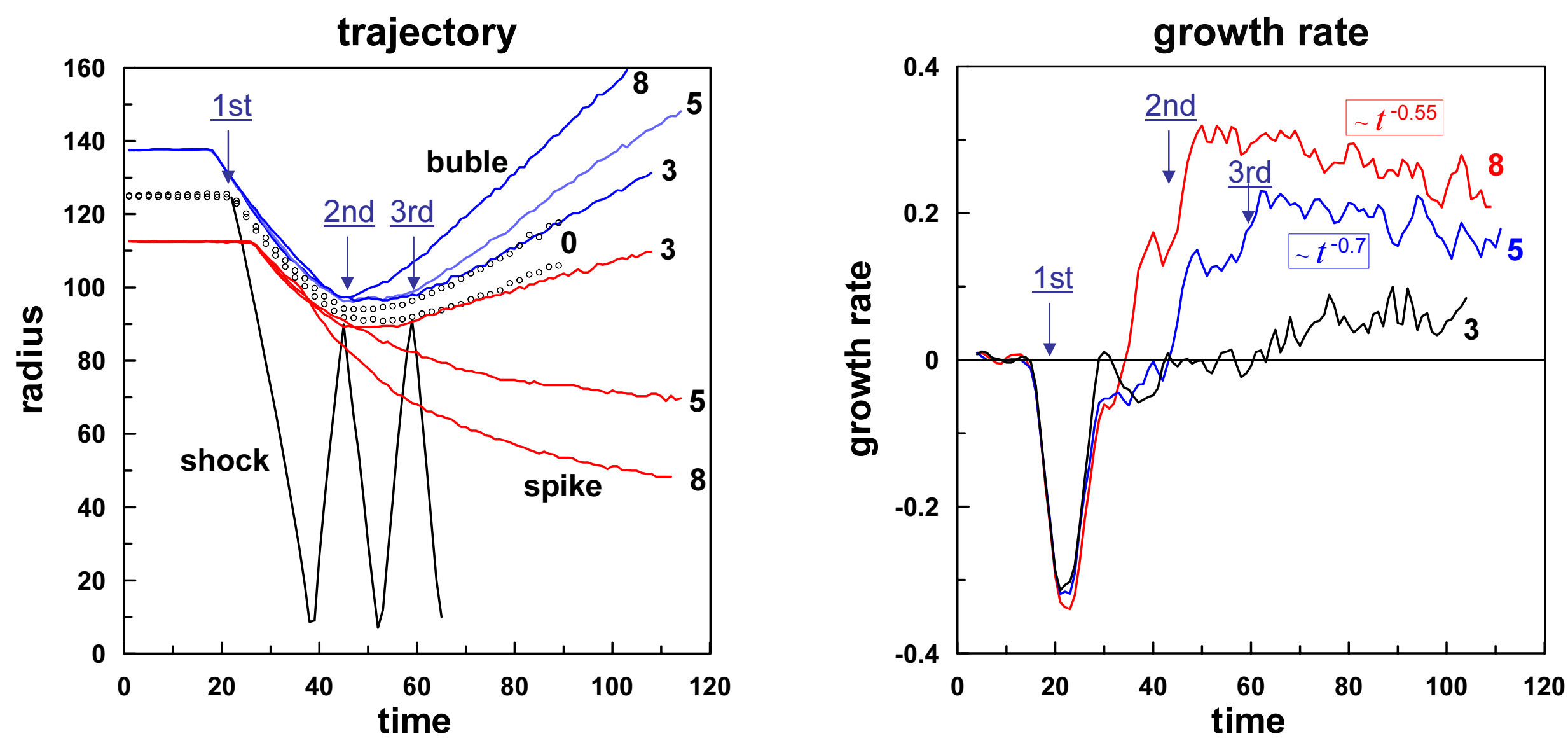
Richtmyer-Meshkov instability in planar geometry

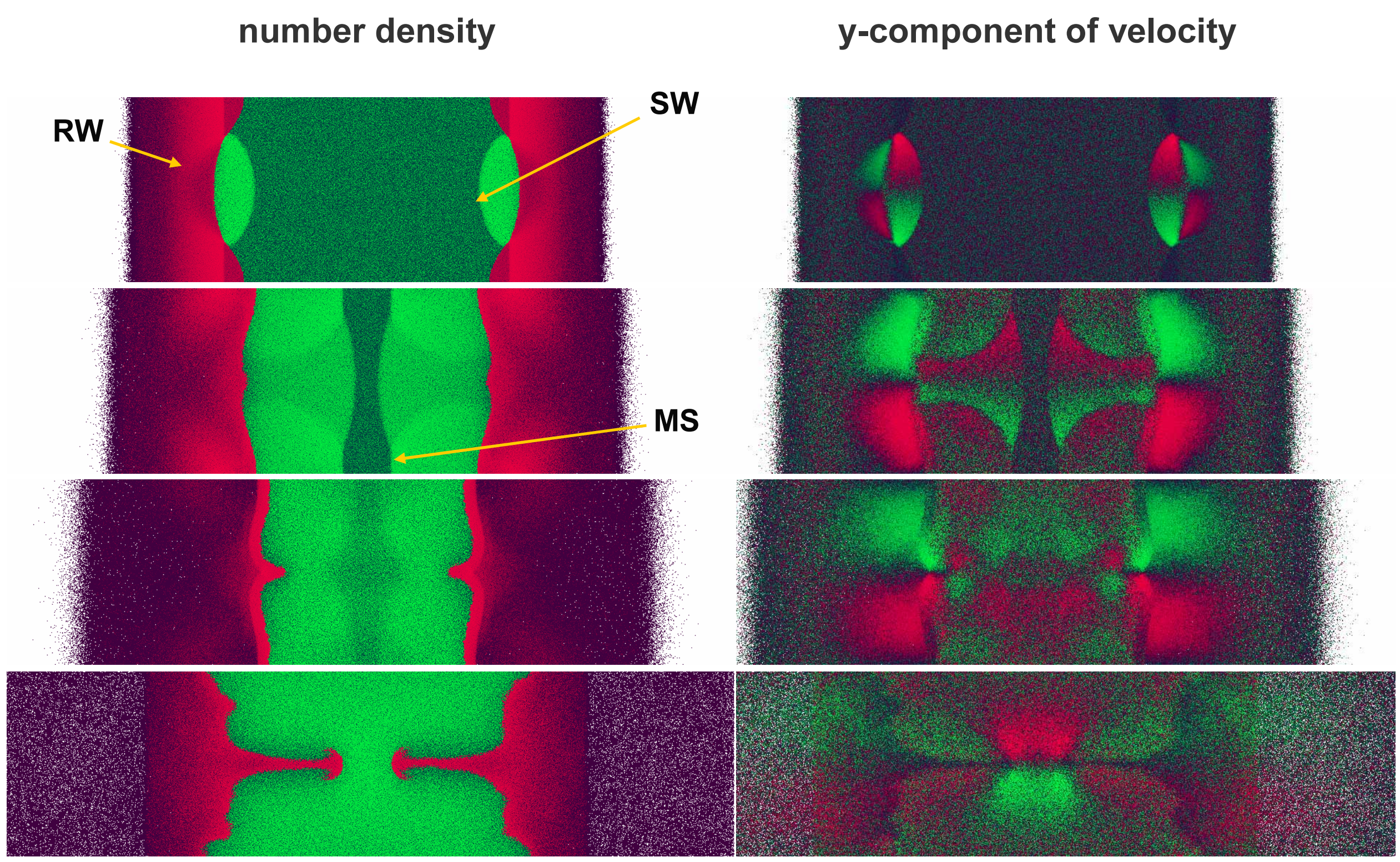


Growth of mixing zone in cylindrical RMI is much larger than in planar RMI

In both cases shocks hit interface repeatedly and growth rate increases
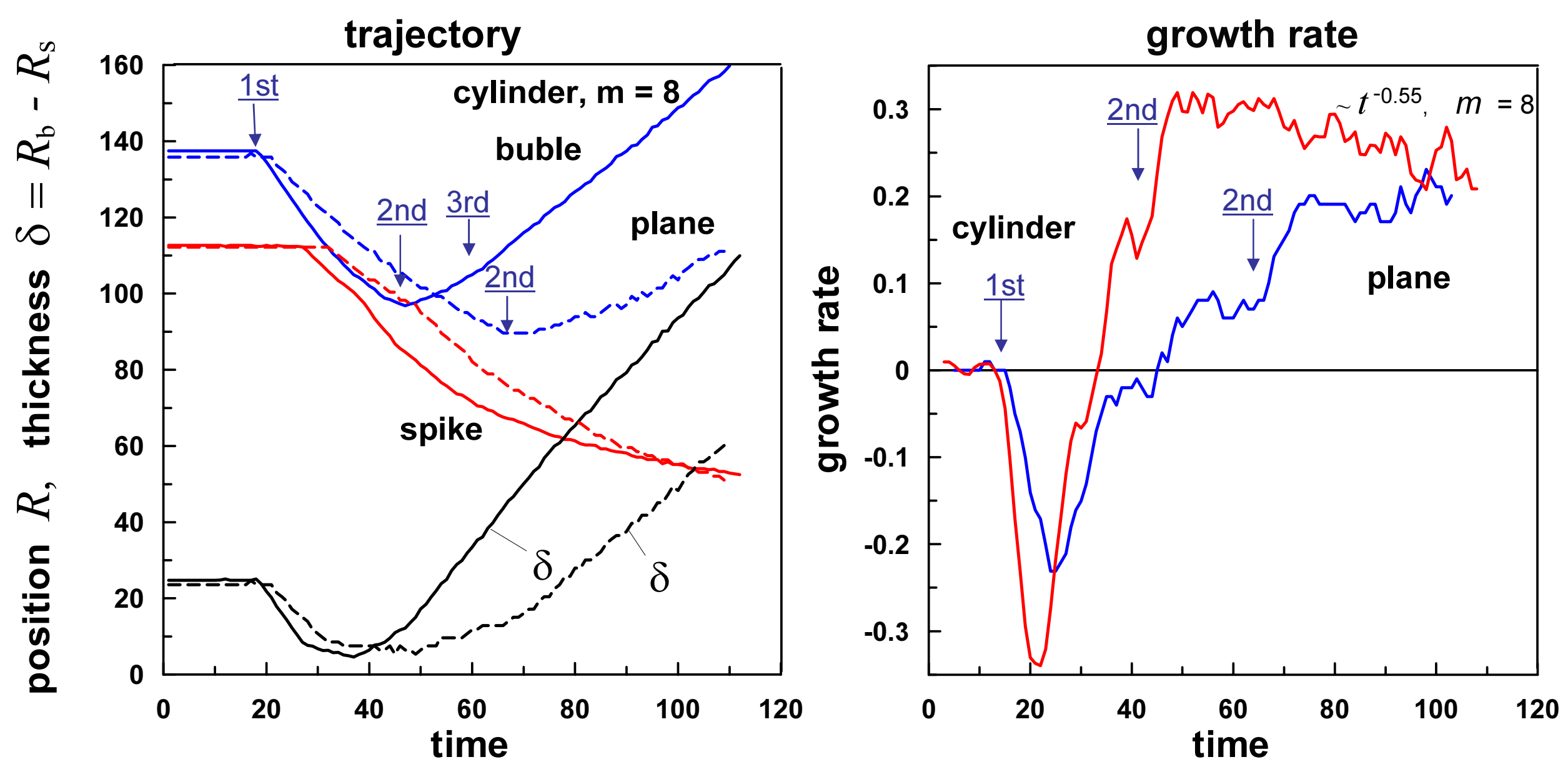


\section{Richtmyer-Meshkov instability at shell surfaces}

Many higher mode appear on inner surface.

Thickness of mixing zone is thinner than that on single interface.

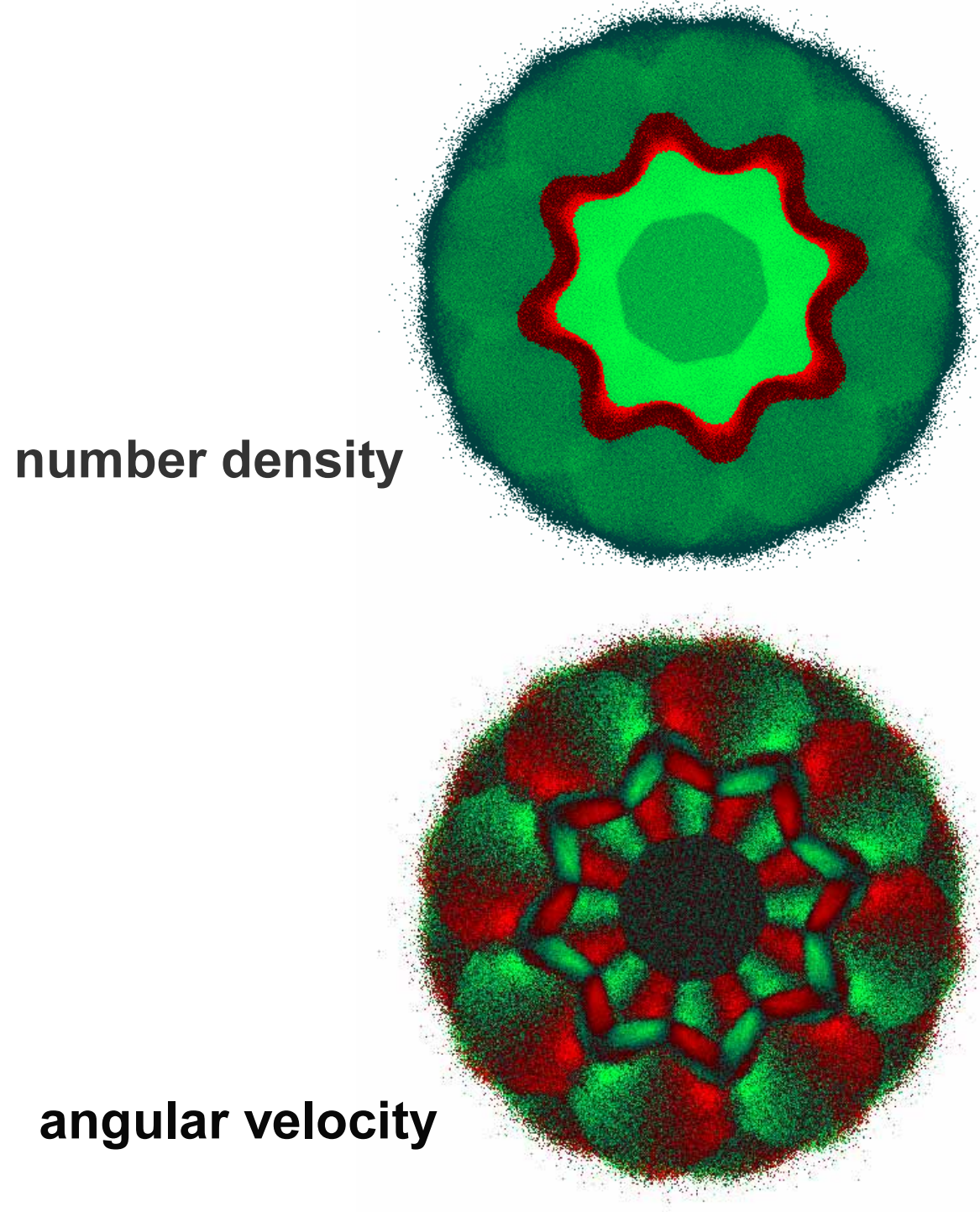

number density of heavy fluid

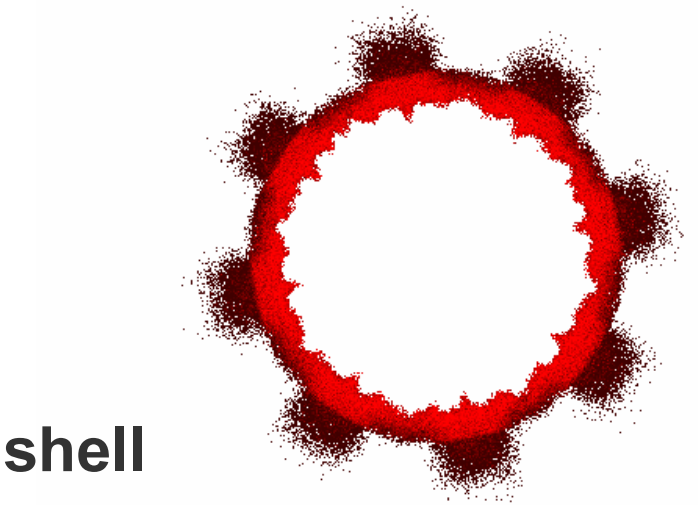

single interface

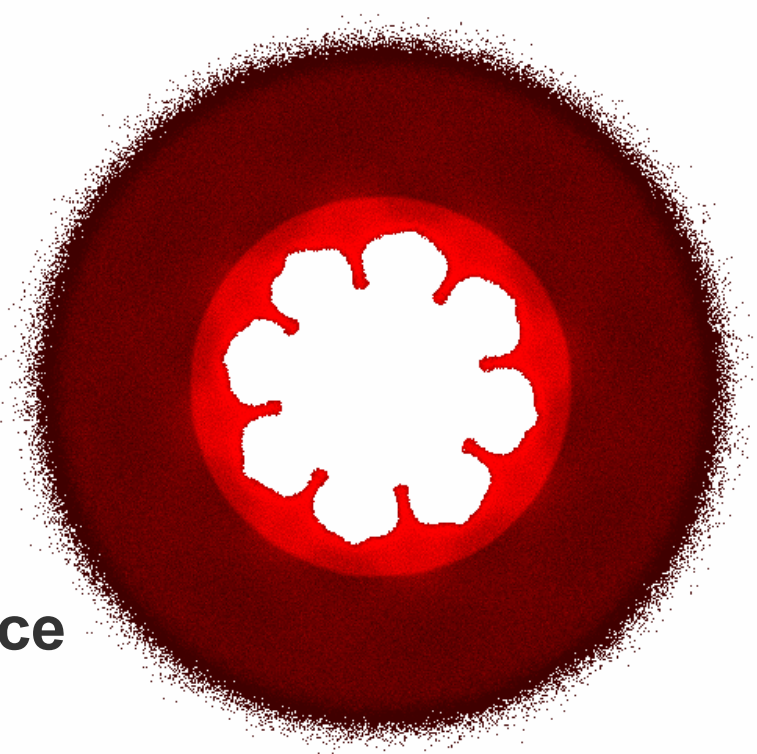




\section{Conclusion}

- MD simulation provides a new tool for a study of hydrodynamic instabilities, when CFD fails.

- Mode dependence of nonlinear growth is investigated for cylindrical RMI. Higher mode decays slower.

- Growth of mixing zone in cylindrical RMI is much larger than that in planar RMT (shocks hit interface repeatedly).

- In a shell case (two interfaces), many higher modes appear on its inner surface, and thickness of mixing zone is thinner than that on single interface.

- www.ile.osaka-u.ac.jp/research/TSI/Vasilii 
The qas curtain setup (1997)
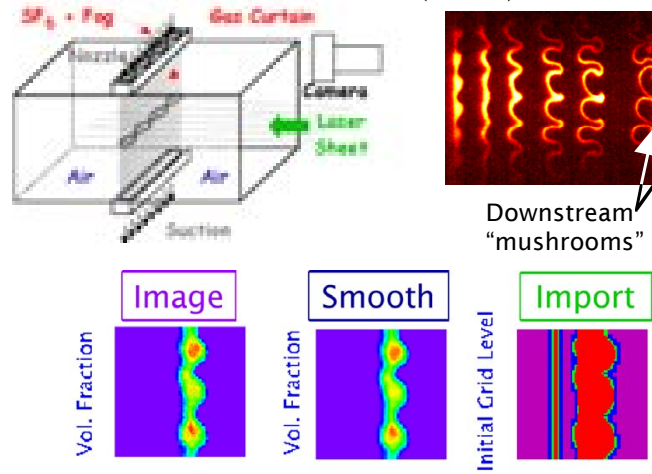

IC 6qus 12aus 20aus 28ăs 40qus 52qus

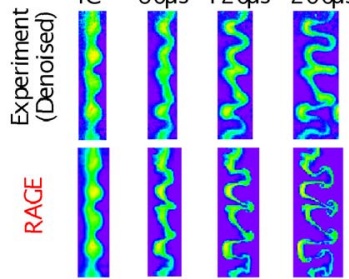

The
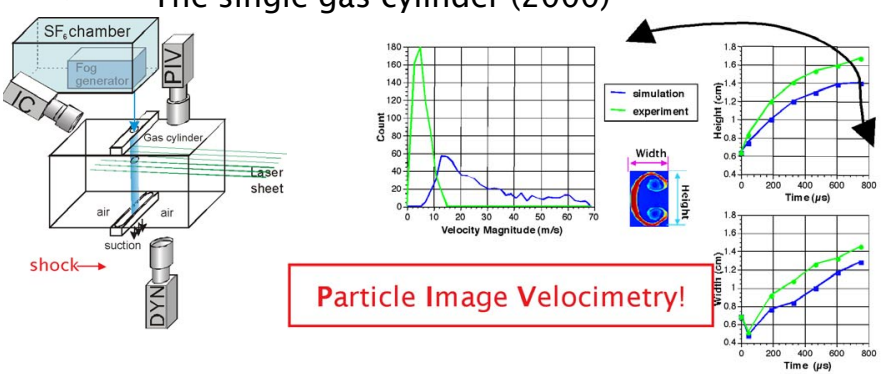

The double gas cylinder (2001)
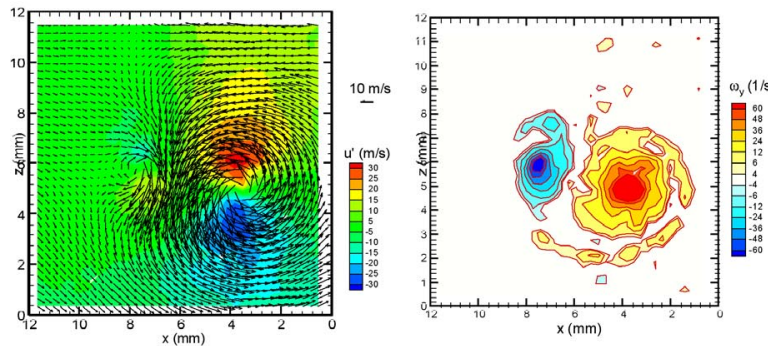

\section{Double Cylinder Experiment ICs}

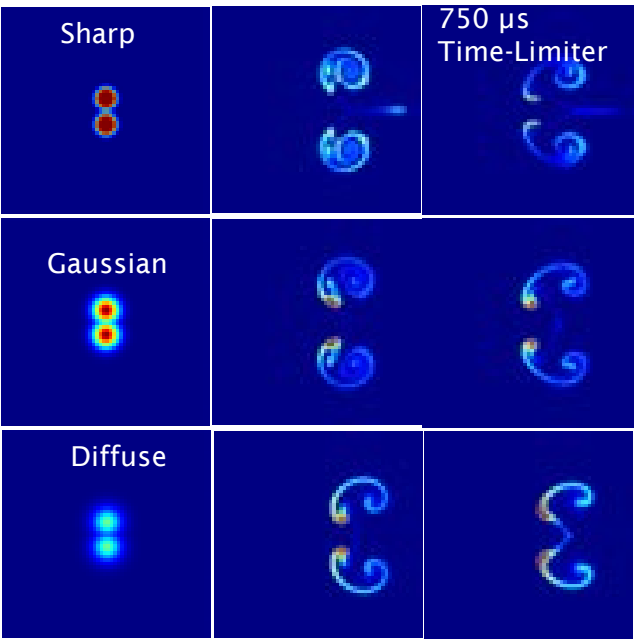

The initial conditions have not been imaged for the double cylinder. Here, we test various initial conditions with two schemes, the standard method and a modified method. With a sharper IC and the modified scheme, the most consistent results are found.

Initial PIV shows 5:1 difference in velocities between the experiment and calculation.

The integral scales also do not match!

New PIV shows 5:3difference in velocities between the experiment and calculation. The air and SF6 have both been seeded giving the complete velocity field.

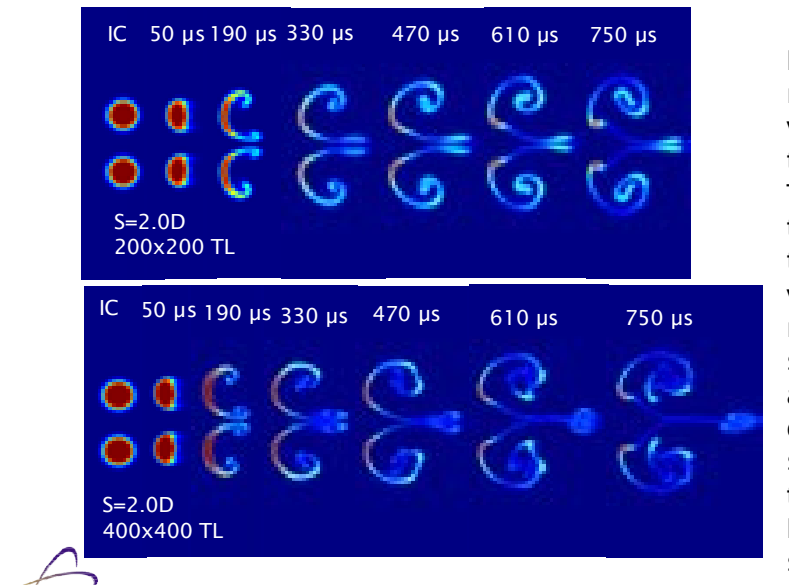

In general the results are not as variable with $\mathrm{S}$ as the experiments. This related to the strength of the inner vortices. The TL method does show less jetting and greater qualitative similarity than the standard MUSCL type scheme.
Double Cylinder Experiments

The behavior of the cylinders shows considerable variation with spacing with a spacing of $1.5 \mathrm{D}$ being a critical value in the behavior.

$\mathrm{C} \overbrace{}^{50 \mu \mathrm{s}} \overbrace{}^{190 \mu \mathrm{s}} \overbrace{}^{330 \mu \mathrm{s}} \overbrace{}^{470 \mu \mathrm{s}}$ $\mathrm{S}=1.2 \mathrm{D}$

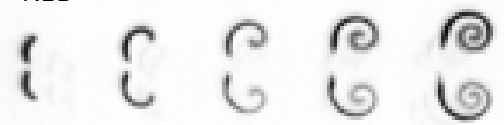
$S=1.4 \mathrm{D}$
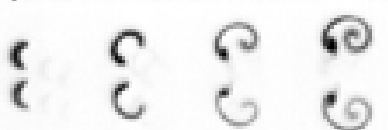

$S=1.6 \mathrm{D}$

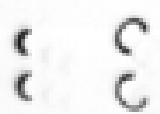

$\mathrm{S}=1.8 \mathrm{D}$

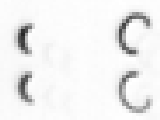

$S=2.0 \mathrm{D}$

\section{Double Cylinder Simulations}

The simulations do not show the experimental variability
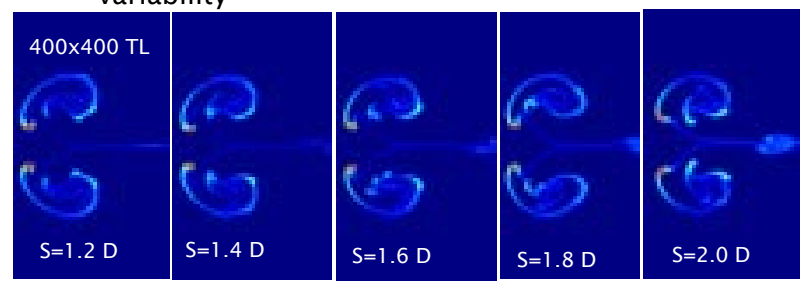

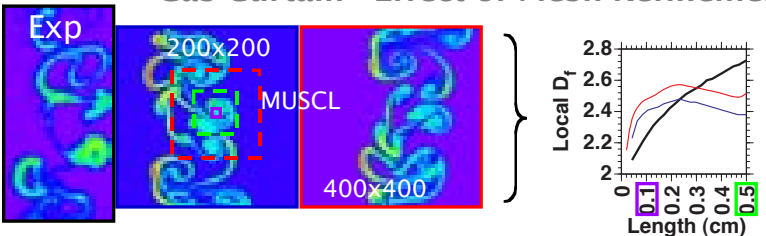

TVD Scheme (Lax\&Litu) Results
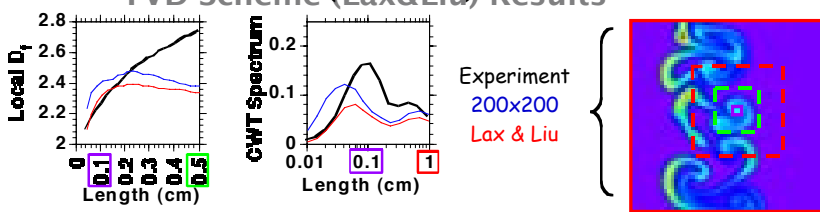

All standard modern schemes tested (MUSCL, WENO, PPM,

TVD, ...showed similar results. All results with these schemes were not consistent with the scaling exhibited by the experimental data. Mesh refinement does not provide improved results, but first-order methods show more consistent scaling (below). We also show a modified method (time-limiter) that has second-order accuracy while providing scaling consistent with the experimental data.

Ideal| Gas Gylinder - 750 $\mathrm{\mu S}$

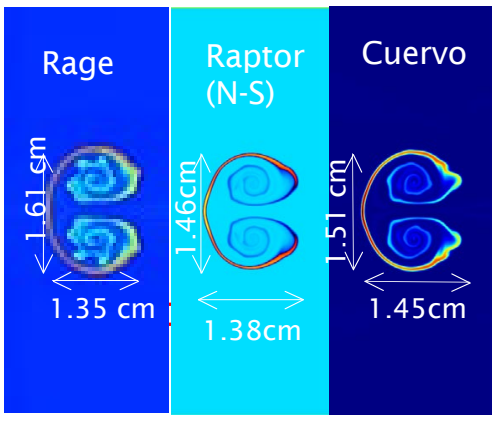

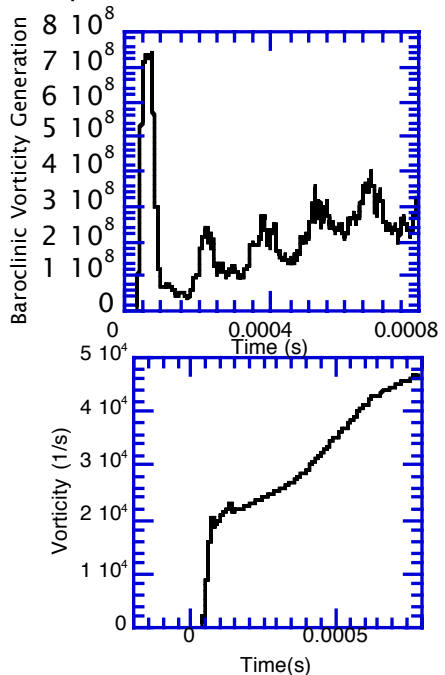

Experimentall \& Ideal Ey/inder Analysiss ?

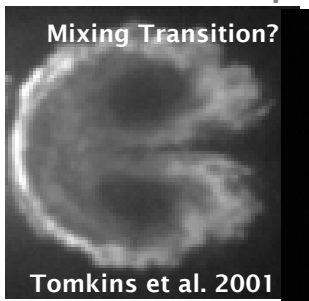

$\tau=9.4, \operatorname{Re} \approx 24,000$

$U=10^{-2} \mathrm{~cm} / \mu \mathrm{s}$

$\mathrm{D}=0.794 \mathrm{~cm}$

$\mathrm{t}=750 \mu \mathrm{s}$

2.8

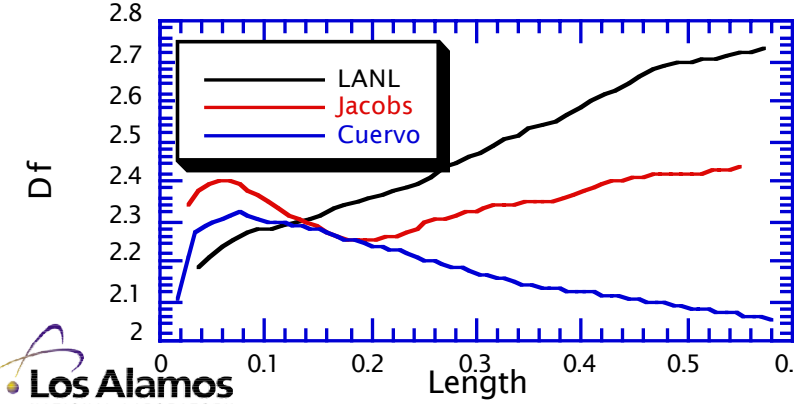

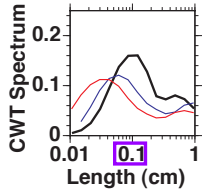
. 


\section{Large Eddy Simulation of Strong-shock Richtmyer- Meshkov Instability}

R. Samtaney
D. I. Pulin
T. Voelkl
D. J. Hill

Graduate Aeronautical Laboratories

Caltech
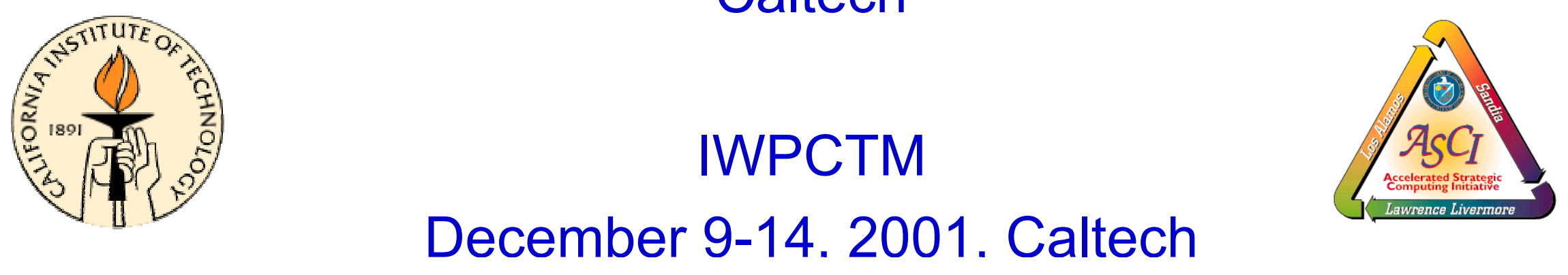


\section{Acknowledgement}

- ASCI Compressible Turbulence Group

- P. E. Dimotakis

- A. Leonard

- D. Meiron

- B. Kosovic

- ASCI/ASAP subcontract no. B341492 of DOE contract W-7405-ENG-48.

- Computational resources: LLNL Blue Pacific, LANL nirvana. 


\section{Outline}

- Objectives and physical problem setup

- Equations and numerical method

- Subgrid scale model description

- Stretched vortex SGS model for LES

- Decaying isotropic turbulence test

- comparison between Pade and WENO

- modified wave number behavior

- RM Simulation results

- Plane averages and rms quantities

- mixing width (with and w/o SGS models)

Conclusion 


\section{Strong-shock Richtmyer-Meshkov Instability (RMI)}

- Objectives:

- Pseudo-DNS of Richtmyer-Meshkov flow with strong shocks

- shocks not resolved (requires shock-capturing method)

- numerical method reverts to high-order in regions away from shocks

- LES with the stretched-vortex model of same flow

- Requirements:

- Shock-capturing method with good resolution characteristics in the high-wavenumber range (not only formally high-order)

- WENO (Shu et al.)

- Hybrid (Pade + WENO) (Adams and Shariff)

- Spectral methods for compressible flows (Gottlieb et al.)

- Numerical method compatible with AMR

- SGS-Model applicable to flows with strong shocks

- Stretched Vortex SGS (Pullin and co-workers) 


\section{RM instability: Setup}

- Strong shocks $(M=10)$

- Density ratios

- light to heavy (fast/slow) (5/1)

- heavy to light (slow/fast)

\section{Shock reflects off end}

- Periodic boundary conditions in transverse directions

- homogeneous turbulence in cross-plane

\section{Incident shock}

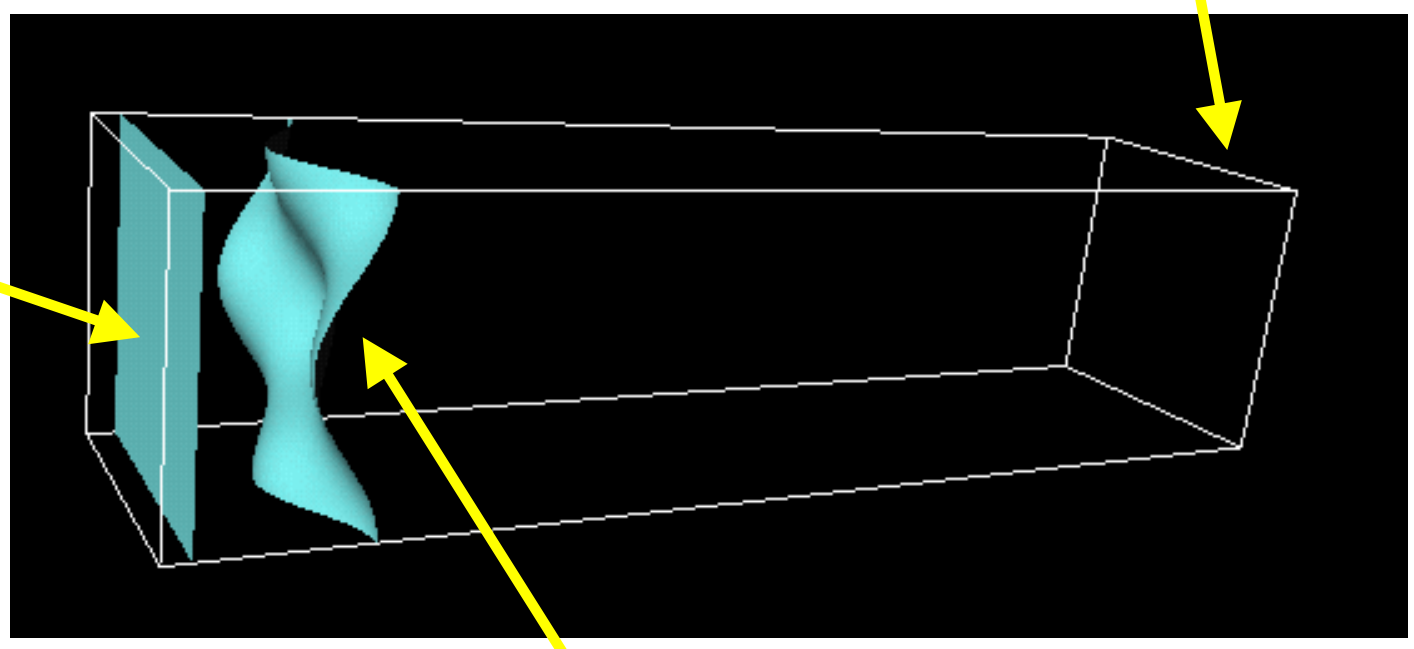

Interface (multiple harmonic perturbationn) 


\section{Favre filtered NS equations}

- Favre average: $\widetilde{q}=\bar{\rho} q / \bar{q}$

- Continuity

$$
\frac{\partial \bar{\rho}}{\partial t}+\frac{\partial \bar{\rho} \widetilde{u}_{i}}{\partial x_{i}}=0
$$

- Momemtum

$$
\frac{\partial \bar{\rho} \widetilde{u}_{i}}{\partial t}+\frac{\partial\left(\bar{\rho} \widetilde{u}_{i} \widetilde{u}_{j}+\bar{p} / \gamma M^{2} \delta_{i j}\right)}{\partial x_{j}}=R e^{-1}\left(\frac{\partial \widetilde{\sigma}_{i j}}{\partial x_{j}}\right)-\frac{\partial T_{i j}}{\partial x_{j}}
$$

where

$$
T_{i j}=\bar{\rho}\left(\widetilde{u_{i} u_{j}}-\widetilde{u}_{i} \widetilde{u}_{j}\right) \equiv \bar{\rho} \tau_{i j} \quad \text { Subgrid stress }
$$




\section{Favre filtered NS equations}

Energy

$$
\begin{aligned}
& \frac{\partial \bar{E}}{\partial t}+\frac{\partial\left(\bar{E}+\bar{p} / \gamma M^{2}\right) \widetilde{u}_{i}}{\partial x_{i}}=\frac{1}{\operatorname{Pr} \operatorname{Re}(\gamma-1) M^{2}} \frac{\partial}{\partial x_{i}}\left(k \frac{\partial \widetilde{T}}{\partial x_{i}}\right) \\
& +R e^{-1} \frac{\partial\left(\widetilde{\sigma}_{i j} \widetilde{u}_{j}\right)}{\partial x_{i}} \\
& -\frac{1}{(\gamma-1) M^{2}} \frac{\partial\left(\bar{\rho} q_{i}\right)}{\partial x_{i}}
\end{aligned}
$$

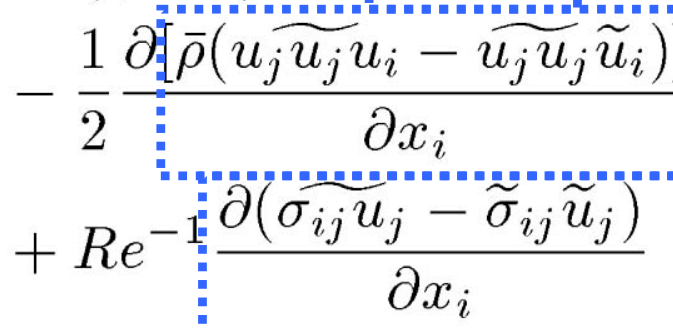

$$
\begin{aligned}
& \bar{E}=\frac{\bar{p}}{(\gamma-1) \gamma M^{2}}+\frac{1}{2} \bar{\rho}\left(\widetilde{u}_{j} \widetilde{u}_{j}\right)+\frac{\bar{i}}{2} \bar{\rho}\left(\widetilde{u_{j} u_{j}}-\widetilde{u}_{j} \widetilde{u}_{j}\right) \\
& q_{i}=\widetilde{T u_{i}}-\widetilde{T} \widetilde{u}_{i} \\
& \bar{p}=\bar{\rho} \widetilde{T}
\end{aligned}
$$




\section{Numerical method: WENO}

- Finite difference formulation WENO (Jiang \& Shu) for inviscid fluxes in the governing equations

- Conservative approximation of flux derivatives

$$
\frac{1}{\Delta x_{i}}\left[\hat{f}_{n}\left(x_{i+1 / 2}\right)-\hat{f}_{n}\left(x_{i-1 / 2}\right)\right]=\partial f_{n}\left(x_{i}\right) / \partial x+O\left(\Delta x^{k}\right)
$$

- Fluxes calculated in characteristic coordinates

- Characteristics -eigenvalues and eigenvectors evaluated using Roe state

- Runge-Kutta (TVD) time discretization 


\section{Prevention of Instabilities}

- "H-correction" by Sanders, Morano \& Druguet adapted for FD-WENO

$$
\begin{aligned}
& \hat{\lambda}_{p ; i+1 / 2 ; j}^{*}=\max \left(\hat{\lambda}_{p ; i+1 / 2 ; j}, \eta_{i+1 / 2 ; j}^{H}\right) \\
& \text { where }
\end{aligned}
$$

$$
\eta_{i+1 / 2, i j}^{H}=\max \left(\eta_{i+1 / 2 ; j}, \eta_{i ; j+1 / 2}, \eta_{i ; j} 1 / 2, \eta_{i+1 ; j+1 / 2}, \eta_{i+1 ; j} 1 / 2\right)
$$

$$
\eta_{i+1 / 2 ; j}=\frac{1}{2} \max _{p}\left(\left|\lambda_{p}\left(\boldsymbol{U}_{i+1 ; j}, \boldsymbol{e}_{x}\right)-\lambda_{p}\left(\boldsymbol{U}_{i ; j}, \boldsymbol{e}_{x}\right)\right|\right)
$$

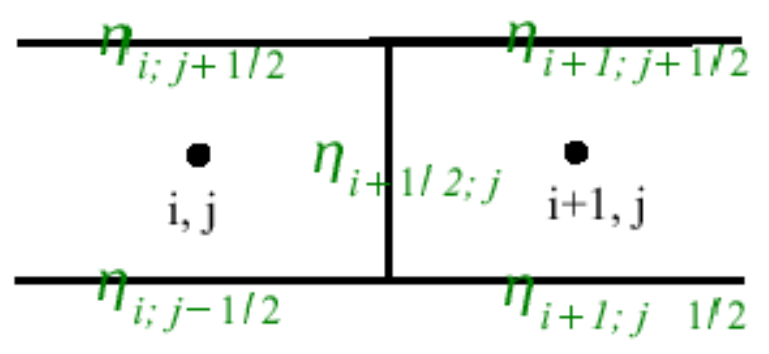




\section{LES Model - Pullin SGS vortex model}

- Extension of stretched vortex sub-grid stress model (Misra \& Pullin 1997) to compressible turbulence

- Structure-based approach

- Subgrid motion represented by nearly axisymmetric vortex within each cell.

- Subgrid stresses are:

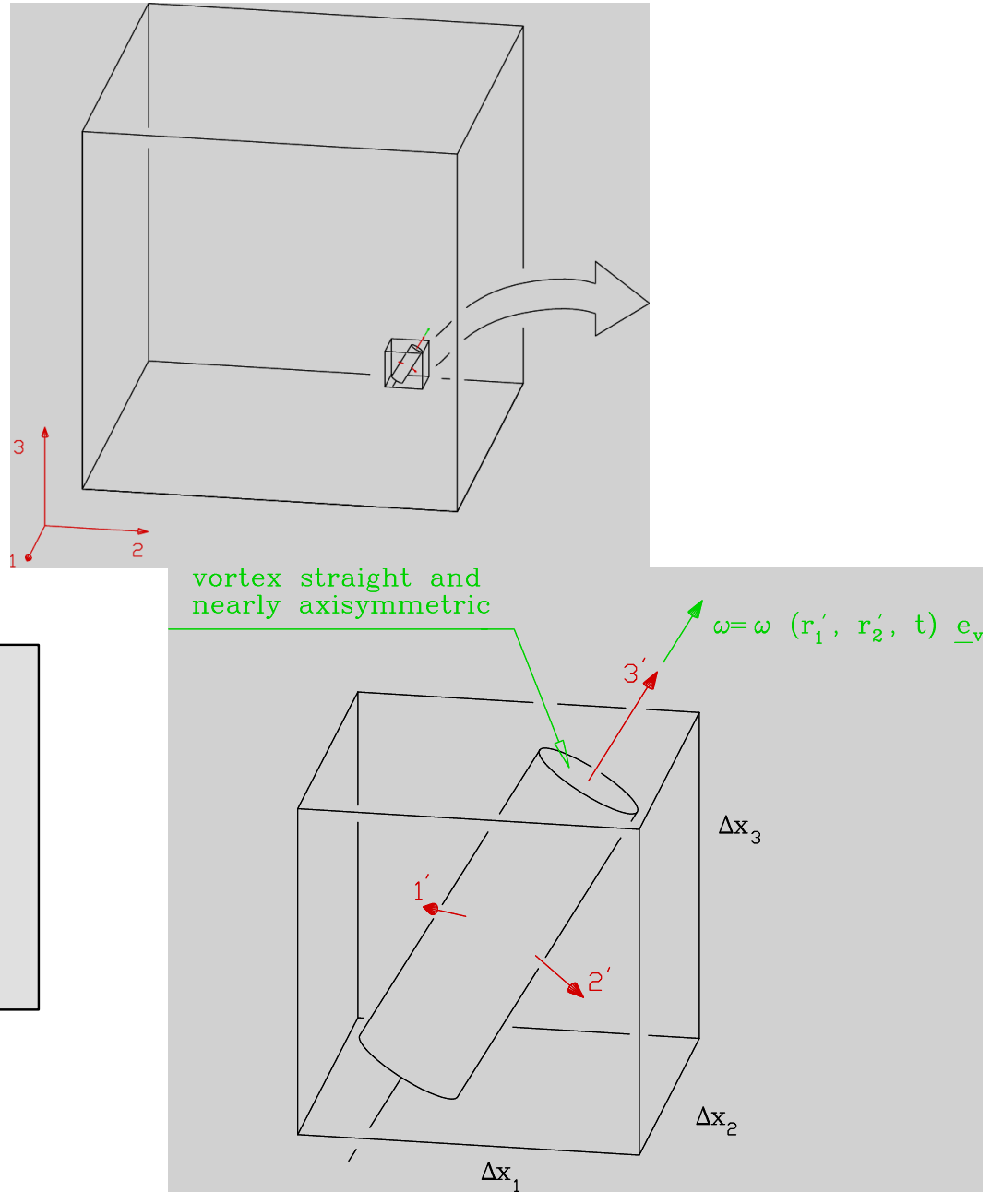

$T_{i j}=K\left(\delta_{i j}-e_{i} e_{j}\right)$,

$K=\int_{k_{c}}^{\infty} E(k) d k$. 


\section{Pullin SGS vortex model}

- Lundgren form assumed for subgrid energy spectrum:

$$
\frac{\mathcal{K}_{0} \epsilon^{2 / 3} k^{-5 / 3} \exp \left(-2 k^{2} \nu /(3|a|)\right)}{a=\tilde{S}_{i j} e_{i}^{v} e_{j}^{v}}
$$

- PDF for vortex orientation in each cell

$$
\begin{gathered}
P(\mathbf{e})=\mu \wp\left(\mathbf{e}^{\mathbf{v}} \mid \tilde{\mathbf{e}}_{3}\right)+(\mathbf{1}-\mu) \wp\left(\mathbf{e}^{\mathbf{v}} \mid \tilde{\mathbf{e}}_{\omega}\right) \\
\mu=\frac{\lambda_{3}}{\lambda_{3}+\|\tilde{\boldsymbol{\omega}}\|}
\end{gathered}
$$

- Subgrid temperature flux (analytical solution for the winding of the local resolved temperature by the elemental vortex)

$$
\left[q_{i}\right]_{m o d}=\frac{1}{2} \Delta K^{1 / 2}\left(\delta_{i j}-\mathrm{e}_{i}^{v} \mathrm{e}_{j}^{v}\right) \frac{\partial \tilde{T}}{\partial x_{j}}
$$




\section{Pullin SGS vortex model}

- $\mathcal{K}_{0} \epsilon^{2 / 3}$ estimated locally by matching local resolved flow 2'ndoraer velocity structure function to local subgrid estimate

- Stretched-vortex model is not an eddy-viscosity model

- allows "back scatter"

- Elements of subgrid stress tensor and subgrid energy calculated directly

- Important for scalar and other subgrid quantities

- No explicit filtering

- No explicit treatment for shock

- verified using aposteriori tests with DNS of decaying isotropic turbulence in the presence of shocklets at modest turbulent Mach numbers (0.3-0.5)

Plug-in model: ease of implementation 


\section{Comparison of DNS with LES + SGS}

Decay of turbulent kinetic energy using

Pullin stretched-vortex SGS model

("SGS modeling for LES of compressible turbulence" Kosovic, Pullin and Samtaney.

To appear in Phys. Fluids)

$$
M_{t}=0.488 R e_{\lambda}=175 O\left(h^{10}\right) 256^{3} \quad \text { IC4 }
$$
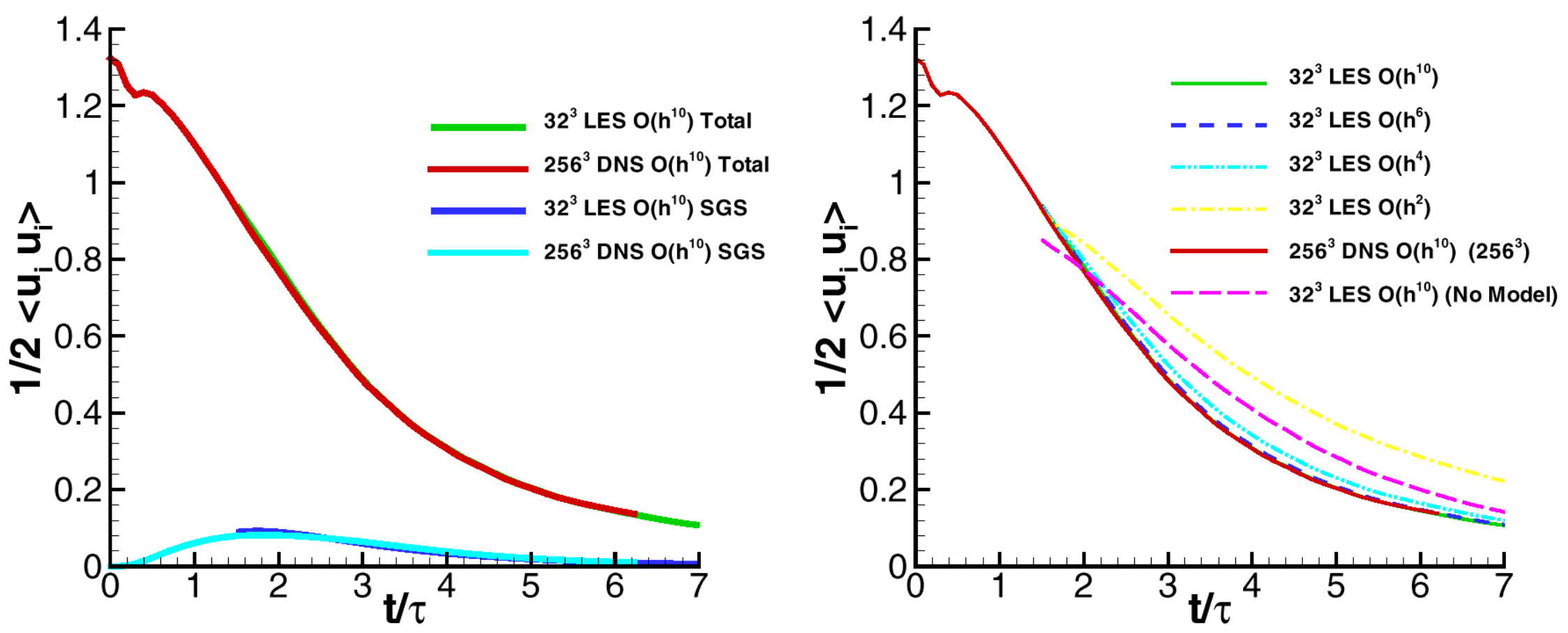

"DNS of decaying isotropic turbulence" - Samtaney, Pullin, Kosovic in Phys. Fluids, May 2001 


\section{Comparison of spectra (LES vs. DNS)}

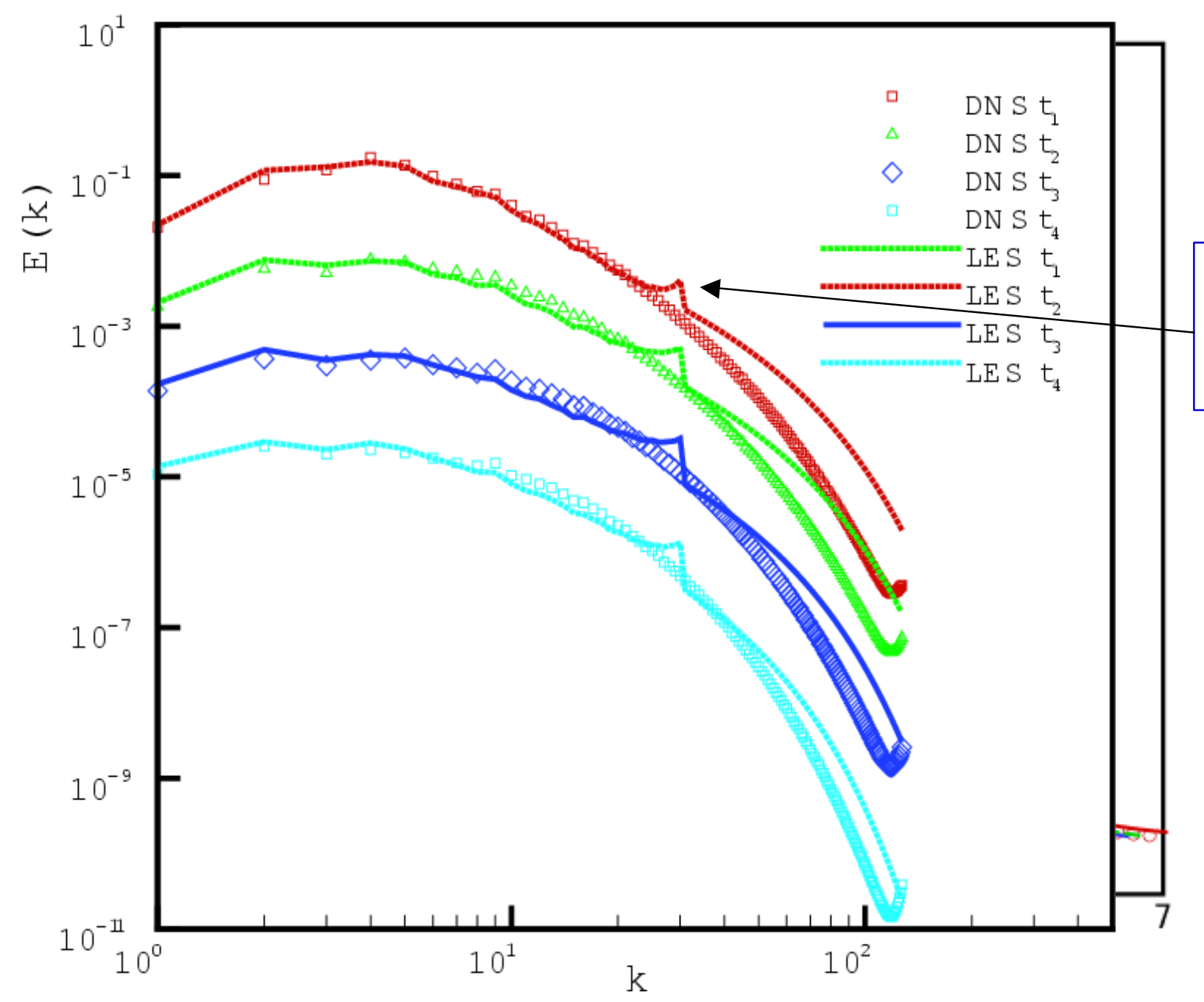

Pile-up is due to aliasing 


\section{Pade vs. WENO}

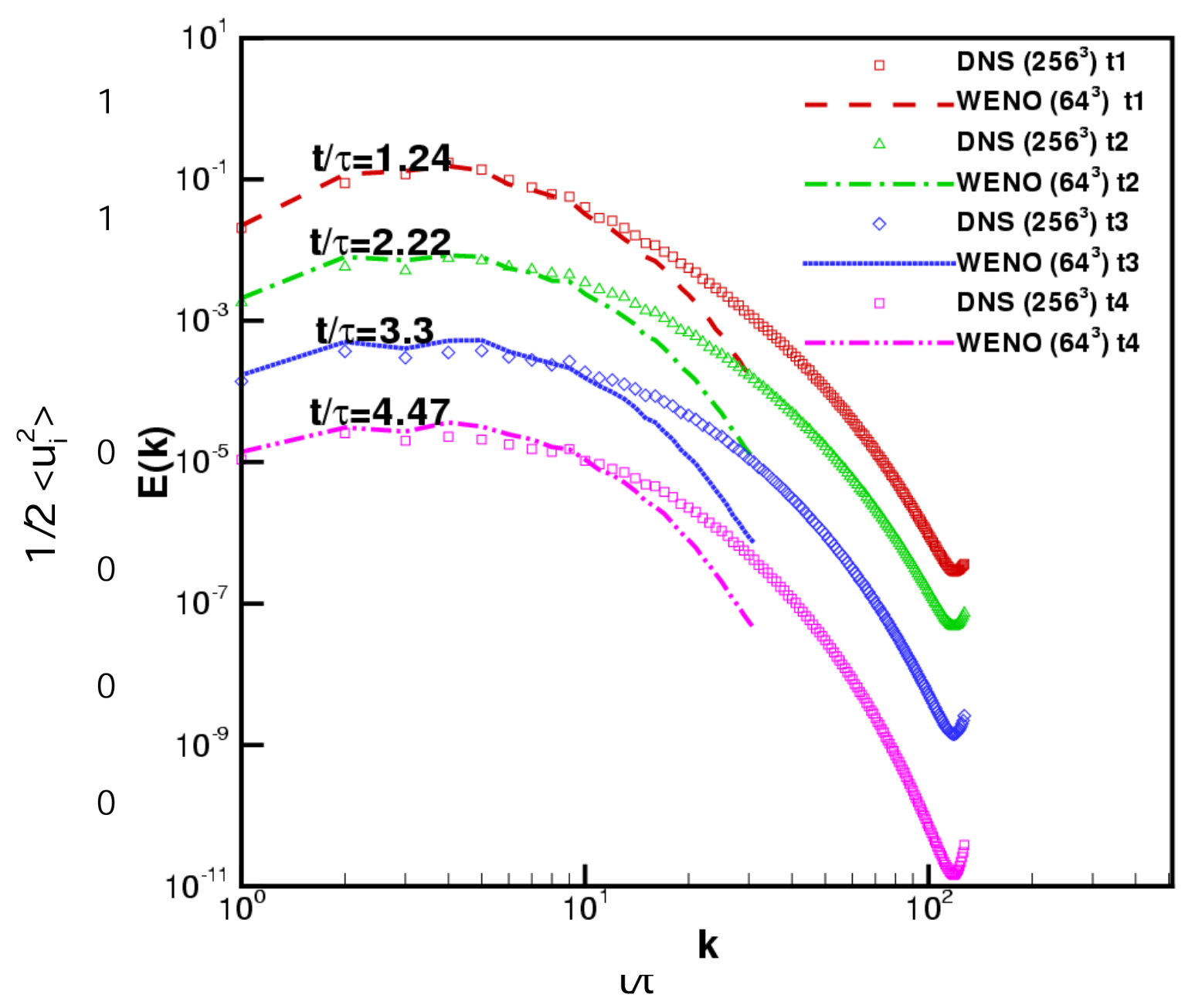




\section{Pade vs. WENO (Modified Wave number)}

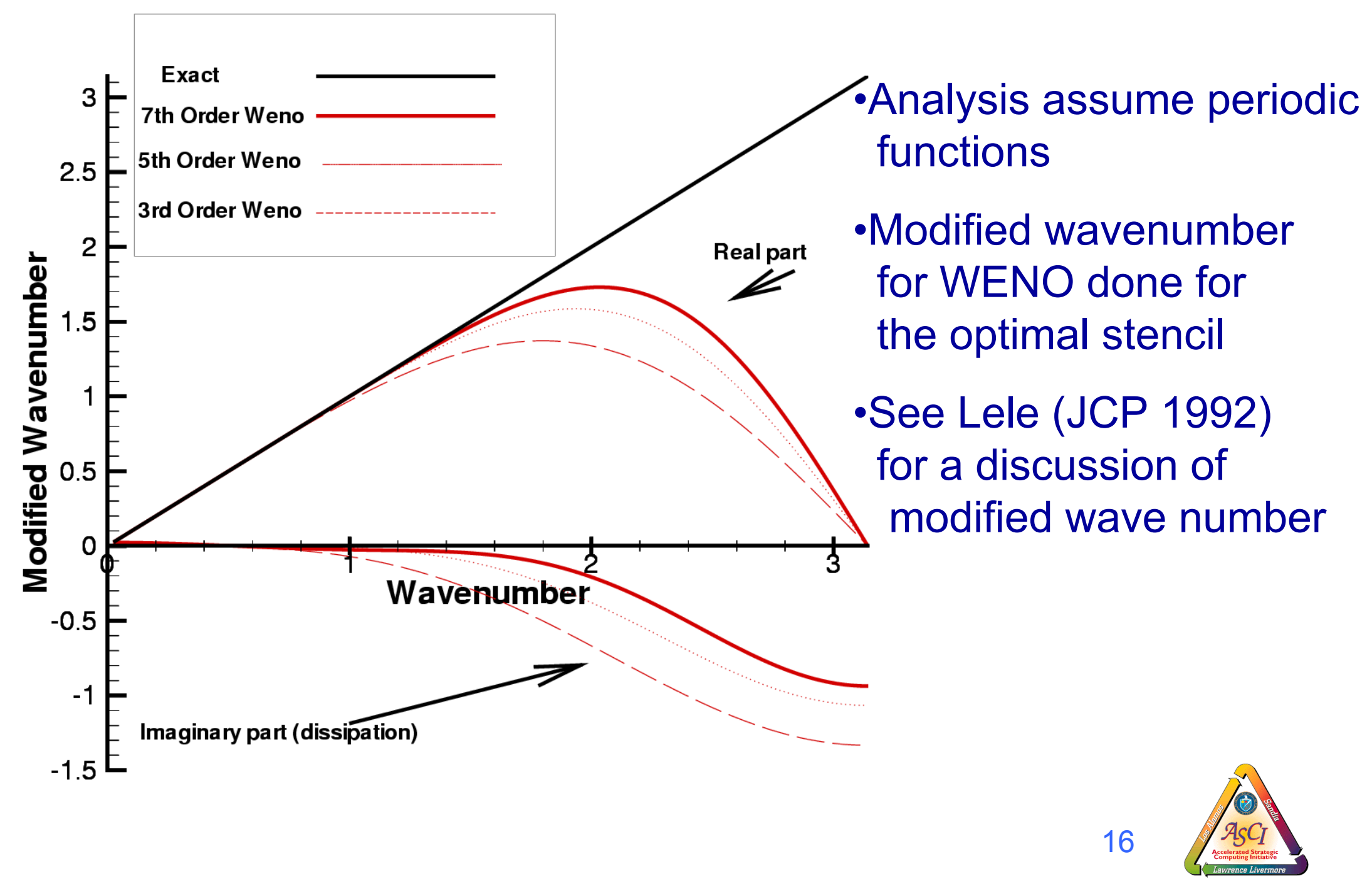




\section{WENO-RMI: Run Parameters}

- Shock Mach number $\mathrm{M}=10$

- Density ratio 1:5

- Interface Initial condition: Multi-mode perturbation with random phases and prescribed spectrum

- BC: Inflow (left), Reflecting (right), Periodic (transverse)

- Physical Domain $16 \pi \times 2 \pi \times 2 \pi$

- 7th-order (formally) WENO with H-correction

- Three runs

- (A) $1024 \times 128 \times 128$ (No SGS model)

- (B) $512 \times 64 \times 64$ (No SGS model)

- (C) 512x64x64 (SV SGS model)

- Simulations on ASCI Blue Mountain (nirvana)

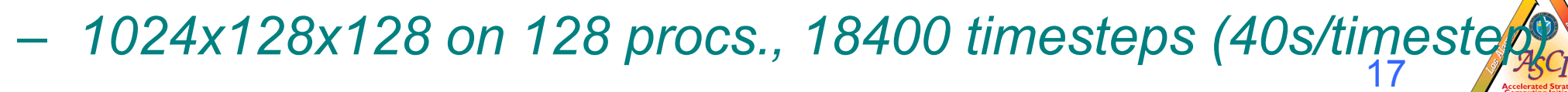
- 512x64x64 on 64 procs., 10000 timesteps (20s/timestep) 


\section{WENO- RMI simulation: Initial Condition}
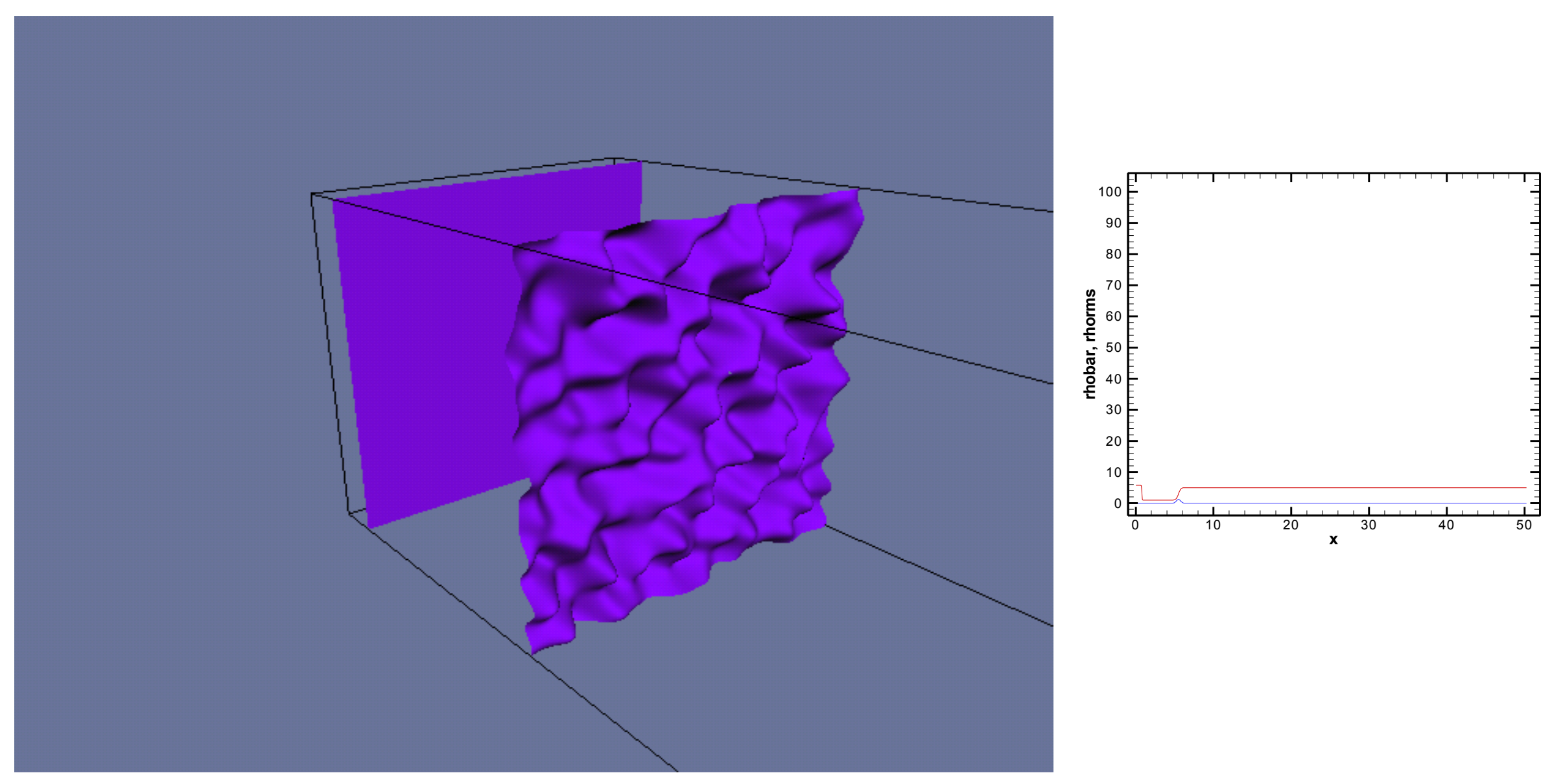

$$
\rho=3
$$




\section{RMI: Before reshock (Run A)}
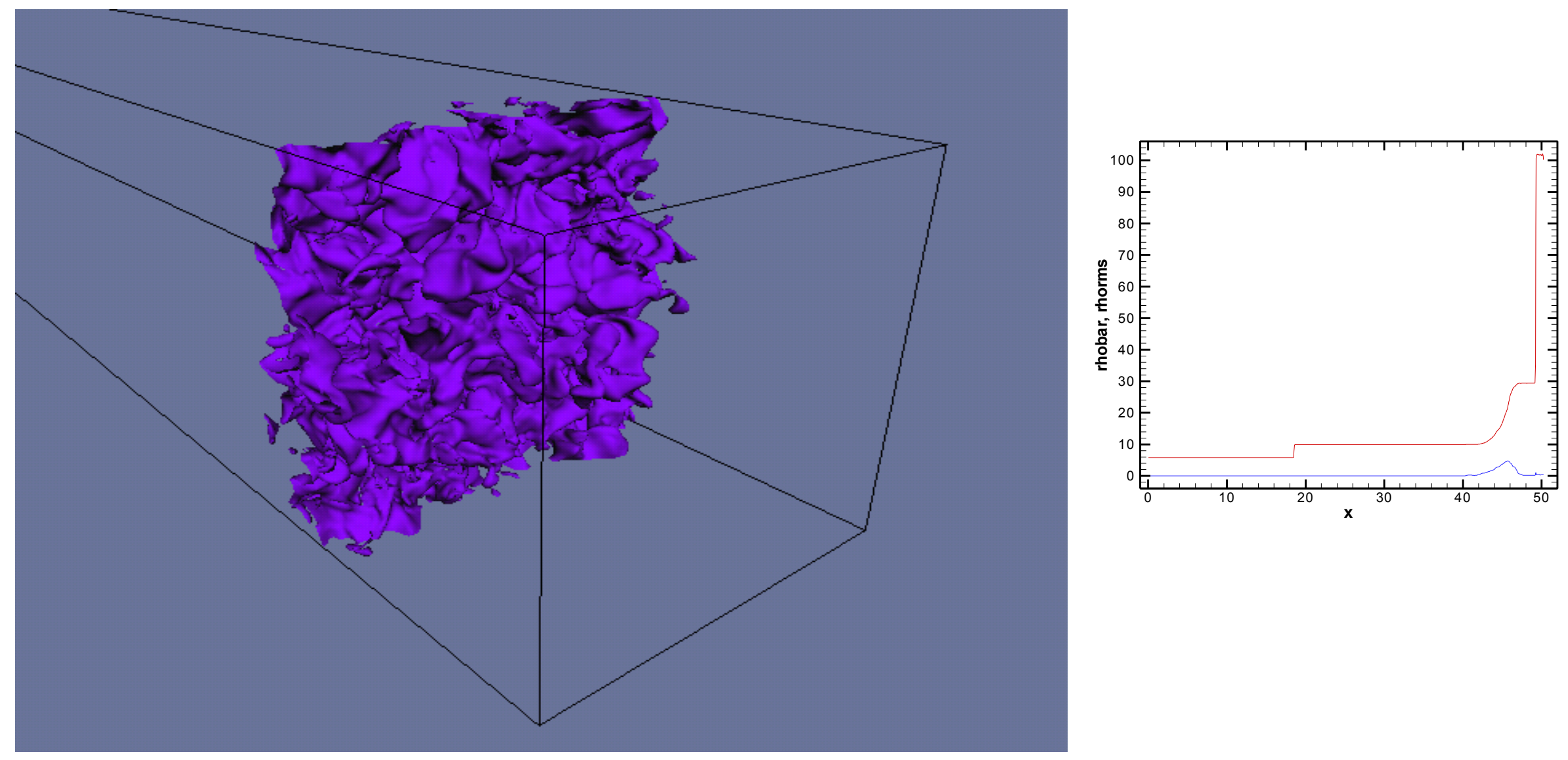

$$
\rho=20
$$




\section{RMI: After reshock (Run A)}
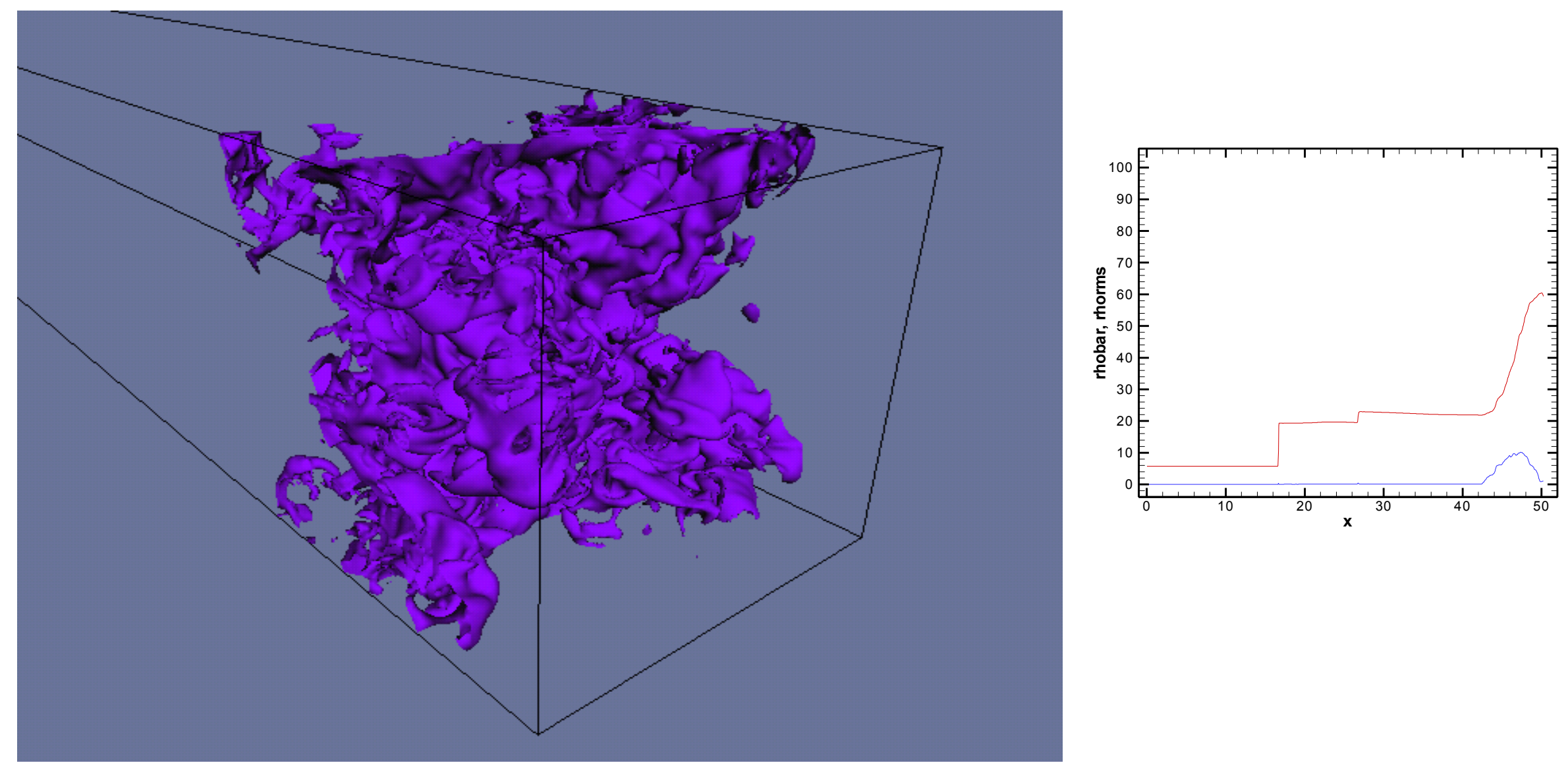

$$
\rho=42
$$




\section{RMI: Spectra (Run A)}

- 2D spectra in planes normal to shock-propagation direction

- Location of plane determined by zero crossing of $\bar{\phi}$

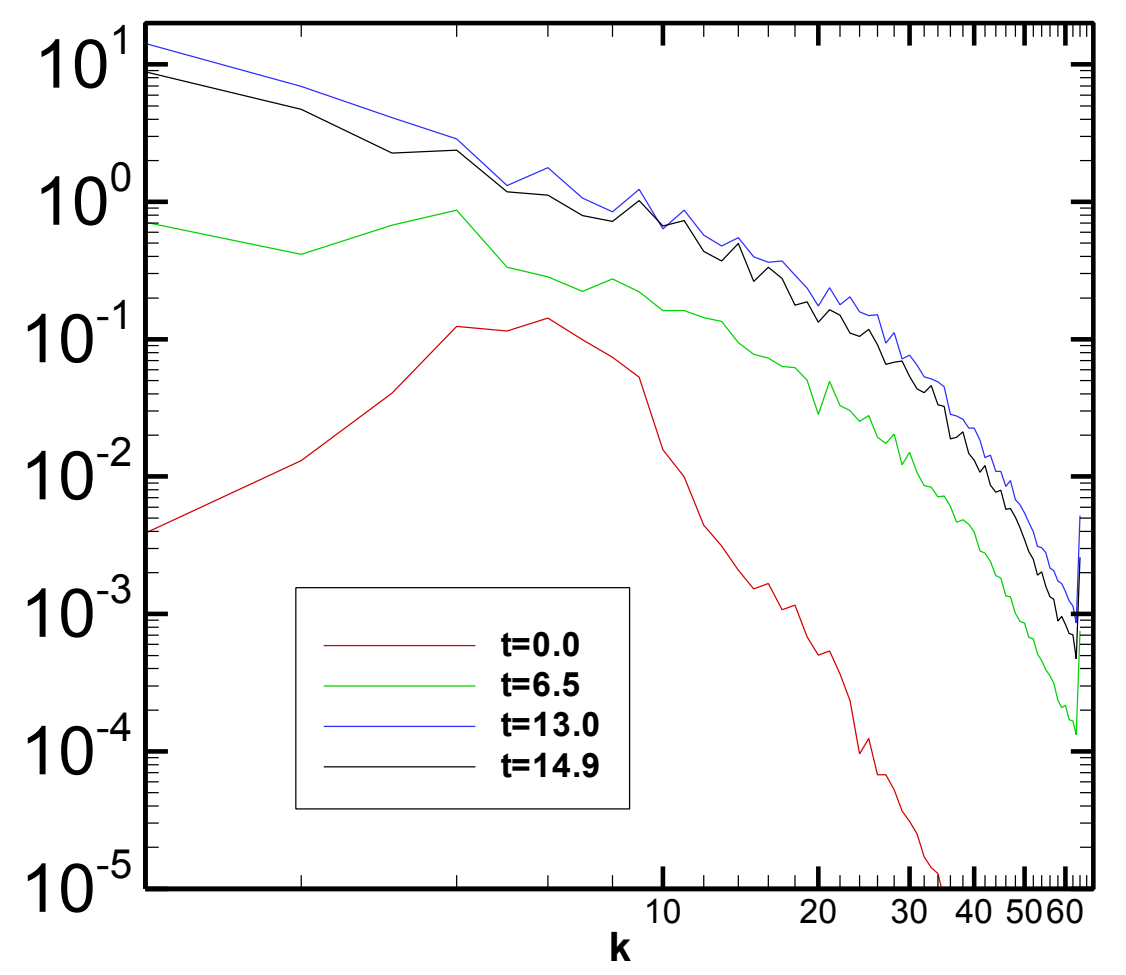

density spectra

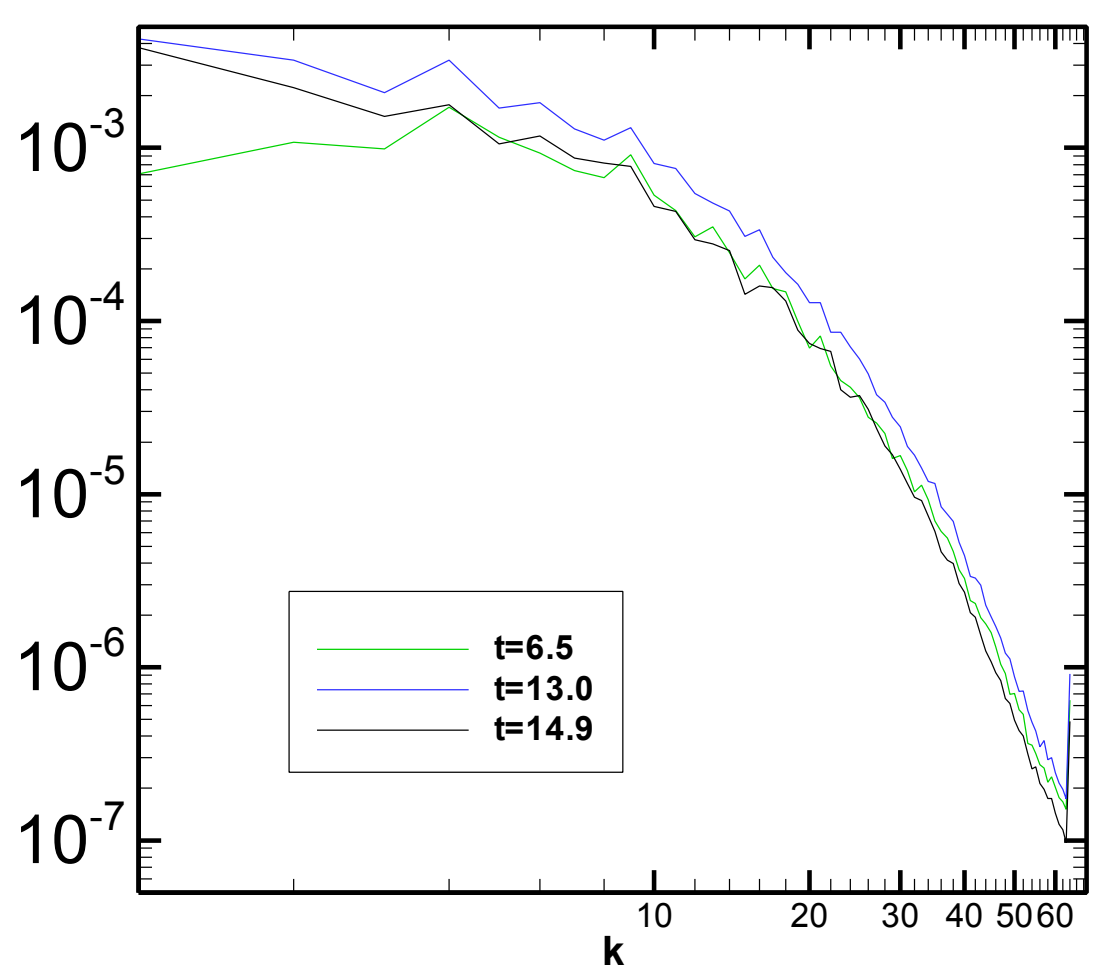

velocity spectra 


\section{RMI: Density plane averages and rms}
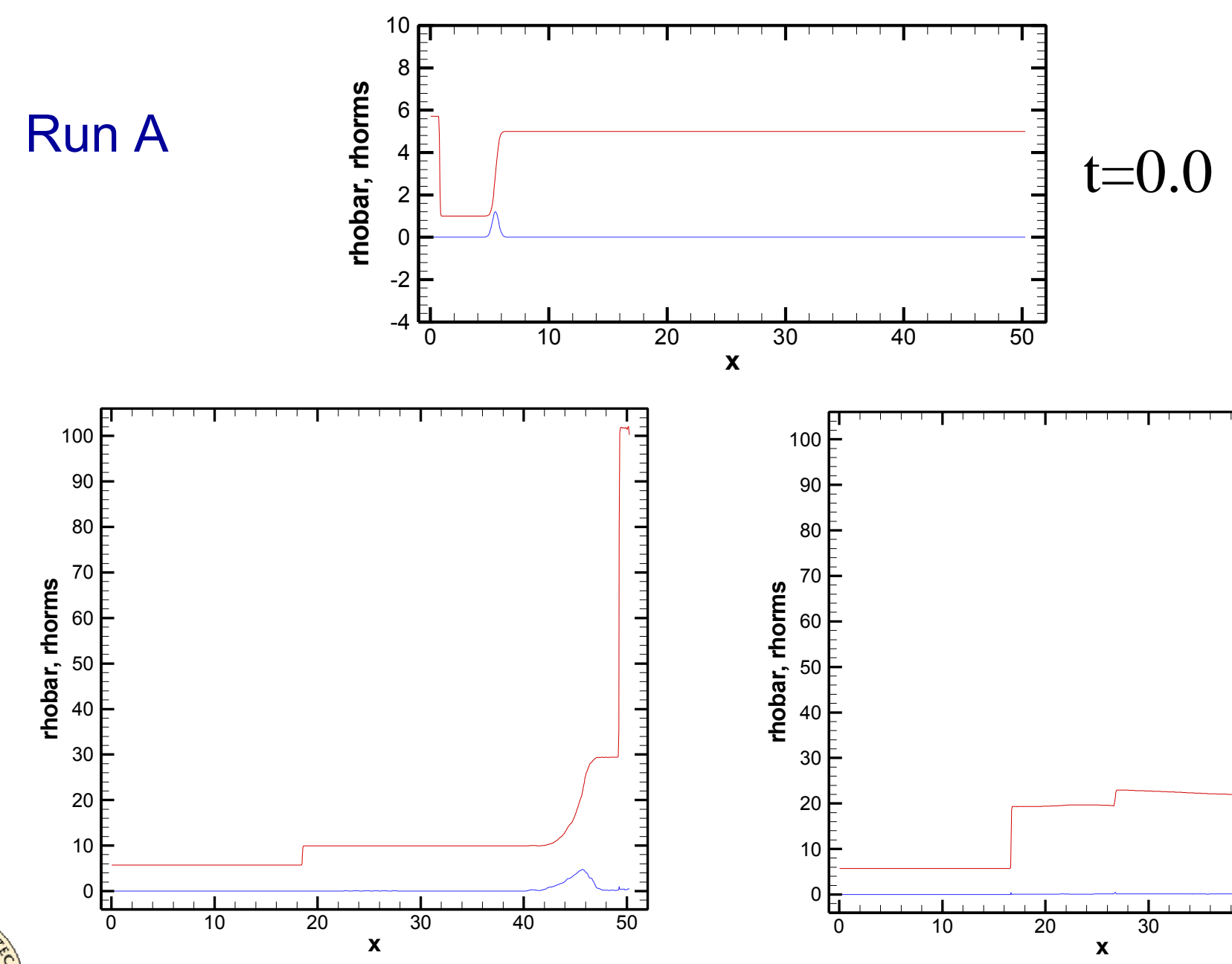

$t=6.5$

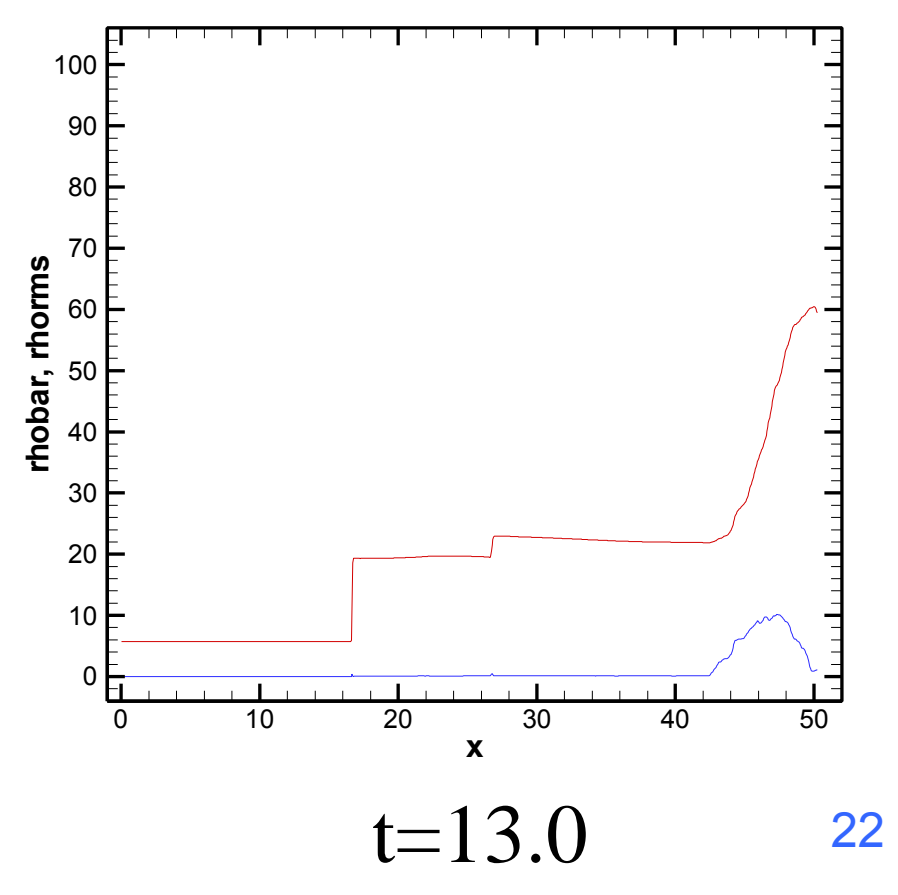




\section{RMI: Plane averages and rms (Run C)}
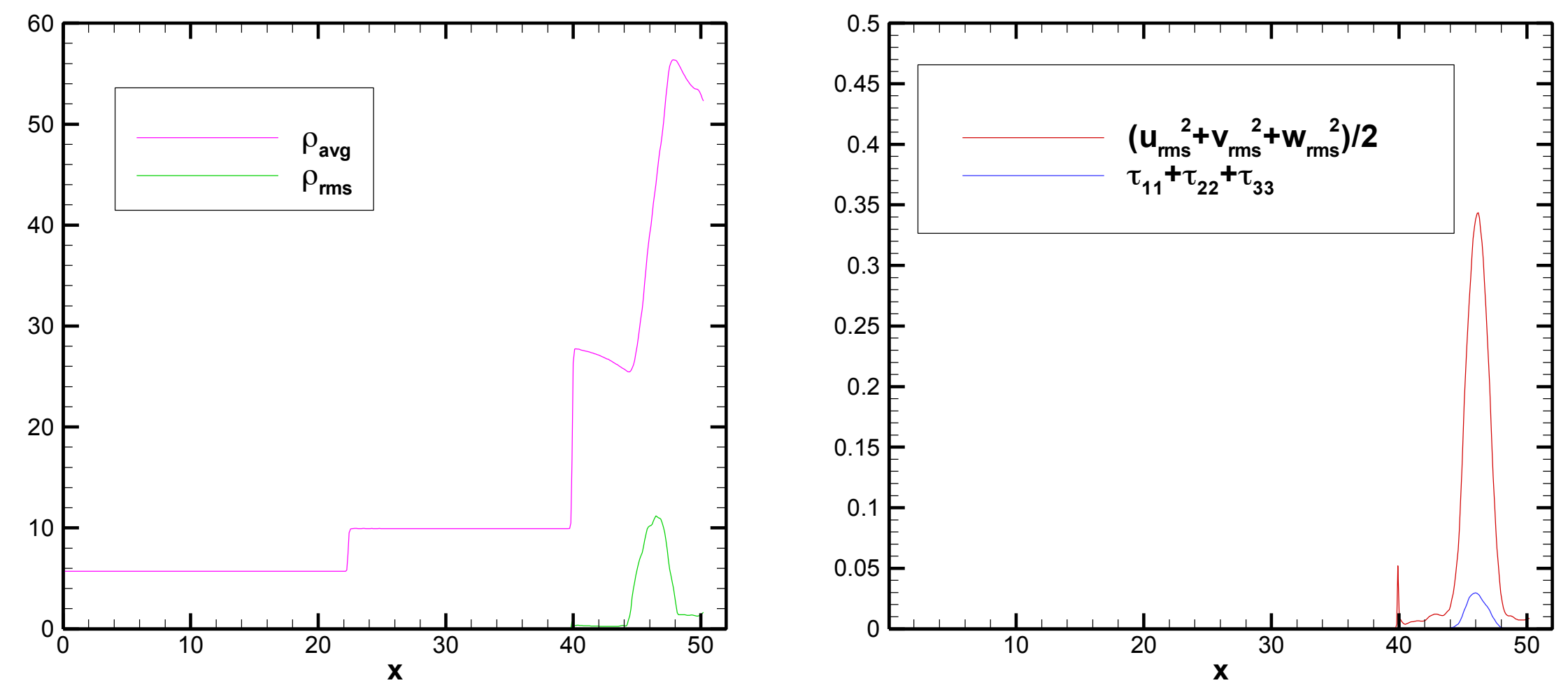

$\mathrm{t}=8.3$ (after reshock) 


\section{RMI: Mixing width (Integral Measure)}

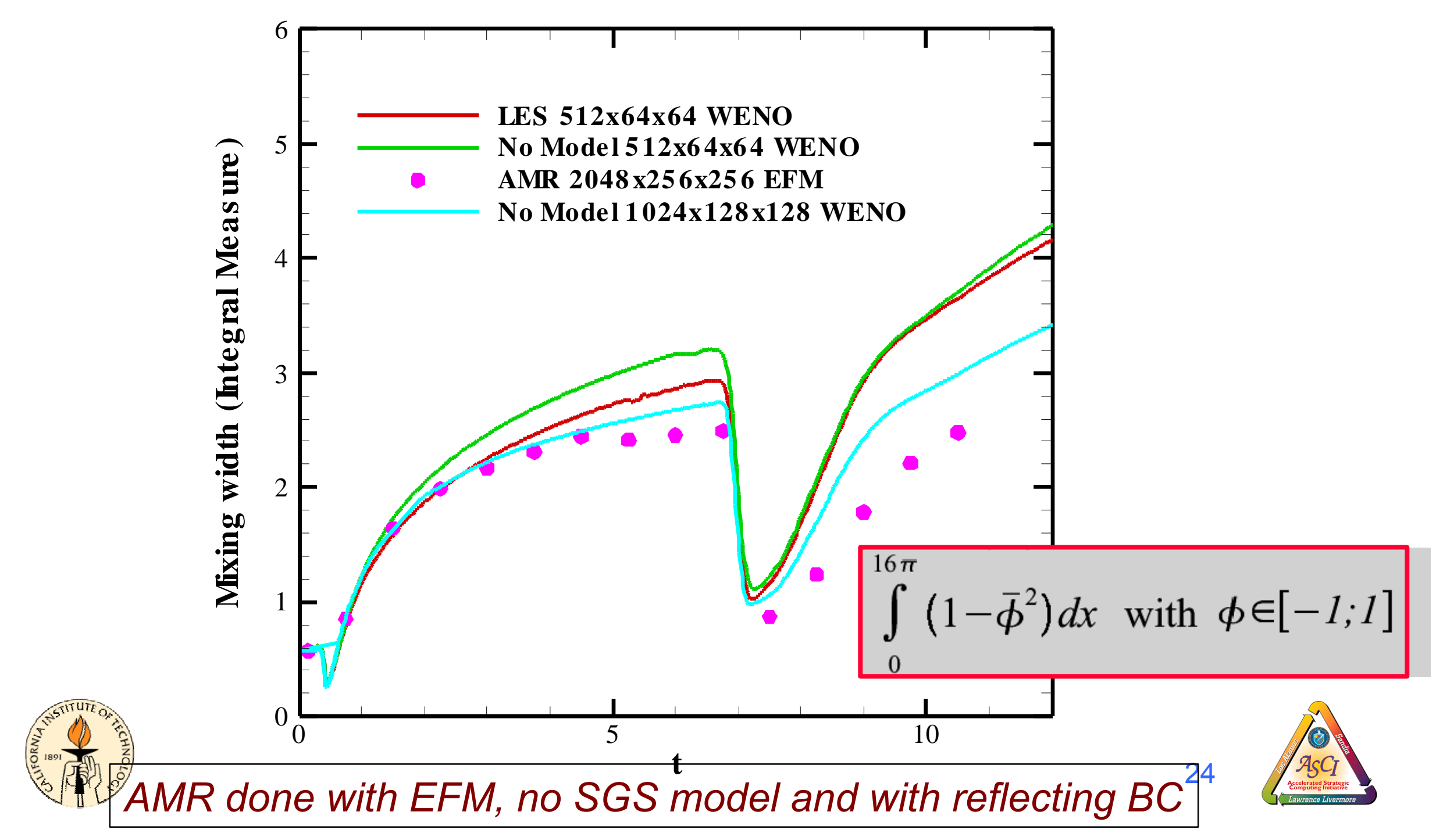




\section{RMI: Width of density interface}

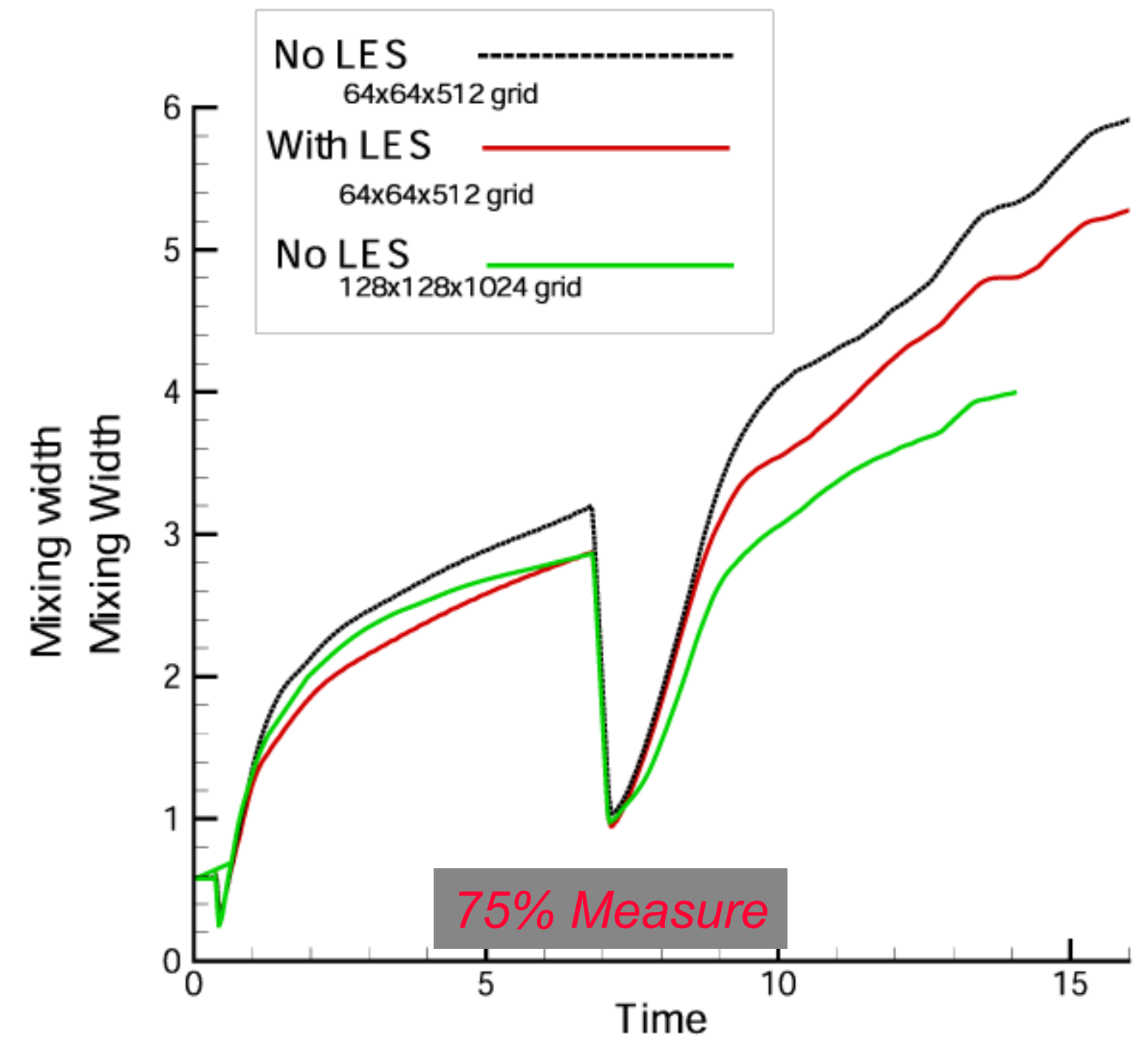




\section{Conclusion}

- Requirement of shock-capturing and higher-order is difficult to achieve in practice

- WENO schemes investigated

- Compared with Pade schemes for decaying isotropic turbulence

- High modified wavenumber behaviour not favourable

- Require "Carbuncle fix" to stabilise the shock

- LES of strong-shock RM performed using the stretched vortex SGS model

- SV - SGS model implemented in the WENO code

- works as a plug-in

- no explicit filtering

- SGS model is robust (I.e., no. numerical stability issues)

- Compared LES with SV model and LES with no model

- SGS model active but subgrid TKE is a small fraction of the totalTKE ( 10\%)

- Small differences in the "mixing width" with and w/o SGS mod(aA 


\title{
Numerical Investigation of a Laser Induced Turbulent Mixing Zone
}

\author{
P. Seytor and M. Legrand \\ CEA/DAM lle de France, BP12, 91680, Bruyères le Châtel, France
}

We have used high Mach number $(\mathrm{M} \approx 20)$ mix instability experiments which have been conducted using the NOVA laser system to investigate the growth of the Richtmyer-Meshkov instability resulting from a strong shock wave. The initial nonlinear single-mode two dimensional perturbation was machined into a brominated plastic ablator (density : $1.22 \mathrm{~g} / \mathrm{cc}$ ) adjacent to a low density carbon foam $(0.10 \mathrm{~g} / \mathrm{cc})$. We compared the experimental results with LLNL simulations (CALE code) and with our own numerical simulations (FCI2 code). A non linear model (Ramshaw [1]) has been previously used to analyze the growth of a mixing zone in this experiment (Farley et al. [2]). We propose here to consider the possibility of a long turbulent phase in this type of experiment. We found mix width results to be in good agreement with the $k-\varepsilon$ statistical model included in a $1 \mathrm{D}$ code when the transition time to turbulence is correctly estimated (high Mach number effects).

The first section of this talk describes the experimental setup. The instability phases of the flow are discussed in section II. The Ramshaw non linear model is described in section III and the necessary conditions for turbulence in section IV. High Mach number effects on the transition time to turbulence are discussed in section V (HESIONE 2D eulerian code) and $k-\varepsilon$ computations are described in section VI. This is followed by a summary in section VII.

[1] J. D. Ramshaw

Simple model for linear and non linear mixing at unstable fluid interfaces with variable acceleration

Phys. Rev. E, 58, N5, 1998

[2] D. R. Farley et al.

High Mach number mix instability experiments of an unstable density interface using a single mode, nonlinear initial perturbation

Physics of plasmas, 6, N11, 1999 


\section{Experimental Setup}

Farley et al.

\section{Front View}

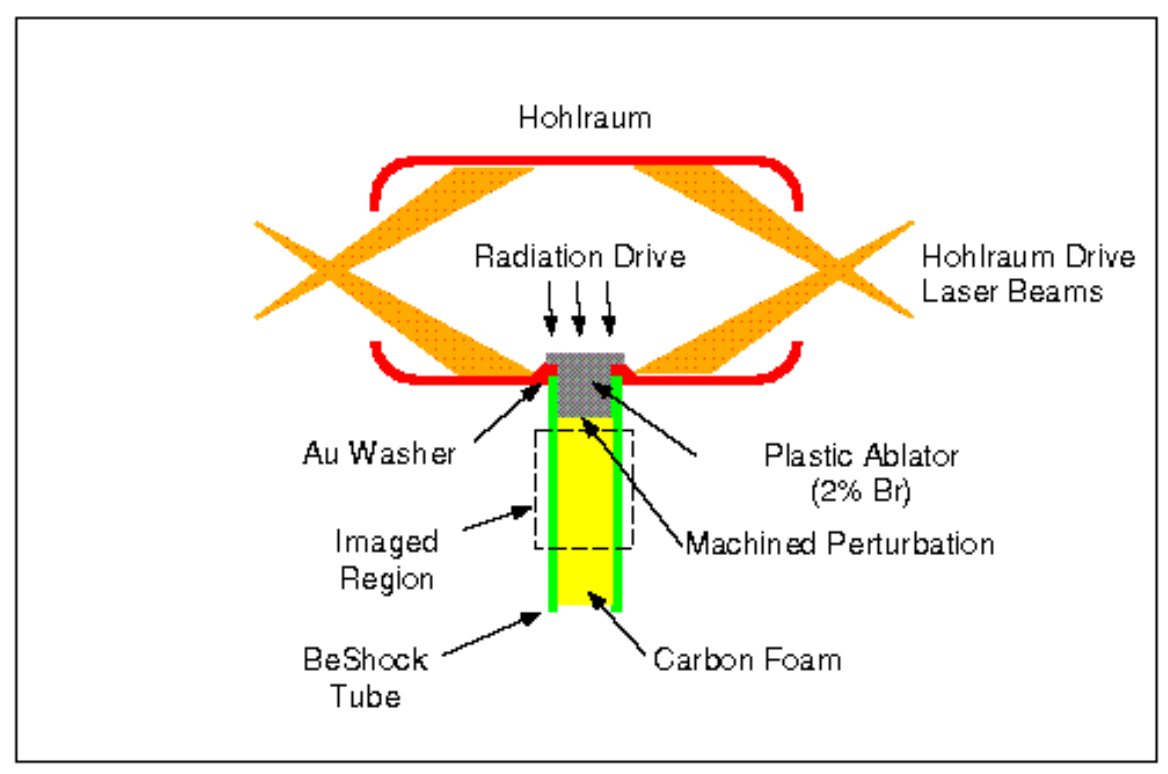

Hohlraum : $3080 \mu \mathrm{m}$ long and $1600 \mu \mathrm{m}$ diameter hollow cylinder

Shock tube : $2200 \mu \mathrm{m}$ long beryllium cylinder outer diameter : $700 \mu \mathrm{m}$ inner diameter : $500 \mu \mathrm{m}$

ablator : $300 \mu \mathrm{m}$ long $(\rho=1.22 \mathrm{~g} / \mathrm{cm} 3)$

carbon foam : $1900 \mu \mathrm{m}$ long $(\rho=0.1 \mathrm{~g} / \mathrm{cm} 3)$

Hohlraum drive : 1ns square pulse $(\approx 20 \mathrm{k}\rfloor)$

Radiation drive : peak flux temperature $=230 \mathrm{eV}( \pm 10 \mathrm{eV})$ 


\section{Instability phases}

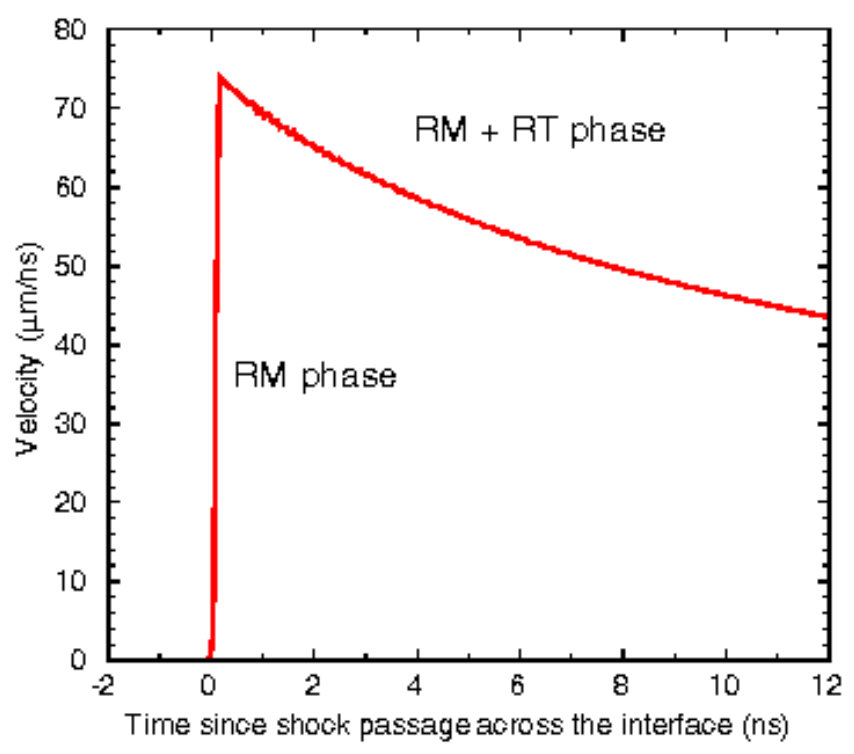

$\square$ A Richtmyer-Meshkov Instability (RMI) is induced by the shock passage across the interface

$\square$ This RM phase is followed by a slow decay of velocity which generates a Rayleigh-Taylor Instability (RTI)

$\square$ These results obtained with our 1D Radiation Hydrodynamics code are very close to Farley's (CALE 1D, [1])

[1] D. R. Farley et al.

High Mach number mix instability experiments of an unstable density interface using a single mode, nonlinear initial perturbation

Physics of Plasmas, 6, N11, 1999 


\section{Nonlinear Model}

- Ramshaw [1] uses a Lagrangian formulation to derive an ODE for the growth of a perturbed density interface subjected to an arbitrary acceleration $\mathrm{g}(\mathrm{t})$ in uncompressible fluids :

$$
\mathrm{b}|\mathrm{a}| \dot{\mathrm{a}}+\frac{\mathrm{ba}}{2|\mathrm{a}|} \dot{\mathrm{a}}^{2}+2 \pi \mathrm{c}|\mathbf{a}| \dot{\mathrm{a}}-2 \pi \mathrm{Aag}(\mathrm{t})=0
$$

$a$ is the amplitude of the perturbation, $A$ is the Atwood number, $b$ is a nondimensional constant related to the RT $\alpha$ parameter and to the RM power law exponent $\theta\{b=\pi \theta / \alpha(2-\theta)\}$ and $c$ is a dissipation factor of order unity.

- Farley et al. [2] use this model to fit the experimental results of the laser experiment.

\section{[1] J. D. Ramshaw}

Simple model for linear and non linear mixing at unstable fluid interfaces with variable acceleration.

Phys. Rev. E, 58, N5, 1998

[2] D. R. Farley et al.

High Mach number mix instability experiments of an unstable density interface using a single mode, nonlinear initial perturbation.

Physics of Plasmas, 6, N11, 1999 


\section{Nonlinear model \\ Farley et al.}

$\square$ The amplitude (experiment) is equal to mix half-width

$\square$ The Ramshaw model is uncompressible and the experimental results have to be decompression-corrected in order to be compared to modelization.

$\square$ The initial values of a and da/dt are given by a 2D eulerian computation (CALE code)

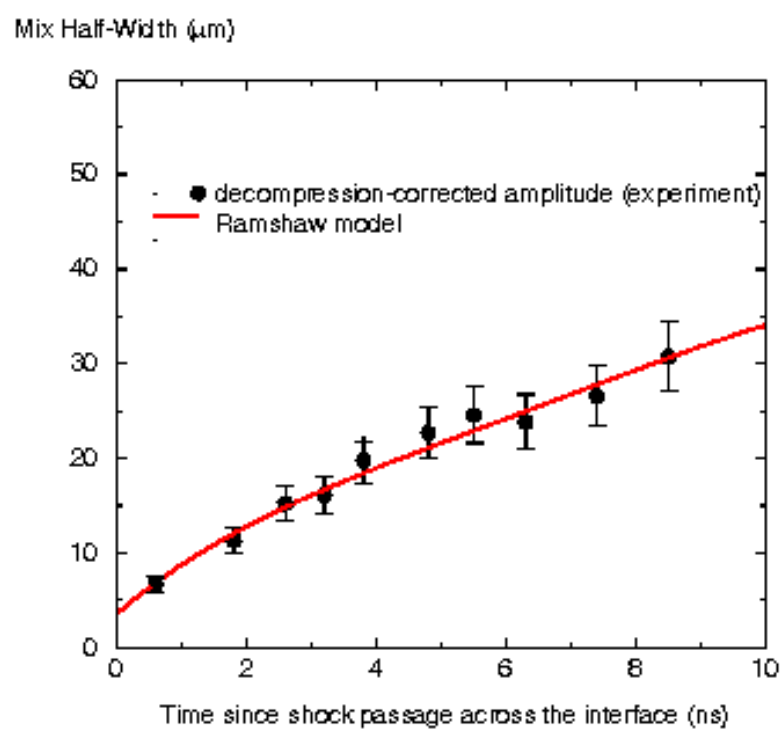

\section{The authors obtain a very reasonable fit of experimental data}


Necessary conditions for turbulence

$\square$ The Reynolds number of the flow must be enough

- Reynolds number estimation

$\square$ The experiment duration must be greater than the transition time to turbulence

- Transition time model 


\section{Necessary conditions for turbulence Reynolds number estimation}

$$
\begin{gathered}
\operatorname{Re}=\frac{\mathrm{ul}}{v} \\
v=0.001 \mu \mathrm{m}^{2} / \mathrm{ns}[1]
\end{gathered}
$$

We propose [2] the following definitions for $\mathrm{u}$ and I

$$
\begin{gathered}
\mathrm{u}=\dot{\mathrm{a}}\left(\mathrm{t}_{\mathrm{s}}\right)=10 \mu \mathrm{m} / \mathrm{ns} \\
1=\lambda=23 \mu \mathrm{m} \\
\operatorname{Re} \approx 10^{5}(\approx \text { Farley's result [1]) }
\end{gathered}
$$

Grégoire [2] founds the following result for three classical shock tubes :

$$
\text { 5. } 10^{3}<\operatorname{Re}<8.10^{4}
$$

The laser induced instability can lead to turbulence

[1] D. R. Farley et al.

High Mach number mix instability experiments of an unstable density interface using a single mode, nonlinear initial perturbation

Physics of Plasmas, 6, N11, 1999

[2] O. Grégoire

CEA/DIF internal report, 2000 


\section{Necessary conditions for turbulence Transition time model [1]}

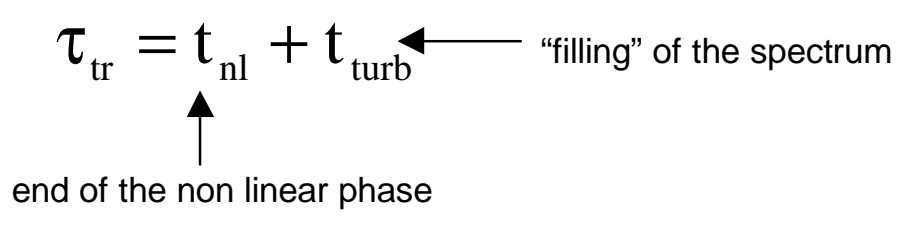

$\square t_{n l}$

$$
\mathrm{a}(\mathrm{t})=\mathrm{a}_{0}^{+}+\dot{\mathrm{a}}_{\mathrm{IM}}\left(\mathrm{t}-\mathrm{t}_{0}\right)
$$

linear phase, Impulsive model (IM)

$$
\mathrm{t}_{\mathrm{as}}(\mathrm{a})=\mathrm{t}-\mathrm{t}_{0}=\frac{\mathrm{a}-\mathrm{a}_{0}^{+}}{\dot{\mathrm{a}}_{\mathrm{IM}}} \quad(\mathrm{d}=\mathrm{ka}<1)
$$

End of the linear phase :

Heuristic geometrical argument : $a\left(t_{n l}\right)=\lambda / 2 \quad(d=\pi)$

$$
\mathrm{t}_{\mathrm{nl}}=\frac{\pi-\mathrm{d}_{0}^{+}}{\mathrm{k} \dot{\mathrm{a}}_{\mathrm{IM}}}
$$

Grégoire [1] uses Vandenboomgaerde's impulsive model [2] valid for low Mach numbers to evaluate $\dot{\mathrm{a}}_{\mathrm{IM}}$

$$
\mathrm{t}_{\mathrm{nl}}=\frac{2}{\mathrm{k}}\left\{\frac{\pi-\mathrm{d}_{0}^{-}\left(1-\frac{\Delta \mathrm{U}}{\mathrm{W}}\right)}{\Delta \mathrm{Ud}_{0}^{-}\left[\mathrm{A}^{+}\left(1-\frac{\Delta \mathrm{U}}{\mathrm{W}}\right)+\mathrm{A}^{-}\right]}\right\}
$$

[1] O. Grégoire

CEA/DIF internal report, 2000

[2] M. Vandenboomgaerde et al.

Phys. Rev. E, 58 (2), 1874 - 1882, 1998 


\section{Necessary conditions for turbulence Transition time model [1]}

$\square t_{\text {turb }}$ : the initial spectrum is a $\delta\left(k-k_{0}\right)$ distribution. $t_{\text {turb }}$ is the time necessary to establish the Kolmogorov cascade :

$$
\begin{gathered}
\mathrm{t}_{\text {turb }}=\frac{\theta_{\mathrm{T}}}{\mathrm{k} \sqrt{\mathrm{K}}} \quad(\mathrm{K} \text { is the fluctuating kinetic energy) } \\
\theta_{\mathrm{T}}=2.1 \quad \text { (Clark et al. [2]) } \\
\mathrm{K} \approx \frac{1}{2} \dot{\mathrm{a}}_{\mathrm{t}=\mathrm{t}_{\mathrm{nl}}}^{2} \quad \text { (Grégoire [1]) } \\
\mathrm{t}_{\text {turb }}=\frac{2}{\mathrm{k}}\left\{\frac{\theta_{\mathrm{T}} \sqrt{2}}{\Delta \mathrm{Ud}_{0}^{-}\left[\mathrm{A}^{+}\left(1-\frac{\Delta \mathrm{U}}{\mathrm{W}}\right)+\mathrm{A}^{-}\right]}\right\}
\end{gathered}
$$

$\square$ We will use an alternate expression for $\dot{\mathrm{a}}_{\mathrm{IM}}$ (Meyer et al. [3]):

$$
\dot{\mathrm{a}}_{\mathrm{IM}}=\mathrm{kA}^{+} \mathrm{a}_{0}^{*} \Delta \mathrm{U} \quad \text { with } \quad \mathrm{a}_{0}^{*}=\frac{1}{2}\left(\mathrm{a}_{0}^{-}+\mathrm{a}_{0}^{+}\right)
$$

$\tau_{\mathrm{tr}}=0.44 \mathrm{~ns}$ after shock passage across the interface in the laser experiment (valid for low Mach number)

[1] O. Grégoire. CEA/DIF internal report, 2000

[2] T. T. Clark et al. Symmetries and the approach to statistical equilibrium in isotropic turbulence. Physics of Fluids, 10 (11),, 2846-2858,1998

[3] Meyer et al. Numerical investigation of the stability of a shock-accelerated interface between two fluids. Phys. Fluids 15, 753-75 


\section{D eulerian computations (HESIONE code) Initial setup}

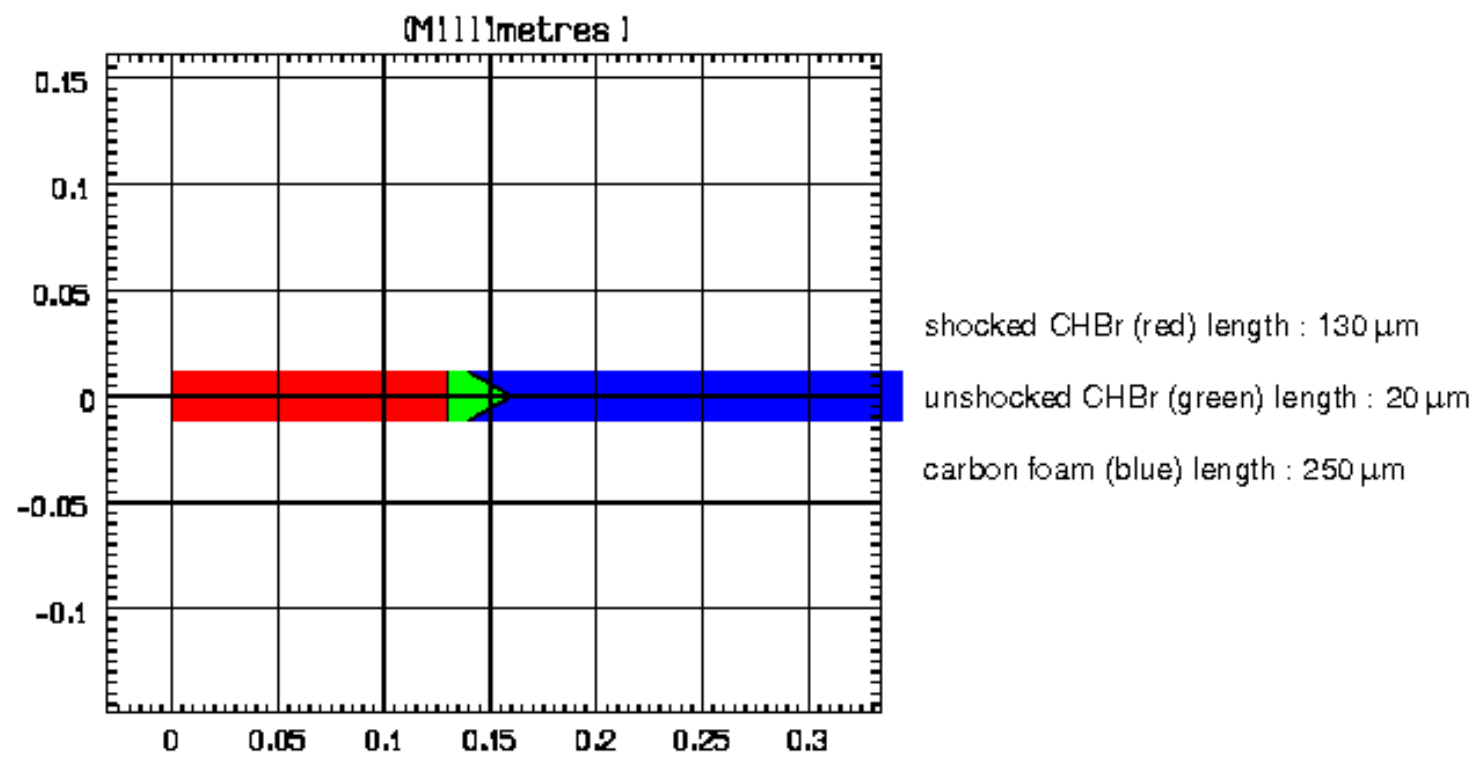

$\square$ Pure RMI computation (35 Mbar incident shock)

$\square$ Planar geometry

$\square$ Rigid walls computation box

$\square$ No radiation effects

$\square$ Standard EOS

ㅁ 100 zones/wavelength

$\checkmark$ Computations valid till $\approx 2 \mathrm{~ns}$ (non physical rarefaction waves effects) 


\section{D eulerian computations (HESIONE code) Interface history}

$\square \vee x$ map + interface

$\square$ Time is referenced to when the shock completes the interface traversal for $\mathrm{a} 0=10 \mu \mathrm{m}$

$$
\mathrm{a} 0=1 \mu \mathrm{m}
$$

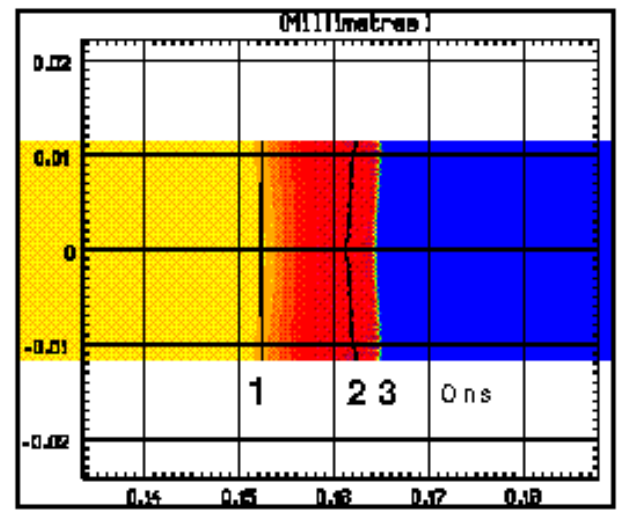

1 : artifact; 2 : interface; 3 : shock

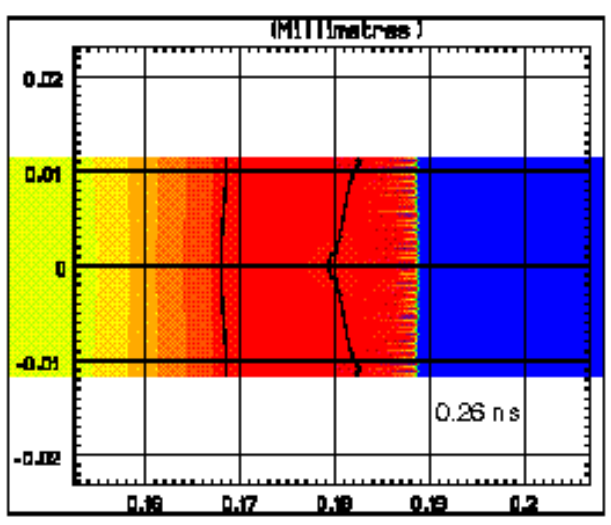

$\mathrm{a} 0=10 \mu \mathrm{m}$

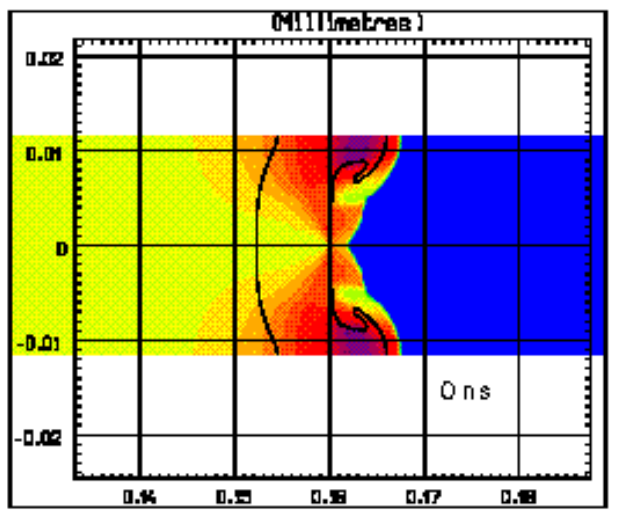

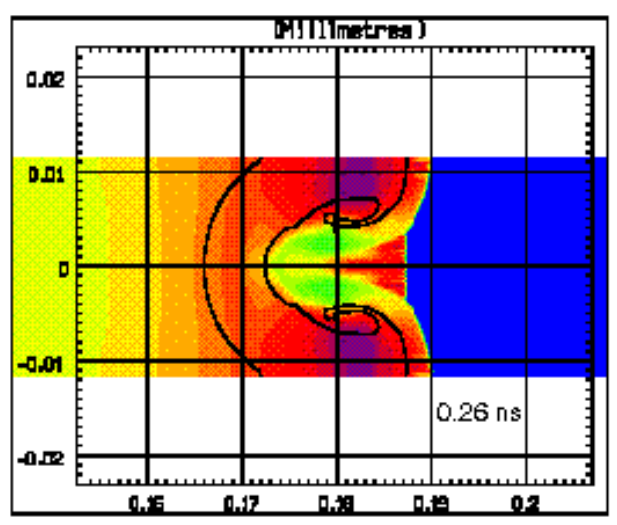

The spike tip cannot overtake the transmitted shock (this penetration is energetically prohibitive)

$\Rightarrow \mathrm{da} / \mathrm{dt} \leq \mathrm{st}$ - uc (st : transmitted shock speed; uc : interface speed) 


\section{D eulerian computations (HESIONE code) Amplitude and growth rate history of the perturbation}

The following analysis was proposed by Holmes et al. [1]
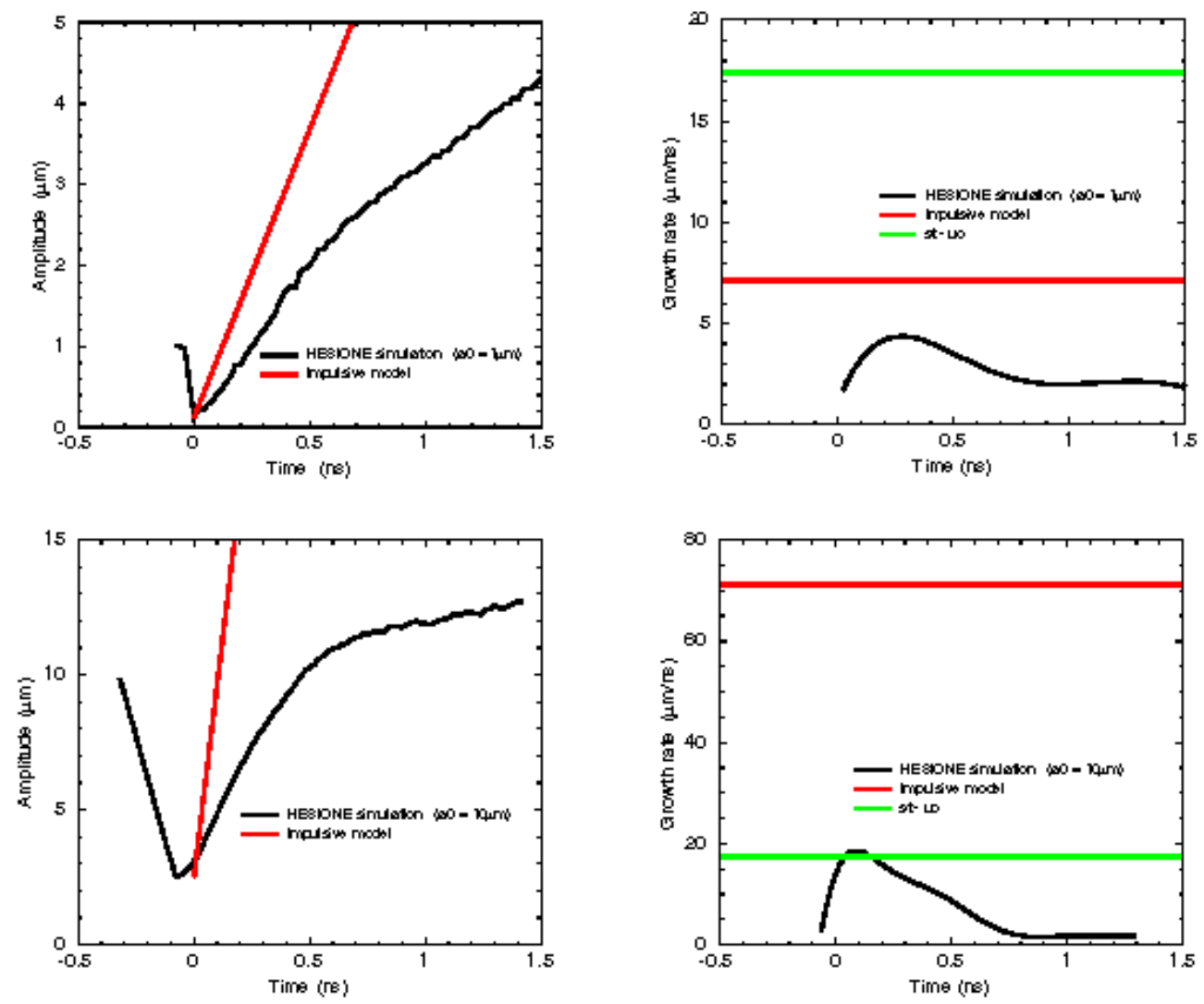

$\square$ Time is referenced to when the shock completes the interface traversal for each of the computations.

$\square$ For $\mathrm{a} 0=1 \mu \mathrm{m}$ the impulsive model leads to a growth rate lower than st-uc. $\Rightarrow$ The maximum growth rate given by HESIONE is of the order of $60 \%$ of the growth rate given by the impulsive model.

$\square$ For a0 $=10 \mu m$ the growth rate given by the impulsive model is four times higher than st-uc which is the limiting factor (the maximum growth rate given by HESIONE is of the order of st-uc).

[1] R. L. Holmes et al.

Richtmyer-Me shkow Instability growth : experiment, simulation and theory

J. Flu id Mech ., vol. 389 , pp. 55-79 (1999) 


\section{D eulerian computations (HESIONE code)}

Saturation of the growth rate

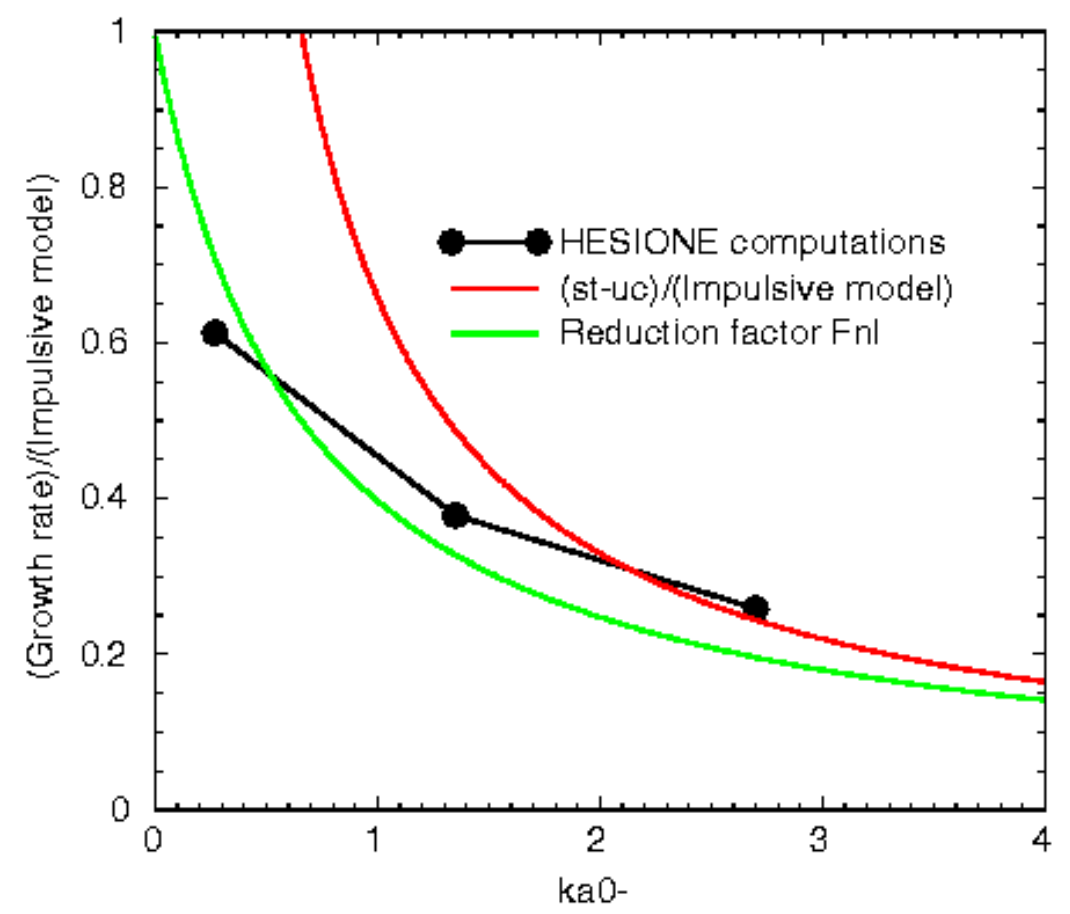

The ratio of the actual growth rate to the IM growth rate (IMgr) is lower than 1 and lower than (st-uc)//Mgr

$\square$ Holmes et al. [1] propose a heuristic reducing factor Fnl which gives the IM growth rate in the linear limit:

$$
\mathrm{Fnl}=1 /\{1+\mathrm{IMgr} /(\mathrm{st}-\mathrm{uc})\}
$$

$\square$ Our results $(M \approx 20)$ are similar to Holmes $(M=15.3)$

[1] R. Holmes et af.

Richtmyer-Meshkov inst ability growth : experiment, simulation and theory

J. Fluid Mech., vol. 389, pp. 55-79 (1999) 


\section{D eulerian computations (HESIONE code)}

Estimation of the transition time

Modifications of the Gregoire's model :

$\square$ The IM growth rate is replaced by the maximum growth rate given by the simulation to calculate tnl and tturb (low value of $\tau$ tr)

$\square$ The growth rate at thl can be used instead of the maximum growth rate to calculate tturb (high value of $\tau$ tr)

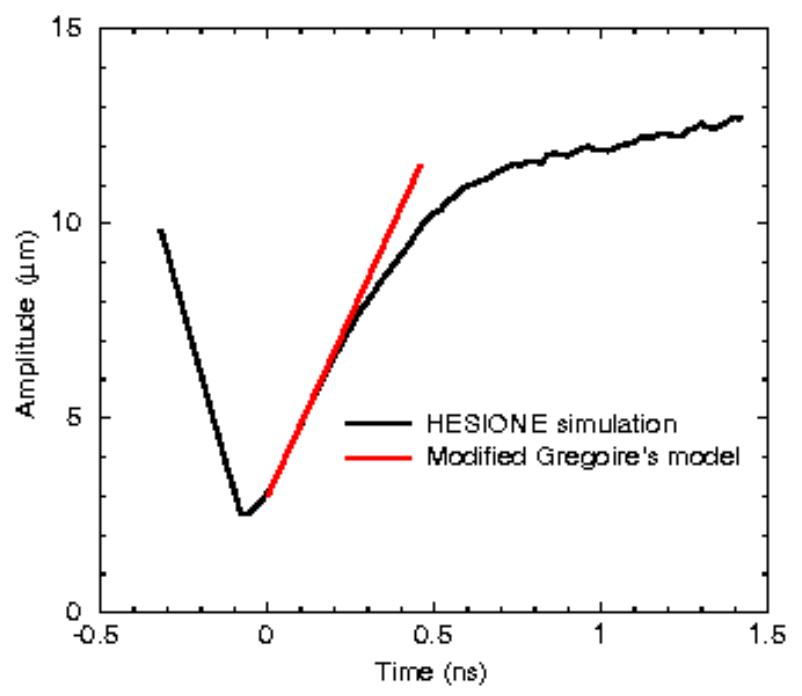

$$
1.2 \mathrm{~ns}<\tau \operatorname{tr}<1.9 \mathrm{~ns}
$$

(after shock passage across the interface) 


\section{k- $\varepsilon$ computations [1] Initial conditions}

- The $k-\varepsilon$ model is a two equations statistical model (one equation for $k$, the turbulent kinetic energy and one equation for $\varepsilon$, its dissipation rate)

$\square \mathrm{k}$ and $\varepsilon$ have to be defined at $\tau_{\mathrm{tr}}$

a $k$ and $\varepsilon$ have the same extension $L$ at $\tau_{\mathrm{tr}}$ (the mixing zone width at $\tau_{\mathrm{tr}}$ ) and have a symmetric triangular shape

$$
\begin{aligned}
& \mathrm{L}=2 \alpha(1+\beta) \mathrm{A}^{+} \Delta \mathrm{U} \tau_{\text {tr }} \text { (Mikaelian [2]) } \\
& \mathrm{k}_{\text {max }}=\frac{4}{3} \alpha\left(\mathrm{A}^{+} \Delta \mathrm{U}\right)^{2} \\
& \varepsilon_{\text {max }}=\mathrm{C} \frac{\mathrm{k}_{\text {max }}^{3 / 2}}{\mathrm{~L}}
\end{aligned}
$$

This simple model has been widely used in classical shock tubes computations.

[1] S. Gauthier et al.

A $k-\varepsilon$ model for turbulent mixing in shock tube flows induced by Rayleigh-Taylor instability

Phys. Fluids. A 2 (9) 1685-1694, 1990

[2] K. O. Mikaelian

Turbulent mixing generated by Rayleigh-Taylor and Richtmyer-Meshkov instabilities Physica D, 36, 343, 1989 


\section{k- $\varepsilon$ computations}

Transition time estimation

k- $\varepsilon$ computations were carried out for three arbitrary values of $\tau \operatorname{tr}(0.5,1.0$ and $1.5 \mathrm{~ns})$

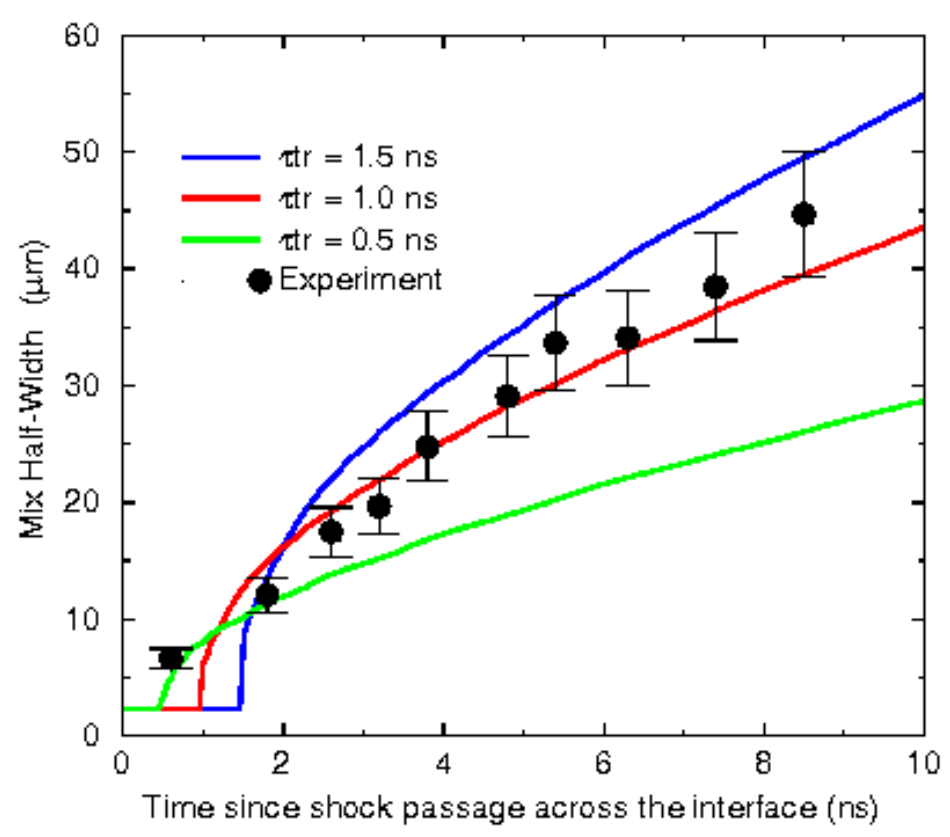

$\square$ The amplitude (Mix half-Width) history for $\tau \operatorname{tr} \approx \tau \operatorname{trlM}$ ( $\tau$ tr $=0.5 \mathrm{~ns}$ ) doesn't fit the experimental data

$\square$ A reasonable fit for the long term amplitude is obtained for $1.0 \mathrm{~ns}<\tau \mathrm{tr}<1.5 \mathrm{~ns}$

$\square$ This $\tau$ tr range reasonably agrees with the Gregoire's model applied to HESIONE computations :

$1.2 \mathrm{~ns}<\tau \mathrm{tr}<1.9 \mathrm{~ns}$ 


\section{k- $\varepsilon$ computations}

Ramshaw model [1] and k- $\varepsilon$ model
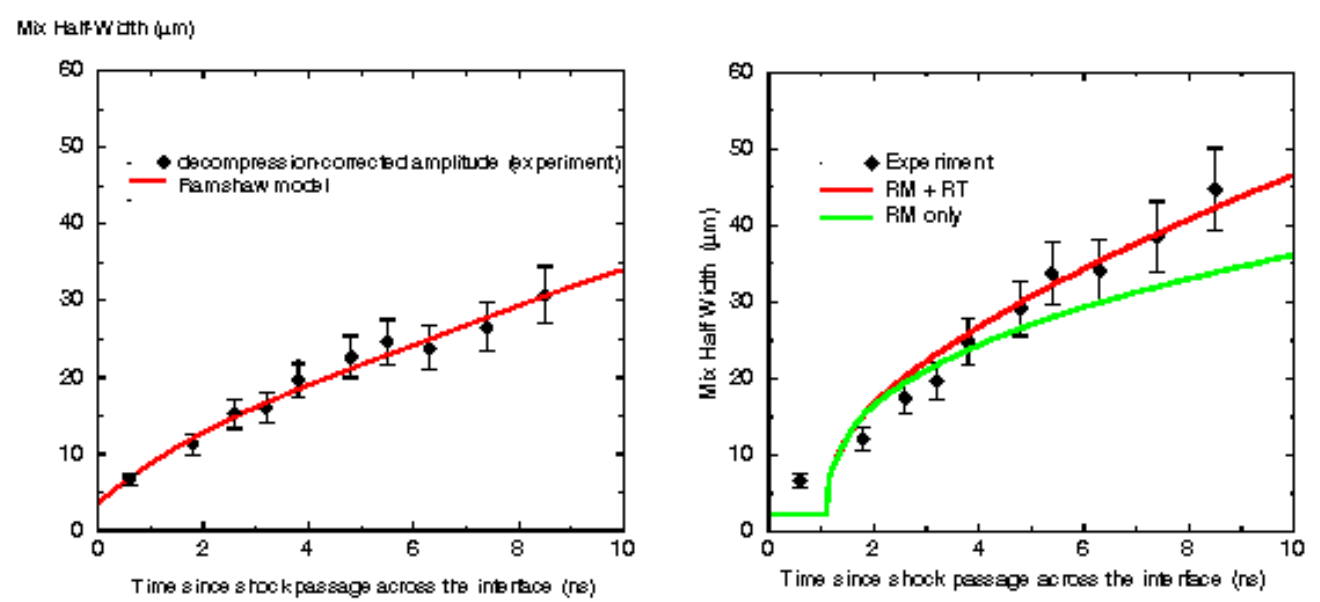

$\square$ A reasonable fit for the long term amplitude is obtained either with the Ramshaw model or with the $k-\varepsilon$ model $(\tau t r=1.125 \mathrm{~ns})$

$\square$ The k- $\varepsilon$ transient phase doesn't permit a satisfactory description of the beginning of the perturbation growth

$\square$ The pure RM case has also been considered in [1] (aRM/aRM+RT $=0.81$ at $10 \mathrm{~ns}$ ) and the $\mathrm{k}-\varepsilon$ model leads to a similar result (aRM/aRM+RT $=0.78$ at $10 \mathrm{~ns}$ )

[1] D. R. Farley et al.

High Mach number mix instability experiments of an unstable density interface using a single mode, nonlinear initial perturbation.

Physics of Plasmas, 6, N11, 1999 


\section{Summary}

$\square$ The Reynolds number of the flow induced by laser in the experiment analyzed here is of the order of magnitude of the Reynolds number obtained in classical shock tube experiments

- The transition time related to the Impulsive Model growth rate is very small as compared to the duration of the experiment

$\square$ The transition time related to the growth rate evaluated through 2D computations stays very shorter than the duration of the experiment

- The k- $\varepsilon$ computations fit the experimental data in a range of $\tau_{\mathrm{tr}}$ compatible with the former result 


\title{
A Mix-Model for One-Dimensional Simulations of Laser-Driven Implosion Experiments
}

\author{
D. Souffland and F. Renaud \\ (Commissariat à l'Énergie Atomique, DAM Île-de-France) \\ BP 12, 91680 Bruyères-le-Châtel, France
}

Acknowledgments: D. Galmiche, C. Cherfils-Clérouin, A. Grisollet (CEA/DIF) 
MéDiC: A tool to mimic hydrodynamic instability effects on laser targets

- Context:

Design of laser targets for LMJ facility

Studies upon the reduction of implosion efficiency due to the development of interface and front perturbations

Usefulness of $1 \mathrm{D}$ quick parametric studies

- By previous theoretical, experimental or multidimensional simulation studies, temporal evolution of the "mixing zone" is known

$($ thickness $=$ peak-to-valley distance, exchange area, mass, ...)

- Basic hypothesis:

Influence of the interpenetration of matter is modelized through diffusion processes although transition to full turbulent mixing do not occur (short wavenumber perturbations, in weakly non-linear stage, are still dominant) 
Application to the hydrodynamic instabilities at the ablator-fuel interface

- Cumulative effects of various processes: Richtmyer-Meshkov instability, feed-in, Bell-Plesset instability ...

Preliminary stages of the mixing of two separate fluids

Main process: mass transport

- Goal: interpretation of the HEP4 experiments at Omega

- Derivation of the mix model from a classical two-fluid formulation 


\section{Averaging the Two-Fluid Model $\Longrightarrow$}

\section{a mono-fluid model}

Hyp.: The 2 fluids have the same presssure

$\frac{\partial \rho}{\partial t}+\frac{\partial}{\partial x}(\rho u)=0$

$\frac{\partial}{\partial t}(\rho c)+\frac{\partial}{\partial x}\left(\rho c u+\rho c(1-c) u_{r}\right)=0$

$\frac{\partial}{\partial t}(\rho u)+\frac{\partial}{\partial x}\left(\rho u^{2}+P+\rho c(1-c) u_{r}^{2}\right)=0$

$\frac{\partial}{\partial t}(\rho E)+\frac{\partial}{\partial x}\left(\rho E u+P u+\rho c(1-c)\left(h_{1}-h_{2}\right) u_{r}+\rho c(1-c)\left(u u_{r}^{2}+\left(\frac{1}{2}-c\right) u_{r}^{3}\right)\right)=S$

where $\rho, u, P$ and $E$ are the density, the velocity, the pressure and the total energy of the mixture. $c$ the mass fraction of fluid $1, h_{1,2}$ are the partial enthalpies of each fluid and $u_{r}$ the relative velocity between each fluid.

Basic hypothesis: Diffusion type closure $\rho c(1-c) u_{r} \equiv-\rho \mathcal{D} \frac{\partial c}{\partial x} \Longrightarrow$ 


\section{Equations for the mixing in a monofluid 1D lagrangian code}

Extra hypothesis: isothermal mixing $\left(h_{i}=\mathcal{F}\left(\rho_{i}, T\right)\right)$

relative velocity is small $\left(u_{r}<<u\right) \Longrightarrow$ "green terms" neglected

Source terms for internal energies equations: thermal conduction, thermalization and radiative contributions

$\frac{\partial \rho}{\partial t}+\frac{\partial}{\partial x}(\rho u)=0$

$\rho \frac{D c}{D t}=\frac{\partial}{\partial x}\left(\rho \mathcal{D} \frac{\partial c}{\partial x}\right)$

$\rho \frac{D u}{D t}=-\frac{\partial P}{\partial x}$

$\rho \frac{D e_{i}}{D t}=-P \frac{\partial u}{\partial x}+\frac{\partial}{\partial x}\left(\rho\left(h_{1}-h_{2}\right) \mathcal{D} \frac{\partial c}{\partial x}\right)+\frac{\partial}{\partial x}\left(\mathcal{K}_{i} \frac{\partial T_{i}}{\partial x}\right)+$ Therm

$\rho \frac{D e_{e}}{D t}=-P \frac{\partial u}{\partial x}+\frac{\partial}{\partial x}\left(\mathcal{K}_{e} \frac{\partial T_{e}}{\partial x}\right)-$ Therm. + Rad.

with $\frac{D}{D t}$ the Lagrangian derivative, $e_{i}, e_{e}, T_{i}$ and $T_{e}$ are the internal energies and temperatures

+ Multigroupe radiative method

$\equiv$ Modelling of the mass transport by "molecular mixing" with diffusion coefficient $\mathcal{D}$ 


\section{Determination of the diffusion coefficient}

The diffusion coefficient in the mass concentration equation, $\mathcal{D}(t)$, must be adjusted to give the requested mixing zone widening, $L_{m i x}(t)$

- For an interface at constant velocity the exact solution is known: $\mathcal{D}(t)=\frac{1}{8 \xi^{2}} L_{m i x} \frac{d L_{m i x}}{d t}(t)$, with $\xi=1.163$ for a $5-95 \%$ concentration profile

- For an homogeneous compression in spherical geometry, the flow is defined by the evolution of the interface location $\Lambda(t)$ A good approximation is given by $\mathcal{D}(t)=\frac{1}{8 \xi^{2}} L_{m i x}\left[\frac{d L_{m i x}}{d t}-\frac{L_{m i x}}{\Lambda} \frac{d \Lambda}{d t}\right]$

Moreover, the mass of the mixing zone can be approximate by $M_{m i x} \approx\left(\rho_{h}+\rho_{l}\right) 4 \pi \Lambda^{2} L_{m i x} / 2$ As, in these ideal flows, we have $\rho(t) \propto \Lambda^{-3}$, we get $M_{m i x}(t) \propto \frac{L_{m i x}}{\Lambda}(t)$

If we know $M_{m i x}(t)$ and $L(t)$, we thus can use the alternative expression $\mathcal{D}(t)=\frac{1}{8 \xi^{2}} L_{\operatorname{mix}}^{2}\left[\frac{1}{M_{\operatorname{mix}}} \frac{d M_{\operatorname{mix}}}{d t}\right]$ 
First results

The mix-model is implemented and validated for simple configurations

(two fluids with the same initial temperature mix without global motion)

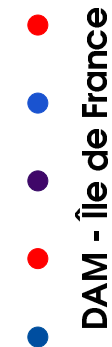

Example of He / DT mixing at $\mathrm{T}=30000 \mathrm{~K}$. Evolution of temperature, density and pressure profiles
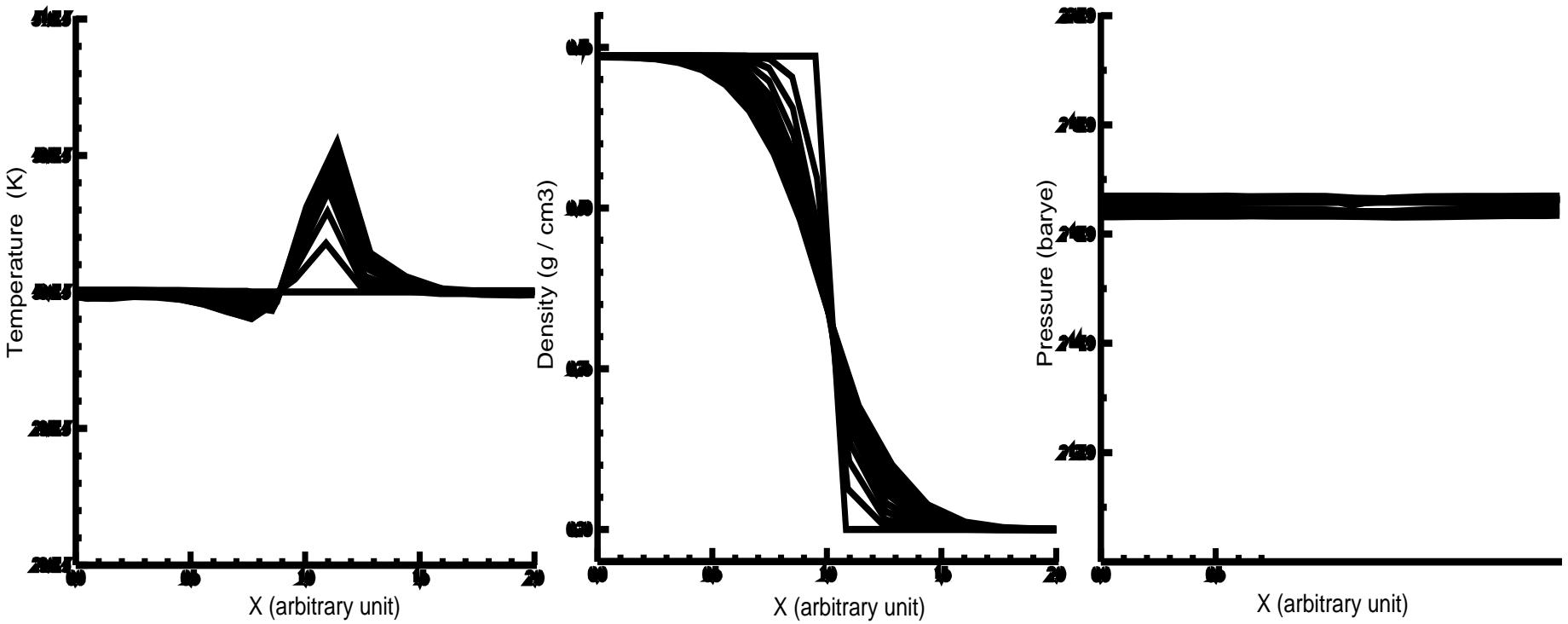

Future work: application to HEP4 experiments 


\section{Application to the hydrodynamic instabilities at the hot spot}

Characteristics:

- Development of an "ablation front like" instability inside the fuel volume between the heavy and cold corona and the light and hot central spot

- Here, the front is not linked to a material interface: its location has to be determined by appropriate criterium

- Partial enthalpy difference transport term is ineffective (same fluid, isothermal mixing)

- Perturbation of the isotherms increase exchange area for thermal conduction 


\section{Perturbation at the edge of the "in progress" hot spot}

In cryogenic capsules, the DT of the hot spot at ignition is made up of the internal part of cryogenic DT corona

During the implosion, an ablation front propagate into the initially cryogenic DT

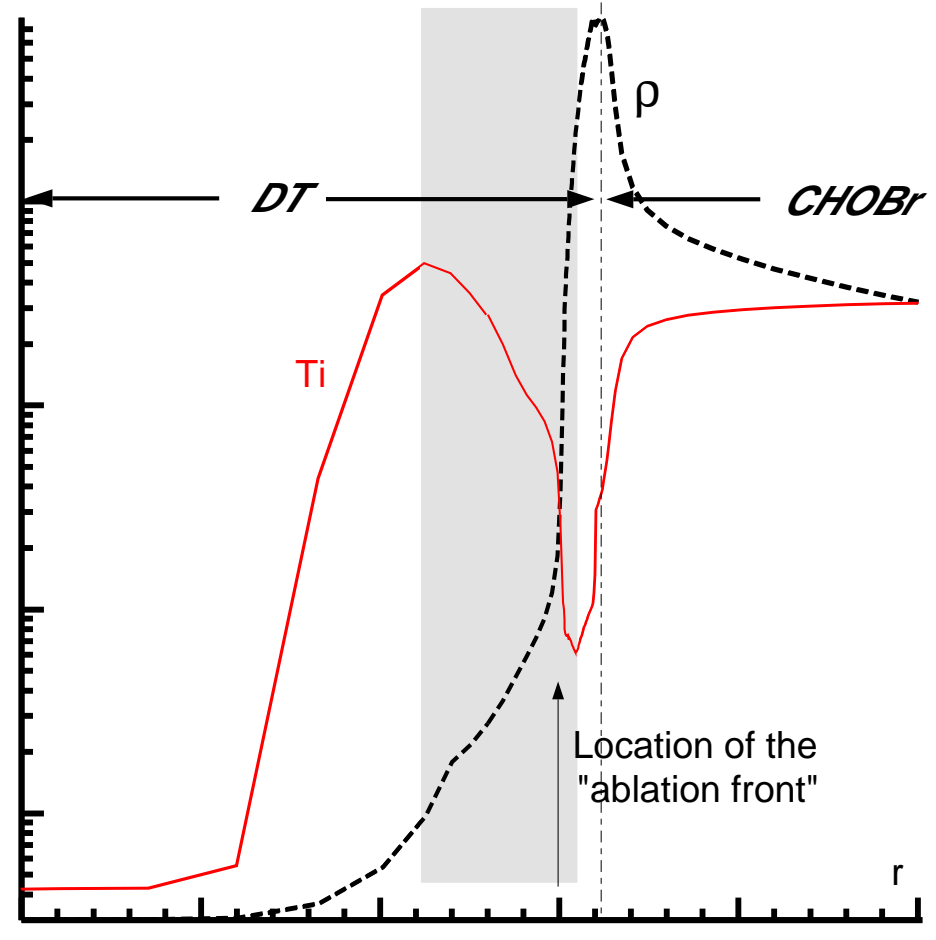

Temperature and density profiles during

We assume identity of hot spot edge, $R_{h s}$, and ablation front location

Ablation front location determination:

$$
\operatorname{Max}\left[2 \frac{\rho^{I+1}-\rho^{I}}{\rho^{I+1}+\rho^{I}} \times\left(T_{i o n}^{I}-T_{i o n}^{I-1}\right)\right]
$$

(based upon R-T instability criterium, first part $\longrightarrow$ local Atwood number)

Good agreement with other definitions of the hot spot, within the last $n s$ before the ignition time (see below)

the implosion (1D unperturbed simulation) 


\section{Modelling the instable front perturbation in a 1D code}

Previous modelling through a "mass transfer" is inappropriate

(perturbations within the DT, where $\Delta h_{i}=0$ : added term do not affect energy balance)

The main process is assumed to be the increase of exchange surface for thermal conduction:

within the perturbated zone $\left(R_{h s}-L_{m i x} / 2<x<R_{h s}+L_{m i x} / 2\right)$

thermal conduction fluxes will be multiplied by the ratio of areas $R a(t)=S_{p e r t u r b} / S_{s p h}$

Modified set of equations (plane geometry):

$\frac{\partial \rho}{\partial t}+\frac{\partial}{\partial x}(\rho u)=0$

$\rho \frac{D u}{D t} t=-\frac{\partial P}{\partial x}$,

$\rho \frac{D e_{i}}{D t}=-P \frac{\partial u}{\partial x}+\frac{\partial}{\partial x}\left(R a \mathcal{K}_{i} \frac{\partial T_{i}}{\partial x}\right)+$ Therm.

$\rho \frac{D e_{e}}{D t}=-P \frac{\partial u}{\partial x}+\frac{\partial}{\partial x}\left(R a \mathcal{K}_{e} \frac{\partial T_{e}}{\partial x}\right)-$ Therm. + Rad.

$R_{h s}$ is calculated through above method; $L_{m i x}, R a(t)$ are deduced from $2 \mathrm{D}$ simulation 


\section{Alternative modelling through a "diffusive model"}

Hypothesis: we add "mix" viscous and conductive terms

within the perturbated zone $\left(R_{h s}-L_{m i x} / 2<x<R_{h s}+L_{m i x} / 2\right)$

"mix viscosity", $\mathcal{D}_{u}$, and "mix conductivity", $\mathcal{D}_{e}$, (homogeneous to $l^{2} / t$ )

are deduced from $\mathcal{D}(t)=\frac{1}{8 \xi^{2}} L_{m i x}^{2}\left[\frac{1}{M_{m i x}} \frac{d M_{m i x}}{d t}\right]$ (see above)

- \& Modified set of equations (plane geometry):

- $\frac{\partial \rho}{\partial t}+\frac{\partial}{\partial x}(\rho u)=0$

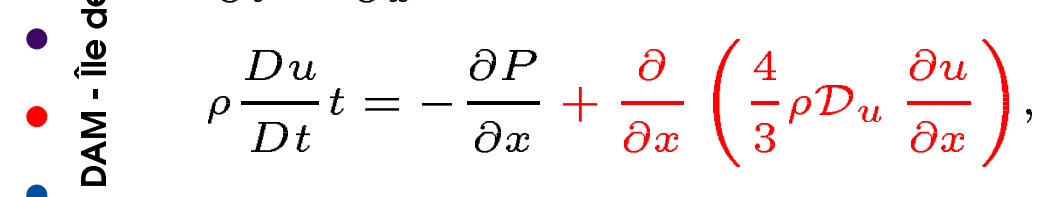

$\rho \frac{D e_{i}}{D t}=-P \frac{\partial u}{\partial x}+\frac{\partial}{\partial x}\left(\rho \mathcal{D}_{e} \frac{\partial e_{i}}{\partial x}\right)+\frac{\partial}{\partial x}\left(\mathcal{K}_{i} \frac{\partial T_{i}}{\partial x}\right)+$ Therm. $+\left(\frac{4}{3} \rho \mathcal{D}_{u} \frac{\partial u}{\partial x}\right) \frac{\partial u}{\partial x}$,

$\rho \frac{D e_{e}}{D t}=-P \frac{\partial u}{\partial x}+\frac{\partial}{\partial x}\left(\rho \mathcal{D}_{e} \frac{\partial e_{e}}{\partial x}\right)+\frac{\partial}{\partial x}\left(\mathcal{K}_{e} \frac{\partial T_{e}}{\partial x}\right)-$ Therm. + Rad. 


\section{Example: simulations of the L1215 capsule}

\section{to recover nonlinear multimode $2 D$ simulations}

Description of the configuration and simulations (Cherfils et al., APS 43rd Annual Meeting, Long Beach CA, 2001):

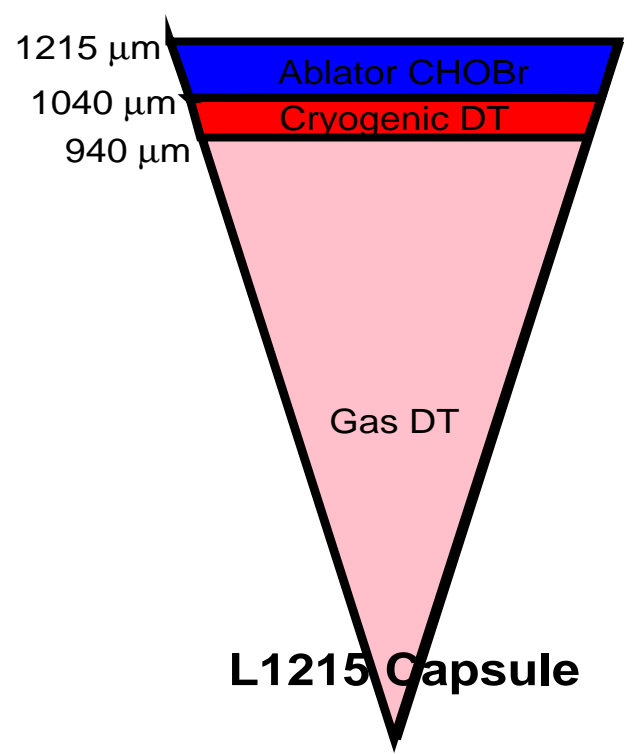

Half-spheres simulations

FCI2 code ("driven" ALE method)

$t_{i}=17.45 n s$,

$V_{D T}^{\max }=3.910^{7} \mathrm{~cm} / \mathrm{s}$
Multimode initial perturbations:

Ablation surface $r m s(l \geq 10)=10 \mathrm{~nm}$,

Cryogenic DT surface $r m s(l \geq 10)=1 \mu m$

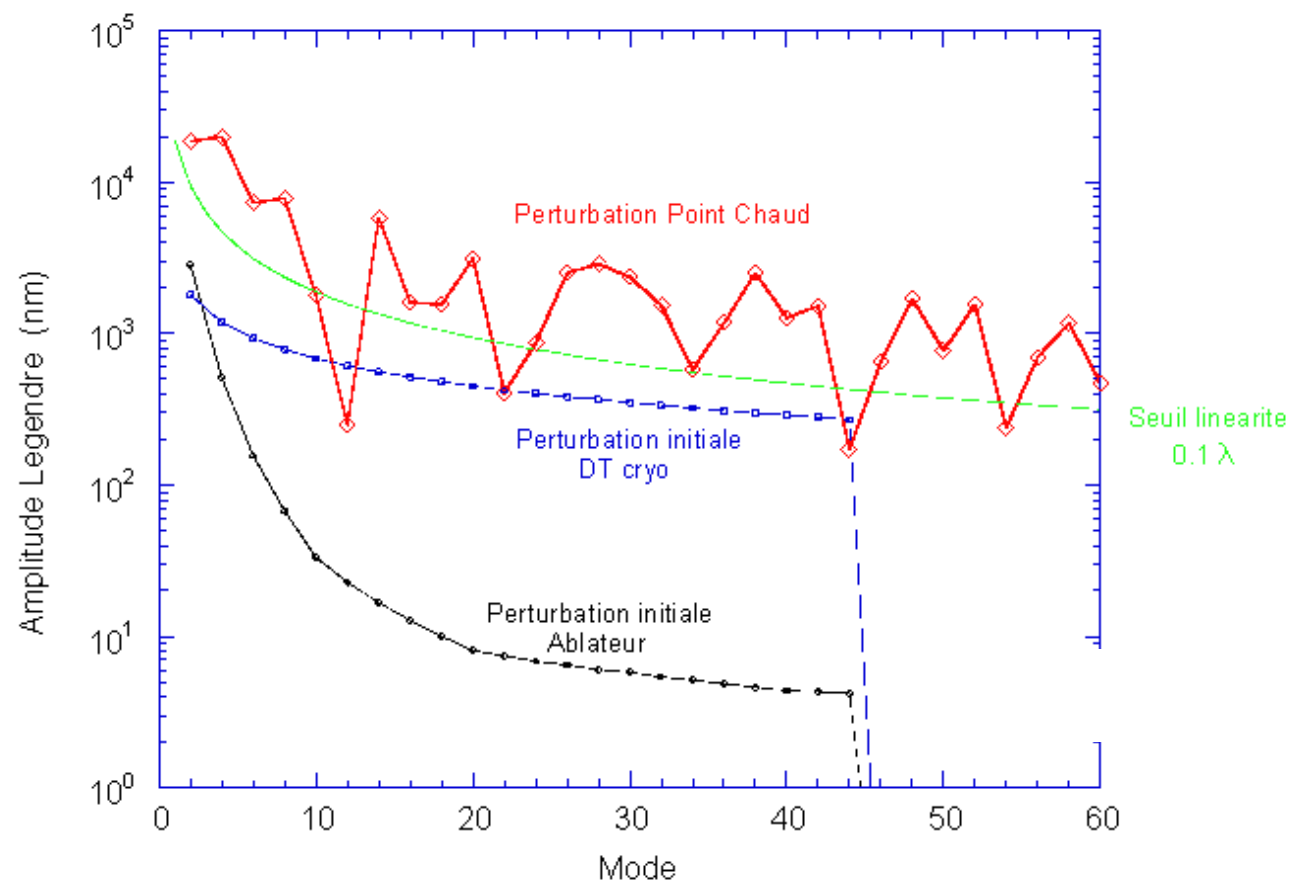




\section{Hot Spot front tracking}

3 ways to track the Hot Spot front:

1) Search the isotherm $T_{\text {ion }}=50 \mathrm{MK}(-)$.

2) Search the isotherm $T_{i o n}=\operatorname{Max}\left(T_{i o n}\right) / 10(-)$.

3) Search the location of the instable front:

$\left[2 \frac{\rho^{I+1}-\rho^{I}}{\rho^{I+1}-\rho^{I}} \times\left(T_{i o n}^{I}-T_{i o n}^{I-1}\right)\right]$ maximum, for each angle $\theta(-)$

this last method can be applied even before the converging time of the shock waves

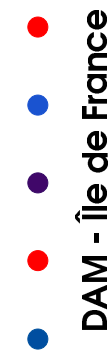
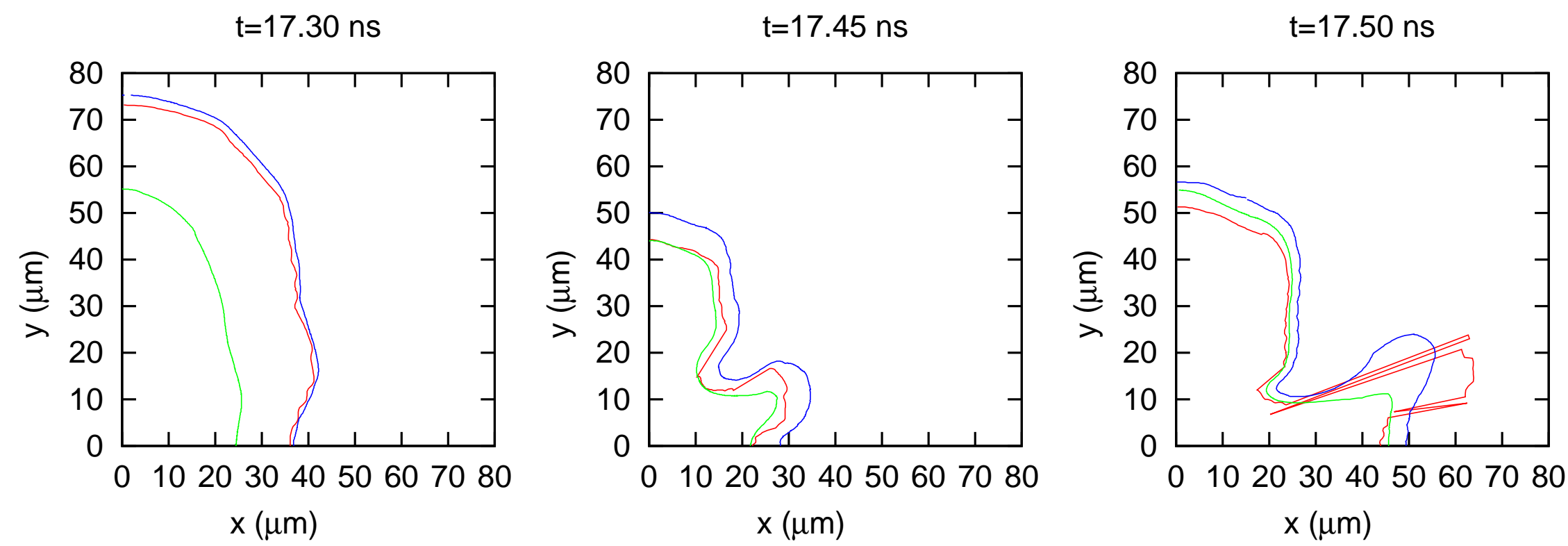

Comparison of Hot Spot fronts for 2D simulation around the ignition time 


\section{Post-processing 2D simulation results}

From the instable front contour

Minimal radius and maximal radius of the Hot Spot front

$\Longrightarrow 1 \mathrm{D}$ mixing length $L_{\text {mix }}$ and mean radius $R_{\mathrm{hs}}$

Integration of the Hot Spot front

$\Longrightarrow 1 \mathrm{D}$ thermal exchange surface in the mixing zone $S_{\text {perturb }}$

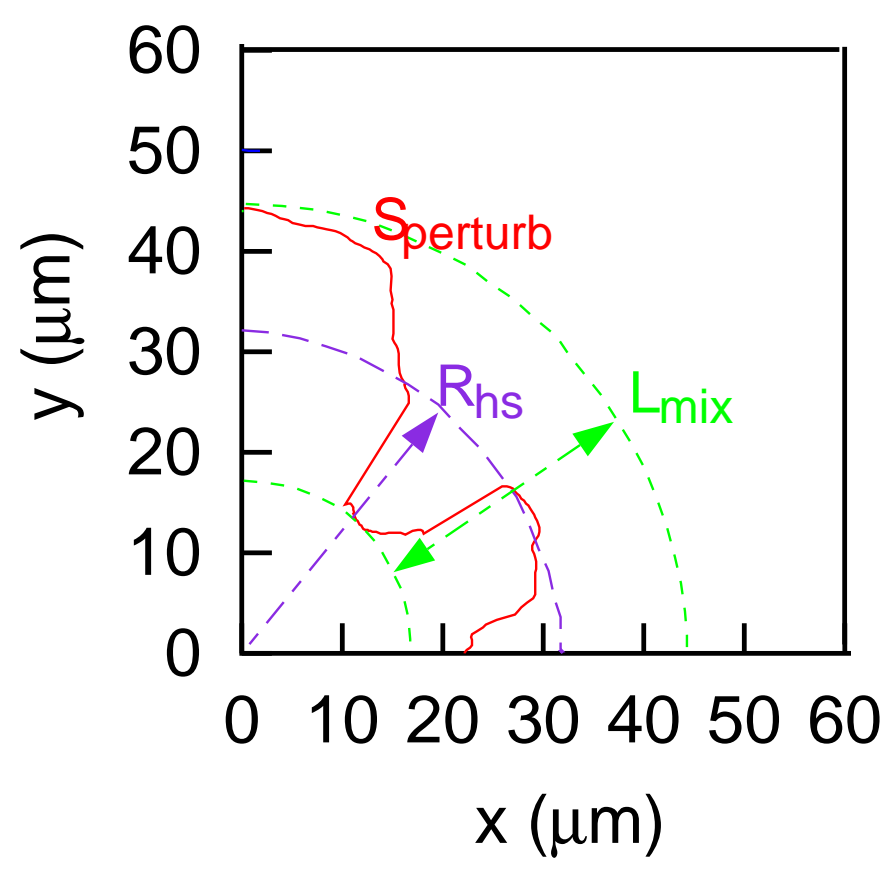


Satisfactory description of the mean hot spot characteristics

The evolution of the mass of DT within the hot spot

and its mean ionic temperature are simulated in 1D calculations
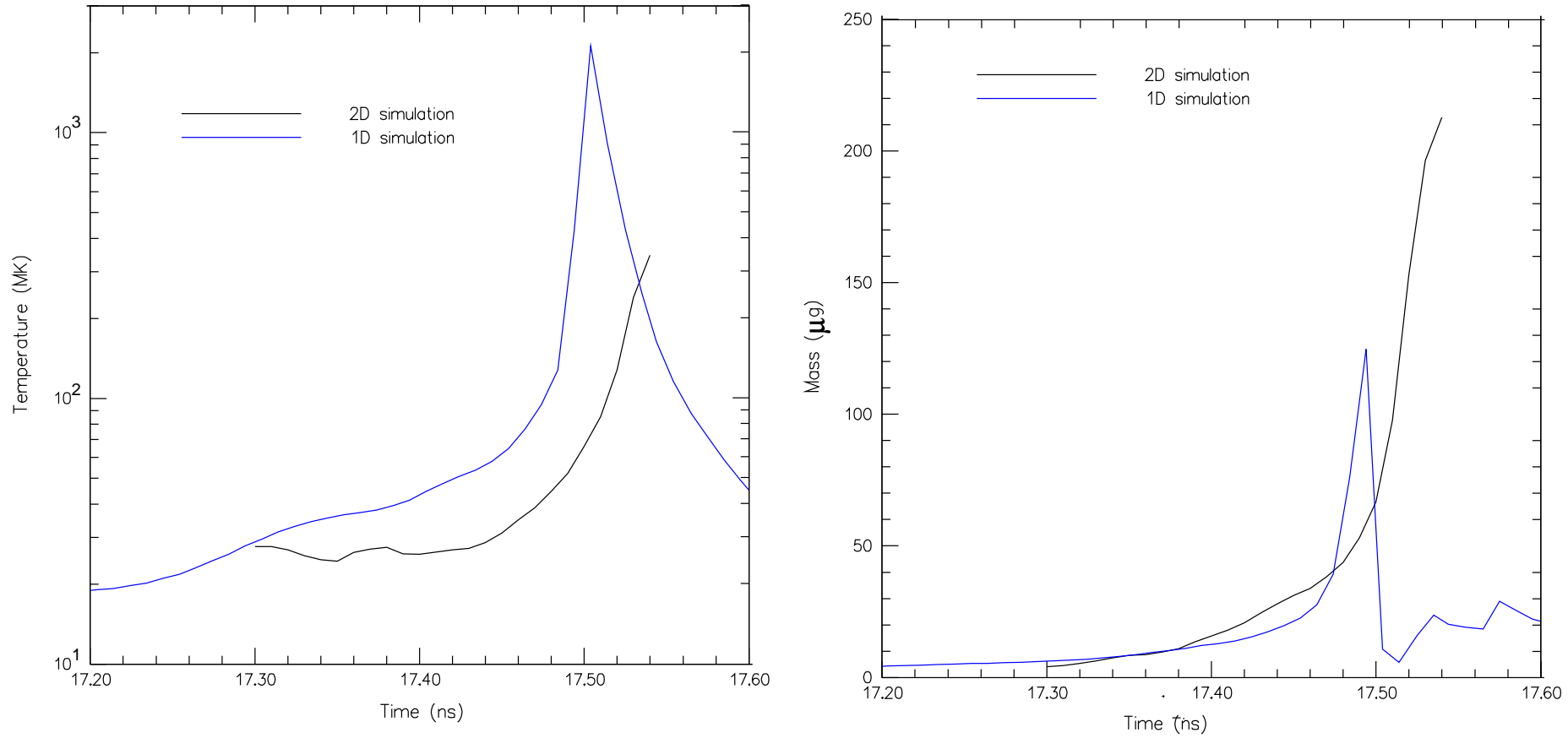

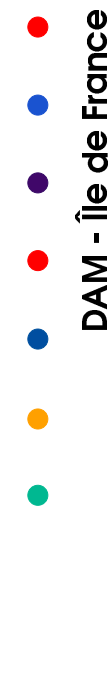




\section{D models fail to represent all 2D flow complexity}

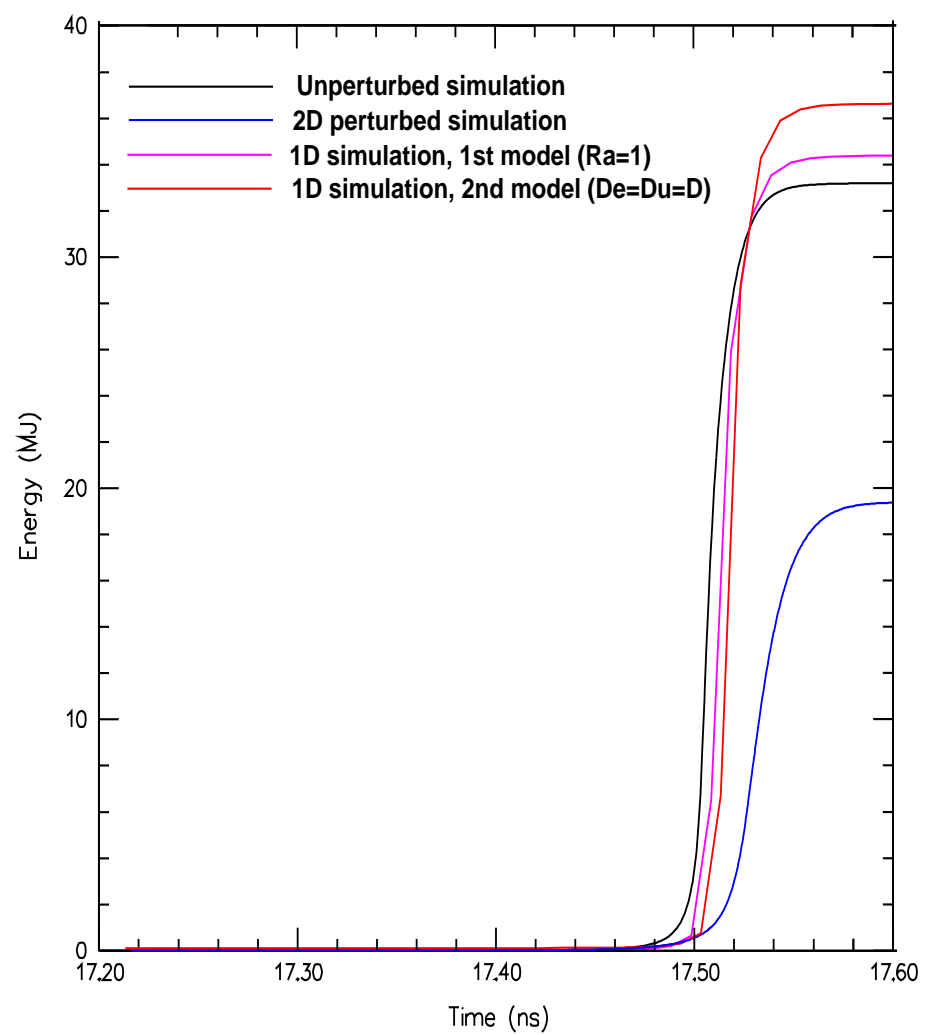

Comparison of energy evolution
With the two mix models,

the relative delay to ignition is underestimated

The implosion is more efficient but closer to the threshold

The non-sphericity of perturbed 2D implosion explain the spread of the energy production 

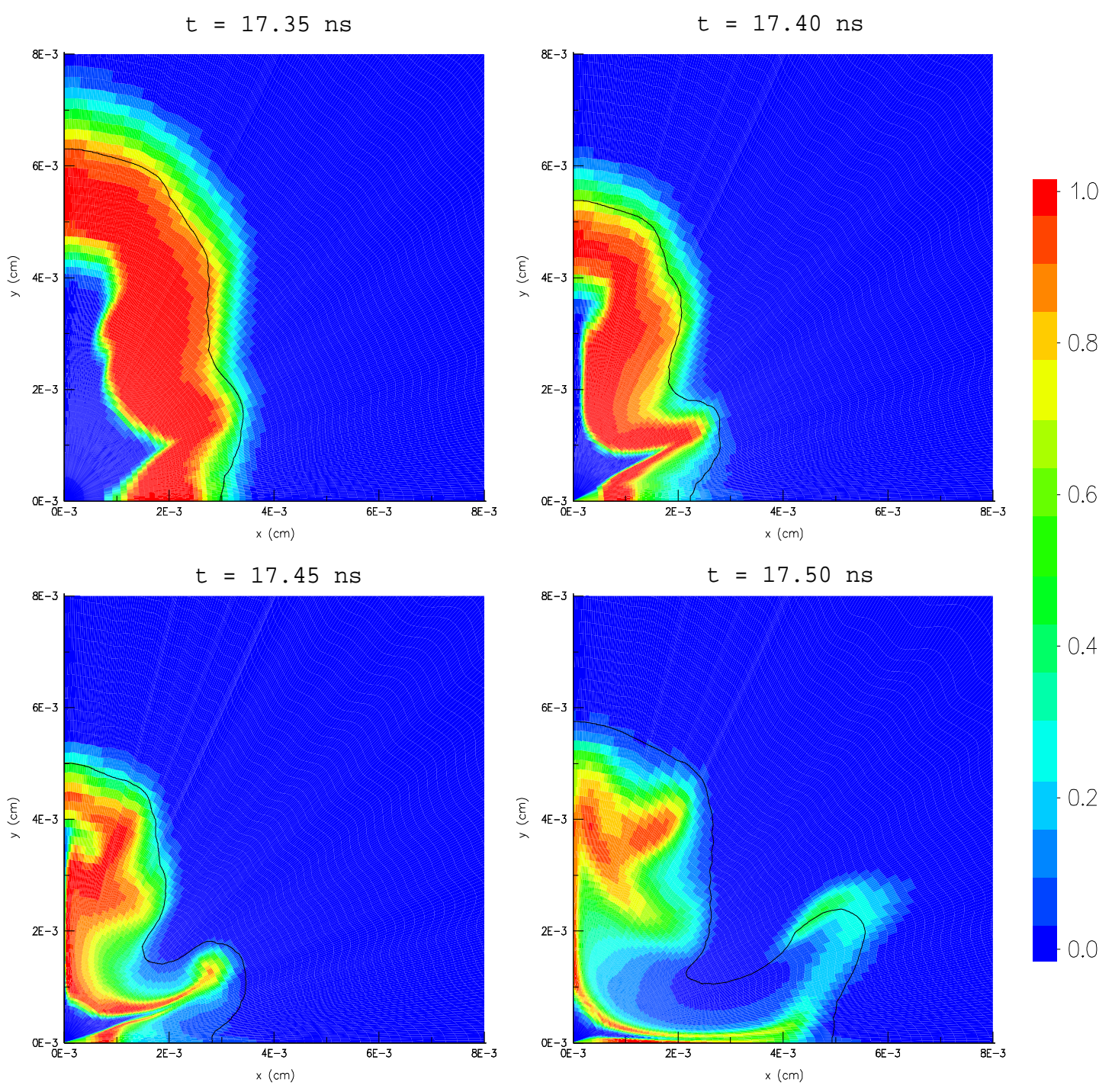

Comparison of the Hot Spot contours $\left(T=\left(\operatorname{Max} T_{i o n}\right) / 10\right)$ with the mass concentrations of the cryogenic DT entering in the Hot Spot at the ignition time.

Iso-concentration contours are more perturbed than Iso-temperature contours 


\section{Simulation of the ignition threshold}

$2 \mathrm{D}$ specific behavior (low mode deformation of the Hot Spot contours, possible mean viscous effects, ) is not recovered by nominal values of $1 \mathrm{D}$ models

We realize parametric studies on $R a$ and $\mathcal{D}_{e} / \mathcal{D}$ to obtain ignition threshold

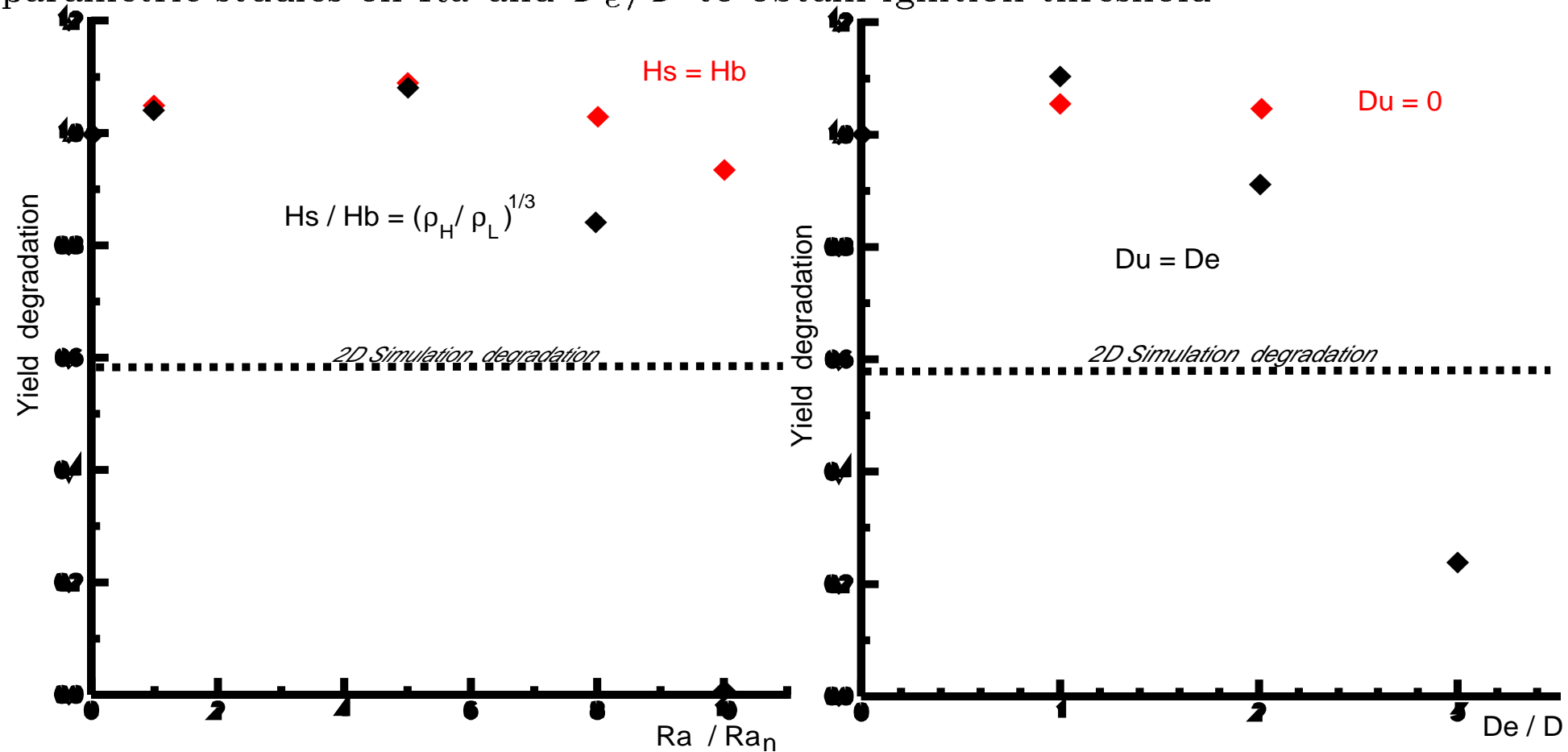

To recover the $2 \mathrm{D}$ simulation degradation we need to multiply by 8 the nominal ratio area

$R a_{n} \equiv S_{\text {perturb }} / S_{s p h}$ (with an asymetric development of "spikes" and "bubbles")

The simulation with the "diffusive model" show the importance of the added term in velocity equation 


\section{Prospects}

- These preliminary results open several ways

- For Hot Spot perturbations we envisage to model separately low modes and high modes

- The modelling of an "average viscous effect" on the velocity profiles seems essential

- The modelling of hydrodynamic instabilities at interfaces could take advantage of the above ideas 


\section{Modeling Turbulent Mixing in Inertial Confinement Fusion Implosions}

Y. Srebro, D. Kushnir, Y. Elbaz and D. Shvarts

Nuclear Research Center - Negev, Israel.

Ben-Gurion University, Beer-Sheva, Israel.

Hebrew University, Jerusalem, Israel. 


\section{Experiments}

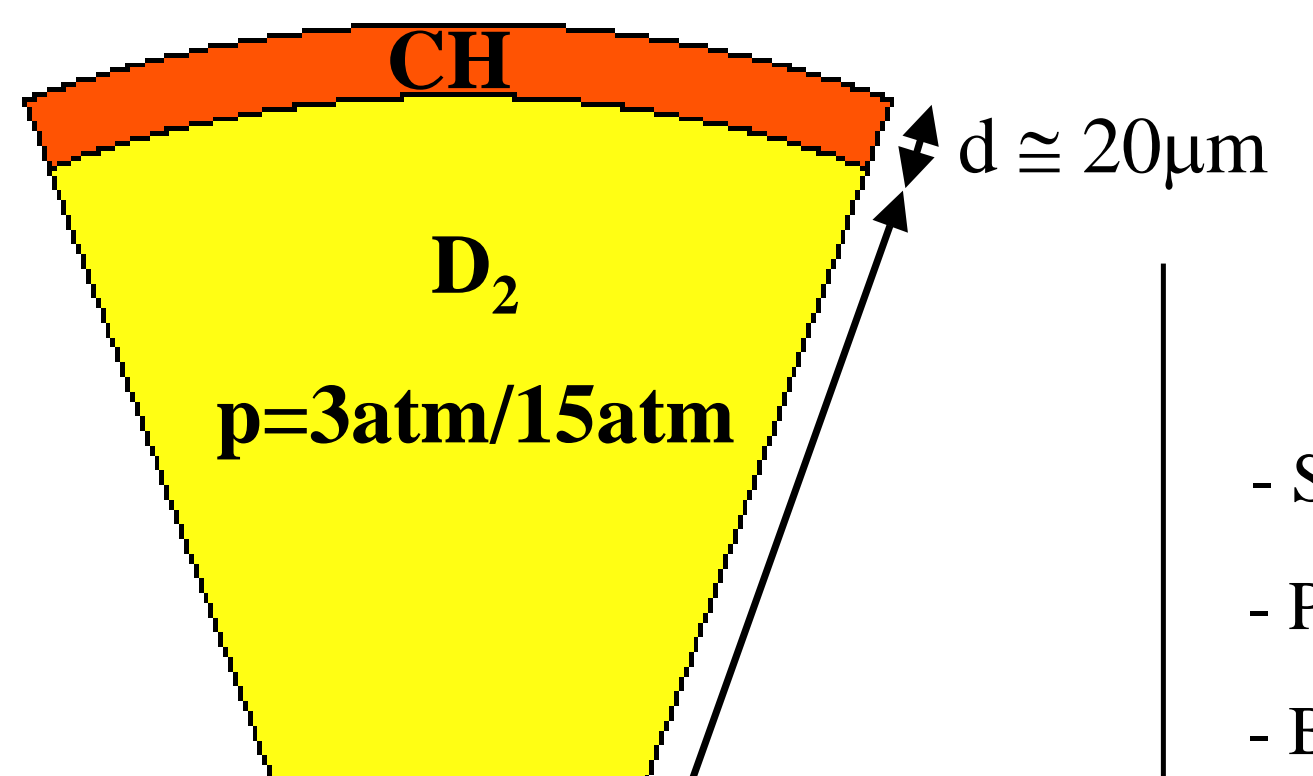

\section{Laser: \\ $\mathrm{E} \cong 20 \mathrm{~kJ} \quad \mathrm{t} \cong 1 \mathrm{~ns}$ \\ Perturbations :}

- Surface roughness (outer and inner)

- Power imbalance between beams

- Beam nonuniformity (1THz 2D-SSD)

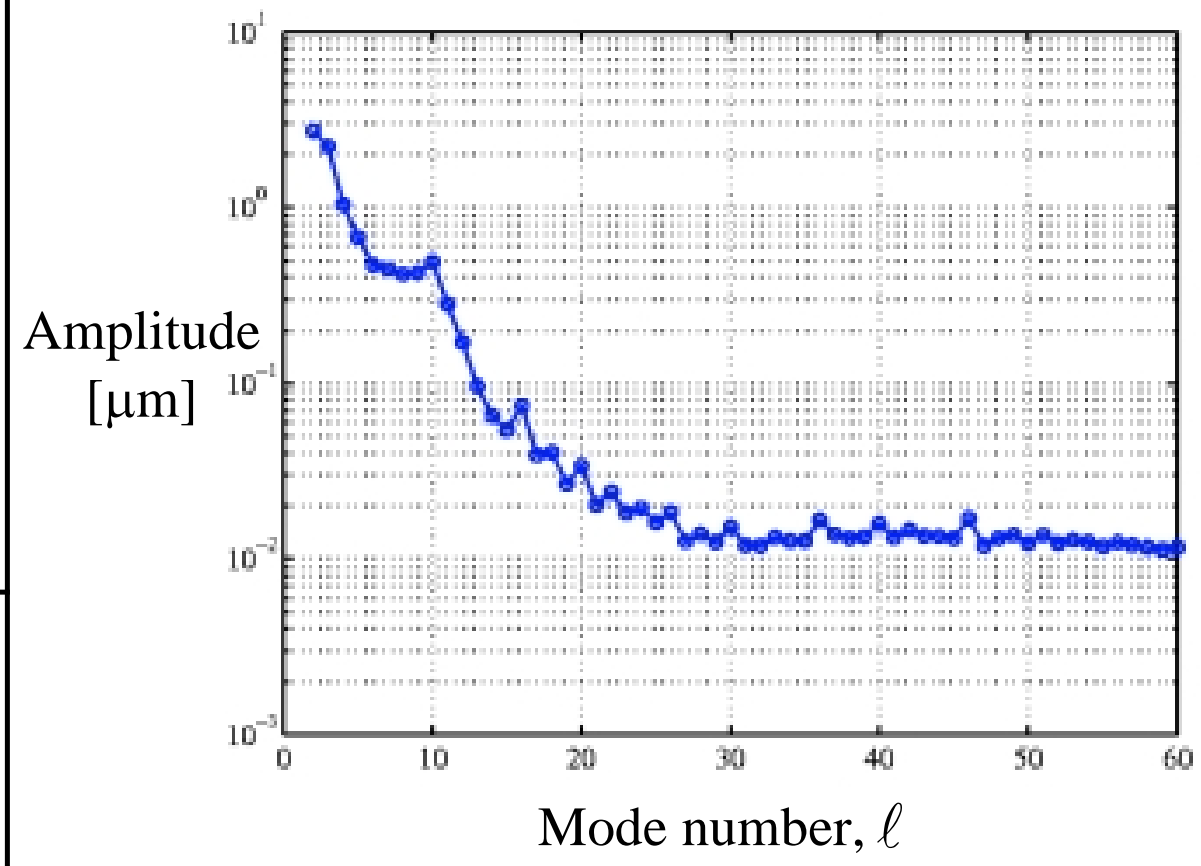

Experiments held at Laboratory for Laser Energetics, U. of Rochester

Mode number, $\ell$ 


\section{Experimental results}

Y.O.C. $=\frac{Y_{\exp }}{Y_{1 D}}$
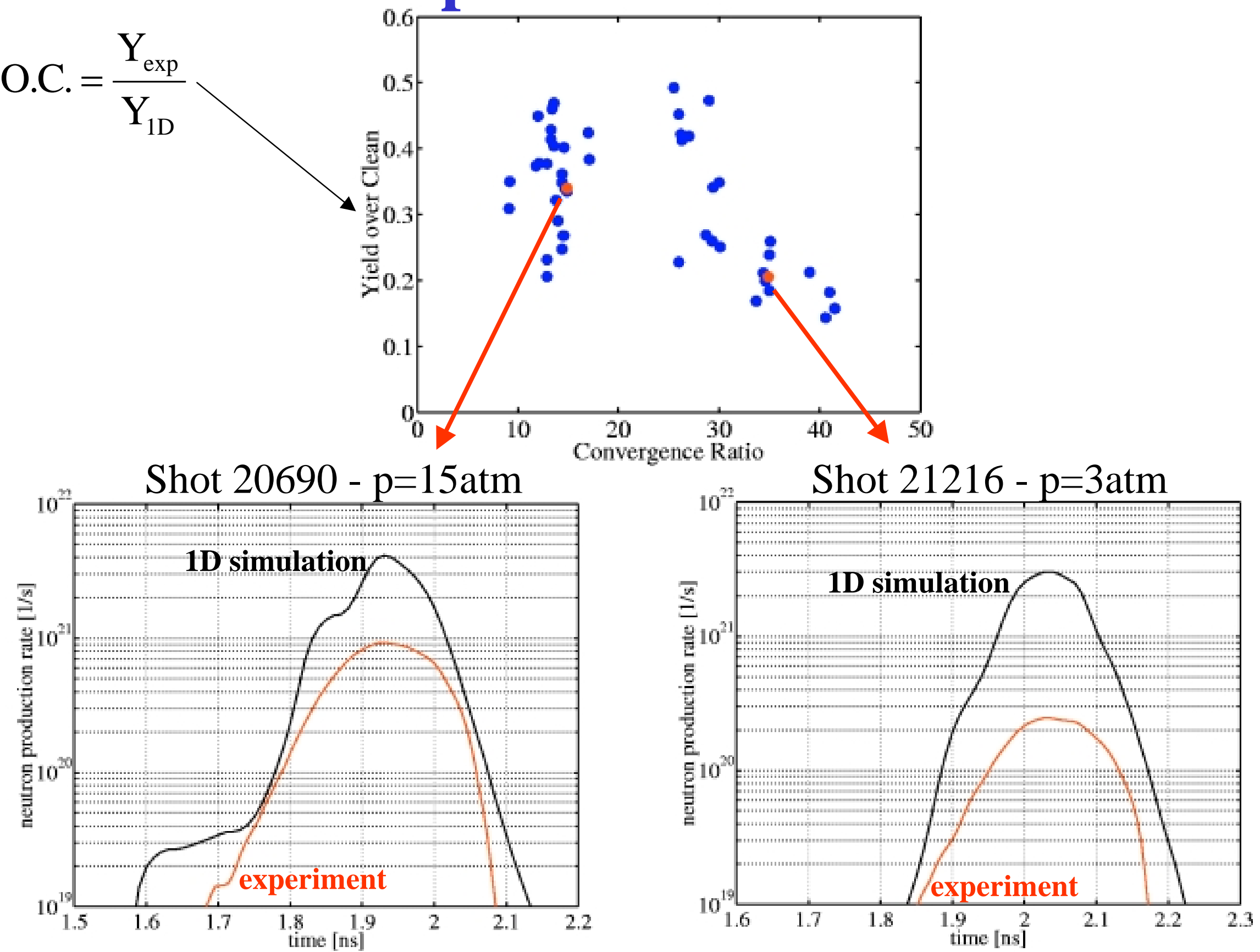


\section{D Simulation}

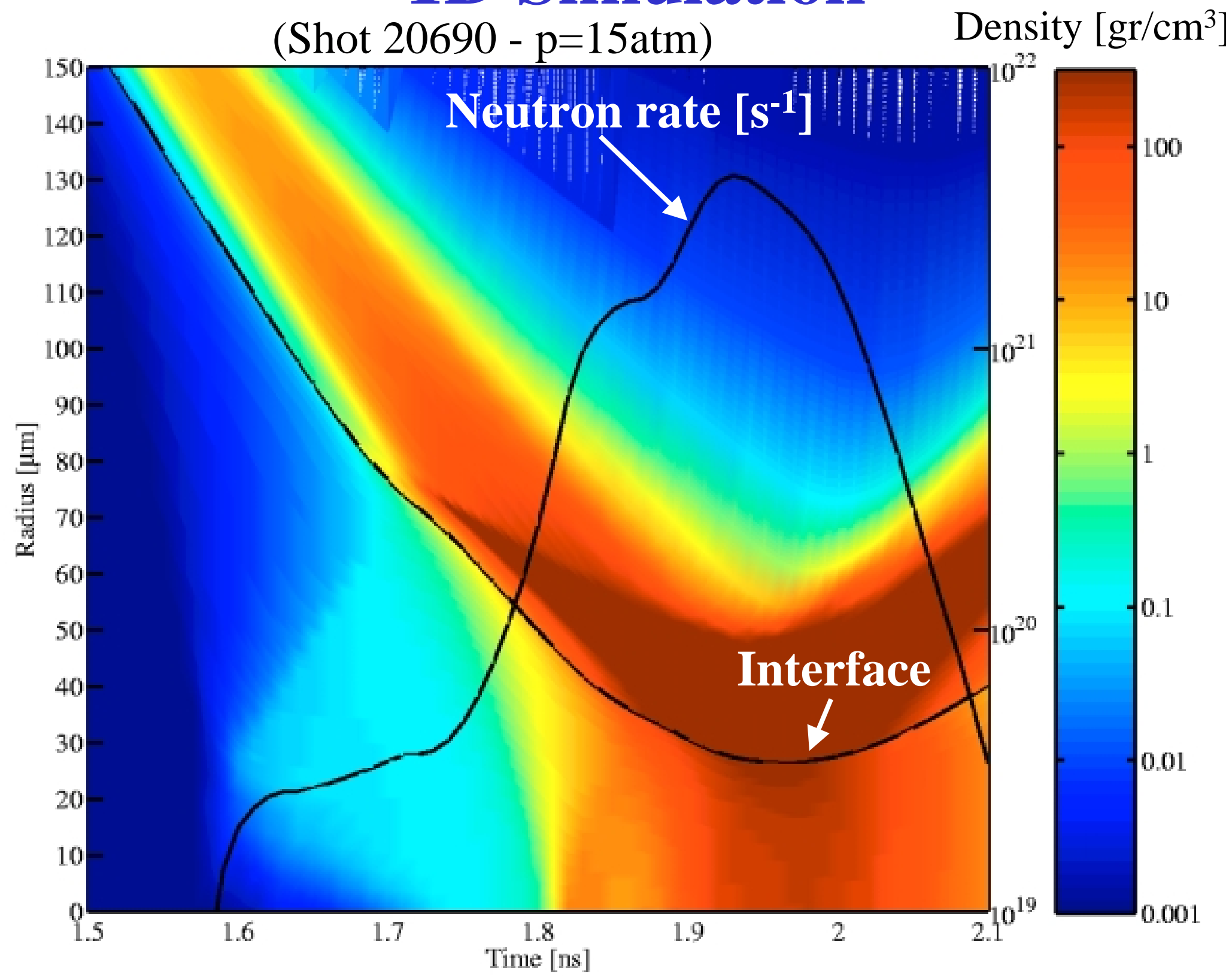

Fusion rate is dominated by shock dynamics 


\section{D multi-mode simulation}

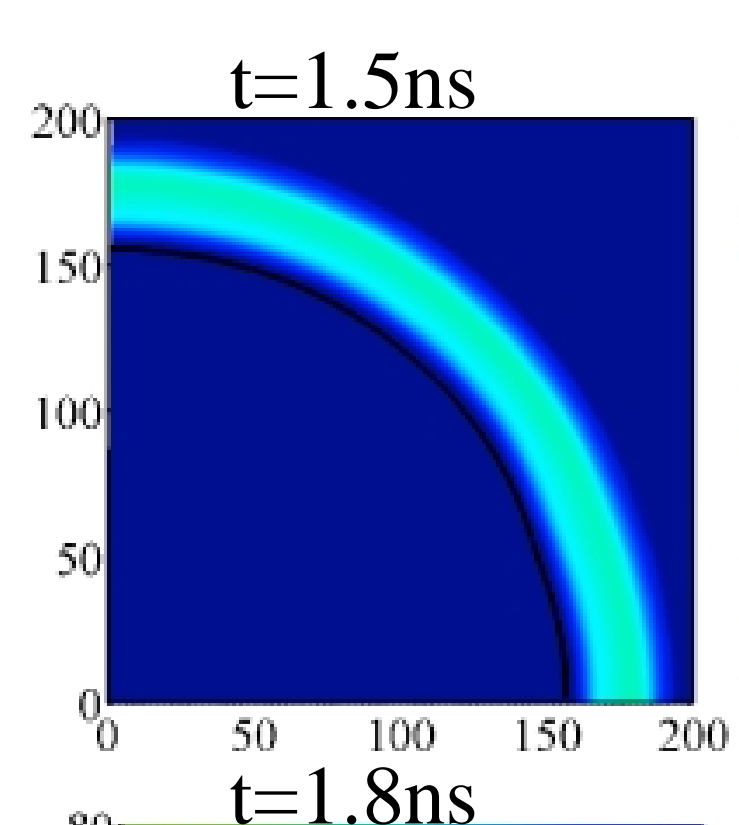

(Shot $20690-\mathrm{p}=15 \mathrm{~atm}$ )

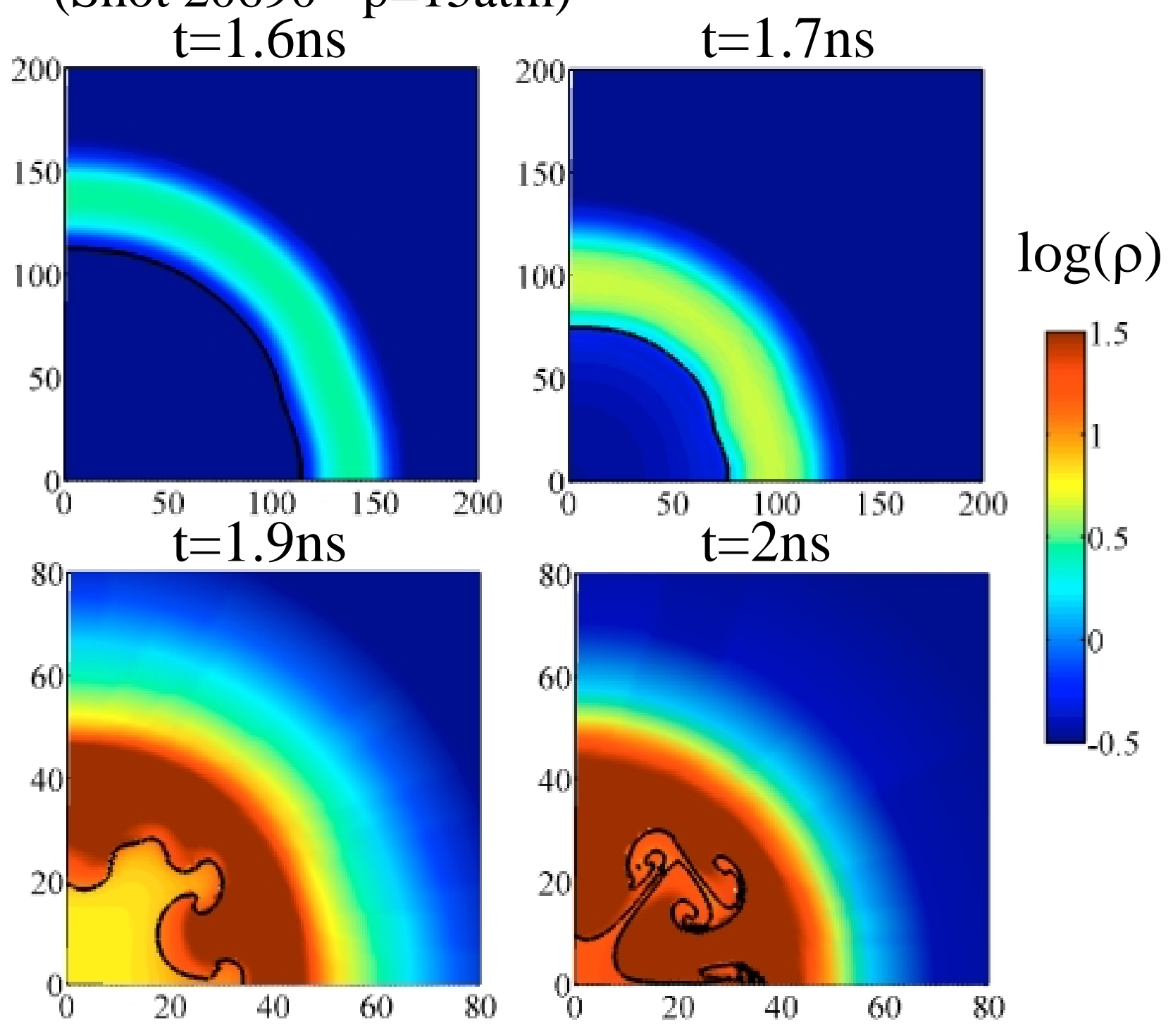

Perturbation dominated by power imbalance $(\ell \approx 6)$ 


\section{Neutron yield}

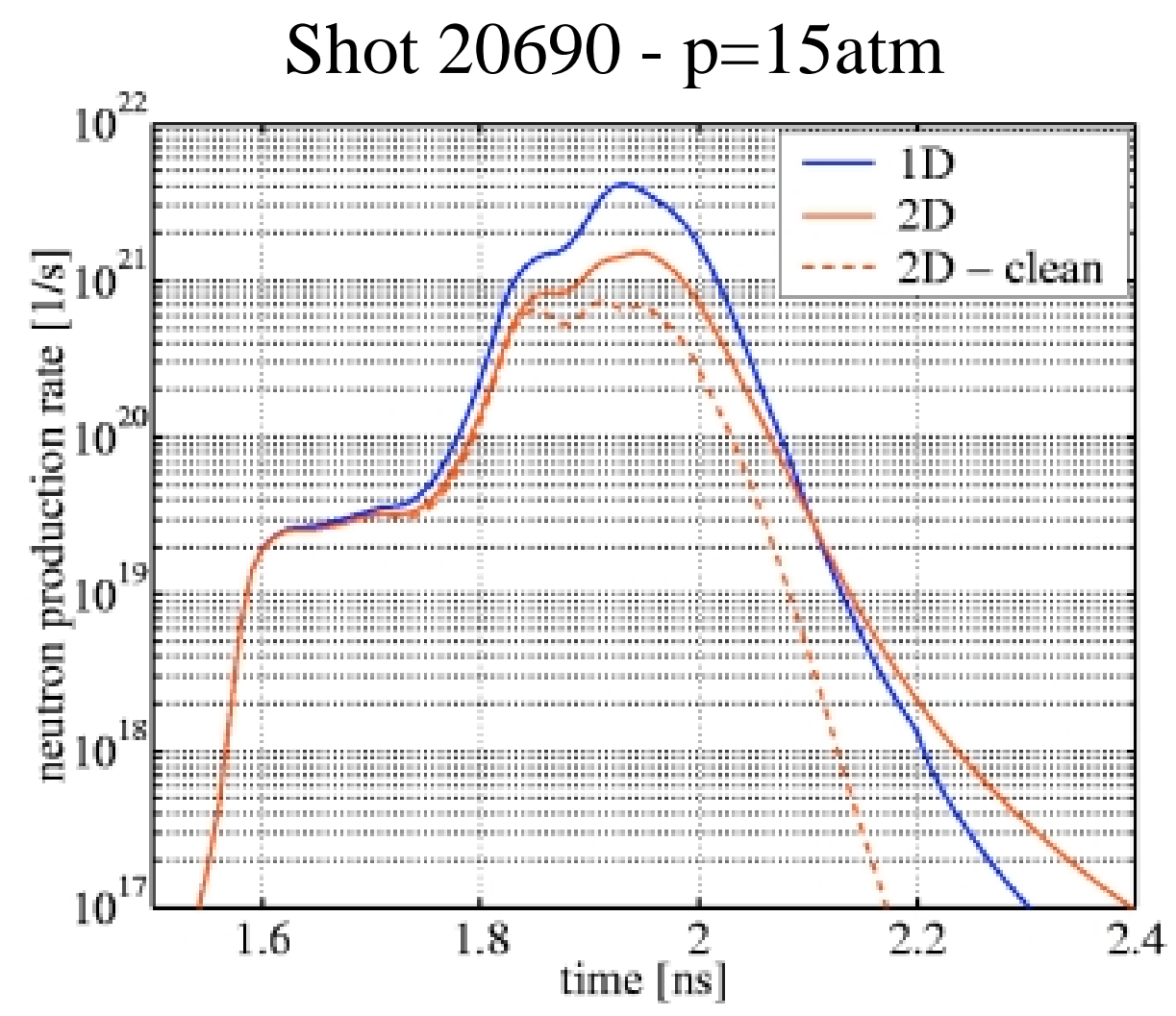

Shot $21216-p=3 a t m$

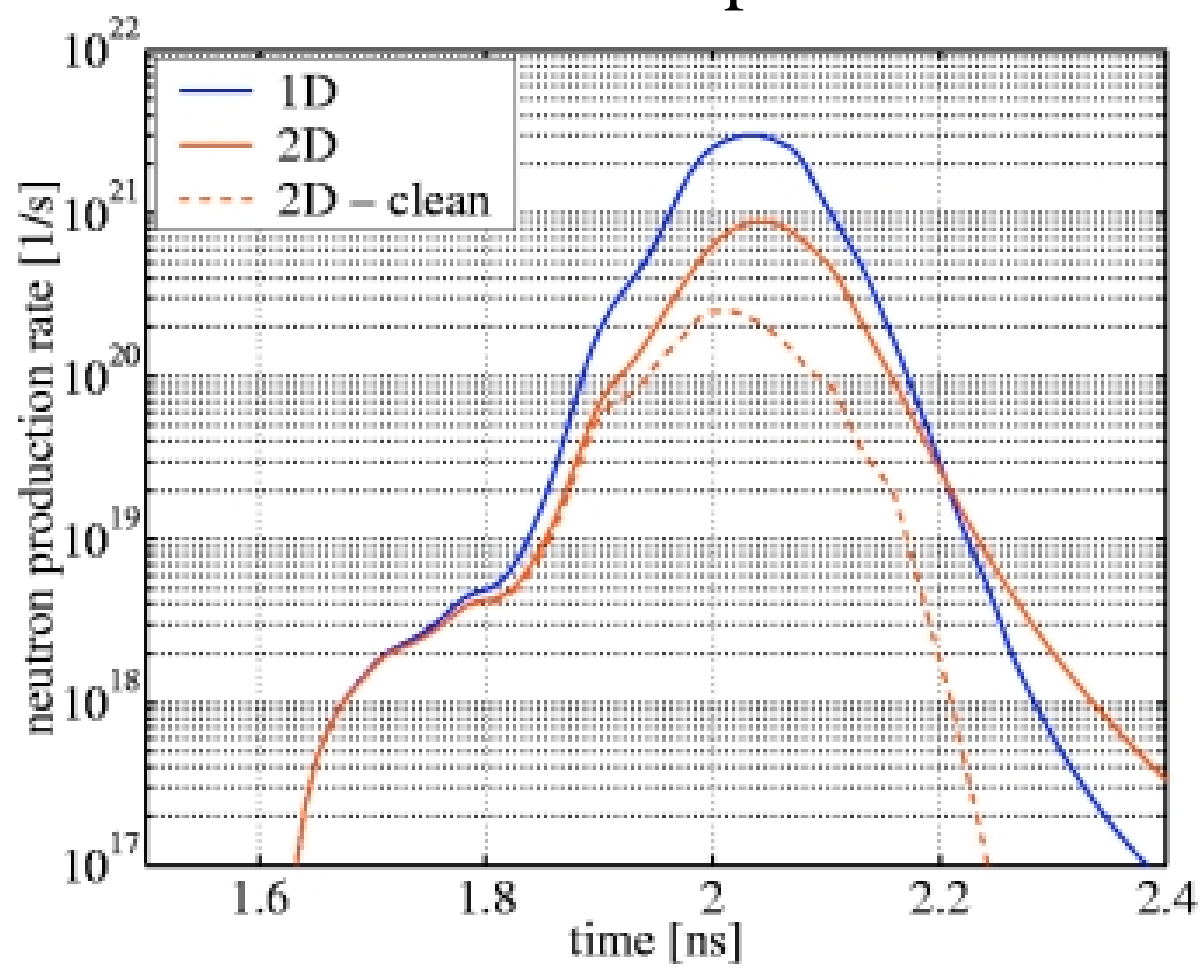

-2D yield lower by factor 2-3 from 1D.

- Fully developed turbulent mixing: Worst case - fusion only in clean zone defined by most penetrating spike.

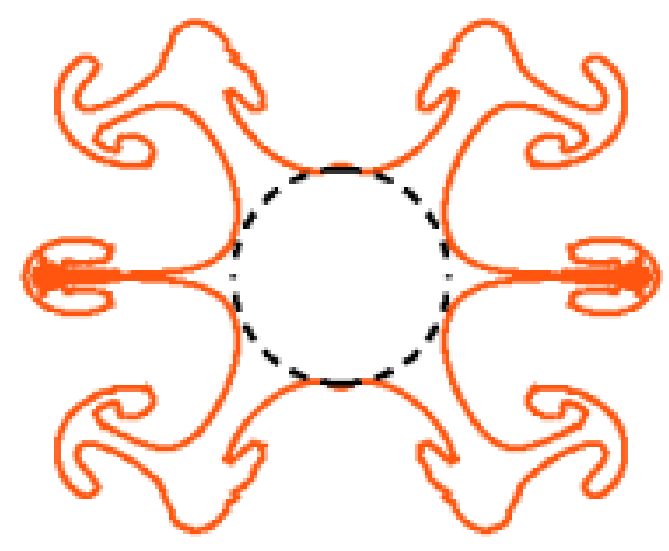




\section{Neutron yield degradation}

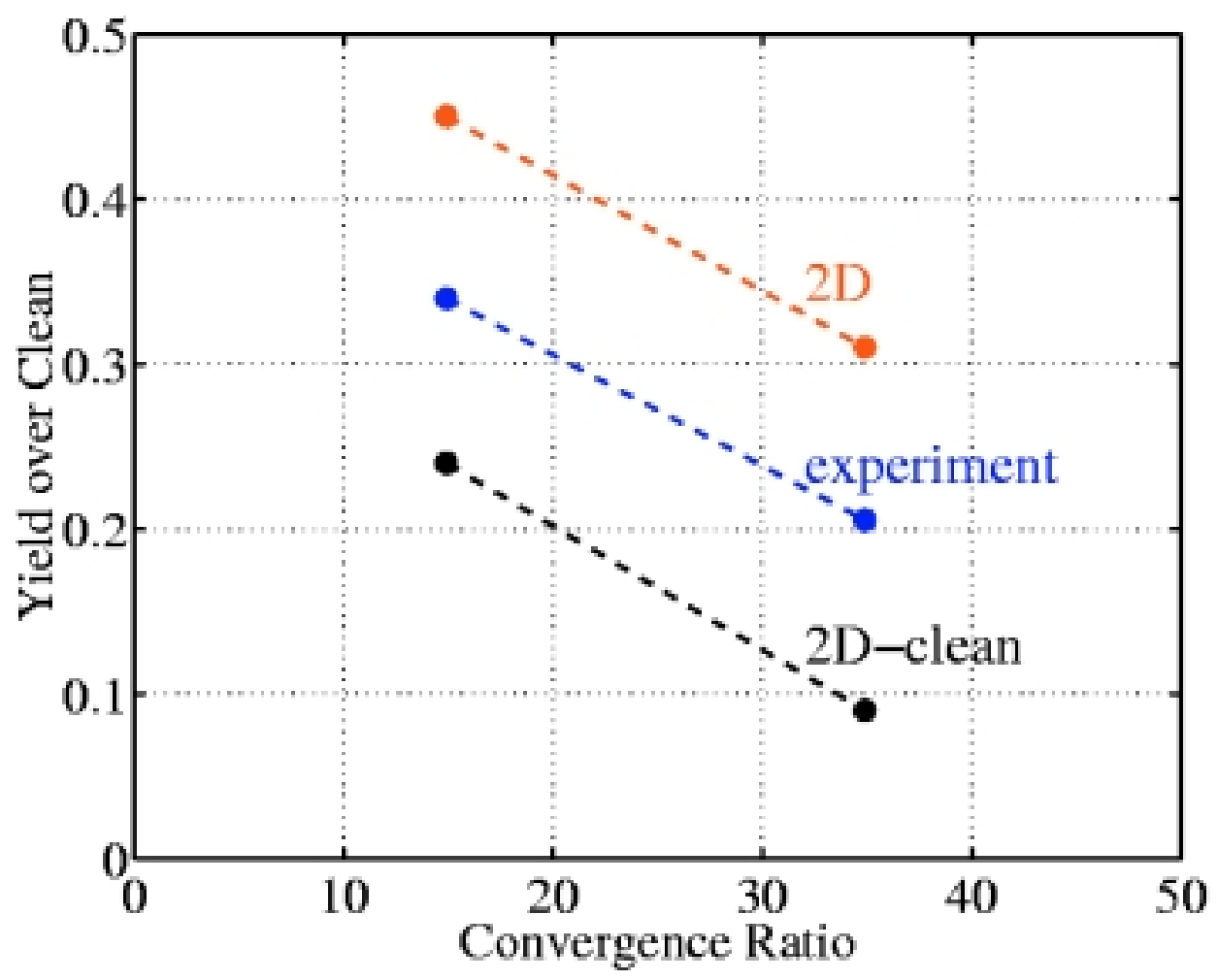

-2D simulations underestimate degradation.

- Assuming fusion only in clean area overestimates degradation. 


\section{Cumulative fusion rate $\quad\left(N(R)=\int_{0}^{R}(r) d^{3} r\right)$ (shot $20690-p=15$ atm)}
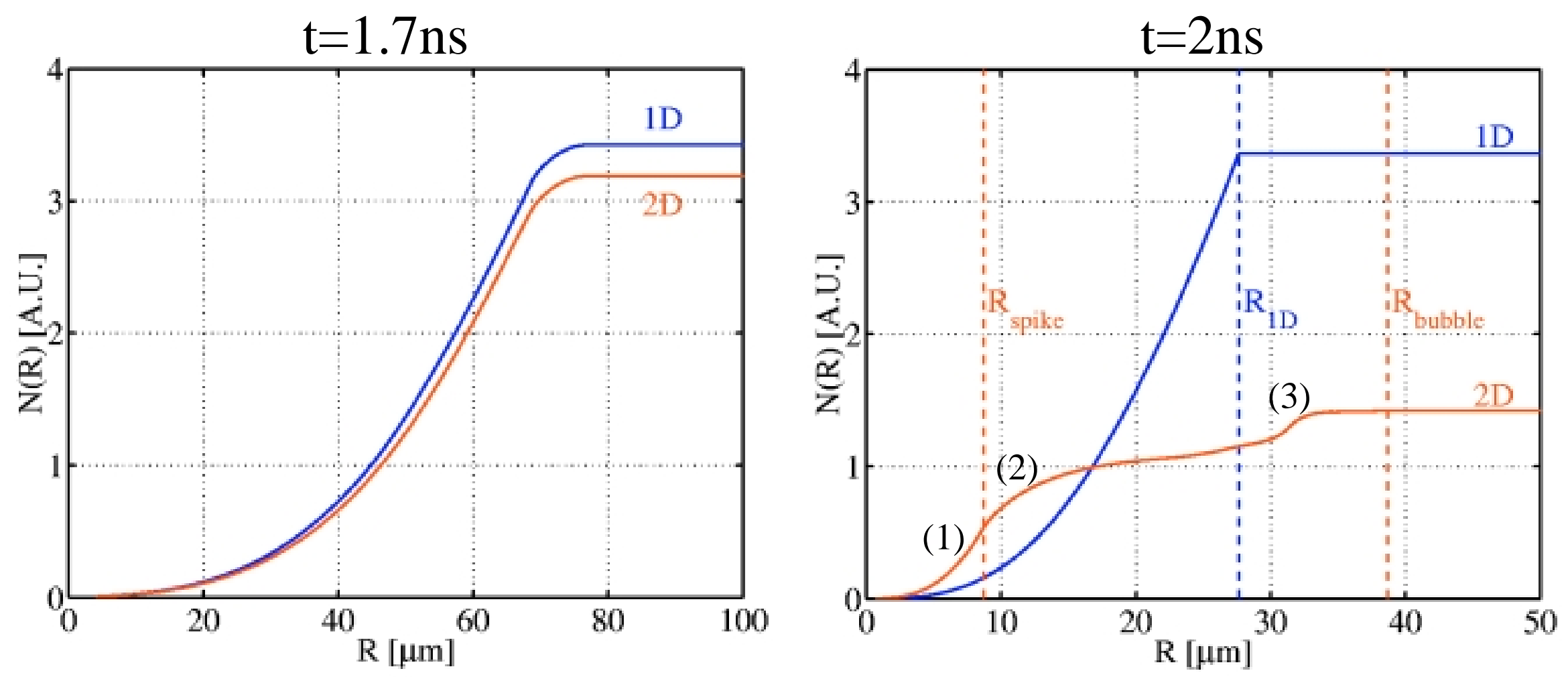

1) Higher $2 \mathrm{D}$ yield from central region.

2) Slight redefinition of $R_{\text {clean }}$ will significantly change yield.

3) Contribution of bubbles to fusion yield. 


\section{Difference in yield from central region}

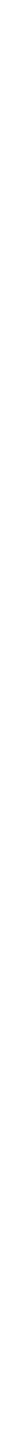

2D central density is higher due to differences in shock dynamics. 


\section{Redefinition of clean region}

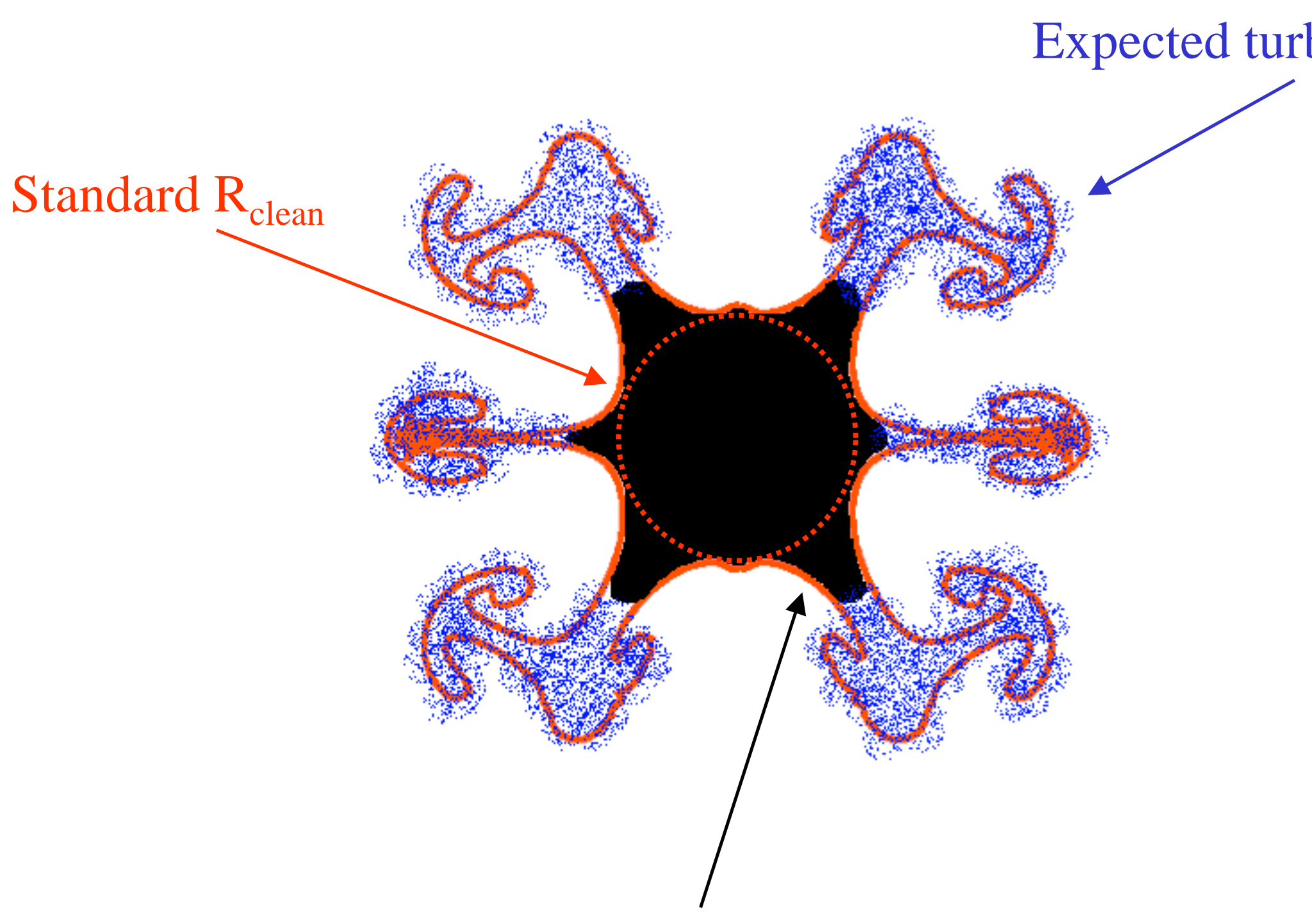

Expected clean region 


\section{Redefinition of $\mathbf{R}_{\text {clean }}$}

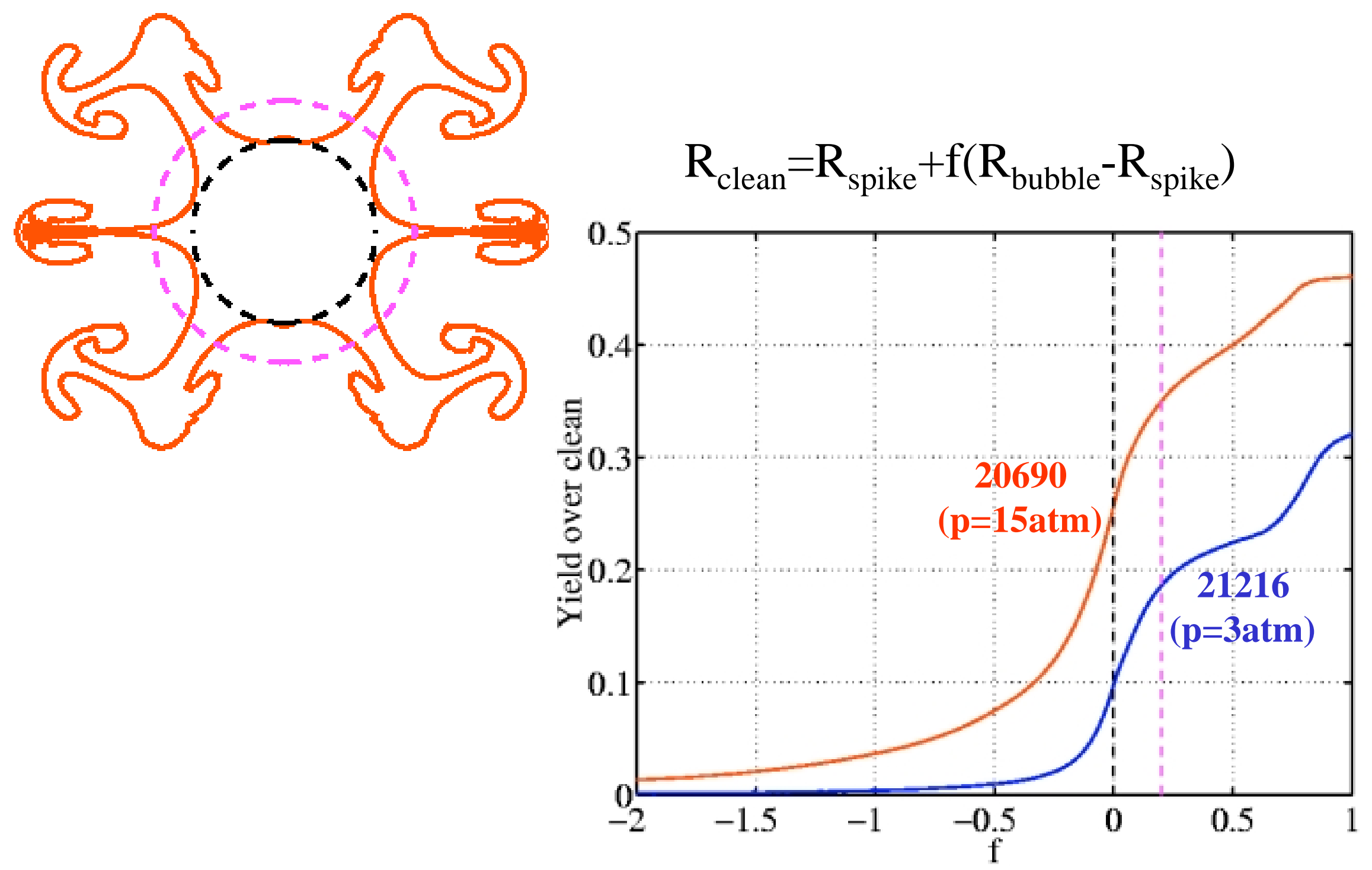

Sharp rise ends at $f=0.2$, coinciding with clean region boundaries. 
Re-definition of clean region improves agreement with experimental results

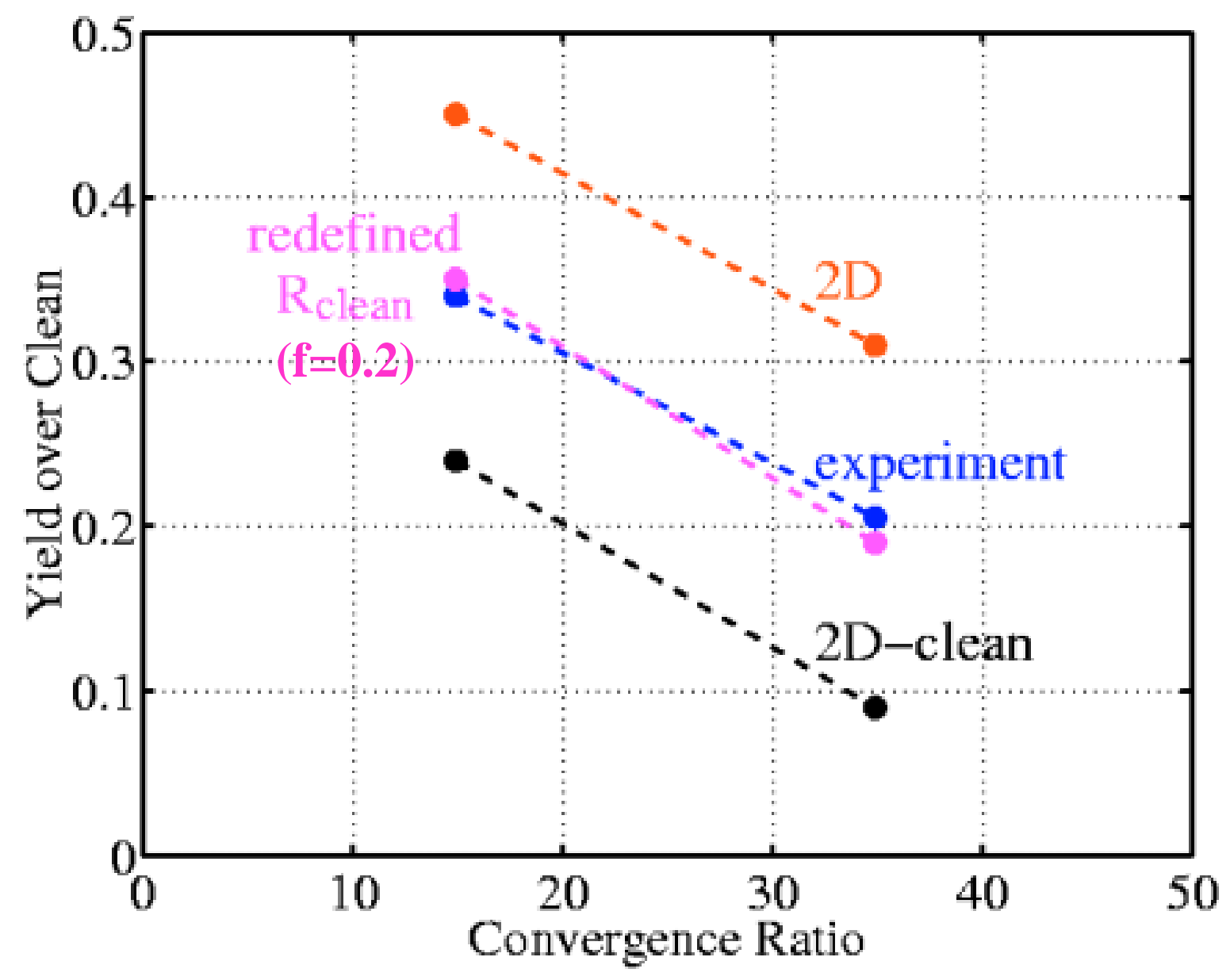




\section{Conclusions}

- Recent ICF experiments have been analyzed by comparing full 2D and 1D numerical simulations.

- Assuming no mixing, bubbles raise fusion yield above experimental results.

- Differences in central pressure, density and fusion rate at high perturbation amplitudes imply that mix effects are not limited to the mix region, hence full 2D simulations are needed.

- Regions slightly beyond $\mathrm{R}_{\text {clean }}$ contribute significantly to fusion yield. New definition for $\mathrm{R}_{\text {clean }}$ improves agreement with experimental results. 


\title{
Turbulent Mixing Nuclear Burning in Type Ia Supernova Explosion based on Bubble Statistical Mechanics
}

\author{
H. Takabe, S. Yamada, K. Kobayashi, A. Mizuta, and K. Nomoto* \\ Institute of Laser Energetics and Graduate School of Science, Osaka University \\ *Department of Astronomy, Graduate School of Science, University of Tokyo \\ E-mail: takabe@ile.osaka-u.ac.jp
}

\begin{abstract}
(This viewgraph was prepared for "the 8th International Workshop on the Physics of Compressible Turbulent Mixing (8th IWPCTM) “ held at the California Institute of Technology (Caltech) in Pasadena, California, USA, from December 9-14, 2001. The Workshop will be held on campus in the Beckman Institute Auditorium, and is hosted by Lawrence Livermore National Laboratory (LLNL).)
\end{abstract}

It is well known that Type Ia supernovae explode when the masses of white dwarfs become close to the Chandrasechar's limiting mass. This is the reason why the Type Ia explosion is used as a standard candle in the universe to determine, for example, the Hubble constant and dark energy. The scale of explosion has been well studied with one-dimensional code with some mixing model; however, the physical mechanism has not determined from the first principle, yet. There are many works to understand the physics with large scale computing based on hydrodynamics in two-dimension or mostly three-dimension in these days[1]. It seems, however, that the smaller scale fluctuation appears, the smaller the grid size, and it is still open question how the instability grows and evolves into nonlinear stage and enhance the energy release by nuclear reactions.

In the present report, we would like to model the growth of the Rayleigh-Taylor instability coupled with the Landau-Darrieus instability. In the nonlinear stage, we consider the statistical mechanics of the bubbles following the way developed by Don Shvart's group[2] and estimate the increase in the nuclear burning rate due to the increase in the surface area of the burning wave in the form of fractal structure. This model is coupled with the multidimensional explosion code to predict the scale of explosion. Such work is expected to be used to identify the physical mechanism of the time evolution of the burning wave, which may change from deflagration wave to detonation wave. 


\section{Reference:}

[1] W. Hillebrandt and J. Niemeyer, Ann. Rev. Astron. Astrophys. 38, 191-230 (2000)

[2] D. Shvarts et al, Physics of Plasmas 2. 2465 (1995).

\section{Introduction}

- We are promoting a new scientific field of "Laser Astrophysics".

- Many issues regarding hydrodynamics instabilities and turbulent mixings.

- Recent review of LA.

H. Takabe, "Astrophysics with intense and ultra-intense lasers -Laser Astrophysics- ”, Progress of Theoretical Physics Suppl. No. 143, 202-265 (2001).

- Many works on hydrodynamic instabilities and mixings have been done in the case of Type-II supernova explosion, especially SN1987A, in the framework of LA. (B. Remington, P. Drake et al.)

- More sophisticated physics scenario should be studied in the case of Type-Ia SN explosion.

\section{What is Type-Ia Supernova ?}

- Type II

- Massive star ( $20 \mathrm{M}_{\text {Sun }}$ for SN1987A), Single

- $\quad \mathrm{H}_{\alpha}$ line emission. 


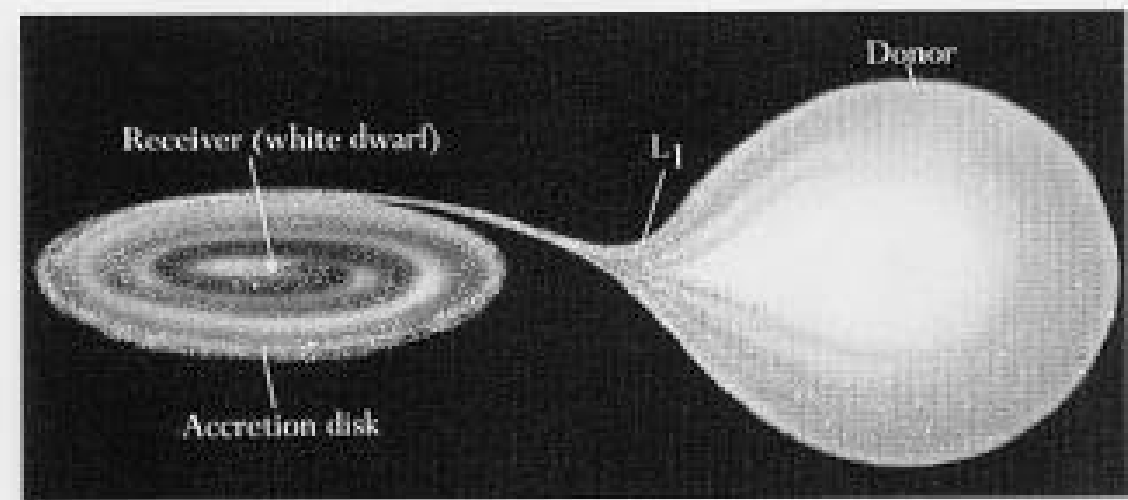

Finure 7. 11. In a cboos double star, fides can cause the larger of the cumponents in fill an enckesed yolume at whose surface the gravity of the sars is equalixod, allasing nass to flox outa and through

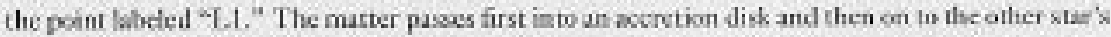

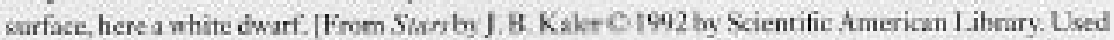
with permisian of W: H Froenzan and Campany.]

Fig. 1 (J. Kaler, "Extreme Stars" (Cambridge, 2001) p.158)

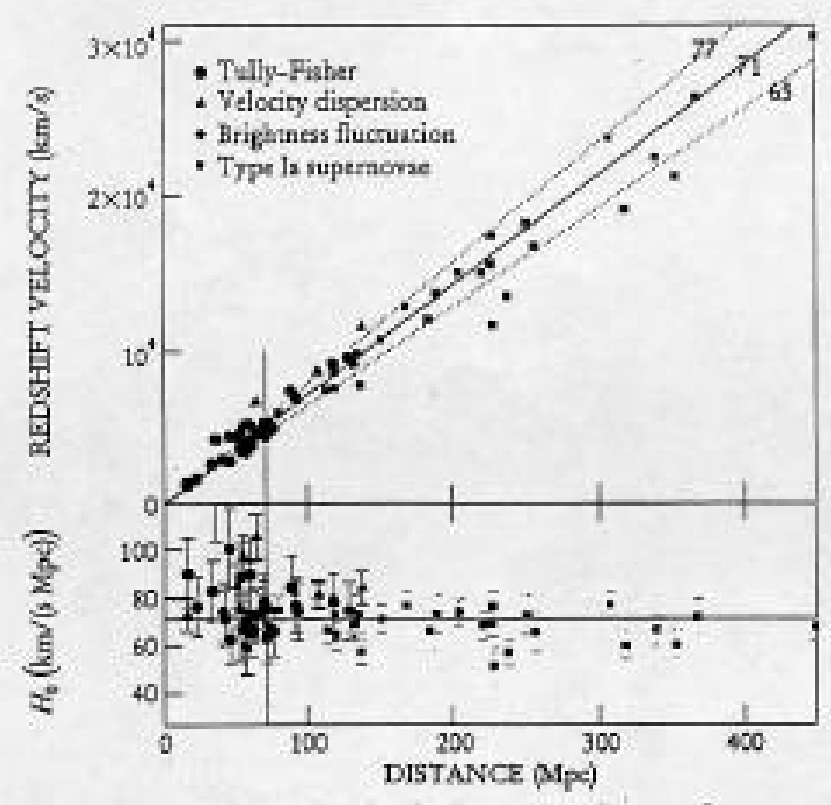

Fig. 2 (Physics Today, August (1999) p.20)

- Type Ia

- White Dwarf, Binary system, Chandrasekhar-mass $\left(\sim 1.4 \mathrm{M}_{\text {Sun }}\right) \quad$ See Fig. 1.

- $\quad \mathrm{No} \mathrm{H}_{\alpha}$ line emission. 
- Light curve of Type Ia SNe.

- Why important?

- Precise determination of Hubble constant

$$
\begin{aligned}
& \mathrm{H}_{0}=(\text { velocity }) /(\text { distance }) \quad \text { for } \mathrm{Z}<0.1 \\
& \text { See Fig. } 2
\end{aligned}
$$

- Einstein constant is finite? Dark energy. For $\mathrm{Z}<1.0$

$$
\left(\mathrm{cf:} \mathrm{Z}=\left(\lambda-\lambda_{0}\right) / \lambda_{0}, \quad \mathrm{~L}=1 \mathrm{~B}-\mathrm{ly} \text { for } \mathrm{Z}=0.1\right)
$$

\section{Hydrodynamic Instability in Laser Implosion}

- Mixing reduces neutron yields --- Degrades thermonuclear reaction and burn. [cf: H. Takabe et al, Phys. Fluids 31, 2884 (1988)]

- Multi-dimensional simulation with surface-tracking is necessary. See Fig. 3

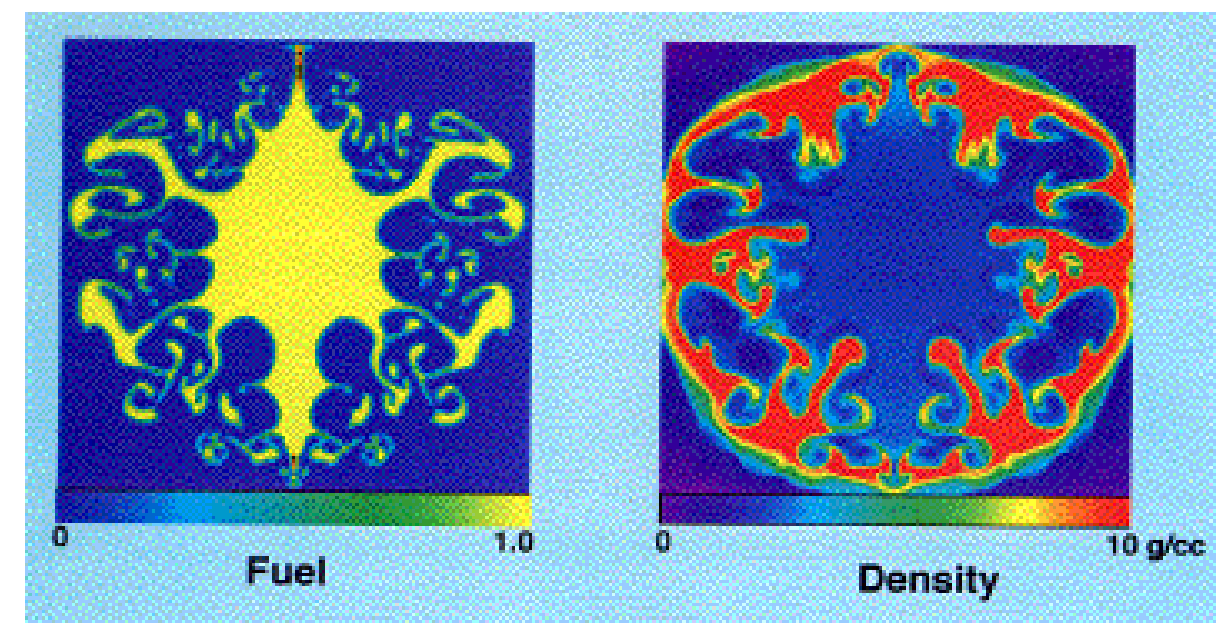

Fig. 3 (2D simulation of laser implosion. by H. Takeuchi and H. Takabe) 


\section{Spherical Symmetry Scenario of Nuclear Burning Wave Propagation in Chandrasekhar-mass WD.}

- Very sharp wave front [(thickness $)<\mathrm{mm}$ ] compared to the size of WD $(\sim 10000 \mathrm{~km})$. See Fig. 4

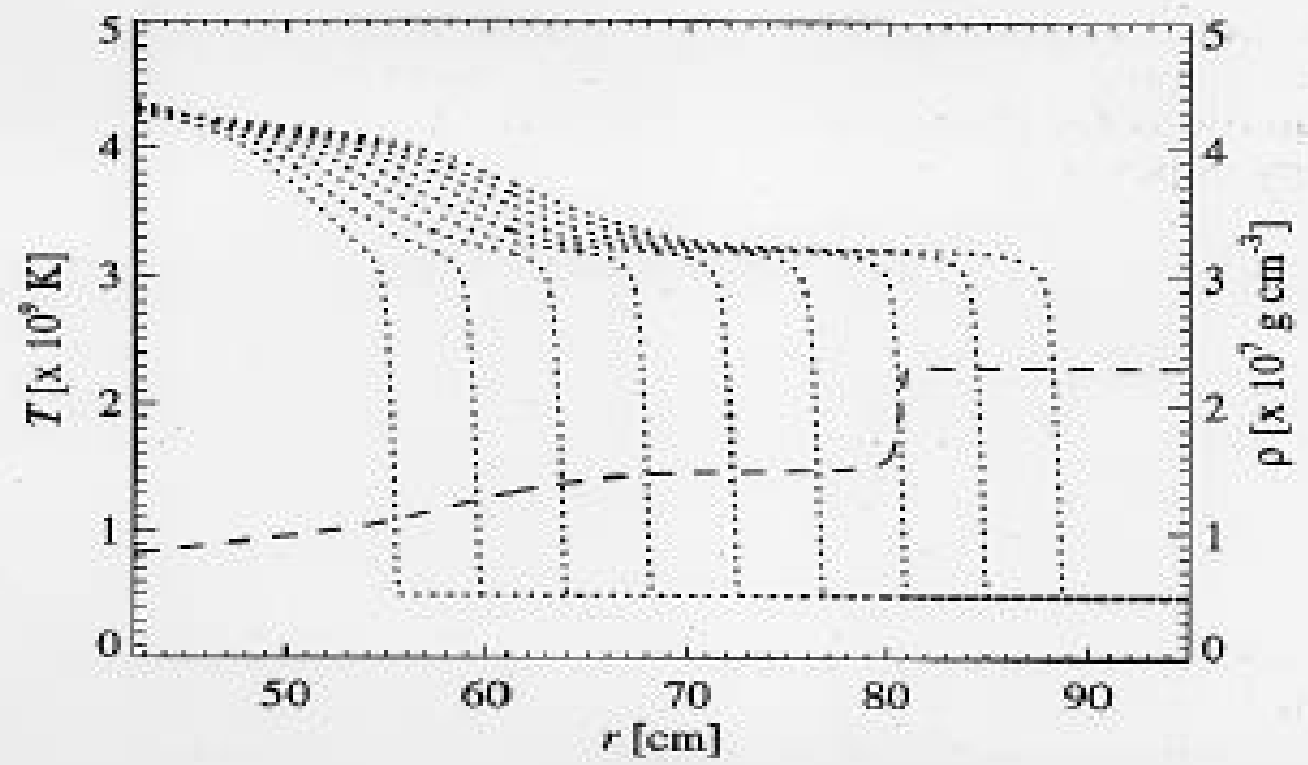

Fio. 2-Propagation of an $\mathrm{X}\left({ }^{12} \mathrm{C}\right)=\mathrm{X}\left({ }^{16} \mathrm{O}\right)-0.5$ conductive flame. The sequential temperature profiles (dotred fines) are shown at times $t=1.3 \times 10^{-4}, \quad 3.0 \times 10^{-4}, \quad 6.5 \times 10^{-4}, 8.3 \times 10^{-4}, 1.01 \times 10^{-3}$, $1.19 \times 10^{-3}, 1.36 \times 10^{-5}$, and $1.51 \times 10^{-3}$ a. The dashed line is the dansity profile at $t-1.19 \times 10^{-3} \mathrm{~s}$. The carbon flame already moves in a steady state, while the temperature rise at the left-band side comes from oxygen burning ass a result of the energy stored at the initial state of the simulation.

Fig. 4 [A. M. Lisewski et al., ApJ 537, 405 (2000)]

- Very week deflagration wave or combustion wave (M $\sim 0.01$ ).

- No explosion:

(Released fusion energy) $<-($ Gravitational binding energy).

- Multi-dimensional physics scenario is necessary. 
- Unstable to R-T instability.

- Unstable to Landau-Darius instability

- $\quad \mathrm{K}-\mathrm{H}$ instability is also important.

\section{Fractal Dimension of the Nuclear Burning Wave Front.}

- Effective area and velocity of the burning front (F. X. Timmes, ApJ, 1994)

$$
\begin{aligned}
& \mathrm{A}_{\text {eff }}=\left(l_{\text {max }} / l_{\text {min }}\right)^{\mathrm{D}-2} \mathrm{~A}_{0} \\
& \mathrm{~V}_{\text {eff }}=\left(l_{\text {max }} / l_{\text {min }}\right)^{\mathrm{D}-2} \mathrm{~V}_{\text {conduction }} \\
& \text { D: Fractal dimension }(\mathrm{D}>2)
\end{aligned}
$$

- Fractal dimension (S. I. Blinnikov, PRE, 1996).

$$
\left(l_{\max } / l_{\min }\right)^{\mathrm{D}-2} \sim 7 \quad \begin{gathered}
\text { Compare: we need } \sim 100 ! \\
\text { See Fig. } 5
\end{gathered}
$$

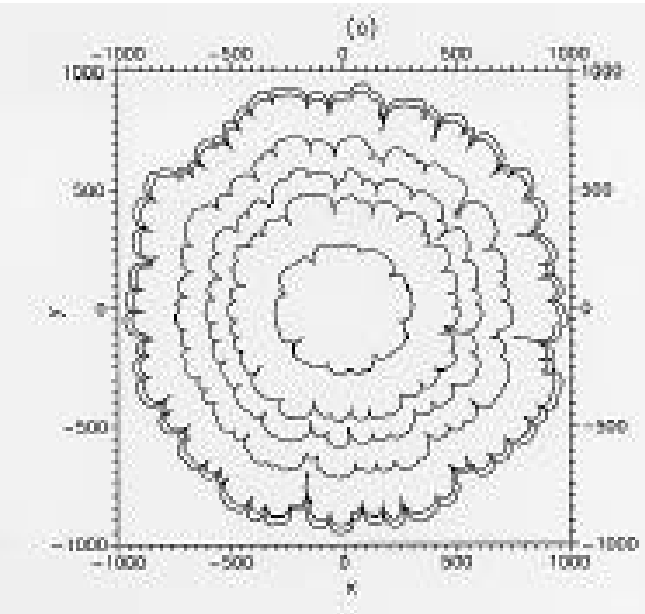

Figure 6.2: Flame front for several moments of time for $\eta=0,8$

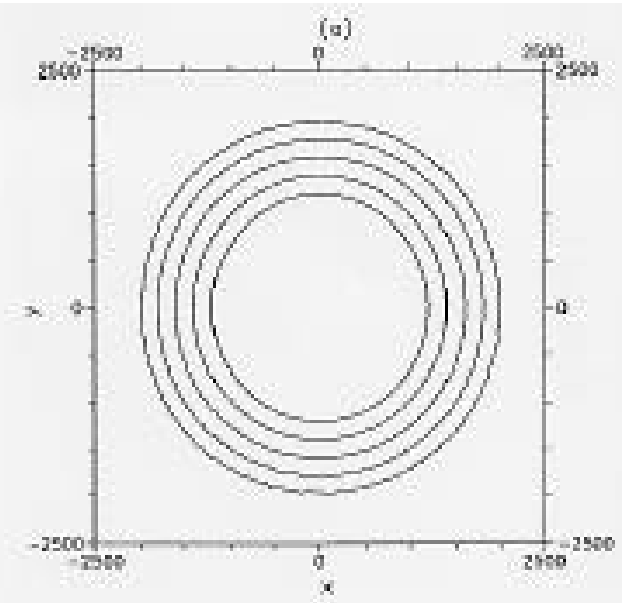

Figure 6.3: The front for several moments of time for $3=0.4$

Fig. 5 (S. I. Blinnikov, Phy. Rev. E, 1996) 


\section{Multi-Dimensional Simulation (LES-like)}

- Intensive works by W. Hillebrandt group (MPA). References:

1. W. Hillebrandt and J. C. Niemeyer, Annu. Rev. Astron. Astrophys. 38, 191 (2000).

2. A. M. Lisewski et al., ApJ 537, 405 (2000).

3. M. Reinecke et al., A\&A 347, 739 (1999).

4. M. Reinecke et al., astro-ph/0111473, 26 Nov (2001).

5. M. Reinecke et al., astro-ph/0111475, 26 Nov (2001).

- Central ignition

- Off-center ignition

- Mesh-size dependence in 2-D simulation

- 3-D simulation See Fig. 6
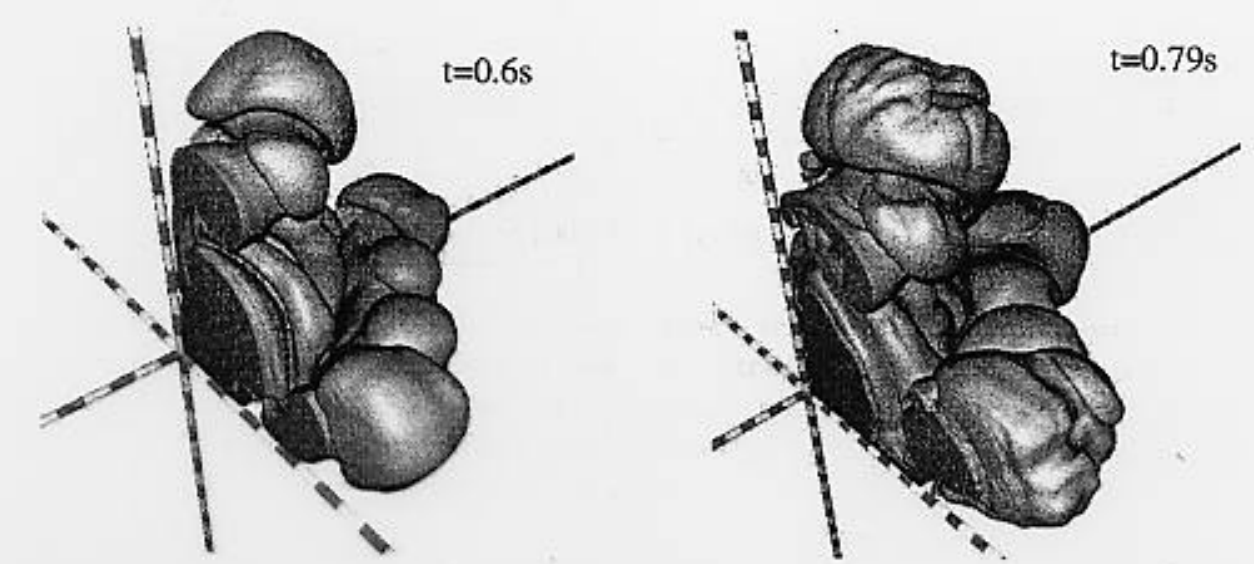

Fig. 3. Burning front geometry evolution of model $c 3.3 \mathrm{~d}-256$. One ring on the axes corresponds to $10^{7} \mathrm{~cm}$.

Fig. 6 [M. Reinecke et al., astro-ph/0111473, 26 Nov (2001)] 


\title{
7. Statistical Bubble Model is applicable to this case?
}

\author{
- Large-Eddy-type simulation based on the spectra by U. \\ Alon et al. (PRL, 74, 534 (1995)). Possible?
}

- Review by Don Shvarts in this morning session.

\section{Summary}

We have started numerical study to see more detail and complete explosion scenario of Type Ia supernovae. They are unstable to Rayleigh-Taylor instability and Landau-Darrius instability. The increase of the surface of the nuclear burning front determines and enhances the rate of thermonuclear energy release. It determines the fraction of the production of ${ }^{56} \mathrm{Nn}$, which finally nuclear decays to ${ }^{56} \mathrm{Fe}$. This is the origin of iron in the universe.

Type Ia is so bright, the peak luminosity is $10^{11}$ time the solar luminosity, and the luminosity is thought to be almost constant, so that it can be used to discuss not only to determine the Hubble constant precisely but also to know the existence of cosmological term in Einstein equation..

The project is not so simple. As summarized in Fig. 7, we have to start from one-dimensional code with realistic equation of state (EOS) in which Fermi pressure in relativistic regime should be modeled. The calculation of thermonuclear burn requires to calculate the equations of concentrations for original element $(\mathrm{C} / \mathrm{O})$ and burn product $(\mathrm{Fe})$. Two dimensional simulations have been carried out by many authors, while the numerical resolution was a big issue. Prof. I. Hachisu, our collaborator at University of Tokyo, thinks that 2D is enough and no sub-grid model is necessary. He is think of usage of Earth simulator, the world-highest-speed vector-parallel computer at Yokohama.

The authors think of developing our original sub-grid model based on the accomplishment in relation to laser implosion physics. The simulation will be done in $2 \mathrm{D}$ and finally $3 \mathrm{D}$. To check the sub-grid model we need to do a variety of local 3D simulations. We hope to relate such explosion simulation to the observable light curve.

Final point of this project is to find a new mixing model to be installed in 1D code so that many-parameter study is able to be done by supernova observation groups. Very tough job but very attractive, isn't it. 


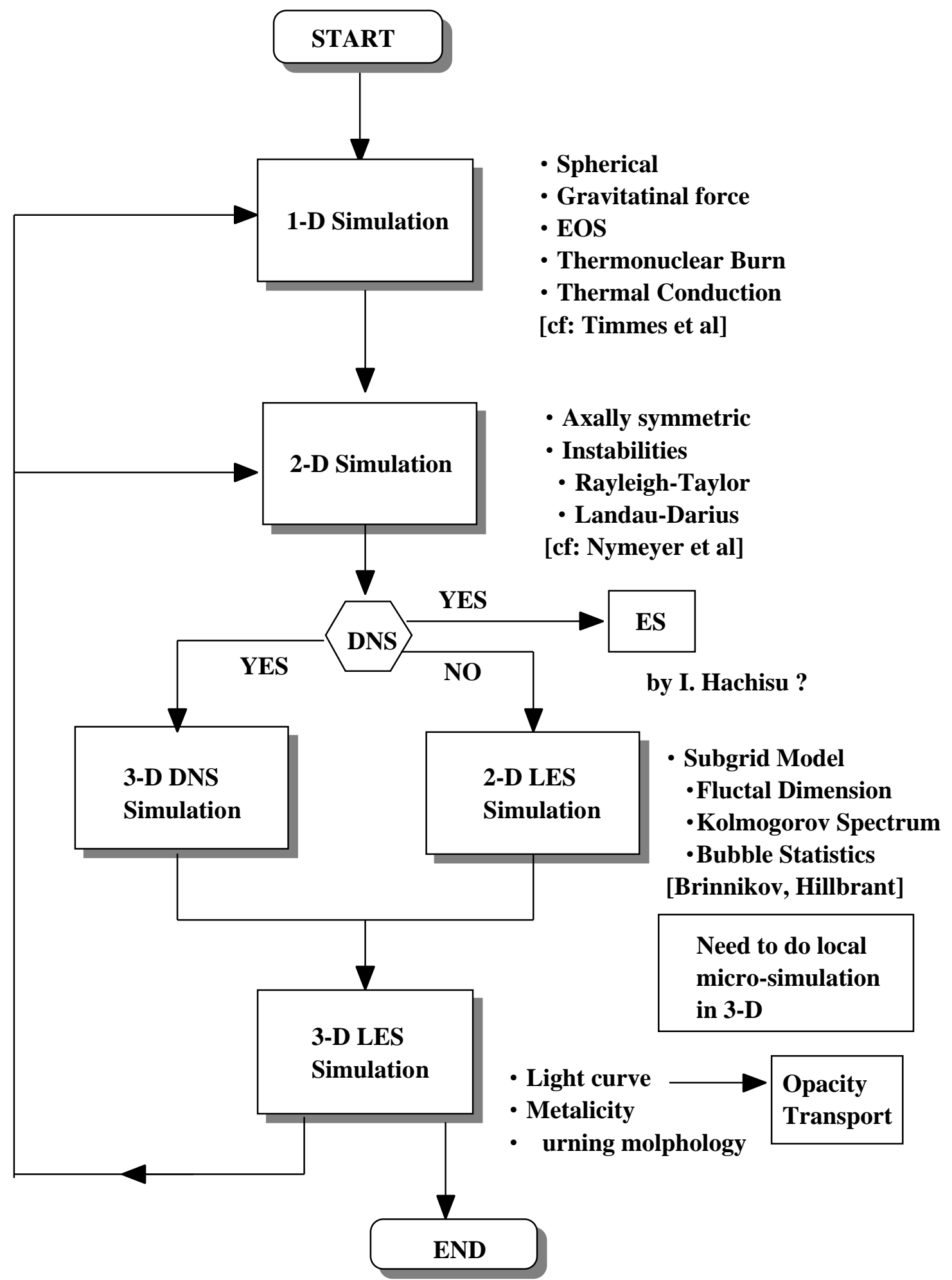

Fig. 7 Flow Diagram of the Present Project (H. Takabe) 


\section{Turbulent diffusion in solar type star}

How may be trapped light elements as lithium in differential rotation zones of solar type star

Nathalie Toqué

Departement of Physics of the University of Montreal, Canada 


\section{Setup of the study}

Tachocline: Differential rotation zone of about

$35000 \mathrm{~km}$ thickness between the convective and radiative parts

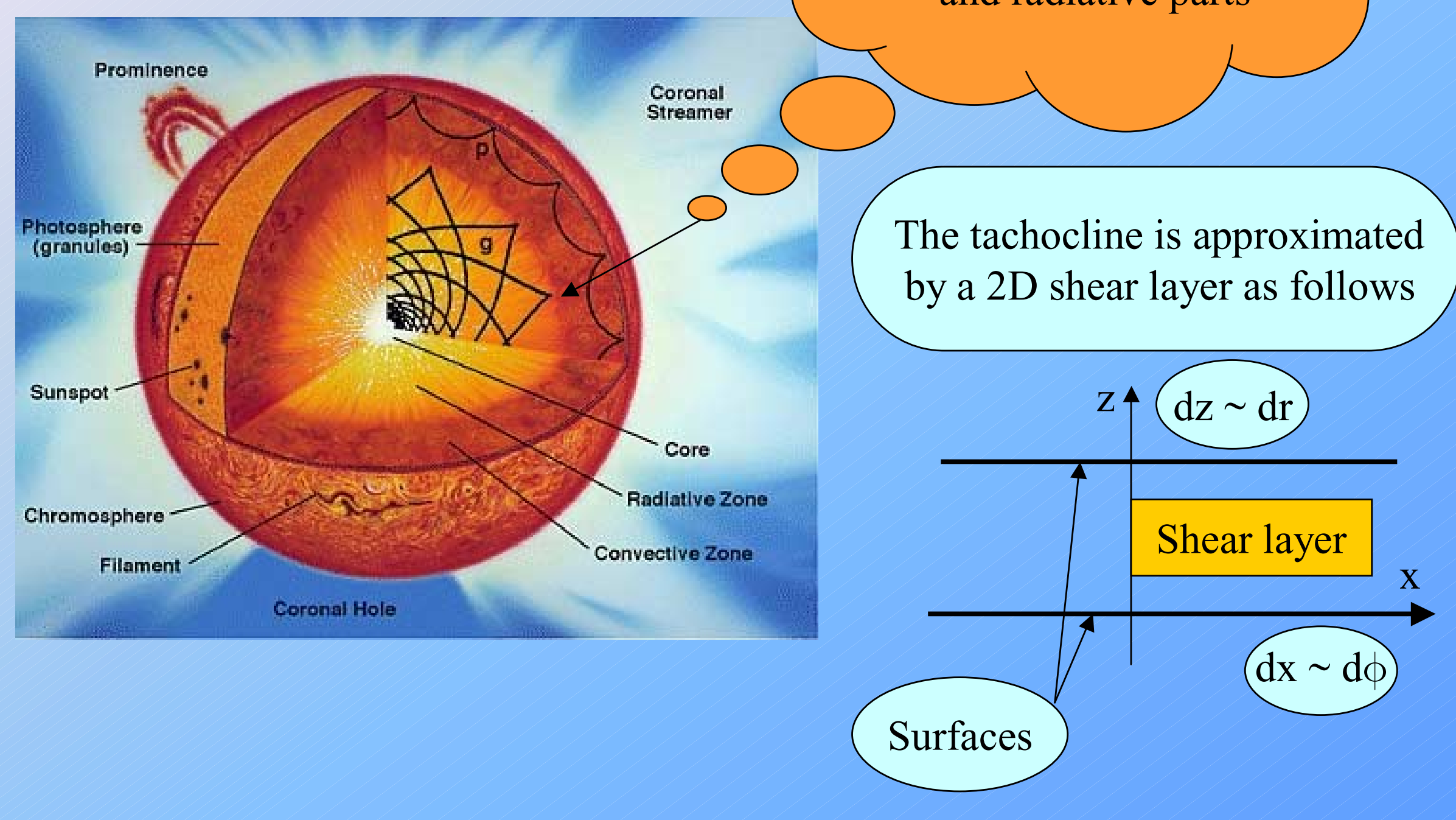




\section{Hypothesis of the model}

Contribution from convective and radiative zones reduced to a forcing in velocity along the $\mathrm{x}$ direction and depending on the solar latitude coordinate

Perfect gas approximation for the fluid

Uncompressible and isentropic flow

No Boussinesq approximation, no thermic dissipation, kinetic and hydrostatic pressure gradients

Free-slip conditions on the surfaces, otherwise periodic

A concentration with an initial gaussian profile versus the $z$ coordinate is convected by the flow. Its diffusion is studied for different values of the Reynolds number depending on the intensity of the forcing 


\section{Theory}

$\left\{\begin{array}{l}\partial_{x} V_{x}+\partial_{z} V_{z}=0 \\ \partial_{t} V_{x}=-\partial_{x} P-R_{x}+\frac{1}{R e}\left\{\partial_{x}^{2} V_{x}+\partial_{z}^{2} V_{x}\right\}+\Phi \\ \partial_{t} V_{z}=-\partial_{z} P-R_{z}+\frac{1}{R e}\left\{\partial_{x}^{2} V_{z}+\partial_{z}^{2} V_{z}\right\} \\ \partial_{t} C=-\partial_{x}\left(V_{x} C\right)-\partial_{z}\left(V_{z} C\right)+\frac{1}{P e}\left\{\partial_{x}{ }^{2} C+\partial_{z}{ }^{2} C\right\}\end{array}\right.$

with $\left\{\begin{array}{l}R_{x}=V_{z}\left(\partial_{z} V_{x}-\partial_{x} V_{z}\right) \\ R_{z}=V_{x}\left(\partial_{x} V_{z}-\partial_{z} V_{x}\right)\end{array}\right.$

Re is the Reynolds number

$\mathbf{P e}$ is the Peclet number

$\boldsymbol{\Phi}$ is the forcing along the horizontal direction

\section{$\left(\partial_{z} V_{x}-\partial_{x} V_{z}\right):$ Vorticity component perpendicular to the domain of study}

The theoretical equations are solved in spectral space
Written in spectral space with the following decompositions,

$$
\left\{\begin{array}{l}
C(t, x, z)=\sum_{\mathbf{k}} \hat{C}(t, \mathbf{k}) e^{i k_{x} x} \sin \left(k_{z} z\right) \\
V_{z}(t, x, z)=\sum_{\mathbf{k}} \hat{V} z(t, \mathbf{k}) e^{i k_{x} x} \sin \left(k_{z} z\right) \\
V_{x}(t, x, z)=\sum_{\mathbf{k}} \hat{V} x(t, \mathbf{k}) e^{i k_{x} x} \cos \left(k_{z} z\right)
\end{array}\right.
$$

The equations under study become

$$
\left\{\begin{array}{l}
\mathbf{i} k_{x} \hat{V}_{x}+\mathbf{i} k_{z} \hat{V}_{z}=0 \\
\partial_{t} \hat{V}_{x}=-\mathbf{i} k_{x} \hat{P}-\hat{R}_{x}-\frac{\left(k_{x}{ }^{2}+k_{z}{ }^{2}\right)}{R e} \hat{V}_{x}+\hat{\Phi} \\
\partial_{t} \hat{V}_{z}=-\mathbf{i} k_{z} \hat{P}-\hat{R}_{z}-\frac{\left(k_{x}{ }^{2}+k_{z}{ }^{2}\right)}{R e} \hat{V}_{z} \\
\partial_{t} \hat{C}=-\mathbf{i} k_{x}\left(\widehat{V_{x} C}\right)-\mathbf{i} k_{z}\left(\widehat{V_{z} C}\right)-\frac{\left(k_{x}{ }^{2}+k_{z}{ }^{2}\right)}{P e} \hat{C}
\end{array}\right.
$$




\section{Subgrid Model}

The time iteration is made with

(M.Lesieur\&R.Rogallo, 1989)

the implicit Cranck-Nicholson method for the diffusion terms

the explicit Leapfrog scheme for the advection terms

The time iteration is divided in two steps for the velocity components. After the first step, the pressure is calculated with the condition :

$$
\widehat{\nabla \cdot \mathbf{V}}=0
$$

The contribution of the small scales of turbulence is carried out by a subgrid model

$$
\nu_{t}\left(k / k_{c}\right)=\left(A+B e^{C\left(k_{c} / k\right)}\right)\left[E\left(k_{c}\right) / k_{c}\right]^{1 / 2}
$$

$$
\mathbf{A}=0.267, \mathbf{B}=9.21 \text { and } \mathbf{C}=-3.03
$$

An extra-diffusion term is added to the equations governing the velocity components and the concentration as follows :

$$
\left\{\begin{array}{l}
\hat{V}_{x}^{n+1}=\hat{V}_{x}^{n+1}-2 d t \nu_{t} k^{2} \hat{V}_{x}^{n+1} \\
\hat{V}_{z}^{n+1}=\hat{V}_{z}^{n+1}-2 d t \nu_{t} k^{2} \hat{V}_{z}^{n+1} \\
\hat{C}^{n+1}=\hat{C}^{n+1}-2 d t \nu_{t} k^{2} \hat{C}^{n+1}
\end{array}\right.
$$




\section{Simulation philosophy}

Given a Reynolds number, a simulation is made in two steps with a forcing on several layers

\section{First step : Stationarity of the anisotropy}

The purpose of the forcing is to create a strong sheared flow with a constant mean value of the anisotropy defined as follows

$$
A(t)=\sqrt{\frac{\left\langle V^{2}(x, z, t)\right\rangle-\left\langle V_{x}(x, z, t)\right\rangle^{2}}{\left\langle V^{2}{ }_{z}(x, z, t)\right\rangle-\left\langle V_{z}(x, z, t)\right\rangle^{2}}}
$$

\section{Second step : Vertical diffusion of the concentration}

The aim of the forcing is to produce the vertical diffusion of the concentration in the flow with a constant anisotropy.

The concentration is named after $\mathbf{C}$ 


\section{Quantities measuring the turbulent diffusion}

\section{Anisotropy}

Turbulent diffusion coefficient

\section{Flux}

\section{$x$ averaged variancy}

$\mathrm{z}$ averaged variancy

$$
\left\{\begin{array}{l}
A(t)=\sqrt{\frac{\left\langle V^{2}(x, z, t)>-<V_{x}(x, z, t)\right\rangle^{2}}{\left\langle V^{2}(x, z, t)>-\left\langle V_{z}(x, z, t)\right\rangle^{2}\right.}} \\
D_{T}(z, t)=\frac{\left\langle V_{z}(x, z, t) \times C(x, z, t)\right\rangle_{x}}{\left\langle\frac{d C(x, z, t)}{d z}\right\rangle_{x}} \\
\left.F(z, t)=<V_{z}(x, z, t) \times C(x, z, t)\right\rangle_{x} \\
\sigma^{2}{ }_{x}(z, t)=<\left(C(x, z, t)-<C(x, z, t)>_{x}\right)^{2}>_{x} \\
\left.\sigma^{2}{ }_{z}(x, t)=<(C(x, z, t)-<C(x, z, t)\rangle_{z}\right)^{2}>_{z}
\end{array}\right.
$$

After averages on time and space coordinates, it involves one value for $\mathbf{A}, \mathbf{D}_{\mathbf{T}}, \mathbf{F}_{\text {max }}, \sigma^{\mathbf{2}}{ }_{\mathbf{x}}$ and $\sigma^{\mathbf{2}}{ }_{\mathbf{z}}$ 


\section{$\mathbf{D}_{\mathbf{T}}, \mathbf{F}_{\max }$ versus the anisotropy}

$$
\begin{aligned}
& d x=d z=1.2 \times 10^{-2} \\
& d t=10^{-5} \text { and } P e=1
\end{aligned}
$$

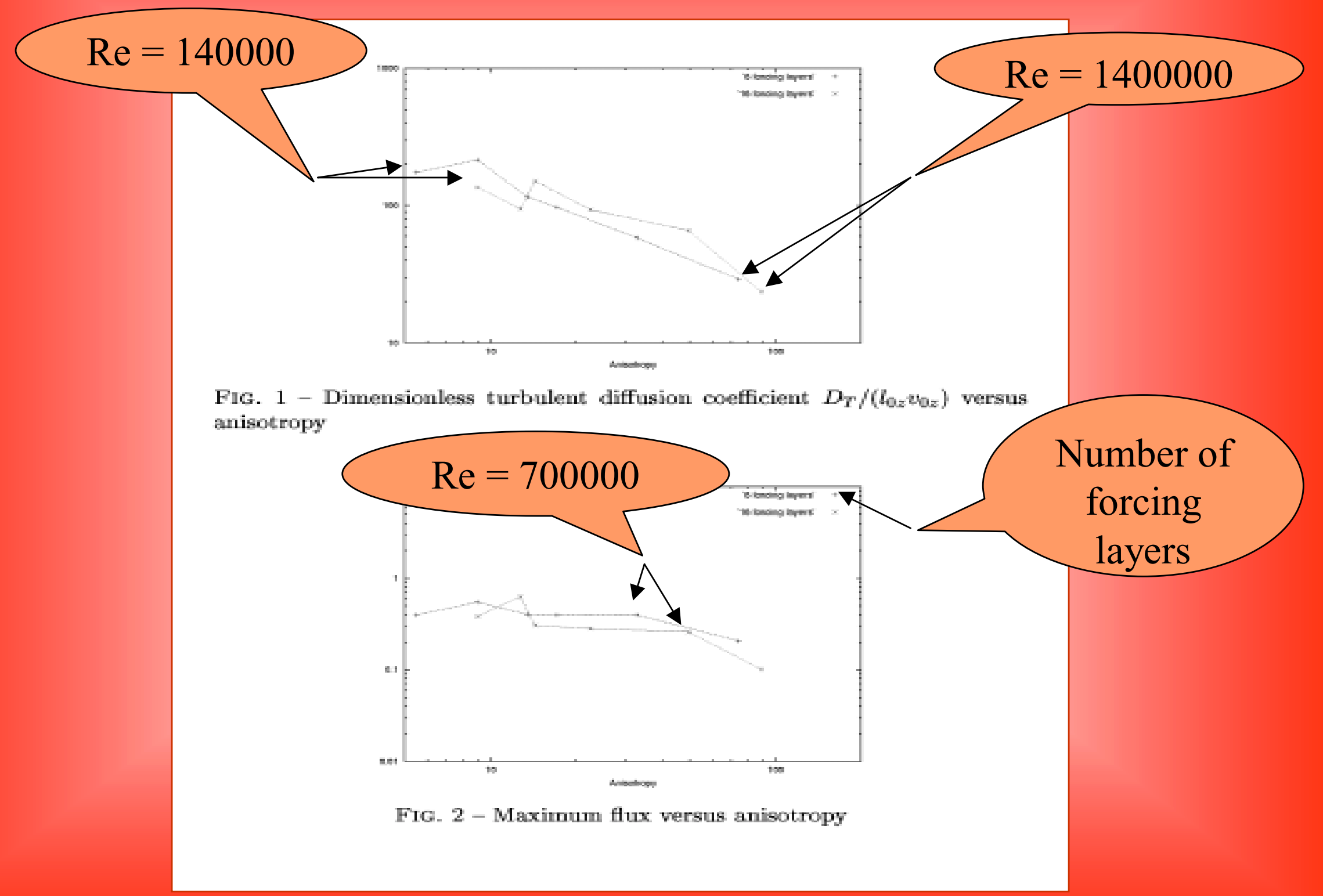




\section{$\sigma^{2}$ and $\sigma_{z}^{2}$ versus the anisotropy}

$d x=d z=1.2 \times 10^{-2}$

$d t=10^{-5}$ and $P e=1$

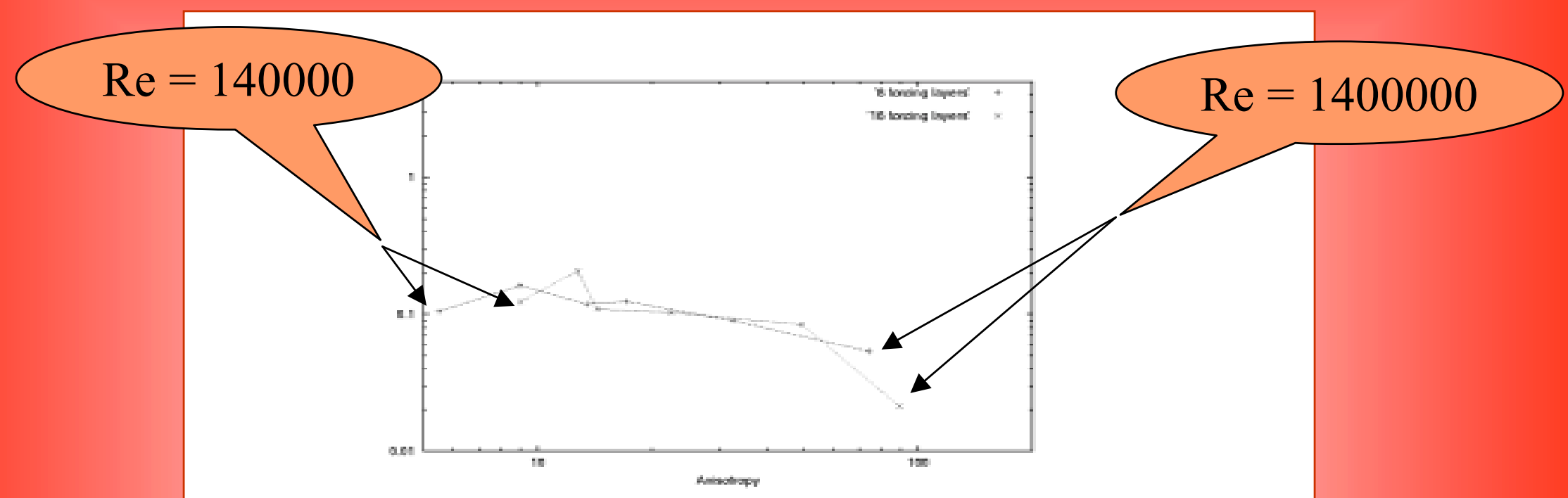

FIG. 3 - Variancy $\sigma^{2}$ versus anisotropy

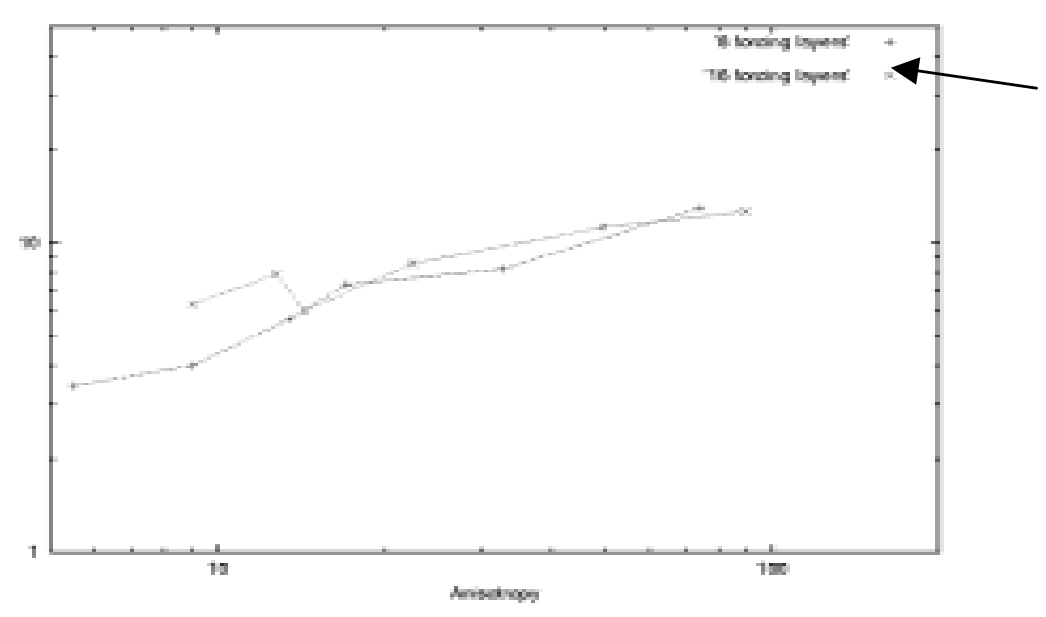

Number of forcing

FIG. 4 - Variancy $\sigma^{2}$ versus anisotropy 


$$
\begin{aligned}
& d x=d z=1.2 \times 10^{-2} \\
& d t=10^{-5} \text { and } P e=1
\end{aligned}
$$

16 forcing layers

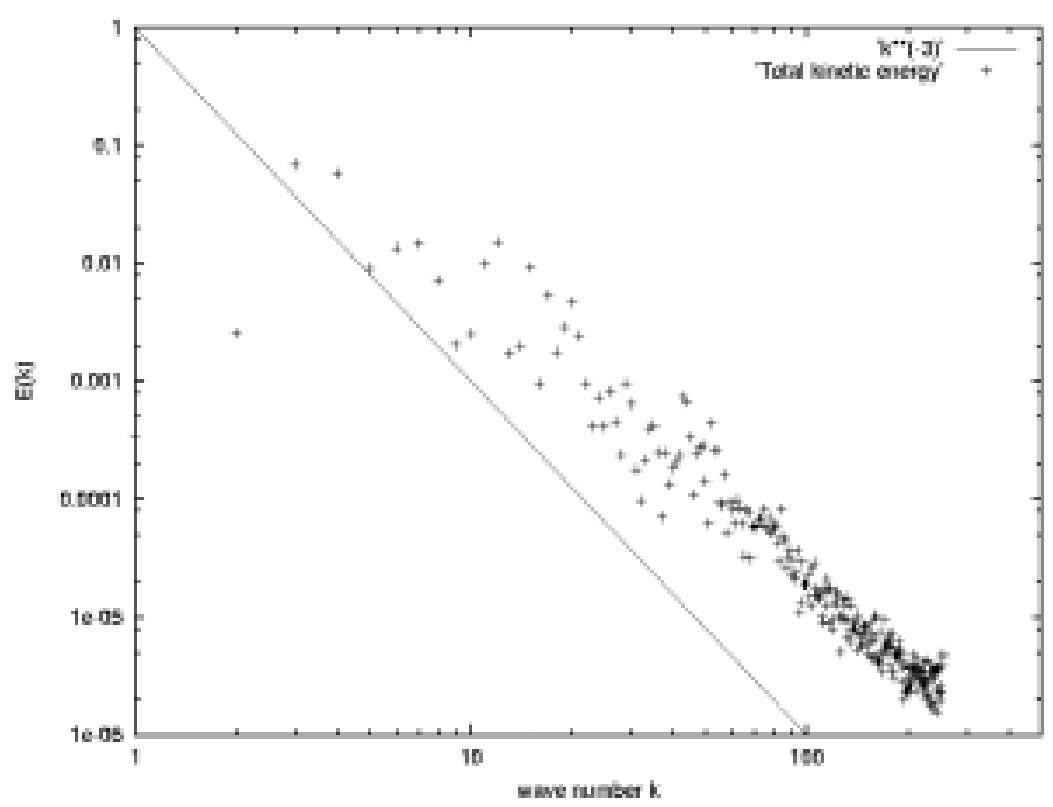

FIG. 5 - Total kinetic energy at the time of maximum vertical flux of the concentration in the flow 
The curves 1 to 4 show that for increasing Reynolds numbers, the turbulent diffusion coefficient, the maximum flux and the variancy $\sigma^{2}{ }_{x}$ are growing down for anisotropy values upper than 40 , and the variancy $\sigma_{z}^{2}$ is growing up

More forcing layers move down the maximum flux of the concentration in the flow because the integral scale and the quadratic fluctuation of vertical velocity are lower

The variances seem not influenced by the number of layers. They measure the turbulent homogenization in the two directions. For a conservative flow, less kinetic energy in the vertical direction involves more in the horizontal direction. So, the turbulent homogenization is better in the $\mathrm{x}$ direction than in the $\mathrm{z}$ direction. For increasing Reynolds numbers, the variancy $\sigma^{2}{ }_{x}$ decays and the variancy $\sigma^{2}$ grows up.

The total kinetic energy evolves as $k^{-3}$ as shown on the diagram 5 
Vorticity component perpendicular to the domain of study, $\operatorname{Re}=700000$ 16 forcing layers

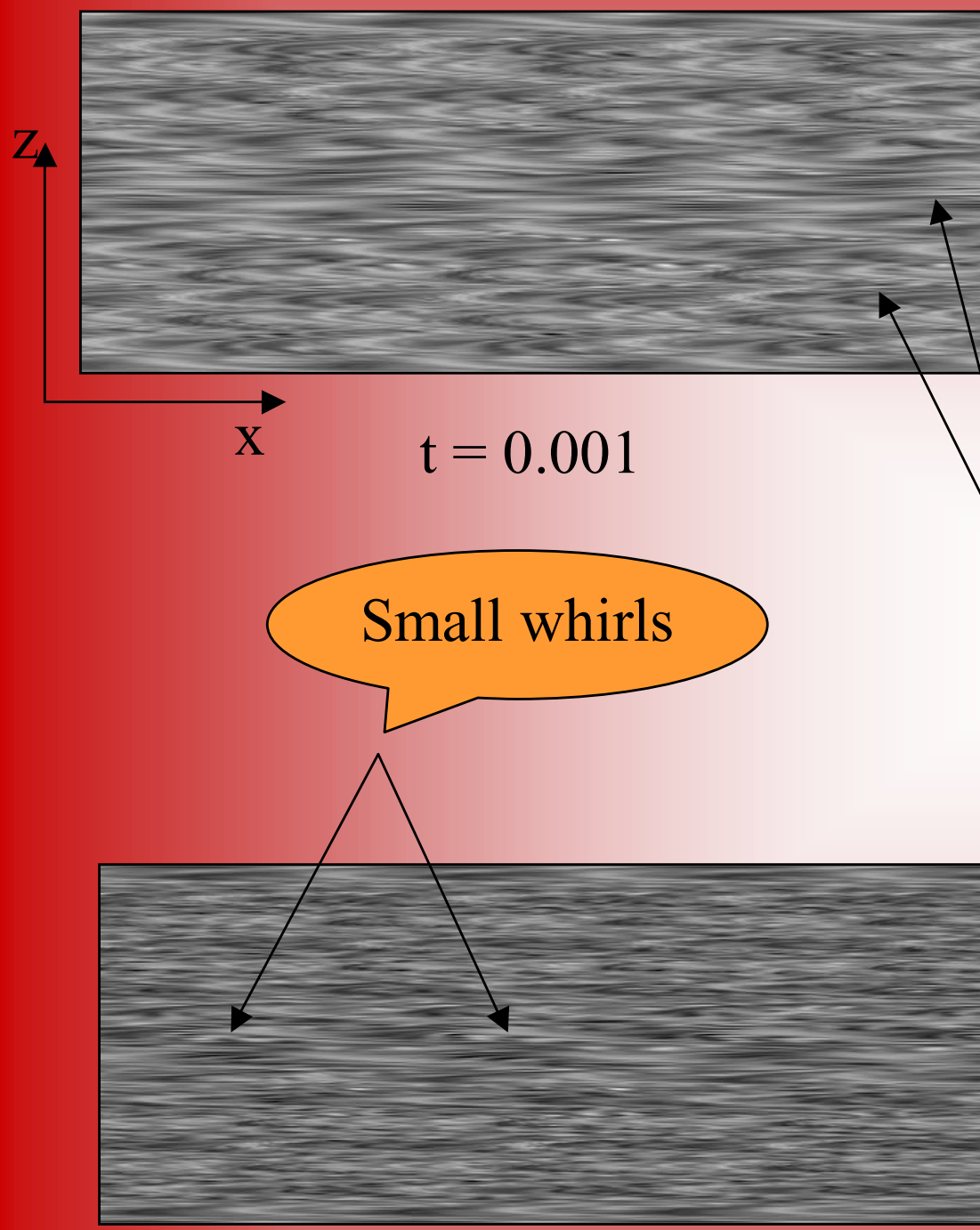

$t=0.003$

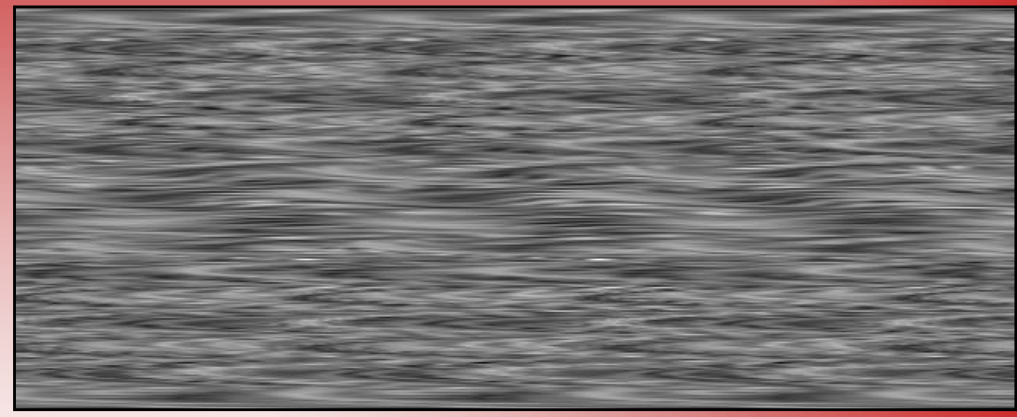

$\mathbf{t}=\mathbf{0 . 0 0 2}$ Time of maximum vertical flux of the concentration
An unhomogeneous flow at the beginning of the simulation

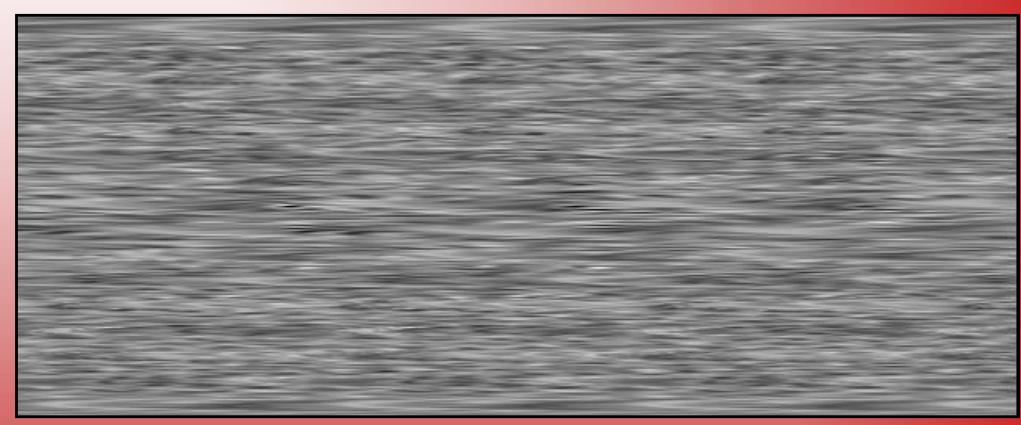

$$
t=0.004
$$


Vorticity component perpendicular to the domain of study, $\operatorname{Re}=\mathbf{7 0 0 0 0 0}$ 16 forcing layers
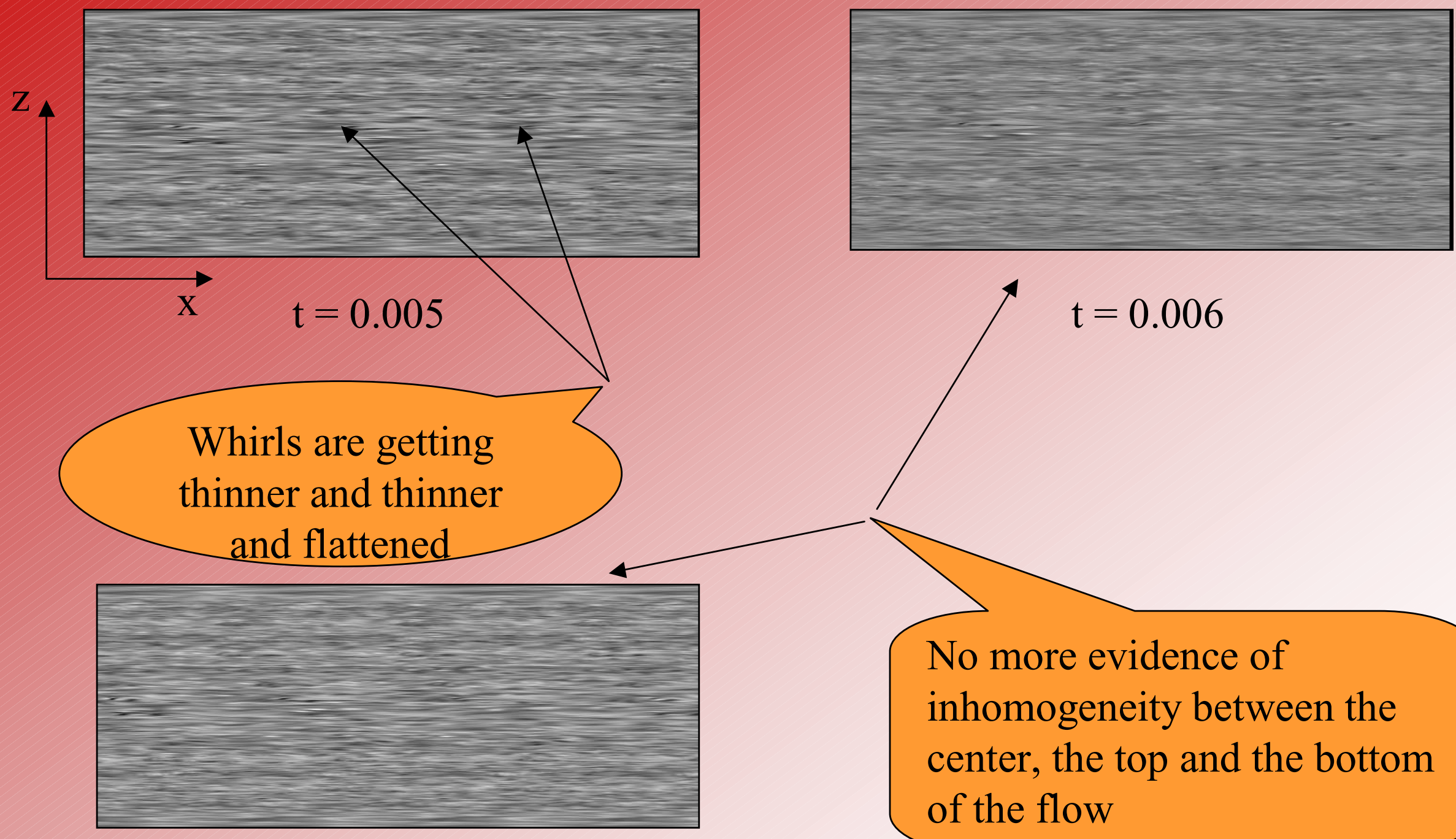

$$
\mathrm{t}=0.007
$$

No more evidence of inhomogeneity between the center, the top and the bottom of the flow 


\section{Continuation of the study}

Simulations with different values of the Peclet number

Boussinesq approximation to take into account a temperature stratification

Forcing with internal waves to quantify their part on the turbulent diffusion in differential rotation zones

3D compressible simulations 


\title{
Recent Computational Simulations of Rayleigh-Taylor Mix Layer Growth with a Multi-fluid Model
}

\author{
Erik Vold \\ Los Alamos National Laboratory \\ w/ acknowledgements to A.J.Scannapieco, \\ Tim Clark, John Grove, Chuck Cranfill, et.al.
}

Presented at the 8th International Workshop on The Physics of Compressible Turbulent Mixing

Pasadena, CA, Dec.10-14, 2001 


\section{disclaimer}

This is a partial sub-set of the viewgraphs presented at the 8th IWCTM.

The work on 'resolved scale' simulations is currently being prepared for publication, to be submitted to Physics of Fluids.

The work on 'sub-grid drift flux' simulations will be prepared for publication soon, to be submitted to a journal TBD. 
Erik Vold

Los Alamos National Laboratory

\section{Abstract - LA-UR-01-2562}

Recent results of computational simulations of the Rayleigh-Taylor mix layer are presented and discussed. Our previous work is summarized briefly comparing mix layer growth characteristics observed in different simulation modes including single fluid with initial density discontinuity, two-fluids with interface reconstruction and in a full multi-fluid dynamic approach. Recent comparisons under varying compressibility are presented showing negligible influence of compressibility on the mix layer growth rate. Using spectral analyses, perturbations intentionally introduced in the initial conditions are compared to long wave length perturbations introduced inadvertently in these initial conditions. The influence of these initial conditions on late time growth and growth rate are explored. The compressible multi-fluid model allows each fluid to have its own 'drift velocity' relative to the mass averaged fluid velocity. This can be applied in several ways within the mix layer to represent a real molecular mixing, a turbulent enhanced diffusive mixing, or an individual species 'sub-grid' convective drift flux. Examples of these in the Rayleigh-Taylor mix layer are discussed. Finally, we consider the combination of these factors which best matches the experimental results for mixing layer growth rates in incompressible experiments, and how these results may apply to compressible fluids.

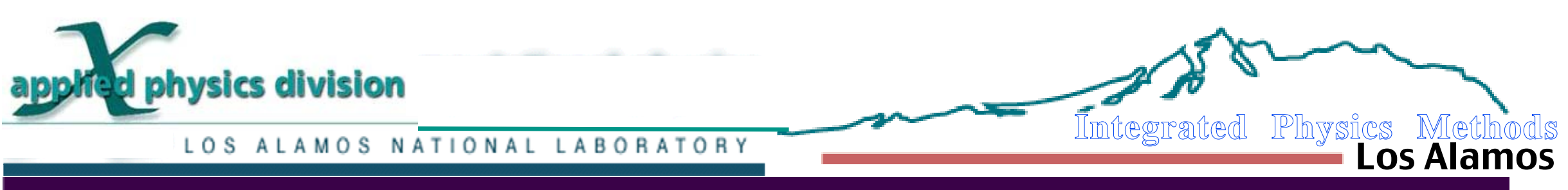




\section{Introduction}

- Goals:

- simulate Rayleigh-Taylor mix layers accurately to predict atomic / molecular mixing (e.g., a reactive R-T mixing front) in macroscopic geometries.

- use 'resolved simulations' to model mix layer growth and use drift flux (subgrid) simulations to model the mix layers atomically mixing components.

- match experimental 'alpha' $(\alpha), h=\alpha A_{t} g t^{2}$

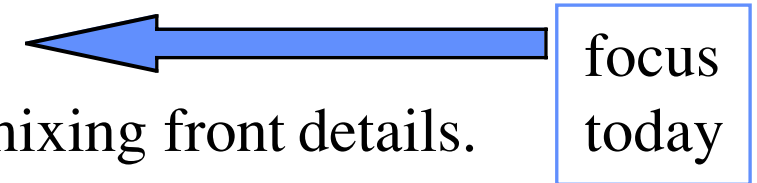

- Central Issues

- numerical mixing must be small enough to have a negligible effect on mix layer growth rates so that 'sub-grid' mixing can be represented realistically.

- Hypothesis: the growth rate seen in computations, which have no subgrid mixing and small numerical diffusion, should equal or exceed the experimental value IF the experiment contains small scale dissipation which reduces the growth rate in the experiment. 


\section{Summary of Relevant Work}

- Experimental

- wide range of experiments (mostly incompressible) show a mix layer growth rate which closely approximates the scaling, $\mathrm{h}=\alpha \mathrm{A}_{\mathrm{t}} \mathrm{g} \mathrm{t}^{2}$

- alpha bubble, $\alpha_{b} \sim 0.06-0.07$ (earlier work, e.g., Youngs and Read et.al., ) - and $\sim 0.05$ (recent work, e.g., Dimonte, Schneider, et.al.)

- Computational

- alpha bubble results range from $\sim 0.03-\sim 0.1$

- many 3-D methods (compressible or incompressible) trending towards low end, $\alpha_{b} \sim 0.03 \sim$ half experimental mean

- front tracking w/ 2 distinct fluids ('Frontier code', Glimm, et.al.) at higher end, $\sim 0.07-0.08$

- large variance in alpha just due to random seed in initial perturbation

- $(\sim 0.05+/-20-50 \%$, in 2-D compressible isothermal fluids, T. Clark, 2001)

- 2-D results $\sim 15 \%$ greater than 3-D results (Youngs, 1994) 


\section{Summary of Our Methods}

- Methods

- 2-D multi-fluid Eulerian AMR formulation

- compressible Euler equations in appropriate limit to recover incompressible approximation, supplemented with fluid volume fractions

- ideal gas equation of state for each fluid

- advection of fluid volume fractions in mixed cells at the interface

- mixed cell treatment (Bowers and Wilson,1991)

- interface reconstruction (D.Youngs, 1984, 1989)

- high-order, monotonic Van Leer advection of fluid quantities

- each fluid has its own density, internal energy and pressure in its fluid volume fraction within the 'mixed cells' (containing the interface)

- in 'drift flux' representation of sub-grid mixing, each fluid has its own 'drift momenta' relative to the mass average which can be adjusted to represent realistic molecular diffusion or a range of assumed turbulent flux forms 


\section{Summary of Our Previous Results}

- Previous Results

- alpha bubble was found on a 128 x 128 grid with no sub-grid mix model, to be $\sim 0.08-0.1$, somewhat larger than experiments.

- interface algorithm does not alter the growth rates significantly.

- molecular mixing (by the drift flux) does not influence the mix layer growth rate but does create a unique distribution of molecularly mixed materials controlled primarily by the volume fraction of the lighter material.

- drift flux mixing significantly above the molecular diffusion level reduces the mix layer growth rate (for the set-up in these results, a drift flux $\sim 50$ times greater matches experimental range $\left(\alpha_{\text {bub }} \sim 0.055\right)$ 
- Numerical

- grid resolution $\left[128^{2}\right.$ or $256^{2}$ or $\left.512^{2}\right]$

- Interface treatment [mix cell volume fraction advection w/ Young's interface reconstruction]

- Differencing schemes [high order monotonic Van Leer like scheme]

- 2-D vs. 3-D [2-D only]

- Initial Conditions

- initial perturbation magnitudes, [volume fractions, Vf, set to match interface perturbation]

- perturbation on density $\left[\rho=\rho_{1} \mathrm{Vf}_{1}+\rho_{2}\left(1 .-\mathrm{Vf}_{1}\right)\right]$

- perturbation on internal energy $\left[\varepsilon=\varepsilon_{1} \mathrm{Vf}_{1}+\varepsilon_{2}\left(1 .-\mathrm{Vf}_{1}\right)\right]$

- wavelength spectrum, [30 modes, mode numbers 30 - 60, random phase, unit amplitude]

- hydrostatic equilibrium by e(z), or $\rho(z)\left[e(z), w / \rho=\rho_{o}\right]$

- Physics

- compressible or incompressible formulation (w/ or w/o internal energy ) [compressible]

- degree of compressibility [varied $\mathrm{Ma}^{2}$ by 2 orders of magnitude]

- fluid equations: Euler, viscid, internal or total energy [Euler using internal energy w/ optional multi-fluid drift flux for 'species momenta' relative to mass averaged single fluid velocity.]

- Interface physics: surface tension, slip or traction, molecular diffusion, sub-grid mixing 
- Los Alames rt $256 b c 4$ den at $t=20,40,60,80$

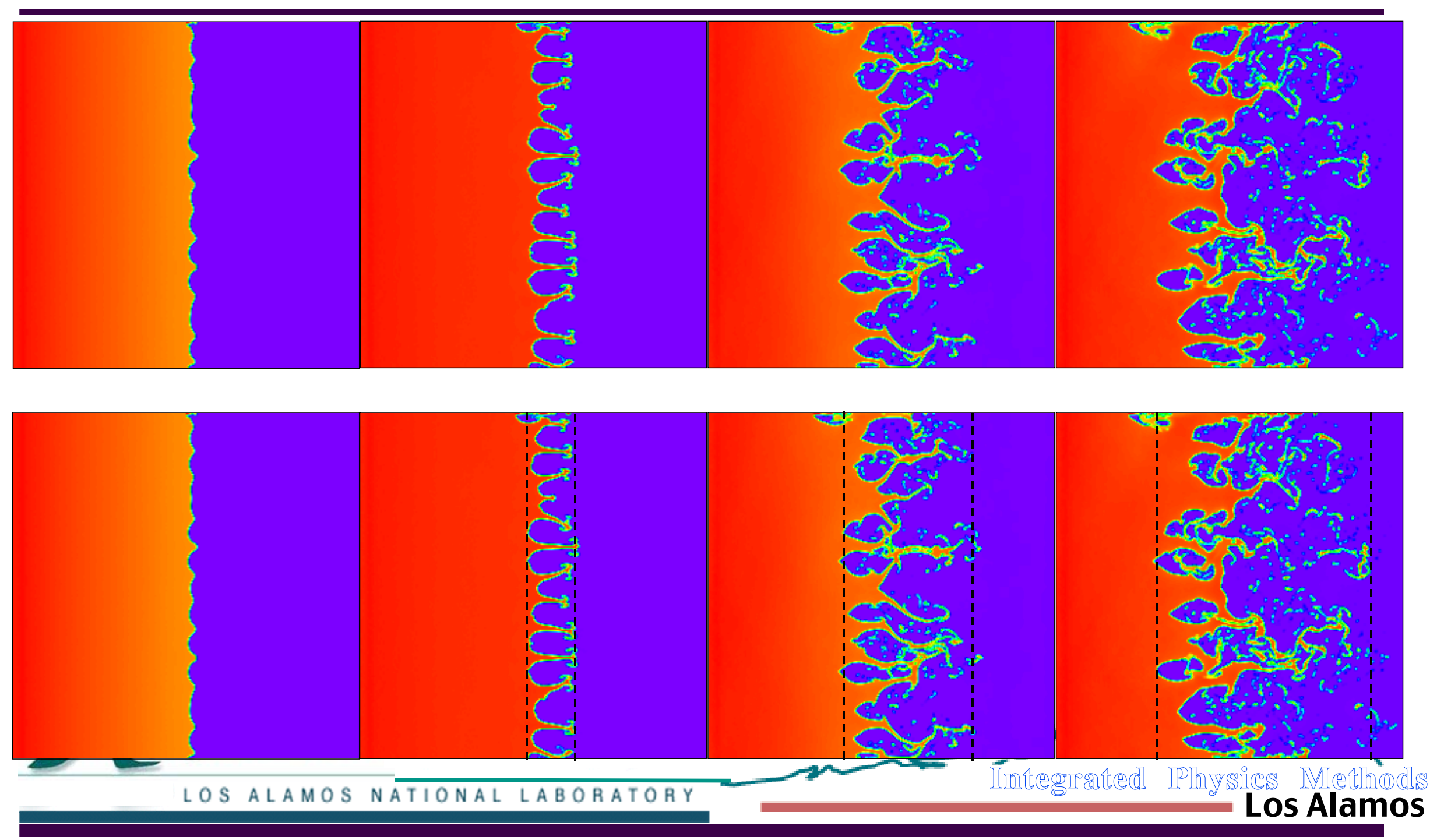




\section{mix layer width, $\mathrm{h}$}

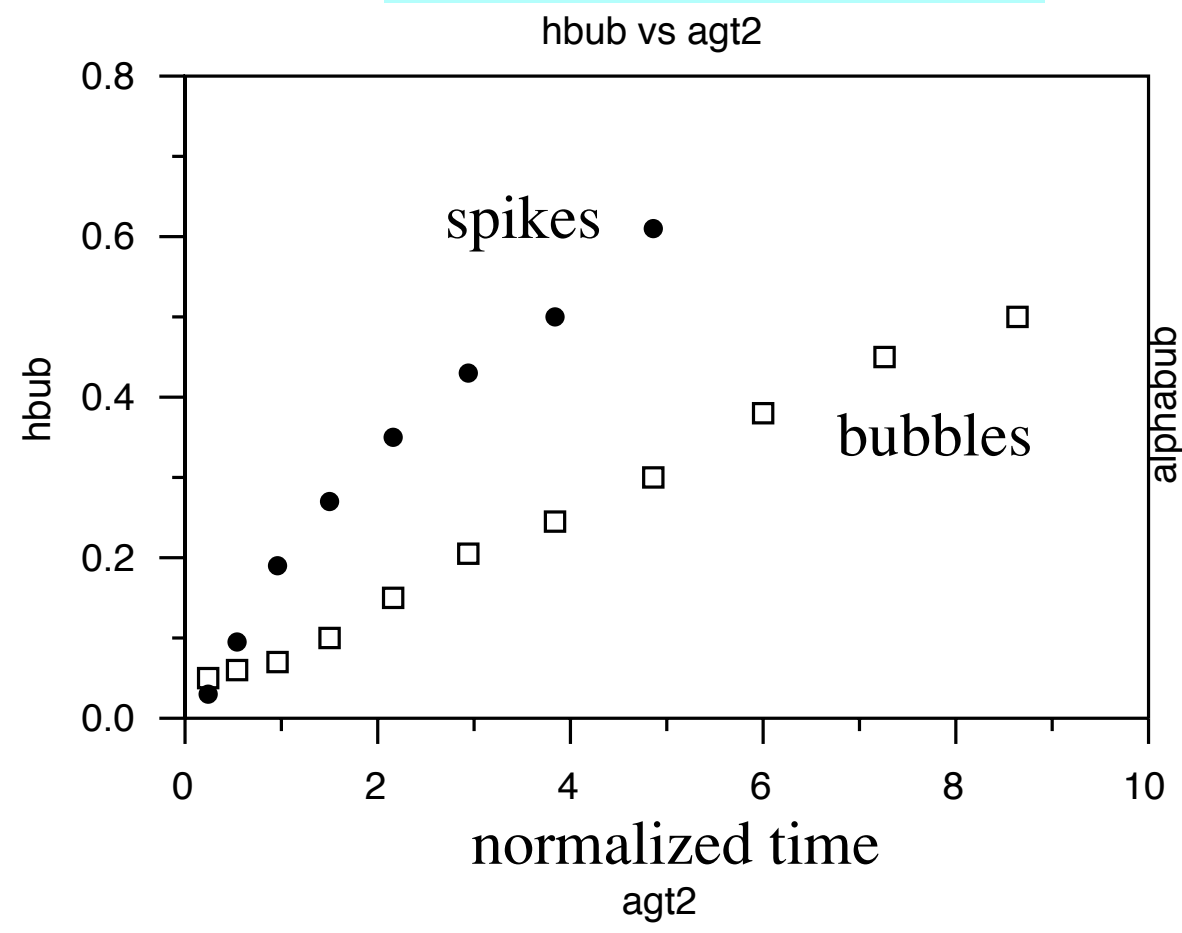

mix width growth coef, $\alpha$

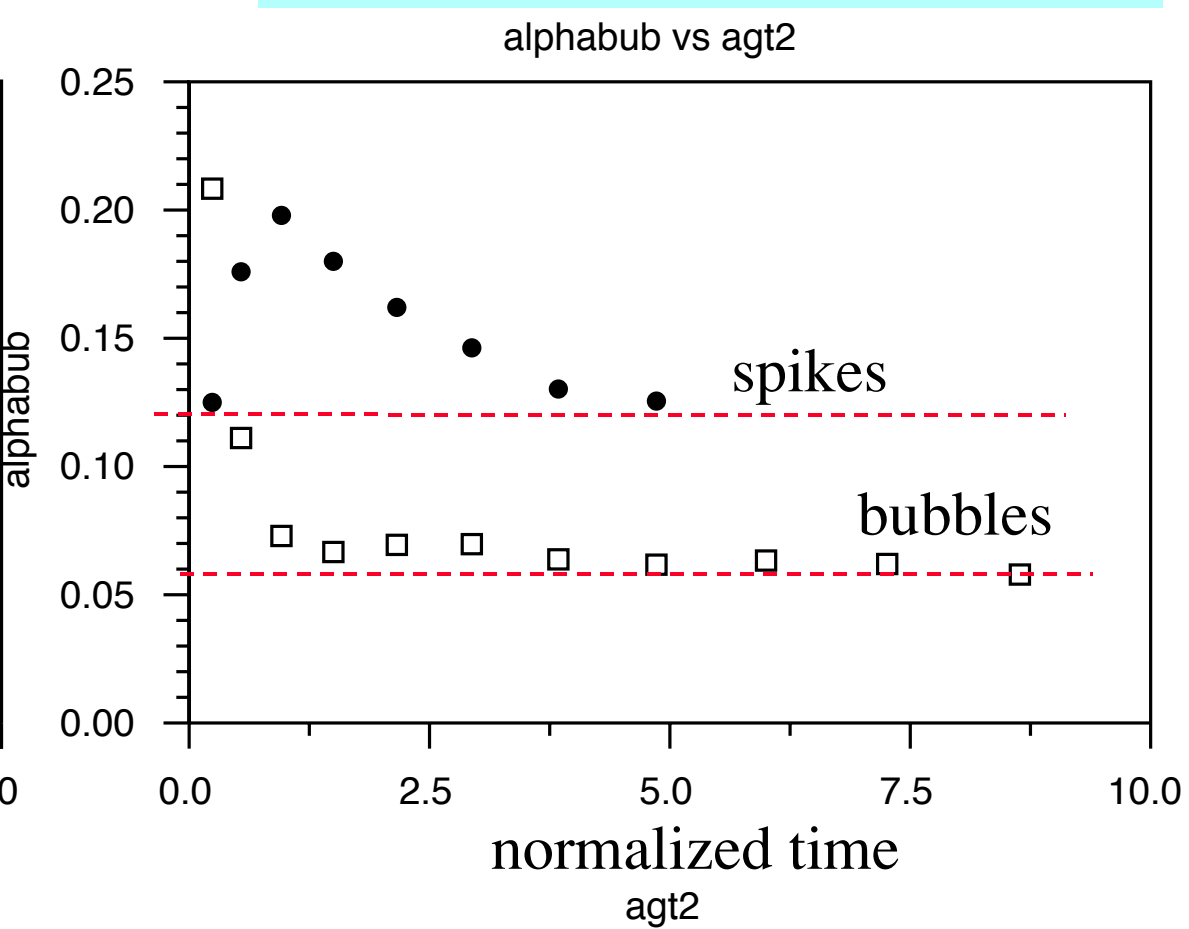




\section{RT mix width for varying compressibility}

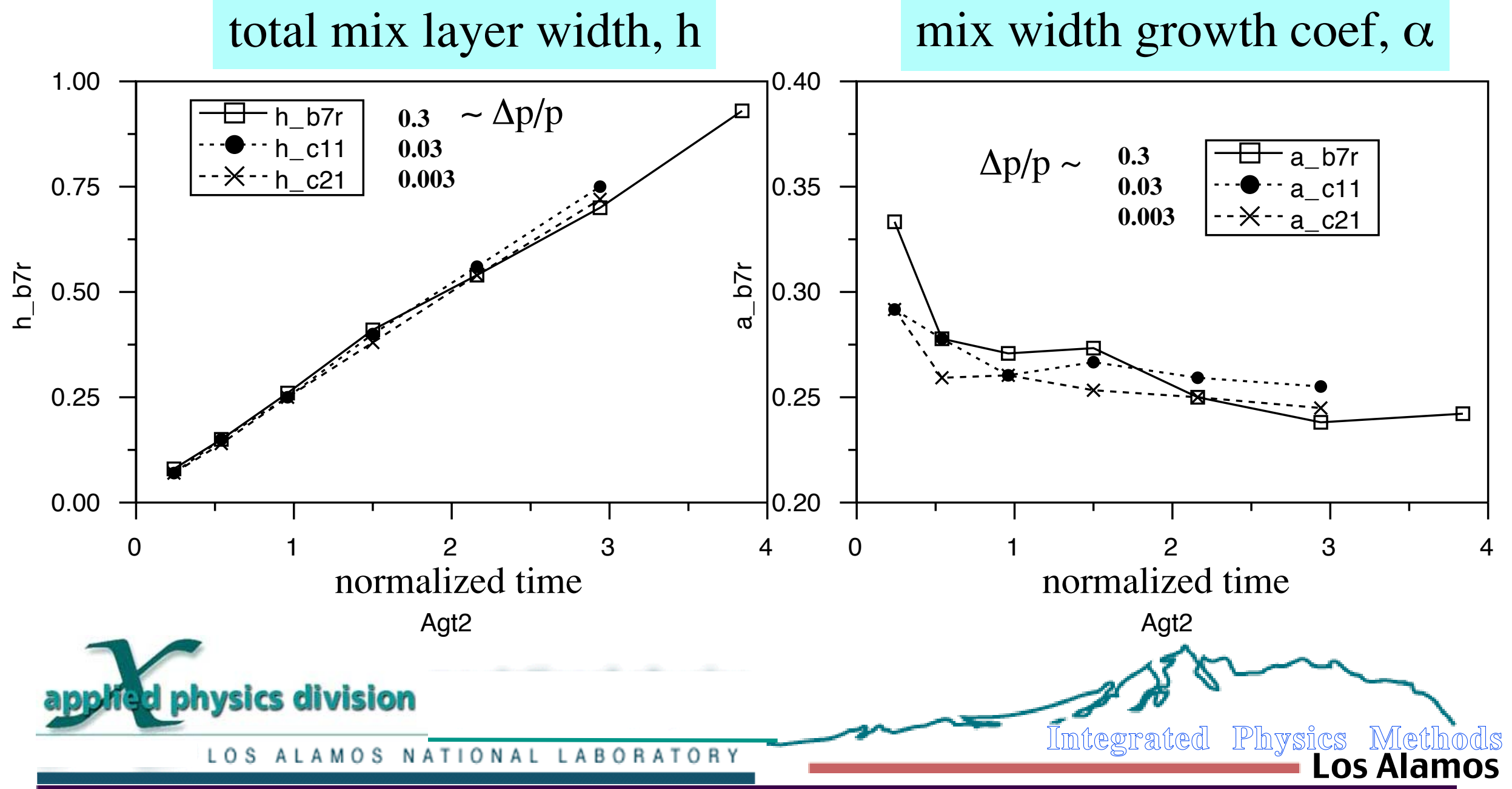


Los Alamos BC4-Zint(IC) fixed grids $128^{2}, 256^{2} \& 512^{2}$ - each grid result on $512^{2}$

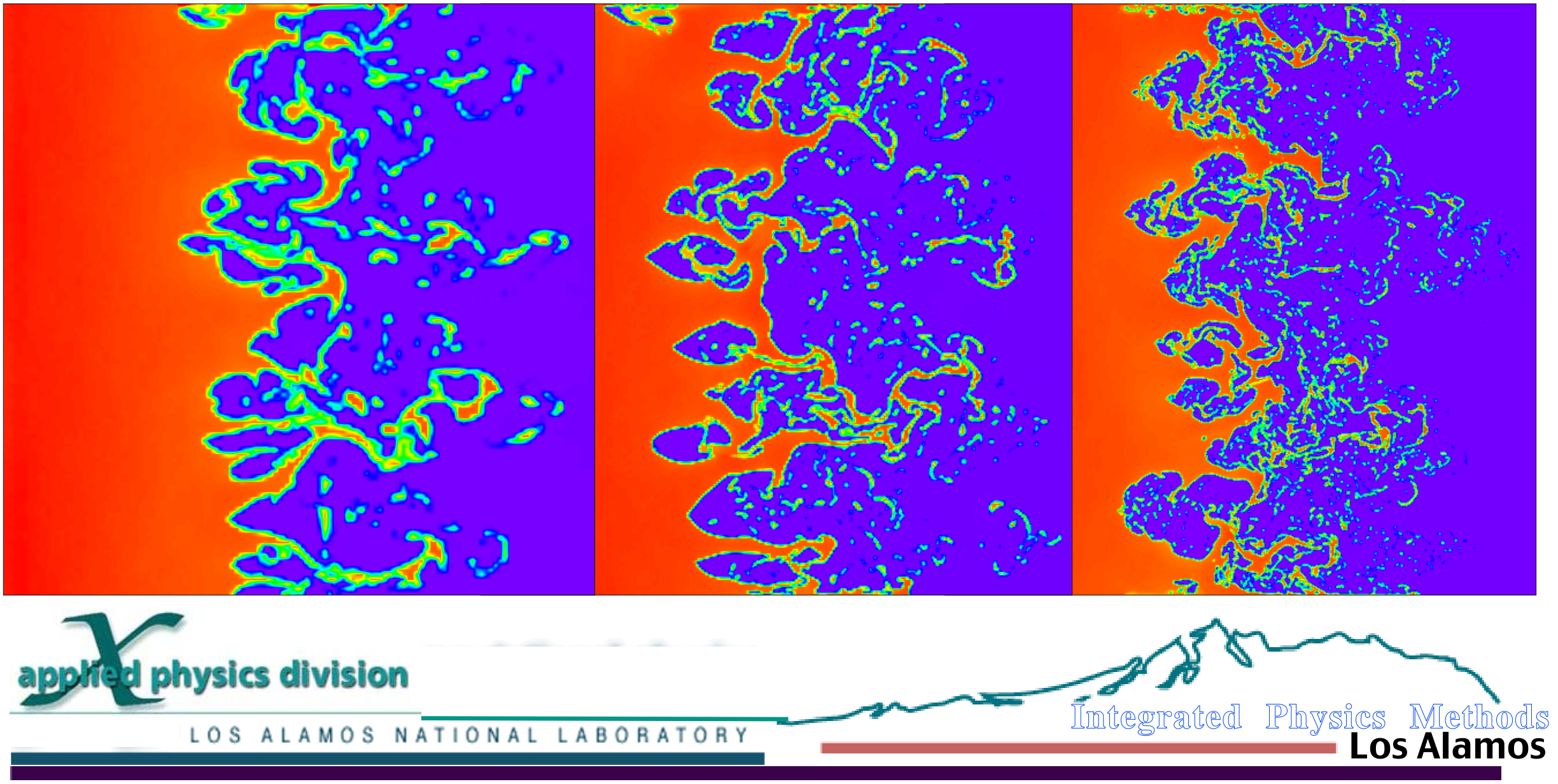


Los Alamos BC4- Zint(IC) fixed grids $128^{2}, 256^{2} \& 512^{2}$ - actual grid results

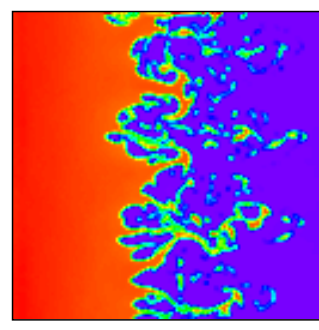

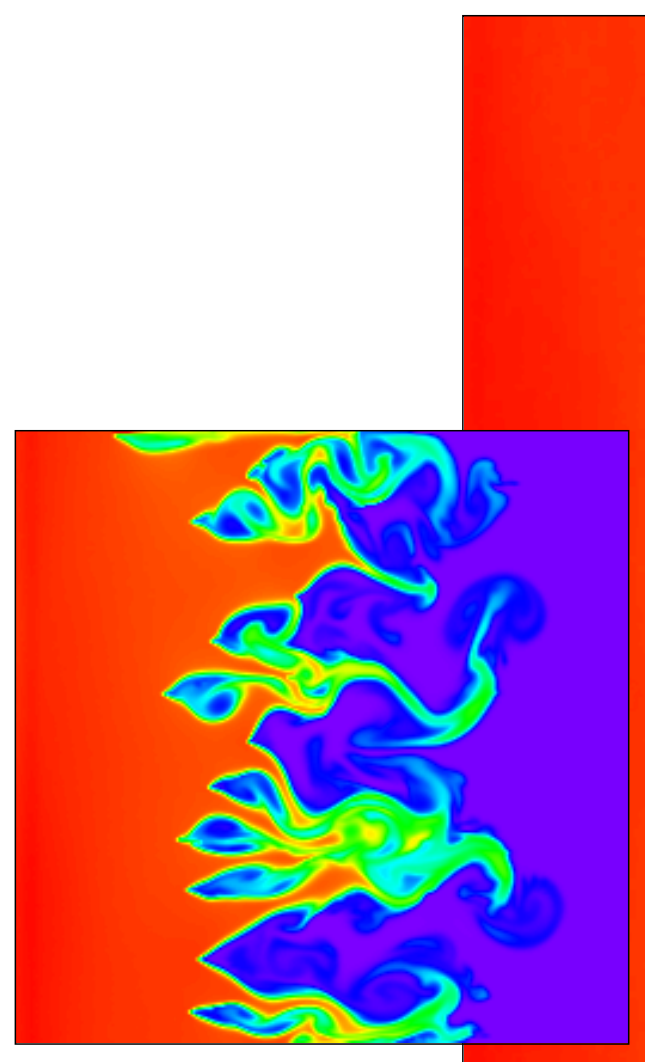

w/o Intrf.Recon.
W/ Intrf.Recon. appolted physics division

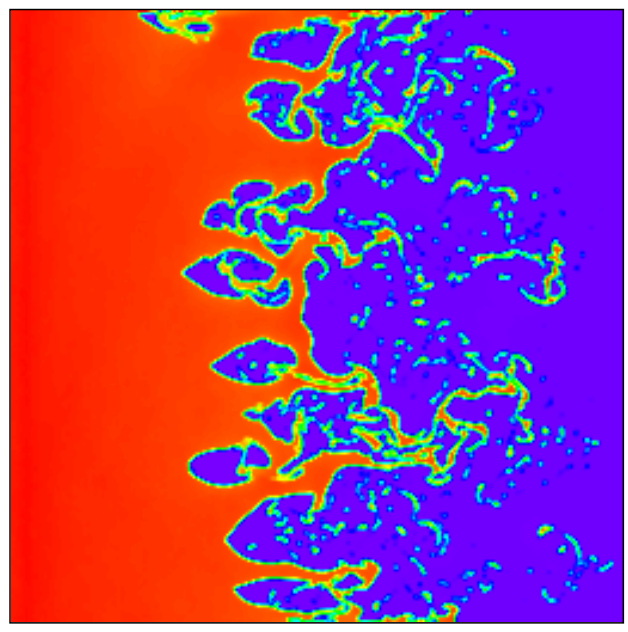

LOS ALAMOS NATIONAL LABORATORY

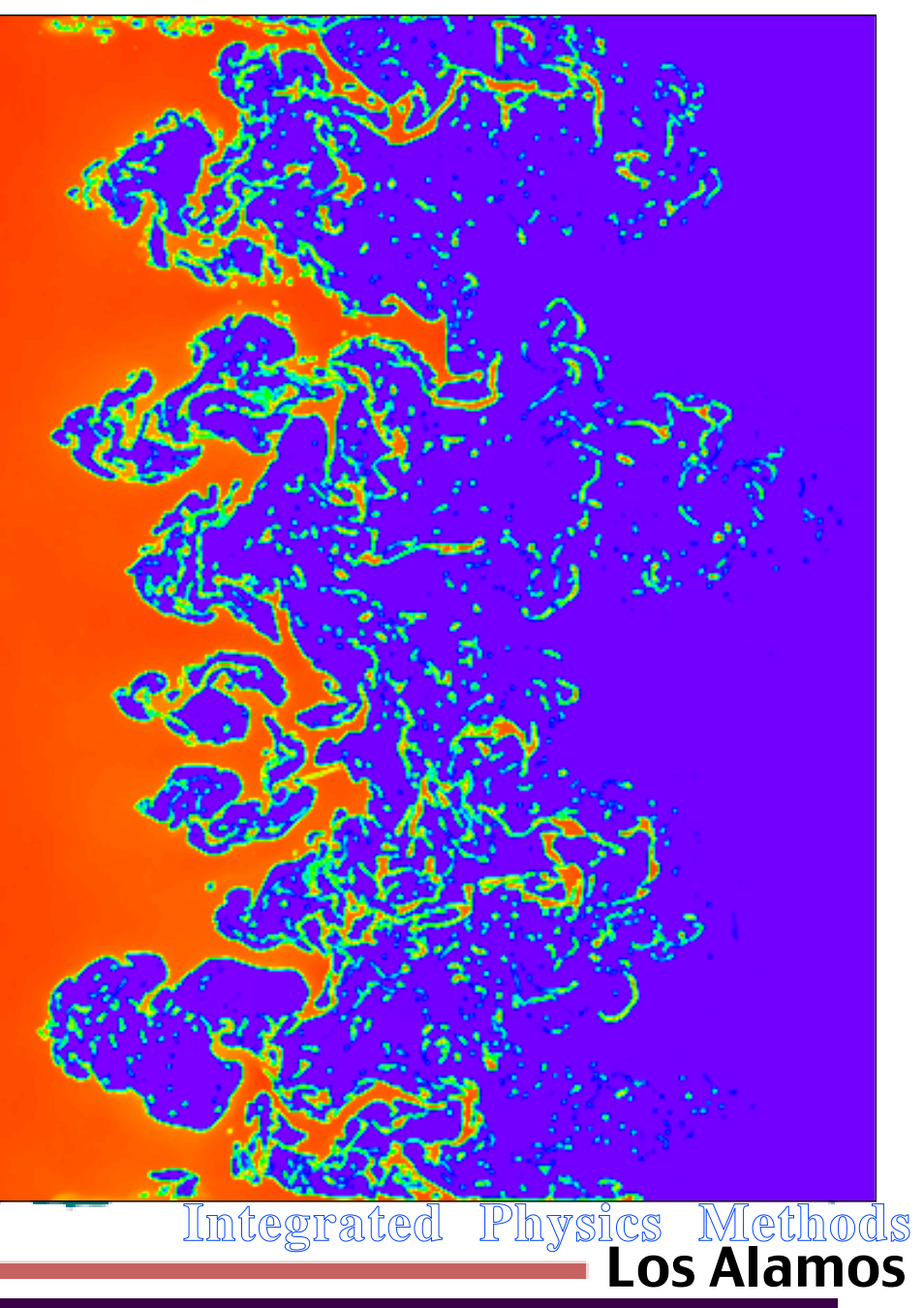




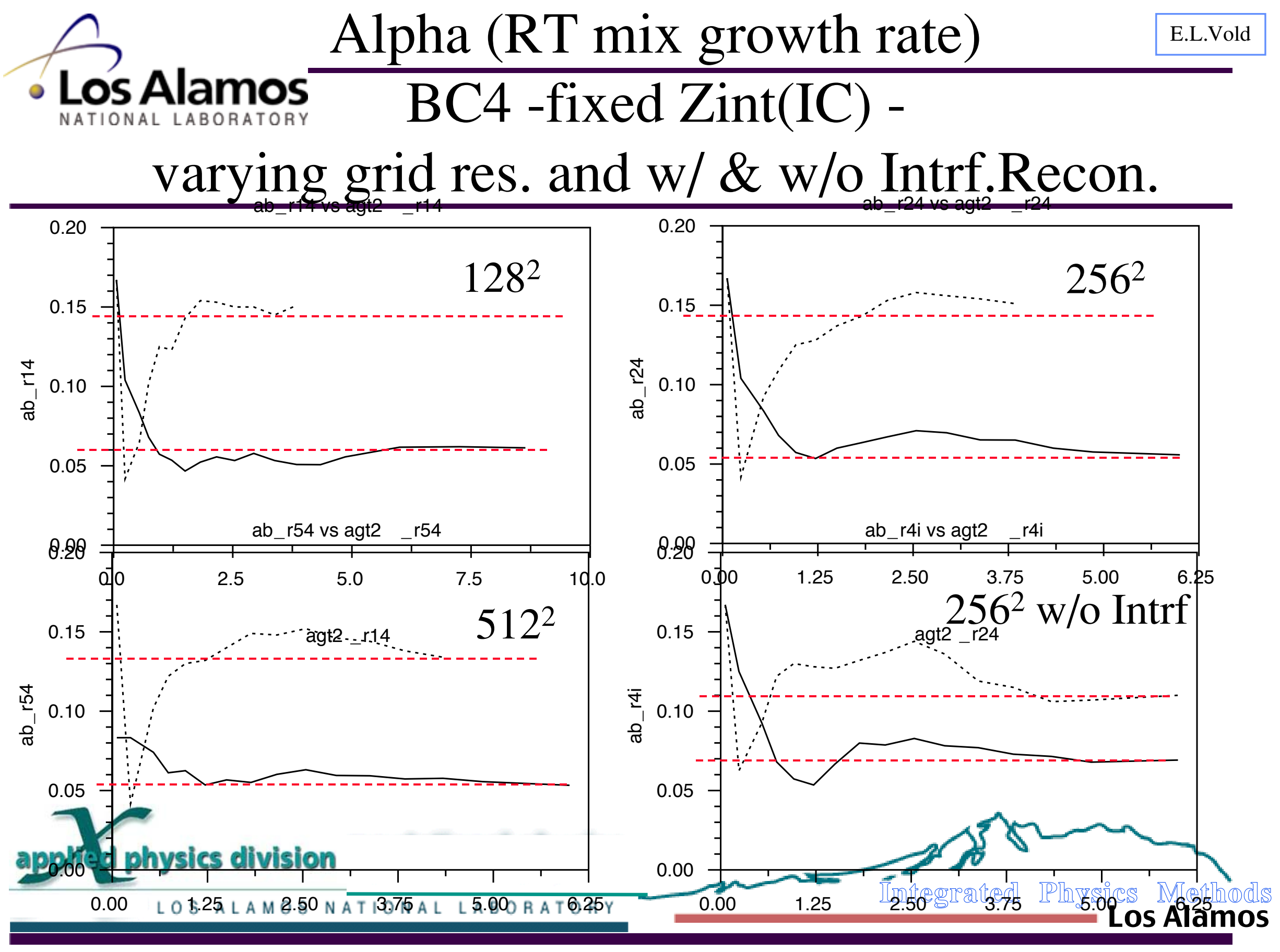




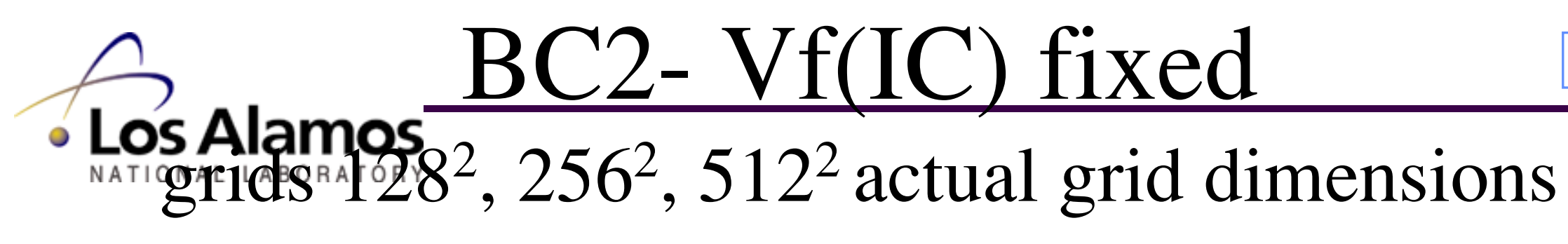

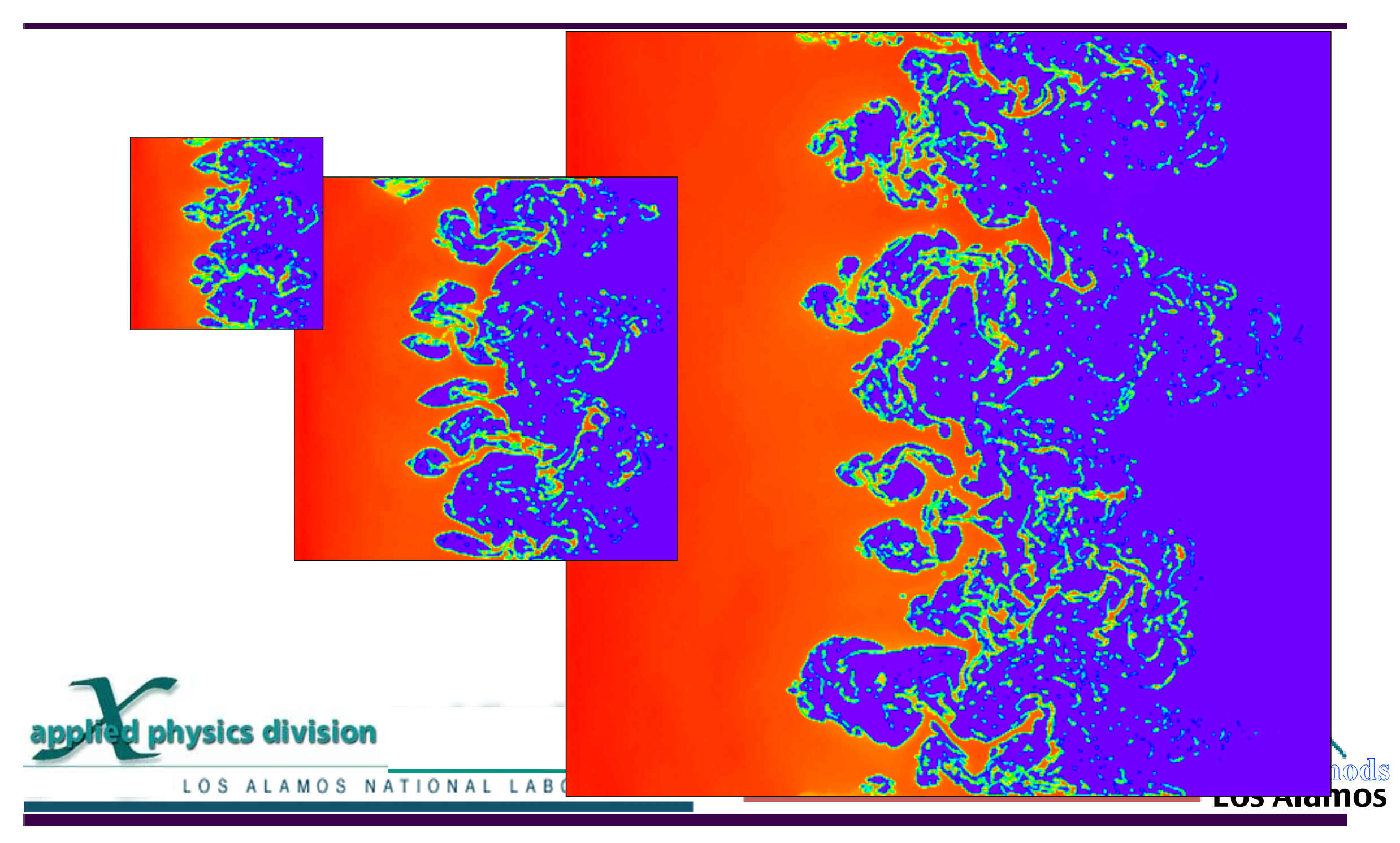




\section{Alpha (RT mix growth rate)}

BC2 -fixed $V_{f}(I C)$ -

varying grid res. and w/ \& w/o Intrf.Recon.

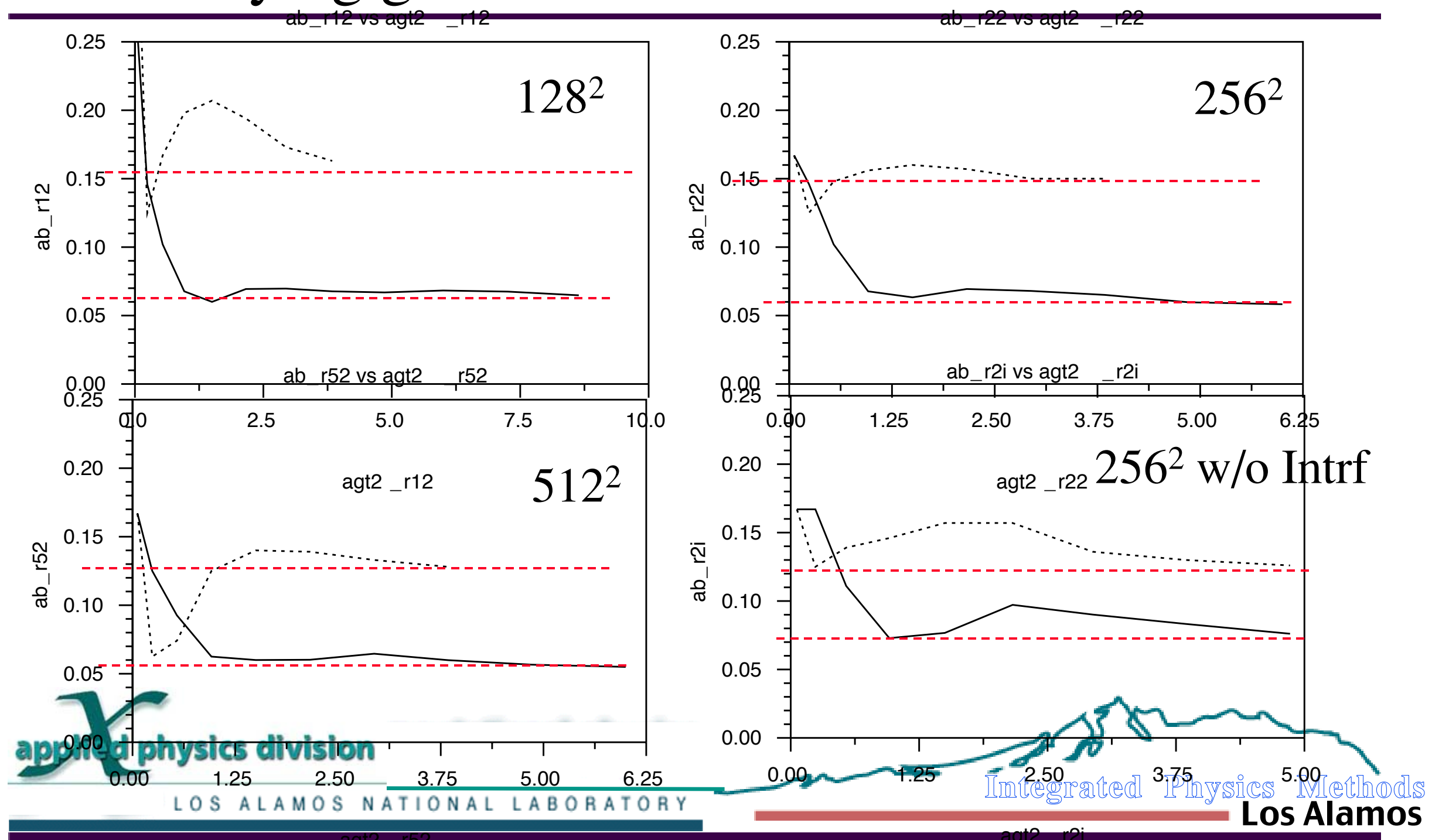


BC2 (fixed VF(IC)) vs. BC4 (fixed Zint(IC) $)^{\text {EL.Vold }}$ - Los A Aamos

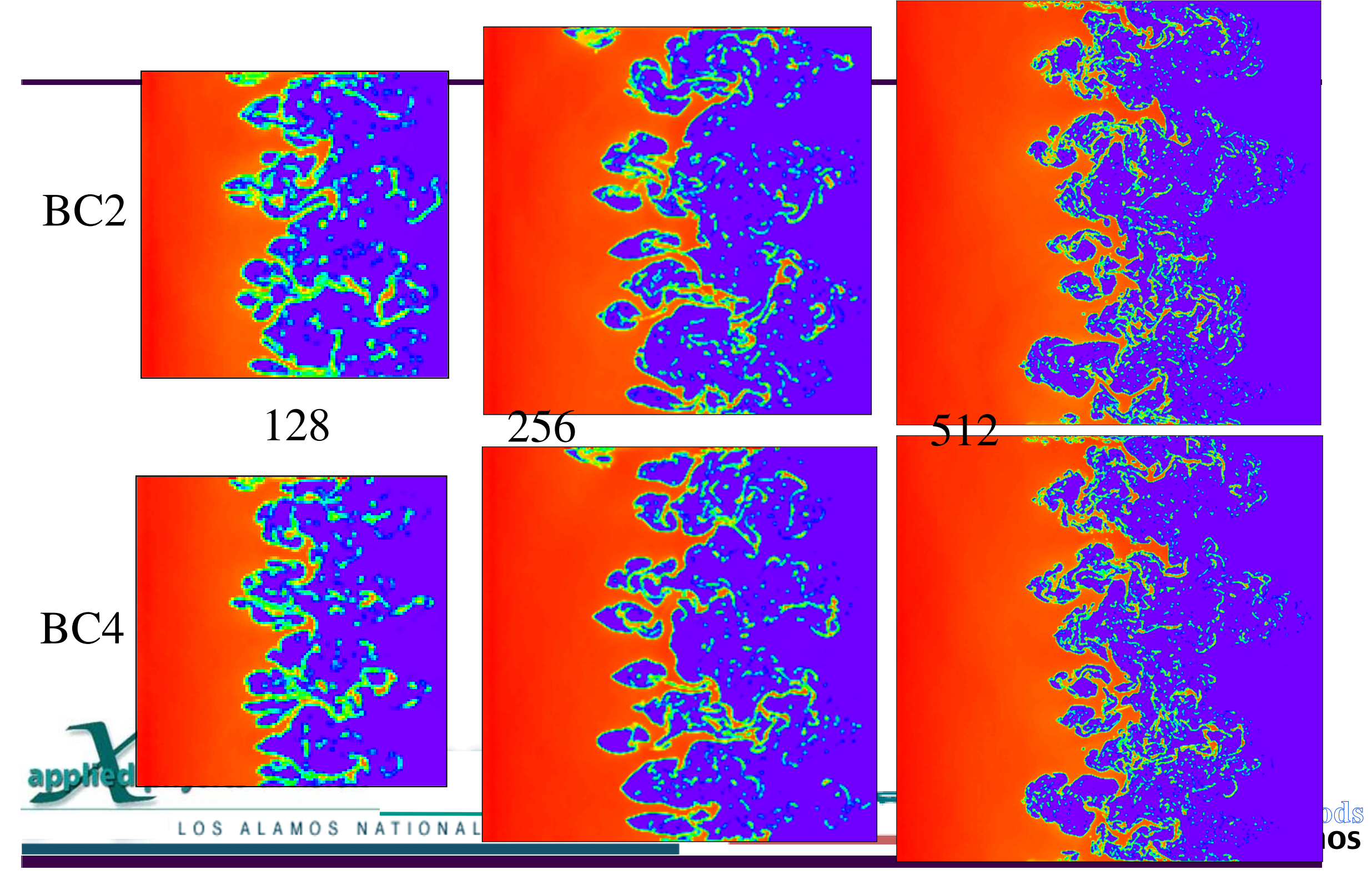




\section{RT resolved simulation cases}

\section{alpha summary for $\mathrm{At}=0.8$}

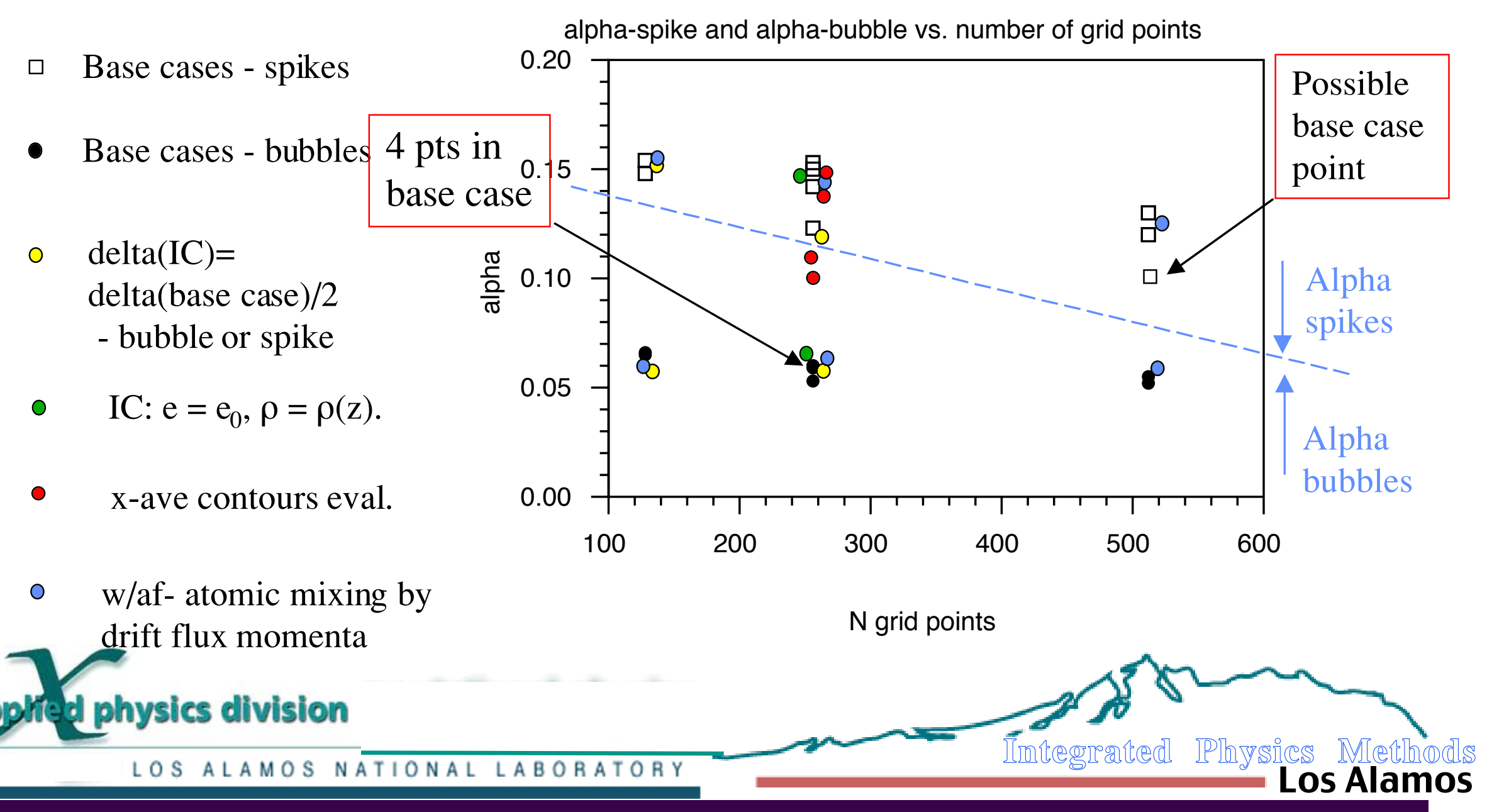




\section{Analytic model compared to fluid equations}

Analytic model:

$$
\begin{gathered}
\frac{\partial h}{\partial t}=u \\
\frac{\partial u_{i}}{\partial t}+C_{D i} \frac{u_{i}^{2}}{\left(\delta_{o}+\alpha_{o} h_{i}\right)}=\beta_{i} A g
\end{gathered}
$$

Fluid model:

$$
\frac{\partial h_{m}}{\partial t} \sim u_{g}
$$

$\frac{\partial u}{\partial t}+u \cdot \nabla u=a \sim A g-\frac{\nabla p}{\rho}$

OR: $\quad \frac{\partial u}{\partial t}+\frac{\nabla u_{s}^{2}}{2}-u \times \omega=a$

alpha result $\left(\mathrm{h} / \mathrm{Agt}^{2}\right)$

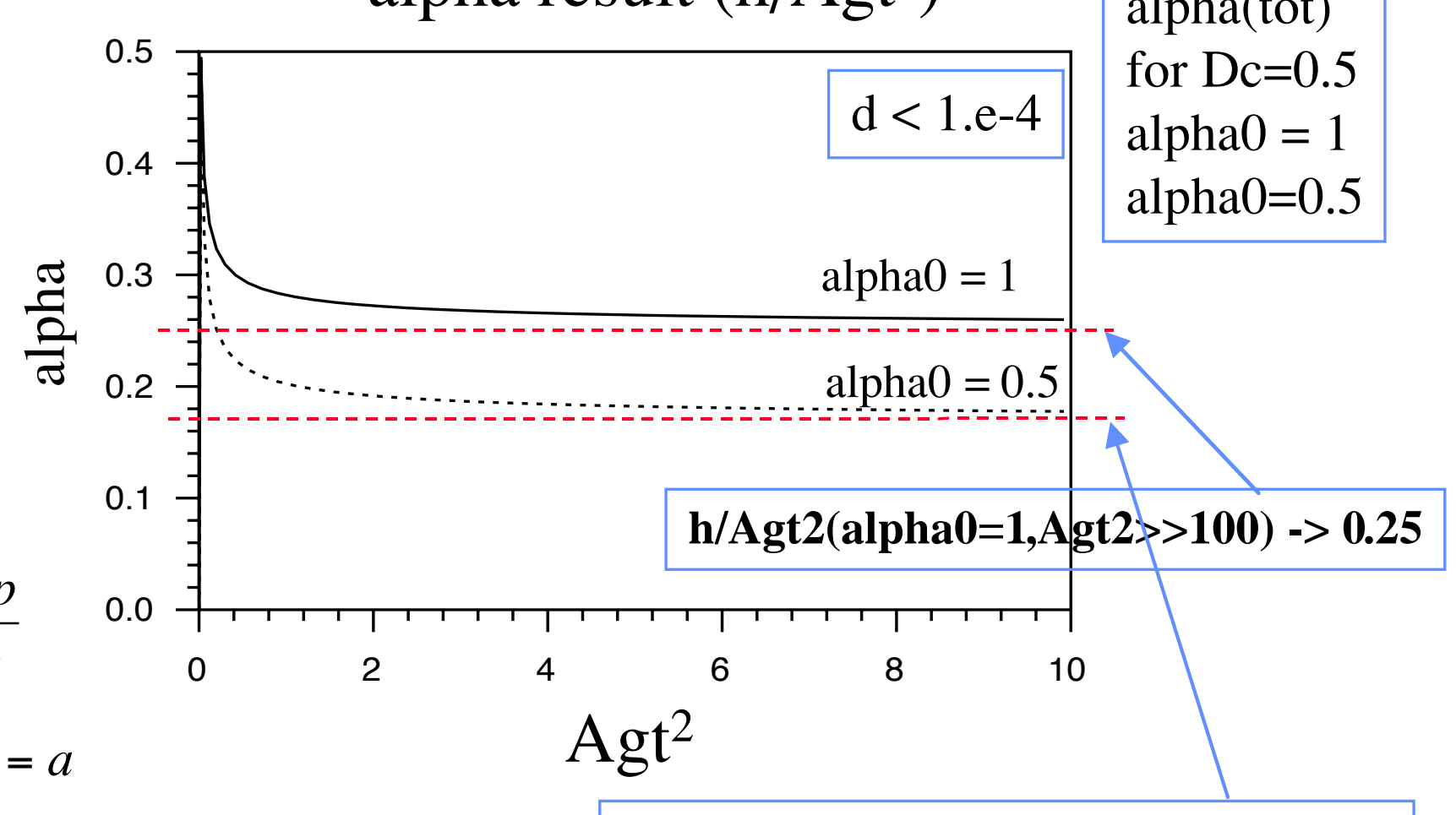

h/Agt2(alpha0=0.5,Agt2 $>>100) ~->0.17$

$$
\text { OR: } \frac{\partial u}{\partial t}+\frac{u_{x}^{2}}{2 L_{x}}+\frac{u_{y}^{2}}{2 L_{x}^{-}}-u \times \omega \sim a
$$

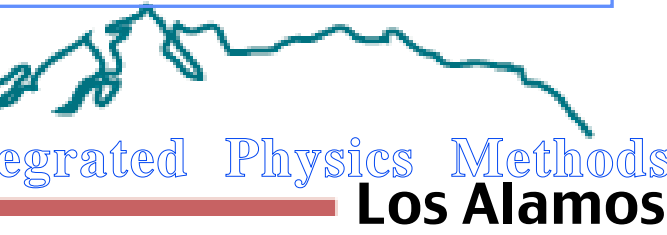




\section{$\mathrm{h} /$ Agt 2 as $\mathrm{f}[$ delta0]}

v determined as:

hoh0_hintvt:

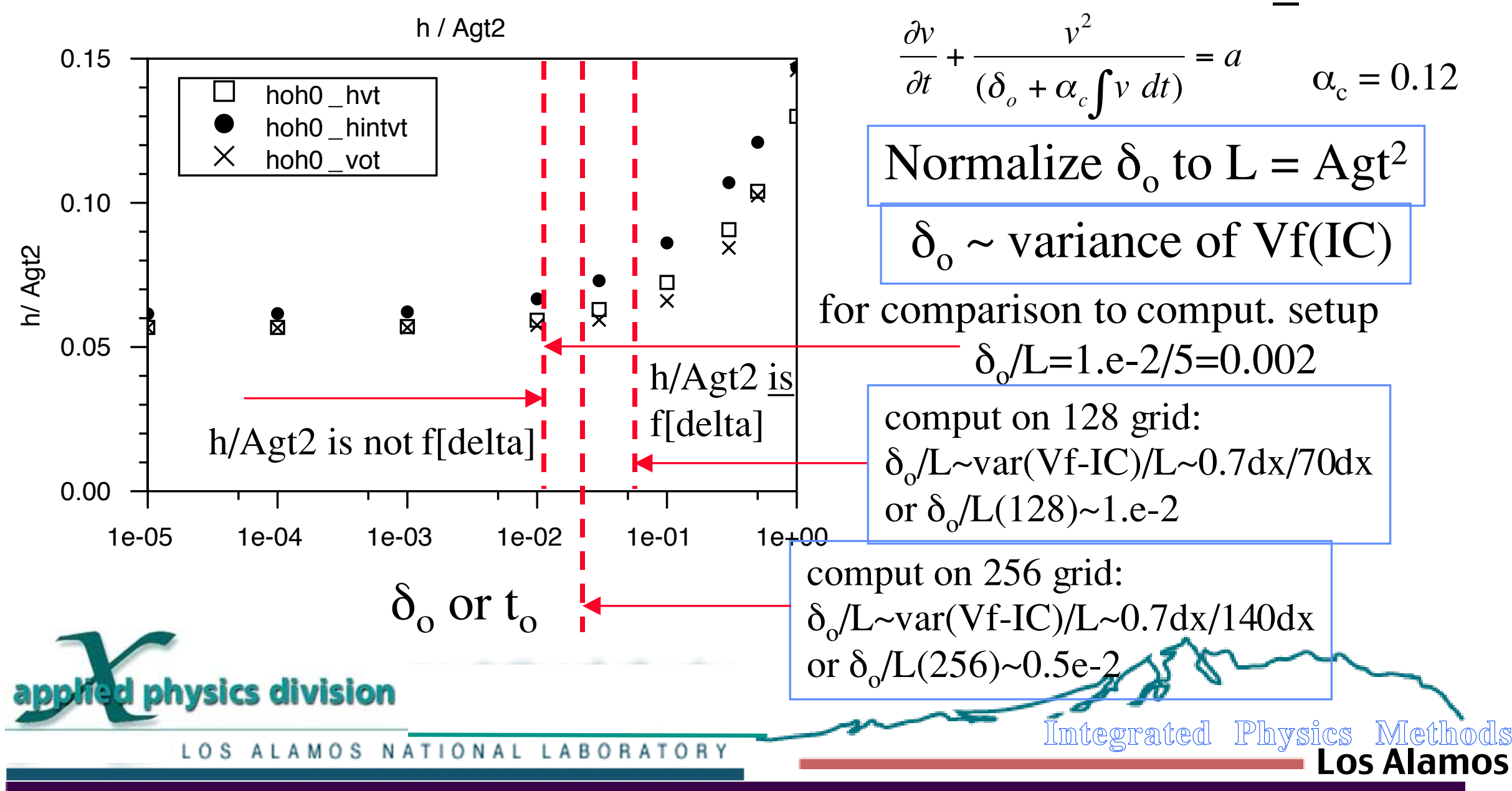


- Ios Alamos-

RT spectral density for IC perturbation

E.L.Vold w/ modes k=30-60: $\mathrm{z}_{\text {int }}(\mathrm{t}=0) \sim \mathrm{Vf}$
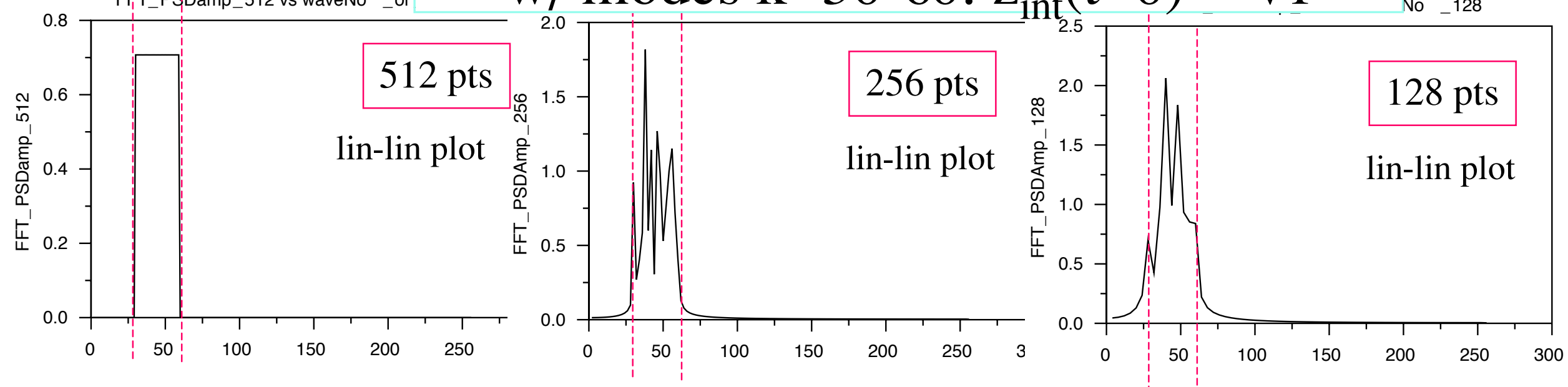

waveNo_or_freq_512

waveNo_256

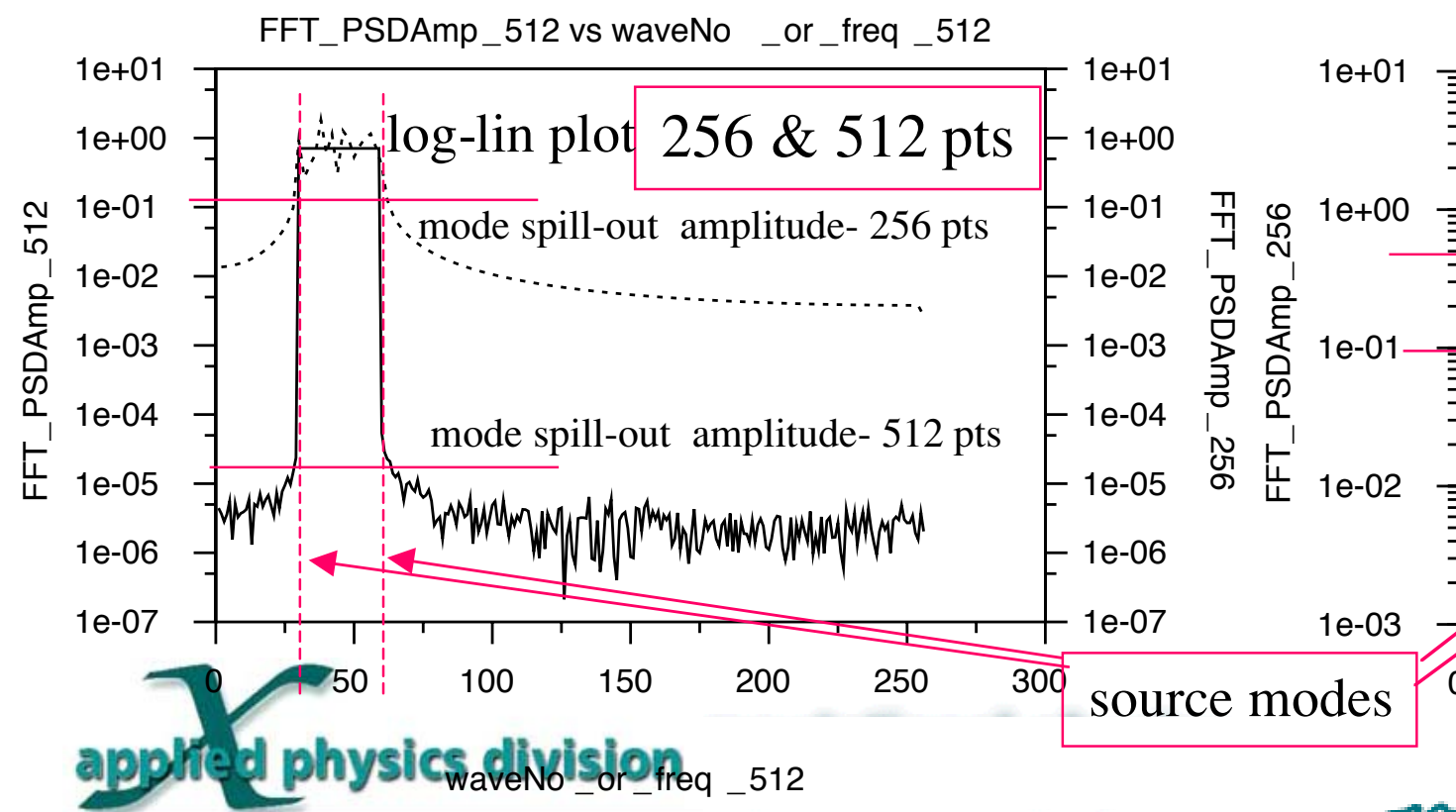

waveNo_128

FFT_PSDAmp_256 vs waveNo_ 256 


\section{Los Alamps ations for FFTs at t80}

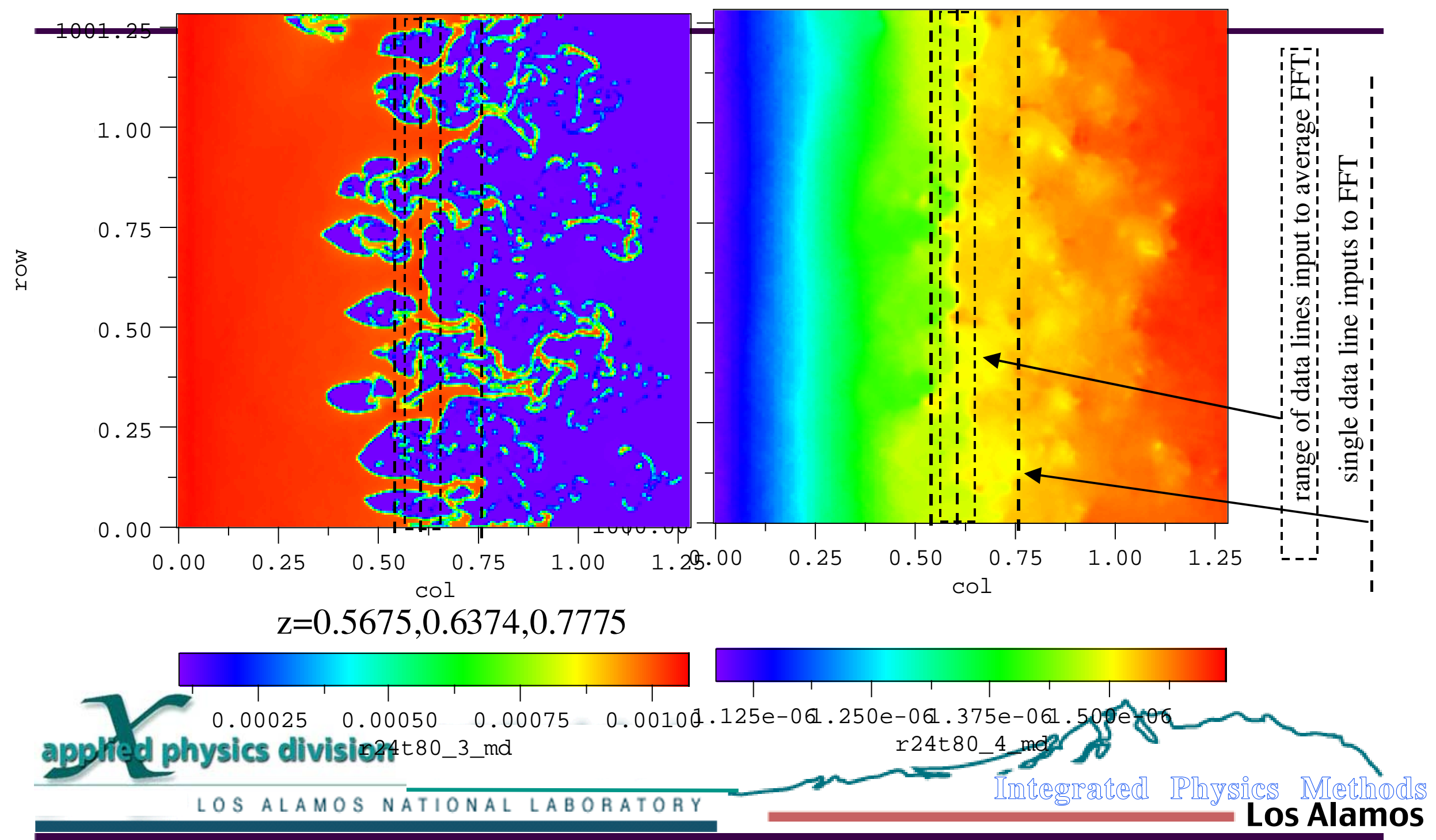




\section{FFTs p-t80 $128 \& 512$}
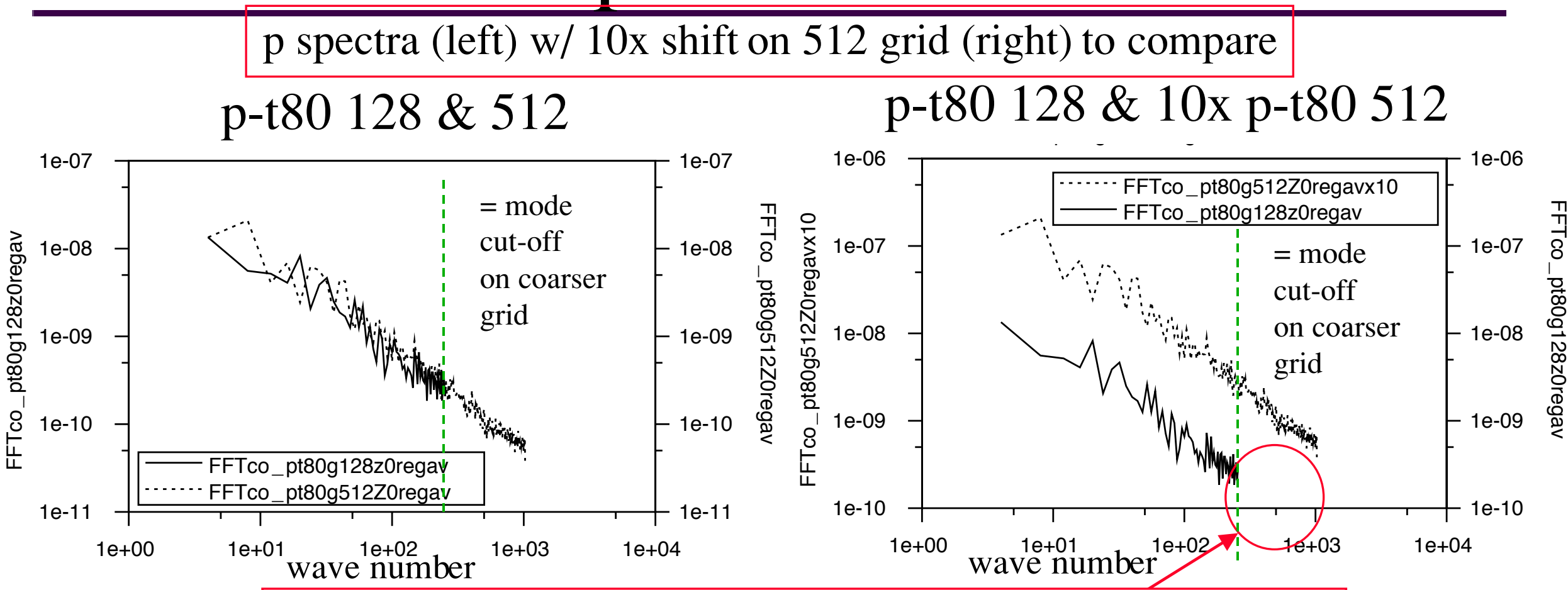

Therefore, unresolved components on 128 grid appear to be irrelevant to small $\mathrm{k}$, long wavelength mode growth**.

** alternative explanation: numerical errors on 128 grid and resolved high mode numbers on 512 grid both have the same effect on the small $\mathrm{k}$, long wavelength mode growth***. 
Los Alamos FFTs p-t80, grids compared

$128 \& 512,256 \& 512,128 \& 256$

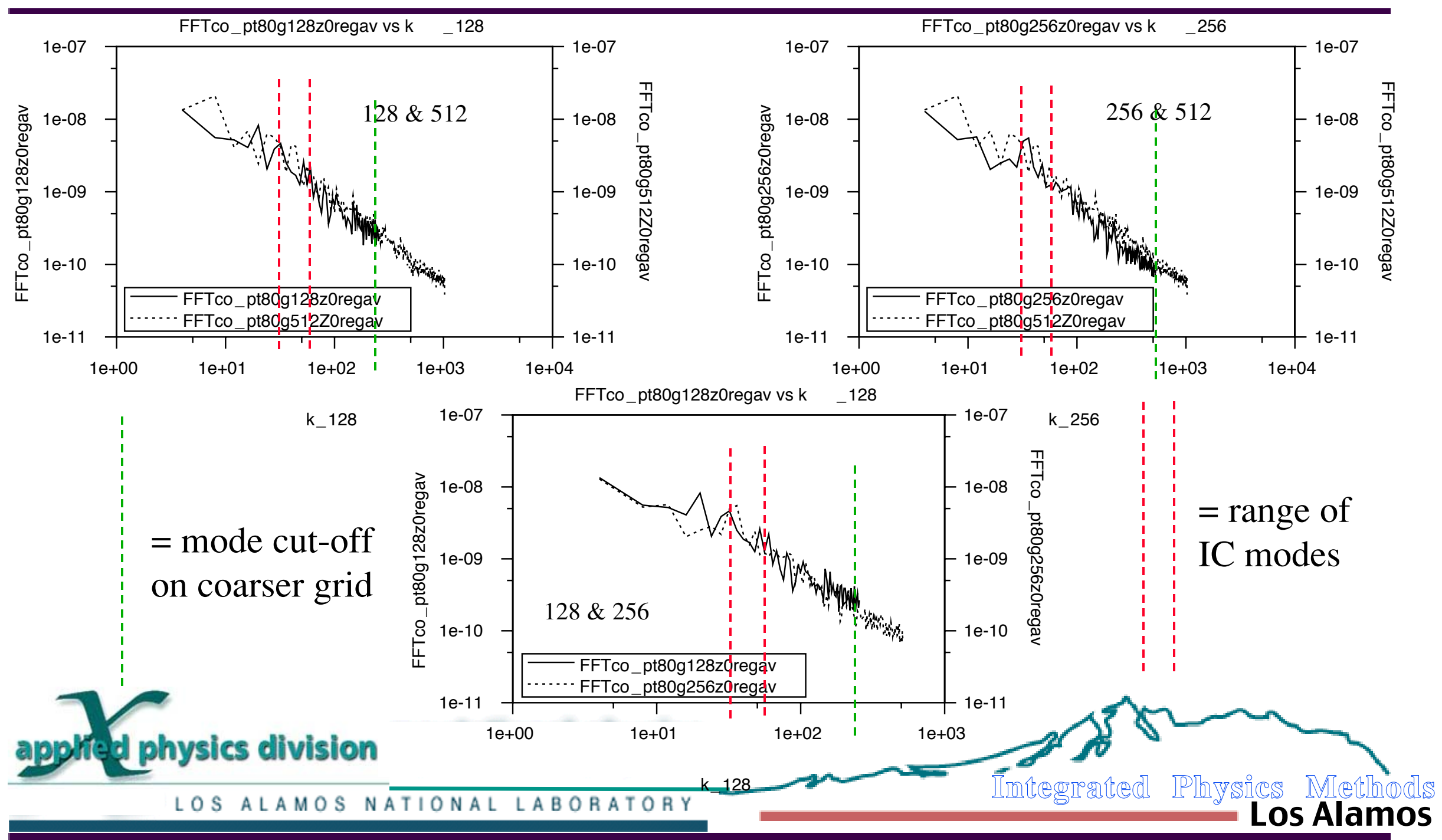


- Los AlamokT mix at late time -varying Atw

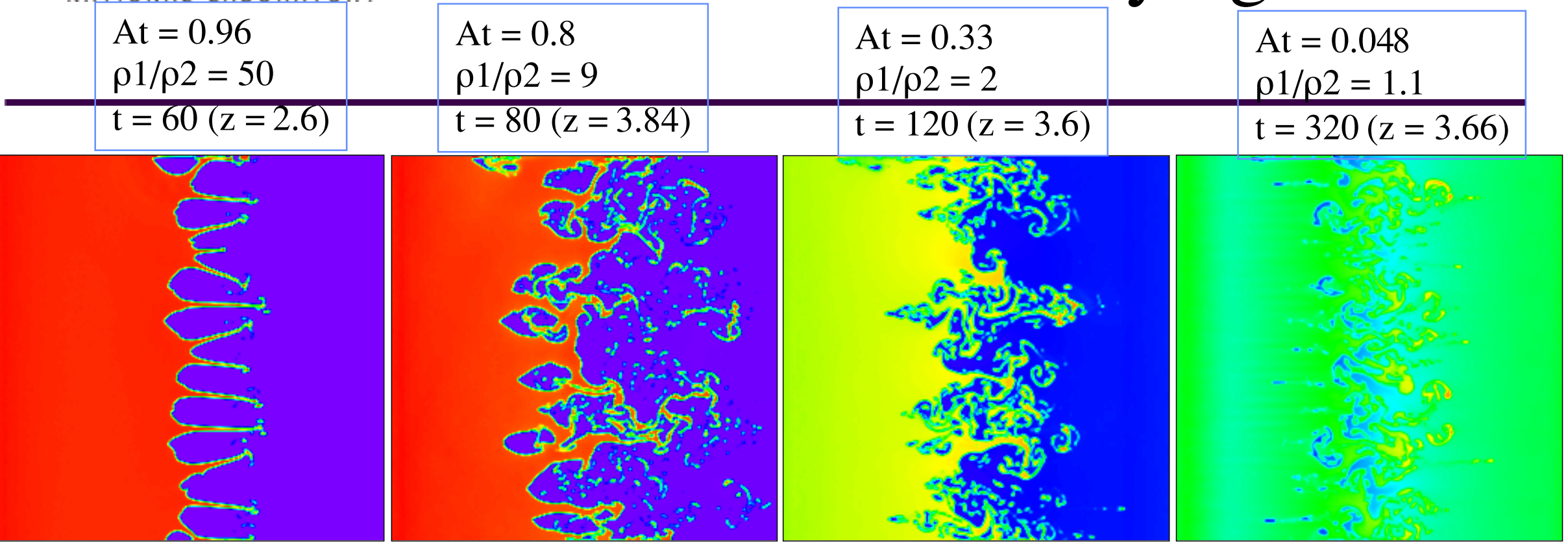

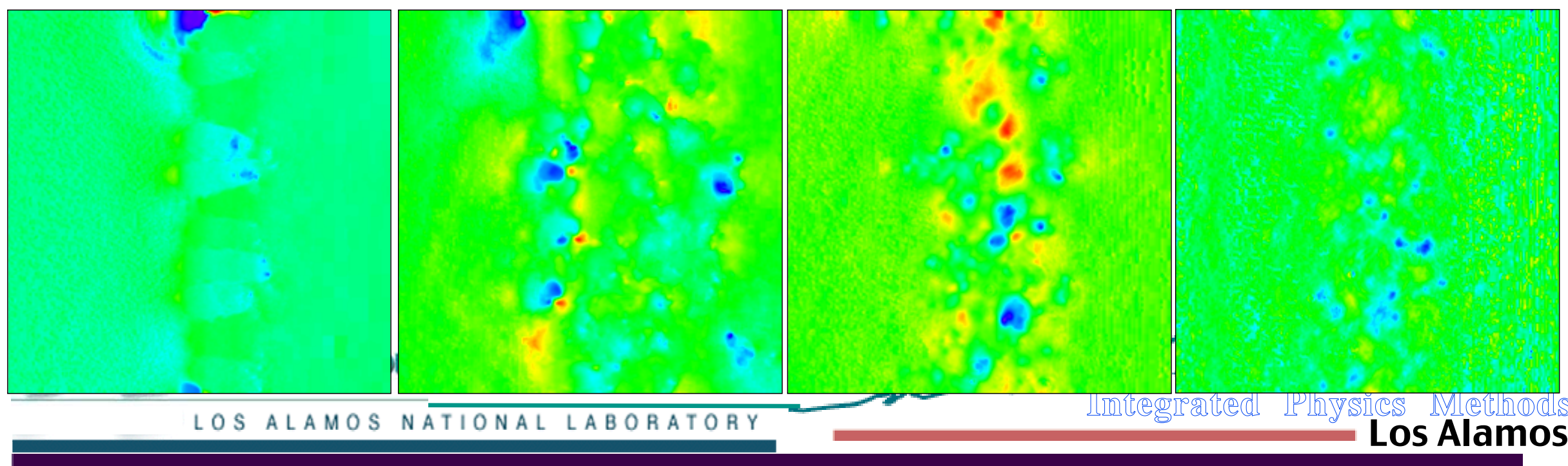


NATIONAL LABORATORY

\section{Alpha (RT growth rate)}

for varying Atwood number and w/ \& w/o Interface Recon.

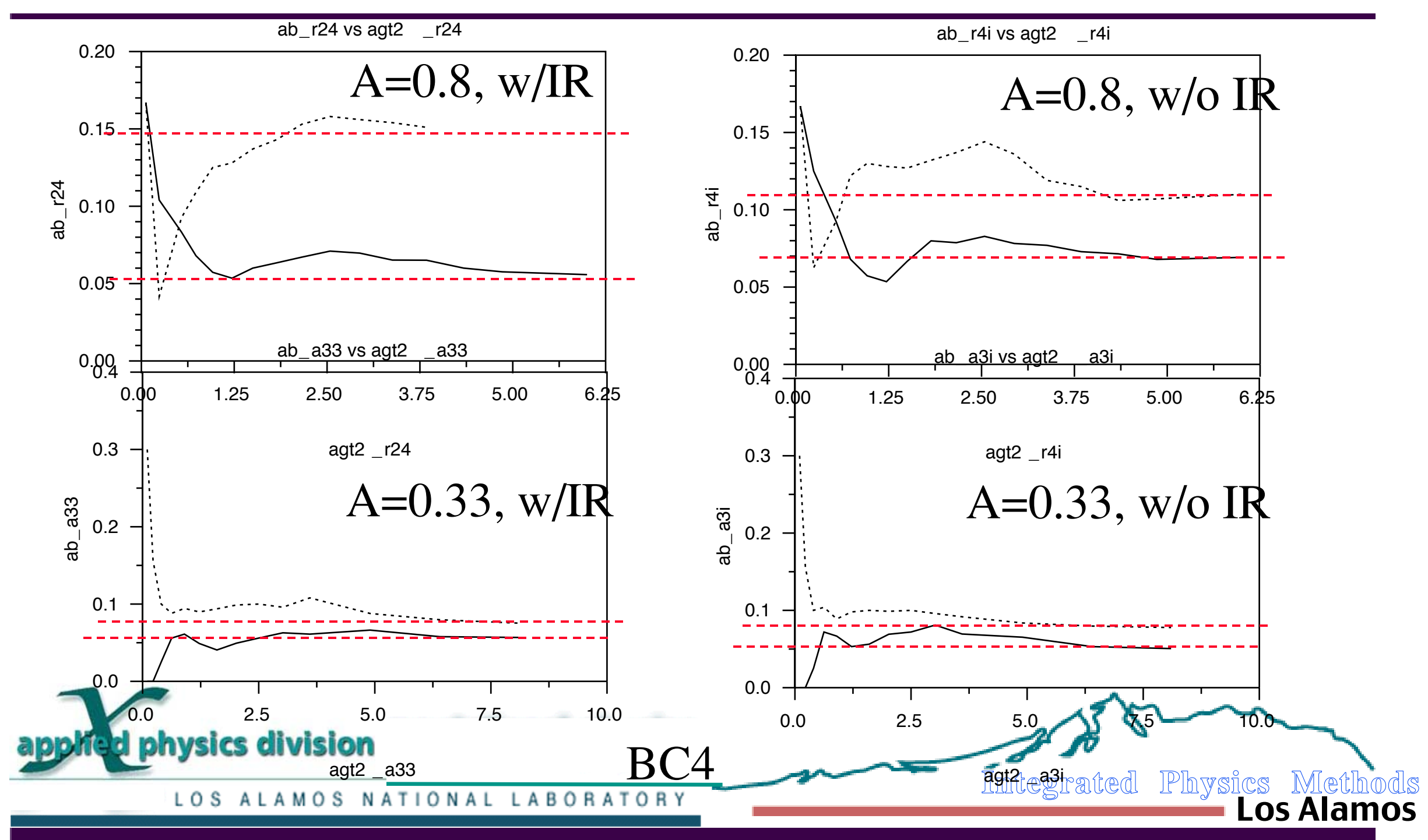


Los Alamos Alpha (RT growth rate) for varying Atwood number and w/ \& w/o Interface Recon.

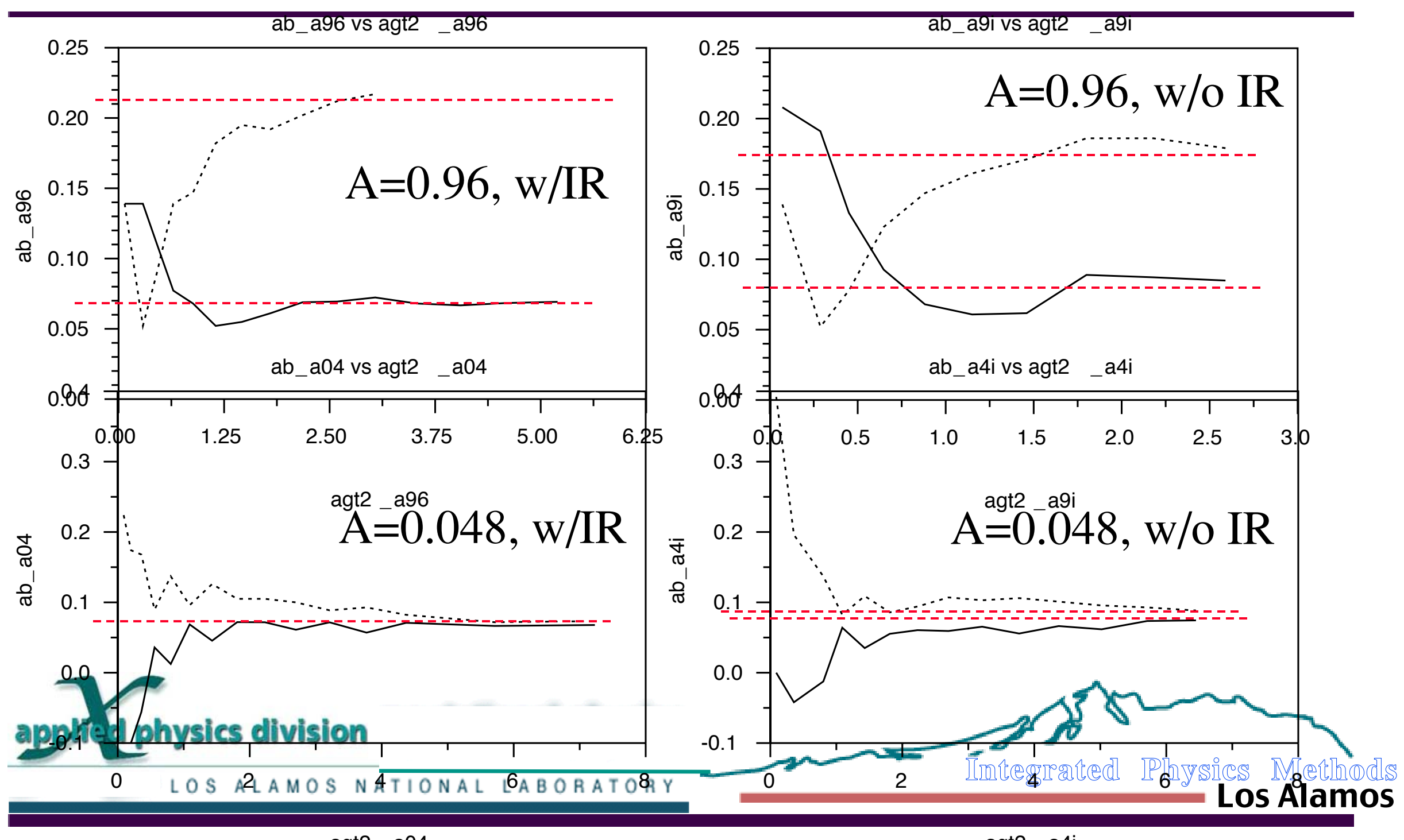




\section{Conclusions: R-T Mix Layer Growth}

- $\quad$ Results apply to 2-D multi-mode-IC simulations for $\mathrm{At}=0.8$

- Grid convergence is good for alpha bubbles - less certain for alpha spikes.

- Alpha bubble computed here, $\sim 0.05-0.065$, agrees with experimental data.

- Discrepancy with other computations predicting lower alpha $(\sim 0.03)$

- may be mostly due to treatment of internal energy discontinuity at interface and/or the internal energy in the long wavelength IC which contributes to growth rate through energy fluctuations.

- a smaller difference ( $15 \%)$ is expected between 2-D and 3-D.

- It is shown to be unlikely that discrepancy is related to compressibility, hydrostatic equilibrium form, IC mode amplitudes or IC mode spectra details, or front evaluation methods..

- Internal energy fluctuations dominate over density fluctuations where $\left(e_{0} / \rho_{0}\right)$ is sufficiently small in the mix layer -

- this occurs in the heavier fluid even in limit as compressibility becomes 'negligible'.

- $\mathrm{e}_{\mathrm{o}}$ is irrelevant in 'ideal' incompressible fluid, so only density fluctuations matter.

- Transition from early time IC dominated regime to later time self-similar solutions is evident and agrees with analytic results.

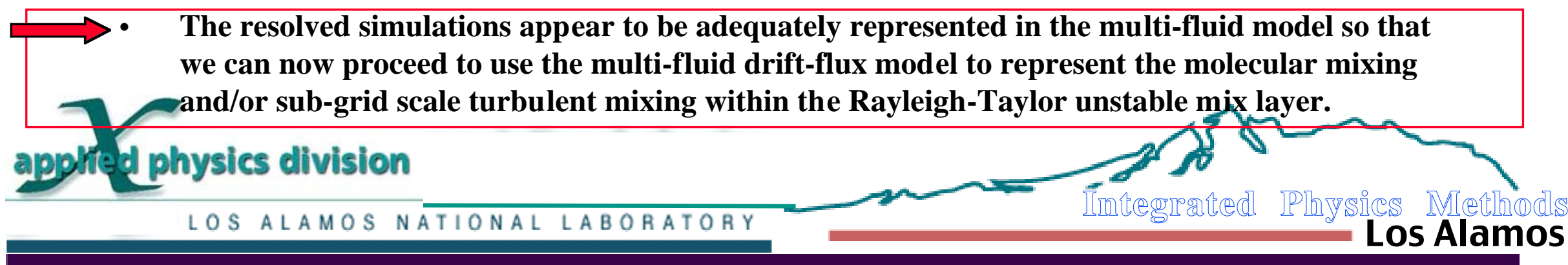




\section{An Efficient and High Resolution Solver for the Two-Dimensional \\ Numerical Simulation of the Richtmyer-Meshkov Instability}

University of Wisconsin - Madison, Fusion Technology Institute

Key features of the Solver

The solver based on the model developed byWang et al. (2001), which uses a thermodynamically

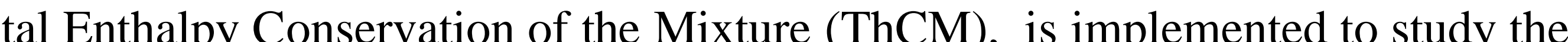
Richtmyer-Meshkov instability. This method utilizes a high resolution Godunov-type scheme based upon a fast exact Riemann solver and the Piece-wise Spline Method (PSM) for data ThCM Model

In the two-dmensional simulation of the Richtmyer-Meshkov instability, the hyperbolic conservation laws

with the ThCM model are given by:

S.P. Wang, M.H. Anderson, J.G. Oakley and R. Bonazza

\section{where $H$, the total enthalpy per unit volume, is given by: \\ the model, the pressure is calcul

$$
p=\frac{(H-p)-\frac{1}{2} \rho\left(u^{2}+v^{2}\right)}{x-1}
$$ \\ Where $\chi$ is introduced in the ThCM model to} $x=\frac{\gamma}{\gamma-1}=\alpha_{1} x_{1}+\alpha_{2} x_{2}$ where $\mathbf{U}$ are the conservative variables and $\mathbf{F}$ and $\mathbf{G}$ are the conservative fluxes in the $x$ and $y$ directions

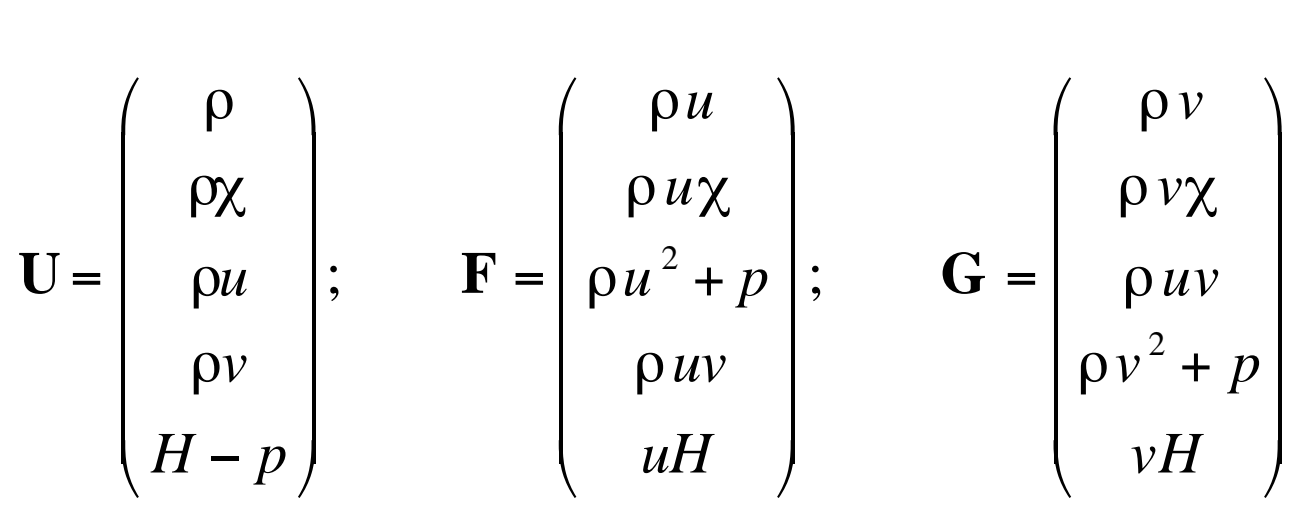

Simulations of the Wisconsin RM Shock Tube Experiments

A series of two Richtmyer-Meshkov instability experiments were conducted
in the University of Wisconsin's shock tube and have been simulated with the numerical model. In these experiments, the distance from the interface to the

yy the retryetion of a sinusidal copper plate which resulted in a Rayleid instability. The RT formed initial condition just prior $(<10 \mathrm{~ms})$ before the shock interaction was recorded, and a sine series representation of the initial condition was used as the input initial condtion for the simulation. The parameters of each
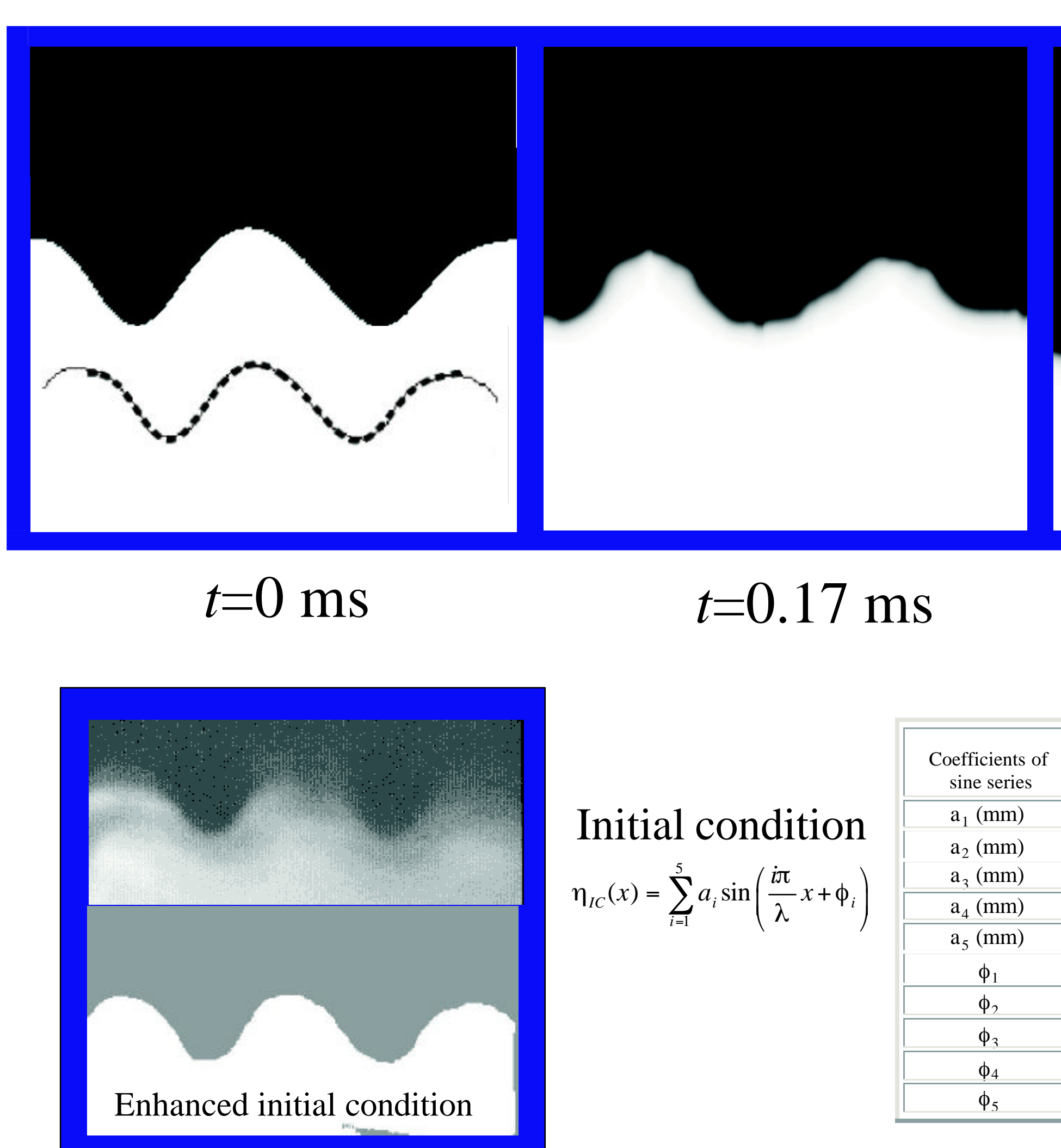

$t=0.17 \mathrm{~ms}$

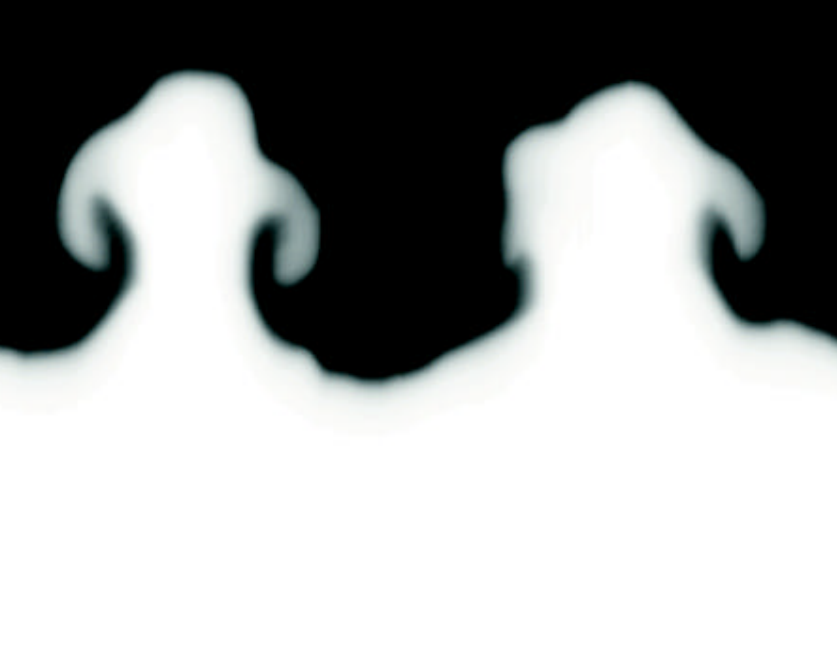

$\begin{array}{lr}\text { Total Length } & 9.2 \mathrm{~m} \\ \text { Driven Length } & 6.8 \mathrm{~m}\end{array}$ $\begin{array}{lr}\text { Inner square cross section } & 0.25 \mathrm{~m} \\ \text { Maximum Driver Pressure } & 100 \mathrm{ath}\end{array}$

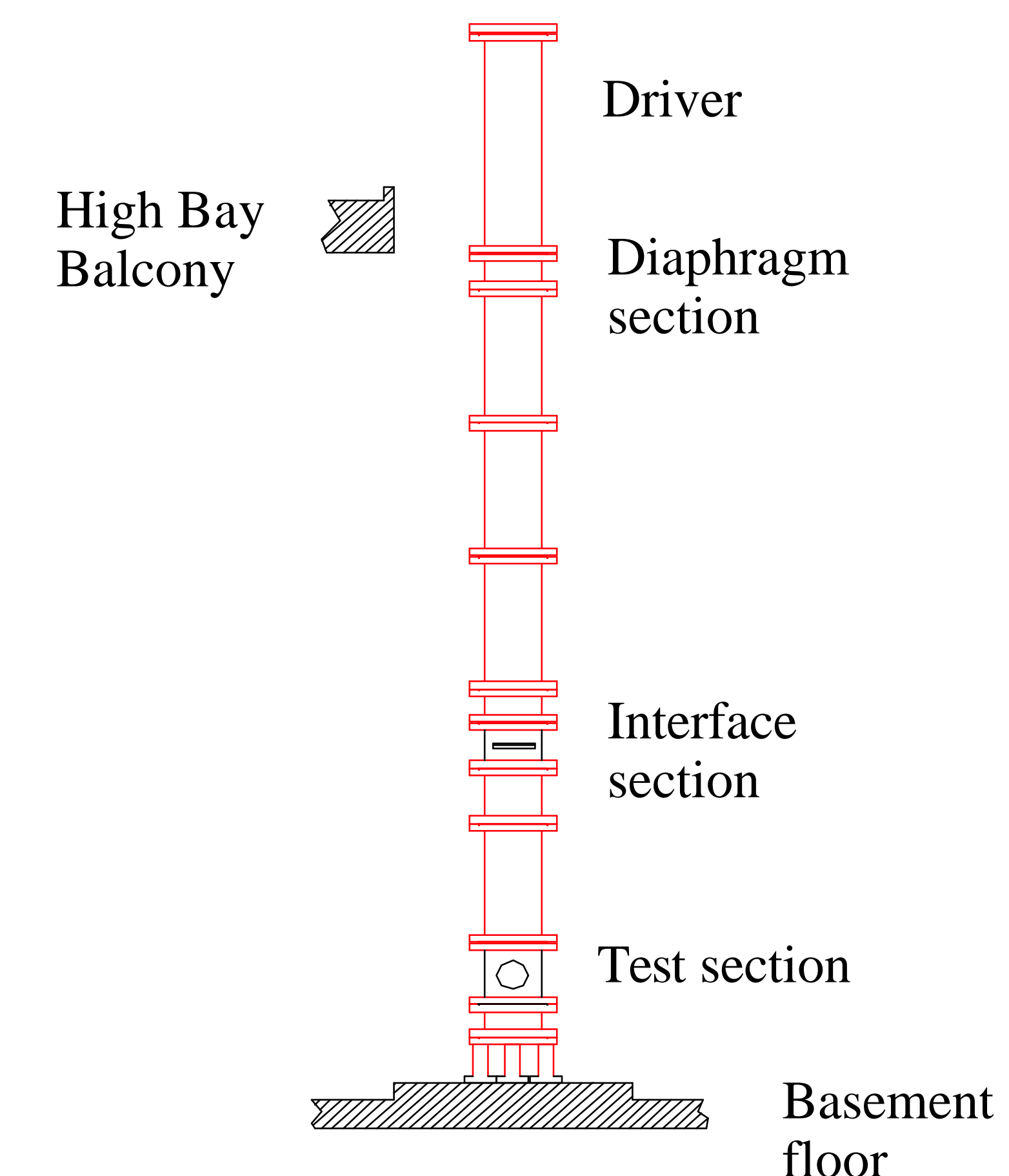

Driven cross section of shock tube

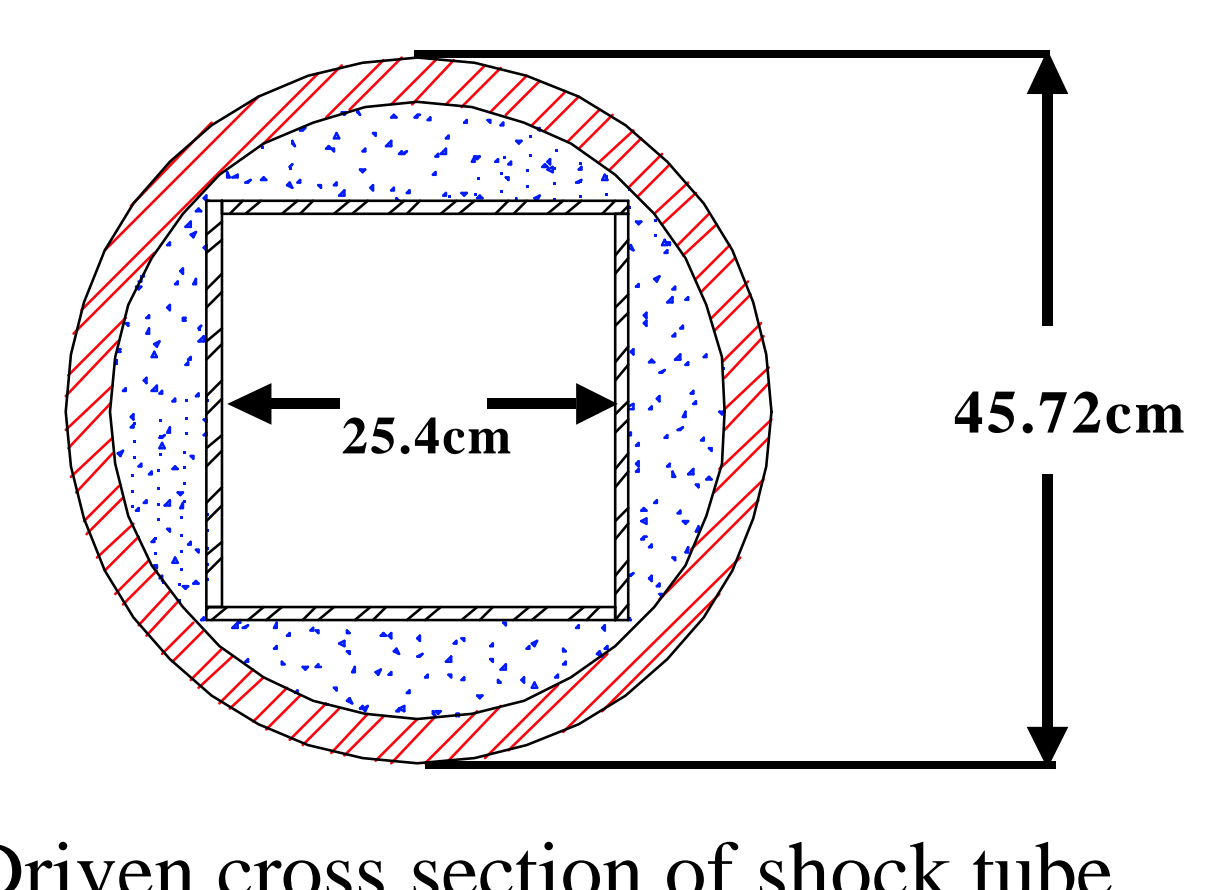

$t=0.47 \mathrm{~ms}$

$t=0.72 \mathrm{~ms}$

$t=0.87 \mathrm{~ms}$
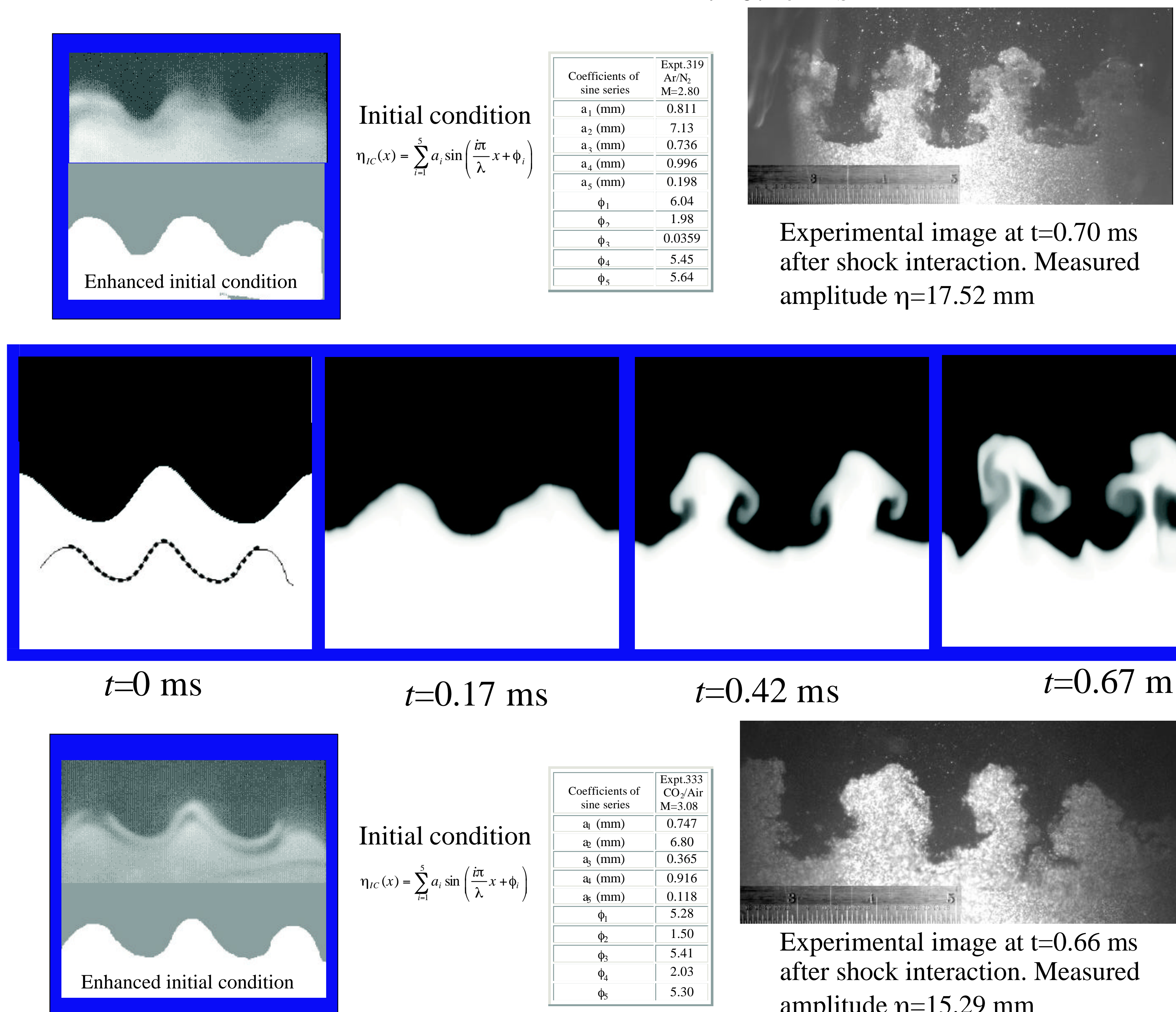

Experimental image at $\mathrm{t}=0.70 \mathrm{~m}$ ter shock interaction. Measure

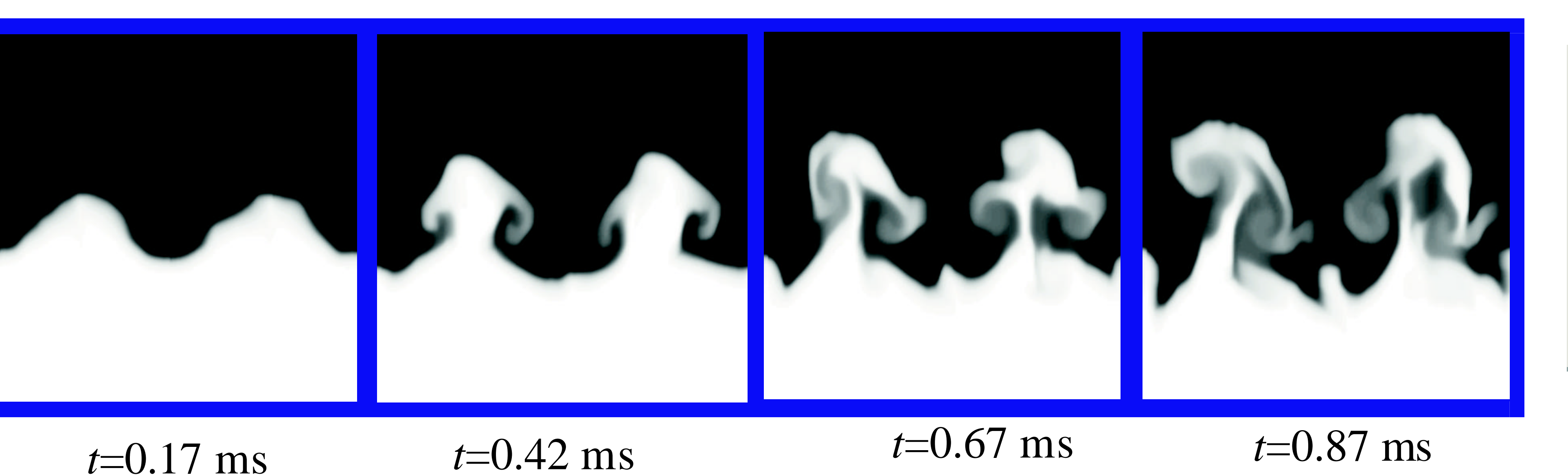

$t=0.17 \mathrm{~ms}$

$t=0.42 \mathrm{~ms}$

$t=0.67 \mathrm{~ms}$

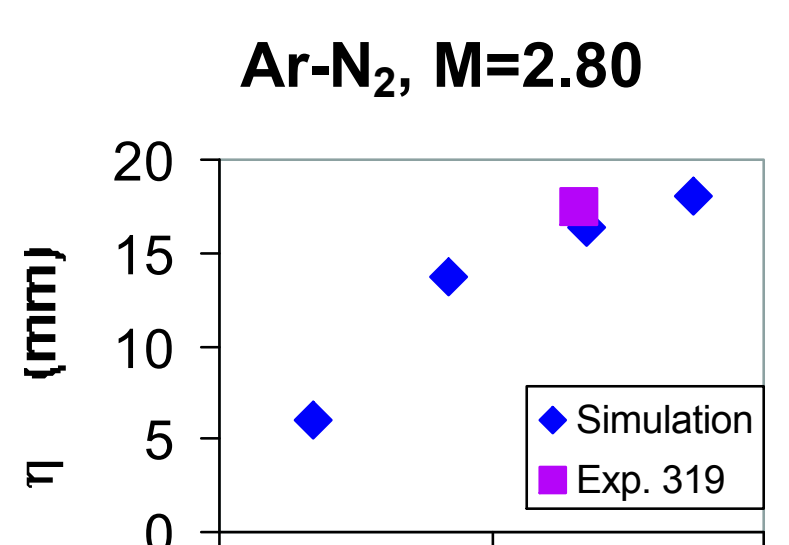

0.5

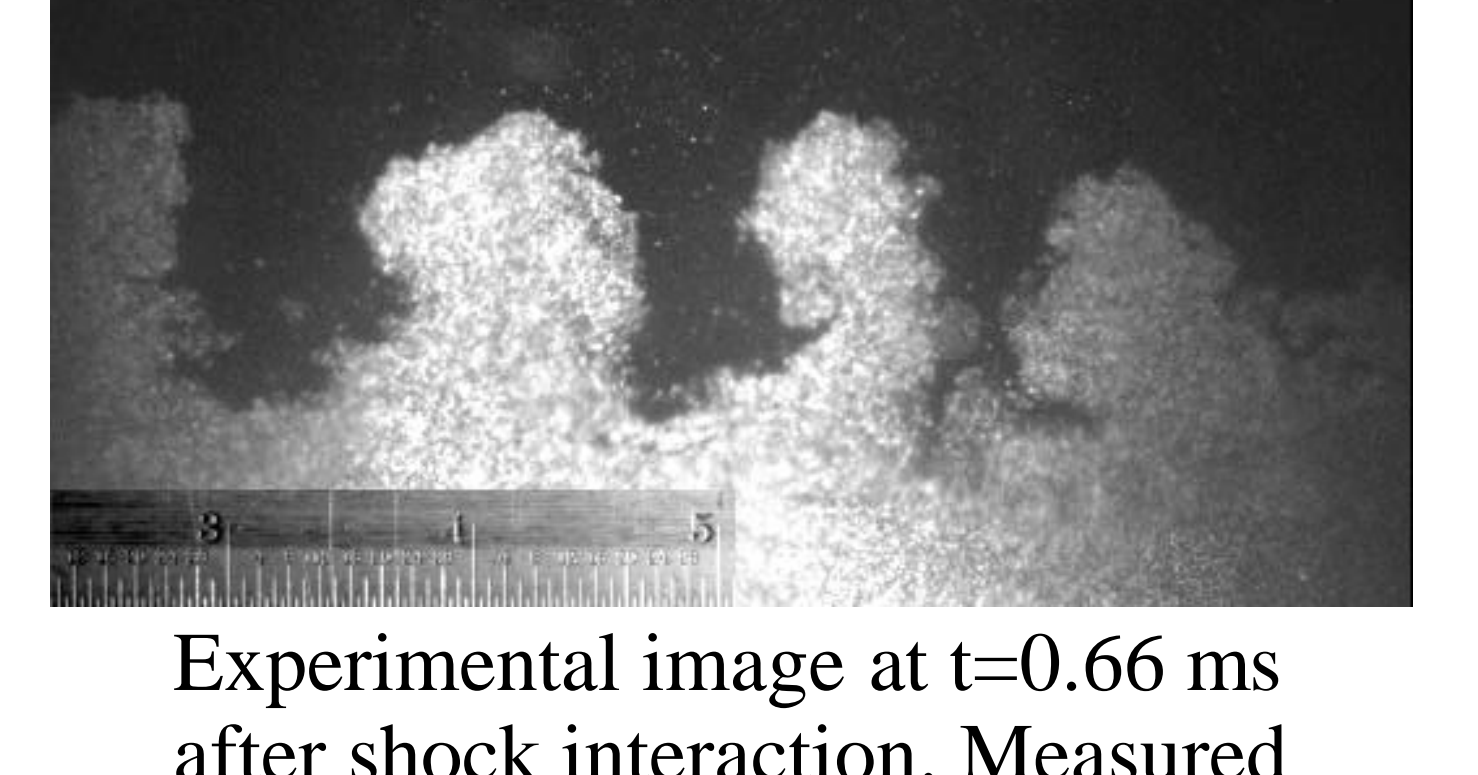

action. Measure

amplitude $\eta=15.29 \mathrm{~mm}$ $t=0.87 \mathrm{~ms}$

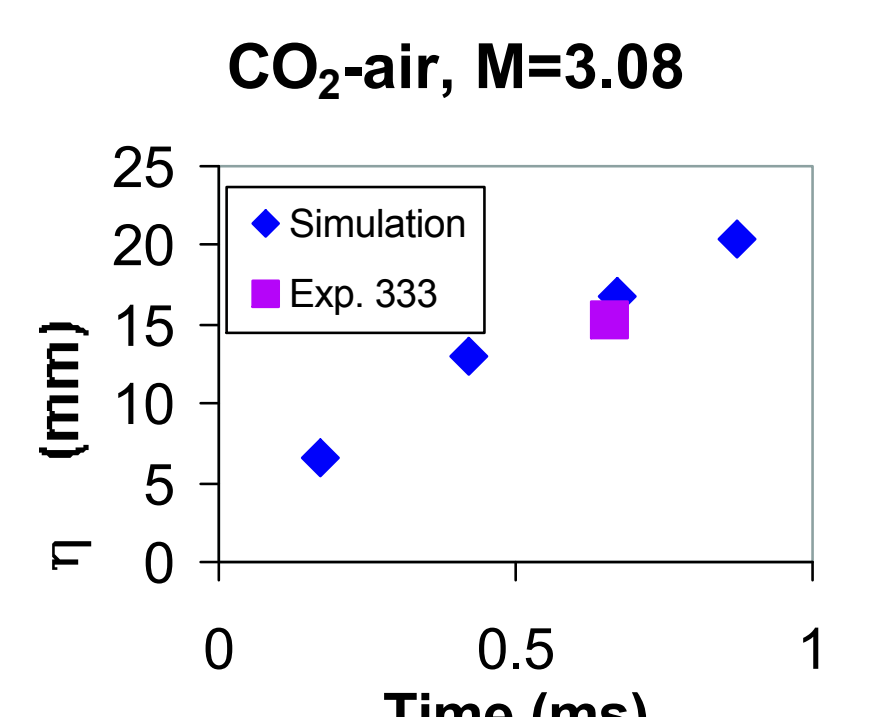

Pentium 4 $9.4 \mathrm{~m} / \mathrm{s}$.

at the time of the experiment is
A splitting scheme is employed for the system in two spatial dimensions defined as follows:

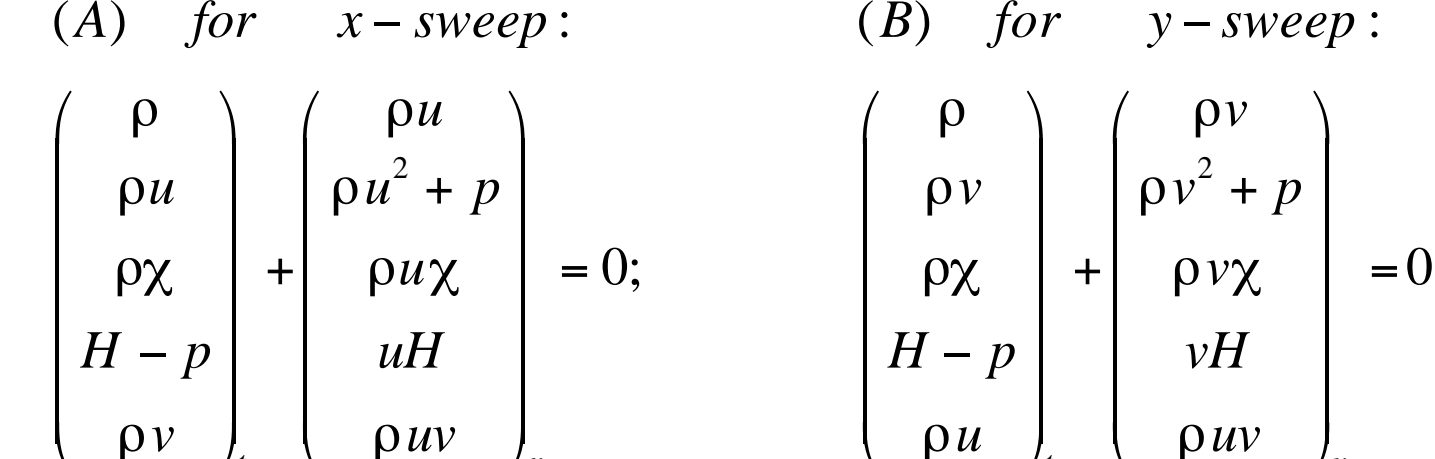

The tangential velocity component $v(u)$ in the $x$-sweep(y-sweep) is passively advected with the norma using a Godunov scheme:

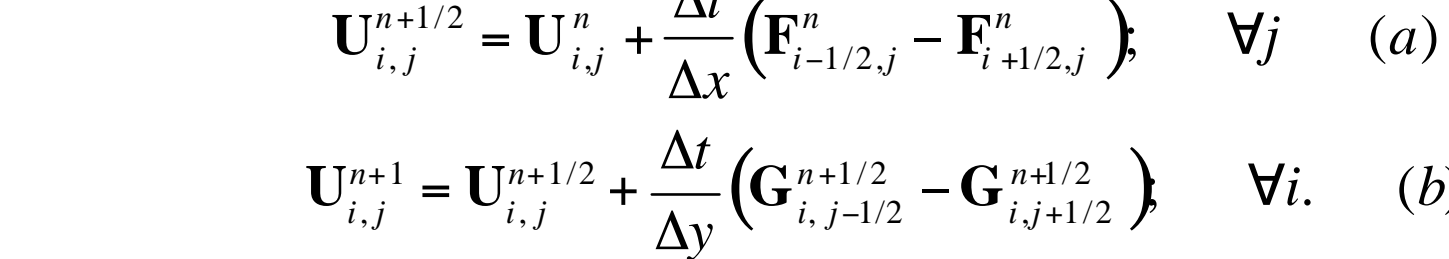

The PSM and following slope limiters, similar to those developed by Ren et al. (1996), are used for the

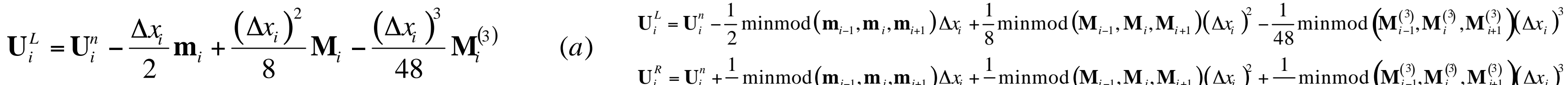

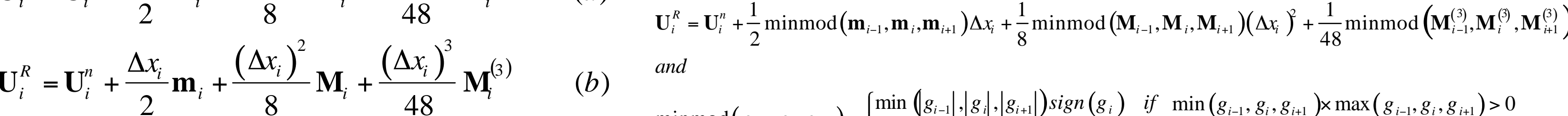

Simulation of Test Problem \#1

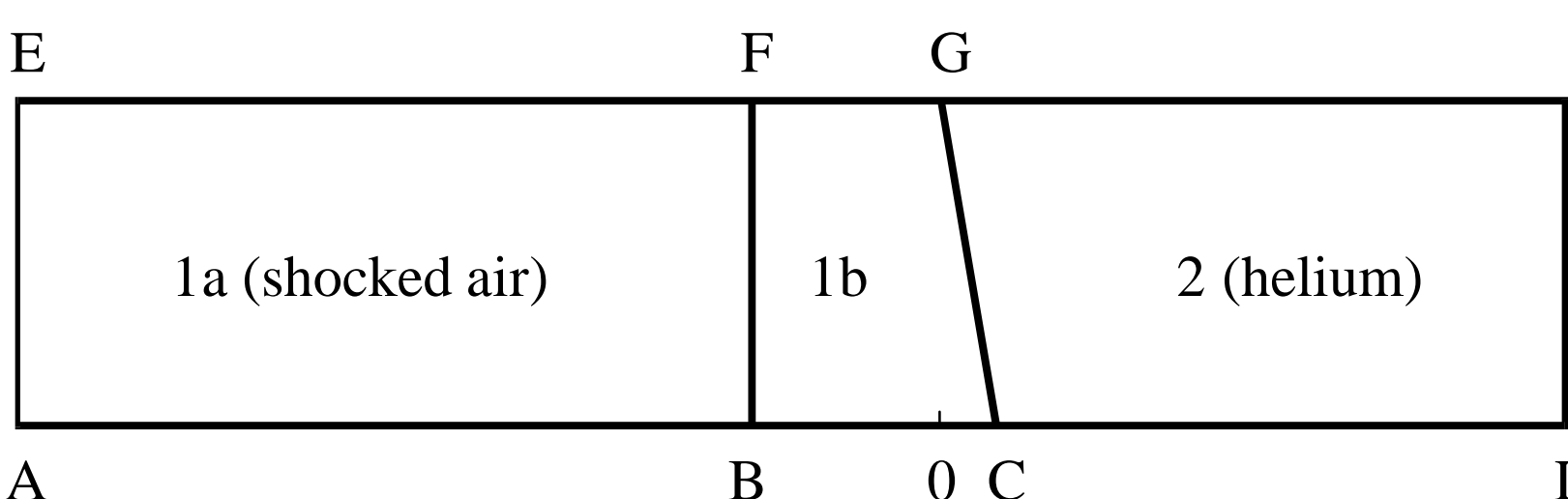

boundary and other boundaries are rigid walls.

Mesh

The mesh is square
per region width.

$A B=38 \mathrm{~cm}, \mathrm{BC}=3.2 \mathrm{~cm}, 0 \mathrm{D}=20 \mathrm{~cm}, \mathrm{AE}=12 \mathrm{~cm}, \mathrm{FG}=0.8 \mathrm{C}$

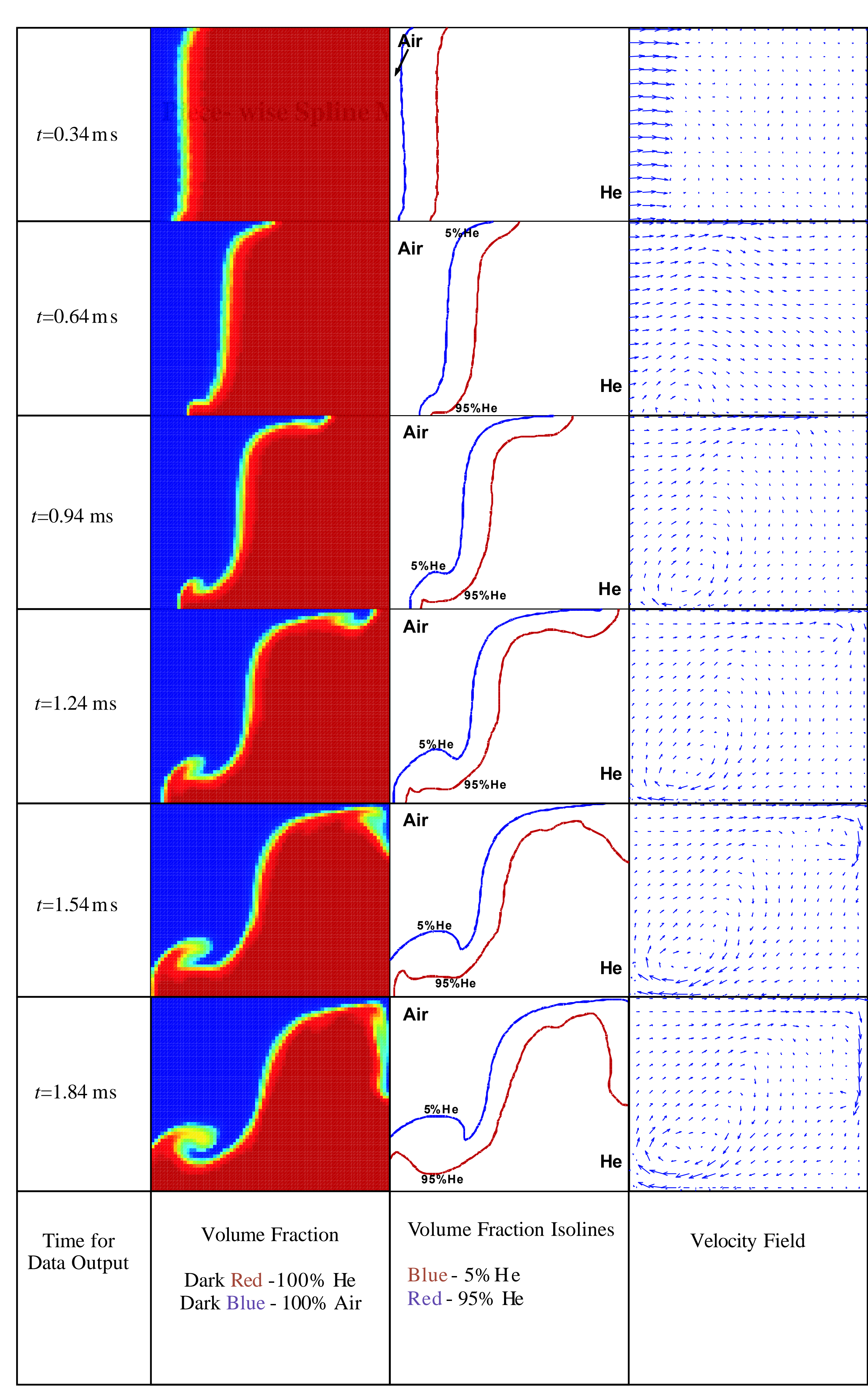

Initial conditions

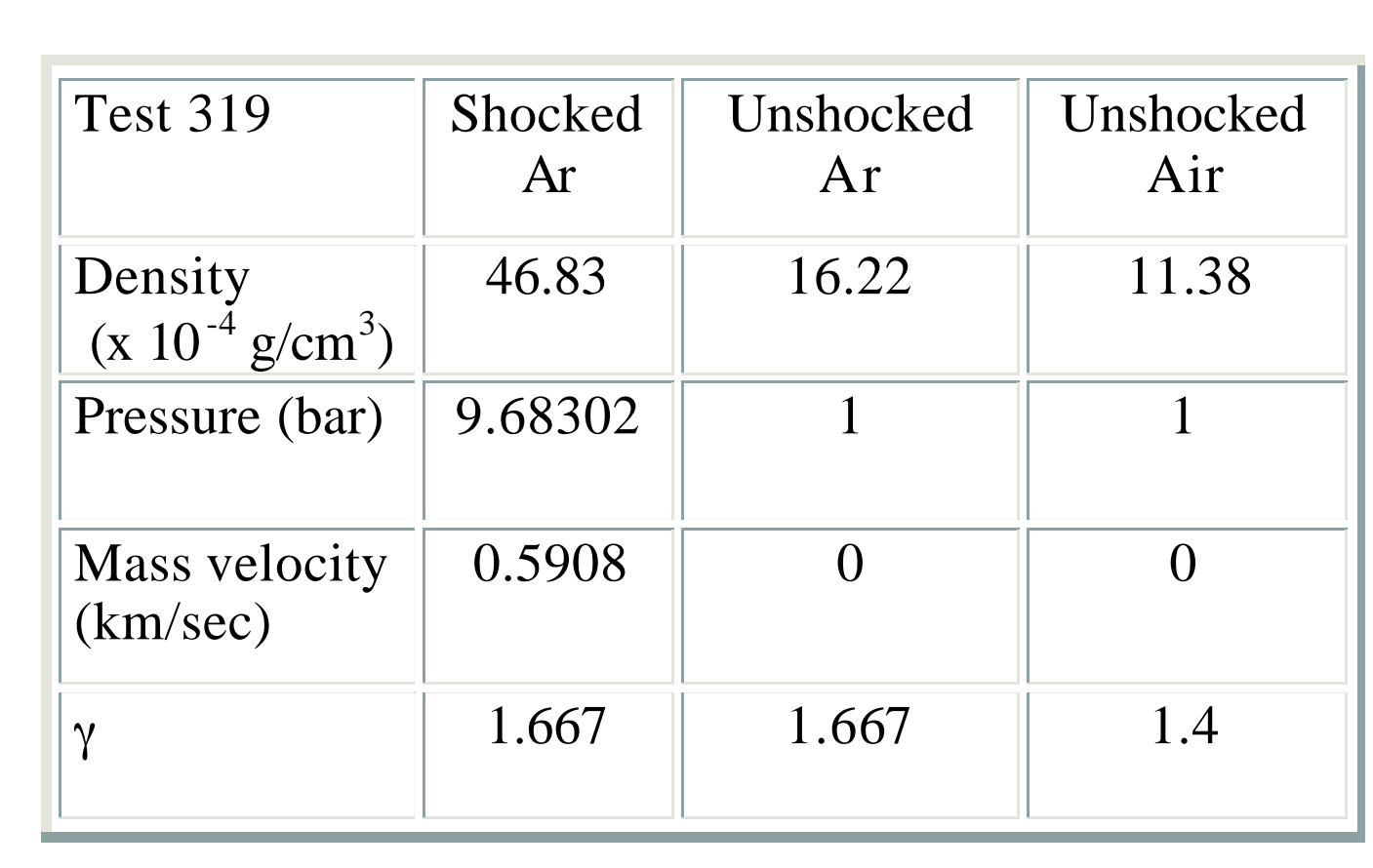

Numerical Domain 2480x200 cells with a spatial resolution of $0.381 \mathrm{~m}$

The growth rate from the simulation

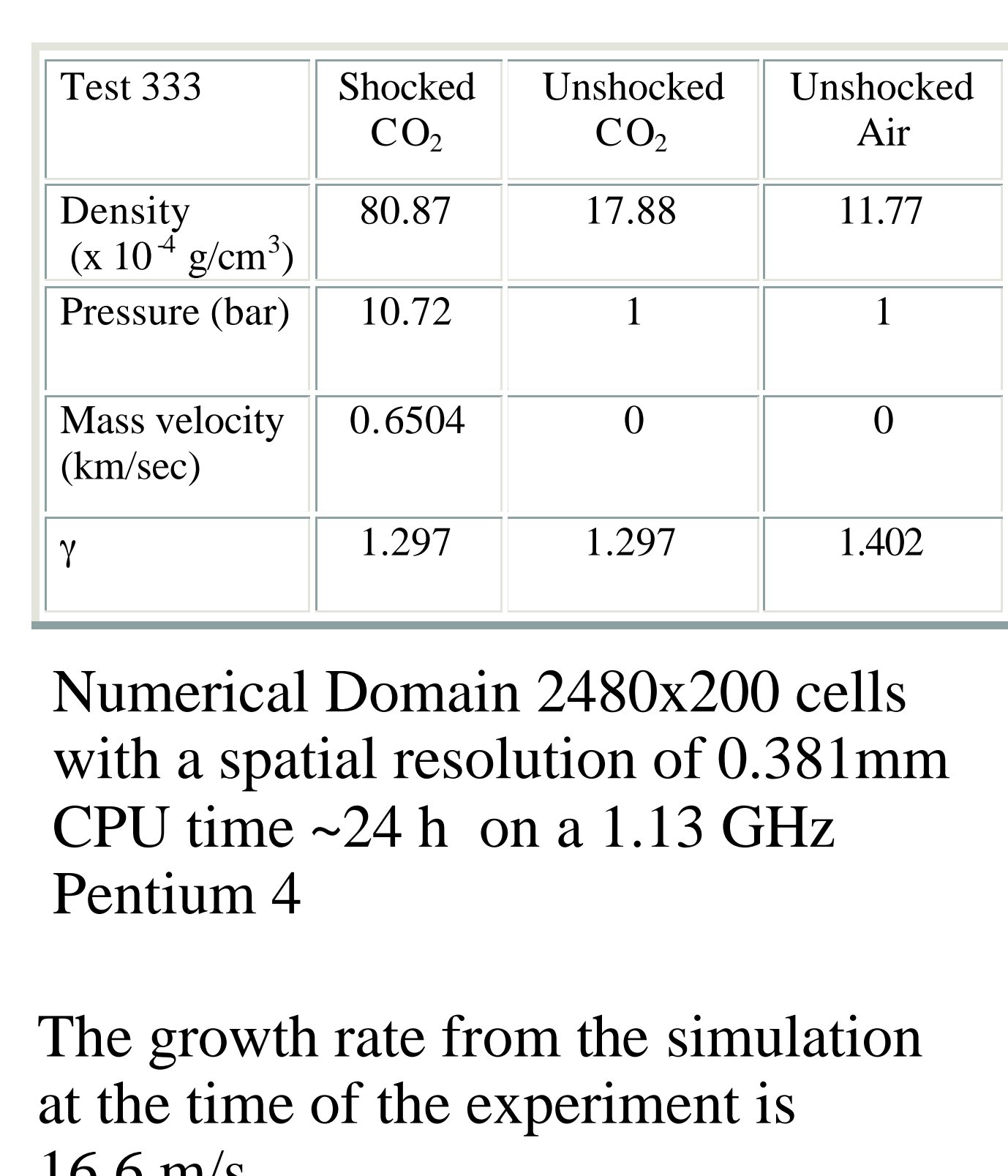

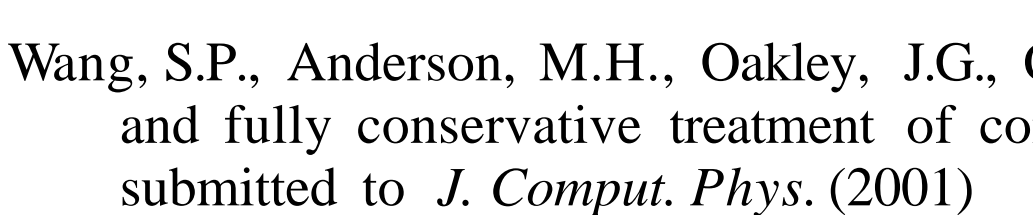

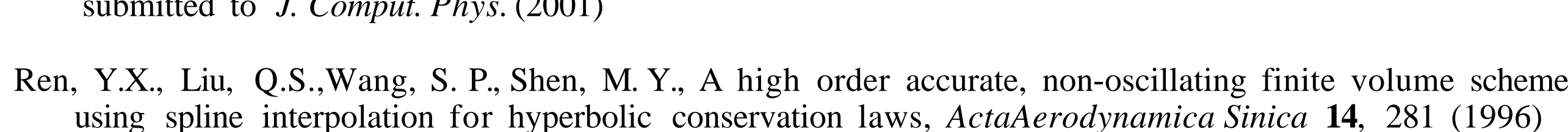

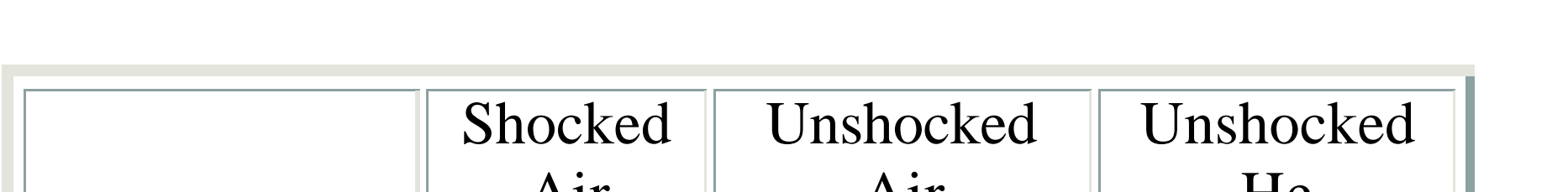

\begin{tabular}{|c|c|c|c|}
\hline & Air & Air & He \\
\hline 18.2641 & 12.05 & 1.67 \\
\hline
\end{tabular}

$\begin{array}{lll}\text { Pressure (bar) } & 1.804997\end{array}$

\begin{tabular}{l|l} 
Mass velocity 0.15076
\end{tabular}

$(\mathrm{km} / \mathrm{sec})$

Results

. The center set of images are $5 \%$ and $95 \%$ local velocity vectors

The graph below shows the total mass of each material fraction). The points on the graph correspond to the

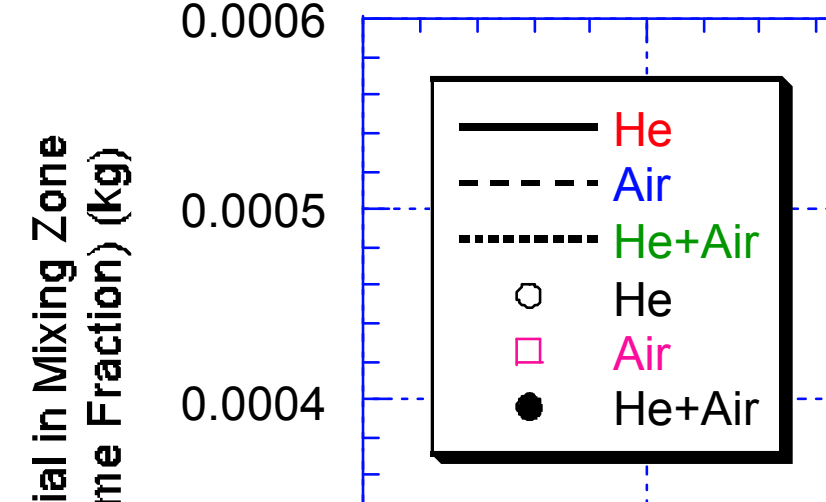

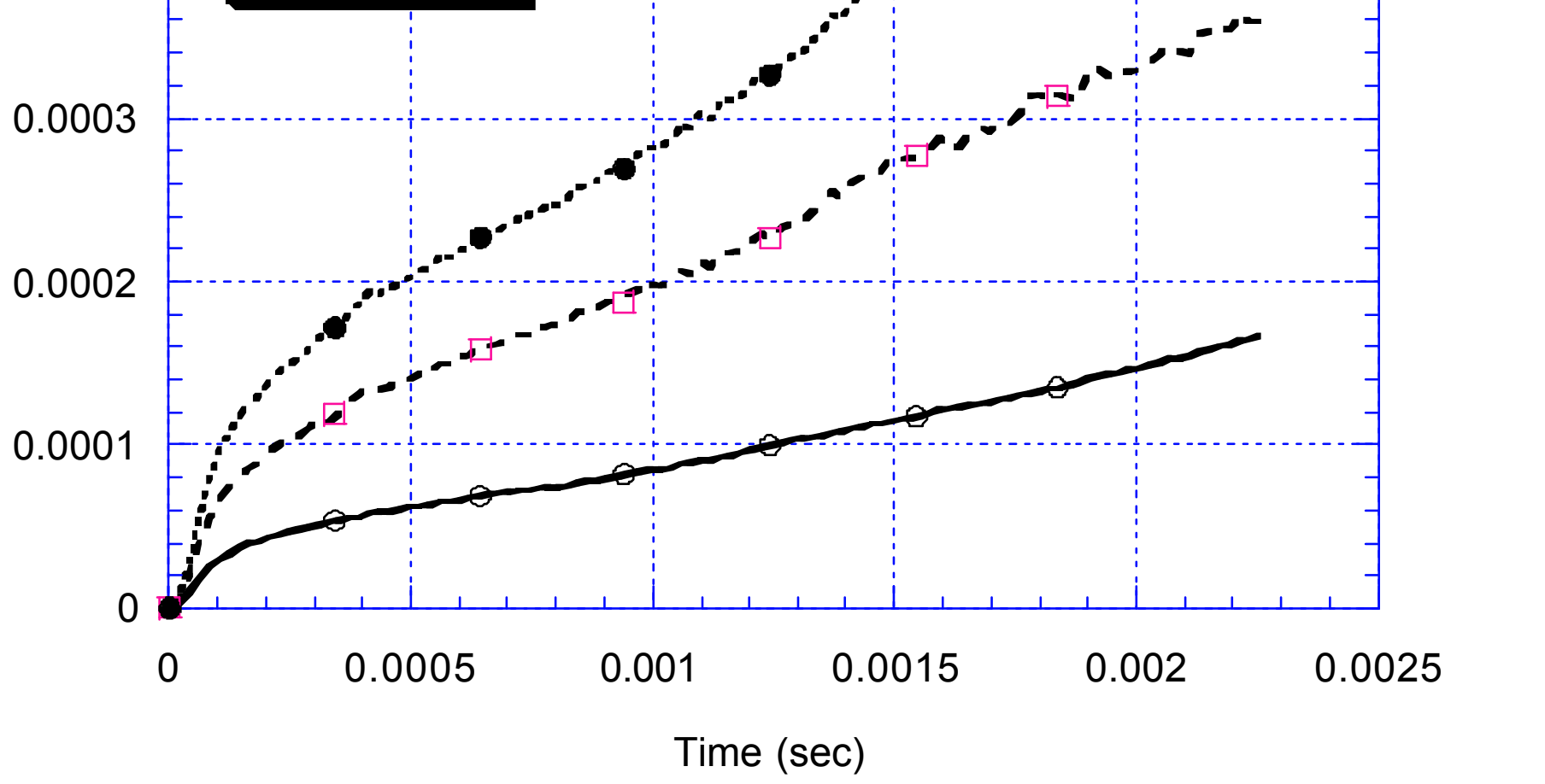




\section{ALE Simulations of Turbulent Rayleigh- Taylor Instability in 2-D and 3-D}

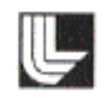

S. V. Weber, G. Dimonte, and M. M. Marinak

Lawrence Livermore National Laboratory

2001 International Workshop on the Physics

of Compressible Turbulent Mixing

Pasadena, CA

December 10-14, 2001 
We have simulated the " $\alpha$ - group" turbulent RayleighTaylor problem in 2-D and 3-D with the ALE code, Hydra

- Weakly compressible RT with a random initial surface spectrum restricted to short wavelength modes (4-8 zones $/ \lambda$ )

- Hydra is a 3-D parallel ALE code with interface reconstruction

- Zoning convergence is suspect

- Simulated linear growth rates for the seed modes are as low as $15 \%$ of the correct classical value

- In 3-D, modes with $k$ aligned with the mesh grow faster than others

- We use a initial surface comprised of cosine modes to avoid aliasing due to reflection span-wise boundary conditions

- 2-D simulations show weak dependence on zoning up to $1024 \times 2048$

- 3-D simulations with interface reconstruction predict $\alpha_{b} \sim 0$.03$0.055, \alpha_{s} \sim 0.06$

- Simulations without interface reconstruction show only slightly lower penetration but much more small-scale mixing 
Initial configuration \& adiabatic equilibrium

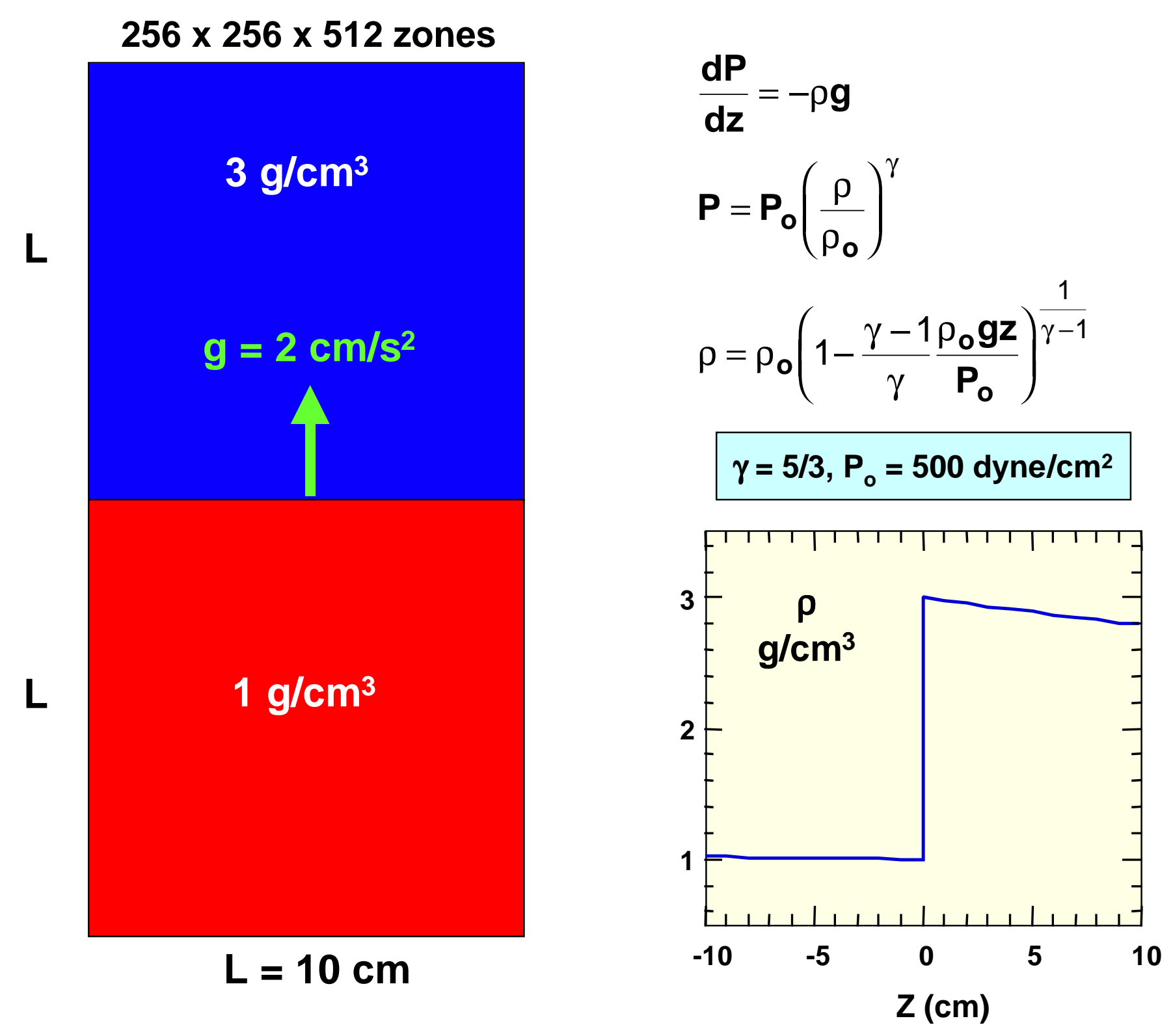


Initial conditions (David Youngs) are random \& distributed in wavenumber shell (modes 32-64) close to Nyquist limit
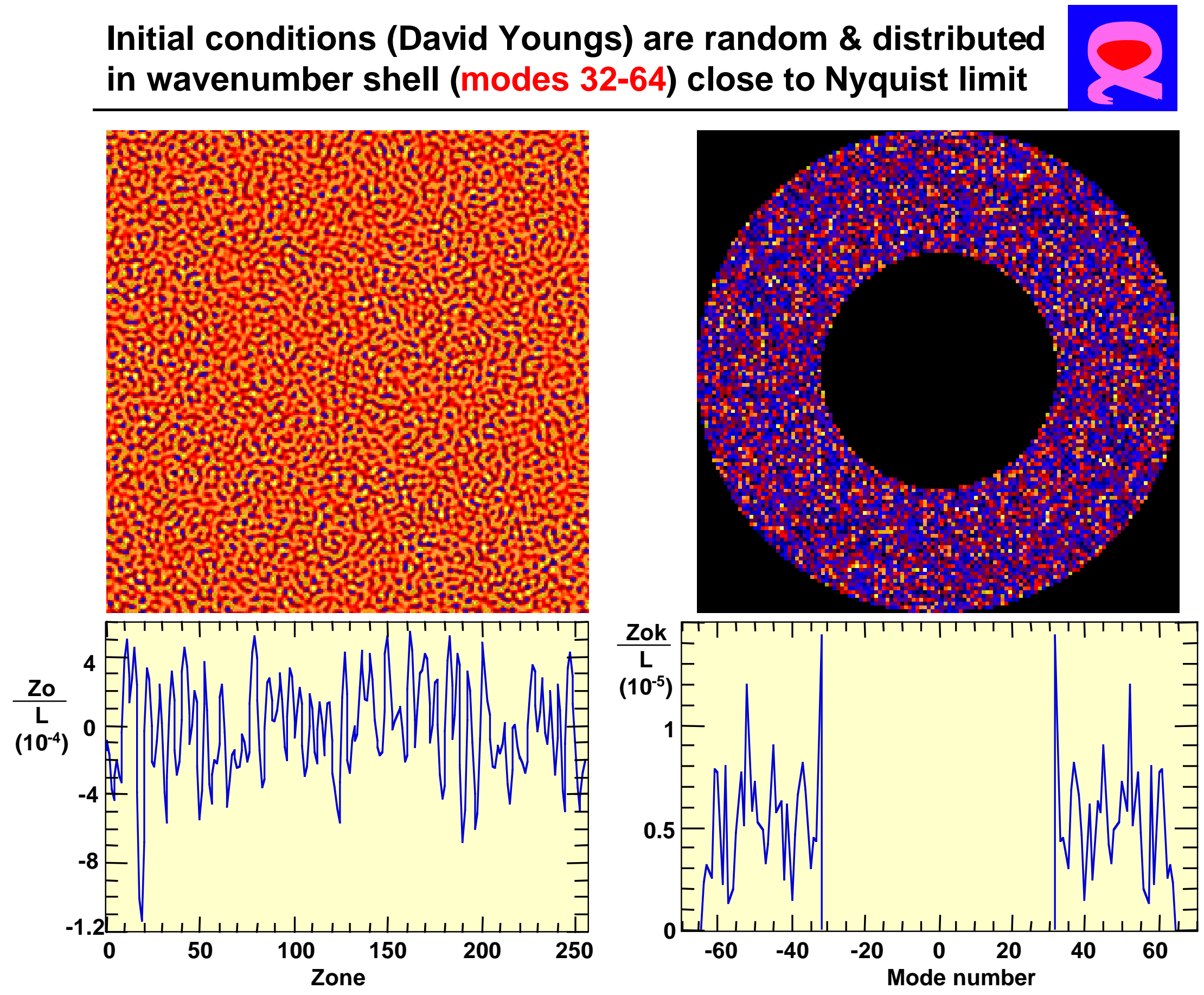

below classical due to under-resolution

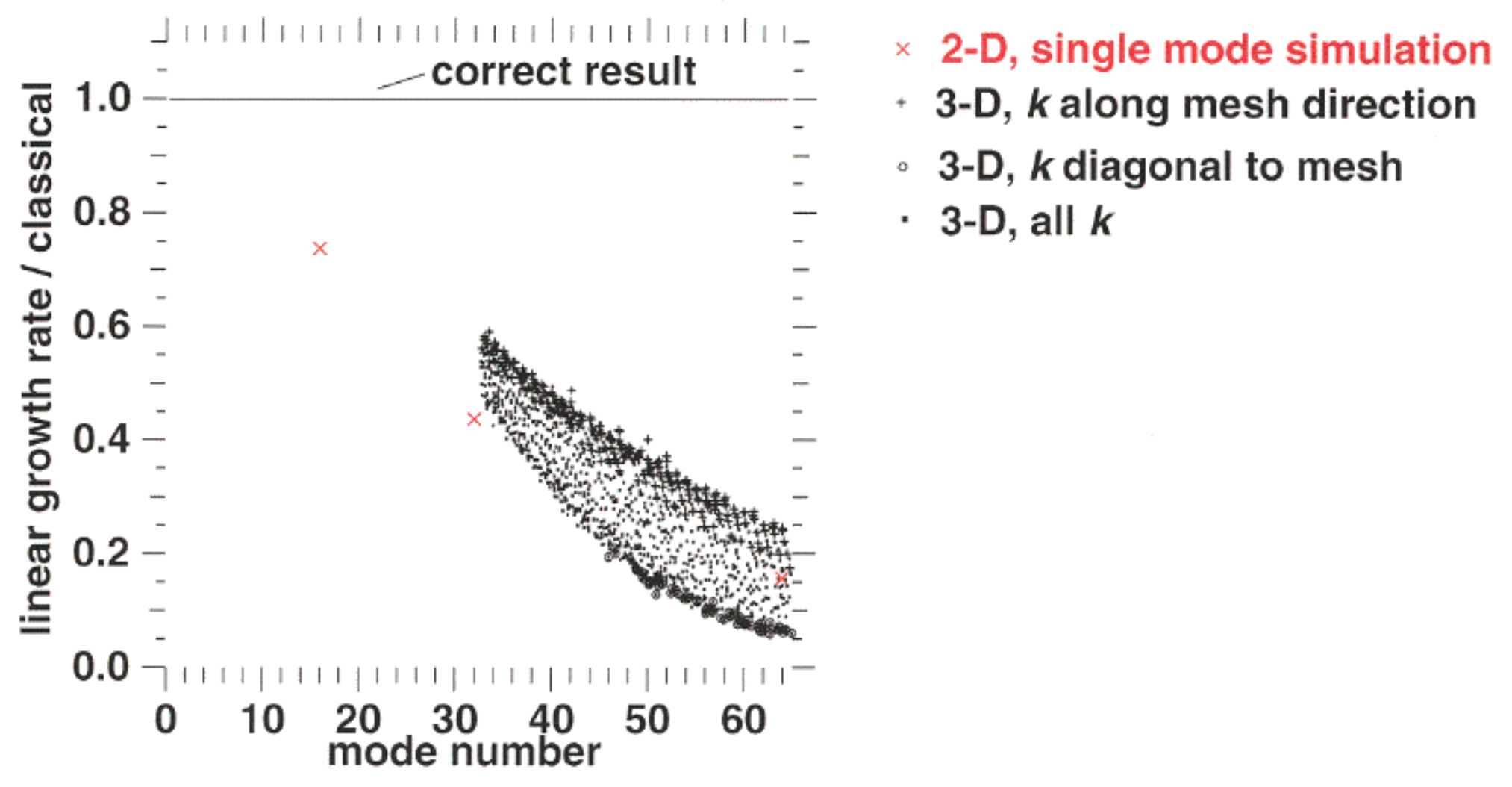

- If the simulation were converged, all modes would grow at the classical rate

- Also note that the simulated growth rate depends upon the orientation of the wave vector, $k$, to the mesh 


\section{In 2-D, the mix extent at late times does not change much with zoning refinement}

2-D 256×512 (1x)

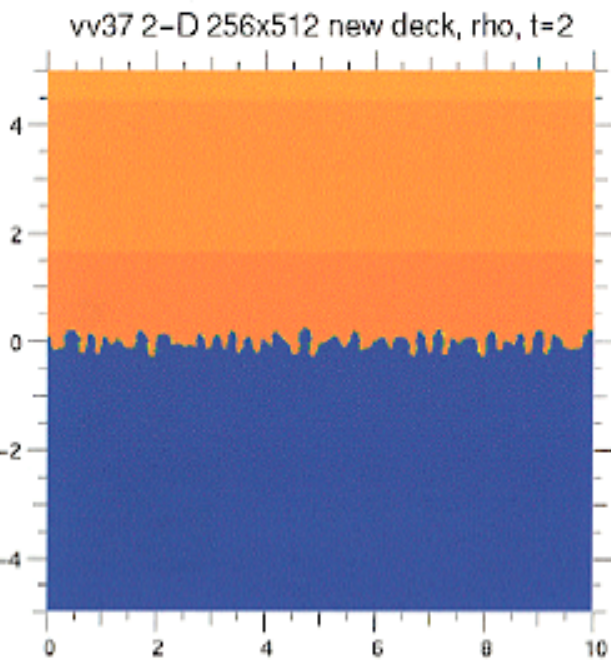

wv60 2-D 1024×2048 rho, t-2

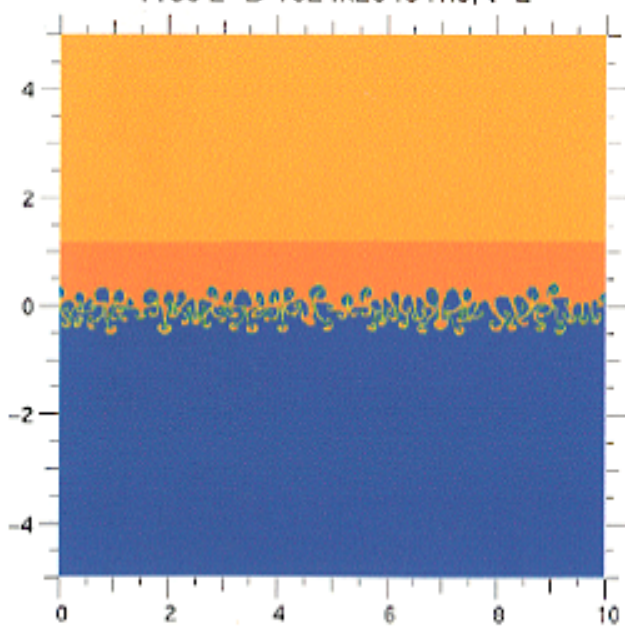

- The seed modes are clearly under-resolved with nominal zoning ry37 2-D 256x512 new deck, rho, $\mathrm{t}=6$

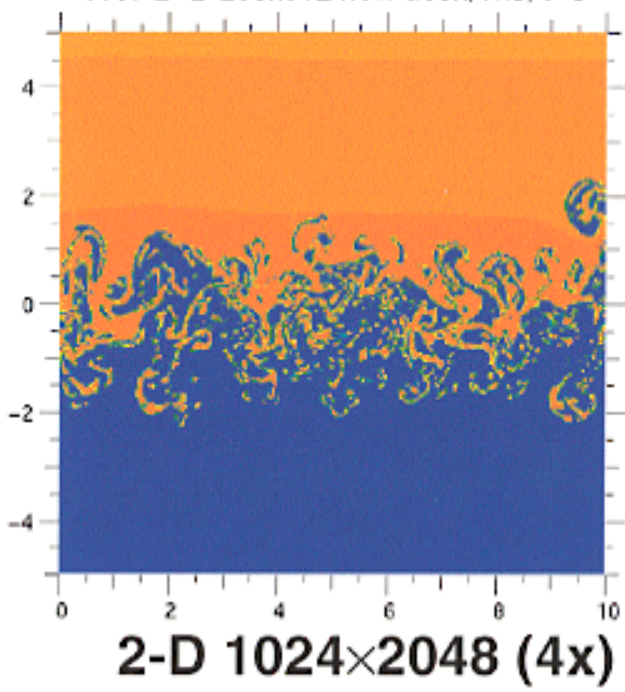

wv60 2-D 1024×2048 tho, t-6

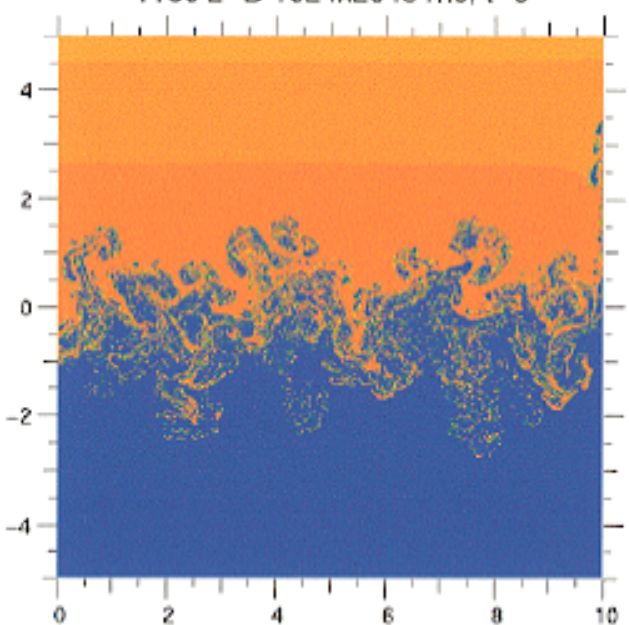

VY37 2-D 256x512 rho t-10

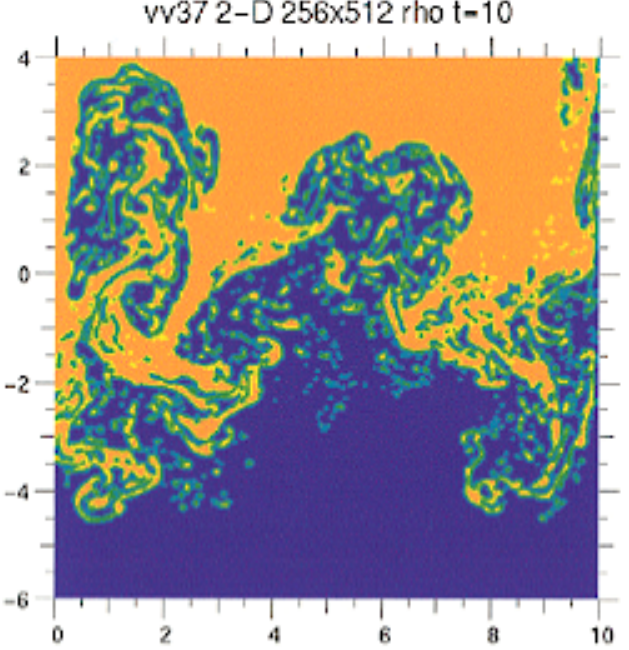

vv60 2-D 1024×2048 rho t=10

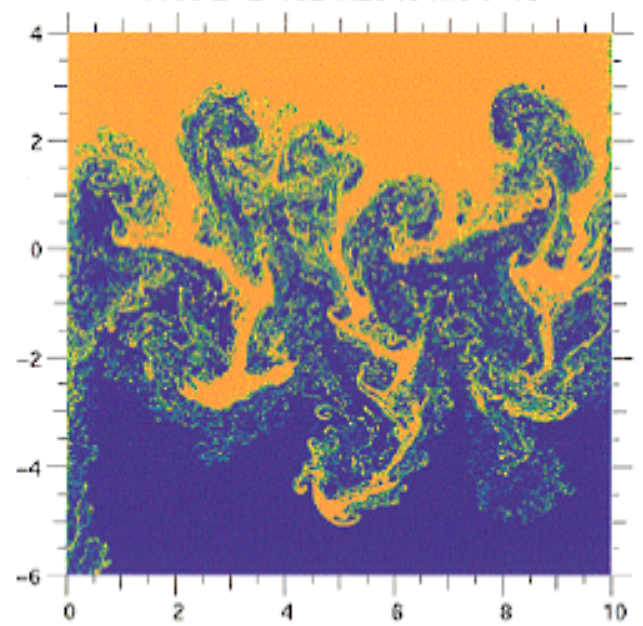




\section{Zoning refinement beyond the nominal resolution does not significantly affect the mix extent in 2-D runs}
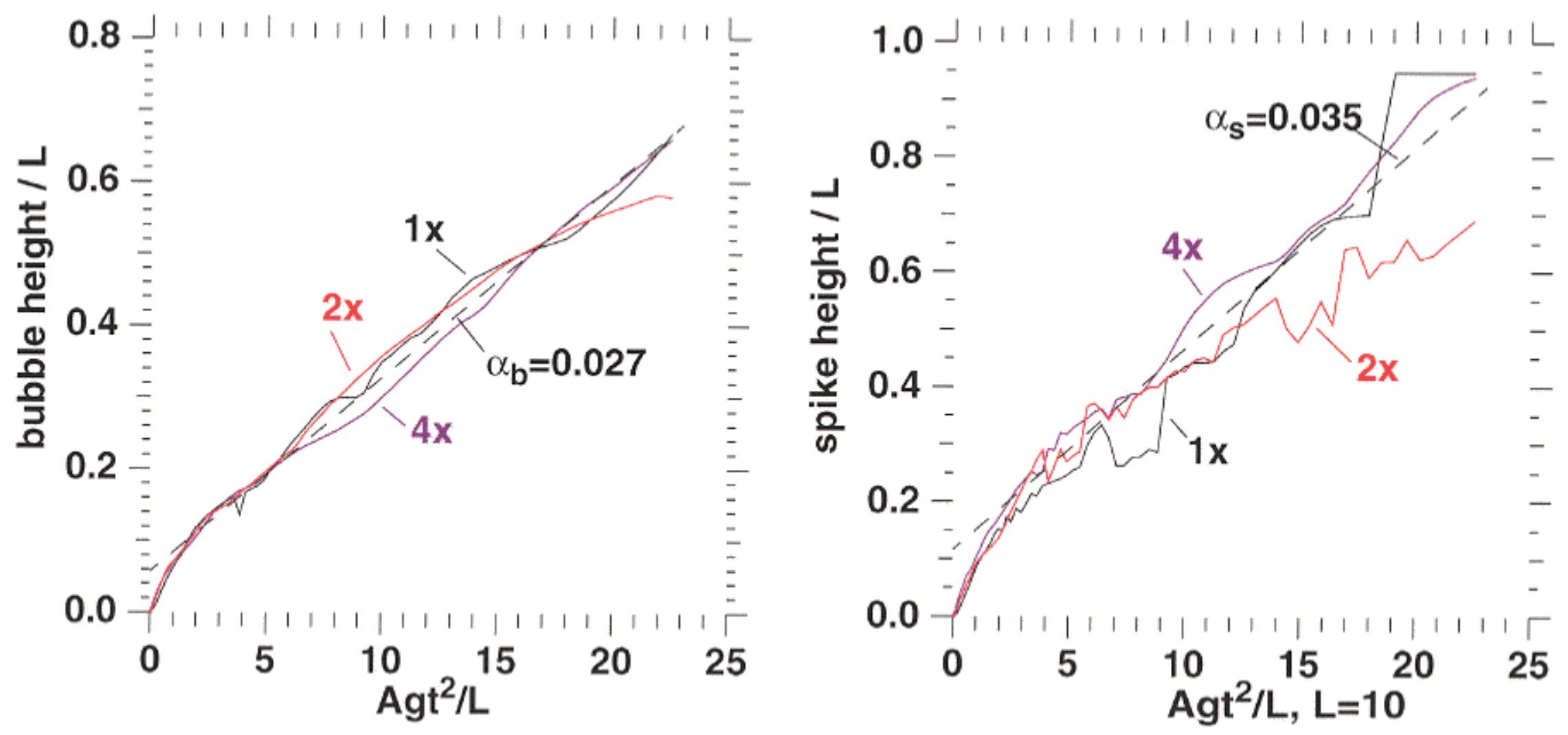

- $1 \mathrm{x}$ - nominal $256 \times 512,2 \mathrm{x}-512 \times 1024,4 \mathrm{x}-1024 \times 2048$

- Bubble and spike limits are taken at $1 \%$ volume fraction within $2<x<8$

- The small number of large bubbles or spikes at later times introduces uncertainty due to selection statistics 


\section{We have performed $128^{2} \times 512$ 3-D simulations in one quadrant of the nominal $\alpha$ problem}

- We use $1 / 4$ of the specified initial interface, a $5 \times 5 \mathrm{~cm}$ square

- The zone size, initial amplitude, initial wavelengths, and $\mathrm{g}$ are preserved from the nominal problem (not scaled by $L$ )

- This preserves three of the four dimensionless length ratios of the problem

$-\lambda_{0} l l$

$-\mathrm{a}_{0} / l$

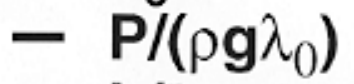

- L/l not preserved

- $l$ - zone size, $\mathrm{a}_{0}$ - initial amplitude, $\mathrm{L}$ - box size

- Results are expected to be nearly the same as for the full problem until the mix extent becomes comparable to the box width

- This will occur earlier in time by a factor $\sim 2^{1 / 2}$ than for the full problem

- We have recently completed the full size $256^{2} \times 512$ problem without interface reconstruction 
Under-resolution of the initial perturbations results in mesh imprinting - a numerical artifact
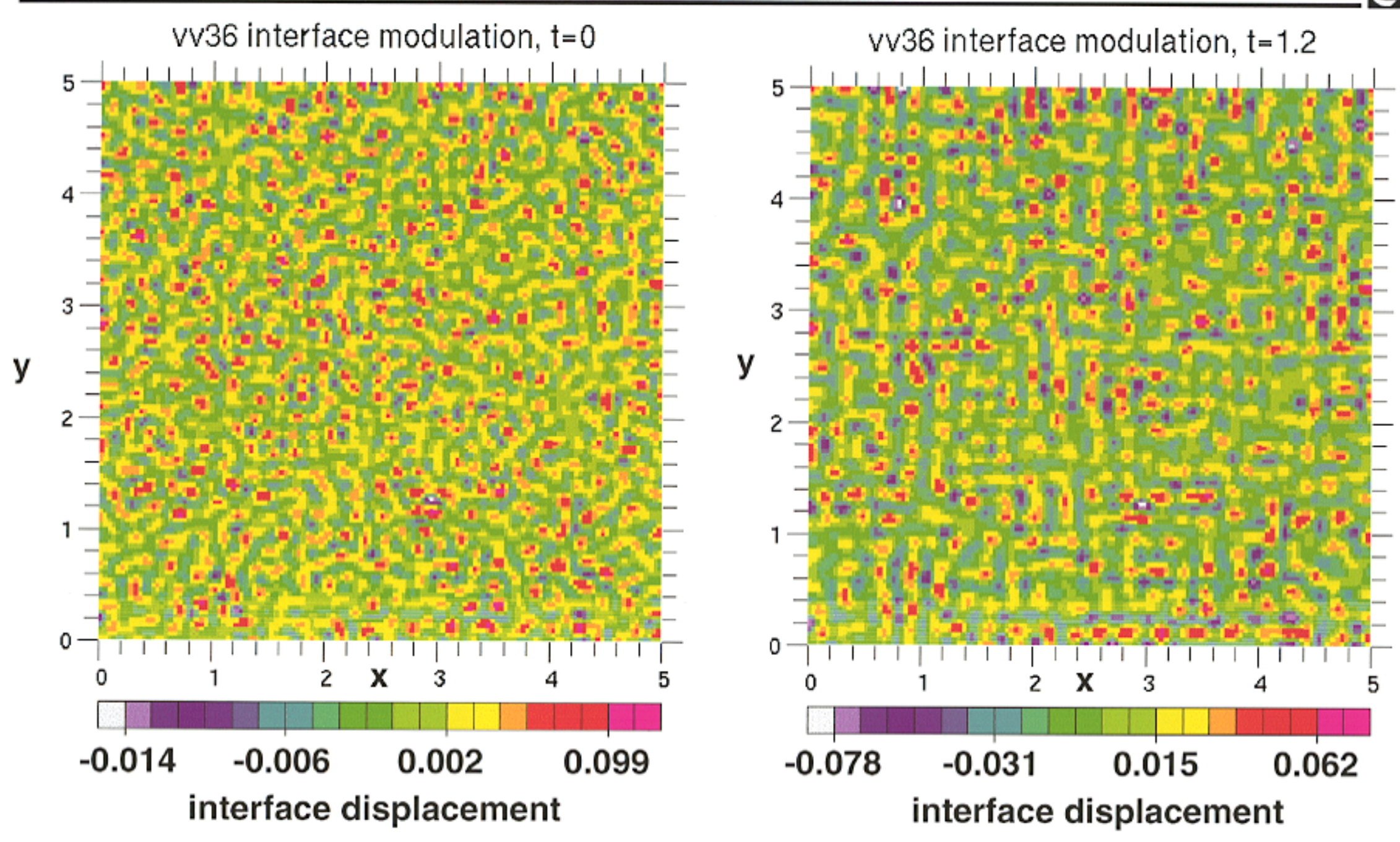

- The random isotropic initial surface evolves into a "checker board" pattern because of more rapid linear growth of modes aligned with the mesh 
The surface finish definition including sine and cosine modes should not be used with reflection boundary conditions

- Reflection boundary conditions support a spectrum consisting of integer and half-integer cosine modes

- Sine modes in the original surface definition are aliased into half-integer cosine modes. A "sine" mode with reflection bc has a cusp ot the boundary resulting in excessive growth near the boundary even in the linear phase

- Therefore, we used a surface finish obtained from Youngs containing only cosine modes, but which looks very similar to the original surface

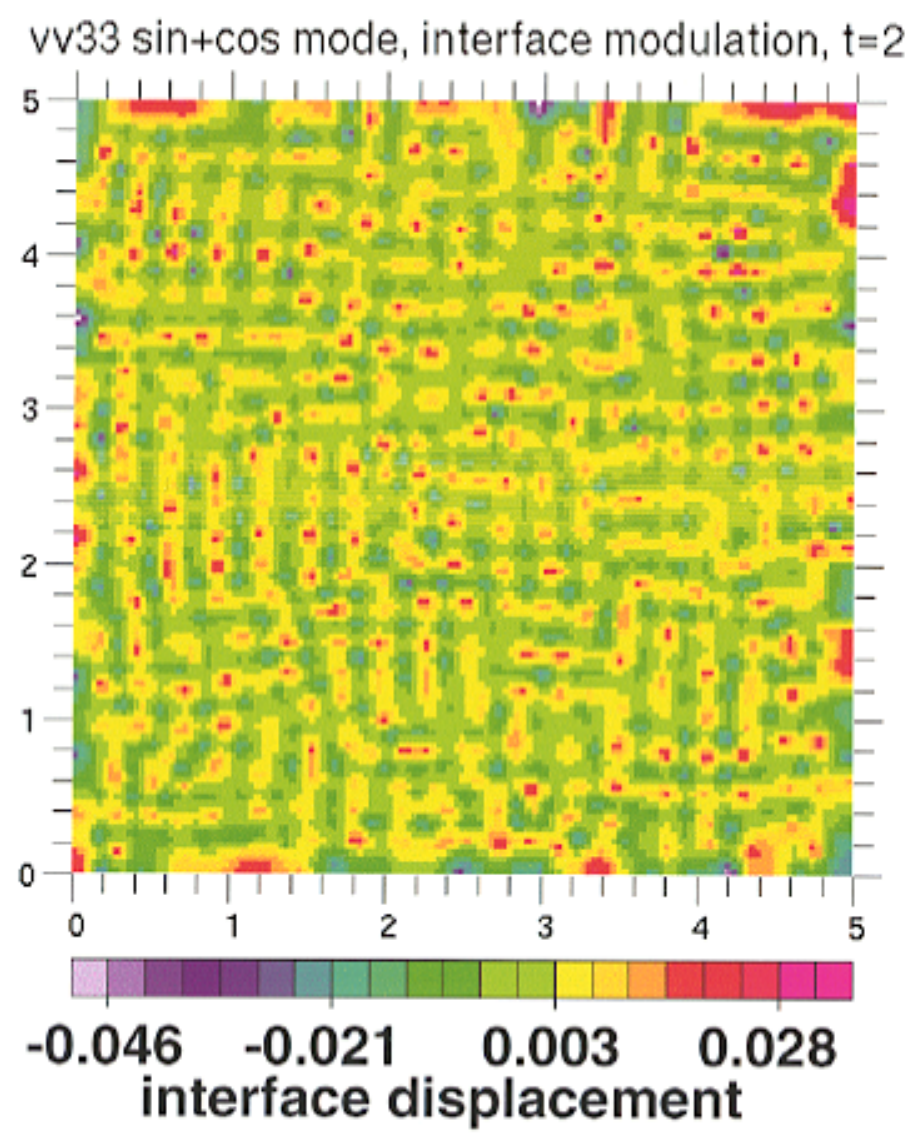


Spurious enhanced penetration at faces and corners is probable a consequence of reflection boundary conditions
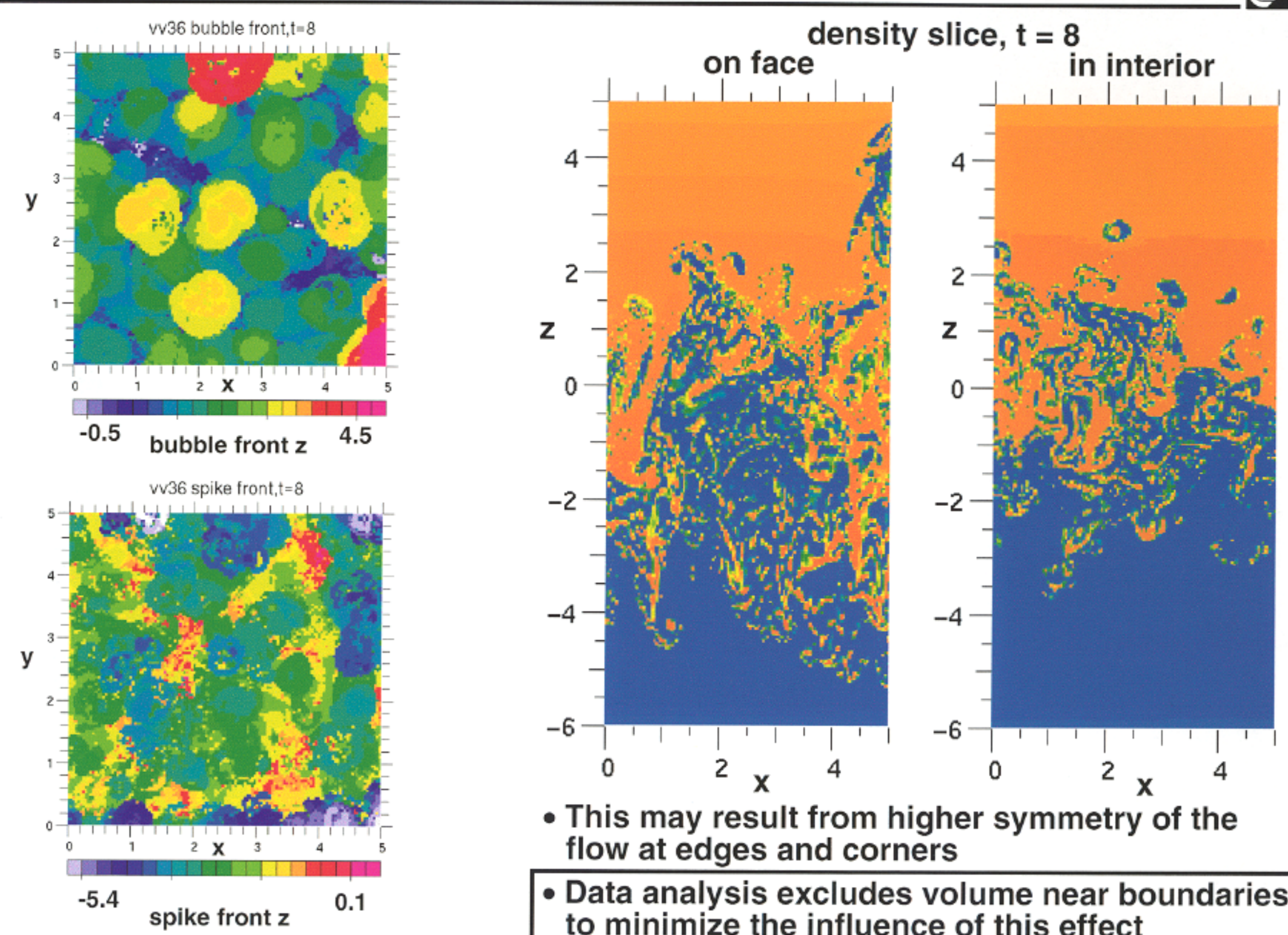

- This may result from higher symmetry of the flow at edges and corners

- Data analysis excludes volume near boundaries to minimize the influence of this effect 


\section{The simulation with interface reconstruction shows breakup into many small drops at late times}

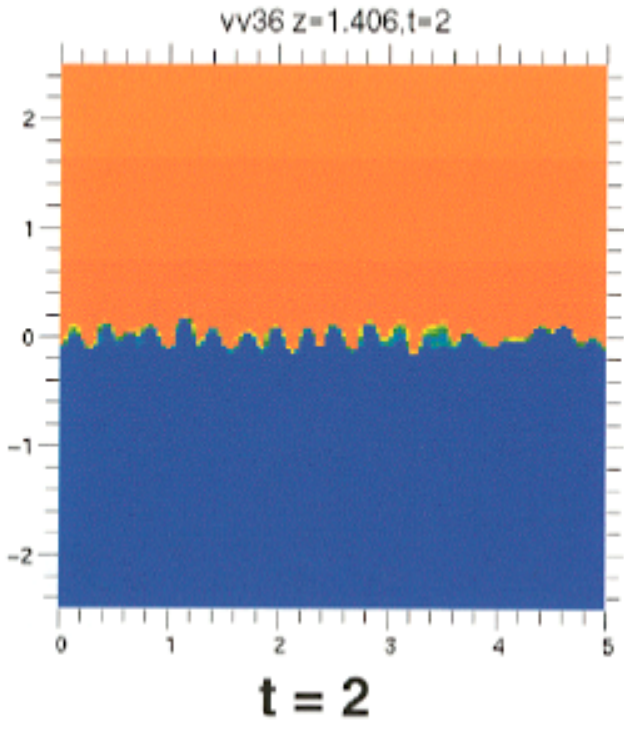

- Sequence of density cuts of 3-D 1282x512 simulation in the interior (away from a face)
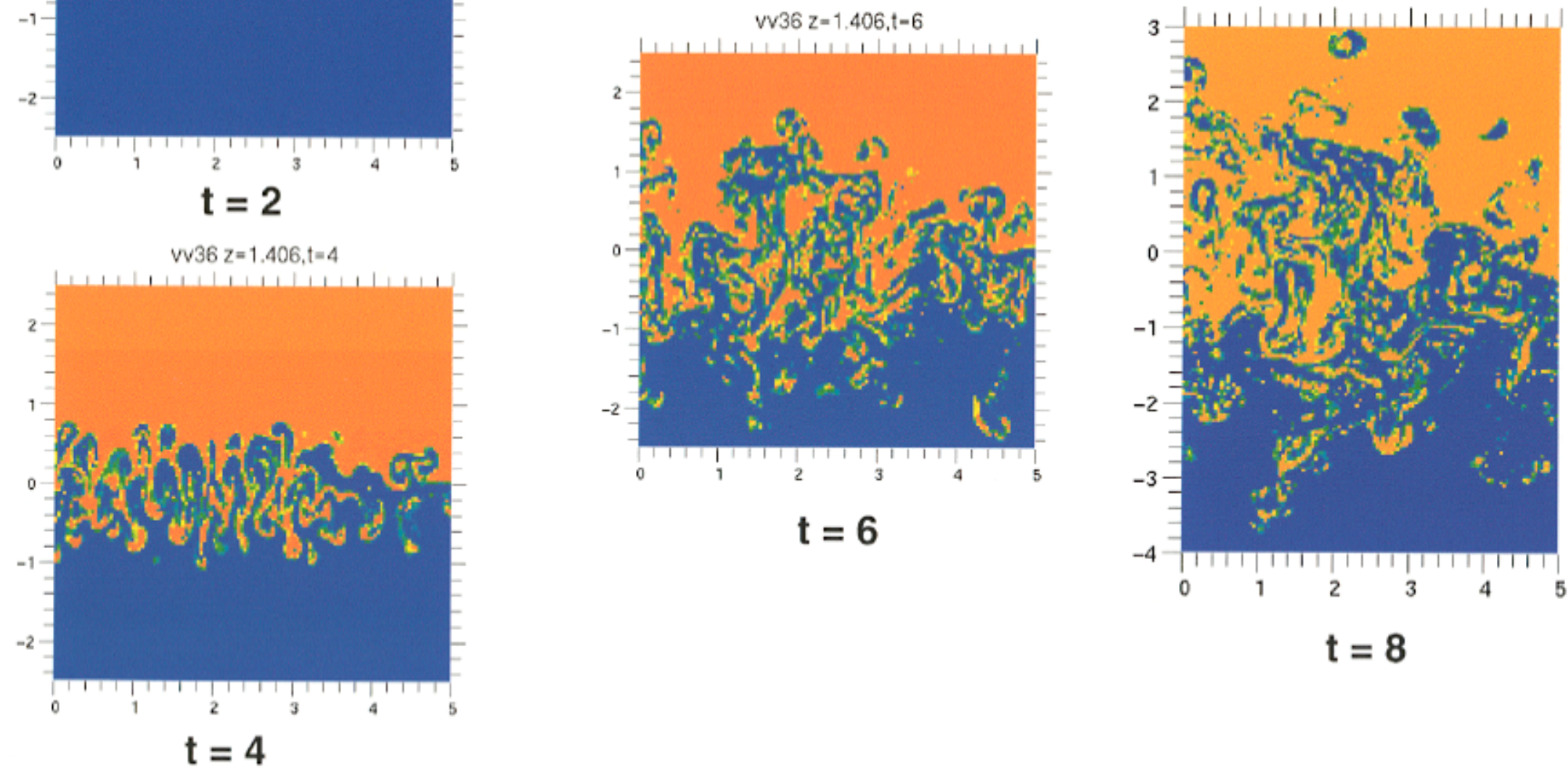
Simulations without interface reconstruction predict a mix extent only slightly lower, but show much more small scale mixing

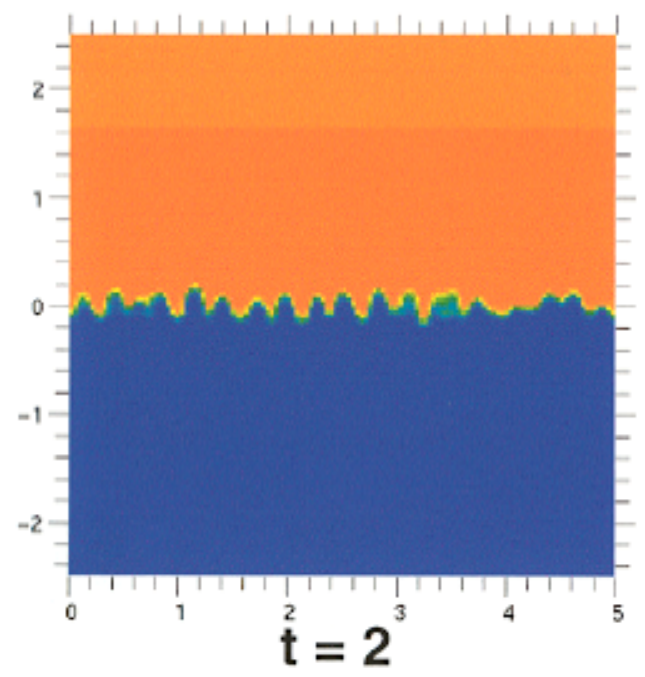

density cuts in $3-D 128^{2} \times 512$ simulation without interface reconstruction
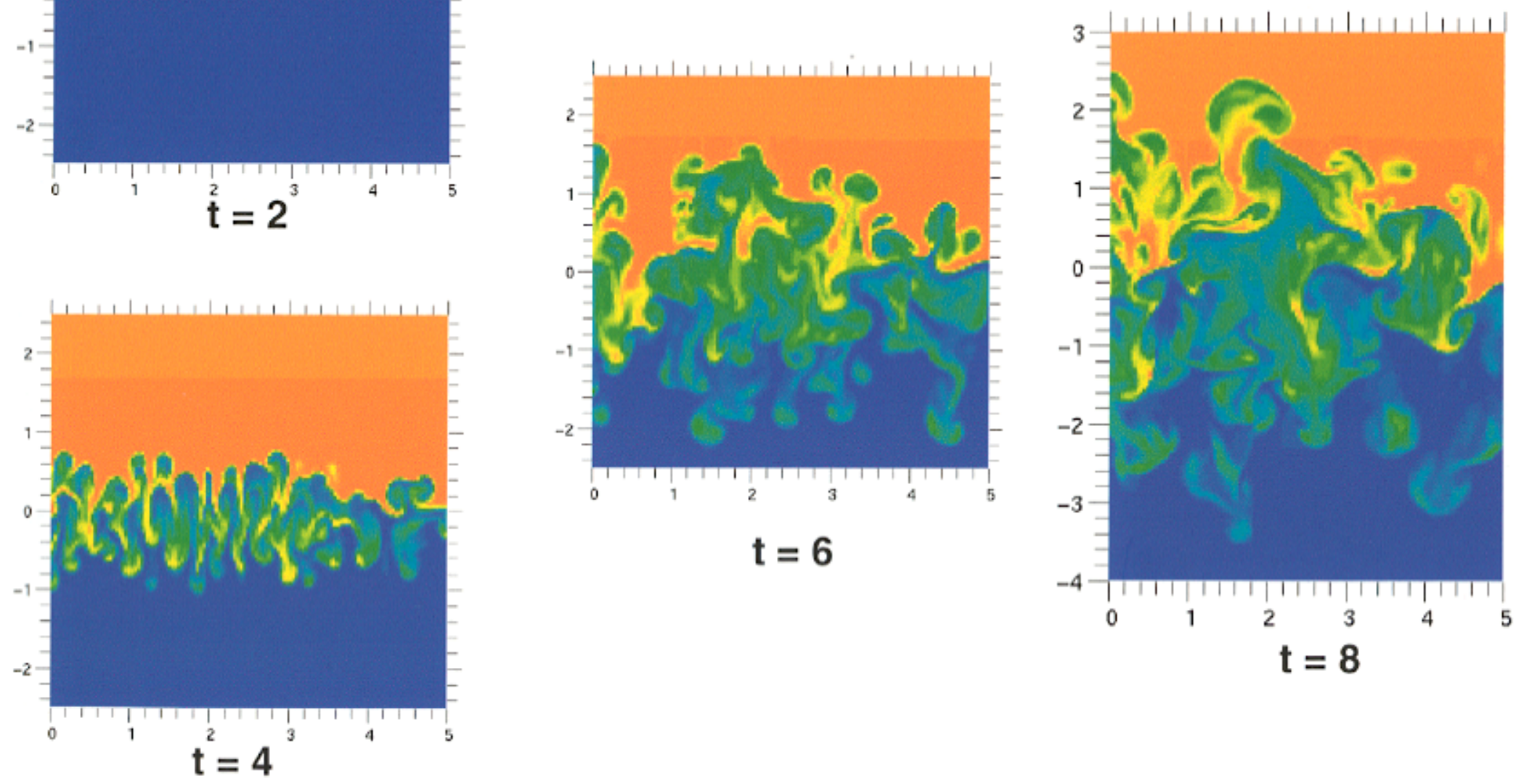
The 3-D simulation shows the expected transition to turbulence, followed by bubble merger

visualization of interface, identified as $\rho=2$ contour

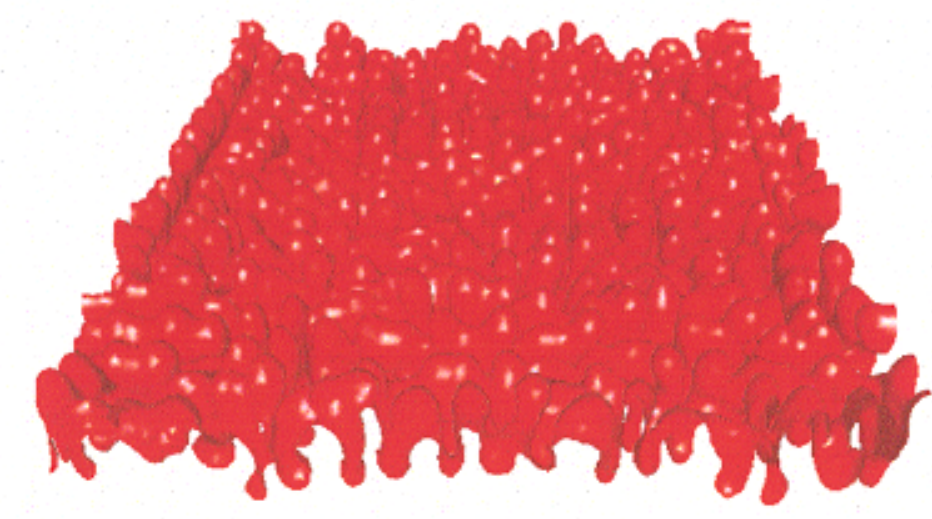

$t=2$
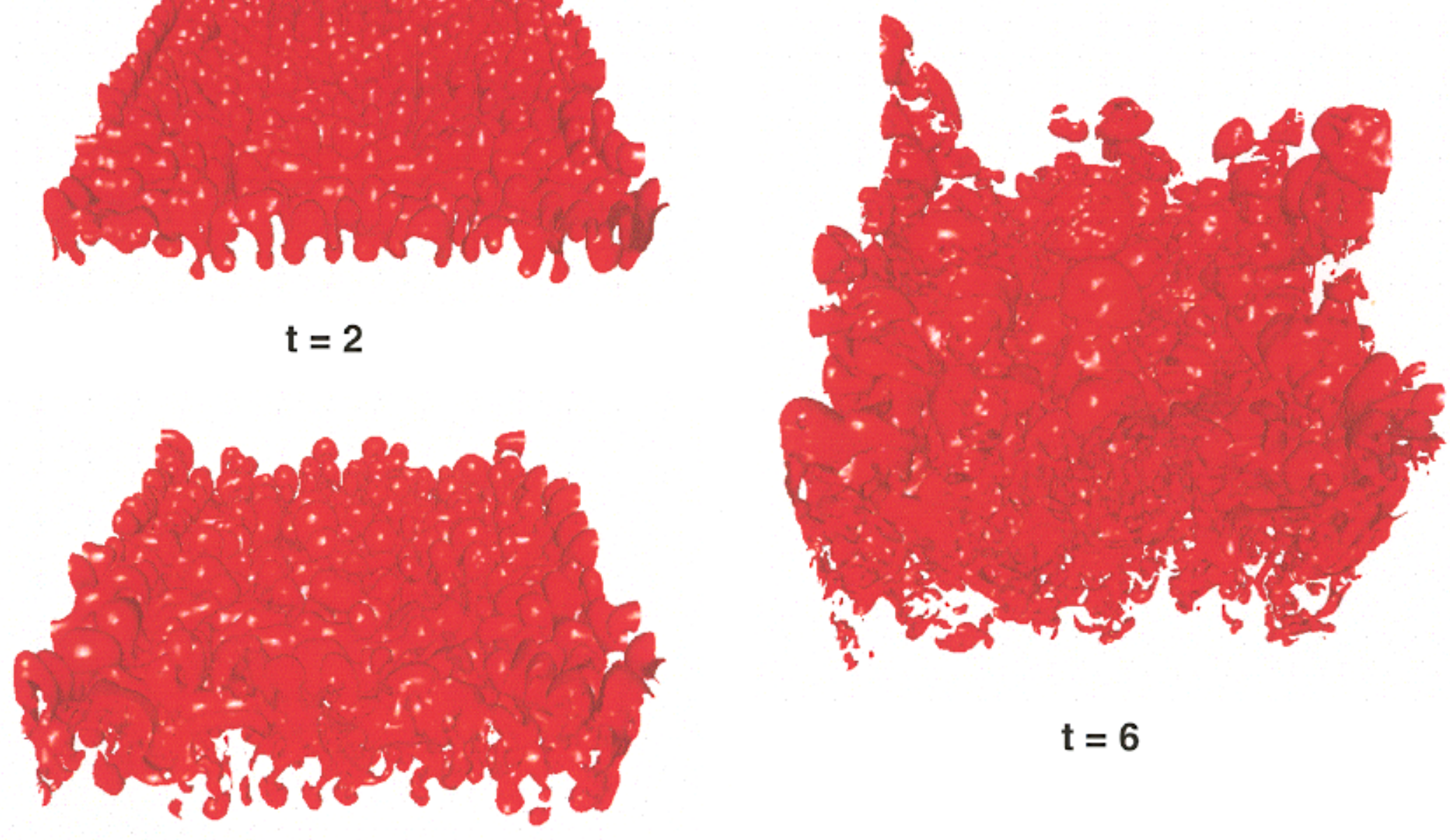

$t=4$ 


\section{It is not apparent that the mix extent ever reaches an asymptotic $\mathrm{t}^{2}$ growth}

bubble height

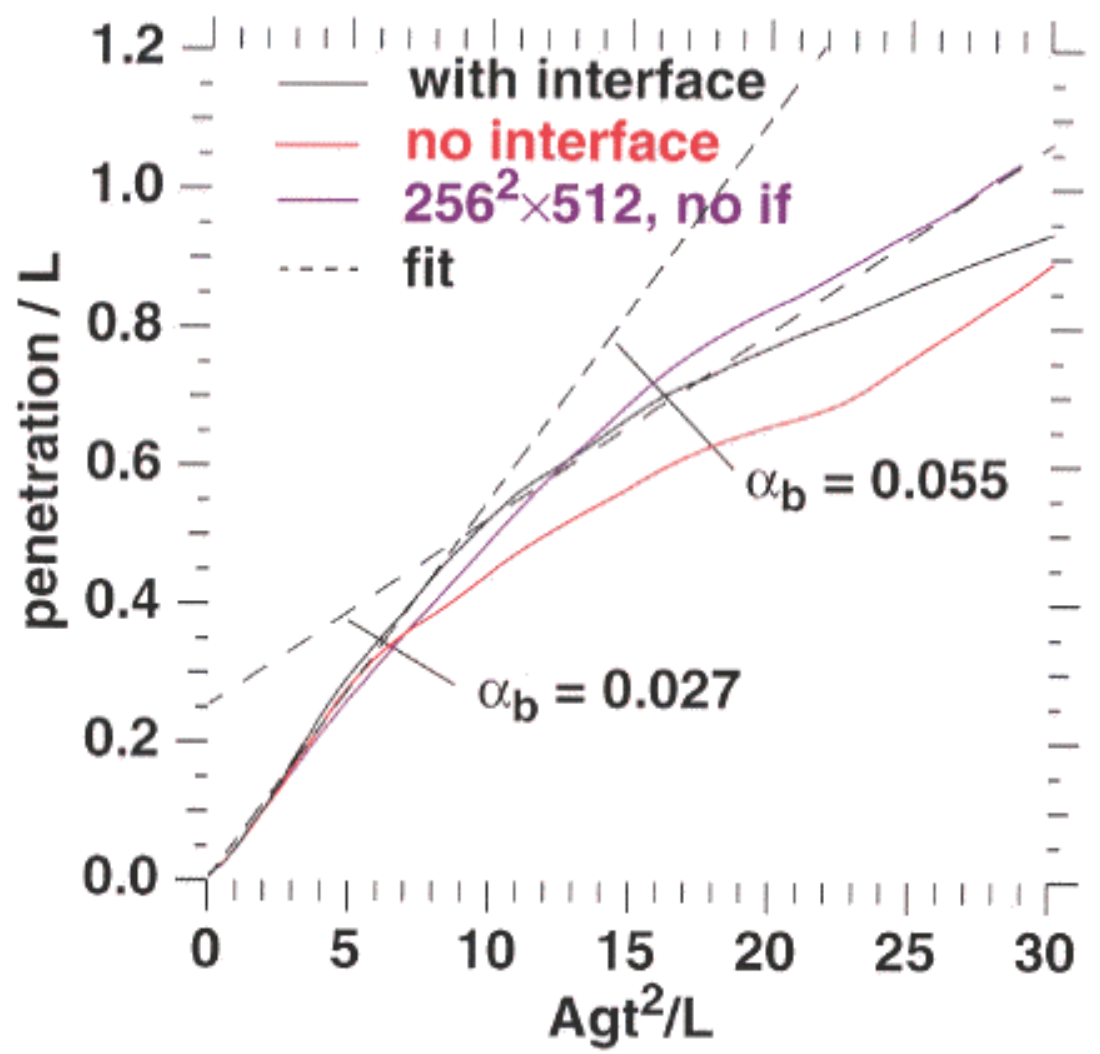

spike height

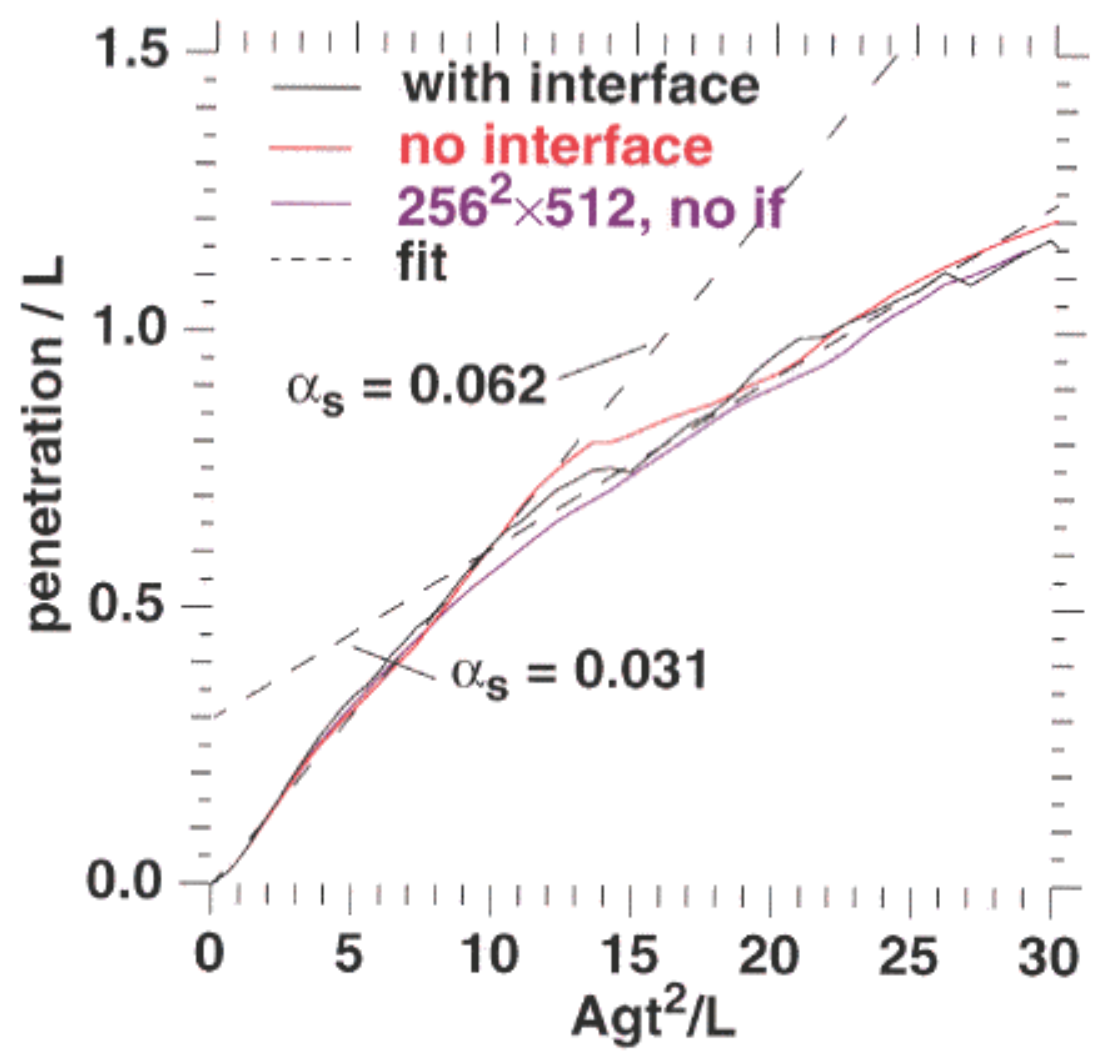

- "fit" lines are drawn by eye

- Interface reconstruction gives only modestly higher growth, only on the bubble side

- All cases show the slope, $\alpha$, decreasing with time

- On the bubble side, the full scale problem retains a higher growth rate later in time as it can grow bigger bubbles (all curves are scaled as $L=5$ )

- The growth of the spikes does not change with problem size 


\section{The total mix width and integral width measure also exhibit decreasing slope with time}

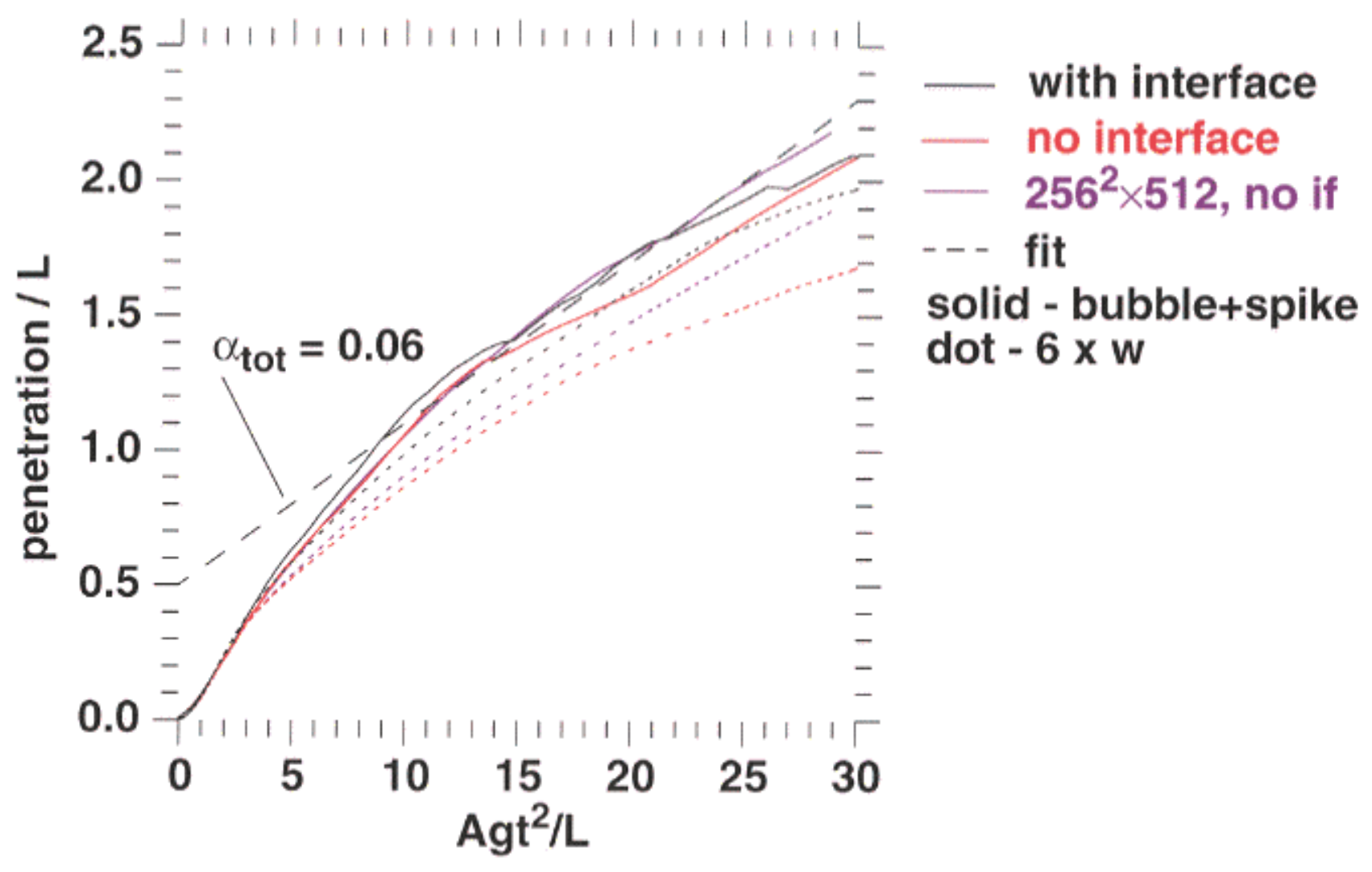

- w - Andrews and Spalding integral width

- The full scale and one quadrant simulations nearly overlay when scaled with the same $L$ (5 here) as the initial amplitude and wavelength were not scaled with L

- We believe that the box size affects the growth only by limiting the size of the largest bubbles late in time, which is a breakdown of self-similarity rather than a feature of the scaling 
Simulations lie below the LEM data when scaled by the box size; however, the slope is about right
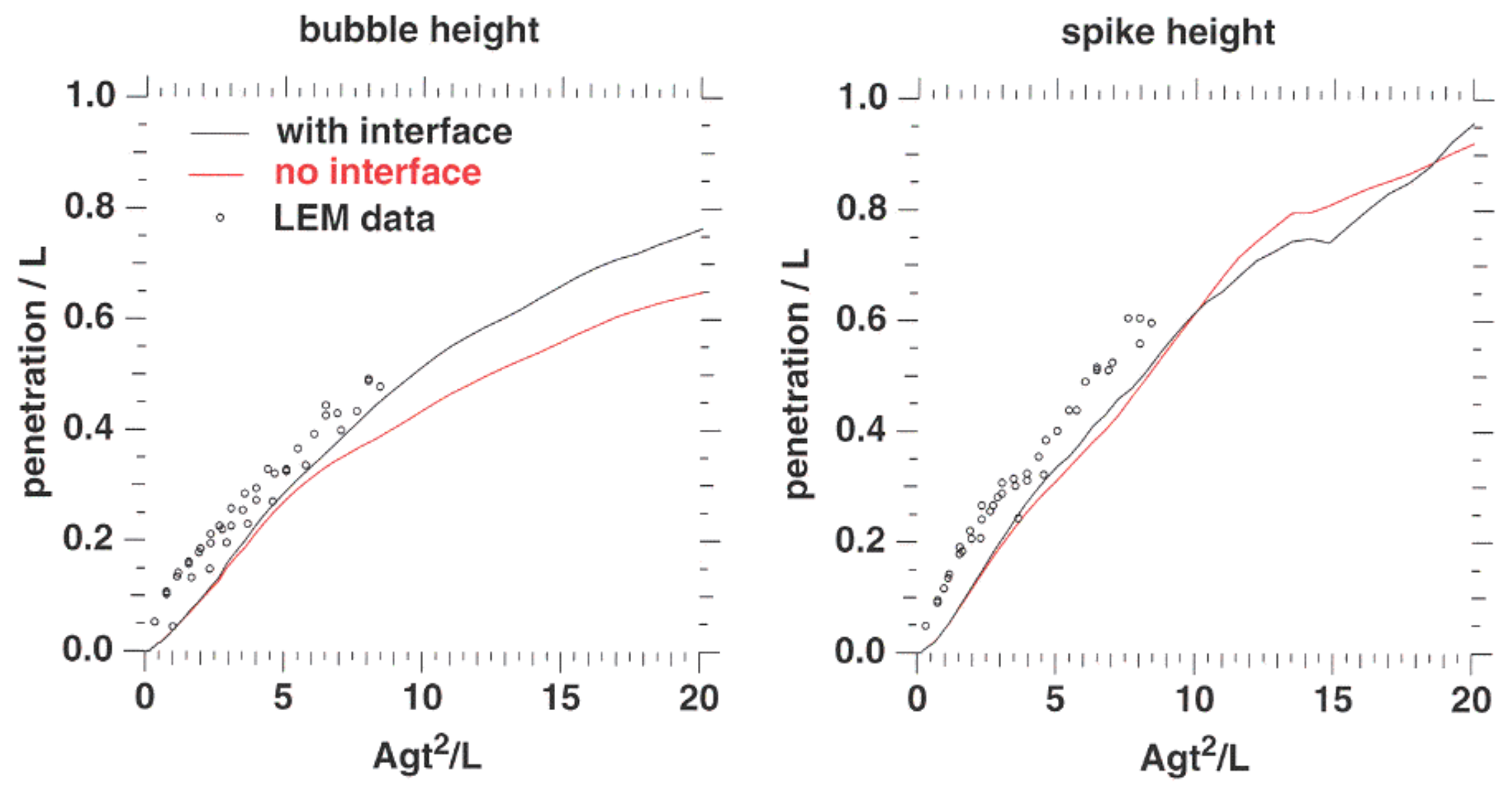
The simulation without interface reconstruction gives atomic mixing $\sim 0.7$, but similar dissipation to the run with tracking
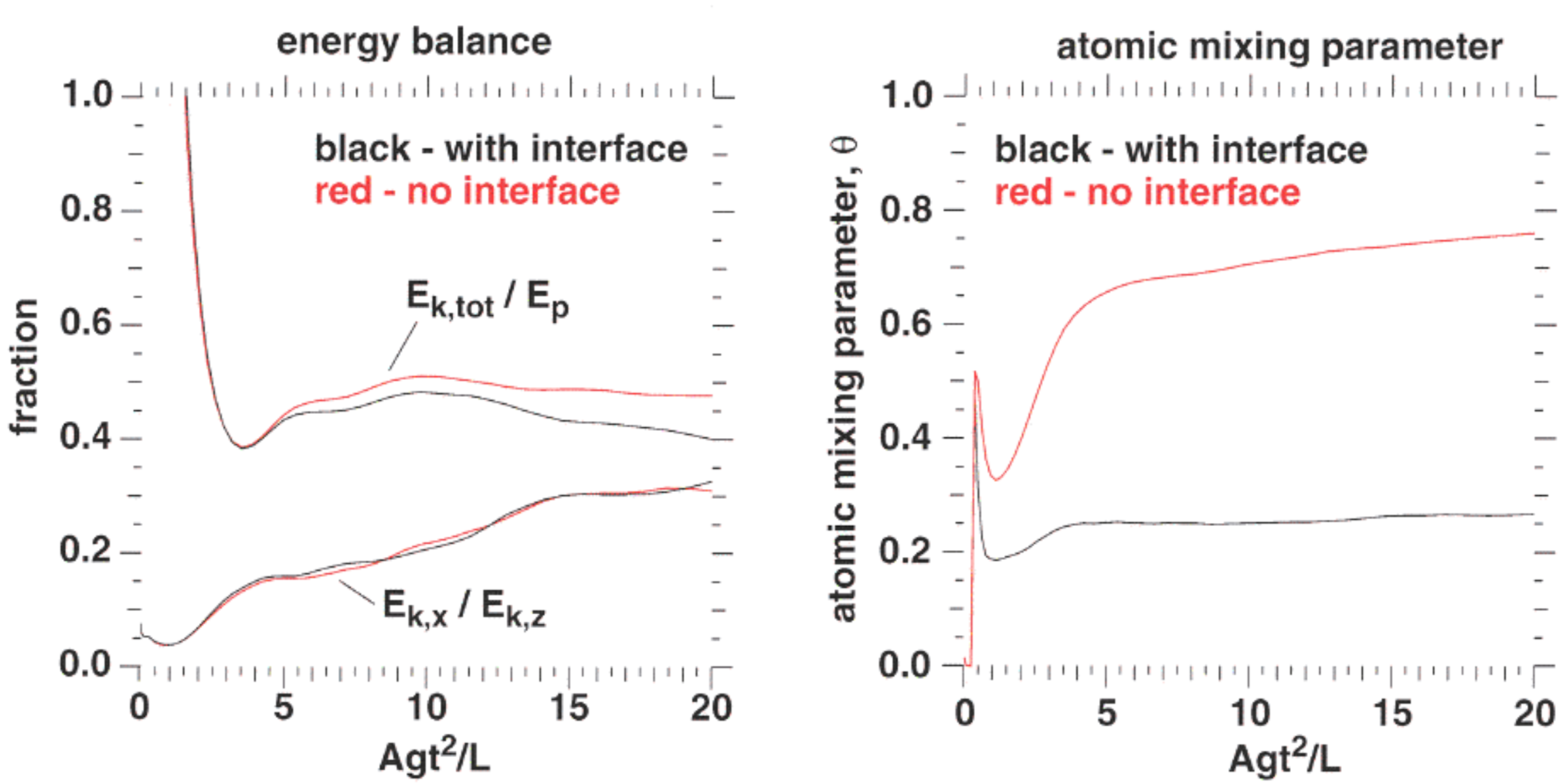

- $E_{p}$ - potential energy drop, dissipated energy, $E_{k, z}$ kinetic energy in streamwise direction, $E_{k, x}$ kinetic energy in one spanwise direction, $E_{k, \text { tot }}$ - total kinetic energy

- The atomic mixing parameter for the interface reconstruction run is an upper limit because post-processing does not account for the interface reconstruction and treats zones through which the interface passes as mixed 


\section{Volume fraction histograms are also very similar with}

or without interface reconstruction

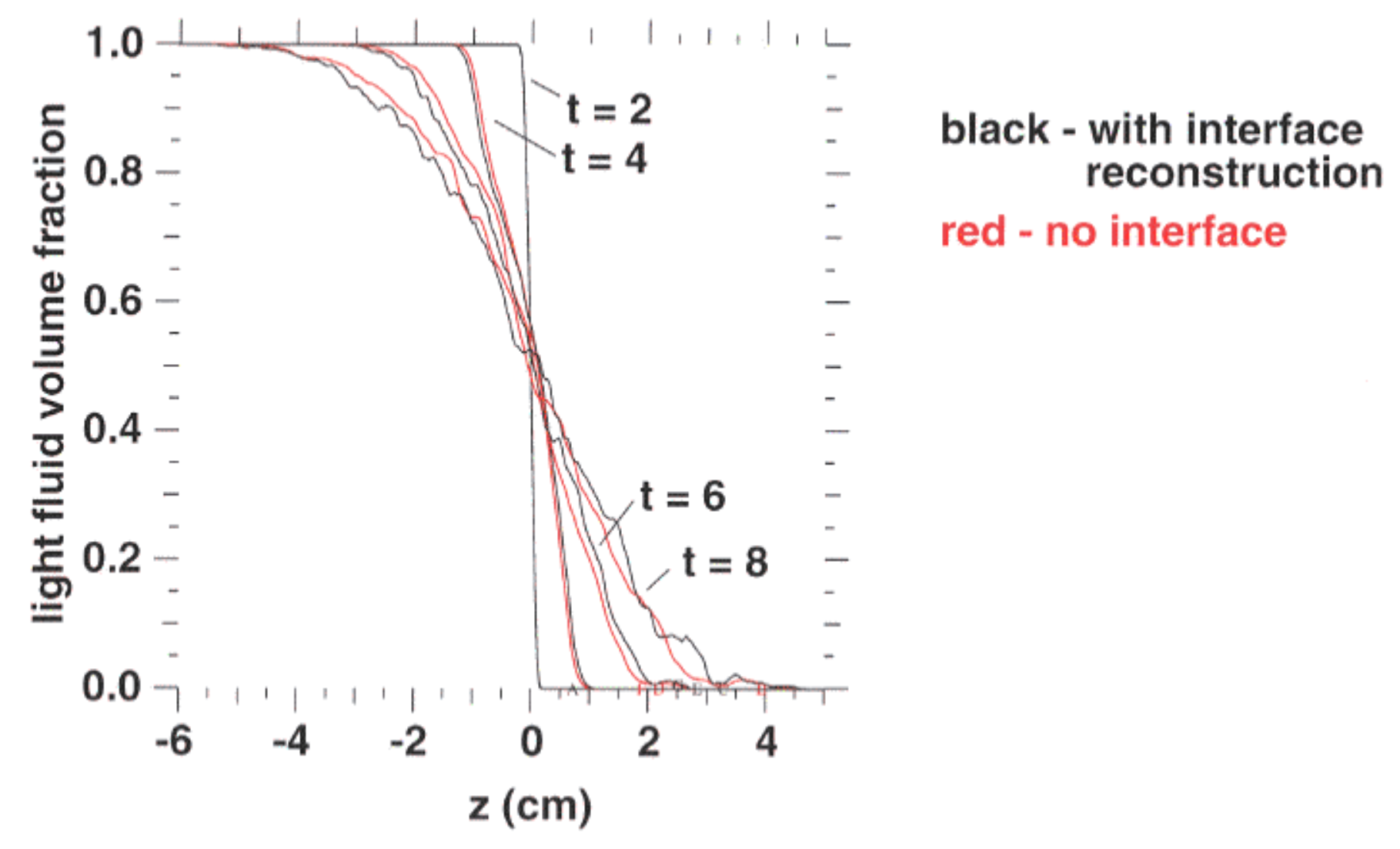




\section{Conclusions}

- Under-resolution of the initial perturbations introduces numerical artifacts, but it is uncertain if the simulated mix extent is compromised

- 2-D runs show no significant change in mix extent with zoning refinement

- Interface reconstruction has only a modest effect on mixing extent

- However, internal structure of the mixing zone is affected

- These runs do not appear to specify a definite value of $\alpha$

- 3-D simulations of one quadrant of the nominal $\alpha$ problem show monotonically decreasing $\alpha$ with increasing time

$-\alpha_{b}$ drops from $\sim 0.055$ at early times to $\sim 0.03$ later in time

$-\alpha_{b}$ remains high longer for the full scale problem

$-\alpha_{s} \sim 0.06$ at early times; also appears to drop later in time

- Simulations lie below the LEM bubble, spike penetration data

- slope of spike penetration and early time slope of bubble penetration are close to measured slopes of penetration vs. $t^{2}$ 


\title{
STUDY OF GRAVITATIONAL TURBULENT MIXING AT LARGE DENSITY DIFFERENCES USING DIRECT 3D NUMERICAL SIMULATION
}

\author{
Yu.V.Yanilkin, V.P.Statsenko, S.V.Rebrov, O.G.Sin'kova, A.L.Stadnik
}

Paper to be presented at the $8^{\text {th }}$ International Seminar on Turbulent Mixing of Compressible Matter ( $8^{\text {th }}$ IWPCTM, Pasadena, USA)

The problem of turbulent mixing under action of a constant gravity force (constant acceleration) on a plane interface of two gases was numerically studied by a number of papers $[1,2]$, however the studies are essentially missing for large density differences in mixing materials.

This paper makes an attempt of direct 3D numerical simulation of the above problem with code TREK [3] for gases of different densities, such that $\mathrm{n}=\rho_{2} / \rho_{1}=3-40$. With these density differences, the numerical simulations involve severe difficulties in achievement of the self-similar regime of a turbulent flow, so the difference scheme and the number of the computational cells were varied in the computations. A large number of the computations were conducted, it is impossible to demonstrate results of all the computations, however, note that the results agree well with each other. Below are results of computations by the same difference scheme and on the same computational grid.

Numerical arrays of hydrodynamic quantities from 3D computations are used to find moments of the quantities (Reynolds tensor, turbulent flows, profiles of density and its mean square pulsation). Besides, they are also used for construction of one-point concentration probability density function (PDF). Spectral analysis of velocity and density pulsations in TMZ was conducted: the effect on the approximation to the Kholmogorov spectrum for the quantities was studied.

The computed data is compared to known experimental data [4-9]. The analysis of the computed and experimental data suggests that the flow is not self- 
similar at the initial quite long stage, and the stage should be excluded to when determining the well-known self-similar constant $\alpha$. In view of this it seems to us that most experimental data need to be corrected.

\section{Setting up TREK computations}

The problem is formulated similar to ref. [1]: at the initial time two halfspaces separated with plane $\mathrm{z}=\mathrm{Z}_{\mathrm{c}}=0$ are filled with ideal gases in rest having densities $\rho_{1}=1$ и $\rho_{2}=n(n=3,10,20,40)$. The initial geometry is presented in Fig.1. The gravitational acceleration, $g_{z}=-1 \equiv-g$, is directed from the heavy material to the light. At the initial time, at the interface (in a layer one cell thick), a random-number generator gives random density perturbations $\delta \rho= \pm \rho_{1} \delta$, where $\delta=0.1$.

Gas dynamics equations for ideal two-component medium (with zero molecular viscosity and heat conduction) are solved. The computational domain is a parallelepiped with height $\Lambda=2$. Its horizontal face is a square with side $\mathrm{L}_{\mathrm{x}}=\mathrm{L}_{\mathrm{y}}=1$.

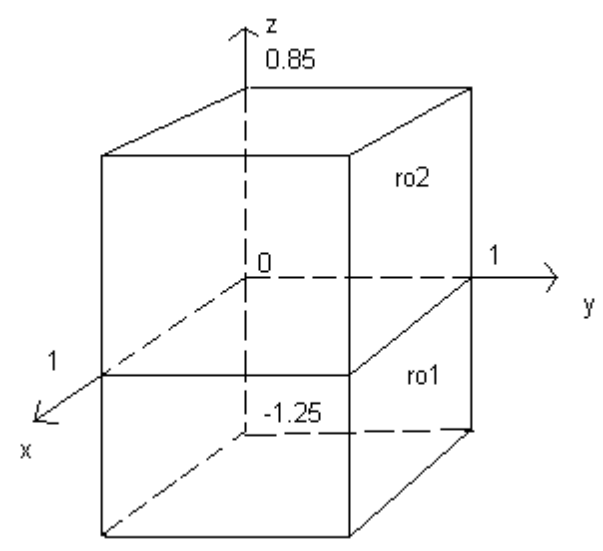

Fig.1. Initial geometry

The initial pressure profile was given using the hydrostatic equilibrium condition $p(z)=p_{0}-\int_{z_{2}}^{z} \rho(z) \cdot g \cdot d z$. Here the coordinate of the upper face is $\mathrm{z}_{2}=0.85$, that of the lower face $\mathrm{z}_{1}=-1.15, \mathrm{p}_{0}=100$. Note that the pressure $\left(\mathrm{p} \approx \mathrm{p}_{0}\right)$ 
is such, that the following non-compressibility condition was met well for the turbulent flow $\mathrm{k}=\xi \mathrm{L}_{\mathrm{t}} \mathrm{g}<<\gamma \mathrm{p} / \rho$, where $\xi=$ const $<<1, \mathrm{~L}_{\mathrm{t}}<\Lambda, \mathrm{L}_{\mathrm{t}}$ is turbulent mixing zone (TMZ) width, $\mathrm{k}$ is turbulent energy.

The equation of state is ideal gas with adiabatic constant $\gamma=1.4$. The computational grid is uniform, having $\mathrm{N}_{\mathrm{x}}=200, \mathrm{~N}_{\mathrm{y}}=200, \mathrm{~N}_{\mathrm{z}}=400$ cells. The "rigid wall" type condition was posed on all the computational domain boundaries.

\section{Results of 3D computations, integral characteristics}

The flow evolution observed in all the computations is similar on the whole to the previous computations [2] for small density differences: whirl enlargement with time and change over to the self-similar regime are observed.

The latter shows up for this problem, in particular, as transition to the linear dependence of $\mathrm{TMZ}$ width function $\mathrm{L}_{\mathrm{t}}(\mathrm{t})$ :

$$
F \equiv \frac{1}{t_{L}} \sqrt{\frac{L_{t}}{A g}}
$$

Here the unit of measurement for time is $t_{L} \equiv \sqrt{\frac{L_{x}}{g}}$ and $\mathrm{L}_{\mathrm{t}}(\mathrm{t})$,

$$
\mathrm{L}_{\mathrm{t}} \equiv \mathrm{Z}_{2}-\mathrm{Z}_{1} \text {, }
$$

is TMZ width along $\mathrm{z}$ determined by points $\mathrm{z}_{1}, \mathrm{z}_{2}$, at which quite small value of $\varepsilon$ of a hydrodynamic quantity, for example, concentration, is achieved. Next, assume that $\mathrm{c}_{2}\left(\mathrm{z}_{1}\right)=\varepsilon, \mathrm{c}_{2}\left(\mathrm{z}_{2}\right)=1-\varepsilon$, where $\mathrm{c}_{2}$ is mass fraction of the material whose initial density was $\rho_{2}=n$.

The angle of inclination, $\mathrm{dF} / \mathrm{dt}$, therewith determines the value of coefficient $\alpha_{a}=(\mathrm{dF} / \mathrm{dt})^{2}$ in the relation for the TMZ width at the self-similar stage:

$$
\mathrm{L}_{\mathrm{ta}}=\alpha_{\mathrm{a}} \mathrm{Agt}^{2} \text {. }
$$

Fig. 2 plots $\mathrm{F}(\mathrm{t})$ from the computations using relations (1) and (2).

For $\mathrm{n}=8.5-29$, the $\alpha_{a}$ obtained in the experiments [7] range from 0.15 to 0.2 and slightly increase with increasing $\mathrm{n}$. The relevant straight lines also appear in Fig.2. 
As seen from Fig.2, there are two flow segments differing in the angle of inclination $\mathrm{dF} / \mathrm{dt}$, at the initial segment this is larger than in the experiment, at the second segment this is, on the contrary, less. Prior to us, similar results were obtained by Youngs [1]. A comprehensive analysis of the flow suggests that at the first segment there is no flow self-similarity, although the inclination $\mathrm{dF} / \mathrm{dt}$ is close to constant. The self-similarity takes place only at the second stage, therefore it is this segment that the self-similar constant $\alpha$ should be measured at, where it proves less than the known experimental data. We assume, that it is caused by that at processing the specified data was not excluded initial nonselfsimilar a stage.

What this can result in can be seen from the experimental data of Kucherenko et al., which we analyzed.

Fig.3 plots function $\mathrm{F}_{2}(\mathrm{t}) \equiv \frac{1}{t_{L}} \sqrt{\frac{z_{2}-z_{c}}{A g}}$ versus time for coordinate $\mathrm{z}_{2}$ of the penetration of the heavy liquid into the light. At the final stage, where the self-similar regime is achieved, our computation for $n=40$ results in the angle of inclination $\mathrm{dF}_{2} / \mathrm{dt}$ close to the observed: Fig. 3 presents minimum and maximum quantities measured in [9] for $n=36.5$. Note that by $\alpha_{2}=0.078\left(\alpha_{2}=\left(\mathrm{dF}_{2} / \mathrm{dt}\right)^{2}\right)$ taken in [9] the angle of inclination is larger than the observed. Apparently, this value was obtained without exclusion of the initial segment, at which the self-similarity also does not take place.

A similar dependence for smaller density differences is shown in Fig.4. Apparently, the self-similar regime is not achieved in the experiment (for $n=3.65)$ : the angle of inclination decreases about in the same manner as in our computation (for $n=3$ ) at the initial stage, where the self-similar regime is not also achieved. In [9] $\alpha_{2}=0.078$, this is significantly higher than the observed and corresponds just to the initial (non-self-similar) stage.

Fig. 5 plots the scaled TMZ width:

$$
\alpha(t)=\frac{L_{t}}{A g\left(t-t_{0}\right)^{2}},
$$


that follows from our computations. Here $t_{0}$ corresponds to the intersection of the extrapolated self-similar segment of curve $F(t)$ estimated by the data of Fig. 2 with the abscissa axis.

In the computations with $\mathrm{n}=10,20,40, \alpha$ approaches approximately constant values (which corresponds to the self-similar stage): $\alpha_{\mathrm{a}} \approx 0.11,0.15,0.16$, respectively, which is somewhat less than the experimental quantities [8]. Note that the self-similar stage is achieved earlier with increasing $\mathbf{n}$, but its duration becomes shorter.

Processing similar to (4) was performed for coordinates $z_{1}, z_{2}$ of the penetration of the heavy liquid into the light and the light into the heavy, respectively. Their scaled values are

$$
\alpha_{1} \equiv \frac{z_{c}-z_{1}}{A g\left(t-t_{0}\right)^{2}}, \quad \alpha_{2} \equiv \frac{z_{2}-z_{c}}{A g\left(t-t_{0}\right)^{2}}
$$

The results are plotted in Fig.6. On the whole, $\alpha_{1}(t), \alpha_{2}(t)$ behave like $\alpha(t)$. In so doing, as might be expected, TMZ grows faster toward the light material. Asymmetry increases with increasing $\mathbf{n}$ and reaches $\approx 2$ for $n=40$.

For this problem the self-similar regime also shows up by the fact that the following quantity becomes time-independent:

$$
E(t) \equiv \frac{k_{m}}{L_{t} g}
$$

where $\mathrm{k}_{\mathrm{m}}(\mathrm{t}) \equiv \max (<\mathrm{k}>(\mathrm{z}, \mathrm{t}))$ is maximum averaged turbulent energy over the TMZ width:

$$
k(z)=\frac{\left\langle\left(u_{j}\right)^{2}\right\rangle-\left\langle u_{j}\right\rangle^{2}}{2},
$$

the averaging (denoted with $<>$ ) is over the entire horizontal section $z=$ const.

As seen from Fig.7, at the initial stage E(t) is large enough [at this stage, there is a small number of computational cells per TMZ, which leads to insufficiently correct values of $E(t)]$ and increases with increasing $\mathbf{n}$ in all the computations. At a later stage this quantity approaches approximately constant $E=E_{a}$ in the computations with $n=3, n=10$, and $n=20$. The $E_{a}$ also increases with increasing $\mathbf{n}$. 
To draw a more justified conclusion about whether or not the self-similar regime is achieved, other turbulent quantities have to be considered.

The TMZ maximum value, $\mathrm{R}_{\mathrm{m}}$, of density pulsation function $\mathrm{R}$ versus time is plotted in Fig.7,

$$
\mathrm{R}_{\mathrm{m}} \equiv \max (\mathrm{R}), \quad \mathrm{R} \equiv \frac{<\rho^{\prime 2}>}{\rho^{2}} .
$$

From the figure it is clear that at the self-similar stage the $\mathrm{R}_{\mathrm{m}}$ quite confidently approaches approximately constant $\mathrm{R}_{\mathrm{m}} \approx \mathrm{R}_{\mathrm{a}}$ in all the computations. The $\mathrm{R}_{\mathrm{a}}$ increases with increasing $\mathbf{n}$.

Fig.9 plots the TMZ maximum turbulent mass flow versus time:

$$
R_{z m} \equiv \max \left(\widetilde{R}_{z}\right), \widetilde{R}_{z} \equiv \frac{R_{z}}{\sqrt{L_{t} g}}, R_{z} \equiv<\rho^{\prime} u_{z}^{\prime}>
$$

Like for the $\mathrm{R}_{\mathrm{m}}$, from Fig.9 it follows that at the self-similar stage the $\mathrm{R}_{\mathrm{zm}}$ approaches approximately constant $\mathrm{R}_{\mathrm{zm}} \approx \mathrm{R}_{\mathrm{zma}}$ increasing with increasing $\mathbf{n}$.

Thus, the analysis shows that at the initial flow stage the self-similar regime is not established and this segment should be excluded when considering self-similar flow characteristics. This is also true for the experiments, in which initial perturbations able to affect the flows under study for long are always present.

\section{Velocity and density pulsation spectra}

The computed data was used as a basis to study the velocity pulsation spectrum according to relation

$$
\begin{aligned}
& E_{i i l}^{(s)}(x, y)=\left\langle u_{i}^{2}\right\rangle_{\mid l, x, y, z}-\left\langle u_{i}\right\rangle_{\left.\right|_{l, x, y, z}}^{2} \quad ; \quad s=1,2 \ldots N_{z} \\
& E_{i i l}(z)=E_{i i l}^{(s)}=\left\langle E_{i i l}^{(s)}(x, y)\right\rangle \quad ; l=r h, \quad r=2,3 \ldots N_{x} .
\end{aligned}
$$

Here averaging \langle\rangle$_{\mid 1, x, y}$ is performed in the s-layer (over $z$ ) in a square with side $1(\mathrm{l}=\mathrm{rh}, \mathrm{h}$ is computational cell size), whose center coordinates are $\mathrm{x}, \mathrm{y}$, and then averaging $(\langle\rangle)$ is made over all possible values $\mathrm{x}, \mathrm{y}$ of the squares with the 1 in the entire s-th layer. In (10) there is no summation over $\mathbf{i}$. 
Next: $\quad \mathrm{E}_{1}=\sum_{\mathrm{i}=1}^{\mathrm{i}=3} \mathrm{E}_{\mathrm{iil}}$

Fig. 10 plots the results of the computation for $t=2, n=40$ (where $K=2 \pi / 1)$ along with Kolmogorov spectrum

$$
\lg E_{\mathrm{l}}=-2 \mathrm{~K} / 3+\text { const. }
$$

Inside the $\mathrm{TMZ}\left(|\zeta| \leq 0.3 \div 0.4 ; \quad \zeta \equiv \frac{\mathrm{z}-\mathrm{z}_{\mathrm{c}}}{\mathrm{L}_{\mathrm{t}}}\right.$, here $\mathrm{L}_{\mathrm{t}}$ was computed by model [2]), the computed spectrum of total energy $E_{1}$ approaches the $3 \mathrm{D}$ Kolmogorov spectrum, with this being less confidently for highest n. Fig. 10 also shows that at small space scales (large $\mathrm{K}$ ) the velocity pulsations become isotropic: all components $\mathrm{E}_{\mathrm{iil}}$ approach each other.

A similar quantity is calculated for the squared density pulsations:

$$
\begin{aligned}
& \rho_{l}^{s}(x, y)=\left\langle\rho^{2}\right\rangle_{\mid l, x, y, z}-\langle\rho\rangle_{\mid l, x, y, z}^{2} \quad ; \quad s=1,2 \ldots N_{z} \\
& \rho_{l}(z) \equiv\left\langle\rho_{l}^{s}(x, y)\right\rangle \quad ; l=r h, \quad r=2,3 \ldots N_{x} ;
\end{aligned}
$$

this is depicted in Fig. 11. As seen, at the TMZ center the spectrum of net $\rho_{1}$ at small scales approaches the Kolmogorov spectrum. In contrast to the velocity pulsation spectrum, the density pulsation spectrum is closer to the Kolmogorov spectrum with increasing $\mathbf{n}$.

\section{Concentration probability density function}

The one-point heavy material mass concentration probability density function was determined by the computed data:

$$
F\left(c_{2}, z, t\right)=\frac{N\left(c_{1}^{(i k)}(z) \geq c_{2}\right)-N\left(c_{2}^{(i k)}(z) \geq\left(c_{2}+\Delta c\right)\right)}{N_{0} \Delta c},
$$

here $\mathrm{N}\left(\mathrm{c}_{2}^{(\mathrm{ik})}(\mathrm{z}) \geq \mathrm{c}_{2}\right)$ is the quantity of points in a given horizontal plane $\mathrm{z}$, at which current concentration $c_{2}^{(i k)}$ is higher than $\mathrm{c}_{2}, \mathrm{~N}_{\mathrm{o}}(\mathrm{z})$ is the total number of the points in the plane. The $\mathrm{c}_{2}$ runs through a sequence of $\mathrm{M}$ numbers:

$$
c_{2}=(0, \quad 1, \quad 2, \quad \ldots ., \quad M-1) \Delta c,
$$

where $\mathrm{M} \Delta \mathrm{c}=1$; in our computations, $\mathrm{M}=100$. 
When the initial concentration array is used, because of circuit features of calculation of concentration in separately taken cell the maximal values $F\left(c_{2}\right)$ are achieved basically (inside TMZ) near to borders of an interval of values of concentration, that is, at $\mathrm{c}_{2} \approx 0$ or $\approx 1$. Thus inside an interval $0<\mathrm{c}<1$ values $\mathrm{F}\left(\mathrm{c}_{2}\right)$ are small, that is, the integral is gathered basically at edges of an interval. Near to border TMZ, adjoining to heavy substance, $F\left(c_{2}\right)$ looks like $\delta$-function concentrated at $\mathrm{c}_{2} \approx 1$.

However, with use of averaging of an initial concentration array on squares with the side $\mathrm{n}_{0}$, in calculation for variant $\mathrm{N}=1.6107, \mathrm{n}=10$ with increase $\mathrm{n}_{\mathrm{o}}$ from 2 up to 4 it is observed (as shows rice 12) fast approach to certain established kind $\mathrm{F}\left(\mathrm{c}_{2}\right)$, not dependent from $\mathrm{n}_{\mathrm{o}}$. Its characteristic feature is almost uniform distribution on $\mathrm{c}_{2}$ inside $\mathrm{TMZ}$, that is, $\mathrm{F}\left(\mathrm{c}_{2}\right) \approx 1-$ and the integral is gathered almost uniformly on all interval.

The form of the function is similar for other $\mathbf{n}$.

\section{References}

1. Youngs D.L., Numerical simulation of mixing by Rayleigh-Taylor and Richtmyer-Meshkov instabilites, Laser and Particle Beams, vol 12, no. 4, pp. 725-750 (1994).

2. Sinkova O.G., Stadnik A.L., Statsenko V.P., Yanilkin Yu.V., Zhmailo V.A., Three-Dimensional Direct Numerical Simulation of Gravitational Turbulent Mixing, $6^{\text {rd }}$ Inter. Workshop on the Physics of Compressible Turbulent Mixing, Marseille France, pp.470-479 (1997).

3. Stadnik A.L., Shanin A.A., Yanilkin Yu.V. Eulerian code TREK for computing 3D gas-dynamic multicomponent medium flows// VANT. Ser. Mat. Modelir. Fiz. Protsessov, No.4 (1994).

4. Read, K.I. Experimental investigation for turbulent mixing by RayleighTaylor instability //Physica D12, 45 (1984).

5. Youngs, D.L. Modeling turbulent mixing by Rayleigh-Taylor instability //Physica D37, 270 (1989). 
6. Yu.A.Kucherenko, L.I.Shibarshov, V.I Chitajkin at al. Experimental study of the gravitational turbulent mixing self-similar mode //3-rd International Workshop on the physics of compressible turbulent mixing, pp.345-356 (1991).

7. Linden P.F., Redondo J.M., Youngs D.L., Molecular mixing in RayleighTaylor instability, J. Fluid Mech, vol.265, pp.97-124, (1994).

8. Youngs, D.L., Three-dimensional numerical simulation of turbulent mixing by Rayleigh-Taylor instability, Phys. Fluids, A3, 1312 (1991).

Kucherenko Yu.A., Shestachenko O.E., Piskunov Yu.A., Sviridov E.V., Medvedev V.M., Baishev A.I. Experimental study for self-similar mode of different-density gas mixing in the Earth's field gravity. Paper presented at the $6^{\text {th }}$ Zababakhin Scientic Talks, 24-28September 2001, Snezhinsk, Russia.

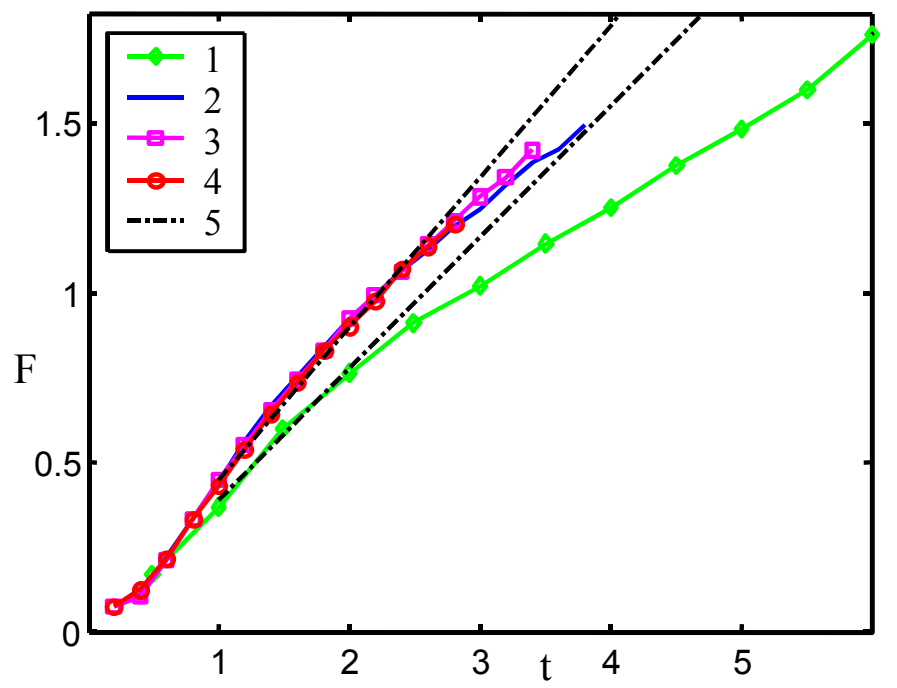

Fig. 2. Function F of TMZ width $L$ versus $t$; our computations: $1-n=3,2-n=10,3-n=20,4-n=40,3-$ by $\alpha$ taken in [7] 


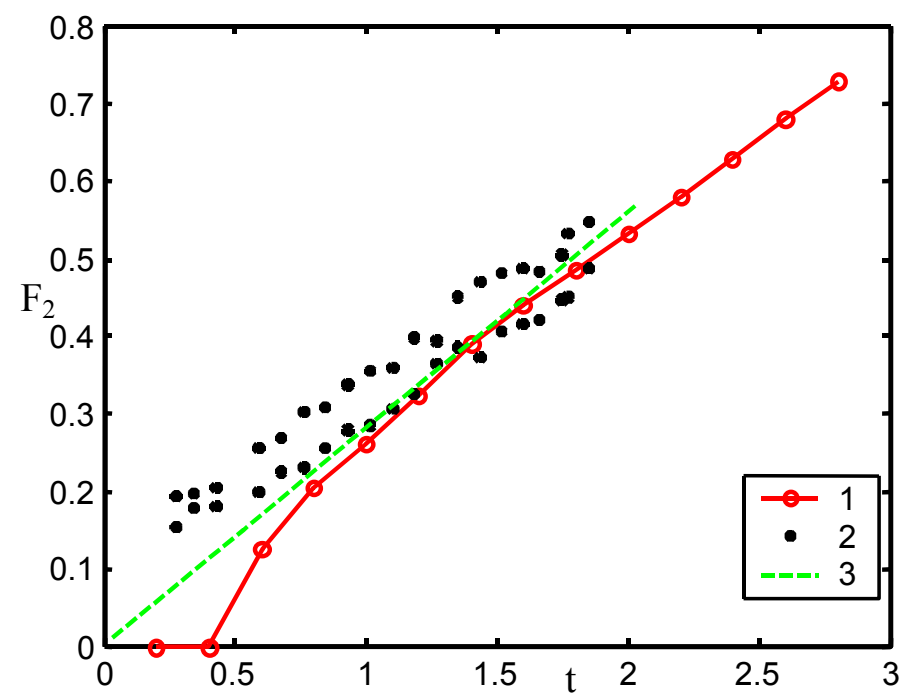

Fig. 3. Function $F_{2}$ of coordinate $z_{2}$ of heavy liquid penetration into light liquid versus time $\mathrm{t}$ : 1 - our computation for $\mathrm{n}=40,2$-minimum and maximum values measured in ref. [9] for $\mathrm{n}=36.5,3-$ by $\alpha_{2}=0.078$ taken in [9]

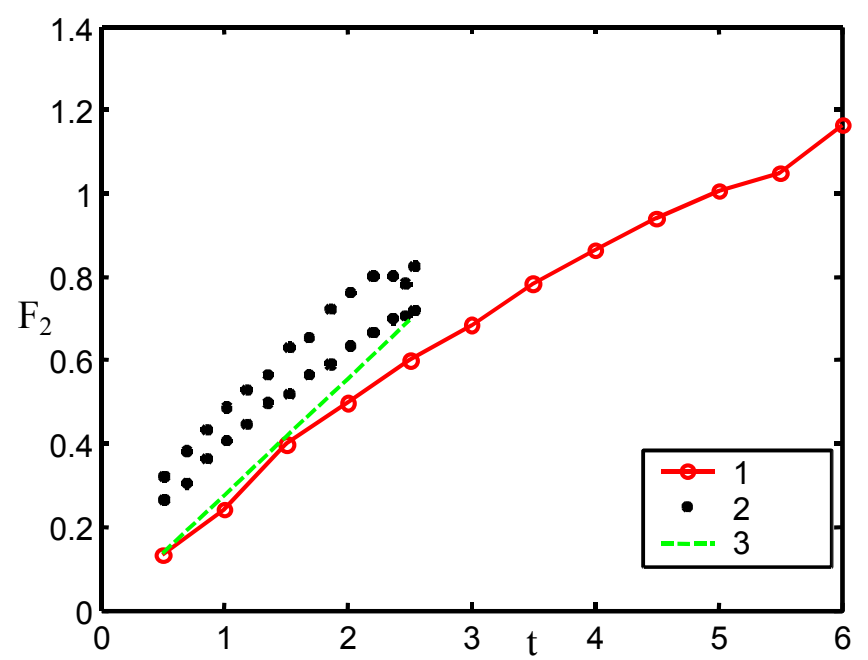

Fig. 4. Function $F_{2}$ of coordinate $z_{2}$ of heavy liquid penetration into light liquid versus time $\mathrm{t}: 1$ - our computation for $\mathrm{n}=3,2-$ minimum and maximum values measured in ref. [9] for $\mathrm{n}=3.65,3-$ by $\alpha_{2}=0.078$ taken in [9] 


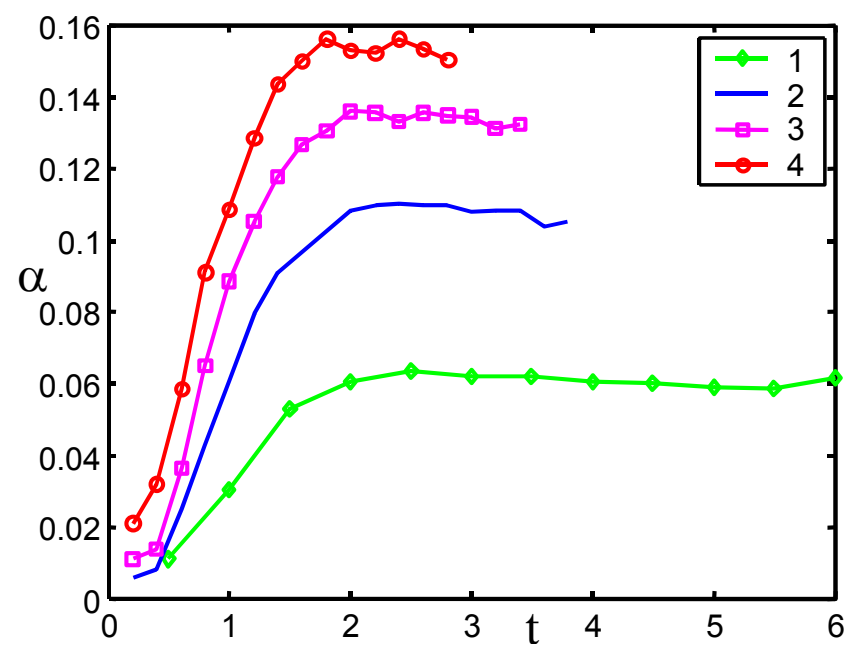

Fig. 5. Scaled TMZ width versus time. The notations are like those in Fig.2
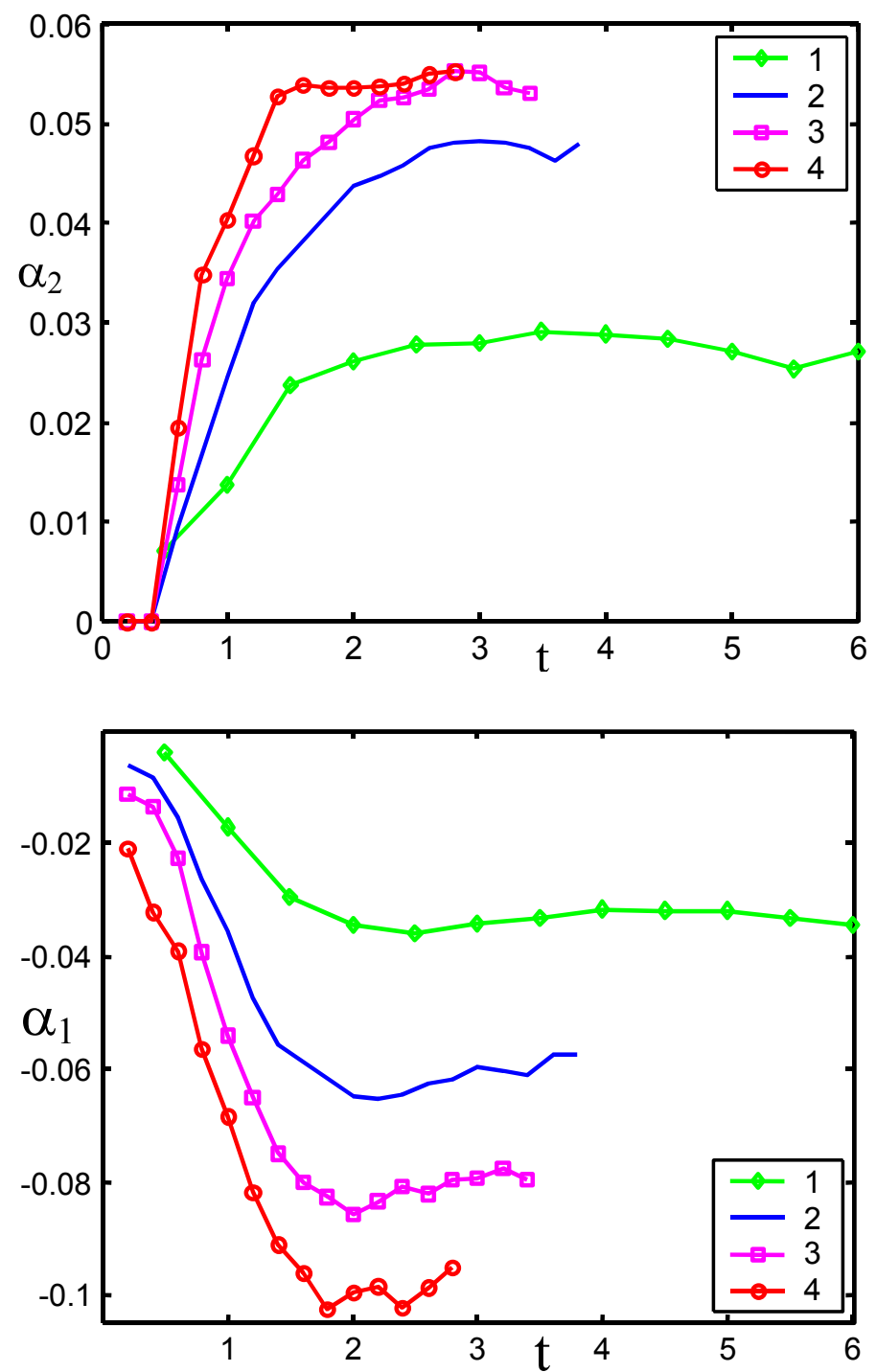

Fig. 6. Scaled TMZ boundary coordinate in light material $\left(\alpha_{1}\right)$ and heavy material $(\alpha 2)$ versus time. The notations are like those in Fig. 2 


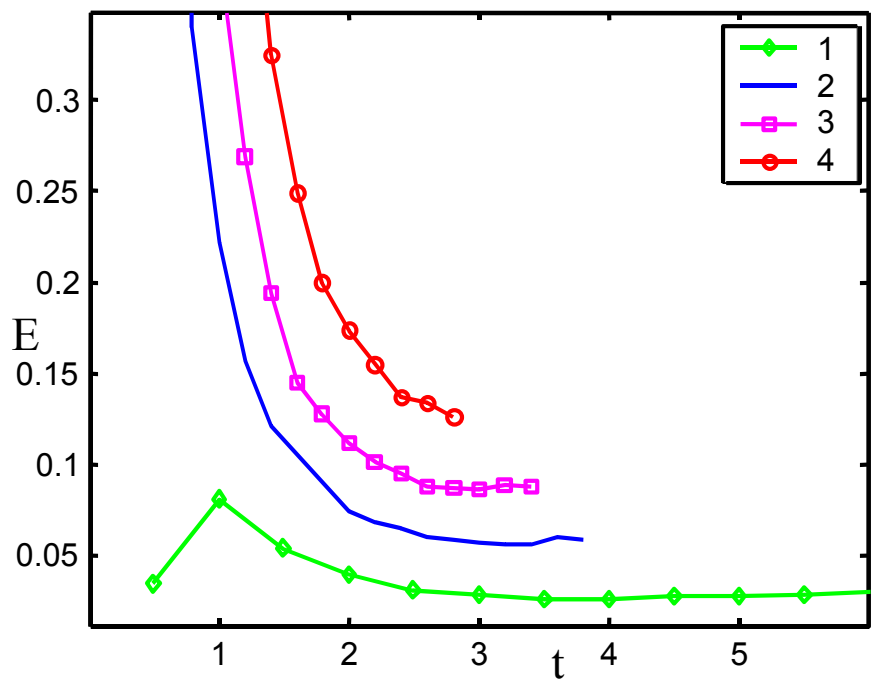

Fig. 7. TMZ maximum scaled turbulent energy versus time. The notations are like those in Fig.2

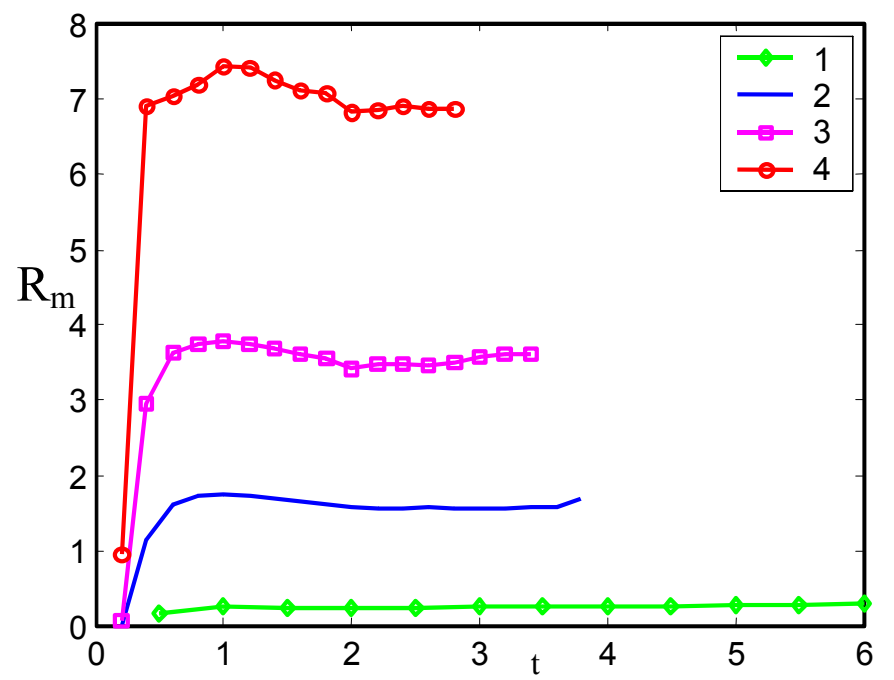

Fig. 8. TMZ maximum density pulsation function versus time.

The notations are like those in Fig.2 


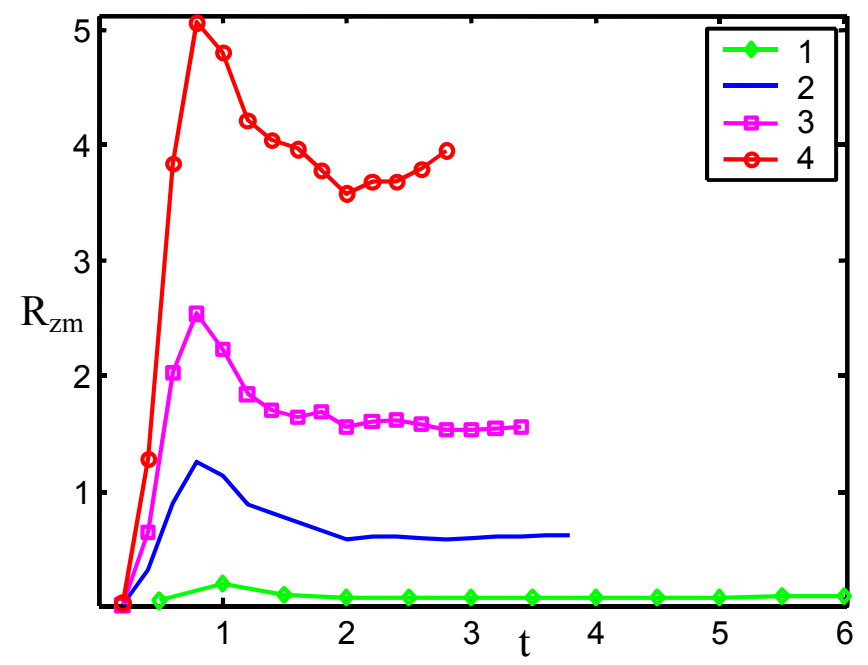

Fig. 9. TMZ maximum turbulent mass flow versus time. The notations are like those in Fig.2
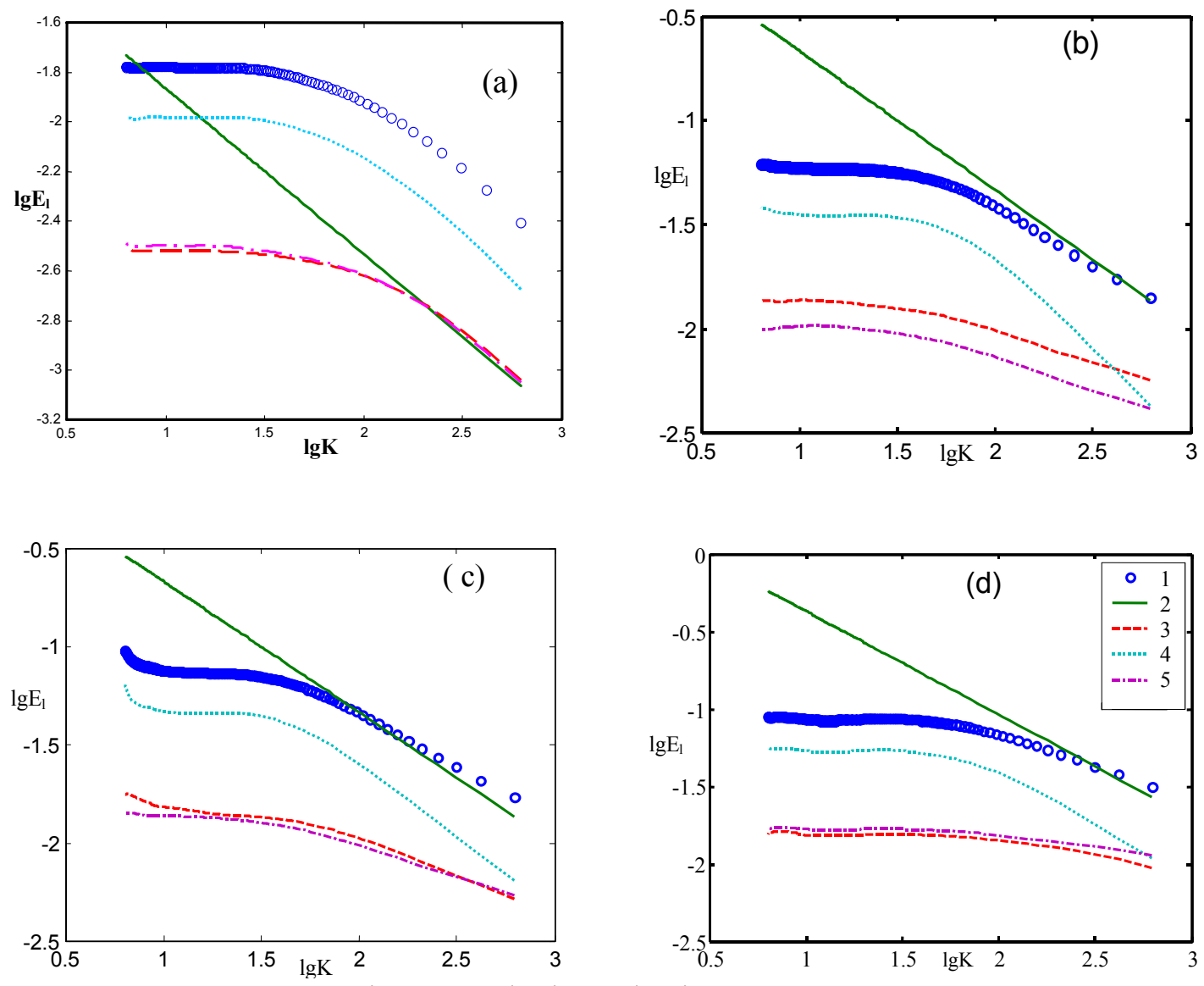

Fig. 10. Velocity pulsation spectrum,

(a) $\mathrm{n}=3, \mathrm{t}=3.5, \zeta=-0.076$, (b) $\mathrm{n}=10, \mathrm{t}=2.5, \zeta=-0.076$, (c) $\mathrm{n}=20, \mathrm{t}=2.5, \zeta=0.055$, (d) $\mathrm{n}=40, \mathrm{t}=2, \zeta=-0.36 ; \quad 1-\mathrm{E}_{\mathrm{l}}, 3-\mathrm{E}_{\mathrm{xxl}}, 4-\mathrm{E}_{\mathrm{zz}}, 5-\mathrm{E}_{\mathrm{yyl}} ; 2-$ Kolmogorov spectrum. 

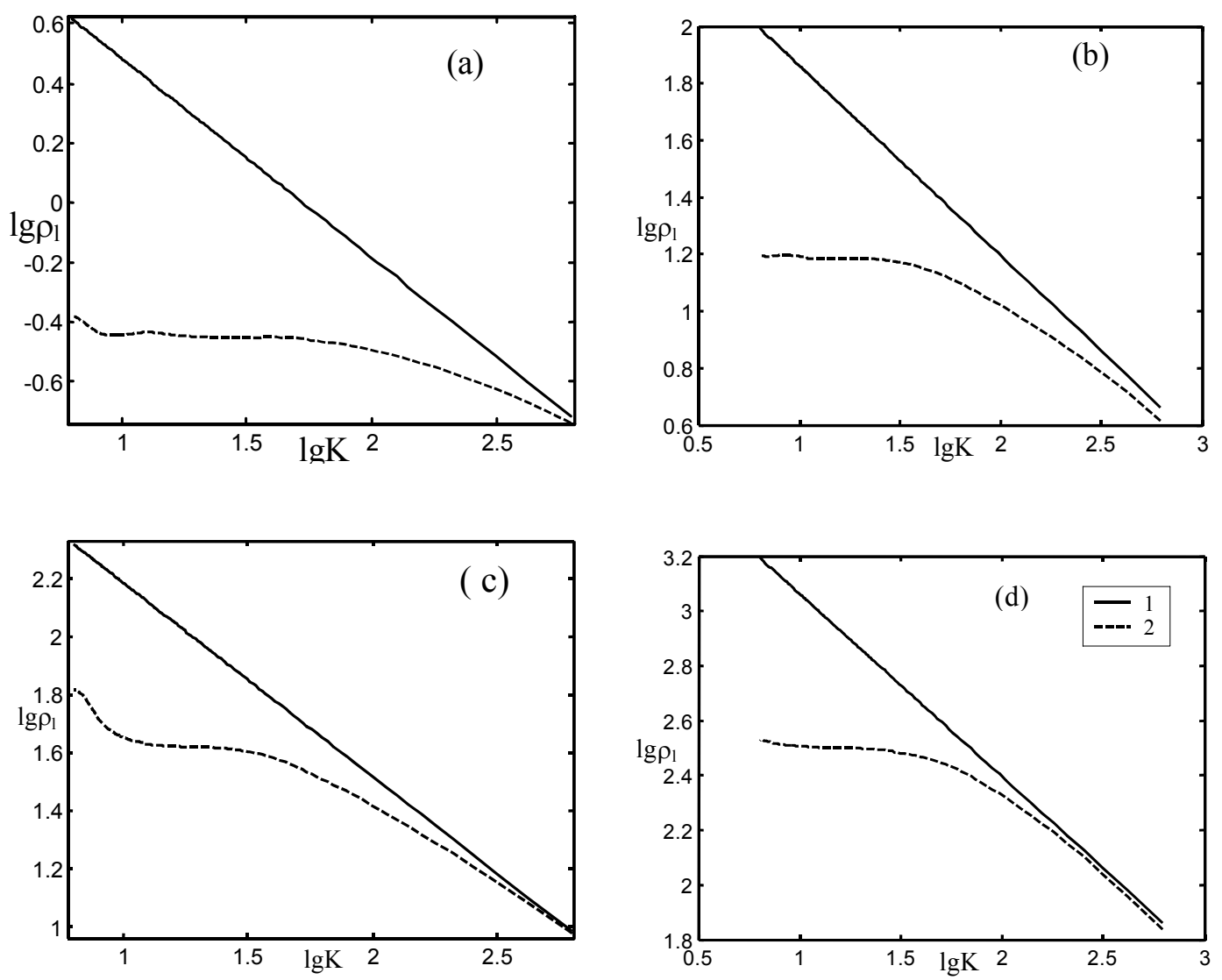

Fig. 11. Density pulsation spectrum,

(a) $\mathrm{n}=3, \mathrm{t}=4.5, \zeta=0.137$, (b) $\mathrm{n}=10, \mathrm{t}=2.5, \zeta=0.236$, (c) $\mathrm{n}=20, \mathrm{t}=2.5, \zeta=0.22$, (d) $\mathrm{n}=40, \mathrm{t}=1.8, \zeta=0.19$; $1-\rho_{\mathrm{l}}, 2$ - Kolmogorov spectrum.
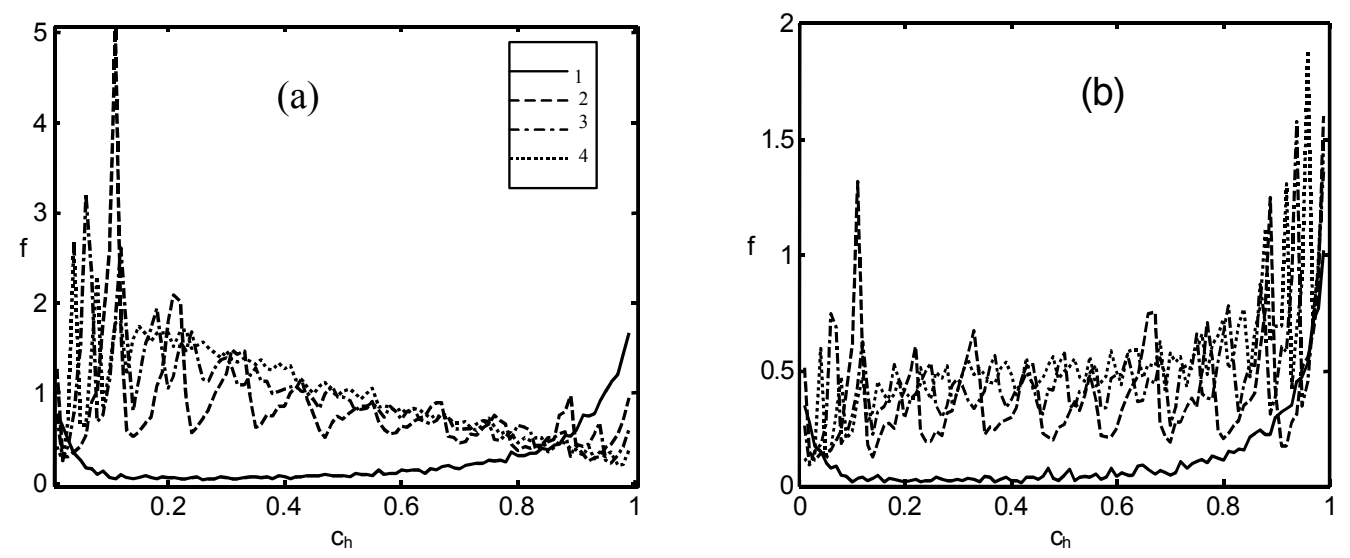

Fig. 12. Heavy material mass concentration probability density function, $\mathrm{n}=10, \mathrm{t}=2$; $\mathrm{M}=100.1-\mathrm{n}_{\mathrm{o}}=1,2-\mathrm{n}_{\mathrm{o}}=2,3-\mathrm{n}_{\mathrm{o}}=3,4-\mathrm{n}_{\mathrm{o}}=4$; a) $\zeta=-0.187$, b) $\zeta=0.41089$. 


\title{
DEVELOPMENT AND VALIDATION OF A 2D TURBULENT MIX MODEL
}

\author{
David L Youngs
}

AWE, Aldermaston

\author{
UK \\ 8th IWPCT M \\ Pasadena, December 2001
}




\section{Problems of interest}

- Compressible flow with interfaces between fluids of widely differing densities.

- High Reynolds No.

- Turbulent mixing at interfaces. Due to RT instability, RM instability. KH instability also important.

Need to model turbulent mixing in flows which are on average 1D or 2D.

Strategy for Numerical Simulation

- 3D Large Eddy Simulation (LES) impractical for real complex applications but can be successfully applied to simplified problems.

- High-resolution 3D LES is applied to simplified problems for which experimental results are available.

- A combination of 3D simulation results and experimental data is then used to set model coefficients used in a turbulence model (RANS model).

- The turbulence model is used to calculate the average mixing behaviour in 1D and 2D numercial simulations of complex real applications. 
TURMOIL 3D

- Simple 3D compressible Eulerian hydrocode used for turbulent mixing studies (LES).

- Same numerical method as the AWE 2D and 3D Eulerian production codes, but without interface tracking.

- Moving mesh option:-

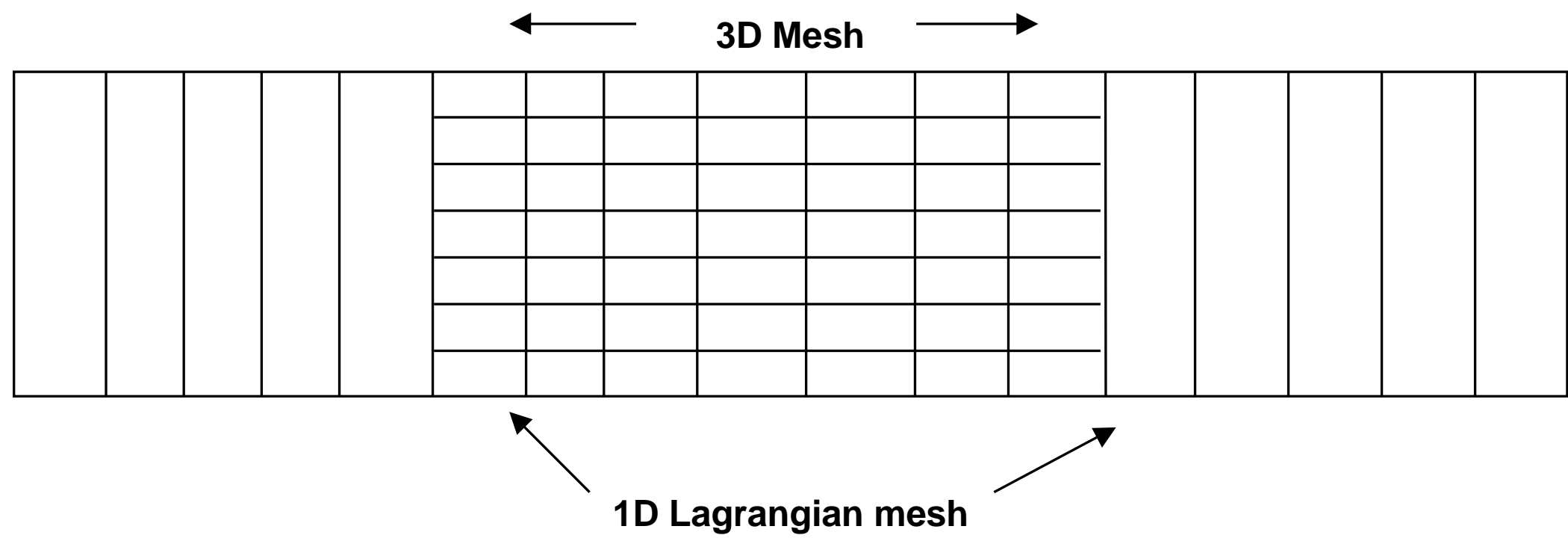

Used for shock tube applications

- Explicit numerical method ideally suited to parallelisation. Low Mach no. calculations used to approximate incompressible flow. 
- Lagrangian phase - non-dissipative except in the presence of shocks. Quadratic artifical viscous pressure used.

- Rezone or Advection phase.

Monotonic advection method of Van Leer used for all fluid variables.

- Monotonic advection considered essential for problems considered here with shocks and initial density continuities.

$\Rightarrow$ non-linear dissipation at high wave numbers.

- Example of MILES (Monotone Integrated Large Eddy Simulation).

- No need for an additional sub-grid dissipation model. 
THE 2D TURBULENCE MODEL (RANS MODEL)

Implemented in a 2D Eulerian hydrocode (which also has the moving mesh option)

Novel form of turbulence model - based on modelling the dynamics of the large scale structures (bubbles of light-fluid, drops of heavy fluid) rather than 1st or 2nd order closure assumptions for the fluctuating quantities)

Combines three basic ideas

1. Mixing induced by a pressure gradient or shock on fluids of different density.

2. Turbulent diffusion in the presence of concentration gradients.

3 Exchange of mass between the initial fluids is used to model the decay of concentration fluctuations [2].

Uses multiphase flow equations with turbulent diffusion terms added [1].

Bouyancy - drag model used to calculate initial behaviour - approximate representation of the initial conditions[3].

References: 1. D.L.Youngs, Laser and Particle Beams, vol 12, p725 (1994)

2. D.L. Youngs, Proceedings of 5th IWPCTM, Stony Brook(1995)

3. J.C.V. Hansom et al., Laser and Particle Beams, vol 8, p51(1990) 
The simple incompressible RT problem $\left(\rho_{1}, \rho_{2}, \mathbf{g}\right.$ constant $)$ is the key problem for fixing the turbulence model coefficients.

Loss of memory of initial conditions tends to occur $\rightarrow$ self-similar mixing with length scale $\mathrm{gt}^{2}$ :

Bubble penetration $\mathbf{h}_{1}=\alpha \frac{\rho_{1}-\rho_{2}}{\rho_{1}+\rho_{2}} \mathbf{g t}^{2}$

$\alpha \sim 0.05$ to 0.06

(AWRE Foulness, LLNL (LEM), Chelyabinsk 70)

TURMOIL3D calculations with short-wavelength initial perturbations (growth purely by mode coupling) give $\alpha \sim 0.03$, less than observed.

Need to add long wavelength initial perturbations:-

$$
\sigma^{2}=\int_{0}^{\infty} \mathbf{P}(\mathbf{k}) \mathbf{d k}
$$

where $\left\{\int_{2 \pi / \lambda}^{\infty} \mathbf{P}(\mathbf{k}) \mathbf{d k}\right\}^{\frac{1}{2}}=\varepsilon \lambda$

$\varepsilon=0.0005$ gives self-similar growth with $\alpha \sim 0.05$. It is assumed that this corresponds to a typical experimental situation. 
Model coefficients are chosen to fit the key quantities for RT mixing

$\alpha$ : growth rate coefficient

D . turbulence KE dissipated

$\bar{P}:$ Loss of potential energy

$\theta$ : molecular mixing fraction

$$
\frac{\int \overline{\mathbf{f}_{1} \mathbf{f}_{2}} \mathbf{d z}}{\int \overline{\mathbf{f}}_{1} \cdot \overline{\mathbf{f}}_{2} \mathbf{d z}}
$$

Values used are based on TURMOIL3D calculations ( $800 \times 400 \times 400$ zones, $\varepsilon=0.0005)$ : -
$\alpha \quad=\quad 0.05$
$\mathrm{D} / \mathrm{P}=0.4$ (no experimental data)
$\theta \quad=\quad 0.7$ (some experimental confirmation)

Still leaves one key degree of freedom

$\Delta=\frac{\Delta \mathbf{u}_{\mathrm{D}}}{\Delta \mathbf{u}_{\mathrm{D}}+\Delta \mathbf{u}_{\mathrm{p}}}$

$\Delta u_{D}=$ mixing velocity due to turbulent diffusion

$\Delta \mathbf{u}_{\mathrm{p}}=$ mixing velocity induced by pressure gradient

Results shown here for $\Delta=\mathbf{0 . 4}$ 
TYPICAL 2D TURBULENCE MODEL APPLICATION

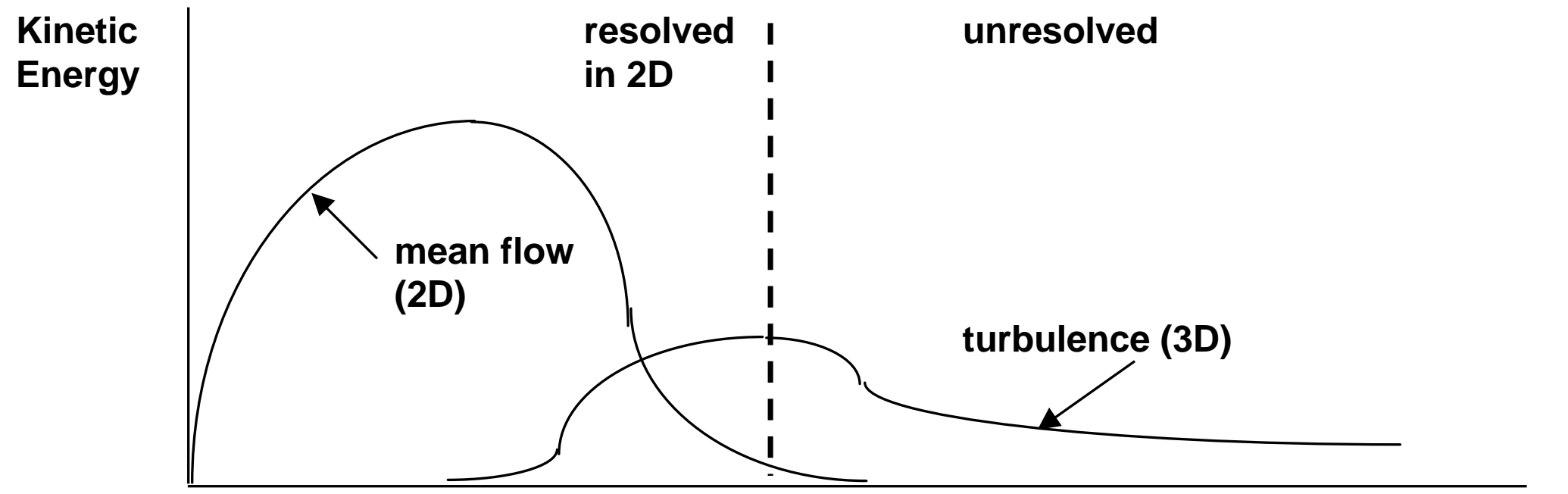

Mean flow (2D) : calculated

wave number

Turbulence (3D) : modelled

Points of concern

(a) Likely to be some overlap between the mean flow scales and the turbulence scales is double counting an issue?

(b) Some of the turbulence scales are resolved (but only in 2D). Does this matter?

(c) Does the turbulence model give the correct spatial distribution in a complex 2D situation. 

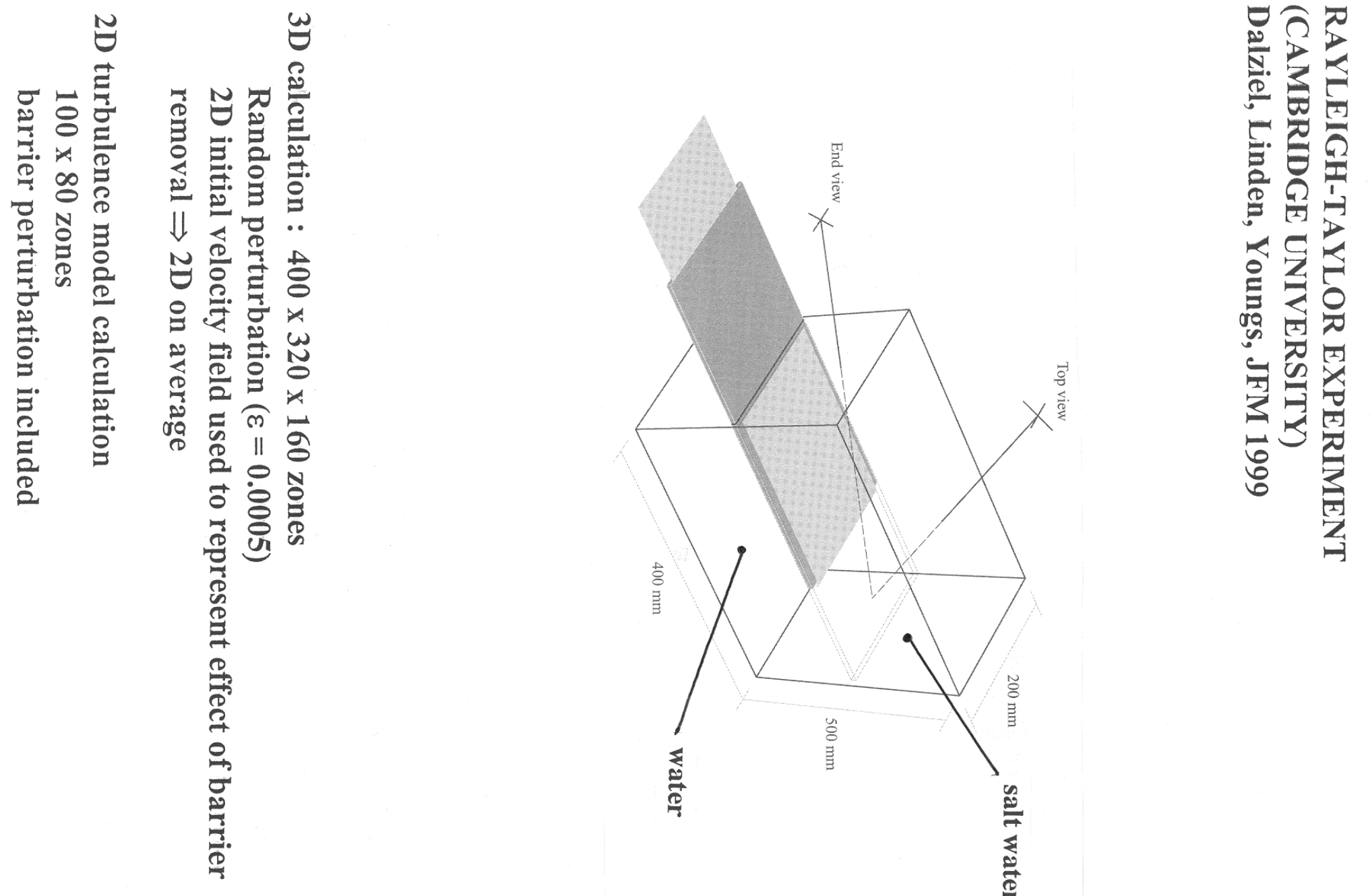


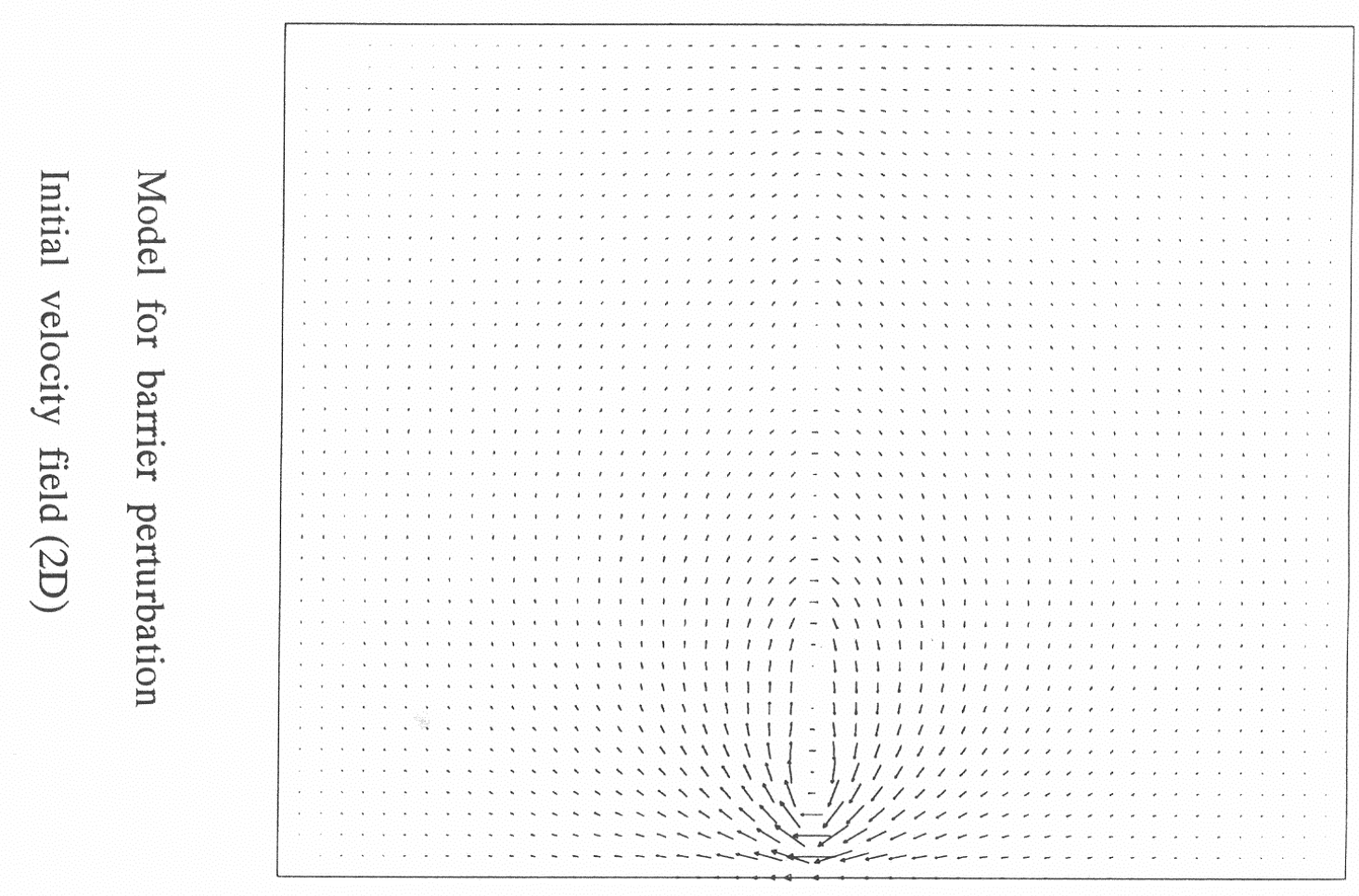



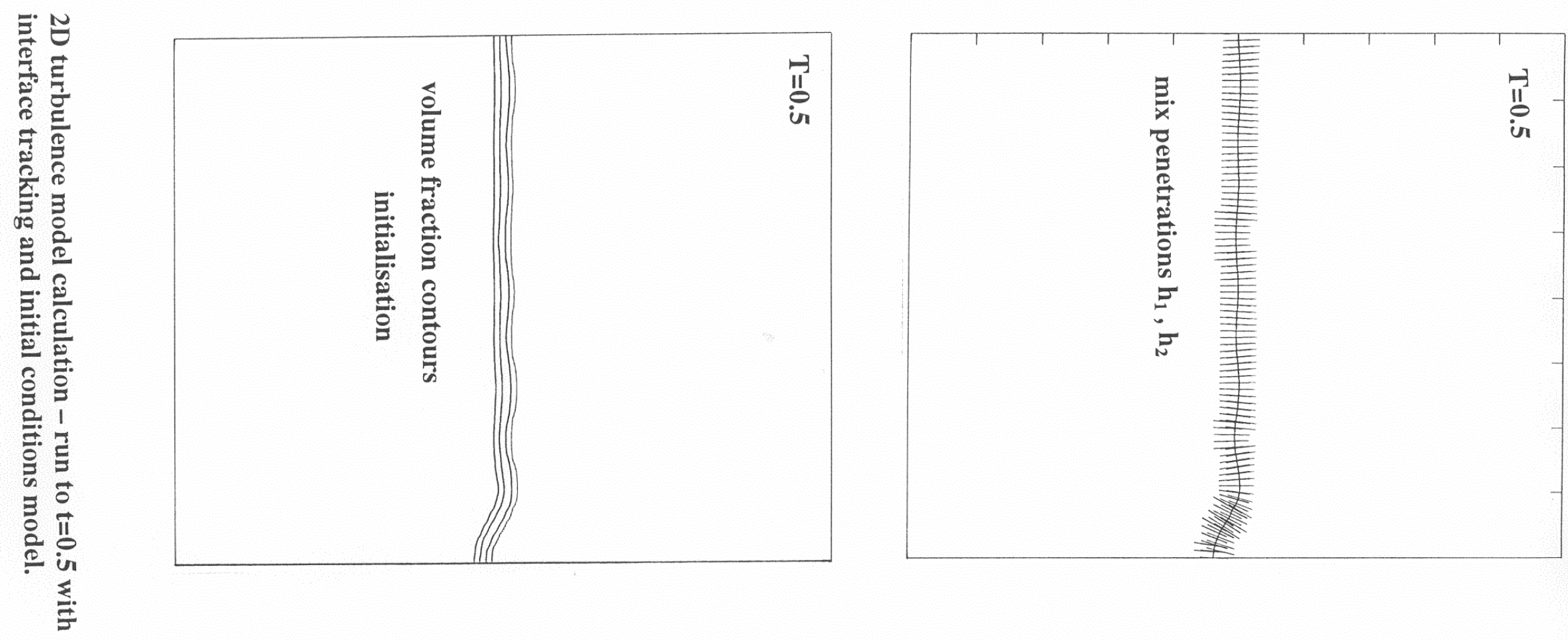

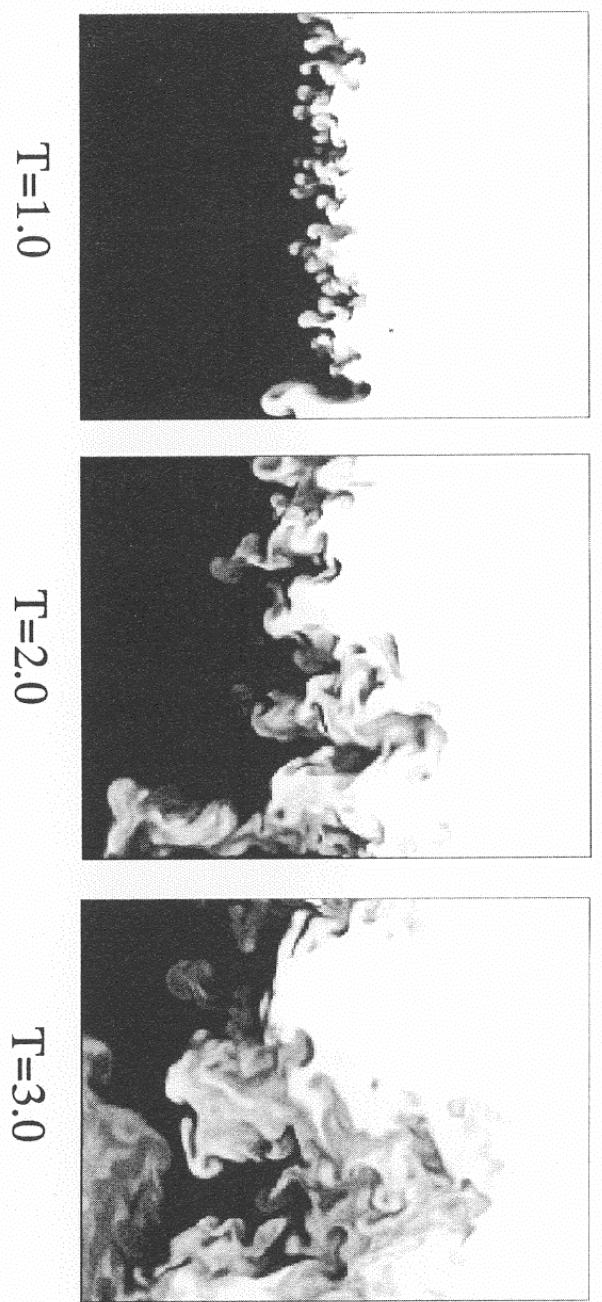

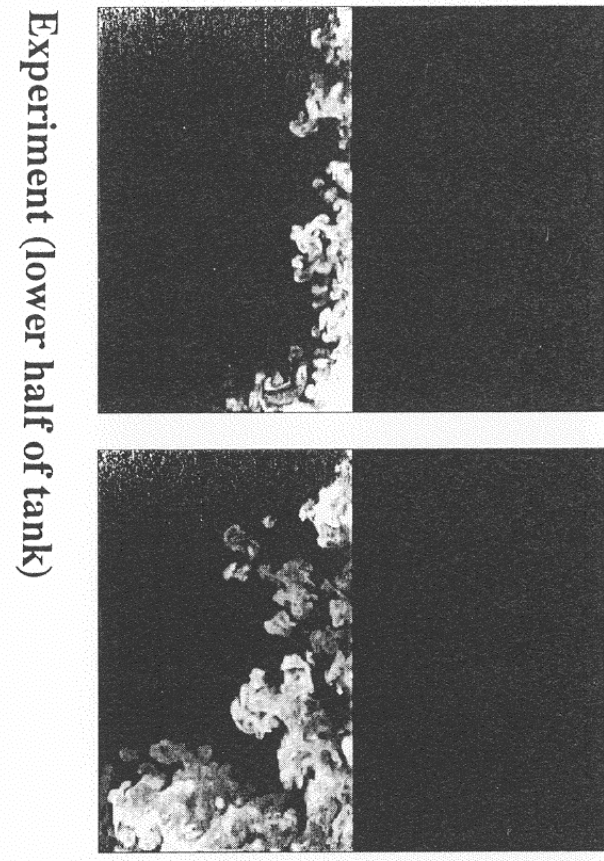

इ

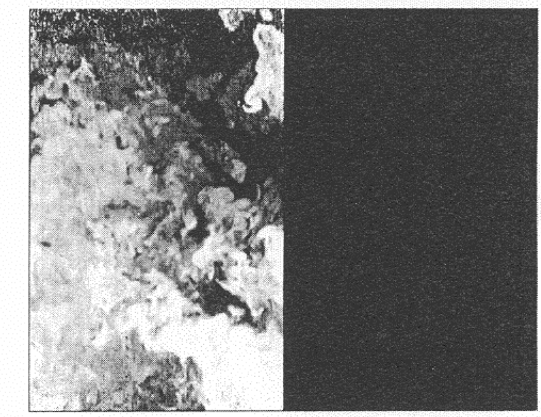




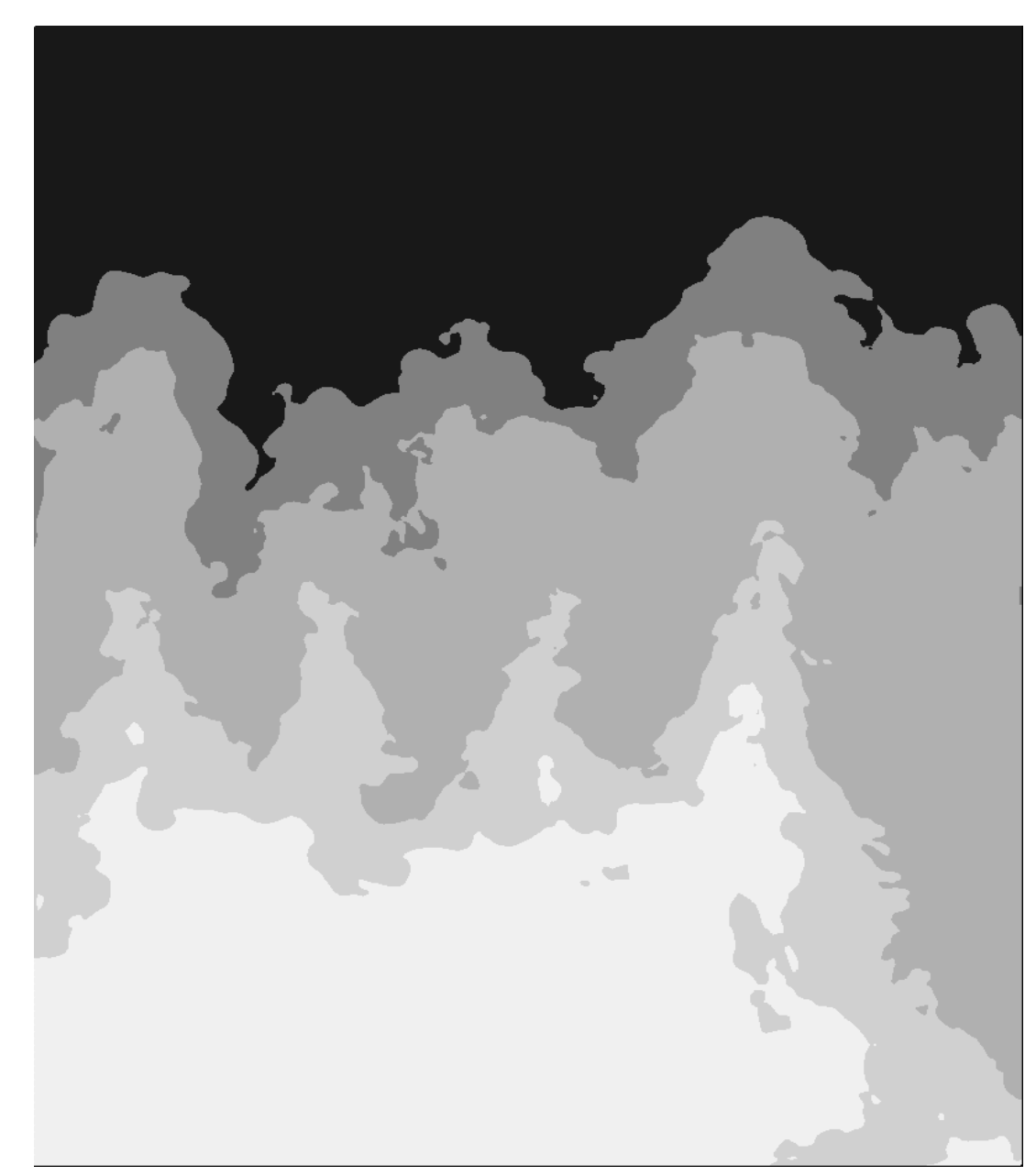

$3 \mathrm{D}$ simulation at $\mathrm{t}=2.0$

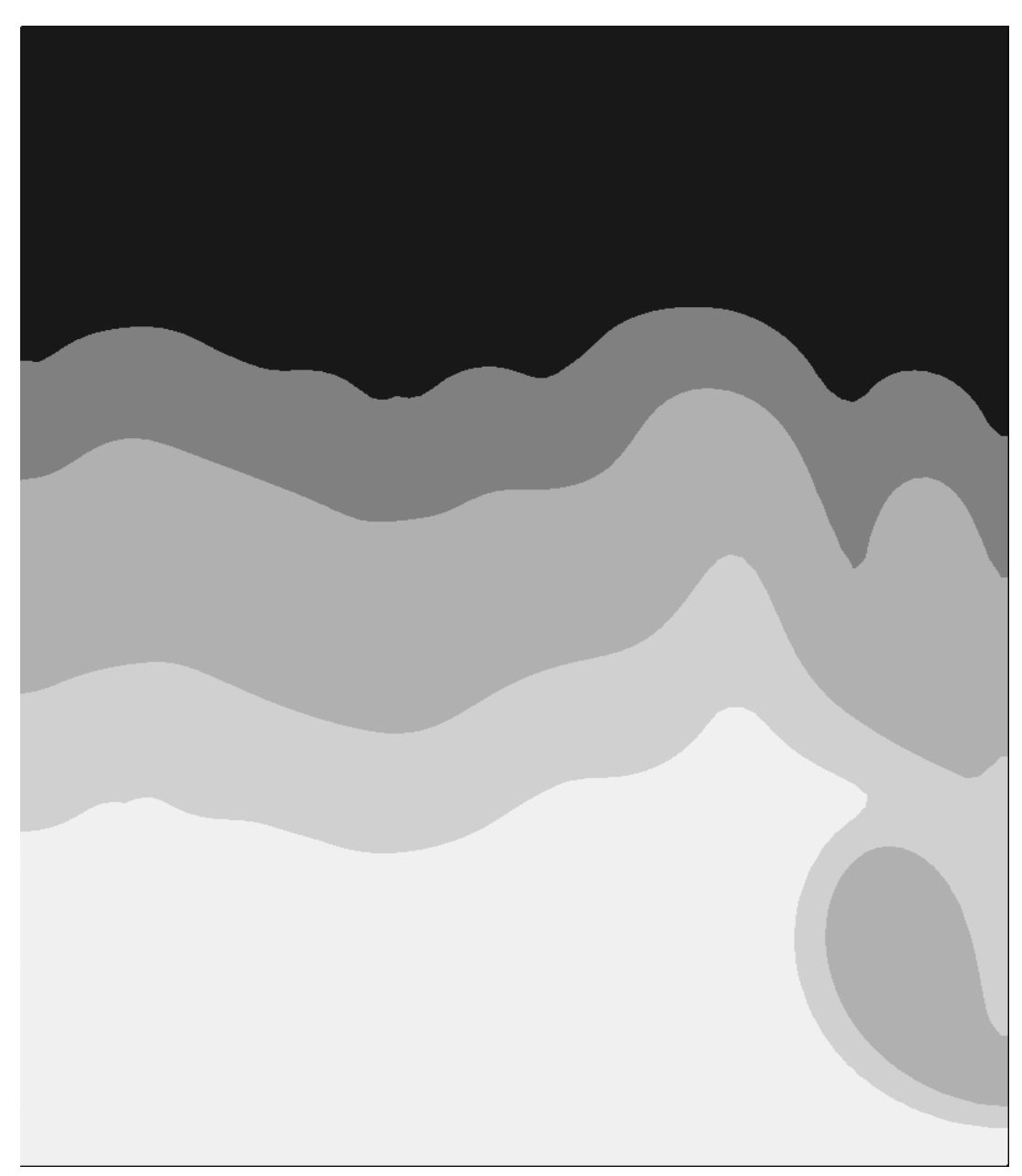

2D turbulence model at $\mathrm{t}=2.0$

Mean volume fraction contours: $0.05,0.25,0.75,0.95$ 

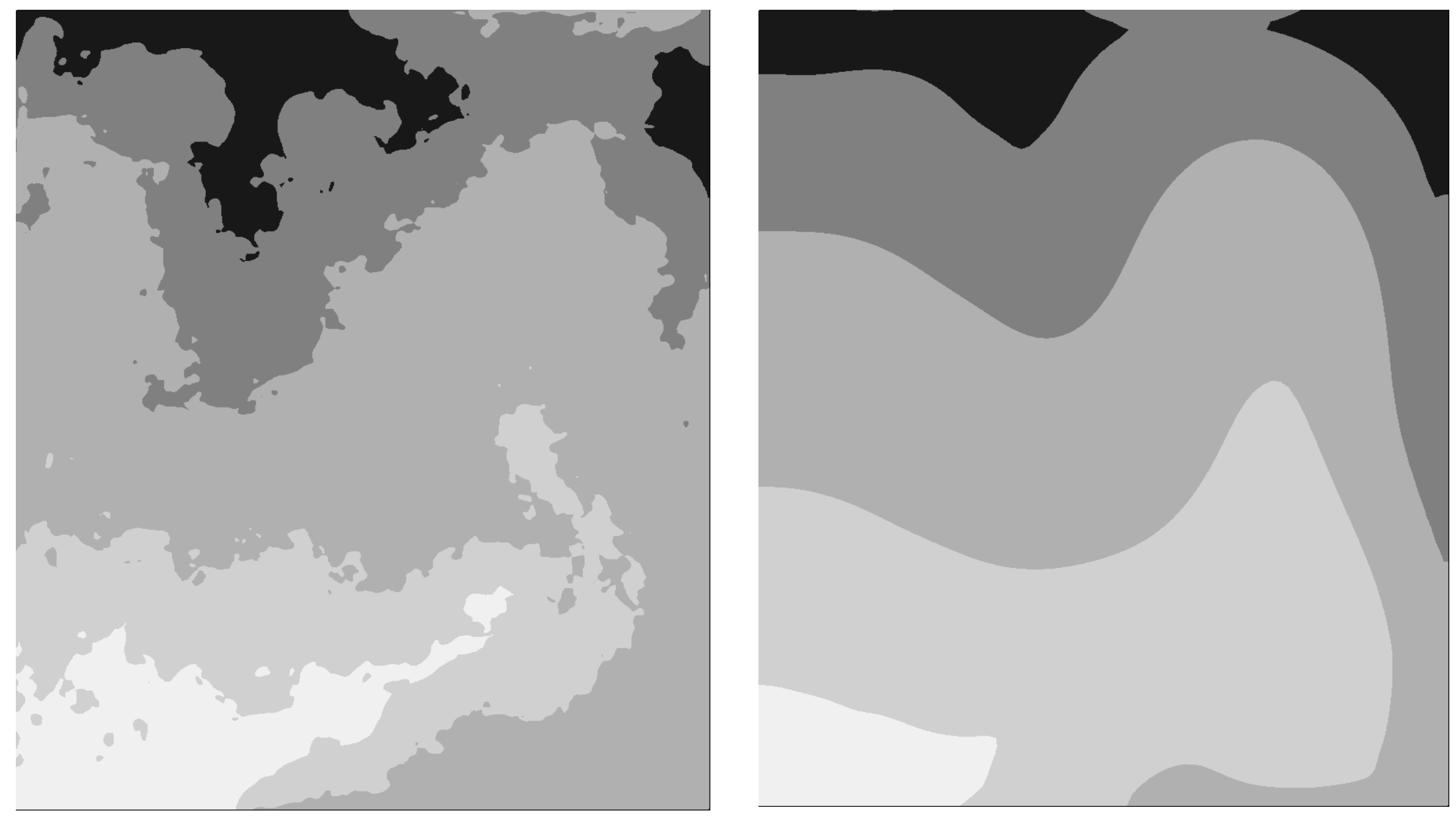

$3 \mathrm{D}$ simulation at $\mathrm{t}=3.0$

$2 \mathrm{D}$ simulation at $\mathrm{t}=3.0$ 
SHOCK TUBE EXPERIMENT (AWE)

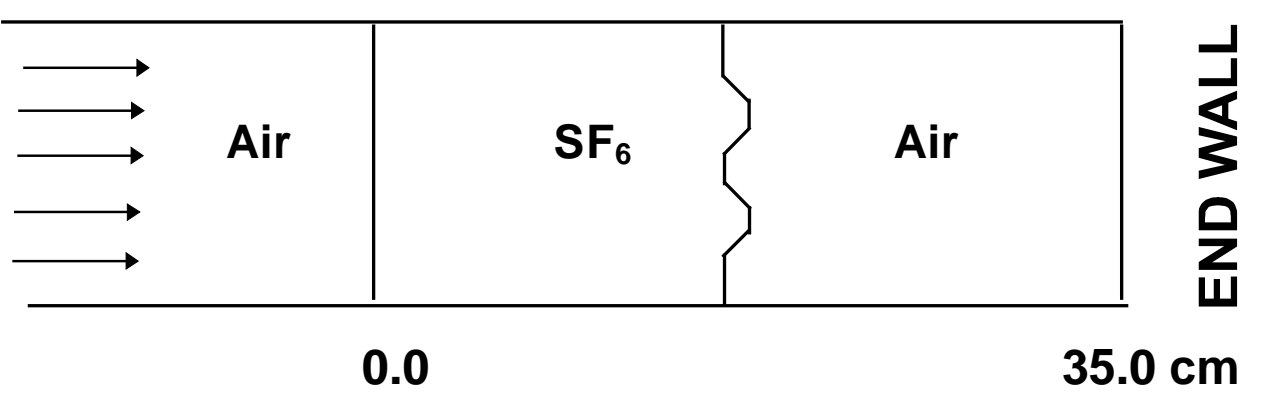

Cross-section $20 \times 10 \mathrm{~cm}$

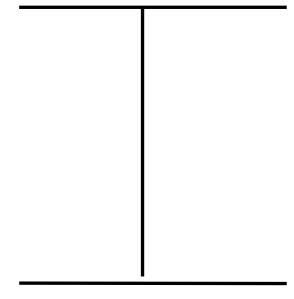

(a) flat

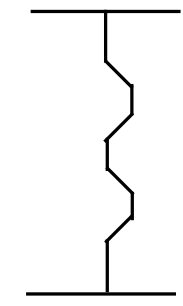

(b) double bump

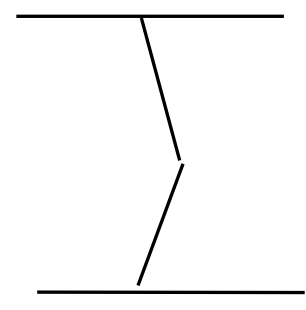

(c) chevron

Moving mesh option used (semi-Lagrangian calculations)

3D LES:

$400 \times 320 \times 160$ zones

random interface perturbations (models effect of membrane rupture)

wavelengths

0.5 to $5 \mathrm{~cm}$

s.d

$0.01 \mathrm{~cm}$

2D turbulence model calculation

$200 \times 160$ zones

initial conditions model

$\mathrm{a}_{\mathrm{o}}=0.02 \mathrm{~cm}, \lambda_{0}=0.5 \mathrm{~cm}$ 

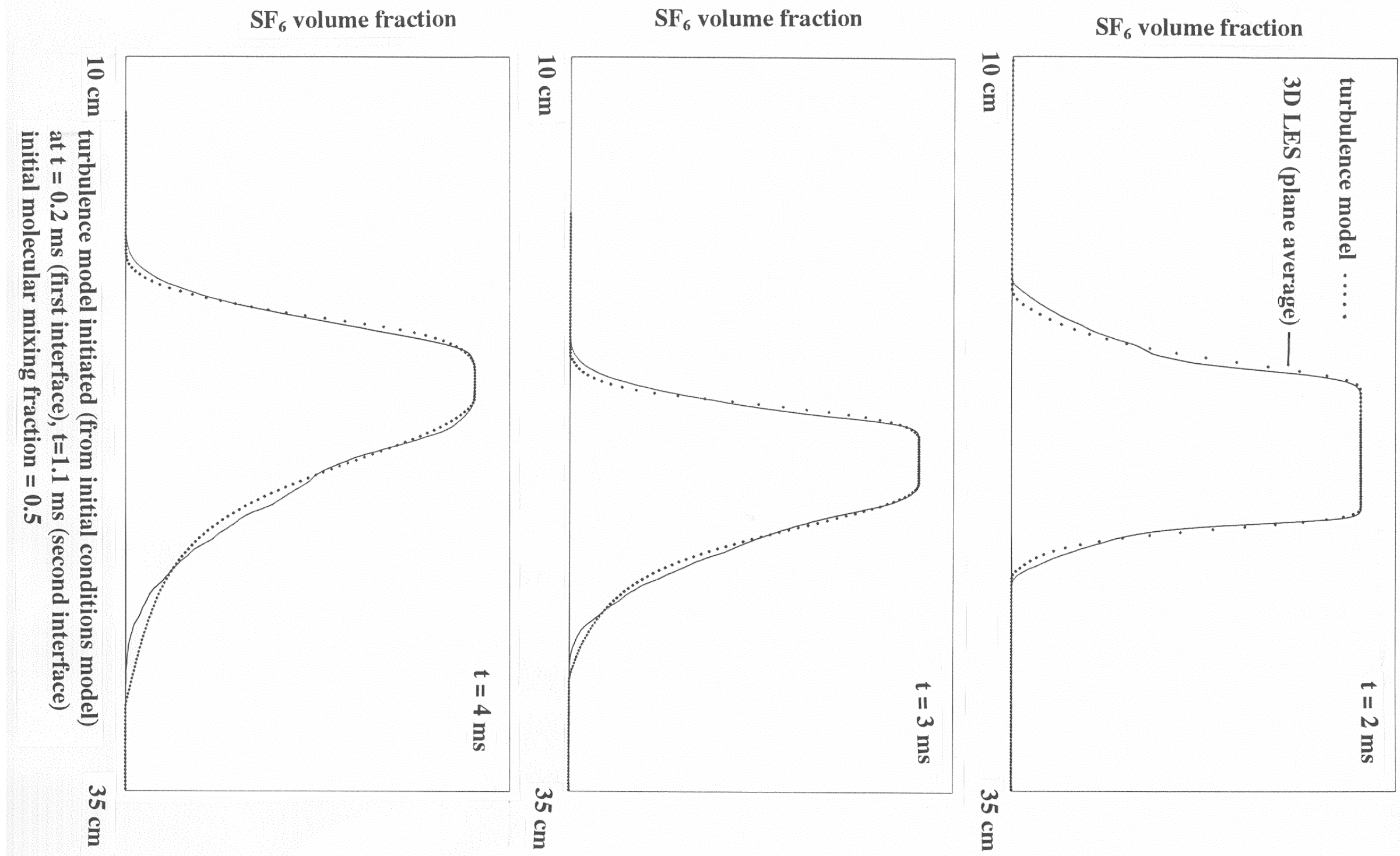

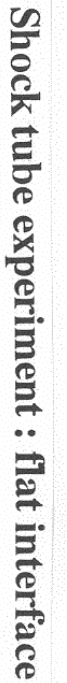




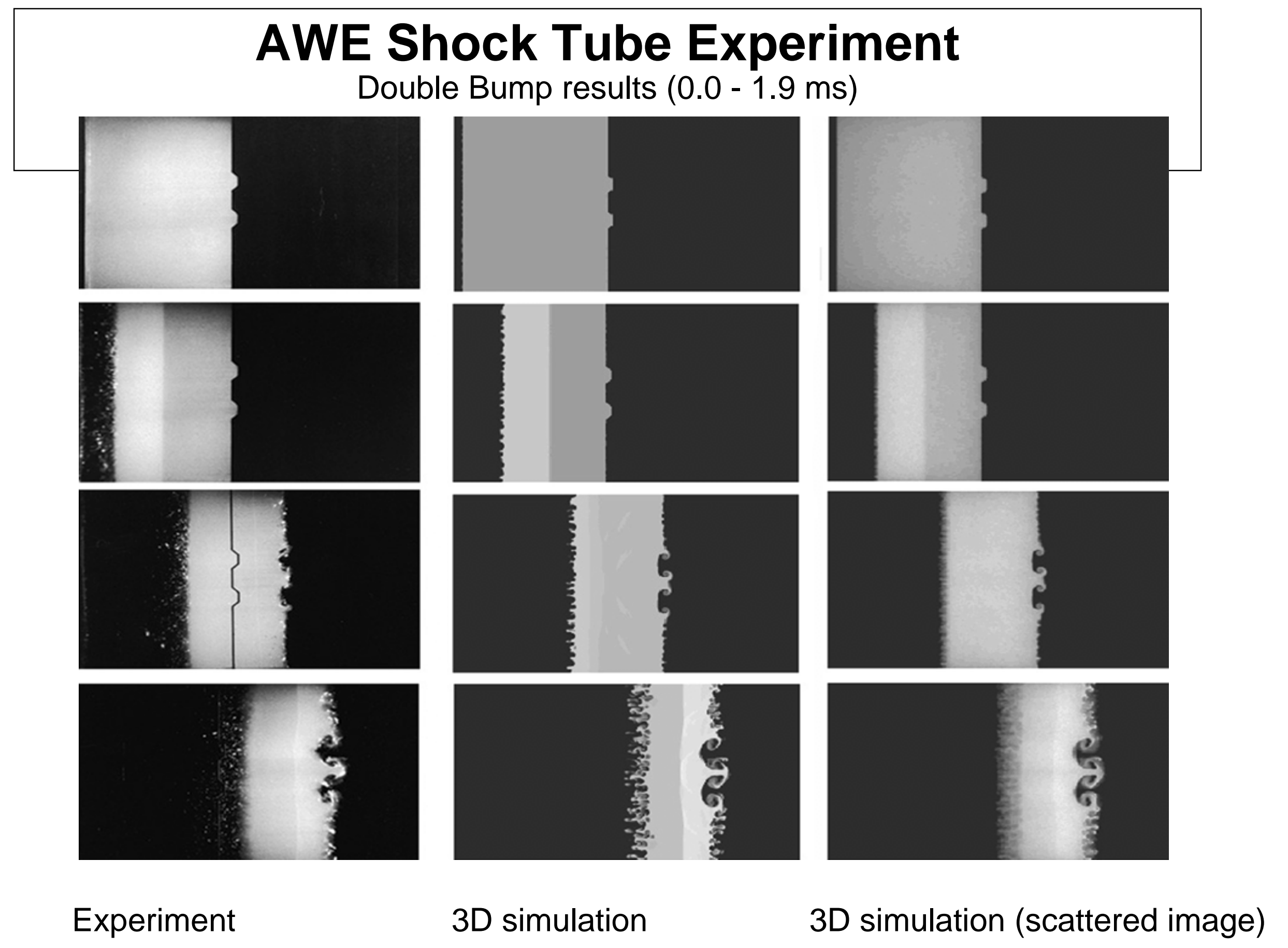




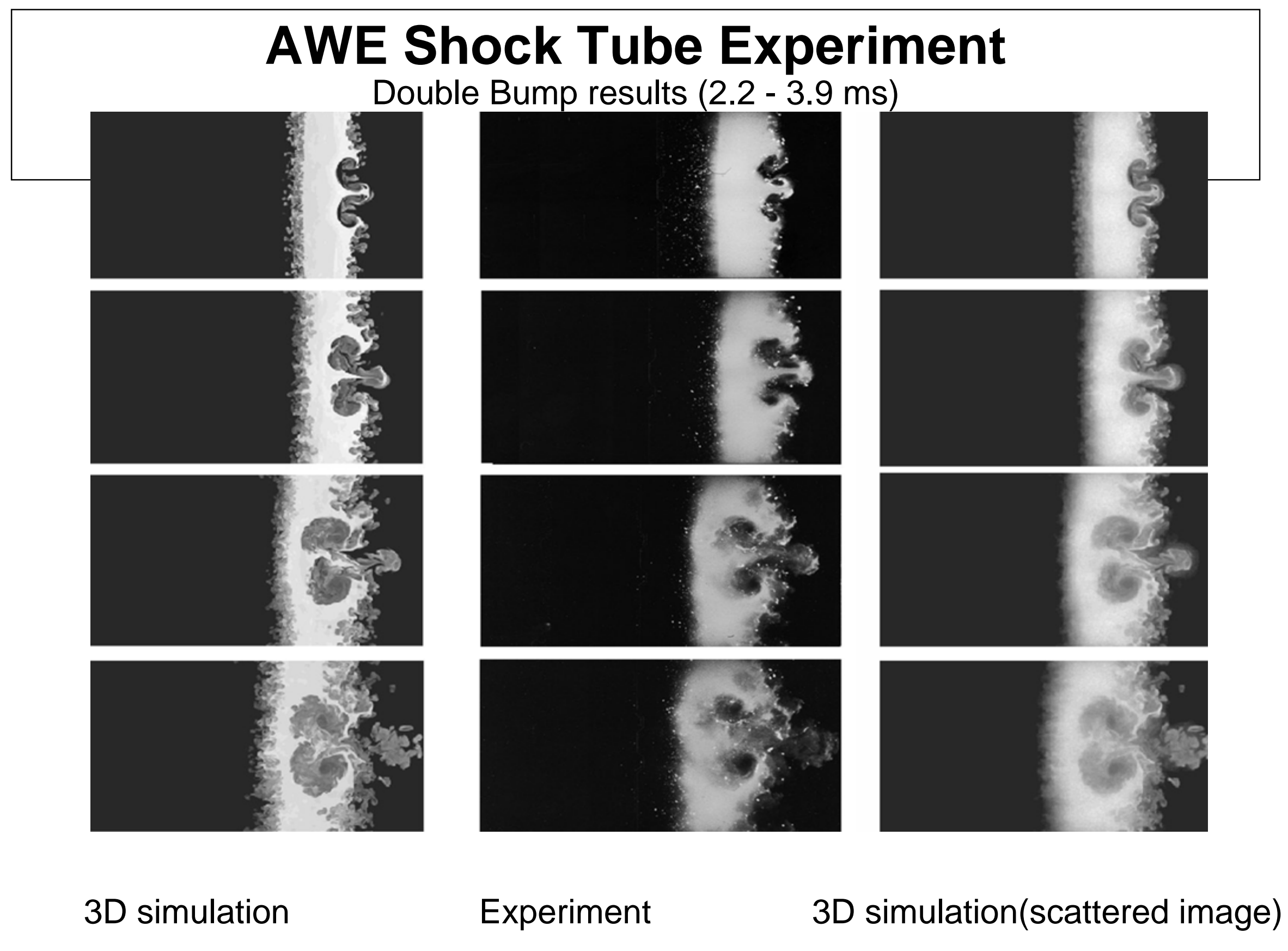



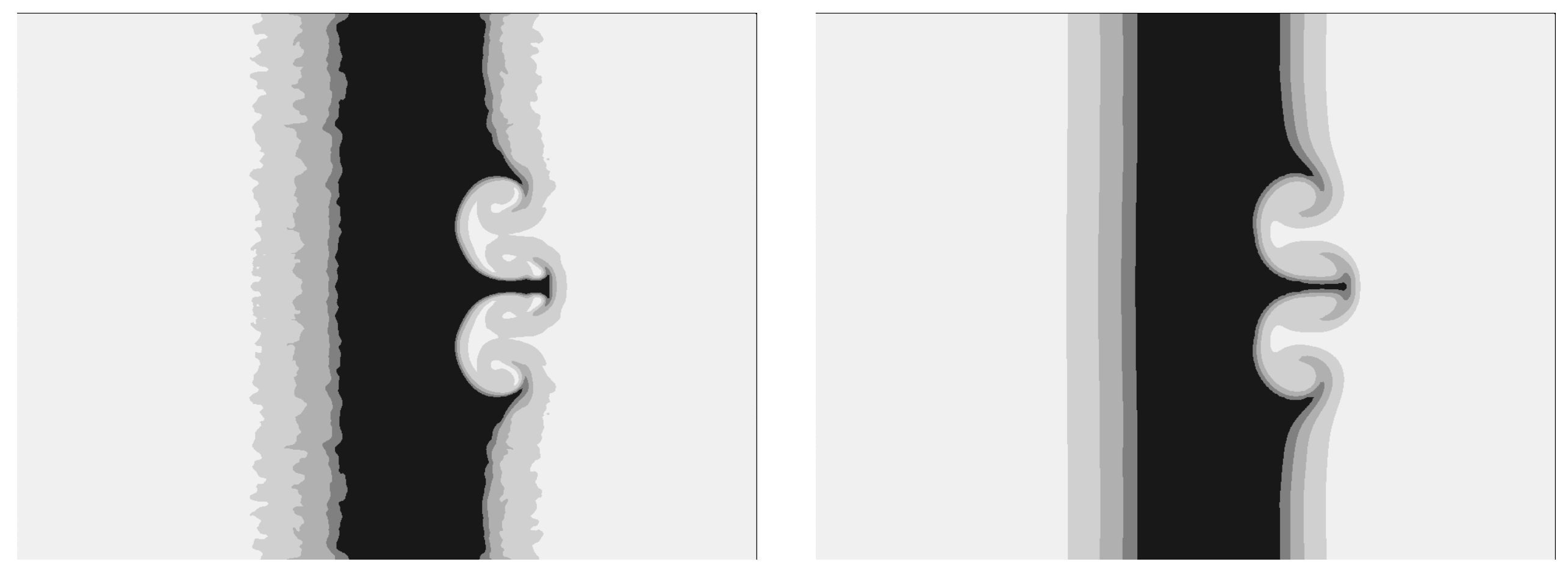

$3 \mathrm{D}$ simulation at $\mathrm{t}=2.0 \mathrm{~ms}$

2D turbulence model at $\mathrm{t}=2.0 \mathrm{~ms}$

Double bump experiment mean volume fraction contours: $0.05,0.3,0.7,0.95$ 

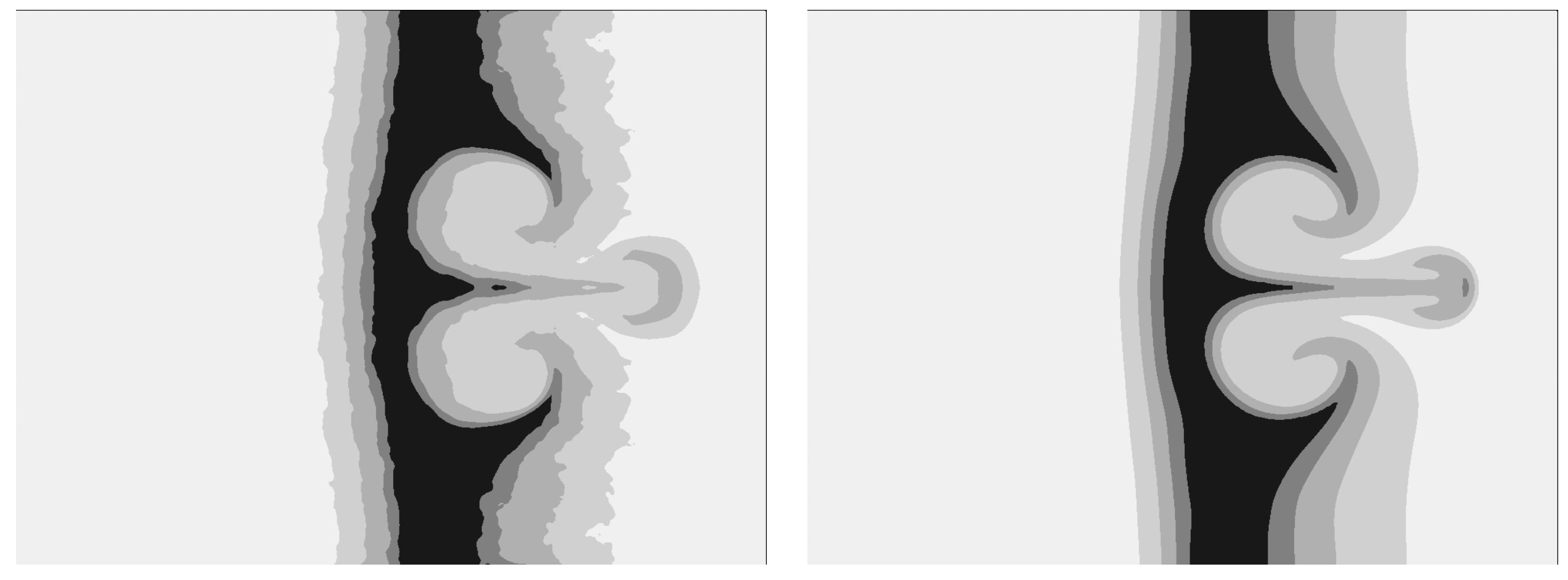

$3 \mathrm{D}$ simulation at $\mathrm{t}=3.0 \mathrm{~ms}$

2D turbulence model at $\mathrm{t}=3.0 \mathrm{~ms}$ 

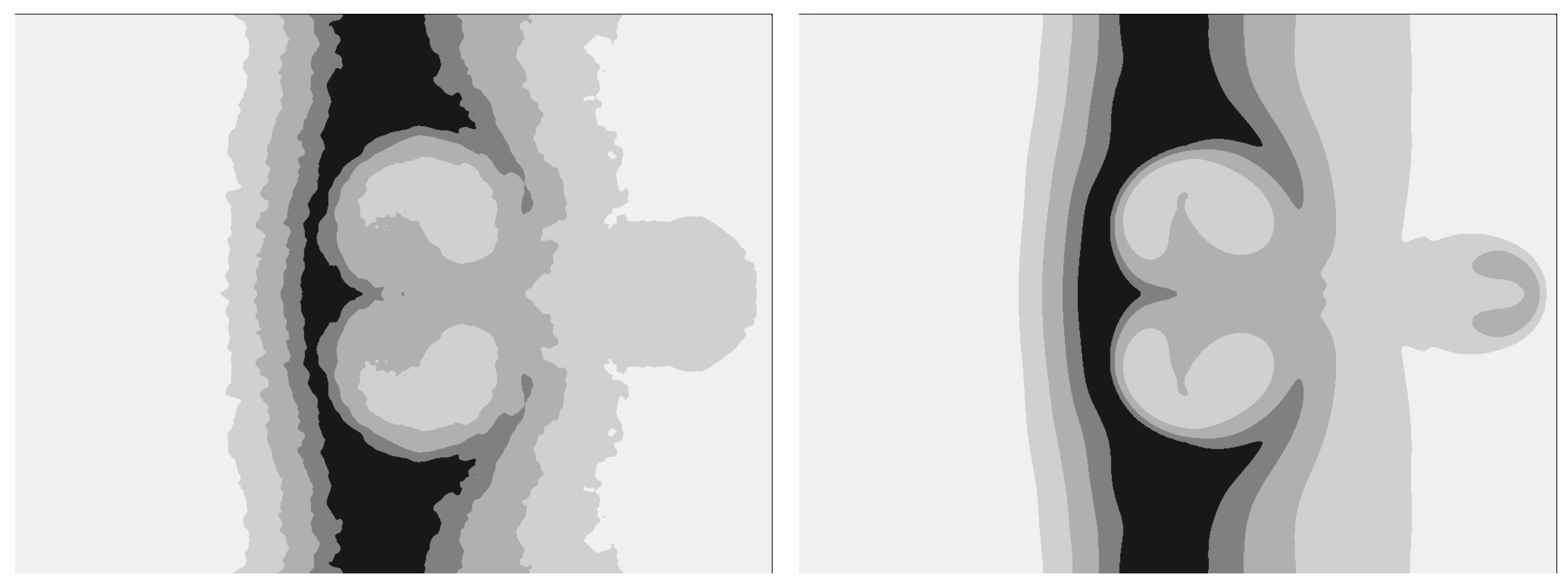

$3 \mathrm{D}$ simulation at $\mathrm{t}=4.0 \mathrm{~ms}$

$2 \mathrm{D}$ turbulence model at $\mathrm{t}=4.0 \mathrm{~ms}$ 


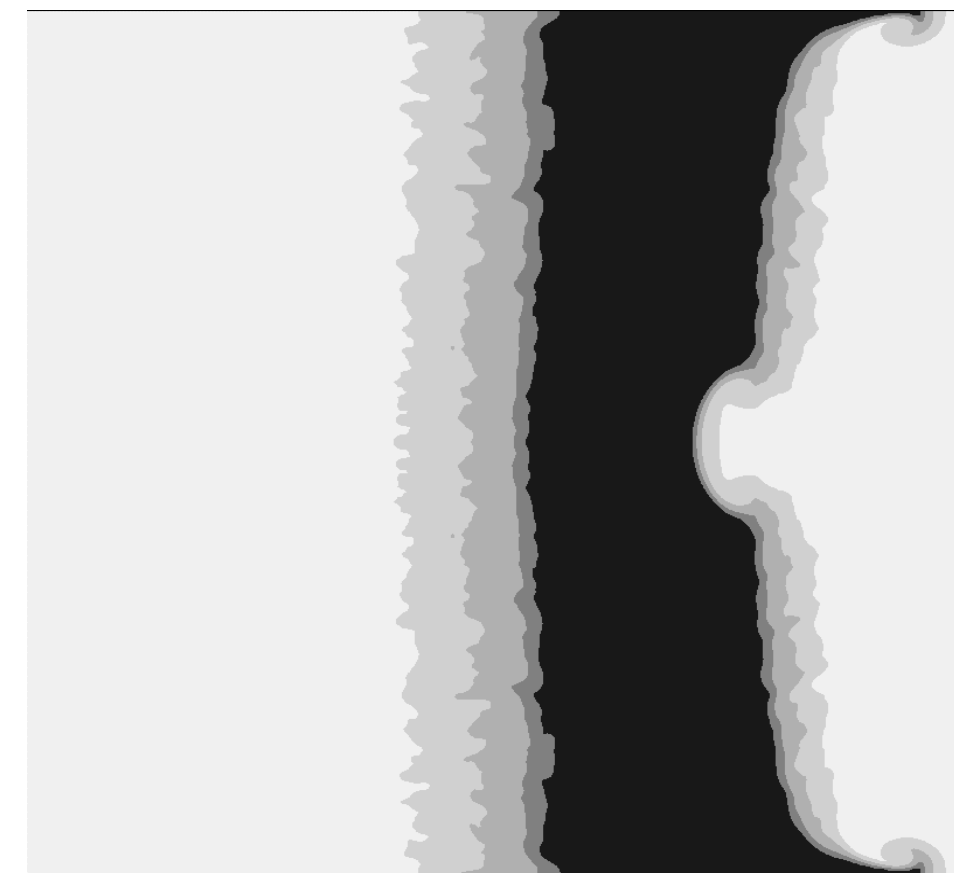

$3 \mathrm{D}$ simulation at $\mathrm{t}=2.0 \mathrm{~ms}$
2D turbulence model at $\mathrm{t}=2.0 \mathrm{~ms}$

mean volume fraction contours: $0.05,0.3,0.7,0.95$

Chevron experiment 

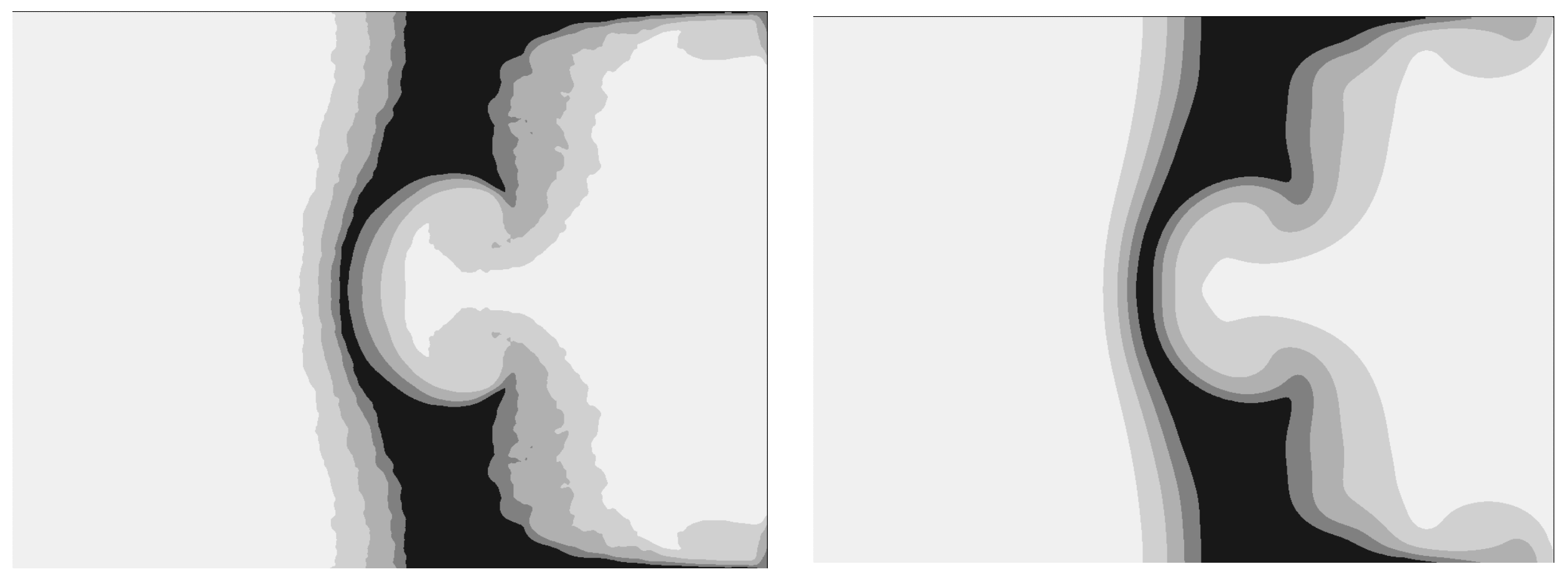

$3 \mathrm{D}$ simulation at $\mathrm{t}=3.0 \mathrm{~ms}$

2D turbulence model at $\mathrm{t}=3.0 \mathrm{~ms}$ 

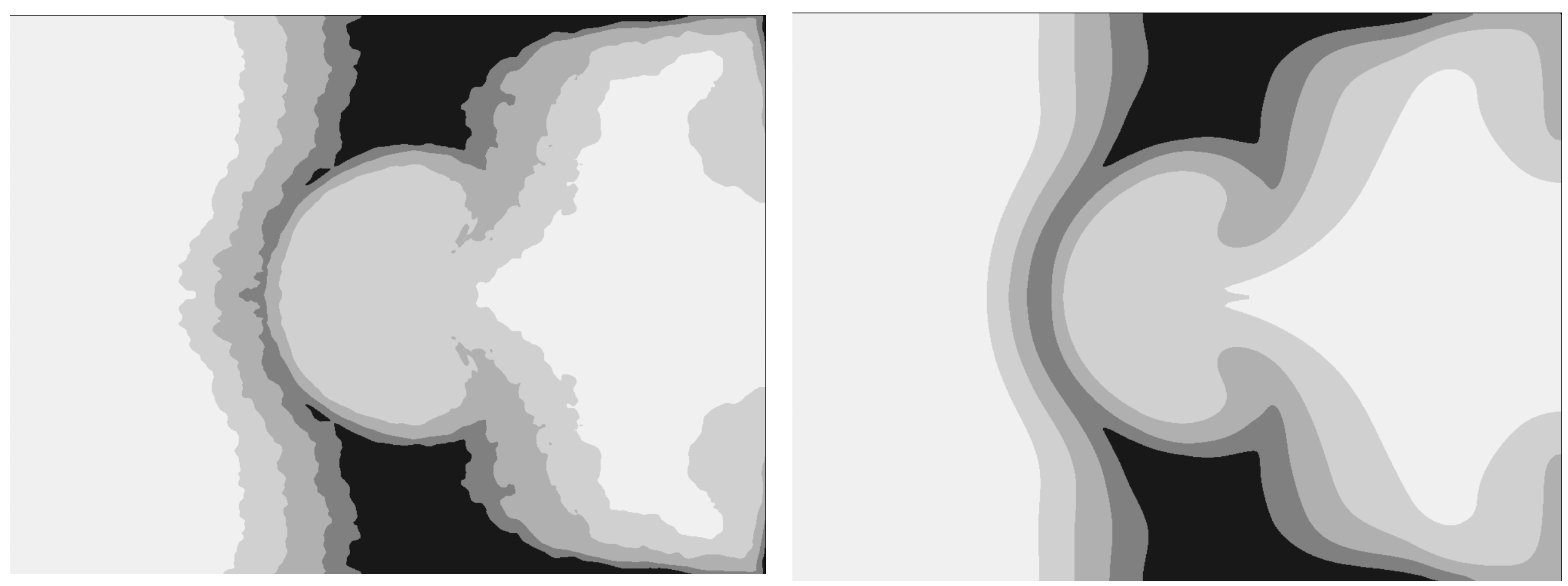

$3 \mathrm{D}$ simulation at $\mathrm{t}=4.0 \mathrm{~ms}$

2D turbulence model at $\mathrm{t}=4.0 \mathrm{~ms}$ 


\section{FINAL REMARKS}

- The 2D turbulence model based on the equations of multiphase flow, using a single set of model coefficients has given satisfactory results for

RT self-similar mixing

A 2D RT experiment

The double-bump shock tube experiment

The chevron shock tube experiment

- 3D LES for simplified problems, in conjunction with experimental data, is making a very valuable contribution to the validation of the turbulence model.

- In the near future (after AWE's next supercomputer procurement) a more detailed comparison, (including 2D distributions of $k$ and $\theta$ ) will be made between the 2D turbulence model results and higher resolution LES. 


\title{
PRELIMINARY RESULTS OF \\ LES SIMULATIONS OF SELF-SIMILAR VARIABLE ACCELERATION RT MIXING FLOWS
}

\author{
D L YOUNGS \\ AWE \\ ALDERMASTON, UK
}

\author{
A LLOR \\ CEA \\ BRUYĖRES-LE-CHÂTEL, FRANCE
}


The importance of self-similar variable acceleration RT flows (SSVARTs) for the design and calibration of turbulent mixing models is shown in the presentation by Antoine Llor at this workshop.

Because experimental results on SSVARTs are not, and will probably not be available in the near future, we are currently investigating such flows by means of LES.

In these incompressible flows the acceleration has the form

$$
\mathbf{g}=\mathbf{k t}^{\mathbf{n}}
$$

and for self-similar mixing the mixing zone width grows in proportion to $\mathrm{t}^{\mathrm{n}+2}$.

Preliminary results, using low-resolution LES are given for $\mathbf{n}=\mathbf{0 , 1}$ and -1 , using the TURMOIL3D code.

Results are compared with simple theoretical models. 


\section{THE TEST PROBLEM}

This is based on the test problem proposed by Guy Dimonte (see presentation at this workshop).

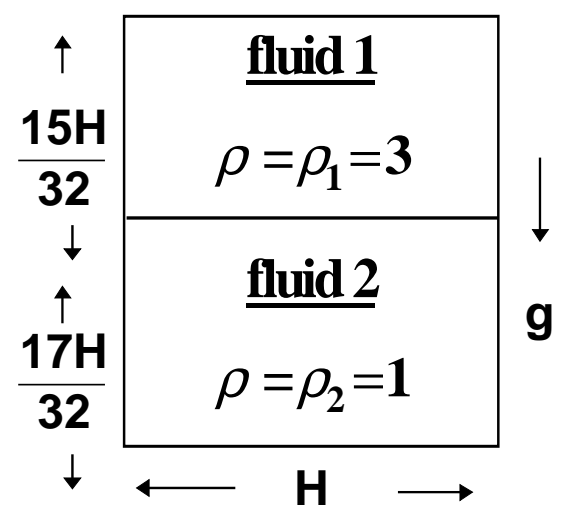

Computational domain

$: \quad \mathrm{H} \times \mathrm{H} \times 2 \mathrm{H}$

Zoning

$\quad 128 \times 128 \times 256$

Dimension

: $\quad H=1$

Acceleration

$\begin{array}{lll}: & \text { (a) } & g=2 \\ \text { (b) } & g=t \\ \text { (c) } & g=9 /(4 t)\end{array}$

Run to $\mathrm{t}=4.5$, when for each case

$$
\left(\int \sqrt{\mathrm{g}} \mathrm{dt}\right)^{2}=40.5
$$

Compressible calculation with Mach number $<0.2$ 
The major calculational problem is the treatment of variable $\mathrm{g}$ within a compressible calculation.

For incompressible flow, the pressure distribution adjusts at each instant of time to maintain div $\underline{\mathbf{u}}=0$. If no mixing occurs this implies hydrostatic equilibrium.

$$
\frac{\partial \mathbf{p}}{\partial \mathbf{z}}=\rho \mathbf{g}
$$

In the compressible simulations an appropriate pressure gradient is maintained by adding an internal energy source.

Initially adiabatic hydrostatic equilibrium (uniform entropy/neutral stability within each fluid) is assumed:-

$$
\begin{aligned}
& \frac{\partial \mathbf{p}_{0}}{\partial \mathbf{z}}=\rho_{0} \mathbf{g}_{0} \\
& \mathbf{p}_{0}=\mathbf{k} \rho_{0} \gamma \quad, \quad \text { in each region } \\
& \rho_{0}= \begin{cases}3 & \text { just above the interface } \\
1 & \text { just below the interface }\end{cases} \\
& \gamma=\frac{5}{3} \text { for both fluids }
\end{aligned}
$$


If $g_{n+\frac{1}{2}}$ is the value of $\mathbf{g}$ for the $\mathrm{n}$-th time step, at the start of the time step

the internal energy is scaled:-

$$
\varepsilon^{\prime}=\varepsilon \cdot \frac{\mathbf{g}_{\mathrm{n}+\frac{1}{2}}}{\mathbf{g}_{\mathrm{n}-\frac{1}{2}}}
$$

This maintains hydrostatic equilibrium outside the mixing zone and uniform entropy within each fluid.

The acceleration history needs to be modified slightly to give finite nonzero $\mathrm{g}$ at $\mathrm{t}=0$ :-

$$
\begin{array}{lll}
\text { case (b) } & \mathbf{g}= & \max \{0.01, \mathbf{t}\} \\
\text { case }(c) & \mathbf{g}= & \min \{50,9 /(4 \mathrm{t})\}
\end{array}
$$

The initial interface pressure is chosen high enough to ensure that the Mach no. of the flow remains small $(M<0.2)$ at all times and also high enough to give small $(<4 \%)$ variation in the initial density of each region. 


\section{THE INITIAL PERTURBATION}

RT experiments with constant g give bubble penetration

$$
\mathbf{h}_{1}=\alpha \frac{\rho_{1}-\rho_{2}}{\rho_{1}+\rho_{2}} \mathbf{g t}^{2} \quad, \text { with } \alpha \sim 0.05 \text { to } 0.06
$$

TURMOIL3D calculations with short wavelength initial perturbations (growth purely by mode coupling) give $\alpha \sim 0.03$.

Need to assume long wavelength initial perturbations with amplitude $\propto$ wavelength (as proposed by Inogamov [1]) to give self-similar growth with $\alpha \sim 0.05$.

Perturbation used $\xi(\mathbf{x}, \mathbf{y})=\xi_{\mathrm{S}}+\xi_{\mathrm{L}}$

$$
\begin{aligned}
& \xi_{\mathrm{S}} \quad \text { : } \quad \text { wavelengths } \quad 4 \Delta x \text { to } 8 \Delta x \\
& \text { s.d }=0.02 \Delta x \\
& \xi_{\mathrm{L}} \quad \text { : } \quad \text { power spectrum } \quad \mathbf{P}(\mathbf{k}) \\
& \sigma_{\lambda}=\left\{\int_{2 \pi / \lambda}^{\infty} \mathbf{P}(\mathbf{k}) \mathbf{d} \mathbf{k}\right\}^{\frac{1}{2}}=\varepsilon \lambda \\
& \Rightarrow P(k) \infty \mathbf{1} / \mathbf{k}^{3} \\
& \varepsilon=0.0005 \\
& \text { wavelengths in the range } 4 \Delta x \text { to } \frac{H}{2}
\end{aligned}
$$




\section{RESULTS SHOWN}

$f_{1}, f_{2} \quad: \quad$ volume fractions of fluids 1 and 2

$\overline{f_{1}}, \overline{f_{2}} \quad: \quad$ plane averaged values

$W=\int \bar{f}_{1} \bar{f}_{2} d z$ integral mix width

$h_{1} \quad: \quad$ bubble penetration - measured to point where $\overline{f_{1}}=0.99$.

Approximation used here : $h_{1}=3.3 \mathrm{~W}$

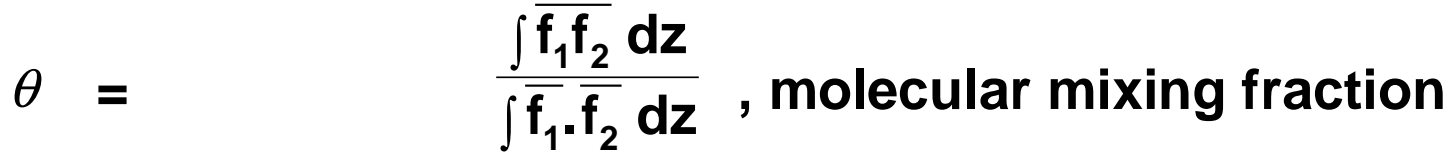

$\frac{D}{P}=\quad$ KE dissipated

$\overline{\mathbf{P}}=\quad$ KE production

$\mathbf{S}=\frac{\rho_{1}-\rho_{2}}{\rho_{1}+\rho_{2}}\left(\int \sqrt{\mathbf{g}} \mathbf{d t}\right)^{2}$

Fig $1 \quad: \quad$ initial long wavelength perturbation

Fig 2 : isosurfaces for the case $g=k$

Fig $3:$ : profiles of $\bar{f}_{1}$

Fig $4: \quad$ plots of $h_{1}$

Fig 5 : $\quad$ plots of $\theta$

Fig $6 \quad$ : $\quad$ plots of D/P 
Figure 1
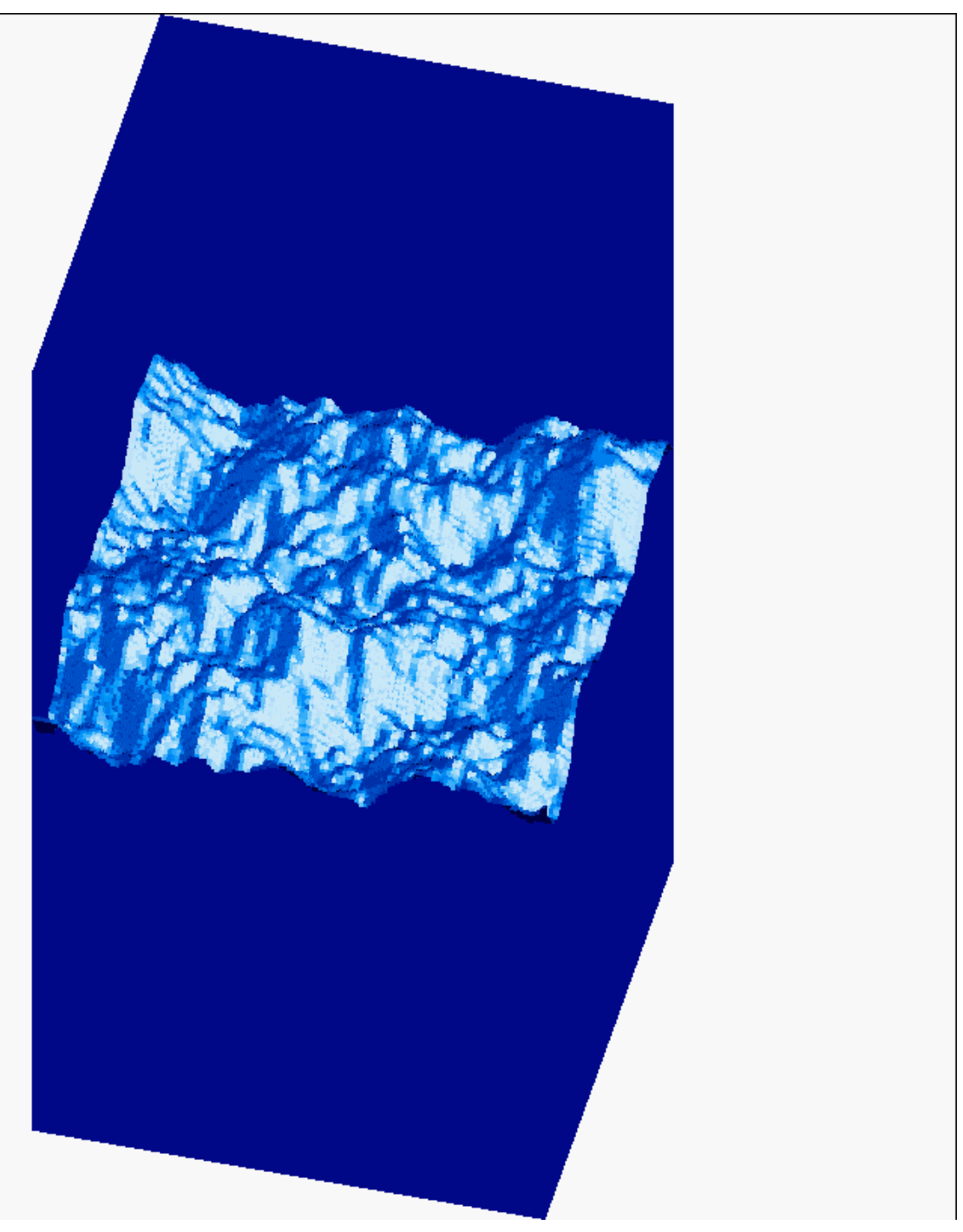

$\varepsilon=0.005$, initial perturbation $\times 200$ 
Figure2: Isosurfaces $\left(f_{1}=0.99\right)$ for $g=k$
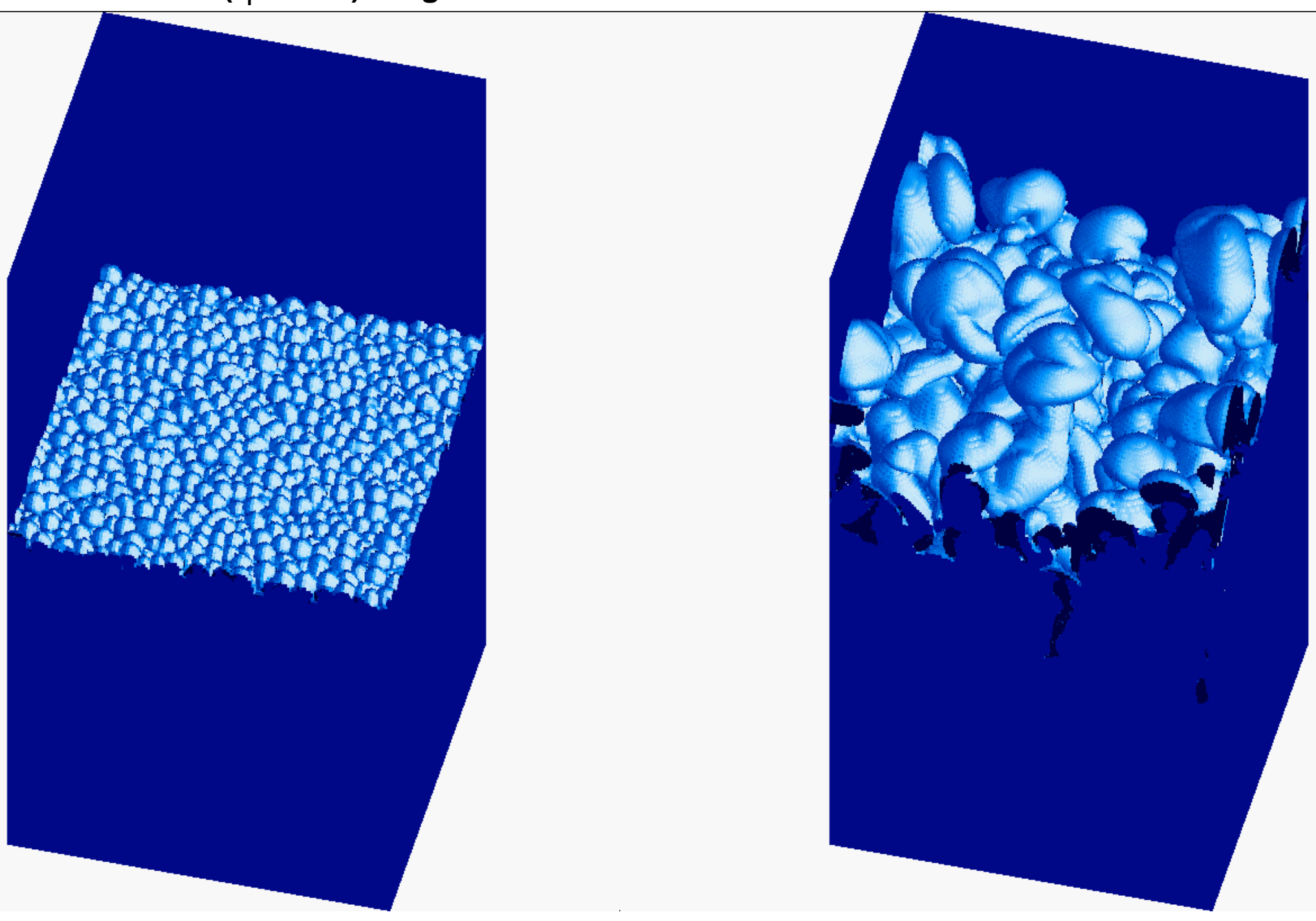

$$
t=0.9
$$$$
\mathrm{t}=\mathbf{3 . 5}
$$ 


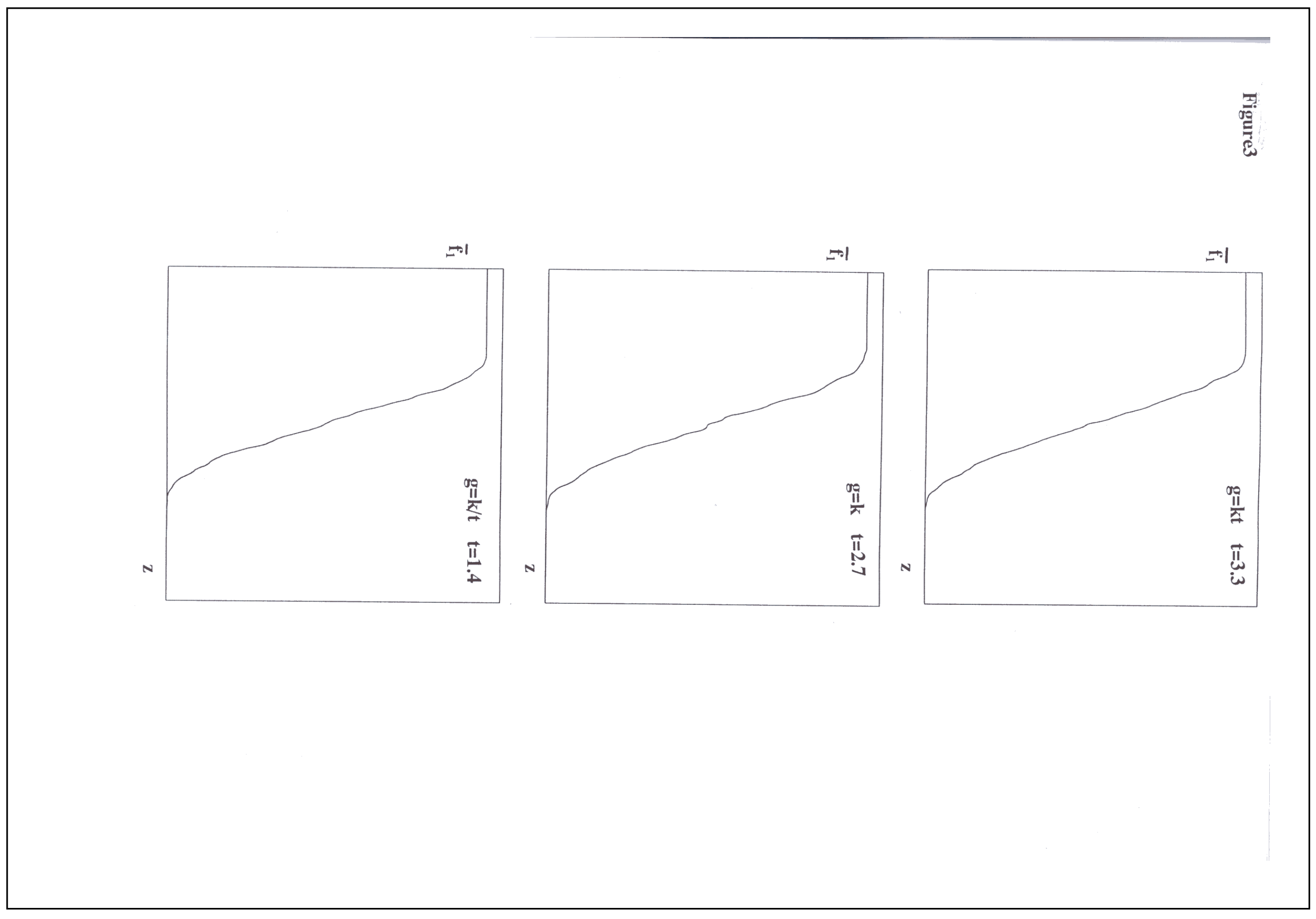




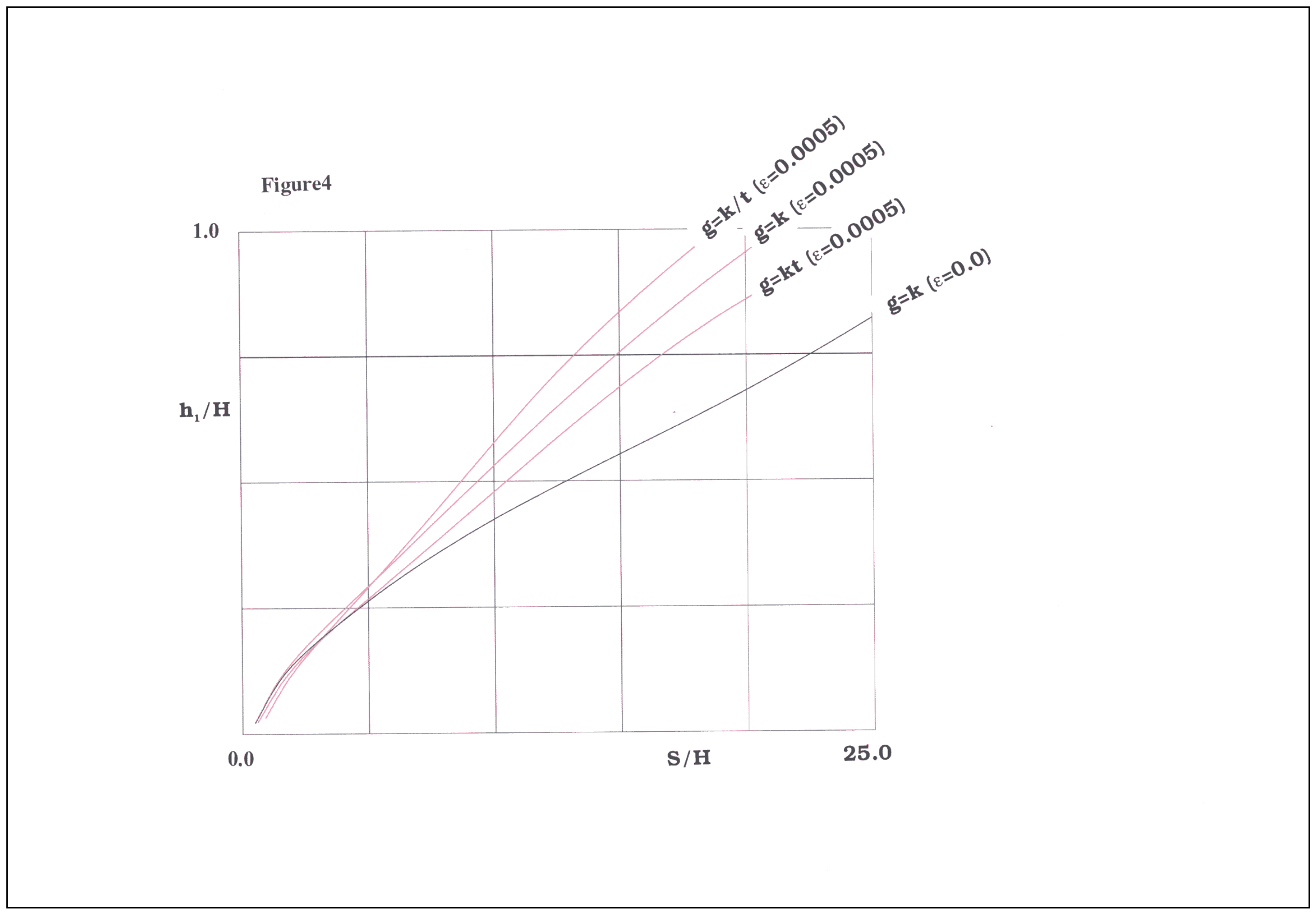


Figure5

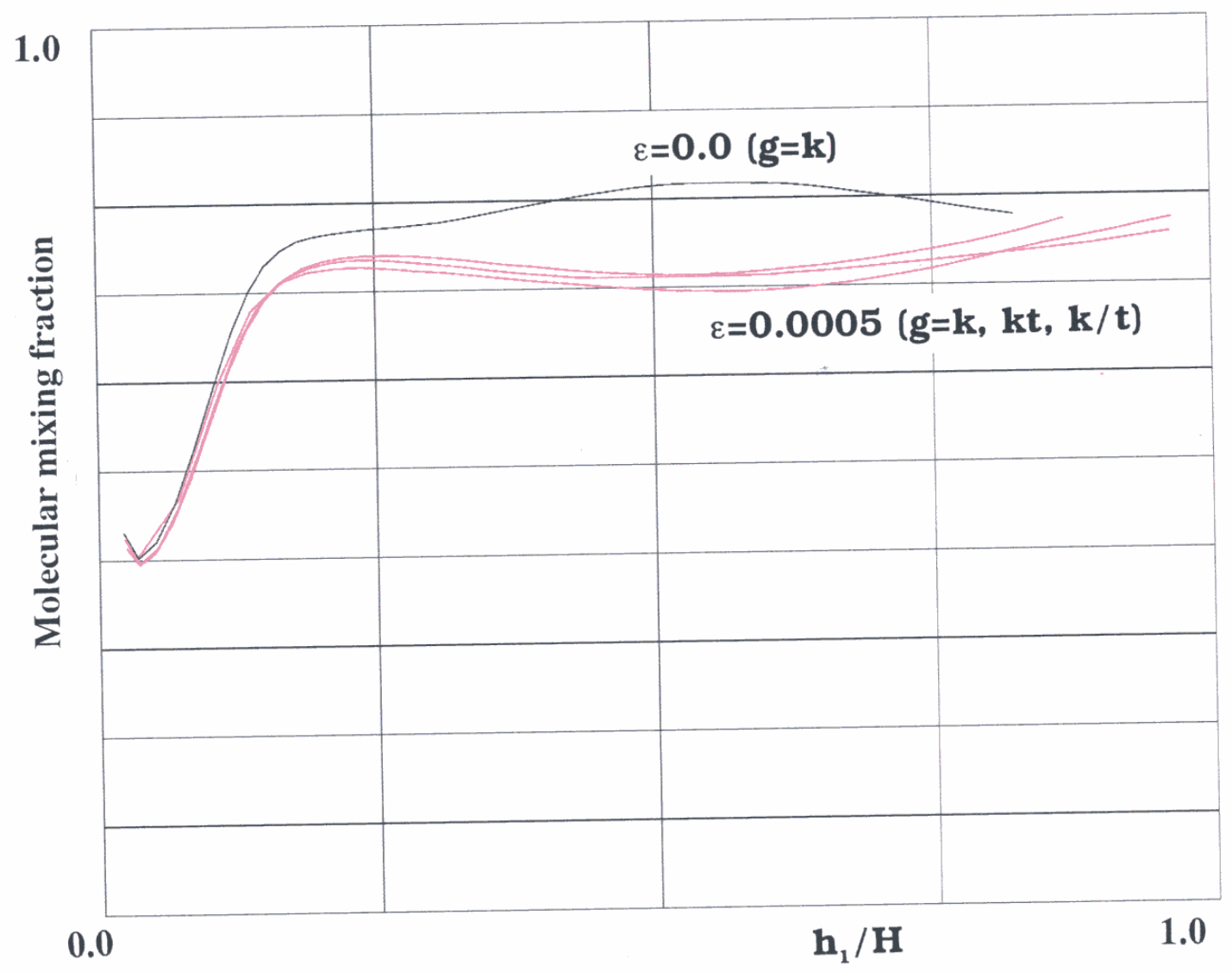


Figure6

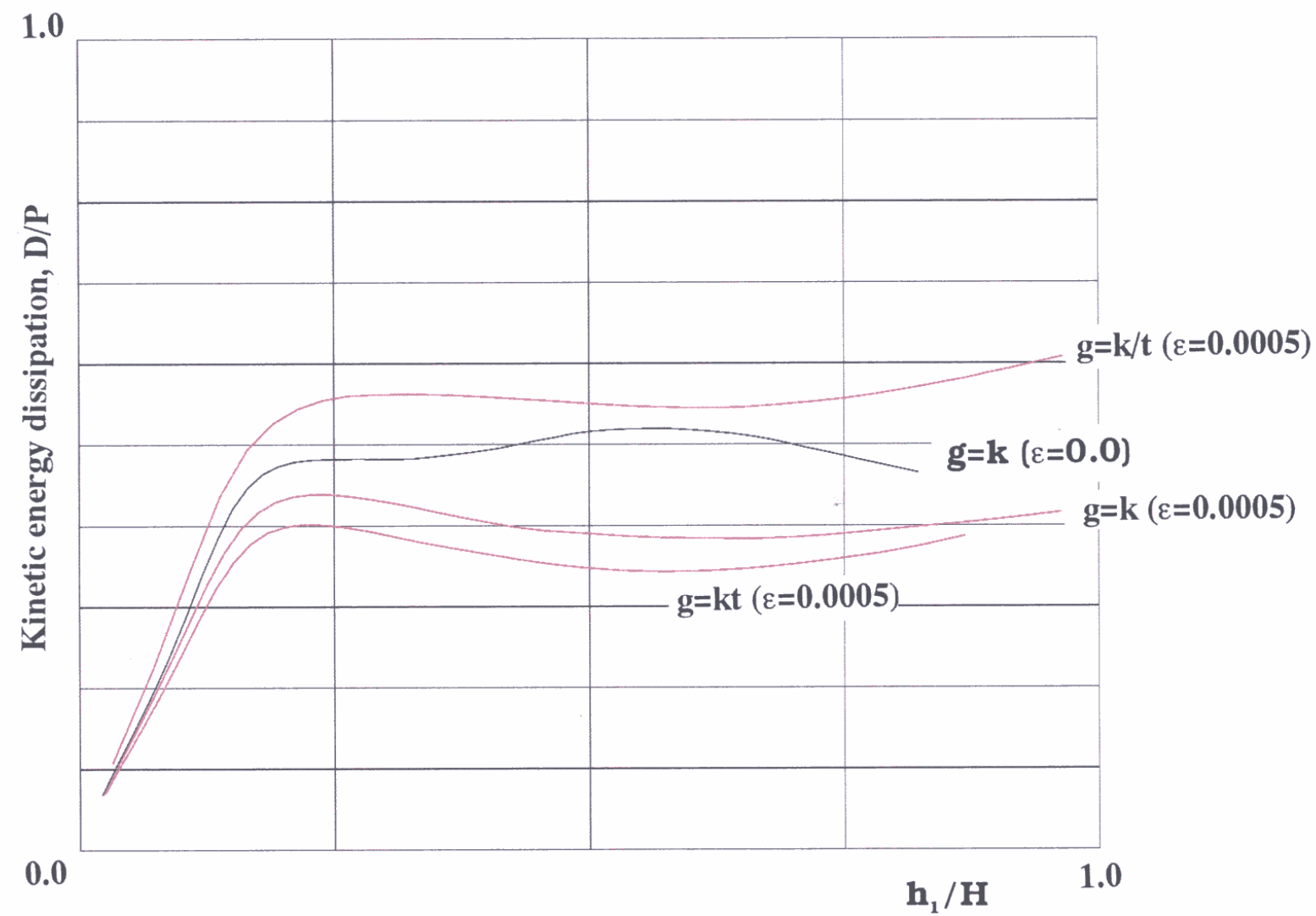




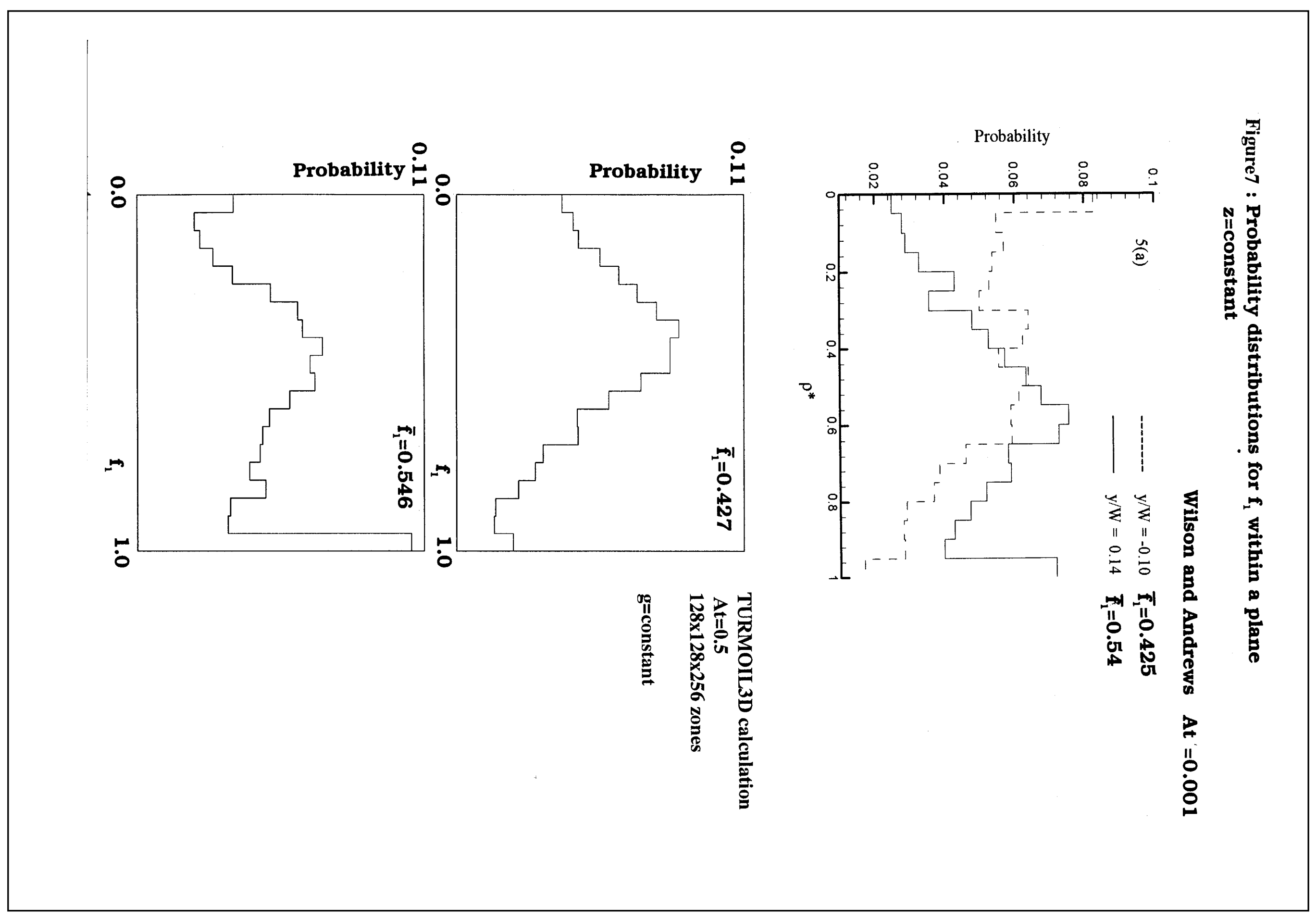


COMPARISON OF RESULTS WITH SIMPLE MODELS

(A) The Simplest Model

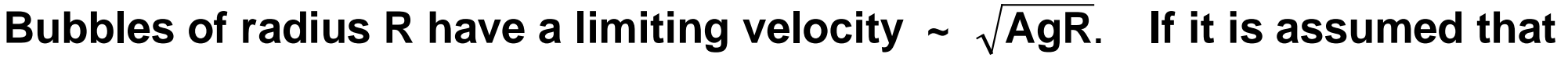
$R \sim h_{1}$, then

$$
\dot{\mathrm{h}}_{1}=\mathrm{c} \sqrt{\mathrm{Agh}_{1}}
$$

ie $\mathbf{h}_{1}=\alpha \mathbf{A}\left\{\int_{0}^{\mathbf{t}} \sqrt{\mathbf{g}\left(\mathbf{t}^{\prime}\right)} \mathbf{d \mathbf { t } ^ { \prime }}\right\}^{2}=\alpha \mathbf{S}$

Figure 5 shows plots of $h_{1}$ vs $S$. The slopes of the curves (for the range $h_{1} / H=0.25$ to 0.75$)$ give; for $\varepsilon=0.0005$

$\begin{array}{lllll}\mathbf{n}=0 & , & \alpha & = & 0.0464 \\ \mathbf{n}=1 & , & \alpha & = & 0.0415 \\ \mathbf{n}=-1 & , & \alpha & = & 0.0559\end{array}$

The model works surprisingly well, but there is some variation of $\alpha$ with $\mathbf{n}:-$

$$
\begin{aligned}
& \frac{\alpha_{1}}{\alpha_{0}}=0.89 \\
& \frac{\alpha_{-1}}{\alpha_{0}}=1.20
\end{aligned}
$$


(B) A Buoyancy - Drag Model

A model of this type, based on a modified form of Layzer's equation for a bubble rising in a cylindrical tube, was used by Hansom et al [2].

$$
\ddot{h}_{1}=\operatorname{Ag}-\frac{C_{D}\left(\dot{h}_{1}\right)^{2}}{h_{1}}
$$

acceleration $=$ buoyancy - drag

For constant $\mathrm{g}, \mathrm{C}_{\mathrm{D}}=\mathbf{4 . 5}$ gives $\alpha=0.05$. Then if $\alpha$ is defined as in equation (1):-

$$
\begin{aligned}
& \frac{\alpha_{1}}{\alpha_{0}}=0.97 \\
& \frac{\alpha_{-1}}{\alpha_{0}}=1.11
\end{aligned}
$$

This is closer to the TURMOIL3D results than taking $\alpha$ independent of $\mathbf{n}$ but the change is not large enough.

Dimonte and Schneider [3] include a factor $\beta<1$ in front of the $\mathrm{Ag}$ term in equation (2). This improves agreement with the 3D simulations. 
(C) An Energy Balance Model

This is version of the model proposed by Ramshaw [4], but with different settings for the model coefficients.

$$
\begin{array}{lll}
\text { Let } \mathbf{K} & = & \frac{1}{2}\left(\rho_{1}+\rho_{2}\right) \mathbf{h}_{1} \mathbf{V}^{2}=\text { kinetic energy within the mixing layer. } \\
\dot{\mathbf{P}} & = & \text { KE production rate (loss of potential energy) } \\
\dot{\mathbf{D}} & = & \text { dissipation rate }
\end{array}
$$

Then the model equations used are

$$
\begin{aligned}
& \dot{\mathrm{K}}=\dot{\mathbf{P}}-\dot{\mathbf{D}} \\
& \dot{\mathbf{P}}=\mathbf{c}_{1}\left(\rho_{1}-\rho_{2}\right) \mathbf{g} \mathbf{h}_{1} \dot{\mathrm{h}}_{1} \\
& \dot{\mathbf{D}}=\mathbf{c}_{2} \frac{1}{2}\left(\rho_{1}+\rho_{2}\right) \mathbf{V}^{3} \\
& \dot{\mathbf{h}}_{1}=\mathbf{c}_{3} \mathbf{V}
\end{aligned}
$$

The coefficient $c_{1}$ depends on the shape of the volume fraction profile. For a linear distribution $c_{1}=1 / 3$. For the TURMOIL3D profiles (figure 4) $c_{1}=0.30$, and this is the value used here.

$$
\begin{aligned}
& \text { If for constant } g \text { we assume, } \alpha=0.05 \text { and } D / P=0.4 \text { (figure } 7 \text { ) then } \\
& c_{2}=1.4055 \\
& c_{3}=1.0541
\end{aligned}
$$


The equation $\dot{K}=\dot{P}-\dot{D}$ gives

$$
\begin{aligned}
\ddot{h}_{1} & =c_{1} c_{3}^{2} A g-\frac{c_{2}+c_{3}}{2 c_{3}} \frac{\left(\dot{h}_{1}\right)^{2}}{h_{1}} \\
& =\frac{1}{3} A g-\frac{7}{6} \frac{\left(\dot{h}_{1}\right)^{2}}{h_{1}}
\end{aligned}
$$

This has the same form as the buoyancy - drag model (2). The coefficient in front of the Ag term is less than unity as in Dimonte and Schneider [3].

This choice of the coefficients gives

$$
\begin{aligned}
& \frac{\alpha_{1}}{\alpha_{0}}=0.91 \\
& \frac{\alpha_{-1}}{\alpha_{0}}=1.43
\end{aligned}
$$

$$
\begin{aligned}
& \text { also } \mathrm{D} / \mathrm{P} \quad=\quad 0.40 \text { assumed for } \mathrm{n}=0 \\
& D / P \quad=\quad 0.36 \text { for } n=1 \\
& \mathrm{D} / \mathrm{P} \quad=\quad 0.57 \text { for } \mathrm{n}=-1
\end{aligned}
$$

$\alpha_{1} / \alpha_{0}$ agrees with the 3D simulations

$\alpha_{-1} / \alpha_{0}$ appears to be somewhat too high. However, the variation of D/P with $\mathrm{n}$ (see fig. 6) is represented very well. 


\section{FUTURE PLANS}

- Values of $n$ outside the range $[-1,1]$

- Higher resolution TURMOIL3D calculations

- Use of the SSVARTs results to differentiate between various types of RANS models.

\section{REFERENCES}

1. N A Inogamov et al, Proceedings of the 3rd IWPCTM, Royaumont (1991)

2. J C V Hansom et al, Laser and Particle Beams, Vol 8, p 51 (1990)

3. G Dimonte and M Schneider, Phys Rev E54, p 3740 (1996)

4. J D Ramshaw, Phys Rev E58, p 5834 (1998) 


\section{Shock - planar curtain interactions}

Strong Secondary baroclinic deposition and emergence of coherent and random vortex projectiles (VPs), and decaying stratified turbulence

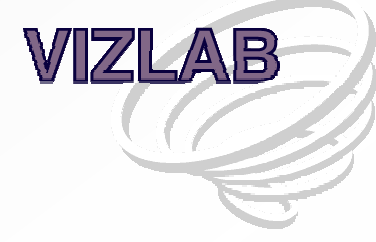

Shuang Zhang and Norman J. Zabusky

Laboratory of Visiometrics and Modeling

Department of Mechanical and Aerospace Engineering

Rutgers, The State University of New Jersey

Rutgers 


\section{Brief Review}

Common geometries for studying accelerated inhomogeneous flows (aifs)

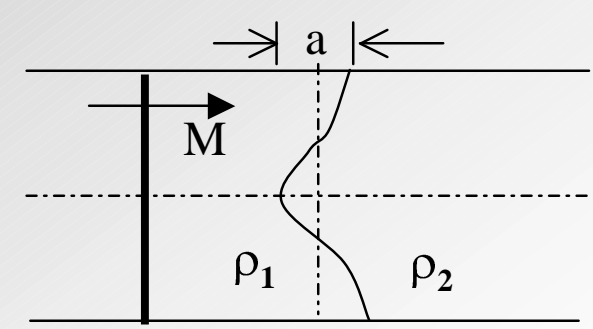

Linear, Nonlinear \&

Vortex: growth rate

... å ...

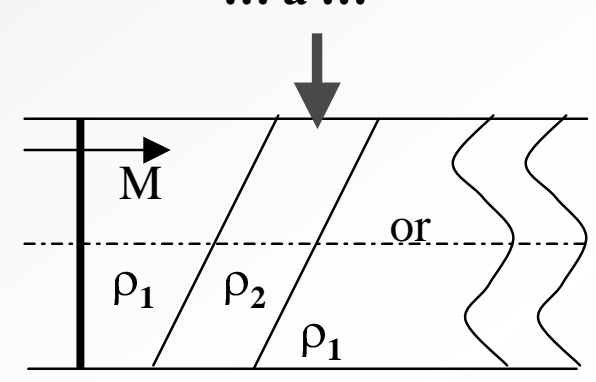

Curtain:

inclined or perturbed

(Sturtevant, 87;

Benjamin et al, 84)

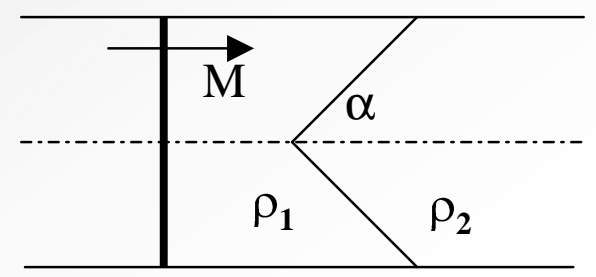

Self-similar Solution;

Quantification on:

Circulation, baroclinic

effects \& convergence

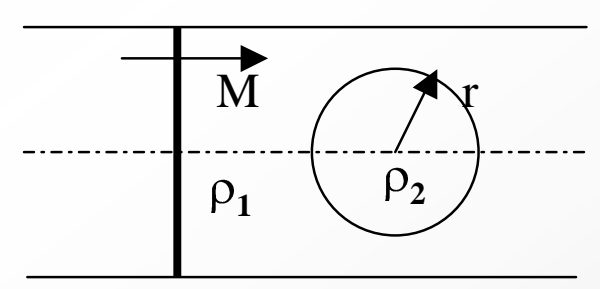

Circular Cylinder or Sphere (axisymmetric or 3D):

Vortex separation \& vortex projectiles (VPs)

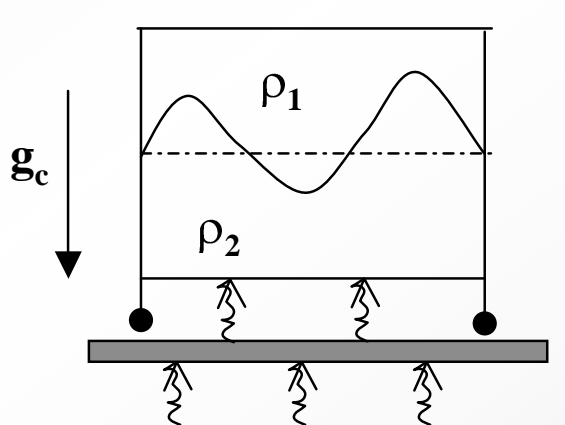

Reaccelerated tank (Jacobs \& Niederhaus, 97)

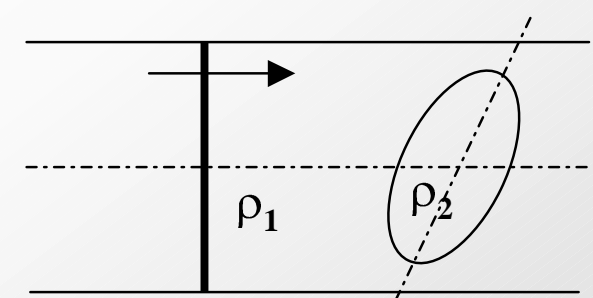

Elliptical cylinder or ellipsoid: Cavity implosion, vortex separation \& vortex projectiles (VPs)

\section{Rutgers}




\section{Motivations}

Visualize, Quantify and Model the vortex dominated physical process, including:

1. Baroclinic vorticity

- Main baroclinic vorticity deposition by the shock wave: vortex double layers (VDL)

- Secondary baroclinic vorticity deposition by vortex interaction

- Scaling with regard to Mach number

2. Emergence, evolution and modeling of "Vortex Projectiles" (VP) or coherent dipolar vortex structures

3. Turbulent mixing and rapid turbulization at high Mach number

4. Study of 3D effects 


\section{Computational Domain}

1. Schematic (following [Yang, Zabusky \& Chern, 1990]):

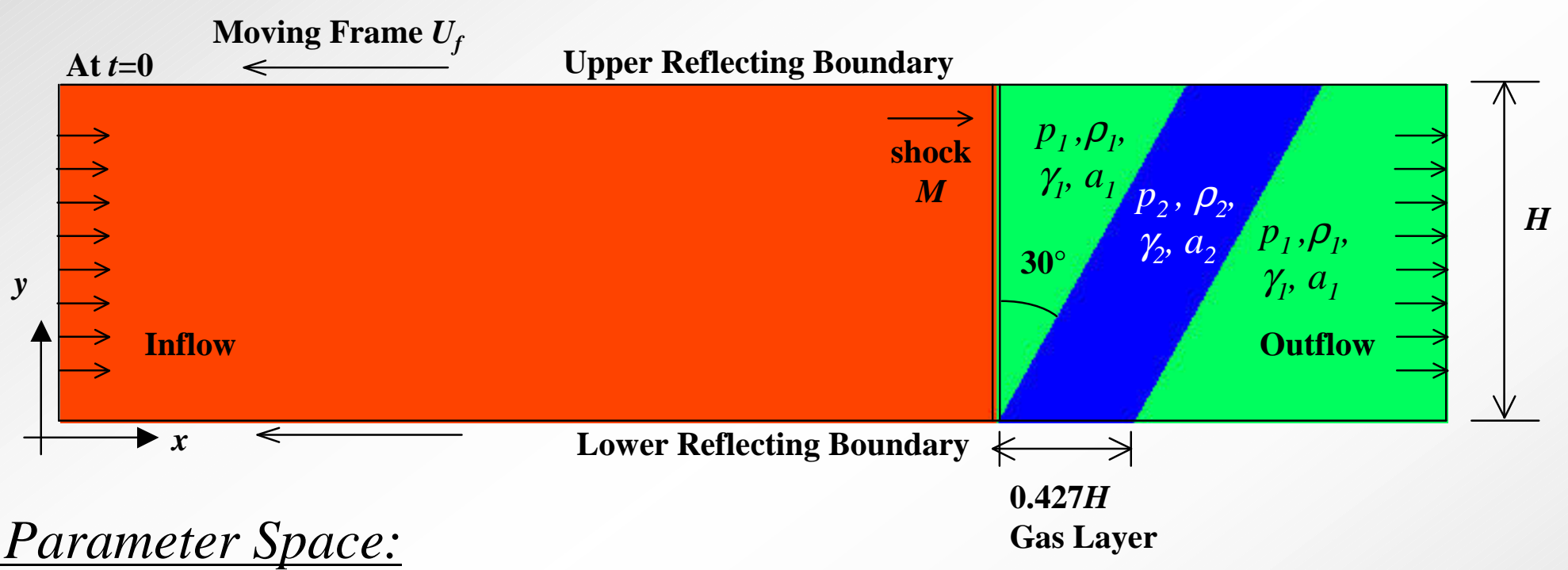

2. Parameter Space:

- Density ratio: $\eta=\rho_{2} / \rho_{1}$ (Slow/Fast/Slow (0.14)

- Mach \#: 1.5, 2.0, 5.0

3. Numerical Method:

- Compressible Euler Equation

- Piecewise Parabolic Method [Woodward \& Colella, 1984]
- Resolution(2D \& 3D):

$800 \times 160(H)$

$200 \times 80 \times 80$

Rutgers 


\section{Global Quantification I: Evolution of normalized circulation}

Normalization factors:

- $M=1.5$ circulation:

$\max \Gamma_{+\mid M=2.0}\left(\max \Gamma_{+\mid M=1.5} \approx 1.4\right.$

- $M=5.0$ circulation:

$\max \Gamma_{+\mid M=2.0} \max \Gamma_{+\mid M=1.5} \approx 0.39$,

- $t_{n}$ : a plain shock traveling

through length of the curtain

thickness horizontally without

inhomogeneity.

\section{$\underline{\text { Note }}$}

- The time epoch: according to

$M=2.0$ run, good for most of $M=1.5$ run

- Separation point of eIIa and eIIb, characterized by the time $V P l$ hit the upper boundary, $\bullet$ for $M=2.0$ and + for $M=1.5$, are identified via negative circulation for $\mathrm{M}=2.0$ and positively circulation for $\mathrm{M}=1.5$ respectively.

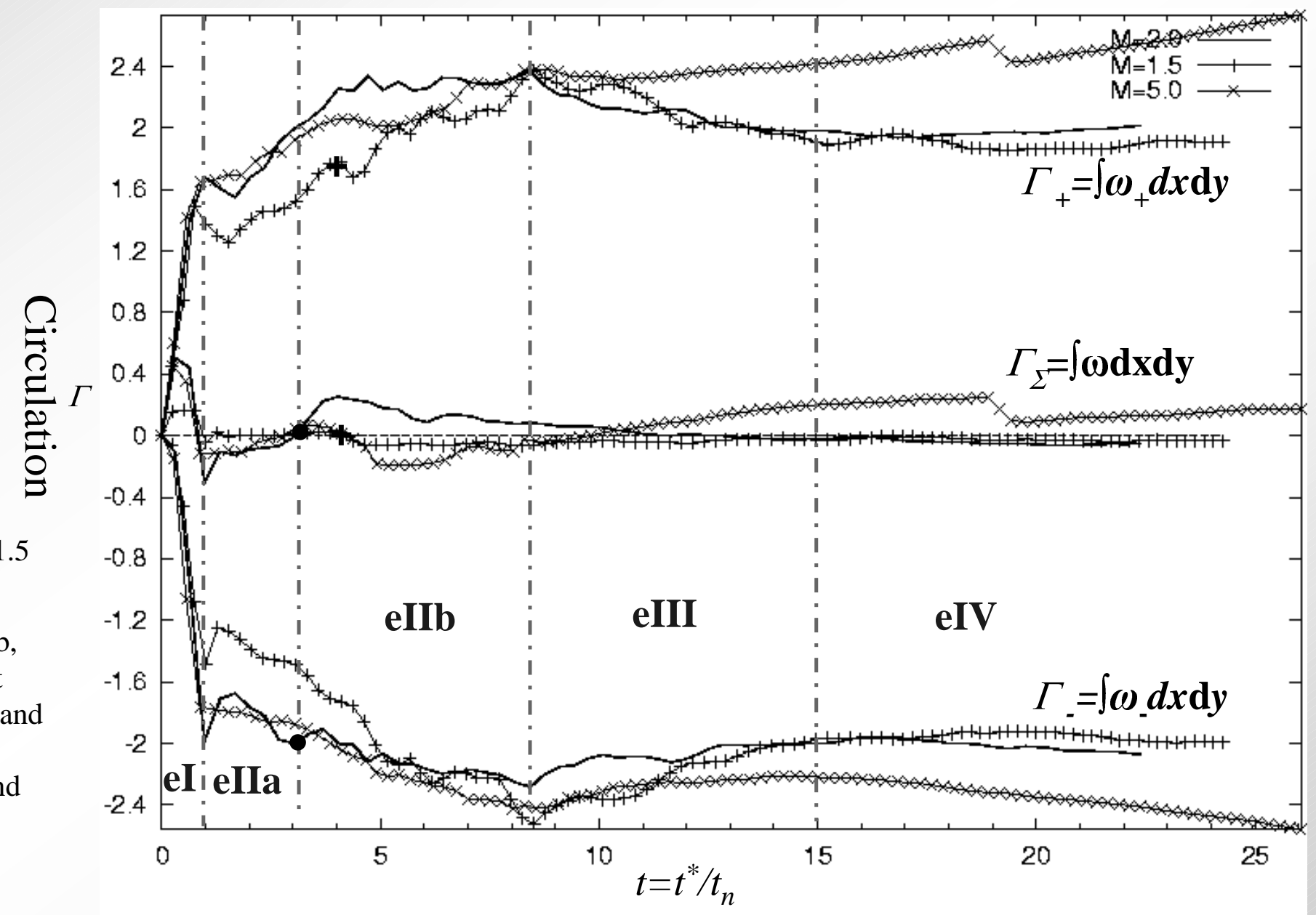

\section{Rutgers}




\section{Time Epoch, Feature Phenomena and Physical Processes}

\begin{tabular}{|c|c|c|c|}
\hline \multirow{2}{*}{$\begin{array}{l}\text { Time } \\
\text { Epochs }\end{array}$} & \multicolumn{2}{|c|}{ Feature Phenomena } & \multirow{2}{*}{$\begin{array}{l}\text { Physical } \\
\text { Processes }\end{array}$} \\
\hline & Density & Vorticity & \\
\hline e(1) & $\begin{array}{l}\text { Shock wave passes the curtain: } \\
\text { reflection (upstream expansion } \\
\text { wave), refraction and } \\
\text { transmission. }\end{array}$ & $\begin{array}{l}\text { Opposite sign vortex sheet } \\
\text { deposition and approach; } \\
\text { Circulation increases with } \\
\text { significant rate. }\end{array}$ & $\begin{array}{l}\text { Primary } \\
\text { baroclinic } \\
\text { effects }\end{array}$ \\
\hline e(2) & $\begin{array}{l}\text { Curtain "breakthrough"; } \\
\text { Mushroom structure hits the } \\
\text { upper boundary; Secondary } \\
\text { reflected shock wave hits the } \\
\text { deformed curtain }\end{array}$ & $\begin{array}{l}\text { Collision of vortex layers; "Vortex } \\
\text { binding" of oppositely signed; } \\
\text { Formation of complex VP1; VP1 } \\
\text { hits the boundary Circulation } \\
\text { increases with lower rate but longer } \\
\text { term (total amount almost the } 50 \% \\
\text { as e(1) }\end{array}$ & $\begin{array}{l}\text { Secondary baroclinic } \\
\text { effect due to mutual } \\
\text { acceleration of } \\
\text { inhomogeneity and } \\
\text { vortex interaction }\end{array}$ \\
\hline e(3) & $\begin{array}{l}\text { Complete formation of upstream } \\
\text { and downstream bubbles (VP's); } \\
\text { Translating dipolar structures; } \\
\text { Transition to turbulence }\end{array}$ & $\begin{array}{l}\text { Separation of VPs; } \\
\text { Vortex binding with image; } \\
\text { Circulation decreasing }\end{array}$ & $\begin{array}{l}\text { Vortex } \\
\text { merging; and } \\
\text { turbulent } \\
\text { mixing }\end{array}$ \\
\hline e(4) & $\begin{array}{l}\text { Emergence of dipolar VP's } \\
\text { which are well isolated; and } \\
\text { intermediate turbulent interval }\end{array}$ & $\begin{array}{l}\text { Approach to nearly stationary } \\
\text { configuration of VPs and } \\
\text { intermediate turbulent domain; } \\
\text { nearly constant circulation }\end{array}$ & $\begin{array}{l}\text { Dissipation and } \\
\text { baroclinic re- } \\
\text { acceleration }\end{array}$ \\
\hline
\end{tabular}




\section{Simulations}

Early time snapshots for $M=1.5$ : $\quad$ Juxtaposition of density, vorticity, baroclinic term

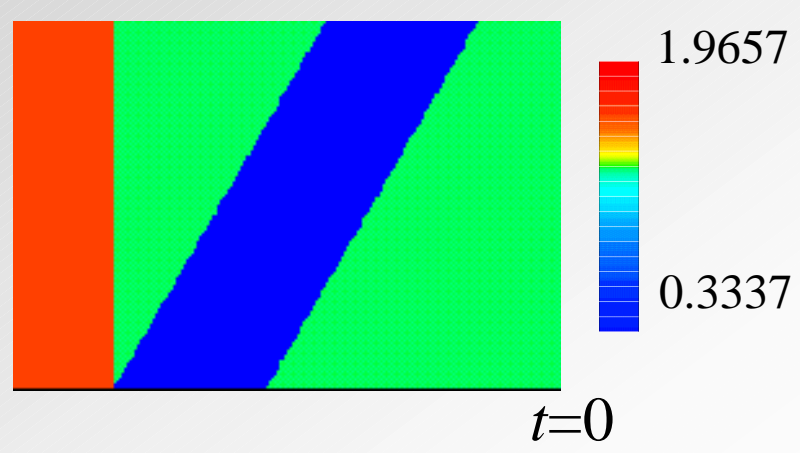
$\nabla \rho \times \nabla p / \rho^{2}$, and dilatation $\nabla \bullet \mathbf{u}$

- $V D L$, shock wave patterns, breaking through process and dominant $V P 1$

- Following our previous work [Yang, Zabusky \& Chern, 1990]
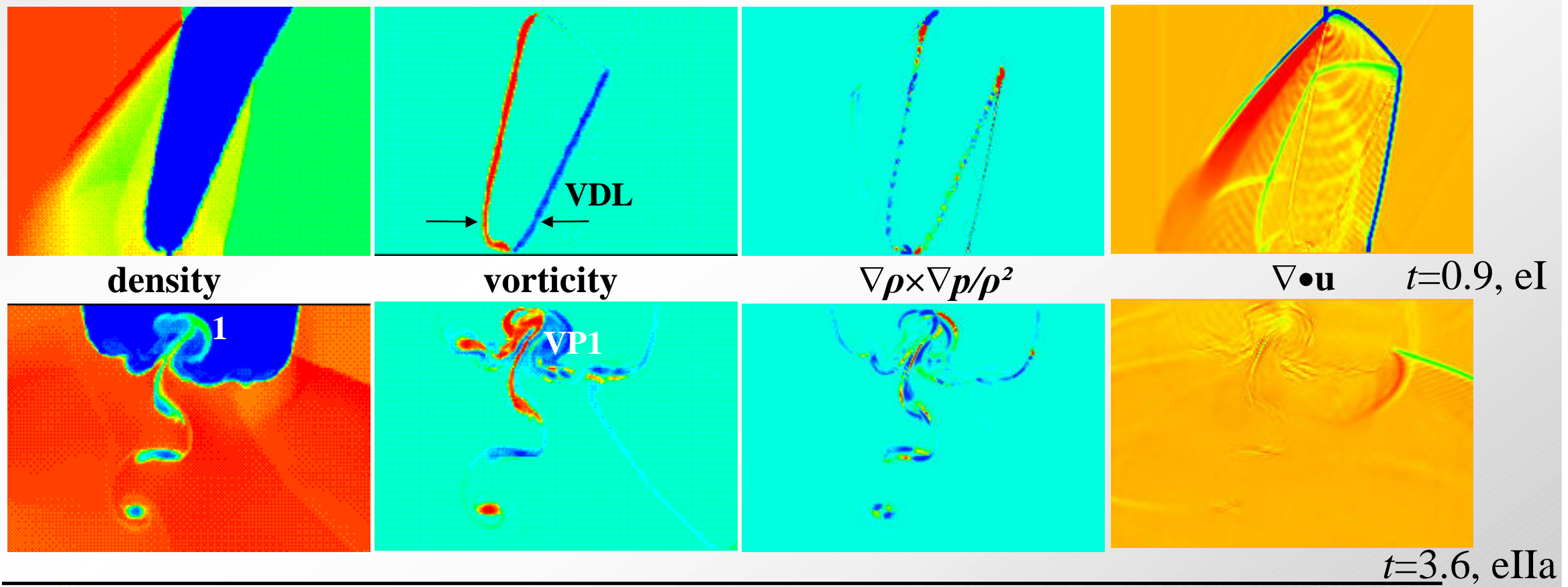

Rutgers 


\section{Early time snapshots for $M=\mathbf{2 . 0}$}

- Similar to M=1.5: VDL, breaking through process, and dominant VP1

- More generic VP configuration

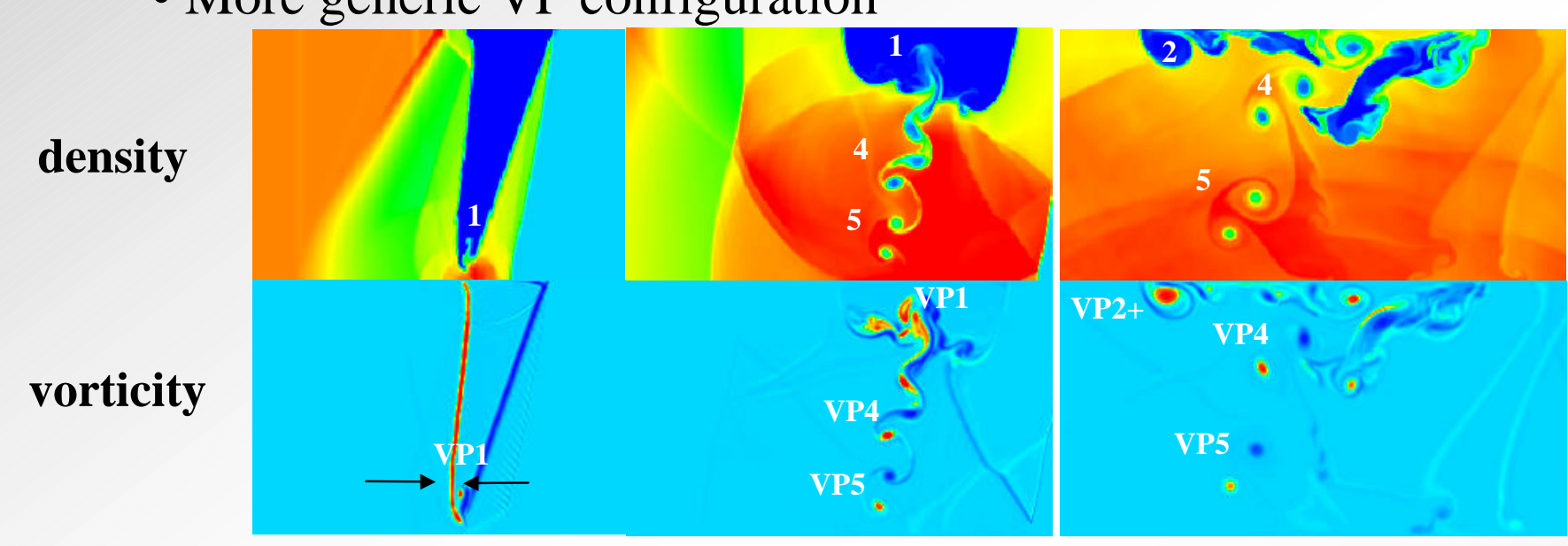

$\begin{array}{lll}\text { (a) } t=0.95 \text {, eIIa begins } & \text { (b) } t=2.62 \text {, during eIIa } & \text { (c) } t=7.25 \text {, during eIIb }\end{array}$

density

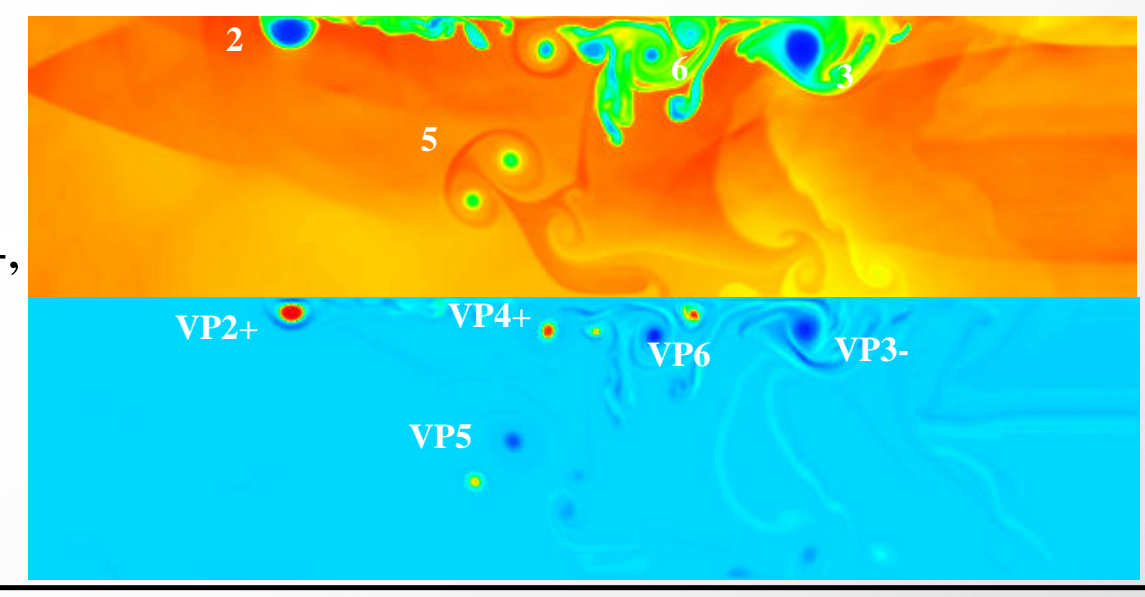

(d) $\mathrm{t}=12.54$ during eIII

vorticity 
Simulation Cont.

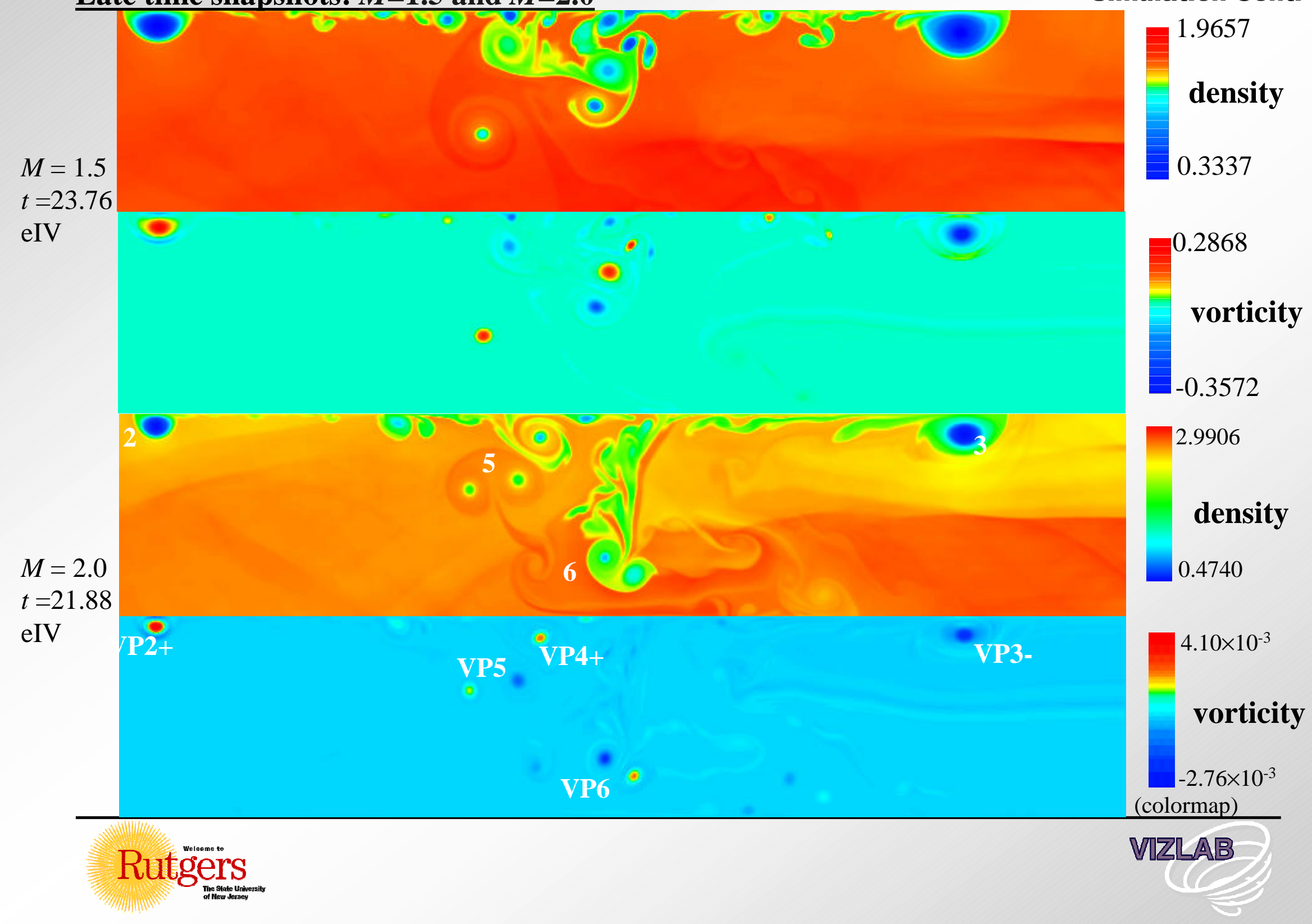




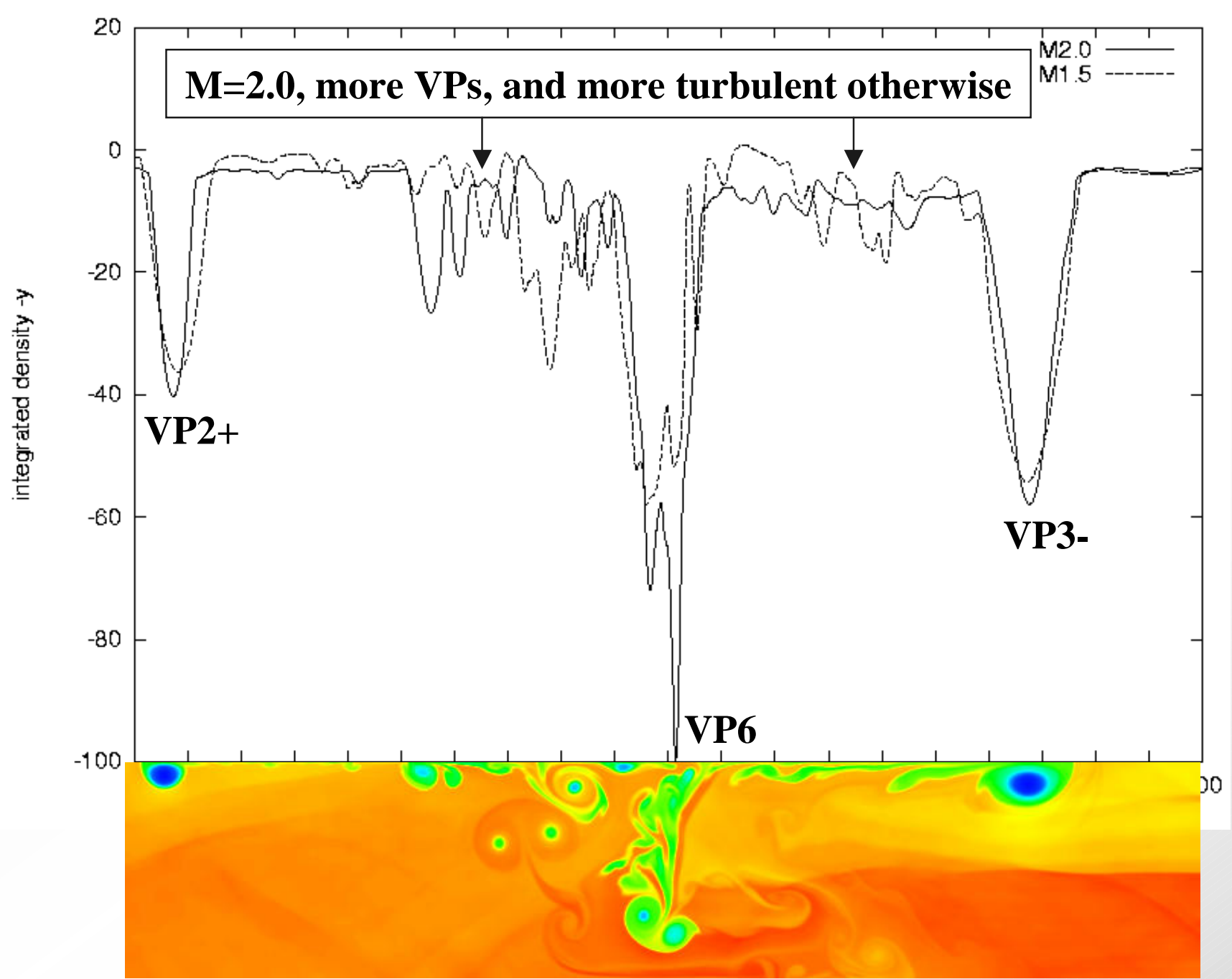

Y-integrated density (filtered the ambient density)

Rutgers 


\section{IWPCTM 8 ORAL C48}

S. Zhang \& N. Zabusky, 12/12/2001

Simulation Cont.

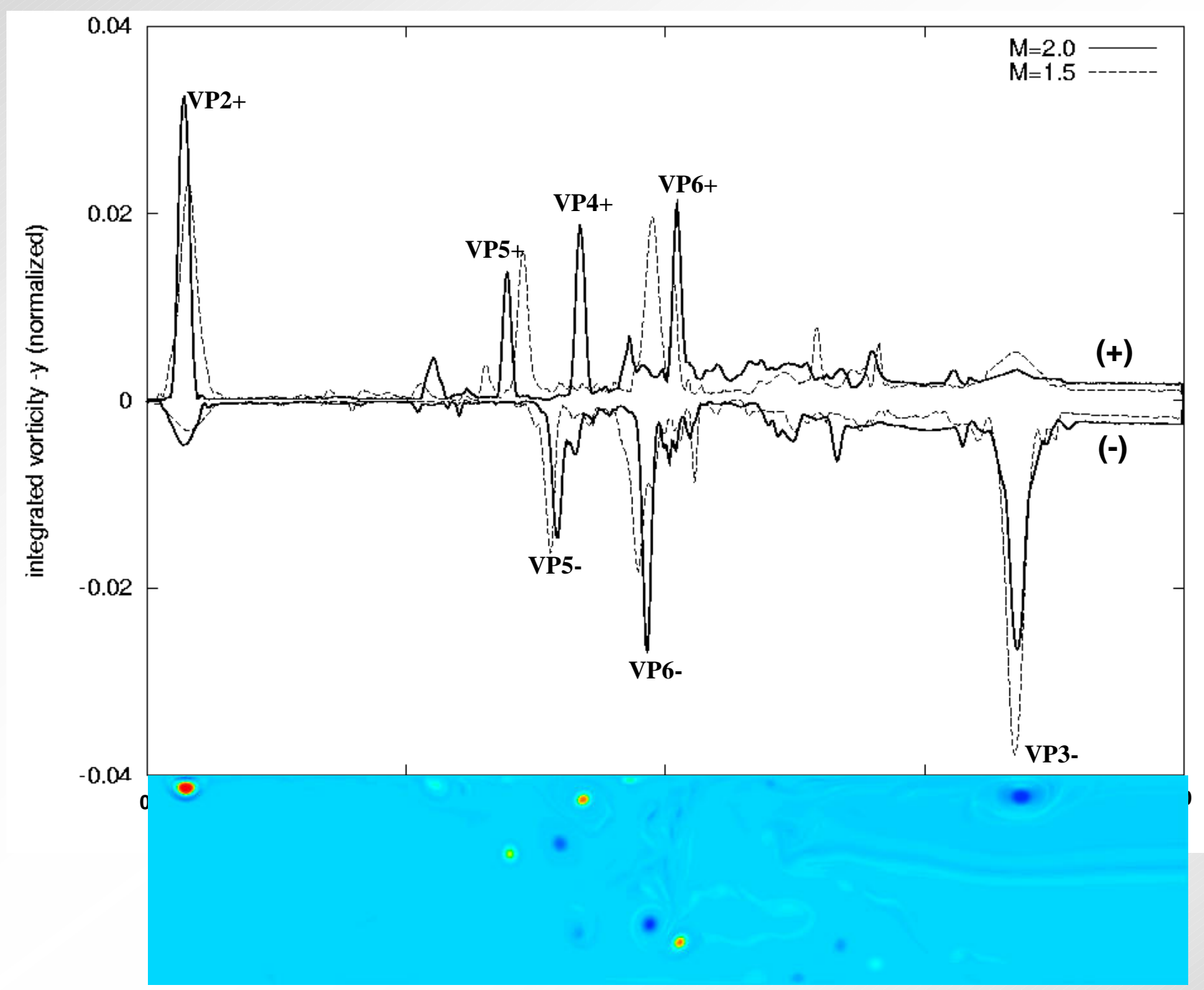

Y-integrated vorticity

Rutgers 


\section{Global Quantification II: Integrated enstrophy evolution equation}

- Emphasizes larger magnitude vortex domains;

- Normalization factor $E_{n}$ : enstrophy at the end of eI.

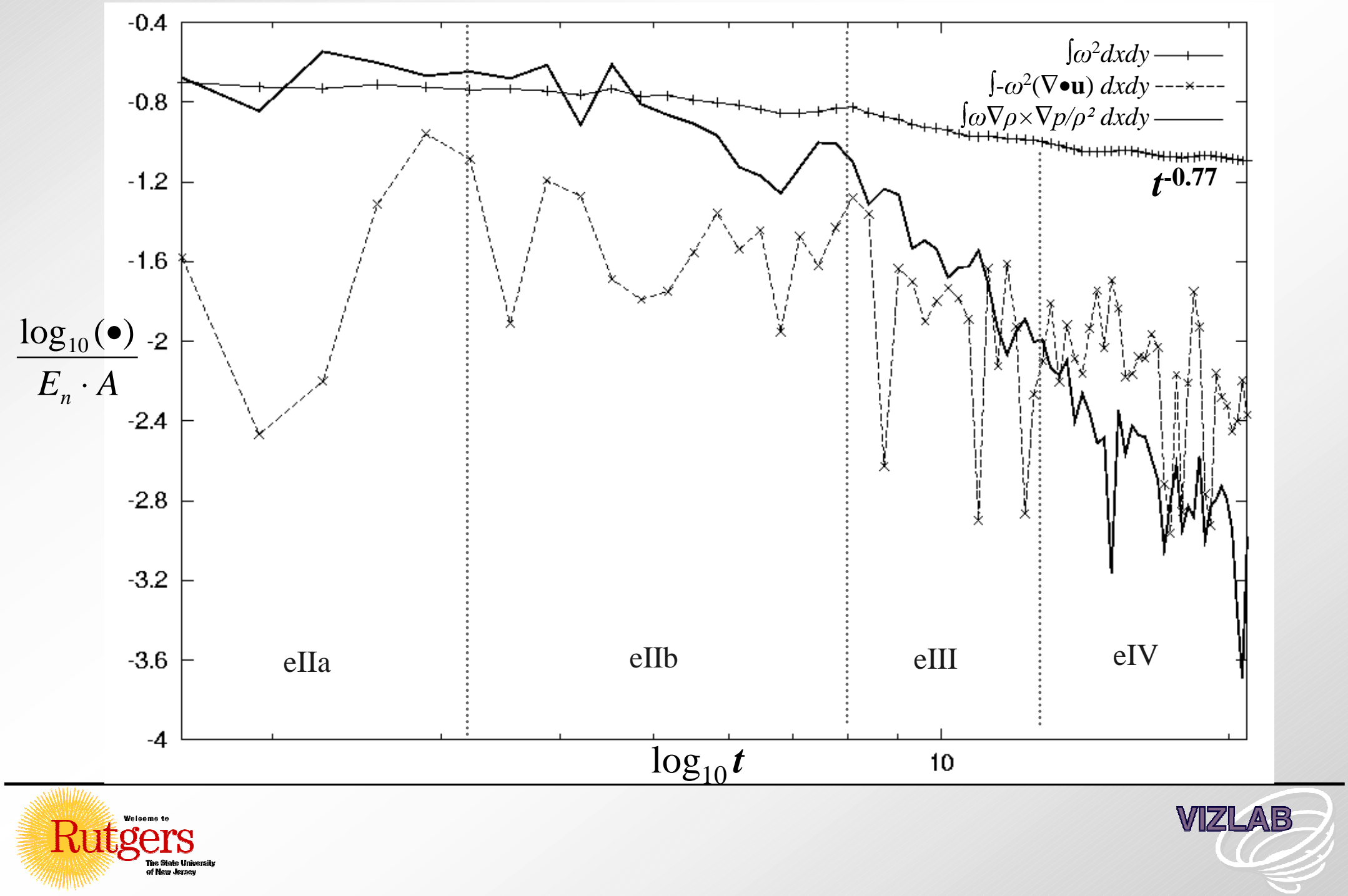




\section{IWPCTM 8 ORAL C48}

\section{Local Structures' Quantification}

\section{Quantification of vortex projectiles}

-VP2+ for upstream and VP3- for downstream, respectively

- $M=1.5$ and $M=2.0\left(\left|\Gamma_{V P 2+}\right|+\left|\Gamma_{+V P 3-}\right|+\Gamma_{a f f i} \mid=30 \%\left(\left|\Gamma_{-}\right|+\left|\Gamma_{+}\right|\right)\right.$

- Extraction threshold: $4 \%$ of the extremum of vorticity

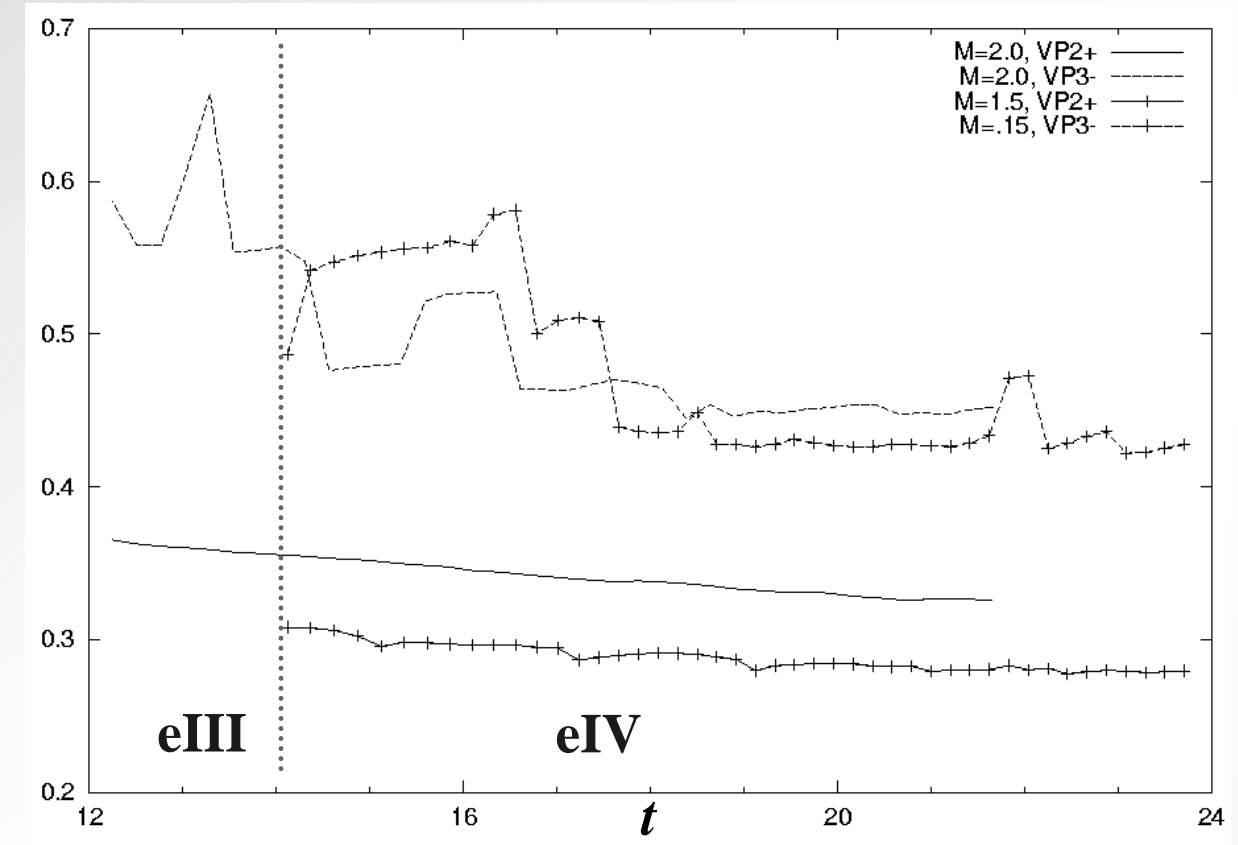

(a) Evolution of local circulation

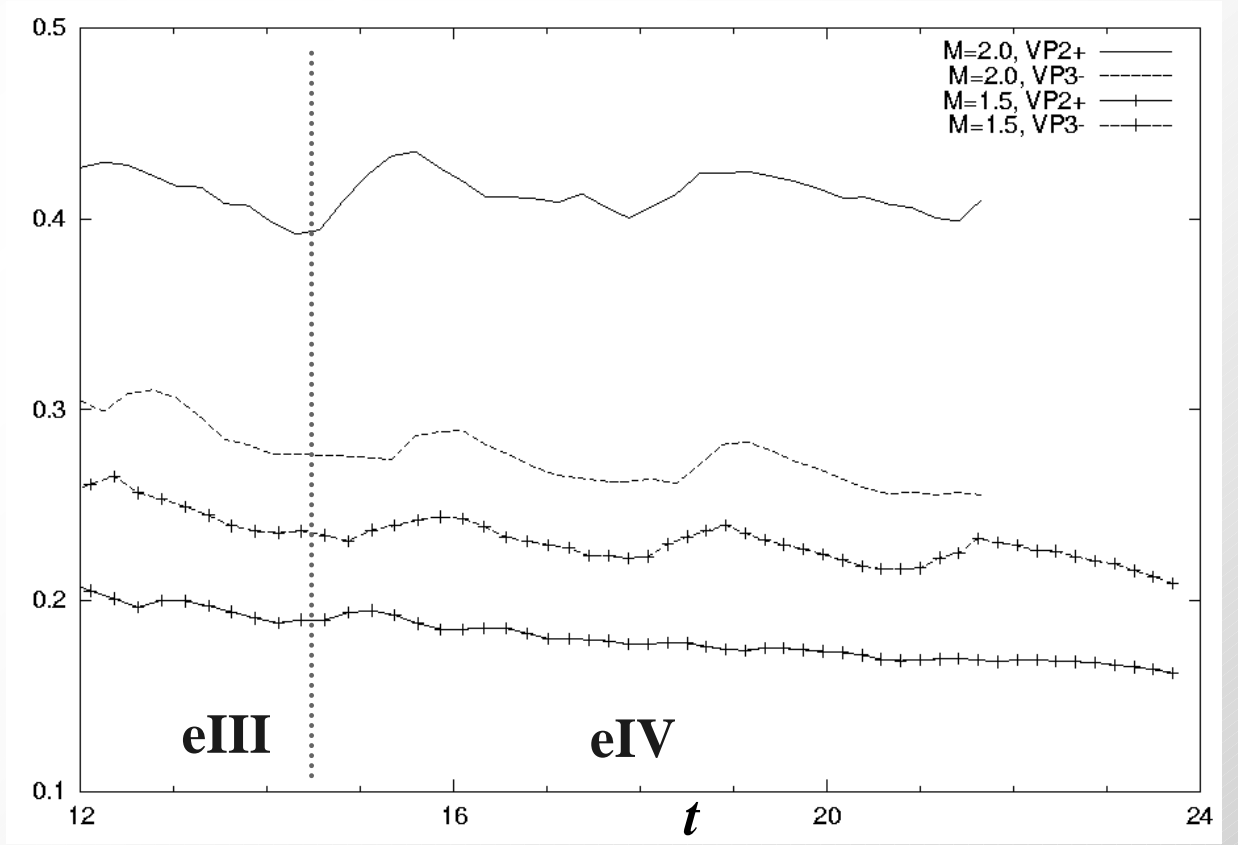

(b) Evolution of local vorticity maxima

Rutgers 


\section{Point vortex modeling $(M=2.0)$}

Modeled translational velocity: $u_{x}=\frac{\Gamma}{2 \pi d}$

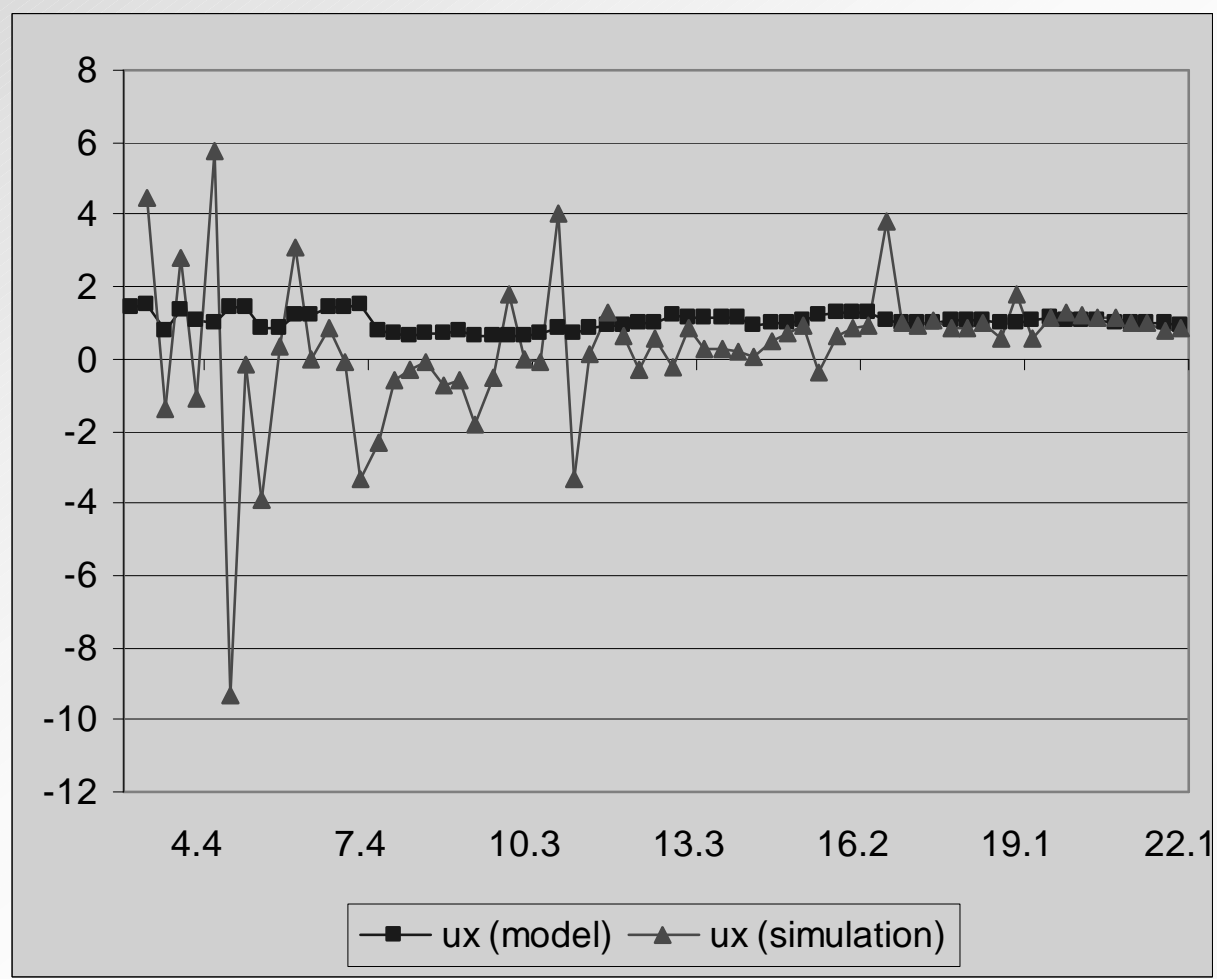

Downstream VP3-

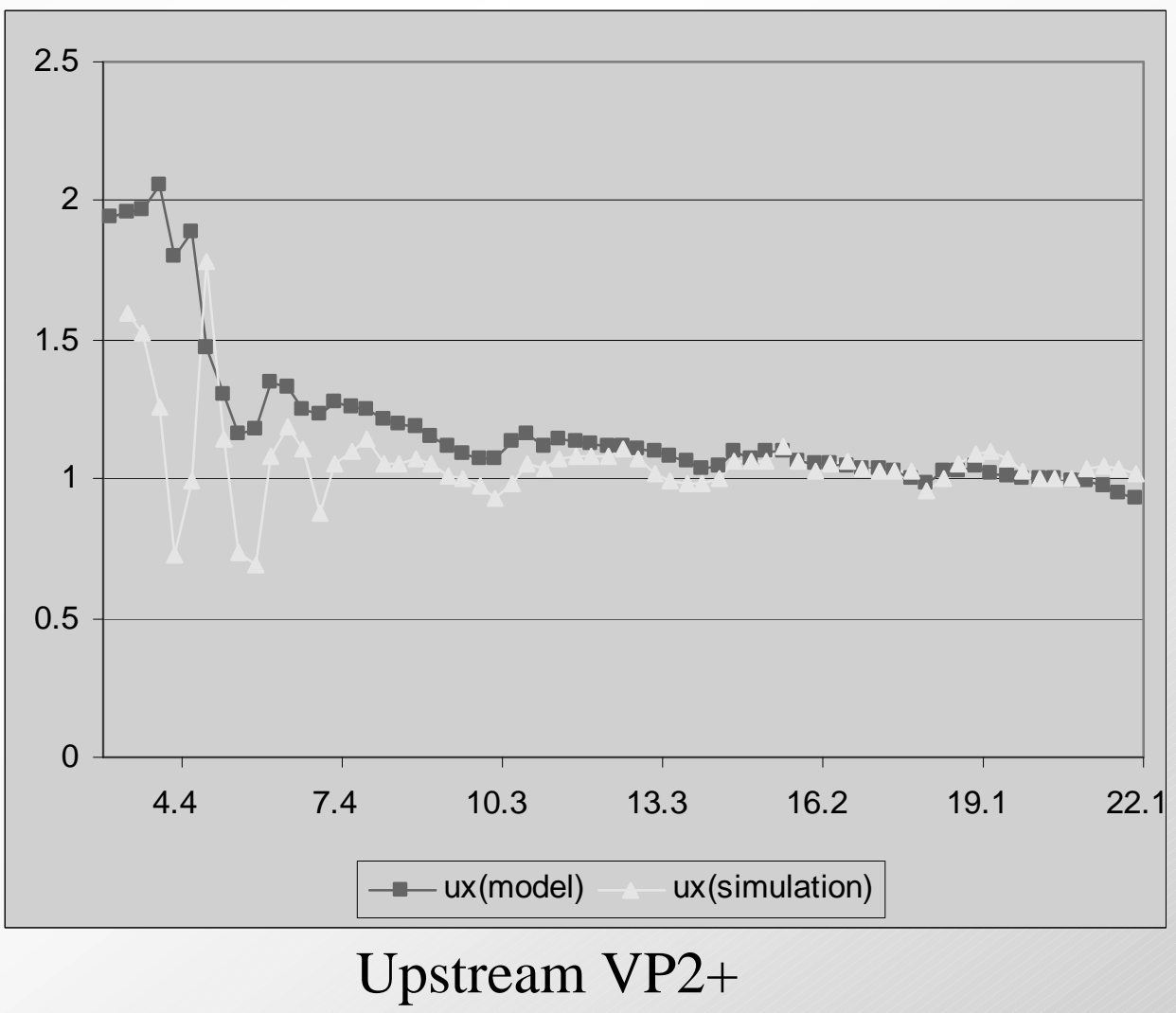

VIZZLA

Rutgers 


\section{Density distribution}

\section{Turbulent mixing}

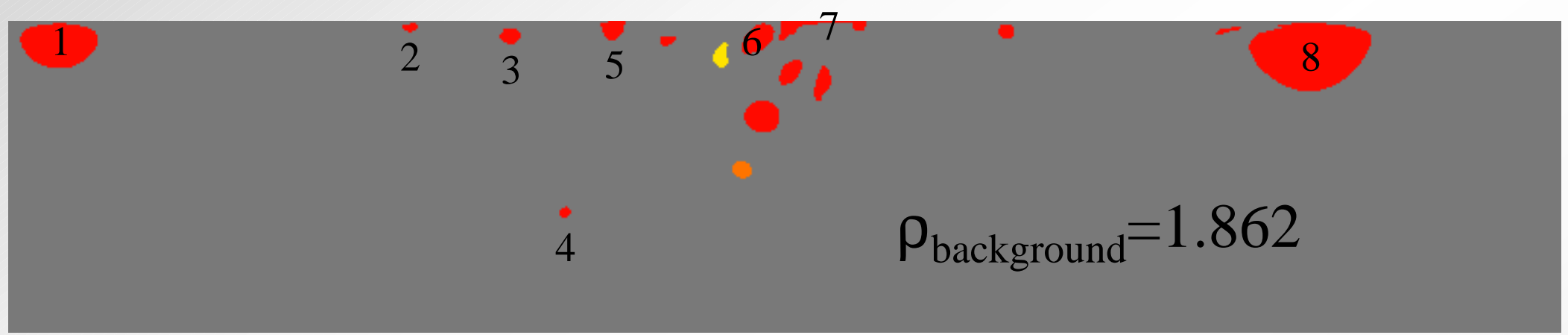

- Initial Density Ratio: 0.14
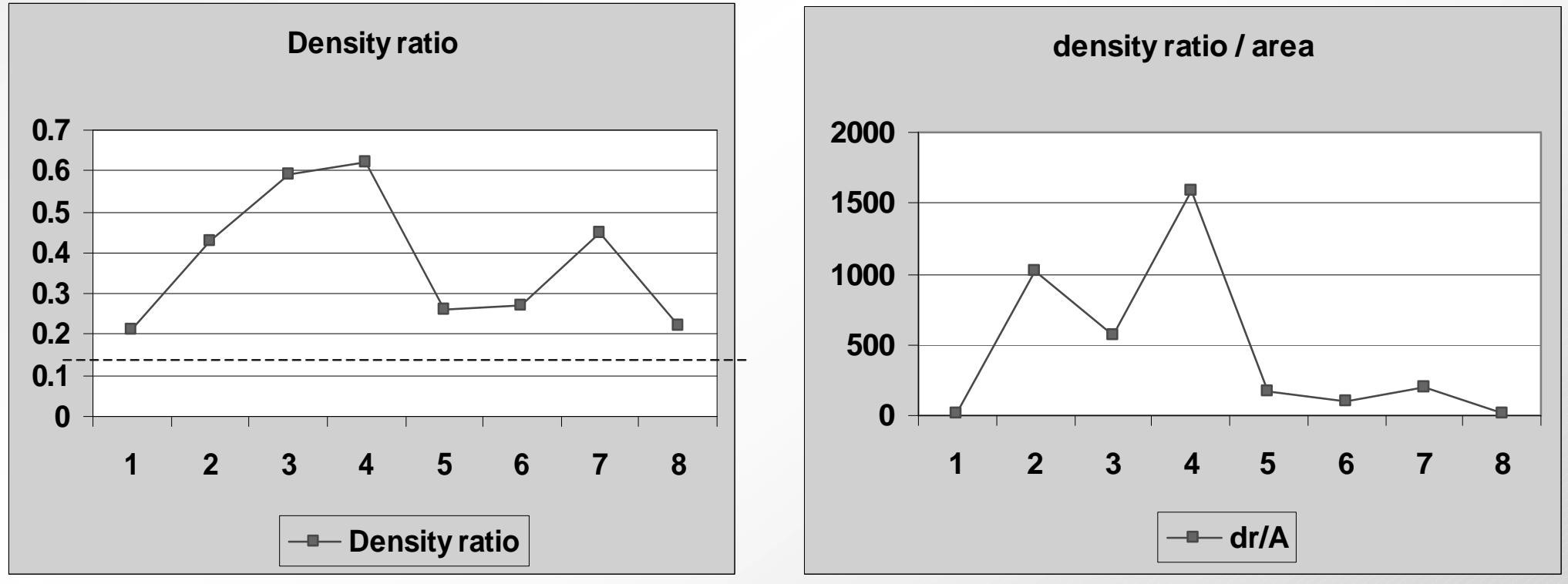

Rutgers 


\section{$\underline{\text { Rapid Turbulization at High Mach number }(M=5.0)}$}

Turbulent mixing cont.
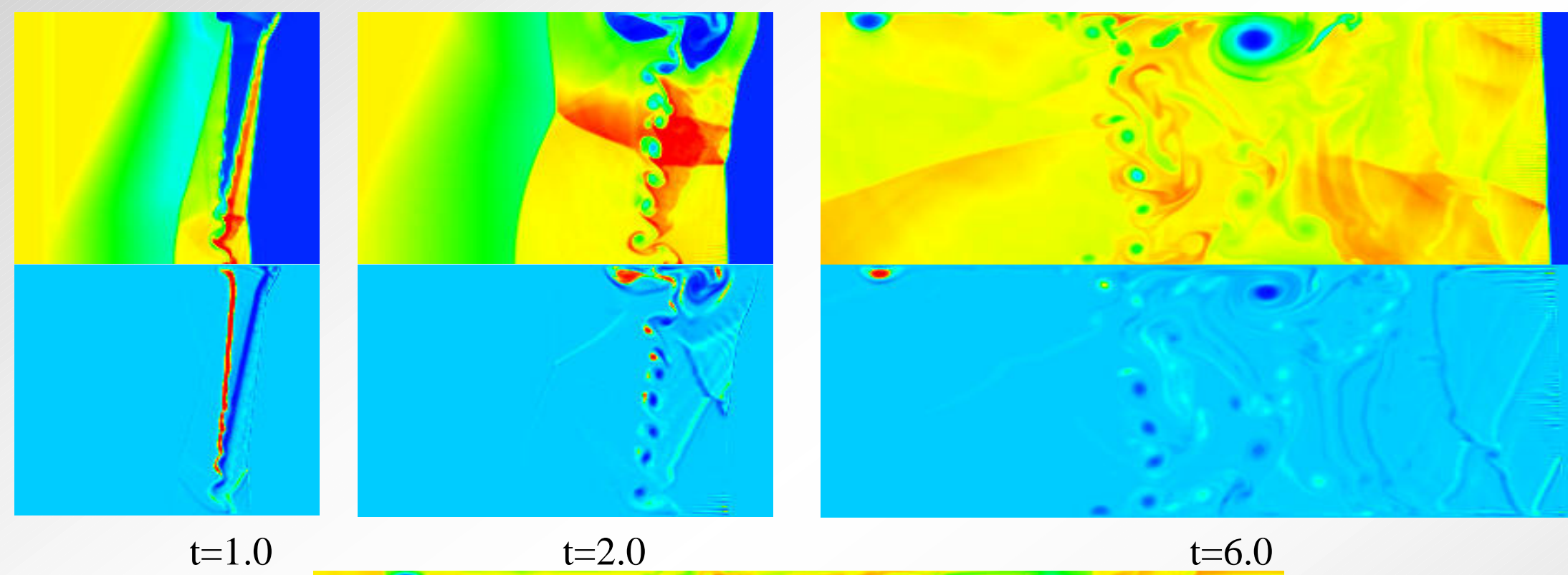

$\mathrm{t}=2.0$

$\mathrm{t}=6.0$
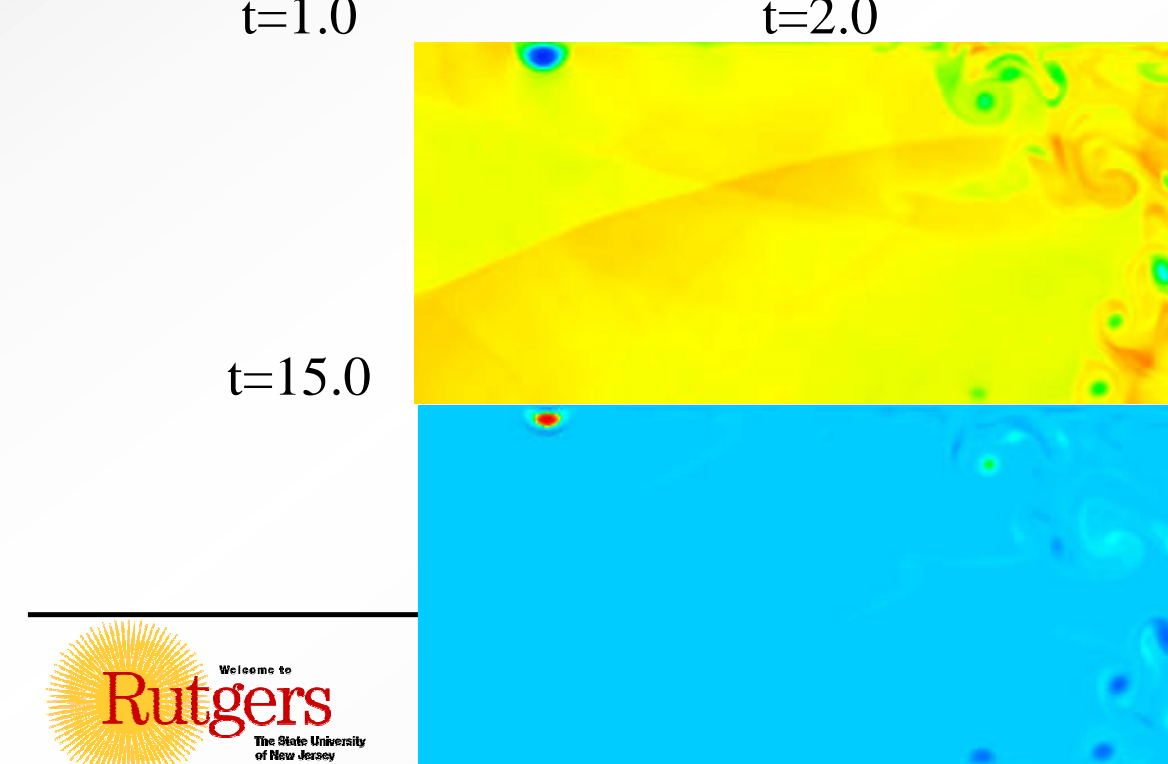

density

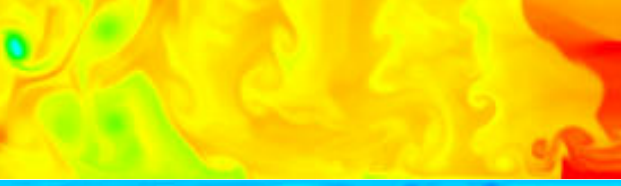

vorticity 


\section{D Curtain study}

Main approaches: Simulation with adaptive mesh; quantification via data projection;

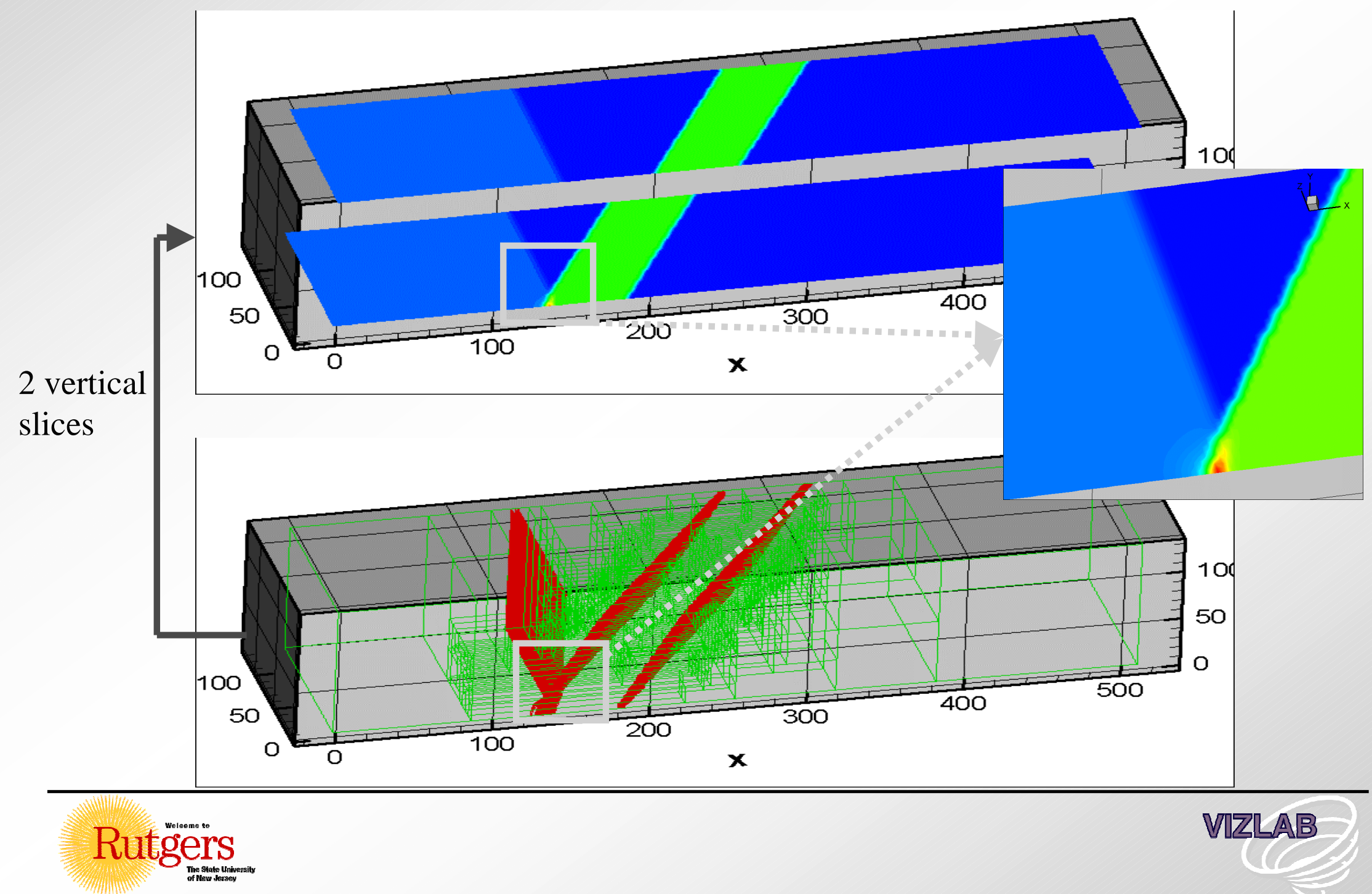


Data Projection: quasi-2D (Resolution: $200 * 80 * 80$ ) 3D curtain cont.

Slice $1(j=72)$

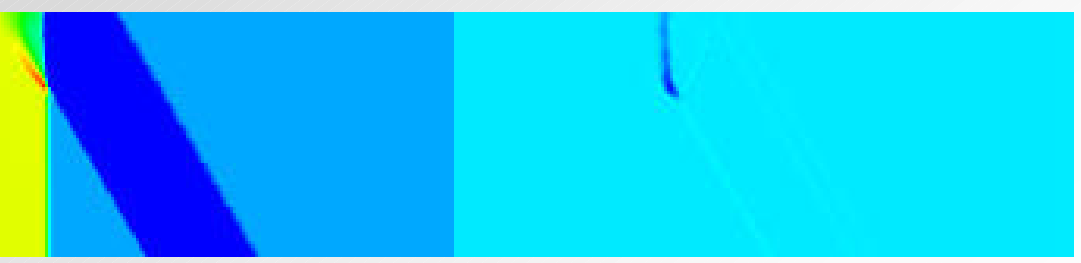

Step 8

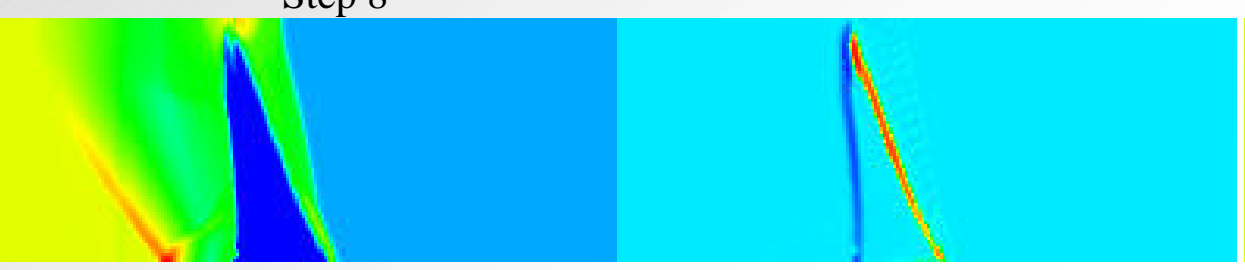

Step 13

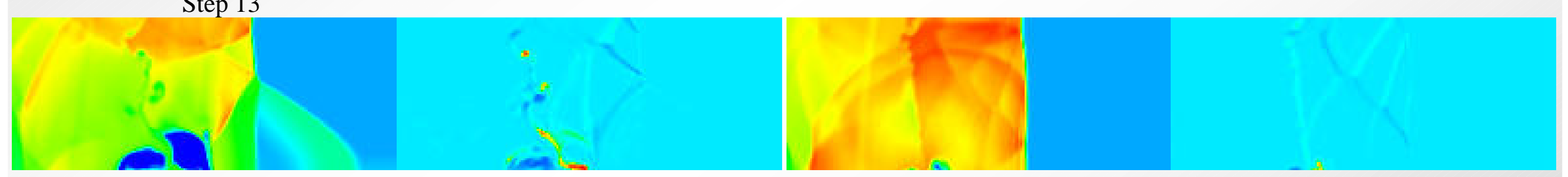

Step 25

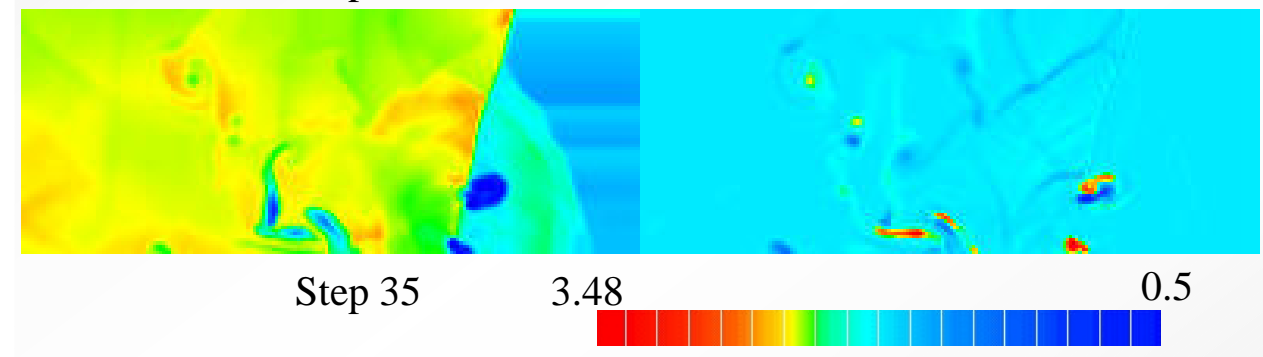

Density colormap
Slice $2(j=40)$
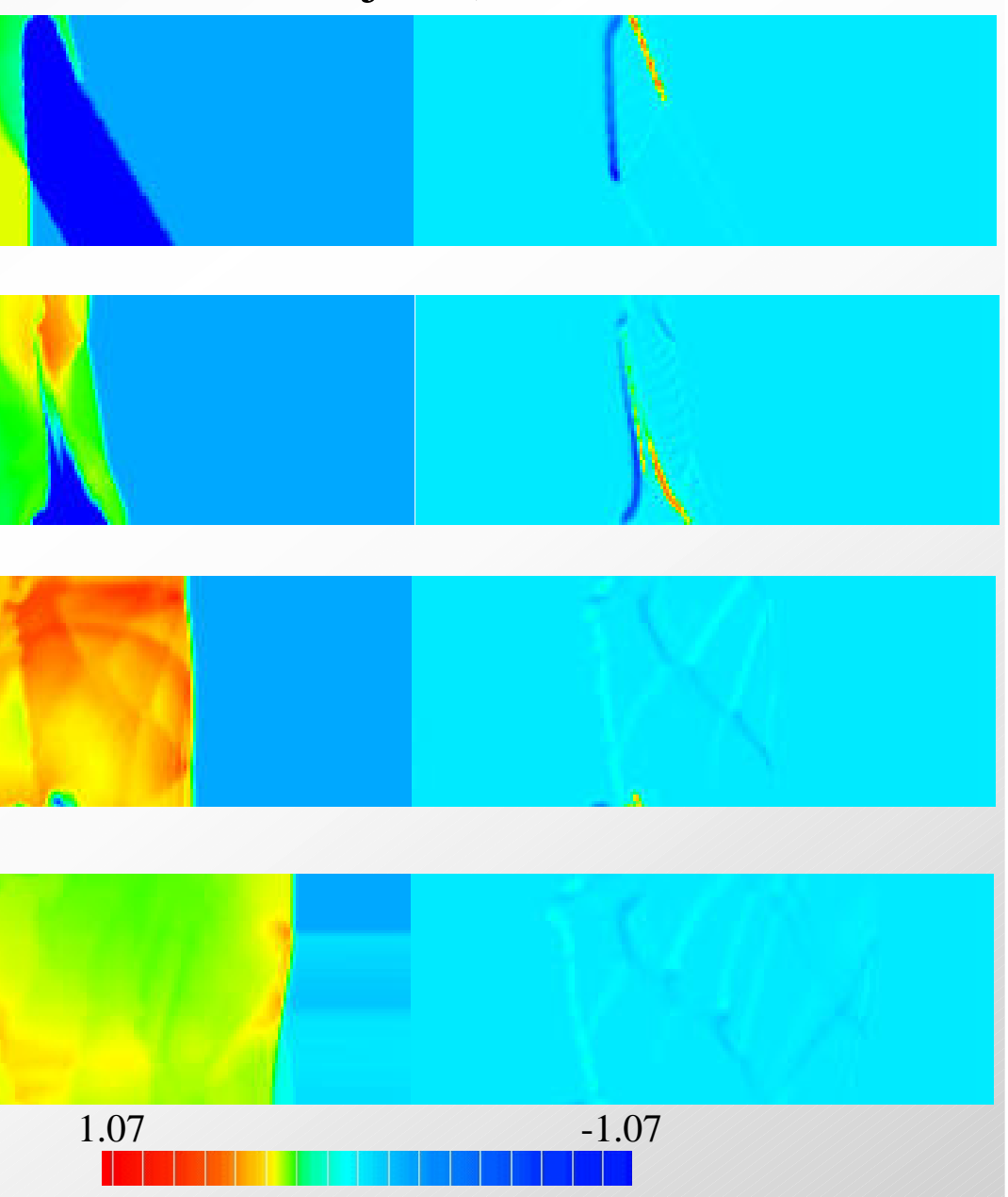

1.07

Vorticity colormap

Rutgers 


\section{D effects: juxtaposition of quasi-2D and 2D global circulation}

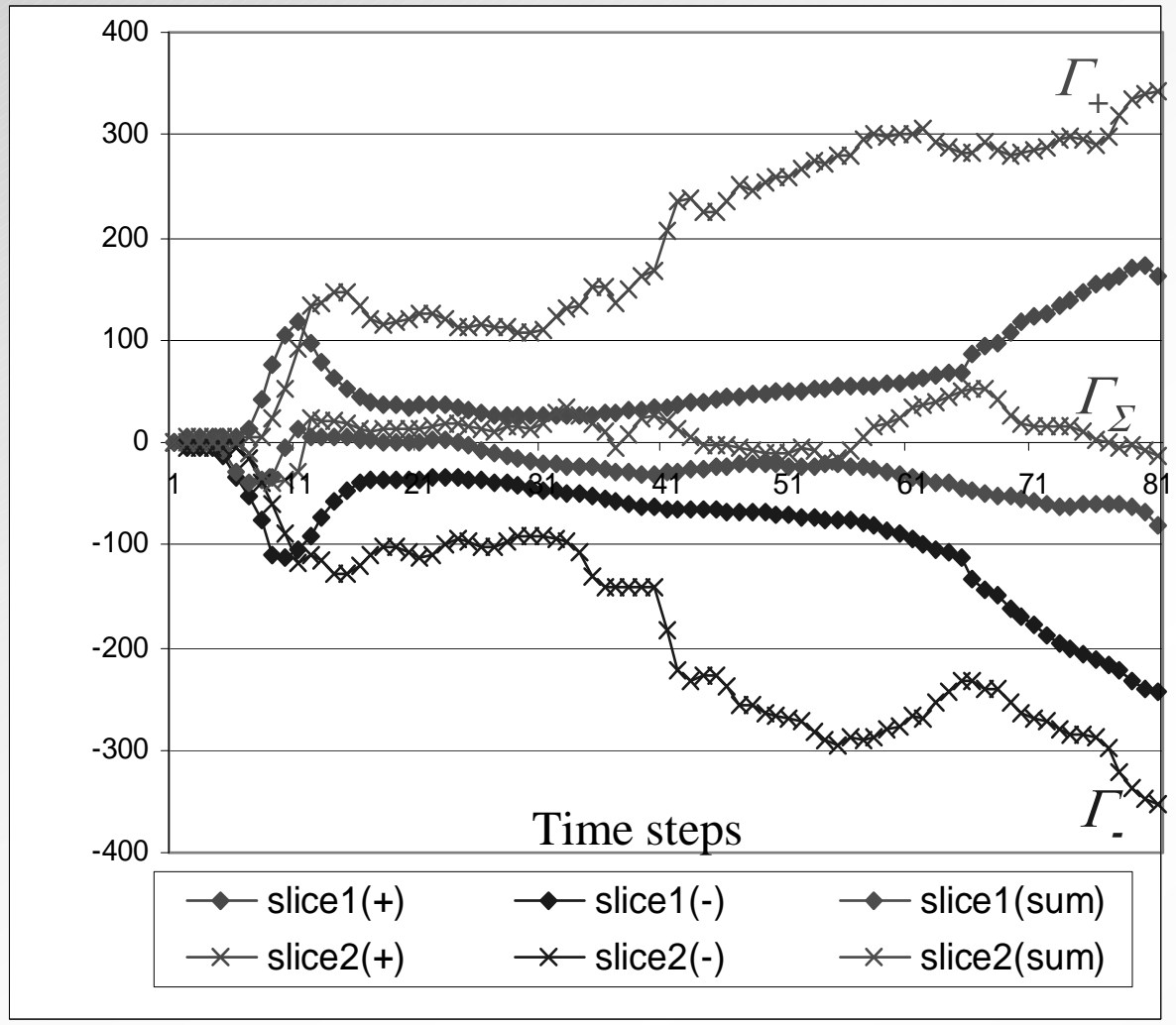

2 of the $3 D$ slices slice $1(y=8)$, slice $2(y=40)$

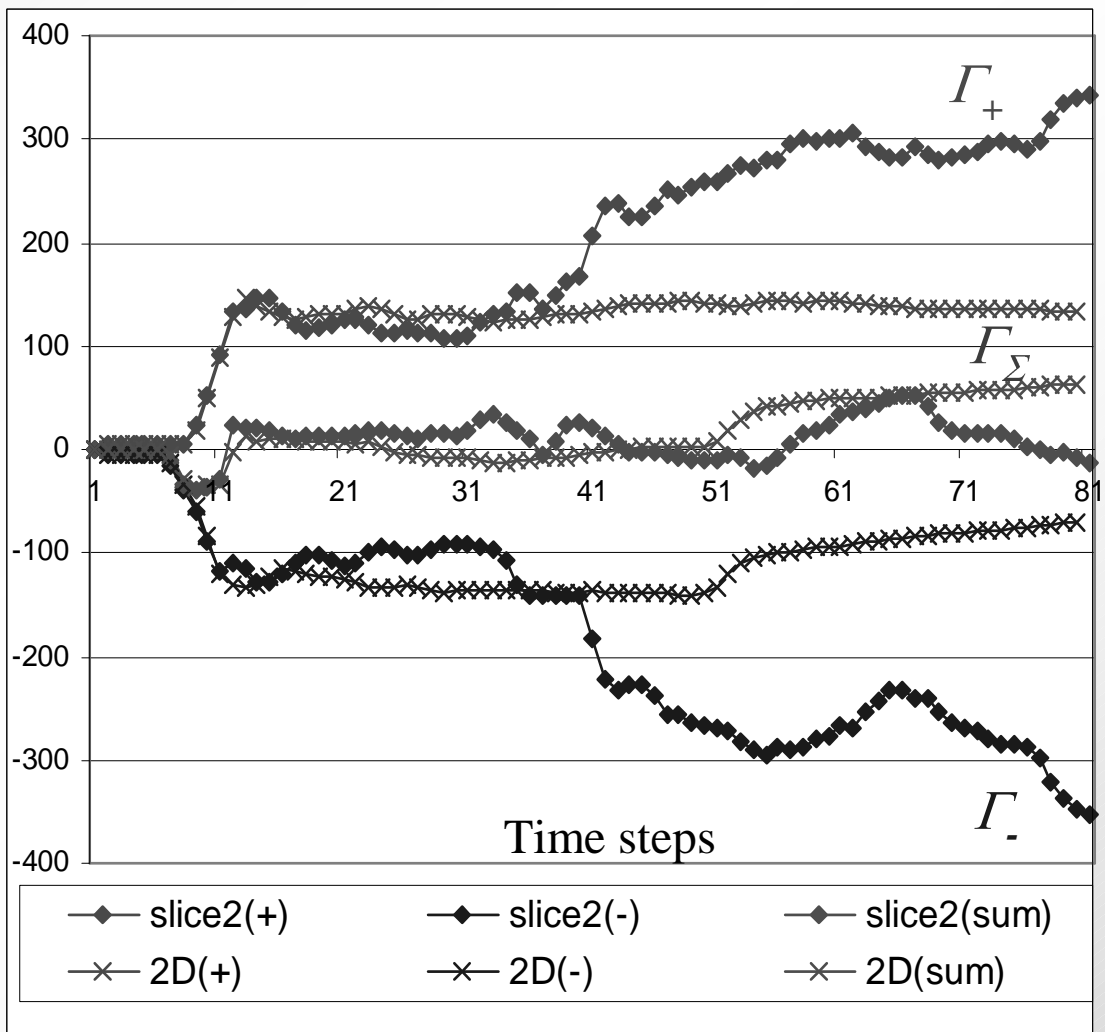

$3 \mathrm{D}$ center slice $(\mathrm{y}=40)$ and $2 \mathrm{D}$

Note:

1). Resolution: $200 * 80 * 80 ; 2$ ). Early time, the curtain behaves similar to $2 \mathrm{D}$ - No obvious $3 \mathrm{D}$ effects;

3). The slice near the boundary influenced by 3D early; 4). 3D effects become important and dominant when vortex structures localized

\section{Rutgers}




\section{Summary \& Future Directions}

- Simulations of s/f/s curtain with $\mathrm{M}=1.5$ to 5.0 ;

- Vortex double layers and large baroclinic circulation evolution;

- Visiometrics of vortex "layer" collisions and binding; Strong secondary baroclinic deposition,;

- Vortex projectiles emergence: quantitative study and modeling;

- Preliminary study of stratified turbulence domain;

- Preliminary adaptive mesh simulation of 3D planar curtain evolution; quantification via juxtaposition of slices. 


\section{Rapid Turbulization Arising from Vortex Double Layers in Interactions of "Complex" Blast Waves and Cylindrical/Spherical Bubbles}

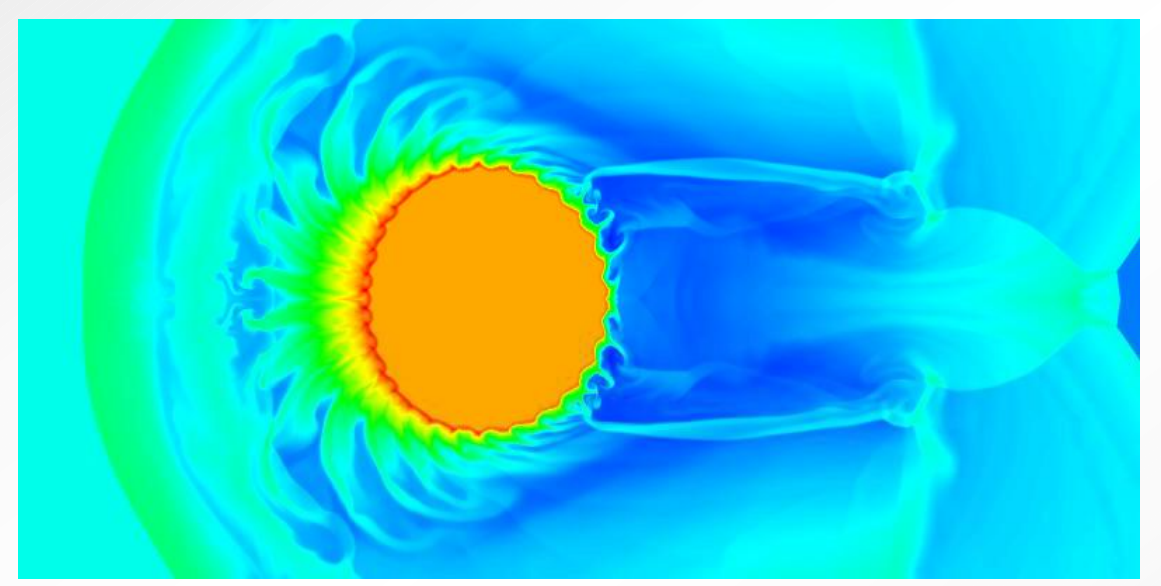

S. Zhang', N. J. Zabusky', K. Nishihara², and Y-G. Kang²

\section{VIZZ4A}

${ }^{1}$ Lab of Visiometrics and Modeling, Dept. of Mechanical \& Aerospace Engineering, Rutgers University, USA

${ }^{2}$ Institute of Laser Engineering, Osaka University, Japan

Rutgers 


\section{Motivations}

1. Supernova 1987A: Hydrodynamics of complex blast wave (shock-contact-shock) and ring interaction;

2. Upstream-ring erosion [Borkowski, Blondin \& McCray, 1997];

3. Emergence and significance of vortex double layer, vortex projectiles due to the primary and secondary deposition of baroclinic vorticity;

4. Numerical simulations (PPM) at extremely high density ratio (up to 5500) and Mach numbers (up to 70);

5. Compare simulation with laser experiment[Kang et al, 2001];

6. Prediction of the late time behavior 


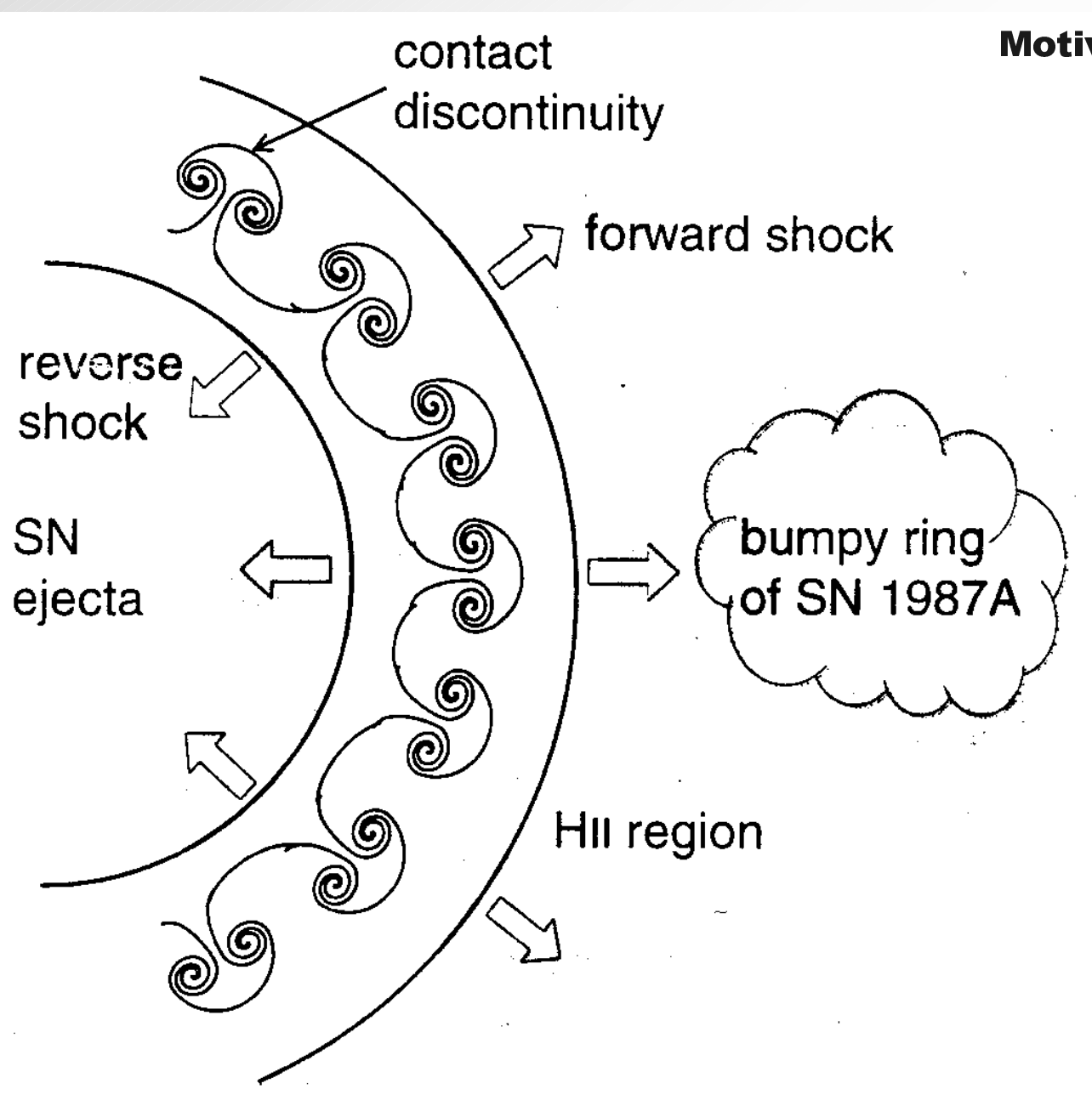

Fig.1. Turbulent contact discontinuity of the circumstellar region and the bumpy ring of SN 1987A: a more realistic situation 


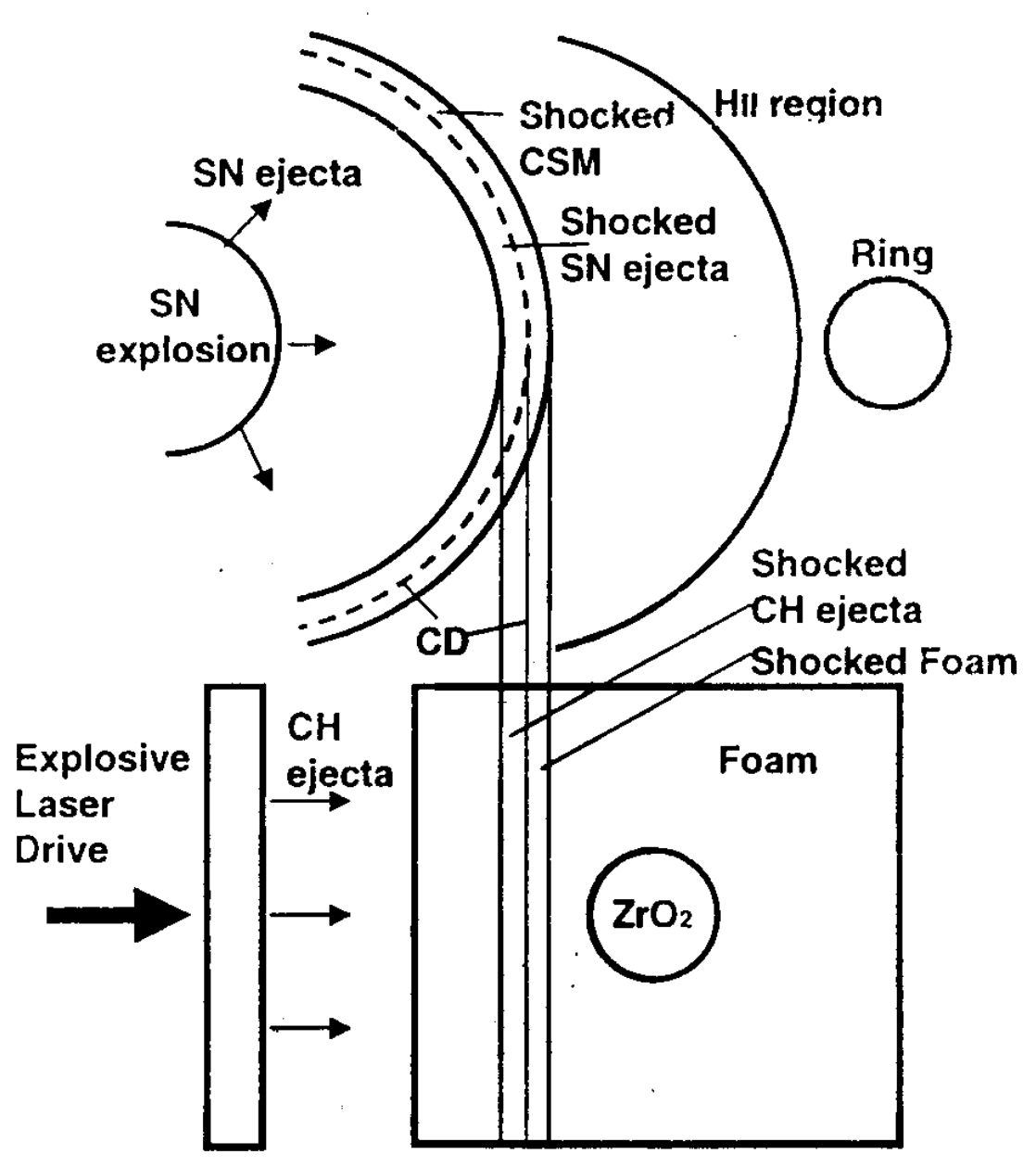

Motivations Cont.

Fig. 2. Schematic structure of the shocked region, the circumstellar region and the ring of SN1987A with an laboratory experiment. The ejected material ( $\mathrm{SN}$ ejecta and the $\mathrm{CH}$ ejecta) and the low density medium (CSM and the foam) are separated by a contact discontinuity (CD).

\section{Rutgers}




\section{Part I: Simulations ---- Cylindrical bubble}

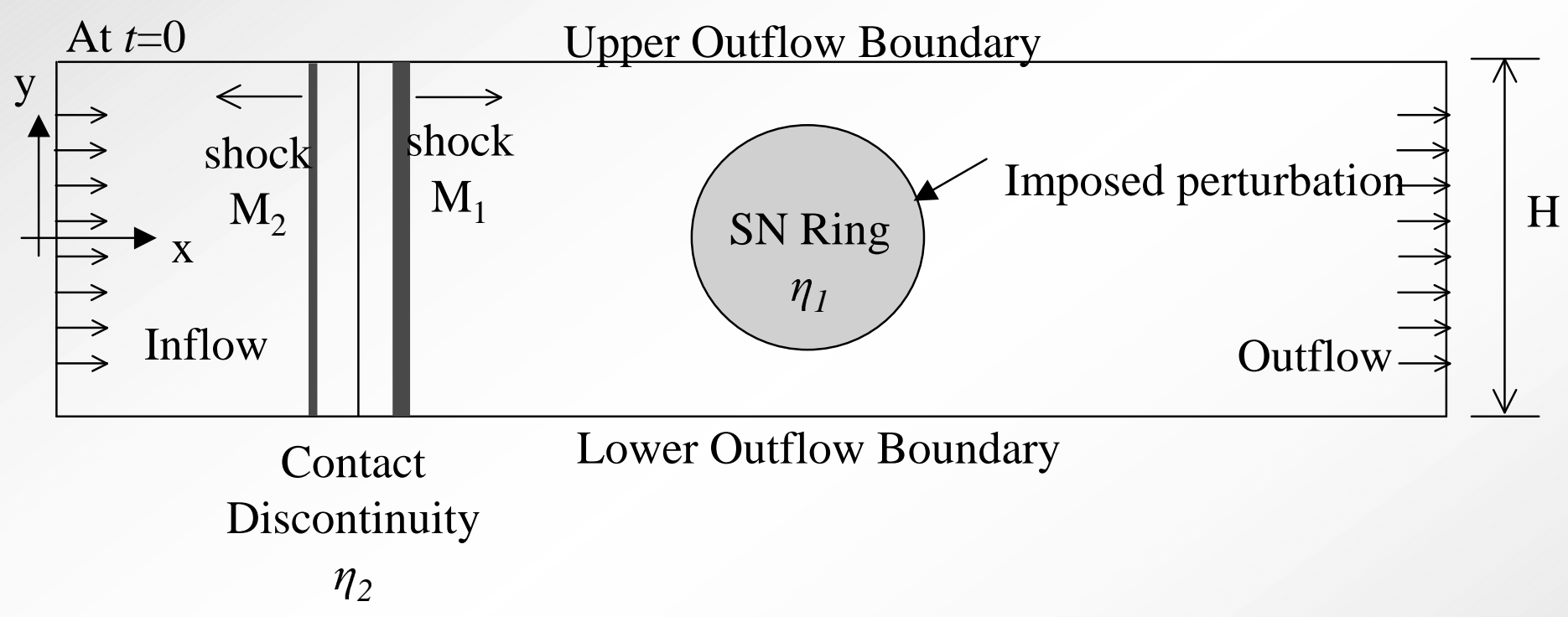

Fig. 3 Schematic of the computational domain and initial condition: Blast wave/SN Ring 


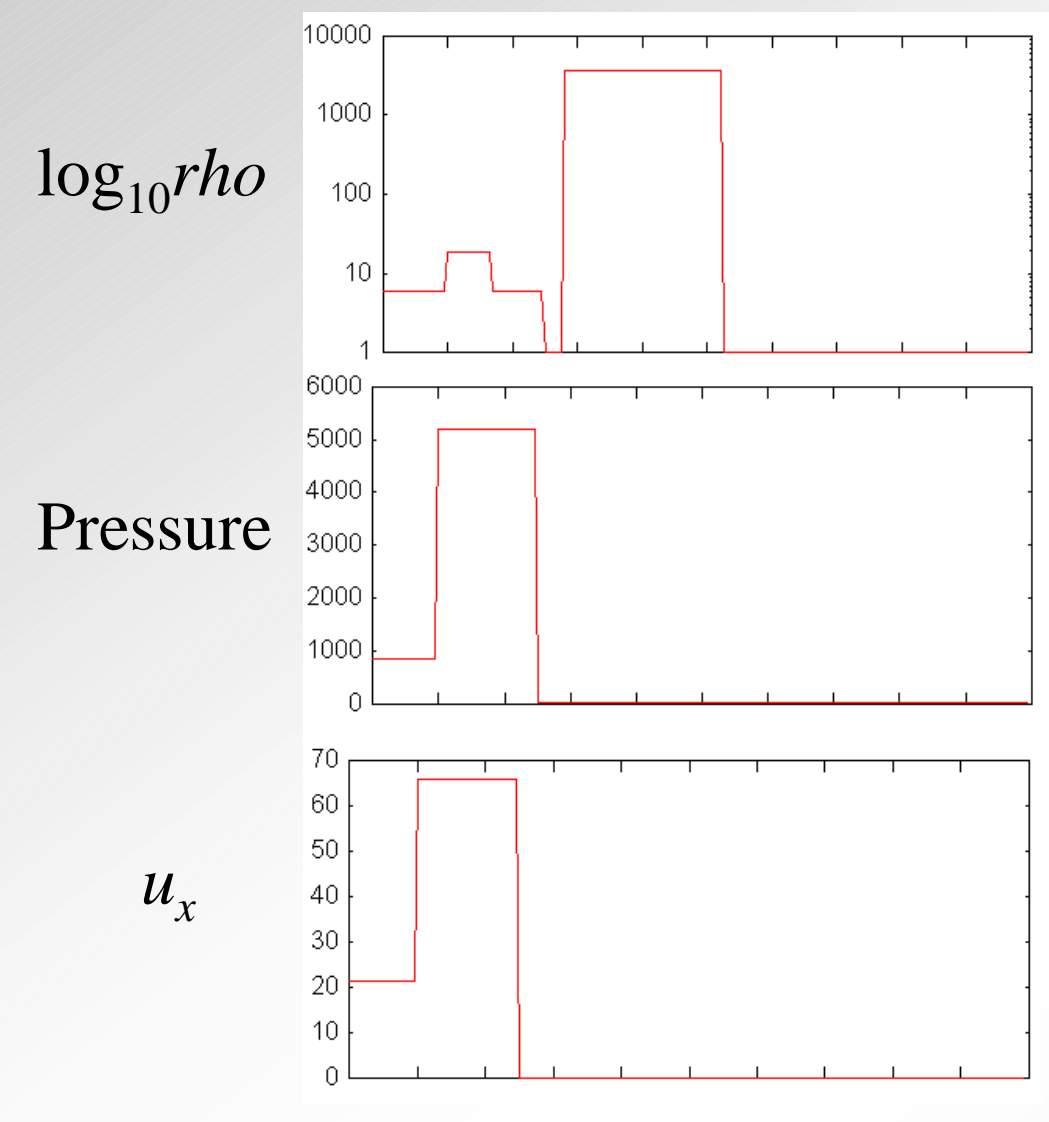

Fig. 4 Initial profiles of density (log scale), pressure, and $\mathrm{x}$-velocity

\section{Part Is Simulations Cont.}

Parameter Space:

$$
\begin{aligned}
& \cdot M_{1}=66.77 \\
& \cdot \eta_{1}=5000 \\
& \cdot \eta_{2}=4.0
\end{aligned}
$$

\section{$\underline{\text { Numerical Method: }}$}

- Compressible Euler Equation

- Piecewise Parabolic Method [Woodward \& Colella, 1984] 


\section{Global quantification:}

Part I: Simulations Cont.

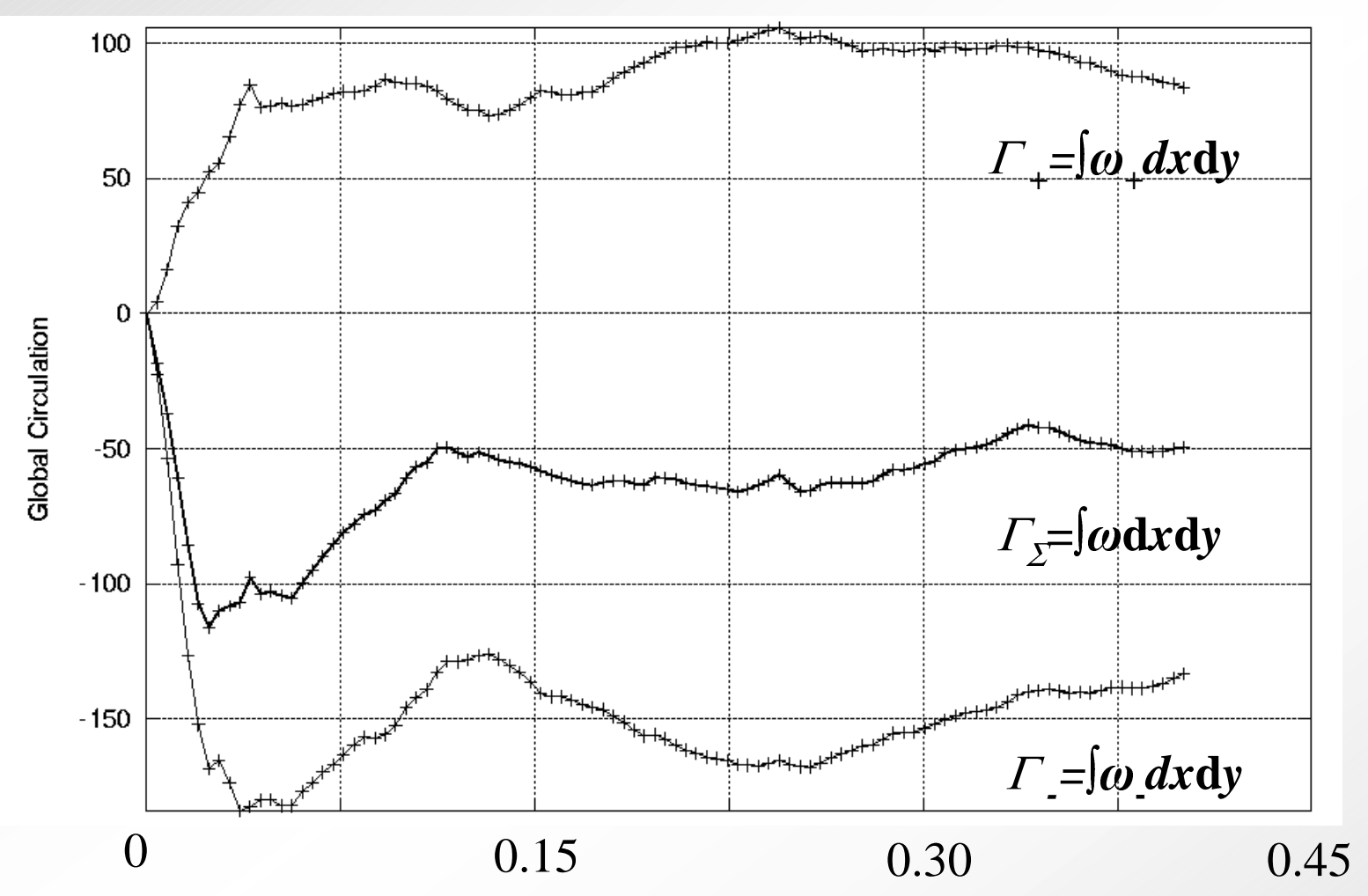

Fig. 5 Evolution of global circulation (positive, negative and sum integrated over the whole domain), $t=0.01$ corresponds approximately 4 years 
Simulation Snapshots I: Early time, juxtaposition of density (log scale), vorticity and $\operatorname{div}(u)$, vortex double layers, vortex projectiles, and upstream erosion
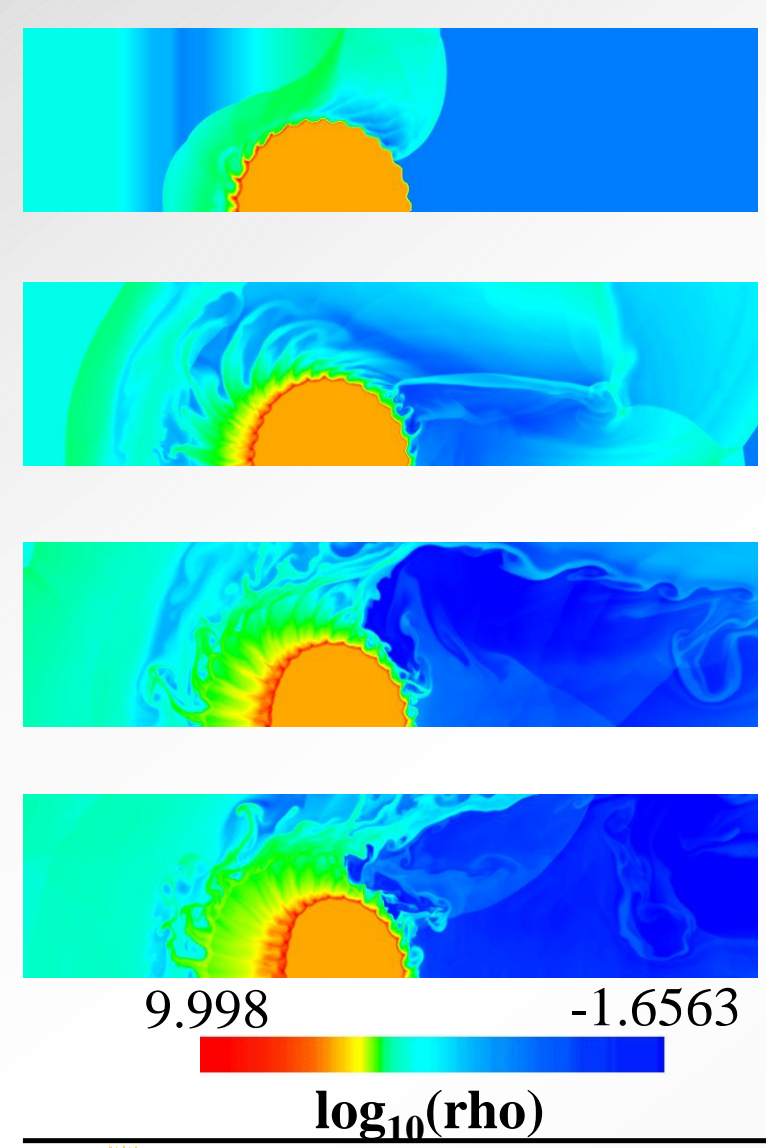

Rutgers
Part I: Simulations Cont.

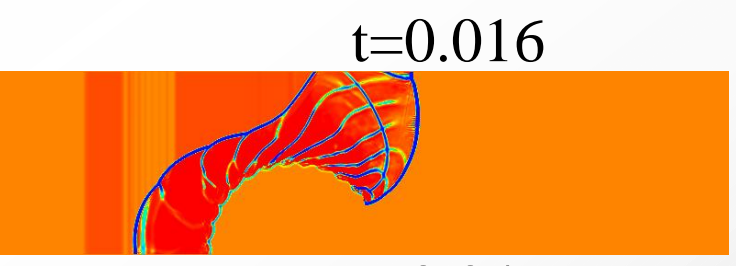

$$
\mathrm{t}=0.04
$$
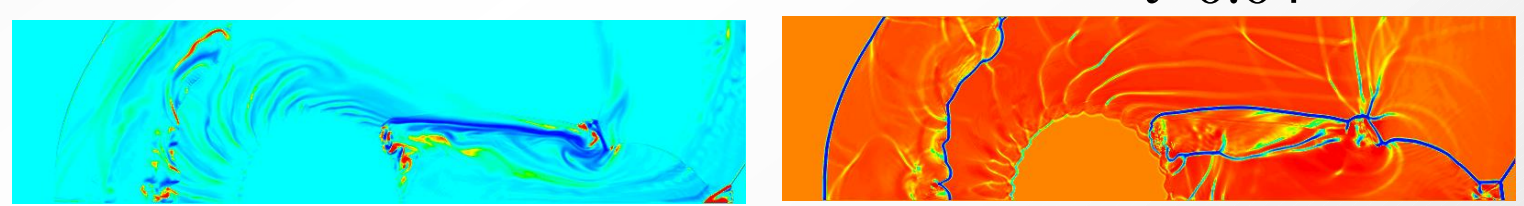

$$
\mathrm{t}=0.08
$$
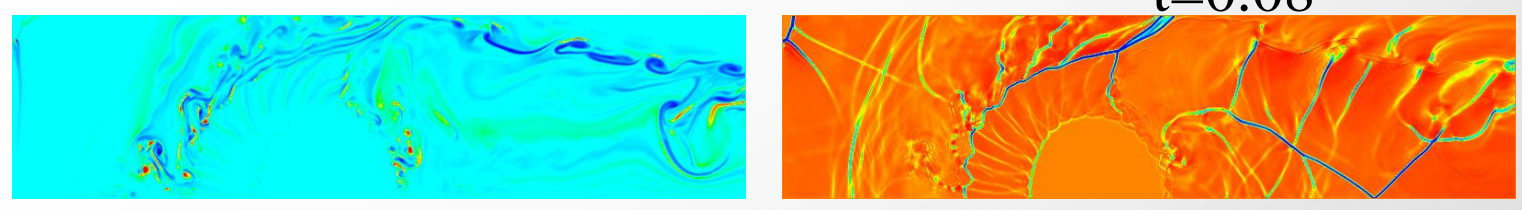

$$
\mathrm{t}=0.12
$$
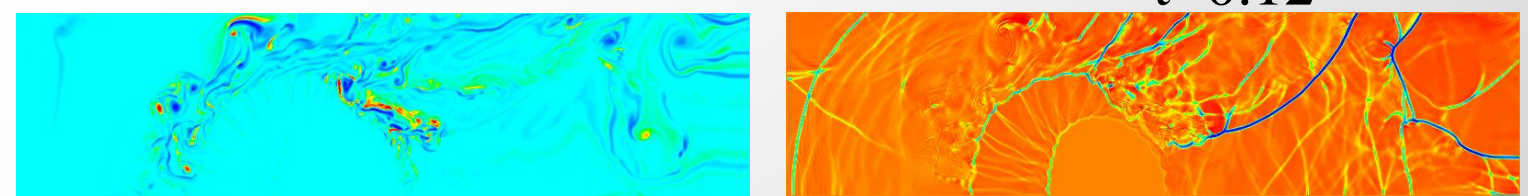

$$
10.52 \quad-10.55
$$

$\nabla \bullet \mathbf{u}$ 


\section{IWPCTM 8 POSTER C49}

\section{Upstream Erosion:}

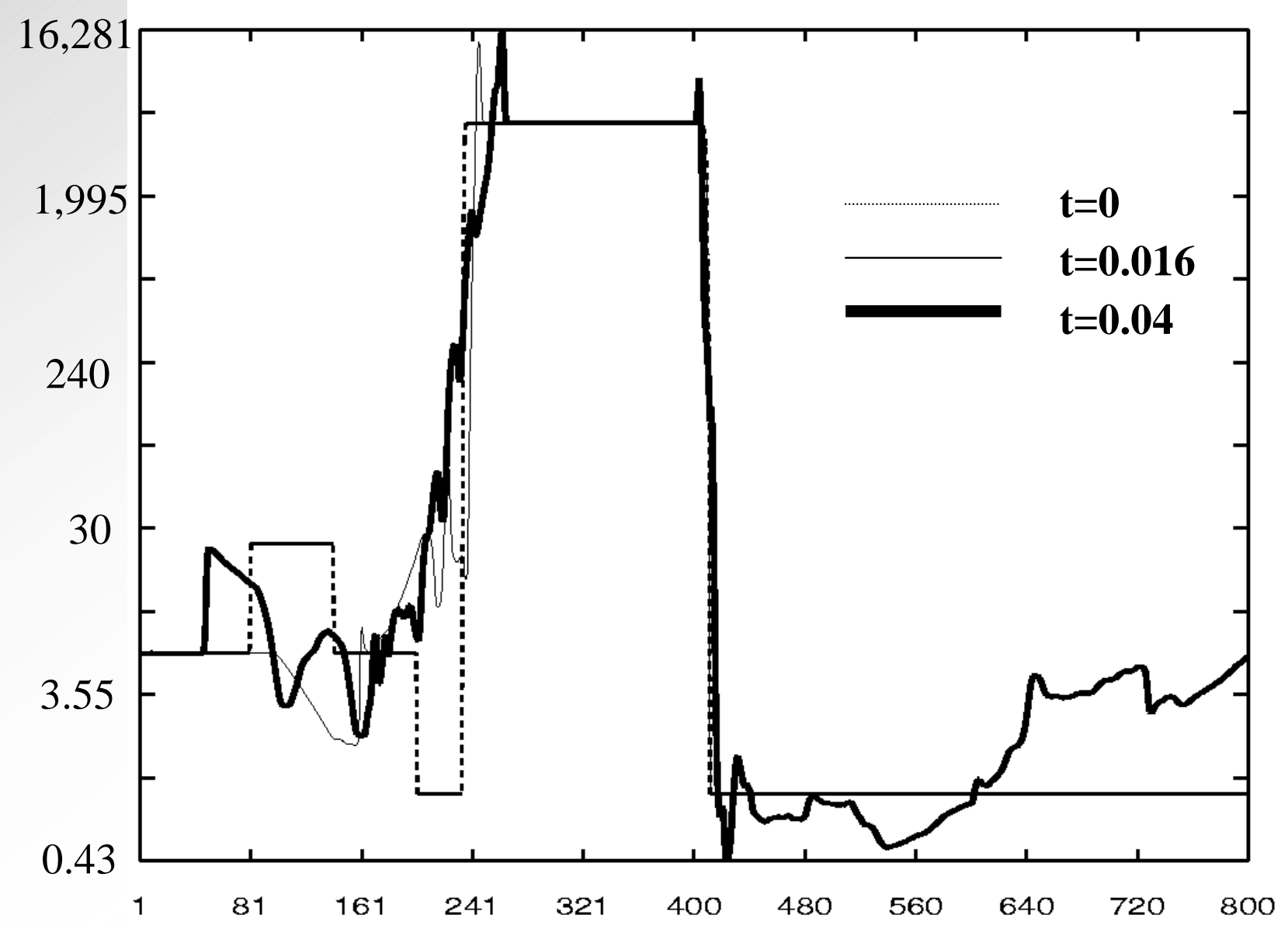

Fig. 6. Evolution of density (log scale) Slice at $j=10$ 


\section{Late time predictions}

Part Is Simulations Cont. $\mathrm{t}=0$
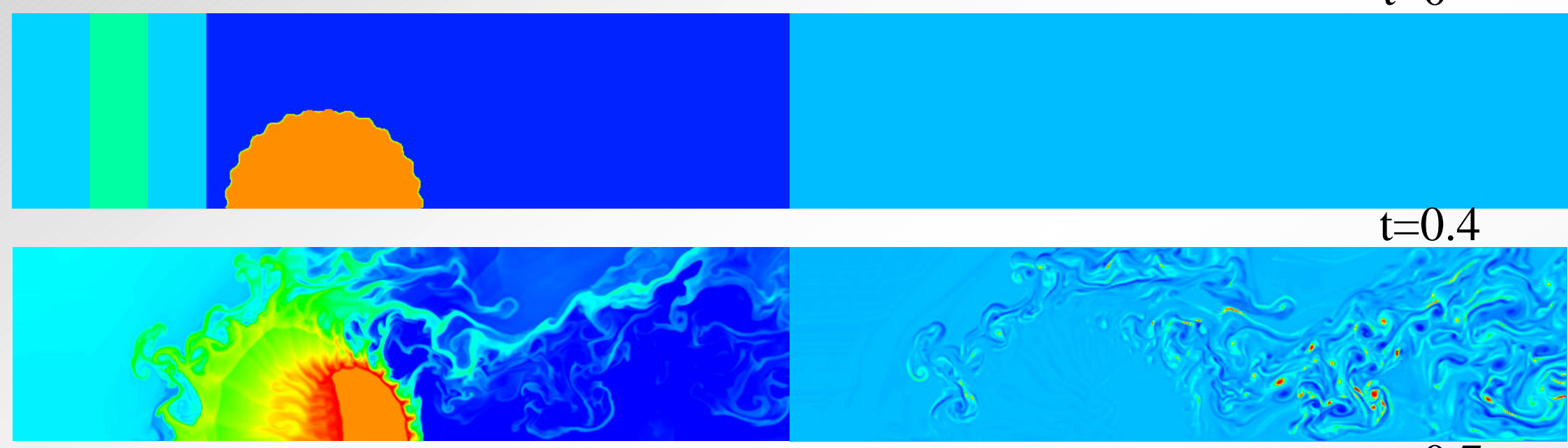

$$
\mathrm{t}=0.7
$$

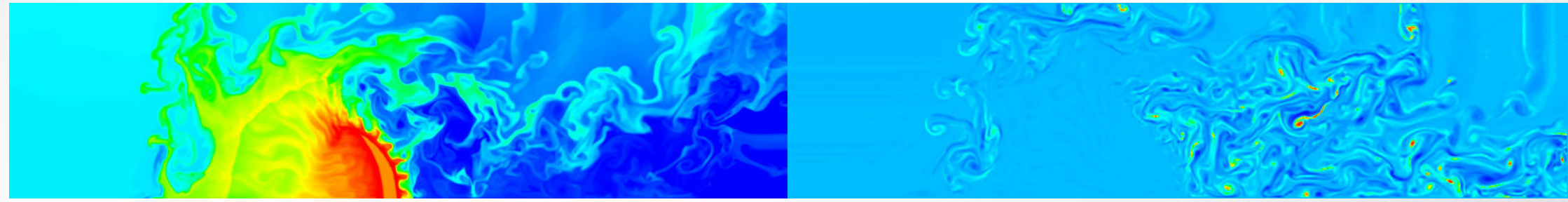

$$
\mathrm{t}=1.0
$$

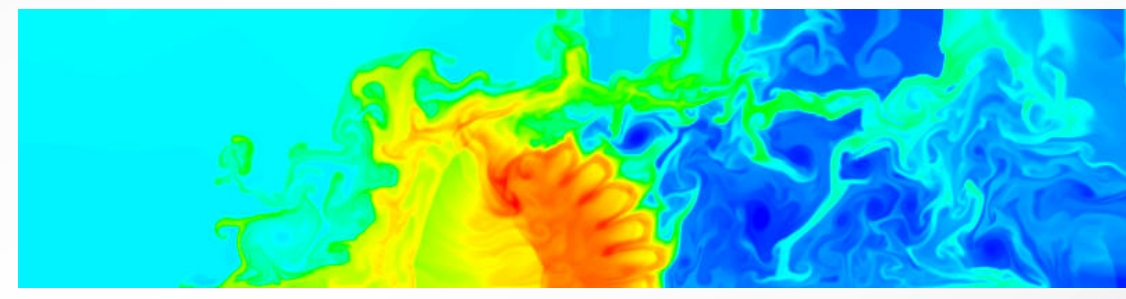

Rutgers 
Part Is Simulations Cont.

$\mathrm{t}=1.4$
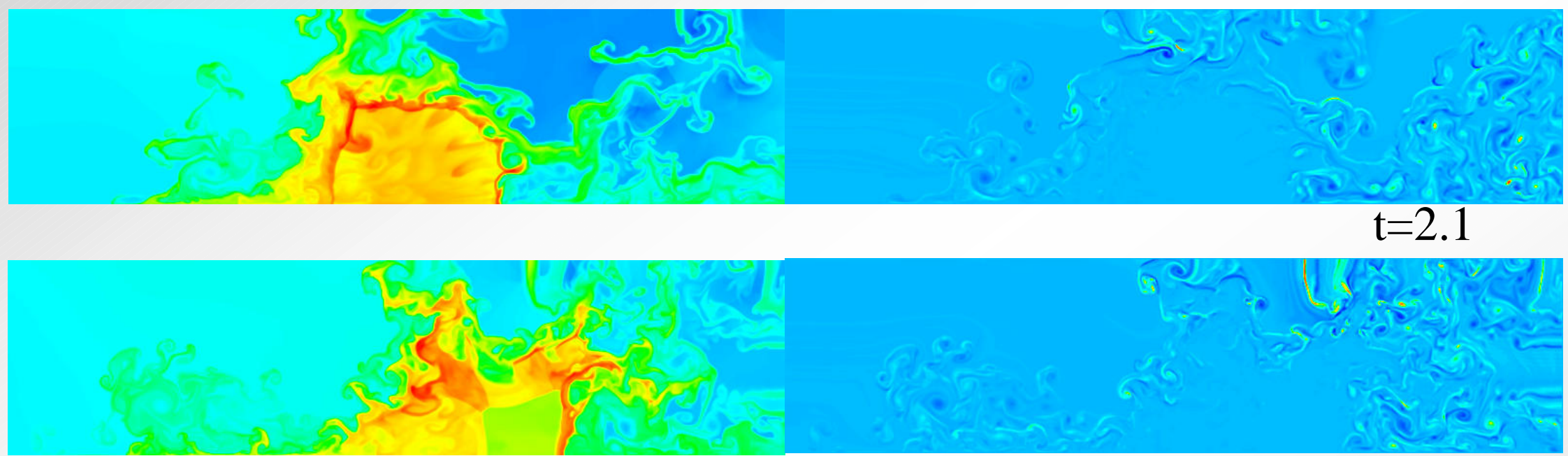

$$
\mathrm{t}=2.9
$$

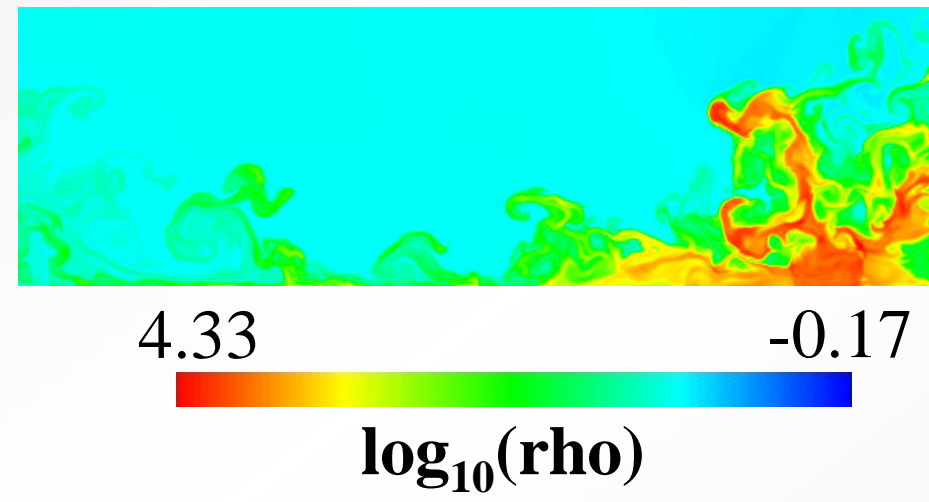

Rutgers

6.26

$-4.85$

vorticity

Rutgens 


\section{Conclusions}

1. We simulate a complex blast wave - cylindrical bubble interaction at $\mathrm{M}=66.77$ and $\eta=5000$ with PPM;

2. We observe strong early time positive and negative circulation and vortex double layers due to complex blast wave structure;

3. We study the upstream erosion, which is consistent with [Borkowski et al 1997];

4. At intermediate time, we observe the primary vorticity deposition separates from the interface in an elongated vortex layer

5. We observe late time cavity collapse and re-expansion and corresponding fragmentation of the $\mathrm{SN}$ ring. 


\title{
Laser shock tube for the study of supersonic gas flows and the development of hydrodynamic instabilities in layered media. Lebo I.G. ${ }^{1), 2)}$ Zvorykin V.D. ${ }^{2)}$
}

1) Technical University-MIREA, Russia, Moscow, Vernadsky prospect, 78,

2) Lebedev Physical Institute, Russia, Moscow, Leninsky prospect, 53, Fax: (095)- 132-11-96, e-mail: lebo@ sci.lebedev.ru

\begin{abstract}
.
The design of miniature laser shock tube for the study of a wide range of hydrodynamic phenomena in liquids at pressures greater than $10 \mathrm{kbar}$ and supersonic flows in gases with large Mach numbers (greater than 10) is discussed. A substance filling a chamber of quadratic cross section, with a characteristic size of several centimeters, is compressed and accelerated due to local absorption of 100-ns, 100-J KrF laser pulses ("GARPUN" installation, Lebedev Phys. Institute, Moscow) near the entrance window. It is proposed to focus a laser beam by a prism raster, which provides a uniform intensity distribution over the tube cross section. The system used to study the hypersonic flow past objects of complex shape and the development of hydrodynamic instabilities in the case of a passage of a shock wave or a compression wave through the interfaces between different media. The numerical simulations are used to model the laser shock tube experiments.
\end{abstract}

\section{Introduction.}

The stability of an interface between two media found in the field of constant or pulsed acceleration is a fundamental problem of fluid and gas mechanics.

In the first case, the contact surface is unstable if the gradients of pressure and density have opposite directions (Rayleigh-Taylor instability) /1/.

In the case of pulsed acceleration caused by the passage of a shock wave through a contact surface between two liquids or gases or a sharp deceleration of a substance, which previously moved, the interface is unstable for any arrangement of layers (Richtmyer-Meshkov instability-RMI) /2,3/.

The study of the evolution of hydrodynamic instabilities is a problem of great importance in inertial fusion, physics of high energy densities, cosmology and astrophysics. The passage of strong shock waves through contact surfaces of two gases with different densities causes the formation and development of complex vortex structures, which are of interest for present-day nonlinear hydrodynamics and for studying the problem of a change from an order state to chaos. Another problem, which is important for the development of modern aerospace engineering and protecting the Earth from collisions with space objects, is the study of supersonic flow past bodies of complex shape at large Mach numbers. The problems mentioned above are studied by numerical methods using $2 \mathrm{D}$ and $3 \mathrm{D}$ codes, in laboratory experiments with shock tubes $/ 3-5 /$, special rocket and gun facilities /6,7/ using high -power lasers /8,9/ and explosions /10,11/.

Lasers are used to study hydrodynamic flows and instabilities in plane, cylindrical, and spherical geometries, both in the case of direct target heating and in the case of converting laser radiation to x-rays. However, the multichannel and multielement laser facilities used for this 
purpose, such as ISKRA-5 (Russia), VULCAN (Great Britain), GEKKO-XII (Japan),

NIKE, NOVA and OMEGA (USA) with radiation energy of order of $10 \mathrm{~kJ}$ are extremely expensive. In typical laser experiments, the radiation intensity $q$ in focal spot of size (0.1-1) $\mathrm{mm}$ is $10^{13}-10^{15} \mathrm{~W} / \mathrm{cm}^{2}$ and the pulse duration is of order of $1-10 \mathrm{~ns}$. In this time, a shock wave travels in a solid path 1-10 $\mu \mathrm{m}$ long, which determines the characteristic scale of a phenomenon being studied, because the unloading wave rapidly weakens the shock wave after the termination of a laser pulse. The development of instability in condensed media, as a rule, is studied with complex and expensive x-ray techniques, which often give only time- and space integrated information.

We propose a new technique for exciting shock waves in gases and compression waves in liquids by a pulsed KrF-laser with considerably lower radiation energy $(\sim 100 \mathrm{~J})$ for modelling a wide range of hydrodynamic phenomena mentioned above at a spatial scale of $1-10 \mathrm{~mm}$ in a range of microsecond duration. The idea is based on two known facts:

(1) lasers with pulse duration of 10-100 $\mathrm{ns}$, when irradiating the surface of a solid coated with a thin transparent layer, which hampers a rapid unload of the evaporated substance, produce pressure jumps with amplitudes more then $10 \mathrm{kbar}$ at moderate radiation intensities $10^{8}-10^{9}$ $W / \mathrm{cm}^{2}$;

(2) unloading target excites strong shock waves with Mach number more then 10 in the atmospheric air surrounding it.

\section{Design of laser shock tube.}

The design of such a laser shock tube is based on the use of following basic components: a miniature shock tube chamber, a powerful $\mathrm{KrF}$ laser, a laser focusing system and 2D numerical codes. The shock tube chamber is shown in Fig.1a,b. 
Figure 1. Schematic of laser shock tube for investigation of RMI (a) and hypersonic flow past objects of complex shape (b).

a)

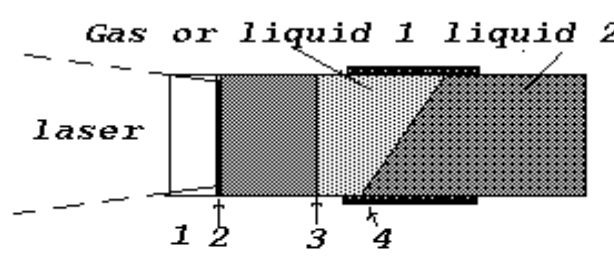

1 transparent plate

2 opaque thin layer

3 shock vave front

4 piezoelectric ceramics b)

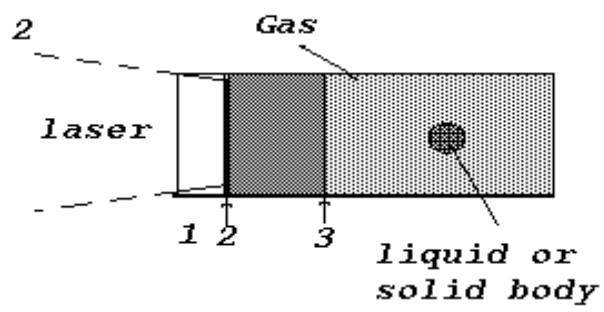

Laser radiation enters through a transparent window (1) inside a chamber, which is filled with a liquid or gas. Radiation is absorbed in a thin layer (2) adjacent to the window, resulting in a compression wave travelling in both directions from the energy release region.

Initial perturbations on the contact surface between two liquids or a liquid and gas can be produced by a piezoceramic transducer (4) attached to the chamber wall or with help of a thin shaped film. One can place liquid drops or solid particles of different shape inside the chamber which is filled with a gas (see Fig.1b). Because the liquids and gases under study are transparent to probing visible radiation, one can use conventional high-speed shadow and schlieren photography. In detail it is possible to find the description of laser shock tube in $/ 12 /$.

\section{Numerical simulation of the propagation of pressure waves in laser shock tube.}

The numerical calculations modeling the formation and propagation of a pressure wave were made using 2D Lagragian code "ATLANT" /13/ and 2D Euler code "NUTCY" /14/ in cylindrical geometry. A laser beam traveled along z-axis. Laser shock tube has length of $12 \mathrm{~cm}$ and contains three (or four) subregions: transparent layer of silica glass with density $2.5 \mathrm{~g} / \mathrm{cm}^{3}$, aluminium foil with thickness of $2 \mu \mathrm{m}$ and $X e$-gas filled chamber with initial density $5.4 \mathrm{mg} / \mathrm{cm}^{3}$. Laser radiation was incident from the right, passed through a transparent layer, and was totally absorbed in aluminium layer. A KrF laser pulse had a trapezoidal form, with leading and trailing edges $20 \mathrm{~ns}$ long and a region of constant intensity $3.1 \times 10^{8} \mathrm{~W} / \mathrm{cm}^{2} 60 \mathrm{~ns}$ long. Because of heating and evaporation of a thin Al-foil, a pressure jump with the amplitude about 10 kbar occurred and compression wave in glass and shock wave in gas was produced. Fig.2 shows the results of calculation modeling the propagation of a shock wave in Xe-gas filled chamber. The sound speed in xenon and glass was $174 \mathrm{~m} / \mathrm{s}$ and $3.7 \mathrm{~km} / \mathrm{s}$, respectively. One can see that strong shock wave 
with Mach numbers $\mathrm{M} 40$ is formed in gas, and this wave gradually damps during its position. By a moment of $700 \mathrm{~ns}$, it travels a $4 \mathrm{~mm}$ path, but its velocity remains rather high and corresponds to $M \geq 20$.

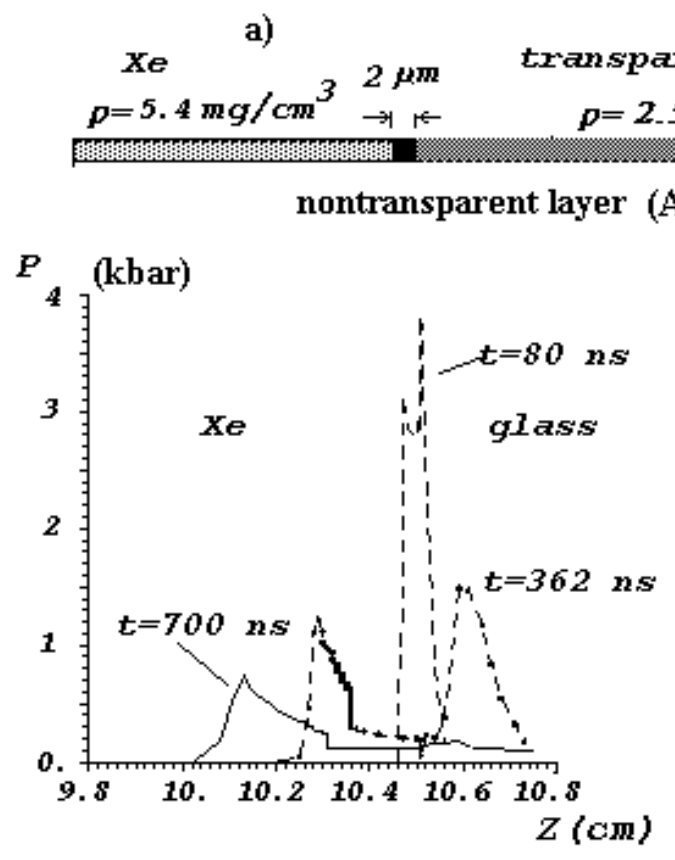

b)

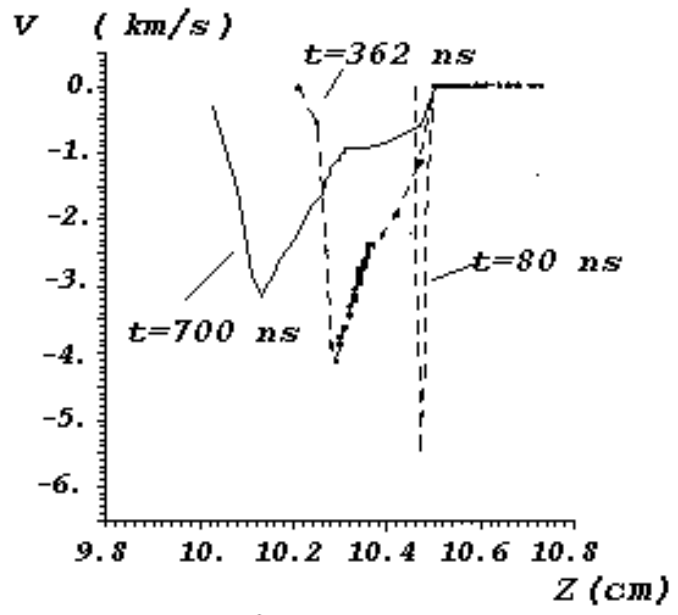

c)

Figure 2. Results of calculation. Schematic of the experiment (a); the distributions of pressure (b), and velocity (c) along the $z$-axis at the moments of time $t=80(1), 362(2)$, and 700

(3) ns.

\section{Preliminary experiments.}

The experiments have been performed at GARPUN electron-beam pumped $\mathrm{KrF}$ laser installation in Lebedev Physical Institute (Moscow) /15/. Laser beam was focused on the targets by an optical system consisting of the prism raster and the lens. Overlapping of 25 individual beamlets provided non-uniformity less then few percents across the square spot. By moving the lens we could vary a spot size from $20 \times 20$ to several $\mathrm{mm}$. For a fixed $7 \times 7 \mathrm{~mm}$ focal spot laser intensities were changed in the range of $0.1-1 \mathrm{GW} / \mathrm{cm}^{2}$ by attenuating incident laser energy. The targets were set inside an evacuated chamber filled with an air, which pressure being varied in the range of $p_{0}=0.0001-1$ bar. Gasdynamic processes that developed under laser-target interaction were studied with the help of high-speed optomecanical camera in combination with schlieren or shadow technique /16/. Slit scanning images of the self-luminescence laser-produced plasma were combined with images of flying foil targets and shock wave (SW) propagating in surrounding air.

It was observed that during laser pulse action plasma front propagated towards an incident radiation together with the SW front. The velocities in dependence on laser intensity (Fig.3) and air pressure (Fig.4) are shown. Solid lines in Fig.3 correspond to calculated velocities of laser- 
supported detonation wave (LSDW) /17/, dashed lines are the results of numerical simulations using “ATLANT_C”-code /18/.

The most part of laser radiation (up to $90 \%$ ) penetrated to the target. At lower air pressures $p_{0}<0.1$ bar (Fig.4) the absorption in air reduced and did not influence on SW velocity $(\cong 30 \mathrm{~km} / \mathrm{s})$.

Air Pressure 1 bar; Fe Target

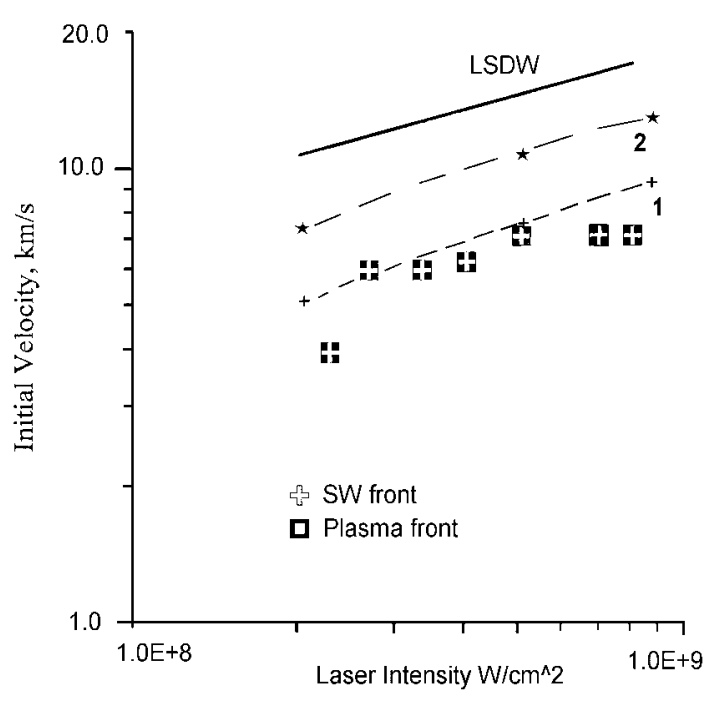

Fig.3. The dependence of plasma front velocity from laser intensity. Dashed lines the results of numerical simulations with allowance for ionization processes (1) and when $\mathrm{Z}=$ const $(2)$.

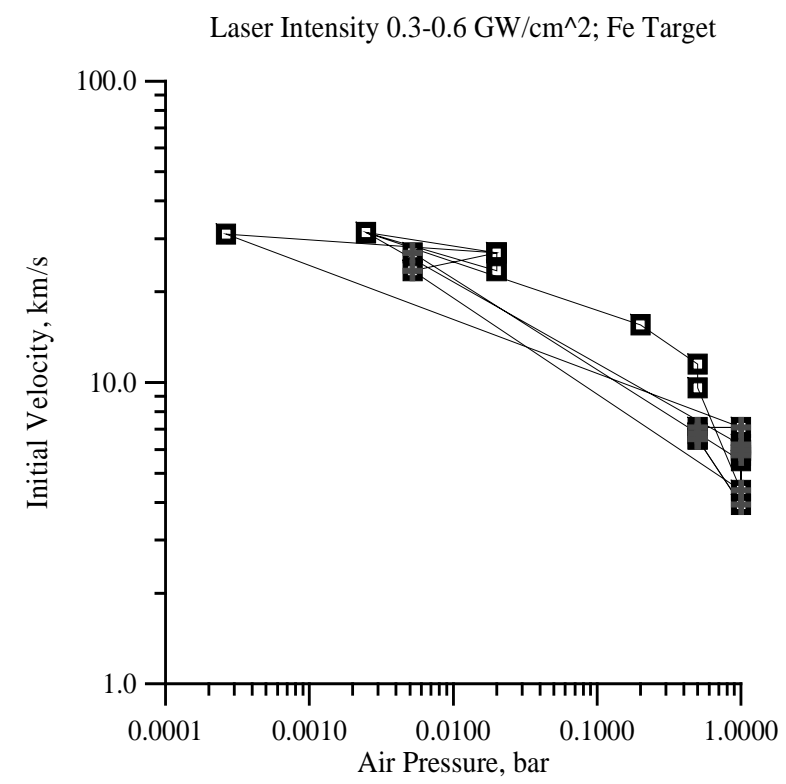

Fig.4. The dependence of plasma front velocity from gas pressure. Laser intensity is $0.3-0.6 \mathrm{GW} / \mathrm{cm}^{2}$.

\section{Conclusions.}

The technique proposed here for exiting shock waves in gases and compression waves in liquids by $\mathrm{KrF}$ laser radiation has some advantages in comparison with the conventional technique used in experiments with nanosecond laser pulses. The typical scale of gas-dynamic flow is of the order of $1 \mathrm{~mm}$, and the duration of processes reaches several microseconds. This enables one to use conventional optical techniques to observe the spatial and time evolution of instabilities at the interfaces between different media and to study the supersonic gas flow past objects of complex shape. One can also study the effect of a repeated initiation of acceleration by several shock waves, which are produced by a tandem of laser pulses.

The advantages of laser shock tube in comparison of ordinary shock tube are 1) the generation of large pressure pulse (as a result high Mach number $\geq 20$ shock wave) and 
2) the economy of noble gases and other supplies.

The preliminary experiments show that in contrast of $\mathrm{CO}_{2}$ laser-plasma experiments the radiation of $\mathrm{UV} \mathrm{KrF}$ laser comes through atmosphere and reach condense target. It allows to carry out experiments with solid targets and background gases with the initial pressure is about 1 bar.

\section{Acknowledgments.}

We are grateful to Prof.V.F.Tishkin and Dr.A.B.Iskakov for help of numerical modeling of laser plasma jet formation and shock wave expansion in Air (see Fig.3).

This work was supported by the Russian Foundation for Basic Research, project N 01-01-00023.

\section{References.}

[1] G.Taylor Proc. Roy. Soc., A201, 192, (1950)

[2] R.D.Richtmyer, Commun. Pure Appl. Math.,13, 297, (1960)

[3] E.E.Meshkov, Izv. Akad. Nauk SSSR, Mekh. Zhidk. Gasa, 5, 151, (1969)

[4] J.Haas, B.J.Sturtevant. Fluid Mech., 1281, 41, (1987)

[5] A.N. Aleshin, E.V.Lazareva, S.G.Zaytsev et al. Dokladi Akademii Nauk SSSR, 310, 1105, (1990)

[6] K.I.Read, Physica D, 12, 45, (1984)

[7] Yu.A. Kucherenko et al., In: Proc. of 3rd International Workshop on Physics of Compressible Turbulent Mixing at Royaumont, France, 1991, p.427

[8] C.J.Pawlew et al. Phys. Plasmas, 4, 1969, (1997)

[9] D.H.Kalantar et al., Phys. Plasmas, \{4\}, 1985, (1997)

[10] R.F.Benjamin. In: Proc. of 3rd International Workshop on Physics of Compressible Turbulent Mixing at Royaumont, France, (1991)

[11] A.Lebedev et al., In: Proc. of 5th International Workshop on Physics of Compressible Turbulent Mixing at Stony Brook, USA, 1995, p.213

[12] V.D.Zvorykin, I.G.Lebo Quantum Electronics, 30, 540, (2000)

[13] I.G.Lebo et al. J. Russ. Laser Research, 15, 136, (1993)

[14] I.G. Lebo et al., In: Proc. of 6th International Workshop on Physics of Compressible Turbulent Mixing at Marcellie, France, 1997, Institute Universitaire des Systemes Thermiques Industriels 1997, p.312

[15] V.D.Zvorykin, I.G.Lebo, Laser and Particle Beams, 17, 69, (1999)

[16]. V.A.Danilychev, V.D.Zvorykin. In: Trudi Fiz. Insit. P.N.Lebedeva Akad. Nauk SSSR, $\{142\}$, 117, (1983)

[17] Yu.P.Raizer Sov. Phys.-JETP, 21,1009, (1965) 
[18] A.B.Iskakov, I.G.Lebo, V.F.Tishkin. J. Russ. Laser Research, 21,247, (2000) 


\section{Simulations of a Shock-Accelerated Gas} Cylinder and Comparison with Experimental Images and Velocity Fields

\section{Cindy A. Zoldi}

(Los Alamos National Laboratory and SUNY at Stony Brook)

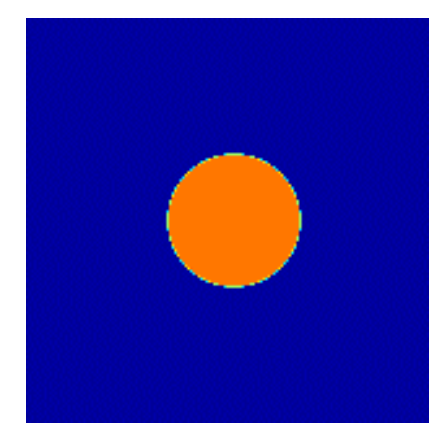

8th IWPCTM

California Institute of Technology

Pasadena, California

December 9-14, 2001

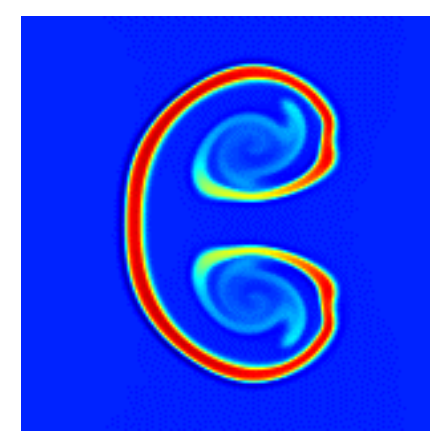




\title{
Collaborators
}

\section{Experimenters:}

Kathy Prestridge (LANL, DX-3) Bob Benjamin (LANL, DX-3)

Paul Rightley (LANL, DX-3) Peter Vorobieff (UNM)

Chris Tomkins (LANL, P-22/DX-3) Mark Marr-Lyon (LANL, DX-3)

\section{Computational Scientists:}

\author{
RAGE: Mike Gittings (LANL/SAIC, X-2) \\ Mike Steinkamp (LANL, X-3) \\ Cuervo: Bill Rider (LANL, CCS-2) \\ Jim Kamm (LANL, CCS-2) \\ CHAD: Barbara Devolder (LANL, X-5) \\ Manjit Sahota (LANL, T-3)
}

\section{Thesis Advisors:}

$$
\text { James Glimm (Stony Brook) David Sharp (LANL, T-3) }
$$




\section{Outline}

- Purpose of research

- Experimental apparatus

- Simulation setup

- Qualitative and quantitative comparisons

- Future work 


\section{Our View of Scientific Modeling}

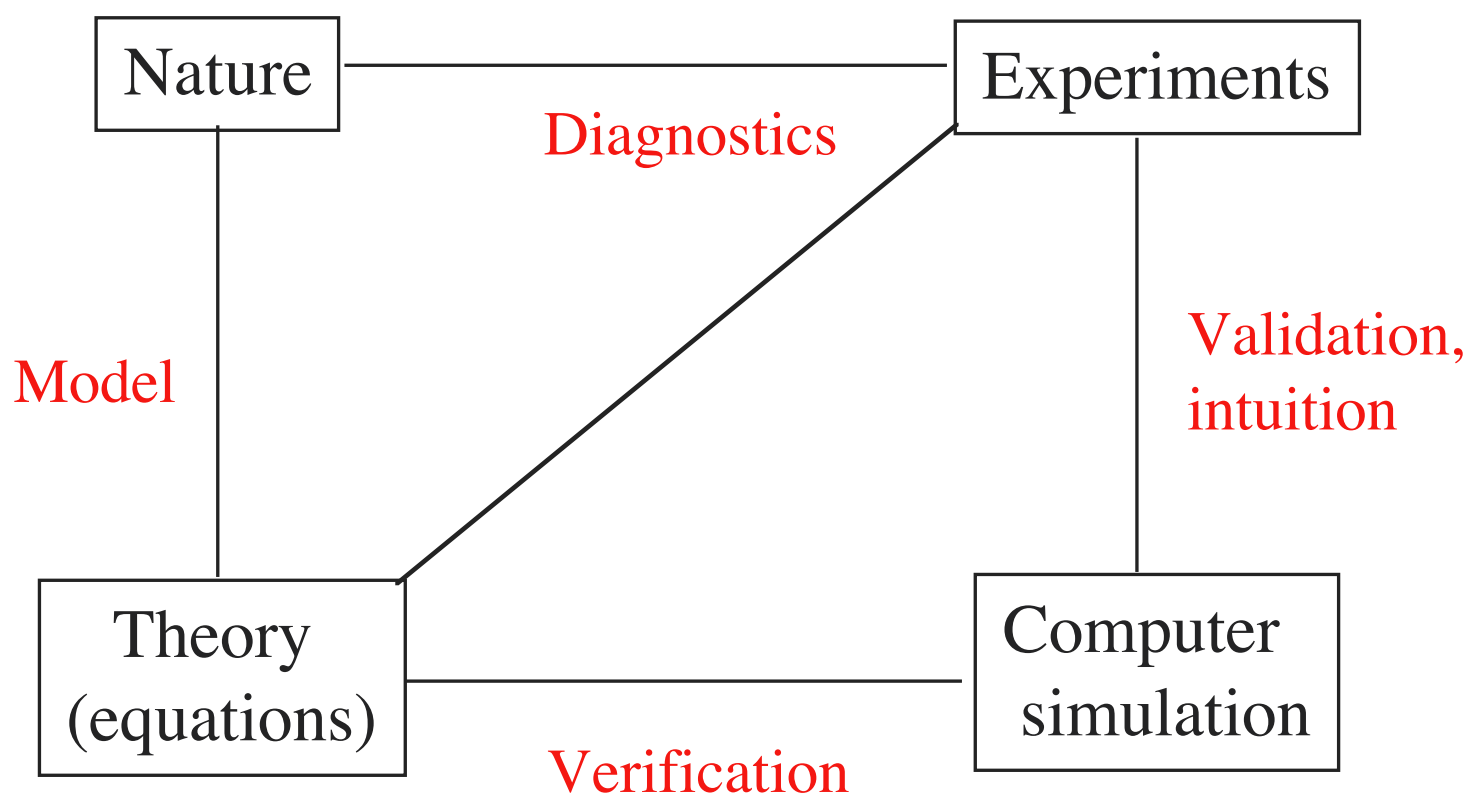

How well do computer simulations approximate nature? 


\section{Richtmyer-Meshkov Instability}

What is the Richtmyer-Meshkov instability?

It occurs when a shock wave collides with an interface between two different materials causing perturbations on the interface to grow.

Example: Shock moving from air into $\mathrm{SF}_{6}$ gas $\left(\right.$ Note: $\left.\rho_{\text {air }}<\rho_{\mathrm{SF} 6}\right)$
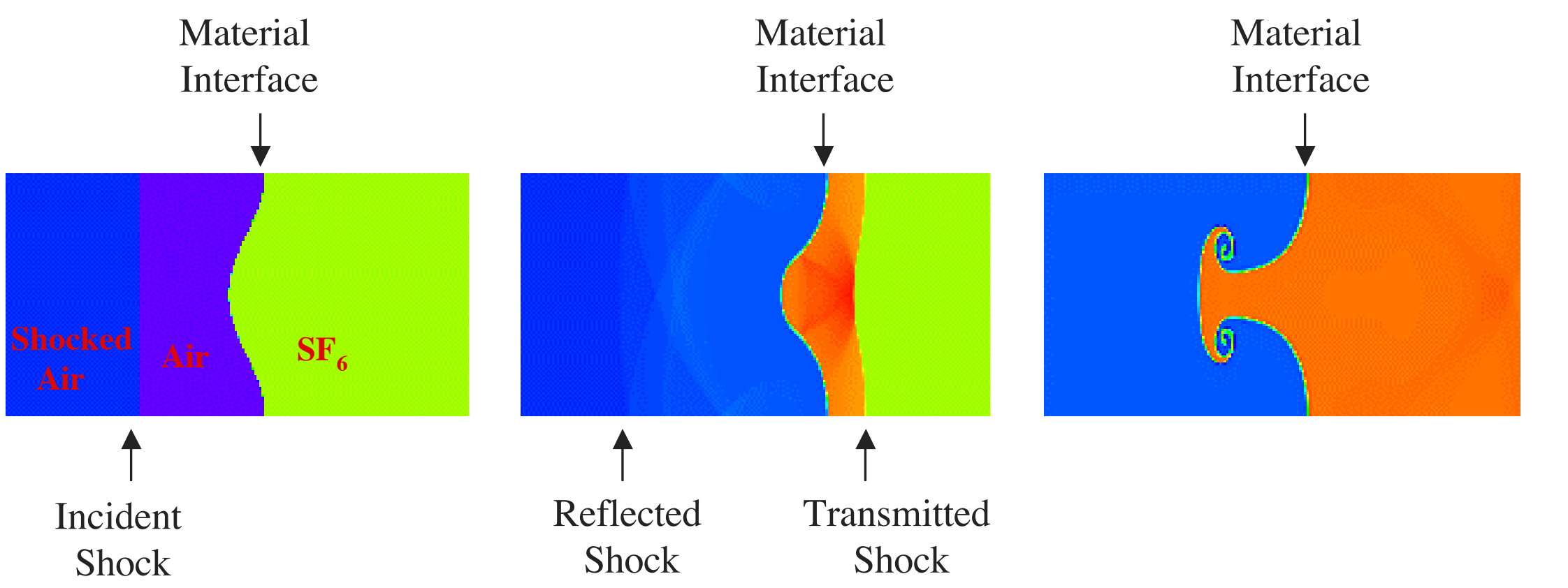


\section{DX-3 Gas Shock Tube}

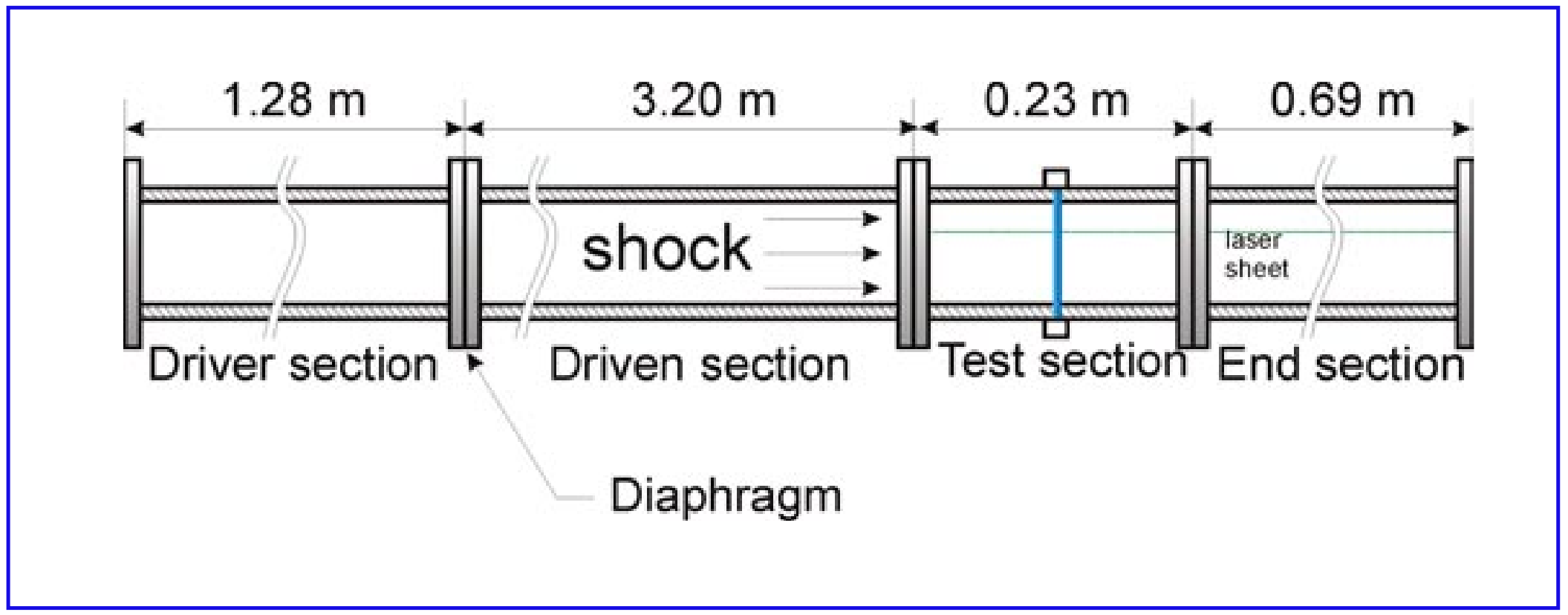

- Gas cylinder composed of $\mathrm{SF}_{6}$ and surrounded by ambient air

- $\mathrm{SF}_{6}$ seeded with glycol droplets to aid in visualizing the flow and to enable the PIV capability

Consult the following paper for more information on the experimental setup: P. M. Rightley, P. Vorobieff, and R. F. Benjamin. Evolution of a shock-accelerated thin fluid layer. Phys. Fluids, 9(6):1770-1782, 1997. 


\section{Test Section of the Shock Tube}

- 2 lasers:

- Customized, frequency doubled Nd:YAG

- $10 \mathrm{~Hz}$ 'New Wave' at $532 \mathrm{~nm}$

- 3 cameras:

- Intensified CCDs, 1134x468

- Initial Conditions (IC), Dynamic (DYN), and PIV

- 8 pulses:

- 7 pulses for ICs and dynamic images with $\Delta \mathrm{t}=140 \mu \mathrm{s}$

- 8th pulse for PIV

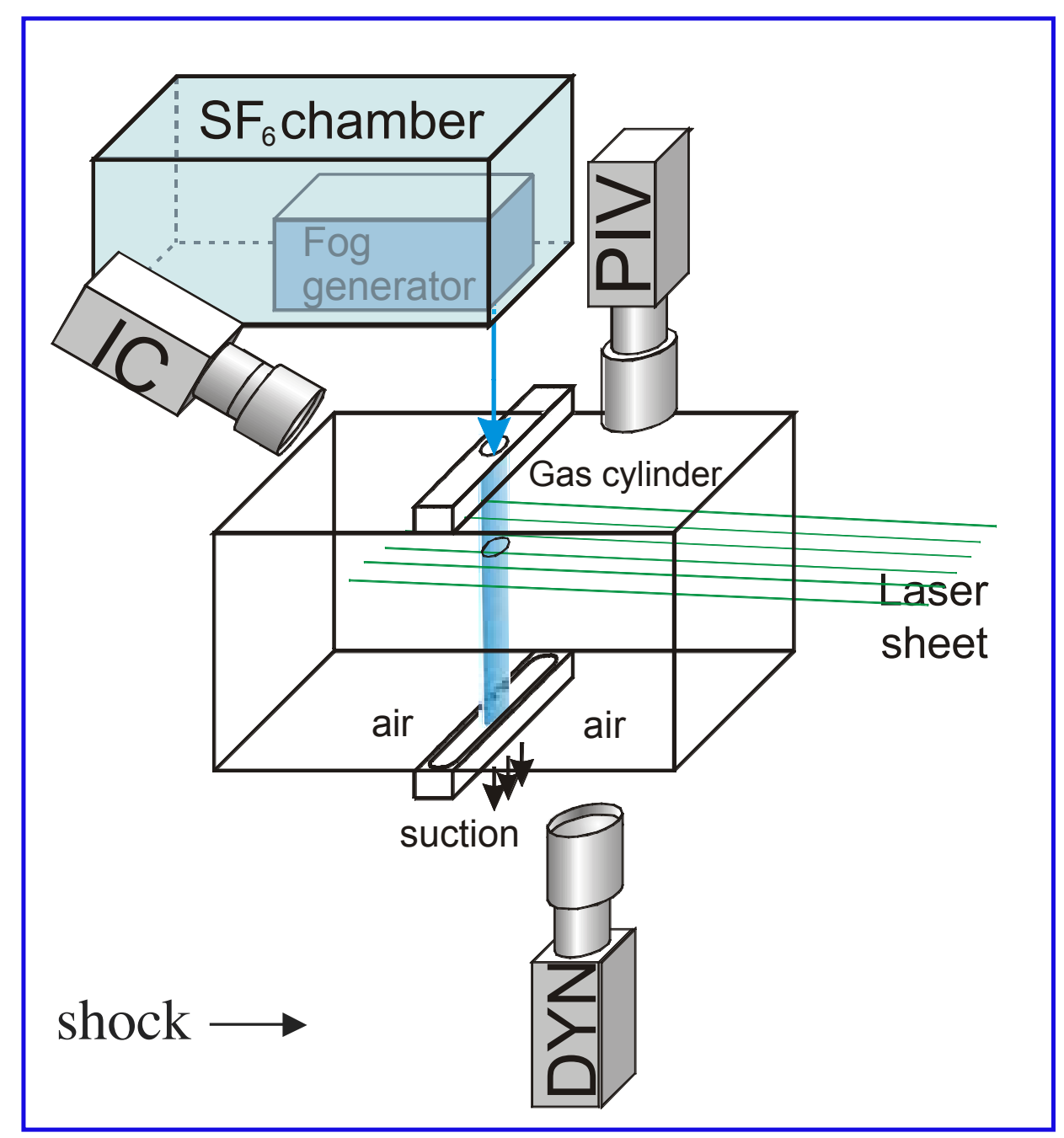




\section{RAGE: $\underline{\text { Radiation }}$ Adaptive $\underline{\text { Grid }}$ Eulerian Code}

- Multi-dimensional Eulerian hydrodynamic code

- Directionally-split second order Godunov scheme

- Continuous adaptive mesh refinement (CAMR)

- Each cell can be coarsened or refined by a factor of two in each timestep

- Only one level of refinement change possible between adjacent cells

- Refinement decisions can be modified for each material or defined for regions of computation

- Running in parallel on ASCI machines (Blue Mountain)

- Substantial validation has been performed on shocked interface problems

- Shocked curtain, single mode RMI, NOVA experiments

RAGE was originally developed by Michael L. Gittings
Initial grid -- level 1

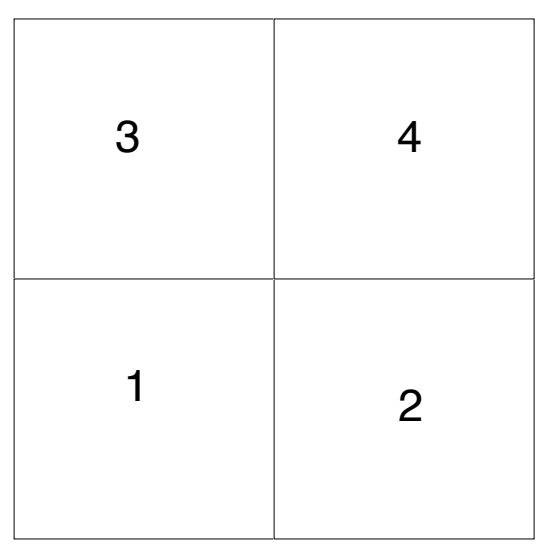

Subdivided cells 2 and 6

\begin{tabular}{|c|c|c|}
\hline 3 & \multicolumn{2}{|c|}{4} \\
\hline \multirow{2}{*}{1} & 7 & 8 \\
\hline & 5 & \begin{tabular}{|l|l|l|}
11 & 12 \\
9 & 10
\end{tabular} \\
\hline
\end{tabular}




\section{Cylinder Simulation Setup}

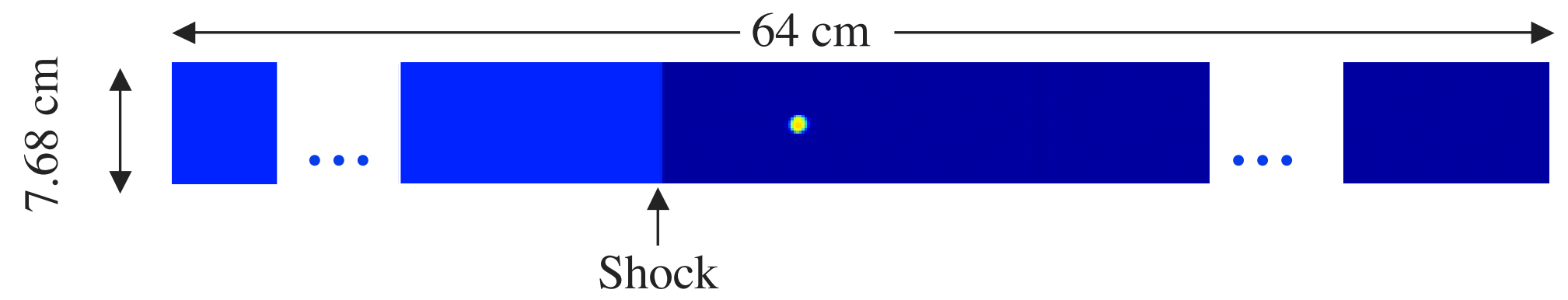

$$
\text { Air } \quad \begin{aligned}
& \rho=0.95 \mathrm{e}-3 \mathrm{~g} / \mathrm{cc} \\
& \mathrm{P}=0.8 \mathrm{Bar}
\end{aligned}
$$

\section{Shock}

- Mach 1.2 shock in air hitting a cylinder of $\mathrm{SF}_{6}$

- Ideal gases: $\gamma_{\mathrm{SF} 6}=1.09 \quad \gamma_{\mathrm{air}}=1.4$

- RAGE grid: level $1=0.64 \mathrm{~cm}$

$$
\text { level } 7=0.01 \mathrm{~cm}
$$

(approx 80 zones across the diameter of the initial cylinder) 


\section{Comparison Between Experimental and Computational Images

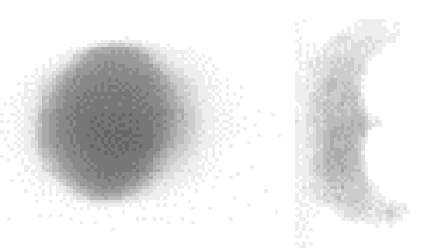

$\mathrm{ICs}$
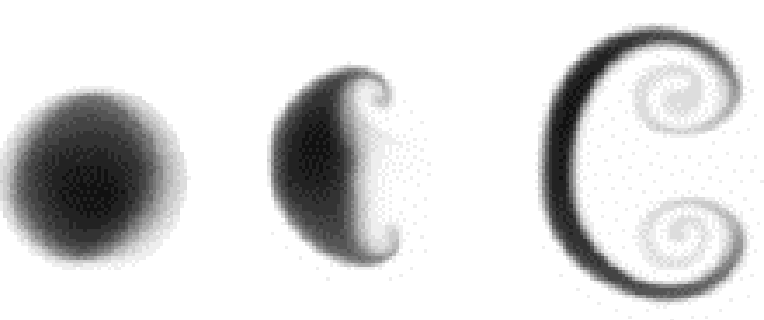
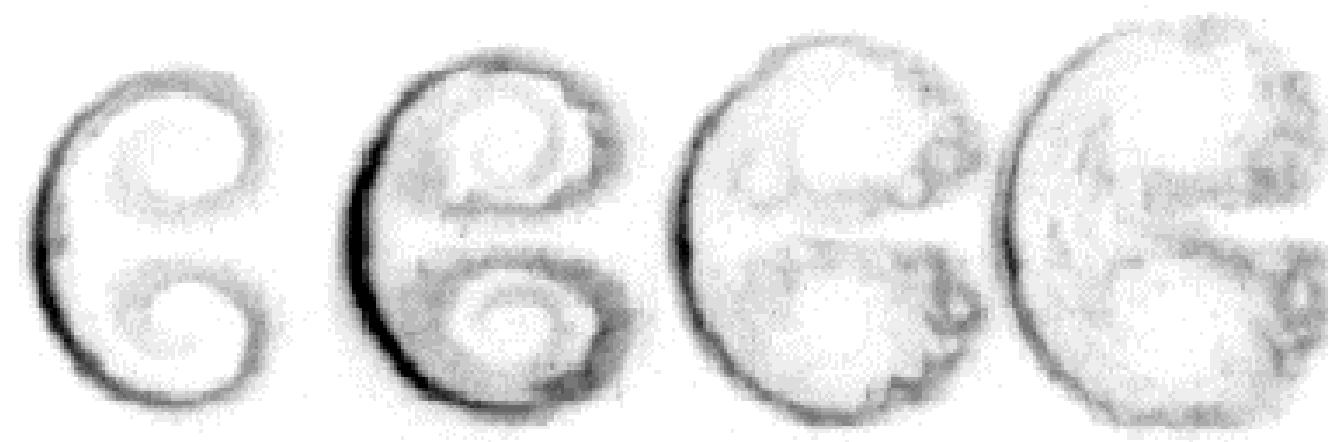

$330 \mu \mathrm{s}$

$470 \mu \mathrm{s}$

$610 \mu \mathrm{s}$

$750 \mu \mathrm{s}$
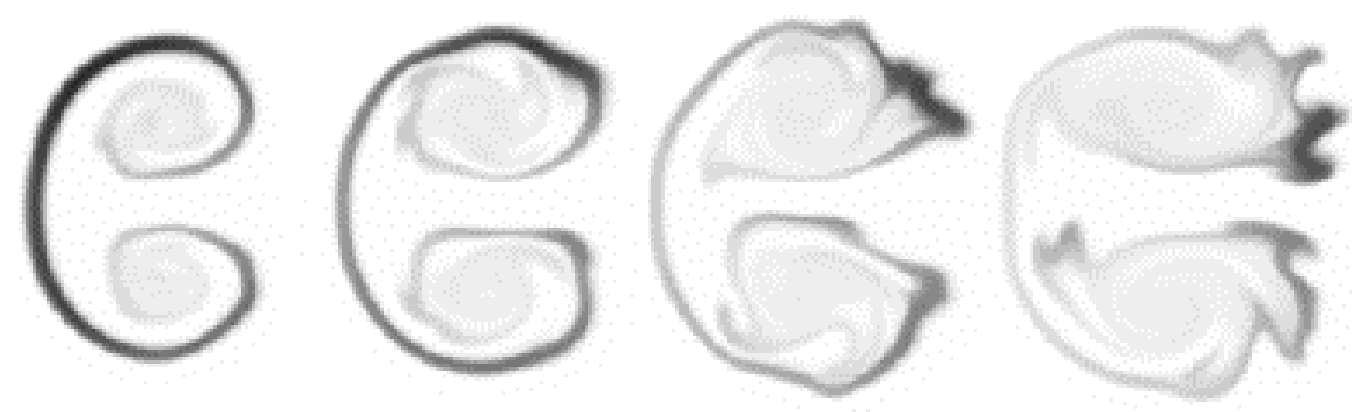

Shock $\longrightarrow$ 


\section{Quantitative Measurements}
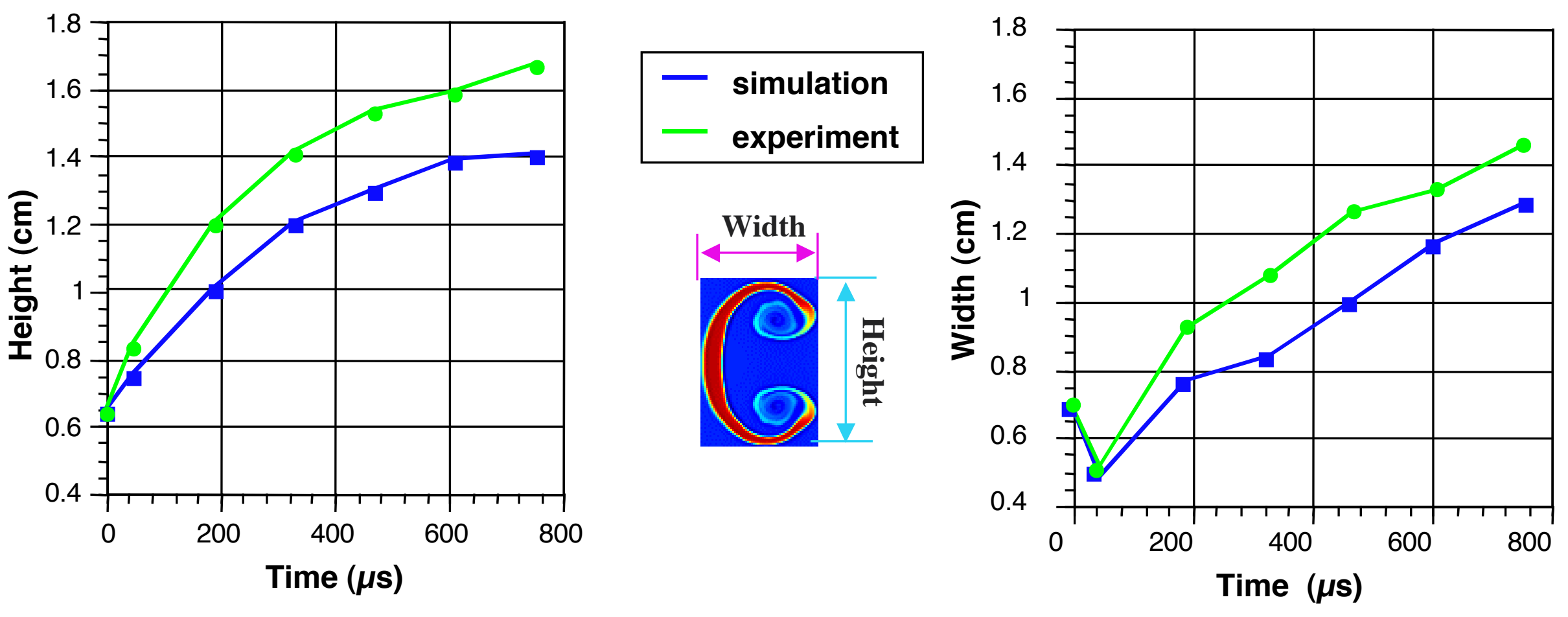

The height and width of the evolving cylinder are $15 \%$ larger in the experiment than in the simulation 


\section{Velocity Fields}

\section{Experiment}
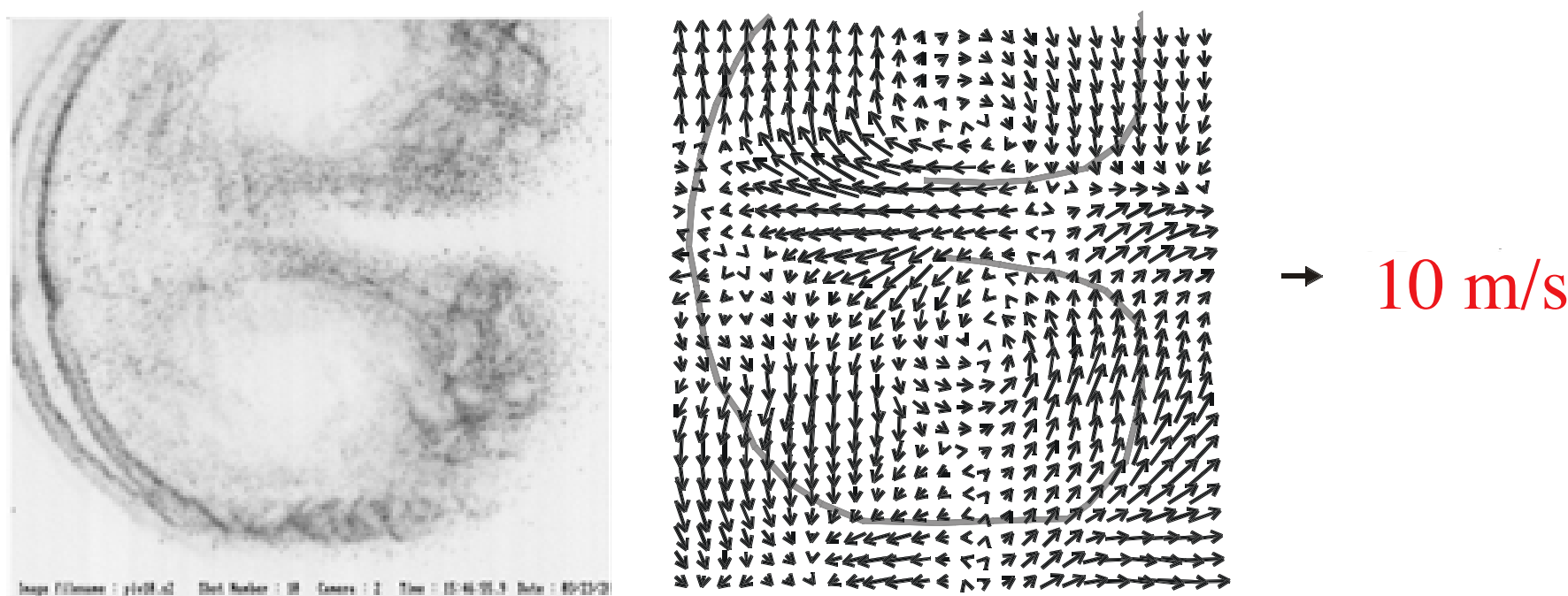

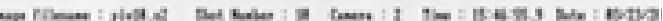

Simulation

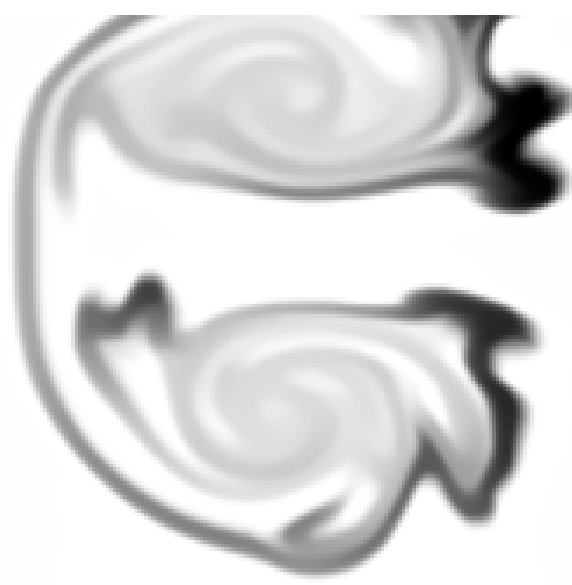

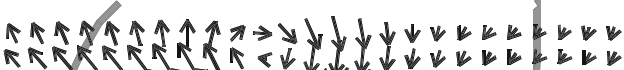
スス下KK RRर रTR 5. TKK $\leftarrow \leftarrow \leftarrow \leftarrow \leftarrow \leftarrow \leftarrow \leftarrow \leftarrow \leftarrow$

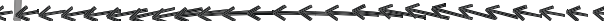

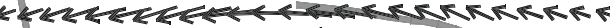

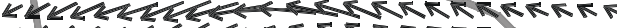
KLLKL1 Y L LUKடKL L L K

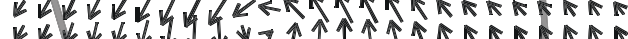
L v manaraman

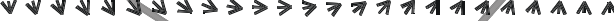

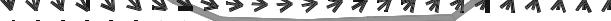
U

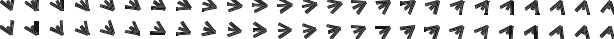




\section{Varying Peak $\mathrm{SF}_{6}$ Concentration}

STONY BROAK

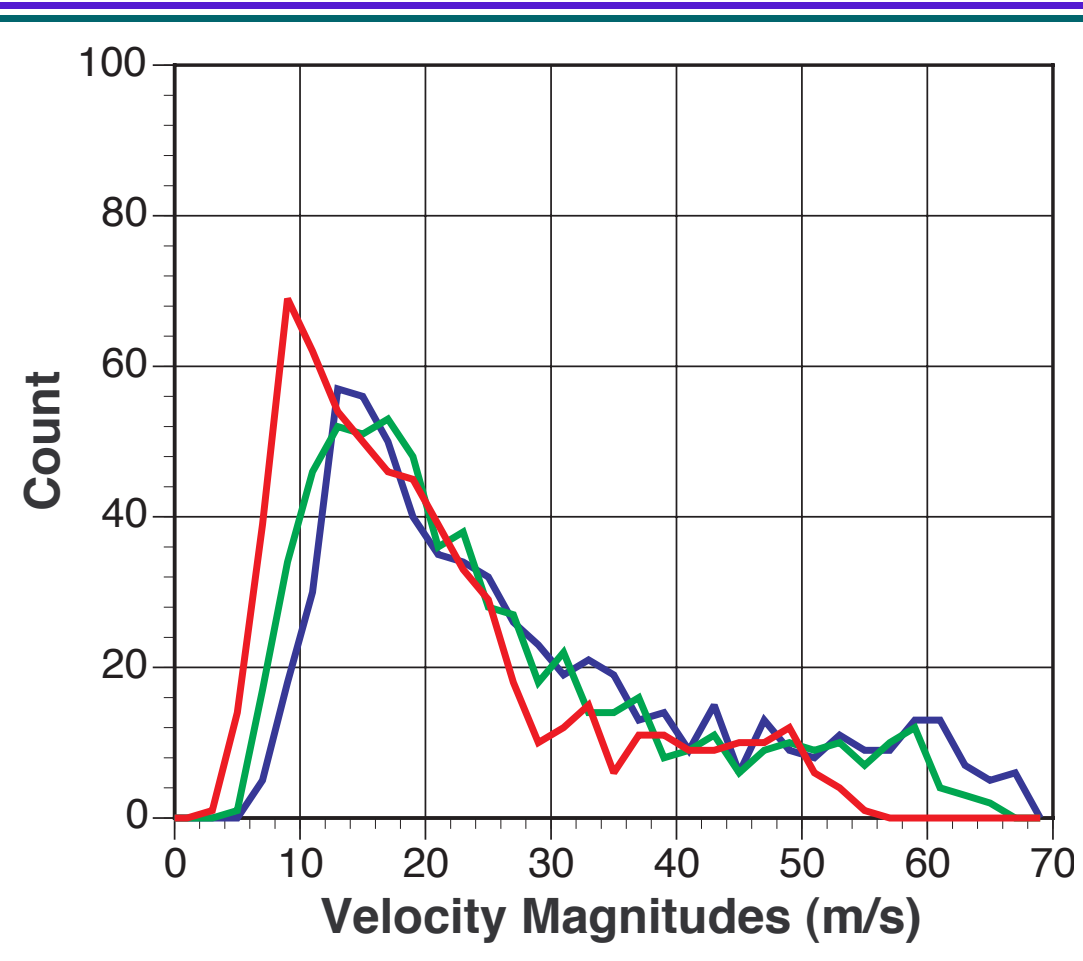

- $100 \%$ peak

- $80 \%$ peak

- $60 \%$ peak

- experiment

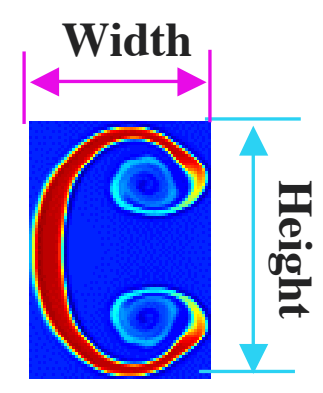

Smaller peak $\mathrm{SF}_{6}$ concentrations result in smaller velocities and smaller lengths
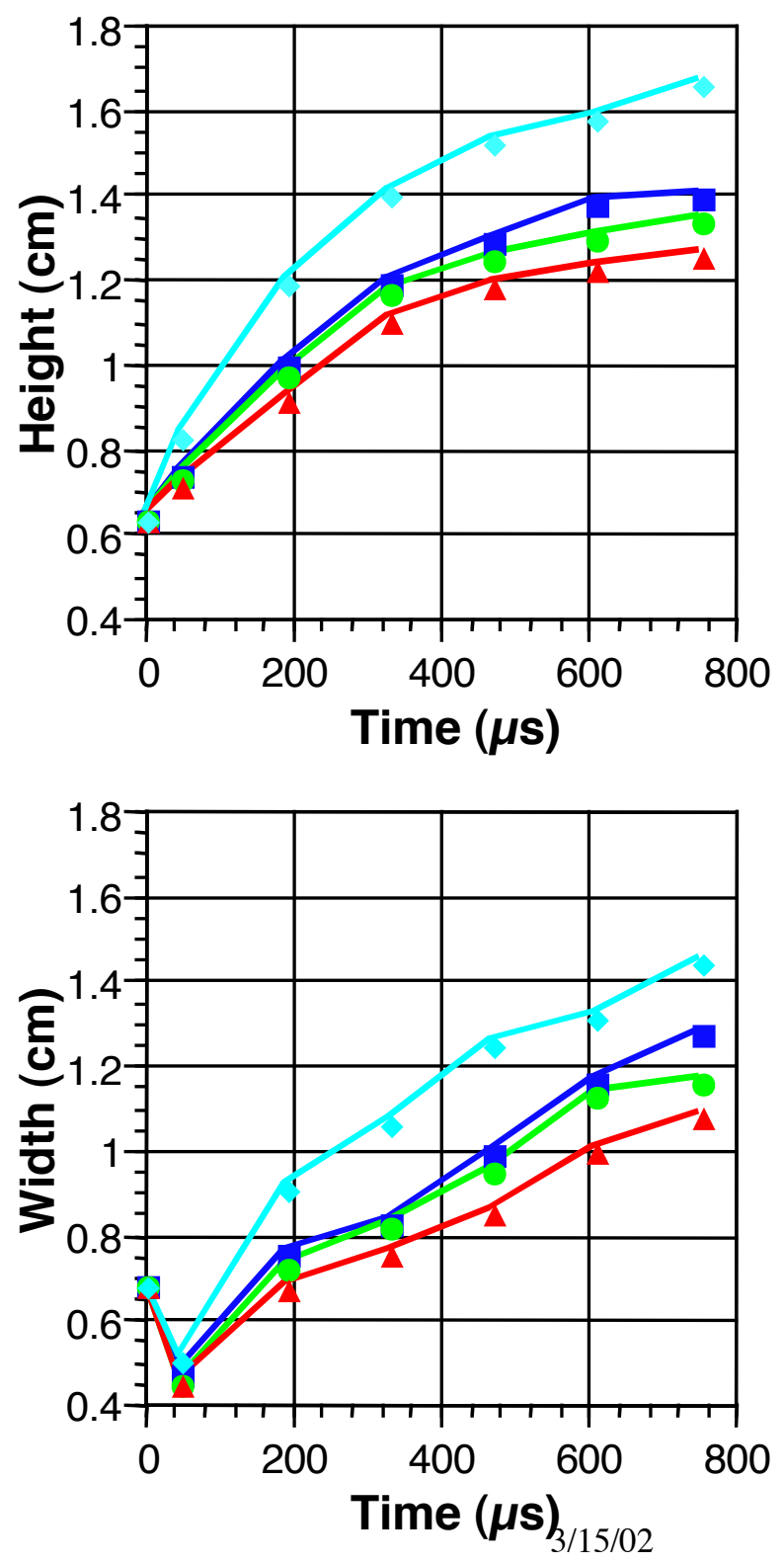


\section{Varying Density Gradient at the Air/ $\mathrm{SF}_{6}$ Interface}

Experiment

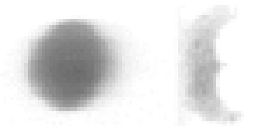

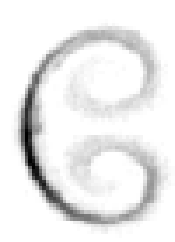

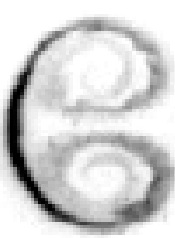

Experimental Initial Conditions
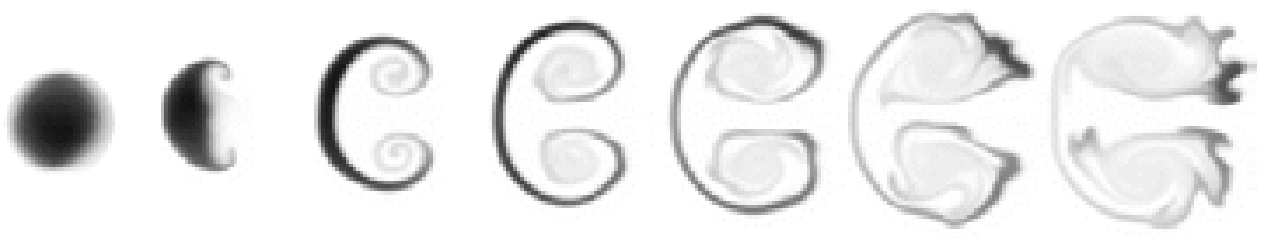

Sharp Interface

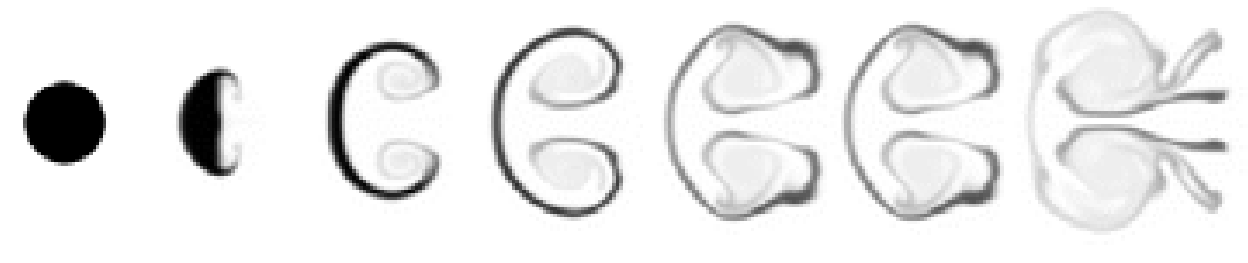

Diffuse Interface

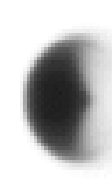

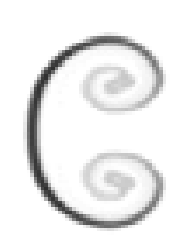

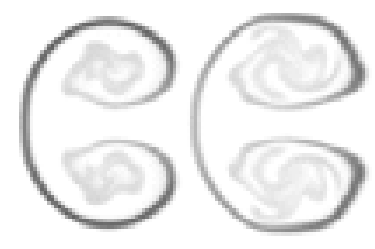

- Differences are visible in the density images with the initially diffuse interface producing the best visual agreement with the experiment

- No significant differences exist in the heights/widths and velocities

How well characterized are the experimental initial conditions? 


\section{Mesh Refinement}

Experiment
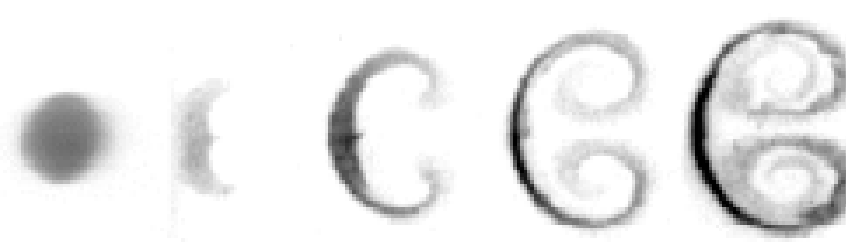

Diffuse Interface - fine $\Delta \mathrm{x}=0.01$
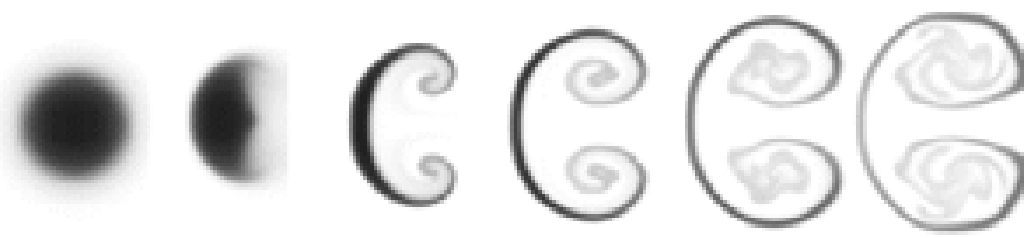

Diffuse Interface - coarse $\Delta \mathrm{x}=0.02$
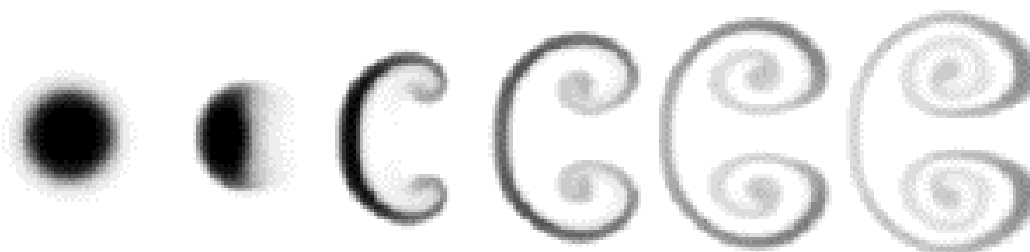

A coarser simulation shows "better" visual agreement with the experiment

Jet velocity:

coarse simulation: $62 \mathrm{~m} / \mathrm{s}$ fine simulation: $69 \mathrm{~m} / \mathrm{s}$

Coarser resolution:

- less rollup in vortex

- less evidence of secondary instability

- smaller jet velocity 


\section{New Velocity Measurements}

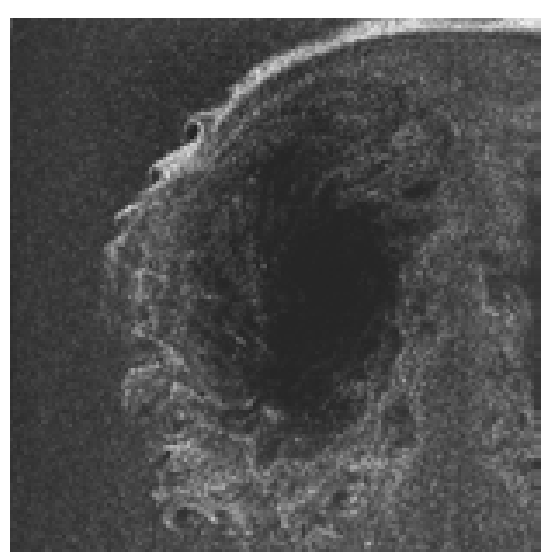

PIV image

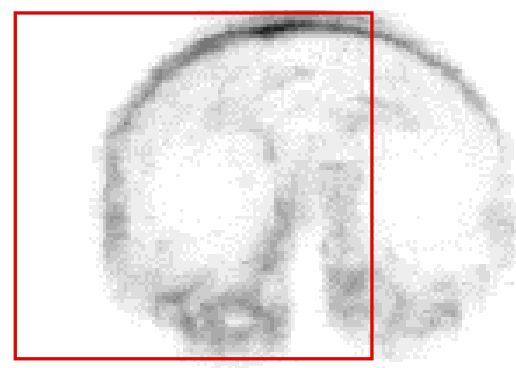

Last dynamic image

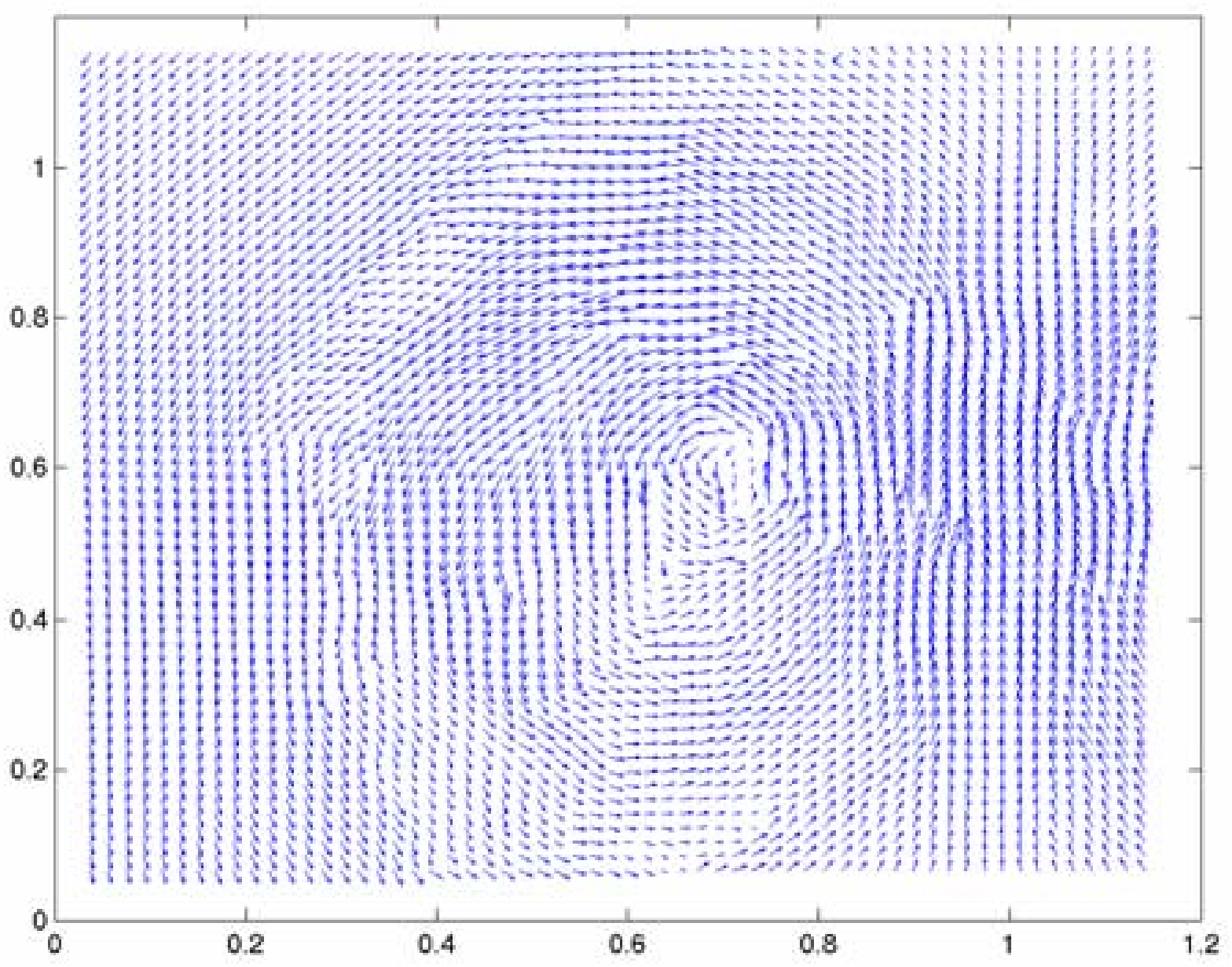

The new velocity field has vectors every $187 \mu \mathrm{m}$ compared to every $537 \mu \mathrm{m}$ obtained previously. 


\section{Location of Velocity Magnitudes}

Experiment
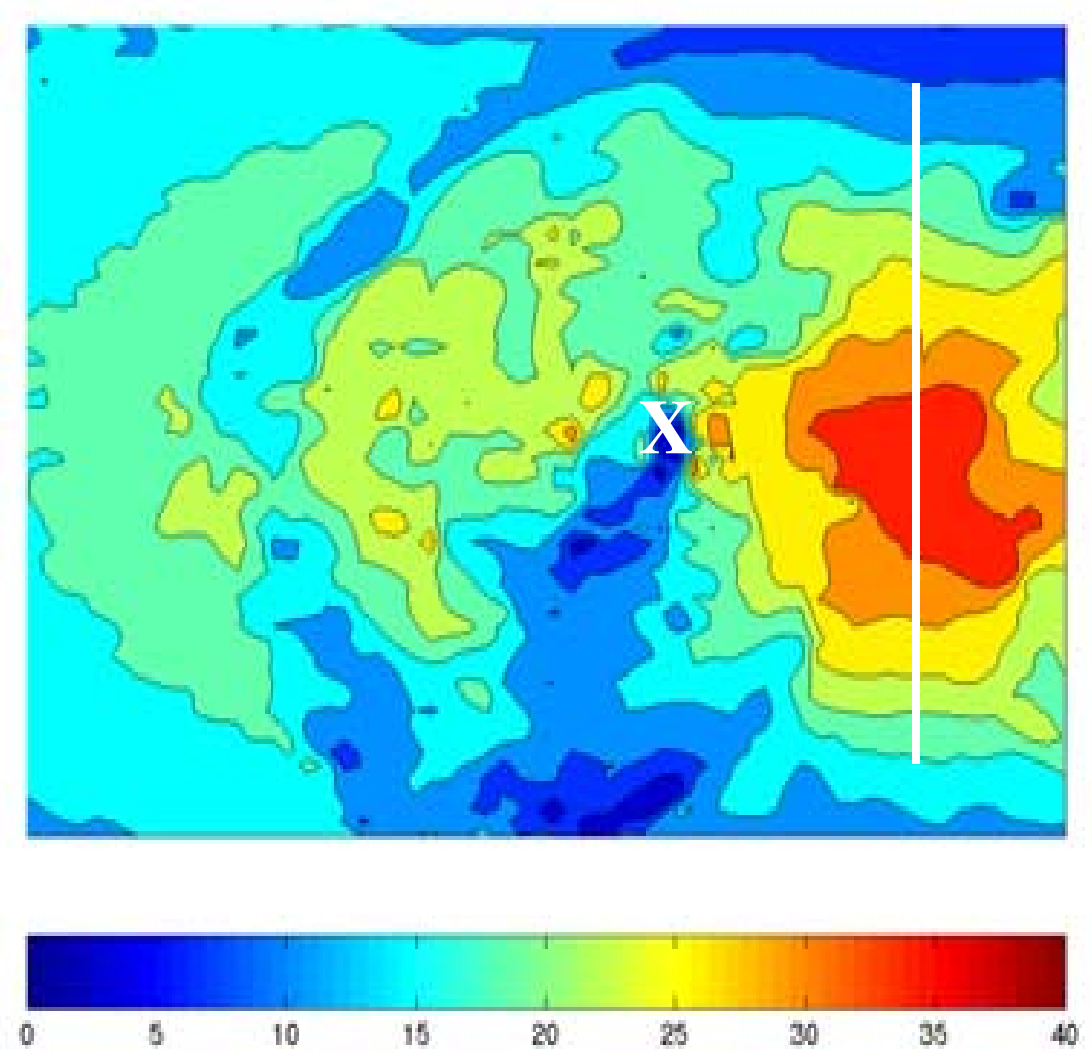

\section{Simulation}
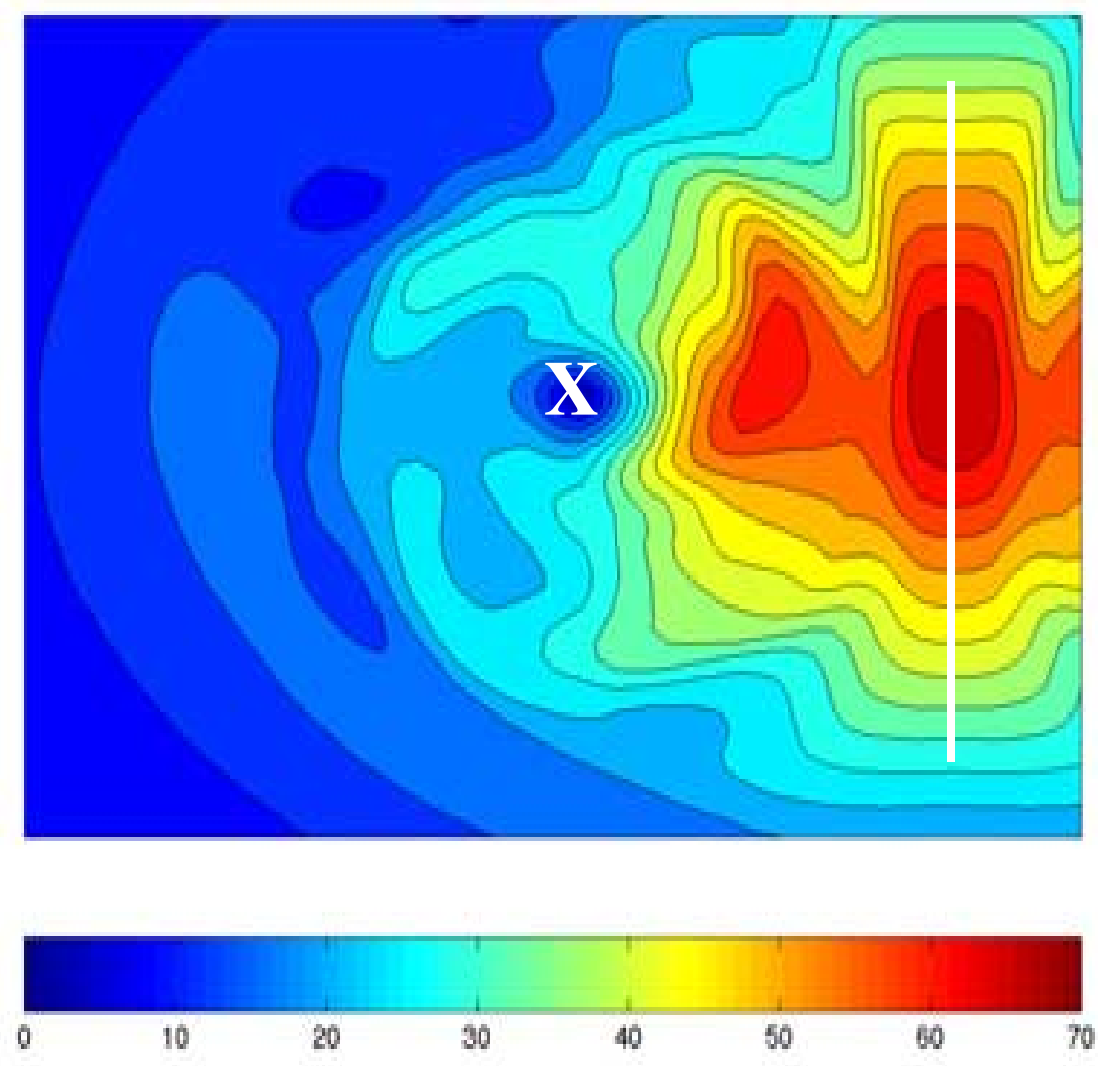

Largest velocities occur in the back-flow area and the smallest velocities occur in the vortex core 


\section{Comparison of Experimental and Computational Velocity Magnitudes}

Experiment

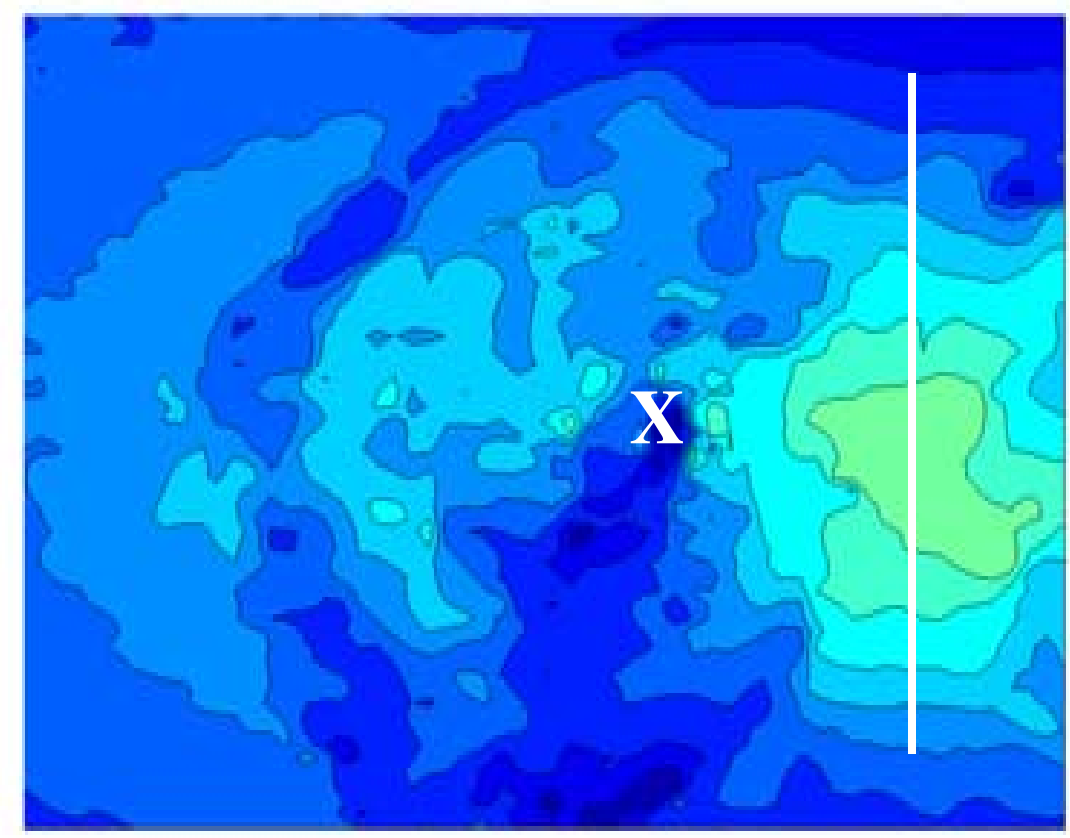

Simulation
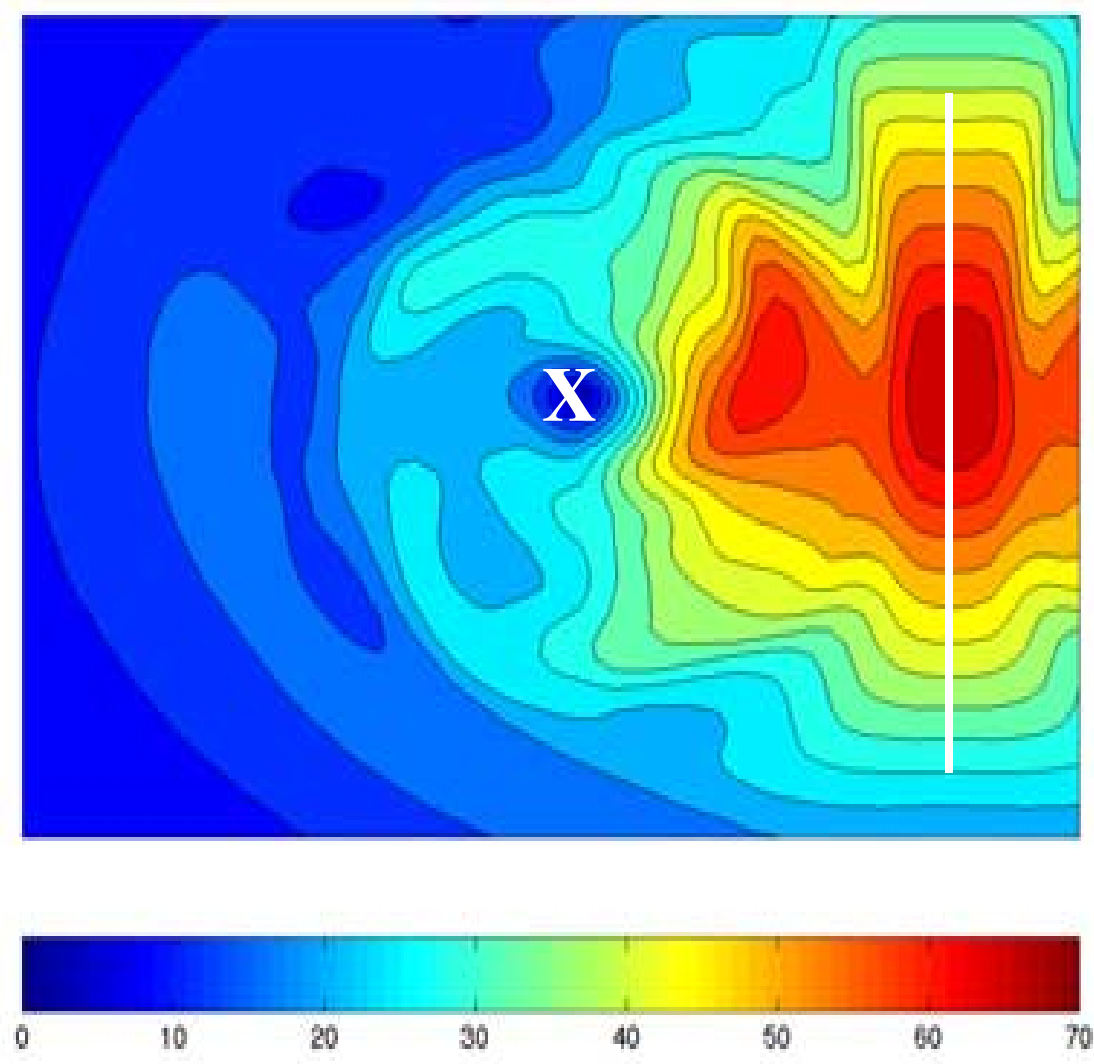

The experiment and the computation have similar velocities in the vortex core 


\section{Histogram of Velocity Magnitudes}

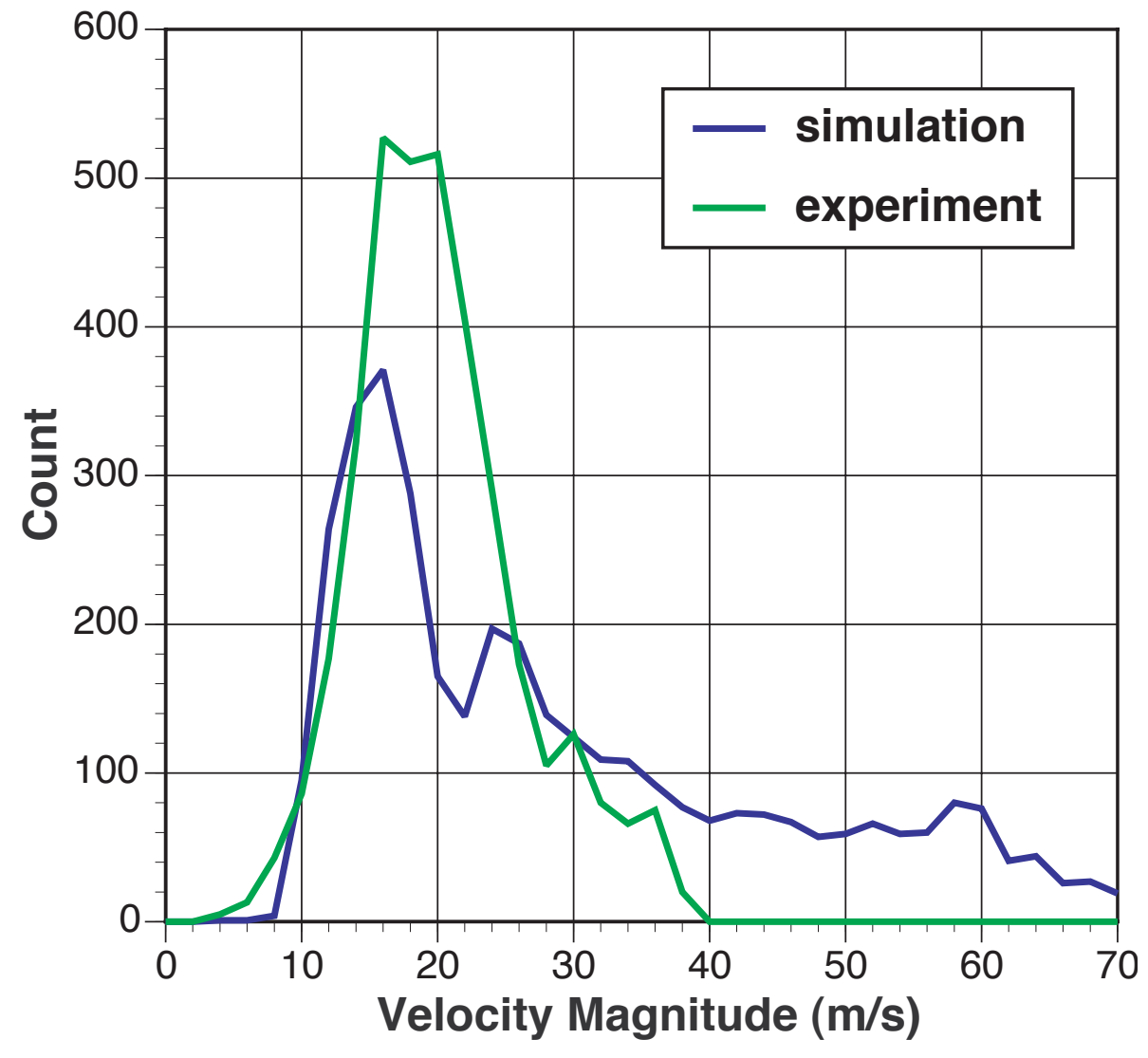

Both the experiment and the computation have a peak velocity of $15 \mathrm{~m} / \mathrm{s}$.

The magnitudes of the back-flow velocities form the tail of the histogram.

Large disagreement still exists between the experimental and computational back-flow velocities. 


\section{Jet Velocity of a Vortex Pair}

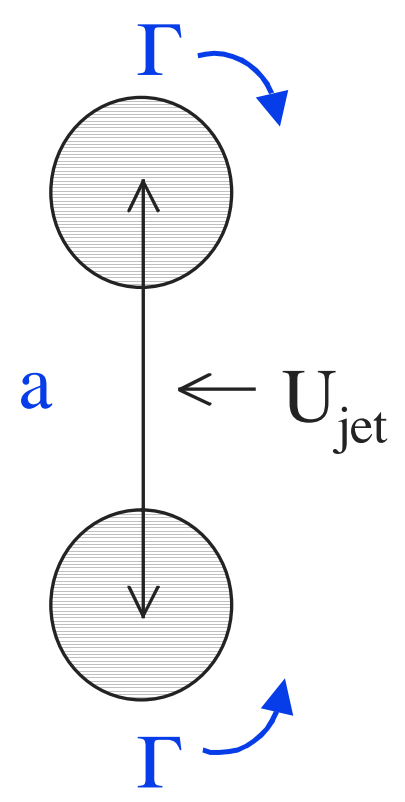

Model the evolving cylinder as a vortex pair composed of two idealized incompressible rectilinear vortices with equal and opposite circulations

For steady state flow (i.e., vortices stationary), the jet velocity $\mathrm{U}_{\text {jet }}$ between the two vortices is equal to*:

$$
\mathrm{U}_{\mathrm{jet}}=3 \Gamma / 2 \pi \mathrm{a}
$$

Simulation: $U_{\text {jet }}=59 \mathrm{~m} / \mathrm{s}$ (predicted) Experiment: $U_{\text {jet }}=37 \mathrm{~m} / \mathrm{s}$ (predicted) $\mathrm{U}_{\text {jet }}=69 \mathrm{~m} / \mathrm{s}$ (observed) $\quad \mathrm{U}_{\text {jet }}=36 \mathrm{~m} / \mathrm{s}$ (observed)

Are the predicted velocities qualitatively consistent with the circulation and vortex spacings measured in the experiment and the simulation?

*L. Prandtl and O.G. Tietjens. Fundamentals of Hydro- and Aeromechanics, McGraw-Hill Book, 1934. 


\section{Vortex Spacing}

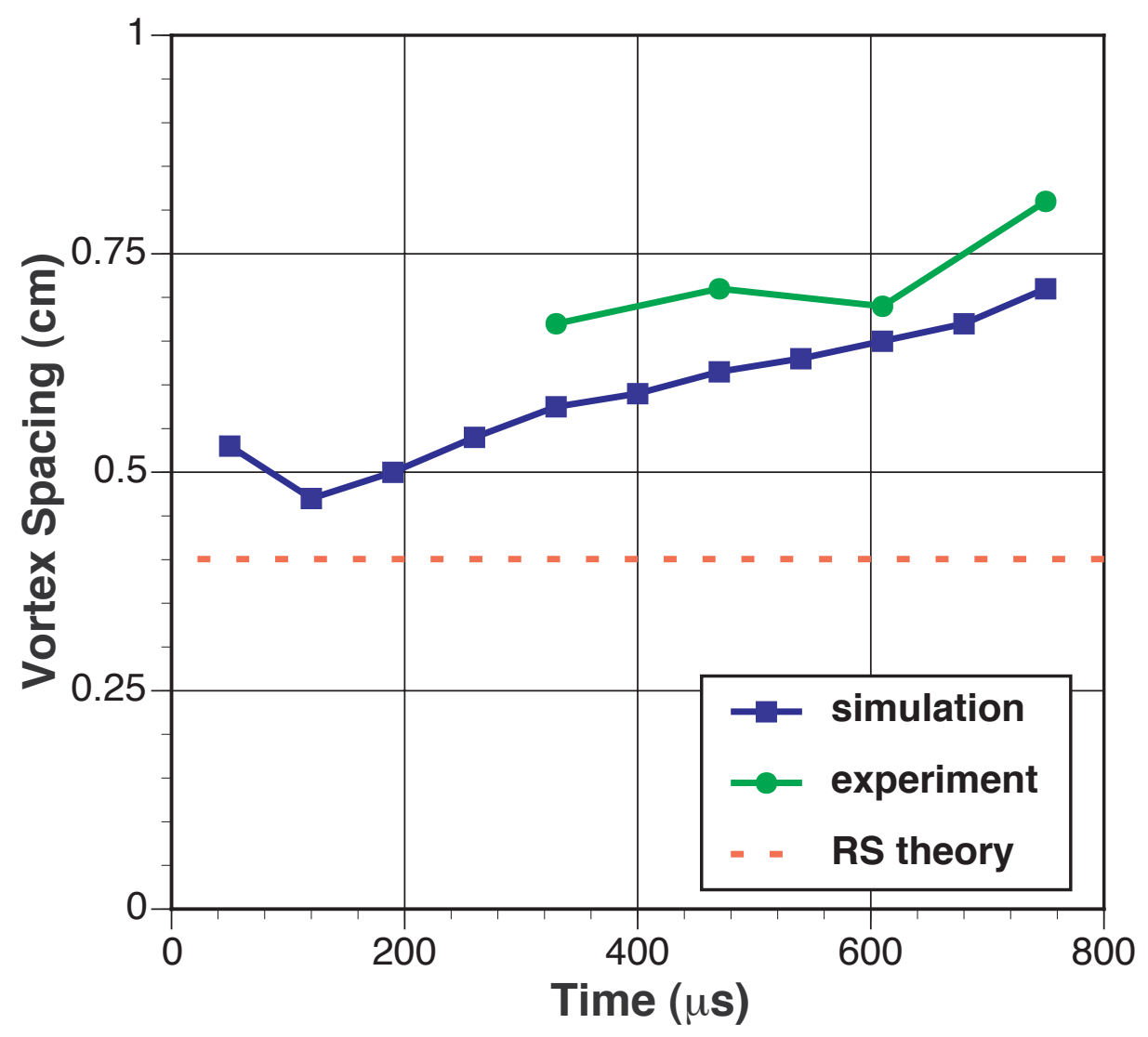

The experiment has larger vortex spacings compared to the simulation

The experimental and computational vortex spacings are in the range of Jacobs' measurements*

Note: The vortex spacing is determined using flow visualization

*J. W. Jacobs. The dynamics of shock accelerated light and heavy gas cylinders. Phys. Fluids A, 5(9):2239, 1993. 


\section{Circulation Values}

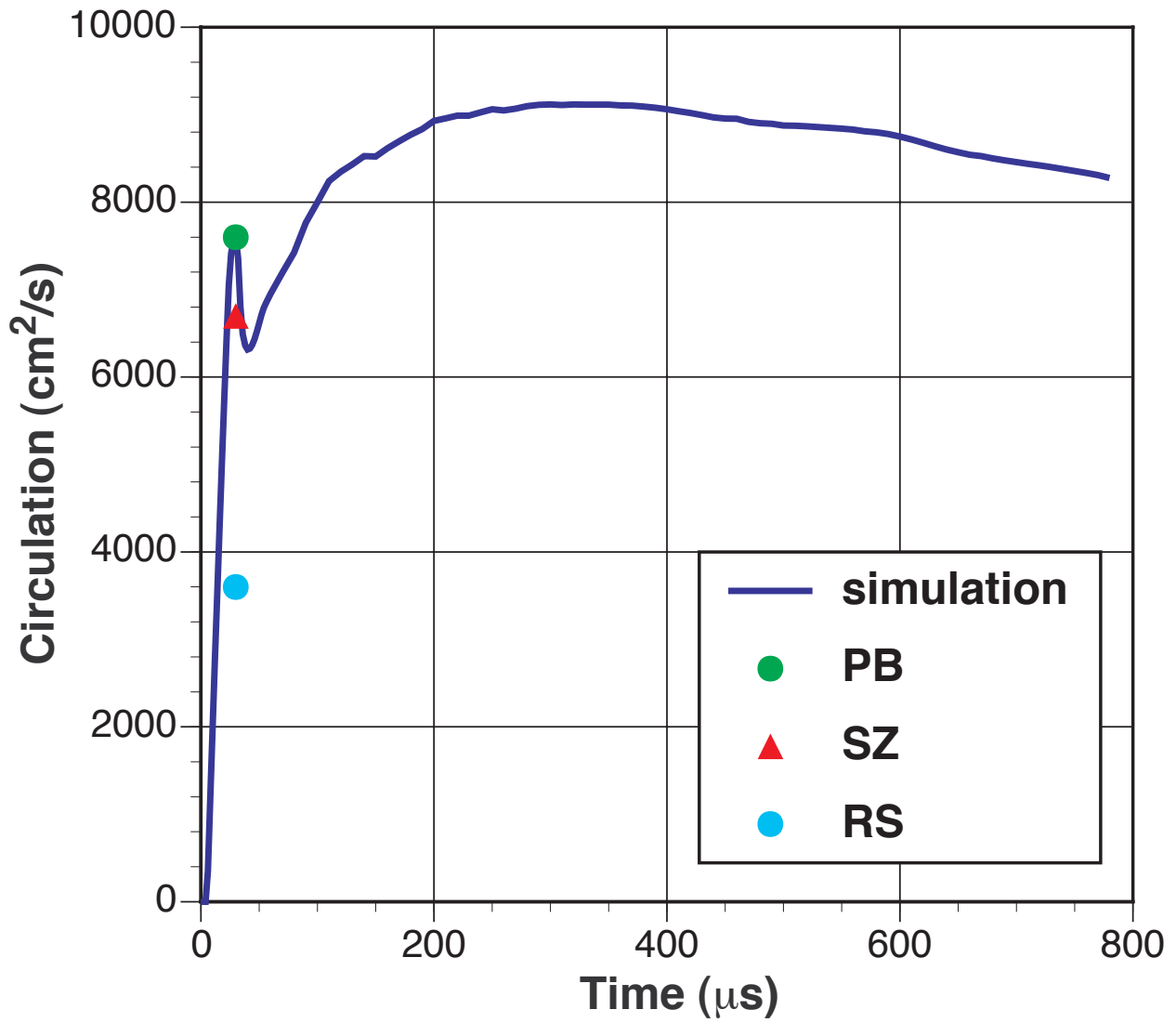

Predictions of circulation: RS: Rudinger \& Somers (1960) PB: Picone and Boris (1988) SZ: Samtaney \& Zabusky (1994)

The computational circulation value right after shock passage agrees well with the theoretical predictions of $\mathrm{PB}$ and SZ.

We need early-time PIV to determine the corresponding experimental circulation value.

Using the PIV results at $750 \mu \mathrm{s}$ we find that: $\Gamma_{\text {experiment }}<\Gamma_{\text {simulation }}$ 


\section{Summary}

- Higher experimental velocities are observed with the improved PIV diagnostic, resulting in better agreement with the computational velocities

- The experiment and the simulation have similar velocities in the vortex core

- The computational jet velocity is approximately twice the value of the experimental jet velocity

- The differences in the jet velocities may be resolved by:

- Examining the early-time shock-cylinder interaction in the experiment

- Comparing the RAGE simulations with other hydrodynamics codes 


\section{Future Work}

- Continue to investigate the length and velocity differences between the experiment and the simulation

- Redesign the experimental hardware to allow for high-resolution PIV at early time

- Obtain a better characterization of the experimental initial conditions

- Examine the effects of mix on the cylinder development using the new mix model added to the RAGE code

- Perform simulations using different computer codes

- Cuervo (Bill Rider, Jim Kamm)

- CHAD (Barbara Devolder, Manjit Sahota)

- Perform statistical analysis of the experimental and computational images (Bill Rider, Jim Kamm) 


\title{
SIMULATIONS OF TURBULENT MIXING OF TWO FLUIDS WITH VARIABLE ACCELERATION LAWS
}

\author{
A.N. Razin, V.I. Kozlov, I.V. Sapozhnikov \\ (RFNC - VNIIEF)
}

Dimonte's experiments have been simulated with the VIKHR technique. The progress of the turbulent mixing zone has been considered resulting from Raleigh-Taylor instability at the interface between two immiscible fluids. The calculations were performed with variable initial turbulent zone width and a sequence of refined grids for increasing, decreasing, pulse and constant accelerations. The turbulent mixing zone growth rate was obtained as a function of the path and initial zone width. It has been shown that the numerical results are in good agreement with the test data.

\section{INTRODUCTION.}

At present, the VIKHR technique [1] with V.V. Nikiforov's turbulent mixing (TM) model is widely used in VNIIEF for numerical simulations of flows with turbulent mixing. The Nikiforov's model has been tested many times against TM tests with gases [2-5], including shock tube tests.

Shock tube tests on TM at an interface of two gases with different densities have a number of specific features. First, in these tests, the gases are initially separated by a thin film, which prevents their diffusion before the mixing starts. After the film is ruptured by a shock wave, an initial turbulent mixing zone forms. In this case, the film's mechanical properties have a considerable effect throughout the TM zone evolution. In particular, TM zone width after the test may vary considerably with the film's thickness and strength. Therefore, a physical model that would treat film rupture and the initial phase of TM zone evolution is required for testing turbulent mixing calculation techniques. As such model does not exist, in VIKHR calculations we set initial fluctuations of density. Their values are specified so that to describe the measurements adequately.

Second, TM zone in shock tube tests is formed by means of intense interaction of shock waves and turbulent eddies. In numerical simulations of turbulent flows with 
shocks, gas-dynamic gradients at a shock front are inversely proportional to numerical point size. As a result, solutions of TM difference equations are highly dependent on a numerical grid, since gas-dynamic gradients are included in turbulence generation terms of the TM equations.

This is the reason why tests where these uncertainties are absent or insignificant are especially interesting for testing TM numerical techniques. Dimonte's experiments [6] belong to these tests.

In Dimonte's experiments two immiscible fluids were placed in a sealed ampoule: freon $\left(\rho=1.57 \mathrm{~g} / \mathrm{cm}^{3}\right)$ at the bottom and water $\left(\rho=1 \mathrm{~g} / \mathrm{cm}^{3}\right)$ at the top. The interface here is planar, except for the menisci at the ampoule walls due to surface tension that are small compared to the ampoule size. Initial perturbations of the interface are low and result from thermal fluctuations. Interface acceleration resulting into a Raleigh-Taylor instability is caused by the ampoule acceleration by a so-called electric motor (a conductor moving along guide electrodes under the influence of a magnetic field). Four acceleration laws have been obtained by varying the current and magnetic induction in [6]: constant, decreasing, increasing and pulse accelerations. The acceleration was directed from the heavy fluid into the light one.

This paper presents numerical results obtained for the tests [6] with four ampoule acceleration laws. The calculations were performed with the VIKHR technique with the incompressible fluid assumption (as the maximum ampoule velocity is within $\sim 31 \mathrm{M} / \mathrm{c}$, the resulting flow inside the ampoule is definitely subsonic). Surface tension effects were not taken into account.

\section{PROBLEM DEFINITION.}

The numerical geometry of the tests [6] is given in the fig. 1. The domain 1 $(0 \leq r \leq 10 \mathrm{~cm})$ is filled with water with the density $\rho_{1}{ }^{0}=1 \mathrm{~g} / \mathrm{cm}^{3}$, the domain 2 $(10 \leq r \leq 20 \mathrm{~cm})$ has liquid freon with the density $\rho_{2}{ }^{0}=1.57 \mathrm{~g} / \mathrm{cm}^{3}$. The boundary conditions at the left and right are: rigid wall. In our calculations we used the following equation of state for weakly compressible fluids:

$$
P=1000+25\left[\left(\rho / \rho_{c}\right)^{3}-1\right] / 3,
$$


where $\rho_{\mathrm{c}}=\rho_{1}^{0} \rho_{1}^{0} /\left(c_{1} \rho_{2}{ }^{0}+c_{2} \rho_{1}^{0}\right), c_{1}$ and $c_{2}$ are the mass fractions of water and freon respectively. In all calculations, the TM zone width was found from the mass fraction levels: $c_{1}=0.02$ for the right boundary, $c_{2}=0.02$ for the left boundary.

Numerical and experimental results are given as a function $h(Z)$, where $h$ is the depth to which the light fluid penetrates into the heavy one, $Z=\int_{0}^{t} d t^{t^{\prime}} \int_{0} d t^{\prime \prime} g\left(t^{\prime \prime}\right)$ is the ampoule path $(g(t)$ is the ampoule's variable acceleration). In our calculations we assumed that at small density difference the mixing occurs nearly symmetrically (in [7], for instance, the mixing asymmetry of $\approx 3 \%$ was obtained for $\rho_{2} / \rho_{1} \approx 1.5$ ), so the light-intoheavy fluid penetration depth was found as $h=0.5\left(r_{1}-r_{2}\right)$, where $r_{1}$ and $r_{2}$ are the coordinates of the right and the left boundaries of the TM zone. For the constant acceleration we also used $S=Z=0.5 g t^{2}$ for the ampoule's path.

\section{NUMERICAL RESULTS}

All calculations were performed with a uniform distribution of numerical points across the domains. The following notation is used in the figures: the first value in brackets is the number of points in the first domain, the second value gives that in the second domain; the notation $1+1$ corresponds to initial density fluctuations set in one point at the left and one point at the right from the interface, the notation $2+2$ corresponds to initial density fluctuations set in two points to the left and two points to the right from the interface, an so on. The initial density fluctuation in all calculations was set to $R_{0}=0.05$, the initial width of the TM zone was $L_{0}$ (the length of the interval where the density fluctuations were set to the non-zero value).

\section{Constant acceleration.}

The fig. 2.a and 2.b depict the simulation results for the constant acceleration of
in tertace the ampoule, the data from [6] are also presented. The fig. 2.a shows that when the grid is refined (which makes the initial TM zone width smaller) the calculations give smaller light-to-heavy fluid penetration depth. So, a twofold increase of the number of points in the differencing grid reduces $h$ at the end of a calculation by about $8 \%$. If we increase the number of points 3-fold, $h$ at the end of a calculation becomes about $12 \%$ smaller, and a 
4-fold increase makes $h$ at the same time about $14 \%$ smaller. The influence of $L_{0}$ and the cell linear size is pronounced throughout the calculation, though the ratio $\Delta h / h$ decreases with time.

At the initial phase of TM zone evolution (to $Z \leq 10$ ) there is a discrepancy between the numerical data and the experiments. In our opinion, this discrepancy can be accounted for by the fluid's interface surface tension, which was not taken into consideration in the calculations (this is also true for the other acceleration laws).

The fig. 2.b present $h$ as a function of $\mathrm{L}_{0}$. One can see that the dependence of the TM zone growth rate $d h / d S$ on $\mathrm{L}_{0}$ is most pronounced at the initial phase of the perturbation evolution. With the developed turbulence at $20<\mathrm{Z}<80$ (in the interval where the ampoule's acceleration is constant) $d h / d S$ shows almost no dependence on $\mathrm{L}_{0}$ :

$$
d h / d S \approx 0.0235 \approx 0.11 \mathrm{~A}
$$

where $A=\left(\rho_{2}-\rho_{1}\right) /\left(\rho_{2}+\rho_{1}\right)$ is the Atwood number.

In the references $[7 \div 9]$ the zone growth rate is to a certain extent dependent on the density level at the left and right boundaries of the TM zone that are used to measure the experimental zone width. For example, the following dependencies are presented in [7] where the TM zone width was found from the dimensionless density profile $\delta=\left(\rho(x)-\rho_{1}\right) /\left(\rho_{2}-\rho_{1}\right):$

$$
\begin{gathered}
d h / d S=(0.14 \pm 0.005) A \text { at } \delta=1, \\
d h / d S=(0.11 \pm 0.01) A \text { at } \delta=0.98
\end{gathered}
$$

(2) and (3) yield at $A=0.222$ (for the system of water and freon): $d h / d S \approx 0.0244 \div 0.031$, which is higher than the value (1), produced by the VIKHR calculation.

The numerical result (1) and the test data [7:9] show considerable discrepancies with the dependence derived by Nikiforov [10]:

$$
d h / d S=0.325 \mathrm{~A},
$$

which in our case at $\mathrm{A}=0.222$ yields a noticeably higher $\mathrm{TM}$ growth rate $d h / d S \approx 0.072$. The correlation (4) was obtained in TOGA technique calculations and was first given in [10]. The VIKHR calculations performed in the standard manner produced a similar result. According to Nikiforov [11], the reason for these discrepancies between the law (4) and the test data is that the turbulence energy dissipation mechanism, which takes 
place when two molecularly immiscible fluids are mixed, is different from that specific for gases.

VIKHR calculations have shown that satisfactory agreement with the test data can be achieved if we introduce a restriction on the function $f_{1}=\overline{\rho^{\prime 2} / \rho} / \rho R$ at the initial phase of a calculation, while preserving the model parameters (all the results, given in this paper were obtained with the restriction on this function). The function $f_{1}$ in the Nikiforov's model is a measure of deviation of liquid particles' acceleration from the gasdynamic acceleration $\nabla P / \rho$, and it is represented as $f_{1}=\left(1+R-D_{t} \nabla R / W\right)^{-1}$. For a heterogeneous mixture of two immiscible fluids there is an analytical expression for this function: $f_{1}=\delta[\delta-(\delta-1) c]^{-2}$, where $\delta=\rho_{h} / \rho_{l}, \rho_{h}$ and $\rho_{l}$ are the densities of the heavy and the light fluid, $c$ is the mass fraction of the light fluid. The value of the function $f_{1}$ for an arbitrary turbulent mixture cannot be higher than its heterogeneous value. Meanwhile, the representation of $f_{1}$, used in the model at the beginning of TM calculations can result into overestimated $f_{1}$. For this reason, a restriction on $f_{1}$ is used in calculations, which is equivalent to a restriction on the archimedian forces' work to move the liquid particles. As a result, the turbulence kinetic energy and the turbulent diffusivity decrease, which, in turn, leads to smaller growth of TM zone width. The restriction on the structural function can thus be interpreted as effective consideration of the turbulence energy conversion to the interface surface energy.

\section{Increasing acceleration.}

The fig. 3.a, 3.b and 3.c present the numerical results for the increasing acceleration of the ampoule. The fig. 3.a shows that when the grid is refined (which results into a decrease of the initial TM zone width) the light-to-heavy fluid penetration depth slightly decreases. And throughout the calculation $h$ depends on $\mathrm{L}_{0}$, though the relative change of $h$ decreases with time.

The influence of $\mathrm{L}_{0}$ on the dependence $h(Z)$ on a fixed grid is shown in the fig. 3.b and 3.c (the data in the fig. 3.c were obtained on a grid with a doubled number of points compared to the fig. 3.b). One can see that when $\mathrm{L}_{0}$ is changed, it changes $h(Z)$ by a constant value $\Delta h$, that is almost independent on the ampoule's path $Z$. Indeed, the fig. 3.b 
shows that at $20<Z<70$ the rate of penetration of the light fluid into the heavy one $d h / d Z$ decreases from 0.0314 for $\mathrm{L}_{0}=0.02778 \mathrm{~cm}$ to 0.0308 for $\mathrm{L}_{0}=0.1111 \mathrm{~cm}$. If the grid has 2 times more cells (see the fig. 3.c) at $20<Z<70$ the value of $d h / d Z$ does not depend on $L_{0}$ and equals to 0.0298 . This result can be approximated by the dependence

$$
d h / d Z \approx 0.135 A \text {. }
$$

\section{Decreasing acceleration.}

The numerical results for the decreasing acceleration of the ampoule are given in the fig. 4.a and 4.b. The fig. 4.a shows that an increase of numerical point size results into an increase of TM zone width. At the end of the calculations the light-to-heavy fluid penetration depth is: $h \approx 2.143 \mathrm{~cm}$ on the grid with $\mathrm{L}_{0}=0.0833 \mathrm{~cm}, h \approx 1.922 \mathrm{~cm}$ on the grid with $\mathrm{L}_{0}=0.0417 \mathrm{~cm}, h \approx 1.831 \mathrm{~cm}$ on the grid with $\mathrm{L}_{0}=0.02778 \mathrm{~cm}$ и $h \approx 1.775 \mathrm{~cm}$ on the grid with $\mathrm{L}_{0}=0.02083 \mathrm{~cm}$.

The influence of $\mathrm{L}_{0}$ on the dependence $h(Z)$ on a fixed grid is shown in the fig. 4.b. One can see that when $\mathrm{L}_{0}$ increases it results into an increase of the the light-to-heavy fluid penetration depth by a constant value $\Delta h$, which weakly depends on the ampoule's path $Z$.

\section{Pulse acceleration.}

The numerical results with the ampoule's pulse acceleration are shown in the fig. 5.a and 5.b. The fig. 5.a shows that if the number of points in the differencing grid is increased by a factor of 1.5 (respectively, $\mathrm{L}_{0}$ decreases by a factor of 1.5 ) $h$ at the end of calculations decreases by about $5.6 \%$, and a twofold increase results into $h$ at the end decreasing by about $16 \%$. In this case there is a slight dependence of the growth rate $d h / d Z$ on $\mathrm{L}_{0}$ through out the calculation.

The fig. 5.b shows the influence of $L_{0}$ on the dependence $h(Z)$ on a fixed grid. One can see that the most pronounced dependence of $d h / d Z$ on $\mathrm{L}_{0}$ is observed at the beginning of TM calculations. At the phase of the developed TM the influence of $L_{0}$ becomes weaker. Comparing the results obtained for the constant, decreasing, increasing and pulse accelerations, one can note that the influence of $\mathrm{L}_{0}$ on the dependence $h(Z)$ is the most pronounced for the pulse acceleration. 


\section{CONCLUSION.}

Numerical simulations of Dimonte's experiments[6] on Raleigh-Taylor mixing of two molecularly immiscible fluids have been performed with the VIKHR technique. The calculations have been performed for four ampoule acceleration laws. There is satisfactory agreement with the data [6]. Thus, we can state that V.V.Nikiforov's TM model provides adequate treatment of turbulent flow evolution in absence of shock waves.

\section{REFERENCES.}

1. V.A. Andronov, V.I. Kozlov, V.V. Nikiforov, A.N. Razin, Yu.A. Yudin Turbulent mixing calculation technique for 1D flows (the VIKHR technique) // Voprosy Atomnoy Nauki i Techniki. Series: Mathematical modeling of physical processes. 1994, issue 2, p. 59-64.

2. V.A. Andronov, V.V. Nikiforov Turbulence evolution calculations with an axially symmetric strain // Voprosy Atomnoy Nauki i Techniki. Series: Theoretical and applied physics. 1990 , issue 1, p. 34-36.

3. V.A.Andronov, S.M.Bakhrakh, E.E.Meshkov, V.V.Nikiforov, A.V.Pevnitsky, A.I.Tolshmyakov An experimental study and numerical simulations of turbulent mixing in 1D flows // Doklady AN SSSR. 1982, vol. 264, № 1, p. 76-82.

4. V.V.Nikiforov, V.A.Andronov, A.N.Razin Turbulent mixing zone evolution under shock impact // Doklady AN SSSR. 1995, vol. 343, \# 3, p. 323-325.

5. V.A.Andronov, S.A.Bel'kov, A.V.Bessarab et.al. Investigation of turbulent mixing in thin layers with different densities during laser acceleration of plane multilayer targets at the ISKRA-4 facility// JETF, 1997, vol. 111, issue 3, p. 882-888.

6. Dimonte Guy, Schneider Marilyn. Turbulent Rayleigh-Taylor instability experiments with variable acceleration // Physical Review E, 1996, v. 54, № 4, p.3740-3743.

7. Kuchtrenko Yu.A., Shibarshov L.I., Chitaikin V.I., Balabin S.I., Pylaev A.P. Experimental study of the gravitational turbulent mixing self-similar mode. $3^{\text {rd }}$ International Workshop on the physics of compressible turbulent mixing (Abbey of Royaumont, France), 1991, p. 427. 
8. D.L. Youngs, Physica 12 D, 32, 1984 .

9. K.I. Read Experimental investigation of turbulent mixing by Rayleigh-Taylor instability, Part 1. // Atomic Weapons Research Establishment, Foulness, England, 1984.

10.V.V.Nikiforov Turbulent mixing at an interface of different-density fluids. // Voprosy Atomnoy Nauki i Techniki. Series: Theoretical and applied physics. 1985, issue 1, p. $3 \div 8$.

11.V.V.Nikiforov Calculations of gravitational turbulent mixing in non-self-similar flows // Voprosy Atomnoy Nauki i Techniki. Series: Theoretical and applied physics. 1993, issue 1, c. 3-13. 


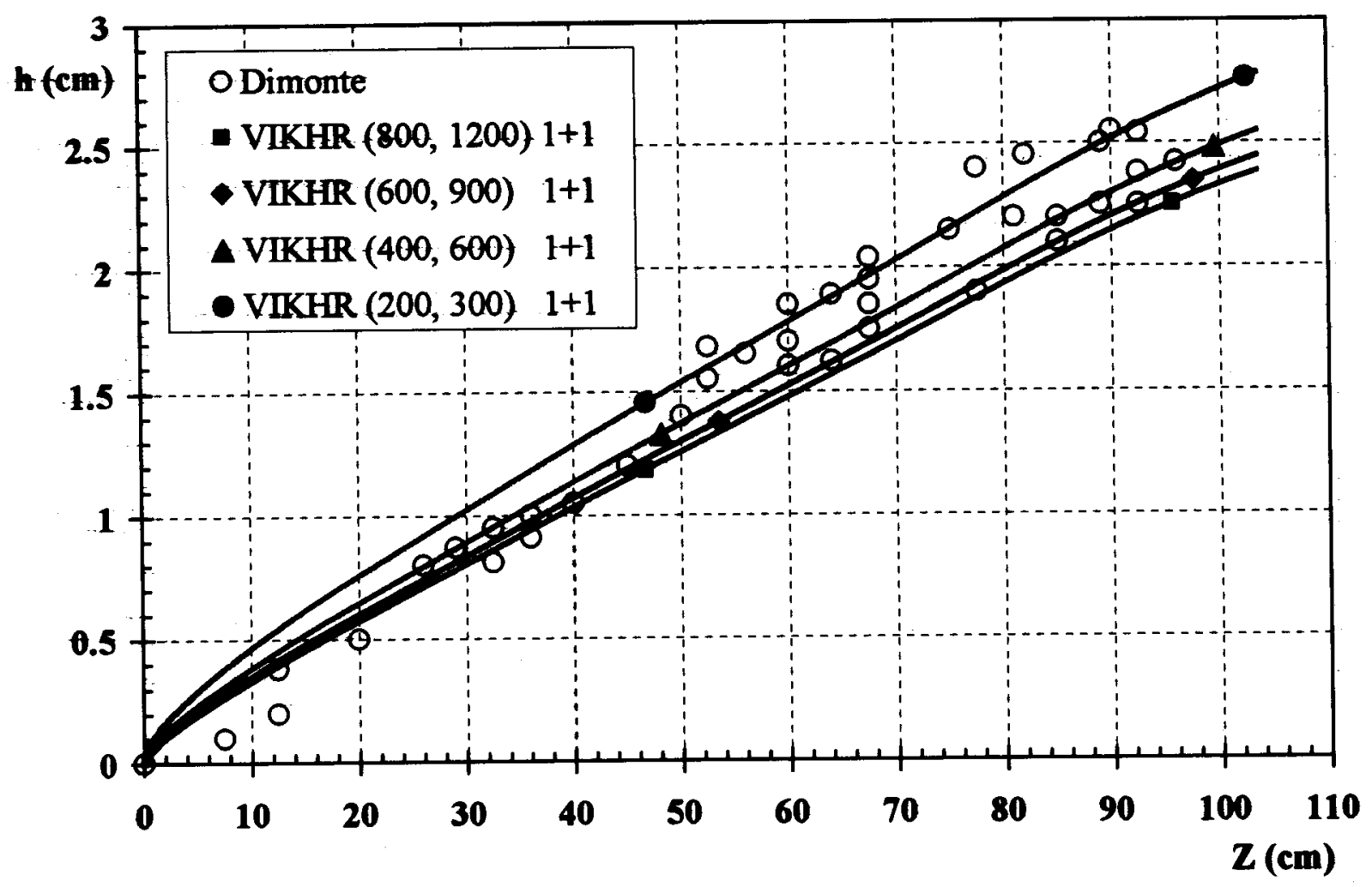

Fig. 2,a. Constant acceleration: calculations with a sequence of refined grids. (Initial density fluctuations are set in one point to the left and one point to the right of the interface.)

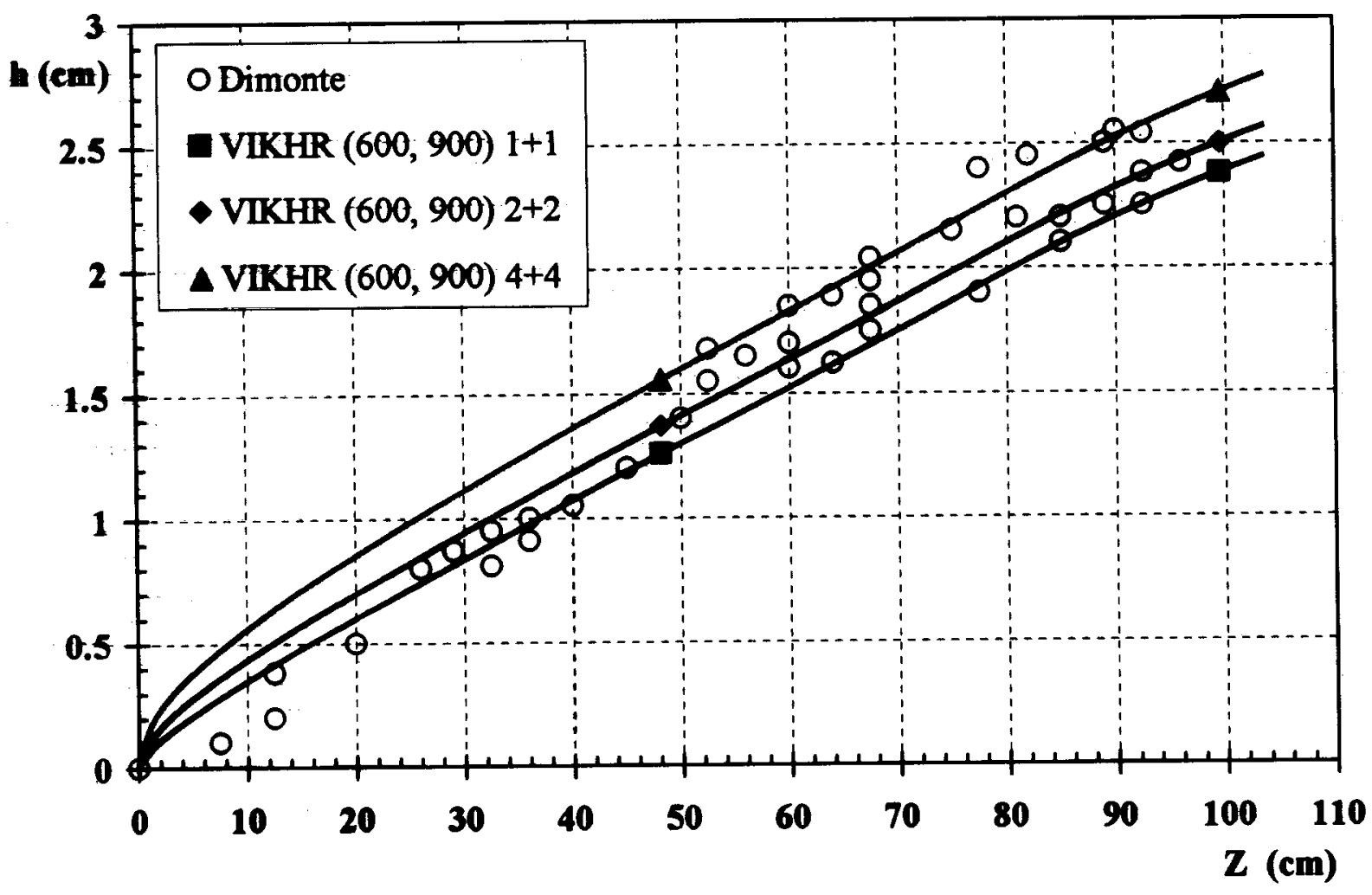

Fig. 2,b Constant acceleration: calculations with variable imitial zone width $L_{0}$ on a fixed grid. ( $\mathrm{L}_{0}$ is given by the number of numerical points to the left and to the right of the interface where the initial density fluctuations are set.) 


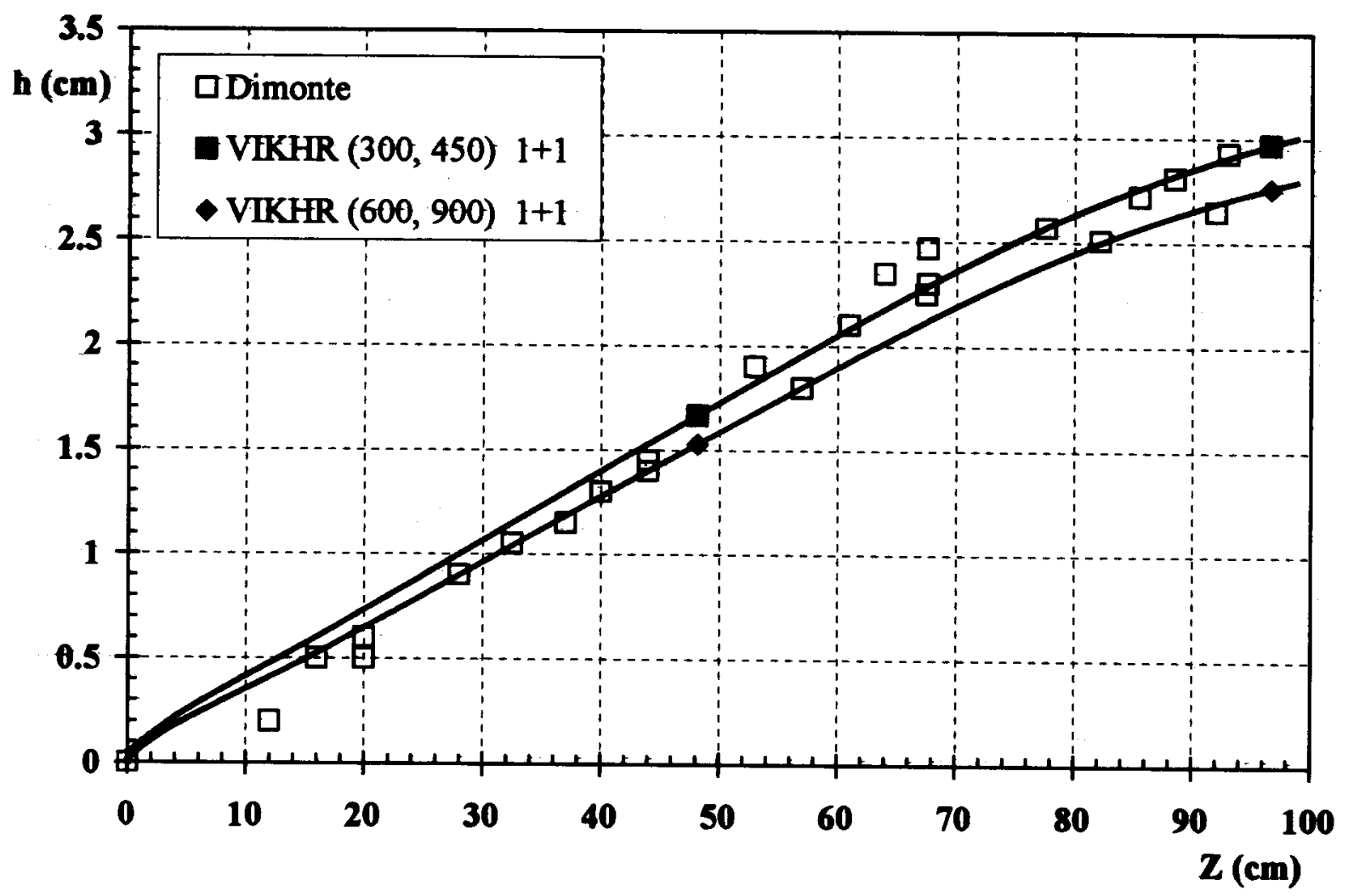

Fig. 3,a. Increasing acceleration: calculations with a sequence of refined grids. (Initial density fluctuations are set in one point to the left and one point to the right of the interface.)

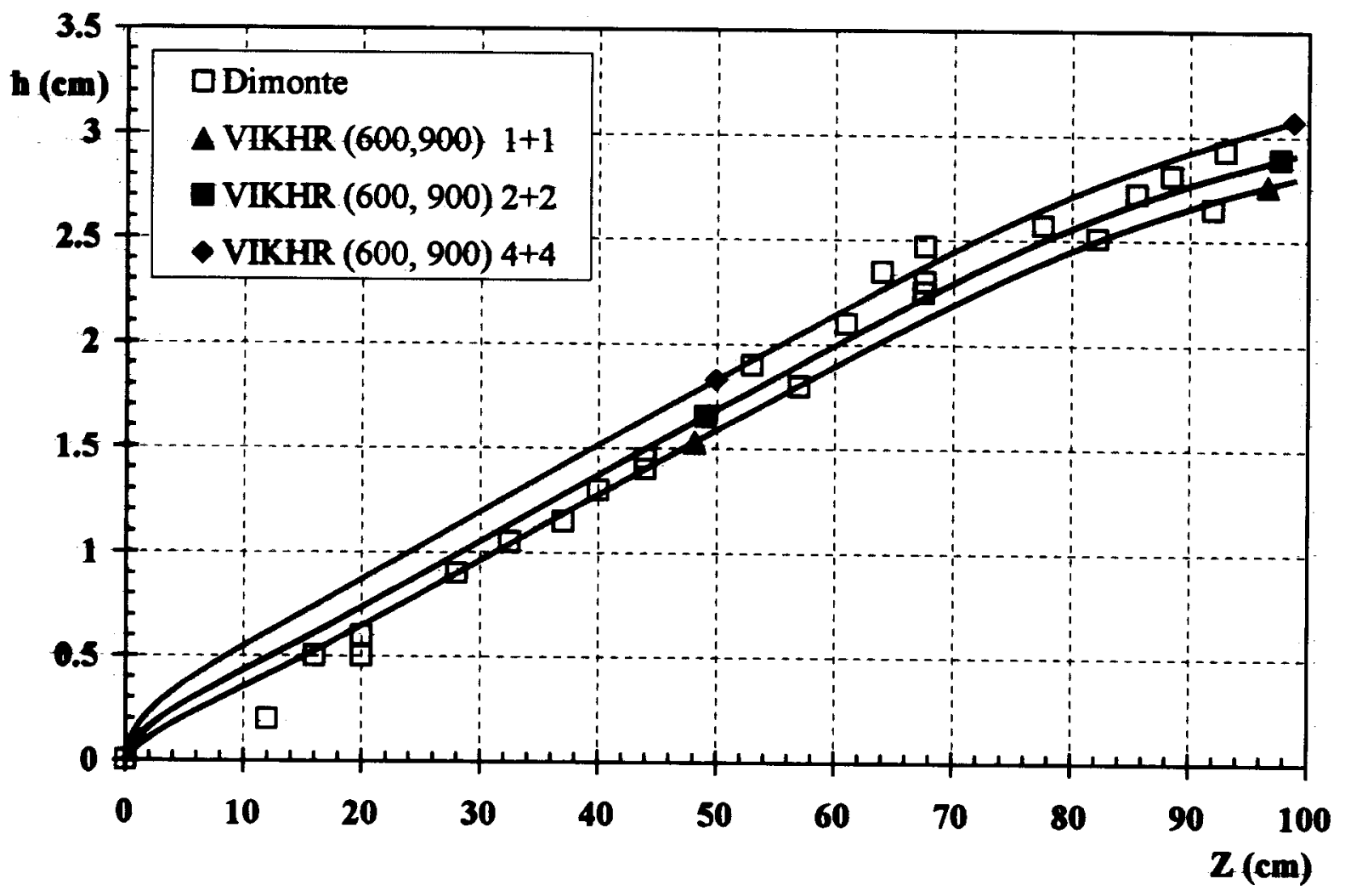

Fig. 3,b. Increasing acceleration: calculations with variable initial zone width LO on a fixed grid. ( $\mathrm{L}_{0}$ is given by the number of numerical points to the left and to the right of the interface where the initial density fluctuations are set.) 


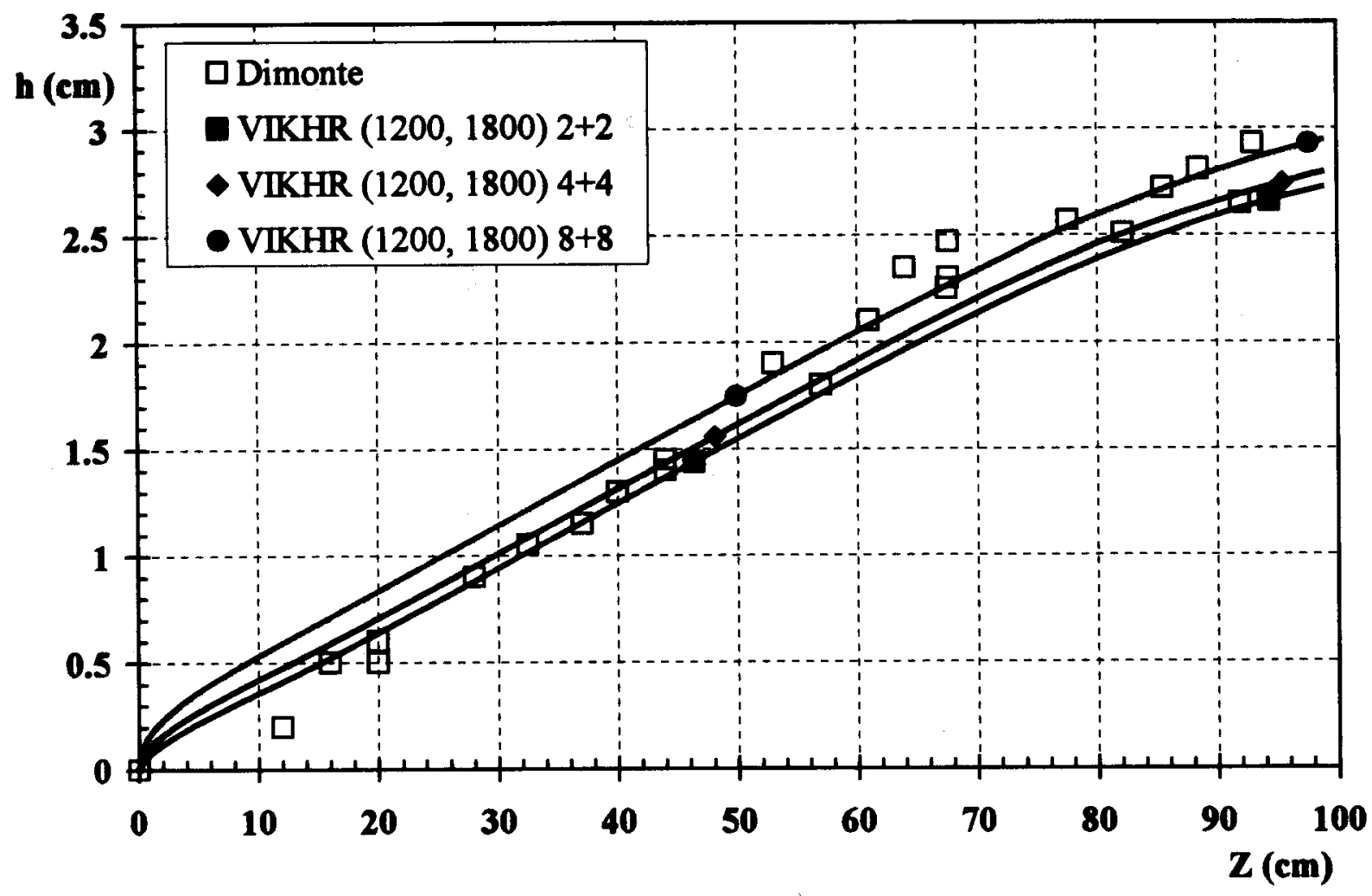

Fig. 3,c. Increasing acceleration: calculations similar to those shown in the fig. 3.b, but the number of numerical points increased twofold. 


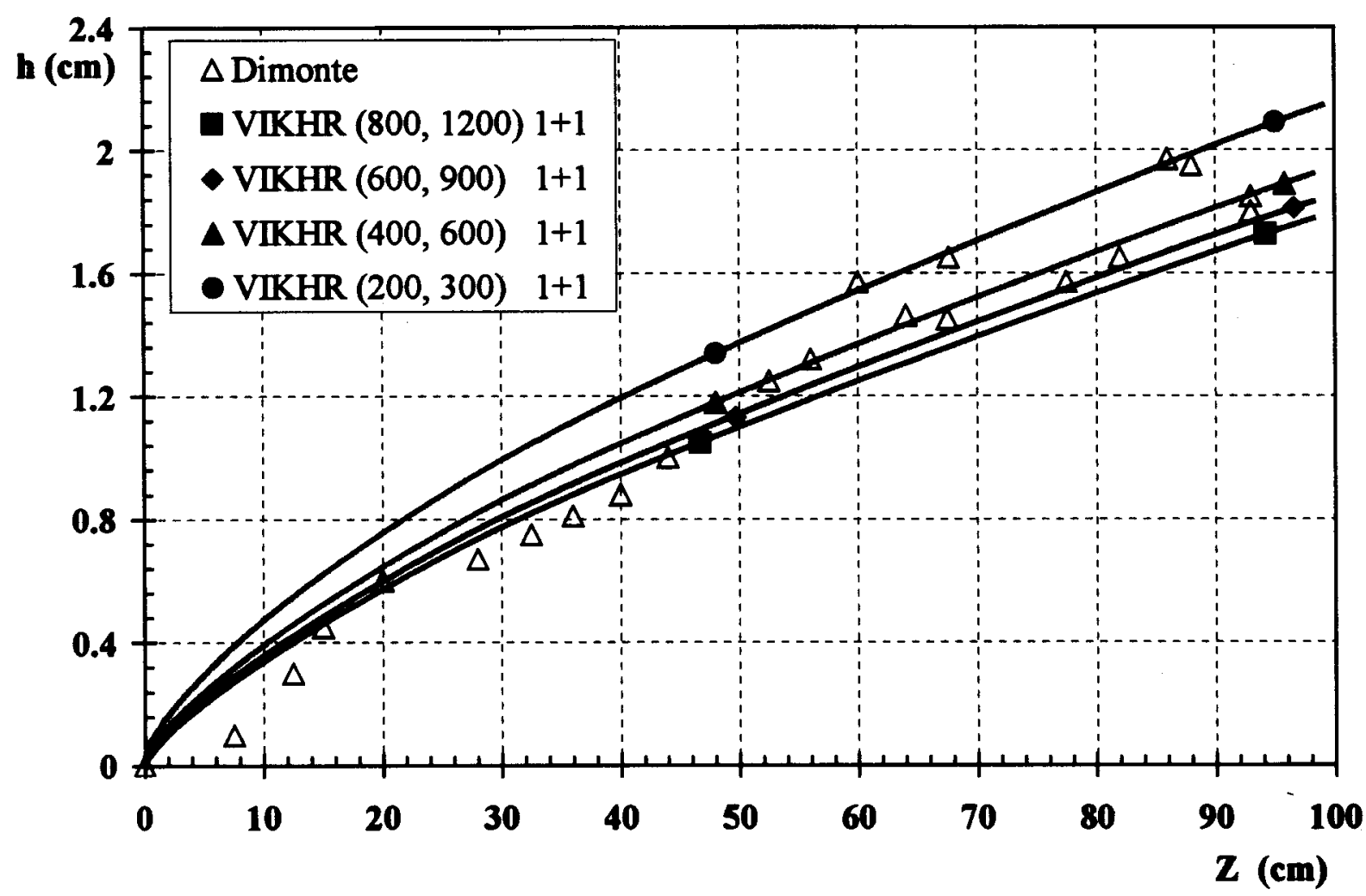

Fig. 4,a. Decreasing acceleration: calculations with a sequence of refined grids. (Initial density fluctuations are set in one point to the left and one point to the right of the interface.)

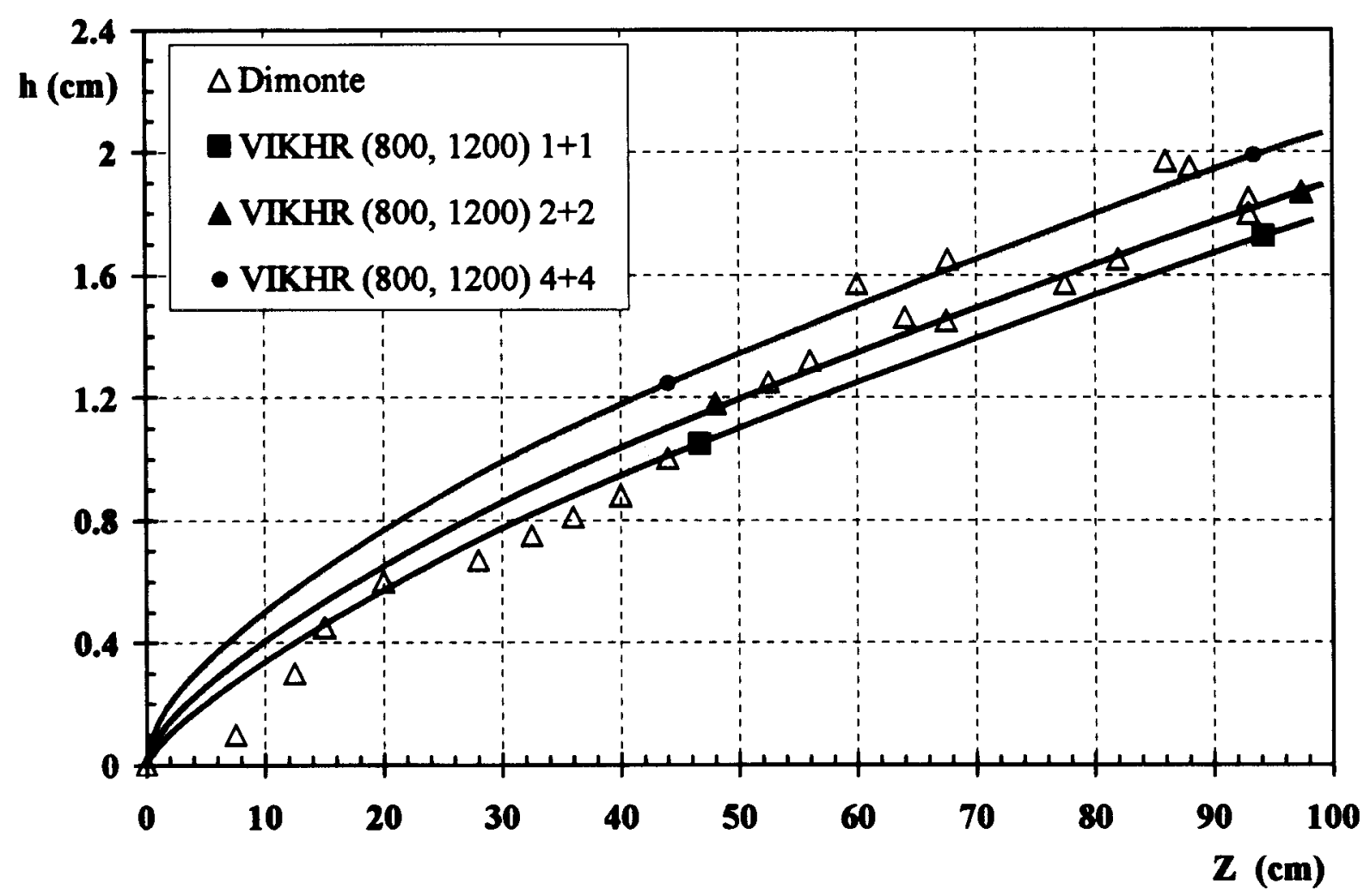

Fig. 4,b. Decreasing acceleration: calculations with variable initial zone width $\mathrm{L}_{0}$ on a fixed grid. ( $\mathrm{L}_{0}$ is given by the number of numerical points to the left and to the right of the interface where the initial density fluctuations are set.) 


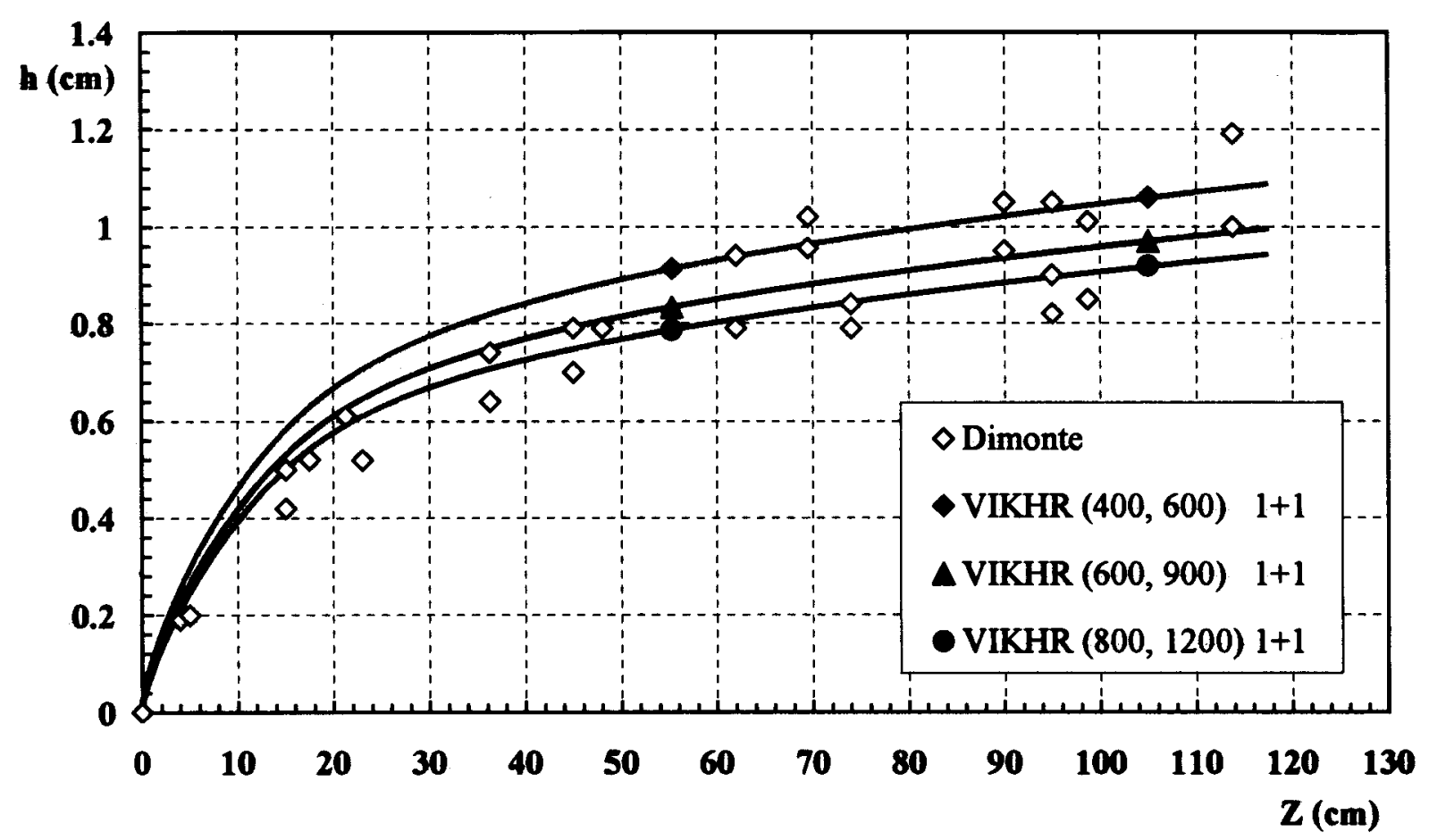

Fig. 5,a. Pulse acceleration: calculations with a sequence of refined grids. (Initial density fluctuations are set in one point to the left and one point to the right of the interface.).

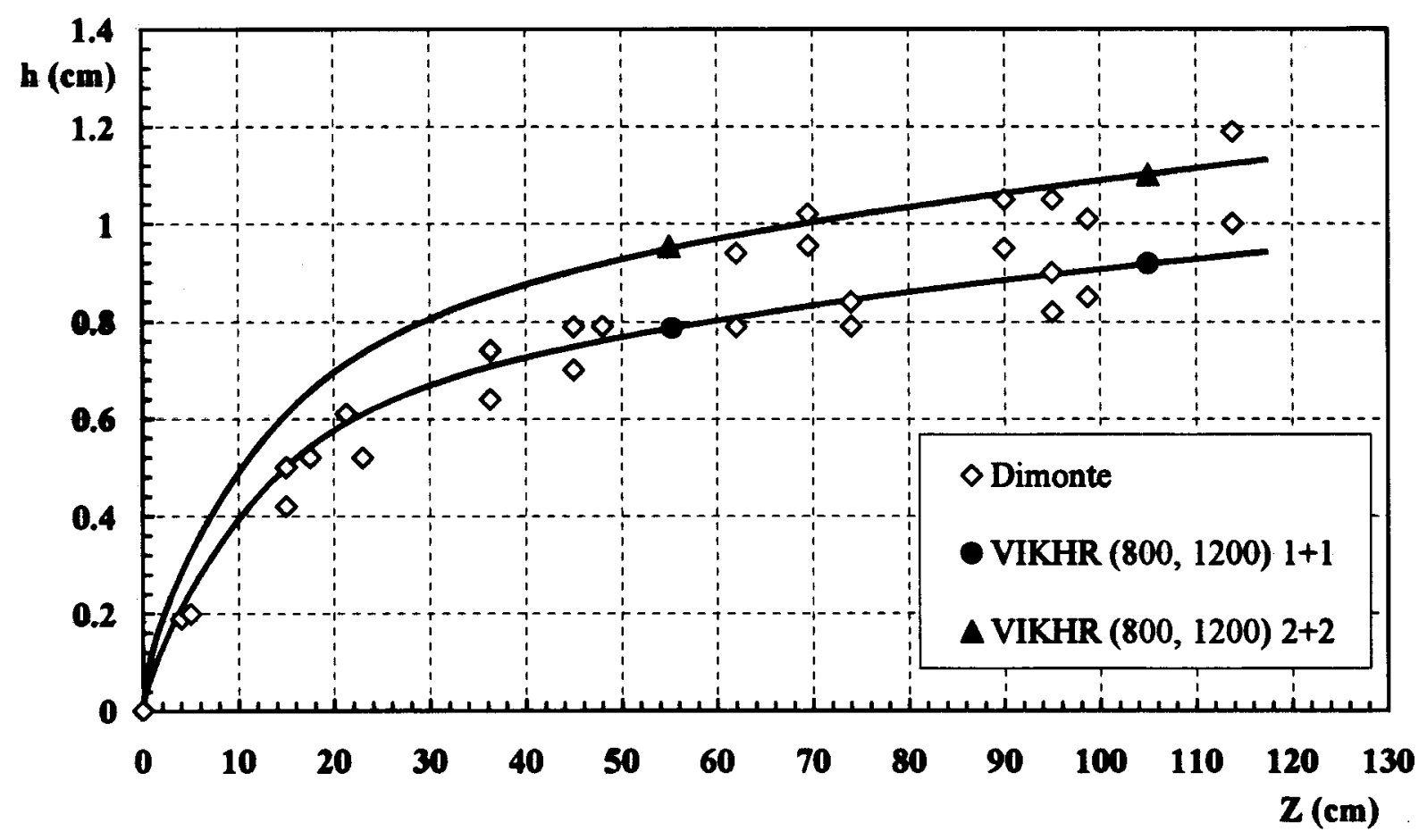

Fig. 5,b. Pulse acceleration: calculations with variable initial zone width $L_{0}$ on a fixed grid. $\left(\mathrm{L}_{0}\right.$ is given by the number of numerical points to the left and to the right of the interface where the initial density fluctuations are set.) 


\title{
$A X J A L$
}

\section{THE BEHAVIOR OF VARIANCE RESULTING FROM TURBULENT MIXING ZONE INTERACTION WITH SHOCKS}

\author{
V.I. Kozlov, A.N. Razin \\ (RFNC - VNIIEF)
}

We have compared numerical and experimental data on turbulent mixing of two gases in a shock tube induced by Richtmeyer-Meshkov's instability. The calculations were performed with the 1D technique VIKHR. This technique implements Nikiforov's semi-empirical model. We compared both the mixing zones and the average variances of the velocity component parallel to the tube axis. There was no special algorithm for calculations of turbulent quantities at the shock front.

\section{INTRODUCTION.}

Nikiforov suggested the turbulent mixing model [1] to treat transient turbulent flows stipulated by Raleigh-Taylor and Richtmeyer-Meshkov instabilities. This model has been implemented in the difference form with the VIKHR technique [2]. Numerical simulations of various tests has shown that the model [1] provides satisfactory treatment of turbulent flows of multicomponent fluids without changing the model's constants $[3 \div 5]$.

Basing on $[3 \div 5]$ we can conclude that the information given by shock tube tests that only measure TM zone width as a function of time and the distribution of average density across this zone is incomplete. In fact, the width is an integral characteristic of the TM zone, and just this quantity taken alone is not sufficient to study in detail the interaction of the TM zone with shocks. On the other hand, TM models based on quite different principles can yield similar average density distributions without noticeable changes of TM zone width as a function of time. Still these model treat the TM zone "fine" structure (the distributions of turbulence intensity, turbulent energy dissipation rate, density fluctuations intensity, etc.) differently. If there is only one shock passing through the TM zone, the fine structure may have no considerable effects on the zone evolution (apparently, this accounts for the fact that different models yield similar results 
for simple flows). Complex flows often have several TM zones that evolve simultaneously under the influence of multiple shocks, and different zones can join. More detailed information about TM zone structure and the mechanism of interaction with shocks is required for such flows.

New results in this direction have been obtained by French researchers in their tests [6]. These tests, which were arranged similarly to Meshkov's shock tube tests, were the first to systematically measure instantaneous flow velocities in the TM zone. The evolution of fluctuations of the axial mass flow velocity (the velocity component parallel to the tube axis) in the TM zone before and after it interacted with shocks reflected from the tube dead-end has been studied by means of Doppler's laser anemometry. Mean square fluctuations of the axial velocity have been calculated in five Eulerian points by statistical averaging of the multiple measurements.

This paper gives VIKHR numerical results on the experiments [6]. On the whole, there is good agreement with the experiments of both the TM zone width as a function of time and the axial variance $\overline{u_{1}^{\prime 2}}$ as a function of time. Meanwhile, we must note that the calculation considerably overestimates (nearly twofold) the maximum $\overline{u_{1}^{\prime 2}}$ when the TM zone interacts with the first shock reflected from the tube dead-end (this is the most intensive shock). In addition, the calculations have shown faster attenuation of $\overline{u_{1}^{\prime 2}}$ with time compared to the experiments.

At present we are working on further development of the TM physical model implemented in the VIKHR technique. In particular, we are improving the correlations for turbulent transport and dissipative processes, and study the mechanism of TM zone interaction with shocks in more detail. The results calculated with the modified VIKHR will be presented upon completion of this work.

\section{NUMERICAL RESULTS}

$\checkmark$ The numerical domain of the problem is shown in the fig. 1. The initial gas pressure is equal to the atmospheric pressure of 1 bar. The initial air density is $0.0012 \mathrm{~g} / \mathrm{cm}^{3}$, that of $\mathrm{SF}_{6}-0.006 \mathrm{~g} / \mathrm{cm}^{3}$. Air and $\mathrm{SF}_{6}$ were treated as perfect gases, the adiabatic index of air was $\gamma=1.4$, that of $\mathrm{SF}_{6}$ was $\gamma=1.094$. The shock tube dead-end has 
the co-ordinate $0 \mathrm{~cm}$, the dead end being regarded as a rigid wall. The interface coordinate is $30 \mathrm{~cm}$. At the right boundary $(120 \mathrm{~cm}$ from the shock tube dead end) a constant pressure of 2.15 bar is set, which causes a shock in $\mathrm{SF}_{6}$ with the front velocity of $197 \mathrm{~m} / \mathrm{s}$.

The comparison of the experimental data and the numerical data calculated with the VIKHR code is given in the fig. $2 \div 4$. Note that $2 e_{1}$ from VIKHR corresponds to the axial variance $\overline{u_{1}^{\prime 2}}$.

The fig. 2 shows the R-t diagram of the TM zone boundaries. The start of the calculation is the reference time (the time when the shock reaches the interface is $\mathrm{t} \approx 4.55 \mathrm{~ms}$ ), and the coordinate center is at the shock tube dead end (rigid wall). One can see that the experimental and numerical data are, on the whole, in satisfactory agreement. The R-t diagram of the finite-difference shock center calculated with the algorithm $[7]$ is also given here. The fig. 2 shows that three shocks reflected from the dead end pass through the TM zone. In the calculation, however, only two waves can be regarded as shocks. As a matter of fact, an analysis of calculations with a sequence of refined grids has shown that the gas-dynamic gradients at the fronts of the first two wayes are inversely proportional to the $\backslash$ numerical point size, with much less pronounced dependence for the third wave. Thus, the third fipite-difference wave can be regarded as a compression wave.

The fig. 3 shows the numerical (solid line) and experimental (step line) axial variance. The coordinates of the Eulerian points (transducers) where the velocity was measured, are given in $\mathrm{mm}$ from the initial interface location, and the time (ms) is counted from the moment when the shock reaches the interface.

The fig. 3 shows that before the first reflected shock enters the TM zone, the numerical and the experimental variance are in satisfactory agreement (according to the profes two transducers nearest to the interface: $51 \mathrm{~mm}$ and $125.5 \mathrm{~mm}$ ). Note that when the TM zone passes through these transducers, there are no shocks in the TM zone.

As for the third transducer $(161 \mathrm{~mm})$, the numerical variance is considerably higher than the experimental data. The fig. 2 shows that when the TM zone passes through this transdueer, the most intensive (the first) reflected shock is within the TM zone. 


$$
\text { pwhe }
$$

On the fourth transducer $(169 \mathrm{~mm})$ the numerical variance satisfactorily agrees with the experimental data. The fig. 2 shows that when the TM zone passes through this proke transdticer, there are no shocks in the TM zone.

For the fifth the numerical variance is lower than $(178.5 \mathrm{~mm})$ the experimental data, though the numerical data are close to the experimental data statistical scatter range. When the TM zone passes through this trastreer two last reflected shocks are crossing the TM zone.

For the detailed analysis of the variance on the fifth transducer, numerical variance has been calculated as a function of time in three extra Eulerian points in the vicinity of the fifth transducer. The results are shown in the fig. 4 (the co-ordinates of the Eulerian points are counted from the dead-end).

According to the fig. 2, the left TM zone boundary (the one closer to the tube's dead-end) passes through the transducer-with $R=12 \mathrm{~cm}$ earlier than it crosses the transducers with $\mathrm{R}=11.5 \mathrm{~cm}$ and $\mathrm{R}=11 \mathrm{~cm}$. The second shock reflected from the rigid wall passes through the traber with $R=12 \mathrm{~cm}$ later than it passes through those with $\mathrm{R}=11.5 \mathrm{~cm}$ and $\mathrm{R}=11 \mathrm{~cm}$. It is shown in the fig. 4 as gradual increase of the signal recording time from $\mathrm{R}=12 \mathrm{~cm}$ to $\mathrm{R}=11 \mathrm{~cm}$ and decreasing maximum variance until the second reflected shock enters the TM zone. As the second shock, when it crosses the proter transducers, is in different Lagrangian points of the TM zone with different turbulence intensity, the peaks due to shock-TM zone interaction have different amplitudes at
profes different transducers.

It is shown in the fig. 2 that the third reflected shock passes through the TM zone at $6.95 \mathrm{~ms}<\mathrm{t}<7.2 \mathrm{~ms}$. In the fig. 4 , one can see an increase of variance at the transducer with $\mathrm{R}=11 \mathrm{~cm}$ during this time interval, while the other two transducers do not show any increase of the variance. The main reason for this is, apparently, that the third reflected shock (which is the weakest) interacts with the TM zone in Lagrangian points with different turbulence intensities,'the turbulent intensity being the lowest at the transducers-with $\mathrm{R}=11.5 \mathrm{~cm}$ and $\mathrm{R}=12 \mathrm{~cm}$ (the corresponding Lagrangian points are near the right boundary of the zone).

On the whole, we can state satisfactory agreement between the numerical results and experimental data, except for the transducer located at $161 \mathrm{~mm}$ from the shock tube 
dead end. The numerical peak value for this transdueer $2 \varepsilon_{1}$ is about 2 times higher than the experimental measurement $\overline{u_{1}^{\prime 2}}$ (for the other transducers-the numerical results are

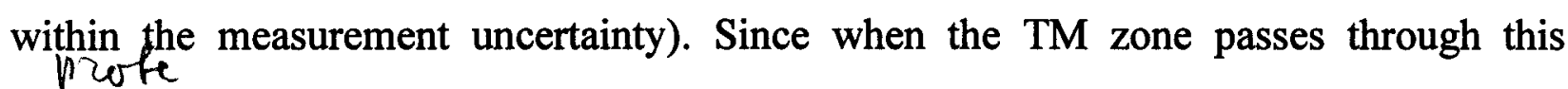

transducer, the most intensive first reflected shock is in the TM zene, we can conclude that one of the reasons for this discrepancy can be that the model [1] unsatisfactorily describes the mechanism of shock-TM zone interaction. (As a matter of fact, the closure correlations of the model [1] have been developed for finite, rather small gas-dynamic gradients. Meanwhile, shocks in turbulent flows are discontinuity surfaces, like those in perfect fluids. This results from the fact that the minimum turbulent eddy size from the dissipative (Kolmogorov's) interval of spectrum is at least an order of magnitude higher than the interatomic distance).

\section{REFERENCES.}

1. V.V.Nikiforov Turbulent mixing at an interface of different-density fluids. // Voprosy Atomnoy Nauki i Techniki. Series: Theoretical and applied physics. 1985, issue 1, p. 3-8.

2. V.A.Andronov, V.I.Kozlov, V.V.Nikiforov, A.N.Razin, Yu.A.Yudin Turbulent mixing calcualtion technique for 1D flows (the VIKHR technique). // Voprosy Atomnoy Nauki i Techniki. Series: Mathematical modeling of physical processes. 1994, issue 2, s. 59-64.

3. V.A.Andronov, S.M.Bakhrakh, E.E.Meshkov et.al. An experimental study and numerical simulations of turbulent mixing in 1D flows // Doklady AN SSSR, 1982. vol. 264, № 1, p. 76-82.

4. V.V.Nikiforov Calculations of gravitational turbulent mixing in non-self-similar flows. // Voprosy Atomnoy Nauki i Techniki. Series: Theoretical and applied physics. 1993, issue 1, p. 3-12.

5. V.V.Nikiforov, V.A.Andronov, A.N.Razin Evolution of a turbulent mixing zone under shock impact // Doklady AN SSSR, 1995. vol. 343, № 3. p. 323-325. 
6. Francoise Poggi, Marie-Helene Thorembey, Gerard Rodriguez. Velocity measurements in turbulent gaseous mixtures induced by Richtmyer-Meshkov instability. Physics of Fluids, v. 10, n. 11, 1998, pp. 2698-2700.

7. A.N.Razin An algorithm for finite-difference shock center location. // Voprosy Atomnoy Nauki i Techniki. Series: Theoretical and applied physics. 2001, issue 1, p. 43-50 . 


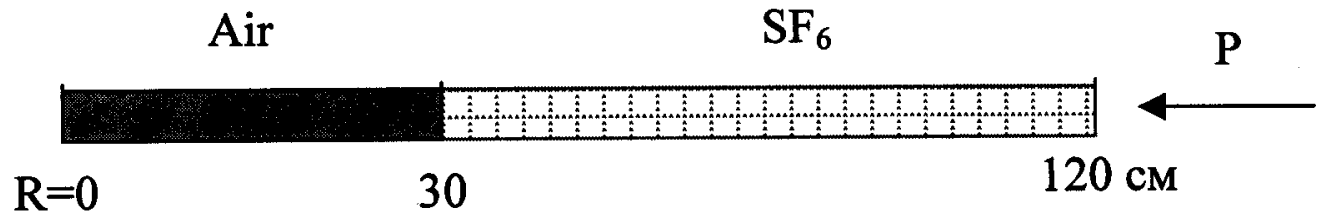

Fig. 1 Numerical geometry.

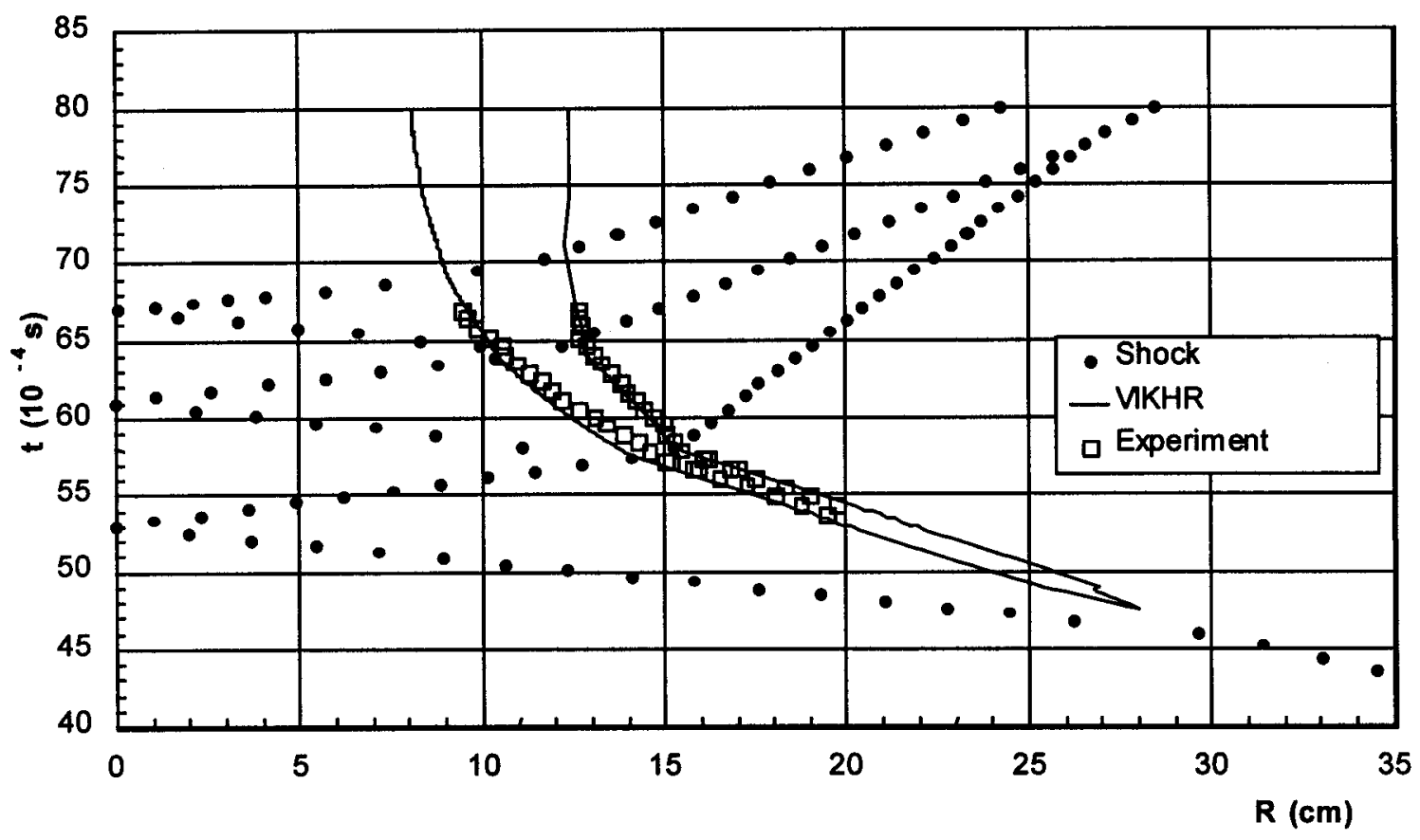

Fig. 2 R-t diagrams of the TM zone boundary and the shock 


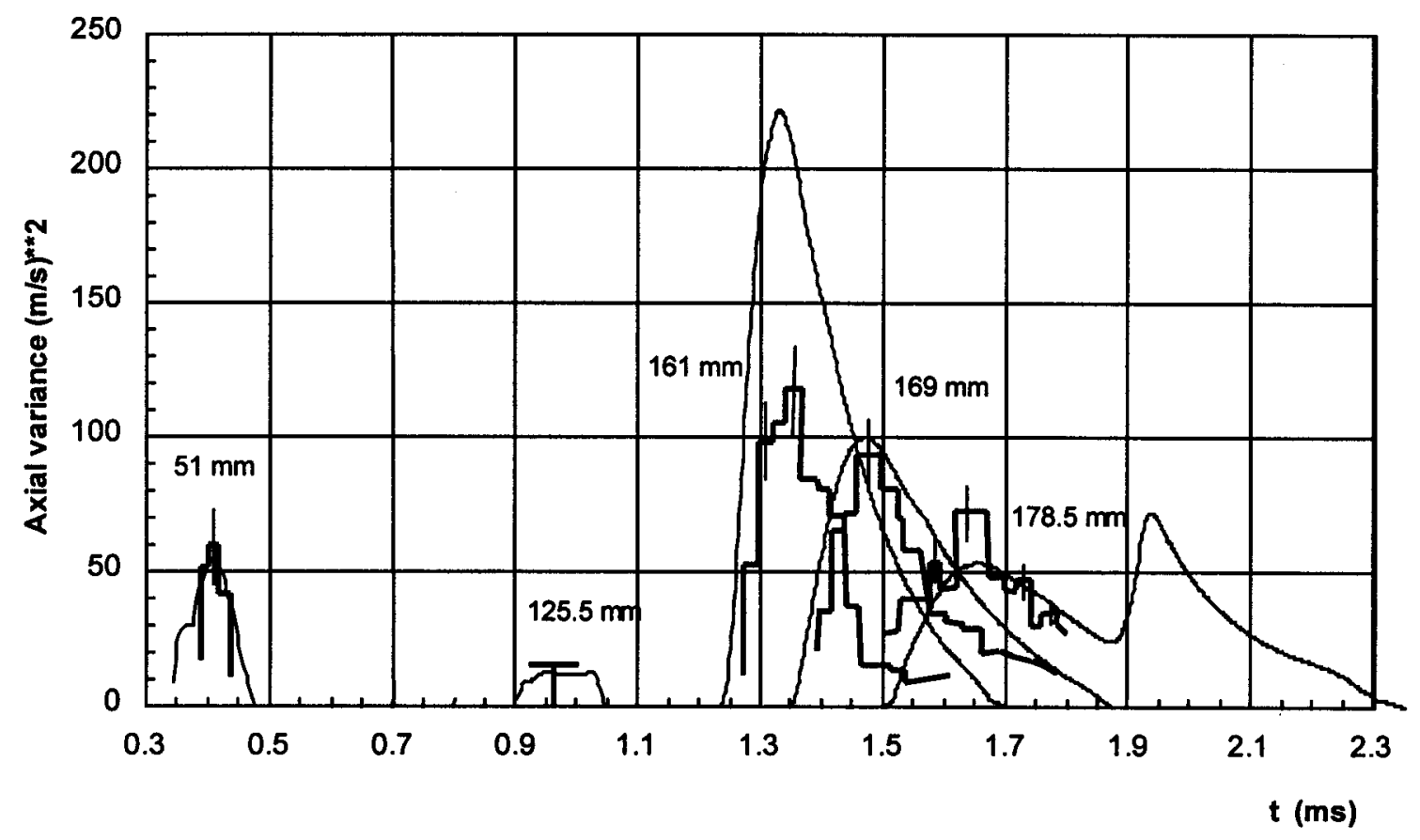

Fig. 3 Variance as a function of time given by different transducers

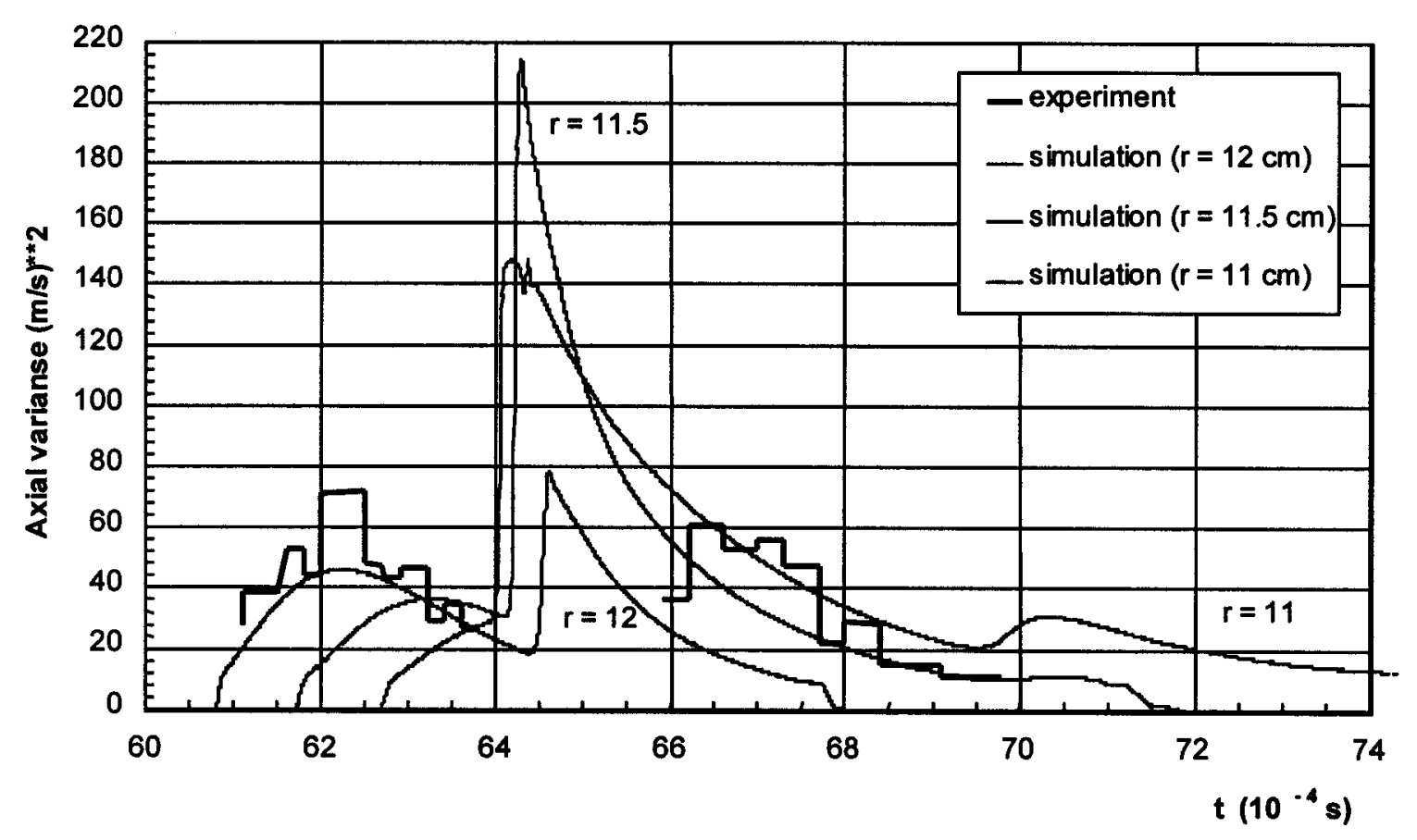

Fig. 4 Variance as a function of time in the vicinity of hte $5^{\text {th }}$ transducer 


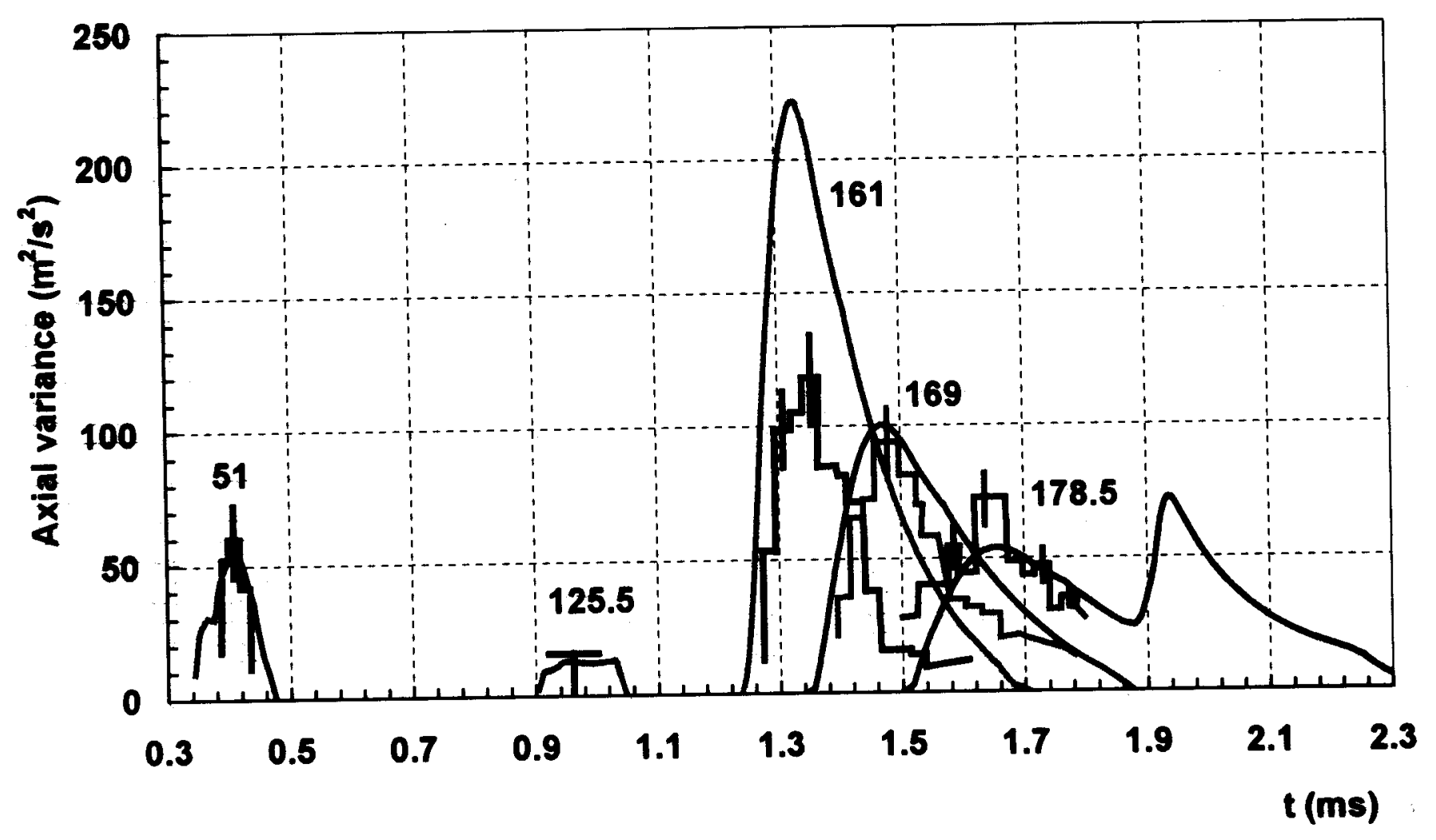

Fig. 3 Variance as a function of time given by different LDA probes.

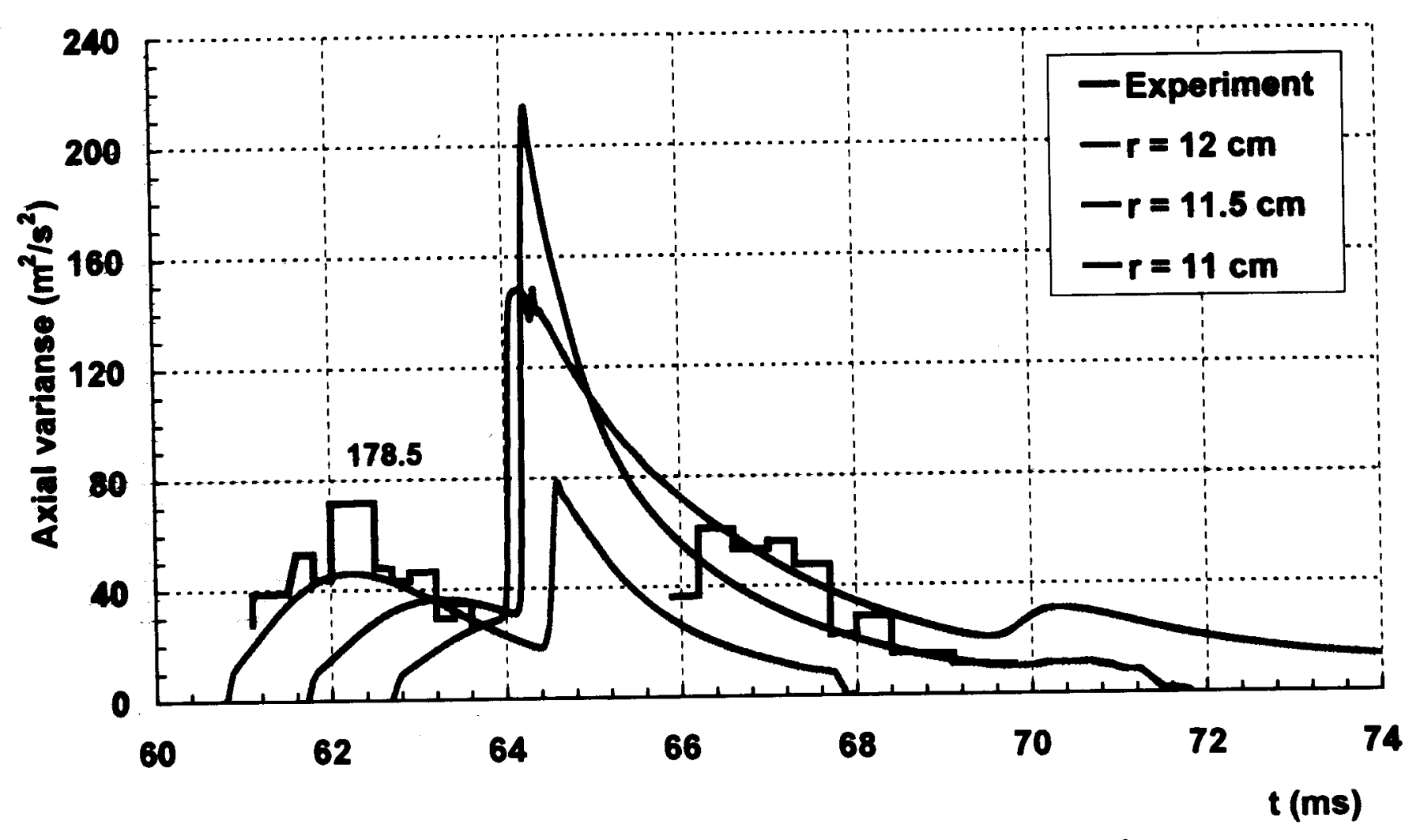

Fig. 4 Variance as a function of time in the vicinity of the $5^{\text {th }}$ LDA probe. 


\section{NIKIFOROV'S MODEL OF TURBULENT MIXING (slab geometry)}

$\frac{d \rho}{d t}=-\rho \operatorname{div} U ; \quad \frac{d U}{d t}=-\frac{1}{\rho} \nabla\left(P+P_{t}\right) ; \quad P_{t}=2 \rho \frac{e_{1}}{e} \Phi$

$\frac{d E}{d t}=-\frac{P}{\rho} \operatorname{div}(U-W)+\frac{1}{\rho} \operatorname{div}(\rho D \nabla E)+\frac{Q}{e} \Phi ; \quad \frac{d c_{i}}{d t}=\frac{1}{\rho} \operatorname{div}\left(\rho D \nabla c_{i}\right)$

$\frac{d e_{1}}{d t}=\operatorname{div}\left(3 D_{t} \nabla e_{1}-W e_{1}\right)-e_{1} \operatorname{div} W-2 V_{11} e_{1}+\left(1-\frac{\alpha k}{5}\right) f_{1} G W-\gamma \frac{Q}{e}\left(e_{1}-\frac{1}{3} e\right)-\frac{Q}{3}$

$\frac{d e_{2}}{d t}=\operatorname{div}\left(D_{t} \nabla e_{2}-W e_{2}\right)-e_{2} \operatorname{div} W+\frac{\alpha k}{10} f_{1} G W-\gamma \frac{Q}{e}\left(e_{2}-\frac{1}{3} e\right)-\frac{Q}{3} ;$

$\frac{d \Phi}{d t}=\frac{1}{\rho} \operatorname{div}\left[\rho\left(3 D_{t} \nabla \Phi-W \Phi\right)\right]-2 \frac{V_{11} e_{1}}{e} \Phi-W \frac{d U}{d t}-\frac{Q}{e} \Phi$

$\frac{d Q}{d t}=\operatorname{div}\left(\frac{5}{3} D_{t} \nabla Q-W Q\right)-Q \operatorname{div} W-\frac{4}{3}\left(1+\frac{\gamma}{2} \frac{e_{1}-e_{2}}{e}\right) V_{11} Q+\frac{f_{1} G W}{3 e_{1}} Q+$

$$
\beta\left(\frac{\alpha k}{5} f_{1} G W+\frac{2 \gamma}{3} \frac{e_{1}-e_{2}}{e} Q\right) \frac{A W}{R}(1+R)-2 \frac{Q^{2}}{k e}
$$

$\frac{d W}{d t}=\frac{1}{\rho} \operatorname{div}\left[\rho\left(2 D_{t} \nabla W-W^{2}\right)\right]-V_{11} W+2 A e_{1}+f_{1} G R-k_{1} \frac{Q}{e} W$

$\frac{d R}{d t}=\frac{1}{\rho^{2}} \operatorname{div}\left[\rho^{2}\left(D_{t} \nabla R-W R\right)\right]+R \operatorname{div} W+2 A W-\frac{3}{2} \frac{Q}{e} R$.

Here: $\nabla \equiv \frac{\partial}{\partial r} ; \quad \operatorname{div} \equiv \frac{\partial}{\partial r} ; \quad V_{11}=\frac{\partial}{\partial r}(U-W) ; \quad A=\frac{\nabla P}{\gamma_{a d} P}-\frac{\nabla \rho}{\rho} ; G=\frac{1}{\rho} \nabla P$;

$e=e_{1}+2 e_{2} ; \quad k=\frac{3 e_{2}}{e} \sqrt{\frac{3 e_{1}}{e}} ; \quad e_{1}=\overline{u_{1}^{\prime 2}} / 2 ; \quad e_{2}=\overline{u_{2}^{\prime 2}} / 2 ; \quad R=\overline{\rho^{\prime 2}} / \rho^{2} ;$

$W=\overline{\rho^{\prime} u_{1}^{\prime}} / \rho ; \quad \Phi=e+\overline{\rho^{\prime} u_{k}^{\prime 2}} / 2 \rho ; \quad f_{1}=\left(1+R-\frac{D_{t}}{W} \nabla R\right)^{-1}$

$D=\left\{\begin{array}{cc}W+A & \text { if } A W>0 \\ 0 & f A W \leq 0\end{array} ; \quad D_{t}=\frac{16 \alpha_{1} e_{1}}{k_{3}+b} ; \quad k_{3}=2 \frac{Q}{e}\left(\gamma+\frac{e}{3 e_{1}}+3\right)\right.$

$b=\alpha_{1}(\xi+|\xi|) ; \quad \xi=\frac{2 A W}{R}-k_{2} \frac{Q}{e}$. 
LDA probe positions from interface:

$5.1 \mathrm{~cm}, 12.55 \mathrm{~cm}, 16.1 \mathrm{~cm}, 16.9 \mathrm{~cm}, 17.85 \mathrm{~cm}$

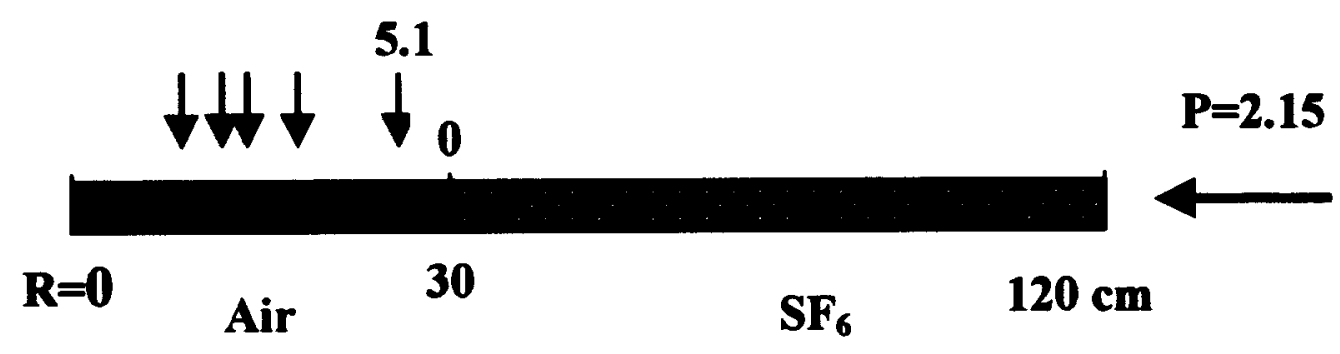

Fig. 1 Numerical geometry.

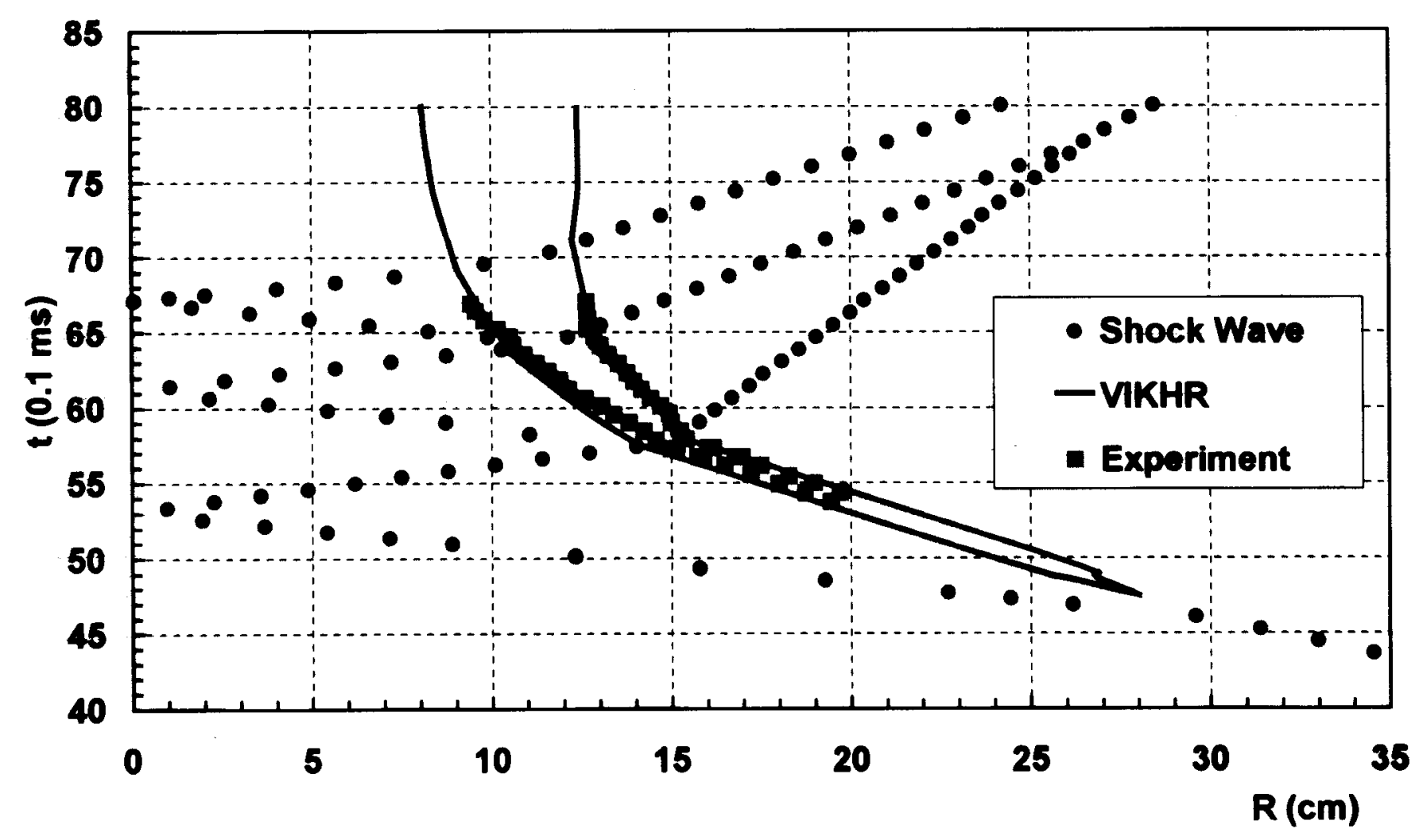

Fig. 2 R-t diagrams of the TM zone boundary and the shock 


\section{A New Two-Scale Mix Model: Towards a Multi- Component Model of Turbulent Mixing*}

\section{Presented to the 8th International Workshop on the} Physics of Compressible Turbulent Mixing

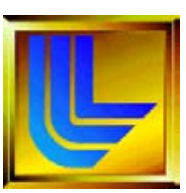

Donald E. Eliason, William H. Cabot, and Ye Zhou AX-Division, Lawrence Livermore National Laboratory

\section{December 2001}

\footnotetext{
*This work was performed under the auspices of the U.S. Department of Energy by the University of California Lawrence
} Livermore National Laboratory under contract No. W-7405-Eng-48. 


\section{Abstract}

Turbulent mixing of the fluids in a multicomponent system is of interest in situations such as inertial confinement fusion (ICF) and core-collapse supernovae ${ }^{1}$. We report results of a project to include a model of turbulent mixing in a multi-component hydrodynamics and physics model called KULL, which is used for ICF. Because KULL is a complex, multi-dimensional model, we have developed a simplified, onedimensional version called sKULL to speedup the development of the turbulent mixing model.

Of primary interest in the development of a turbulent mixing model for a multicomponent fluid is the question of whether it is necessary to allow each component of the fluid to retain its own velocity. Generally a multi-component, multi-velocity turbulent mixing model should allow separate velocities for each component of the fluid ${ }^{2}$. However, the necessity to carry separate velocities for each component of the fluid greatly increases the memory requirements and complexity of the computer implementation. In contrast, we present a new two-scale formulation of the K- $\varepsilon$ turbulent mixing model, with production terms based on a recent scaling analysis ${ }^{3}$, which treats all components of the fluid as if they had the same velocity. We also show that our new method for the initial conditions of the uncoupled two-scale K- $\varepsilon$ model yields asymptotic growth. Future work will compare the results of using this single velocity model with those from a more complete multi-velocity formulation of turbulent mixing, to decide whether the multi-velocity formulation needs to be used in KULL. 


\section{The goal of this work is to develop a turbulent mixing}

model for the ICF code called KULL

- Turbulent mixing of the fluids in a multi-component system is of interest in situations such as inertial confinement fusion (ICF) and core-collapse supernovae ${ }^{1}$

- We report results of a project to include a model of turbulent mixing in a multi-component hydrodynamics and physics model called KULL, which is used for ICF

- Because KULL is a complex, multi-dimensional code, we have developed a simplified, one-dimensional version called sKULL to speed-up the development of the turbulent mixing model

${ }^{1}$ Remington, B.A., D. Arnett, R.P. Drake, and H. Takabe, Modeling Astrophysical

Phenomena in the Laboratory with Intense Lasers, Science 284, 1488 (1999). 


\section{Three areas of this research are highlighted}

- sKULL reproduces KULL's multi-component hydrodynamics and numerics

- A single velocity, multi-component, two-scale $\mathrm{K}-\varepsilon$ turbulent mixing model has been developed within sKULL

- A new method for the uncoupled two-scale K- $\varepsilon$ initial conditions yields asymptotic growth 


\section{We have an appropriate path to develop a turbulent mixing model for KULL}

Classic KULL:

ALE Hydrodynamics

The most general model of turbulent mixing is multicomponent and multi-velocity
Single sKULL:

Duplicates

KULL's

ALE

Hydrodynamics
Multi-sKULL:

Multi-

Component

and

Multi-Velocity 


\section{sKULL is the right platform in which to develop a turbulent mixing model for KULL}

- sKULL duplicates KULL's hydrodynamics

- Side-by-side runs of KULL and sKULL on the Sod shock produce the same results

- We tested the Lagrangian, Eulerian, and ALE capabilities of sKULL to ensure they matched KULL's

- The simplified nature of sKULL, due both to 1-D and no additional physics, allows it to run more quickly

- Faster run times lead to shorter turn-around times for testing turbulent mixing models 


\section{Side-by-side runs of KULL and sKULL on the Sod shock problem produce the same results}

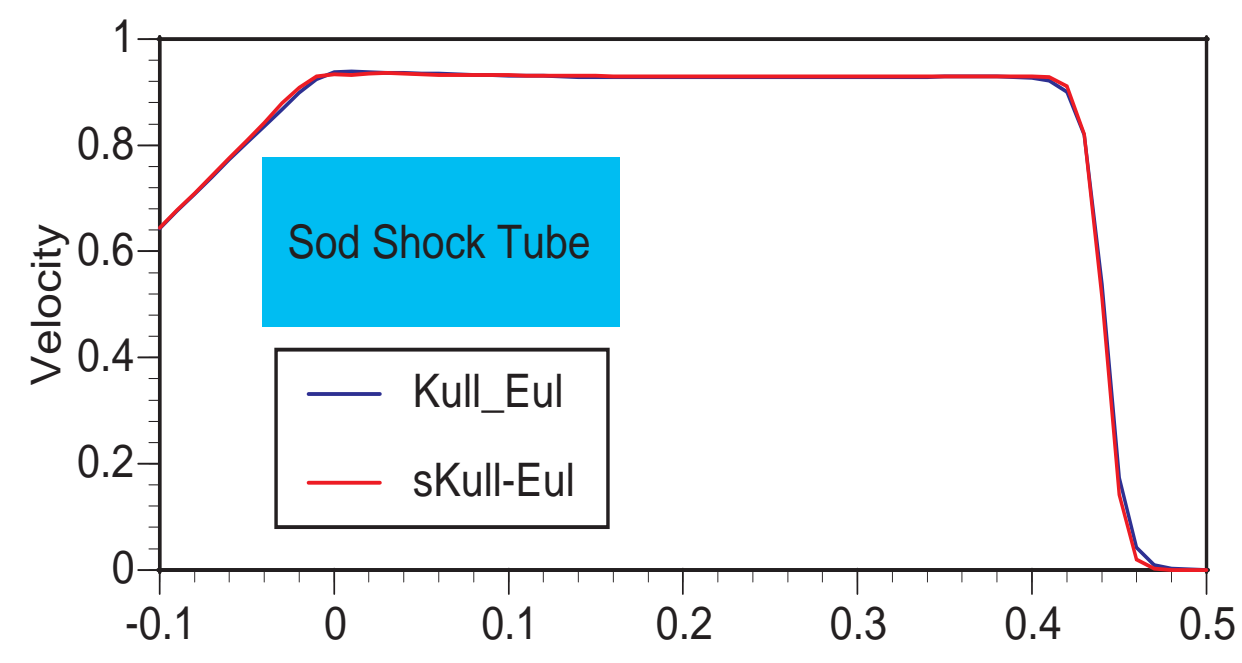

- Duplication of KULL results on selected problems w/ sKULL verifies that we've duplicated KULL's numerics

- Sod (1978) shock tube problem:

\begin{tabular}{|l|l|}
\hline$\rho=1$ & $\rho=0.125$ \\
$p=1$ & $p=0.1$ \\
$u=0$ & $u=0$ \\
\hline
\end{tabular}

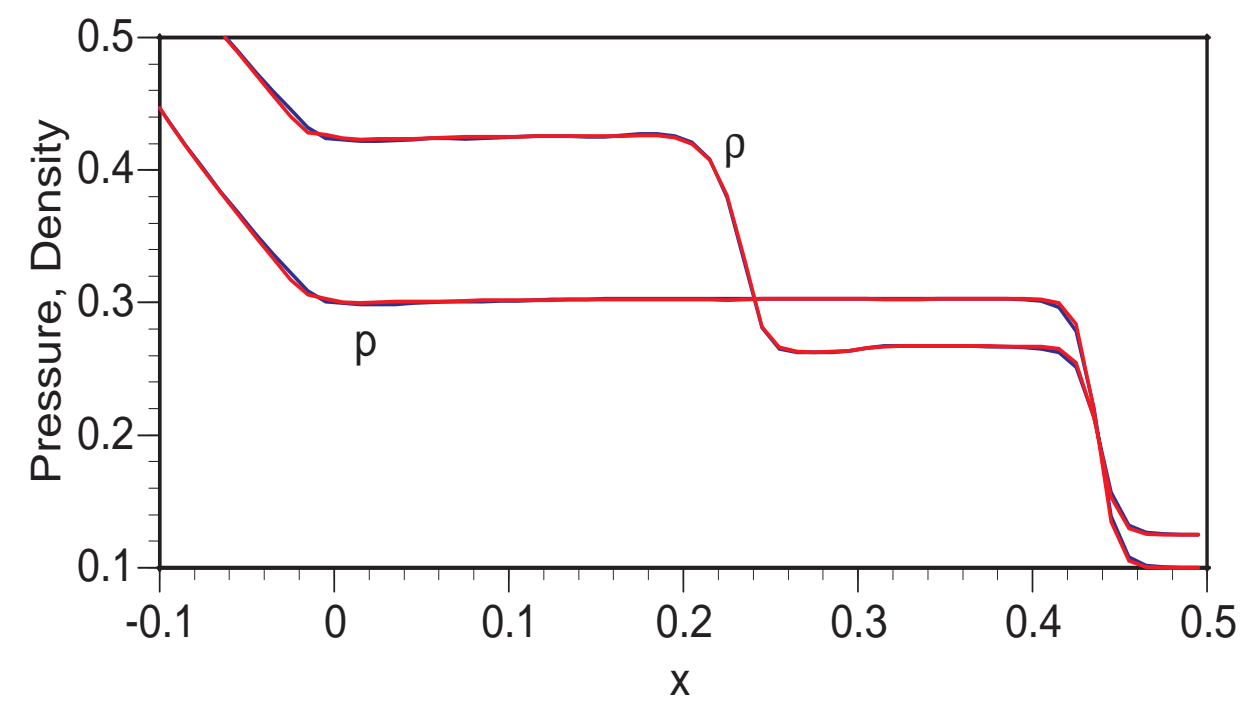

- Standard test problem

- Compared Lagrangian, Eulerian, and ALE results to ensure that the results from the two codes agreed 


\section{SKULL MG-1V's simulation of the Benjamin air-SF 6}

\section{shock tube agrees well with the exact solution}

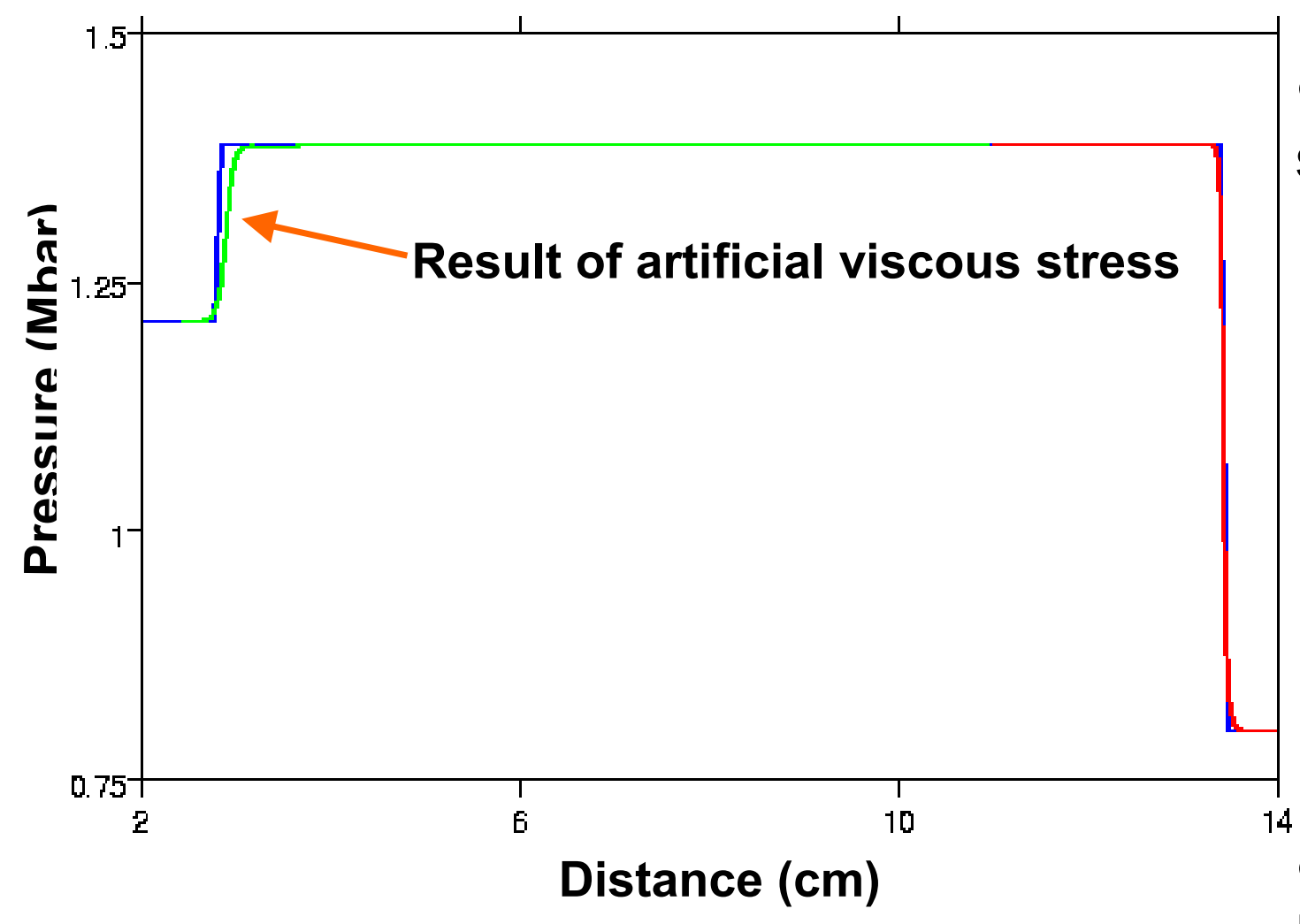

- Benjamin et al. (1993) air-SF shock tube:

\begin{tabular}{|l|l|}
\multicolumn{1}{c|}{ Air* } & \multicolumn{1}{c}{ SF $_{6}$} \\
\hline$\rho=1.27 \times 10^{-3}$ & $\rho=4.85 \times 10^{-3}$ \\
$\mathbf{u}=1.05 \times 10^{4}$ & $u=0$ \\
$p=1.21 \times 10^{6}$ & $p=8.00 \times 10^{5}$ \\
\hline Shock (Ma=1.2)
\end{tabular}

- Pressure results from the MC-1V Lagrangian simulation versus exact solution at time $232 \mu$ s show good agreement 


\section{A multi-component, multi-velocity (MC-MV) approach needs to be considered for the turbulent mixing model}

- In RTI/RMI, zones may contain more than one component, each with its own velocity

- Component interactions (e.g., drag) can lead to mixing

- From the rocket rig experiments, this led David Youngs (AWE) to create his MC-MV mixing model ${ }^{2}$

- The MC-MV equations add a great deal of complexity - Carrying separate velocities increases the memory requirement

- The drag term may require an implicit treatment

${ }^{2}$ Youngs, D.L., Laser \& Particle Beams 12, 725 (1994). 


\section{sKULL will be used to test multi-velocity versus single}

velocity based turbulent mixing models

Because of sKULL's simplified nature it is faster and cheaper than KULL

- The extra memory requirement of MC-MV will be manageable

- Additional computation for interactions will be do-able

- Different numerical treatments of the drag term can be tested (explicit vs. implicit vs. iterated)

Faster and cheaper makes sKULL the ideal platform to test whether MC-MV might be needed in KULL 


\section{The MC-MV equations (Youngs ${ }^{2}$ ) add a great deal of complexity}

ALE: "Grid Velocity" $u=\left\{\begin{array}{l}u_{r} \text {, Lagrangian } \\ 0, \text { Eulerian }\end{array}\right.$

$$
\begin{gathered}
\frac{\partial x}{\partial t}=u \\
\frac{\partial\left(f_{r} \rho_{r}\right)}{\partial t}=-\frac{\partial}{\partial x}\left[f_{r} \rho_{r}\left(u_{r}-u\right)\right]-f_{r} \rho_{r} \frac{\partial u}{\partial x} \\
\frac{\partial\left(f_{r} \rho_{r} u_{r}\right)}{\partial t}=\frac{\left.\frac{\partial}{\partial x}\left[f_{r} \rho_{r} u_{r}\left(u_{r}-u\right)\right]-f_{r} \rho_{r} u_{r} \frac{\partial u}{\partial x}-f_{r} \frac{\partial P}{\partial x}+f_{r} \rho_{r} g\right]}{+\sum_{s}\left(D_{r s}+M_{r s}\right)-m_{r} \frac{\partial \tau}{\partial x}} \\
\frac{\partial\left(f_{r} \rho_{r} e_{r}\right)}{\partial t}=\frac{-\frac{\partial}{\partial x}\left[f_{r} \rho_{r} e_{r}\left(u_{r}-u\right)\right]-f_{r} \rho_{r} e_{r} \frac{\partial u}{\partial x}-h_{r} P_{r} \frac{\partial \bar{u}}{\partial x}}{+\frac{\partial}{\partial x}\left(f_{r} \rho_{r} v_{r} \frac{\partial e_{r}}{\partial x}\right)+f_{r} \varepsilon}
\end{gathered}
$$

“ALE"-like,

$\mathrm{g}=\mathbf{0}$ if shock tube, Interactions, 
Because of the MC-MV equations' complexity, we've first developed a single velocity version, MC-1V

\begin{tabular}{|l|}
\hline sKULL MC-1V \\
Lagrangian \\
equations with a \\
new two-scale K- $\varepsilon$ \\
mixing model
\end{tabular}

$$
\frac{d x}{d t}=u
$$$$
\frac{d V_{r}}{d t} \approx h_{r} \frac{d V}{d t}, V=\sum_{r} V_{r}
$$

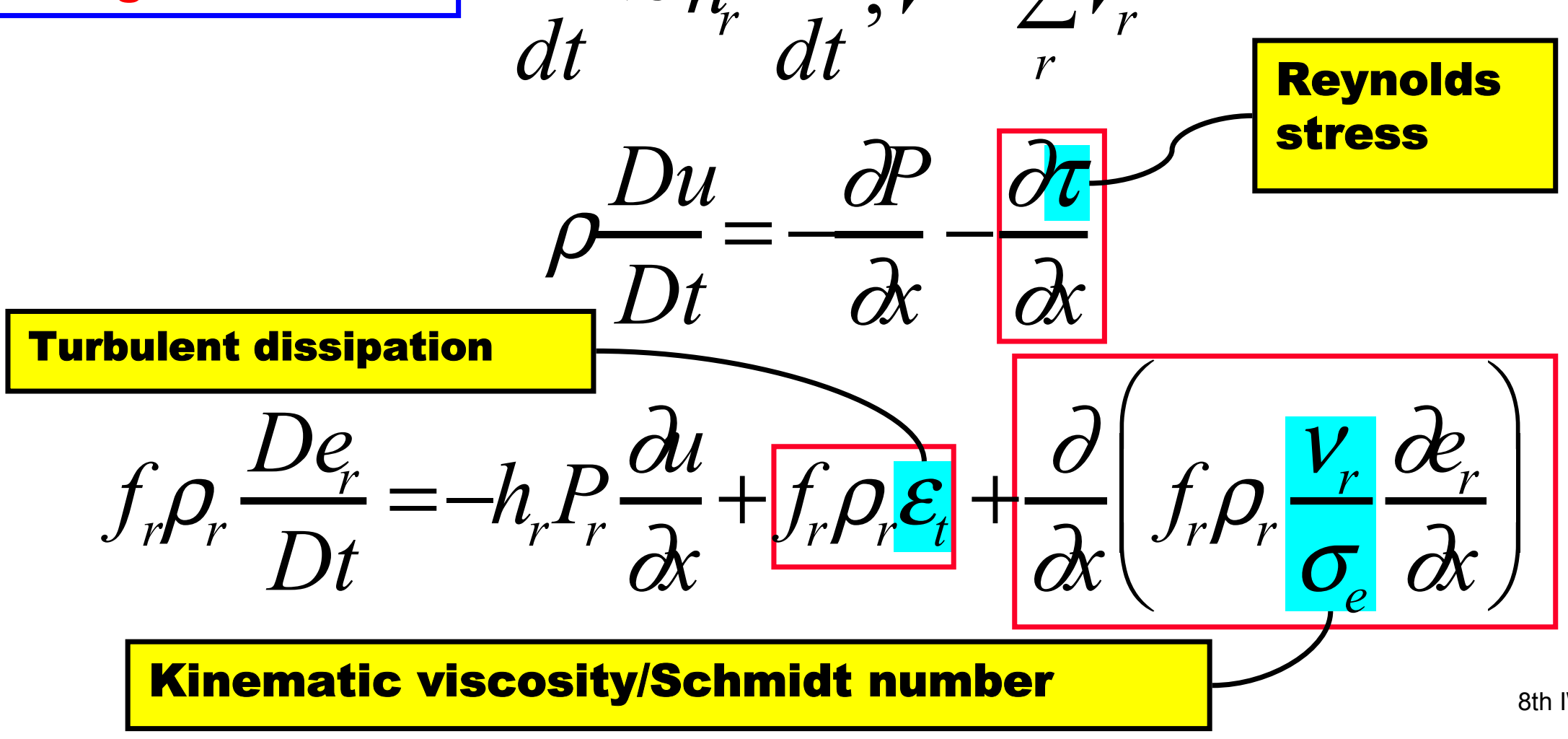


The use of the compressibility in the effective pressure allows the simplification to single velocity

$$
\begin{aligned}
& \sum P_{r} f_{r} K_{r} \\
& P=\frac{r}{\sum f K_{r}}, P_{r}=p_{r}+q_{r} \\
& \sum f_{r} K_{r} \\
& \text { Effective pressure } \\
& \text { (includes artificial } \\
& \text { viscosity) } \\
& K_{r}^{-1}=\left.\rho_{r} \frac{\partial P_{r}}{\partial p_{r}}\right|_{e_{r}} \\
& +\left.\frac{P_{r}}{\rho_{r}} \frac{\partial P_{r}}{\partial e_{r}}\right|_{\rho_{r}}, h_{r}=\frac{f_{r} K_{r}}{\sum_{s} f_{s} K_{s}}
\end{aligned}
$$

For an ideal gas and $\mathbf{q}_{\mathbf{r}}=\mathbf{0}, \mathbf{K}_{\mathbf{r}}^{-1}=\gamma_{\mathrm{r}} \mathbf{p}_{\mathbf{r}}$ (adiabatic compres-sibility), and $h_{r}=\left[f_{r} /\left(\rho_{r} c_{r}^{2}\right)\right] /\left[\sum_{s} f_{s} /\left(\rho_{s} c_{s}^{2}\right)\right] \quad\left(Y_{\text {oungs }}^{2}\right)$ 
The viscosity for energy diffusion and Reynolds stress comes from the two-scale K-E model

$$
\begin{gathered}
\tau=\frac{2}{3} \rho K_{p}-\frac{4}{3} \rho v_{p} \frac{\partial u}{\partial x} \text { Reynolds stress } \\
v_{\alpha}=v_{0}+v_{T \alpha} \quad \text { Kinematic visc. }
\end{gathered}
$$

Equations for $\mathbf{K}_{\alpha}$ and $\varepsilon_{\alpha}$ are needed for closure 
The two-scale K-E equations describe evolution of the production and turbulence scales
$\underline{D K_{p}}$
$\partial v_{p} \partial K_{p}$
$\tau \partial u$
$D t=P_{R^{*}}-\varepsilon_{p}+\frac{\partial}{\partial x} \sigma_{K} \partial x \quad \frac{\partial}{\partial x}$ scale
Production
$\frac{D K_{t}}{D t}=\varepsilon_{p}-\varepsilon_{t}+\frac{\partial}{\partial x} \frac{v_{t}}{\sigma_{K}} \frac{\partial K_{t}}{\partial x}$
Turbulence scale
$\frac{D \varepsilon_{p}}{D t}=C_{p 1} \frac{\varepsilon_{p}}{K_{p}} P_{R^{*}}-C_{p 2} \frac{\varepsilon_{p}^{2}}{K_{p}}+\frac{\partial}{\partial x} \frac{v_{p}}{\sigma_{\varepsilon}} \frac{\partial \varepsilon_{p}}{\partial x}$
$\frac{D \varepsilon_{t}}{D t}=C_{t 1} \frac{\varepsilon_{p} \varepsilon_{t}}{K_{t}}-C_{t 2} \frac{\varepsilon_{t}^{2}}{K_{t}}+\frac{\partial}{\partial x} \frac{v_{t}}{\sigma_{\varepsilon}} \frac{\partial \varepsilon_{t}}{\partial x}$ 


\section{The production terms $P_{R^{*}}$ for the two-scale $K-\varepsilon$} equations parameterize mixing caused by RTI or RMI

Based on a recent scaling analysis ${ }^{3}$ of $R T$ and $R M$ instabilities, the production term may be written as

Rayleigh-Taylor

$$
P_{R T}=4 C_{R T} \varepsilon_{p}^{1 / 2}(g A)^{3 / 4}\left(k_{0}^{-1 / 4}-k_{1}^{-1 / 4}\right)
$$

\section{Richtmyer-Meshkov}

$$
P_{R M}=2 C_{R M} \varepsilon_{p}^{1 / 2}(A \Delta u)^{3 / 2}\left(k_{1}^{1 / 2}-k_{0}^{1 / 2}\right)
$$

${ }^{3}$ Zhou, Y., A scaling analysis of turbulent flows driven by Rayleigh-Taylor and Richtmyer-Meshkov instabilities, Phys. Fluids 13, 538-543 (2001). 


\section{Wave numbers $k_{0}$ and $k_{1}$ for the production terms evolve with the flow}

Initially $k_{0}$ and $k_{1}$ are set by the initial perturbation scales, but thereafter evolve according to the computed production and turbulence scales

Production scale

Rayleigh-Taylor

$$
k_{0}=\left[\frac{\frac{4}{7} C_{R T} \varepsilon_{p}^{1 / 2}(g A)^{1 / 4}}{\frac{4}{7} C_{R T} \varepsilon_{p}^{1 / 2}(g A)^{1 / 4} k_{1}^{-3 / 4}+K_{p}}\right]^{4 / 3}
$$

$$
k_{0}=\frac{4 C_{R M}^{2} \varepsilon_{p} A \Delta u}{\left[2 C_{R M}\left(\varepsilon_{p} A \Delta u\right)^{1 / 2} k_{1}^{-1 / 2}+K_{p}\right]^{2}}
$$

Turbulence scale

$$
k_{1}=\varepsilon_{t}\left(\frac{3}{2} C_{K} / K_{t}\right)^{3 / 2} \mathbf{R T} \text { or } \mathbf{R M}
$$


The change in total energy is due to production minus dissipation and surface fluxes

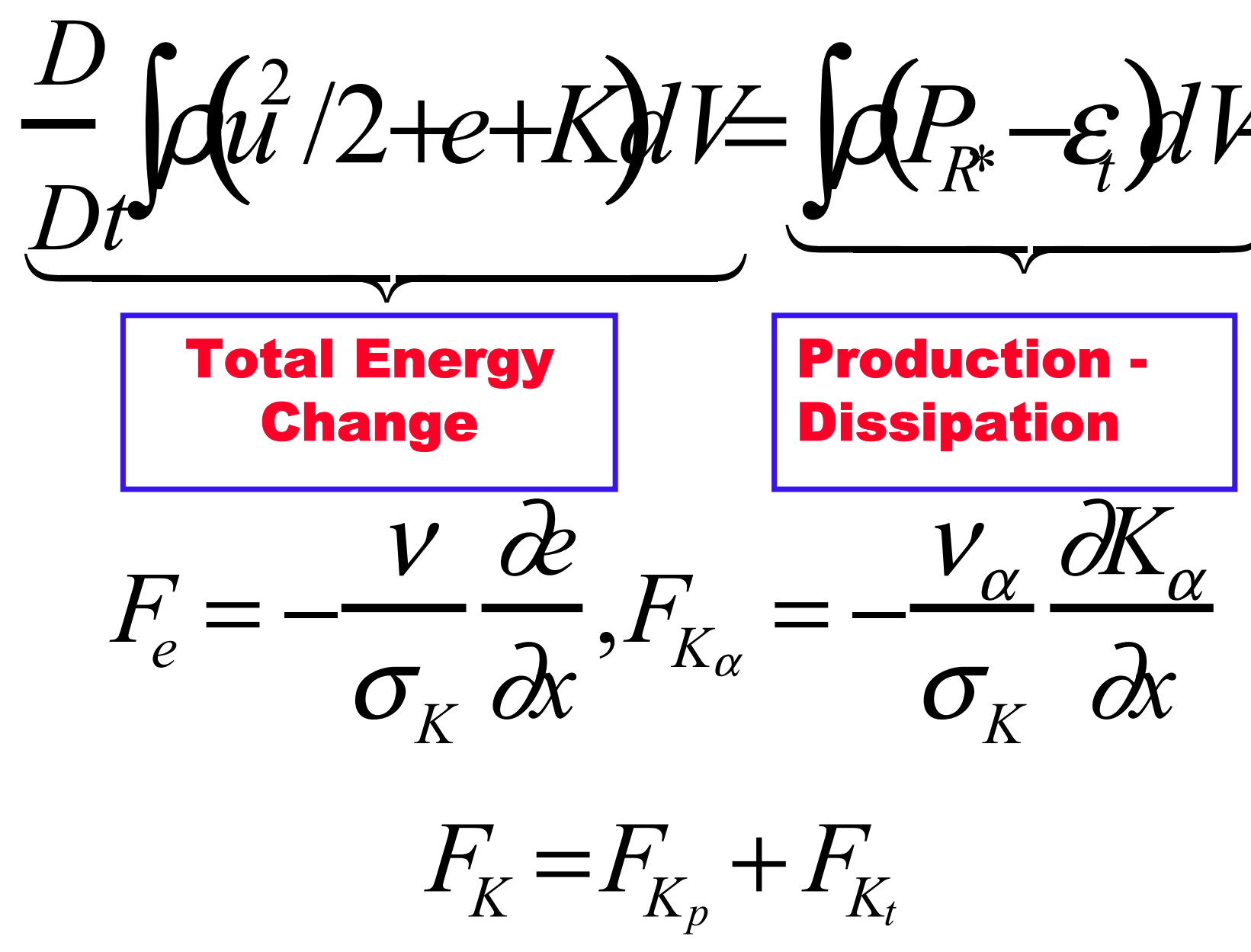

Diffusive fluxes of internal and turbulent kinetic energies 


\section{Results from Orszag and Speziale will be used to provide lCs for the two-scale turbulent mixing model}

- Steve Orszag's work for the ASCI Turbulence Group:

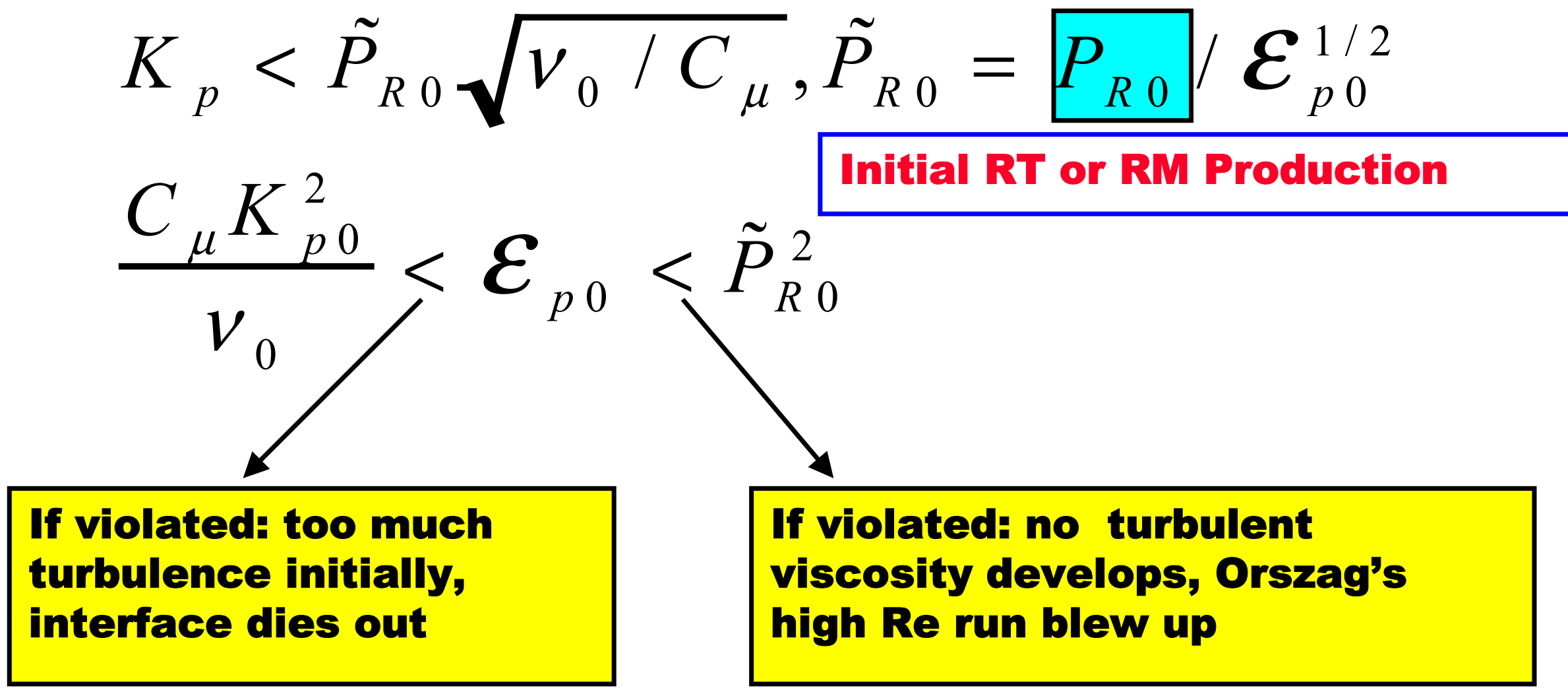

- Is this result consistent $w /$ Speziale's fixed point analysis? 


\section{The result using Orszag's approach is consistent with} Speziale's fixed point analysis

- The "fixed points" from Speziale's analysis ${ }^{4}$ act as attractors

- Initialize with fixed points that are consistent with desired long-term behavior

Leads more quickly to the desired long-term state

- Speziale's analysis yields the following fixed points:

$$
\begin{gathered}
\mathcal{E}_{p f}=f_{p} P_{R 0}, \mathcal{E}_{t f}=f_{t} \mathcal{E}_{p f} \\
f_{p}=\left(C_{p 1}-1\right) /\left(C_{p 2}-1\right), f_{t}=\left(C_{t 1}-1\right) /\left(C_{t 2}-1\right) \\
\text { suggesting } \mathcal{E}_{p 0}=f_{p}^{2} \tilde{P}_{R 0}^{2} \\
\downarrow \text { consistent with Orszag's approach if } f_{\mathrm{p}}<1
\end{gathered}
$$

${ }^{4}$ Speziale, C.G., and N. Mac Giolla Mhuiris, On the prediction of equilibrium states in homogeneous turbulence, J. Fluid Mech., 209, 591-615 (1989). 
The Orszag-Speziale ICs yield asymptotically growing solutions for the two-scale turbulent mixing model

- Current recommended values $C_{p 1}=1.5, C_{p 2}=2$ give $f_{p}=1 / 2$

$\downarrow$ The result using Orszag's approach is consistent with Speziale's fixed point analysis

$\downarrow$ Also consistent with constraints $C_{*_{2}}>1$ and $C_{*_{2}}>C_{*_{1}}>1 / 2$ needed to get asymptotically growing solutions

- Solution of the two-scale turbulent mixing model equations as ODEs using the Orszag-Speziale ICs produced the expected asymptotically growing results

$\downarrow$ The Orszag-Speziale ICs will be used in the two-scale turbulent mixing model for sKULL MC-1V 


\section{A test of the Orszag-Speziale ICs w/ the uncoupled two-scale K-E model yjelds asymptotic growth}

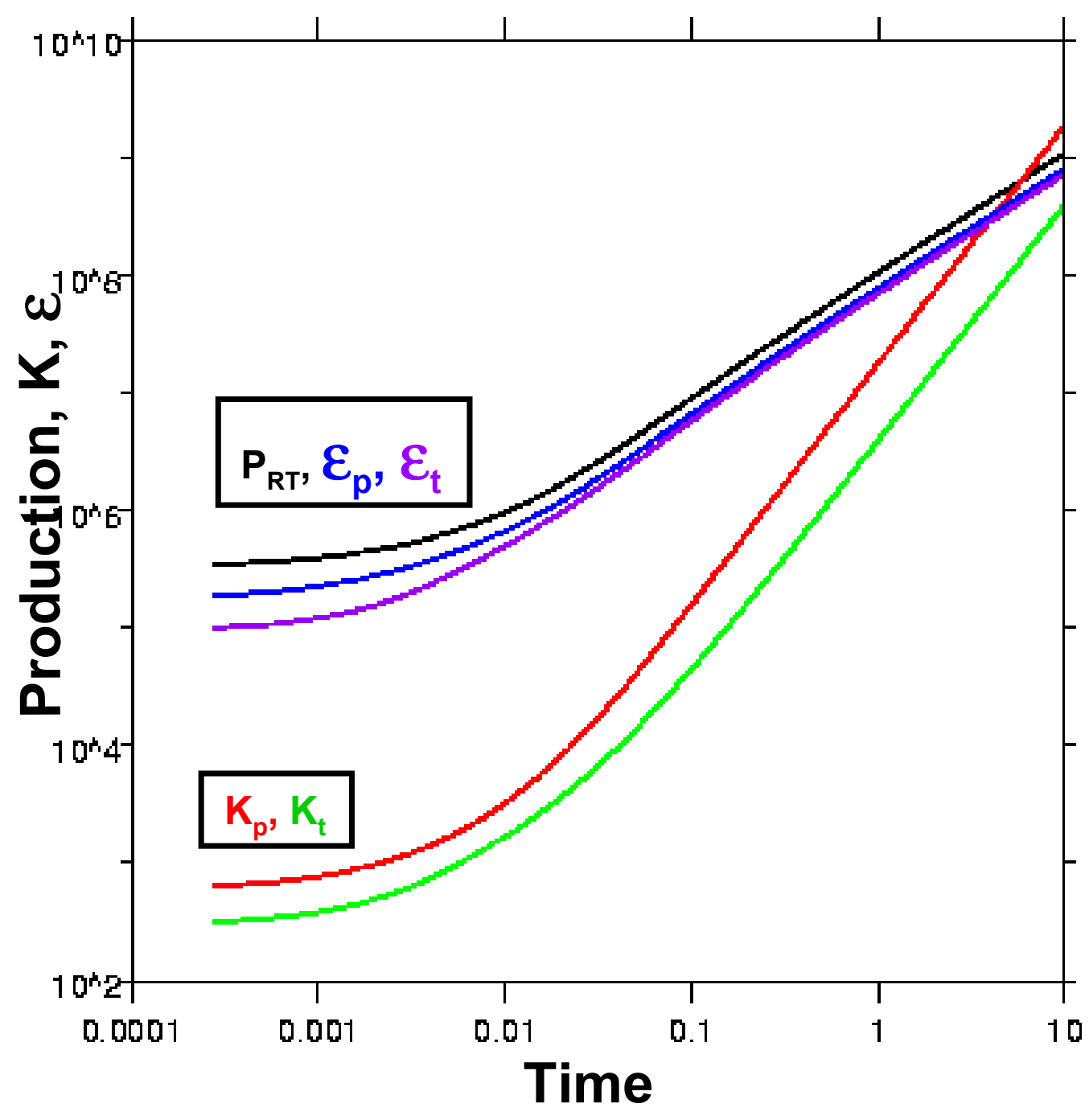

\section{Rayleigh-Taylor Test}

- $\mathrm{gA}=\mathbf{4 0 0 0}$

- $\mathrm{k}_{1}(0)=10 \mathrm{k}_{0}(0)=20 \pi$

- $\mathrm{C}_{\mathrm{K}}=\mathrm{C}_{\mathrm{RT}}=1.5$

- $C_{p 1}=1.5, C_{p 2}=2.0$

- $\mathrm{C}_{\mathrm{t} 1}=1.08, \mathrm{C}_{\mathrm{t} 2}=1.15$

- Constraints for asymptotic growth are satisfied, and asymptotic growth achieved

Consistency with a stand-alone ODE solution demonstrates that the uncoupled two-scale K- $\varepsilon$ equations have been correctly implemented in sKULL 


\section{Summary and Future Work}

- A simplified version of the ICF code KULL has been developed which reproduces KULL's multi-component hydrodynamics

- The purpose of simplified KULL, sKULL, is to serve as a test-bed for implementation of multi-component turbulent mixing models

- Tests show that sKULL faithfully duplicates KULL's numerics

- A single velocity, multi-component, two-scale K- $\varepsilon$ turbulent mixing model has been developed within sKULL

- A new method for the uncoupled two-scale K- $\varepsilon$ initial conditions yields asymptotic growth

- Future work will compare these single velocity results with those from a more complete multi-velocity formulation of turbulent mixing, to decide whether the multi-velocity formulation needs to be used in KULL 


\section{Table of Symbols \#1}

\begin{tabular}{|l|l|}
\hline Quantity & Description \\
\hline$D_{r s}$ & Drag force on fluid $\mathbf{r}$ due to fluid $\mathbf{s}$ \\
\hline$e_{r}$ & Specific internal energy of fluid $\mathbf{r}$ \\
\hline $\boldsymbol{\varepsilon}_{p}$ & Dissipation at production scale \\
\hline $\boldsymbol{\varepsilon}_{t}$ & Dissipation at turbulence scale \\
\hline$f_{r}$ & Volume fraction of fluid $\mathbf{r}$ \\
\hline$g$ & Acceleration (e.g., gravitational) \\
\hline$h_{r}$ & Relative compressibility of fluid $\mathbf{r}$ \\
\hline$K_{p}$ & Turbulence kinetic energy at production scale \\
\hline$K_{t}$ & Turbulence kinetic energy at turbulence scale \\
\hline$m_{r}$ & Mass fraction of fluid $\mathbf{r}$ \\
\hline
\end{tabular}




\section{Table of Symbols \#2}

\begin{tabular}{|l|l|}
\hline Quantity & Description \\
\hline$M_{r s}$ & Added mass effect fr fluid $\mathbf{r}$ due to fluid $\mathbf{s}$ \\
\hline$v_{0 r}$ & Molecular viscosity of fluid $\mathbf{r}$ \\
\hline$v_{T}=v_{p}+v_{t}$ & Total turbulent viscosity \\
\hline$v_{p}$ & Turbulent viscosity, production scale \\
\hline$v_{t}$ & Turbulent viscosity, turbulence scale \\
\hline$v_{r}=v_{0 r}+v_{T}$ & Total viscosity of fluid $\mathbf{r}$ \\
\hline$P_{r}=p_{r}+q_{r}$ & Effective pressure of fluid $\mathbf{r}$ \\
\hline$P=p+q$ & Effective mean pressure \\
\hline$p=\sum h_{r} p_{r}$ & Mean pressure \\
\hline$p_{r}=p_{r}\left(\rho_{r}, e_{r}\right)$ & Pressure of fluid $\mathbf{r}$ from EOS \\
\hline
\end{tabular}




\section{Table of Symbols \#3}

\begin{tabular}{|l|l|}
\hline Quantity & Description \\
\hline$q=\sum f_{r} q_{r}$ & Møn atificial viscous stess \\
\hline$q_{r}$ & Artificial viscous stress of Luid $\mathbf{r}$ \\
\hline$\rho=\sum f_{r} \rho_{r}$ & Møn density \\
\hline$\rho_{r}$ & Density of fluid $\mathbf{~}$ \\
\hline$t$ & Time \\
\hline$u$ & Møn velocity \\
\hline $\bar{u}=\sum_{r} f_{r} \bar{u}_{r}$ & Vdume-weighted mean velocity \\
\hline $\bar{u}_{r}=u_{r}+\frac{v_{r}}{\rho_{r}} \frac{\partial \rho_{r}}{\partial x}$ & $\begin{array}{l}\text { Vdume-weighted velocity of } \\
\text { fluid } \mathbf{r}\end{array}$ \\
\hline$u_{r}$ & Velocity offluid $\mathbf{r}$ \\
\hline$x$ & Positionat time $\mathbf{t}$ \\
\hline
\end{tabular}




\title{
High order methods for 2D Richtmyer-Meshkov Instability
}

\author{
Wai Sun Don, David Gottlieb, Chi-Wang Shu \\ Division of Applied Mathematics \\ Brown University \\ Leeland Jameson \\ Lawerance Livermore National Laboratory \\ University of California
}

This research is supported under the auspices of the US Department of Energy by the University of California Lawerance Livermore National Laboratory. 


\section{Richtmyer-Meshkov Instability}

- Shock induced instability of the interface between fluids of different density (Theoretized by Richtmyer and experimented by Meshkov.)

- Growth of the interface amplitude and secondary shear instability promote the onset of turbulence mixing.

- Applications included but not limited to mixing enhancement and inertial confinement fusion (ICF). 


\section{Richtmyer-Meshkov Instability (Cont.)}

The two-dimensional Euler equations,

$$
\mathbf{Q}_{t}+\mathbf{F}_{x}+\mathbf{G}_{y}=0 .
$$

The state vector $\mathbf{Q}$ is

$$
\mathbf{Q}=(\rho, \rho u, \rho v, E)^{\mathrm{T}} .
$$

The inviscid fluxes $\mathbf{F}$ and $\mathbf{G}$ are given by

$$
\begin{aligned}
& \mathbf{F}=(\rho u, \rho u u+P, \rho u v,(E+P) u)^{\mathrm{T}}, \\
& \mathbf{G}=(\rho v, \rho u v, \rho v v+P,(E+P) v)^{\mathrm{T}} .
\end{aligned}
$$

where

$$
P=(\gamma-1)\left(E-\frac{1}{2} \rho \mathbf{U} \cdot \mathbf{U}\right) .
$$




\section{Richtmyer-Meshkov Instability (Cont.)}

\section{Initial Condition :}

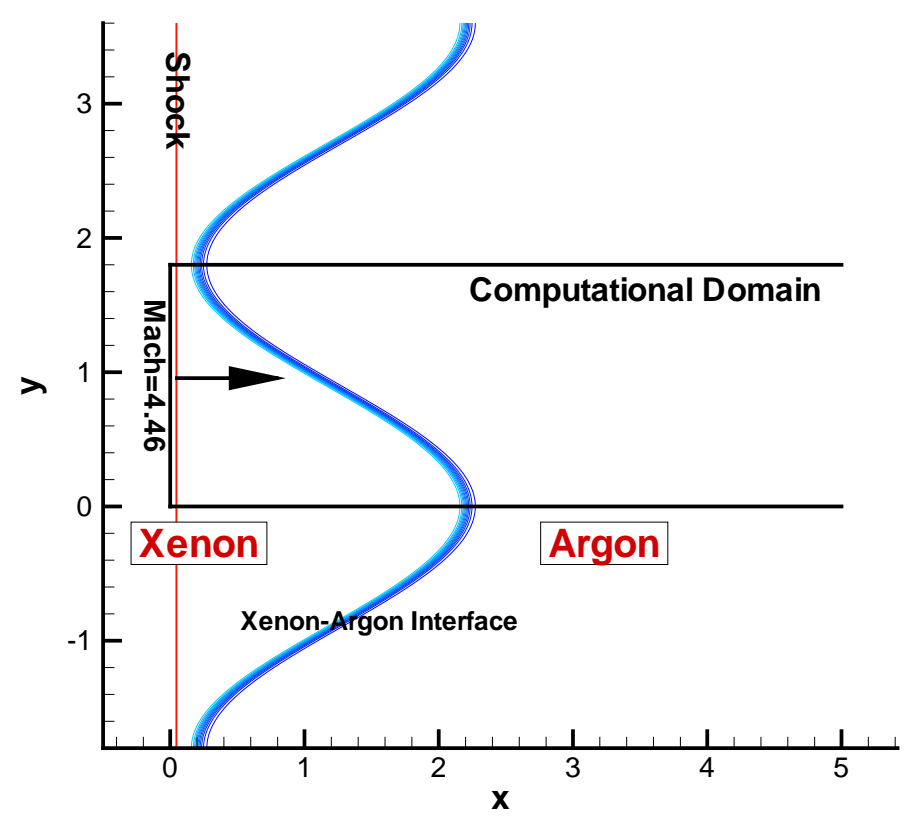

- Hugoniot-Rankine condition for the shock

- Pre-Shock Temperature $T=296 K$

- Pre-Shock Pressure $P=0.5$ atm

- Xenon and Argon density are $\rho_{X e}=2.90 \times$ $10^{-3} \frac{\mathrm{g}}{\mathrm{cm}^{3}}$ and $\rho_{A r}=0.89 \times 10^{-3} \frac{\mathrm{g}}{\mathrm{cm}^{3}}$ respectively, at half of the normal atmospheric pressure

- Specific heat ratio $\gamma=\frac{5}{3}$

- Atwood number $A t=0.54$

- Mach number $M=2,4.46,8,10$

- Wave Length $\lambda=36 \mathrm{~mm}$

- Amplitude $a=1,2.5,5,10,15 \mathrm{~mm}$ 


\section{Richtmyer-Meshkov Instability (Cont.)}

Xenon-Argon interface definition :

$$
S(x, y)=\exp \left(-\alpha|d|^{\beta}\right) \quad 0<d<1
$$

where

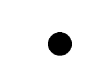

$$
d=\frac{\left(x_{i}+a \cos (2 \pi y / \lambda)+\delta\right)-x}{2 \delta}
$$

- $\delta=a / 5$ is the interface thickness,

- $\beta=8$ is the interface order,

- $\alpha=-\ln \epsilon$ with $\epsilon$ being the machine zero. 


\section{Richtmyer-Meshkov Instability (Cont.)}

\section{Regions of Interest :}

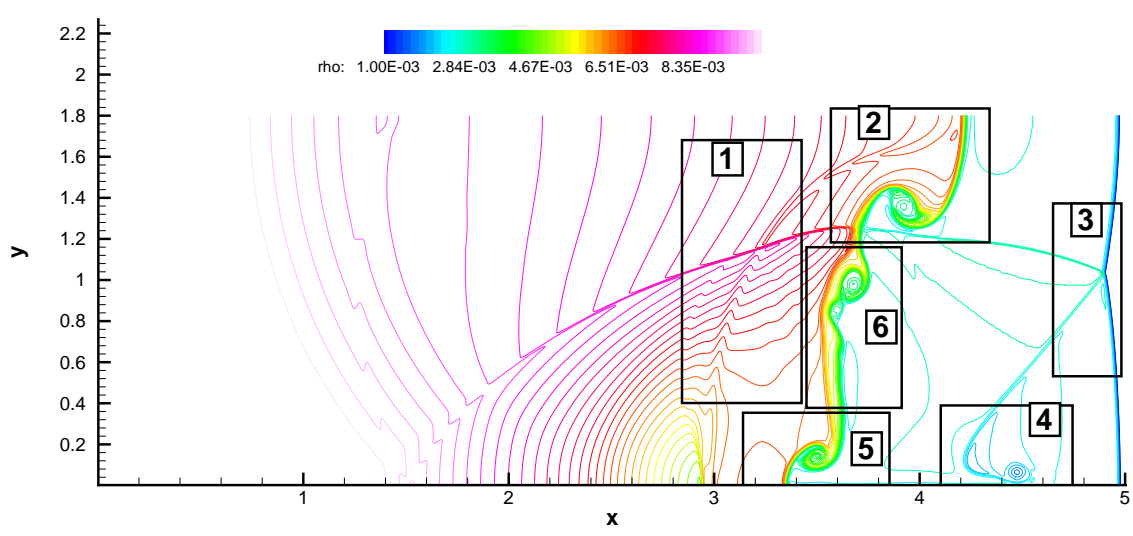

1. Reflected shock generated by the shock refraction;

2. The penetration of the heavy $(\mathrm{Xe})$ to light (Ar) fluid forms the Spike;

3. Triple point on the transmitted shock;

4. A small jet and its vortical structure. The Kelvin-Helmholtz instability caused the instability along the contact discontinuity in long time simulation;

5. The penetration of the light (Ar) to heavy (Xe) fluid forms the Bubble;

6. Vortical rollups of the gaseous interface. 


\section{Richtmyer-Meshkov Instability : Algorithms}

- Spatial Algorithm : (Spectral)

1. Combined Chebyshev $(x)$ and Fourier collocation method $(y)$

- Differentiation and Smoothing operations are done via an optimized library PseudoPack (Costa \& Don);

- a. $10^{\prime}$ th and 9 'th order exponential filter used for the differentiation and solution smoothing respectively.

- Incremental domain size in $x$ as shock moved downstream.

- Parallelized with OpenMP.

2. WENO fifth order finite difference scheme (WENO) with Lax-Frederick flux.

3. Symmetry property in $y$ is utilized to reduce the cost of computation.

- Temporal Algorithm :

Third order TVD Runge Kutta method (Shu and Osher). 


\section{Spectral Methods - Introduction}

The solution of an equation is assumed to be in a space $\mathcal{B}_{\mathcal{N}}$ spanned by smooth basis functions $\left\{\phi_{k}\right\}$.

- For periodic problems

$$
\phi_{k}=e^{i k x}
$$

- for non-periodic problems in finite intervals

- Either

$$
\phi_{k}=T_{k}(x)
$$

where $T_{k}$ are the Chebyshev polynomials.

$-\mathrm{Or}$

$$
\phi_{k}=L_{k}(x)
$$

where $L_{k}$ are the Legendre polynomials. 


\section{Spectral Methods - Introduction (Cont.)}

The approximation is obtained in one of two ways:

- Galerkin projection:

$$
\mathcal{P}_{\mathcal{N}} F(x)=\sum_{k=0}^{N}\left(F, \phi_{k}\right) \phi_{k}(x)
$$

where

$$
\left(F, \phi_{k}\right)=\int \omega(x) F(x) \phi_{k}(x) d x
$$




\section{Spectral Methods - Introduction (Cont.)}

- The Pseudo-spectral approximation

$$
\mathcal{I}_{\mathcal{N}} F(x)=\sum_{k=0}^{N}\left(F, \phi_{k}\right)_{N} \phi_{k}(x)
$$

where

$$
\left(F, \phi_{k}\right)_{N}=\sum_{i=0}^{N} \omega_{i} F\left(x_{i}\right) \phi_{k}\left(x_{i}\right)
$$

$-x_{i}$ and $\omega_{i}$ are the Gauss-Lobatto Quadrature nodes and weights respectively.

- Thus

$$
\int f(x) \omega(x) d x=\sum_{i=0}^{N} \omega_{i} f\left(x_{i}\right) \phi_{k}\left(x_{i}\right)
$$

when $f(x)$ is a polynomial or trigonometrical polynomial of degree $2 N-1$. 


\section{Spectral Methods - Introduction (Cont.)}

- Alternatively

$$
\mathcal{I}_{\mathcal{N}} F(x)=\sum_{i=0}^{N} F\left(x_{i}\right) g_{i}(x)
$$

where

$$
-g_{i}(x) \text { is a polynomial of degree } N \text { such that } g_{i}\left(x_{k}\right)=\delta_{i, k} \text {. }
$$

This leads naturally to the formula:

$$
\frac{d}{d x} \mathcal{I}_{\mathcal{N}} F\left(x_{k}\right)=\sum_{i=0}^{N} F\left(x_{i}\right) g_{i}^{\prime}\left(x_{k}\right)
$$

and defines the Differentiation Matrix

$$
\mathcal{D}=g_{i}^{\prime}\left(x_{k}\right)
$$


Approximation Results

$$
\begin{aligned}
\left\|F-\mathcal{P}_{N} F\right\| & \leq K \frac{\|F\|_{s}}{N^{s}} \\
\left\|F-\mathcal{I}_{N} F\right\| & \leq K \frac{\|F\|_{s}}{N^{s}}
\end{aligned}
$$




\section{Filtering}

- To solve most nonlinear PDE, for example Burgers' Equation, some form of dissipation (natural or numerical) is generally required for stability by damping the growth of the high modes.

- Spectral methods is a conservative method.

- Stabilization of the spectral methods requires Low Pass Filter. 


\section{Filtering (Cont.)}

\section{Filters in the Transform Space}

Force the Fourier/Chebyshev coefficient $a_{k}$ decays faster.

Replace

$$
f_{N}(x)=\sum_{k=0}^{N} a_{k} e^{i k x}
$$

by

$$
\hat{f}_{N}(x)=\sum_{k=0}^{N} \sigma\left(\frac{k}{N}\right) a_{k} e^{i k x}
$$




\section{Filtering (Cont.)}

\section{Definition of Filters}

A filter of $p$ order $\sigma(x)$ is defined as

$$
\begin{aligned}
\sigma(0) & =1 \\
\sigma^{l}(0) & =0 \quad 1 \leq l \leq p-1 \\
\sigma^{l}(1) & =0 \quad 1 \leq l \leq p-1
\end{aligned}
$$

Theorem (Vandeven 1989)

$$
\left|f(x)-\hat{f}_{N}(x)\right| \leq C_{p} N^{1-p} d(x)^{1-p}|| f||
$$

where $d(x)$ is the distance from the discontinuity.

If $p$ is proportional to $N$, accuracy can be recovered away from the discontinuity. 


\section{Filtering (Cont.)}

\section{Exponential Filter}

$$
\sigma\left(\frac{k}{N}\right)=\exp \left(-\alpha\left(\frac{k}{N}\right)^{\gamma}\right)
$$

where $\alpha=-\ln \epsilon, \epsilon$ is the machine zero.
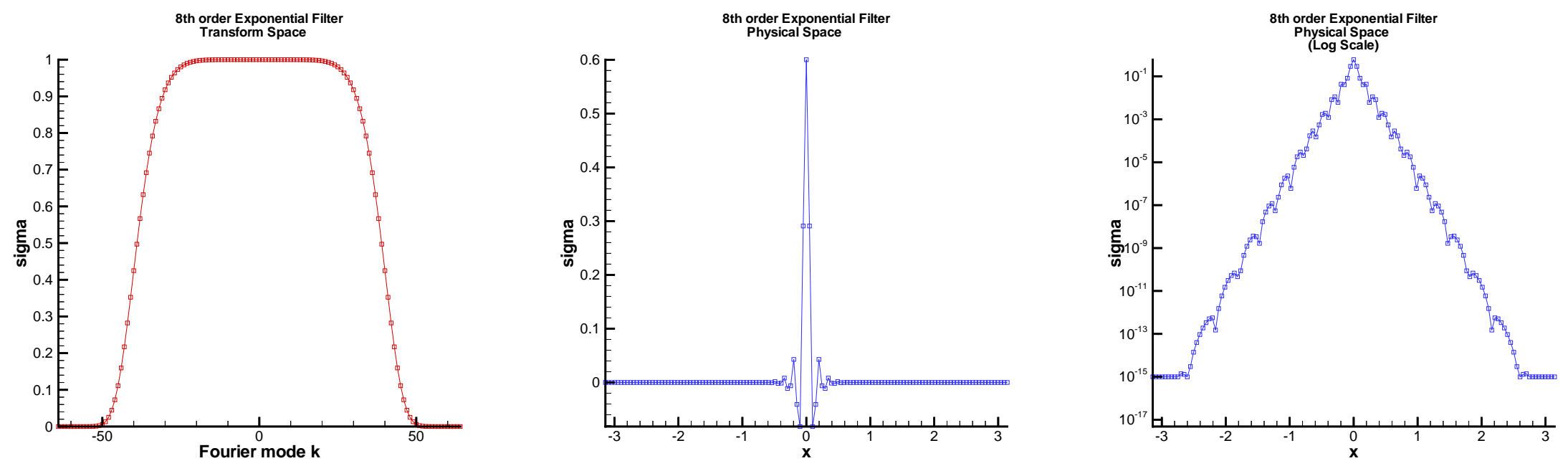


\section{Time Stepping Scheme}

The algorithm employs the third order TVD Runge-Kutta scheme (Shu \& Osher) to solve the system of ODE's produced by the spatial differencing.

It has the form of

$$
\begin{aligned}
\vec{U}^{1} & =\vec{U}^{n}+\Delta t L\left(\vec{U}^{n}\right) \\
\vec{U}^{2} & =\frac{1}{4}\left(3 \vec{U}^{n}+\vec{U}^{1}+\Delta t L\left(\vec{U}^{1}\right)\right) \\
\vec{U}^{n+1} & =\frac{1}{3}\left(\vec{U}^{n}+2 \vec{U}^{2}+2 \Delta t L\left(\vec{U}^{2}\right)\right) .
\end{aligned}
$$

$L$ is the spatial operator.

$\vec{U}^{n}$ and $\vec{U}^{n+1}$ are the data arrays at the $n$-th and $(n+1)$-th time step, respectively. $\vec{U}^{1}$ and $\vec{U}^{2}$ are two temporary arrays at the intermediate Runge-Kutta stages.

The scheme is stable for CFL $<1$. 


\section{Mapping for Chebyshev Method}

Consider the scalar hyperbolic equation

$$
\begin{aligned}
U_{t} & =U_{x} \quad-1 \leq x \leq 1 \\
U(1, t) & =0
\end{aligned}
$$

In the semi-discreet form

$$
\frac{\partial}{\partial t} U_{N}(t)=D U_{N}(t)
$$

where $D$ is the Chebyshev differentiation matrix with the first row and column removed.

\section{$\underline{\text { Disadvantages : }}$}

1. Roundoff Error grows as $O\left(N^{2 k}\right)$.

2. Restrictive CFL conditions for explicit time stepping scheme with $\Delta t \approx O\left(N^{-2 k}\right)$. with $k$ being the number of derivatives taken. 


\section{Mapping for Chebyshev Method (Cont.)}

\section{$\underline{\text { Kosloff-Tal-Ezer Mapping }}$}

$$
x=g(\xi, \alpha)=\frac{\sin ^{-1}(\alpha \xi)}{\sin ^{-1} \alpha}
$$

where $\xi_{j}$ and $x_{j}$ are the original and mapped Chebyshev collocation points respectively.

If $\alpha \in[0,1]$ is chosen as

$$
\alpha=\alpha(N, \epsilon)=\operatorname{sech}\left(\frac{|\ln \epsilon|}{N}\right),
$$

then the approximation error is roughly $\epsilon$. Note that $\alpha$ is not a constant but a function of $N$.

By choosing $\epsilon$ to be machine zero, the error of the coordinate transformation is essentially guaranteed to be harmless.

Under the mapping, the differentiation matrix $D$ becomes

$$
\mathcal{D}=\mathcal{M} D
$$

where $\mathcal{M}$ is a diagonal matrix with element $\mathcal{M}_{i i}=g^{\prime}\left(\xi_{i}, \alpha\right)^{-1}$. 


\section{Mapping for Chebyshev Method (Cont.)}
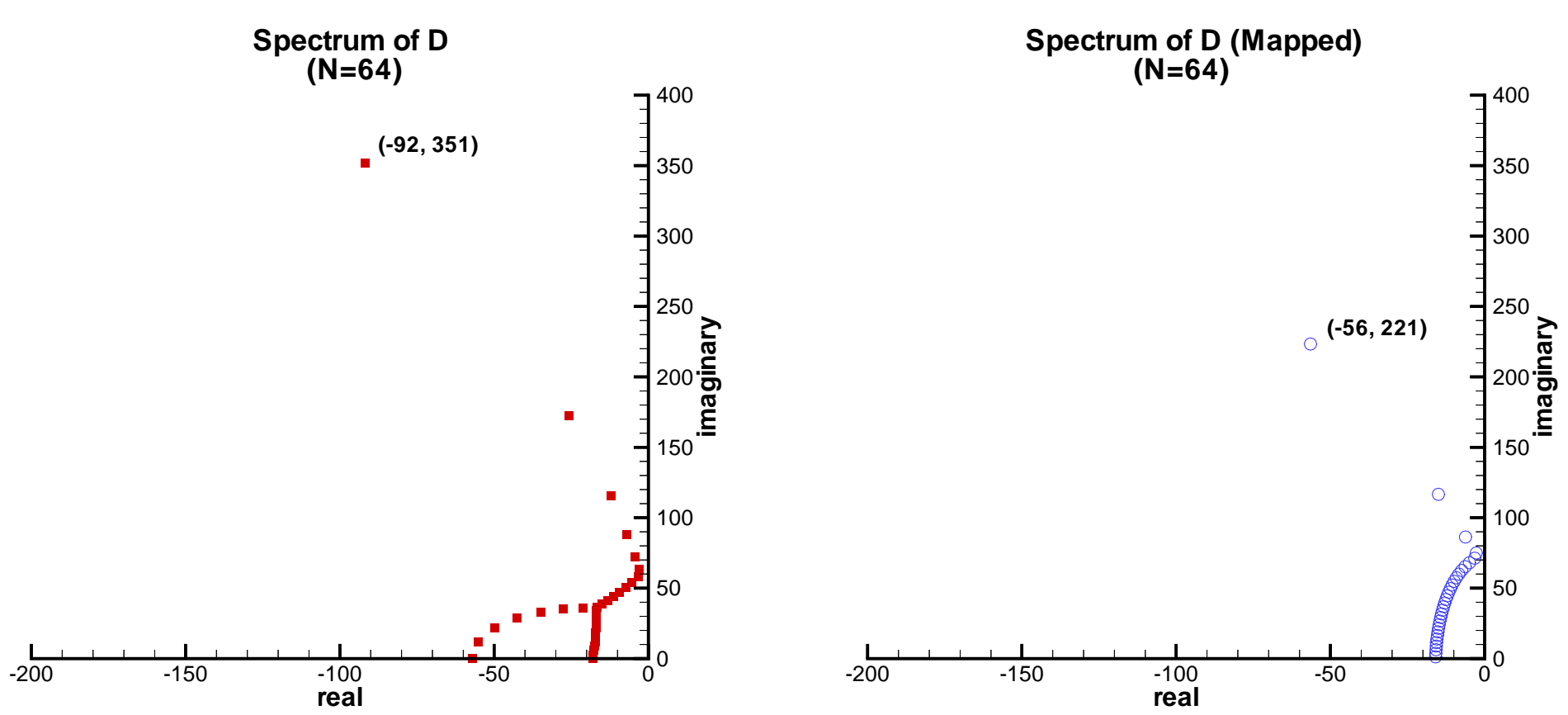


\section{Mapping for Chebyshev Method (Cont.)}

\section{Advantages}

- Reduction of roundoff error from $O\left(N^{2 k} \epsilon\right)$ to $O\left(N^{k} \epsilon\right)$.

\begin{tabular}{|c||c|c|}
\hline$N$ & No Mapping & with Mapping \\
\hline 32 & $0.47 \mathrm{E}-09$ & $0.20 \mathrm{E}-09$ \\
64 & $0.62 \mathrm{E}-08$ & $0.20 \mathrm{E}-08$ \\
128 & $0.71 \mathrm{E}-07$ & $0.13 \mathrm{E}-07$ \\
256 & $0.35 \mathrm{E}-05$ & $0.21 \mathrm{E}-06$ \\
512 & $0.98 \mathrm{E}-05$ & $0.33 \mathrm{E}-06$ \\
1024 & $0.13 \mathrm{E}-02$ & $0.21 \mathrm{E}-05$ \\
\hline
\end{tabular}

Table I: Absolute maximum error for the second derivative of $\sin (2 x)$. 


\section{Mapping for Chebyshev Method (Cont.)}

- Reduction of the spectral radius of the differentiation matrix from $O\left(N^{2 k}\right)$ to $O\left(N^{k}\right)$ asymptotically for $D$ and $\mathcal{D}$ respectively.

\begin{tabular}{|r||r|c||r|c|}
\hline$N$ & $\lambda(D)$ & $\begin{array}{c}\text { Growth } \\
\text { Rate }\end{array}$ & $\lambda(\mathcal{D})$ & $\begin{array}{c}\text { Growth } \\
\text { Rate }\end{array}$ \\
\hline \hline 32 & 91.6 & & 80.8 & \\
64 & 263.8 & 2 & 230.4 & 1.50 \\
128 & 1452.7 & 2 & 555.4 & 1.27 \\
256 & 5808.4 & 2 & 1219.1 & 1.13 \\
512 & 23231.3 & 2 & 2553.5 & 1.07 \\
1024 & 92922.8 & 2 & 5225.8 & 1.03 \\
\hline
\end{tabular}

Table II: The spectral radius $\lambda$ of $D$ and $\mathcal{D}$.

$k$ is the order of differentiation. 
Richtmyer-Meshkov Instability

Results 


\section{Richtmyer-Meshkov Instability (Cont.)}

$(M=4.46, a=10 \mathrm{~mm}, \delta=6 \mathrm{~mm}, t=50 \mu \mathrm{s}):$ Density

Spectral

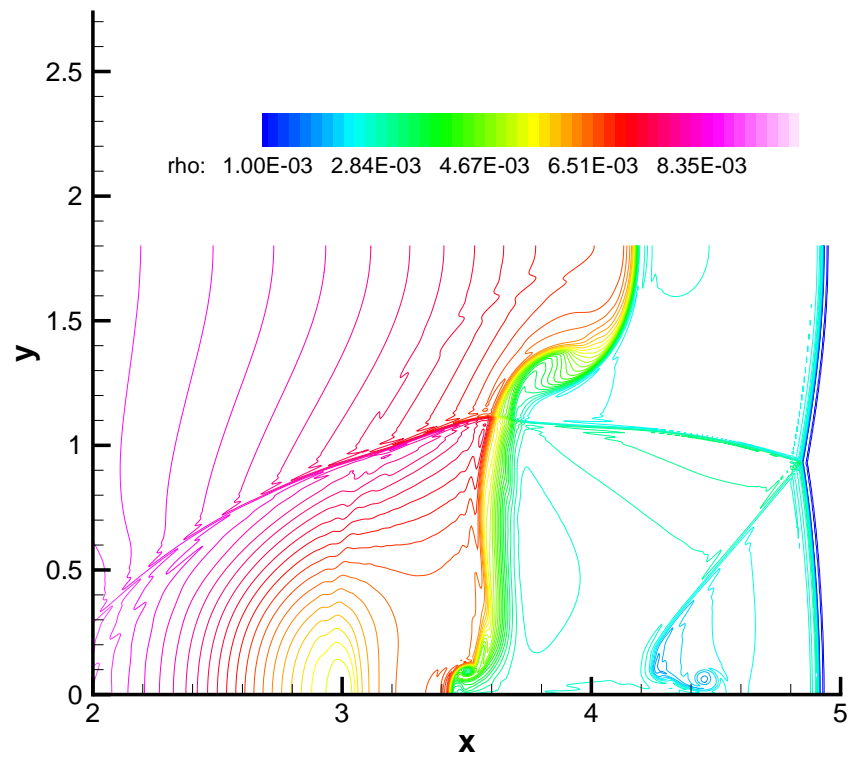

WENO

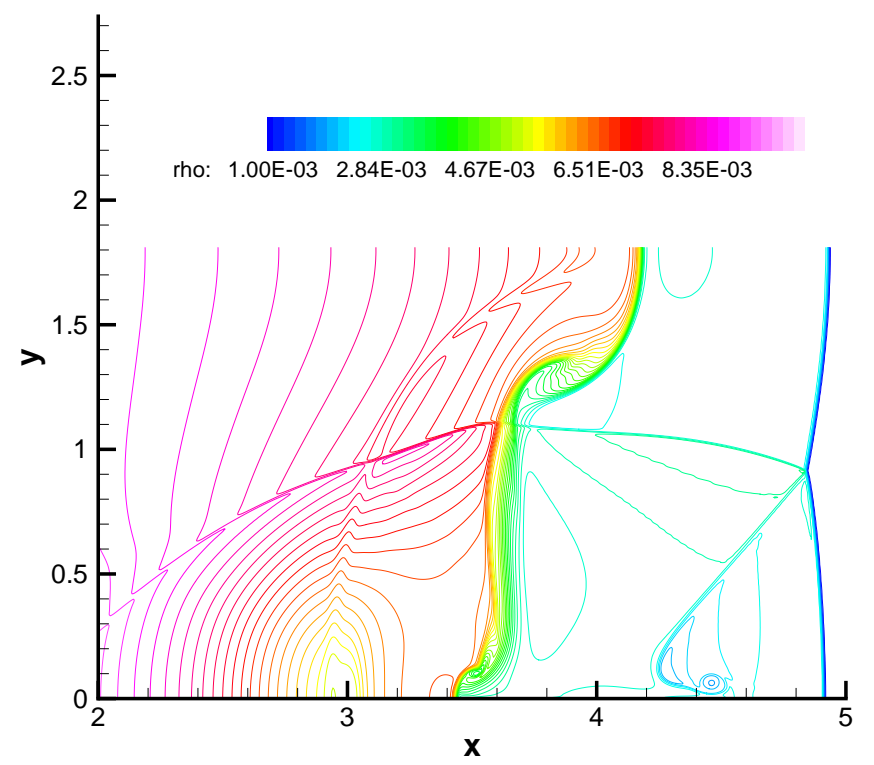

- Grid size for the Spectral and WENO schemes are 1024x512. 


\section{Richtmyer-Meshkov Instability (Cont.)}

$(M=4.46, a=10 \mathrm{~mm}, \delta=6 \mathrm{~mm}, t=50 \mu s): \mathrm{V}$-Velocity

Spectral

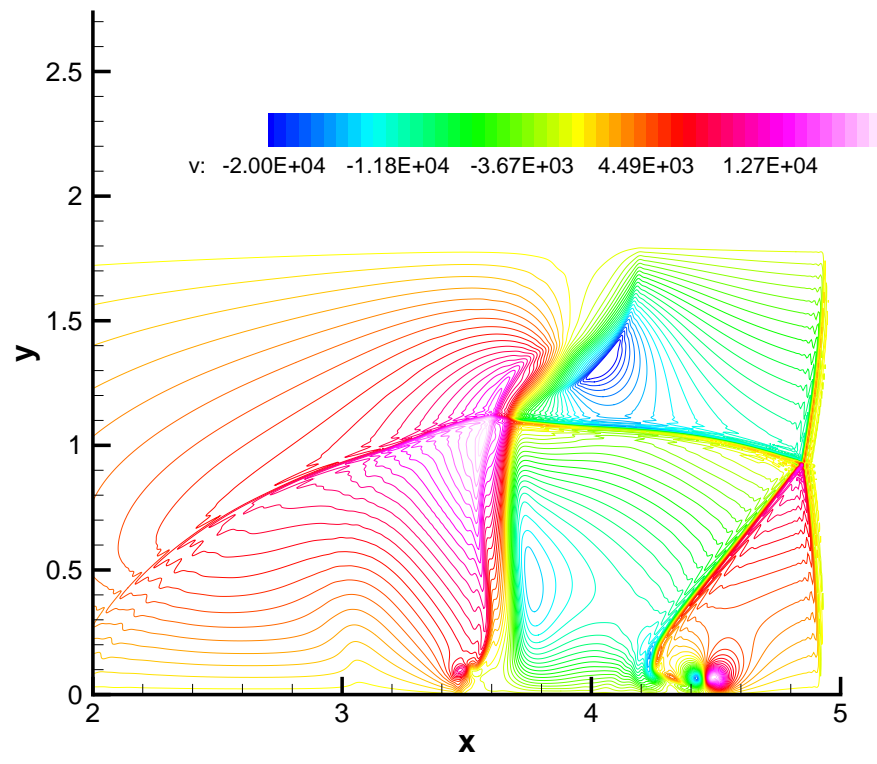

WENO

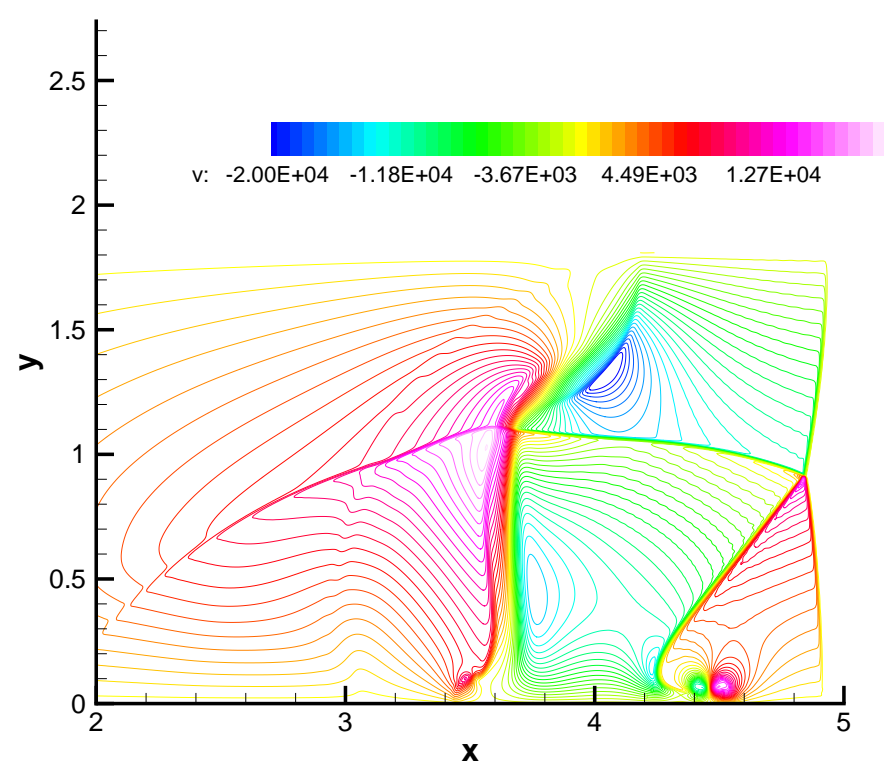

- Grid size for the Spectral and WENO schemes are 1024x512. 


\section{Richtmyer-Meshkov Instability (Cont.)}

$(M=4.46, a=10 \mathrm{~mm}, \delta=2 \mathrm{~mm}, t=50 \mu s):$ Density

Spectral

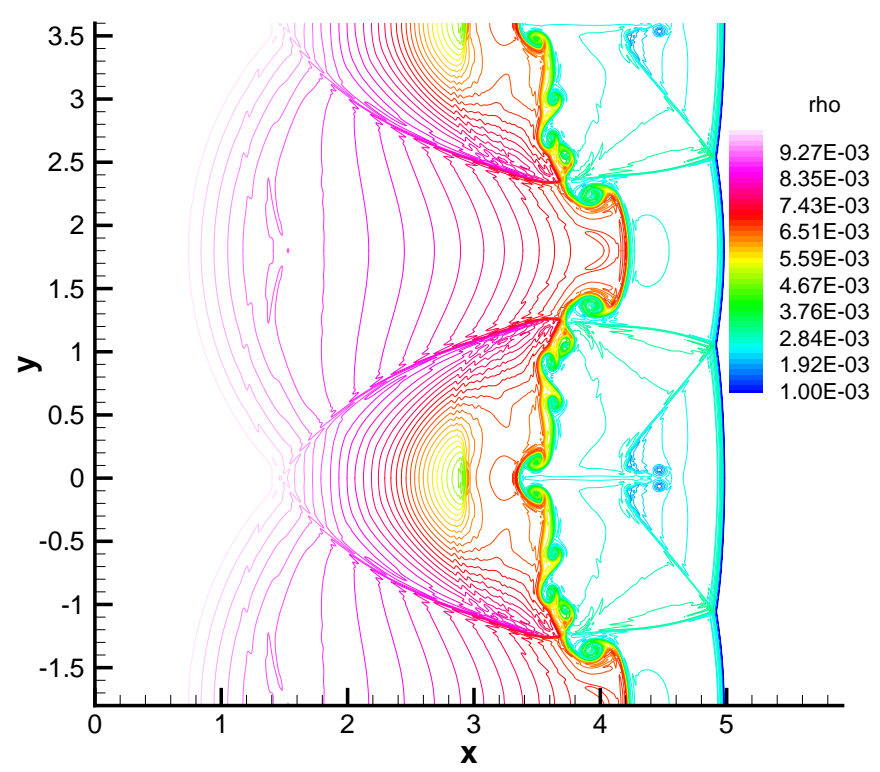

WENO

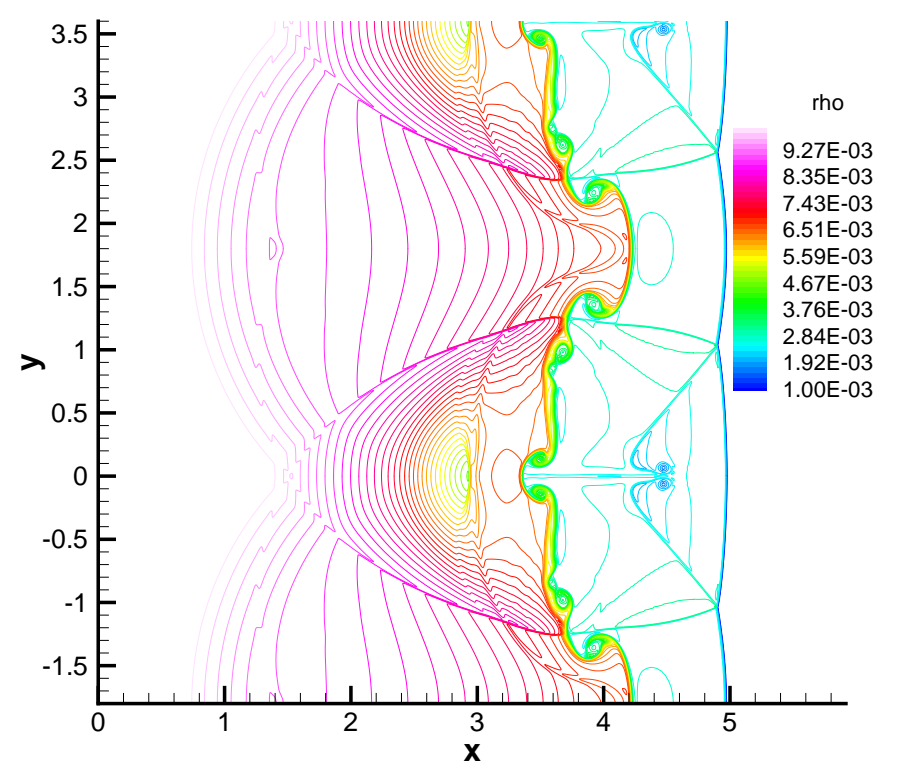

- Grid size for the Spectral and WENO schemes are 1024x256 and 1024x512 respectively. 


\section{Richtmyer-Meshkov Instability (Cont.)}

$(M=4.46, a=10 \mathrm{~mm}, \delta=2 \mathrm{~mm}, t=50 \mu s): \mathrm{V}$-Velocity

Spectral

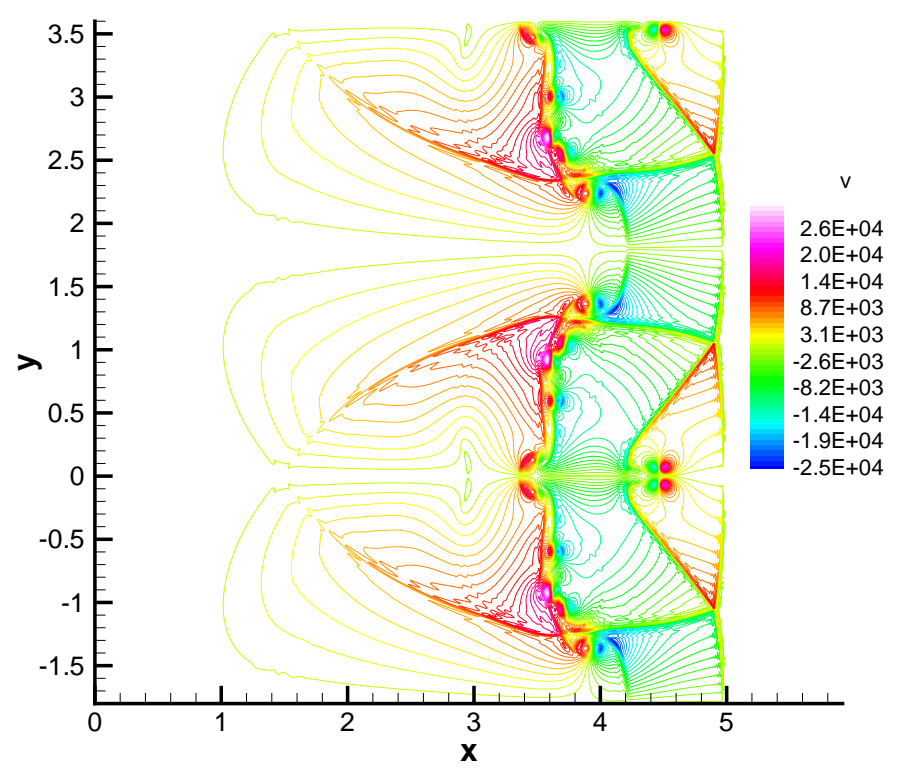

WENO

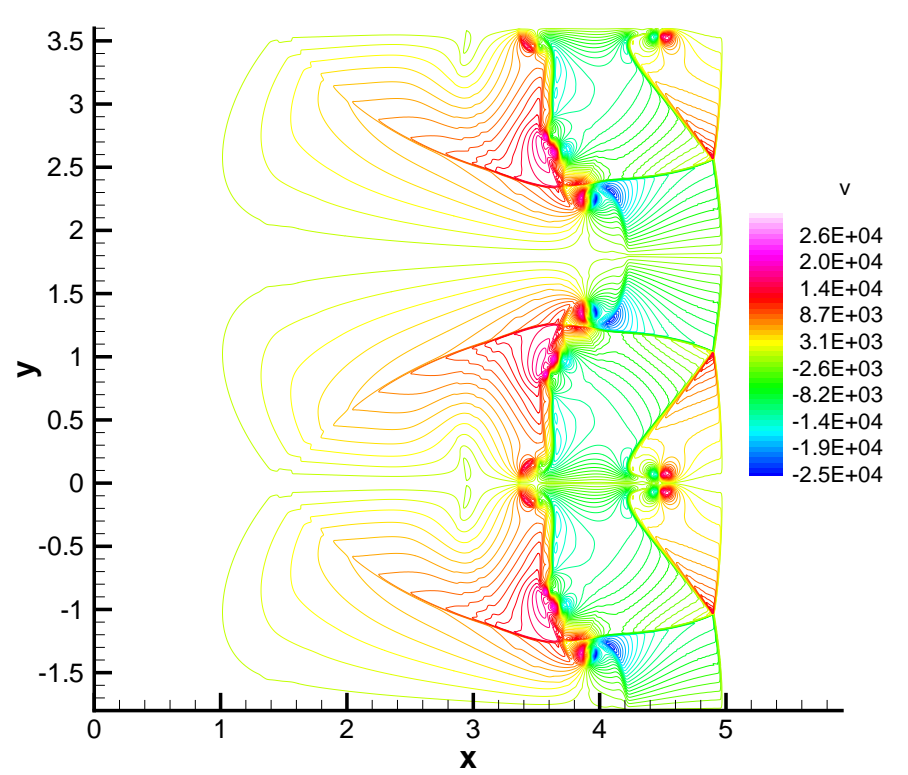

- Grid size for the Spectral and WENO schemes are 1024x256 and 1024x512 respectively. 


\section{Richtmyer-Meshkov Instability (Cont.)}

Long time Case $(M=4.46, a=10 \mathrm{~mm}, \delta=2 \mathrm{~mm}, t=124 \mu \mathrm{s})$ : Density

Spectral

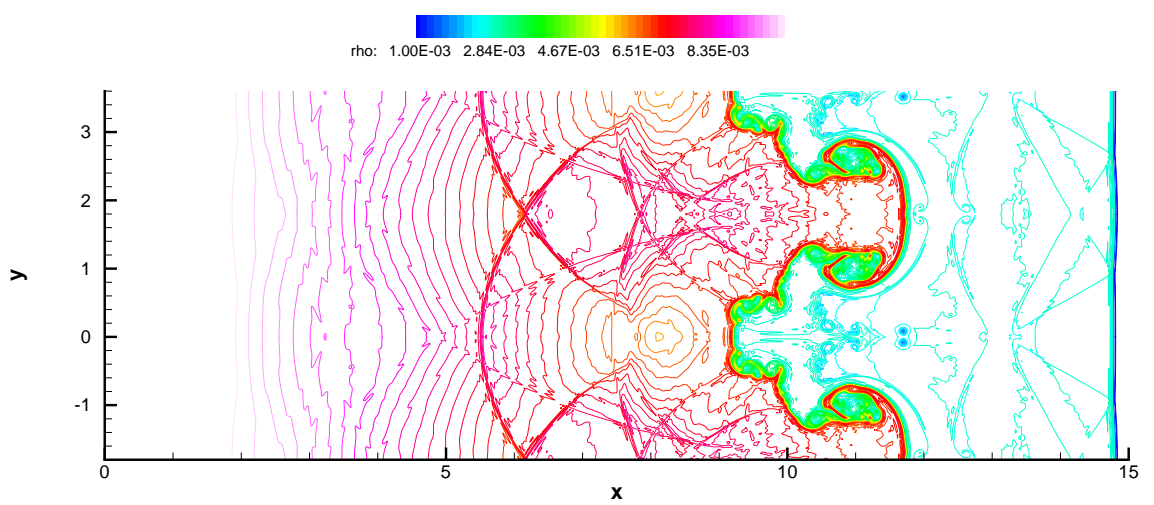

WENO

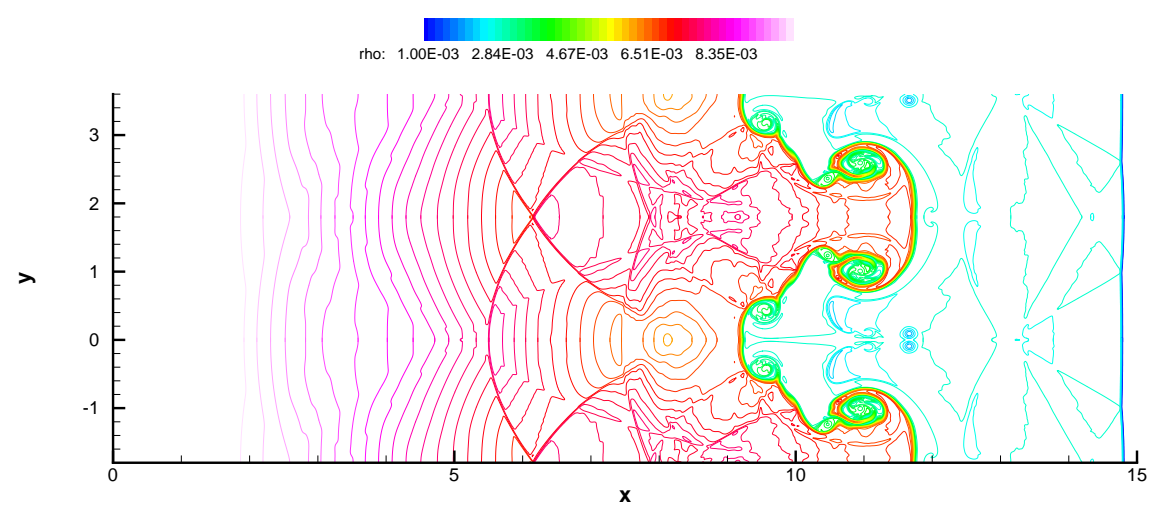




\section{Richtmyer-Meshkov Instability (Cont.)}

Long time Case $(M=4.46, a=10 \mathrm{~mm}, \delta=2 \mathrm{~mm}, t=124 \mu \mathrm{s})$ : V-Velocity

Spectral

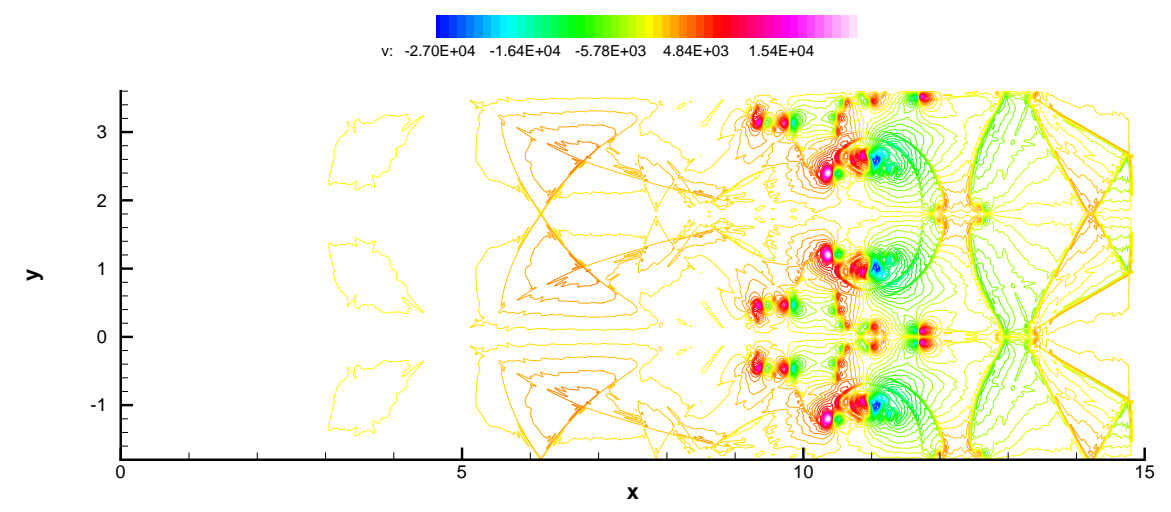

WENO

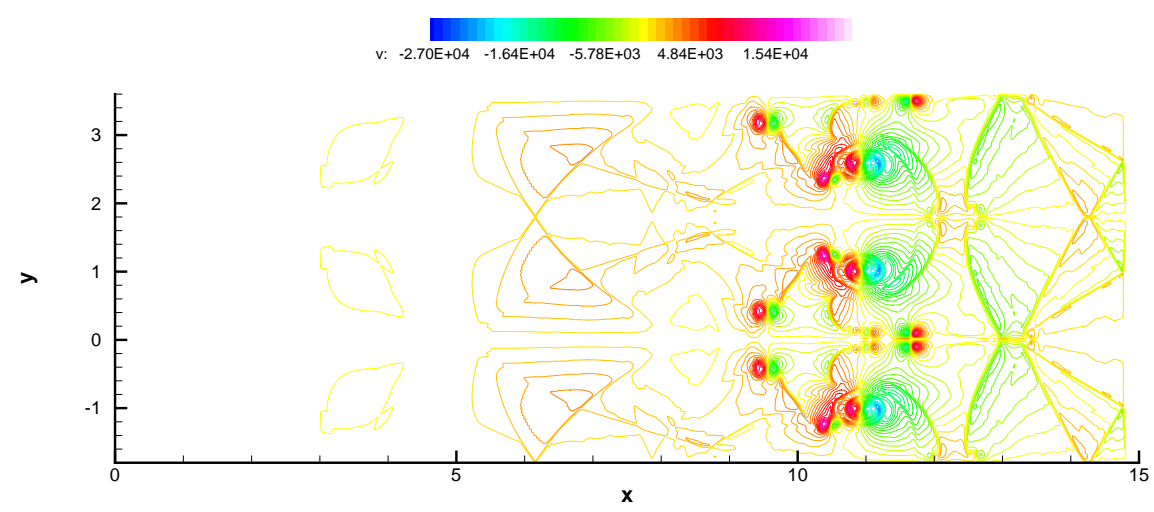




\section{Richtmyer-Meshkov Instability (Cont.)}

Large Domain Case $(M=4.46, a=10 \mathrm{~mm}, \delta=2 \mathrm{~mm}, t=237 \mu \mathrm{s})$ : Spectral scheme

Density

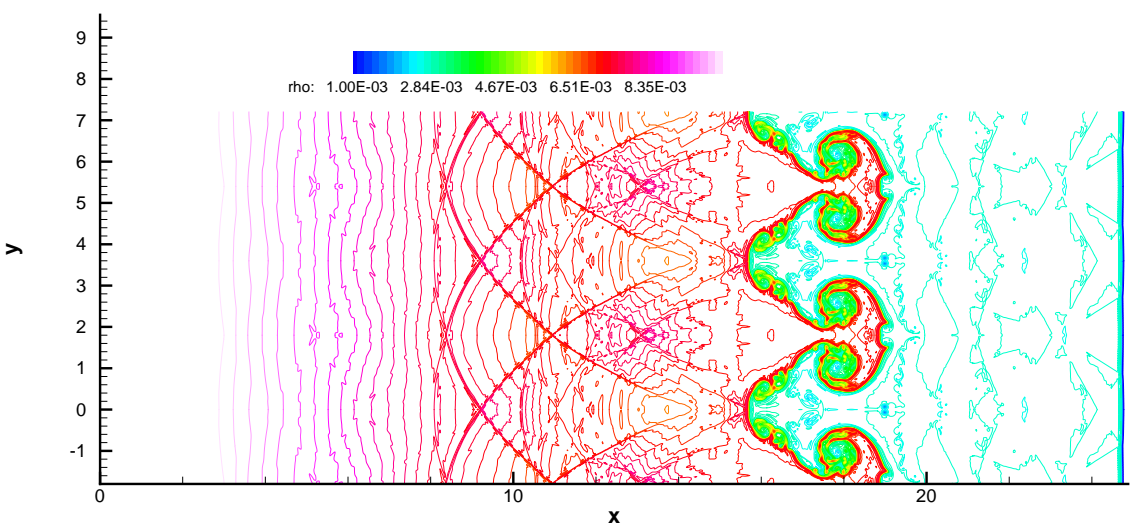

V-Velocity

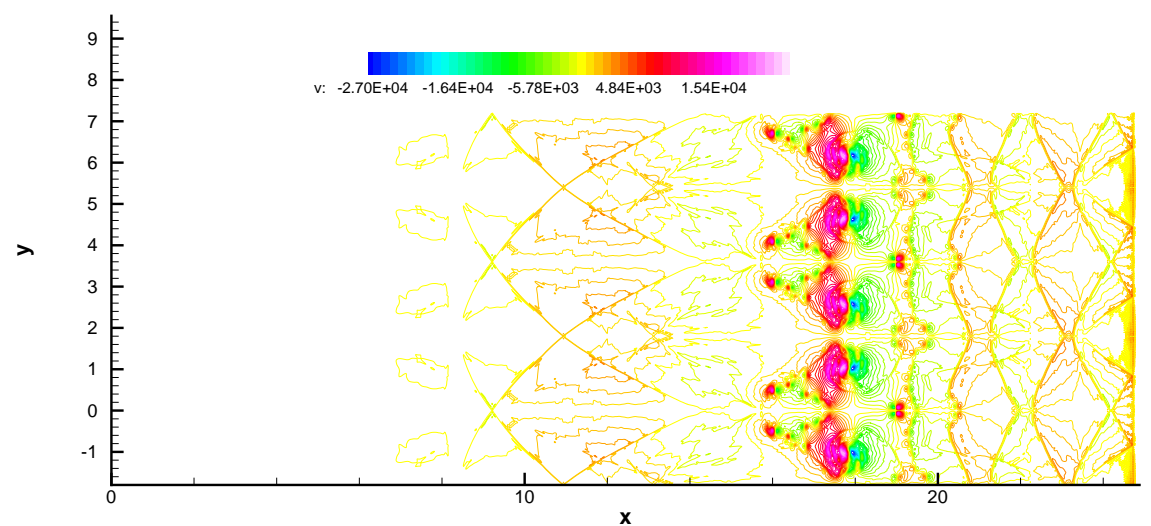




\section{Richtmyer-Meshkov Instability (Cont.)}

High Mach Number $(M=8, a=10 m m, \delta=2 m m, t=200 \mu s)$ : Spectral scheme

Density

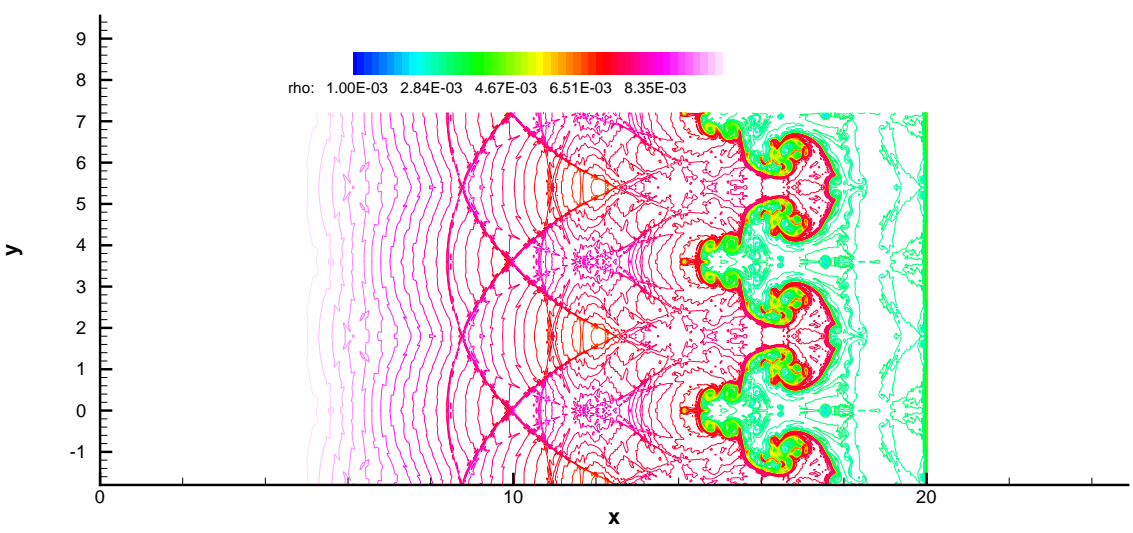

V-Velocity

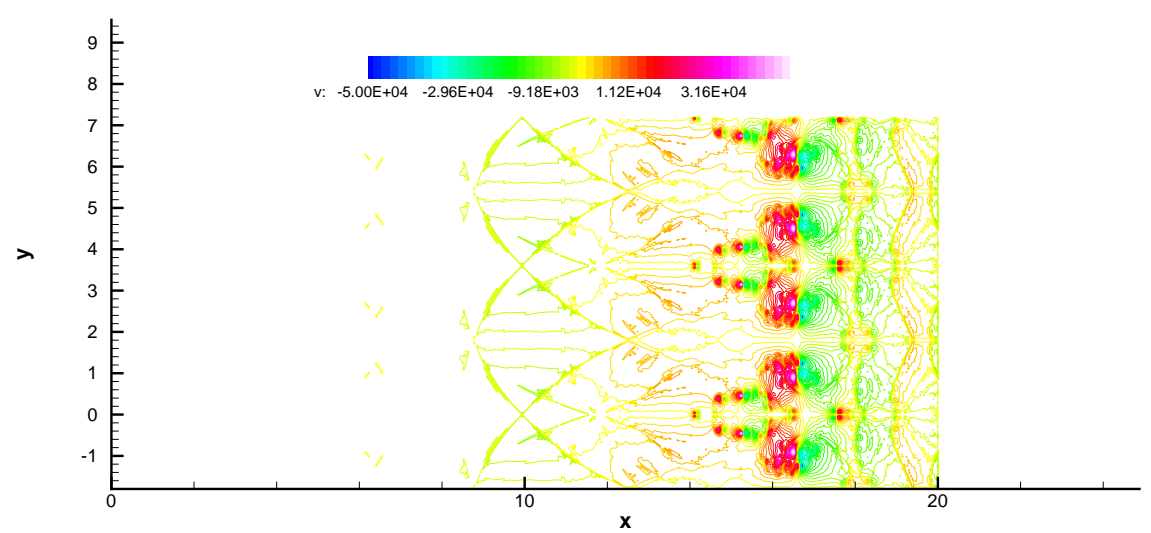




\section{Richtmyer-Meshkov Instability (Cont.)}

\section{Amplitudes vs. Displacement}
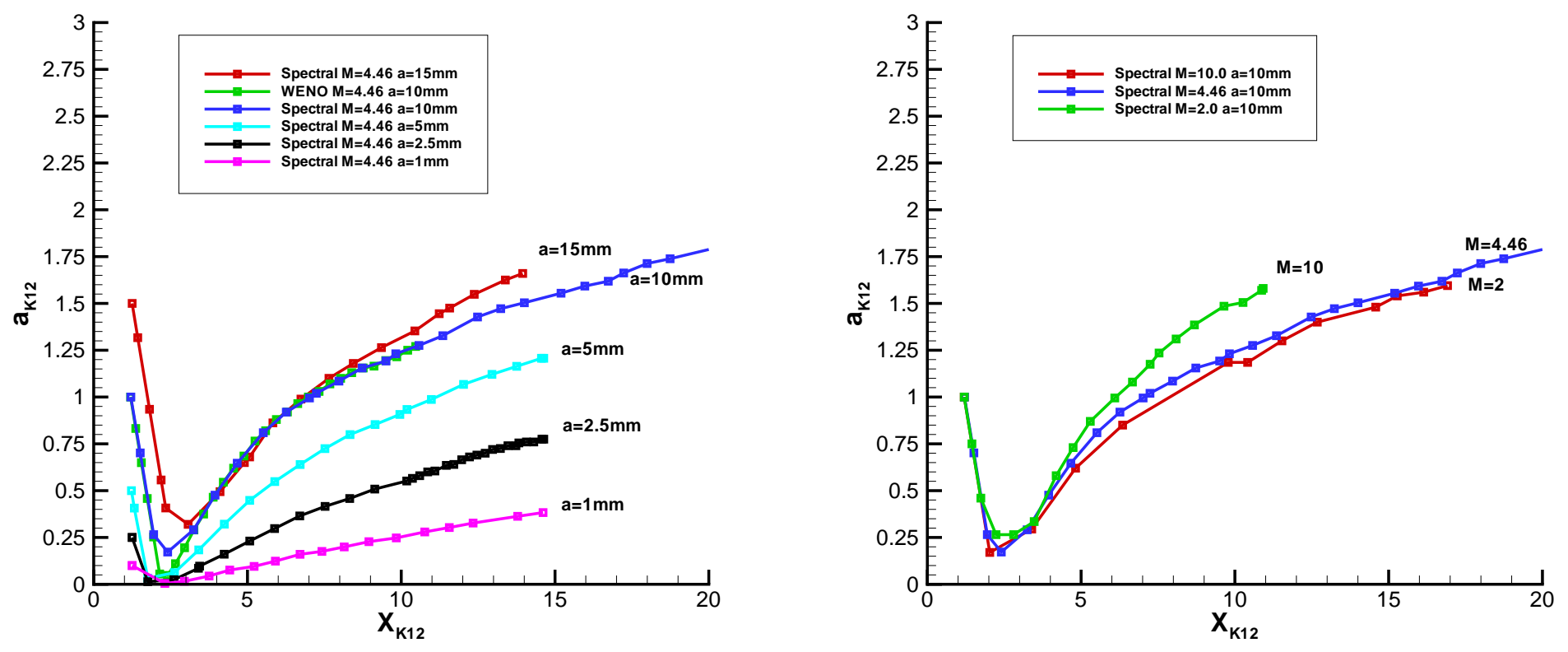

- Consistent with the experimental observations by Aleshin et al.

1. Linear growth of $a$ within "soft regular" regime;

2. Decrease growth of $a$ within the irregular regime. 


\section{Richtmyer-Meshkov Instability : Conclusion}

\section{Summary :}

- Good agreement of the global large and medium features between the Spectral scheme and the WENO scheme.

- Some discrepancy of the fine scale vortical structures along the gaseous interface as expected for simulations of this sensitive nature to small perturbation (physically and/or numerically).

- Good agreement with the experimental data (Aleshin et al.) 


\section{Examination of Rayleigh-Taylor $\alpha$ in Three Cases: PPM}

Simulations, Vasilenko et al. Shock Tube Experiment, and Meshkov-Nevmerzhitsky Accelerated Tank Experiment

\section{A.M. Dimits \\ Lawrence Livermore National Laboratory}

Acknowledgments: E.W. Burke, A.W. Cook, G. Dimonte, J.A. Greenough, D. Klem T.A. Peyser, D.H. Porter, D. Youngs, Y. Zhou. 


\section{Abstract}

Several aspects of mixing due to the Rayleigh-Taylor (RT) instabilityare investigated.

Analysis of 3D multimode simulations using the PPM code [D.H. Porter and P.R. Woodward, Astrophys. J. Suppl. 93, 309 (1994), and references therein.] show that there are regions of the parameter space of the initial conditions in which the growth rate is independent of variations in the initial conditions. The simulated growth rates are found to increase as the Navier-Stokes viscosity is increased. It is investigated whether this couterintuitive result is due to the suppression of material mixing at the molecular level for larger viscosities.

Analyses of two RT experiments, one in which water is accelerated by a compressed gas (E.E. Meshkov and N.V. Nevmerzhitsky, Proc. $3^{\text {rd }}$ Int. Wkshp. on the Physics of Compressible Turbulent Mixing, 1991) and one in which an interface between gases of different density is decellerated in the post-shock region of a shock in an electromagnetic shock tube (A.M. Vasilenko et al., ibid.), are presented. Direct compressibility effects on the RT growth are shown to be negligible in the former. Various effects of the expansion of the gases in the region of the interface on the RT growth rates are investigated for the latter experiment, both analytically and with 1D simulations. These effects are found to be insufficient to reconcile the growth rates observed in the Vasilenko et al. experiments with some other experimental and simulation results. 
- Nonlinear Rayleigh-Taylor evolution arises in

- astrophysics - collapsed stars, supernovae

- performance degradation of inertial fusion capsules

- Rayleigh-Taylor evolution is of general basic interest as a paradigm for phenomena in

- atmospheric and space sciences

- magnetic fusion

- combustion 


\section{High-Resolution 3D PPM simulations of Rayleigh- Taylor instability give the following results.}

- Lower growth rates $(\alpha)$ than experiments and many other simulations.

- $\alpha$ increases with Navier-Stokes viscosity $v$.

- $\alpha$ is insensitive to changes in system size and spatial scale of initial perturbations.

- There appears to be a single large scale of the evolution; i.e.,

- amplitudes from concentration thresholds and profile overlap, and perpendicular integral correlation length scales agree for most cases.

- Cases with very different a have very similar atomic mix fraction profiles. 


\section{THE PPM CODE IS AN ESTABLISHED HYDRO CODE USED EXTENSIVELY IN ASTROPHYSICS}

- PPM = Piecewise Parabolic Method

- Godunov-type code

- Uses piecewise parabolic interpolation

- Woodward-Porter version

- Lagrange + remap to Euler mesh

- 3D; Directionally split

- Has Navier-Stokes dissipation

- Robust for high-Mach-number flows

- Runs well on large parallel computers 


\section{Baker, Meiron, and Orszag RT cases were used as validation tests of PPM.}

- G.H. Baker, D.I. Meiron, and S.A. Orszag, Phys. Fluids 23, 1485 (1980).

- $\mathrm{A}=0.33$ case

- PPM and Baker et al. agree well for bubble and spike amplitudes

- Qualitative appearance of interface is similar, apart from discrete polygon nature of Baker et al. results, and slightly more roll up and some evidence of residual secondary Kelvin-Helmholtz notches in the PPM case

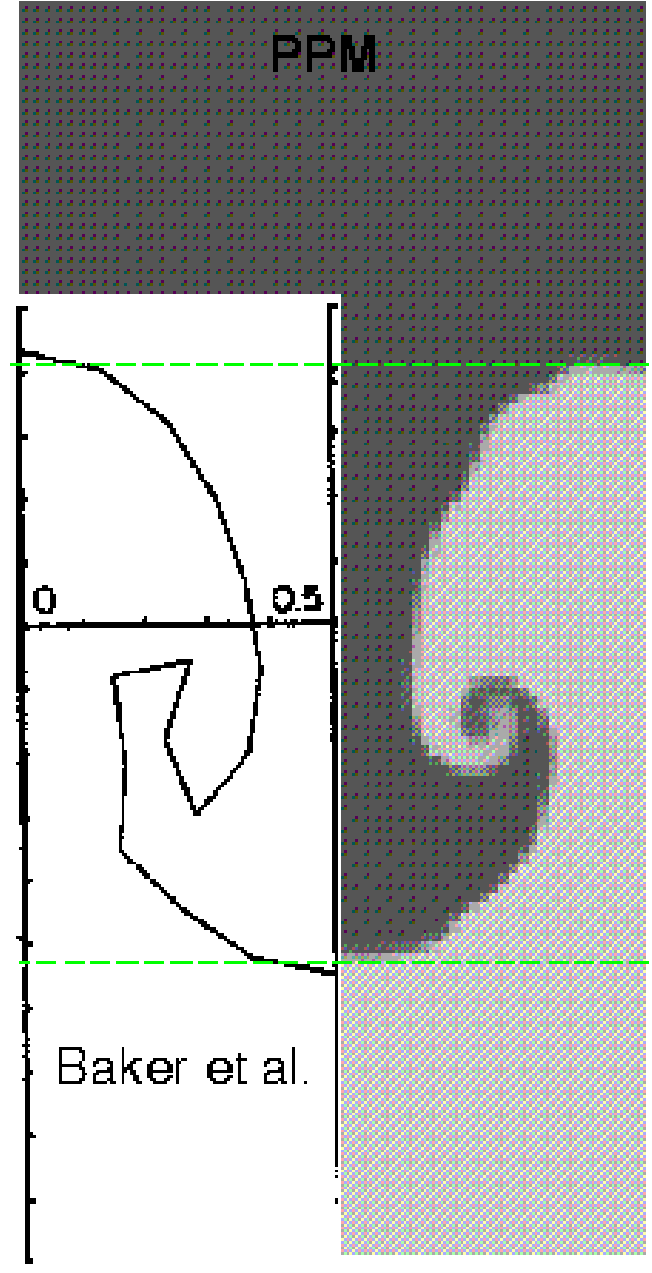




\section{For $\mathbf{A}=\mathbf{0 . 0 4 8}$, PPM gives amplitudes slightly lower than Baker et al.}

- Qualitative appearance of interface is similar, apart from discrete polygon nature of Baker et al. results and slightly more roll up in the PPM case.

PPM W=1

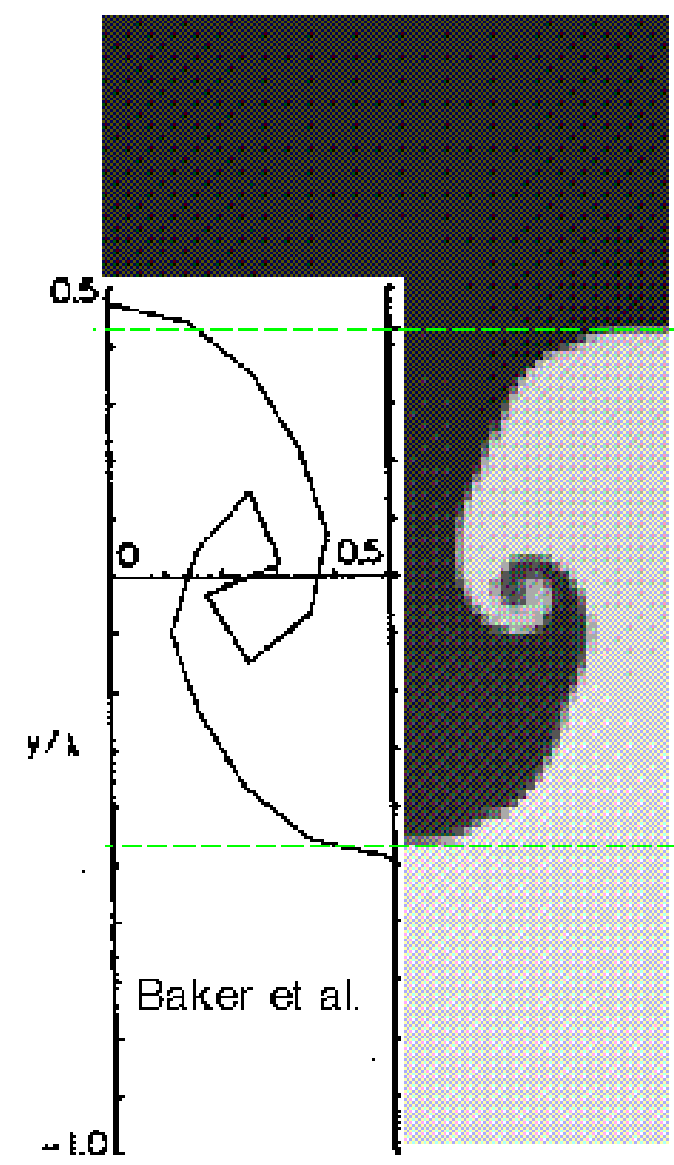

$P P M W=2$

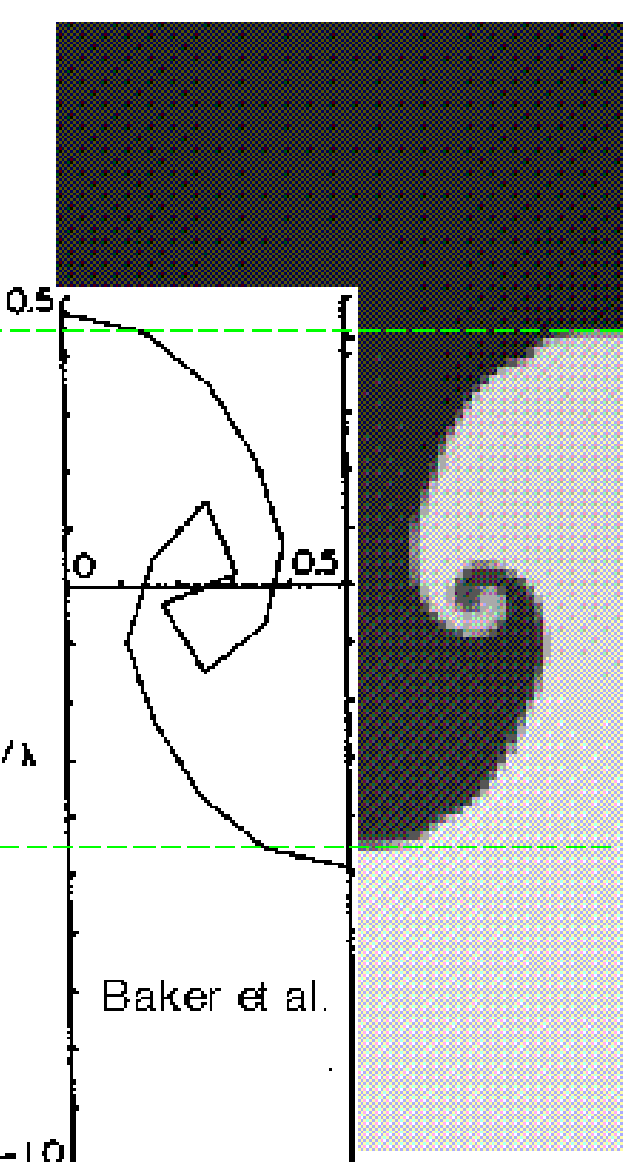


THE SETUP MOST USED HERE HAS UNIFORM TEMPERATURES ABOVE AND BELOW THE INTERFACE
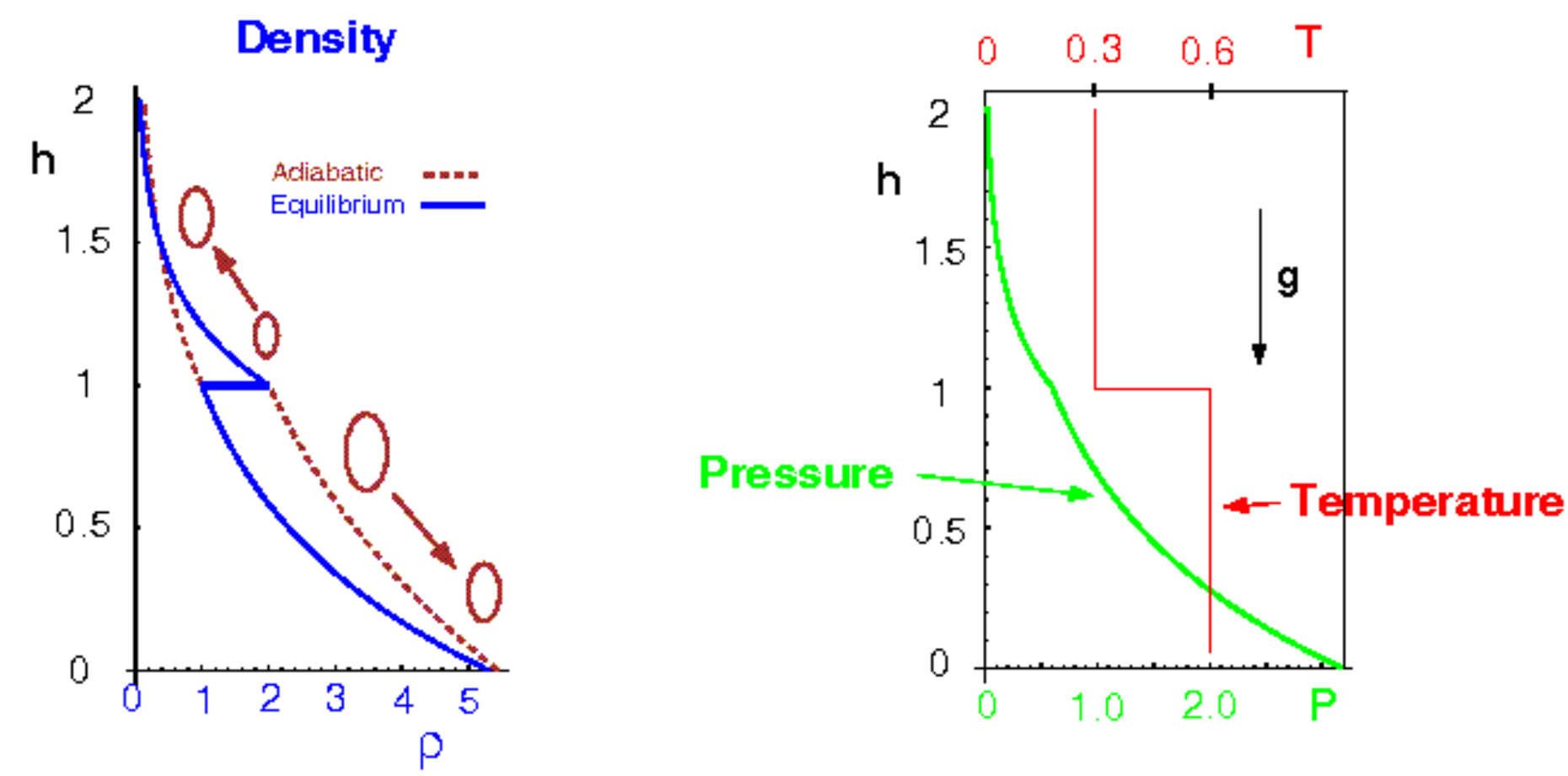

- ideal, single- $\gamma(=5 / 3)$ gas

- density discontinuity supported by a discontinuity in the temperature or the molecular mass

- exponential density and pressure profiles above and below interface

- near-incompressible low-level initial velocity perturbations

- mult-mode - quasi-Gaussian with a specified peak k 
A RANGE OF RESOLUTIONS, NAVIER-STOKES DISSIPATION VALUES, AND SYSTEM SIZES IN THE NEAR-INCOMPRESSIBLE REGIME WERE USED

- $\rho_{\mathrm{h}} / \rho_{\mathrm{l}}=2-3(\mathrm{~A}=1 / 3-1 / 2)$

- $v=\kappa=0,1 / 6.5,1 / 2.3 \mathrm{~g} \Delta^{3 / 2}$

- $\mathrm{L}=0.02,0.04,0.08 \mathrm{c}^{2} / \mathrm{g}$

- $256^{3}, 256^{2} \times 512,512^{3}$

- Low-level initial velocity perturbations

- Peak wavenumber varied
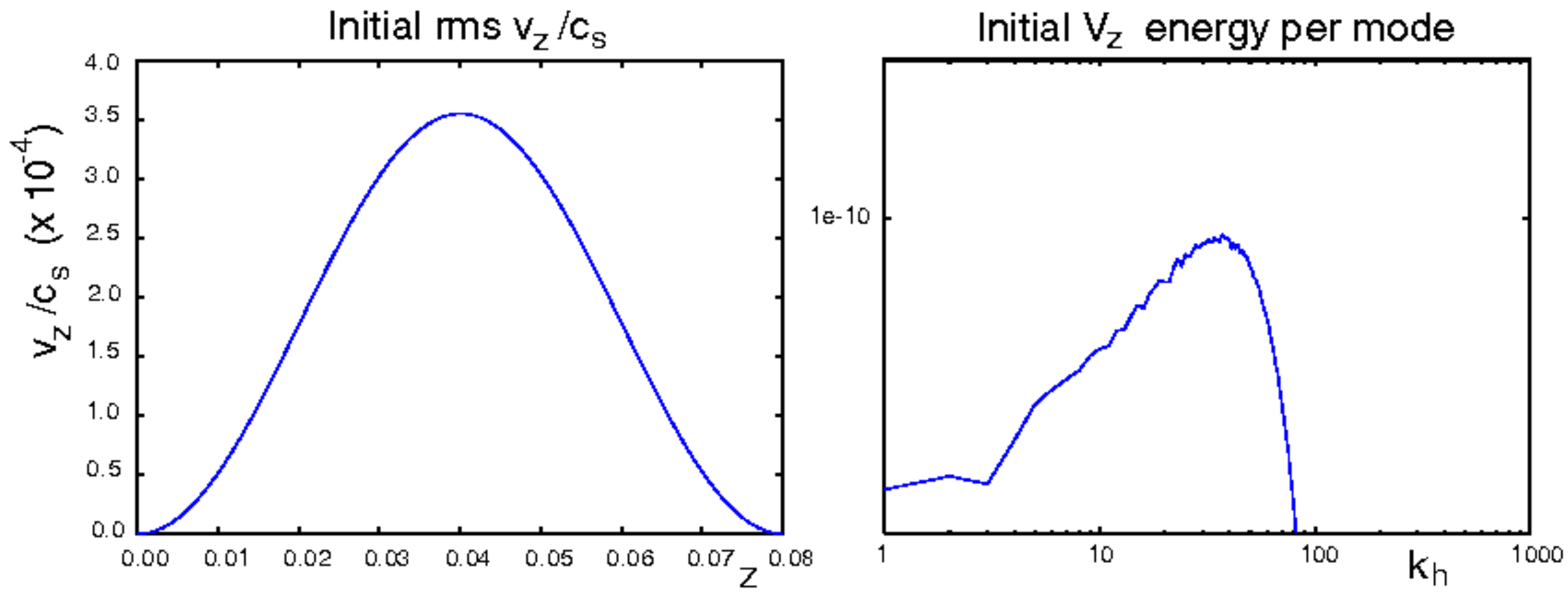
PPM AND PPM+NS SIMULATIONS OF RAYLEIGHTAYLOR EVOLUTION SHOW BUBBLES, SPIKES, AND ROBUST "BUBBLE MERGER"

$\mathrm{t}=0.1 \mathrm{c} / \mathrm{g}$

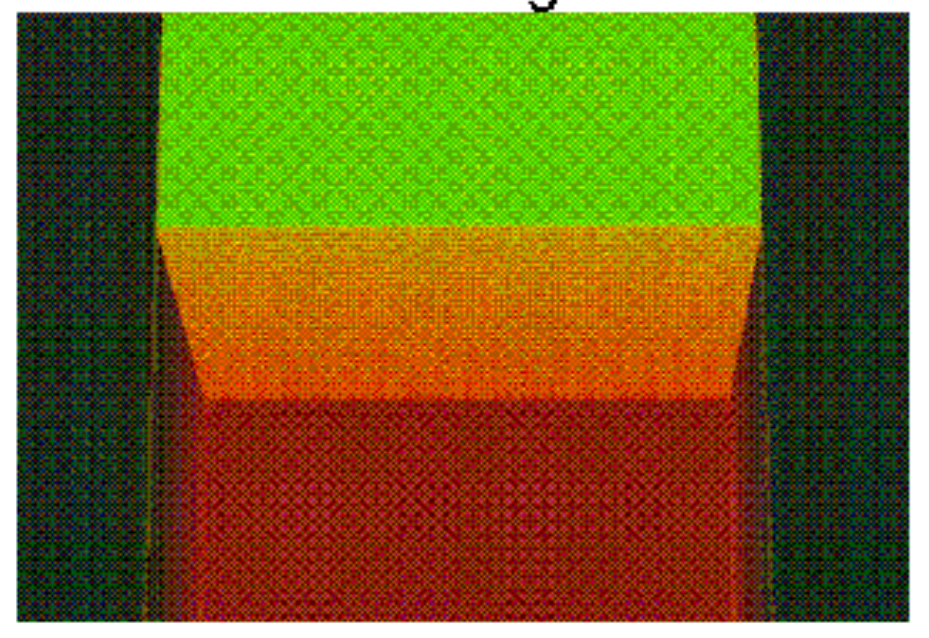

$\mathrm{t}=0.35 \mathrm{c} / \mathrm{g}$

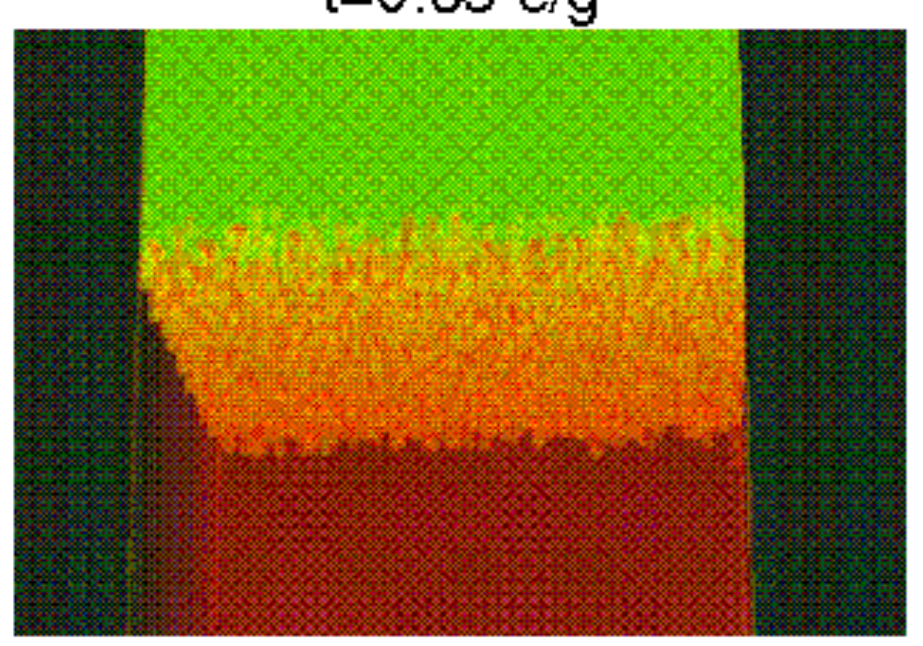

$\mathrm{t}=1.125 \mathrm{c} / \mathrm{g}$

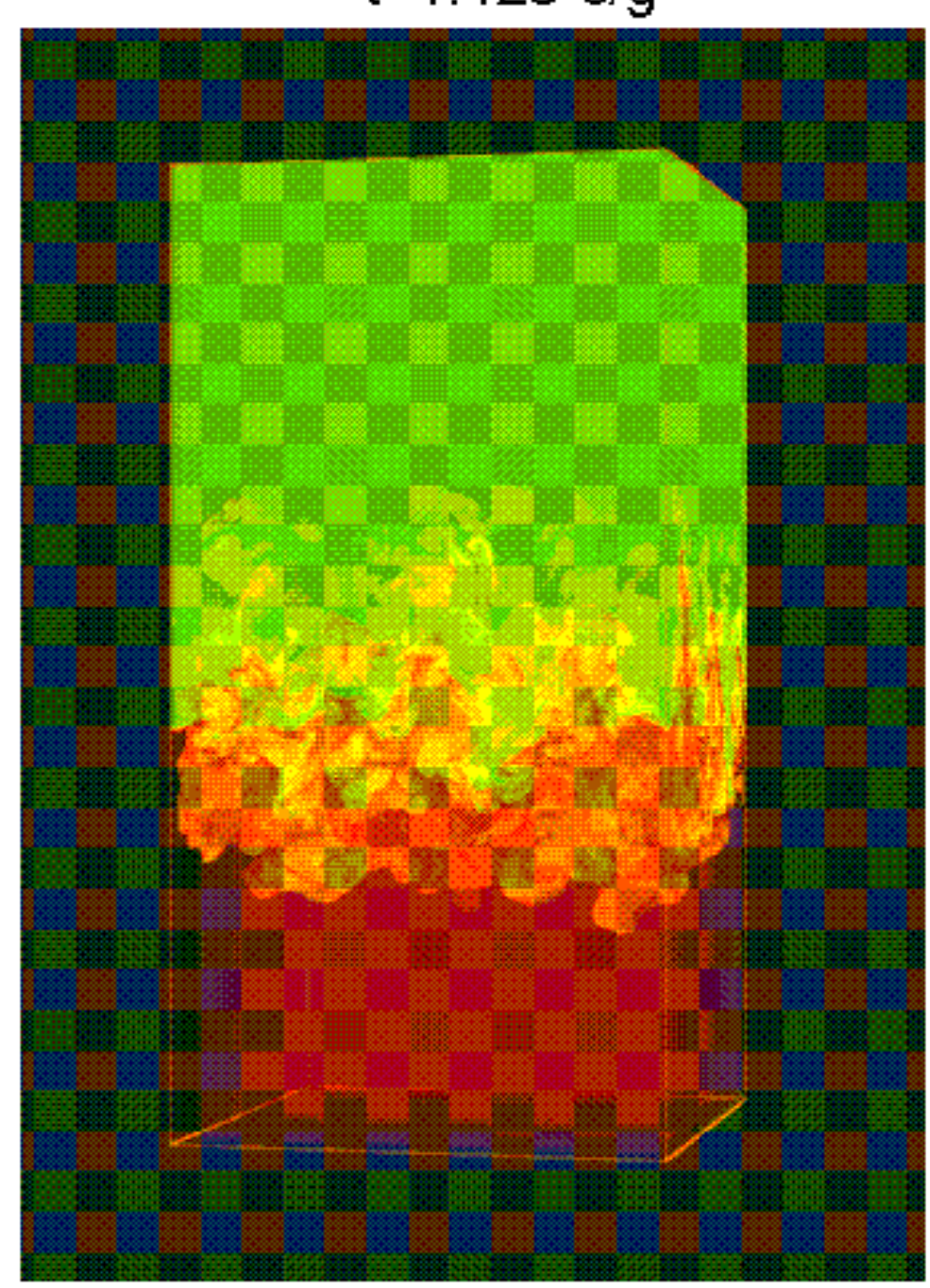


- All spatial scales $/$ grow as $l(t)=F(f) \cdot g \cdot\left(t-t_{0}\right)^{2}$, where $f$ is the density ratio, and $t$ is a "movable" time origin.

- Common characterization:

- bubble heights have $F(f)=\alpha \cdot A$

- $A=(f-1) /(f+1)$ is the "Atwood number"

- $\alpha$ bubble is a key parameter for comparisons.

- $I_{\text {bubble }}$ is obtained as the height at which the volume fraction of fluid originating below the interface is $1 \%$.

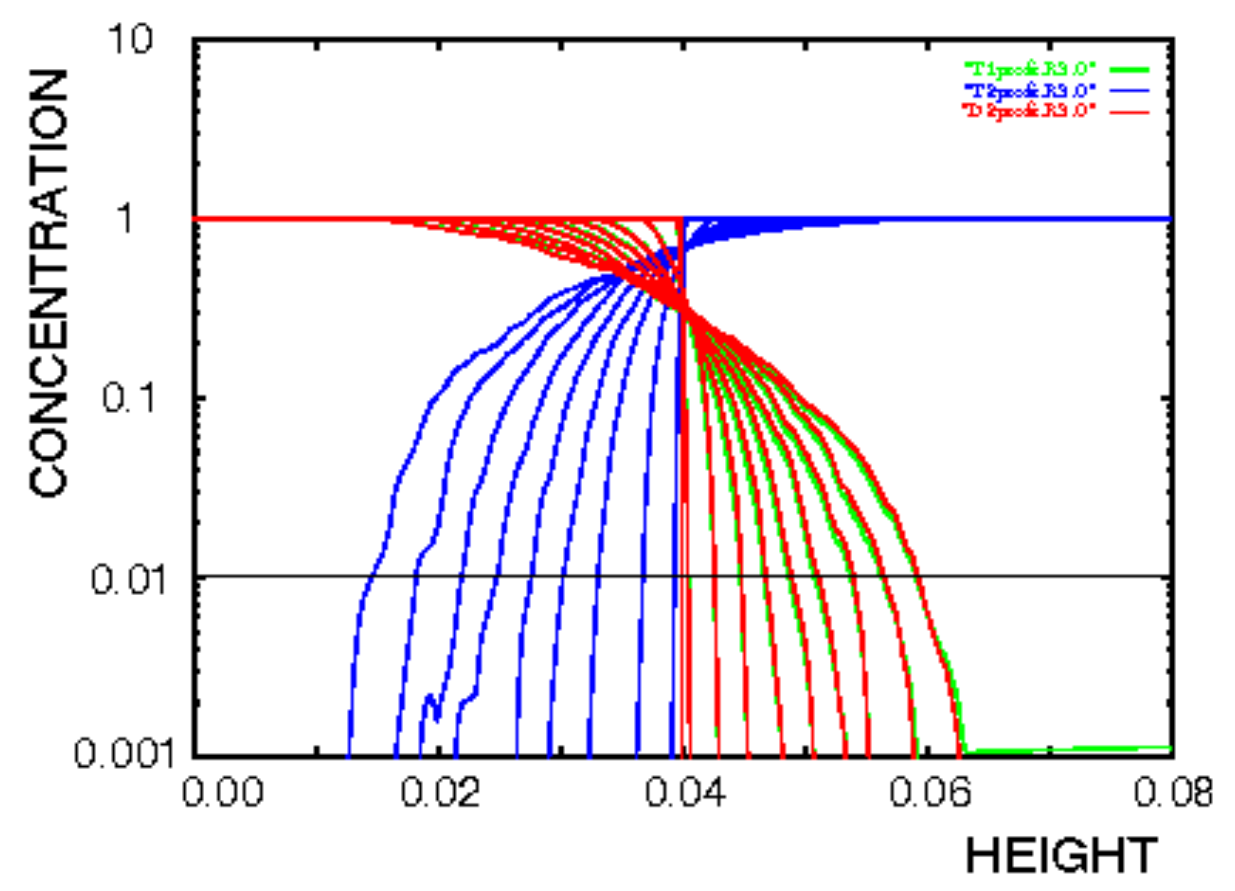




\section{AT LATE TIME (>0.4), PPM-NS CALCULATIONS ARE IN THE $\mathrm{t}^{2}$ ASYMPTOTIC REGIME}

- $\alpha$ is sensitive to the criterion for determining the envelope of the mixing layer.

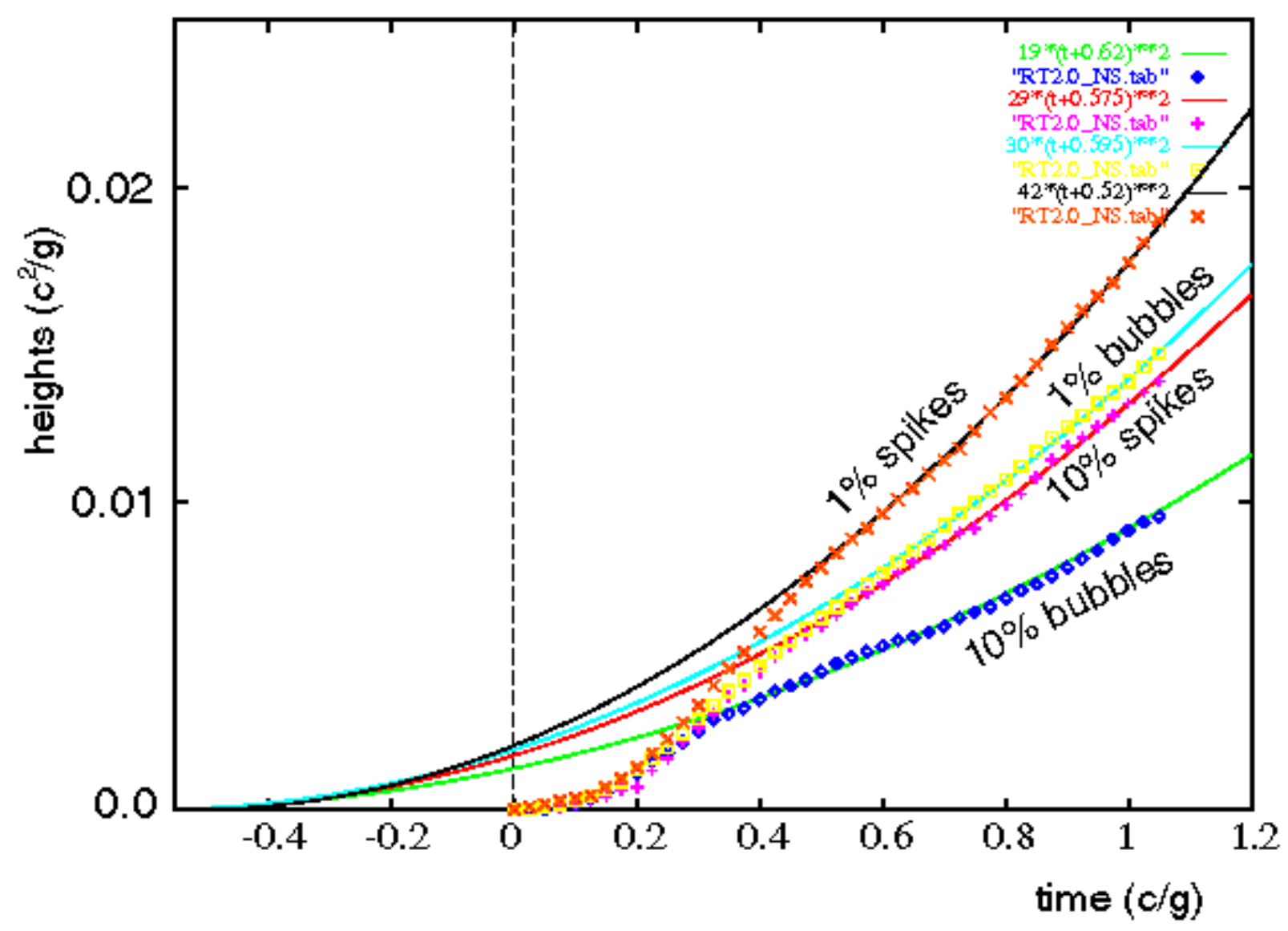


3D PPM SIMULATIONS GIVE LOWER VALUES OF a, THAN EXPERIMENTS AND OTHER CODES

\begin{tabular}{|c|c|c|c|c|c|}
\hline \multicolumn{2}{|c|}{ Code/Exp. Author } & $A$ & Resolution & $y^{\frac{1}{2}} \Delta^{\frac{3}{2}} / t$ & the \\
\hline Turmoil & Youngs & 0.50 & $\leq 10 \pm \overline{2} \times \bar{y}^{2} 2^{2}$ & (x) VitrI Lerr & $0.02 \overline{3}-0.028$ \\
\hline Turmoil & Youngs & 0.20 & $210 \times 160^{2}$ & $\infty$ & 0.027 \\
\hline Turrmoil & Yourigs & 0.90 & $210 \times 160^{2}$ & $\infty$ & 0.029 \\
\hline PPM-NS & & $0.33-0.5$ & $256^{*}-512^{*}$ & $2: 3-18.1$ & $0.012-0.020$ \\
\hline PPM & & 0.33 & $250^{\circ}$ & $\infty-$ PPM & $0.011-0.013$ \\
\hline rocket & Read & 0.23 & na & & 0.06 \\
\hline LEM & Dimonte & 0.22 & na & & 0.06 \\
\hline
\end{tabular}

- References:

- Simulations by D.L. Youngs [Laser and Particle Beams L2, 725 (1994)].

- Experinents by K.I. Read [Pliysica D 12, 45 (1984).], G. Dimonte et al., [Phys. Rev. E54 3740 (1996).] 


\section{$\alpha_{b}$ in PPM+NS simulations increases with $v$}

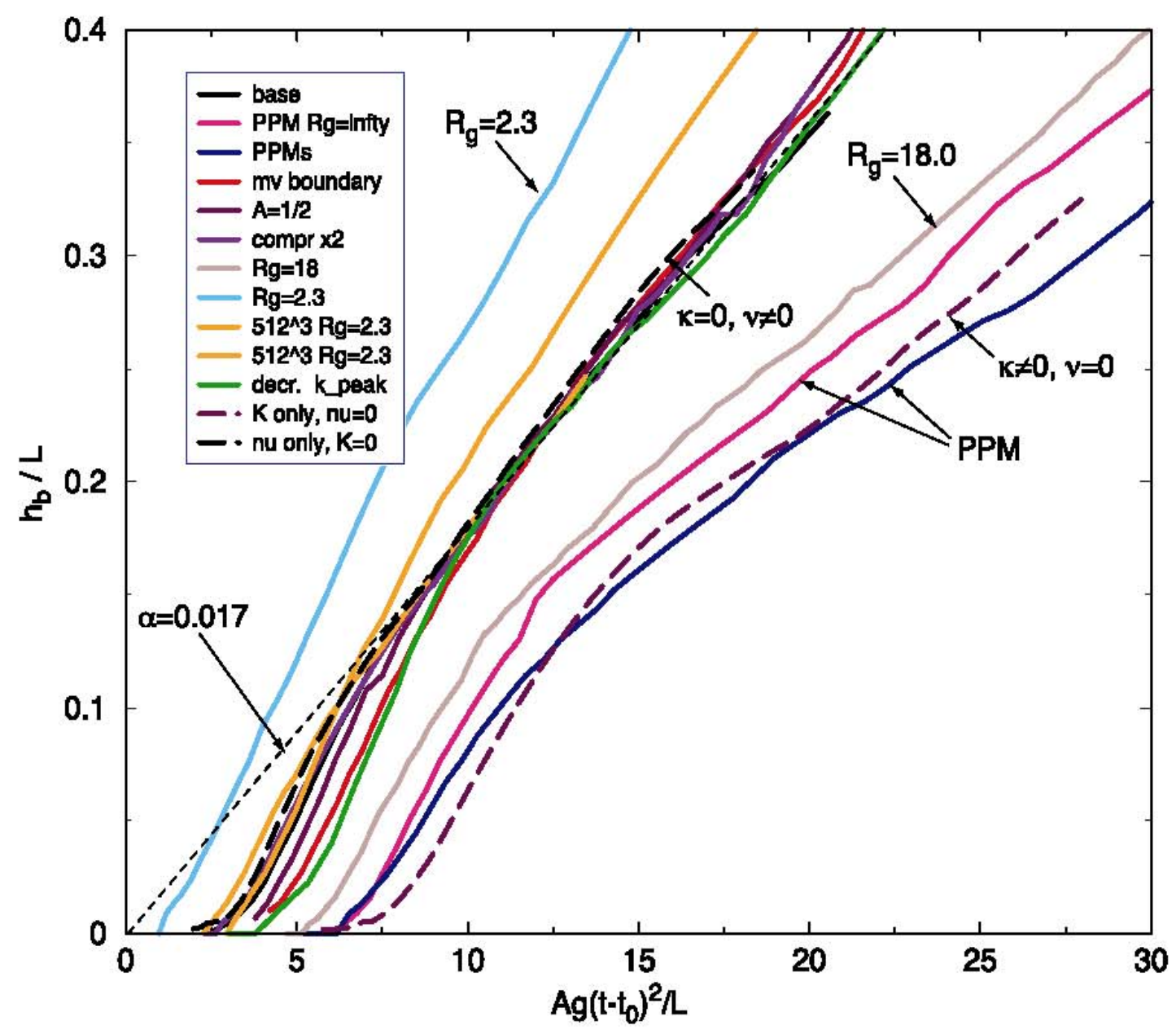

$\alpha_{b}$ is insensitive to - boundary position

- A

- compressibility

- $k_{h}$ of IC spectrum 
CHANGING THE BOX SIZE AND $A$ DOES NOT CHANGE $a, b$ in 3D PPM SIMULATIONS

\begin{tabular}{|c|c|c|c|c|c|}
\hline Model & Comment & $A$ & Resolution & $y^{\frac{1}{2}} \Delta^{\frac{2}{2}} / y$ & $t_{\mathrm{b}}$ \\
\hline PPM-NS & & 0.33 & $256^{3}, k_{1}-32$ & 6.5 & 0.018 \\
\hline PPM-NS & incr. $L_{i}$ & 0.33 & $256^{2} \times 512$ & 6.5 & 0.018 \\
\hline PPM-NS & change $A$ & 0.5 & $250^{3} \times 512$ & 6.5 & $0.0 \perp 8$ \\
\hline PPM-NS & lower $k_{p}$ & 0.33 & $296^{3}, k_{p}-16$ & 6.5 & $0.0 \perp 8$ \\
\hline PPM-NS & $L_{z}-0.08$ & 0.33 & $256^{3}$ & 6.5 & 0.018 \\
\hline PPM-NS & incr. $V, k$ & 0.33 & $256^{3}$ & 2.3 & 0.028 \\
\hline PPM-NS & decr. $\nu_{r} k$ & 0.33 & $256^{3}$ & 18.4 & 0.012 \\
\hline PPM-NS & incr. res. & 0.33 & $5 \perp 2^{3}$ & 2.3 & $0.022 *$ \\
\hline PPM & no NS & 0.33 & $256^{3}, k_{p}-32$ & $\infty-$ PPM & 0.0125 \\
\hline PPM & & 0.33 & $256^{i}, k_{1}-61$ & - PPM & 0.011 \\
\hline
\end{tabular}

- Increasing (decreasing) Navier-Stokes dissipation raises (lowers) a $a$ b

- Increasing compressibility has no effect on $x_{k r-}$

- Run with lower initial $k_{\mathrm{r}}(=16)$ - $\alpha$ unaffected. 


\section{PPM simulations of RT show a single large scale.}

- In RT studies, the large scales commonly diagnosed are measures of the vertical scale, e.g.,

- amplitudes from threshold concentration levels,

- integral measures of overlap of concentration profiles.

- In much of fluid dynamics "integral" correlation length scales are used.

- Integral scales measured at the position the interface would have in the absence of RT instability provide measures of the transverse scale.

- In high resolution 3D multimode PPM simulations of RT, these scales usually maintain a proportionality with ratios independent of time. 

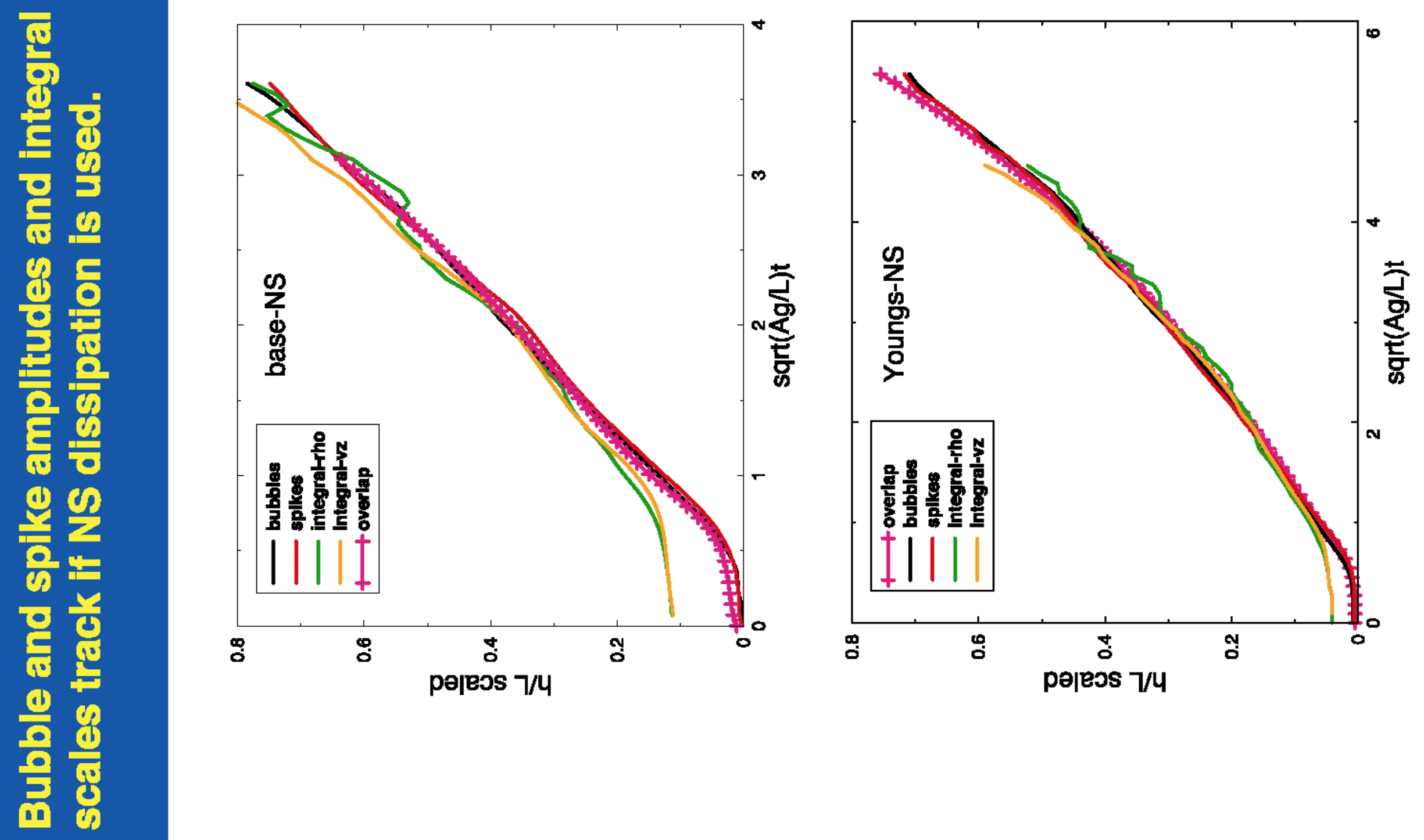

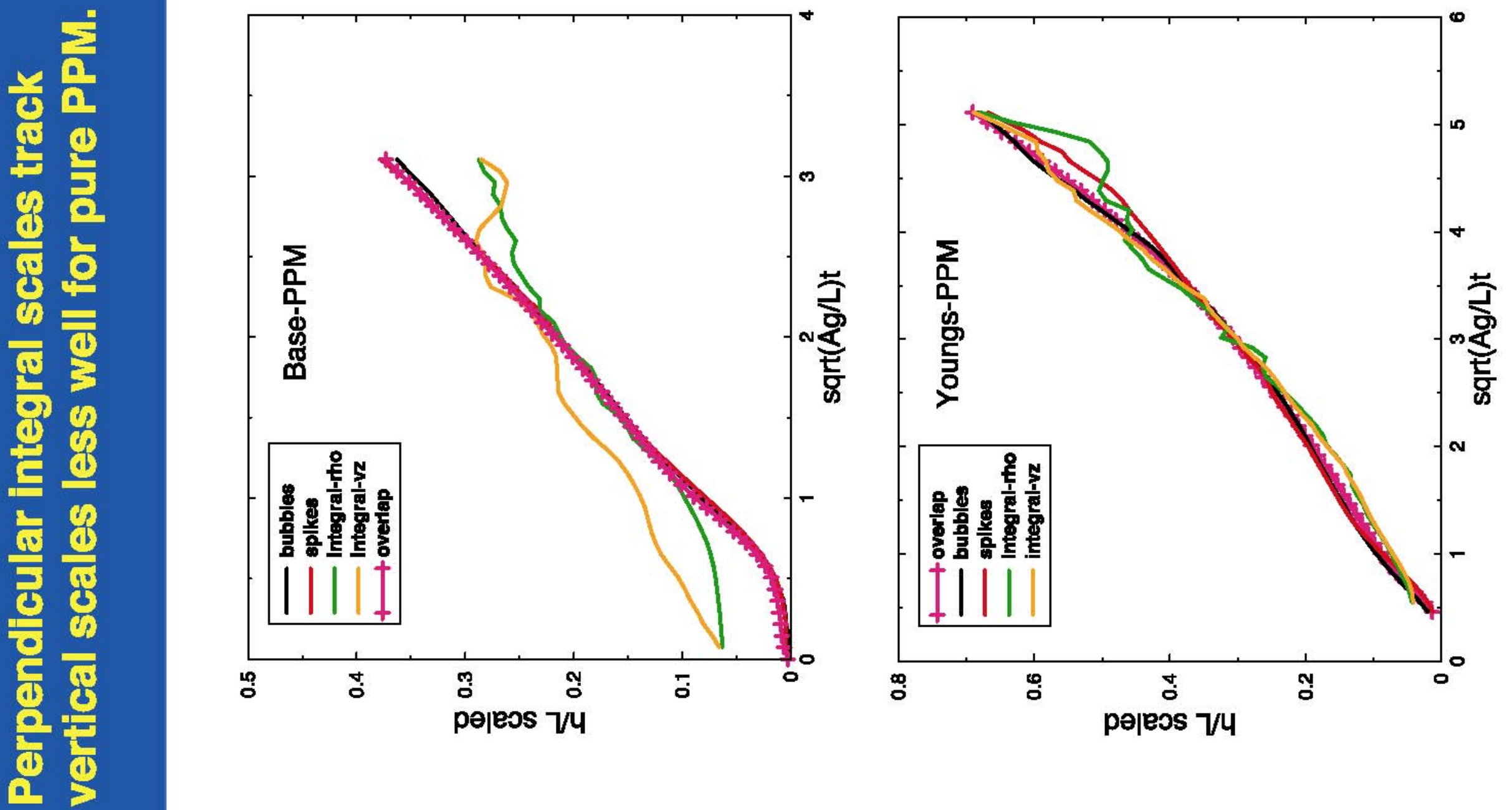


\section{A hypothesis that low $\alpha^{\prime} s$ are associated}

with hight atomic mix fraction was tested.

- If the mixing fraction is large, bubbles and spikes are impure and experience small buoyancy relative to the surrounding fluid.

- This might be expected to result in low growth rates.
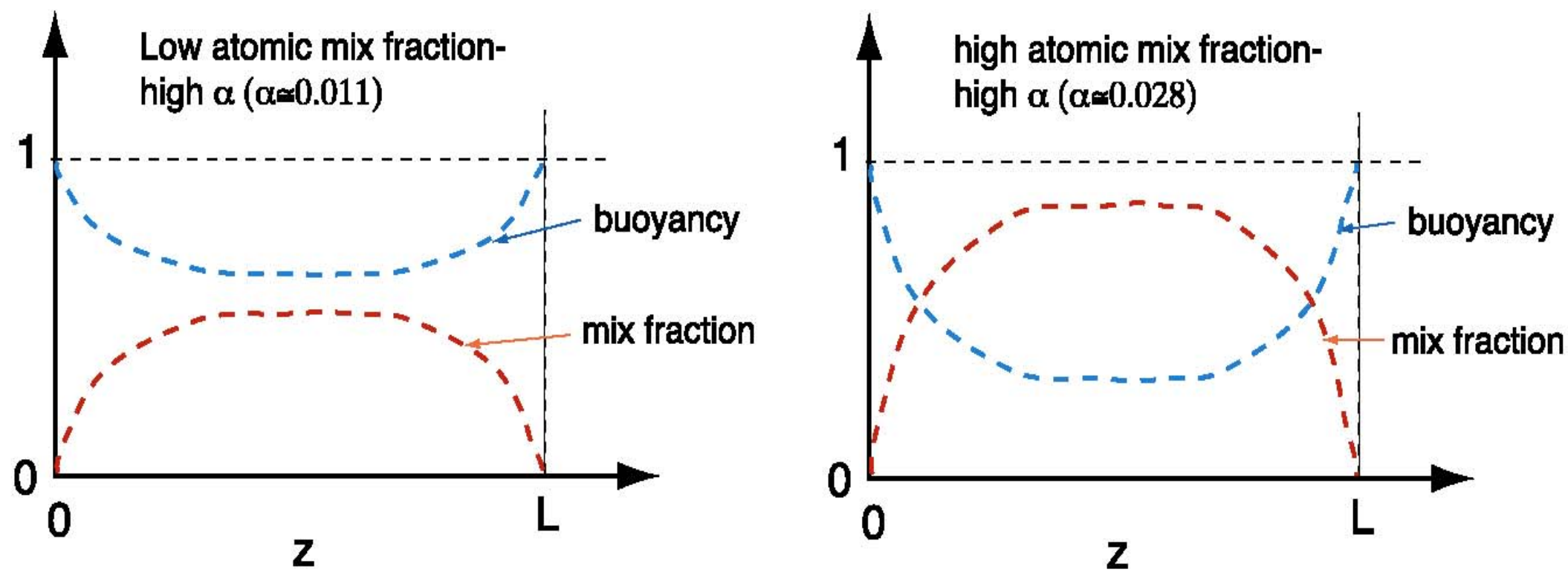


\section{Cases with very different $\alpha$ show very similar atomic mix fraction profiles.}

- PPM and $R_{g}=6.5$ have similar mix fraction profiles, different $\alpha\left(\alpha_{b}=0.013,0.018\right)$.

- $\mathrm{R}_{\mathrm{g}}=2.3$ has slightly lower mix fraction, highest $\alpha$ $\left(\alpha_{b}=0.028\right)$.

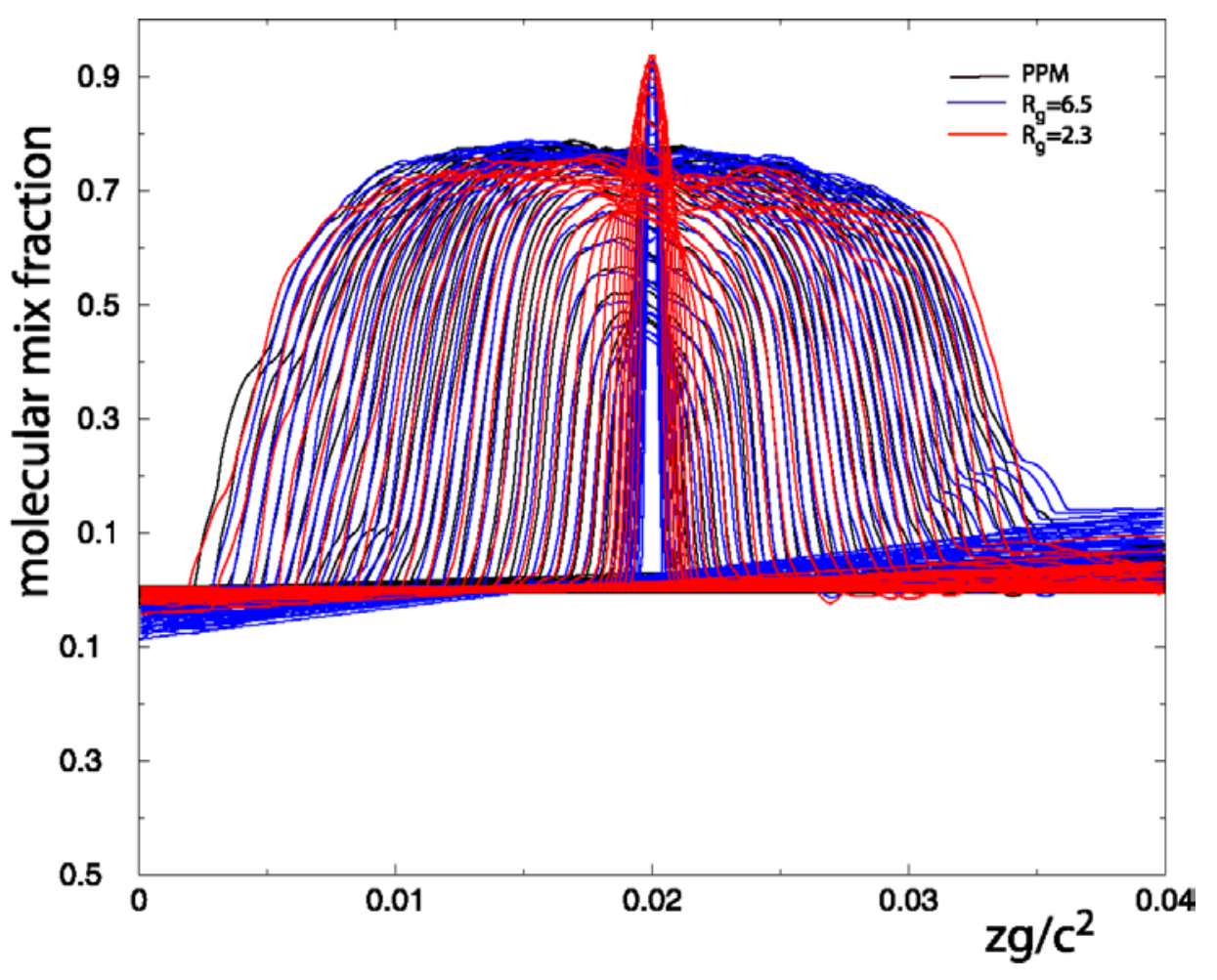




\section{Summary of Results From High Resolution 3D PPM Simulations of Rayleigh-Taylor Instability Evolution}

- Lower growth rates $(\alpha)$ than experiments and many other simulations.

- $\alpha$ increases with Navier-Stokes viscosity $v$.

- $\alpha$ is insensitive to changes in system size and spatial scale of initial perturbations.

- There appears to be a single large scale of the evolution; i.e.,

- amplitudes from concentration thresholds and profile overlap, and perpendicular integral correlation length scales agree for most cases.

- Cases with very different $\alpha$ have very similar atomic mix fraction profiles.

- Demonstrated that simulations are in a regime where compressibility is negligible. 


\section{The experiments by E.E. Meshkov and N.V. Nevmerzhitsky}

were reexamined because they appeared to give large

\section{values of $\alpha$ and an apparent increase of $\alpha$ with acceleration.}

- Rayleigh-Taylor experiment (3rd. IWPCTM, France, 7/91.)

- Water accelerated by compressed air or Helium

- Atwood number $A \cong 1$

- Membrane breaks; plywood plate detaches; water and plate accelerated downwards by compressed air

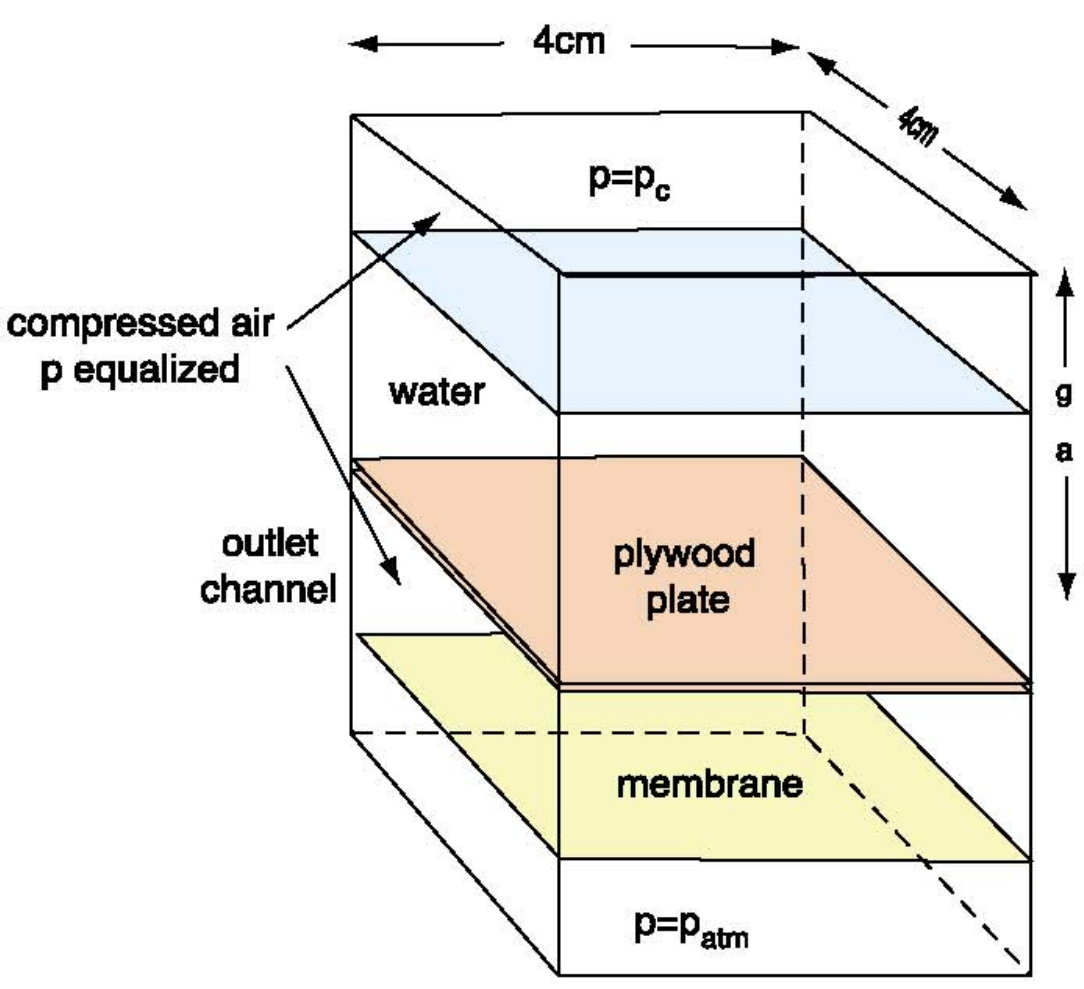

- Authors conclude that $\alpha$ increases with $p_{c}$ 


\section{The results shown in the Meshkov-Nevmerzhitsky '91 IWPCTM paper do not demonstrate a systematic increase in $\alpha$ due to compressibility.}

- Data shown for expts \#422 and \#446.

- Different curve fitting to \#422 data can revise $\alpha_{b}$ down slightly from 0.128 to $\approx 0.1$.

- $\alpha_{b}$ for \#446 has large uncertainty.

- Compressibility effects are highest, but negligible, in \#422. 

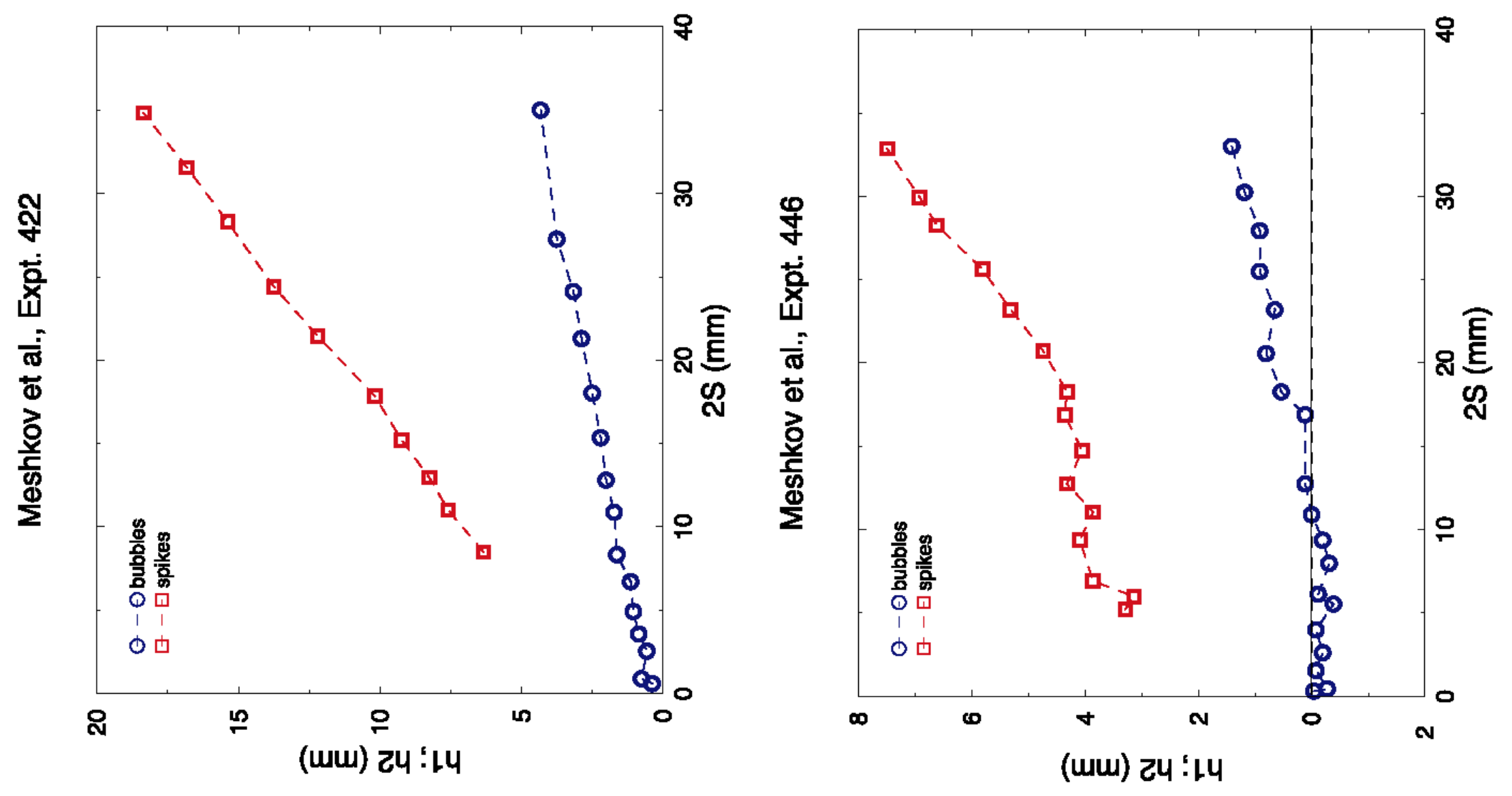


\section{Compressibility is negligible in Meshkov - Nevmerzhitsky experiment \#422.}

- Subsonic

$$
-M_{\text {bubbles }} \leq 0.04, M_{\text {spikes }} \leq 0.13 \text {. }
$$

- Bubble expansion due to change in relative pressure small

- $\delta \mathrm{I}_{\text {exp }} / 1 \leq 0.03$ for isolated bubble

- $\delta \mathrm{l}_{\text {exp }} / \mathrm{l}$ negligible for open-cell bubble structure

- Change in drag due to bubble expansion results in $<2 \%$ change in bubble height. 


\section{Conclusions on Meshkov - Nevmerzhitsky Experiments}

- Expt. \#422 has higher $\alpha_{b}$ than experiments by Read and by Dimonte and Schneider (but has higher density ratio).

- Difference not explained by compressibility

- more likely connected with initial conditions.

- For the other experiments, $\alpha_{b}$ is highly uncertain (e.g., \#446) or data is insufficient to demonstrate a trend. 
The experiments of Vasilenko et al. were reexamined because o's were found that were much larger than in other experiments and simulations.

- Miscible (gas) Rayleigh-Taylor experiment

- $S_{c}(\equiv v / D)=O(1)$ (>> 1 for liquids)

- $W=\alpha A g t^{2} ;\left(\alpha=\alpha_{\text {bubble }}+\alpha_{\text {spike }}\right)$

- $\alpha=0.29-0.34,\left(\right.$ for $\left.\rho_{2} \rho_{1}=1.4-20\right)$

- Conflict with other data:

- experiment: $\alpha_{\text {bubble }} \approx 0.06, \alpha_{\text {spike }} / \alpha_{\text {bubble }} \approx 1+A$

- simulation: $\alpha_{\text {bubble }} \approx 0.02-0.06$ 


\section{Experiment by A.M. Vasilenko, V.I. Olhovskaya, O.V. Buryakov and V.G. Yakovlev}

- Rayleigh-Taylor experiment (3rd. IWPCTM, France, 7/91.)

- Electromagnetic shock tube

- Impulsive shock with rarefaction passing through 3 noble gases (e.g., $\mathrm{He}-\mathrm{Ar}-\mathrm{He}, \mathrm{He}-\mathrm{Kr}-\mathrm{He}, \mathrm{He}-\mathrm{Xe}-\mathrm{Kr}$ )

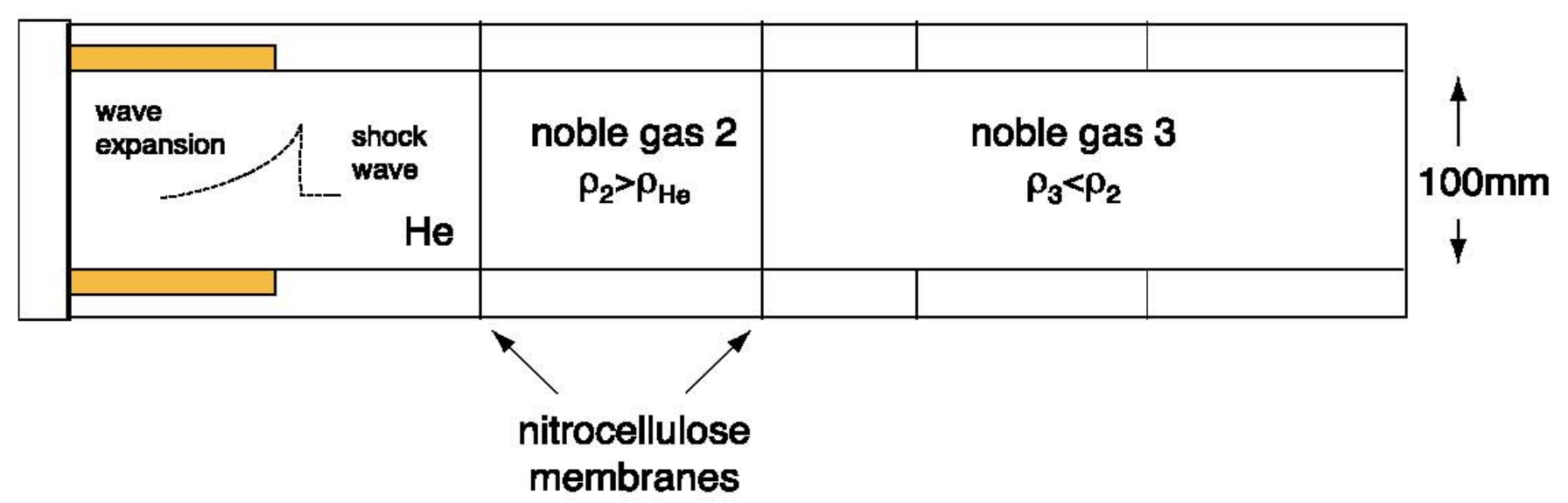




\section{Expansion behind the shock front does not directly account for differences in $\alpha$.}

- $A<<1$ - analytical estimate of expansion effect using 1D Sedov wave based model

- General $A$ : 1D HAMR computations show that propagation of a Sedov wave through light-heavy-light gases can be modeled as a shift in origin of Sedov wave.

- Same analysis applies for bounds on expansion effect.

- Analysis by Zhou and Dimits (Zhou et. al, this meeting) finds that experiments do not reach turbulence transition.

- Expect significant influence of initial conditions. 


\section{Effect of expansion on layer width for $A<<1$}

- Approximate shock/rarefaction as 1D Sedov wave.

- $\mathrm{u} \approx \mathrm{u}_{0} \xi / \xi_{0} ; \xi \equiv r\left[\rho_{0} /\left(\mathrm{E} \mathrm{t}^{2}\right)\right]^{1 /(2+\mathrm{d})}$ (supported by 1D simulations); $\mathrm{r}_{0}(\mathrm{t}) \equiv$ shock position; $\mathrm{u}_{0} \cong \mathrm{CV}_{\mathrm{s}} ; \mathrm{C} \cong(\gamma-1) /(\gamma+1) ; \mathrm{V}_{\mathrm{s}} \equiv$ shock speed.

- Then $u \approx 2 C /(2+d)(r / t)$ for $0 \leq r \leq r_{0}$

- Define $r_{1}(t) \equiv$ Lagrangian trajectory of fluid element that coincides with shock at $t=t_{1} \quad\left[i . e ., r_{1}\left(t_{1}\right)=r_{0}\left(t_{1}\right)\right]$.

- Then $r_{1}(t) / r_{0}\left(t_{1}\right)=\left(t / t_{1}\right)^{2 C /(2+d)}$ 


\section{Effect of expansion for $\mathrm{A}<<1$}

- Define decceleration path:

$-R_{a}(t) \equiv u_{0} \cdot\left(t-t_{1}\right)-\left[r_{1}(t)-r_{1}\left(t_{1}\right)\right]$.

- Suppose layer width $h \approx \alpha R_{a}(t)$.

- Contribution of expansion to $h^{\prime}$ is $h^{\prime}{ }_{\exp }=h \partial u / \partial r$.

- Then $h_{\text {exp }} / h^{\prime}$ obtainable analytically. 


\section{RT growth enhancement by expansion is less than $\approx 1.3$}

- $\mathrm{h}_{\text {exp }}^{\prime} / \mathrm{h}^{\prime} \leq 0.2 \Rightarrow$ less than factor 1.3 direct enhancement of layer width by expansion.

- Indirect effects? - need 2D or 3D simulation.

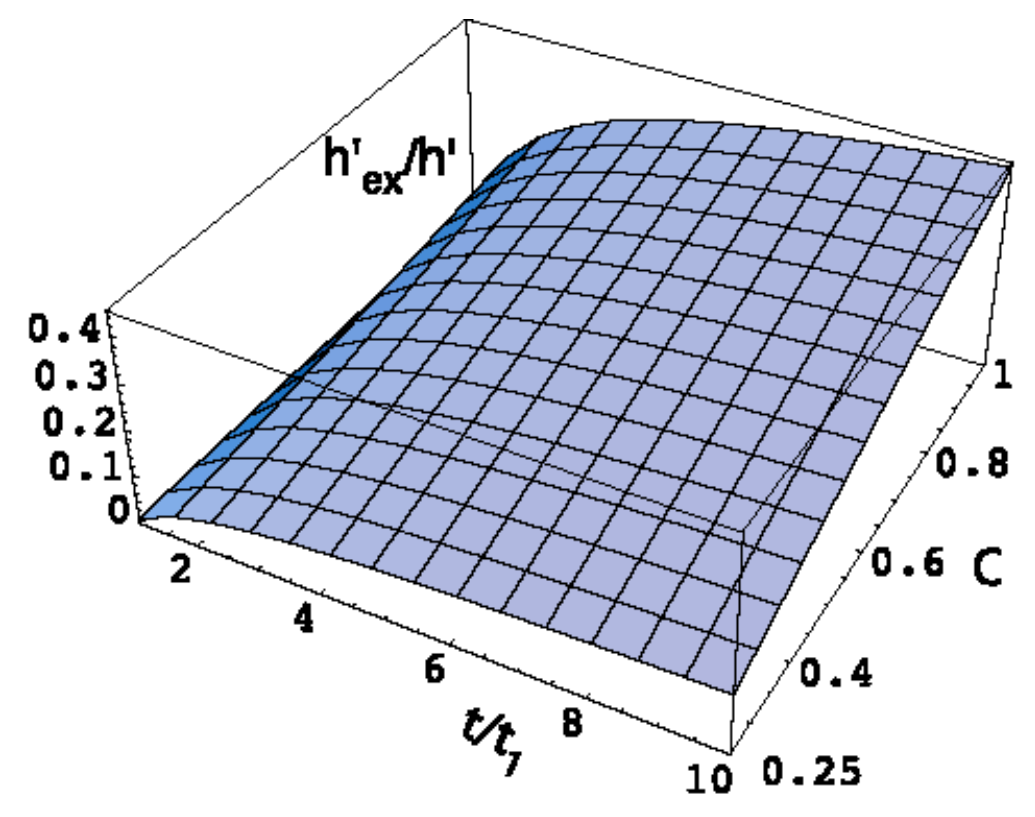




\section{D HAMR simulations support the application of the A $<1$}

analytical model to cases where $A<1$ does not hold.

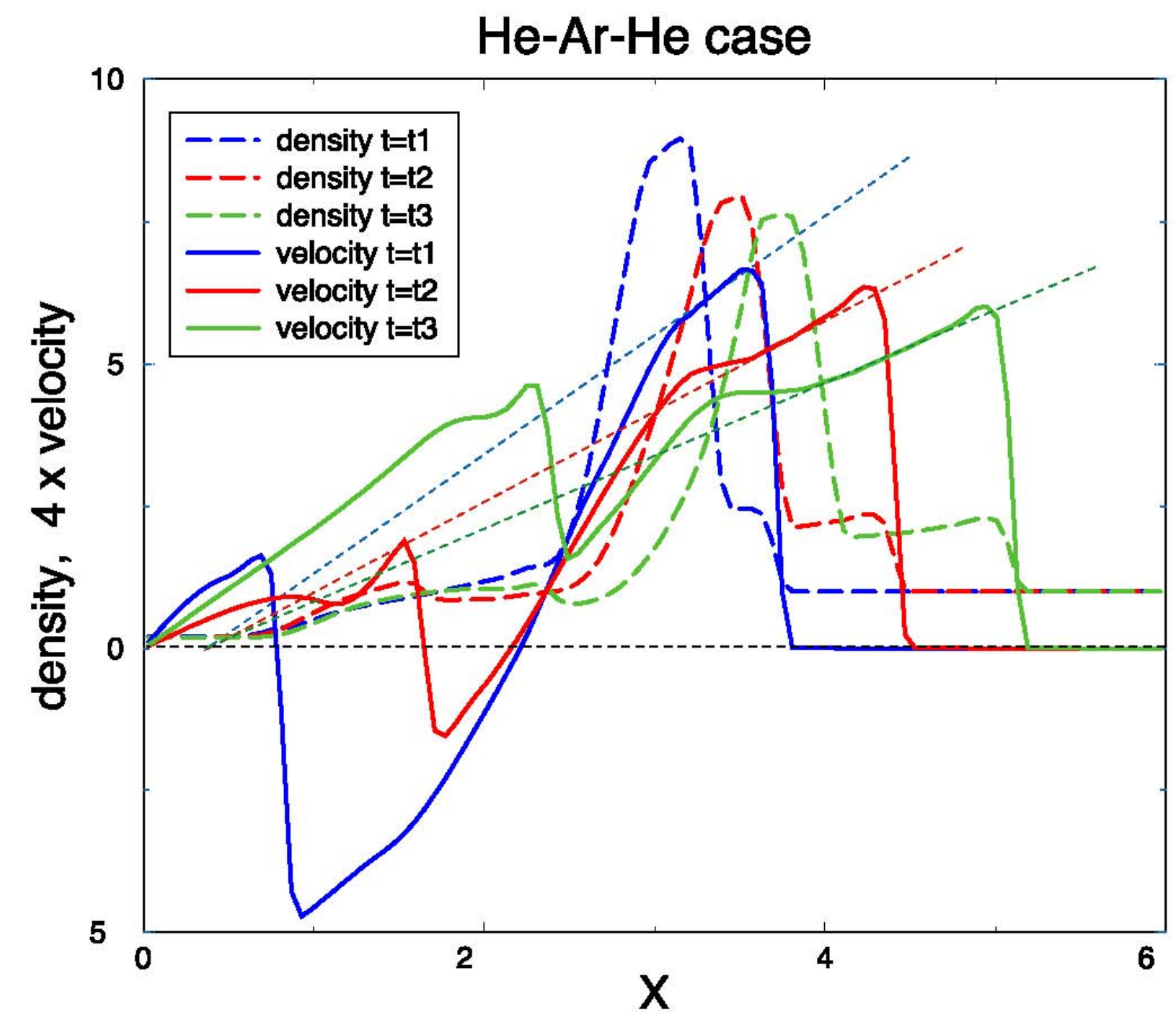



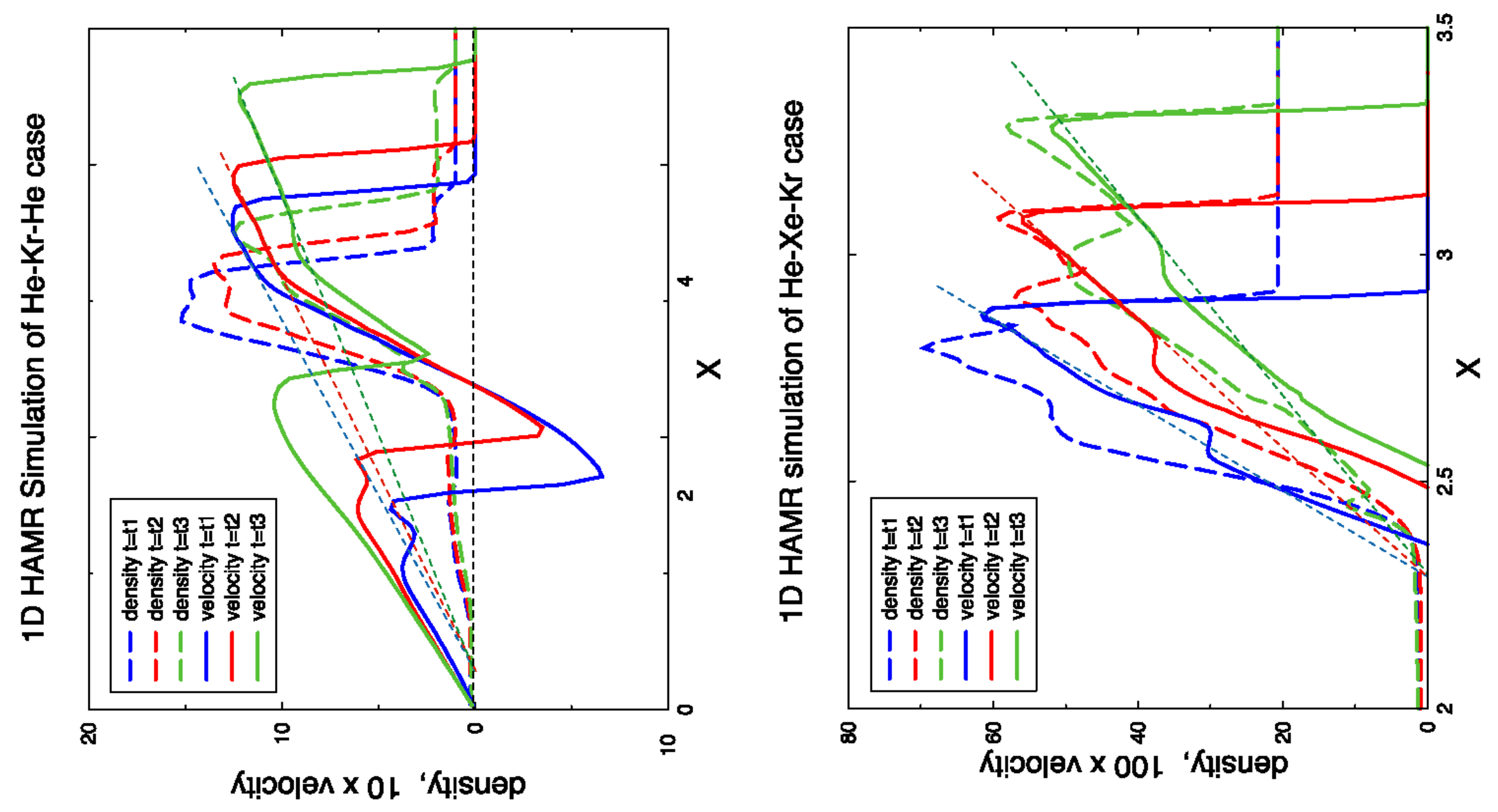


\section{Summary an Conclusions Concerning Experiments by Vasilenko et all.}

- Expansion behind the shock front does not account for differences in $\alpha$ between Vasilenko et al. experiments and other results.

- Estimated effect of expansion on $\alpha$ using a 1D Sedov-wave based model, valid for $A<<1$.

- 1D HAMR computations support the extension and application of this model to cases where $A<<1$ does not hold.

- Turbulence-transition analysis by Zhou and Dimits (Zhou et al., this meeting) suggests a significant influence of initial conditions on $\alpha$ as a possible explanation of the discrepancy. 


\section{Overview}

- Three 256x256x1024-point and one 512x512x2040-point Direct Numerical Simulations (DNS) were performed of Rayleigh-Taylor instability with different initial conditions.

- The smaller jobs executed on 128 CPU's of ASCI Blue-Pacific (IBM SP2) at LLNL. Simulation times varied from 200 to 300 machine hours.

- The larger job executed on 1024 CPU's and took roughly 50 machine days.

- Small and large simulations attained outer-scale Reynolds numbers of 3000 and 5500, respectively.

- Mixing-zone growth exhibits an initial diffusion stage, followed by a nonlinear stage.

- Growth and mixing are sensitive to initial conditions for duration of simulations.

- For many statistics, height of mixing zone (h) is a better progress variable than time $(\mathrm{t})$. 


\section{Governing equations}

$$
\begin{aligned}
& \begin{array}{l}
\frac{\partial \rho}{\partial t}+u_{j} \frac{\partial \rho}{\partial x_{j}}=-\rho \frac{\partial u_{j}}{\partial x_{j}}=\frac{\rho}{\operatorname{Re} S c} \frac{\partial}{\partial x_{j}}\left(\frac{1}{\rho} \frac{\partial \rho}{\partial x_{j}}\right) \\
\frac{\partial \rho u_{i}}{\partial t}+\frac{\partial \rho u_{i} u_{j}}{\partial x_{j}}=-\frac{\partial p}{\partial x_{i}}+\frac{1}{\operatorname{Re}} \frac{\partial \tau_{i j}}{\partial x_{j}}-\frac{\rho \delta_{i 3}}{F r^{2}}
\end{array} \\
& \tau_{i j}=\frac{\partial u_{i}}{\partial x_{j}}+\frac{\partial u_{j}}{\partial x_{i}}-\frac{2}{3} \delta_{i j} \frac{\partial u_{k}}{\partial x_{k}} \\
& \frac{\partial \rho Y}{\partial t}+\frac{\partial}{\partial x_{j}}\left(\rho u_{j} Y\right)=\frac{1}{\operatorname{Re} S c}+\frac{\partial}{\partial x_{j}}\left(\rho \frac{\partial Y}{\partial x_{j}}\right) \\
& X=\frac{\rho-\rho_{1}}{\rho_{2}-\rho_{1}} \\
& \rho Y=\rho_{2} X \\
& \rho=\text { density } \\
& \mathrm{u}_{\mathrm{i}}=\text { velocity vector } \\
& \mathrm{p}=\text { pressure } \\
& \tau_{i j}=\text { viscous stress tensor } \\
& Y=\text { heavy fluid mass-fraction } \\
& X=\text { heavy fluid mole-fraction } \\
& \rho_{1}=\text { density of light fluid } \\
& \rho_{2}=\text { density of heavy fluid } \\
& \operatorname{Re}=\rho_{1} I U / \mu=(2 \pi / / \mathrm{N})^{-4 / 3} \\
& \mathrm{Sc}=\mu / \rho_{1} \mathrm{D}=1 \\
& \mathrm{Fr}=\mathrm{U} /(\mathrm{g} l)^{1 / 2}=1 \\
& \mathrm{~N}=\text { number of } \mathrm{x} \text { grid points } \\
& l=\text { scaling length } \\
& \mathrm{U}=\text { scaling velocity } \\
& \mathrm{g}=\text { acceleration }
\end{aligned}
$$




\section{Numerical method}

- Spatial derivatives:

- Spectral in $\mathrm{x}$ and $\mathrm{y}$

- Eighth-order compact in z

- Time integration of density equation:

-3rd-order, Adams-Bashforth-Moulton method

- Time integration of momentum equation:

- (1) account for advection and diffusion

- (2) solve Poisson equation for pressure

- (3) add pressure and acceleration effects

- Boundary conditions:

- periodic in $\mathrm{x}$ and $\mathrm{y}$

- walls in z (Neumann pressure, no through flow) 


\section{Initial conditions}

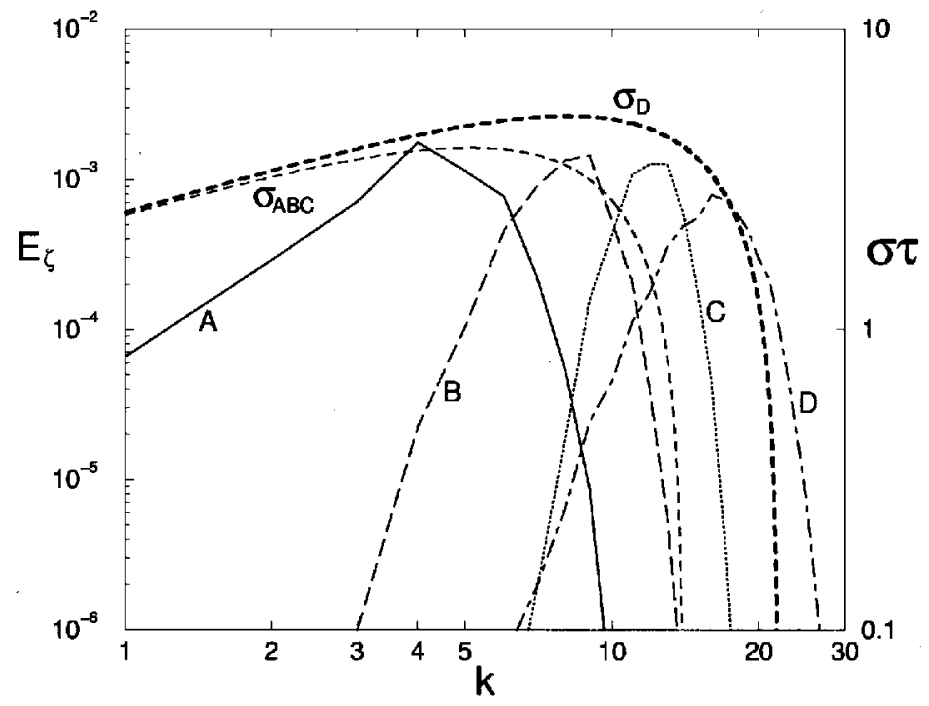

- $E_{\zeta}(k)=$ spectrum of initial interfacial perturbations

- $k=2-D(x-y)$ wavenumber

- $\sigma \tau=$ nondimensional growth rate from Linear Stability Theory (LST)

- High wavenumber perturbations are initially damped by diffusion 
Time-evolution of R-T instability for DNS Case C

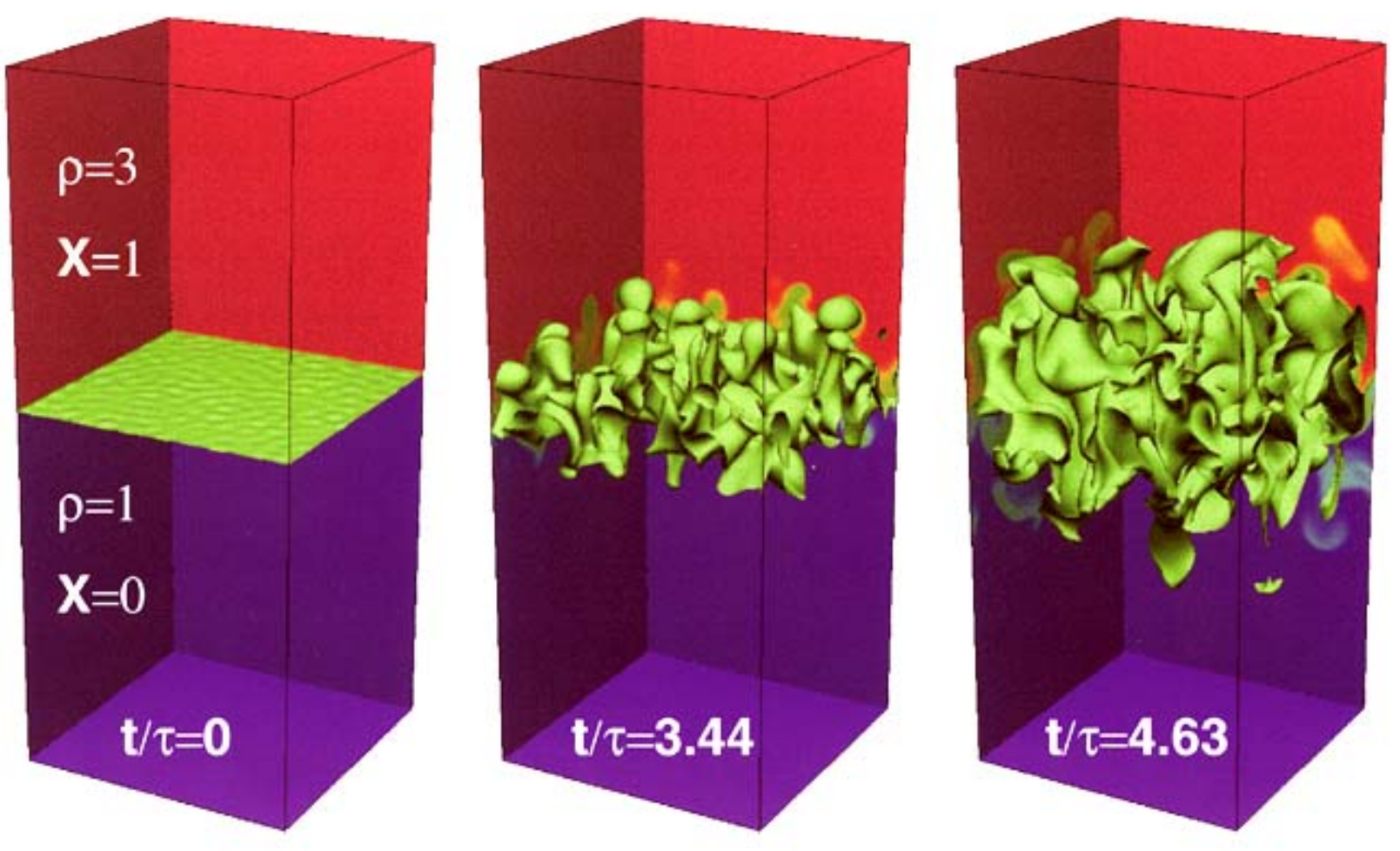




\section{Time-evolution of horizontally-averaged mole- fraction profiles for Case C}
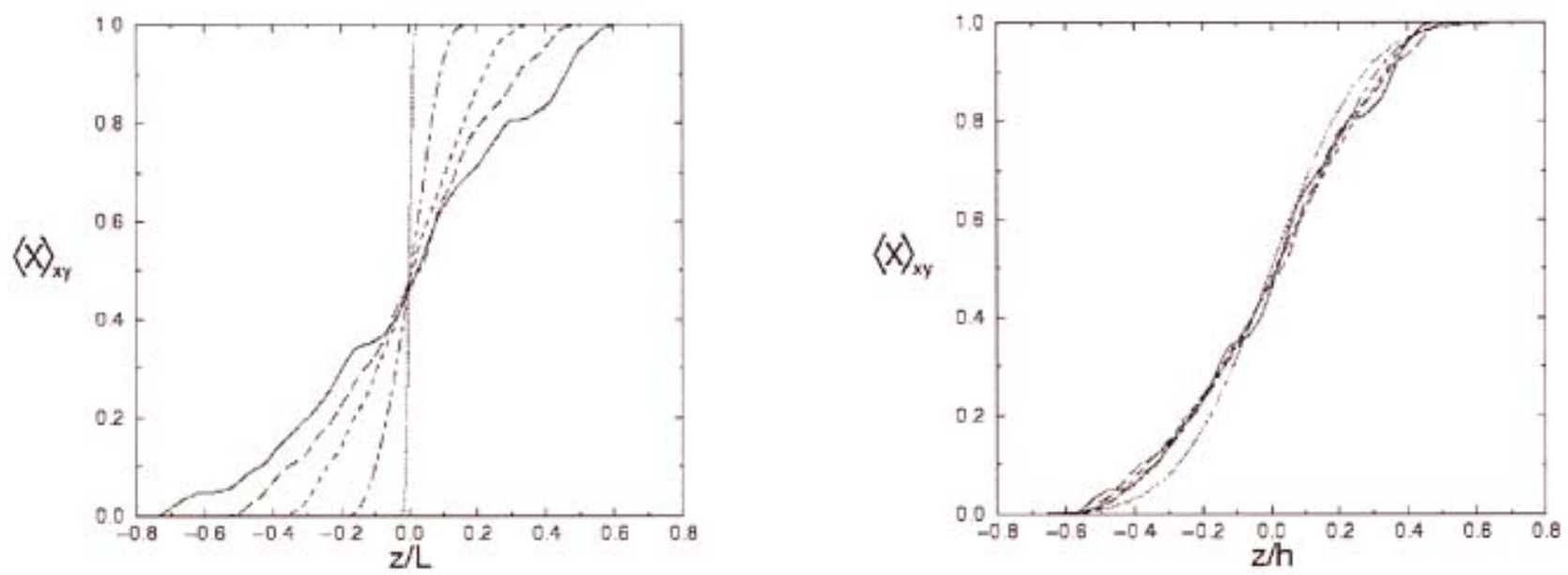

Dotted line: $t / \tau=0.0$, Short-long dashed line: $t / \tau=2.26$, Short dashed line: $t / \tau=3.40$, Long dashed line: $t / \tau=3.95$, Solid line: $t / \tau=4.52$

- Mix height $h$ defined as range of $z$ for which: $0.01<X<0.99$

- Profiles collapse when plotted vs. z/h 


\section{Growth rates of bubbles and spikes}
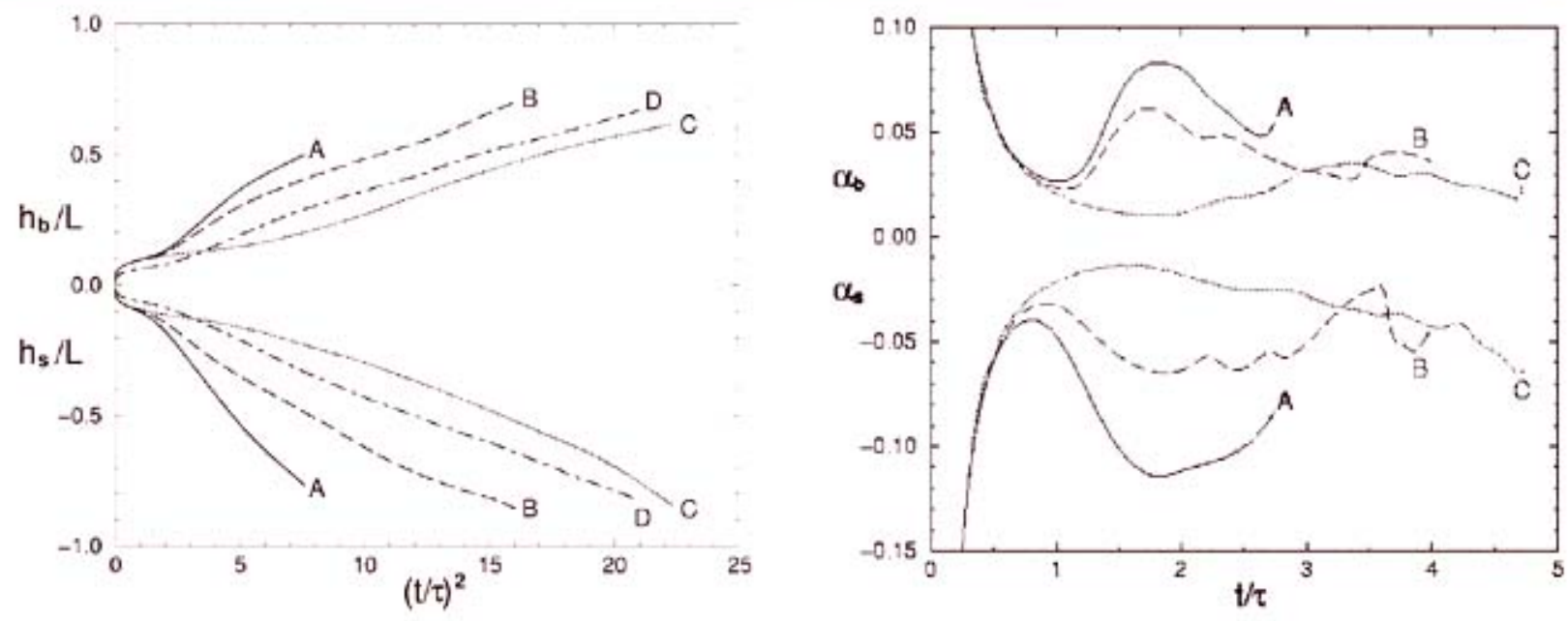

$h_{b}=$ height of bubbles, $h_{s}=$ height of spikes, i.e., $h=h_{b}-h_{s}$

$\alpha_{b}=$ growth coefficient of bubbles, $\alpha_{s}=$ growth coefficient of spikes, i.e., $h_{i}=\alpha_{i} A g t^{2}$

- Growth rates can vary by factor of 2 depending on initial conditions

- $\alpha_{b}$ and $\alpha_{s}$ do not approach constant values 


\section{Growth of mixing layer}

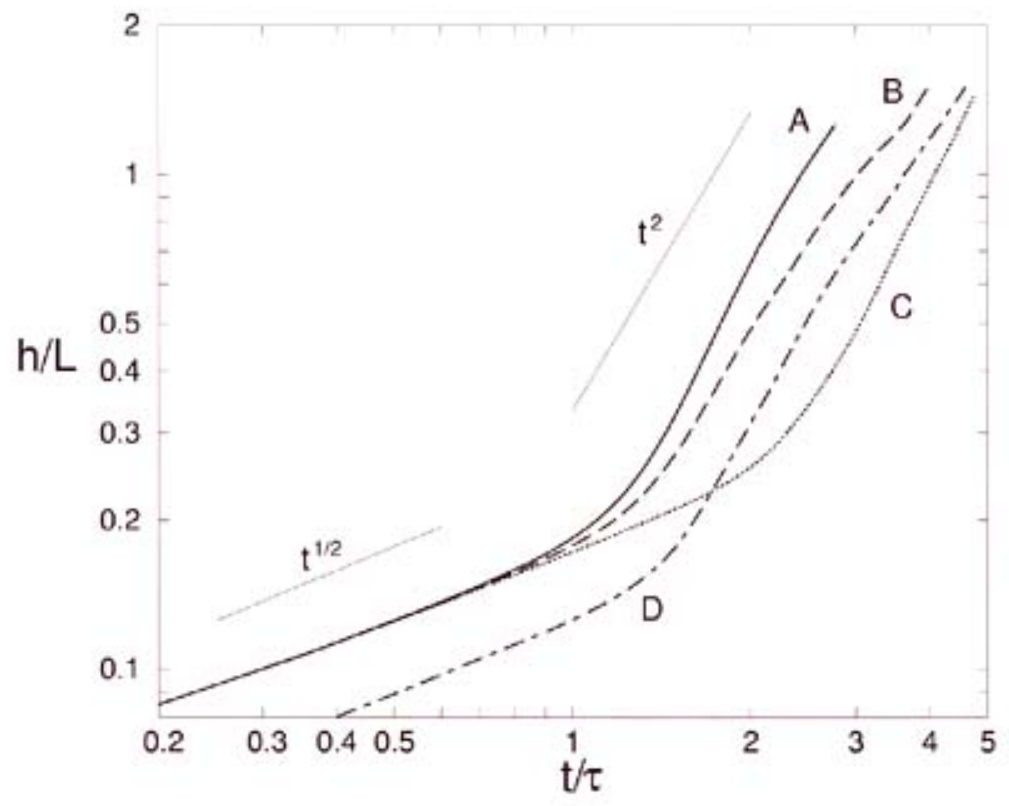

$\mathrm{h}=$ height of mixing layer

$\mathrm{L}=2 \pi l=$ width of flow domain

$\mathrm{t}=$ time

$\tau=(\mathrm{L} / \mathrm{Ag})^{1 / 2}$

$A=$ Atwood number

$\mathrm{g}=$ gravity

Early growth is diffusive, i.e., $\mathrm{t}^{1 / 2}$; later, growth $\propto \mathrm{t}^{2}$ 


\section{Bubble merger from initial perturbations}

\section{A \\ B \\ C}
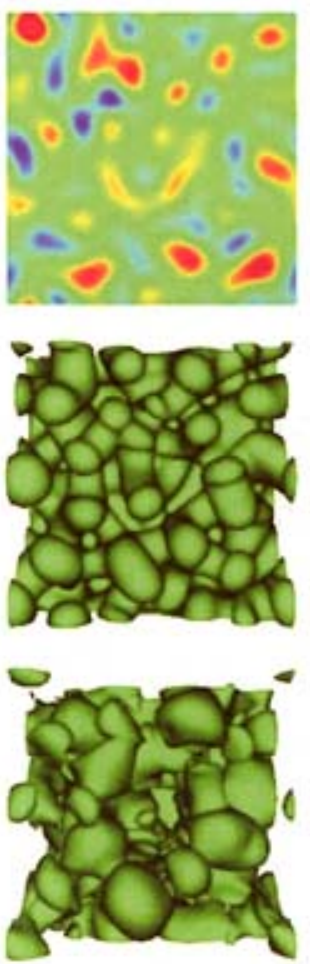
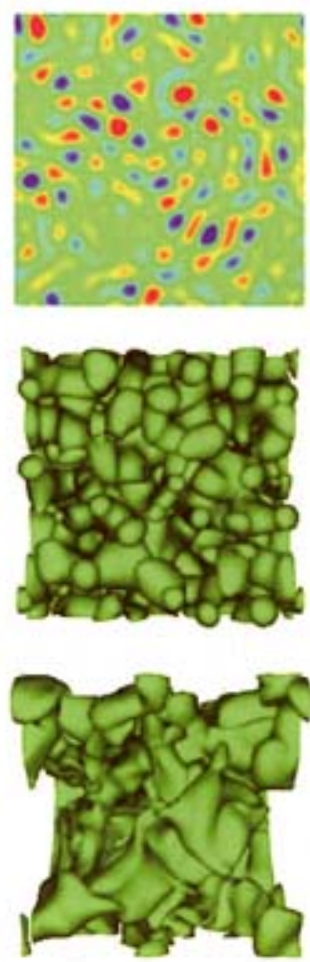
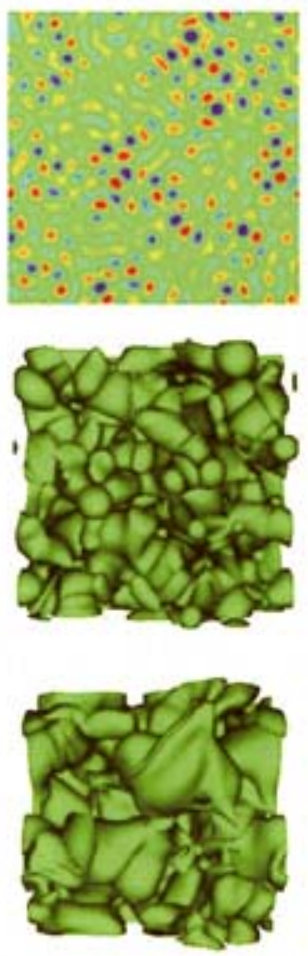

- Top Row: Initial perturbations at $z=0$, blue: $X=0.35$, green: $X=$ 0.5 and red: $X=0.65$

- Middle Row: $X=0.5$ isosurface when $h / L=0.6$

- Bottom Row: $X=0.5$ isosurface when $h / L=1.25$

- Case A: bubbles rise more or less independently

- Case B: bubbles begin to merge when $0.6<\mathrm{h} / \mathrm{L}<1.25$

- Case C: significant bubble merger for both $\mathrm{h} / \mathrm{L}=\mathbf{0 . 6}$ and $\mathrm{h} / \mathrm{L}=1.25$ 


\section{Outer-scale Reynolds numbers}
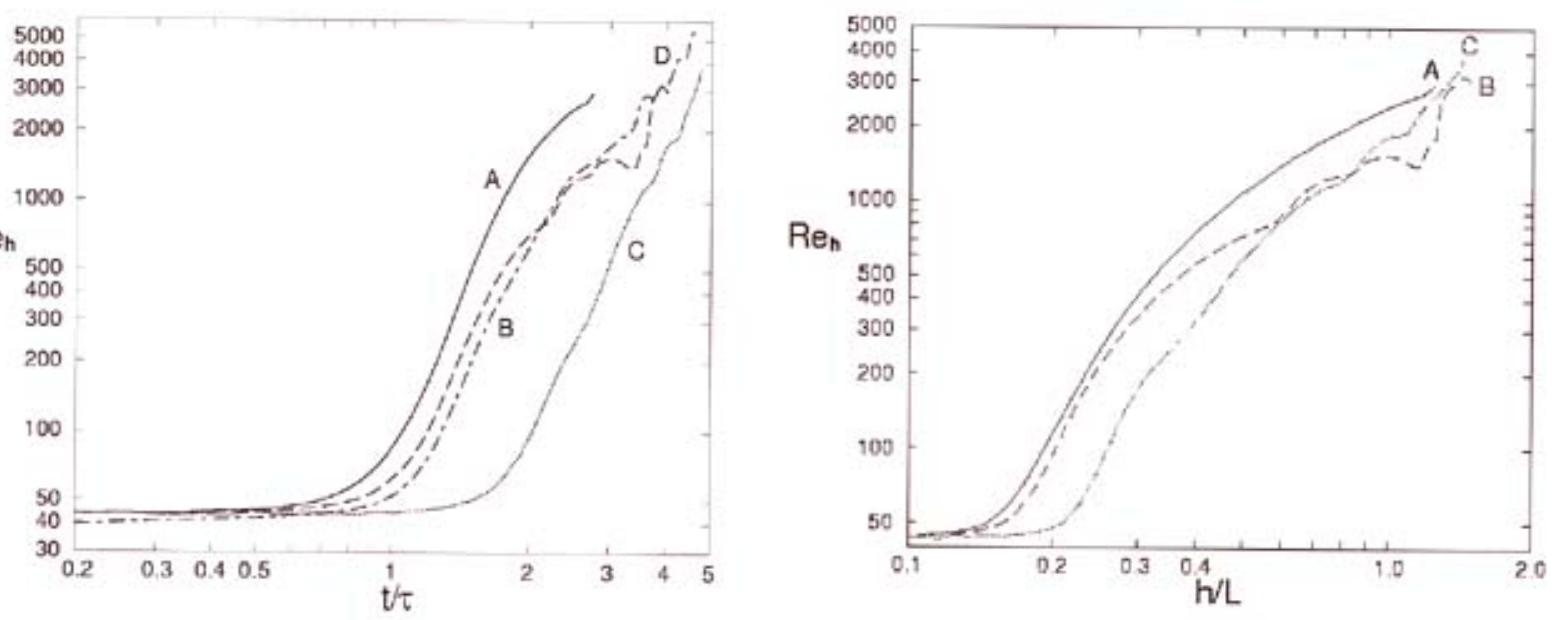

$\operatorname{Re}_{\mathrm{h}}=\bar{\rho} \mathrm{hh} / \mu$, where $\bar{\rho}=\left(\rho_{1}+\rho_{2}\right) / 2$

- Cases reach outer-scale Reynolds numbers of 3000-5500.

- Improved collapse achieved by replacing $\mathrm{t}$ with $\mathrm{h}$ as progress variable. 


\section{Mixing}

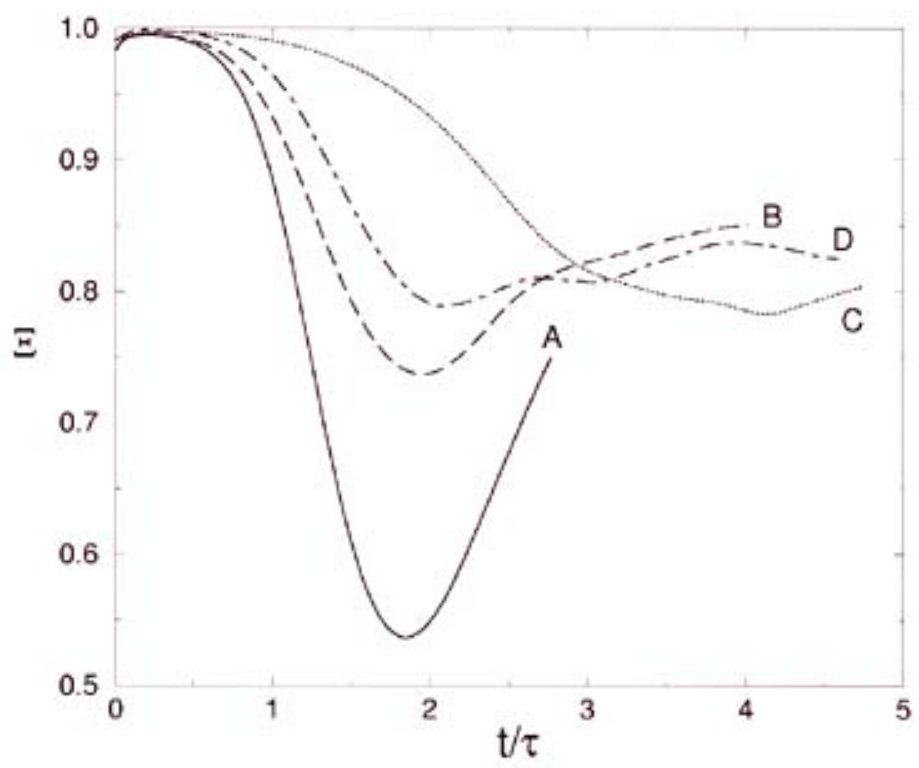

- Considering an equilibrium chemical reaction between fluids (stoichiometric mole ratio: $\left.X_{s}=1 / 2\right), \Xi$ is the ratio of maximum possible product to actual product.

- $\Xi=1$ corresponds to complete mixing within layer, whereas $\Xi=0$ indicates no mixing (immiscible case).

- Rates of mixing and entrainment do not come into balance within timespan of simulations. 


\section{Spectra}

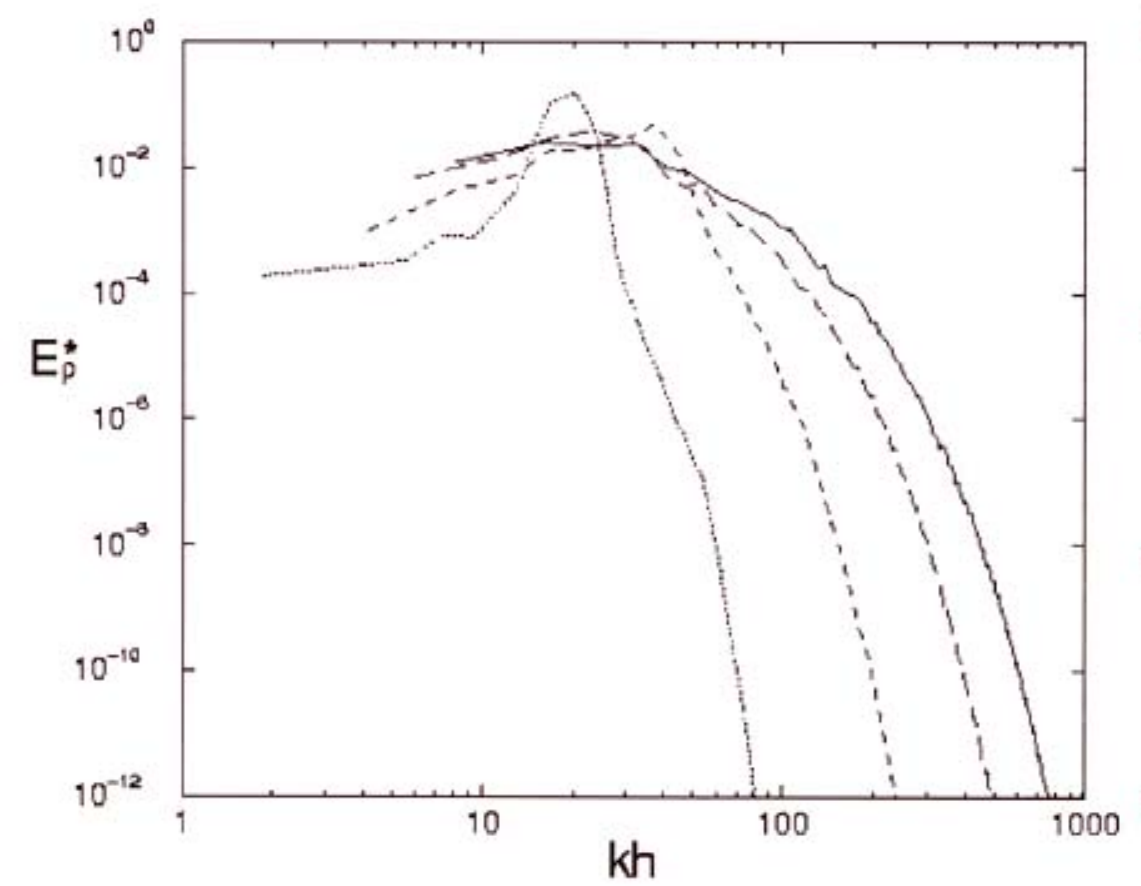

- Normalized 2-D density spectra in $\mathrm{z}=0$ plane for Case C. Lines of increasing solidity denote increasing time.

- Beginning of a collapse at low wavenumber is evident towards end of simulation.

- Low-(kh) collapse indicates large-scale structures scale with mixing zone height.

- Full spectrum is still evolving, however, due to low Reynolds number. 


\section{Taylor microscales}
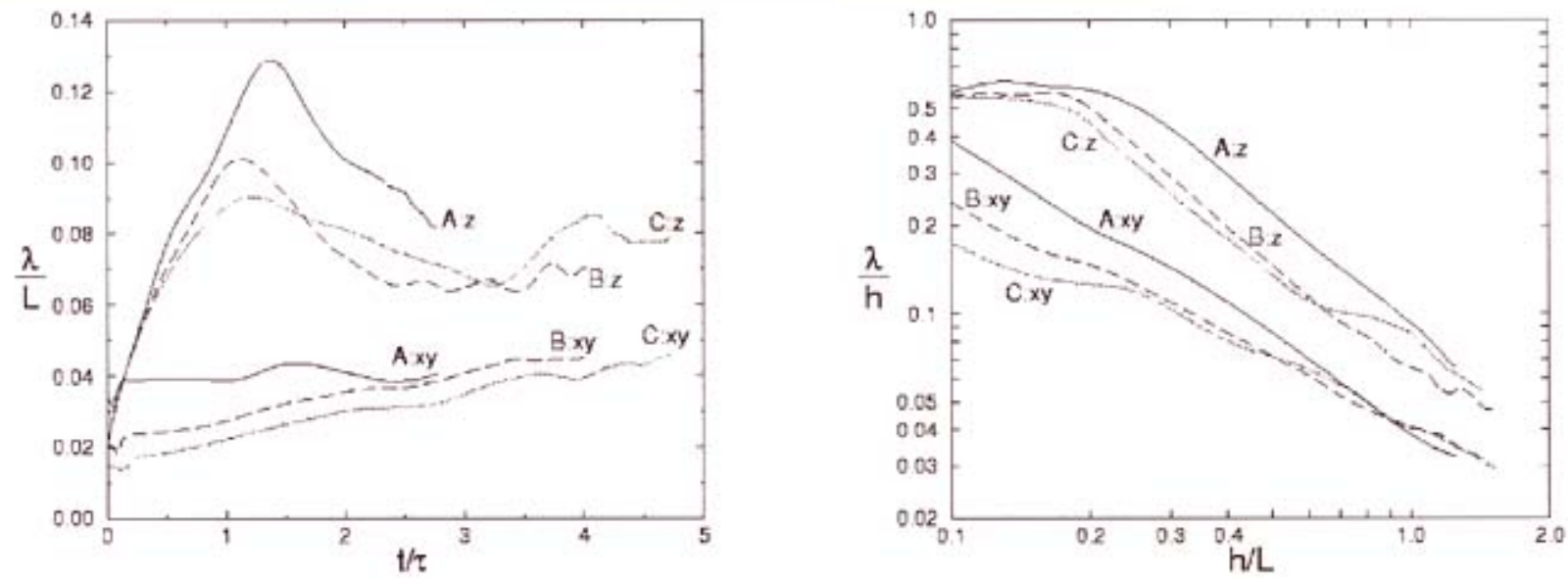

- Initial values for horizontal microscales are ranked in order of peaks of initial perturbation spectra.

- Vertical microscales grow much faster than horizontal scales due to directed body force.

- Flows exhibit strong anisotropy between horizontal and vertical microscales for duration of simulations. 


\section{Taylor Reynolds numbers}
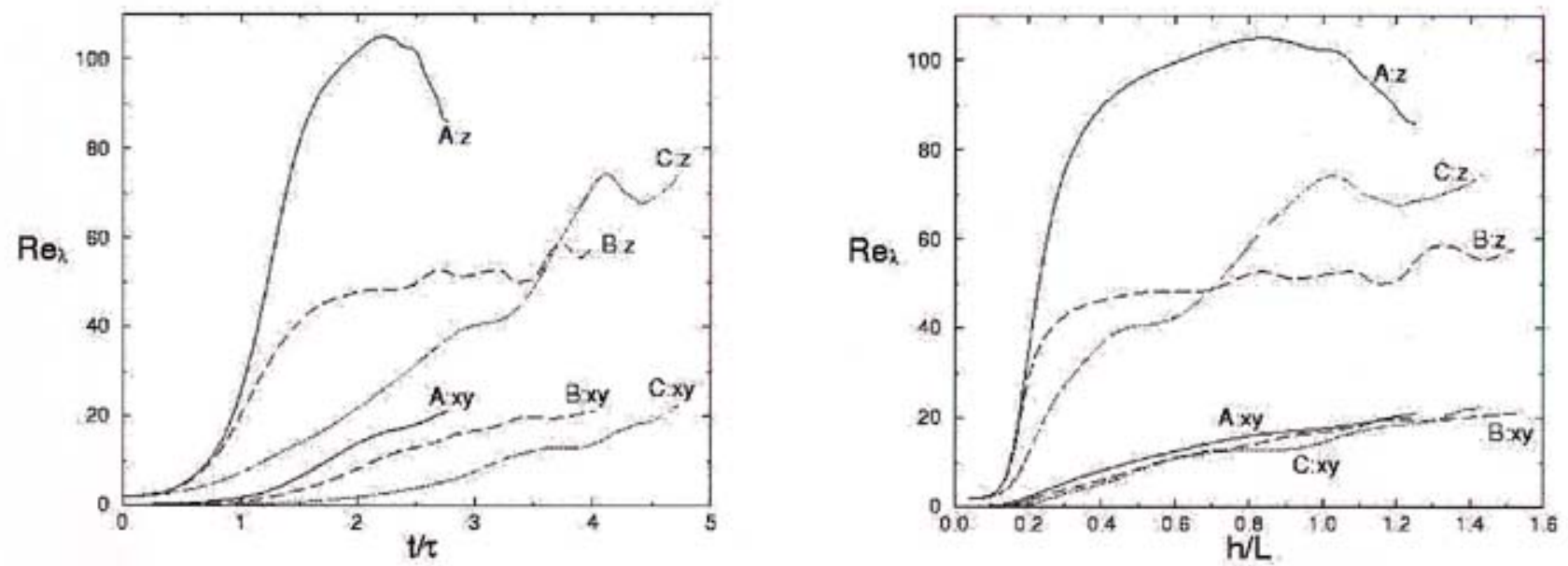

- Anisotropy in microscales also manifest in Taylor Reynolds numbers

- Vertical Taylor Reynolds numbers extremely sensitive to initial conditions with no collapse for either independent variable

- Near perfect collapse of horizontal Reynolds numbers when plotted vs. $h / L$ 


\section{Conclusions}

- Growth of R-T mixing layer depends sensitively on initial conditions.

- Ranking of growth rates is in same order as suggested by Linear Stability Theory.

- Mixing exhibits greater sensitivity to initial conditions than growth rates.

- Density-fluctuation spectra in interior of mixing zone indicate that imprint of the initial perturbation spectra is eventually lost; furthermore, the spectra exhibit good collapse at low wavenumbers by late simulation time.

- Taylor microscales and Reynolds numbers are also sensitive to initial conditions, nonmonotonic in their temporal evolution and reflect considerable and persistant anisotropy in the flow.

- Mixing zone height is a better progress variable than time, i.e., improved collapse occurs if statistics are compared at the same value of $h$ and if spatial scales are nondimensionalized in terms of $h$. 


\title{
CALE simulation of Richtmyer- Meshkov instability experiments at high Mach number
}

\author{
A. Miles ${ }^{1,2}$, J. Edwards' ${ }^{1}$, G. Glendinning ${ }^{1}$ \\ ${ }^{1}$ Lawrence Livermore National Laboratory \\ ${ }^{2}$ University of Maryland at College Park
}

8th International Workshop on the

Physics of Compressible Turbulent Mixing

Pasadena, CA

Dec 9-14, 2001 


\section{Experiment:}

- Omega laser used to generate a strong shock $(\mathrm{M} \approx 10)$ in a plastic pusher

- Shock drives a corrugated plastic - foam interface $(\mathrm{ka}=0.92)$ at near constant velocity

- Richtmyer-Meshkov instability produces growth of the initial perturbation

Simulation:

- C-based Arbitrary Lagrangian-Eulerian (CALE) used to simulate the experiments

-Discrepancies between experiment and simulation are investigated 
This experiment uses an $11 \mathrm{~ns}$ laser drive to create a steady shock incident on a modulated interface

\section{Drive beams}

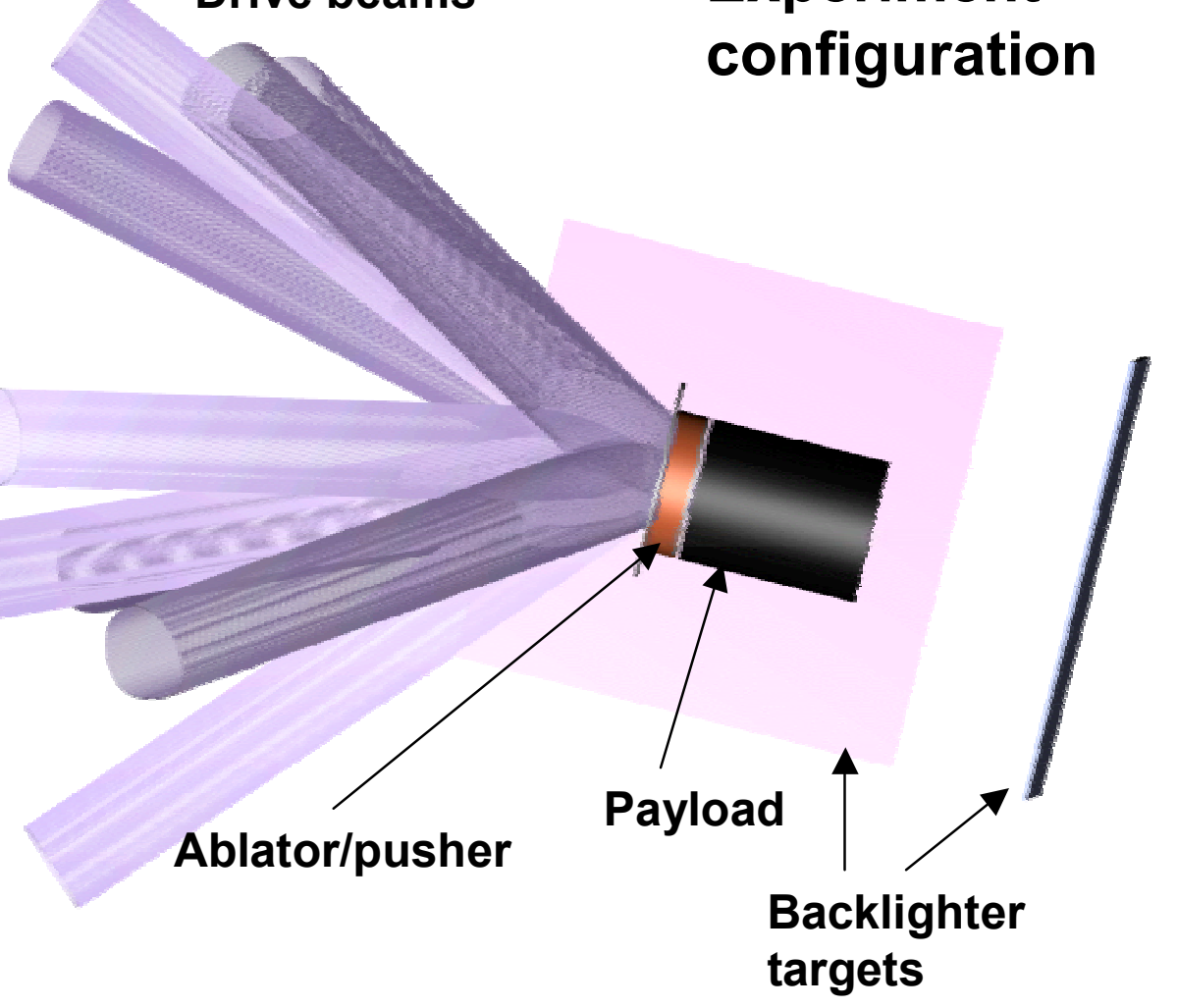

Target package

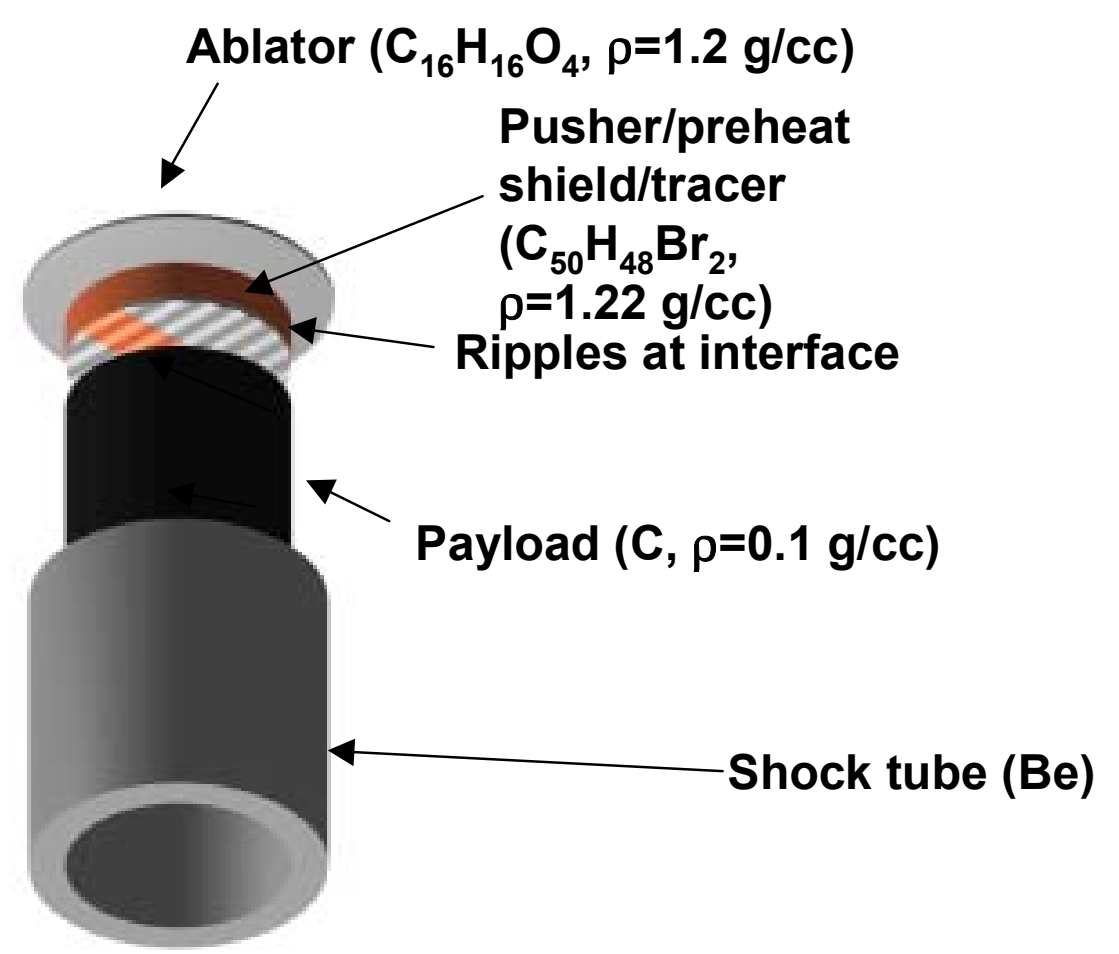

- Backlighting is done on two axes, along target axis and perpendicular to modulations

- Target package is encased in a beryllium shock tube

- The incident shock and interface velocities are constant within $\pm 5 \%$ RMS

- The shock is incident on a 12:1 density contrast

- The interface position is measured by side-on radiography 


\section{Computational Parameters (CALE)}

- $\rho_{\text {plastic }}=1.1 \mathrm{~g} / \mathrm{cc}$

- $\rho_{\text {foam }}=0.1 \mathrm{~g} / \mathrm{cc}$

- $\rho_{\text {void }}=0.001 \mathrm{~g} / \mathrm{cc}$

- $\mathrm{a}=22 \mu \mathrm{m}$

- $\lambda=150 \mu \mathrm{m}$

- Computational grid dimensions: $\lambda / 2$ x $700 \mu \mathrm{m}$

- With EOP EOS:

$-\mathrm{c}_{\text {plastic }}=1.84 \mu \mathrm{m} / \mathrm{ns}$

$-\mathrm{c}_{\text {foam }}=0.11 \mu \mathrm{m} / \mathrm{ns}$

- $\mathrm{P}_{\text {plastic }}=728$ barr

$-\mathrm{P}_{\text {foam }}<1$ barr 


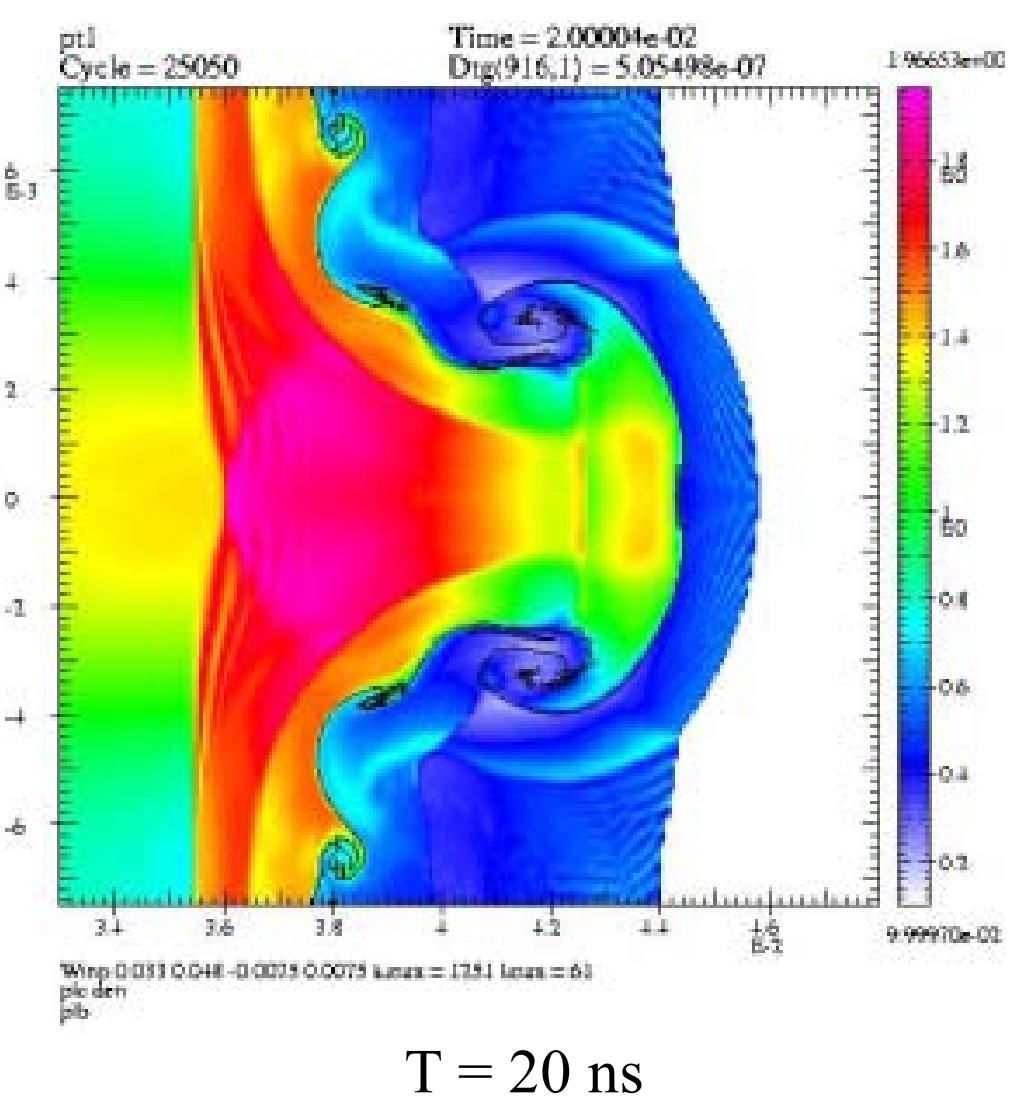

Discrepancies between experiment and simulation:

1. Post-shock amplitude $\mathrm{a}^{*}$ is much too large in simulation - $9 \mu \mathrm{m}$ instead of $5 \mu \mathrm{m}$.

2. Growth rate from $14-18 \mathrm{~ns}$ is higher in simulation than experiment.

3. The standoff distance of the shock from the interface is larger in the simulation than in the experiment.
Typical side-on data at $20 \mathrm{~ns}$
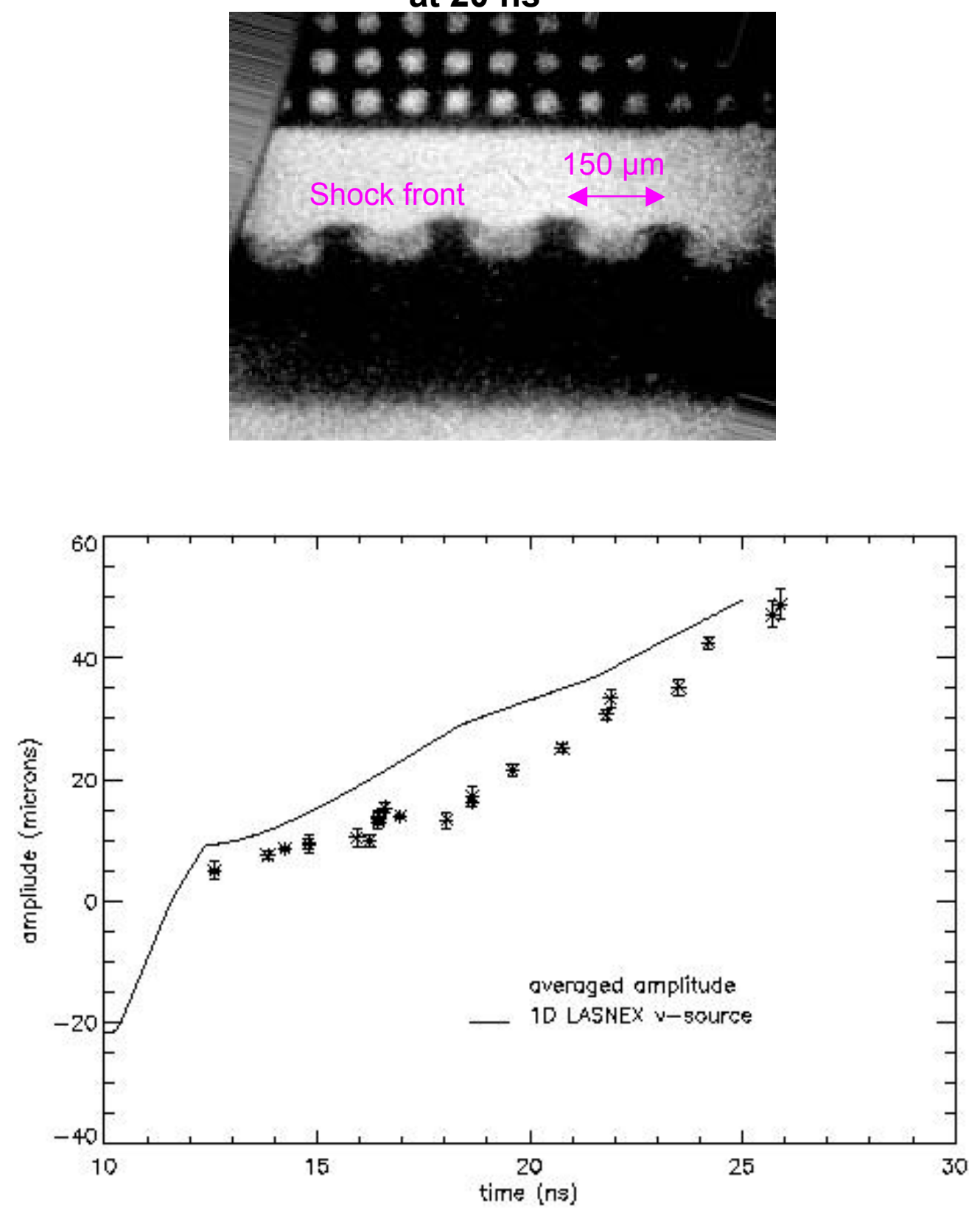


\section{Zoning}

\section{Two grid configurations used:}

- Rectangular grid

Typical parameters:

- 120 cells/wavelength

$1.25 \mu \mathrm{m} /$ cell

- Cell dimensions:

$1.25 \mu \mathrm{m} \times 0.5 \mu \mathrm{m}$

- Conforming mesh

Typical parameters:

- 120 cells/wavelength

- Plastic and foam "interface" $68 \%$ mass-matched

- $0.1 \mu \mathrm{m} /$ cell at plasticvoid interface

- $0.75 \mu \mathrm{m} /$ cell at voidfoam interface
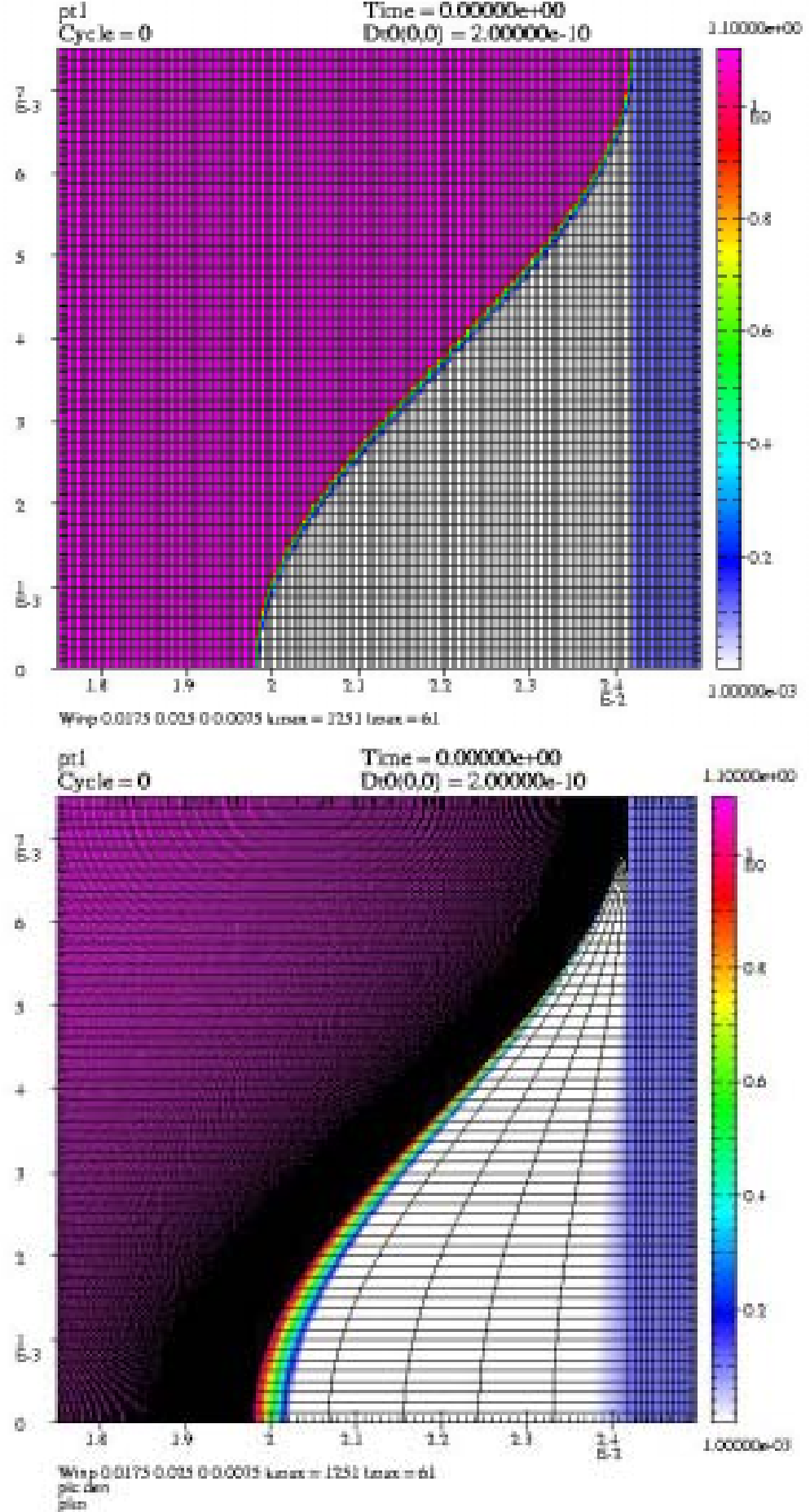


\section{Zoning Effects* I: Conforming Mesh}

Cells/wavelength $\quad$ Cell width across interface $(\mu \mathrm{m}) \quad \mathrm{a}^{*}(\mu \mathrm{m}) \quad \mathrm{a}(25 \mathrm{~ns})(\mu \mathrm{m})$

$0.1-\Delta_{\text {void }} / 5$ - 0.75
120

When the number of cells/wavelength $\leq 240$, a "dimple" forms in the center of the spike.This yields a smaller amplitude, which is defined as half the distance from bubble tip to spike tip. A further increase of cells/wavelength to 480 looks identical to the 240 case, suggesting convergence by 240 . 


\section{Zoning Effects II: Rectangular Grid}

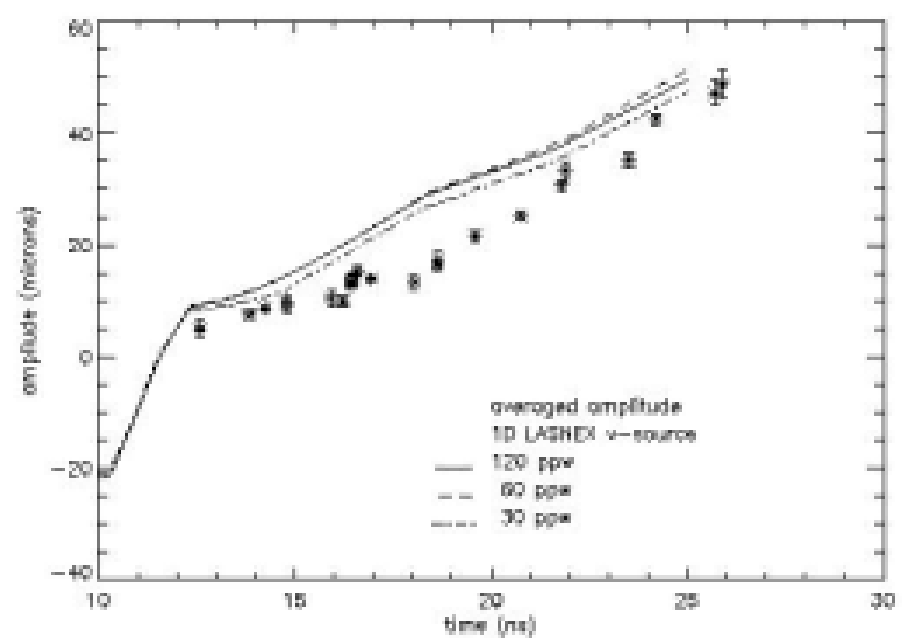

Cells/wavelength

Cell dimensions $(\mu \mathrm{m})$

120

$1.25 \times 0.5$

60

$2.50 \times 1.0$

30

$5.00 \times 2.0$

Further increase of cells/wavelength to 240 looks identical to the 120 case, suggesting convergence by 120 .

Beyond $15 \mathrm{~ns}$, the converged conforming and rectangular zoning schemes show virtually identical growth rates. The post shock amplitude $\mathrm{a}^{*}$ is about $1.5 \mu \mathrm{m}$ lower here than in the converged conforming mesh case, and the growth rate at early times is slightly smaller. In what follows, the above grid is used unless otherwise specified.

A one-dimensional study of the effects of cell size for zones mass-matched across the plastic-foam interface shows virtually no change in interface position $z_{i}(t)$ when the cell size in the plastic at the plastic-void interface is varied from $0.1-0.001 \mu \mathrm{m}$. Over this range, $z_{i}(t)$ is also shown to be insensitive to whether CALE is run in pure lagrangian mode or lagrangian-eulerian hybrid mode. 


\section{Comparison of simulations of Aleshin experiment}
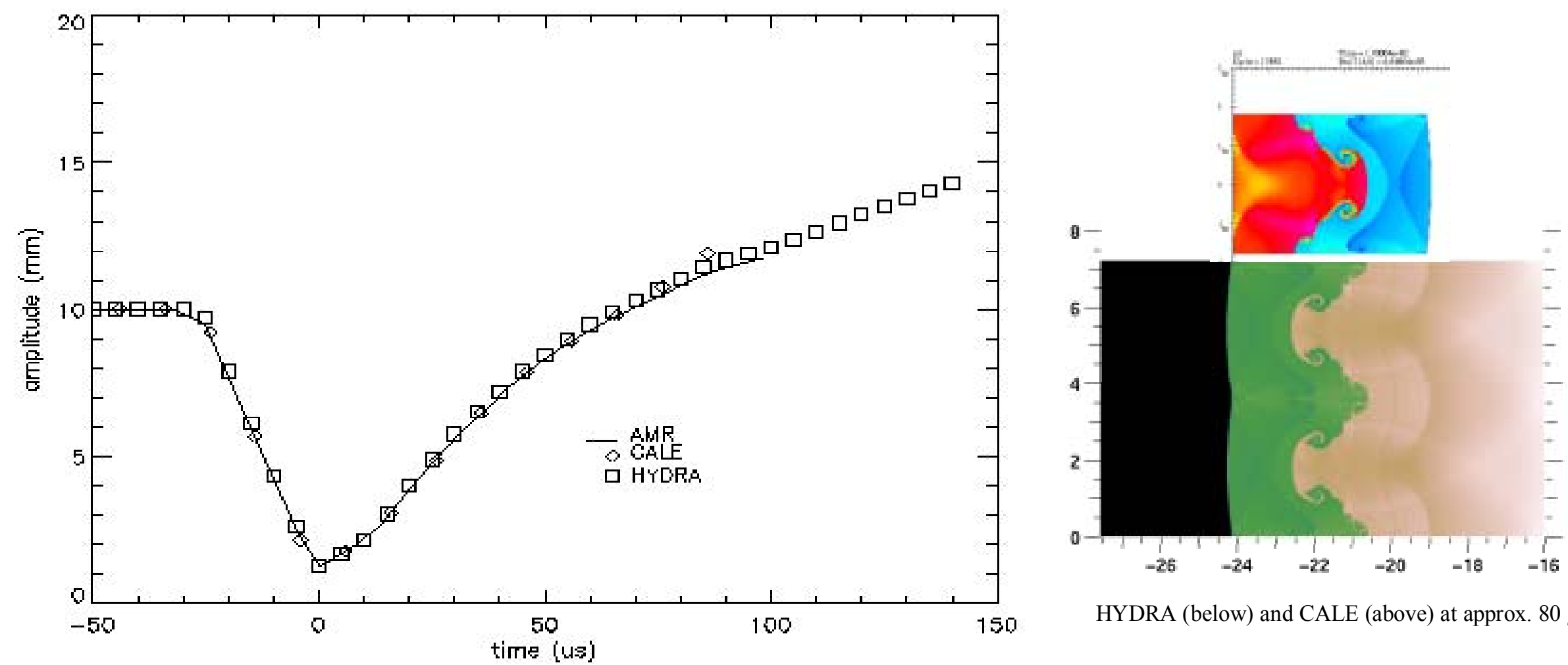

HYDRA (below) and CALE (above) at approx. $80 \mu \mathrm{s}$

Gas-tube experiment with $\mathrm{M}=4.5$ shock across Xe-Ar interface with $\rho_{\mathrm{Xe}}=2.95 \mathrm{~g} / \mathrm{l}$,

$\rho_{\mathrm{Ar}}=2.95 \mathrm{~g} / \mathrm{l}\left(\rho_{\mathrm{Xe}} / \rho_{\mathrm{Ar}}=0.30\right), \mathrm{ak}=1.75$

- $\quad$ AMR (Greenough, 2001) and ALE (CALE and HYDRA (Weber, 2001)) simulations of the Aleshin experiment (Aleshin et al., 1996).

- The RAPTOR and HYDRA simulations include 2560 and 512 cells/wavelength, respectively. The CALE simulation was run with only 120 cells/wavelength.

- Despite differences in coarseness, the three simulations show very good agreement in their predictions of amplitude history, and all three are in good agreement with the experimental data (not shown).

- The number of cells/wavelength and the initial ratio of cell dimensions (cell width along / perpendicular to the unperturbed interface) in the above CALE simulation is 2.5 - the same as in simulations of the Omega experiments. 


\section{$\underline{\text { Modified Velocity Source I }}$}
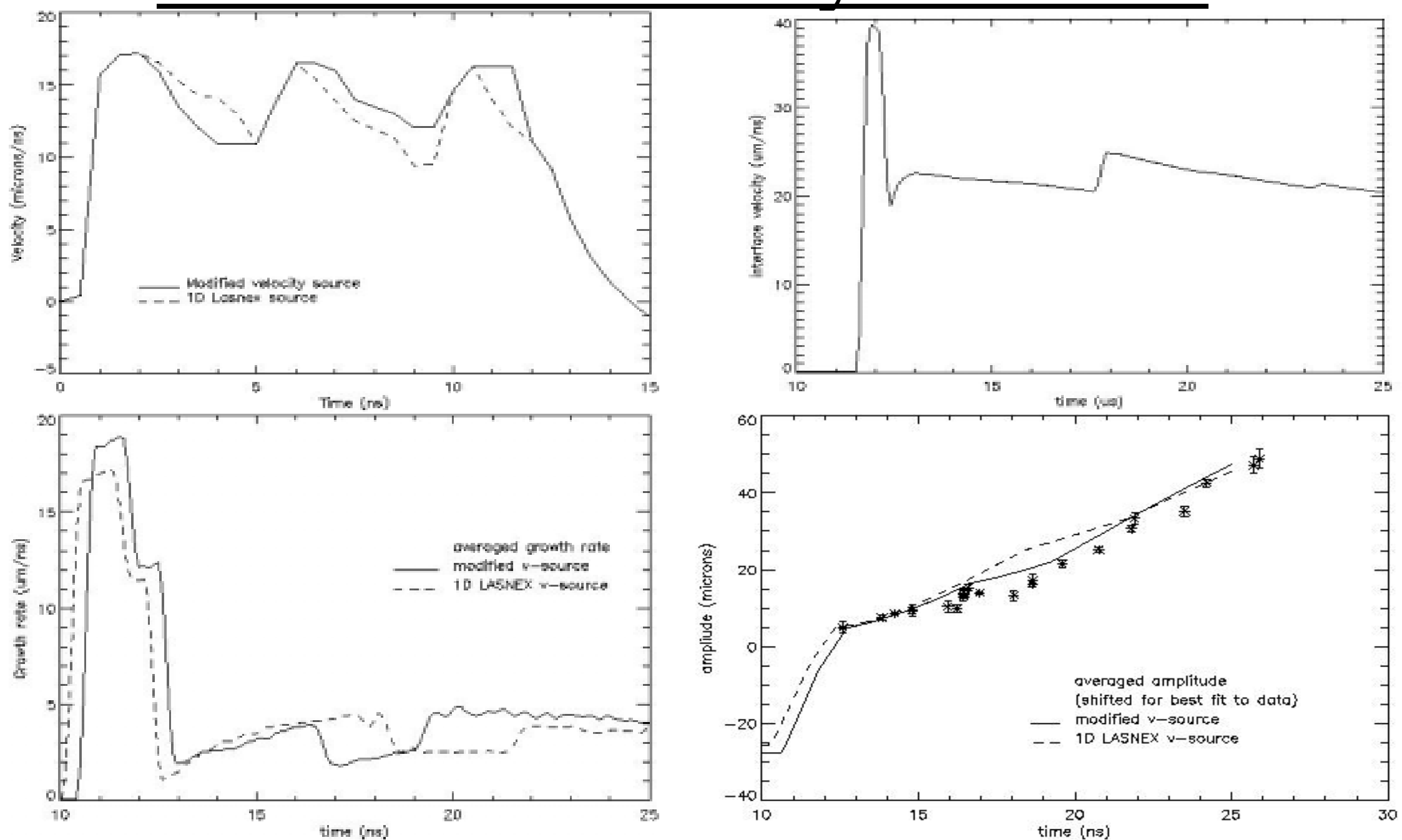

Small changes in the velocity source strengthen the second shock and decrease its arrival time. The resulting amplitude history more closely matches the experimental data.

However, the post-shock amplitude is further increased to $11 \mu \mathrm{m}$ (from $9 \mu \mathrm{m}$ ). 


\section{D LASNEX Velocity Source I}
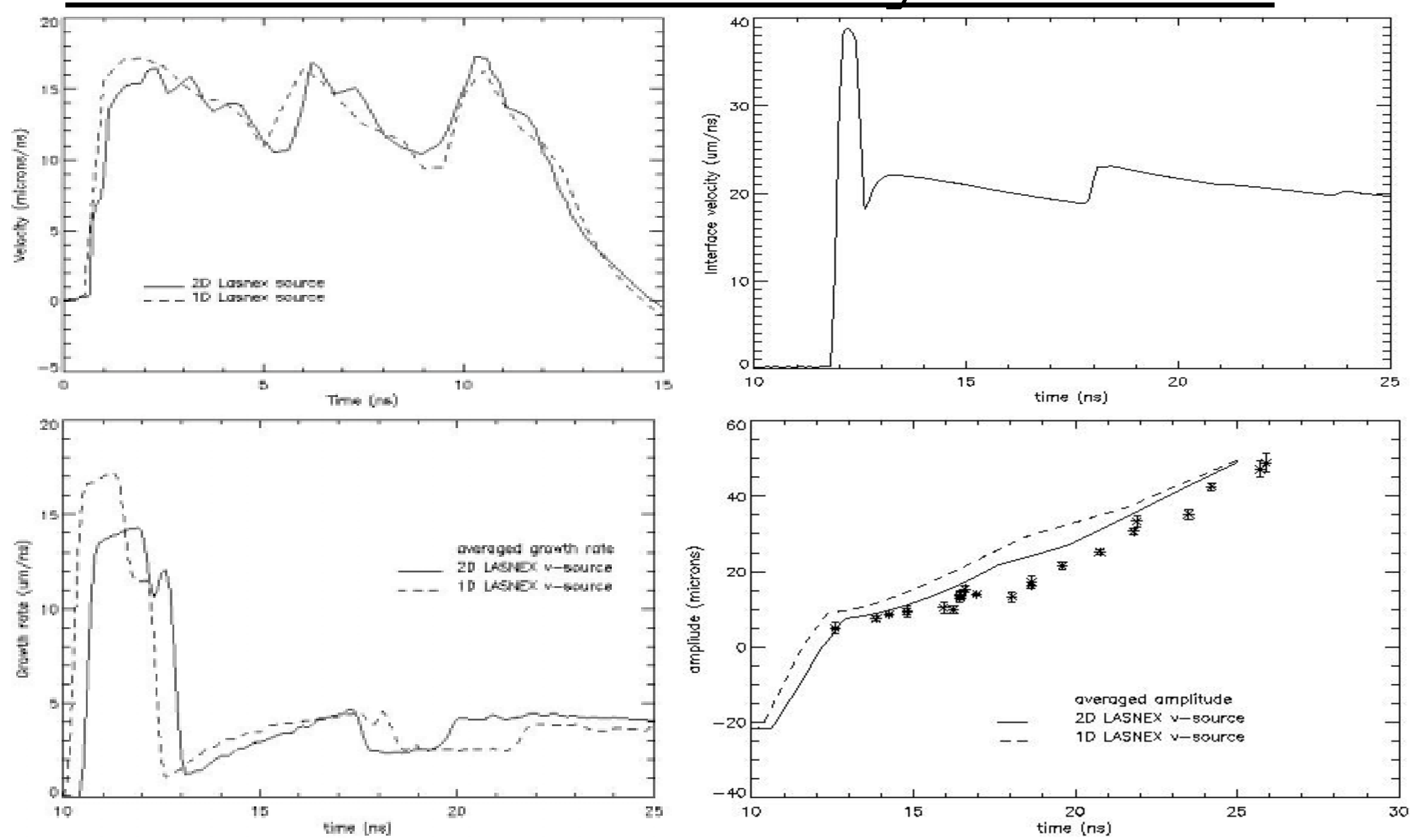

A 2D LASNEX simulation produces a velocity source that differs slightly from the 1D LASNEX prediction. The average interface velocity is increased from $20.4 \mu \mathrm{m} / \mathrm{ns}$ to $21.0 \mu \mathrm{m} / \mathrm{ns}$. The resulting amplitude history more closely matches the experimental data. Notably, the post-shock amplitude is decreased to $7.7 \mu \mathrm{m}$ (from $9 \mu \mathrm{m}$ ). 


\section{D LASNEX Velocity Source II}

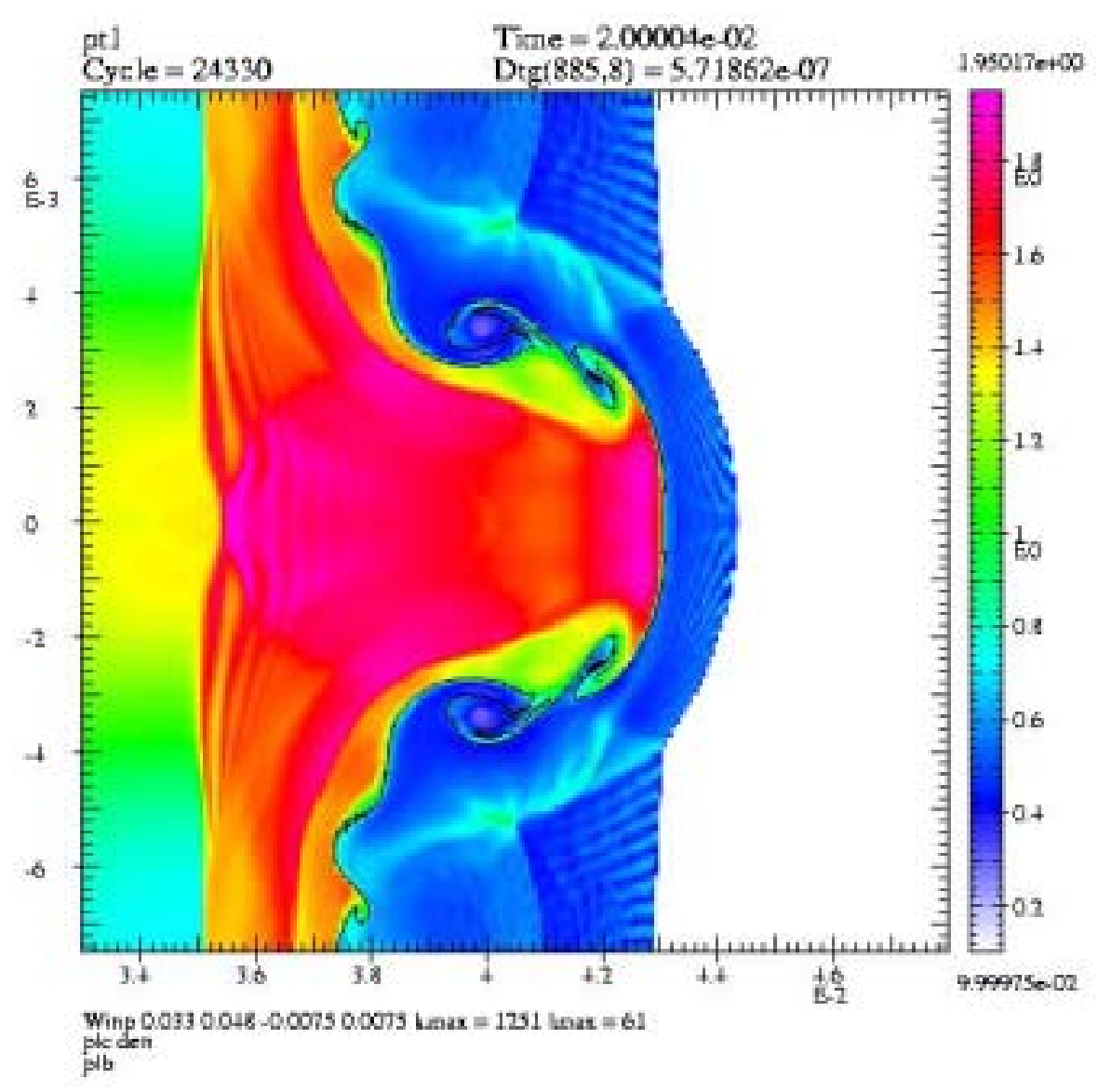

Density plot at $20 \mathrm{~ns}$.

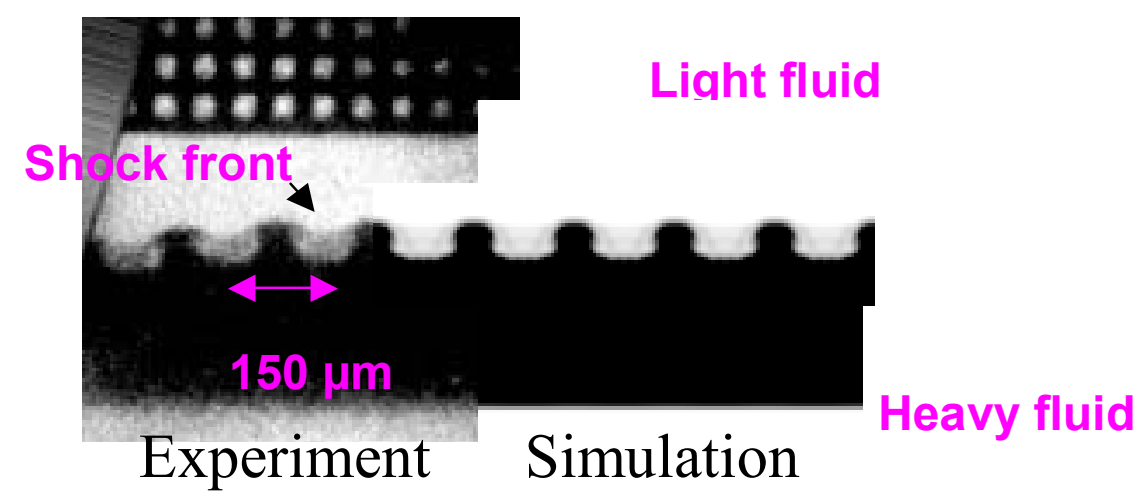

Comparison of simulated $\mathrm{x}$-ray radiograph with experimental x-ray snapshot at 20 ns shows good agreement. However, the CALE-predicted shock is flatter than in the experiment. 


\section{D LANSEX Velocity Source III}
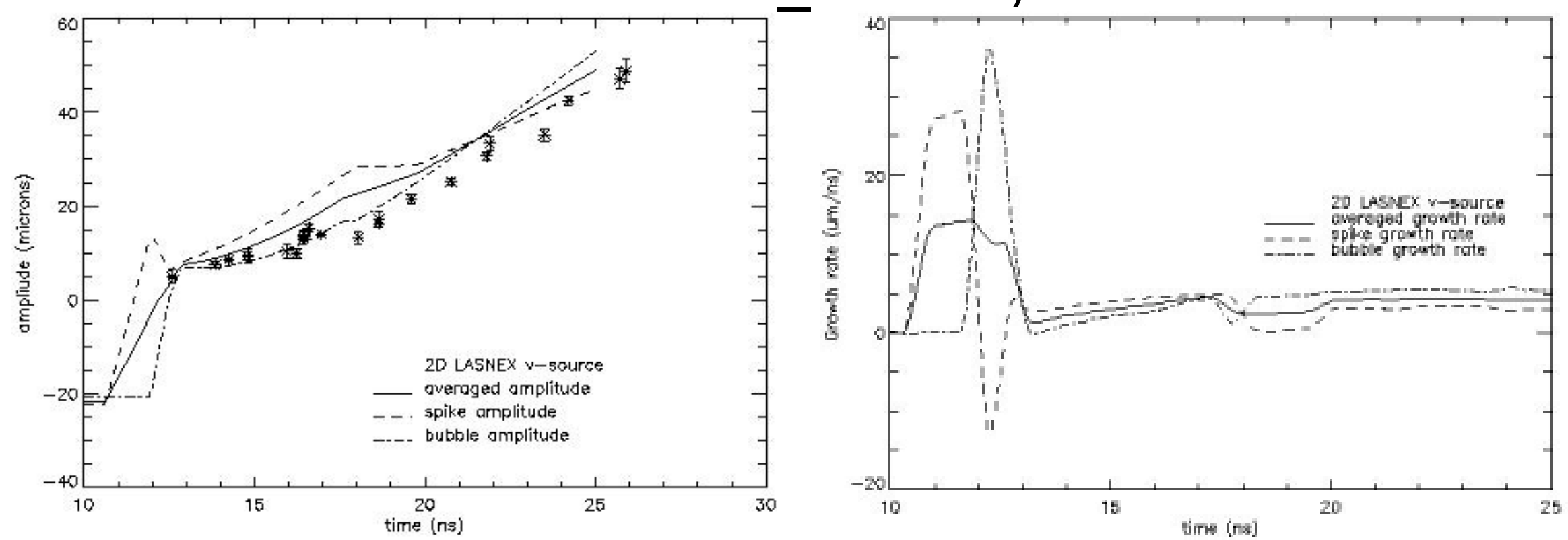

Separate spike and bubble amplitudes and growth rates. The reference timedependent interface position is obtained from a 1D simulation including a $22 \mu \mathrm{m}$ gap separating the plastic and foam regions.

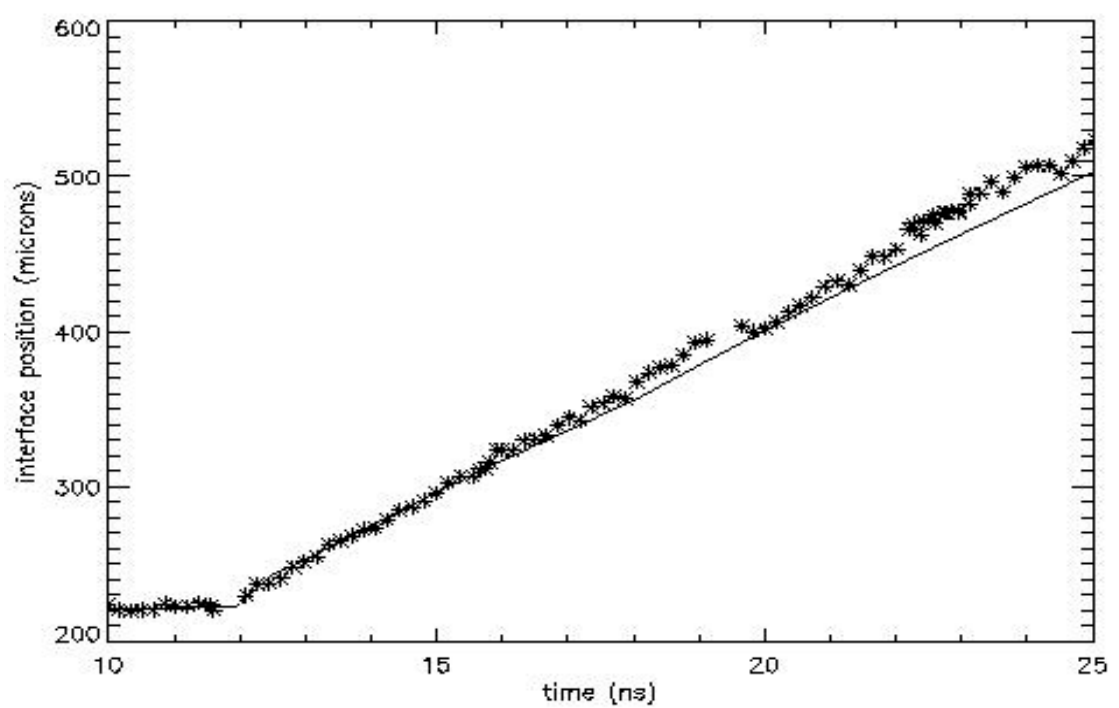

The time-dependent interface position (from 1D CALE simulation) is shown to agree well with experimental measurements at early times. After $15 \mathrm{~ns}$, however, the interface velocity is too low. 


\section{D LASNEX Velocity Source IV}

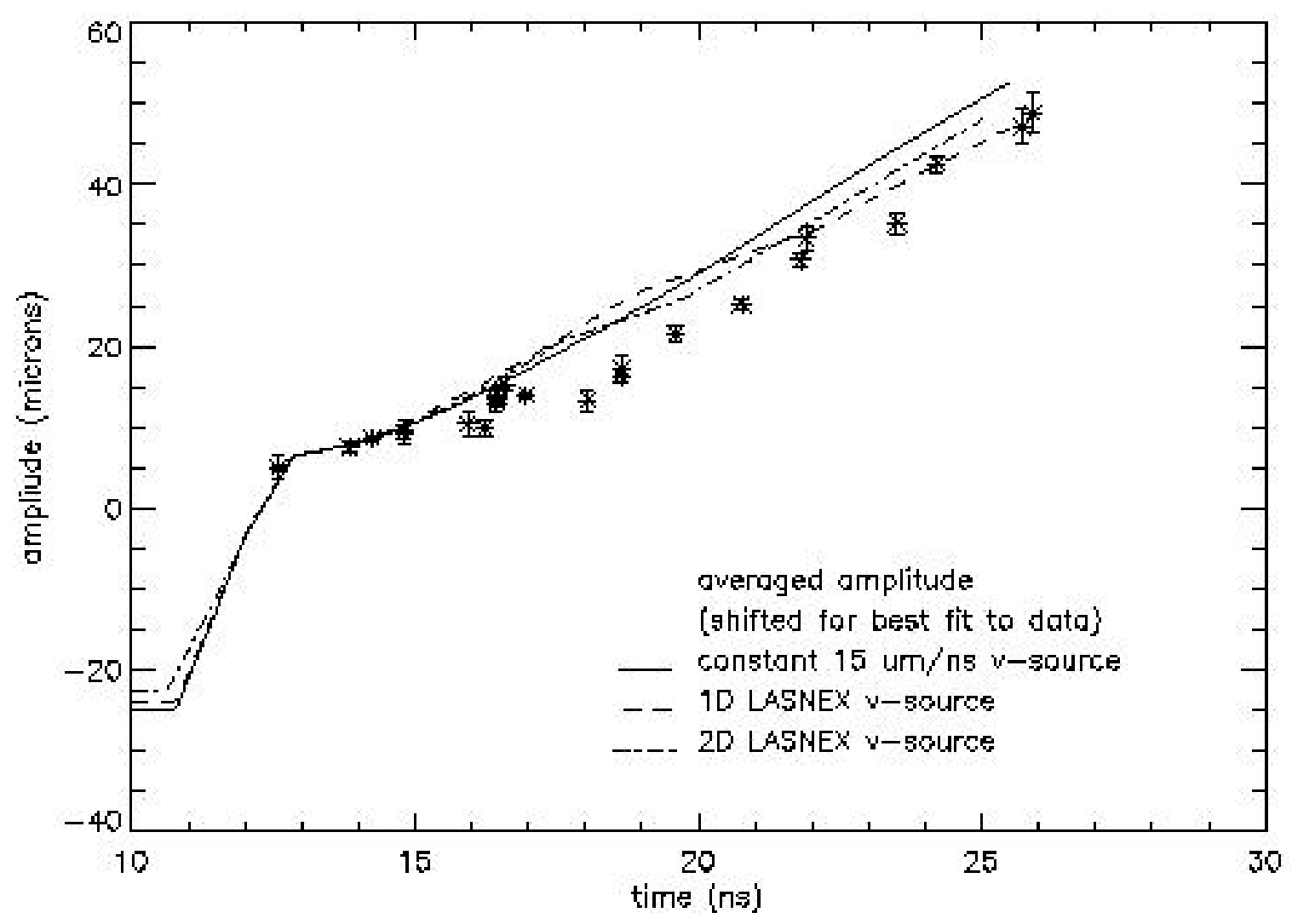

2D LASNEX: $\mathrm{t}$, a $-1.0 \mu \mathrm{m}$; 1D LASNEX: $\mathrm{t}+0.5 \mathrm{~ns}$, a $-2.5 \mu \mathrm{m}$; Flat source: $\mathrm{t}+0.5 \mathrm{~ns}, \mathrm{a}-3.5 \mu \mathrm{m}$

Aside from a shifts in time and amplitude, the constant velocity $15 \mu \mathrm{m} / \mathrm{ns}$ velocity source provides early time instability growth that agrees well with the 2D as well as 1D LASNEX v-source. 


\section{Effect of Pusher Velocity}

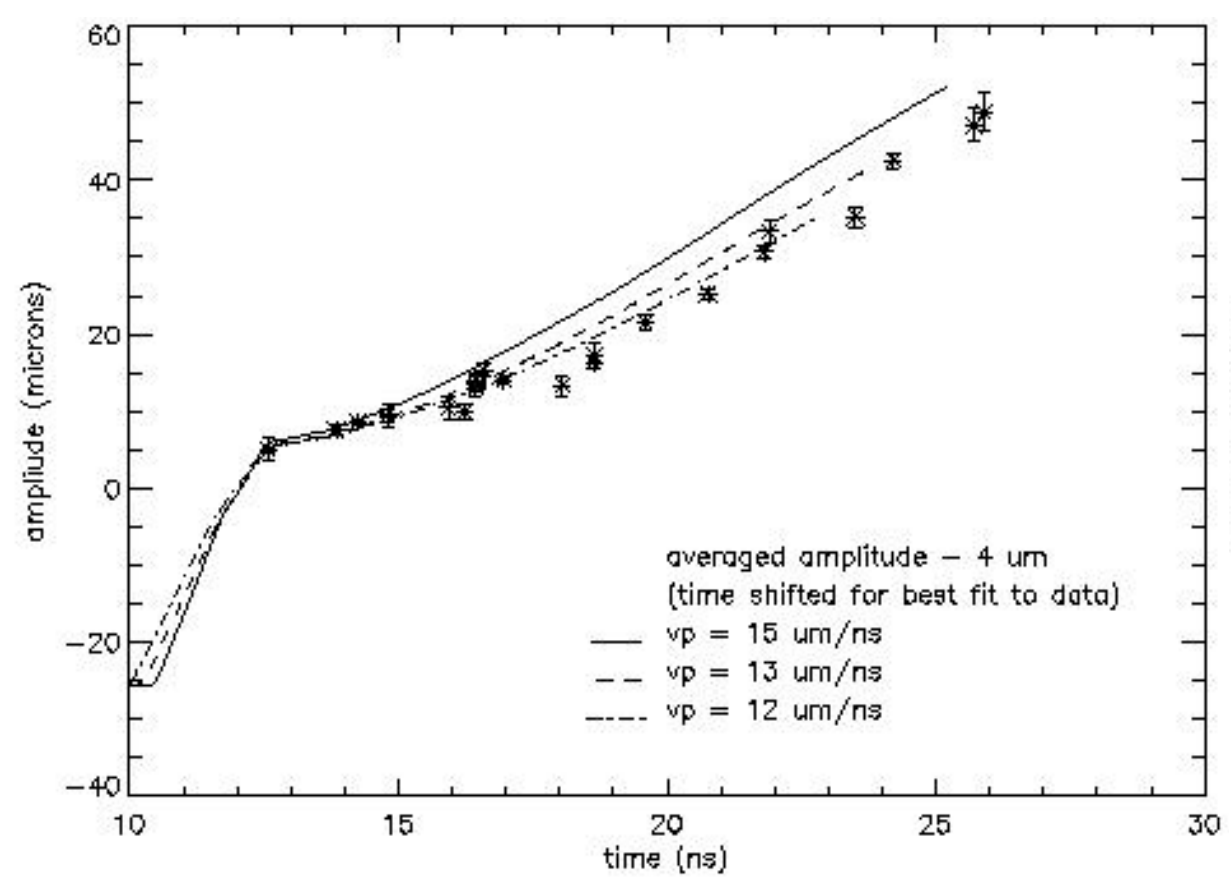

$22 \mu \mathrm{m}$ initial amplitude

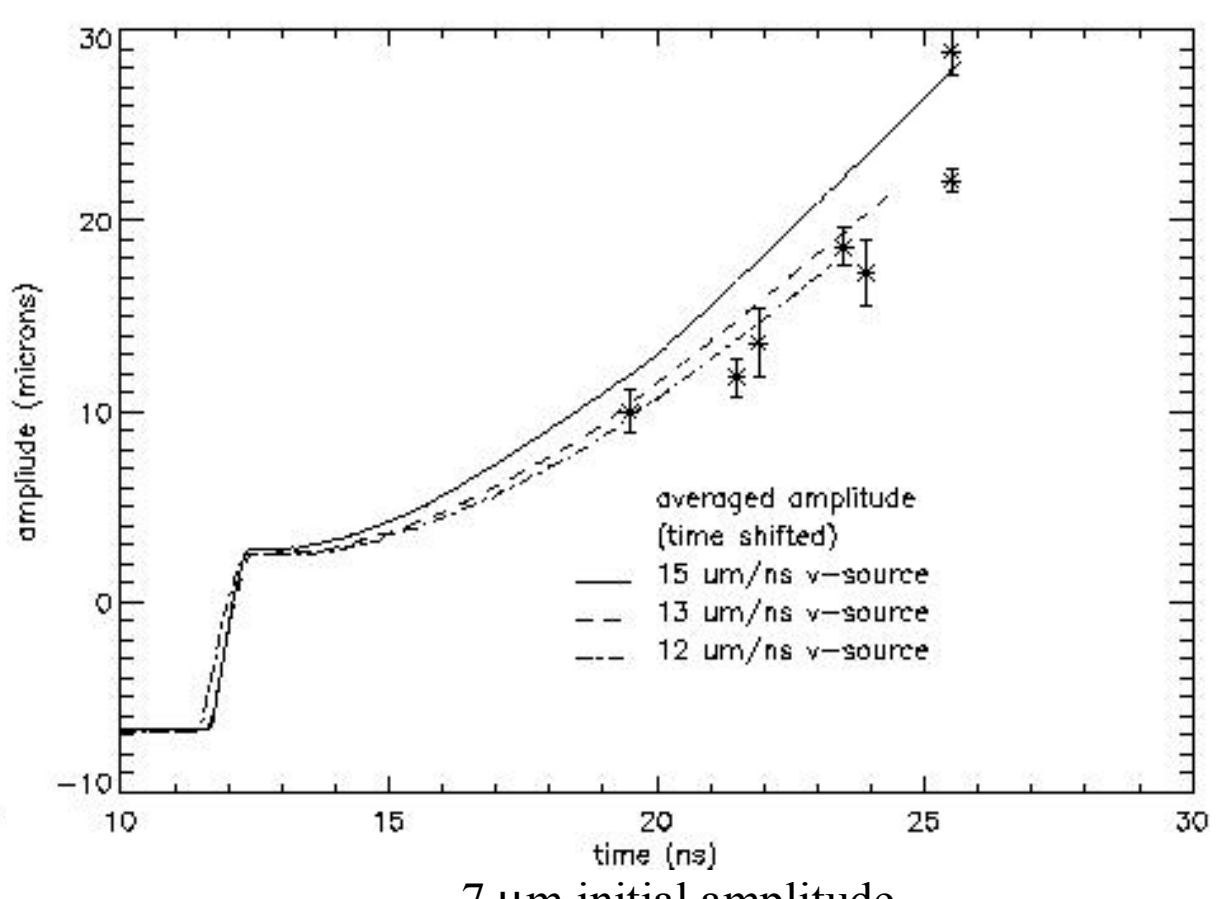

$7 \mu \mathrm{m}$ initial amplitude

The post-shock amplitude is insensitive to the piston velocity over a range of several $\mu \mathrm{m} / \mathrm{ns}$. For comparison, the average velocities of the 1 and 2D Lasnex v-sources from $1-11 \mathrm{~ns}$ are 14.0 and 13.9 $\mu \mathrm{m} / \mathrm{ns}$, respectively. The analogous averages taken from the first to the second peak in the velocity profiles are 15.0 and $14.1 \mu \mathrm{m} / \mathrm{ns}$. 


\section{D LASNEX Velocity Source V}
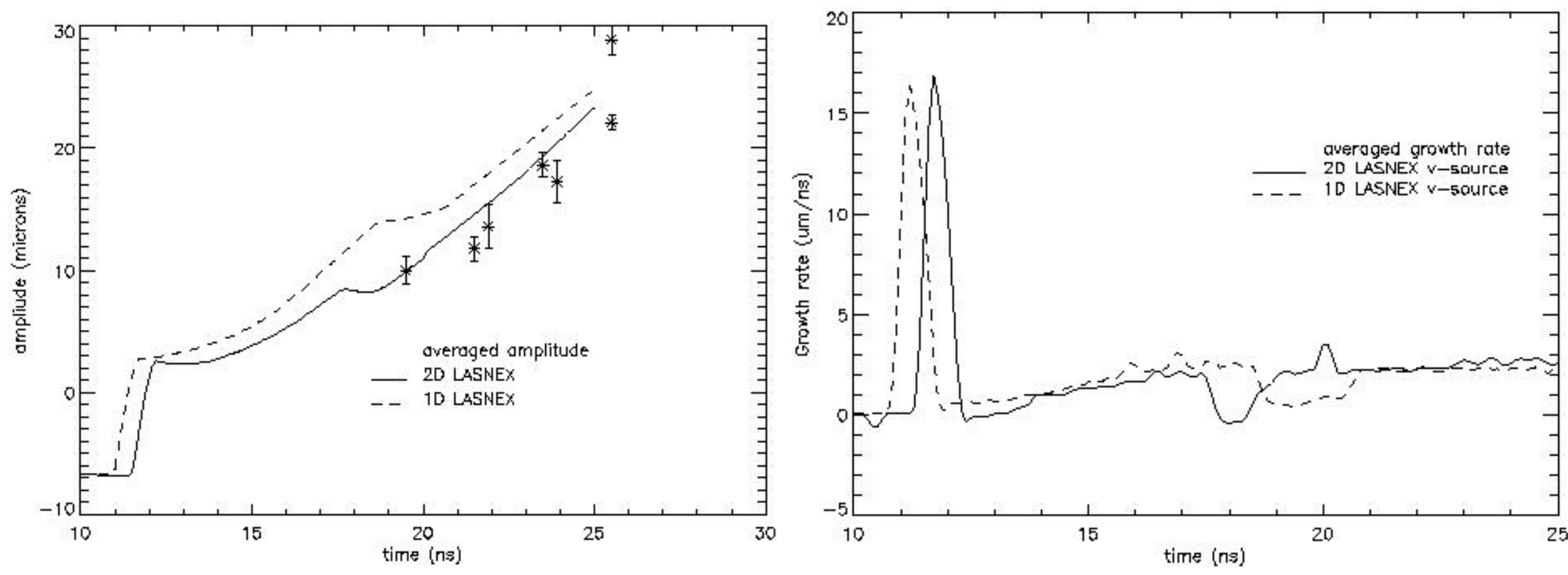

The simulation with the 2D

LASNEX velocity source is also

in closer agreement with

experimental data obtained from

experiments with small initial

perturbation amplitude $(\mathrm{a}=7 \mu \mathrm{m}$,

$\mathrm{ka}=0.29)$. 


\section{Equation of State I:}

Effect on basic parameters, post-shock amplitude, and early-time growth rate All lengths are in $\mu \mathrm{m}$, times in ns.

\begin{tabular}{|c|c|c|c|c|c|c|c|c|}
\hline & $\begin{array}{l}\mathrm{V}_{1} \\
\text { (ave 2-8 ns) }\end{array}$ & $\gamma_{1}{ }^{\text {eff }}$ & $\mathrm{u}_{\mathrm{i}}$ & $\mathrm{v}_{2}\left(\mathrm{v}_{2}{ }^{\mathrm{rel}}\right)$ & $\gamma_{2}{ }^{\text {eff }}$ & $a^{*}$ & $\begin{array}{c}\mathrm{da} / \mathrm{dt} \\
\operatorname{ave}\left(\mathrm{t}^{*}-18 \mathrm{~ns}\right)\end{array}$ & $\mathrm{da} / \mathrm{dt}^{\dagger \dagger}$ \\
\hline Exp & $22.0 \pm 0.2$ & $1.93 \pm 0.03$ & $21.9 \pm 1$ & $26.1 \pm 0.5(4.2)$ & 1.38 & 5.0 & $\approx 1.9 \pm 0.1$ & \\
\hline EOP-1D ${ }^{+}$ & 20.9 & 1.80 & 20.4 & $25.1(4.7)$ & 1.46 & 9.0 & 3.4 & 4.3 \\
\hline EOP- $2 D^{+}$ & 20.8 & & 21.0 & $26.1(5.1)$ & & 7.7 & 3.0 & 4.5 \\
\hline EOP & 19.6 & & 22.2 & $27.0(4.8)$ & & 10.5 & 2.8 & 4.0 \\
\hline LEOS & 18.9 & 1.67 & 22.9 & $29.4(6.5)$ & 1.66 & 13.0 & 3.1 & 4.5 \\
\hline$\gamma / \mathrm{EOP}$ & & 1.5 & & & & 13.5 & & 2.1 \\
\hline$\gamma / \mathrm{EOP}$ & & 1.9 & & & & 11.0 & & 4.9 \\
\hline$\gamma / \mathrm{EOP}$ & & 2.0 & & & & 7.0 & & 5.0 \\
\hline$\gamma / \mathrm{EOP}$ & & 2.2 & & & & 7.0 & & 5.5 \\
\hline $\mathrm{EOP} / \gamma$ & & & & & 1.1 & 11.0 & & 3.2 \\
\hline $\mathrm{EOP} / \gamma$ & & & & & 1.2 & & & 3.5 \\
\hline $\mathrm{EOP} / \gamma$ & & & & & 1.3 & & & 3.8 \\
\hline $\mathrm{EOP} / \gamma$ & & & & & 1.4 & & & 3.9 \\
\hline $\mathrm{EOP} / \gamma$ & & & & & 1.5 & 10.5 & & 4.0 \\
\hline
\end{tabular}

${ }^{+}$Simulation run with drive from LASNEX calculation - all others run with modified v-source.

${ }^{\dagger+}$ Peak growth rate over interval from $\mathrm{t}^{*}$, defined by $\mathrm{a}\left(\mathrm{t}^{*}\right)=\mathrm{a}^{*}$, to instant where second shock reaches the bubble front. $\mathrm{v}_{2}{ }^{\text {rel }}$ is shock speed in interface frame 


\section{Equation of State II: Shock Proximity}
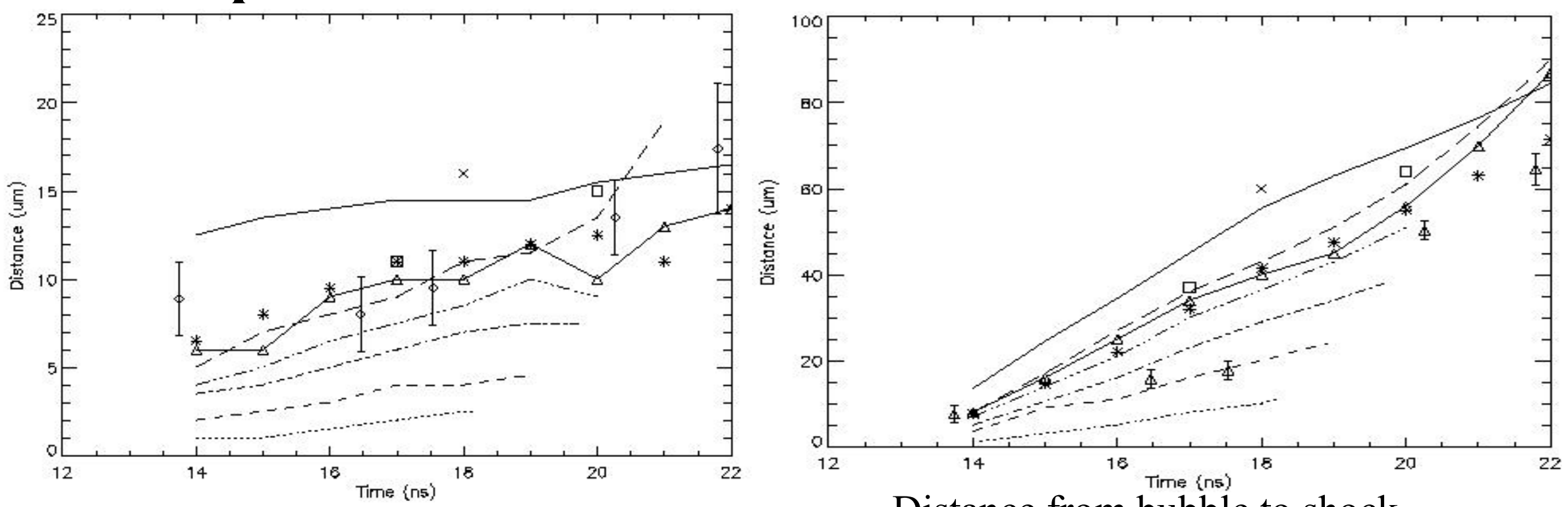

Distance from spike to shock

Modified v-source and EOP EOS unless otherwise specified $-\Delta-$ EOP

$\mathrm{X}$ LEOS

$\square \quad$ 1D LASNEX v-source

* 2D LASNEX v-source 1D LASNEX v-source, foam-filled gap

$$
\begin{array}{ll}
\cdots \cdots \cdots \cdots & \gamma_{\text {fom }}=1.1 \\
-\cdot-\cdot- & \gamma_{\text {fom }}=1.2 \\
-\cdots-1.3 \\
\gamma_{\text {fom }}=1.4 \\
\gamma_{\text {fom }}=1.5
\end{array}
$$

Shock proximity and distortion compared for various EOS models and velocity source profiles. No one combination of EOS model and v-source matches all of the data. The discrepancy between data and experiment on the bubble proximity is generally consistent with the discrepancy in perturbation amplitude.
Distance from bubble to shock

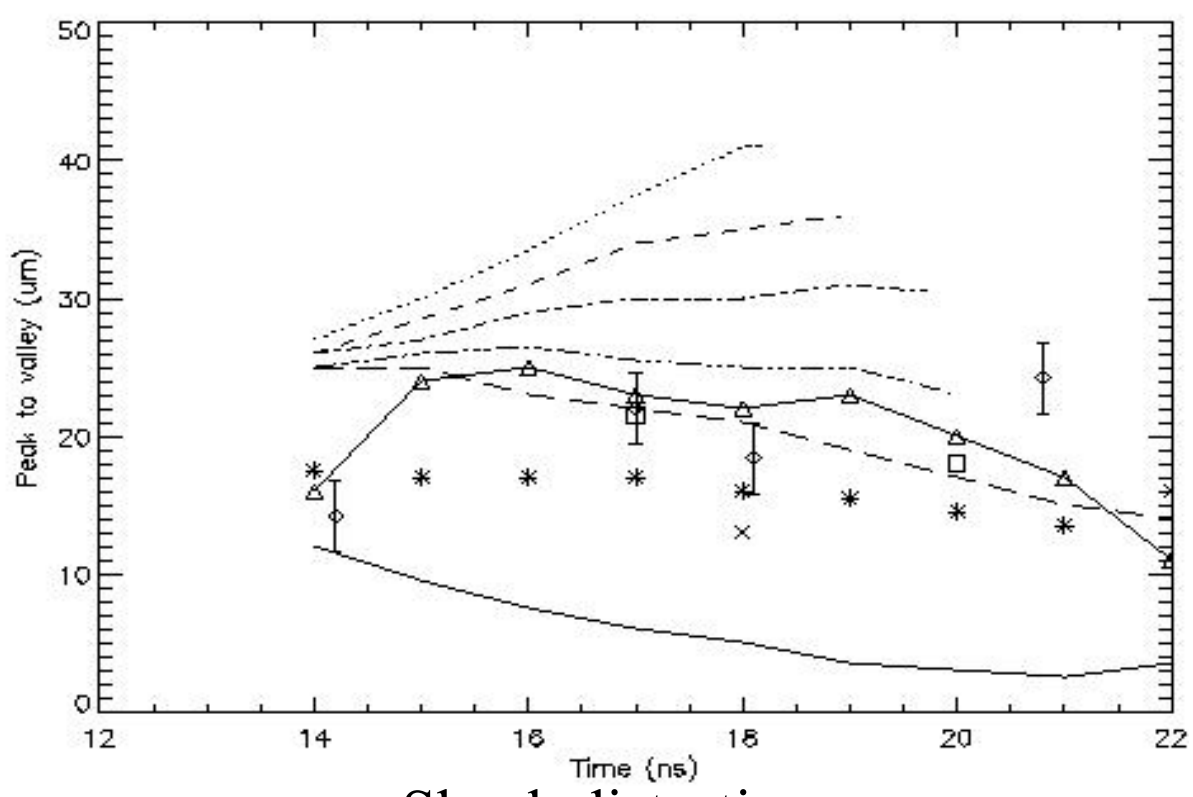

Shock distortion 


\section{Shock-Proximity vs Amplitude}

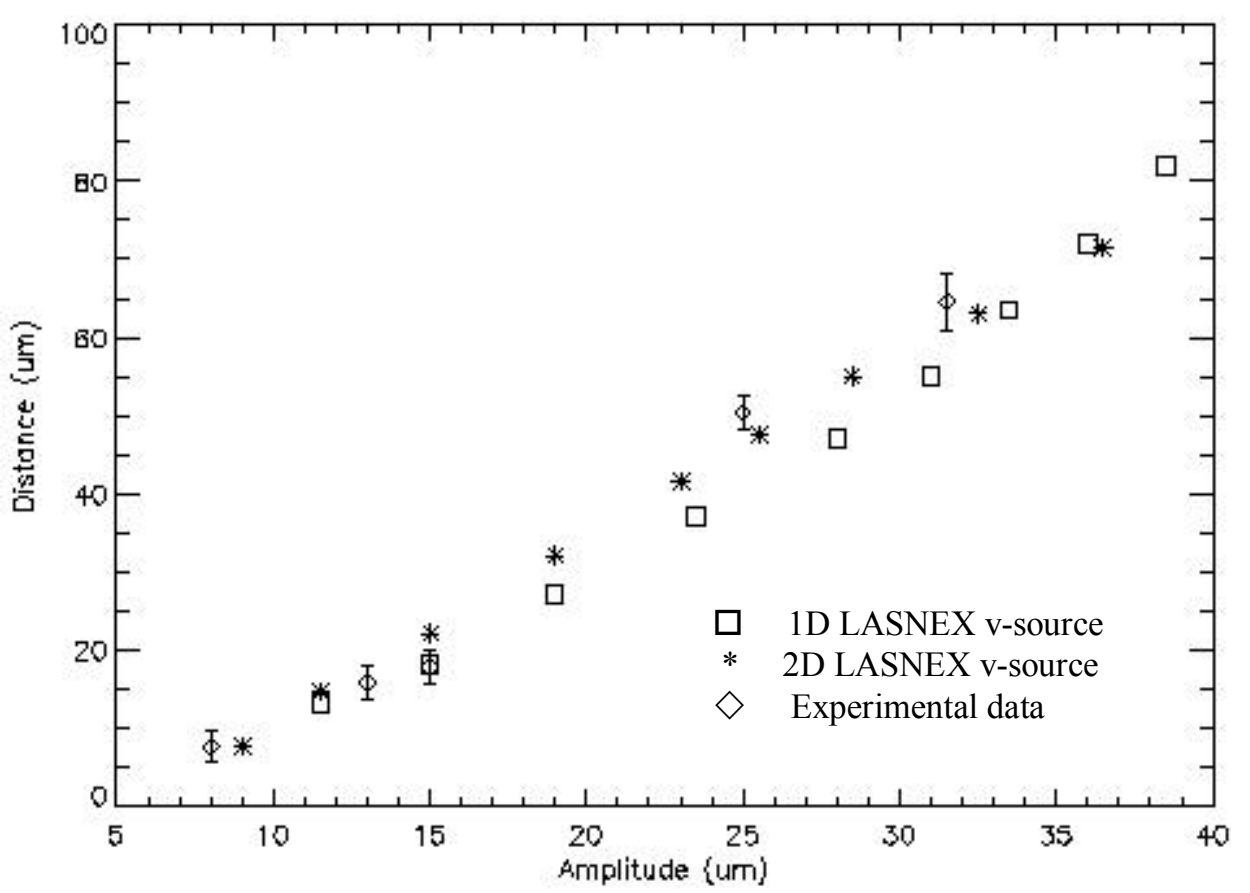

Distance from bubble to shock

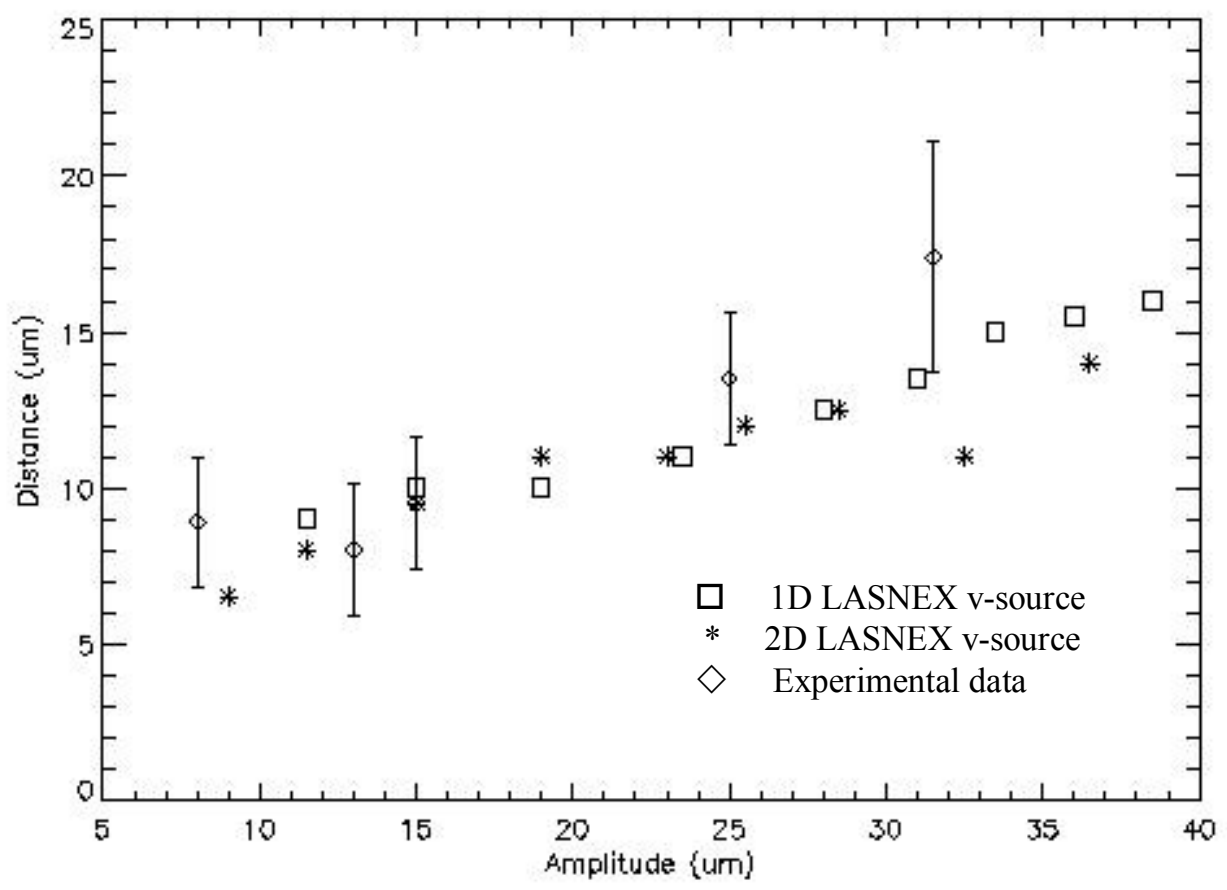

Distance from spike to shock 


\section{$\underline{\text { Post-shock amplitude }}$}

- $\mathrm{a}^{*}$ has been shown to exhibit only weak dependence on EOS and zoning issues.

- $a^{*}$ is defined by half the bubble to spike distance at the instant the shock completely crosses the interface, and is determined by the compression factor:

$$
\frac{a^{*}}{a}=u_{i} \frac{1}{v_{1}}-\frac{1}{u_{g}}
$$

Where $u_{i}$ is the interface velocity, $v_{1}$ is the shock speed in the plastic, and $u_{g}$ is the interface velocity in the gap. This reduces to the usual expression in the absence of a gap, when $u_{g}=u_{i}$.

- For the reported experimental amplitude and velocity values, this requires $\mathrm{u}_{\mathrm{g}}=28.5 \pm 1.3 \mu \mathrm{m} / \mathrm{ns}$. If the incident shock and interface velocities at the time of interaction are used (rather than their average values), we find $u_{\mathrm{g}}=26.5 \mu \mathrm{m} / \mathrm{ns}$. A rough measurement from the data suggests $u_{\mathrm{g}}=20 \pm 10 \mu \mathrm{m} / \mathrm{ns}$. The analogous calculation for the simulations with an EOP EOS gives $u_{\mathrm{g}}=33.9 \mu \mathrm{m} / \mathrm{ns}$ (modified $\mathrm{v}$-source), $\mathrm{u}_{\mathrm{g}}=36.0 \mu \mathrm{m} / \mathrm{ns}$ (1D LASNEX v-source), and $\mathrm{u}_{\mathrm{g}}=31.8 \mu \mathrm{m} / \mathrm{ns}$ (2D LASNEX v-source), while, for LEOS, $u_{\mathrm{g}}=36.9 \mu \mathrm{m} / \mathrm{ns}$ (modified v-source). The actual values from the three simulations are $37 \pm 1,34 \pm 1,28 \pm 1$ and $40 \pm 1 \mu \mathrm{m} / \mathrm{ns}$, respectively. 


\section{Post-shock Amplitude II: Modified gap}
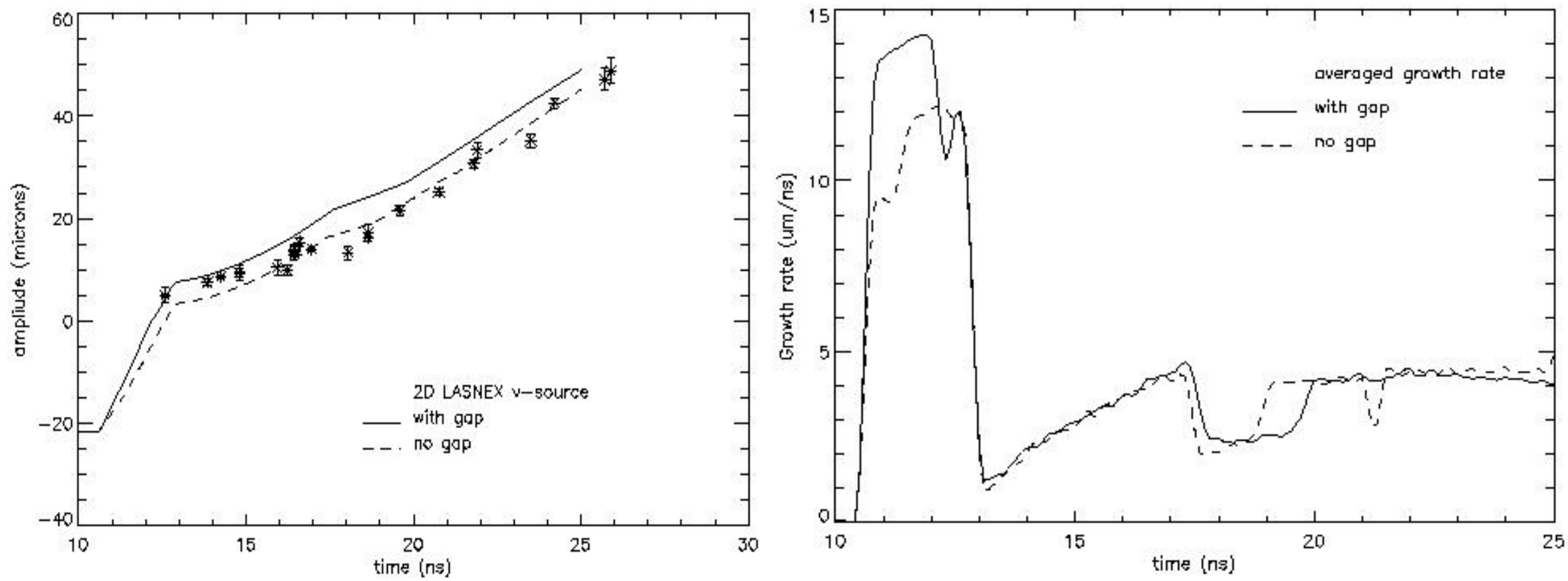

When the gap is "filled in" with foam, the breakout speed of the interface into the gap region is reduced. The speed of the interface in the gap is one-half the instability growth rate until the moment that the incident shock reaches the position of the unperturbed interface. The reduction in breakout speed produces a corresponding reduction in post-shock amplitude. 


\section{Conclusions I}

- CALE simulations of high Mach number RM experiments have been performed.

- Results obtained using computational grids initially rectangular or conforming agree with one another provided the number of zones/wavelength is sufficiently large. Convergence is obtained more rapidly for rectangular grid.

- Although the general features of the experiments are correctly predicted by he simulations, there are discrepancies.

- The discrepancy between the experimental and simulation $a^{*}$ does not appear to appear to be caused by EOS issues. It has been shown most sensitively dependent on the details of: the composition of the gap region, the shock-gap dynamics, and the velocity source. 


\section{Conclusions II}

- Relatively small details of the velocity source can significantly affect the post-shock amplitude and the instability growth rate. CALE simulations in which the input velocity source was obtained from a 2D rather than 1D Lasnex simulation show improved agreement of a* and $\mathrm{da}(\mathrm{t}) / \mathrm{dt}$ with the experimental data. This is true for both both the large and small amplitude cases.

- The discrepancy between experimental and simulation $\mathrm{da}(\mathrm{t}) / \mathrm{dt}$ from 17

- 20 ns does not appear to be caused by zoning or EOS issues. Simulations with a modified v-source suggest that, in the experiment, the second shock (observed with both Lasnex velocity sources) reaches the perturbation about $1 \mathrm{~ns}$ earlier than in the CALE simulation with 2D Lasnex v-source. This in turn suggests that further refinement of the velocity source will be required for more accurate simulations of the experiment.

- The long-term shock proximity observed in the experiments suggests that the foam may more compressible than predicted by the EOP tables, and is certainly more compressible than predicted by the LEOS tables. The degree of shock proximity remains the most significant discrepancy between the experiments and simulations. 
LA-UR-01-6602 (Rev)

\section{Improvements to Convergent Cylindrical Plasma Mix Experiments using Laser Direct Drive}

Cris W. Barnes ${ }^{\star}$, Steven H. Batha ${ }^{*}$, A. M. Dunne ${ }^{\#}$, N. E. Lanier ${ }^{*}$, Glenn R. Magelssen*, Thomas J. Murphy*, Kenneth W. Parker\#, Stephen Rothman ${ }^{\#}$, John M. Scott ${ }^{\star}$, David Youngs ${ }^{\#}$ *Los Alamos National Laboratory, Los Alamos, NM USA \#AWE, Aldermaston, UK 


\section{Abstract- LA-UR-01-2545}

- Experiments studying mix in a compressible, convergent, miscible, plasma system are being conducted on the OMEGA Laser at the Laboratory for Laser Energetics at the University of Rochester. Thin-walled polystyrene cylinders $2.25-\mathrm{mm}$ long and $0.86 \mathrm{~mm}$ inner diameter with foam inside are directly illuminated with 351-nm wavelength light from 50 laser beams in a 1-ns square laser pulse. The turbulence driven by the Richtmyer-Meshkov instability by shock passage across a density discontinuity mixes marker material that is radiographically opaque. Initial work using a high-density, highopacity marker layer of gold between the plastic ablator and foam clearly demonstrated significant measurable mix width. However, the high opacity of the gold prevented determination of a density profile in the mix region, and it was also overly sensitive to hydrodynamic effects at the end of the marker layer. Use of lower opacity marker material will be described and its impact on end effects and the measurements of mix density profile described.

- C. W. Barnes et al., Rev . Sci. Instrum. 70 (1999) 471.

- C. W. Barnes et al., submitted to Physical Review Letters (2001). 


\section{Why use direct-drive cylindrical implosions to study mix?}

- Purpose

- Study Richtmyer-Meshkov (RM) instability in compressible, convergent, miscible, plasma system

- Method

- Implode cylinder with an unstable interface and measure resulting mix

- Diagnostic advantages, fewer ends to affect experiment, convergent

- Strategy

- Compare results from wide variations in initial conditions

"Smooth Cl vs. Rough Au interfaces (no mix vs. lots of mix)

"Smooth Au vs. Rough Au interfaces

"Vary surface roughness

- Improve design (to make less sensitive to small amounts of marker material) using Al marker and epoxy ablator 


\section{We have established a useful, laser- based test bed for mix experiments}

- Implode cylinder with thick ablator with 1-ns square pulse direct laser irradiation

- Hydrodynamically unstable at plastic/Au and Au/foam interfaces

- Backlight with $\mathrm{x}$ rays through cylinder

- Measure radial extent of "mix layer" of Au into adjacent materials

- 1D convergent experiment with Mach number $\approx 20$ (pre-shock; Mach $\approx 5$ post-shock), convergence $\approx 4$, Pressure > 45 Mbars, Reynold's number $\approx 10^{6 *}$

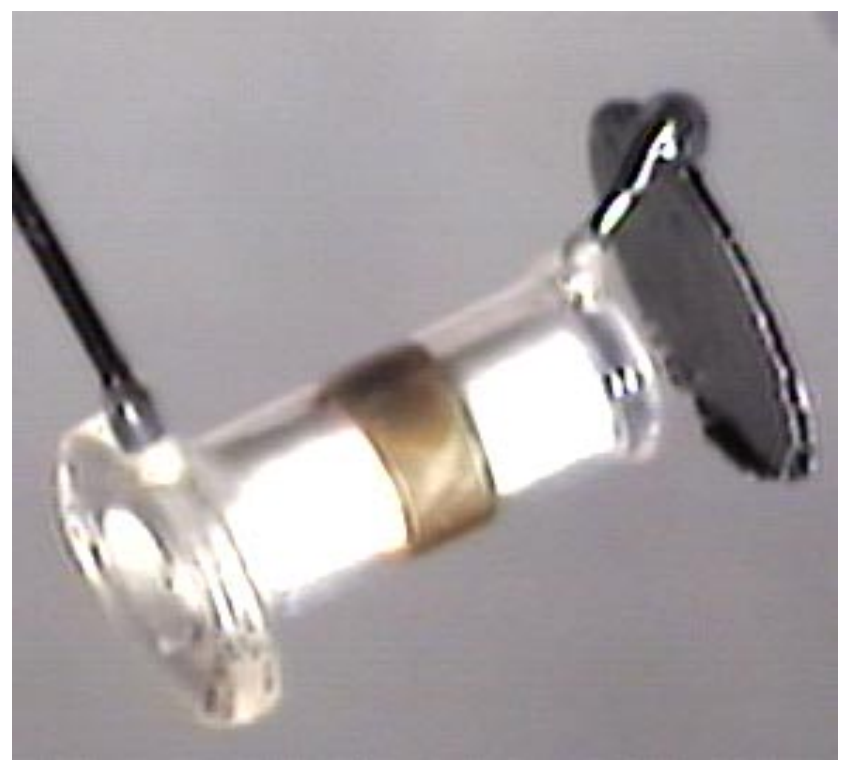

*Galmiche and Gauthier, Jpn. J. Appl. Phys. 35 (1996) 4516
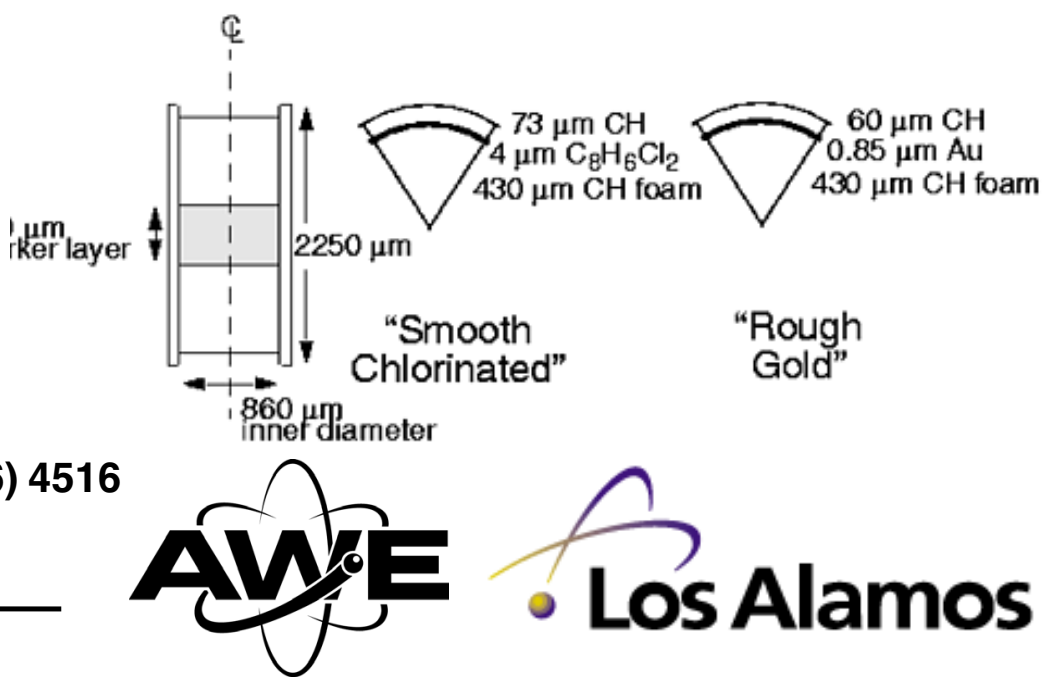


\section{Compressibility and convergence matter to mix}

-What mix width do we expect?

- Planar Meyer-Blewett:

$-\mathrm{dH} / \mathrm{dt} \sim \mathrm{A}^{*} \mathrm{U}<\mathrm{k}><\mathrm{a}_{0}>\approx 140 \mu \mathrm{m}$ in 4ns (estimated using measured surface roughness)

- Incompressible Bell-Plesset:

- Convergence ratio $\approx 4$

-Thickness $\approx 1 / \delta R \approx 4 x$ more than planar

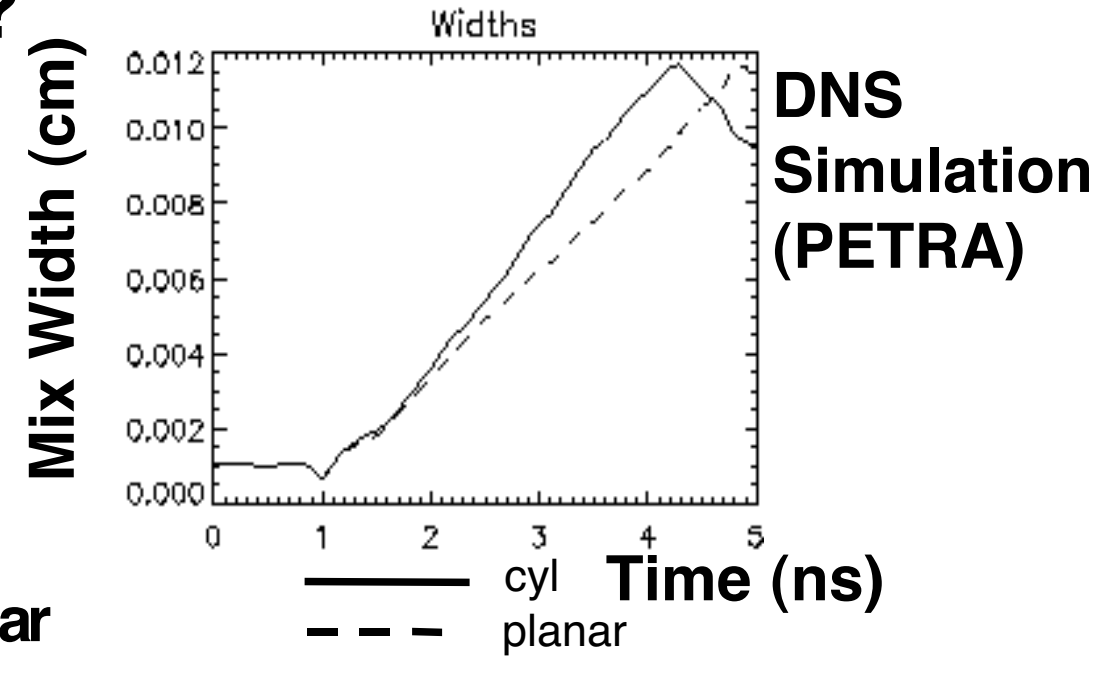

- Would be big effect

- Compressible Bell-Plesset:

- Shock increases density

- Convergence also increases density eventually

- Thickness $\approx 1 / \delta(\rho R) \approx 1.25 x$ planar, consistent with simulations 


\section{Complete Diagnostic Coverage Available}

(example from thin ablator Rayleigh-Taylor experiments)

XRFC4 Axial Radiography

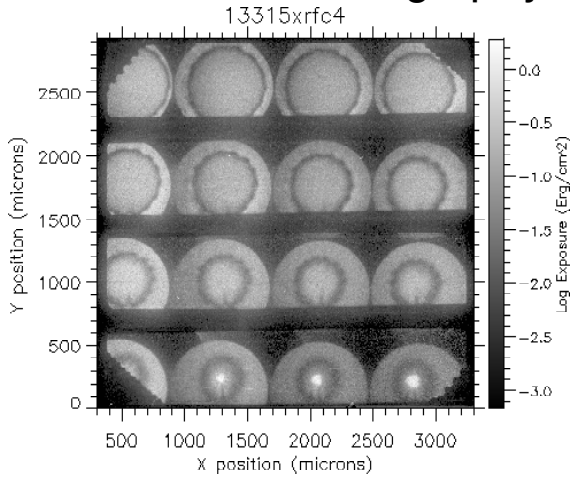

XRFC3 Transverse View of Self-Emission

LA-UR-01-6602
Imaging X-Ray Streak Camera

(1)

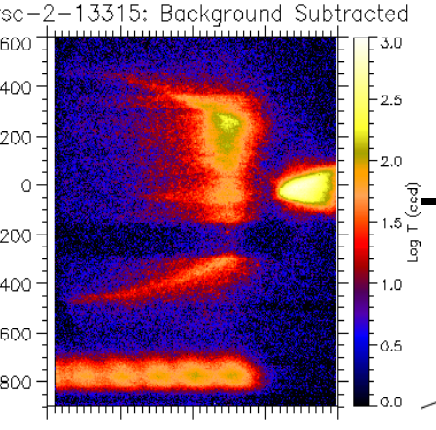

\section{Shot 13315 \\ Diagnostics for}

SSC1 Streak of Backlighter

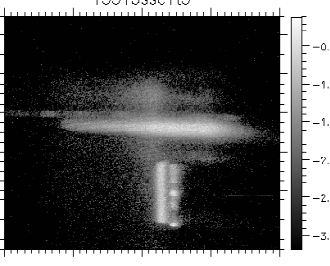

$\underset{\substack{\text { Allin the } \\ \mathrm{P}-\mathrm{PT}-\mathrm{Pl} \text { plane }}}{\text { XRFC1 View of Backlighter }}$
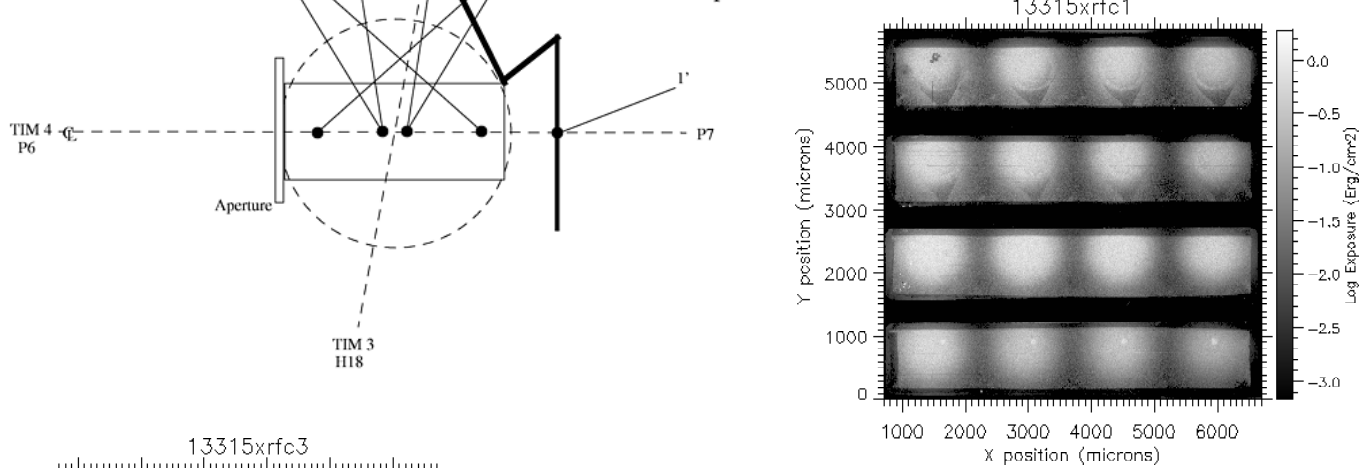

LLE X-Ray Spectrometer

Streak of Chlorine Emission

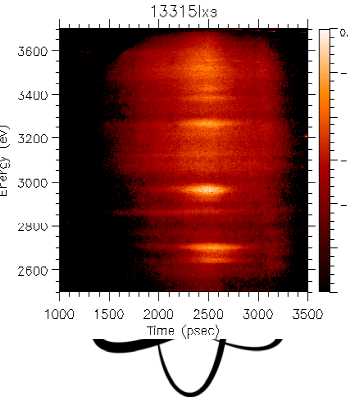

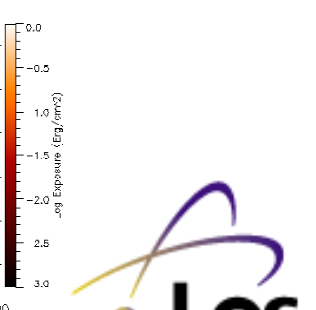

- Los Alamos 


\section{Extremes of mix/no-mix measured, demonstrating principle of experiment}

- Different surface roughness

- Different marker layer density

- Did not change the Atwood number significantly

- Changed the behavior at the end of the marker
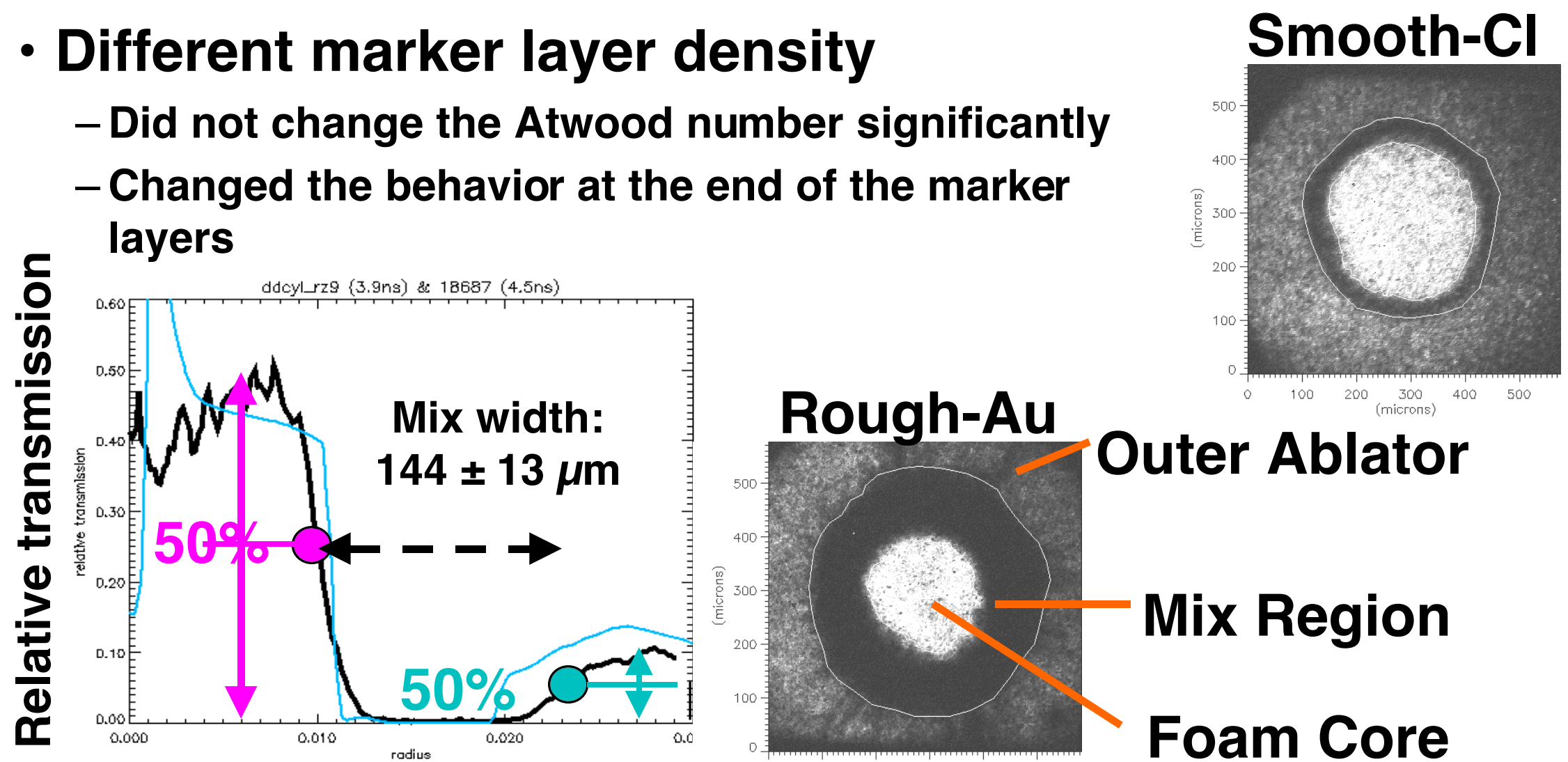

Rough-Au

Outer Ablator

Radius (cm) 


\section{Creation of vorticity at ends of marker layer can increase apparent marker layer width}

Experimental Set-Up

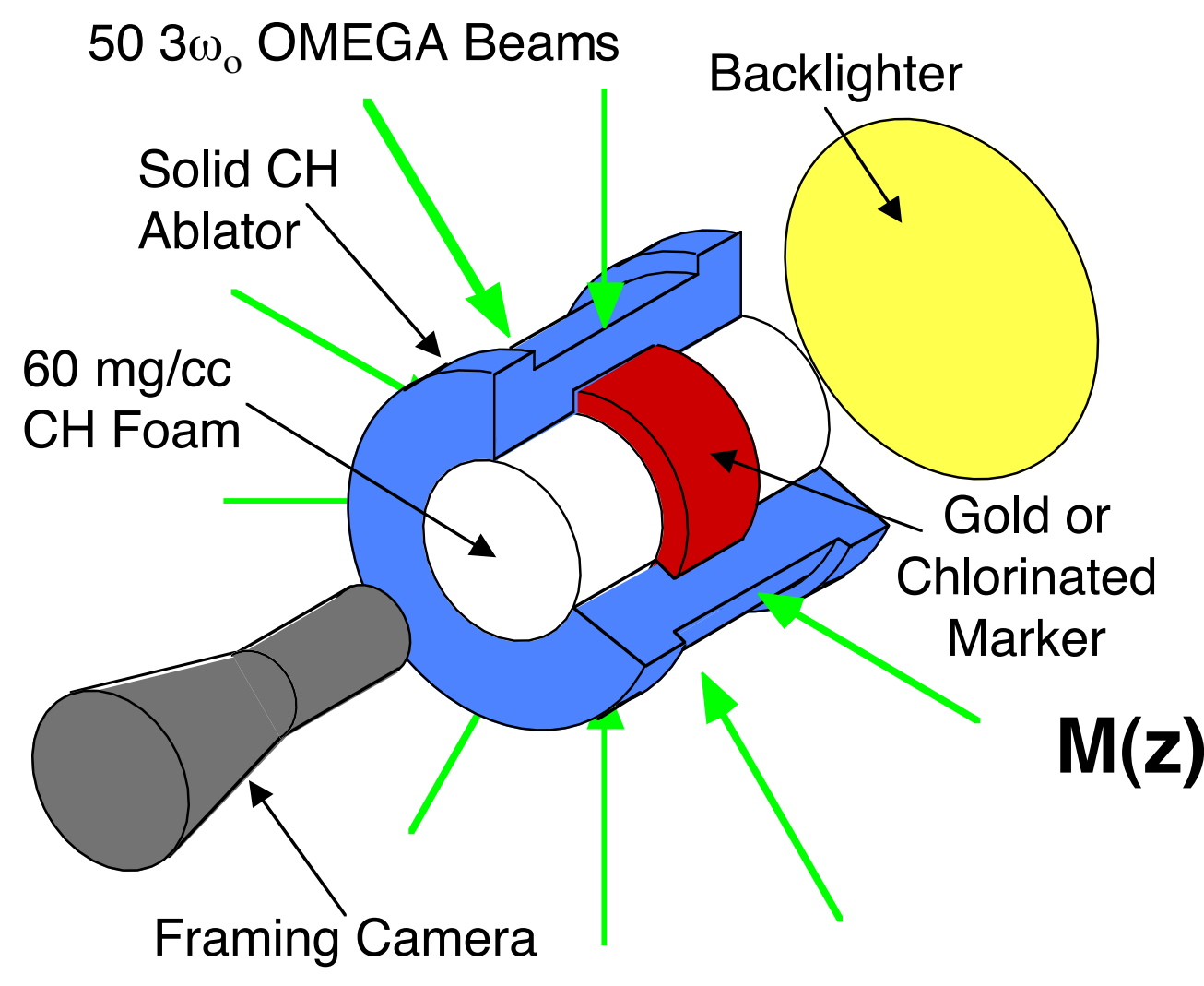

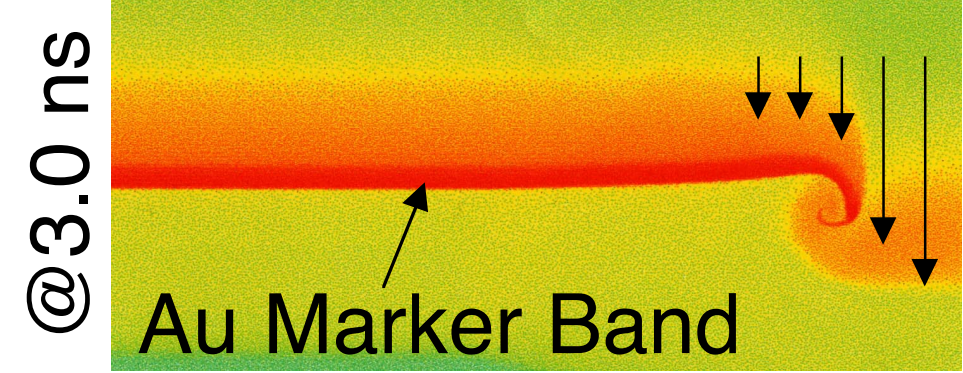

$M(z)=\int_{0}^{\infty} \rho(r, z) d r$

Mass step at ends

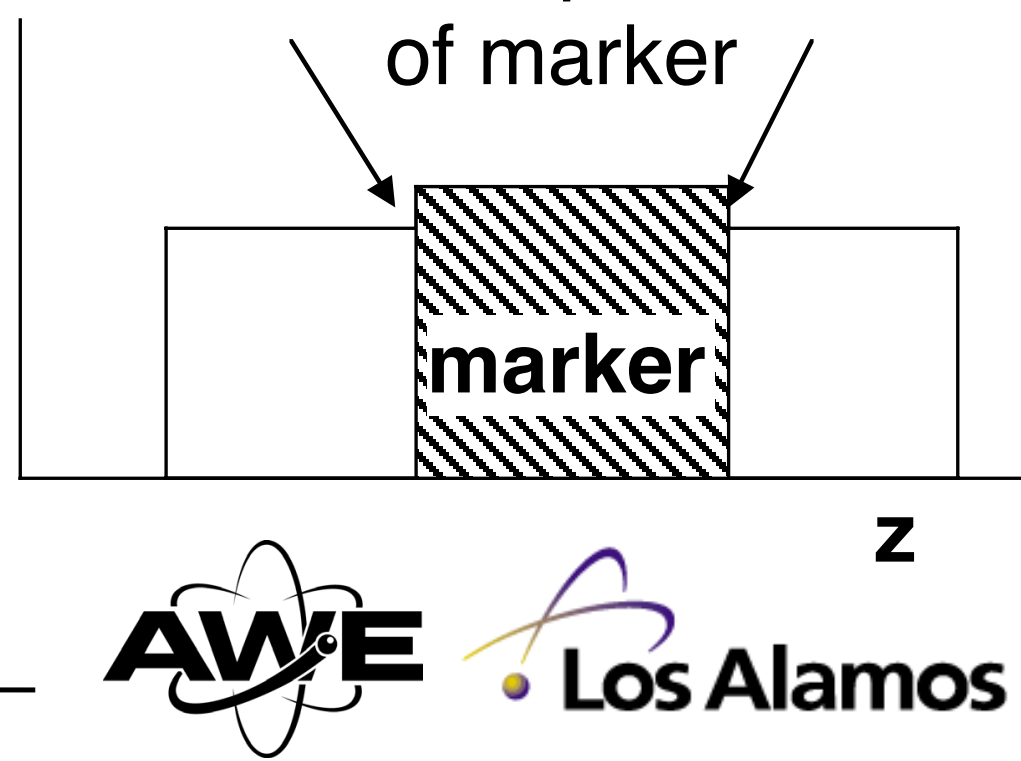




\section{Smoother surfaces don't show as much mix}

- Same material

- Same Atwood number and end effects

- Smooth mix width: $\approx 95 \mu \mathrm{m}$

- Rough mix width: $144 \mu \mathrm{m}$

- Mixing from larger surface roughness overwhelms end effect, which dominates smooth case

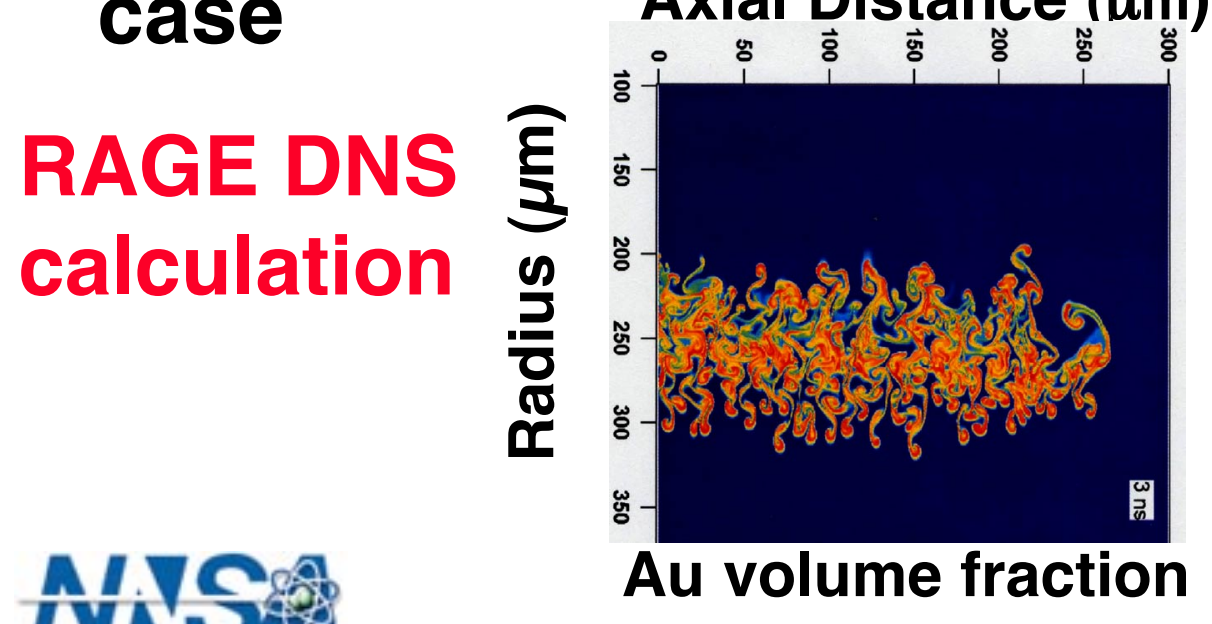

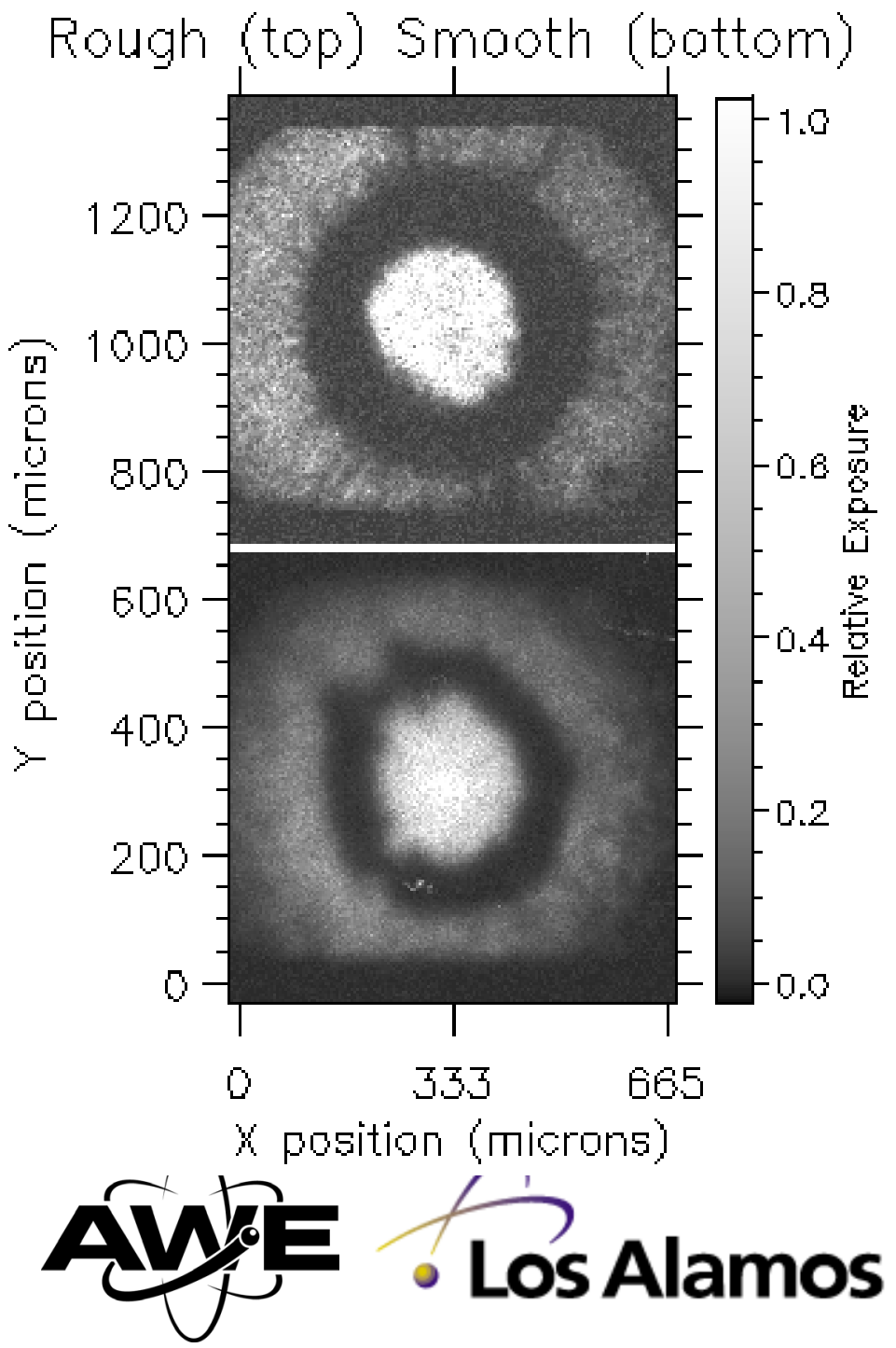




\section{Numerical simulations help uncover the important physics in mix}

A wide range of simulations are underway at LANL and AWE

-SOXO : 1D Lagrangian + dynamic mix model

-PETRA : 2-D R- $\Theta$ and R-Z semi Eulerian

- TURMOIL : 2D \& 3D perfect fluid code

- LASNEX : 2D R-Z

- RAGE : 2D R-Z and R- $\Theta$

Can use measured surface roughness 


\section{RAGE does highly resolved simulations*}

- RAGE is an Eulerian, radiation-hydrodynamics code with continuous adaptive mesh refinement

- Unlike LASNEX, RAGE can calculate Rough surfaces

- All calculations included:

- One group (grey) radiation diffusion used in smooth cases

- Radiation transport was turned 'off' for rough simulations

»Similar radiographs are produced with radiation transport 'On'

- Sesame equation-of-state and opacities

- LTE

- Caveat :

- Rage lacks 3-D Laser raytrace package

" Laser energy is deposited directly into cylinder surface with an energy source

${ }^{*}$ See poster by John Scott 


\section{Calculated axial transmission profiles from more detailed simulations agree with experiments}

-PETRA and RAGE (short dashed lines) predictions in general agreement with observations (solid curve) from initially rough gold markers.

-Simulations with smooth initial conditions (as with LASNEX, long dashed lines) predict much smaller mix widths

-Some modeling uncertainties remain:

- Absorbed energy affects zero-order hydro

$-\mathrm{R}-\mathrm{Z}$ and $\mathrm{r}-\Theta$ calculations differ at $7 \%$ level
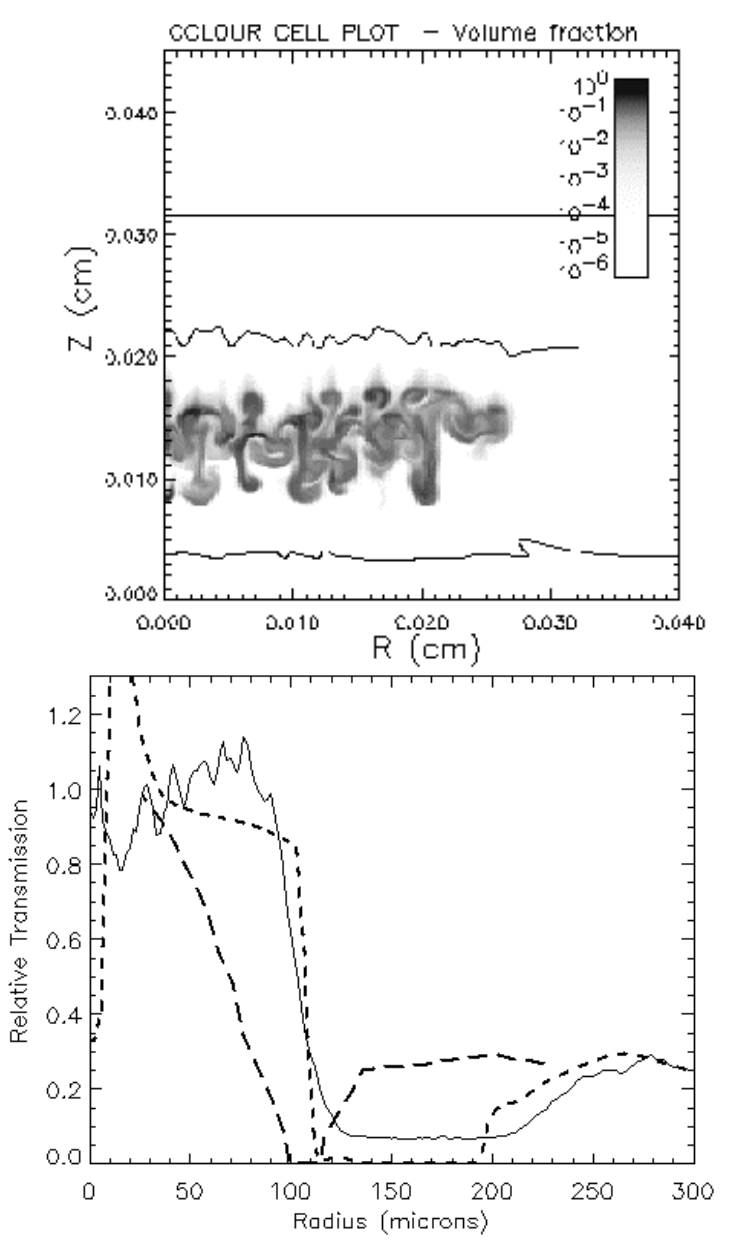


\section{RAGE can simulate as shot Rough interfaces, in both $R-Z$ and $R-\Theta$ geometries}

- Initial R-Z and R-Q setups with as-shot rough surface finish
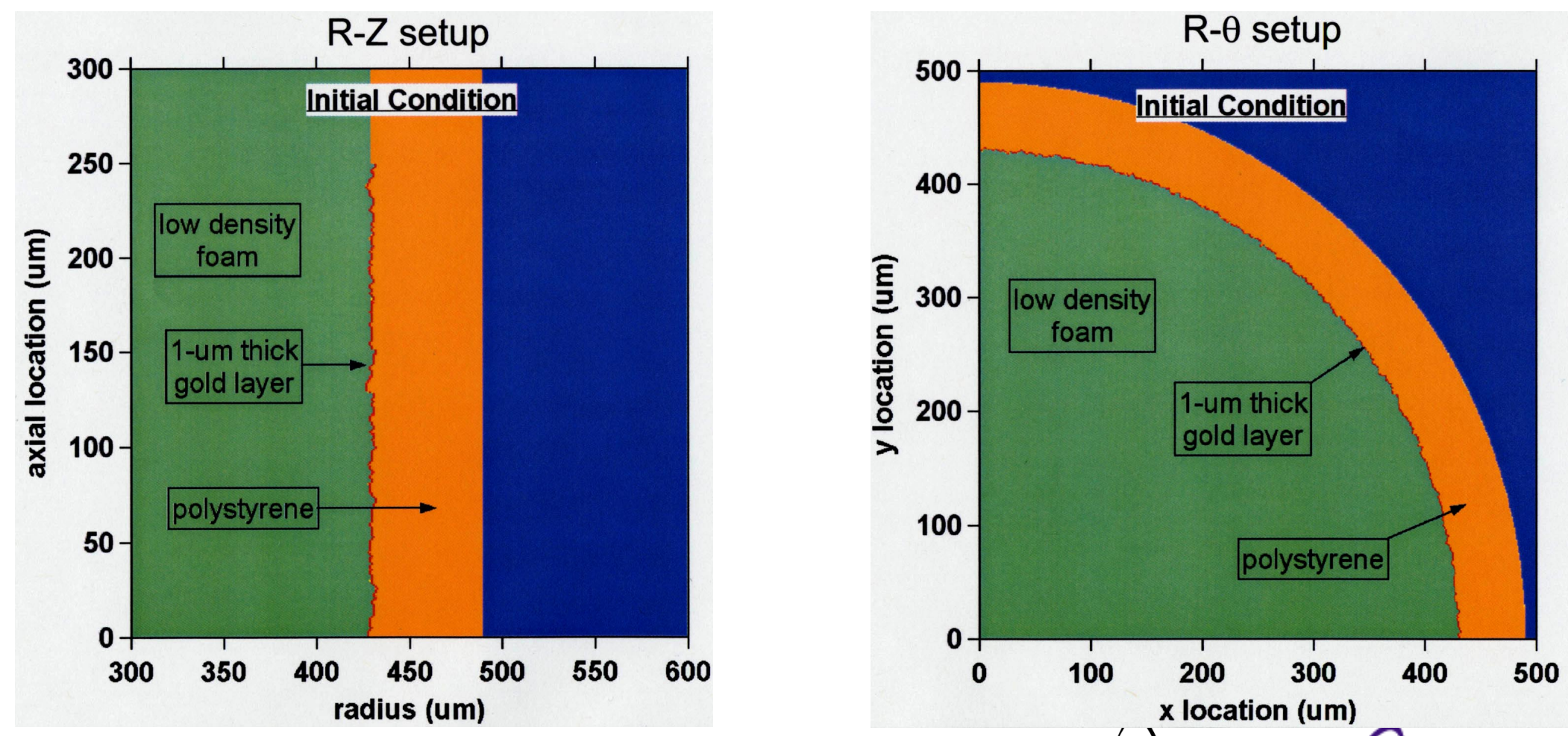


\section{RAGE predicts mix from Rough surfaces overwhelms the filigree}
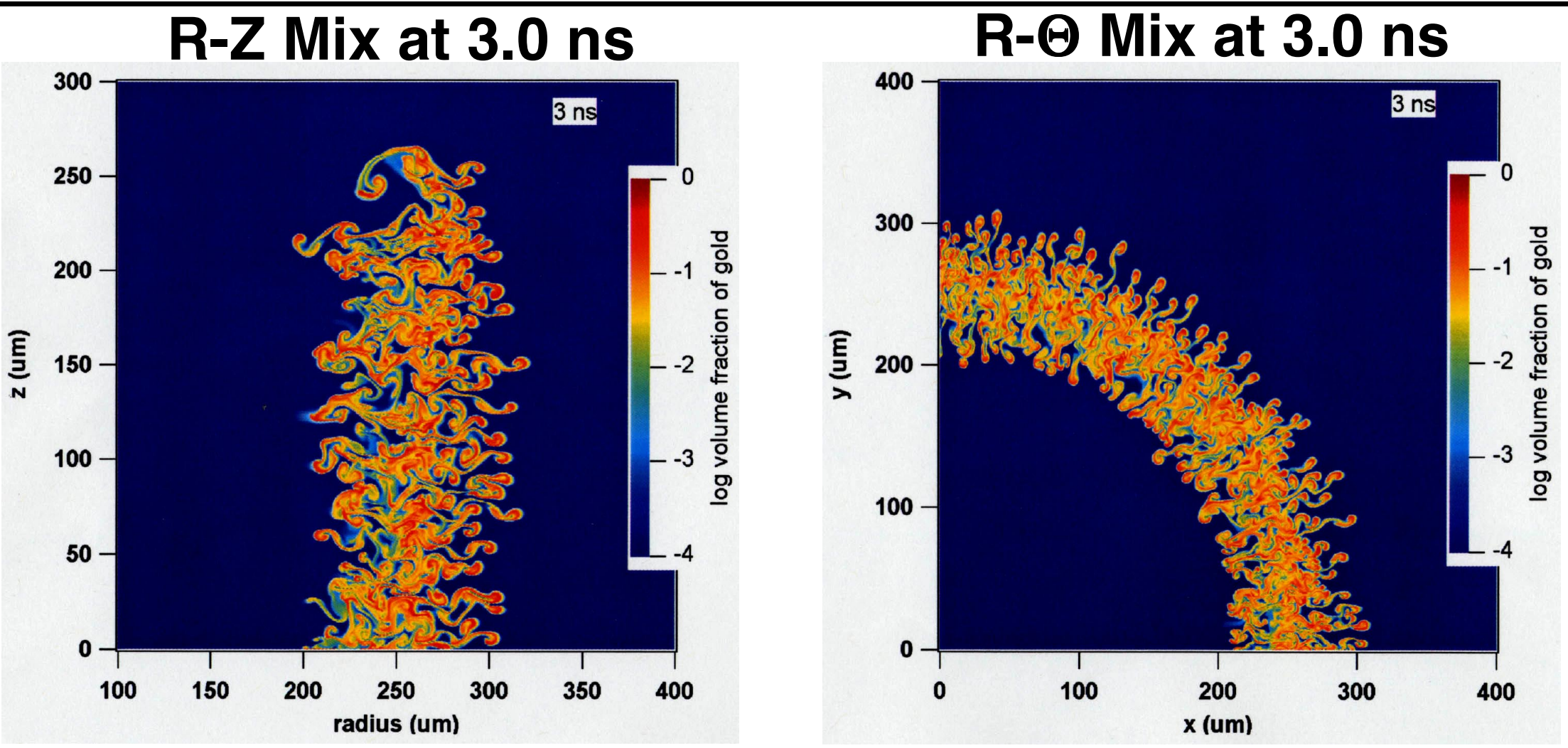

- Even at 3.0 ns, predicted mix is significant

- Of order $\sim 100 \mu \mathrm{m}$ 


\section{$R-\theta$ and $R-Z$ calculations in RAGE agree in implosion hydro and volume fraction}

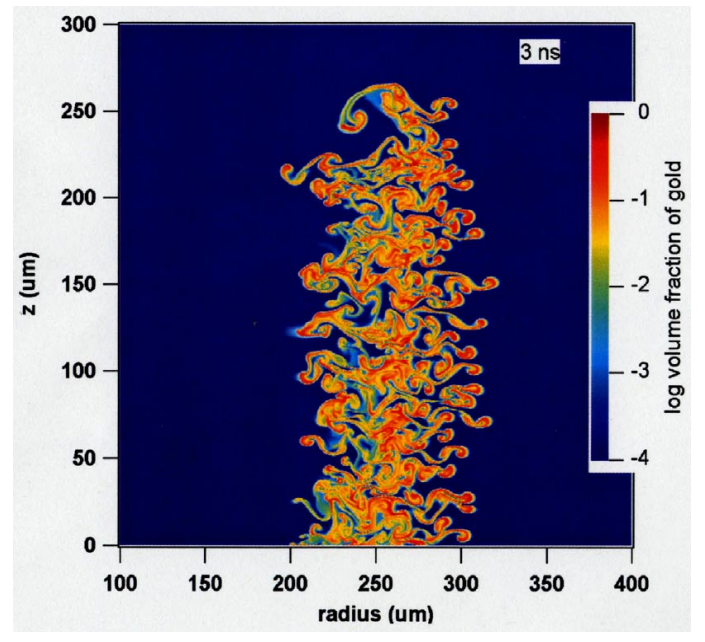

-The zeroth-order hydro is the same in RAGE for $\mathbf{R}-\mathbf{Z}$ and $\mathbf{R}-\boldsymbol{\theta}$ calculations (same implosion for same energy boundary condition)

-The volume fraction profiles are the same -The mix width of simulated radiography transmission profiles in $\mathbf{R}-\boldsymbol{\theta}$ calculations is greater than in $\mathbf{R}-\mathbf{Z}$ calculations.
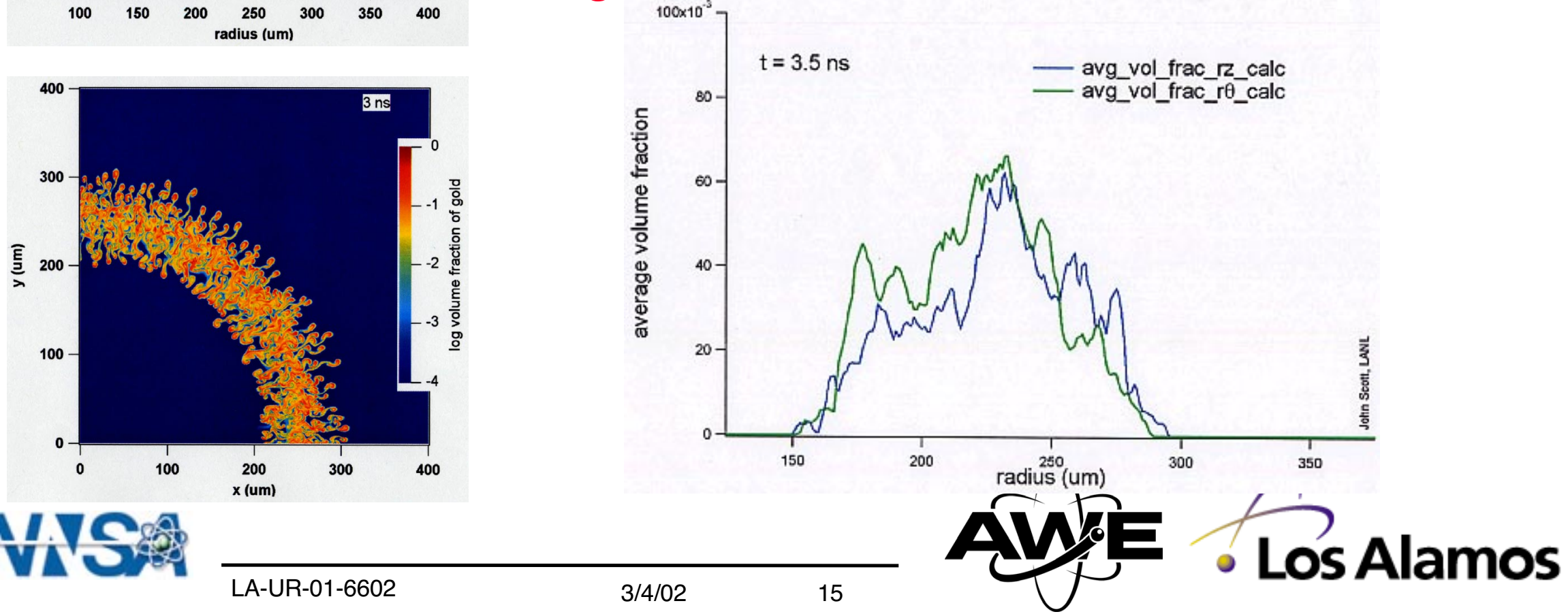


\section{What kind of temporal evolution is expected?}

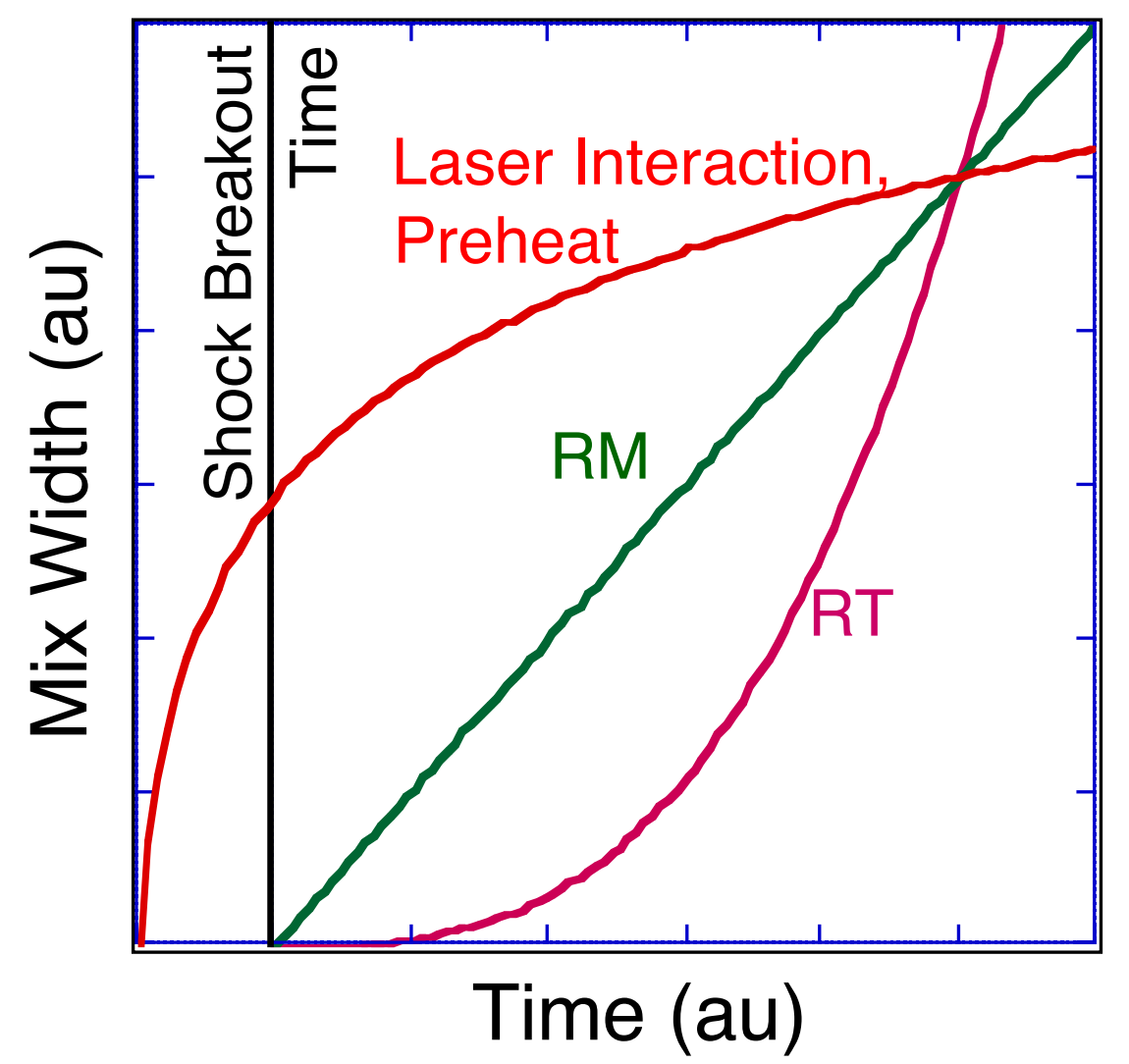

- Preheat effects or strong laser interaction with marker, could make marker initially very wide with little or no subsequent evolution.

- Late-time Rayleigh-Taylor from deceleration could make marker grow quadratic or faster late in time.

- Linear evolution would be consistent with Richtmyer-Meshkov growth.

Multiple measurements in time required to discern cause of mixing. 


\section{Time dependent measurements of compressible mix in a convergent geometry have been made}
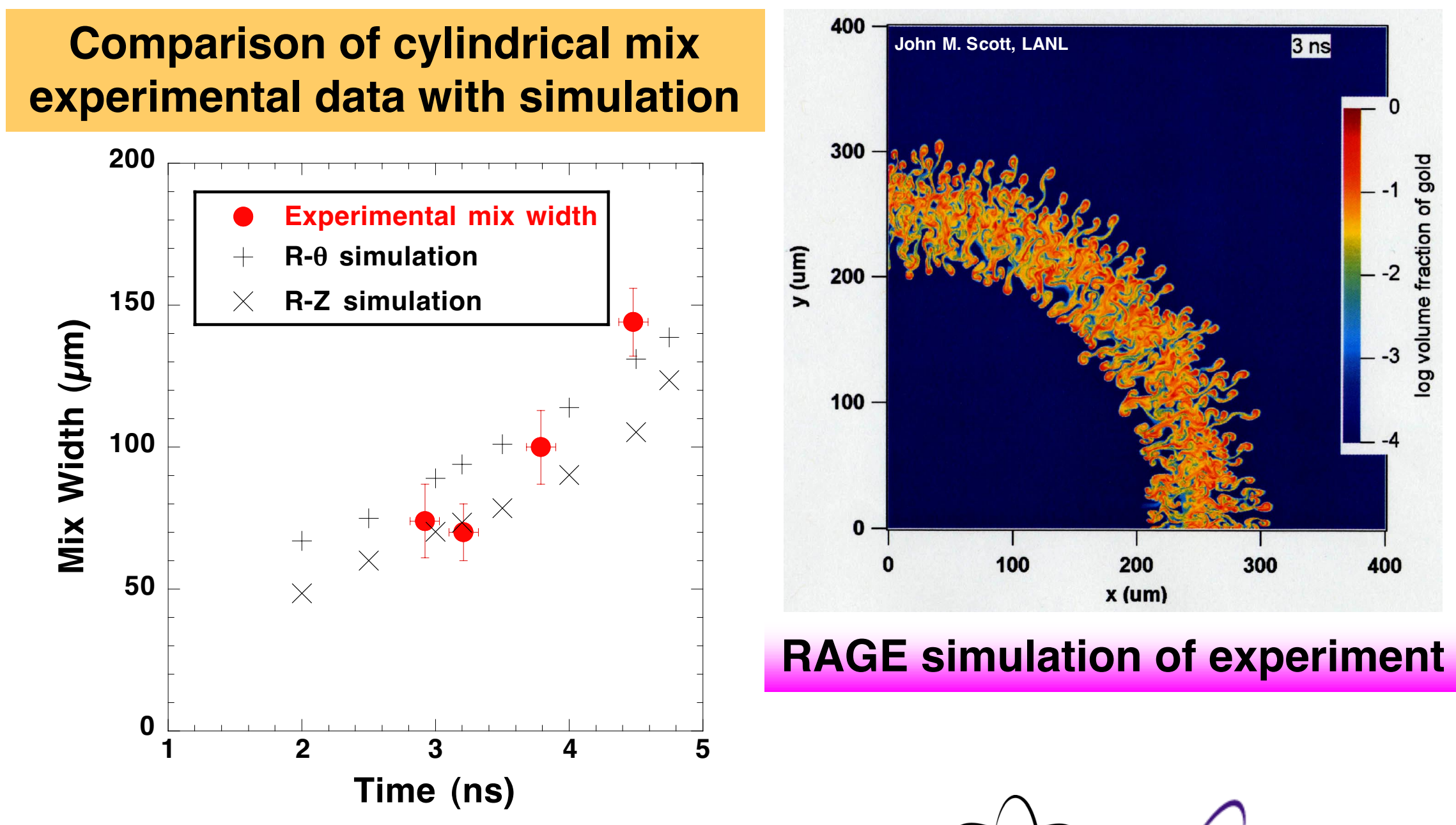

RAGE simulation of experiment 


\section{D and fully 3D TURMOIL calculations predict similar mixing profiles}

2D results

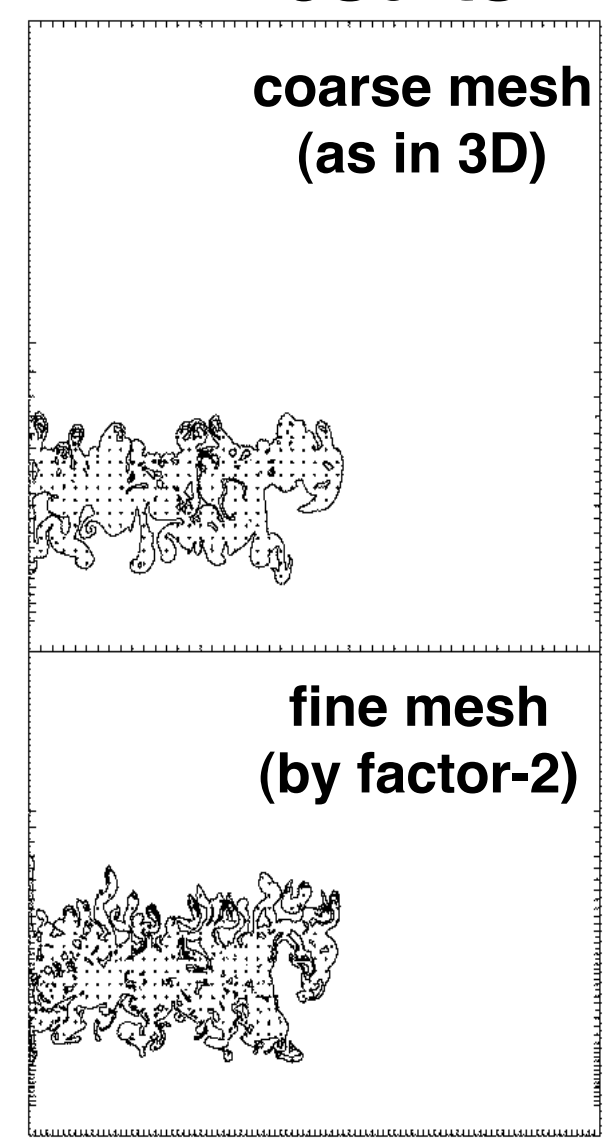

3D results

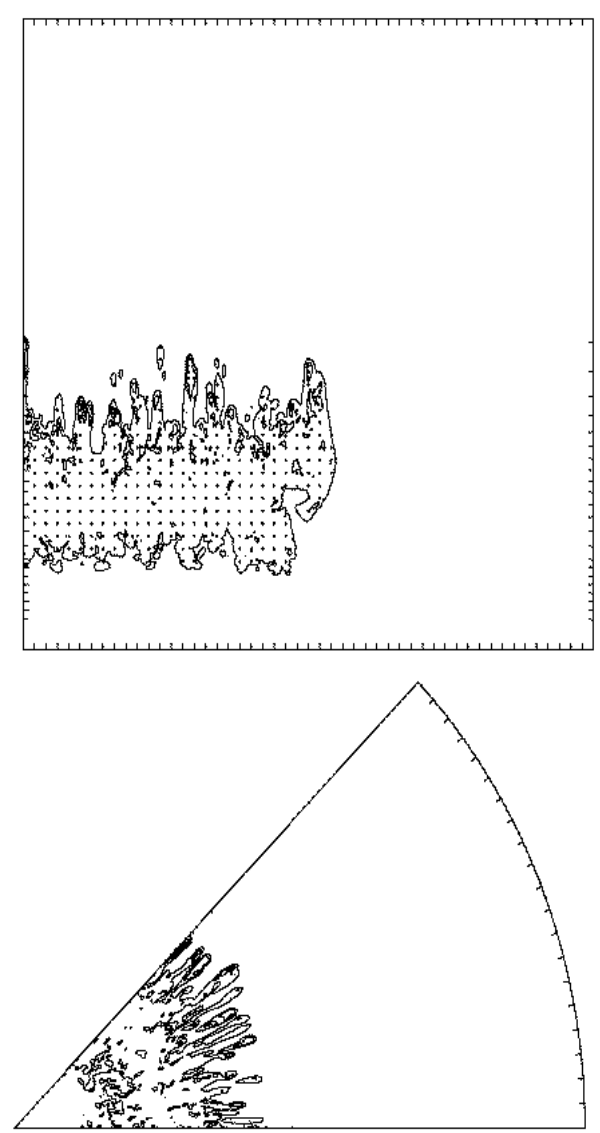

3D code calculates shortwavelength streamers penetrating to high radius (as seen in experiment?)

Overall mix profile similar
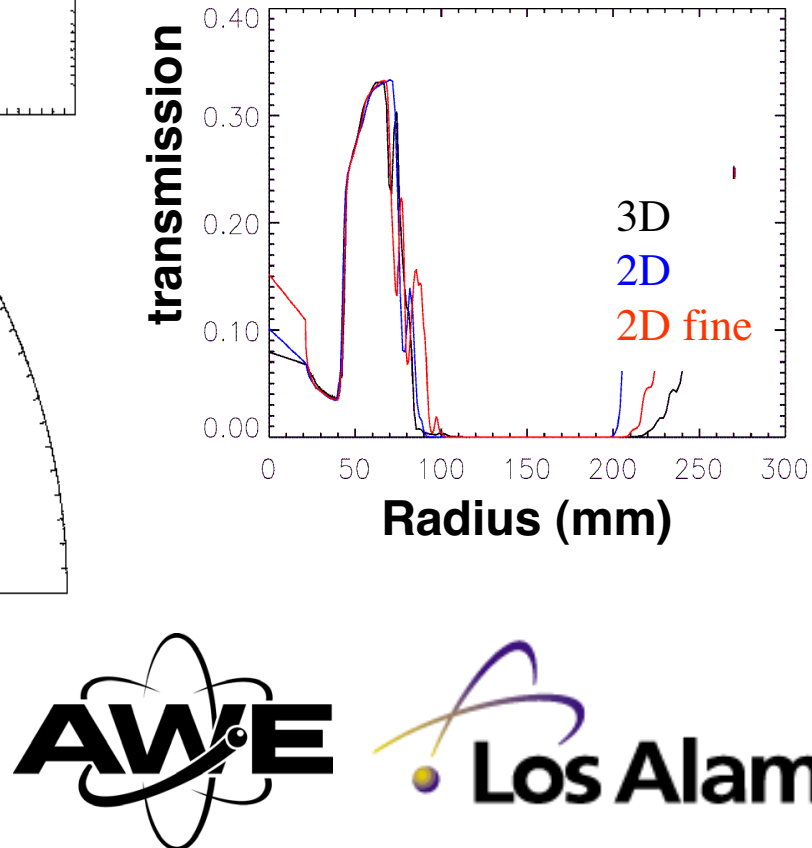

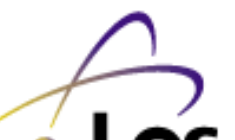




\section{Conclusions}

-We have established a useful, laser-based test bed for convergent, compressible, plasma mix experiments

- With sufficiently rough initial surfaces, we see measurable mix which increase close to linearly in time

- Results are in agreement with 2-D (in r-Z and $r-\theta$ ) and 3-D fully resolved direct numerical simulations

- Future improvements: A lower opacity marker layer will eliminate the end-effect vortical structures 


\section{Mixing Between Two Compressing Cylinders}

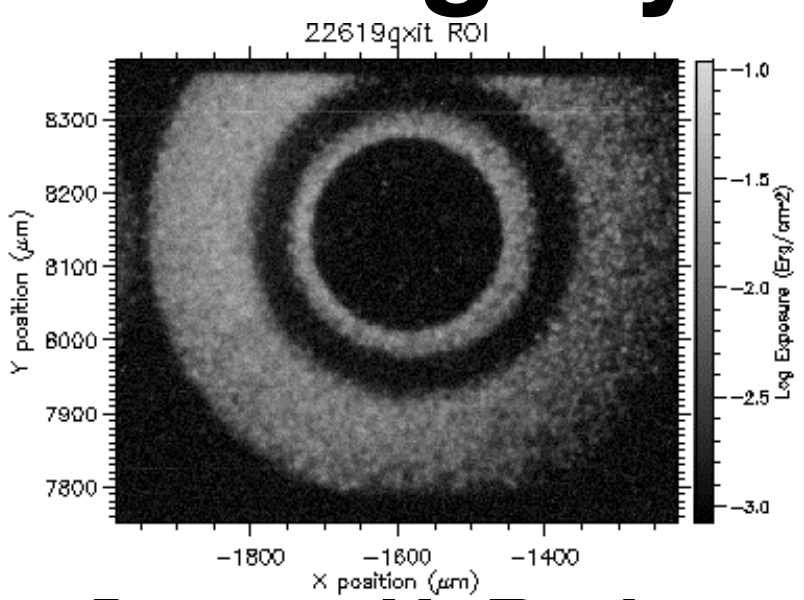

Steven H. Batha

Los Alamos National Laboratory

K. W. Parker, ${ }^{\#}$ C. W. Barnes, ${ }^{*}$ A. M. Dunne, ${ }^{\#}$ N. E. Lanier, ${ }^{*}$ G. R. Magelssen, ${ }^{*}$ T. J. Murphy, ${ }^{\star}$ S. D. Rothman, ${ }^{*}$ J. M. Scott, ${ }^{*}$ and D. L. Youngs ${ }^{\#}$

*LANL, "AWE

8th International Workshop on Compressible Turbulence and Mix

Poster E5; Pasadena, CA December 11, 2001 


\section{Abstract}

- Foam-filled cylinders have been imploded by the OMEGA laser at the University of Rochester. A marker layer of heavier material is placed between the foam and the outside ablator. The marker layer is hydrodynamically unstable when a strong shock passes through both these interfaces and the marker layer material mixes into the foam and the ablator.

- These experiments thus measure mix in the compressible, convergent, miscible, strong-shock regime. These experiments are being extended by placing a solid cylinder at the center of the foam, forming a set of concentric cylinders separated by foam. The initial shock converges on the central cylinder and then rebounds and expands. The shock is predicted to create even more mixing of the marker layer as it traverses the previously mixed region. We present experimental measurements of this configuration.

- LA-UR-01-2575: This document was produced by the Los Alamos National Laboratory under the auspices of the United States Department of Energy under contract no. W-7405-ENG-36.

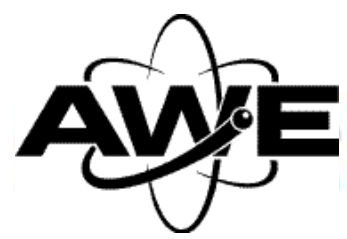




\section{Why direct-drive cylindrical implosions?}

- Purpose

-Study Richtmyer-Meshkov (RM) instability in compressible, convergent, miscible systems undergoing strong shocks

- Examine mixing due to reflectance of a shock from the center (reshock)

- Method

- Implode cylinder with an unstable interface and measure resulting mix

- Diagnostic advantages, fewer ends to affect experiment, convergent

- This poster presents initial experimental results

- See Kenny Parker's poster for design information

- "Computational Modeling of 2 - Shell Cylindrical Implosions with Mix" for design information

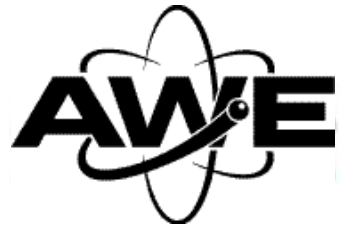




\section{We have established a useful, laser- based test bed for mix experiments}

- Implode cylinder with thick ablator with 1-ns square pulse direct laser irradiation

- Hydrodynamically unstable at plastic/Au and Au/foam interfaces

- Backlight with $\mathrm{x}$ rays through cylinder

- Measure radial extent of "mix layer" of Au into adjacent materials

- 1D convergent experiment with Mach number $\approx 20$ (pre-shock; Mach $\approx 5$ post-shock), convergence $\approx 4$, Pressure > 45 Mbars, Reynold's number $\approx 10^{6 *}$
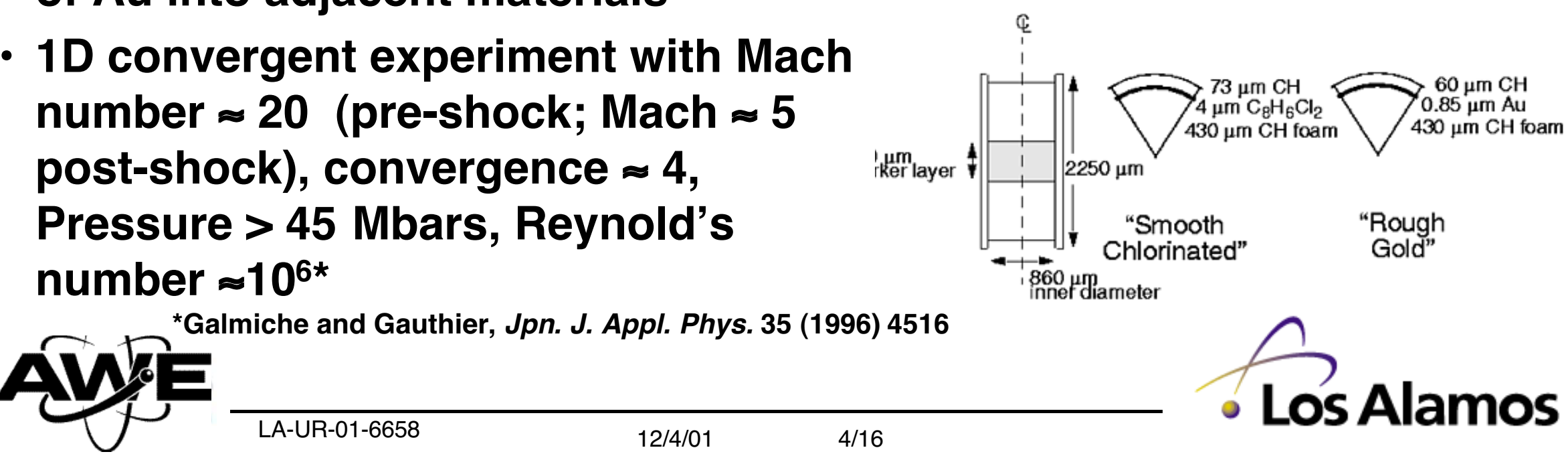

Galmiche and Gauthier, Jpn. J. Appl. Phys. 35 (1996) 4516

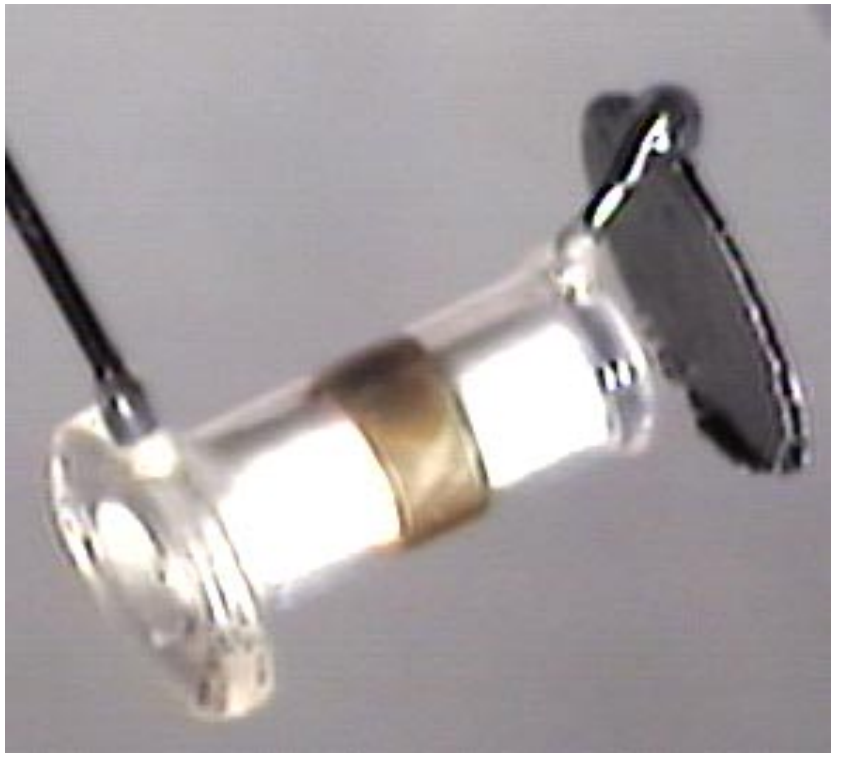




\section{We use the Omega laser at the University of Rochester}

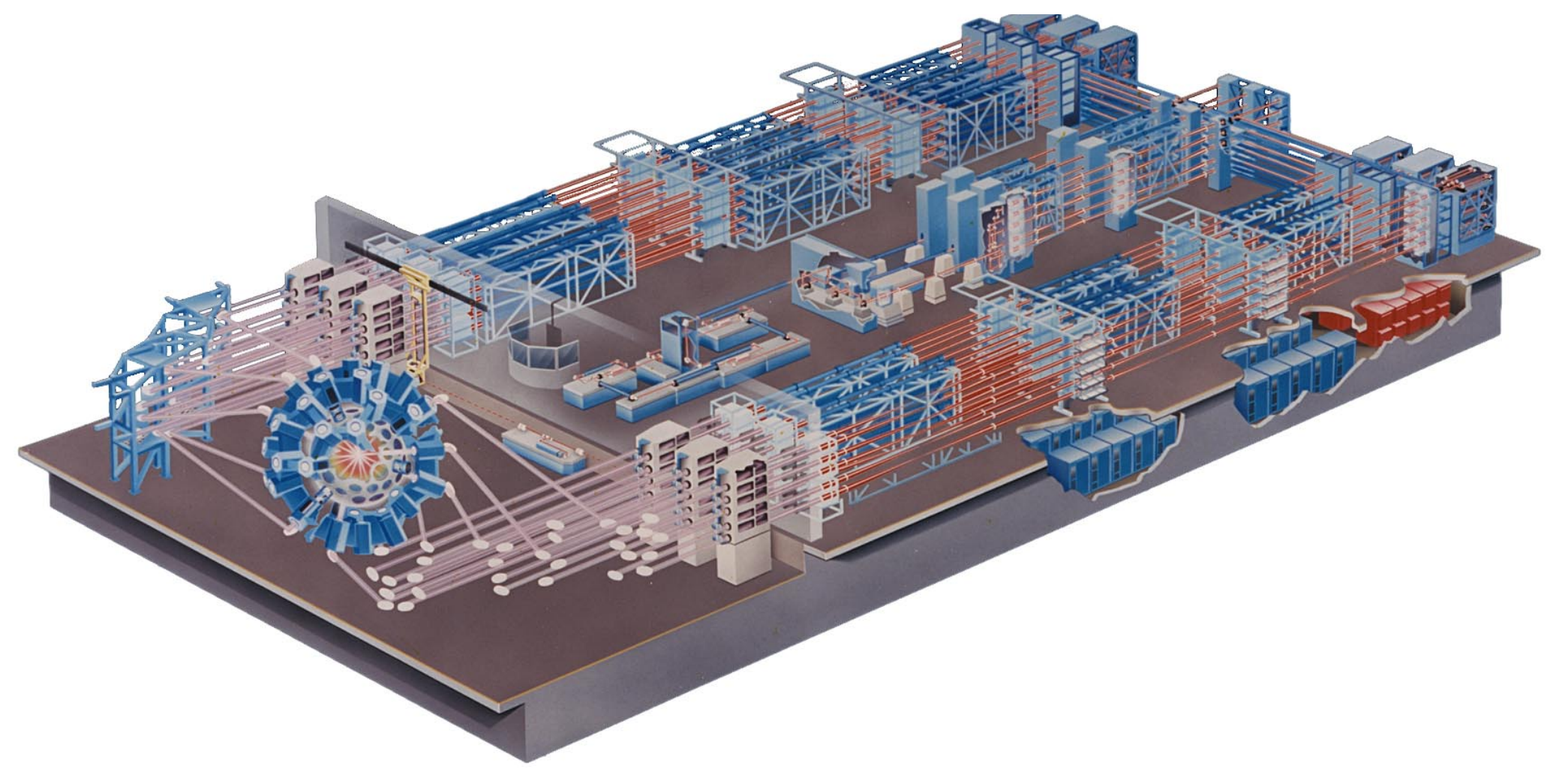

- 60 beams

- >30 kJ UV on target

- 1\%-2\% irradiation nonúniformity

- Flexible pulse shaping

- Short shot cvcle (1 h) 


\section{Complete Diagnostic}

Coverage Available

- Diagnostics for shot 13315

XRFC4 Axial Radiography

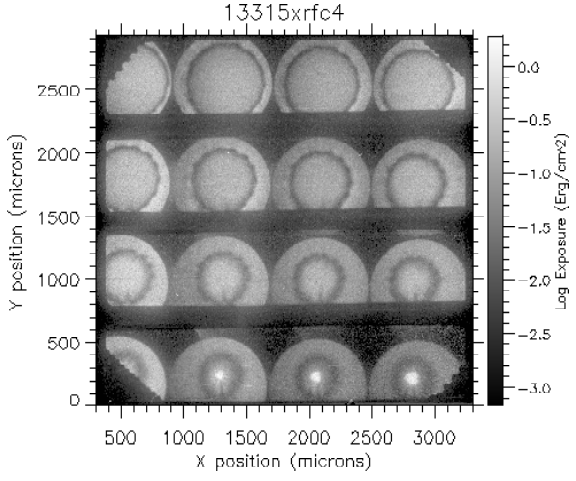

XRFC3 Transverse View of Self-Emission

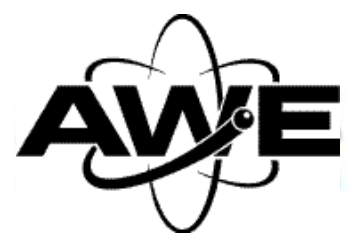

LA-UR-01-6658

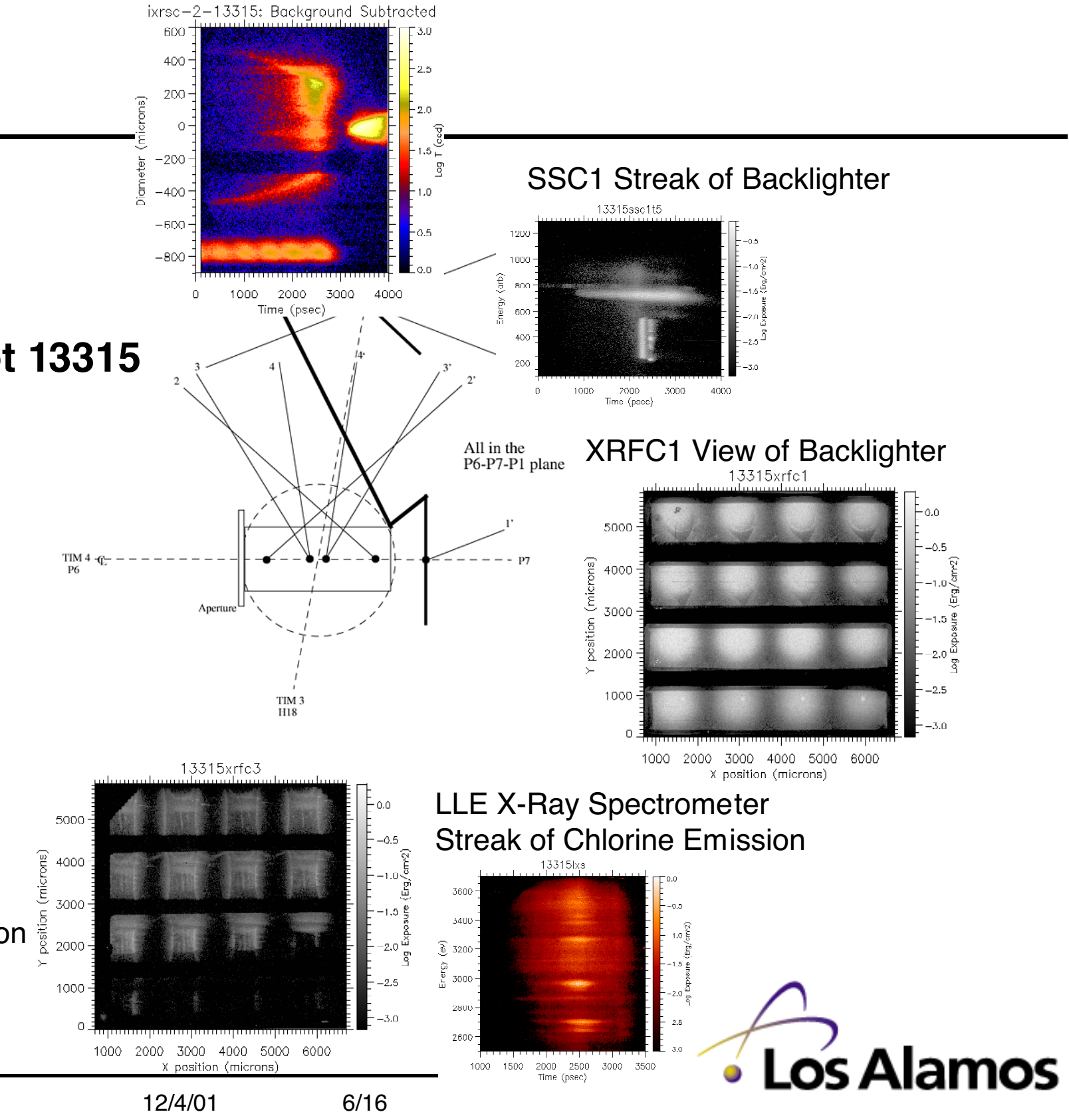

LLE X-Ray Spectrometer

Streak of Chlorine Emission
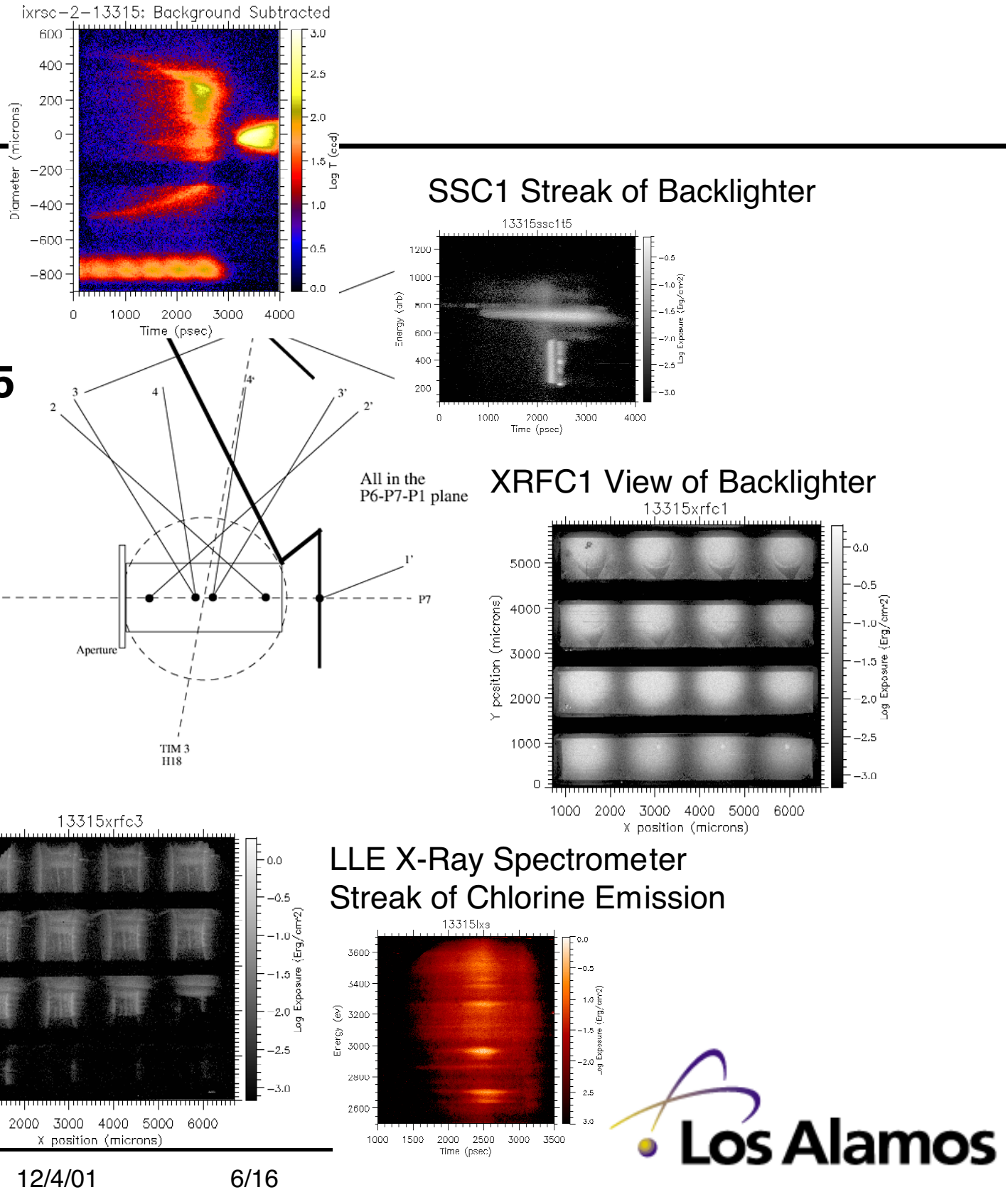

- Los Alamos 


\section{Double cylinder adds an inner shell}

- Al or Cu wire in center provides a hard reflector for the main shock

-Wire: $700 \mu \mathrm{m}$ long

- Marker layer: $\mathbf{5 0 0} \boldsymbol{\mu \mathrm { m }}$ long

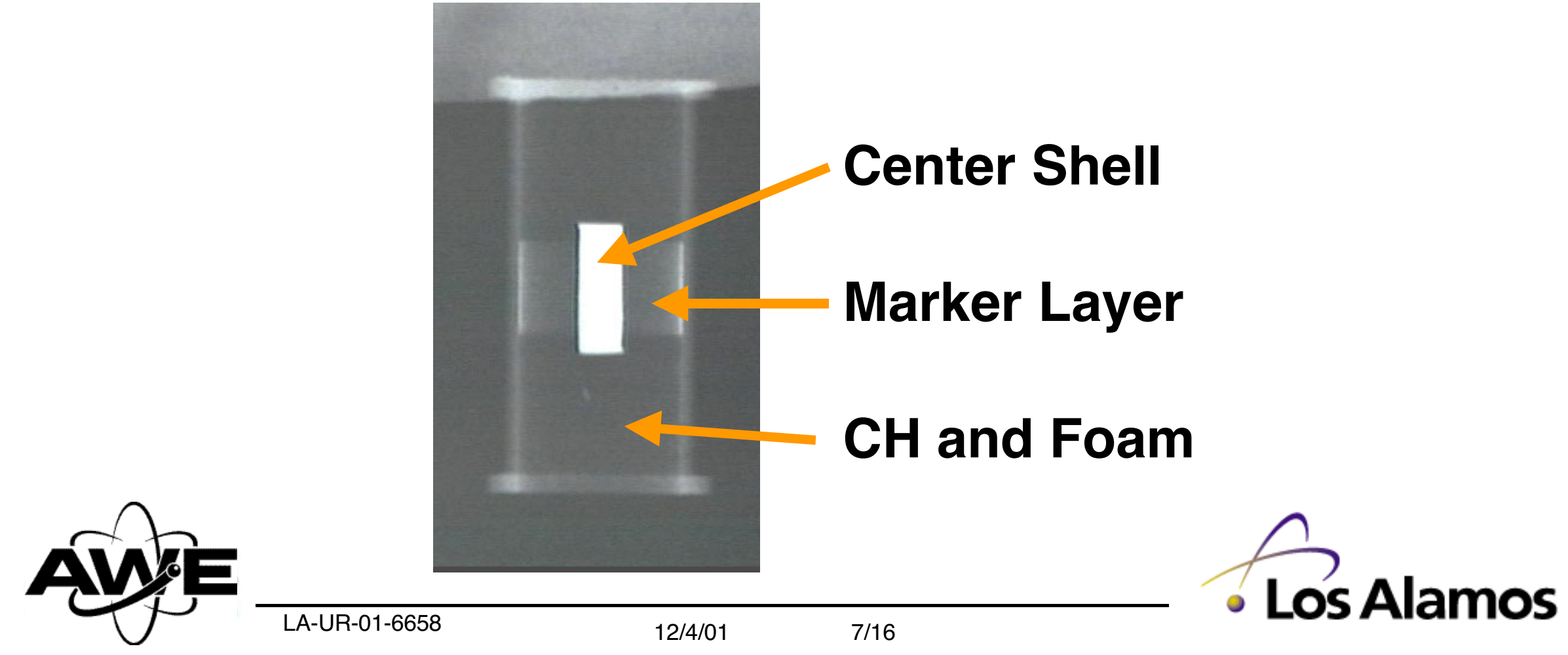




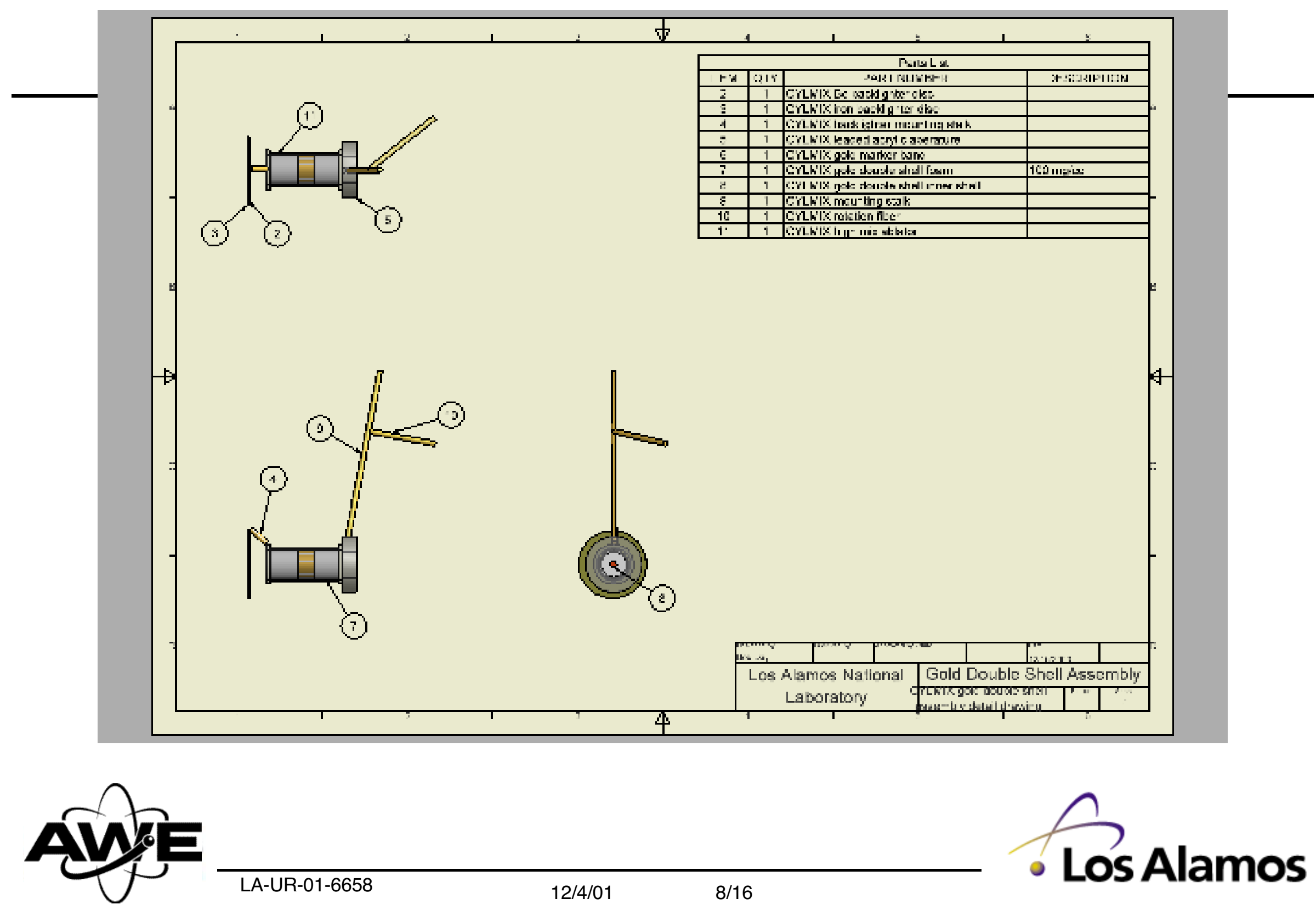




\section{Intershell region undergoes strong shocks and mix}
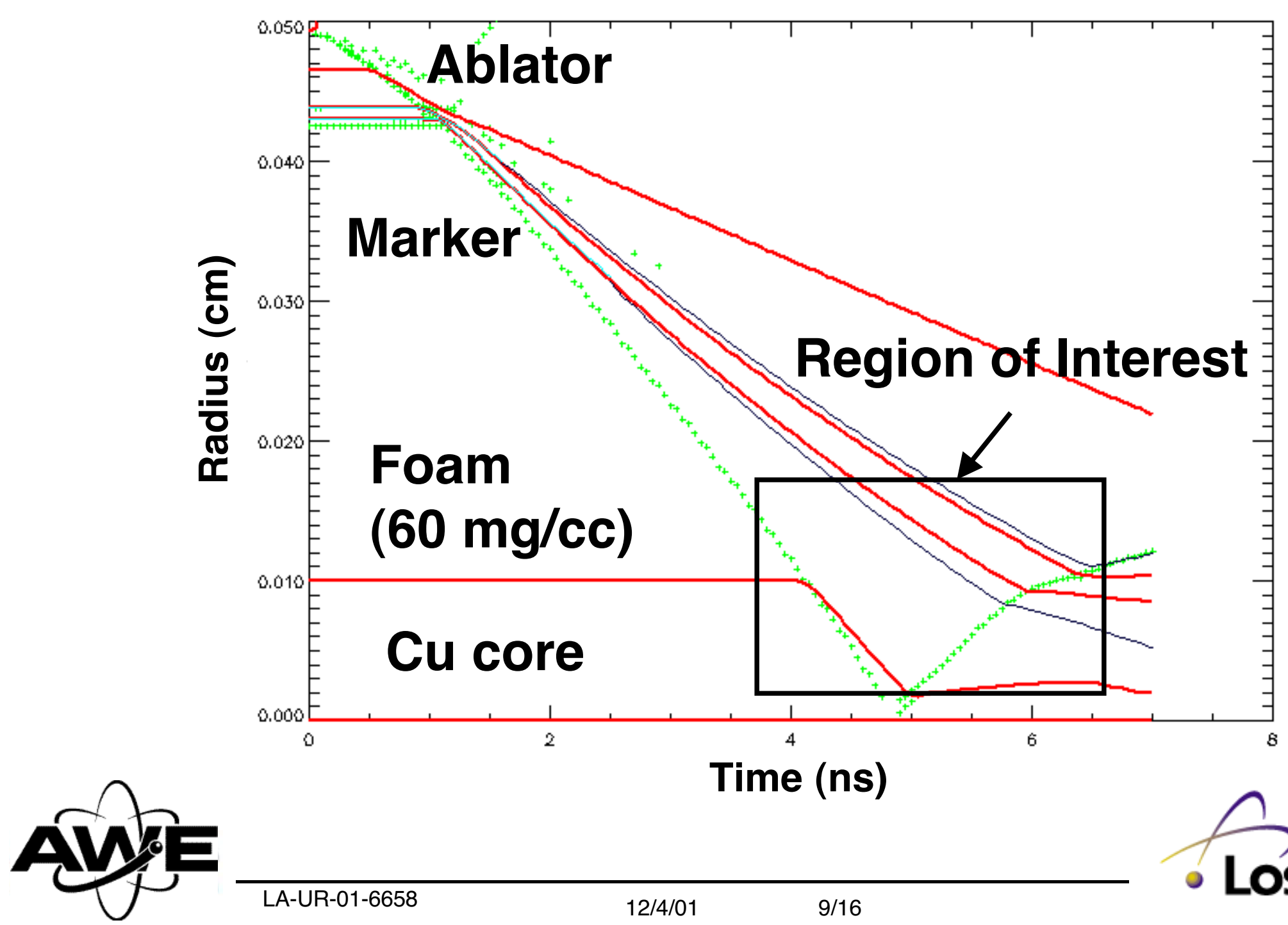


\section{Time/space regions of interest}

- Proof-of-principle experiment based on low-mix design

-Solid, centered Al "shell"

- Want to diagnose mix between shells

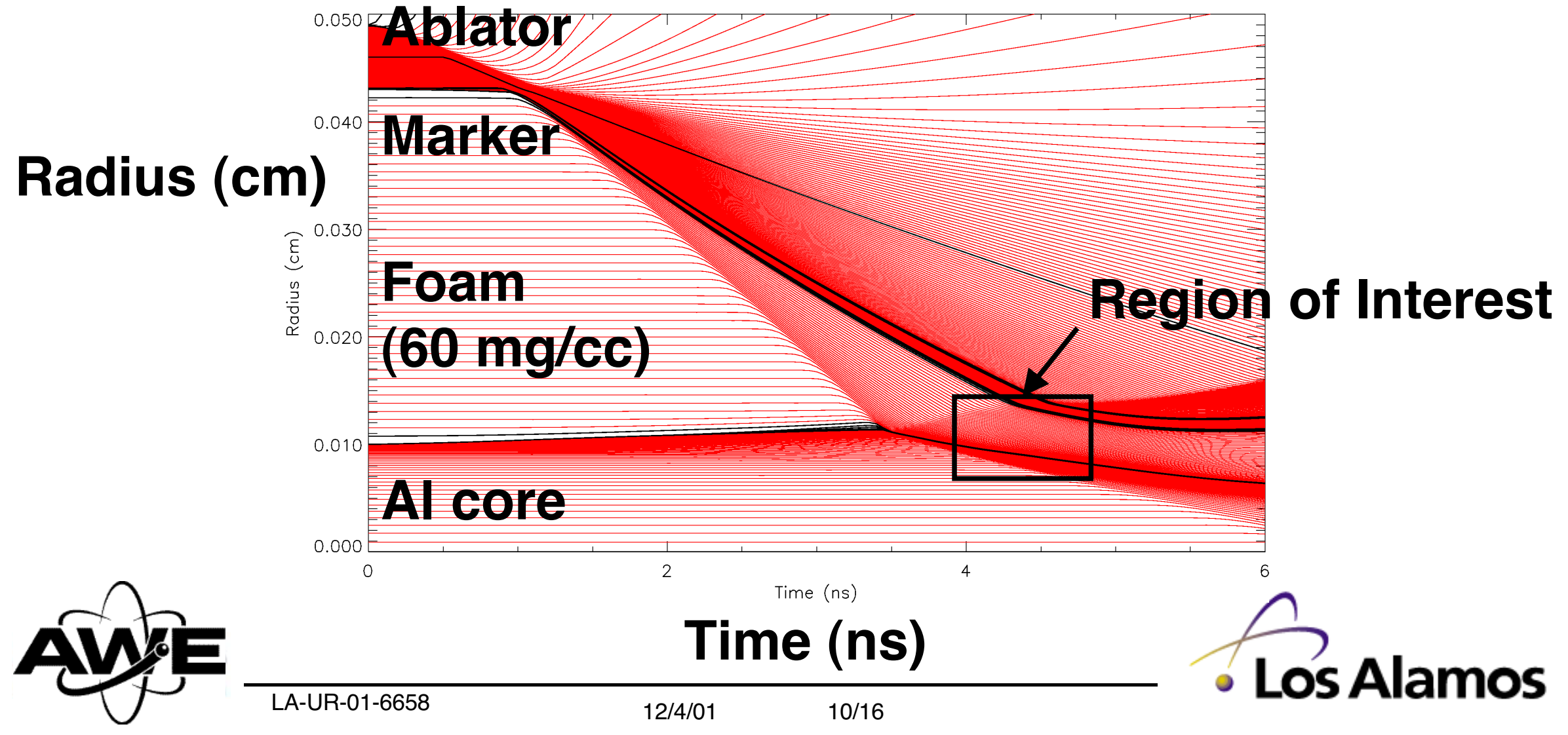




\section{Intershell region clearly visible}

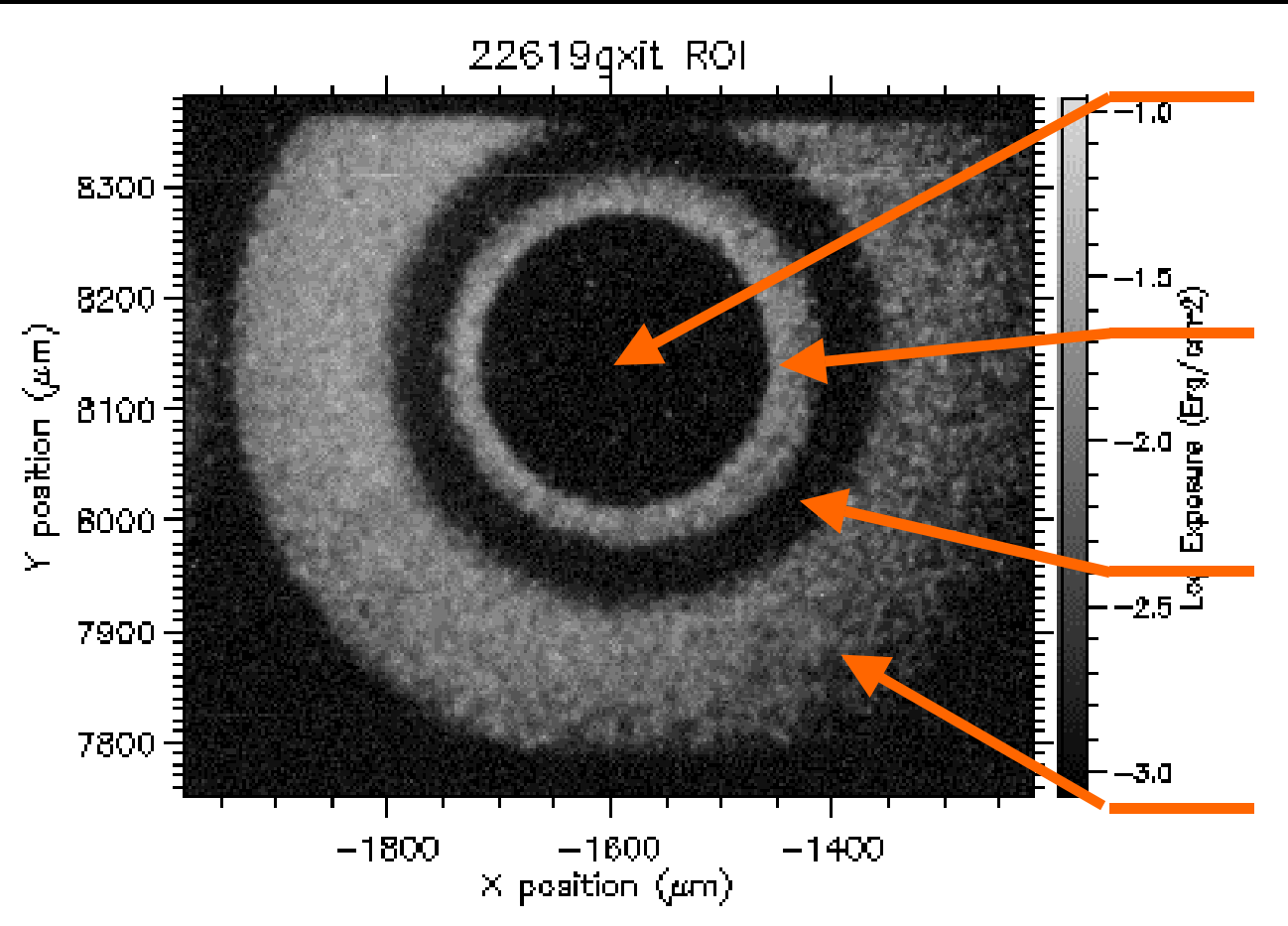

Cu center shell (opaque)

Foam between shells (transparent)

Ni marker layer (opaque)

Plastic ablator

(transparent)

- Marker mixes into foam and ablator

- Radiograph at $6.9 \mathrm{keV}$ (Fe K-shell)

- Backlighter intensity varies smoothly across image

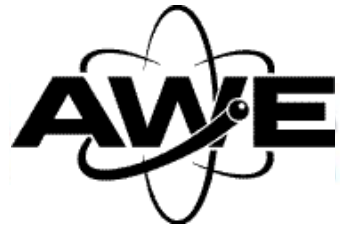




\section{Radial lineout shows different regions}

- Average radial transmission profile

Cu center shell (opaque)

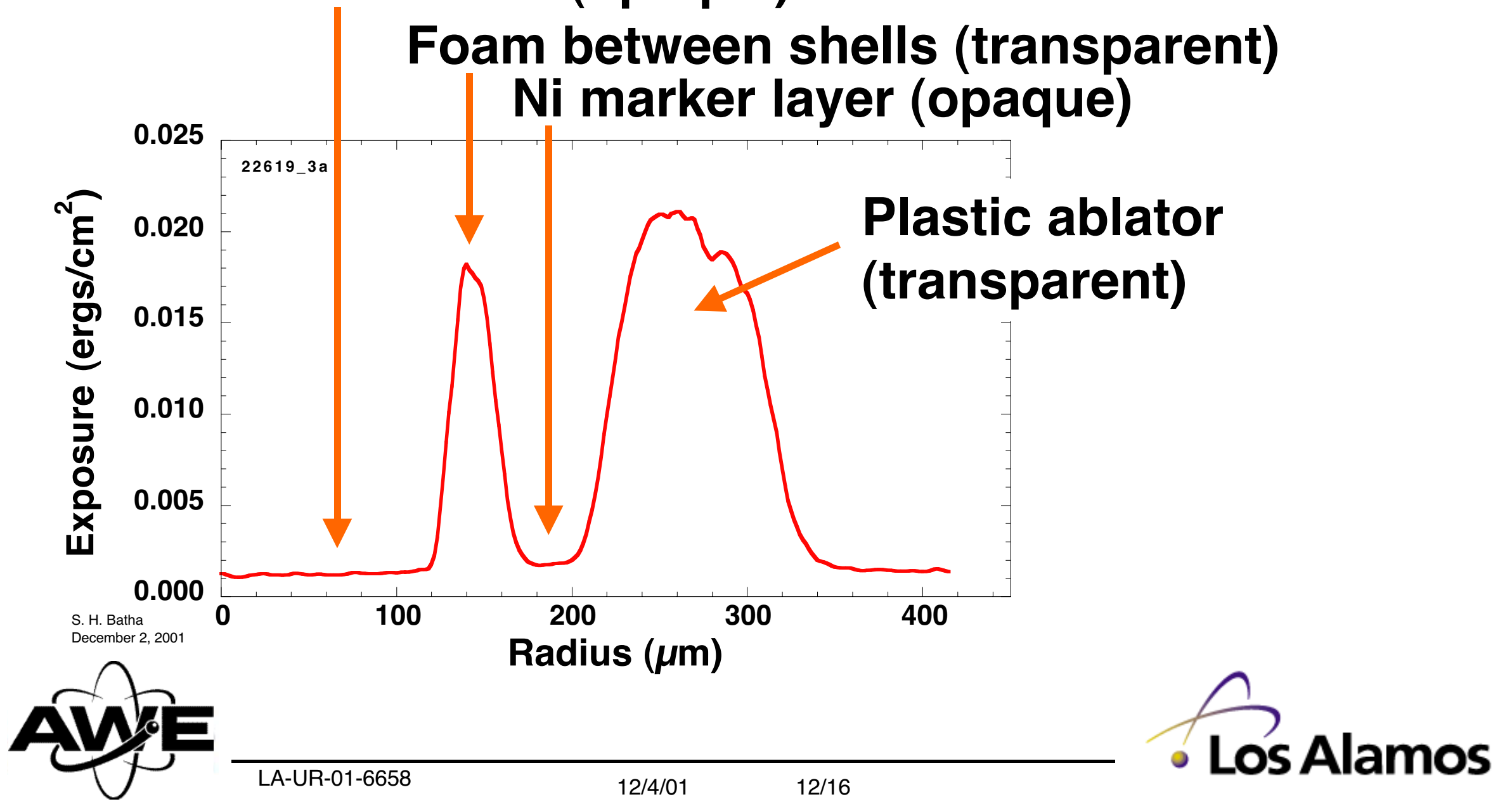




\section{Compare double to single cylinders}

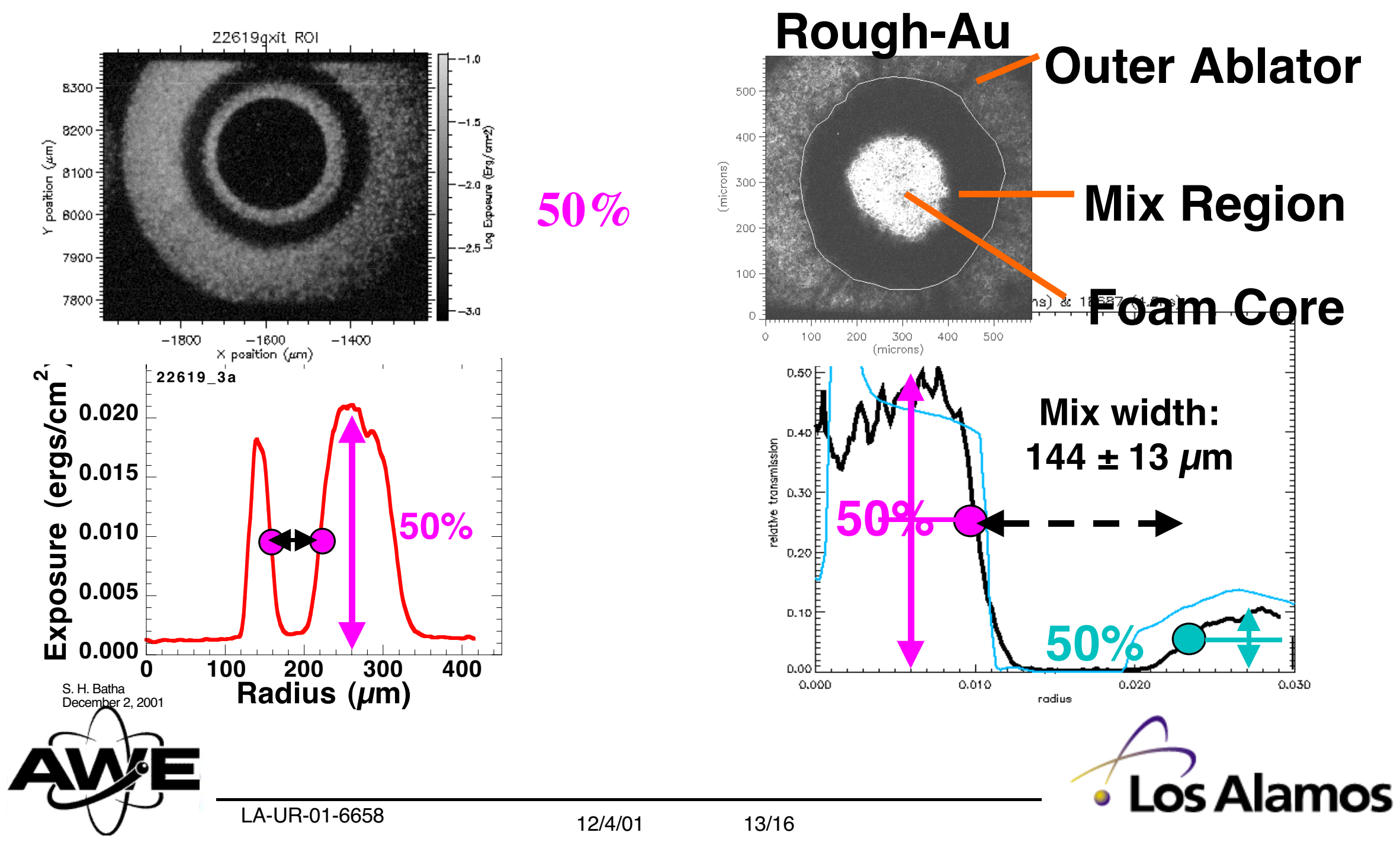




\section{Marker layer has expanded near peak compression}

- Marker/inner shell separation initially $290 \mu \mathrm{m}$

-4- $\mu$ m-thick Ni marker

- Mix width $\approx 65 \mu \mathrm{m}$

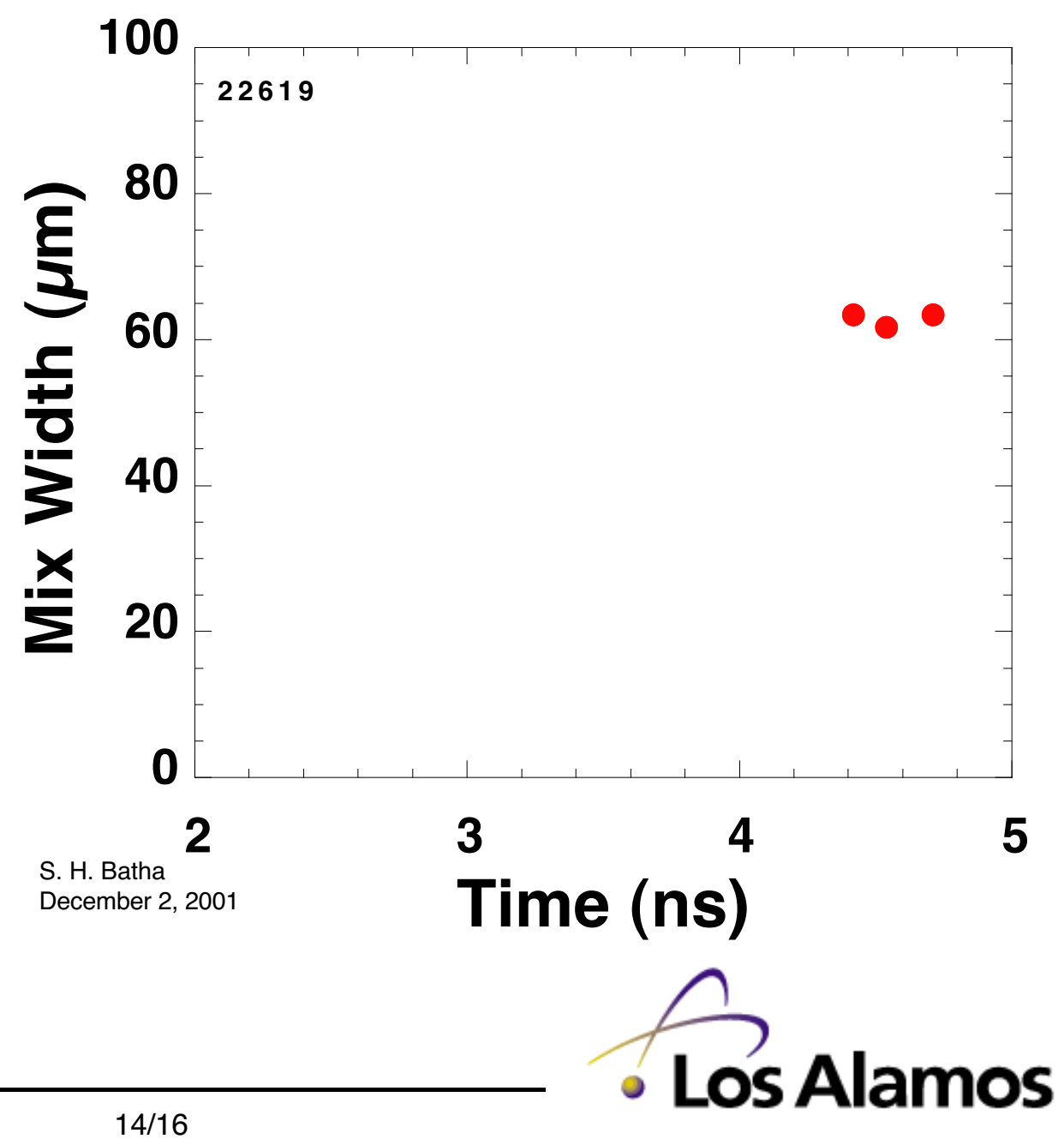




\section{Dynamics of implosion as expected}

- Little change in outershell radius seen over 400 ps

- Central shell has not expanded (initial radius $\approx 140 \mu \mathrm{m}$ )
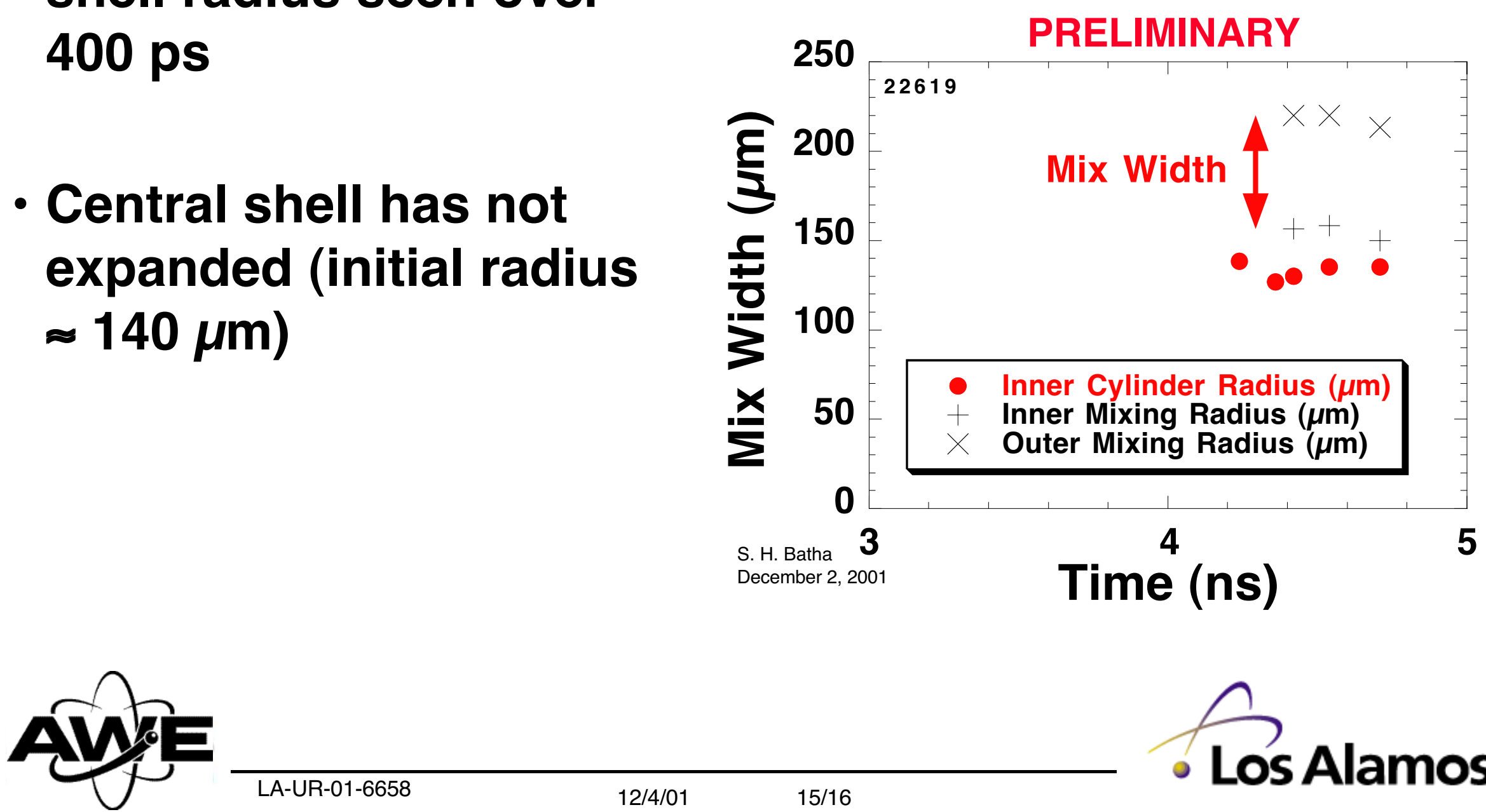


\section{Conclusions}

- Double-cylinder targets can be built and fielded

- Excellent radiographic data of intershell region obtained

- Central shell does not expand during experiment

- Mixing observed

- See Kenny Parker's poster (C28)

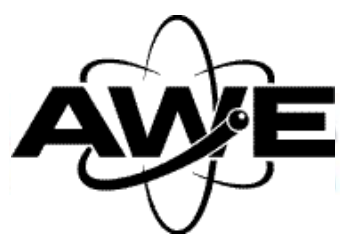




\title{
DEVELOPMENT OF THE METHOD FOR INTERACTION BETWEEN SHOCK WAVE AND FLAME
}

\author{
M.Bliznetsov ${ }^{1}$, V.Dudin ${ }^{1}$, S.Gerasimov ${ }^{1}$, L.Houas $^{3}$, G.Jourdan $^{3}$, A.Logvinov $^{1,2}$, \\ E.Meshkov ${ }^{1,2}, Y u$. Vlasov $^{1}$ \\ ${ }^{1}$ Russian Federal Nuclear Center - Institute of Experimental Physics (VNIIEF), 607190, Sarov,Russia \\ ${ }^{2}$ Sarov Physics Technical Institute, 607190, Sarov, Russia \\ ${ }^{3}$ IUSTI/CNRS UMR 6595, Universite de Provence, Technopole de Chateau-Gombert, 13013, Marseille, France
}

\section{Introduction.}

Wild fires are disastrous events damaging the environment. Numerous examples show that extinguishing the wild fires is a hard line, which does not ever finish successfully. Let us remember, for example, the fire in the Los Alamos region in spring, 2000.

There is a method of extinguishing the wild fires by means of exploding of longitudinal charge located before the flame front $[1,2]$. Here, there is observed the amplifying of the shock wave after it passing through the flame zone. The effectiveness of the method was experimentally demonstrated [3]. However, it is not put into practice.

According to the authors' hypothesis [3] this extinguishing is due to blowing a flame off by a shock. Amplifying the shock is considered in terms of additional explosion of a mixture consisting of air and pyrolysis products (PP) from crown fragments (pine needles, small branches, and leaves). This additional explosion is supposed to follow a shock from initial explosion.

There is another hypothesis [4], which considers the process is due to growing the hydrodynamic instabilities.

On explosion of a cylindrical longitudinal charge, an expanding non-steady shock is generated. The substance accelerates on the shock wave front, and, then decelerates. When the shock wave passes through the flame front, this corresponds to its propagation from a substance with a more high density (PP-air mixture) into another of lower one (flame). As a result, we have conditions for occurrence of instability induced by the shock wave [5,6], and then, the conditions of Rayleigh-Taylor instability arise for the flow behind the front of shock wave because the acceleration directs from light to heavy substance $[7,8]$.

Thus, the instability of flame front grows, and an intense mixing between flame and PP-air mixture should arise, so it should effect the sharp combustion of this mixture. A rapid (or possibly, explosive) combustion of the mixture should result in generation of a compression wave (or series of compression waves) overrunning and amplifying the shock wave generated by the charge explosion.

Experimental research with a real wild fire is rather complex, dangerous, and expensive. That is why the new methods for study the interaction between non-steady shock with a flame front in cases close to a crown forest fires are of actual interest.

The results obtained at developing such method are presented below.

The most complex problem here is to create a model even qualitatively related to peculiarities of crown fire propagation:

- $\quad$ generation of gaseous explosive mixtures before flame front due to its high temperature

- $\quad$ high rate for the flame front propagation

For study the interaction between non-steady shock with a flame front we suggest a spatial model consisting of parallel (or diverging from a point) threads or rods along which an inflammable substance able to be fume before a flame front is distributed.

A non-steady shock could be generated towards the flame front propagating through the model; so, one can observe the process of the shock-flame interaction. The means for generating the non-steady shock could be different for different scales:

- electrical explosion of a wire tightened before the lame front;

- $\quad$ shock tube with compressed gas or gaseous explosive mixture as a driver [9];

- $\quad$ explosion of a longitudinal HE (for example, detonation flex).

\section{Experimental research for the spatial thread model.}

First stage dealt with laboratory experiments using a small spatial model consisting of threads diverging from a point.

At first we performed experiments with one and two threads. The threads were saturated with combustible liquid. Such liquids were alcohol, acetone, solvent 646, and kerosene. As it turned out kerosene was the most suitable. Kerosene as well as PP is a limiting hydrocarbon.

The flame propagation was visualized by video.
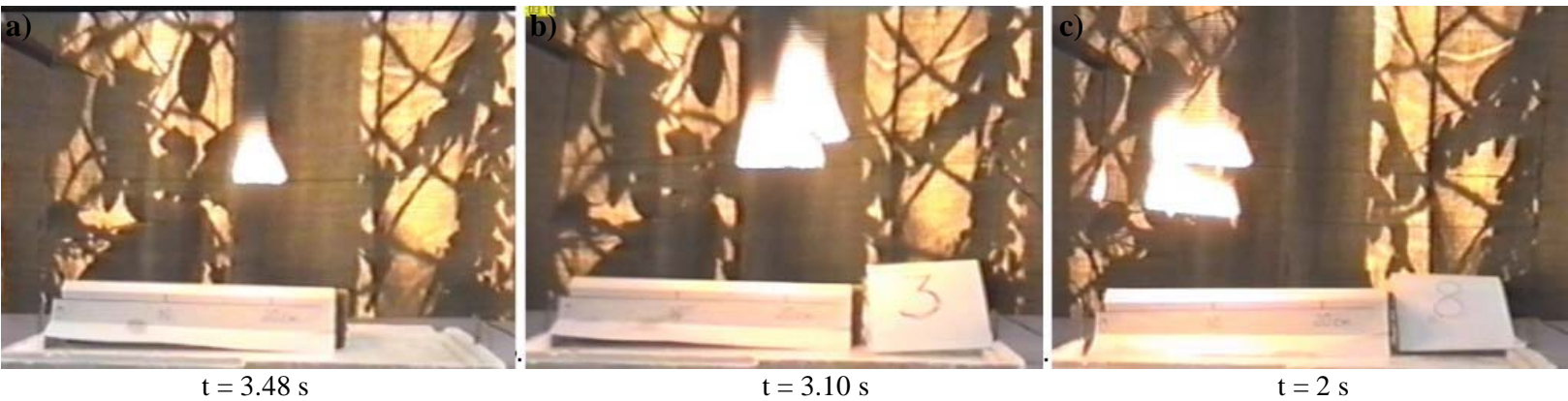

Figure 1. Flame propagation along a) a thread saturated with kerosene, b) two threads diverging from a point, c) two parallel threads 
Figure 1 presents the frames of the flame propagation a) along a thread, b) along two threads diverging from a point, and c) along two parallel threads. The frames show that in b) case the upper flame overruns the low - it was due to the dependence of flame rate on slope tangent. We performed a series of tests varying the slope tangent. The results obtained were handled (Figure 2). Ought to note the linear character for the dependence.

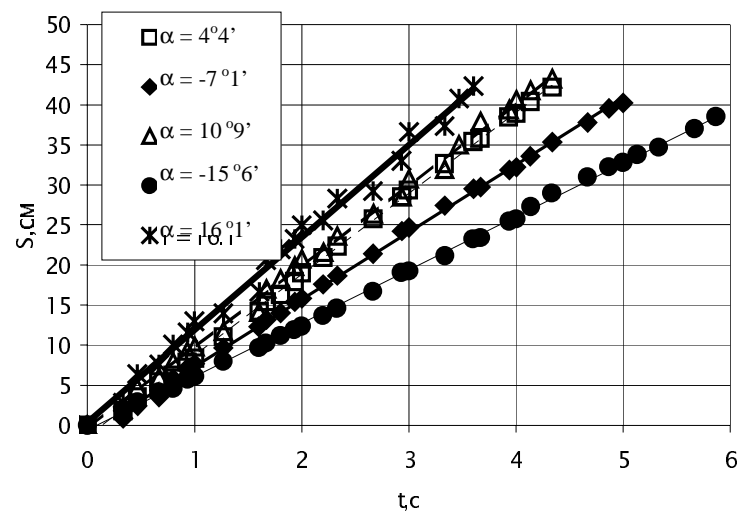

Figure 2. Dependence in time $t$ of the flame front propagation $S$ along a thread on its slope tangent $\alpha$. The angle is counted from skyline, positive direction at propagation from below.

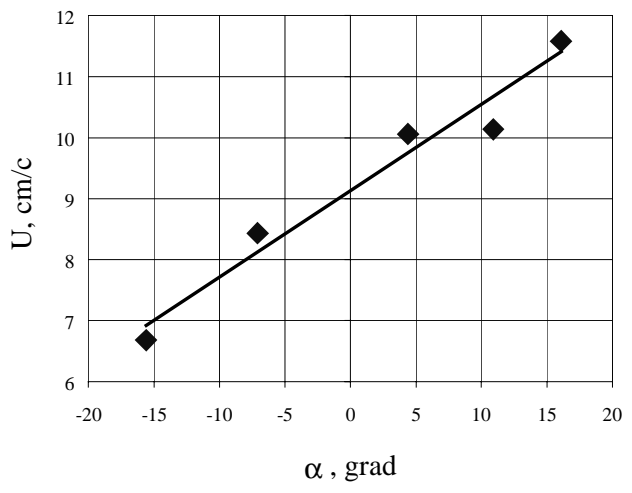

Figure 3. Dependence of rate of flame propagation $U$ along a thread against its slope tangent $\alpha$.

The measured rate of flame propagation for the tests is presented in Figure 3.

Figure 4 shows the frames for flame propagation in a model consisting of 25 threads diverging from a point. The ends of threads were fixed in knots of a grid $(5 \times 5)$ with a cell of $2 \mathrm{~cm}$. The threads were saturated with kerosene.

Figure 5 shows the results of measurements for this model.

It should be noted that at recording the process from side one could find a) a weak acceleration of the flame front and b) overrunning for the second thread from the top (Figure 4,a a frame at $3.15 \mathrm{~s}$.) but not for the upper. Simultaneously, at recording from above one could observe the overrunning in the middle region (Figure 4,b).

These effects could be related to the heating of the threads before the flame front and evaporating of the combustible liquid from the threads. A more intensive heating of the threads should be realized in the middle region of the front. Due to the more intensive heating a more intensive evaporation of the combustible liquid is realized that corresponds to a more rapid propagation of the flame front.

These effects are weakly found here owing to the small sizes of the model; apparently, increasing the scale of these effects should follow increasing the model sizes.

\section{Shock wave and flame interaction. Preliminary tests.}

We have performed a few preliminary tests for the shock wave and flame interaction. There were two types of the tests:

1. The shock wave was induced by electrical explosion of a thin metal wire (nickel-chrome, $0.05 \mathrm{~mm}$ ) through which there was realized a discharge from a capacity of $0.1 \mathrm{mcF}$ at $60 \mathrm{kV}$. It was followed by a cylindrical shock wave.

The shadow pattern of the interaction between the shock wave and the flame propagating along a thread saturated with kerosene was visualized with a high-speed camera.

The thread and wire were located in the same plane; the axis of the thread was normal to the wire's.

The frames for the interaction process are presented in Figure 6. One can find the following stages of the process:

- $\quad$ some displacement of the flame under shock wave

- generation of a part of the shock wave passed through the flame and overrunning the main part of the front $\left(\mathrm{ShW}_{1}\right)$

- growth of perturbations on the initially smooth, laminar flame front with its further turbulence. In this test the flame was more probably extinguished due to blowing it off. But it is a fact that the flame being initially laminar with a rather smooth front got unstable and turbulent.

2. In test (Figure 8$)$ the shock wave was generated through detonation of a layer $(8 \times 8 \times 2 \mathrm{~cm})$ of gaseous explosive mixture of acetylene and oxygen. Initially, the mixture filled the volume of a shock tube [9] with rigid lateral and back walls; the output window was closed with a thin mylar film of $0.9 \mathrm{~mm}$ - thickness.

In this case the flame was generated on a spatial model with 25 parallel threads saturated with kerosene. Figure 8 shows the frames of shadow pattern for the interaction process. The shock wave was stronger (2-3 Mach) in this case. The flame was extinguished with the wave. There were observed both the perturbation growth and sufficient displacement of the flame under the wave. 
a)
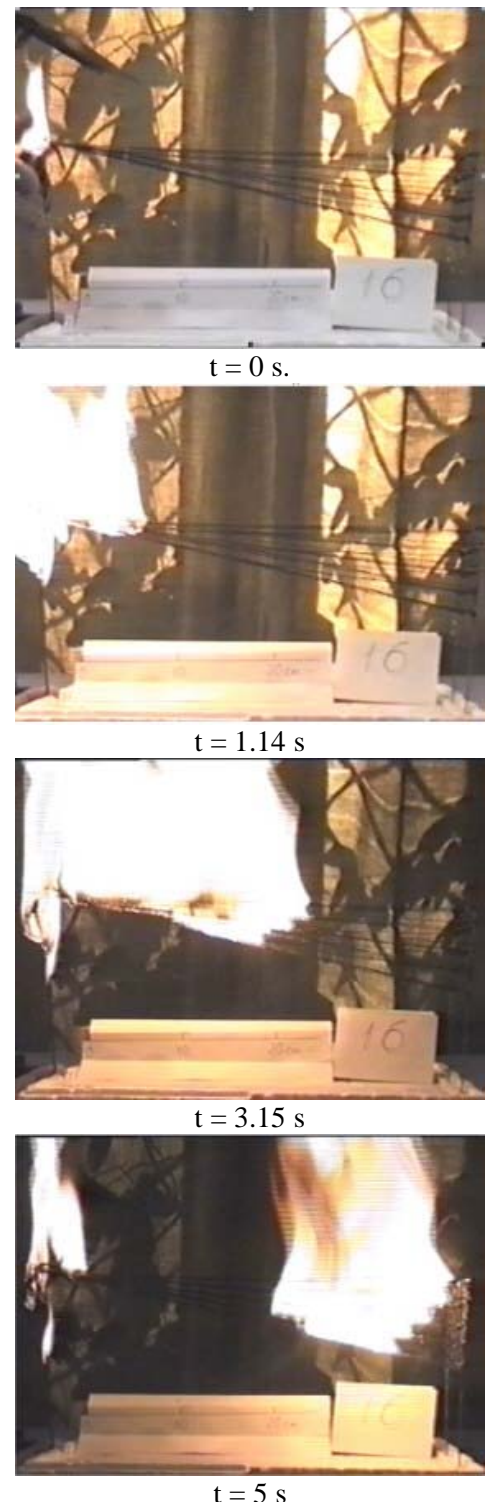

b)
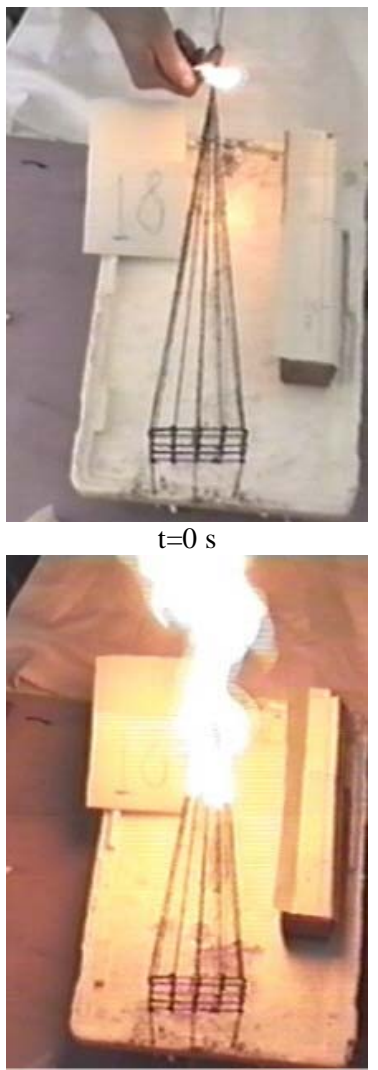

$\mathrm{t}=2.15 \mathrm{~s}$

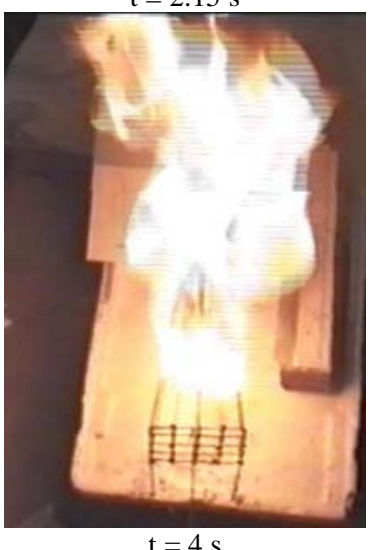

$\mathrm{t}=4 \mathrm{~s}$

Figure 4. Flame propagation along spatial model consisting of $5 \times 5$ threads diverging from a point a) side view, b) from above view (test N16 and 18)

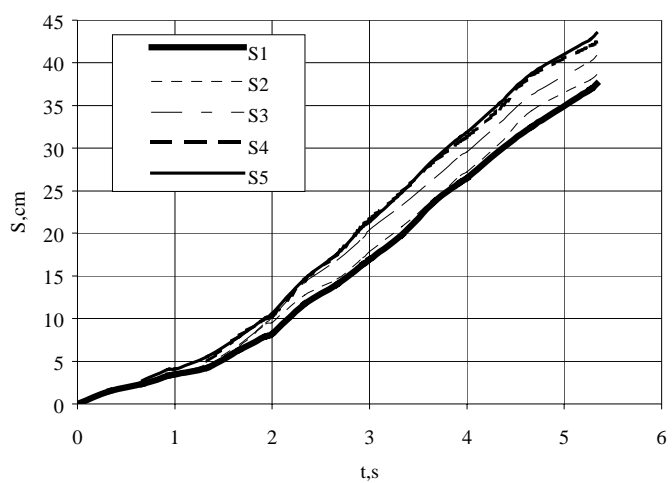

Figure 5. Dependence of flame propagation $S$ along diverging threads saturated with kerosene on time $t$ 


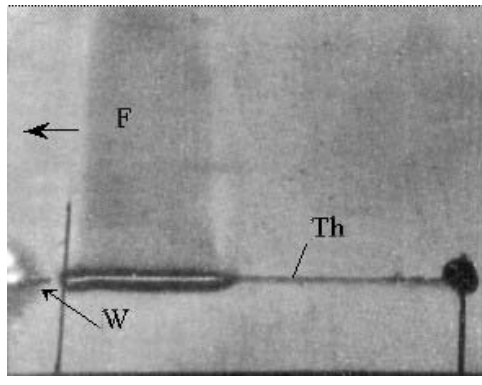

$\mathrm{t}=32 \mu \mathrm{s}$

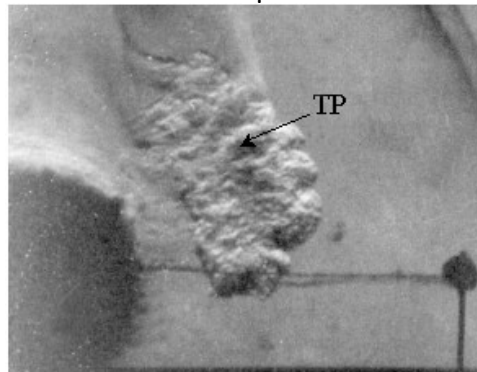

$\mathrm{t}=355 \mu \mathrm{s}$

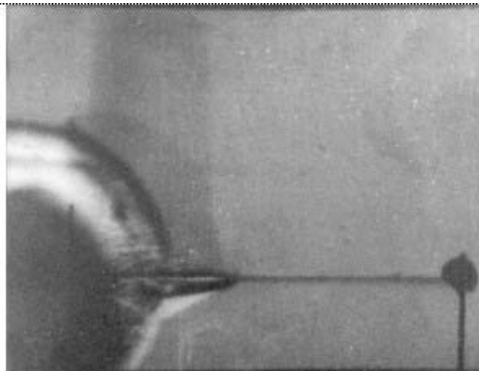

$\mathrm{t}=97 \mu \mathrm{s}$

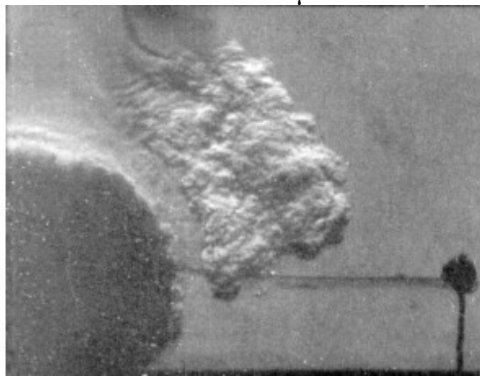

$\mathrm{t}=614 \mu \mathrm{s}$

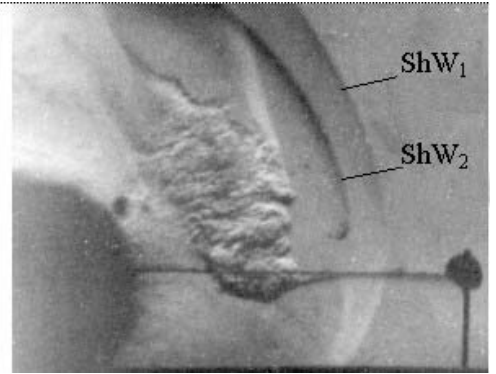

$\mathrm{t}=226 \mu \mathrm{s}$

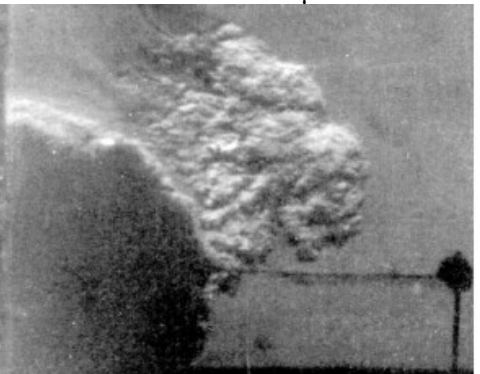

$\mathrm{t}=937 \mu \mathrm{s}$

Figure 6. Frames for interaction between a shock wave generated by electrical explosion of a thin wire and flame propagating along a thread saturated with kerosene. Notations: F - flame; W - electrically exploded wire (its axes is normal to plane of picture); $\mathrm{ShW}_{1}-$ shock wave, that passed region of flame; $\mathrm{ShW}_{2}$ - that spread in air (outside flame); $\mathrm{Th}$ - thread; $\mathrm{TP}$ - turbulized products of burning. Time is counted from the moment of wire explosion.

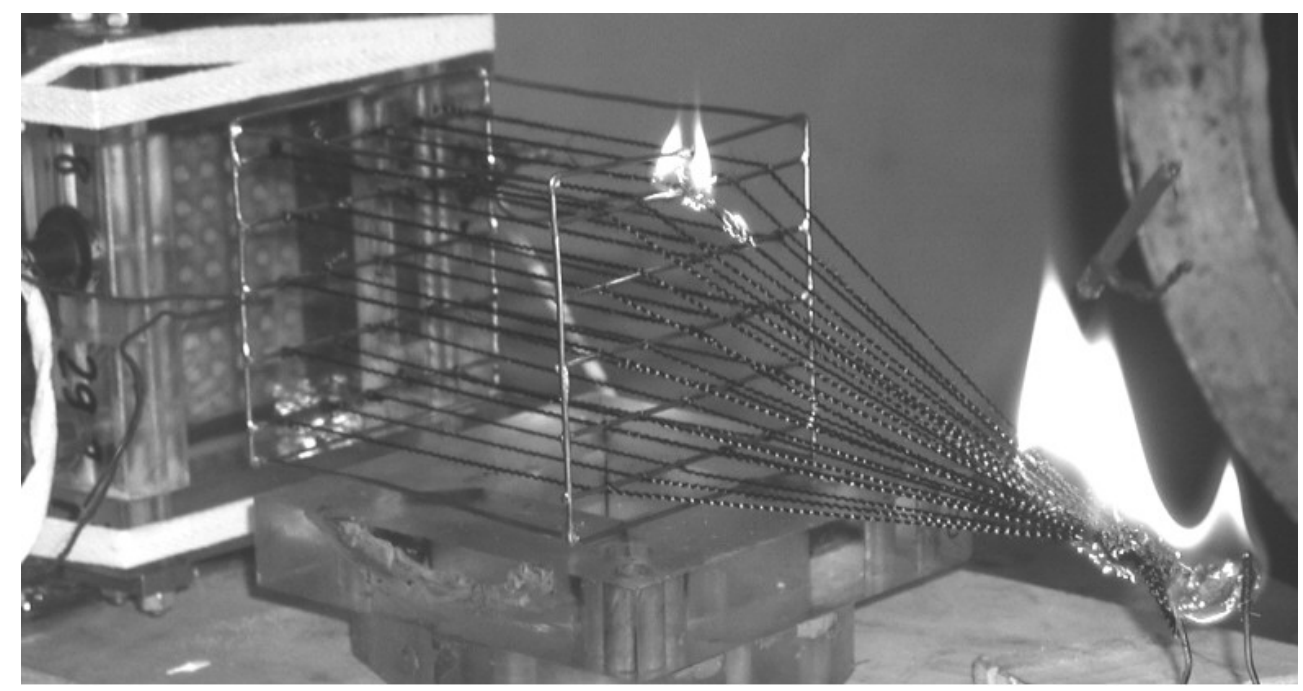

Figure 7 Experimental assembly 


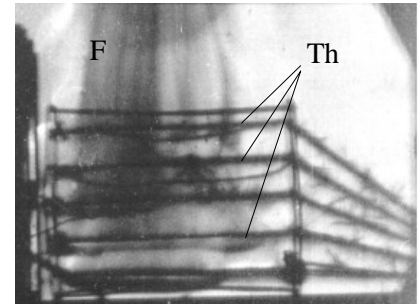

$\mathrm{t}=0 \mu \mathrm{s}$

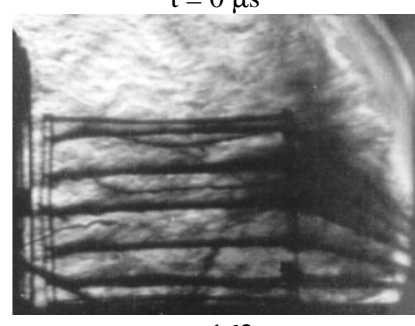

$\mathrm{t}=162 \mu \mathrm{s}$

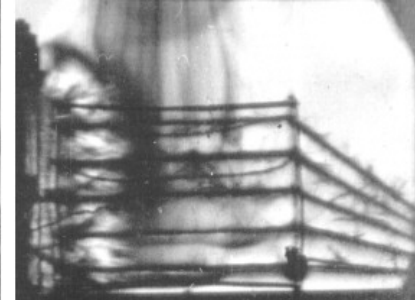

$\mathrm{t}=65 \mu \mathrm{s}$

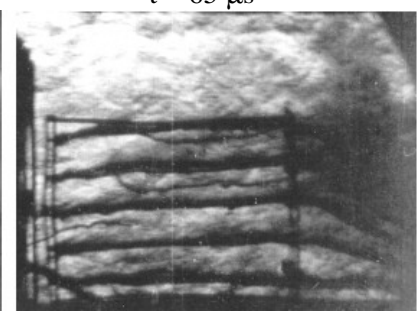

$\mathrm{t}=323 \mu \mathrm{s}$

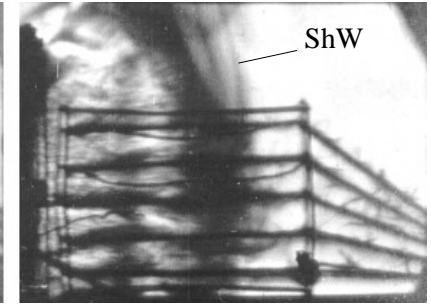

$\mathrm{t}=97 \mu \mathrm{s}$

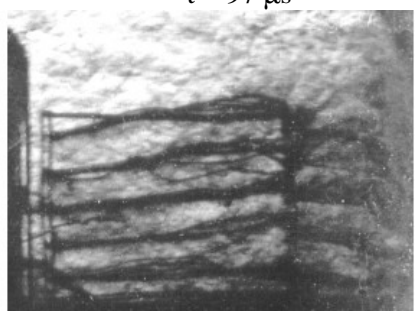

$\mathrm{t}=517 \mu \mathrm{s}$

Figure 8. Frames for interaction between shock wave and flame propagating through spatial model of 25 parallel threads saturated with kerosene. Notations: F - flame; ShW - shock wave; Th - thread. Time is counted from the moment of gas explosive mixture detonation.

\section{Summary and conclusion} progress.

A method for study the interaction between non-steady shock wave and the flame front at conditions close to crown fire is on

An experimental, laboratory model for generating a quasi-flat flame front propagating along a spatial structure of threads saturated with combustible liquid (kerosene), was supposed.

A series of experiments for study the flame propagation both along 1-2 threads and along a spatial structure of $5 \times 5=25$ threads diverging from a point, was performed.

A series of experiments for the interaction between flame front and the non-steady shock generated by a) electrical explosion of a wire and b) explosion of a thin layer of a gaseous explosive mixture was performed.

The obtained results for the shock-flame interaction show:

- the flame displacement under shock wave

- a part of shock, that has passed through the flame, overruns the main front

- a flame front being initially smooth gets turbulent

Further steps in developing this method should be done by increasing the model scale and applying the improved methods of recording. The development of a model with a grid of 1-2 $\mathrm{m}$ size is supposed to be the next step; in this case the most suitable generation of a shock could be done with a longitudinal HE. In the case of favorable results with using the model it could be done a device with characteristic sizes close to real - of several tens of meters.

The development of the method for study the interaction between shock and flame could develop new methods for fighting the forest fires or could improve the existing ones.

Besides, the study in terms of the described above models could be used for checking the corresponding numerical methods.

This work is partially supported by Conseil Generale de Bouche du Rhone (France) and Joint Intersectorial Science - Technical Program of Minatom and Ministry of Education of Russia (grant 749/01).

\section{References.}

1. A.M.Grishin Physics of wild fires. Tomsk. Iss. by Tomsk Univer., 1994, p.218

2. A.M.Grishin, V.P.Zima, A.Ya.Mashovich, V.I.Samoilov Experimental study between shock generated with point charges and crowns. Proceedings of international conference "Common problems of mechanics and ecology". Tomsk, Iss. by Tomsk University, 2000, pp 83-85

3. A.M.Grishin, Yu.Kovalev, Experimental and theoretical study of action of explosion on a crown wild fire. FGV, 1989, №6, pp 7279

4. E.E.Meshkov Turbulent mixing associated with hydrodynamic instabilities in modern problems. Proceedings of international conference "Common problems of mechanics and ecology". Tomsk, Iss. by Tomsk University, 2000, pp 156-158

5. Richtmyer Taylor instability in shock acceleration of compressible fluids. Commun.Pure Appl.Math., 1960, v.13,p.297.

6. E.E.Meshkov Instability of interface between two gases accelerated by a shock. MZhG, 1969, N5, 151-158

7. Lord Rayleigh Proc.London Math Soc, 1983, v.14, p.170

8. G.I.Taylor The instability of liquid surfaces when accelerated in a direction perpendicular to their planes. I. Proc.Roy.Soc., 1950, v.A201,p.192

9. Yu.A.Vlasov, S.I.Gerasimov, E.B.Gubkov, V.A.Dudin, V.P.Kopyshev, E.E.Meshkov, A.A.Nikulin, V.P.Ryabov, V.A.Til'kunov Shock tube with GEM as a driver. Sarov, preprint VNIIEF, 1996, 47-96 
Influence of Scales of Initial Perturbations on Rayleigh-Taylor Instability Growth on GasLiquid Interface

M.V.Bliznetsov, E.E.Meshkov, N.V.Nevmerzhitsky, A.A.Nikulin, E.D.Sen'kovsky, E.A.Sotskov

RFNC-VNIIEF, Sarov, Russia

$8^{\text {th }}$ IWPCTM, Pasadena, USA

\begin{abstract}
Results on the influence of scales of periodical 3D perturbations on the turbulent mix growth associated with the Rayleigh-Taylor (RT) instability evolution at the gas-liquid interface, are reported. A layer of low-strength, dissolved-in-water- gelatin was employed for modeling the liquid driven with compressed helium. The perturbations given on an obviously unstable surface were 4-hedral pyramids with a height $\Delta$ equal to the perturbation wavelength $\Delta=\lambda_{0}$ $=0.25,1,2,2.86 \mathrm{~mm}$. The results obtained in RT experiments with no initial perturbations are also presented. The width of the turbulent mix zone was found growing with increasing the perturbation scales.
\end{abstract}

\title{
Introduction
}

The dynamics of turbulent mix (TM) growth caused by RT instability [1] is known to be in relation with a series of parameters such as surface tension, viscosity, initial perturbations on the interface $[2,3,4]$.

According to current recognition, viscosity and surface tension of liquid at considerable acceleration $g$ of the layer does not practically affect the TM growth. More complex case is the relation between initial perturbations and TM growth.

Self-similar turbulence occurs, in particular, at "forgetting" the initial perturbations.

As it has been shown in theoretical-numerical work [4], the rate and how the rate goes to self-similar form depend on perturbation density within the initial transition zone. Moreover, this work has shown, that TM (TMZ) does not occur when initial perturbations are lacking.

Currently, in experimental as well as in theoretical plane there are some questions of interest: what are initial given perturbations on the interface to be further "forgotten", when it occurs, and whether they are "forgotten" in terms of the experimental data we have or for all practically significant cases.

Jelly technique [5] intended for studying non-stationary hydrodynamic flows allows us to answer partly the questions. The core of the technique is modeling a liquid layer by a lowstrength gelatin dissolved in water. For example, the jellies, using gelatin dissolved in water at 
the concentration of $\mathrm{C} \approx 4.4 \%$, posses some strength $(\sigma \approx 0.005 \mathrm{MPa})$, and they are capable of conserving their shapes initially imposed on. When these jellies are driven by compressed gas at about 10 atm pressure, they would show behavior similar to that of incompressible liquids [5]. Any perturbation can be imposed on the surface of the jelly; further, its evolution could be recorded during dynamic experiment [6-8].

The subject matter of the paper is experimental results for the influence of the scales of $3 \mathrm{D}$ periodical perturbations given on unstable surface of a plane jelly driven by a compressed gas on TM evolution.

\section{Experimental}

Schematically, the experiment setup is shown in Fig.1. By this technique, a section of the acceleration channel of $40 \times 40 \mathrm{~mm}$ sectional area, was placed on a matrix which surface had the following 3D perturbations a) of height (amplitude) $\Delta=0.25 \mathrm{~mm}$ and wavelength $\lambda_{0}=0.1 \mathrm{~mm}$, b) $\Delta=1 \mathrm{~mm}, \lambda_{0}=1 \mathrm{~mm}$, c) $\Delta=2 \mathrm{~mm}, \lambda_{0}=2 \mathrm{~mm}$, d) $\Delta=2.86 \mathrm{~mm}, \lambda_{0}=2.86 \mathrm{~mm}$. (The perturbations had a regular shape of 4-hedral pyramid. Apex angle was directed to the jelly. Accuracy of manufacturing the perturbations using matrix was of about $0.07 \mathrm{~mm}$.) The section of the acceleration channel (which wall surfaces were greased thinly with vaseline) was filled with gelatin solution $(\mathrm{C}=4.4 \%)$ above which was placed a $4 \mathrm{~mm}$ thick plywood to prevent the jelly from sagging. On soliciting, the matrix was removed, so the free surface of the jelly had perturbations being mirror images of ones imposed on the matrix. We also performed experiments with jellies without artificially given perturbations on their unstable surfaces. In this case, the jellies were manufactured in a thin transparent container (Fig.1, variant1). Unstable surfaces of such jellies had roughness with convexity $\approx 0.05 \mathrm{~mm}$ high due to vaseline. The mass of a jelly was about $47-52 \mathrm{~g}$.

The section with jelly was put in the acceleration channel. An end of the channel was tightly sealed with a cap, another one was closed with a $0.1 \mathrm{~mm}$ thick millar diaphragm. The acceleration channel (on either side of the jelly) was filled with compressed helium of $P=13$ atm. The diaphragm was broken with the electrical explosion of a nichrome wire glued on to it, so that the gas under the jelly would rapidly flow out to the atmosphere, and the jelly accelerated. The motion of jellies was visualized using high-speed frame filming in transmitted light. 


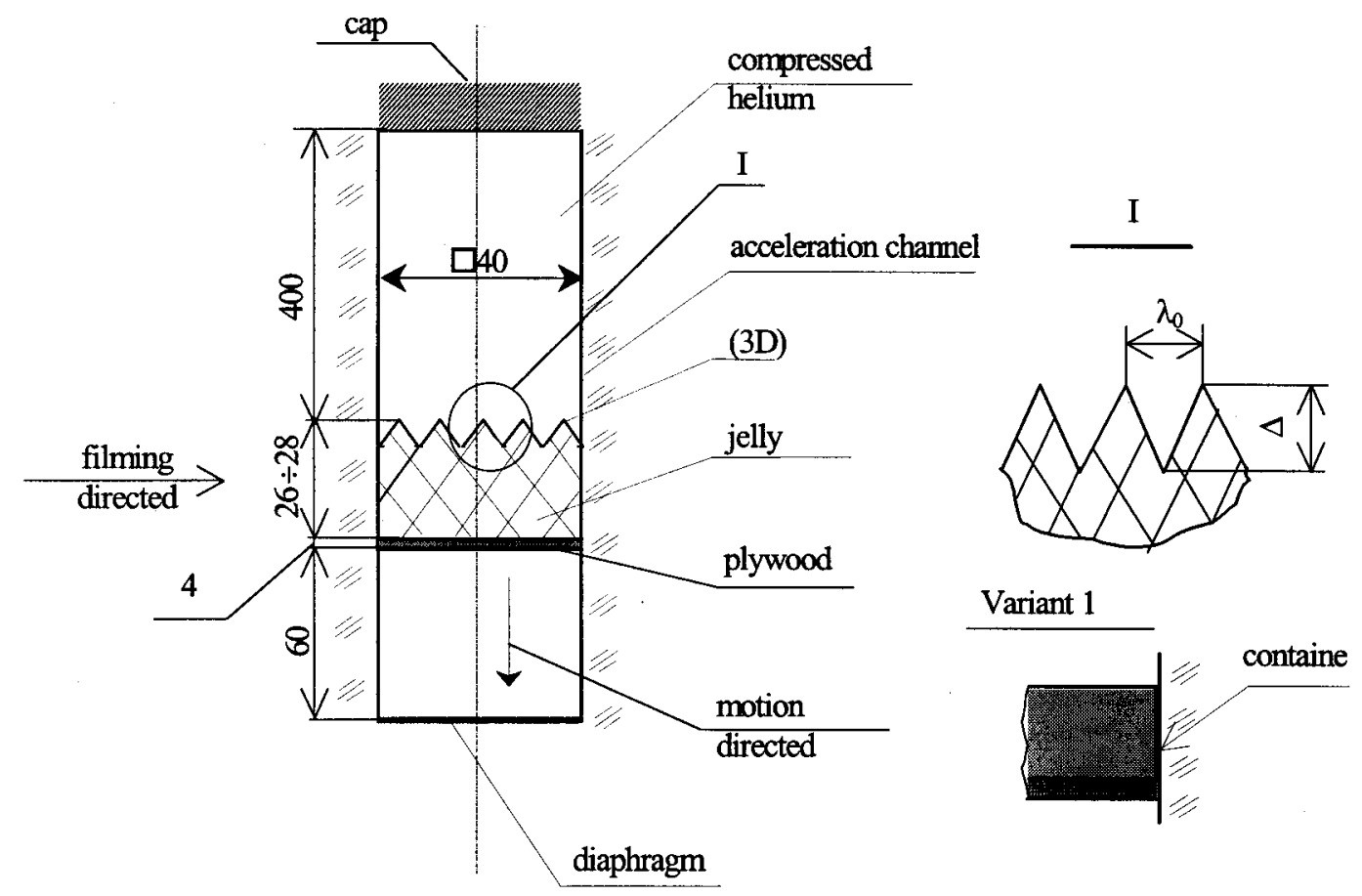

Fig.1 Schematic of the experiment setup

\section{Experimental results. Analysis.}

Fig. 2 shows separate frames for the experiments performed with jellies without artificially given initial perturbations on their unstable surfaces and for jelly experiments with the perturbations: $\lambda_{0}=\Delta=0.25 \mathrm{~mm},: \lambda_{0}=\Delta=1 \mathrm{~mm}, \lambda_{0}=\Delta=2 \mathrm{~mm}, \lambda_{0}=\Delta=2.86 \mathrm{~mm}$.

Accelerations for all the experiments were almost equal and constant $\left(\mathrm{g} \approx 3 \cdot 10^{4} \mathrm{~m} / \mathrm{s}^{2}\right)$.

Fig. 3 presents the handling results for these experiments:

- the dependence of the averaged penetration depth (bubbles into jelly) $h_{\mathrm{lh}}$ on double jelly displacement $\mathrm{S}$;

- the dependence of the averaged penetration depth (jelly(jets) into gas) $h_{h 1}$ on double jelly displacement $S$;

- the dependence of the total width of mixing zone $\mathrm{H}$ on double jelly displacement $\mathrm{S}(\mathrm{H}=$ $\left.h_{l h}+h_{h l}\right)$.

At $S>20 \mathrm{~mm}$, the dependencies are close to be linear. The error in measuring the front $h_{1 h}$ was about $\pm 0.5 \mathrm{~mm}$. The error in measuring the front $h_{h l}$ was estimated as $\pm 0.5 \mathrm{~mm}$ for the initial stage of the TM growth and as $\pm 2 \mathrm{~mm}$ for the final stage of recording. Such range is due to jet character for penetration of heavy material into a lighter - jet front is hardly processed from photos. 
a)
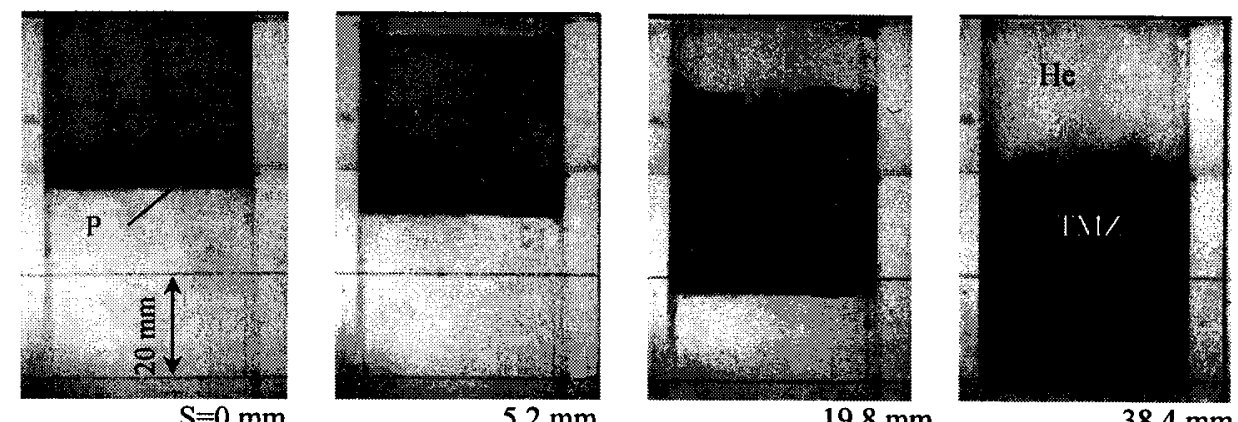

b)
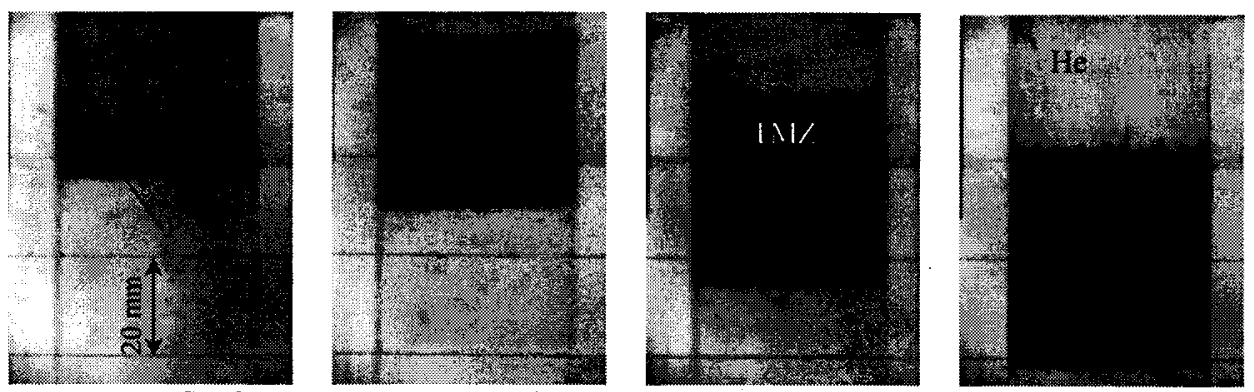

$5.4 \mathrm{~mm}$

$20.1 \mathrm{~mm}$

$37.4 \mathrm{~mm}$

c)
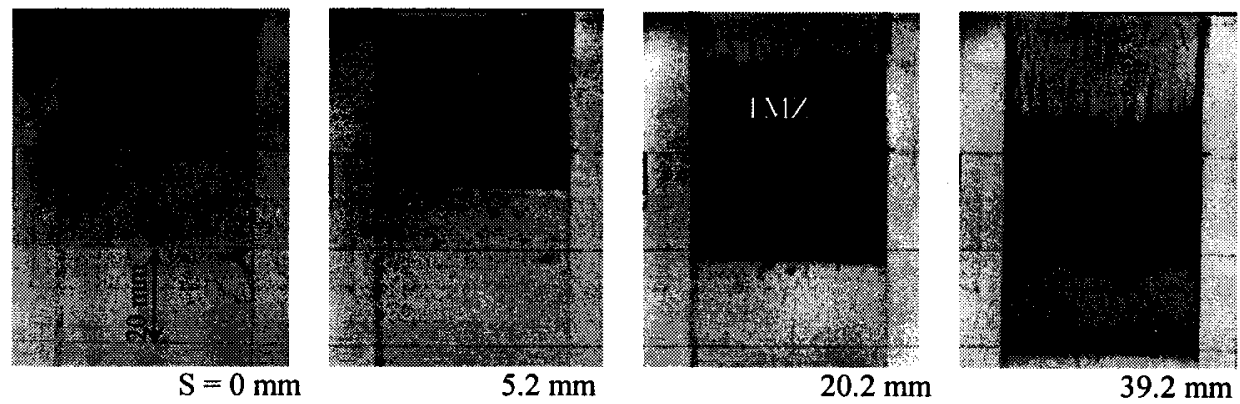

d)
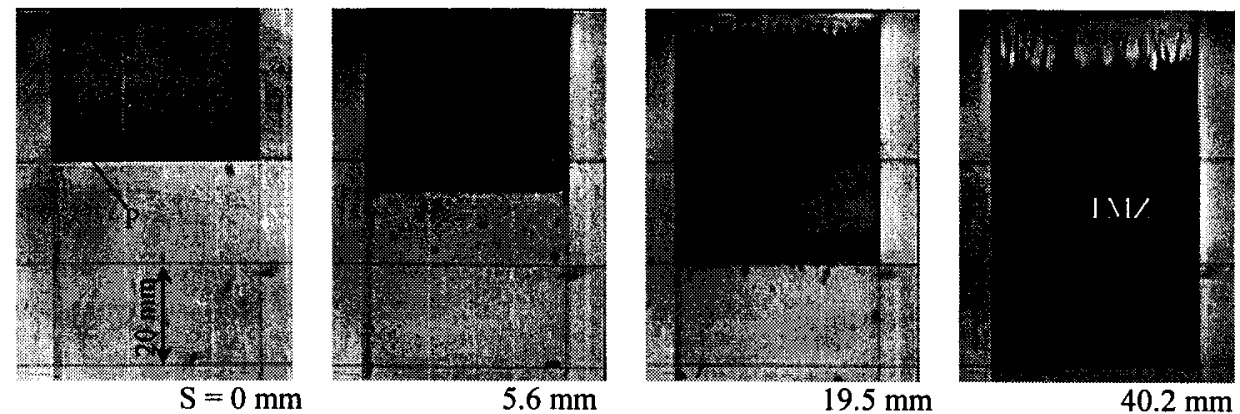

e)
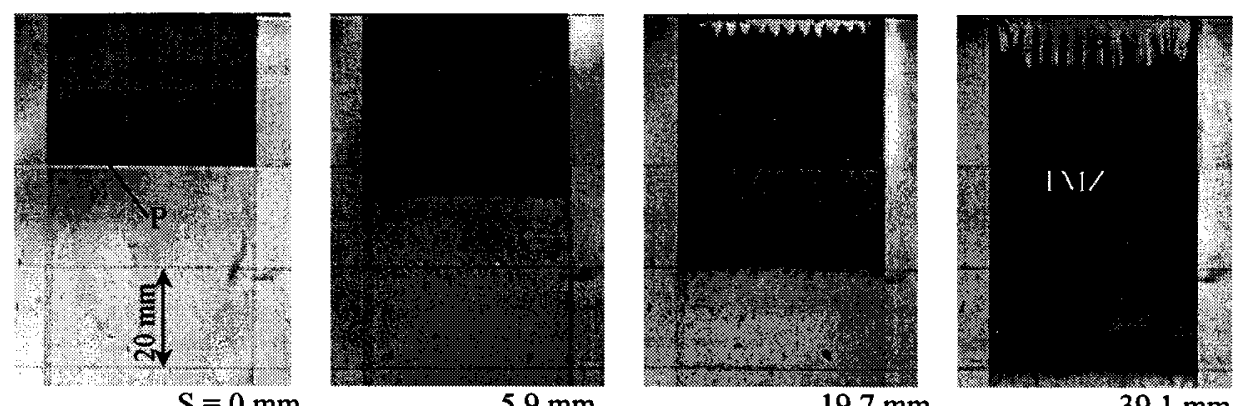

$39.1 \mathrm{~mm}$

Fig.2. Frames for evolution of the TM zone on unstable surface of jellies with different initial perturbations a) without perturbations, b) $\lambda_{0}=\Delta=0.25 \mathrm{~mm}$, c) $\left.\left.\lambda_{0}=\Delta=1 \mathrm{~mm}, \mathrm{~d}\right) \lambda_{0}=\Delta=2 \mathrm{~mm}, \mathrm{e}\right) \lambda_{0}=\Delta=2.86 \mathrm{~mm}$. Notations: J-jelly; He-compressed helium, TMZ-turbulent mix zone, $\mathrm{P}$ - plywood, $\mathrm{S}$ - displacement 
a)

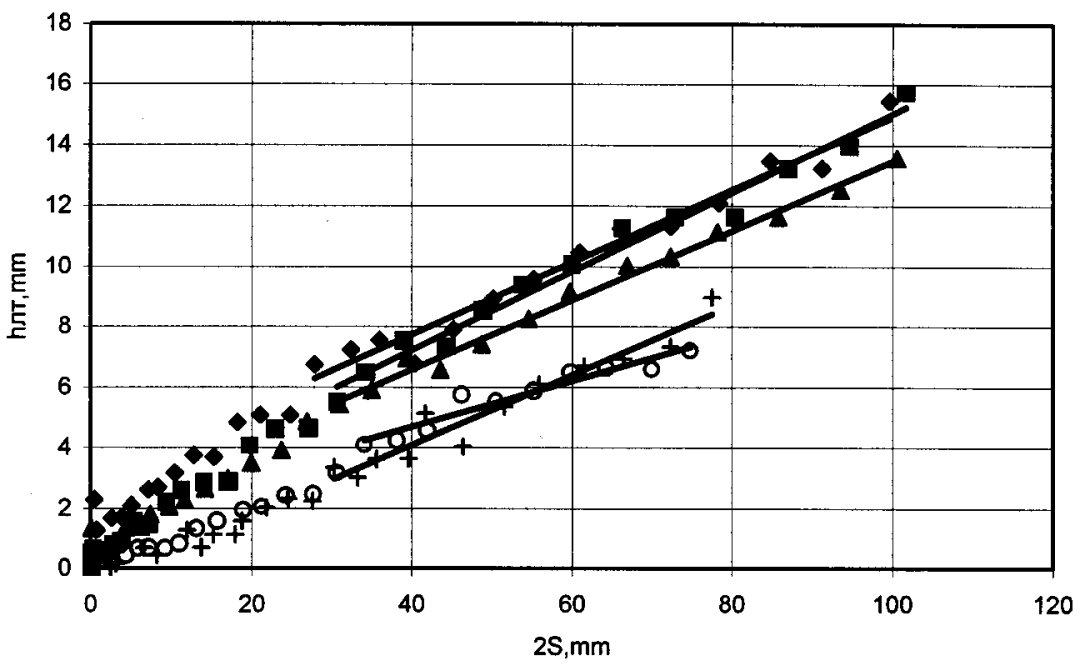

b)

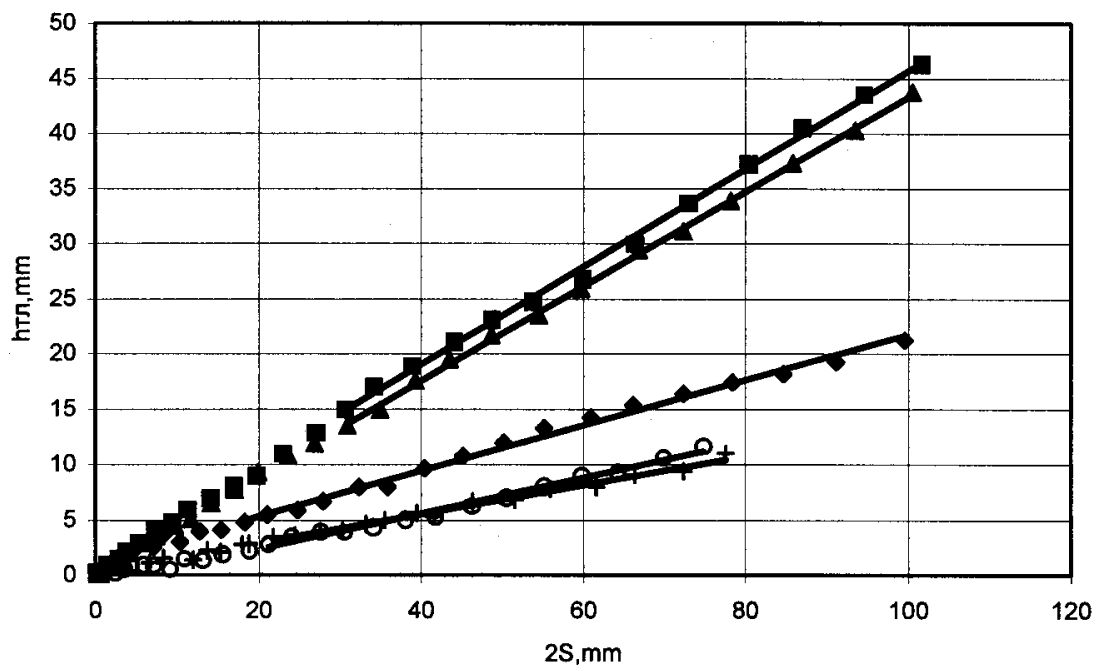

c)

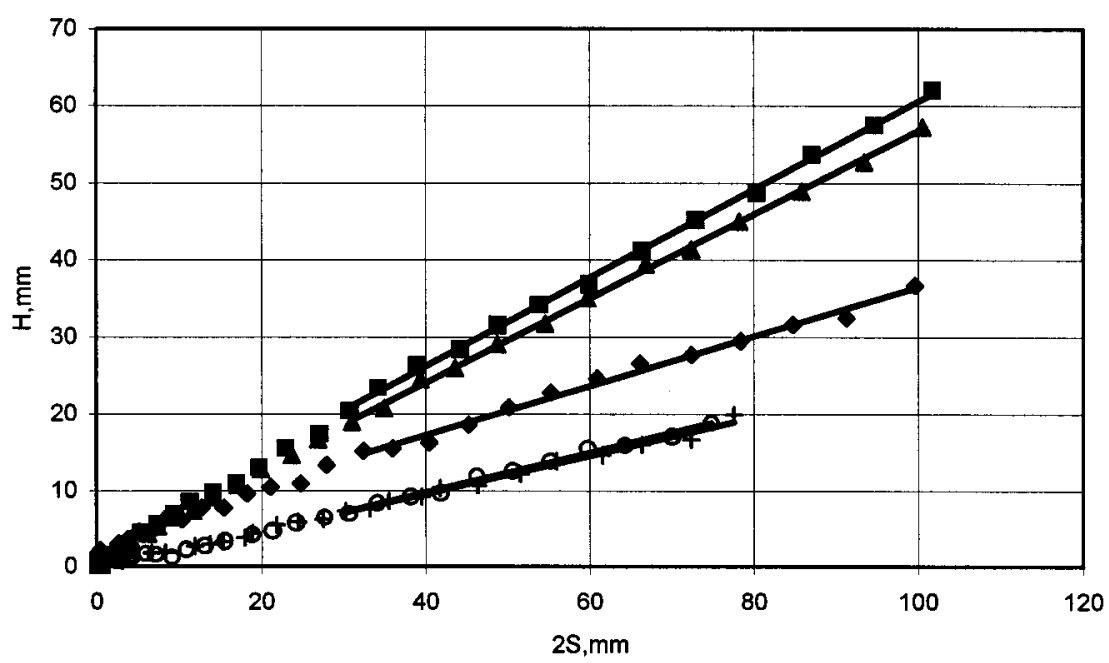

Fig.3. Processed results: a) dependence of penetration depth of gas into jelly hlh versus double displacement $2 \mathrm{~S}$; b) dependence of penetration depth of jelly into gas hhl versus double displacement $2 \mathrm{~S}$; dependence of total width of turbulent mix zone $\mathrm{H}$ versus double displacement 2S. Notations: $-\lambda_{0}=\Delta=1 \mathrm{~mm}, \boldsymbol{\Delta}-\lambda_{0}=\Delta=2 \mathrm{~mm}, \boldsymbol{\square}-\lambda_{0}=\Delta=2.86 \mathrm{~mm}$, +-without perturbations, $\mathrm{o}-\lambda_{0}=\Delta=0.25 \mathrm{MM}$. 


\section{Particular attention is given for the following:}

- $\quad$ somewhat unusual long stage of the non-linear jet growth; the perturbation amplitude increases fifteen times while the laminar flow takes place

- There is a delay in mixing at unstable interface for the jelly experiments without given initial perturbations with respect to ones with given initial perturbations.

- At $0<\mathrm{S} \leq 20 \mathrm{~mm}$, the front of bubbles is more smooth for jellies with $\lambda_{0}=\Delta=1,2,2.86$ $\mathrm{mm}$ with respect to jellies without perturbations and with $\lambda_{0}=\Delta=0.25 \mathrm{~mm}$. It may be assumed that, for this range of $S$ and for jellies with $\lambda_{0}=\Delta=1,2,2.86 \mathrm{~mm}$, the TM growth is determined, in general, by given perturbations; i.e. random perturbations are, in some extent, suppressed.

- Intensity of the light-into-heavy penetration) $\beta_{\mathrm{lh}}$, determined for a linear stage, is higher than the presented above dependencies; $\beta_{\mathrm{lh}}=\mathrm{dh} \mathrm{hh}_{\mathrm{h}} / \mathrm{d}(2 \mathrm{~S}), \beta_{\mathrm{lh}} \approx 0.12$ for jellies with $\Delta \approx 0.05 \mathrm{~mm}$ and $\Delta \approx 0.25 \mathrm{~mm}$; with $\Delta$ increases from 1 to $2.86 \mathrm{~mm}, \beta_{\mathrm{lh}}$ grows from 0.1 to 0.14 . However, this growth lies within measurement error which is $\delta \beta_{1 \mathrm{~h}}=0.025$. So, due to low accuracy $(\approx 20 \%)$, we can't unambiguously say about "forgetting" these perturbations for the recorded jelly displacement.

- Intensity of the heavy-into-light penetration $\beta_{\mathrm{hl}}$, determined for a linear stage in the same manner as $\beta_{\mathrm{lh}}$, increases from $\approx 0.13$ to $\approx 0.42$ (i.e. threefold) with increasing the $\Delta$ from 0.05 to 2.86 . Such growth is much more than the value of errors in measuring, thus, the initial perturbations are not "forgotten" during the penetration of heavy material into a lighter (at least, for the $\mathrm{S} \leq 50 \mathrm{~mm}$ ).

- The total width of the TM zone H grows with the scale of initial perturbations; this growth is, in general, due to high intensity of the heavy-into-light penetration.

\section{Conclusion}

The experimental results show that the width of turbulent mix zone grows with the scale of initial, periodical 3D perturbations.

The data obtained could be used for testing numerical techniques.

\section{References}

1. G.I.Taylor. The instability of liquid surfaces when accelerated in a direction perpebdicular to their planes.Proc.Roy.Soc.,VA2016P.192,1950.

2. D.H.Sharp. An Overview of Rayleigh-Taylor Instability.Physica 12D,3-8, 1984

3. Yu.A.Kucherenko, G.G.Tomashev, L.I.Shibarshov. Experimental study of gravitational turbulent mix for self-similar regime. VANT, is. Th.and Ap. Ph., 1,p.13,1988 
4. V.V.Nikiforov. Turbulent mixing on an interface between media different in density VANT, is. Th.and Ap. Ph., 1,p.3,1983

5. O.I.Volchenko, I.G.Zhidov, B.A.Klopov, E.E.Meshkov et al. A mean for modeling nonstationary flows of incompressible liquid A.S.1026154,MKI 09 B23/12

6. M.V.Bliznetsov, E.E.Meshkov,N.V.Nevmerzitsky, P.I.Nizovtsev, E.D.Sen'kovsky, E.A.Sotskov, L.V.Tochilina. On possibility of modeling some cases of RT instability in strength media VANT, is. Th.and Ap. Ph., 3,p.54-57,2000

7. S.T.Weir, E.A.Chandler and B.T.Goodwin. Rayleigh-taylor instability growth experiments in a cylindrically convergent geometry. The Proc. of 6th IWPCTM, Marseilles, 1997,(France)

8. E.E.Meshkov, N.V.Nevmerzhitsky,V.G.Rogachev,I.G.Zhidov. The use of jelly technique to study hydrodynamic perturbation evalution at unstable plane and cylindrical surfaces. The Proc. of 5th IWPCTM, Stony Brook, 1995, (USA). P.243-250 
Formation and propagation of shock-generated vortex rings

Martin Brouillette and Christian Hébert

Laboratoire d'ondes de choc

Université de Sherbrooke

Sherbrooke (Québec) CANADA 


\section{Outline}
1. Introduction
2. Experimental considerations
3. Vortex propagation
4. Vortex formation - Circulation standpoint
5. Other features - Shock formation by vortex
6. Summary 


\section{Introduction}

Explore compressible turbulence via the standpoint of compressible vorticity and its building blocks.

Compressible vorticity is also important from both fundamental and practical standpoints, in:

- Blade-vortex interaction, including sound generation, for rotary wing aircraft applications.

- Shock-vortex interaction, including sound generation, for jet noise applications. 


\section{Objectives}

Experimental study of isolated vortices as building blocks of compressible turbulence.

For example, in the Richtmyer-Meshkov instability:

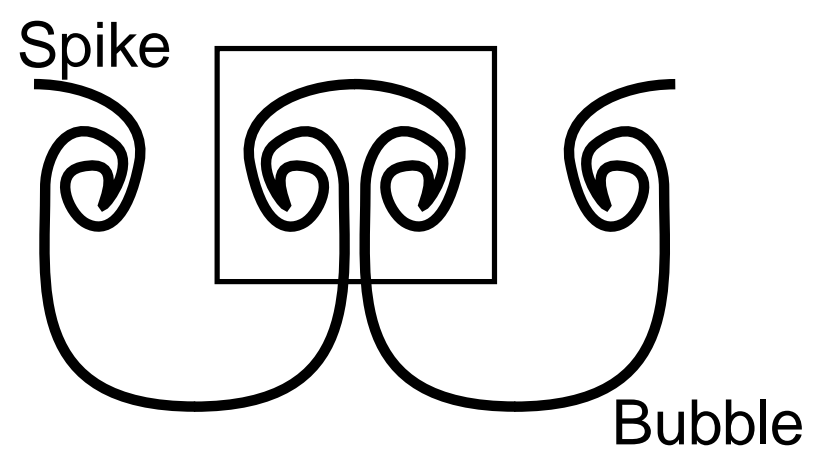

In particular,

- What exactly are compressible vortices?

- How are they different from incompressible vortical structures? 


\section{Specific experimental objectives}

- Characterize the effects of the generator on the production and propagation of compressible vortices.

- Examine the effects of compressibility and scale on these properties.

- Compare with incompressible results. 


\section{Experimental considerations}

Experiments are performed with a modified shock tube:

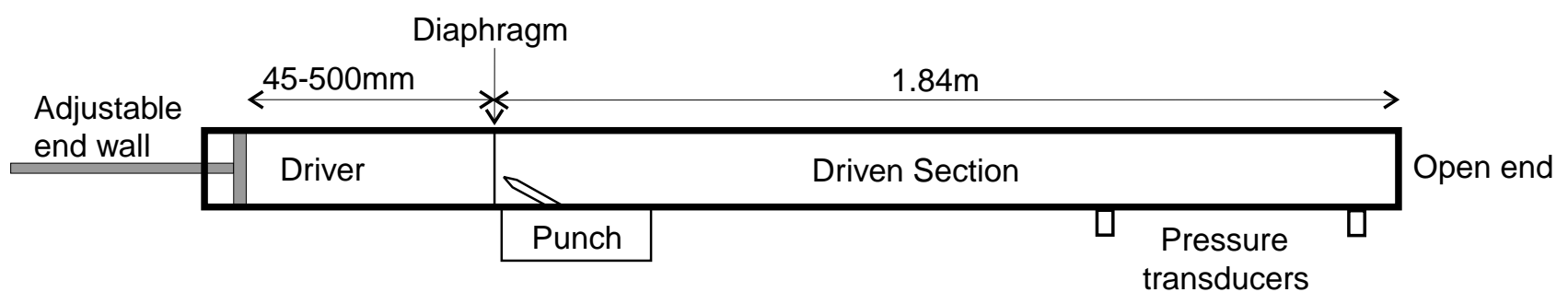

Open driven end, with 3 different exit nozzle diameters: $6.4,12.7$ and $25.4 \mathrm{~mm}$

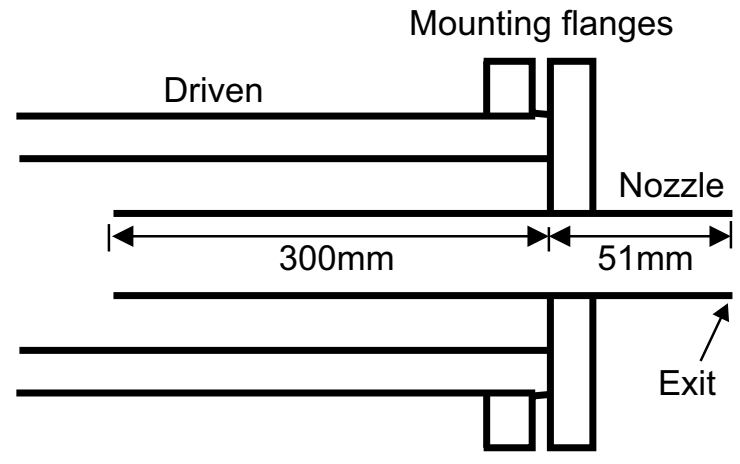


Features of this setup:

- Produce shear-driven vortices (Kelvin-Helmoltz instability) as opposed to baroclinically-driven vortices (Rayleigh-Taylor or Richtmyer-Meshkov instabilities)

- High vorticity production rates.

- Fluid piston analogous to high speed spike in RMI. 
Effect of driver length on fluid ejection history

Standard (= long) driver
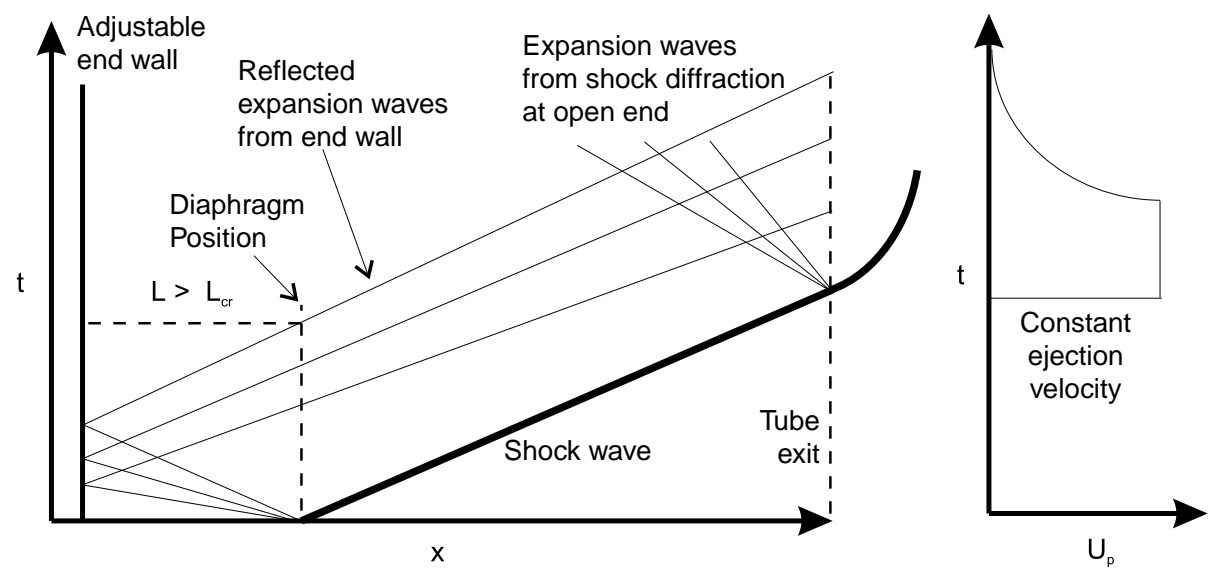

Analogous to RMI followed by RTI. 
Tuned driver

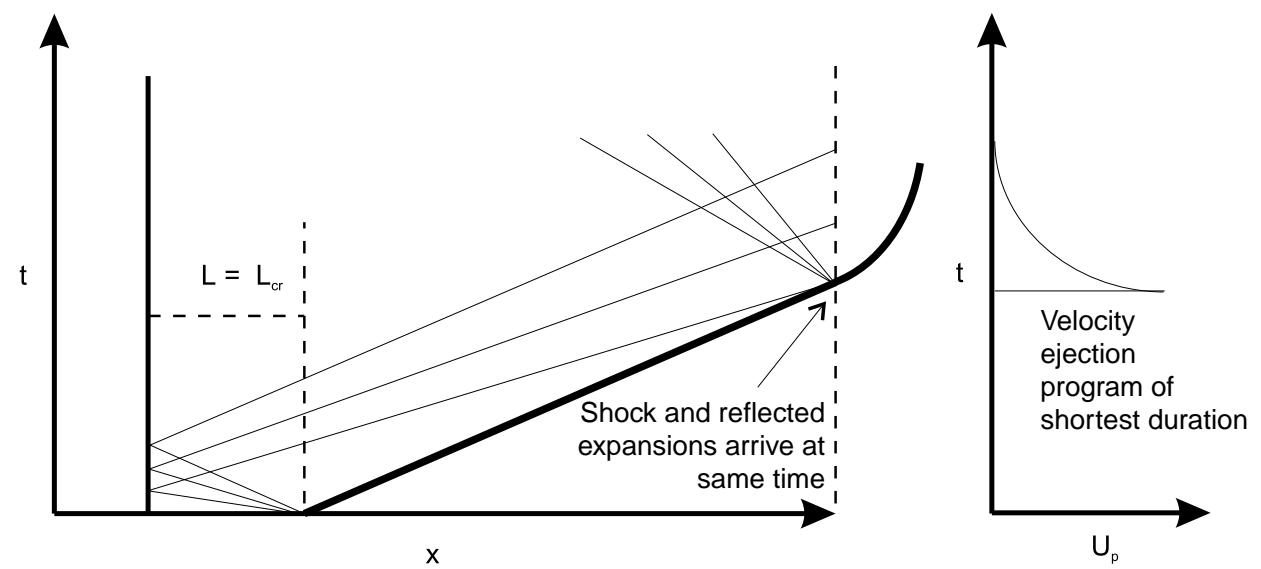

Analogous to "almost only" RMI. 


\section{Flow diagnostics}

- Piezoelectric pressure transducers.

- Flow visualization (shadowgraph, schlieren, holographic interferometry). 


\section{Vortex propagation}

Three regimes of propagation

Low shock Mach number $\rightarrow$ regime 1

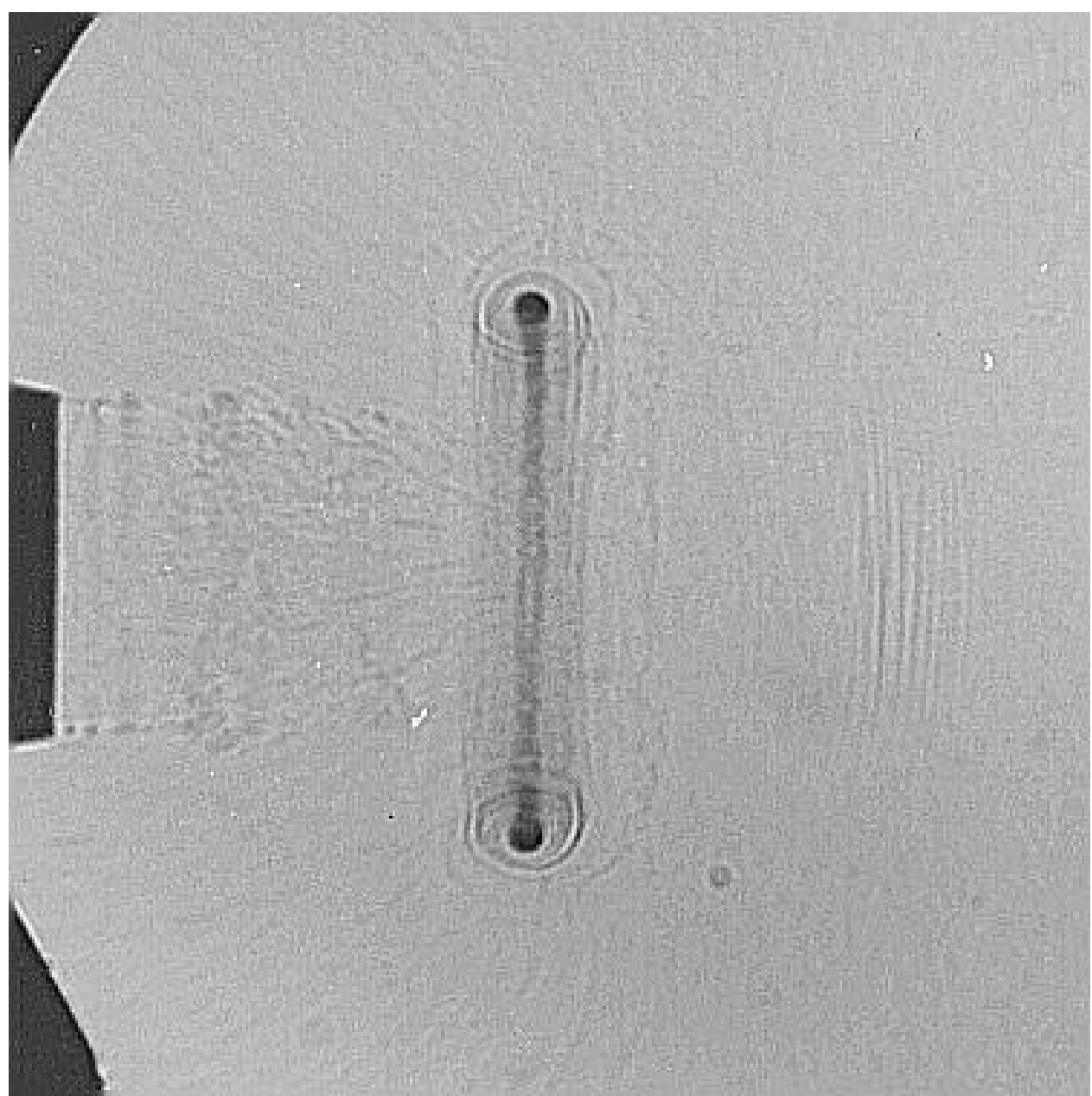


Regime 1 - Development of circonferential instabilities (oblique view)

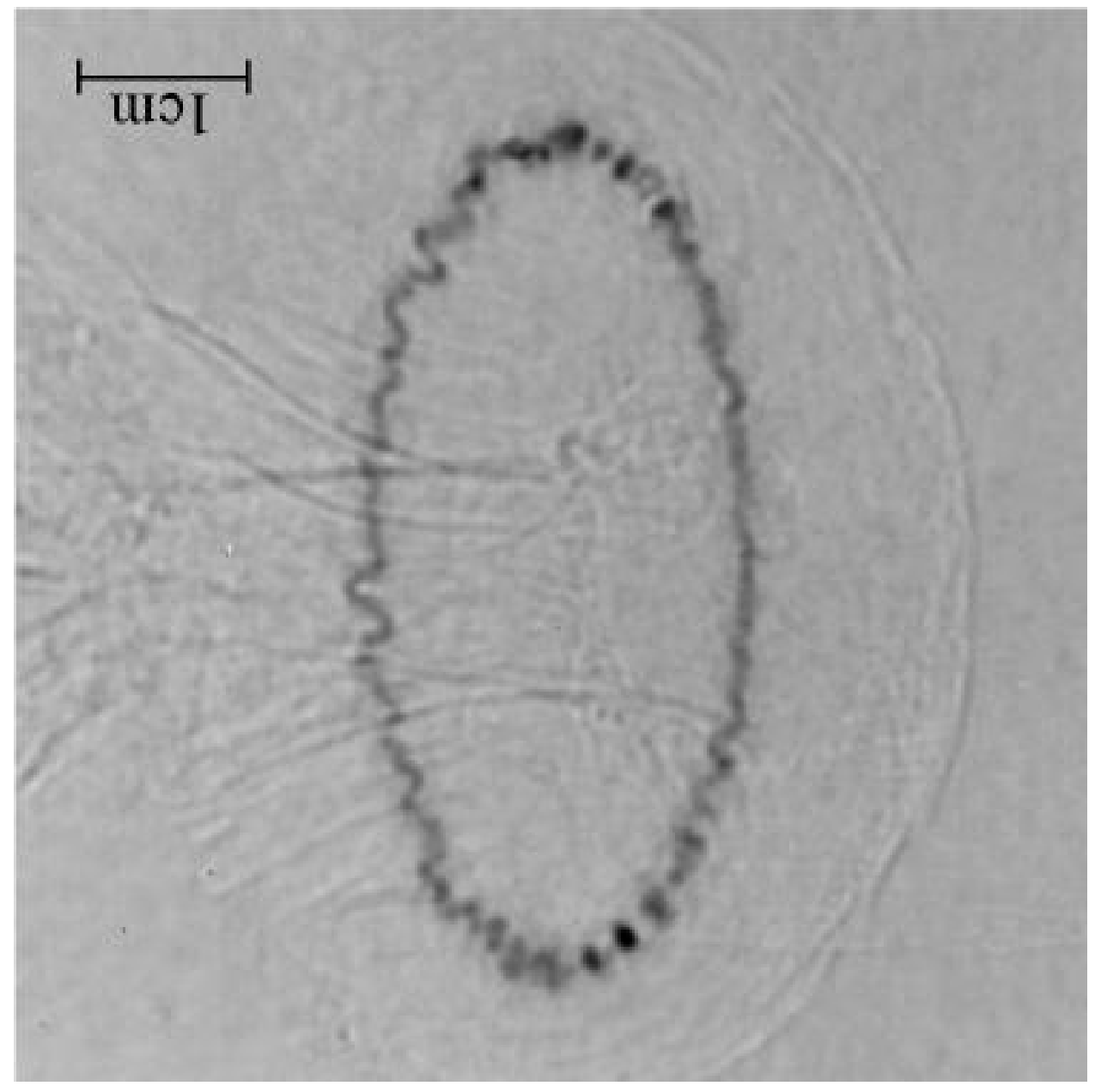

Oblique spark shadowgraph, $M_{s}=1.32, D_{p}=38 \mathrm{~mm}: t=1.42 \mathrm{~ms}, x / D_{p}=$ 3.30 
As $M_{s}$ is increased:

Regime 2 - Appearance of shocks

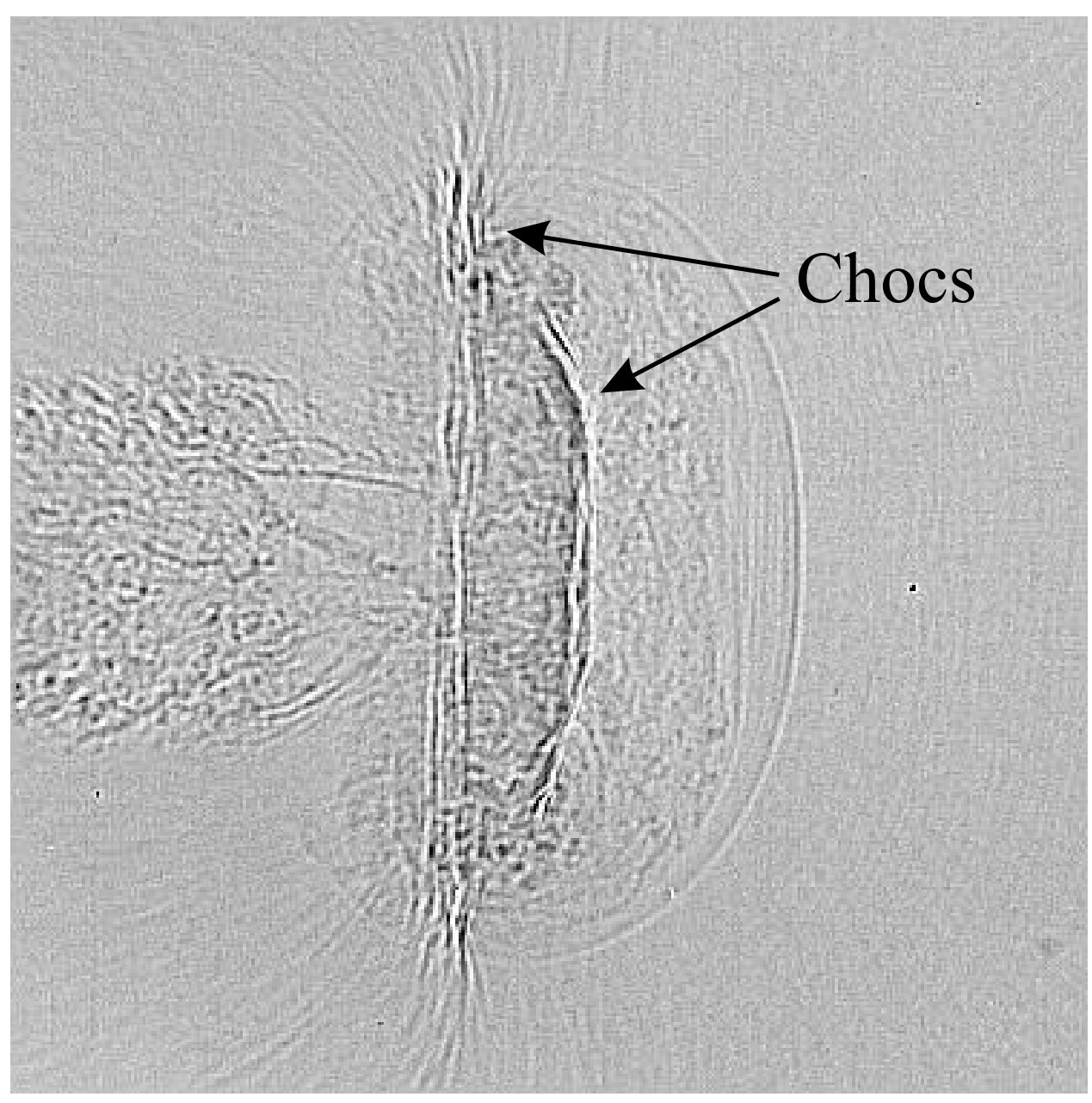


As $M_{s}$ is further increased:

Regime 3 - Secondary vorticity generation

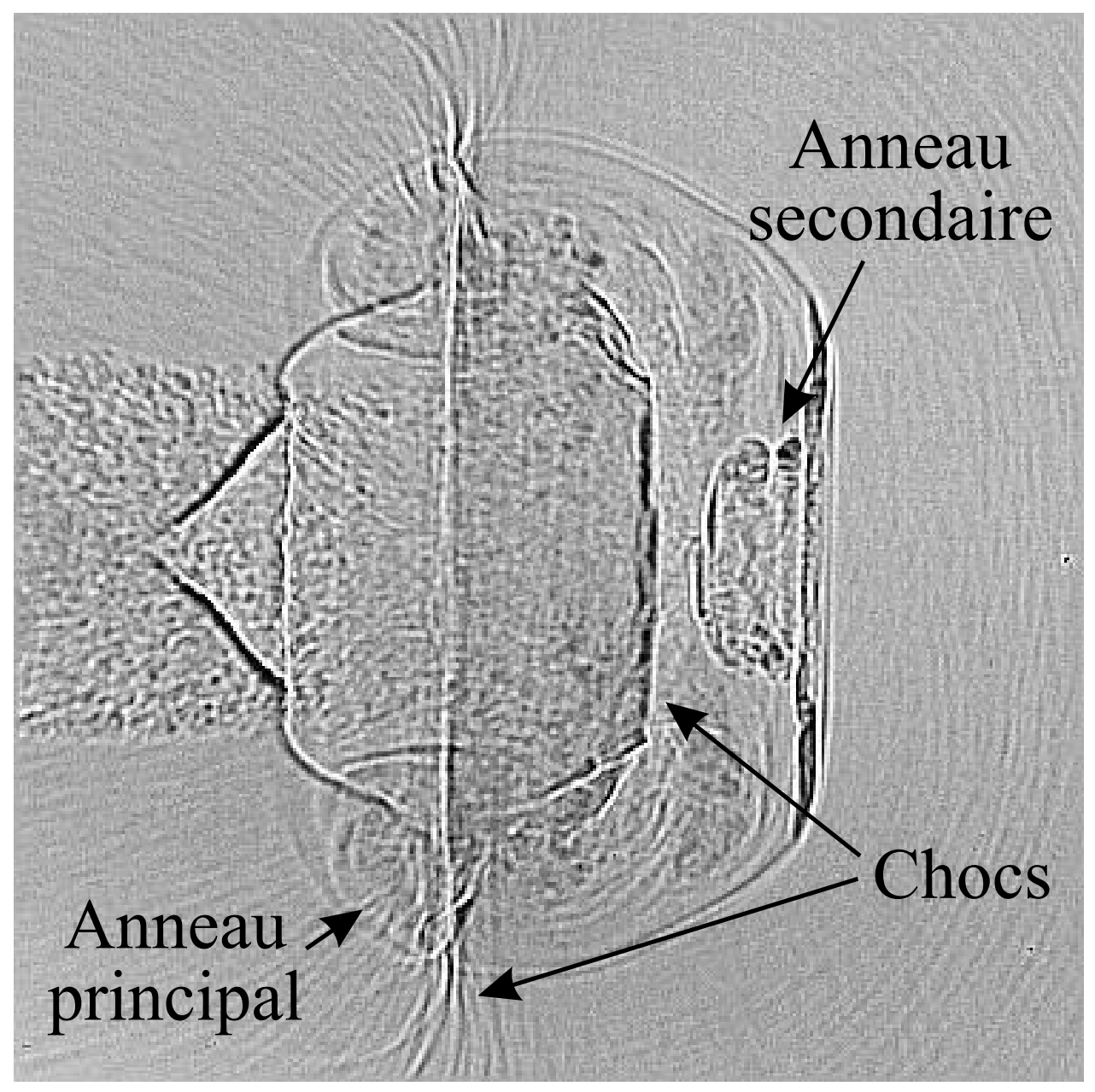


Vortex propagation (37 $\mathrm{mm}$ orifice)

$\begin{array}{ll}\text { Standard driver } & \text { Tuned driver } \\ 1<M_{s}<1.34 & 1<M_{s}<1.44 \\ 1.34<M_{s}<1.45 & 1.44<M_{s}<1.60 \\ M_{s}>1.45 & M_{s}>1.60\end{array}$

We know that for the same shock Mach number, impulse is larger for standard driver.

Regimes appear at lower Mach numbers for the standard case. 


\section{Vortex propagation - Position vs time}

Results normalized with orifice diameter $D_{p}$ and maximum fluid velocity $U_{p}$ as:

$$
x^{*}=\frac{x}{D_{p}} \quad t^{*}=\frac{t U_{p}}{D_{p}}
$$

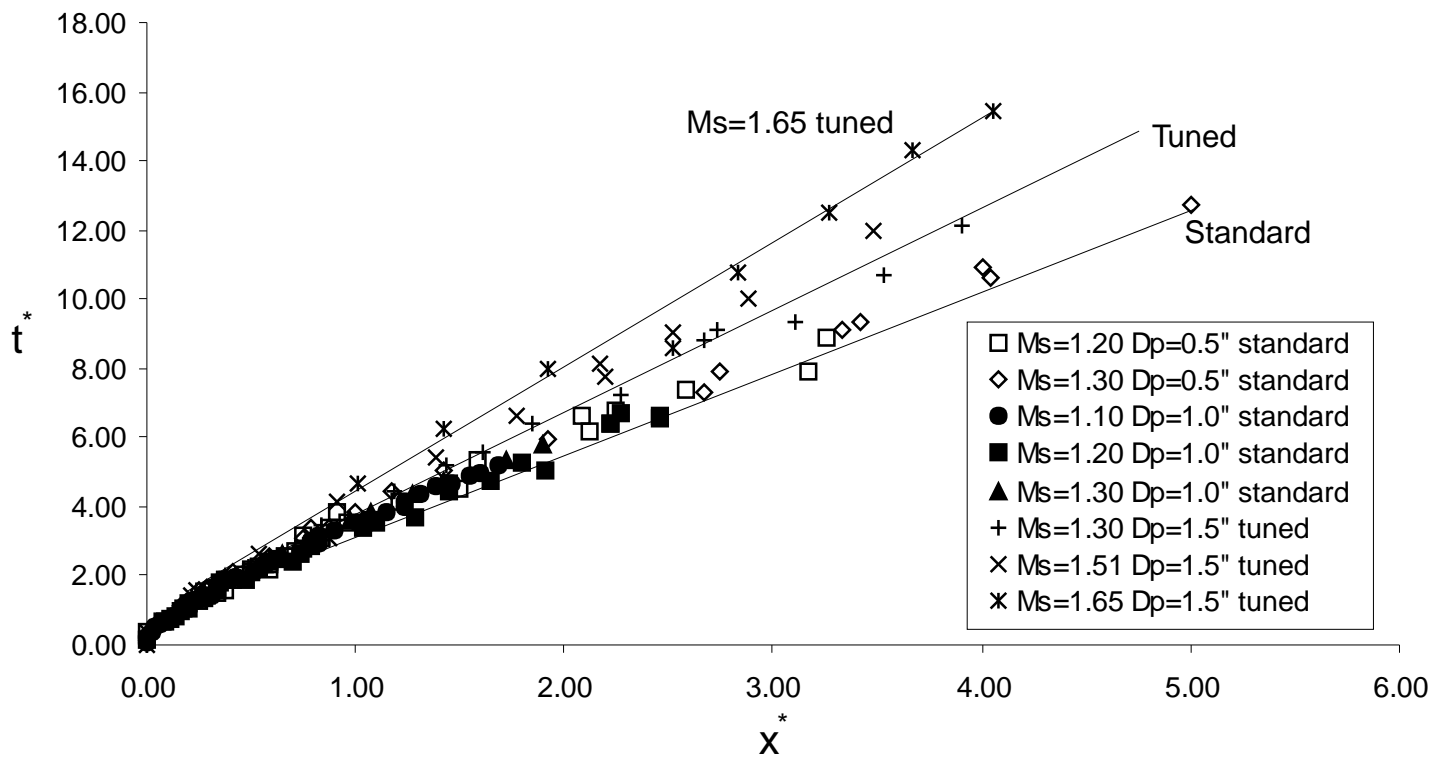




\section{Observations:}

- Speed of vortex rings increases with shock strength.

- Rings produced with the tuned driver propagate slower $U^{*} \approx 0.34$ than with the standard driver $U^{*} \approx 0.42$.

- Within experimental error, not possible to detect compressibility effects. 


\section{Vortex formation}

In incompressible experiments, typically use a piston to eject a slug of fluid (liquid).

- Ejection Mach number near zero.

- Normalized ejected slug length relatively much smaller than in the present study.

- Vortex propagation mostly free from the effects of the generating jet.

Examine vortex formation in terms of circulation deposition history:

Use a normalized circulation

$$
\Gamma^{*}=U^{*} d^{*}
$$


Normalized circulation vs normalized time

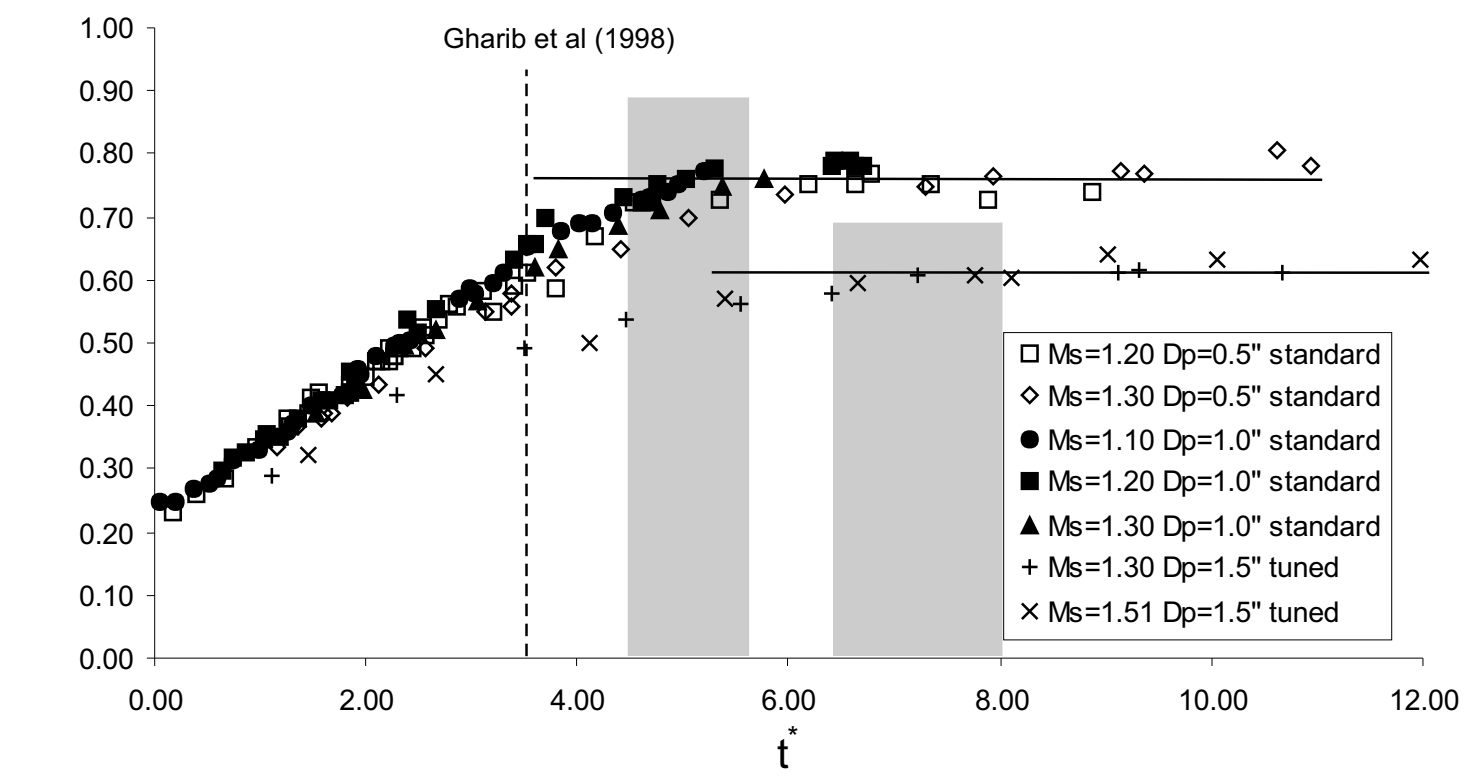




\section{Observations:}

- Vortex ring is formed when a vorticity saturation threshold is reached.

- Concept of vortex formation number (Gharib et al. 1998).

- Formation number higher for compressible rings.

- Maximum circulation similar between incompressible results and standard driver results.

- Lower circulation with tuned driver.

- Non-zero "initial" circulation (purely impulsive ejection history). 


\section{Other features — Shock formation by vortex}

Onset of appearance of shock wave within recirculating region:

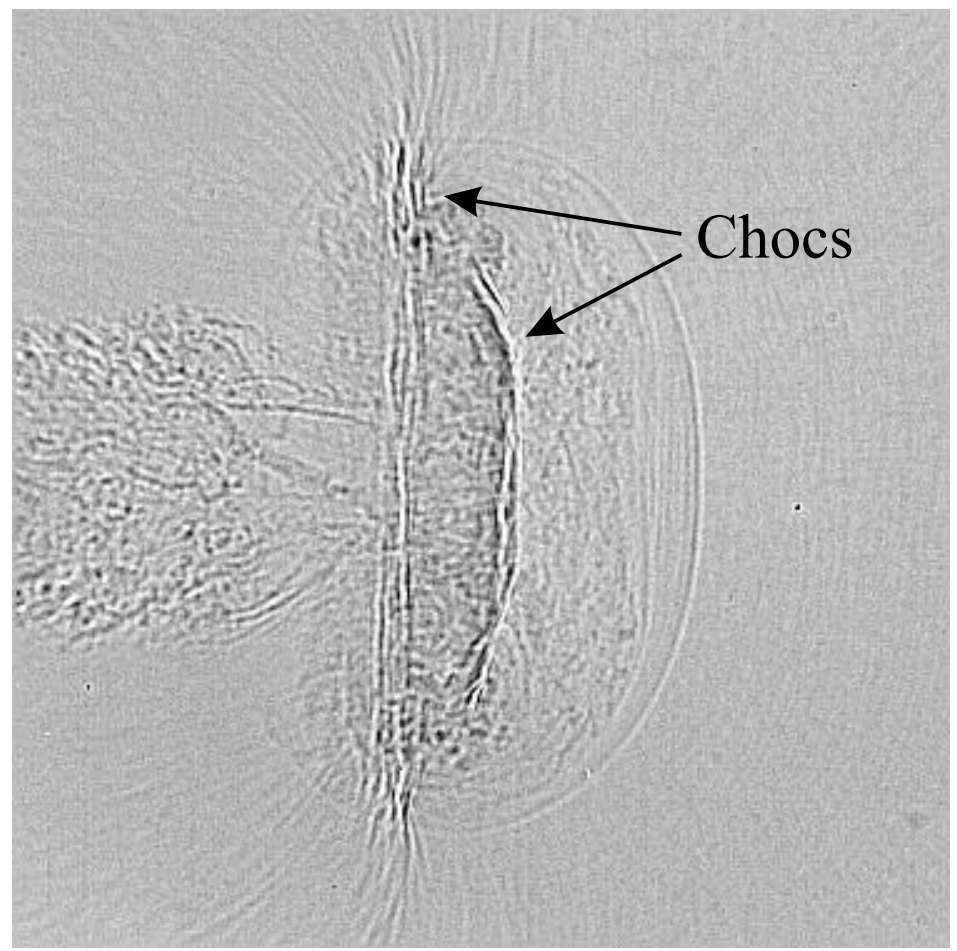

For the standard driver, this shock appears at $M_{s}=1.34\left(U_{p}=339 \mathrm{~m} / \mathrm{s}\right)$.

For the tuned driver, this shock appears at $M_{s}=1.44\left(U_{p}=425 \mathrm{~m} / \mathrm{s}\right)$. 
This threshold is reached when flow velocities within ring recirculating region become sonic $u / c=1$.

But since $u \sim \Gamma / d$ this threshold occurs when:

$$
\frac{\Gamma}{d c}=1
$$

With $\Gamma \sim U d$, then $\Gamma=\Gamma^{*} U_{p} D_{p}$. and this threshold can then be expressed as:

$$
\frac{\Gamma^{*} U_{p} D_{p}}{d c}=1
$$

If this criterion is satisfied for both tuned and standard cases, then:

$$
\frac{\Gamma_{\text {tuned }}^{*} U_{p_{\text {tuned }}} D_{p_{\text {tuned }}}}{d_{\text {tuned }} c_{\text {tuned }}}=\frac{\Gamma_{\text {std }}^{*} U_{p_{s t d}} D_{p_{s t d}}}{d_{s t d} c_{s t d}}
$$

For identical test gases $c_{\text {tuned }}=c_{s t d}$, for identical orifices $D_{p_{\text {tuned }}}=D_{p_{s t d}}$ and we observe that $d_{\text {tuned }}=d_{\text {std }}$. Therefore

$$
\frac{\Gamma_{\text {tuned }}^{*}}{\Gamma_{\text {std }}^{*}}=\frac{U_{p_{\text {std }}}}{U_{p_{\text {tuned }}}}
$$

is satisfied if postulate is correct! 


\section{Experimental data:}

Standard driver: $\Gamma_{\text {std }}^{*}=0.76$

Tuned driver : $\Gamma_{\text {tuned }}^{*}=0.61$

$$
\frac{\Gamma_{\text {tuned }}^{*}}{\Gamma_{\text {std }}^{*}}=0.80
$$

Standard driver: transition at $M_{s}=1.34 U_{p_{s t d}}=339 \mathrm{~m} / \mathrm{s}$

Tuned driver: transition at $M_{s}=1.44 U_{p_{\text {tuned }}}=425 \mathrm{~m} / \mathrm{s}$.

$$
\frac{U_{p_{s t d}}}{U_{p_{\text {tuned }}}}=0.80
$$

at the onset of appearance of the shock within the recirculating region.

Postulate appears satisfied!

- For a given size, shock appears at a given ring circulation.

- The estimation of ring circulation rests on solid ground. 


\section{Consequences:}

In a compressible turbulent flow, shocklets would appear if sufficient vorticity is locally present.

For a purely impulsive (delta function) fluid ejection history, since the maximum vorticity deposition $\Gamma^{*}$ is small, a shock would appear at a very large ejection velocity. Our limited experiments at $M_{s}=2$ support this.

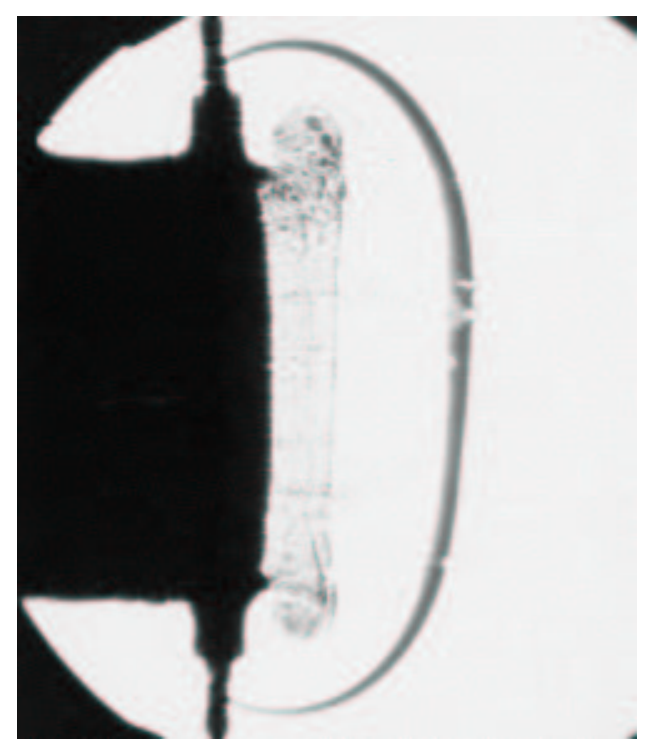




\section{Conclusions}

- The behavior of compressible vortices is somewhat similar to that of incompressible vortices, but they attain circulation saturation slower.

- Vortex rings can only absorb a maximum amount of circulation.

- The most sustained and higher vorticity production rate lead to faster normalized formation and higher circulation.

- Can use this point of view to explain he appearance of shocks within vortical structures. 


\title{
Design of Flyer-Plate-Driven, Compressible-Turbulent-Mix
}

\section{Experiments}

\author{
R. P. Drake
}

University of Michigan

IWPCTM, Pasadena, December 2001

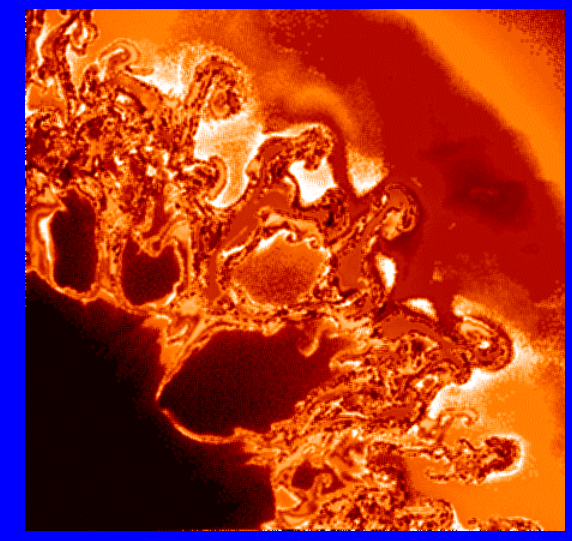

Supernova Simulation

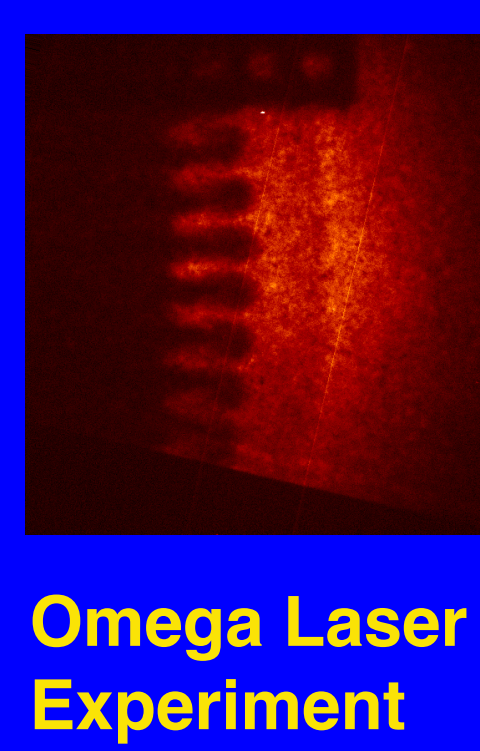

2

Z Flyer Experiment 


\section{$Z$ has opened up new experimental possibilities}
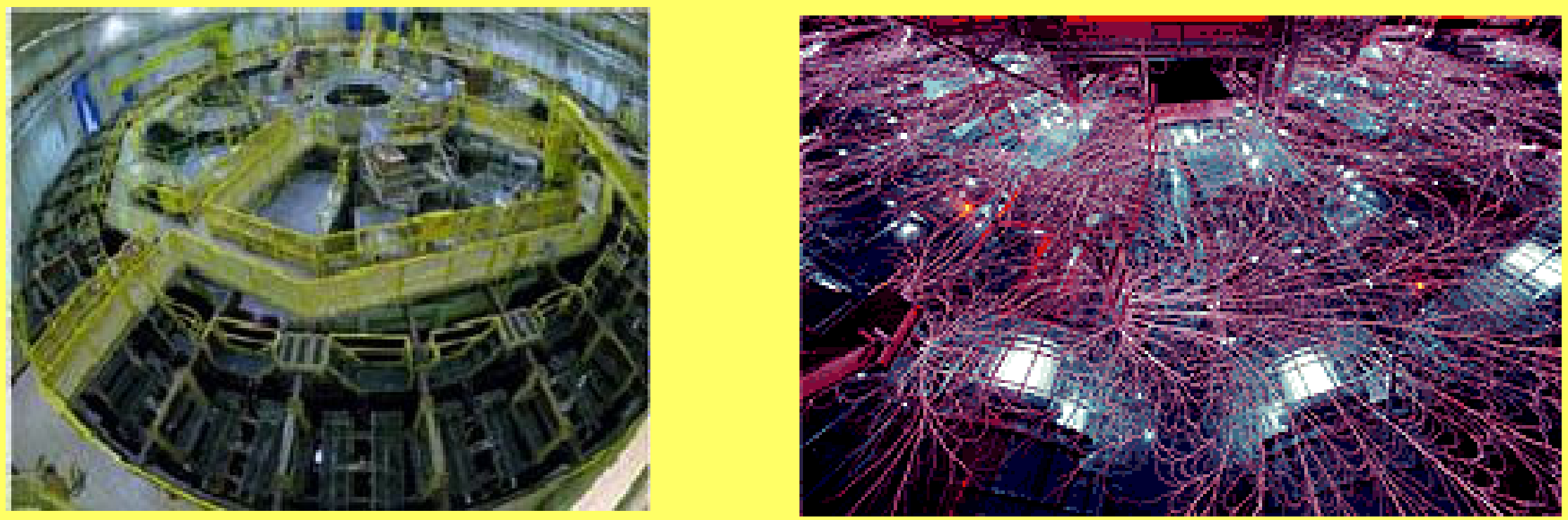

Shown above are pictures of the Z-pinch: (left) prior to firing (right) during firing 
By imploding hundreds of wires,

$Z$ can make $M J$ of $x$-rays

When the wires collide they produce up to

2 Mega-Joules of X-rays

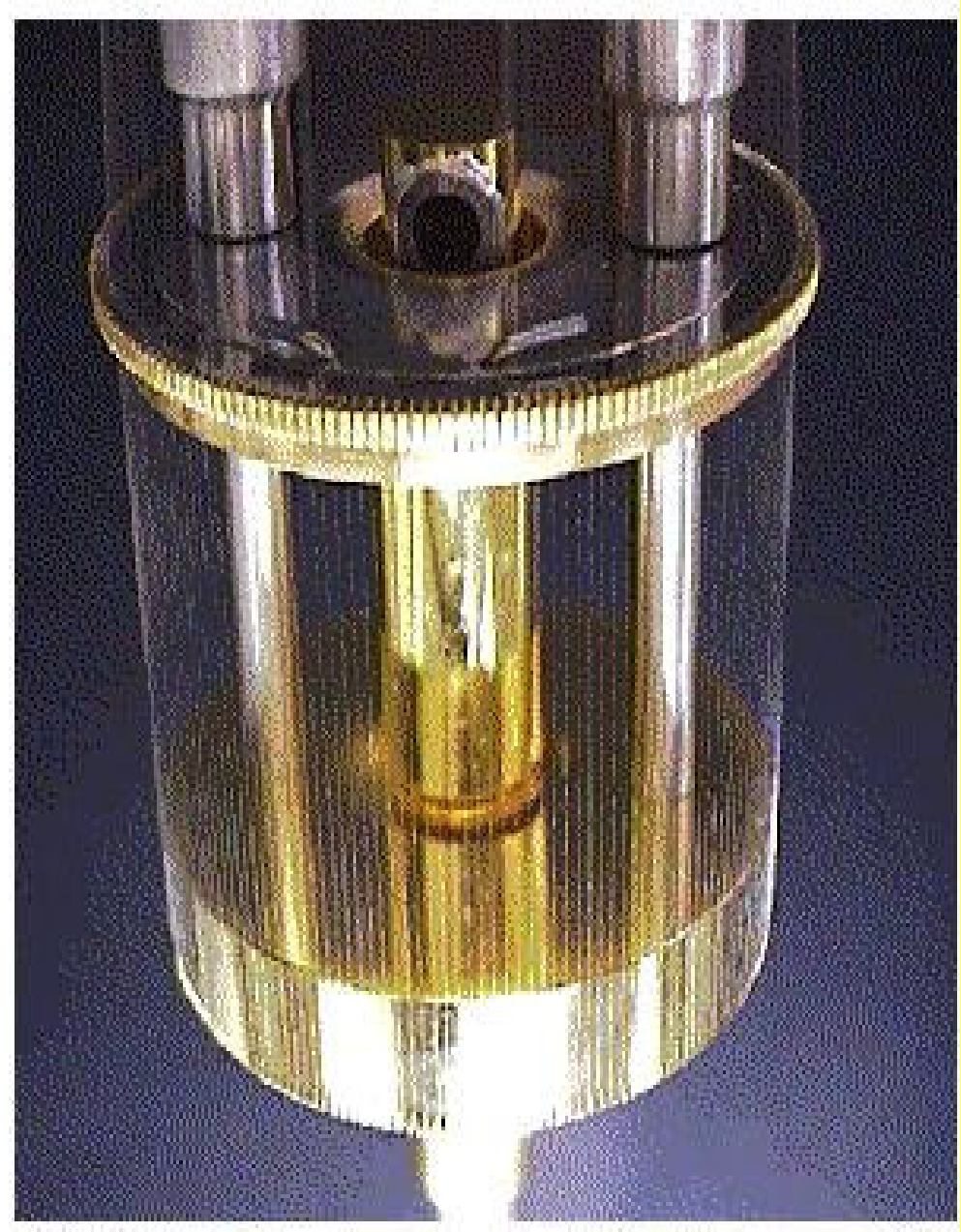
$\mathrm{x}(\mathrm{cm})$ 


\section{What matters here: $Z$ can use $\mathrm{J} X$ B forces to launch Al flyer plates at $>20 \mathrm{~km} / \mathrm{s}$}

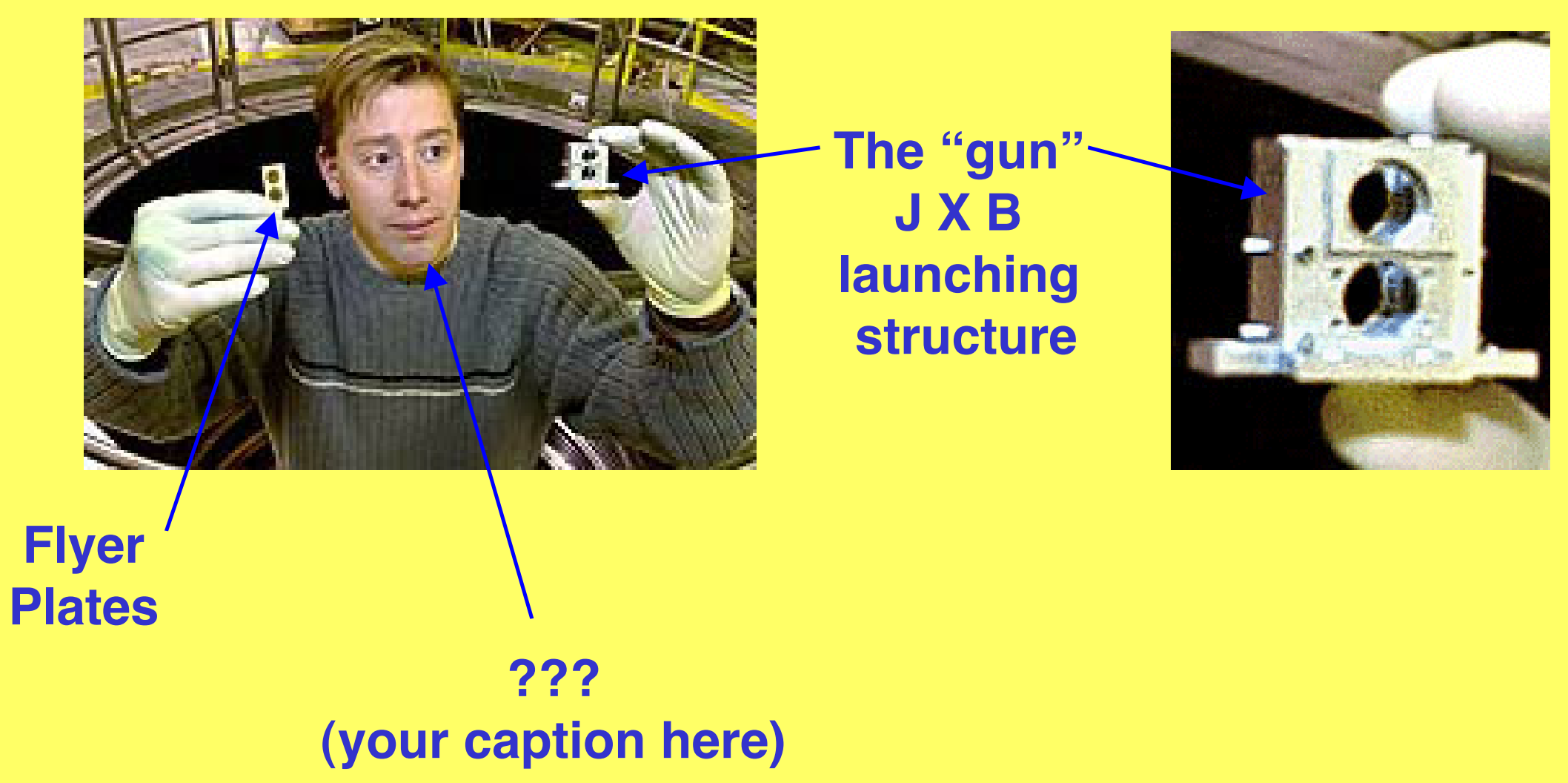

Photo: www.spacedaily.com/news/milspace-tech-01a.html 


\section{Acknowledgements: useful discussions with}

M. D. Knudson, J. R. Asay, C. Deeney

Sandia National Laboratory

S.G. Glendinning, H.A. Robey, J.O. Kane,

Lawrence Livermore National Laboratory 


\section{Flyer-plate mix experiments differ from flyer-plate EOS experiments}

- EOS experiments

- Need rock-steady shock

- Experiment ends when shock exits material

- Mix experiments

- Experiment begins when interface of interest is shocked

- May not want steady shock

- Even steady shock need not meet EOS constraints 


\section{Basic geometry for flyer plate experiments}

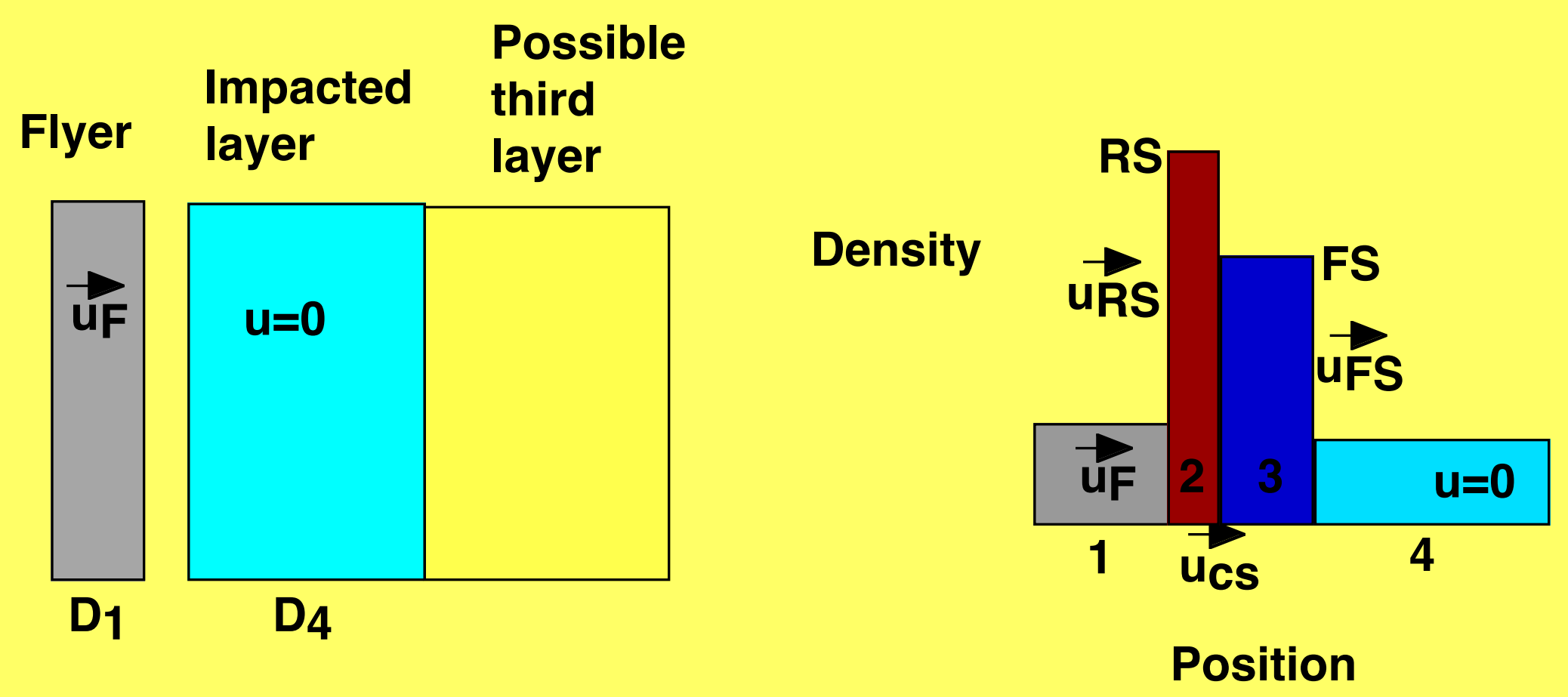

In the following:

Analytic results use $\gamma$-law gas, strong shock equations

Simulations used the HYADES Lagrangian hydrocode with SESAME EOS 


\section{Basic relationships}

\section{for strong shocks in this system}

In lab frame:

$$
\begin{aligned}
& u_{C S}=\frac{1}{1+\sqrt{\rho_{4} / \rho_{1}}} u_{F} \\
& u_{R S}=\left(1-\frac{\gamma-1}{2} \sqrt{\frac{\rho_{4}}{\rho_{1}}}\right) \frac{u_{F}}{1+\sqrt{\rho_{4} / \rho_{1}}}
\end{aligned}
$$

\section{In flyer frame:}

$$
u_{R S}^{\prime \prime}=\frac{-\sqrt{\rho_{4} / \rho_{1}}}{1+\sqrt{\rho_{4} / \rho_{1}}} \frac{\gamma+1}{2} u_{F}
$$

Sound speeds:

$$
\begin{gathered}
c_{3}=\sqrt{\frac{\gamma(\gamma-1)}{2}} u_{C S}=\sqrt{\frac{\gamma(\gamma-1)}{2}} \frac{u_{F}}{1+\sqrt{\rho_{4} / \rho_{1}}} \\
c_{2}=\sqrt{\frac{\gamma(\gamma-1)}{2}}\left(u_{F}-u_{C S}\right)=\sqrt{\frac{\gamma(\gamma-1)}{2}} \frac{\sqrt{\rho_{4} / \rho_{1}}}{1+\sqrt{\rho_{4} / \rho_{1}}} u_{F}
\end{gathered}
$$




\section{In mix experiments one will often not use an Aluminum impact layer}
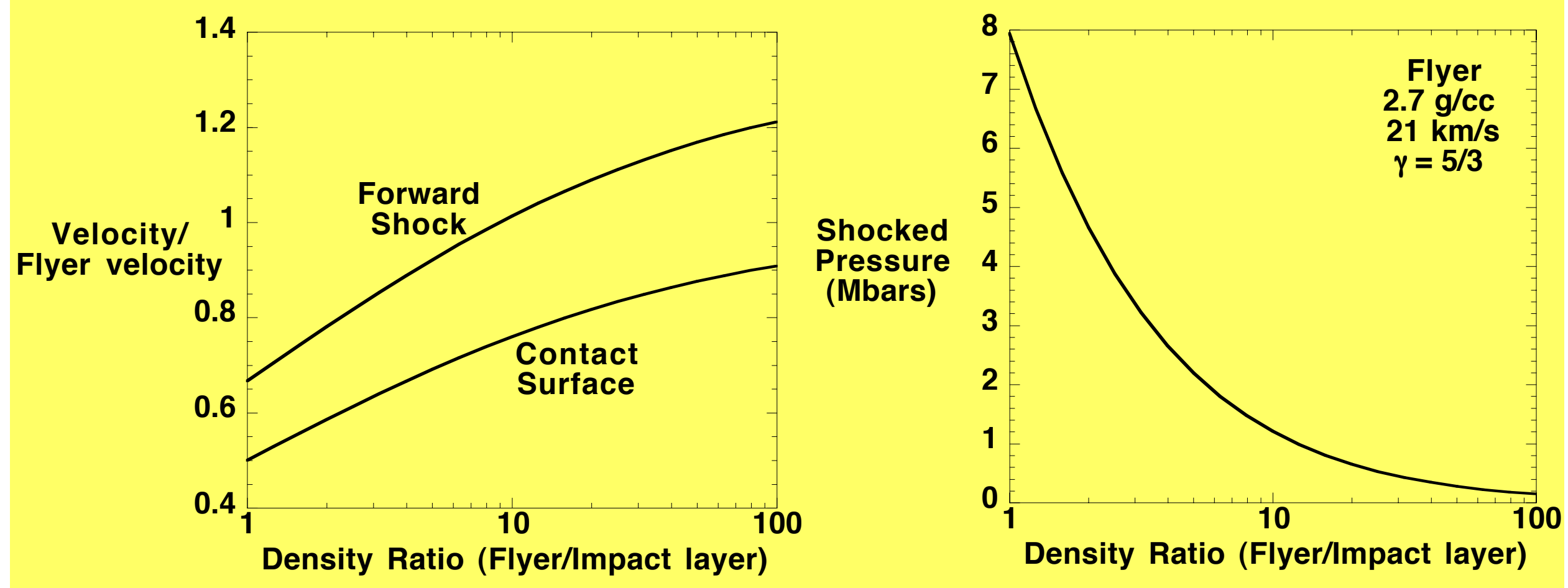


\section{At lower impact layer density, one can drive steady shocks over larger distances}

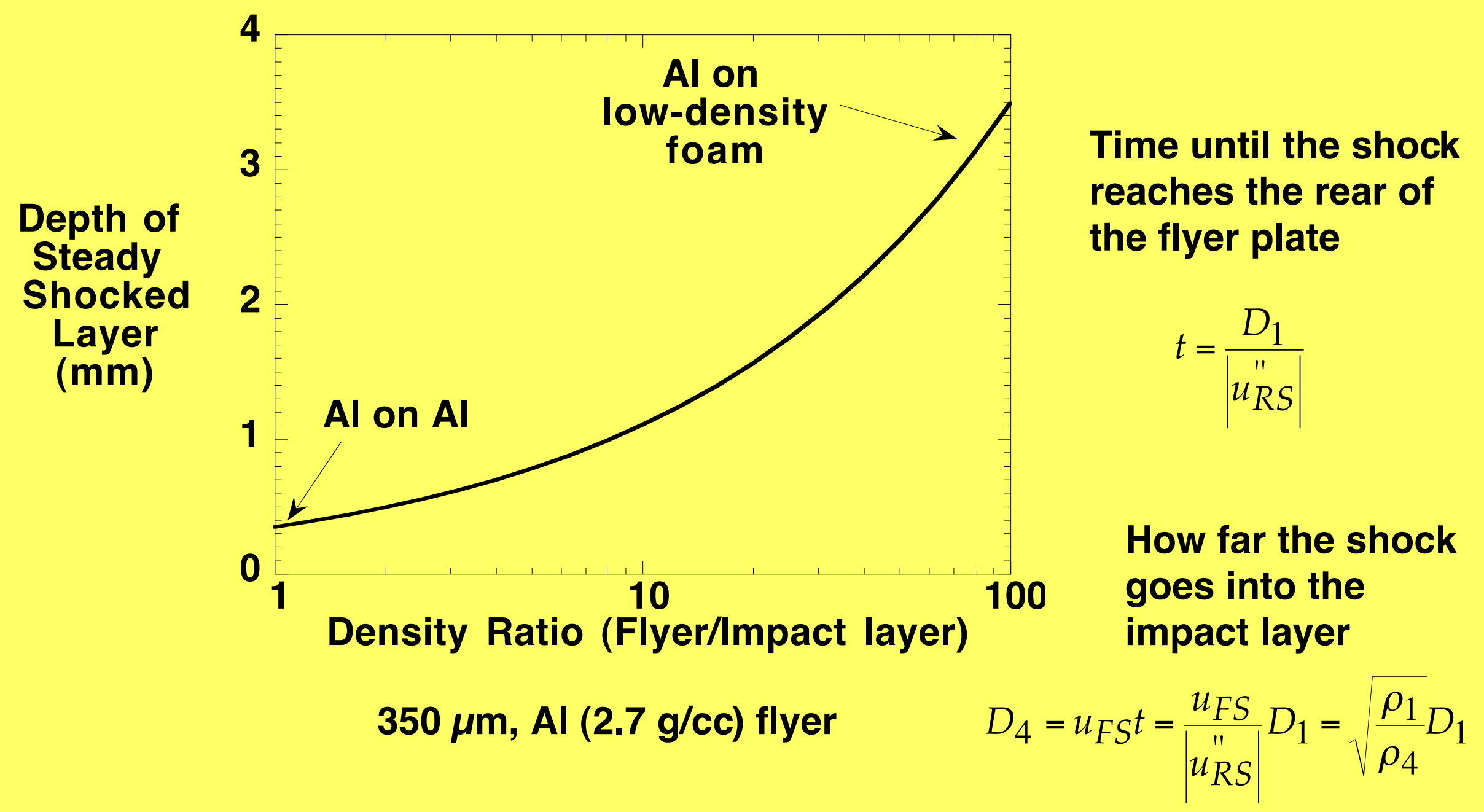




\section{For RT experiments, one creates a blast wave}

$350 \mu \mathrm{m}$ thick, $21 \mathrm{~km} / \mathrm{s}$ flyer

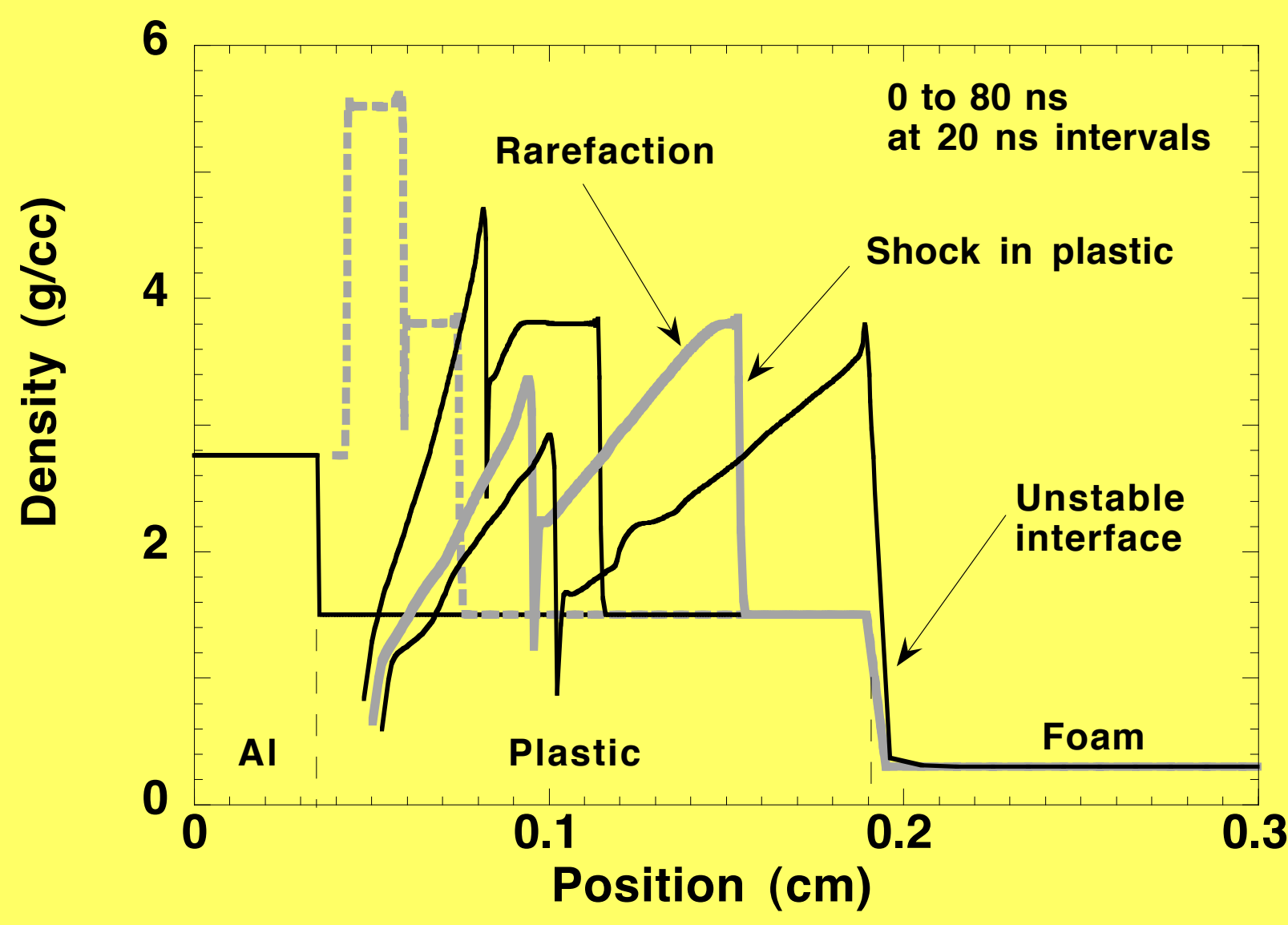

Goal: waste none of the deceleration

$$
\begin{gathered}
t_{4}=\frac{D_{4}}{u_{F S}}=\frac{D_{4}}{u_{F}} \frac{2\left(1+\sqrt{\rho_{4} / \rho_{1}}\right)}{\gamma+1} \\
t_{1}=\frac{D_{1}}{\left|u_{R S}^{\prime \prime}\right|}+\frac{\gamma-1}{\gamma+1} \frac{D_{1}}{c_{2}}+\frac{\gamma-1}{\gamma+1} \frac{D_{4}}{c_{3}} \\
\boldsymbol{t}_{\mathbf{4}}=\boldsymbol{t}_{\mathbf{1}} \text { so }
\end{gathered}
$$

$\frac{D_{4}}{D_{1}}=\sqrt{\frac{\rho_{1}}{\rho_{4}}} \frac{(1+\sqrt{(\gamma-1) / 2 \gamma})}{(1-\sqrt{(\gamma-1) / 2 \gamma})}$ 


\section{The unstable interface moves several $\mathrm{mm}$}
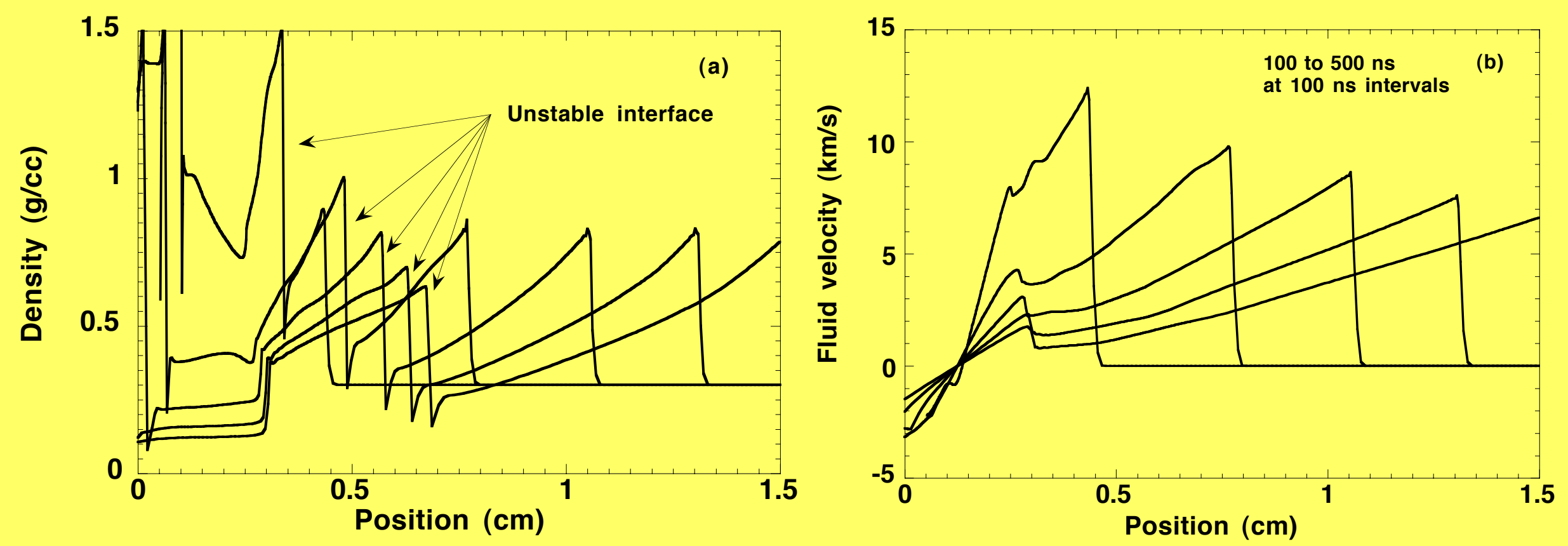


\section{This graph shows the interface behavior}

- The linear growth exponent is $\sim 30$ by $1.5 \mu \mathrm{sec}$

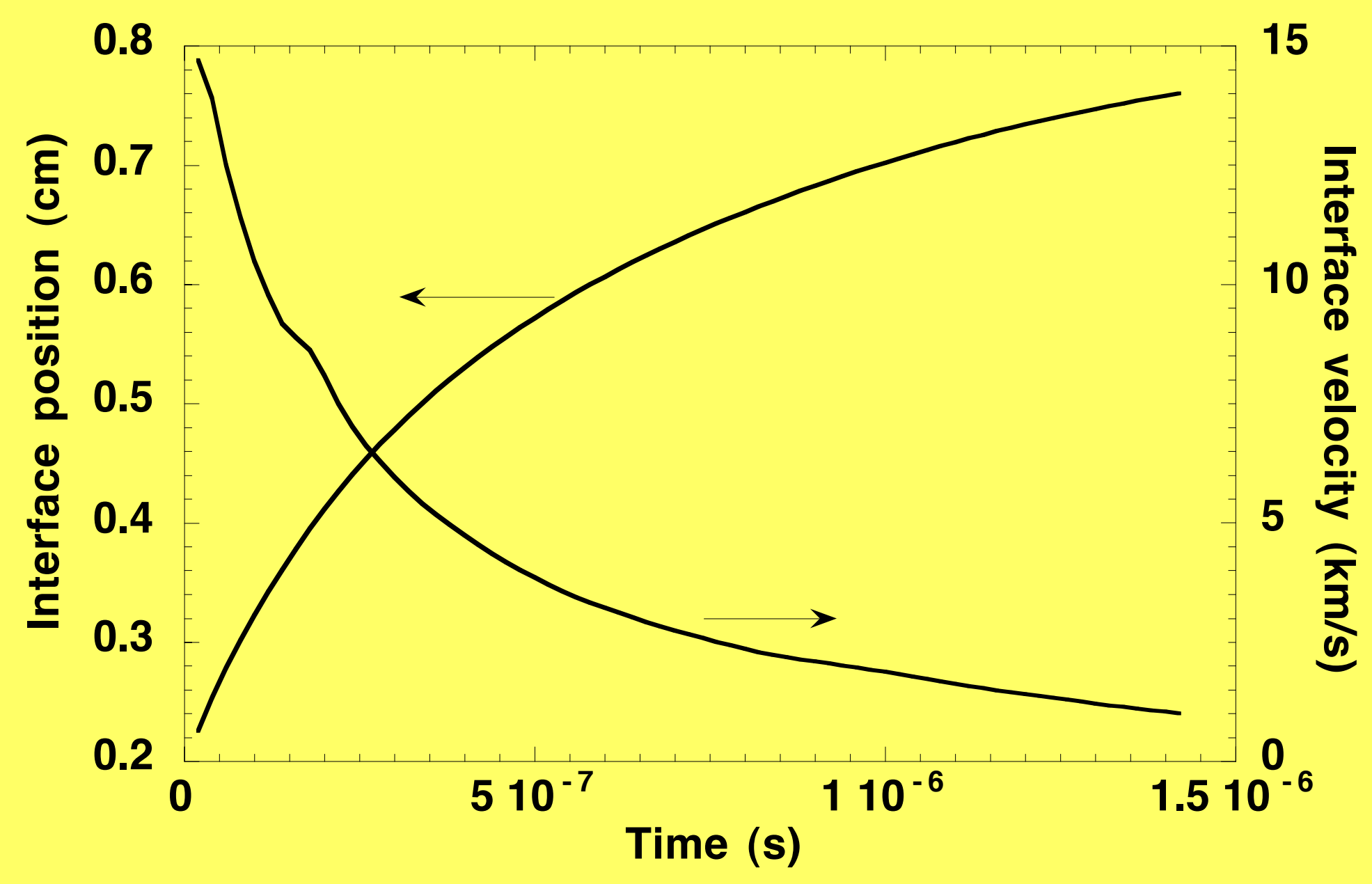




\section{Richtmyer-Meshkov experiments are harder}

Goal: maximum steady post-shock motion of interface

Means: make rarefactions in flyer and impact layer meet at contact surface

$$
\begin{gathered}
t_{1}=\frac{D_{1}}{\left|u_{R S}^{\prime \prime}\right|}+\frac{\gamma-1}{\gamma+1} \frac{D_{1}}{c_{2}}=\frac{D_{1}}{u_{F}} \frac{\left(1+\sqrt{\rho_{4} / \rho_{1}}\right)}{\sqrt{\rho_{4} / \rho_{1}}}\left(\frac{2}{\gamma+1}+\sqrt{\frac{2}{\gamma(\gamma-1)}}\right) \\
t_{4}=\frac{D_{4}}{u_{F S}}+\frac{\gamma-1}{\gamma+1} \frac{D_{4}}{c_{3}}=\frac{D_{4}}{u_{F}}\left(1+\sqrt{\rho_{4} / \rho_{1}}\right)\left\{\frac{2}{\gamma+1}+\sqrt{\frac{2}{\gamma(\gamma-1)}}\right\} \\
\boldsymbol{t}_{\mathbf{4}}=\boldsymbol{t}_{\mathbf{1}} \text { so } \\
D_{4} / D_{1}=\sqrt{\rho_{1} / \rho_{4}}
\end{gathered}
$$




\section{An example where the rarefactions meet}
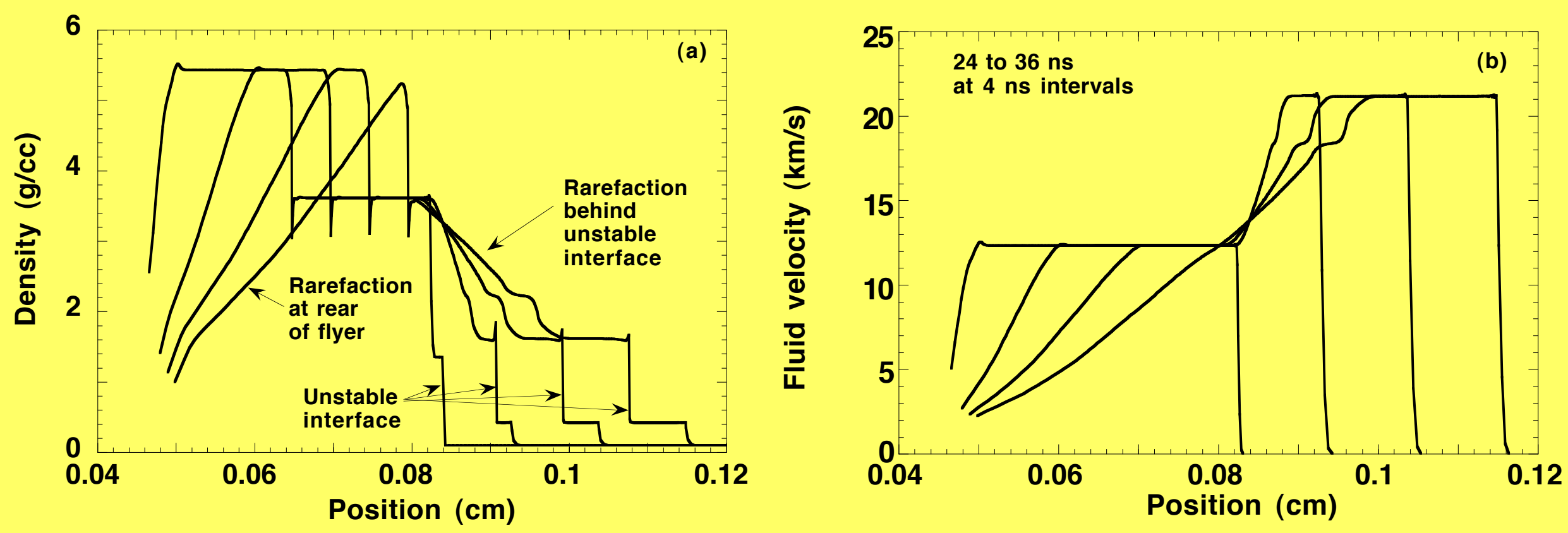

$350 \mu \mathrm{m}$ thick, $21 \mathrm{~km} / \mathrm{s}$ flyer 


\section{The unstable interface moves steadily}

\section{for $\sim 1 \mathrm{~mm}$}
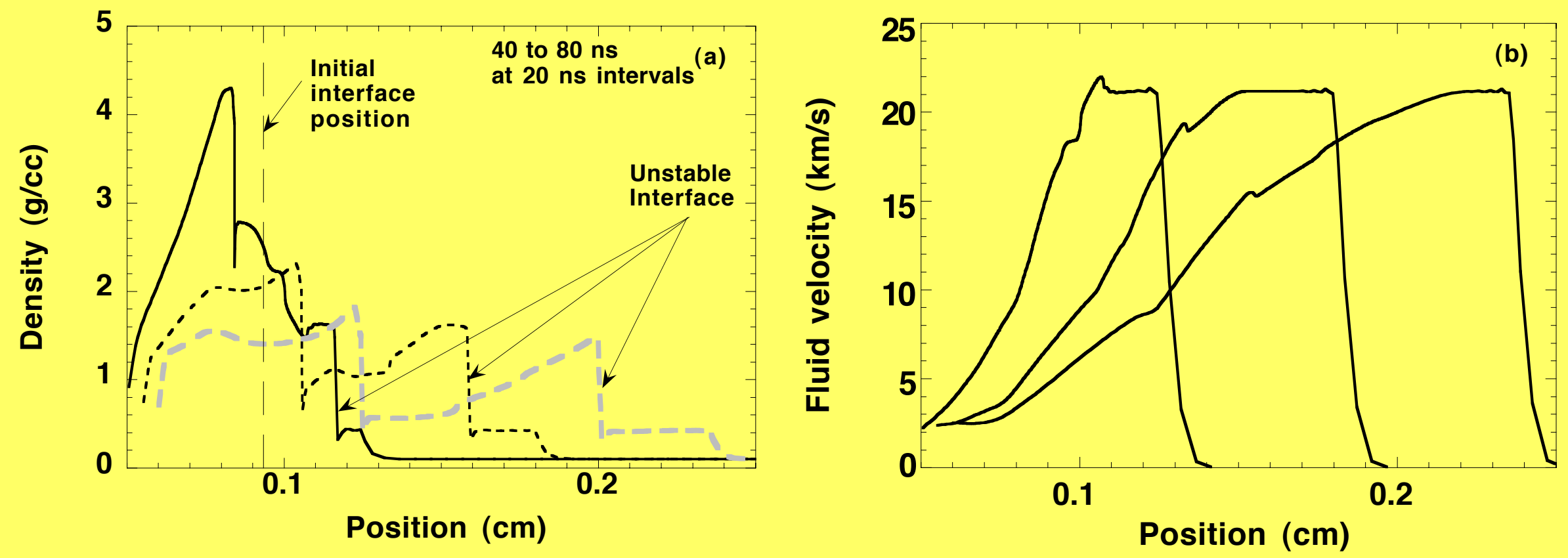


\section{Conclusions}

- This poster has described design approaches for flyerdriven RT and RM experiments on Z

- The advent of the $\mathbf{Z}$ backlighter makes these timely

- Z should be able to accomplish very interesting compressible turbulent mix experiments
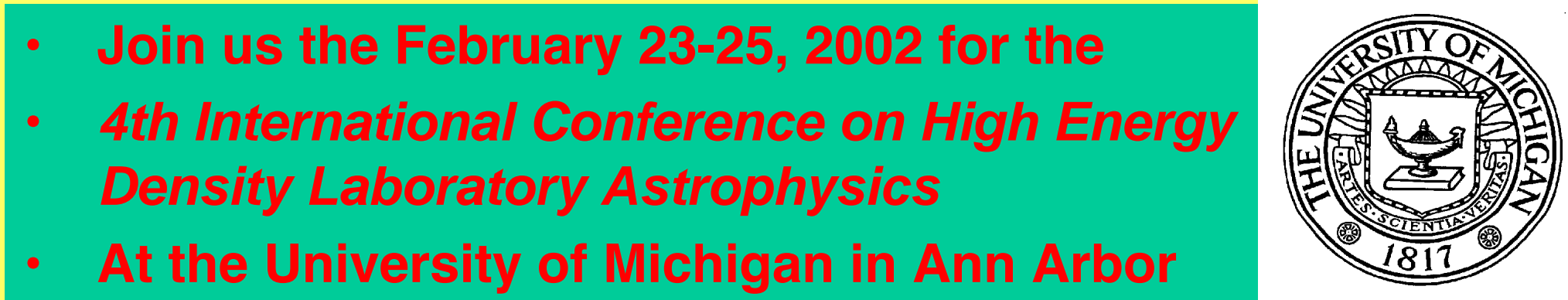


\section{Compressible Hydrodynamics on the}

Omega Laser: Motivated by Astrophysics

R. P. Drake, P. Keiter, K.E. Korreck, K. Dannenberg University of Michigan

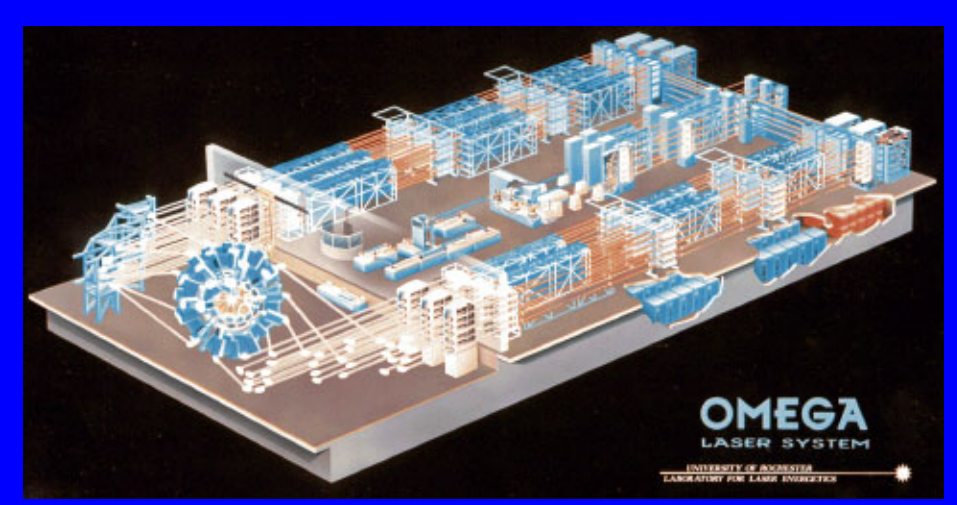

H.A. Robey, T.S. Perry, J.O. Kane, O.A. Hurricane, B.A. Remington, D.D. Ryutov, R.J. Wallace

Lawrence Livermore National Laboratory

J. Knauer, University of Rochester

R. Teyssier, CEA Saclay, France

A. Calder, B. Fryxell, R. Rosner, U. Chicago

Y. Zhang, J. Glimm, SUNY Stony Brook

J. Grove, Los Alamos National Laboratory

D. Arnett, University of Arizona

R. McCray, Univ. of Colorado

N. Turner, J. Stone, Univ. of Maryland

IWPCTM, Pasadena, CA, December 2001

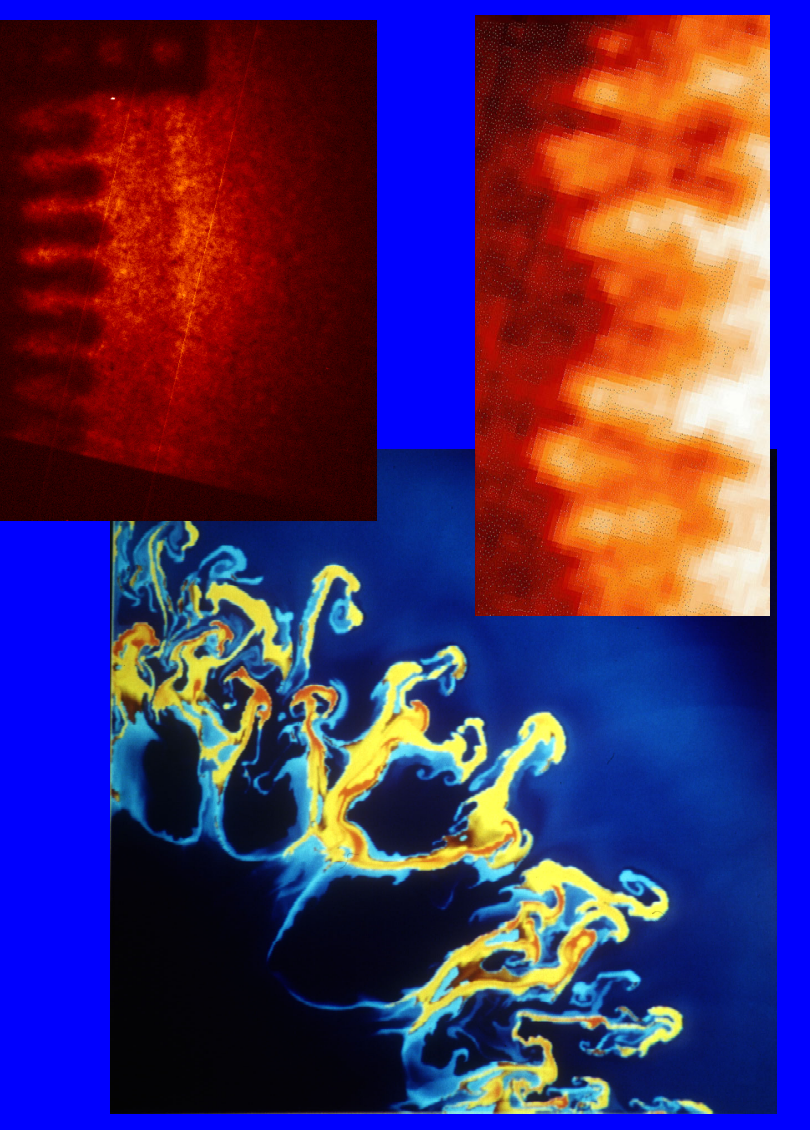


We use the Omega laser to explore phenomena that matter for astrophysics

- Our goal is to experimentally ground the understanding of important mechanisms

- Theory or simulation suggests explanations for astrophysical data

- The important mechanisms have often never been observed anywhere

- We produce and observe these mechanisms in scaled experiments

- This tests the theory and simulations
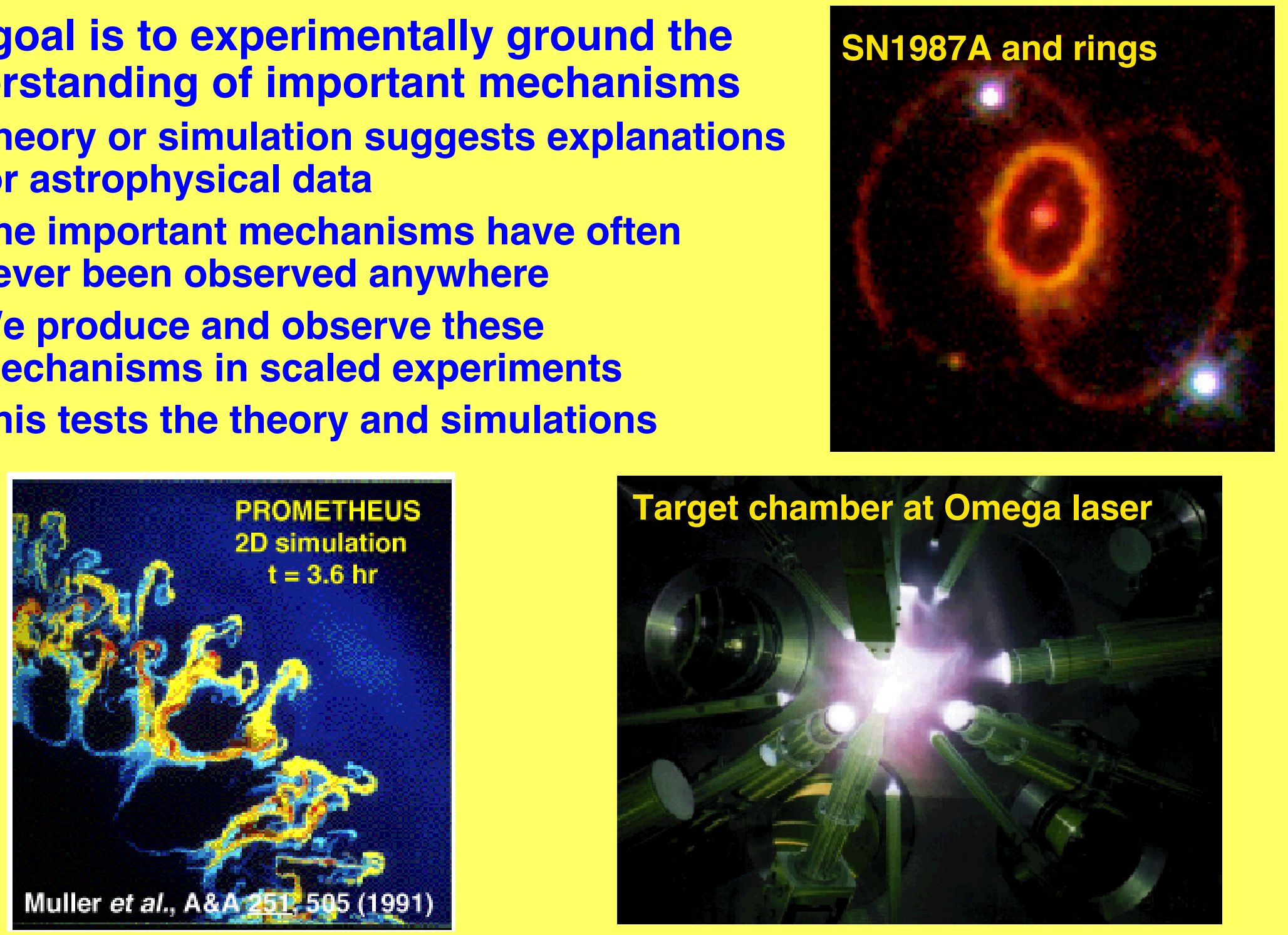


\section{Astrophysical systems provide strong motivations for hydrodynamic studies}

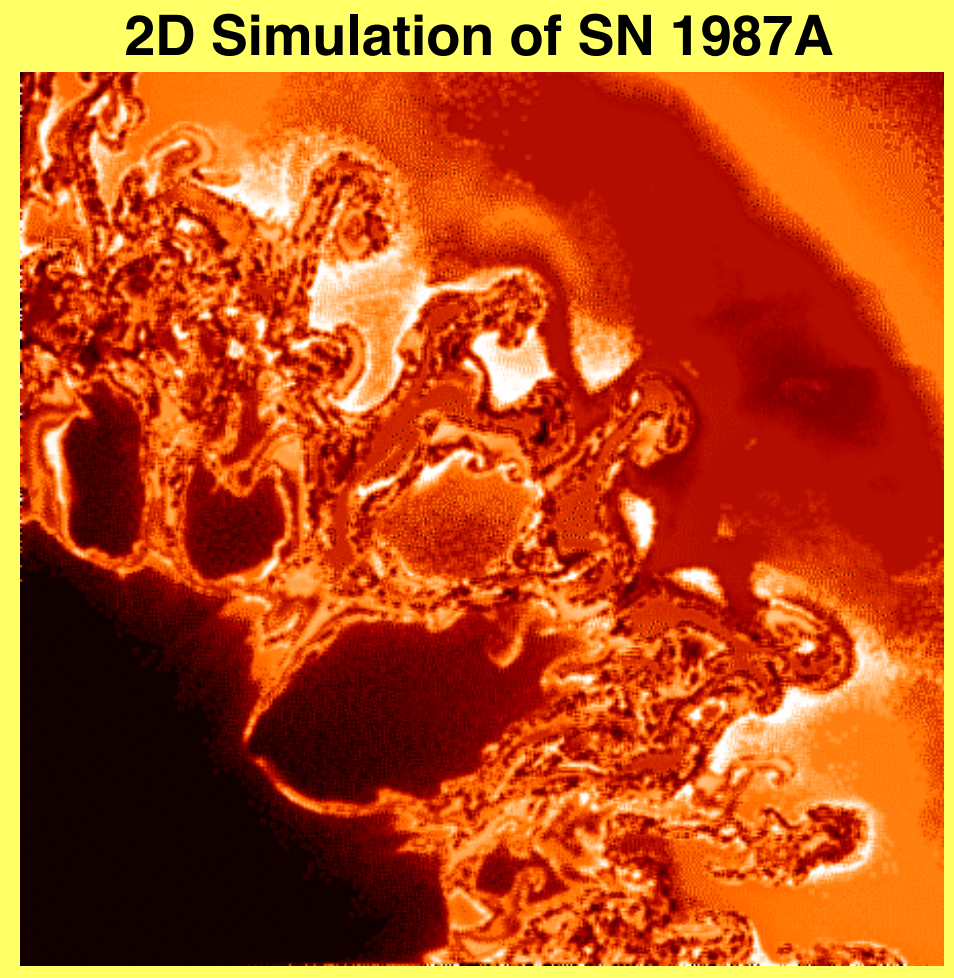

Muller, Fryxell, and Arnett (1991)

The velocities of the heavy element remain larger than predicted.
SN 1987A provided compelling evidence that hydrodynamic instabilities are essential to supernovae

- Light curve

- Spectra

Compressible turbulent mixing is also present in other astrophysical systems

- Supernova Remnants

- Jets

- Shocks into clouds

Well-scaled experiments are feasible: D.D. Ryutov, et al. ApJ 518, 821 (1999) ApJ Suppl. 127, 465 (2000) Phys. Plasmas 8, 1804 (2001) 


\section{We often must explain to astrophysicists that we don't understand hydrodynamics}

- We understand the EQUATIONS of hydrodynamics

- But NOT numerical simulations

- They are like series solutions to differential equations without the ability to quantify errors

- Vanishing metrics add complications

- No stochastic backscatter in current simulations (Leith ‘90, Piomelli ‘91)

- Also no full turbulence

Codes disagree about asymptotic growth rates [Glimm et al. 2001]

Simulation RT growth factor, $\alpha$

'91 Youngs $\quad 0.04-0.05$

'91 Youngs $\quad 0.03$

'99 S.Y. Chen $\quad 0.043$

'99 Dimits et al. $\quad 0.016$

'99 Cheng et al. $\quad 0.08$

'99 Glimm et al. $\quad 0.07$

'99 Oparin $\quad 0.075$

Codes disagree about structure

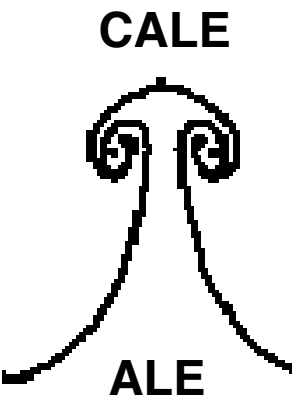

Tabular

EOS
PROMETHEUS

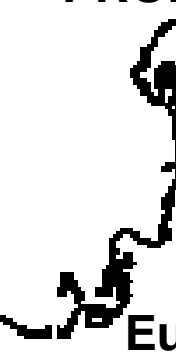

Eulerian Ideal gas EOS
CALE

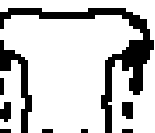

.

$:$ :

Eulerian

Ideal gas

EOS

- This can matter for astrophysics

- Examples:

- Size of mix layers is uncertain

- Small details affect production of "hydrodynamic bullets" or other important structures 


\section{Our hydrodynamic experiments all use similar target packages}

CAD drawing of target \& beams

\section{Photograph of target}
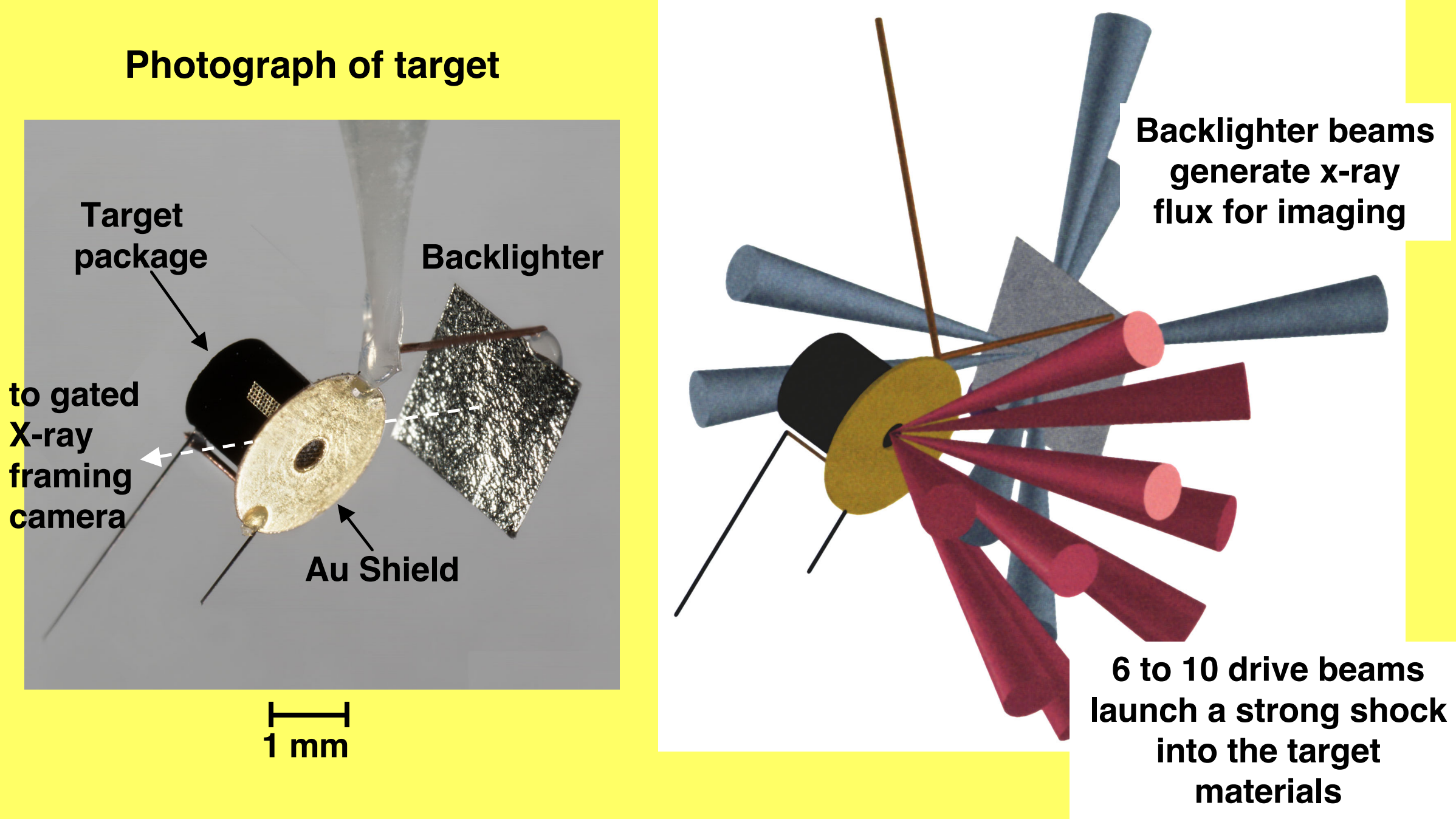


\section{Our experiments have been aimed at addressing the obvious first question:}

- THE FIRST QUESTION: Can the codes follow the evolution of such hydrodynamics deep into the nonlinear regime? 


\section{Our experiments at Omega have addressed this question while probing several mechanisms present in supernova explosions}

\section{Spherical divergence}

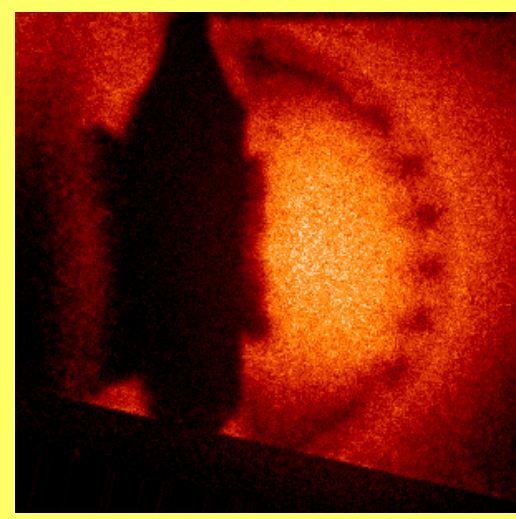

Drake et al., ApJ Jan. '02

Multi-interface coupling

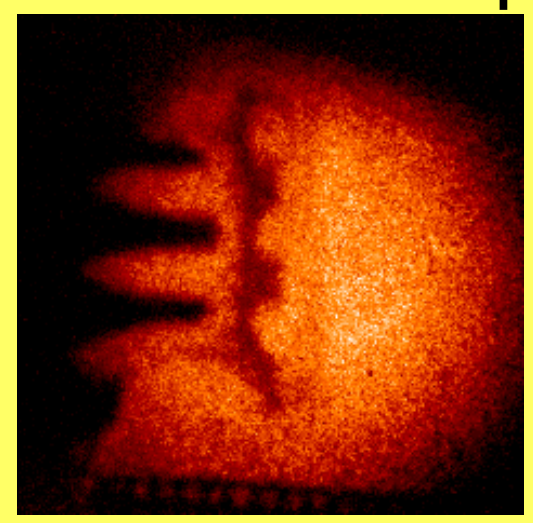

Kane et al., PRE 63, 55401 (2001)

October, 2001
2D simulation of SN1987A Muller, Fryxell, and Arnett (1991)<smiles>C1CCCC1</smiles><smiles>C1CCCC1</smiles>
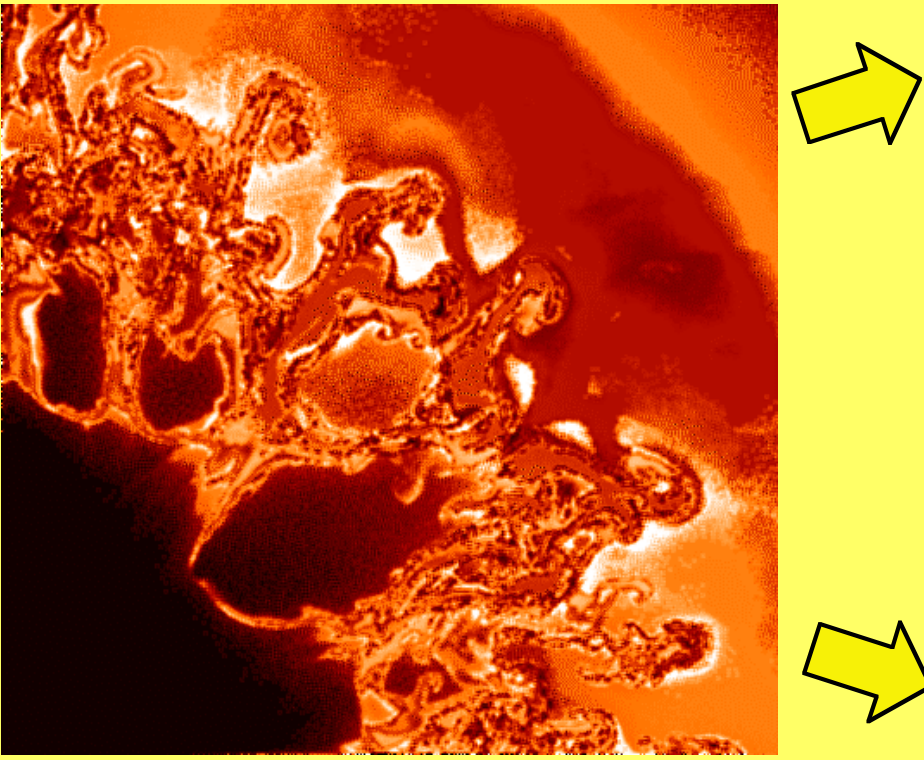

$Y$
2D vs. 3D instability

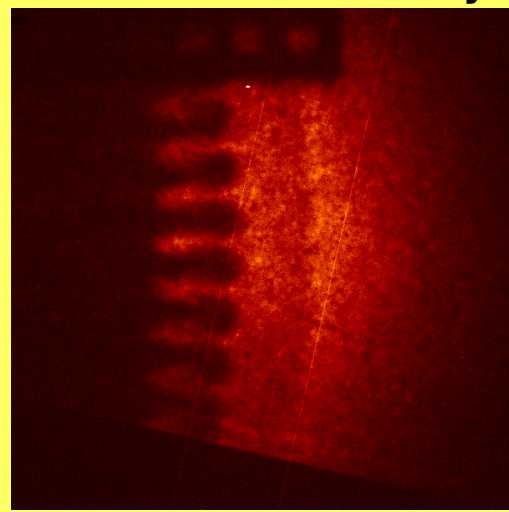

Finished in September

Multi-mode instability

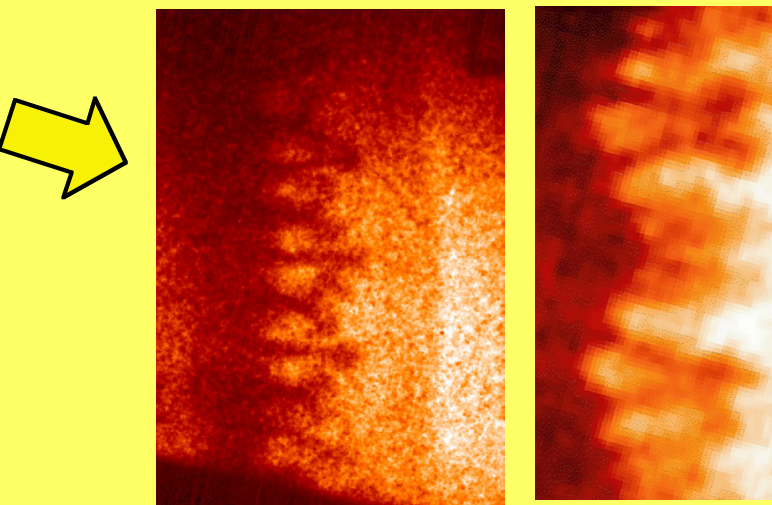

Ongoing experiments 
Coupling between spatially separated interfaces in a SN is studied with a multi-layer target

Exploded view of multi-layer target

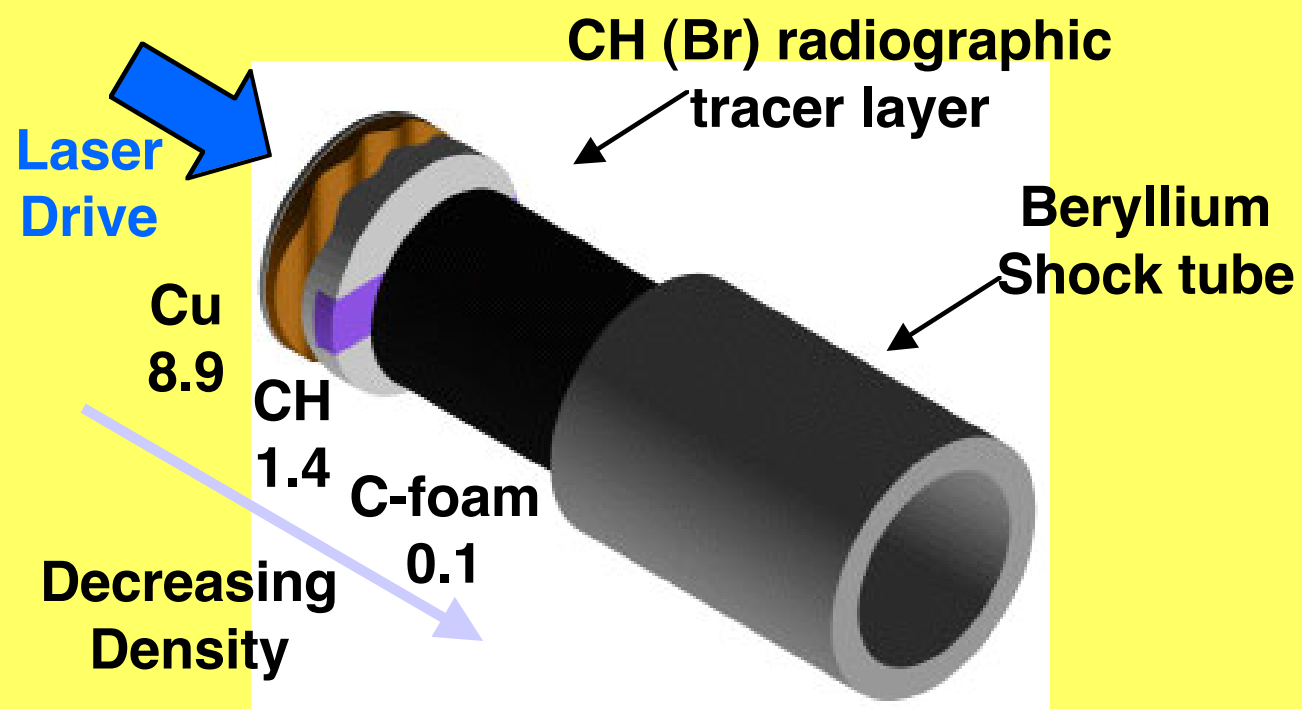

Simulation of this experiment by the ASCI code FLASH at Chicago

$\log 10$ Density $\left(\mathrm{g} / \mathrm{cm}^{3}\right)$

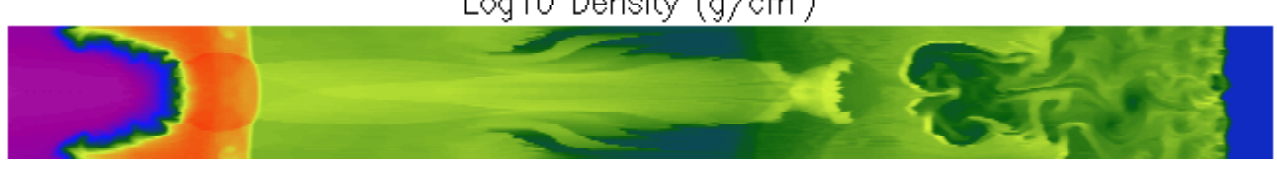

October, 2001
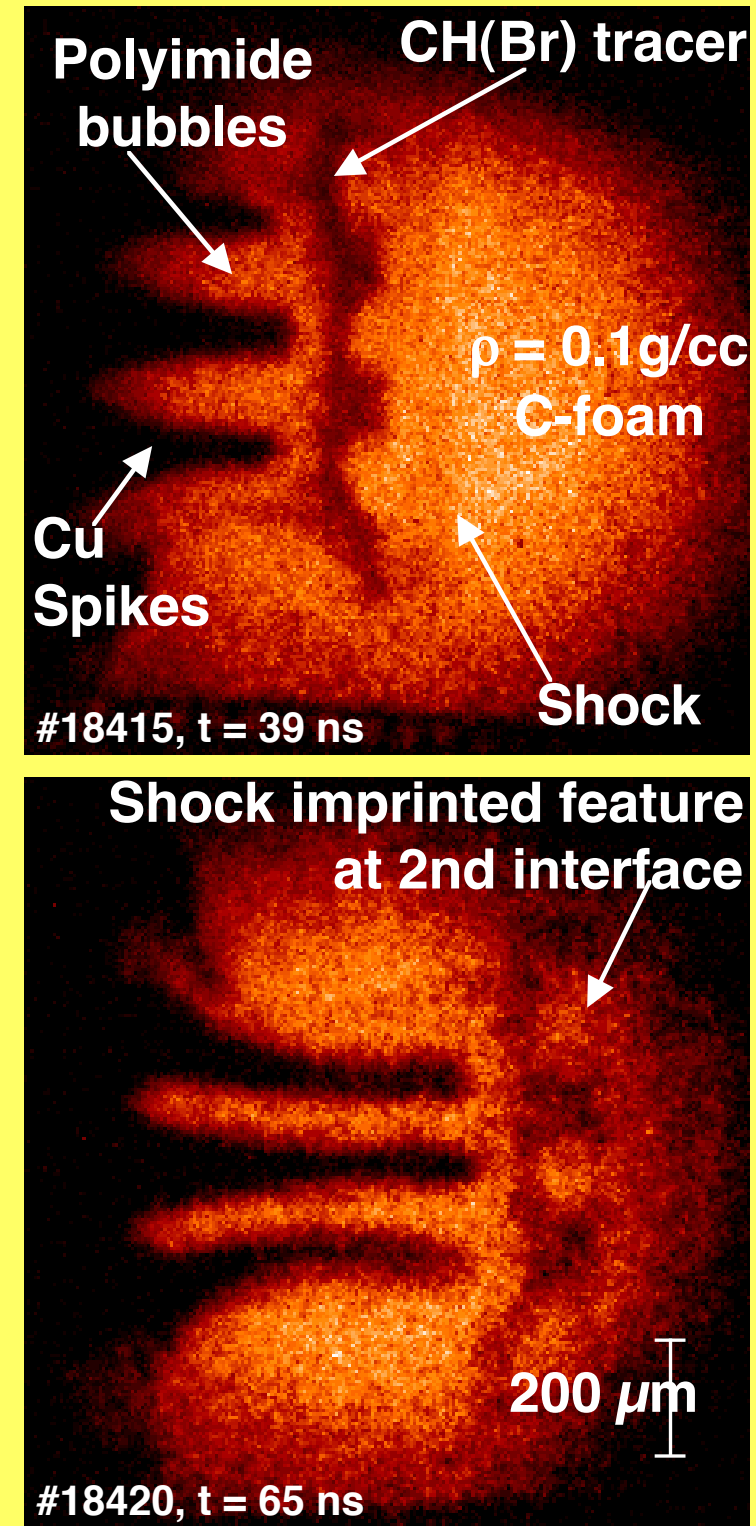


\section{The interface coupling experiments are typical of what we have found}

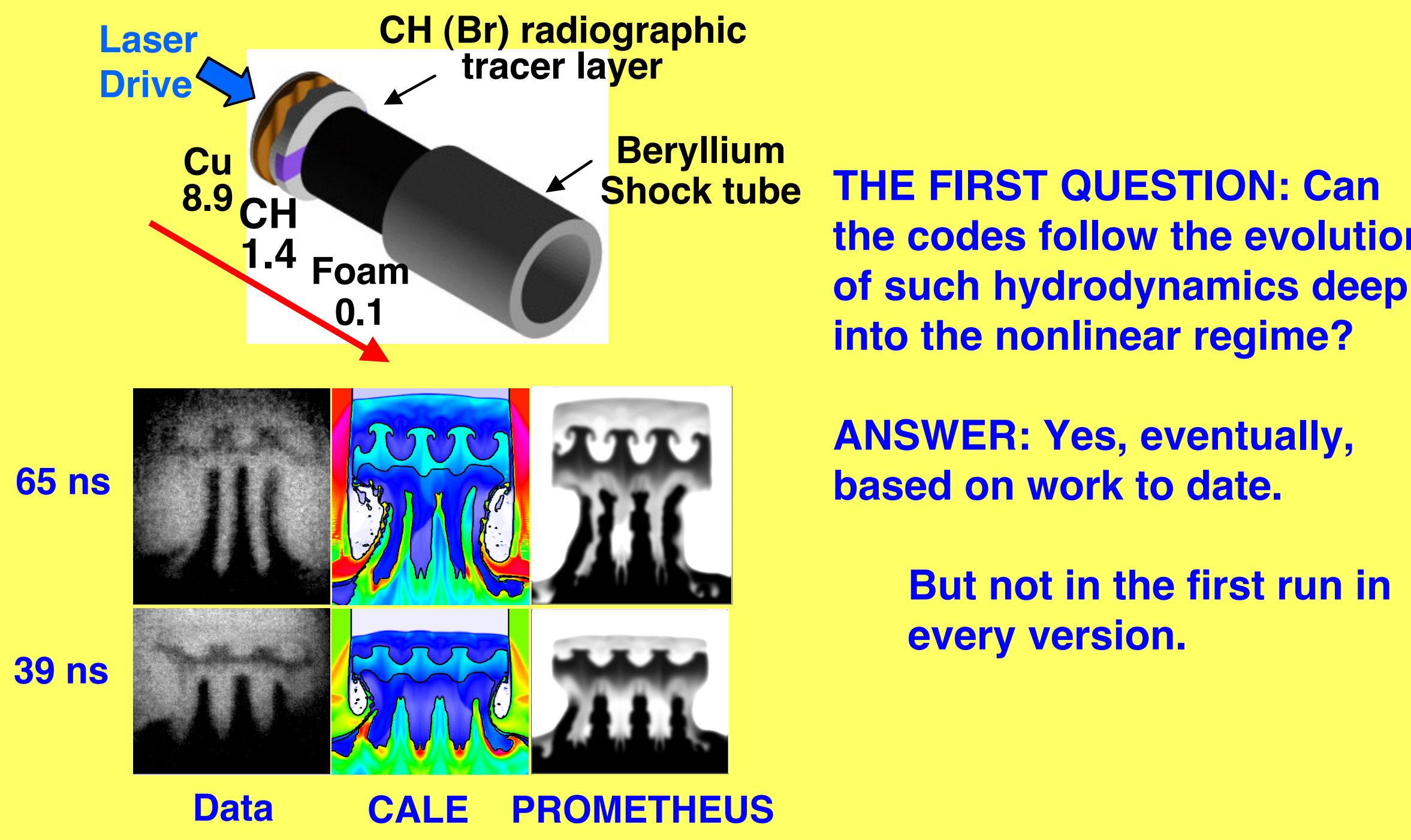




\section{Another example: effect of spherical divergence is studied}

by laser illumination of a perturbed hemispherical shell

Laser Drive
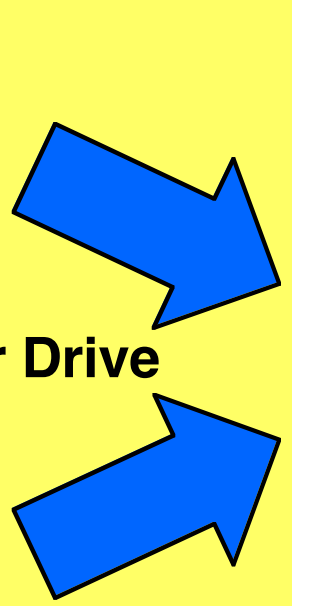

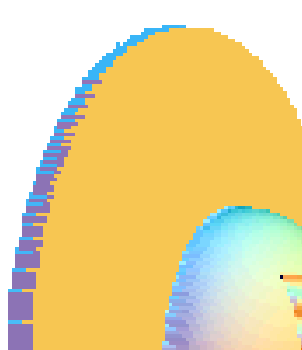

Perturbed $\mathrm{CH}(\mathrm{Ge})$ shell with low density foam cylinder removed

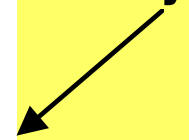

Au shield prevents propagation of a planar shock

- The unperturbed hemispherical shell remains intact indicating no significant perturbation from the laser drive

- The perturbed shell $\left(\lambda=70 \mu \mathrm{m}, \mathrm{a}_{\mathrm{p}-\mathrm{v}}=10 \mu \mathrm{m}\right)$ breaks up due to $R-T$ and $R-M$ instability
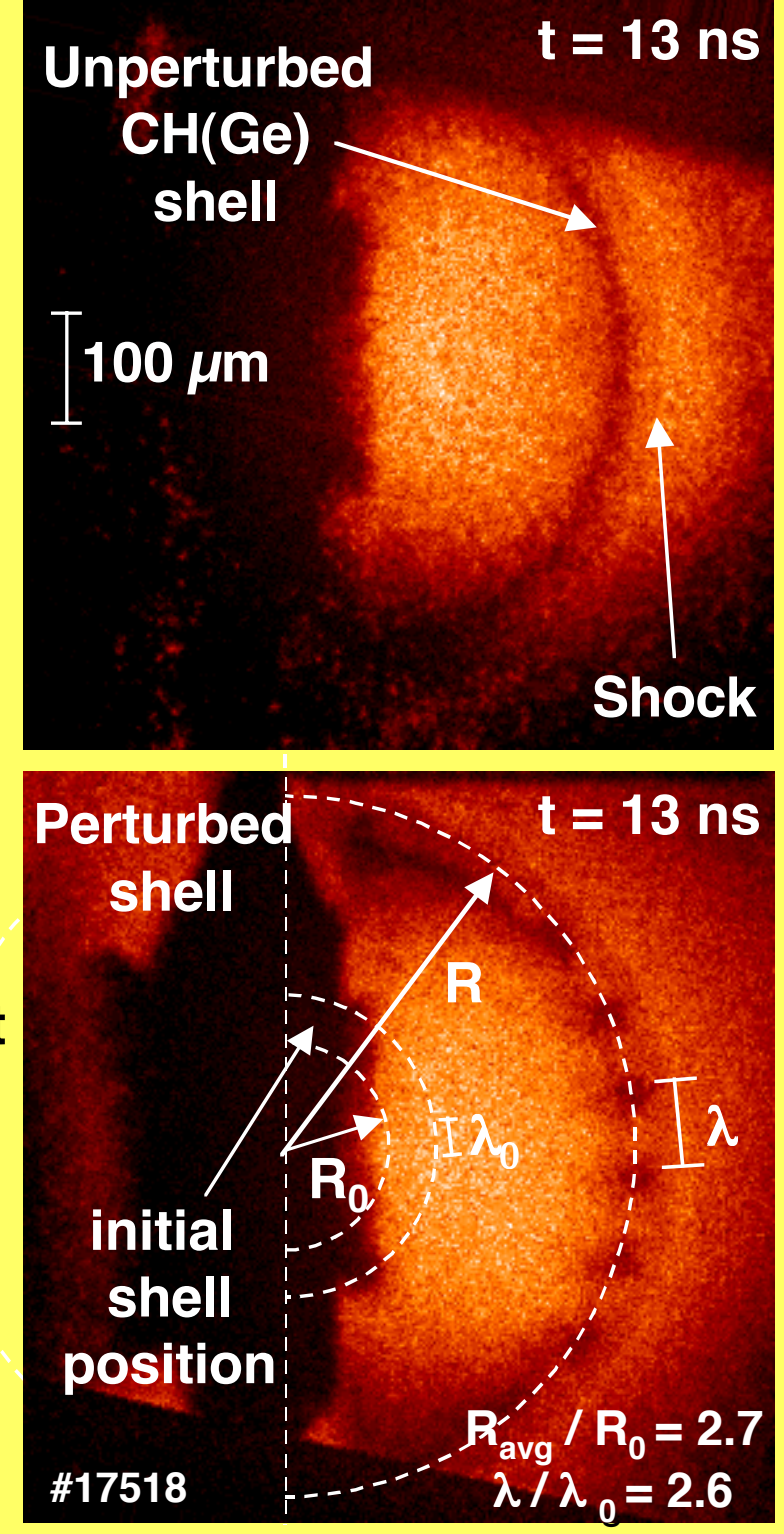


\section{Perturbation growth in spherical geometry is observed to be significantly smaller than in planar geometry}

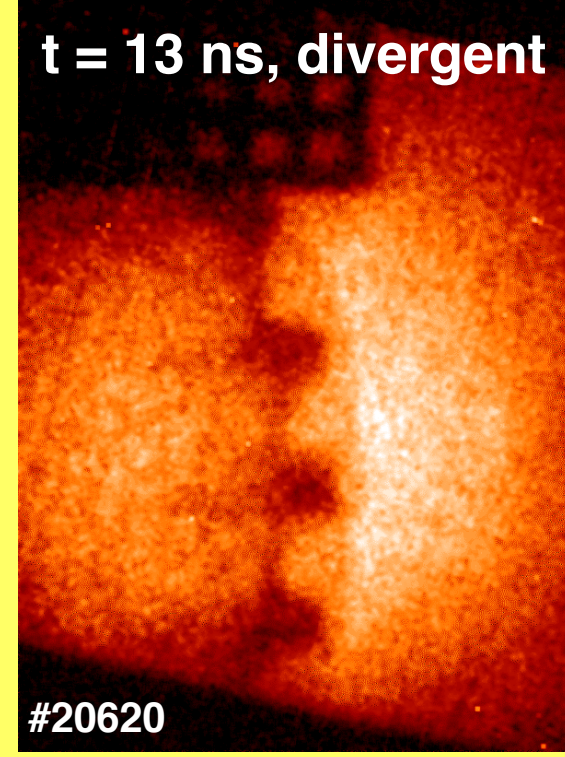

$t=13$ ns, planar

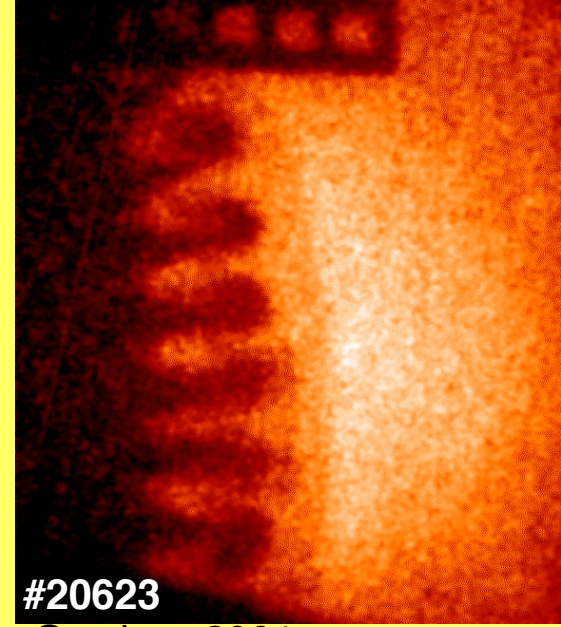

October, 2001
CALE vs. data

Comparison of spherical vs. planar

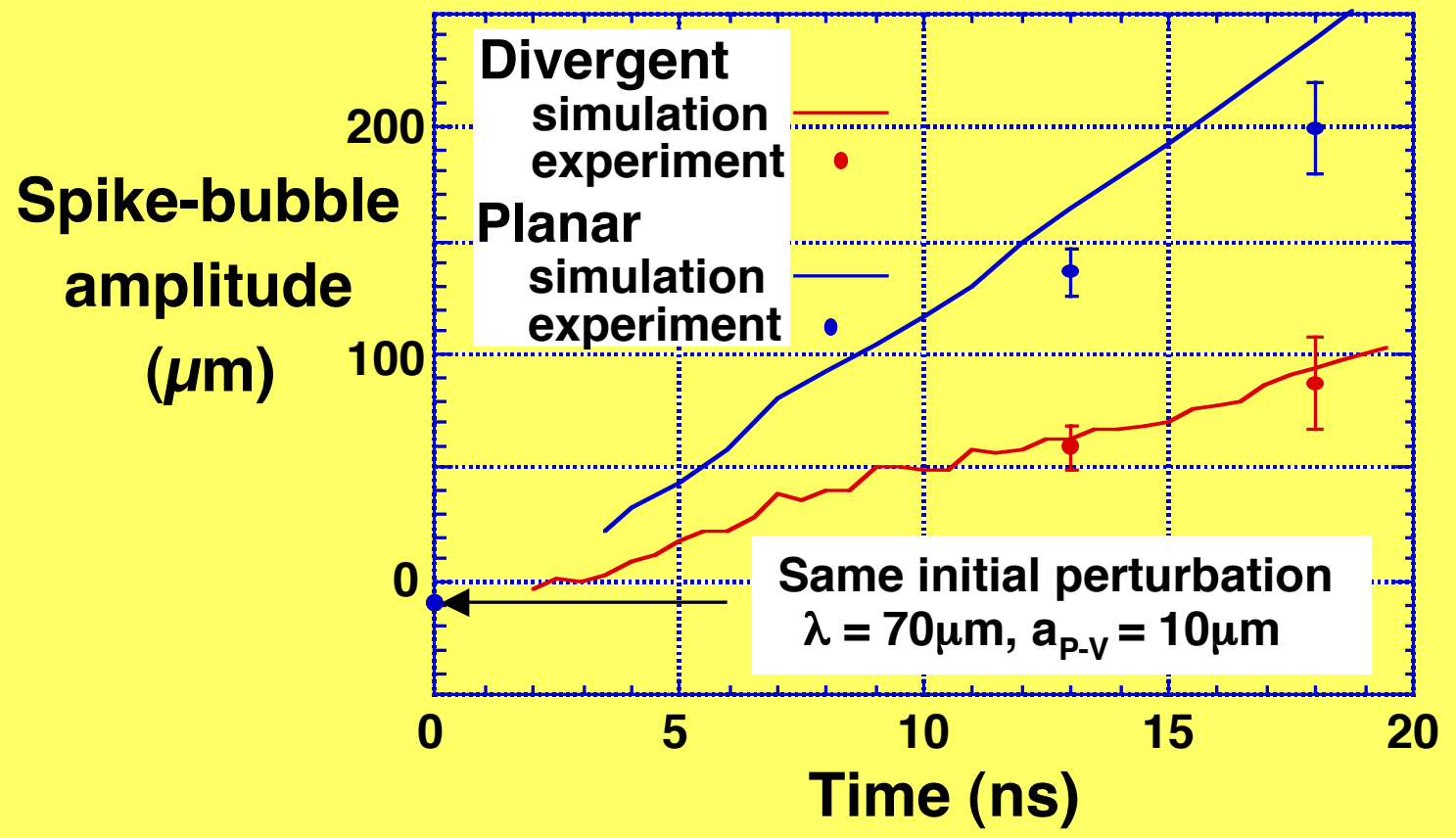

Growth in spherical geometry is reduced due to :

- Wavelength increase due to divergence

- Possible effect of shock proximity 


\section{CALE results show spike development}
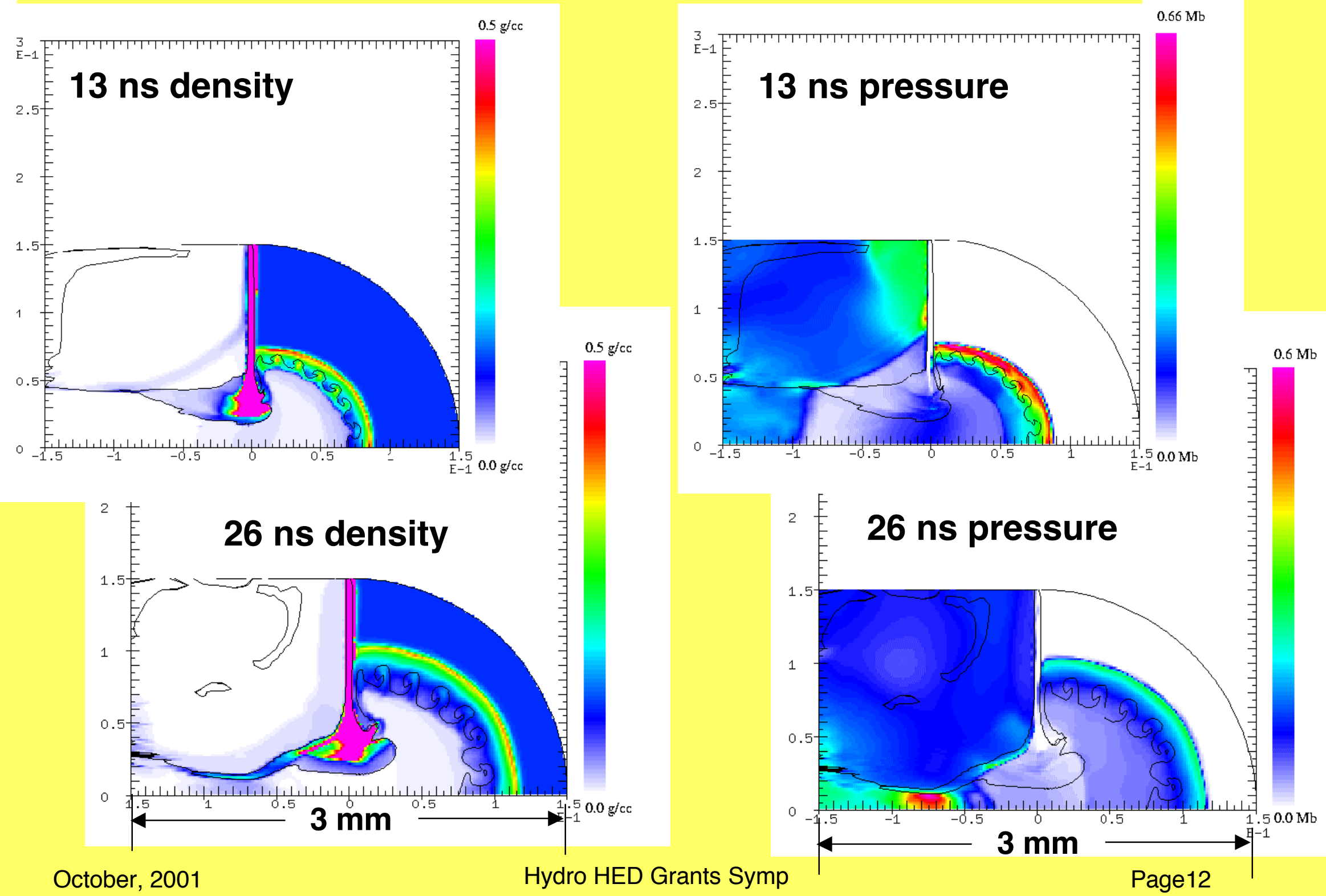


\section{We used FronTier to demonstrate mapping to a purely hydrodynamic code}

- FronTier

- Front tracking by independent updating on the two sides of the discontinuity: there is never finite differencing across the front
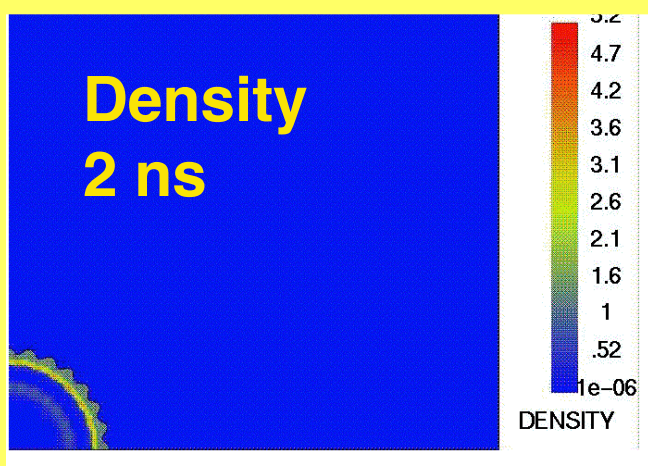

- The common MUSCL (Monotonic Upstream-centered Scheme for Conservation Laws) approach is used to advance the hydro normal to the front and elsewhere

- For this problem, outgoing boundary conditions are used within the capsule and at the outer boundaries; the remainder of the axis of symmetry is a reflecting boundary

- Results shown on $1.5 \mathrm{~mm} \mathrm{x}$ $1.5 \mathrm{~mm}$ scale

October, 2001
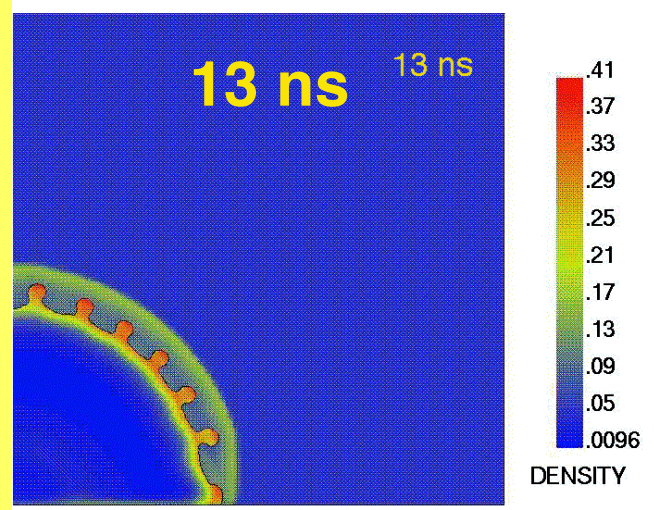

\section{Pressure \\ 2 ns}

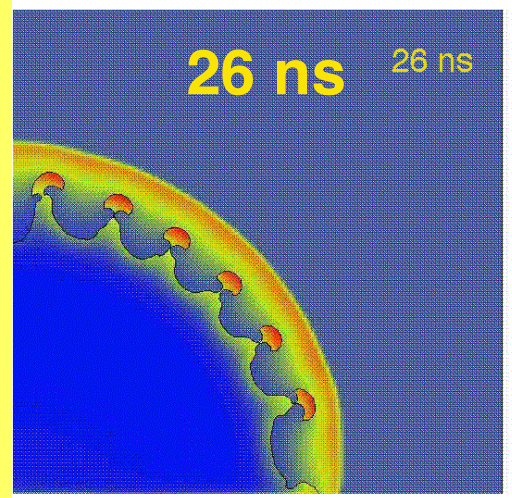

Hydro HED Grants Symp
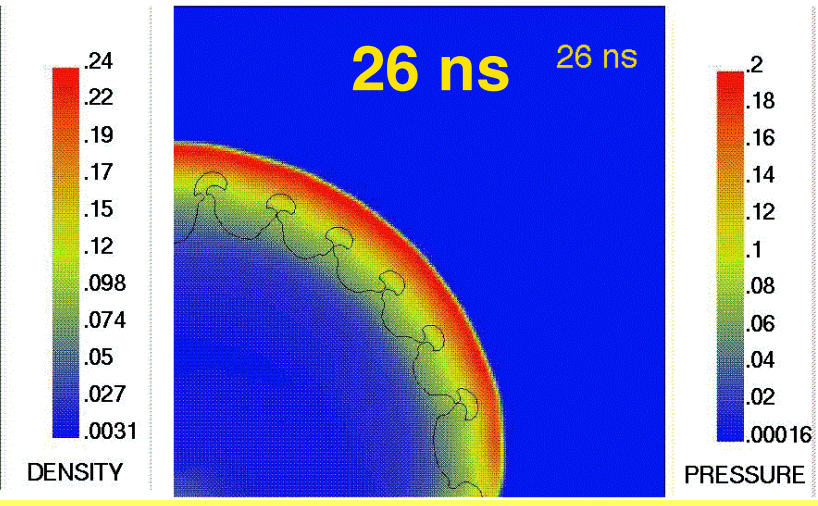


\section{Our hydrodynamic experiments are ready to ask the second question}

- THE SECOND QUESTION: Does this medium (plasma) exhibit a transition to turbulence like that seen in fluids?

- A: In ongoing experiments, we are working to find out.

- If so, this will be a much bigger challenge for simulations.

- It will probably also mean that much published astrophysical hydrodynamics is wrong.

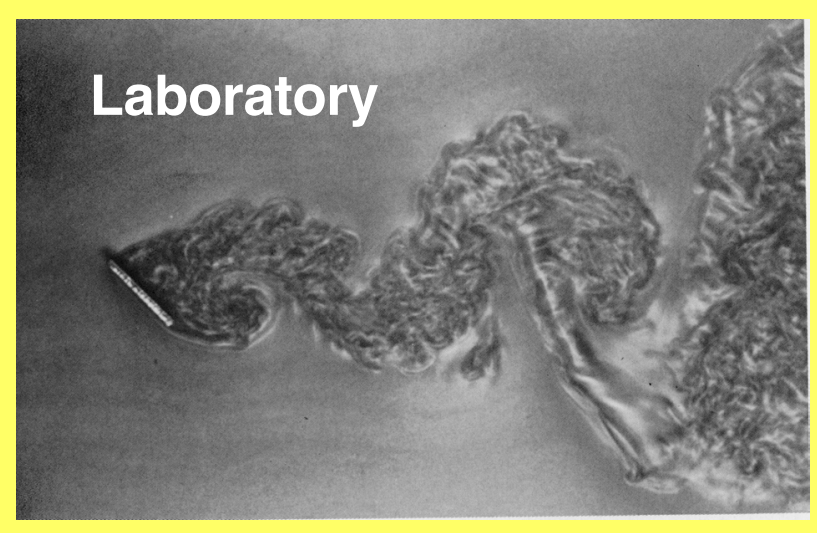

$\mathrm{Re}=4300$ Cantwell, Ann. Rev. Fluid Mech., 13, 457 (1981)

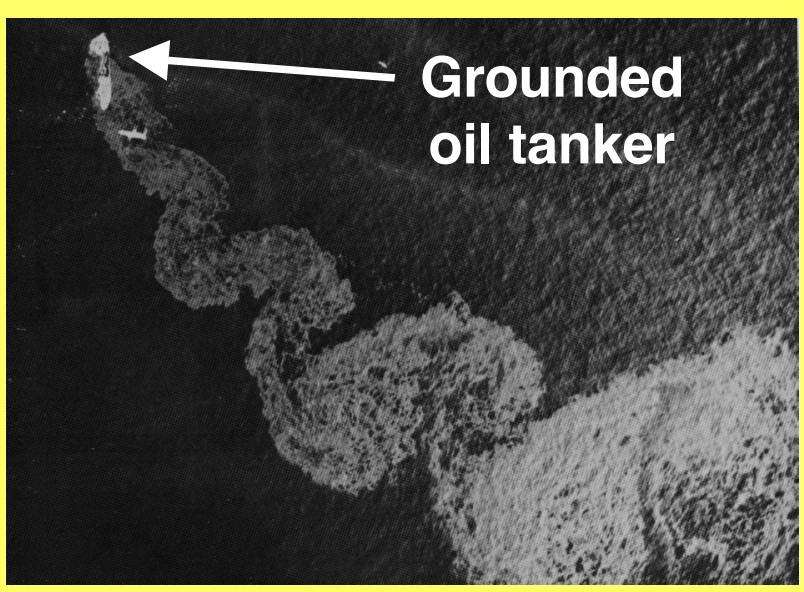

Re $=10^{7}$ Van Dyke, "An Album of Fluid Motion”, Parabolic Press, p.100 (1982) 


\section{We are excited about our progress and look forward to forthcoming experiments}

- We've made demanding tests of deep nonlinear hydrodynamics at high energy density

- Next: Push high energy density systems into the "fully turbulent" regime
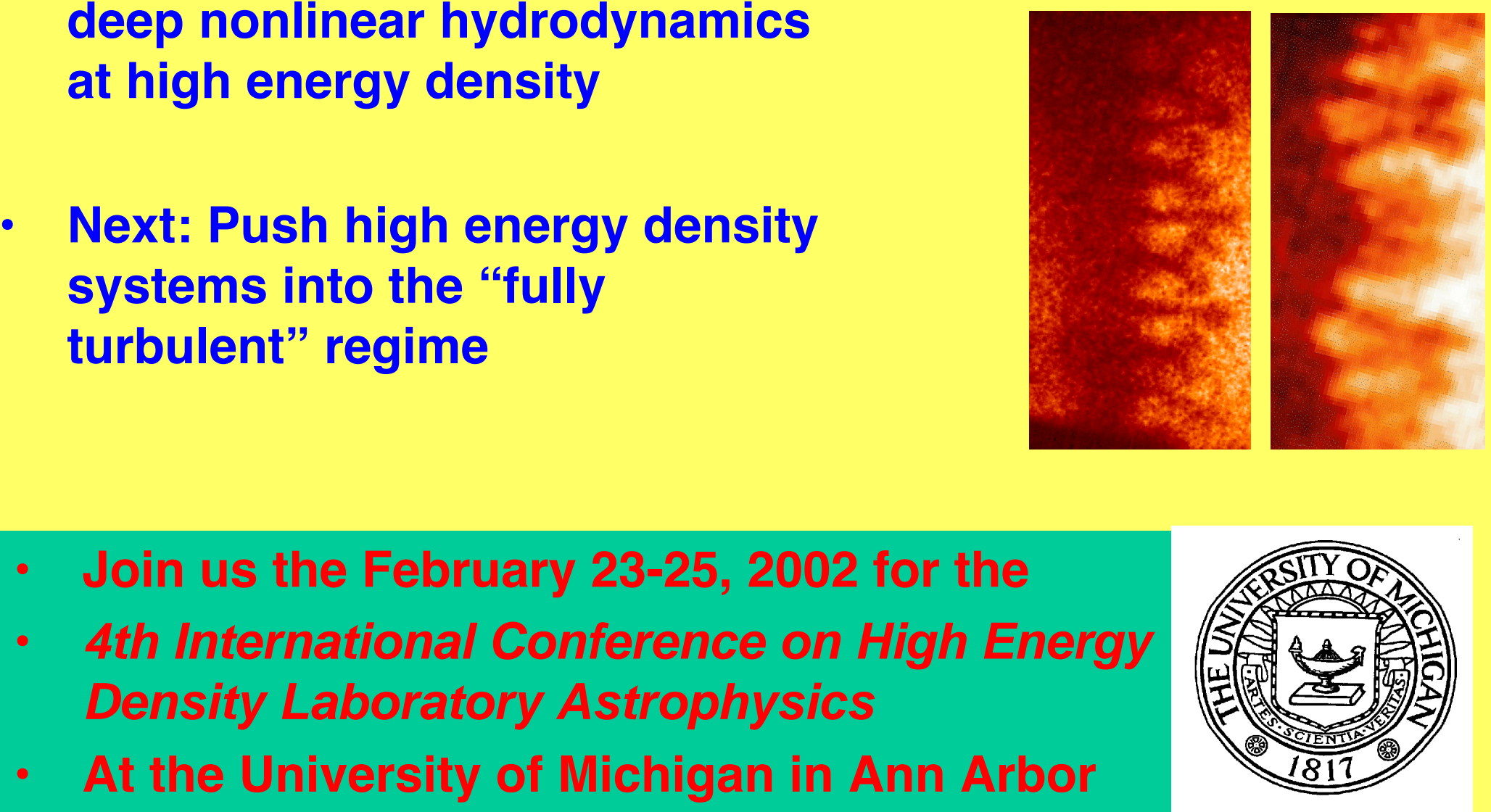


\section{An experimental study of the effect of shock proximity on the Richtmyer-Meshkov instability at high Mach number}

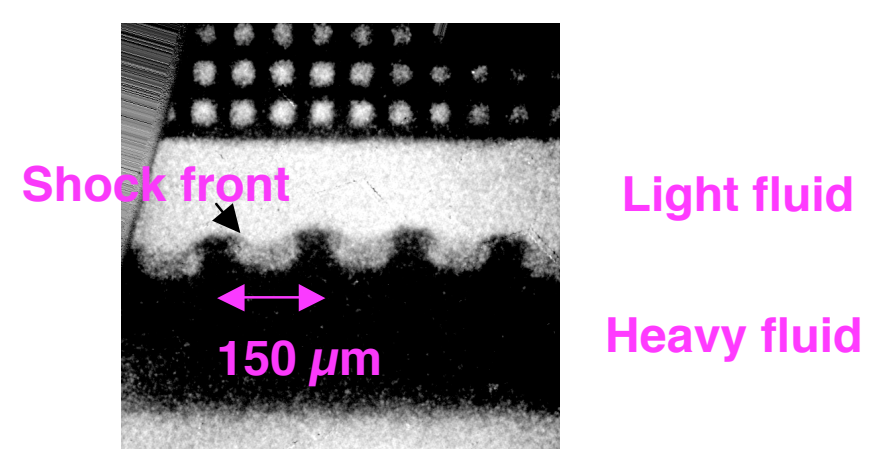

S. G. Glendinning, D.G.Braun , M.J.Edwards, W.W.Hsing, B.F.Lasinski, H.Louis, A. Miles, J.Moreno, T.A.Peyser, B.A.Remington, H.F.Robey, E.J.Turano, C.P.Verdon, Y.Zhou

LLNL
Presented to: 8th International Workshop on the Physics of Compressible Turbulent Mixing Pasadena, CA

\section{December, 2001}

This work was performed under the auspices of the U. S. Department of Energy by the University of California, Lawrence Livermore National Laboratory under Contract No. W-7405-Eng-48. 


\section{Summary}

- We have used the Omega laser to generate a nearly steady interface velocity for Richtmyer-Meshkov experiments

- The interface is a heavy-to-light (12:1) density step

- The incident shock Mach number is $\sim 10$

- The shock velocity is only about $20 \%$ higher than the interface velocity

- An initially sinusoidal perturbation with $\square=150 \mu \mathrm{m}, \square_{0}=7 \mu \mathrm{m}\left(\mathrm{k} \square_{0}=0.3\right)$ grows according to incompressible models

- The growth of with $\square=150 \mu \mathrm{m}, \square_{0}=22 \mu \mathrm{m}\left(\mathrm{k} \square_{0}=0.9\right)$ is about half that predicted from incompressible models

- The shock remains very close to the spike tips as the perturbation grows

- An analytical model which accounts for the effect of the shock proximity predicts the reduced growth 


\section{The Richtmyer-Meshkov instability occurs at an interface impulsively accelerated by a shock}

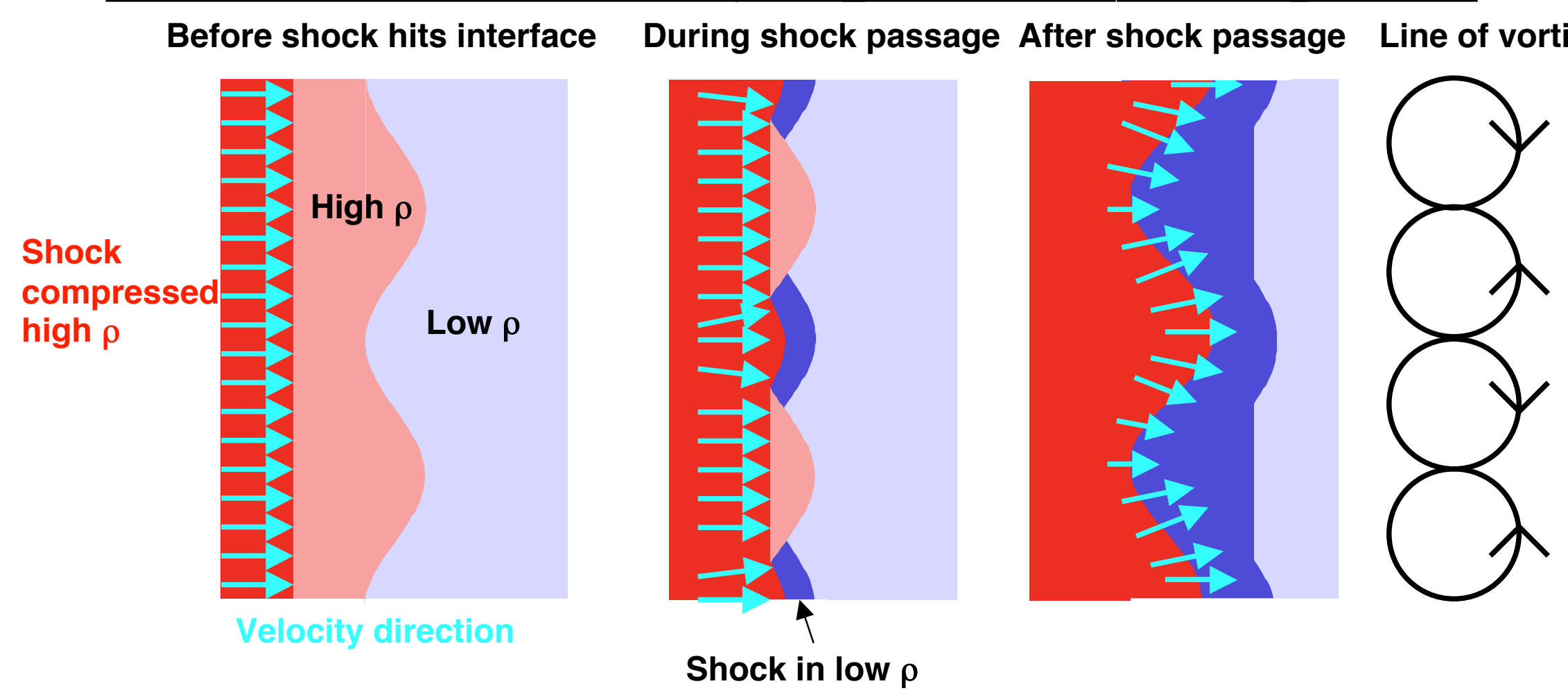

- The interface may be at a density decrease or increase in the propagation direction

- These experiments are at a density decrease

- A perturbation at the interface creates a velocity perturbation (vorticity field)

- The perturbation grows linearly as $\square(\mathrm{t})=\mathrm{kA} A^{*}=\frac{\square^{*}+\square_{0}}{2} \mathrm{u}_{\mathrm{c}} \mathrm{t}$ (Meyer and Blewett, 1972) 


\section{Laser experiments are important to understand effects of compressibility (high Mach number)}

- Compressibility results in a shock front which remains close to the interface

- $\mathrm{u}_{\mathrm{c}} \sim \mathrm{s}_{\mathrm{f}}$, where $\mathrm{u}_{\mathrm{c}}$ is the interface speed and $\mathrm{s}_{\mathrm{f}}$ is the receding shock speed

- Incompressible models (Meyer-Blewett, 1972; Sadot, 1998) predict that spike tip moves faster than shock

- Various models predict reduction in growth rate due to shock proximity:

- Holmes et al., (1999) $\square=\square_{\mathbb{M}} /\left[1+\square_{M} /\left(s_{f} \square u_{c}\right)\right]$, where $\square_{M}$ is $\mathrm{ku}_{\mathrm{c}} \mathrm{A}^{*}\left(\square^{*}+\square_{0}\right) / 2$.

- Hurricane et al., (2000) $\square=u_{c}\left(1 \square u_{c} / s_{f}\right) \tanh \left[\square_{M} / u_{c}\left(1 \square u_{c} / s_{f}\right)\right]$

- Laser experiments at Mach 15 (Dimonte, 1996; Holmes, 1999; Farley 1999) may show large amplitude effects rather than compressibility effects (BenDor et al., 2001)

- $\mathrm{k} \square_{0}=2$ (Dimonte/Holmes), $\mathrm{k} \square_{0} \sim 2.7$ (Farley)

- Rikanati et al. (2000) predicts $\square / \square_{\mathrm{M}}$ at Mach 15 of $\sim 0.9$ for $\mathrm{k} \square_{0}=0.9$, 0.65 for $\mathrm{k} \square_{0}=2$

- On Omega we have investigated this with $\mathrm{k} \square_{0}=0.9, \mathrm{u}_{\mathrm{c}}=21.9 \mu \mathrm{m} / \mathrm{ns}, \mathrm{s}_{\mathrm{f}}=$ $26.1 \mu \mathrm{m} / \mathrm{ns}$ 


\section{A model of vortex evolution (Rikanati, 1998) was proposed for low Atwood number RMI}

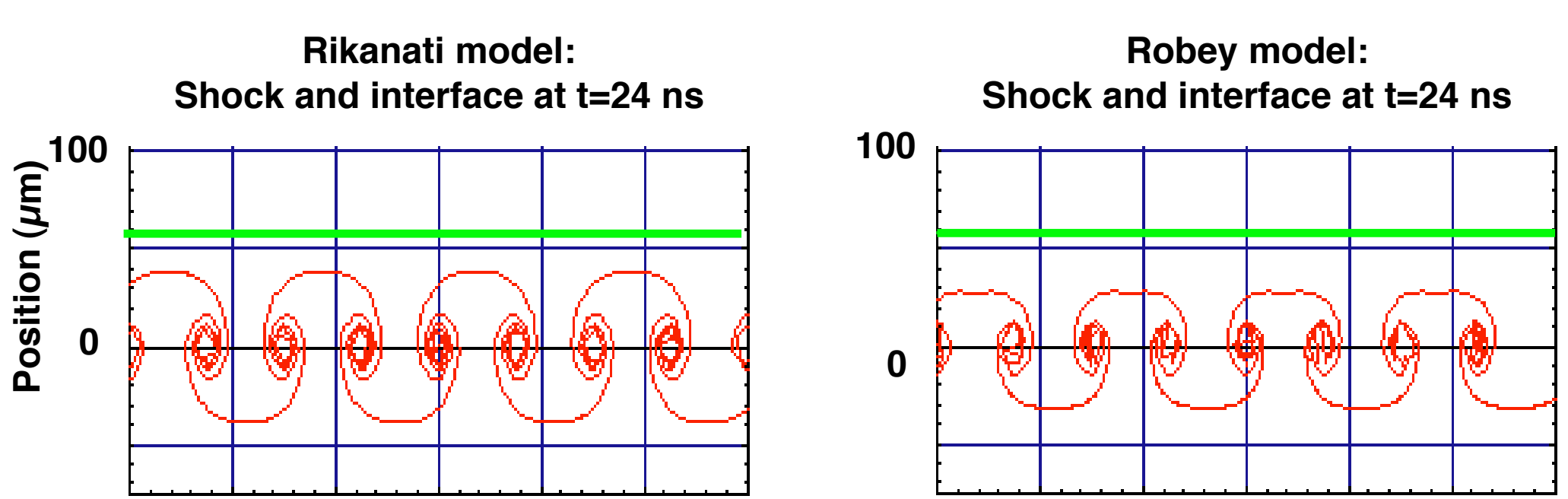

- This model calculates growth rates from analytical solutions to vortex flow problem

- An extension of this model (Robey, 2001) constrains the shock front to be flat by introducing mirror image vortices

- However, the shock front is not in reality flat

- Robey's is the only model which predicts an increase in growth rate after initially slow growth 


\section{This experiment uses an $11 \mathrm{~ns}$ laser drive to create a steady shock incident on a modulated interface}

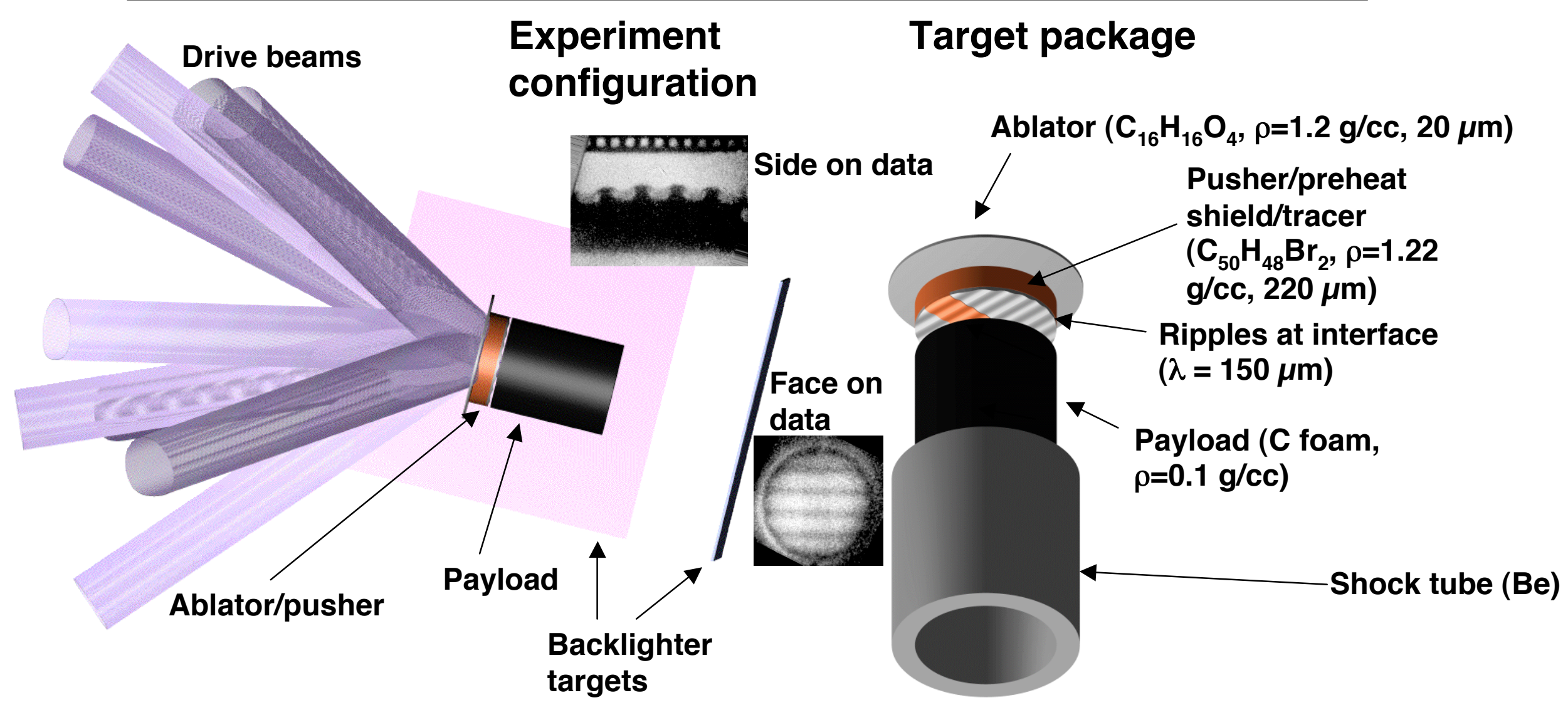

- Radiography is done on two axes, along target axis and perpendicular to modulations

- Target package is encased in a beryllium shock tube 


\section{The incident shock and interface velocities are constant within $\pm 5 \%$ RMS}
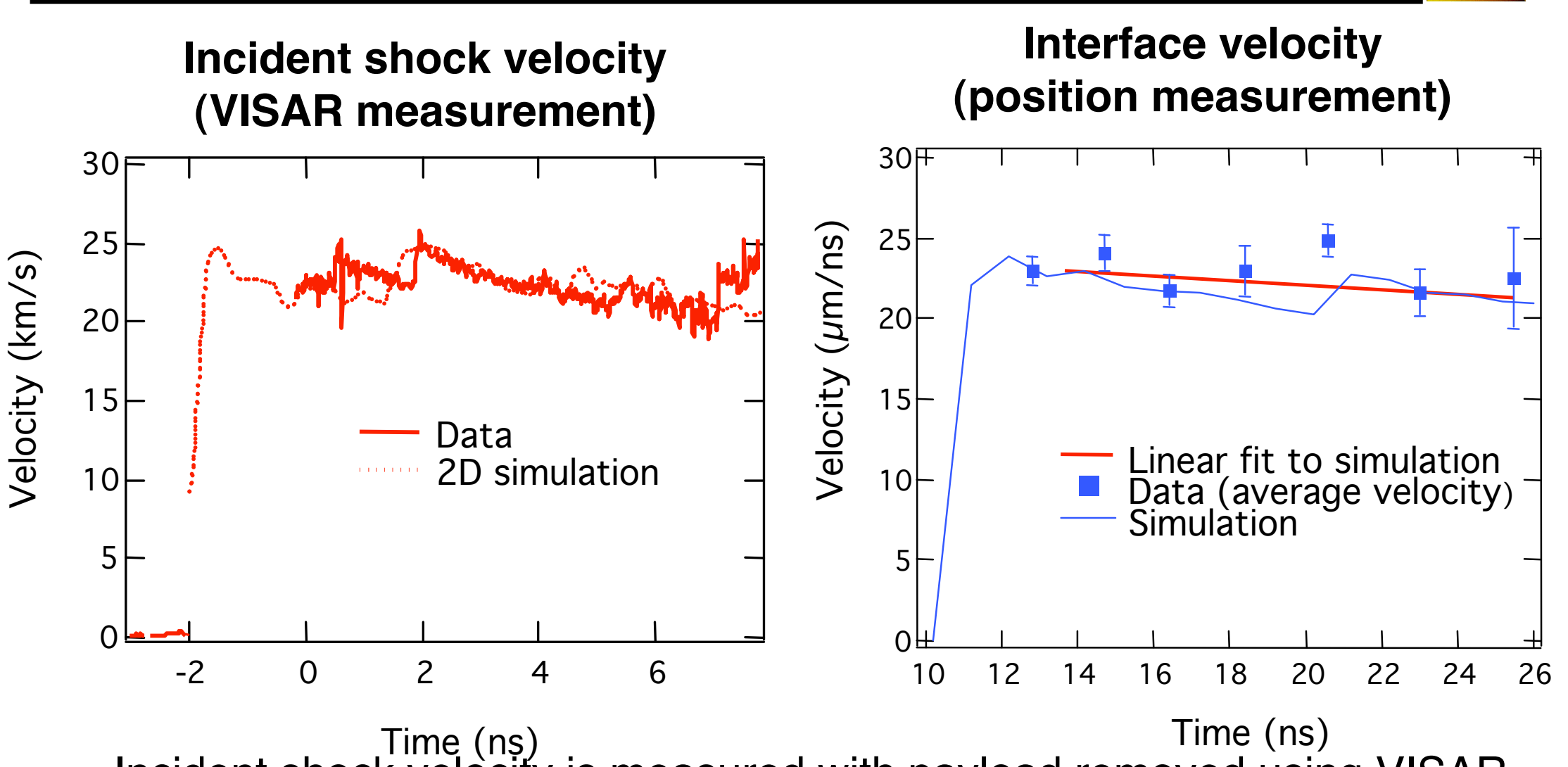

- Incident shock velocity is measured with payload removed using VISAR

- Result $22.0 \pm 0.2 \mu \mathrm{m} / \mathrm{ns}, \pm 5 \%$ (RMS) variations

- The shock is incident on a 12:1 density contrast

- The interface position is measured by side-on radiography

- Average interface velocity $21.9 \pm 1.0 \mu \mathrm{m} / \mathrm{ns}$

- Transmitted shock velocity $26.1 \pm 0.5 \mu \mathrm{m} / \mathrm{ns}$ 


\section{At low initial amplitude results show no effect of shock proximity and little nonlinearity}

Side-on radiographs, $\square_{0}=7 \mu \mathrm{m}$

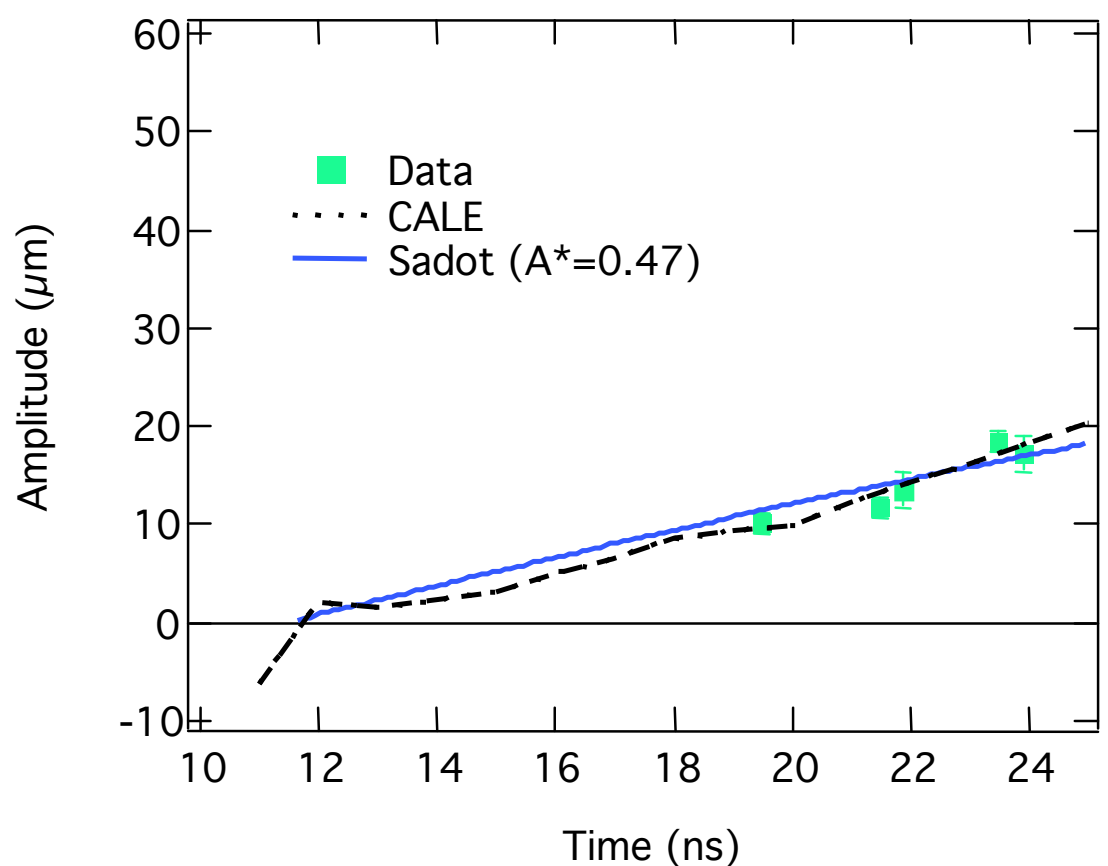

Face-on radiographs, $\square_{0}=7 \mu \mathrm{m}$

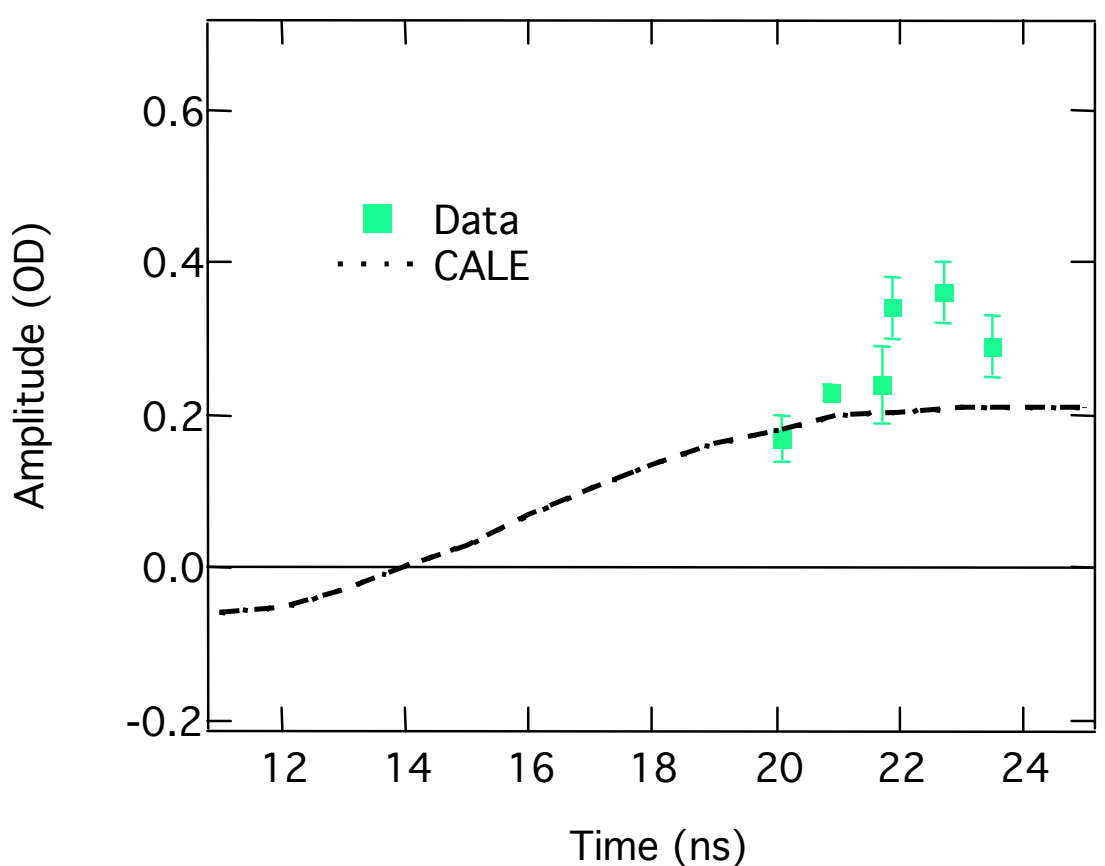

- The nonlinear, incompressible model of Sadot (1998) was used to describe the side-on data with an inferred post-shock Atwood number of 0.47

- Atwood number of 0.47 agrees with one-dimensional simulations

- Linear growth rate is $1.5 \mu \mathrm{m} / \mathrm{ns}$

- CALE simulations agree with the data 


\section{Larger initial amplitude results show reduced growth due to shock proximity}
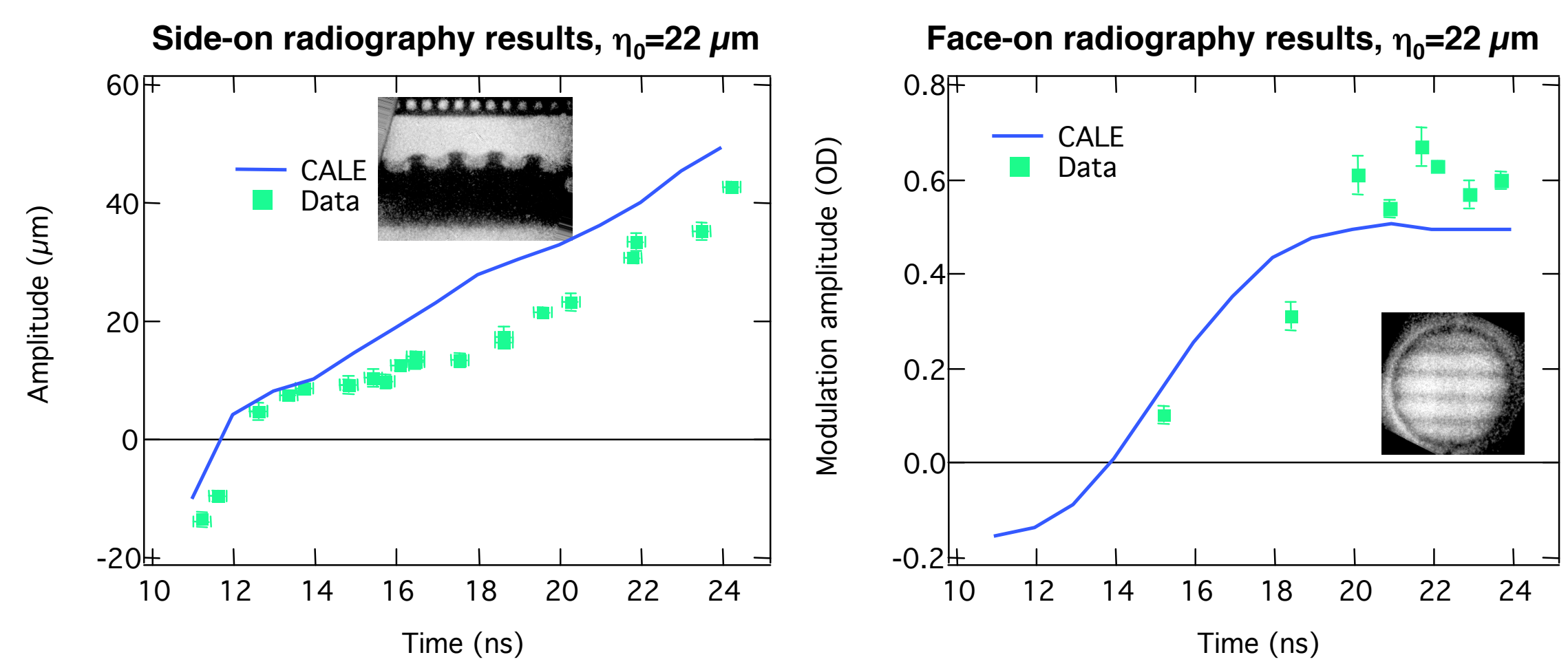

- Linear growth rate would be $4.8 \mu \mathrm{m} / \mathrm{ns}$

- The average ${ }^{\prime} \mathrm{is} 2.4 \pm 0.1 \mu \mathrm{m} / \mathrm{ns}$

- Before $18 \mathrm{~ns}$ is about $1.9 \pm 0.1 \mu \mathrm{m} / \mathrm{ns}$

- The CALE simulation gives a growth rate of $3.9 \mu \mathrm{m} / \mathrm{ns}$ before $18 \mathrm{~ns}, 3.7$ $\mu \mathrm{m} / \mathrm{ns}$ average $12-24 \mathrm{~ns}$ 


\section{We may constrain the CALE simulations to come closer to the modulation growth at $\square=150, \square_{0}=22$ by changing the drive}

Growth at $\square=150 \mu \mathrm{m}$ vs. time
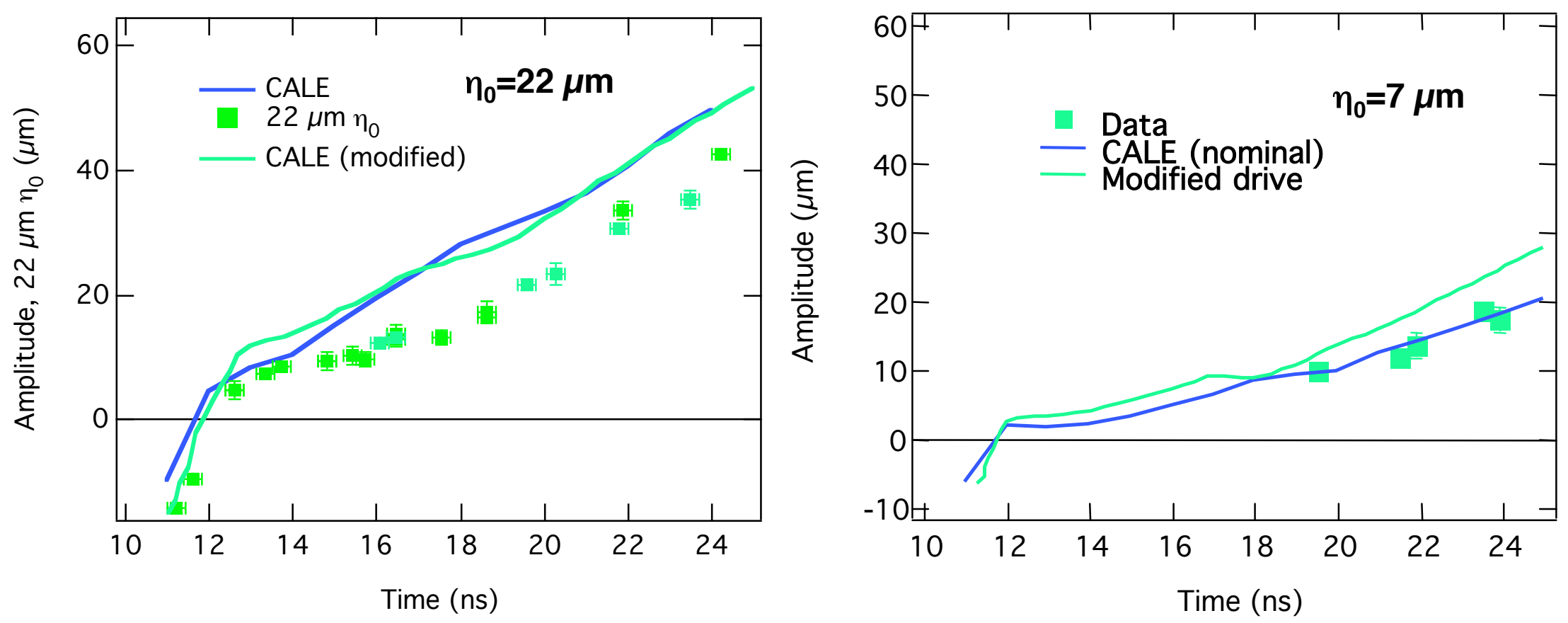

- The modified drive gives a growth rate at early time of $3.0 \mu \mathrm{m} / \mathrm{ns}$

- The modified drive does not predict the $\square=150 \mu \mathrm{m}, \square_{0}=7 \mu \mathrm{m}$ data

- We are currently investigating EOS issues

- The discrepancy between CALE and the data is currently not understood 


\section{The vortex model of Robey does predict growth very much like that seen}

Side-on radiography results

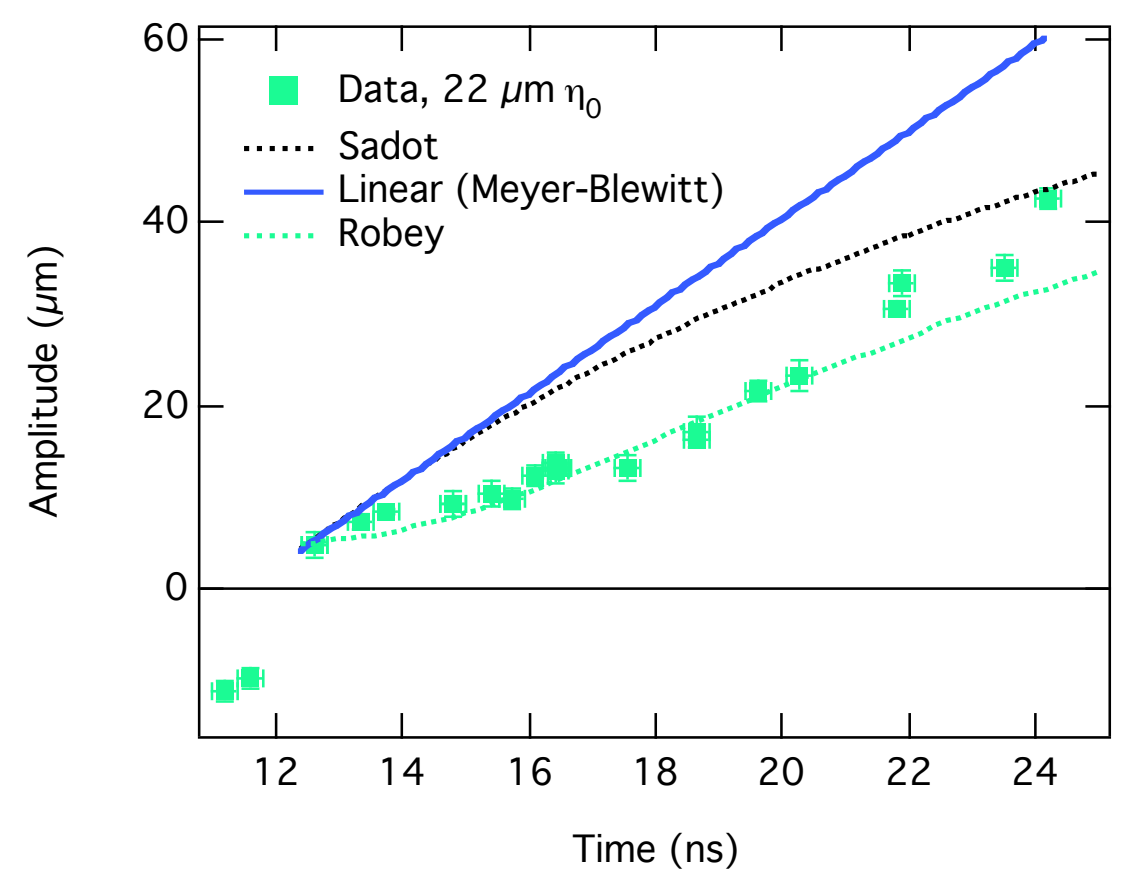

- The model predictions are offset to the first observed data point 


\section{The data may be compared with the shock tube data of Aleshin et al.}

Time normalized to Meyer-Blewett growth

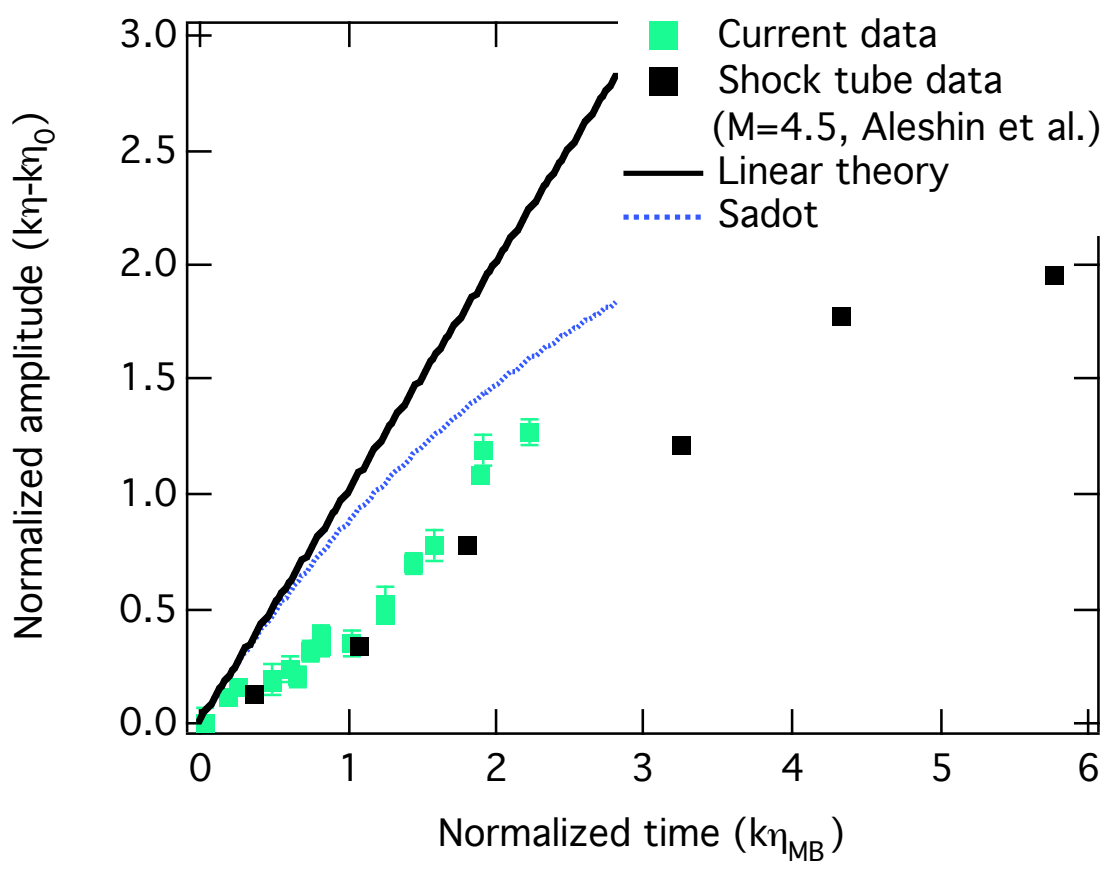

Time normalized to shock recession

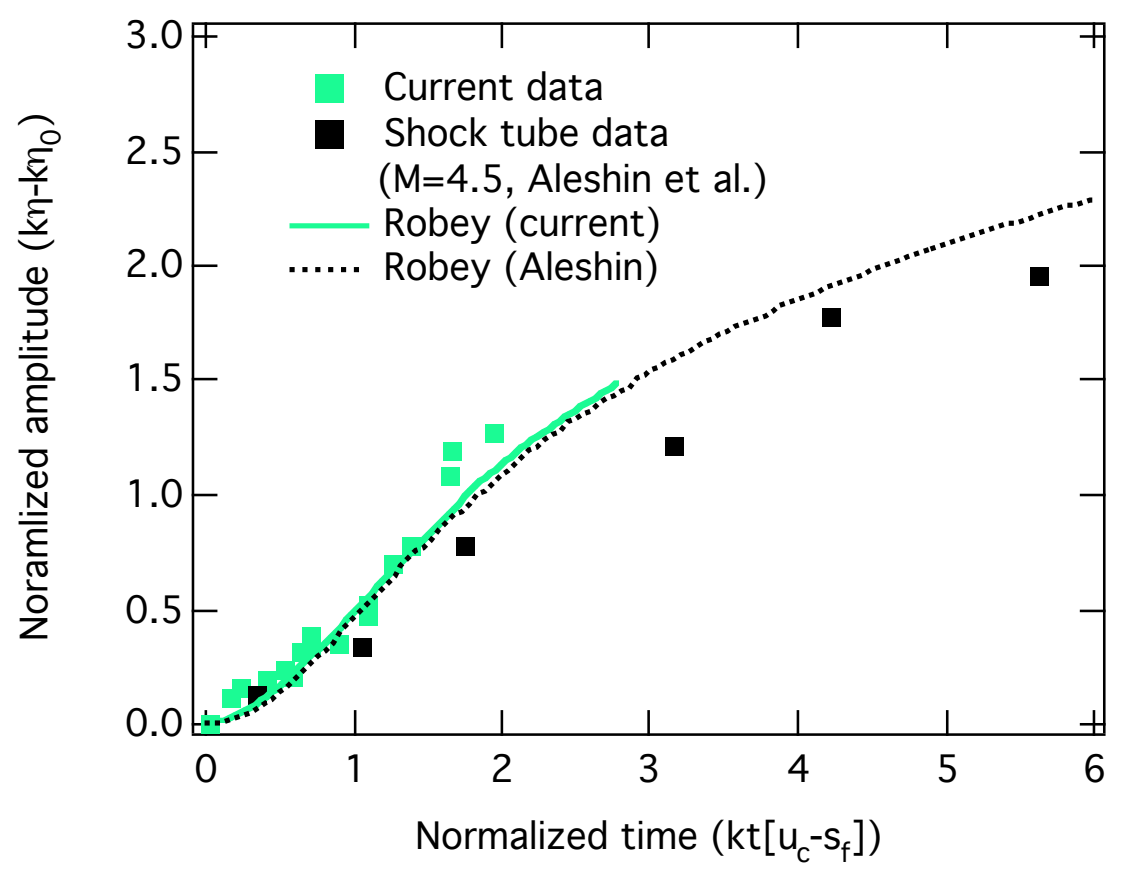

- One normalization is $\mathrm{k} \square$ vs. $\mathrm{k} \square_{\mathrm{IM}}$ (Meyer-Blewett, shows nonlinearity)

- Another normalization is $k \square v s k^{*}\left(u_{c}-s_{f}\right)^{*} t$ (shows shock proximity) 


\section{The initial growth rate is much lower than linear or large-initial-amplitude models predict}

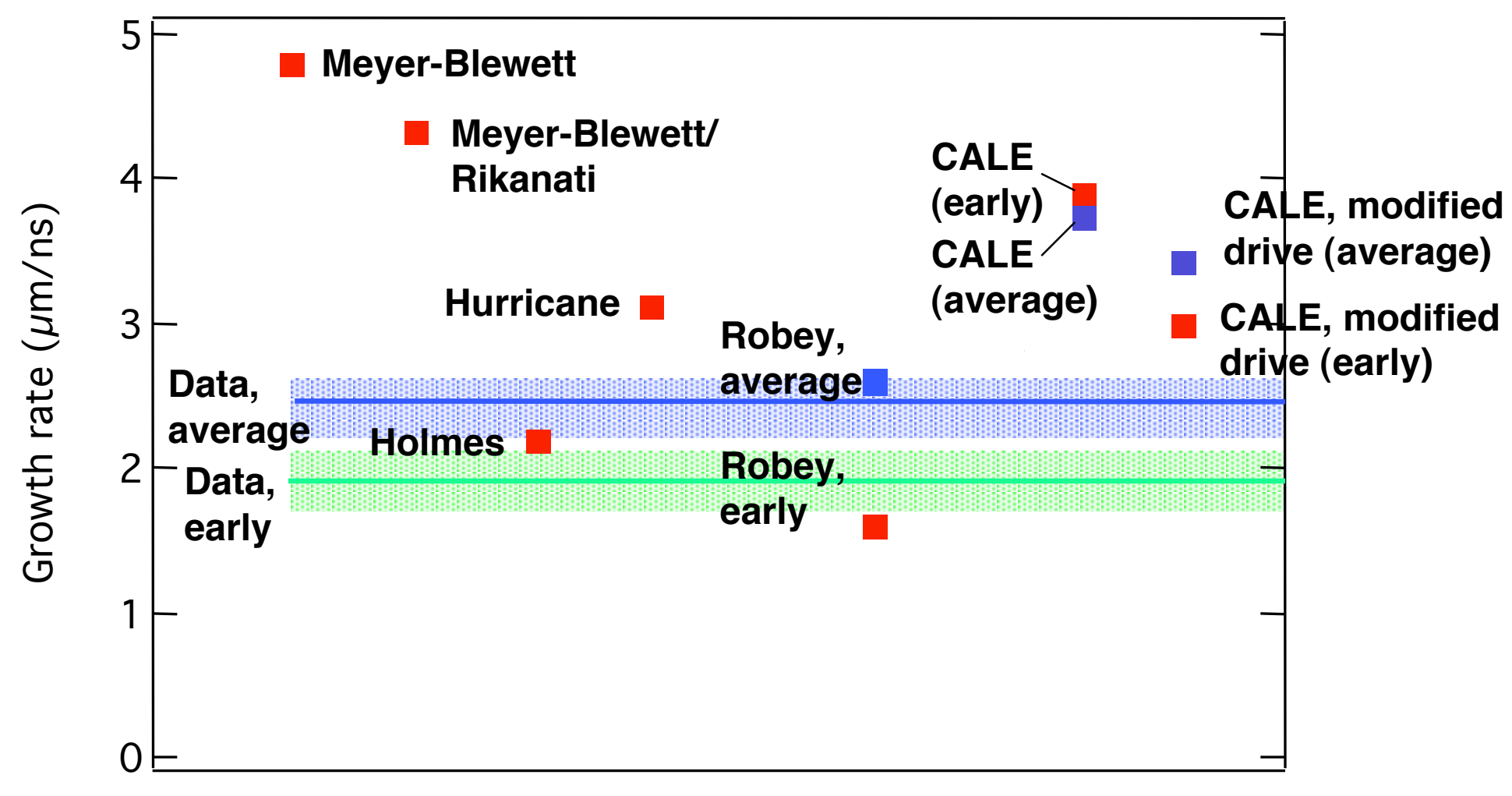

- Only the Robey model predicts an increase in velocity later in time (as the shock recedes) 


\section{Summary}

- We have used the Omega laser to generate a nearly steady interface velocity for Richtmyer-Meshkov experiments

- The interface is a heavy-to-light (12:1) density step

- The incident shock Mach number is $\sim 10$

- The shock velocity is only about $20 \%$ higher than the interface velocity

- An initially sinusoidal perturbation with $\square=150 \mu \mathrm{m}, \square_{0}=7 \mu \mathrm{m}\left(\mathrm{k} \square_{0}=0.3\right)$ grows according to incompressible models

- The growth of with $\square=150 \mu \mathrm{m}, \square_{0}=22 \mu \mathrm{m}\left(\mathrm{k} \square_{0}=0.9\right)$ is about half that predicted from incompressible models

- The shock remains very close to the spike tips as the perturbation grows

- An analytical model which accounts for the effect of the shock proximity predicts the reduced growth 


\title{
Mix Experiments using a Two-Dimensional Convergent Shock Tube
}

\author{
D A Holder, C J Barton, A V Smith, D L Youngs
}

AWE Aldermaston UK 


\section{Principle of Design}

Compressed Gas

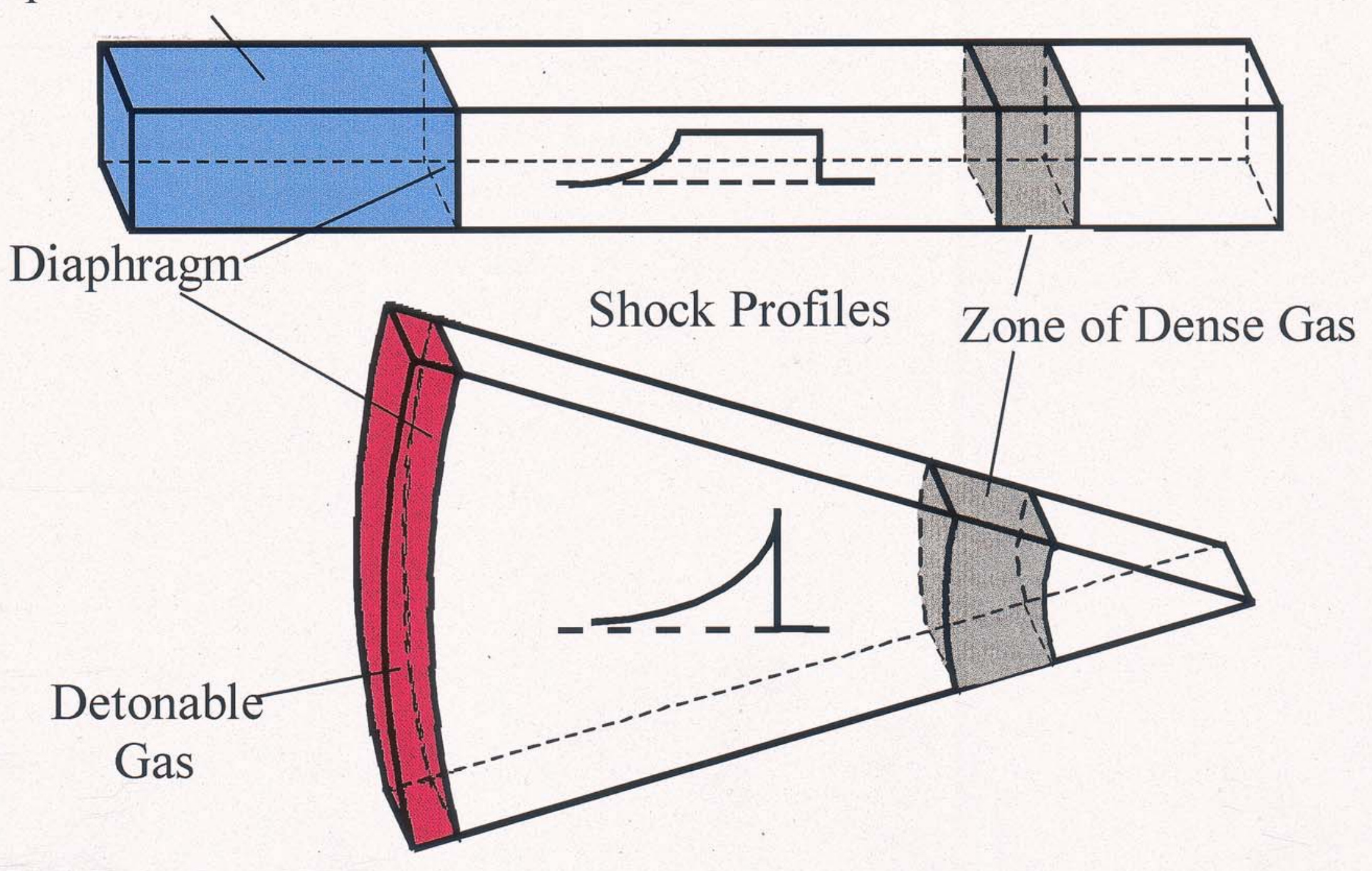




\section{Experiments performed}

Basic cylindrical

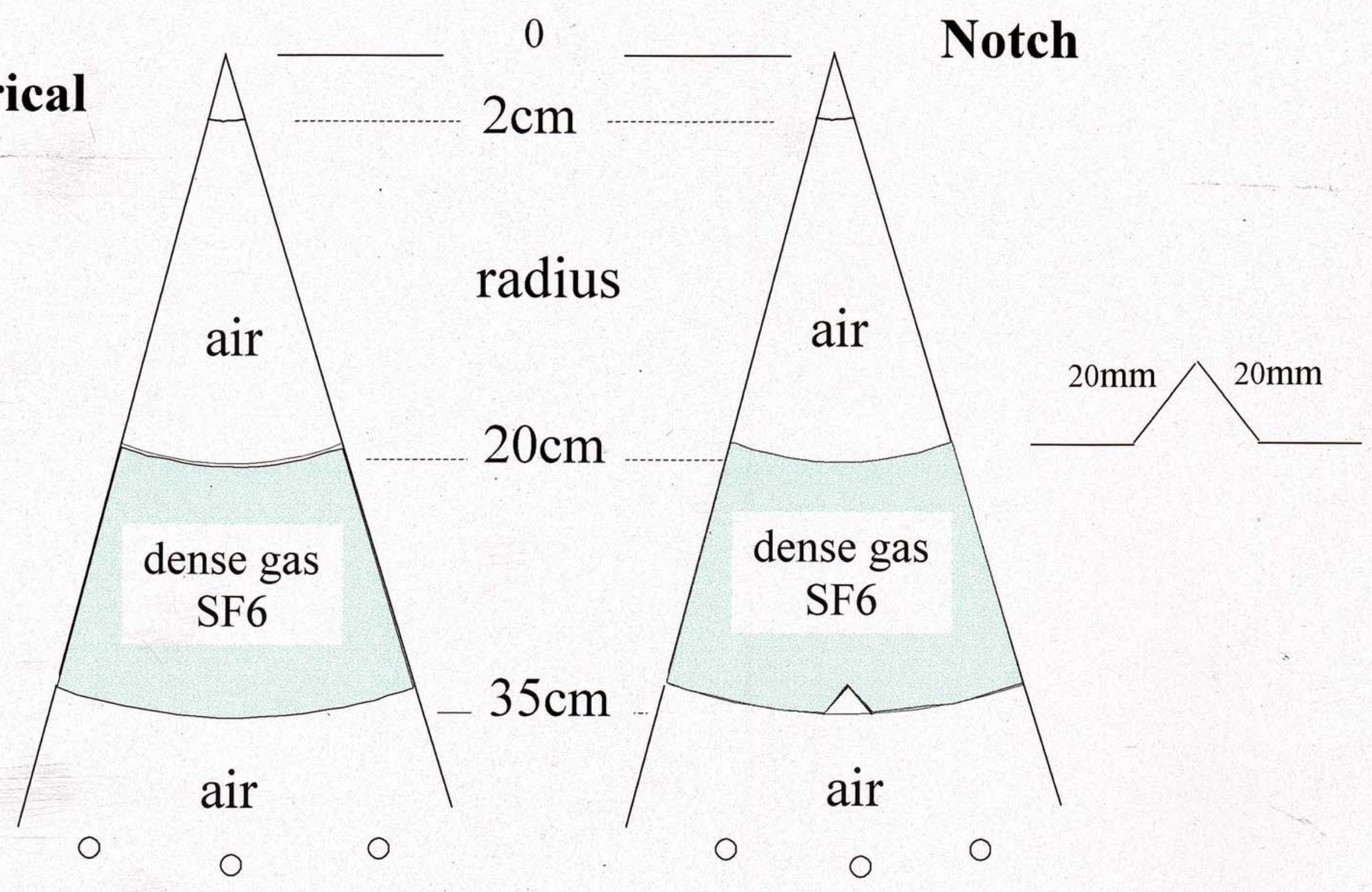




\section{Shock Pressure Waveform}

\section{Radius $50 \mathrm{~cm}$}

radius $50 \mathrm{~cm}$

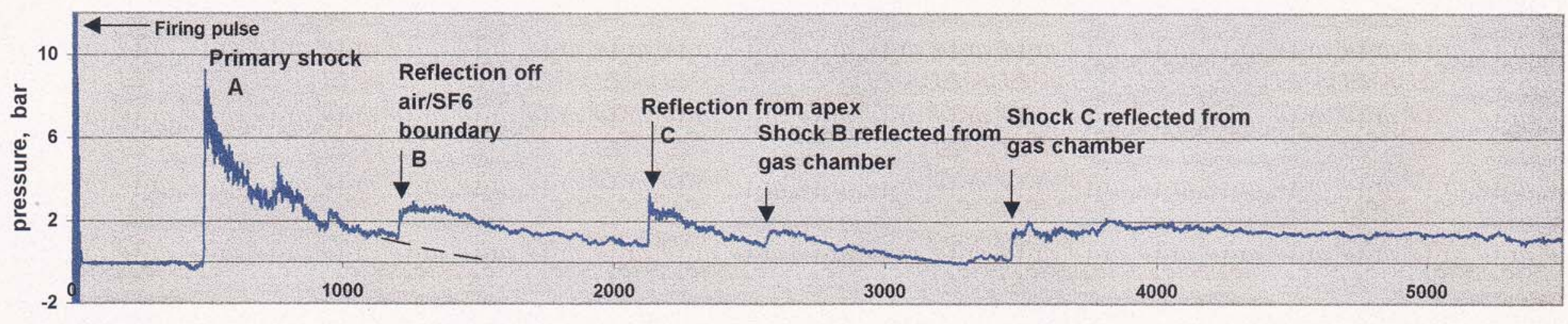

time, microsec 


\section{Details of the Dense Gas Containment}

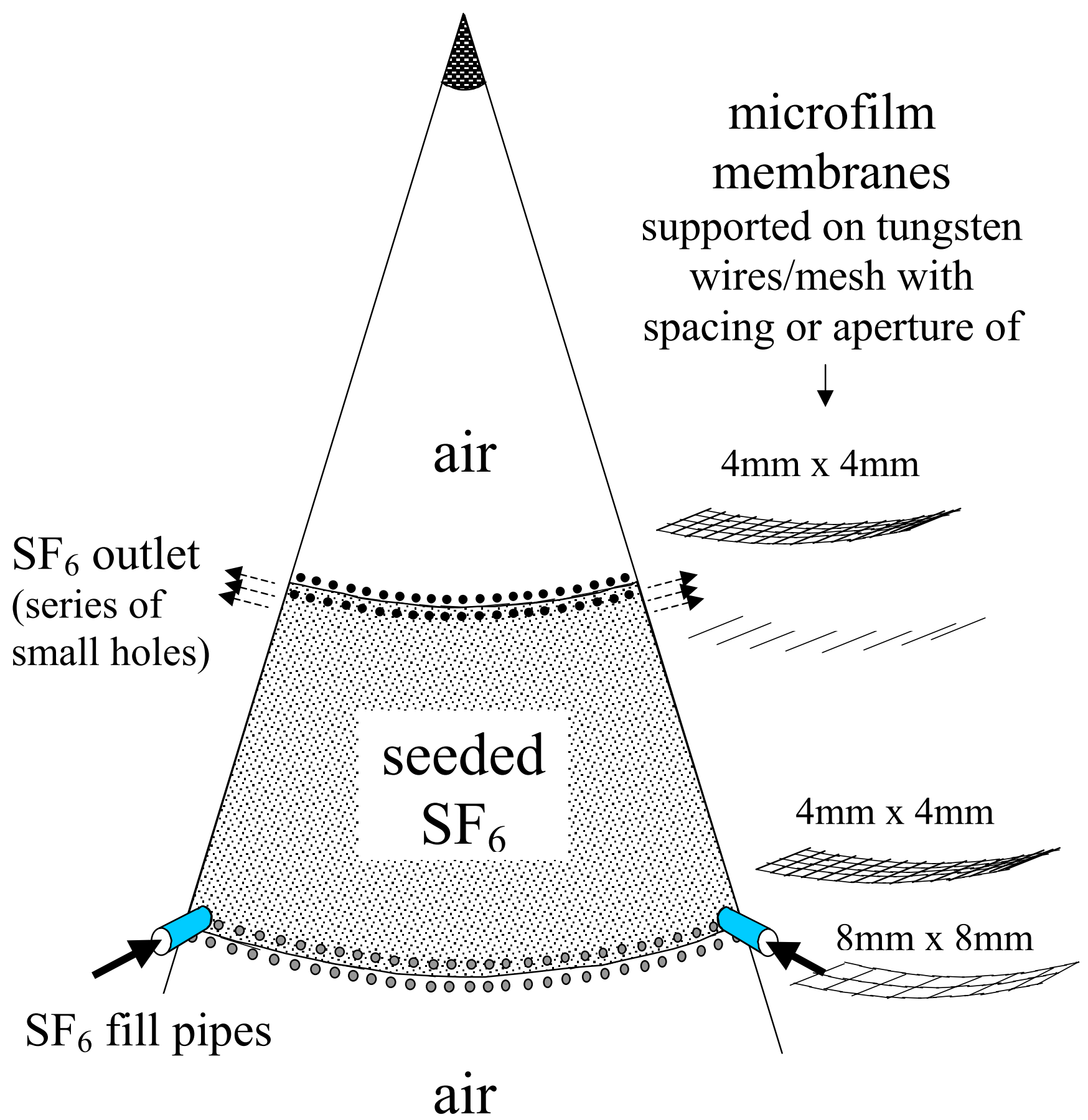


Detonable Gas Chamber

Ante

$\therefore 000000000$ 


\section{Oxy-acetylene gas chamber}

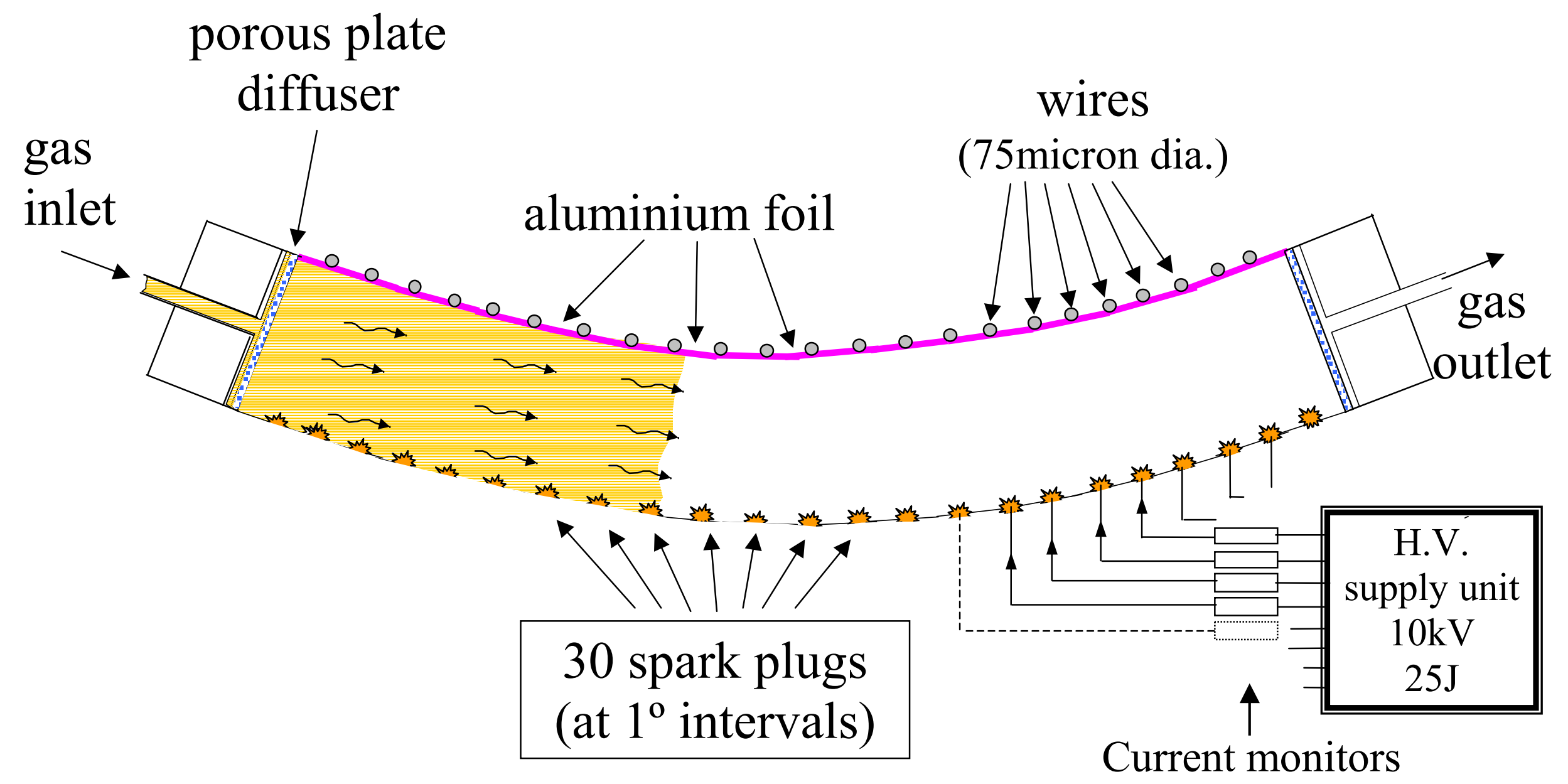




\section{Small Detonation Test-cell}

to check for simultaneity of multi-point detonation

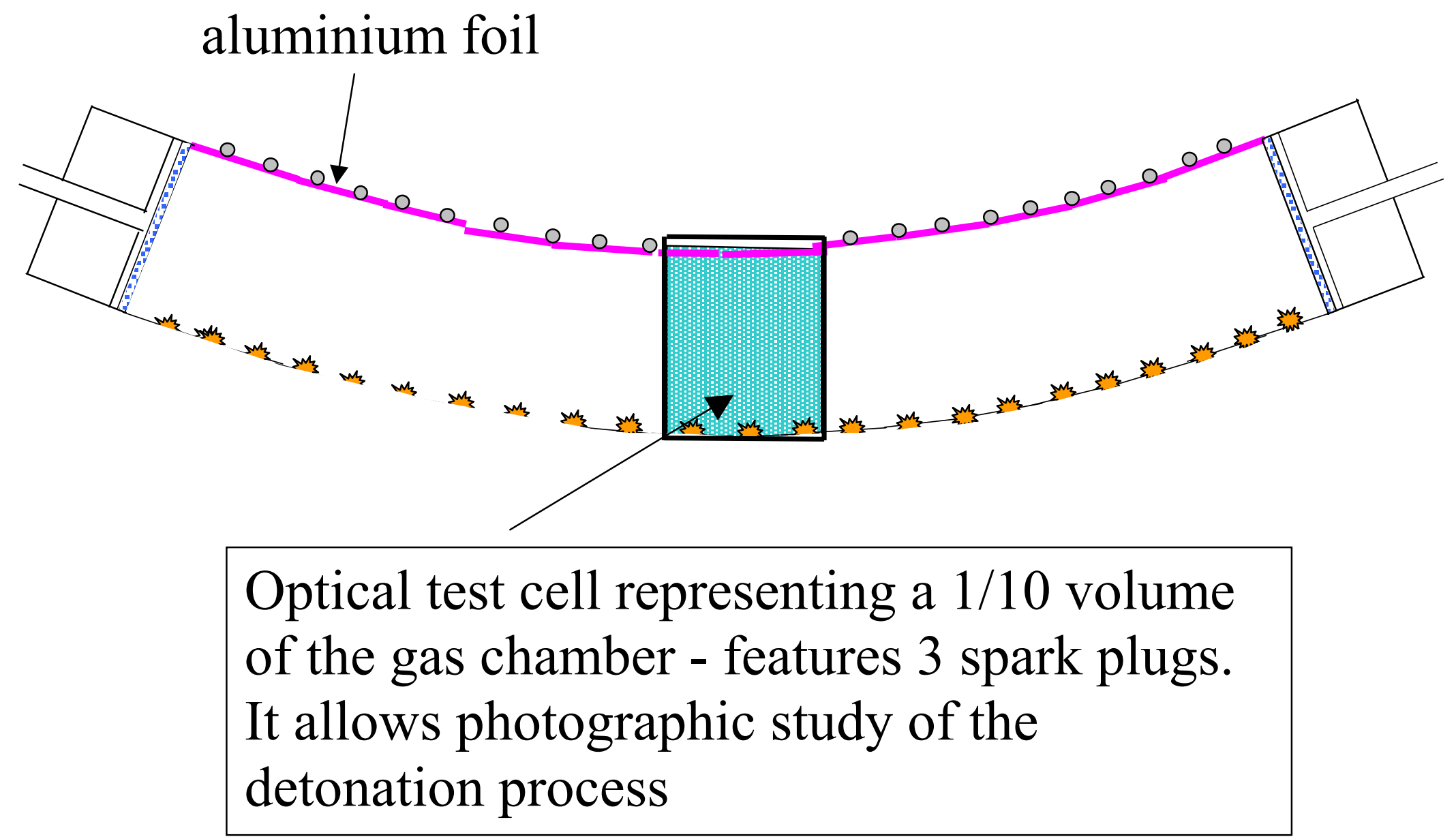




\section{Small Detonation Test Cell - Sample Images}

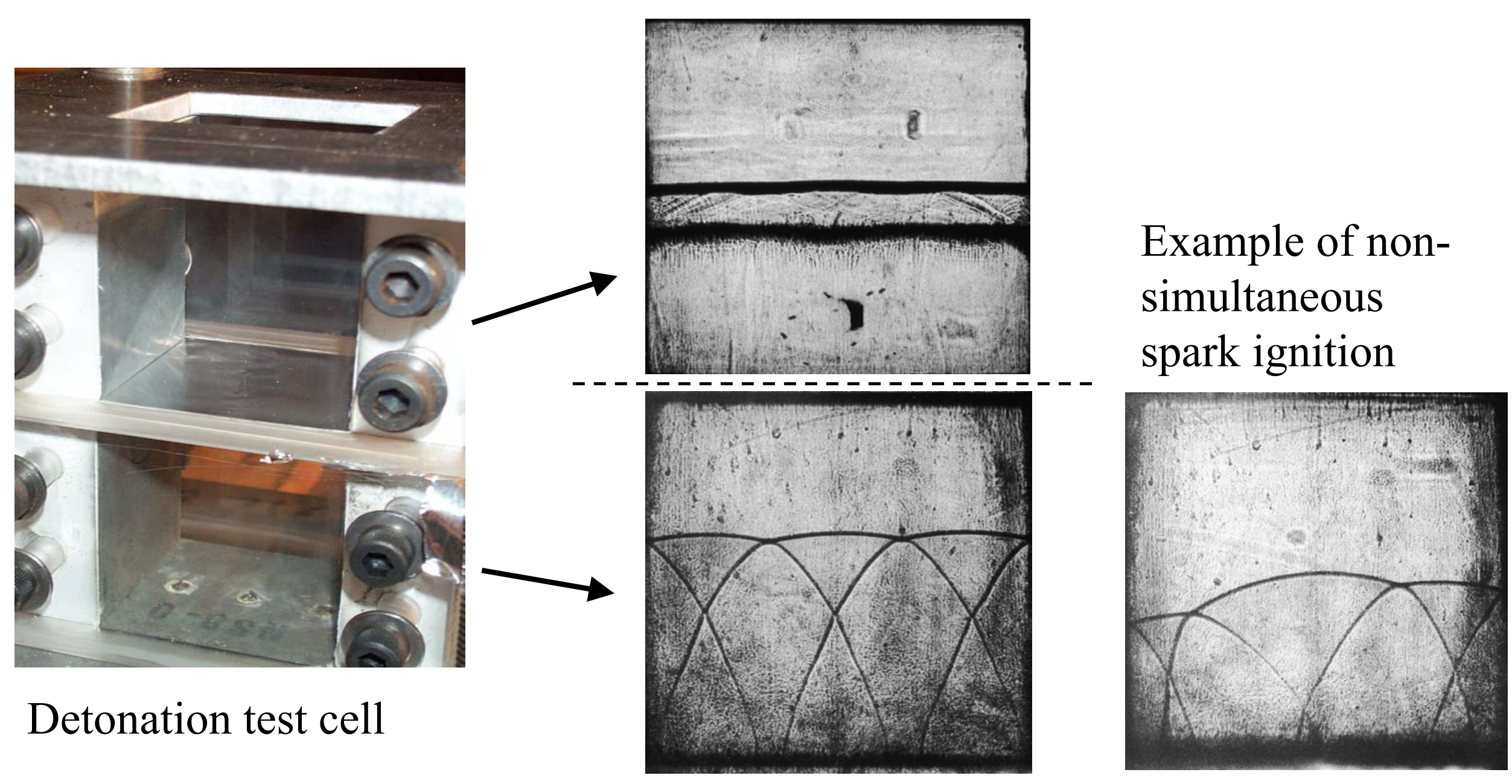




\section{Shadowgraph Images}

(air / $\mathrm{SF}_{6} /$ air cylindrical experiment)
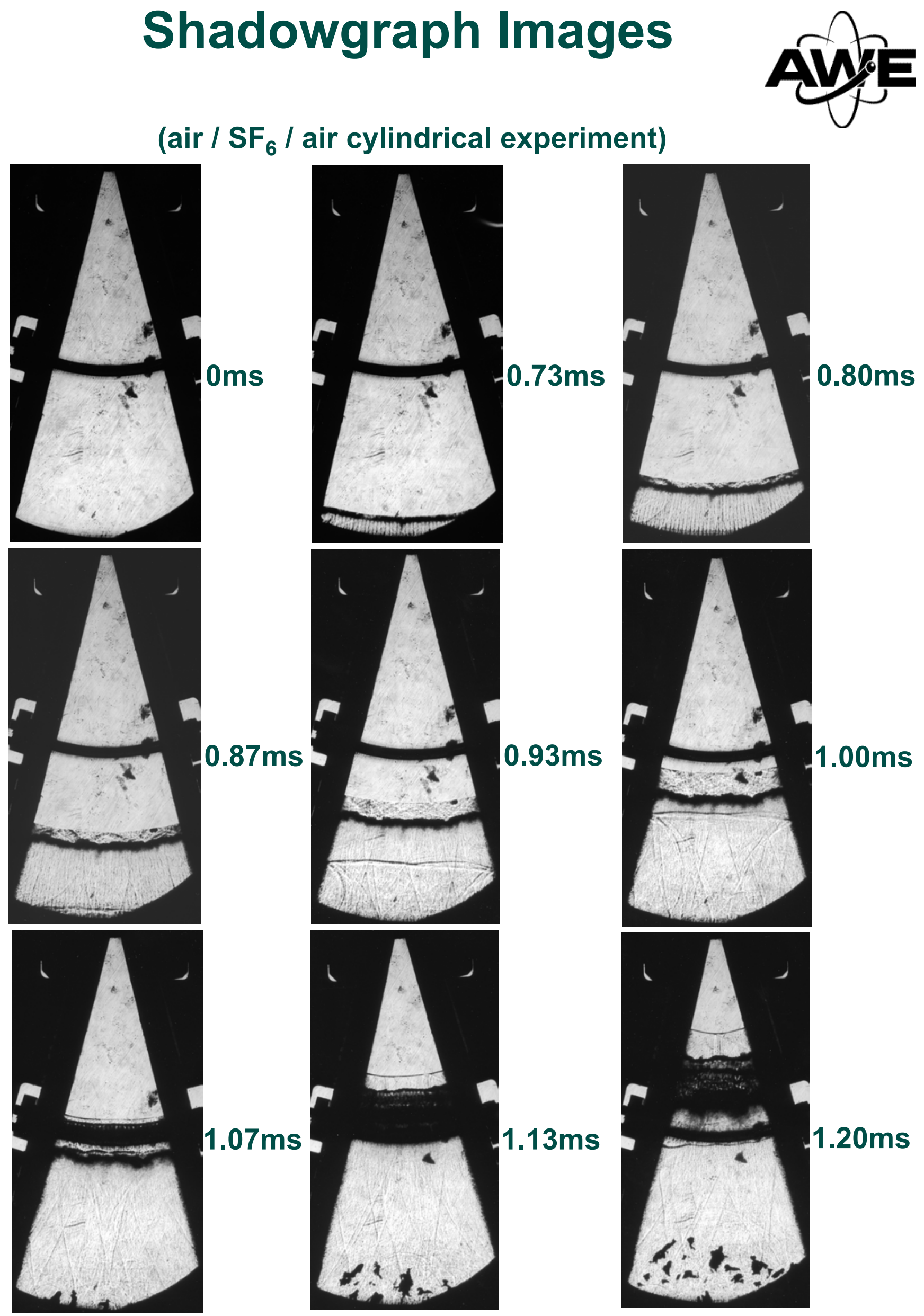


\section{Shadowgraph Images}

(air / $\mathrm{SF}_{6} /$ air cylindrical experiment)
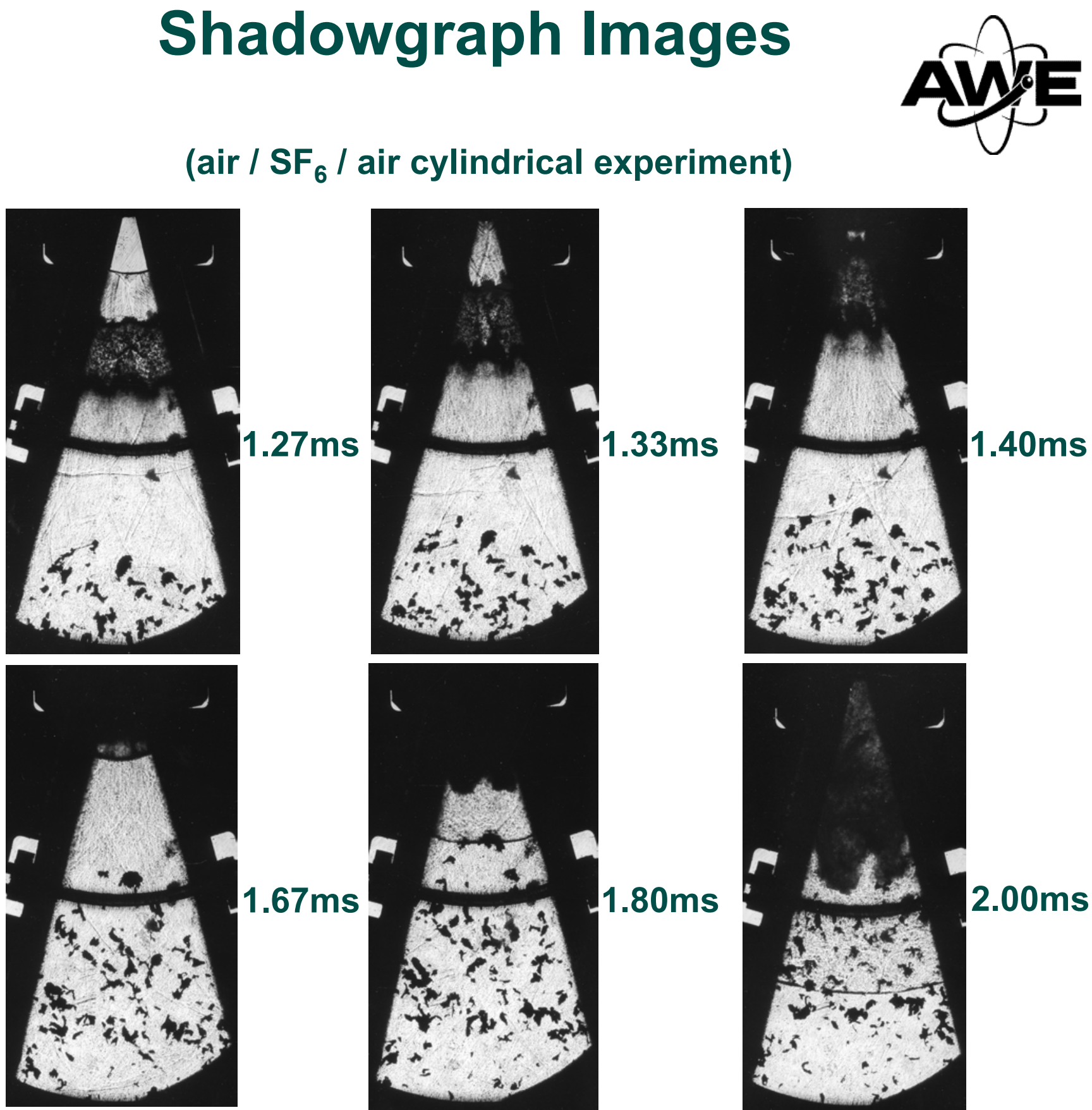

$1.80 \mathrm{~ms}$
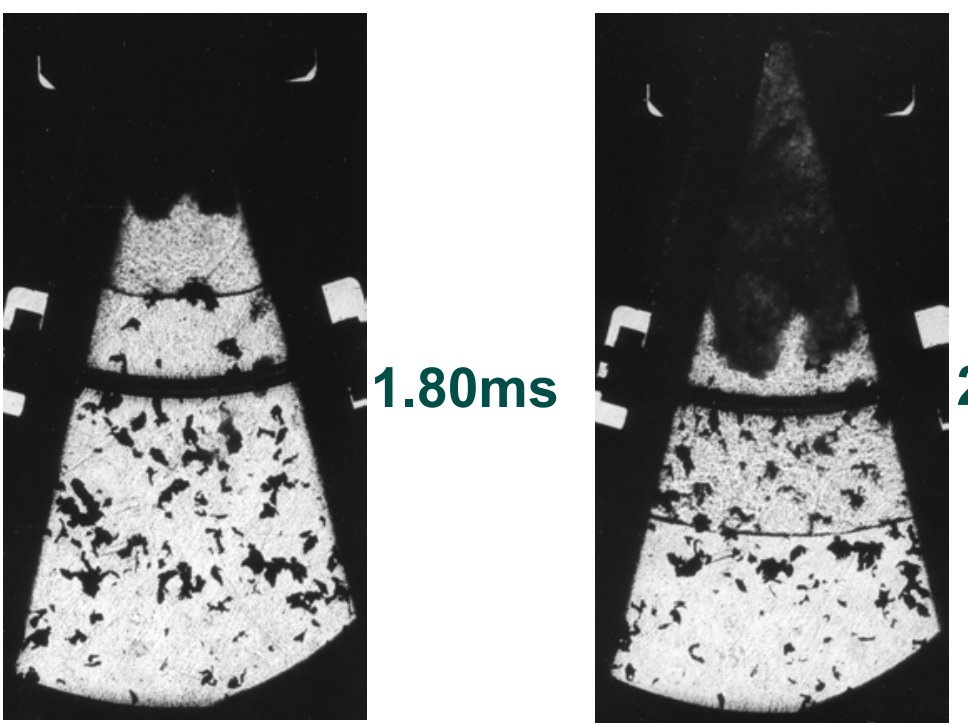

$2.00 \mathrm{~ms}$

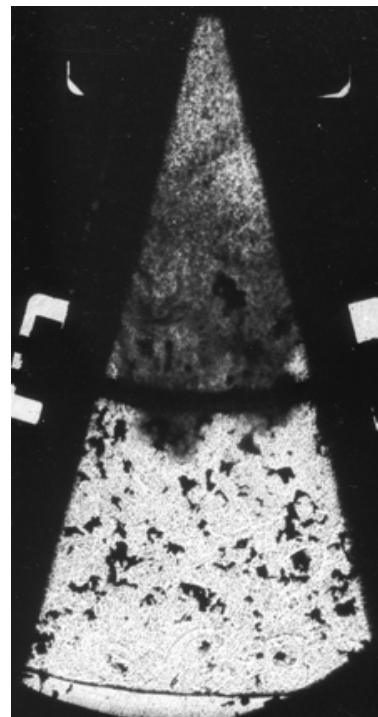

$2.13 \mathrm{~ms} 2.27 \mathrm{~ms}$

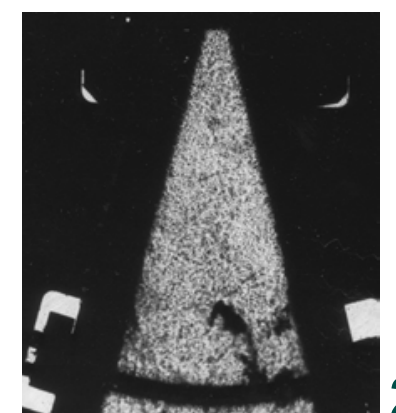

$2.40 \mathrm{~ms}$

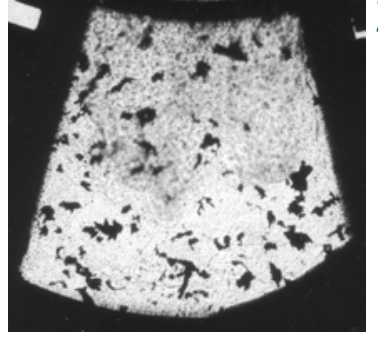




\section{Shadowgraph Images}

(air / $\mathrm{SF}_{6} /$ air cylindrical experiment)
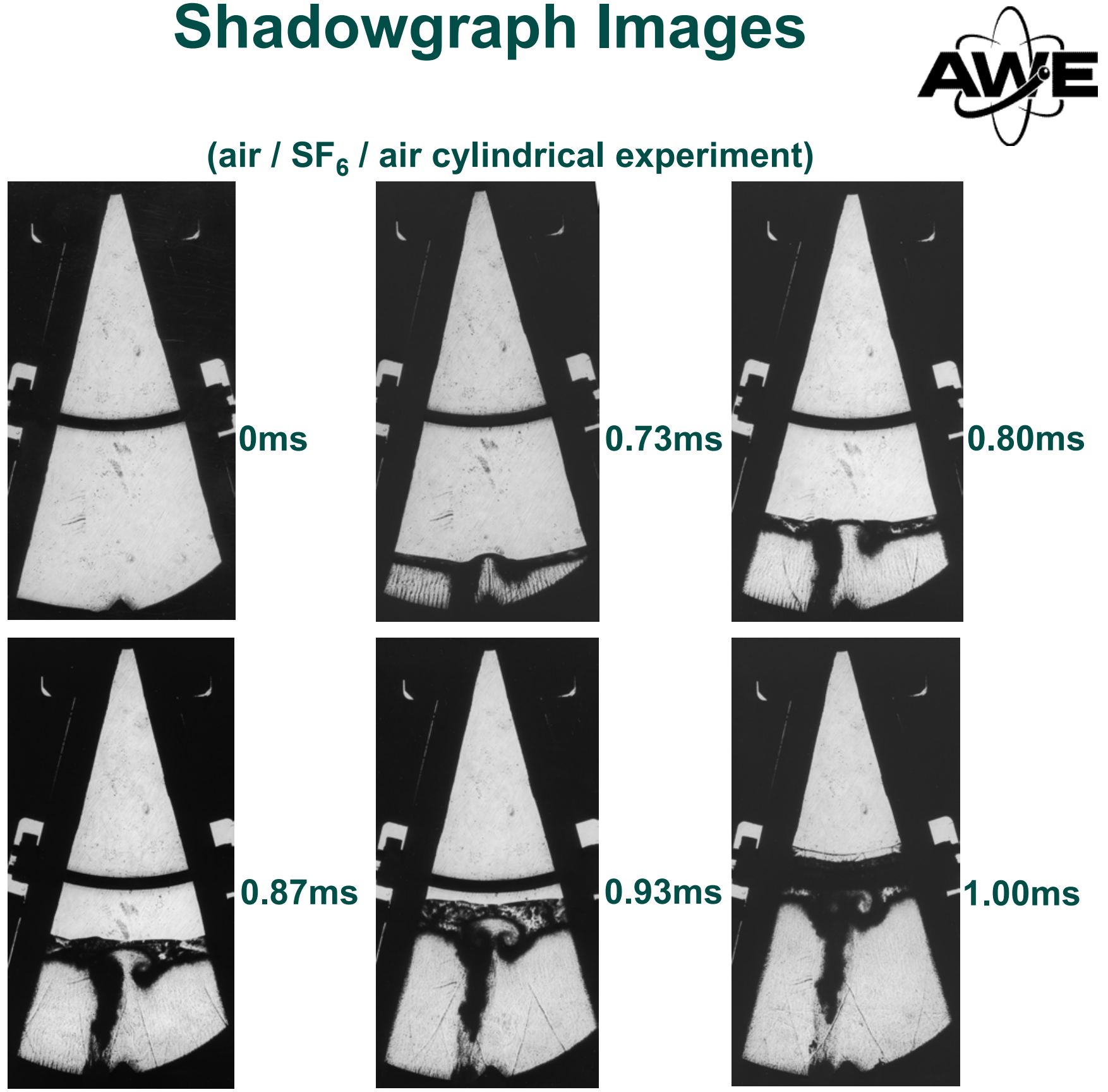

Oms

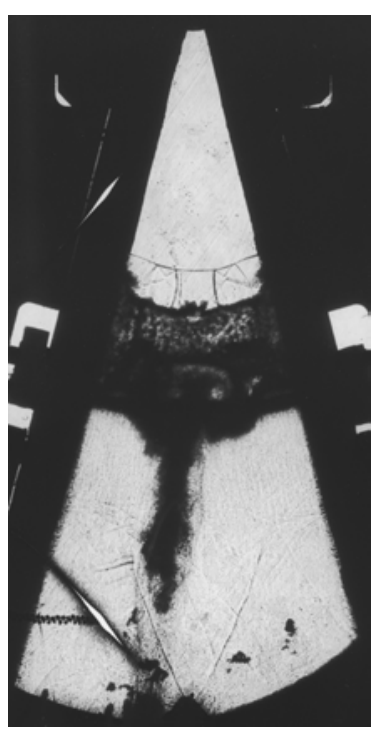

$-0.87 \mathrm{~ms}$

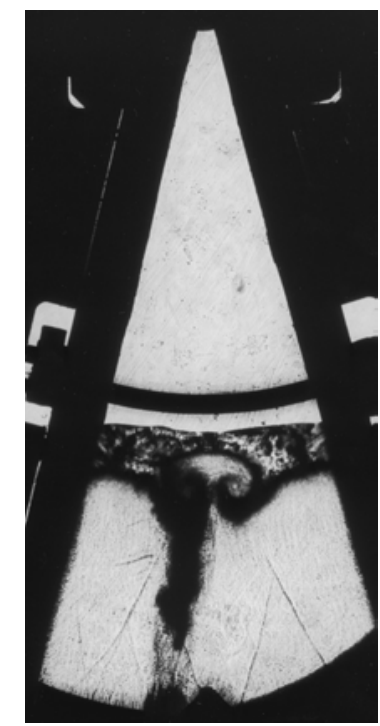

$0.73 \mathrm{~ms}$
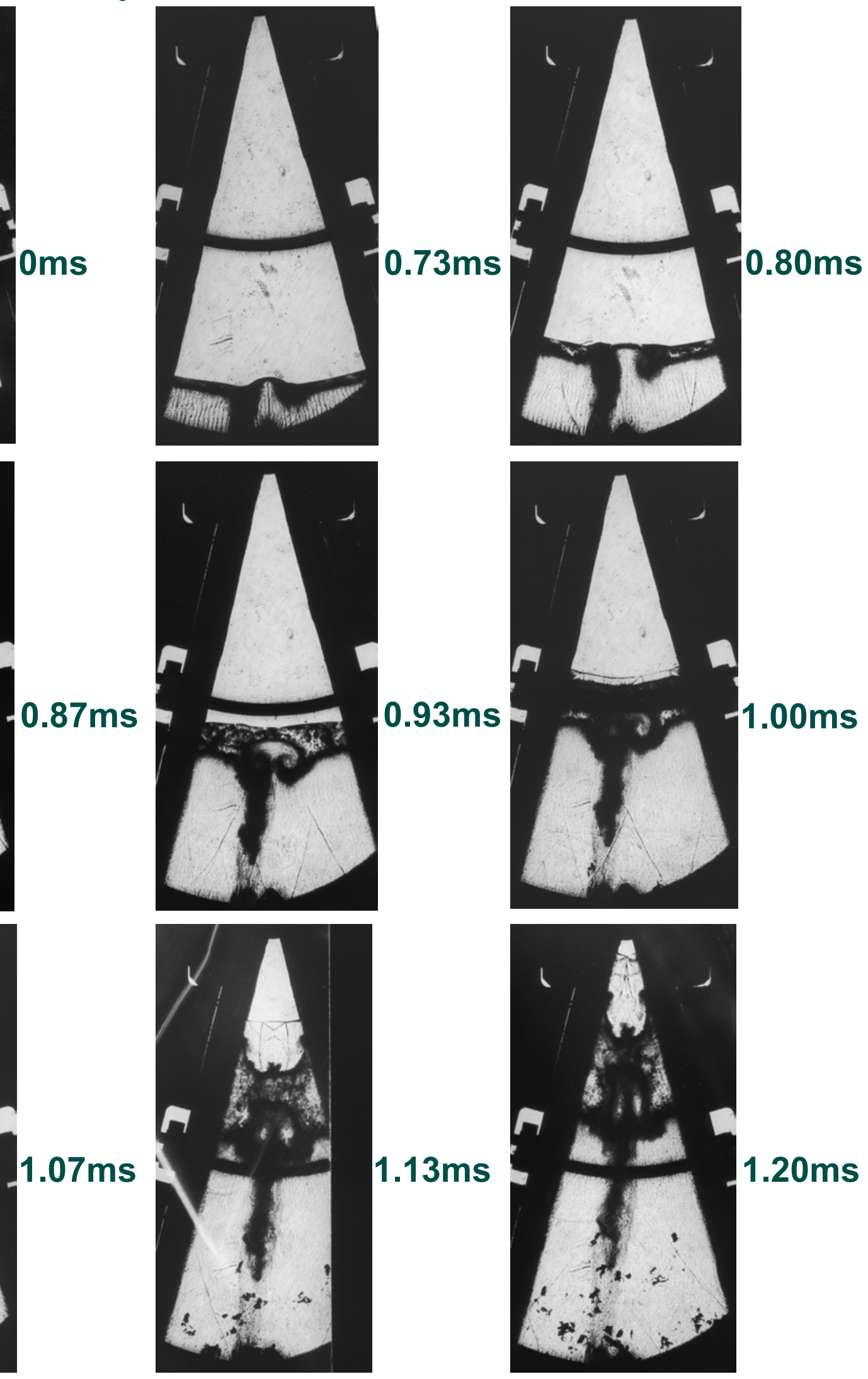


\section{Shadowgraph Images}

(air / $\mathrm{SF}_{6}$ / air cylindrical experiment)
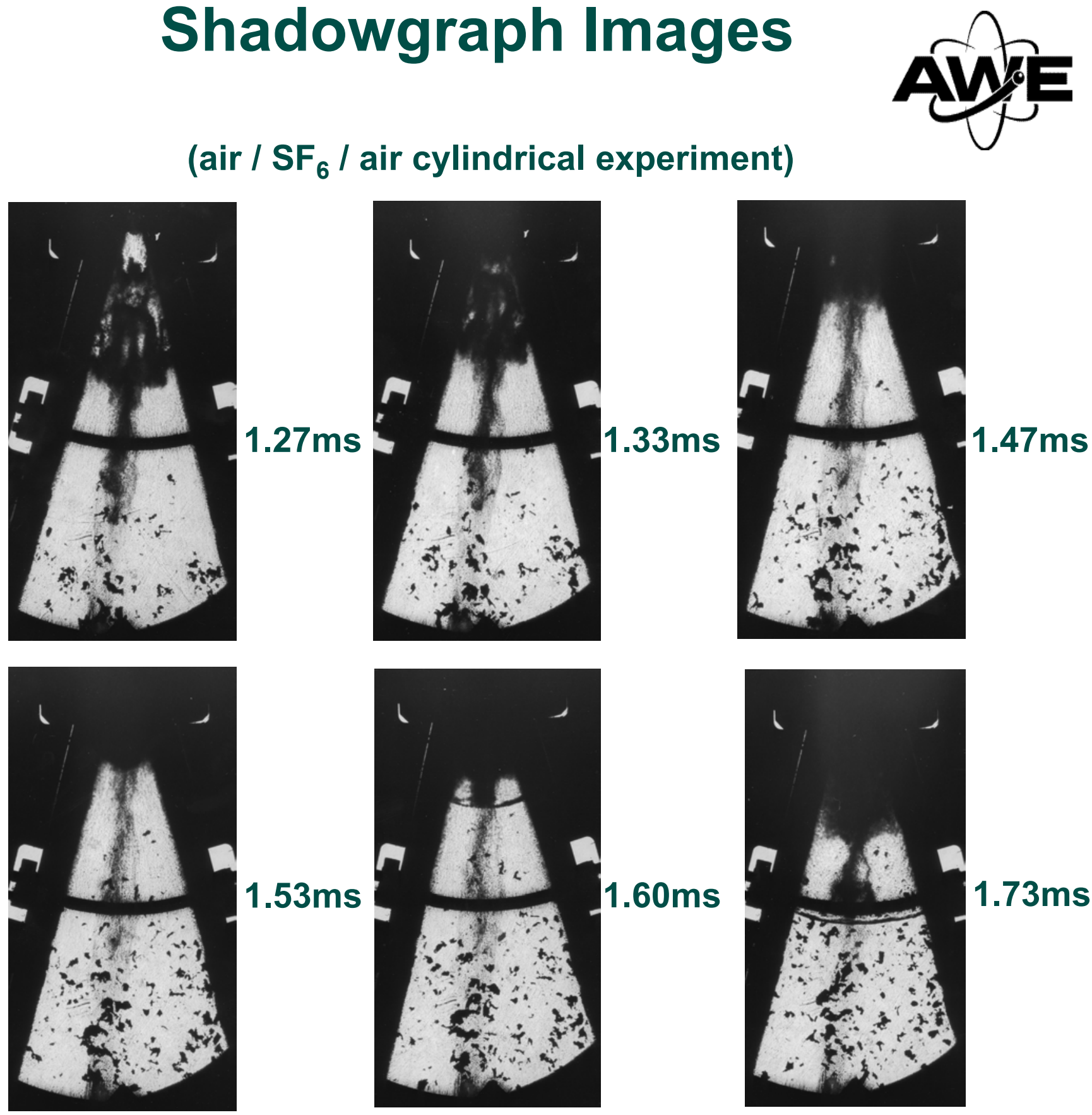

$1.73 \mathrm{~ms}$
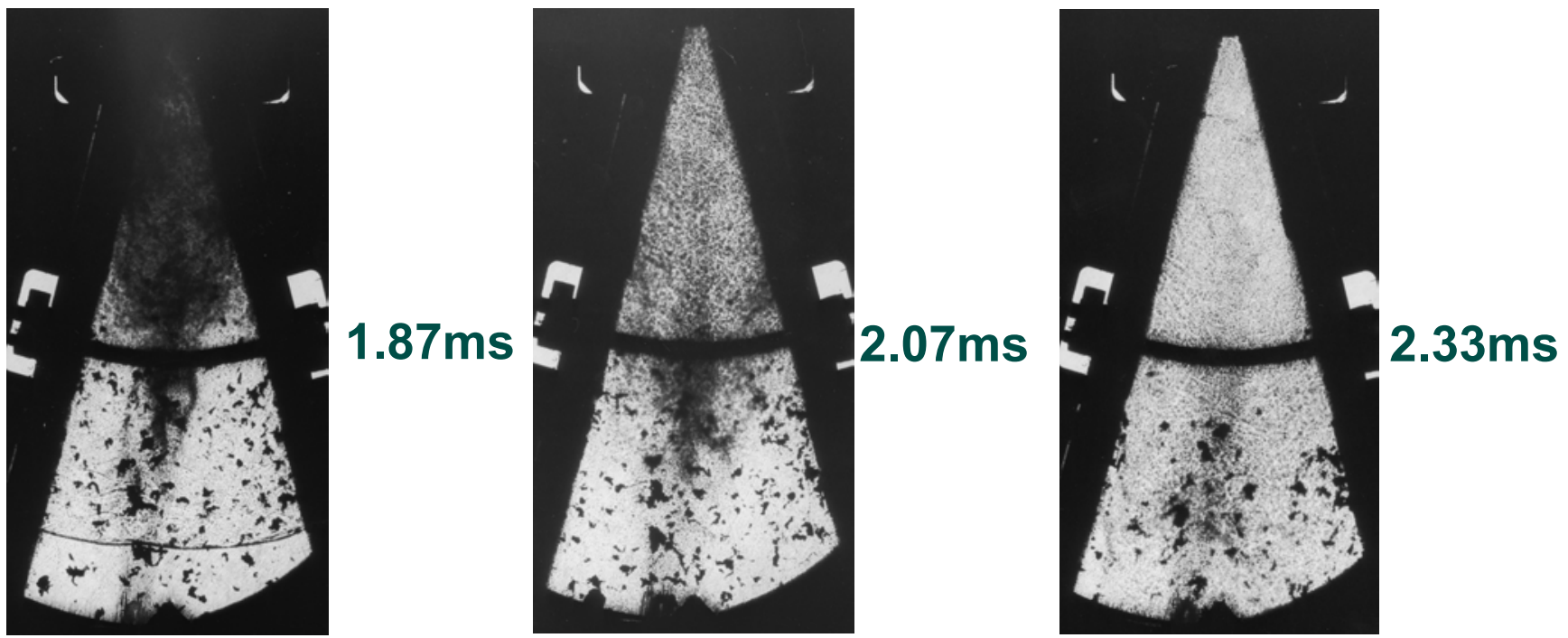


\section{TURMOIL 3D Simulation Ange}

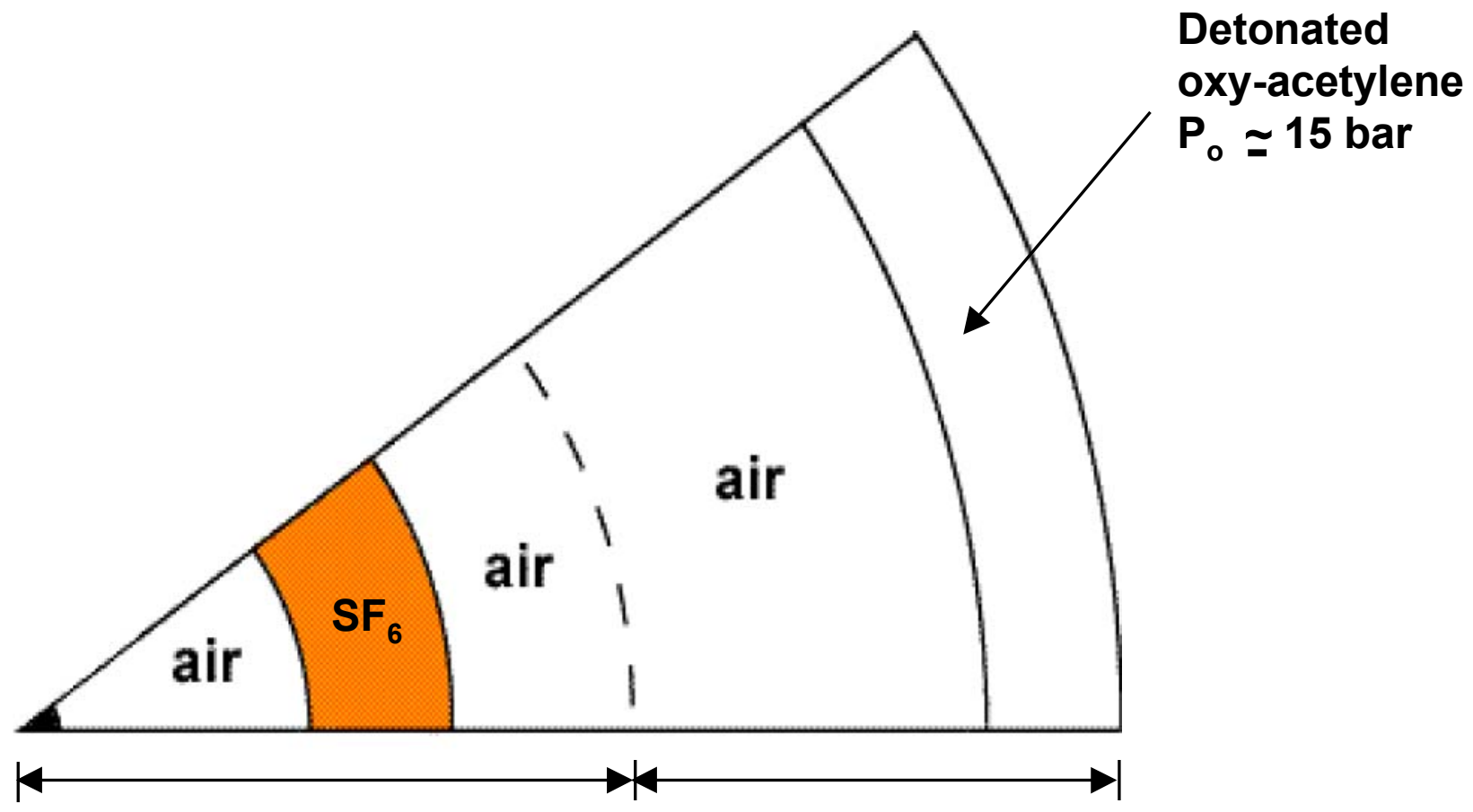

3D region

1D Lagrangian

region

Cylindrical polar mesh.

Semi-Lagrangian calculation - $r$ - direction mesh moves with the mean fluid velocity.

Mesh used in the 3D region $(r, \theta, z): 344 \times 200 \times 140$.

Random pertubations imposed at each interface:

Wavelengths: 0.5 to $5.0 \mathrm{~cm}$

s.d: $0.01 \mathrm{~cm}$ 


\section{Notch Experiment}

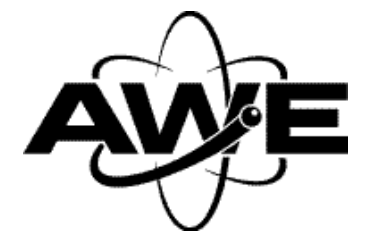

Experiment

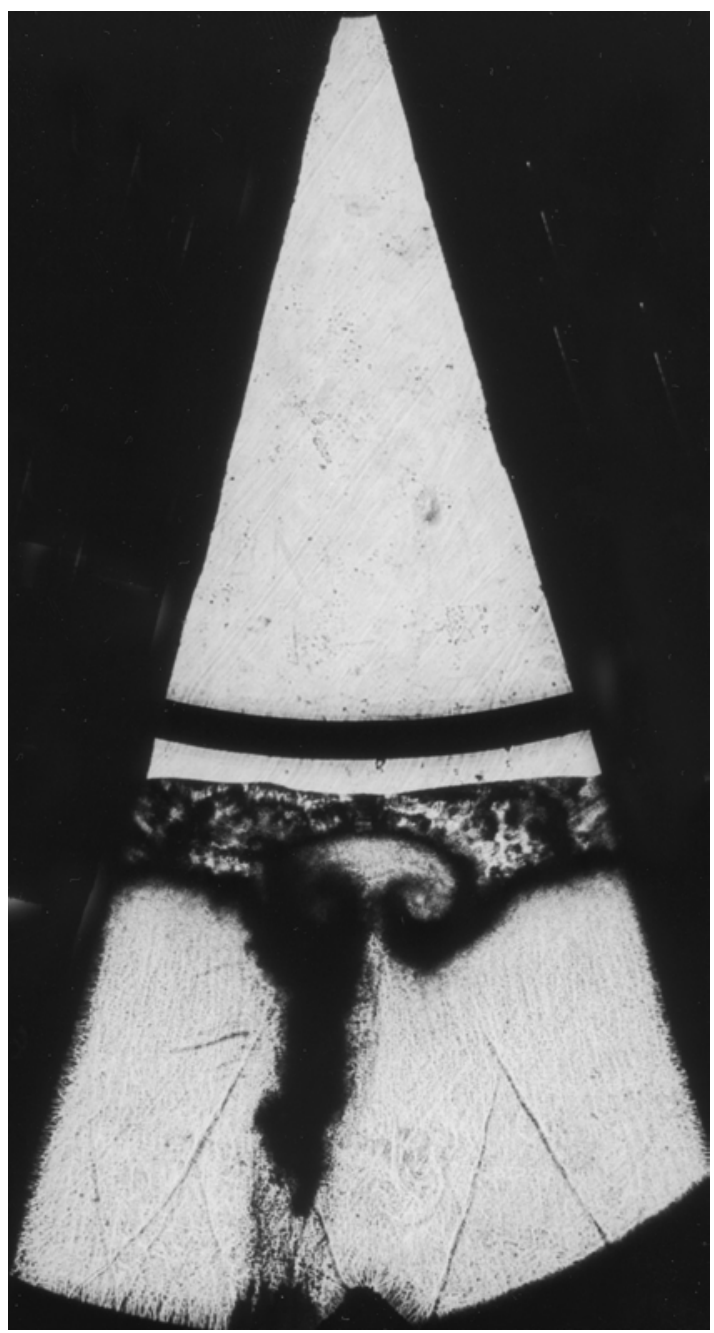

Turmoil 3D code

$0.93 \mathrm{~ms}$

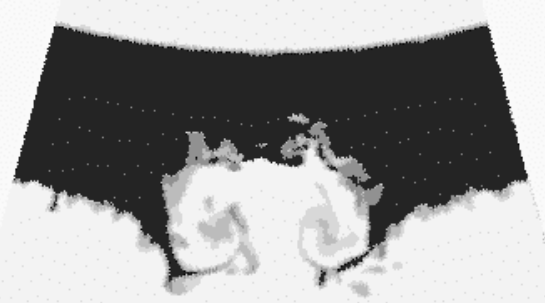

$0.9 \mathrm{~ms}$ 


\section{Notch Experiment}

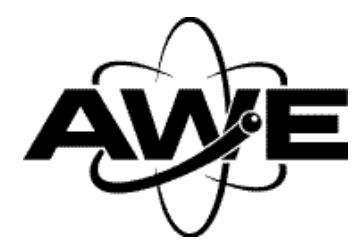

Experiment

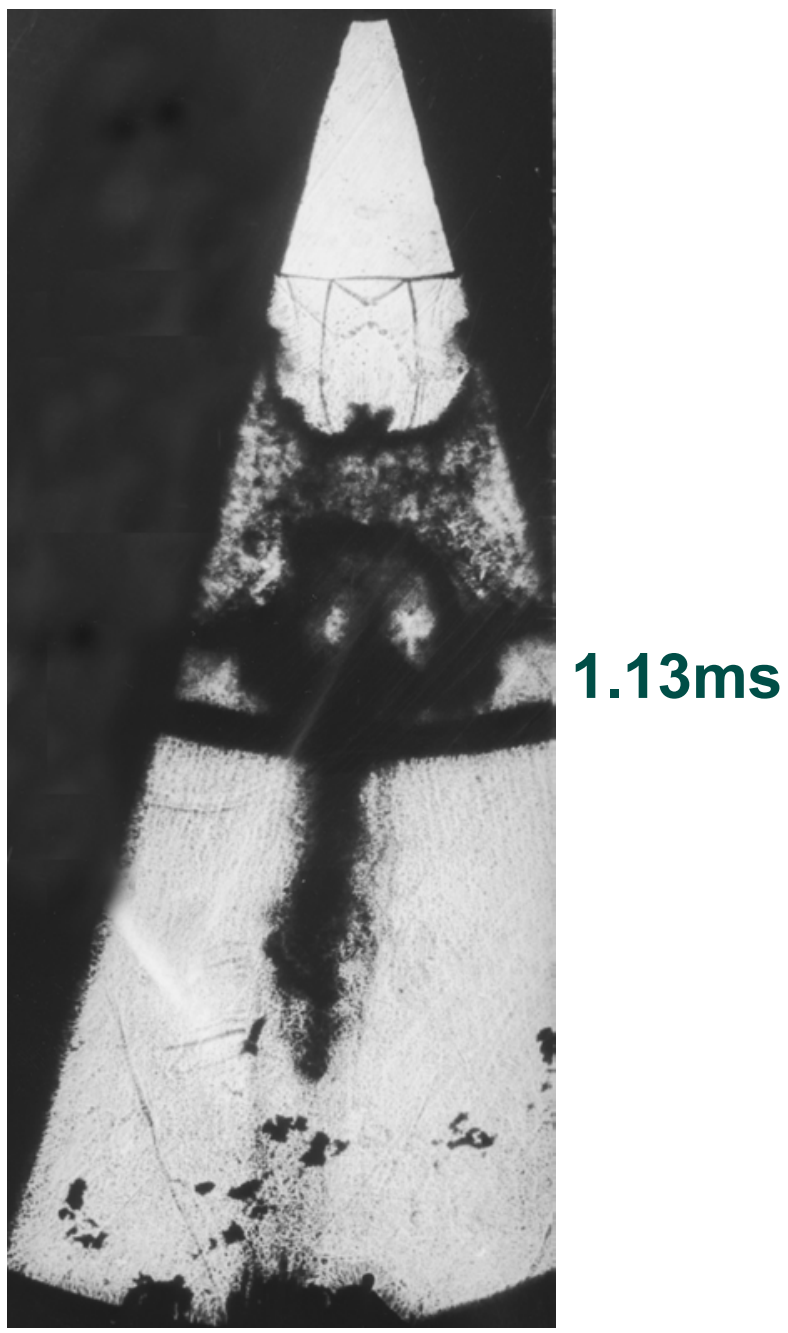

Turmoil 3D code

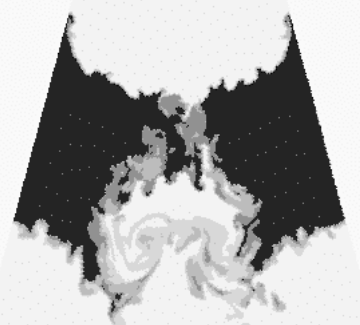

$1.05 \mathrm{~ms}$ 


\section{Notch Experiment}

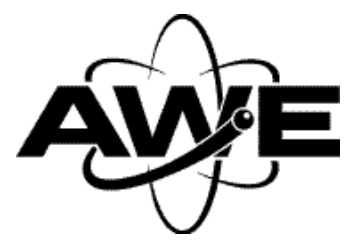

Experiment

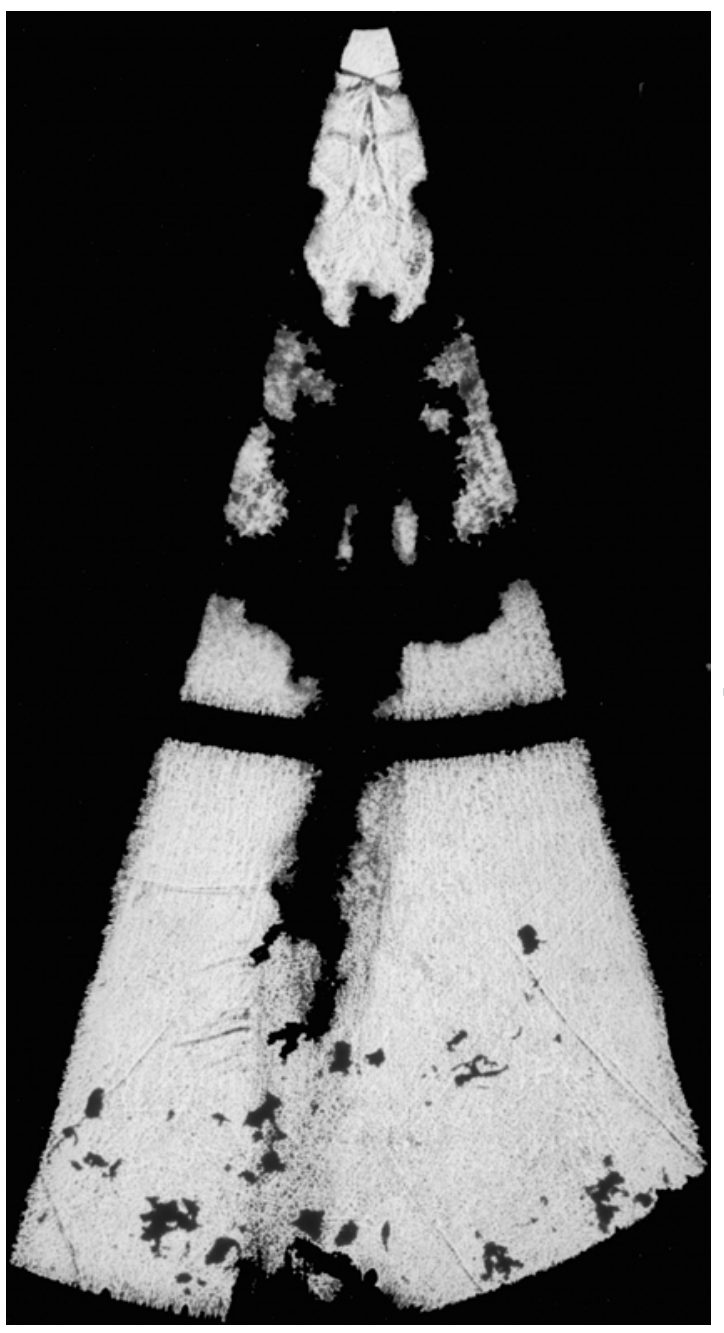

Turmoil 3D code

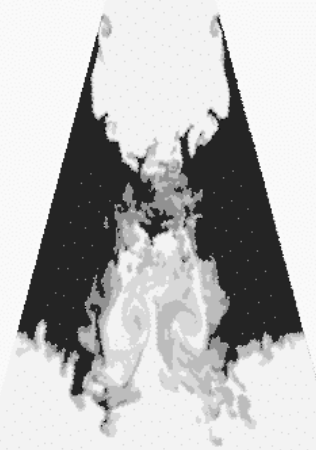

$1.20 \mathrm{~ms}$

$1.15 \mathrm{~ms}$ 


\section{Notch Experiment}

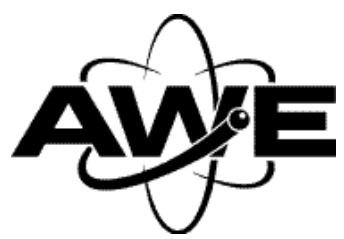

Experiment

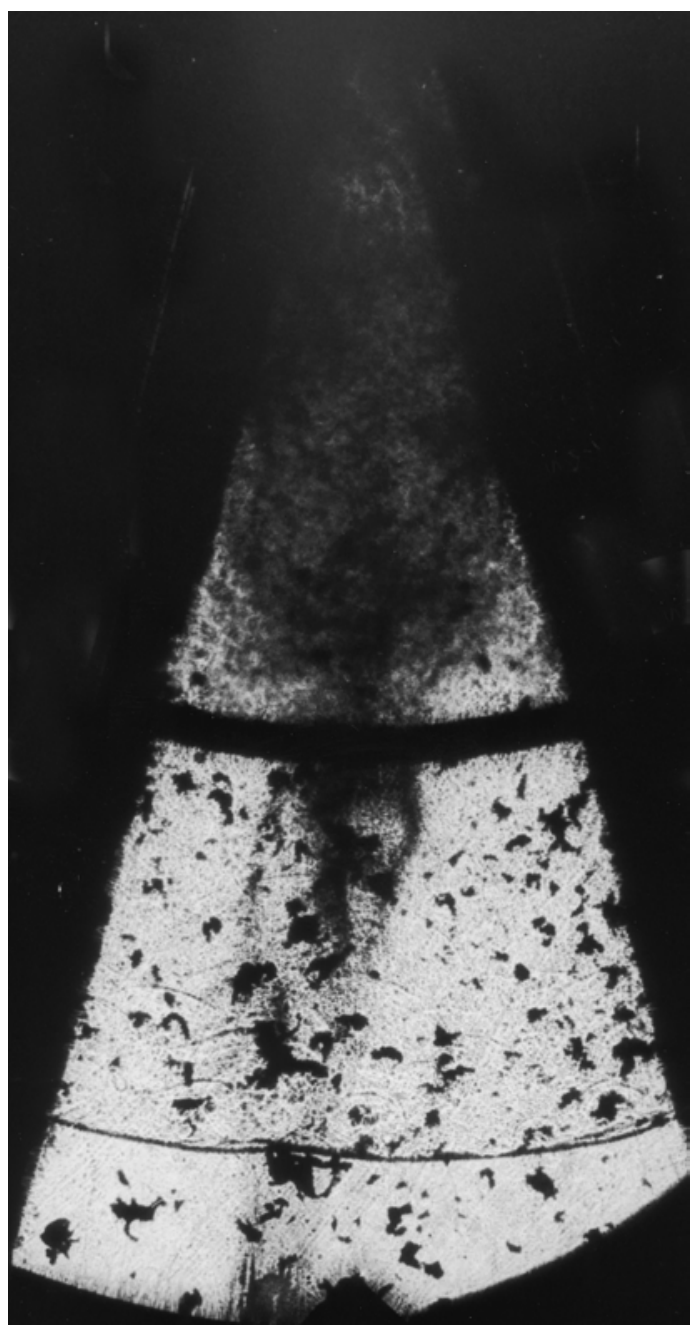

Turmoil 3D code

$1.87 \mathrm{~ms}$

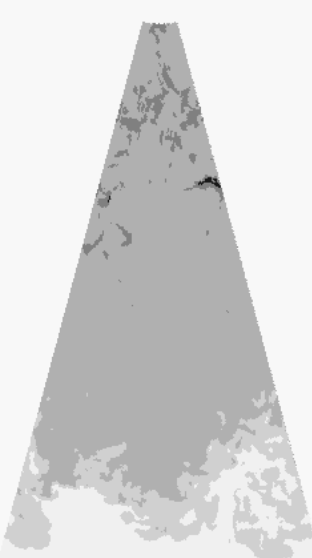

$1.75 \mathrm{~ms}$ 


\section{Turmoil 3D Calculations}

\section{Ange}

Cylindrical Experiment

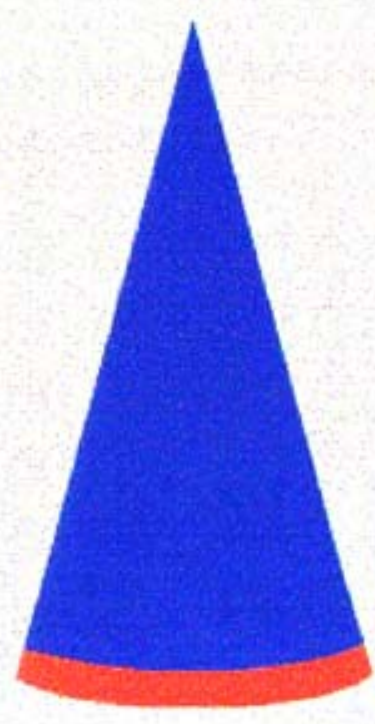

0

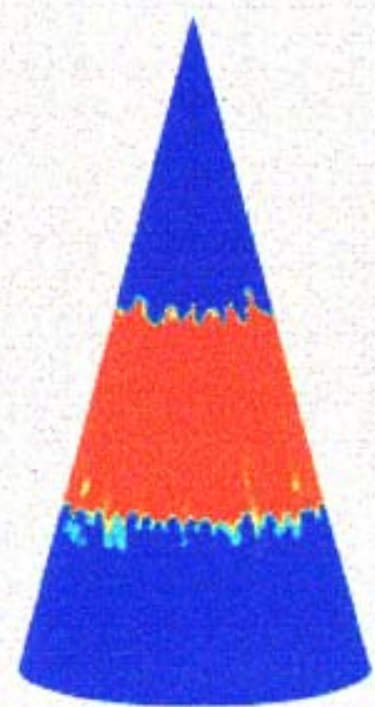

$1.2 \mathrm{~ms}$

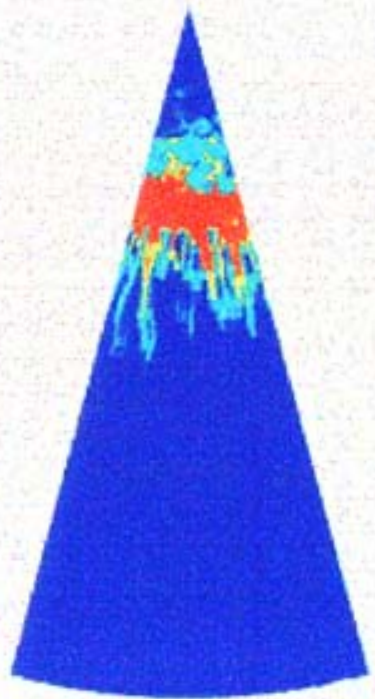

$1.5 \mathrm{~ms}$

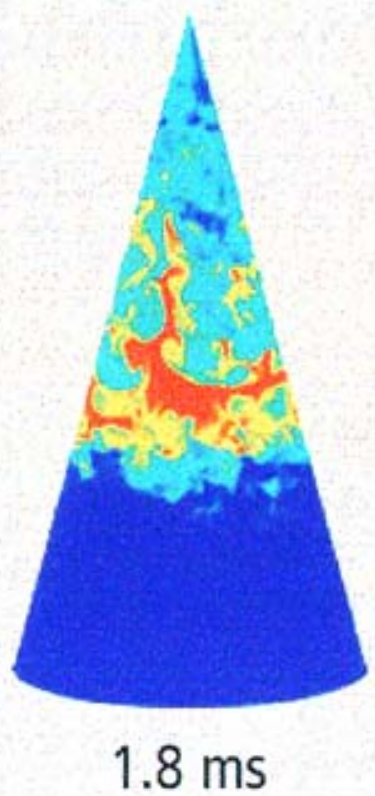

$1.8 \mathrm{~ms}$ 


\section{Conclusions}

1. Successfully demonstrated suitability of a Convergent Shock Tube for performing R-M experiments with gases in 2D geometry

2. Achieved compressions of dense gas of typically $25: 1$ using shock Mach No. $\sim 3$

3. Achieved good understanding of design requirements for constructing a new improved Convergent Shock Tube 


\section{Future Work}

- Construct new Convergent Shock Tube which operates with the laser sheet diagnostic (and variants)

- Establish seeding with fluorescent gas suitable for:-

- use of notch filter to 'remove' laser light scattered from membrane fragments

- seeding at high gas compression

- Continue experiments with different perturbation profiles

- Substitute Xe gas for $\mathrm{SF}_{6}$

- Establish calibration technique for gas data analysis of laser sheet images

- [Consider 'inverse' experiment to check the influence of the side walls ] 


\title{
Rayleigh-Taylor instability at a tilted interface
}

\section{in incompressible laboratory experiments and compressible numerical simulations}

\author{
Joanne M. Holford ${ }^{\dagger}$ \\ Stuart B. Dalziel ${ }^{\dagger}$ \\ David Youngs*
}

${ }^{\dagger}$ DAMTP, University of Cambridge, Silver Street, Cambridge CB3 9EW, UK ${ }^{\ddagger}$ AWE Aldermaston, Reading, Berkshire, RG7 4PR, UK 


\section{Outline}

- Introduction

RT instability at a tilted interface

Mixing, available energy and mixing efficiency

- Laboratory experiments

At DAMTP, in the Fluid Dynamics Laboratory

Incompressible water, $\mathrm{NaCl}$ to create density contrast

- Numerical simulations

At AWE, using Turmoil3D (with David Youngs)

Compressible code, for a mixture of two ideal gases

- Conclusions and further work 


\section{Introduction}

- RT instability

Instability of dense fluid accelerated into less dense fluid

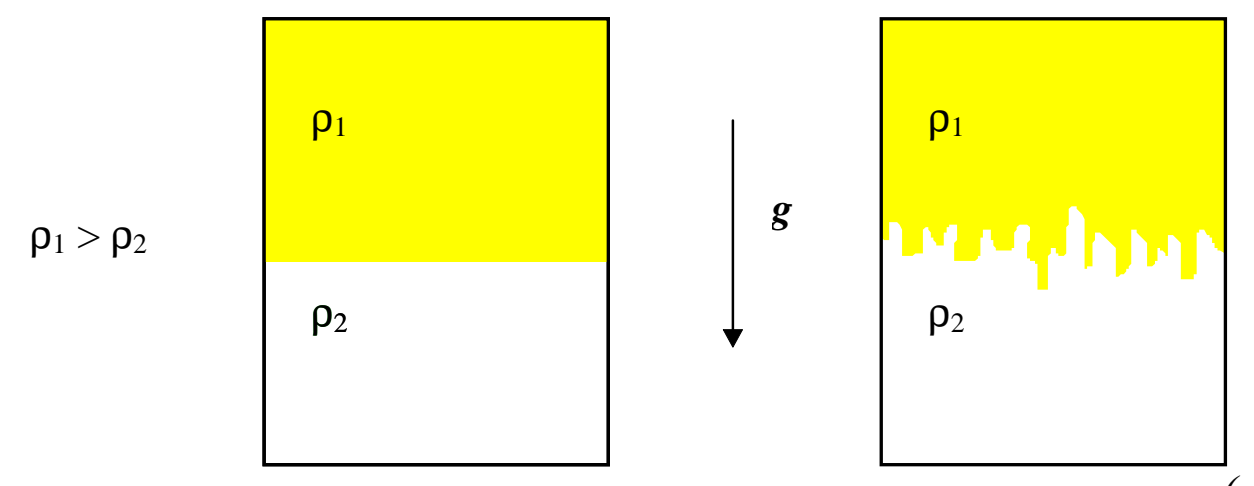

Non-dimensional parameter Atwood number $A=\left(\frac{\rho_{1}-\rho_{2}}{\rho_{1}+\rho_{2}}\right)=\frac{g^{\prime}}{2 g}$

For an external lengthscale $H$, timescale $\tau=\sqrt{\frac{H}{A g}}$

Much more efficient mixing than other mechanisms (shear instability, mechanical stirring)

An important mixing process within larger-scale flows (3D instability of 2D shear billows)

In environment, R-T instability has non-ideal initial conditions At a tilted interface, there is competition between local instability and large-scale overturning

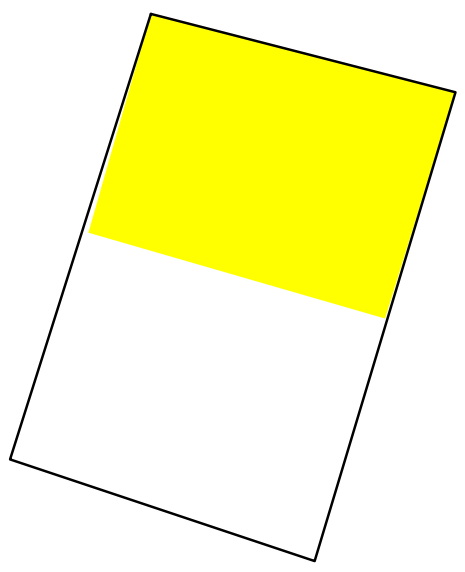

DAMTP, University of Cambridge

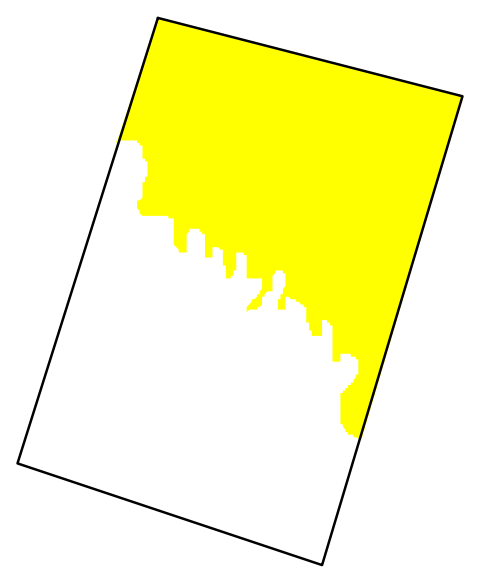

Rayleigh-Taylor instability 


\section{- Definitions of mixing}

Distinguish between reversible and irreversible mixing:

Reversible mixing - interleaving of fluid with different properties - "reversible mixing = stirring"

Irreversible mixing - homogenisation of fluid properties at the molecular scale - "irreversible mixing = stirring + diffusion"

Irreversible mixing is important for

- chemical reactions

- removal of available energy when mixing density gradients across a gravitational field

\section{- How do we measure mixing?}

Mixing can be measured by a molecular mixing fraction

For two fluids, volume fractions $f$ and $(1-f)$ :

$$
\vartheta(\boldsymbol{x}, t)=f(\boldsymbol{x}, t)(1-f(\boldsymbol{x}, t))
$$

Alternatively, for fluids of varying density in a gravitational field, can measure the mixing efficiency $\eta$

For a fluid at rest, stirred by an energy input and returning to rest,

$$
\eta=\frac{\text { increase in potential energy }}{\text { amount of energy added }}
$$

fraction of energy lost to fluid motion doing work against gravity 


\section{- Mixing in R-T instability}

Measurements of $\eta$ in laboratory experiments high values with some dependence on $A$

Linden \& Redondo (1991)

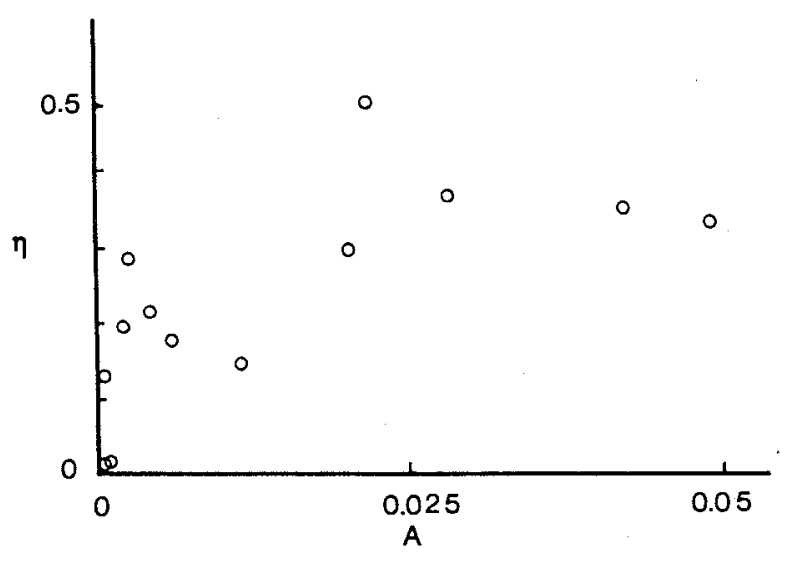

Numerical simulations show sensitivity to initial conditions Linden, Redondo \& Youngs (1991), Cook \& Dimotakis (2001)

- Diffusion and viscosity in incompressible fluids

Mechanical energy density per unit volume $E_{v}=\frac{1}{2} \rho|u|^{2}+\rho g z$

$$
\begin{gathered}
\frac{\partial}{\partial t} E_{v}(\boldsymbol{x}, t)+\nabla \cdot \boldsymbol{f}_{v}(\boldsymbol{x}, t)=-\varepsilon_{v}(\boldsymbol{x}, t), \\
\boldsymbol{f}_{v} \text { energy flux } \\
\boldsymbol{\varepsilon}_{v} \text { energy dissipation }
\end{gathered}
$$

Water/salt system $-v=1.0 \times 10^{-2} \mathrm{~cm}^{2} \mathrm{~s}^{-1} \quad$ kinematic viscosity

$$
\kappa=1.4 \times 10^{-5} \mathrm{~cm}^{2} \mathrm{~s}^{-1}
$$

diffusivity

concentration fluctuations persist at smaller scales than velocity fluctuations $-\operatorname{Pr}=\frac{\nu}{\mathrm{K}}=700$

$\underline{\text { Turbulent flows }}$ - eddy viscosity = eddy diffusivity effectively $\operatorname{Pr}=1$ 


\section{- Available energy in incompressible flow}

Mechanical energy in whole fluid $E=\int_{V} E_{v} d V$ decomposes:

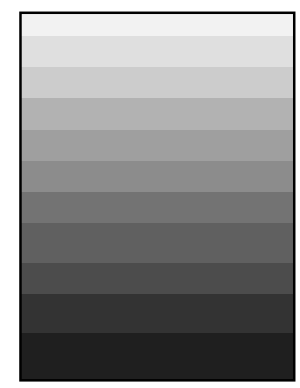

$P E_{\text {back }}$

$E_{\text {back }}$

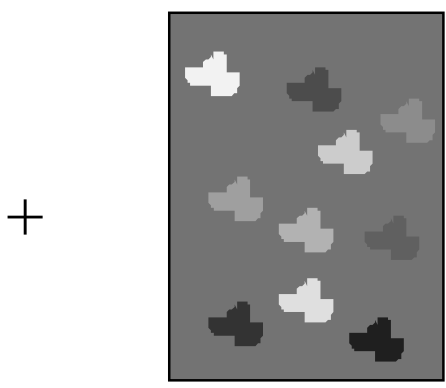

$P E_{\text {avail }}$

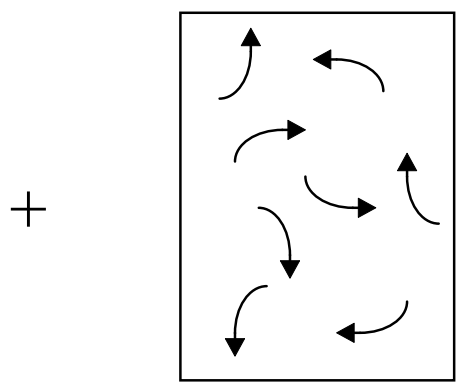

$K E$

Lorenz (1955), Thorpe (1977), Winters et al. (1995)

In unforced, decaying flow

$$
\begin{aligned}
& \frac{d}{d t}\left(E_{\text {back }}+E_{\text {avail }}\right)=-\varepsilon \text { loss of } E \text { due to } \\
& \text { turbulent dissipation } \\
& \frac{d}{d t}\left(E_{\text {back }}\right)=q \text { gain in } E_{\text {back }} \text { due to } \\
& \text { molecular mixing }
\end{aligned}
$$

Define cumulative mixing efficiency

$$
\eta_{\text {cumulative }}=\frac{\int_{t_{0}}^{t} q d t}{\int_{t_{0}}^{t} q+\varepsilon d t}=\frac{\Delta P E_{\text {back }}}{-\Delta E_{\text {avail }}}
$$

and instantaneous mixing efficiency

$$
\eta_{\text {instantaneous }}=\frac{q}{q+\varepsilon}=\frac{\delta P E_{\text {back }}}{-\delta E_{\text {avail }}}
$$


- Available energy in compressible flow

Now concerned with total energy (mechanical + internal) so $E_{v}=\frac{1}{2} \rho|u|^{2}+\rho g z+\rho e, e$ internal energy. In whole fluid:

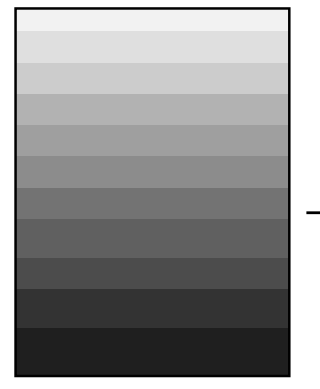

$P E_{\text {back }}$

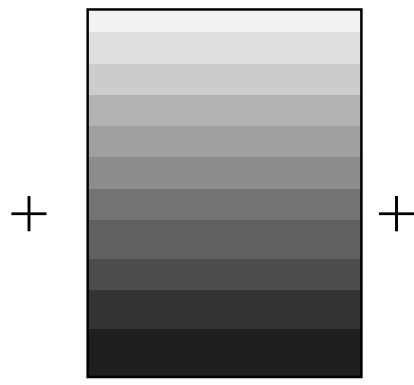

$I E_{\text {back }}$

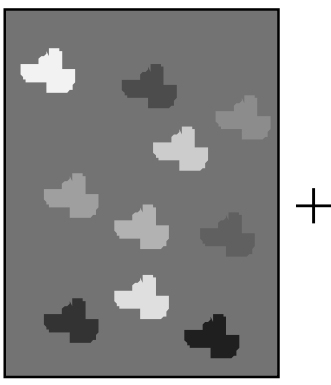

$P E_{\text {avail }}$

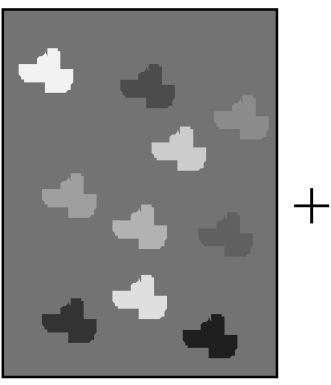

$I E_{\text {avail }}$

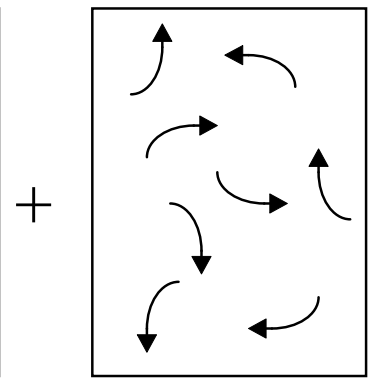

$K E$
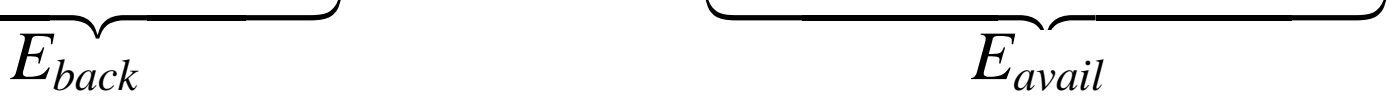

Lorenz (1955), Andrews (1981), Shepherd (1993)

In unforced, decaying flow

$$
\begin{aligned}
& \frac{d}{d t}\left(E_{\text {back }}+E_{\text {avail }}\right)=0 \\
& \frac{d}{d t}\left(I E_{b a c k}\right)=\varepsilon \\
& \text { gain in } I E_{b a c k} \text { due to } \\
& \text { turbulent dissipation and } \\
& \text { molecular mixing } \\
& \frac{d}{d t}\left(P E_{\text {back }}\right)=q \\
& \text { gain in } P E_{\text {back }} \text { due to } \\
& \text { molecular mixing }
\end{aligned}
$$

Same definitions of mixing efficiency apply, so $\eta$ is still fraction of energy lost to fluid motion (reduction in $E_{\text {avail }}$ ) doing work against gravity (gain in $E_{b a c k}$ ) 


\section{Laboratory Experiments}

\section{- Configuration}

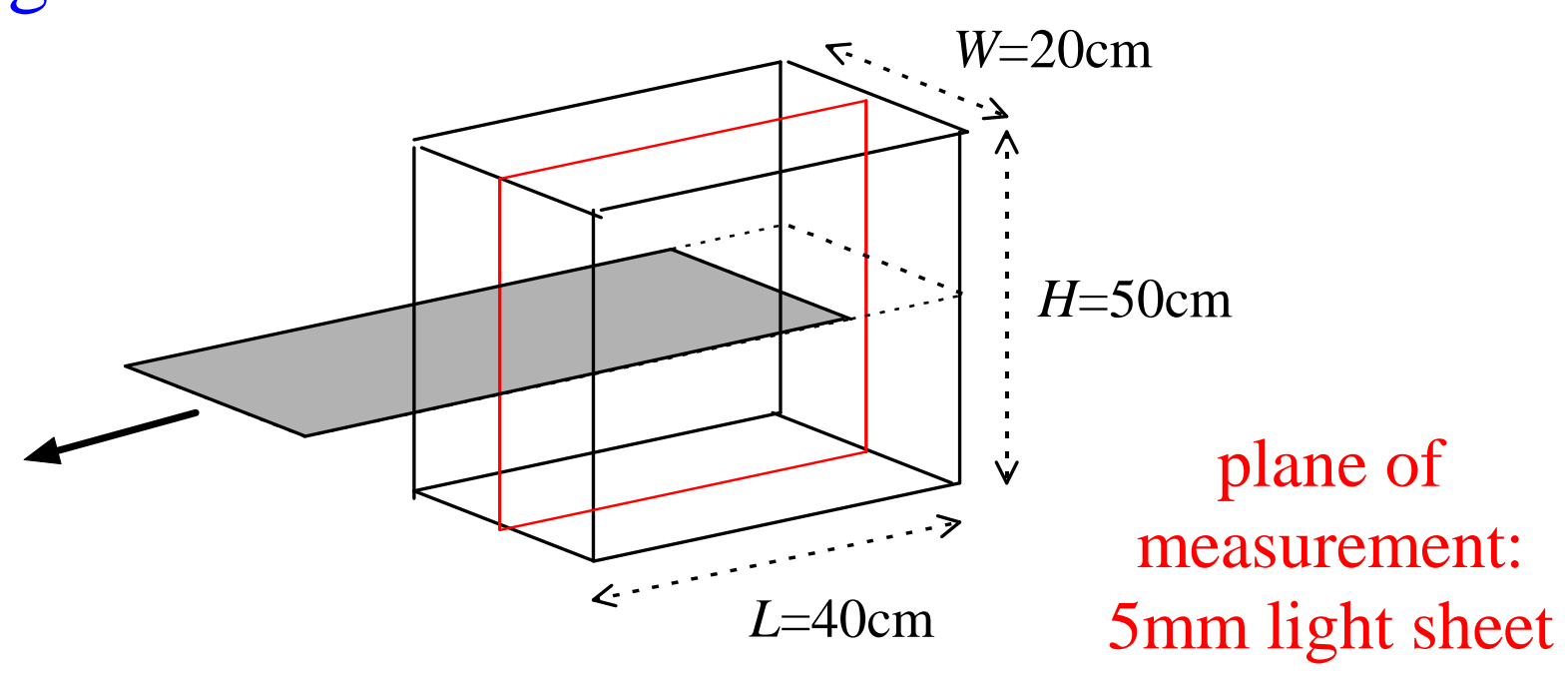

- Initial conditions

A solid barrier introduces significant shear

Reduced shear barrier:

Dalziel, Linden \& Youngs (1999)

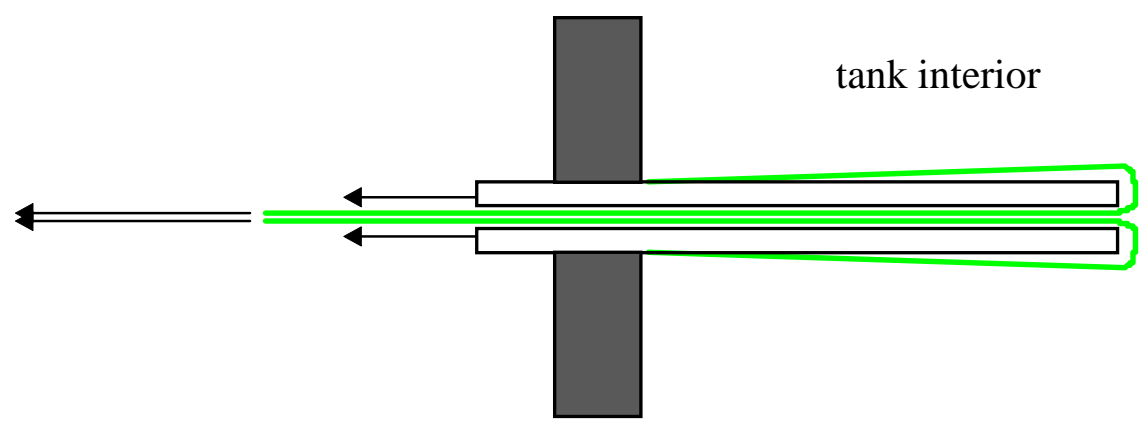

SIDE VIEW

fabric

Removal of finite thickness barrier causes initial velocity field
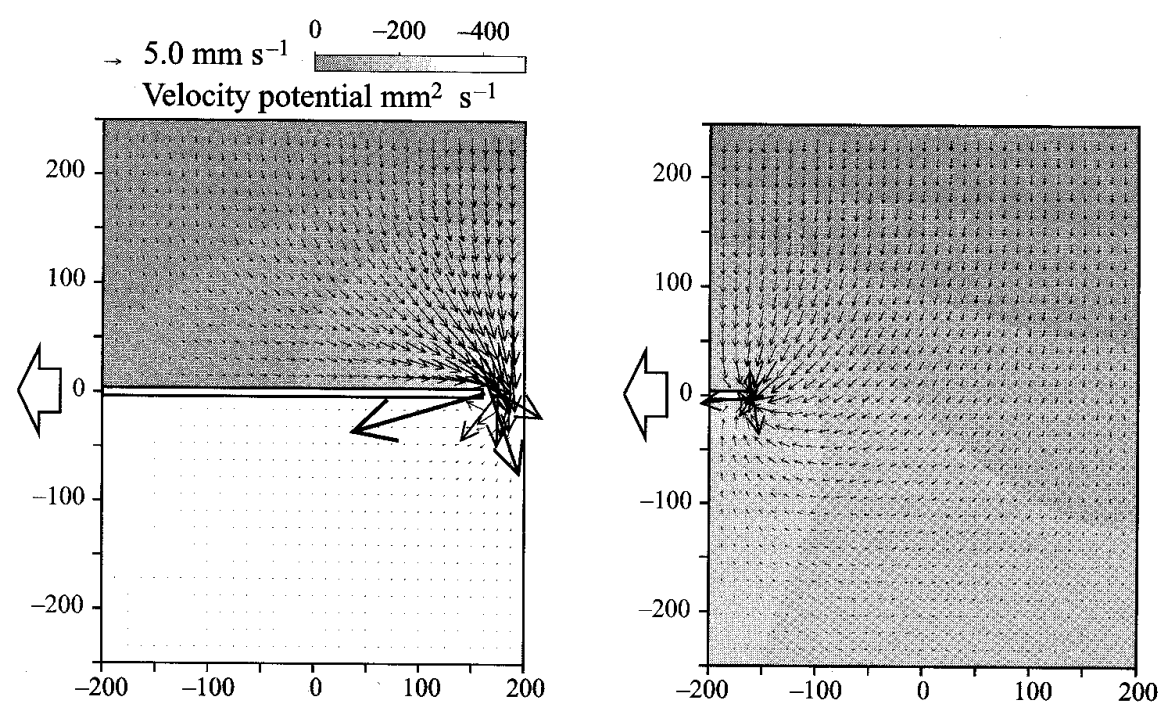


\section{- Diagnostic measurements}

Image analysis: $\quad$ spatial resolution 1 pixel $\cong 0.1 \mathrm{~cm}$ temporal resolution $25 \mathrm{~Hz}$

Assume statistical homogeneity across tank

Add propanol to fresh water to match refractive index

\section{Density measurement}

Dense fluid dyed with fluorescent dye

Images corrected for divergence of light sheet and attenuation

\section{Velocity measurement}

Fluid seeded with $400 \mu \mathrm{m}$ neutrally-buoyant particles

Lagrangian tracks for particles from tracking a frame sequence Interpolating onto a grid gives Eulerian velocities

Gridded at two scales: $1 \mathrm{~cm}$ - resolved velocity

$$
3 \mathrm{~cm} \text { - mean velocity }
$$

(overcomes lack of similarity between experiments)

Assume isotropy at small scales $\Rightarrow$ estimate of total KE

- Parameters
Atwood number
$0.5 \times 10^{-3}<A<2.5 \times 10^{-3}$
$\Rightarrow$ Boussinesq
Timescale
$10 \mathrm{~s}>\tau>4.5 \mathrm{~s}$
RMS velocity
$0.8 \mathrm{cms}^{-1}<|\boldsymbol{u}|<2 \mathrm{cms}^{-1}$
Integral lengthscale
$1.8 \mathrm{~cm}<l<2.5 \mathrm{~cm}$
Reynolds number
$150<\operatorname{Re}<500$ 
divergence

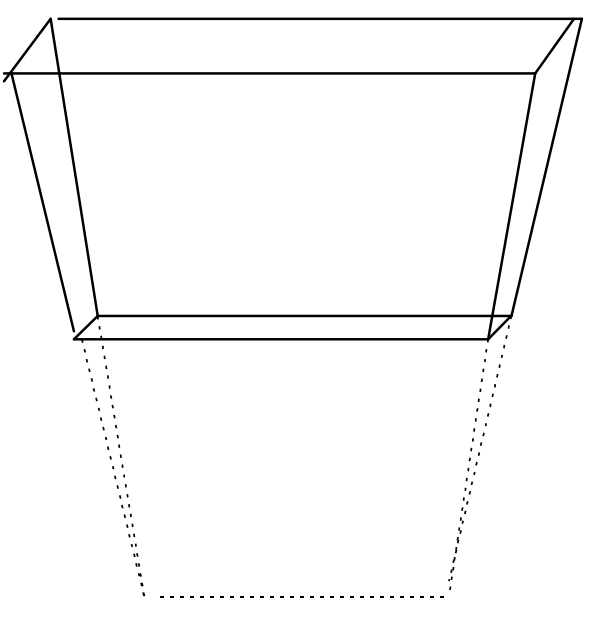

light source

attenuation

(of incident, not fluoresced light) light rays

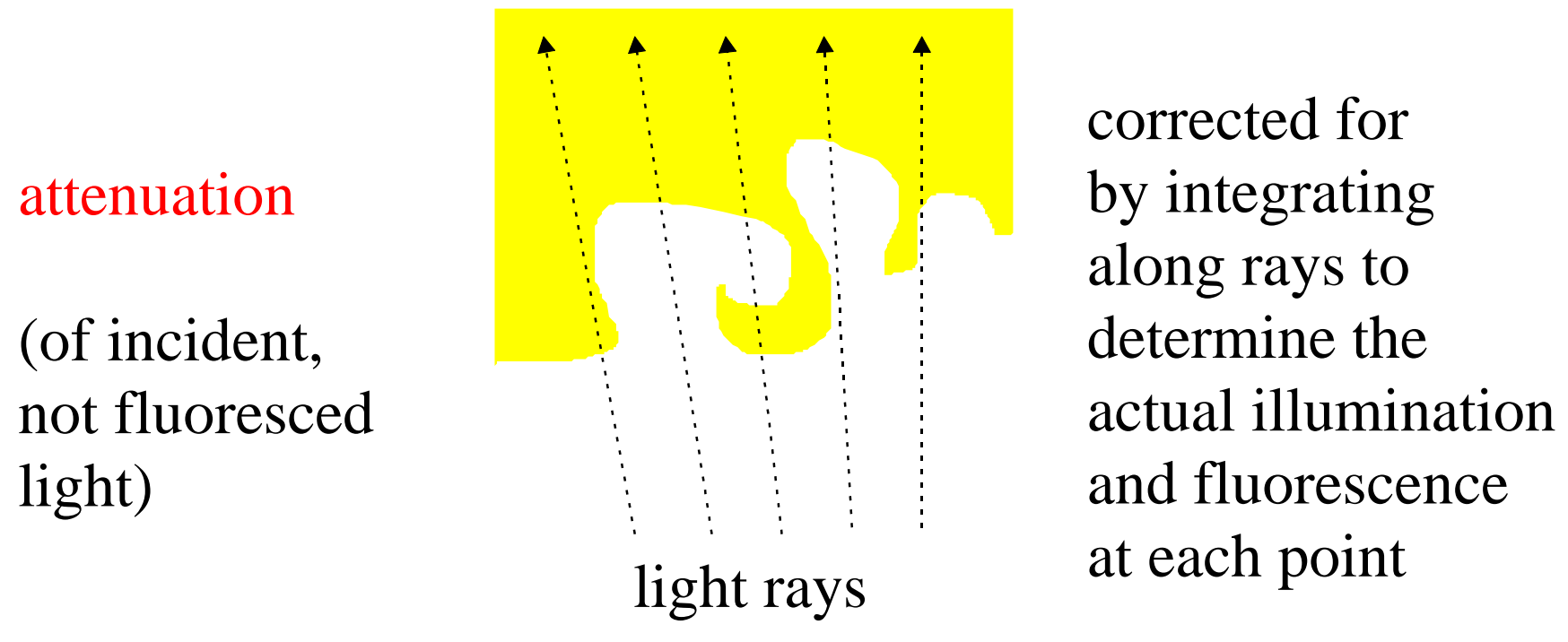

corrected for by comparing fluorescence pattern of image with uniform dye concentration 
Density field
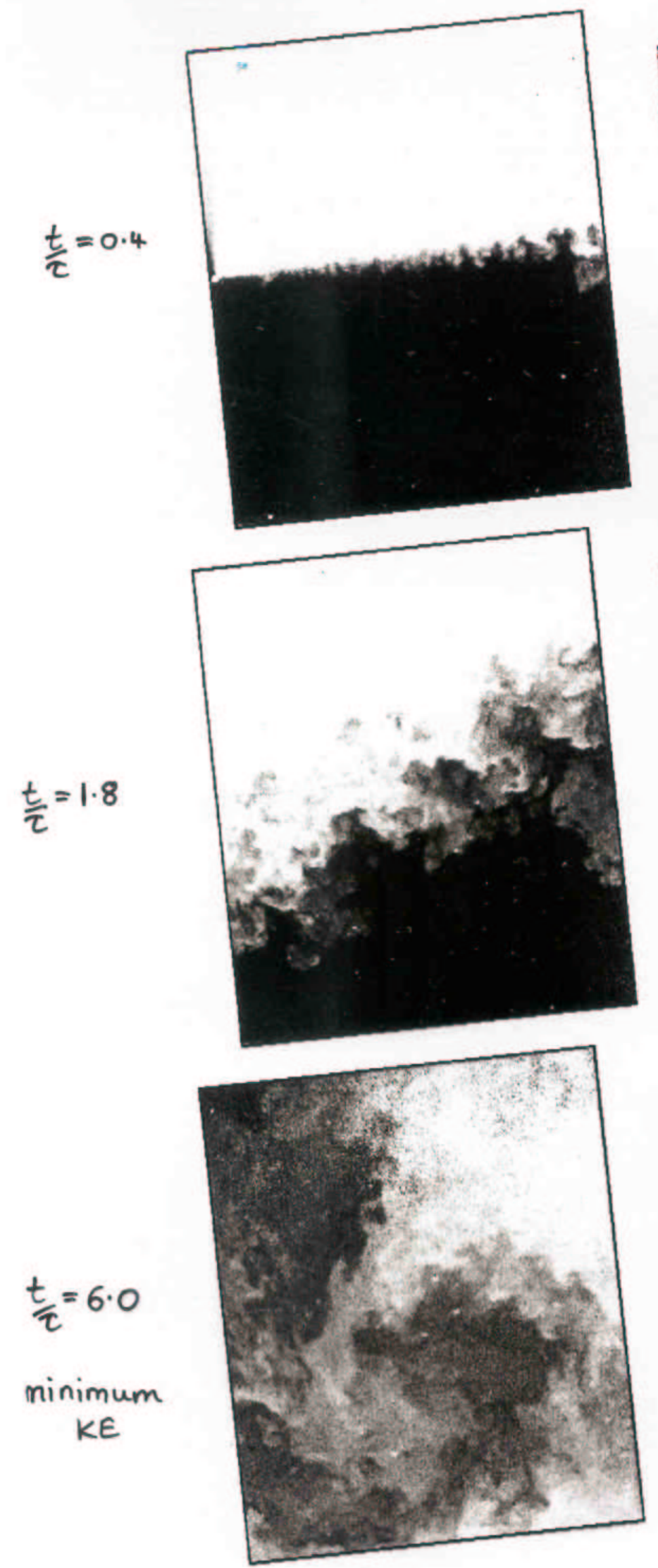

$A=2.6 \times 10^{-3}$
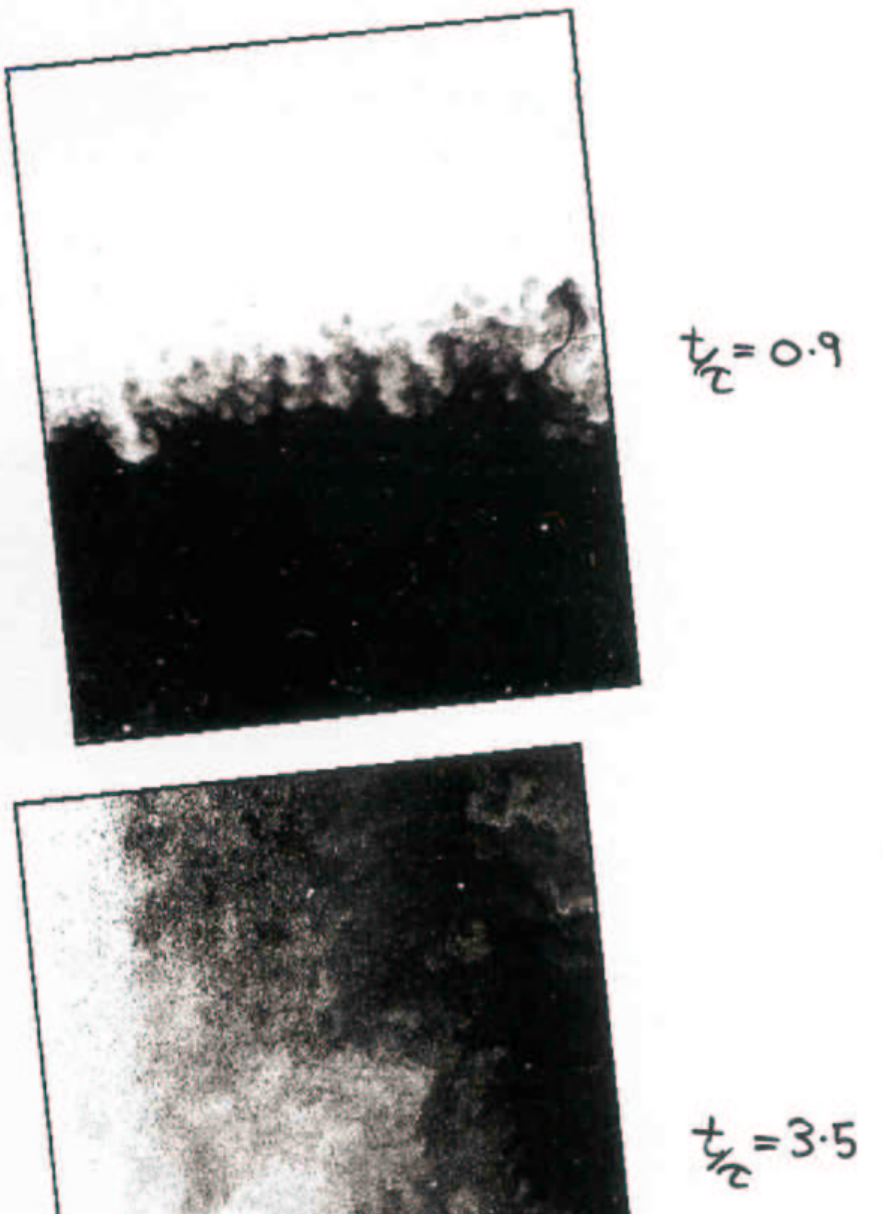

maximum KE

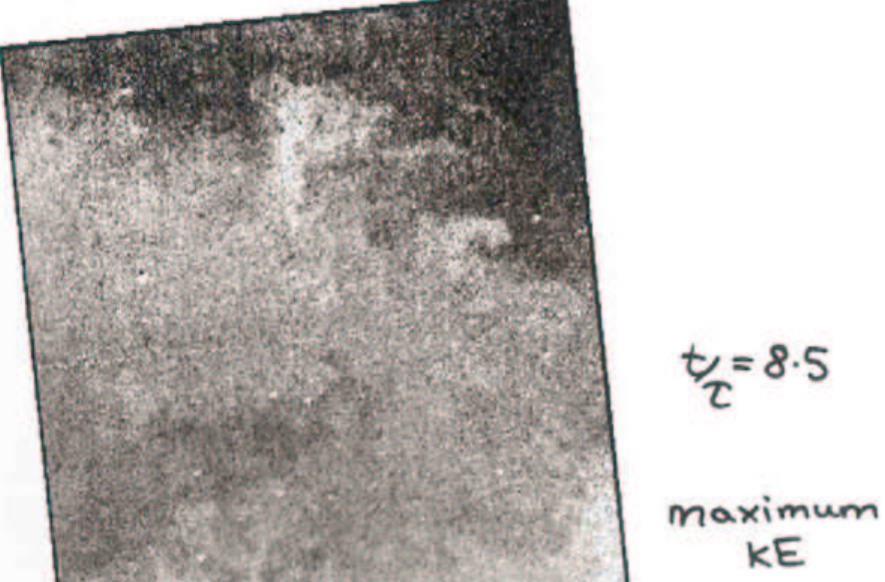




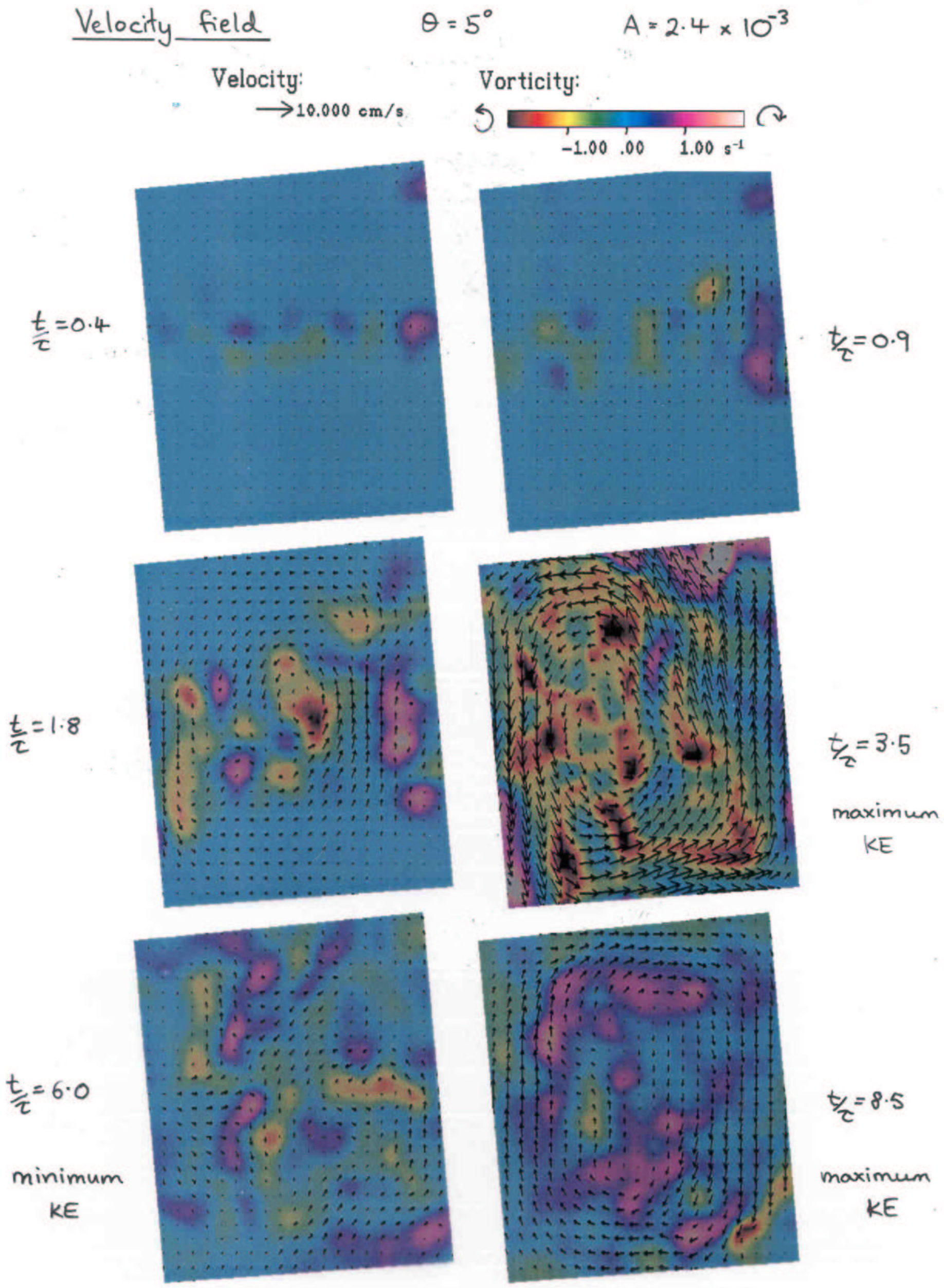


Energy evolution $\quad \theta=5^{\circ}$
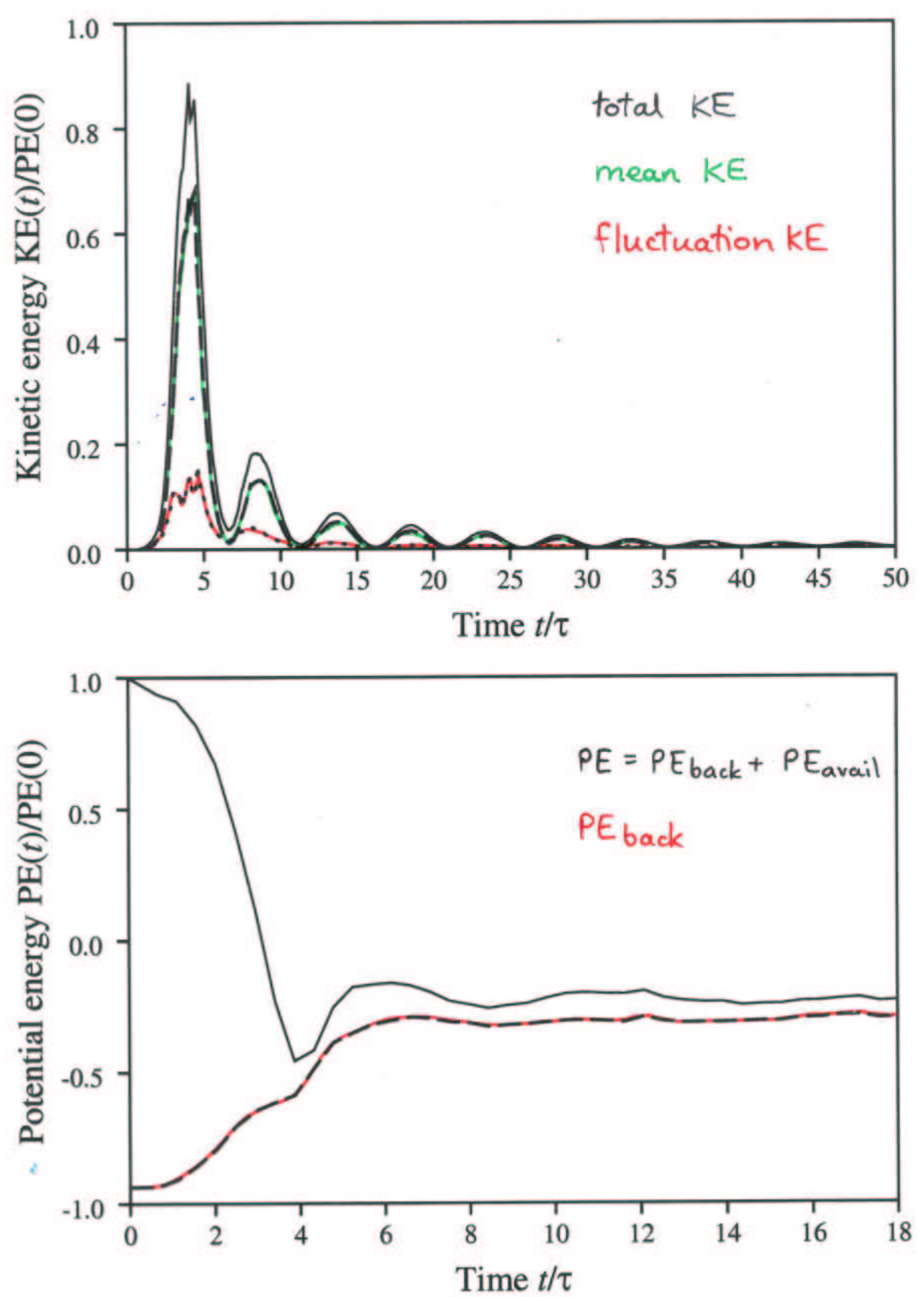
Energy evolution $\quad \theta=0^{\circ}$
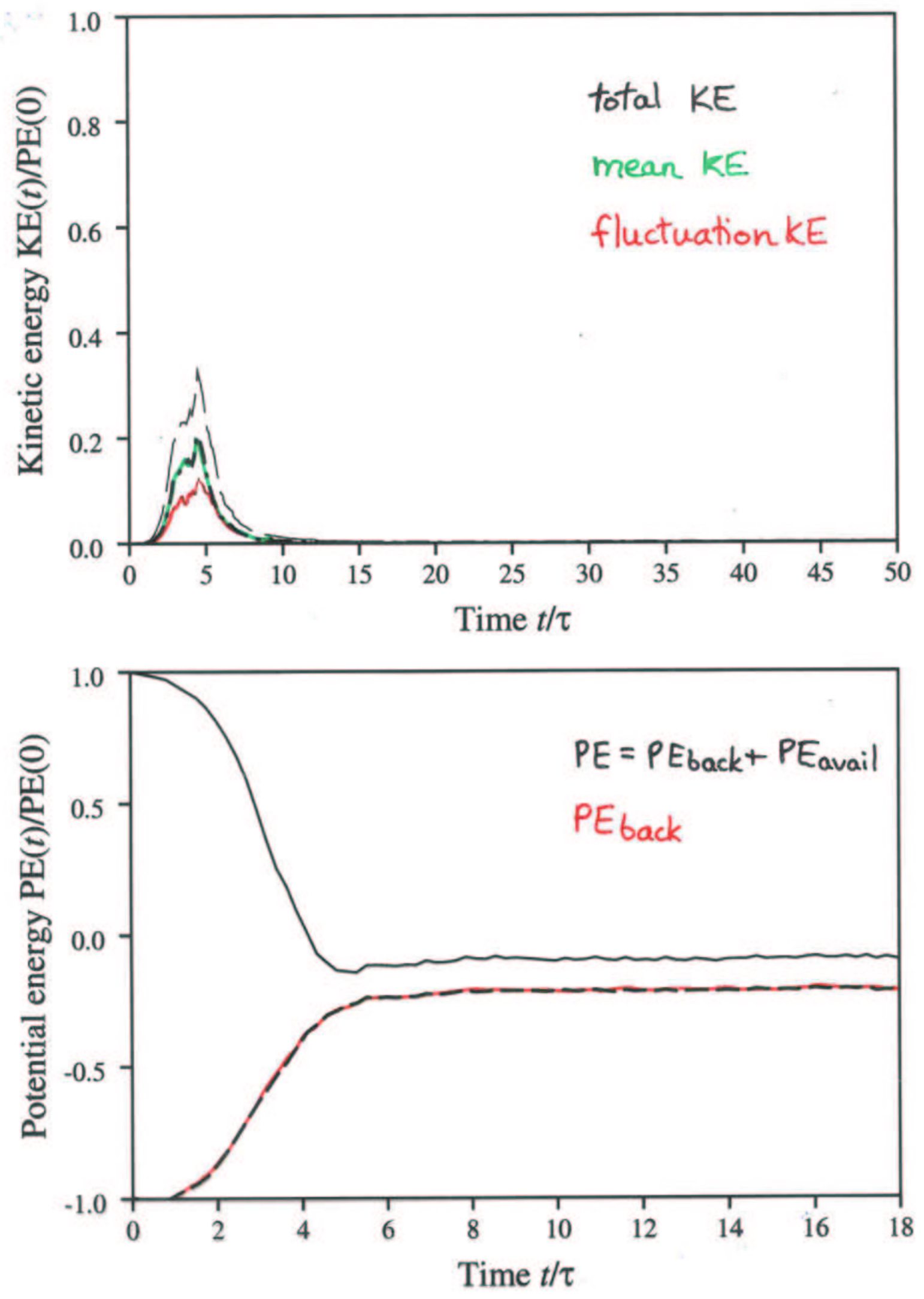


\section{Numerical Simulations}

- Code type

Semi-Lagrangian finite volume code

(conservation of fluid masses and momentum)

Two ideal gases $(\gamma=5 / 3)$

Typical simulation: $3 \mathrm{D}$ at resolution $200 \times 160 \times 80$

\section{- Viscosity and diffusion}

Loss of resolution at grid scale $\Rightarrow$ diffusion-like behaviour

for mass fractions, analogous to molecular mixing

for $\mathrm{KE}$, analogous to dissipation, and added to IE

In some runs, an explicit viscosity was added

- Approximating an incompressible fluid

Normalisation: choose $H=1, A g=1, \rho_{1}=1$

Non-dimensional parameters (ideally small):

Density ratio

$$
B=\frac{\Delta \rho}{\rho_{0}}=\frac{2}{g} \quad \approx 0.18
$$

Mach number

$$
M=\sqrt{\frac{A g H \rho}{p \gamma}} \approx \sqrt{\frac{3}{5 p_{0}}} \approx 0.08
$$

Incompressibility ratio

$$
I=\frac{g H \rho^{2}}{5 p \Delta \rho} \approx \frac{g^{2}}{10 p_{0}}
$$

Compromise $g=11, p_{0}=100$ 


\section{- Initial conditions - basic distribution}

Away from interface:

Since $\boldsymbol{u} \approx 0$, require $\frac{\partial p}{\partial z}=-\rho g$.

Require neutral stability, buoyancy frequency

$N^{2}=\frac{g}{T}\left(\frac{\partial T}{\partial z}+\frac{g}{c_{p}}\right)=0 \Leftrightarrow$ isentropic fluid $p=k(s) \rho^{\gamma}$.

At interface:

Choose specific heats at constant volume, $c_{v 1}$ and $c_{v 2}$.

Require temperature continuous $\Leftrightarrow c_{v 1} \rho_{1}=c_{v 2} \rho_{2}$.

Everywhere:

Pressure field cannot be entirely hydrostatic.

Require $\frac{\partial}{\partial t}(\nabla \cdot \boldsymbol{u})=0$.

Ignoring terms of $\mathrm{O}\left(u^{2}\right)$, require

$$
\nabla \cdot\left(\frac{1}{\rho} \nabla p\right) \propto \nabla \cdot\left(k^{1 / \gamma} \nabla p^{(\gamma-1) / \gamma}\right)=0,
$$

with $\frac{\partial p}{\partial n}=-\rho g \hat{\boldsymbol{n}} . \hat{z}$ on boundaries with outward normal $\hat{\boldsymbol{n}}$.

\section{- Initial conditions - perturbations}

$2 \mathrm{D}$ velocity field with vorticity at interface models experimental barrier withdrawal.

3D random perturbation to interface position, wavelengths $\frac{L}{40}<\lambda<\frac{L}{20}$, rms amplitude $\sigma=\frac{H}{2500}$. 

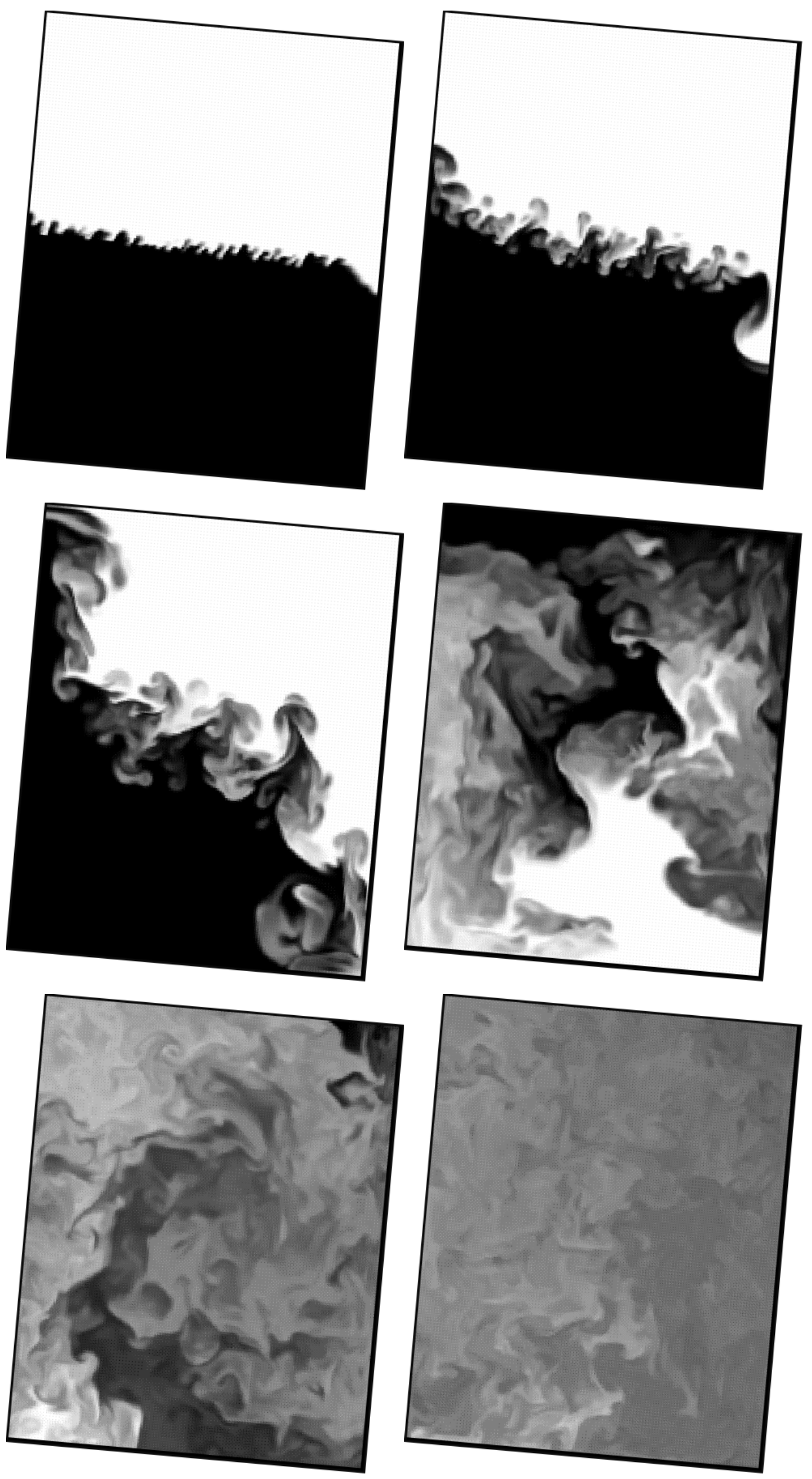

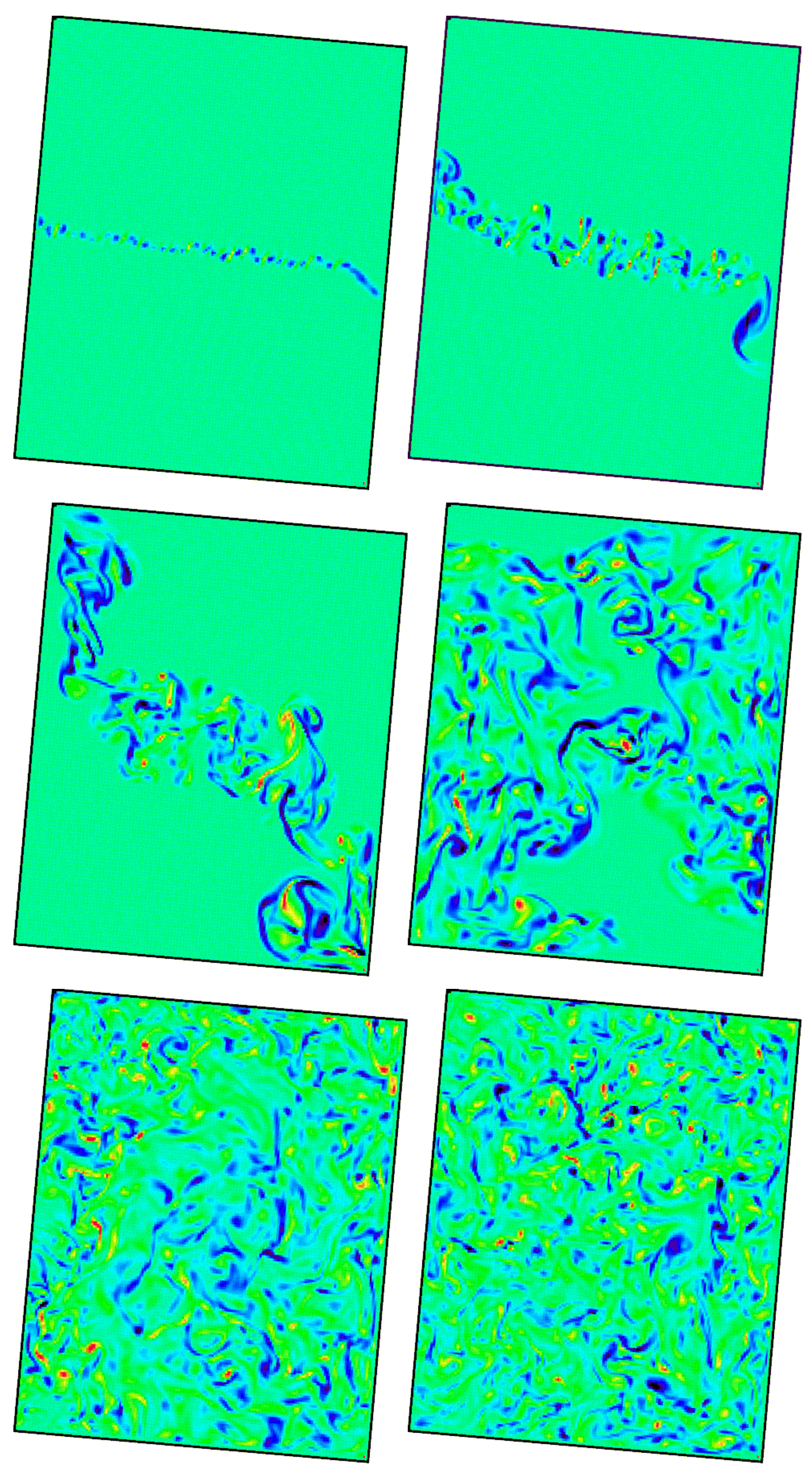


\section{Energy budget}

Numerical results $\eta \approx 0.48$

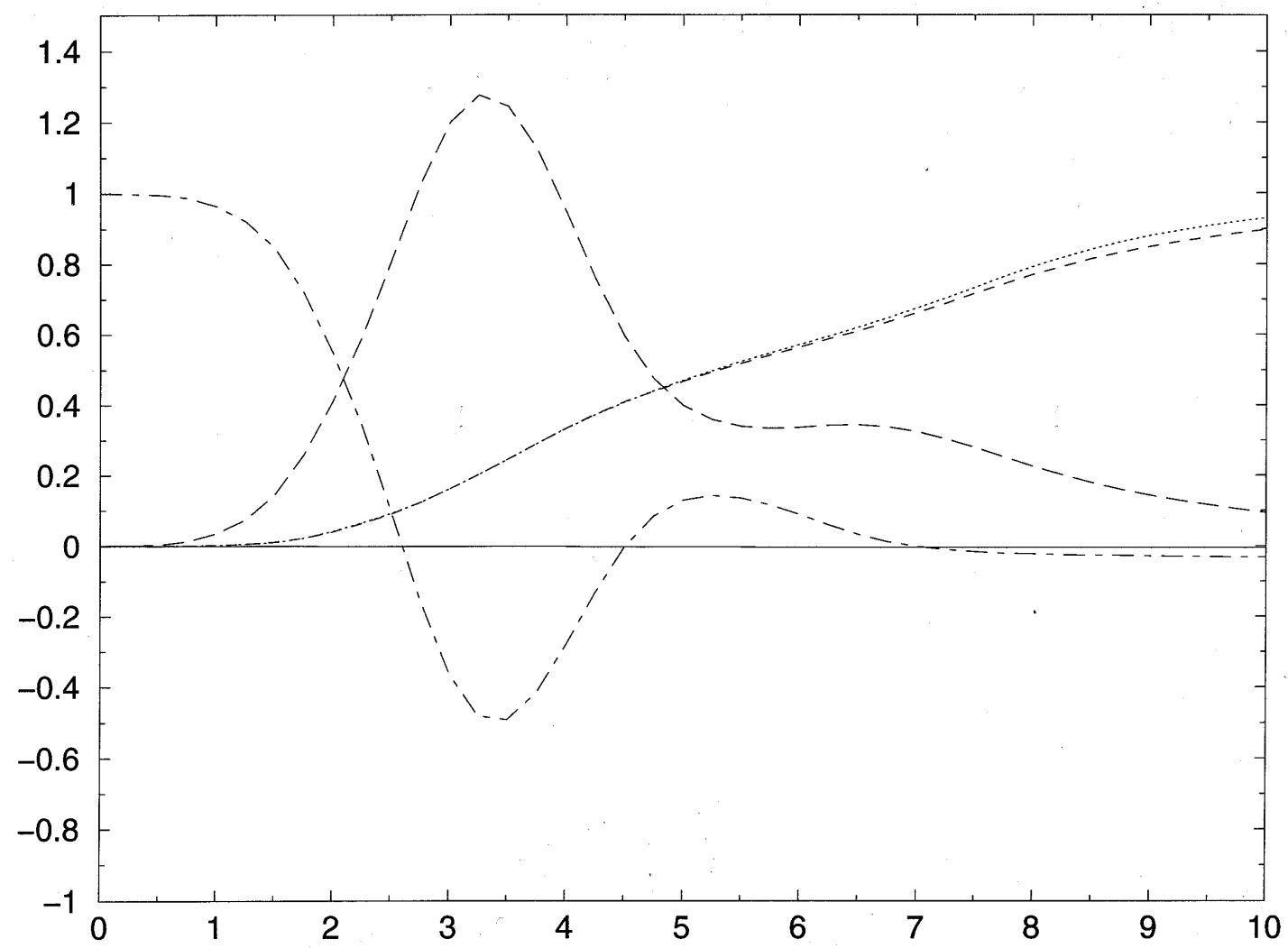

\section{Typical experimental results $\eta \approx 0.38$}

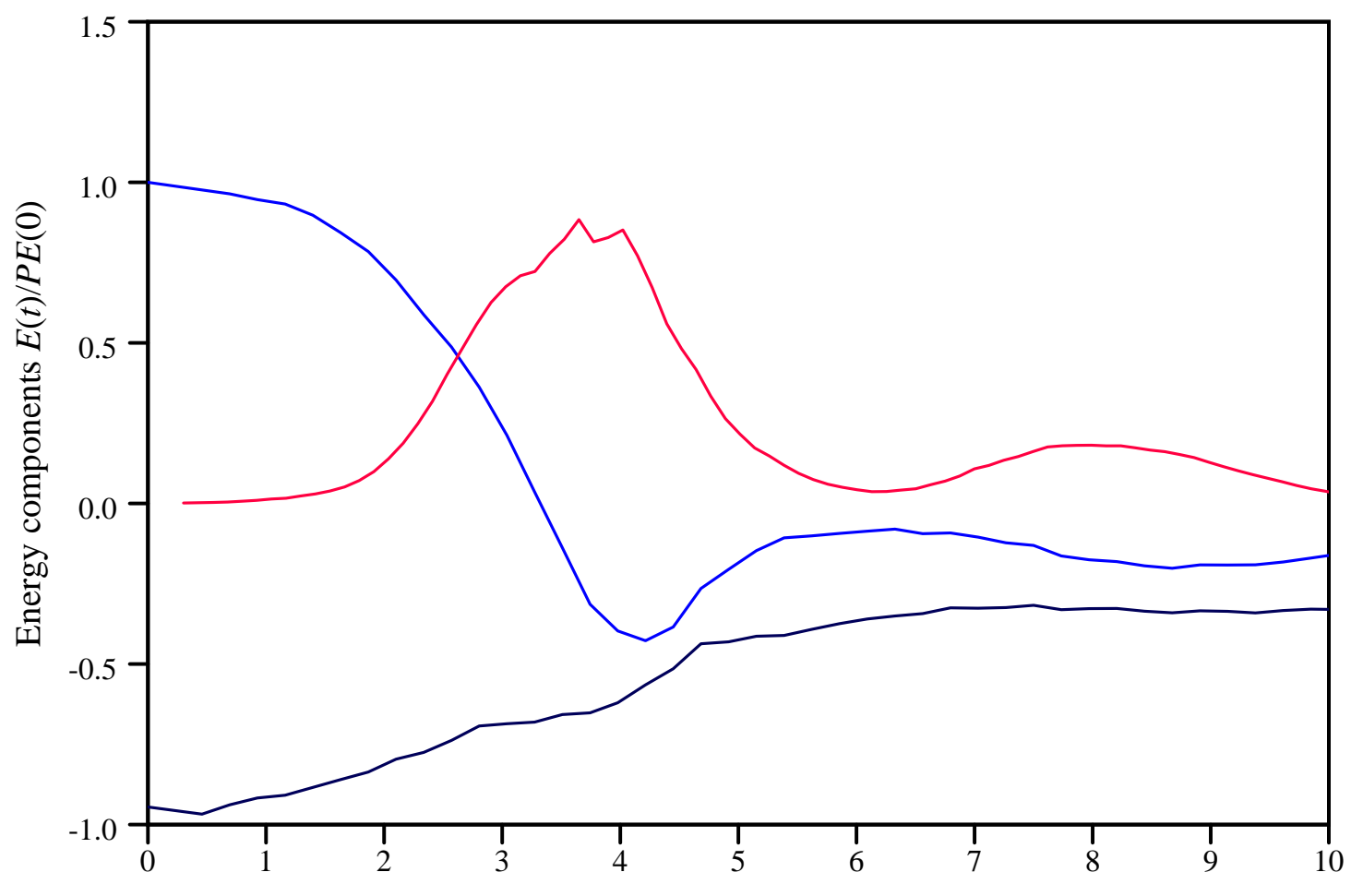

Time $t / \tau$ 
-Why the difference in energy budgets?

Numerical diffusion not $\nabla^{2}$

$2 \mathrm{D}$ advection test pattern

Numerical viscosity acts preferentially at small scales and is resolution and velocity-dependent

Total dissipation is unaffected by ratio of explicit/numerical viscosity until explicit viscosity dominates

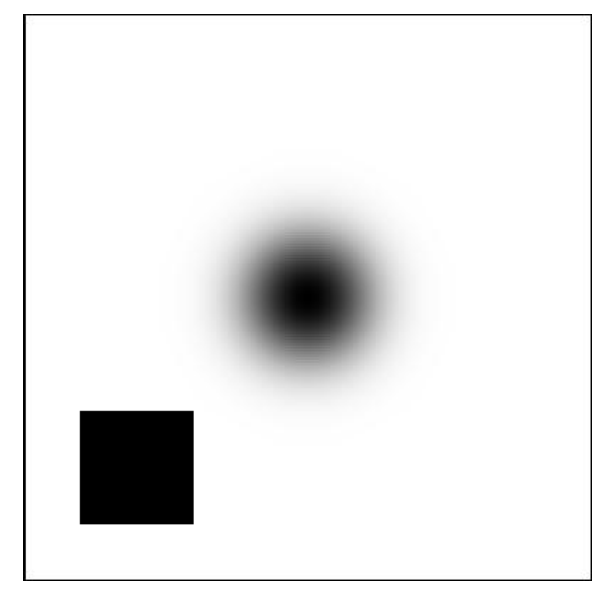

Re of experiments is low

But experiments do not show Re dependence

Energy conservation

Small departures from energy conservation in stable waves

$\underline{\text { Sensitivity to initial conditions }}$

But there is no change when $\lambda_{\text {random }}$ increased by 4

Different molecular Pr

Does small-scale dynamics adjust to forcing from larger scales? 
Instantaneous mixing efficiency

$$
\theta=0^{\circ}
$$

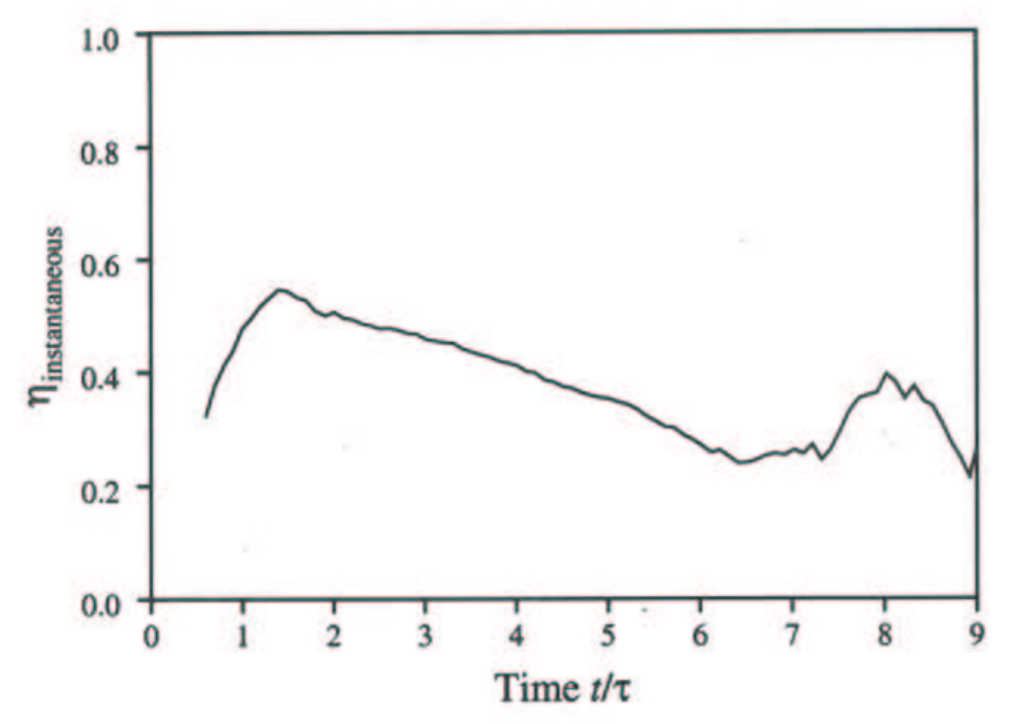

peak values

instantaneous 0.5 around

$$
t / \tau=1
$$

for $\theta=0^{\circ}, 5^{\circ}$

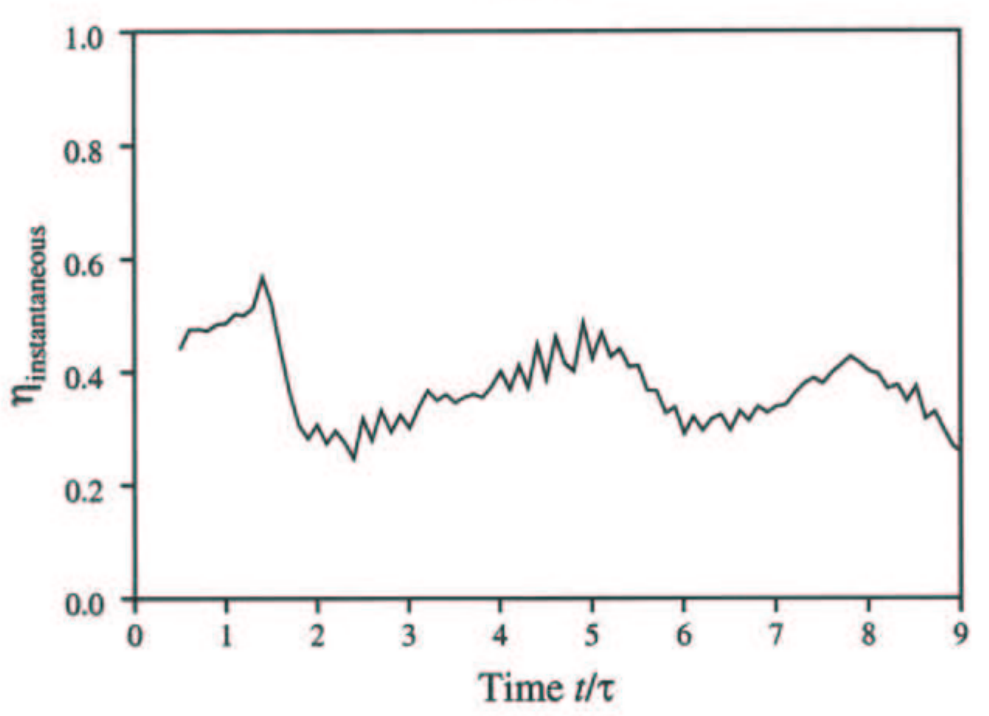

typical values $0.2-0.4$

$$
\theta=10^{\circ}
$$

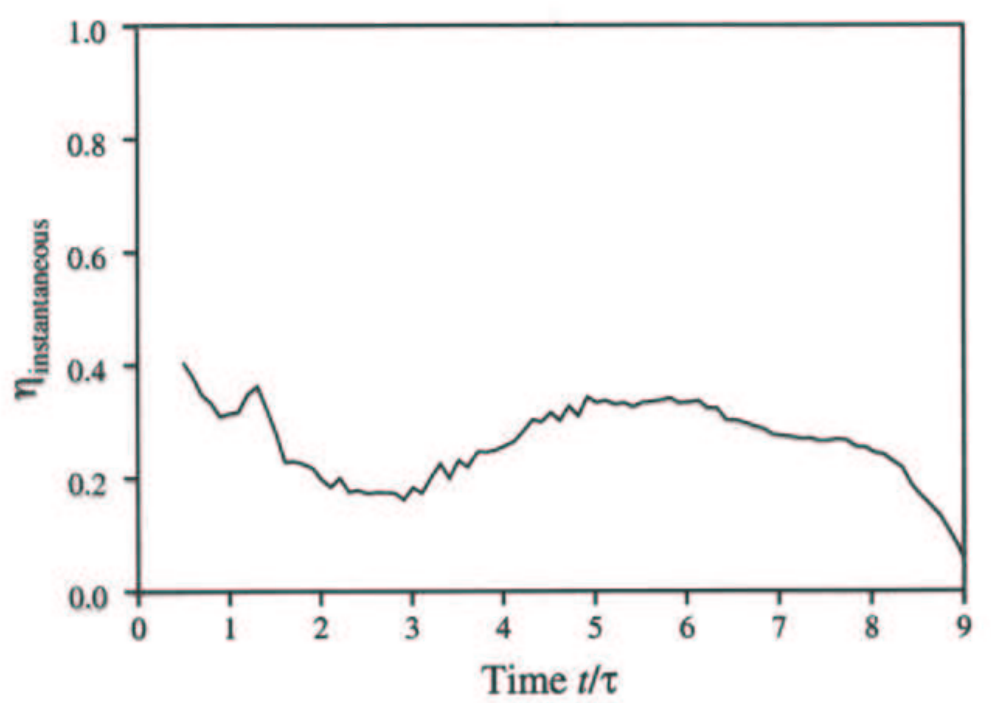

Dominant contribution to cumulative when Eavail decreases most rapidly - during overturning

DAlI I

Kayleıg- I taylor instability 


\section{Cumulative mixing efficiency}

theoretical maximum
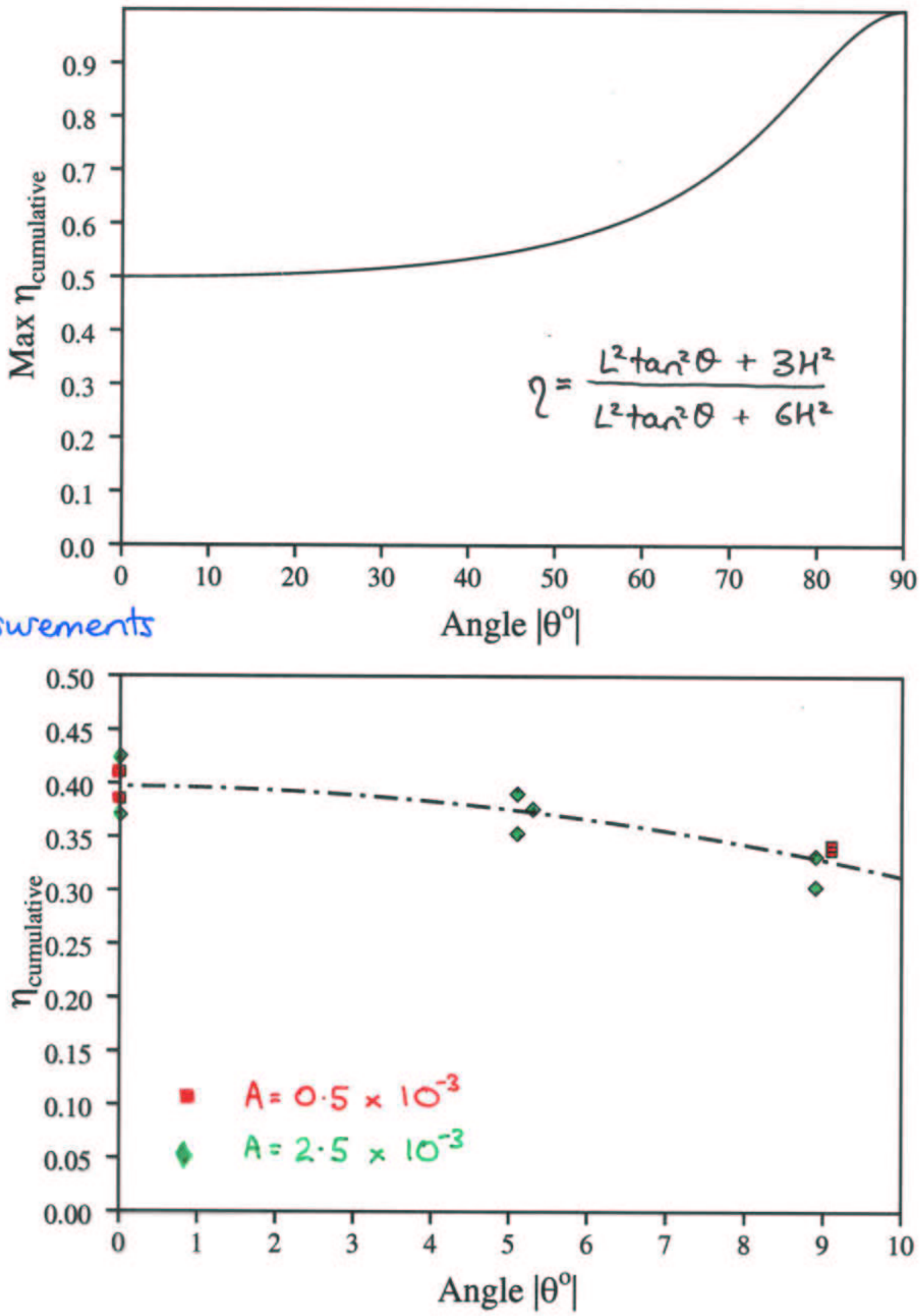


\section{Conclusions and further work}

- Laboratory experiments

$* \theta=0^{\circ}-\eta_{\text {cumulative }} \approx 0.4$

* As $\theta \uparrow, \eta_{\text {cumulative }} \downarrow$

$*$ For $\theta \leq 5^{\circ}, \eta_{\text {instantaneous }} \approx 0.5$

- Numerical simulations

* Models experiments at suitable parameters

* Good agreement in large-scale overturning

- Further work

* Investigate sensitivity of mixing to various factors

* Investigate instability at higher angles - up to limiting case:
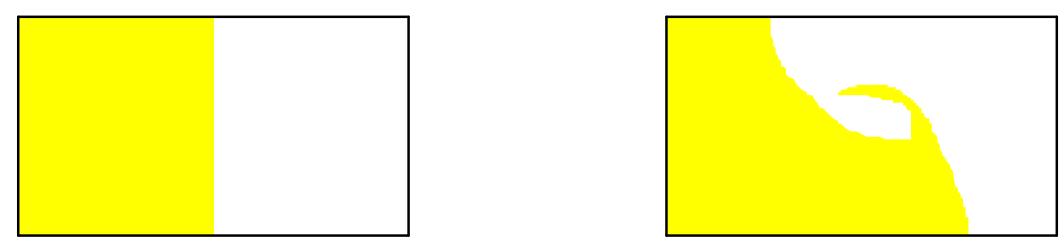

* Extend study of mixing efficiency to more complex stratifications

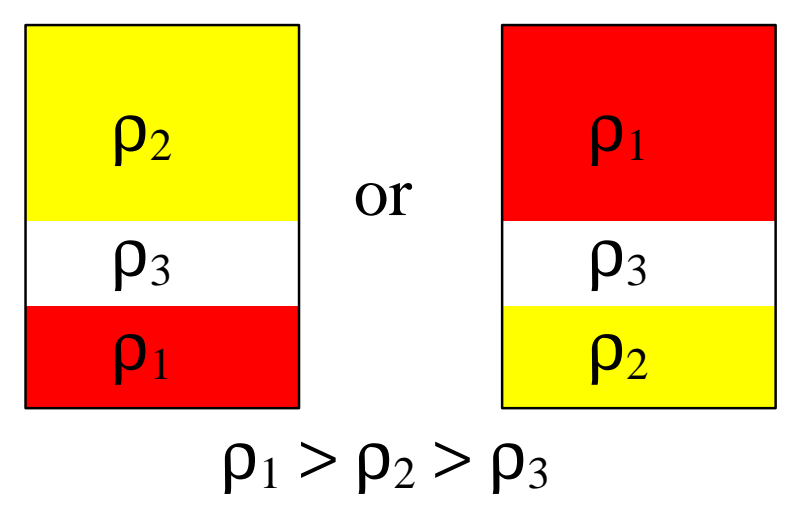

Dalziel \& Jacobs (2000) 
The 8th International Workshop on the Physics of Compressible Turbulent Mixing Dec. 9-14, 2001, Pasadena, CA

\title{
Production of diverging and converging spherical shock waves and eccentric interaction of converging shock waves with cylindrical interfaces
}

\author{
S.H.R. Hosseini and K. Takayama
}

Shock Wave Research Center, I.F.S., Tohoku University, Sendai, Japan 


\section{Introduction}

- Upon focusing of spherical or cylindrical shock waves, high pressures and temperatures created at the center of convergence and have been used for various scientific and industrial applications.

- It is not necessarily easy in laboratories to produce uniformly converging shock waves.

- Applications of R-M instability appearing in converging spherical and cylindrical geometries, such as inertial confinement fusion, supersonic combustion, and astrophysics, made it of considerable interest.

- In the present research, results of recent experiment of R-M instability will be reported. 


\section{Aspheric spherical test section}

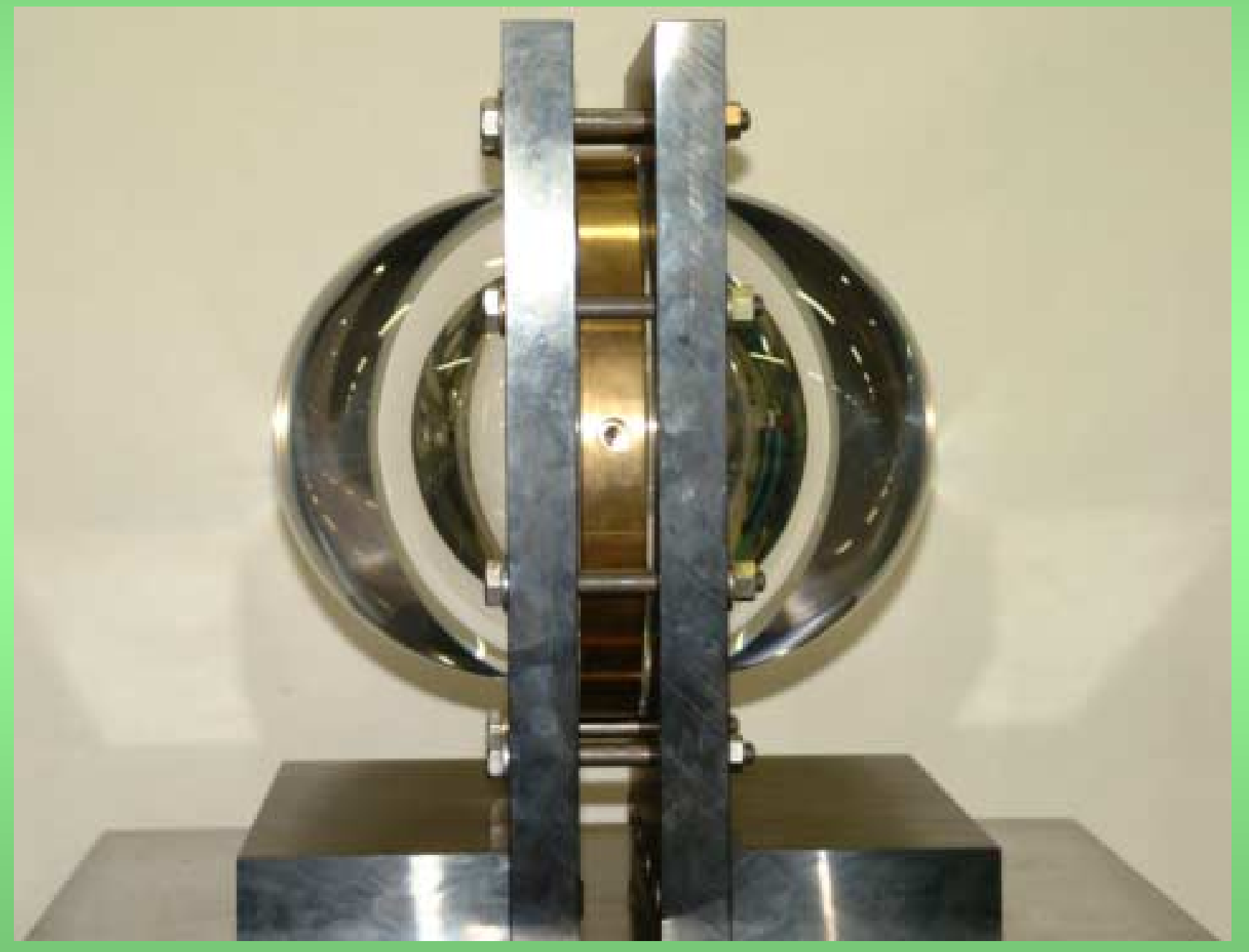

Shock Wave Research Center, I. F. S., Tohoku University 


\section{Front view of the aspheric spherical test section}

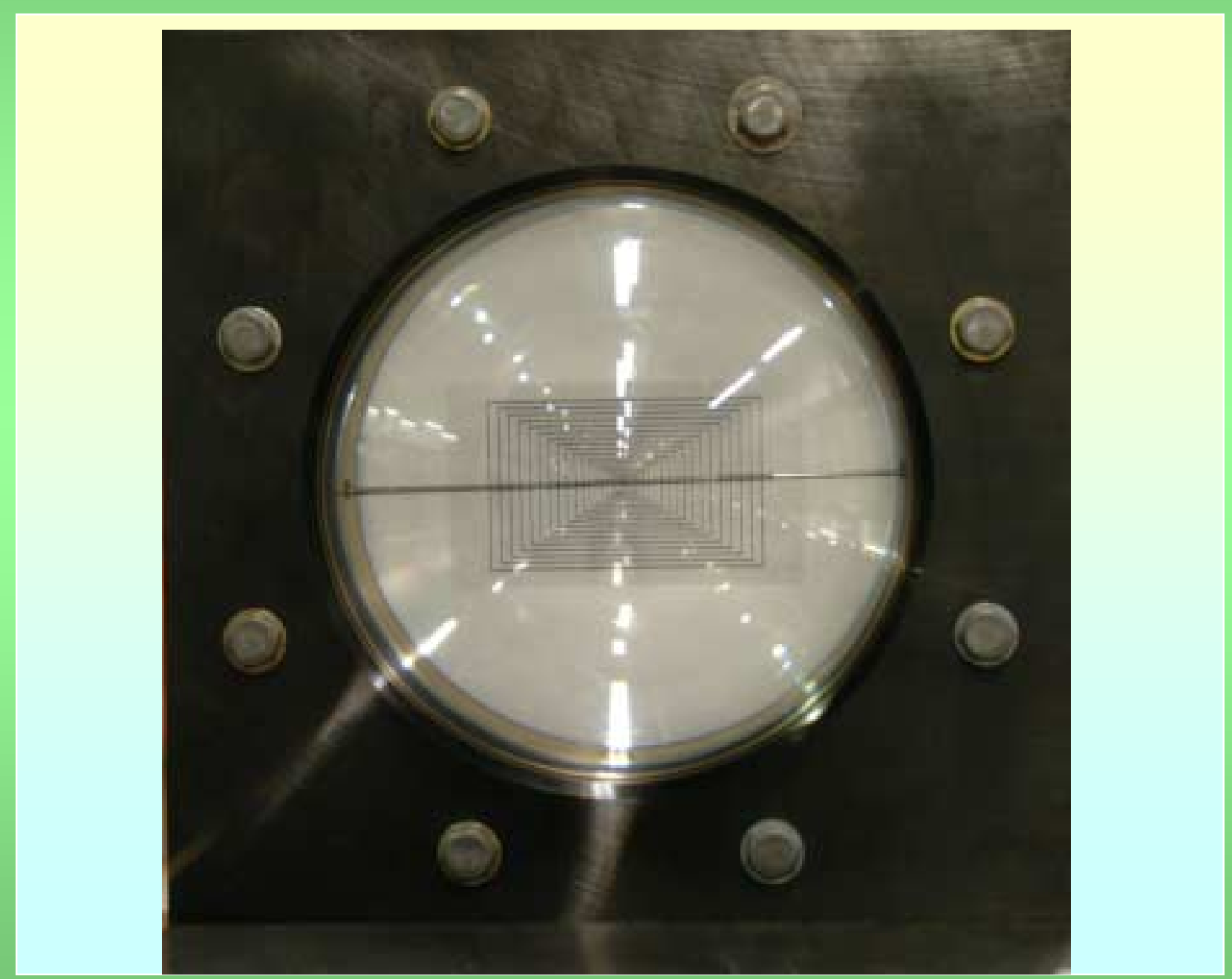

Shock Wave Research Center, I. F. S., Tohoku University 


\section{Laser light rays in the aspheric test section}

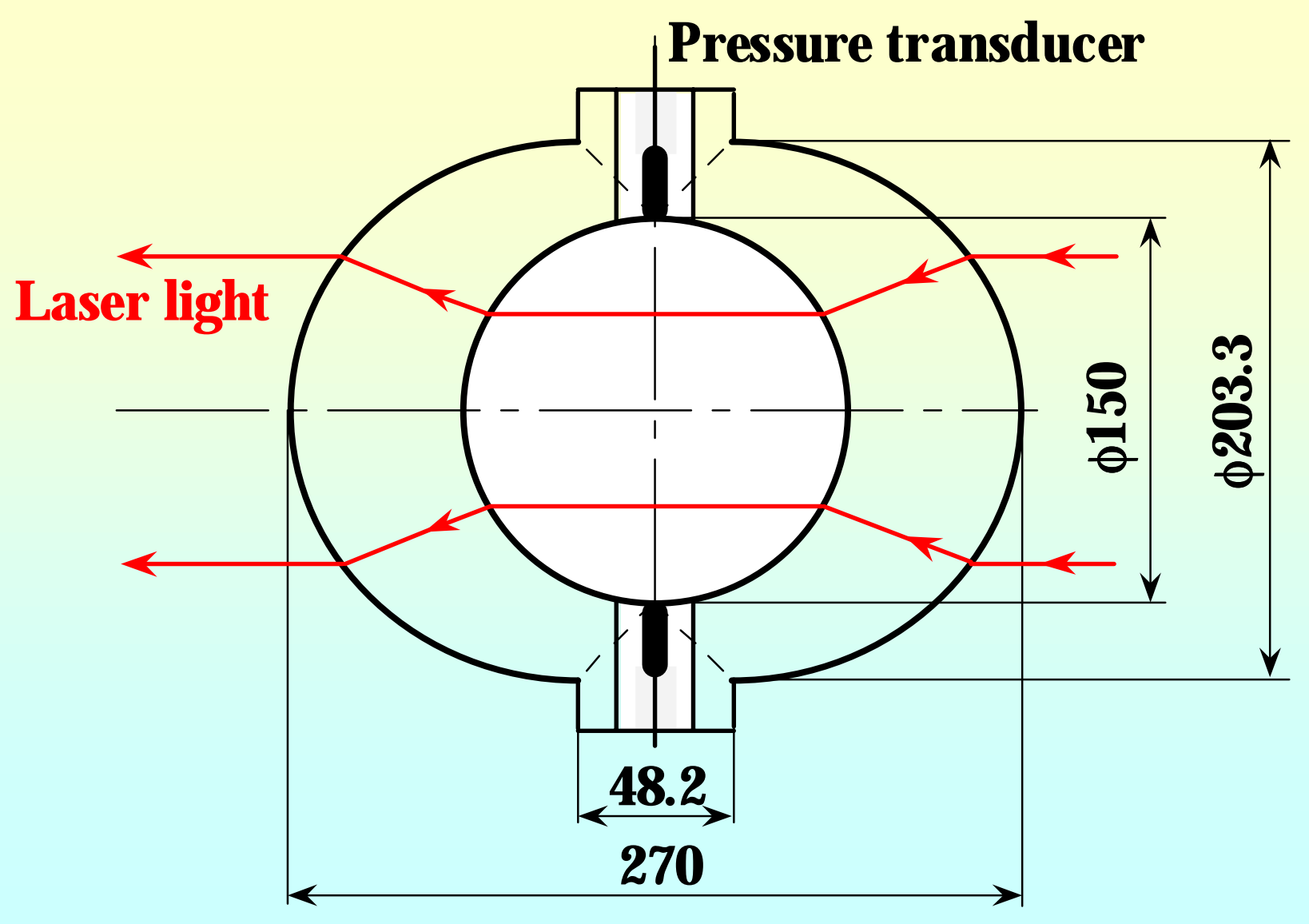

Shock Wave Research Center, I. F. S., Tohoku University 


\section{Double exposure holographic interferometric optical set-up}

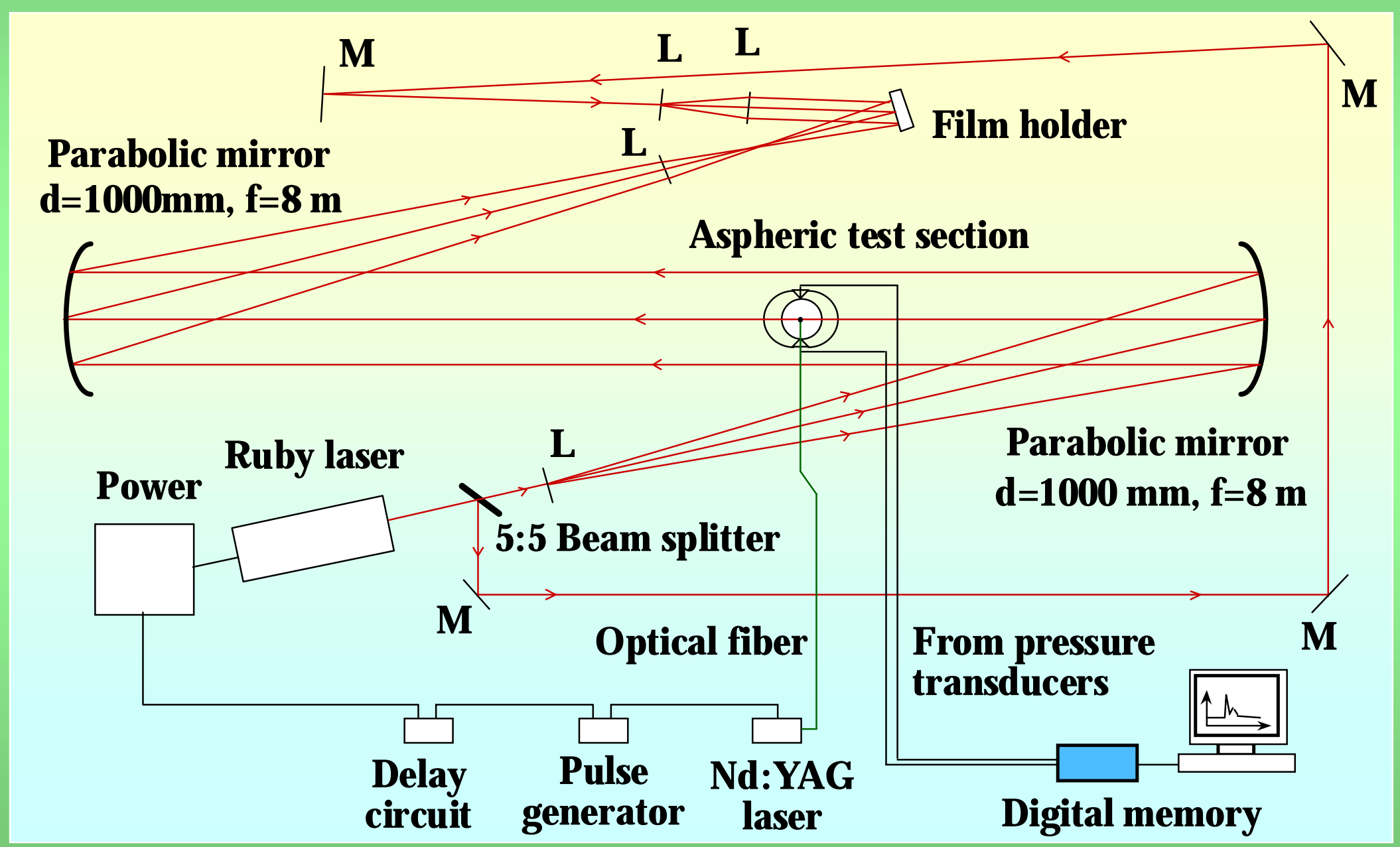

Shock Wave Research Center, I. F. S., Tohoku University 


\section{Structure of vertical diaphragmless shock tube}

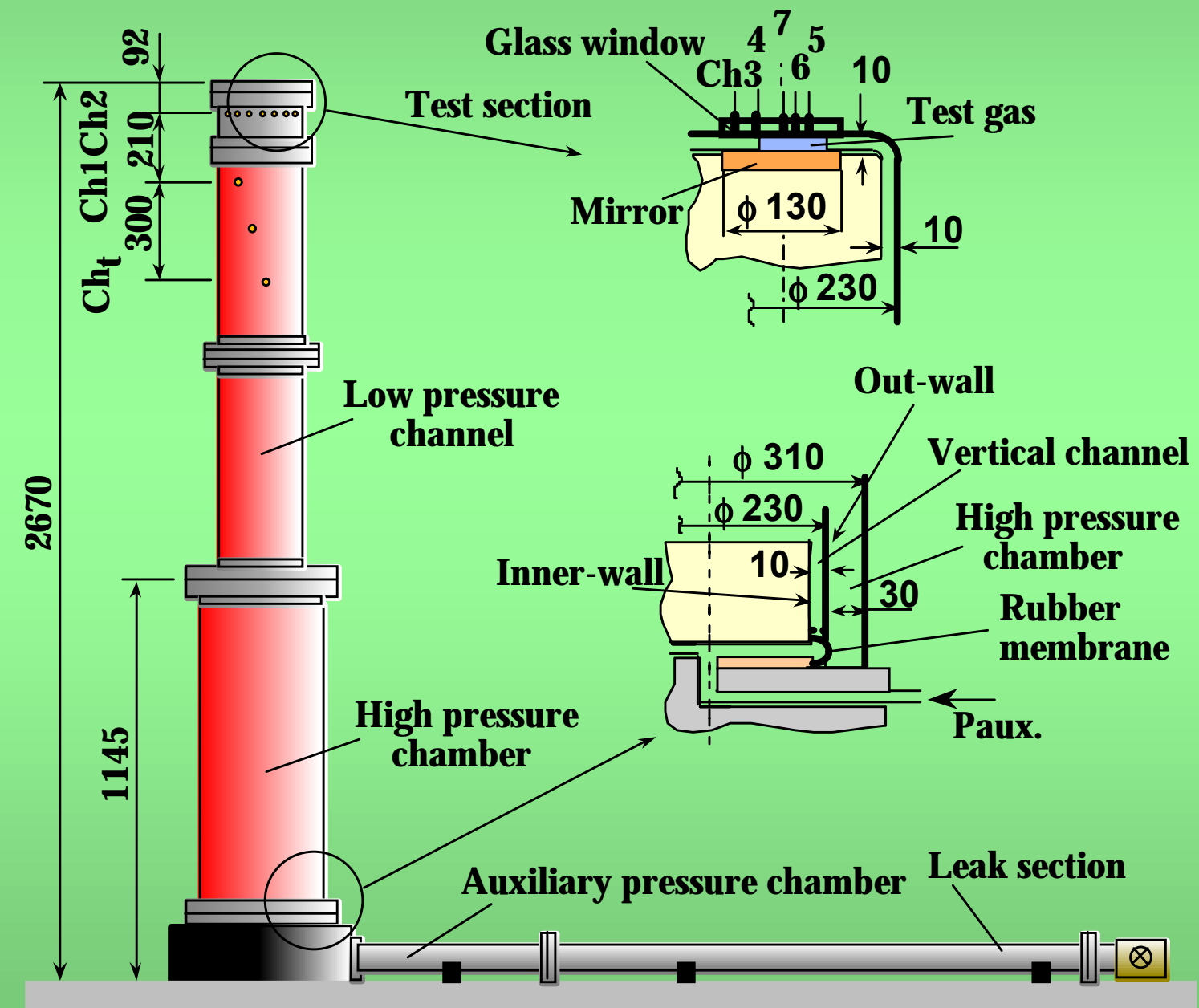

Shock Wave Research Center, I. F. S., Tohoku University 


\section{Double exposure holographic interferometry}

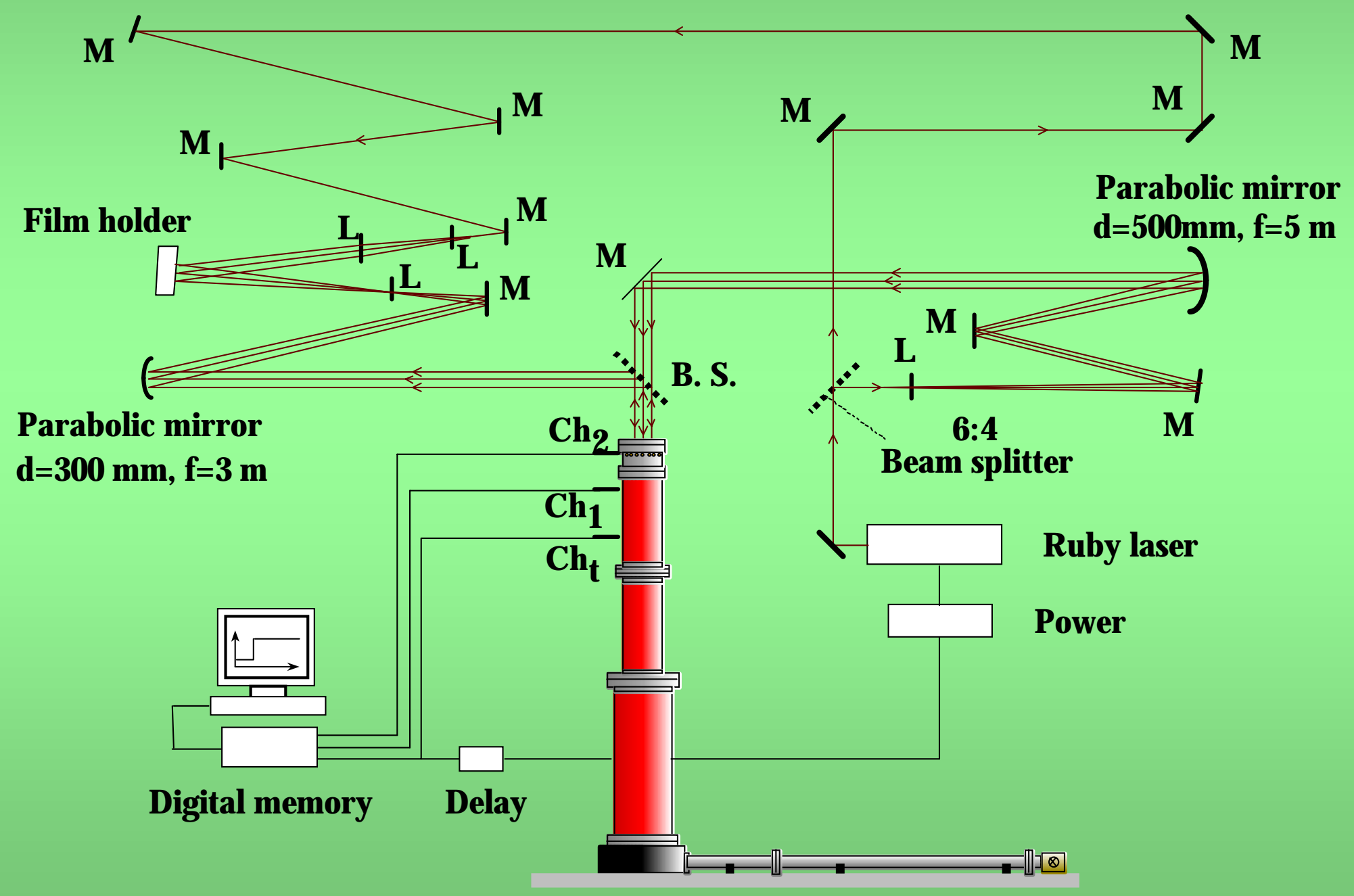

Shock Wave Research Center, I. F. S., Tohoku University 


\section{Test section with cylindrical bubble}

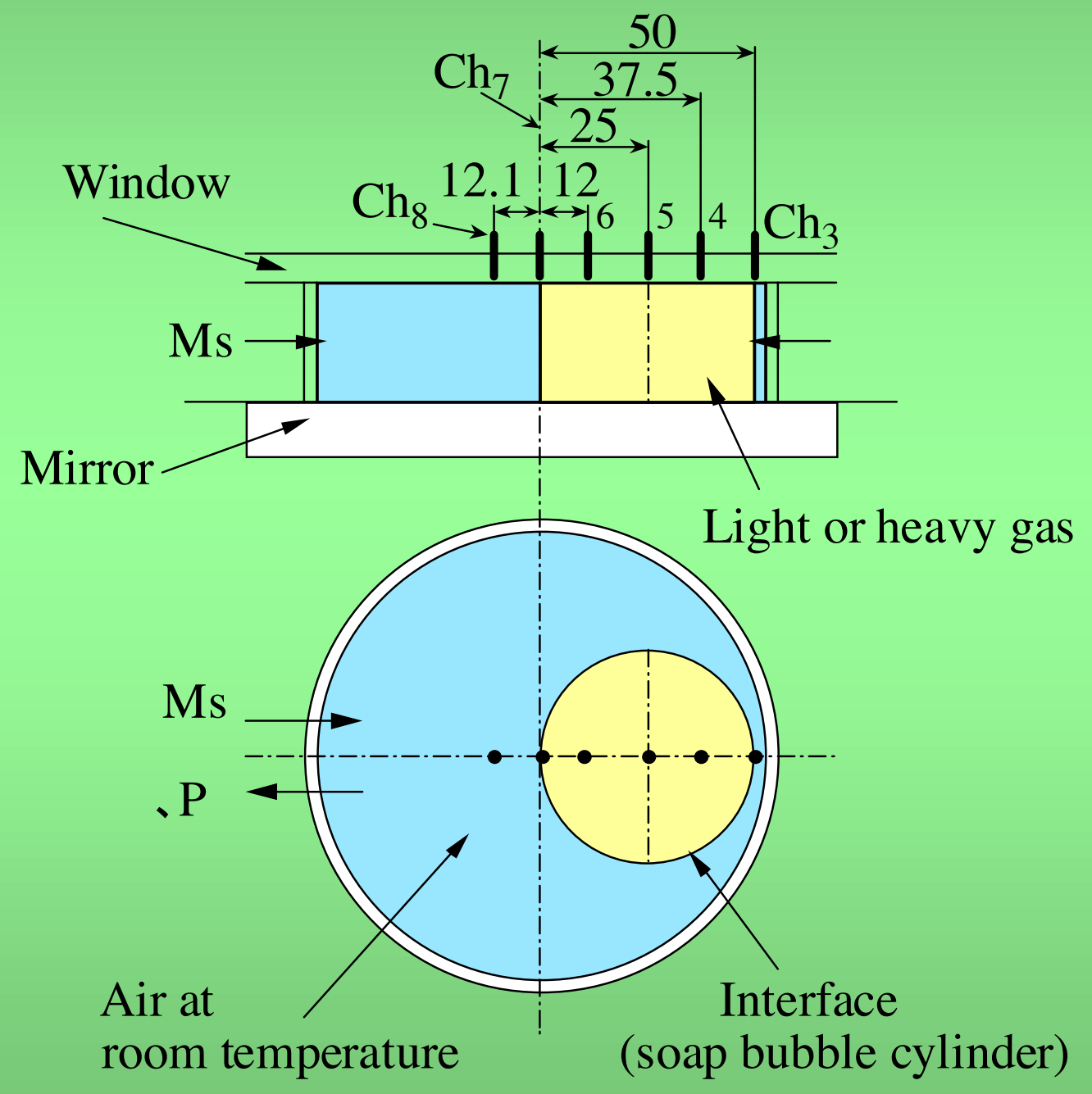

Shock Wave Research Center, I. F. S., Tohoku University 
Pressure histories at the test section for eccentric interaction of cylindrical shock wave with cylindrical $\mathrm{SF}_{6}$ bubble, $\mathrm{Ms}_{\mathrm{i}}=1.18, \mathrm{P}_{0}=101.13 \mathrm{kPa}$

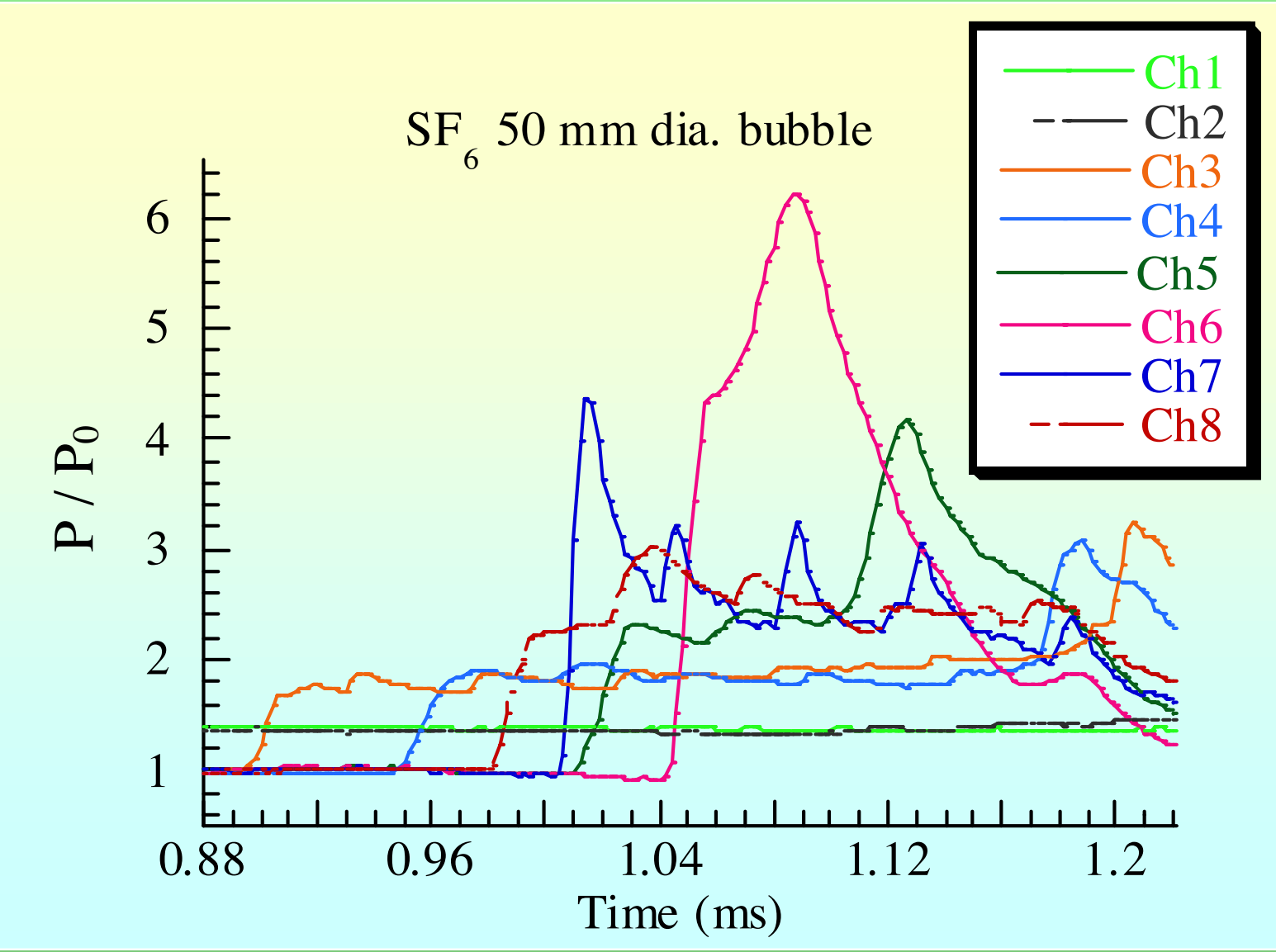

Shock Wave Research Center, I. F. S., Tohoku University 


\section{$\mathrm{Ms}_{\mathrm{i}}=1.18$ in air, $\mathrm{P}_{0}=100.3 \mathrm{kPa}, \mathrm{D}_{\mathrm{SF}_{6}}=50 \mathrm{~mm}$}

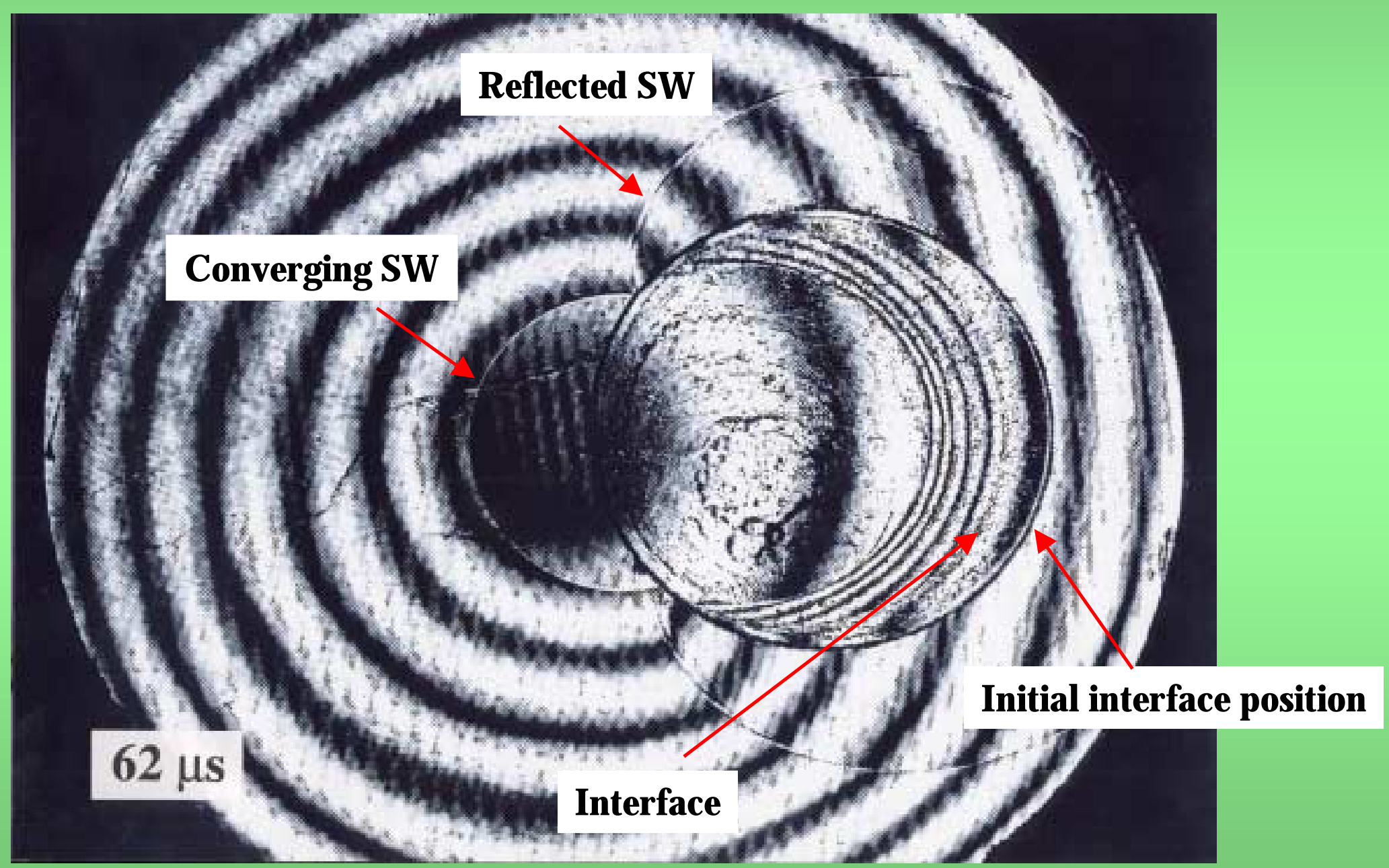

Shock Wave Research Center, I. F. S., Tohoku University 


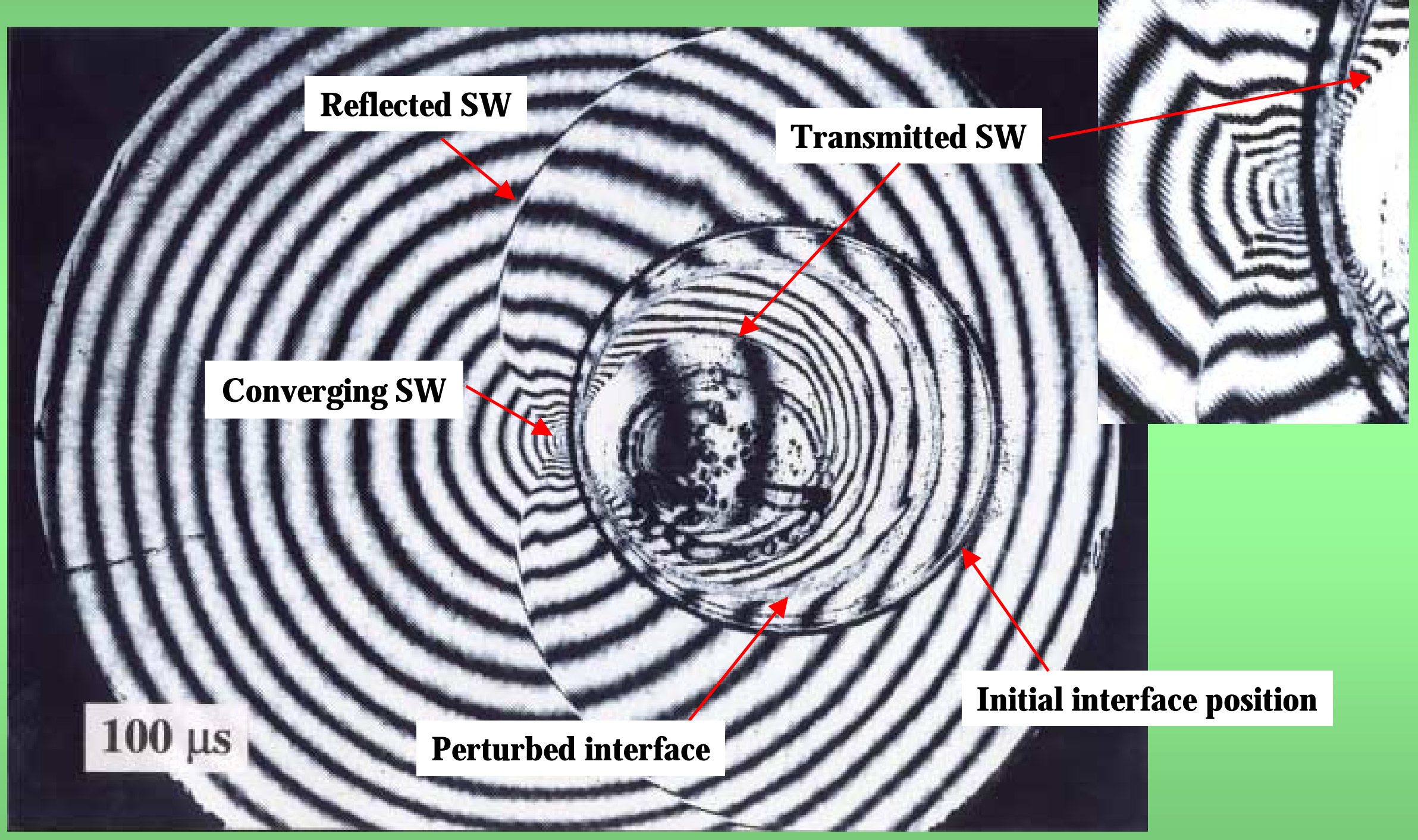

Shock Wave Research Center, I. F. S., Tohoku University 


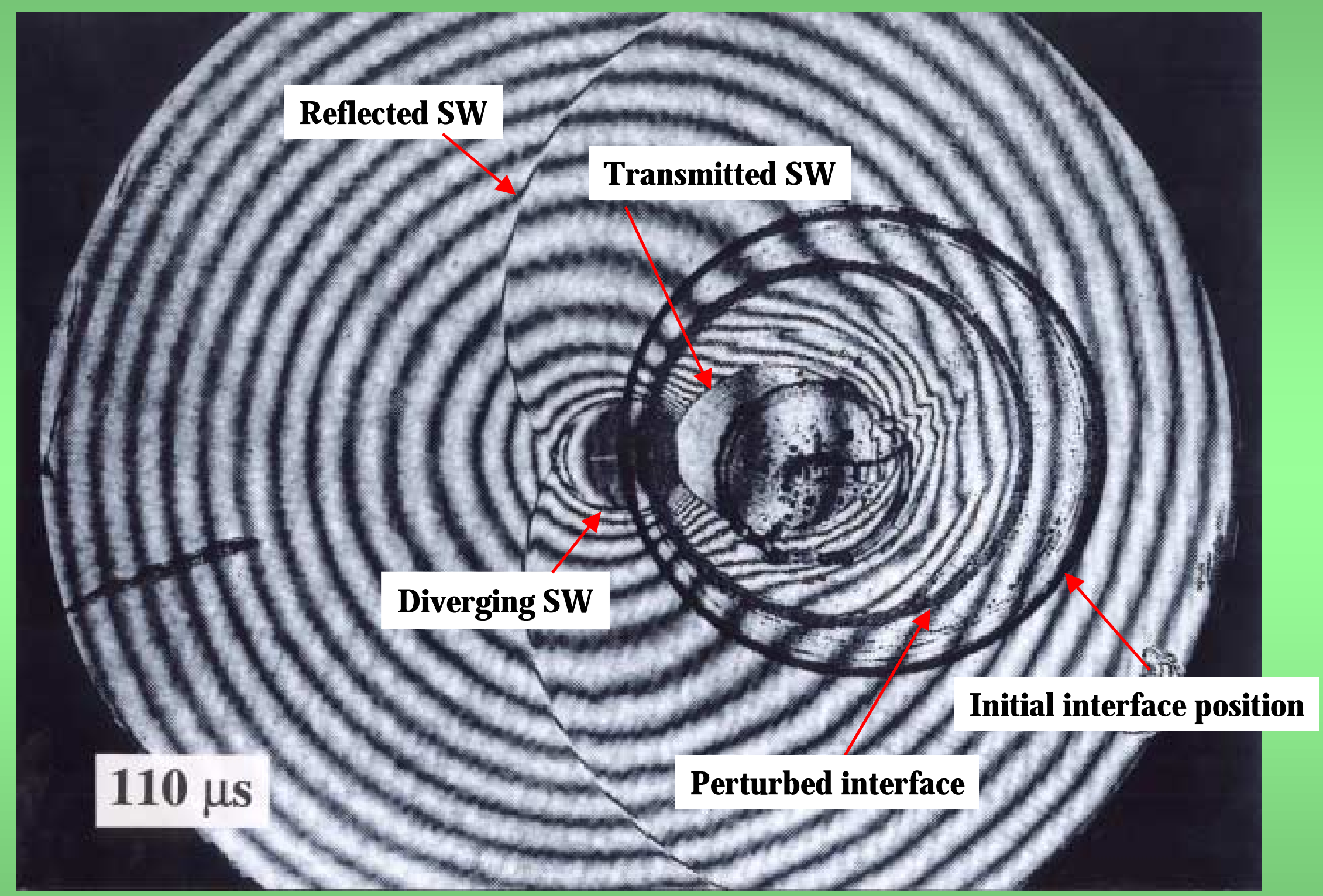

Shock Wave Research Center, I. F. S., Tohoku University 


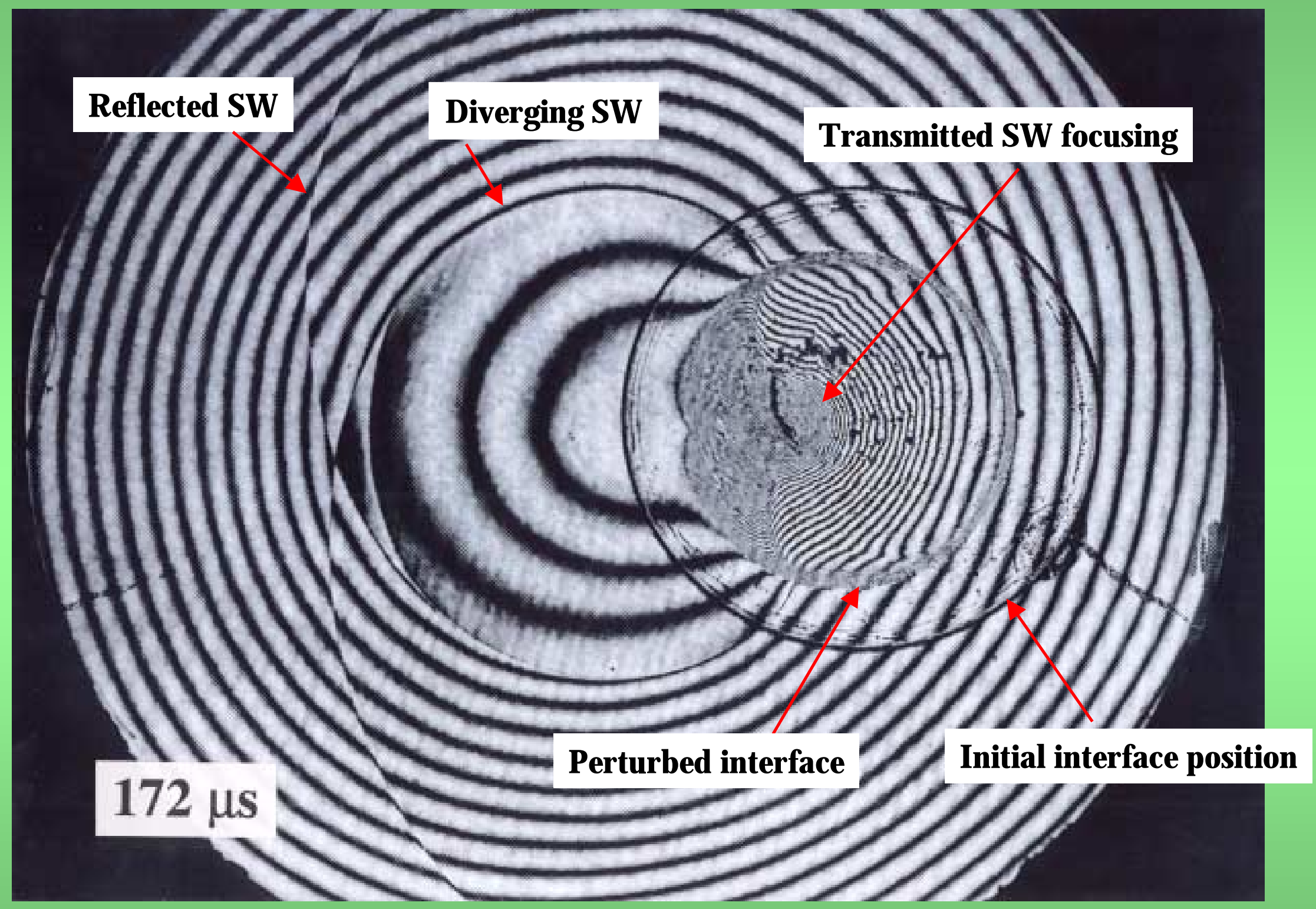

Shock Wave Research Center, I. F. S., Tohoku University 


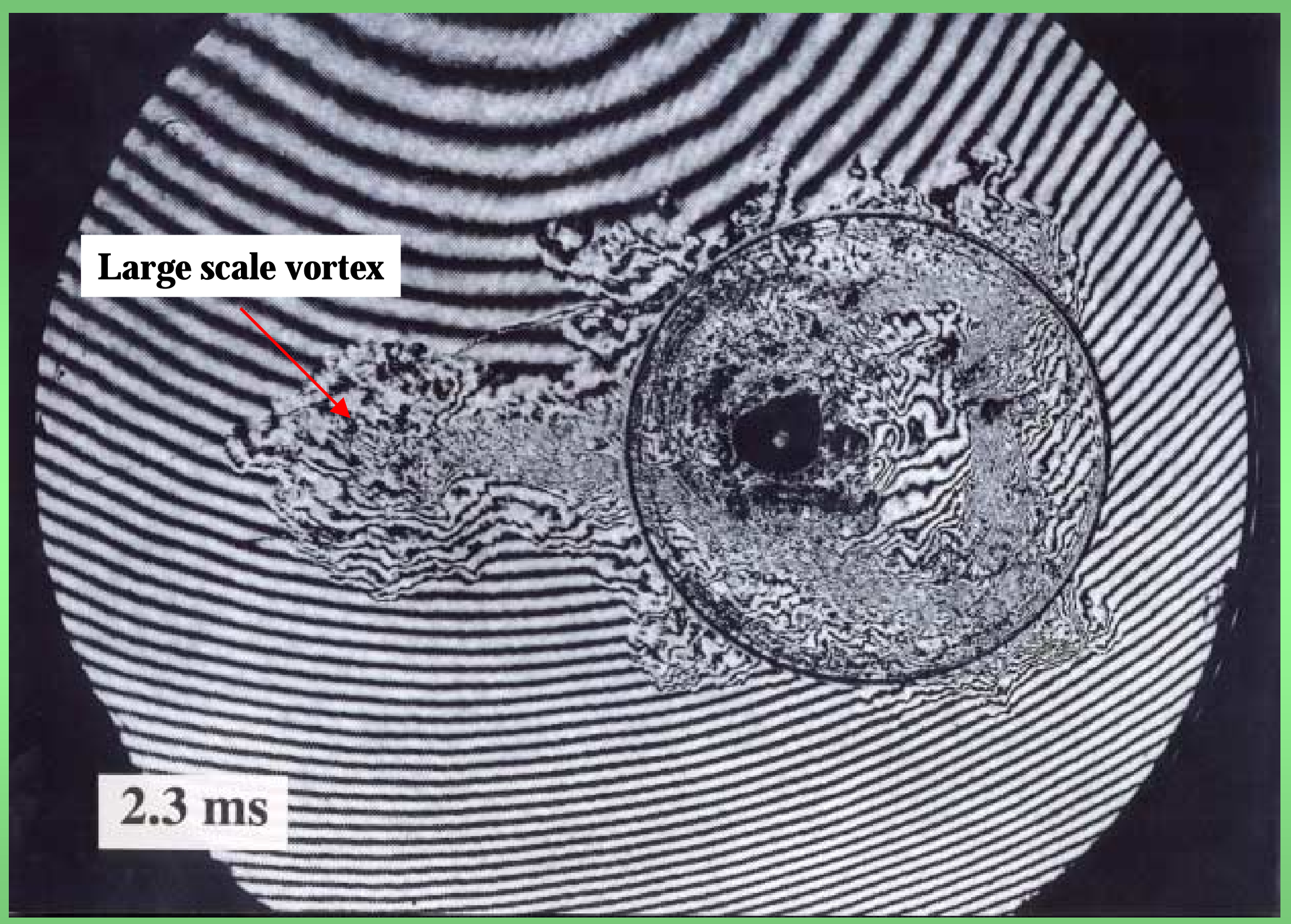

Shock Wave Research Center, I. F. S., Tohoku University 


\section{Time variation of average $\mathrm{SF}_{6}$ jet velocity in air}

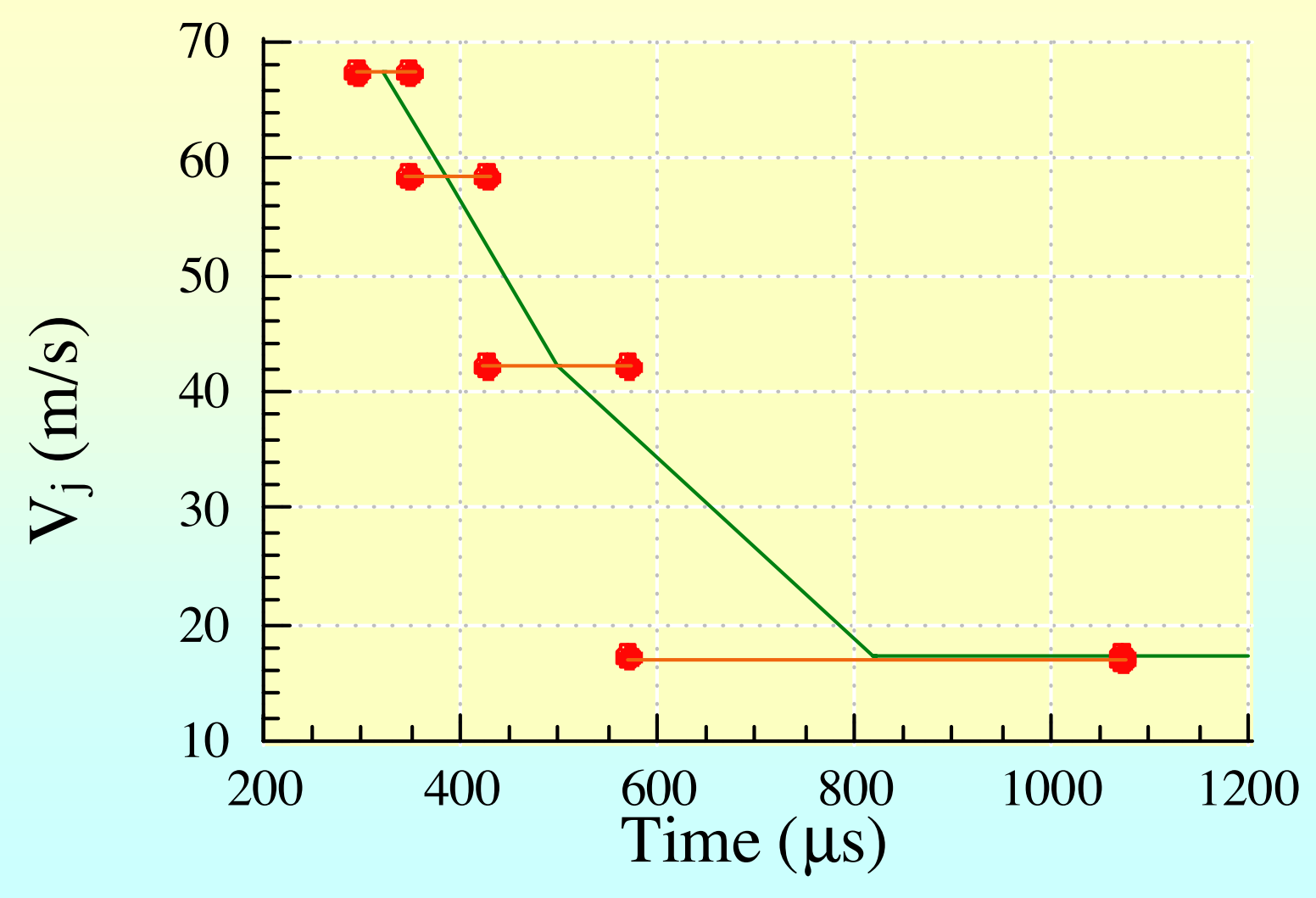

Shock Wave Research Center, I. F. S., Tohoku University 


\section{Summary}

1) Converging spherical shock waves and their interaction with micro-explosive product gases were investigated by using a spherical transparent test section.

2) Using double exposure holographic interferometry, the interactions of converging shock waves with light/heavy cylindrical gaseous interface were quantitatively visualized. A relatively strong secondary shock wave focusing in $\mathbf{S F}_{6}$ heavy gas bubble resulted a strong $\mathrm{SF}_{6}$ jet in air, which made the final distortion of the bubbles to be different from planar shock wave loading. 
LINEAR AND NON-LINEAR STAGES OF THE RICHTMYER-MESHKOV INSTABILITY DEVELOPMENT IN A LARGE CROSS SECTION SHOCK-TUBE

$\log =0$

LHOUAS*, G JOURDAN* L SCHWAEDERLE* and E E MESHKOV*

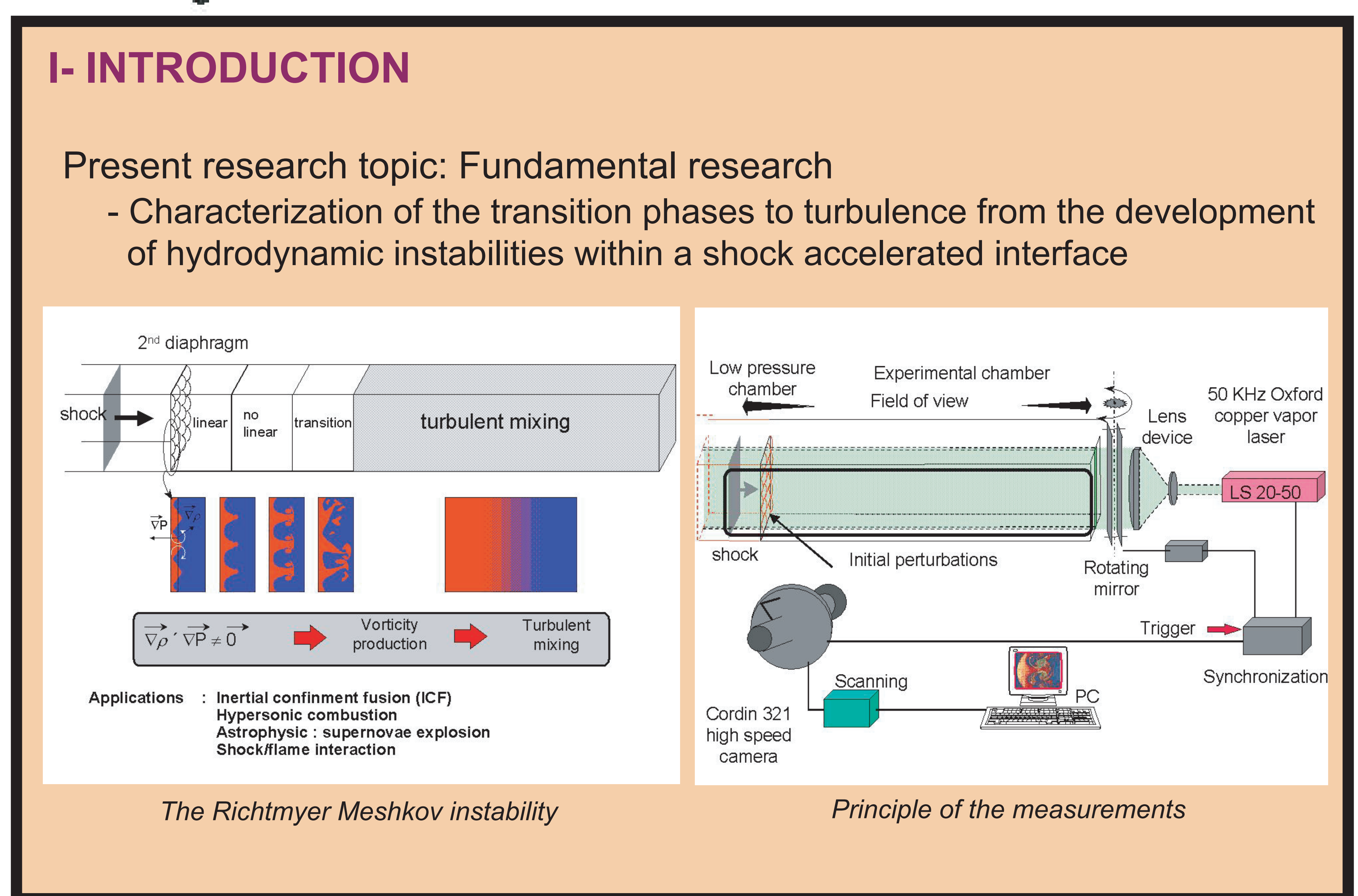

II- THE NEW LARGE CROSS SECTION SHOCK TUBE OF IUSTI

High pressure chamber

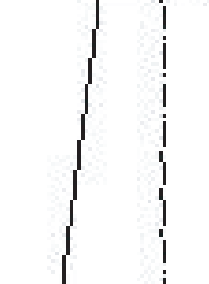

Characteristics and usual performances

- Square cross section: $20 \times 20 \mathrm{~cm} 2$

- Length: 7.04 meters

1.1 to 3

- Initial pressure: from $0.5 \mathrm{~atm}$ to $1 \mathrm{~atm}$

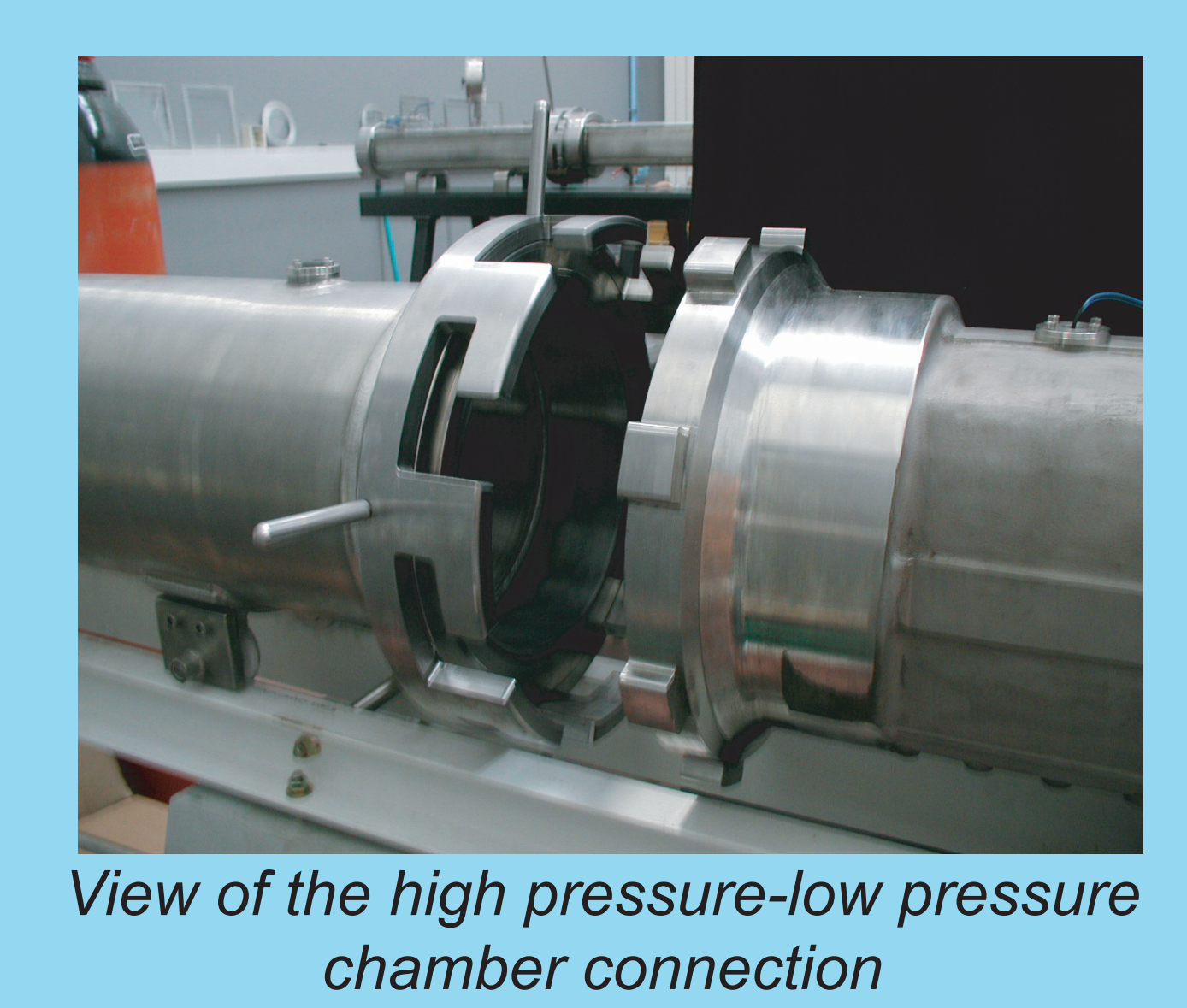

Control of the initial conditions

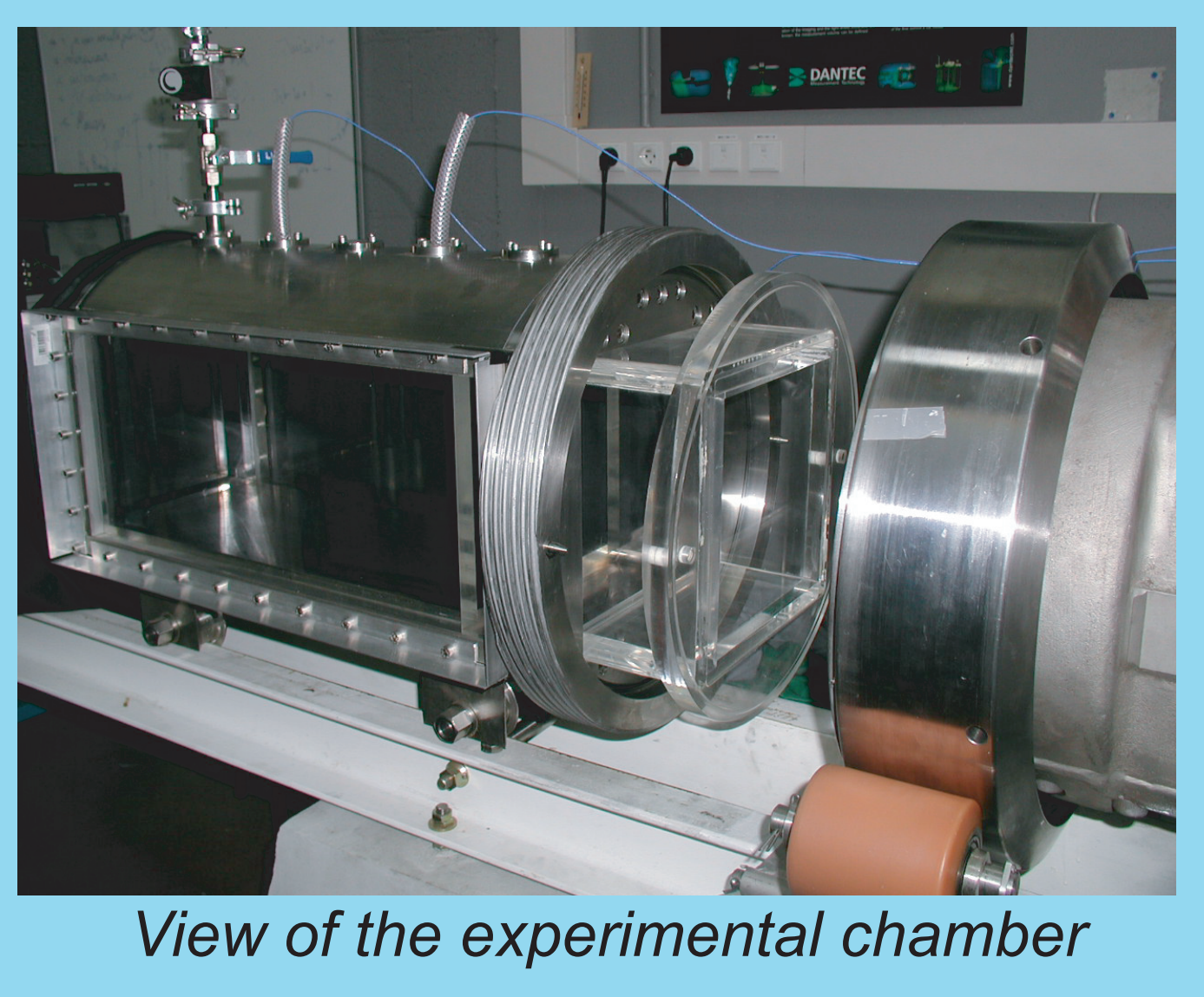

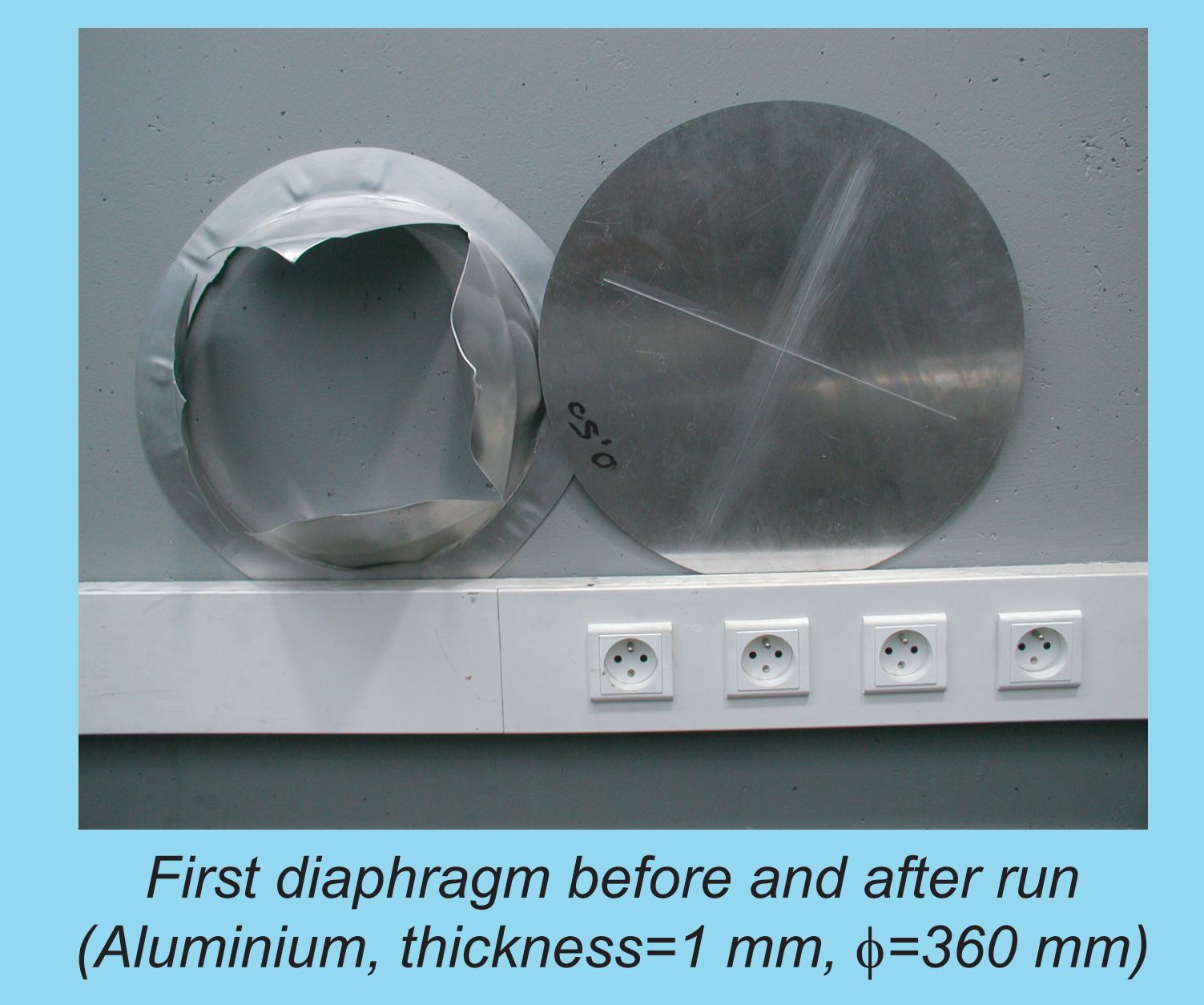

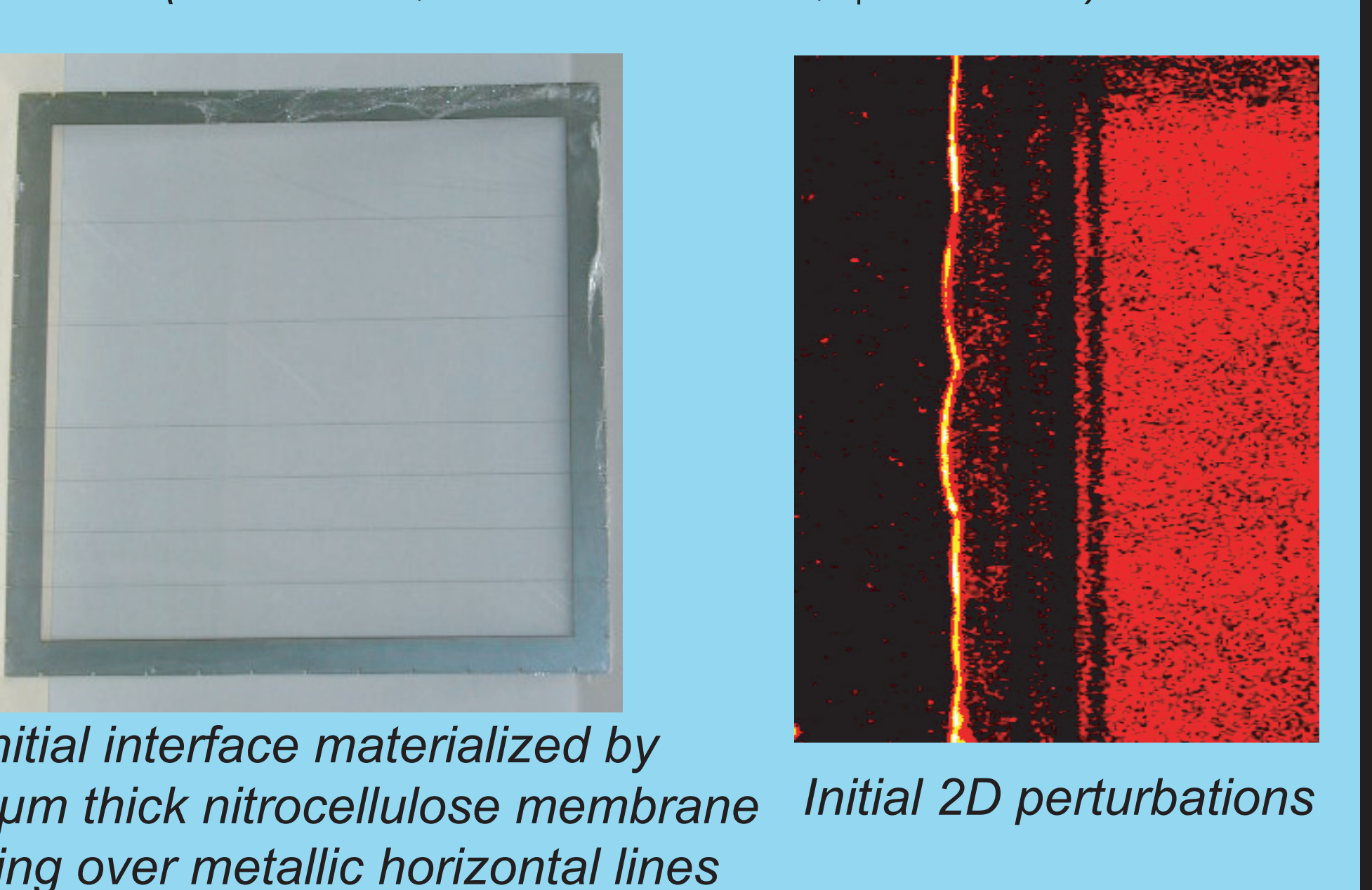

Université de Provence, Technopôle de Château-Gombert, 5 rue Enrico Fermi, 13453 Marseille Cedex 13, FRANCE

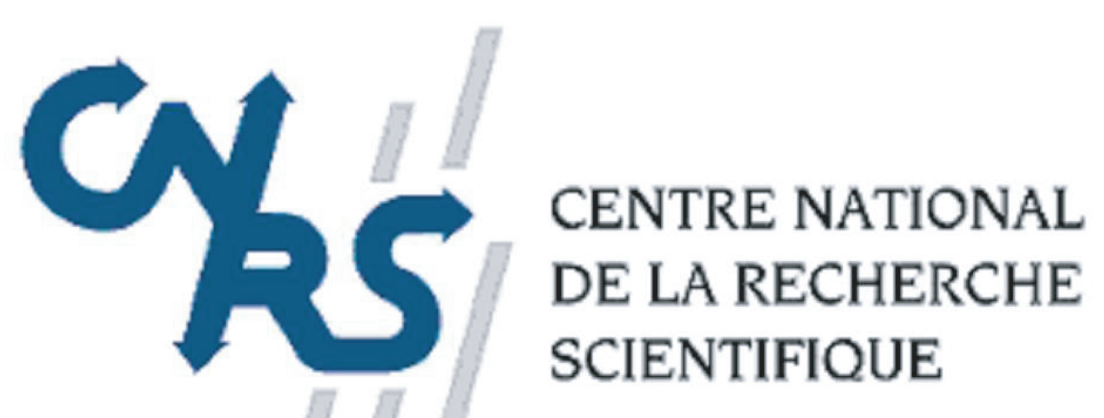

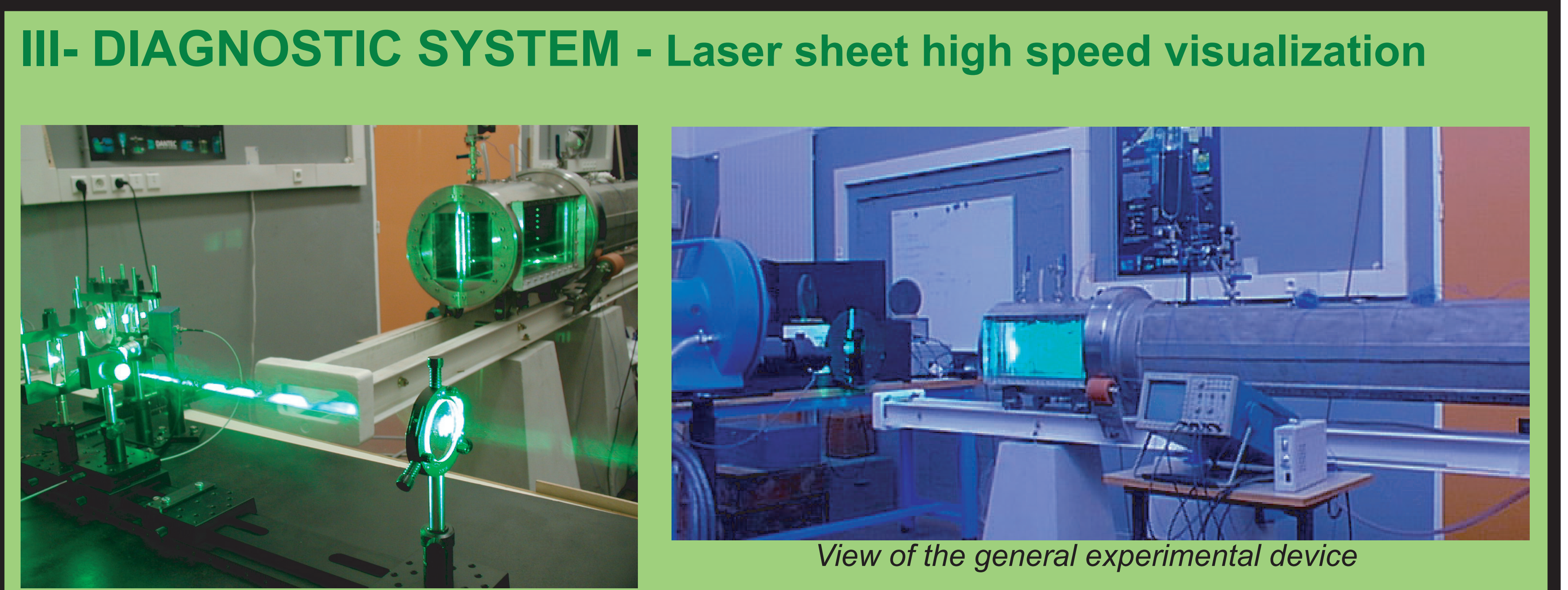

- Source: from 2 to $50 \mathrm{kHz}$ pulsed copper vapour Oxford laser

- Camera: 321 Cordin streak camera

- External shutter: Electro-optical shutter Response time: $\sim 1 \mu \mathrm{s}$

- Acquisition device: 720 Tektronix scope 4 channels

- Trigger: signal from PCB pressure gauge
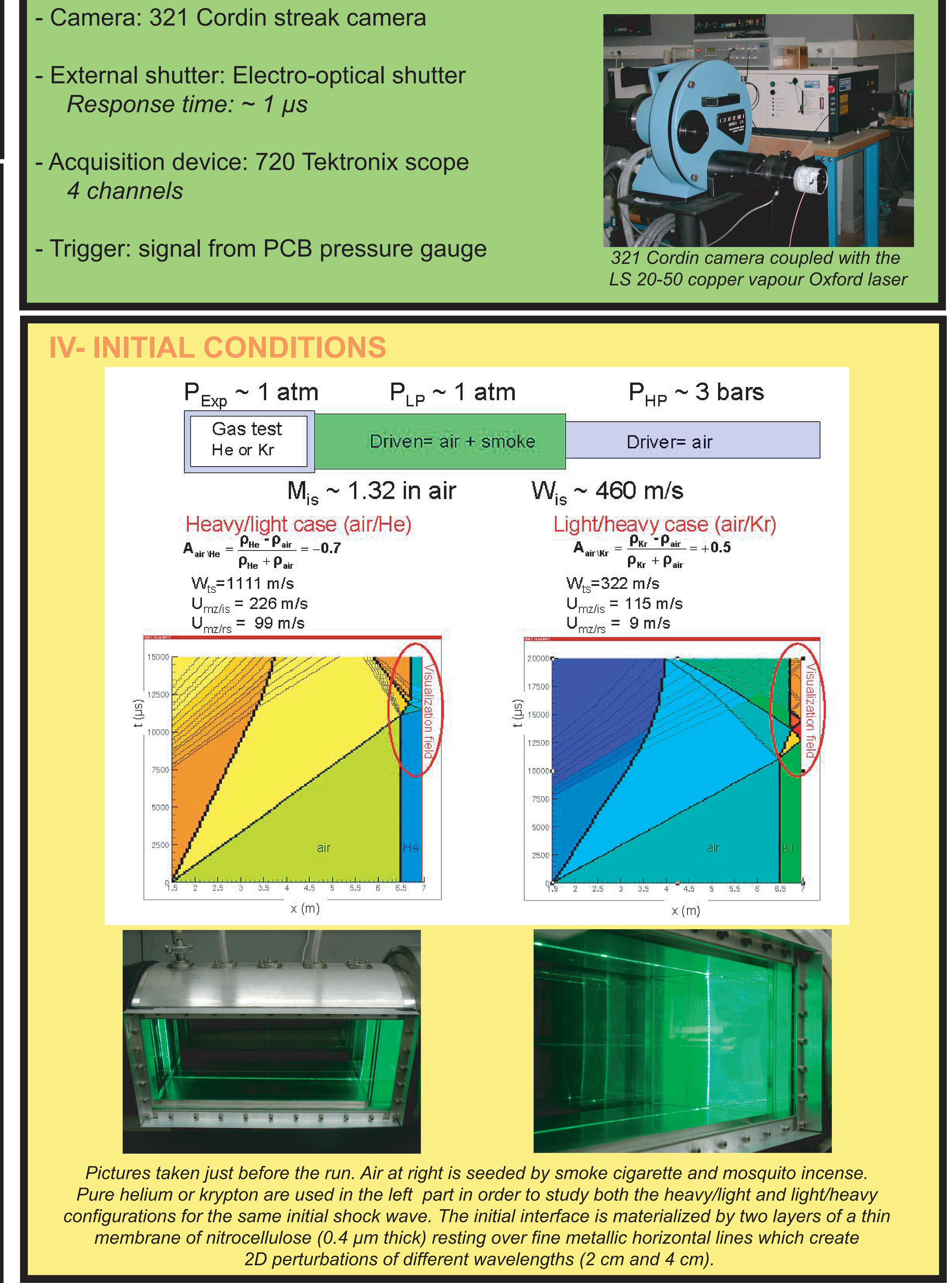
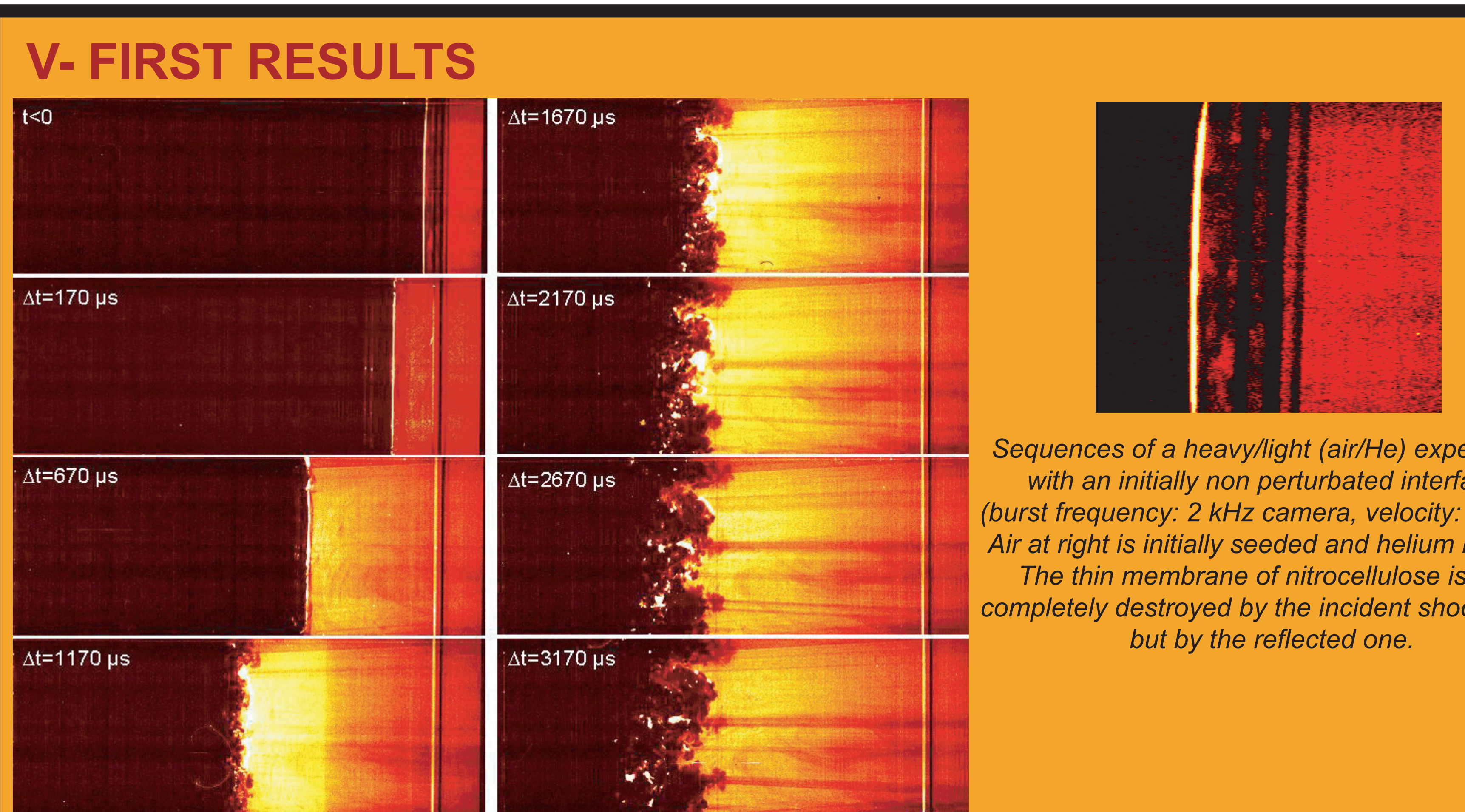

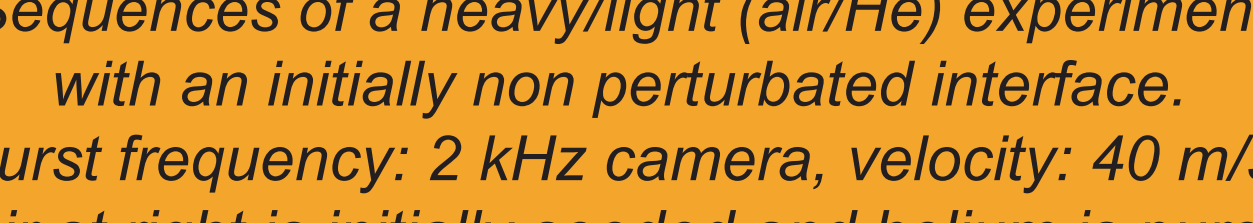
the thin membrane of nitrocelllulose is is not
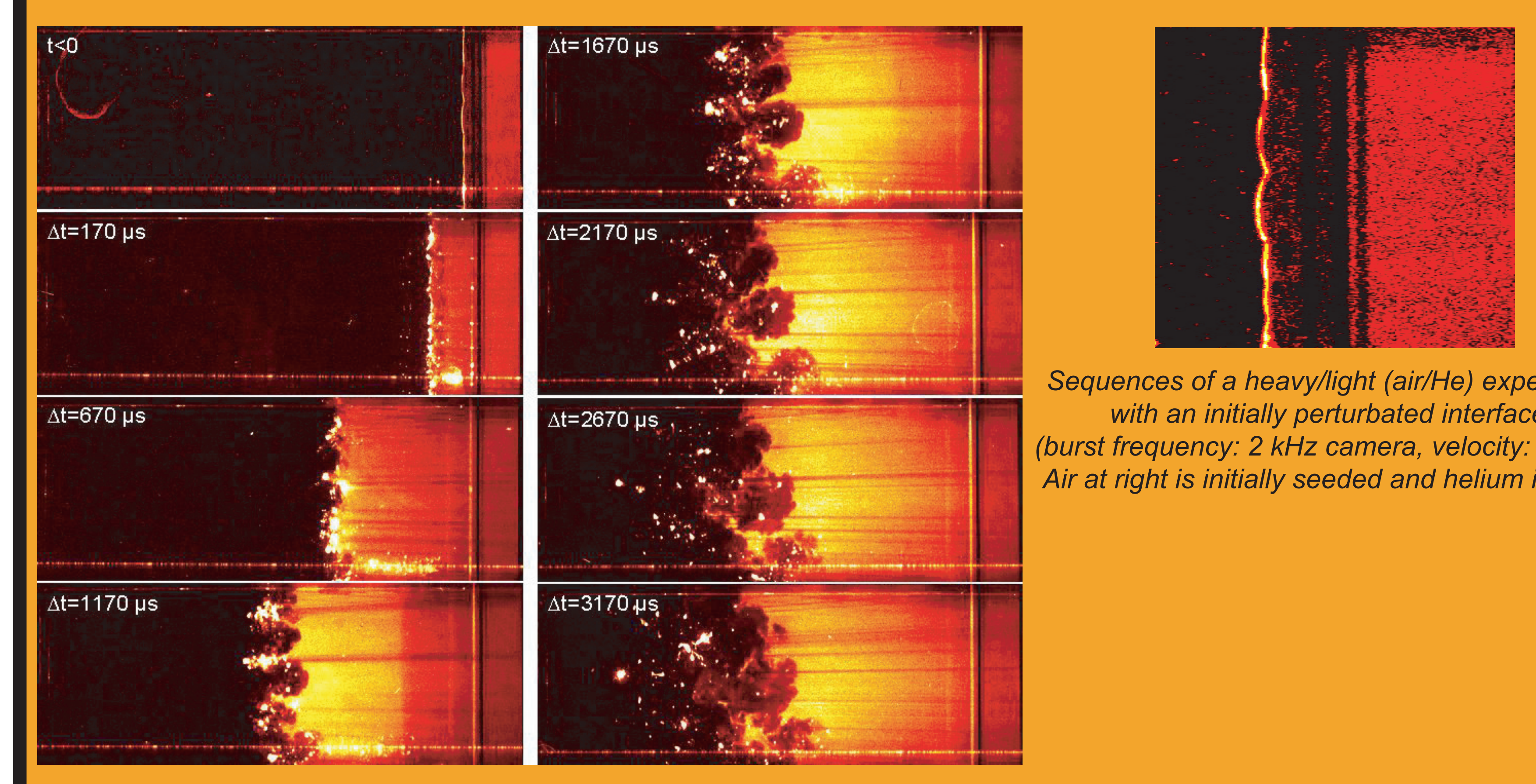

With an in intially perturustated int interface

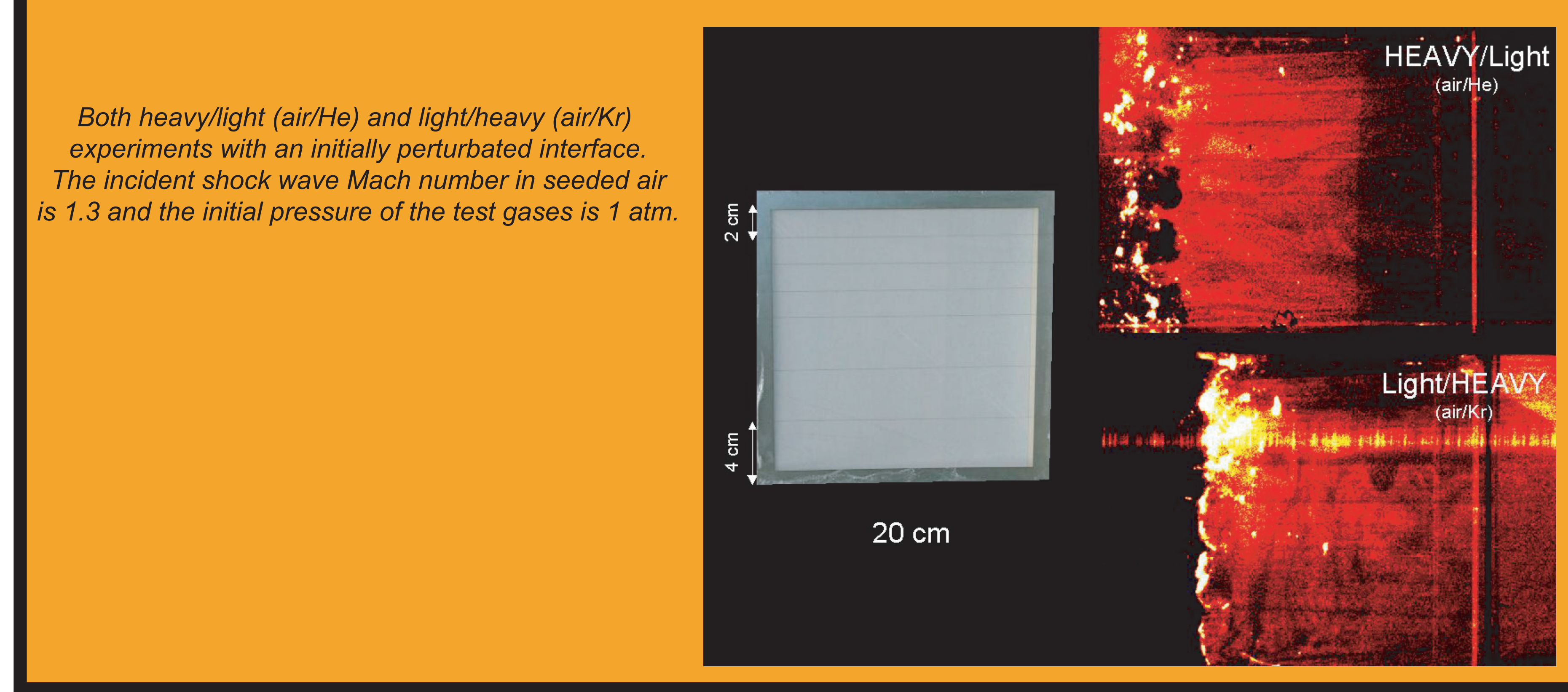

VI- CONCLUSIONS AND PERSPECTIVES

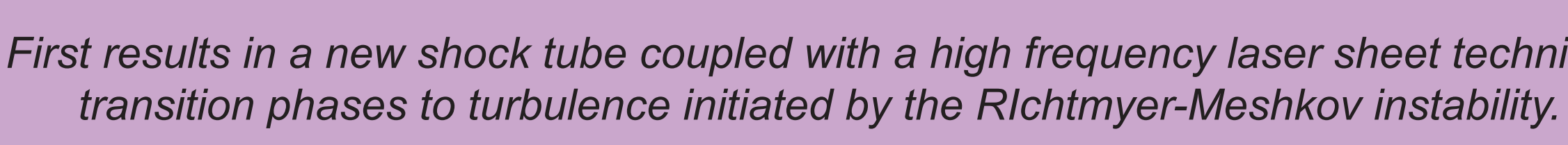

-The $20 \mathrm{~cm}$ marge suare sross shock tube prevents
of the intitial condifitions is successully avaliable.

- The growing up of the initial perturbations at the interface and the Rlchtmyer

- The 


\title{
Experimental Investigation of Richtmyer- Meshkov Instability after a Second Interaction with a Reflected Shock Wave
}

\author{
J.W. Jacobs and V.V. Krivets
}

Department of Aerospace and Mechanical Engineering University of Arizona

Supported by:

Lawrence Livermore National Laboratory 


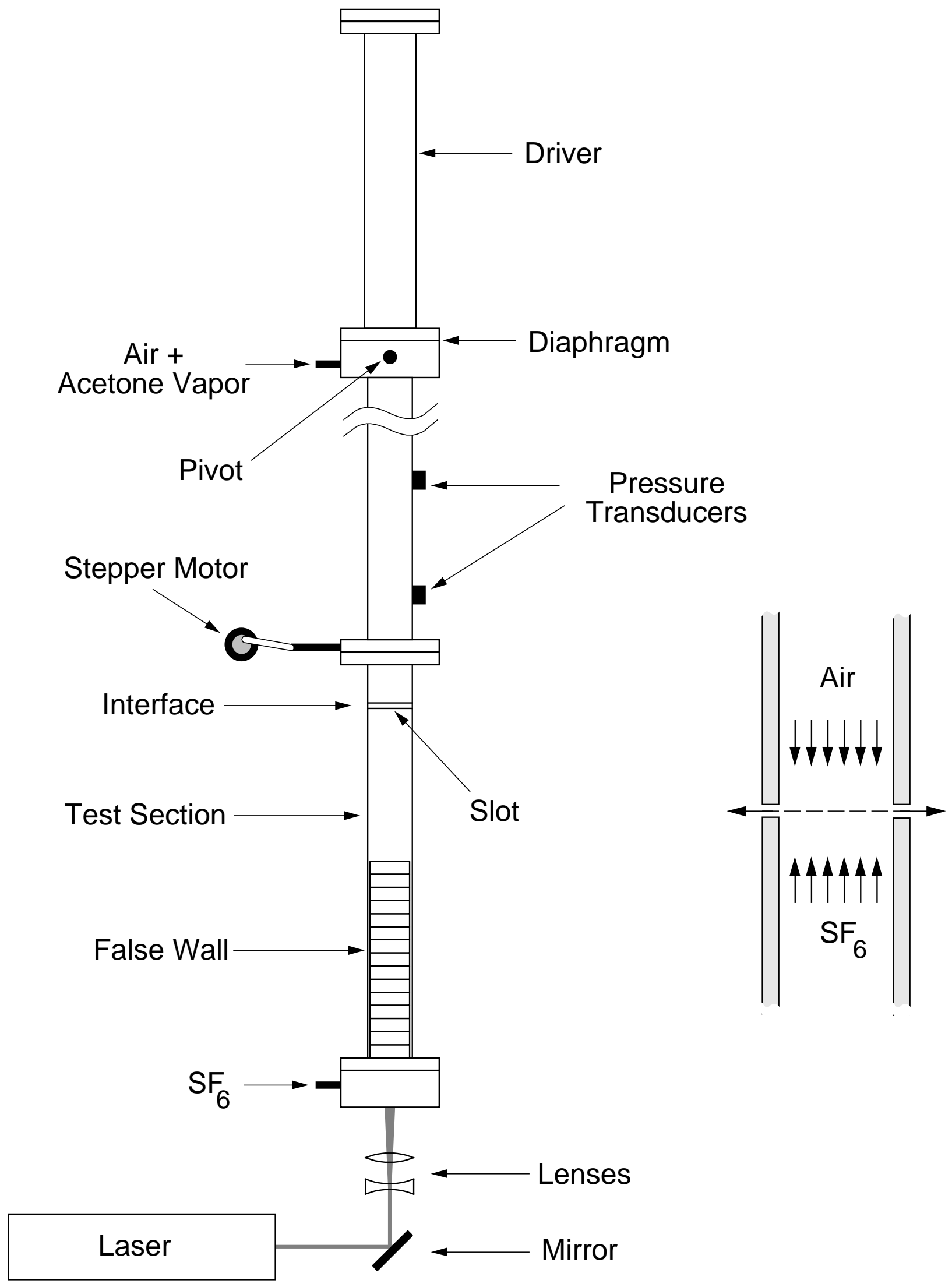




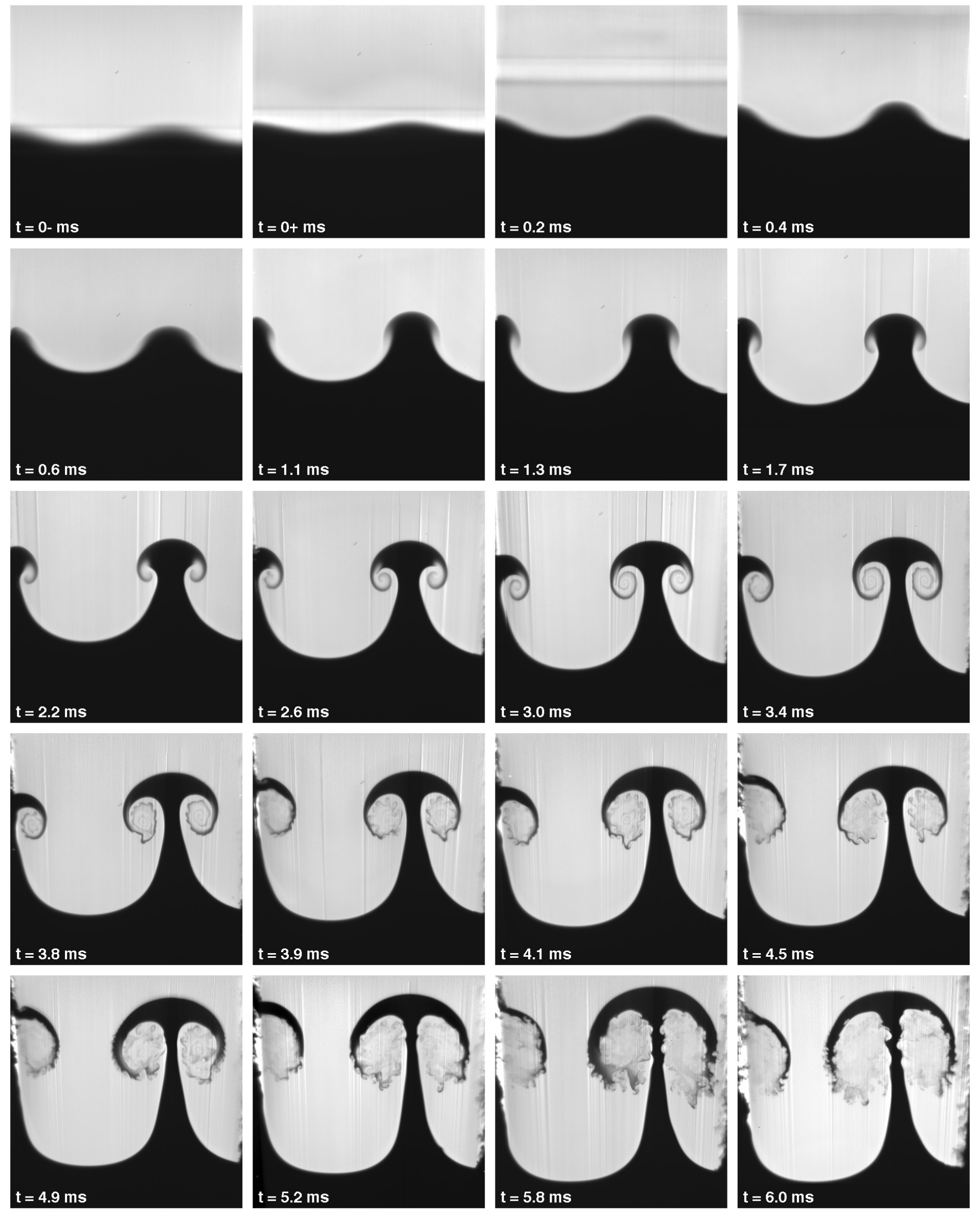



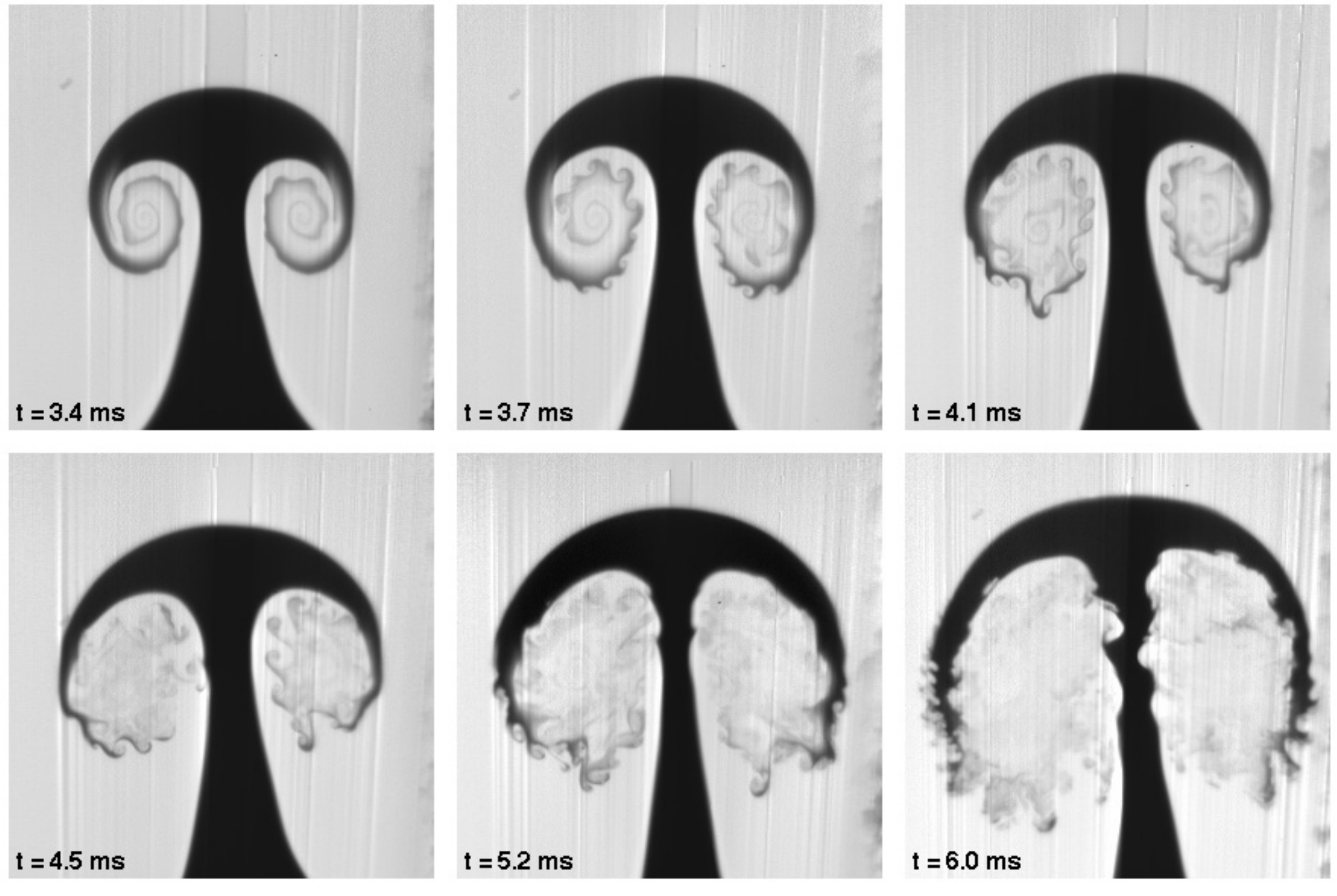


\section{Reynolds Number}

$$
\operatorname{Re}=\frac{\mathrm{U} \ell}{\mathrm{v}}
$$

Let, $\quad \ell \sim \mathrm{h}=2 \mathrm{a}, \mathrm{U} \sim \frac{\mathrm{dh}}{\mathrm{dt}}, \quad \mathrm{v}=$ average viscosity

At time of transition,

$$
\operatorname{Re} \sim \frac{\mathrm{h} \dot{\mathrm{h}}}{\mathrm{v}} \approx 50,000
$$

Alternatively let,

$$
\operatorname{Re}=\frac{\Gamma}{v}
$$

Linear stability theory gives, $\Gamma=\frac{2}{\pi} \lambda \dot{\mathrm{a}}_{0}$

Then,

$$
\operatorname{Re}=\frac{2}{\pi} \frac{\lambda \dot{\mathrm{a}}_{0}}{\mathrm{v}} \approx 42,000
$$




\section{Nonlinear Models}

\section{Zhang \& Sohn (1997)}

$$
\begin{aligned}
& \mathrm{v}_{\mathrm{b} / \mathrm{s}}= \frac{\mathrm{v}_{0}}{1+\mathrm{v}_{0} \mathrm{a}_{0} \mathrm{k}^{2} \mathrm{t}+\max \left[0, \mathrm{a}_{0}{ }^{2} \mathrm{k}^{2}-\mathrm{A}^{2}+\frac{1}{2}\right] \mathrm{v}_{0}{ }^{2} \mathrm{k}^{2} \mathrm{t}^{2}} \\
& \mp \frac{\mathrm{Av}_{0}{ }^{2} \mathrm{kt}}{1+2 \mathrm{k}^{2} \mathrm{a}_{0} \mathrm{v}_{0} \mathrm{t}+4 \mathrm{k}^{2} \mathrm{v}_{0}{ }^{2}\left[\mathrm{a}_{0}{ }^{2} \mathrm{k}^{2}+\frac{1}{3}\left(1-\mathrm{A}^{2}\right)\right] \mathrm{t}^{2}}
\end{aligned}
$$

when $\mathrm{A}^{2} \leq \frac{1}{2}+\mathrm{a}_{0}{ }^{2} \mathrm{k}^{2}$

$$
\mathrm{v}=\frac{1}{2}\left(\mathrm{v}_{\mathrm{b}}+\mathrm{v}_{\mathrm{s}}\right) \rightarrow \frac{\mathrm{v}_{0}}{\left[\mathrm{a}_{0}{ }^{2} \mathrm{k}^{2}-\mathrm{A}^{2}+\frac{1}{2}\right] \mathrm{v}_{0}{ }^{2} \mathrm{k}^{2} \mathrm{t}^{2}} \propto \frac{1}{\mathrm{t}^{2}} \quad \text { as } \quad \mathrm{t} \rightarrow \infty
$$

\section{Sadot el al. (1998)}

$$
\mathrm{v}_{\mathrm{b} / \mathrm{s}}=\frac{\mathrm{v}_{0}\left(1+\mathrm{v}_{0} \mathrm{kt}\right)}{1+(1 \pm \mathrm{A}) \mathrm{v}_{0} \mathrm{kt}+\left[\frac{1 \pm \mathrm{A}}{1+\mathrm{A}}\right\rceil \frac{1}{2 \pi \mathrm{C}} \mathrm{v}_{0}{ }^{2} \mathrm{k}^{2} \mathrm{t}^{2}}
$$

where

$$
\begin{gathered}
\mathrm{C}= \begin{cases}1 / 3 \pi & \mathrm{A} \geq 0.5 \\
1 / 2 \pi & \mathrm{A} \rightarrow 0\end{cases} \\
\mathrm{v} \rightarrow\left(\frac{1}{1-\mathrm{A}}\right) \frac{\mathrm{C} \lambda}{\mathrm{t}} \propto \frac{1}{\mathrm{t}} \text { as } \mathrm{t} \rightarrow \infty
\end{gathered}
$$




\section{Late Time Growth}

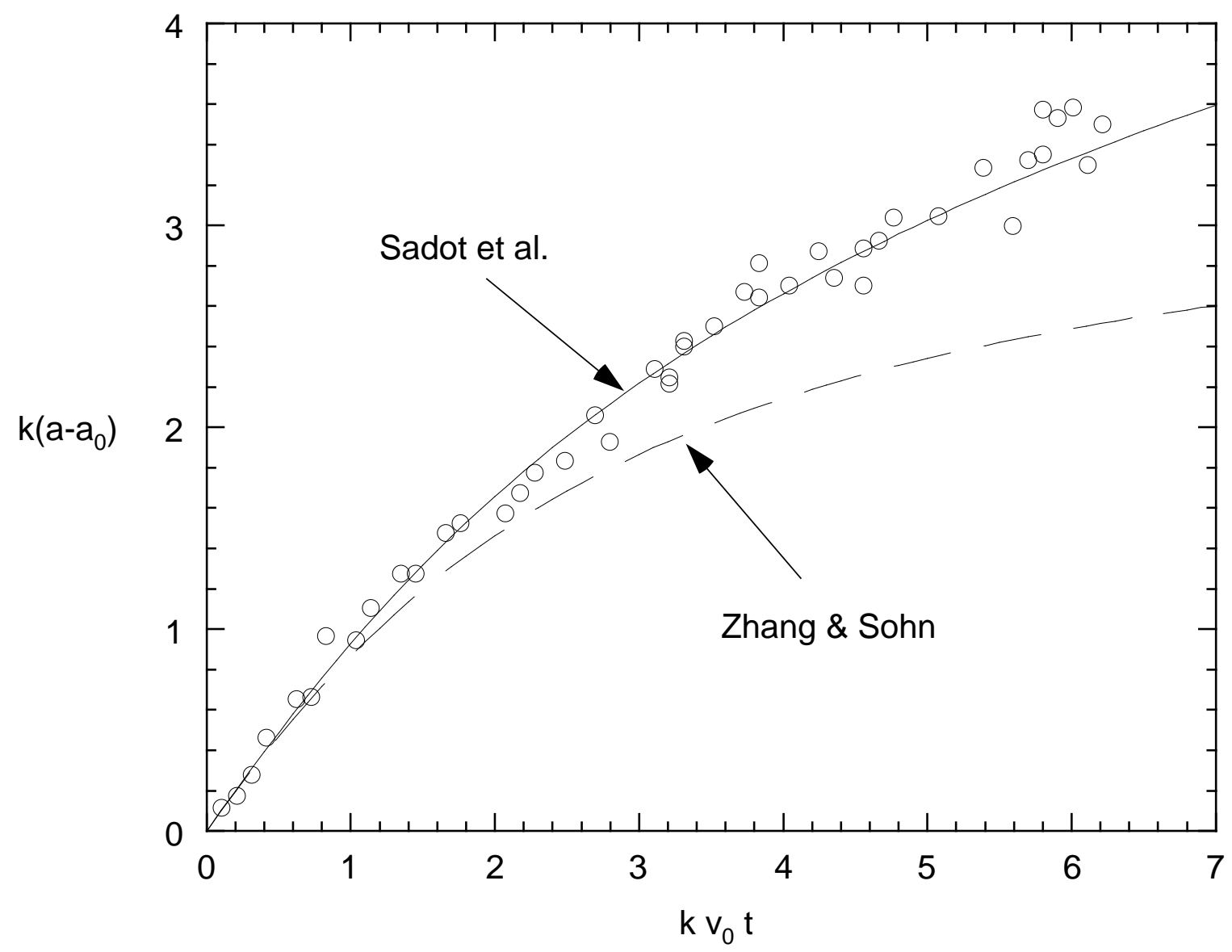


Bubble and Spike Amplitudes

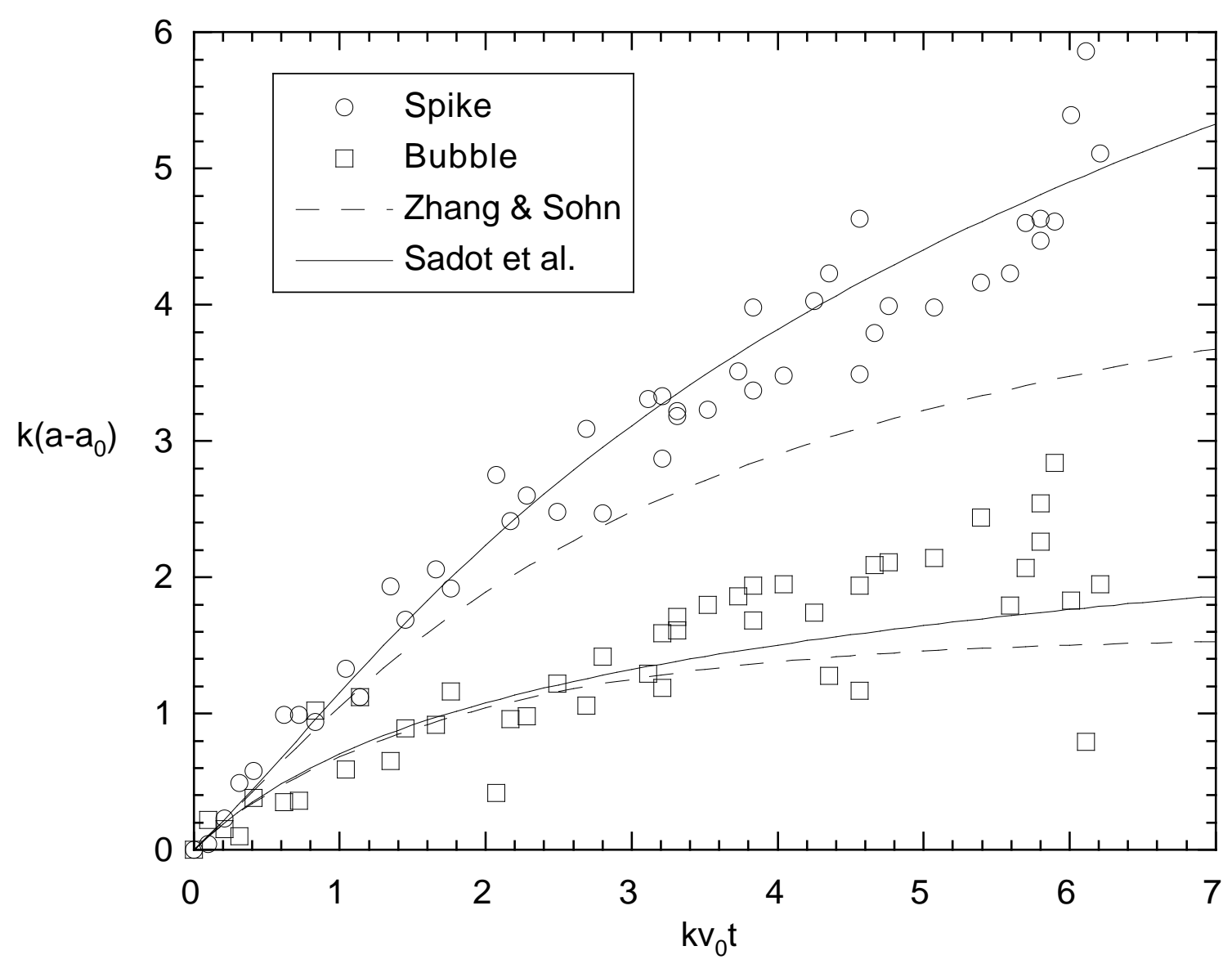




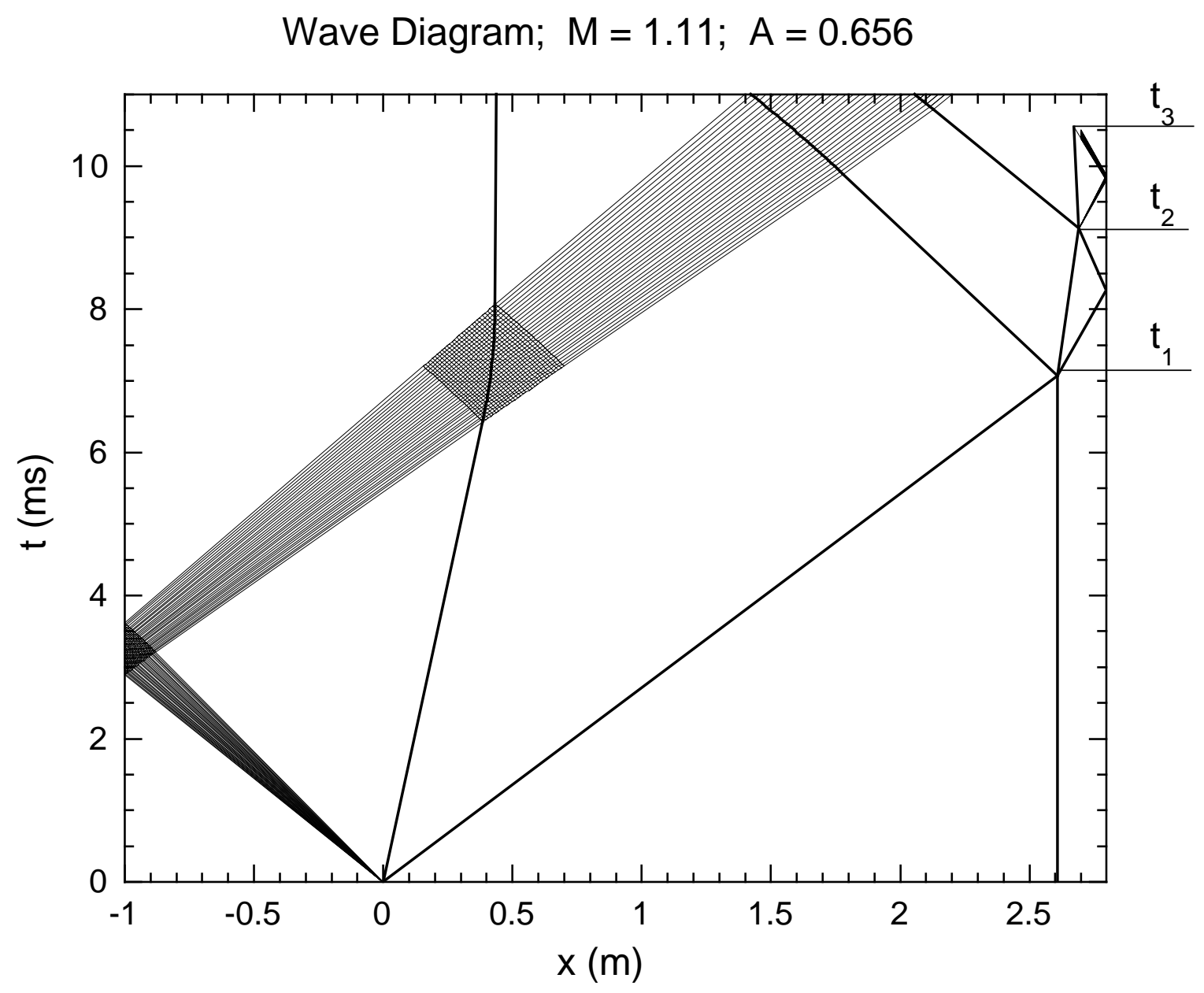



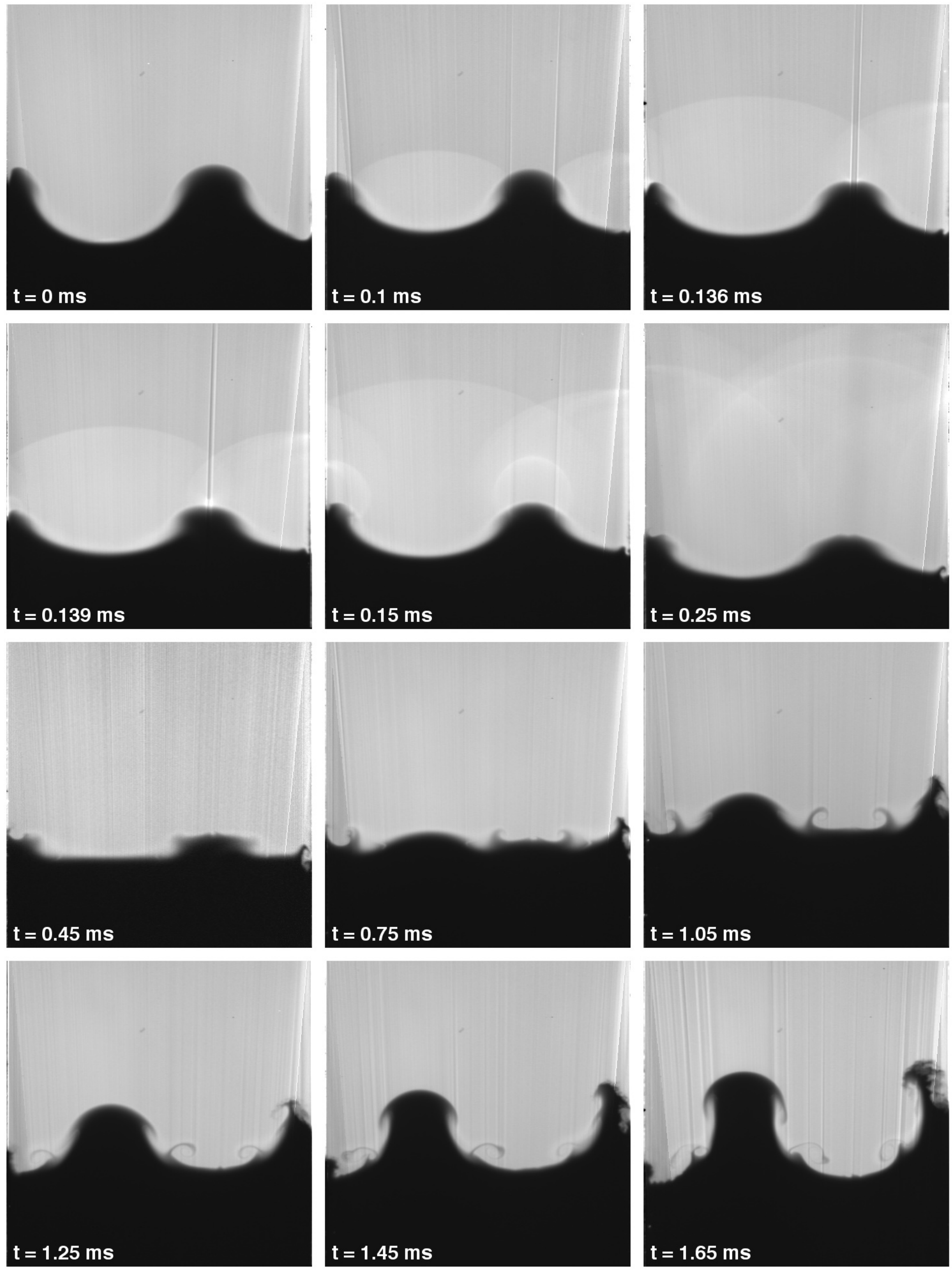

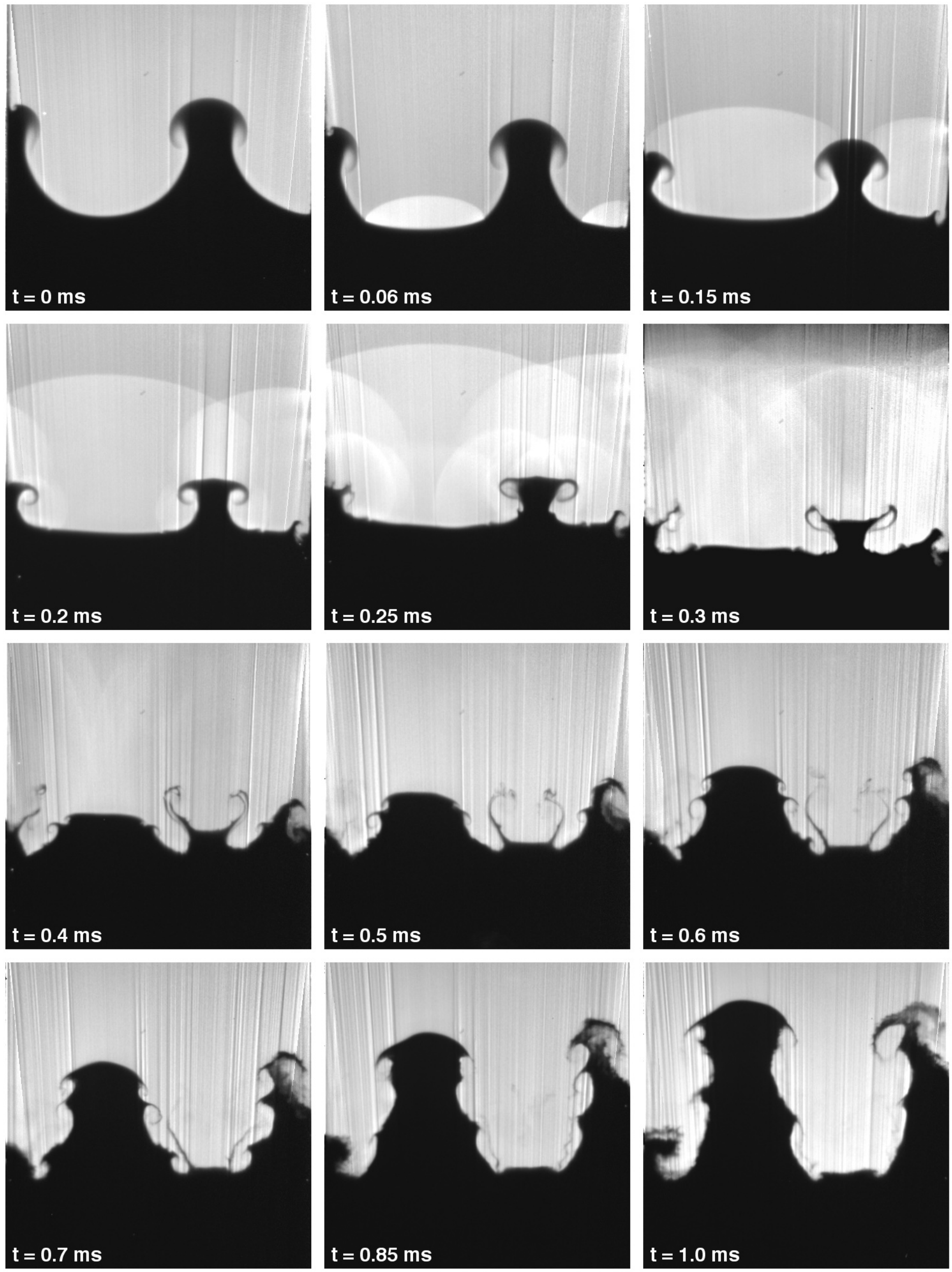

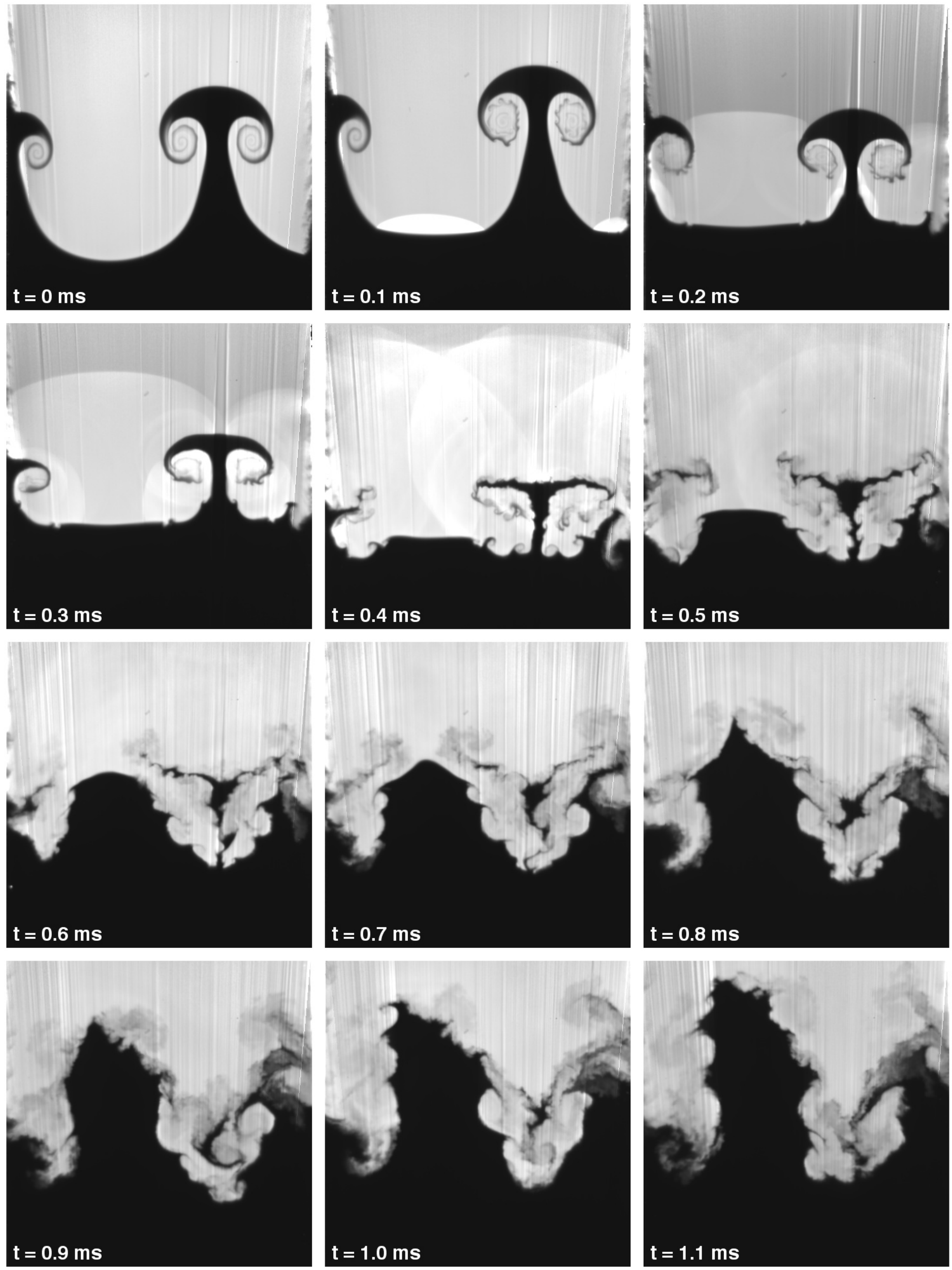
Reshock Amplitude

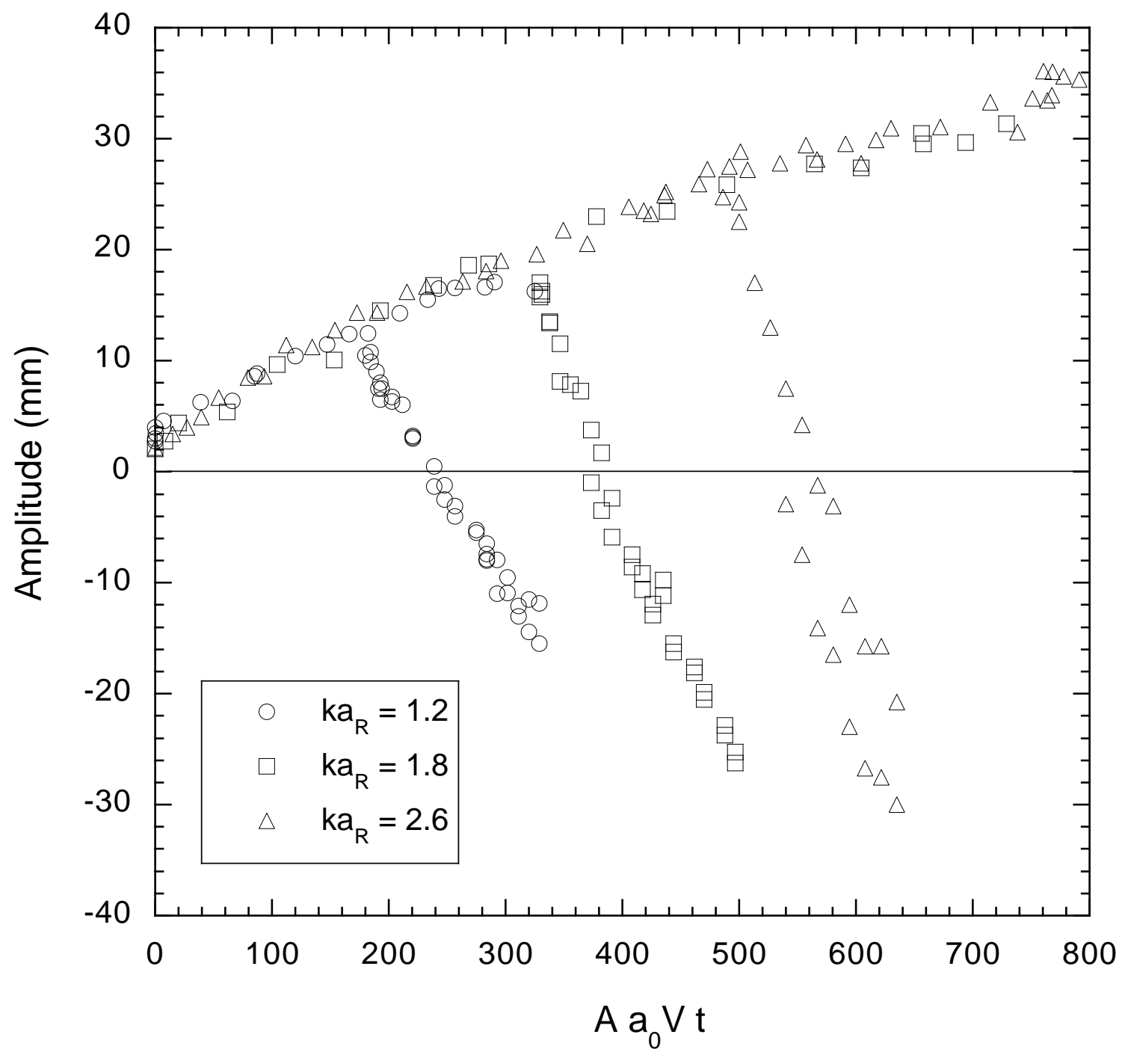




\section{Growth Rate after Reshock}

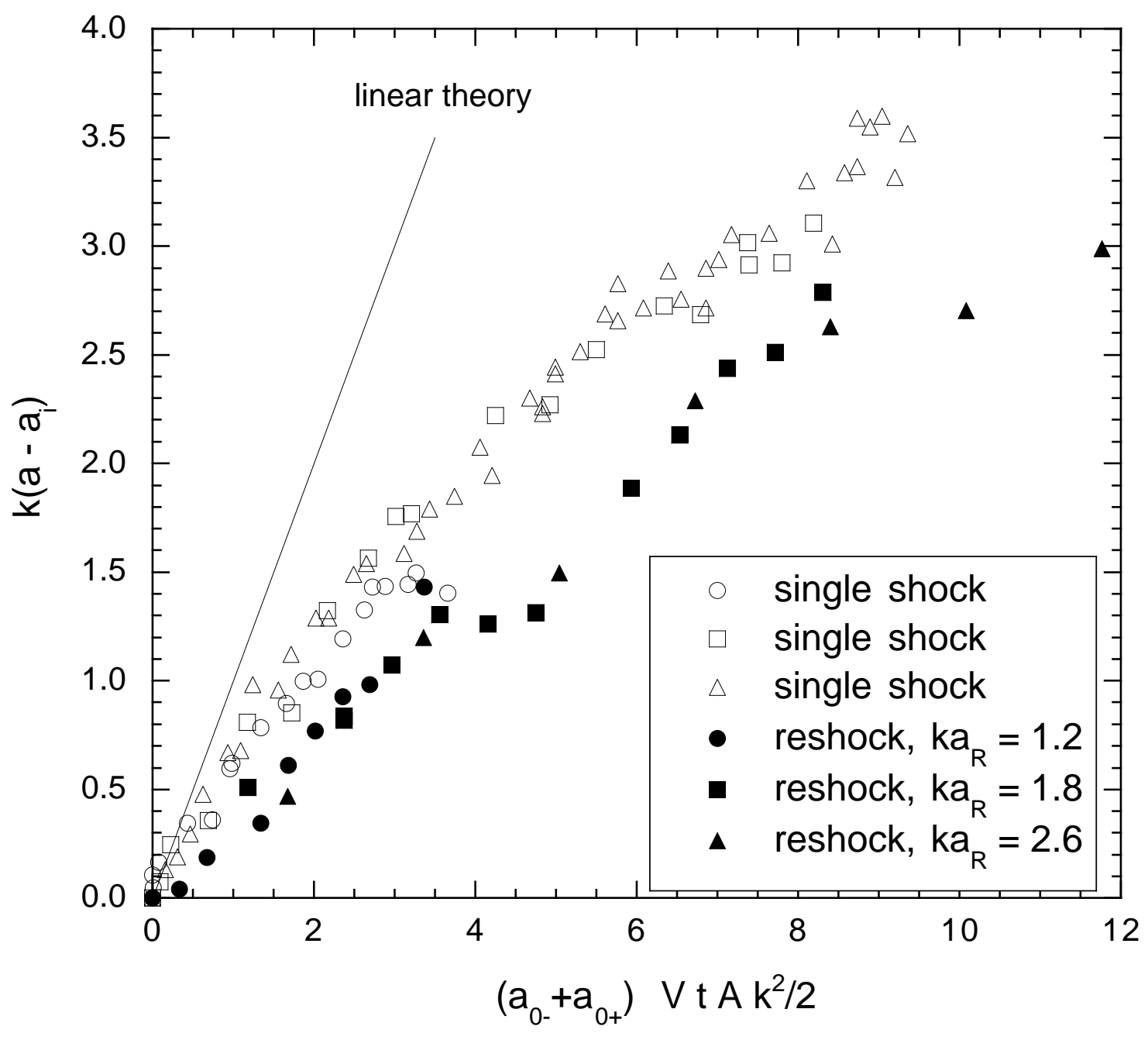


$10 \%$ to $70 \%$ Mixed Fraction

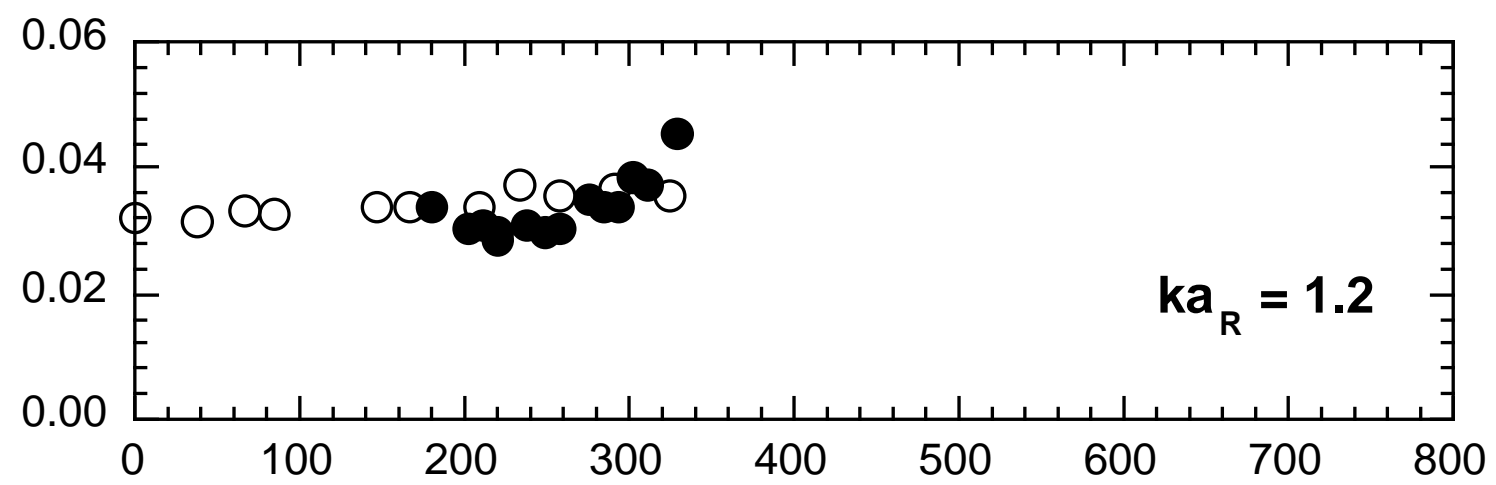

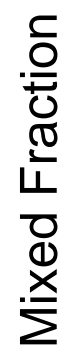
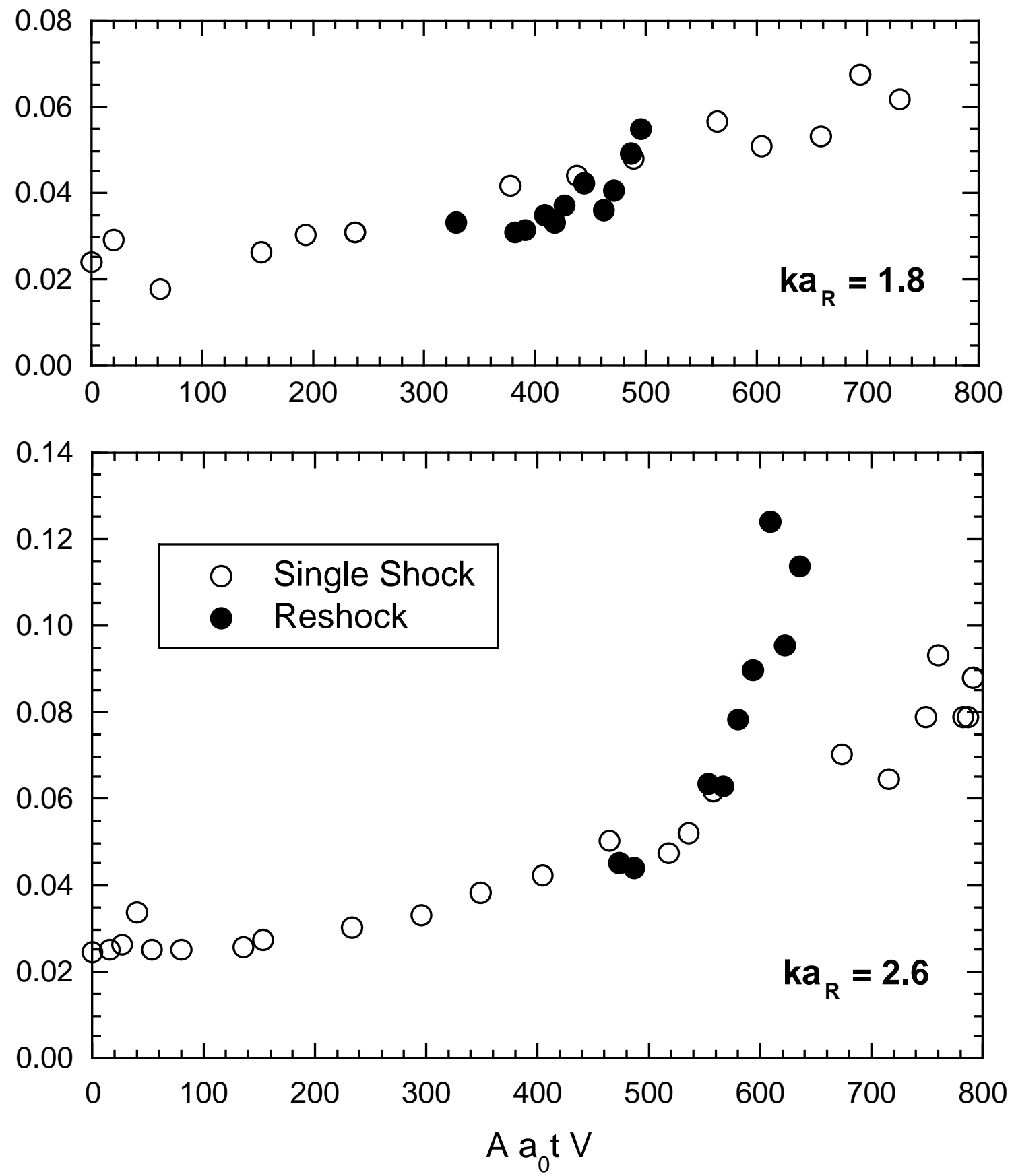


\section{Conclusions}

Single-mode RM experiments show a transition to turbulence in the vortex cores at $\operatorname{Re} \approx 50,000$.

Late-time amplitude measurements show excellent agreement with the model of Sadot et al. (1998).

Penetration depth measurements for the three reshock conditions collapse when plotted in dimensionless form and have a growth rate approximately $1 / 3$ that given by linear stability theory.

Reshock at early stages of the instability show similar evolution to that of single interaction with little increase in the mixing rate.

Reshock at late stages of the instability produces a complex interface pattern and a significant increase in the mixing rate. 


\section{Laser-based high-pressure, \\ high-straingrate materials-experiments}

Daniel H. Kalantar - Lawrence Livermore National Laboratory

Presented by Thomas Lorenz

$8^{\text {th }}$ International Workshop on the Physics of Compressible Turbulent Mixing

Pasedena, CA - December 9-14, 2001

J. Belak, J. D. Colvin, M. Kumar, K. T. Lorenz, K. O. Mikaelian, S. Pollaine, B. A. Remington,

S. V. Weber, L. G. Wiley (LLNL),

J. S. Wark, A. Loveridge, A. M. Allen (University of Oxford), M. A. Meyers, M. Schneider (UCSD)

This work was performed under the auspices of the US DOE by UC LLNL under contract No. W-7405-48-Eng. Supported in part through the Science Use of Nova Program, DOE Grants program, and NLUF program (OMEGA). 


\section{Outline}

- Introduction

- Solid-state experiments at high pressure on a laser

- High pressure strength

- RT instability in solid Al at high pressure to infer $Y(P)$

- Dynamic material response

- Dynamic X-ray diffraction of the lattice level response in $\mathrm{Si}$ and $\mathrm{Cu}$

- Wave profile and residual deformation

- VISAR measurement, sample recovery and characterization 


\section{The high pressure response of materials is of interest for many reasons; lasers provide a way to access high pressures and strain rates}

- The core of the earth is Fe at $3 \mathrm{Mbar}$, both solid and liquid

- Long time scale, diamond anvil experiments

- Survivability of passengers in a car crash depend on the material response of the car

- ms- $\mu s$ time scale, Hopkinson bar and gun experiments

- Space station wall integrity from space debris, dust, micro-asteroids

- $\mu$ s time scale, gun and high explosives experiments

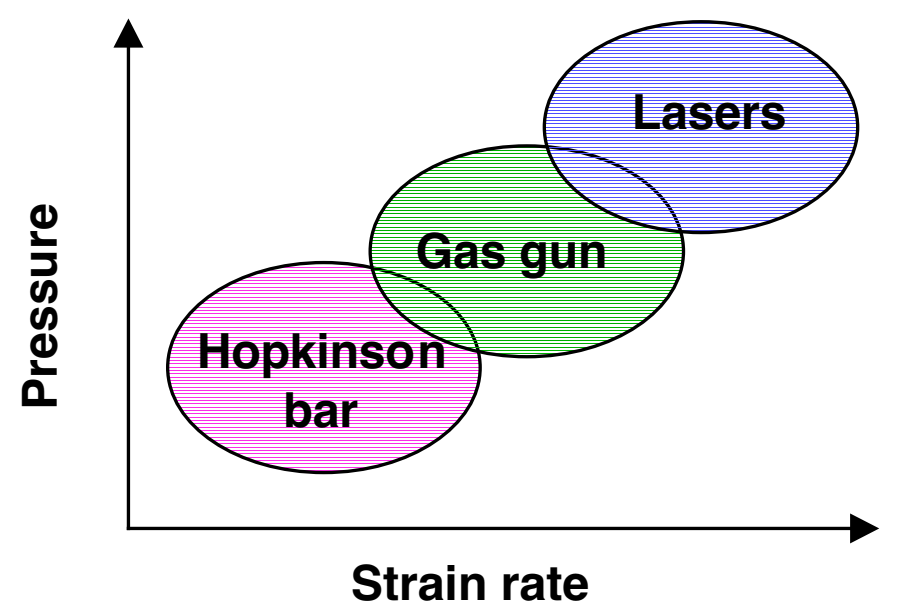

Lasers access unique high pressure, high strain rate regime of material response to test the limits of theories and scaling laws 


\section{Example - strength measurements at high pressure using a high explosive drive and modulated Al plate}

- Shockless HE drive used to compress and accelerate a plate with preimposed modulations

- Pre-imposed modulations grow by the Rayleigh-Taylor instability

- The growth is reduced from classical (fluid) due to material strength

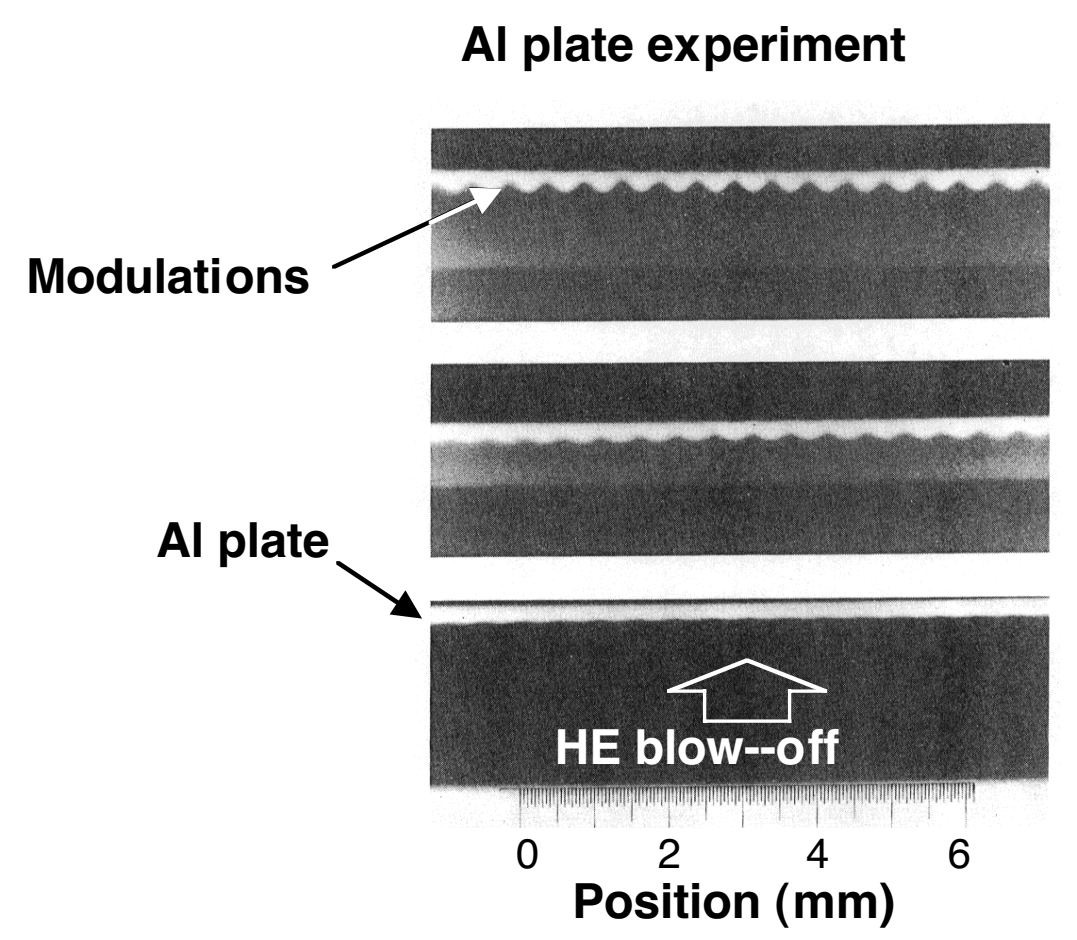

Growth is reduced from fluid

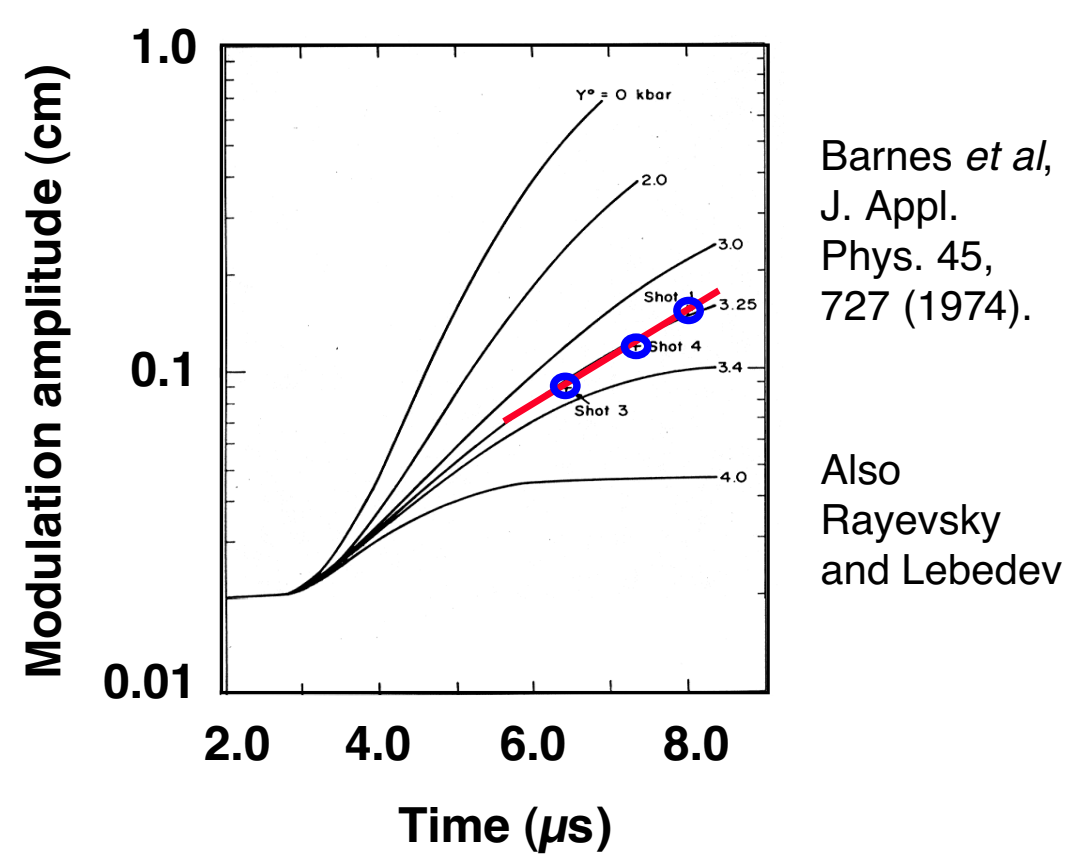




\section{Solid state RT instability experiment}

- An internally shielded hohlraum is used to shock compress an Al-6061 metal foil at high pressure

- Internal shields block hard x-rays from preheating package

- A shaped laser pulse generates a series of gentle shocks for nearly isentropic compression

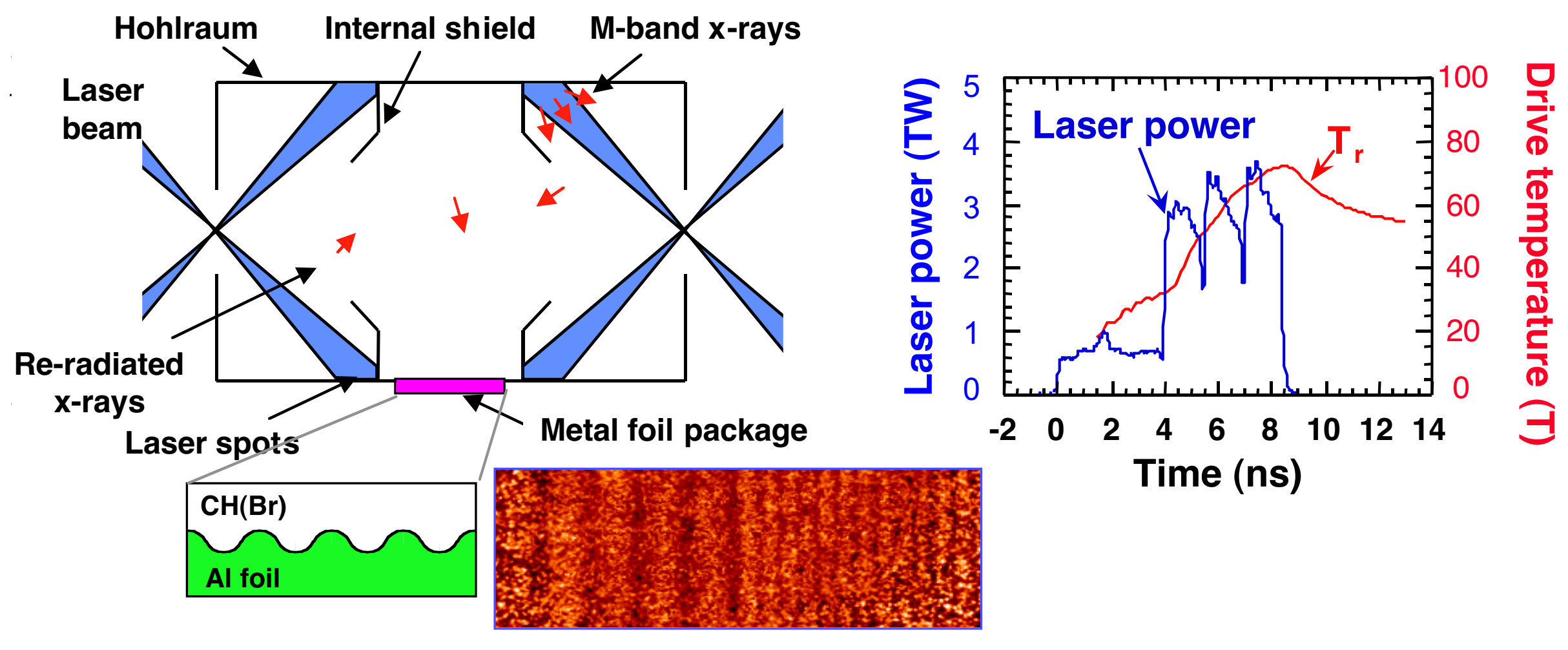




\section{Detailed simulations predict that the Al foil remains solid throughout the experiment}

- The Al remains below the melt curve

- The foil trajectory is nearly isentropic to $1.8 \mathrm{Mbar}$

$$
\text { Lindemann melt model } \quad T_{m}=T_{m_{o}} e^{2 a(1-\eta)} \eta^{2\left(\gamma_{o}-a-1 / 3\right)}
$$

Pressure and temperature at the embedded interface

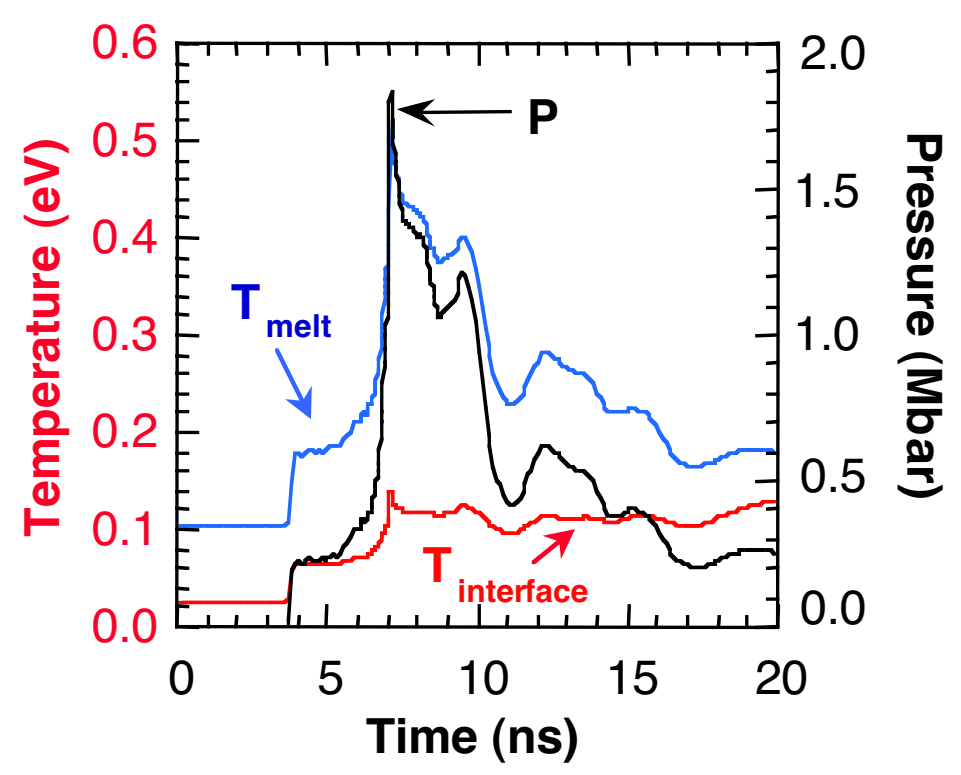

Calculated internal energy trajectory

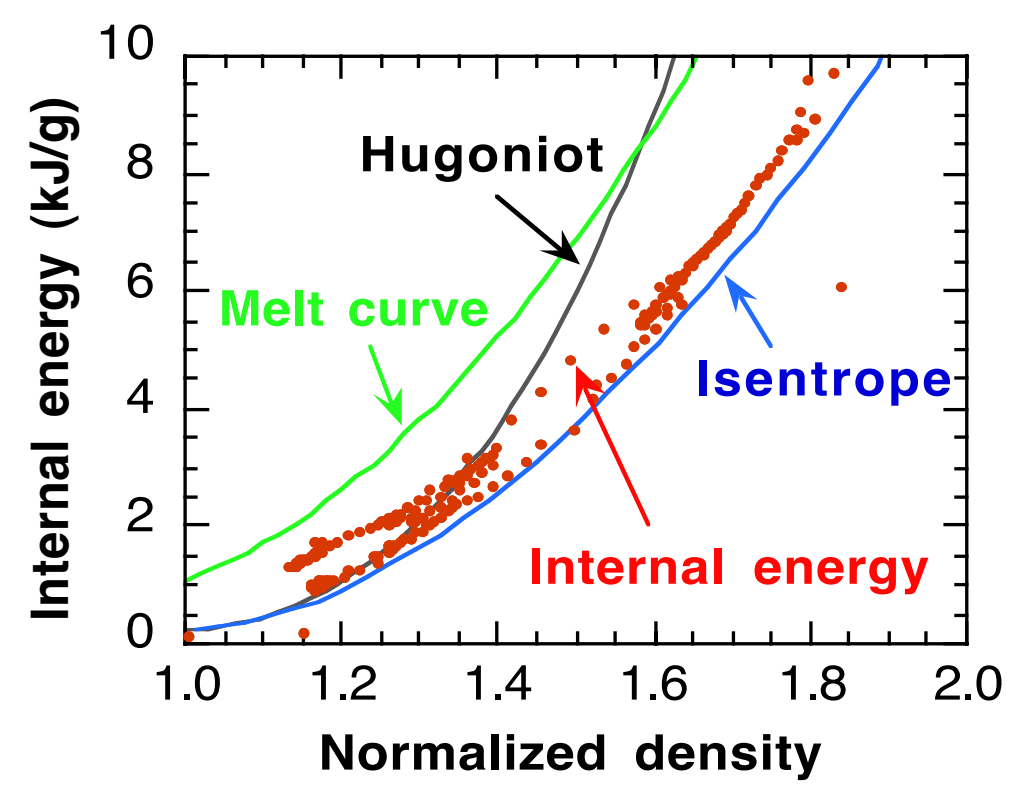




\section{Simulations of the instability growth demonstrate sensitivity to the strength of the Al}

- Growth rates with strength are expected to be reduced from classical (fluid)

Steinberg-Guinan constitutive model

$$
\begin{aligned}
& Y=Y_{o}\left(1+\beta\left(\varepsilon+\varepsilon_{i}\right)\right)^{n} \frac{G(P, T)}{G_{o}} \\
& G=G_{o}\left(1+\left(\frac{G_{P}}{G_{o}}\right) \frac{P}{\eta^{1 / 3}}-\left(\frac{G_{T}}{G_{o}}\right)(T-300)\right)
\end{aligned}
$$

Predicted growth factors $\lambda=20 \mu \mathrm{m}$

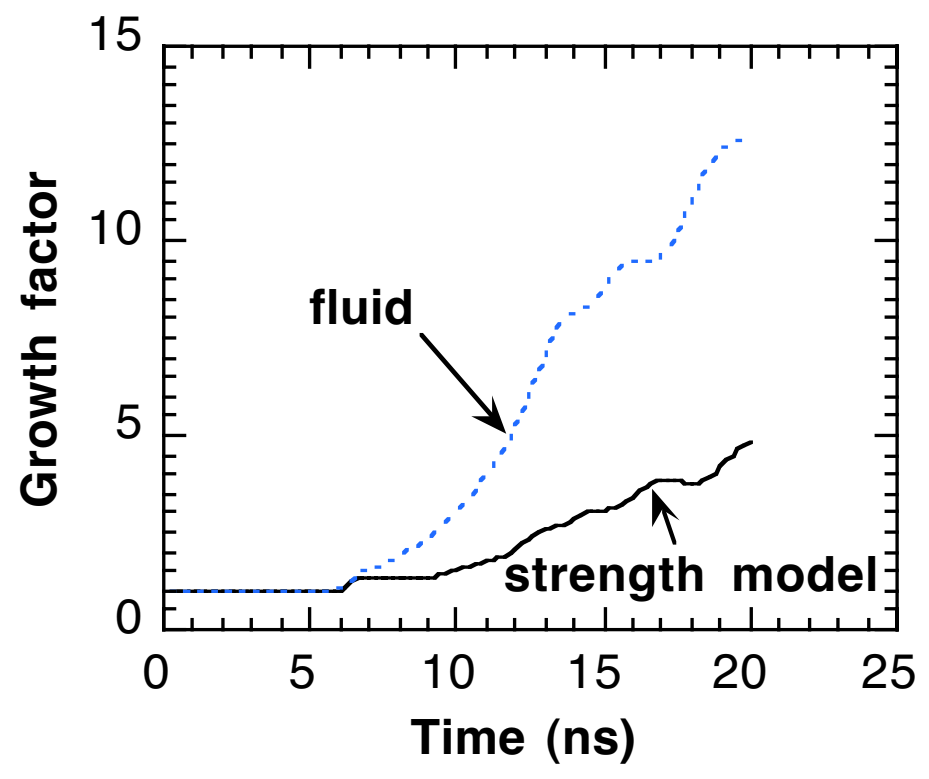

Rayevsky Stability boundary formula

$$
\begin{gathered}
\eta_{c}=\eta_{D}\left[1-0.86 e^{-\frac{2 \pi H}{\sqrt{3} \lambda}}\right]\left\{\left[1-e^{-\frac{2 \pi H}{\sqrt{3} \lambda}}\right]^{2}-\left[\frac{\lambda}{\lambda_{M}}\right]^{2}\right\} \\
\eta_{D}=\frac{2 Y}{\rho g} \quad \lambda_{M}=\frac{4 \pi G}{\rho g}
\end{gathered}
$$

Stability curve (Nizovtsev and Rayevsky, 1991)

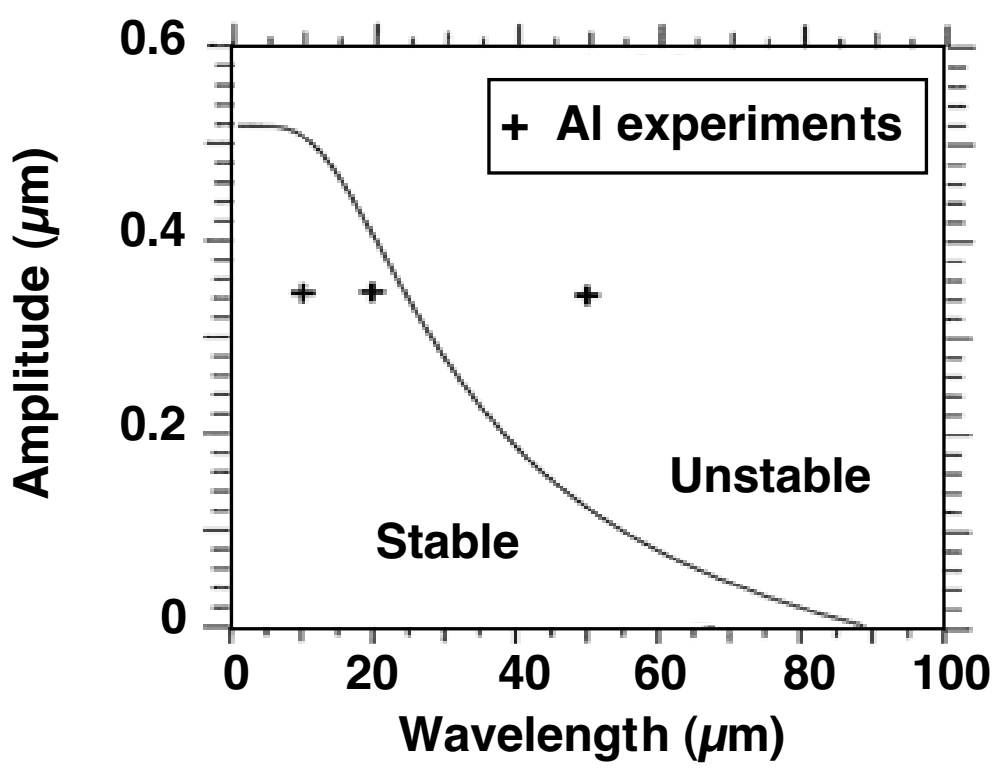




\section{The RT growth is nearly fluid at early times, but it is suppressed at later times}

- Experiments were conducted with 10, 20 and $50 \mu \mathrm{m}$ wavelengths

- Modeling was done assuming the following:

- Fluid

- Nominal Steinberg-Guinan

- Fluid until $13 \mathrm{~ns}$, then S-G with theoretical maximum $\mathrm{Y}=\mathrm{G} / 10$
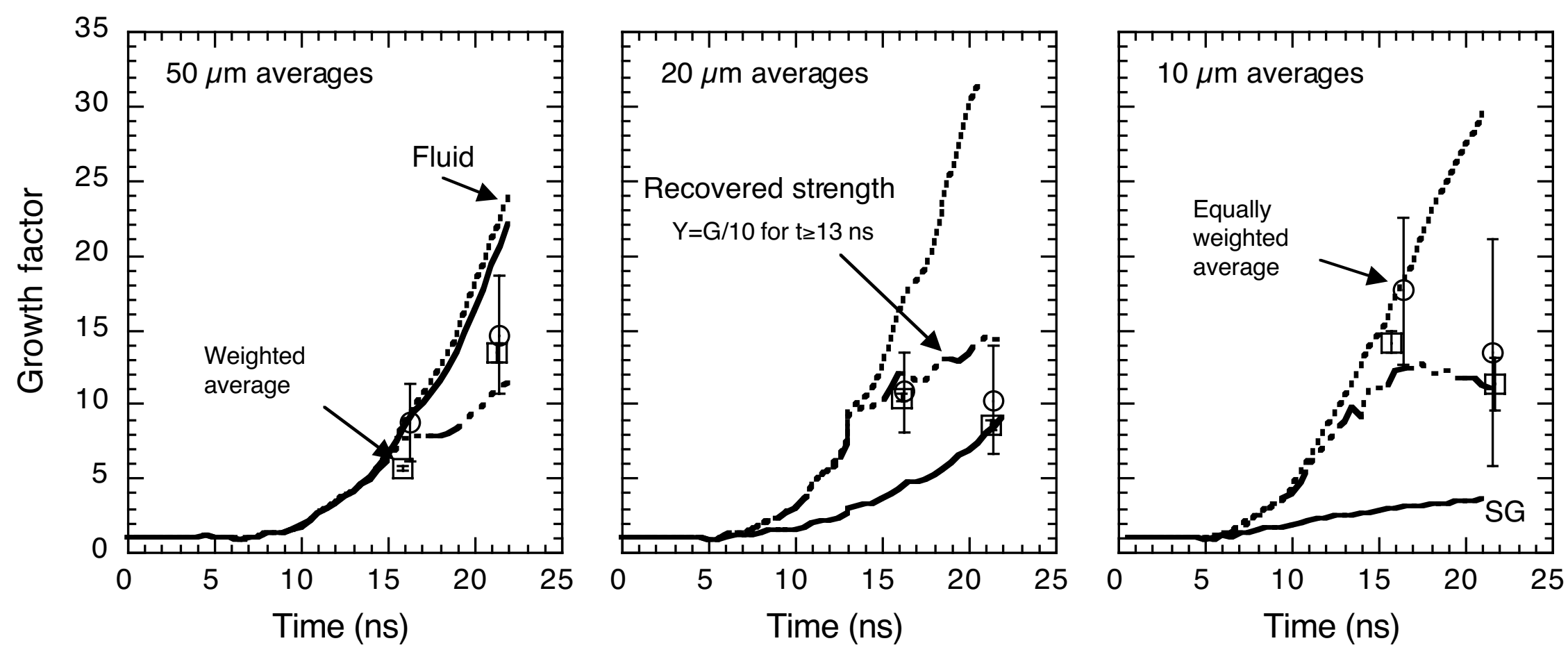


\section{The RT growth is nearly fluid at early times, but it is}

suppressed at later times; suggestive of model from Grady/Asay and data by Rayevsky and Lebedev

- High pressure strain causes localized heating and softening in shear bands; bulk Al flows as fluid due to localized deformation

- As heat conducts into the bulk material, the metal regains bulk solid strength and continued growth is inhibited

Optical emission from shocked quartz

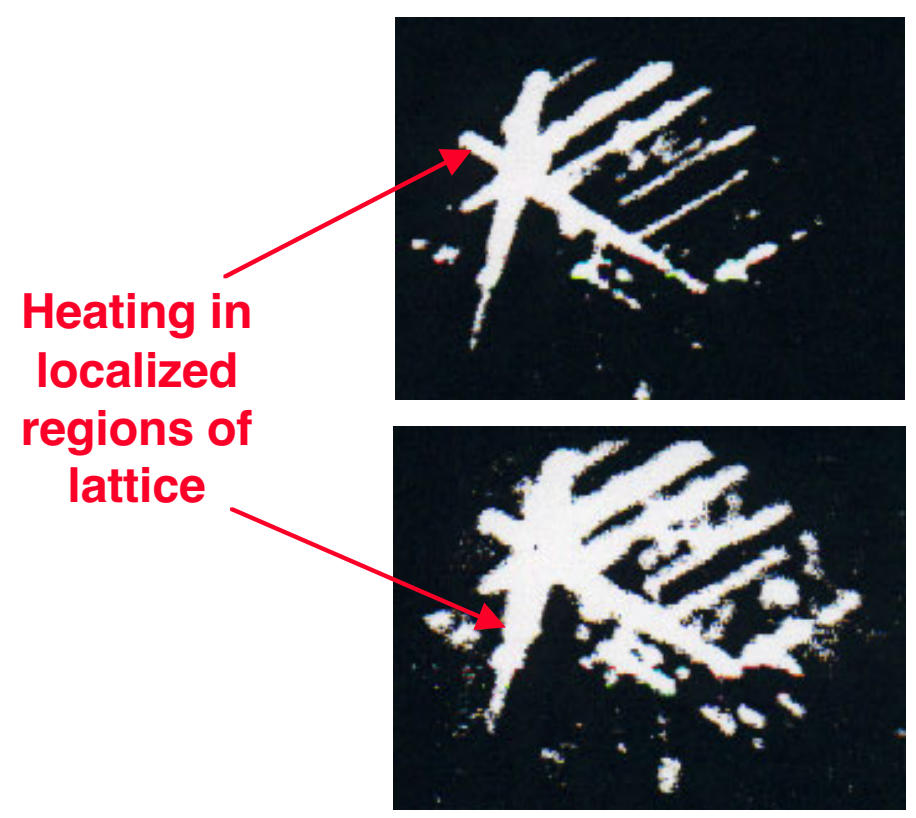

Brannon et al, SCCM 1983.
Shocked HE experiments show fluid-like response with saturation

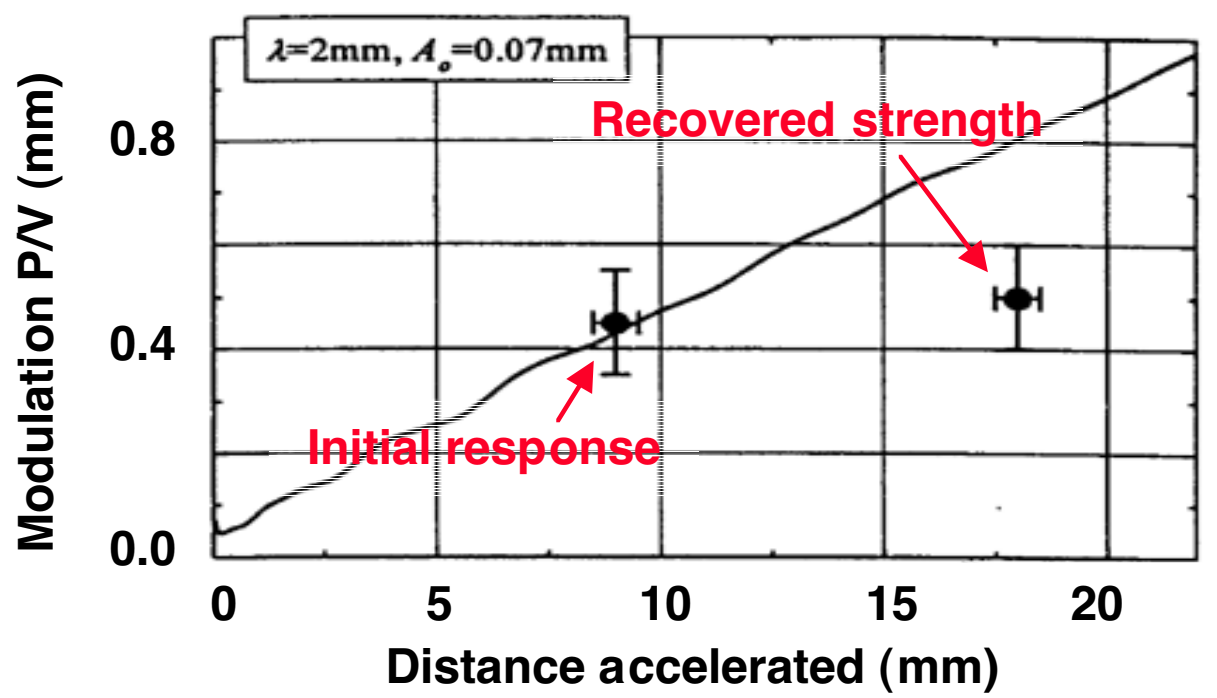

Rayevsky et al, IWPCTM, 1999. 


\section{The late time images show features that may be due to hydrodynamic imprinting of the grain structure}

- The spatial scale of the late-time modulation is similar to initial grain structure

- 2D simulations incorporating the grain boundaries start to show effects at $t=18 \mathrm{~ns}$, 3D simulation has been started

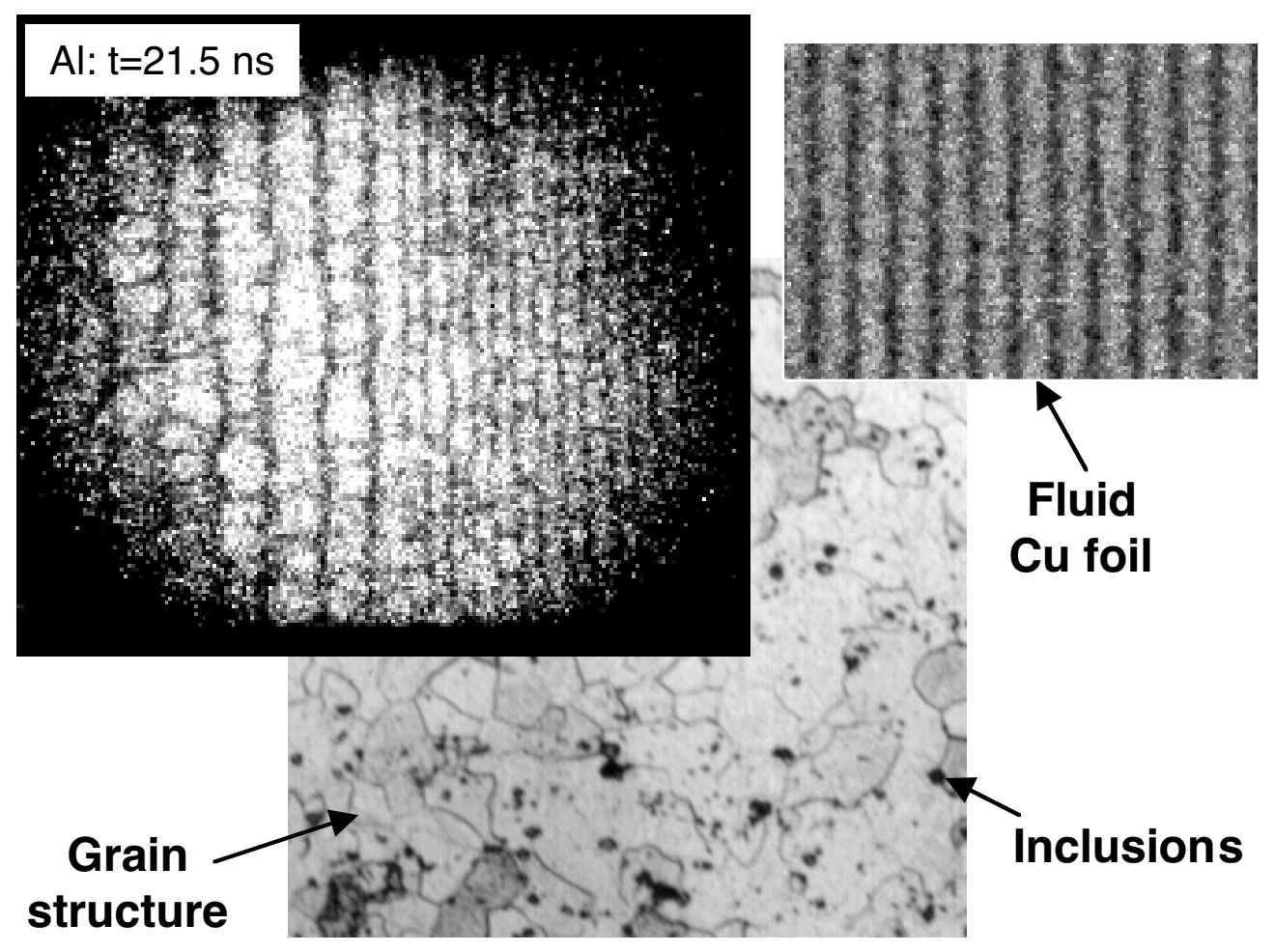

2D simulation including grain structure

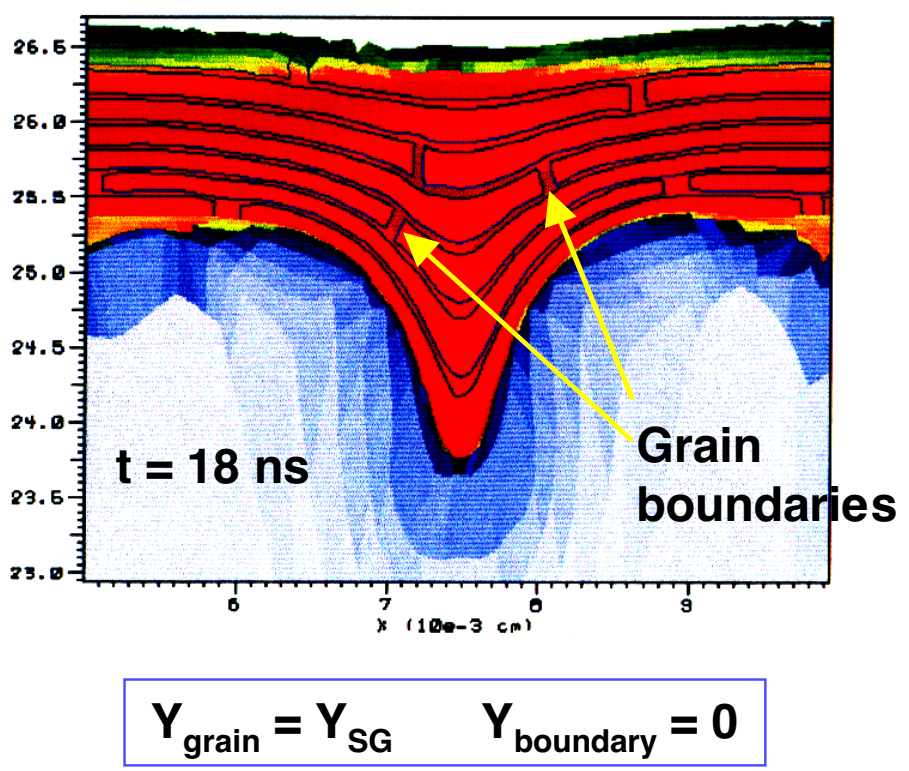




\section{Dynamic x-ray diffraction}

- In situ x-ray diffraction probes the long range lattice order under shock compression

- Shock pressure generated using a hohlraum x-ray drive or by direct laser irradiation

- Time-resolution with x-ray streak cameras provides information on dynamic lattice response

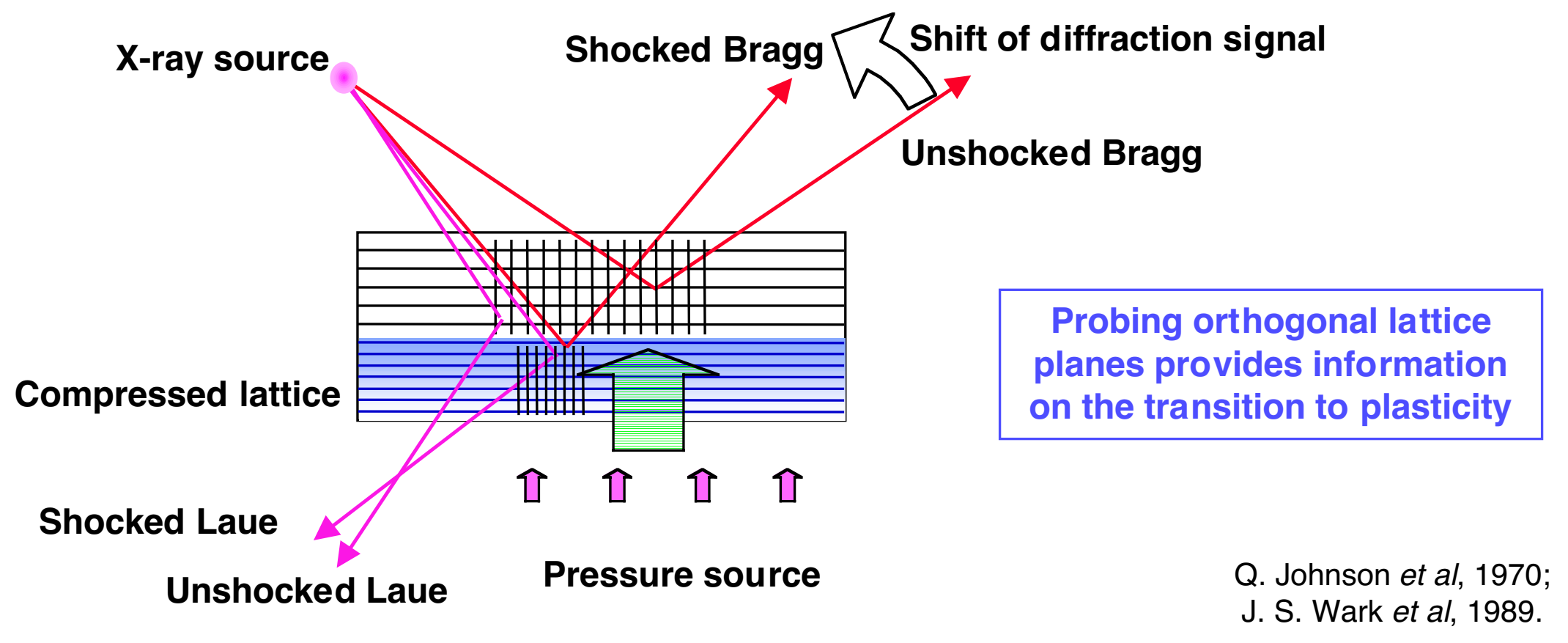




\section{Simultaneous measurements of orthogonal planes indicates Si responds uniaxially on a ns time scale}

- $40 \mu \mathrm{m}$ thick Si shocked along (100) axis

- $P=115-135$ kbar; HEL=84 kbar
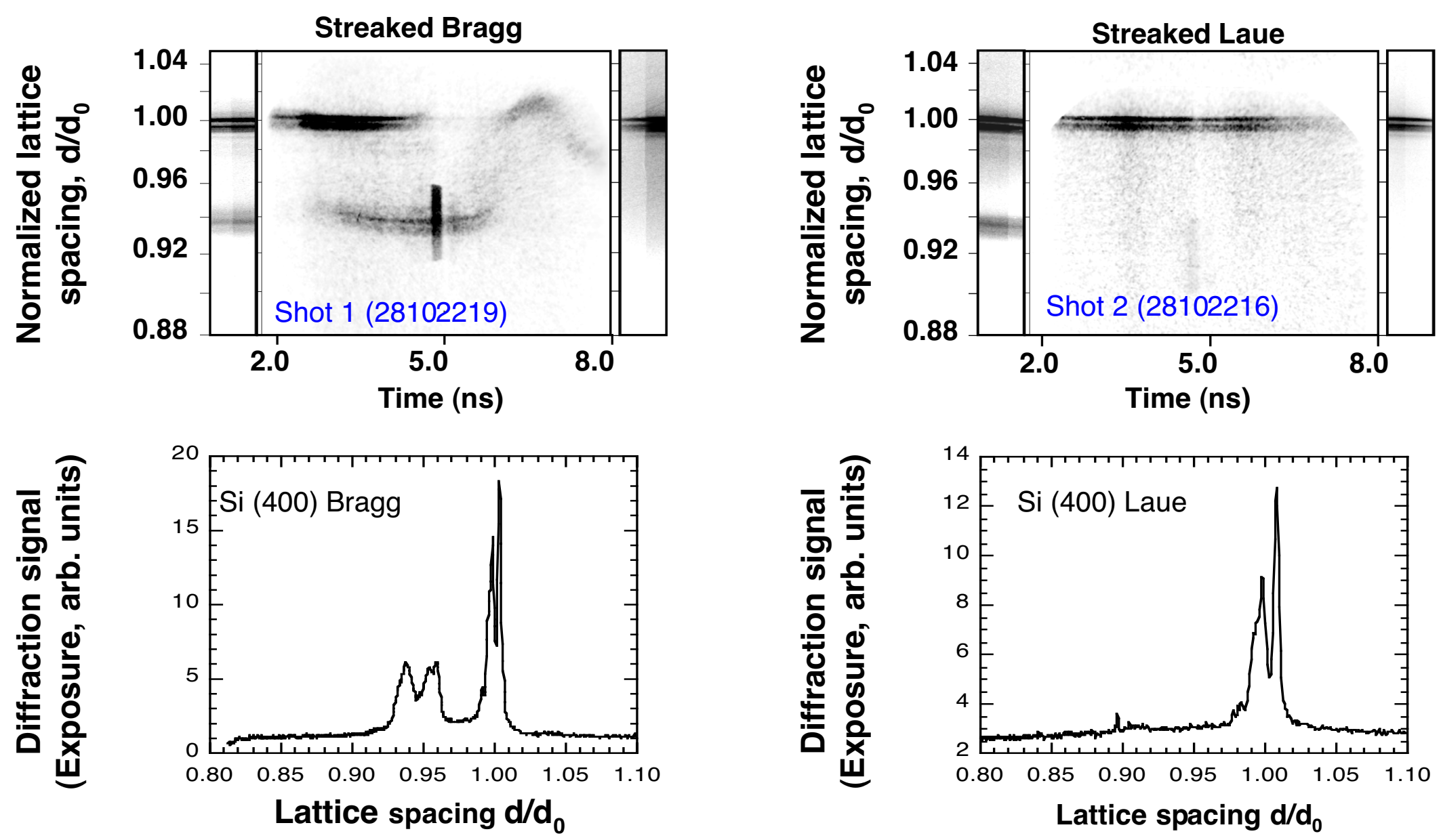


\section{Cu undergoes a transition to 3D lattice compression at high pressure}

- $8 \mu \mathrm{m}$ single crystal Cu shocked along (100) axis

- $\mathrm{P}=180$ kbar; HEL 2 kbar

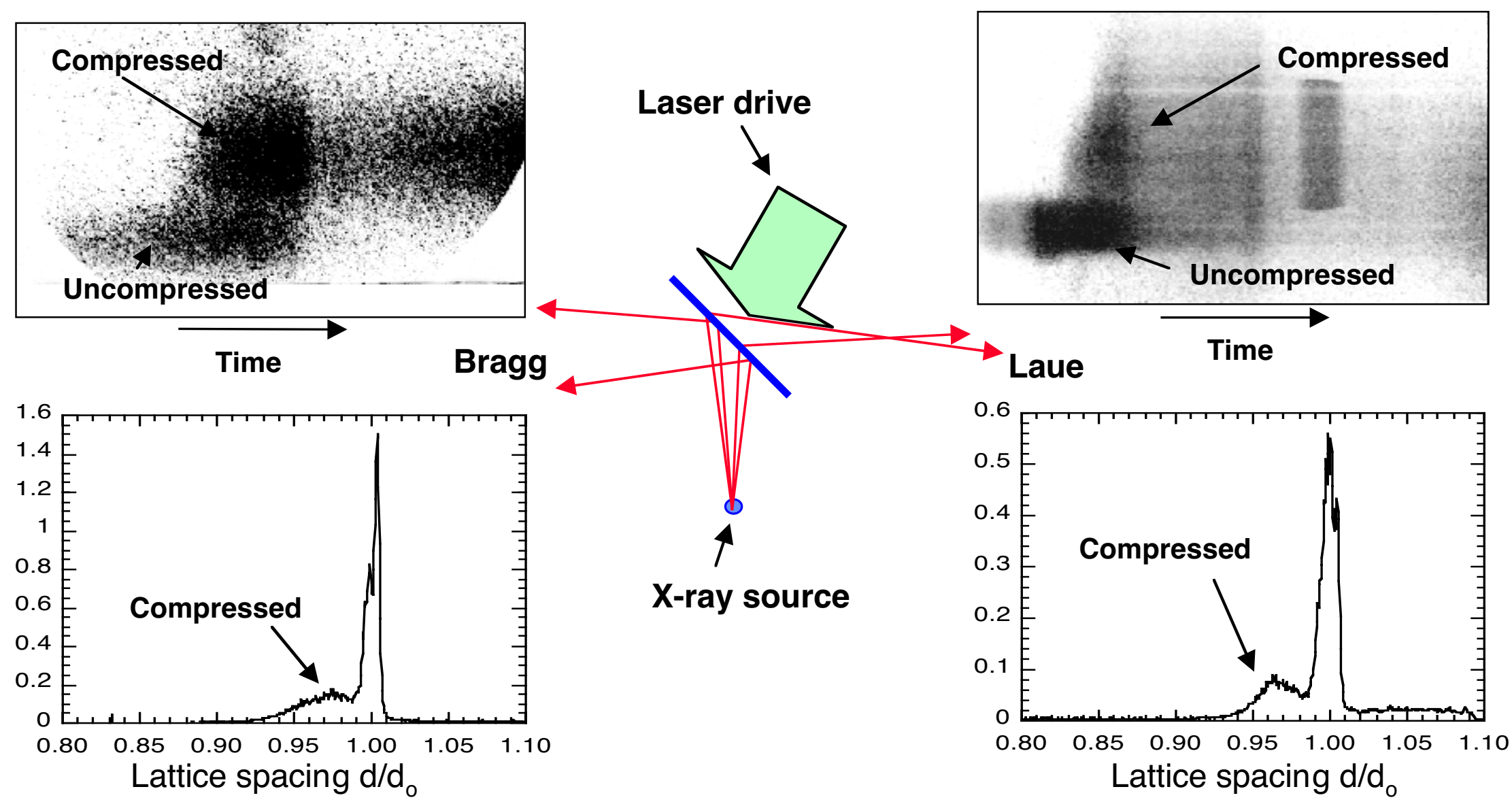




\section{The timescale for plastic deformation in $\mathrm{Si}$ is much longer than for Cu based on Orowan's equation}

Orowan equation: $\frac{\Delta \varepsilon}{\Delta t}=N|b| \mathbf{v}$

\section{Silicon}

- $\Delta t>1 \mu s$ : dislocations do not move

$-5 \%$ strain, dislocations separated by at least the Burger's vector (3.8 $\AA$ ) (diffraction linewidth indicates $N<10^{14} \mathrm{~m}^{-2}$ )

- Linear extrapolation of dislocation velocity in $\mathrm{Si}(0.1 \mathrm{~mm} / \mathrm{s})$

\section{Copper}

- $\Delta \mathrm{t}<\mathbf{1 0}$ ps : dislocations do move

$-5 \%$ strain, dislocations separated by at least the Berger's vector $(2.5 \AA)$

- Velocity of dislocations calculated to be $400 \mathrm{~m} / \mathrm{s}$ in MD simulations

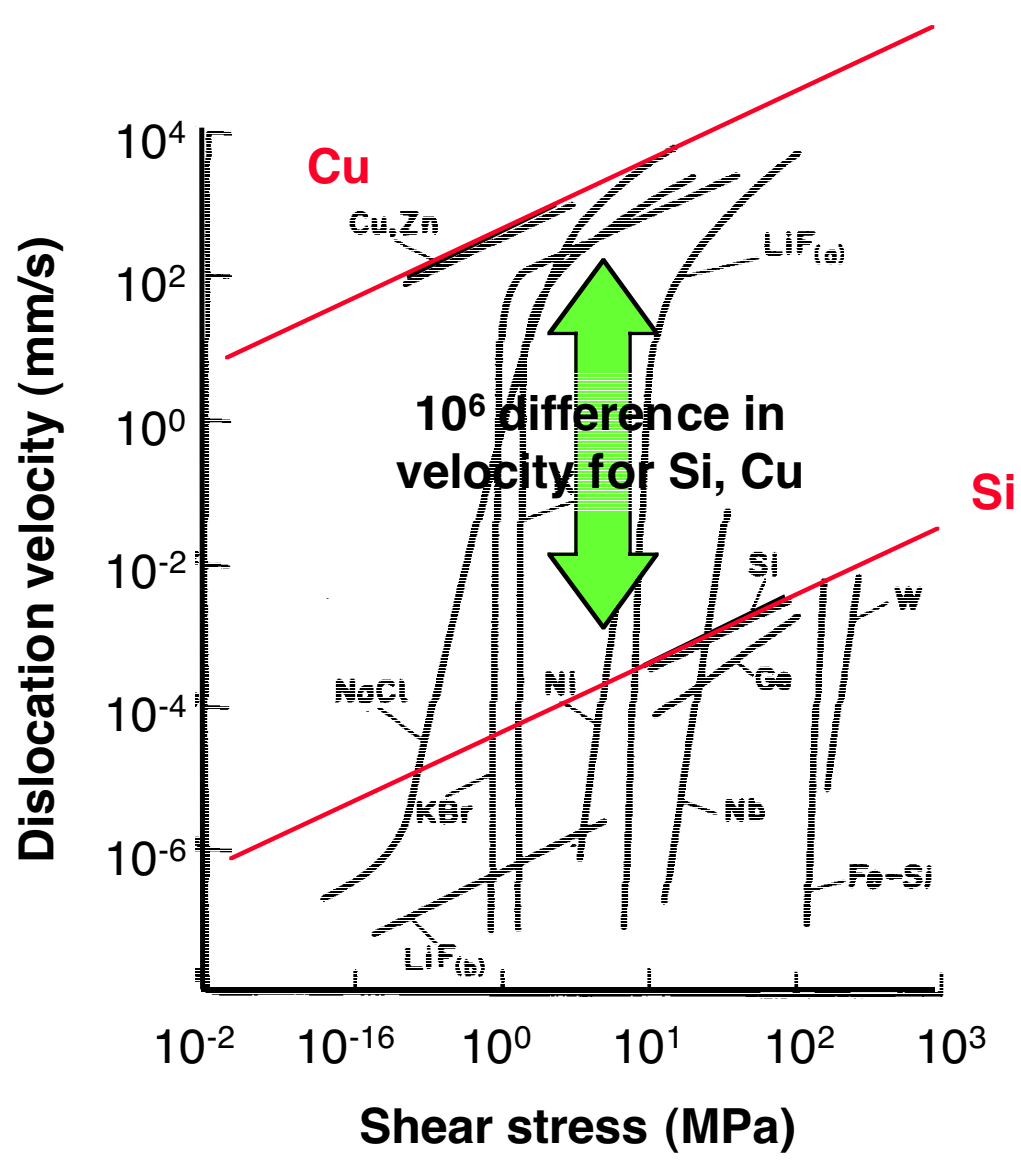

Introduction to Dislocations, Hull and Bacon (Fig. 3.12) 
The time scale for plastic deformation in $\mathrm{Si}$ is much longer than for $\mathrm{Cu}$, due to its high Peierls barrier and activation energy

- For $\sigma<\mathrm{Y}_{\mathrm{P}}$ : thermal activation regime, and

$$
t=t_{\text {wait }}=\frac{1}{v_{\text {attempt }} \exp \left[\frac{-\Delta F}{k T}\left(1-\frac{\sigma}{Y_{P}}\right)^{2}\right]}
$$

- For $\sigma>\mathrm{Y}_{\mathrm{p}}$ : phonon drag regime, $t=t_{r u n}=\frac{a B}{\sigma b}$

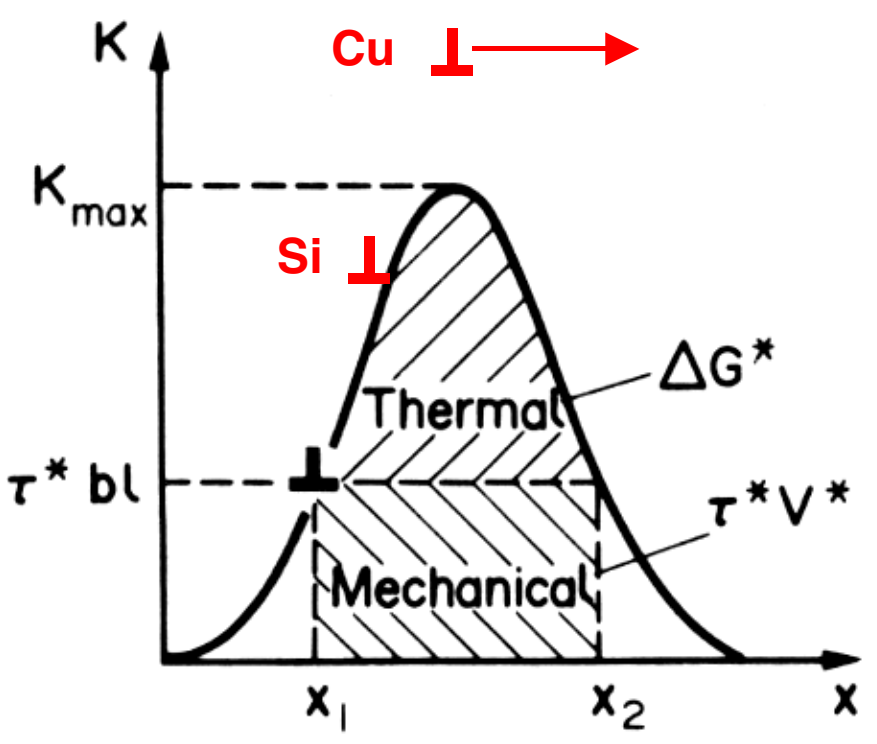

Assume that $\sigma=3 \mathrm{GPa}$, and $\mathrm{kT}=0.05 \mathrm{eV}$ :

\section{For Si:}

$Y_{P}=0.07 G_{0}\left(1+A P / \eta^{1 / 3}\right)>0.07 G_{0}$ giving $Y_{P}>0.07(63.7 \mathrm{GPa})=4.5 \mathrm{GPa}$, $\Delta \mathrm{F}=0.2 \mathrm{~Gb}^{3}=0.2(63.7 \mathrm{GPa})(3.83 \mathrm{~A})^{3}=4.5 \mathrm{eV}$

So $\sigma \ll Y_{P}$, and $k T \ll \Delta F$ : thermal activ. regime

Assume $v_{\text {attempt }}=v_{\text {Debye }} / 100=10^{11} \mathrm{~s}^{-1}$, So $1 / t_{\text {wait }}=\left(10^{11} \mathrm{~s}^{-1}\right) \exp \left[-(4.5 / .05)(1-3 / 4.5)^{2}\right]$

Giving $t_{\text {wait }}>\sim 150$ ns, meaning slow

\section{For Cu:}

$Y_{P}=\left(6.3 \times 10^{-3}\right) G_{0}\left(1+A P / \eta^{1 / 3}\right)=0.42 \mathrm{GPa}$ So $\sigma>Y_{P}$, meaning phonon drag regime

Assume $\mathrm{B}=10^{-10} \mathrm{MPa} \cdot \mathrm{s}$, and $\mathrm{a} / \mathrm{b}<10^{3}$,

So $t_{\text {run }}<\left(10^{3}\right)\left(10^{-10} \mathrm{MPa} \cdot \mathrm{s}\right) /(3 \mathrm{GPa})=30 \mathrm{ps}$ : fast 


\section{A new wide-angle film detector is used to record many more lattice planes}

- X-rays diffracted from orthogonal lattice planes are recorded with 2 xray streak cameras

- A segmented film assembly records $x$-rays diffracted over a $\pi$-solid angle from many more lattice planes

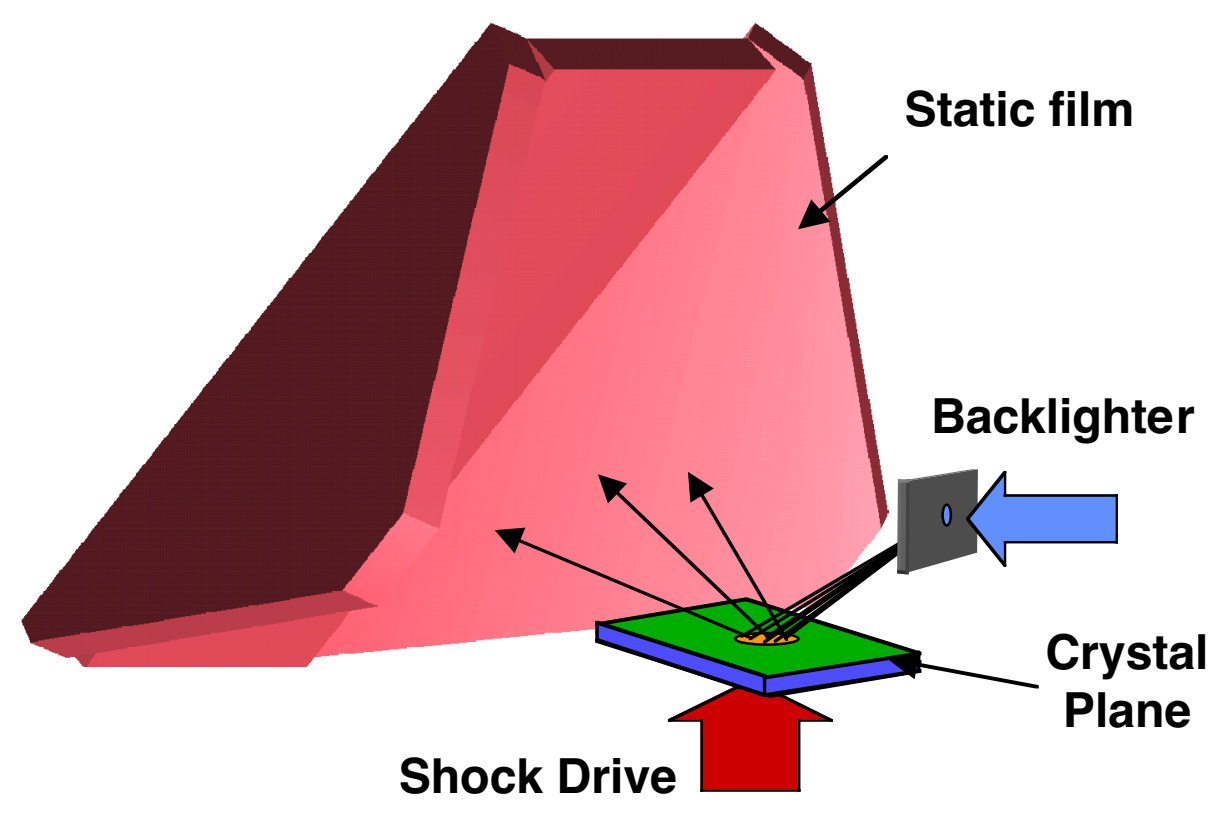

Calculated diffraction pattern from static $\mathrm{Cu}$

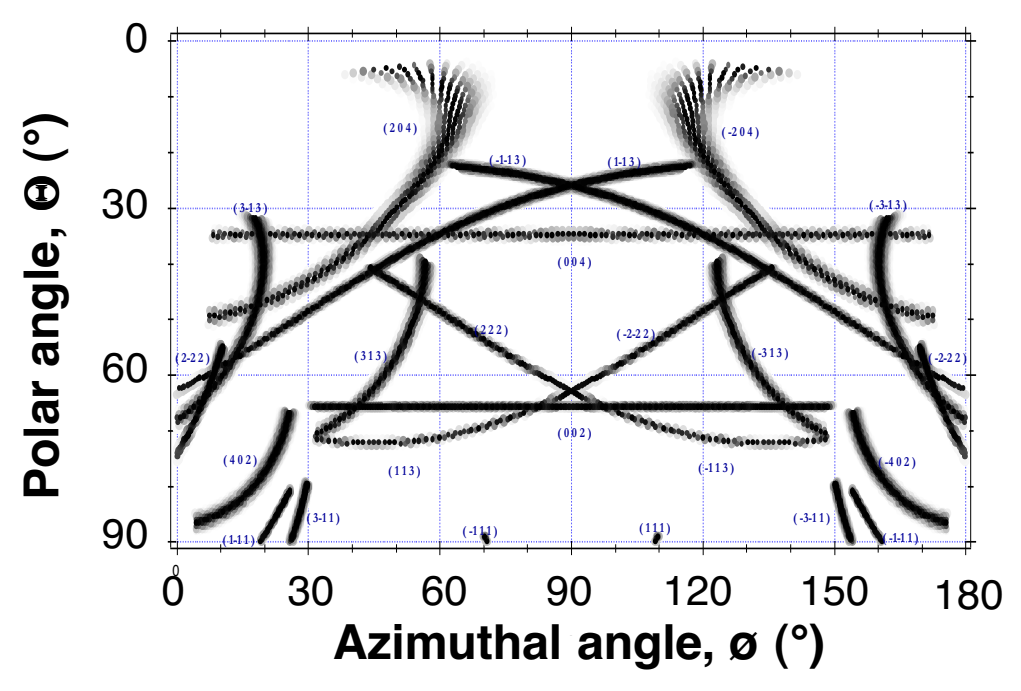




\section{Detailed response of the lattice is better understood by recording diffraction from other lattice planes}

- Large angle detector has been fielded on Si shock experiments

- Shift of many different lines is observed; details are being studied

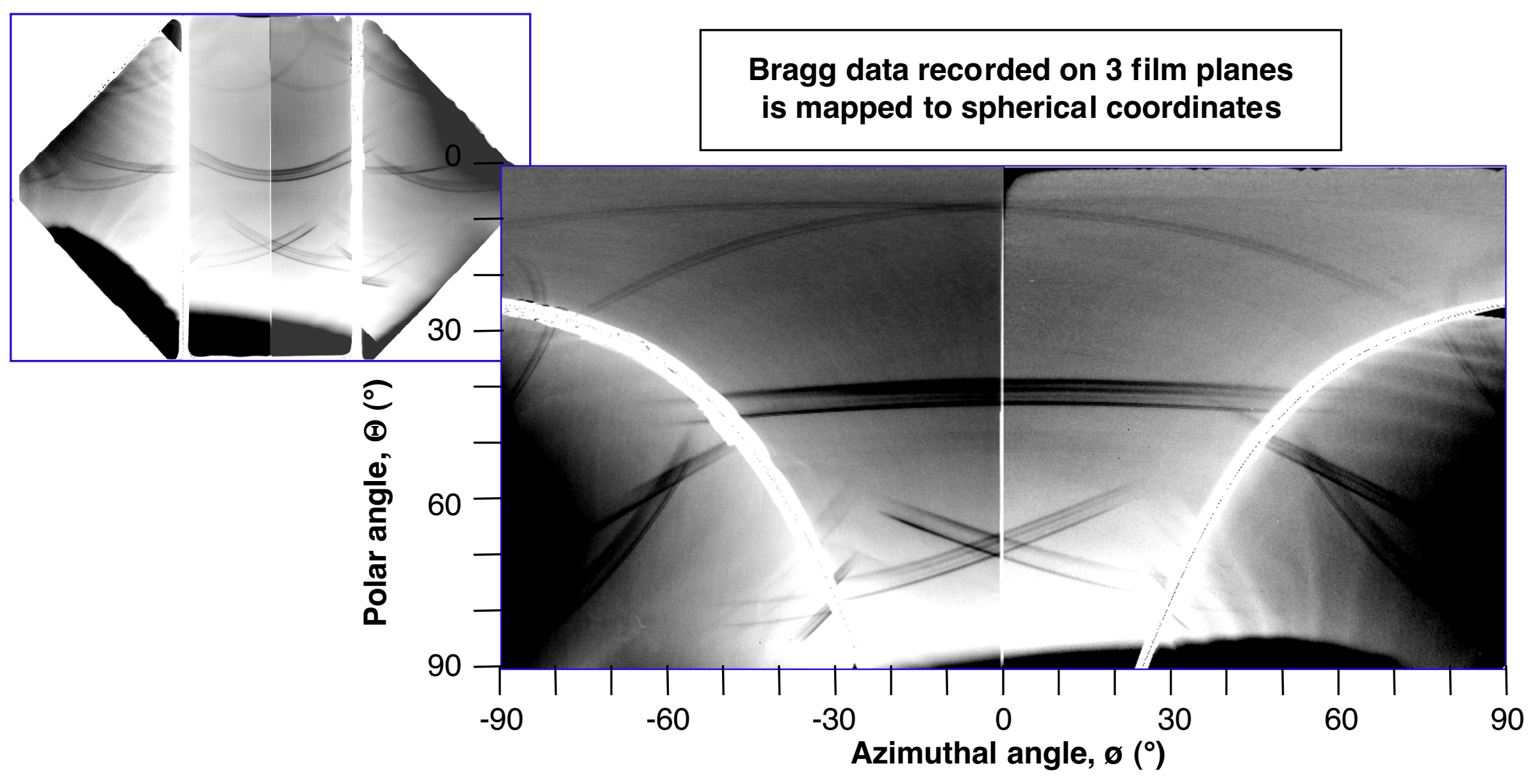




\section{VISAR wave profiles and sample recovery}

- Al-6061 wave profile measurements show elastic-plastic response with spall on release

- Fitting the shock breakout wave profile provides best-fit strength parameters
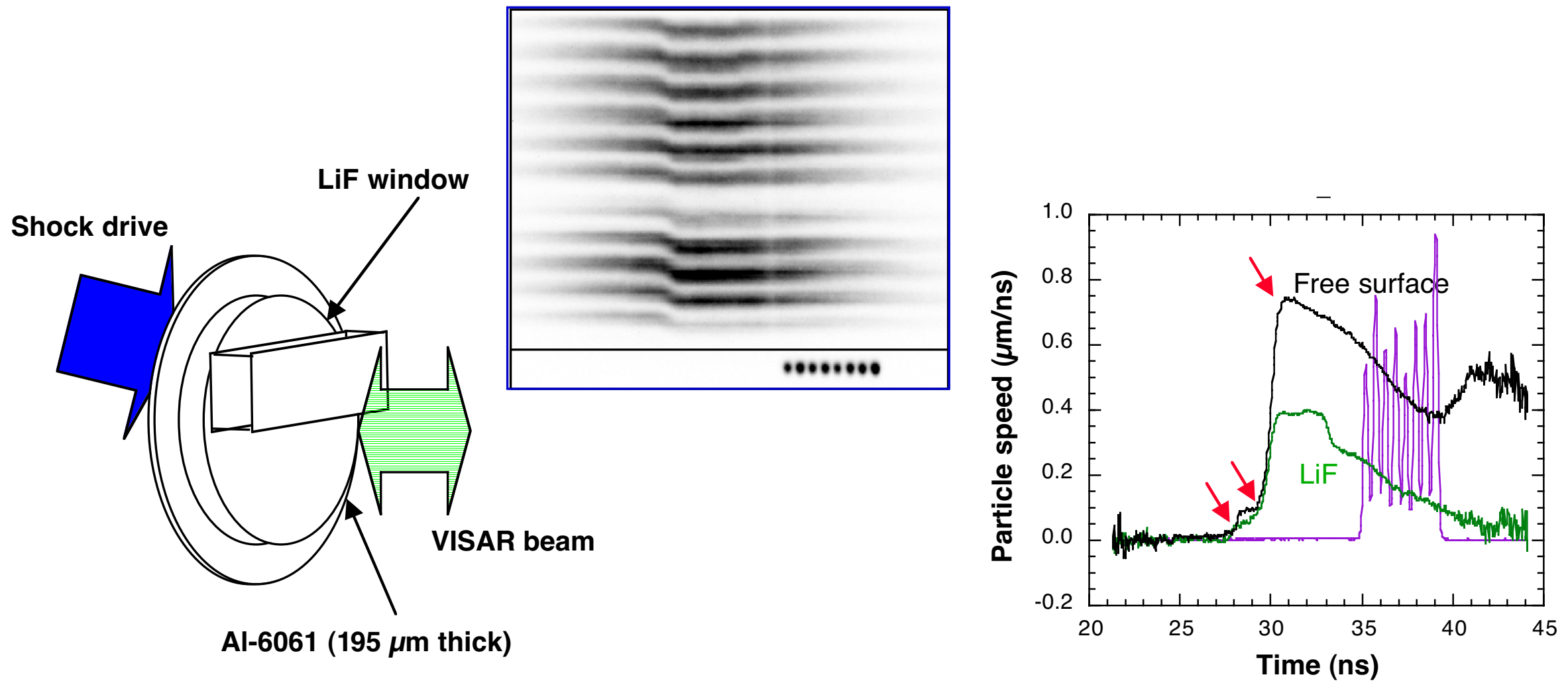


\section{VISAR wave profiles provide information on the strength parameters for the shocked metal}

- The wave profile is sensitive to the constitutive model parameters for the metal foil

- Best-fit wave profile provides model parameters:

- Shear modulus G=320 kbar (276)

- Bulk modulus K=794 kbar (742)

- Yield strength $Y=4.27$ kbar (2.9)
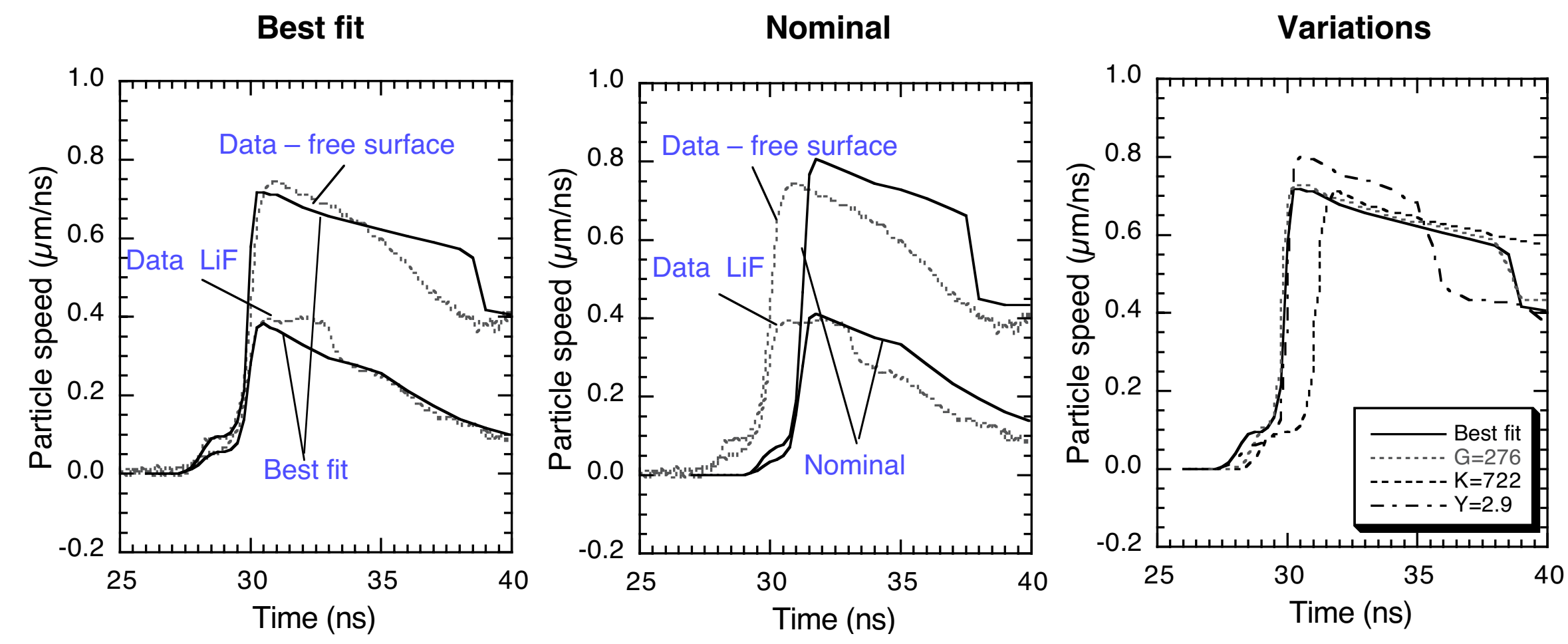
Transmission electron and optical microscope analysis shows residual structure that depends on the drive conditions

- Shocked samples are recovered in a low density foam-filled tube

- Preliminary tests done at OMEGA; shock pressure is $\sim 400 \mathrm{kbar}$, decays to $\sim 25 \mathrm{kbar}$ at the rear surface

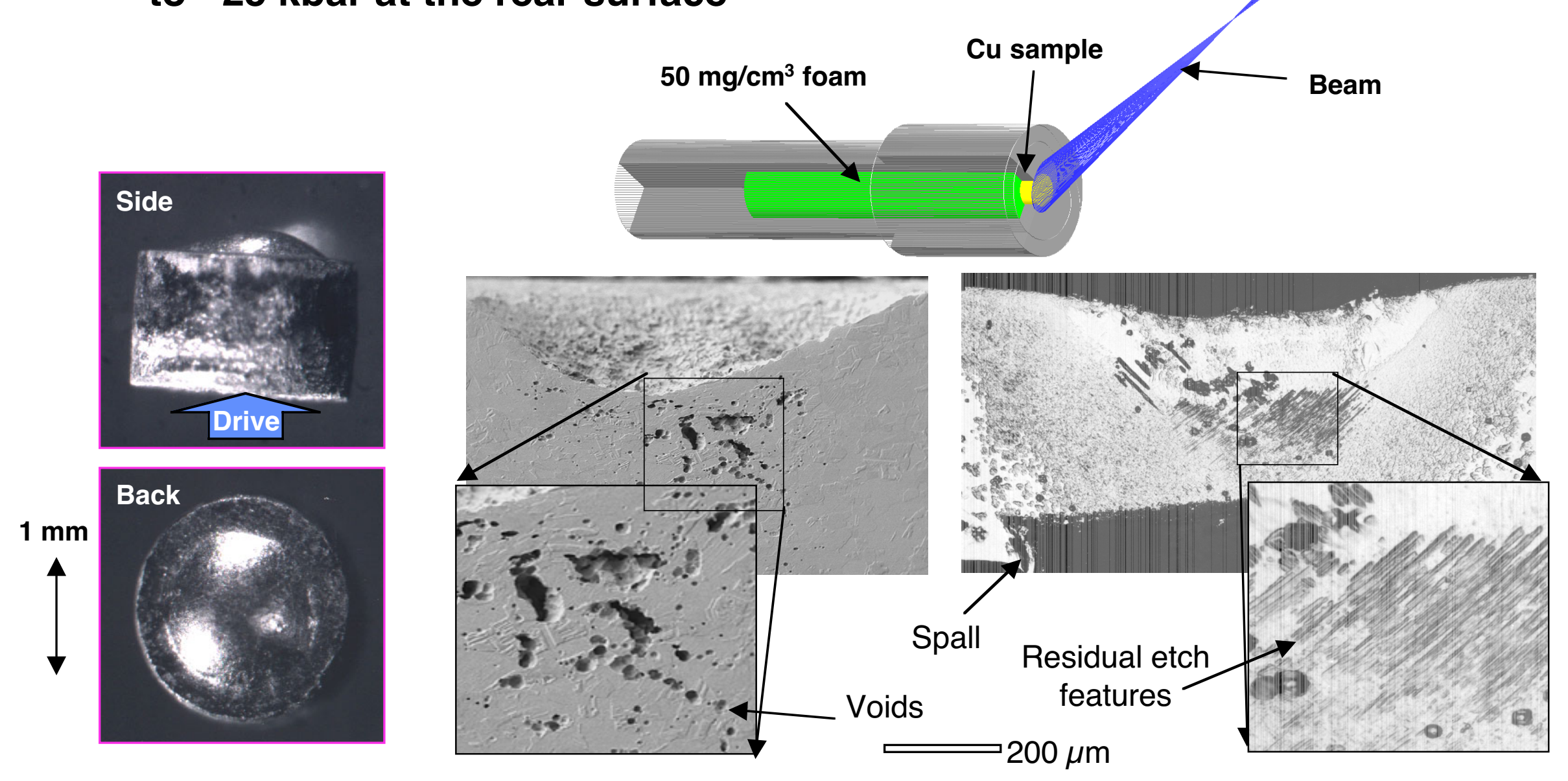




\section{TEM analysis of recovered $\mathrm{Cu}$ shows the residual microstructure}

- Residual microstructure of recovered single crystal Cu samples

- Higher pressures show twinning

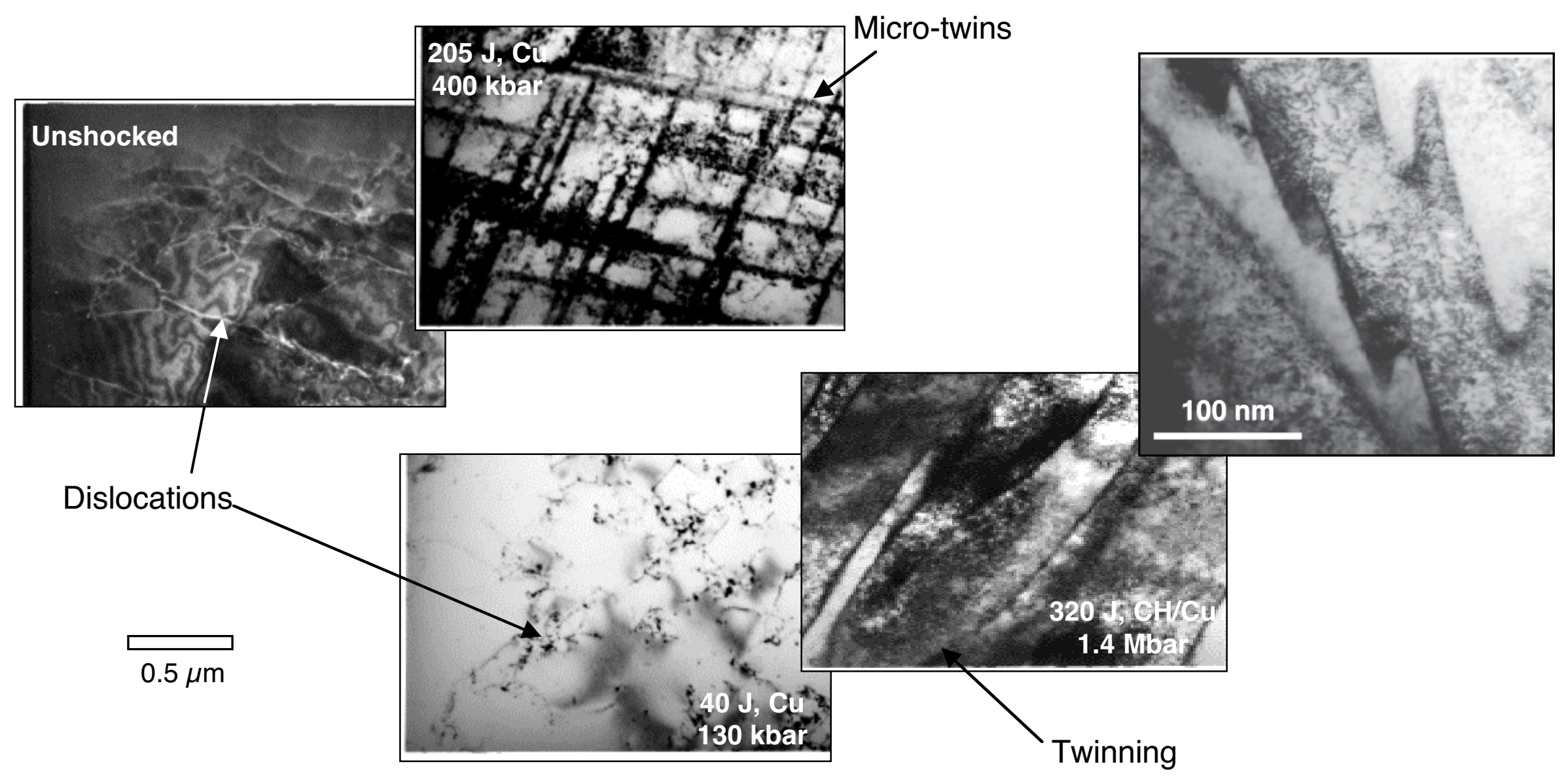




\section{Summary}

- Solid state hydrodynamic instability

- RT instability in Al to infer $Y(P)$

- There is possible imprinting due to the initial grain structure

- In situ dynamic x-ray diffraction

- Time-resolved diffraction relates the lattice behavior to the macroscopic response of $\mathrm{Si}$ and $\mathrm{Cu}$ under shock loading

- Si responds uniaxially, Cu deforms plastically

- Shock/recovery experiments

- Residual deformation structure in Cu depends on the shock pressure 
$8^{\text {th }}$ International Workshop on Physics of Compressible Turbulent Mixing

Pasadena, USA, December 9 - 14, 2001

\title{
RFNC-VNIITF MULTIFUNCTIONAL SHOCK TUBE FOR INVESTIGATING THE EVOLUTION OF INSTABILITIES IN UNSTATIONARY GASDYNAMIC FLOWS
}

\author{
Yu. A. Kucherenko, O. E. Shestachenko, S. I. Balabin, and A. P. Pylaev \\ Russian Federal Nuclear Center - Academician E. I. Zababakhin \\ All-Russian Research Institute of Technical Physics (RFNC-VNIITF) \\ 456770 Snezhinsk, Chelyabinsk region, P.O.245, Russia \\ E-mail: kucherenko@five.ch70.chel.su
}

\begin{abstract}
Parameters of flows in the RFNC-VNIITF shock tube at its operation under three modes are given. In the first, ode a stationary shock wave is formed. This makes it possible to investigate the evolution of the Richtmyer-Meshkov instability and the turbulence induced by it. In the second mode, in the shock tube a nonstationary shock wave is formed that makes it possible to investigate the behaviour of the contact boundaries of different density gases when the conditions for the evolution of the RichtmyerMeshkov and Rayleigh-Taylor instability are realized. In the third mode a compression wave is formed that makes it possible to investigate the evolution of the Rayleigh-Taylor instability and the turbulence induced by it.
\end{abstract}

\section{Introduction}

In spite of a great number of investigations performed with a view to study the evolution of hydrodynamic instabilities and turbulent mixing associated with them in gases, there are many questions hat have not been studied as a result of the experimental technique imperfection.

When studying the turbulence induced by the action of the Richtmyer-Meshkov instability, a great uncertainty takes place, which is associated with the parameters of diaphragms separating the different density gases at the initial instant of time. At the same time, the influence of the diaphragms is such that the obtained turbulent flow "does not forget" the initial conditions. This leads to the turbulent flow structure distortion and, as a consequence, to great errors when measuring the turbulence parameters.

When studying the turbulence induced by the action of the Rayleigh-Taylor instability, the role of the separating diaphragms is also rather important. This problem is especially significant when studying the self-similar mode of mixing at which it is important to have the self-similar spectrum of perturbations at the contact boundary of different density gases. A great uncertainty is, possibly, associated with this circumstance in the determination of the non-dimensional rate of different density gases in the self-similar mode. This mode is characterized by the constant rate of the mixing zone width growth, at the same time, the mixing zone width $\mathbf{L}$ depends only on the density ratio of miscible media $\mathbf{n}$ or Atwood number $\mathrm{A}=(\mathbf{n}-1) /(\mathbf{n}+1)$, the contact boundary acceleration $\mathbf{g}_{\mathbf{1}}$ and time $\mathbf{t}$ :

$$
L \sim A g_{1} t^{2}
$$

The self-similar mode of the gravitational turbulent mixing is everywhere used both for the calibration of the semiempirical models of mixing and for the mathematical modeling of mixing processes due to the minimum number of parameters determining this mode. The proportionality coefficient in the relation (1), which represents the non-dimensional rate of mixing, is determined in experiments and is estimated at the numerical modeling.

It is known [1] that the gravitational turbulent mixing process of different density media is processed of the definite asymmetry which consists in the fact the fronts of the penetration of the light medium into the heavy one and the heavy medium into the light one are spreading with different 
velocity. Historically the non-dimensional rate $\alpha_{b}$ of spreading the light medium front into the heavy one is assumed to be the characteristic of the gravitational turbulent mixing [2]. Denoting the light medium penetration front coordinate counted off from the contact boundary as $\mathrm{L}_{12}$, it is possible to write down

$$
\mathrm{L}_{12}=2 \alpha \mathrm{AS}
$$

where $\mathbf{S}=\mathbf{g t}^{2} / 2$

Results obtained in experiments with different density liquids [1- 4] give the value of $\boldsymbol{\alpha}$ being found in the range of

$$
\alpha=0.06-0.07
$$

At the same time, the results obtained in the work [5] with different density gases give the magnitude of this value, which exceeds the above one shown more than by a factor of two. The reasons of such a difference have not been elucidated up to now. It may be proposed that in the work [5] either the conditions of self-similarity were not satisfied in the set-up of experiments or the measurements were made a the nonlinear stage of the Rayleigh-Taylor instability evolution, when the initial experimental conditions "were not yet forgotten". The last argument is supported by the absence of the direct control of the initial conditions when performing experiments in this work. Moreover, the factor of compressibility can exert an influence on the result in the work [5]. However, the investigations performed in the work [6] with compressible media have shown that the values of $\alpha_{b}$ for different combinations of gases are found in the range of

$$
\alpha=0.052-0.098 \text {. }
$$

In the works $[7,8]$ the numerical three-dimensional modeling of the gravitational turbulent mixing evolution has been carried out by means of different mathematical codes. In the work [7] the value of

$$
\alpha \approx 0.052
$$

was obtained, but in work [8] this value is in the range of

$$
\alpha=0.04-0.06 \text {. }
$$

Thus, it is seen that the results obtained in the work [5] for gases are contradictory. This contradiction is, most likely, associated with the experimental technique imperfection. The study of the turbulence induced by the successive action of the Richtmyer-Meshkov and Rayleigh-Taylor instabilities has not yet been performed up to now. However, this situation is rather often realized when studying the operation of laser targets in the problem of the inertial thermonuclear fusion. The absence of such work being set up under laboratory conditions is, apparently, associated with the absence of the appropriate experimental technique.

The multifunctional shock tube (MST) being developed at present in RFNC-VNIITF will make it possible to solve a number of fundamental problems of nonstationary turbulence which were described above. In the present work three modes of the MST operation associated with the shown problems are described. This development has been the result of the RFNC-VNIITF and LLNL collaboration and initially it has been known as the Project "BIZON". 


\section{Multifunctional shock tube with driver I}

The physical scheme of MST with driver I is presented in Fig 1.

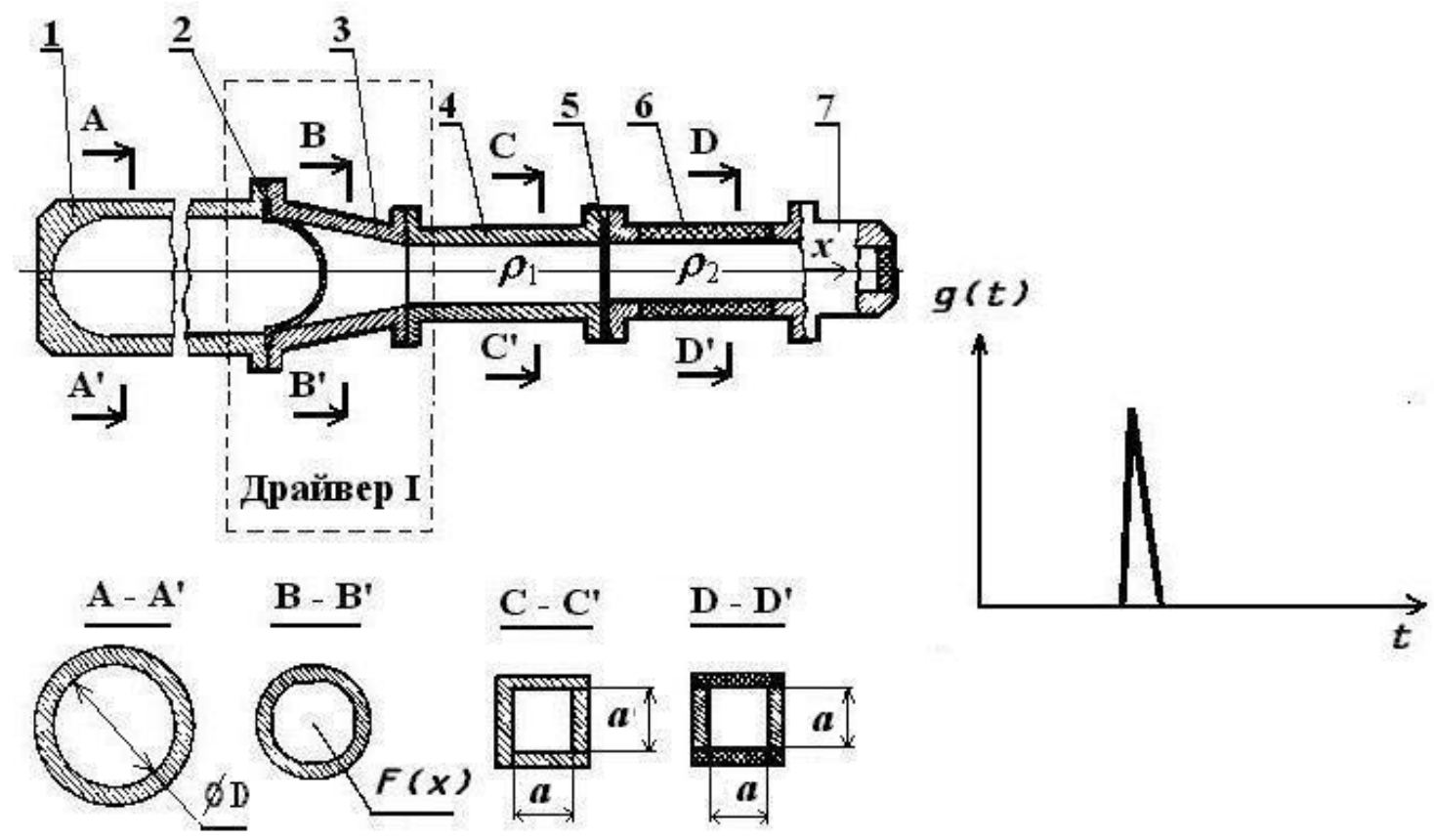

Fig. 1. The physical scheme of MST with driver I

This driver intends to be used for studying the Richtmyer-Meshkov instability and the turbulent mixing induced by it. One of the investigated gases with density $\rho_{1}$ is located in the measuring section I (4), second gas with density $\rho_{2}$ - in the measuring section II (6). At the initial instant of time a separating membrane (5) is found between gases. The composition of driver I includes a high pressure chamber (1), a high pressure membrane (2), a transitional section (3) and a part of the measuring section I. Driver I operates as follows. Gas is forced into the high-pressure chamber up to such pressure $\mathbf{P}_{0}$, at which the high-pressure membrane is opened. The gas flow rushes into the transitional section and then into the measuring section I creating a shock wave (SW). The function of the transitional section consists in coordinating the round cross-section A-A of the high-pressure chamber with the square cross-section C-C of the measuring section I. The cross-section A-A of the highpressure chamber is chosen to be round proceeding from the considerations of its strength and technology to mount the high-pressure membrane on it. C-C and D-D cross-section of the measuring sections were chosen to be right-angled (square), proceeding from the convenience to register the turbulent mixing parameters by the light techniques. The cross-section of the other form would nduce difficulties associated either with taking into account the additional refraction of light beams or with mounting the plane transparent windows on the non-planar walls of the measuring sections. The transitional section along the axis $\mathrm{x}$ is of a variable cross-section $\mathbf{F}(\mathbf{x})$ which changes from the round cross-section to the square one. At the same time, the gas flow form is smoothly changed. The intensity of the shock wave (SW) being created is determined by the value of pressure $\mathbf{P}_{\mathrm{o}}$. The part of the measuring section I is used to generate a stationary SW propagating through a low-pressure gas. The required length of the stationary SW determines the length of this part of the measuring section. As a result of the SW passage through the contact boundary of gases, the contact boundary undergoes he impulsive acceleration whose character is shown in the right part of Fig.1. Mach number of the stationary SW generated by the driver I amounts to $\mathbf{M} \leq 5$. 
The separating membrane (5) performs two functions. Firstly, it stabilizes the contact boundary $(\mathrm{CB})$ of different density gases while preventing the mixing of gases prior to the SW arrival at CB. Secondly, by means of this membrane, at the initial instant of time, the zone of initial perturbations with specified parameters is created at the contact boundary. Such a membrane has, actually, been developed and has been named the "specter-diaphragm". Its distinctive features are its initiation from the external force and its disappearance just after the creation of the initial perturbations zone.

The length of the measuring section II is chosen depending on the problem being solved. If a single passage of SW through the zone of mixture is required, then the outlet section (7) is used for this purpose, which prevents from the creation of the reflected shock waves.

\section{Multifunctional shock tube with driver II}

The physical scheme of MST with driver II is presented in Fig.2.

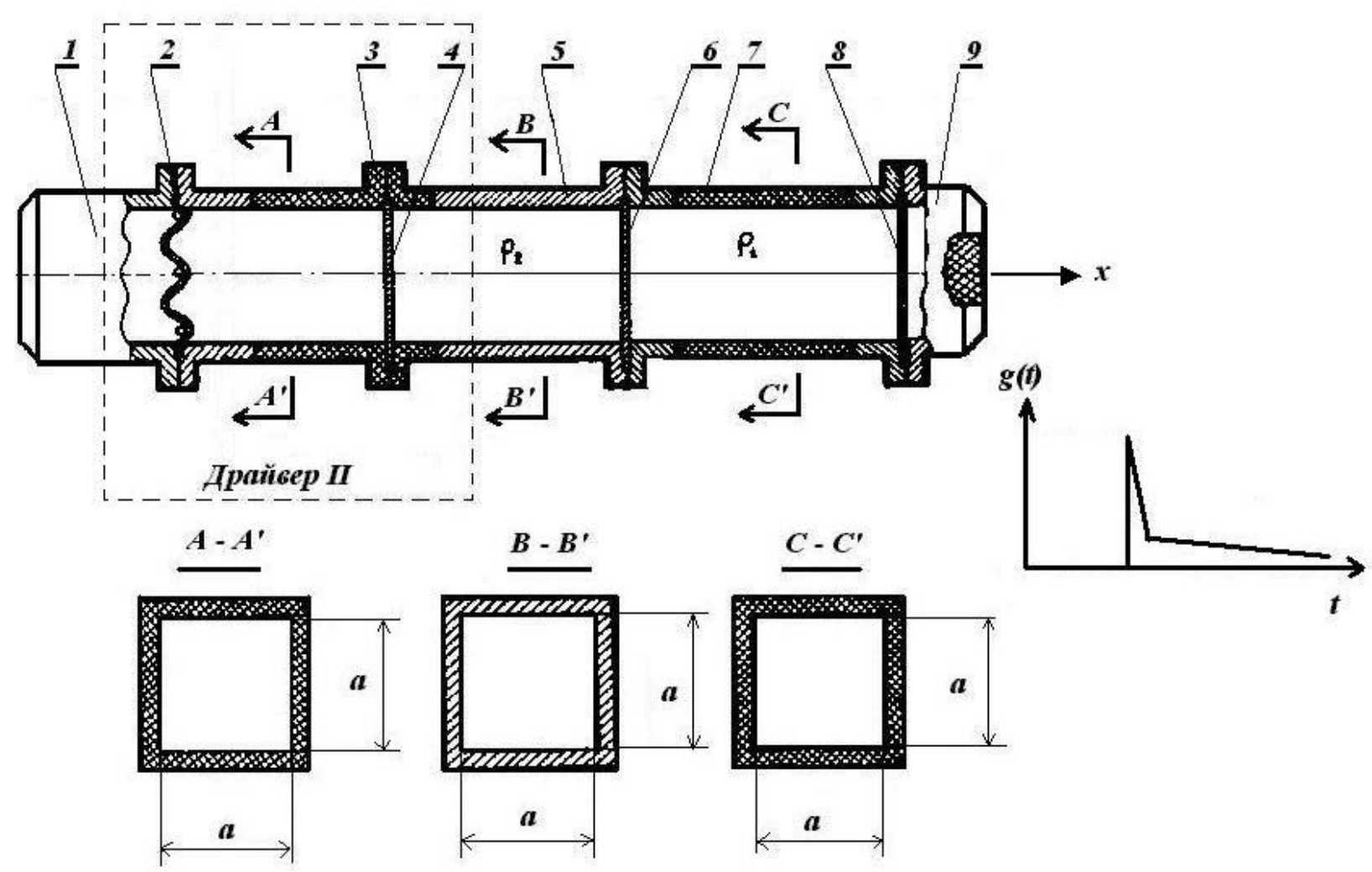

Fig.2. The physical scheme of MST with driver II

This driver intends to be used for investigating the successive action of the RichtmyerMeshkov and Rayleigh-Taylor instabilities and the turbulent mixing induced by them. One of the gases (heavier one) being investigated and having density $\mathrm{p} 2$ is placed into the measuring section I (5), but the lghter gas of density $p$ is placed into the measuring section II (7). At the initial instant of time the separating membrane (5) separates gases. This membrane performs the same function as in the case with driver I. Driver II includes a vacuum section (1), a restraining membrane (2), a section of the electrically exploded foil (EEF) (3) and the electrically exploded metal foil (4). The metal foil separates the measuring sections I from the section of EEF. The restraining membrane separates the section of $\mathrm{EF}$ from the vacuum section. At the moment of the metal foil blasting a shock wave (SW) is formed which propagates into both sides. When reaching the restraining membrane (2), AW ruptures 
it, and gas moving behind SW begins to flow into vacuum. As a result, a rarefaction wave is formed which propagates toward the side of the measuring section I. This rarefaction wave overtakes SW, which propagate along the measuring section I. As a result, a nonstationary SW with pressure sharply dropping at the back front flls on the contact boundary. Pressure at the leading front of SW can reach $5^{*} 10^{5} \mathrm{~Pa}$ at the distance of $500 \mathrm{~mm}$ from the point of the electrically exploded foil location. Thus, the leading front of the shock wave creates the Richtmyer-Meshkov instability after passing through the contact boundary of gases and then the different density gases underwent the action of the RayleighTaylor instability in the region of the contact boundary, because the gradients of pressure and density are directed in the opposite sides. For MST with the driver II the contact boundary acceleration dependence on time is shown in the right part of Fig.2. At first the contact boundary is accelerated in the pulsed mode, then it moves with almost constant acceleration. In case of MST vith driver II all the section have the same square cross-section.

\section{Multifunctional shock tube with driver III}

The physical scheme of MST with driver III is presented in Fig.3.

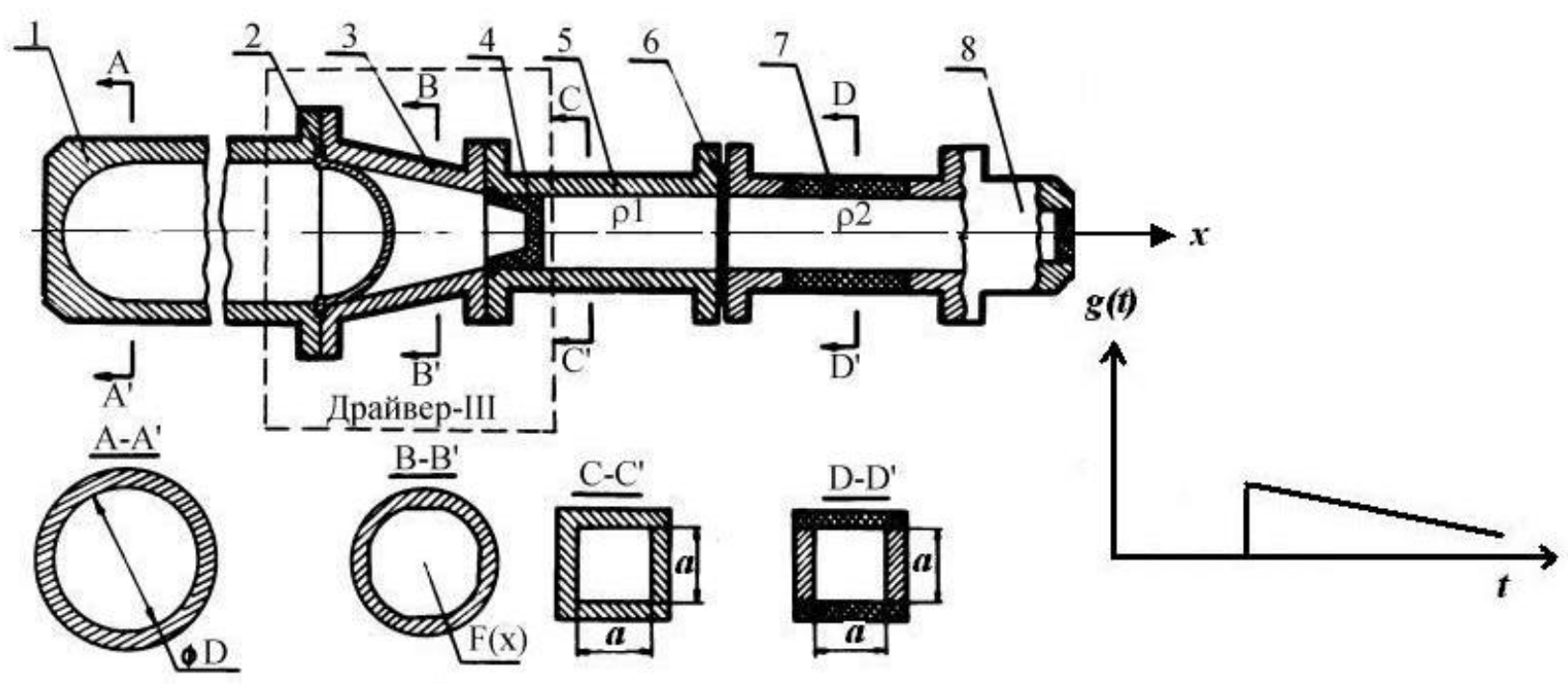

Fig.3. The physical scheme of MST with driver III

This driver intends to be used for studying the Rayleigh-Taylor instability and the turbulent mixing induced by this instability. The Lighter gas of density $\mathrm{p}_{1}$ is placed into the measuring section I (5), the other heavier gas of density $\mathrm{p}_{2}$ is located in the measuring section II (7). The separating membrane (6) separates gases. This membrane performs the same functions as in two preceding cases with drivers II and I. Driver III includes a high-pressure chamber (1), a high-pressure membrane (2), a transitional section (3) and a piston (4). The high-pressure chamber, the high-pressure membrane (2) and the transitional section of the driver III perform the same functions as in the driver I. However, here the gas flow does not form the stationary shock wave after passing through the transitional section, but accelerates the light piston. Then piston moving with acceleration creates a compression wave before itself, which is transformed into a shock wave in time. In the case of driver III the separating membrane and, consequently, the contact boundary of gases should be arranged at some distance from the initial position of the piston in order that by the moment of the arrival at the contact boundary the compression wave could not be transformed into SW. The pressure profile in the 
compression wave is such that pressure drops in the positive direction of the axis $\mathbf{x}$. Therefore, the gradients of pressure and density in the region of the contact boundary of different density gases will be directed to the opposite sides, therefore, the contact boundary of gases will be found under condition of the Rayleigh-Taylor instability. This boundary will move along the axis $\mathbf{x}$ with almost constant acceleration whose dependence on time is shown in the right part of Fig.3. The maximum value of the contact boundary acceleration may reach the value of $\mathrm{g}_{1}=10^{5} \mathbf{g}$, where $\mathbf{g}$ is the acceleration of the Earth's gravitational field. The registration of the evolution process of the instability and the turbulent mixing of gases in the region of the contact boundary during its motion with acceleration is completed in the measuring section II before the piston will reach this region. Subsequently, the piston will get into the outlet section (8) where its deceleration takes place. For the same reasons which are shown when describing MST with driver I, the high pressure chamber crosssection is chosen to be round, but the cross-section of the measuring sections - to be square one. The transitional section is of the variable cross-section $\mathbf{F}(\mathbf{x})$ that ensures the smooth change of the form of the gas flow at its transition from the high pressure chamber to the measuring section I.

\section{Conclusion}

The multifunctional shock tube, which has been developed in RFNC-VNIITF in collaboration with colleagues from Lawrence Livermore National Laboratory and has made it possible to realize three different modes of nonstationary gas dynamic flows, will give possibility to accomplish applied tasks in the interests of solving the inertial thermonuclear fusion problems and for the development of different cumulative devices.

The multifunctional shock tube provides the following parameters of gas dynamic flows:

- $\quad$ in the mode with driver I a stationary shock wave with Mach number $\mathbf{M} \leq 5$ is generated;

- in the mode with driver II a nonstationary shock wave is generated whose initial pressure at the front is $5^{*} 10^{6} \mathrm{~Pa}$ with the acceleration of the contact boundary of different density gases behind the wave front $\mathbf{g}_{1} \leq 10^{6} \mathbf{g}$, where $\mathbf{g}$ is the acceleration of the Earth's gravitational field;

- in the mode with driver III a compression wave is generated which ensures the acceleration of the contact boundaries of different density gases $\mathbf{g}_{1} \leq 10^{5} \mathbf{g}$.

In all three modes the gases with density ration $\mathrm{p}_{2} / \mathrm{p}_{1} \leq 34$ may be used.

\section{References}

1. Yu. Kucherenko et al. "Experimental Study of the Gravitational Turbulent Mixing in Self-Similar mode". The $3^{\text {rd }}$ Int. Workshop on Physics of Compressible Turbulent Mixing. Abbey of Royamont, France, 1991.

2. K. Read. "Experimental Investigation of Turbulent Mixing by Rayleigh-Taylor Instability". Physica 12D, 1984.

3. D. Youngs. "Experimental Investigation of Turbulent Mixing by Rayleigh-Taylor Instability". Advances in Compressible Turbulent Mixing.

W. P. Dannevik, A. C. Buckingham, C. E. Leith Editors, 1992.

4. Yu. Kucherenko, G. G. Tomashev, L. I. Shibarshov . "Experimental Investigation of the Gravitational Turbulent Mixing in Self-Similar mode". Rus. J. VANT, Series Theoretical and Applied Physics, Issue 1,1988. 
5. A. Vasilenko, V. Olhovskaya, O. Buryakov, V.Yakovlev . "Experimental Investigations for Turbulent Mixing of Gases at the Interface under the Influence of the Decelerating shock wave". The $3^{\text {rd }}$ Int. Workshop on Physics of Compressible Turbulent Mixing. Abbey of Royaumont, France, 1991.

6. S. G. Zaitsev et al. "Evolution of the Rayleigh-Taylor Instability in Compressible Media". Report under ISTC Project ${ }^{1}$ 96-029.

7. N. Anuchina, V. Gordeichuk, N. Es`kov, O. Ilyutina, O. Kozyrev, V. Volkov. "Three-dimensional Numerical Simulation of Rayleigh-Taylor Instability by MAH-3 Code". The $6^{\text {th }}$ Int. Workshop on Physics of Compressible Turbulent Mixing. Marseilles, France, 1997.

8. D. Youngs. "Three-dimensional Numerical Simulation of Turbulent Mixing by Rayleigh-Taylor Instability". Phys. Fluids A3 (5), 1991., 


\section{HYDRODYNAMIC INSTABILITIES

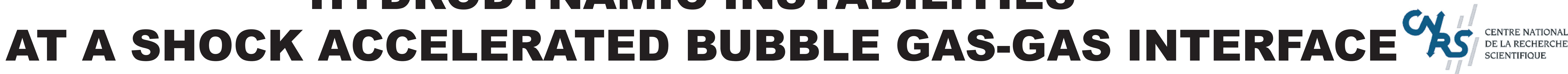

G. LAYES*, G. JOURDAN*, P. ROUALDES * and L.HOUAS*

*IUSTI, UMR CNRS 6595, Université de Provence, Technopôle de Château-Gombert, 5 rue Enrico Fermi, 13453 Marseille Cedex 13, FRANCE *Centre d'Etudes de Gramat, 46500 Gramat, FRANCE
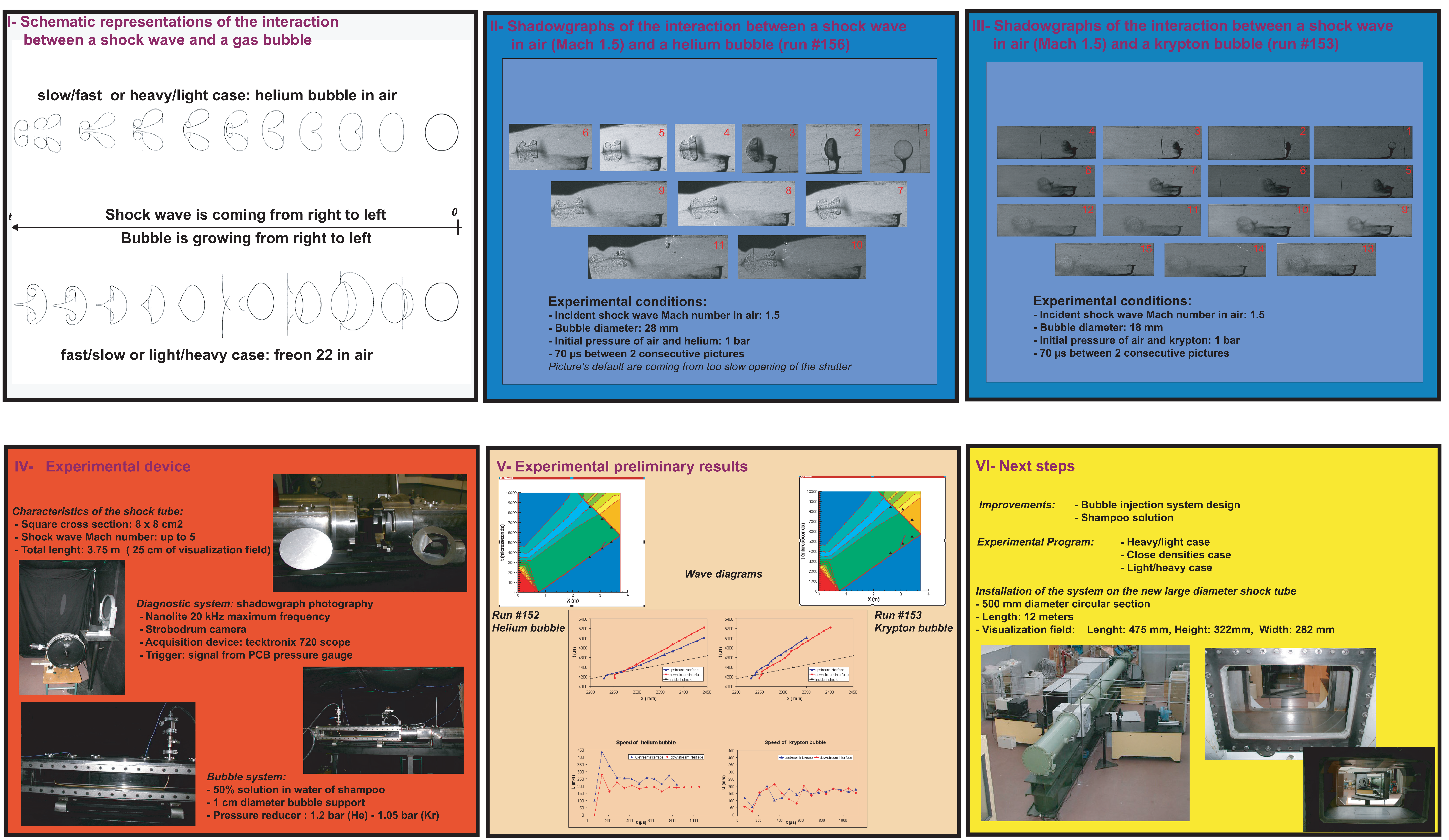


\title{
Scaling in the Shock-Bubble Interaction
}

\author{
K. Levy ${ }^{(1,2)}$, O. Sadot ${ }^{(1,2)}$ A. Rikanati ${ }^{(1,2)}$, \\ D. Kartoon ${ }^{(1,2)}$, Y. Srebro(1,2), A. Yosef-Hai ${ }^{(1,2)}$, \\ G. Ben-Dor ${ }^{(1)}$, D. Shvarts ${ }^{(1,2)}$
}

1) Ben-Gurion Univeristy, Beer Sheva, Israel

2) Nuclear Research Center Negev, Israel 


\section{Introduction}

The phenomenon of shock-bubble interaction is of importance in several differently scaled situations, from fragmentation of gallstones or kidney stones by shock waves (Gracewski et al. 1993) to the interaction of supernovae shock waves with interstellar clouds (Klein et al. 2000). Therefore the scalability of the interaction is important. The passage of a shock wave through a spherical bubble results in the formation of a vortex ring. In the present study, simple dimensional analysis shows that the circulation is linearly dependent on the surrounding speed of sound $c_{s}$ and the initial bubble radius $R$. In addition, it is found that the velocities characterizing the flow field are linearly dependent on the speed of sound, and are independent of the initial bubble radius. The dependence of the circulation on the shock wave Mach number $M$ is derived by Samtaney \& Zabusky $(1994)$ as $\left(1+1 / M+2 / M^{2}\right)(M-1)$. Using the dimensional analysis the velocities are shown to have a similar dependence. Full numerical simulations and experiments were conducted for slow/fast (air-helium) and fast/slow (air- $\mathrm{SF}_{6}$ ) interactions. Good agreement was achieved. From the results it is seen that in both cases, according to the proposed scaling, the vortex ring velocity is bubble radius independent. The numerical rosults for the slow/fast interaction show that the proposed Mach scaling is valid for $\mathrm{M}<2$. Above $\mathrm{M} \cong 2$ the topology of the bubble changes due to a competition between the upstream surface of the bubble and the incident shockwave.

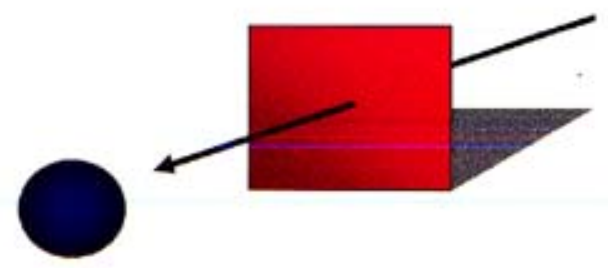




\section{Computational method}

- Leeor-2D*: ALE, interface-tracking, finite differences

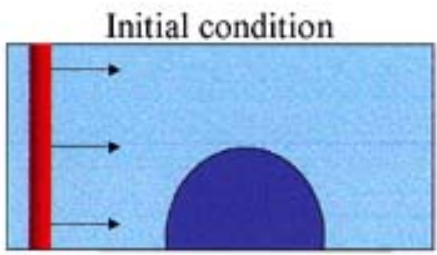

\section{Computational grid $(450 \times 100)$}

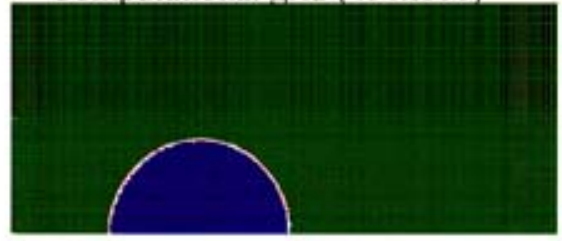

- Ambient gas - air, $E=(\gamma-1) \cdot P / \rho$

- Heavy bubble (f/s) - $\mathrm{Sf}_{6}, \eta=5.034, r_{0}=1.3 \mathrm{~cm}$

- Light bubble (s/f) - Helium, $\eta=0.138, r_{0}=1.63 \mathrm{~cm}$

- Mach range - 1.05 to 4

- Boundary conditions - free slip

- Shock tube width - $4 \mathrm{~cm}$

$$
\eta=\rho_{\text {bubble }} / \rho_{\text {ambient_gas }}
$$

*D. Shvarts et al., Phys. Plasma 2, 2465 (1995) 


\section{Experimental Apparatus}

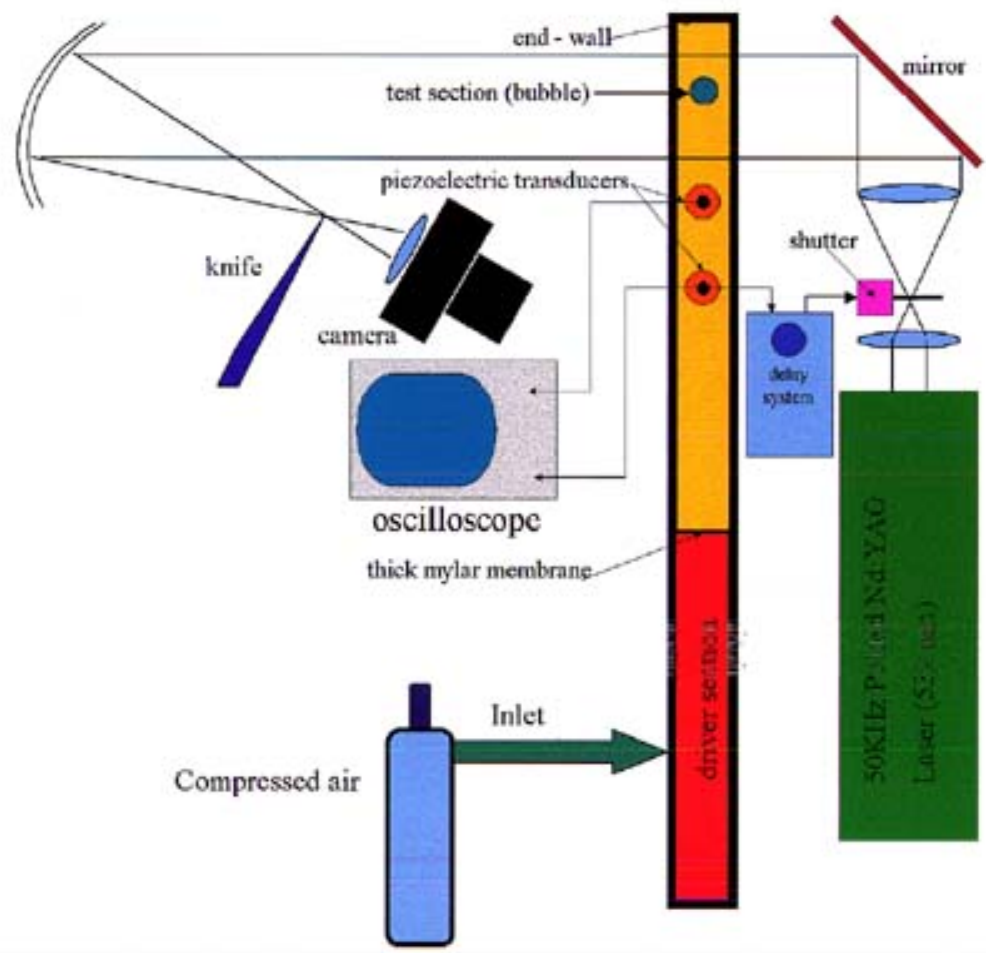




\section{Experiment/simulation comparison slow/fast (air-helium), $M=1.22$}
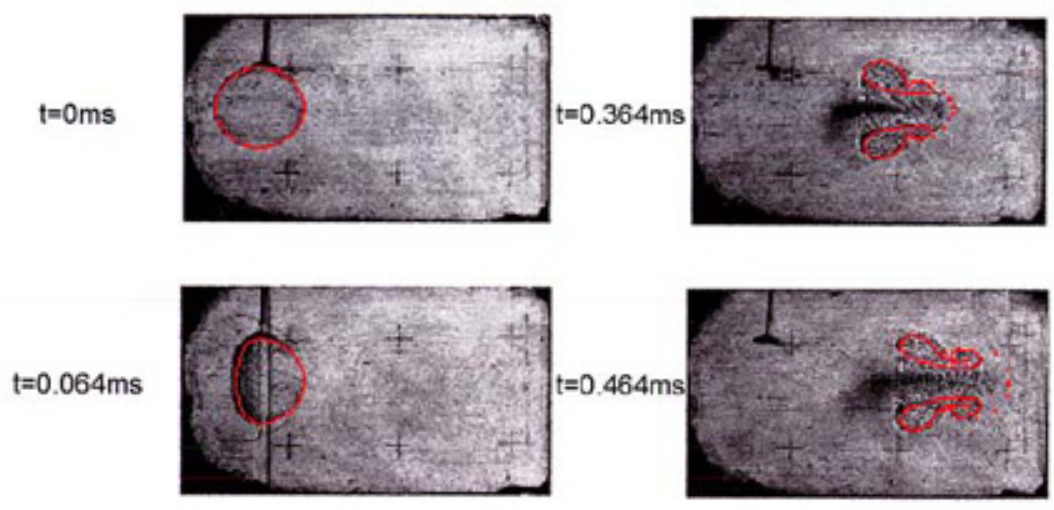

- Schliren images of the experiment

- Red contour: gas interface from the simulation

- Good agreement is found during the early and late times of the interaction.

$t=0.264 \mathrm{~ms}$
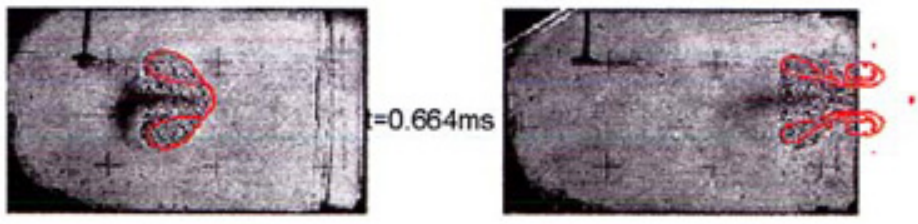


\section{Experiment/simulation comparison}

fast/slow (air-SF 6 ), $M=1.17$

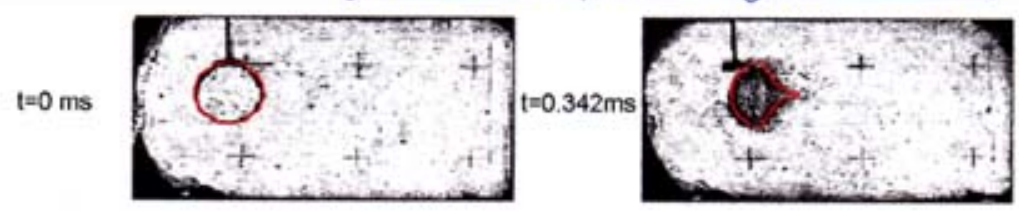

- Schliren images of the experiment

- Red contour:
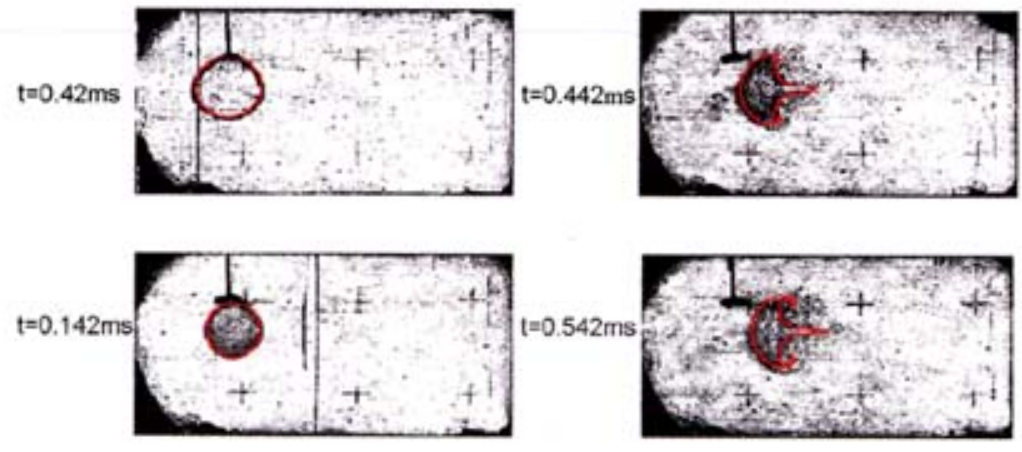
gas interface from the simulation

- Good agreement is found during the early and late times of the interaction.

- Emerging jet is highly sensitive to resolution

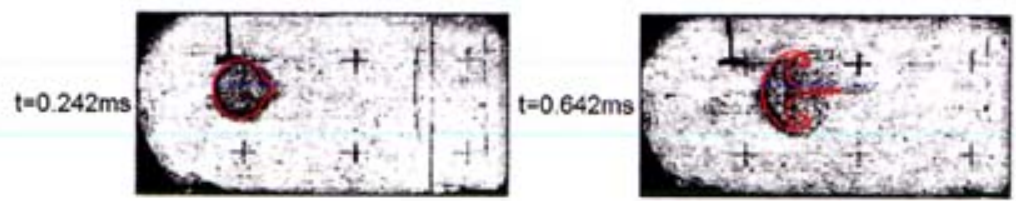
changes. 


\section{Circulation Dimensional Analysis}

- Circulation: $[\Gamma]=[\text { length }]^{2} /[$ time $]$

- Flow parameters: $c_{s}$-speed of sound, $R$ - bubble radius, dimensionless parameters: $M$ - Mach number, $\eta$-density ratio, $\gamma$ - ratio of specific heats of both gases

$$
\Rightarrow \Gamma=f(M, \gamma, \eta) \cdot R \cdot c_{s}
$$

- Using a vorticity deposition model ${ }^{*}$ :

$$
\Gamma=\left(1+\frac{\pi}{2}\right) \cdot \frac{2 \gamma^{1 / 2}}{1+\gamma}\left(1-\eta^{-1 / 2}\right)\left(1+\frac{1}{M}+\frac{2}{M^{2}}\right)(M-1) \cdot R \cdot c_{s}
$$




\section{Velocity Dimensional Analysis}

- Velocity: $[\mathrm{U}]=[$ length $] /[$ time $]$

- Since the flow velocities are determined by the vorticity distribution in the flow:

$\longrightarrow U_{i}=g_{i} \cdot \frac{\Gamma}{R}=g_{i} \cdot f(M, \eta, \gamma) \cdot c_{s}$

$g_{i}$ - is a constant representing the influence of the topology of the problem, the boundary conditions and the specific velocity $U_{i}$.

For example: the velocity of an ideal vortex ring*:

$$
U_{\text {ring }}=\frac{1}{4 \pi}\left\{\log \left(\frac{8 R}{\delta}\right)-1 / 4\right\} \frac{\Gamma}{R}
$$

$\delta$ - radius of core section (or average of ellipse axes) 


\section{Typical simulation}

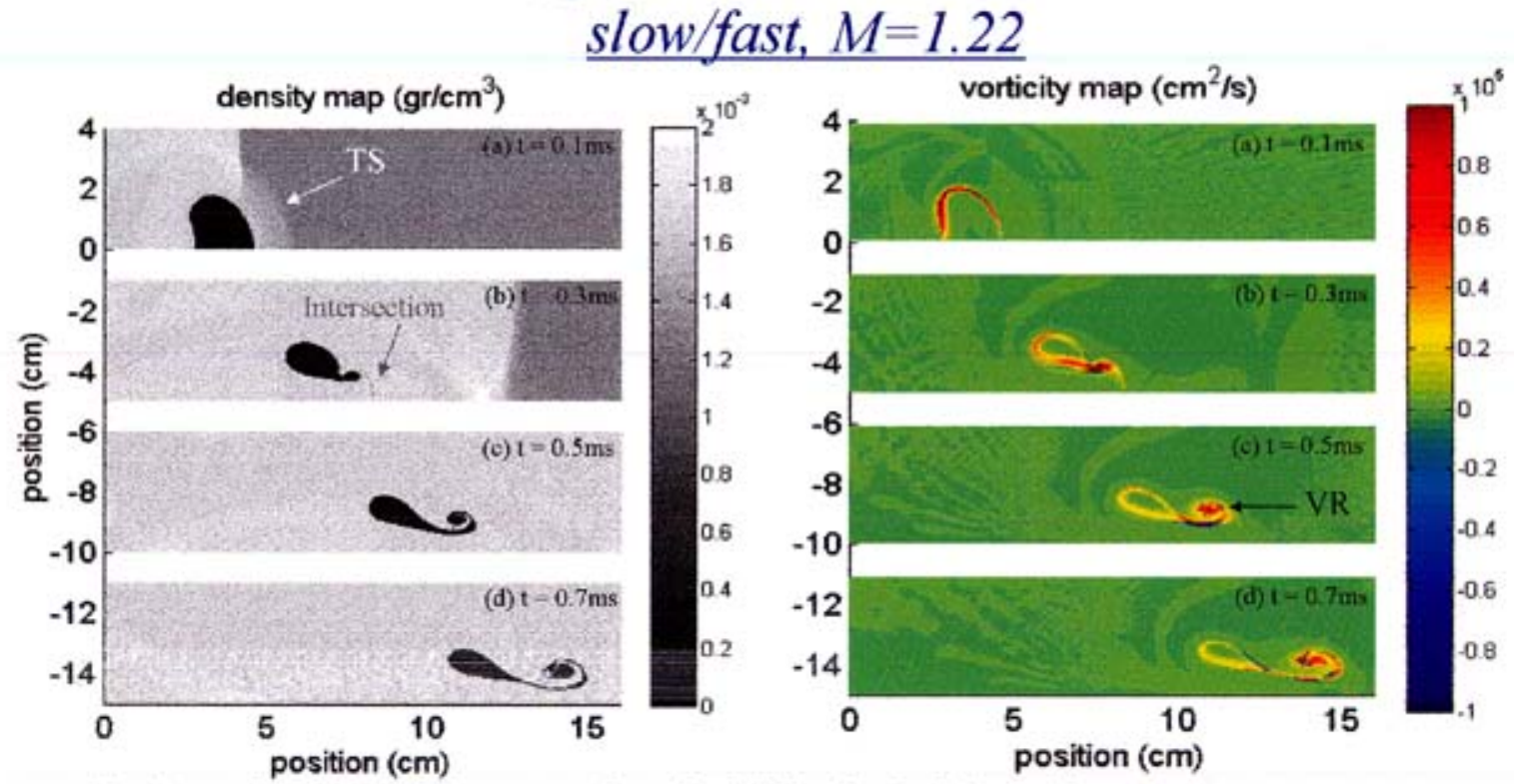

(a) - Transmitted wave (TS) emerges from the bubble ahead of the incident wave.

(b) - Upstream side of bubble reaches the downstream side. There is vorticity on the inner side of the bubble because of the shear velocity.

(c) - The vortex ring (VR) has formed at the downstream side.

(d) - The vortex ring is moving at a steady speed. The vortex ring separates from the upstream remains of the bubble. 


\section{Vortex ring formation - velocity field low/fast, $M=1.22$}

(a) $\mathrm{t}=0.064 \mathrm{~ms}$

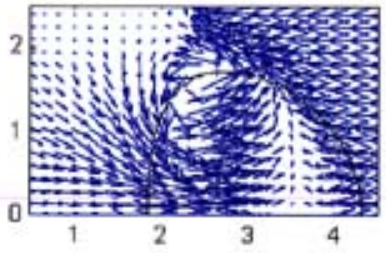

(c) $\mathrm{t}=0.264 \mathrm{~ms}$

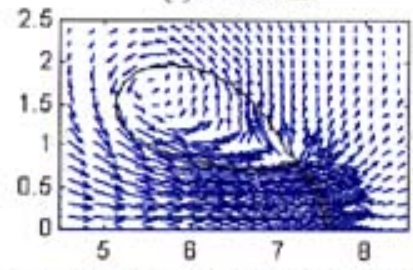

(b) $\mathrm{T}=0.164 \mathrm{~ms}$

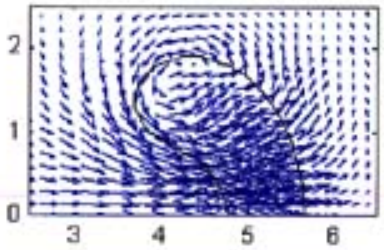

(d) $\mathrm{t}=0.364 \mathrm{~ms}$

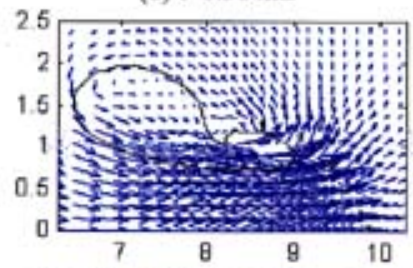

The velocity field is drawn in a coordinate moving with the shocked air

(a) The upstream interface of the bubble is initially accelerated and a shear velocity is formed leading to rotational flow.

(b) The ambient gas moves in to fill the space left by the bubble.

(c) The bubble is penetrated through the center creating a torus-like shape

(d) The vortex ring is formed 


\section{Vortex ring velocity definition}
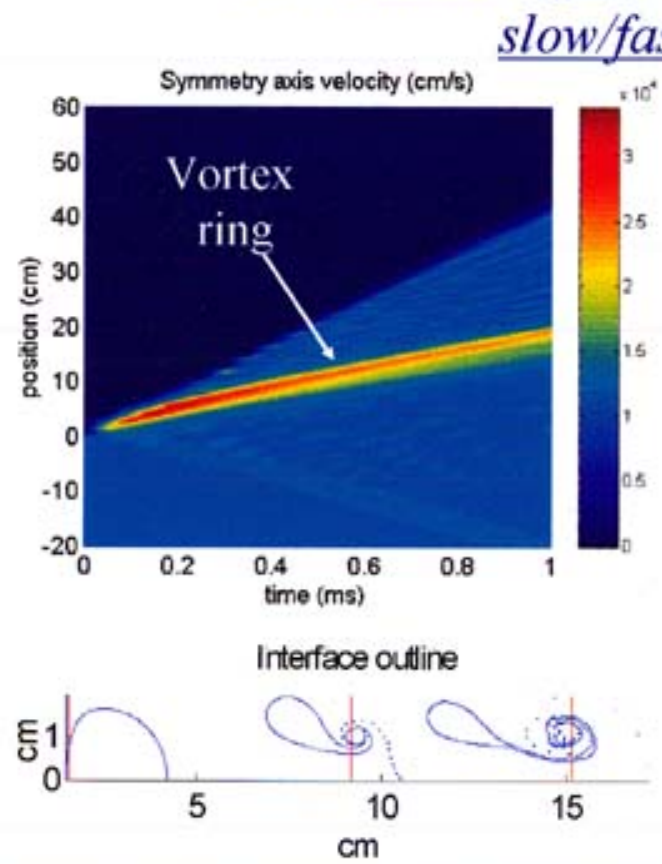

Vertical lines represent position of maximum velocity

- The motion of the vortex ring is characterized by the region of high velocity.

- The maximum velocity on the symmetry axis is located at the center of the vortex ring.

- The vortex ring velocity is derived from the movement of this point.

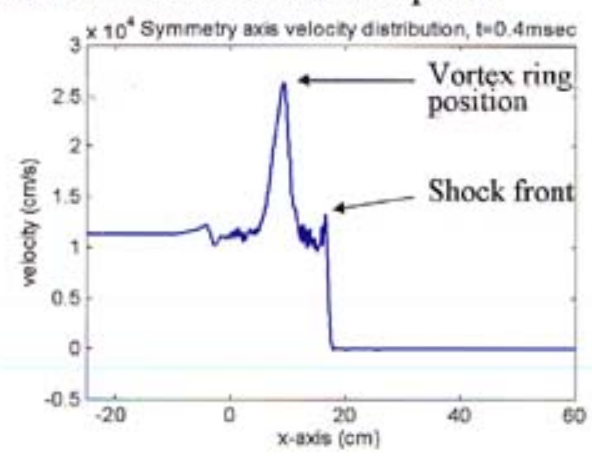




\section{Vortex ring velocity development}

\section{slow/fast, $M=1.22$}
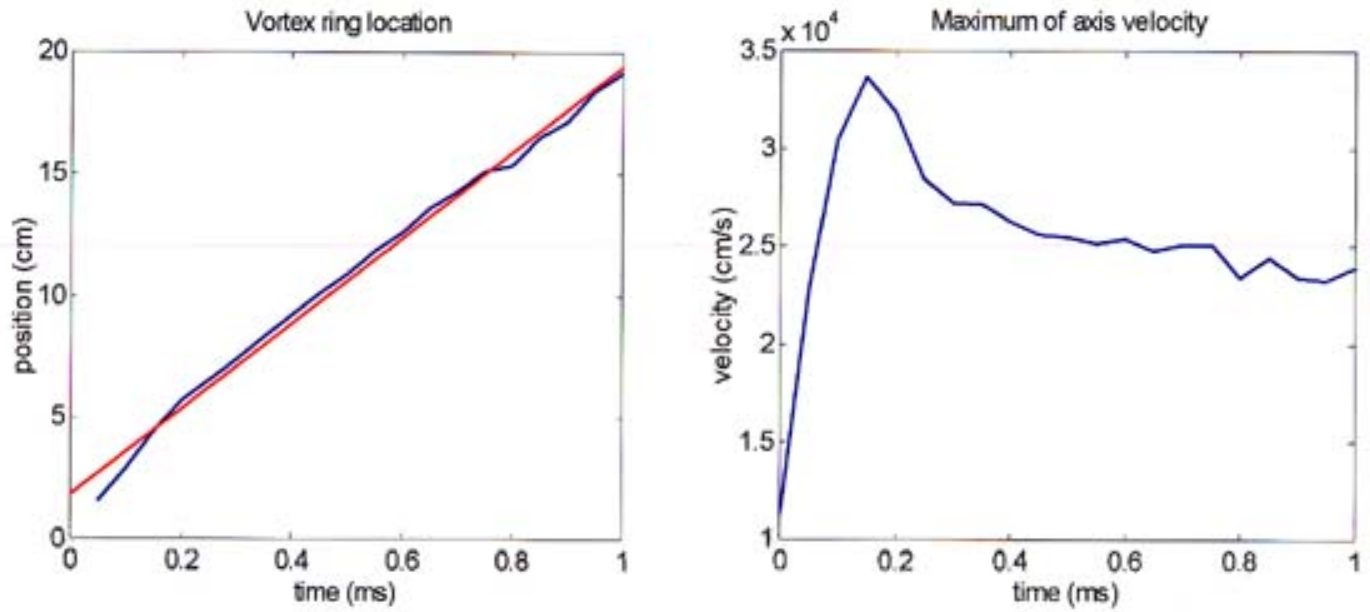

- From $t=0.2 \mathrm{~ms}$ the maximum axis velocity and the vortex ring velocity stabilize.

- Resulting vortex ring velocity is approximately $176 \mathrm{~m} / \mathrm{s}$. 


\section{Circulation \\ slow/fast, $M=1.22$}

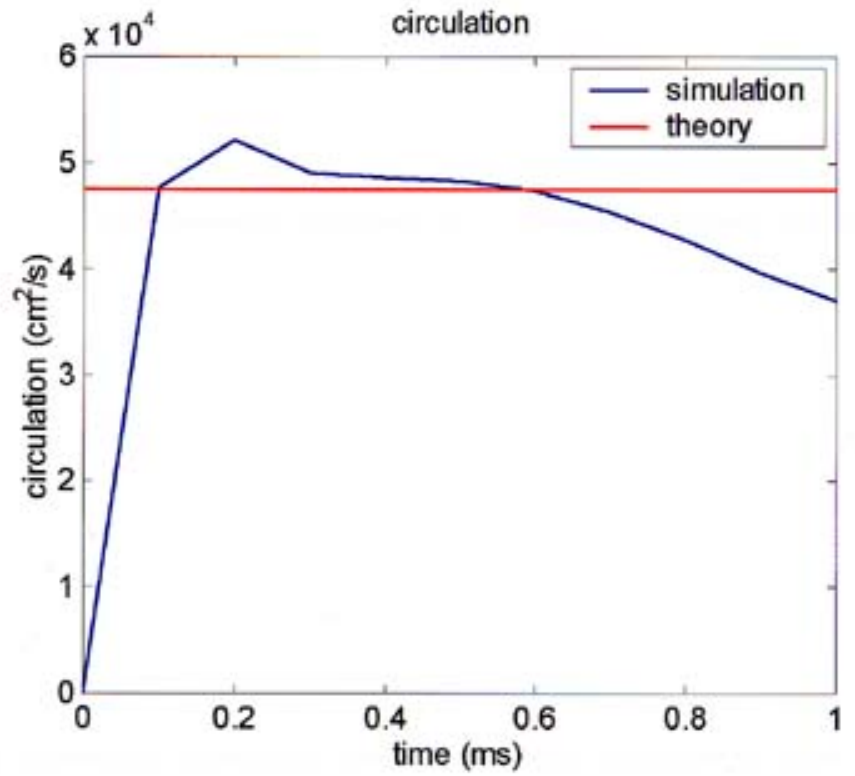

Circulation from simulation:

$$
\Gamma=\int_{A} \omega \cdot d A
$$

where $\omega$ is the vorticity:

$$
\omega=\frac{\partial v}{\partial x}-\frac{\partial u}{\partial y}
$$

- The circulation is in good agreement with Zabusky's model in early times.

- The decrease after $\mathrm{t}=0.6 \mathrm{~ms}$ is a result of numerical viscosity 


\section{Radius scaling}

slow/fast, $M=1.22$
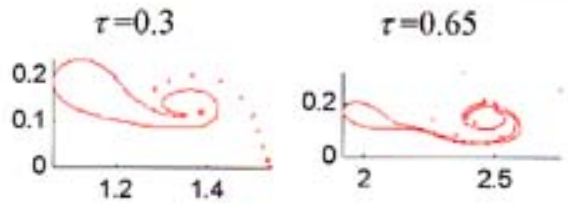

$\tau=1$
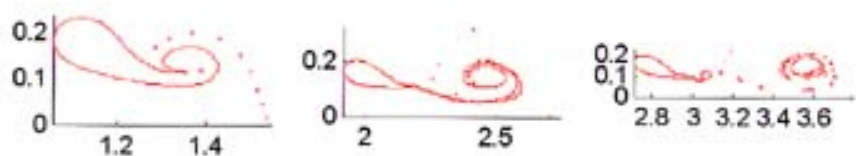

$\mathrm{R}_{1}=1 \mathrm{~cm}$

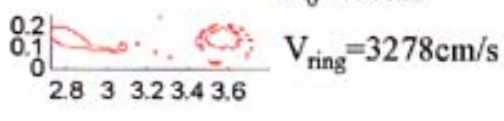

Scaling:

$$
\begin{aligned}
& \tau=t \cdot R_{0} / R_{i} \\
& \zeta=x \cdot R_{0} / R_{i} \\
& \xi=y \cdot R_{0} / R_{i}
\end{aligned}
$$
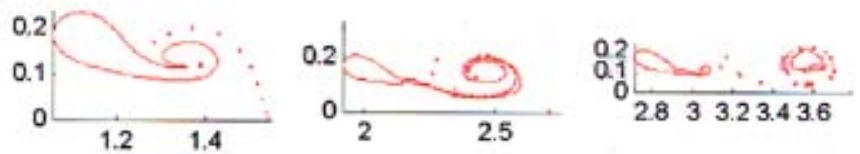

$\mathrm{R}_{2}=5 \mathrm{~cm}$

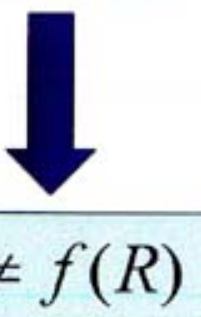

The ring velocity in the three cases differs by less than $1 \%$. Furthermore, the full evolution of the ring is also velocity independent. 


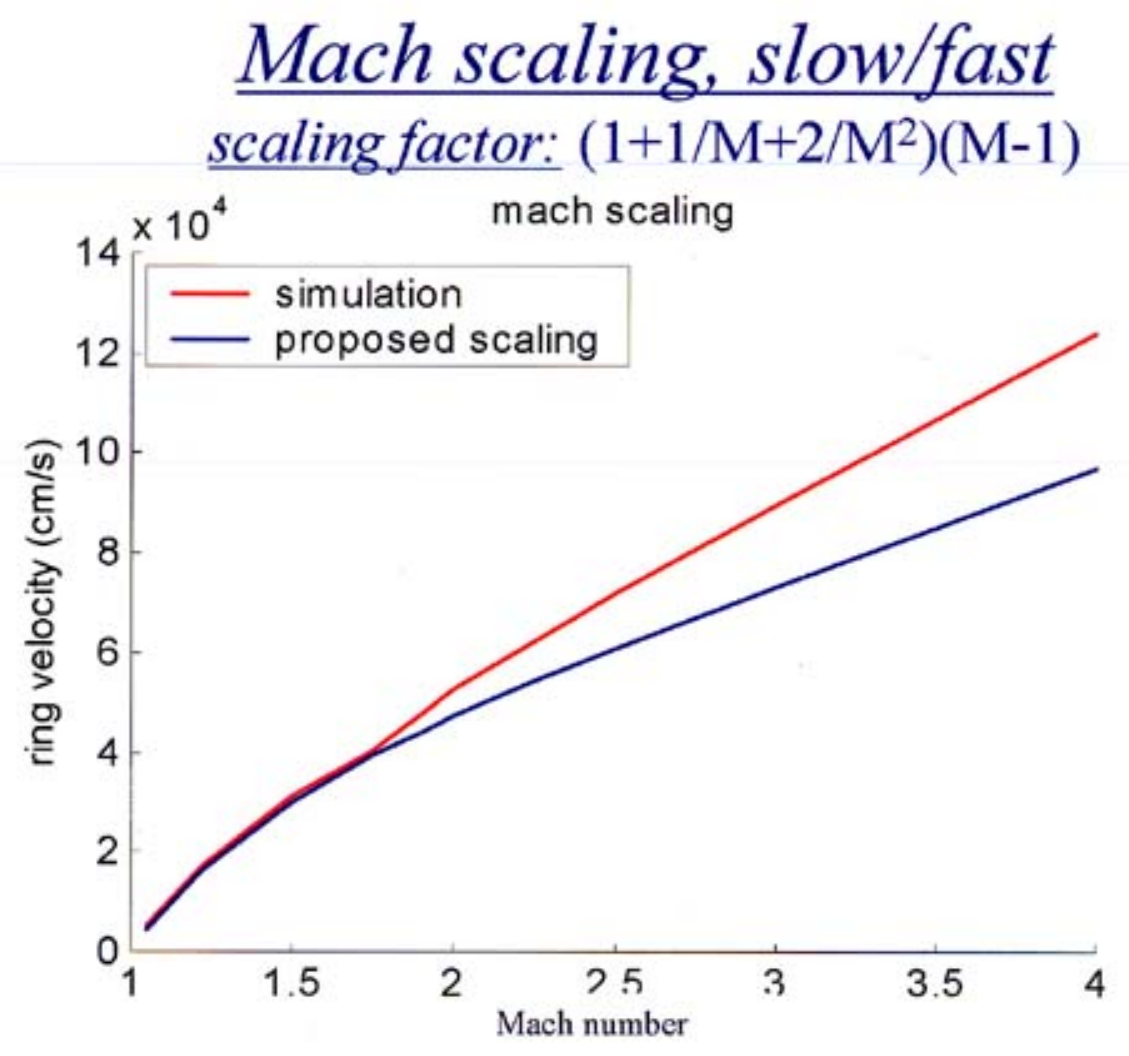

- Good agreement is found for $\mathrm{M}<2$. 


\section{Early shock/upstream interface competition}

\section{pressure contours}

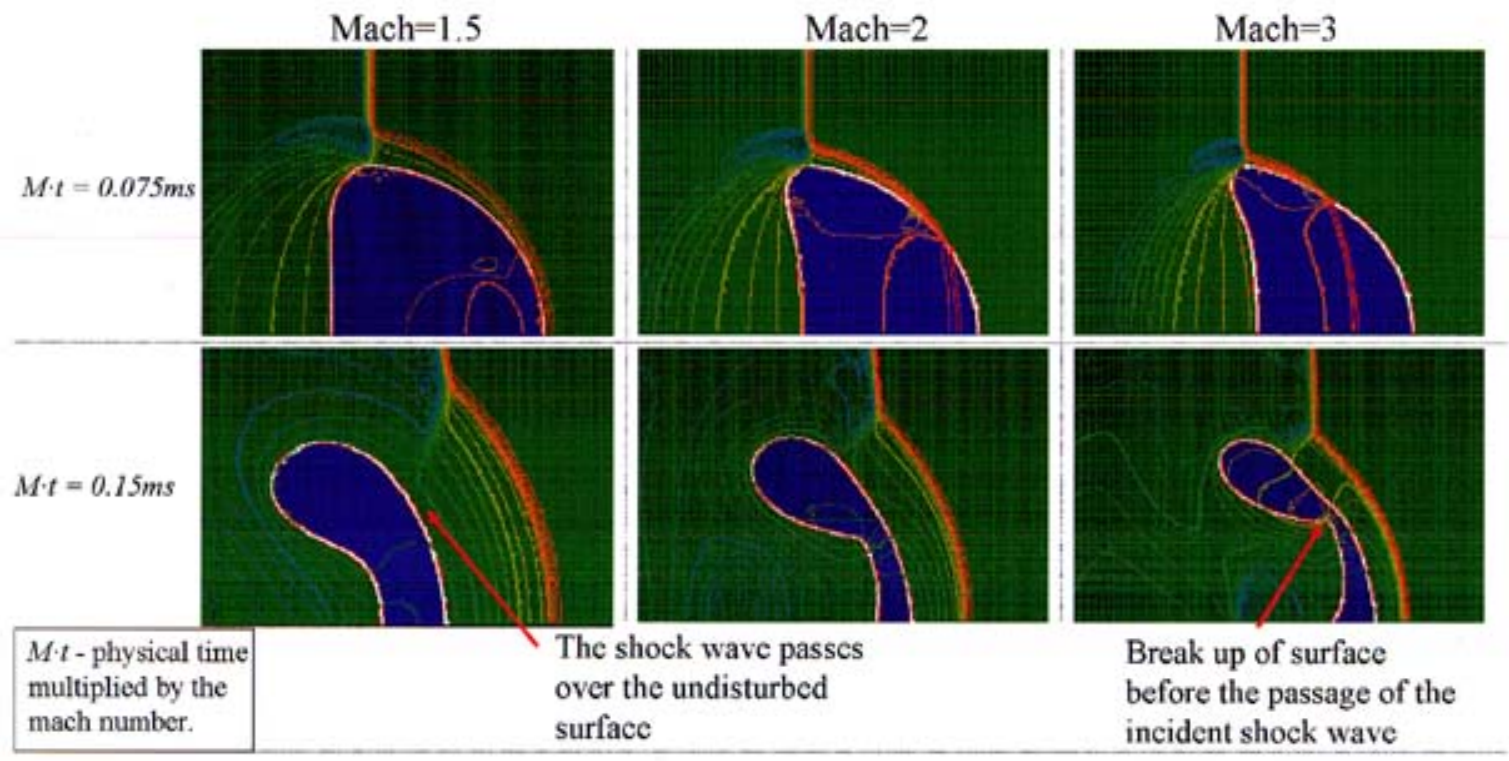

The results show that for $\mathrm{M}>2$ the upstream surface reaches the downstream side of the bubble before the incident shock wave. In this case the incident wave deposits the vorticity on an interface different from the $\mathrm{M}<2$ case. This phenomenon is not considered in the scaling rendering it invalid for $\mathrm{M}>2$. 
Late shock/upstream interface competition vorticity maps $(1 / \mathrm{s})$
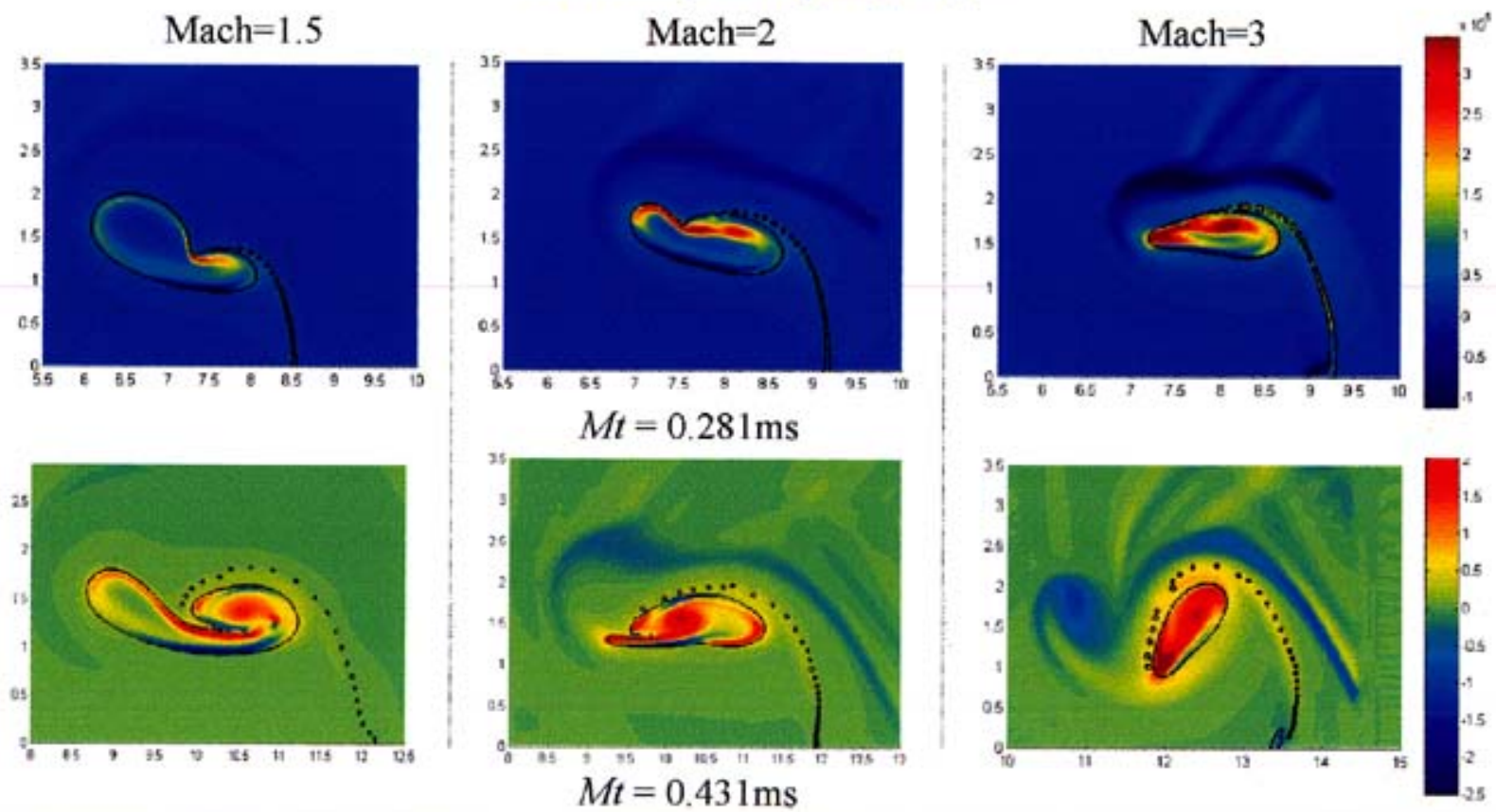

The main difference between the results is that for higher mach numoers tne vorucity is more concentratea at the vortex ring, whereas for mach=1.5 there is a distribution of the vorticity on the remains of the upstream side of the bubble. In addition, for $\mathrm{M}=3$ the vortex ring is spinning around its axis. These differences are a possible cause for the increase in velocit. 


\section{Summary}

- The phenomenon of a shock wave bubble interaction was investigated using shock tube experiments and simulations. A comparison of the bubble interface shows very good agreement.

- Using dimensional analysis and a previously suggested Mach scaling, a new velocities scaling of the shock bubble interaction is proposed.

- The velocities in the interaction were found to be independent of the initial bubble radius.

- For slow/fast interactions (air/helium) the mach scaling factor was found to be valid for $\mathrm{M}<2$.

- It is shown that the scaling is invalid for $M>2$ due to a change in the topology of the ring evolution. 


\section{Single-Mode Incompressible Richtmyer-Meshkov Instability Experiments}

C. E. Niederhaus ${ }^{1}$ and J. W. Jacobs University of Arizona

${ }^{1}$ Currently at NASA Glenn Research Center
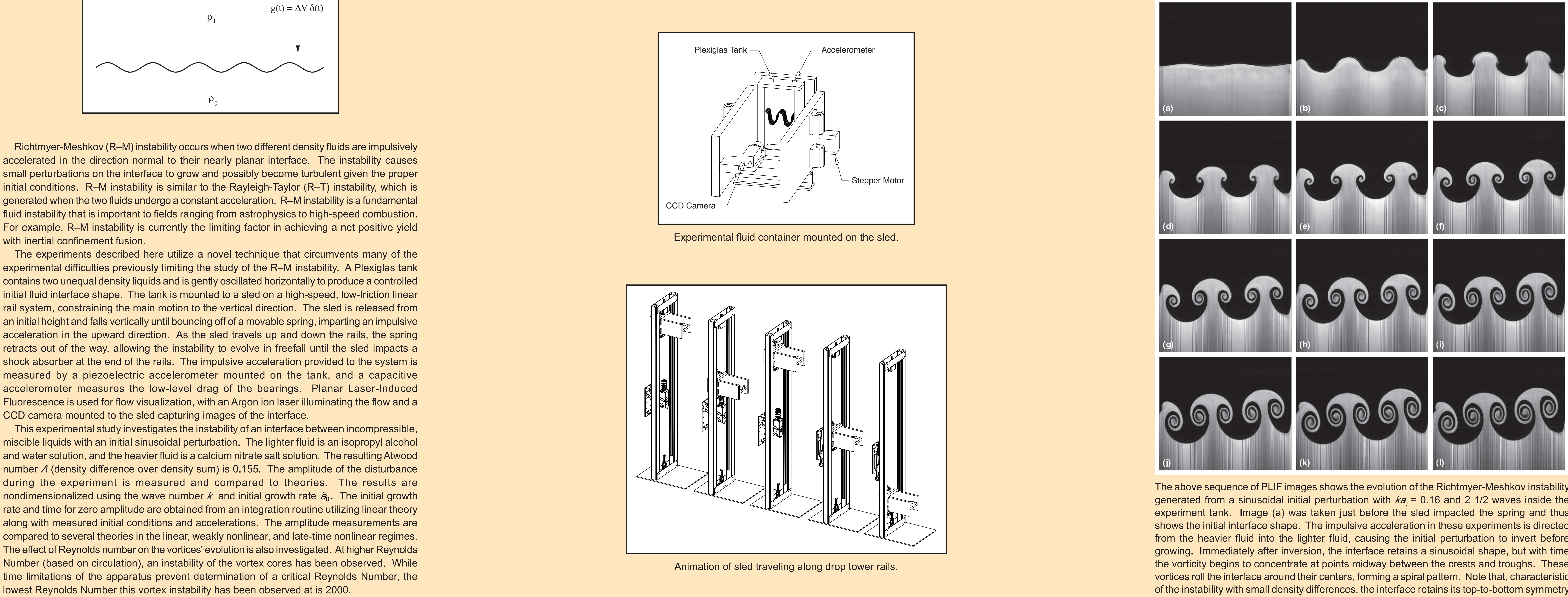

The above sequence of PLIF images shows the evolution of he Richmyer-Meshkovinstabl
generated from a sinusoidal initial perturbation with $k a,=0.16$ and 212 waves inside the experiment tank. Image (a) was taken just before the sled impacted the spring and thus
shows the initial interface shape. The impulsive acceleration in these experiments is directed growing. Immediately after inversion, the interface retains a sinusoidal shape, but with time the vorticity begins to concentrate a points midway between the crests and troughs. These
vortices roll the interface around their centers, forming a spiral pattern. Note that, characteristic of the instability with small densiy
well into the nonlinear regime.
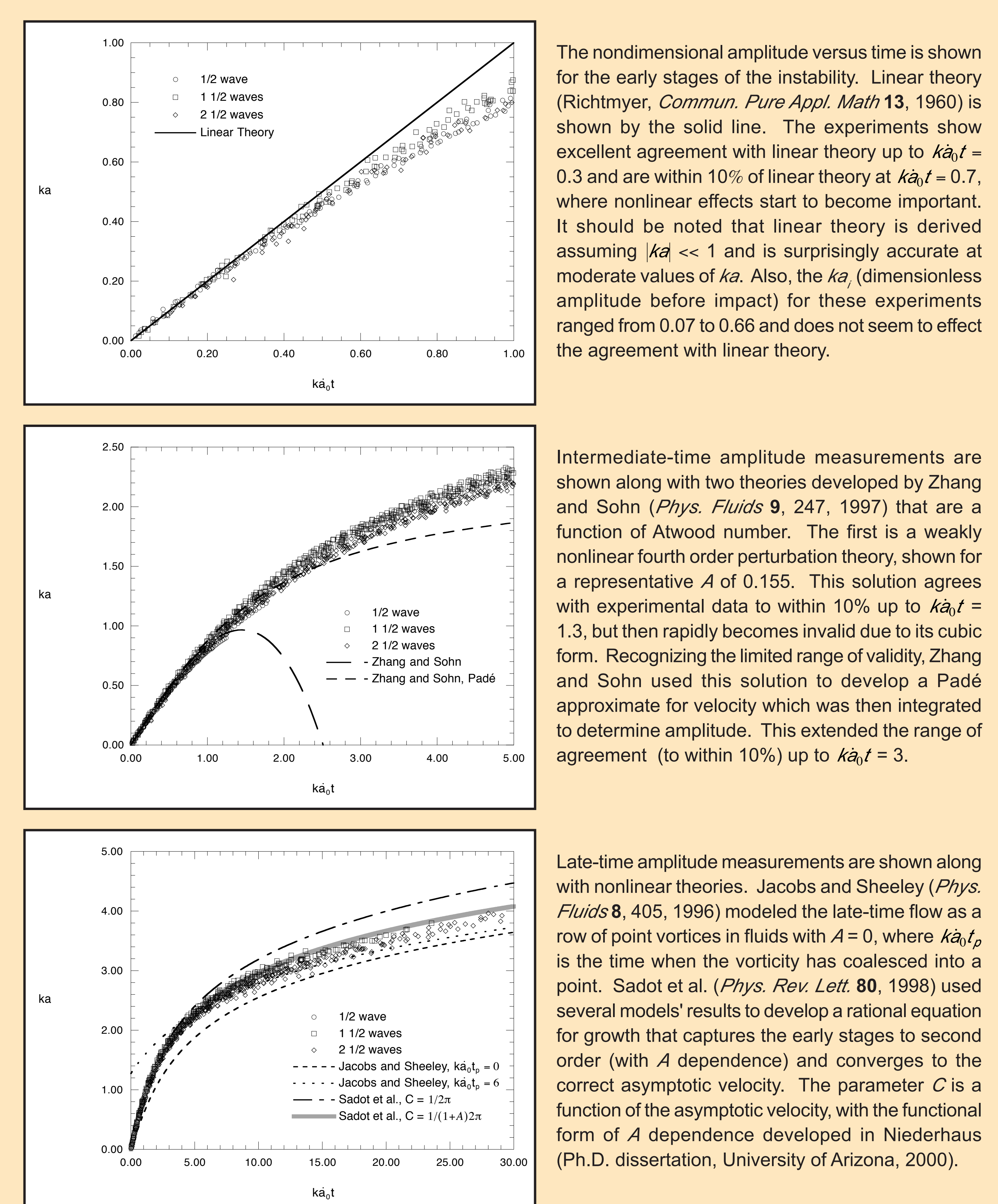
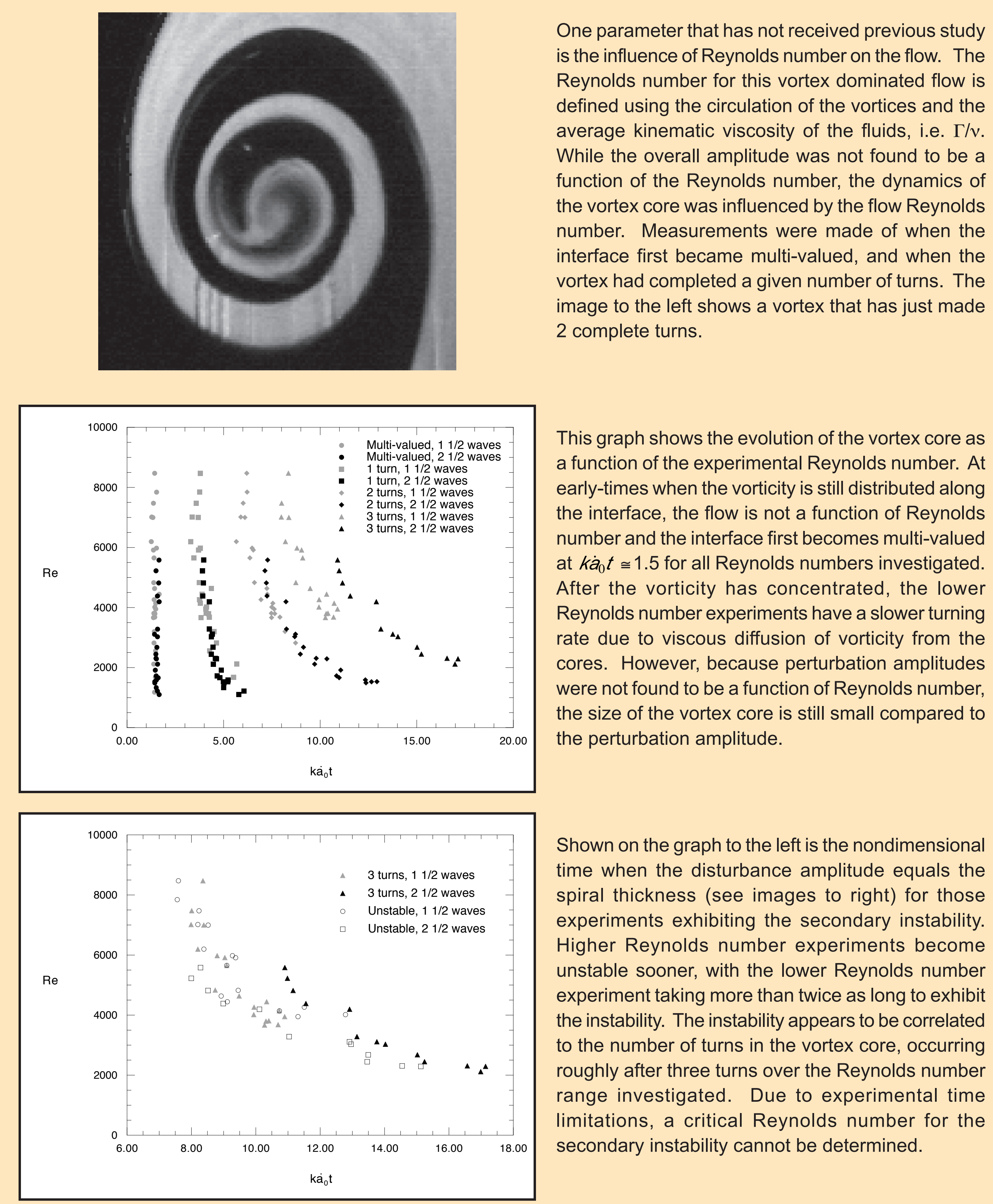

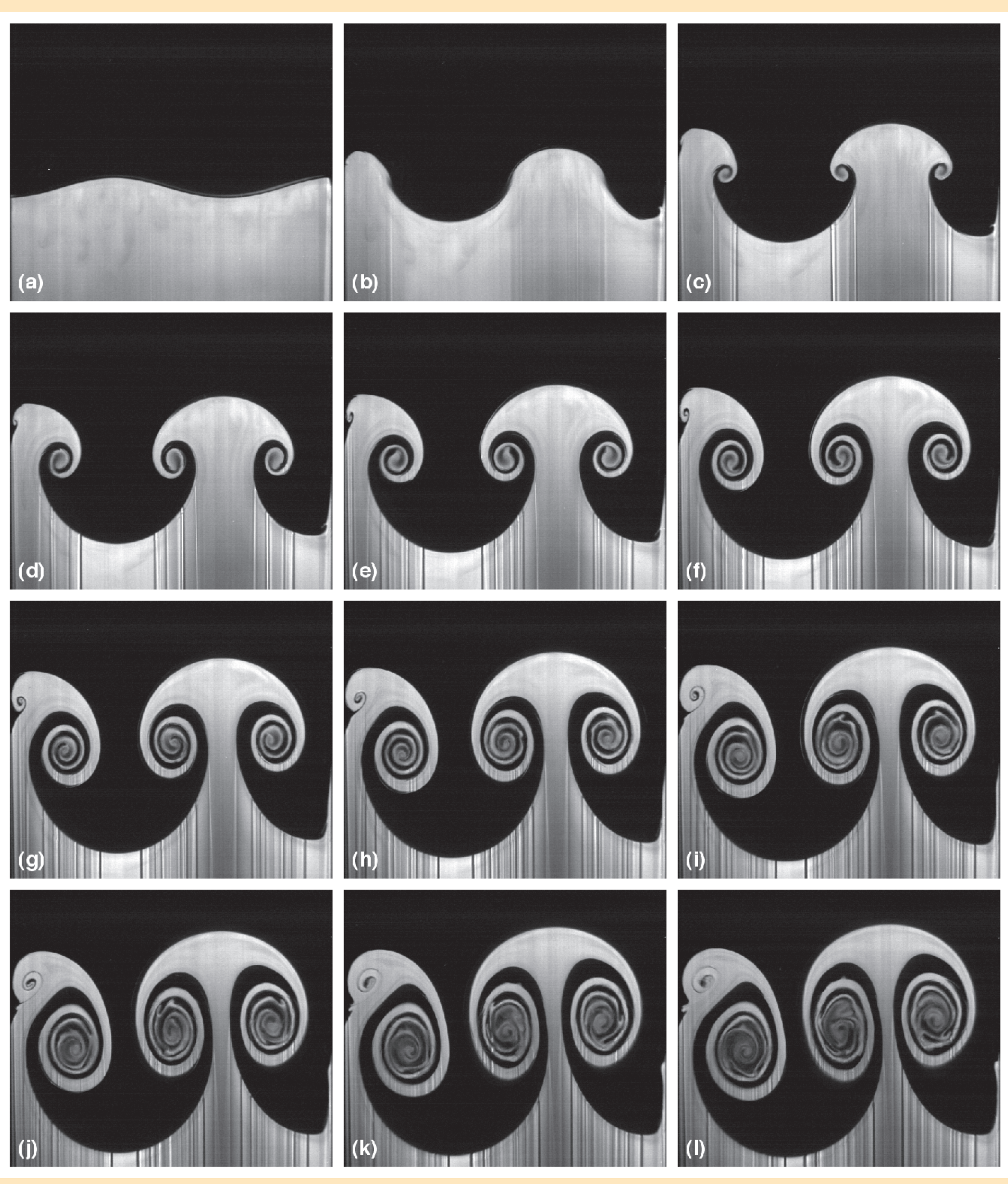

Shown above is a sequence of images from an experiment with $k a=0.29$ and $11 / 2$ waves
nside the tank. The Reynolds number (based on circulation) of the experiment is 4830 . Intially the instability develops very similarly to the lower Reynolds number cases. Starting a
frame (h), however, one can see the start of a secondary instability in the core of the vortex 政 The waves start near the center of the core and grow in size and extent unth all layers of the and it appears
smaller scale. 


\section{Experimental Study of a Strongly Shocked Gas Interface with Visualized Initial Conditions}

8th International Workshop on the Physics of

Compressible Turbulent Mixing

California Institute of Technology,

Pasadena, California, USA, Dec. 9-14, 2001

Mark Anderson,

Jason Oakley,

Bhalchandra Puranik,

Riccardo Bonazza

Department of Engineering Physics

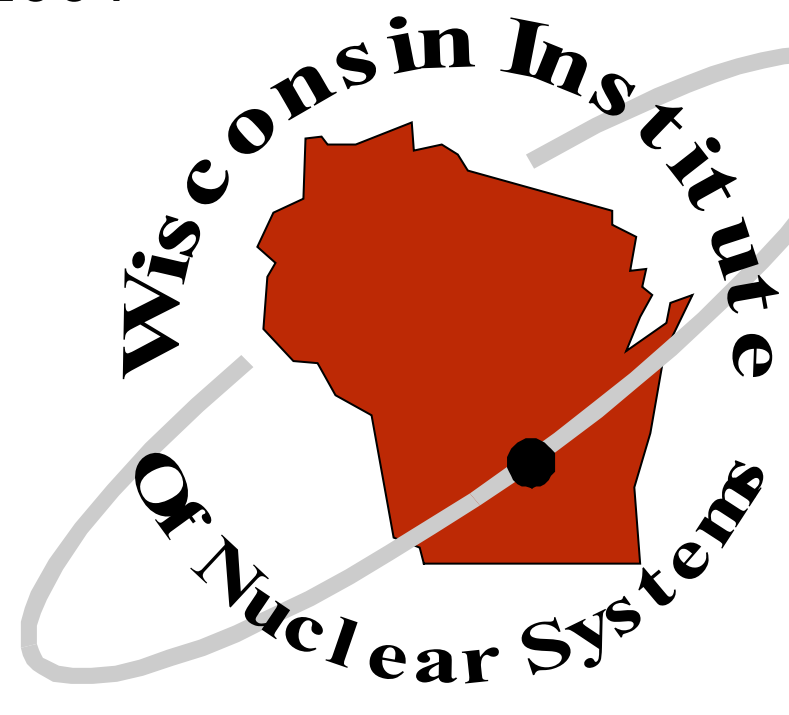
University of Wisconsin-Madison 


\section{Outline}

- University of Wisconsin Shock-Tube Laboratory (WiSTL)

- Interface preparation

- Shocked interfaces

- Comparisons with non-linear theories

- Conclusions 


\section{WiSTL (Wisconsin Shock Tube Laboratory)}

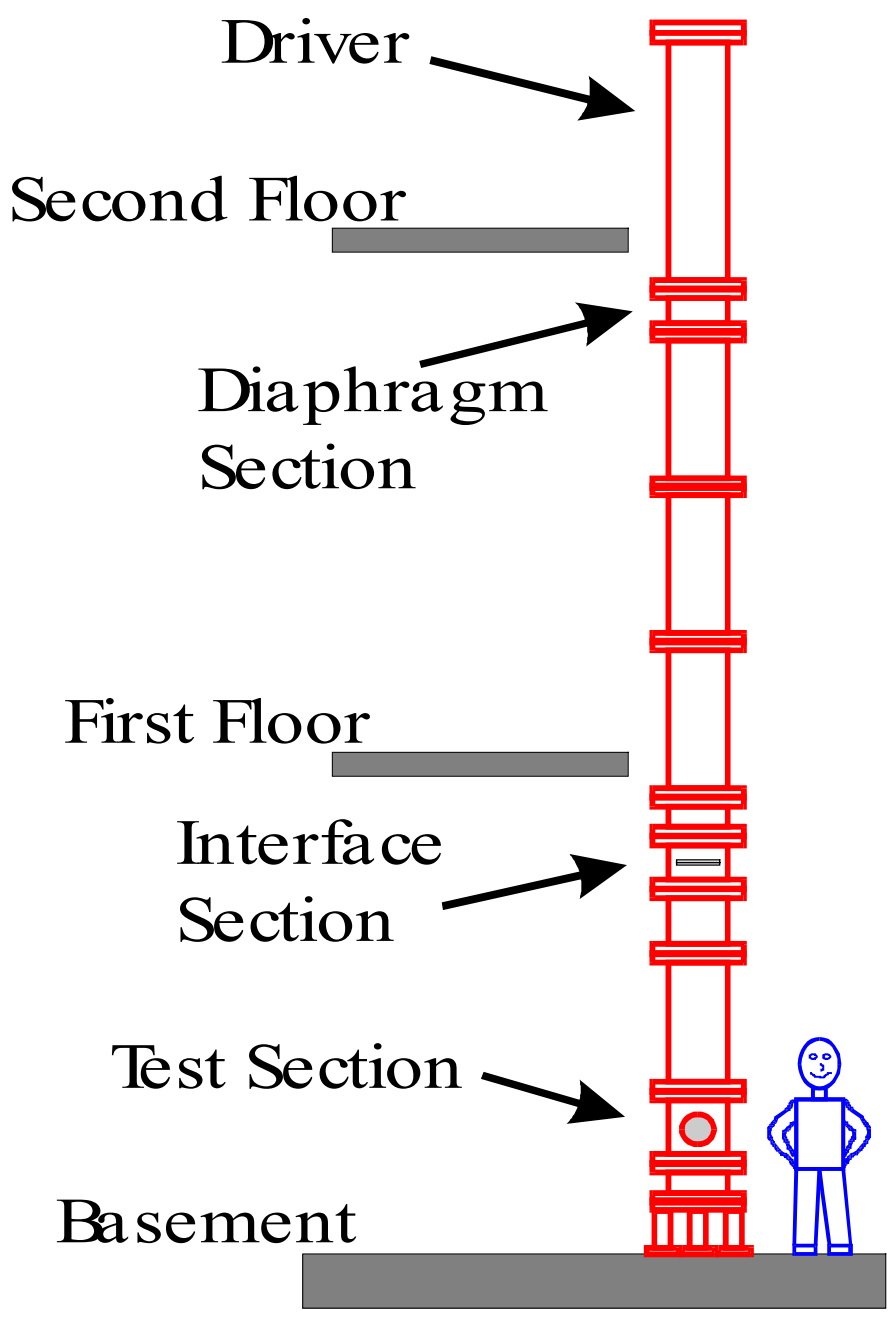

- Vertical Orientation

- Large Internal Square

Cross-Section ( $25 \mathrm{~cm}$ square)

- Total Length $=9.2 \mathrm{~m}$

Driven Length $=6.8 \mathrm{~m}$

- Structural Capacity $20 \mathrm{Mpa}$

- Modular Construction

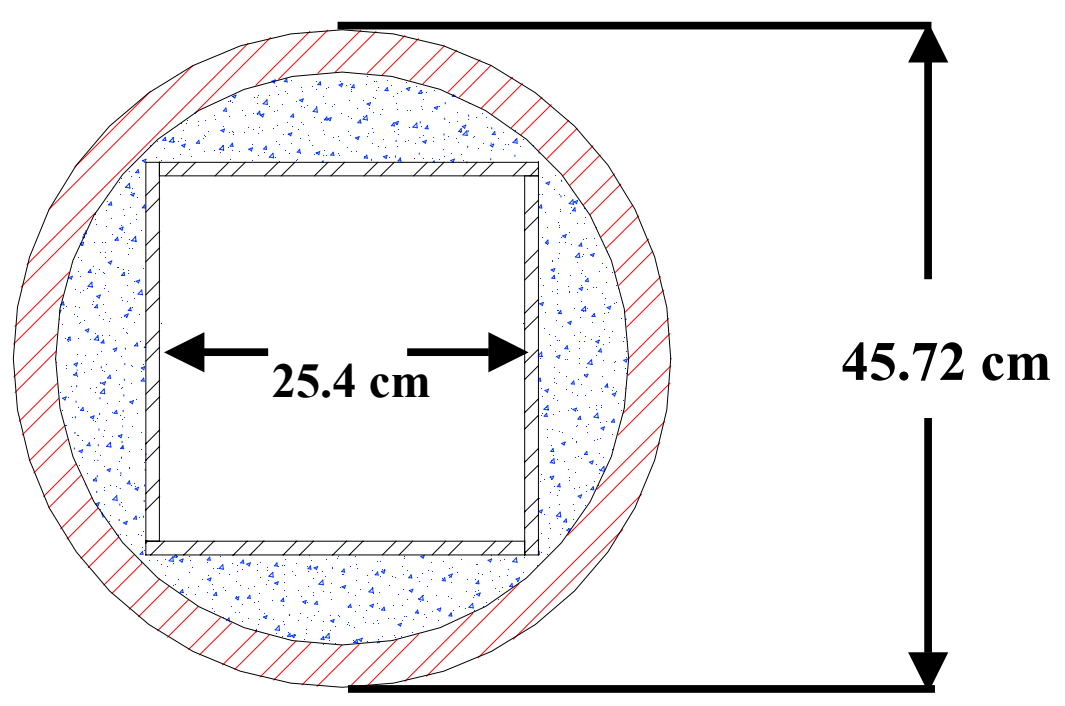




\section{Interface Preparation}

- Use of a retractable metal plate formed into a sinusoidal shape

- Copper plate, $0.6 \mathrm{~mm}$ thick

- Plastic deformation by rolling operation

- Sine wave parameters:

- Amplitude $=3.18 \mathrm{~mm}$

- Wavelength $=38.1 \mathrm{~mm}$

- $\eta_{d} / \lambda=0.083$

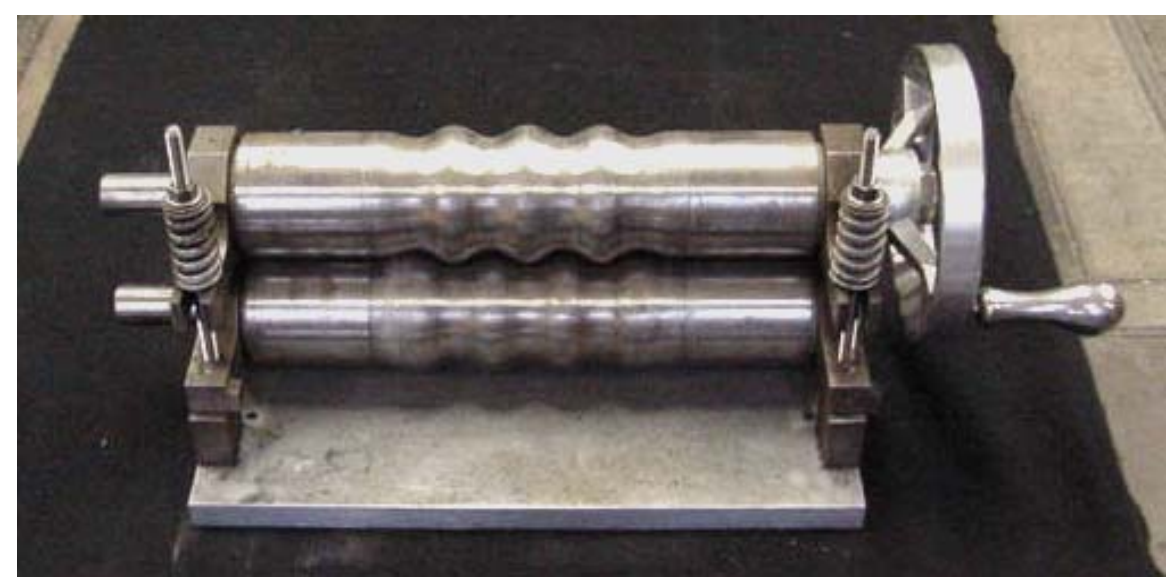

Rollers

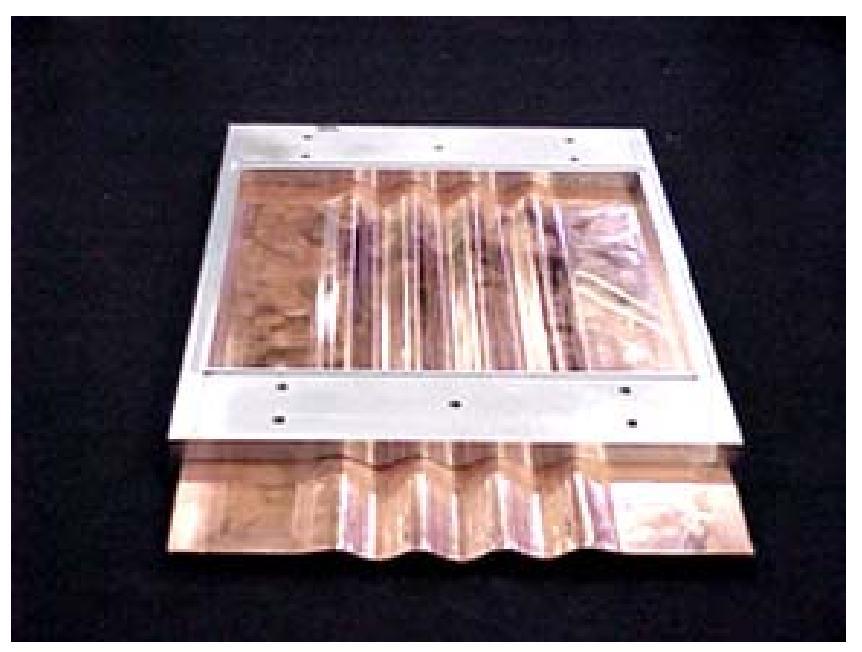

Formed plate 


\section{Study of initial conditions}

- $P_{\text {initial }}=1 \mathrm{~atm}, T_{\text {initial }}=298 \mathrm{~K}$

-Ar-ion laser@ $@ \lambda=514$ and 488 nm, CW

- Planar Mie scattering visualization

- CCD camera: 256 x 256 pixel array, 8 bit/pixel

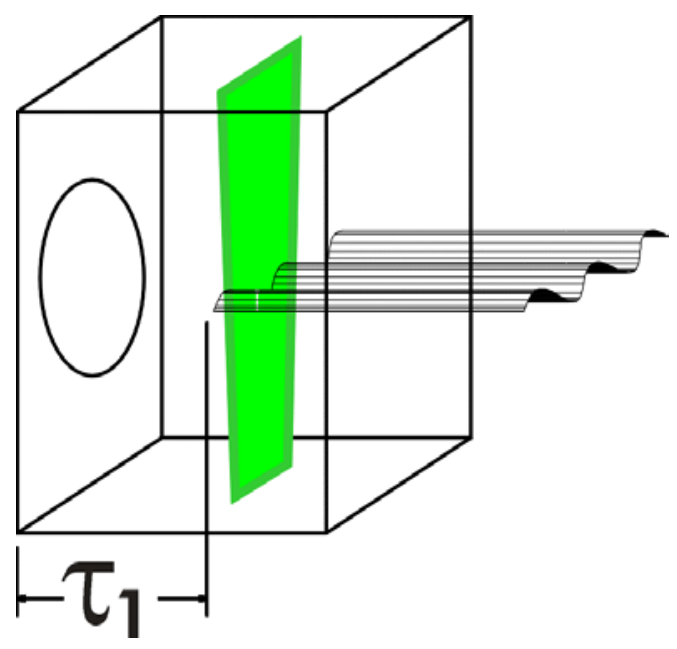

- Two-stage retraction $\left(\tau_{1} \sim 250 \mathrm{~ms}, \tau_{2} \sim 80 \mathrm{~ms}\right)$

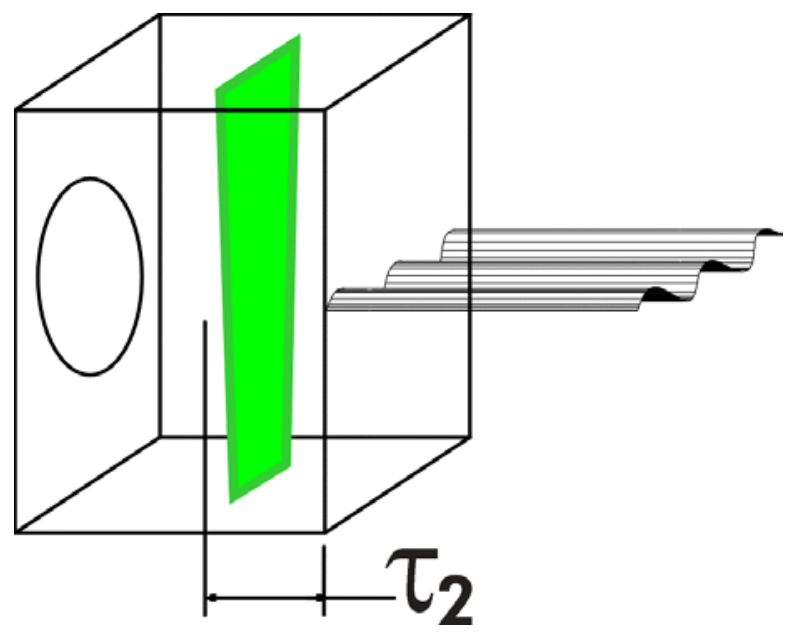




\section{RT Unstable Interface $\left(\mathrm{CO}_{2} / \mathrm{Air}\right)$}

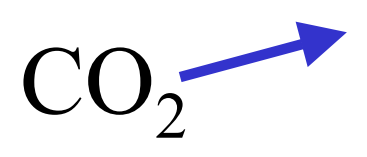

\section{Air seeded with smoke}

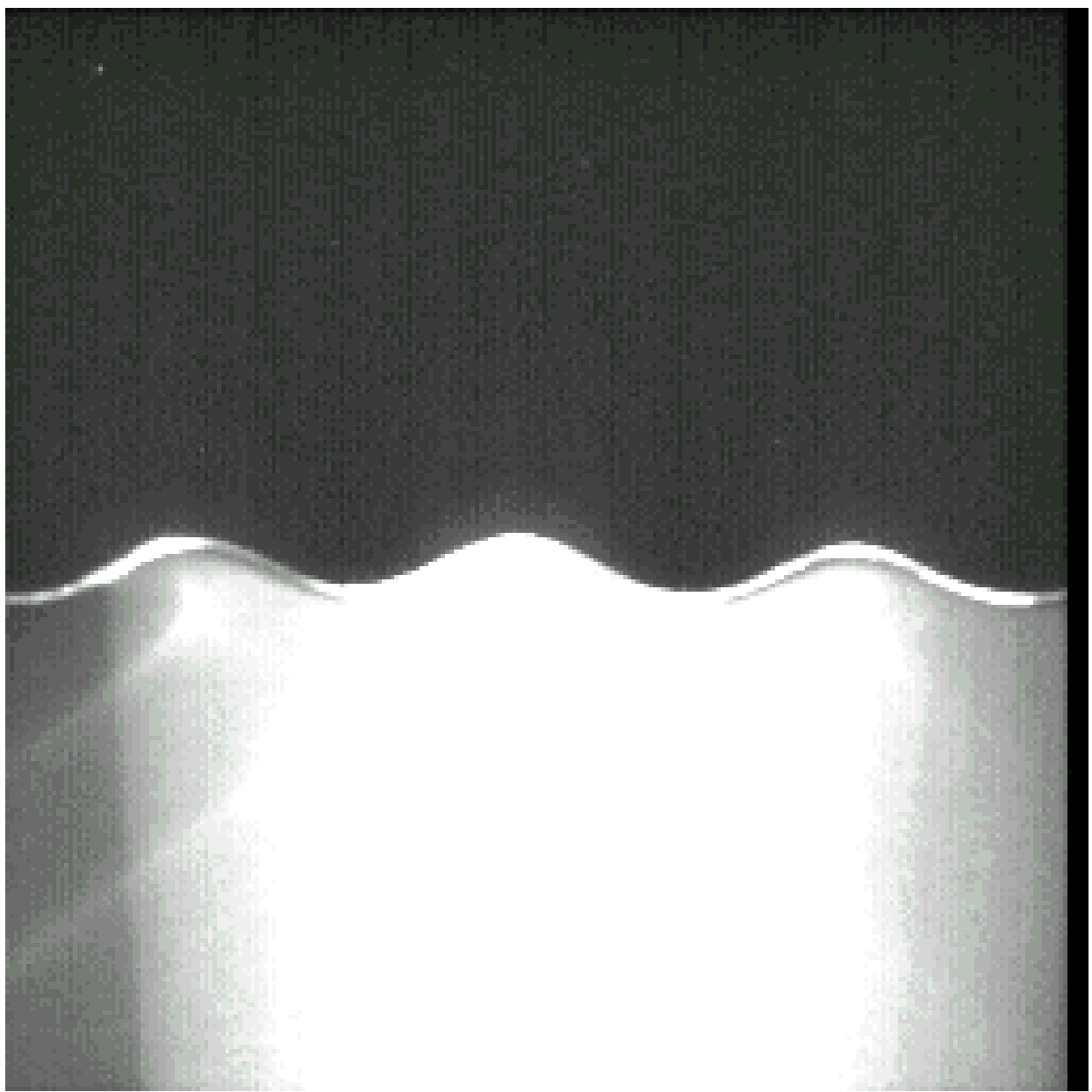




\section{Desired $\tau_{\mathrm{RT}}<120 \mathrm{~ms}$ for RM Initial Condition}
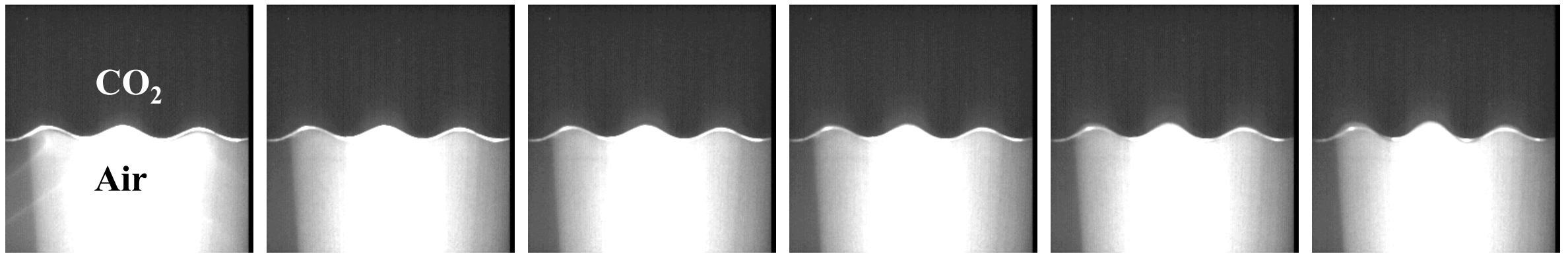

$0 \mathrm{~ms}$

$10 \mathrm{~ms}$

$20 \mathrm{~ms}$

$30 \mathrm{~ms}$
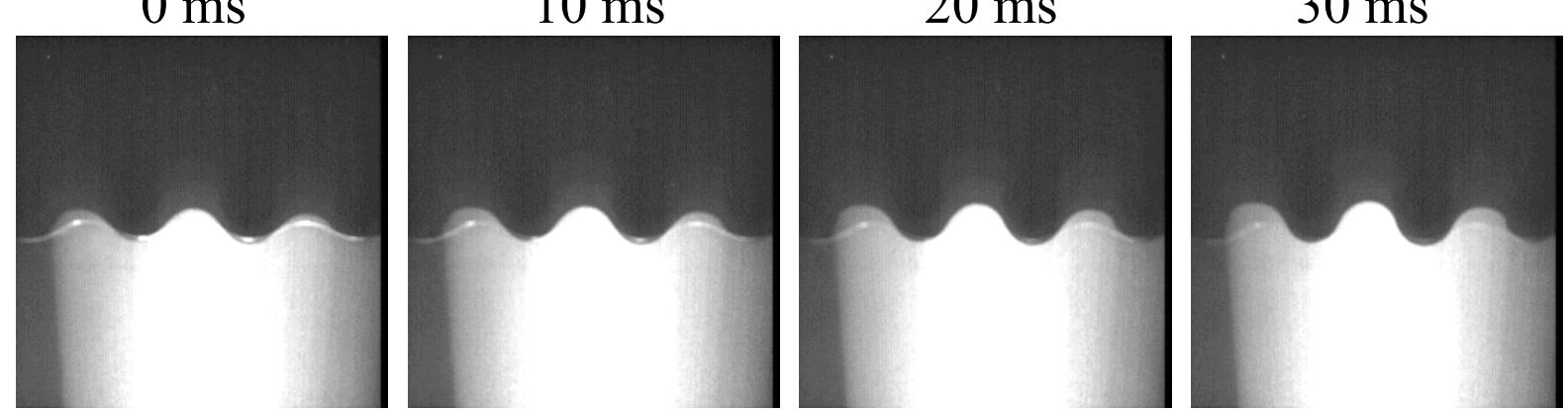

$40 \mathrm{~ms}$

$50 \mathrm{~ms}$

$60 \mathrm{~ms}$

$70 \mathrm{~ms}$

$80 \mathrm{~ms}$

$90 \mathrm{~ms}$

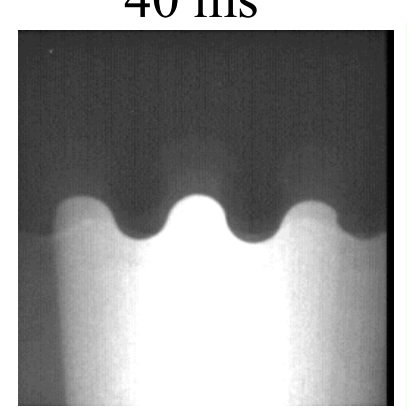

$100 \mathrm{~ms}$
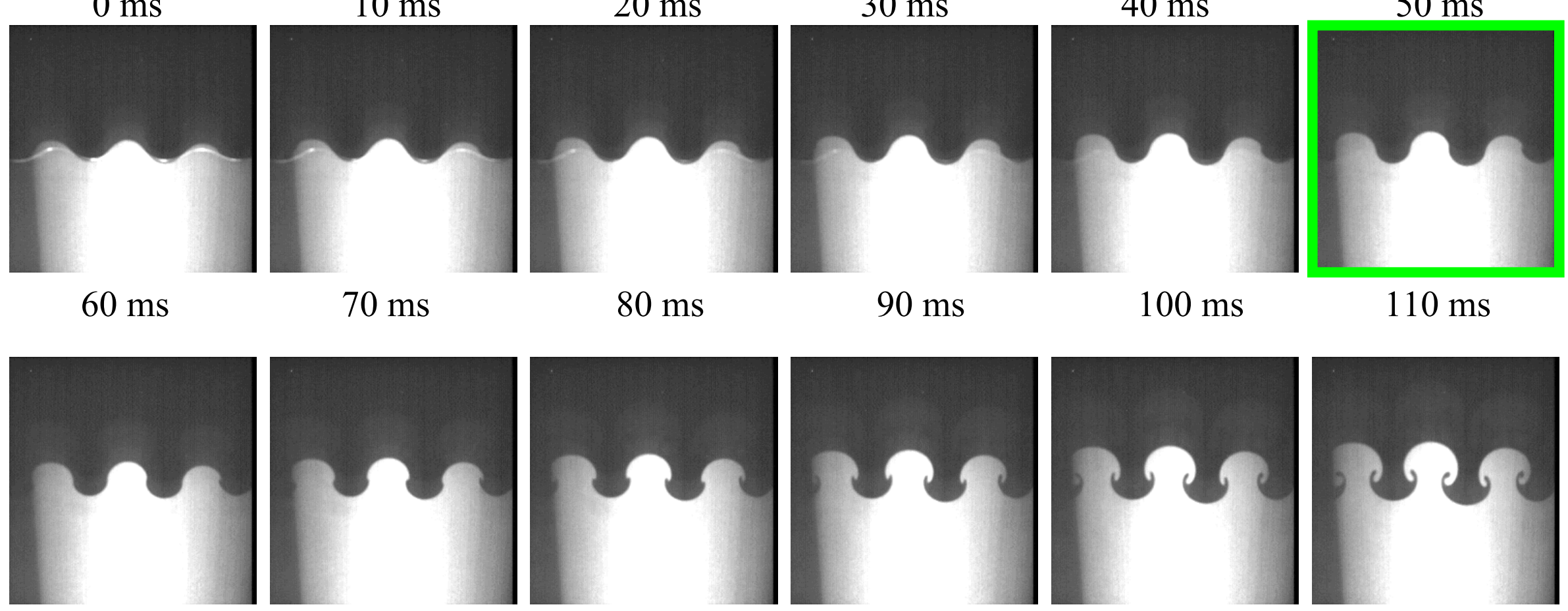

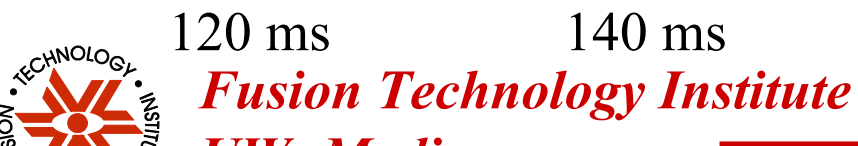

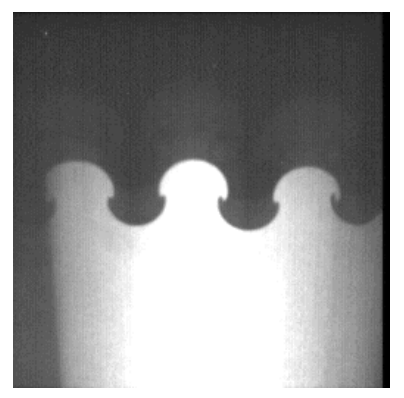

$160 \mathrm{~ms}$

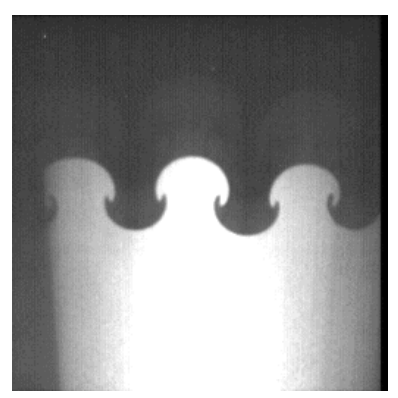

$180 \mathrm{~ms}$

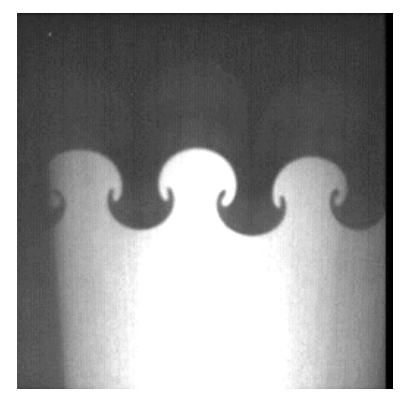

$110 \mathrm{~ms}$ 


\section{R-M instability visualization results}

- $\mathrm{CO}_{2} /$ Air, $\mathrm{A}_{\text {post }}=0.246, \mathrm{~A}_{\text {pre }}=0.206$

-Very early interaction of the $\mathrm{M}=3.06$ shock wave with the sinusoidal interface

- Development of phase reversal (heavy/light configuration)

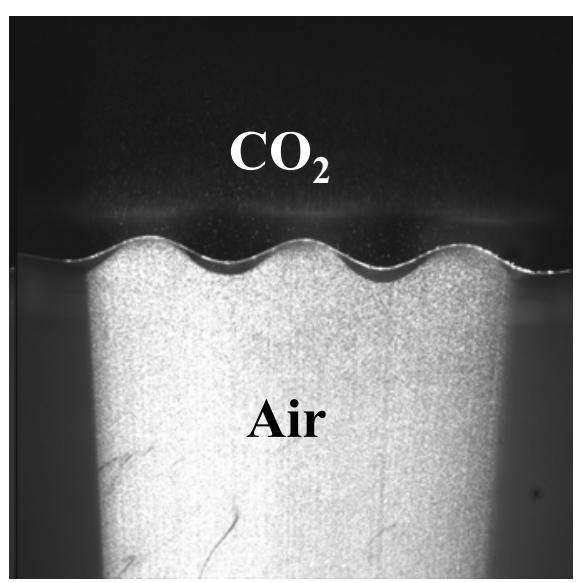

(a)

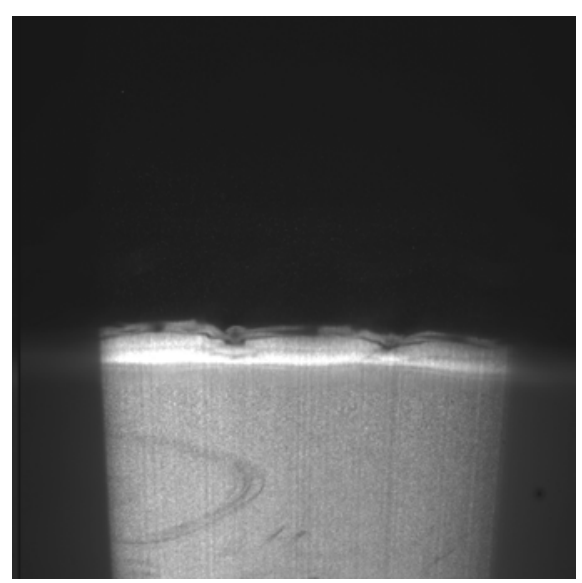

(b)

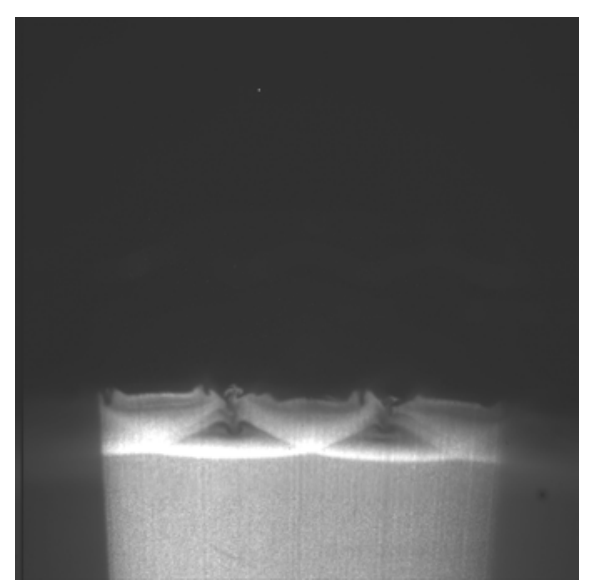

(c)

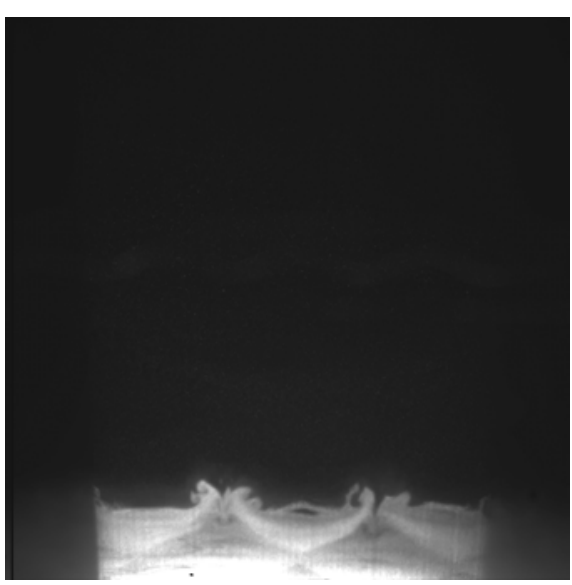

(d)

- (a): Pre-shocked interface (Note the location of peaks and troughs)

- (b): Shocked interface $\sim 5 \mu$ s after initial shock acceleration

- (c): Shocked interface $\sim 36 \mu$ s after initial shock acceleration

- (d): Shocked interface $\sim 39 \mu$ s after initial shock acceleration 


\section{R-M instability visualization results (Cont'd)}

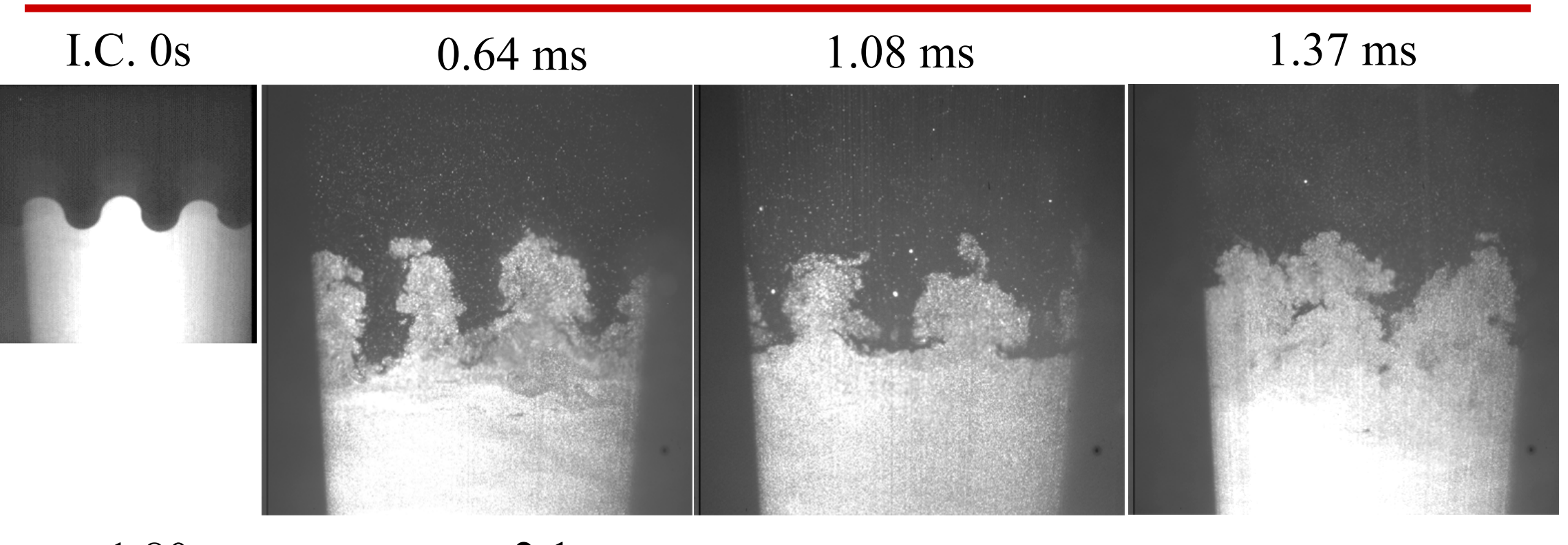

$1.80 \mathrm{~ms}$

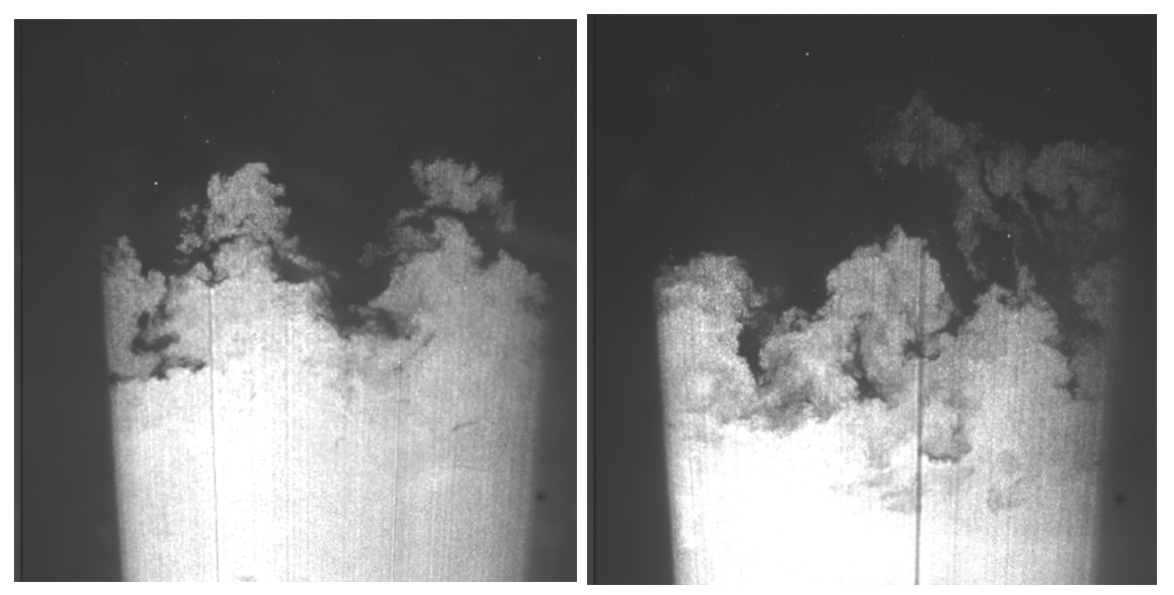

Wisconsin Institute of Nuclear Systems UW-Madison
- Evolution of interface growth for the same nominal initial condition.

- Each image was taken in a separate experiment with a $\mathrm{M} \sim 3.06$ shock.

- Initial condition inferred from time of shock interaction and RT experiments. 


\section{Experiments: Image Analysis}

- Images

- Initial condition: 3 peaks, 2 troughs

- Shocked image: 1-4 peaks, 1-3 troughs

- Median filter

- Excess noise removed in driven and test gases manually

- Convert to black and white, then apply Sobel operator to detect edge

- Perturbation amplitude:

$$
\eta=\frac{1}{2}\left(\bar{P}_{P I X}-\bar{V}_{P I X}-1\right) P_{D I M}
$$

$\bar{P}_{P I X}=$ average pixel row number of perturbation peaks

$\bar{V}_{P I X}=$ average pixel row number of perturbation valleys

$P_{D I M}=$ pixel dimension $(\mathrm{mm} / \mathrm{pixel})$

- Error less than 2 pixels: $0.8 \mathrm{~mm}$ for initial condition, $0.4 \mathrm{~mm}$ for shocked interface 


\section{Analytic theories}

Richtmyer (1960) impulsive model: $\eta(t)=k\left[u_{p}\right] A \eta_{0} t$

Sadot et al. (1998) nonlinear theory: $\left(\frac{d \eta}{d t}\right)=\left(\frac{d \eta}{d t}\right)_{L I N}\left(\frac{1+B t}{1+D t+E t^{2}}\right)$

$$
\begin{aligned}
& D_{b / s}=\left(1 \pm A^{\prime}\right)\left(\frac{d \eta}{d t}\right)_{i m p} k \quad E_{b / s}=\left[\left(1 \pm A^{\prime}\right) /\left(1+A^{\prime}\right)\right] \times(1 / 2 \pi C)\left(\frac{d \eta}{d t}\right)_{i m p}^{2} k^{2} \\
& C=1 / 2 \pi \text { for low } A^{\prime}
\end{aligned}
$$

Zhang and Sohn (1997) nonlinear theory:

$$
\left(\frac{d \eta}{d t}\right)_{\text {total }}=\frac{\left(\frac{d \eta}{d t}\right)_{\text {lin }}}{1+\left(\frac{d \eta}{d t}\right)_{\text {lin }} \eta_{0}^{\prime} k^{2} t+\max \left\{0, \eta_{0}^{\prime 2} k^{2}-A^{\prime 2}+1 / 2\right\}\left(\frac{d \eta}{d t}\right)_{\text {lin }}^{2} k^{2} t^{2}}
$$




\section{Comparison with Theories}

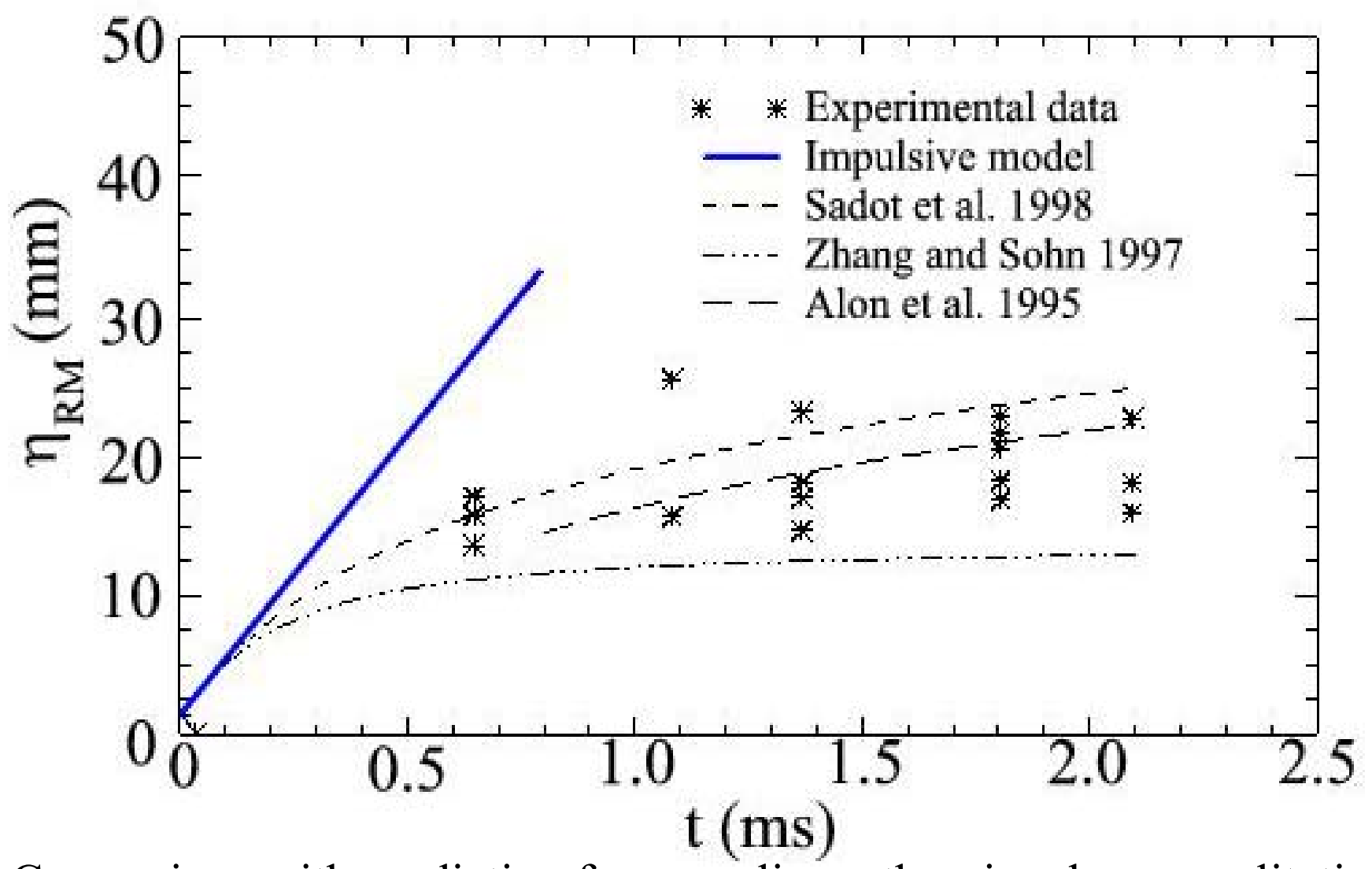

- Comparison with prediction from nonlinear theories shows qualitative agreement - - - Sadot et al. theory overpredicts at late times

-... Zhang and Sohn theory underpredicts at all times 


\section{Experiment: Combined Imaging Setup}

Previously, the RM initial condition Argon lon Continuous Wave Laser Sheet was inferred from a reference set of RT experiments.

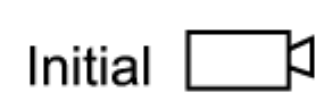
Conditions

Dynamic imaging of the interface, prior to being shocked, provides interfacial initial condition data for each RM experiment.

Provides the interface geometry of the initial condition which may be used in a numerical simulation.
Imaging

Camera

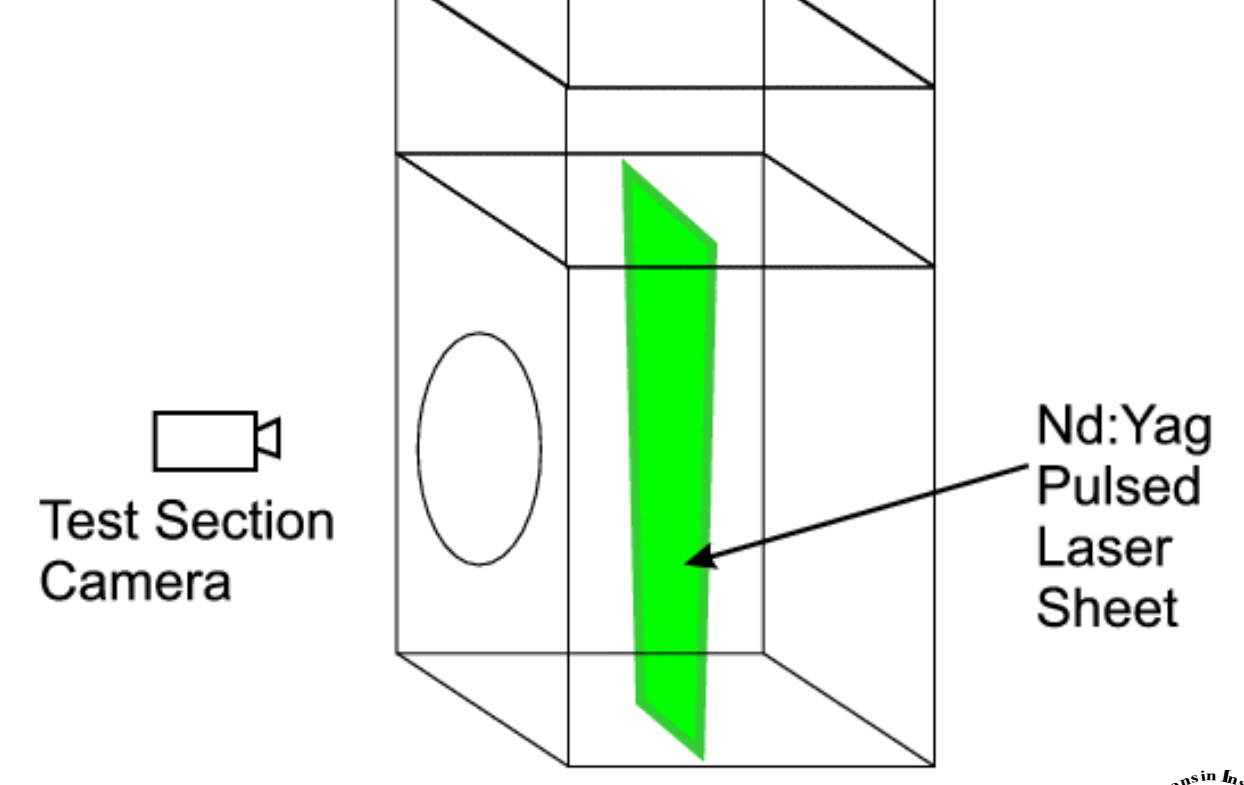

Sine Wave

$\rightarrow$ Plate

Retraction

Direction

Nd:Yag Pulsed Laser Sheet 


\section{Experimental conditions}

-Incident shock wave: $M=2.90$, in $\mathrm{CO}_{2}$

- $P_{\text {initial }}=1 \mathrm{~atm}, T_{\text {initial }}=300 \mathrm{~K}$

-Post-shock $A^{\prime}=0.245\left(A=0.206, A=\left(\rho_{1}-\rho_{2}\right) /\left(\rho_{1}+\rho_{2}\right)\right)$

- Planar Mie scattering visualization, smoke particles

-Two-stage retraction ( $\tau_{1} \sim 250 \mathrm{~ms}, \tau_{2} \sim 80 \mathrm{~ms}$ )

-Interface section

- Ar+ laser@ $\lambda=488$ nm, continuous wave

- CCD camera, 256 x 256 pixel array, 8 bit/pixel, framing@ 100 fps

-Test section

- Nd:YAG laser@ $\lambda=532$ nm, 10 ns pulse

- CCD camera: 1024 x 1024 pixel array, 16 bit/pixel, one shocked image per experiment 


\section{Experiment: $\mathrm{CO}_{2}$-air $M=2.90$}

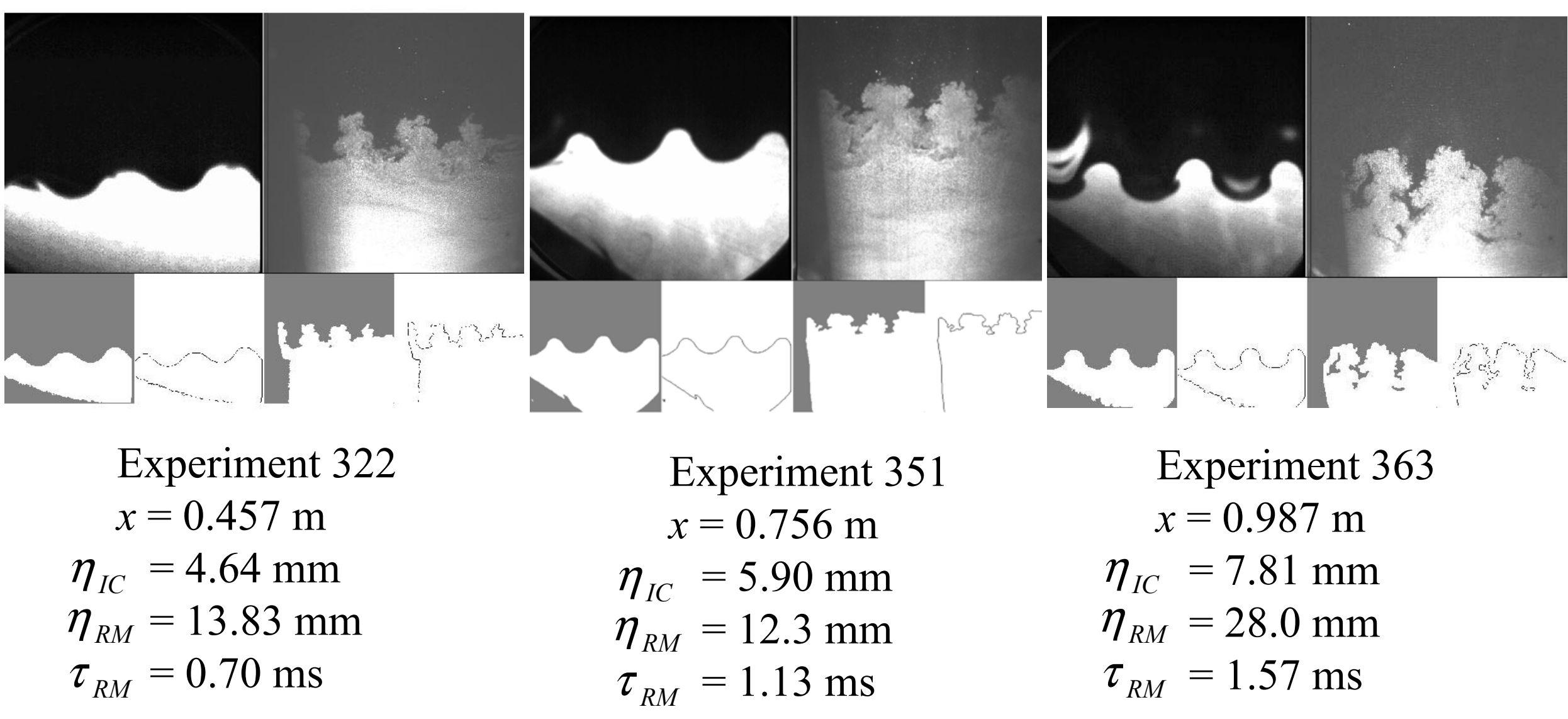

- Initial condition well into nonlinear regime $\left(\eta_{0} / \lambda>0.2\right)$

- Phase inversion of shocked interface 


\section{Comparison with Theories}

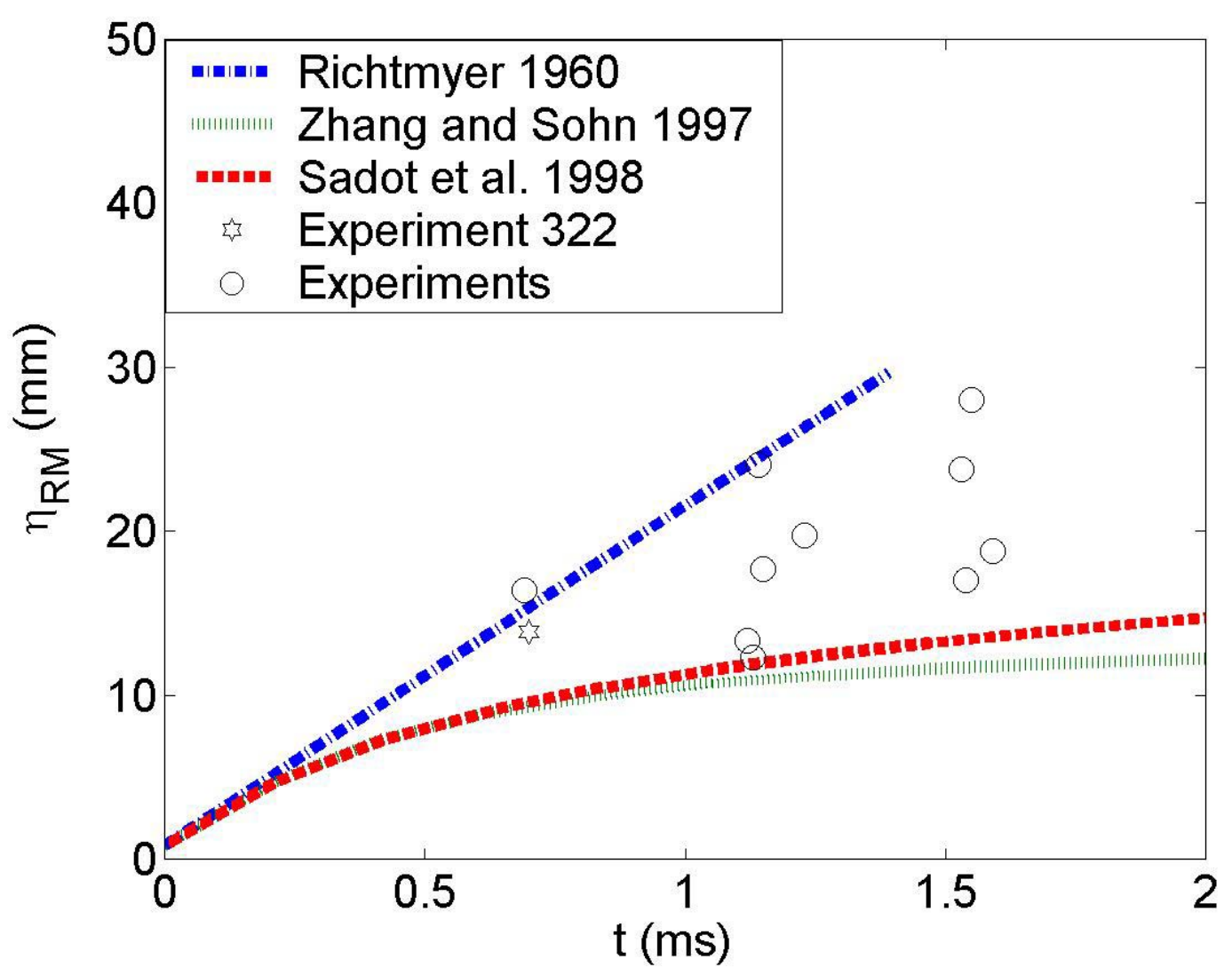

Comparison with prediction from theories shows qualitative agreement and experimental data bounded by the linear (upper) and nonlinear theories (lower) 


\section{Conclusions}

- Two dimensional gas-gas interface without a membrane

- Strongly shocked interface ( $\mathrm{CO}_{2}$-air, $M$ up to 3.06)

- Initial condition geometry imaged for each experiment

- Scatter in data attributed to extreme sensitivity to initial conditions

- Results are similar to existing linear theories

- Needed improvements

- Better retraction mechanism for more repeatable initial condition

- Diagnostic upgrade to obtain more than one shocked image per experiment 


\section{Lower Mach \# Experiment: $\mathrm{CO}_{2}$-air $M=1.41$}

$A=0.2061, A^{\prime}=0.2242, \mathrm{~A} 130030.508 \mathrm{~mm}$ diaphragm

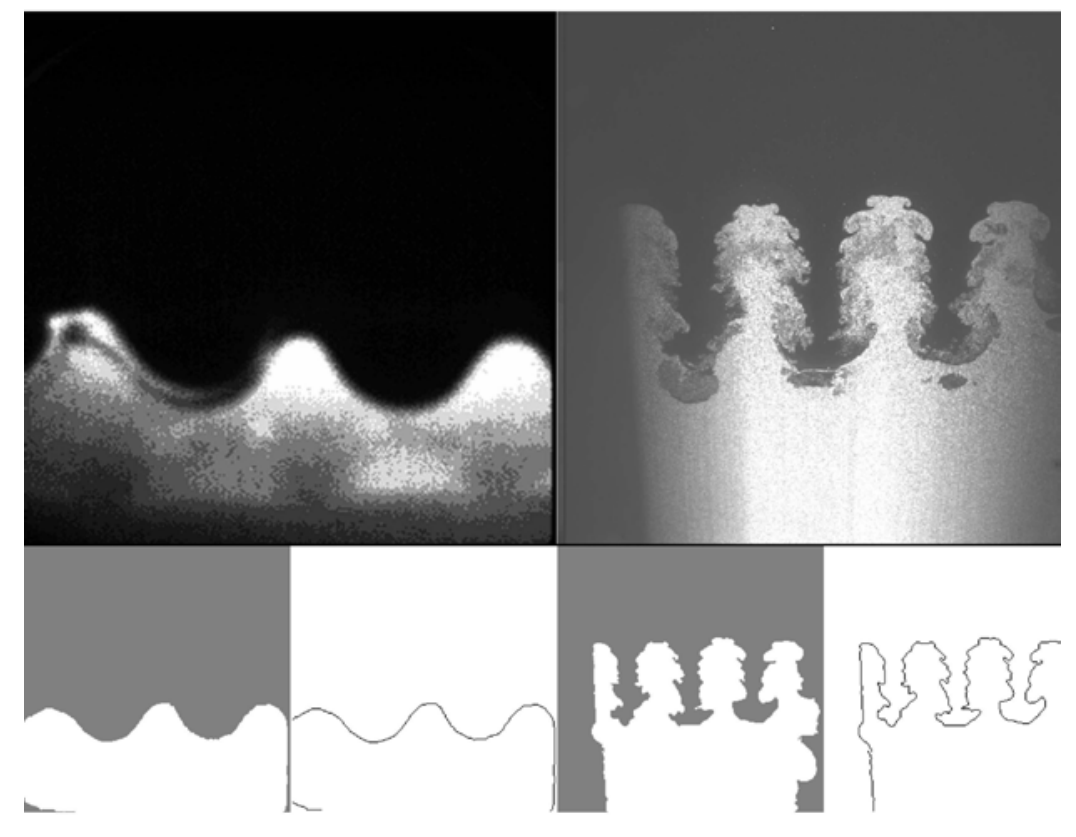

Experiment 327

$$
\begin{aligned}
x & =0.457 \mathrm{~m} \\
\eta_{I C} & =6.12 \mathrm{~mm} \\
\eta_{R M} & =25.3 \mathrm{~mm} \\
\tau_{R M} & =2.60 \mathrm{~ms}
\end{aligned}
$$

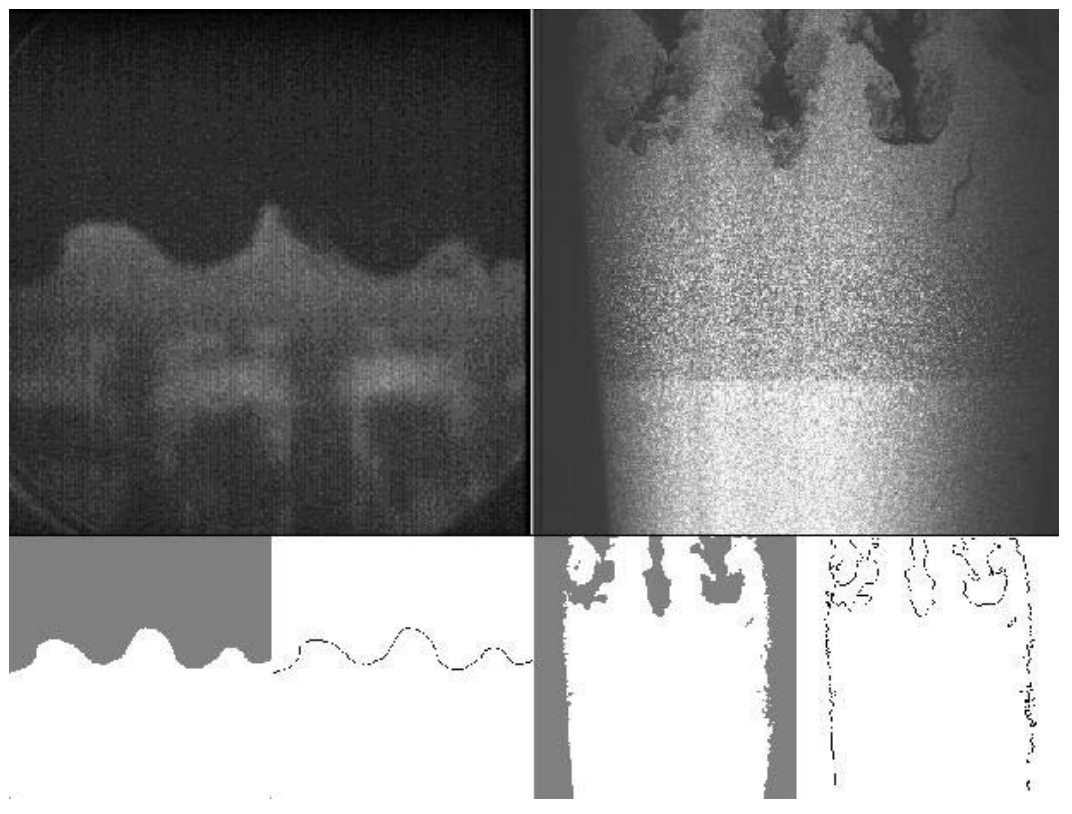

Experiment 343

$$
\begin{aligned}
x & =0.756 \mathrm{~m} \\
\eta_{I C} & =5.45 \mathrm{~mm} \\
\eta_{R M} & =23.0 \mathrm{~mm} \\
\tau_{R M} & =3.97 \mathrm{~ms}
\end{aligned}
$$

Fusion Technology Institute UW-Madison 


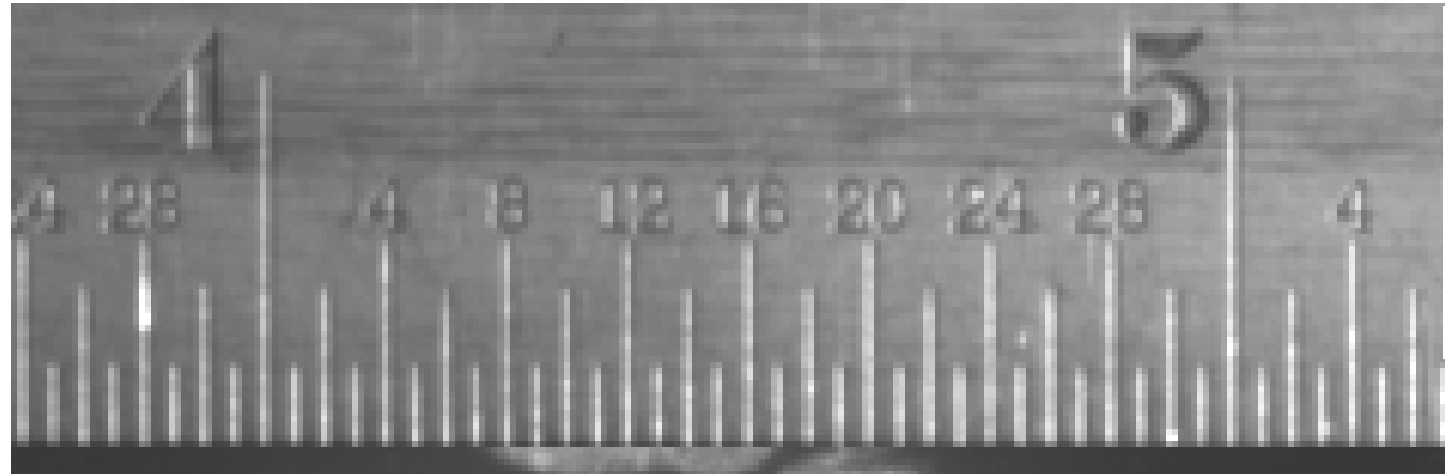

Magnified image of one peak from test 327 , the scale above the instability is in inches.

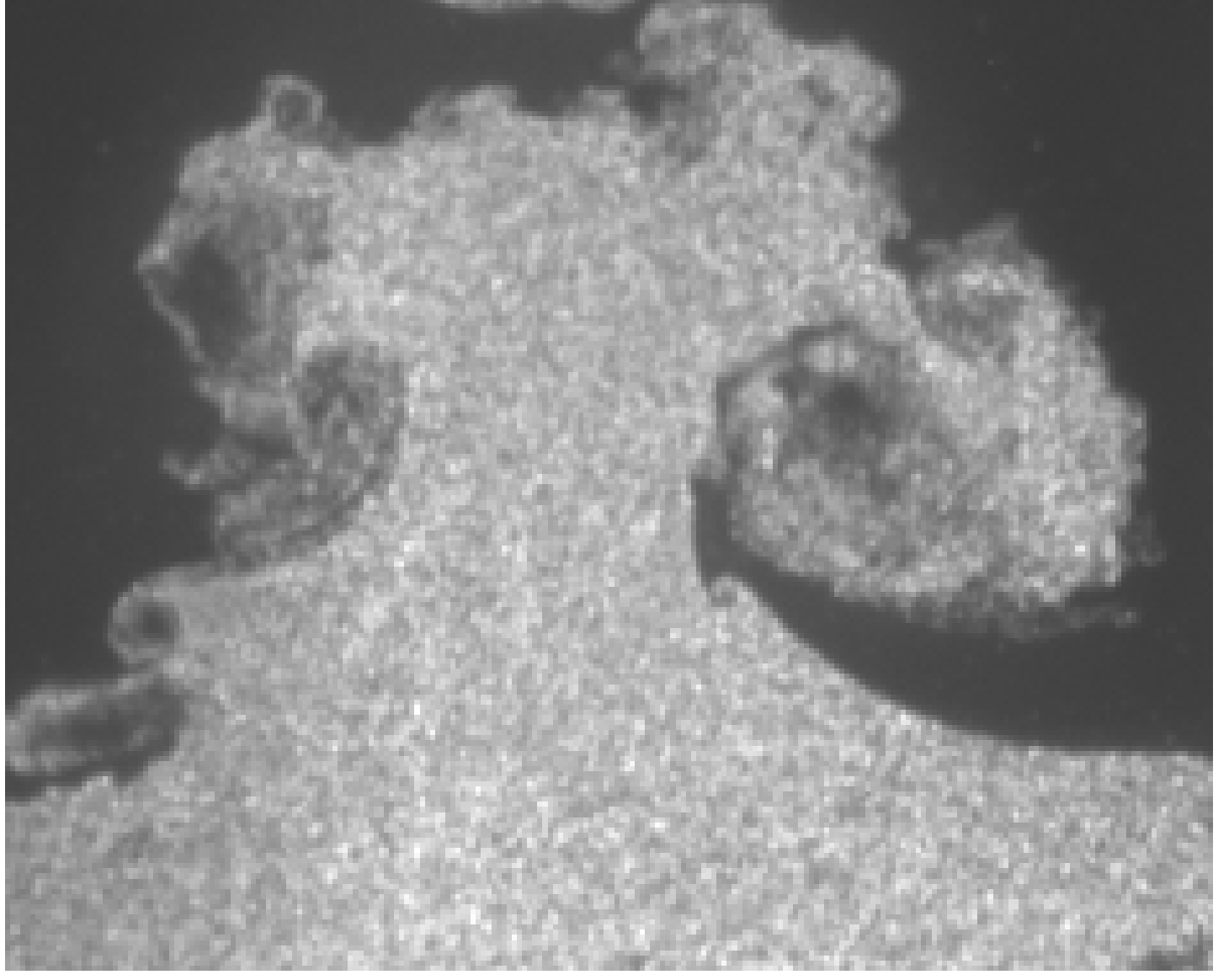


Paper at VIII International Workshop on the Physics

of Compressible Turbulent Mixing

California Technological Institute, Pasadena, California, USA

\title{
Experimental Investigation into the Evolution of Turbulent Mixing of Gases by Using the Multifunctional Shock Tube
}

\author{
Yu.A.Kucherenko, O.E.Shestachenko, Yu. A. Piskunov, E.V. Sviridov, \\ V.M.Medvedev, A.I.Baishev
}

Russian Federal Nuclear Center - Academician E. I. Zababakhin

All-Russian Research Institute of Technical Physics

456770 Snezhinsk, Russia

At the initial instant of time, different density gases being investigated are found in the multifunctional shock tube and are separated by a "specter-membrane". Then the specter-membrane is destroyed into small-scale fragments by the external force. The contact boundary of gases is accelerated by means of a compression wave, which is formed in the shock tube. At the same time, at the contact boundary of different density gases, the Rayleigh-Taylor instability arises and the unstationary zone of the gravitational turbulent mixing forms. On the basis of the experimental results the dependence of the turbulent mixing zone width on the contact boundary displacement has been constructed, and the gravitational turbulent mixing constant alpha has been determined.

\section{INTRODUCTION}

In most of problems associated with the fast compression processes of a matter the situation arises when a matter of less density (a light matter) and a more dense matter (a heavy matter) have a surface of their contact (a contact surface) and are moving with acceleration. In case of constant acceleration the contact surface is said to be subjected to the action of the Rayleigh-Taylor instability (RTI). If the pulsed acceleration (for example, at the passage of shock waves) takes place, then any contact surface is unstable, because the Richtmyer-Meshkov instability (RMI) arises.

The instability means that any small perturbation has a tendency to an unlimited growth, the mutual penetration of media and the destruction of structures under the action of shear turbulence take place, the turbulent mixing zone (TMZ) arises. The evolution of instabilities on the contact surface of different density media exerts an influence on the dynamics of the compression, restricts the limiting value of compression and the dynamics of subsequent processes.

The determining parameter to take into account the gravitational turbulent mixing influence is the turbulent mixing zone width which depends on the density ratio of different density media, the time of the unstable situation existence, etc. In a number of problems, taking into account the compressibility media being found along the different sides of the contact surface becomes important. 
Under laboratory conditions the investigation of RTI and arising gravitational turbulent mixing (GTM) is performed with using different density liquid and gaseous media at the installations EKAP and SOM. The installation OSA makes it possible to investigate different kinds of instability (RTI, RMI) by using three replaceable drivers for these purposes. The distinctive feature of the experiments is the usage of the controlled separating membrane making it possible to form the evolution process of GTM of different gases with preset initial conditions.

The aim of the present work is to perform experiments by using the shock tube OSA creating RTI and to apply the controlled separating membrane for these investigations.

\section{SET- UP OF EXPERIMENTS}

For performing experiments regarding the gravitational turbulent mixing investigation the scheme presented in Fig. 1 was used. The $0<\mathrm{x}<\mathrm{x}_{1}$ region is filled up with the compressed gas and represents a high pressure chamber. From the rest of the shock tube part the chamber is separated by a light piston which is found at the point $\tilde{\mathrm{o}}=\tilde{\mathrm{o}}_{1}$. The $\tilde{\mathrm{o}}_{1}<\tilde{\mathrm{o}}<\tilde{\mathrm{o}}_{2}$ region is filled up with a light working gas $\mathbf{1}$ of density $\rho_{1}$ and represents the low pressure chamber. The $\tilde{\mathrm{o}}>\tilde{\mathrm{o}}_{2}$ region is filled up with a heavy working gas $\mathbf{2}$ of density $\rho_{2}$ and represents a measuring chamber by which the mixing process registration is carried out. In the point $\tilde{\mathrm{o}}=\tilde{\mathrm{o}}_{2}$ the separating membrane is found which prevents from the mixing of working gases during the experiment preparation. At the specified instant of time the separating membrane is destroyed into pieces of definite size by the external force. The installation operates as follows. At the instant of time $\mathrm{t}=\mathrm{t}_{0}$ the piston begins to move with constant acceleration in the positive direction of the axis $\mathrm{X}$ under the action of the compressed gas in the high pressure chamber. From the piston a compression wave begins to propagate in the

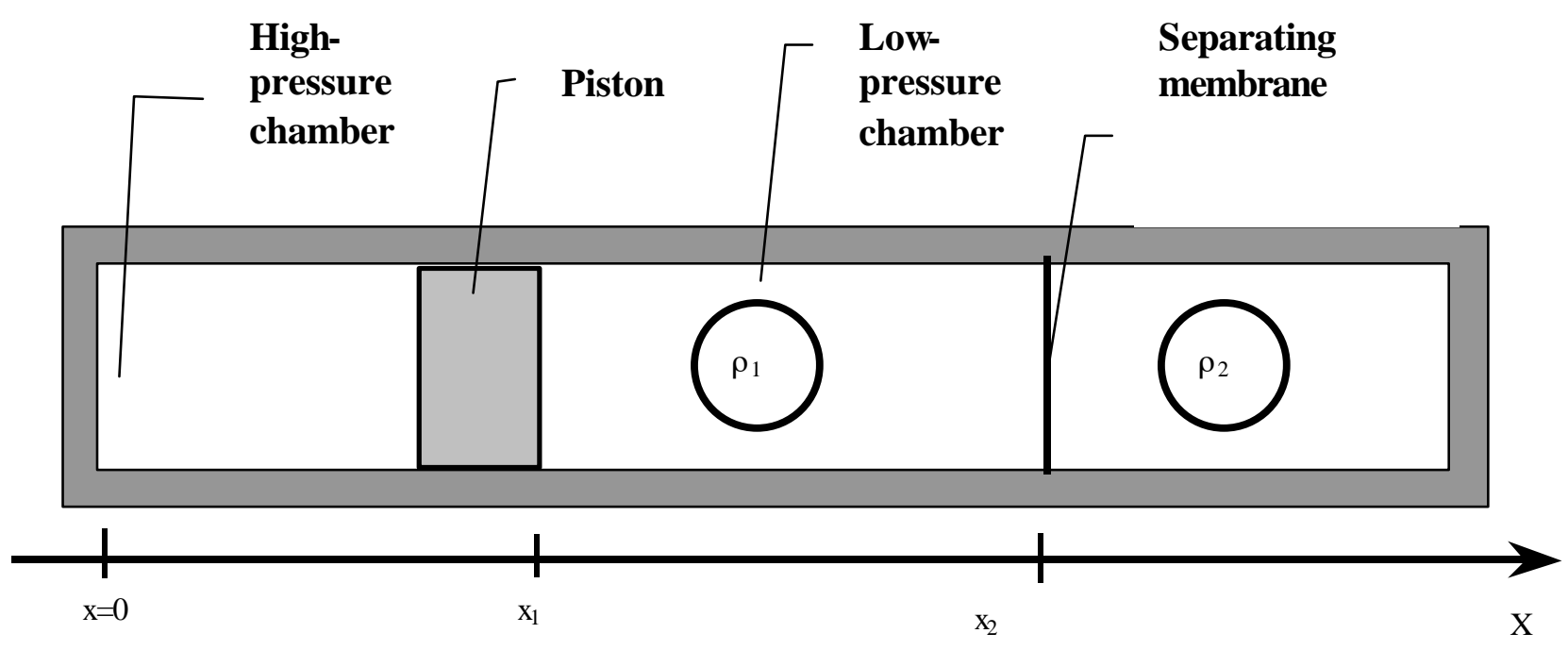

Fig. 1 Physical scheme of the experiment 
positive direction of the axis $X$ with velocity $\tilde{N}_{1}$, where $\tilde{N}_{1}$ - sound velocity in the working gas $\mathbf{1}$. At the instant of time $t=\left(\mathrm{x}_{2}-\mathrm{x}_{1}\right) / \mathrm{C}_{1}$ the compression wave arrives at the interface of gases $\tilde{\mathrm{o}}=\tilde{\mathrm{o}}_{2}$. At the same time, the external destructive force is applied to the separating membrane, and the contact boundary between gases begins to be accelerated. As the contact boundary acceleration profile is slightly falling and the pressure gradient in the compression wave is directed oppositely to the density gradient at the contact boundary, then the conditions are created for the RTI occurrence.

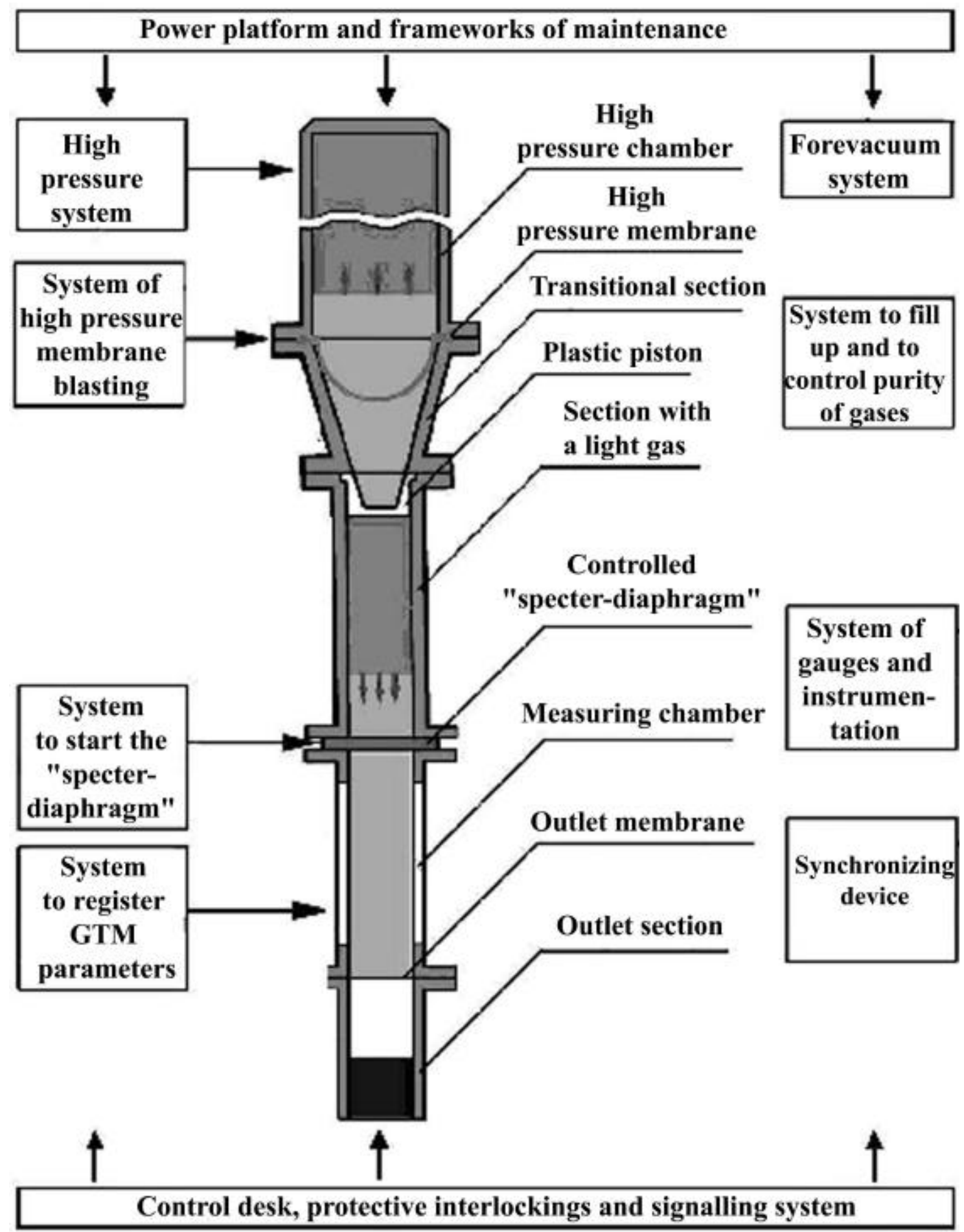

Fig.2. Functional scheme of the installation OSA 
Fig.2 shows the functional scheme of the installation OSA. The total height of the installation amounts to $\approx 5 \mathrm{~m}$. In the upper part of the installation the high pressure chamber is located. It represents a thick-walled vessel consisting of three parts connected among themselves by flanges. The operating pressure in the chamber is up to $2 \mathrm{MPa}$. At the upper flange of the high pressure chamber there is the emergency valve of pressure drop, pipelines for gas inlet and outlet. From the rest of the shock tube part the high pressure chamber is separated by the aluminum membrane. The membrane thickness amounts to $1 \mathrm{~mm}$ or $0.5 \mathrm{~mm}$ and determines the limiting pressure of gas in the reservoir. For the membrane destruction at the specified instant of time, a strong electric explosion is used. A sliding contact in the form of a metal needle touches the membrane in the center. The needle is connected to the positive pole of the capacitor bank by means of cables, but the membrane - to the negative pole. At the instant of time $t=t$ the pulse of current burns through the aluminum membrane in the center. Gas begins to flow out of the reservoir and opens the membrane completely. The gas flow passes through the conical part of the transitional section and begins to push a plastic piston. In the compression wave the pressure profile and amplitude depend on the piston mass. Under the action of the compressed gas the piston is moving with acceleration along the section with a light gas. The section with a light gas is filled up with gas of density $\rho_{1}$. The internal crosssection of the light gas section is equal to $138 \times 138 \mathrm{~mm}^{2}$, but the length amounts to $500 \mathrm{~mm}$. The contact boundary acceleration profile depends on the light gas section. The further the contact boundary of gases from the piston is, the more the acceleration differs from the constant one and approaches to the delta-shaped one.

Prior to the experiment performing the section with a light gas is filled up with the working gas of density $\rho_{1}$ through the gas inlet system. Simultaneously, the measuring chamber is filled up with the working gas of density $\rho_{2}$ through the gas inlet system. The gas inlet system controls the extent of purity of working gases. Between the light gas section and the measuring chamber the controlled separating membrane is located. It is designed to prevent from the mixing of working gases during the experiment preparation.

The controlled measuring membrane represents the interweaved grid of microconductors, $20 \mu \mathrm{m}$ in diameter and $4 \mathrm{~mm}$ in step. To this grid the liquid film of the soapy solution is applied. The film thickness amounts to $\approx 1 \mu \mathrm{m}$. At the specified instant of time the electric current is passed through the grid. Microconductors are heated, and the liquid film begins to be destroyed into the pieces with the typical scale $\lambda \approx 4 \mathrm{~mm}$ in the places of contact with microconductors. Then the surface tension forces tighten the pieces of liquid film into small balls which act as initial perturbations at the contact boundary of working gases. When the compression wave reaches the boundary between gases, the contact boundary begins to be accelerated. As acceleration is directed from the light gas to the heavy one, the conditions are created for the gravitational turbulent mixing zone evolution. 

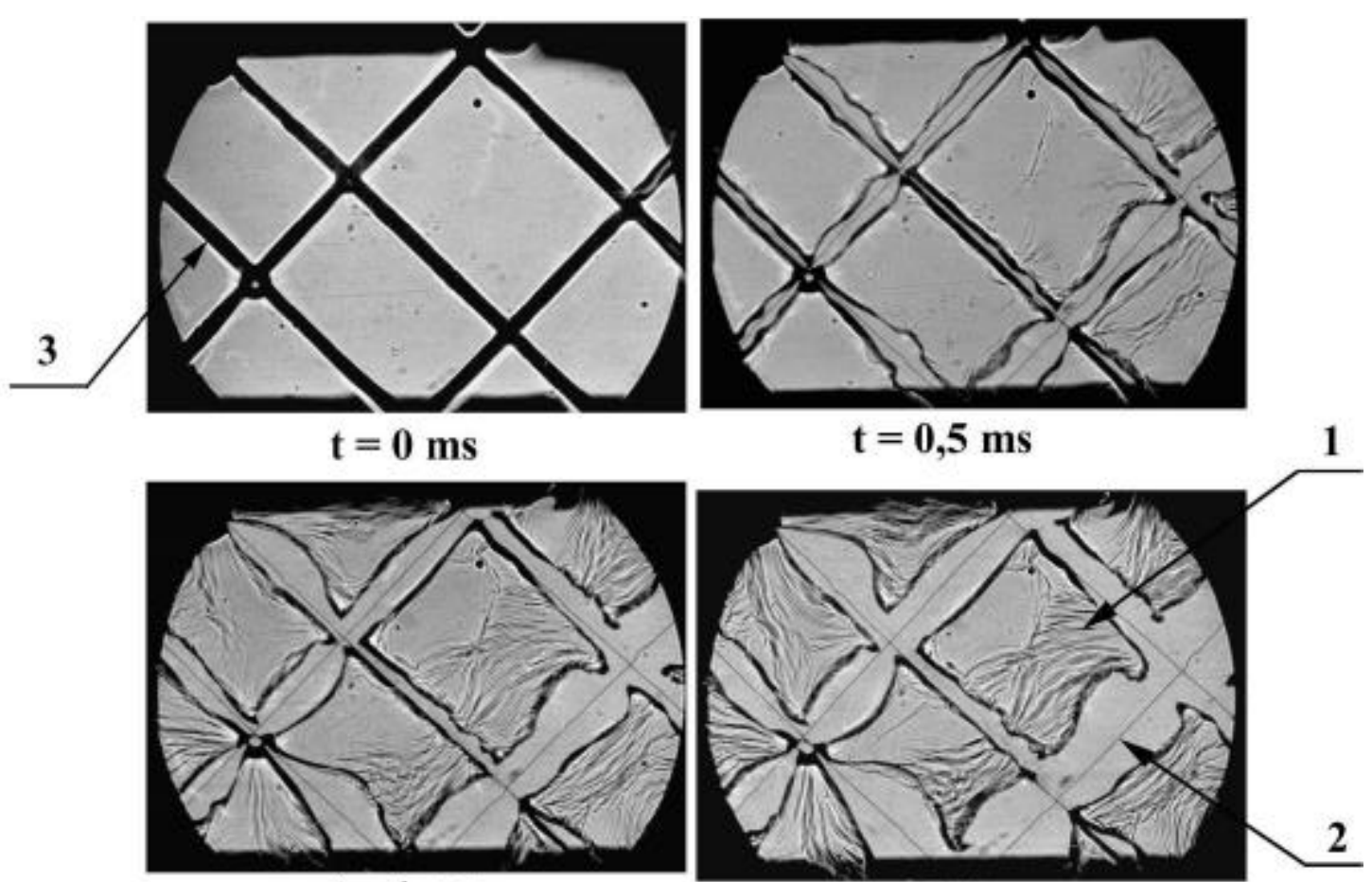

$\mathrm{t}=1 \mathrm{~ms}$

$$
\mathrm{t}=1,5 \mathrm{~ms}
$$

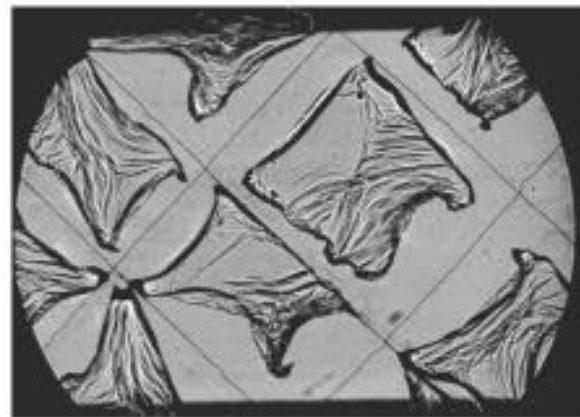

$\mathbf{t}=\mathbf{2} \mathbf{m s}$

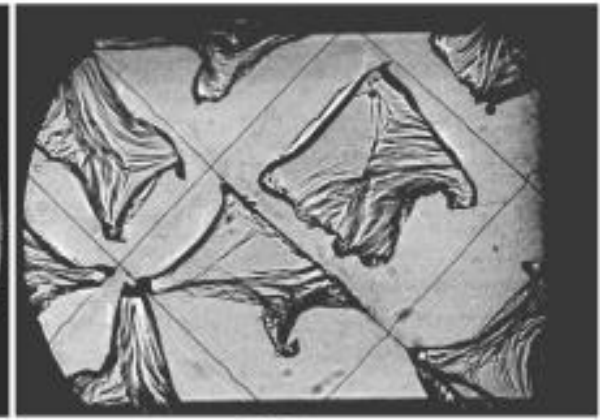

$\mathrm{t}=\mathbf{2 , 5} \mathrm{ms}$

Fig. 3 Characteristic photographic images of the liquid film destruction process.

Fig. 3 shows the characteristic photographic images of the separating membrane destruction process at different instants of time. In this figure microconductors are denoted by number $\mathbf{1}$, liquid film pieces - by number $\mathbf{2}$, the microconductor with the liquid film around it - by number $\mathbf{3}$. From this figure it is seen that after applying the current pulse to the grid the liquid film begins to separate from microconductors and then, under the action of the surface tension forces, it is tightened into a drop.

After the block with the controlled separating membrane, the measuring section is located. It is filled with the working gas of density $\rho_{2}$. The measuring chamber has two transparent walls of high-quality optical glass. This makes possible to perform the photographic record of the turbulent mixing zone by means of a schlieren (photograph) technique. If it is necessary to investigate the late stages of the turbulent mixing process, then the additional chamber of low pressure is mounted between the block of the controlled separating membrane and the measuring section. 
After the measuring chamber the low pressure chamber, $260 \mathrm{~mm}$ in length, is found. It is also filled up with the working gas of density $\rho_{2}$.

This chamber is necessary so that the reflected compression wave does not exert an influence on the photographic record of the turbulent mixing zone. After terminating the photographic record and passing of the piston into the low pressure chamber, it is necessary to slow down the piston. For this purpose the special outlet section is designed. When the piston passes into the low pressure chamber its velocity is $\approx 100 \div 150 \mathrm{~m} / \mathrm{sec}$. Gas being pushed by the piston ruptures the outlet membrane which is located between the measuring chamber and the outlet section. Gas pressure before the piston becomes gradually higher than the pressure behind the piston, and the latter begins to be decelerated. When moving the piston along the outlet section the pressure of the decelerating gas increases. In order to decrease the acceleration of retardation and to prevent the reverse motion of the piston, it is necessary to release gas from the inlet section into atmosphere. For this purpose in the outlet section there are exhaust windows in which the membranes of lavsan are found. When reaching the ultimate strength the membranes are ruptured and gas gets into atmosphere. The rubber shock - absorber located at the bottom of the outlet section is designed to cancel the residual speed of the piston.

The registration of flow arising at the contact surface (CS) of two different density gases after its acceleration was carried out through the peepholes in the measuring section by the schlieren device IAB-451. The device is consistent with the high-speed camera VFU, operating in the mode of a time magnifier. Illuminating was carried out by a flash lamp. The light pulse duration was equal to $4 \mathrm{msec}$. The flash lamp, the camera VFU-1 and the phenomenon itself were synchronized is such a way that the phenomenon registration was performed at the required stage of evolution. The optical method to register transparent inhomogeneities is based on the dependence of the refractive index of gases on density.

\section{Discussion of results}

In the given work argon $\mathrm{Ar}$ (density $\rho=1.78 \mathrm{~kg} / \mathrm{m}^{3}$ ) and $\mathrm{Kr}$ gas (density $\rho=3.7 \mathrm{~kg} / \mathrm{m}^{3}$ ) were used as working gases. The density ratio for the given pair of gases $n=2.1$, but the Atwood number $A=\frac{\rho_{2}-\rho_{1}}{\rho_{2}+\rho_{1}}$ for the given pair of gases was equal to $\grave{A}=0.35$. For these experiments the acceleration of the contact boundary of gases amounted to $\mathrm{g} \approx 40000 \mathrm{~m} / \mathrm{sec}^{2}$. The photographic record of the turbulent mixing process was carried out when changing the parameter $\mathrm{S}=\mathrm{gt}^{2} / 2$ from $0.1 \mathrm{~m}$ to $0.5 \mathrm{~m}$.

Fig. 4 shows the characteristic photographic records of the gravitational turbulent mixing process. The turbulent mixing zone is seen in photos in the form of a wide dark band. It is seen that the zone width is increased with time, but the zone itself is mixing downwards. For the correct determination of the image scale, reference bench marks are set before the measuring chamber glasses. 


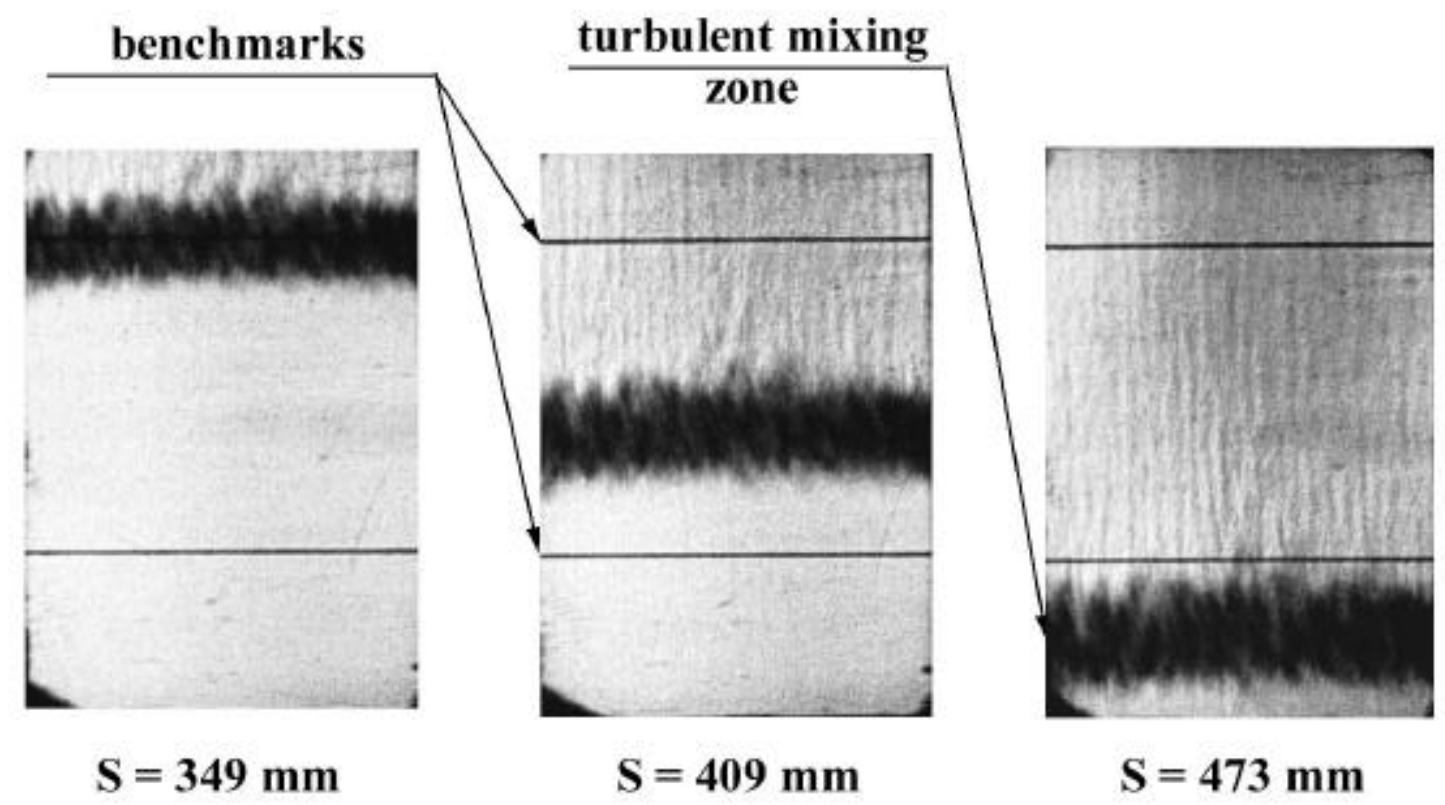

Fig. 4 Characteristic photographic images of the gravitational turbulent mixing process

Fig.5 shows the dependence of the turbulent mixing zone width on the parameter $\mathrm{S}$ for the pair of gases $\mathrm{Ar}-\mathrm{Kr}$. The conditions of self-similarity for the given experiment

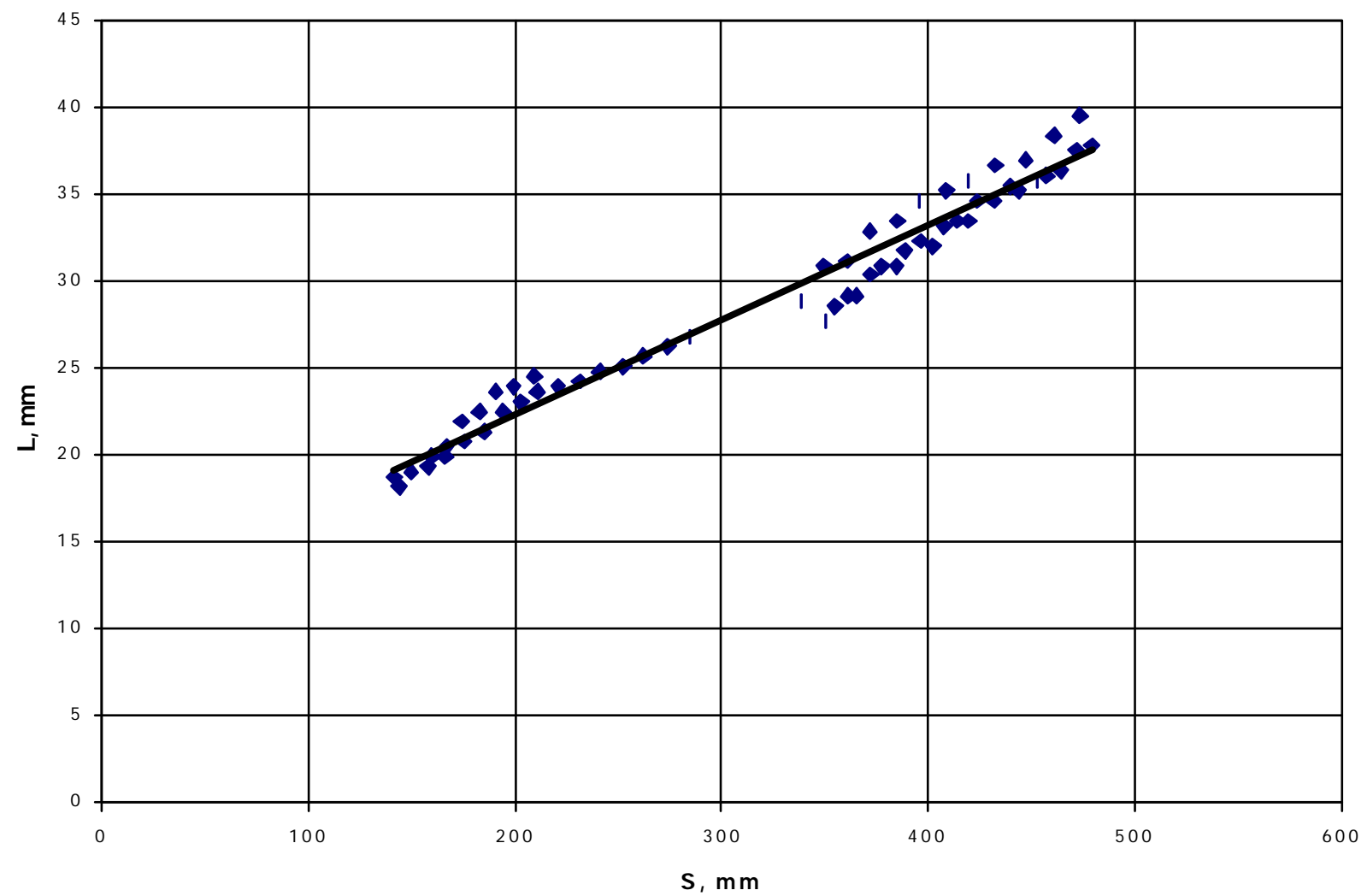

Fig.5. Dependence of the turbulent mixing zone width on the parameter $\mathrm{S}$ for the pair of gases $\mathrm{Ar}-\mathrm{Kr}$. 
are carried out at $\mathrm{S} \geq 100 \mathrm{~mm}$. It means that the turbulent mixing zone growth does not depend on the initial conditions on the contact boundary of gases. All the totality of experimental points at $S \geq 100 \mathrm{~mm}$ can be described by the formula $\mathrm{L}=2 \alpha \mathrm{AS}$, where the parameter $S=\mathrm{gt}^{2} / 2$ is the contact boundary displacement, $A=\frac{\rho_{2}-\rho_{1}}{\rho_{2}+\rho_{1}}$ is Atwood number and $\alpha$ is the dimensionless velocity of turbulent mixing. On the basis of experimental results the constant $\alpha=0.04$ has been determined.

\section{Conclusion}

In the given work the Rayleigh-Taylor instability for gaseous media was studied. Ar and $\mathrm{Kr}$ were used as working gases. Density ratio $\rho_{2} / \rho_{1}$ was taken to be equal to 2.1, but Atwood number $A=\left(\rho_{1}-\rho_{2}\right) /\left(\rho_{1}+\rho_{2}\right)$ was equal to 0.35 .

At the initial instant of time, different density gases being investigated were located in the multifunctional shock tube and were separated by the controlled separating membrane. Then the separating membrane was destroyed by the external force into small - scale fragments with the typical size $\lambda \approx 4 \mathrm{~mm}$.

The contact boundary of gases was accelerated by means of a compression wave, which was generated by the piston being accelerated. The initial acceleration of the contact boundary of gases amounted to $\approx 40000 \mathrm{~m} / \mathrm{sec}^{2}$.

According to the results of experiments, the dependencies of the turbulent mixing zone width on the contact boundary displacement $S$ have been constructed. The parameter $\mathrm{S}$ was changed from $100 \mathrm{~mm}$ to $500 \mathrm{~mm}$. At the self-similar stage of the turbulent mixing evolution the dimensionless velocity of turbulent mixing $\alpha$ was determined to be equal to 0.04 . 


\section{Modeling Laser Material Strength Experiments}

\section{Steve Pollaine \\ David Petersen \\ Lawrence Livermore National Laboratory}

\section{8th IWPCTM December 10-14, 2001}

D. Kalantar, B. Remington, J. Belak, J. Colvin,

M. Kumar, T. Lorenz, S. Weber

Lawrence Livermore National Laboratory

J. Wark, A. Loveridge, A. Allen

University of Oxford

M. Meyers

University of California, San Diego 


\section{Outline of poster}

-Material strength model

-Elastic-plastic flow

-Steinberg-Guinan and Steinberg-Lund models

-VISAR velocity measurement

-Experiment

-Model

-Diffraction

-Experiment

-Model

-Sample recovery

-Experiment

-Decay of shock strength

-Summary and future developments 
The constituitive properties of metals is of general scientific interest

Laser experiments give us access to new regimes

High pressures

High strain rates

How materials deform at strain rates $>108 / \mathrm{s}$ is unknown

Relevant for impact of micrometeorites on space hardware Diagnostics

VISAR

X-ray diffraction

Recovery

Infer properties such as EOS, K, G, Y

Recovery

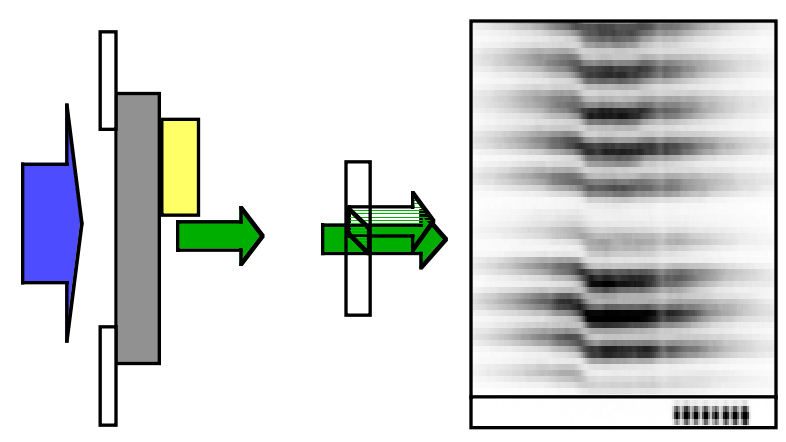

VISAR

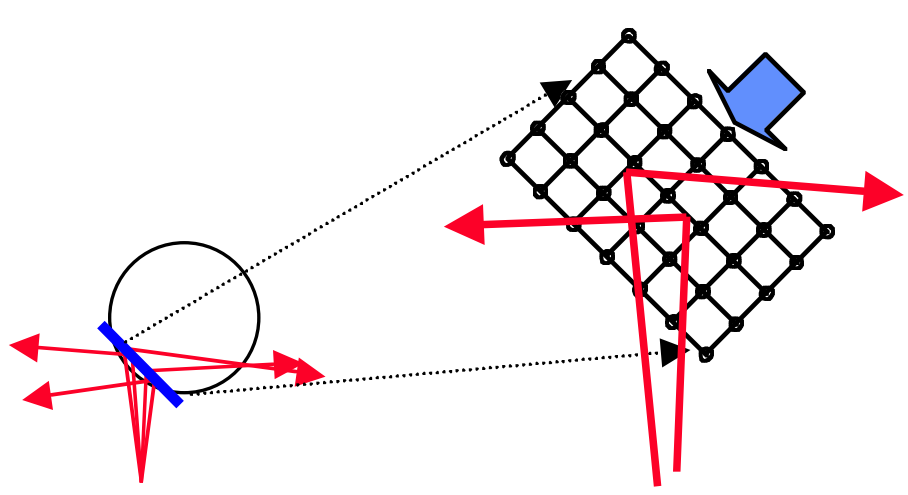

X-ray diffraction 


\section{Moderate shocks show both elastic and plastic waves}
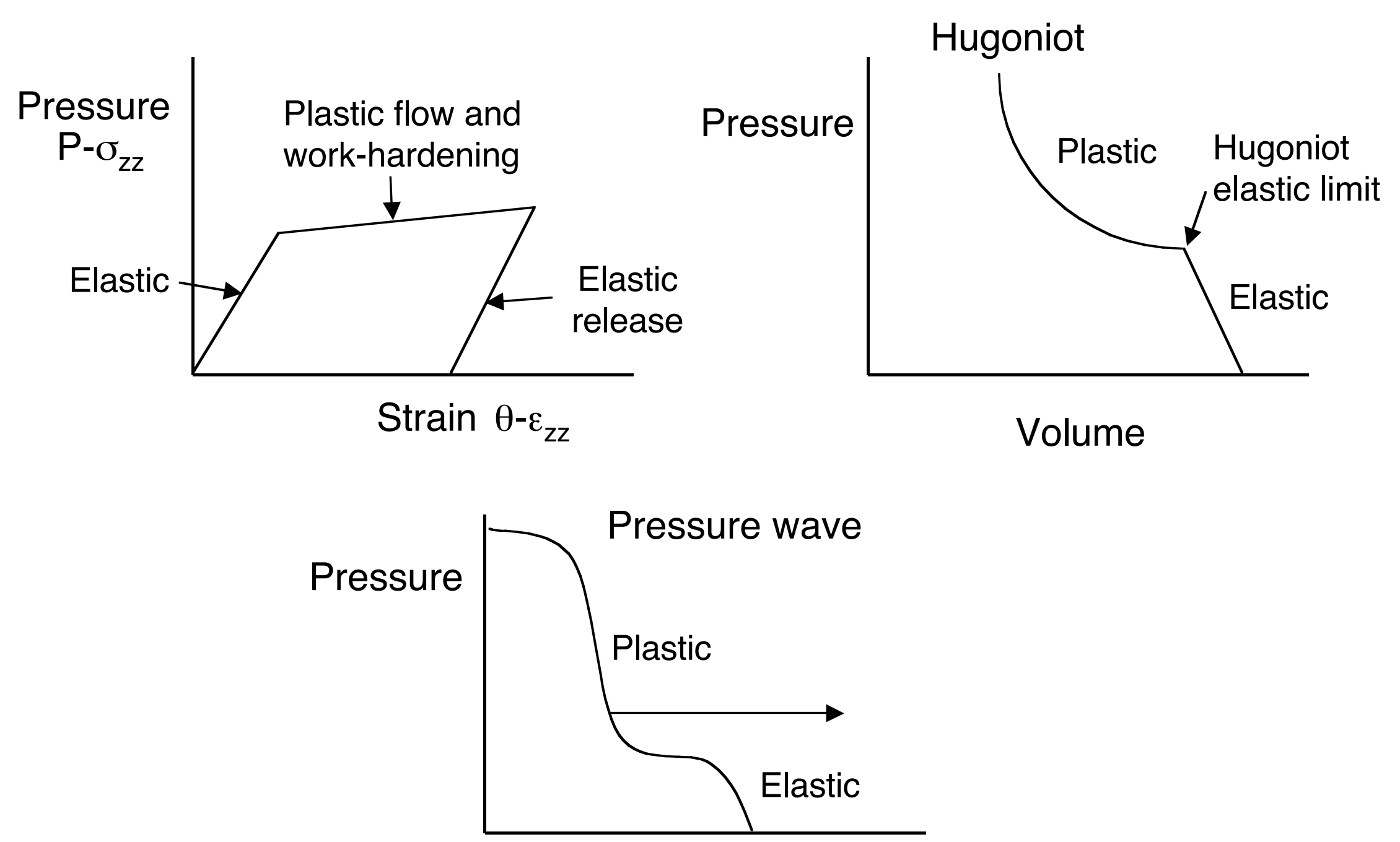

Distance 


\section{We use a material strength package in our code}

Newton's law

$\rho \alpha_{r}=-\frac{\partial}{\partial r}\left(P-\sigma_{r r}\right)+\frac{\partial}{\partial z} \sigma_{r z}+\frac{1}{r}\left(2 \sigma_{r r}+\sigma_{z z}\right)$

$\rho \alpha_{z}=-\frac{\partial}{\partial z}\left(P-\sigma_{z z}\right)+\frac{\partial}{\partial r} \sigma_{r z}+\frac{1}{r} \sigma_{r z}$

\section{Definition of strain}

$\forall=\frac{\partial v_{r}}{\partial r}+\frac{\partial v_{z}}{\partial z}+\frac{v_{r}}{r}$

$\mathbb{E}_{r r}=\frac{1}{3}\left(2 \frac{\partial v_{r}}{\partial r}-\frac{\partial v_{z}}{\partial z}-\frac{v_{r}}{r}\right)$

$\mathbb{E}_{z z}=\frac{1}{3}\left(2 \frac{\partial v_{z}}{\partial z}-\frac{\partial v_{r}}{\partial r}-\frac{v_{r}}{r}\right)$

$\mathcal{\delta}_{r z}=\frac{1}{2}\left(\frac{\partial v_{r}}{\partial z}+\frac{\partial v_{z}}{\partial r}\right)$ $\rho=$ density

$\mathrm{v}=$ velocity

$\mathrm{P}=$ hydrodynamic pressure

$\sigma=$ deviatoric stress

$\theta=$ hydrodynamic strain

$\varepsilon=$ deviatoric strain

$\mathrm{K}=$ bulk modulus

$\mathrm{G}=$ shear modulus
EOS with strain

$P^{x}=-K \otimes-P^{\text {inelassic }}$

$\mho_{r r}=2 G \mathcal{\mho}_{r r}-\mho_{r r}^{\text {inelastic }}+2 \sigma_{r z} \omega+\left(\sigma_{z z}-\sigma_{r r}\right) \omega^{2}$

$\boldsymbol{\alpha}_{z z}=2 G \mathcal{\mho}_{z z}-\sigma_{z z}^{\text {inelastic }}-2 \sigma_{r z} \omega-\left(\sigma_{z z}-\sigma_{r r}\right) \omega^{2}$

$\boldsymbol{\sigma}_{r z}=2 G \boldsymbol{\delta}_{r z}-\boldsymbol{\sigma}_{r z}^{\text {inelastic }}+2\left(\sigma_{r z}-\sigma_{r r}\right) \omega-2 \sigma_{r z} \omega^{2}$

$\omega=\frac{1}{2}\left(\frac{\partial v_{r}}{\partial z}-\frac{\partial v_{z}}{\partial r}\right)$ 


\section{We use a von Mises yield criterion for the onset of plastic flow}

Deviatoric strain invariant $J=\sqrt{\frac{4}{3}\left(\sigma_{r r}^{2}+\sigma_{z z}^{2}+\sigma_{r z}^{2}+\sigma_{r r} \sigma_{z z}\right)}$

Effective pressure $P_{e}=P-\sqrt[3]{\left(\sigma_{r r}+\sigma_{z z}\right)\left(\sigma_{r z}^{2}-\sigma_{r r} \sigma_{z z}\right) / 16}$

When $\mathrm{J}>\mathrm{Y}\left(\mathrm{P}_{\mathrm{e}}\right)$, the elastic limit is exceeded and plastic flow begins

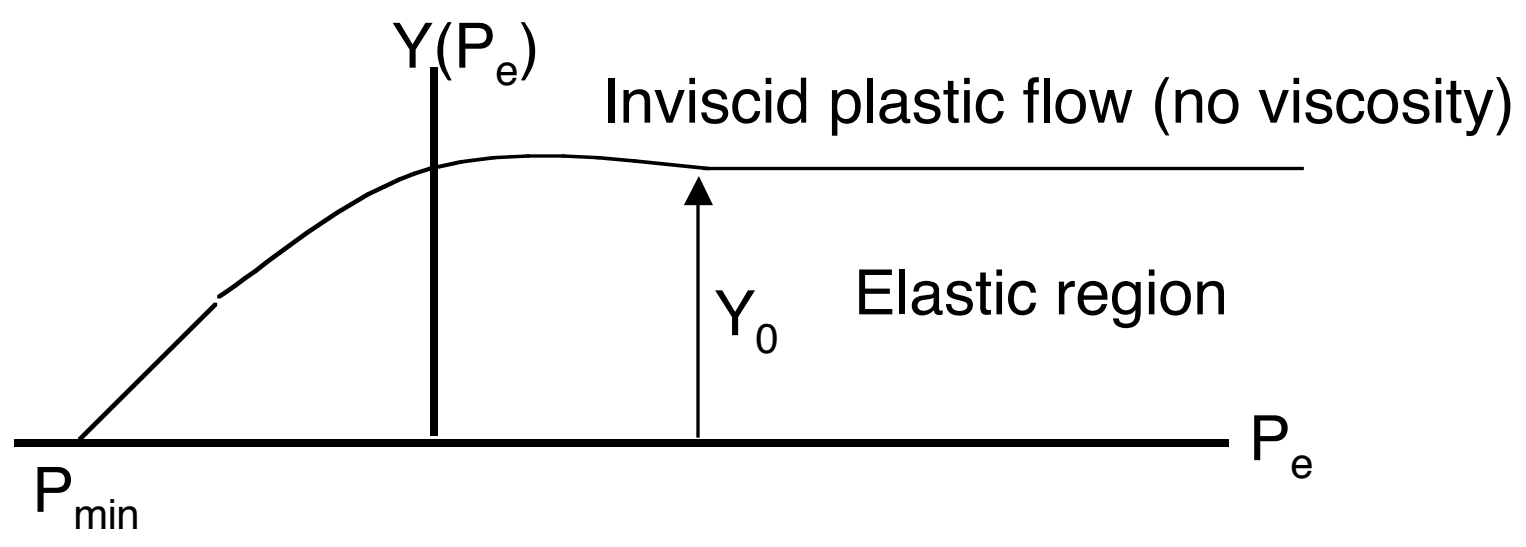

von Mises, Z. Angew. Math. U. Mech. 8 (1928),

translated in UCRL Trans. 872 


\section{Uniaxial strain equations}

$$
\begin{aligned}
& \rho \aleph_{z}=\frac{\partial}{\partial z}\left(-P+\sigma_{z z}\right) \\
& \theta=\frac{\partial v_{z}}{\partial z} \\
& \varangle_{z z}=\frac{2}{3} \frac{\partial v_{z}}{\partial z} \\
& P^{x}=-K \theta^{*}-P^{x \text { inelassic }} \\
& \mho_{z z}=2 G \mho_{z z}-\mho_{z z}^{\text {inelastic }} \\
& P_{e}=P-\frac{1}{4} \sigma_{z z} \\
& J=\left|\sigma_{z z}\right|
\end{aligned}
$$

Sound speed $\mathrm{c}_{11}=\sqrt{\left(K+\frac{4}{3} G\right) / \rho}$ 


\section{Steinberg-Guinan Model}

$$
\begin{aligned}
& G(P, T)=G_{0}\left(1+\frac{1}{G_{0}} \frac{\partial G}{\partial P} \frac{P}{\eta^{1 / 3}}-\frac{1}{G_{0}} \frac{\partial G}{\partial T}(T-300)\right) \\
& Y=Y_{0} f\left(\varepsilon_{p}\right) G(P, T) / G_{0} \\
& Y_{0} f\left(\varepsilon_{p}\right)=Y_{0}\left(1+\beta\left(\varepsilon_{p}+\varepsilon_{i}\right)\right)^{n} \leq Y_{\max } \\
& T_{\text {melt }}=T_{0} \exp \left(2 a\left(1-\frac{1}{\eta}\right) \eta^{2\left(\gamma_{0}-a-1 / 3\right)}\right), \quad \eta=\frac{\rho}{\rho_{0}}
\end{aligned}
$$

D.J. Stenberg, S.G. Cochran and M. W. Guinan, J. Appl. Phys. 51, 1498 (1980)

D.J. Steinberg, UCRL-MA-106439 (1991) 


\section{Steinberg-Lund Model}

$$
\begin{aligned}
& Y=\left\{Y_{T}\left(\mathcal{E}_{p}, T\right)+Y_{A} f\left(\varepsilon_{p}\right)\right\} G(P, T) / G_{0} \\
& \mathcal{\mho}_{p}=\left\{\frac{1}{C_{1}} \exp \left[\frac{2 U_{K}}{k T}\left(1-\frac{Y_{T}}{Y_{P}}\right)^{2}\right]+\frac{C_{2}}{Y_{T}}\right\}^{-1} \\
& Y_{A} f\left(\varepsilon_{p}\right)=Y_{A}\left(1+\beta\left(\varepsilon_{p}+\varepsilon_{i}\right)\right)^{n} \leq Y_{\max } \\
& Y_{T} \leq Y_{P}
\end{aligned}
$$

D.J. Stenberg and C.M. Lund, J. Appl. Phys. 65, 1528 (1989) 


\section{VISAR measures the surface velocity history}

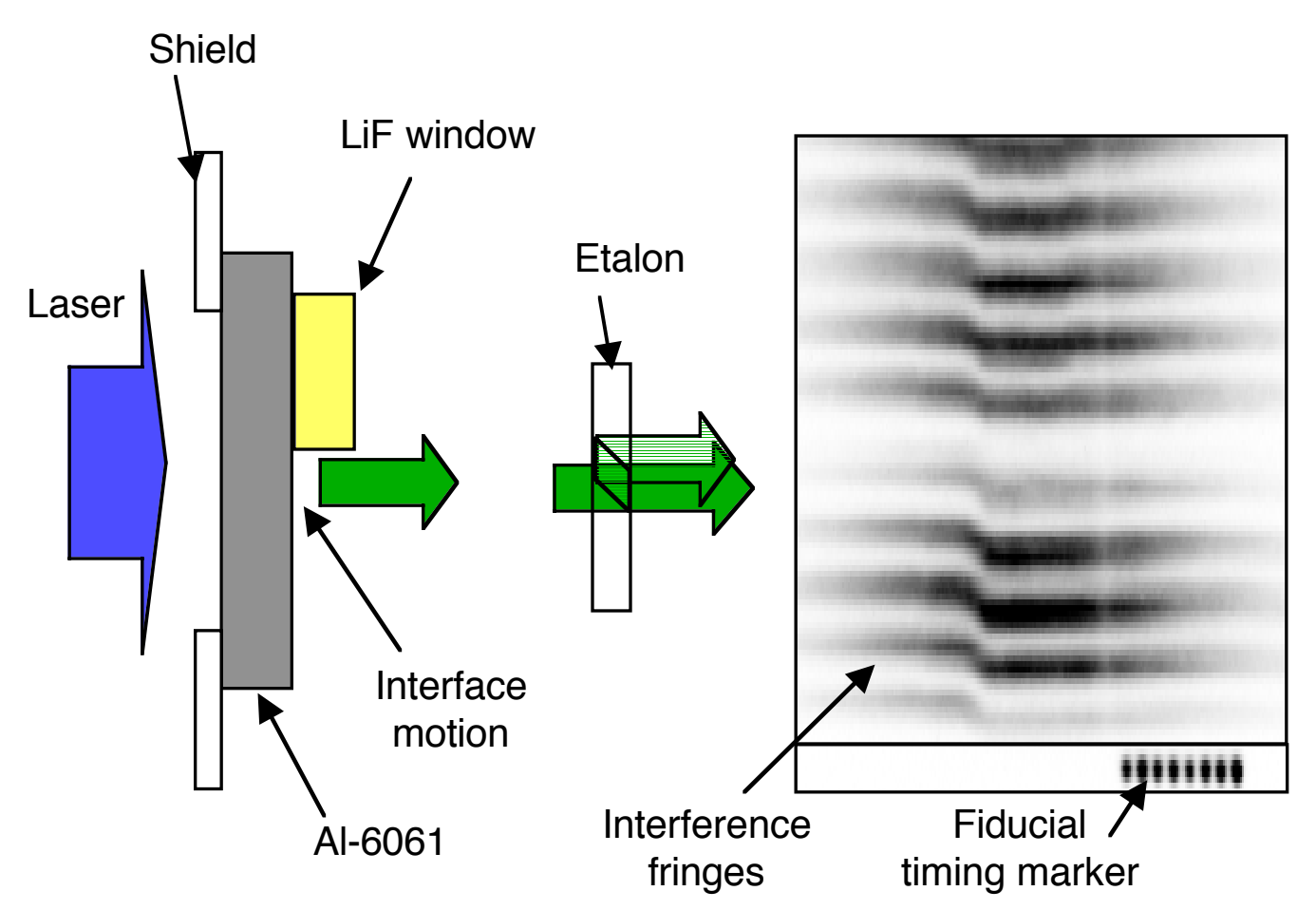

-An optical laser pulse is reflected from the free surface of the foil and injected into an interferometer

-The phase of the fringe is proportional to the velocity of the free surface -Spatial resolution of the VISAR system provides data on the rearsurface motion with and without the LiF window 


\section{VISAR measurement of elastic-plastic wave breakout in Al-6061}

- $195 \mu \mathrm{m}$ Al-6061, LiF over half of the rear surface

- Omega shot \#21382 - $19 \mathrm{~J}$ on target
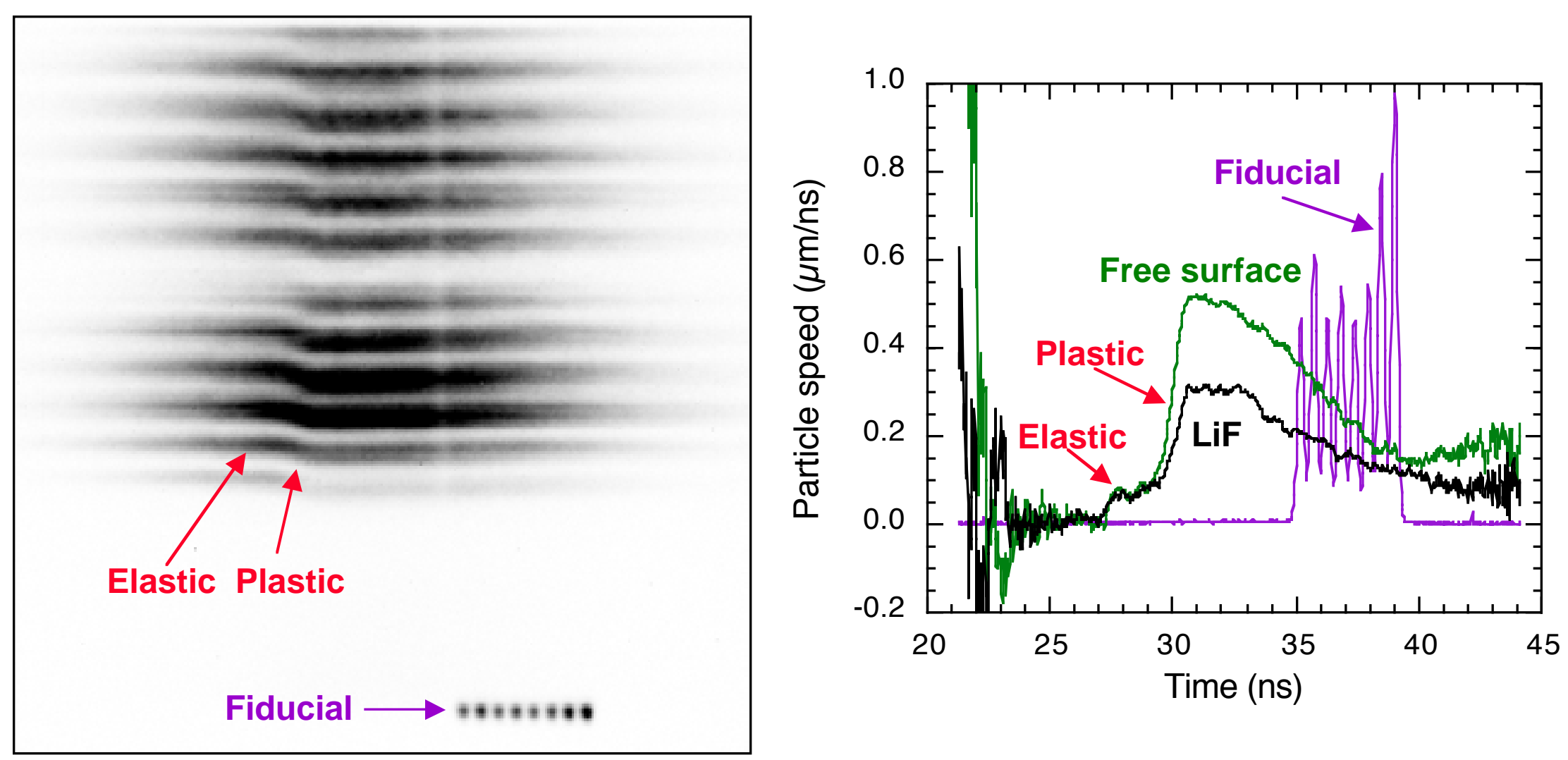


\section{The wave profile shows a pull-back at higher drive}

pressure

- $195 \mu \mathrm{m}$ Al-6061, LiF over half of the rear surface

- Omega shot \#21384 - $33 \mathrm{~J}$ on target
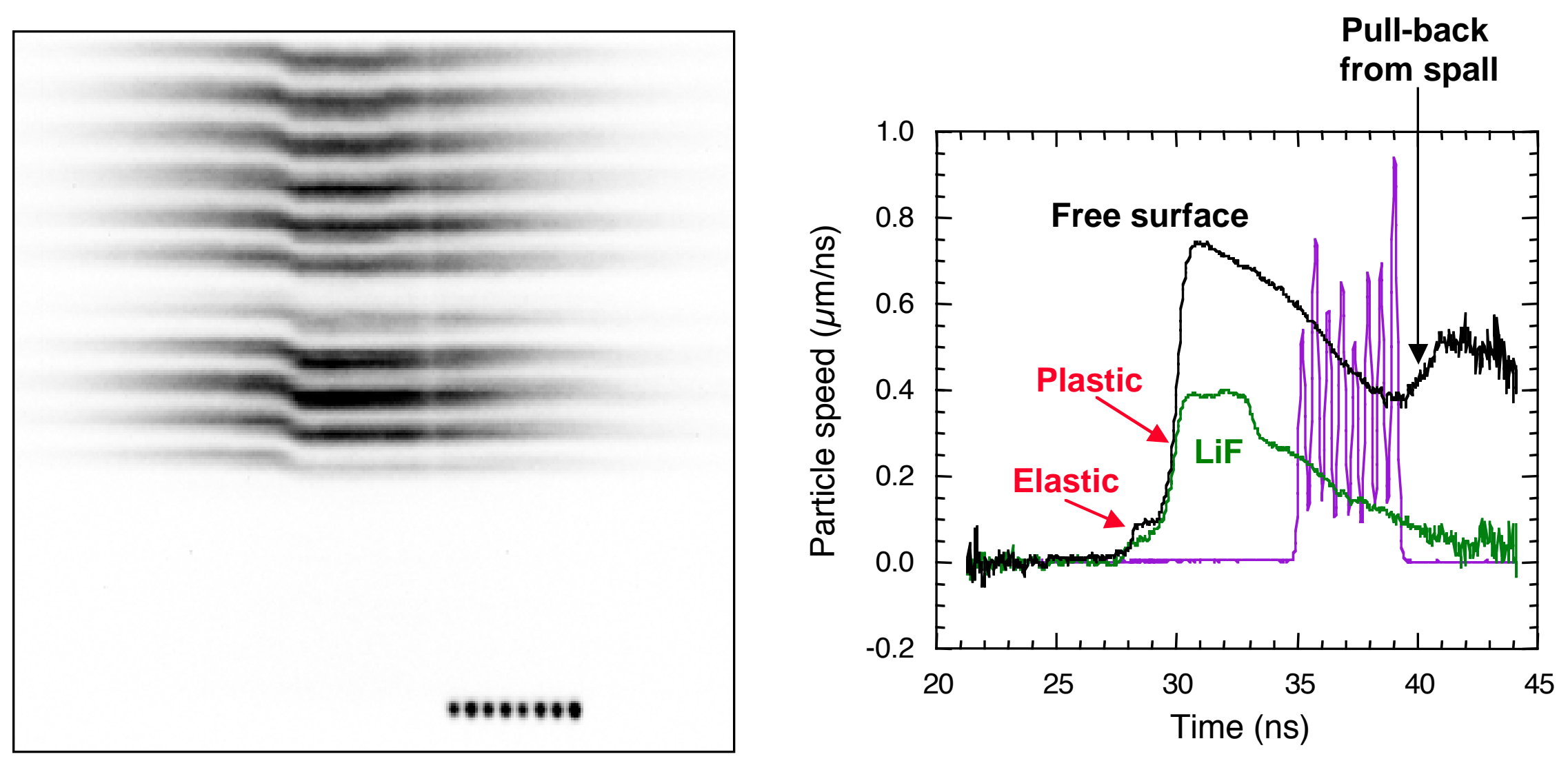


\section{We use VISAR data to determine the shear modulus, bulk modulus and yield strength}

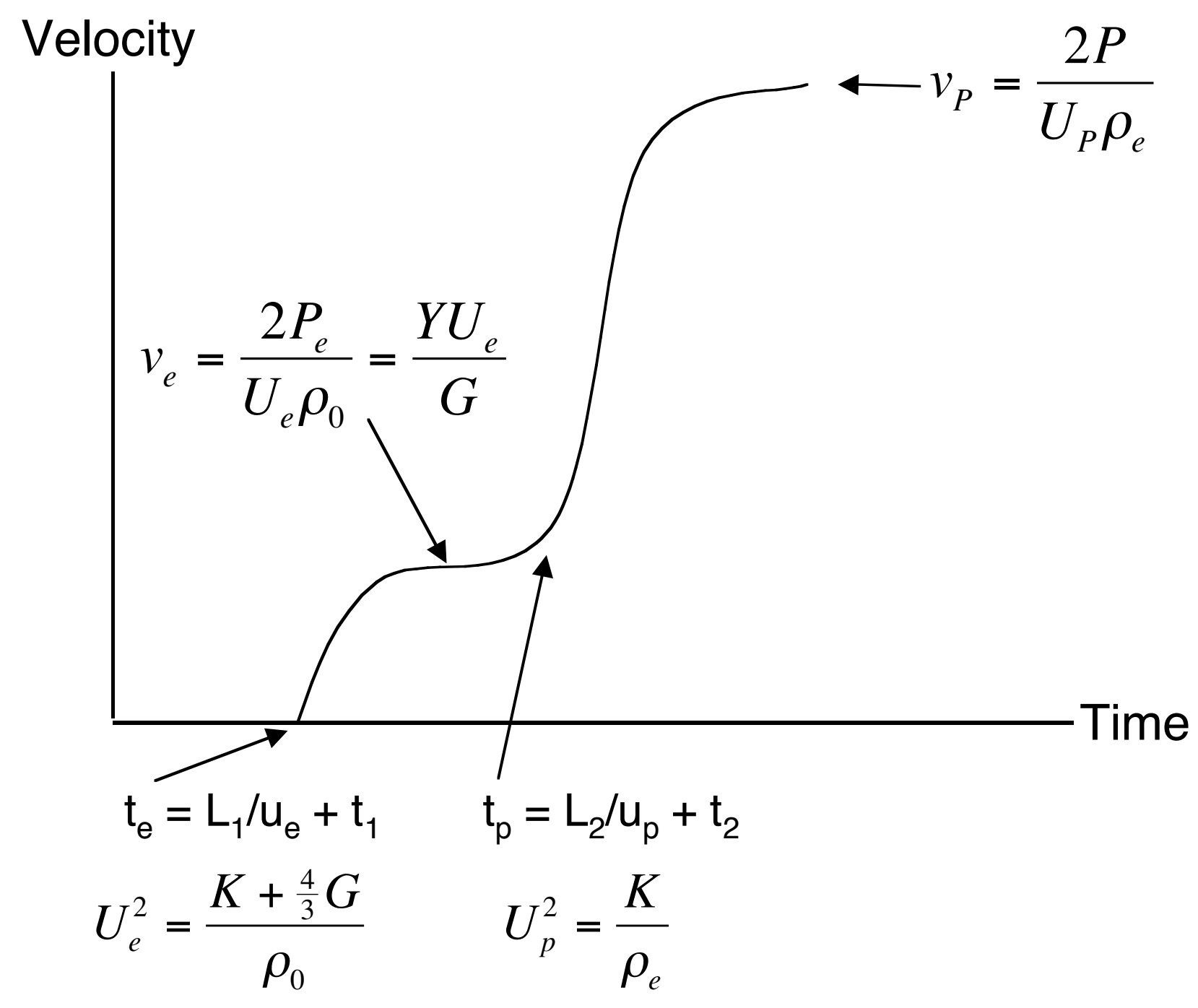




\section{Shocks lose strength as they propagate}

$$
\left.\left.\frac{d P}{d x}\right)_{\text {shock }}=-\frac{d P}{d x}\right)_{\text {rarefaction }}\left(\frac{u_{\text {material }}+c_{s}}{U_{\text {shock }}}-1\right)
$$

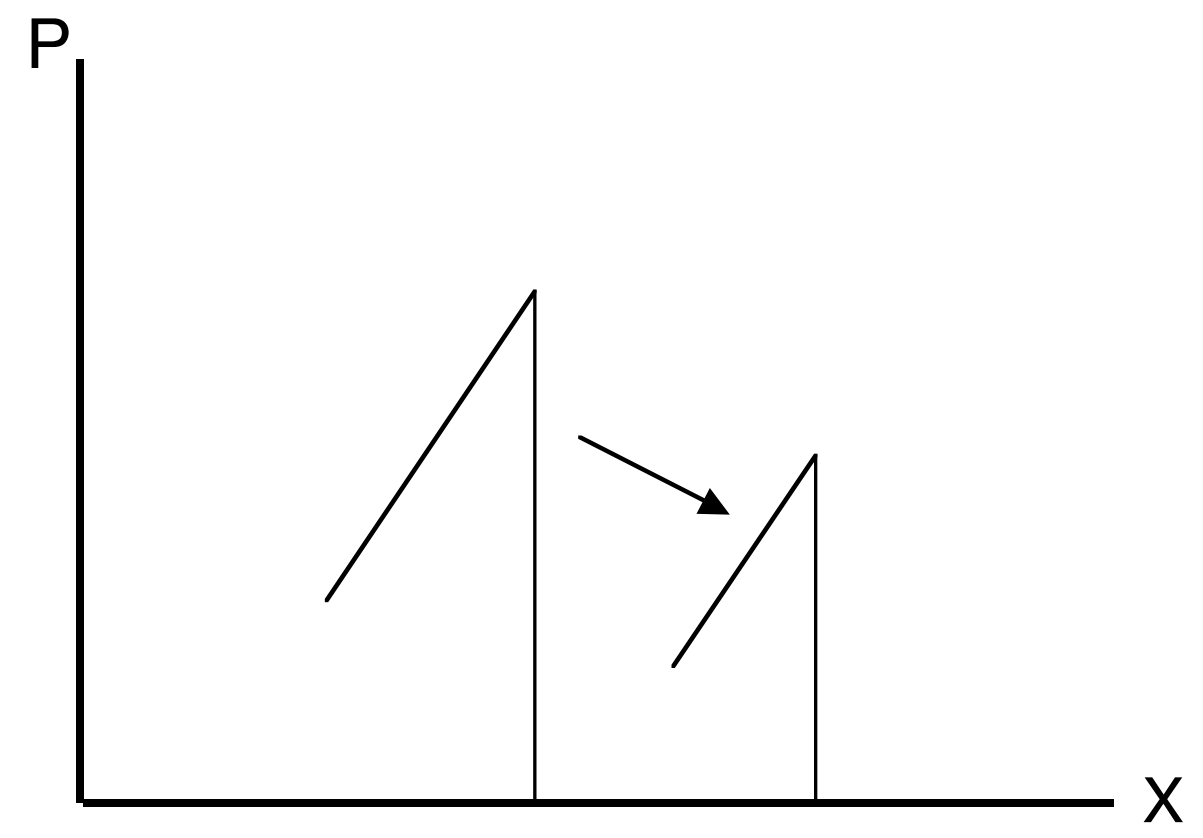


The shear modulus, bulk modulus and yield strength affect the rise time and velocity of the VISAR data

Change shear modulus Change bulk modulus

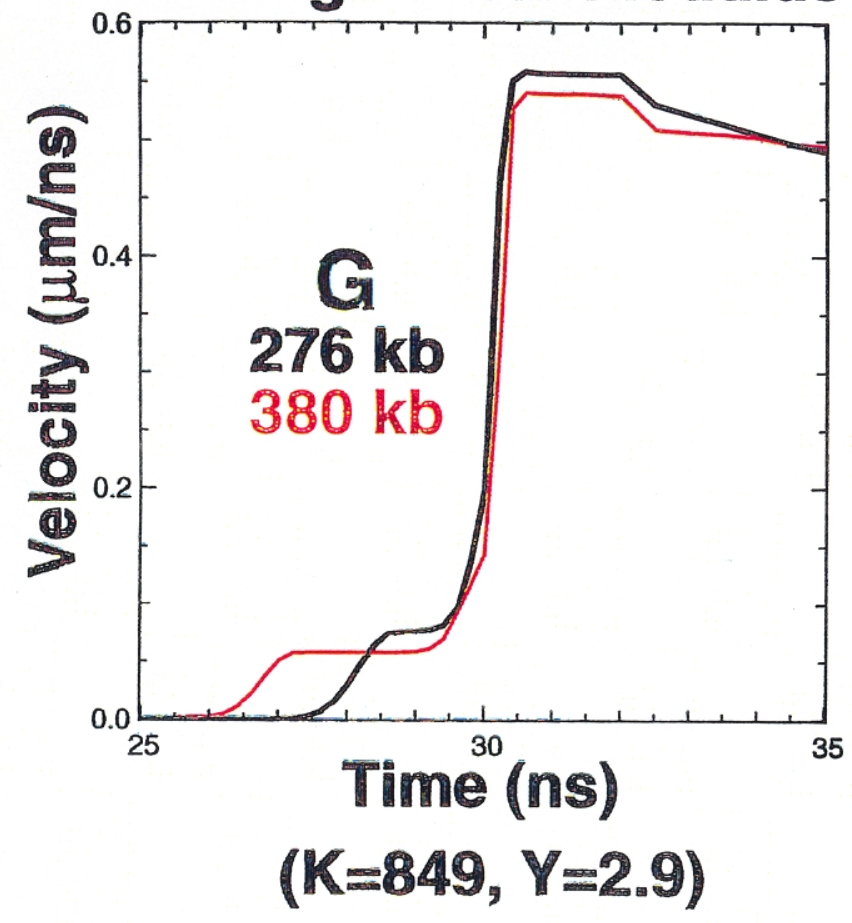

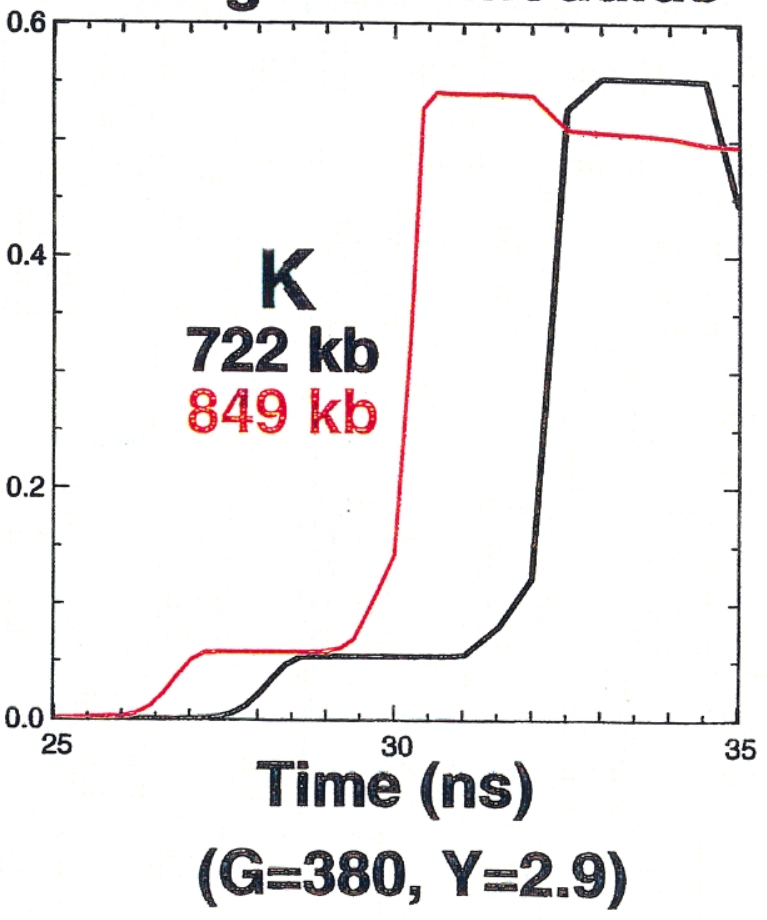

Change yield strength

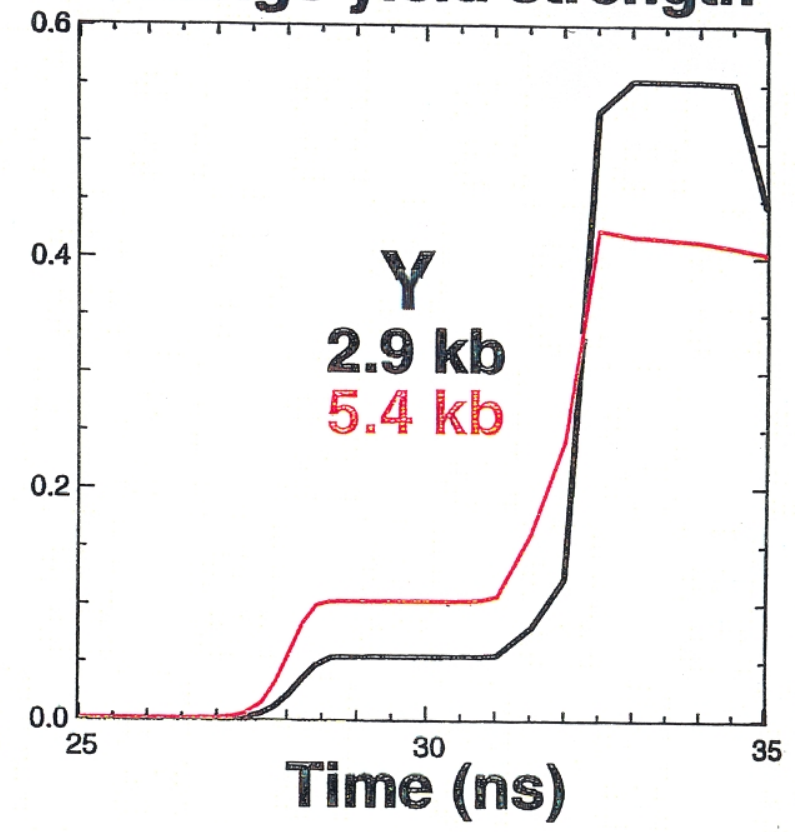

$(\mathrm{G}=380, \mathrm{~K}=722)$ 
The shock strength decreases more rapidly with increasing yield strength
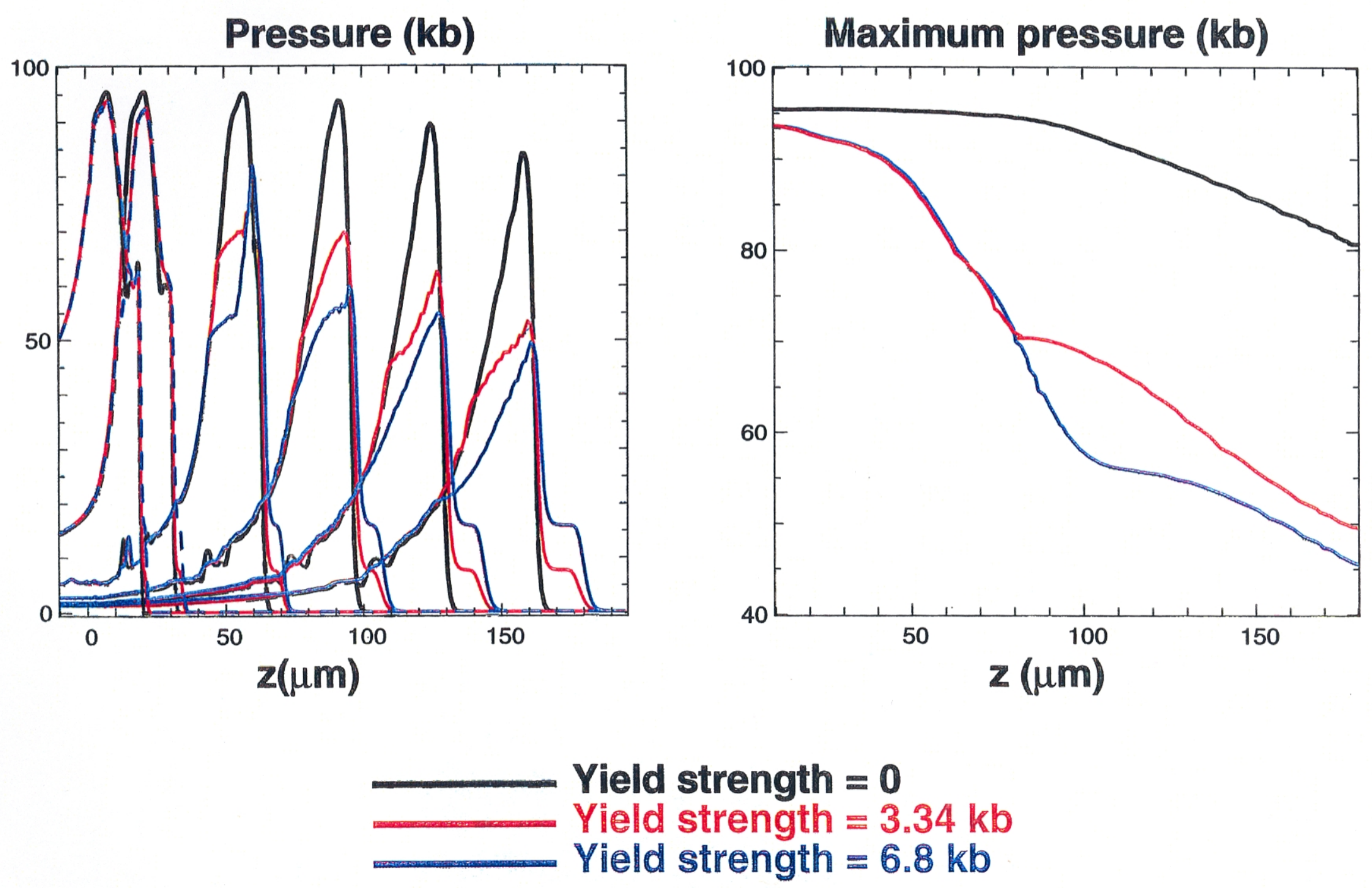


\section{We can match rise times and velocities by varying bulk modulus, shear modulus and yield strength}

$$
\mathrm{G}=320, \mathrm{~K}=866, \mathrm{Y}=3.34 \mathrm{~kb}
$$

VISAR, shot 21382

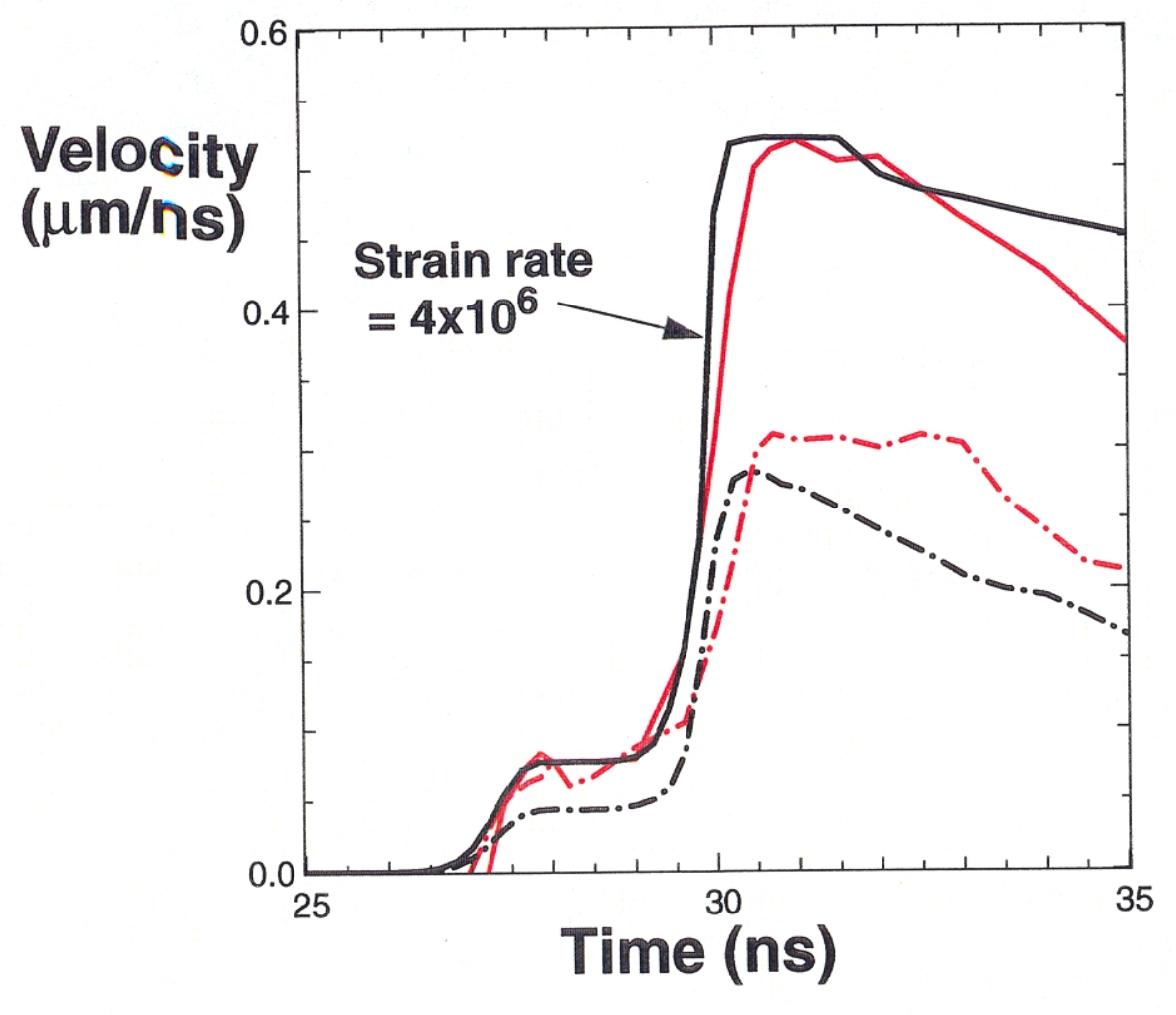

Data Simulation

$$
\begin{gathered}
G=320, K=794, Y=4.27 ~ k b \\
\text { VISAR, shot } 21384
\end{gathered}
$$

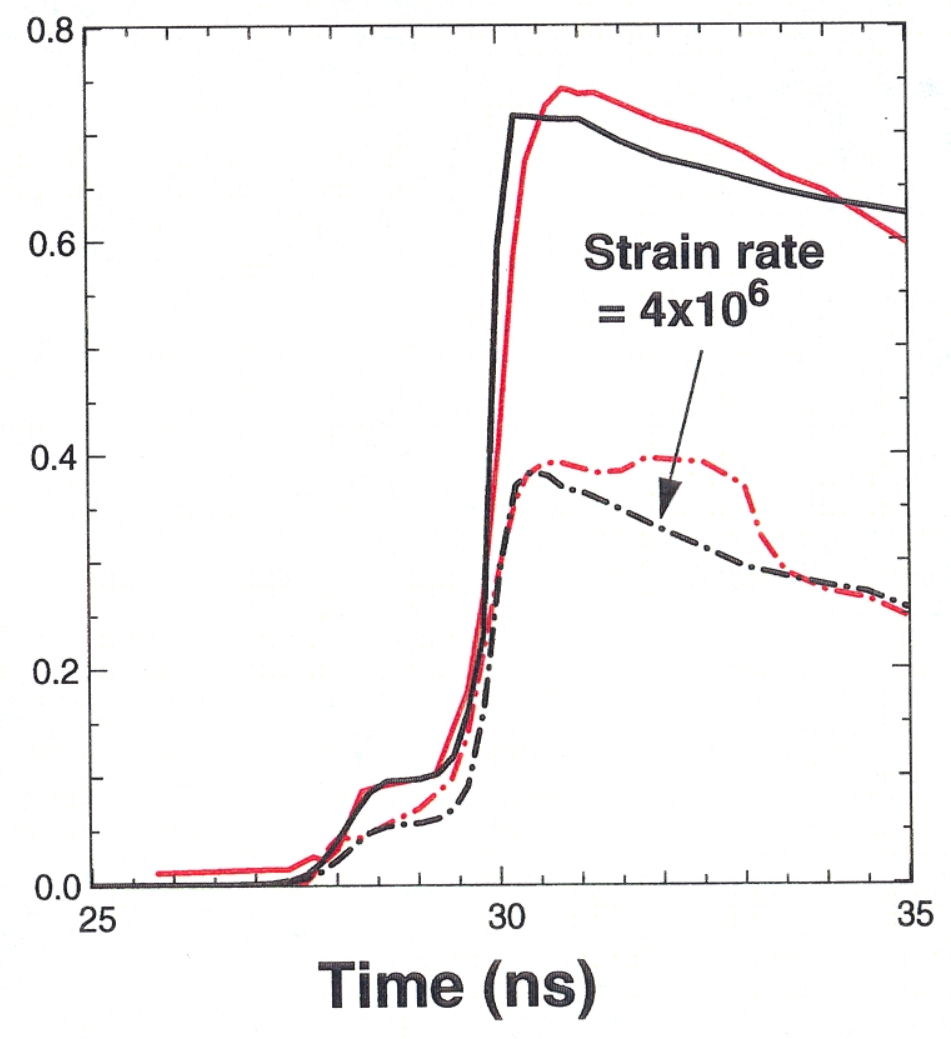

Free surface

- - - - - - With LiF 


\section{The Steinberg-Guinan model by itself gives a spall time that is too late compared to the data}

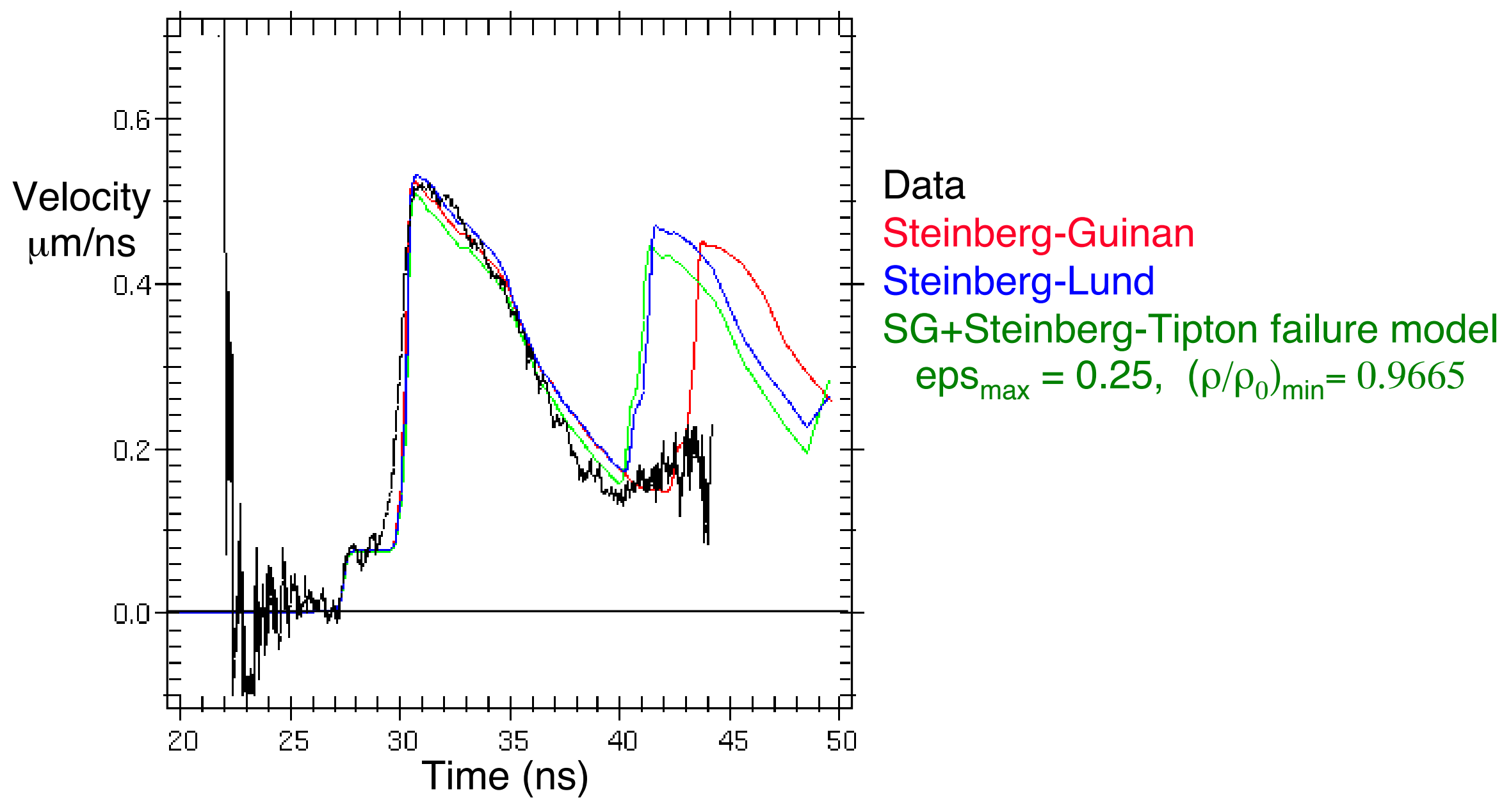




\section{Steinberg-Tipton Failure Model}

Damage ranges from 0 to 1

Broken material: $Y_{b}<P, G_{b} / G_{0}=Y_{b} / Y_{0}$

$\{P, G, Y\}=$ damage $^{\star}\left\{P_{0}, G_{0}, Y_{0}\right\}+\left(1\right.$-damage ${ }^{\star}\left\{P_{b}, G_{b}, Y_{b}\right\}$

$\frac{d}{d t}$ Damage $= \begin{cases}\frac{\mathrm{RC}_{\mathrm{s}}}{\Delta \mathrm{X}_{\mathrm{zone}}} & \sum_{i} \max \left(0, \frac{f_{i}}{\text { fmax }_{i}}\right)^{2}>1 \\ 0 & \sum_{i} \max \left(0, \frac{f_{i}}{\text { fmax }_{i}}\right)^{2}<1\end{cases}$

$$
\mathrm{C}_{\mathrm{s}}=\sqrt{\frac{4 \mathrm{G}_{0}}{3 \rho}} \quad \mathrm{f}_{\mathrm{i}}=\left\{\text { eps, } \rho / \rho_{0}-1, \mathrm{P}, \sigma, \Delta \sigma\right\}
$$




\section{Parameters}

-Steinberg-Guinan

- $p_{\min }=-30 \mathrm{~kb}$

- $\rho / \rho_{0}=0.9665$

- $K=940$ kb

- $\mathrm{G}_{0}=325 \mathrm{~kb}$

$\cdot Y=3.335 \mathrm{~kb}$

-eps $_{\text {max }}=2.0$

-Steinberg-Tipton

- $\rho / \rho_{0}-1=-.0335$

-eps $=.25$

- $R=10^{20}$

-Steinberg-Lund

$\cdot Y=1.5 \mathrm{~kb}$

$\cdot c_{1}=.71$

$\cdot c_{2}=.12$

$\cdot \mathrm{u}_{\mathrm{k}}=.31$

$\cdot \mathrm{y}_{\mathrm{prl}}=1.9 \mathrm{~kb}$ 


\section{Dynamic x-ray diffraction measures density and crystal structure}

- In situ x-ray diffraction allows us to probe the material state by providing information on the lattice under compression

- Technique applied on laser experiments at Nova and elsewhere (Janus, Vulcan, Trident, OMEGA) and powder and gas gun facilities

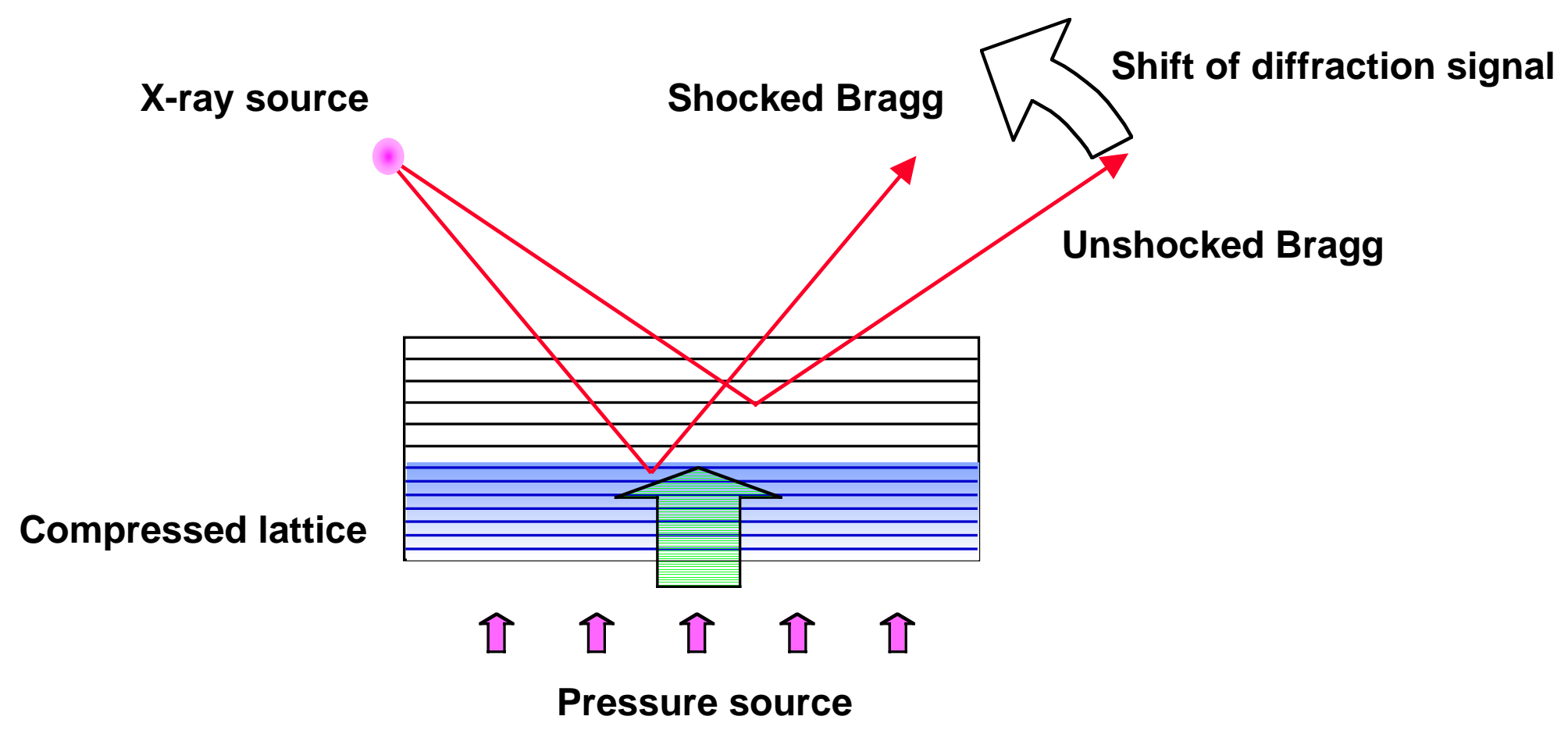

Q. Johnson, A. Mitchell, R.N. Keeler, L. Evans, Phys Rev Lett 25, 1099 (1970)

J..S Wark, R.R. Whitlock, A.A. Hauer, J.E. Swain, P.J. Solone, Phys Rev B 40, 5705 (1989) 


\section{Diffraction from shock compressed Si has been demonstrated on Nova}

- Low intensity square laser pulse generates a single shock drive

- Displacement of the diffraction signal indicates a compression of the lattice spacing

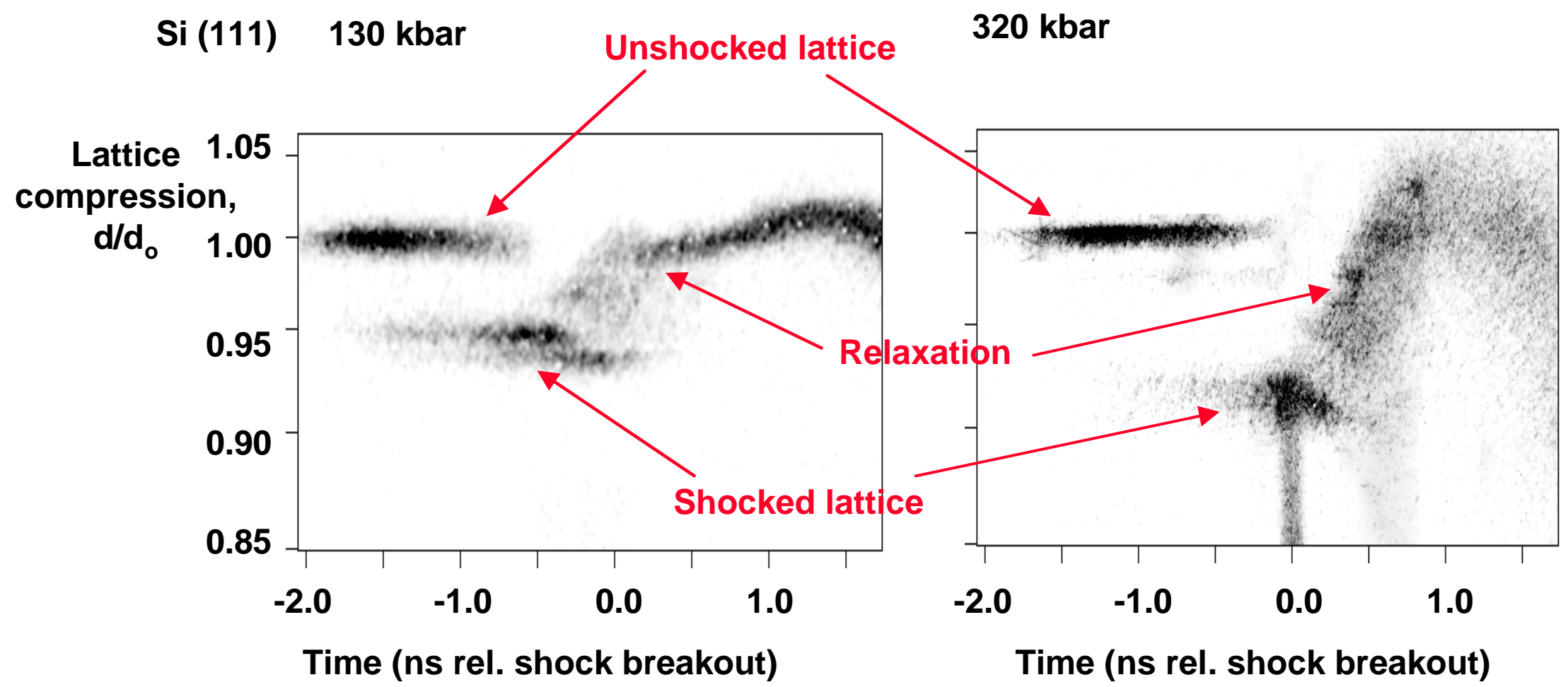




\section{Diffraction from orthogonal lattice planes provides information on the transition to plasticity}

- Simultaneous measurements are made of compression of orthogonal lattice planes

- Shock compression above the HEL for Si and Cu show very different behavior on the ns time scale ${ }^{1}$

- Si responds uniaxially

- Cu shows plastic deformation

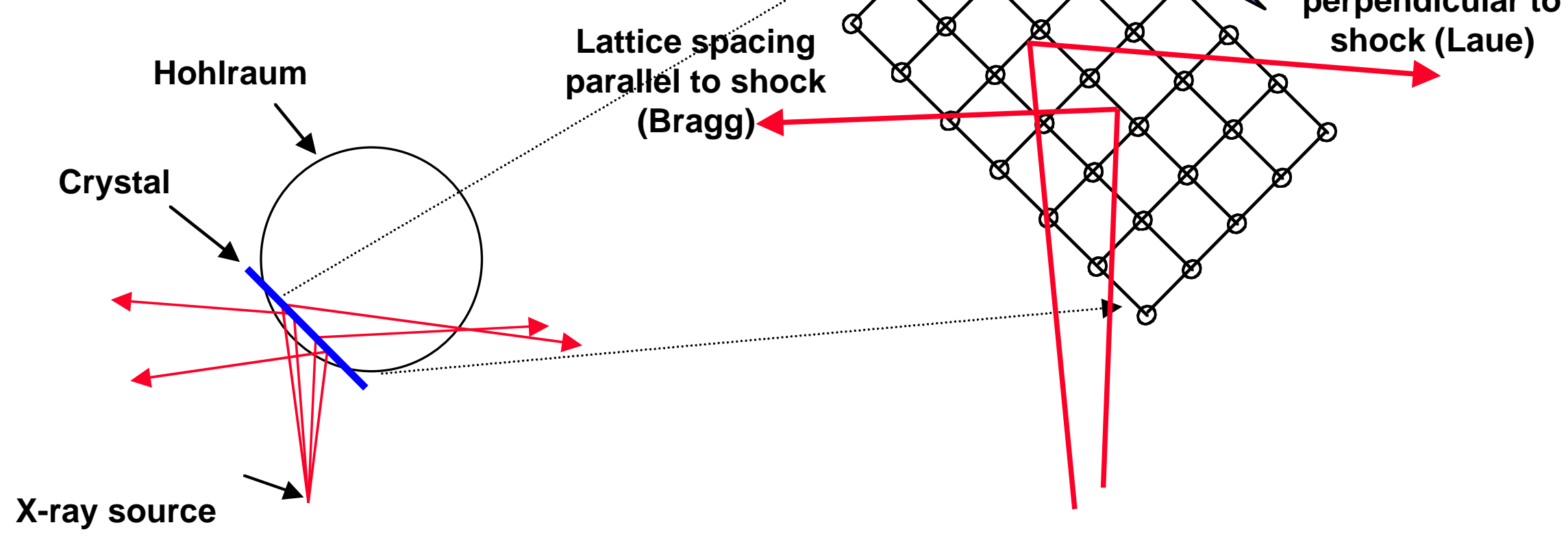

[1] A. Loveridge et al, "Anomalous elastic response of silicon to uniaxial shock compression on nanosecond timescales", Phys. Rev. Letters 86, 2349 (2001) 


\section{Simultaneous measurements of orthogonal planes indicate Si responds uniaxially on a ns time scale}

- Si shock compressed along (400); probed along (400), (040)

- $P=115-135$ kbar; HEL = 84 kbar, $40 \mu \mathrm{m}$ thick Si

- Simultaneous measurements of Bragg and Laue diffraction
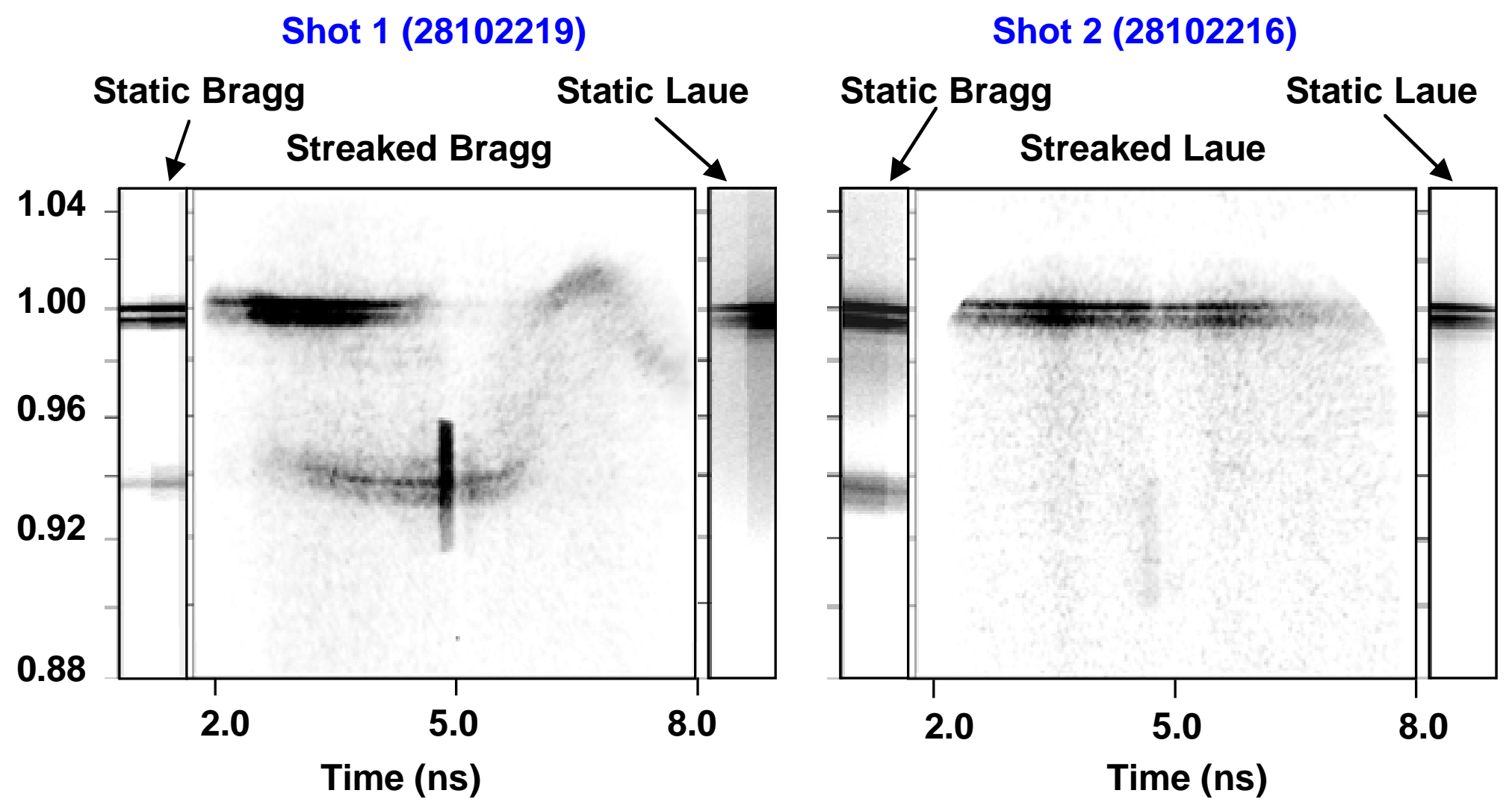

1-D compression in $\mathrm{Si}$ is due to high Peierls barrier 


\section{X-ray diffraction of $40 \mu \mathrm{m}$ Si shows density features that vary with drive temperature}

Temperature $(\mathrm{eV})$

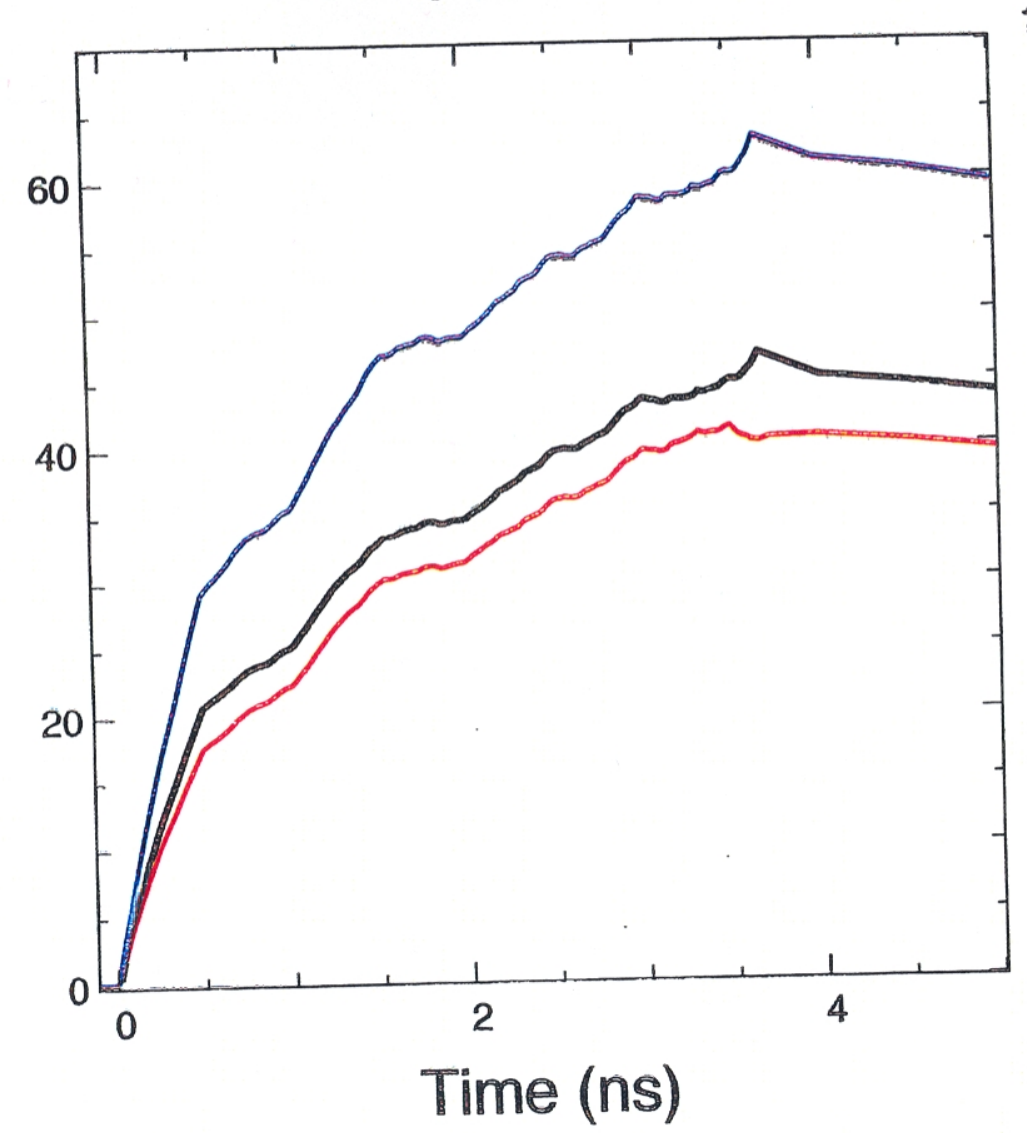

Relative mass per density

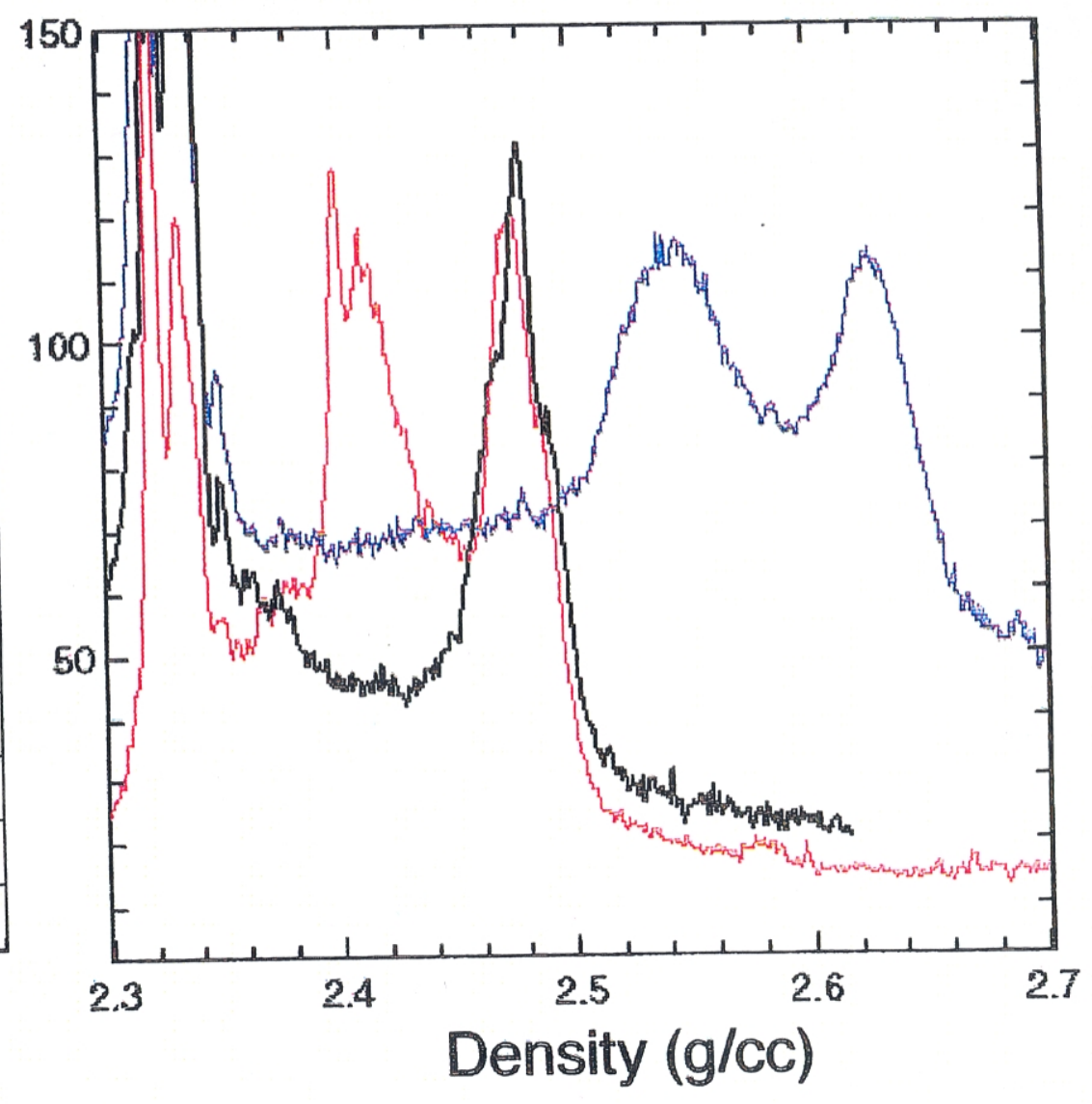




\section{Molecular dynamic simulations show that the Si longitudinal stiffness increases with pressure}

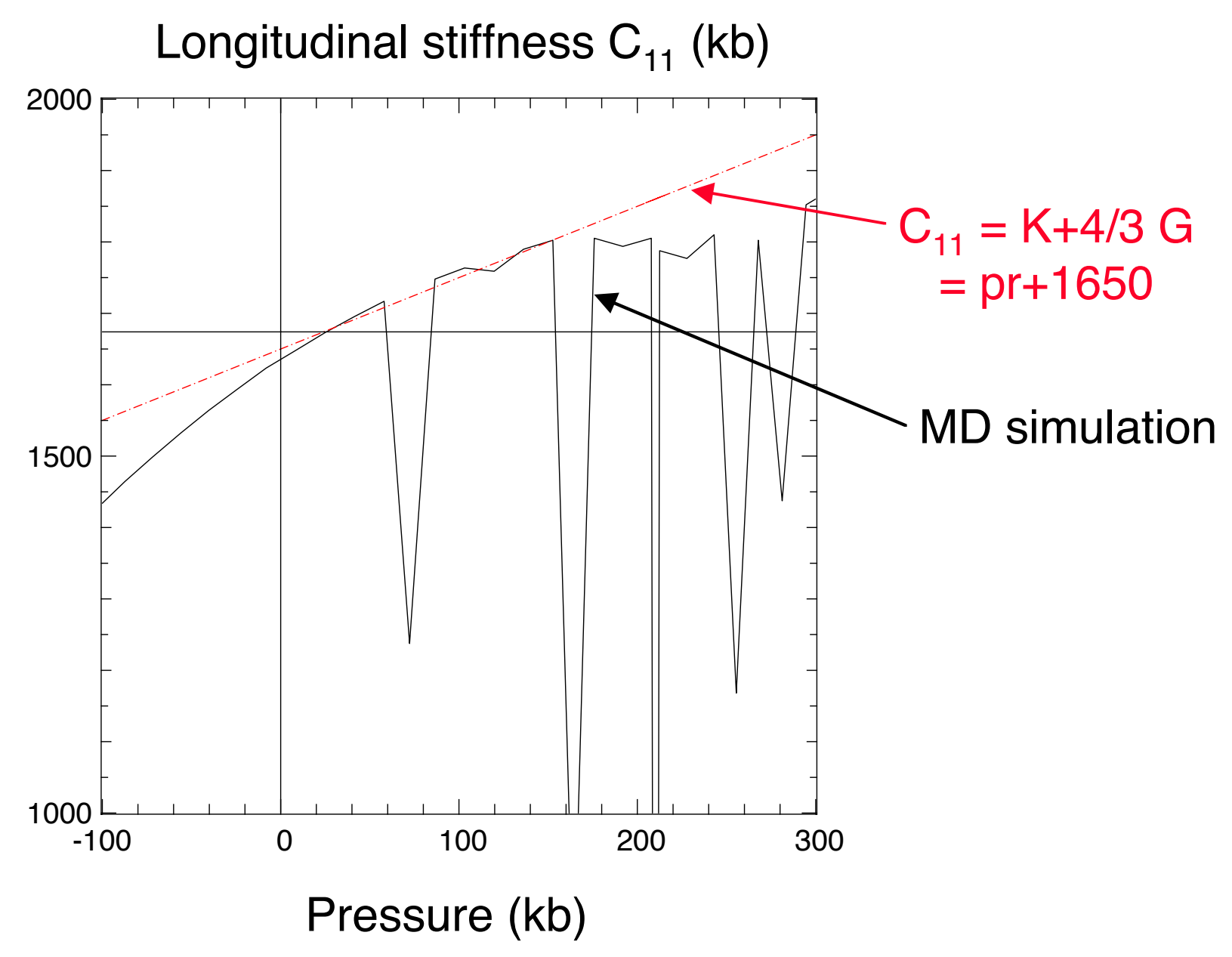

Simulation done by D. J. Roundy 
The density structure depends in a complicated way on the drive temperature

Temperature (eV)

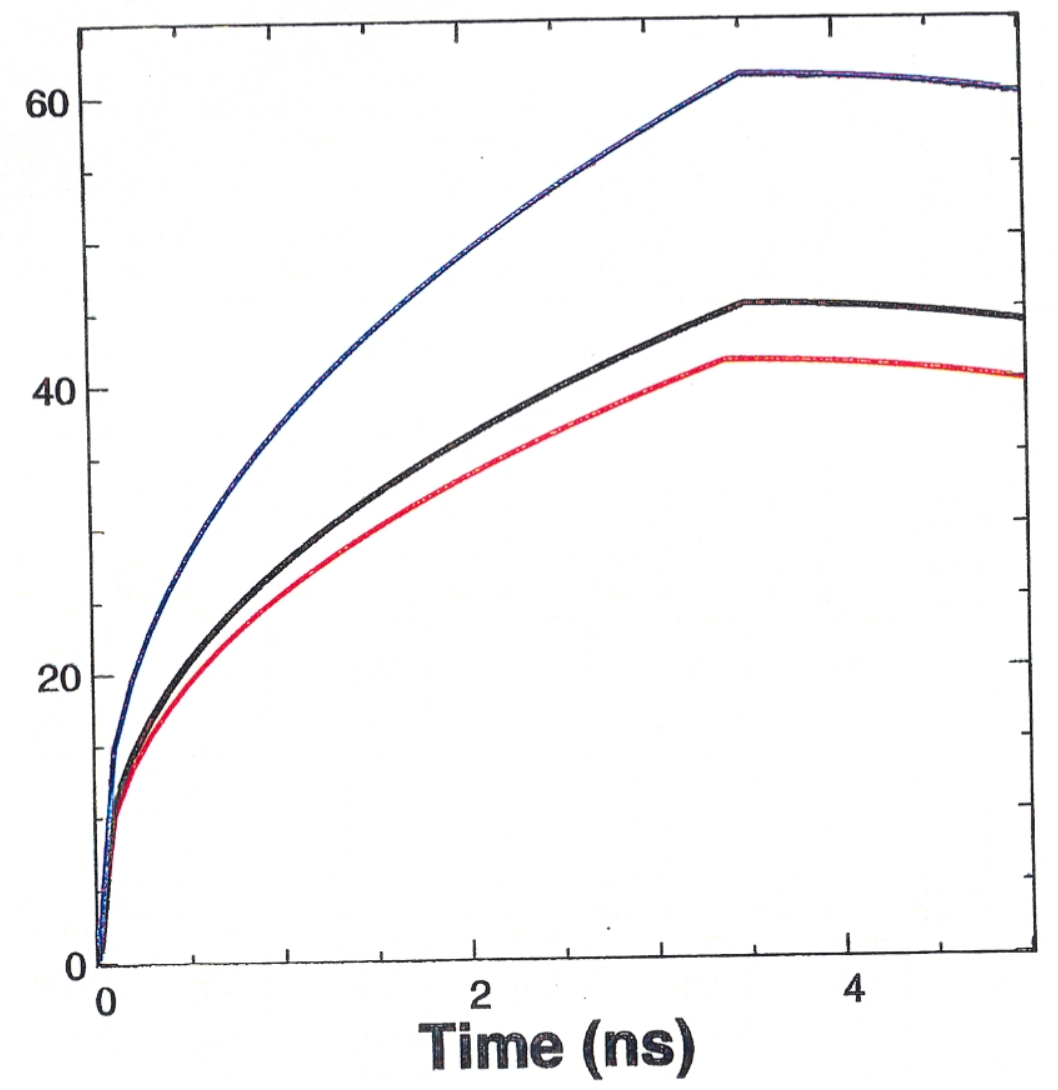

Mass per density interval

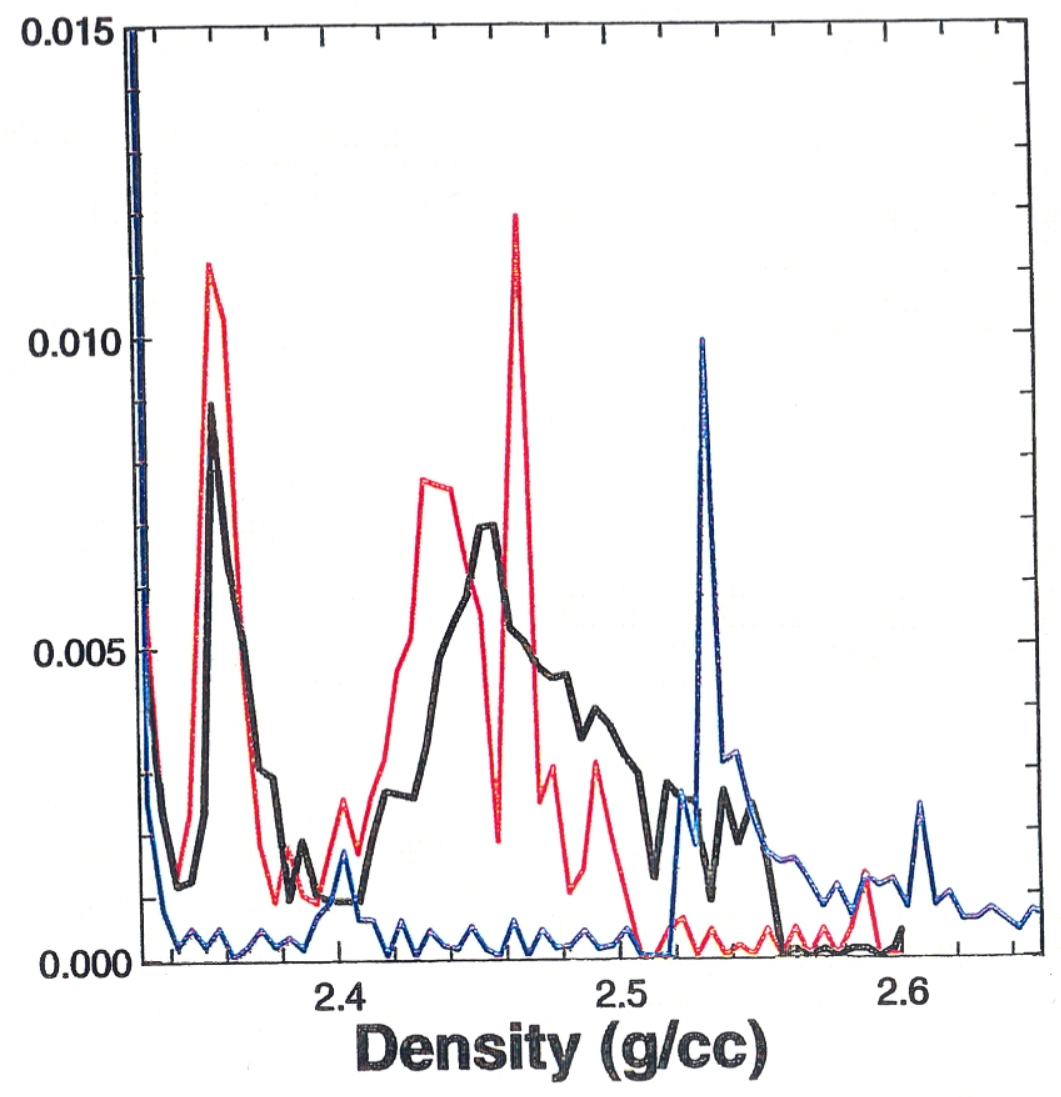




\section{By increasing the drive, we can match part of the data}
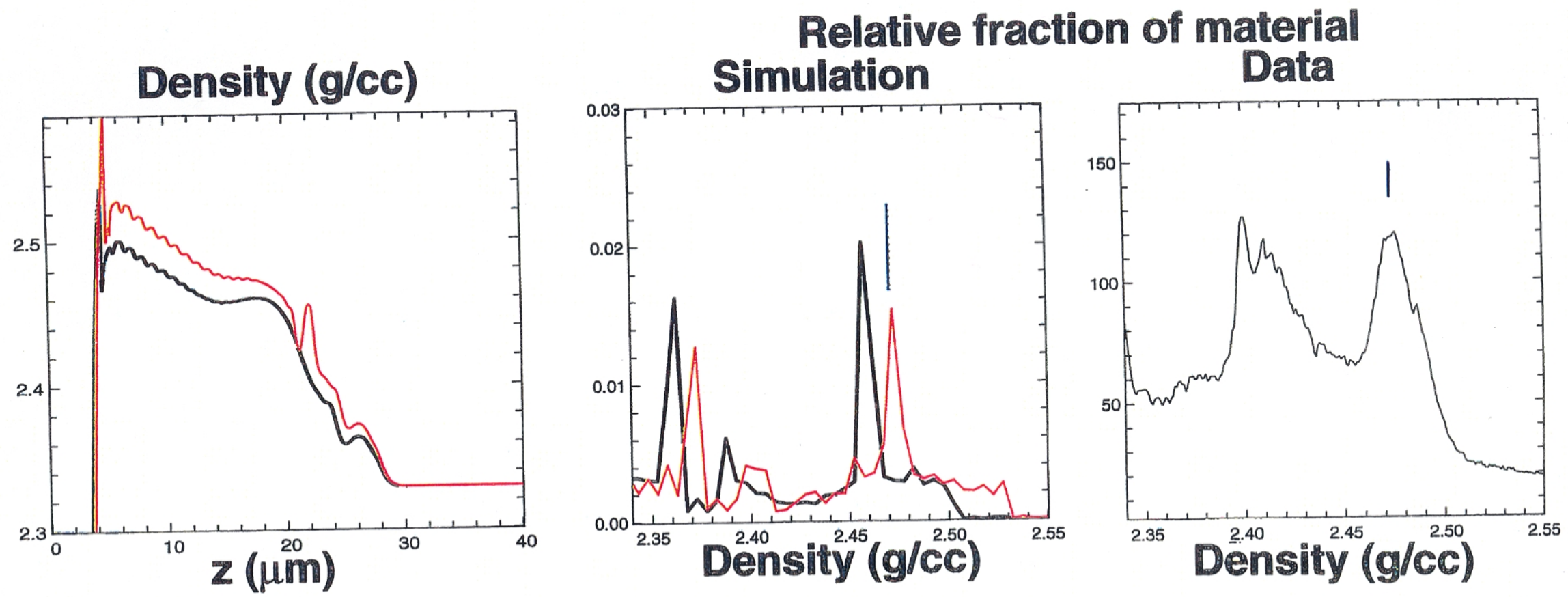

Nominal drive

Tr x 1.06 


\section{We have recovered samples to study the residual effects due to these high strain rate laser experiments}

- Single crystal Cu samples were shocked by direct laser irradiation and captured in a foam-filled cavity

- Preliminary tests done at OMEGA; shock pressure is $>1$ Mbar, decays to $\sim 50 \mathrm{kbar}$ at the rear surface
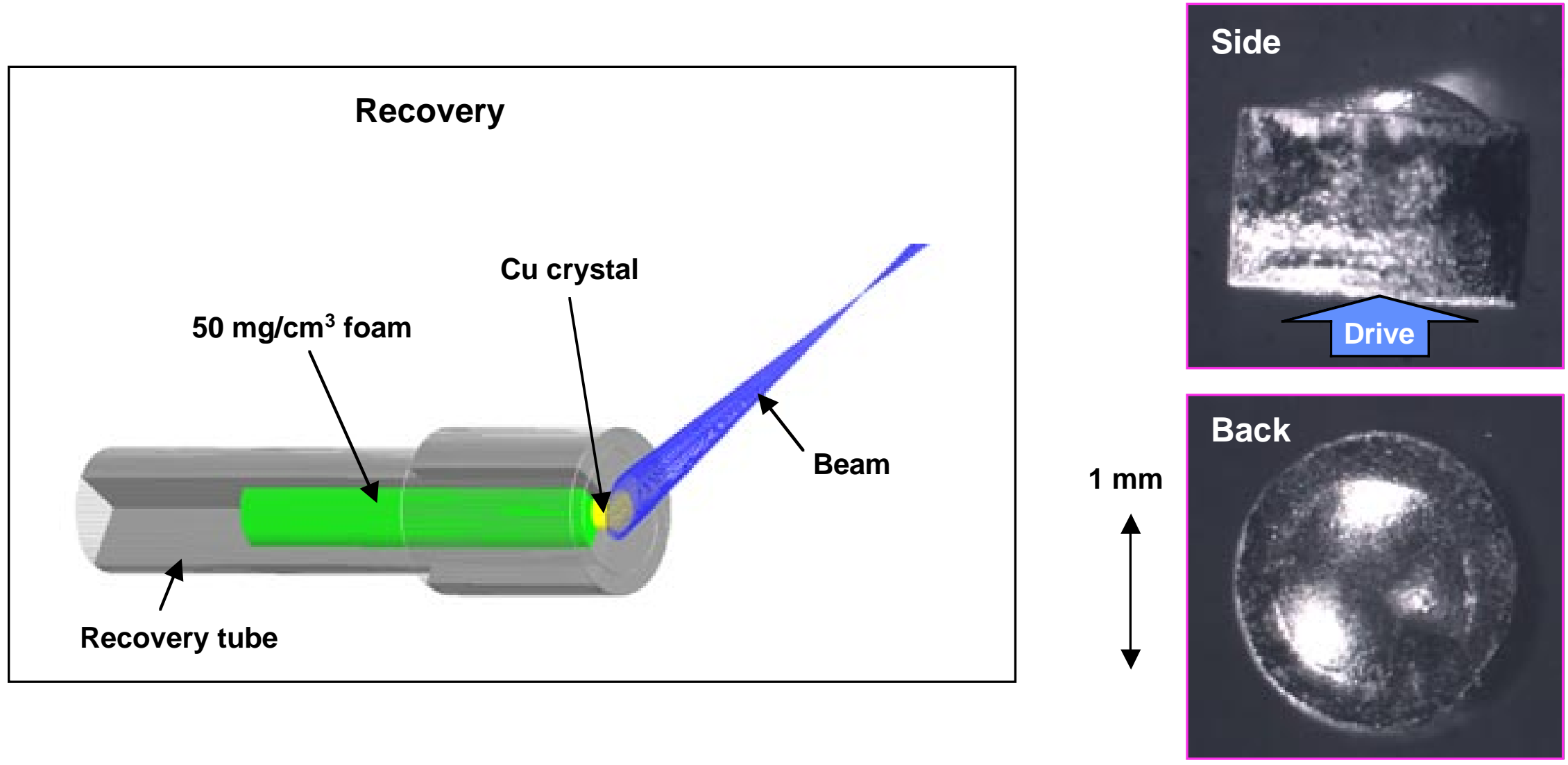
We see spall on a Cu sample driven by Janus

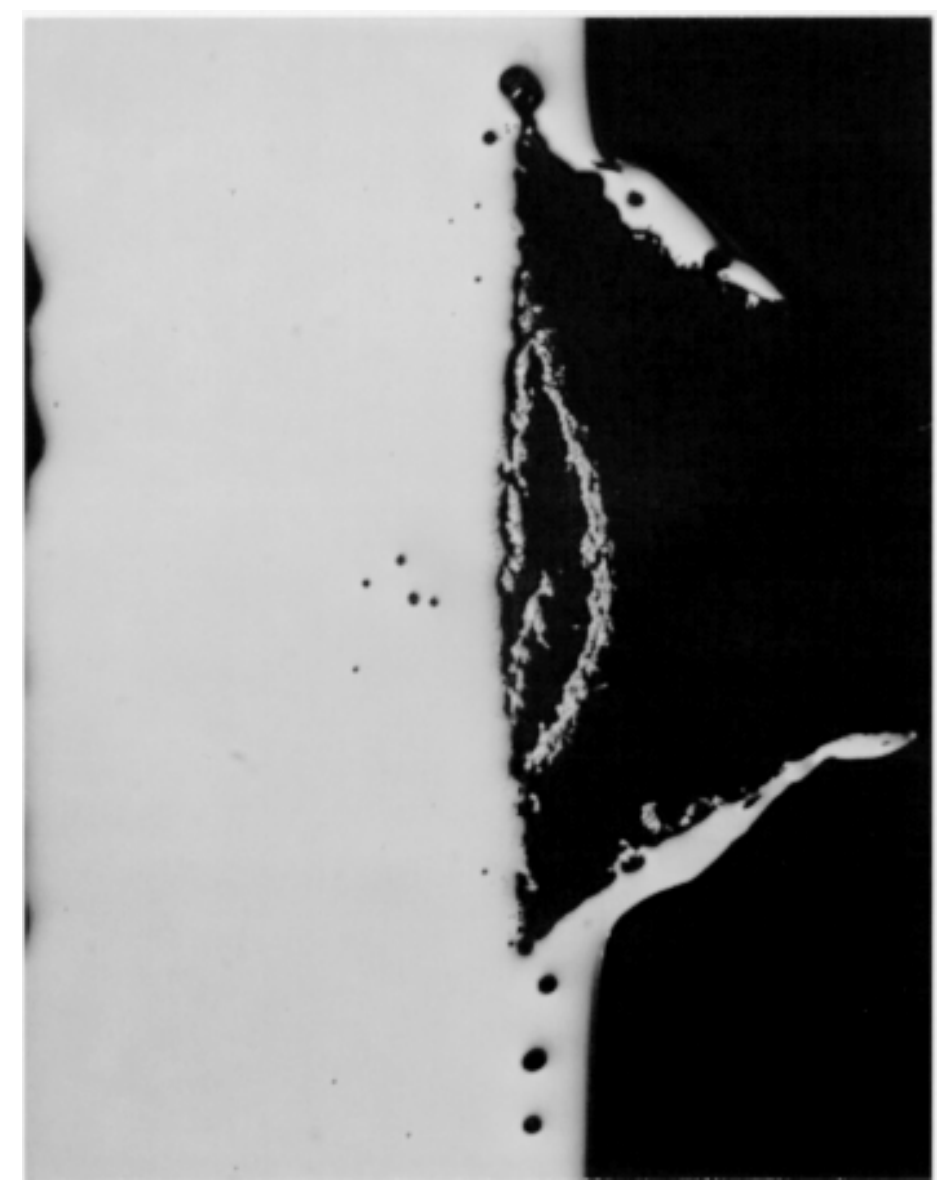




\section{Shock strength falls roughly as distance ${ }^{-3 / 4}$ in $\mathrm{Cu}$}
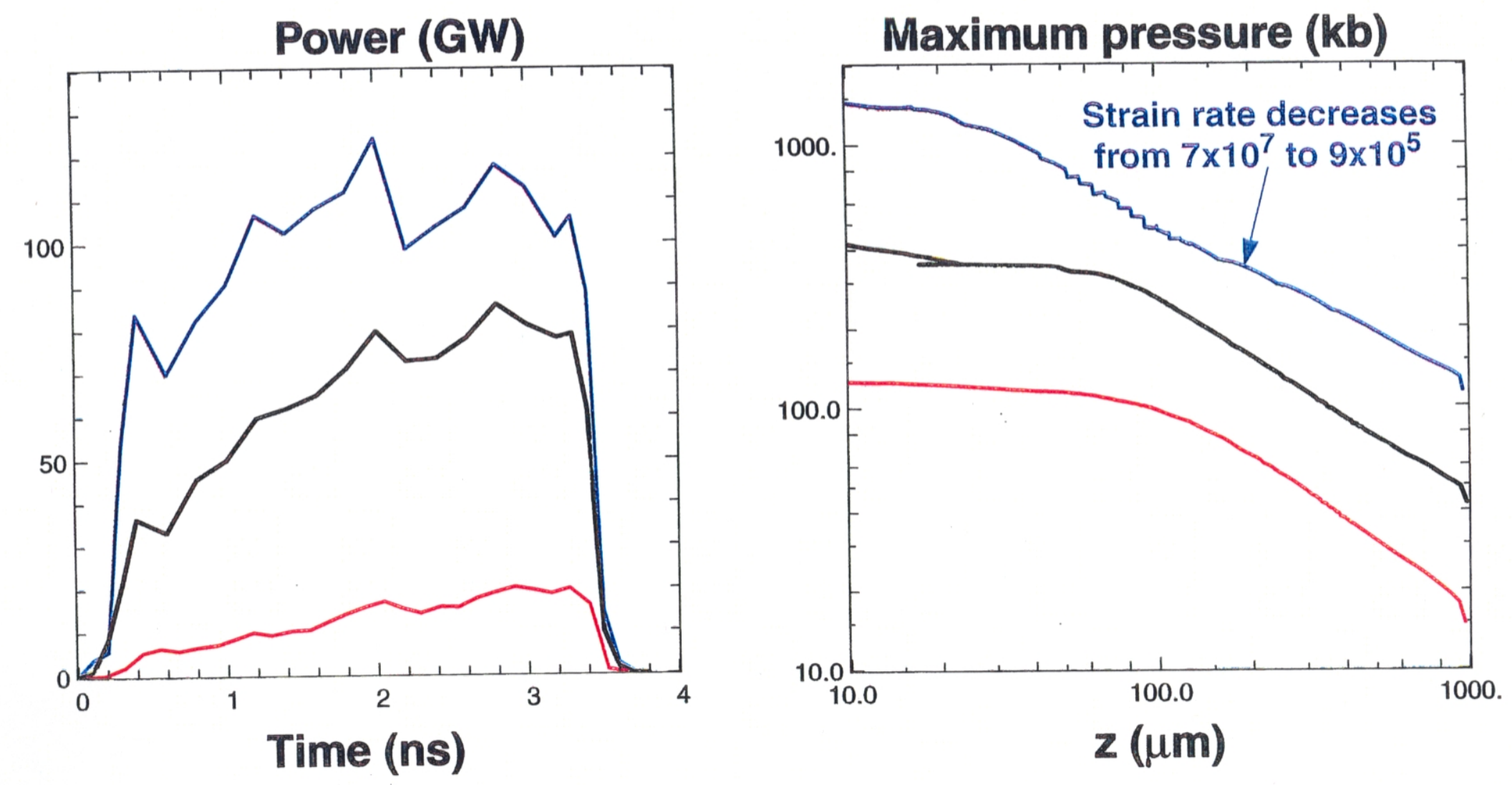


\section{Summary and future work}

-VISAR provides free surface velocity history

- Gives shear modulus, bulk modulus and yield strength

- Gives information on fracture model and spall

-X-ray diffraction provides information about lattice deformation

-Future work

-Correlate VISAR with x-ray diffraction

-Relate VISAR with post-shock recovery and residual deformation of structure 
K. Prestridge', C. Tomkins', C. Zoldi',2, M. Marr-Lyon', P. Vorobieff', P. Rightley', R. Benjamin'

1 Los Alamos National Laboratory 2 SUNY Stony Brook

3 University of New Mexico 
Shock Tube Facility

\section{Shock-accelerated gas cylinder}

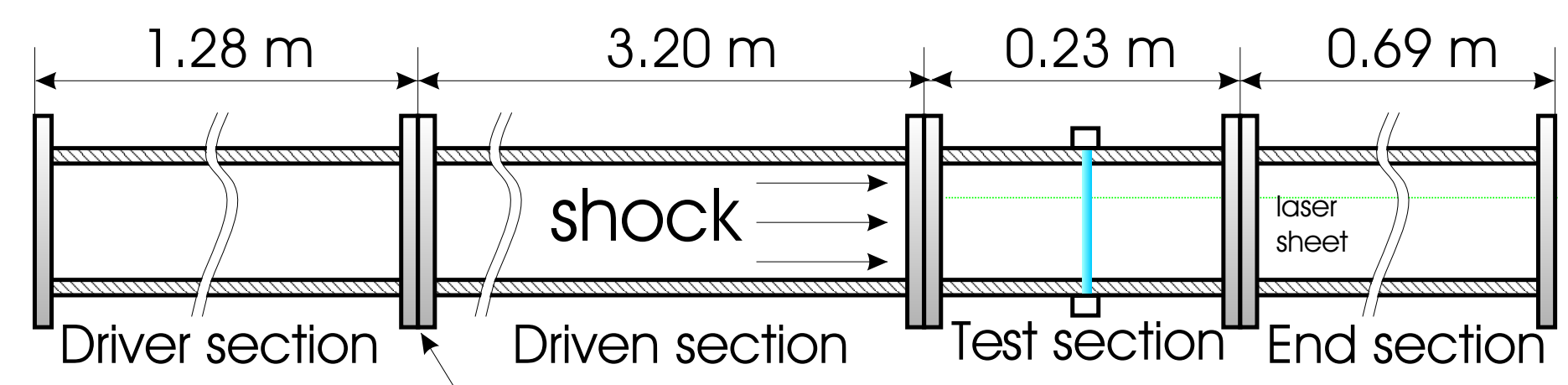

- Comparison between experimental density-field images and density-field images from a 2-D adaptive-mesh Eulerian code simulation reveal that the simulation has smaller lengths in the spanwise and streamwise directions.

- When velocity fields are compared at one instant in time, the higher magnitudes appear in the backflow region and the lower magnitudes appear in the vortex cores for both experiment and simulation. The overall magnitudes of the velocities in the simulation are higher than those of the experiment.

Diaphragm
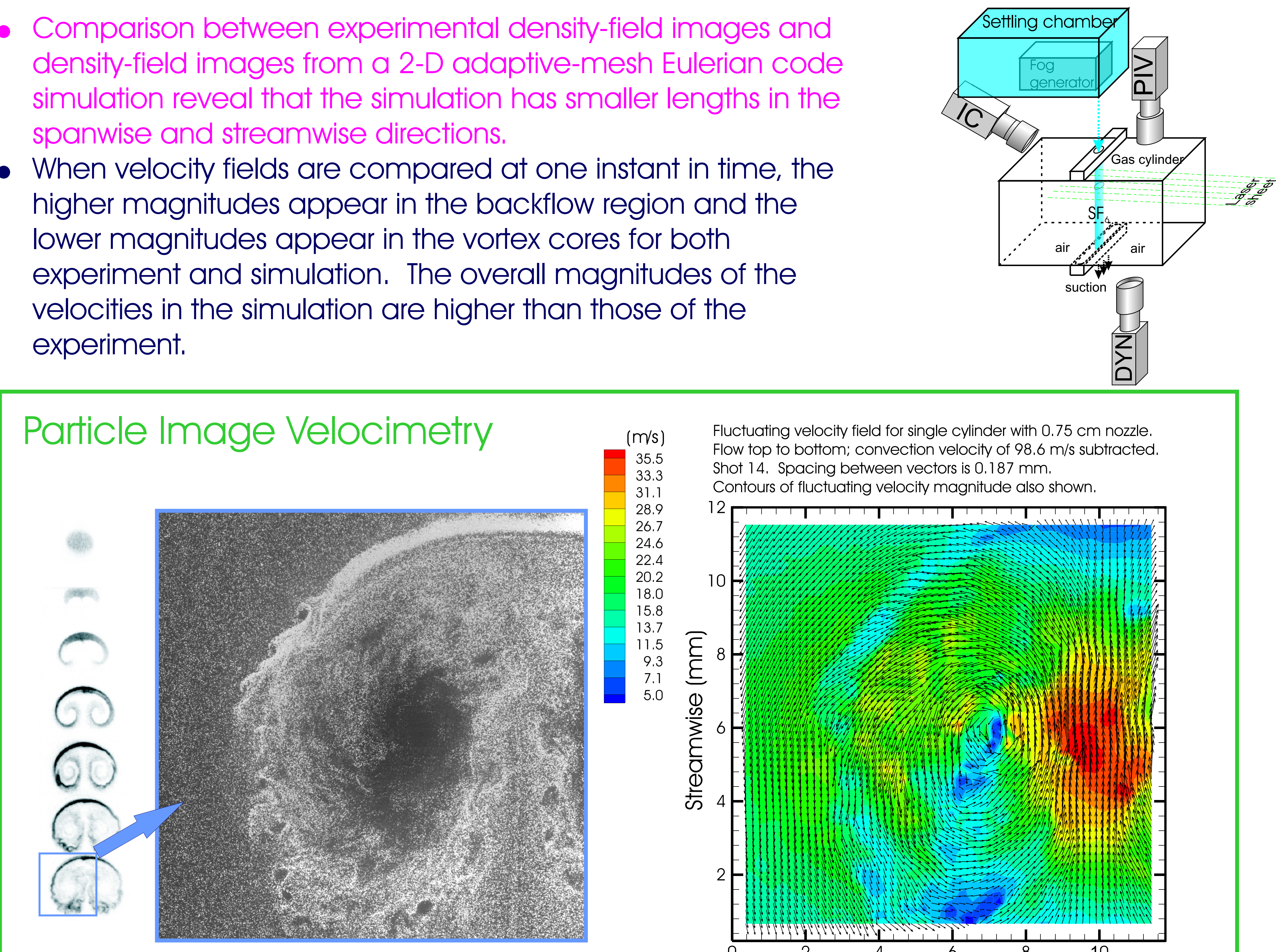

Fluctuating velocity field for single cylinder with $0.75 \mathrm{~cm}$ nozzle.
Flow top to bottlom; convection velocity of $98.6 \mathrm{~m} / \mathrm{s}$ subtracted.

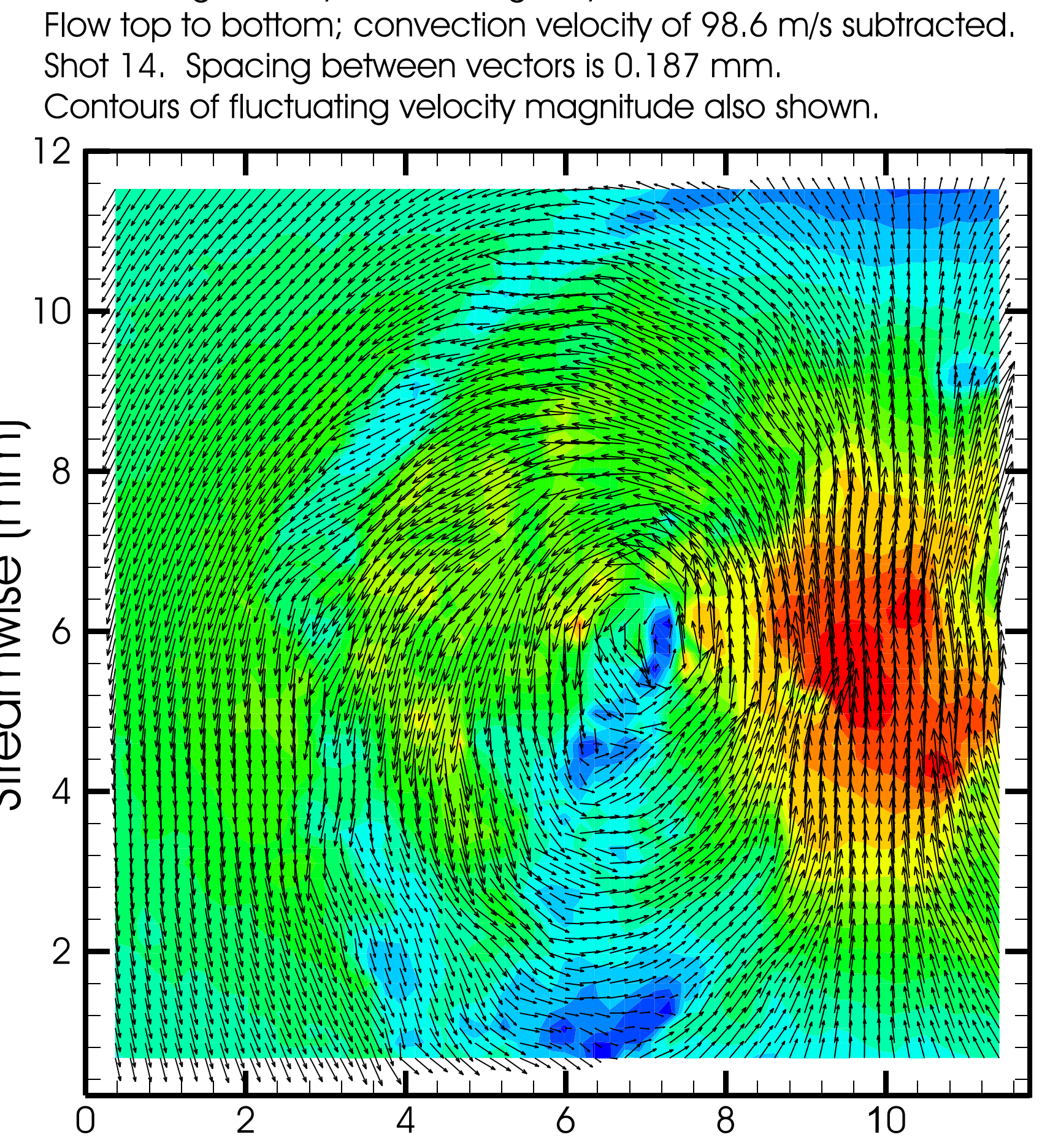

Spanwise $(\mathrm{mm})$

\section{Density Fields}

Experiment

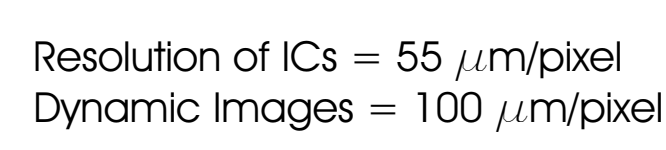

$t=0 \quad 50 \quad 190 \quad 330 \quad 470 \quad 610 \quad 750 \mu s$

- с 6668 Simulation (using experimental ICs) Time of PIV
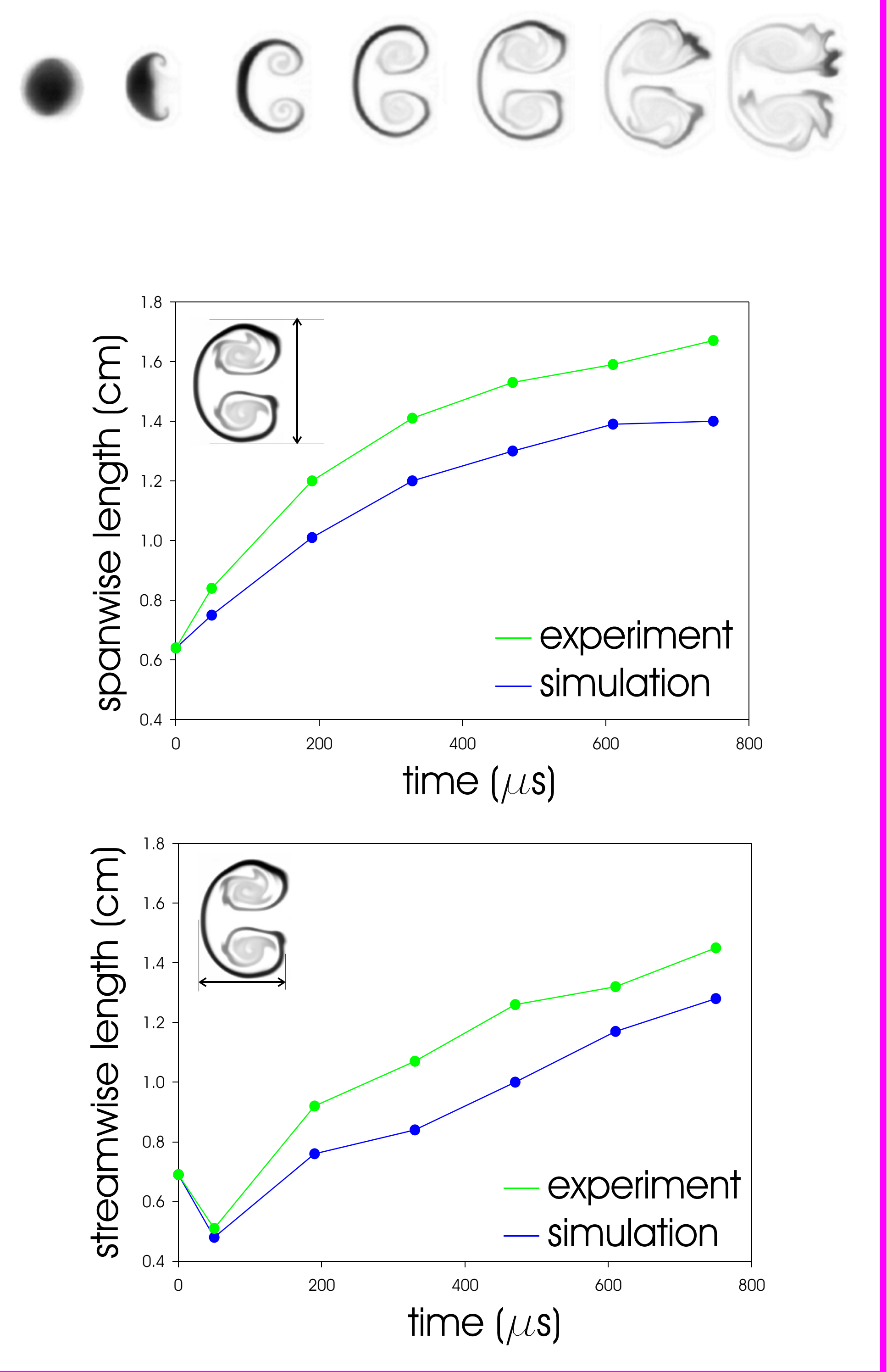

Velocity Magnitudes

Experiment

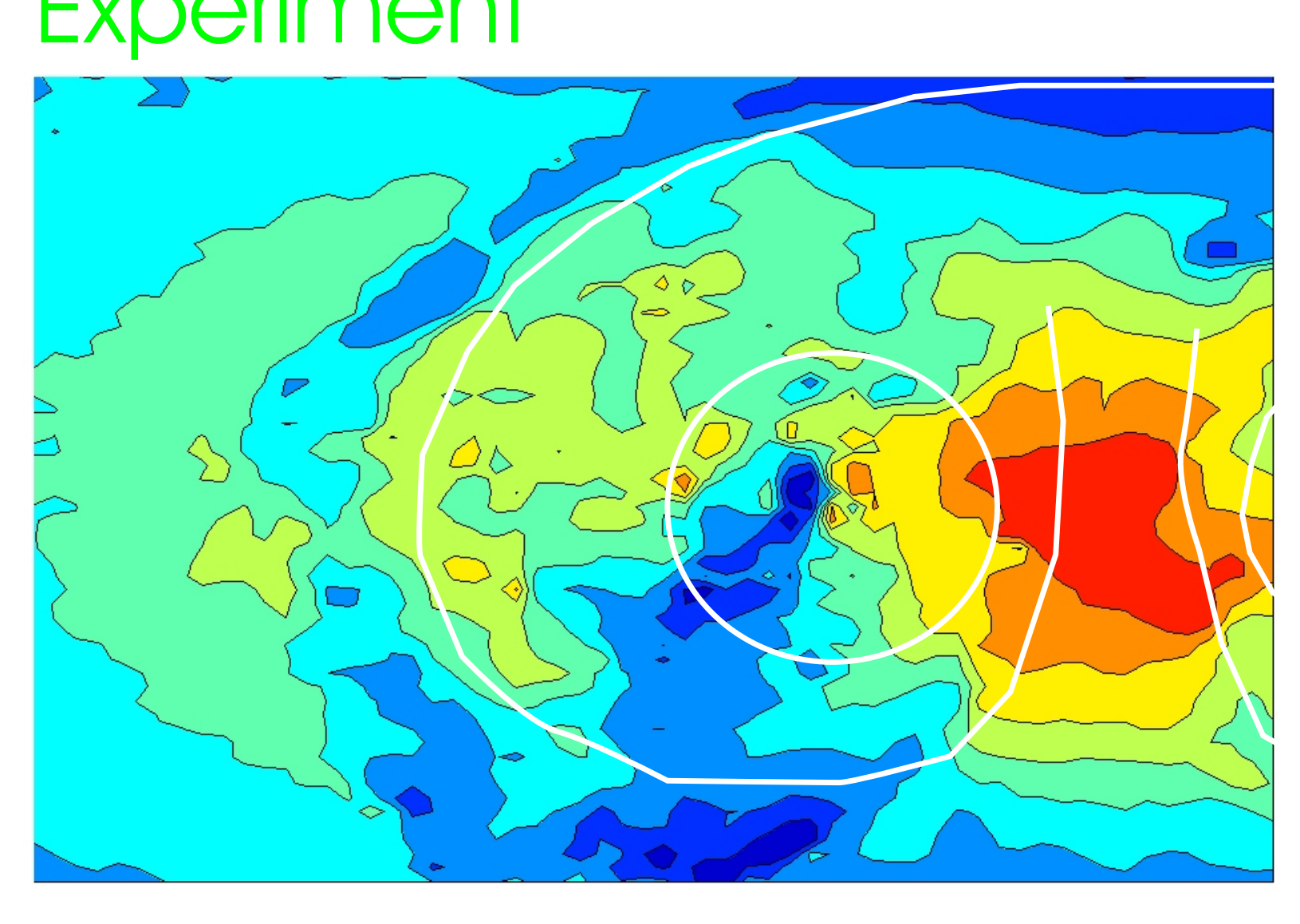

${ }^{5}{ }^{5}{ }^{10}{ }^{15}$
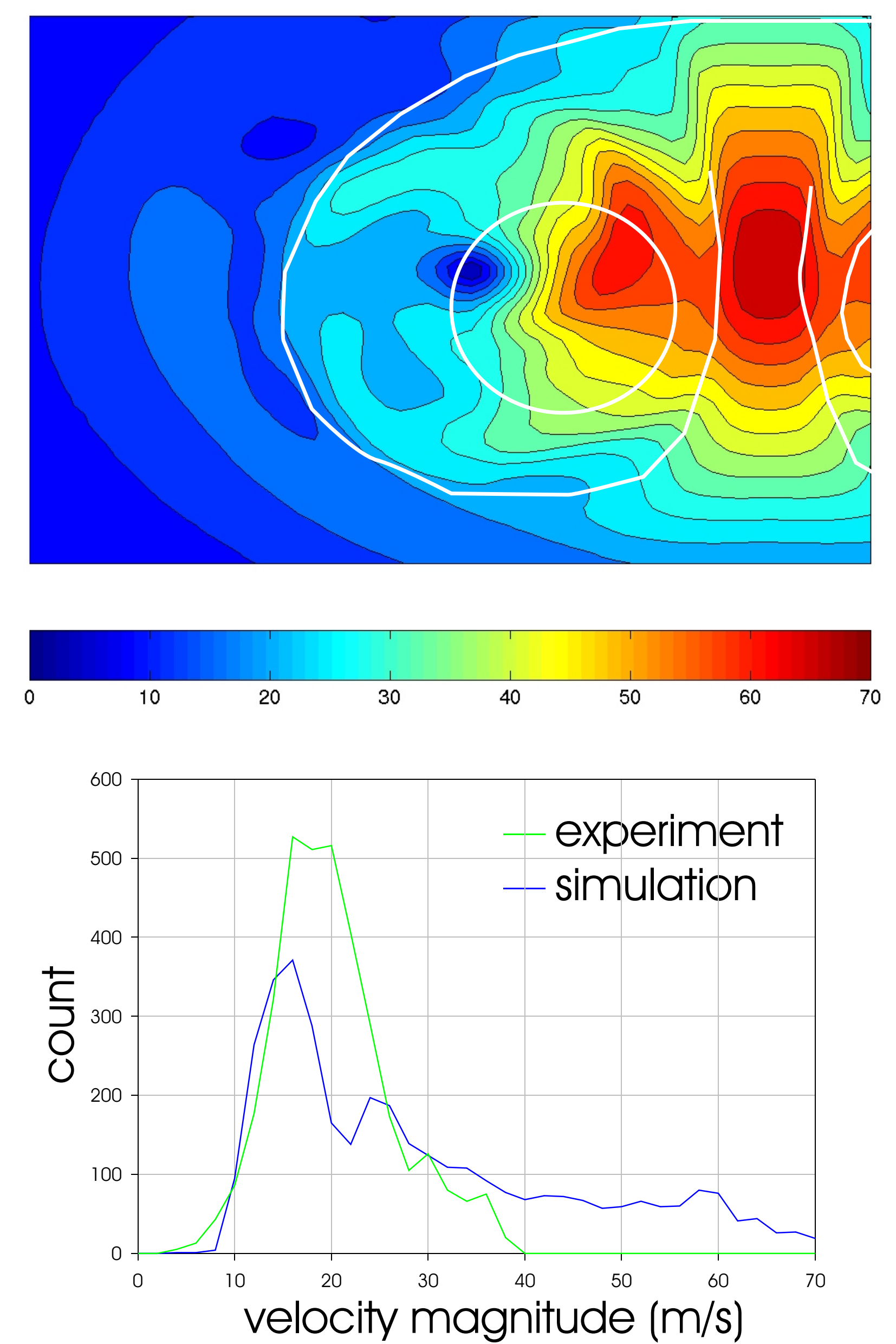
Pairs of cylinders

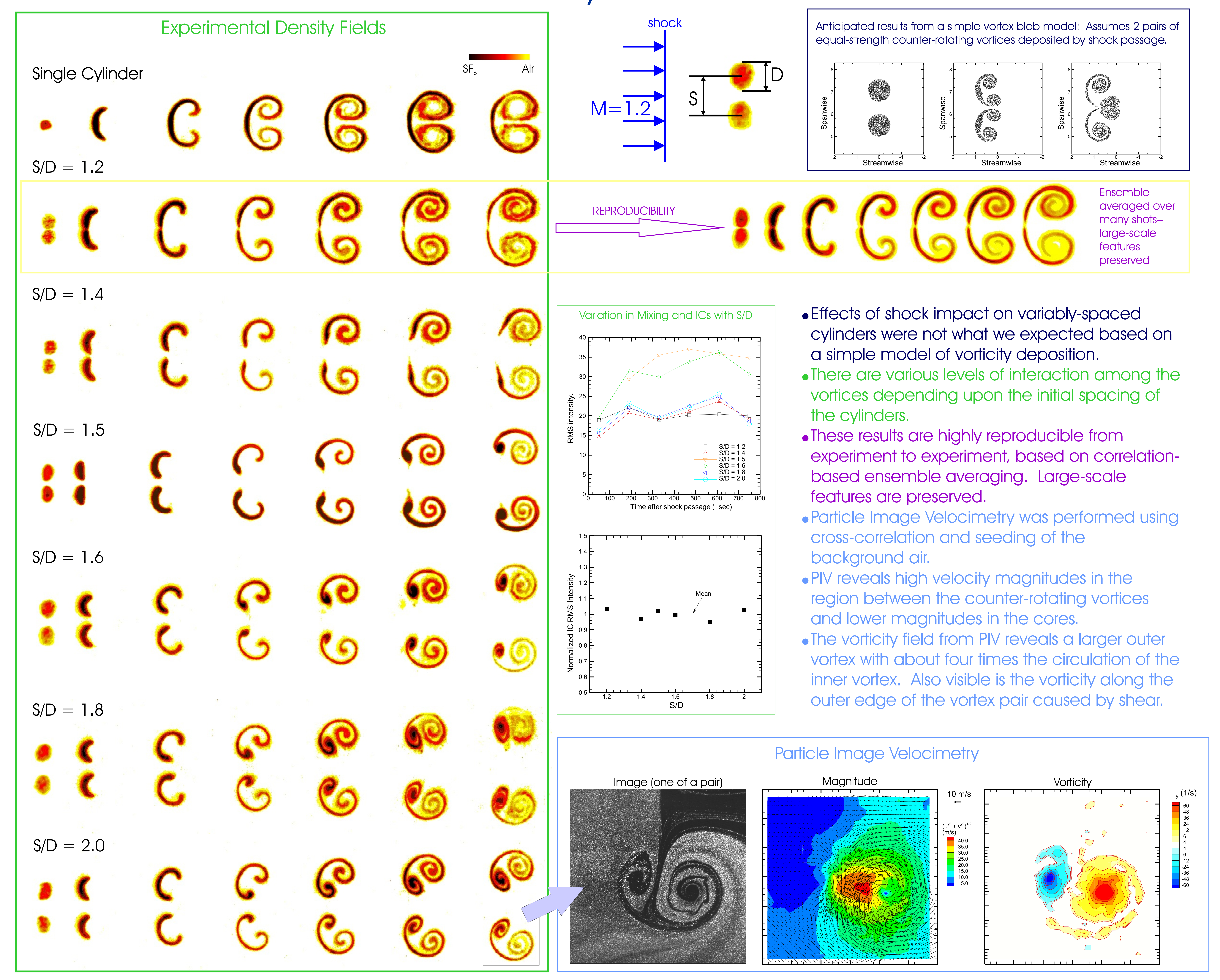


The $8^{\text {th }}$ International Workshop on the Physics of Compressible Turbulent Mixing

\title{
Experimental study into Rayleigh-Taylor turbulent mixing zone heterogeneous structure
}

\author{
Yu. A. Kucherenko, A. P. Pylaev, V. D. Murzakov, A. V. Belomestnih, V. N. Popov, A. A. Tyaktev \\ Academician E. I. Zababakhin Russian Federal Nuclear Center - All-Russian Research Institute of Technical Physics \\ P. O. 245, Snezhinsk, Chelyabinsk region, 456770, Russia \\ E-mail: kucherenko@five.ch70.chel.su
}

\begin{abstract}
The heterogeneous structure study has been performed by means of a "light-sheet" technique at the SOM gas-dynamic accelerator. The investigated system consisted of three layers of different density liquids. For leading out the information from the mixing zone inner region illuminated by the "light-sheet", visualizing particles were seeded into one of the liquids. The visualizing particles, which got into the "light-sheet", diffused light, and at the same time photo images of the liquid fragments, contained the visualizing particles, were formed by a light-sensitive receiver. For the error reduction, refractive indexes of all the three liquids were equalized. A special test has been conducted for determining of measurements inaccuracy. Experiments have been performed for two values of acceleration of artificial field of gravity. Distributions of liquid fragments sizes are showed in the form of bar charts for different moments of time.
\end{abstract}

\section{Introduction}

Up to the present, the gravitational turbulent mixing heterogeneous structure is insufficiently known though it has been made considerable efforts for solution of this problem. There had been made an attempt to value fragments scales of different density miscible liquids at their gravitational mixing by an electro contact technique in experimental work [1]. In this work, a qualitative result had been obtained. According to this result, mixing at the unstable stage occurred by large fragments but at the turbulent mixing stage fragment sizes amounted $\sim 1 \mathrm{~mm}$. In work [2] structure of the Richtmyer-Meshkov turbulent mixing of different density gases had been studied by a "laser knife" technique. In this work, there had been obtained photo images of non-uniformities in inner sections -of the mixing zone that gave an idea of the gases mixing character. In experimental \& numerical works $[3,4]$ the structure of gravitational turbulent mixing of miscible liquids with educing of molecular component had been studied for low Atwood numbers. Molecular part evaluations of mixing and density fluctuations were obtained in these works. In experimental \& numerical work [5] fractal dimension evaluations of constant concentration contours had been obtained for the Rayleigh-Taylor turbulent mixing of liquids for low Atwood numbers. In work [6] density profiles of mixing liquids had been obtained from photo images of mixing zone sections by a "laser sheet" technique.

In the present work, an attempt of direct determination of immiscible liquids fragments sizes at their Rayleigh-Taylor turbulent mixing has been made. A "light sheet" technique has been employed for this study. It is known that for immiscible liquids the smallest size of fluid elements, which are in result of fragmentation, depends on relation between inertial forces determining by acceleration of a system and resistant forces determining by surface tension of given couple of liquids (Kolmogorov's criterion). A. V. Polionov offered the following relation for evaluation of the minimum size of fluid elements:

$$
d \approx 4,3\left(\frac{\sigma / \rho}{A g_{1}}\right)^{4 / 7} \frac{1}{L^{1 / 7}}
$$


Here $\boldsymbol{\sigma}$ is surface tension value, $\rho$ is density, $A=\frac{\rho_{2}-\rho_{1}}{\rho_{2}+\rho_{1}}$ is Atwood number, $g_{1}$ is acceleration of artificial field of gravity, $\boldsymbol{L}$ - is turbulent mixing zone size. So the minimum size of fluid elements for chosen experimental system containing immiscible liquids depends on acceleration value $\boldsymbol{g}_{1}$. Therefore experiments have been performed for two essentially different accelerations.

\section{Experimental technique}

Experiments were performed at the SOM installation described in work [7]. The measuring module of the installation represents a vertical channel, in upper part of which an ampoule containing studied liquids is placed at initial moment of time. The ampoule is accelerated by a gas flow, and a liquid system placed inside of the ampoule becomes unstable because of the acceleration is directed from a heavy liquid to a light one in the coordinate system connected with the ampoule. Owing to unstable a turbulent mixing arises at the contact boundary of the liquids. In the present work the measuring module was equipped with 14 horizontal light channels located with a step of $56 \mathrm{~mm}$. Each channel contained the "light sheet»-forming block. The sketch of a horizontal section of the light channel is shown in Fig. 1.

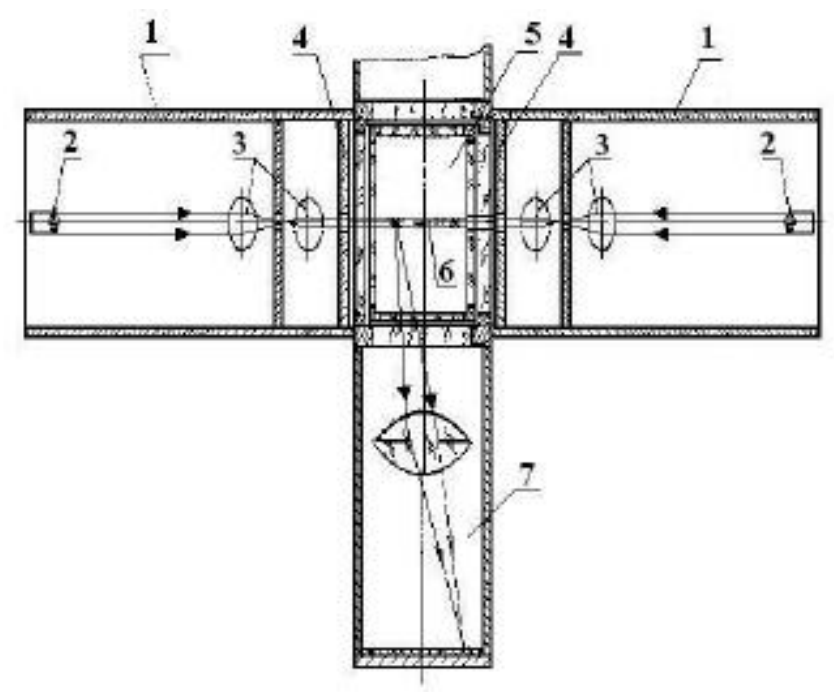

Fig. 1. Sketch of a horizontal section of the light channel

Light radiated by a pair of impulse sources (2), which is located in case (1), transforms by means of cylindrical optics (3) and diaphragms (4) to a luminous flux having a form of "light sheet" of thickness $\sim 1.5 \mathrm{~mm}$. The "light sheet" comes into the ampoule (5) from two sides and illuminates chosen section of the mixing zone. Scheme with two-side coming of the "light sheet" is chosen from consideration of uniform illumination of chosen section along the ampoule length.

Visualizing particles inserts into one of the liquids. Light scattered by the visualizing particles, which are in the "light sheet" section, finds itself in the photo recorder (7) where a photo image of the mixing zone section forms. This photo image is some set of fragments of that liquid which contains visualizing particles.

\section{Sensitivity of the technique}

The light channel sensitivity, i. e. the least registered size of non-uniformities, depends on a set of factors, so that it was determined by the most direct method - with using some models. There 
used jets specified size and form as models of non-uniformities. The jets formed by special formers two of which is shown in Fig. 2.

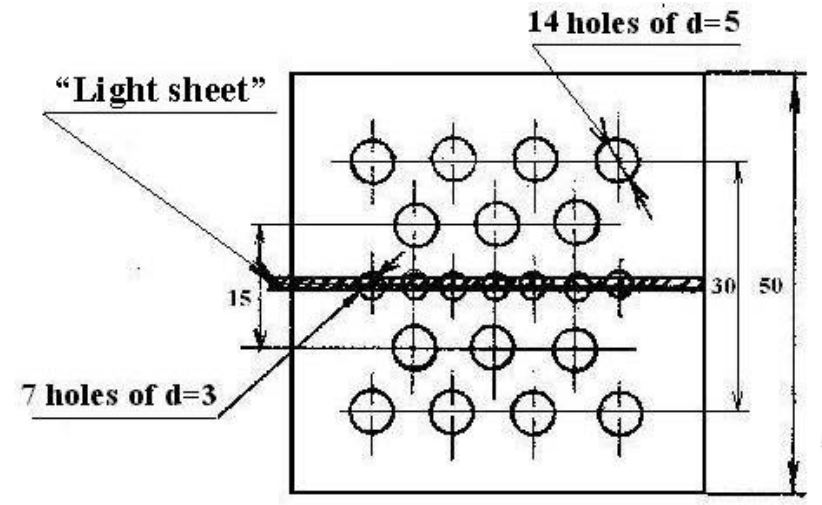

a)

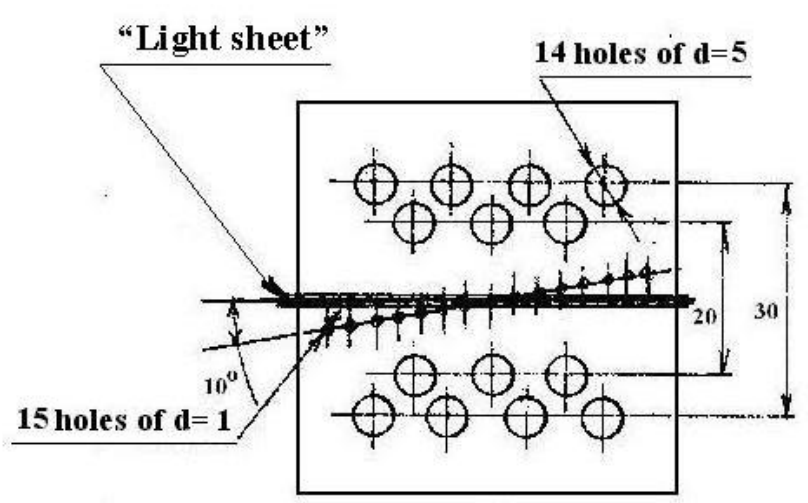

b)

Fig.2. Formers for jets forming

The formers were located inside the ampoule at the contact boundary of the liquids which were aqueous solution of glycerin and benzine with the density ratio $\boldsymbol{n}=1.6$. Visualizing particles were in the aqueous solution of glycerin. At moving a former down, the heavy liquid containing visualizing particles passes through the holes producing jets, form and diameter of which corresponds to the form and diameter of the holes in the former. The photo recorder only takes those images for which jets find themselves in the "light sheet" section. Photo images of the jets are shown in Fig. 3. In Fig. 3a, there are distinctly seen four jets of diameter $5 \mathrm{~mm}$ found themselves in the "light sheet" section and not seen other jets not found themselves there. In Fig. $3 b$, there are distinctly seen seven jets of diameter $3 \mathrm{~mm}$ formed with applying the former shown in Fig. $2 a$. In Fig. $3 c$ and Fig. $3 d$, there are seen by four jets of diameter correspondingly $2 \mathrm{~mm}$ and $1 \mathrm{~mm}$. These jets were formed with the former shown in Fig. $2 b$. Holes of that former were placed on an angle to the "light sheet". It is seen that the images only correspond to those jets which found themselves in the "light sheet" section. Jets of diameter less than $1 \mathrm{~mm}$ do not practically have images. Obtained results give a possibility to assert that:

1. Concentration of visualizing substance is enough for sharp image acquisition of nonuniformities of sizes not less than $1 \mathrm{~mm}$;

2. Those non-uniformities, which do not find themselves in the "light sheet" section, do not have photo images.

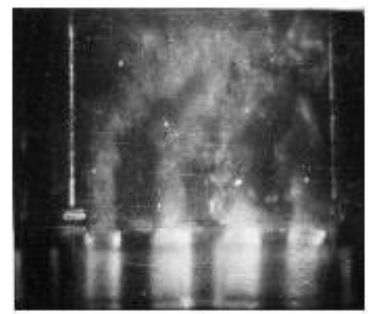

a)

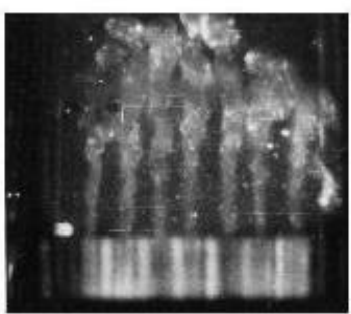

b)

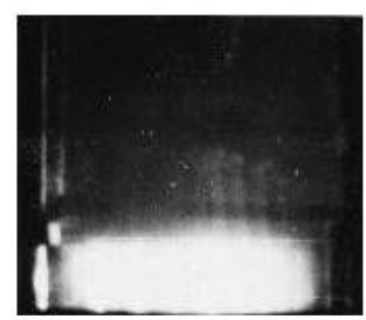

c)

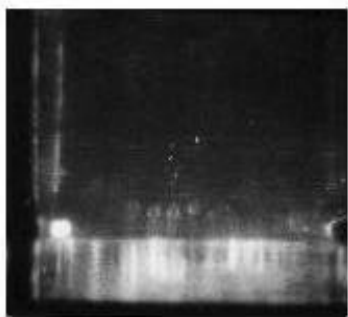

d)

Fig. 3. Photo images of jets taken with special formers 


\section{Set up of experiments}

Scheme of set up of experiments is shown in Fig. 4.

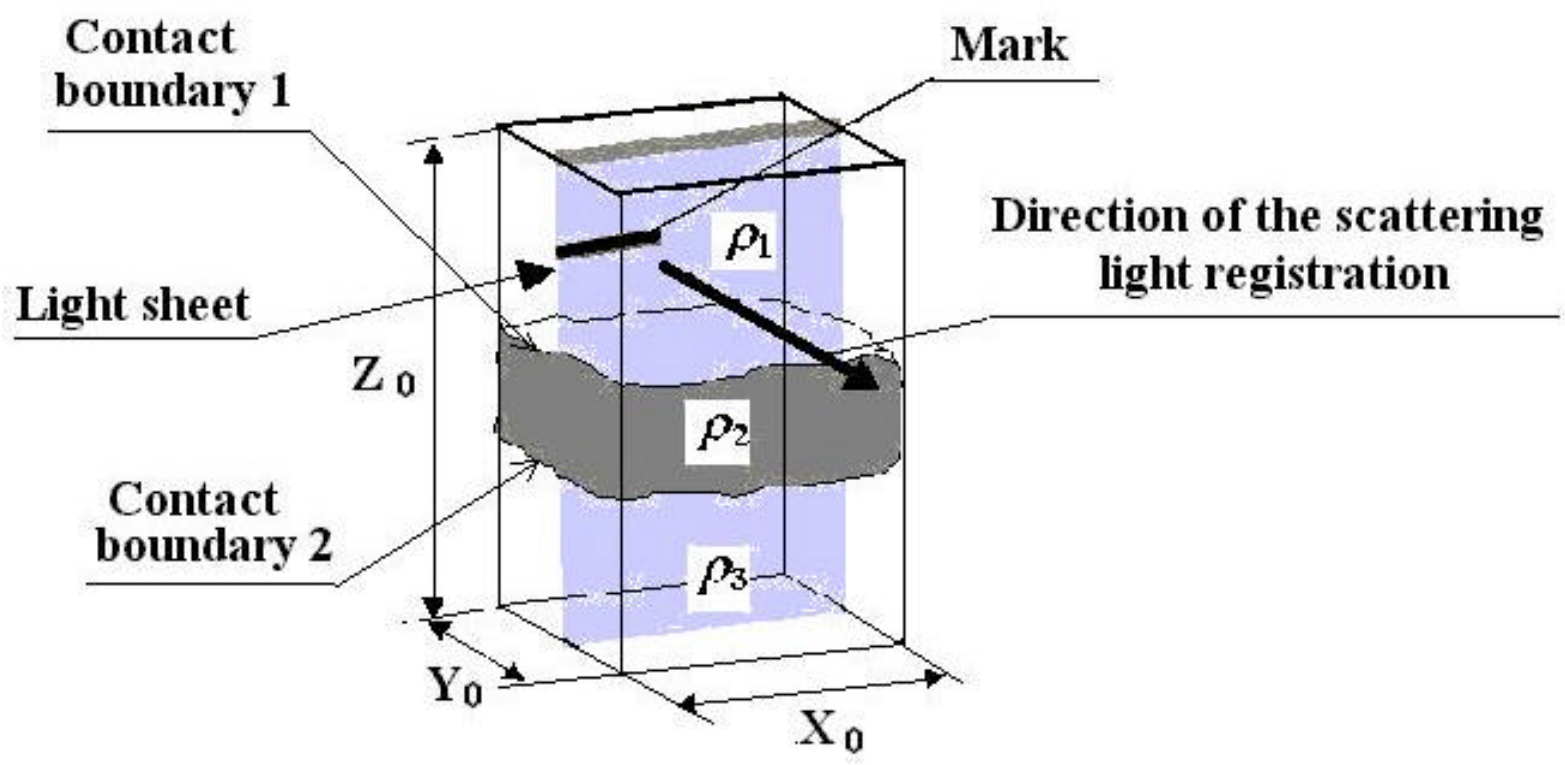

Fig.4. Scheme of set up of experiments

Experiments were performed with the system consisted of three layers of different density liquids placed inside of a hermetically ampoule. The lower layer was an aqueous solution of sodium hyposulfite $\left(\mathrm{Na}_{2} \mathrm{SO}_{3}\right)$ with adding of glycerin of density $\rho_{3}=1.24 \mathrm{~g} / \mathrm{cm}^{3}$ and viscosity $\mathrm{v}_{3}=1.95$ CSt. The middle layer of thickness $15 \mathrm{~mm}$ was a mixture of water and glycerin of density $\rho_{2}=1.10$ $\mathrm{g} / \mathrm{cm}^{3}$ and viscosity $v_{2}=4.5 \mathrm{CSt}$. The upper layer was benzine of density $\rho_{1}=069 \mathrm{~g} / \mathrm{cm}^{3}$ and viscosity $v_{1}=0.77 \mathrm{CSt}$. Gelatin as a visualizing substance was added in the mixture of water and glycerin. Mass concentration of gelatin was $2 \%$. Glycerin was only used for matching of indexes of refraction of all three layers. Optimum concentration of visualizing substance was determined by a photoelectric pickup having recorded intensity of transmitted and scattered laser light. Surface tension at the contact boundaries between aqueous solution of sodium hyposulfite and benzine, and between the glycerin and water mixture and benzine amount to $20-30$ dyne $/ \mathrm{cm}$.

The inner sizes of working volume of the ampoule were $X_{0}=64 \mathrm{~mm}, Y_{0}=54 \mathrm{~mm}, Z_{0}=120$ mm. Impulse luminous flux in the form of "sheet" illuminated the central section of the ampoule (the "light sheet" coordinate is $y=27 \mathrm{~mm}$ ). There was a fixed mark inside the ampoule in the light sheet plane for determining of the contact boundaries initial positions and mixing fronts coordinates. A scale grid was placed inside the ampoule in the light sheet plane for preliminary determining the light channels enlargement. Initial perturbations at the contact boundaries of the liquids were created by blow with a special striker made of fluoroplastic of mass $40 \mathrm{~g}$ upon the ampoule cover. Specific mass of the equipped ampoule was $\boldsymbol{m}=23.9 \mathrm{~g} / \mathrm{cm}^{2}$.

There were performed two groups of experiments differed by initial acceleration $g_{1}$. In the first group of experiments acceleration was $g_{1}=350 \mathrm{~g}$, in the second one - $g_{1}=100 \mathrm{~g}$, where $g-$ acceleration of the Earth's gravity. When the ampoule has passed distance $784 \mathrm{~mm}$, in the first group of experiments acceleration becomes $230 \mathrm{~g}$ and in the second group - $66 \mathrm{~g}$. In its turn every group consisted of two series of experiments differed by the range of recording. The reason was connected with availability of CB2 formed by miscible liquids with not great Atwood number $\grave{A}=$ 0.11 . Because of that turbulent mixing at that contact boundary started after some delay and was developing not enough intensive for heterogeneous structure measuring in that range. It had to enlarge the ampoule displacement along the measuring channel by $500 \mathrm{~mm}$ to measure the 
heterogeneous structure. So that the ampoule displacement was $S=784 \mathrm{~mm}$ in the first series of experiments and $S=1284 \mathrm{~mm}-$ in the second one.

\section{Experimental results}

Each group consisted of 20 experiments. Characteristic photo images captured in the $1^{\text {st }}$ group of experiments are shown in Fig. 5. Photo images for the $2^{\text {nd }}$ group are presented in Fig. 6.

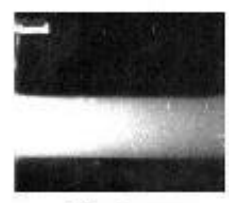

$\mathbf{S}=\mathbf{0}$

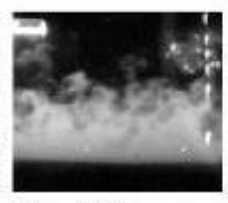

$\mathrm{S}=\mathbf{4 4 8 \mathrm { mm }}$

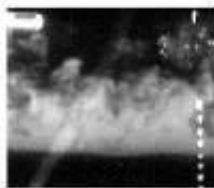

$\mathrm{S}=560 \mathrm{~mm}$

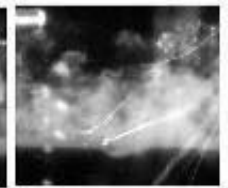

$S=672 \mathrm{~mm}$
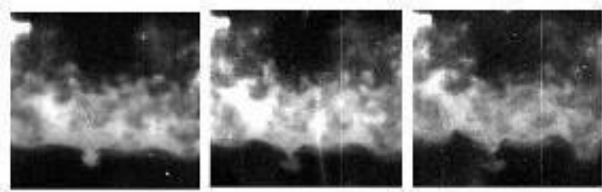

$S=836 \mathrm{~mm} S=948 \mathrm{~mm} \quad S=1060 \mathrm{~mm} \quad S=1172 \mathrm{~mm}$

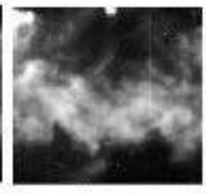

Fig. 5. Characteristic photo images of the mixing zone structure for the $1^{\text {st }}$ group of experiments

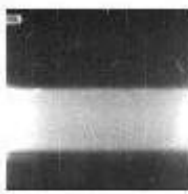

$\mathbf{S}=\mathbf{0}$

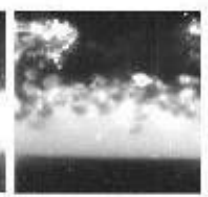

$\mathrm{S}=\mathbf{4 4 8 \mathrm { mm }}$

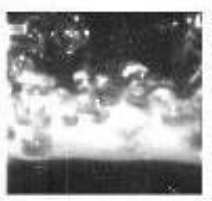

$\mathrm{S}=560 \mathrm{~mm}$
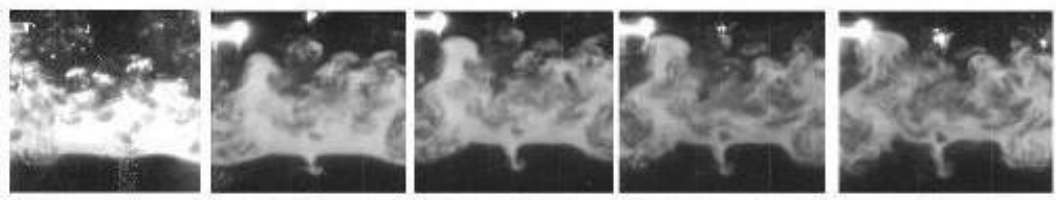

$\mathrm{S}=672 \mathrm{~mm}$

Fig. 6. Characteristic photo images of the mixing zone structure for the $2^{\text {nd }}$ group of experiments

It is seen from the photo images that the turbulent mixing first develops at the contact boundary $\mathrm{CB} 1$ and some time later - at the contact boundary CB2.

Photo images were processing the following manner. Two-dimensional $(X-Z)$ matrix of the film blackening intensities was produced at scanning each photo image. For arbitrary section $Z=Z_{0}$ the dependence of the film blackening density on coordinate $X$ was built. Next this dependence was processing according to a special developed algorithm, which gave a possibility to obtain sizes of liquid fragments having found themselves in the light sheet section by computer. The program was developed so that it determined fragments sizes of one of the liquids, namely that liquid whose fragments at positive image were light. For determining fragments sizes of another liquid (not containing visualizing substance) it was processing negative image of the frame at which fragments images of that liquid were light. All the data obtained for the same moment of time were referred to the same statistical population. Bar charts of liquid fragments distribution at their sizes were built as the result of processing.

At each photo image, determination of liquid fragments sizes in the mixing zone was produced at the following sections (along $Z$-coordinate): at the section where the initial contact boundary $\mathrm{CB} 1$ was placed $Z=0$ ), and at the sections $Z= \pm 4 \mathrm{~mm}, Z= \pm 8 \mathrm{~mm}$. Inaccuracy of measurements of liquid fragments sizes was obtained with using of photo images of the jets, produced in model experiments. Transversal sizes of the jets were determined in ten sections along Z-coordinate by both a handle method and machine one. As a result of this measuring the maximum inaccuracy is $\eta=15 \%$.

Bar charts of sizes $\boldsymbol{d}$ distributions of both light liquid fragments (of density $\rho_{1}=0.69 \mathrm{~g} / \mathrm{cm}^{3}$ ) and heavy ones (of density $\rho_{2}=1.23 \mathrm{~g} / \mathrm{cm}^{3}$ ) built at considering all fragment sizes of each liquid obtained for all displacements $S$ and all sections $\boldsymbol{Z}$ as the same statistical population in each group of experiments are shown in Fig. 7. 

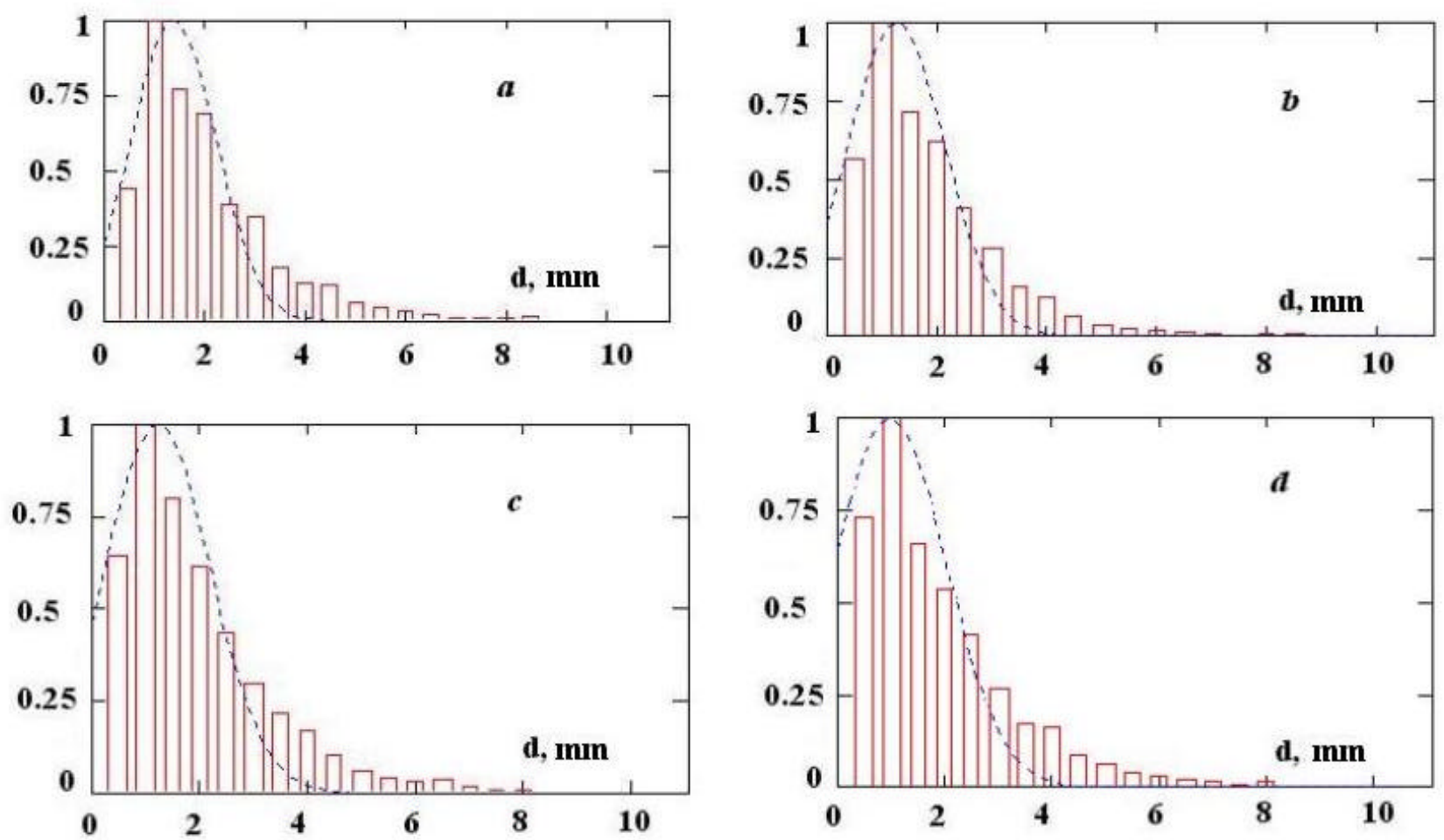

Fig. 7. Bar charts of distributions of liquids fragments sizes in the mixing zone $\boldsymbol{a}$ - light liquid of density $\boldsymbol{\rho}_{1}$, the $1^{\text {st }}$ group of experiments; $\boldsymbol{b}$ - heavy liquid of density $\boldsymbol{\rho}_{2}$, the $1^{\text {st }}$ group of experiments; $\boldsymbol{c}$ - light liquid of density $\boldsymbol{\rho}_{1}$, the $2^{\text {nd }}$ group of experiments; $\boldsymbol{d}$ - heavy liquid of density $\boldsymbol{\rho}_{2}$, the $2^{\text {nd }}$ group of experiments

It is seen from the bar charts that in the $1^{\text {st }}$ group of experiments the maximum of the distribution is $\grave{a}_{1}=1.39 \mathrm{~mm}$ for the liquid of density $\boldsymbol{\rho}_{1}$, and $\grave{a}_{2}=1.26 \mathrm{~mm}$ for the liquid of density $\rho_{2}$. In the $2^{\text {nd }}$ group of experiments the maximums of the distributions are $\grave{a}_{1}=1.23 \mathrm{~mm}$ and $\grave{a}_{2}=0.98 \mathrm{~mm}$ for the light and heavy liquids correspondingly.

\section{Conclusion}

Performed experiments showed that in developed turbulent flow produced by the Rayleigh-Taylor instability sizes of most part of fragments of immiscible liquids are in the range from $1 \mathrm{~mm}$ to 1.5 $\mathrm{mm}$. Evaluations of minimum sizes of liquid fragments by the Polionov's relation (1) show that they are in the range from $0.5 \mathrm{~mm}$ to $1.1 \mathrm{~mm}$ for the light liquid and from $0.36 \mathrm{~mm}$ to $0.8 \mathrm{~mm}$ for the heavy one. These evaluations are in qualitative agreement with the experiment.

\section{Literature}

1. I. K. Kikoin, D. I. Voskoboinik, V. A. Dmitrievsky, V. I. Stefanov. Experimental study of turbulent mixing of liquids in an acceleration field. LIPAN Report, p.2, 1953.

2. E. E. Meshkov, A.I. Tolshmyakov. Study into turbulent mixing zone structure by scattered light. RFNC-VNIIEF Report, 1982.

3. P. F. Linden and J. M. Redondo. Molecular mixing in Rayleigh-Taylor instability. Part I: Global mixing. Phys. Fluids, A3 (5), 1991.

4. P. F. Linden, J. M. Redondo and D. L. Youngs. Molecular mixing in Rayleigh-Taylor instability. J. Fluid Mech., v. 265, 1994. 
5. S. B. Dalziel, P. F. Linden and D. L. Youngs. Self-similarity and Internal Structure of Turbulence Induced by Rayleigh-Taylor Instability. Proceedings of the Sixth International Workshop on the Physics of Compressible Turbulent Mixing. Marseilles, 18 -21 June 1997.

6. M. Schneider, G. Dimonte and B. Remington. Structure of Rayleigh-Taylor Mixing from Laser Induced Fluorescence on the Linear Electric Motor (in typescript).

7. Yu. A. Kucherenko, A. P. Pylaev, V. D. Murzakov, V. N. Popov, O. R. Komarov, V. E. Savel'ev, R. Cherret, and J.-F. Haas. Experimental study into the Asymptotic Stage of the Separation of the Turbulized Mixtures in Gravitationally Stable Mode. Proceedings of the $5^{\text {th }}$ International Workshop on Compressible Turbulent Mixing. Stony Brook, 18 - 21 July 1995. 


\section{Measurements of Turbulence Correlations in Low Atwood Number Rayleigh-Taylor Mixing}

Malcolm Andrews \& Praveen Ramaprabhu

Department of Mechanical Engineering

Texas A\&M University

College Station, TX 77845

Tel: (979) 847 8843; e-mail: mandrews@mengr.tamu.edu

International Workshop on the Physics of

Compressible Turbulent Mixing

CalTech, Pasadena, CA

December 9-14, 2001

DOE Grant\# DE-FG03-99DP00276/A000 


\section{Overview}

- Detailed measurements of turbulent Rayleigh-Taylor have been taken in support of mix models for the description and understanding of hydrodynamic instabilities that develop during the implosion phase of ICF capsules.

- Highlights include: extensive collection of data and development of the PIV-S method.

- The intent is data to aid in the development of statistical turbulence models

- What follows is a selection of our results. 


\section{ATM \\ Schematic of experiment}

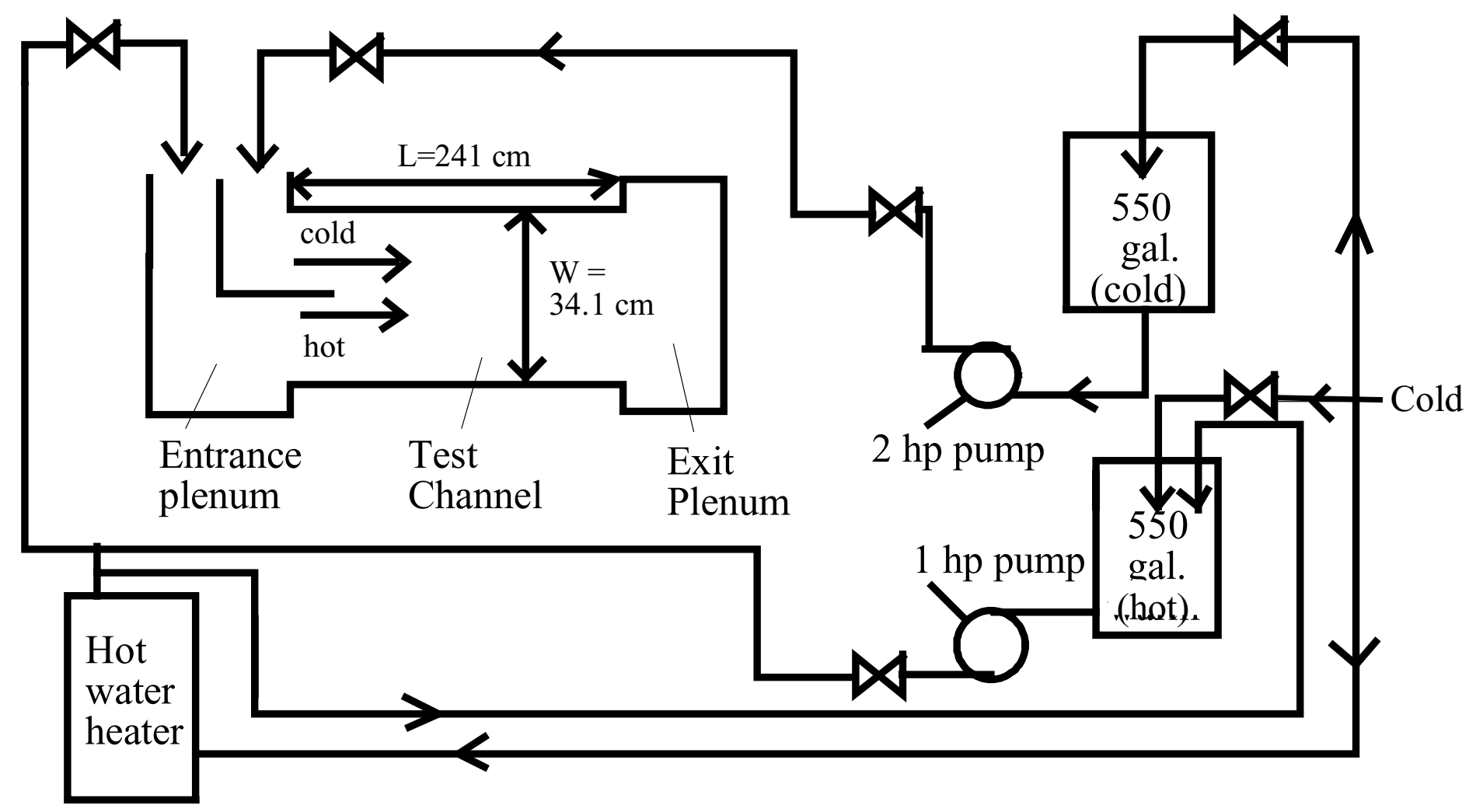

Malcolm Andrews 


\section{Experimental details}

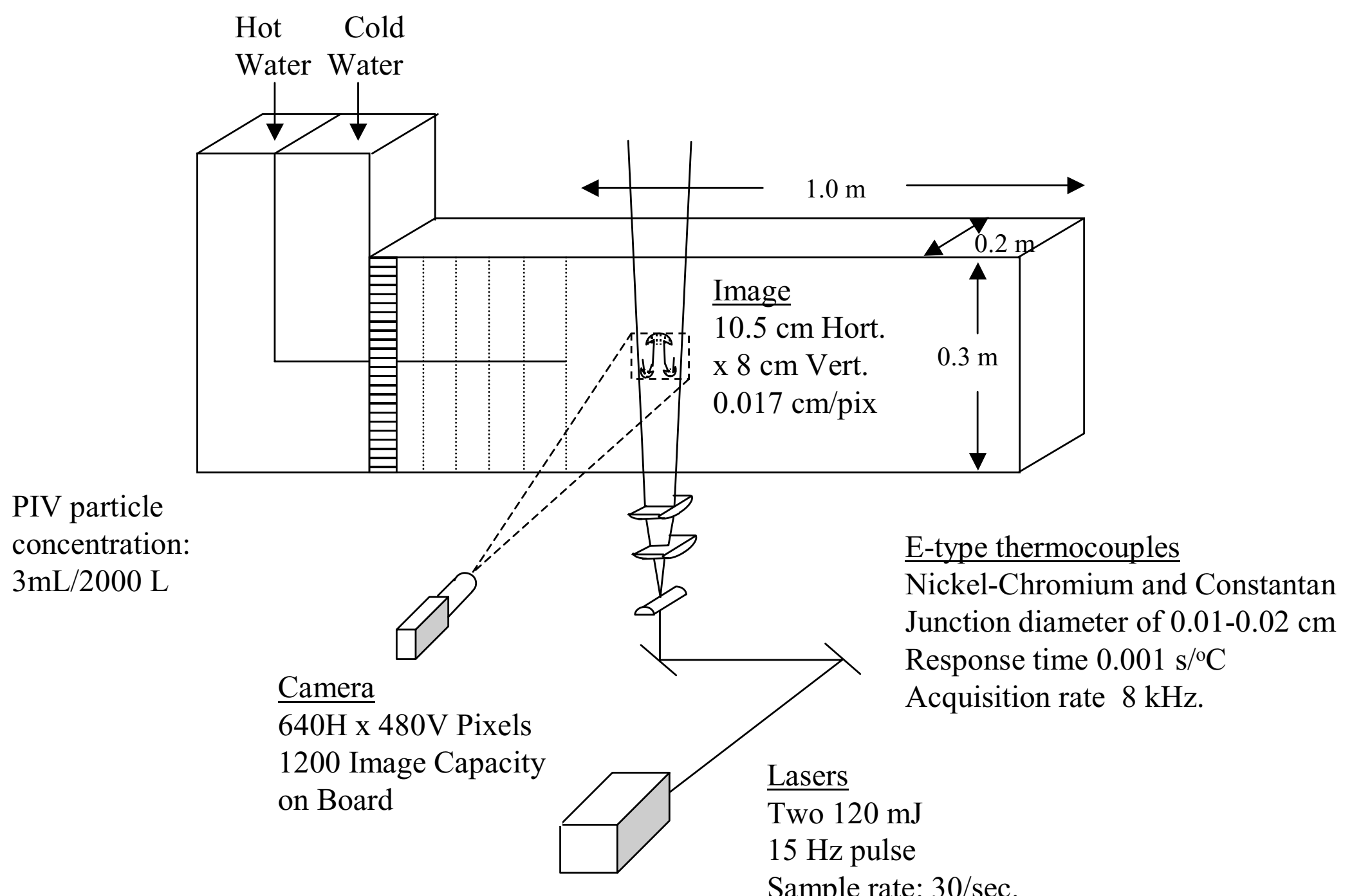




\section{Photograph from experiment}

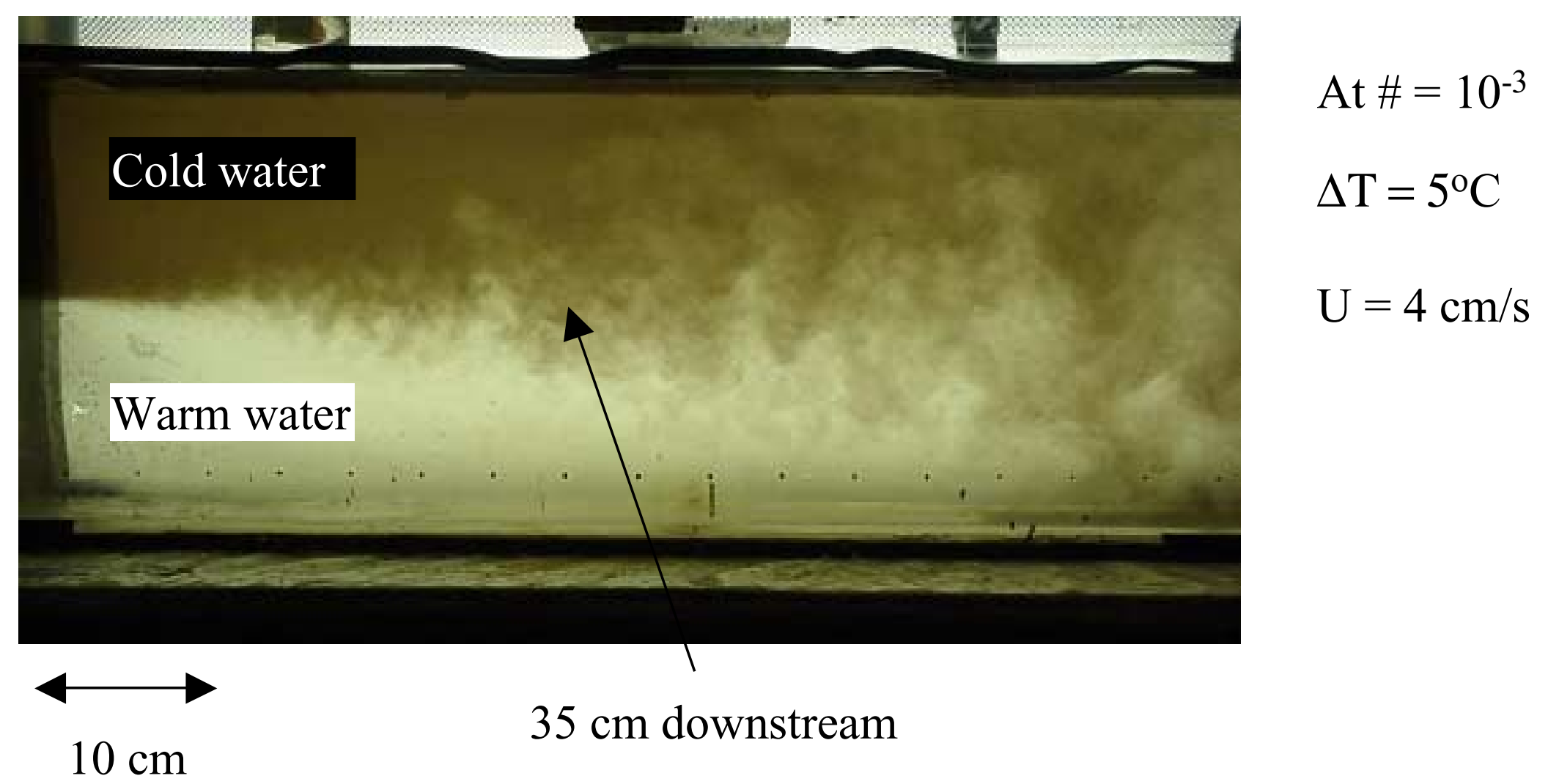




\section{Summary of data collected}

At Atwood numbers of $10^{-3}$ and $5 \times 10^{-4}$ :

- Density profiles across mix; width quadratic growth rate, $\alpha$

- Ensemble averaged measurements of turbulence R-T mixing correlations:

$$
\overline{\rho^{\prime 2}} \overline{u^{\prime 2}}, \overline{v^{\prime 2}}, \overline{u^{\prime} v^{\prime}} \text {, and } \overline{\rho^{\prime} u^{\prime}}, \overline{\rho^{\prime} v^{\prime}}
$$

- Turbulence density fluctuation energy spectra.

- Molecular mix fraction, $\theta$

- Anisotropy tensor 


\section{Parameter definitions}

$$
\begin{gathered}
\mathrm{B}_{0}=\lim _{\mathrm{T} \rightarrow \infty} \frac{1}{\mathrm{~T}} \int_{0}^{\mathrm{T}}(\rho-\bar{\rho})^{2} \mathrm{dt} / \Delta \rho^{2}=\lim _{\mathrm{T} \rightarrow \infty} \frac{1}{\mathrm{~T}} \int_{0}^{\mathrm{T}}\left(\rho^{\prime}\right)^{2} \mathrm{dt} / \Delta \rho^{2} \\
B_{2}=\overline{\rho^{*}}\left(1-\overline{\rho^{*}}\right)=f_{1}\left(1-f_{1}\right) \quad \theta \equiv 1-B_{0} / B_{2} \\
\rho^{*}=\frac{\left(\rho-\rho_{\min }\right)}{\left(\rho_{\max }-\rho_{\min }\right)} \quad \overline{\rho^{*}}=\frac{\sum_{1}^{n} \rho_{\mathrm{i}}^{*}}{\mathrm{n}} \quad B_{0}=\frac{\mathrm{n} \sum_{1}^{n} \rho_{\mathrm{i}}^{* 2}-\left(\sum_{1}^{n} \rho_{\mathrm{i}}^{*}\right)^{2}}{\mathrm{n}(\mathrm{n}-1)} \\
\mathrm{B}_{0}\left(\omega_{\mathrm{n}}\right)=\frac{2 \delta \mathrm{t}}{\mathrm{N}} \mid \sum_{i=0}^{N-1}\left(\rho_{i}^{*}\right)^{\prime} \mathrm{e}^{2 \pi j \omega_{n} t_{i} \mid}
\end{gathered}
$$




\section{Mean density profiles}

ATM

$2.4 \mathrm{~cm}$ downstream

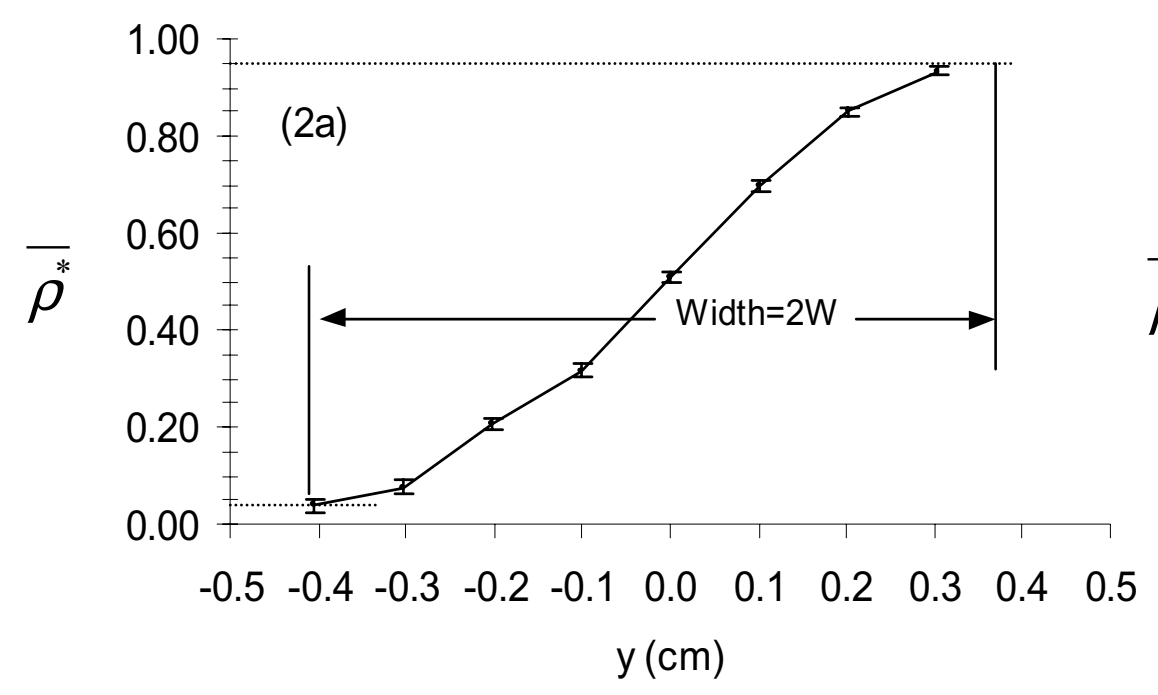

$30 \mathrm{~cm}$ downstream

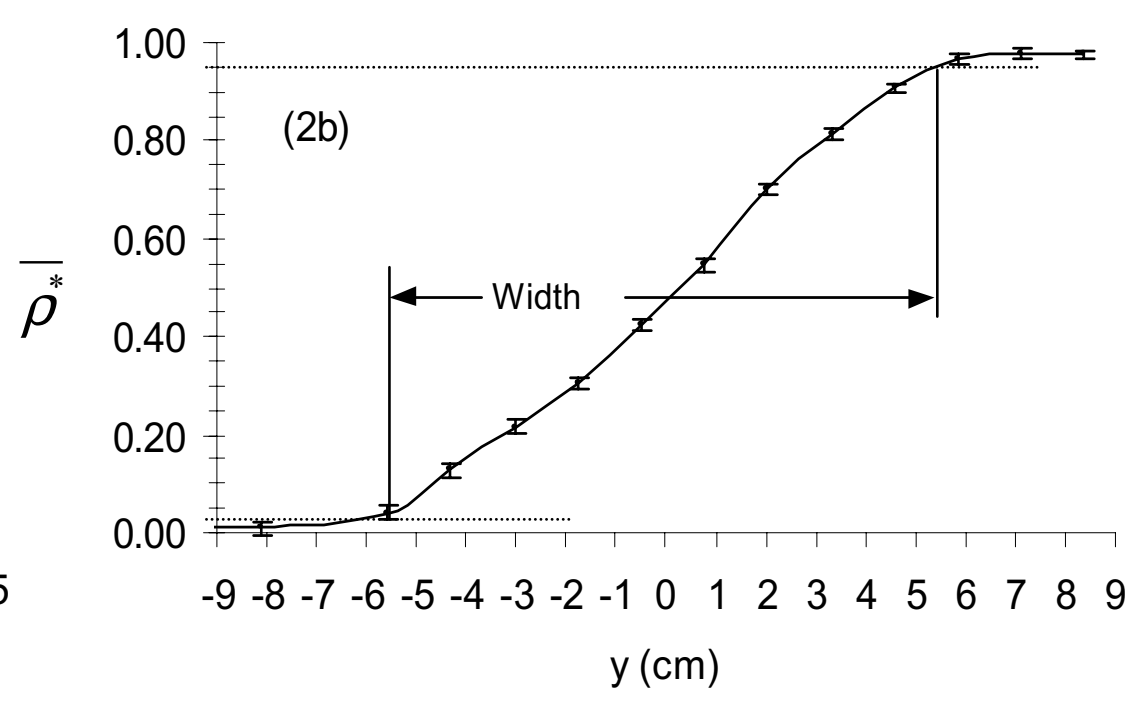

Mean density profile taken with thermocouple measurements, and showing error bars. 


\section{Density fluctuation power \\ ATM spectra}
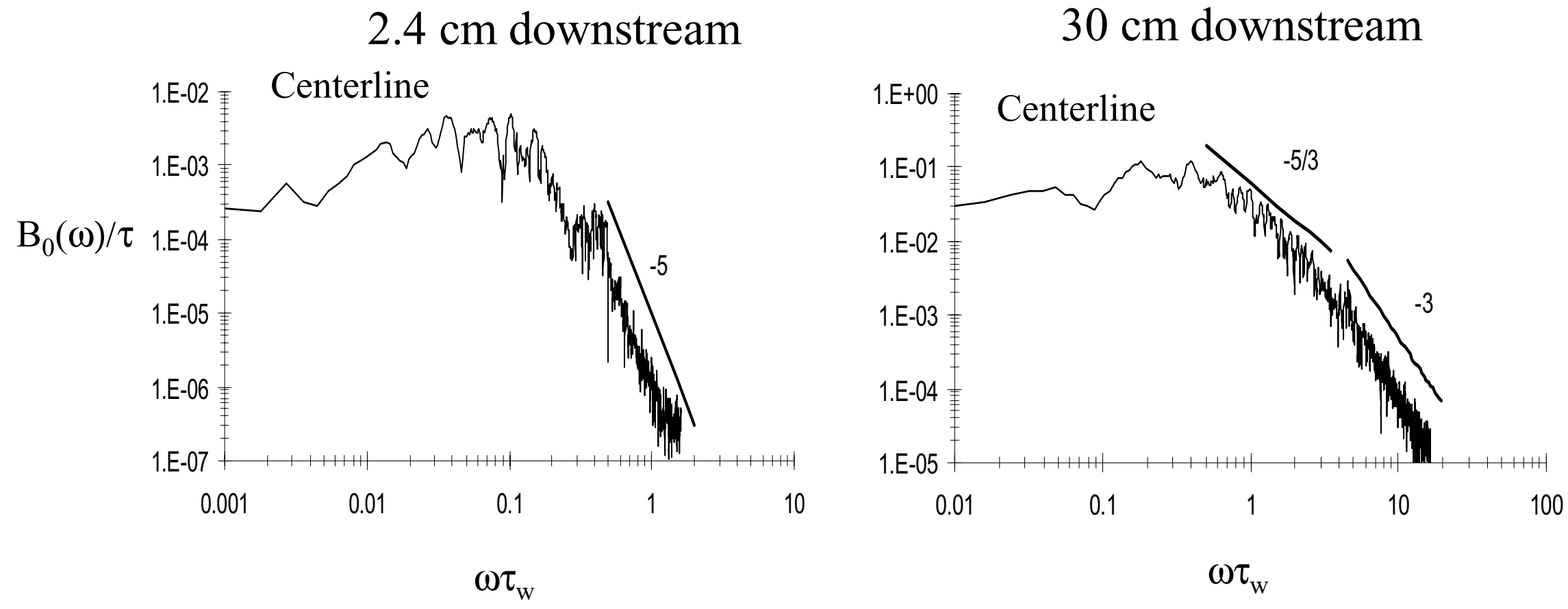


\section{More power spectra ATM}

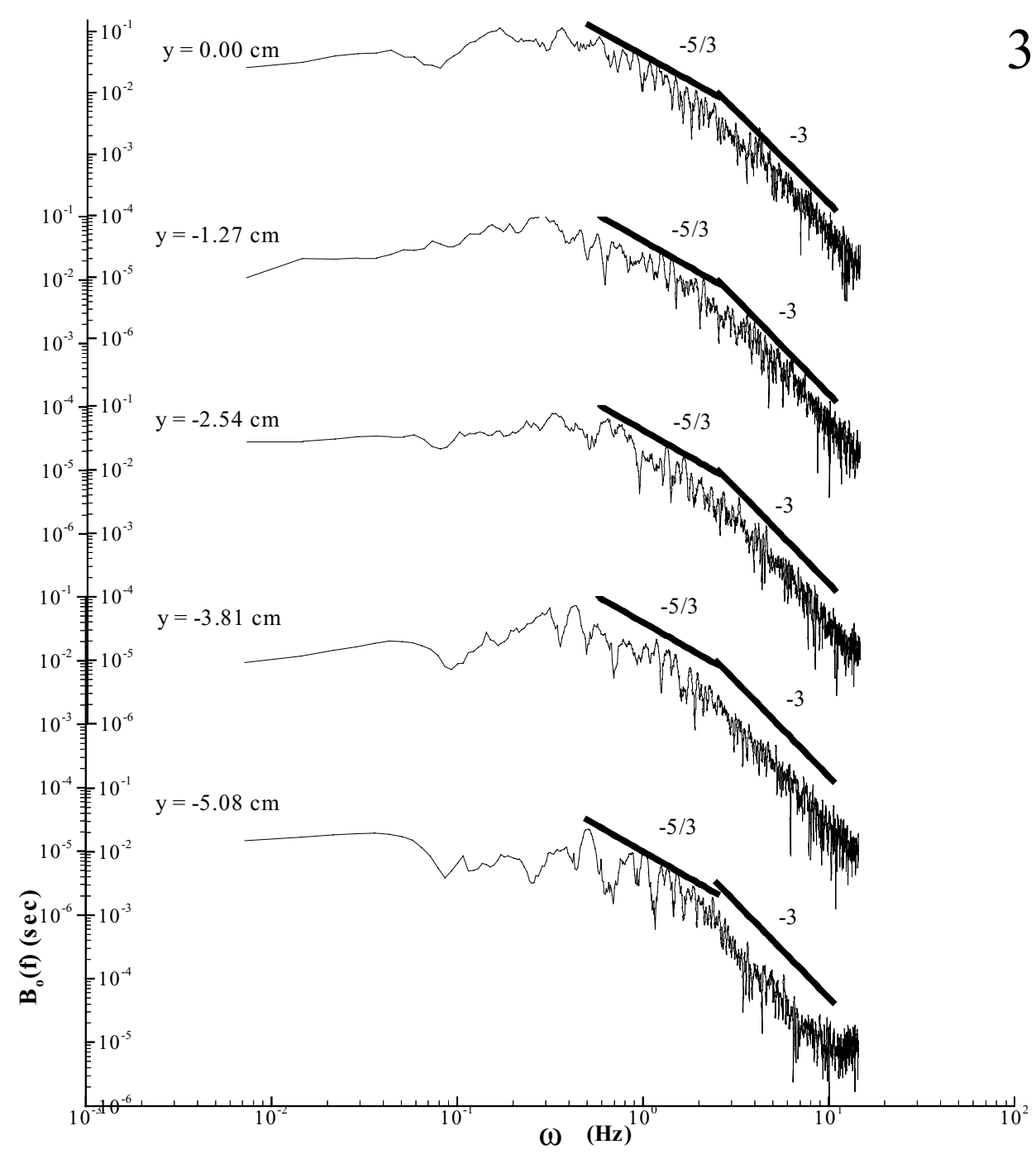

$30 \mathrm{~cm}$ downstream

Centerline

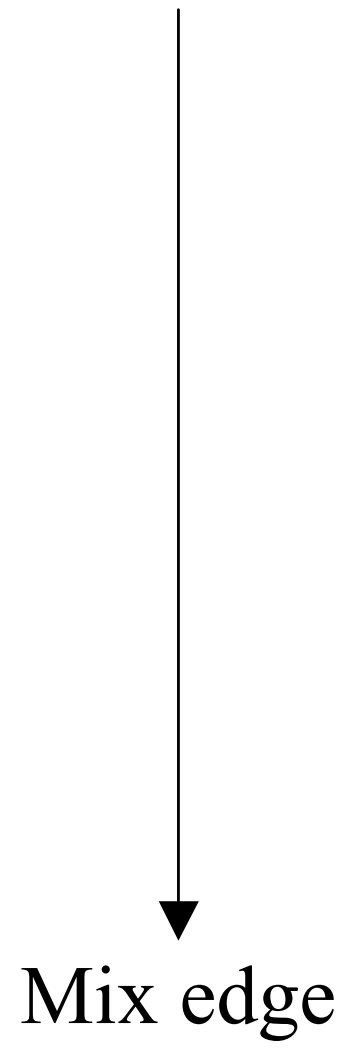

Malcolm Andrews 


\section{PIV-S}

ATM
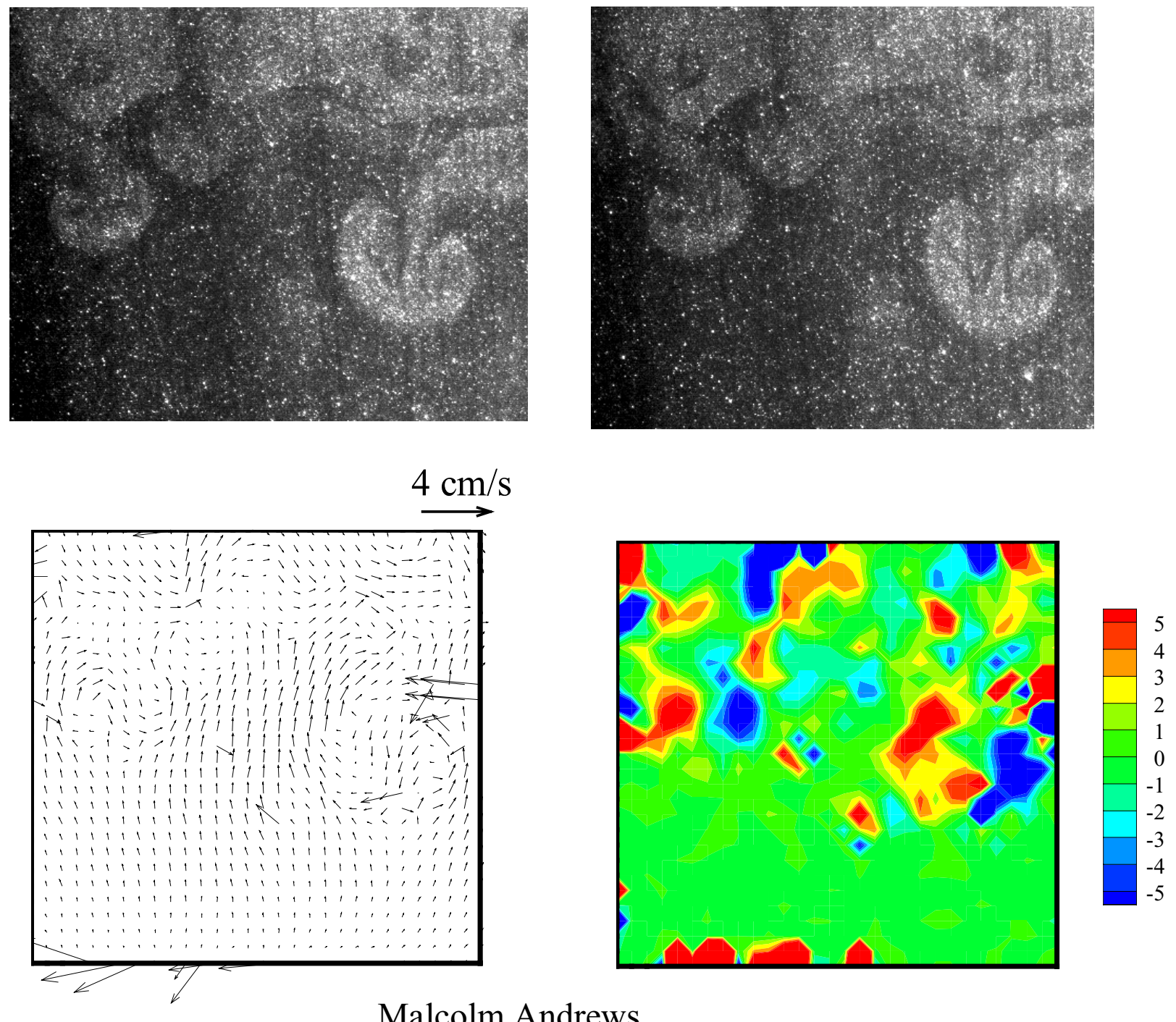


\section{Density fluctuations}

$\mathrm{ATM}$

$\div$

$B_{0}$ from Thermocouple

- $B_{2}$ from PIV-S

$\triangle \quad B_{2}$ from Thermocouple
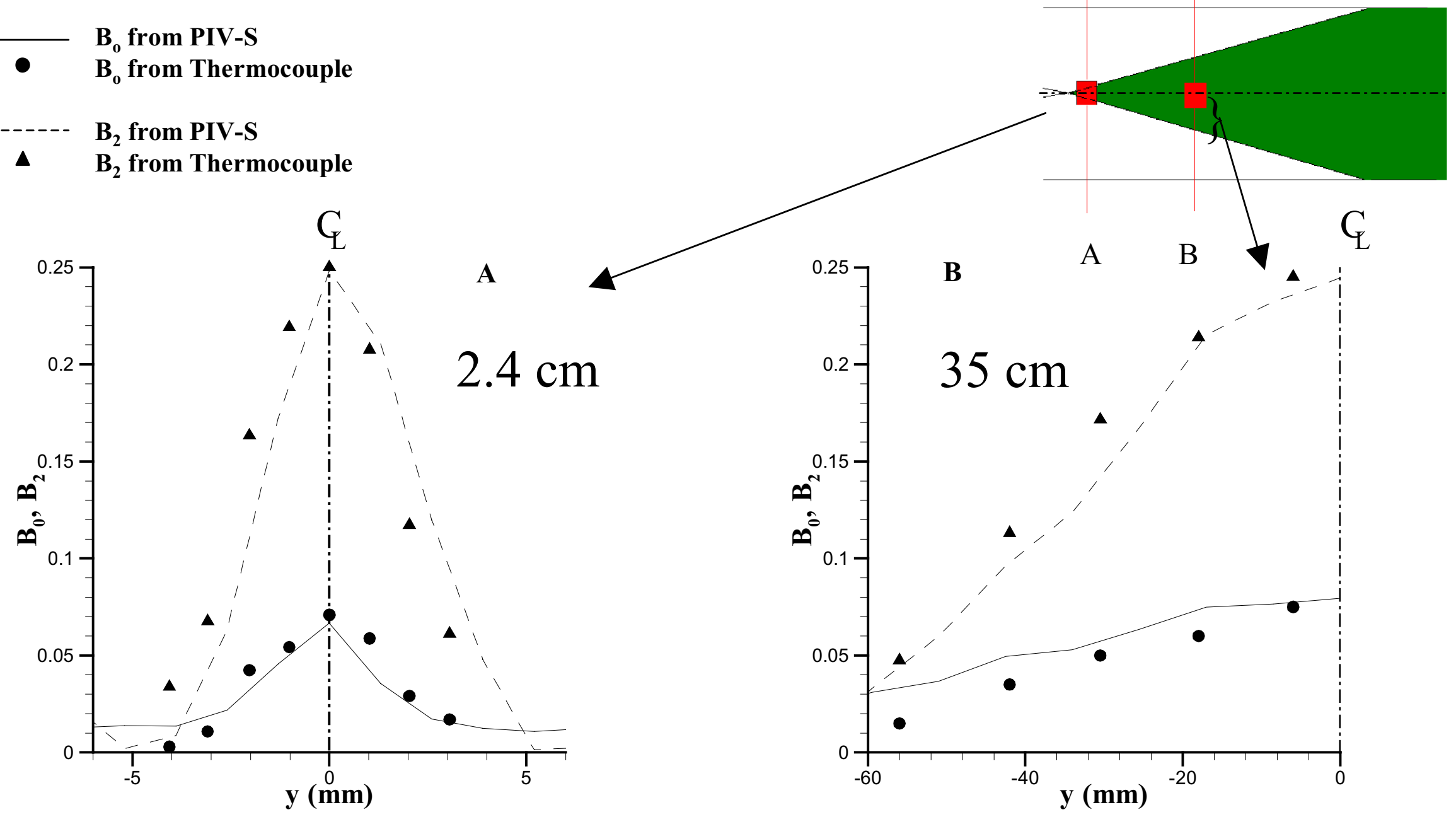

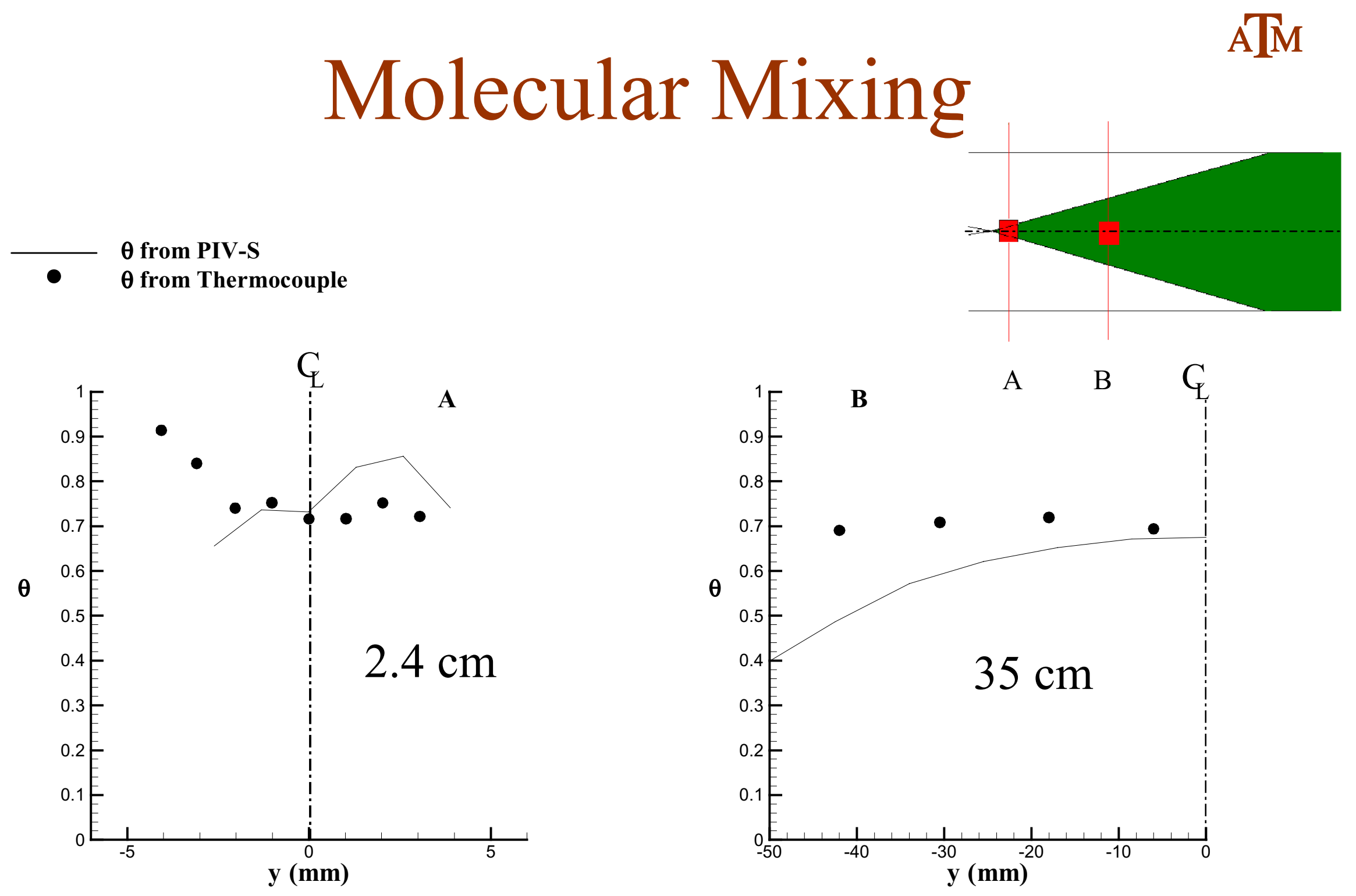

Malcolm Andrews 


\section{Density/Velocity Correlations ATM}
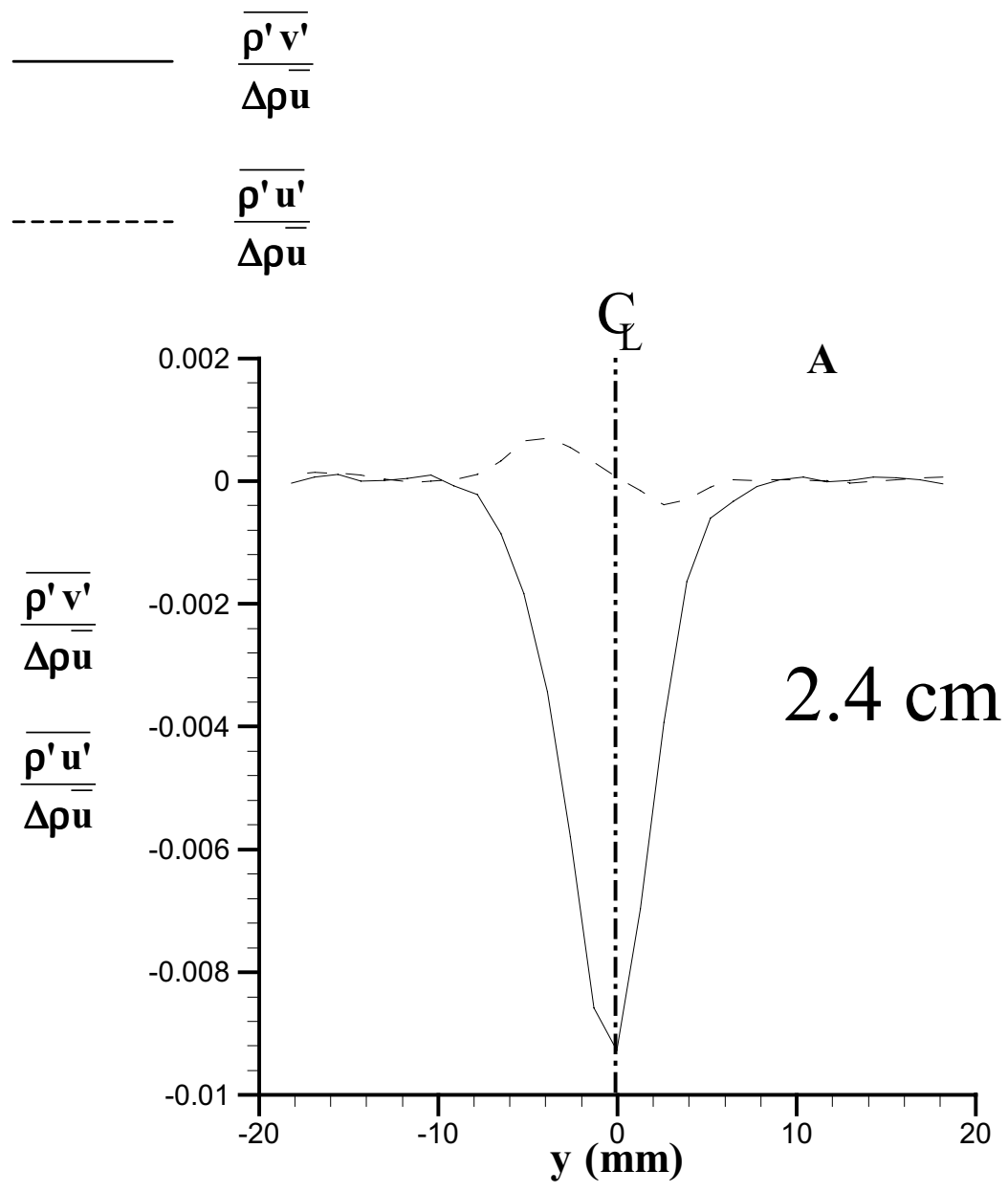

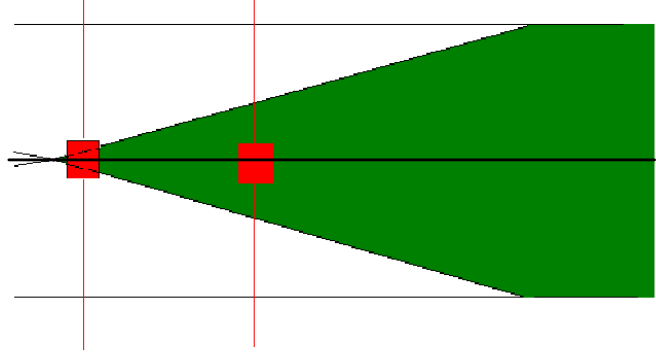

A B

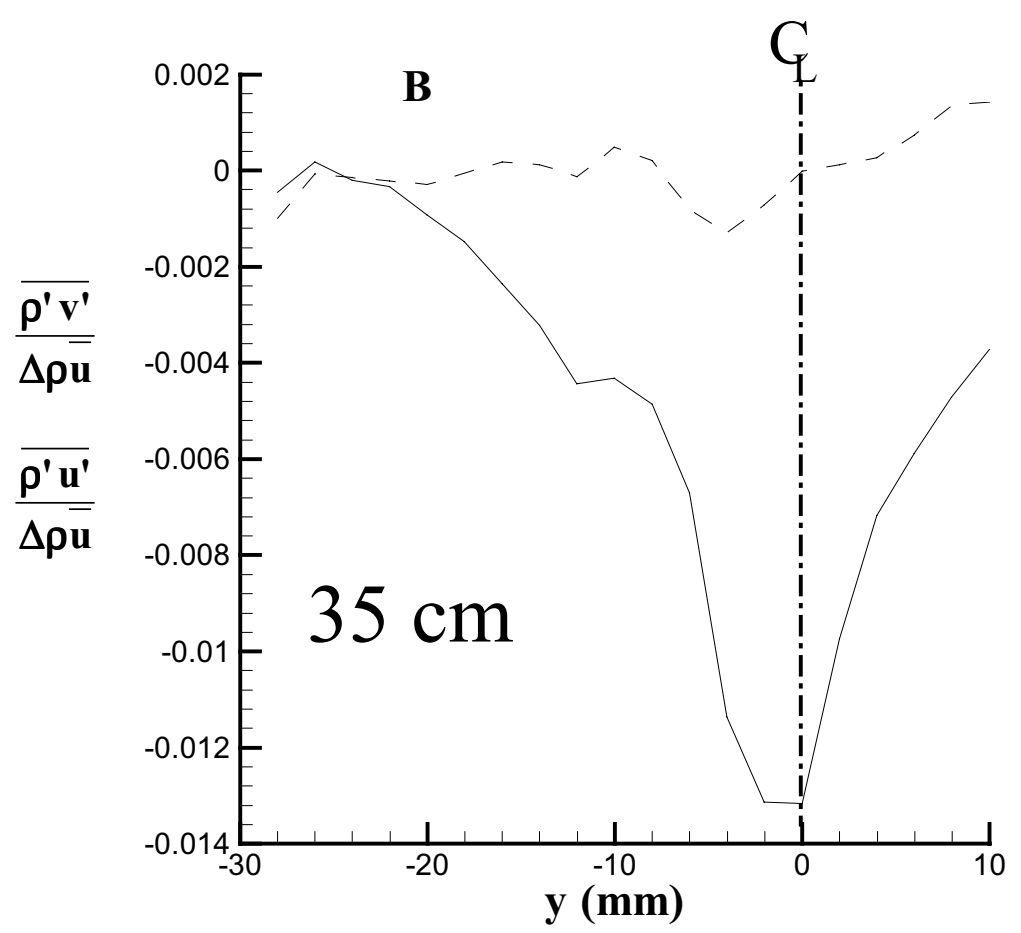

Malcolm Andrews 


\section{Scatter plots of velocity \\ ATM}

\section{fluctuations}

Taken at centerline
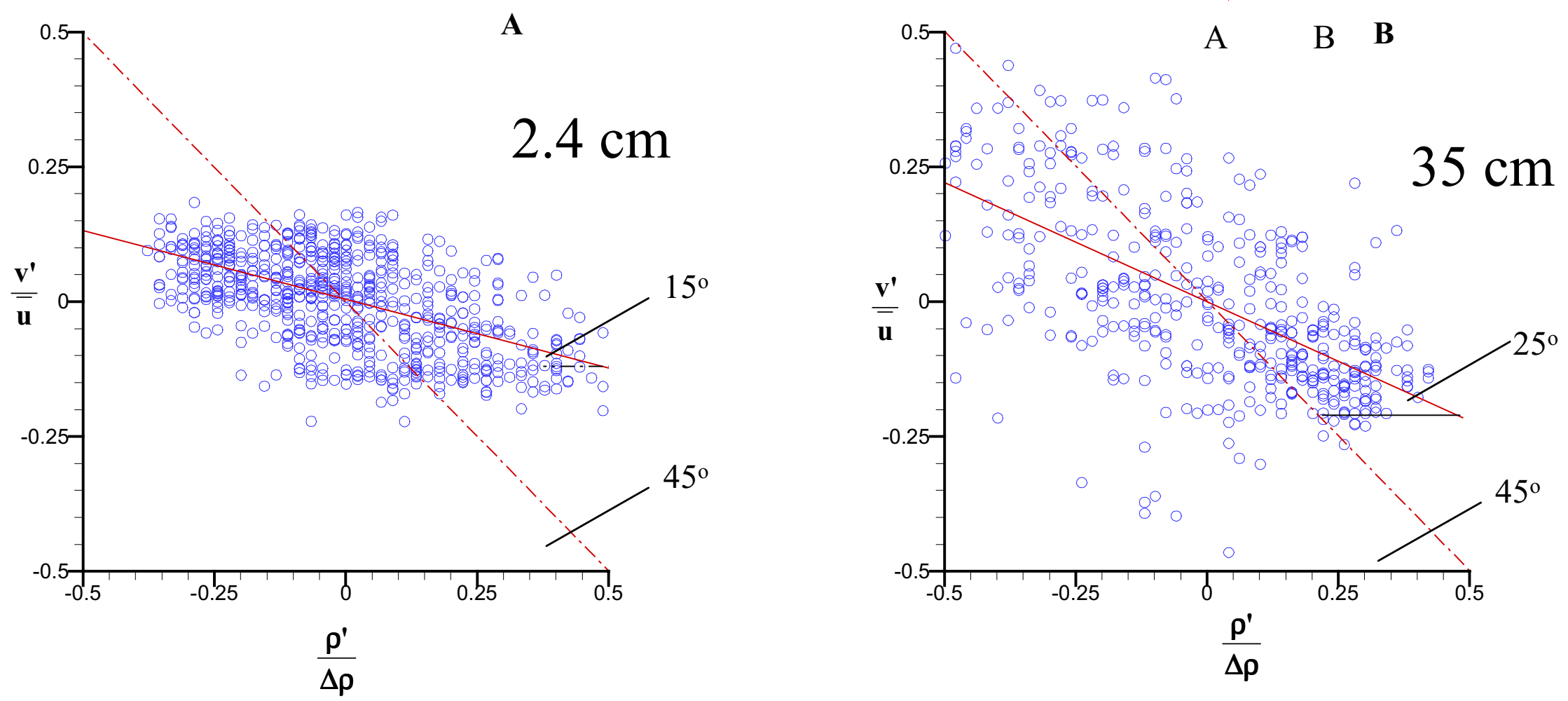


\section{Velocity Fluctuations $(35 \mathrm{~cm})$}

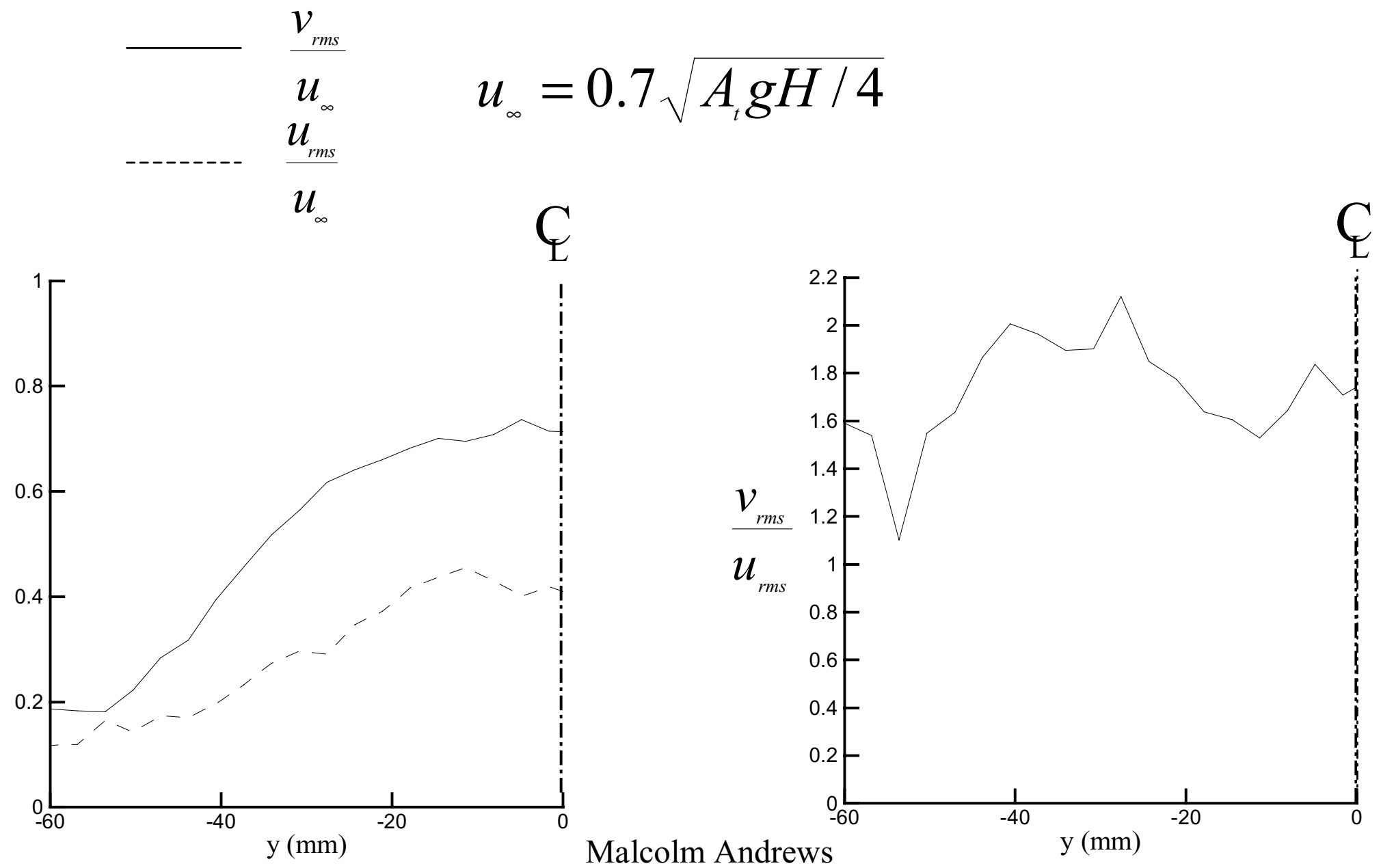




\section{Anisotropy Tensor}

$$
\begin{aligned}
& b_{i j}=\frac{<u_{i}^{\prime} u_{j}^{\prime}>}{<u_{k}^{\prime} u_{k}^{\prime}>}-\frac{1}{3} \delta_{i j} \\
& <u_{i}^{\prime} u_{j}^{\prime}{ }_{j}>=0 \quad \text { if } \mathrm{i} \neq \mathrm{j}
\end{aligned}
$$

where

$$
<u_{k}^{\prime} u_{k}^{\prime}>=\overline{u^{\prime 2}}+\overline{v^{\prime 2}}+\overline{w^{\prime 2}}
$$

$$
\approx 2 \overline{u^{\prime 2}}+\overline{v^{\prime 2}}
$$

Isotropy $\Rightarrow b_{i i}=0$ 


\section{Anisotropy Tensor}

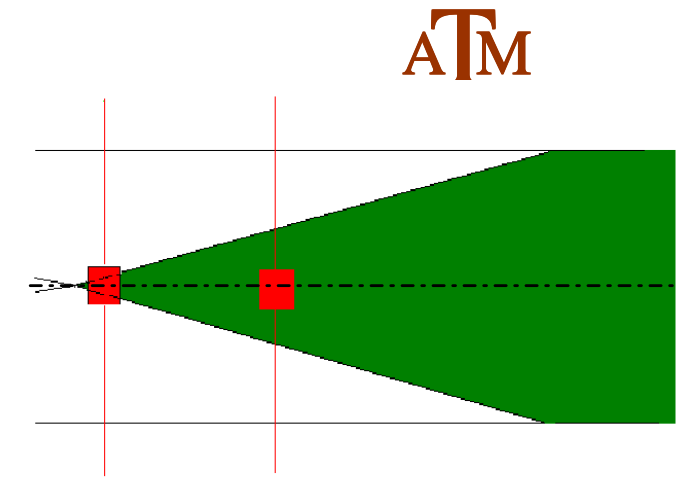

A B
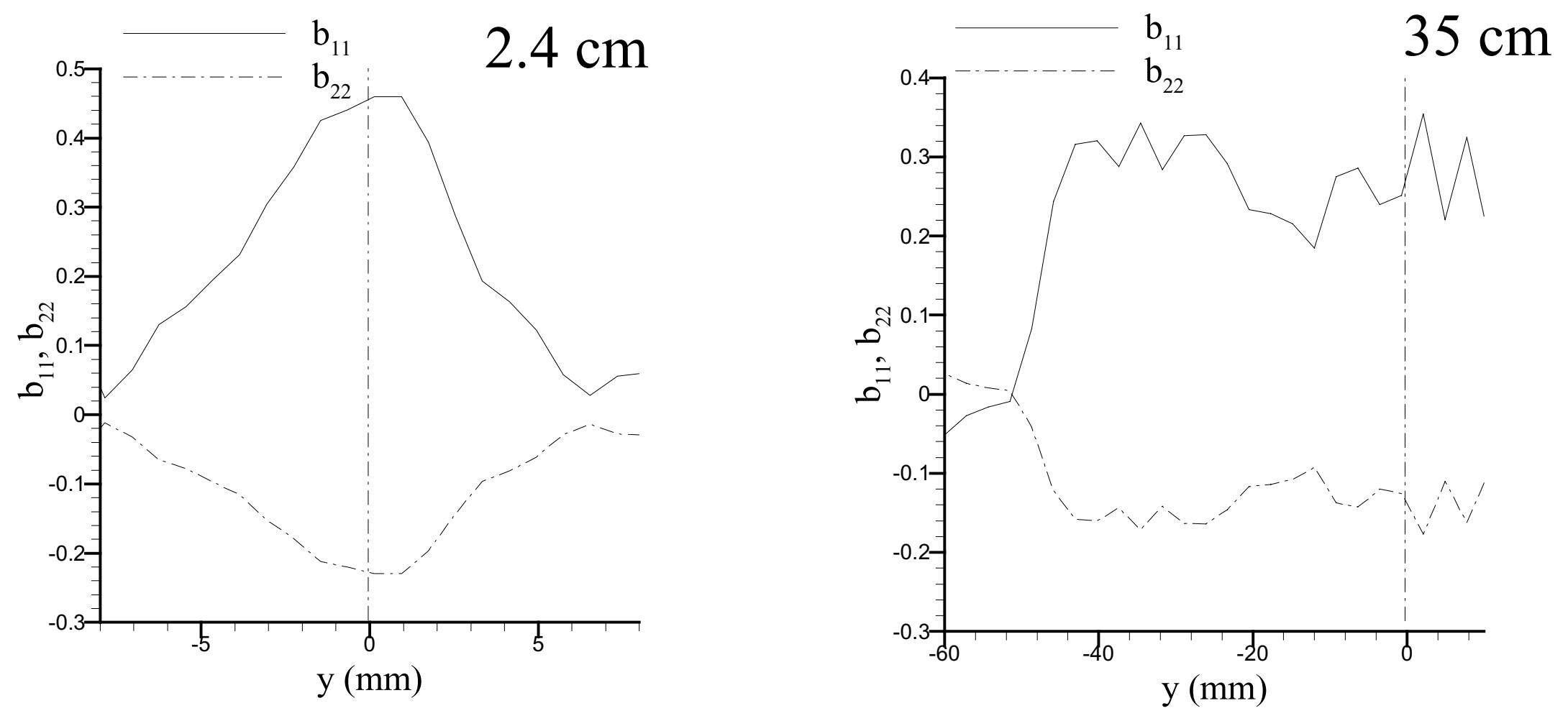

Malcolm Andrews 


\section{Future work}

-Buoyancy and shear

-Non-equilibrium strained configurations:

Contractions

Obstacles

-Modeling

-Large density differences 


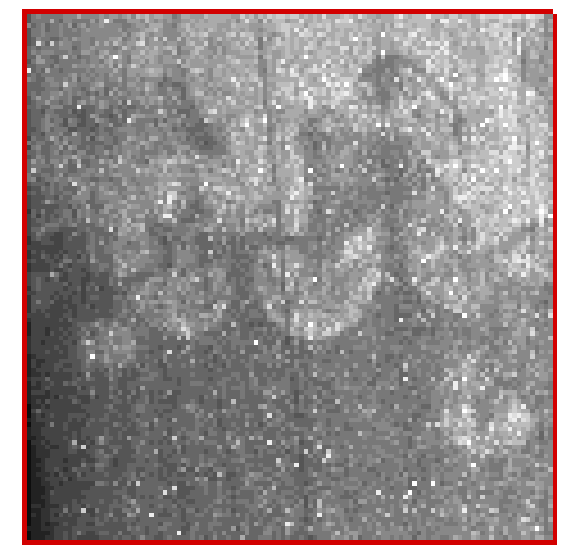

Photographs

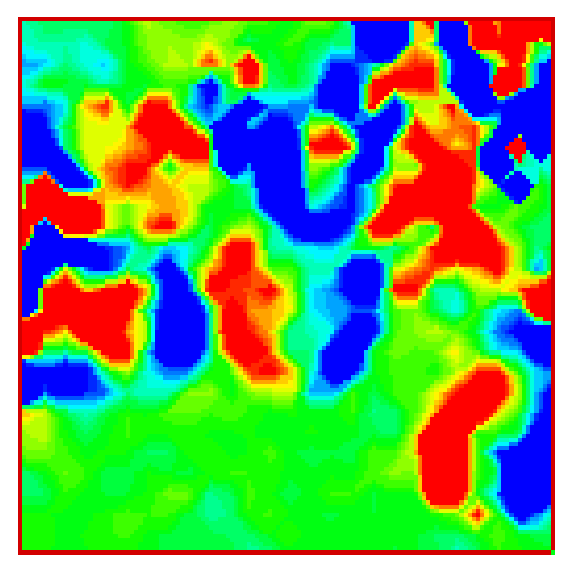

Vorticity 


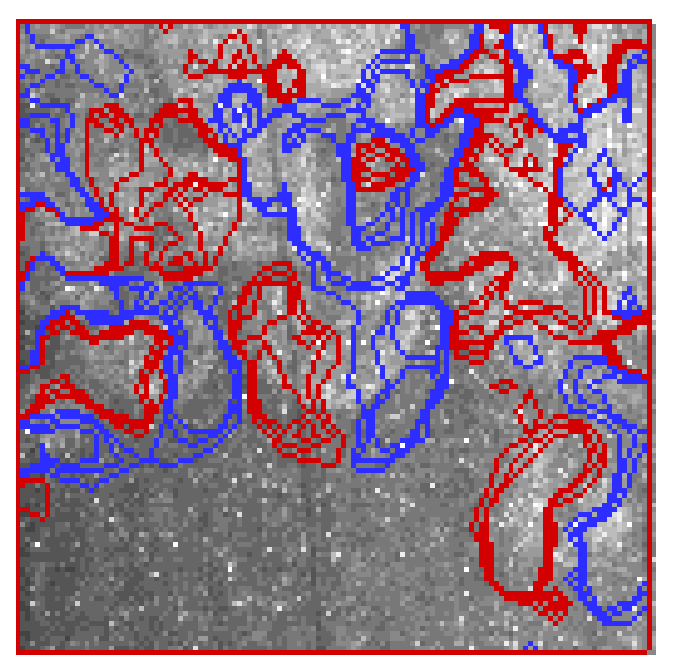

\section{Photographs overlaid with vorticity}




\section{Experimental study of the interaction of a strong shock with a spherical density inhomogeneity}

H. F. Robey ${ }^{1}$, T. S. Perry ${ }^{1}$, R. I. Klein ${ }^{1,2}$, J. A. Greenough ${ }^{1}$, H. Louis ${ }^{1}$, P. Davis ${ }^{1}$, J. O. Kane ${ }^{1}$, T. R. Boehly ${ }^{3}$

${ }^{1}$ Lawrence Livermore National Laboratory, Livermore, California 94550

${ }^{2}$ U. C. Berkeley, Department of Astronomy

${ }^{3}$ Laboratory for Laser Energetics, University of Rochester, Rochester, NY

Presented at the $8^{\text {th }}$ Meeting of the

International Workshop on the Physics of Compressible Turbulent Mixing

Pasadena, CA

December 9-14, 2001

This work was performed under the auspices of the U. S. Department of Energy by the University of California, Lawrence Livermore National Laboratory under Contract No. W-7405-Eng-48. 


\section{Summary}

- Experiments have been conducted on the Omega Laser to study the interaction of a strong shock $(M>10)$ with a spatially localized density inhomogeneity (Cu sphere)

- The interaction is diagnosed with x-ray radiography simultaneously from two orthogonal directions

- The evolution of the shocked sphere is observed to proceed as an initial roll-up into a double vortex ring structure followed by the appearance of an azimuthal instability which ultimately results in the three-dimensional breakup of the sphere.

- Numerical simulations are performed in both two and threedimensions, and results are in good agreement with experiment. 


\section{Outline}

- Background / motivation

- Omega Experimental Results

- Numerical simulations

- Conclusions 


\section{These experiments recreate in a controlled setting the interaction of a strong shock with a dense molecular cloud}

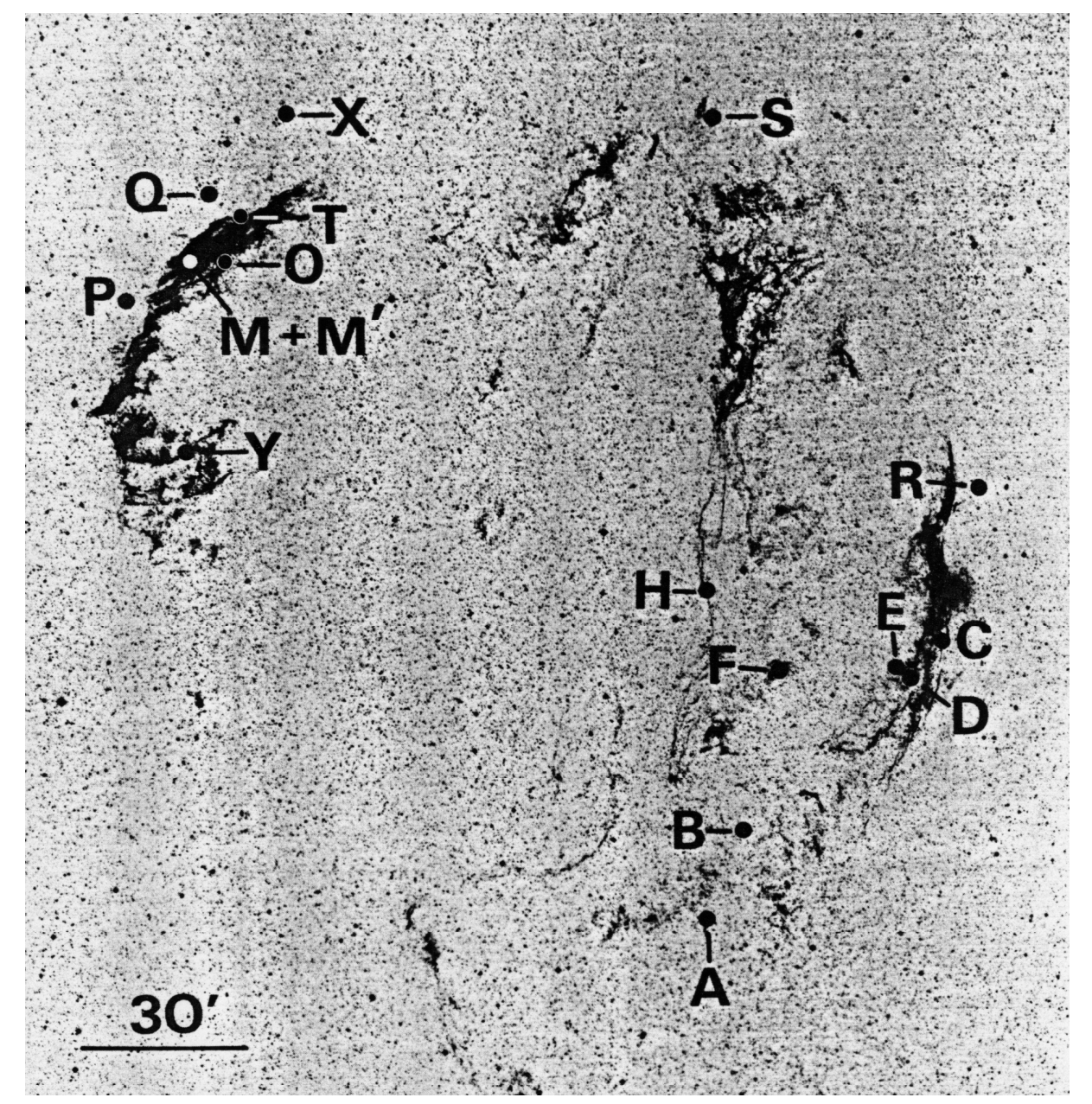

From Fesen el al., Ap.J. 262, 171 (1982):

"The Cygnus Loop is the classic example of a moderately old supernova remnant (SNR). its structure and physical properties are the result of a supernova-generated shock wave interacting with the surrounding interstellar medium."

"Comparisons with published shock models indicate significant differences between the models and observations ..." 
The interaction of a shock with a dense spherical inhomogeneity has previously been studied only at low mach number
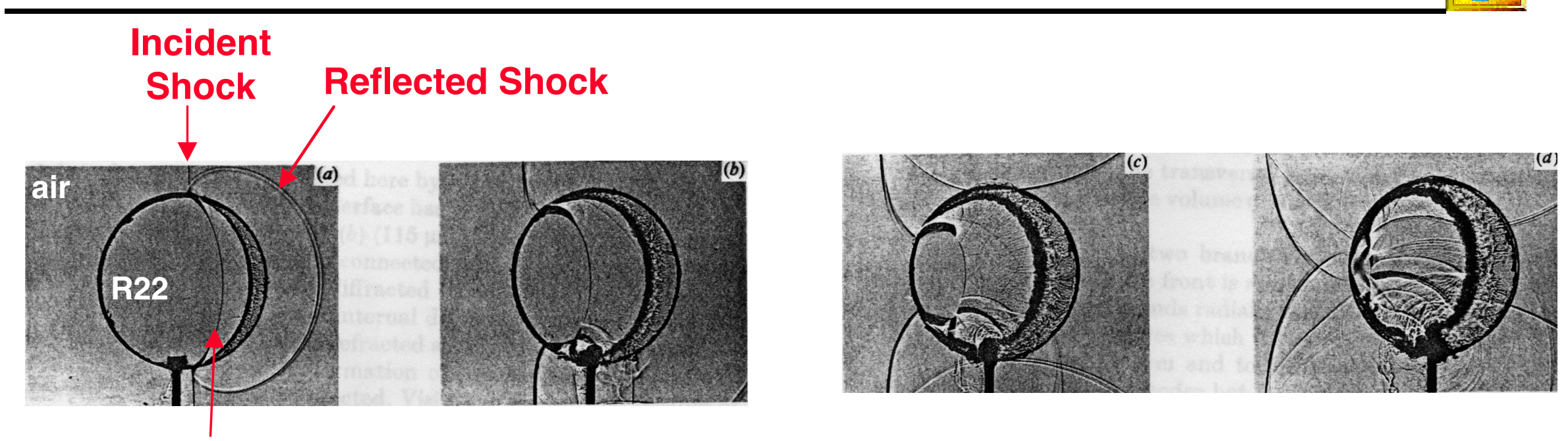

Refracted Shock
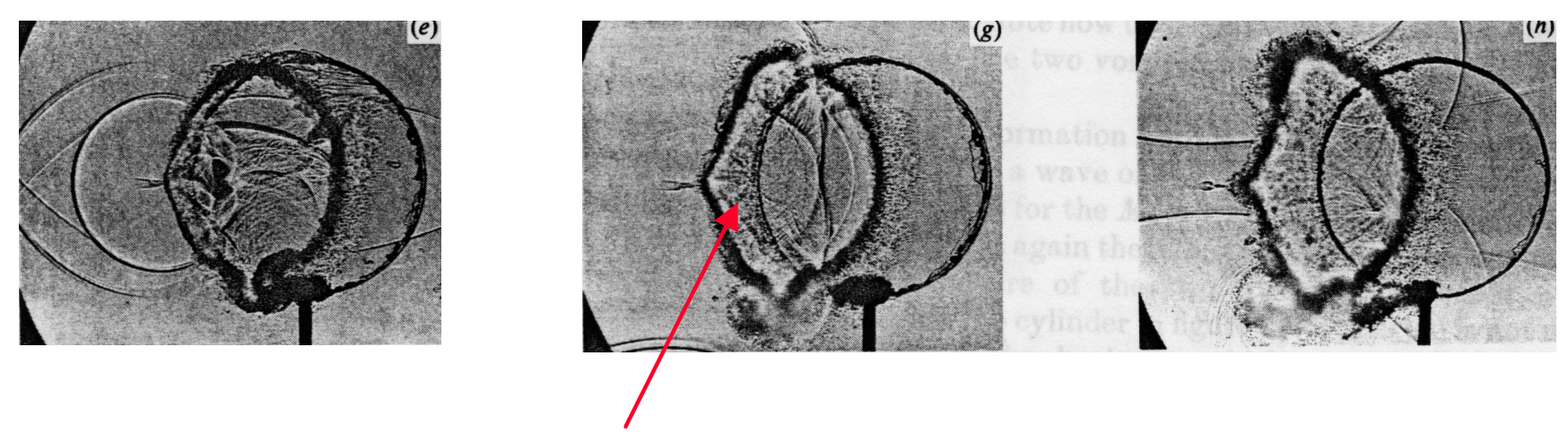

Shocked R22

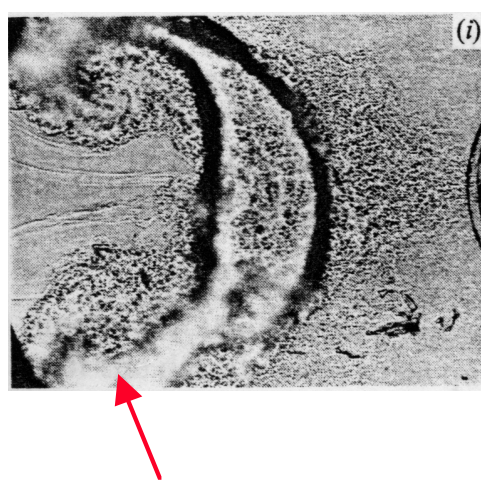

Vortex ring

From $M=1.2$ shock tube experiments of Haas \& Sturtevant, JFM 181, 41 (1987) 
Once formed, a vortex ring is subject to a 3D azimuthal bending mode instability

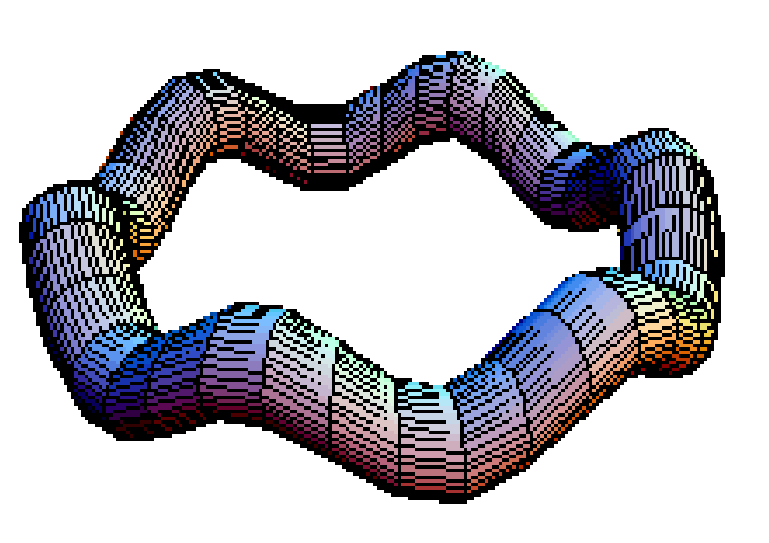

time
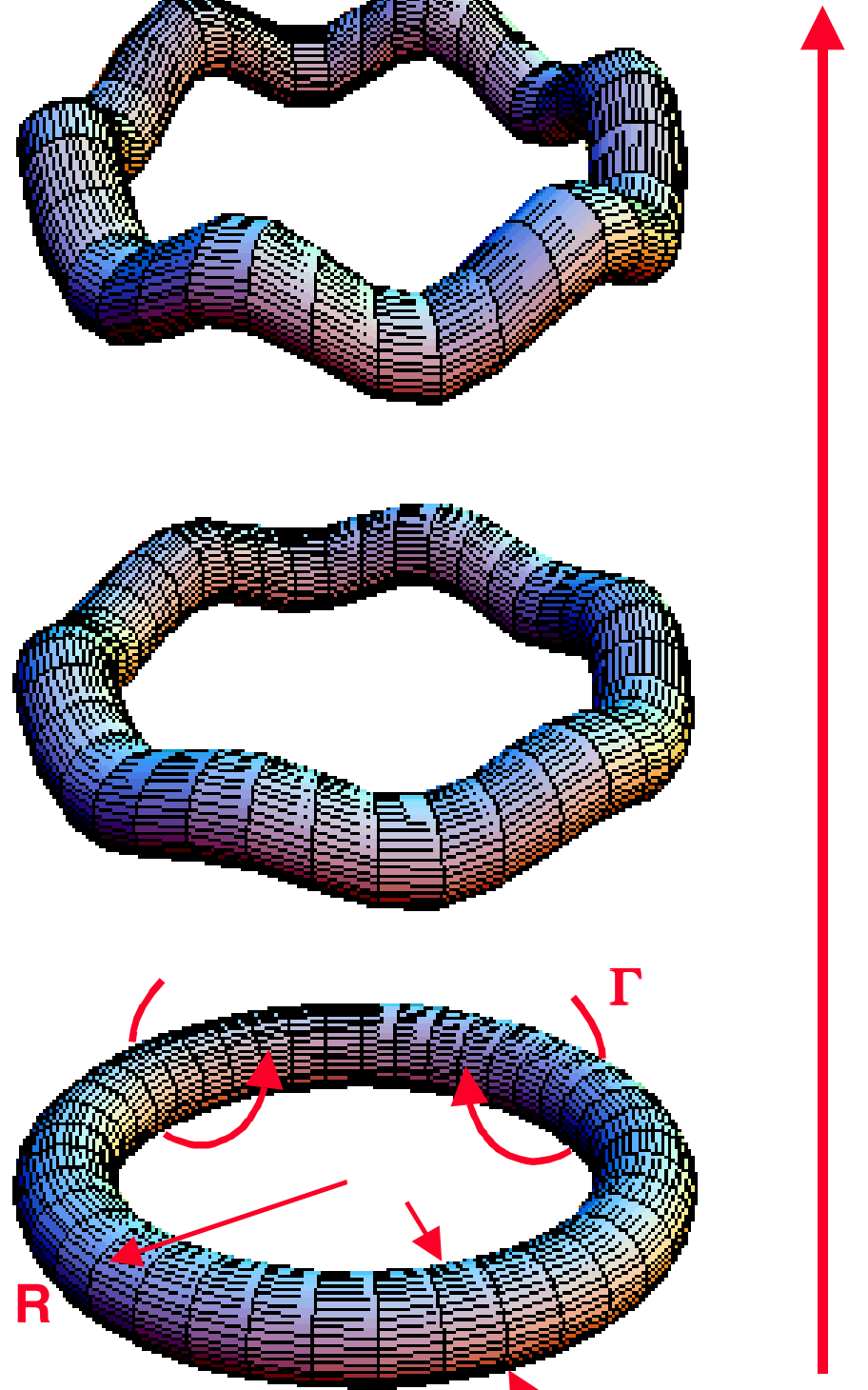

Mode number, $\mathbf{n}$ vs. non-dimensional ring translation velocity, $\widetilde{v}$

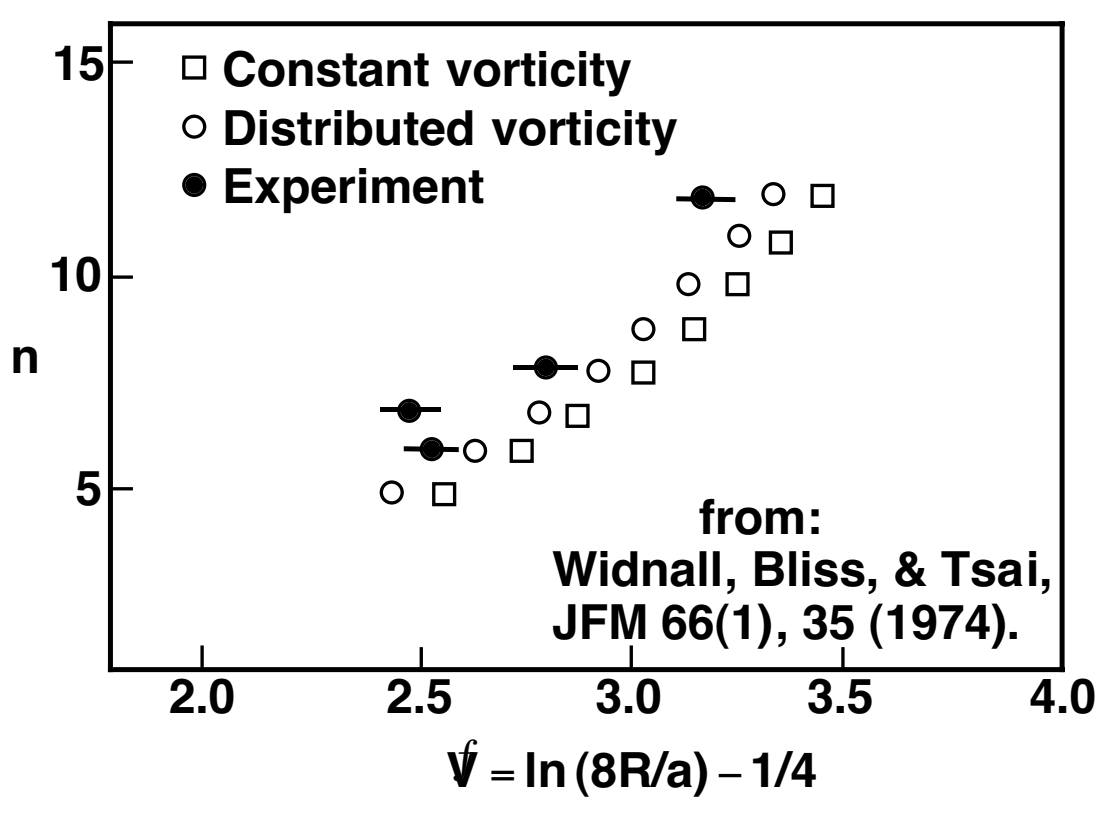

The mode number is a function of the ring radius $R$ and thickness a 


\section{Outline}

- Background / motivation

- Omega Experimental Results

- Numerical simulations

- Conclusions 
The Omega experiments are conducted in a very small Beryllium shock tube

2D slice through target

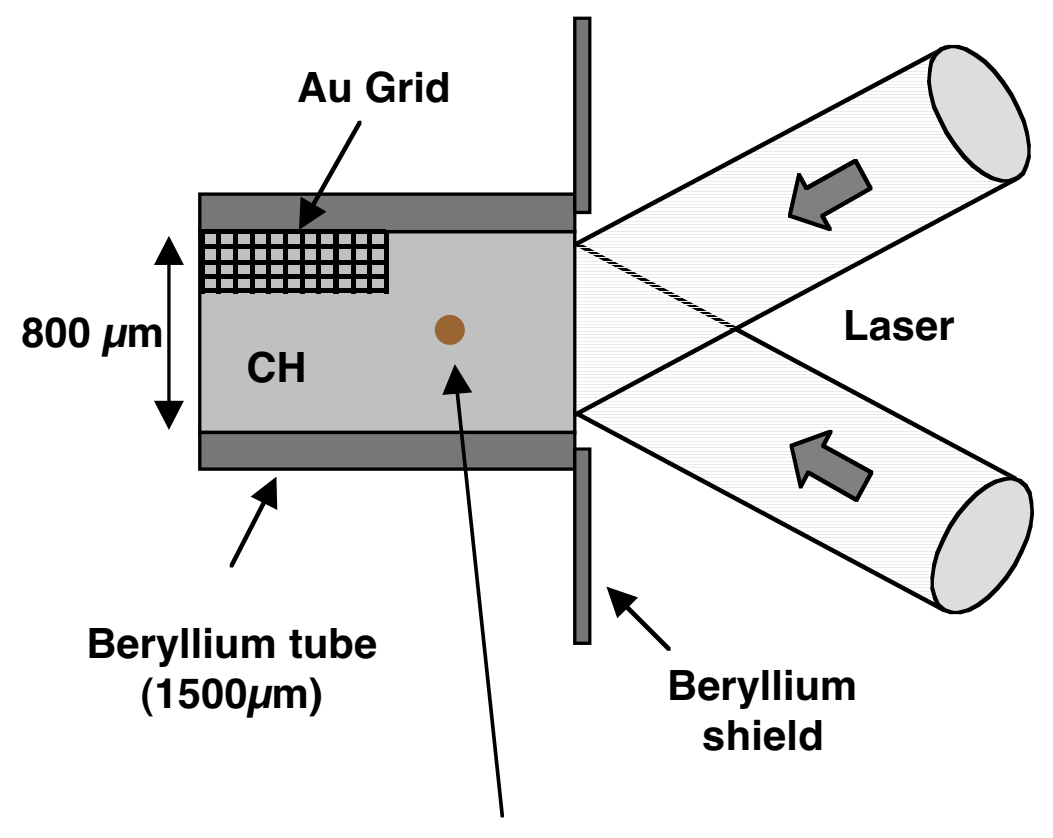

Cu sphere

(120 $\mu \mathrm{m}$ diameter)
3D view of target

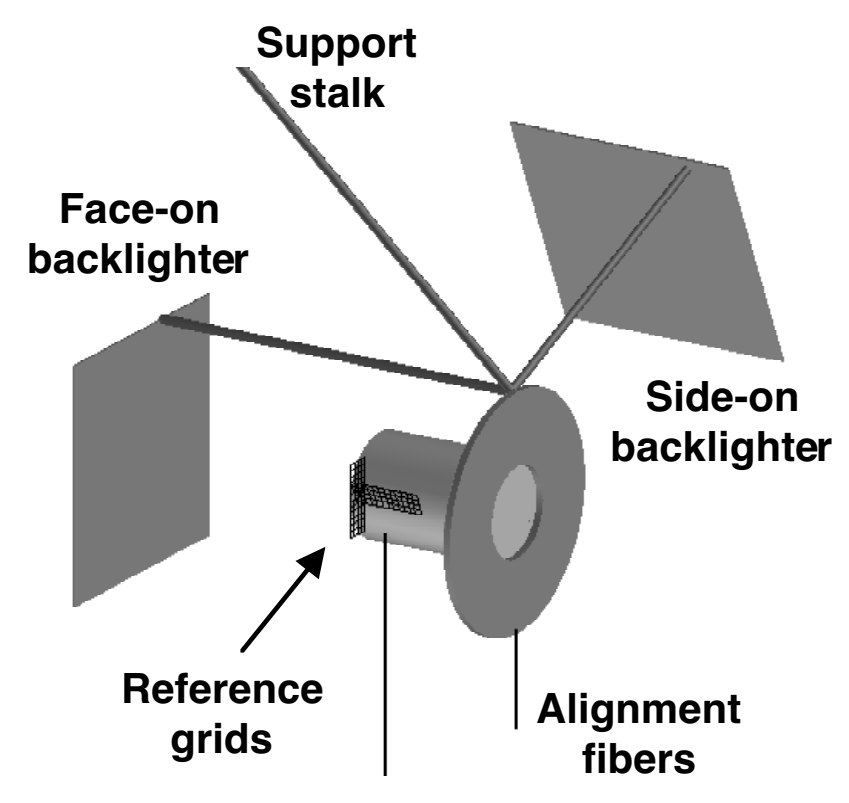


Multiple beams of the Omega laser are used to both drive the strong shock and diagnose the interaction

Target CAD drawing with

Omega beam orientations

Side-on backlighter beams

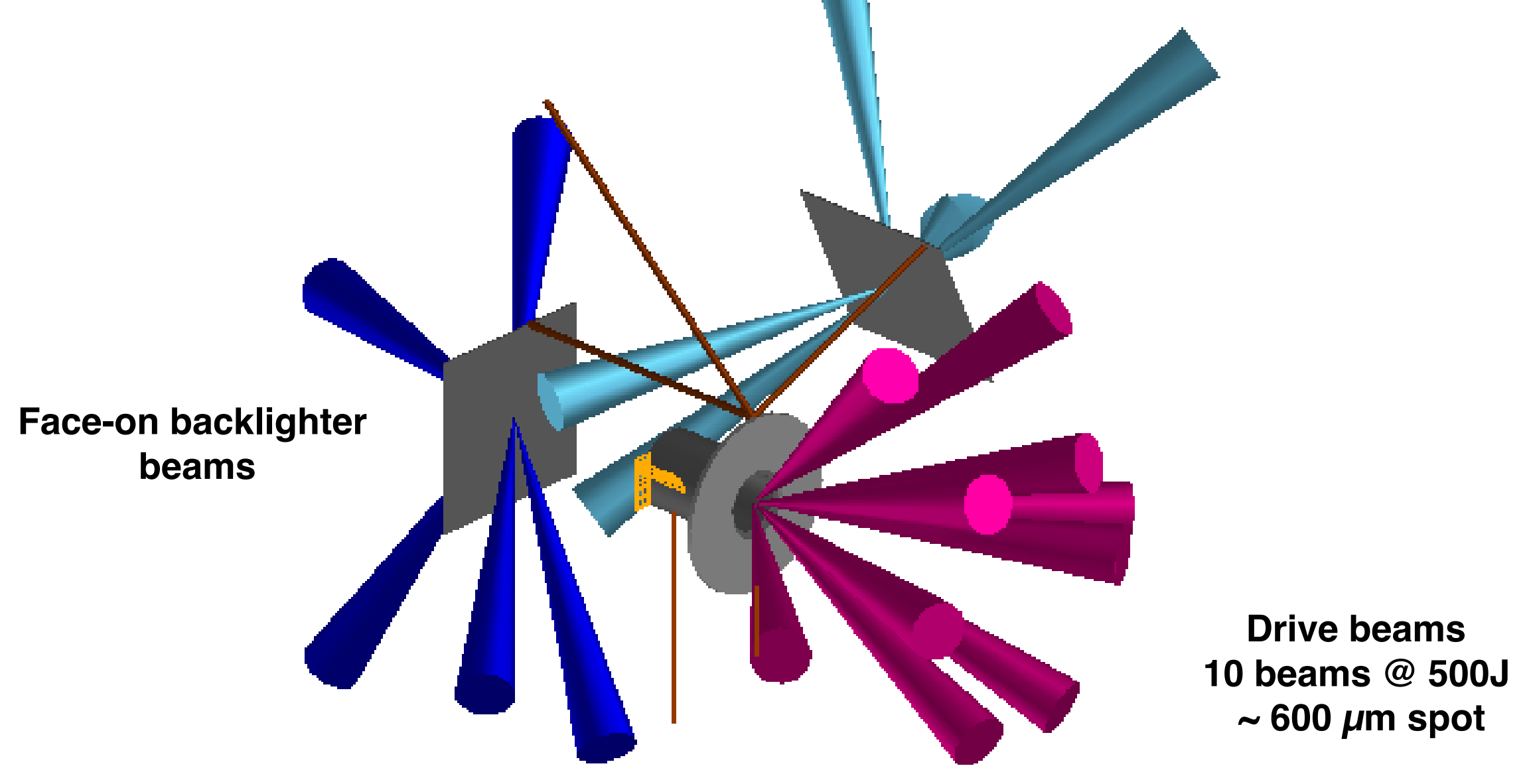


Simultaneous side-on and face-on images of shock / sphere interaction with $120 \mu \mathrm{m}$ diameter Cu sphere
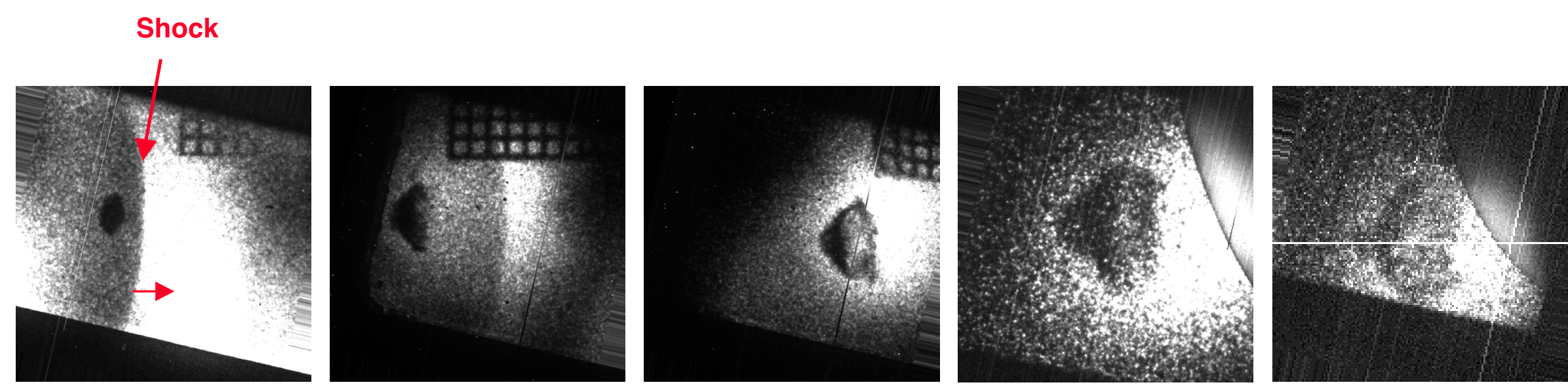

\# 19728

\# 19736

\# 19732

\# 20637

\# 20645
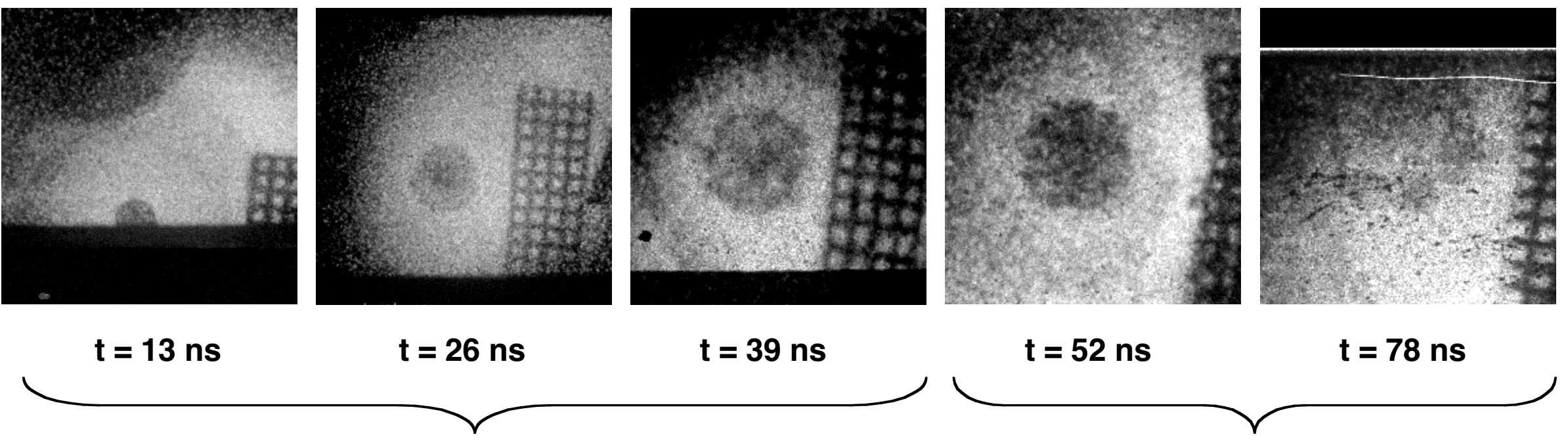

Omega data of April, 2000

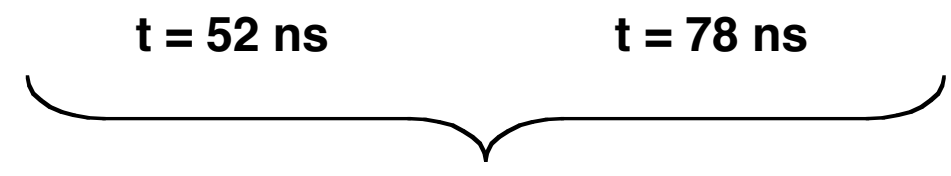

Omega data of Aug 2-3, 2000 
Simultaneous side-on and face-on images of shock / sphere interaction with $240 \mu \mathrm{m}$ diameter Cu sphere
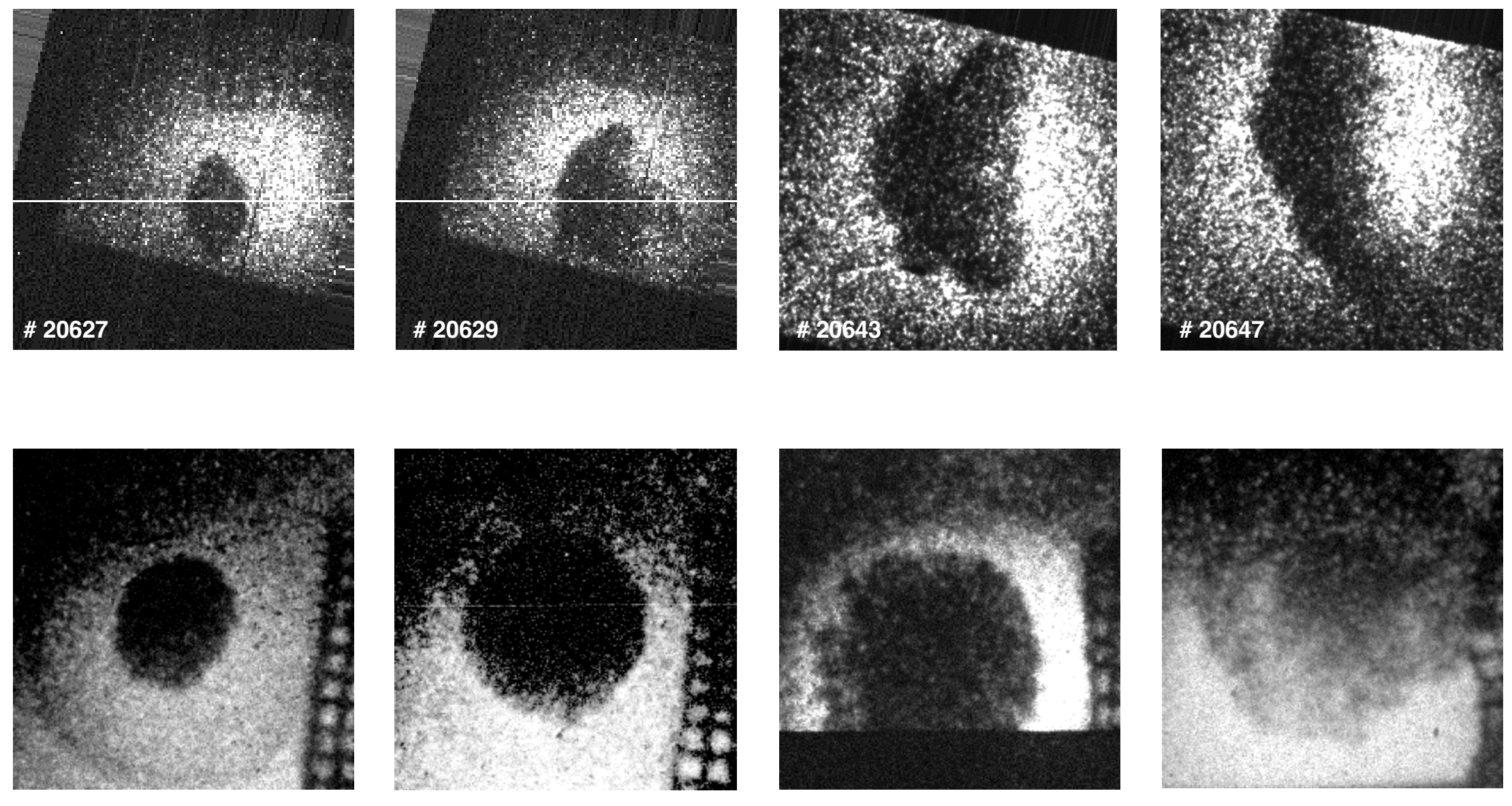

$\mathbf{t}=\mathbf{2 7}$ ns

$\mathbf{t}=\mathbf{5 4} \mathrm{ns}$

$\mathrm{t}=78 \mathrm{~ns}$

$\mathrm{t}=105 \mathrm{~ns}$

Omega data of Aug 2-3, 2000 
Large-scale features appear repeatable from shot-to-shot, but small-scale details differ

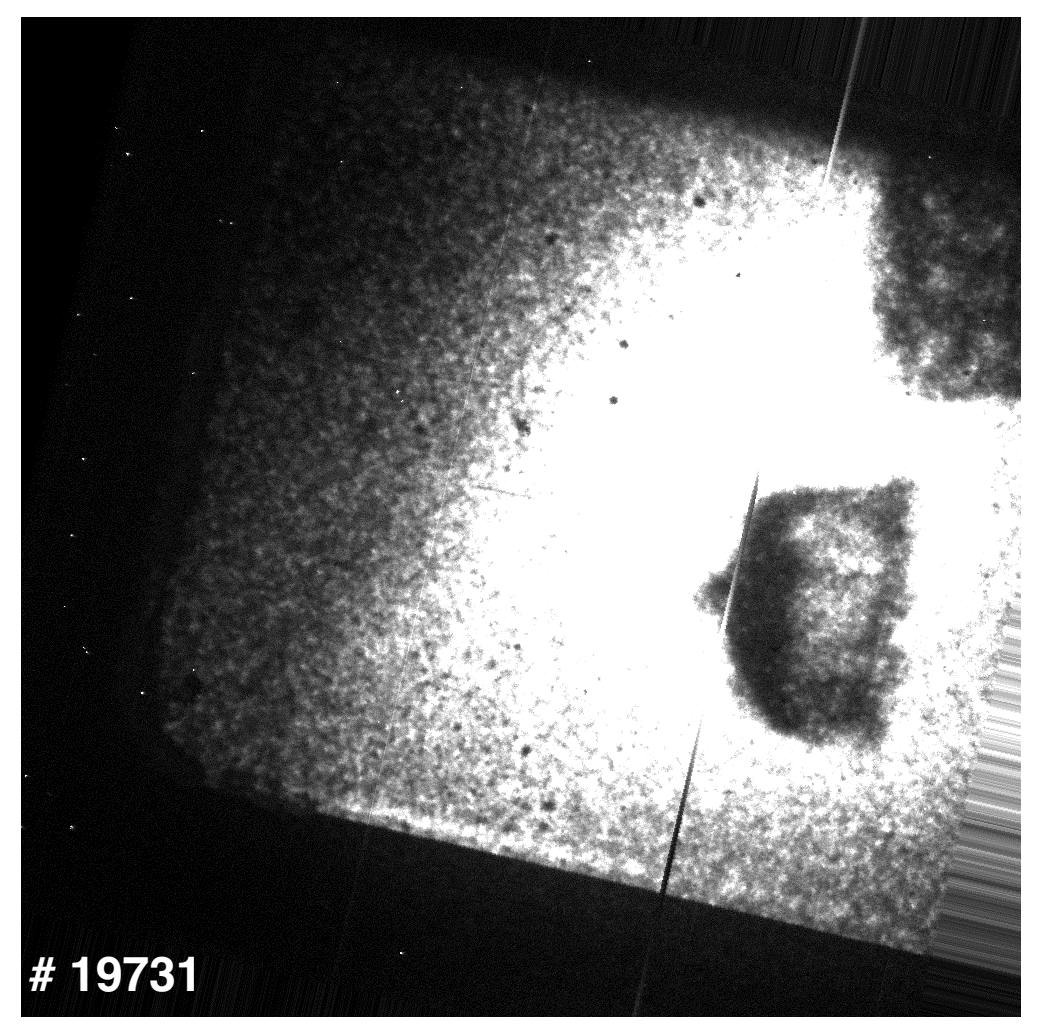

$\mathrm{t}=39 \mathrm{~ns}$

V-backlighter

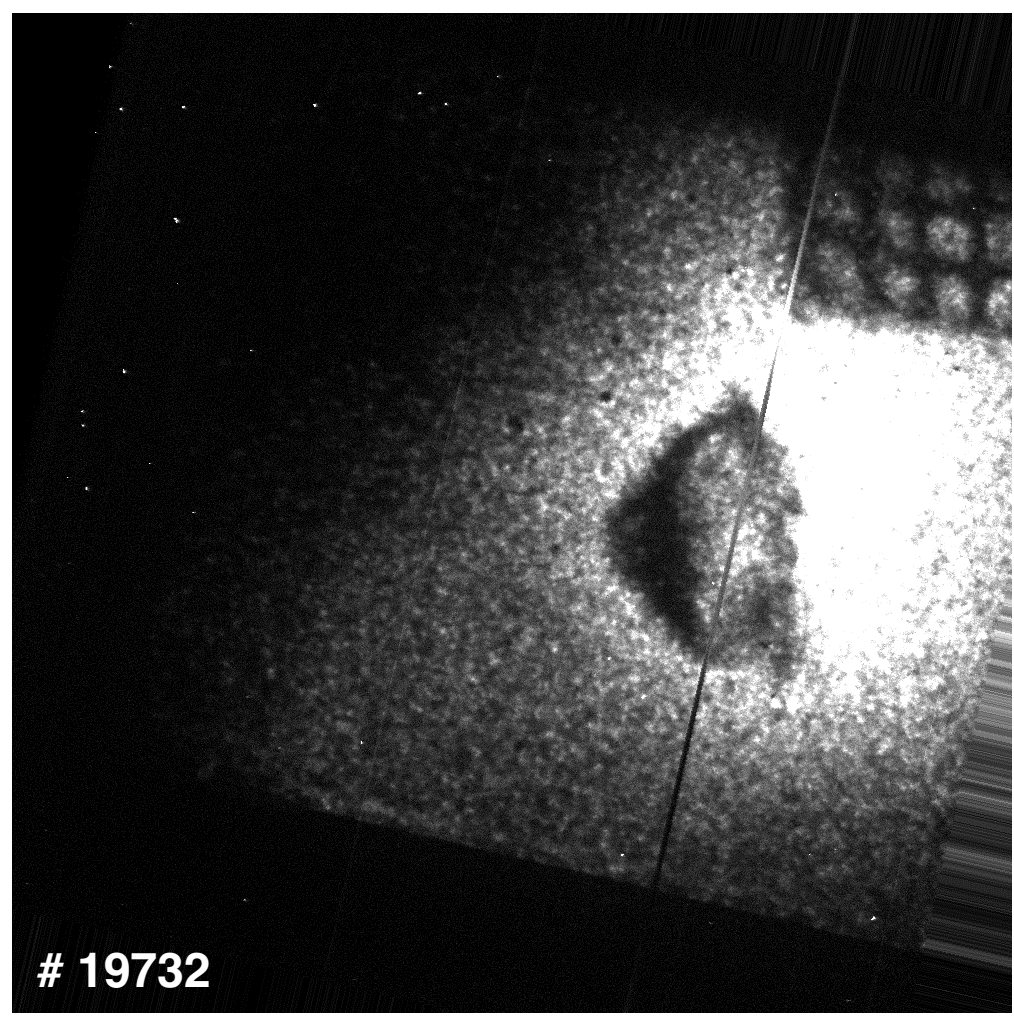

$\mathrm{t}=39 \mathrm{~ns}$

Fe-backlighter 
The two orthogonal diagnostic views help to reveal the 3D morphology of this flow

Illustration of 3D morphology
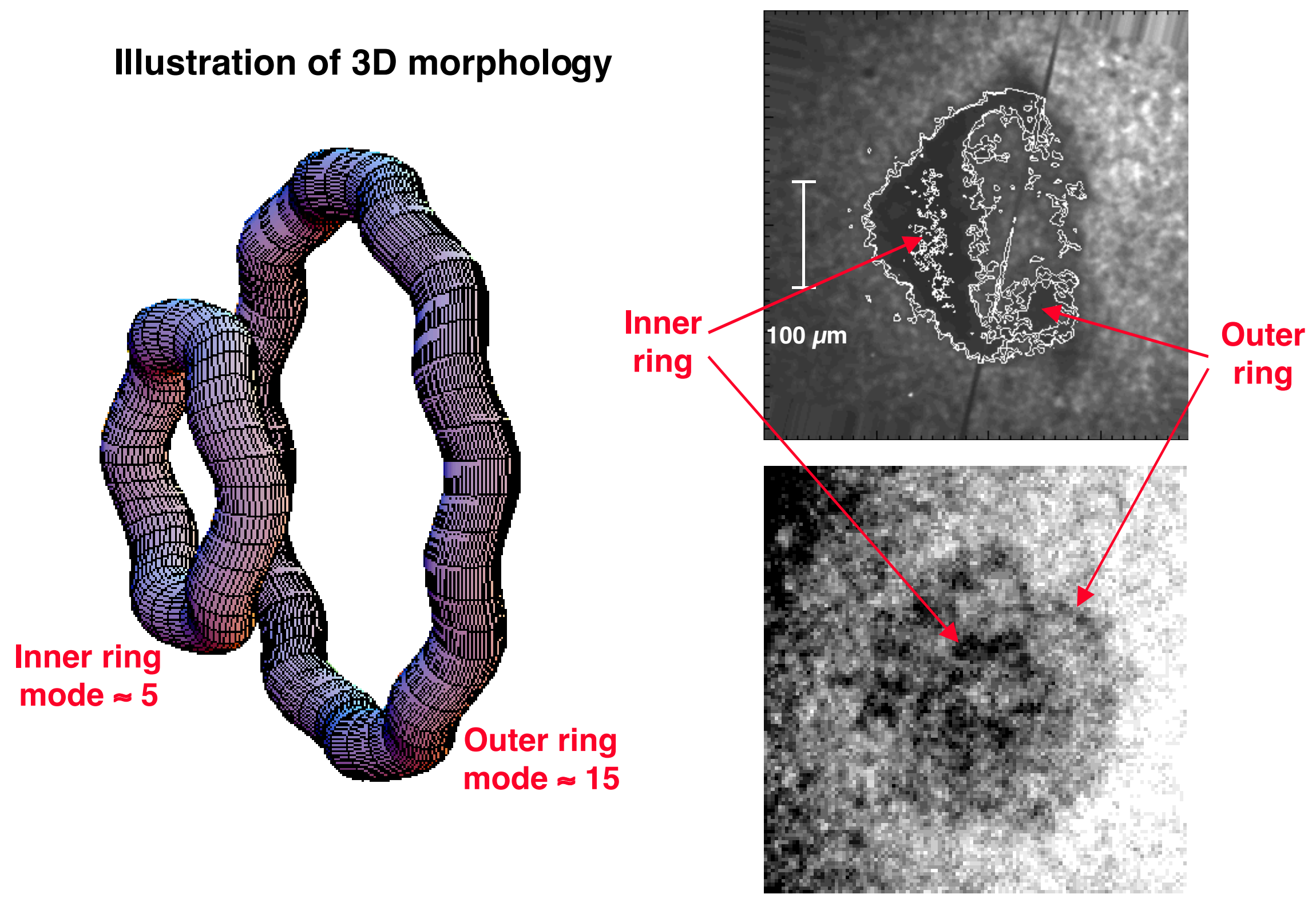
Analysis of Omega shock / sphere data quantifies the three-dimensional instability and breakup of the sphere

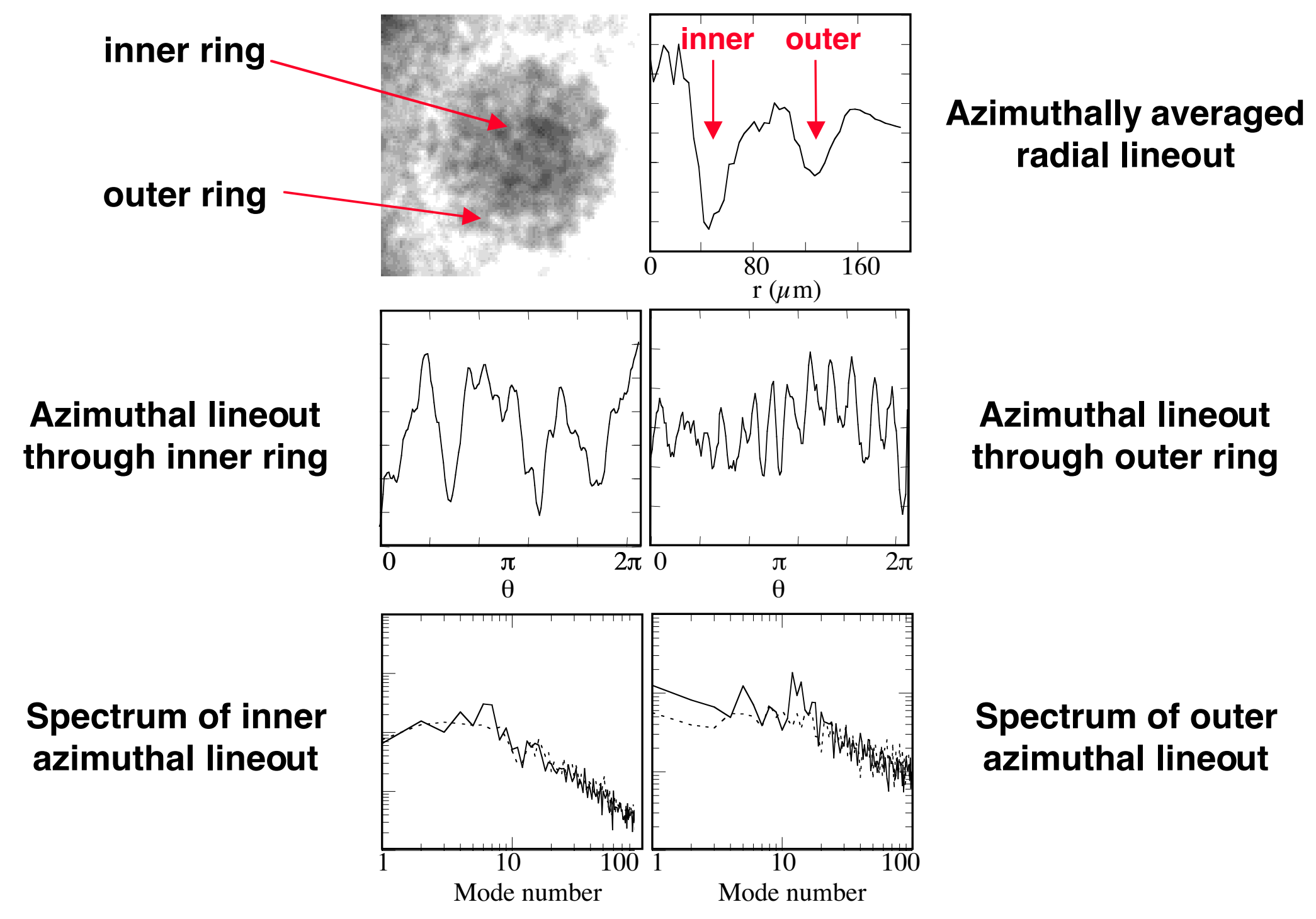

From Robey et al., submitted to PRL (May, 2001) 


\section{Mode number spectra from face-on images of shock / sphere interaction reveal a dominant azimuthal mode}
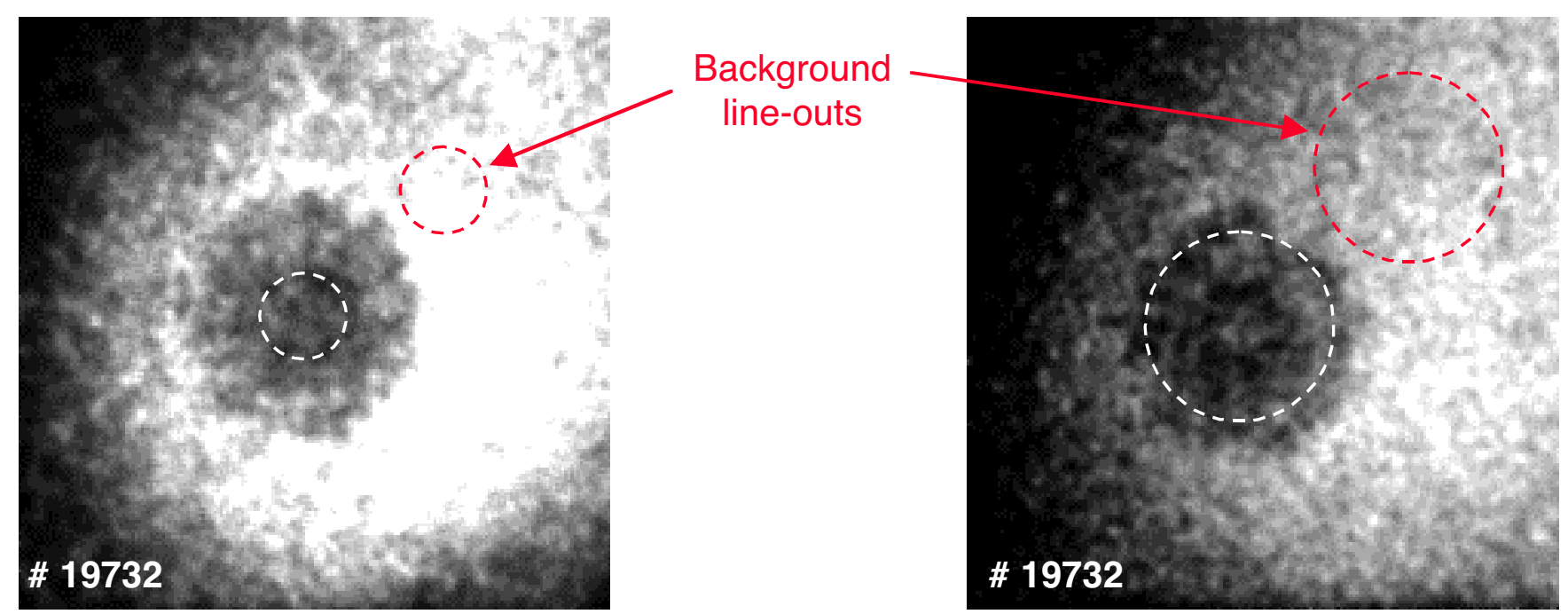

Power spectrum of circular line-out

Power spectrum of circular line-out through central feature $(\mathrm{r}=50 \mu \mathrm{m})$
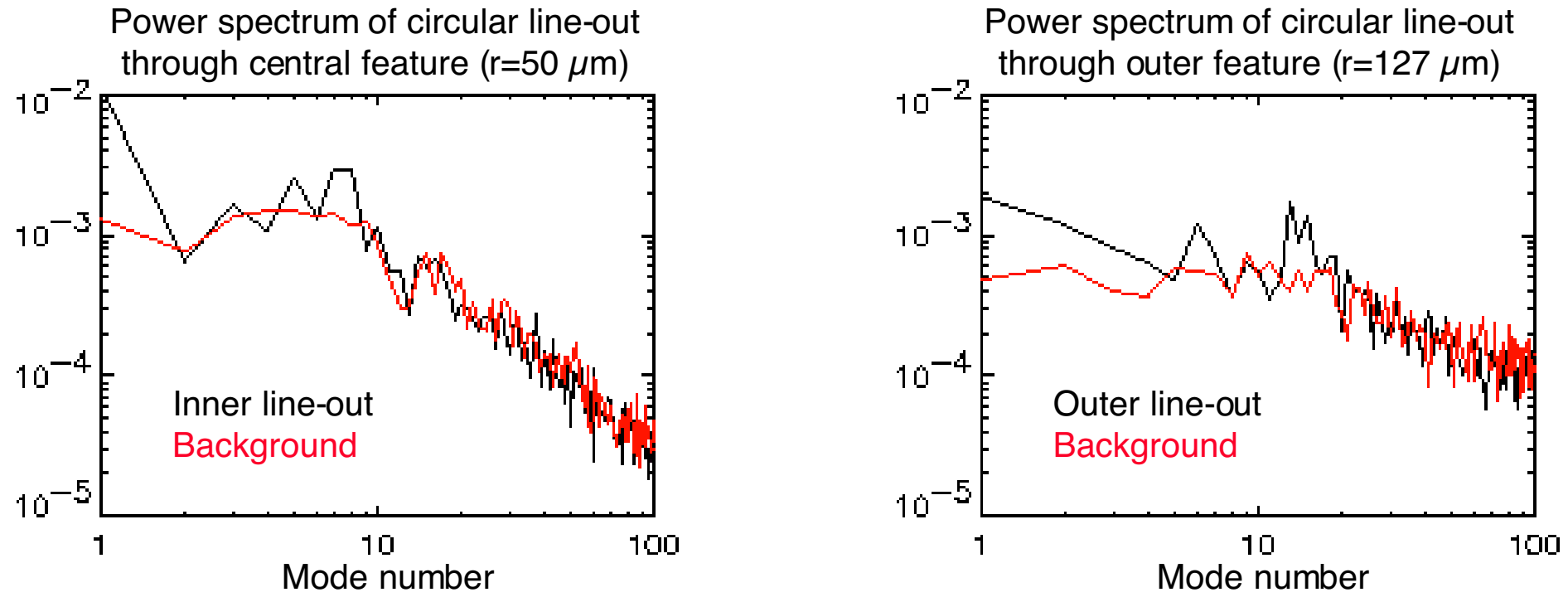


\section{The observed azimuthal mode number agrees well with the prediction from Widnall's theory}

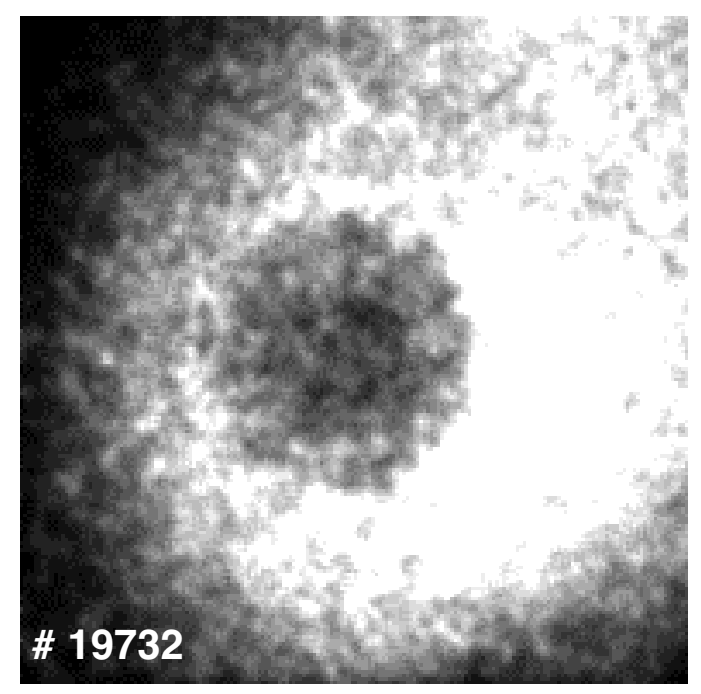

Power spectrum of circular line-out through outer feature $(r=127 \mu \mathrm{m})$

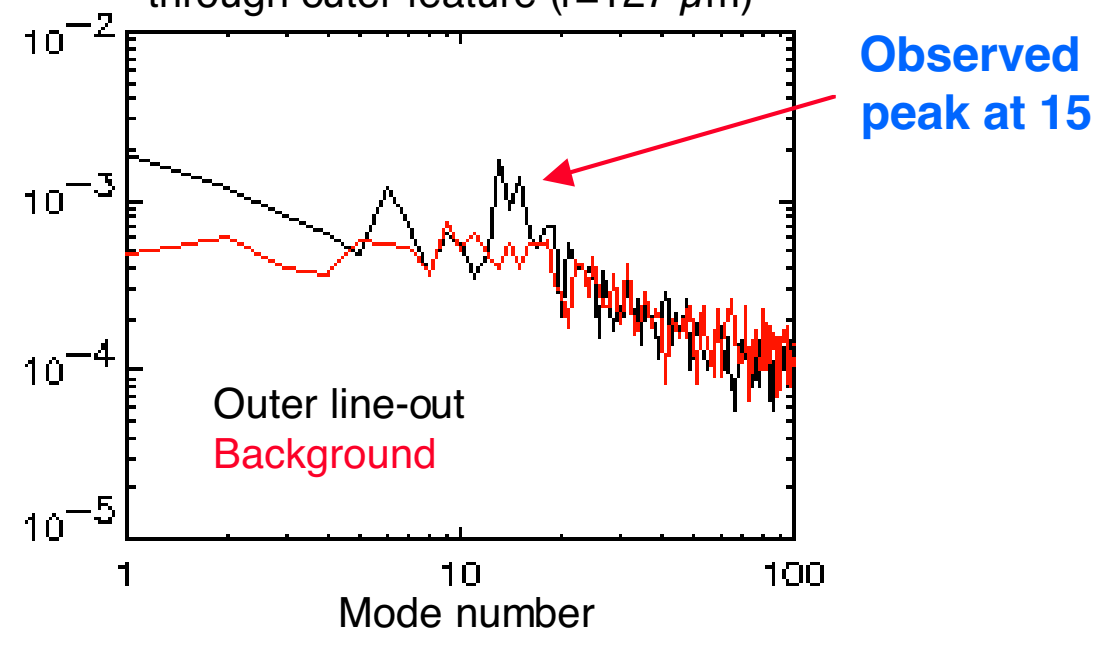

Mode number, $\mathbf{n}$ vs. non-dimensional ring translation velocity, $\widetilde{\mathbf{V}}$

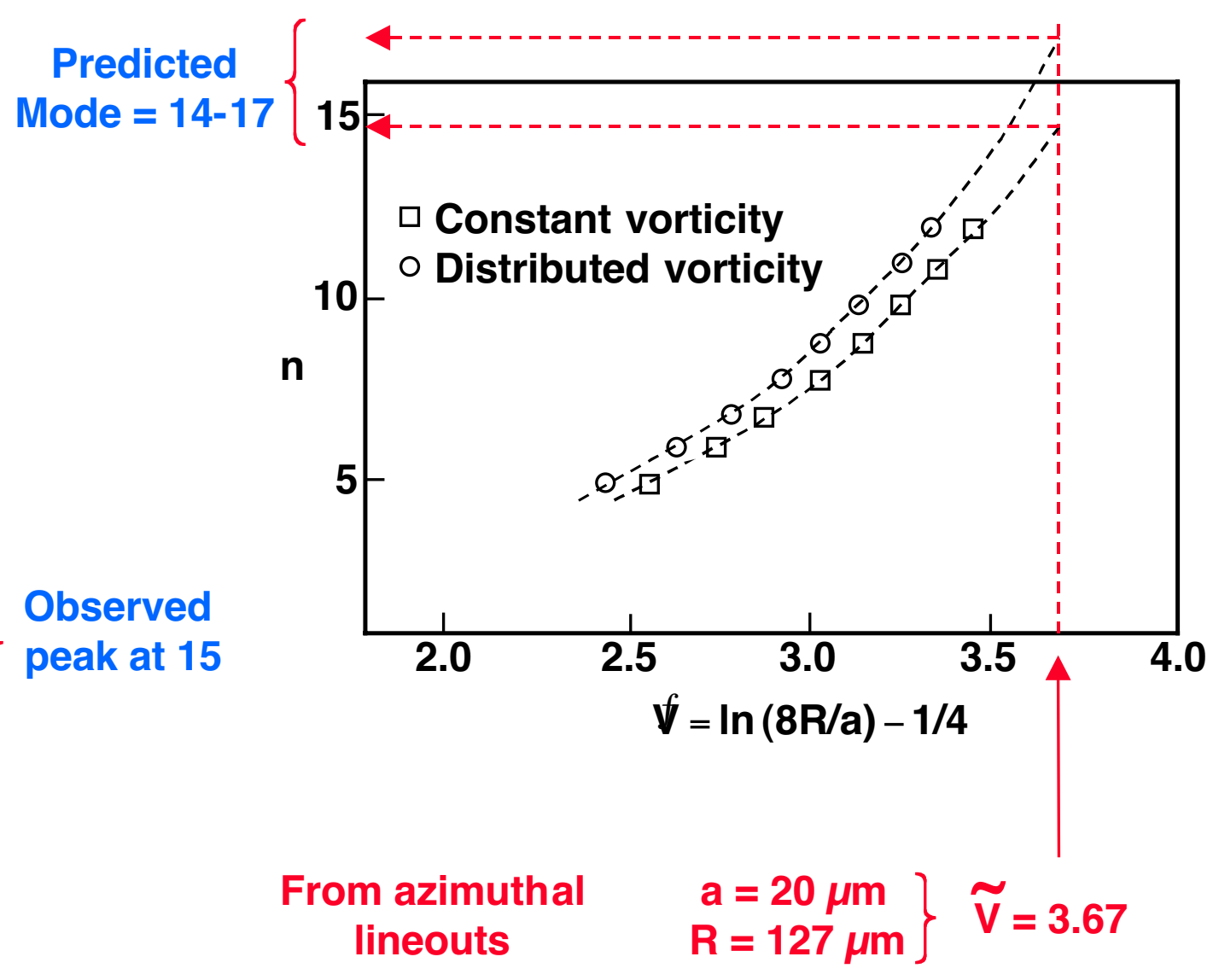


SNR should be greatly improved using a backlit pinhole due to greatly decreased pinhole-to-target distance

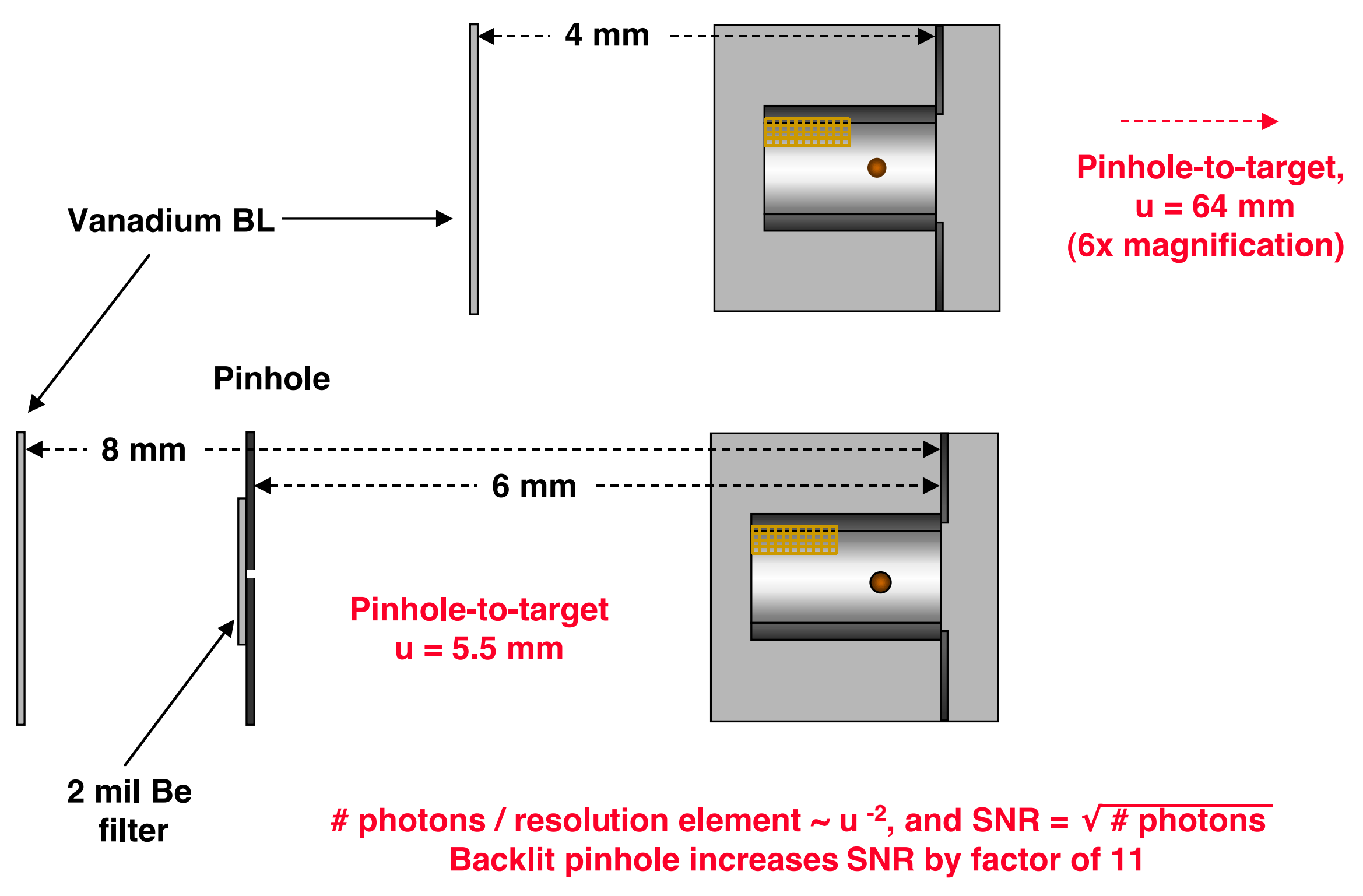




\section{We have begun investigating the ability to seed the azimuthal instability with machined initial perturbations}

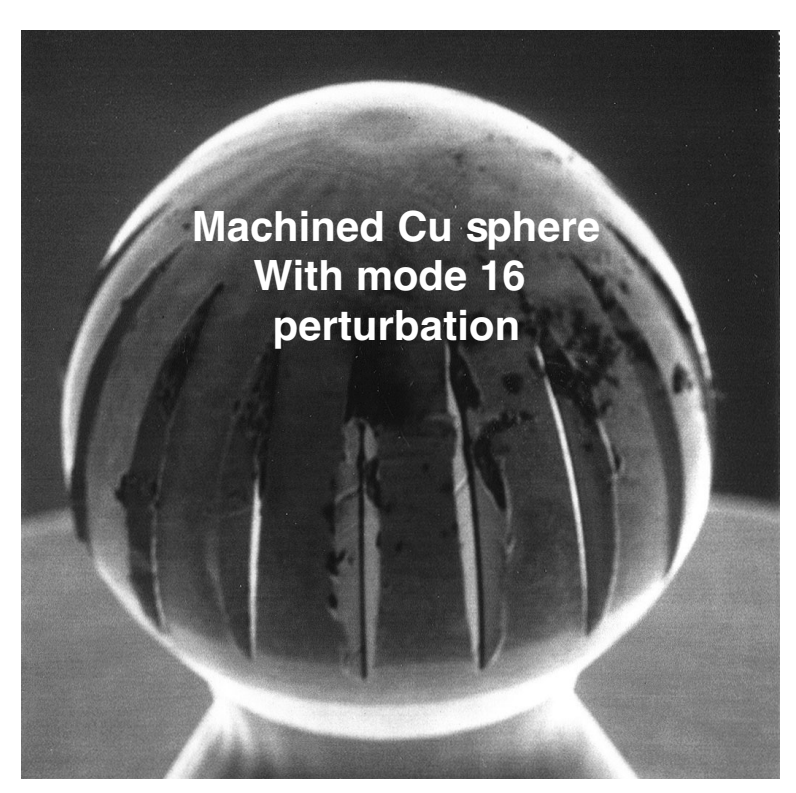

Face-on view using point projection backlighting

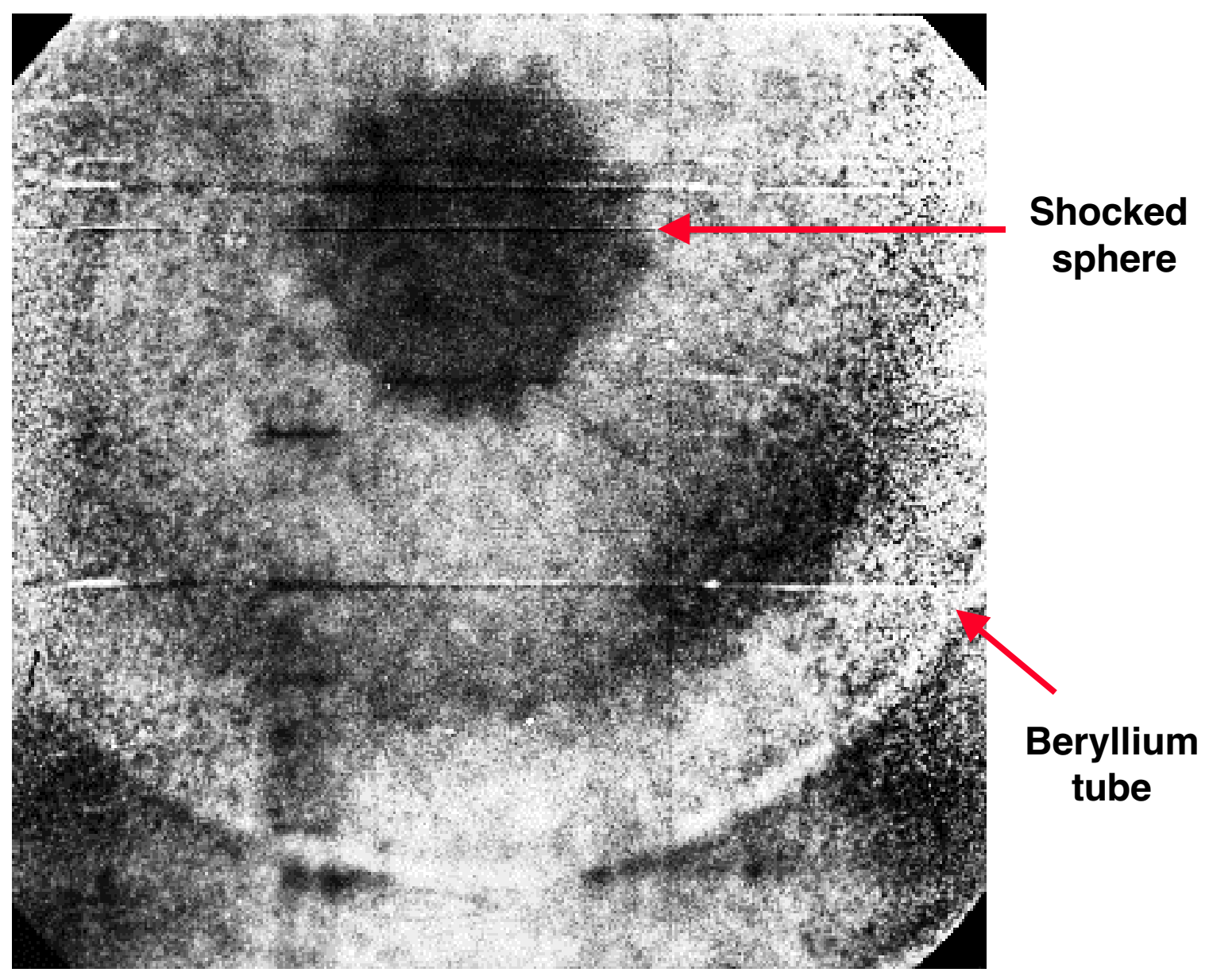

Shot \#24527

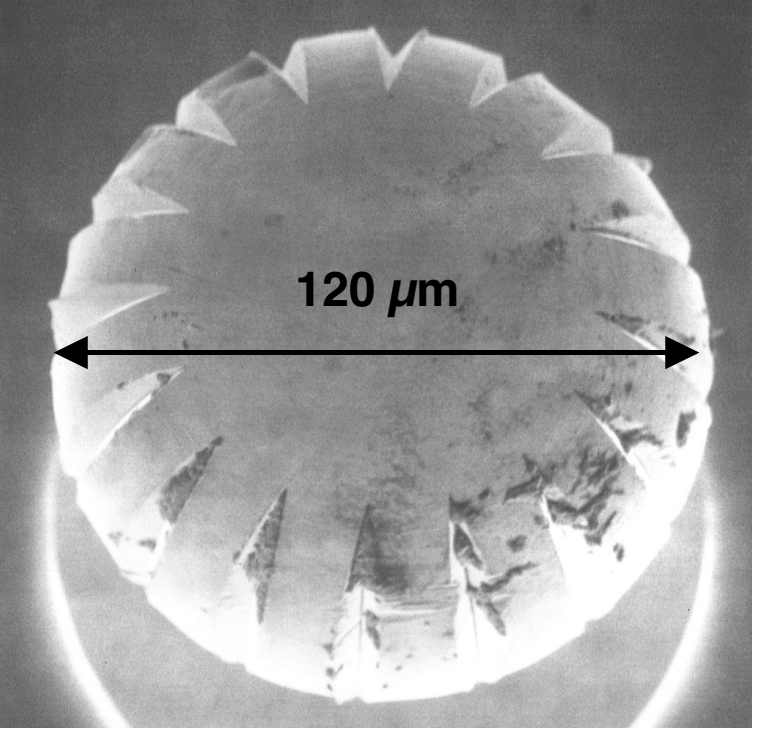




\section{Outline}

- Background / motivation

- Omega Experimental Results

- Numerical simulations

- Conclusions 


\section{D simulations of the experiment performed with CALE}

predict the basic evolution of the sphere into a vortex ring

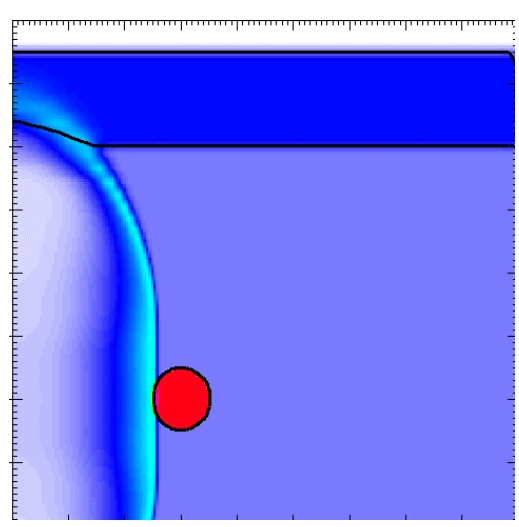

$\mathrm{t}=\mathbf{1 0} \mathrm{ns}$

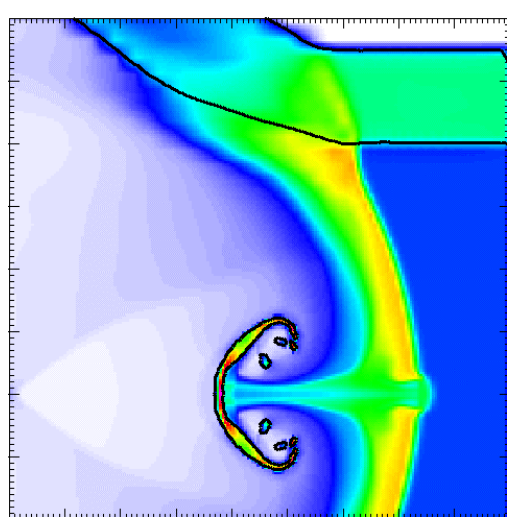

$\mathrm{t}=40 \mathrm{~ns}$

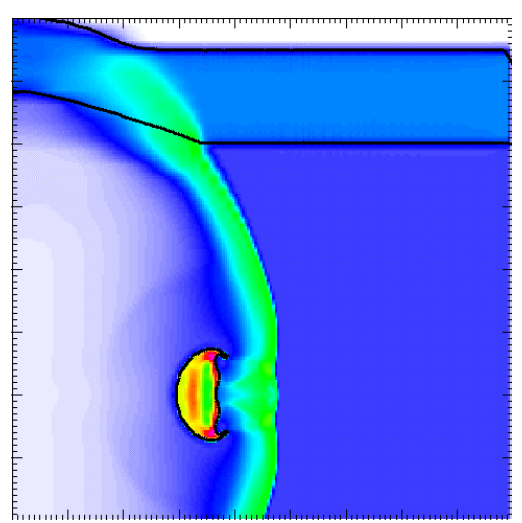

$\mathbf{t}=\mathbf{2 0}$ ns

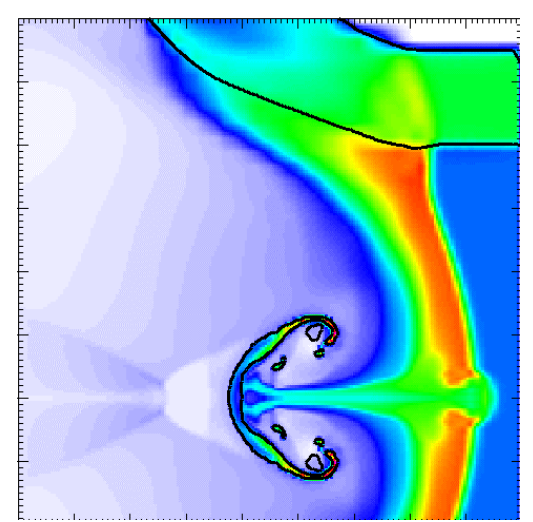

$\mathrm{t}=\mathbf{5 0} \mathrm{ns}$

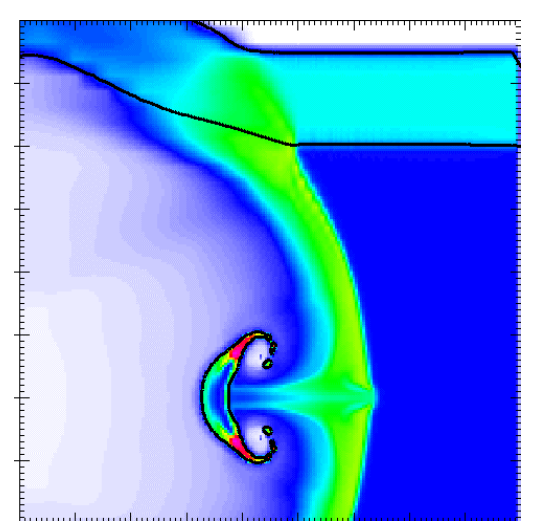

$\mathrm{t}=\mathbf{3 0} \mathrm{ns}$

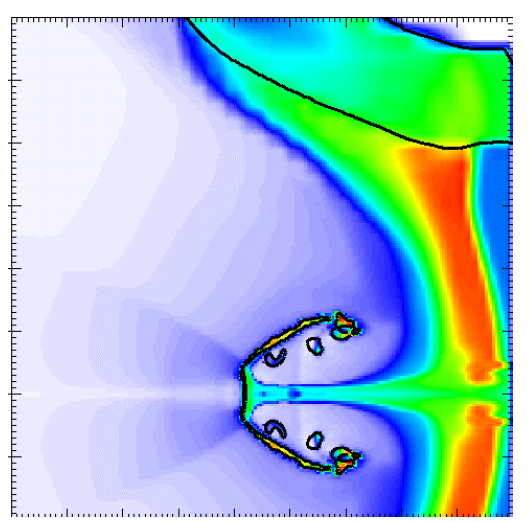

$\mathrm{t}=60 \mathrm{~ns}$ 
3D simulations of the experiment have been performed with an AMR code

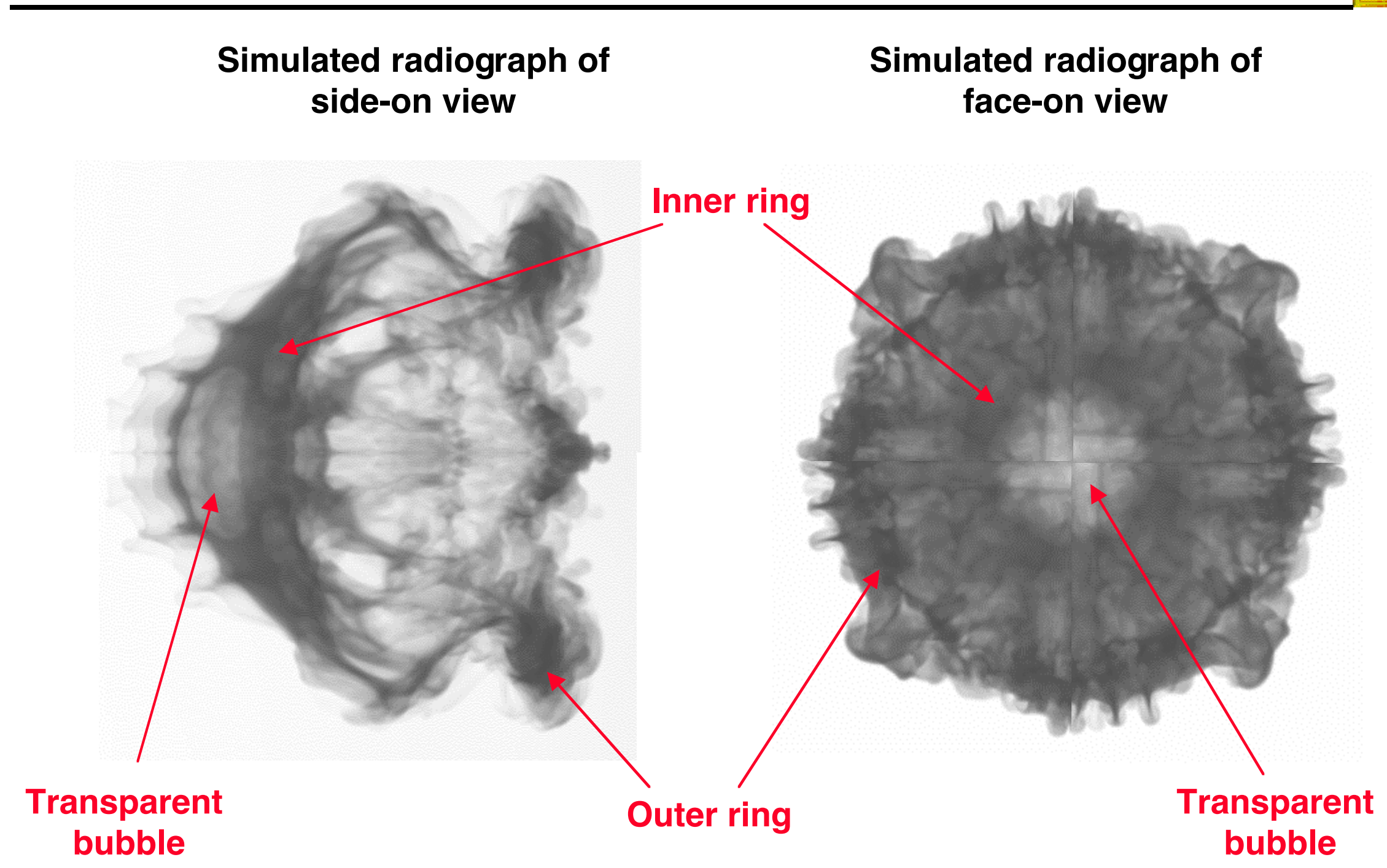

Simulations by J. A. Greenough 
Mode number spectra of the experimental and the AMR face-on images are in good agreement

\section{Experiment}

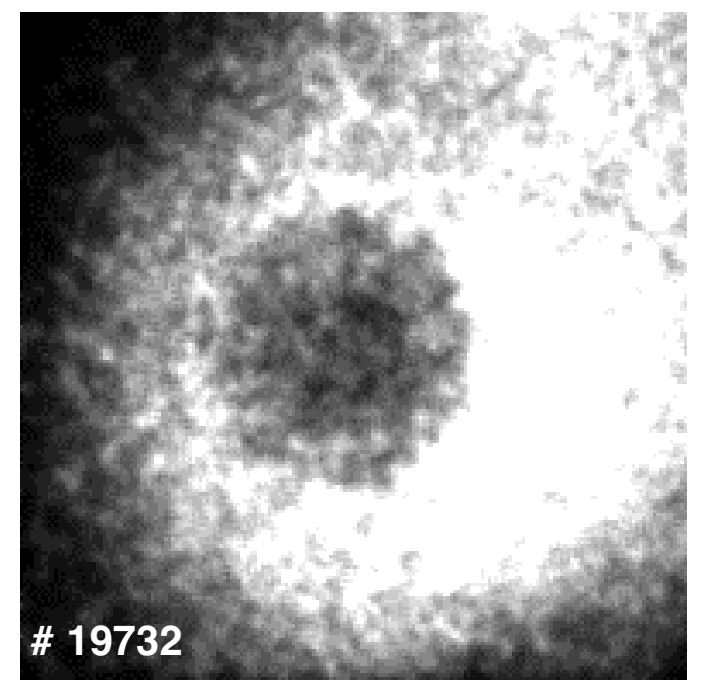

Power spectrum of circular line-out through outer portion of ring

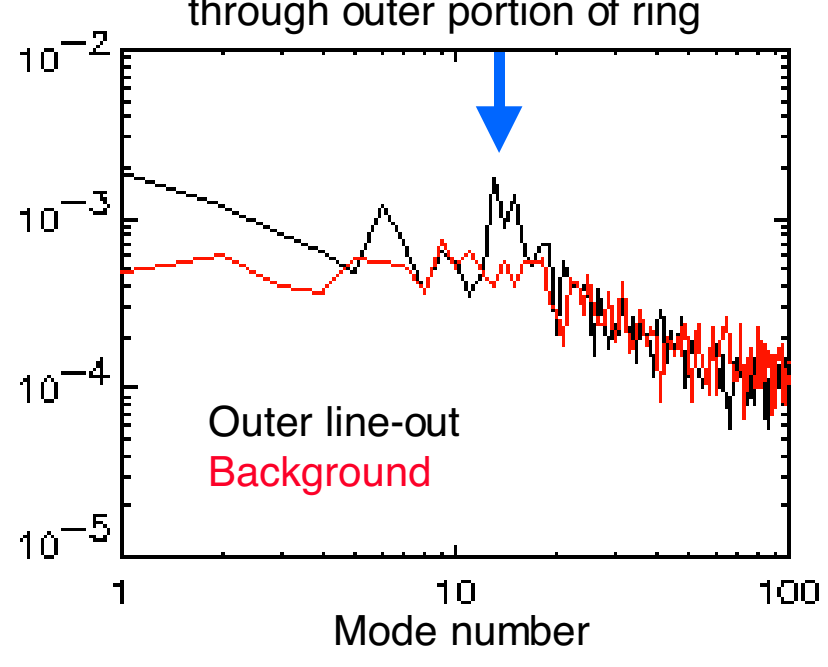

\section{AMR simulation}
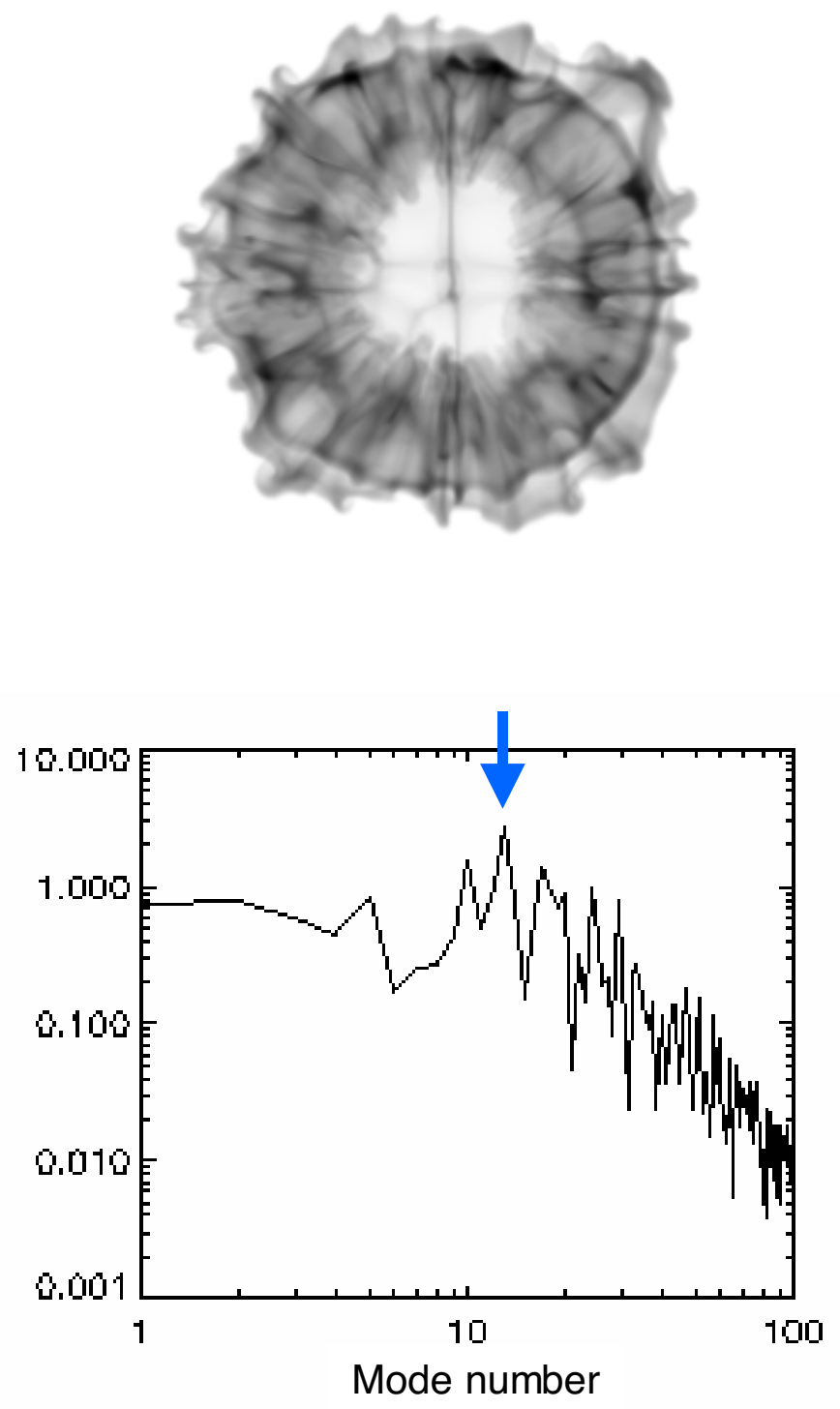


\section{Conclusion}

- Experiments have been conducted on the Omega laser to explore the interaction of a strong shock with a dense sphere

- The experiment has been diagnosed simultaneously from two orthogonal directions

- The experimentally observed azimuthal mode number is in good agreement with both incompressible theory of Widnall and 3D numerical simulations.

- Future work will focus on shock interaction with less-dense objects and interactions with multiple objects 


\title{
Turbulent transition in a high Reynolds number, Rayleigh-Taylor unstable plasma flow
}

\author{
H. F. Robey, Y. K. Zhou, A. C. Buckingham, P.Keiter, \\ B. A. Remington, and R. P. Drake \\ Lawrence Livermore National Laboratory \\ Livermore, California 94550
}

Presented at the $8^{\text {th }}$ Meeting of the

International Workshop on the Physics of Compressible Turbulent Mixing

Pasadena, CA

December 9-14, 2001

This work was performed under the auspices of the U. S. Department of Energy by the University of California, Lawrence Livermore National Laboratory under Contract No. W-7405-Eng-48. 


\section{Summary}

- The transition to turbulence in a high Reynolds number, RayleighTaylor unstable plasma flow is studied.

-1D numerical simulations (HYADES) are used to determine the plasma flow parameters $(P, \rho, T, Z)$ from which the kinematic viscosity is then determined.

- The Reynolds number is determined using the experimentally measured perturbation amplitude and growth rate together with the plasma kinematic viscosity determined from the $1 \mathrm{D}$ numerical simulations.

- It is observed that the Reynolds number is sufficiently greater than the mixing transition threshold of Dimotakis (i.e. Re $>2 \times 10^{4}$ ) for much of the experiment, yet the flow has not transitioned to turbulence.

- An extension of the Dimotakis mixing transition to non-stationary flows of short time-duration is presented. 


\section{Outline}

- Experimental setup and results of Omega laser experiment

- Results from 1D HYADES simulation of the experiment

Basic plasma flow parameters $(P, \rho, T, Z)$

Derived flow parameters $(v, D)$

Estimation of the Reynolds number

- Extension of Dimotakis mixing transition to non-stationary flows of short time-duration

- Conclusions 


\section{The experiments are conducted on the Omega laser in a very small Beryllium shock tube}

\section{Schematic of target}

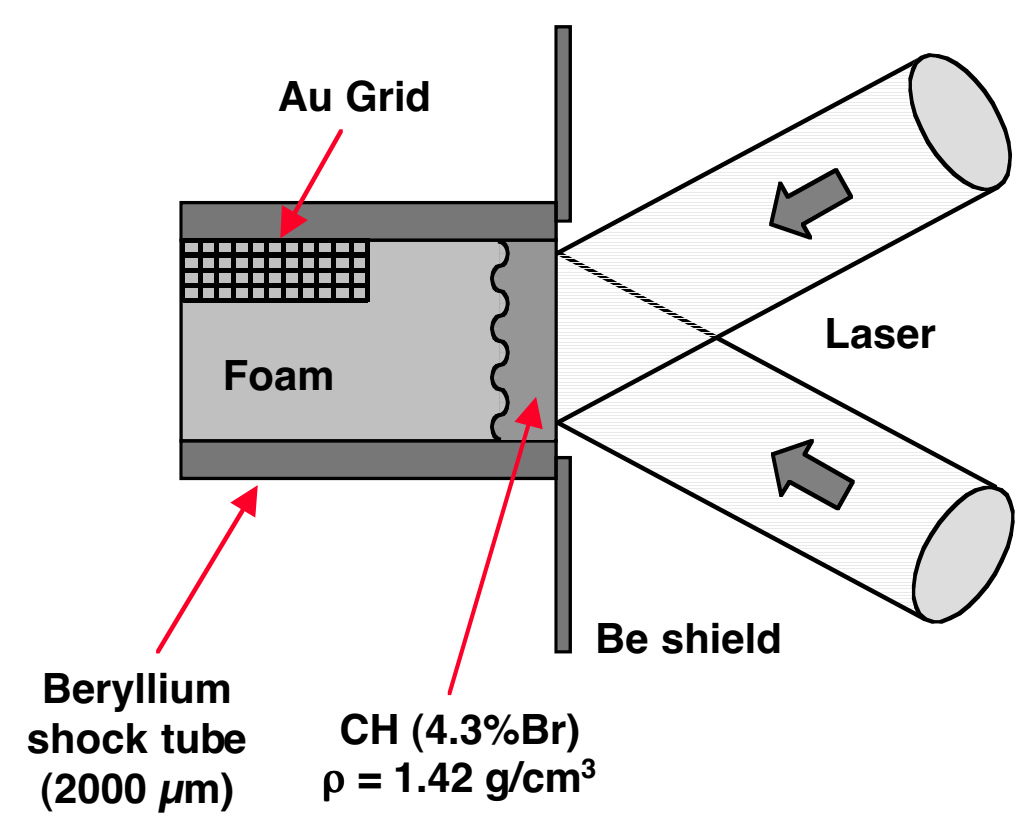

3D CAD rendering of target

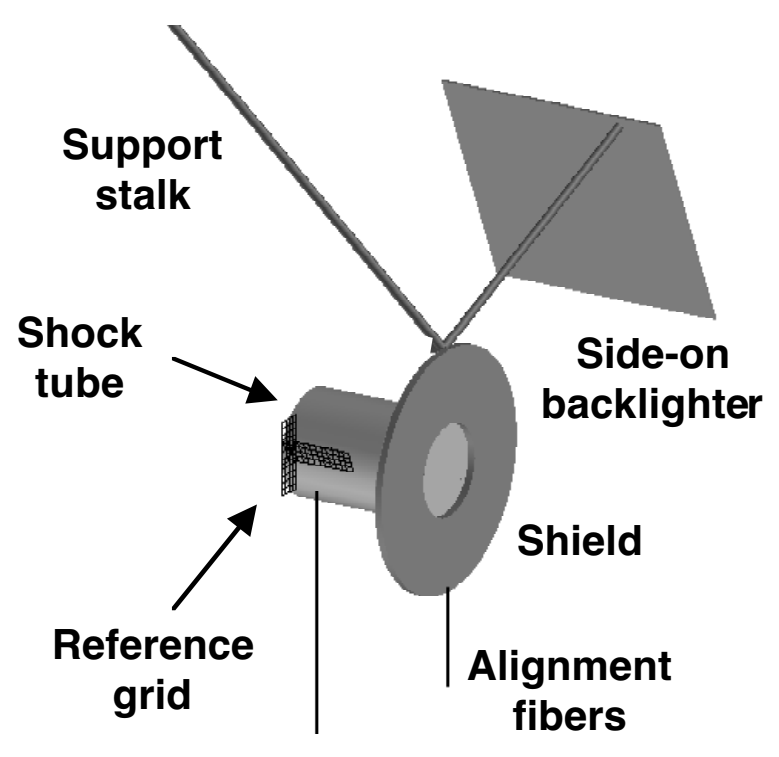

\section{Face-on view of target}

The target has a radiographic tracer strip which is density matched to the surrounding material

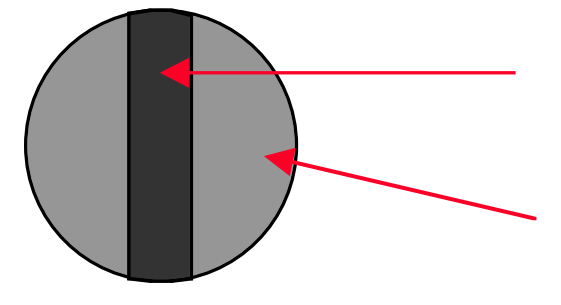

$1.42 \mathrm{~g} / \mathrm{cm}^{3} \mathrm{CH}(4.3 \% \mathrm{Br})$ tracer

$1.41 \mathrm{~g} / \mathrm{cm}^{3}$ polyimide 
Multiple beams of the Omega laser are used to both drive the strong shock and diagnose the interaction

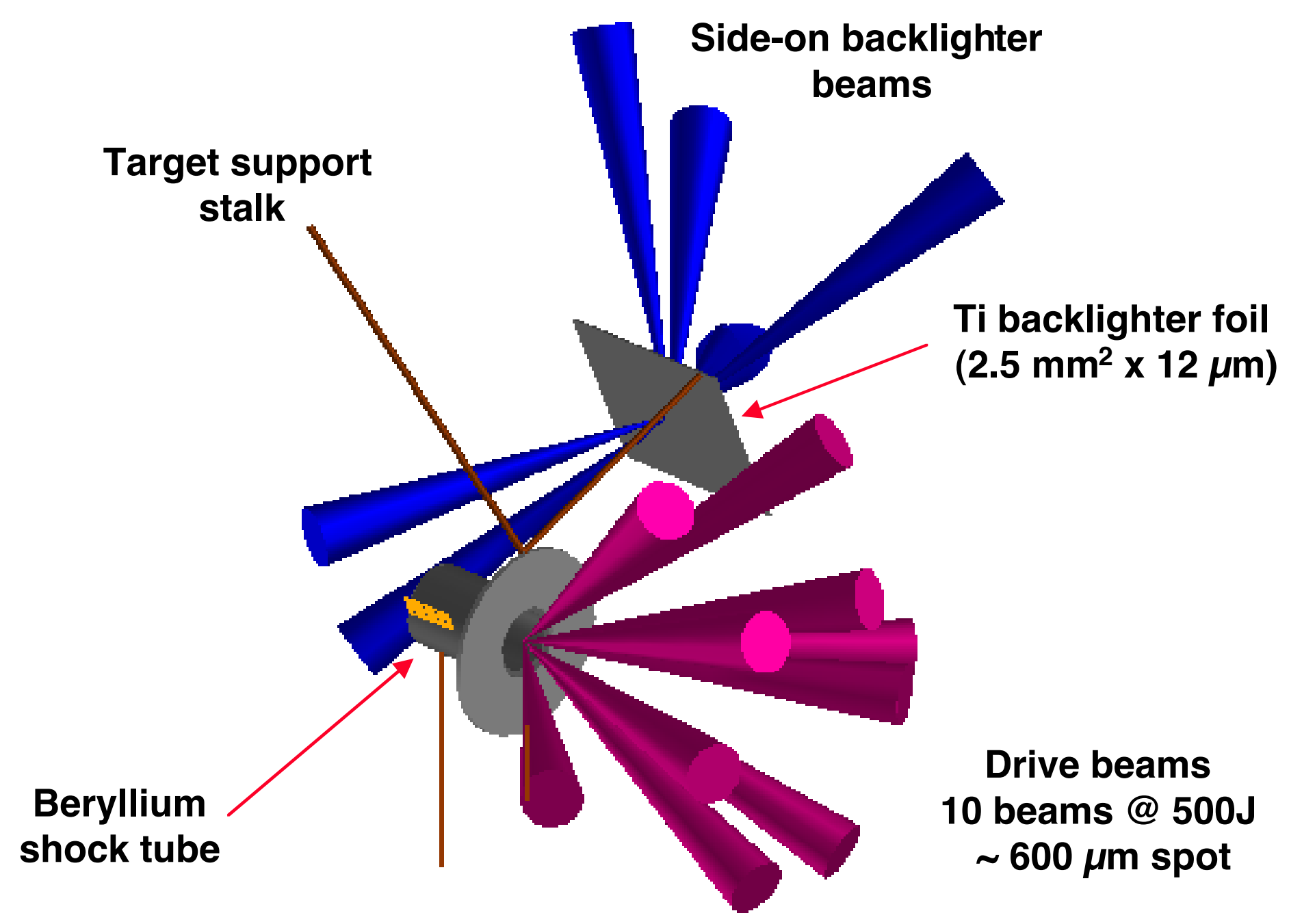


The evolution of a 2D single-mode perturbation $(\lambda=50 \mu \mathrm{m}$, $a_{0}=2.5 \mu \mathrm{m}$ ) is observed with $x$-ray radiography

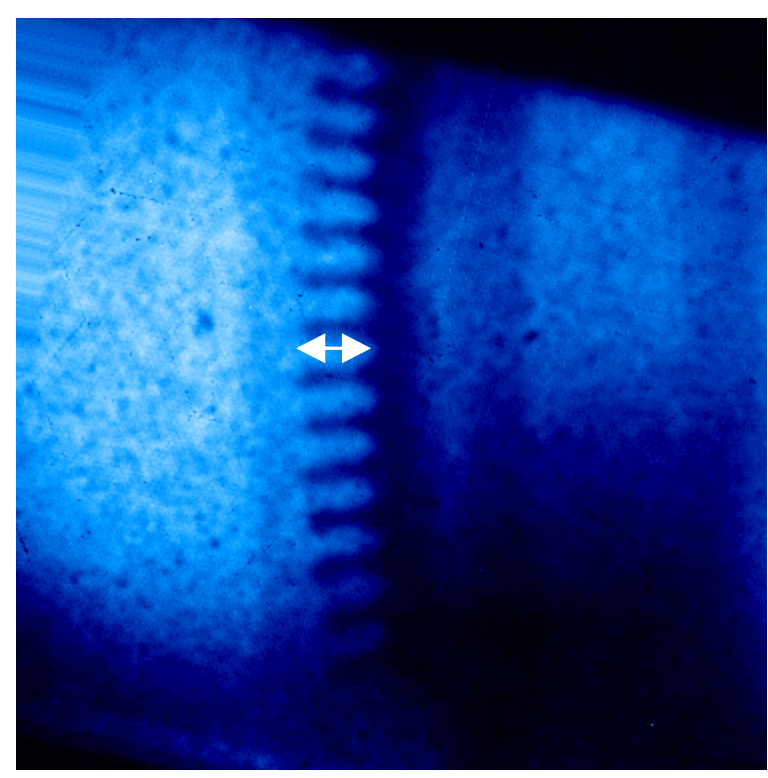

$\mathbf{t}=\mathbf{8}$ ns

$\mathrm{a}_{\mathrm{P}-\mathrm{v}}=\mathbf{8 3} \boldsymbol{\mu \mathrm { m }}$

$a_{\mathrm{P}-\mathrm{v}}=121 \mu \mathrm{m}$

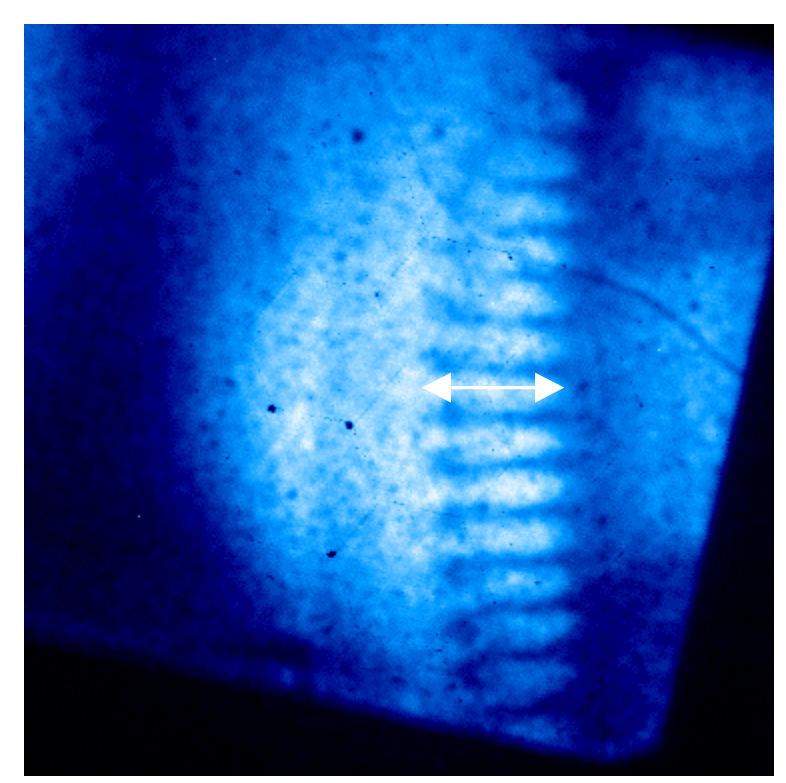

$t=14$ ns

$a_{\mathrm{P}-\mathrm{v}}=157 \mu \mathrm{m}$

Radiographic images obtained with 4.7keV Ti He- $\alpha$ x-rays imaged onto a gated $x$-ray framing camera 


\section{Results from 1D numerical simulation of the experiment}
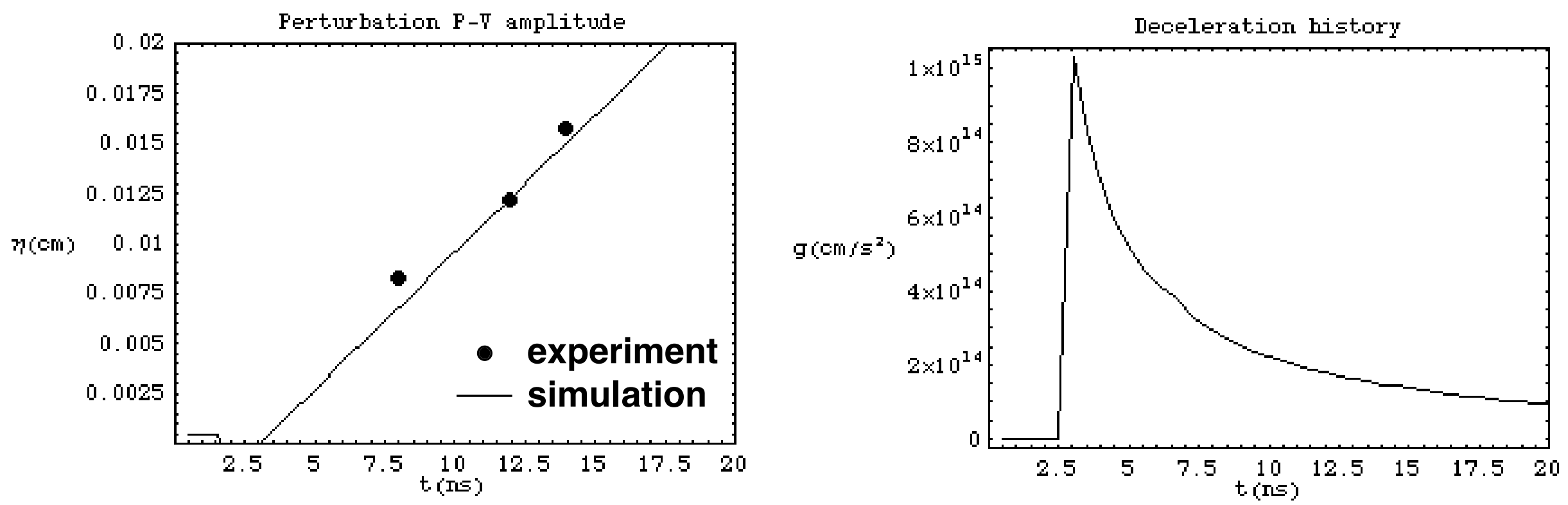

The effect of decompression of the interface has been taken into account 


\section{Outline}

- Experimental setup and results of Omega laser experiment

- Results from 1D HYADES simulation of the experiment

Basic plasma flow parameters $(\mathbf{P}, \rho, T, Z)$

Derived flow parameters $(v, D)$

Estimation of the Reynolds number

- Extension of Dimotakis mixing transition to non-stationary flows of short time-duration

- Conclusions 


\section{Time dependent values of the basic flow parameters}

(pressure, density, temperature, and degree of ionization)
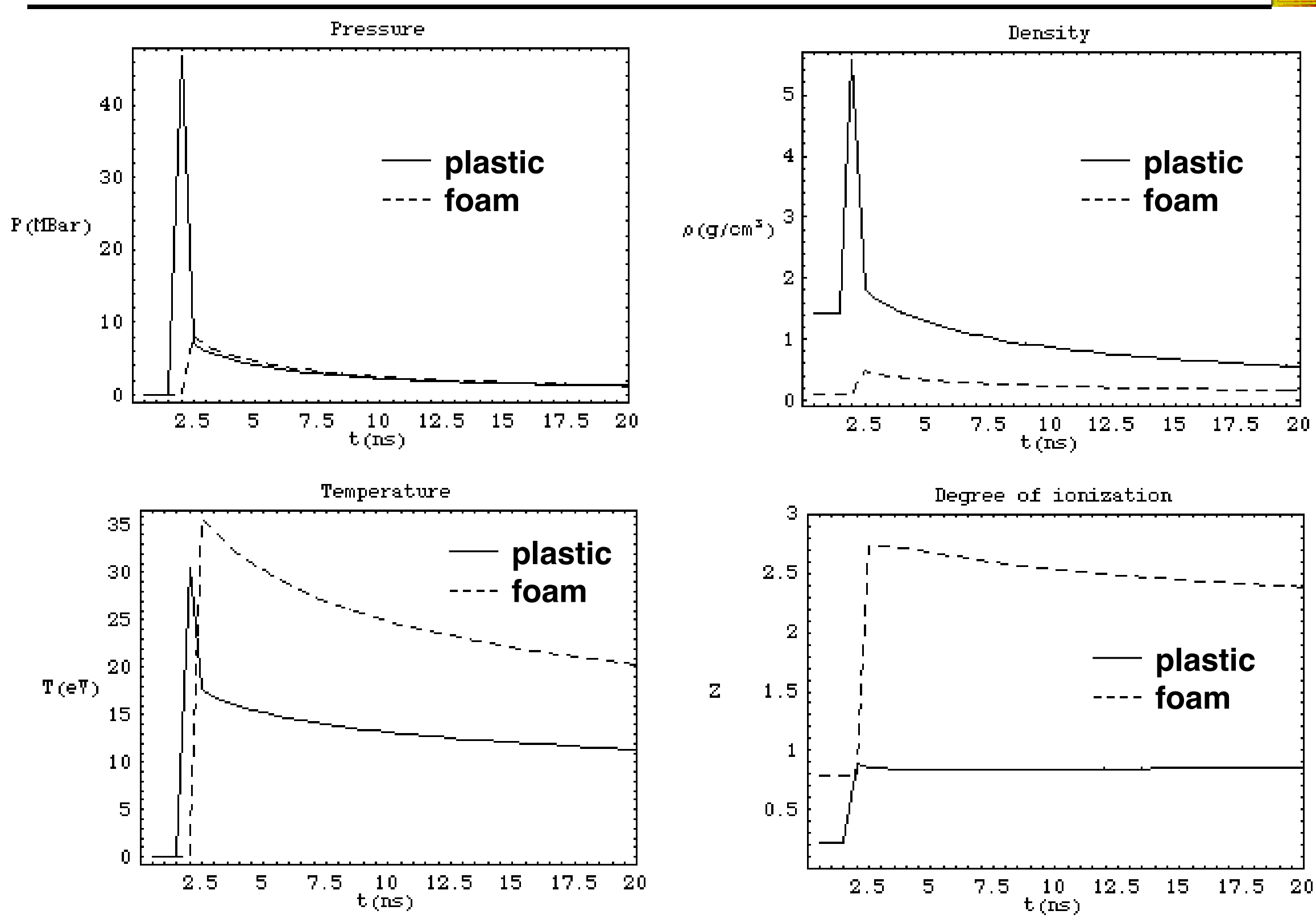
Time dependent values of related flow quantities (Atwood number, adiabatic index, and Mach number)
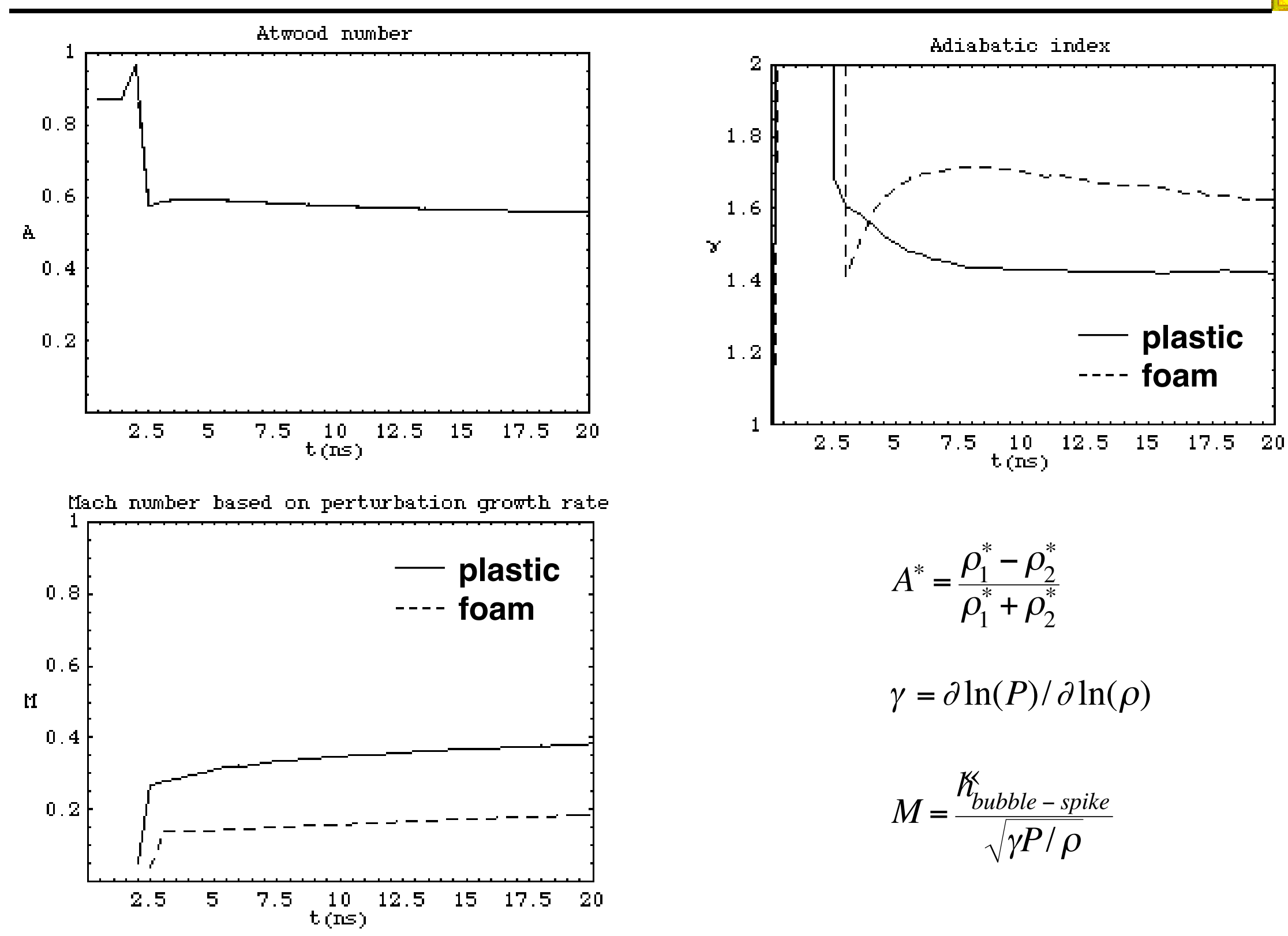

$$
\begin{aligned}
& A^{*}=\frac{\rho_{1}^{*}-\rho_{2}^{*}}{\rho_{1}^{*}+\rho_{2}^{*}} \\
& \gamma=\partial \ln (P) / \partial \ln (\rho) \\
& M=\frac{K_{\text {bubble }- \text { spike }}^{K}}{\sqrt{\gamma P / \rho}}
\end{aligned}
$$


Time dependent values of the plasma coupling parameter, $\Gamma$

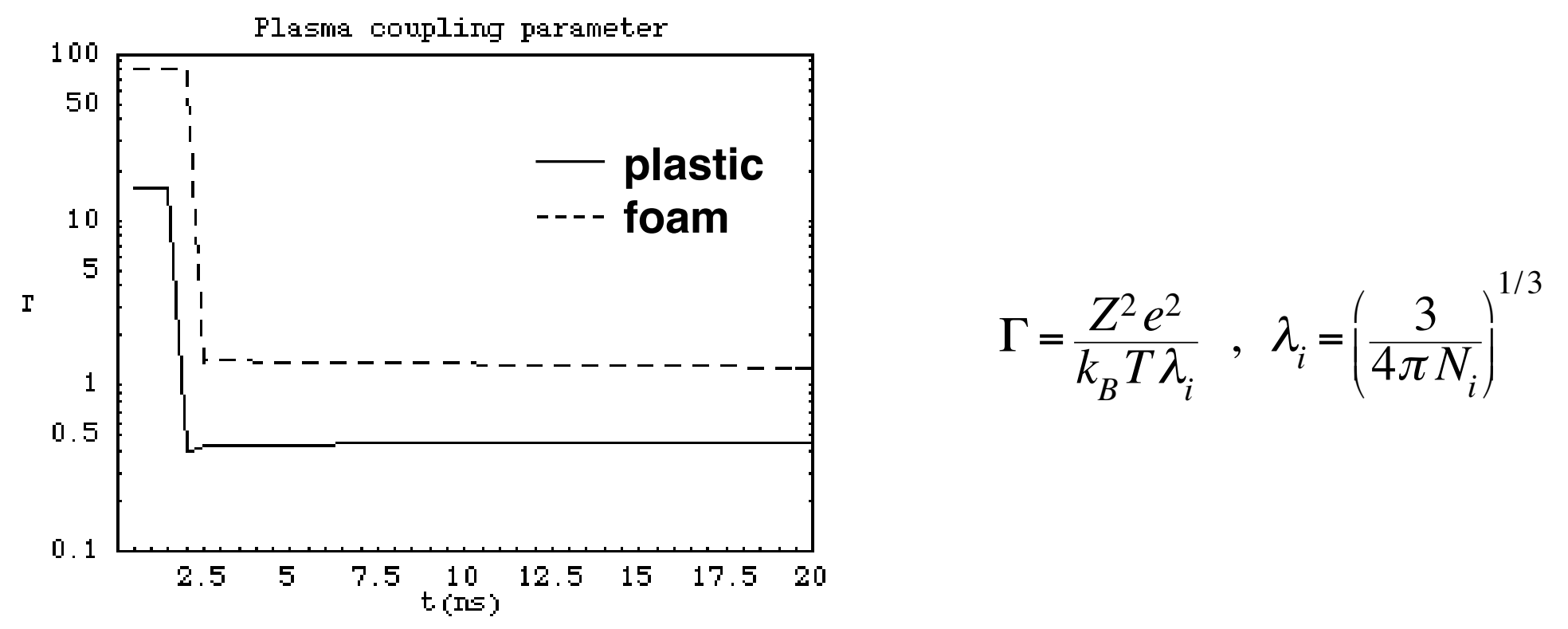

The plasma coupling parameter is in the "uncomfortable" range,

i.e neither weakly coupled $(\Gamma<1)$ where kinetic theory applies nor strongly coupled $(\Gamma \gg 1)$ where molecular dynamics simulations can provide rigorous transport properties 
Time dependent values of the kinematic viscosity, $v$

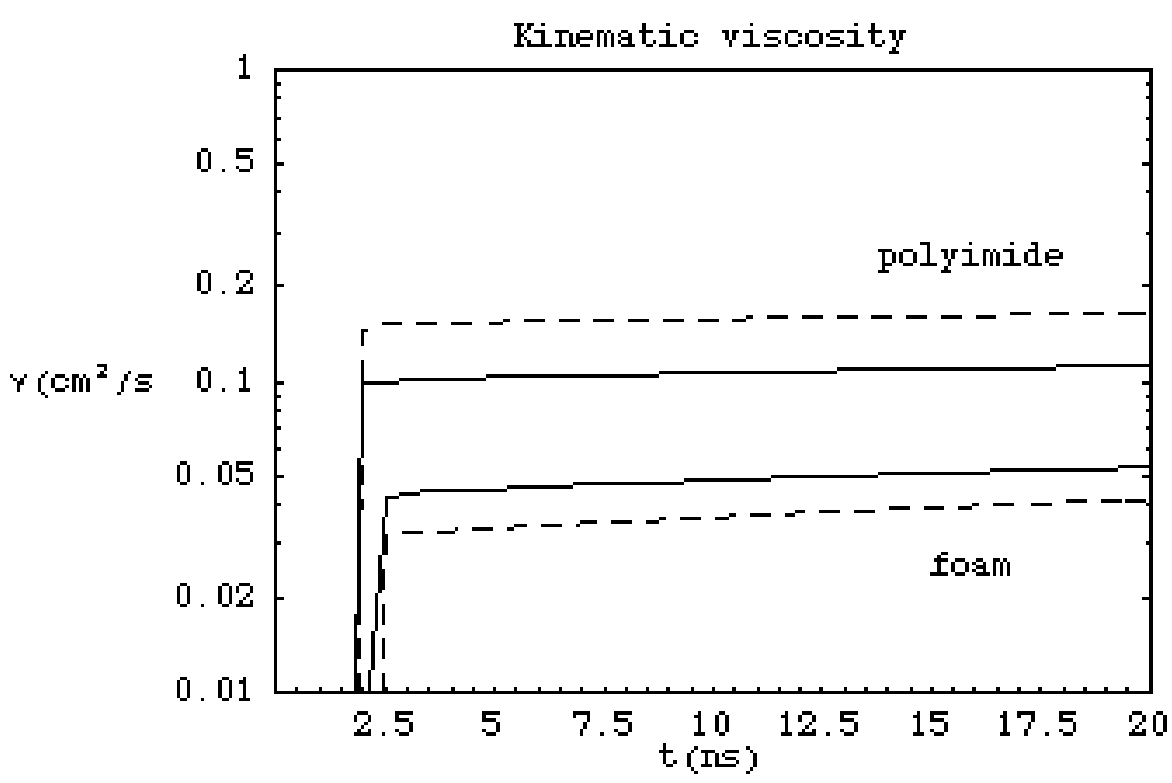

-.-.- S.I. Braginskii, in Reviews of Plasma Physics, New York, Consultants Bureau (1965).

J.G. Clerouin, M.H. Cherfi, and G. Zerah, EuroPhys. Lett. 42, 37 (1998).

- The kinematic viscosity is relatively constant throughout the experiment

- The value differs by more than a factor of 2 across the interface

- The Braginskii and Clerouin models show significant differences 


\section{Time dependent values of the Reynolds number}

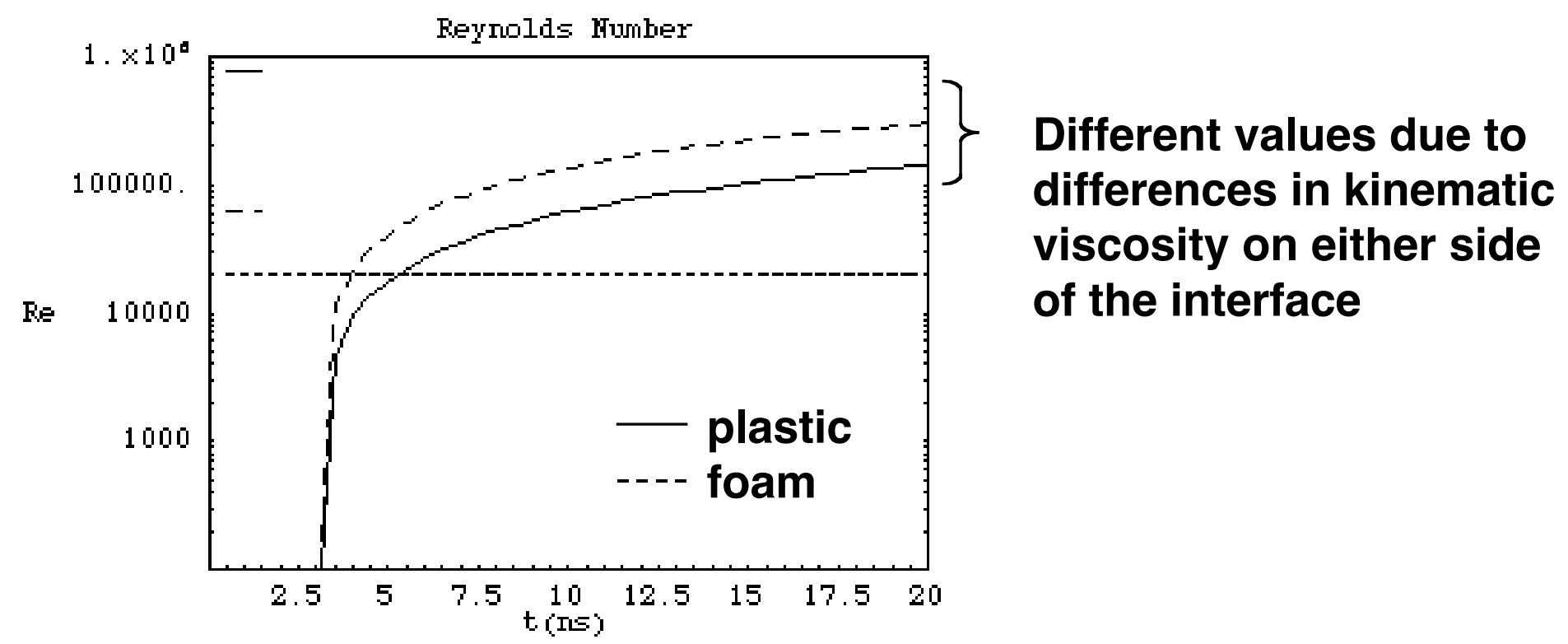

The Reynolds number exceeds the mixing transition threshold of Dimotakis ${ }^{\star}\left(\mathrm{Re}_{\text {crit }}=2 \times \mathbf{1 0}^{4}\right)$ on both sides of the interface for $t>5 \mathrm{~ns}$.

*P.E. Dimotakis, JFM 409, 69 (2000) 
The binary mass diffusivity at the interface and the Schmidt number have been calculated as well
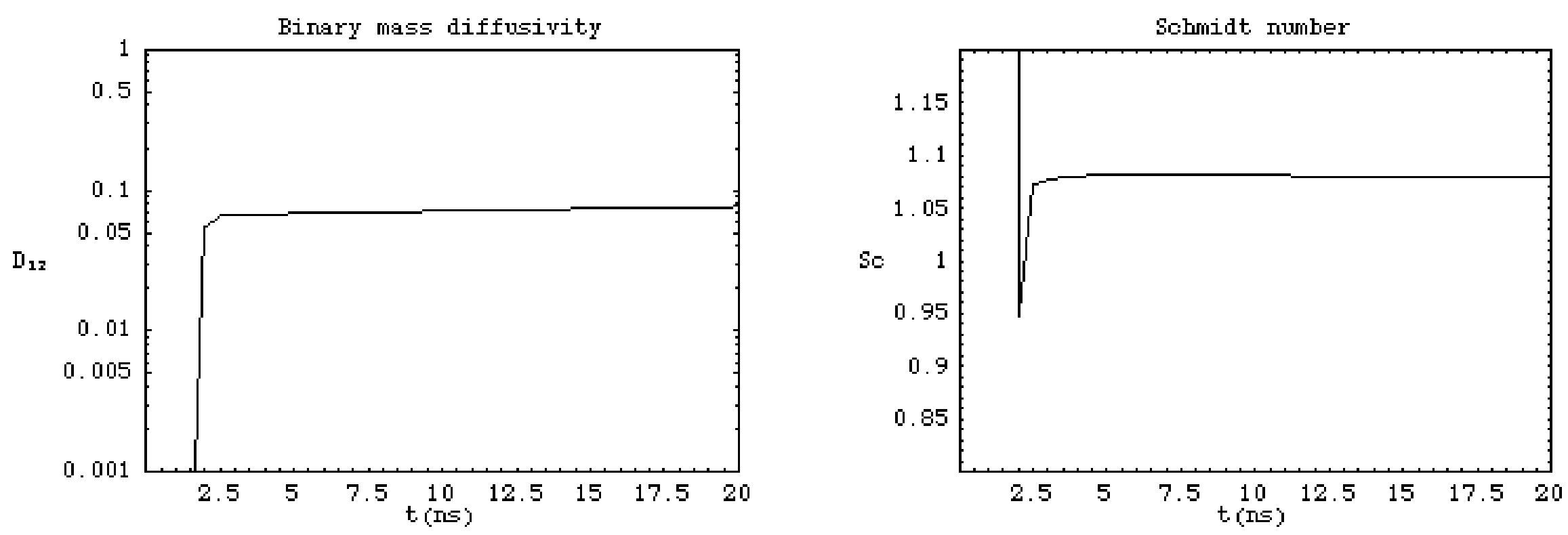

Binary mass diffusivity calculation follow the method outlined in :

C. Paquette et al., Astrophys. J. Suppl.Ser. 61, 177 (1986). 
From the kinematic viscosity $v$ and mass diffusivity $D$, the Rayleigh-Taylor growth rate dispersion curve can be calculated
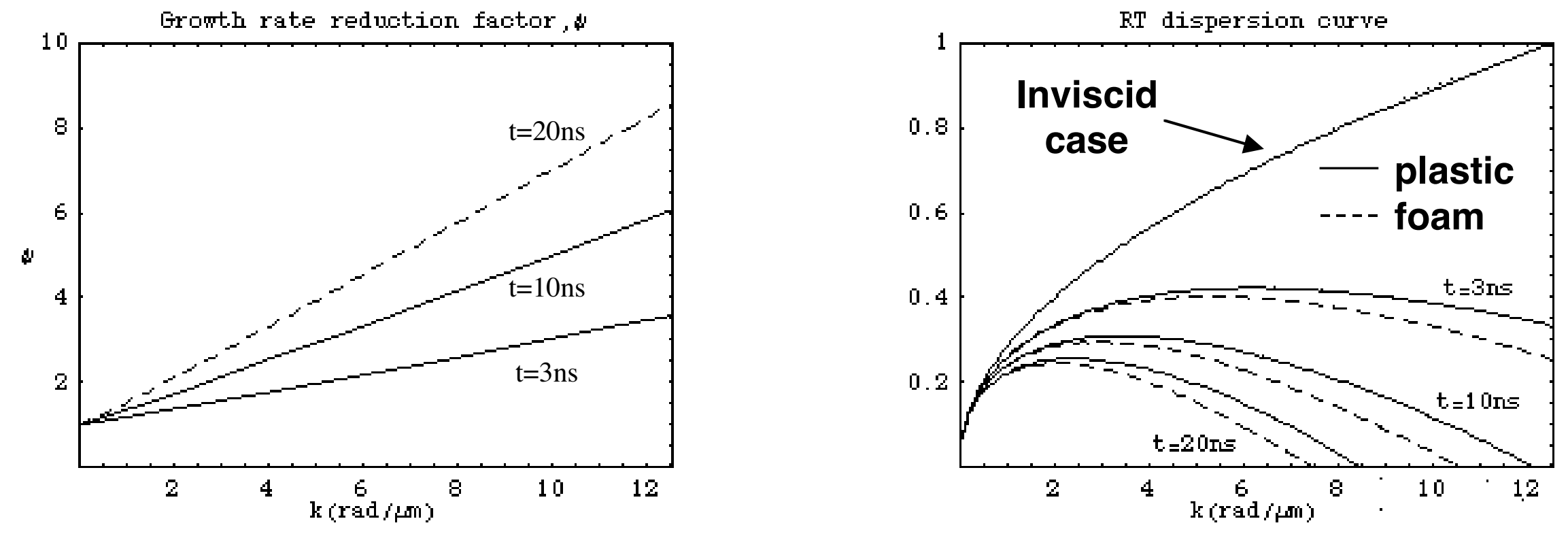

The Rayleigh-Taylor dispersion curve is : $\quad r_{v, D}=\sqrt{\frac{A k g}{\psi(k, t)}+v^{2} k^{4}}-(v+D) k^{2}$

where $\Psi(\mathbf{k}, \mathbf{t})$ is the growth rate reduction factor due to a finite density gradient and is found as the solution of the following eigenvalue equation :

$$
\frac{d}{d z}\left(\rho \frac{d w}{d z}\right)=w k^{2}\left(\rho-\frac{\psi}{A k} \frac{d \rho}{d z}\right)
$$




\section{A sufficient range of Rayleigh-Taylor unstable scales exists to populate a turbulent spectrum}

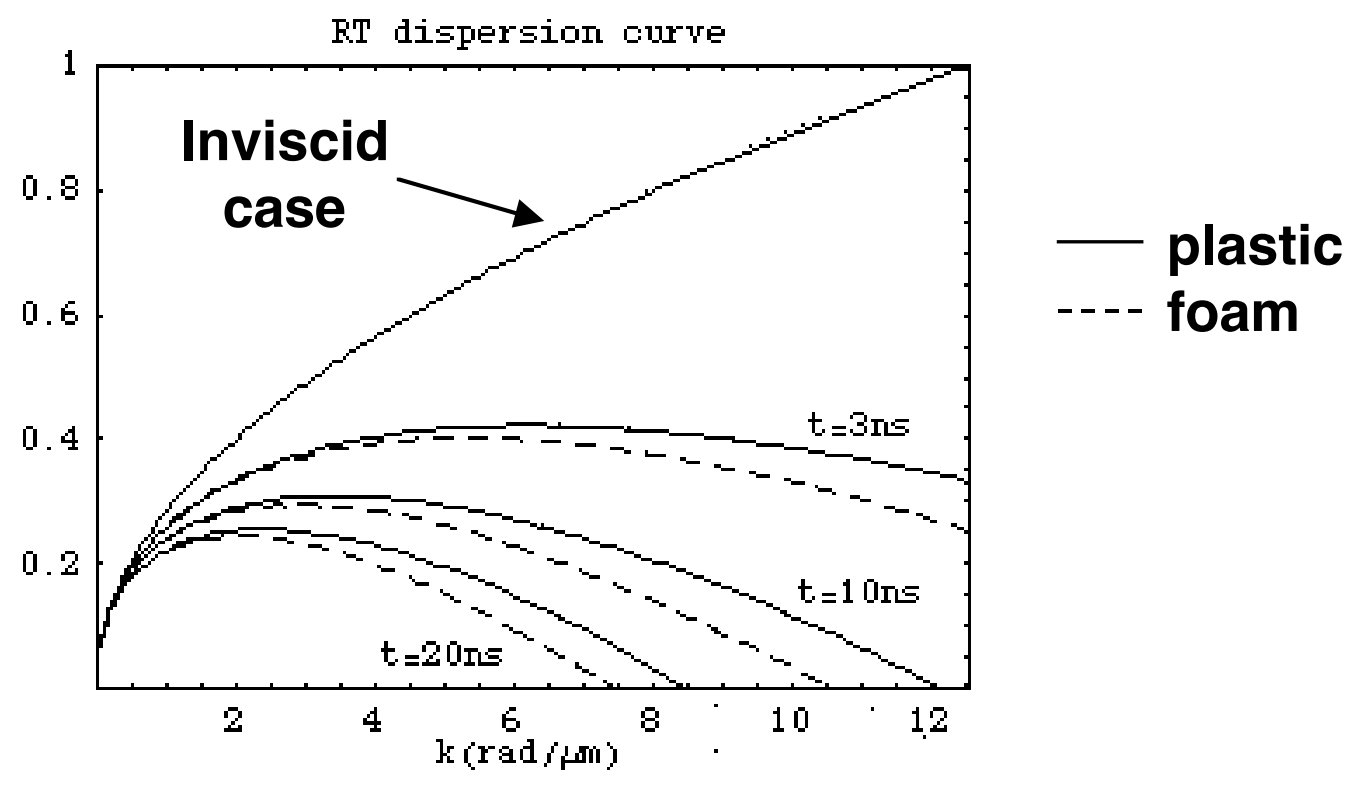

- The initially imposed perturbation has wavelength $\lambda=50 \mu \mathrm{m}$, or $\mathrm{k}=0.126 \mathrm{rad} / \mu \mathrm{m}$.

- At $\mathrm{t}=20 \mathrm{~ns}$, perturbations with $\mathrm{k}>8 \mathrm{rad} / \mu \mathrm{m}(\lambda<1.3 \mu \mathrm{m})$ are completely stablized.

- At $\mathrm{t}=20 \mathrm{~ns}$, the peak growth rate occurs at $\mathrm{k}=2.5 \mathrm{rad} / \mu \mathrm{m}(\lambda=2.5 \mu \mathrm{m})$

- A sufficient range of scales exists, subject to RT instability which can populate a turbulent spectrum 


\section{Outline}

- Experimental setup and results of Omega laser experiment

- Results from 1D HYADES simulation of the experiment

Plasma flow parameters $(P, \rho, T, Z)$

Derived flow parameters $(v, D)$

Estimation of the Reynolds number

- Extension of Dimotakis mixing transition to non-stationary flows of short time-duration

- Conclusions 


\section{Dimotakis has identified a critical Reynolds number at which a rather abrupt transition to a well mixed state occurs}

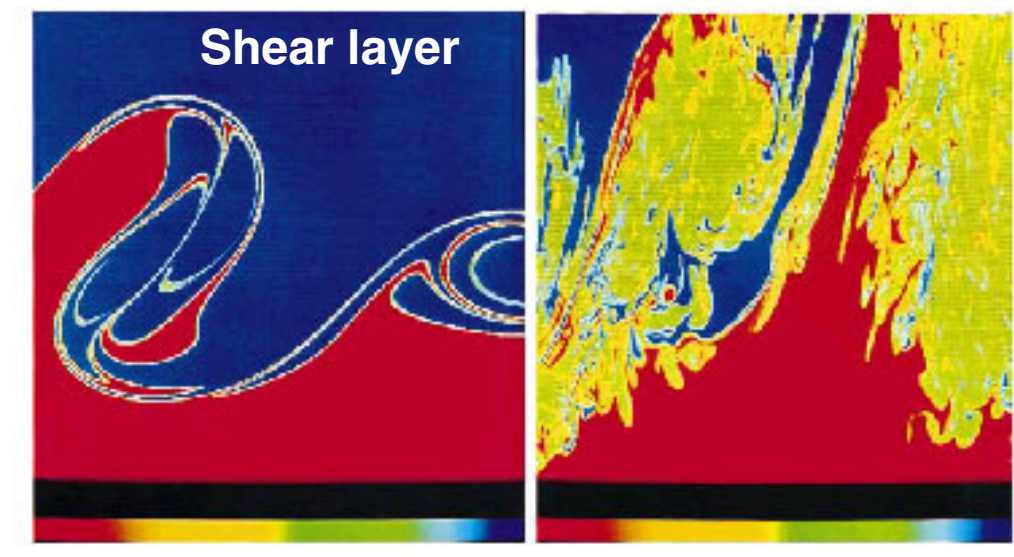

FiGune 2. Laser-induced fluorescence streak images of the scalar field in a liquid-phase sbear layer for $R e \simeq 1.75 \times 10^{\prime \prime}(a)$ and $R e \simeq 23 \times 10^{\circ}(b)$. From $\mathrm{KD} 86$, figures $7 b$ and 9 , respectively.

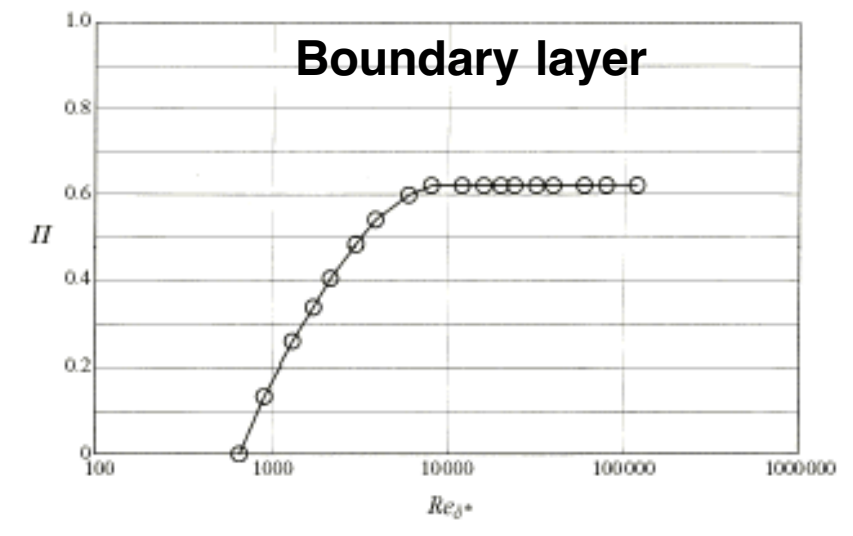

Ficure 12. Turbulent boundary-layer $I$ parameter $\mathbf{s} . R e_{n}$. Skin-friction-law fit data (Collins et al. 1978, table 4)

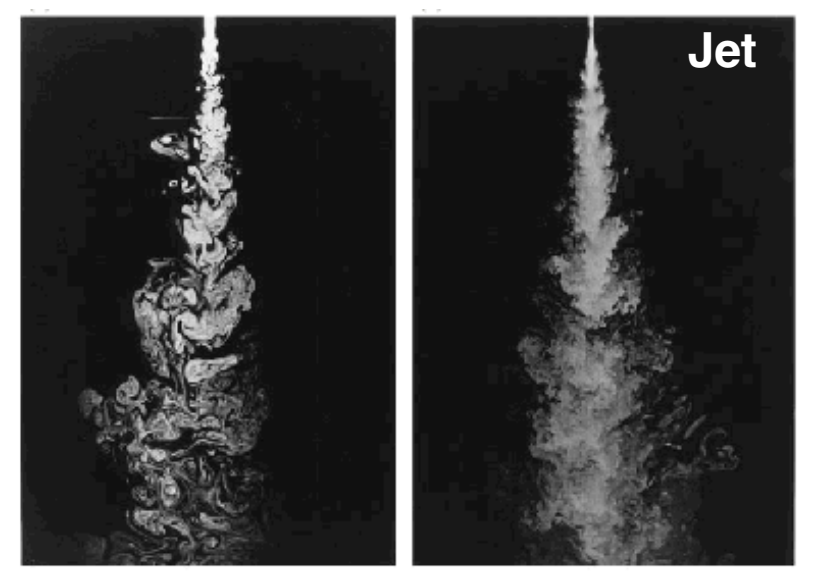

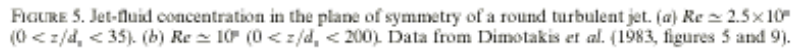

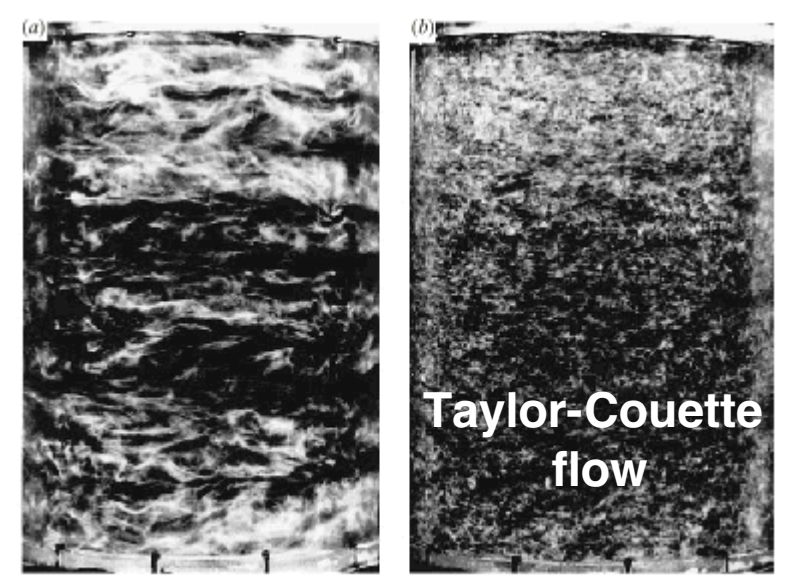

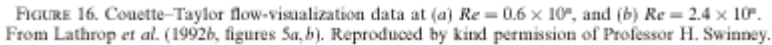

This mixing transition at $\mathrm{Re} \approx 2 \times 10^{4}$ is observed to occur in a very wide range of stationary flows 
This transition is co-incident with the appearance of a range of scales decoupled from both large-scale and viscous effects

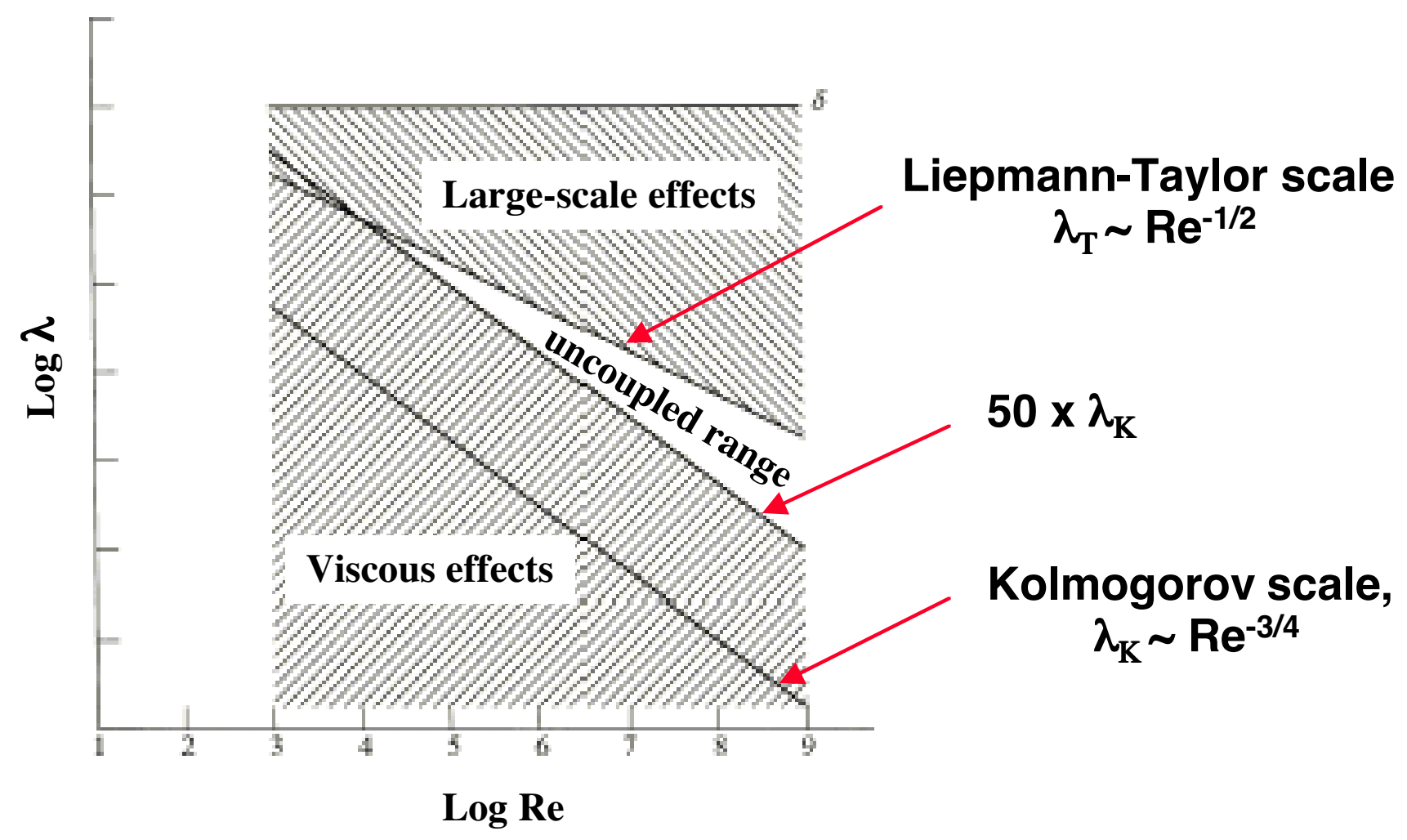

Figure 19. Reynolds number dependence of spatial scales for a turbulent jet

Figure 19 from P.E. Dimotakis, JFM 409, 69 (2000) 
In high Re flows of short time duration, the Taylor microscale may not have sufficient time to reach its asymptotic value

The Taylor microscale (for stationary, homogeneous, isotropic flows) depends on the integral scale $\delta$ and the Reynolds number as :

$$
\lambda_{T} \sim \delta \operatorname{Re}_{\delta}^{-1 / 2}
$$

This dependence is analogous to the development of a laminar viscous boundary layer on a flat plate :

$$
\lambda_{v} \sim x \mathrm{Re}_{x}^{-1 / 2}
$$

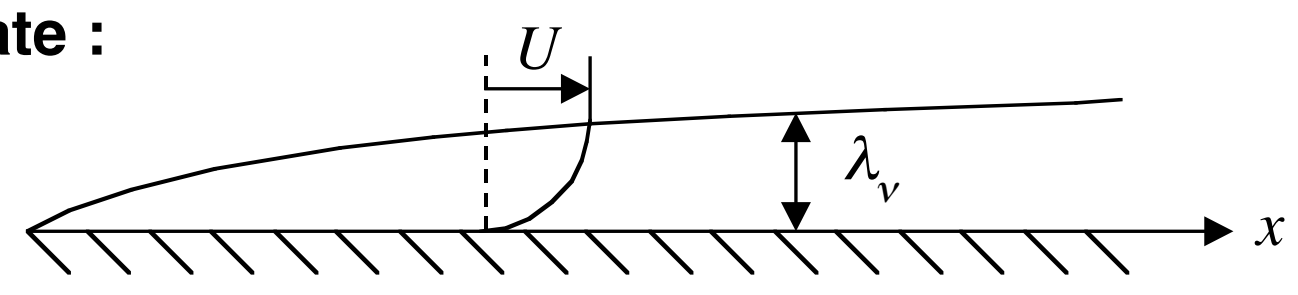

For an impulsively accelerated plate, however, the boundary layer development will initially grow as :

$$
\lambda_{v}(t) \sim \sqrt{v t}
$$

We propose a modification to the mixing transition as the time at which the smaller of the Taylor microscale and the viscous diffusion scale exceeds the dissipation scale (50 x Kolmogorov scale) :

$$
\operatorname{Min}\left(\sqrt{v t}, \lambda_{T}\right)>50 \lambda_{K}
$$


Time dependent values of the Taylor microscale, Kolmogorov scale, and viscous diffusion scale
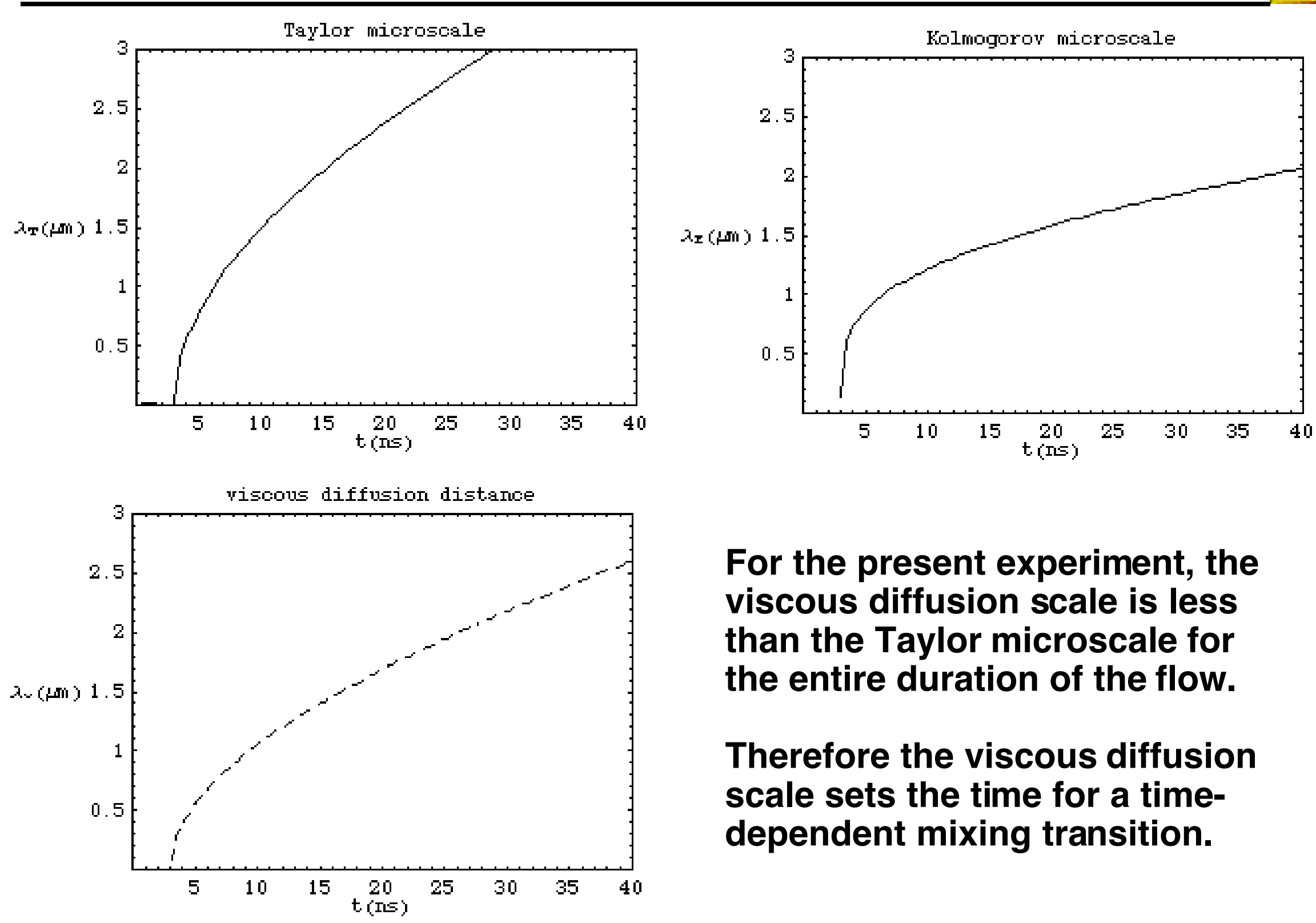

For the present experiment, the viscous diffusion scale is less than the Taylor microscale for the entire duration of the flow.

Therefore the viscous diffusion scale sets the time for a timedependent mixing transition. 
A comparison of viscous length scales shows the appearance of a decoupled range of scales for $t>17 \mathrm{~ns}$

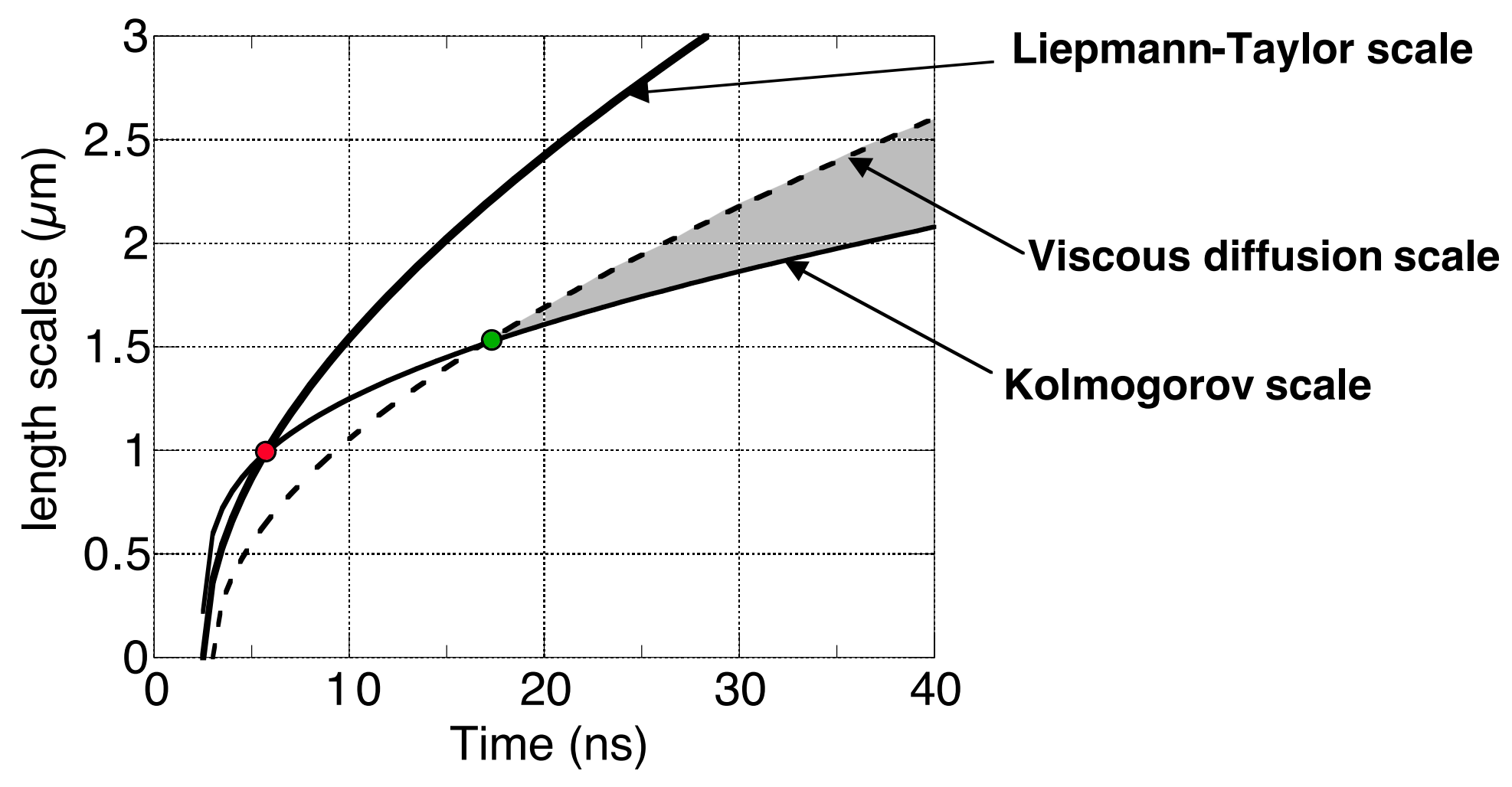

- The red dot indicates the Dimotakis criterion for transition in a stationary flow. This occurs at $\mathrm{t} \approx 5.5 \mathrm{~ns}$ or $\mathrm{Re} \approx 2 \times 10^{4}$.

- The green dot indicates the present criterion for transition in a temporally-limited flow. This occurs at $t \sim 17 \mathrm{~ns}$ or $\operatorname{Re} \approx 10^{5}$. 


\section{This method has been applied to estimate the turbulent transition time in the LANL gas curtain experiment *}
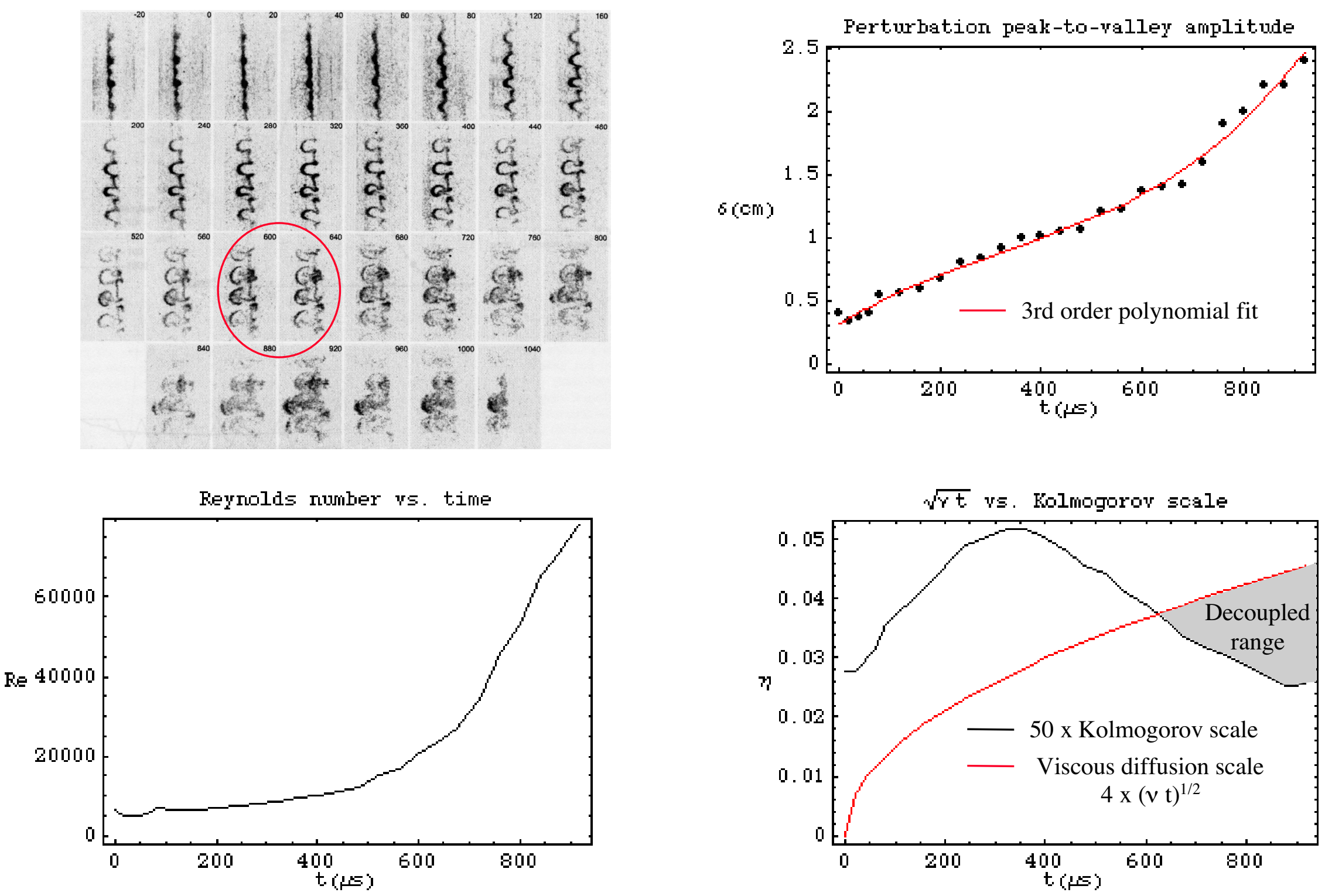

* From Rightly, Vorobieff, Martin, \& Benjamin, Phys. Fluids 11(1), 186 (1999) 
Current and future work on Omega will focus on the role of modal content and dimensionality of the initial perturbation

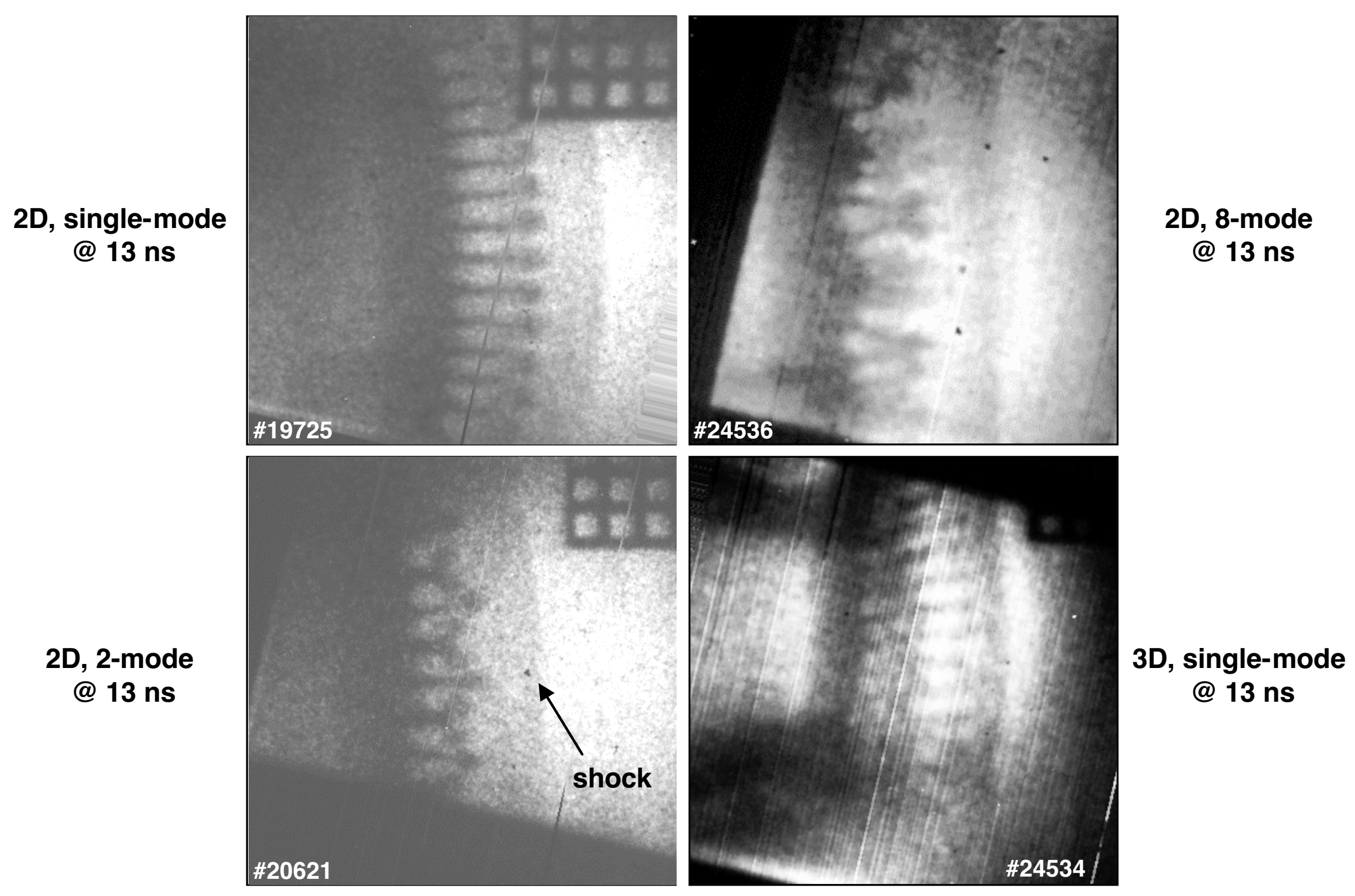


Conclusions

The transition to turbulence in a high Reynolds number, RayleighTaylor unstable plasma flow has been studied experimentally.

The following observations are made :

- The Reynolds number exceeds the mixing transition threshold of Dimotakis (i.e. Re $>2 \times 10^{4}$ ) for much of the experiment, yet no transition to turbulence is observed.

- An extension of the Dimotakis mixing transition to non-stationary flows of short time-duration is presented. This method illustrates that the temporal duration of the present flow is insufficient to allow for the appearance of a mixing transition. 


\title{
High Mach Number and High Initial Amplitude Effects on the Evolution of the Single-Mode Richtmyer-Meshkov Instability An Experimental Study
}

\author{
O. $\operatorname{Sadot}^{(1,2)}$, A. Rikanati(1,2), D. Oron ${ }^{(1)}$, A. Yosef-Hai ${ }^{(2)}$, \\ G. Ben-Dor ${ }^{(2)}$, D. Shvarts ${ }^{(1,2)}$
}

1) Nuclear Research Center Negev, ISRAEL.

2) Ben-Gurion University of the Negev, ISRAEL. 


\section{Theory: The case of a single-mode RM instability}

\section{Small initial amplitude: (Richtmyer Impulsive model)}

Using:

1) Incompressible flow after the shock passes the interface.

2) Modeling the shock as:

$$
g(t)=U_{0} \delta(t)
$$

Leads to a linear growth:

$$
U_{\text {bubble }}=U_{0} \cdot k \cdot \frac{\rho_{1}-\rho_{2}}{\rho_{1}+\rho_{2}} a_{+}
$$

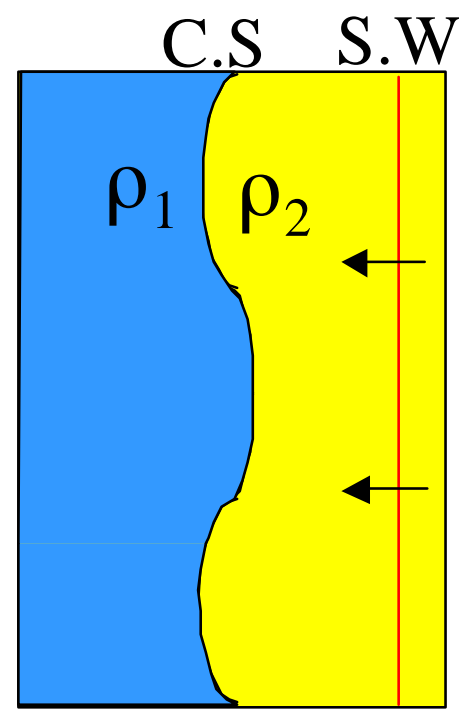

$\mathrm{k}$ wave-number

$\mathrm{U}_{0}$-shock wave induced velocity of the contact surface

$a_{+}$- initial post shock amplitude

$\rho_{1}, \rho_{2}$ - densities ahead and behind the contact surface $\mathrm{t}$-time 


\section{Reduction from the impulsive model}

Aleshin et al. (1997) $\quad \mathrm{M}=2.5-4.5$

(t)

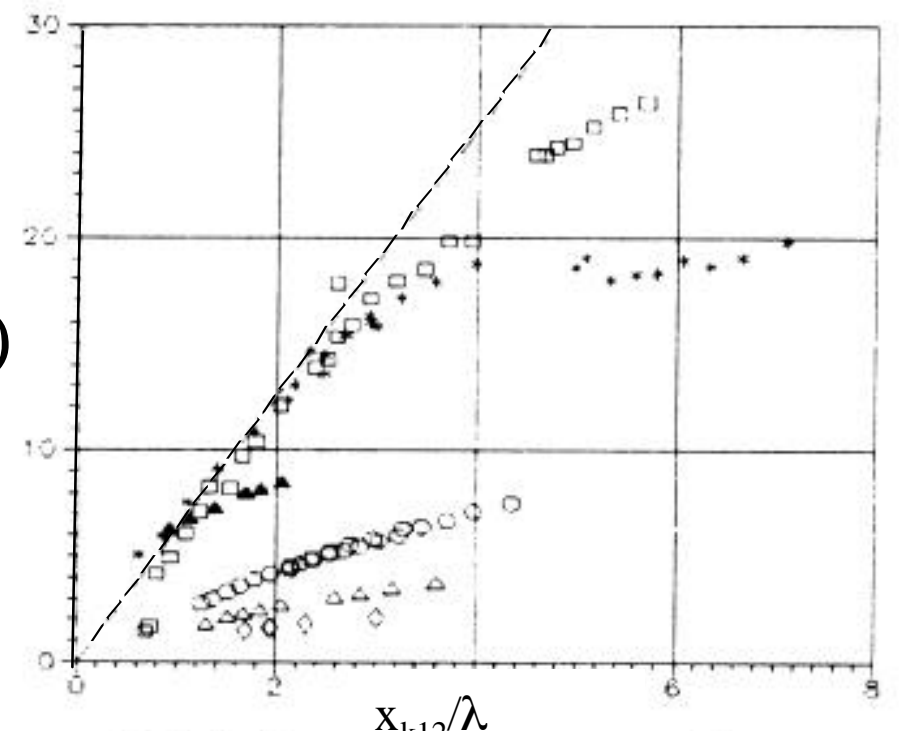

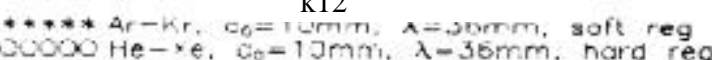

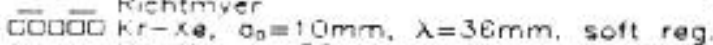

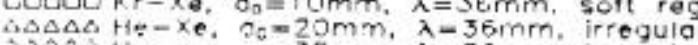

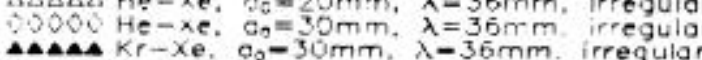

$\widetilde{\mathrm{a}}(\mathrm{t})=\left(\mathrm{a}_{\mathrm{k} 12}\left(\mathrm{x}_{\mathrm{k} 12}\right)-\mathrm{a}(0)_{\mathrm{k} 12}\right) /\left(\dot{\mathrm{a}}_{\mathrm{k} 12} \mathrm{At}\right)$

At high Mach numbers:
Dimonte et al. (1996) $\quad \mathrm{M}=15.3$

Holmes et al. (1997) $\quad a_{\mathrm{k}}=0.63$

$$
\mathrm{U}=\min \left(\mathrm{U}_{\text {richtmyer }}, \mathrm{U}_{\text {Transmitted }}-\mathrm{U}_{\text {Interface }}\right)
$$

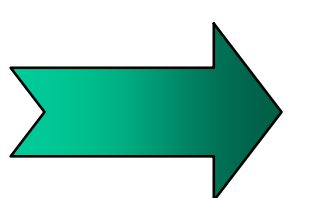

The reduction is a high Mach number effect 


\section{Experimental results: reduction from the impulsive model}

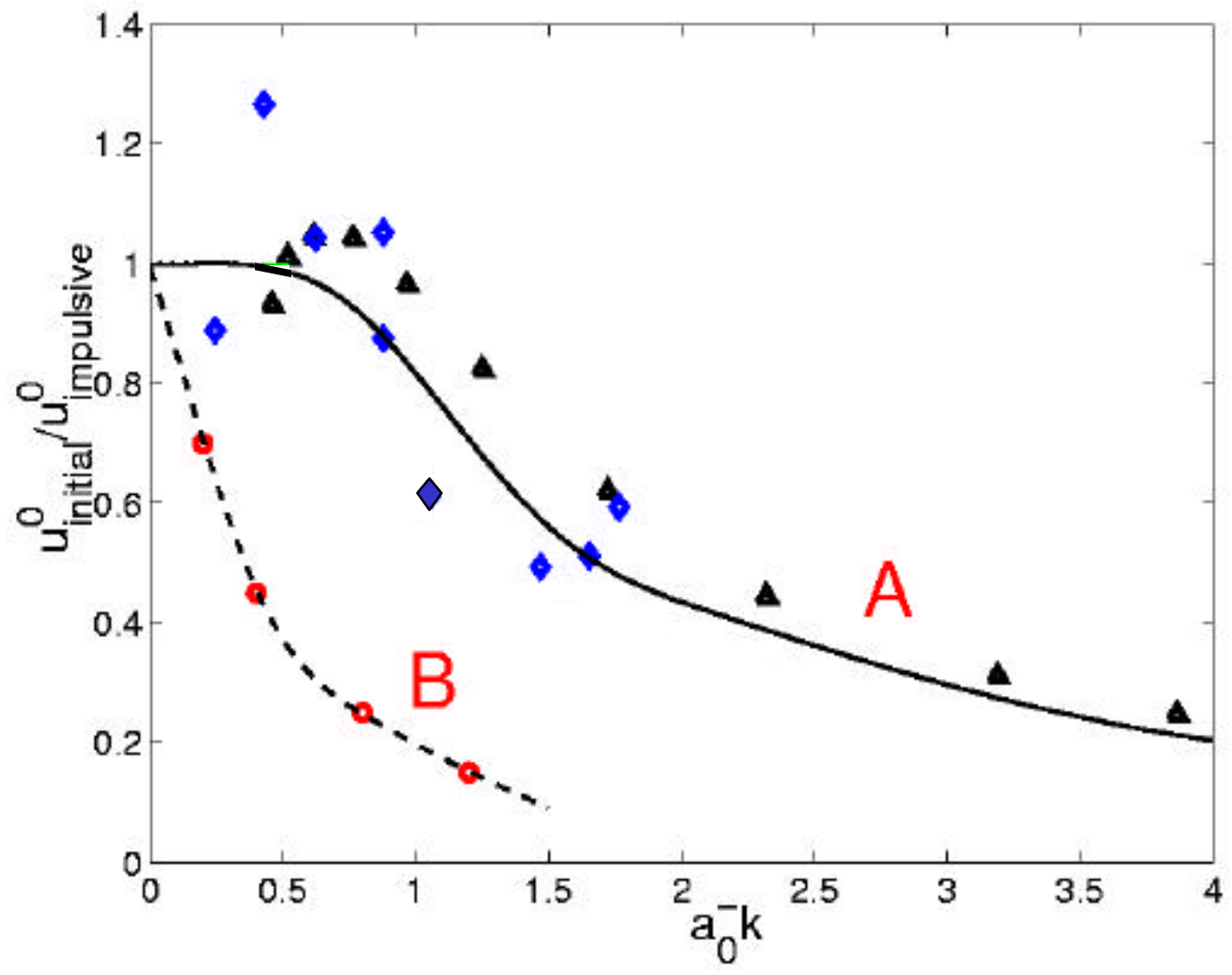

$\checkmark$ Dimonte $\mathrm{Be} \rightarrow$ Foam $(\mathrm{M}=15.3)$

- Aleshin $\mathrm{Ar} \rightarrow \mathrm{Xe}(\mathrm{M}=2.5)$

- Aleshin $\mathrm{He} \rightarrow \mathrm{Xe}(\mathrm{M}=2.5)$ 


\section{Experimental Apparatus}

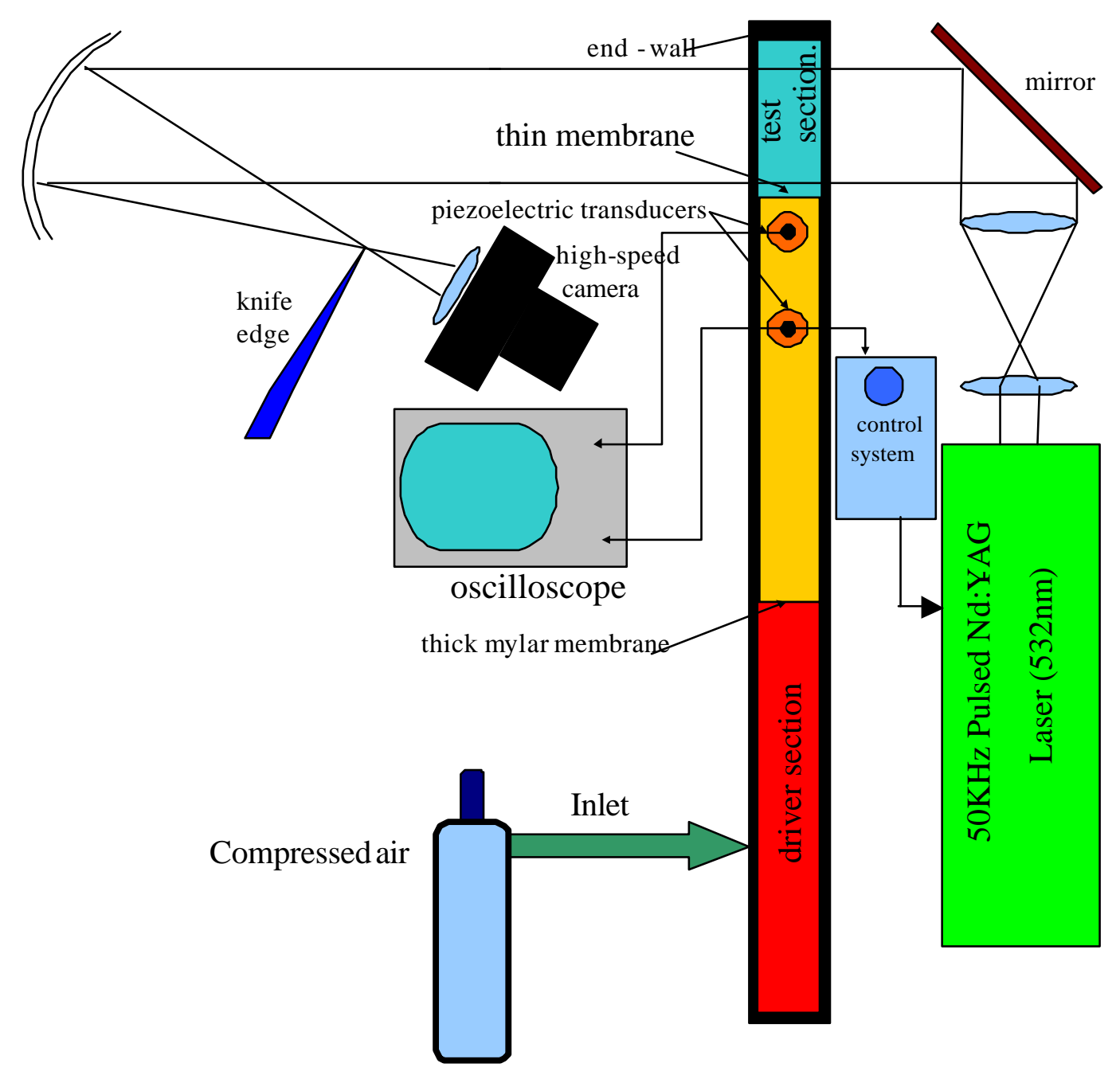




\section{$\underline{\text { Control and diagnostic }}$}

Digital oscilloscope

Inputs:

$\Delta \mathrm{t}$ (minimum 20us), Delay time,

Lightning duration

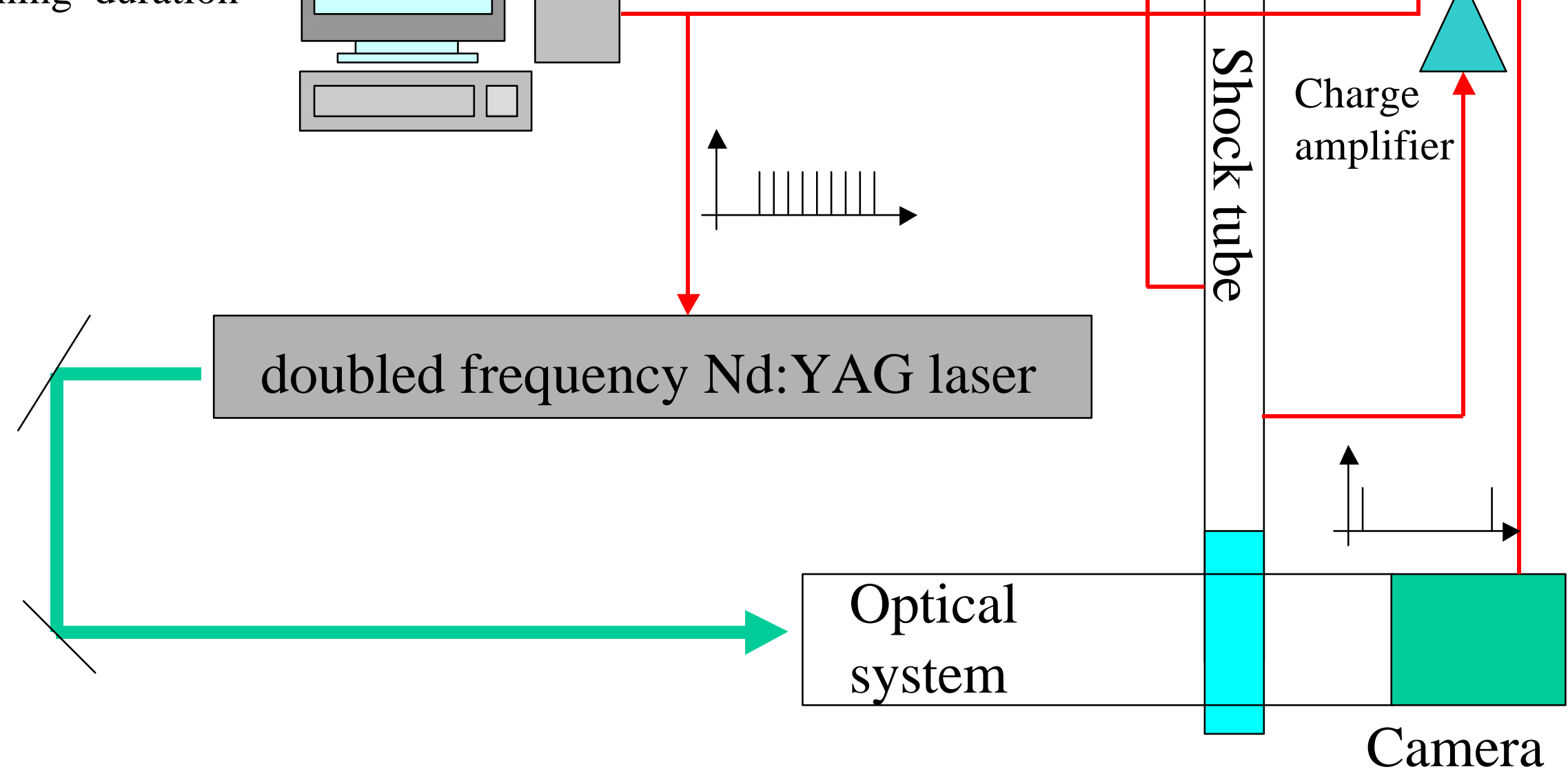




\section{Experimental apparatus The membrane}

Mach number $\mathrm{M}=1.2$

\begin{tabular}{|c|c|c|}
\hline$\lambda$ & $\mathrm{a}_{-}$ & $\mathrm{a}_{-} \mathrm{k}$ \\
\hline $80 \mathrm{~mm}$ & $40 \mathrm{~mm}$ & 3.14 \\
\hline $26 \mathrm{~mm}$ & $10 \mathrm{~mm}$ & 2.45 \\
\hline $40 \mathrm{~mm}$ & $12 \mathrm{~mm}$ & 1.9 \\
\hline $80 \mathrm{~mm}$ & $20 \mathrm{~mm}$ & 1.57 \\
\hline $40 \mathrm{~mm}$ & $7 \mathrm{~mm}$ & 1.1 \\
\hline $40 \mathrm{~mm}$ & $3 \mathrm{~mm}$ & 0.5 \\
\hline
\end{tabular}

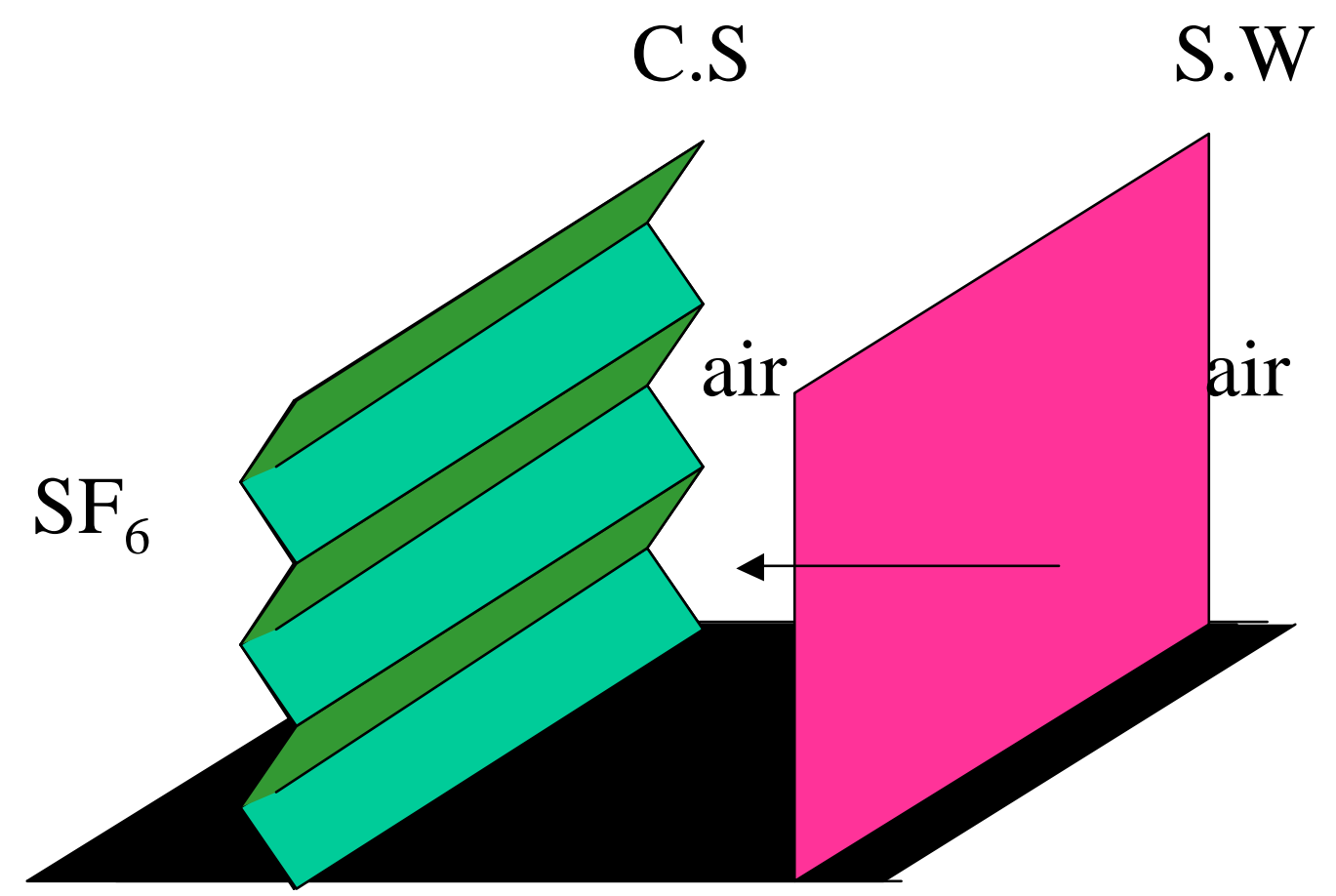




\section{Experimental results $(M=1.2)$}

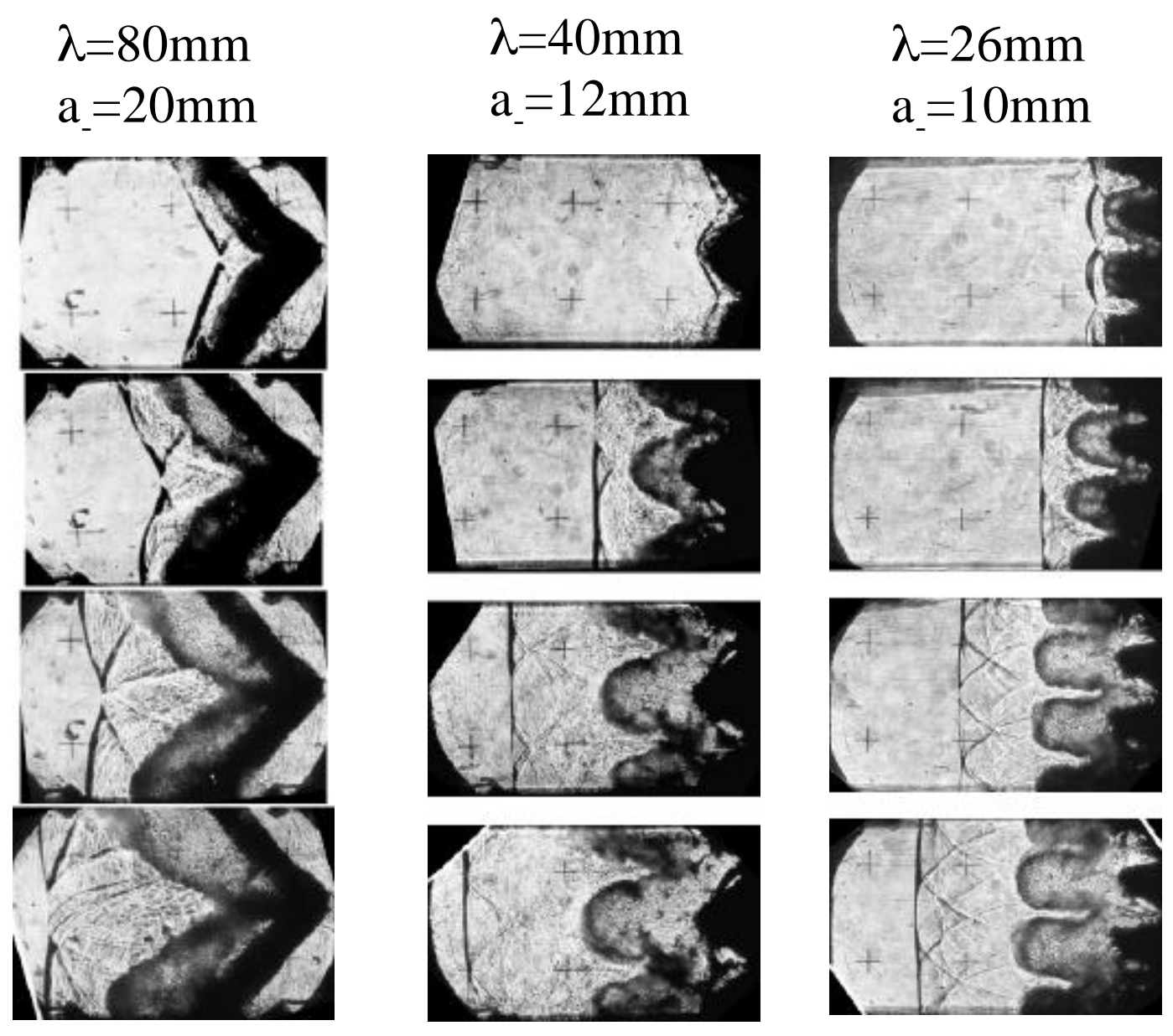




\section{Numerical simulation}

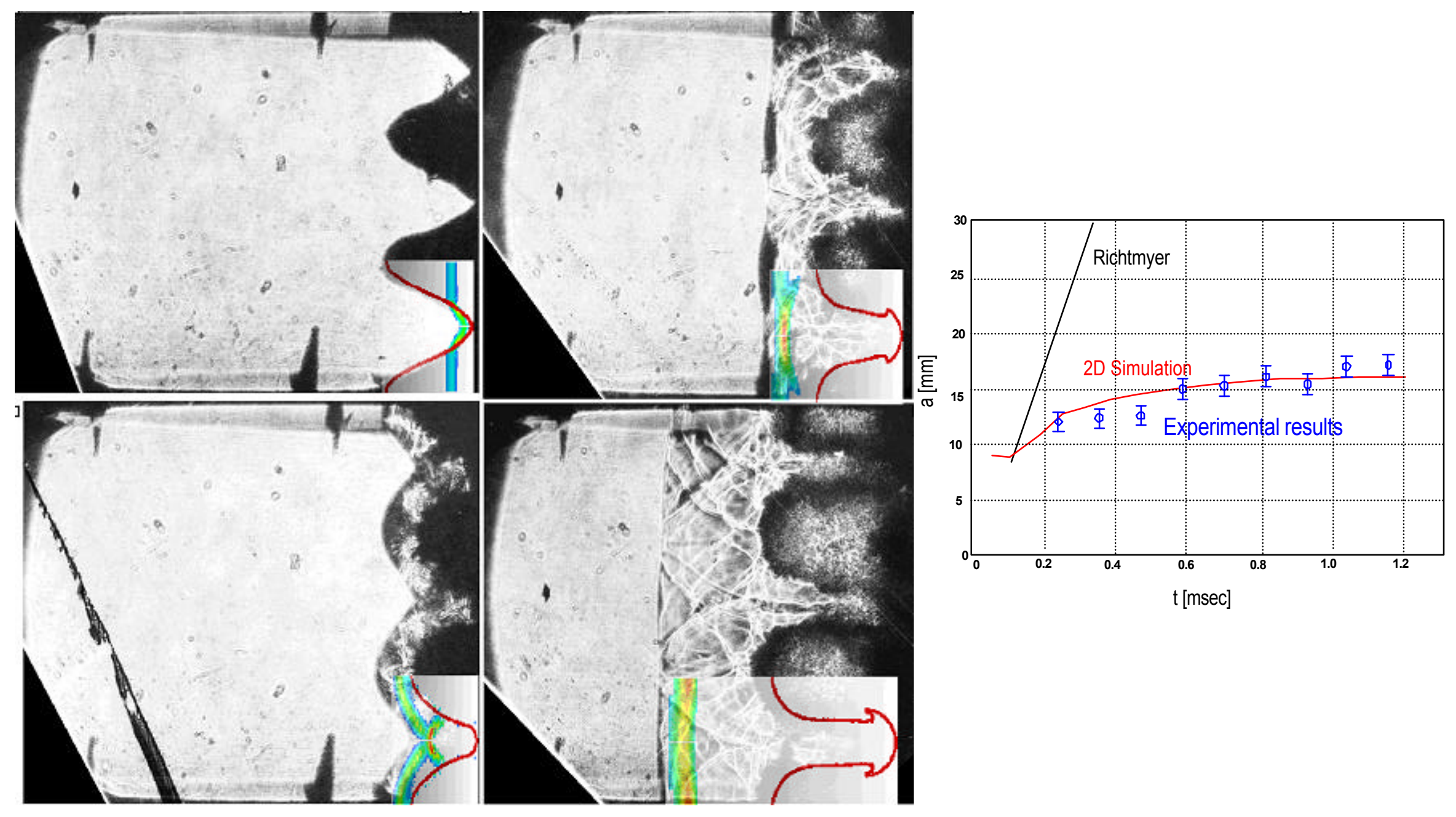

The initial velocity was found from the simulation 


\section{Reduction from the impulsive model: \\ Results of experiment, model and simulation}

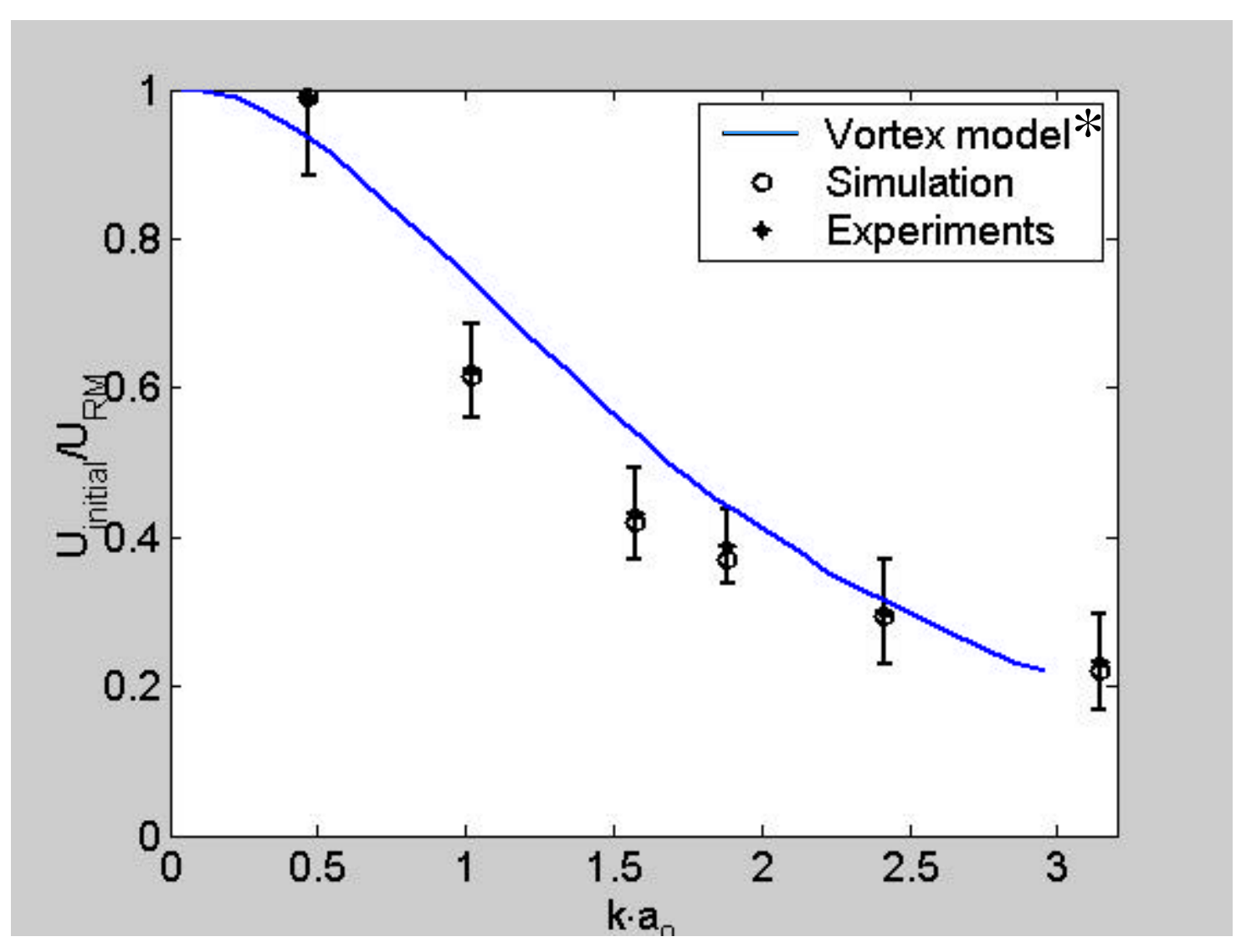

* See Rikanati Thursday T23. 


\section{Experimental results: reduction from the impulsive model}

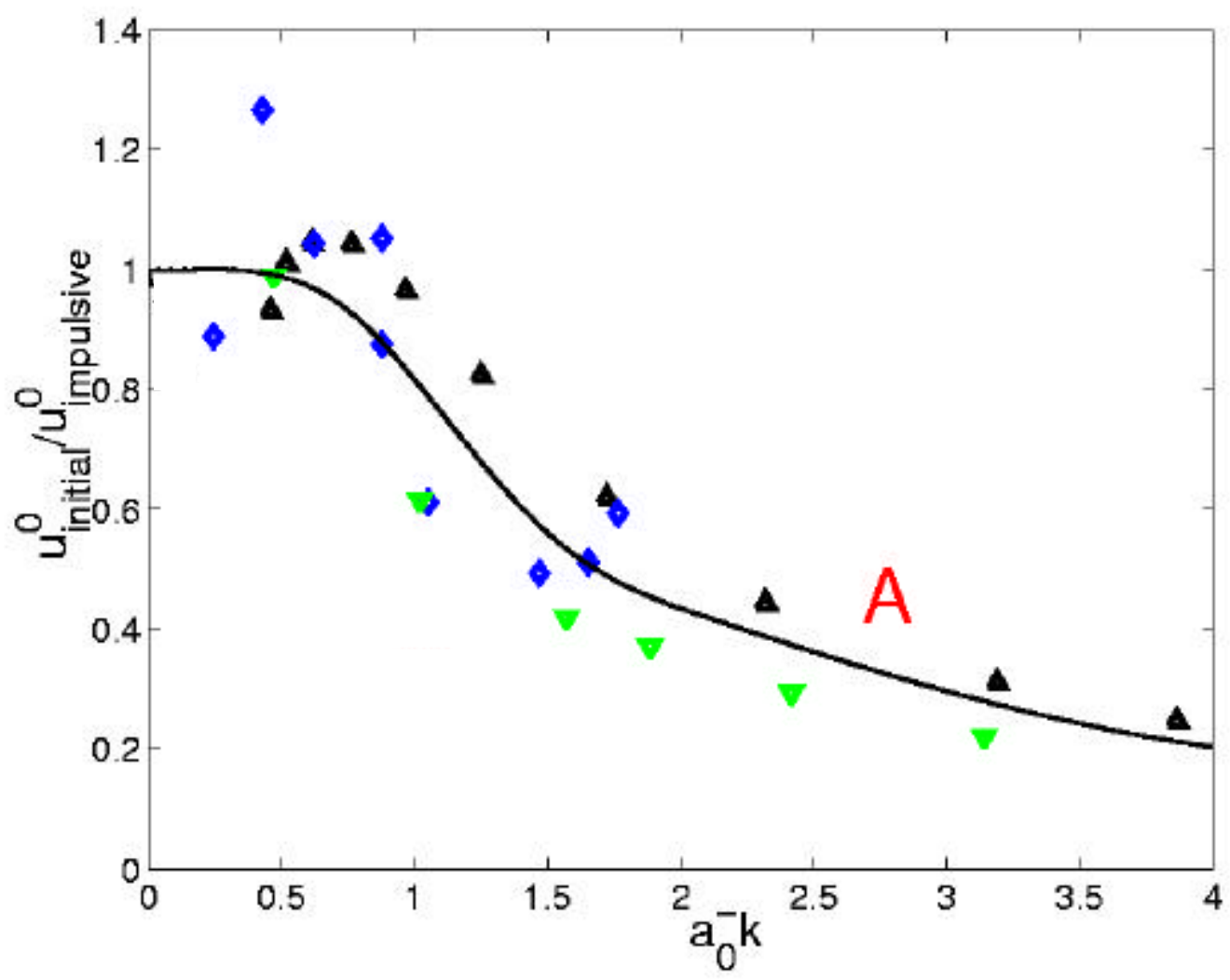

$\checkmark \quad$ Dimonte $\mathrm{Be} \rightarrow$ Foam $(\mathrm{M}=15.3)$

- Aleshin $\mathrm{Ar} \rightarrow \mathrm{Xe}(\mathrm{M}=2.5)$ Sadot Air $\rightarrow \mathrm{SF}_{6}(\mathrm{M}=1.2)$

High initial amplitude effect 


\section{Bubble late time evolution in the}

large amplitude experiment
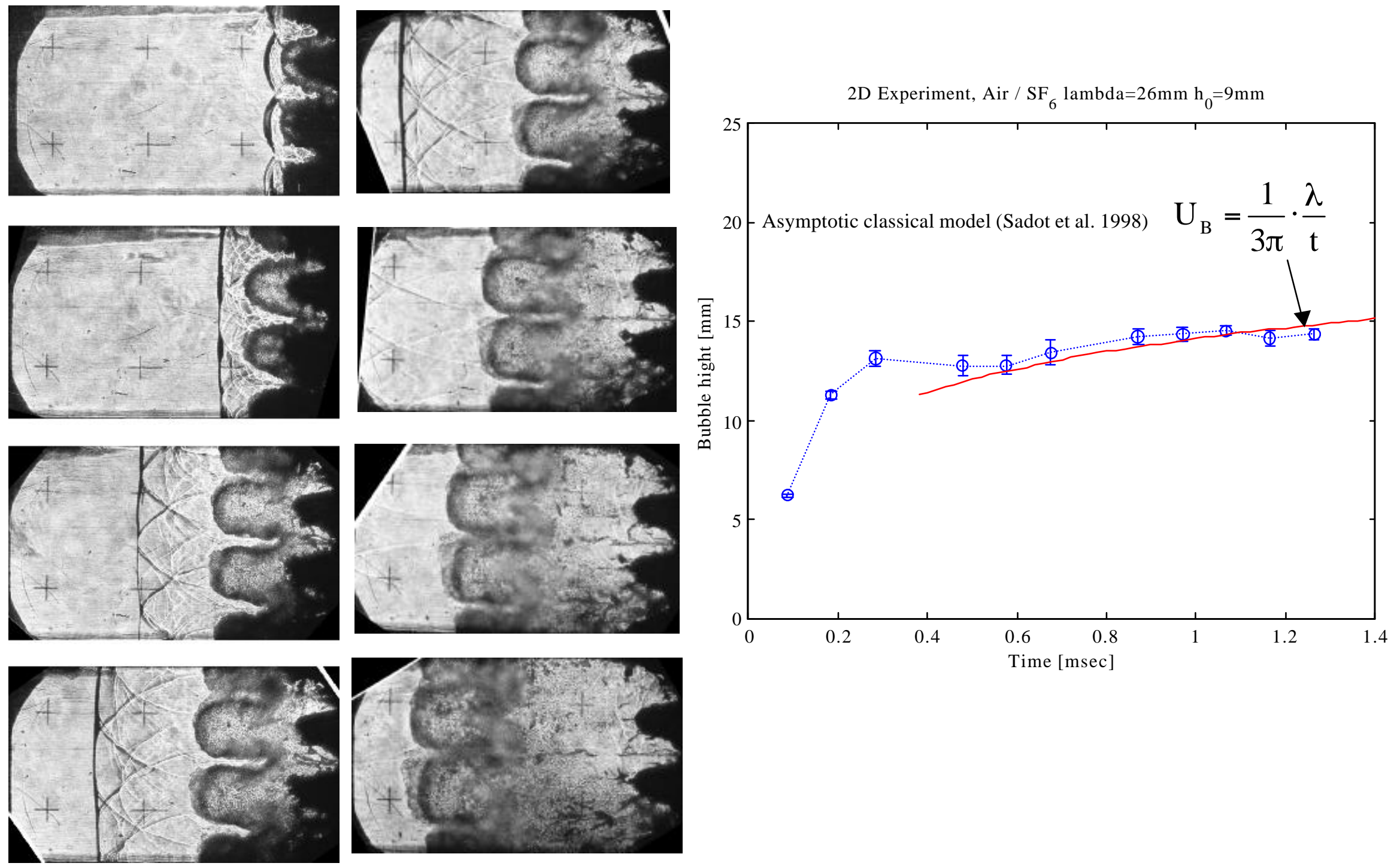


\section{Late time reduction from the classical models due to high Mach number effects}

$\mathrm{a}_{0}=3 \mathrm{~mm}, \mathrm{M}=2$

air to $\mathrm{SF}_{6}\left(\gamma_{\mathrm{SF}_{6}}=1.09\right)$

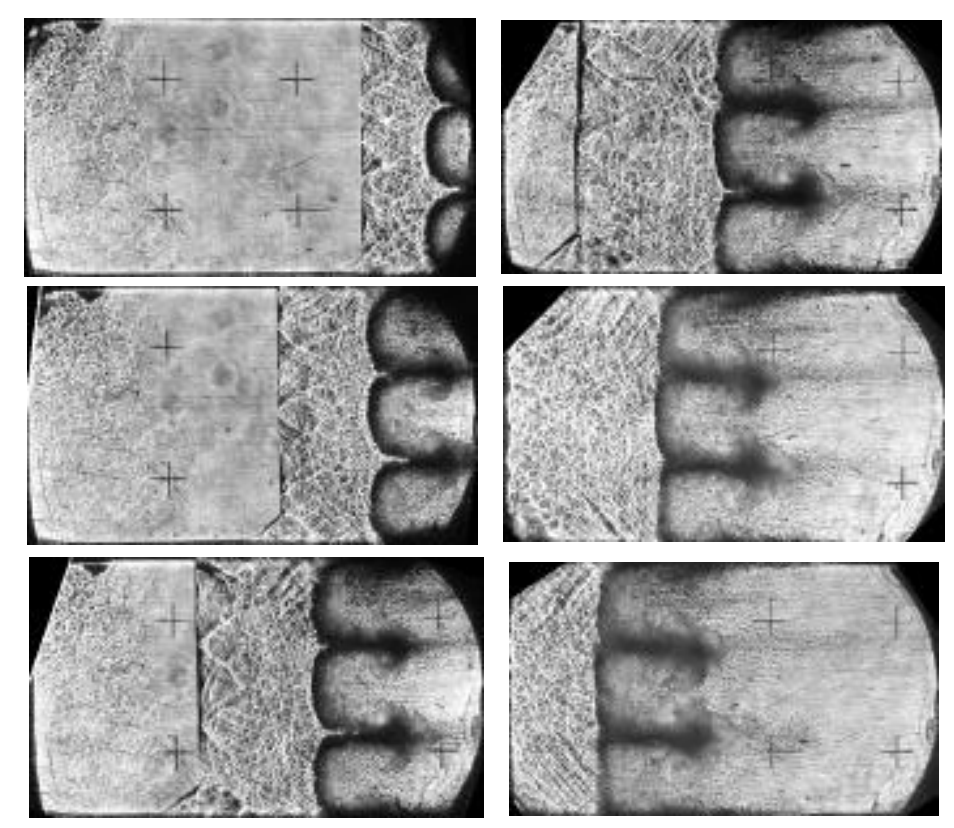

The radius of curvature of the bubble:

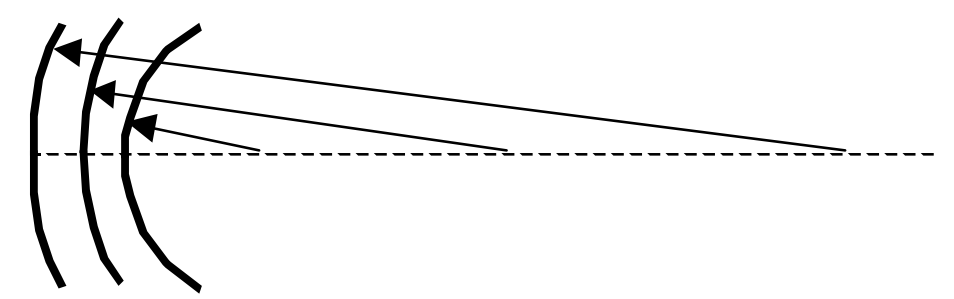

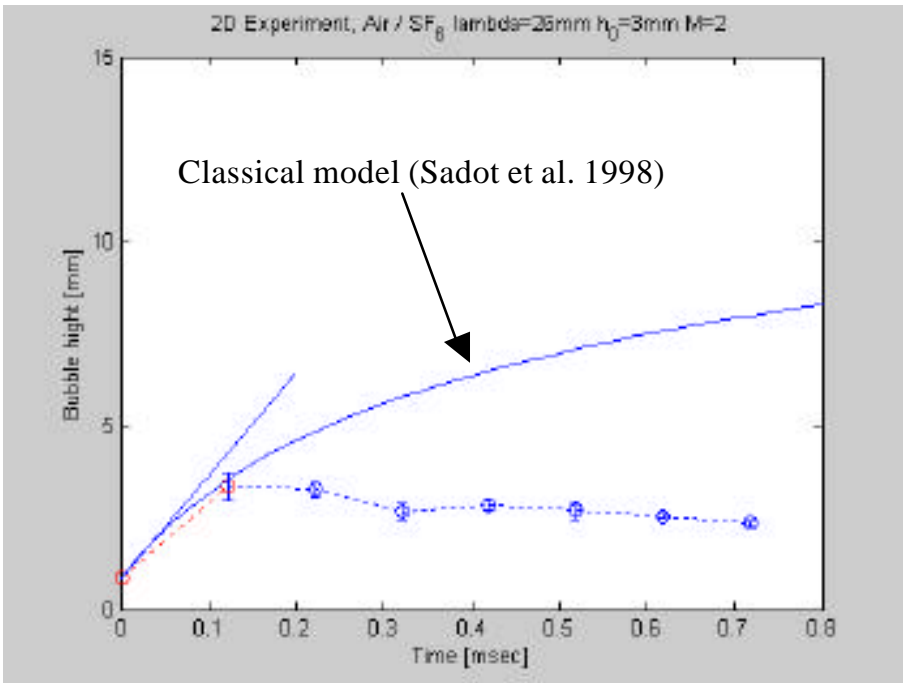

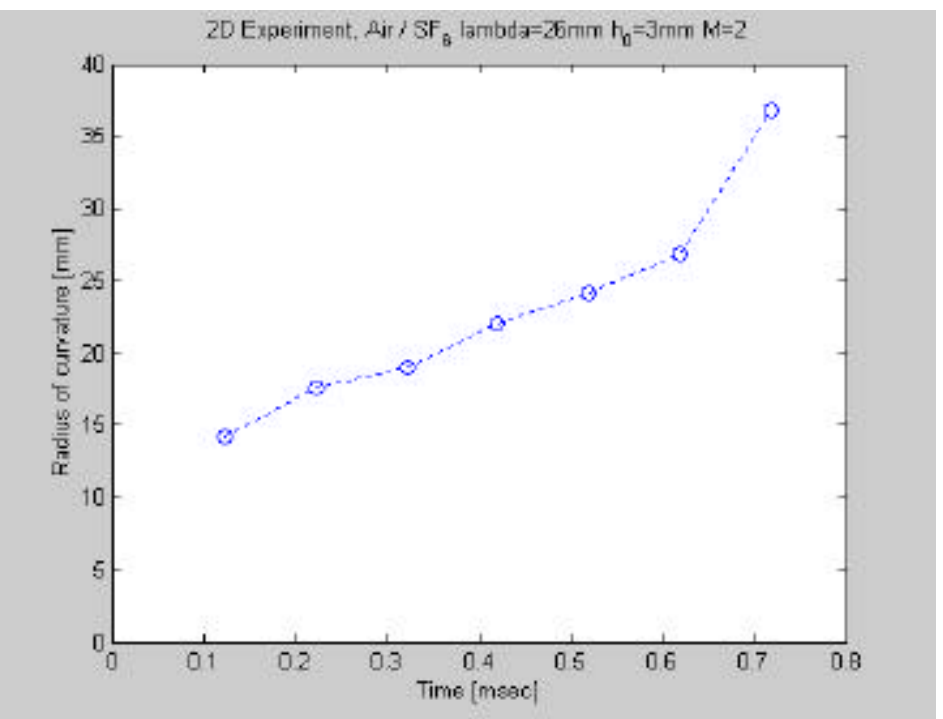




\section{At late time the shock reverberation reduces the}

\section{bubble growth rate}

Shock front
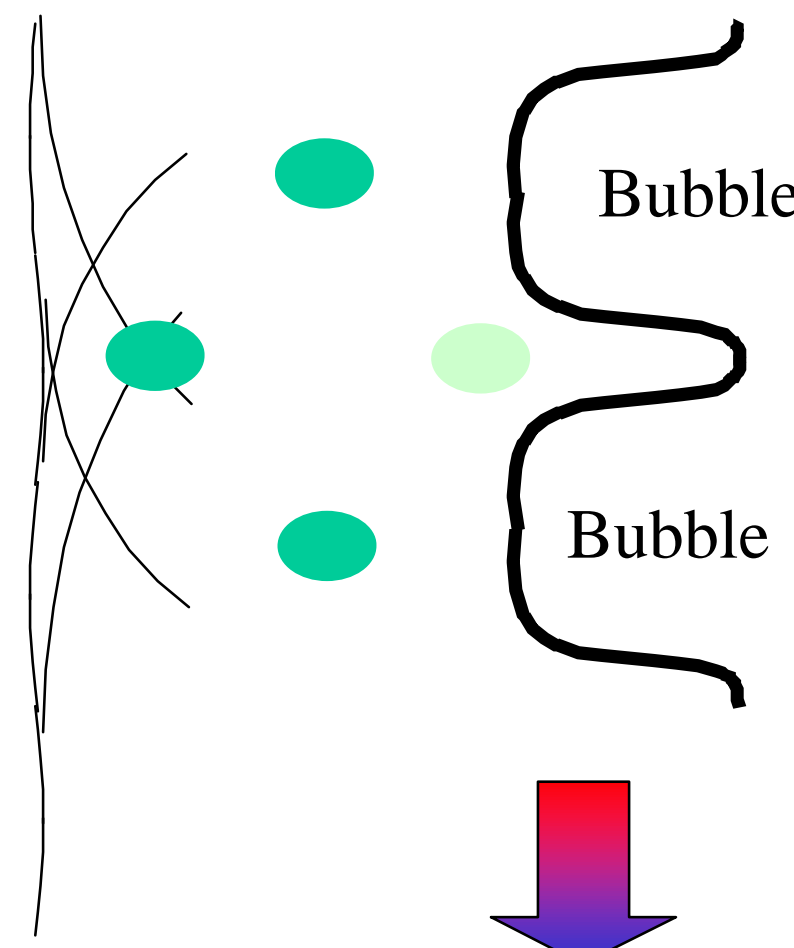

Shock front

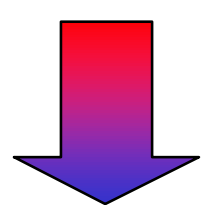

The growth velocity is reduced The radius of curvature is increased

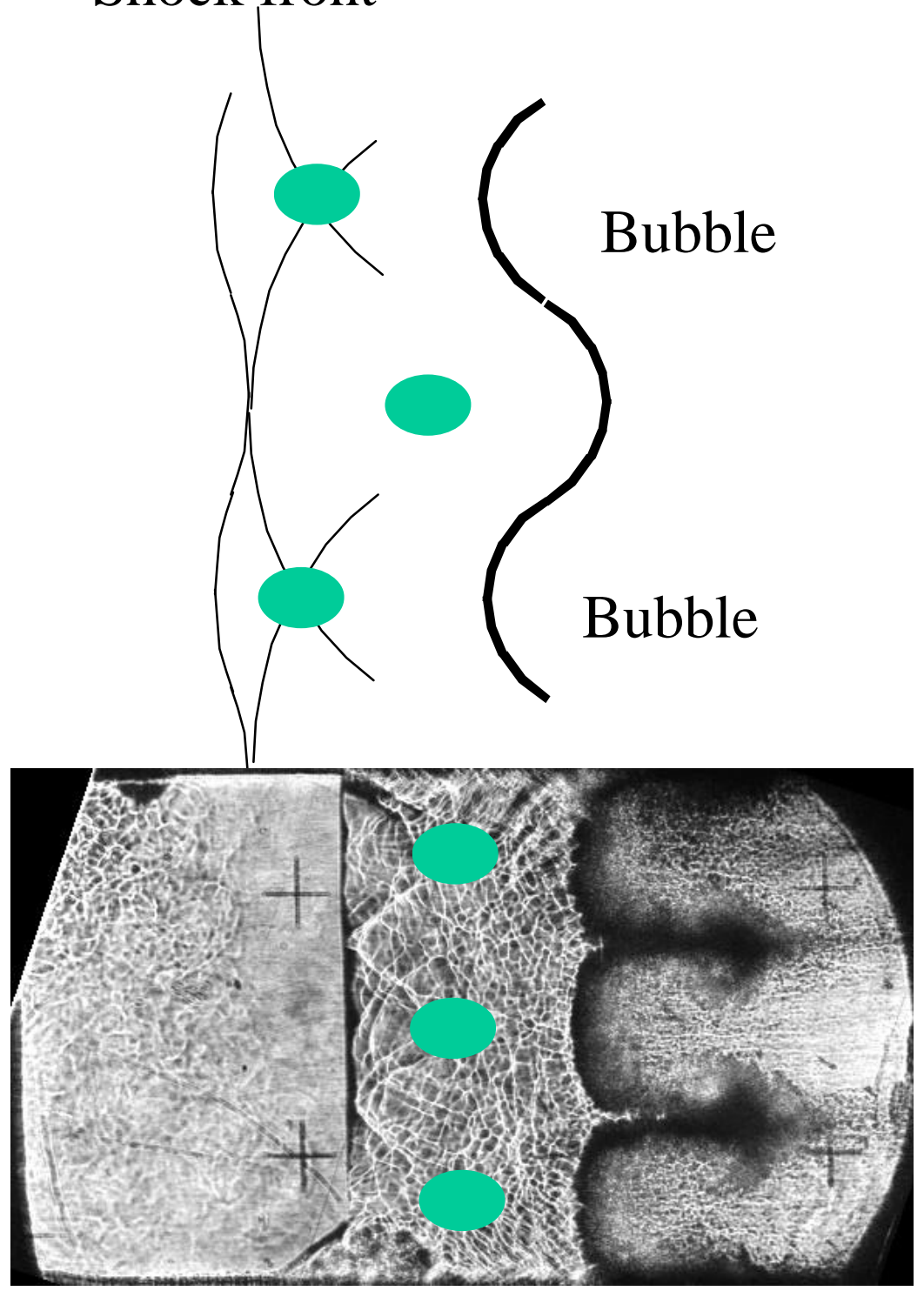




\section{Summary}

Effects of high initial amplitudes were quantified experimentally for the early linear stages of the flow.

The reduction from the Richtmyer initial velocity occur even at low Mach number $(\mathrm{M}=1.2)$.

For the late nonlinear stages of the flow:

$>$ The initial amplitudes effect was forgotten and the bubble evolves as in the classical case (depends only on the wave length).

$>$ New effect was observed for high Mach numbers which decreases the bubble asymptotic velocity. 


\section{MEASUREMENTS WITHIN A RICHTMYER-MESHKOV MIXING ZONE USING A TRIPLE HOT WIRE PROBE TECHNIQUE

*IUSTI, UMR CNRS 6595, Université de Provence, Technopôle de Château-Gombert, 5 rue Enrico Fermi, 13453 Marseille Cedex 13, FRANCE *CEA-DAM, Ile de France, BP 12, 91680 Bruyères-le-Chatel, FRANCE

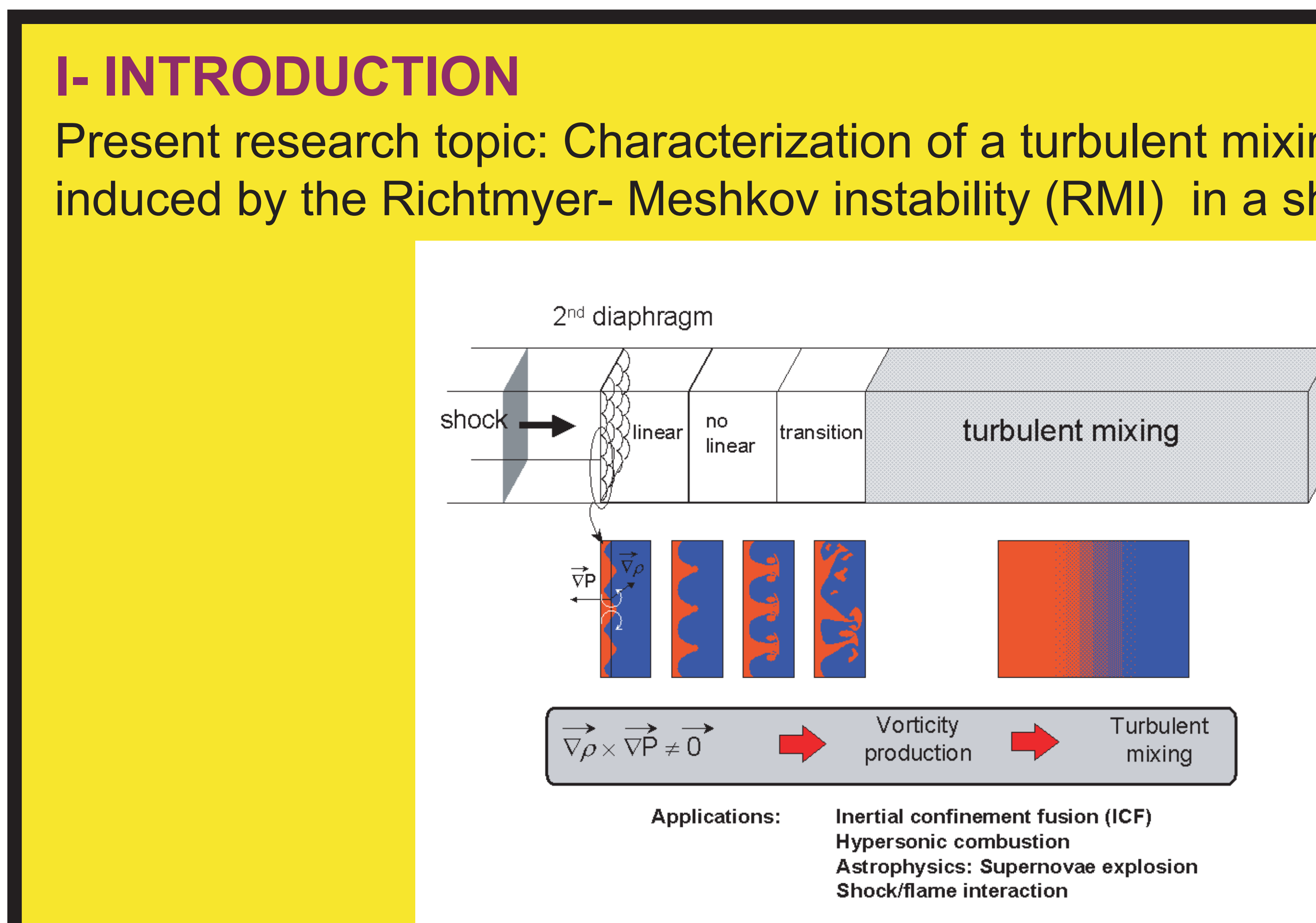

Global characterization : visual structures, thickness

- Time evolution of local measurements (molar fraction and velocity) -Turbulent energy level

\begin{tabular}{lc}
\multicolumn{2}{c}{ Schlieren visualizations } \\
Laser Doppler + & Constant temperature \\
anemometry & hot wire anemometry \\
(CEA/DAM) & (IUSTI)
\end{tabular}

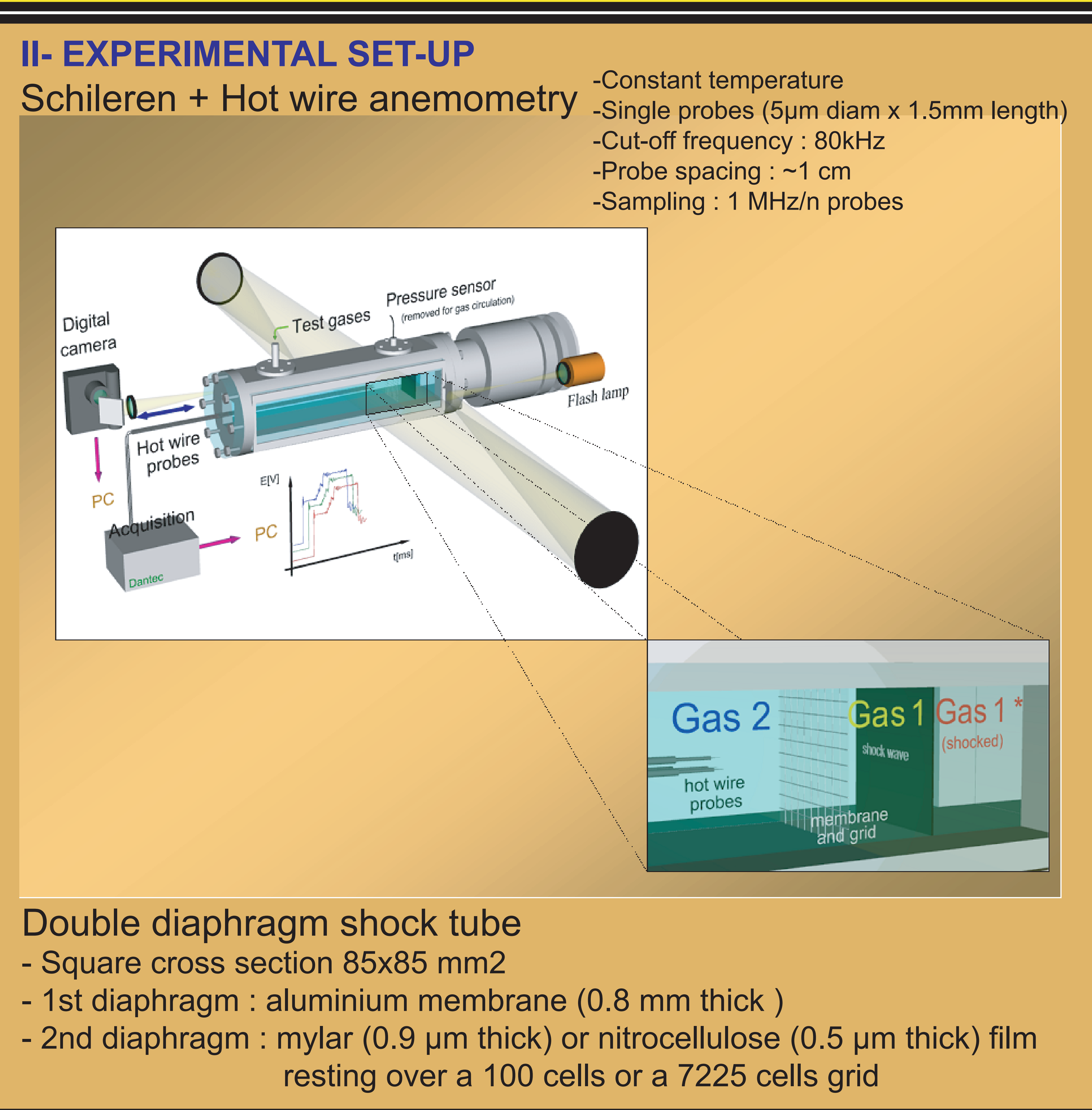

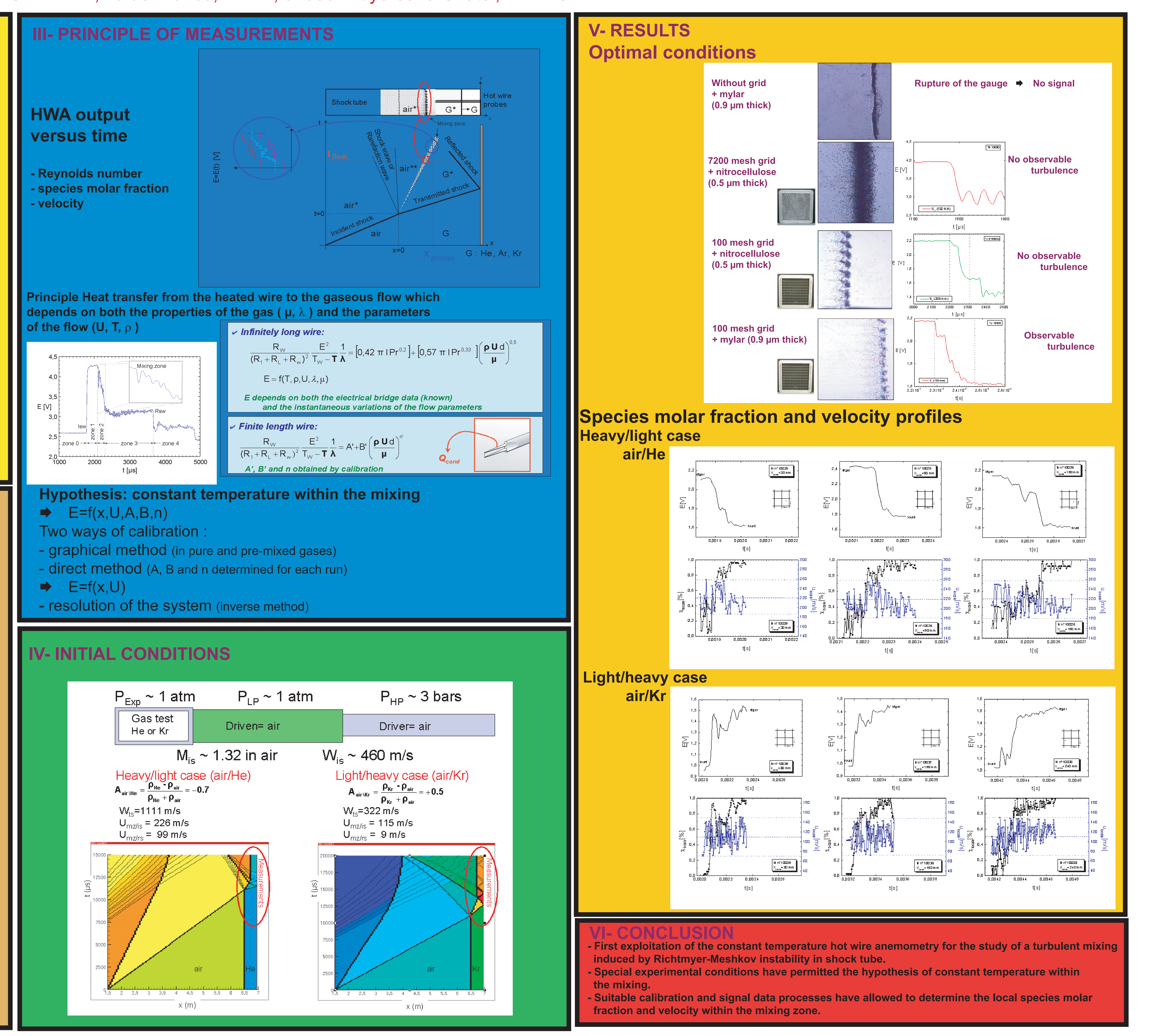


Paper at the VIII International Workshop on Physics of Compressible

Turbulent Mixing

Technological Institute, Pasadena, California, USA

\title{
EXPERIMENTAL INVESTIGATION INTO THE SELF-SIMILAR MODE OF MIXING OF DIFFERENT DENSITY GASES IN THE EARTHS GRAVITATIONAL FIELD
}

\author{
Yu.A.Kucherenko, O.E.Shestachenko, Yu.A.Piskunov, E.V.Sviridov, \\ V.M.Medvedev, A.I.Baishev \\ Russian Federal Nuclear Center - Academician E. I. Zababakhin \\ All-Russian Research Institute of Technical Physics \\ 456770 Snezhinsk, Russia
}

\begin{abstract}
At the installation OSA the experiments on the investigation of the self-similar mixing of different density gases in the Earth's gravitational field have been performed. At the same time, the light gas was found under the heavy one, and the gases were separated by a specter-diaphragm. At some instant of time the specter-diaphragm was ruptures into small-scale fragments by the external force. At the formed contact boundary of two different density gases the Rayleigh-Taylor instability and the unstationary zone of turbulent mixing evolved. For three values of Atwood number the experiments were performed. In the experiments the mixing front trajectories in the light gas and the heavy one were recorded. According to the results of experiments the mixing asymmetry coefficient and the constant $\alpha$ defining the nondimensional rate of mixing have been determined.
\end{abstract}

\section{Introduction}

In many gasdynamic phenomena such situations are widely met when a heavy medium accelerates the light one and vice versa. Depending on the acceleration profile and direction, at the contact boundary the RayleighTaylor or Richtmyer-Meshkov instabilities can arise. At the same time, at the contact boundary of two different density media the unstationary mixing zone arises. The given work is devoted to the investigation of the self-similar mode of different density gases in the Earth's gravitational field. 


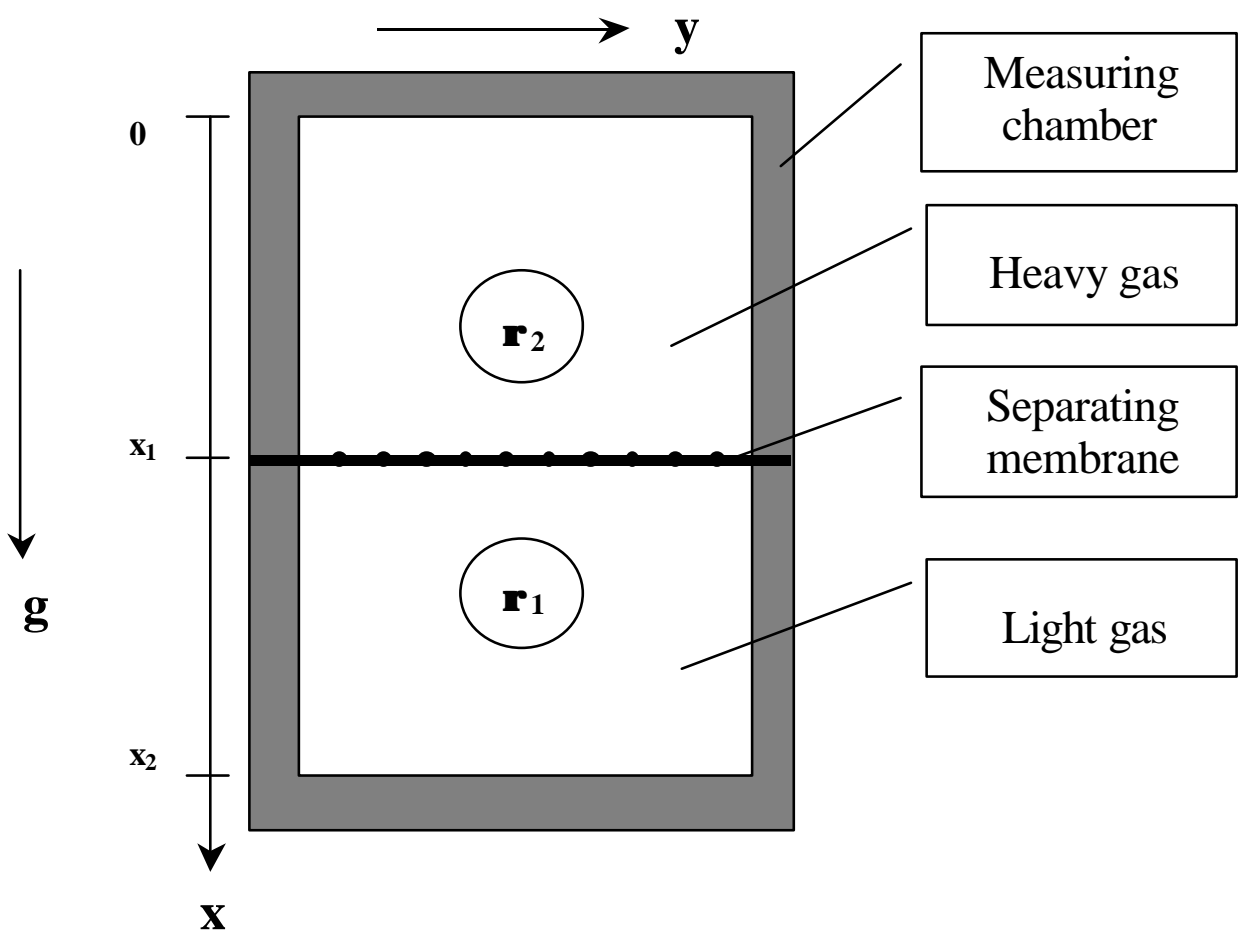

Fig.1 Physical scheme to perform an experiment measuring chamber

The physical scheme of experiments is shown in Fig.1. In the region $0<\mathrm{x}<\mathrm{x}_{1}$ there is gas 2 of density $\rho_{2}$, and in region $\mathrm{x}_{1}<\mathrm{x}<\mathrm{x}_{2}$ there is gas 1 of density $\rho_{1}$. In the point $x=x_{1}$ a separating membrane is placed which prevents from mixing of working gases during the experiment preparation. At the specified instant of time the separating membrane is ruptured into fragments of definite size under the action of the external force, and different density gases begin to interact between themselves. In so far as the heavy gas $\rho_{2}$ is found under light gas $\rho_{1}$ and the Earth's gravitational acceleration is directed from the heavy gas to the light one, then at the contact boundary the Rayleigh-Taylor instabilities arises. The process of the gravitational turbulent mixing zone evolution is visualized by means of schlierentechnique and is recorded on the photographic film.

\section{Set-up of experiment}

In Fig.2 the functional scheme to perform experiments is shown. The gases being investigated were located in the measuring chamber with transparent walls and the internal cross-section equal to $138 \times 138 \mathrm{~mm}^{2}$. The gases are separated by the separating membrane to prevent from the interaction between themselves at the stage of the experiment preparation. 
Filling up with gases was carried out by means of the gas filling system, which supported the pressure drop $\Delta \mathrm{P}<10 \mathrm{~Pa}$ on both sides of the separating membrane. This is necessary to provide the conservation of the separating membrane, which withstands the limiting pressure drop $\Delta \mathrm{P} \approx 40 \mathrm{~Pa}$.

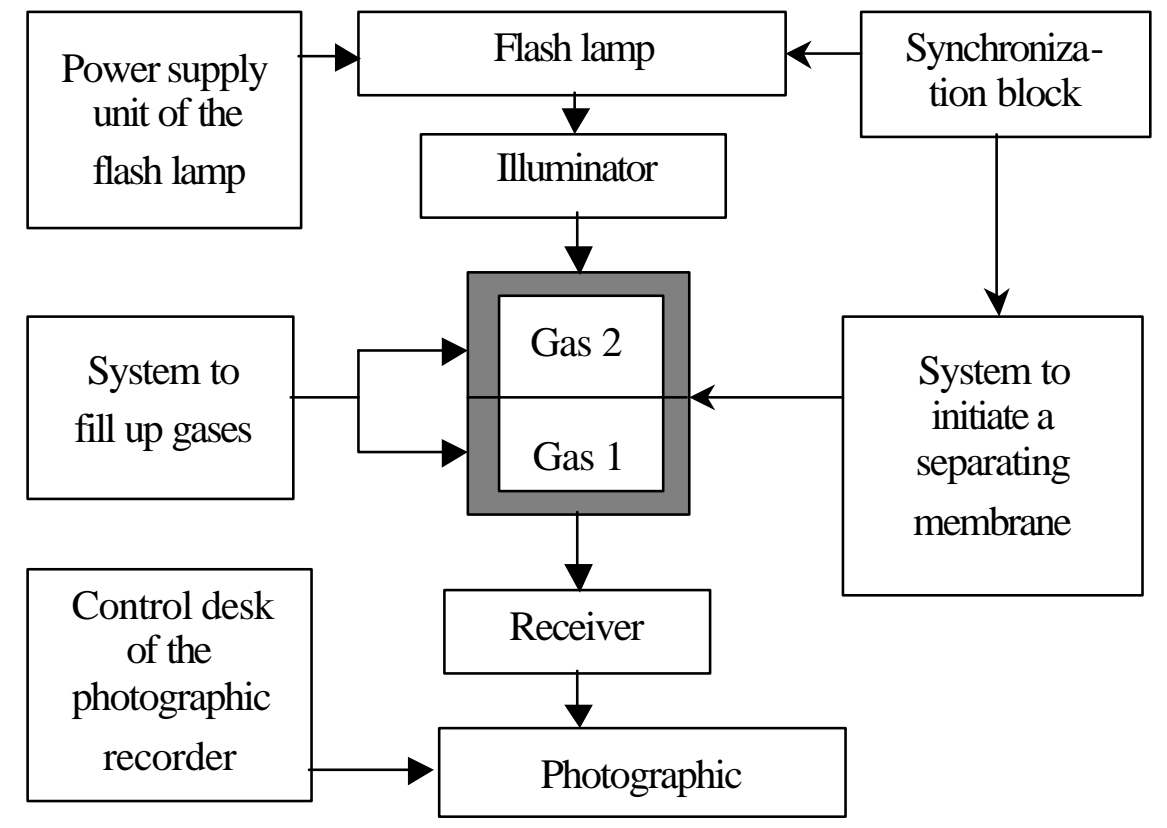

Fig 2 Functional scheme of experiments

At instant of time $t=0$ an electrical pulse is applied to the grid of microconductors from the capacitor bank which is the part of the initiation system of the separating membrane (capacitor bank capacity $\mathrm{C}=0.25 \mu \mathrm{F}$, voltage $\mathrm{U}=12 \mathrm{kV}$ ). At the same instant of time, the flash lamp begins to operate in a stroboscopic mode illuminating the measuring chamber. Optical nonuniformities are visualized by means of the light and shade device IAB 451. The turbulent mixing process evolution is recorded on the photographic film by means of a drum-type photographic recorder. are:

The distinctive features of the given scheme to perform experiments

- Constancy of acceleration at the contact boundary of gases. In the other experiments on gases the contact boundary acceleration is quasi-constant.

- Absence of gases compression and the gravitational turbulent mixing zone during the whole experiment. This makes possible to perform the unambiguous interpretation of the turbulent mixing zone width.

- Constancy of Atwood number on the contact boundary of gases during the whole experiment even for gases with different adiabatic indices. 
- Absence of parietal flows, because the turbulent mixing zone, upon the whole, does not move relative to the measuring chamber walls.

- Absence of the turbulent mixing zone motion as the whole. This makes possible to determine the asymmetry coefficient of the gravitational turbulent mixing for gases.

Real gases are possessed of viscosity and in order that this parameter does not exert any influence on the mixing process, it is necessary to satisfy the condition $\mathrm{g} 1 \gg \mathrm{v}^{2} \cdot \mathrm{L}^{-3}$, where $\mathrm{g}_{1}$ - contact boundary acceleration, $\mathrm{v}-$ viscosity, $\mathrm{L}-$ turbulent mixing zone width. For such a gas as helium $\mathrm{v} \approx 10^{-4}$ $\mathrm{m}^{2} / \mathrm{s}$, and if measurements are made at $\mathrm{L}>5 \mathrm{~mm}$, then for the realization of the above shown inequality it would be sufficient to reach
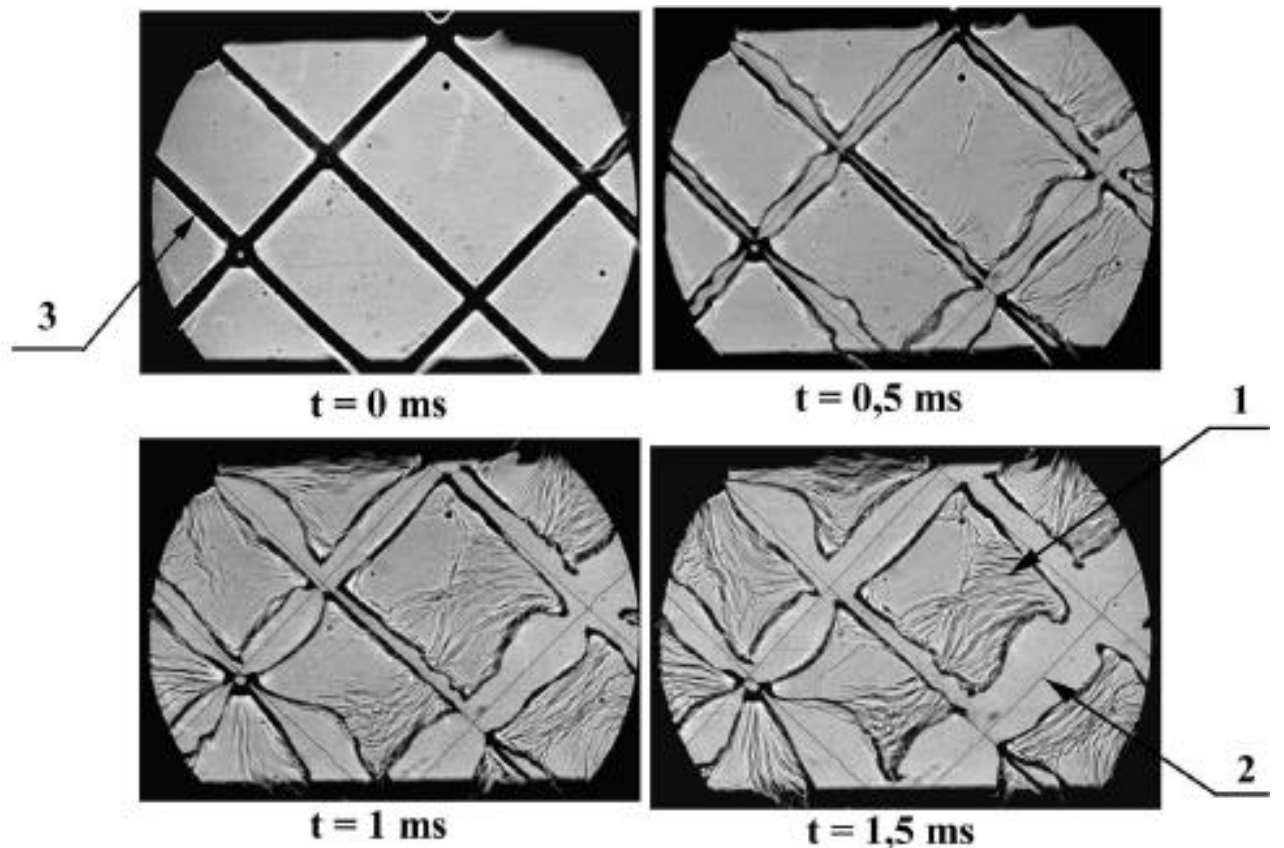

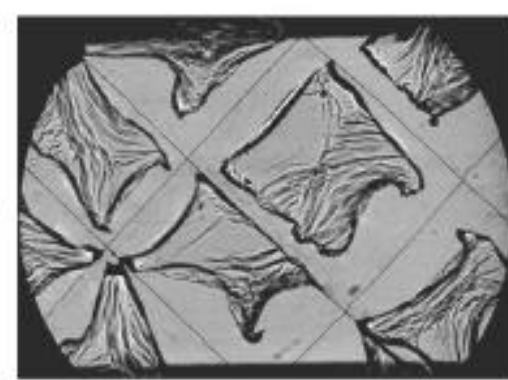

$\mathrm{t}=\mathbf{2} \mathbf{m s}$

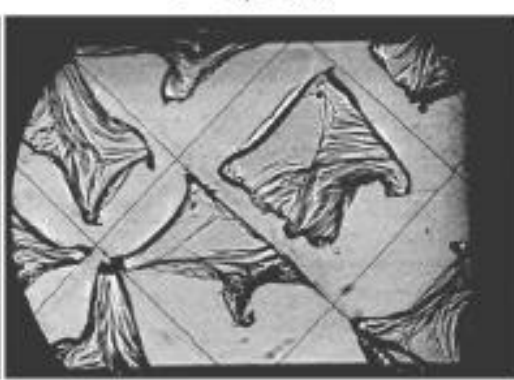

$\mathrm{t}=\mathbf{2 , 5} \mathbf{m s}$

Fig.3 Characteristic photographic images of the rupture process of the liquid film. 
$\mathrm{g}_{1} \gg>0.09 \mathrm{~m} / \mathrm{s}^{2}$. For heavier gases (air, argon, krypton) the shown inequality is satisfied at $\mathrm{L}>1 \mathrm{~mm}$ and $\mathrm{g}_{1}>0.18 \mathrm{~m} / \mathrm{s}^{2}$. Thus, viscosity of gases does not exert any influence on the gravitational turbulent mixing zone evolution at the contact boundary acceleration $\mathrm{g}_{1}=\mathrm{g}_{0}$.

The separating membrane represents an interlaced grid of microconductors, $20 \mu \mathrm{m}$ in diameter, with a $4 \mathrm{~mm}$ spacing. The liquid film of soap solution is applied on this grid. The film thickness is $\approx 1 \mu \mathrm{m}$. At the specified instant of time the electric current is conducted through the grid. Microconductors get warm and the liquid film begins to be ruptured in the places of contact with microconductors. Then the surface tension forces

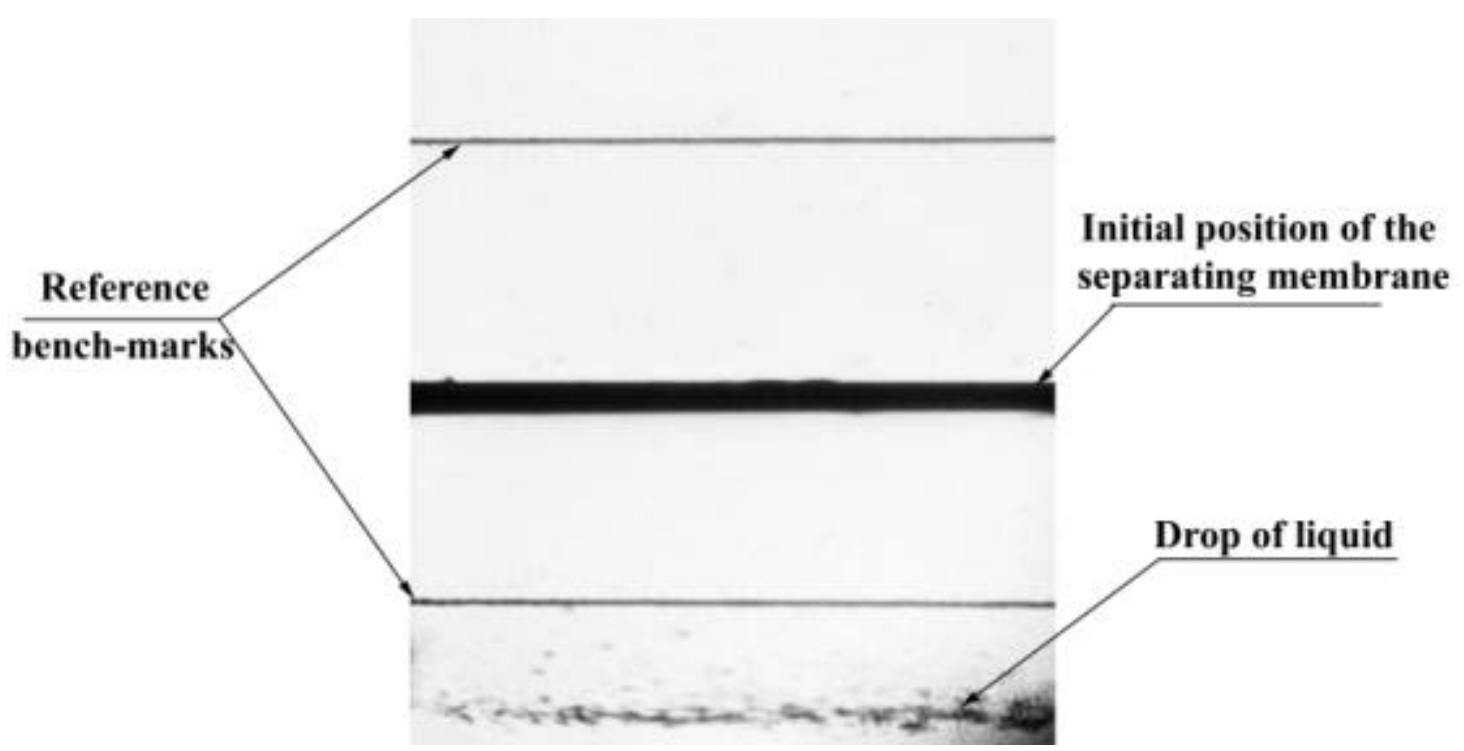

Fig.4 Characteristic photographic images of the separating membrane residues motion.

pull together the liquid film pieces into small balls which under the action of the Earth's gravitational field begins to fall down and do not take part subsequently in the turbulent mixing process.

Fig.3 shows the characteristic photographic images of the rupture process of the separating membrane for different instants of time. Microconductors are denoted by number 1 , liquid film pieces - by number 2 , a microconductor with a liquid film around it - by number 3 . From the figure it is seen that after applying the electric current pulses to the grid the liquid film begins to be separated from the microconductor and then, under the action of surface tension forces, it is tightened into a drop. 
The characteristic photographic images of the separating membrane residues (liquid drops) are shown in Fig. 4. It is seen that the drops of liquid fly in the form of a plane being parallel to that of the separating membrane. Hence, it is possible to conclude that the separating membrane rupture takes place simultaneously all over the plane.

\section{Discussion of results}

In the given work three groups of experiments were performed with different working gases: helium $\mathrm{He}$ (density $\rho=0.178 \mathrm{~kg} / \mathrm{m}^{3}$ ), argon $\mathrm{Ar}$ (density $\rho=1.78 \mathrm{~kg} / \mathrm{m}^{3}$ ), $\mathrm{SF}_{6}$ gas (density $\rho=6.0 \mathrm{~kg} / \mathrm{m}^{3}$ ), krypton $\mathrm{Kr}$ (density $\rho=3.74 \mathrm{~kg} / \mathrm{m}^{3}$ ). In each group eight experiments have been carried out. The relation of densities and Atwood numbers for different groups are shown in Table.

\begin{tabular}{|c|c|c|c|}
\hline Group number & Pair of gases & Relation of densities & Atwood number \\
\hline $\mathbf{1}$ & $\mathbf{S F}_{\mathbf{6}}-\mathbf{A r}$ & $\mathbf{3 , 3 7}$ & $\mathbf{0 , 5 4}$ \\
$\mathbf{2}$ & $\mathbf{S F}_{\mathbf{6}}-\mathbf{H e}$ & $\mathbf{3 3 , 7}$ & $\mathbf{0 , 9 4}$ \\
\hline $\mathbf{3}$ & $\mathbf{K r}-\mathbf{A r}$ & $\mathbf{2 , 1}$ & $\mathbf{0 , 3 5}$ \\
\hline
\end{tabular}

Fig. 5 shows the characteristic photographic images of the gravitational turbulent mixing process in the Earth's gravitational field. Heavy gas is denoted by number $\mathbf{1}$, light gas - by number $\mathbf{2}$. Time $t$ is counted off since the moment of applying the current pulse to the grid of

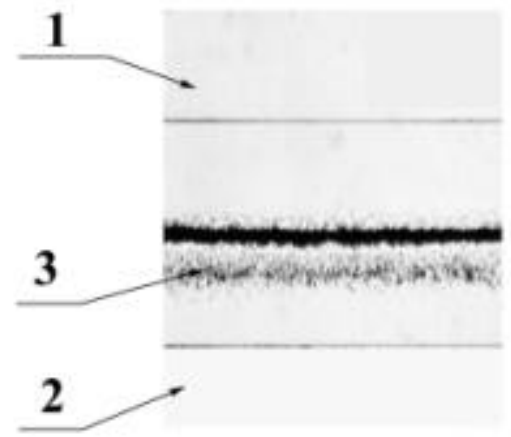

$t_{1}=\mathbf{4 8} \mathrm{ms}$

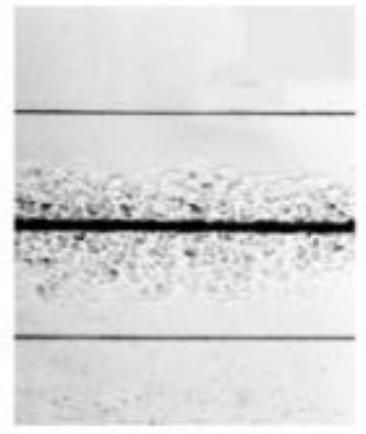

$\mathrm{t}_{2}=148 \mathrm{~ms}$

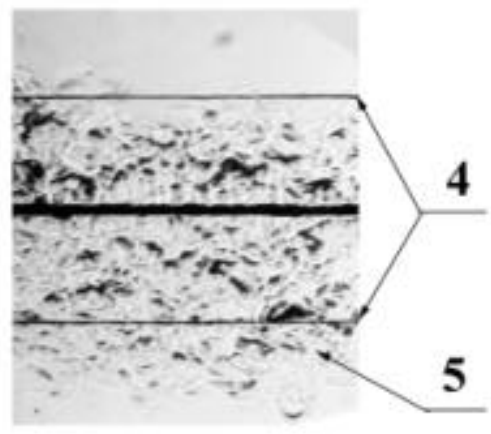

$t_{3}=268 \mathrm{~ms}$

Fig. 5. The characteristic photographic images of the turbulent mixing process. 
microconductors. At the first photo a number of drops $\mathbf{3}$ can be seen which fall down under the action of gravitational forces. At the following two photos the growth of the turbulent mixing zone $\mathbf{5}$ is seen. For the accurate determination of the scale and the coordinates of the mixing front the reference bench-marks $\mathbf{4}$ are set before the measuring chamber glasses.

Fig. $6 \mathrm{a}, 6 \mathrm{~b}$ and $6 \mathrm{c}$ show dependencies of the mixing front coordinate of the light gas into the heavy one on the parameter S. The parameter $\mathrm{S}=\mathrm{gt}^{2} / 2$, where $\mathrm{g}$ is acceleration of the Earth's gravitational field and $\mathrm{t}$ is time since the moment of the separating membrane rupture.

From the presented plots it can be seen that beginning from some value of the parameter $\mathrm{S}$ the mixing front coordinate $\mathrm{L}_{12}$ is growing according to the linear law $\mathrm{L}_{12}=2 \alpha \mathrm{AS}$, where $\mathrm{A}$ is Atwood number and $\alpha$ is constant which determines the nondimensional rate of mixing. If all the experimental points for all three groups of experiments are processed as a single set, then it is possible to obtain the value of $\alpha$ equal to 0.078 .

According to the results of experiments the mixing asymmetry coefficient $\mathrm{k}=\mathrm{L}_{21} / \mathrm{L}_{12}$. For different values of the parameter $\mathrm{S}$ the asymmetry coefficient is changed from 1.1 to 1.7 .

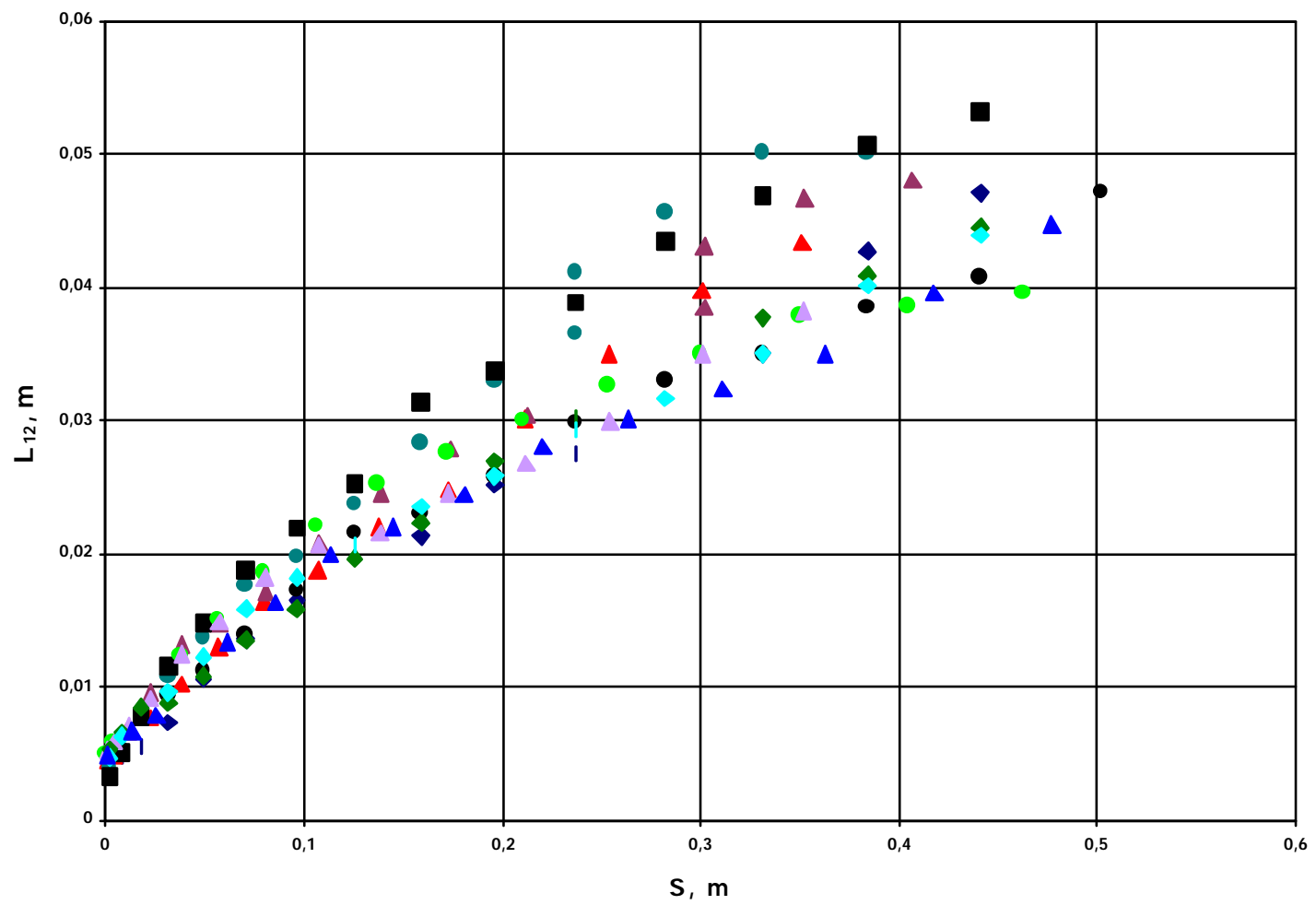

Fig. 6a Dependence of the mixing front coordinate $\mathrm{L}_{12}$ on the parameter $\mathrm{S}$ for the first group of experiments $\left(\mathrm{SF}_{6}-\mathrm{Ar}\right)$. 


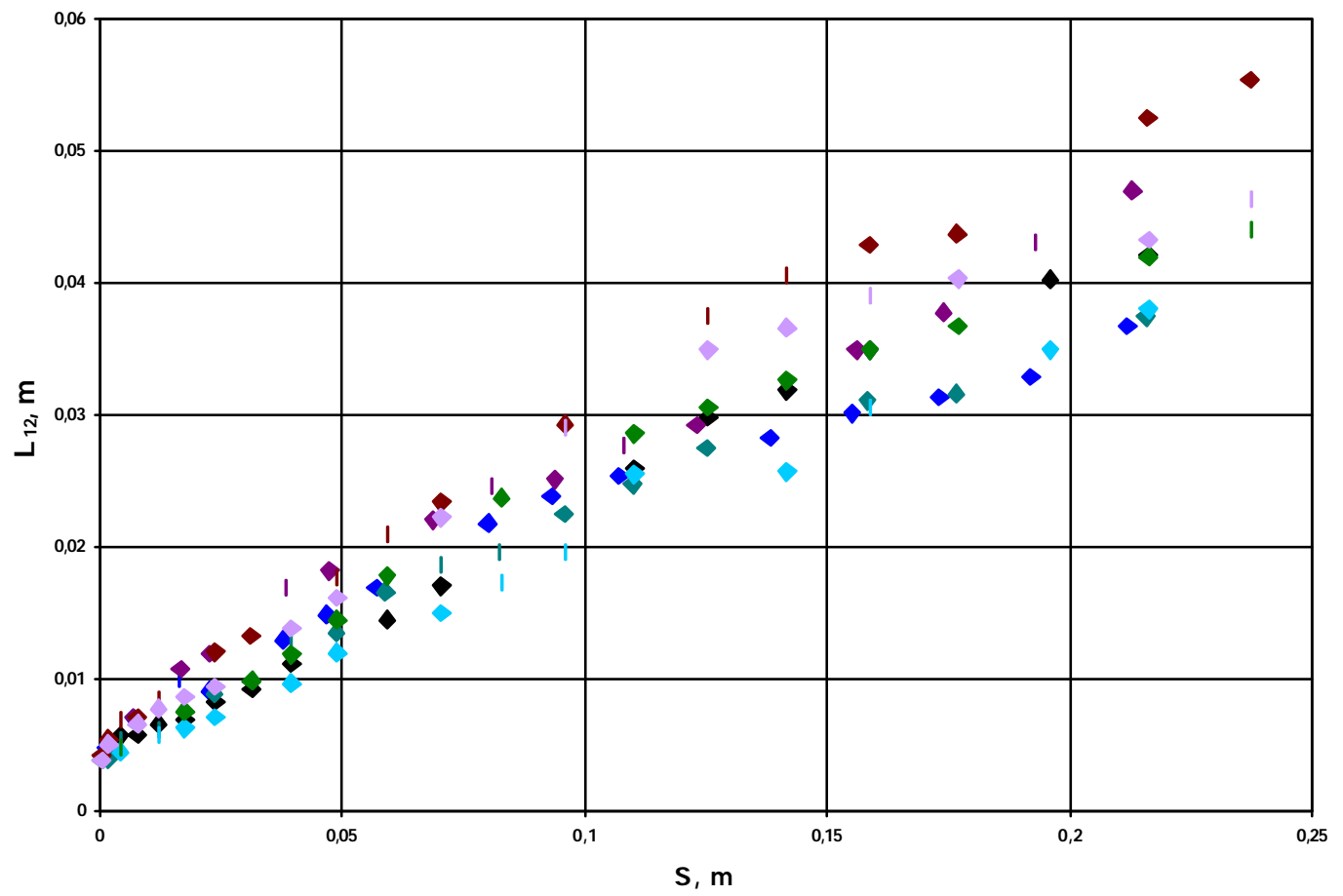

Fig. $6 b$ Dependence of the mixing front coordinate $\mathrm{L}_{12}$ on the parameter $\mathrm{S}$ for the second group of experiments $\left(\mathrm{SF}_{6}-\mathrm{He}\right)$.

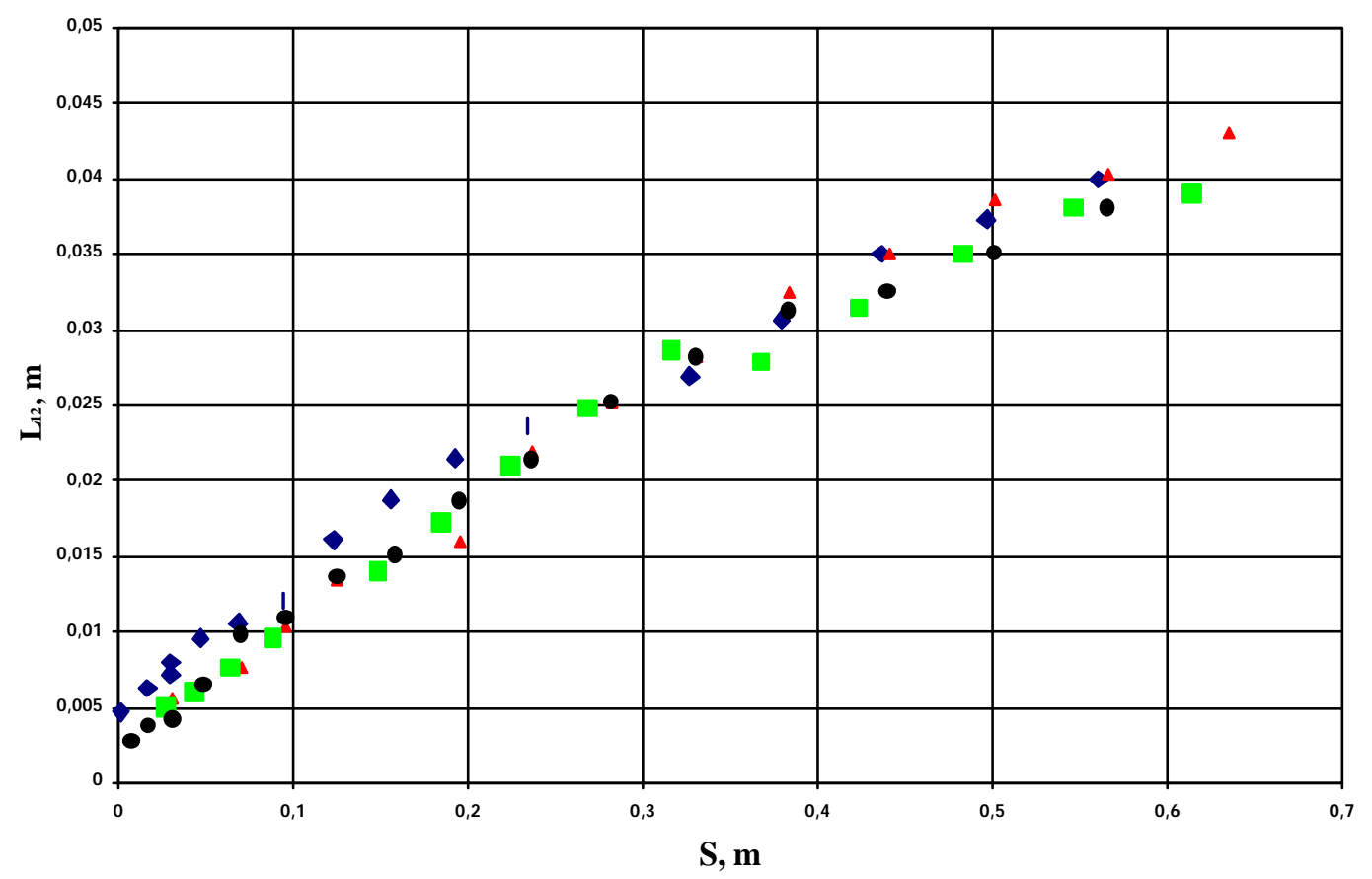

Fig. 6c Dependence of the mixing front coordinate $\mathrm{L}_{12}$ on the parameter $\mathrm{S}$ for the third group of experiments $(\mathrm{Kr}-\mathrm{Ar})$. 


\section{Conclusion}

At the installation OSA the experiments have been performed with respect to the investigation of the self-similar mixing of different density gases in the Earth's gravitational field. In these experiments the controlled separating membrane of a new type with a liquid film was used. This separating membrane made it possible to carry out experiments with gases at the acceleration of the contact boundary $\mathrm{g}_{1}=\mathrm{g}_{0}$. The distinctive feature of a new membrane is its low density. At the specified instant of time the membrane was ruptured under the external force action into pieces whose characteristic size $\lambda \approx 4 \mathrm{~mm}$. At the contact boundary the gravitational turbulent mixing zone growing with time was formed.

Three groups of experiments with different working gases have been performed. The density ratio and Atwood numbers for different groups of experiments are shown in Table.

\begin{tabular}{|c|c|c|c|}
\hline Group number & Pair of gases & Density ratio & Atwood number \\
\hline $\mathbf{1}$ & $\mathbf{S F}_{\mathbf{6}}-\mathbf{A r}$ & $\mathbf{3 , 3 7}$ & $\mathbf{0 , 5 4}$ \\
\hline $\mathbf{2}$ & $\mathbf{S F}_{\mathbf{6}}-\mathbf{H e}$ & $\mathbf{3 3 , 7}$ & $\mathbf{0 , 9 4}$ \\
\hline $\mathbf{3}$ & $\mathbf{K r}-\mathbf{A r}$ & $\mathbf{2 , 1}$ & $\mathbf{0 , 3 5}$ \\
\hline
\end{tabular}

According to the results of experiments, the mixing asymmetry coefficient $\mathrm{k}=1.1 \div 1.7$ (for different values of the parameters $\mathrm{S}$ ) and constant $\alpha=0.078$ defining the nondimensional rate of mixing were determined. 


\section{Shock Tube Experiments on Richtmyer-Meshkov Instability across a Chevron Profiled Interface}

A V Smith, D A Holder, C J Barton, A P Morris D L Youngs

AWE, Aldermaston, Reading, Berkshire, RG 7 4PR, UK

(c) Crown Copyright 2000

This document is of United Kingdom origin and contains proprietary information which is the property of the Secretary of State for Defence. It is furnished in confidence and may not be copied, used or disclosed in whole or in part without prior written consent of the Director Commercial 2, Defence Procurement Agency, Ash 1b \#3110, MoD Abbey Wood, Bristol. BS34 8JH England. 


\section{Content}

- Show experimental mix results from a chevron interface perturbation

- comparable to numerical test problem \#1 and

- similar to experiments of Meshkov et al (5th IWPCTM)

- Compare these to 3D code results

- Illustrate the potential benefits of Intensified CCD imaging to overcome Multiple Scattering

- Future work

- Video of results 


\section{Perturbation}
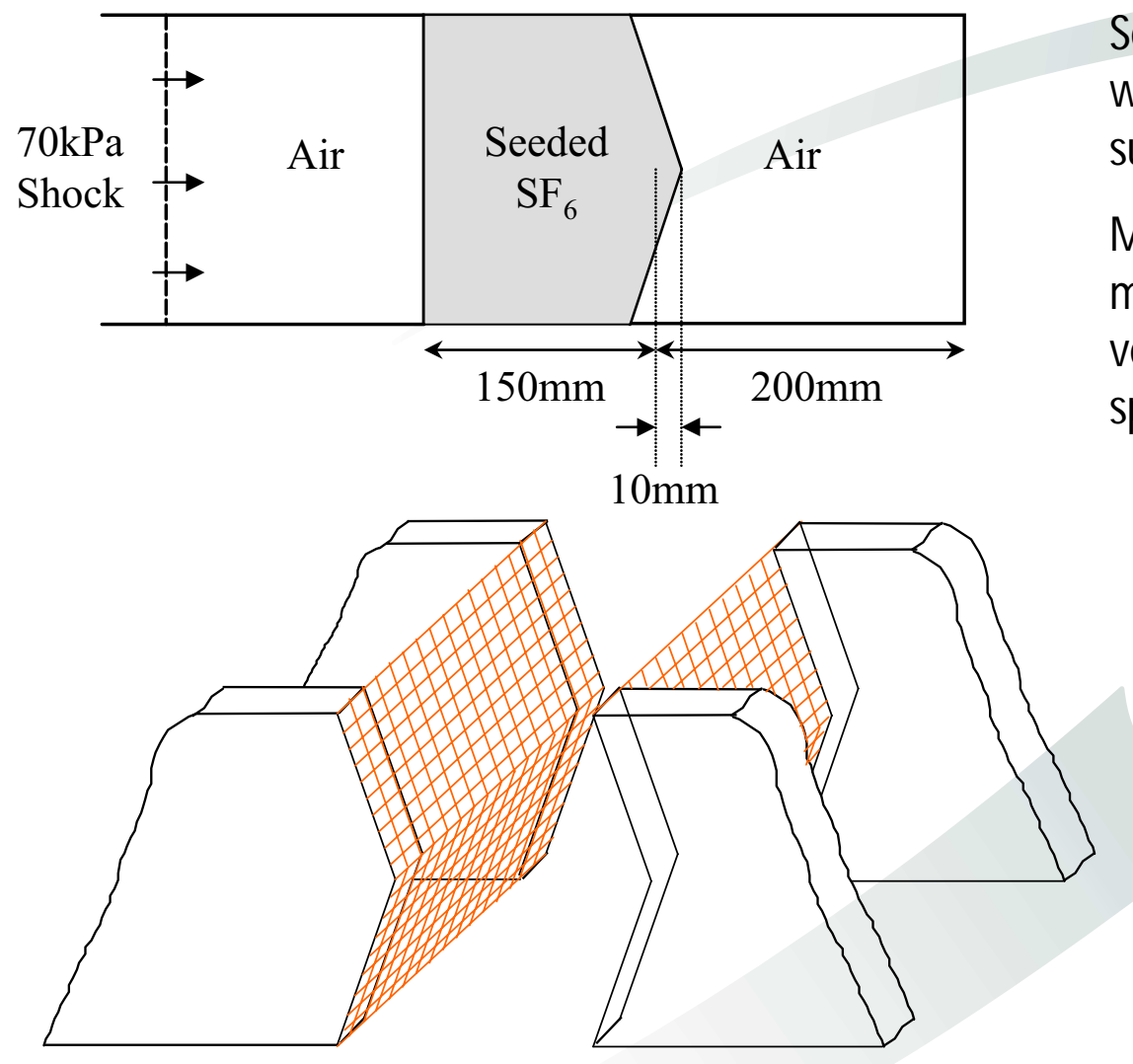

Seeded $\mathrm{SF}_{6}$ region constrained with microfilm membranes supported on wire meshes.

Meshes on both interfaces are made from horizontal and vertical $25 \mu \mathrm{m}$ w ires with $4 \mathrm{~mm}$ spacing.

the total flow blockage is $1.25 \%$ 


\section{Perturbation (cont.)}

The chevron was conceived as a progression from the work of M eshkov et al and Test Problem \#1.
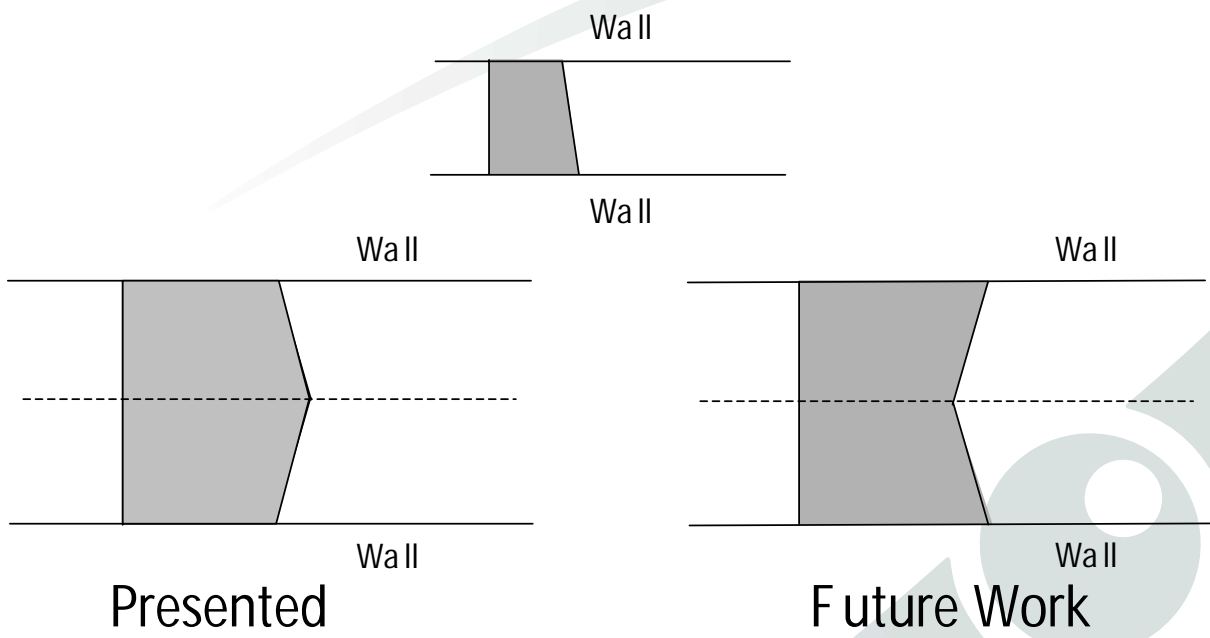

The chevron takes advantage of a larger shock tube to eliminate any possible constraining effect of one wall and also to investigate boundary layer effects.

The two chevron experiments will together complement the previous work 


\section{An $=$}

\section{nes}

Excellegice in ingovatiyerscience \& technology

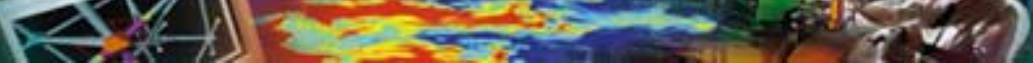

\section{$200 \times 100$ mm Shock Tube}

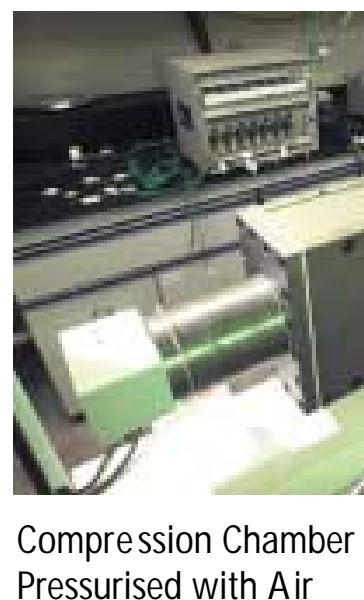

to 2 bar
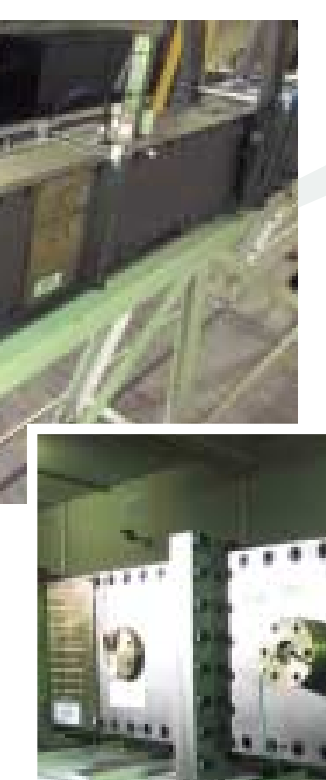
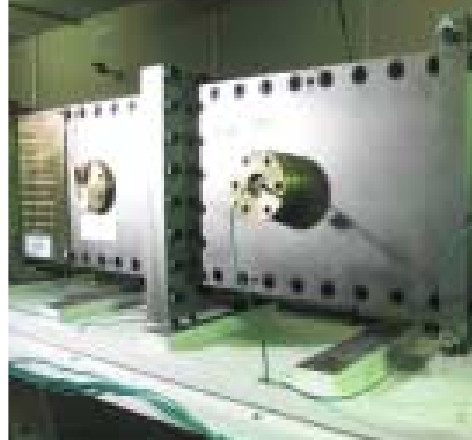

$+\infty$
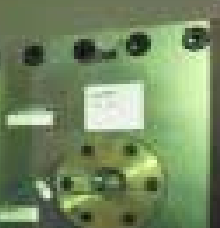

\section{8}

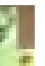

tarna

Test Cell

Chevron Interface Profile $\mathrm{SF}_{6}$ gives Atw ood Number, $A=0.67$
Shock of $70 \mathrm{kPa}$ Overpressure and 7 ms Duration
Shock Mach Number 1.26 


\section{Pressure Profile}

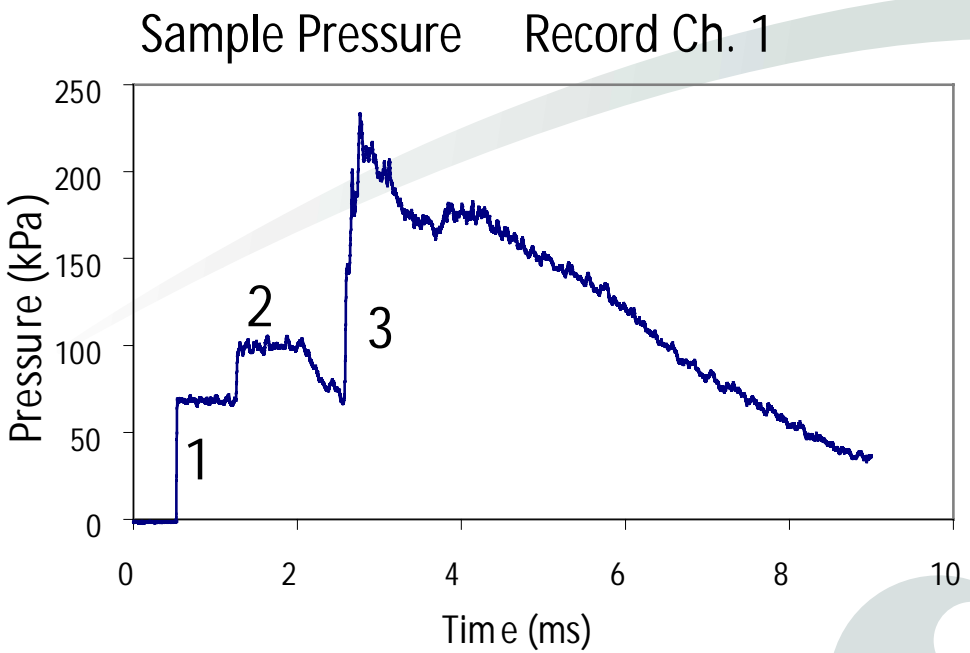

Pressure transducers monitor shock passage and record waveforms. This is a typical waveform from the pressure gauge nearest the test cell.

1. Incident shock

2. First shock reflection from dense gas region

3. First shock reflection from end wall 


\section{Laser Sheet Arrangement}

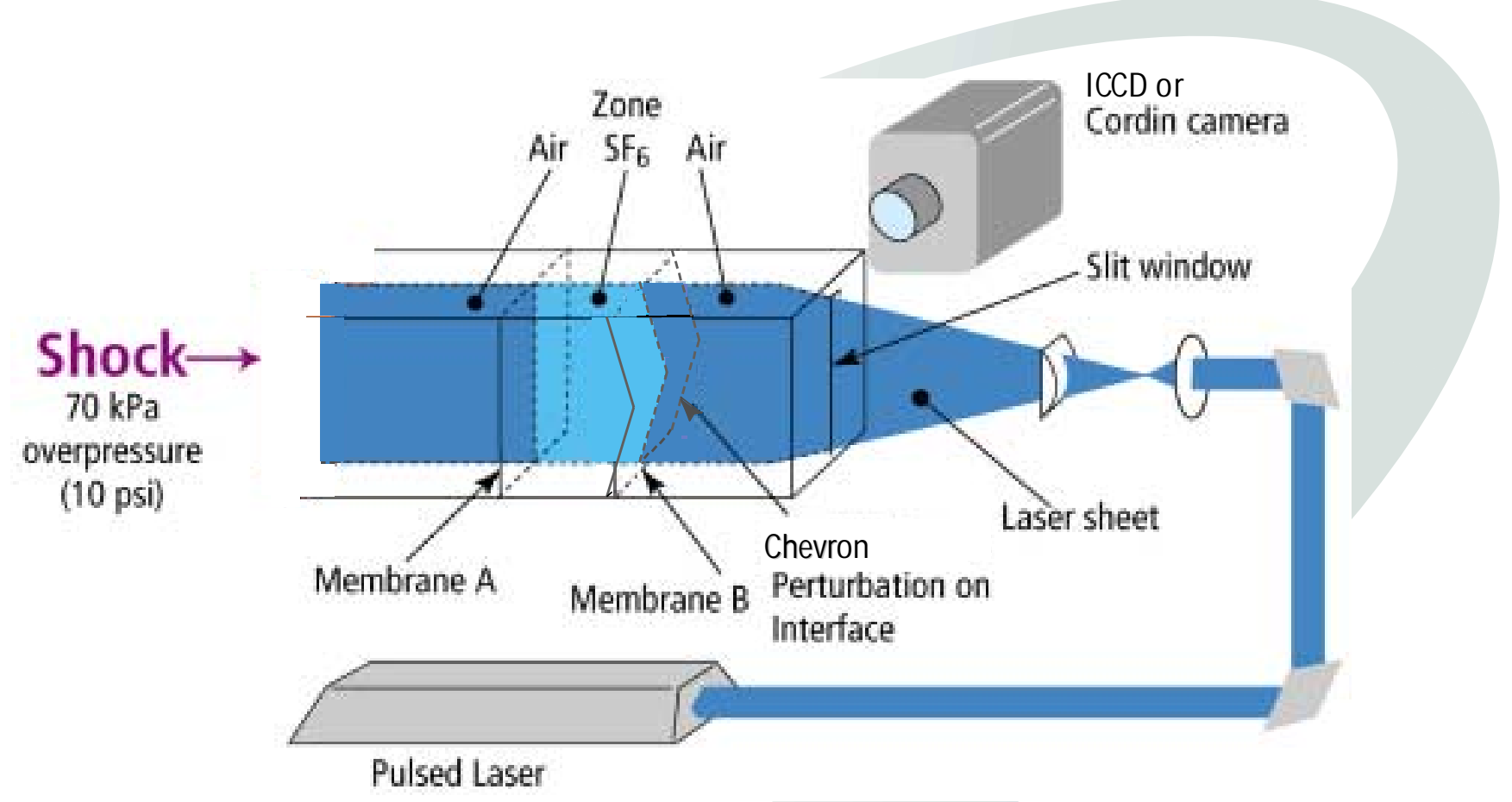




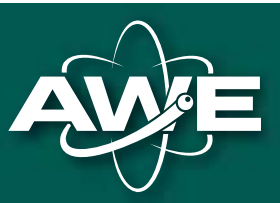

Excellegce in ingovativerscience \& technology

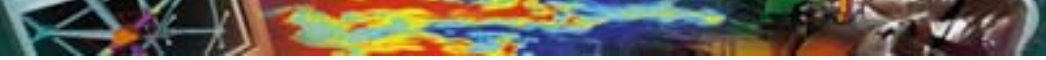

\section{Chevron Experiment}

Sequence of selected images from a single experiment
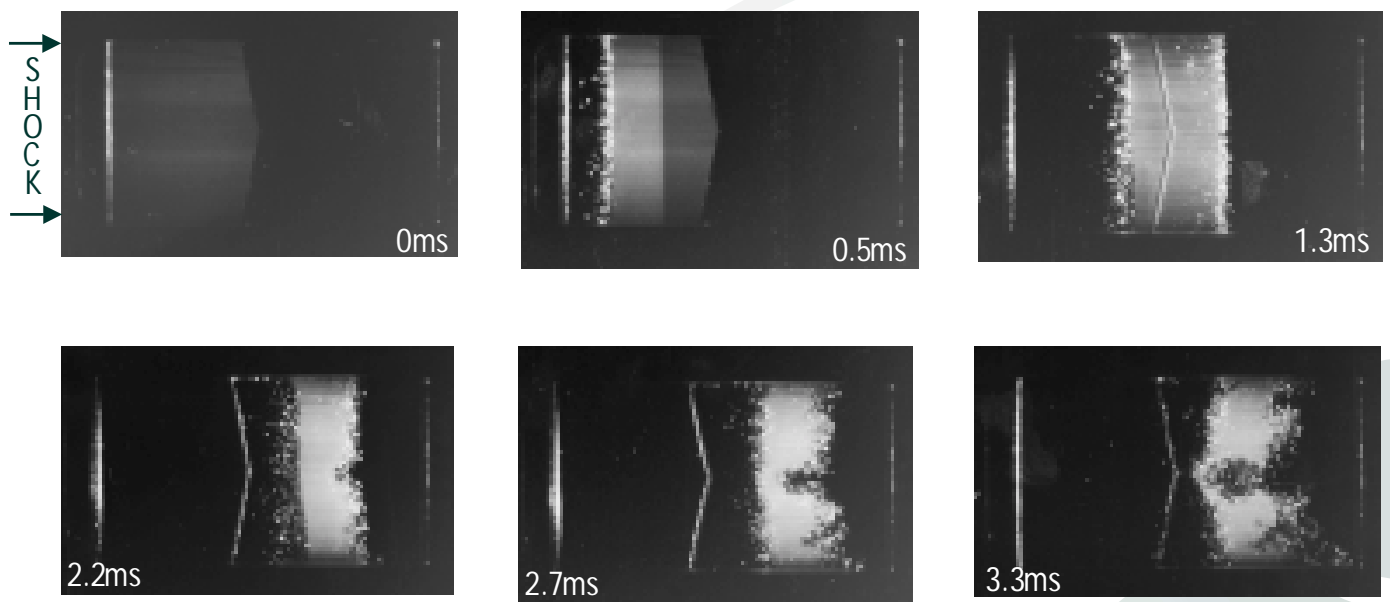
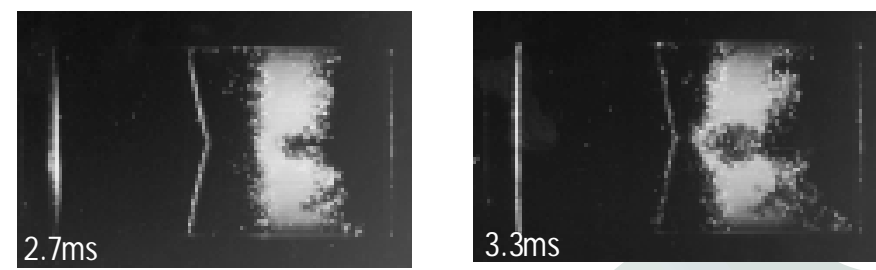
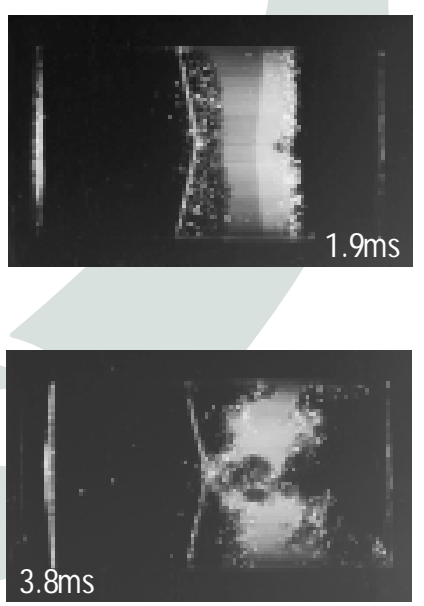


\section{TURMOIL 3D Calculations}

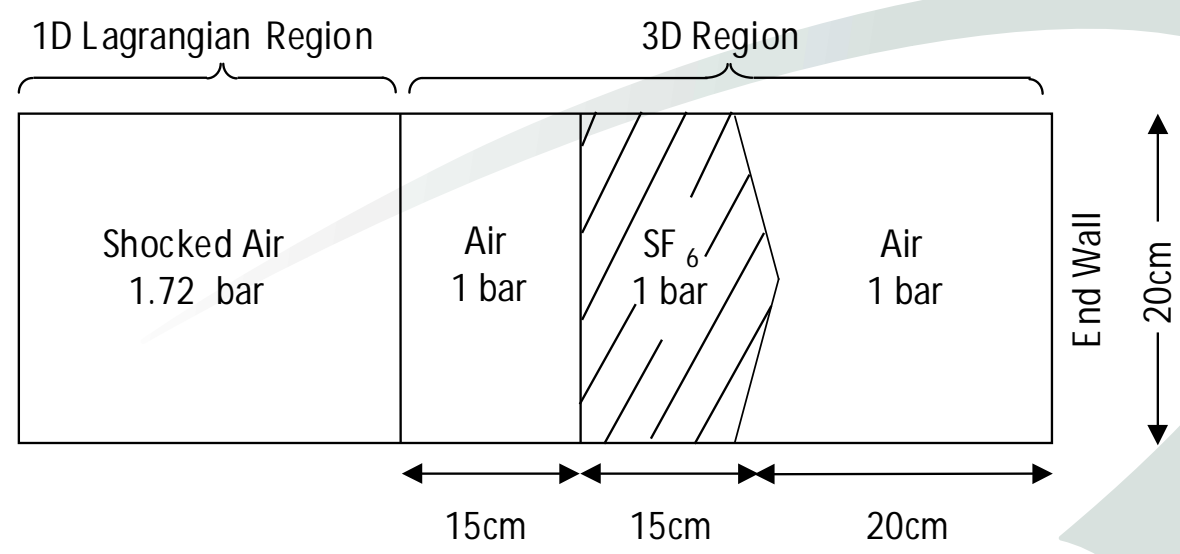

Zoning used in 3D region : $400 \times 320 \times 160$

Semi-Lagrangian calculation : $x$-direction mesh moves with the mean fluid velocity.

Random initial perturbation at air/SF6 interfaces:

Wavelengths $=0.5$ to $5 \mathrm{~cm}$

R.M.S. amplitude $=0.01 \mathrm{~cm}$ 


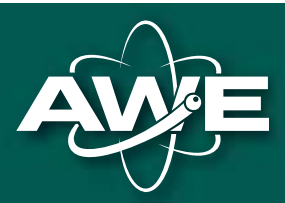

Excellerce rinjunovativescience \& technology siv

\section{Experiment and \\ Code Comparison (2.2 - 3.8ms)}

Experiment
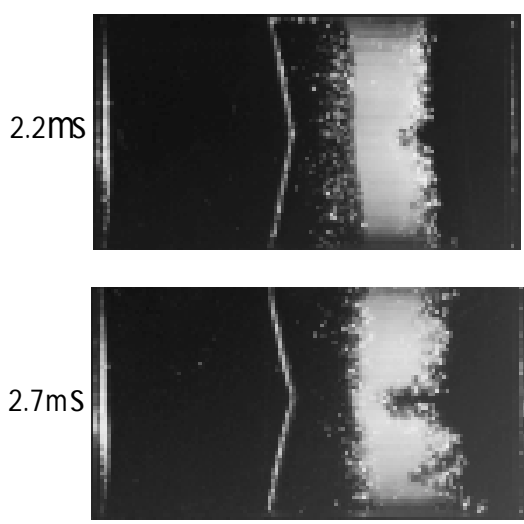

Code
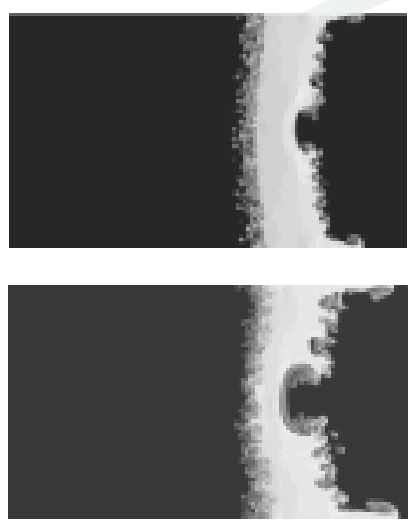

Experiment
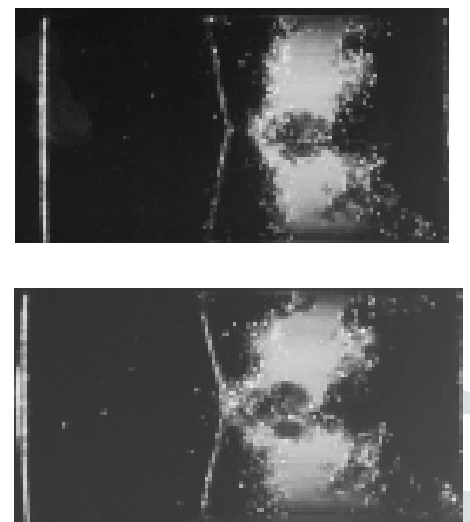

Code

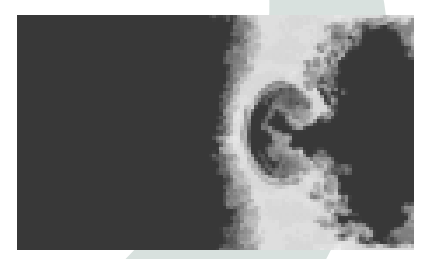

$3.3 \mathrm{~ms}$

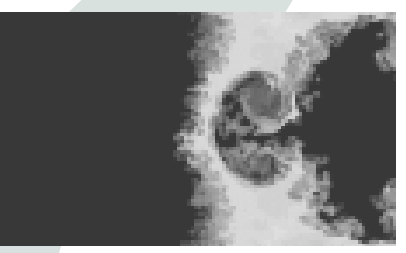




\section{Multiple Scattering}

A M onte Carlo multiple scattering simulation has been devised to successfully 'add' multiple scattering to the mix code results.

Ref: $\quad$ Giddings et.al. $7^{\text {th }}$ IWPC TM Holder et.al. $23^{\text {rd }}$ ISSW

Multiple scattering in experiment

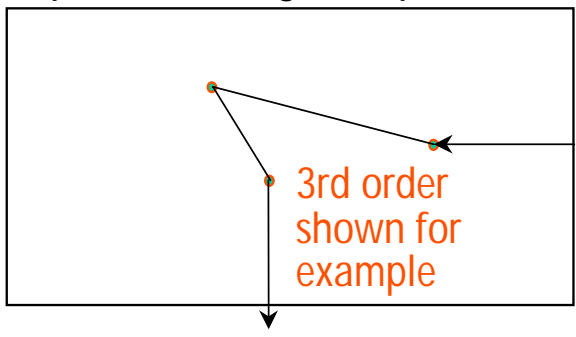

Monte Carlo code simulation

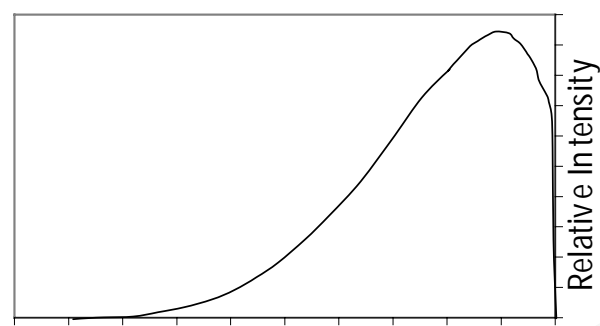

Distance
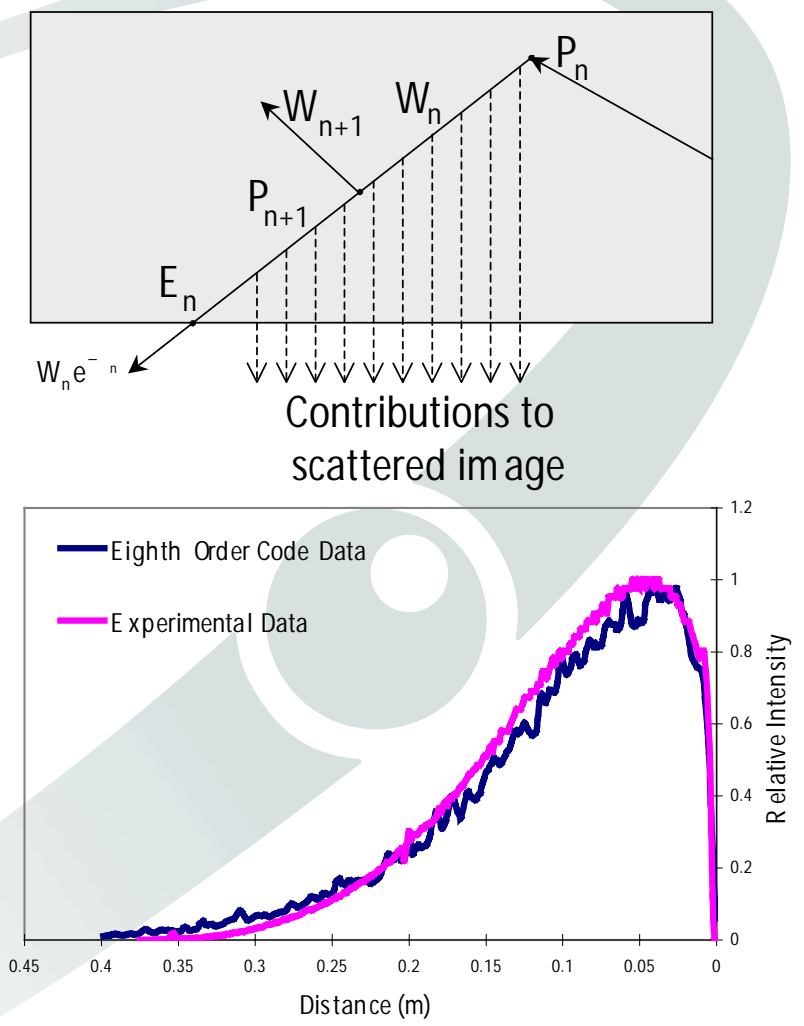


\section{Multiple Scattering Example}

Experiment

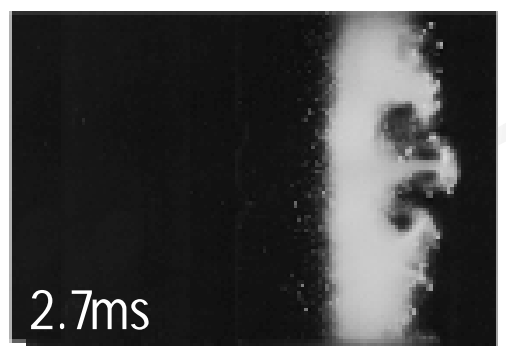

Code

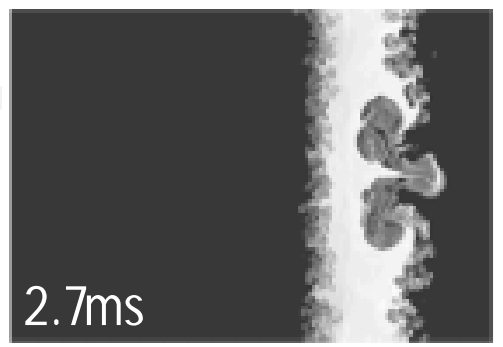

Code + Scattering

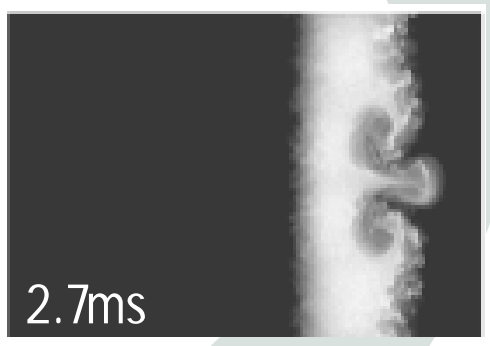

These are images from a single time $(2.7 \mathrm{~ms})$ from a double bump experiment presented to ISSW 23.

These are an experimental image, a plane section through a mix calculation and the same calculation with the M onte Carlo simulation applied.

The M onte Carlo scattering code greatly improves qualitative comparison between experiment and code results. 


\section{Wall Effects}

Code results at 2.2 and $3.3 \mathrm{~ms}$
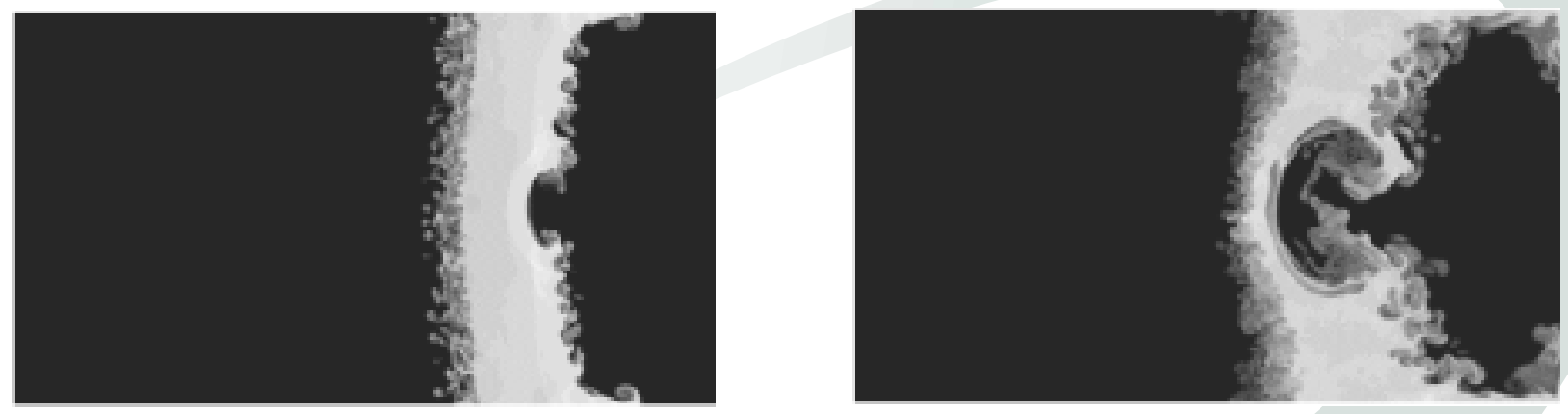

Spikes of dense gas are seen to advance along the top and bottom.

Are these due to the Chevron pert urbation, boundary layer interactions, or a combination of both?

The bubble of air penetrating into the dense gas is not axi-symmetric. Would the presence of a wall, as in numerical Test Problem \#1 prevent this occurring? 


\section{Conclusions}

- Chevron perturbation appears to produce feed-through in the experiments, but not in the code results.

- Acceptable agreement between experiment and code has not yet been achieved. The experiment will have to be repeated.

- Inverse chevron experiment to

- further investigate boundary layer effects

- investigate asymmetric effects

- Further use of ICCD imaging and fluorescent seeding

- allows a reduction in multiple scattering

- promising for future, allowing quantitative analysis

- Chevron experiments are useful to validate test problem calculations in addition to the work of M eshkov et al.. 


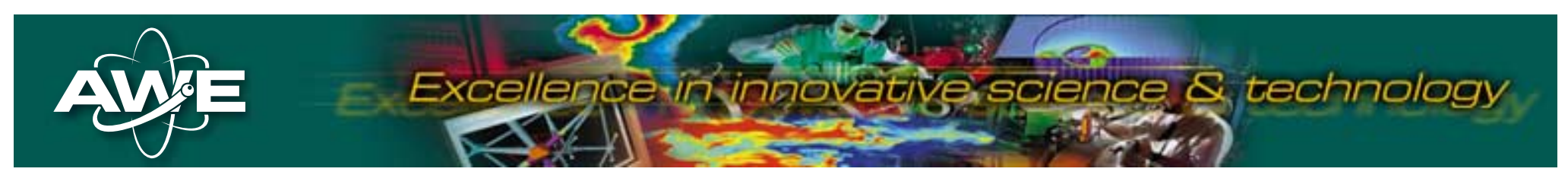

\section{Code Data}

Move cursor over image and click to play 


\section{The evolution and interaction of two shock-accelerated, unstable gas cylinders}

Chris Tomkins, Kathy Prestridge,

Paul Rightley, Mark Marr-Lyon,

Robert Benjamin

Summer students: James Doyle and Michael Schneider Dynamic Experimentation Division, Los Alamos National Laboratory

\section{Peter Vorobieff}

Department of Mechanical Engineering, University of New Mexico

Cindy Zoldi

Applied Physics Division, Los Alamos National Laboratory 


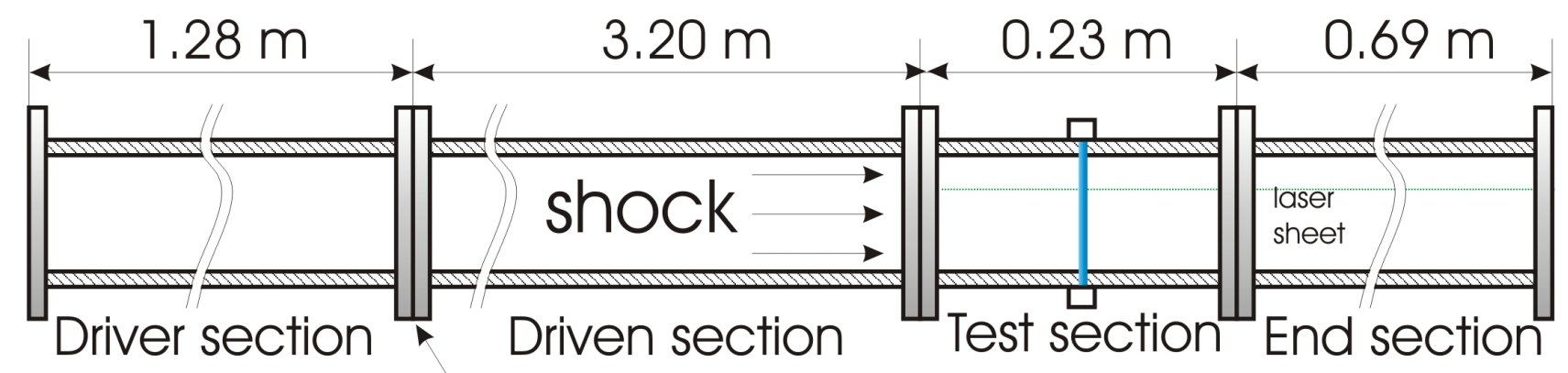

- Diaphragm

\section{Experimental setup: shock tube}

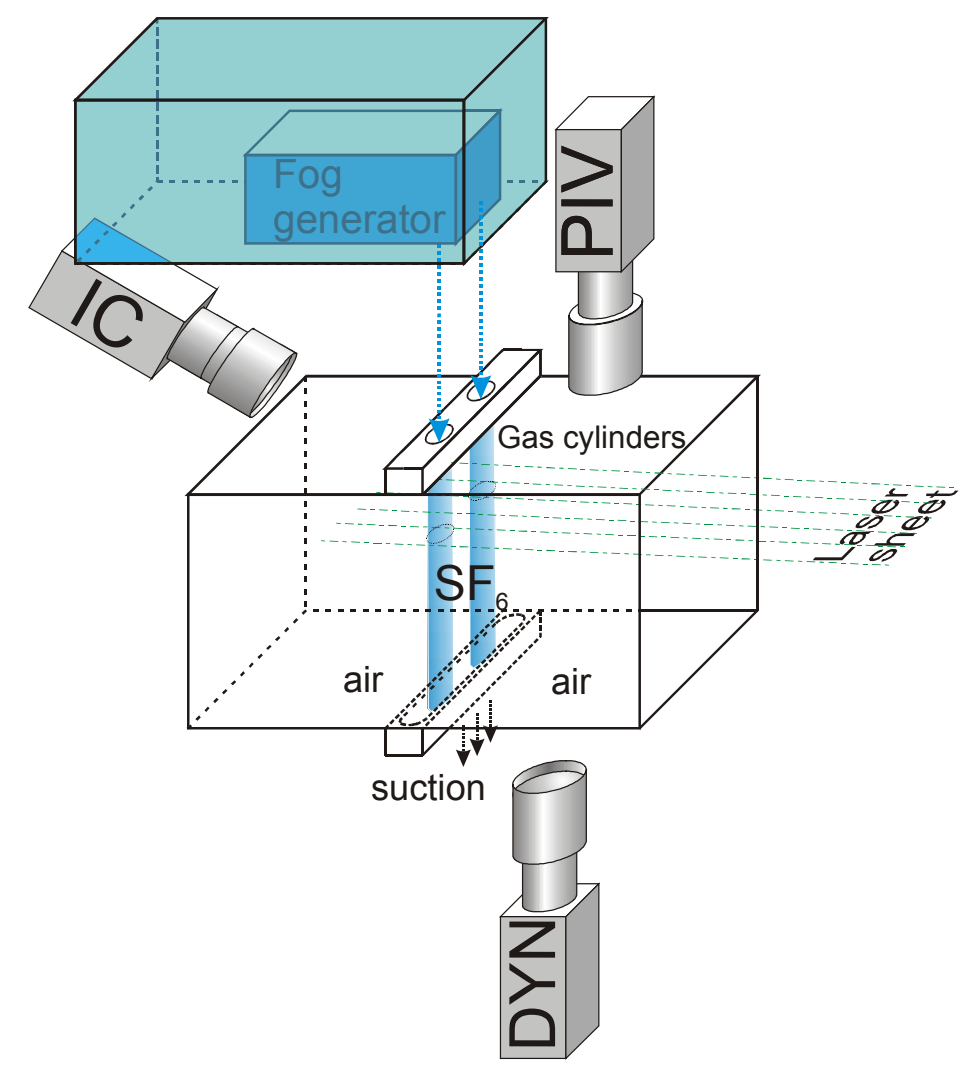




\section{Overview}

- Examine interaction of planar shock with 2 gas cylinders, separated spanwise.

- $\mathrm{S}=1.2 \mathrm{D}$ to $2.0 \mathrm{D}$.

$(\mathrm{D}=$ cylinder diameter $)$

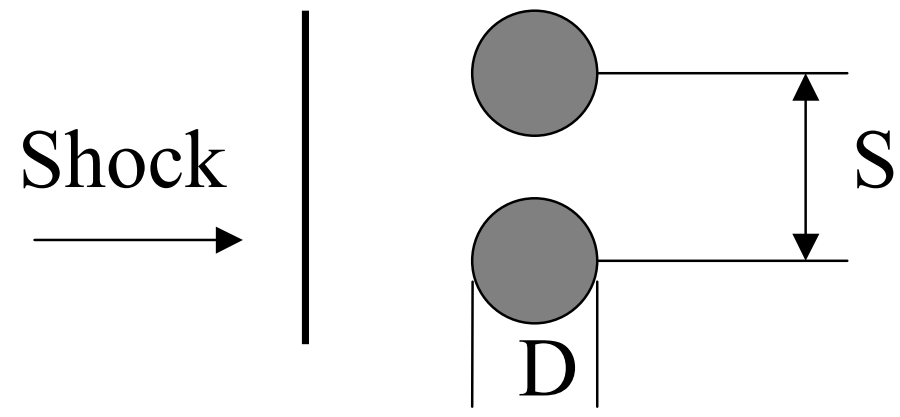

- Goal: Investigate the evolution of the interacting, RM-unstable cylinders. Issues of interest include:

- What is the effect of the interaction on the resulting flow morphologies? On the initial vorticity deposition? On the post-shock vortex development?

- How sensitive is the flow evolution to the initial separation S? 


\section{Single shock-accelerated cylinder}

\section{Double-cylinder "vortex blob" simulation}

(a)

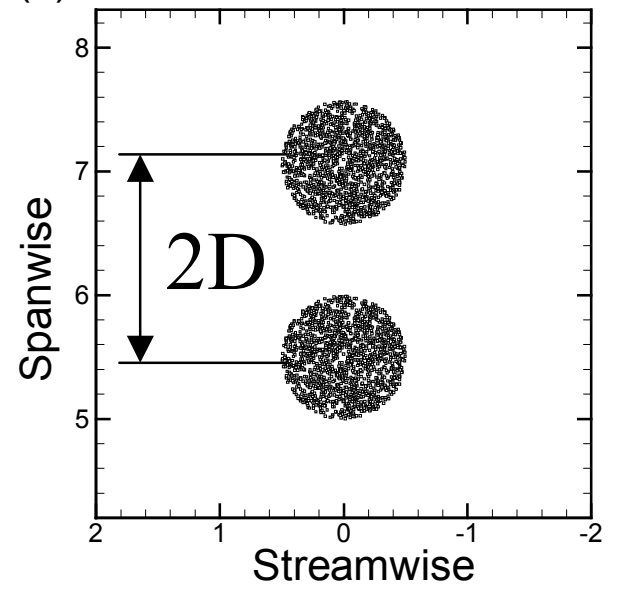

(b)

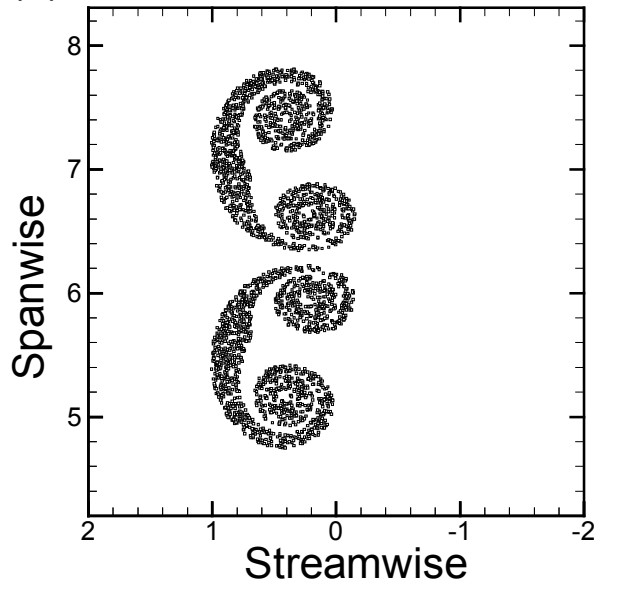

(c)

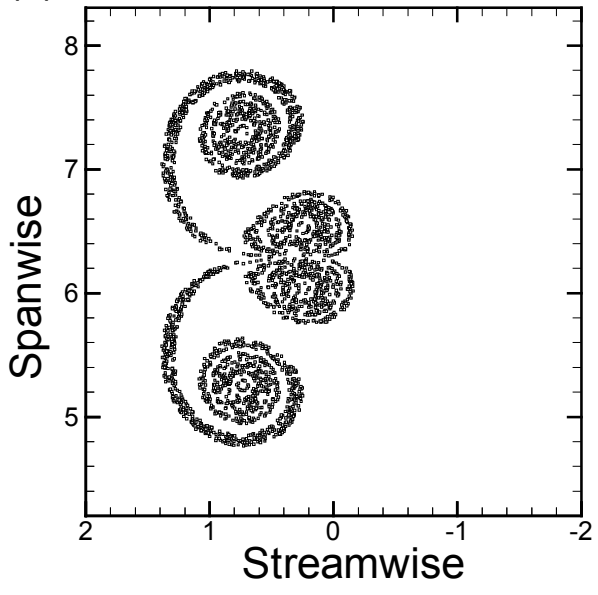




\section{Double-cylinder interaction: weak}

$$
\mathrm{S} \approx 2.0 \mathrm{D}
$$

Shock

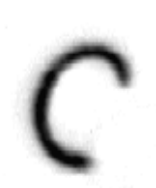

c

$$
\mathrm{S} \approx 1.8 \mathrm{D}
$$
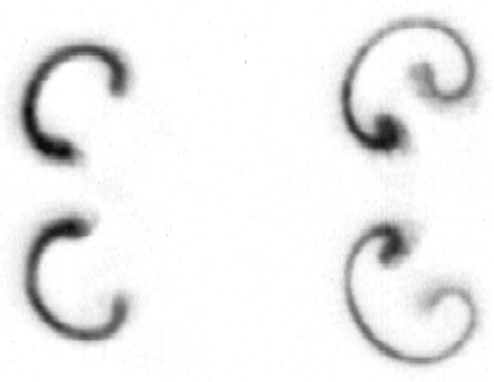

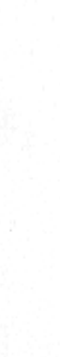
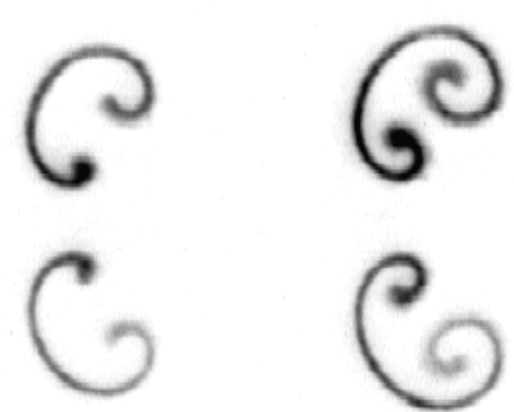

(c)
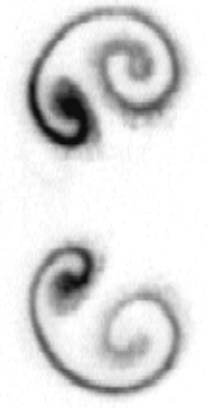

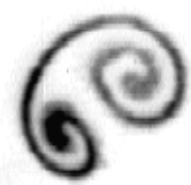

C)

C
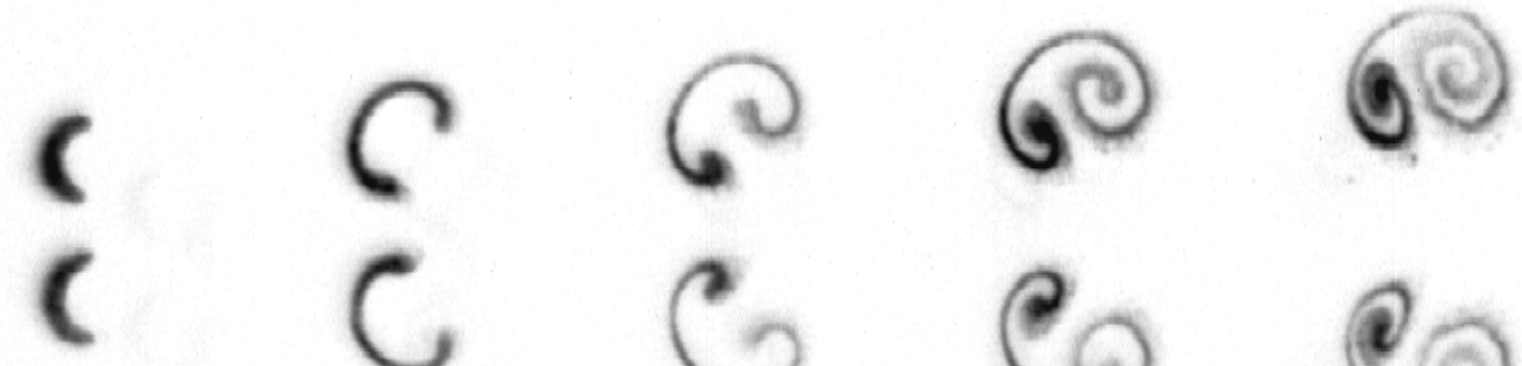
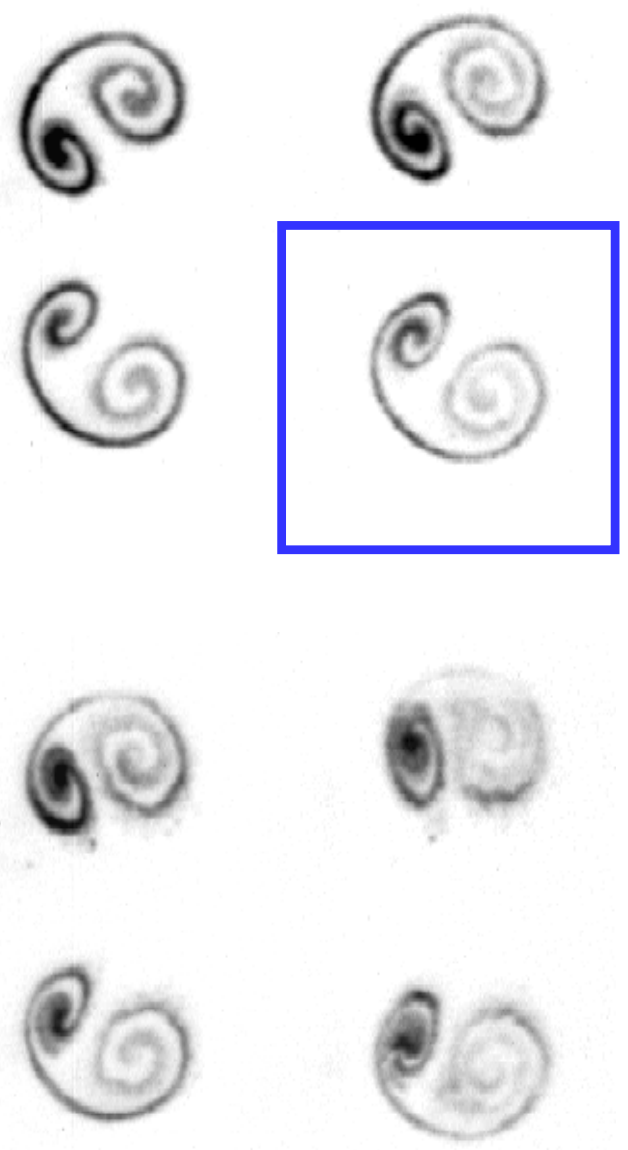


\section{Double-cylinder interaction: moderate}

$\stackrel{\text { Shock }}{\longrightarrow}$

$$
\mathrm{S} \approx 1.6 \mathrm{D}
$$
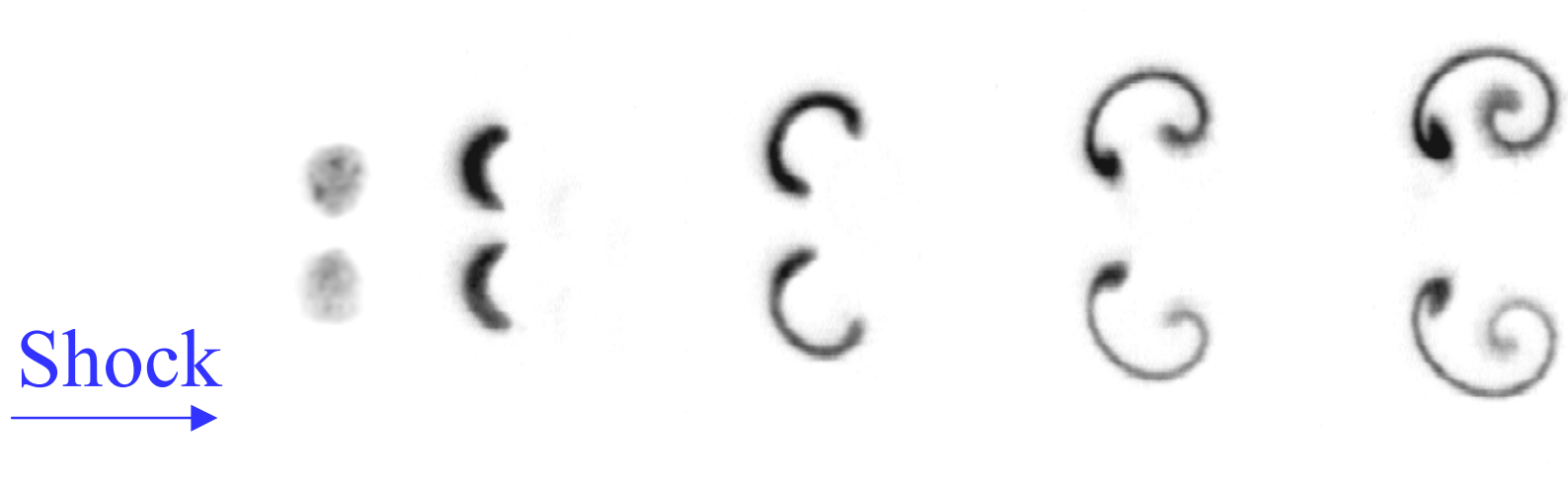

(อ)

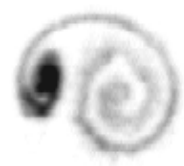

$\mathrm{S} \approx 1.5 \mathrm{D}$

11

○

(5)

(2)

(2)

c

6

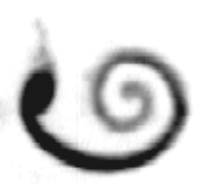

C 


\section{Double-cylinder interaction: strong}

$\stackrel{\text { Shock }}{\longrightarrow}$

$$
\mathrm{S} \approx 1.4 \mathrm{D}
$$
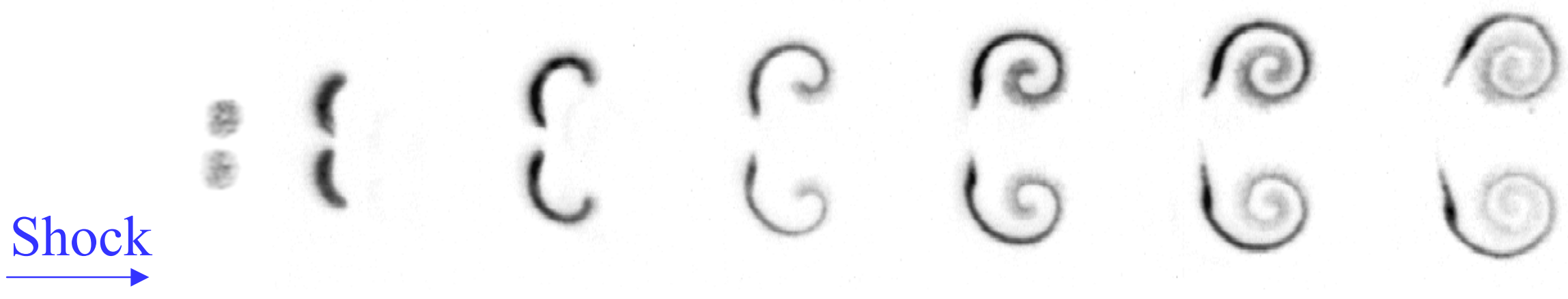

$\mathrm{S} \approx 1.2 \mathrm{D}$
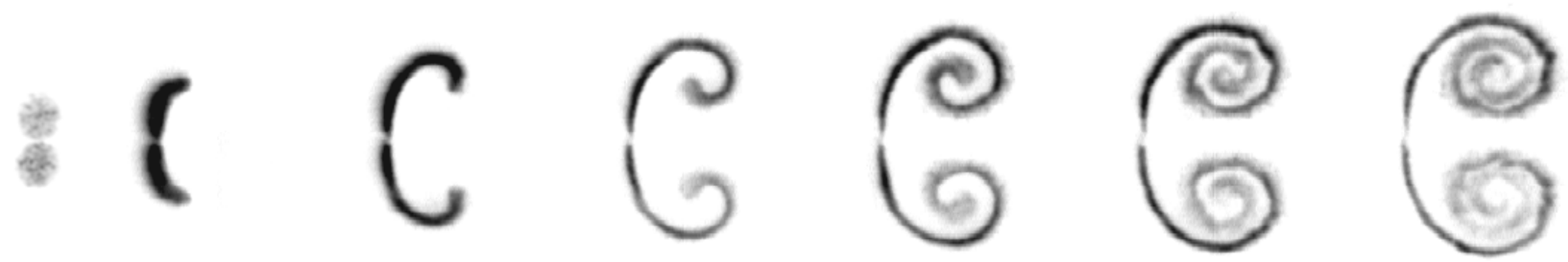


\section{PIV images: double cylinder}

- Two-frame cross-correlation, flow left to right, $6^{\text {th }}$ pulse

- $S=2.0 D$. Note non-uniform seeding.

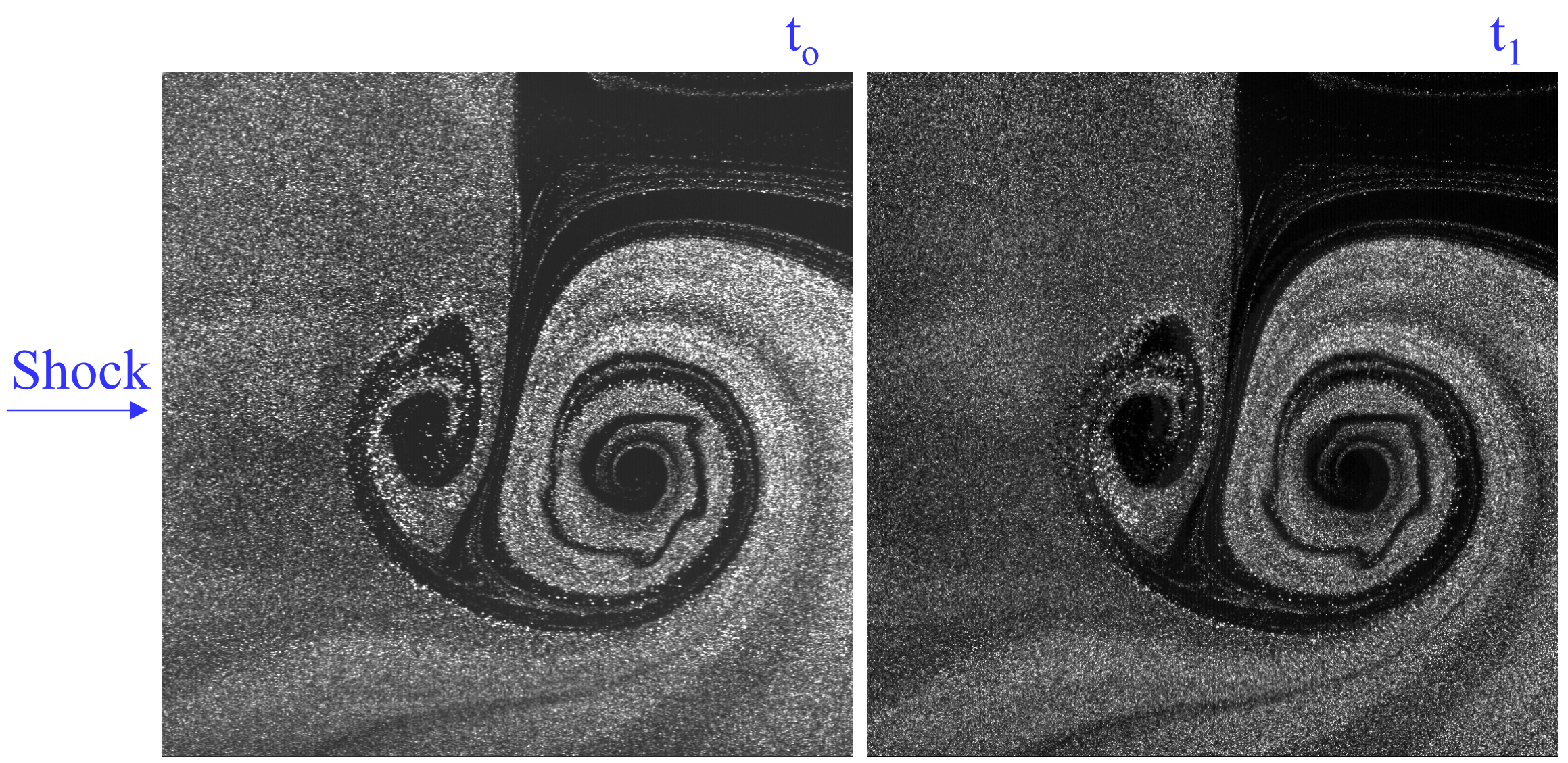




\section{Double-cylinder velocity field: PIV}

$\begin{array}{llll}\text { i } & C & \odot & 6 \\ \text { - Double-cylinder }\end{array}$ data, $6^{\text {th }}$ pulse, $\mathrm{S}=2.0 \mathrm{D}$

- Two-frame crosscorrelation (Christensen et al., 2000)

- Not smoothed

- Contours are fluctuating velocity magnitude

$\stackrel{\text { Shock }}{\longrightarrow}$
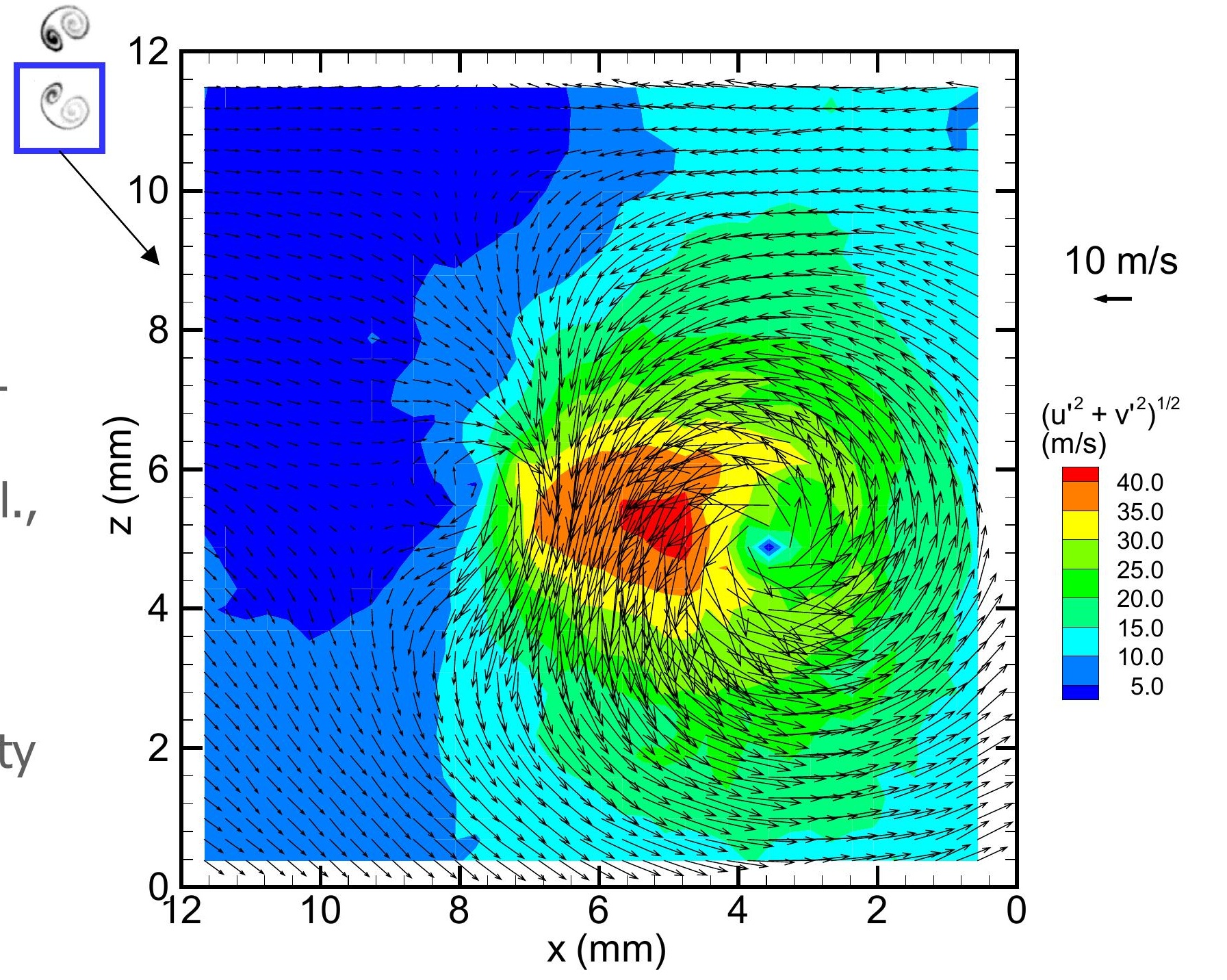


\section{Double-cylinder vorticity field}

- Same realization

- Vorticity contours

- Not smoothed

- And the ratio of circulations is
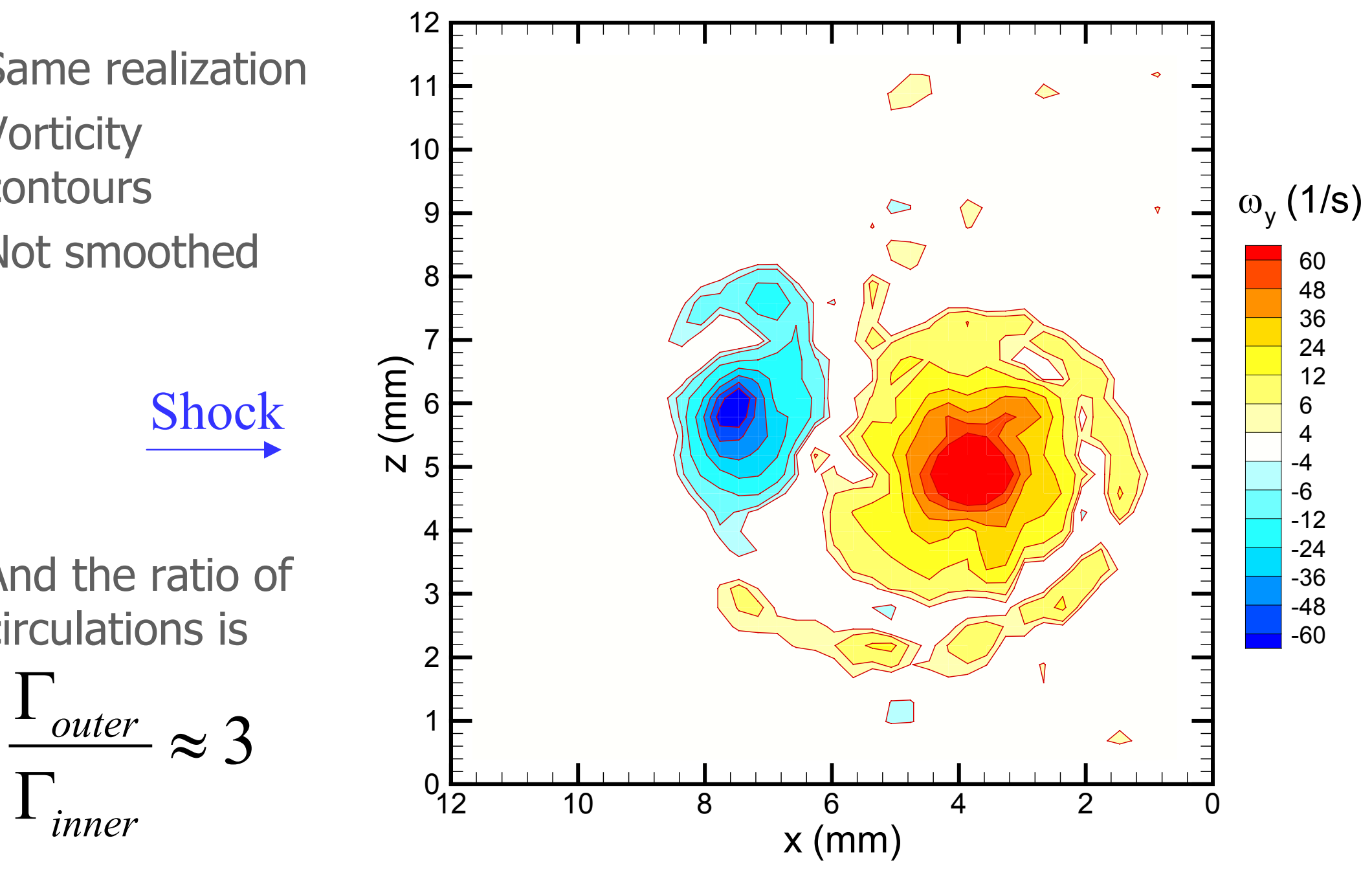


\section{Correlation-based ensemble averaging}

Match one image (template) to each individual realization.

Desire optimum match between template and image, i.e. minimize mean sq. error: $e=\int\left|I(\underline{x})-I_{t}\left(\underline{x}-\underline{x}_{0}\right)\right|^{2} d A$

This requires maximizing $\int_{D} I(\mathbf{x}) \bullet I_{t}\left(\mathbf{x}-\mathbf{x}_{o}\right) d A \quad$ w.r.t. $\mathbf{X}_{o}$

Do for each realization, then extract and average (Soloff, 1997) Yields cond. avg.: $<I\left(\mathbf{x}-\mathbf{x}_{o}\right) \mid \mathbf{x}_{o}>$

This avg. becomes the new template.

Properties:

-Minimizes dependence on initial choice of template.

-Converges quickly.

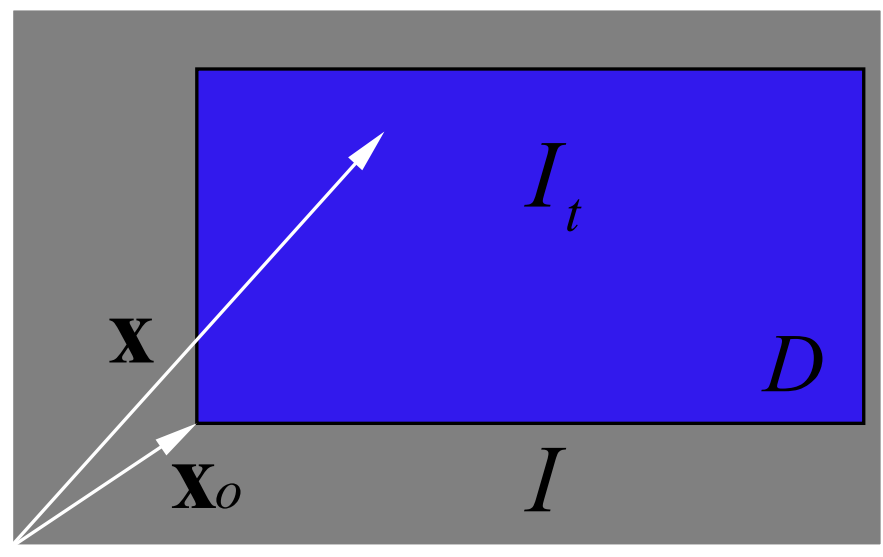




\section{Correlation-based ensemble average}

$\mathrm{S} \approx 1.2 \mathrm{D}$, Ensemble average

Shock
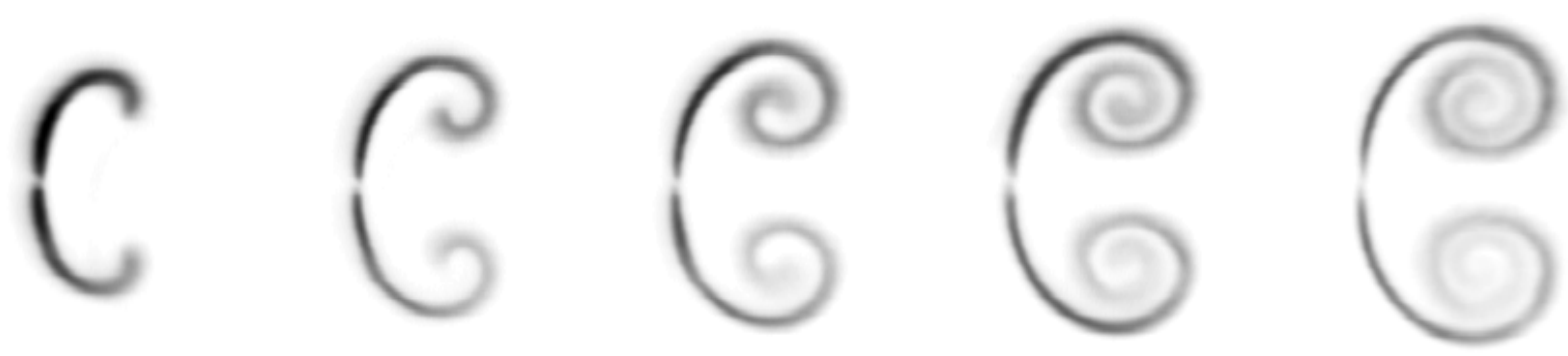

$\mathrm{S} \approx 1.2 \mathrm{D}$, Individual realization
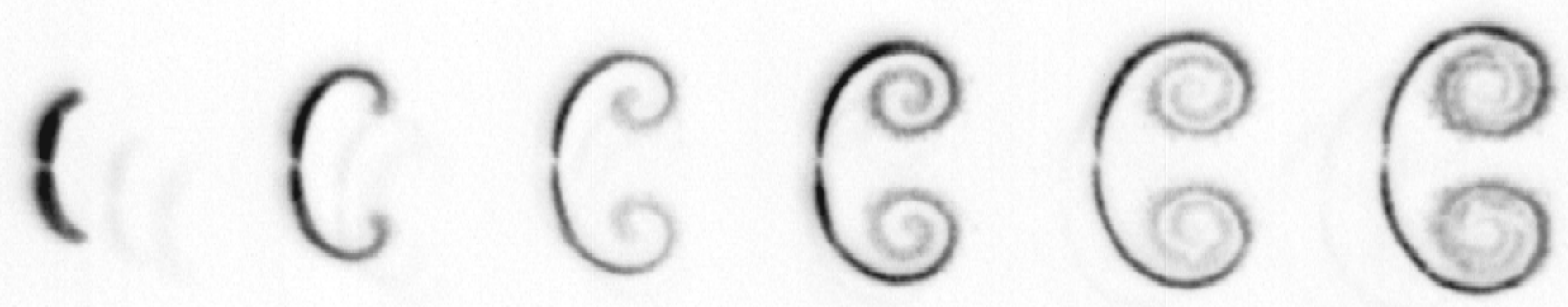


\section{Fluctuating intensity fields, $\mathrm{S}=1.2 \mathrm{D}$}

$$
t=470 \mu s
$$

Total

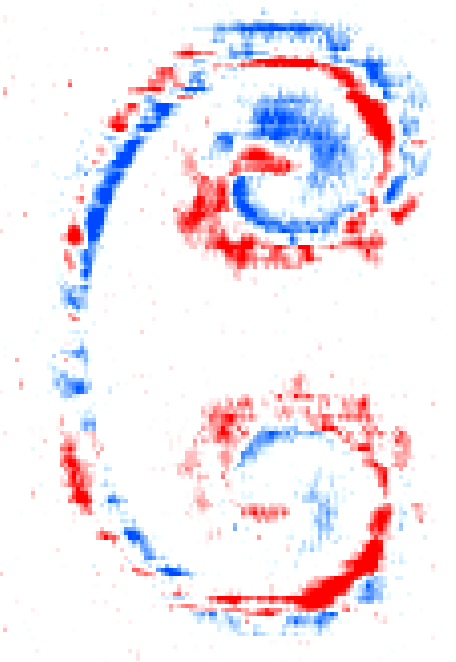

Fluctuating

$$
t=750 \mu \mathrm{s}
$$

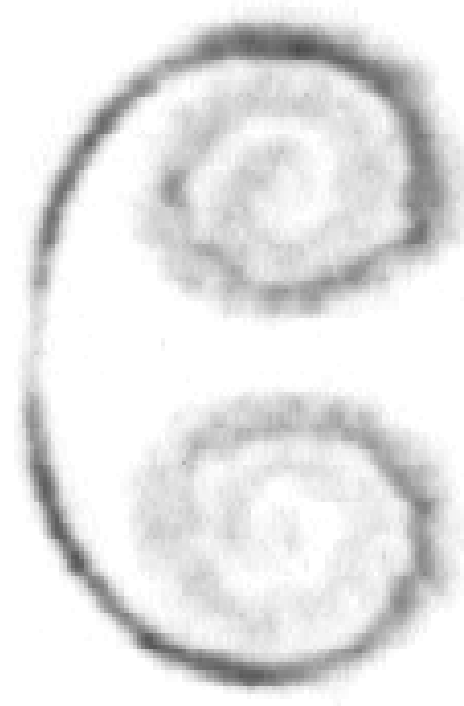

Total

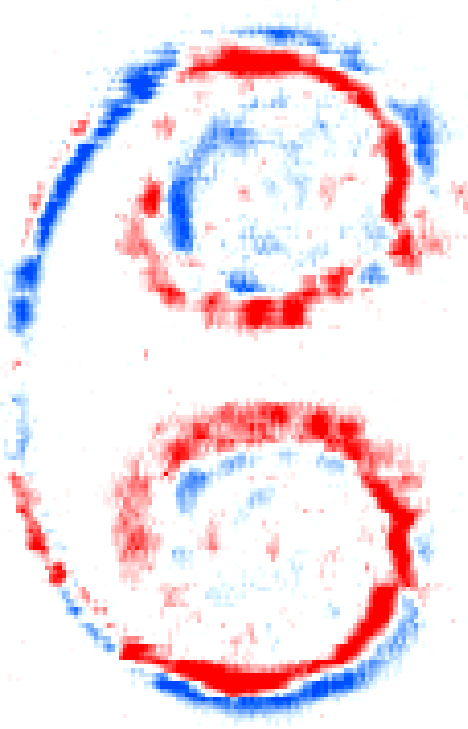

Fluctuating 


\section{RMS of fluctuating intensity}

RMS Intensity vs. Time for several values of $S / D$.

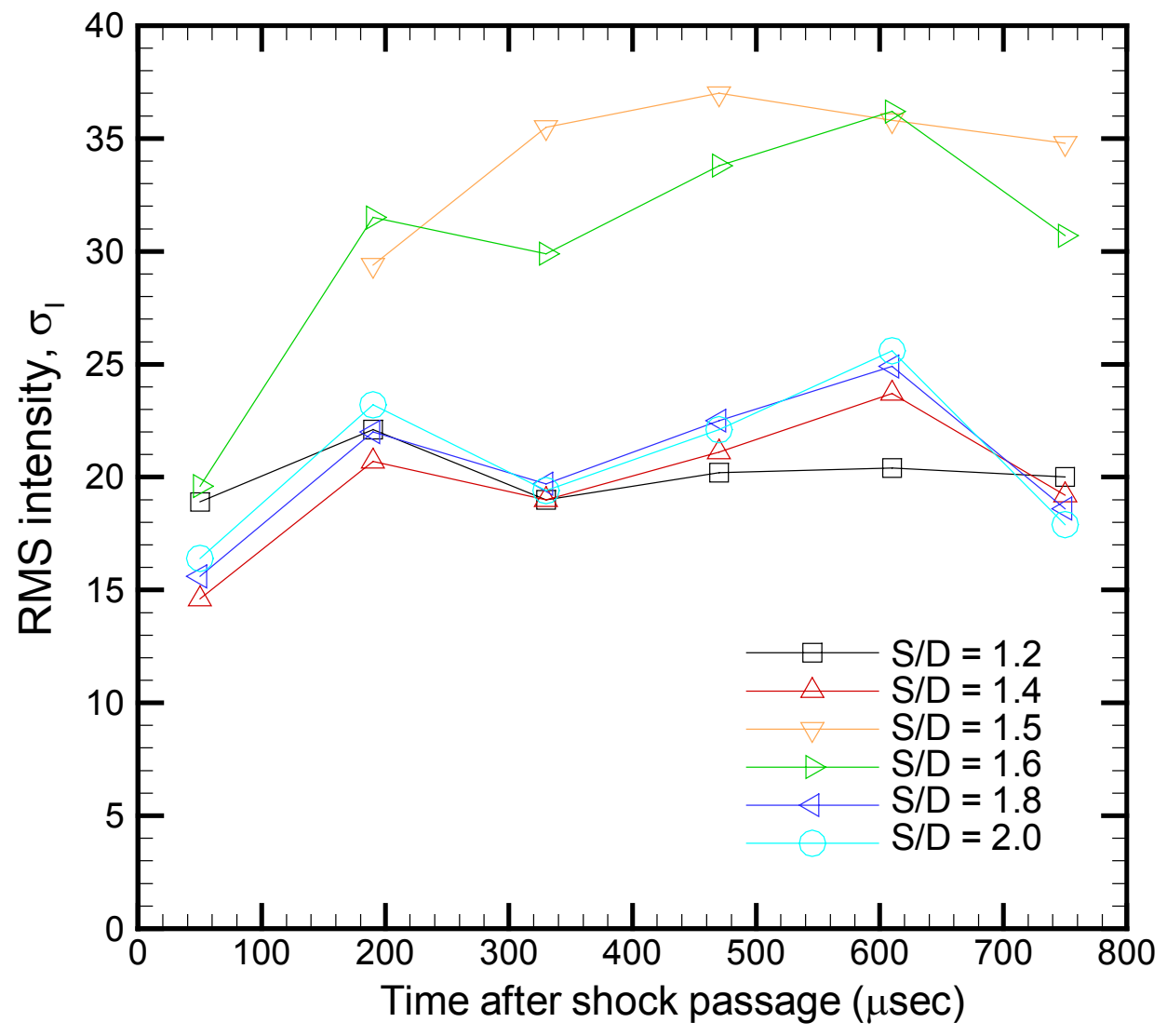

RMS of Initial Conditions

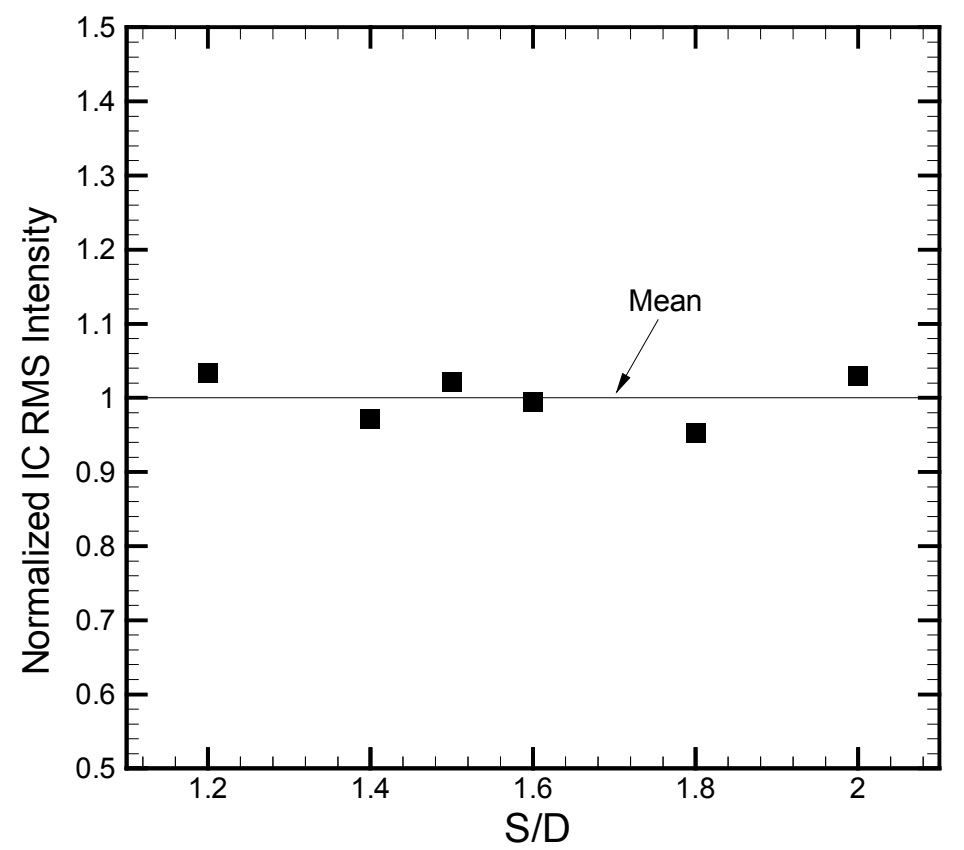




\section{Small-scale activity: single cylinder}

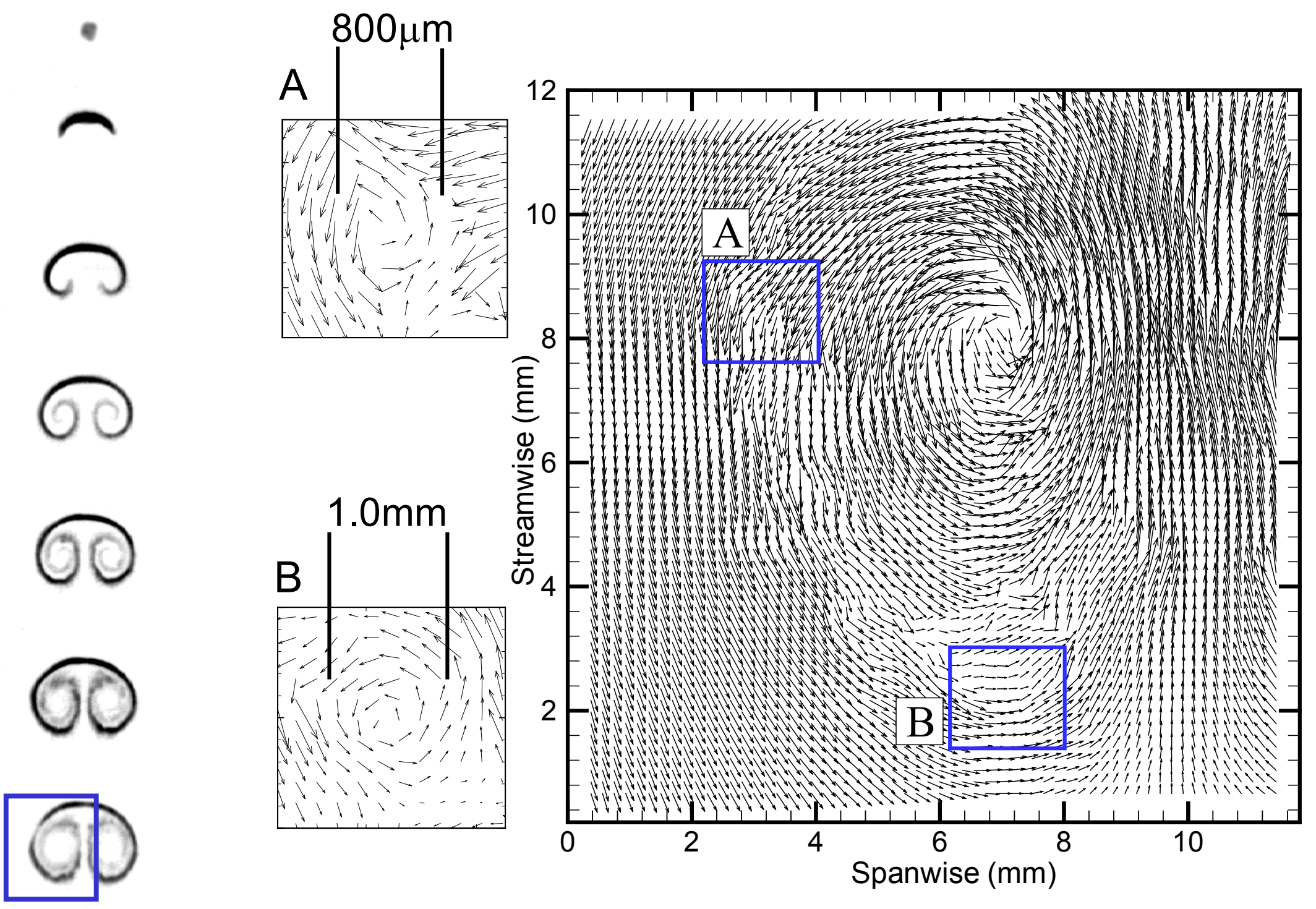




\section{Vorticity and swirling strength}
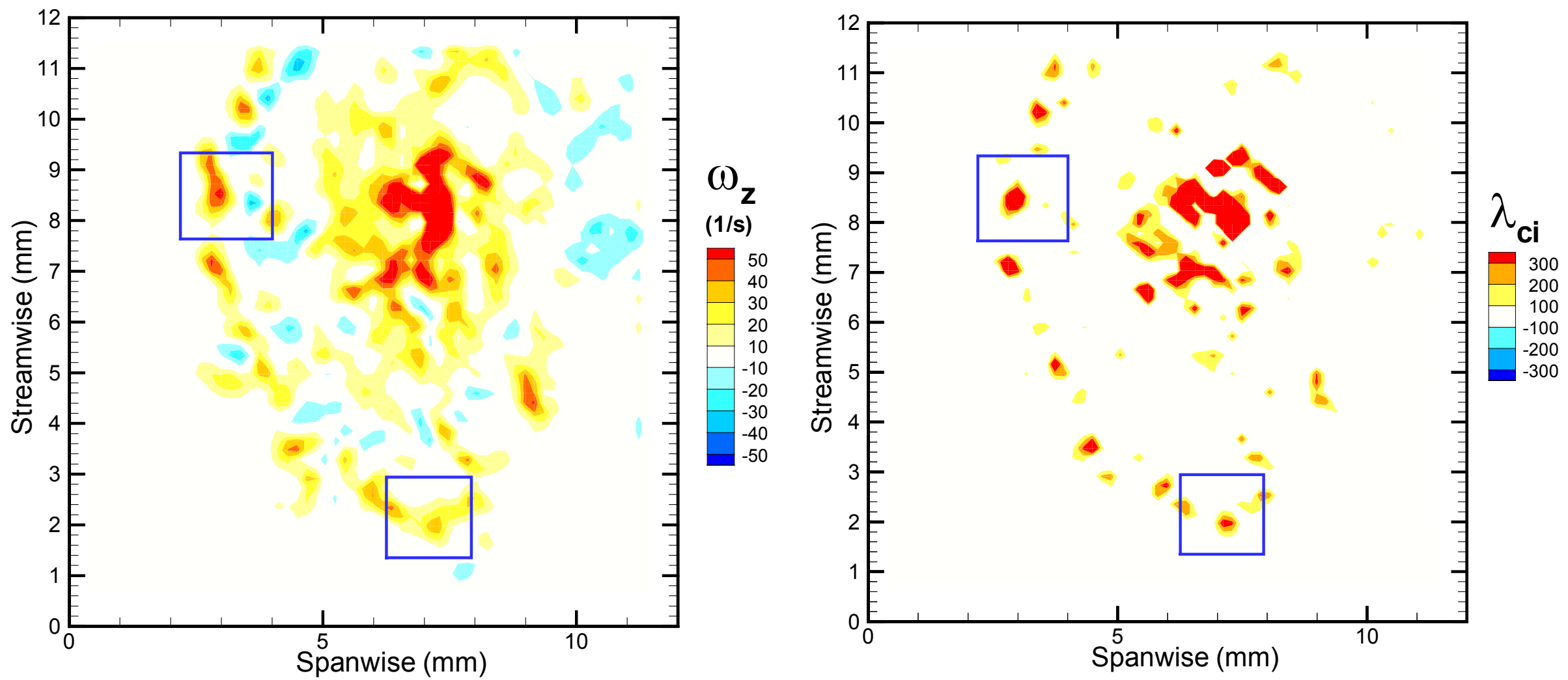


\section{Conclusions}

- The degree of cylinder-cylinder interaction, and hence the resulting flow morphology, is highly sensitive to the initial cylinder separation.

- Different separations may lead to weak, moderate, or strong interactions.

- An idealized "vortex blob" simulation leads to very different flow morphologies than experiment, suggesting that the inner vortices are weakened by interaction.

- Vorticity fields calculated from high-resolution PIV measurements confirm that the inner vortices are significantly weaker, even for $S / D=2.0$ :

$\Gamma_{\text {outer }} / \Gamma_{\text {inner }} \approx 3$ 


\section{Conclusions}

- A correlation-based ensemble averaging procedure effectively captures the large and intermediate scales of the flow, providing confirmation of the experimental repeatability, and permitting decomposition of the density field into mean and fluctuating components.

- The RMS intensity fluctuations based on this decomposition are substantially greater for the case of "moderate" interaction than for the "strong" or "weak" interaction cases, despite comparable initial RMS values.

- High-resolution PIV data resolves mm-scale vortices being convected around the vortex cores. 


\section{The Interaction of Supernova Blast Waves with Interstellar clouds: Experiments on the Omega Laser}

Richard I. Klein

University of California LLNL and

Berkeley, Department of Astronomy

Collaborators

Harry Robey, Ted Perry, Jave Kane, Jeff Greenough, Marty Marinak

Lawrence Livermore National Laboratory

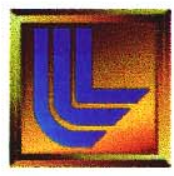




\section{Four Stages of Shock-Sphere Interaction}

- Initial development of reflected, transmitted and diffracted shocks

- Shock compression, convergence and multiple shock interaction $\Rightarrow$ triple point flow and vorticity production

- Re-expansion and shear flow instabilities

- Destruction by instabilities and differential forces 


\section{The interaction of shock waves with clouds in the ISM is a problem of fundamental importance}

Composite image from the ROSAT satellite

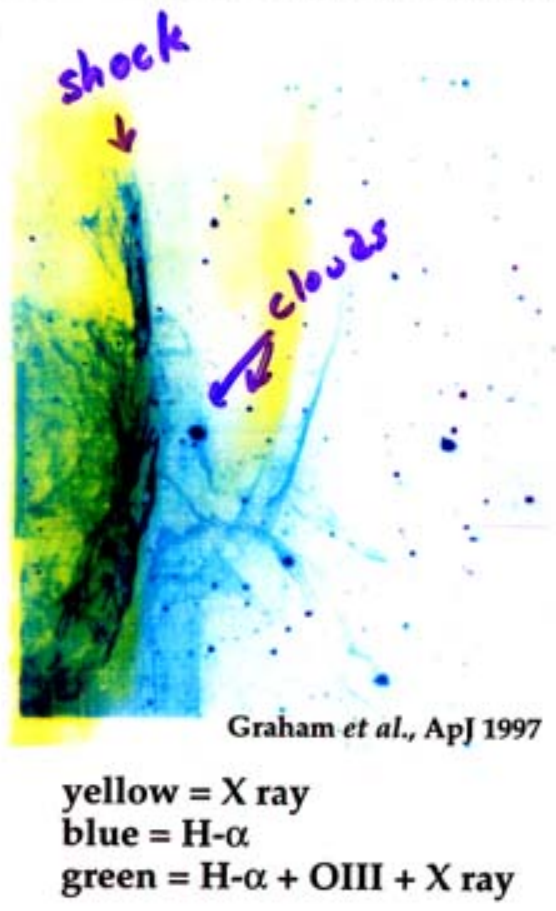

Shock waves from supernovae

shocks heat the coronal phase of the ISM

determine the velocity dispersion of clouds

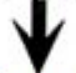

governs the scale height of the ISM

may effectively destroy clouds

$\sqrt{2}$

mixing of clouds with ISM

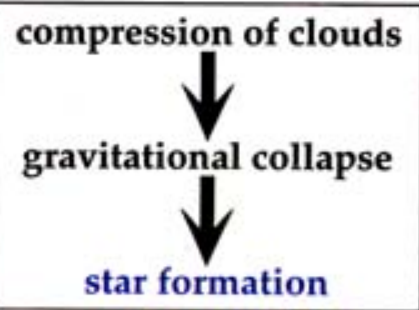

Kimberly S. Budil et al, 1998 Joint APS/AAPT Mecting

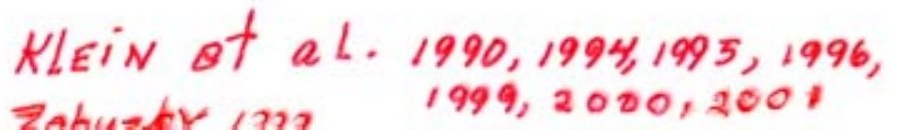




\section{Therę is ample evidence of the aftermath of shock-cloud interations in the Cygnus Loop supernova remnant}

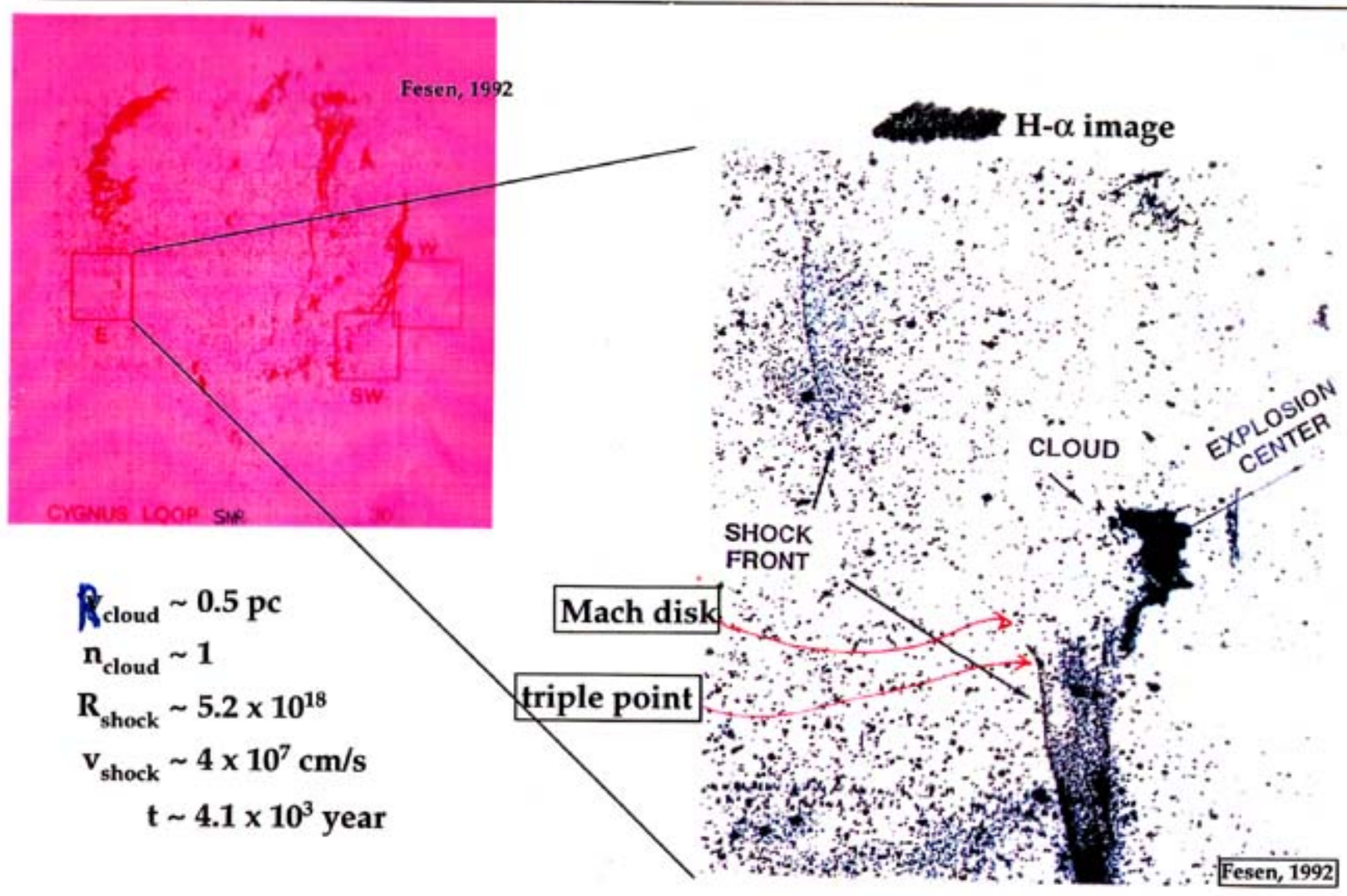


Understanding and Modeling the Evolution of 3D Hydrodynamic Instabilities and Turbulence is Crucial for Code Validation

- The shock-sphere experiments are rich in shock physics relevant to code validation

- Multiple shock structure interactions from reflected, transmitted, diffracted shocks

- Multiple shock-shock interactions with Mach stems, triple points, shear flows

- Non-Linear hydrodynamics instabilities such as R-M, R-T, K-H

- Vortex ring instabilities (bending mode) that cause large-scale vorticity and mixing

- Turbulent flows 


\section{Timescales continued}

Pressures comparable

$$
\begin{aligned}
& V_{s} \approx\left(\rho_{10} / \rho_{c o}\right)^{1 / 2} V_{b} \text { (non radiative) } \\
& \text { if } \dot{\mathrm{V}}_{\mathrm{s}}, \dot{\mathrm{V}}_{\mathrm{b}}<\mathbf{0} \Rightarrow \text { accurate to } \approx 1.5 \\
& \text { if shock is radiative } \rho_{11} \gg \rho_{10} \text { typically } \rho_{11} \gtrsim \rho_{10} \\
& \Rightarrow V_{s} \text { (radiative) } \gg V_{s} \text { (non-radiative) }
\end{aligned}
$$

Timescale for shock to sweep across sphere

$$
t_{i c} \equiv \frac{2 a_{0}}{V_{b}} \quad \begin{aligned}
& \text { Intercloud } \\
& \text { Crossing time }
\end{aligned}
$$

Timescale for sphere to be crushed by shock moving into the sphere

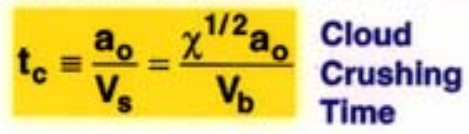

$t_{c}$ is the basic timescale governing the evolution of the sphere $\{\chi, m\}$ characterize evolution $\Rightarrow$ scaling 
After the shock has swept over the sphere, the shocked sphere is subject to Kelvin-Hemholtz and Rayleigh-Taylor instabilities

For $\chi \gg 1$, the time-scale $t_{K H}$ for growth of $K-H$ instabilities for perturbations of wavenumber $k$ parallel to relative $V_{\text {rel }}$ between sphere and surrounding medium is

$$
\begin{aligned}
& \mathrm{t}^{-1} \mathrm{KH}_{\mathrm{H}}=\mathbf{k} \mathbf{v}_{\mathrm{rel}} / \chi^{1 / 2} \\
& \Rightarrow \frac{t_{K H}}{t_{c}}=\frac{V_{b} / V_{\text {rel }}}{k_{0}} \rightarrow K-H \text { growth time }-t c,
\end{aligned}
$$

Shortest wavelength cause the fastest growth

It can also be shown for Rayleigh-Taylor

$\Rightarrow \frac{t_{\mathrm{RT}}}{t_{\mathrm{c}}} \cong \frac{1}{\left(k \mathrm{a}_{\mathrm{o}}\right)^{1 / 2}} \Rightarrow$ sphere undergoes severe instabilities in few $t_{c}$ 


\section{SNR age}

For Sedov-Taylor

Blast Wave

$\mathbf{R}_{\text {blast }} \propto \mathbf{t}^{\alpha}(\alpha=2 / 5)$

$$
t \equiv \frac{\alpha \mathbf{R}_{\mathrm{b}}}{\mathbf{V}_{\mathrm{b}}}=\frac{2}{5} \quad \frac{\mathbf{R}_{\mathrm{b}}}{\mathrm{V}_{\mathrm{b}}} \quad\left(\mathrm{V}_{\mathrm{b}}=\frac{\mathrm{dR}_{\mathrm{b}}}{\mathrm{dt}}=\alpha \mathbf{t}^{\alpha-1}\right)
$$

If $\chi \gg 1 \Rightarrow t_{c c}>t_{i c}$, Clouds are characterized in 3 sizes

Small clouds

$$
\mathrm{t}>\mathrm{t}_{\mathrm{cc}} \Rightarrow \mathrm{a}<<\frac{0.1 \mathrm{R}}{\chi^{1 / 2}}
$$

SNR does not evolve significantly during cloud crushing - cloud is

promptly crushed. $\mathbf{V}_{\mathbf{s}} \sim$ constant pressure on cloud $\approx$ steady.

Medium clouds $\quad t_{c c}>t>t_{i c} \Rightarrow \frac{0.1 R}{\chi^{1 / 2}}<a<0.05 R V_{s}$ decelarated

Blast wave does evolve during the time to crush the cloud.
Large clouds
$\mathrm{t}<\mathrm{t}_{\mathrm{ic}} \Rightarrow \mathrm{a}>0.05 R$

Blast wave weakens significantly over time to cross clouds

Force on clouds impulsive 


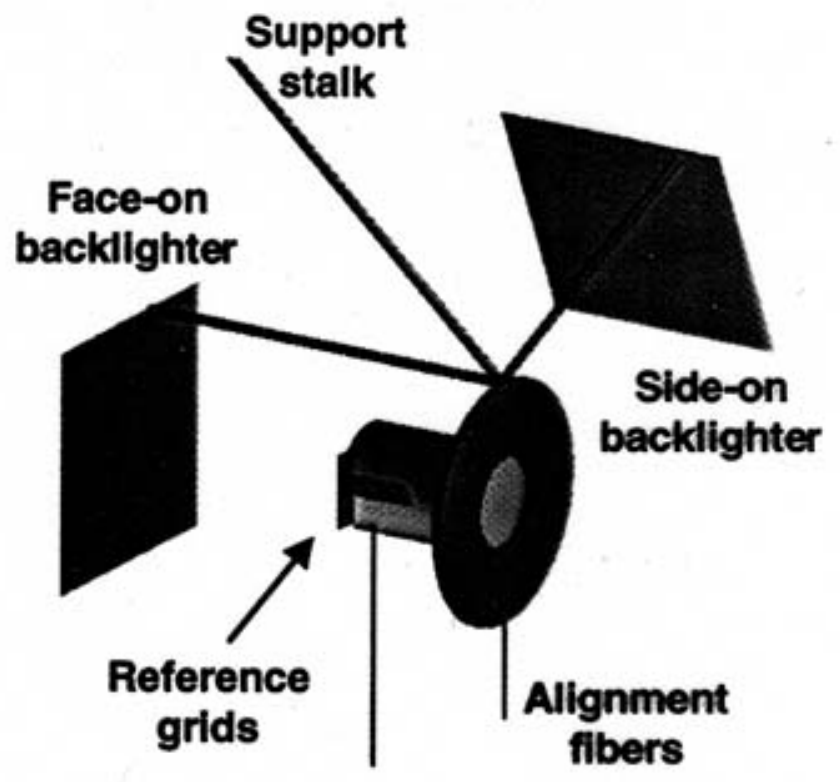

Figure 1(b), Robey, Phys. Rev. Lett. 


\section{Experimental Setup}

- Achieve strong shock conditions with direct 10 beams with $500 \mathrm{~J} /$ beam, flat pulse length $10 \mathrm{~ns}$ and laser wavelength of $0.351 \mu \mathrm{m}$ onto target

- Beams have super-Gaussian spatial intensity

$$
\mathrm{I} / \mathrm{Io}=\exp [-(\mathrm{r} / 412 \mu \mathrm{m})]^{4.7}
$$

- Target Diameter is $800 \mu \mathrm{m}$

- Berryllium shield was used to delay shock propagation around target and generate planar shock propagation.

- Direct -drive laser illumination used to permit face-on imagining of target

$\Rightarrow$ Previous experiments (Klein et al. 2000 - 2001) used indirect-drive 


\section{Experimental Setup - con’t}

- $\mathrm{Cu}$ sphere with $120 \mu \mathrm{m}$ diameter

(representing interstellar cloud) and density $8.95 \mathrm{~g} / \mathrm{cm}^{3}$ embedded in

Beryllium shock tube filled with

polystyrene $(\mathrm{CH})$ at density

$1.044 \mathrm{~g} / \mu^{3}{ }^{3}$ (representing interstellar medium)

- Interaction is imaged with $5.2 \mathrm{KeV} \mathrm{x}$ rays with 7 additional Omega beams 


\section{Experimental Setup - con't}

- Advantages of indirect-Drive $\Rightarrow$ produces a more uniform radiation flux and shocks of greater planarity

- Disadvantages: Plasma blown off the walls prohibits late-time imaging of interaction from face-on direction.

- Direct-Drive maintains face-on access for duration of the experiment 


\section{Experimental Radiographs}

$$
\text { sider on }
$$

FAce-ON

\section{$t=13 \mathrm{~ns}$ \\ shock passes OVER Sphere}

$$
t=26 \mathrm{~ns}
$$

shocks is

well passed. sphere

$$
t=39 \mathrm{~ns}
$$

shock is out of field of viem

(a)

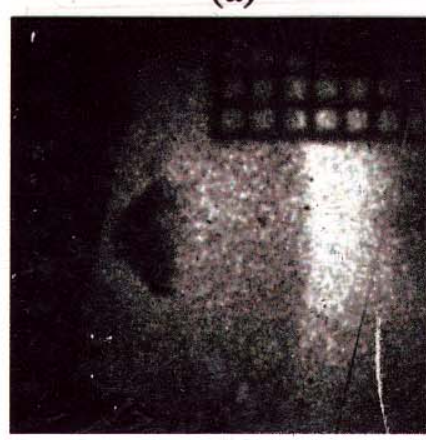

(c)

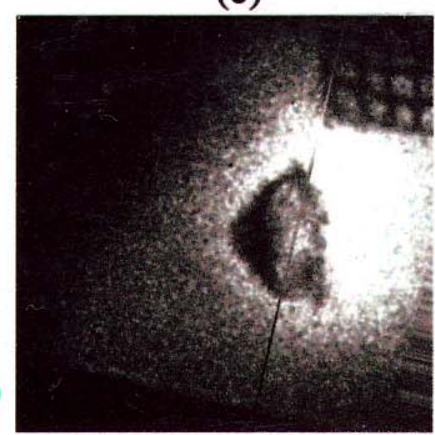

(e)

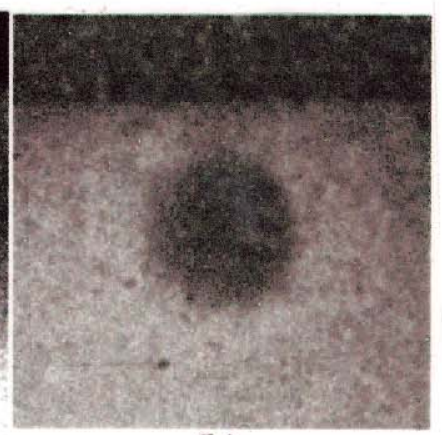

(b)

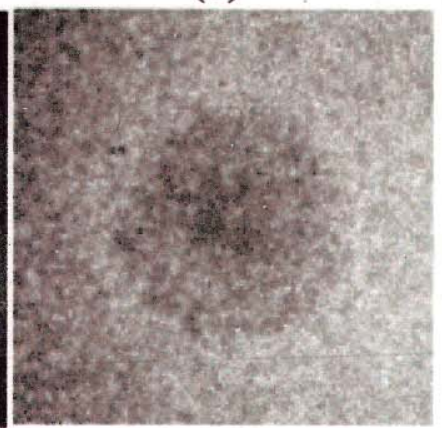

(d)

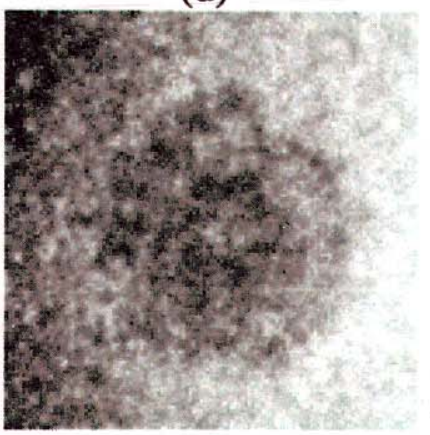

(f)

Figure 2, Robey, Phys. Rev. Lett. 


\section{Experimental Results}

- Experimental radiographs show interaction with "simultaneous" sideon and face-on images

\section{$\underline{\mathrm{t}=12 \mathrm{~ns}}$}

- Sphere compressed in axial direction with little radial compression

\section{$\mathrm{t}=26 \mathrm{~ns}$}

- $\quad$ Sphere undergoes severe axial compression. Baroclinic vorticity generated at sphere- $\mathrm{CH}$ interface

- Vorticity generated during initial passage of shock over spherical cloud 


\section{Experimental Results - con't}

- Vorticity generated during subsequent post-shock flow at cloud-intercloud boundary

- Flow around cloud produces highpressure Mach reflected shock into back side of cloud

- Side shocks weaker than front and aft shocks due to pressure minimum at sides

$\Rightarrow$ Results in strong axial compression sphere begins to pancake 


\section{Experimental Results - con't}

- Emergence of double ring structure seen in face-on radiograph

\section{$\underline{\mathrm{t}=39 \mathrm{~ns}}$}

- Strong vortex roll up noted on sides of sphere

- Face-on view shows distinct double ring structure which tracks development of double vortex ring

- Klein and McKee (1994) showed that 3-D vorticity field of cloud is well tracked by full density field $\Rightarrow$ Vortex Ring Instability 


\section{Azimuthal line-outs from face-on images of shock / sphere interaction at $t=39$ ns}

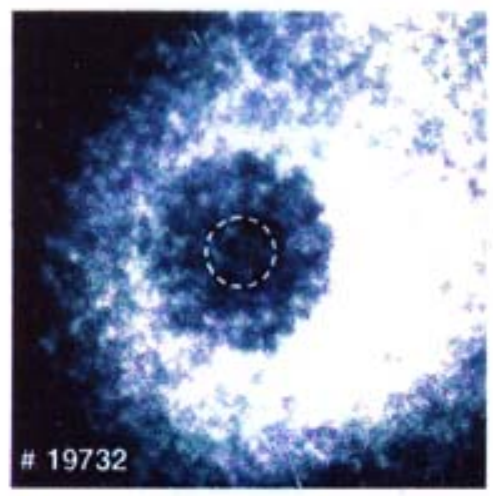

Azimuthal line-out through central feature ( $r=36 \mu \mathrm{m})$

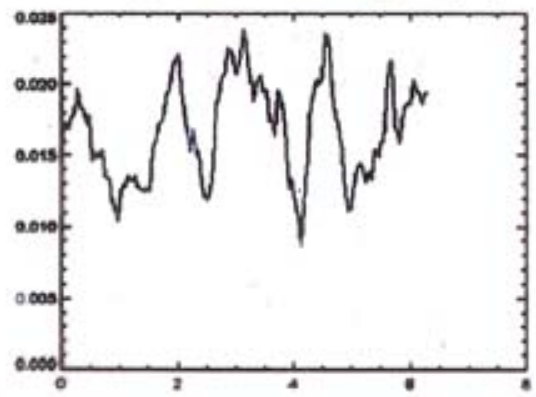

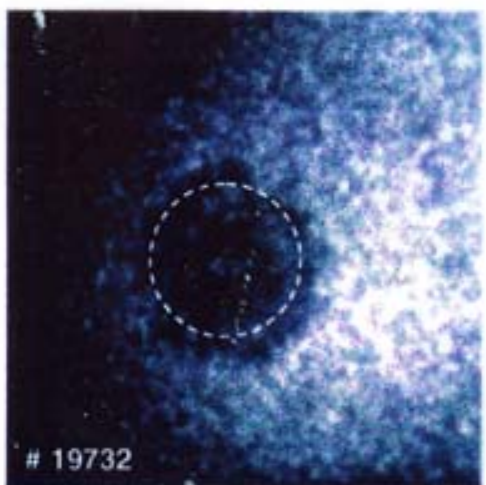

Azimuthal line-out through outer feature $(r=80 \mu \mathrm{m})$

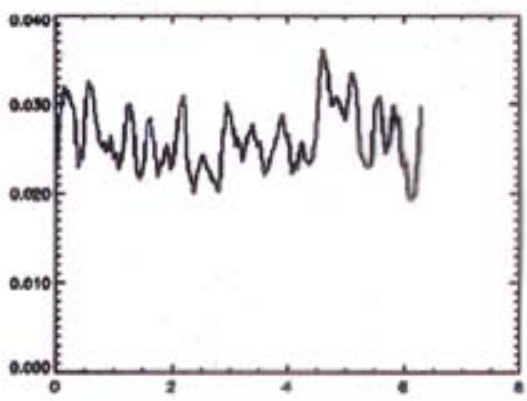


Modal Analysis of Double Vortex pine: At $t=39 \mathrm{~ns}$

Double Vortex Ring structure (a)

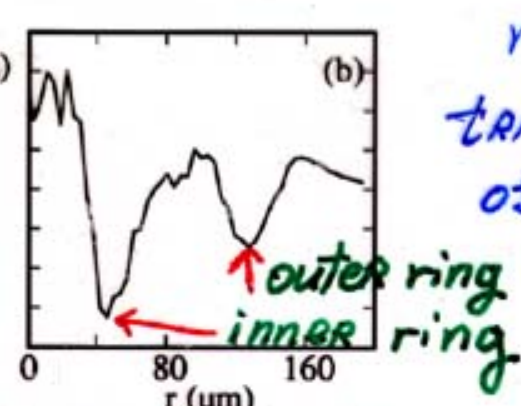

radial transparency of Double. ring.
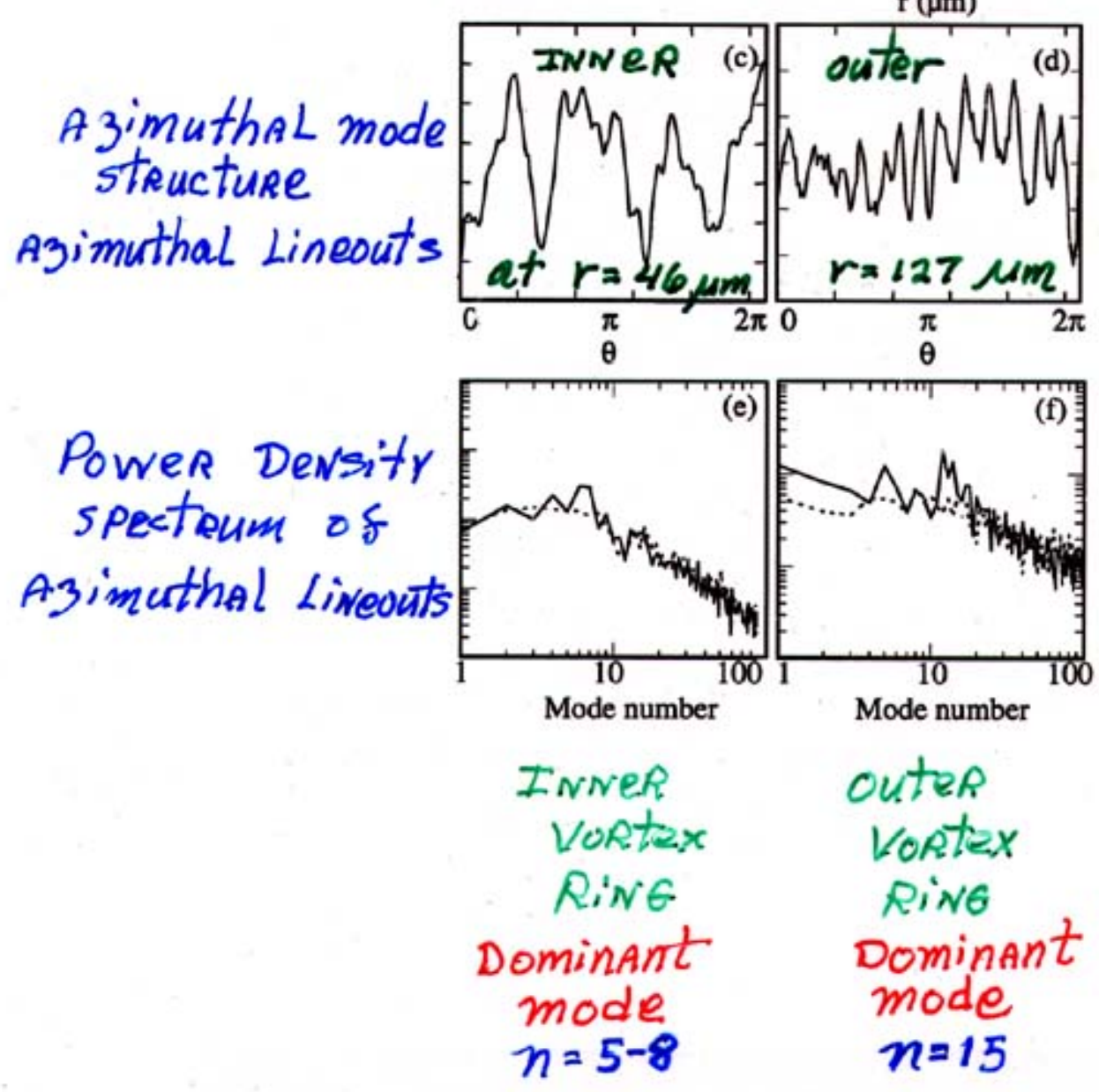

Figure 3, Robey, Phys. Rev. Lett. 


\section{Modal Analysis of \\ Double Vortex Ring}

\section{Structure}

- 3-D simulations show development of

2 Vortex Rings associated with cloud

that undergo bending mode instability

(Klein and McKee, 1994; Marinak,

Klein and Perry 2001)

- Azimuthally averaged radial lineouts of experimental radiograph show

unambiguous double ring structure

- Azimuth lineouts at radial location of each ring show periodic azimuthal modulation suggesting a dominant mode number of 5 for inner ring and higher mode number of $14-16$ for the outer ring 


\section{Modal Analysis of}

\section{Double Vortex Ring}

\section{Structure - con't}

- Power density spectrum of azimuthal

lineouts confirm dominant mode

number of 5-8 for inner ring and

dominant mode number 15 for outer

ring 


\section{Experimental-Theoretical}

Comparison of Vortex

\section{Ring Modal Structure}

- At $t=39 n s$, shocks have left sphere and sphere advects essentially as an incompressible fluid

- Applying incompressible theory to describe an incompressible bending mode instability in vortex ring to experimental face-on radiographs $\Rightarrow$ dominant unstable mode for inner ring $\mathrm{n}=5-7$ and $\mathrm{n}=15-17$ for outer ring

- Theoretically determined dominant modes in non-axisymmetric unstable vortex rings are in remarkable agreement with experimentally determined unstable modes 
Contours showing location of InNer snel outer Vortex Rings

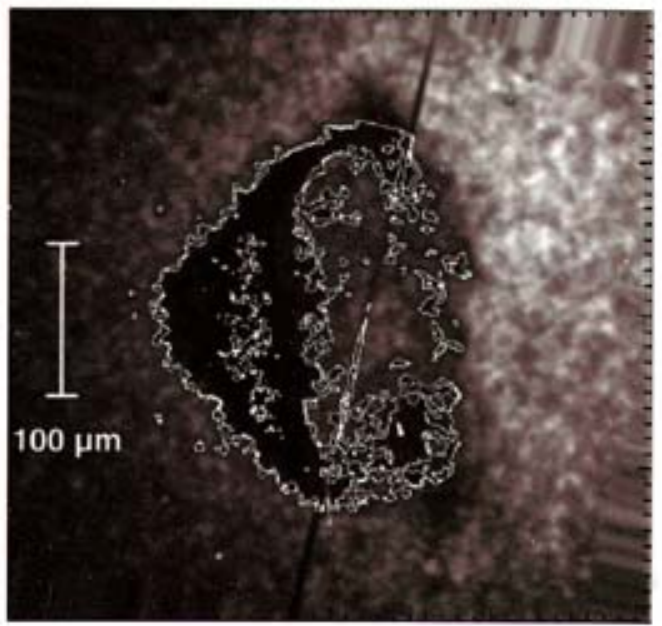

Figure 4, Robey, Phys. Rev. Lett. 


\section{3-D High Resolution}

AMR Simulations

- Side on view of simulation shows well separated front and back part of the sphere due to instabilities

- Side on view models inner transparent bubble seen in experimental radiograph

- Face on view shows clear evidence of inner and outer vortex rings seen in experimental radiograph

- Inner ring shows low mode structure and outer ring shows high mode structure as in radiographs

- Overall morphology of 3-D simulations in excellent agreement with experimental radiographs 


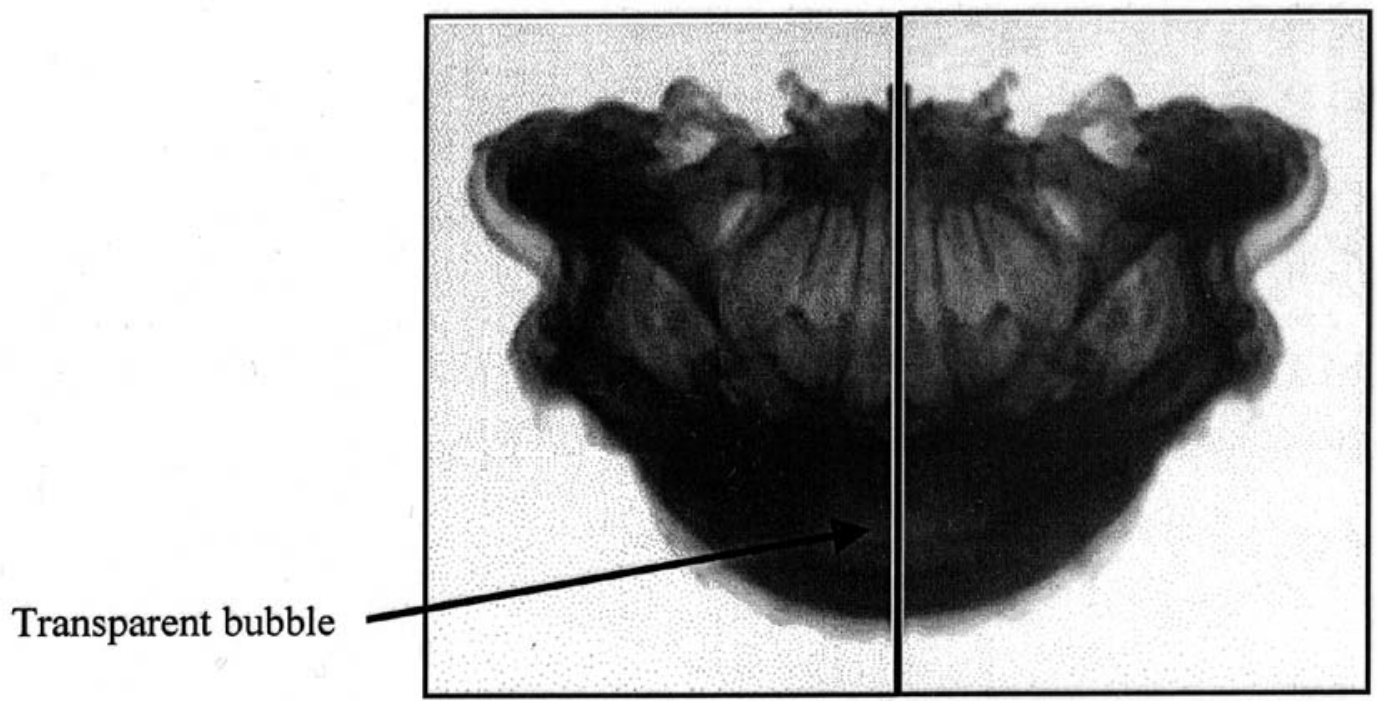

Figure $\mathrm{X}(\mathrm{a})$ 


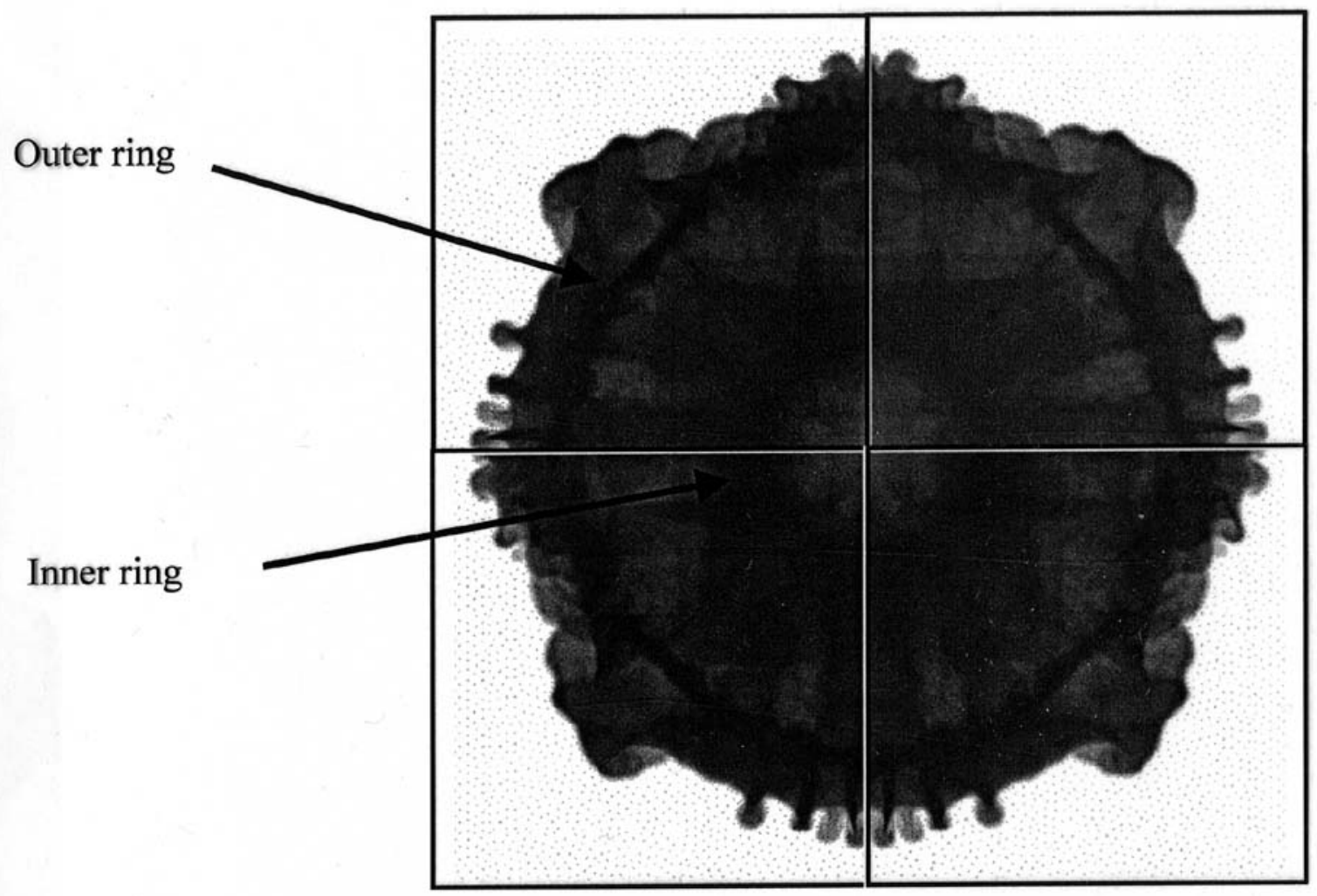

Figure $\mathrm{X}(\mathrm{b})$ 


\section{Conclusions}

- The interaction of a supernova blast wave with interstellar clouds can be well scaled to an HED

Omega laser experiment

- Development of vortex flow gives rise to strong vortex rings unstable to Widnall Bending Mode instabilities. These are shown for the first time in HED laser experiment

- $\quad$ At $39 \mathrm{~ns}\left(\sim 3 \mathrm{t}_{\mathrm{c}}\right)$ hydrodynamic instabilities (R-T, K-H and Widnall) destroy sphere (cloud) resulting in turbulent mixing of cloud with surrounding medium (ISM)

- Power density spectrum of face-on radiographs determine dominant mode of inner and outer vortex rings to be $n=5-8$ and $n=15$

- Incompressible vortex ring theory shows remarkable agreement in predicting dominant unstable modes of vortex rings

- $\quad$ High resolution 3-D AMR simulations capture all essential morphological features of side-on and faceon experimental radiographs including appearance of double vortex rings 


\section{Ablative Rayleigh-Taylor Instability at Short Wavelengths}

Moire Interferometry
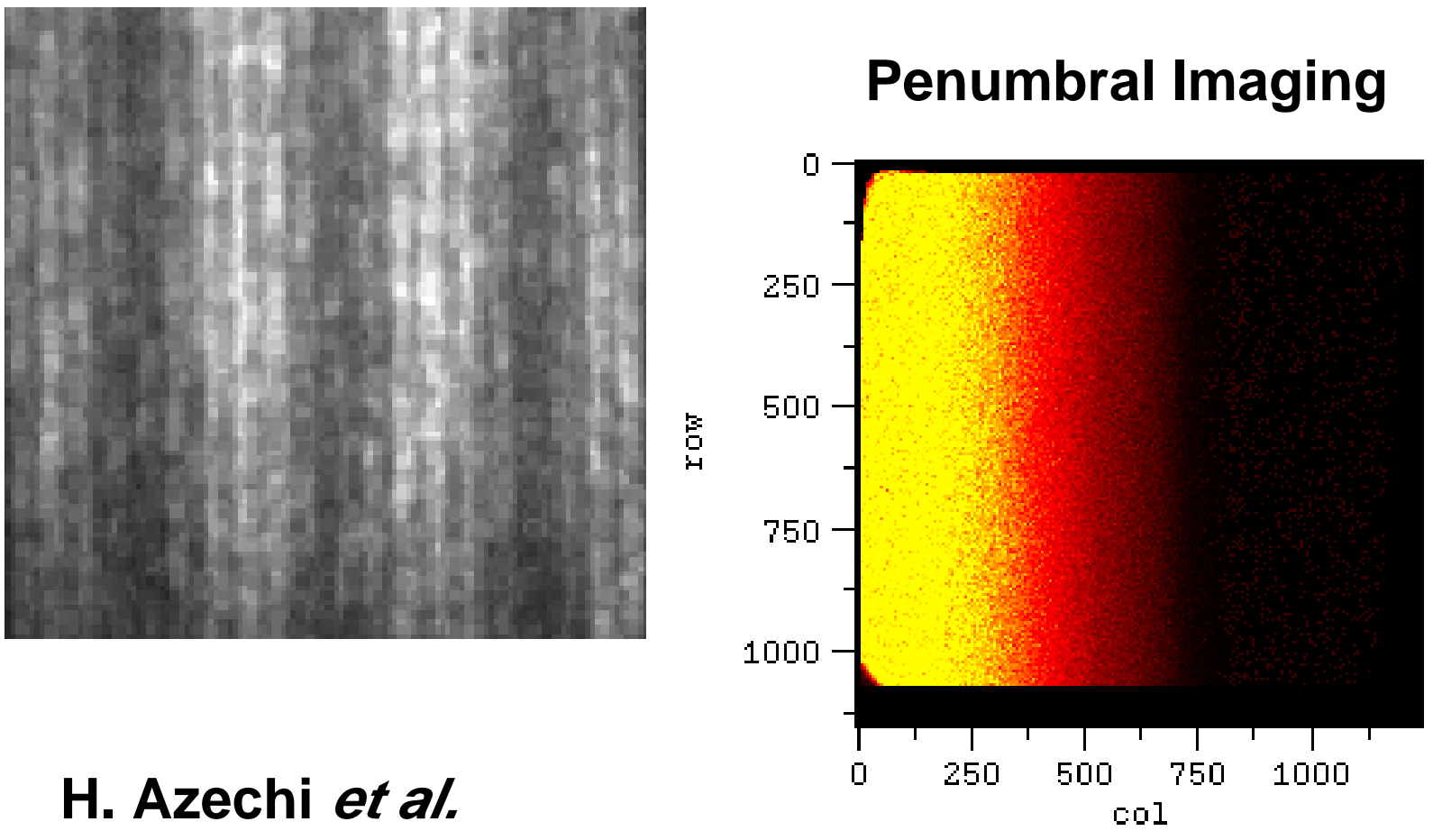

Fresnel Phase Zone Plate

H. Azechi et al.

Institute of Laser Engineerıng

Osaka University

IWPCTM 2001 Paper\# E-45

9-14 Dec 2001,

Pasadena, USA

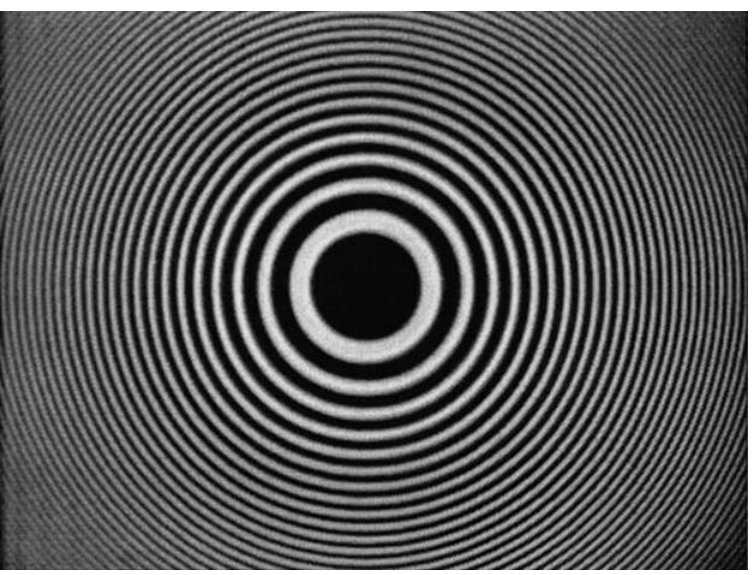




\section{Primary obstacle of IFE is Rayleigh-Taylor instability.}

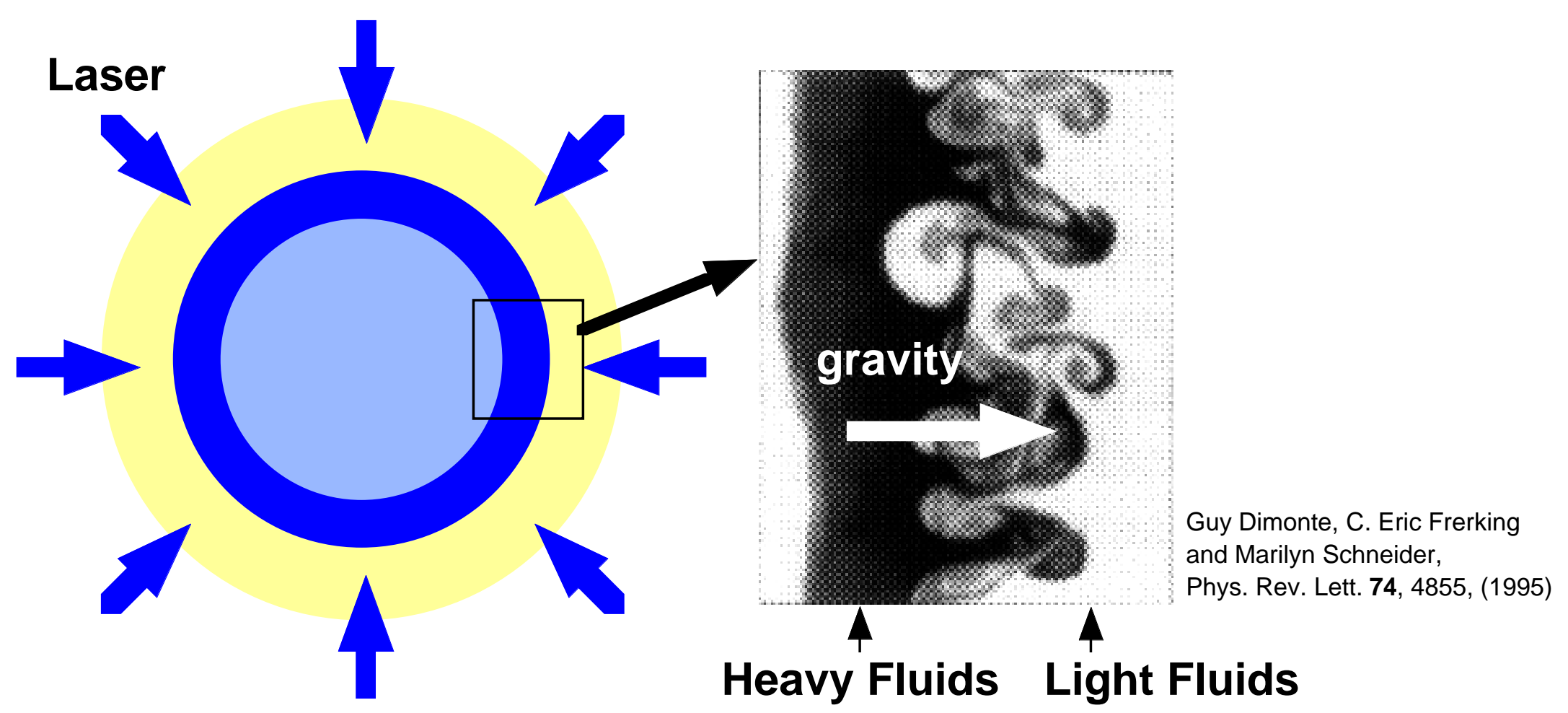

Typical wavelength $=$ several tens $\mu \mathrm{m}$

time scale $=$ ns

High resolution advanced diagnostics are required.

X-ray Moire interferometry

Fresnel phase zone plate

Penumbral imaging 


\section{Ablative Rayleigh-Taylor instabilities}

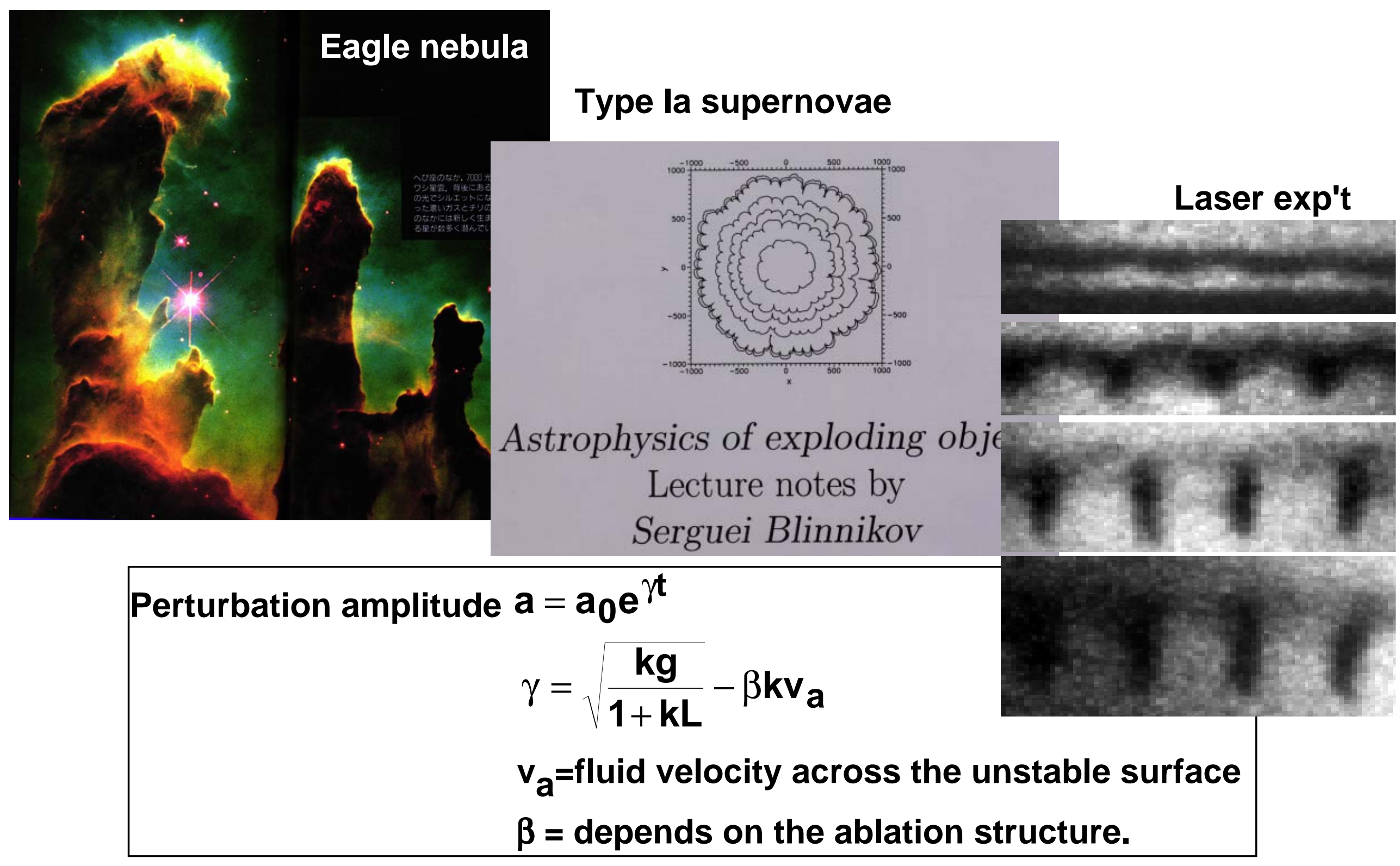




\section{Motivation}

New method is needed for the measurement of short wavelength Rayleigh-Taylor (RT) Growth.

It is necessary to measure short wavelength RT growth in order

to understand the mechanism of the ablative stabilization.

\section{Dispersion curve of the Rayleigh-Taylor instability}

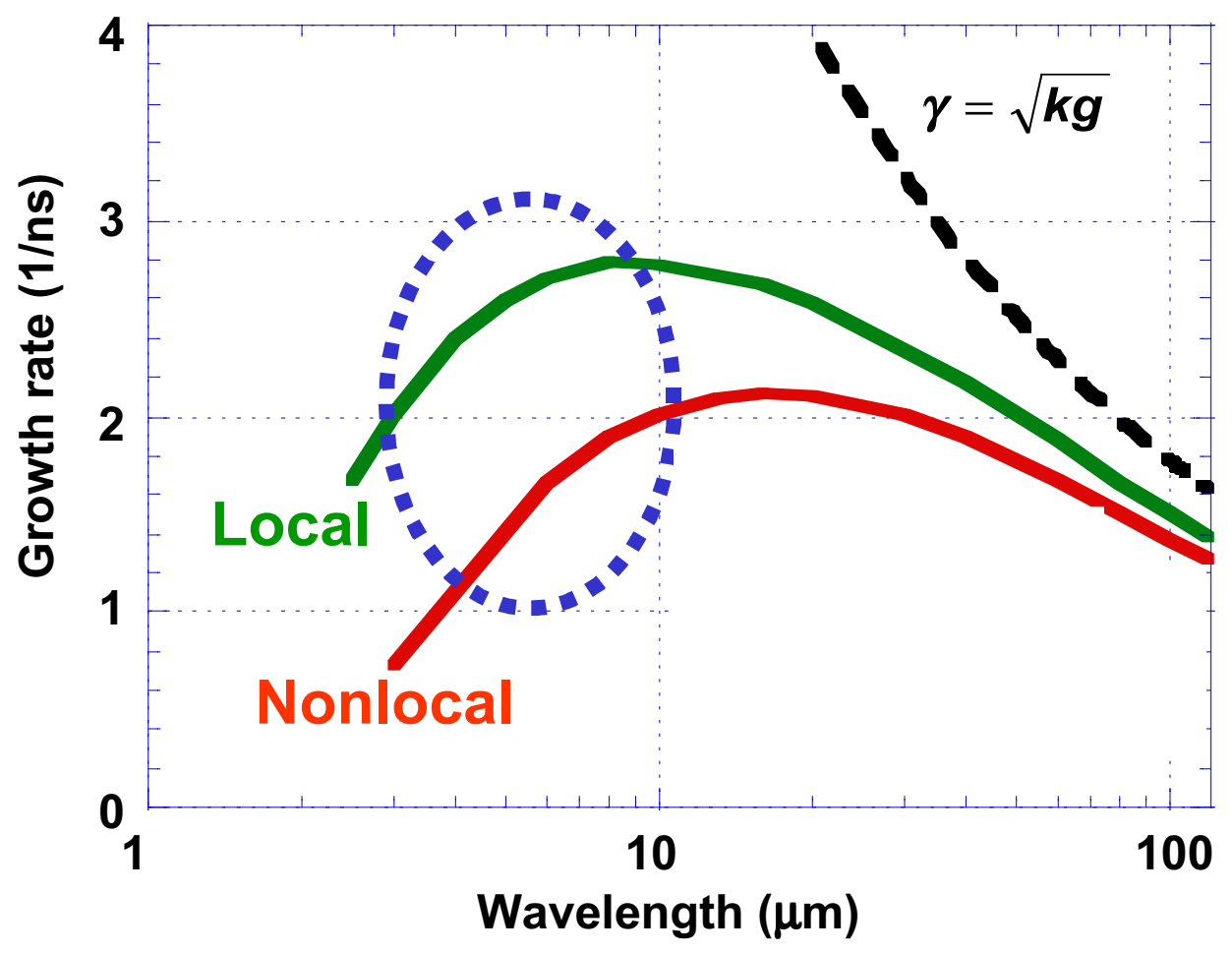

- Short wavelength RT

Moire interferometry

- Independent test

Penumbral imaging

Fresnel phase zone plate 
Moire interferometry / short wavelength Rayleigh-Taylor 


\section{Experimental procedure}

Schematic view of the experimental setup

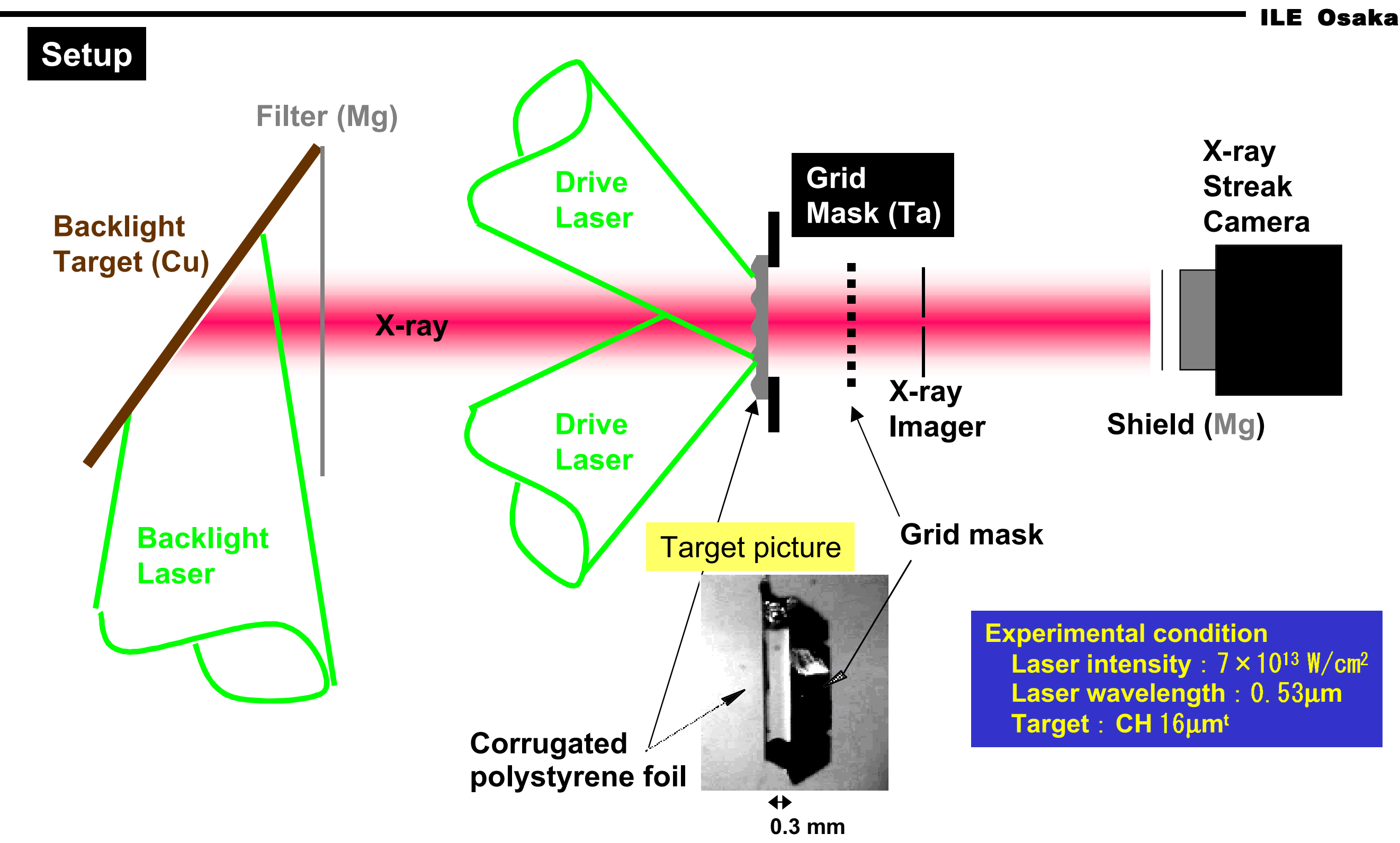




\section{Moiré interferometry}

Moiré interferometry is very useful for measurements of the RT instability at short wavelength.

Principle

Perturbation wavelength

$$
\lambda_{\text {Perturb. }}=12 \mu \mathrm{m}
$$

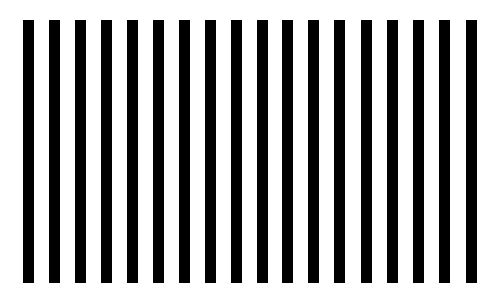

Grid mask

$$
\lambda_{\text {Grid }}=10 \mu \mathrm{m}
$$

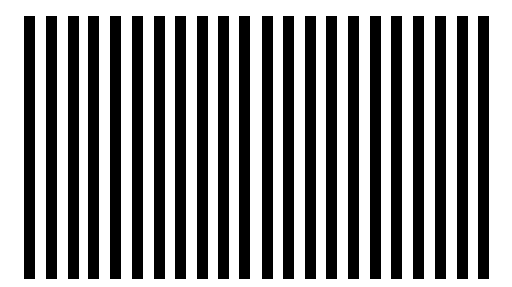

Moiré interferometry

$$
\lambda_{\text {Moiré }}=\underline{60} \mu \mathrm{m}
$$

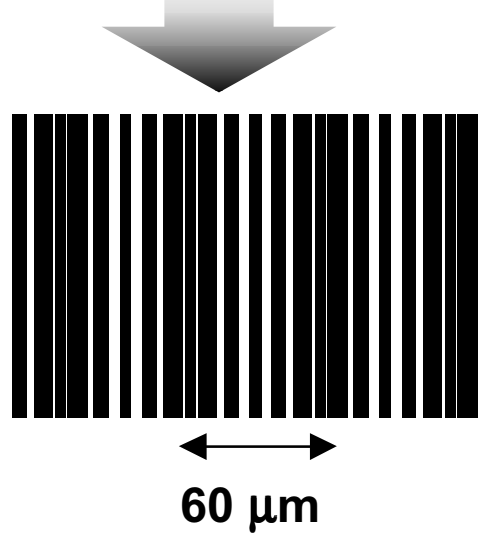

$$
k_{\text {Moire }}=\left|k_{\text {Perturb. }} \pm k_{\text {Grid }}\right|
$$

Due to the moiré interference, the short wavelength perturbation is converted to longer wavelength perturbation.

M. Matsuoka et al., Rev. Sci. Instrum., 70, 637 (1999)

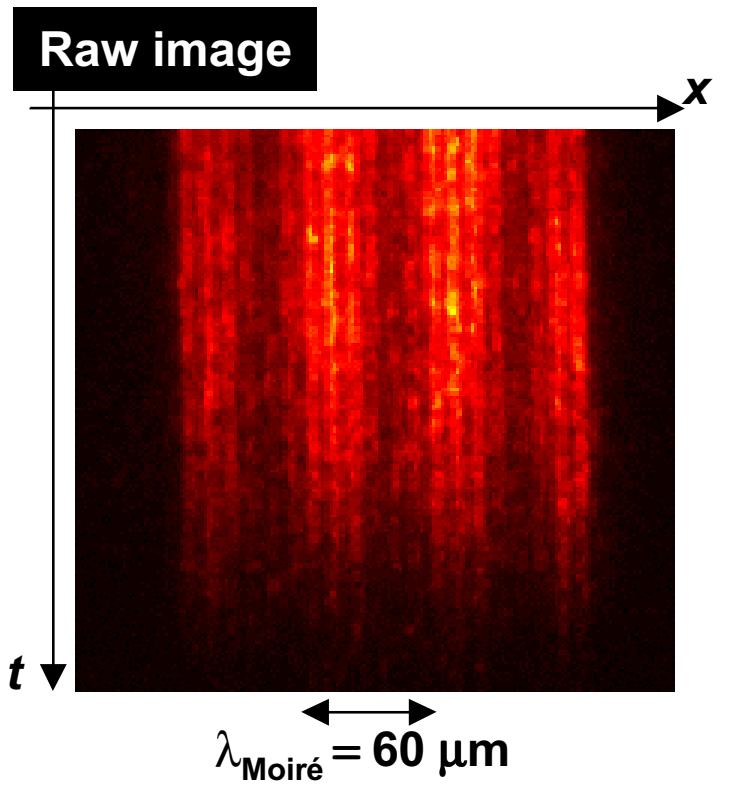




\section{Experimental results}

Raw data of Rayleigh-Taylor instability observed with moiré interferometry
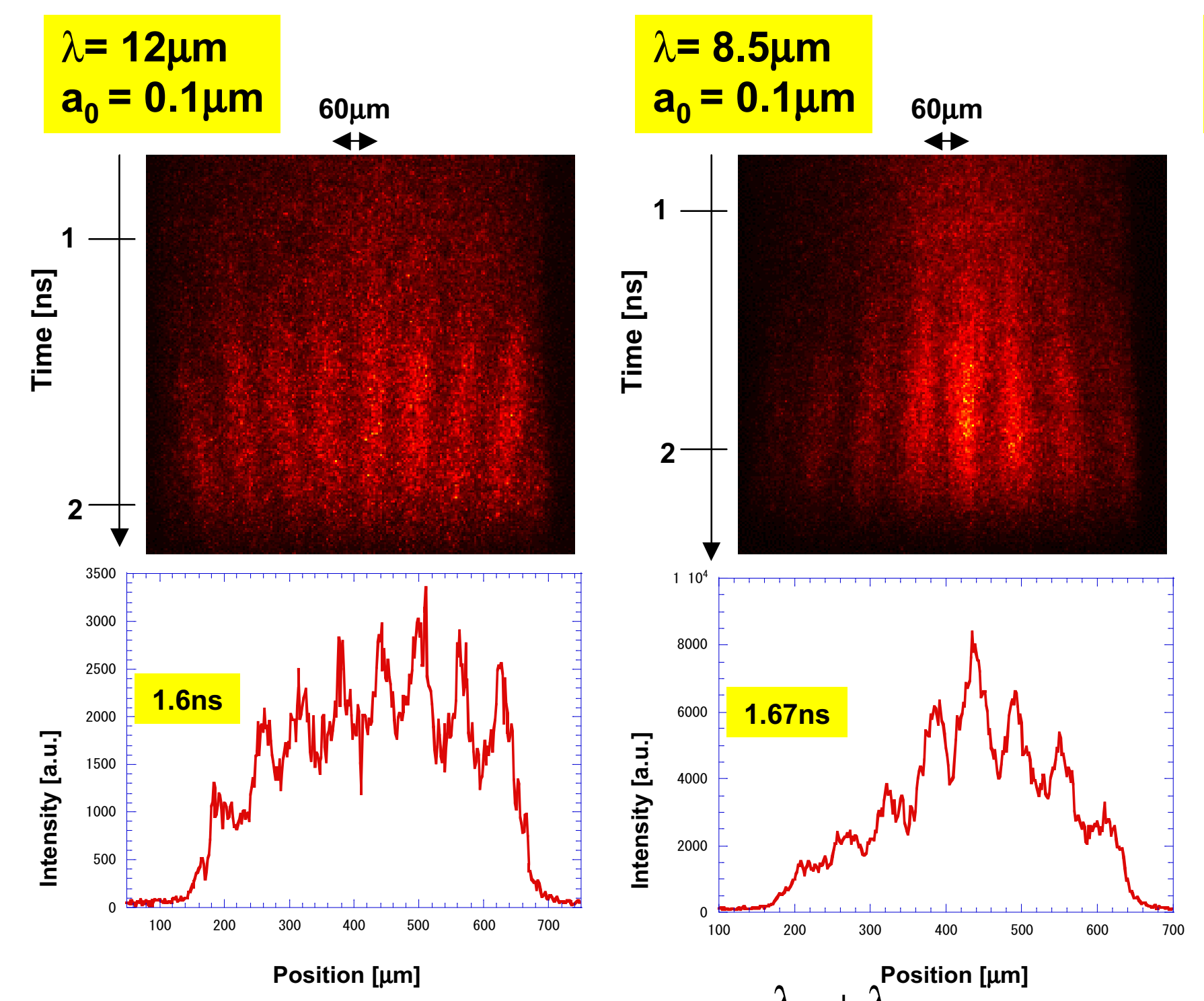

$$
\begin{aligned}
& \lambda=4.7 \mu \mathrm{m} \\
& a_{0}=0.05 \mu \mathrm{m} \quad 80 \mu \mathrm{m}
\end{aligned}
$$
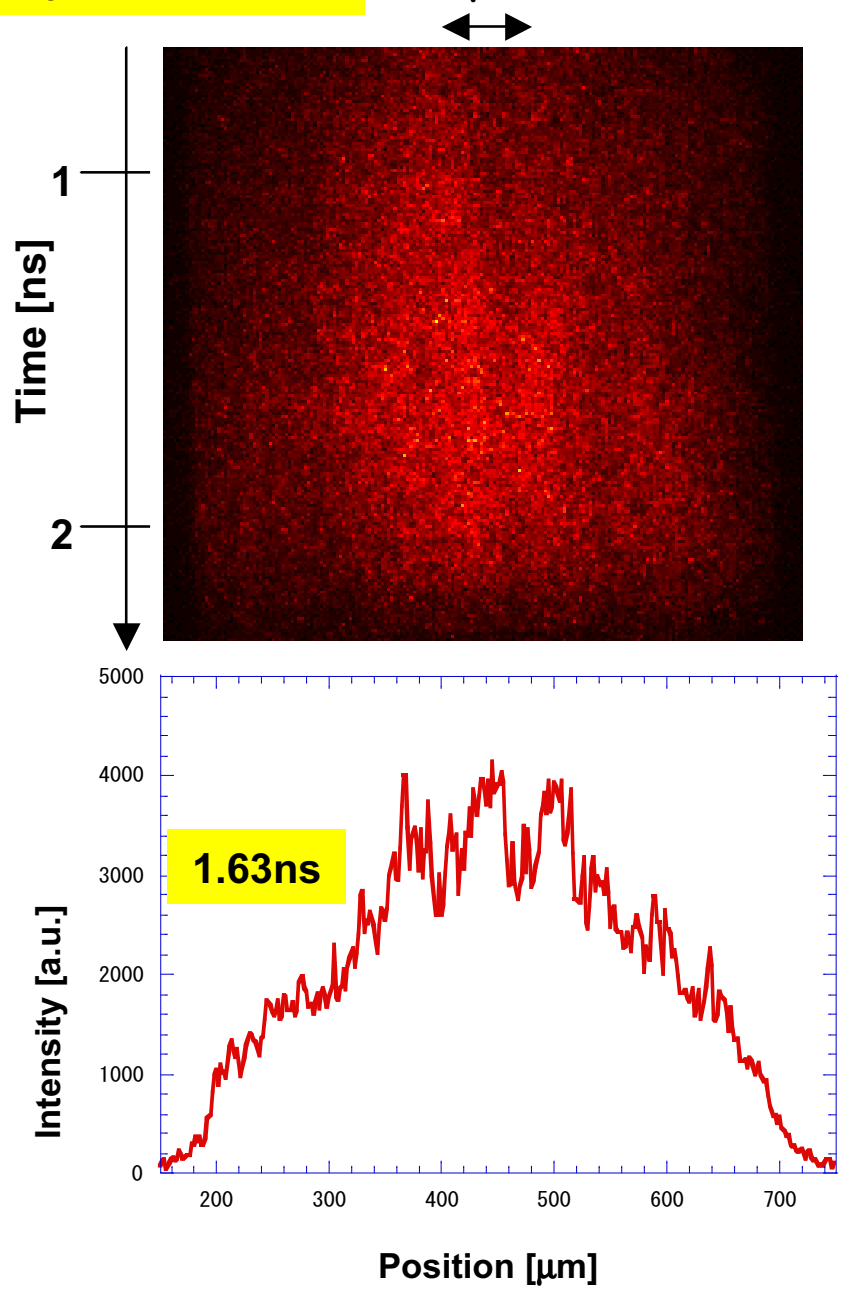

$$
\lambda_{M}=\frac{\lambda_{1}+\lambda_{2}^{\text {Position }[\mu \mathrm{m}]}}{\lambda_{1}-\lambda_{2}} \text { sensitive to } \Delta \lambda
$$




\section{Short Wavelength RT}

Large Rayleigh-Taylor growth was observed up to 5- $\mathrm{mm}$ wavelength.
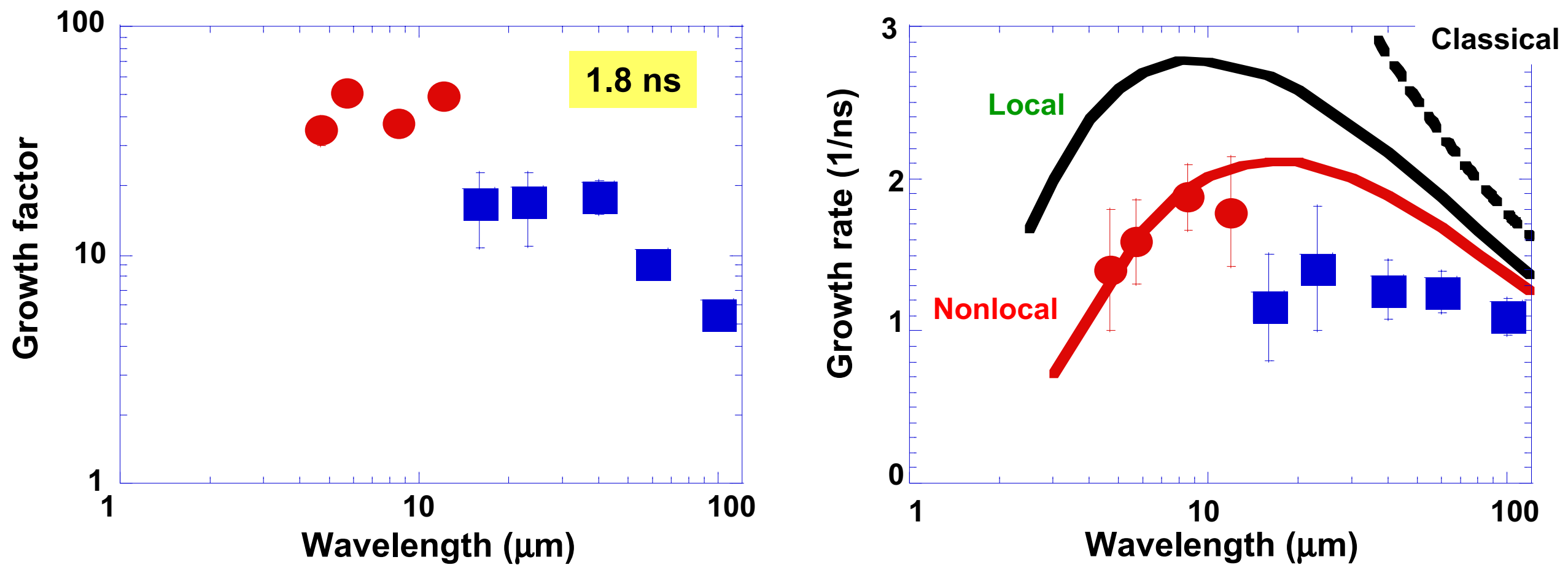

- This exp't suggests that nonlocal heat transport plays a role in ablative stabilization.

- However, for unambiguous clarification, we need to make independent observation. 


\section{Reduction of the target density \\ with nonlocal heat transport}

Density profile at $1.3 \mathrm{~ns}$

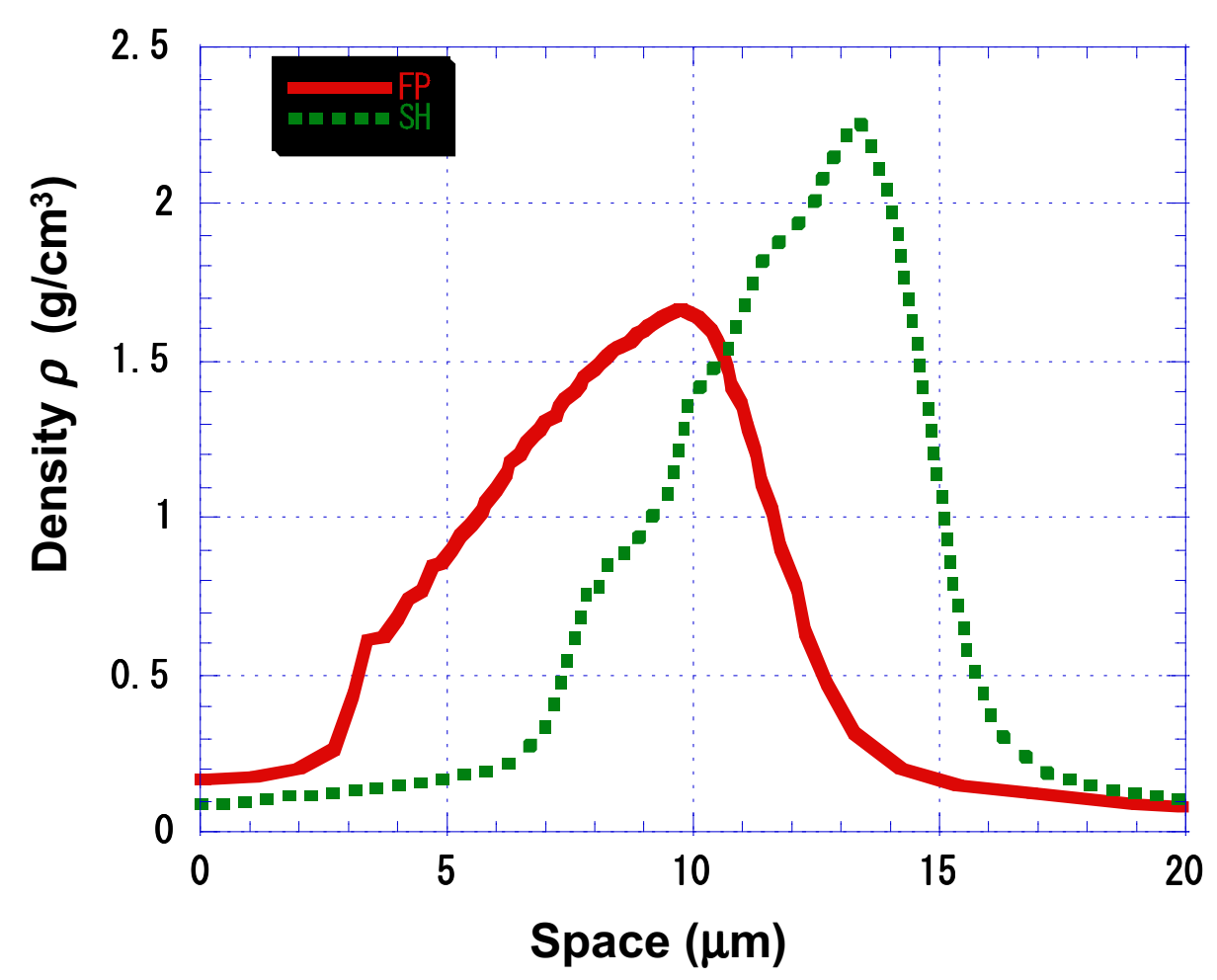

if $m=\rho_{a} v_{a} \cong$ const

A. Sunahara et al., "Nonlocal Electron Transport" (2000)

\section{Spitzer-Härm (SH) \\ Local heat transport :}

- Diffusion approximation of electron thermal conduction

Fokker-Planck (FP)

Nonlocal heat transport :

- High-energy electrons in the tail of Maxwellian distribution penetrate into the target and preheat it.

\section{Target expansion $\rightarrow$ Density reduction}

Ablation velocity increases

\section{Stabilization}




\section{Method of Density Measurement}

Density profile was obtained from the $x$-ray backlighting image of the planar target.
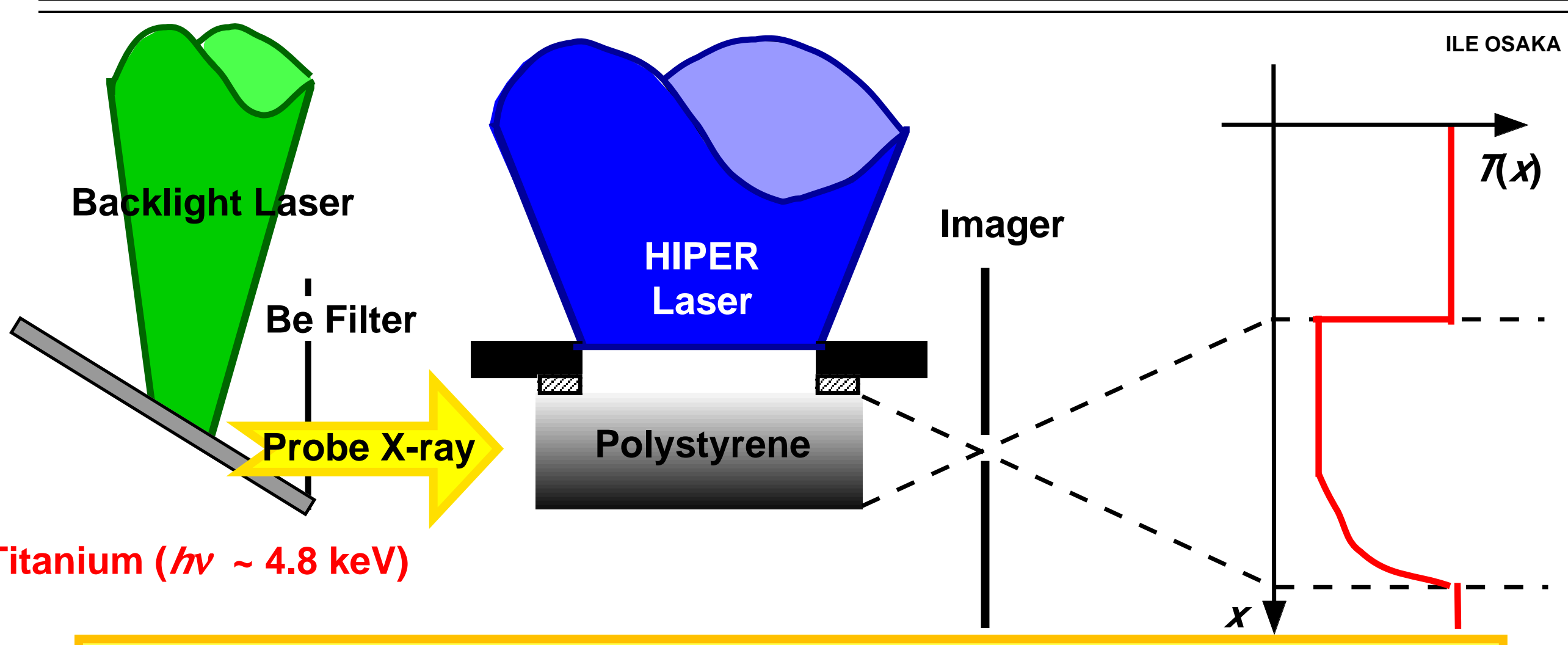

Titanium $(h v \sim 4.8 \mathrm{keV})$

$$
T=\exp (-\mu \rho /)_{\text {I: material thickness, } \rho: \text { density }}^{T: \text { Transmission, } \mu: \text { mass absorption coeff. }}
$$


Fresnel phase zone plate / density profile 


\section{The principle of the FPZP imaging}

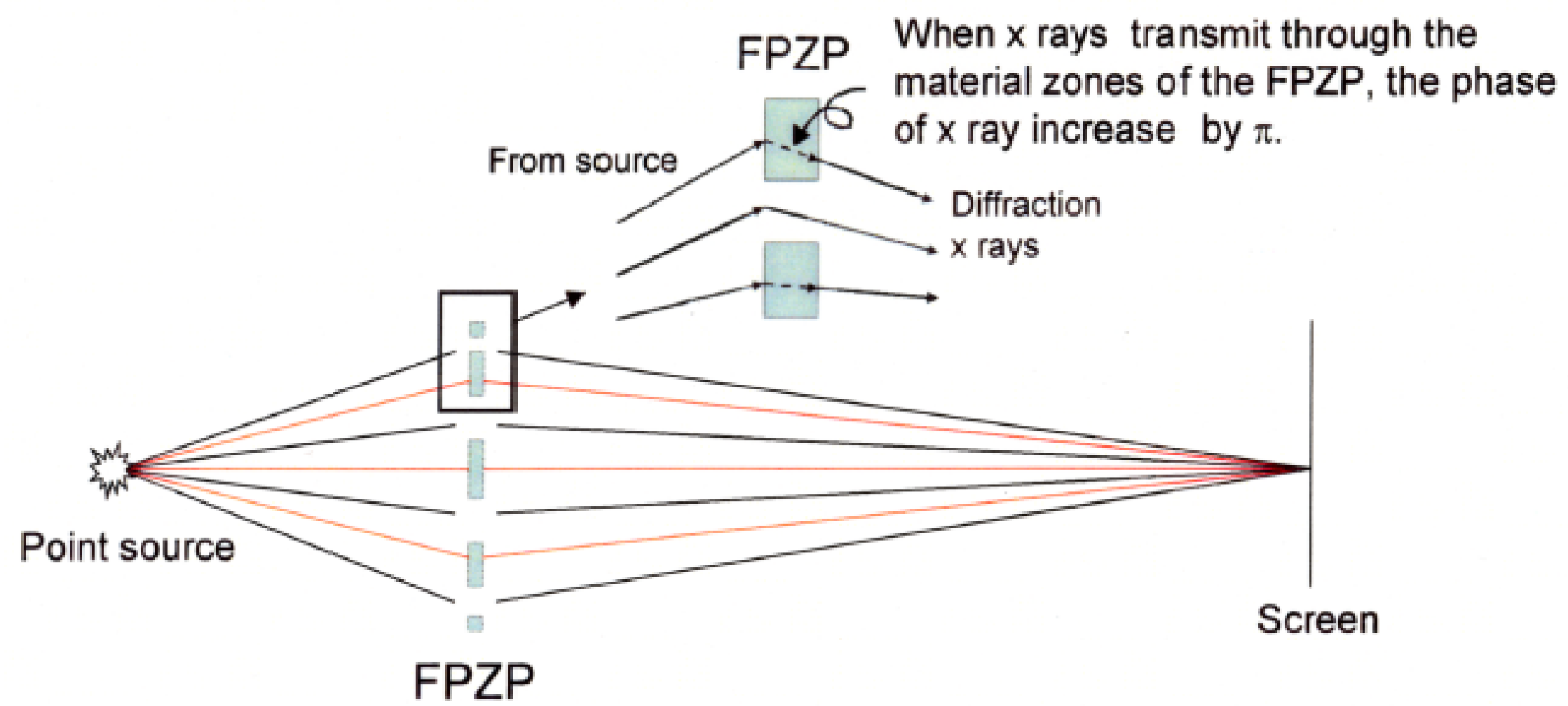

Advantage

- High spatial resolution $2.2 \mu \mathrm{m}$

-Hard x-ray imaging $\quad 4.7 \mathrm{keV}$

Disadvantage

- Chromatic aberration Possible to obtain $\sim \mu \mathrm{m}$ spatial resolution

-Background 


\section{Spatial resolution test of FZP}

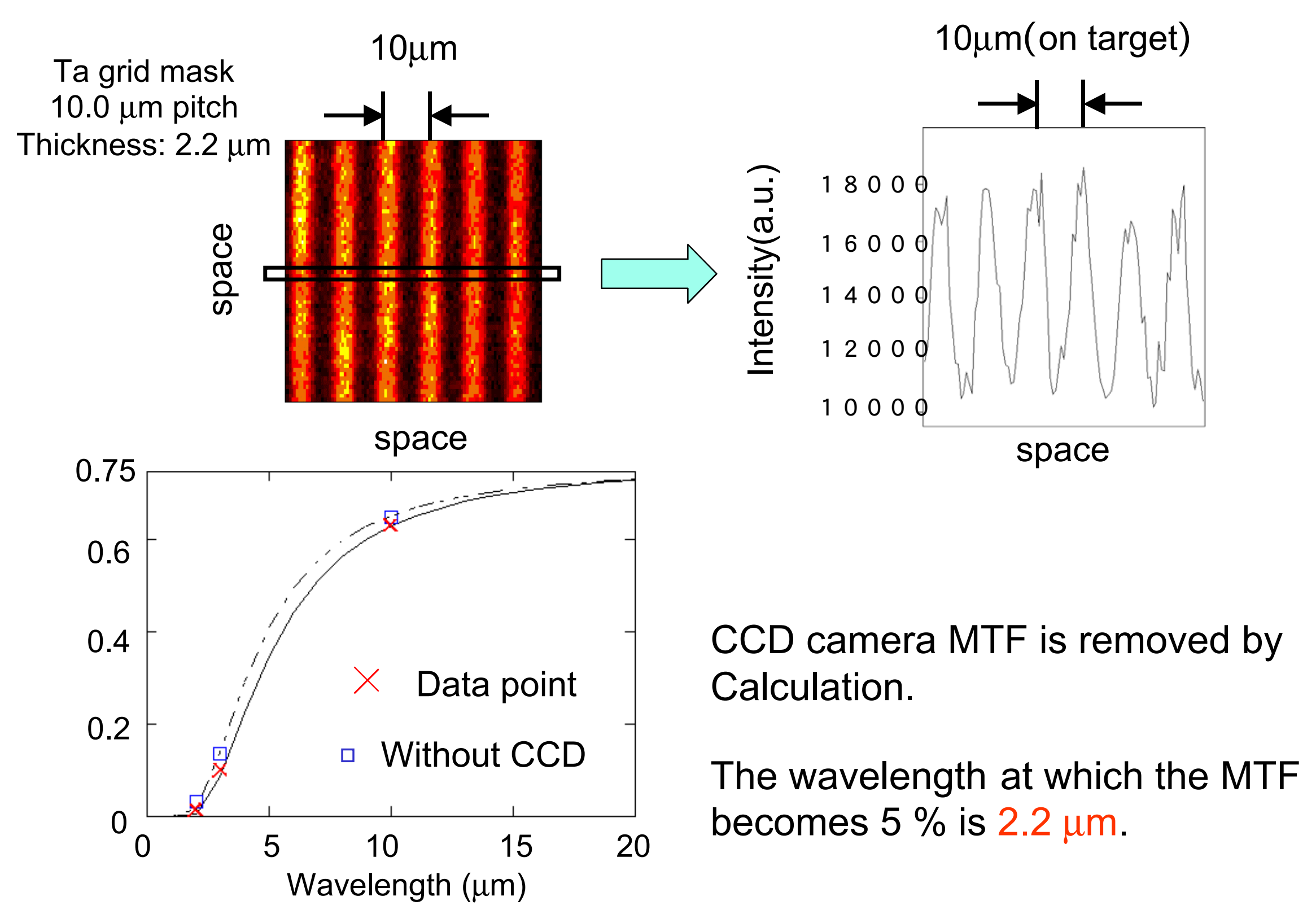




\section{Ablation density profile}

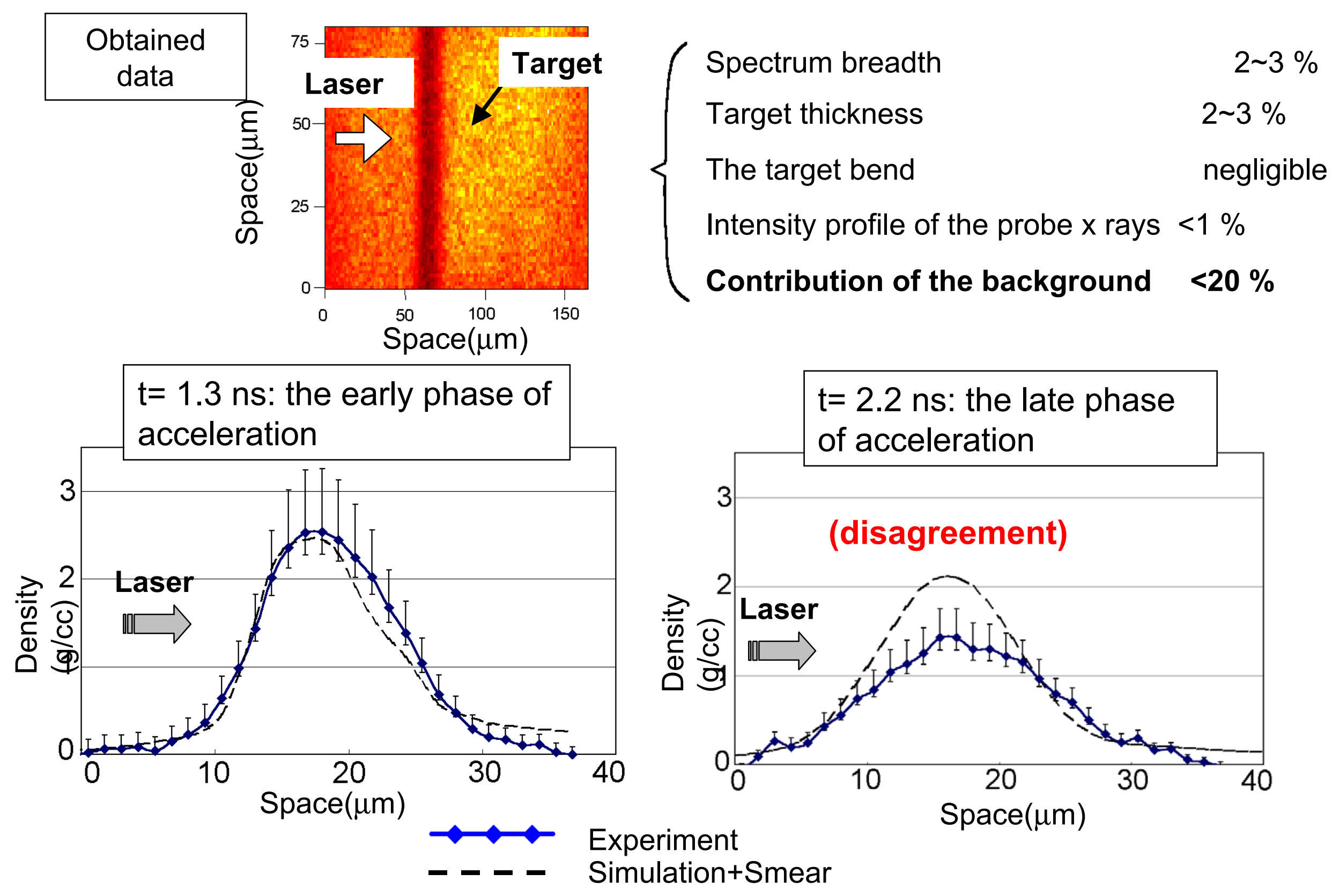




\section{Penumbral Imaging / density profile}




\section{Proof of Principle experiment}

The proper density profile of the laser-undriven polystyrene target was obtained with penumbral imaging coupled with a side-on $\mathrm{x}$-ray backlighting.

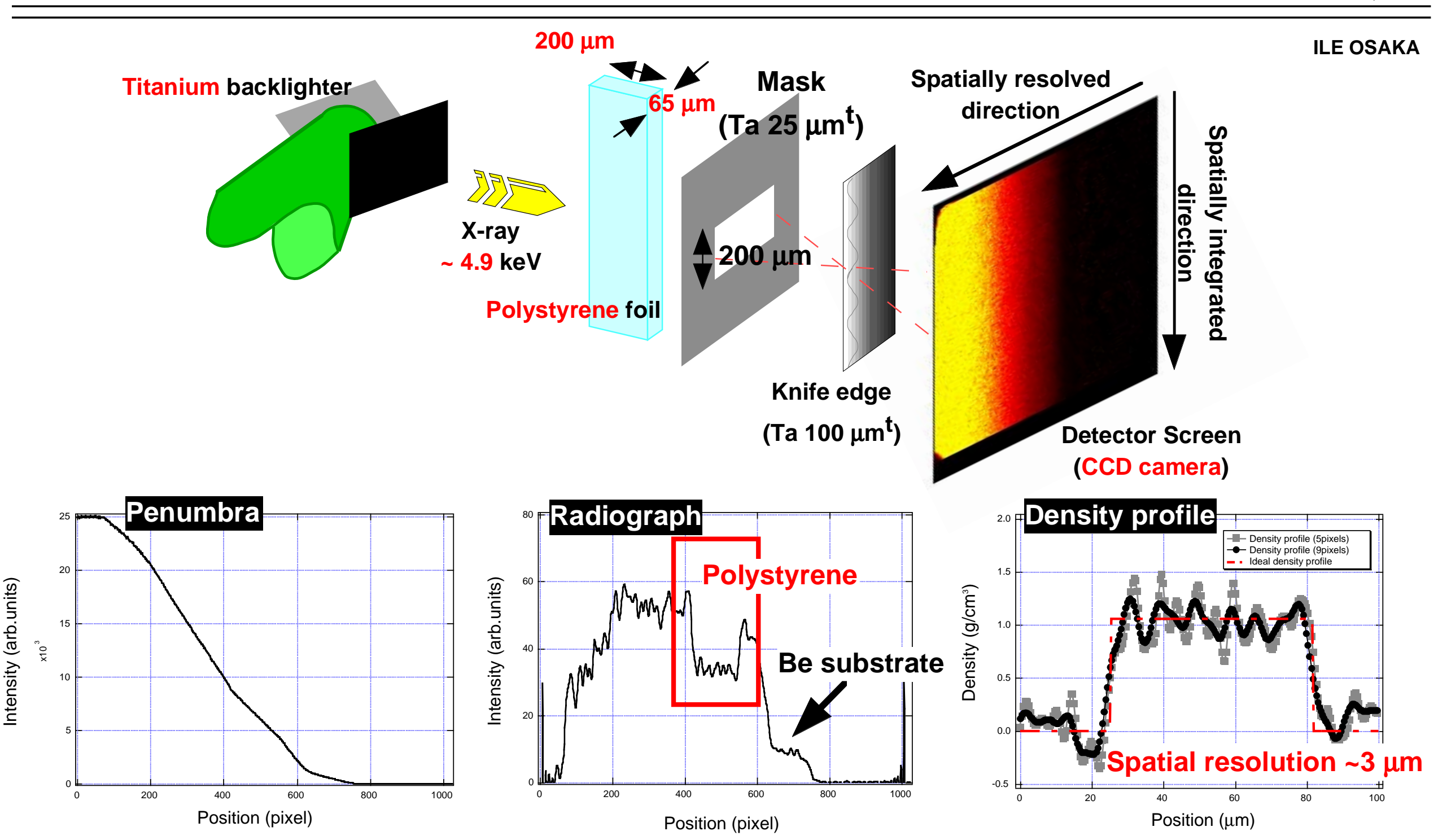


The density profiles in target plasmas driven by the HIPER laser were observed from shock transit to target acceleration.

S. Fujioka (ILE. Osaka)

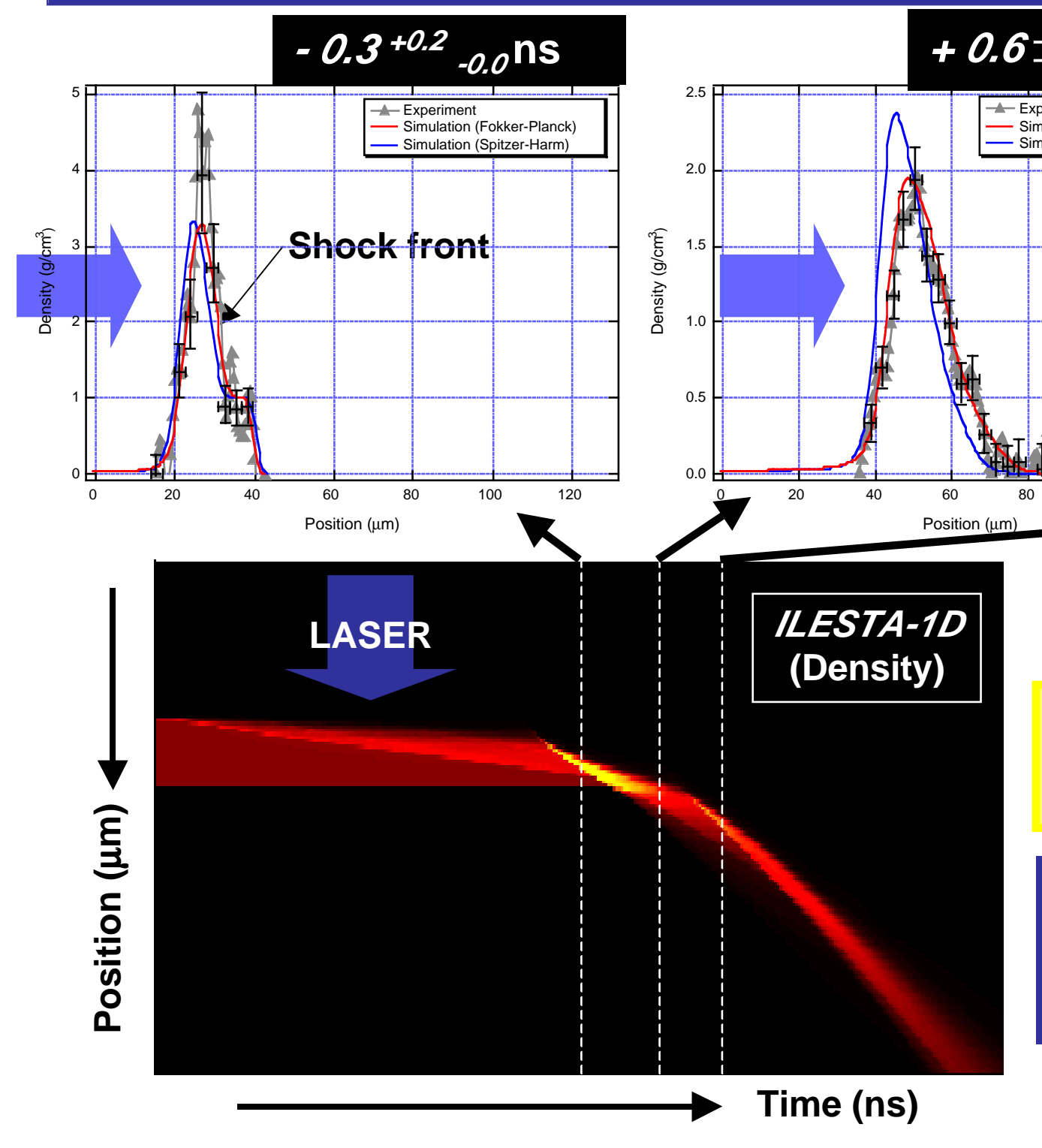

Spatial resolution : 3 - $5 \mu \mathrm{m}$

Temporal resolution : $140-160$ ps

The origin of the time is set to be the time when the shock breaks out at a target rear surface. 
Kinetic effects on electron energy transport are not negligible even in the case of relatively low intensity blue laser irradiation $\left(\Lambda_{L}=0.7 \times 10^{14} \mathrm{~W} / \mathrm{cm}^{2}, \lambda_{L}=0.35 \mu \mathrm{m}\right)$.

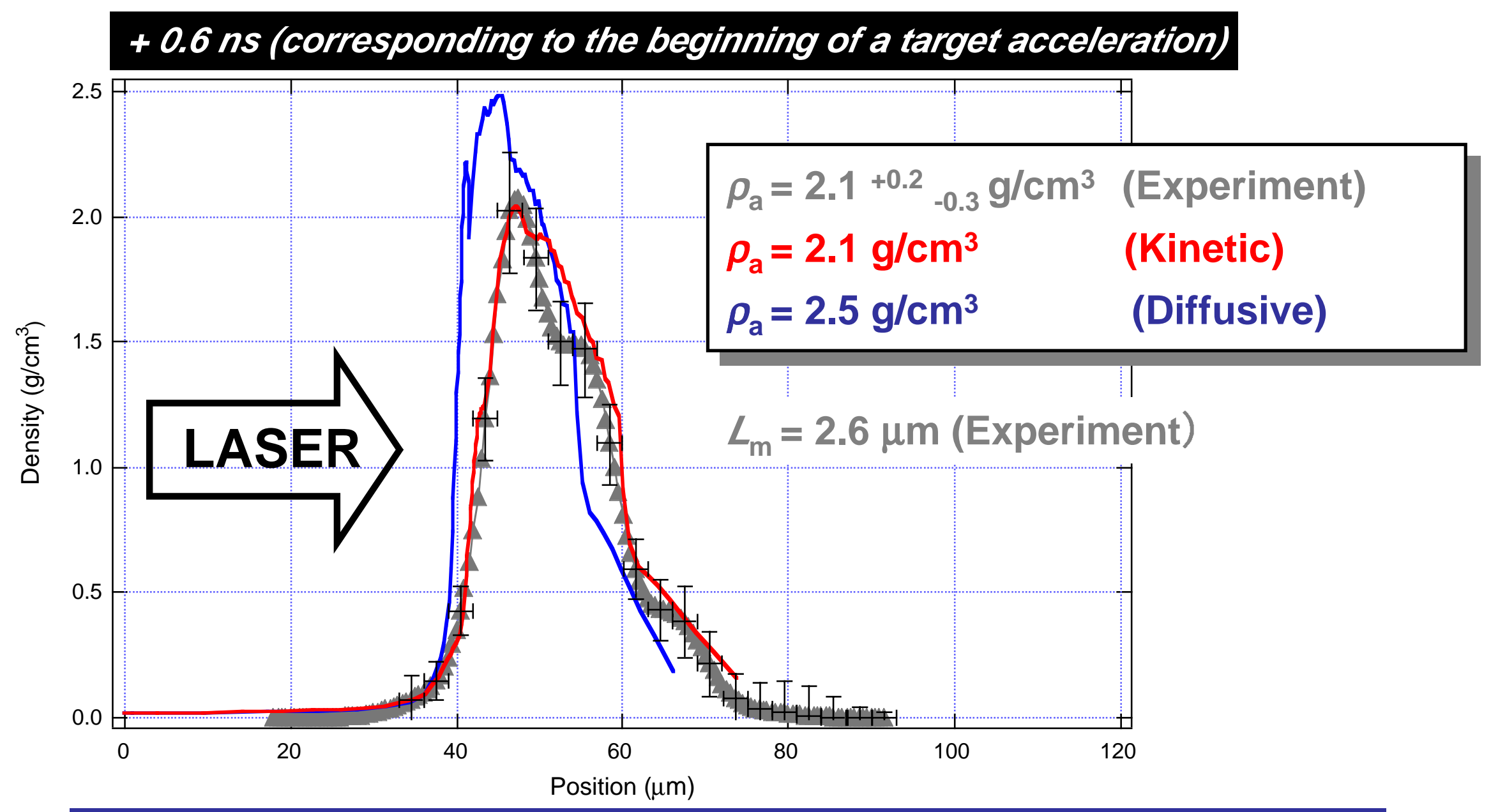

Motion blurring were cleared away by a deconvolution process with measured temporal history of backlight x-rays and velocity of targets. 
With advanced diagnostic techniques, we are approaching to better understanding of the Raylei-Taylor instability.

- Rayleigh-Taylor (RT) is the critical physics for high-gain IFE

- Energy transport can modify the RT growth at short wavelengths.

- Moire interferometry first observed the short wavelength RT growth.

- The observed RT growth suggests that nonlocal transport plays a role in ablative stabilization. But there is some ambiguity due to saturation.

- For independent test of the transport effect, we are measuring the ablation density with high-resolution imaging techniques.

- Initial test result is supportive to the nonlocal transport.

Our strategy is to measure all necessary quantities $\left(\gamma, k, g, m, \rho_{a}, L\right)$ to test various RT theories. 


\section{A Vortex Model for Studying the Effect of Shock Proximity on Richtmyer-Meshkov Instability at High Mach Number}

H. F. Robey, S. G. Glendinning, J. A. Greenough, \& S. V. Weber

Lawrence Livermore National Laboratory

Livermore, California 94550

Presented at the $8^{\text {th }}$ Meeting of the

International Workshop on the Physics of Compressible Turbulent Mixing

Pasadena, CA

December 9-14, 2001

This work was performed under the auspices of the U. S. Department of Energy by the University of California, Lawrence Livermore National Laboratory under Contract No. W-7405-Eng-48. 
Issue: At high Mach number, the transmitted shock can remain in close proximity to an $\mathrm{R}-\mathrm{M}$ unstable interface

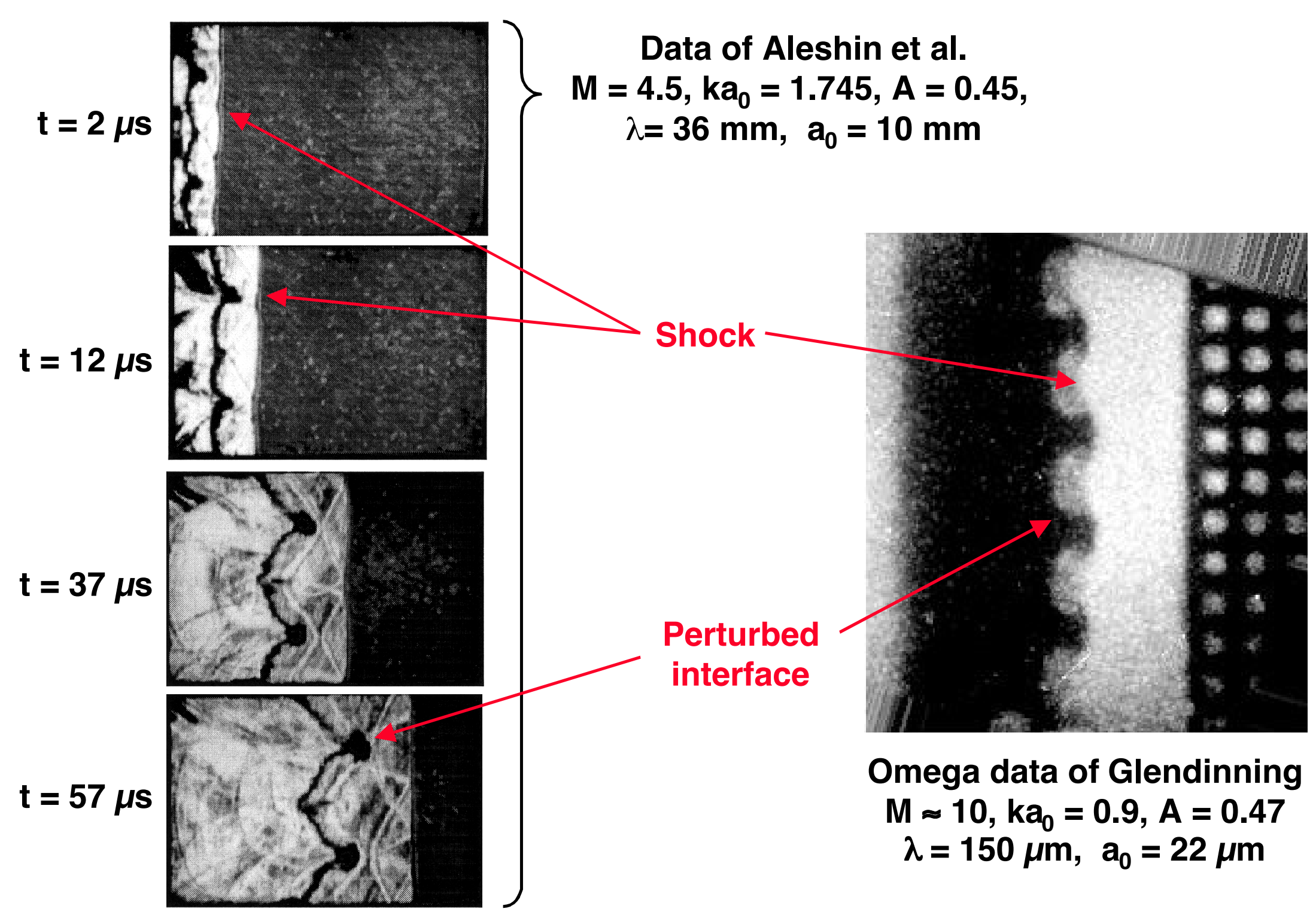


At these high Mach number conditions, the presence of the shock can affect the Richtmyer-Meshkov growth rate

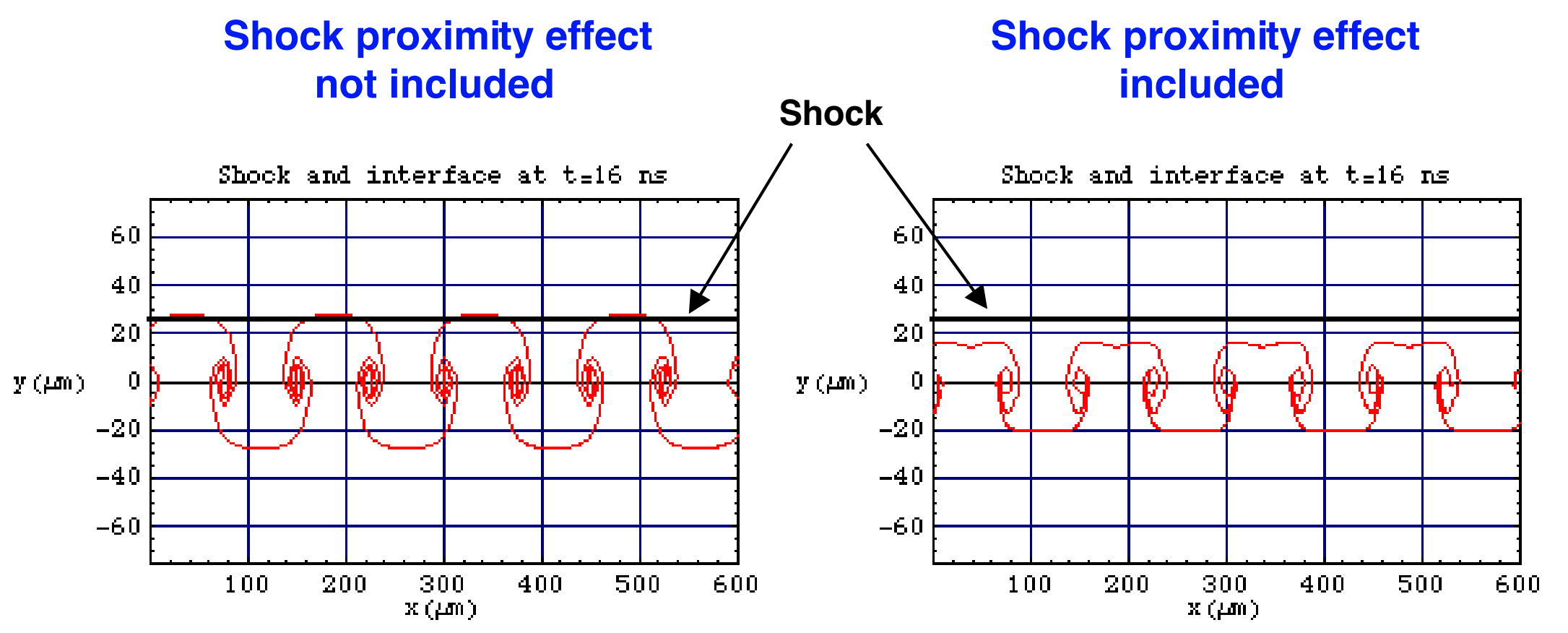

- In certain cases, the predicted linear growth rate can exceed the speed of the transmitted shock relative to the interface

- In this study, point vortex methods are used as a simple means of incorporating the effect of a transmitted shock on the instability growth 
Point vortex methods can be used to approximate the evolution of interfacial perturbations throughout the non-linear regime

Following Jacobs \& Sheeley*, interfacial vorticity is modeled by an alternating array of point vortices of circulation, $\Gamma$ :
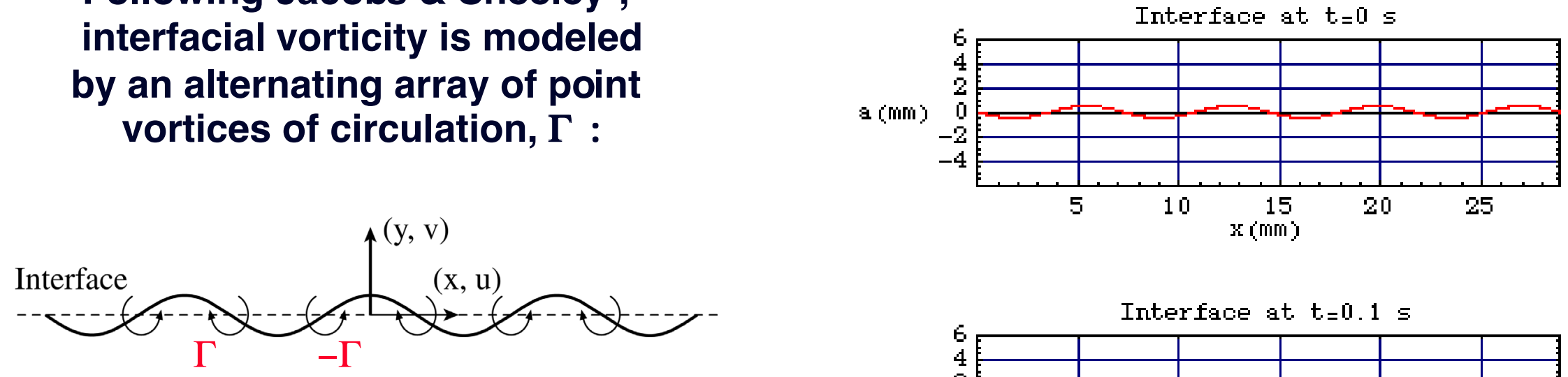

The flow evolution is obtained from

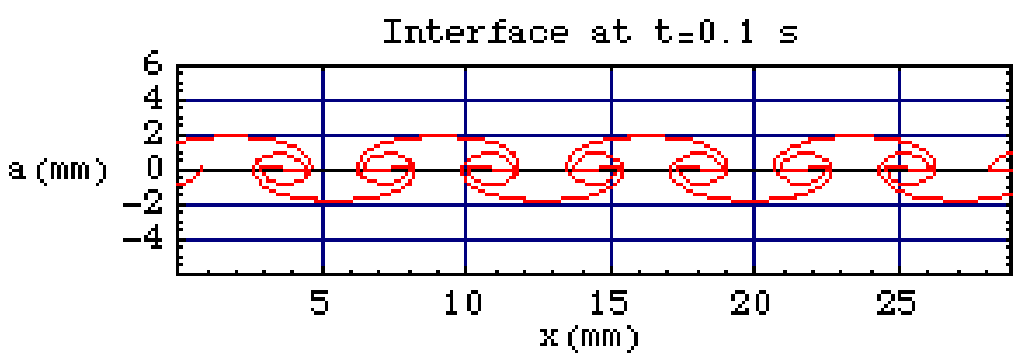

a streamfunction of the form:

$$
\begin{gathered}
\psi=\frac{\Gamma}{4 \pi} \ln \left(\frac{\cosh (k y)+\sin (k x)}{\cosh (k y)-\sin (k x)}\right) \\
u=\partial \psi / \partial y \quad, \quad v=-\partial \psi / \partial x
\end{gathered}
$$

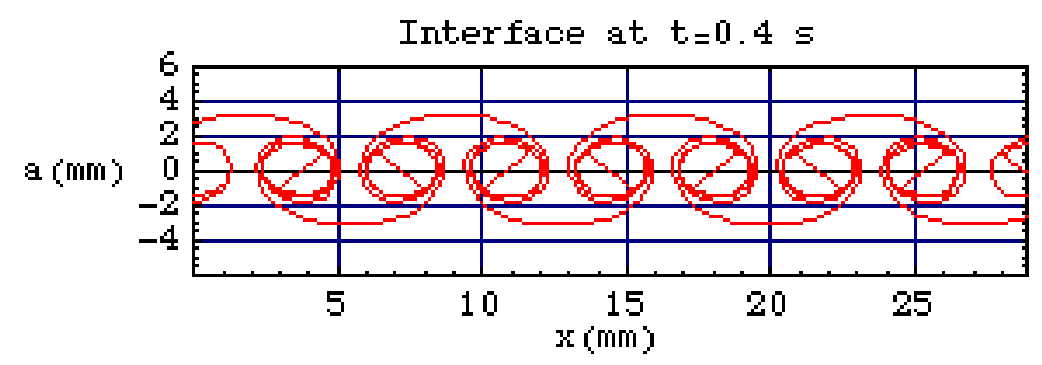

*Phys. Fluids 8(2), 405 (1996) 
The incompressible, $\mathrm{A}=\mathbf{0 . 1 5 5}$ experiments of Jacobs \& Sheeley are well modeled by point vortex methods

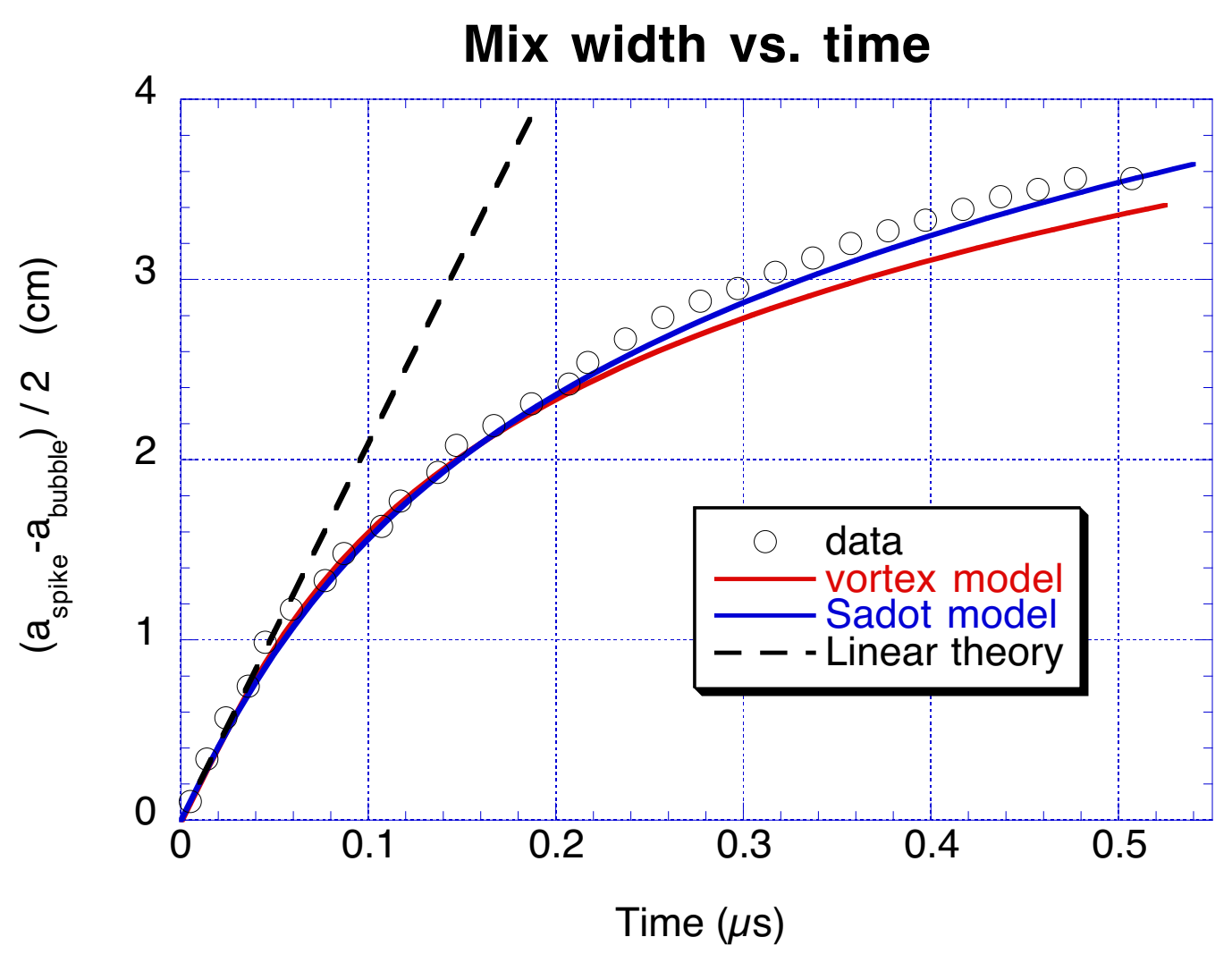

The circulation $\Gamma$ is defined as that required to reproduce the initial linear growth rate :

$$
\Gamma=2 \pi v_{I M} / k
$$

- The model of Sadot et al., PRL 80(8), 1654 (1998) is in excellent agreement with the data

- The vortex model predicts an amplitude slightly below the data at later time, but is within $6 \%$ of the data and the Sadot model. 


\section{An image vortex model can be used to incorporate the effect of a transmitted shock as a downstream boundary condition}

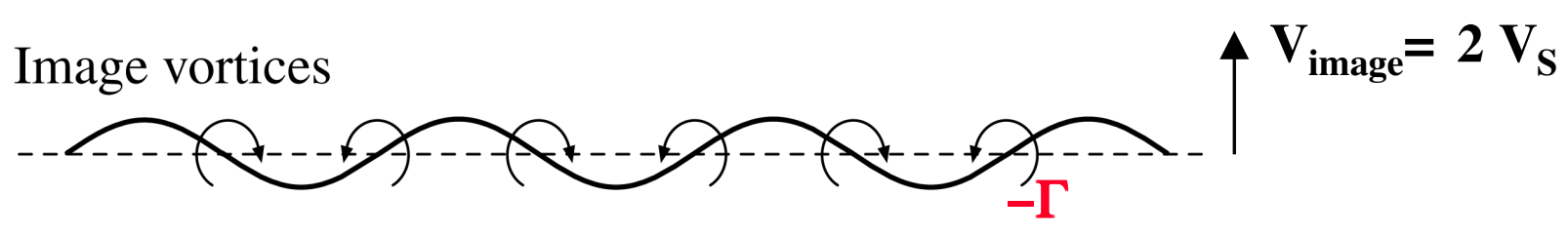

Shock $\uparrow \mathbf{v}_{\mathrm{S}}$

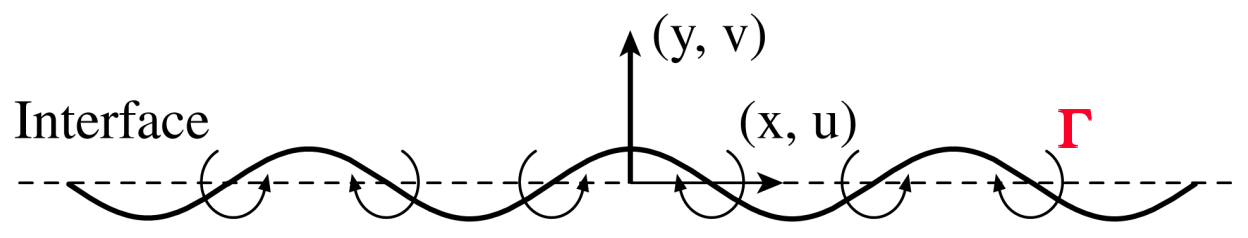

The image vortex array moves away from the interface at twice the shock-to-interface velocity

The streamfunction is now given by :

$$
\psi=\frac{\Gamma}{4 \pi}[\underbrace{\ln (\underbrace{\frac{\cosh (k y)+\sin (k x)}{\cosh (k y)-\sin (k x)}}_{\text {Image vortices }})}_{\text {Interface vortices }}-\ln \underbrace{}_{\ln \left(\frac{\cosh \left(k\left(2\left(a_{0}^{*}+V_{\mathrm{st}} t\right)-y\right)\right)+\sin (k x)}{\cosh \left(k\left(2\left(a_{0}^{*}+V_{\mathrm{st}} t\right)-y\right)\right)-\sin (k x)}\right)}]
$$


Though derived from potential flow theory, this model includes effects due to compressibility and finite Atwood number

Compressibility enters through the circulation which depends on the post-shock Atwood number and compressed perturbation amplitude

$$
\Gamma=\frac{2 \pi v_{I M}}{k} \& v_{I M}=k A^{*} \frac{\left(a_{0}+a_{0}^{*}\right)}{2} u_{C}
$$

wher e the post-shock Atwood number is : $A^{*}=\frac{\rho_{1}^{*}-\rho_{2}^{*}}{\rho_{1}^{*}+\rho_{2}^{*}}$

and post-shock perturbation amplitude is approximated as : $a_{0}^{*}=a_{0}\left(1-\frac{u_{C}}{V_{S I}}\right)$

All model parameters are obtained from the solution of the associated Riemann problem for the unperturbed interface 
Example 1: The image vortex model has been applied to the $M=4.5$ shock tube experiments of Aleshin et al.

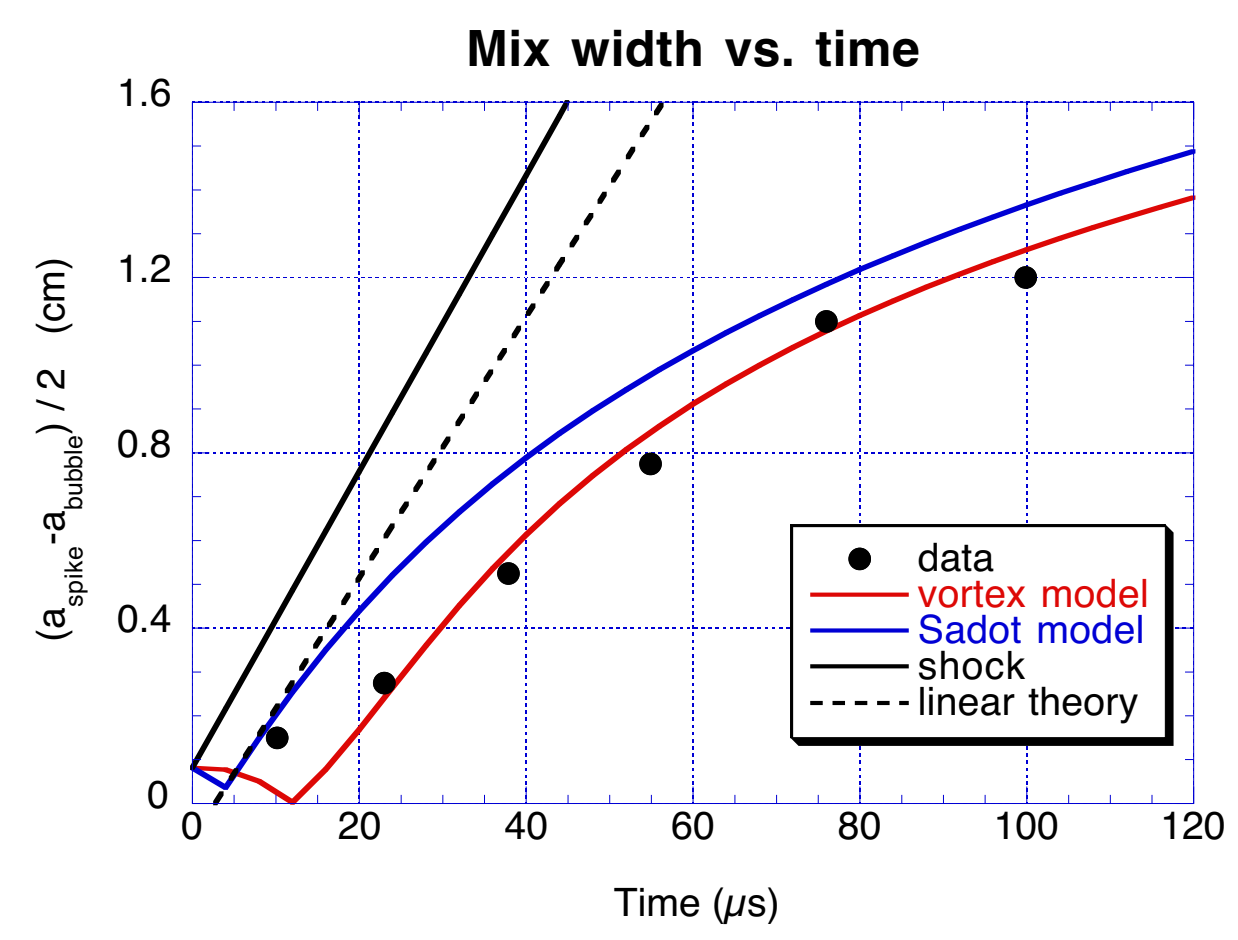

Aleshin et al., run \#630B

$$
\begin{gathered}
\mathrm{Xe} \rightarrow \mathrm{Ar}, \mathrm{M}=4.5, \\
\begin{array}{c}
\mathrm{A}=0.45, \lambda=36 \mathrm{~mm} \\
2 \mathrm{a}_{0}=20 \mathrm{~mm}(\mathrm{P}-\mathrm{V}) \\
\mathrm{ka}_{0}=1.745
\end{array}
\end{gathered}
$$

- The data falls well below the linear theory for the entire experiment

- After phase inversion, the vortex model agrees well with the data

- The Sadot model predicts an amplitude consistently above the data. 
A look at the development of individual spike and bubble amplitudes reveals further differences

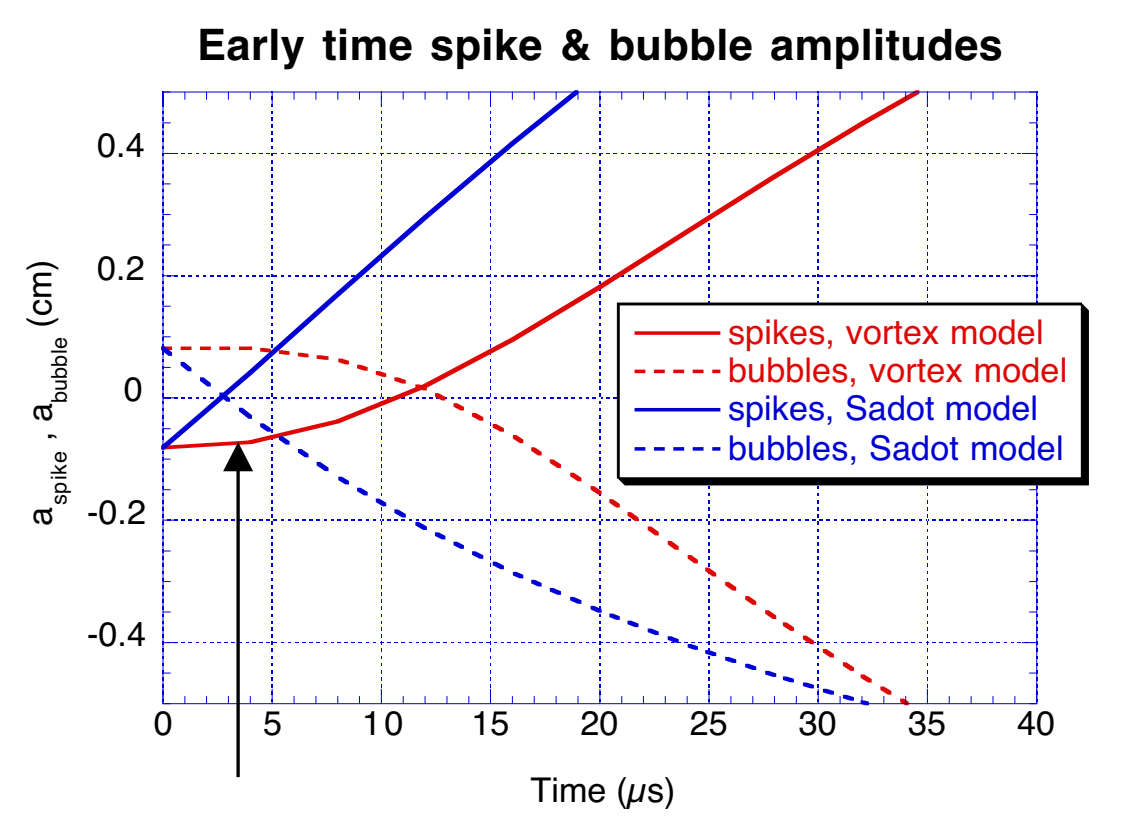

Suppressed growth early in time

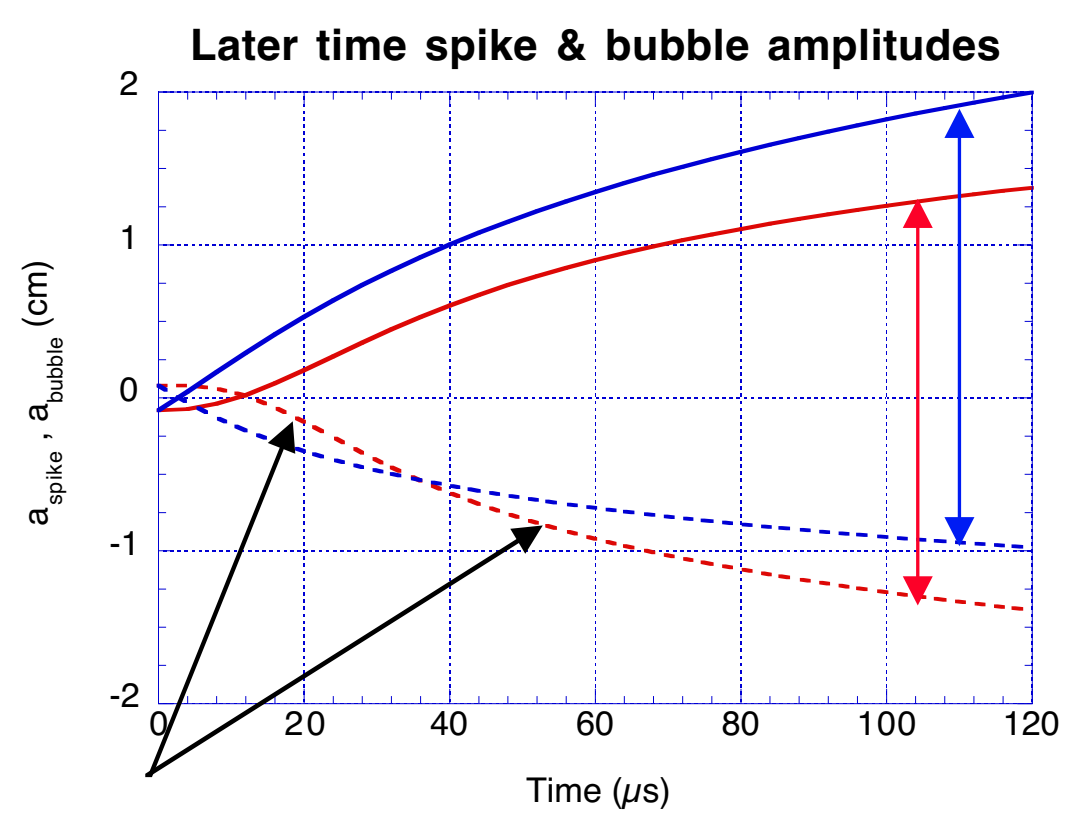

Bubble growth is initially suppressed, but later rebounds

- The vortex model exhibits a suppressed growth early in time when the shock (and therefore the image vortex system) are close to the interface

- Later in time, the spike growth continues to be suppressed since the spikes remain in close proximity to the shock, whereas the bubble growth rebounds. This results in a more symmetrical bubble-to-spike development. 
The spike and bubble growth rates and asymptotic behavior also show the effect of shock proximity
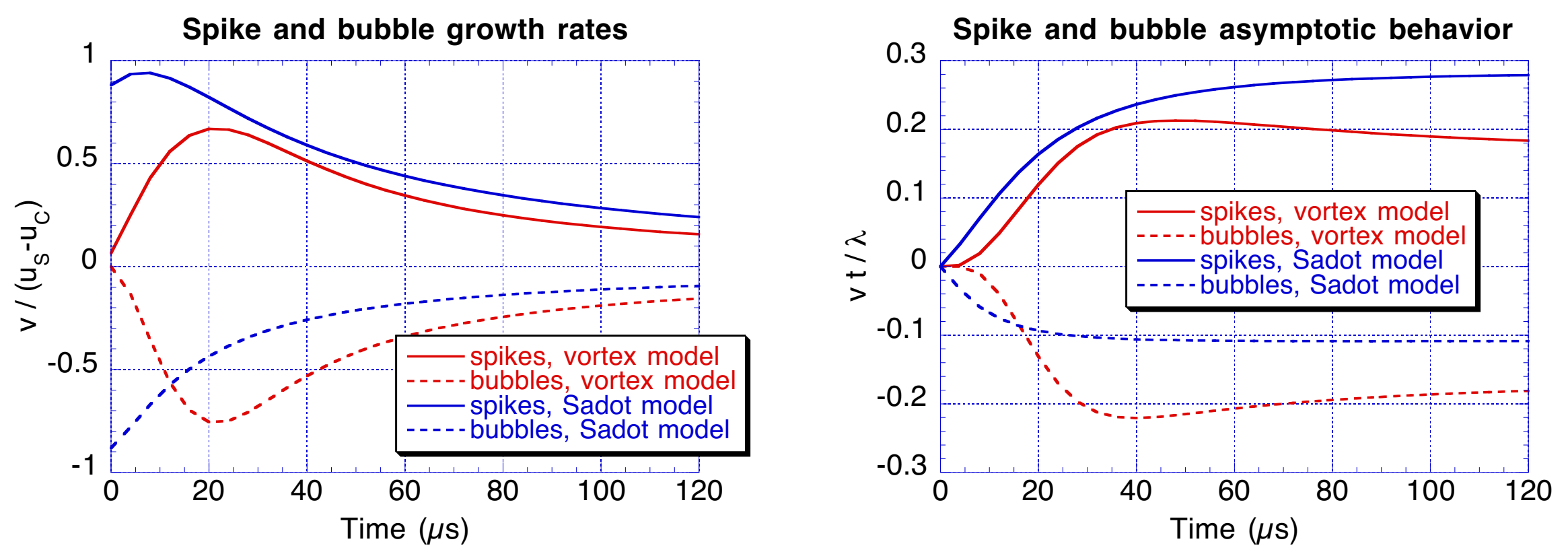

- The growth rate of the vortex model exhibits a peak which is both reduced in magnitude and delayed in time.

- The delayed peak growth is qualitatively consistent with the fully compressible linear theory of Yang, Zhang, \& Sharp, Phys. Fluids 6, 1856 (1994)

- At late time, all models asymptote to a $\mathrm{t}^{-1}$ behavior. 
The ratio of spike-to-bubble amplitudes quantifies a very important difference resulting from shock proximity

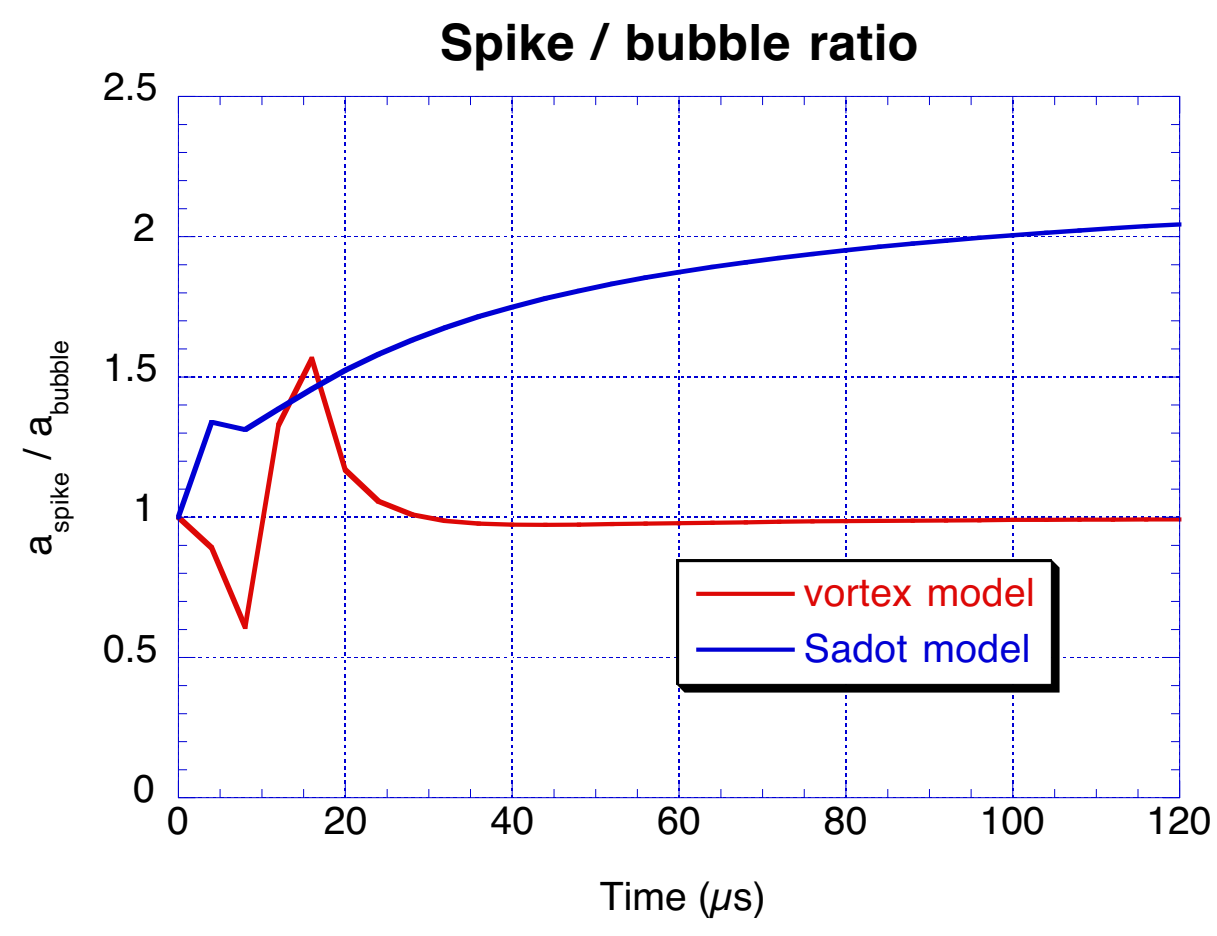

- Clearly, the spike to bubble ratio of the vortex model is due to the single fluid $(A=0)$ assumption and is therefore wrong, right?

- To answer this question, we turn to numerical simulation 
Numerical simulations of Aleshin experiment N630B have been performed using a 2D ALE code, HYDRA

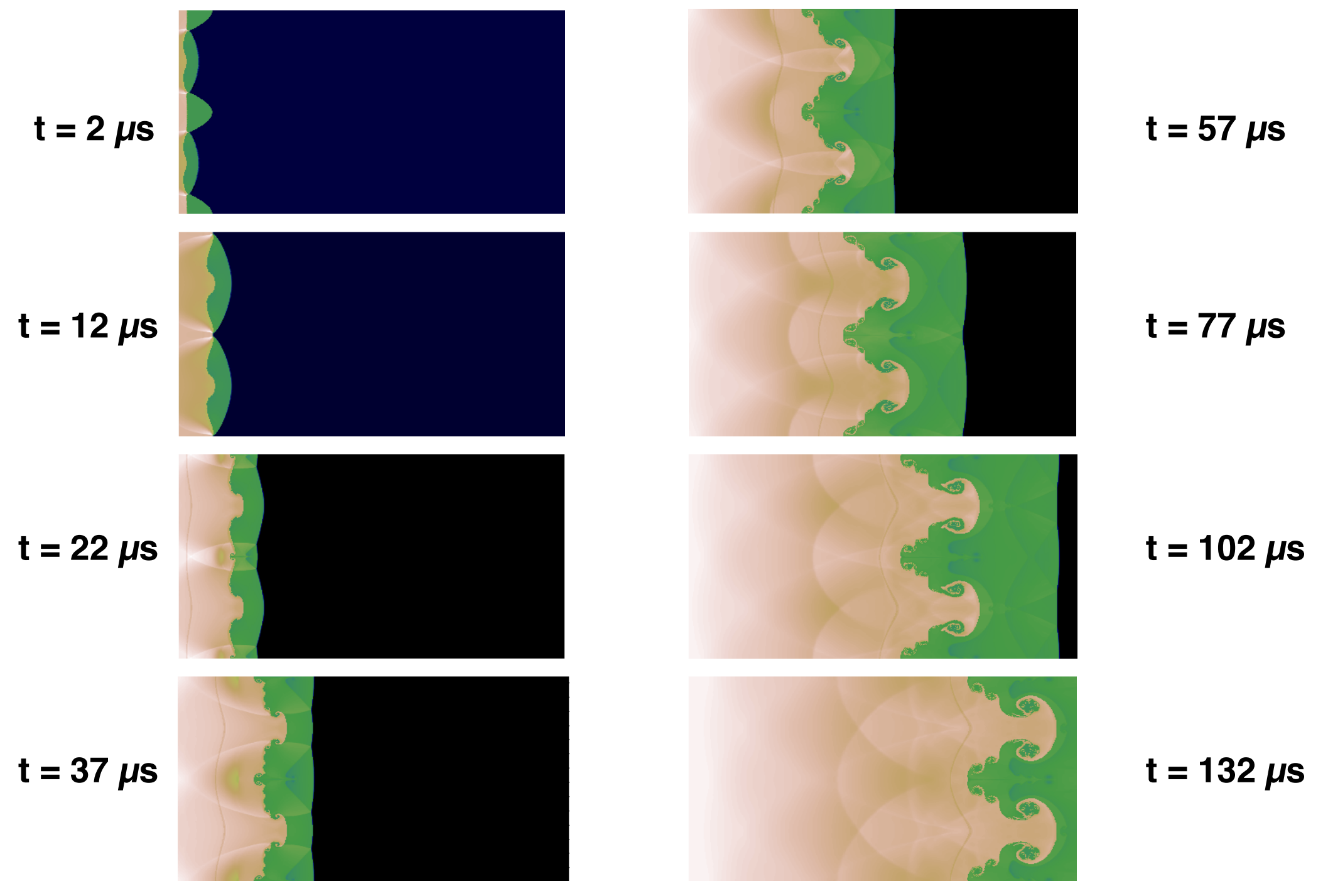

Simulations of S. V. Weber, resolution $=\mathbf{5 1 2}$ zones $/$ wavelength 
Numerical simulations of Aleshin experiment N630B have also been performed using a 2D AMR code
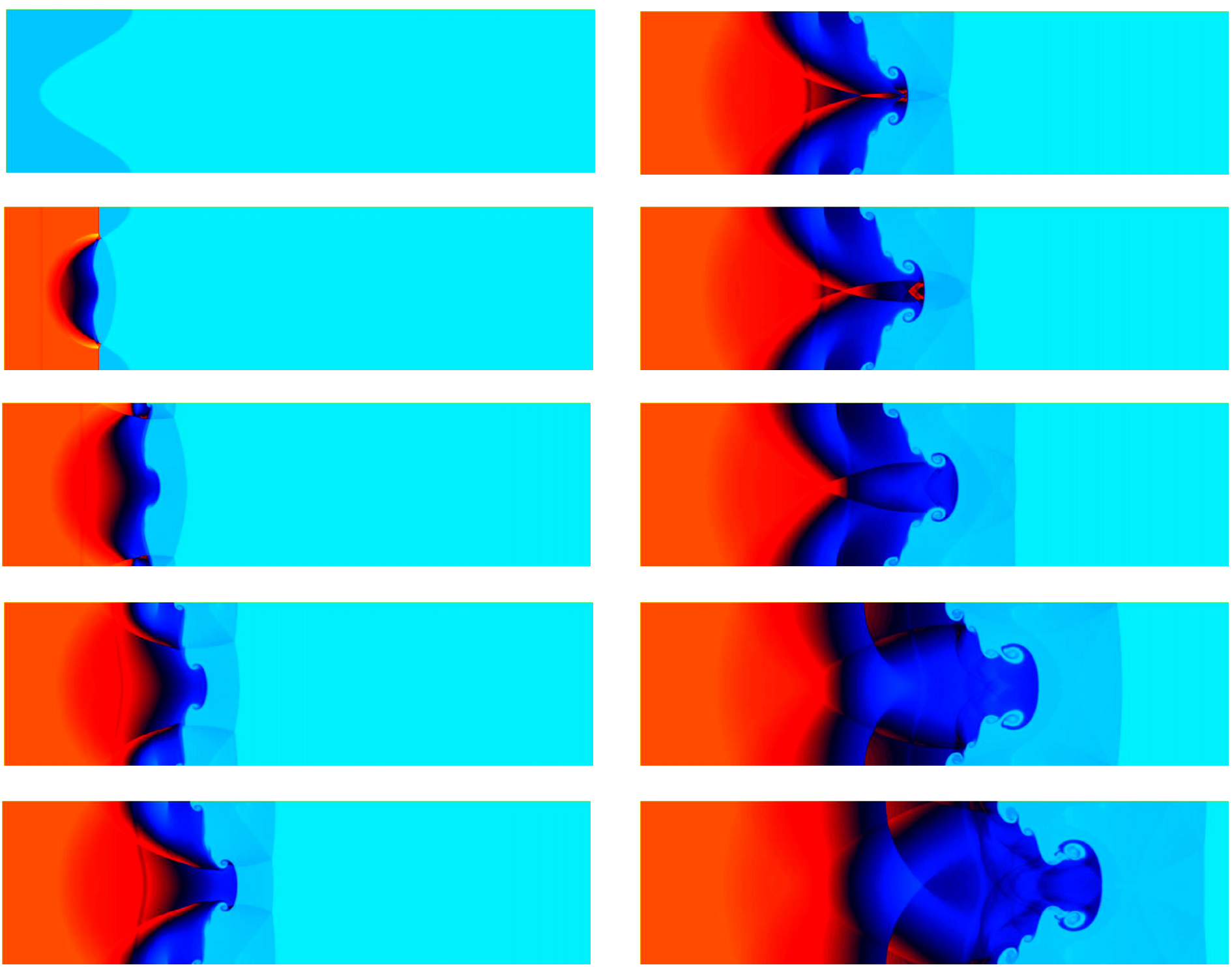

Simulations of J. A. Greenough, resolution $=2560$ zones $/$ wavelength 


\section{Both numerical simulations are in reasonable agreement}

with the image vortex model
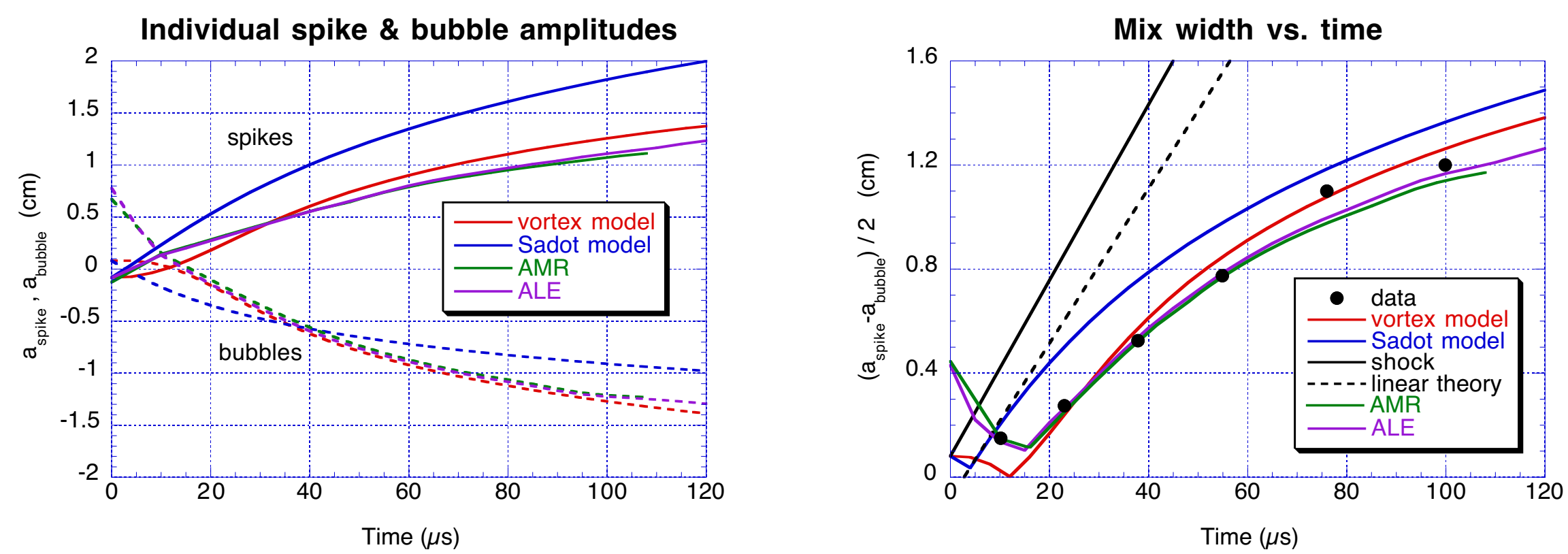

\section{Both simulations show :}

- A delayed phase inversion due to reduced growth early in time

- Reduced spike growth throughout the duration of the experiment

- more symmetrical spike-to-bubble development 
The spike / bubble ratio obtained from the numerical simulations also agrees with the image vortex model

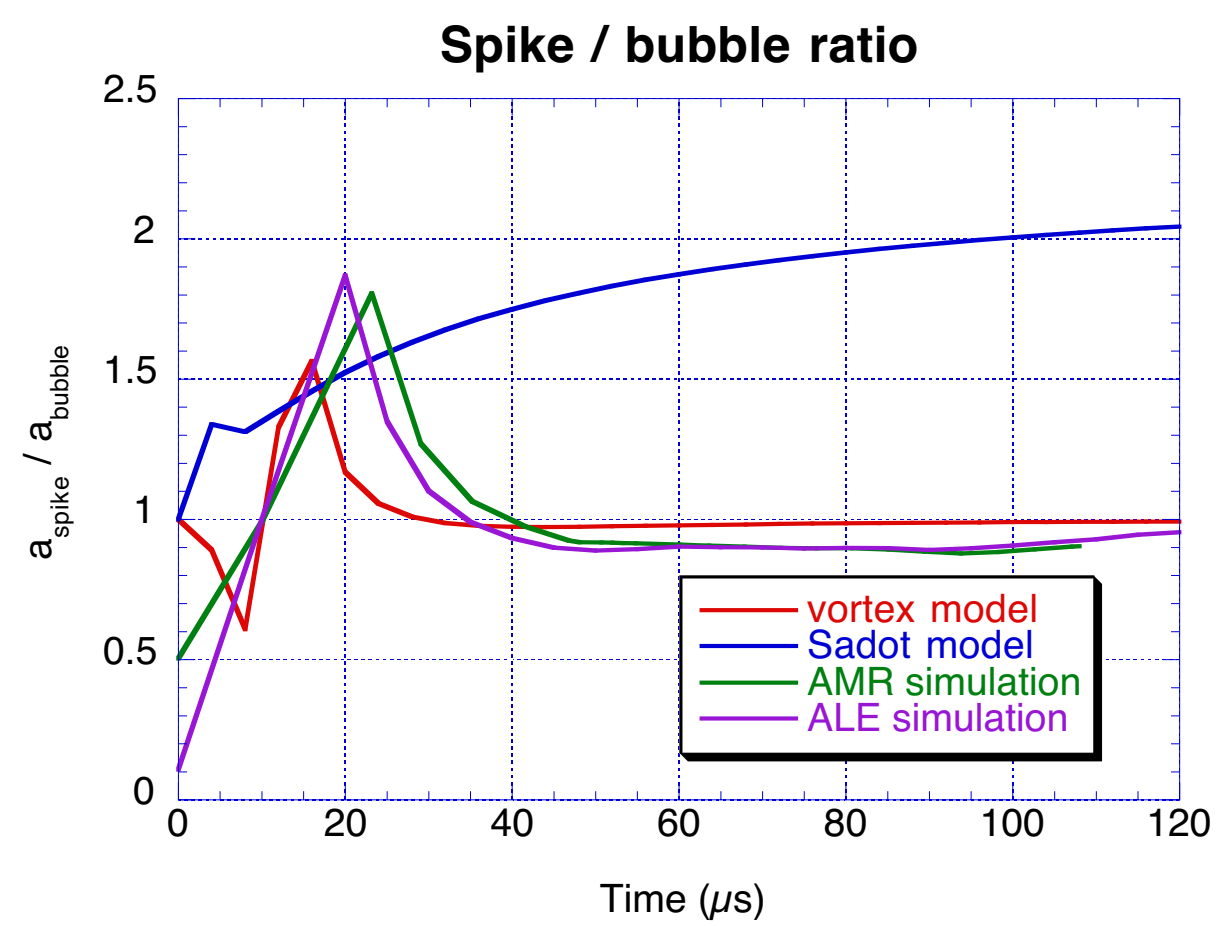

- Differences are again observed at early time as the interface inverts phase, but later the amplitude of the spikes remains less than that of the bubbles.

- This effect is not observed at lower Mach number and is an essential effect due to shock proximity. 


\section{Example 2: The image vortex model has also been applied to the Omega experiments of Glendinning et al.}

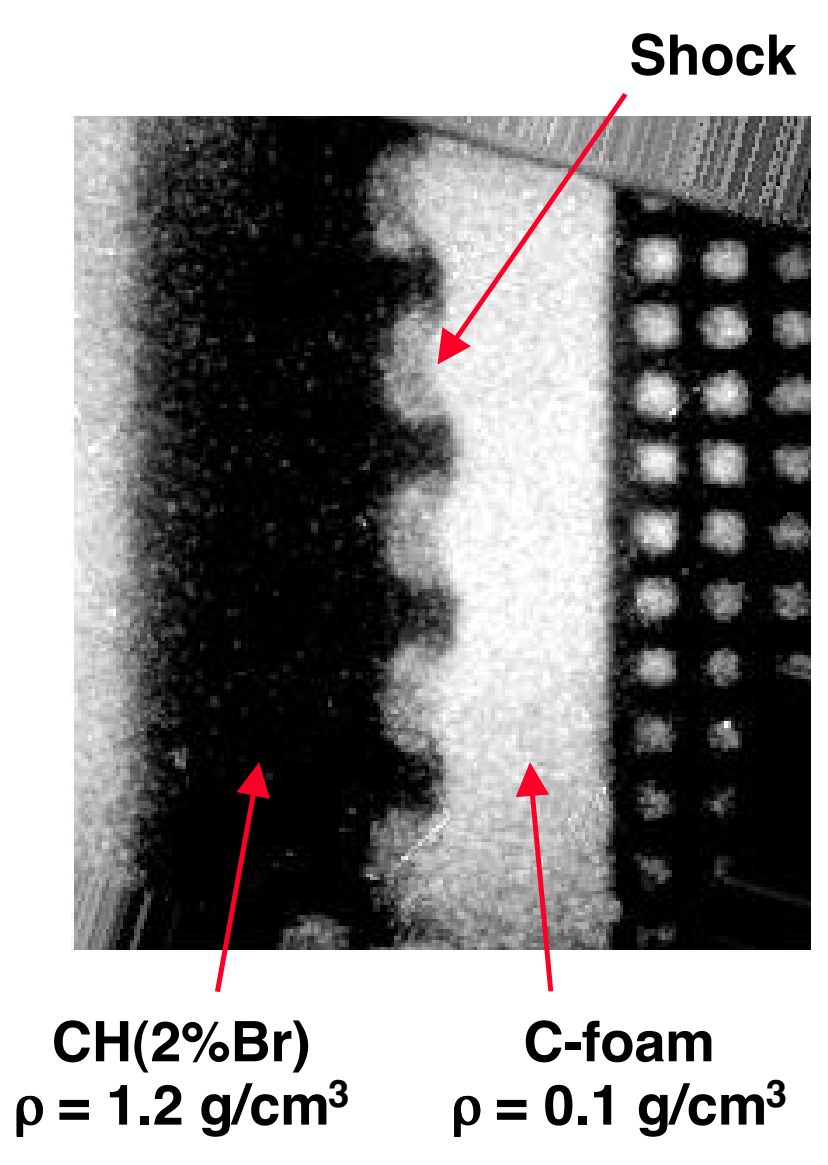

X-ray radiograph

@ $\mathbf{t}=22 \mathrm{~ns}$
This experiment differs from that of Aleshin et al. in the following :

- Higher Mach number, $M \approx 10$

- Lower initial perturbation amplitude $k a_{0}=0.92$ (vs. $\left.k_{0}=1.745\right)$

- Linear theory (Meyer-Blewett) predicts a growth rate which exceeds the shock-to-interface velocity

- Phase inversion of the perturbation is completed by the end of shock refraction

- The effect of shock proximity is more pronounced than before. 
The image vortex model does a reasonable job of predicting the perturbation amplitude vs. time

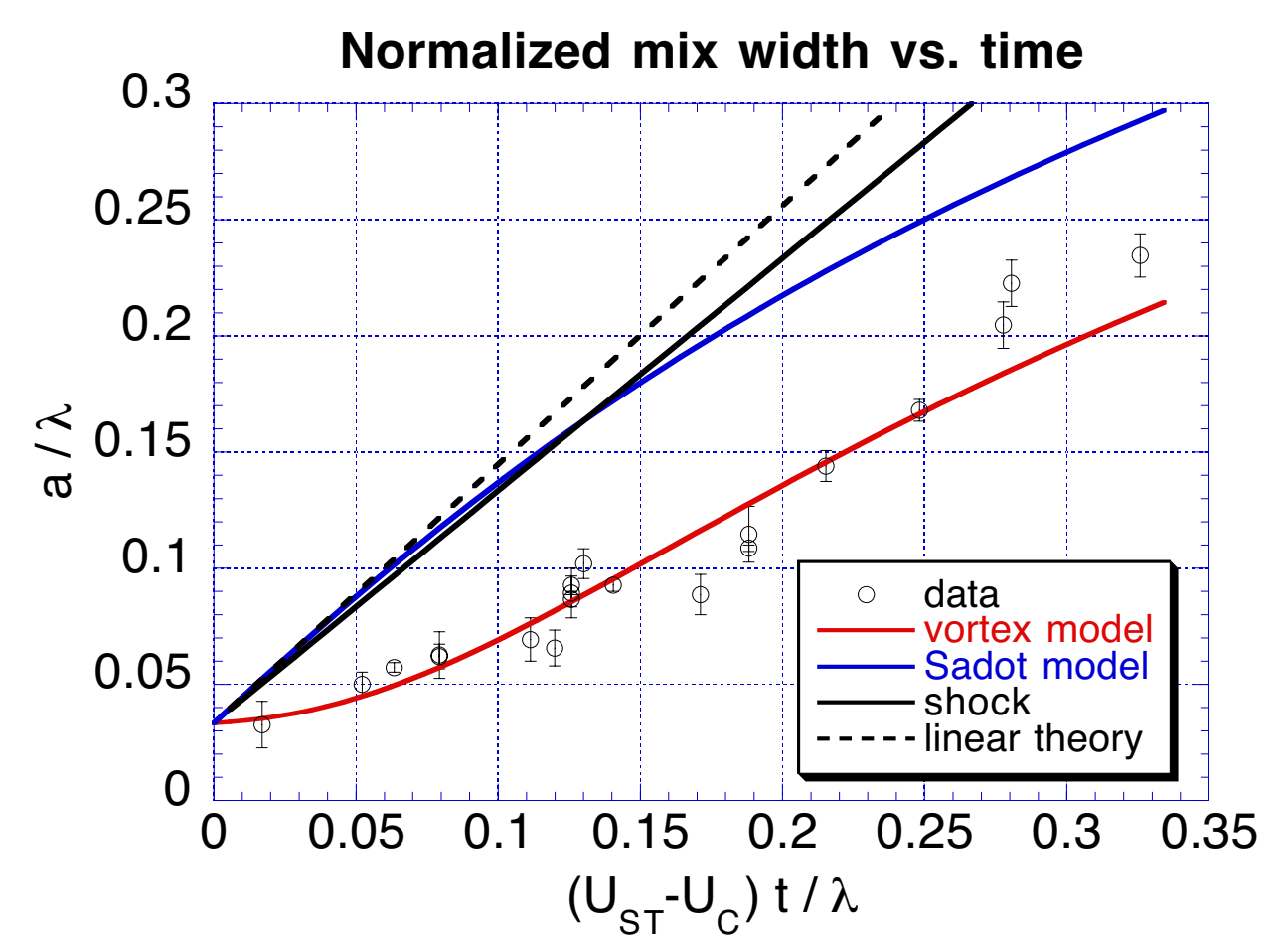

- The data is well below the linear theory at all times.

- The data shows a distinct increase in the growth rate later in time, when the normalized amplitude is small $(a / \lambda=0.1)$

- The shock separation distance from the interface is only $0.33 \lambda$ at the latest time observed in the experiment. 
Large differences are again seen in the spike and bubble growth rates due to shock proximity

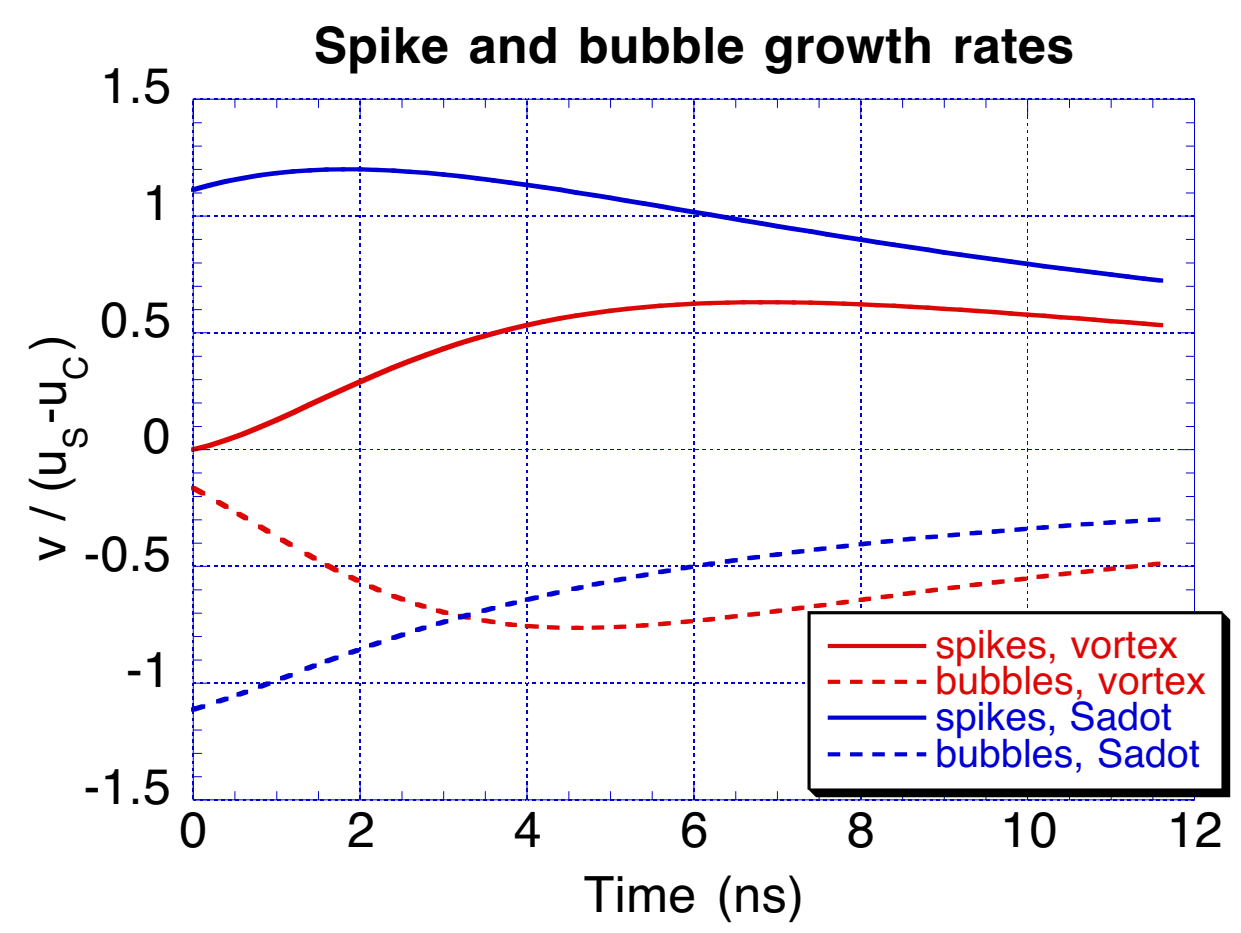

- In this case, the linear theory predicts a spike growth rate which is faster than the velocity of the transmitted shock.

- The vortex model again predicts a spike growth rate which is at all times lower than that of the Sadot model. The peak growth rate does not occur until $\sim 6$ ns after passage of the shock 
The spike / bubble ratio again shows large differences

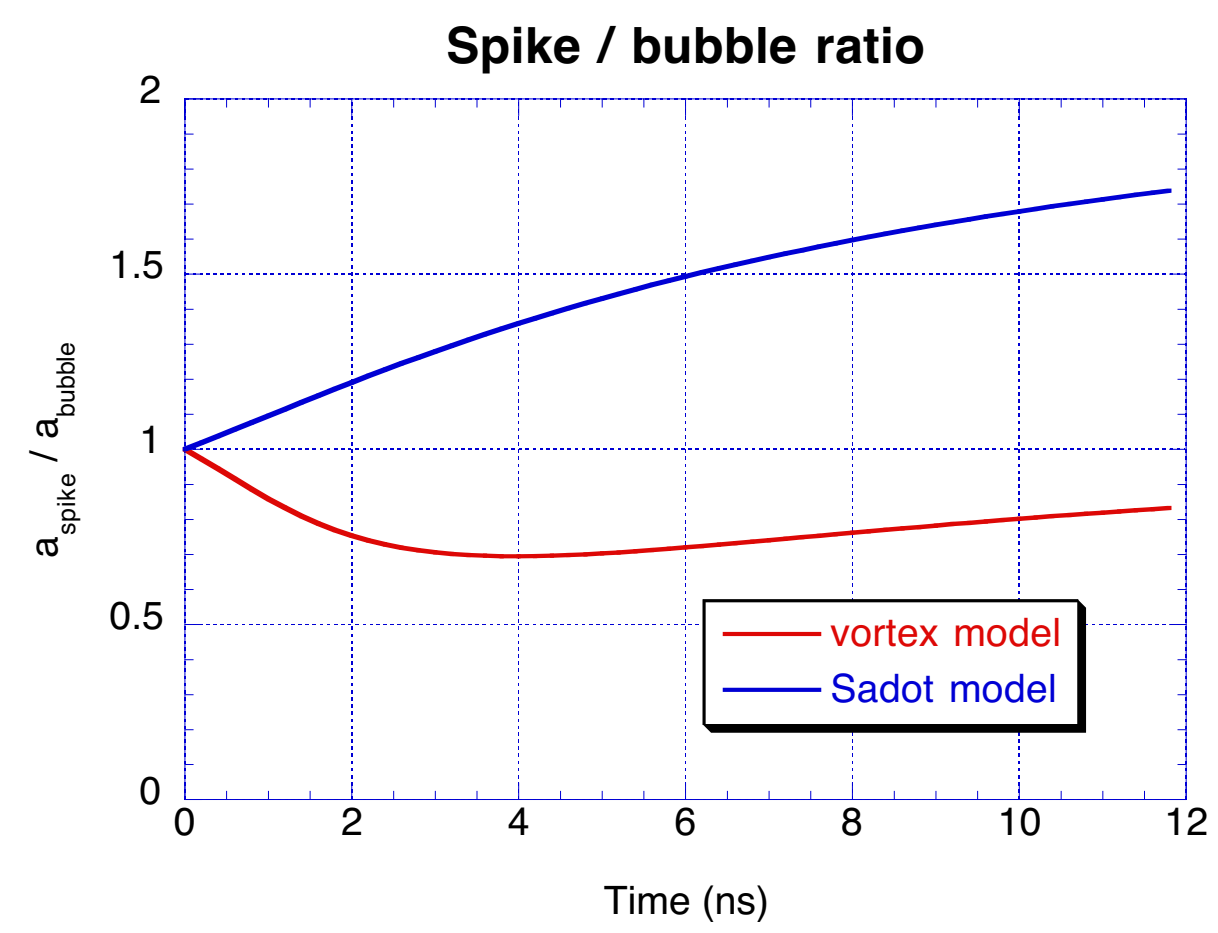

- The large supression of the spike growth results in a spike amplitude which remains considerably lower (20-30\%) than the amplitude of the of the bubbles. 
- An image vortex model has been presented as a simple means of incorporating the effect of a transmitted shock as a downstream boundary condition on the growth of a Richtmyer-Meshkov unstable interface.

- At low Mach number, the vortex model agrees well with the incompressible experiments of Jacobs and Sheeley and also agrees well with the model of Sadot et al.

- At high Mach number, the image vortex model agrees well with shock tube experiments of Aleshin et al. $(M=4.5)$ and laserdriven experiments of of Glendinning et al. $(M>10)$. 
Conclusions, continued

The effect of shock proximity is distinguished from saturation effects due to large perturbation amplitude in the following:

- For shock propagation from heavy to light, the Atwood number dependence observed at lower Mach number is significantly altered due to the presence of the shock boundary. For the two cases discussed, the spike amplitude remains slightly less than that of the bubbles throughout the experiment.

- The perturbation growth immediately following passage of the shock is significantly smaller than that given by the linear theory. As the shock departs from the interface, the growth rate increases. Later in time, as the perturbation amplitude increases, normal growth rate saturation effects are seen. 


\section{Review on RTI, RMI and TM experiments}

\section{J-F Haas CEA/DIF \& S G Zaytsev ENIN}

a discussion based on the analysis of past (5th, 6th \& 7th) workshops and other meetings : 21, 22 \& 23rd ISSW (International Shock Wave Symposia), 51 \& 53rd APS/DFD (American Physical Society, Division of Fluid Dynanics), 43rd APS/DPP (Division of Plasma Physics), recent ECLIM \& IFSA conferences.

Acknowlegements to CEA colleagues with whom some of these meetings were analysed for our official trip reports, recent IWPCTM organizers, contributors of the 23rd ISSW who let us consult their very recent work (July 2001) and colleagues who spontaneouly sent their papers.

Apologies to all contributors for any omissions, distorsions, low quality reproduction of their high quality work and general imperfections for this difficult exercise.

See this as a draft for our contribution to the summary experimental roundtable? 


\section{Outline of this review}

-Introductory statistics

We cite the conclusions of roundtable for experiments at mix 99 and add comments

-Incompressible experiments

-Shock-tube for planar shock waves

-Convergent flows

-Explosively driven flows

- Laser driven flows

There is a wide spectrum of experiments

but we have a clearer view of shock tubes

-Conclusions

2 8th IWPCTM, Pasadena, December 10, 2001 

in CTM workshop series averages $40 \%$

1st Princeton 1988 : ? (misplaced Proceedings)

2nd Pleasanton 1989 : ? (misplaced Proceedings)

3rd Royaumont 1991: poster 6/24, oral 12/25

$$
\text { summary } 18 \exp / 49 \text { total }=37 \%
$$

4th Cambridge 1993: RT 10/17, RM 9/14, KH 2/8, laser 4/4, converging flows $2 / 7$ : summary $27 \exp / 61$ total $=44 \%$ *

5th Stony Brook 1995: $23 \exp / 53$ total $=43 \%$ *

6th Marseille 1997: $44 \exp / 96$ total $=46 \%$ *

7th Saint Petersburg 1999: 53 exp / 126 total $=42 \%$ *

8th Pasadena 2001: poster 25/63, oral 25/66,

$$
\text { summary } 50 \exp / 129 \text { total }=39 \%
$$

* does a proportion above $40 \%$ correlate with stronger russian attendance ? 
mix 99 : summary roundtable for experiments :

incompressible domain (J. Jacobs spokesman),

Interpreted by E. Meshkov, L. Houas \& JF Haas for report in the ERCOFTAC bulletin (2000) and the proceedings of the 7thIWPCTM

It had been pointed out that even if a lot of experiments, for both low Atwood numbers in the miscible and high Atwood numbers in the non-miscible ranges, have been successfully undertaken, the initial conditions generally still remain unknown.

Consequently, it was suggested for future work to carry large Atwood number experiments for miscible liquids, as well as to evaluate the importance of the surface tension in the RTI development.

In any case, the emphasis was made on the fact that the initial conditions must be known. From the other hand, more complex geometry experiments (multi-layer) are welcome for a better understanding of the influence on the exponent a of the mixing zone thickness evolution law.

In a near future, the influence of medium on the 2D-3D transition will also be greatly interesting. 
cold and siow Rayıeıgn- I ayıor experiments allow tne most precise measurements and richest interplay with theory

Ordinary set-ups (as seen by the fluid mechanics community):

- Texas A \& M : stationary mixing of co-flowing liquids under earth gravity @ low Atwood (small DT):

- thermo-couples measurements for (long) time evolution of local density, statistics compared with spectral models (LANL),

- PIV-S (for scalar) for combined instantaneous maps of velocity and density, velocity-density correlations

- continuous mixing of co-flowing gases in curved channels (ancient CalTech work in Roshko's group)

- Some work was in progress at Univ. of Washington (Breidenthal)

- We need to communicate with the « non RTI-induced » mixing community (for instance, in Europe the ERCOFTAC 's Special Interest Group on variable density low speed turbulent flows). 


\section{Conventional Rayleigh-Taylor set-ups}

- Cambridge : great attention to the control \& analysis of perturbations induced by the separating barrier removal, double interface, tilted interface, concentration measurements from fluorescent dye in arc lamp light sheet

-CalTech : RTI for miscible fluids

-Univ. of Arizona : tank accelerated downwards by weights ( $\mathrm{g}$ above gravity)

\section{Non-conventional (i.e. non shock-induced) Richtmyer-Meshkov set-ups}

- Univ. of Arizona : falling tank bouncing on springs with long inertial flight thereafter: laser sheet visualisation of incompressible linear \& nonlinear RMI

-Univ. of Catalunya : similar principle reported by Redondo in 1993 : falling tank suddenly stopped on foam pad.

6 8th IWPCTM, Pasadena, December 10, 2001

Ceal DAM île-de-France 
More vigorous incompressible RTI experiments : a VNIITF specialty

but the Linear Electric Motor facility of LLNL has become famous

At VNIITF, the setups EKAP and SOM have an high acceleration range : 100$1000 \mathrm{~g}$ and provide various acceleration histories (acceleration, braking, inertial)

Vertical gas guns for propelling experimental packages with various liquids :

(various Atwood, unmiscible and soon miscible, various interface geometries)

Various diagnostics : shadowgraph, X-rays, laser sheet soon?

At LLNL, the LEM is characterized by controllable acceleration, state of the art diagnostics and imaginative liquids (yogurt)

Sorry, for this very uncomplete overview, the " alpha » group should take over... 


\section{Planar shock tube experiments (JF Haas et al.)}

There are 12 experimental laboratories involved with the conventional shock tube experiments :

AWE : (Aldermaston), ENIN (Moscow), CEA (France), IUSTI (Marseille), LANL, University of Arizona, University of the Negev (Beer-Sheva, Israel), University of Wisconsin (Madison), CalTech (Pasadena), SWRC (Tohoku University, Sendai), VNIIEF (Sarov) and VNIITF (Snejinsk).

\section{Flow definition}

In a first plot we attempted to classify the various flows to be studied:

-horizontal axis for flow compressibility, connected to the incident shock Mach \# -vertical axis for instability strength : At $\mathrm{k} h$

An issue loosely linked to the issue of deterministic vs. random initial interface 
mix 99 : summary roundtable for experiments (continued).

Planar shock tube experiments (diagnostics)

Schlieren and shadowgraph visualizations, the laser sheet with the following principles: Mye scattering by particles planar laser induced fluorescence (PLIF) using tracer molecules, the Rayleigh diffusion (differential among gases) the particle image velocimetry (PIV), the laser Doppler velocimetry (LDV), the hot wire anemometry (HWA), the $\mathrm{CO}_{2}$ infrared emission and absorption, the X-ray absorption (X-Ray), the interferometry (ENIN, SWRC), differential interferometry (CEA).

In a second plot, we sought to relate the performance of diagnostics to its usefulness for physical understanding, hence modelling and simulation : not so simple !

It should be obvious that an experiment has to be imaginatively designed before spending time and money on sophisticated diagnostics 


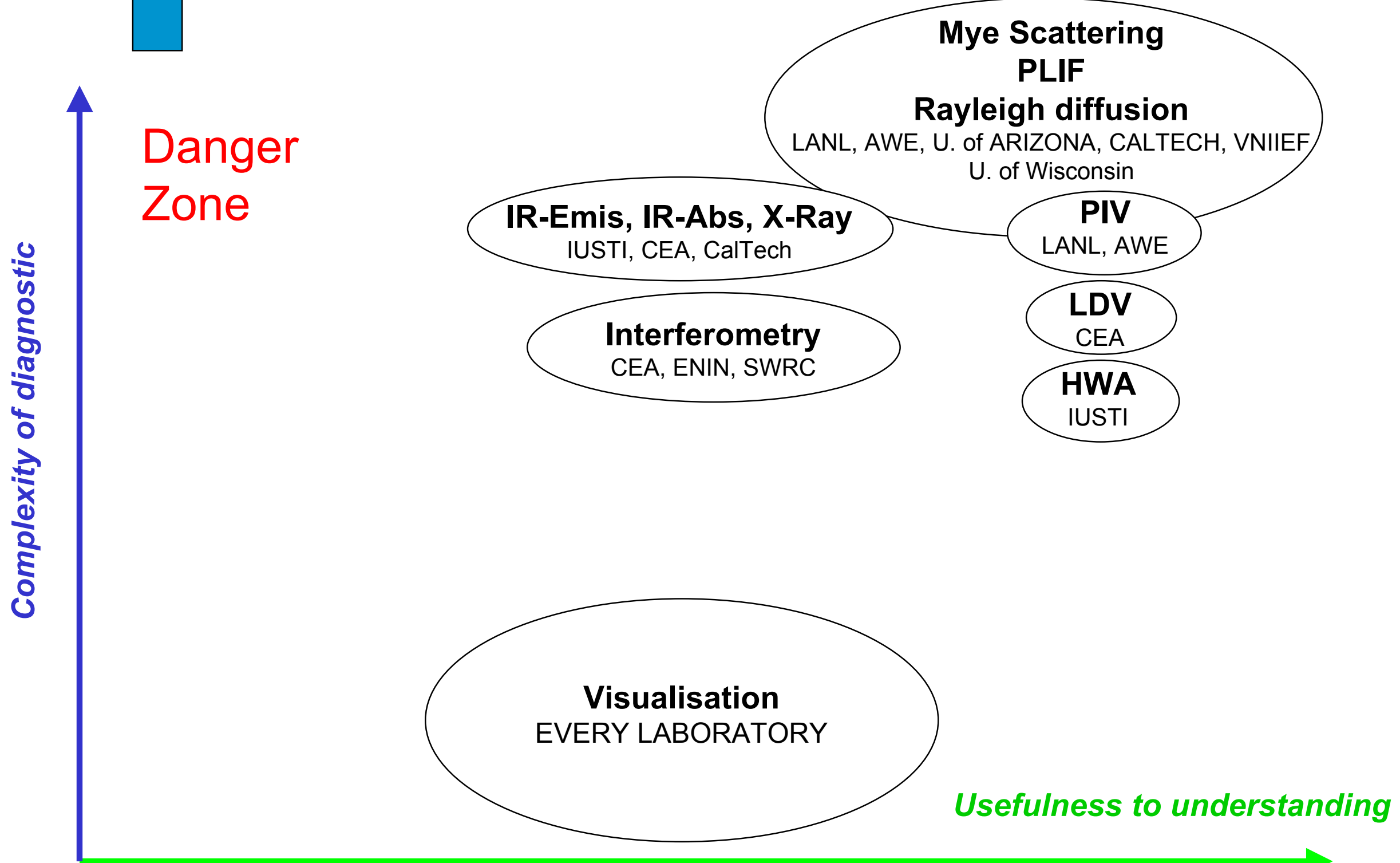


mix 99 : summary roundtable for experiments.

\section{Shock tube experiments (continued)}

The choice of the "usefulness to understanding" axis on the second graph was problematic as no laboratory would like to be positioned on the left side!

As an afterthought, we could have drawn instead a map with two axis, one dedicated to the measurement of the density field, the other for the velocity field.

The distance from the origin could be proportional to the probed volume (local vs. global).

Most of the presently used diagnostics would then be positioned on the density axis, LDV and PIV on the velocity axis and the HWA somewhere in between (probably closer to the density as explained on the poster E37). 
Density versus velocity diagnostics,

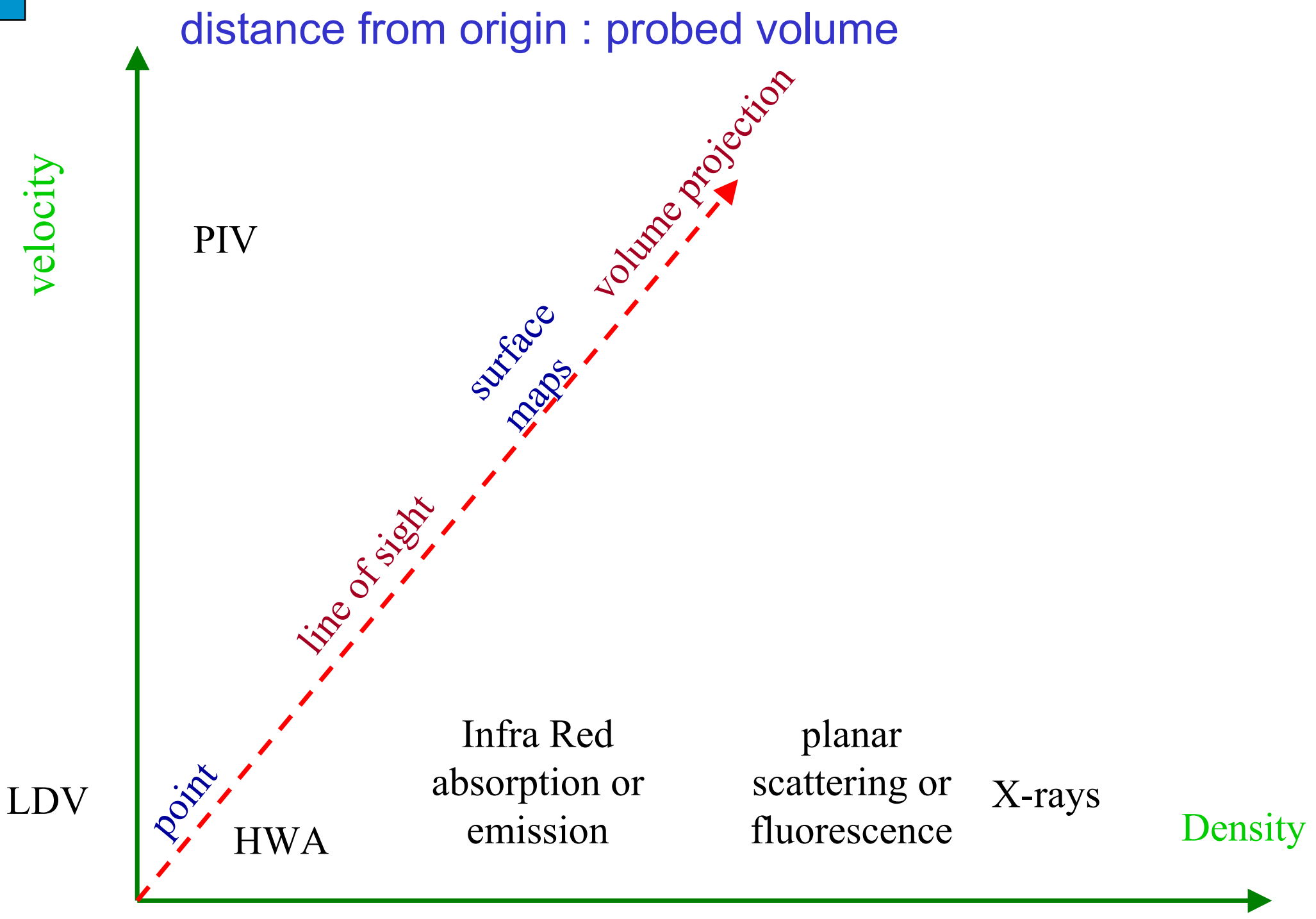




\section{Shock tube experiments (continued)}

The discussion pointed out the parameters such as interface geometry, the shock tube dimension and the initial interface control.

Again, it appears that the initial conditions, both wavelengths and amplitudes of the perturbations and the mechanical properties of the membrane, must be known.

An effort has to be undertaken on this fundamental point of interest for the next conference. 
Richtmyer-Meshkov shock tube experiments : some issues on flow definition

single mode interface (Arizona, Wisconsin, Caltech) deterministic multiple mode (ENIN, Beer-Sheva) simple geometries : transverse curtain, round jet, tilted plane, step, bubbles (LANL, AWE, VNIIEF) undetermined, multimode planar interface (CEA, IUSTI, VNIIEF, VNIITF, ENIN)

continuous or discontinuous interfaces : the killing of small scale turbulence or the membrane problem ?

low / high Mach \# : incompressible / compressible flow

the Reynolds \# : transition to turbulence, boundary layers

shock and reshock (of variable strength, ENIN) 
Review on RT, RM \& TM experiments

The issues on shock tube diagnostics

Shadowgraph \& Schlieren (AWE, Beer Sheva, Marseille)

Interferometry (Sendai, Moscow, CEA)

Flash X-rays (CalTech, CEA-Vaujours)

Laser sheet visualization

Mye scattering (VNIIEF, AWE)

Rayleigh scattering (CalTech \& Los Alamos, Madison)

Planar laser induced fluorescence

Velocity measurements : PIV (Los-Alamos), LDV (CEA)

Combined velocity \& concentration : HWA (Marseille) 

macroscopic perurbations with laser sheet diagnostics

-Excellent measurement of RMI growth rate @ Mach 1.1-1.2 for single mode linear \& non-linear regimes (U. of Arizona) : comparison with analytical models.

-Turbulent mixing soon arising from RMI @ Mach 3 (U of Wisconsin) :

after schlieren observations of discontinuous interfaces (with code comparisons), the initial interface is now controlled by gravity-induced RTI after sinusoidal plate removal and observed using planar Mye scattering.

Precise comparison of experiments of shocked SF6 curtain or cylindrical SF6 jet @ Mach 1.2 with simulations (Los Alamos) :

good agreement for concentration field, but low experimental velocities as compared to code, very detailed discussions about codes : first order « better » than second order, use of turbulence models.

-Archetypal (late 80's) shocked SF6 or Helium gas cylindrical jet at Caltech using PLIF (Jacobs) and Rayleigh scattering (Budzinski) in large shock tube @ Mach 1.1 - 2.0

-Wish from a simulation specialist: good measures of mixed mass of accelerated heavy gas jets ? 
VNIIEF relies on irregular membranes or on burning by combustible gas mixture prior shock VNIITF has invented the MIRAGE membrane with controlled burning

ENIN lets the hot shocked gases burn the membrane

CEA and IUSTI slice the membrane with a very fine wire mesh

An important effort on the analysis of laser sheet based diagnostics :

AWE experiments on shocked SF6 layers with discrete downstream defects and membrane :

good agreement with 2D or 3D code using postprocessor for multiple scattering

(now 10th order, see Holder et al.).

Other important issues were presented by the AWE team in Marseille :

characterisation of photographic film and laser sheet intensity, removal of membrane fragments : a good example for the recently started effort at IUSTI and the one just starting one at CEA. 
VNIIEF(Sarov) : tilted and step interface with cigarette smoke, comparison with 2D or 3D code

IUSTI (Marseille) : multimode air/helium and air/krypton interface using Mye scattering in new large shock tube with pulsed copper vapor laser (as in AWE, see poster E17 by Houas et al.)

CEA (Bruyères) : preliminary shots on planar interfaces using Mye scattering from prehistorical single pulse 2 Joule ruby laser (see abstract E21 by Lassis et al., but poster is not available). 


\section{Other visualization and densitometry methods}

Classical refractive visualisation (shadowgraph, schlieren) still in use : Beer Sheva, IUSTI, ENIN (Moscow), VNIITF, VNIIEF, CEA

Interferometry at ENIN and SWRC (Sendai, Japan)

Differential interferometry, useless in turbulence, just as qualitative as color schlieren (CEA, reported in mix 95, to be revived?)

Flash X-ray densitometry (Caltech, mix 89, CEA, mix 93\&95, to be revived), needs xenon as absorber of low energy $X$-rays but provides an excellent averaged concentration in turbulence

Laser interferometric tomography (Bashurin et al., Sarov) : the principle of LIT was described in Stony Brook and its application to a propane jet in air and to wire explosion in air was shown in Marseille : application to RT\&RM induced mixing?

Infrared emission and absorption was explored at IUSTI from 1985 to 1995. 
-Laser Doppler Velocimetry (LDV): was used on planar air/SF6 interfaces in CEA-Vaujours (Poggi et al., mix 97) : direct measurement of velocity, no detection of mixing zone passage by itself. To be used again in CEA-Bruyères in a larger shock tube (abstract E21). Non intrusive but seeding required, OK for reshock.

-Constant temperature hot wire anemometry (CTA) explored in IUSTI since 1997 : the signal is a fonction of concentration and velocity. Recent analysis (poster E37) shows that the density is better resolved than velocity. Intrusive method, problematic for reshock.

-Simultaneous measurements of LDV and CTA in CEA in 2002 ?

-Small scale resolution : LDV OK, CTA and PIV problematic?

-PIV seems ideal for large structures, long time development of instability. 
The wire is $1.25 \mathrm{~mm}$ long has a diameter of $5 \mu \mathrm{m}$ and breaks too often!

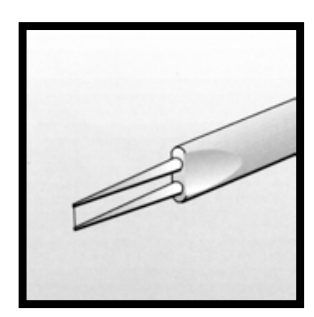

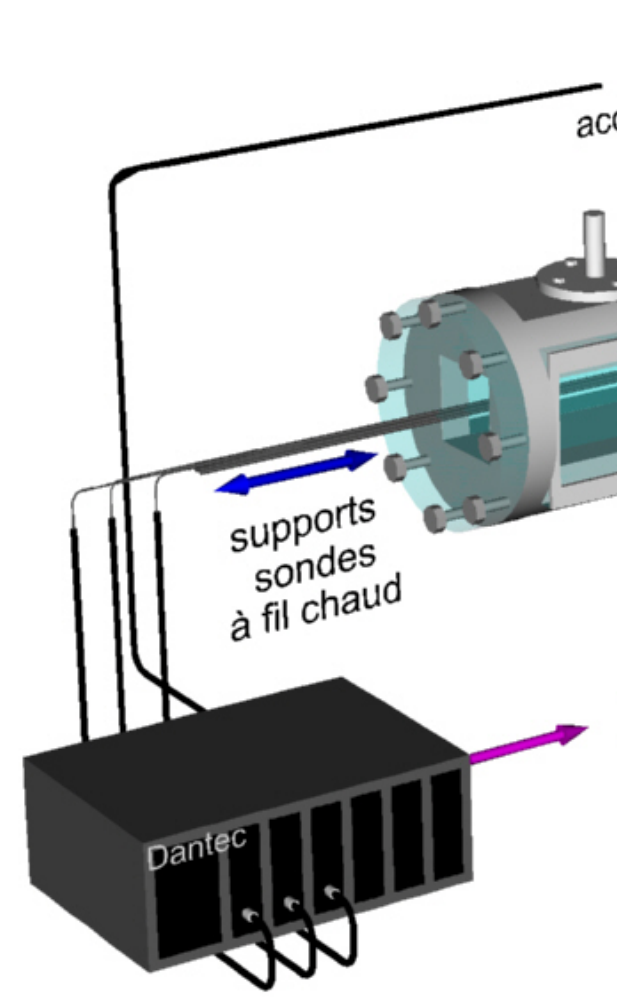

déclenchement declen fil chaud acquisition filchaud chambre EXP chambre BP

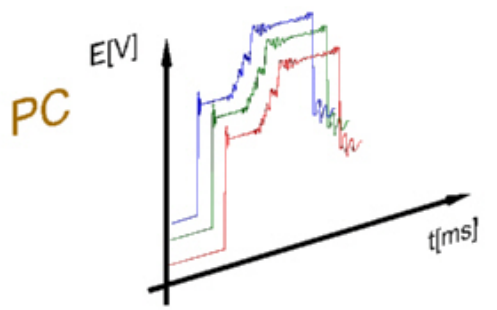

3 probes inserted from the end plate

Borrowed from Laurent Schwaederlé 's presentation for his succesful thesis defense on December 7th 2001 


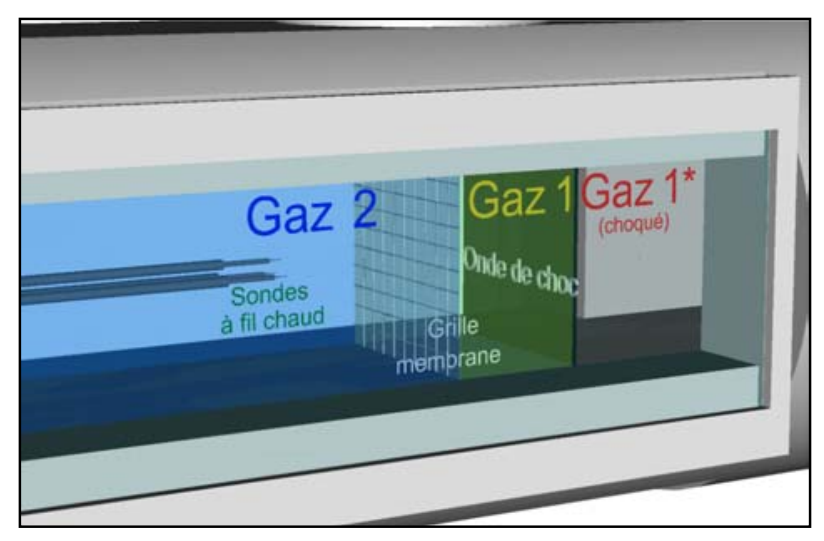

But the probes are intrusive and will disturb the reshock phase
The signal clearly shows the mixing zone passage and yields the local density time evolution

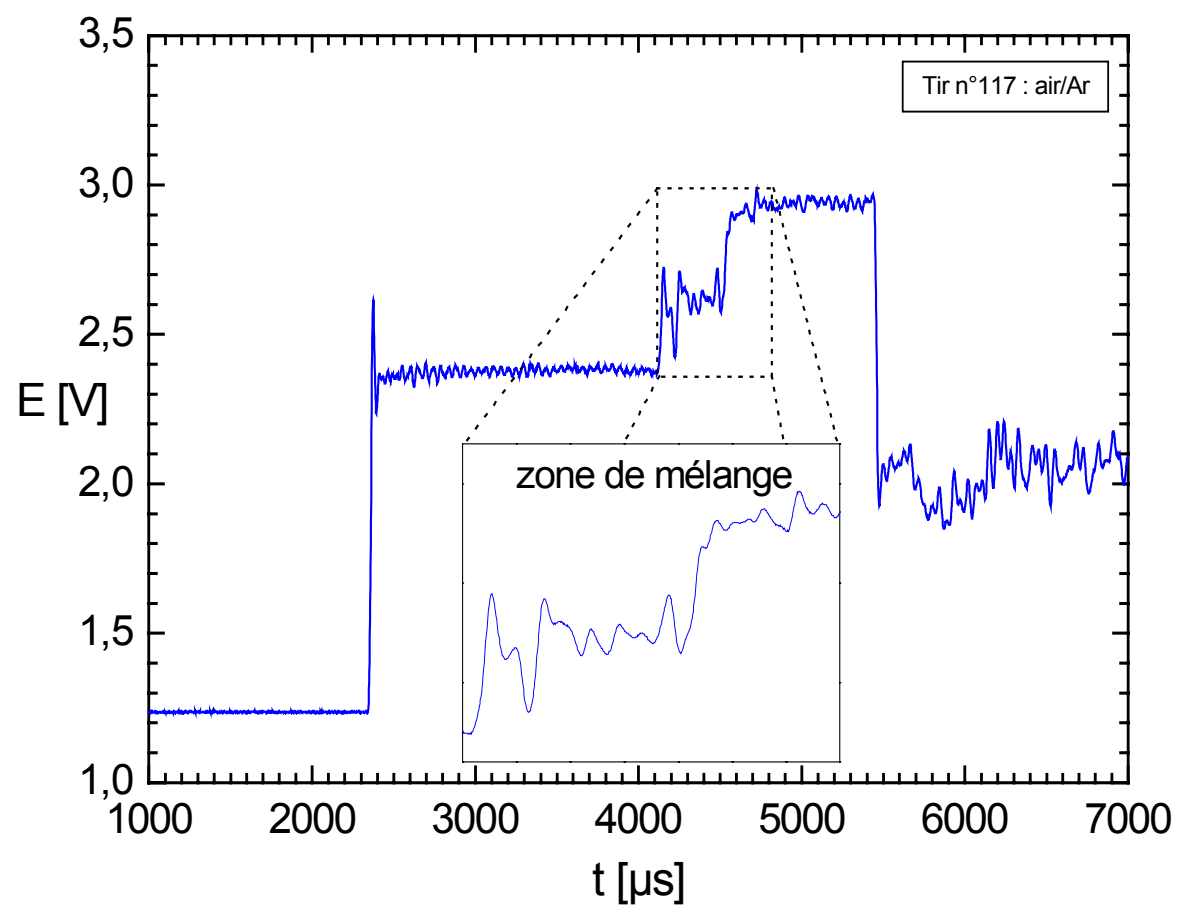

Meet Dr Schwaederlé in front of his poster E37 to learn about the first application of this ancient technique to our difficult flows ! 
Gas detonation driven experiments on jelly layers or gas interfaces : a VNIIEF tradition followed at LLNL

Easiest method to study material with strength and with well defined defects:

-in mix 99 : accelerated layer in square box at VNIIEF (Zhidov)

-applications to security against accidental explosions : protective liquid layers -subject left to be commented by the specialists !

Vertical combustion tube for nonstationary compressible RTI flows listen to review presentation by Zaytsev tomorrow, see poster E43

Ectromagnetic shock tube for Rayleigh-Taylor mixing at high gas speeds in VNIIEF , also multipurpose OSA facility: initial RMI phase due to strong shock, then constant acceleration for stable or unstable RTI phase. 
mix 99 : convergent shock experiments (K. Takayama, spokesman)

The measurement of the behavior of converging shock waves is useful for the study of the evolution of the interface perturbation from the linear to the non-linear (still deterministic) regime. In the last few years, novel converging shock experiments have appeared.

The cylindrical implosion can be obtained from the coalescence into a detonation wave of spherical explosions from a large number of point sources. At AWE : the large chamber $\left(30^{\circ}\right.$ sector, $1 \mathrm{~m}$ radius) is a difficult undertaking but with promising laser sheet diagnostics.

From VNIIEF, we have seen results obtained with smaller, fully circular detonation chamber, with traditional visualisation.

It can also be generated using a diaphragmless vertical shock tube (SWRC, Sendai).

At VNIIEF, spherical chambers have been used with diagnostics from wall mounted pressure transducers (timing and intensity of reflected waves with or without TMZ inside.

A pertinent suggestion was made about the visualization of a spherically converging flow inside a cone by the holographic interferometry technique (SWRC, Sendai). We certainly hope to be able to discuss such experiments during the next workshop. 
High explosive experiments

a russian domain of excellence

Z-pinch experiments

a competitor to laser experiments ?

Laser driven experiments 
At mix 99: Bruce Remington, the spokesman for the laser experiments subgroup brillantly summarized the goals of laser (and all) experiments:

We must demonstrate that we can access to interesting scientific domains: i.e. the linear and non-linear regimes (ICF) of the RT/RM-I as well as to turbulent mixing (supernovae), but also the behavior of liquids (or solids) at high pressure (planetary interior) and the coupled radiation hydrodynamics (photo evaporation front).

But experiments have to be improved to facilitate independence of their results from the facility (an issue of science versus "facility calibration"). For this, we need to derive scaling laws in each domain (test of "scalability") and to develop the required numerous diagnostics. We are not there yet.

The action items for future laser facilities (LIL, NIF, LMJ) are: progress towards longer steady drive, large enough ground facilities allowing turbulent regimes, bright source of hard X-rays combined to spectroscopy, codes to grasp all domains, use of the spallation experiments for demonstrating facility independence, specific diagnostics for astrophysics motivated experiments, spherical effects in explosions, convergence effects in spherical implosions. In any case, we have a long and interesting way to do in this domain which is still full of promises. 
T. Peyser wrote in 1995: « Nova, the world's largest laser, was used to drive one of the world 's smallest shock tube » and obtain various exciting flows :

•non linear RMI growth from sawtooth initial perturbations @ Mach 20 (1995)

-hypervelocity jet from hemispheric defect (1995)

- developped mixing from broad spectrum multimode perturbations (1997)

-reshock @ Mach 15 of a primary shock induced mixing zone (1999)

We will see some careful checks of theories for RMI in the compressible, non-linear regime from experimental results obtained on Omega @ Mach 10 with comparisons with shock tube results from ENIN (@ Mach 3).

Note that mixing experiments in large size and at large Reynolds for Mach 10 to 20 shocks could be obtained in the (intermediate) shock tube section of free piston facilities such as T5 here at CalTech (1998 proposal by Bradford Sturtevant). 


\section{At mix 99 , Evgenij Meshkov insisted on practical and peaceful applications of RT\&RMI \& TM experiments.}

Finally, it is about time for our scientific community to harness turbulent mixing in nature and ordinary technology and try to bring some (more?) contribution to humanity.

Some Sarov colleagues have already pointed out some interesting ways such as the fighting of forest fires by explosive methods (joint project VNIIEF-IUSTI), the conception of new explosive light sources and of protective liquid wall against explosions (VNIIEF, after an idea from the czar Peter the Great ?).

The common point of these three methods is the development of turbulent mixing arising from the evolution of hydrodynamic interface instability. 
Experimental investigators should collaborate with theory and simulation specialists :

by proposing and carrying out imaginative experiments with reasonnable diagnostics

but the diagnostics can also be simulated in code postprocessors

The experiments should be fun (because these flows are exciting)

to be continued in the experimental discussion, tuesday $11,16: 15-16: 45$ 


\section{Summary of the $8^{\text {th }}$ International Workshop on the Physics of Compressible Turbulent Mixing (9-14 December 2001, Pasadena, CA)}

\section{Oleg Schilling}

Chairman of the $8^{\text {th }}$ International Workshop on the Physics of Compressible Turbulent Mixing

University of California, Lawrence Livermore National Laboratory

$$
\begin{gathered}
\text { P.O. Box 808, L-22 } \\
\text { Livermore, CA } 94551 \\
\text { (925) 423-6879, schilling1@IInl.gov }
\end{gathered}
$$

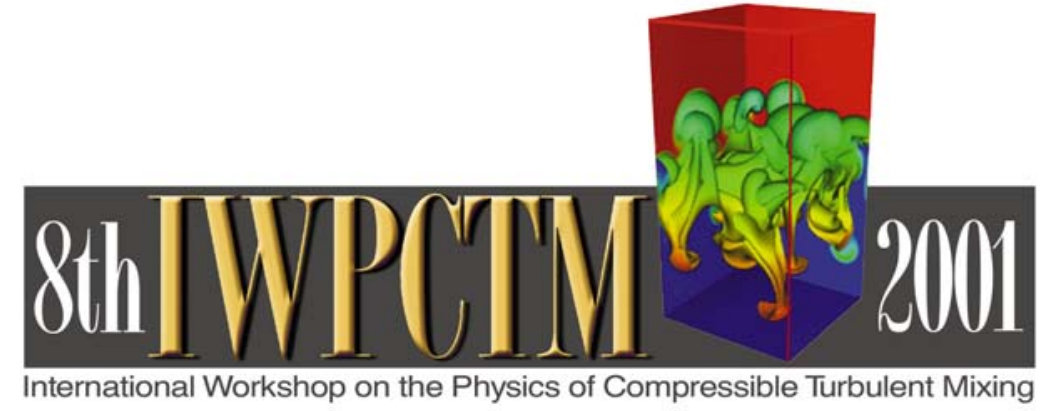

This work was performed under the auspices of the U.S. Department of Energy by the University of California, Lawrence Livermore National Laboratory under Contract No. W-7405-Eng-48

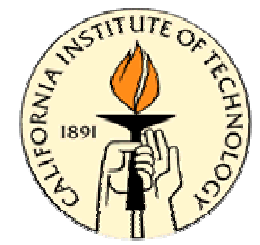




\section{Outline of IWPCTM summary/synthesis}

- Introduction

- Background, previous venues, and 2003 location of the IWPCTM

- Demographics: how many attendees and from where

- Difference in format from previous conferences

- Technical summary

- Experimental research

- Computational research

- Theoretical research

- Observations on the IWPCTM

- Past

- Present

- Future 


\section{The $8^{\text {th }}$ International Workshop on the Physics of Compressible Turbulent Mixing is a biennial conference originally established by LLNL

- Previous venues

1) Princeton, NJ, USA (1988) [LLNL]

2) Pleasanton, CA, USA (1989) [LLNL]

3) Royaumont, France (1991) [CEA]

4) Cambridge, UK (1993) [AWE/Cambridge University]

5) Stony Brook, NY, USA (1995) [SUNY Stony Brook]

6) Marseille, France (1997) [Université de Provênce]

7) St. Petersburg, Russia (1999) [RFNC-VNIIEF]

8) Pasadena, CA, USA (2001) [LLNL]

- Venue for the $9^{\text {th }}$ IWPCTM

- Cambridge, UK (tentatively Spring 2003) [AWE/Cambridge University] 


\section{The $8^{\text {th }}$ IWPCTM was very well attended, with approximately $1 / 3$ of participants from the academic community

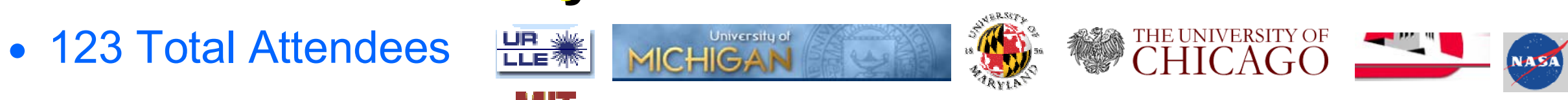

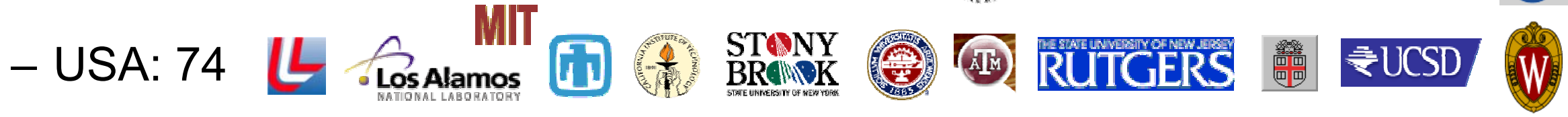 \\ - Russia: 20

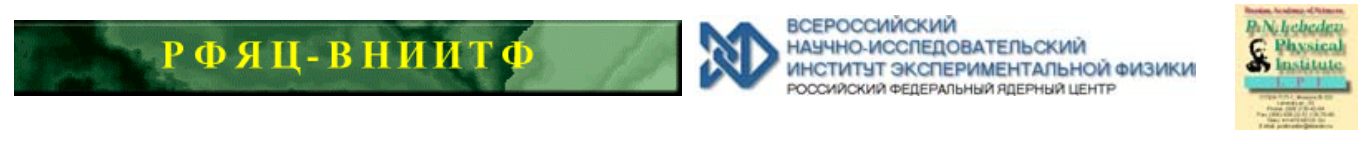

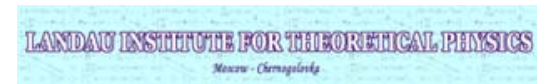 \\ - France: 8

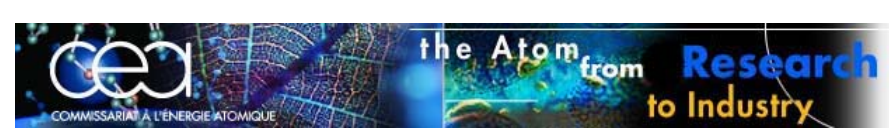 \\ - Israel: 8

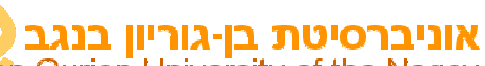 Ben-Gurion University of the Negev \\ Langues \\ - UK: 6

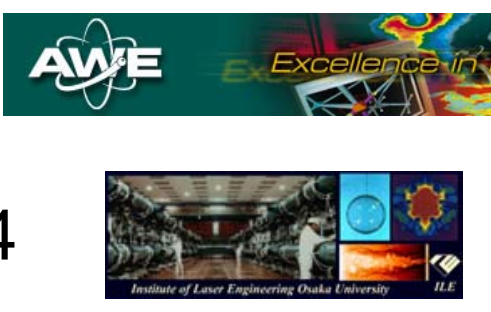

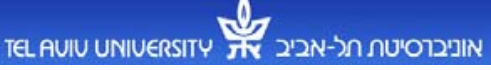 \\ Weizmann Institute of Selence

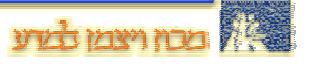 \\ - Japan: 4 \\ - Canada: 2

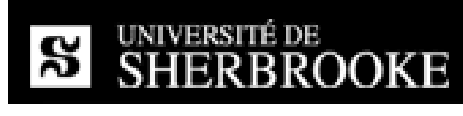 Université th
de Montréal \\ - Spain: 1 त्वCIII}




\section{Invited review talks were presented, and more presentations were given than during past workshops}

- One hour talks given at beginning of Experimental, Computational, and Theoretical sessions to review state-of-the-art

- Experimental: "Review on RTI, RMI and TM Experiments" (Haas \& Zaytsev) and "The Experimental Study of Excitation and Development of the Hydrodynamic Instability in the Mixing Zone Separating Gases of Different Densities at Their Accelerated Motion" (Zaytsev)

- Computational: "Review of Numerical Simulation of Mixing due to RayleighTaylor and Richtmyer-Meshkov Instabilities" (Youngs)

- Theoretical: "Three Dimensional Multi-Mode Rayleigh-Taylor and RichtmyerMeshkov Instabilities at All Density Ratios" (Kartoon et al.)

- This further stimulated the Panel Discussions at the conclusion of each of the three sessions

- 126 total oral and poster presentations

- 67 oral presentations

- 59 poster presentations (staggered format used for more coverage) 


\section{Summary of experimental research}

- "Complex" Rayleigh-Taylor instability experiments

- Combined Rayleigh-Taylor and Kelvin-Helmholtz instability

- "Demixing" experiments

- Helium-driven gelatin experiments

- Diagnostic developments for Rayleigh-Taylor instability experiments

- Scalar PIV

- Wavelet post-processing

- Moiré interferometry, Fresnel phase zone plate/penumbral imaging

- "Complex" Richtmyer-Meshkov instability experiments

- Retractable plate shock tube experiments

- Experiments to study velocity reduction due to large Ma and initial amplitude

- Converging geometry experiments

- Diagnostic developments for Richtmyer-Meshkov instability experiments

- PLIF, PIV

- Hot-wire anemometry, laser sheet visualization, and LDV

- Shadowgraphy

- High-energy density (laser) experiments

- "Laboratory astrophysics" 


\section{"Complex" Rayleigh-Taylor instability experiments with shear and "demixing" were reported}

- Combined effects of Rayleigh-Taylor instability and shear in tilted interface experiments using double-shielded barrier plate withdrawal (Holford, Dalziel \& Youngs E14)

- Similar to Cambridge University experiments reported in JFM in 1999

- Shear results in competition between large $\lambda$ (large-scale) overturning and small $\lambda$ (large $k$ ) Rayleigh-Taylor instability growth

- Turbulence models examining combined shear-buoyancy are under development (Wilson, Andrews \& Harlow T33)

- Chemically-reactive experiments resulting in a fluorescent product envisaged

- "Demixing" experiments in liquids with three different At with sign of acceleration reversed (Kucherenko et al. E2)

- 3D periodic perturbation influence on turbulent mixing using gelatin driven by He compressed to 13 atm with $g \sim 3 \times 10^{6} \mathrm{~cm} / \mathrm{s}^{2}$ giving $\alpha=0.1$ (Bliznetsov et al. E7) 


\section{New diagnostics used in Rayleigh-Taylor instability experiments to measure statistical, in addition to integral (large-scale), properties}

- Splitter-plate experiment similar to classical shear flow experiments (Ramaprabhu \& Andrews E32)

- Small Atwood number $\left(A t \sim 10^{-3}\right)$ in a water channel

- Cold and hot horizontally-moving streams of water achieving $\operatorname{Re} \sim \mathrm{O}\left(10^{3}\right)$

- Scalar PIV with different particle concentrations used to simultaneously measure $\rho$ and $v_{i}$ fields to obtain $\left\langle\rho^{\prime 2}\right\rangle,\left\langle v_{x}^{\prime 2}\right\rangle,\left\langle v_{y}^{\prime 2}\right\rangle,\left\langle v_{x}^{\prime} v_{y}^{\prime}\right\rangle,\left\langle\rho^{\prime} v_{x}^{\prime}\right\rangle,\left\langle\rho^{\prime} v_{y}^{\prime}\right\rangle$ and 2D spectra (needed for DNS, LES, and turbulence models)

- Results in good agreement with previous thermocouple experiments

- Chemically-reactive fluids to diagnose molecular mixing possible also

- Wavelet post-processing of sequential density data from experiments to denoise, compress, and detect patterns (Afeyan, Ramaprabhu \& Andrews T39)

- Moiré interferometry used to diagnose ablative Rayleigh-Taylor instability at small wavelengths and Fresnel phase zone plate/penumbral imaging used for density measurements on lasers (Azechi et al. E45) 


\section{Progress in Richtmyer-Meshkov instability experiments was reported, especially at larger Mach numbers and in convergent geometries

- Retractable Cu plate vertical shock tube experiments at $M a=2.9$ with imposed perturbations in air/ $/ \mathrm{CO}_{2}$ (Anderson et al. E27)

- Heavy $\rightarrow$ light Rayleigh-Taylor followed by Richtmyer-Meshkov instability diagnosed with Mie scattering

- Experiments studying velocity reduction due to large $M a$ and large initial amplitude with $M a=5$ achievable (Sadot et al. E36) and large cross-section shock tube facility (Houas et al. E17)

- Classical and high-energy density convergent experiments reported

- Detonation-driven 2D shock tube experiments with Ma = 2-3 (Holder et al. E13; Hosseini \& Takayama E15, E16)

- "Chevron" (notch) shock tube experiments at $M a=1.26$ diagnosed with Mie scattering (Smith et al. E39)

- Cylindrical direct-drive experiments on OMEGA (Barnes et al. E4; Batha et al. E5; Parker et al. C28)

- Shock/flame interactions studied (Bliznetsov et al. E6)

- Kelvin-Helmholtz instability studied in analysis of impact of oblique metal plates (Bakrakh et al.) 


\section{New diagnostics have also been proposed for better imaging and measuring additional quantities in Richtmyer-Meshkov instability experiments

- "Membraneless" shock tube experiments in air/SF 6 at $M a=1.3$ (Jacobs \& Krivets E18)

- PLIF shows that interface evolution depends strongly on degree of nonlinearity at time of onset of second shock

- Impulsive Richtmyer-Meshkov experiments (Niederhaus \& Jacobs E26)

- PIV used to study interaction of a shock with one cylinder (Prestridge et al. E30) and two cylinders (Tomkins et al. E40)

- Triple probe, constant temperature hot-wire anemometry measurements of $\rho, v_{i}$, and $T$ at $M a=1.25$ (Schwaederlé et al. E37)

- Planar laser sheet visualization and laser Doppler velocimetry (LDV) measurements (Lassis et al. E21)

- Shadowgraphy used to diagnose interaction of a Ma $=1.5$ shock with a $\mathrm{Kr}$ bubble (Layes et al. E22); shock/bubble interactions also simulated using ALE code LEEOR2D (Levy et al. E23)

- Development of a liquid film conductor to rupture membrane with an electric current prior to shock passage (Kucherenko et al. E20, E28, E38)

- Design of novel miniature KrF laser shock tube for experiments with $M a>20$ and $p>10$ kbar in liquids (Lebo \& Zvorykin C25) 


\section{High-energy density experiments on OMEGA continue to be valuable for studying nonlinear Richtmyer-Meshkov instability growth and ICF}

$1952-2002$

- Study of effects of shock proximity on linear/nonlinear instability growth at $M a \sim 10$ in C foam with a polycarbonate pusher (Glendinning et al. E12; Robey et al. E46)

- CALE simulations of $M a \sim 10$ experiments (Miles, Edwards \& Glendinning C57); also Mikaelian T20

- 1D/2D CALE simulations compared to 1D/2D FCl simulations (using a $K-\varepsilon$ model) of $M a \sim 30$ RM instability experiments (Seytor \& Legrand C35; see Souffland \& Renaud C36)

- NIF ignition target development (Haan et al. C22)

- ICF implosion experiments simulated in 2D (Srebro et al. C37)

- High-strain-rate material strength experiments (Kalantar et al. E19; Pollaine et al. E29)

- Rayleigh-Taylor mode-coupling experiments on NOVA simulated using ALE code (Darlington \& Budil C9) 


\section{"Laboratory astrophysics" continues to motivate High-energy density experiments on OMEGA}

- Shock-sphere interaction experiments

- Study vortex structure and evolution (Robey et al. E34; see Brouillette \& Hébert E8 for classical fluid experiments on vortex rings)

- Paradigm for interaction of supernova blast wave interaction with interstellar clouds (Klein et al. E42)

- Simulations of shock-sphere/shock-cylinder interactions using PPM (Peng et al. C29) and shock-planar curtain interactions (Zhang \& Zabusky C48)

- Compressible turbulent mixing experiments in support of astrophysics (Drake et al. E9, E10)

- Compressible MHD turbulence application to radiating molecular clouds discussed (Ryutov T25) 


\section{Summary of computational research}

$1952-2002$

- Differences between DNS, LES, and MILES

- MILES of variable acceleration Rayleigh-Taylor flow

- Code intercomparisons

- Spectral/compact, Godunov, and CENO simulations of Shu-Osher problem, RichtmyerMehskov instability, and Taylor-Green vortex

- RAGE, Cuervo, Raptor simulations of shock/cylinder interaction

- High-order and high-resolution methods

- Filtered spectral and WENO schemes

- PPM scheme

- Sensitivity of Rayleigh-Taylor mixing to initial conditions and studies of self-similarity

- Effects of small scales on large scales

- Stabilizing effects of a transitional layer

- Compressibility effects on supersonic, reacting shear layers

- Spherical combustion layers

- Stability of converging shocks

- Numerical simulations and comparisons to bubble merger models

- Subgrid-scale model development and LES of interfacial instability-driven turbulence 


\section{Systematic comparisons between simulation methods (direct numerical simulation, large-eddy simulation, and monotone integrated LES) are being conducted}

- Differences between DNS, LES and MILES discussed (Youngs)

- Reynolds-Averaged Navier-Stokes models will still continue to be needed (Youngs C46)

- MILES of variable acceleration Rayleigh-Taylor flow (Youngs \& Llor C47)

- Simulations of 1D density-shock interaction, 2D air-acetone/SF ${ }_{6} \mathrm{Ma}=1.2$ Richtmyer-Meshkov instability experiment, and 3D Taylor-Green vortex using spectral/compact, Godunov, and CENO schemes to assess numerical effects (Cook et al. C8)

- Eulerian codes RAGE, Cuervo, and Raptor compared in 2D simulations of $M a=1.2$ shock acceleration of a diffuse, dense gas cylinder (Greenough et al. C16; Rider, Kamm \& Zoldi C31; Zoldi C50)

- 2D simulations of shocked interfaces using high-order and high-resolution methods

- Aleshin et al. Ma $=4.5$ experiment using high-order filtered spectral and WENO schemes (Don et al. C54)

- WENO and PPM schemes (Zabusky et al. C20)

- Interaction of blast waves with cylindrical/spherical bubbles (Zhang et al. C49)

- Efficient solver developed (Wang et al. C41) 


\section{The sensitivity of Rayleigh-Taylor mixing to the numerical algorithm used remains undetermined, and the "determination" of $\alpha$ remains inconclusive

- 3D simulations $\left(256^{2} \times 512\right)$ using different numerical methods (PPM, ALE, etc.) and same initial conditions have not conclusively determined that $\alpha$ is universal (Dimonte et al. C10; see Weber, Dimonte \& Marinak C42 and Dimits C55)

- Mixing (entrainment) changes the effective $A t$ at the mixing layer front

- Now being recognized that the front-tracking result $\alpha=0.07$ may be an overestimate as this method inhibits mixing at the interface, i.e., as in immiscible fluids (Dutta et al. C11)

- Immiscible experiments and simulations inhibit energy cascade to small-scales and dissipation (mixing) at large $R e$, resulting in a more rapid mixing layer growth and larger $\alpha$

- Simulations using highly diffusive algorithms result in excessive (numerical) "mixing", reducing At locally and the mixing layer growth (giving a small $\alpha$ )

- 3D simulations using TREK code for $\rho_{1} / \rho_{2}=3-40$ gave $\alpha=0.06-0.16$ (Yanilkin et al. C44); TREK also used for simulating instabilities in plasma clouds during expansion in a magnetic field (Gavrilova et al. C14)

- A turbulence model was developed for magnetohydrodynamic Rayleigh-Taylor instability evolution at the interface of an accelerated plasma and magnetic field (Gubkov, Zhmailo \& Yanilkin C19) 


\section{The effects of small scales (molecular mixing) on the dynamics of the large scales was an important experimental, numerical, and theoretical theme

- Stabilizing effects of an intermediate, transitional layer due to molecular diffusion studied (Kucherenko et al. E3, E31)

- DNS of moderate Re Rayleigh-Taylor instability examined sensitivity of large-scale properties (mixing widths) and statistics (such as molecular mixing rate) to initial conditions (Cook, Dimotakis \& Mattner C56)

- Reduction of growth rate and other effects of compressibility (increasing $M a_{\mathrm{c}}$ ) studied in DNS of a supersonic, reacting shear layer (Pantano \& Sarkar C27)

- 3D AMR simulations of a spherical combustion layer resulting from a TNT explosion (Kuhl \& Ferguson T37)

- Molecular dynamics simulations to study stability of converging shocks and Richtmyer-Meshkov instability in a dense Lennard-Jones fluid (Nishihara, Zhakhovskii \& Abe C26)

- Mechanisms of turbulent diffusion in solar-type stars studied numerically (Toqué C39) 


\section{Numerical simulations showed qualitative consistency with statistical bubble merger models}

- A general buoyancy-drag model was developed to include shock compression and spherical convergence (Elbaz et al. T11, C12)

- Multi-mode perturbation evolution is described by evolution of a single, effective $\lambda$ (mode)

- Internal density profile of mixing layer studied using a diffusion model

- Bubble merger results in a pure front of low density fluid rising in higher density fluid (Rikanati, Alon \& Shvarts T22)

- Effectively leads to a single bubble of light fluid on top and heavy fluid on bottom

- In this 2D model, mixing occurs at center and bottom of mixing layer due to Kelvin-Helmholtz instabilities generated when falling spikes shear rising bubbles

- 3D ALE simulations of random perturbation growth with different $\rho_{1} / \rho_{2}$ using front-tracking consistent with this bubble merger model (Kartoon et al. T14)

- Thermonuclear burning in a Type IA supernova explosion modeled using this merger model (Takabe et al. C38) 


\section{Limited progress has been made on the}

development/application of explicit subgrid-scale models for interfacial instability-driven turbulence 1952-2002

- Large-eddy simulation (LES) of Richtmyer-Meshkov unstable turbulent flows generated by a $M a=10$ shock (Samtaney et al. C33)

- Used the Pullin stretched-vortex subgrid-scale model

- 5th- and 7th-order WENO schemes

- Evolution of mixing layer, spectra, and statistics studied (with reshock)

- A methodology was extended to study interaction between small and large scales (subgrid-scale dynamics) in Rayleigh-Taylor and Richtmyer-Meshkov instability (Schilling \& Cook T28)

- Applied to a $512^{2}$ x 2040 Rayleigh-Taylor mixing spectral/compact DNS dataset (Cabot, Schilling \& Zhou, submitted to Physics of Fluids)

- Examined transfer dynamics of small and large scales as a function of scale and vertical height

- Extracted eddy viscosity and backscatter 


\section{Summary of theoretical research}

- Development of models

- Bubble merger, two-phase flow, and buoyancy-drag models

- Potential flow, vorticity deposition, and shell models

- One-dimensional turbulence model

- Modeling of nonlinear instability growth and transition to turbulence

- Application of Dimotakis transition criterion at $R e \sim 10^{4}$

- Modeling of combined shear and buoyancy instabilities

- Effects of heat transfer and ablation

- Modified Zhang-Sohn model

- Compressible Rayleigh-Taylor instability

- Stability of converging/diverging shock waves

- Turbulent transport and mixing models

- Two-fluid model for combined Rayleigh-Taylor, Richtmyer-Meshkov, and Kelvin-Helmholtz instabilities

- Two-equation, single-velocity turbulence models

- Two-scale turbulence model

- Simulations using multi-fluid models in ALE and AMR codes 


\section{Progress has been made in the theoretical study of nonlinear instability evolution using bubble merger, two-phase flow, and simple models}

- A model for mixing layer width evolution was described (Cheng, Glimm \& Sharp T7)

- Bubble merger model based on a renormalization group fixed-point

- Center-of-mass model coupling bubble and spike mixing zone edges

- Buoyancy-drag model calibrated against above models

- A buoyancy-drag model was used to model dependence of spatial dimensionality and density ratio on Richtmyer-Meshkov instability evolution (Yosef-Hai et al. T35)

- A vorticity deposition model was developed for single-mode Richtmyer-Meshkov instability growth at large Ma and large initial amplitude (Rikanati et al. T23)

- Extensions of Layzer potential flow model used to study bubble/spike front evolution with modal interactions (Abarzhi T1, T3; Abarzhi, Glimm \& der Lin T2)

- Regular and singular asymptotic solutions studied for $A t<1$

- Group theoretical methods used to study morphology/topology of large-scale structure of periodic bubble/spike arrays (Inogamov et al. T12, T13)

- Layzer-type and shell models including effects of entrainment and diffusion on mixing layer growth used to study Rayleigh-Taylor evolution and examine $\alpha$ (Dalziel T10)

- One-dimensional Turbulence (ODT) model used to study large At, small Ma mixing (Ashurst \& Kerstein C4)

- A model of inhibition of Rayleigh-Taylor mixing was developed (Breidenthal T5) 


\section{Progress has been made in the theoretical study of nonlinearity and the transition to turbulence}

- Dimotakis criteria for transition to turbulence at $R e \sim 10^{4}$ applied as a function of time to:

- Cambridge University experiment and an OMEGA experiment (Zhou et al. T36)

- Rayleigh-Taylor unstable plasma flow (Robey et al. E35)

- Turbulence modeling of combined shear and buoyancy instabilities (Wilson, Andrews \& Harlow T33)

- Effects of heat transfer/ablation on turbulent mixing investigated (Clark \& Harlow T8)

- Predictions of Nikiforov's turbulence model in the VIKHR code was shown to be in good agreement with:

- LEM data (Kozlov, Razin \& Sapozhnikov C51)

- Poggi et al. RM instability data (Kozlov \& Razin C52)

- A modified Zhang-Sohn model was developed for single-mode, nonlinear growth of the Rayleigh-Taylor and Richtmyer-Meshkov instability (Vandenboomgaerde T31; Vandenboomgaerde et al. T32)

- Growth rates of linear (Wouchuk T34) and nonlinear (Nishihara, Matsuoka \& Fukuda T19) Richtmyer-Meshkov instability studied

- Rayleigh-Taylor instability in compressible fluids was studied (Tricottet \& Bouquet T30)

- The stability of diverging shock waves (Ktitorov T15) and of converging shock waves (Ktitorov T16) was studied 


\section{Progress has been made in turbulent transport}

\section{model development for turbulent mixing induced}

by interfacial instabilities

- A 1D two-fluid model incorporating combined Rayleigh-Taylor/RichtmyerMeshkov/Kelvin-Helmholtz instabilities with arbitrary $g(t)$ was developed (Bailly \& Llor T4)

- Mass transfer between fluids and diffusion ( $K-\varepsilon$ model) included

- Self-similarity studied in "OD", i.e., as a function of time only (Llor T18)

- A family of two-equation models was developed for 2D/3D single-velocity, compressible, multi-component flow (K-Z: K- $\varepsilon, K-I, K-\omega, K-\tau)$ (Schilling T26)

- Includes closures for compressibility, reaction terms not previously included

- Special cases studied analytically (decay, shear layer, boundary layer, jet)

- A 1D two-scale $K-\varepsilon$ model was formulated using spectral and scaling concepts, and preliminary validation studies were performed in the ALE code sKULL (Eliason, Cabot \& Zhou C53)

- Youngs multi-fluid turbulence model was implemented in the 2D finite-element ALE code CORVUS (Grieves C17)

- Multi-fluid simulations of Rayleigh-Taylor mixing using 2D AMR code (Vold C40)

- A 1D diffusive mixing model was used to study neutron and charged-particle yields in laser implosion experiments (Epstein et al. C13)

- A $K-\varepsilon$ model was developed for applications to the atmospheric surface layer (Anuchin, Neuvazhayev \& Parshukov C2) 


\section{Concluding observations: past, present, and future}

of the IWPCTM

- In the past, many presentations had limited relevancy to compressibility, turbulence, or mixing

- Experiments and simulations limited to very small $R e$ and $M a$

- Euler simulations generated "numerical mixing" and "numerical turbulence"

- Analytical models were limited to linear and weakly-nonlinear regimes

- Presentations this year were more focused on the subjects of the Waorkshop, with good progress in:

- Experimental designs/diagnostics, especially for classical fluid experiments

- Higher accuracy and higher resolution simulations in 2D and 3D

- Multi-fluid interpenetration, turbulent transport and mixing model development

- Future focus should include:

- Diagnostic methods that can experimentally measure statistical quantities

- Experiments, simulations, and modeling of reacting turbulent flows

- Analysis of DNS data, and development of subgrid-scale models

- LES of compressible turbulent mixing

- Refined turbulence models validated against experiments and LES 
Nonlinear evolution of unstable fluid interface

S.I. Abarzhi

Department of Applied Mathematics and Statistics

State University of New-York at Stony Brook 
misalignment PRESSURE and DENSITY gradients

INSTABILITY

Rayleigh-Taylor instability

Richtmyer-Meshkov instability
TURBULENT MIXING

sustained acceleration (gravity) impulsive acceleration (shock)

- thermonuclear flashes on surface of stars; supernova explosion

- inertial confinement fusion; interaction of laser with matter

Basic objective: reliable description of turbulent mixing

\section{Fundamental issues:}

- the cascades of energy

- the dynamics of small-scale structures

- the dynamics of the large-scale coherent structure

$\underline{\text { Coherent structure }}$

an array of bubble and spikes periodic in the plane normal to the direction of acceleration (shock)

Dynamics of 3D and 2D nonlinear structures in RMI

Properties of the 3D-2D dimensional crossover in RMI 
INTERFACE active regions

Aref $^{1989}$

large-scale coherent motion

spectral approach

Abarzhi $^{1996}$ (RTI) passive regions

large scales

simple advection

scalar fields

group theory

\section{Group theory}

coherent structure

periodicity

group of invariance 17 plane crystallographic symmetry groups

$\boldsymbol{G}$ translations in the plane + rotations + reflections

The COHERENT STRUCTURE is OBSERVABLE

- A significant part of the fluid energy is concentrated in the coherent motion

a DOMINANT mode $\mathbf{K}$ governs macroscopic dynamics

- The structure is stable under modulations
$\boldsymbol{K}+\xi$
$\varphi(\boldsymbol{K}+\xi) \approx \varphi(\boldsymbol{K})+F(\boldsymbol{K}) \xi^{2}$
$\boldsymbol{K} \leftrightarrow-\boldsymbol{K}$
a scalar macroscopic function

$\boldsymbol{G}$ is a symmorphic group with inversion in the plane

3D: p6mm, p4mm, p2mm, cmm, p2

2D: pm11 


\section{LARGE-SCALE COHERENT MOTION}

time $t$ potential $\Phi(x, y, z, t) \quad$ free surface $z^{*}(x, y, t)$

$$
\Delta \Phi=0,\left.\quad \nabla \Phi\right|_{z=+\infty}=0
$$$$
\frac{\partial z^{*}}{\partial t}+\nabla z^{*} \nabla \Phi-\left.\frac{\partial \Phi}{\partial z}\right|_{z=z^{*}}=0,\left.\frac{\partial \Phi}{\partial t}\right|_{z=z^{*}}+\frac{1}{2}(\nabla \Phi)^{2}+\left.g(t) z\right|_{z=z^{*}}=0
$$$$
g \geq 0 \text { - instability: RT } \quad g>0 \quad \text { RM } g=0
$$

Initial conditions: $\quad z^{*}\left(x, y, t_{0}\right) \quad \boldsymbol{v}\left(x, y, t_{0}\right)$

length scale

$$
\lambda\left(\sim \lambda_{\max }\right) \quad \text { time scale } \tau \sim \lambda /\left|v_{0}\right|
$$

Symmetry: periodic, symmorphic + inversion in the plane $(x, y)$

\section{difficulty SINGULARITY interplay of harmonics}

2D RTI: Taylor ${ }^{1950}$, Fermi ${ }^{1952}$, Layzer $^{1955}$, Garabedian $^{1957}$,

Birkgoff $^{1957}$, Zuffiria ${ }^{1986}$, Inogamov ${ }^{1990}$, Tanveer ${ }^{1993}$, Hazak ${ }^{1997}$

3D RTI: Abarzhi ${ }^{1995}$

LOCAL EXPANSIONS

ASYMPTOTIC SOLUTIONS

2D \& 3D RMI: Shvarts ${ }^{1995}$, Inogamov ${ }^{1995}$, Mikaelian ${ }^{1998}$, Zhang $^{1998}$, Abarzhi ${ }^{2000}$

Layzer-type approach single-mode approximation 
- Expansion in terms of orthogonal functions:

$$
\Phi=\sum_{n=1}^{\infty} \Phi_{n}(t)\left(\frac{1}{\gamma_{n}} \exp \left(-\gamma_{n}\left(z-z_{0}(t)\right)+i \sum_{j} \boldsymbol{k}_{j} \boldsymbol{r} n_{j}\right)+c . c\right)
$$

irreducible representations of group $\boldsymbol{G}$ wave-vectors $\boldsymbol{k}, \boldsymbol{G}_{\boldsymbol{k}}$ project operators

Fourier expansion

- Local expansion at a highly symmetric point of the interface $x \approx 0, y \approx 0, z \approx z_{0}(t)$ :

$z^{*}(x, y, t)=z_{0}(t)+\sum_{i+j=1}^{\infty} \zeta_{i j}(t) x^{2 i} y^{2 j}, \quad N=i+j=1,2, \ldots \infty$

\section{Dynamical system of ordinary differential equations}

$$
\begin{gathered}
\sum_{i+j=1}^{\infty} D_{i j}(\dot{M}, M, \zeta) x^{2 i} y^{2 j}=0 \quad \sum_{i+j=1}^{\infty} K_{i j}(\dot{\zeta}, M, \zeta) x^{2 i} y^{2 j}=0 \\
\zeta=\left\{\zeta_{i j}\right\} ; \quad M=\left\{M_{n}\right\} \text { moments } M_{n}=\sum_{m=1}^{\infty} \Phi_{m}(k m)^{n}
\end{gathered}
$$

- Local dynamics, any time $t$; the length scale(s) $\lambda$ is invariable

- Multiple harmonics presentation

- 3D flows with general type of symmetry and 2D flows

- Desired accuracy, $x \approx 0, y \approx 0, z \approx z_{0}(t), N=i+j=1,2, \ldots \infty$ 


\section{REGULAR ASYMPTOTIC SOLUTIONS}

$\sum_{i+j=1}^{\infty} D_{i j}(\dot{M}, M, \zeta) x^{2 i} y^{2 j}=0 \quad \sum_{i+j=1}^{\infty} K_{i j}(\dot{\zeta}, M, \zeta) x^{2 i} y^{2 j}=0$

regular asymptotic solutions

$t / \tau>>1$

Richtmyer-Meshkov bubble:

$v(t) \sim \lambda / t, \zeta(t) \sim 1 / \lambda$

Layzer-type expansion: regular asymptotic solutions are absent in general case

- non-linearity is non-local

- singularities determine the interplay of harmonics

At a fixed length scale(s) $\lambda$, shape of the regular bubble is free and is parameterised by the principal curvature(s) number of the parameters $N_{p}$ symmetry of the 3D (2D) flow

$$
N_{p} \leq 3
$$

2D pm11, 3D p4mm, p6mm $N_{p}=1$

$3 \mathrm{D} \mathrm{p} 2 \mathrm{~mm} N_{p}=2$

$\checkmark$ to capture the interplay of harmonics

$\checkmark$ to show existence and convergence for solutions in the family

$\checkmark$ to involve all bubbles allowed by symmetry of the flow

$\checkmark$ to choose the physically dominant (i.e. the fastest stable) solution 


\section{RICHTMYER-MESHKOV bubbles}

Curvature radius $R \quad\left(\right.$ radii $\left.R_{x, y}\right) \quad k R_{c r} \leq k R \leq \infty$

Velocity $v=L(k, R) / t \quad$ surface variables $\zeta_{n}=\zeta_{n}(k, R)$

Fourier amplitudes $\Phi_{n}=\varphi_{n}(k, R) / t$

$$
\left|\Phi_{n} / \Phi_{1}\right| \sim \exp (-p n)
$$

Asymptotic stability $v-L(k, R) / t \sim t^{\beta-1}, \zeta_{n}(t)-\zeta_{n}(k, R) \sim t^{\beta}$

$$
\beta=\beta(k R) \quad \text { for stable solutions } \operatorname{Re}[\beta]<0
$$

\section{Properties}

1. The physically dominant solution in the family corresponds to a bubble with a flattened surface, $k R \rightarrow \infty$

2. The bubble flattens in time as $k R \sim(t / \tau)^{\left|\beta_{\infty}\right|}$

3. For highly symmetric 3D flows: $k R_{3 D} \sim(t / \tau)^{\left|\beta_{\infty}\right|}, v_{3 D} \sim 4 / k t$

4. The local dynamics of 3D highly symmetric flows is universal; near-circular contour $z^{*} \sim \zeta_{1}\left(x^{2}+y^{2}\right)$

5. 3D anisotropic bubbles tend to conserve a near-circular contour

6. 3D anisotropic bubbles are unstable

7. 3D Layzer-type "square" solution is the point of bifurcation

8. The dimensional crossover is discontinuous, $\beta_{3 D-2 D}>0$

9. NO 2D flows 


\section{Family of regular asymptotic solutions in RMI}

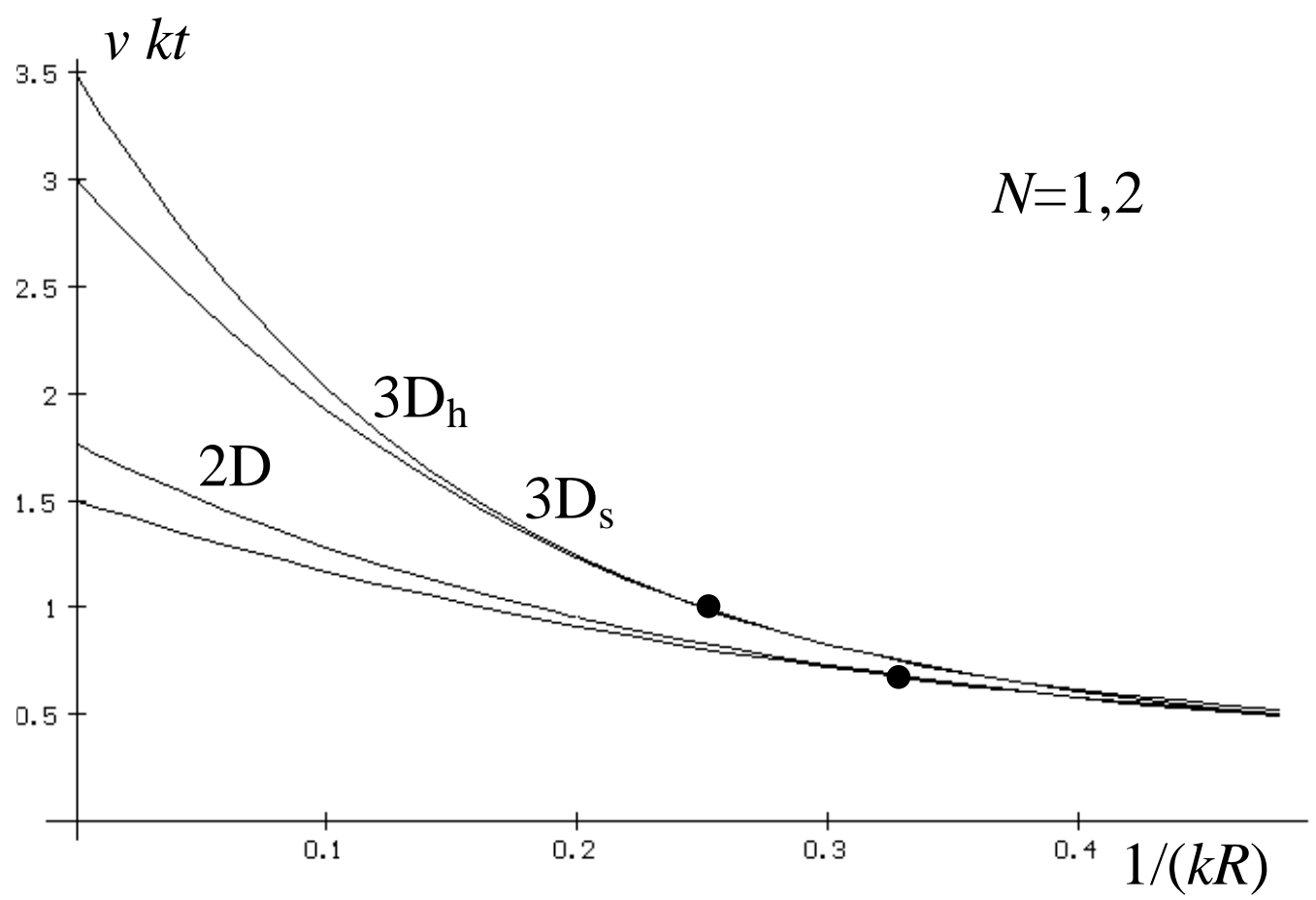

Velocity $v$ as the function on the radius of curvature $R$ Three-dimensional flows with hexagonal $\left(3 \mathrm{D}_{\mathrm{h}}\right)$ and square $\left(3 \mathrm{D}_{\mathrm{s}}\right)$ symmetry and two-dimensional flow (2D); $k$ is the wave-vector, $t$ is time, $N$ is order of approximation. Black circles mark the Layzer-type solutions with $R_{L}=4 / k, v_{L}=1 / k t$ in $3 \mathrm{D}$ and $R_{L}=3 / k, v_{L}=2 / 3 k t$ in $2 \mathrm{D}$. 
Family of regular asymptotic solutions in RMI

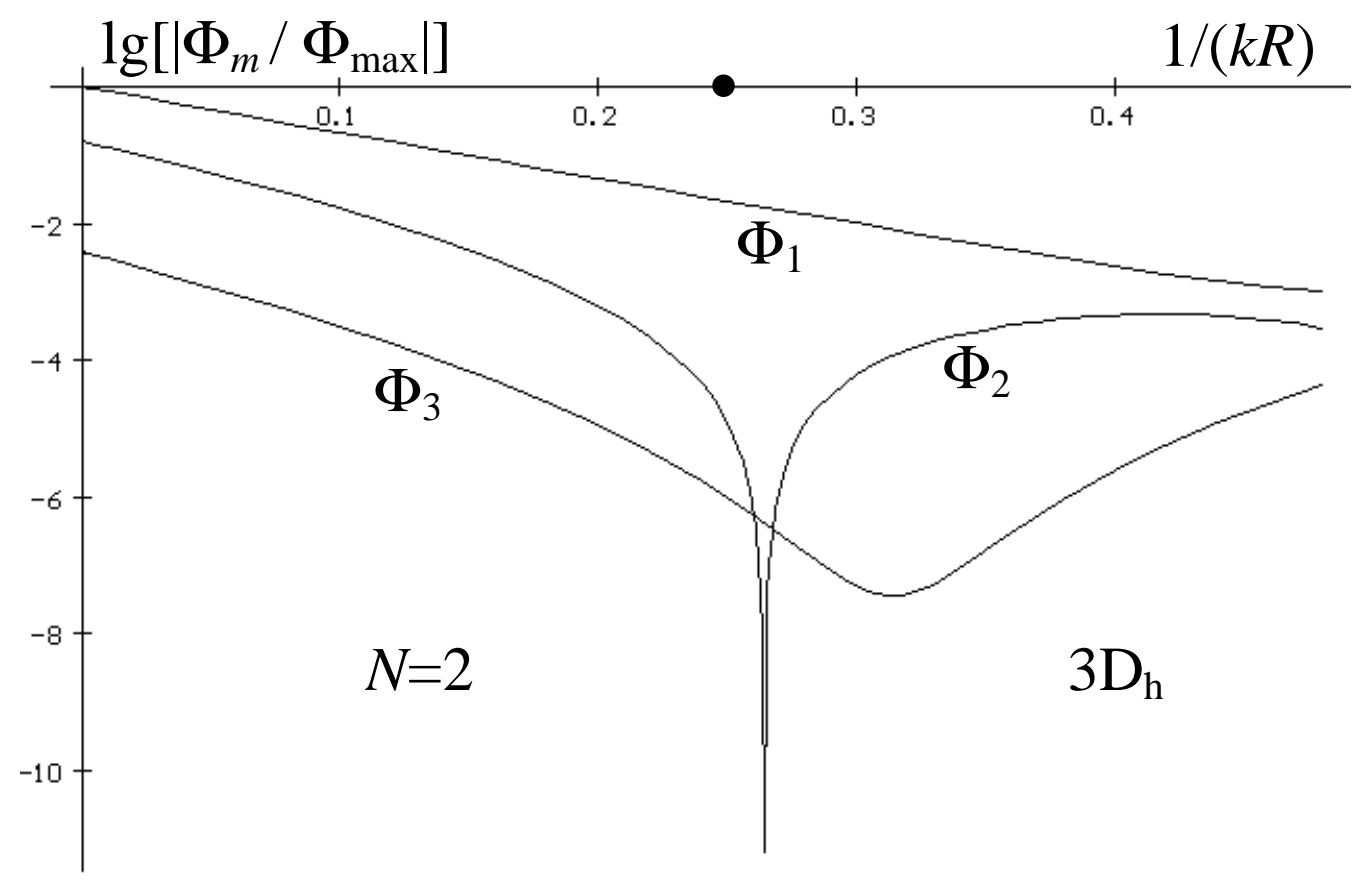

Exponential decay of the Fourier-amplitudes with an increase in their number.

Three-dimensional flows with hexagonal symmetry p6mm $\left(3 \mathrm{D}_{\mathrm{h}}\right)$; $\Phi_{\max }=\Phi_{1}(k R \equiv \infty)$; black circle corresponds to the Layzer-type bubble. 
Family of regular asymptotic solutions in RMI

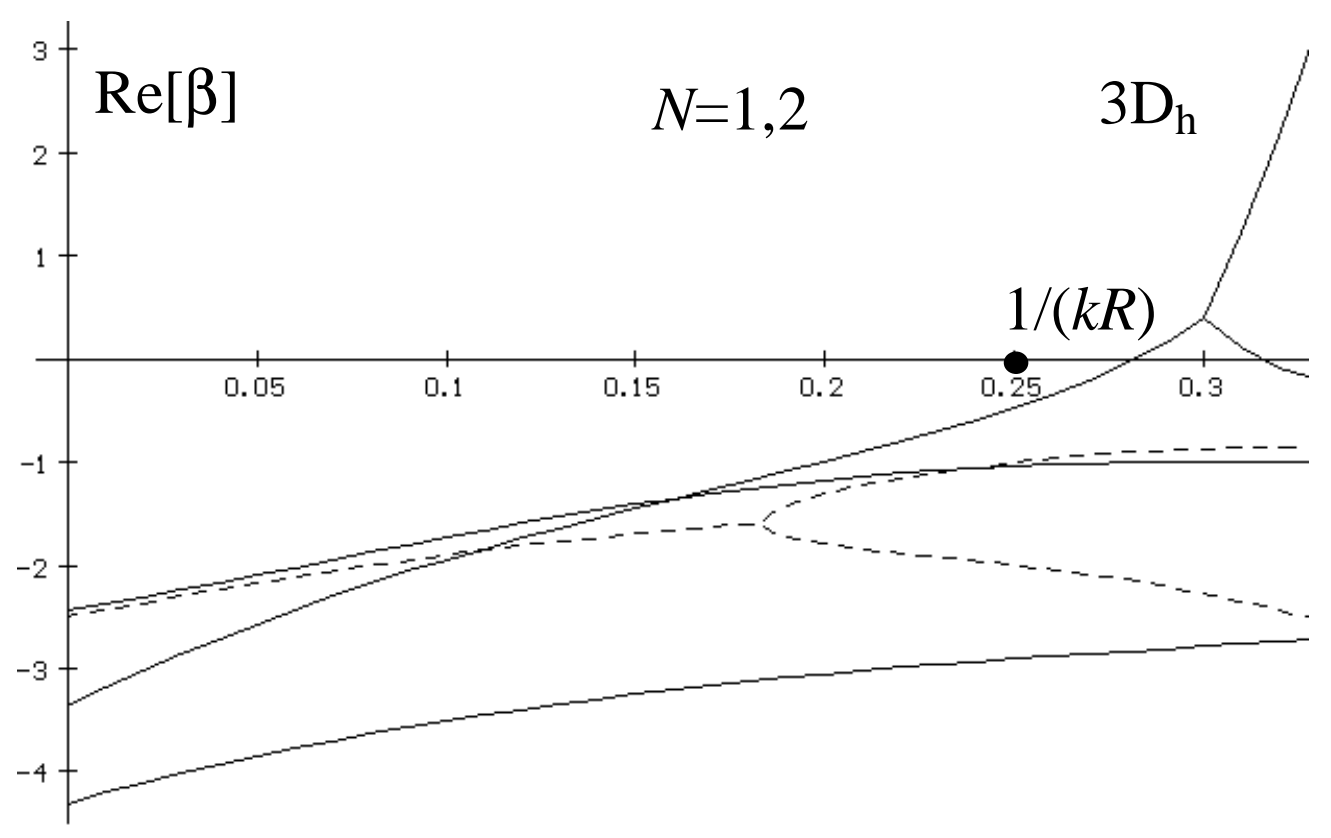

Stability analysis for the family of regular asymptotic solutions Real parts of exponents $\beta$ as functions on the radius of curvature $R$ Dashed lines correspond to $N=1$, solid lines - to $N=2$, black circle corresponds to the Layzer-type solution. 


\title{
Evolution of the bubble front in RMI Highly symmetric 3D and 2D coherent structures
}

\author{
time scale $\tau \sim 1 / v_{0} k$ \\ $t<<\tau$ : curvature $\zeta_{1}(t) \sim-k t / \tau, \quad$ velocity $v(t)-v_{0} \sim v_{0} t / \tau$ \\ $t \sim \tau:$ curvature $\zeta_{1}(t) \sim-k$ \\ $t>>\tau$ : curvature $\zeta_{1}(t) \sim-k(t / \tau)^{-\left|\beta_{\infty}\right|}$, velocity $v(t) \sim C_{\infty} / k t$
}

\section{Dynamic trajectories}

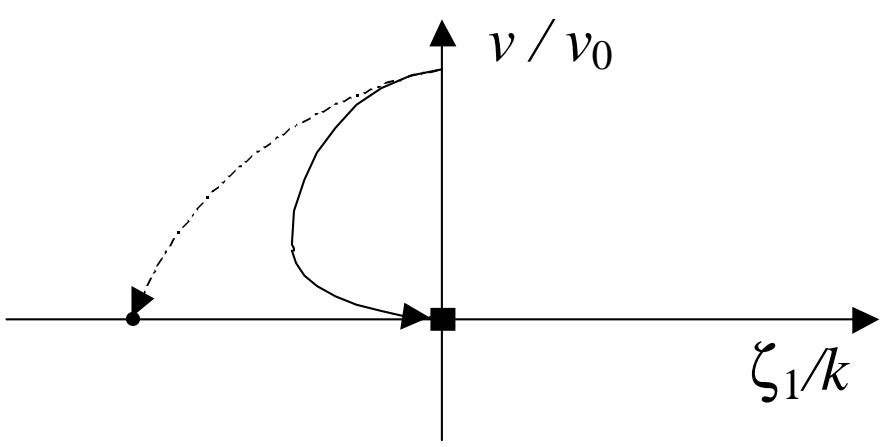

Solid line corresponds to multiple harmonic solution, and black square - to the flattened bubble. Dashed line corresponds to Layzer-type single-mode solution, and black circle - to the Layzertype bubble. 


\section{Evolution of the bubble front in RMI}

\section{RM bubbles flatten $\quad$ RM bubbles decelerate}

- Qualitative agreement with experiments

- Bubble velocity $v_{\infty} \sim C_{\infty} / k t \quad v_{L} \sim C_{L} / k t$

$$
C_{\infty} / C_{L} \sim 3-4 \quad \Delta h \sim C \ln (t / \tau)
$$

- Bubble shape $\zeta_{1}(t) \sim-k(t / \tau)^{-\left|\beta_{\infty}\right|} \quad$ reliable parameter

- Existence of an exact analytical solution

- a rigid body curvature $\sim 1 / R \quad$ drag force $=\rho v^{2} R^{2}$

\section{For a two-fluid system, Atwood number $<1$}

- the Layzer-type approach requires MASS FLUX through the interface

- Flattened RM bubble is a multiple-harmonic solution with NO MASS FLUX through the interface 
Family of regular asymptotic solutions in RMI

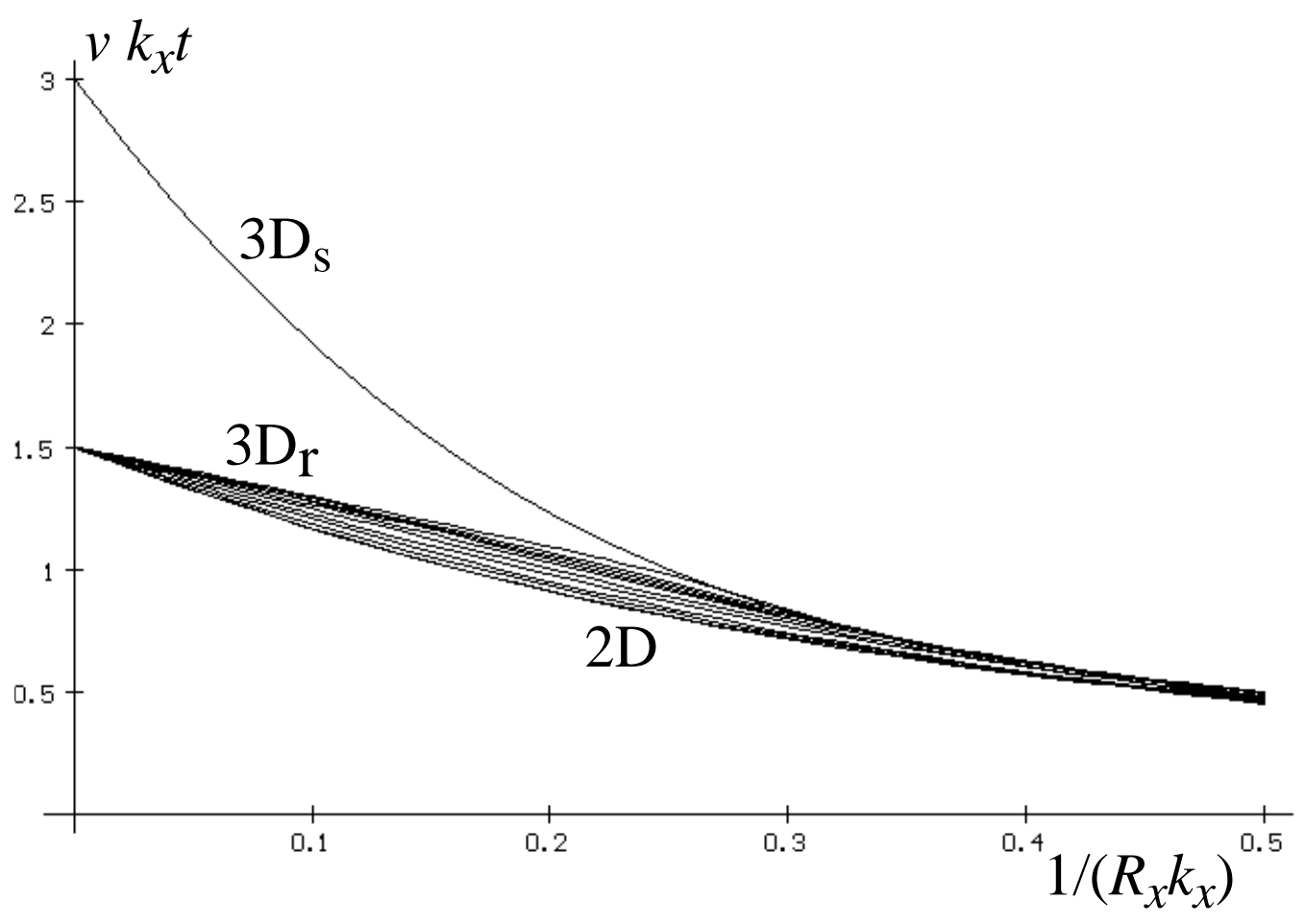

Dependence of velocity $v=L\left(R_{x, y}, k_{x, y}\right) / t$ on the bubble shape

Low-symmetric bubbles with rectangular symmetry $3 \mathrm{D}_{\mathrm{r}}$, twoparameter family; various values of the aspect ratio; the highest curve $3 \mathrm{D}_{\mathrm{s}}$ is the family of solutions for $3 \mathrm{D}$ square bubbles with $R_{y}=R_{x}$ and $k_{x} / k_{y}=1$; the lowest curve $2 \mathrm{D}$ is the family of solutions for 2D bubbles flat in the $y$-direction with $R_{y} \equiv \infty$ 
Family of regular asymptotic solutions in RMI

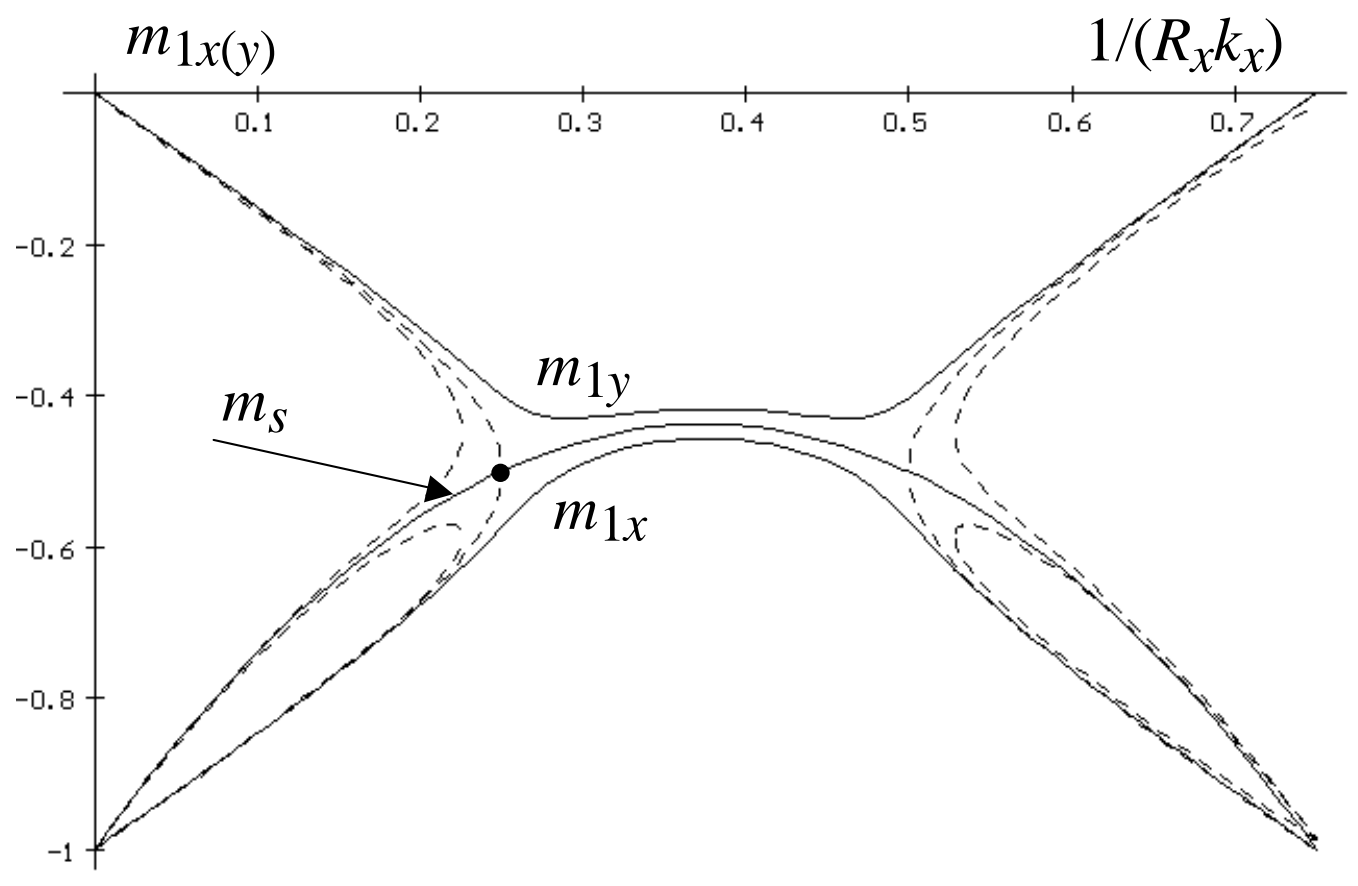

Bifurcation of the Layzer-type square solution (black point) for nearly symmetric flows with $k_{x} \sim k_{y}$ and $R_{x} \sim R_{y}$ 
Family of regular asymptotic solutions in RMI

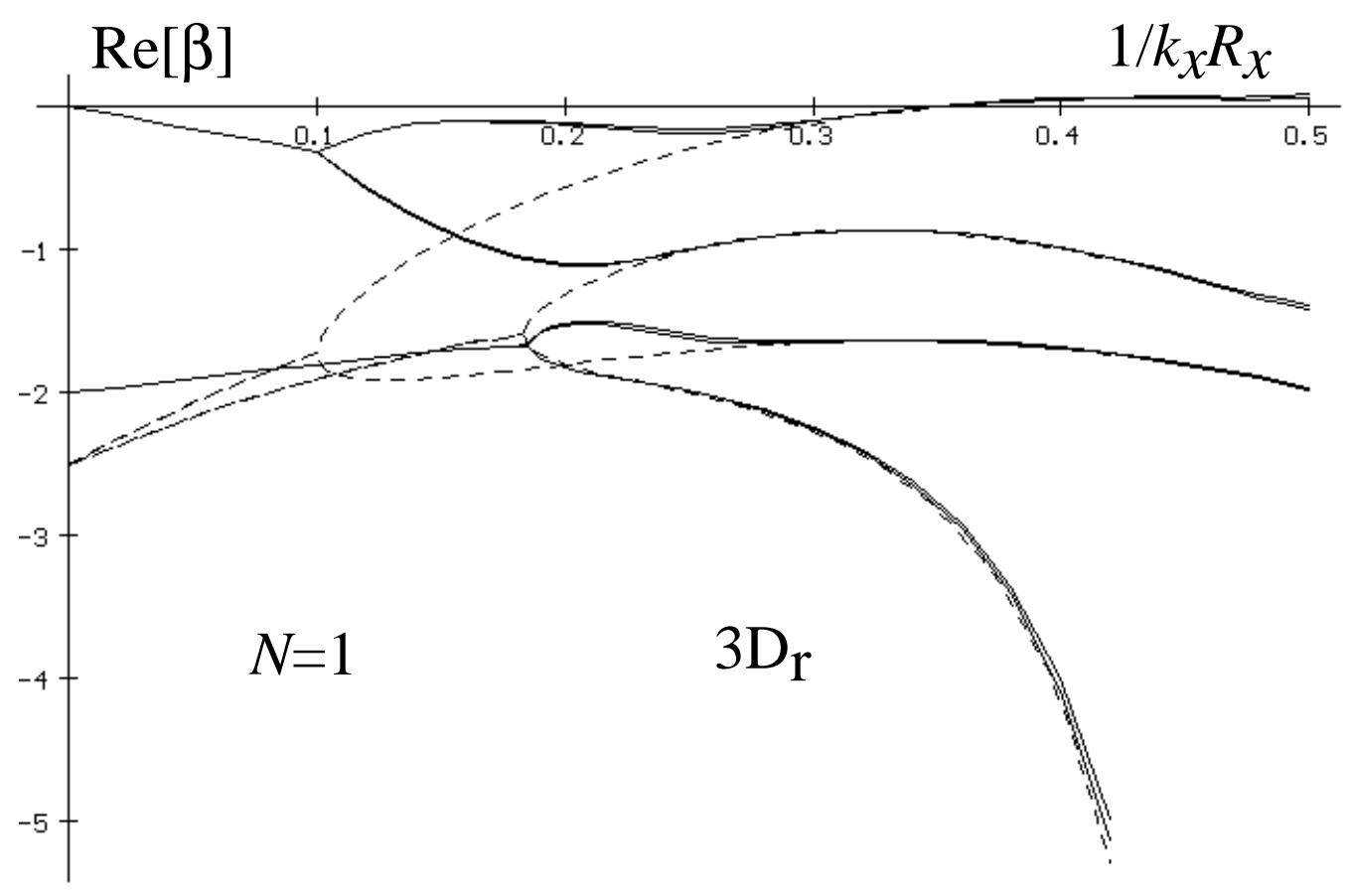

Stability analysis for low-symmetric RM bubbles Dashing lines corresponds to highly symmetric 3D square solutions with $k_{x}=k_{y}$ and $R_{x}=R_{y}$. Solid lines correspond to nearly symmetric solutions with $k_{x} \sim k_{y}$ and $R_{x} \sim R_{y}$. Nonsymmetric solutions are unstable. 


\section{Evolution of the bubble front in RMI}

\section{Low-symmetric 3D coherent structures}

The dimensional 3D-2D crossover

2D bubbles under 3D modulations

$$
\begin{array}{ccc} 
& \text { time scale } \tau \sim 1 /\left|v_{0}\right| k_{x} \\
t<<\tau & \zeta_{1 x}(t) \sim-k_{x} t / \tau & \zeta_{1 y} \sim-k_{y} t / \tau \\
t>> & \zeta_{1 x}(t) \sim-k_{x}(t / \tau)^{-\left|\beta_{\infty}\right|} & \zeta_{1 y} \sim-k_{x}(t / \tau)^{\beta_{3 D-2 D} \mid}
\end{array}
$$

the dimensional crossover is discontinuous, $\beta_{3 D-2 D}>0$

\section{Secondary instabilities}

- Secondary instabilities in RMI are "slow" in contrast to RTI

\section{SINGULAR ASYMPTOTIC SOLUTIONS}

Richtmyer-Meshkov spikes small-scale structure dynamics

Singular asymptotic solutions to dynamical system

$$
\text { Zhang }^{1998}, \text { Abarzhi }^{2000}
$$

$t>>$ : shape $z^{*}(t) \sim \sum_{n=1} C_{n}\left(\exp (t / \tau) r^{2}\right)^{n}$, velocity $v(t) \sim-v_{0}$

- Tanveer 1993, Baker and Meiron 1989, Pullin ${ }^{2001} \ldots$

- For a two-fluid system, Atwood number < 1, the singular asymptotic solutions requires mass flux through the interface 


\section{Conclusion}

$\checkmark$ Large-scale coherent motion in RMI

$\checkmark$ Separation of scales active regions passive regions

$\checkmark$ Group symmetry large-scale coherent motion

$\checkmark$ Local dynamics of regular bubbles and singular spikes

$\checkmark$ Consideration of 3D flows with general type of symmetry

$\checkmark$ Singularity - interplay of harmonics - shape of the bubble

$\checkmark$ Family of regular asymptotic solutions - symmetry of the flow

$\checkmark$ The physically dominant solution in the family

$\checkmark$ Multiple harmonic solution

$\checkmark$ Universality of local dynamics for 3D highly symmetric flows

$\checkmark$ Conservation of a near-circular contour of 3D bubbles

$\checkmark$ Discontinuous 3D-2D dimensional crossover

$\checkmark$ Singular asymptotes

$\checkmark$ Comparison between the local dynamics in RTI and RMI

$\checkmark$ Different types of the bubble front evolution in RTI and RMI

$\checkmark$ Layzer-type bubbles in RTI and RMI

$\checkmark$ New type of the evolution of the bubble front in RMI

$\checkmark$ Integral (velocity) and internal (shape) diagnostic parameters

$\checkmark$ Theory works effectively for a two-fluid system

\section{Discussion}

$? ? ?$

turbulent mixing in RMI and RTI 
Nonlinear asymptotic solutions to Rayleigh-Taylor and

\section{Richtmyer-Meshkov problems for fluids with a finite density contrast}

S. Abarzhi, J. Glimm, A. der Lin

Department of Applied Mathematics and Statistics

State University of New York at Stony Brook, USA 
INSTABILITY of the interface

TURBULENT MIXING of the fluids

Rayleigh-Taylor instability

Richtmyer-Meshkov instability sustained acceleration (gravity)

impulsive acceleration (shock)

\section{Fundamental issues:}

- the cascades of energy

- the dynamics of the large-scale coherent structure

Coherent structure: a spatially periodic array of bubble and spikes basic scales: period $\lambda$, gravity $g$ (RTI), initial velocity $v_{0}$ (RMI) time scale $\quad \tau \sim \sqrt{\lambda / A g}$ (RTI) $\quad \tau \sim \lambda / A\left|v_{0}\right|$ (RMI)

$$
\text { Atwood number } A=\left(\rho_{h}-\rho_{l}\right) /\left(\rho_{h}+\rho_{l}\right)
$$

density ratio is a determining physical factor in RTI/RMI dynamics

Heuristic models
RTI, traditional approach
$g \rightarrow A g \quad$ Sharp 1984
RMI, buoyancy-drag model
$g \rightarrow(2 A /(1+A)) g$ for bubbles
Shvarts 1995
$g \rightarrow(2 A /(1-A)) g$ for spikes

more formal theoretical approach and a systematic study 
INTERFACE active regions small scales intensive vorticity passive regions large scales simply advected

time $t \quad$ coordinates $(x, y, z) \quad$ free surface $z^{*}(x, y, t)$
$z^{*}(x, y, t)<z<+\infty \quad$ density $\rho=\rho_{h} \quad$ velocity $\boldsymbol{v}=\boldsymbol{v}_{h}$
$z^{*}(x, y, t)>z>-\infty \quad$ density $\rho=\rho_{l} \quad$ velocity $\boldsymbol{v}=\boldsymbol{v}_{l}$
scalar function $\quad \theta(x, y, z, t)=z^{*}(x, y, t)-z$

Conservation laws:

$$
\nabla \cdot v=0
$$

momentum $\left.\left(\dot{\boldsymbol{v}}_{h}+\left(\boldsymbol{v}_{h} \nabla\right) \boldsymbol{v}_{h}-\boldsymbol{g}\right) \rho_{h}\right|_{\theta=0}=\left.\left(\dot{\boldsymbol{v}}_{l}+\left(\boldsymbol{v}_{l} \nabla\right) \boldsymbol{v}_{l}-\boldsymbol{g}\right) \rho_{l}\right|_{\theta=0}$

mass

$$
\left.\left(\dot{\theta}+v_{h} \nabla \theta\right) \rho_{h}\right|_{\theta=0}=\left.\left(\dot{\theta}+v_{l} \nabla \theta\right) \rho_{l}\right|_{\theta=0}
$$

no mass flux $\left.\quad\left(\dot{\theta}+v_{h} \nabla \theta\right) \rho_{h}\right|_{\theta=0}=\left.\left(\dot{\theta}+v_{l} \nabla \theta\right) \rho_{l}\right|_{\theta=0}=0$

boundary conditions

$$
\left.\boldsymbol{v}_{h}\right|_{z=+\infty}=\left.\boldsymbol{v}_{l}\right|_{z=-\infty}=0
$$

$g \geq 0$ - instability:

RTI $g>0$,

RMI $g=0$ 
$0<A \leq 1$ no significant energy cascade potential approximation

$$
\boldsymbol{v}_{h(l)}=\nabla \Phi_{h(l)}
$$

- Fourier expansion

$$
\begin{aligned}
& \Phi=\sum_{n=0}^{\infty} \Phi_{n}(t)\left(\frac{1}{\gamma_{n}} \exp \left(-\gamma_{n}\left(z-z_{0}(t)\right)+i \sum_{j} \boldsymbol{k}_{j} \boldsymbol{r} n_{j}\right)+c . c\right) \\
& \Phi_{h} \Rightarrow\left\{\Phi_{n}\right\}, \quad \Phi_{l} \Rightarrow\left\{\tilde{\Phi}_{n}\right\}, \quad \boldsymbol{r}=(x, y), \quad \boldsymbol{k} \text {-wave-vectors }
\end{aligned}
$$

- Spatial expansion at a highly symmetric point of the interface

$$
x \approx 0, y \approx 0, z \approx z_{0}(t) \quad z^{*}(x, y, t)-z_{0}(t)=\sum_{i+j=N=1}^{\infty} \zeta_{i j}(t) x^{2 i} y^{2 j}
$$

Conservation laws

$$
x^{2 i} y^{2 j} \quad i+j=N=1,2, \ldots \infty
$$

are reduced to dynamical system of ODE in terms of

$$
\text { surface variables } \quad \xi_{i j}(t) \quad \text { and }
$$

$$
\begin{gathered}
\text { moments } M_{n}(t)=\sum_{m=0}^{\infty} \Phi_{m}(t)(k m)^{n}, \tilde{M}_{n}(t)=\sum_{m=0}^{\infty} \widetilde{\Phi}_{m}(t)(k m)^{n} \\
\text { with } v=\partial z_{0} / \partial t=-M_{0}=\tilde{M}_{0}
\end{gathered}
$$

- Local dynamics at any time $t$; the length scale $\lambda$ is unchanged

- Multiple harmonics analysis, $m=0,1,2, \ldots \infty$

- Desired accuracy, $i+j=N=1,2, \ldots \infty$

- 3D flows with general type of symmetry and 2D flows 


\section{D Rayleigh-Taylor and Richtmyer-Meshkov instabilities}

$$
\begin{aligned}
& N=1: \quad z^{*}-z_{0} \approx \zeta_{1}(t) x^{2} \quad \text { principal curvature } \zeta_{1}(t) \\
& \left(\dot{M}_{1} / 2+\zeta_{1} \dot{M}_{0}-M_{1}^{2} / 2+\zeta_{1} g\right) \rho_{h}=\left(\dot{\tilde{M}}_{1} / 2-\zeta_{1} \dot{\tilde{M}}_{0}-\tilde{M}_{1}^{2} / 2+\zeta_{1} g\right) \rho_{l} \\
& \left(\dot{\zeta}_{1}-3 \zeta_{1} M_{1}-M_{2} / 2\right) \rho_{h}=\left(\dot{\zeta}_{1}-3 \zeta_{1} \tilde{M}_{1}+\tilde{M}_{2} / 2\right) \rho_{l} \\
& \text { no mass flux: } \quad\left(\dot{\zeta}_{1}-3 \zeta_{1} M_{1}-M_{2} / 2\right) \rho_{h}=\left(\dot{\zeta}_{1}-3 \zeta_{1} \tilde{M}_{1}+\tilde{M}_{2} / 2\right) \rho_{l}=0
\end{aligned}
$$

\section{Layzer-type expansion amplitudes $\Phi_{1}$ and $\widetilde{\Phi}_{1}$}

\section{Regular asymptotic solutions}

$$
\text { A=1 Layzer } 1955
$$

\section{Rayleigh-Taylor bubbles}

$t<<\tau$

$t>\tau$

re-scaling

$$
\begin{aligned}
& v, \zeta_{1} \sim \exp (t / \tau) \\
& \zeta_{1}=\zeta_{L}=-A k / 6
\end{aligned}
$$$$
\text { time scale } \tau=1 / \sqrt{A g k}
$$

$$
v_{L}=\sqrt{A g / 3 k}
$$


Richtmyer-Meshkov bubbles
$t<<\tau$
$\zeta_{1}=-(k / A)(t / \tau)$
$v-v_{0}=-v_{0}(t / \tau)$
$t>\tau$
$\zeta_{1}=\zeta_{L}=-A k / 6$
$v_{L}=\left(1-A^{2} / 3\right) / A k t$

Singular asymptotic solutions

$A=1$

Zhang 1998, Abarzhi 2000

Rayleigh-Taylor spikes time scale $\tau=1 / \sqrt{A g k}$

$t<\tau \quad v, \zeta_{1} \sim \exp (t / \tau)$

$t>>\quad \zeta_{1} \approx k \exp \left((3 / 2)(t / A \tau)^{2}\right) \quad v \approx-g t$

Richtmyer-Meshkov spikes time scale $\tau=1 / A k v_{0}$

$$
\begin{array}{lll}
t<\tau & \zeta_{1}=-(k / A)(t / \tau) & v-v_{0}=-v_{0}(t / \tau) \\
t>\tau & \zeta_{1} \approx k \exp \left(C\left(t / \tau A^{2}\right)\right) & v-C v_{0} \approx \exp \left(C\left(t / \tau A^{2}\right)\right)
\end{array}
$$

finite - time singularities:

Baker, Meiron 1980s, Moore 1980s, Tanveer 1990s

!!!

Layzer-type expansion requires

MASS FLUX through the interface 


\section{NON-LINEAR REGULAR ASYMPTOTIC SOLUTION \\ NO MASS FLUX through the INTERFACE}

1. Non-linearity is non-local

2. Interplay of harmonics bubble shape singularities

3. Multiple harmonics analysis

4. The bubble shape is free principal curvature

5. Family of regular asymptotic solutions with no mass flux through the interface

6. The fastest solution in the Family physically dominant

7. Family of asymptotic solutions at $A=1$ in 2D RTI (Garabedian) and 3D RTI and 3D/2D RMI (Abarzhi)

\section{Family of regular asymptotic solutions}

Rayleigh-Taylor bubbles, 3D/2D:

$t>\tau$

$$
v=v\left(\zeta_{1}, A\right)
$$

the fastest solution in the family $\zeta_{1}=\zeta_{A}$

$v=v_{A}$

Richtmyer-Meshkov bubbles, 3D/2D

$t \gg \tau$

$$
v=v\left(\zeta_{1}, A, t\right)=L\left(\zeta_{1}, A\right) / t
$$

the fastest solution in the family $\zeta_{1}=\zeta_{A}$

$$
v=v_{A}=L_{A} / t
$$

!!! lowest-order harmonics $\quad \Phi_{1}, \widetilde{\Phi}_{1} \quad$ are dominant 
2D Rayleigh-Taylor bubble

$$
\begin{aligned}
& t>\tau \quad \zeta_{A} \quad v_{A} \\
& A \approx 1, \zeta_{A} \approx-(k / 6)(1-(1-A) / 8), v_{A} \approx \sqrt{g / 3 k}(1-3(1-A) / 16) \\
& A \approx 0, \zeta_{A} \approx-(k / 2) A^{1 / 3}, \quad v_{A} \approx(3 / 2)^{3 / 2} \sqrt{A g / 3 k}
\end{aligned}
$$

!!! $\quad$ For $0<A \leq 1$, velocity $v_{A}$ is quite close (10-15\%) to $v_{L}=\sqrt{A g / 3 k} \quad$ traditional empirical approach $v_{D}=\sqrt{2 A /(1+A)} \sqrt{g / 3 k} \quad$ drag model

Bubble curvature is a more sensitive parameter

2D Richtmyer-Meshkov bubble
$t>\tau$
$\zeta_{A}=0$
$v_{A}=3 / 2 A k t$

!!! Agreement with multiple harmonic analysis at $A=1$ (S.A. 2000)

!!! Qualitative agreement with experiments

RM bubbles decelerate

RM bubbles flatten

$$
\begin{gathered}
v_{A} / v_{L}=3 / 2\left(1-A^{2} / 3\right) \\
v_{A}, v_{L} \sim C / k t \quad \Delta h \sim C \ln (t / \tau)
\end{gathered}
$$

!!! Bubble curvature is a more sensitive parameter

!!! $A \rightarrow 0$ and $(t / \tau) \rightarrow \infty \quad v(k t)>>1$ 


\section{SIMULATIONS}

Front Tracking method

FronTier (Glimm, 1988)

- 2D compressible adiabatic Navier-Stokes equation

Euler equations augmented viscous forces and heat flux

- weakly compressible fluids

- contribution of viscous and thermal terms is small to yield a slightly stabilized but nearly inviscid calculations

mesh refinement: 80 x 800, $160 \times 1600, \quad 320 \times 3200$

$A<0.05$ slow evolution no satisfactorily late-time convergence

$A>0.85$ certain numerical restrictions late-time dynamics

$$
0.3<A<0.8
$$

\section{Nonlinear regime}

Bubble: terminal velocity accompanied by slight oscillations

oscillations: $\quad$ small amplitude $\quad$ sensitive to $A$

$A>0.7: \quad$ terminal velocity

Comparison

numerical data quasi-terminal regime for each $A$ averaged values deviations 3-8\% 


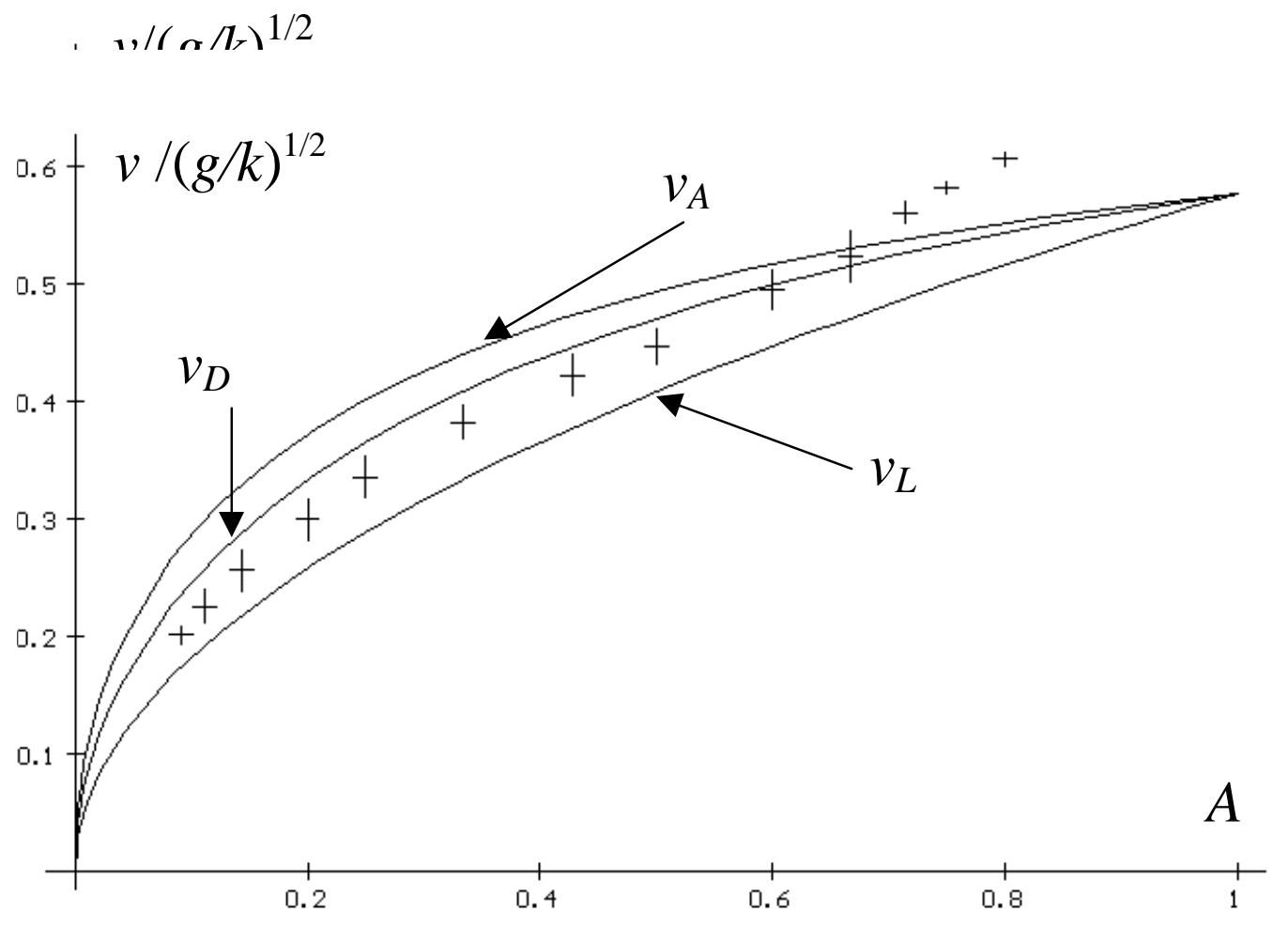

2D RTI

Dependence of the quasi-steady velocity on the Atwood number.

$v_{L}=\sqrt{A g / 3 k}$ is the velocity of the Layzer-type bubble,

$v_{D}=\sqrt{g / 3 k} \sqrt{2 A /(1+A)}$ corresponds to drag model, and

$v_{A}$ corresponds to nonlinear solution with no mass flux through the interface. 


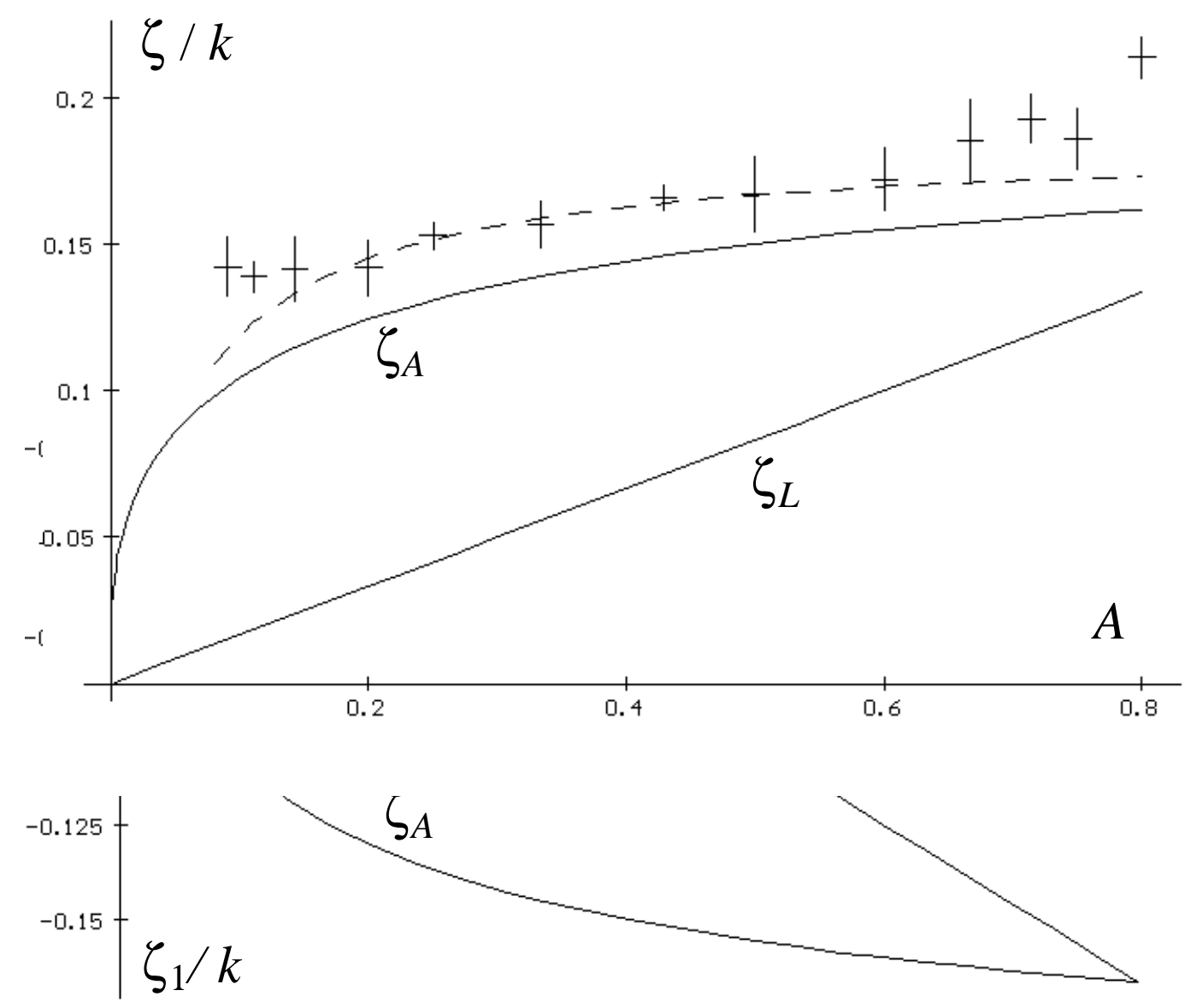

2D RTI:

Dependence of the curvature of the quasi-steady bubble on the Atwood number. The curvature of the Layzer-type bubble is $\zeta_{L}=-A k / 6 ;$ the curvature corresponding to the nonlinear solution with no mass flux is $\zeta_{A}$. 


\title{
LIMITATIONS:
}

NON-LINEAR SOLUTIONS are "QUASI-STEADY"

vorticity energy cascade time-dependence

$$
A \rightarrow 0 \quad \text { applicability of the theory }
$$

\author{
NON-LINEAR SINGULAR ASYMPTOTIC SOLUTIONS \\ $A \approx 1 \quad$ finite-time singularities \\ $A<1 \quad$ vorticity energy cascade \\ small-scale structures dispersive properties of the flow
}

\section{ADVANTAGES:}

3D Rayleigh-Taylor and Richtmyer-Meshkov instabilities for fluids with a finite density contrast

!!! CHAOTIC REGIME
RTI
width of the mixing zone
$h \approx \alpha A g t^{2}$
re-scaling $g \rightarrow A g$
mass flux
$\alpha=\alpha(A)$ 


\section{CONCLUSIONS}

1. Rayleigh-Taylor and Richtmyer-Meshkov instabilities for fluids with a finite density contrast in $3 \mathrm{D}$ and $2 \mathrm{D}$

2. Analytical solutions for the conservation laws

3. Layzer-type solution in RTI/RMI, re-scaling

4. Layzer-type approach requires mass flux through the interface

5. Approximate nonlinear solution with no mass flux

6. Parameters of the RT and RM bubbles

7. RT bubble is curved, RM bubble is flat

8. The bubble curvature is a more sensitive parameter than the bubble velocity

9. Good quantitative agreement between theory and simulations in RTI

10. Comparison with heuristic models

11. Limitations 


\title{
Turbulent mixing in RTI as order-disorder process
}

\author{
S.I. Abarzhi \\ Department of Applied Mathematics and Statistics \\ State University of New York at Stony Brook, USA
}


the misalignment of the PRESSURE and DENSITY gradients the INSTABILITY of the interface TURBULENT MIXING of the fluids
Rayleigh-Taylor instability
sustained acceleration (gravity)
Richtmyer-Meshkov instability impulsive acceleration (shock)

Basic Objective: $\quad$ reliable description of turbulent mixing

\section{Fundamental issues:}

- the cascades of energy

- the dynamics of the large-scale coherent structure

\section{DYNAMICS of the COHERENT STRUCTURE of BUBBLES and SPIKES in 3D RTI / RMI}

to PUT BACK a REGULARITY into the flow 
INTERFACE active regions

small scales

intensive vorticity

large-scale coherent motion

spectral approach passive regions

large scales

simply advected

scalar fields

group theory

\section{GROUP THEORY}

An array of bubbles and spikes periodic in the plane normal to the direction of gravity

- NO travelling waves $\sim e^{i w t}$, NO convection

- The flow dynamics in the direction of gravity is much faster than the evolution of large scales in the normal plane

Periodicity Equivalence of points and directions

17 plane crystallographic symmetry groups

translations in the plane + rotations + reflections

irreducible representations of crystallographic space groups $\boldsymbol{G}$ wave-vectors $\boldsymbol{k}$ in the reciprocal lattice with subgroups $\boldsymbol{G}_{\boldsymbol{k}}$ 


\section{A STRUCTURE is OBSERVABLE \\ Structure $\quad$ group $\boldsymbol{G} \quad$ wave-vector $\boldsymbol{K}$}

- A significant part of the fluid energy is concentrated in the coherent motion

A DOMINANT mode $\boldsymbol{K}$ governs the macroscopic dynamics

- The structure is stable under large-scale modulations

a scalar function of the macroscopic motion

$\boldsymbol{K}+\xi: \quad \varphi(\boldsymbol{K}+\xi) \approx \varphi(\boldsymbol{K})+F(\boldsymbol{K}) \xi^{2}, \quad \boldsymbol{K} \leftrightarrow-\boldsymbol{K}$

$\begin{array}{lll}\text { subgroup } & \boldsymbol{G}_{\boldsymbol{K}} & \text { inversion in the plane }\end{array}$

$G$ is a symmorphic group with inversion in the plane

3D: p6mm, p4mm, p2mm, cmm, p2

2D: pm11

- Rayleigh-Taylor instability: the structure is isotropic in the plane normal to the direction of gravity

$\begin{array}{lll}\text { 3D: } & \text { 2D } 6 \mathrm{~mm}, \mathrm{p} 4 \mathrm{~mm} & \mathrm{Dm} 11\end{array}$ 


\section{TURBULENT MIXING and GROUP THEORY}

inverse cascade transitions with the length scale growth

3D flow symmetry group $\boldsymbol{G}$ representations $\left\{\boldsymbol{k}, \boldsymbol{G}_{\boldsymbol{k}}\right\}$

Transitions between structures with various wave-vectors $\boldsymbol{k}$

Stability under large-scale modulations

$\boldsymbol{k}+\xi \quad \varphi(k+\xi) \approx \varphi(k)+f(\boldsymbol{k}) \xi^{2}, \quad \boldsymbol{k} \leftrightarrow-\boldsymbol{k}, \quad G_{\boldsymbol{k}}$

wave-vectors $\quad \boldsymbol{k} \quad$ subgroup $\boldsymbol{G}_{\boldsymbol{k}} \quad$ inversion in the plane

Lifshits ${ }^{1940} \quad$ order-disorder transitions

2D flow, $\boldsymbol{G}=$ pm11: $\quad \boldsymbol{k}=0, \boldsymbol{G}_{\boldsymbol{k}}=m, \quad$ length scale $\Lambda=\lambda$

$$
\boldsymbol{k}=1 / 2, \boldsymbol{G}_{\boldsymbol{k}}=m, \quad \text { length scale } \Lambda=2 \lambda
$$

2D: scale growing as doubling of the spatial period 
3D flow, $G=$ p6mm:

$\boldsymbol{k}=0, \boldsymbol{G}_{\boldsymbol{k}}=6 \mathrm{~mm} \quad$ length scale $\Lambda=\lambda \quad$ hexagonal p6mm

$\boldsymbol{k}=(1 / 2,0), \boldsymbol{G}_{\boldsymbol{k}}=2 \mathrm{~mm} \quad \vec{\Lambda}=(2 \lambda, \lambda) \quad$ rectangular $\mathrm{p} 2 \mathrm{~mm}$

$\boldsymbol{k}=(1 / 3,1 / 3), \boldsymbol{G}_{\boldsymbol{k}}=3 m \quad 2$ ray star $\quad$ triangular $\mathrm{p} 31 \mathrm{~m}$

$\underline{\text { 3D flow, } \boldsymbol{G}=\mathrm{p} 4 \mathrm{~mm}}$ :

$\boldsymbol{k}=0, \boldsymbol{G}_{\boldsymbol{k}}=4 \mathrm{~mm} \quad$ length scale $\Lambda=\lambda \quad$ square $\mathrm{p} 4 \mathrm{~mm}$

$\boldsymbol{k}=(1 / 2,0), \boldsymbol{G}_{\boldsymbol{k}}=2 \mathrm{~mm} \quad \vec{\Lambda}=(2 \lambda, \lambda) \quad$ rectangular $\mathrm{p} 2 \mathrm{~mm}$

$\boldsymbol{k}=(1 / 2,1 / 2), \boldsymbol{G}_{\boldsymbol{k}}=4 m m \vec{\Lambda}=(2 \lambda, 2 \lambda) \quad$ square $\mathrm{p} 4 \mathrm{~mm}$

4 bubble interaction

special modulation

3D: scale growing

symmetry lowering anisotropy

To keep isotropy of the RT flow, a balance is required

between the inverse and direct cascades merging and splitting

An internal coherent structure with hexagonal symmetry and with the length scale $\Lambda=O\left(\lambda_{\max }\right)$ 


\section{CONCLUSION}

\section{Group theory}

- formulation and general requirements

- structural stability order-disorder

- 3D flow with general type of spatial symmetry

- structural transitions

- isotropy, inverse and direct cascades

- internal coherent structure

??? Concept of self-similarity of turbulent mixing

??? Dynamical symmetry 


\title{
A New Turbulent Two-Fluid RANS Model for $\mathrm{KH}, \mathrm{RT}$ and RM Mixing Layers
}

\author{
Pascal BAILLY, Antoine LLOR \\ Commissariat à l'Energie Atomique \\ B.P.12 91680 Bruyères-le-Châtel Cedex
}

December 14th, 2001

\begin{abstract}
Our aim is to develop an accurate turbulent mixing model for combined RT, RM and $\mathrm{KH}$ types of instabilities, with arbitrarily variable accelerations.

Following the recent analysis of the RT and RM cases by G. Dimonte 2000, and of the self-similar variable acceleration RT flows (SSVARTs) by A. Llor in an other presentation to the present workshop, we have considered as crucial to capture the following physical aspects by using the corresponding model features:

- the directed transport by a two-fluid approach,

- the correct buoyancy force by including mass transfer between the fluids,

- the turbulence diffusion by including most of the standard k-e features,

- the geometrical aspects by consistent closures of the length scales.

Identifying the large scale transport structures and including the above physical aspects, we developped the TTT model (Two-structure Two-fluid Two-turbulence) whose specific and original features will be discussed. Preliminary 1D numerical results of the TTT model will be presented for self-similar RT flows.
\end{abstract}




\section{Introduction}

I.1 Aim and strategy

Our aim is to craft a RANS model applicable to:

- Kelvin-Helmoltz,

- Richtmyer-Meshkov,

- Rayleigh-Taylor,

- and SSVARTs (self-similar variable $g$ RT, see Llor)

and able to capture consistently:

- TMZ growth laws $(L(t))$,

- global energy balance $\left(K_{I}, K_{D}, K_{T}\right)$,

- and length scales

- (Von Kármán numbers $\left.K^{3 / 2} / E L, \mathrm{KH} \approx 0.63, \mathrm{RT} \approx 0.09\right)$.

Our strategy is to combine as much as possible the relevant features from simple and efficient existing models:

Turbulence dynamics (KH and RM) $\longleftrightarrow \quad k-\varepsilon$ model,

Directed transport (RT, SSVARTs) $\longleftrightarrow$ Two-fluid model

(Youngs 1989),

Buoyancy (RT, SSVARTs) $\longleftrightarrow$ Mass exchange

(Youngs 1995).

The model has to be a closure of RANS equations

to permit calibrations using measured correlations $\longrightarrow$ avoid a phenomenological length equation. 


\section{I.2 Starting point}

Young's two-fluid model (1989) as starting point:

- mass conservation, $\alpha^{ \pm} \rho^{ \pm}$,

- 2 eq. momentum conservation, $\alpha^{ \pm} \rho^{ \pm} U^{ \pm}$,

- 1 eq. turbulent kinetic energy, $K$,

- 1 eq. characteristic length scale, $\lambda$, with main closures:

- drag $\propto(\delta U)^{2} / \lambda$,

- turbulent integral length scale $\propto \lambda$,

- length scale production term $\propto\|\delta \vec{U}\|$.

Problems:

- no equation for dissipation, $\varepsilon$,

- no extension of turbulence to pure fluids,

- debatable closures and coefficients,

- $\lambda$ equation not RANS based,

- singular behavior of $\lambda$ at TMZ edges,

- overestimates Von Kármán number in RT by factor 6.5. 
I.3 Preliminary attempt, $k-\varepsilon-\Sigma$

Ideas:

- add $\varepsilon$ equation "as in $k-\varepsilon$ ",

- replace $\lambda$ by $\Sigma \approx \alpha^{+} \alpha^{-} / \lambda$, interfacial area density (RANS),

- add interaction between turbulent and geometrical lengths.

$\longrightarrow$ " $k-\varepsilon-\sum$ " model

(Vallet 2000)

Failed because of incompatible behavior at TMZ edges:

$\lambda_{i} \rightarrow 0$ for standard $k-\varepsilon$,

$\lambda_{d} \rightarrow$ Constant in usual two-fluid models.

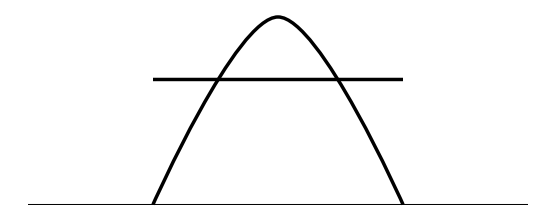

Schematic mean profiles of drag and integral length scales in typical TMZ.

However, raised the basic issues:

What are the transported/transporting structures in TMZ ?

What mechanisms control the length/drag of the structures?

How can length singularities be avoided at edges of TMZ? 


\section{The TTT model}

II.1 The transport structure concept

Large structures

$$
\begin{array}{r}
\approx \text { Large turbulent eddies } \approx \text { Bubbles } \approx \text { Plumes } \approx \ldots \\
\approx \text { Basic building block for transport and mixing. }
\end{array}
$$

Assumption holds because:

- separation of fluids inside large eddies cannot occur

$$
\text { (small size, large drag, high turbulence), }
$$

- experimentally, bubble sizes match Von Kármán number,

(Dimonte 2000...),

- in simulations, visual correlation of turbulence and density, (Dalziel 1999, Inogamov 2001...).

Solves length scale paradox at edges:

$\lambda_{i} / \lambda_{d} \rightarrow$ Constant in structures at edges, but $\left\langle\lambda_{i}\right\rangle \rightarrow 0$ because of intermittency (structures at edges enter laminar zones when TMZ grows).

Simple modeling should be accessible since plumes are appropriatly captured by adapted $k-\varepsilon$ models.

Averaging over the TMZ, modeling demands:

- two velocities (plume shearing),

- mass exchange (entrainment in plumes),

- two turbulence fields (intermittency between plumes). 


\section{II.2 Two-structure RANS equations}

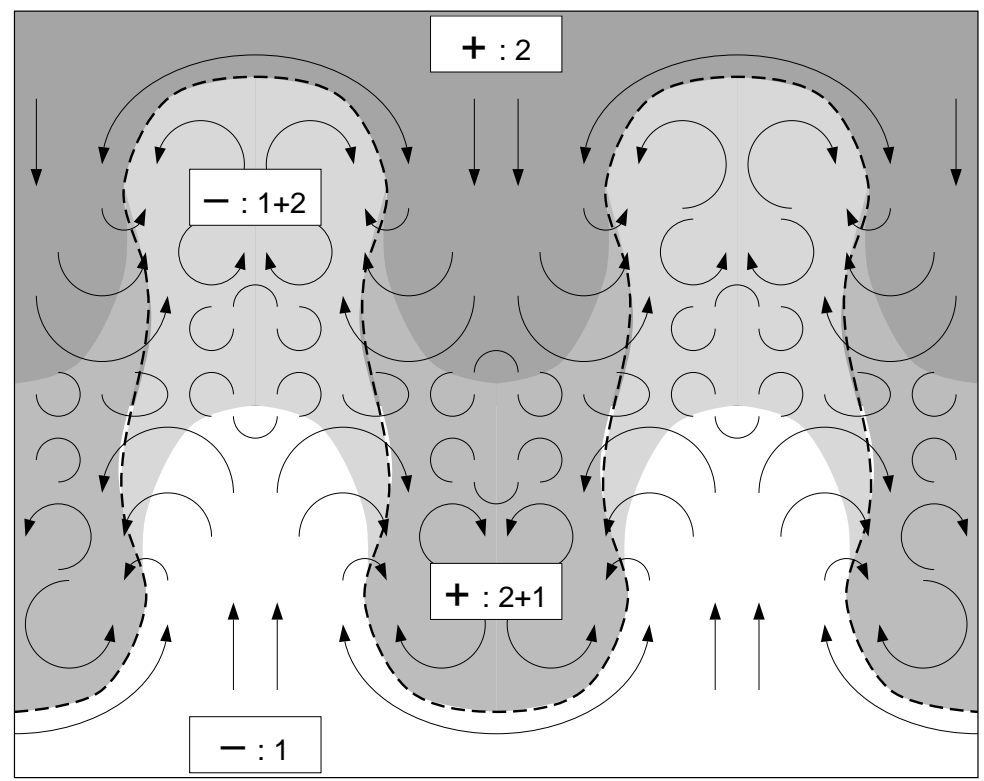

Schematic representation of the Two-structure, Two-fluid, Two-turbulent TMZ; dashed line: $b^{ \pm}$boundary; gray shading: $c^{1,2}$ levels.

Structures + and - defined by presence functions $b^{ \pm}(=0$ or 1$)$.

Structure evolution defined by velocity field $\vec{w}$ for $b^{ \pm}$:

$\frac{\partial}{\partial t} b^{ \pm}+w_{j} b_{, j}^{ \pm}=0$

Evolution equations for hydro quantities $a$ :

$\frac{\partial}{\partial t}(\rho a)+\left(\rho a u_{j}\right)_{, j}=s-\left(\theta_{j}\right)_{, j}$,

where $a=c^{1,2}$ (fluid mass fractions), $u_{i}, k, \varepsilon, e \ldots$

$c^{1}=b^{-}$and $c^{2}=b^{+}$in initial state (separate fluids). 
Conditional averaging by $b^{ \pm}$yields two-structure RANS :

$$
\begin{aligned}
\frac{\partial}{\partial t}\left(\alpha^{ \pm} \rho^{ \pm} A^{ \pm}\right)+\left(\alpha^{ \pm} \rho^{ \pm} A^{ \pm} U_{j}^{ \pm}\right. & , j \\
& =S^{ \pm}-\left(\Theta_{j}^{ \pm}\right)_{, j}-\left(\Phi_{j}^{ \pm}\right)_{, j} \mp X \mp \Psi
\end{aligned}
$$

where:

$$
\begin{array}{ll}
\alpha^{ \pm}=\overline{b^{ \pm}} & \text {structure volume fractions, } \\
\rho^{ \pm}=\overline{b^{ \pm} \rho} / \alpha^{ \pm} & \text {structure densities, } \\
A^{ \pm}=\overline{b^{ \pm} \rho a} /\left(\alpha^{ \pm} \rho^{ \pm}\right) & \text {structure quantities, } \\
U_{i}^{ \pm}=\overline{b^{ \pm} \rho u_{i}} /\left(\alpha^{ \pm} \rho^{ \pm}\right) & \text {structure velocities, } \\
S^{ \pm}=\overline{b^{ \pm} s} & \text { structure source terms, } \\
\Theta_{i}^{ \pm}=\overline{b^{ \pm} \theta_{i}^{ \pm}} & \text {structure fluxes, } \\
\Phi_{i}^{ \pm}=\overline{b^{ \pm} \rho a u_{i}^{ \pm}} & \text {structure turbulent fluxes, } \\
u_{i}^{ \pm}=u_{i}-U_{i}^{ \pm} & \text {structure velocity fluctuations, } \\
X X \overline{b_{, j}^{ \pm} \theta_{j}^{ \pm}} \text {inter-structure flux term ( } \rightarrow \text { buoyancy }+ \text { drag), } &
\end{array}
$$

inter-structure turbulent-flux and exchange term.

Closures of standard terms are adapted from usual single fluid approaches and not discussed here.

Closures of exchange terms $X$ and $\Psi$ are crucial.

Presently, $b^{ \pm}$is loosely defined

to match the turbulent/laminar areas in flow.

Eventually, $b^{ \pm}$will be more precisely given

by analysis of density/velocity fields in simulations.

However, closure of $b^{ \pm}$-involving average terms is possible now. 
II.3 TTT: the closed modeled equations

TTT = Two-structure, Two-fluid, Two- $k-\varepsilon$. "Minimal".

Incompressible version (extension to compressibility and shocks to be published).

Single pressure is assumed (two pressure version also possible, but weak effect).

$$
\left\{\begin{aligned}
&\left(\alpha^{ \pm} \rho^{ \pm}\right)_{, t}+\quad\left(\alpha^{ \pm} \rho^{ \pm} U_{j}^{ \pm}\right)_{, j}=\mp \Psi \\
&\left(\alpha^{ \pm} \rho^{ \pm} C^{m \pm}\right)_{, t}+\left(\alpha^{ \pm} \rho^{ \pm} C^{m \pm} U_{j}^{ \pm}\right)_{, j}=-\Phi_{j, j}^{m \pm} \mp \Psi^{m} \\
&\left(\alpha^{ \pm} \rho^{ \pm} U_{i}^{ \pm}\right)_{, t}+\left(\alpha^{ \pm} \rho^{ \pm} U_{i}^{ \pm} U_{j}^{ \pm}\right)_{, j}=-\alpha^{ \pm} P_{, i}+\alpha^{ \pm} \rho^{ \pm} g_{i} \\
&- R_{i j, j}^{ \pm} \mp D_{i} \mp M_{i} \mp X_{i}^{U} \mp \Psi_{i}^{U} \\
&\left(\alpha^{ \pm} \rho^{ \pm} K^{ \pm}\right)_{, t}+\left(\alpha^{ \pm} \rho^{ \pm} K^{ \pm} U_{j}^{ \pm}\right)_{, j}=+\Pi^{ \pm}+\xi^{ \pm} \Pi^{d}+\chi^{ \pm} \Pi^{\Psi} \\
&-\alpha^{ \pm} \rho^{ \pm} \varepsilon^{ \pm}-\Phi_{j, j}^{K \pm} \mp \Psi^{K} \\
&\left(\alpha^{ \pm} \rho^{ \pm} \varepsilon^{ \pm}\right)_{, t}+\left(\alpha^{ \pm} \rho^{ \pm} \varepsilon^{ \pm} U_{j}^{ \pm}\right)_{, j}= \\
&+C_{\varepsilon 1} \frac{\varepsilon^{ \pm}}{K^{ \pm}}\left(\Pi^{ \pm}+\xi^{ \pm} \Pi^{d}+\chi^{ \pm} \Pi^{\Psi}\right) \\
&-C_{\varepsilon 2} \alpha^{ \pm} \rho^{ \pm} \frac{\left(\varepsilon^{ \pm}\right)^{2}}{K^{ \pm}}-\Phi_{j, j}^{\varepsilon \pm} \mp \Psi^{\varepsilon}
\end{aligned}\right.
$$

Production of $K^{ \pm}$by structure shear, $\Pi^{ \pm}$, drag dissipation, $\Pi^{d}$, and momentum exchange due to mass exchange, $\Pi^{\Psi}$. Weighing of productions on \pm according to $\xi^{ \pm}$and $\chi^{ \pm}$.

Single-fluid $\varepsilon$ equation is assumed valid in each structure: $K^{ \pm}$production and dissipation mirrored with $C_{\varepsilon 1}$ and $C_{\varepsilon 2}$.

Reduces to standard $k-\varepsilon$ for identical structures/fluids. 
II.4 $k-\varepsilon$ like closures

Turbulence characteristics:

$\nu_{t}^{ \pm}=C_{\mu}\left(K^{ \pm}\right)^{2} / \varepsilon^{ \pm}, \quad \sigma^{ \pm}=\varepsilon^{ \pm} / K^{ \pm}, \quad \lambda^{ \pm}=\left(K^{ \pm}\right)^{3 / 2} / \varepsilon^{ \pm}$.

First gradient assumption for turbulent fluxes in structures:

$$
\begin{aligned}
\Phi_{j}^{A \pm} \stackrel{\mathrm{m}}{=} \alpha^{ \pm} \rho^{ \pm}\left(\frac{\nu_{t}^{ \pm}}{\sigma_{a}}+D_{W}\right) A_{, j}^{ \pm}+\text {correction for } \rho_{, j}^{ \pm}, \\
R_{i j}^{ \pm} \stackrel{\stackrel{\mathrm{m}}{=}}{=} \alpha^{ \pm} \rho^{ \pm}\left[\frac{2}{3}\left(K^{ \pm}+\nu_{t}^{ \pm} U_{k, k}^{ \pm}\right) \delta_{i j}-\nu_{t}^{ \pm}\left(U_{i, j}^{ \pm}+U_{j, i}^{ \pm}\right)\right] .
\end{aligned}
$$

Turbulent kinetic energy production by Reynolds stresses:

$$
\Pi^{ \pm}=-R_{i j}^{ \pm}\left(U_{i, j}^{ \pm}-U_{k, k}^{ \pm} \delta_{i j} / 3\right) .
$$

Conpensation of spurious production by turbulent pressure demands presence of momentum exchange term:

$$
\begin{aligned}
X_{i}^{U}=-\frac{2}{3}\left[\alpha _ { , i } ^ { + } \left(\alpha^{+} \rho^{+}\right.\right. & \left.K^{+}+\alpha^{-} \rho^{-} K^{-}\right) \\
& \left.+\left(\alpha^{+} \alpha^{-}\left(\rho^{+} K^{+}-\rho^{-} K^{-}\right)\right)_{, i}\right]
\end{aligned}
$$

here called turbulent buoyancy. 
II.5 Mass exchange and structure growth closure

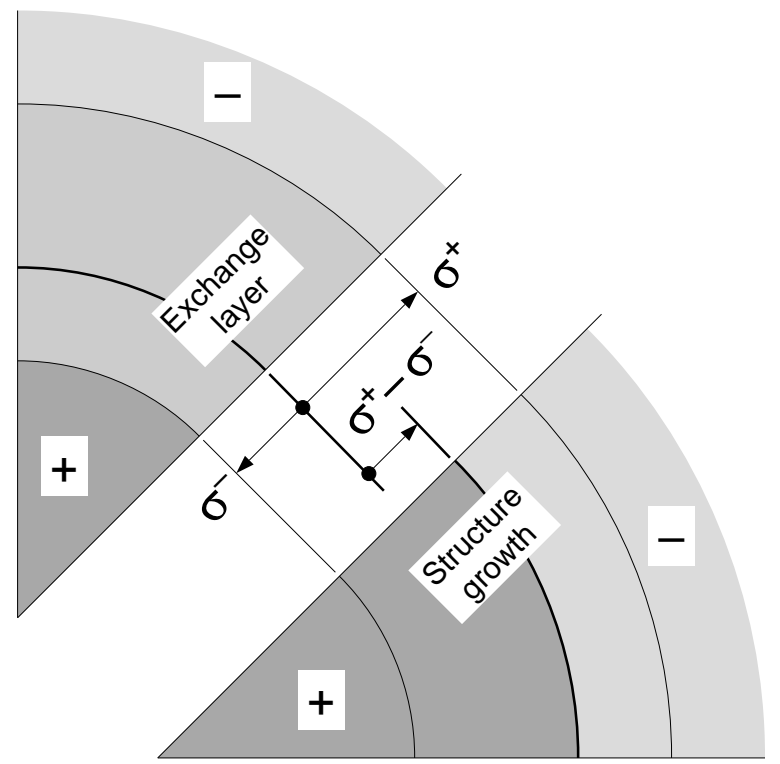

Schematic representation of inter-structure mass transfer and structure growth.

Closure of $\Psi^{a}$ obtained by extension of Youngs' approach (1995).

$\Psi^{a}$ is proportional to interface density $\approx \alpha^{+} \alpha^{-}$.

Affected volume fractions per time unit are proportional to $\sigma^{ \pm}$.

Mixing layer thus defined is assumed homogeneous.

Rate of volume fraction transfer is proportional to $\sigma^{+}-\sigma^{-}$.

Conserved quantities in layer are then attributed to \pm structures. 
Therefore:

$$
\begin{aligned}
& \Psi \stackrel{\mathrm{m}}{=} C_{\psi} \alpha^{+} \alpha^{-}\left(\xi^{-} \sigma^{-} \rho^{+}-\xi^{+} \sigma^{+} \rho^{-}\right) \\
& \Psi^{m} \stackrel{\mathrm{m}}{=} C_{\psi} \alpha^{+} \alpha^{-}\left(\xi^{-} \sigma^{-} \rho^{+} C^{m+}-\xi^{+} \sigma^{+} \rho^{-} C^{m-}\right) \\
& \Psi_{i}^{U} \stackrel{\mathrm{m}}{=} C_{\psi} \alpha^{+} \alpha^{-}\left(\xi^{-} \sigma^{-} \rho^{+} U_{i}^{+}-\xi^{+} \sigma^{+} \rho^{-} U_{i}^{-}\right) \\
& \Psi^{K} \stackrel{\mathrm{m}}{=} C_{\psi} \alpha^{+} \alpha^{-}\left(\xi^{-} \sigma^{-} \rho^{+} K^{+}-\xi^{+} \sigma^{+} \rho^{-} K^{-}\right) \\
& \Psi^{\varepsilon} \stackrel{\mathrm{m}}{=} C_{\psi} \alpha^{+} \alpha^{-}\left(\xi^{-} \sigma^{-} \rho^{+} \varepsilon^{+}-\xi^{+} \sigma^{+} \rho^{-} \varepsilon^{-}\right) \\
& \text {where } \xi^{ \pm}=\sigma^{ \pm} /\left(\sigma^{+}+\sigma^{-}\right) .
\end{aligned}
$$

Dissipation of directed energy by momentum exchange results in total turbulent kinetic energy production:

$$
\begin{aligned}
\Pi^{\psi}=\left[\Psi_{i}^{U}-\Psi\left(U_{i}^{+}+U_{i}^{-}\right) / 2\right] \delta U_{i} \\
\quad=C_{\psi} \alpha^{+} \alpha^{-}\left(\xi^{-} \sigma^{-} \rho^{+}+\xi^{+} \sigma^{+} \rho^{-}\right) \delta U_{i} \delta U_{i} / 2,
\end{aligned}
$$

distributed on \pm structures according to $\xi^{ \pm}$.

Matching the dissipation rate of density fluctuations in homogeneous isotropic turbulence yields $C_{\psi} \approx 1$. 


\section{II.6 Drag closure}

Analogy with $\Pi^{\psi}$ suggest to choose drag as:

$D_{i} \stackrel{\mathrm{m}}{=} C_{d} \alpha^{+} \alpha^{-}\left(\sigma^{+} \rho^{+}+\sigma^{-} \rho^{-}\right)\left(\delta U_{i}-W_{i}\right)$,

where $C_{d} \approx 0.3$ for invicid bubble of size $2 \lambda^{ \pm}$in viscous liquid.

Is not a Newton regime drag in $(\delta \vec{U})^{2}$ !

Is a Stokes regime drag in $\delta \vec{U}$ because structure size and turbulent viscosity yield $\operatorname{Re} \approx 1.7$.

Thus, drag length scale is implicit combination of $\lambda^{+}$and $\lambda^{-}$.

Can be interpreted by assuming that shear due to $\delta U_{i}$ is evenly spread on structures, where structure viscosities induce dissipation.

Total turbulent kinetic energy production by drag, $\Pi^{d}=D_{i} \delta U_{i}$, spread on structures according to:

$\chi^{ \pm}=\sigma^{ \pm} \rho^{ \pm} /\left(\sigma^{+} \rho^{+}+\sigma^{-} \rho^{-}\right)$.

Dispersion drift velocity is given by

$W_{i} \stackrel{\stackrel{m}{=}}{=} D_{W}\left(\frac{\left(\alpha^{+} \rho^{+}\right)_{, i}}{\alpha^{+} \rho^{+}}-\frac{\left(\alpha^{-} \rho^{-}\right)_{, i}}{\alpha^{-} \rho^{-}}\right)$.

Since $D_{W} \propto(\delta \vec{U})^{2}$, drag vanishes if $\delta \vec{U}=\overrightarrow{0}$. 


\section{II.7 Added mass closure}

Various motivations for including added mass energy, $K_{A}$ :

- significant in energy balance since $K_{A} \approx K_{D}$,

- important at TMZ edges (dominant in invicid laminar flow),

- may affect evolution in demixing and SSVARTs at $n \rightarrow-2$,

- makes $k^{ \pm}-k_{a}$ and $\left(U^{ \pm}\right)^{2} / 2+k_{a}$ continuous,

- represents large scale contribution to $k^{ \pm}$spectra,

- provides rationale of drag closure.

In two-field RANS equations, $k_{a}$ is included in $k^{ \pm}$, and should be separated and modeled by evolution equation.

Here, algebraic closure will be assumed:

$K_{A} \stackrel{\mathrm{m}}{=} C_{a} \alpha^{+} \alpha^{-}\left(\rho^{+}+\rho^{-}\right) \delta U_{i} \delta U_{i} / 2$.

where $C_{a} \approx 0.5$ for bubbles in liquid.

Drag can now be interpreted as

dissipation of added mass energy in each structure.

An associated term in momentum equation can be found as:

$$
\begin{aligned}
M_{i} & \stackrel{\mathrm{m}}{=} C_{a} \alpha^{+} \alpha^{-}\left(\rho^{+}+\rho^{-}\right)\left[\left(\frac{\mathrm{d}^{+}}{\mathrm{d} t} U_{i}^{+}-\frac{\mathrm{d}^{-}}{\mathrm{d} t} U_{i}^{-}\right)\right. \\
& \left.-\left(\alpha^{+} U_{i, j}^{+}+\alpha^{-} U_{i, j}^{-}\right)^{S} \delta U_{j}-\left(\frac{\bar{\rho}_{, j}}{\rho^{+}+\rho^{-}}+\alpha_{, j}^{+}\right) \delta U_{j} \delta U_{i} / 2\right]
\end{aligned}
$$

without $M_{i} \delta U_{i}$ dissipation in $K^{ \pm}$equations.

Will be rederived in near future from least action principle. 
II.8 Diffusion from added mass velocity fluctuations

Added mass energy is contained in velocity fluctuations which induce diffusion, described by $D_{W}$ :

$D_{W} \stackrel{\mathrm{m}}{=} C_{W} \alpha^{+} \alpha^{-} \frac{(\delta \vec{U})^{2}}{\alpha^{+} \sigma^{-}+\alpha^{-} \sigma^{+}}$

$D_{W}$ obtained assuming:

- characteristic velocity fluctuations are $\|\delta \vec{U}\|$.

- characteristic "interaction time" is average of $\sigma^{ \pm}$,

- average is weighed by less present structure (avoids singularities at TMZ edges).

Added mass diffusion dominates turbulent diffusion, increases transport slightly, and damps elliptic instabilities.

II.9 Summary of constants

\begin{tabular}{|c|c|c|c|c|c|c|c|c|c|}
\hline \multicolumn{5}{|c|}{ Standard $k-\varepsilon$} & \multicolumn{5}{c|}{ TTT extension } \\
\hline$C_{\mu}$ & $\sigma_{c}$ & $\sigma_{k}$ & $\sigma_{\varepsilon}$ & $C_{\varepsilon 1}$ & $C_{\varepsilon 2}$ & $C_{\psi}$ & $C_{d}$ & $C_{a}$ & $C_{W}$ \\
0.09 & 0.7 & 1.0 & 1.3 & 1.47 & 1.92 & 0.8 & 0.15 & 0.17 & 3 \\
\hline
\end{tabular}

$C_{\psi}, C_{d}, C_{a}$ and $C_{W}$ ajusted on RT to match respectively:

- molecular mixing fraction $\Theta$ (Youngs 1995),

- growth rate coefficient $\mathcal{Y}_{0}$,

- estimated added mass energy in internal shear,

- damping of elliptical modes (Youngs 1989...). 


\section{Results of TTT on SSVARTs}

III.1 Numerical conditions

Used 1D all-purpose test code :

compressible, artificial viscosity, explicit, no splitting, second order RK2, bi-lagrangian + TVD projection (ALE), non-diffusive evolution of TMZ edges,

(P.-H. Cournède 2001)

TTT model adapted for compressibility effects.

Calculation conditions :

$-x \in[0,1]$,

- 100 cells,

$-g(t) \propto t^{n}$

- energy source term to compensate $g(t)$

(see Youngs, this conference)

- $t_{0}$ and $g\left(t_{0}\right)$ ajusted so $L\left(t_{0}\right) \approx 0.4 \Delta x$ and $L(1) \approx 1$ for selected $n$,

- isentropic stratification of perfect gases at $\gamma=5 / 3$,

- maximum Mach number below 5\%,

- At $=0.2$ at interface for $t=t_{0}$,

- Initial turbulence in pure fluids with $\sigma \ll \sigma_{\mathrm{TMZ}}$ and $K \ll K_{\mathrm{TMZ}}$. 
III.2 Typical TTT profiles in RT TMZ
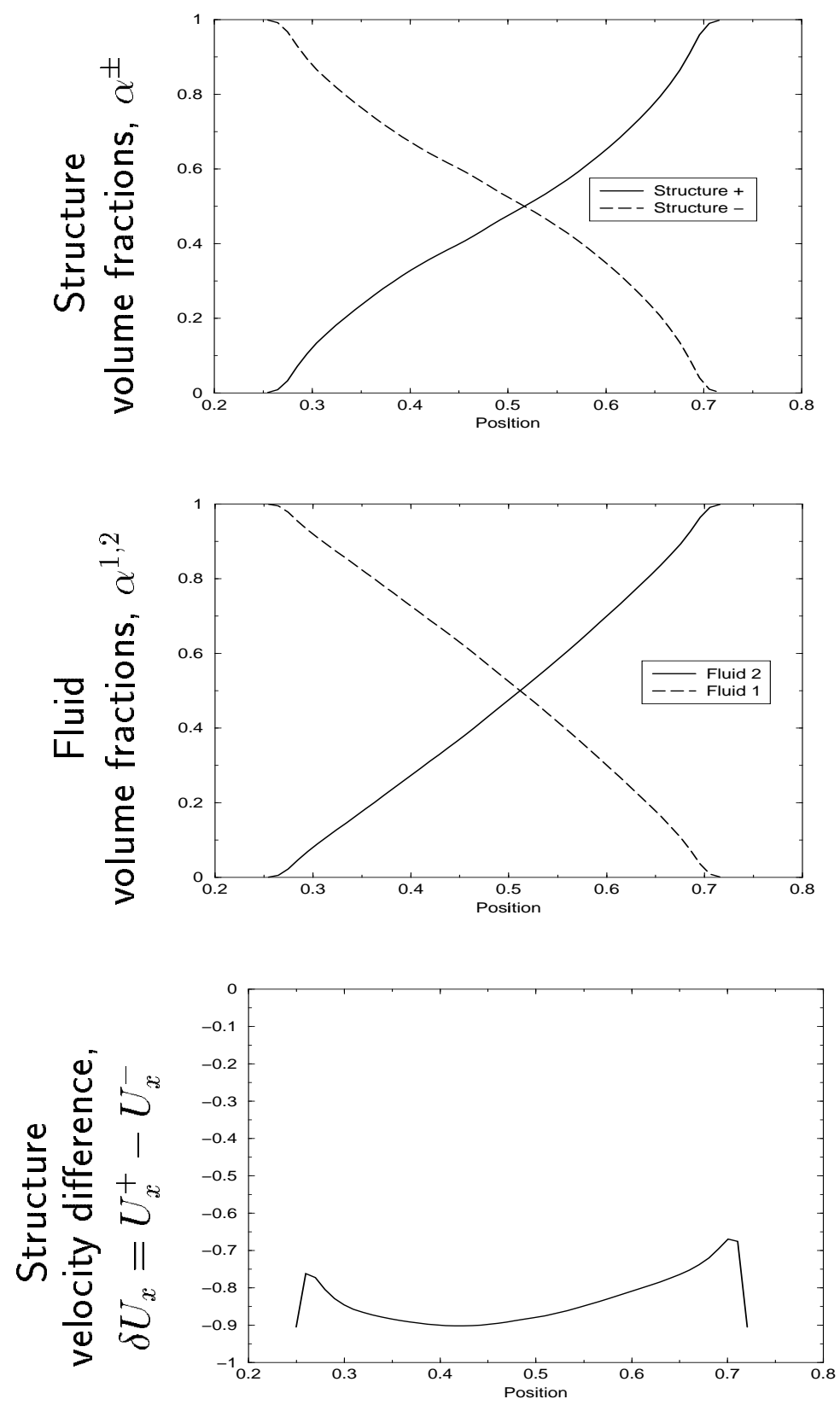

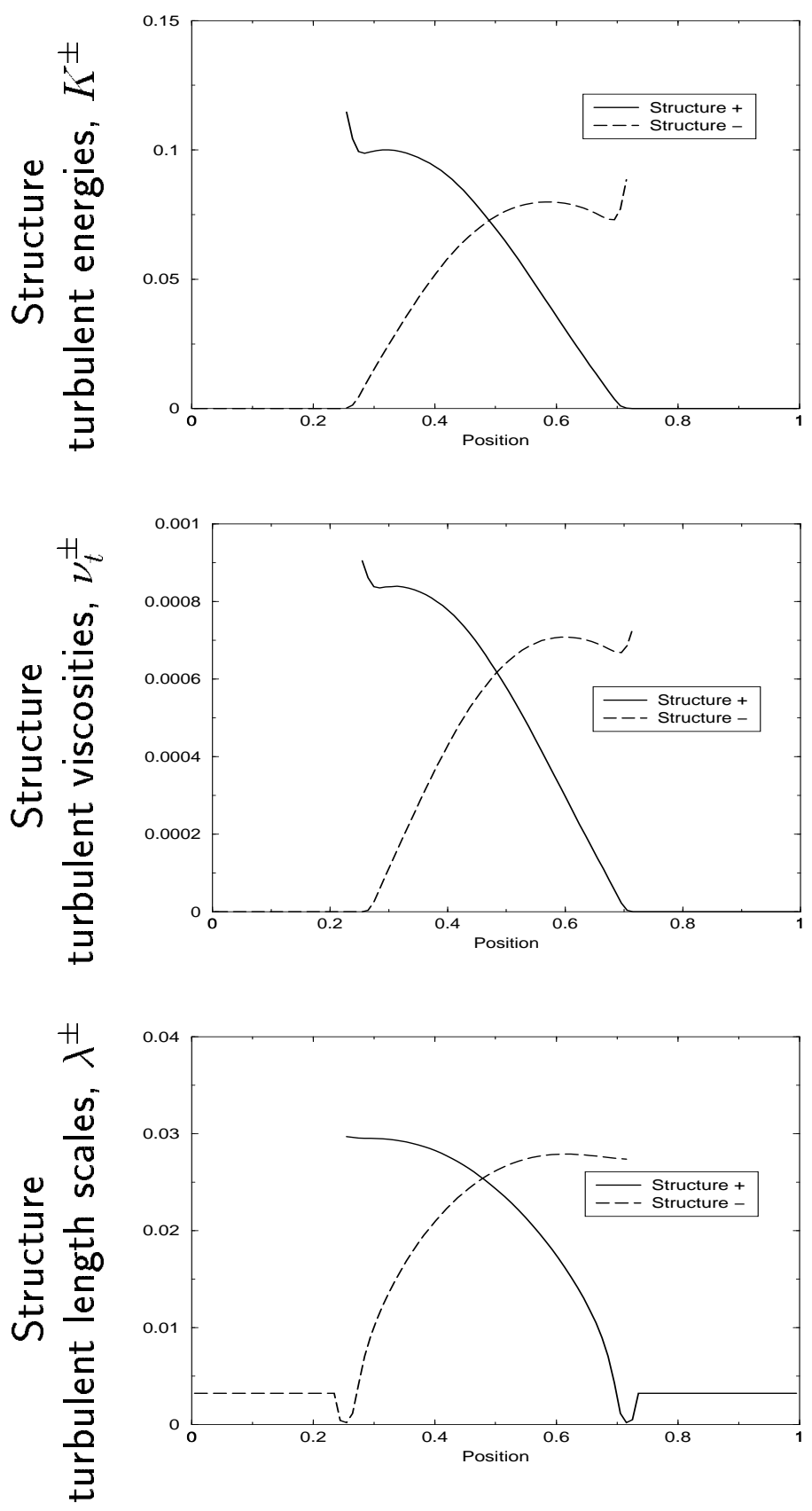

Profiles are very similar for explored SSVARTs at $n=-1$ to 1 .

Slight artifacts at TMZ edges for $\alpha^{ \pm} \rightarrow 0$ are numerical. 
III.3 Typical TTT “OD” parameters in RT TMZ

Three main "OD" parameters :

- growth rate, $\mathcal{Y}_{0} \approx 0.12$,

- molecular mixing fraction, $\Theta \approx 0.83$,

- Von Kármán number, $\kappa \approx 0.13$,

are defined by:

$$
\begin{aligned}
L(t) & =6 \int \alpha^{+} \alpha^{-} d x \\
\Theta(t) & =\int \alpha^{+} \alpha^{2+} \alpha^{1+}+\alpha^{-} \alpha^{2-} \alpha^{1-} d x / \int \alpha^{2} \alpha^{1} d x \\
E(t) & =\int \alpha^{+} \rho^{+} \varepsilon^{+}+\alpha^{-} \rho^{-} \varepsilon^{-} d x / \int \alpha^{+} \rho^{+}+\alpha^{-} \rho^{-} d x \\
K(t) & =\ldots=K^{+}+K^{-}+K_{D}+K_{A} \\
\kappa(t) & =K^{3 / 2} / E L
\end{aligned}
$$

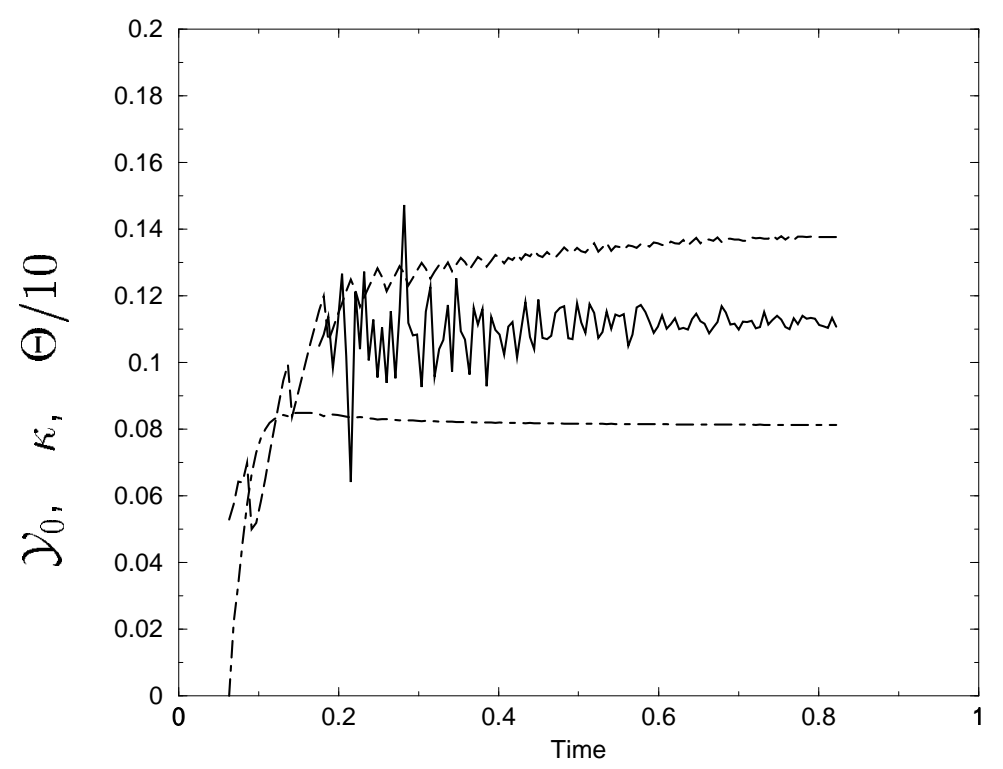

Time evolution of "0D" parameters: $\mathcal{Y}_{0}$ (continuous), $\Theta$ (dot-dashed), and $\kappa$ (dashed).

Von Kármán number captured within $20 \%$ without ajustments.

For Richtmyer-Meshkov, TTT yields $n_{0} \approx 0.3$ as $k-\varepsilon$. 


\section{III.4 Growth rate compared to other models}

Estimate of growth coefficient corrected for transient and relaxation effects:

$L(t)=\mathcal{Y}_{n}$ At $g(t) t^{2} \times\left[1+\frac{\delta t}{t}+\frac{\tau^{2}}{t^{2}}\right]^{n+2}$

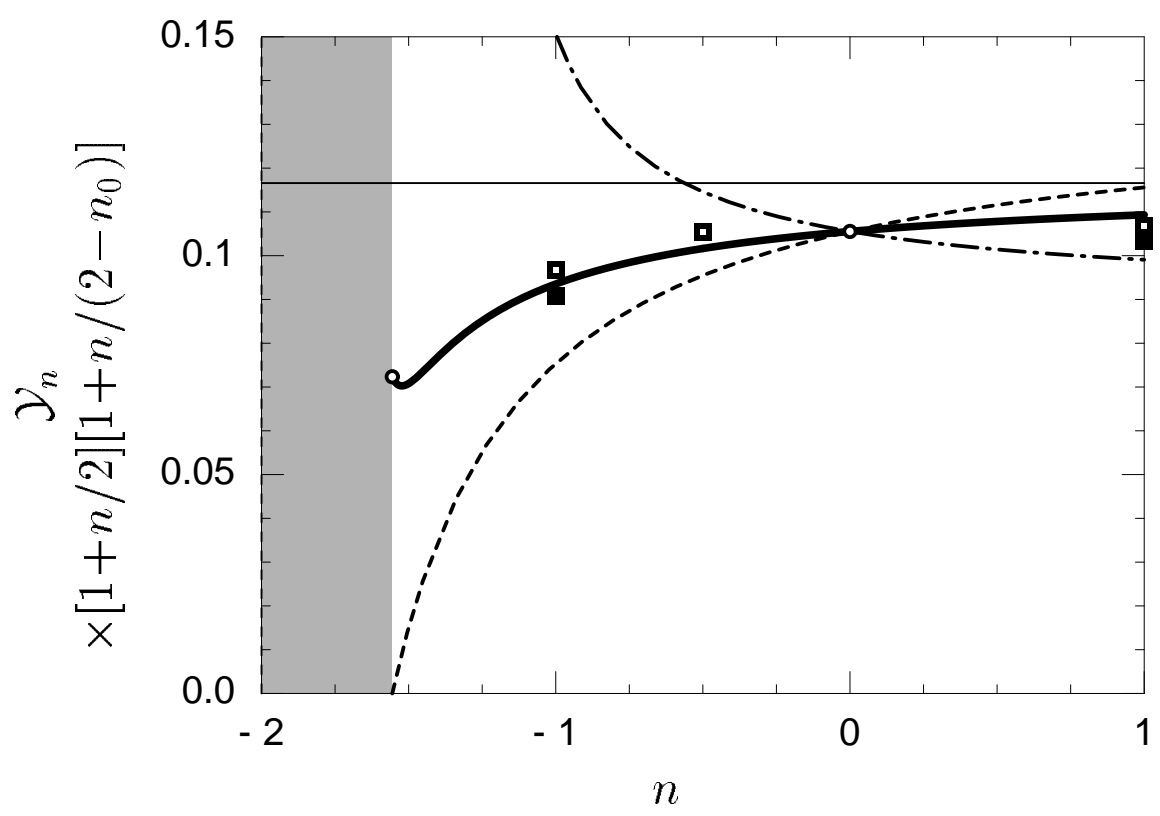

Normalized growth rates of various models: TTT (squares),

Youngs' two-fluid (dot-dashed), free fall (dashed) Read's formula (dotted),

and Youngs' preliminary simulations presented at this conference (filled squares).

Molecular mixing fraction and Von Kármán number, $\Theta_{n}$ and $\kappa_{n}$, are practically independent of $n$ for $-1<n<1$. 


\section{Conclusions}

A two-structure, two-fluid, two- $k-\varepsilon$ model (TTT)

has been developped which:

- derives from two-field RANS closure,

- provides physically consistent picture of RT/SSVARTs TMZs,

- contains single fluid $k-\varepsilon(\rightarrow \mathrm{KH}, \mathrm{RM})$,

- captures Von Kármán numbers,

- is potentially accurate on a range of SSVARTs and demixing,

- involves few unknown ajustable constants.

TTT is currently being improved regarding:

- Atwood number influence,

- added mass closure,

- response to $\mathrm{KH}$,

- dispersion by added mass velocity fluctuations,

- shocks and compressibility effects.

- confirmation of closures from simulated correlations. 


\section{Acknowledgements}

Benoît Dejardins (Commissariat à l'Energie Atomique, Bruyères-le-Châtel, France),

Olivier Simonin, Ariane Vallet

(Institut de Mécanique des Fluides de Toulouse, France),

David L. Youngs (Atomic Weapons Establishment, United Kingdom),

Paul-Henry Cournède (Ecole Centrale de Paris, France), Laurent Chodorge (Commissariat à l'Energie Atomique, Fontenay-aux-Roses, France).

\section{References}

P.-H. Cournède, Thèse de Doctorat, Ecole Centrale de Paris, France (2001).

S.B. Dalziel, P.F. Linden, and D.L. Youngs, J. Fluid Mech. 399, 1 (1999).

G. Dimonte, Phys. Plasmas 7, 2255 (2000).

G. Dimonte, and M. Schneider, Phys. Fluids 12, 304 (2000).

N.A. Inogamov, A.M. Oparin, A.Yu. Dem'yanov, L.N. Dembitskii, and V.A. Khokhlov, J. Exp. Theor. Phys. 92, 715 (2001).

A. Llor, CEA Report in print R-5983, France, 2001.

A. Llor, This conference.

A. Vallet, O. Simonin, and A. Llor, Proceedings of "Trends in numerical and physical modelling for industrial multiphase flows", Carghese, France 27-29/09/00.

D.L. Youngs, Physica D37, 270 (1989).

D.L. Youngs, Laser and Particle Beams 12, 725 (1994).

D.L. Youngs, in Proceedings of the 5 th International Workshop on Compressible Turbulent Mixing, Stony Brook, New York, August 1995, edited by R. Young, J. Glimm, and B. Boston, World Scientific, Singapore, 1995.

D.L. Youngs, and A. Llor, This conference. 


\title{
How to inhibit Rayleigh- Taylor mixing
}

\author{
Robert E. Breidenthal \\ University of Washington
}




\section{Self-similar flow}

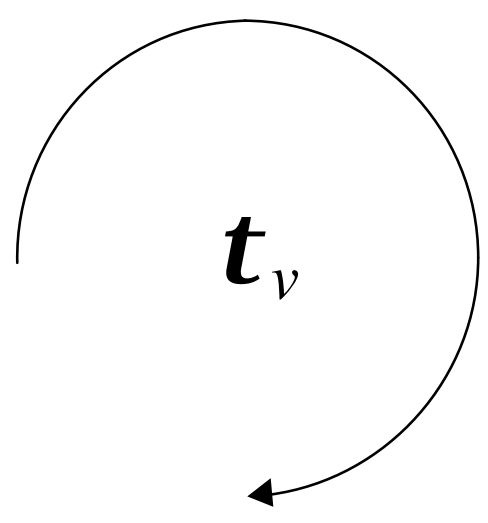

Self-similarity means the next rotation period is always proportional to the current one.

$$
\tau_{v}\left(t+\tau_{v}(t)\right)=\text { const. } \tau_{v}(t) .
$$

In other words, the fractional decrease in

rotation period per rotation is a constant, independent of time,

$$
\left[\tau_{v}(t)-\tau_{v}\left(t+\tau_{v}(t)\right)\right] / \tau_{v}(t)=\text { const. }=\beta \text {. }
$$




\title{
Proof that $\tau_{v}(t)$ must linear if self
}

\author{
similar
}

Assume

$$
\tau_{v}(t)=\tau_{v}(0)+a_{1} t+a_{2} t^{2}+\ldots a_{n} t^{n} .
$$

Then

$\left[-a_{1}\left(\tau_{0}+a_{1} t+a_{2} t^{2}+\ldots a_{n} t^{n}\right)+\right.$ terms up to

$$
\left.\mathrm{t}^{\mathrm{n} 2}\right] /\left(\tau_{0}+\mathrm{a}_{1} \mathrm{t}+\mathrm{a}_{2} \mathrm{t}^{2}+\ldots \mathrm{a}_{\mathrm{n}} \mathrm{t}^{\mathrm{n}}\right)=\beta .
$$

Satisfied iff $n^{2}-n=0$. So $n=0$ or 1 .

Thus $a_{j}=0$ for all $j>1$, and $a_{1}=-\beta$.

The only possible self-similar evolution

$$
\text { is } \tau_{\mathrm{v}}(\mathrm{t})=\tau_{\mathrm{v}}(0)-\beta \mathrm{t}=\tau_{0}-\beta \mathrm{t} \text {. }
$$




\title{
Example - Inertial subrange
}

One eddy 'turnover time'$$
\tau_{\lambda}=\lambda / \mathrm{v}_{\lambda}=\lambda^{2 / 3} / \mathrm{e}^{1 / 3}=(\lambda / \delta)^{2 / 3} \tau_{\delta}
$$ \\ Time interval from scale $\lambda$ to $(\lambda-\mathrm{d} \lambda)$$$
\mathrm{dt}=\operatorname{const} \cdot \lambda^{2 / 3} \mathrm{~d} \lambda /\left(\mathrm{e}^{1 / 3} \lambda\right)
$$ \\ (Kulkarny \& Broadwell) \\ Total elapsed time to reach scale $\lambda$
}

starting from scale $\delta$ is

$$
t(\lambda)=\left[1-(\lambda / \delta)^{2 / 3}\right] \tau_{\delta},
$$

SO

$$
\tau_{\lambda} / \tau_{\delta}=1-\mathrm{t}(\lambda) / \tau_{\delta} \quad \ldots \text { linear }
$$

in $\mathrm{t}$ 



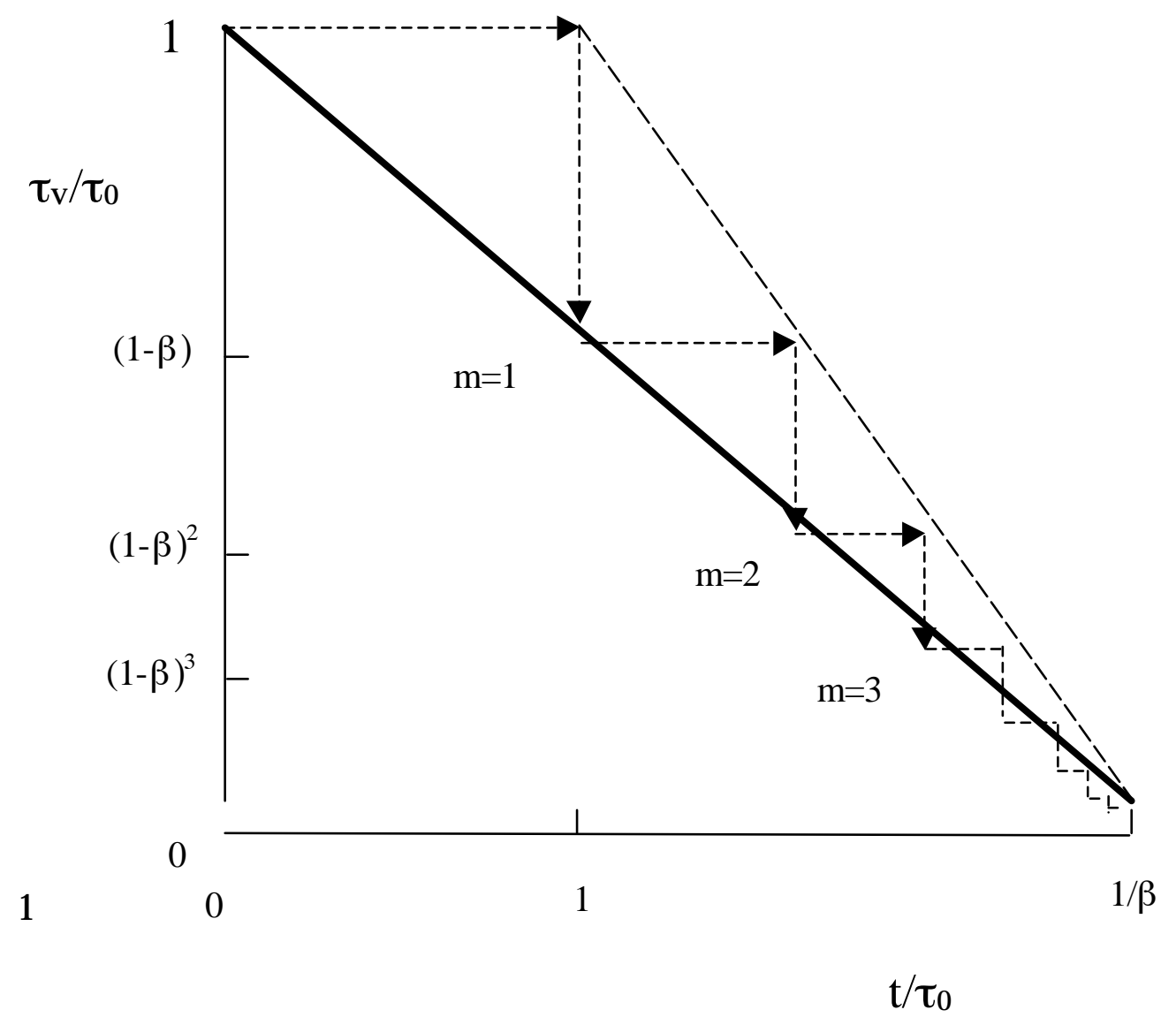

Vortex rotation period $\tau_{\mathrm{v}}(\mathrm{t})$ for $\beta>0$ 


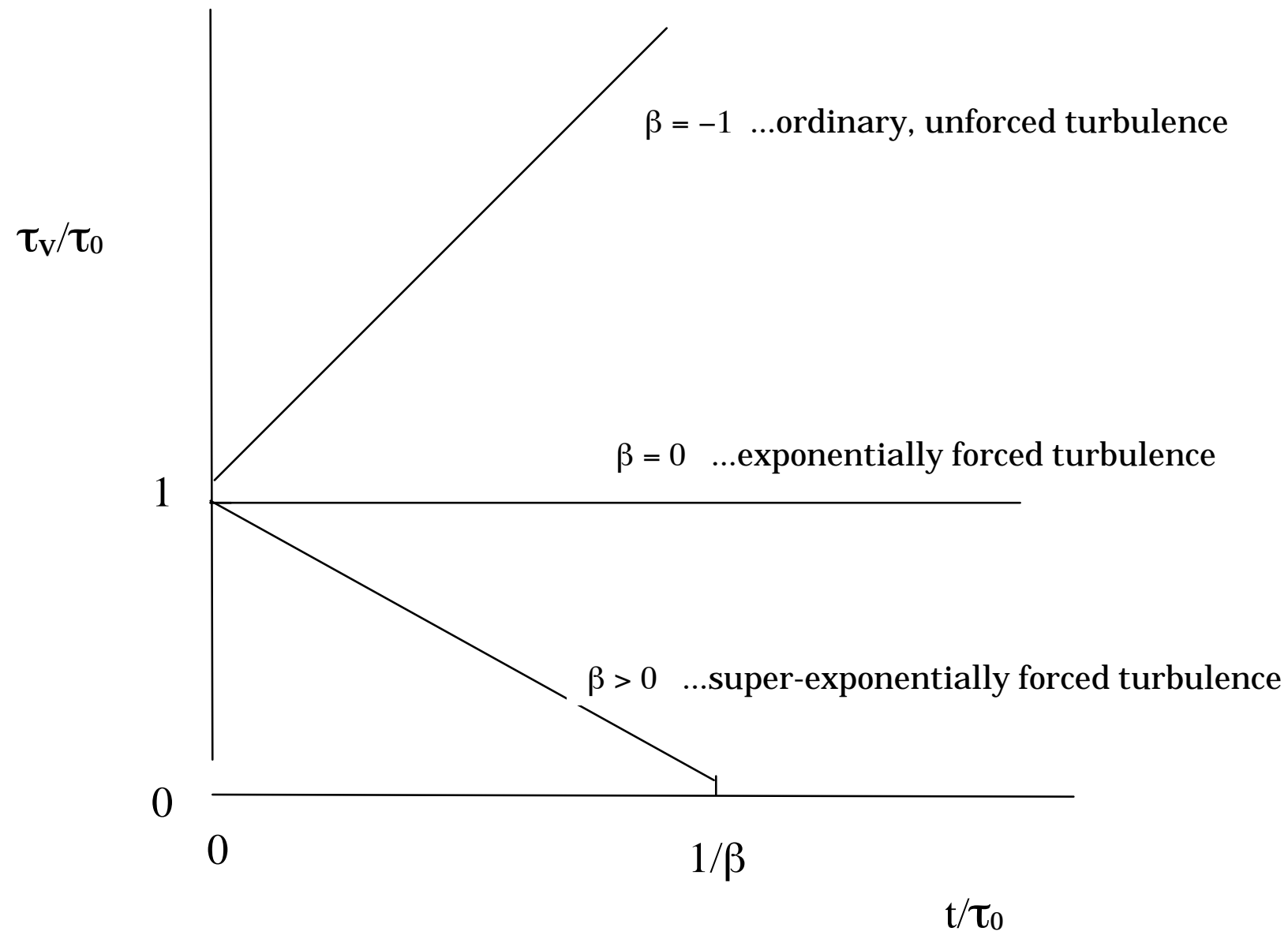

Vortex rotation period $\tau_{\mathrm{v}}(\mathrm{t})$ for all self-similar flows 
Vortex stretching

$\mathrm{D} \omega / \mathrm{Dt}=(\nabla \mathbf{u}) \omega$

Symmetry arguments imply

$$
\operatorname{mag}((\nabla \mathbf{u}) \omega)=\mathrm{c} \omega^{2}
$$

$\mathrm{D} \omega / \mathrm{Dt}=\mathrm{c} \omega^{2}$.

$$
\omega(\mathrm{t}) / \omega(0)=1 /\left(1-\mathrm{ct} / \tau_{\mathrm{v}}(0)\right),
$$

identical to

$$
\tau_{\mathrm{v}}(\mathrm{t}) / \tau_{\mathrm{v}}(0)=1-\beta \mathrm{t} / \tau_{\mathrm{v}}(0)
$$

for $\omega=1 / \tau_{v}$ and $c=\beta$. 



\section{Rayleigh-Taylor}

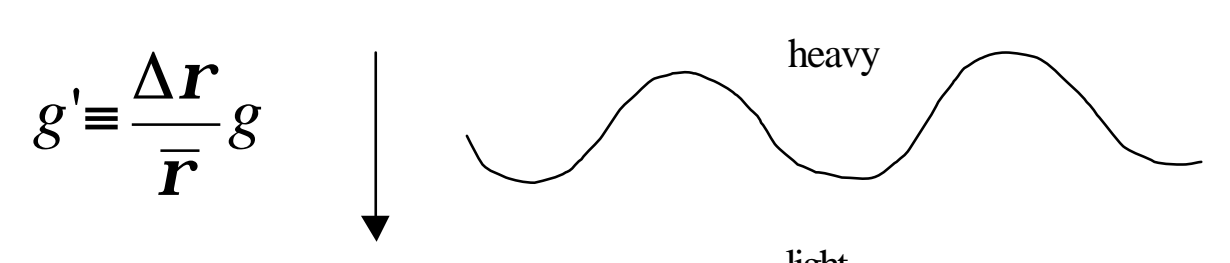

light

Impose an e-folding time scale $\tau_{\mathrm{v}}(\mathrm{t})=\tau_{0}-\beta \mathrm{t}$.

So force with "super-exponential"

acceleration

$$
\frac{g^{\prime}}{g_{0}^{\prime}}=e^{\left(\frac{t}{\tau_{0}-\beta t}\right)} .
$$

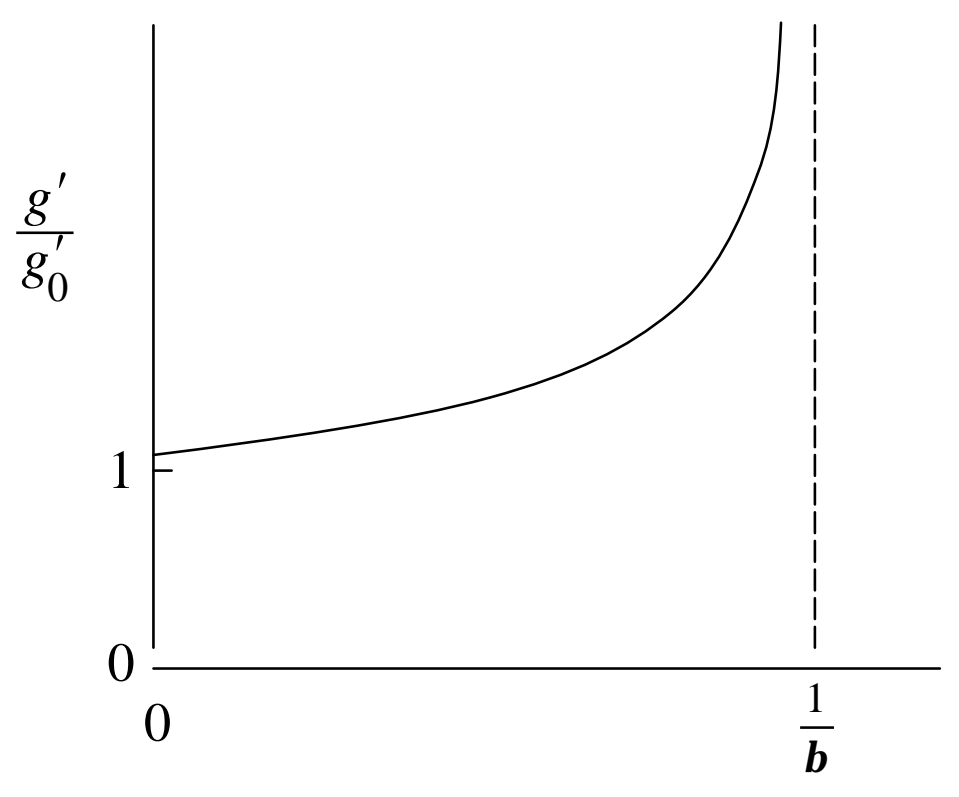




\section{$\frac{t}{\tau_{0}}$ \\ How to inhibit Rayleigh-Taylor entrainment?}

Instead of constant acceleration, use super-exponential, e.g.

$$
\frac{g^{\prime}}{g_{0}^{\prime}}=\exp \left(\frac{t}{\tau_{0}-\beta t}\right) .
$$

As $\beta \rightarrow 1$, entrainment is inhibited for all perturbation wavelengths $\lambda>\mathrm{g}_{0}{ }^{\prime} \tau_{0}{ }^{2}$. 


\title{
2D inviscid simulations
}

\author{
Christian Anitei
}

Randy Leveque's CLAWPACK code (suggested by Hamid Johari)

Derek Bale

James Rossmanith 

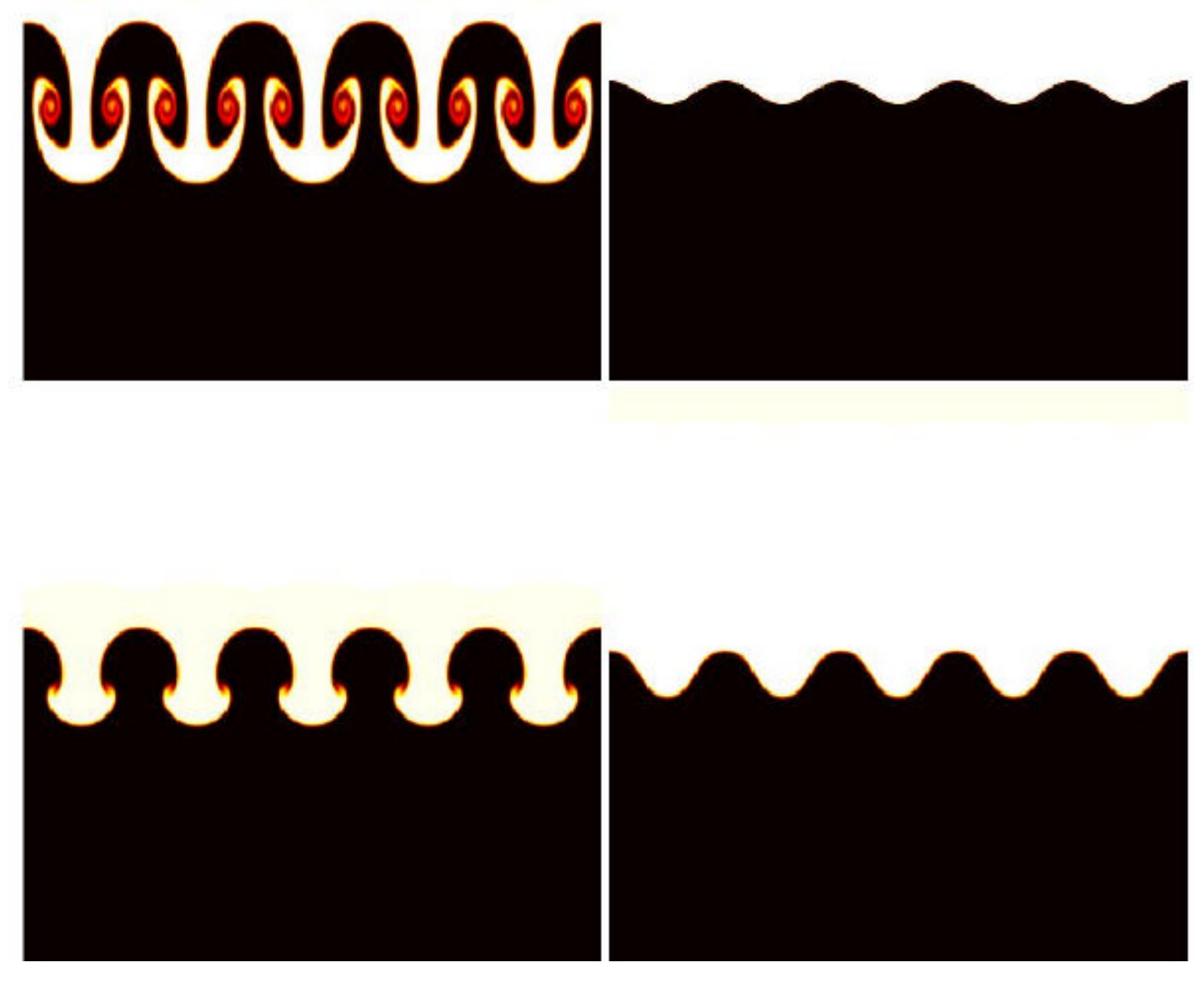

$$
\beta=-\infty
$$

Time progresses clockwise from upper right 

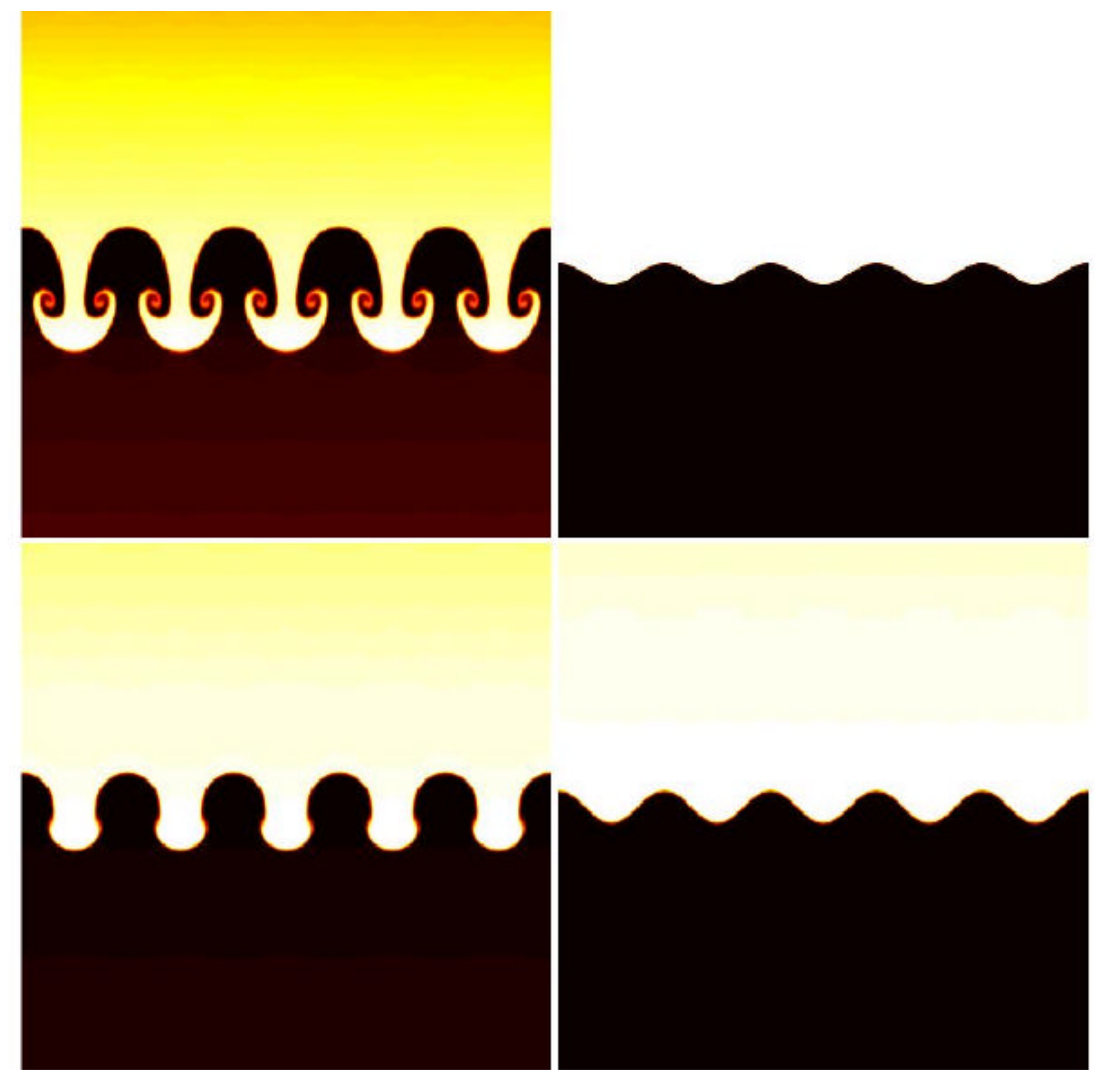

$\beta=0$ 

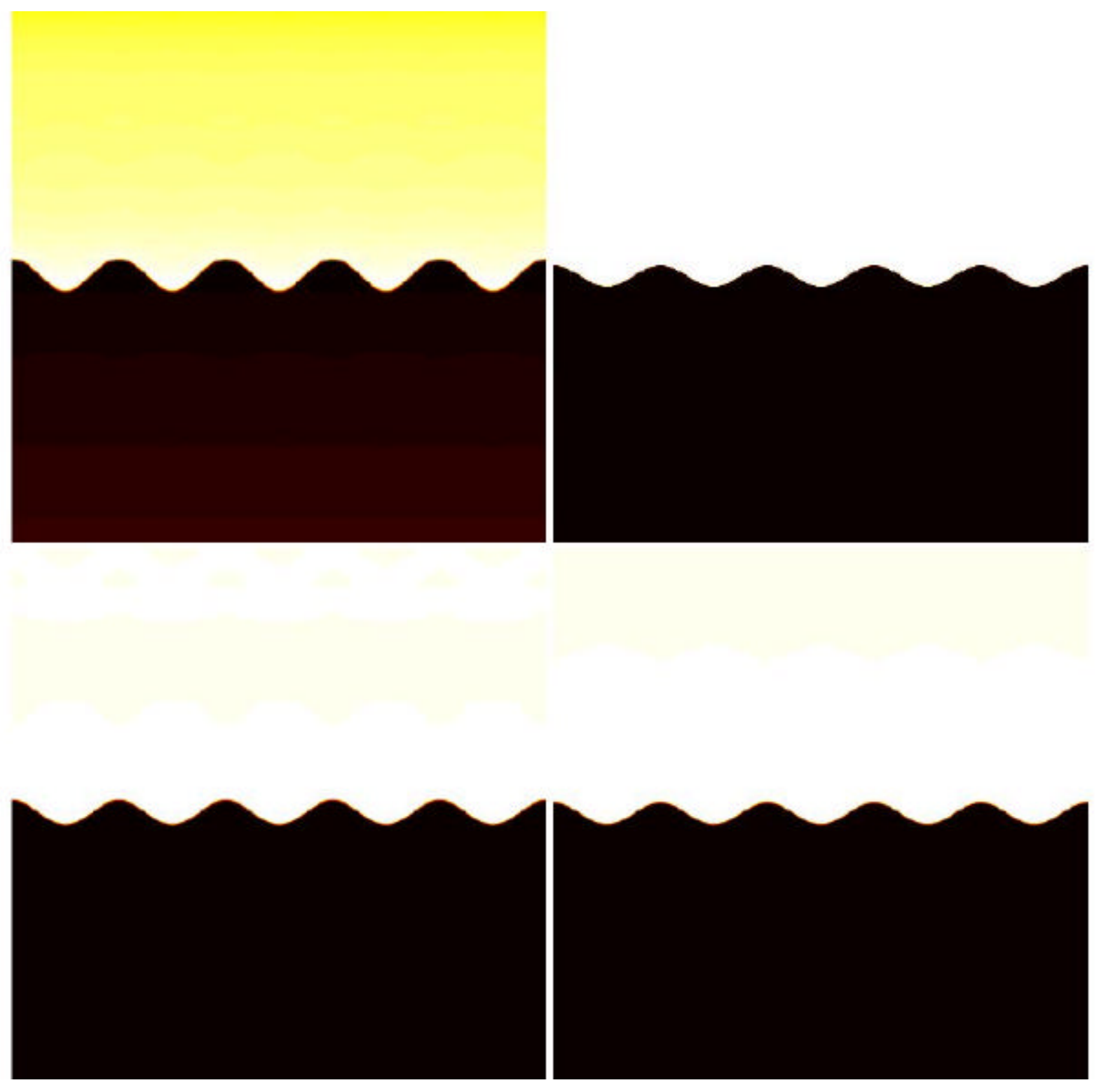

$\beta=1$ 


\section{Physical interpretation}

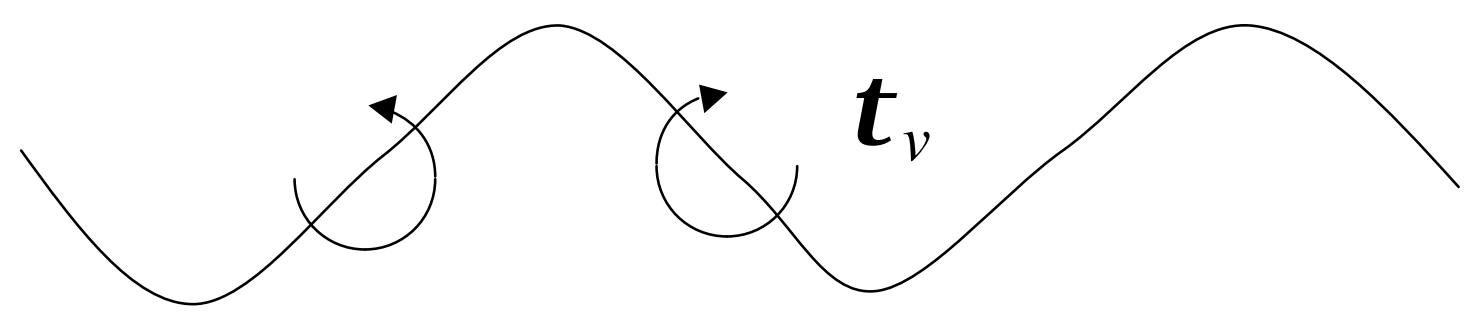

It takes time for a vortex sheet to roll up. If at every instant, the required roll-up time is just equal to the remaining available time $(\beta=1)$, there can be no roll up. Even more that this, the acceleration provides a stabilization. 


$$
\beta=1
$$

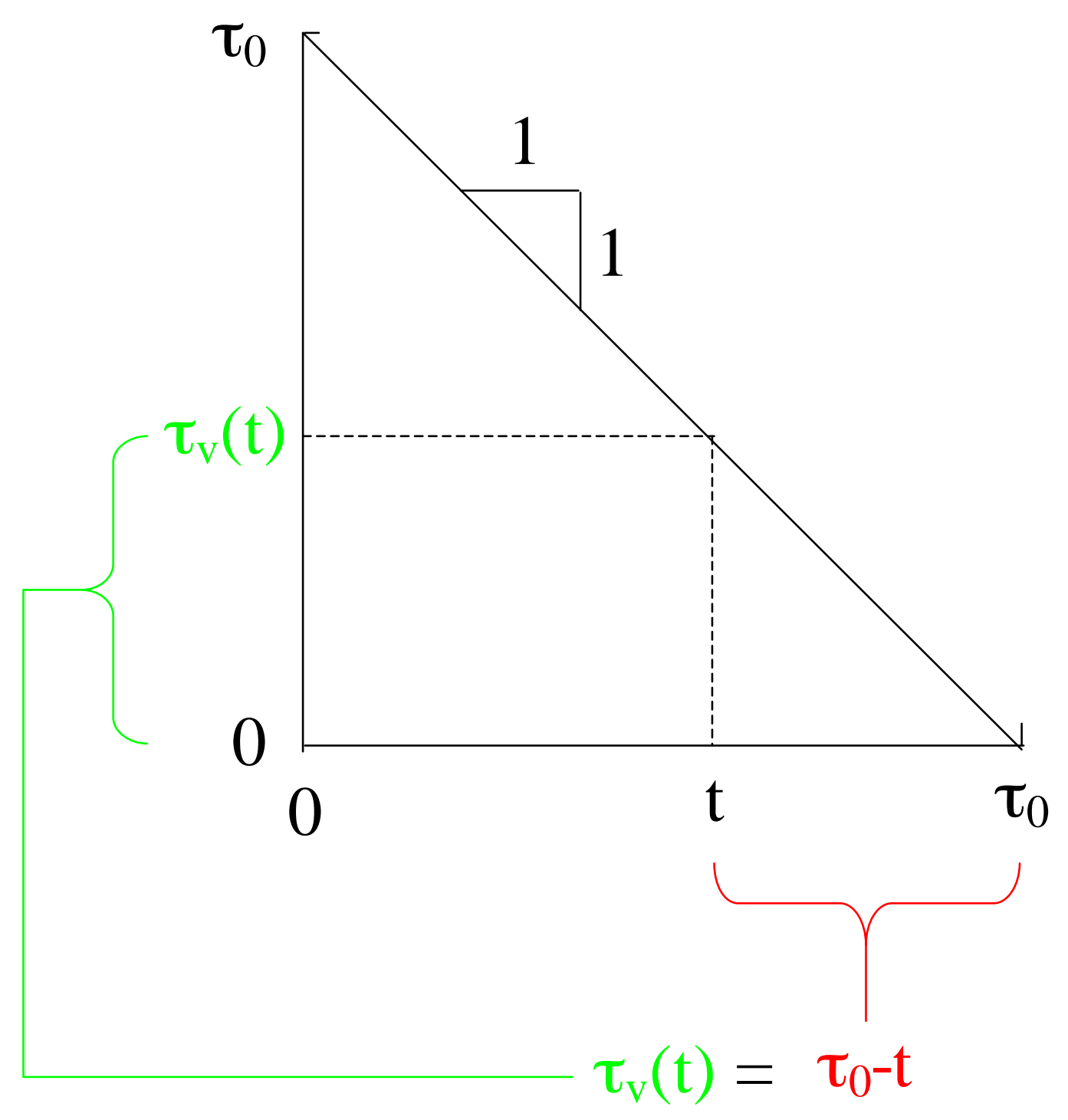

At every instant, the current rotation period is just equal to the remaining time. 


\section{Conclusions}

- For all self-similar turbulence, the vortex rotation period is a linear function of time,

$$
\tau_{\mathrm{v}}=\tau_{0}-\beta \mathrm{t} .
$$

- Entrainment and dissipation are inhibited as $\beta \rightarrow 1$.

- For $\beta=1$, Rayleigh-Taylor entrainment is suppressed for all wavelengths

$\lambda>\mathrm{g}_{0}{ }^{\prime} \tau_{0}^{2}$. 


\section{Theoretical Methods for the Determination of Mix}

\section{Baolian Cheng, James Glimm and David Sharp}

Los Alamos National Laboratory

Los Alamos, NM

Department of Applied Mathematics \& Statistics

State University of New York

Stony Brook, NY 11794-3600

Center for Data Intensive Computing

Brookhaven National Laboratory

Upton, NY 11973-5000

- in collaboration with -

Hyeonseong Jin 


\section{Main Results}

1. Buoyancy drag mixing edge motion equations -

Agree with bubble merger model, experiments, FT simulation and $\mathrm{A}=1$ theory

Spike - bubble coupling (Center of Mass)

All drag coefficients determined

Lower than leading order asymptotics

2. Improved two phase mix model equations -

mathematically stable and thermodynamically

determinate

Closure specified from asymptotic analysis

3. Turbulent diffusivity derived from mix model

Brookhaven Science Associates U.S. Department of Energy 


\section{Comparison of Bubble Merger Model with Experiments, Simulation}

$Z_{b}(t)=$ penetration distance of light fluid into heavy

$$
=\alpha_{b} A g t^{2}
$$

$$
\alpha_{b}=\begin{array}{ll}
0.05-0.077 & \text { (Experiment) } \\
0.05-0.06 & \text { (Theory) } \\
0.07 & \text { (Simulation - tracked) }
\end{array}
$$

Bubble height / bubble width $=3.3$ (experiment)

$$
=23 \text { (theory) }
$$




\section{A Bubble Merger Model}

\section{Statistical Models of Interacting Bubbles}

Bubble Merger Models

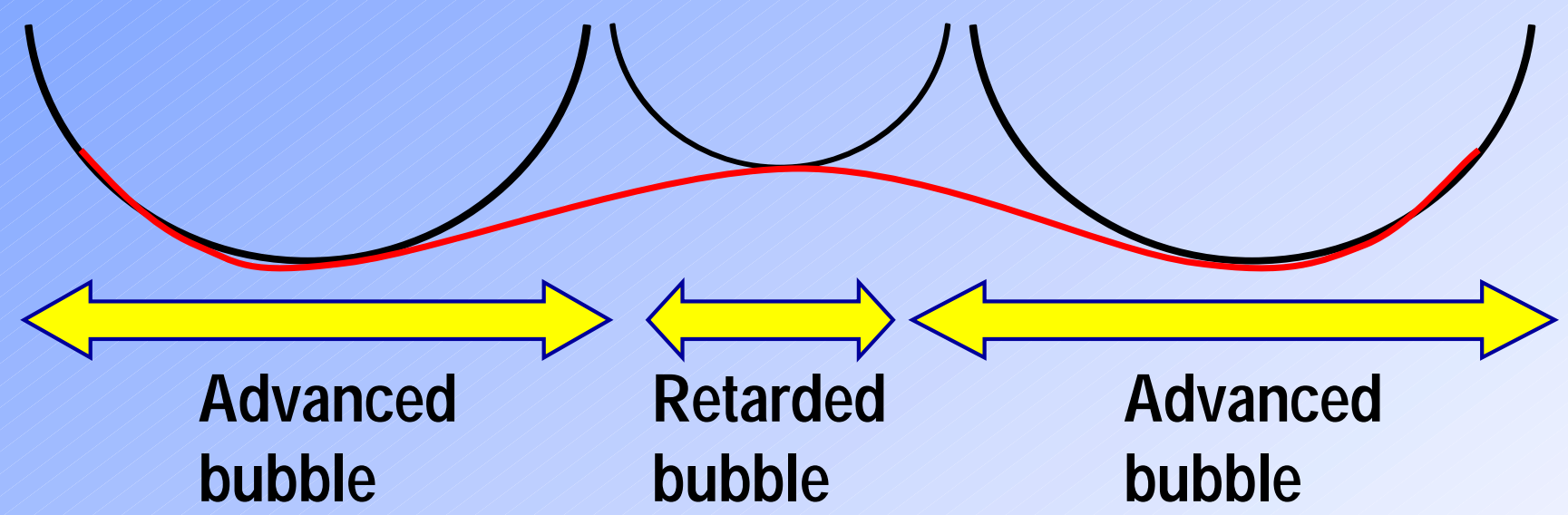

Bubble velocity = single mode velocity + envelope velocity 


\section{Bubble Merger Criterion}

Envelope velocity $>0$

advanced bubble

Envelope velocity $<0$

retarded bubble

Remove bubble from ensemble where velocity = 0: | single mode velocity $|=|$ envelope velocity |

$$
\alpha_{b} \approx 0.5-0.6
$$




\section{Scaled Variables}

\section{$r=$ mean bubble radius}

$t_{m}=$ time to bubble merger

$t_{m}{ }^{\prime}=$ scaled time to merger

$d t^{\prime}=(A g / r)^{1 / 2} d t$

\langle\rangle$_{*}=$ fixed point expectation 


\section{Renormalization group (RNG) fixed point equation for bubble radius}

$d r$

$\frac{d r}{d t}=\Delta r \times$ merger rate $=k r \times$ merger rate

$$
=k r\left\langle\frac{1}{t_{m}}\right\rangle=k \sqrt{A g}\left\langle\frac{1}{t_{m}{ }^{\prime}}\right\rangle r^{1 / 2}
$$

$k=$ fractional increase in radius due to one merger event; $t_{m}=$ time to merger

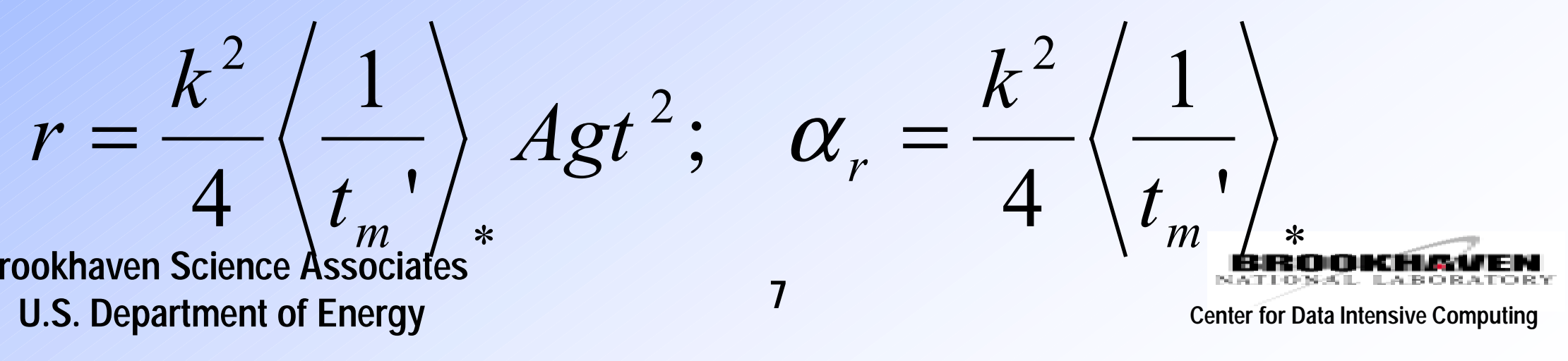




\section{Bubble height variables}

$k=$ geometric factor, $\approx .43$

$\bar{h}=$ mean bubble height

$h_{m}=$ bubble height separation for merger

$h=\bar{h}+h_{m} / 2$

Derive rate equation for $h$ in RNG scaling

Brookhaven Science Associates U.S. Department of Energy
8

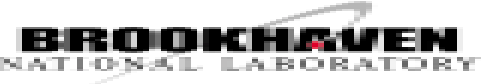

Center for Data Intensive Computing 


\section{RNG Bubble Height Equation}

$$
\alpha_{b}=\frac{1}{2} c_{b} \alpha_{r}^{1 / 2}+\left[\frac{1}{2 k}+\frac{1}{2}\right] \alpha_{h_{m}}
$$

$c_{b}=$ terminal velocity coefficient for single (periodic) bubble

Average of three Smeeton and Youngs experiments:

$$
\begin{aligned}
& \text { US }=0.067 ; \quad \text { RHS }=0.0695 ; \\
& \text { Fixed Point Calculation }=0.056
\end{aligned}
$$




\section{Center of Mass (COM) Hypothesis}

$$
\begin{gathered}
Z_{\mathrm{COM}}=\alpha_{\mathrm{COM}} A g t^{2} \\
\alpha_{\mathrm{COM}}=\frac{7}{60} \alpha_{s} A^{\gamma} ; \quad \gamma=3-17 \\
\approx 0 \text { unless } A \approx 1
\end{gathered}
$$

fits data and theory $(A=1) . \alpha_{s} / \alpha_{b}=$ solution of quadratic equation

$$
\alpha_{s}=\alpha_{s}\left(\alpha_{b}\right)
$$




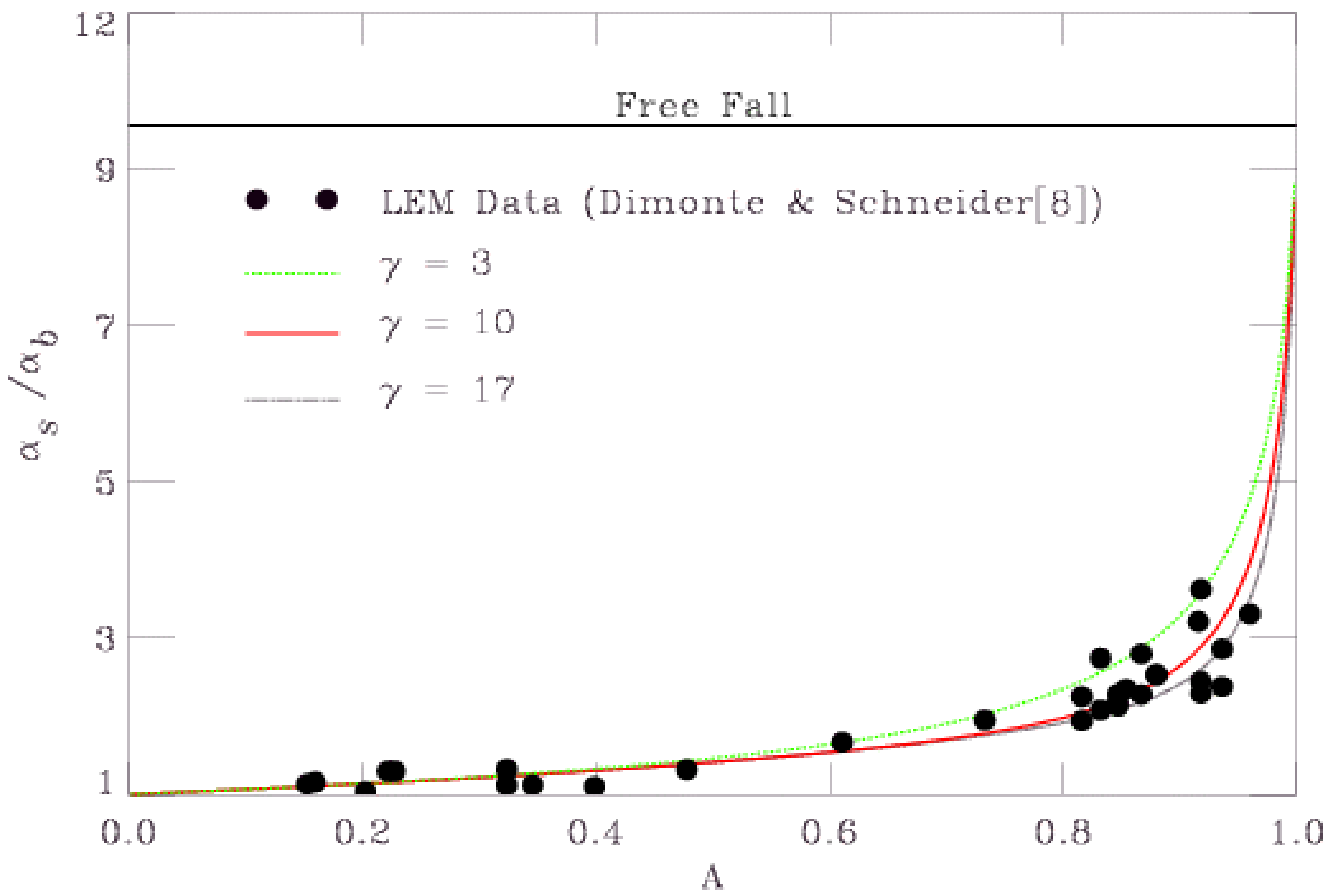

Brookhaven Science Associates

U.S. Department of Energy 


\section{Mixing Zone Edge Models}

$\mathrm{z}_{\mathrm{b}, \mathrm{s}}(\mathrm{t})=\mathbf{h}_{\mathrm{b}, \mathrm{s}}=\alpha_{\mathrm{bs}} \mathrm{Agt}^{2}$ in RT case

Buoyancy Drag equation for $Z_{b, s}(t)$ :

$\left(\rho_{b, s}+k \rho_{s, b}\right) \ddot{Z}_{b, s}(t)=\left(\rho_{b}-\rho_{s}\right) g-\rho_{s, b} C_{b, s} \dot{Z}_{b, s}{ }^{2} / Z_{b, s}$

Determine $\mathrm{C}_{\mathrm{b}, \mathrm{s}}$ from RT edge motion theory.

ODE valid for arbitrary acceleration

$k=1$ from standard fluid dynamics and from bubble geometry

Brookhaven Science Associates U.S. Department of Energy 


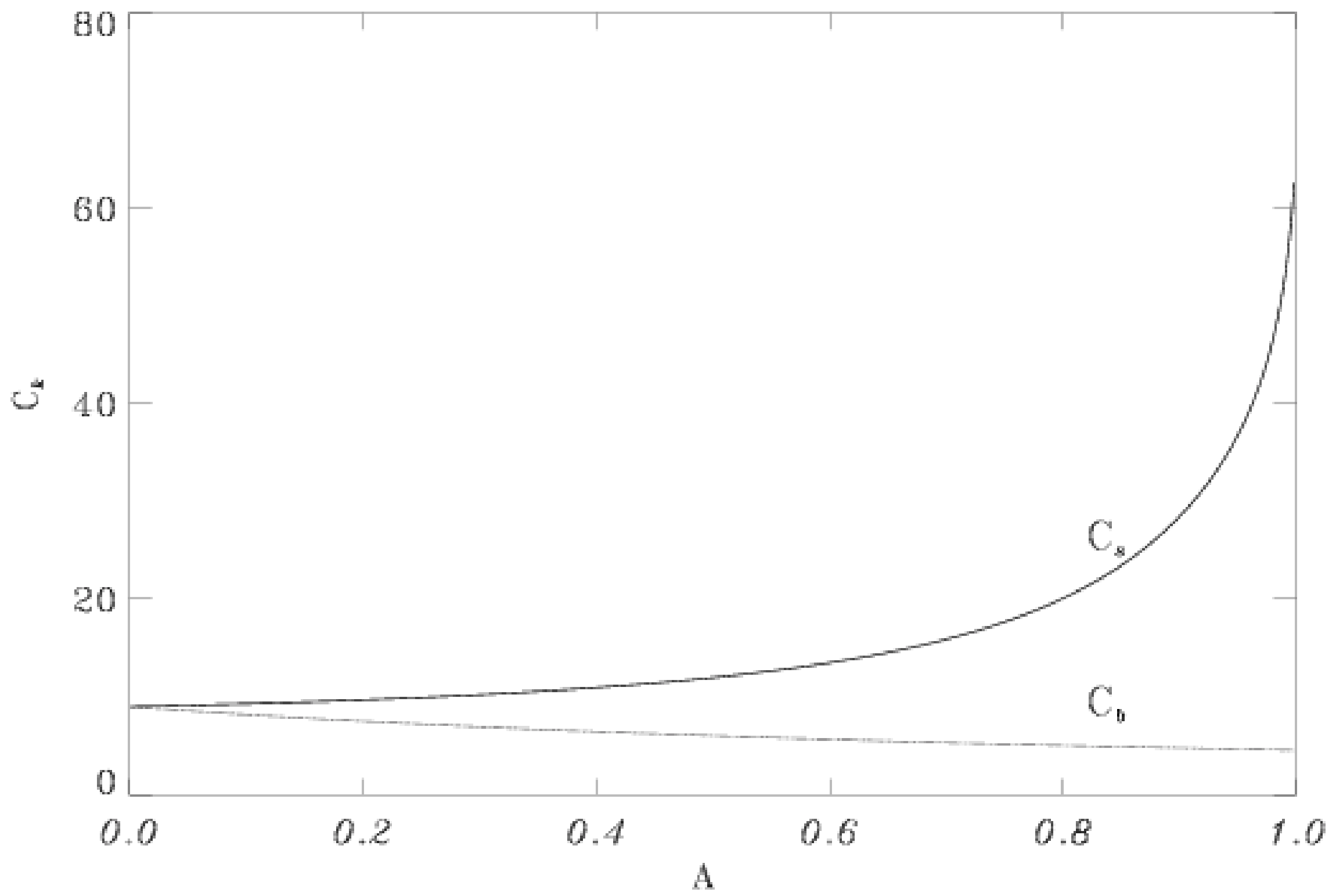

Brookhaven Science Associates U.S. Department of Energy 


\section{Non-leading Order Terms in RT Asymptotics}

$Z(\mathrm{t})=\alpha A g t^{2}+\beta t+\gamma$

$\beta, \gamma$ depend on initial data :

$$
t_{0}, Z_{0}, V_{0}
$$

$\alpha$ does not depend on initial data

Brookhaven Science Associates U.S. Department of Energy 


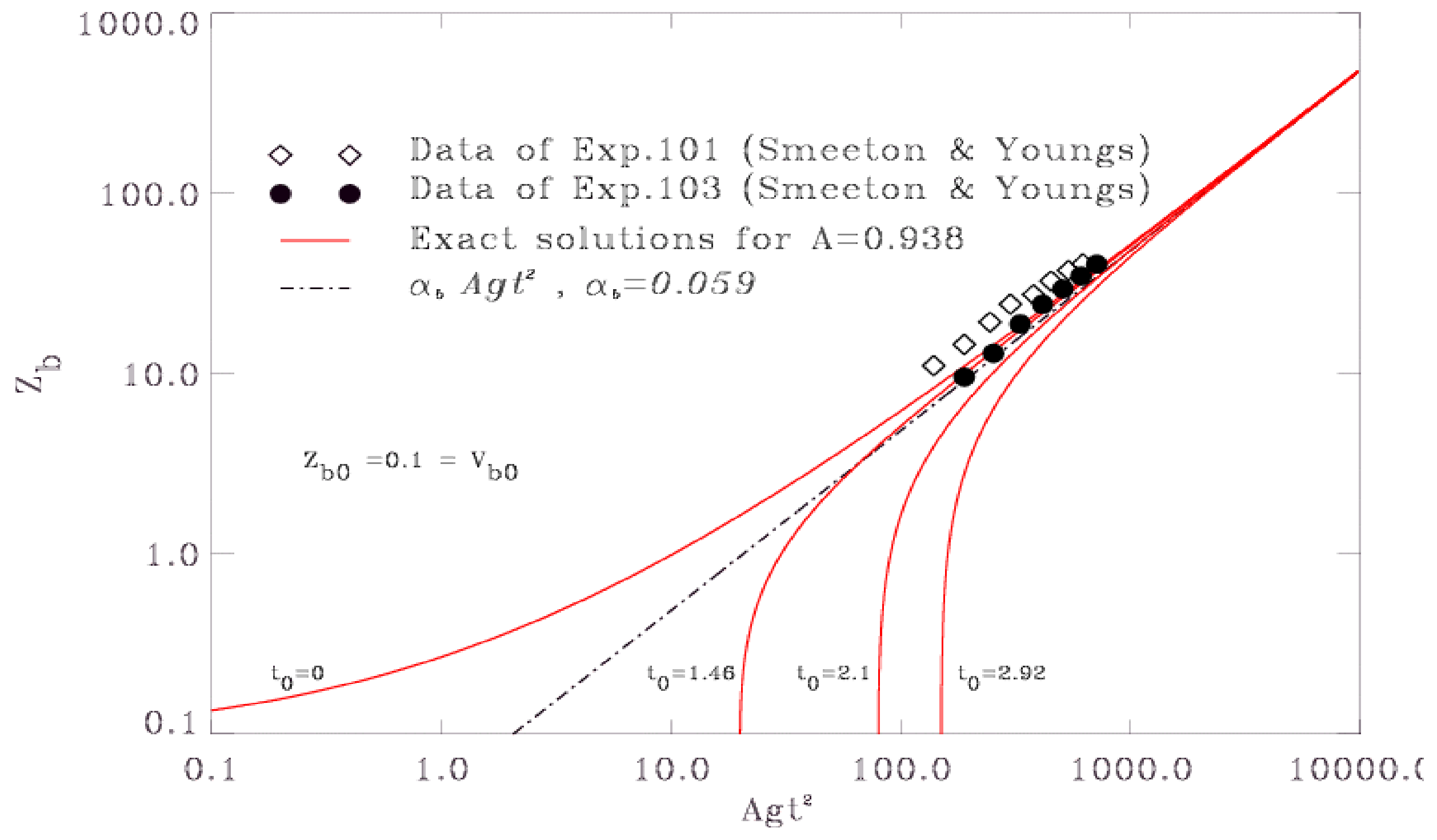




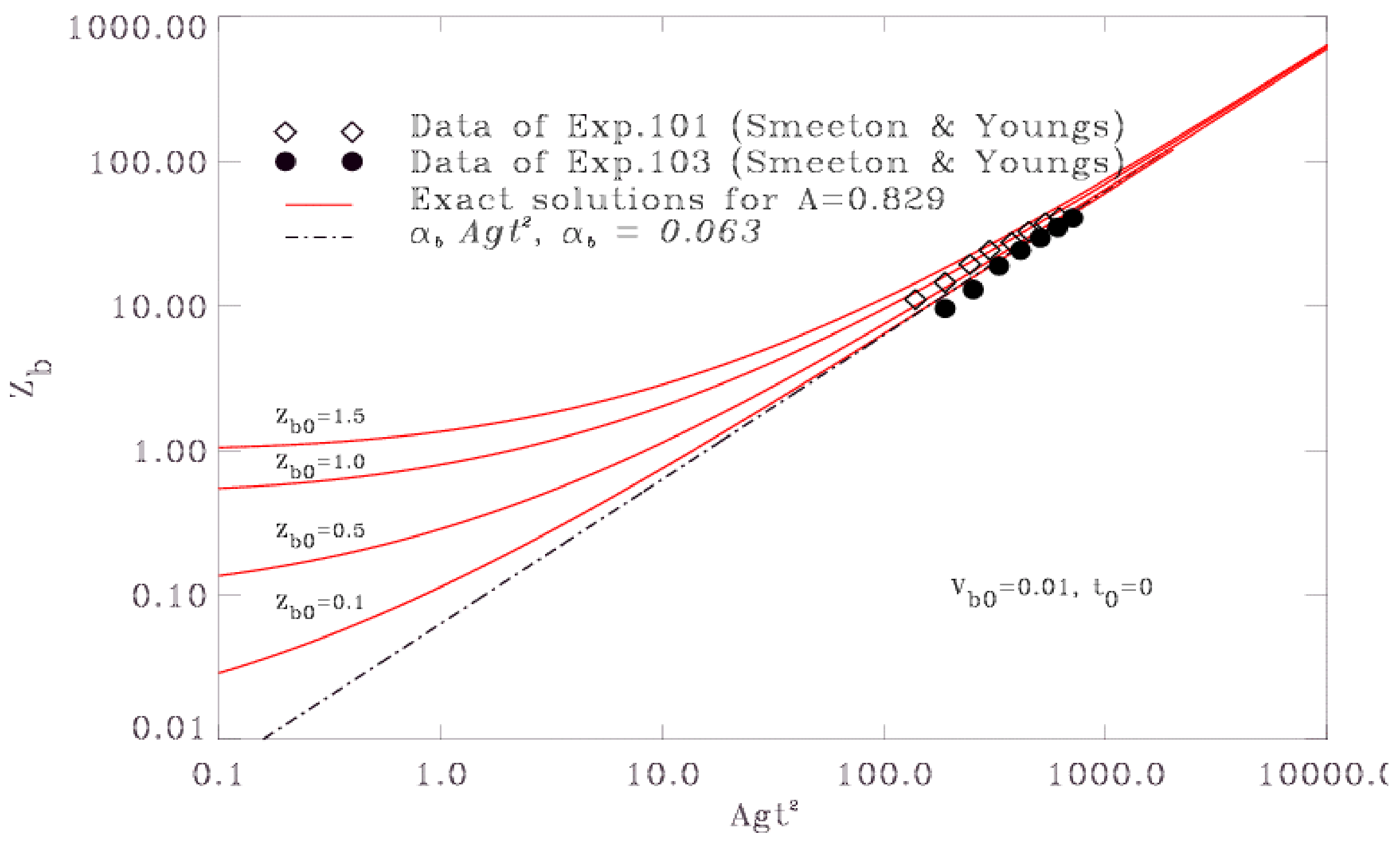

Brookhaven Science Associates

U.S. Department of Energy 


\section{Chunk Mix Model}

- Complete fluid variables for each fluid

- Mathematically stable equations

- Improved physics model for mix

- Pressure difference forces $\sim$ drag

- Thermodynamics is process independent

- New closure proposed and tested

- Zero parameters (incompressible flow)

- Analytic solution for incompressible case 


\section{Multiphase Averaged Equations}

Microphysics: $U_{t}+\nabla F(U)=0$

Macrophysics: $\overline{\mathrm{U}}_{t}+\nabla \overline{\mathrm{F}(\mathrm{U})}=0$

$\overline{\mathrm{F}(\mathrm{U})} \neq \mathrm{F}(\overline{\mathrm{U}})$

$\mathrm{F}_{\text {ren }}(\overline{\mathrm{U}}) \approx \overline{\mathrm{F}(\mathrm{U})}$

$\overline{\mathrm{U}}_{\mathrm{t}}+\nabla \mathrm{F}_{\text {ren }}(\overline{\mathrm{U}})=0$

\section{Closure Problem: Determine $F_{\text {ren }}$}




\section{Ensemble Averages}

Assume two fluids, labeled $k=1$ (light) and $k=2$ (heavy). Define

$$
\mathbf{X}_{\mathbf{k}}(\mathbf{x}, \mathbf{y}, \mathbf{z}, \mathbf{t})=\left\{\begin{array}{l}
1 \text { if }(x, y, z) \text { is in fluid } k \text { at time } t \\
0 \text { otherwise }
\end{array}\right.
$$

Let $\langle\cdot\rangle$ denote (ensemble) average.

Microphysics

$$
\frac{\partial \mathbf{X}_{\mathbf{k}}}{\partial \mathbf{t}}+v \cdot \nabla \mathbf{X}_{\mathbf{k}}=\mathbf{0}
$$

Define $v^{*}$ :

$$
\left\langle v \cdot \nabla \mathbf{X}_{\mathbf{k}}\right\rangle \equiv v^{*} \nabla \boldsymbol{\beta}_{\mathbf{k}}
$$

Brookhaven Science Associates

U.S. Department of Energy
Macrophysics

$$
\begin{aligned}
& \boldsymbol{\beta}_{\mathbf{k}}=\left\langle\mathbf{X}_{\mathbf{k}}\right\rangle \\
& \frac{\partial \boldsymbol{\beta}_{\mathbf{k}}}{\partial \mathbf{t}}+\left\langle v \cdot \nabla \mathbf{X}_{\mathbf{k}}\right\rangle=\mathbf{0}
\end{aligned}
$$

Thus

$$
\frac{\partial \boldsymbol{\beta}_{\mathbf{k}}}{\partial \mathbf{t}}+v^{*} \cdot \nabla \boldsymbol{\beta}_{\mathbf{k}}=\mathbf{0}
$$




\section{Closure}

Assume: $v^{*}$ depends on $v_{1}$ and $v_{2}$ and spatially dimensionless quantities only.

Assume: regularity of $\mathbf{v}^{*}$.

Theorem:

$$
v^{*}=\mu_{2}^{v} v_{1}+\mu_{1}^{v} v_{2}
$$

\section{(convex combination) and related expressions for} $p^{*}$ and $(p v)^{*}$

Assume: all $\mu$ 's depend on $\beta_{k}$ and $t$ only.

Brookhaven Science Associates U.S. Department of Energy 


\section{Explicit Model: Zero Parameters}

Exact calculation: $\mu_{k}^{v}$ is fractional linear. Assume same for $\mu_{k}^{p}$. Assume dependence on $\beta_{k}$ alone. Then

$$
\mu_{k}^{q}=\frac{\beta_{k}}{\beta_{k}+c_{k}^{q} \beta_{k^{\prime}}}
$$

with $\mathbf{k}^{\prime}$ denoting the other fluid index and $c_{1}^{q} c_{2}^{q}=1 \quad \mathrm{q}=v \quad$ or $\quad \mathrm{q}=\mathrm{p}$.

With the mixing zone boundaries $Z_{\mathbf{k}}(t)$, and velocities $V_{k}(t)$,

$$
c_{k}^{v}=\frac{\left|V_{k^{\prime}}\right|}{\left|V_{k}\right|}, \quad c_{k}^{p}=\frac{\rho_{k^{\prime}}}{\rho_{k}}
$$

for incompressible flow. Boundary accelerations $\ddot{Z}_{k}(t)$ must be must be supplied externally to this model.

$$
\ddot{Z}_{k}(t)=\text { Drag + buoyancy }
$$




\section{Analytic Solution: Incompressible Case}

$$
\begin{aligned}
& v_{k}\left(\beta_{k}, t\right)=V_{k} \mu_{k^{\prime}}^{v} \\
& v *\left(\beta_{k}, t\right)=\frac{V_{k} V_{k^{\prime}}\left(V_{k} \beta_{k}^{2}+V_{k} \beta_{k^{\prime}}^{2}\right)}{\left(\left|V_{k} \beta_{k}+\right| V_{k^{\prime}} \beta_{k^{\prime}}\right)^{2}} \\
& z\left(\beta_{k}, t\right)=z_{0}\left(\beta_{k}\right)+\int_{0}^{t} v^{*}\left(\beta_{k}, s\right) d s
\end{aligned}
$$

Let

$$
\begin{aligned}
& \bar{p}=\beta_{1} p_{1}+\beta_{2} p_{2}, \quad p_{\text {diff }}=\frac{p_{2}}{\rho_{2}}-\frac{p_{1}}{\rho_{1}} \\
& \frac{D_{k}}{D_{t}}=\frac{\partial}{\partial t}+v_{k} \frac{\partial}{\partial_{z}} .
\end{aligned}
$$

Then

$$
\begin{aligned}
& \bar{p}(z, t)=p_{2}\left(Z_{1}\right)+\int_{z_{1}}^{z} \sum_{k=1}^{2} \beta_{k} \rho_{k}\left(g-\frac{D_{k} v_{k}}{D t}\right) d z \\
& p_{\text {diff }}=p_{\text {diff }}\left(Z_{1}\right)-\int_{z_{1}}^{z}\left(\frac{D_{2} v_{2}}{D t}-\frac{D_{1} v_{1}}{D t}\right) d z
\end{aligned}
$$




\section{Asymptotic Expansion in Powers of M= Mach Number}

$\mathbf{0}^{\text {th }}$ order $\quad=$ incompressible $\mathbf{v}, \beta$

$1^{\text {st }}$ order $\quad=$ correction $v, \beta$

$2^{\text {nd }}$ order $\quad=$ incompressible $p_{1}, p_{2}$

$+v, \beta$ correction

$2^{\text {nd }}$ order $p_{1}, p_{2}=$ incompressible $p_{1}, p_{2}$ $\Rightarrow$ constraint:

"'missing" incompressible pressure equation Also resolves "missing" compressible closure.

Brookhaven Science Associates U.S. Department of Energy 


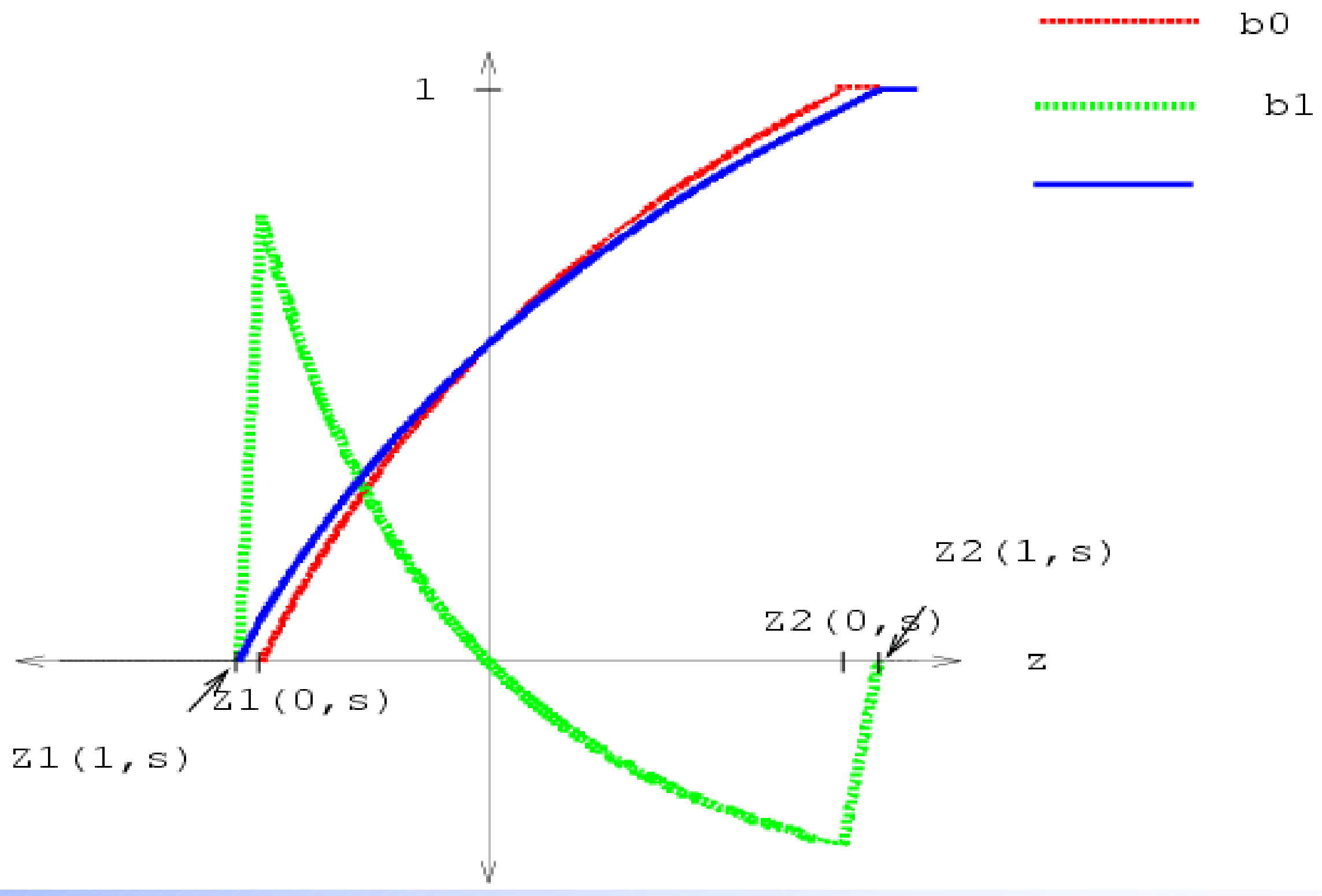




\section{Reduced Models: Equilibrated Pressures and Velocities}

Equilibrated pressures $\left(p_{1}=p_{2}\right)$

requires equilibrated velocities for hyperbolic equations.

Equilibrated velocities requires a diffusion term to move phase particles.

Diffusion can be computed within the Chunk Mix model. 


\section{RT and RM Diffusion Coefficients}

RT diffusion coefficient:

$D=2 A^{2} g^{2} t^{3}\left[\beta_{2} \alpha_{2}^{2}\left(\frac{\alpha_{1} \beta_{1}}{\alpha_{1} \beta_{1}+\alpha_{2} \beta_{2}}\right)^{3}+\beta_{1} \alpha_{1}^{2}\left(\frac{\alpha_{2} \beta_{2}}{\alpha_{1} \beta_{1}+\alpha_{2} \beta_{2}}\right)^{3}\right]$

RM diffusion coefficient ( $s$ = entrainment time, obtained from solution of ODE):

$$
\begin{aligned}
& D=\frac{\alpha_{2}^{2} \theta_{2} \beta_{2} s_{1}^{\theta_{2}}}{1+\tau} t^{\theta_{2}-1}+\frac{\alpha_{1}^{2} \theta_{1} \beta_{1} \tau_{2}^{\theta_{1}}}{1+\tau} t^{\theta_{1}-1} \\
& \tau=\frac{\alpha_{2} \beta_{2} \theta_{2}}{\alpha_{1} \beta_{1} \theta_{1}} t^{\theta_{2}-\theta_{1}}
\end{aligned}
$$

Brookhaven Science Associates U.S. Department of Energy 


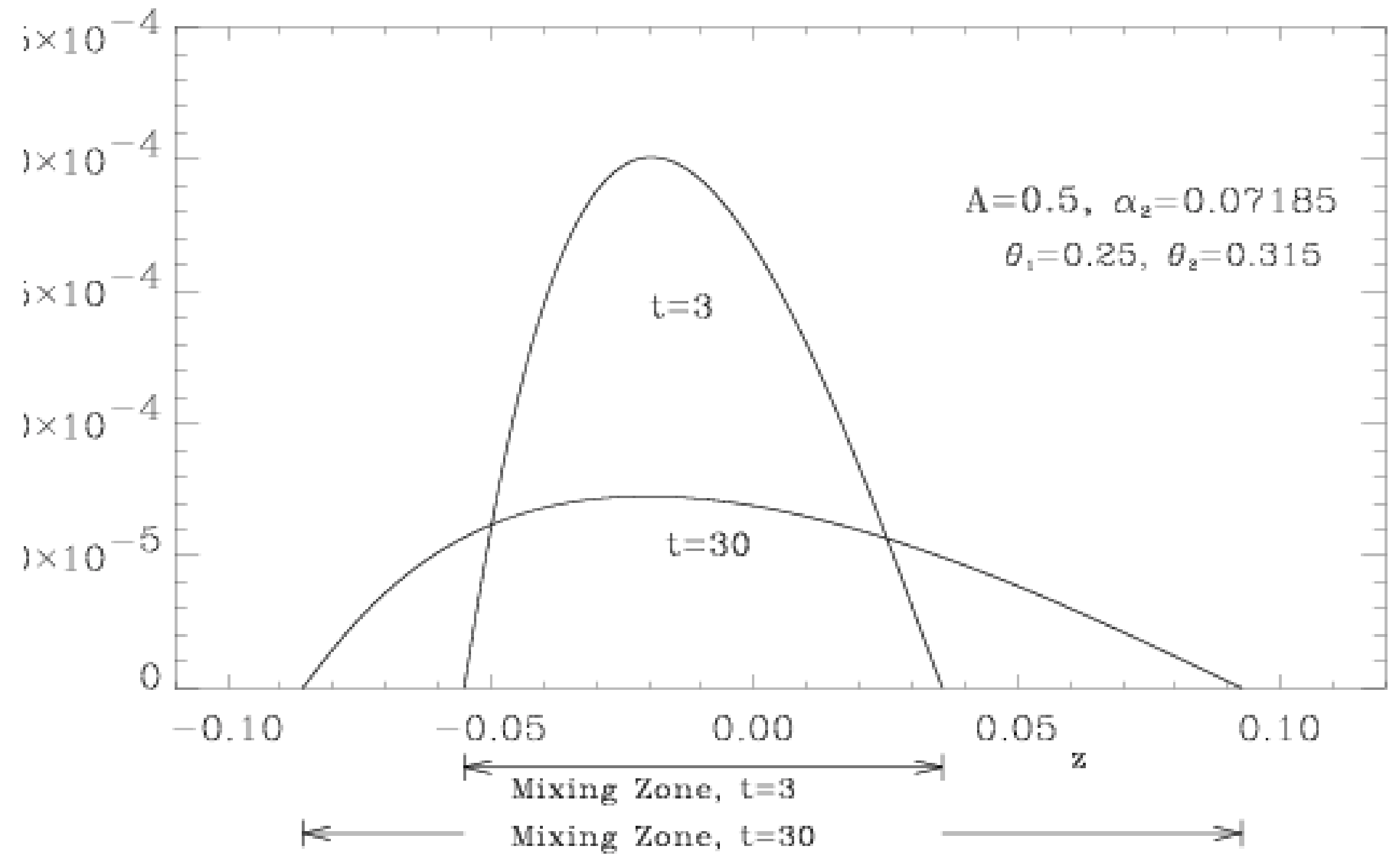




\section{Summary: A Predictive Science for Mix}

Consistent theory, simulation and experiment for 3D Rayleigh-Taylor fluid mixing

Determine the mixing zone edge motions for general accelerations in agreement with experiment and $A=1$ theory

Lower than leading order asymptotics with explicit dependence on intial conditions

Improved mix model equations: Stable mathematically and thermodynamically determinate

Asmptotics defined; closure improved 


\title{
Modeling Radiation Effects in Mixing Layers
}

\author{
Tim Clark (T-13) \\ Frank Harlow (T-3)
}

Theoretical Division

Los Alamos National Laboratory

December, 2001 


\section{Background/Goals}

- Models of Rayleigh-Taylor and Richtmyer-Meshkov mixing layers are relatively sophisticated.

- Multiphase flow models, turbulent models, hybrid models...

- Additional physical phenomena must be included for many practical circumstances.

- Material strength, heat flux, evaporation....

- Goal: To develop a simple model to describe the effects of radiative heat transfer and ablation on a turbulent mixing layer.

- Emphasis is on simplicity--fidelity will be assessed by comparison to experiment or simulations, possibly motivating additional complications.

- Competition between instability growth rate and ablative growth rate; depends on the initial scale of the perturbations. 


\section{Strategy/Problem Formulation}

- Multi-material flow formulation.

- Appropriate for multi-material problems...

- Simple drag model for multi-field interaction.

- May include more sophisticated models later, as needed.

- Radiation diffusion approach.

- For simplicity...

- Simple heat transport model.

- Heat exchange occurs in a thin "skin" of the cold material.

- Heat transfer to cold material leads to ablation, not temperature increase.

- Ablated "cold" material becomes "hot fluid" (e.g. melting ice in water etc.)

- Cold material and hot fluid experience PdV work (and temperature change). 


\section{Heat Transfer Model}

- Prescription for area for heat transfer per unit volume

- Uniform distribution of "spheres" of varying sizes.

- (Similar to simple spherical-particle model for multi-fluid drag models?)

- Quasi-steady state for local heat flux at particle surface.

- Model:

$$
Q=\left(\frac{12 \pi}{7}\right) \frac{\alpha_{1} \alpha_{2} \kappa\left(T_{2}\right)}{\rho_{2} r^{2}\left[1-\left(\frac{3 \alpha_{1}}{4 \pi}\right)^{1 / 3}\right]}\left(T_{2}^{4}-T_{1}^{4}\right)
$$

- We also have an equation for r ("radius"):

$$
\frac{\partial r}{\partial t}+u_{1 n} \frac{\partial r}{\partial x_{n}}=-\frac{r}{3 \rho_{1}}\left\{\frac{d \rho_{1}}{d t}+\frac{1}{\alpha_{1}}\left(\frac{Q}{C_{v 1} T_{c 1}+L_{H 1}}\right)\right\}
$$




\section{Two-Field Continuity Equation}

- Cold material (1) is heated and becomes hot fluid (2).

$$
\frac{\partial \rho_{k} \alpha_{k}}{\partial t}+\frac{\partial \rho_{k} \alpha_{k} u_{k n}}{\partial x_{n}}=(-1)^{k} \frac{Q}{C_{v 1} T_{c 1}+L_{H 1}}
$$

- Material exchange rate is related to heat transfer rate, Q.

- More complicated model could be incorporated, involving temperature increase and then "evaporation."

- Adjustable rate base on $\mathrm{C}_{\mathrm{v} 1} \mathrm{~T}_{\mathrm{c} 1}+\mathrm{L}_{\mathrm{H} 1}$ 


\section{Momentum Equation}

- Momentum equation

- Changes in momentum enter through pressure and changes in mass fractions.

$$
\frac{\partial \rho_{k} \alpha_{k} u_{k i}}{\partial t}+\frac{\partial \rho_{k} \alpha_{k} u_{k i} u_{k n}}{\partial x_{n}}=-\alpha_{k} \frac{\partial P}{\partial x_{i}}+\bar{\rho} \kappa_{D}\left(u_{k^{\prime} i}-u_{k i}\right)
$$

- Drag coefficient is based on a simple spherical particle drag model (courtesy of B. Kashiwa, T-3)(Note Re is very large...).

- We need additional guidance from simulation or experiment to improve this model for ablating materials.

$$
\kappa_{D}=\frac{3 C_{D}}{8 r} \frac{\alpha_{1} \alpha_{2}}{\rho}\left|u_{1}-u_{2}\right| \quad C_{D}=0.44+\frac{24.0}{\operatorname{Re}}+\frac{6}{1+\operatorname{Re}^{1 / 2}}
$$




\section{Equations of State (present example...)}

- Simple equation of state for sample calculation.

- Model does not require a particular thermodynamic EOS.

- May need better EOS for comparison to experiments.

- Hot fluid (2) has radiation pressure contribution.

- May require radiation pressure and heat transfer/temperature increase in cold material if $T_{c}$ is much hotter than $T_{1}$.

- Pressure equilibration between phases.

- Restrictive assumption-- presents difficulties when temperature difference is very large....

$$
\begin{array}{cc}
p_{1}=c_{v 1}\left(\gamma_{1}-1\right) \rho_{1} T_{1} & p_{2}=c_{v 2}\left(\gamma_{2}-1\right) \rho_{2} T_{2}+\frac{4 \sigma}{3 c} T_{2}^{4} \\
I_{1}=c_{v 1} T_{1} & I_{2}=c_{v 2} T_{2}+\frac{4 \sigma}{\rho_{2} c} T_{2}^{4}
\end{array}
$$




\section{Energy Equation}

- Energy equations

$$
\begin{gathered}
\frac{\partial \rho_{1} \alpha_{1} I_{1}}{\partial t}+\frac{\partial \rho_{1} \alpha_{1} u_{1 i} I_{1}}{\partial x_{n}}=+P_{1} \frac{\alpha_{1}}{\rho_{1}} \frac{d \rho_{1}}{d t} \\
\frac{\partial \rho_{2} \alpha_{2} I_{2}}{\partial t}+\frac{\partial \rho_{2} \alpha_{2} u_{2 i} C_{v 2} T_{2}}{\partial x_{n}}=\frac{\partial}{\partial x_{n}}\left\{\frac{\alpha_{2}}{\rho_{2}} \kappa_{2}\left(T_{2}\right) \frac{\partial T_{2}^{4}}{\partial x_{n}}\right\}+P_{2} \frac{\alpha_{2}}{\rho_{2}} \frac{d \rho_{2}}{d t}-Q \\
\text { - Opacity: } \mu\left(T_{2}\right)=\mu_{0}\left(\frac{T_{0}}{T_{2}}\right)^{3}+\mu_{\text {scattering }} \\
\text { - Transmissivity: } \quad \kappa\left(T_{2}\right)=\frac{1}{\mu\left(T_{2}\right)}
\end{gathered}
$$




\section{Turbulence Model?}

- Current formulation does not have a detailed model for turbulence.

- Need more comparison to simulations/experiments for motivation

- We could add hybrid multi-phase model.

- Cranfill's hybrid model, Youngs model etc.

- Modification of turbulence model for ablated materials?

- Fluctuating velocity is not solenoidal, pressure fluctuations tied to radiation (i.e., opacity....), and material transfer, et cetera.

- Such modifications would require simulations and experiment for guidance... 


\section{Sample Problem}

- Problem is statistically one-dimensional.

- Hot fluid (2) is $1.5 \mathrm{keV}$, cold fluid (2) is $0.2 \mathrm{keV}$

- Boundary conditions:

- Hot side (left) is constant temperature, no mass flux or velocity.

- Cold side (right) is simple out-flow boundary $(\mathrm{dp} / \mathrm{dx}=0)$.

- Pressure equilibration in mixed zone requires some artful choices.

- Assume that pressure in mixing zone has a smooth transition from hot side to cold side.

- Choice of constants based on iron properties (and expedience)... 


\section{Sample Problem Parameters}

- Constants:

$$
\begin{aligned}
& \Sigma_{0}=10^{4} \frac{\mathrm{cm}^{2}}{\mathrm{gm}}, \quad \Sigma_{\text {scatter }}=0.2 \frac{\mathrm{cm}^{2}}{\mathrm{gm}} \\
& C_{v 1}=C_{v 2}=6.856 \times 10^{-3} \frac{j}{\mathrm{gm} \mathrm{keV}} \\
& \gamma_{1}=\gamma_{2}=0.6667 \\
& T_{c}=0.2 \mathrm{keV}, \quad L_{H}=0.2 \frac{j}{\mathrm{gm}} \\
& \text { Pressure is in }\left(\frac{j}{\mathrm{~cm}^{3}}\right), \quad \text { Energy, } I_{k} \text { is in }\left(\frac{j}{\mathrm{gm}}\right)
\end{aligned}
$$




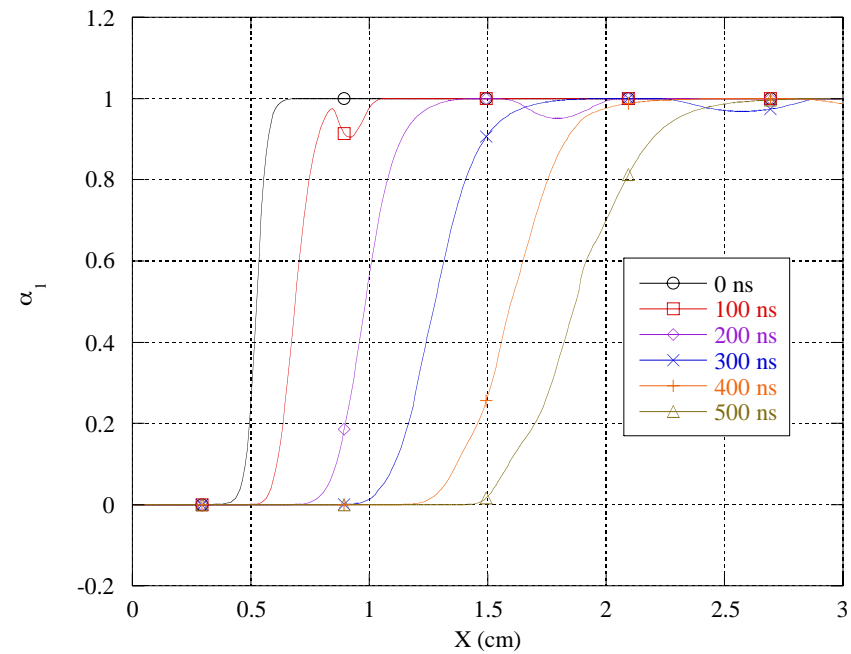

Volume Fraction of Cold Fluid $R_{0}=1.0 \mathrm{~cm}$

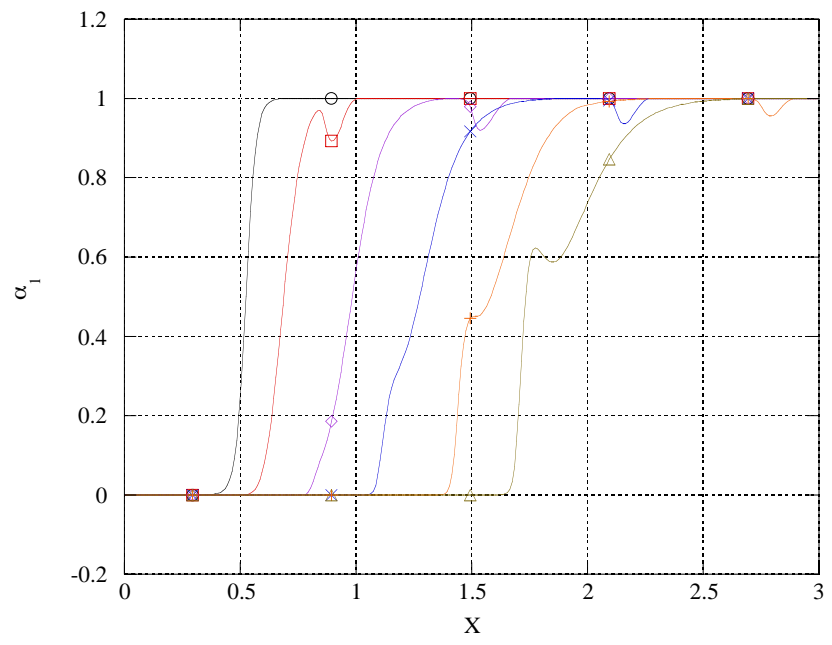

Volume Fraction of Cold Fluid $R_{0}=1.0 \times 10^{-1} \mathrm{~cm}$

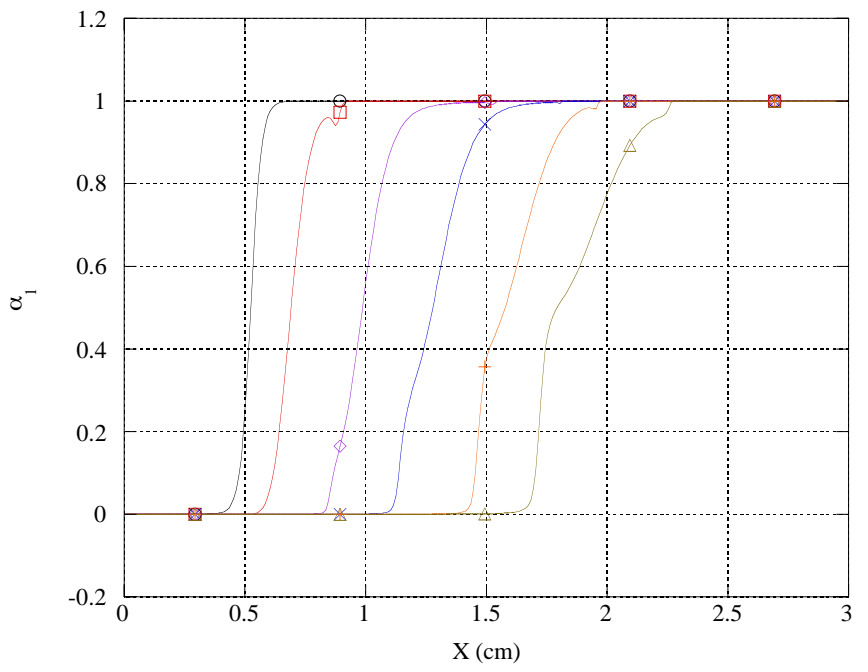

Volume Fraction of Cold Fluid $R_{0}=1.0 \times 10^{-2} \mathrm{~cm}$ 


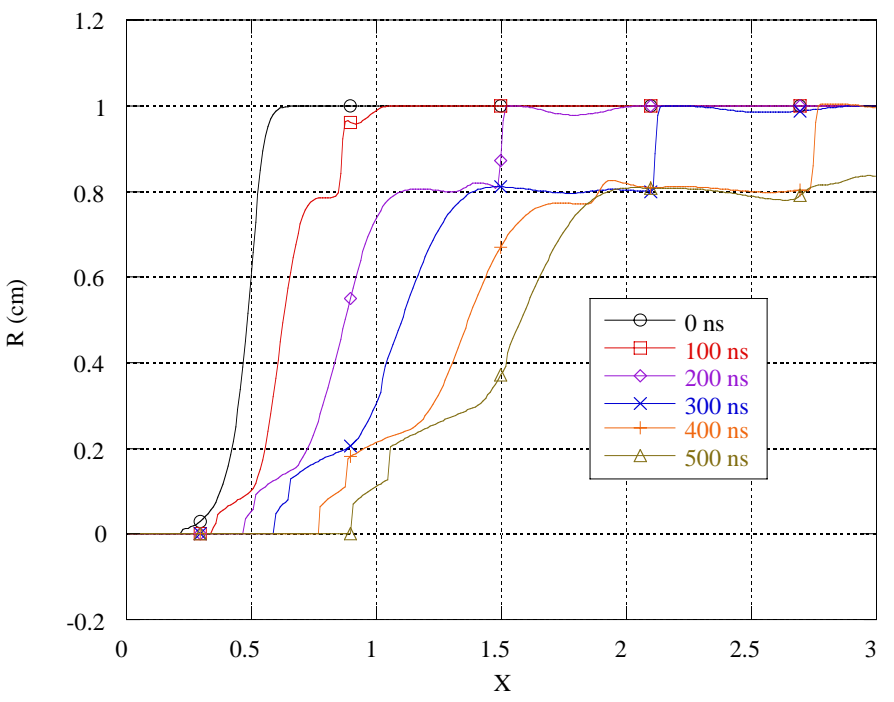

Radius of Characteristic "Droplet"

$R_{0}=1.0 \mathrm{~cm}$

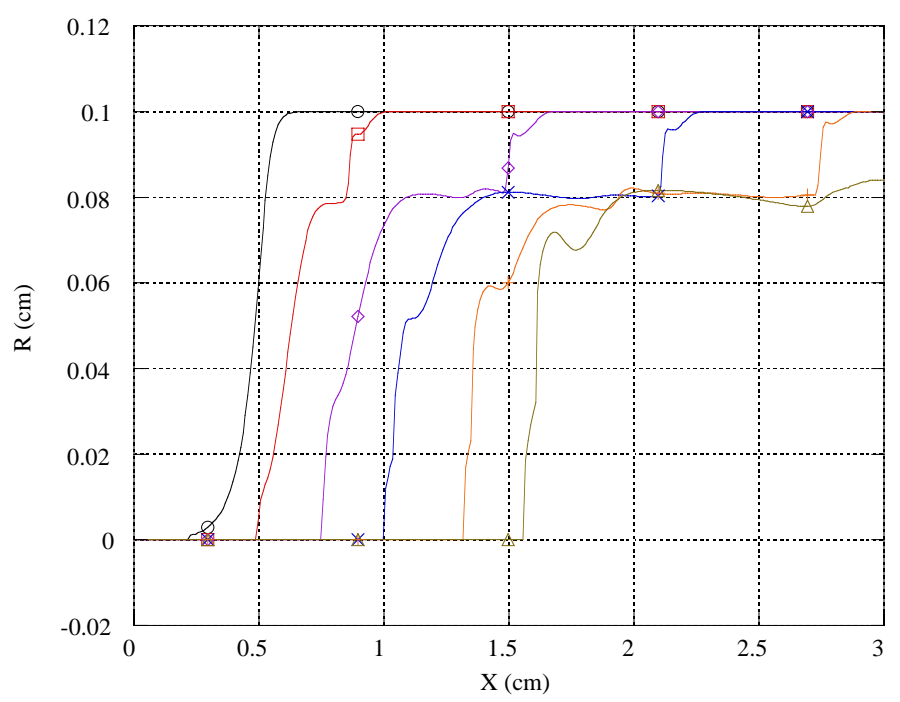

Radius of Characteristic "Droplet"

$$
R_{0}=1.0 \times 10^{-1} \mathrm{~cm}
$$

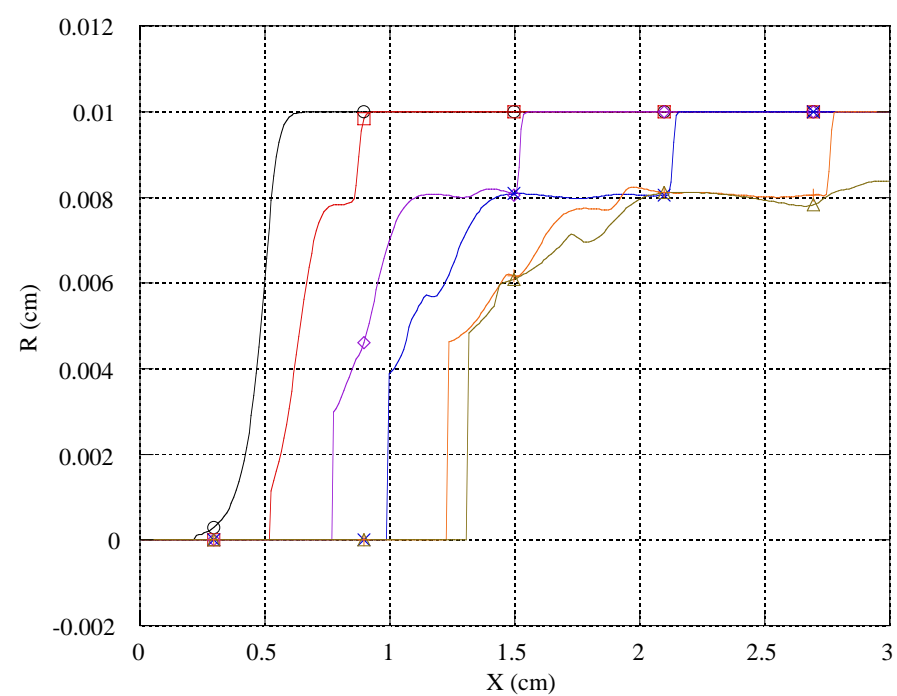

Radius of Characteristic "Droplet"

$$
R_{0}=1.0 \times 10^{-2} \mathrm{~cm}
$$




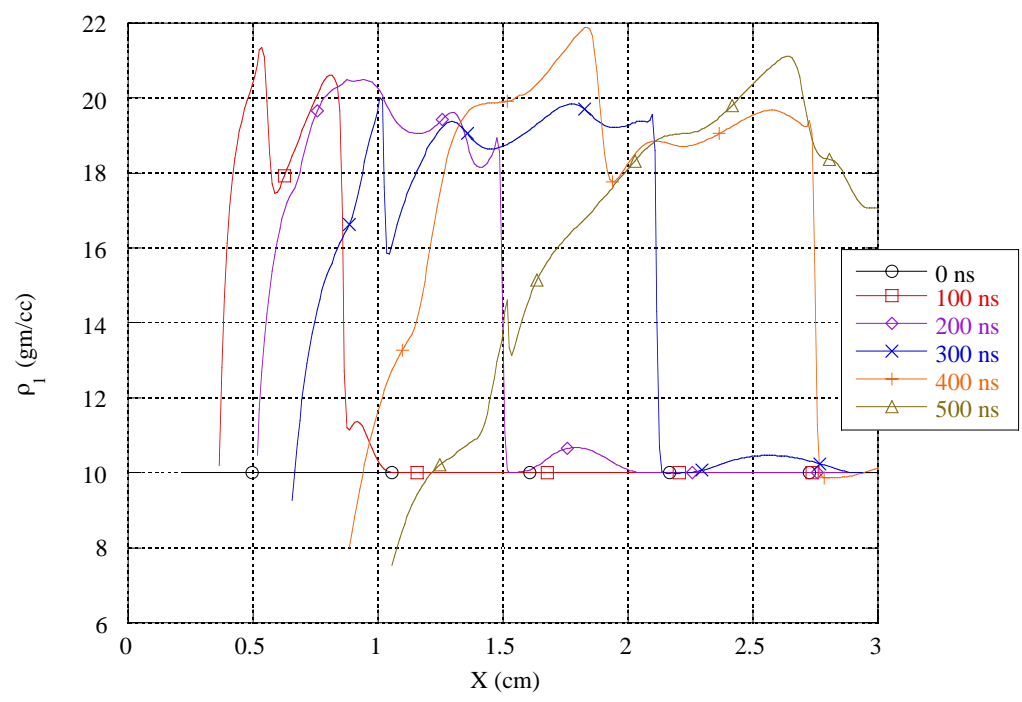

Density of Cold Fluid

$R_{0}=1.0 \mathrm{~cm}$

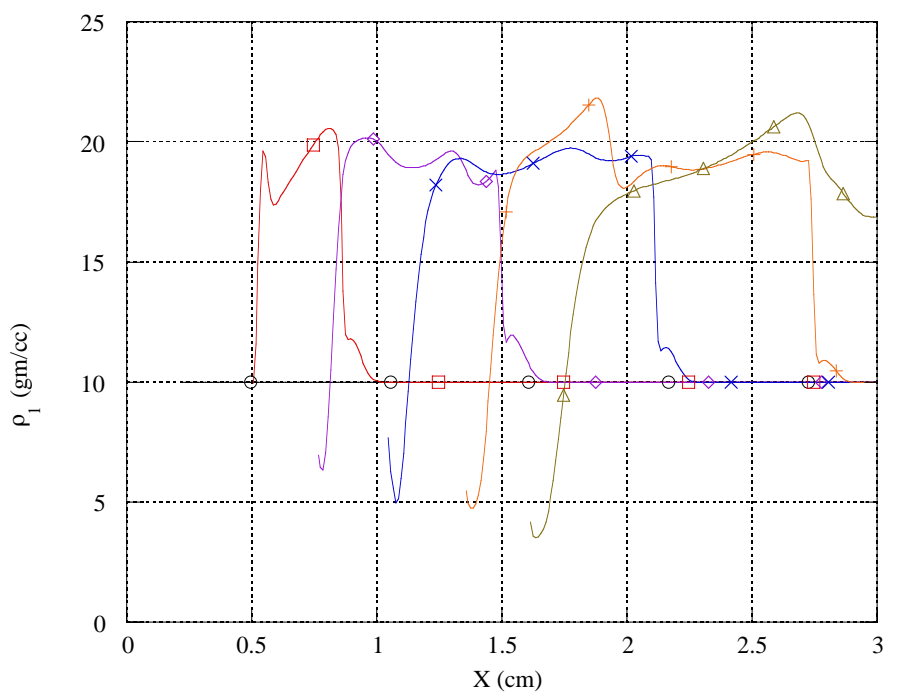

Density of Cold Fluid

$R_{0}=1.0 \times 10^{-1} \mathrm{~cm}$

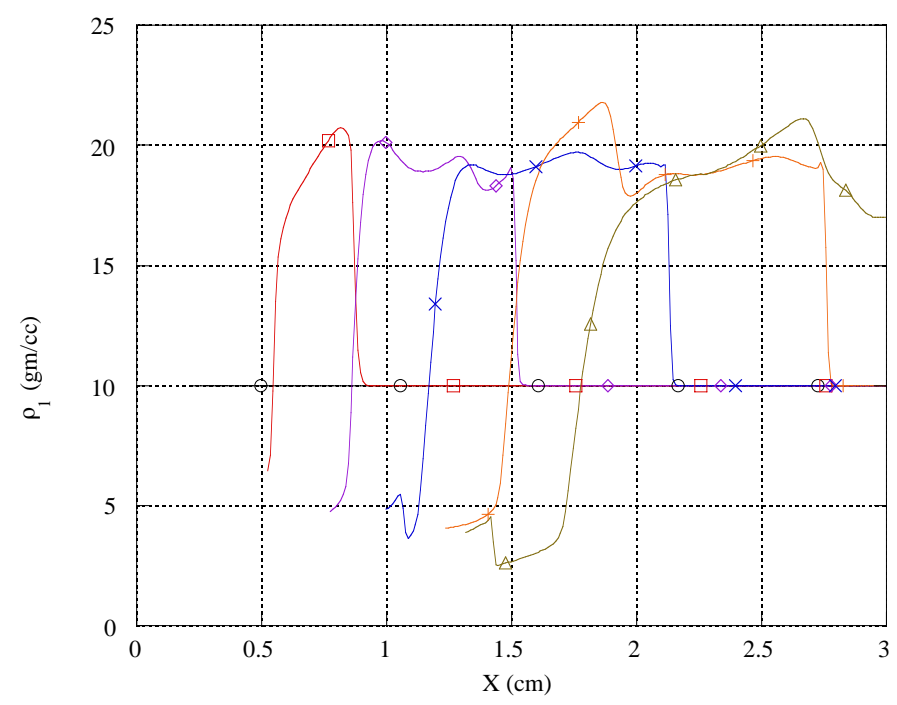

Density of Cold Fluid

$R_{0}=1.0 \times 10^{-2} \mathrm{~cm}$ 


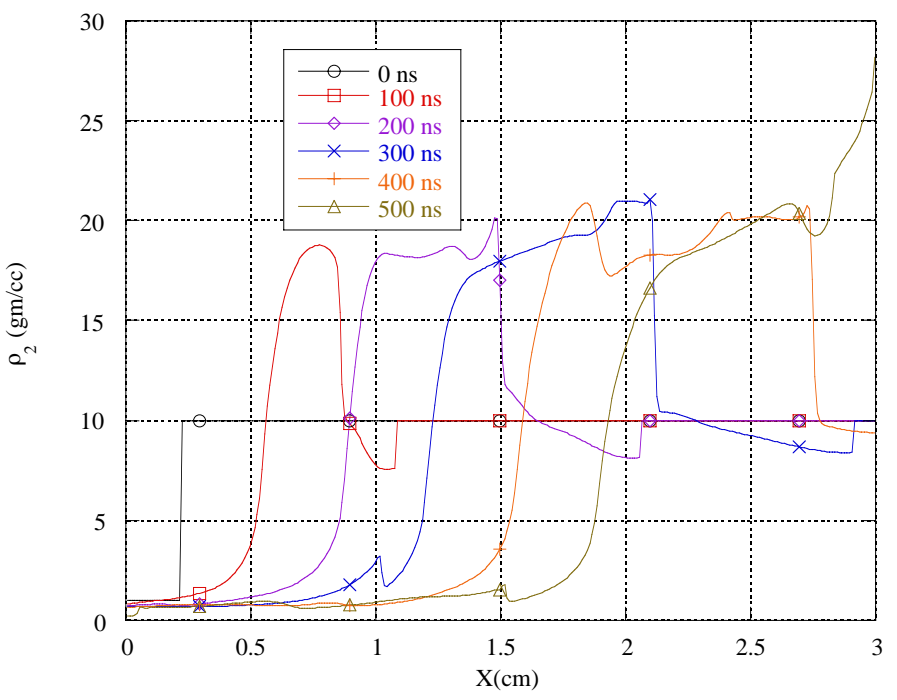

Density of Hot Fluid

$R_{0}=1.0 \mathrm{~cm}$

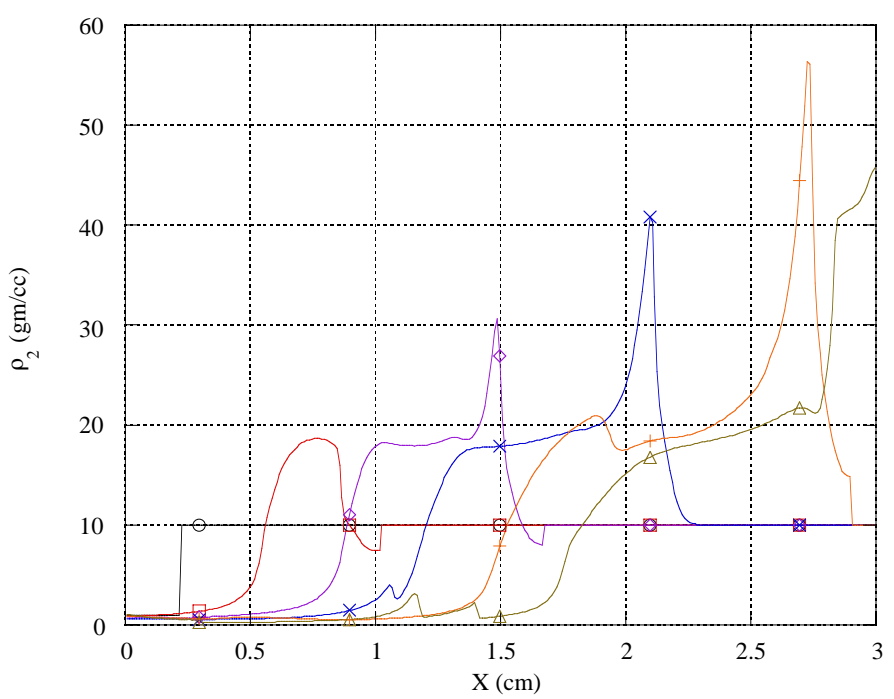

Density of Hot Fluid

$R_{0}=1.0 \times 10^{-1} \mathrm{~cm}$

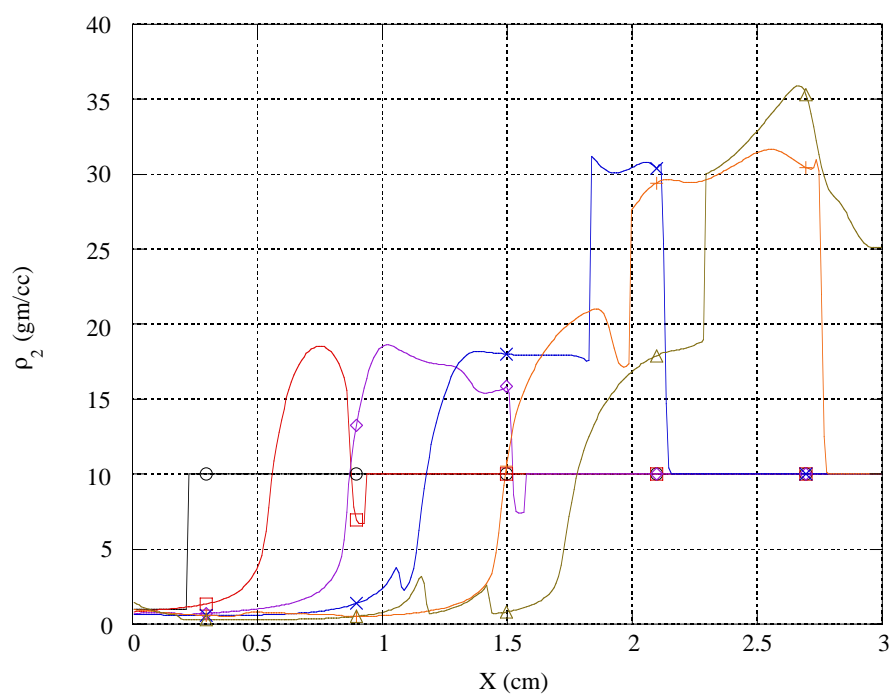

Density of Hot Fluid

$R_{0}=1.0 \times 10^{-2} \mathrm{~cm}$ 


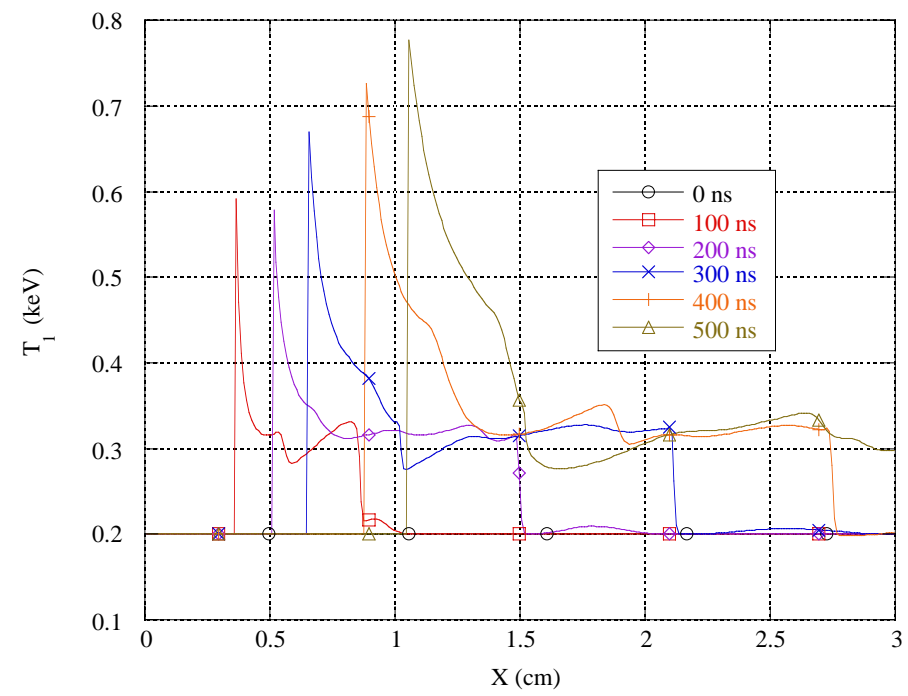

Temperature of Cold Fluid $R_{0}=1.0 \mathrm{~cm}$

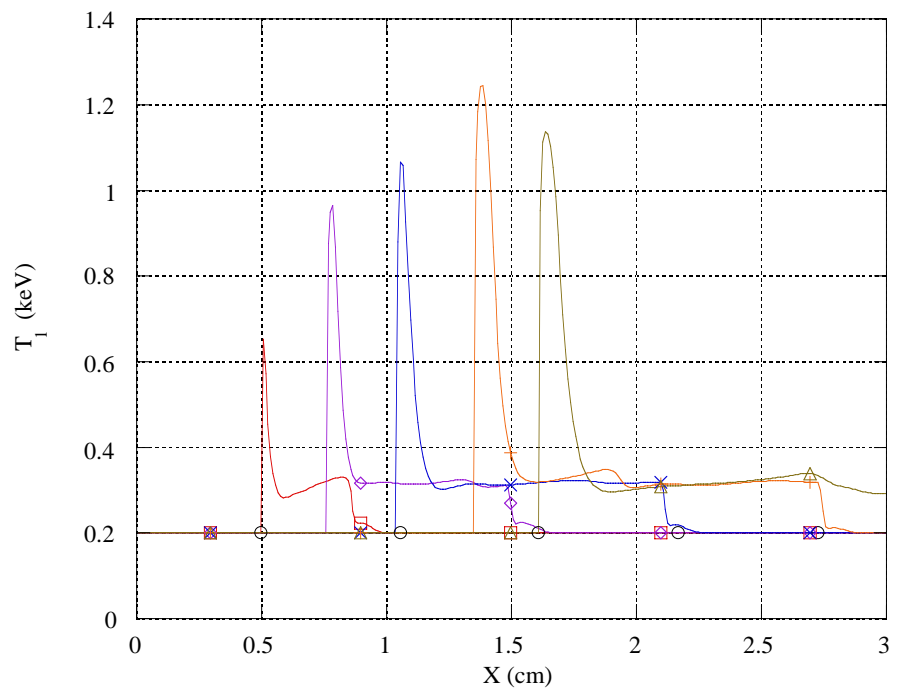

Temperature of Cold Fluid $R_{0}=1.0 \times 10^{-1} \mathrm{~cm}$

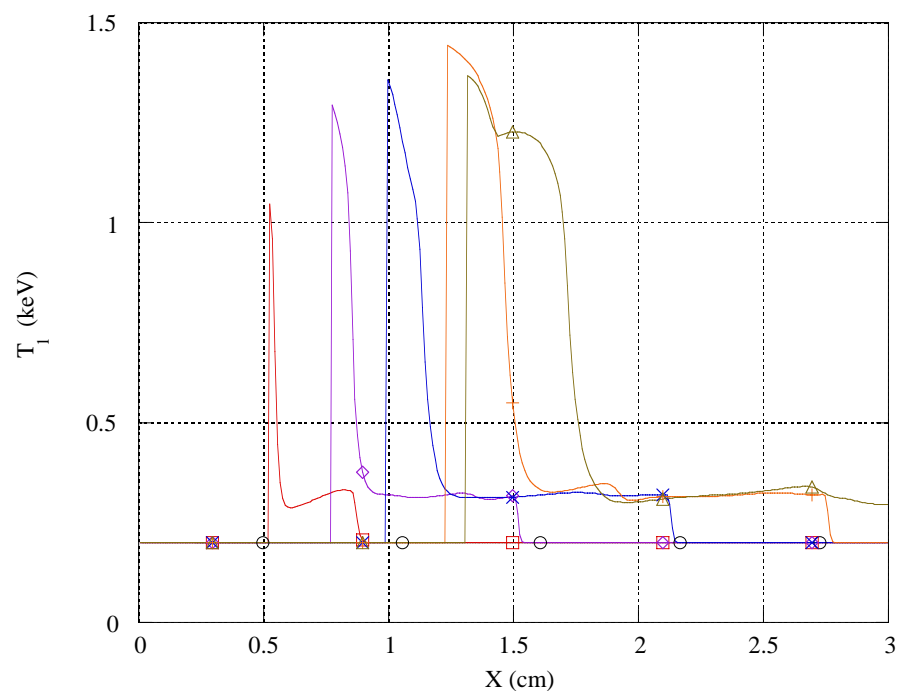

Temperature of Cold Fluid $R_{0}=1.0 \times 10^{-2} \mathrm{~cm}$ 


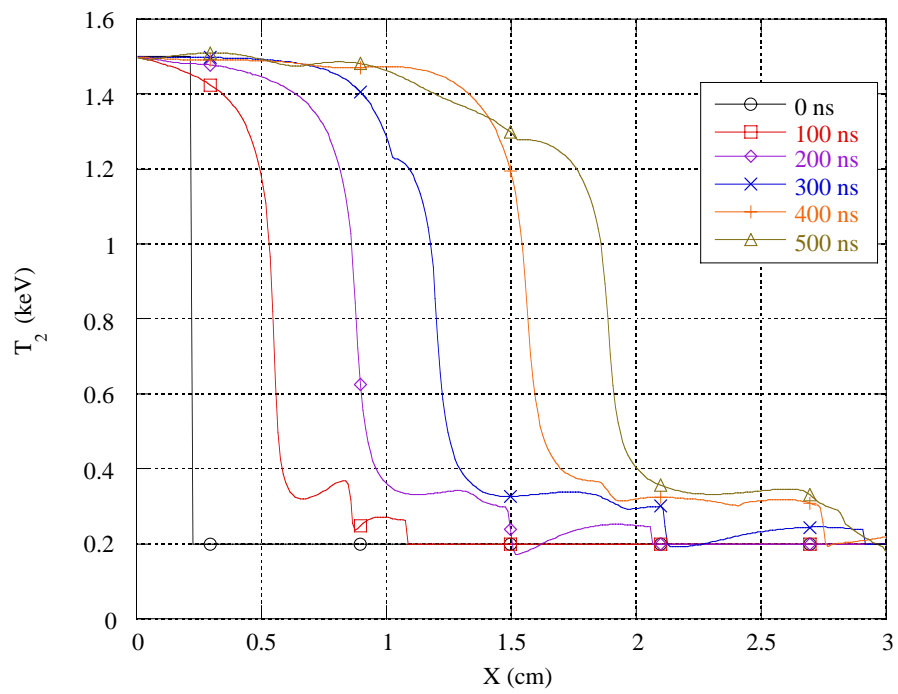

Temperature of the Hot Fluid

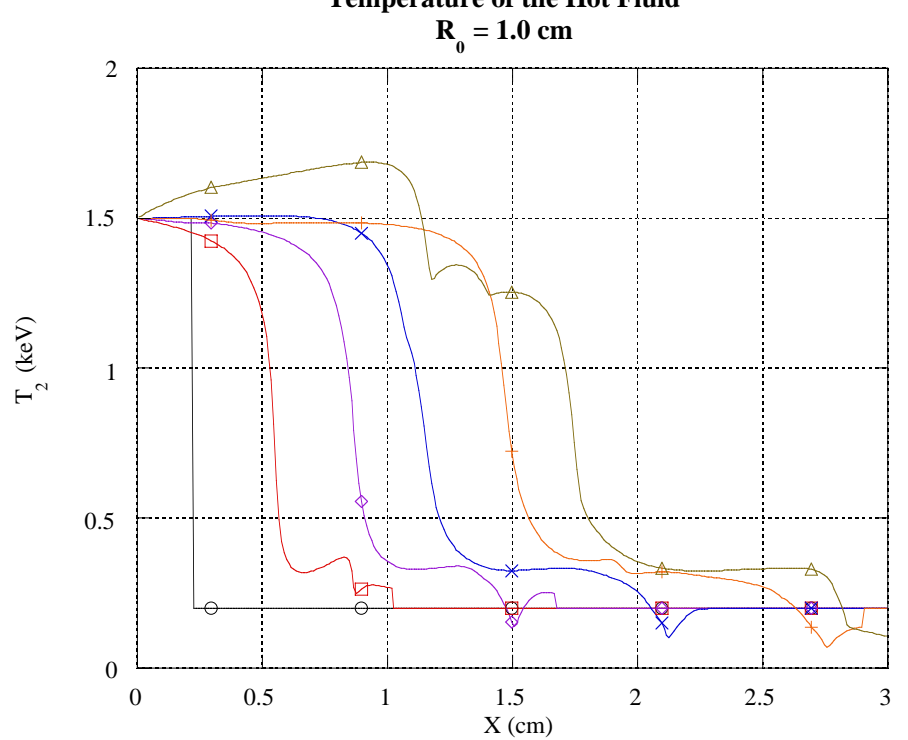

Temperature of the Hot Fluid $R_{0}=1.0 \times 10^{-1} \mathrm{~cm}$

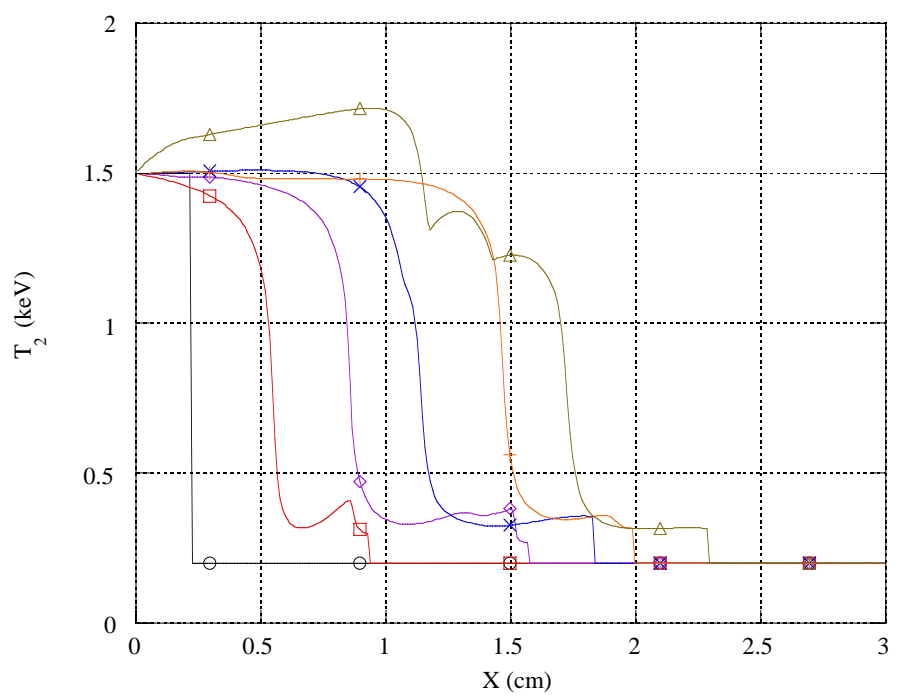

Temperature of the Hot Fluid $R_{0}=1.0 \times 10^{-2} \mathrm{~cm}$ 


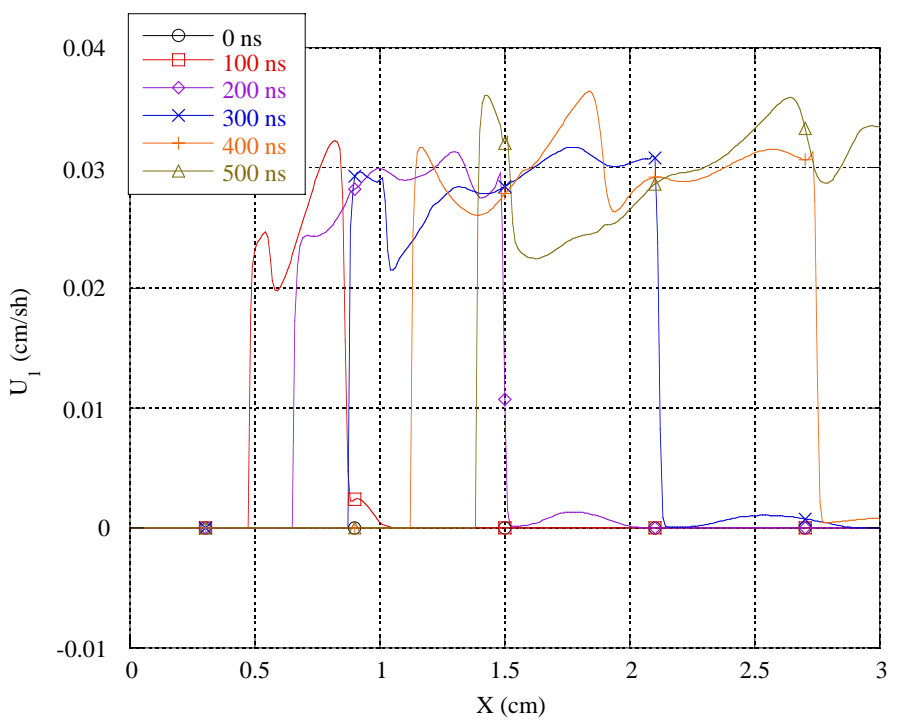

Velocity of Cold Fluid

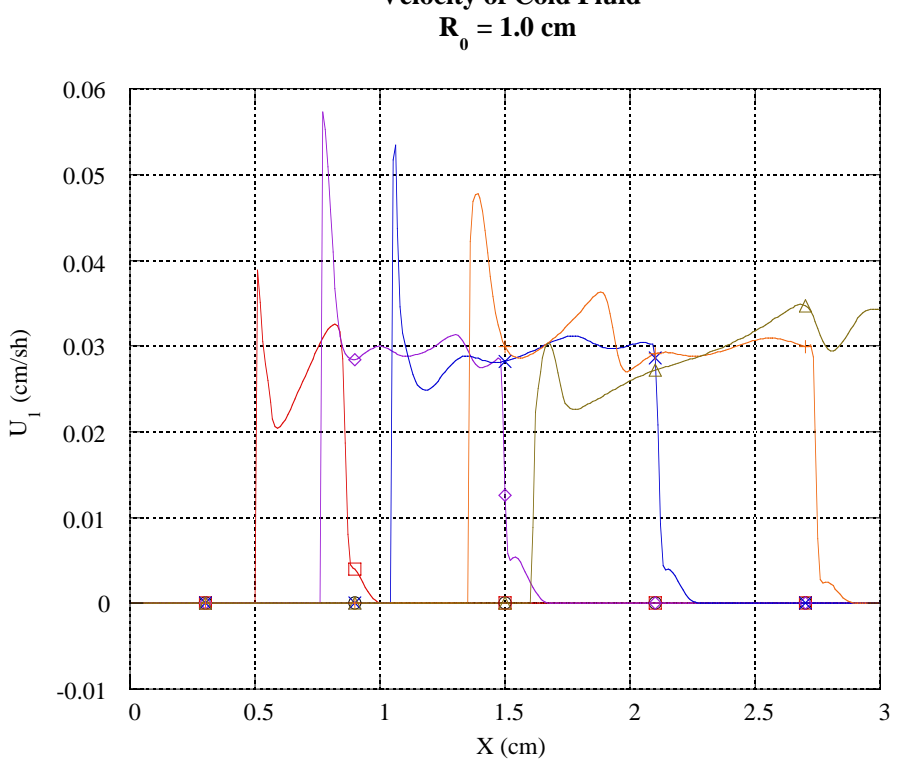

Velocity of Cold Fluid

$R_{0}=1.0 \times 10^{-1} \mathrm{~cm}$

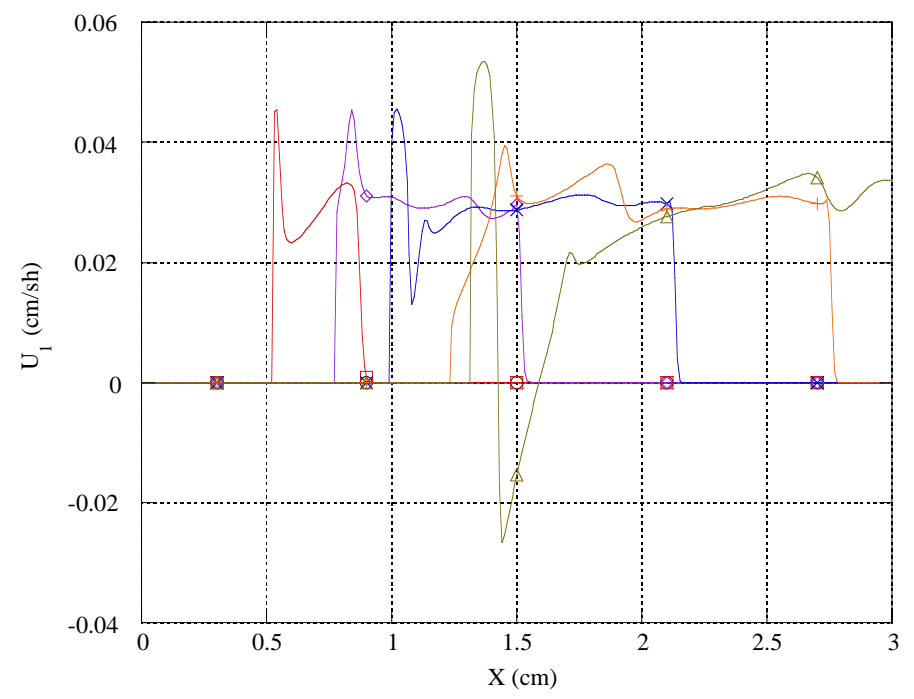

Velocity of Cold Fluid

$R_{0}=1.0 \times 10^{-2} \mathrm{~cm}$ 


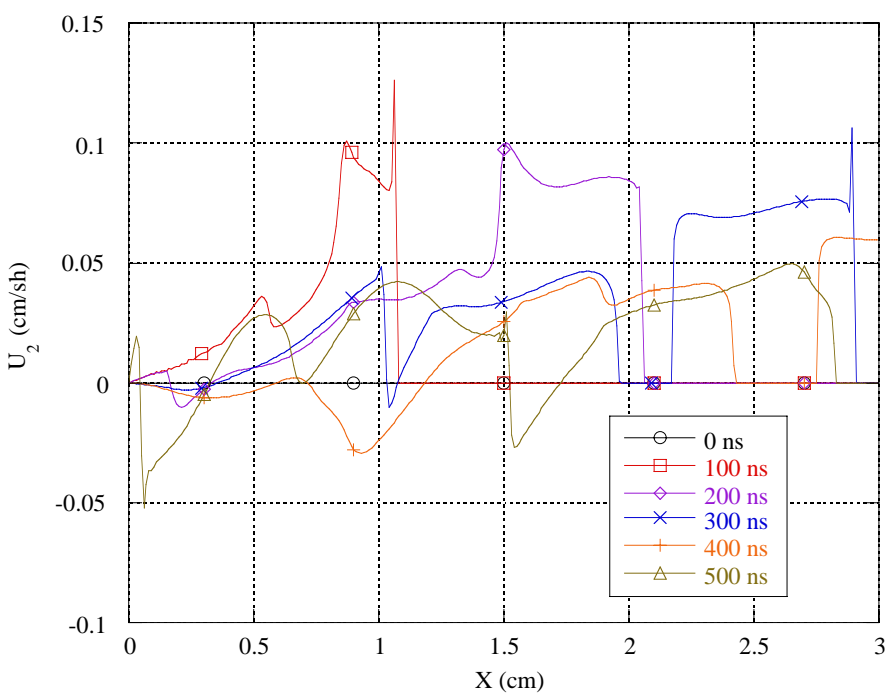

Velocity of Hot Fluid

$R_{0}=1.0 \mathrm{~cm}$

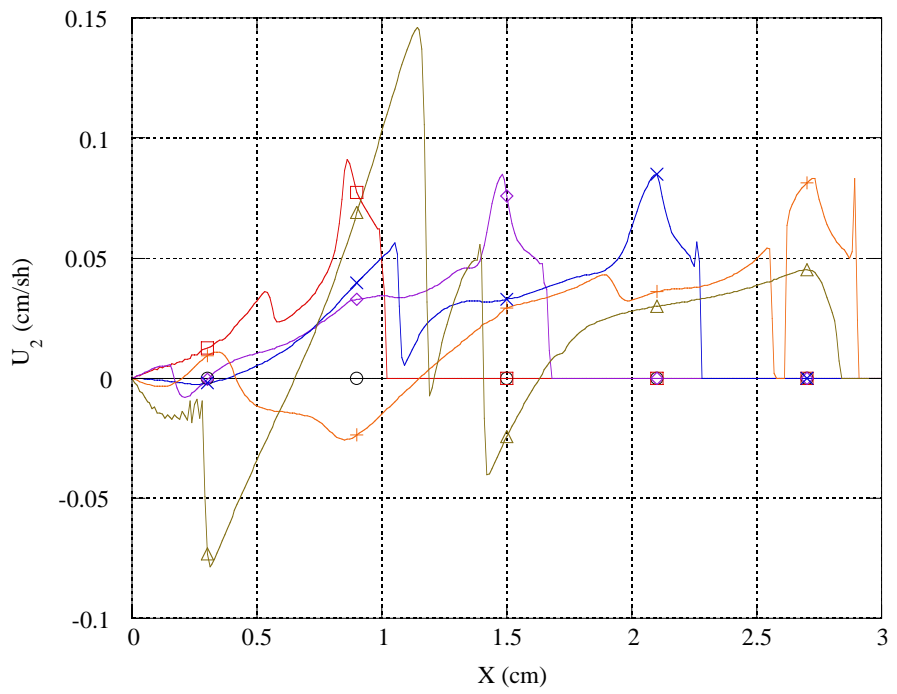

Velocity of Hot Fluid

$R_{0}=1.0 \times 10^{-1} \mathrm{~cm}$

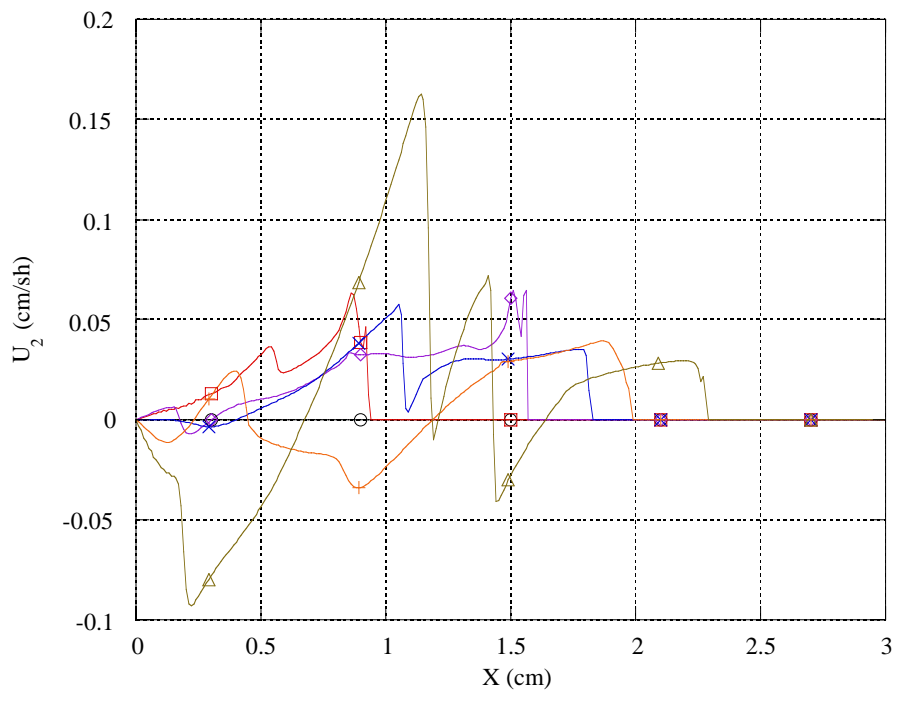

Velocity of Hot Fluid

$R_{0}=1.0 \times 10^{-2} \mathrm{~cm}$ 


\section{Conclusions}

- Model represents a simple, tractable approach to account for radiation and phase change in a two-phase flow.

- Length scale for radiation transfer (spherical model) is consistent with the drag model.

- Can be extended to multiple fluids, and more complicated prescriptions for drag, turbulence, heat exchange.

- Demonstrates ablative phenomenon.

- Have not fully explored parameter regimes.

- We now need detailed comparisons to experiments, simulations and observations.

- Laser-driven flyer plates, Cepheid variables, Computer simulations. 


\title{
Toy models for Rayleigh- Taylor instability:
}

\author{
Stuart Dalziel \\ Department of Applied Mathematics and Theoretical Physics \\ University of Cambridge
}

International Workshop on the Physics of Compressible Turbulent Mixing

14 December 2001

$$
\text { (9:10 - 9:30) }
$$

\author{
with thanks to \\ Joanne Holford (DAMTP) \\ David Youngs (AWE)
}




\section{The growth question:}

$$
h=\alpha A g t^{2}, \text { where } A=\frac{\rho_{1}-\rho_{2}}{\rho_{1}+\rho_{2}}
$$

\section{But what is $\alpha$ ?}

$\diamond 0.10, \ldots 0.07,0.06, \ldots 0.03,0.02 ?$

Timescale:

$$
T=\sqrt{\frac{H}{A g}}
$$

If $\delta=h / H$, and $\tau=t / T$,

then

$$
\delta=\alpha \tau^{2}
$$




\section{Experiments}
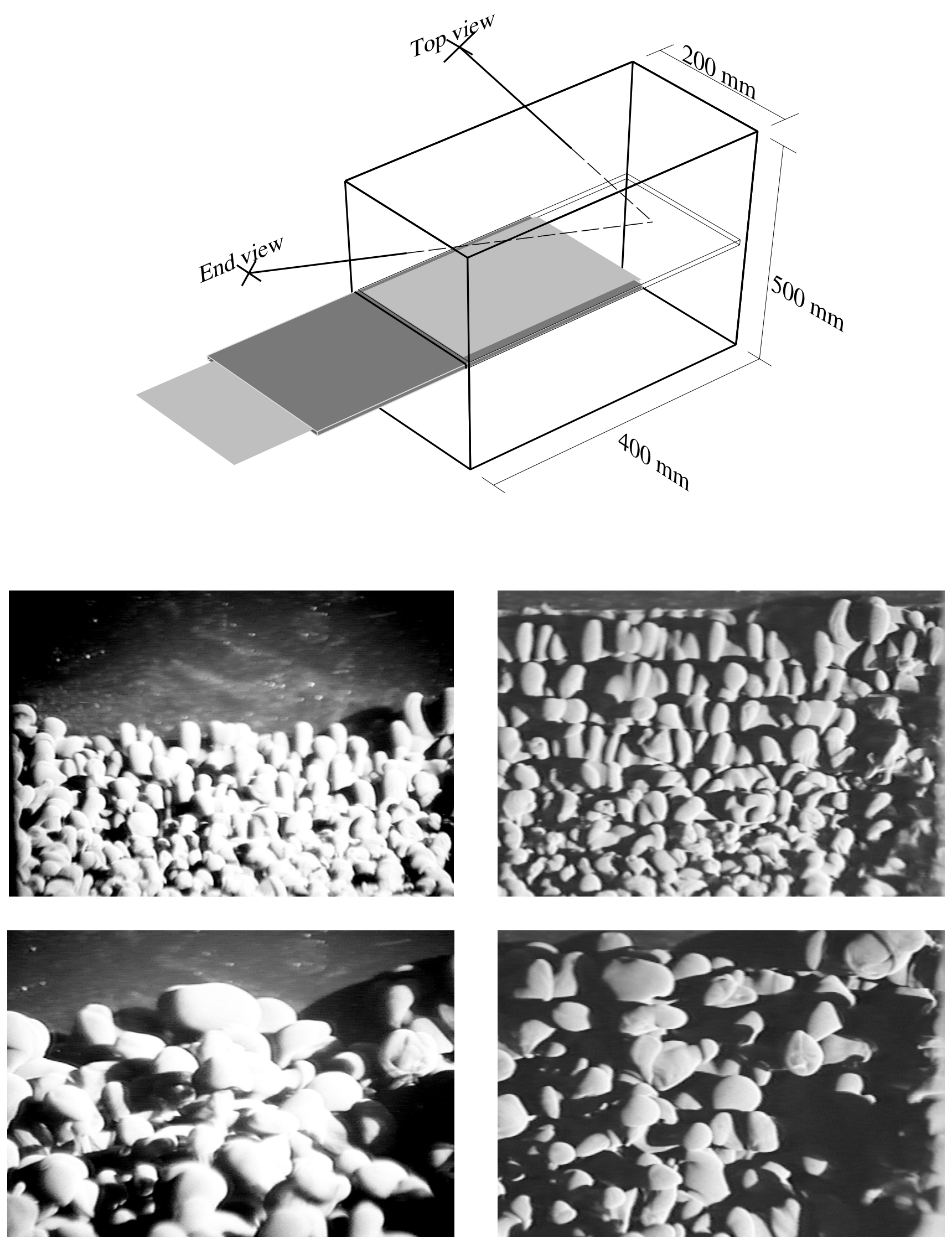
Appropriate modelling (?)
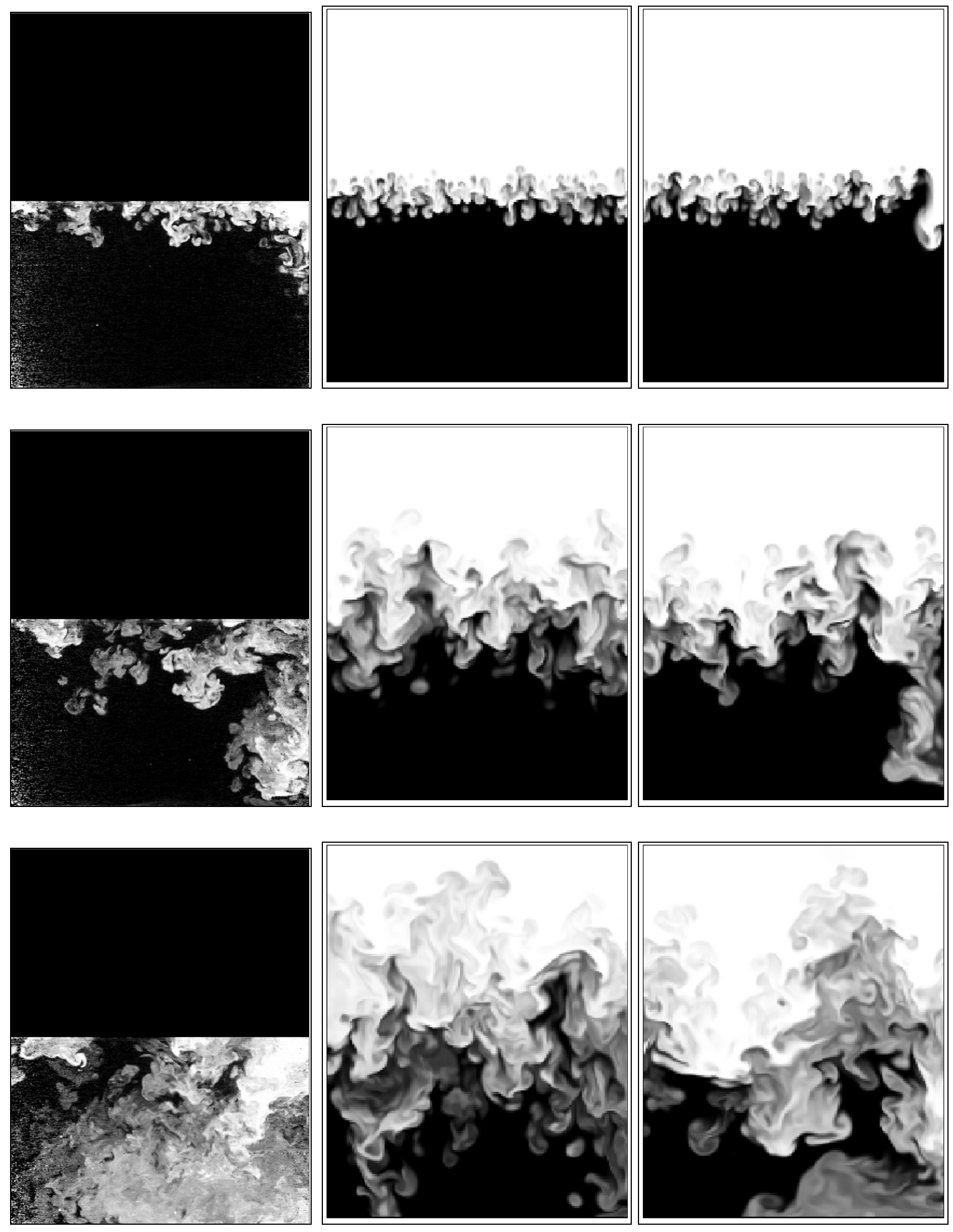


\section{Growth}

\section{Dimensional analysis/similarity theory}

$$
h=\alpha A g t^{2} \text {. }
$$

\section{Single mode}

Layzer (1955)

For

$$
\zeta(x, y)=a_{0} \cos \frac{2 \pi x}{\lambda}
$$

if

$$
\frac{d h}{d t}=w,
$$

then

$$
(2+E) \frac{d w}{d t}=A g(1-E)-C_{D} \frac{w^{2}}{\lambda},
$$

where

$$
E=\exp \left(\frac{-6 \pi h}{\lambda}\right) .
$$

Experimentally $C_{D} \sim 10$

$\diamond$ Does this make sense?

Early time $\rightarrow$ linear theory $\frac{d^{2} h}{d t^{2}}=\frac{2 \pi A g}{\lambda} h$

Late time $\rightarrow$ constant velocity $w_{\infty}=\sqrt{\frac{A g \lambda}{C_{D}}}$

$\Rightarrow h \rightarrow w_{\infty}\left(t-t_{0}\right)$ 


\section{Structure}
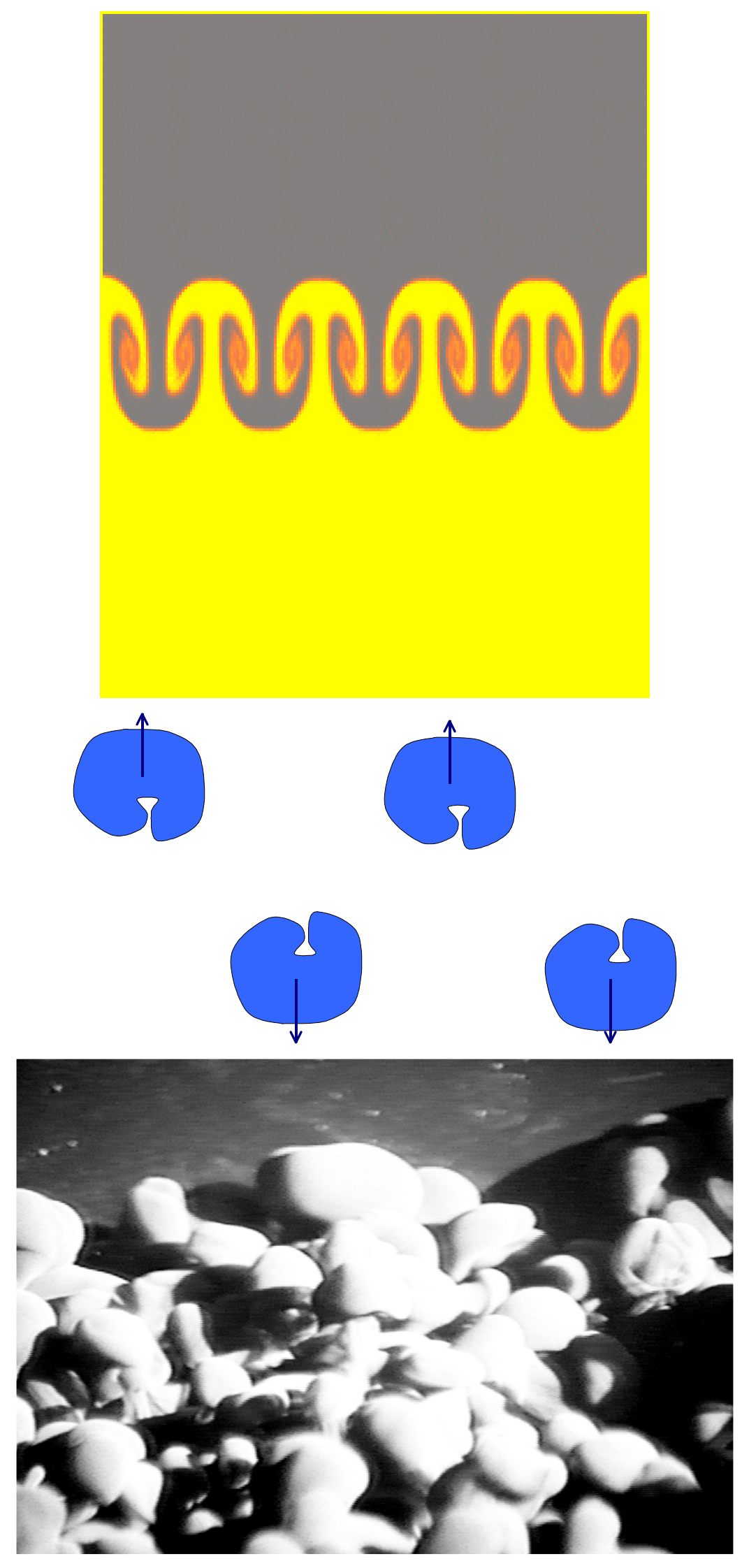

Often described as 'bubbles'...

...but more like 'thermals' in miscible fluids 


\section{Thermals}

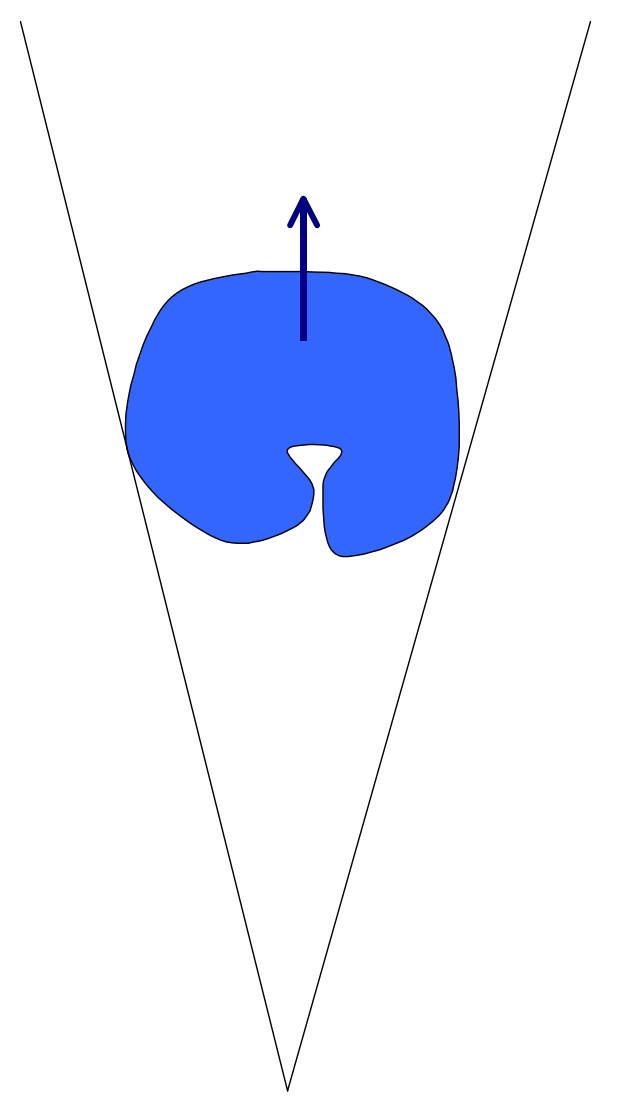

Self-similar

$$
\begin{gathered}
r=\theta z \\
V=\gamma r^{3} .
\end{gathered}
$$

Buoyancy conserved

$$
g^{\prime} V=g^{\prime} \gamma r^{3}=g_{0}^{\prime} V_{0} .
$$

Constant Froude number

$$
F^{2}=\frac{w^{2}}{g^{\prime} r}
$$

Integrating $w=d z / d t$

$$
\frac{\gamma^{1 / 2} \theta}{2 F\left(g_{0}^{\prime} V_{0}\right)^{1 / 2}} z^{2}=t
$$

Experimental results $\rightarrow F \approx 1.2$. 


\section{Rayleigh-Taylor as thermals}
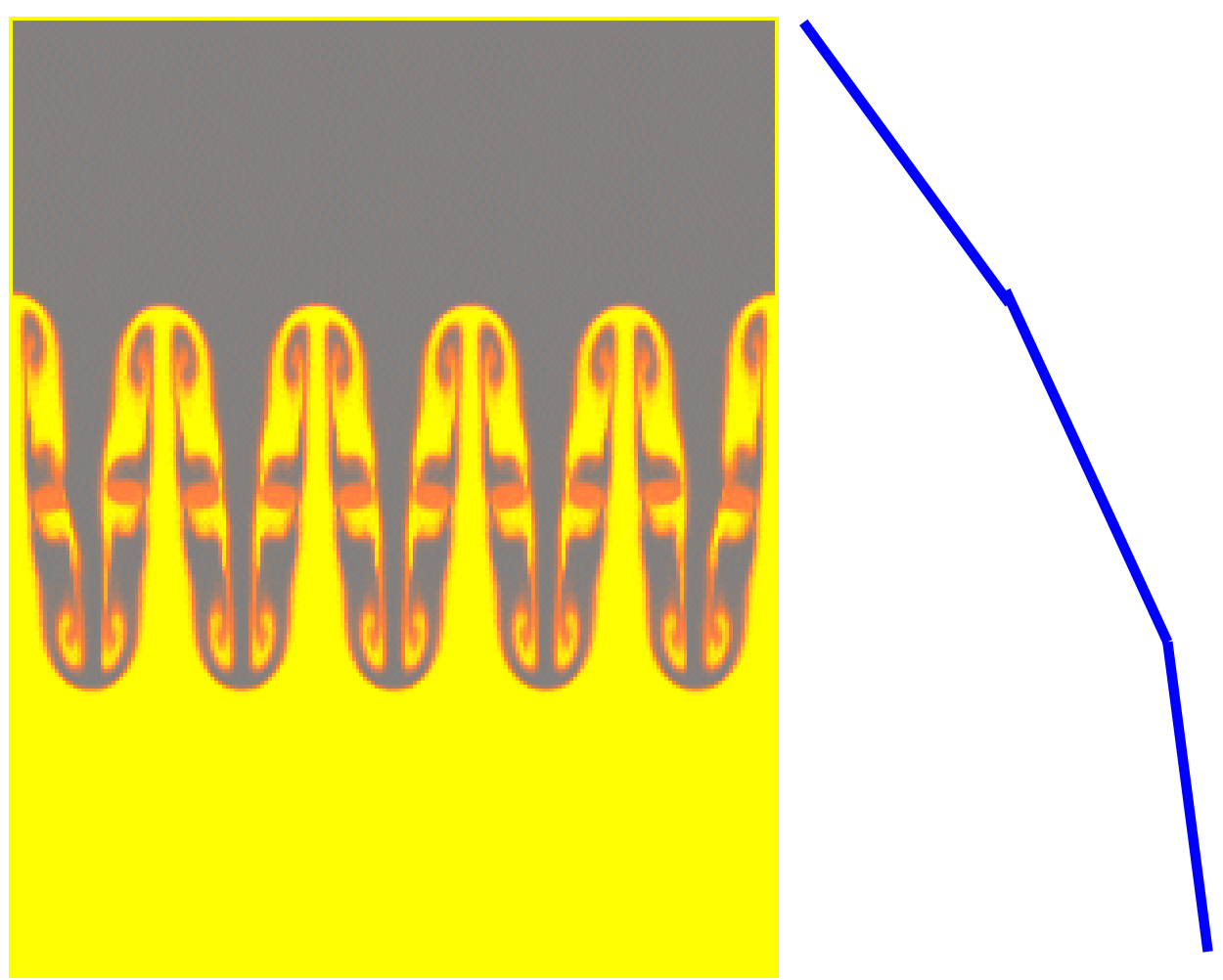

Froude number $\sim 1.2$ (aspect ratio 0.72$) \quad \Rightarrow C_{\text {Thermal }} \approx 1.3$.

Rayleigh-Taylor bubbles a little like thermals $\rightarrow C_{D} \approx 1.3$

But in Rayleigh-Taylor environment

- Density field not hydrostatic in ambient

$\diamond$ Hydrostatic in mean density $\Rightarrow$ halve buoyancy force

$$
\rightarrow C_{D} \approx 2.6
$$

- Flow around bubble affected by bubble moving in opposite direction

$\diamond$ Drag due to twice rise speed of bubble

$\rightarrow C_{D} \approx 10.4$

In agreement with single mode experiments

BUT natural R-T has more than one mode 


\section{Multi-mode}

What happens if $\lambda$ grows with $h$ ?

Let $\lambda=\psi h$

Late times approximation:

$$
\begin{gathered}
\frac{d h}{d t}=\left(\frac{A g}{C_{D}}(1-E) \psi\right)^{1 / 2} h^{1 / 2} \\
h=\frac{A g}{C_{D}}(1-E) \psi\left(t-t_{0}\right)^{2}=\alpha A g\left(t-t_{0}\right)^{2}
\end{gathered}
$$

For $C_{D}=10$ and $\psi=1, \alpha=0.025$.

[Full Layzer growth with $\psi=1$ gives $\alpha=0.023$.]

Growth rate maximised with $\psi \sim 10$ giving $\alpha \sim 0.103$

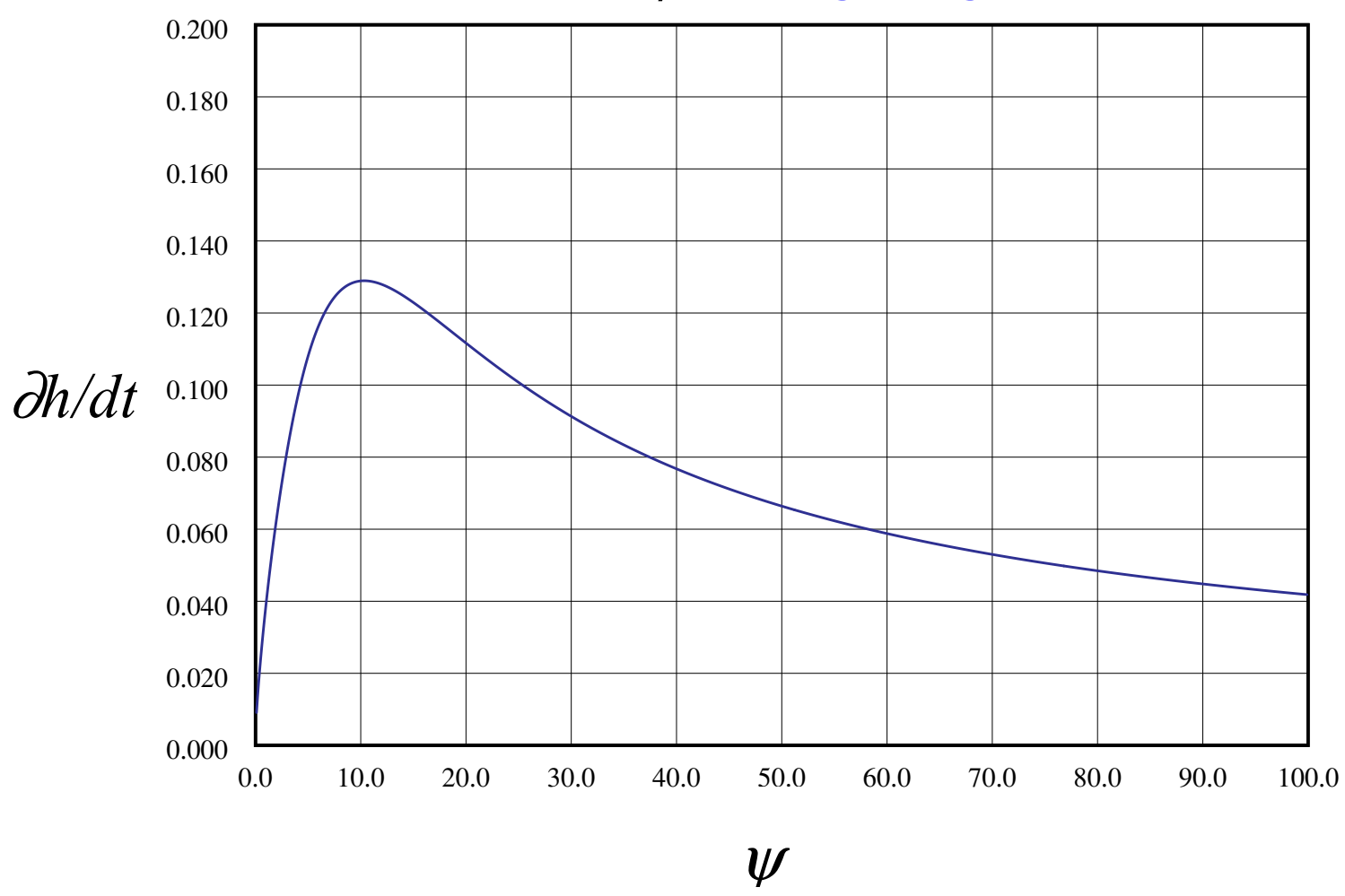


Where do the modes begin? How do they interact?

$\diamond$ Nonlinear interaction?

\section{$\diamond$ Initial perturbation?}

If modes independent and equal amplitude:

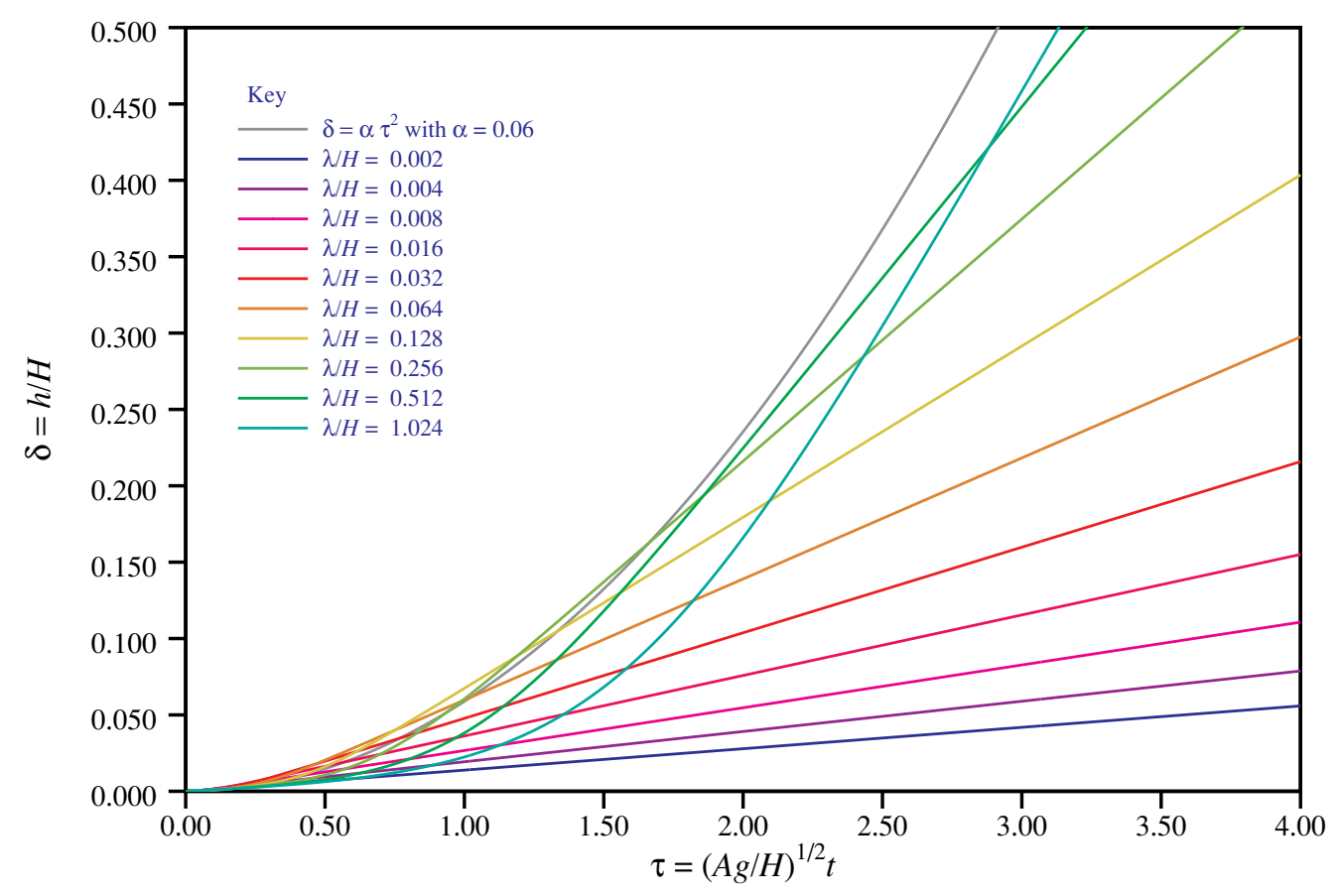

Instantaneous nonlinear mode halving interaction when $h=\lambda$ :

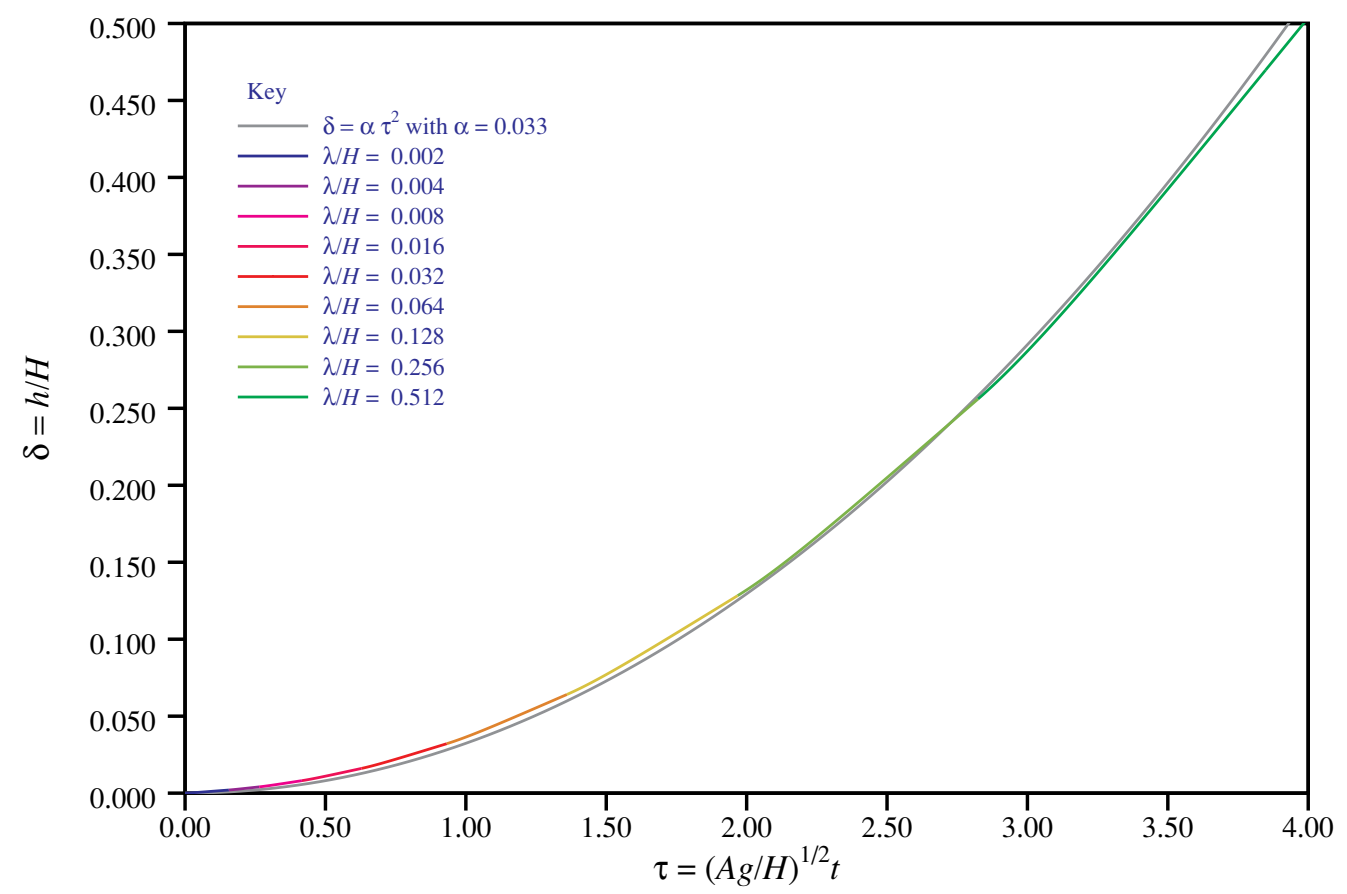

Which is it? 


\section{Mixing}

See talk by Joanne Holford

Energy budget

Can decompose $P E$ into Background PE and Available PE.

$P E_{\text {back }}$ is the minimum energy state that is achieved by adiabatic rearrangement of fluid parcels.

Mixing increases $P E_{\text {back }}-$ it cannot decrease it!

$P E_{\text {avail }}$ is the component of $P E$ that can be converted into $K E$, heat (through dissipation) and, if mixing occurs, into $P E_{b a c k}$.

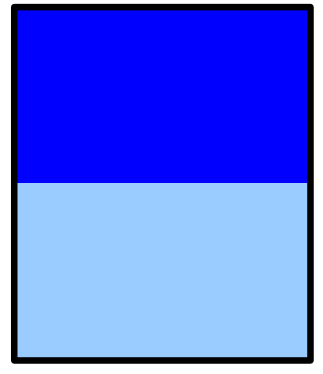

$P E$

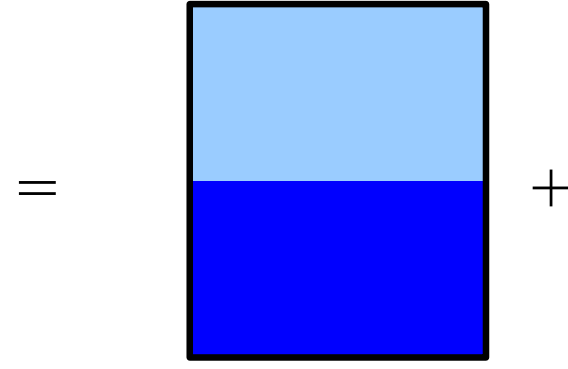

$P E_{\text {Back }}$

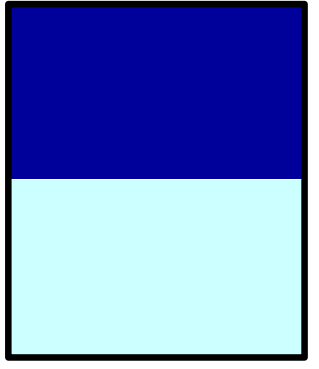

$P E_{\text {Avail }}$

In the absence of external work:

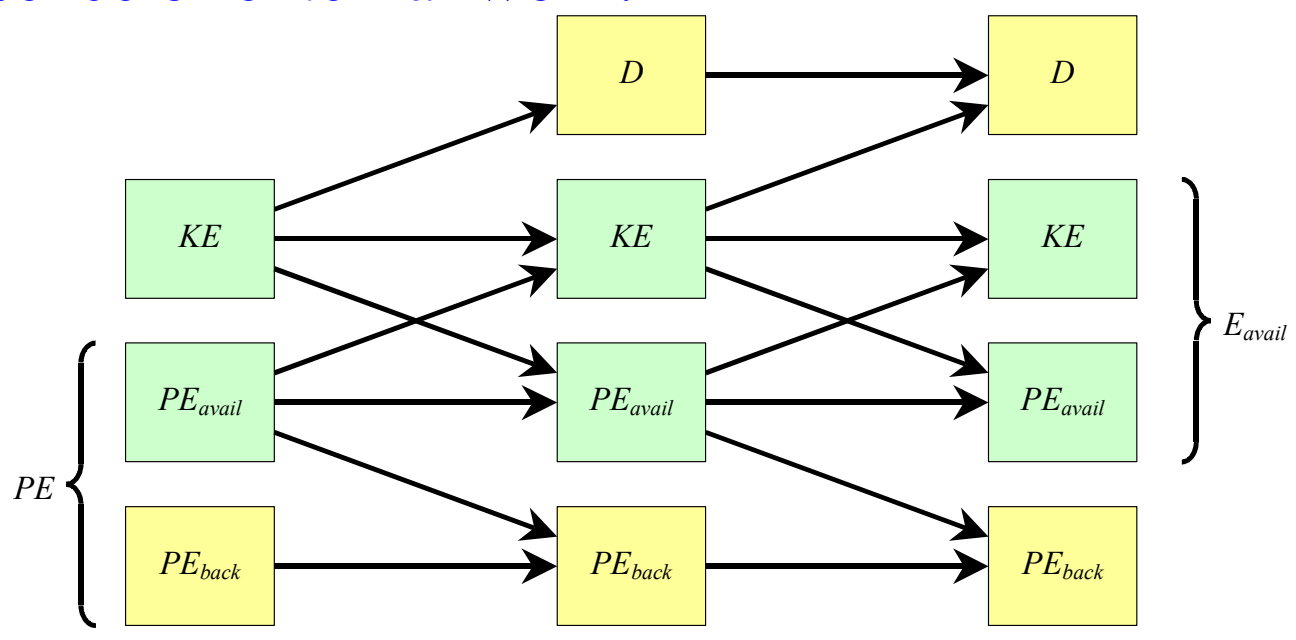


$\eta_{\text {Integral }}=-\frac{\Delta E_{\text {back }}}{\Delta E_{\text {avail }}}=\frac{\Delta P E_{\text {Back }}}{\Delta P E_{\text {Back }}+\int \varepsilon d t}=\frac{\Delta P E_{\text {Back }}}{-\left(\Delta K E+\Delta P E_{\text {Avail }}\right)}$

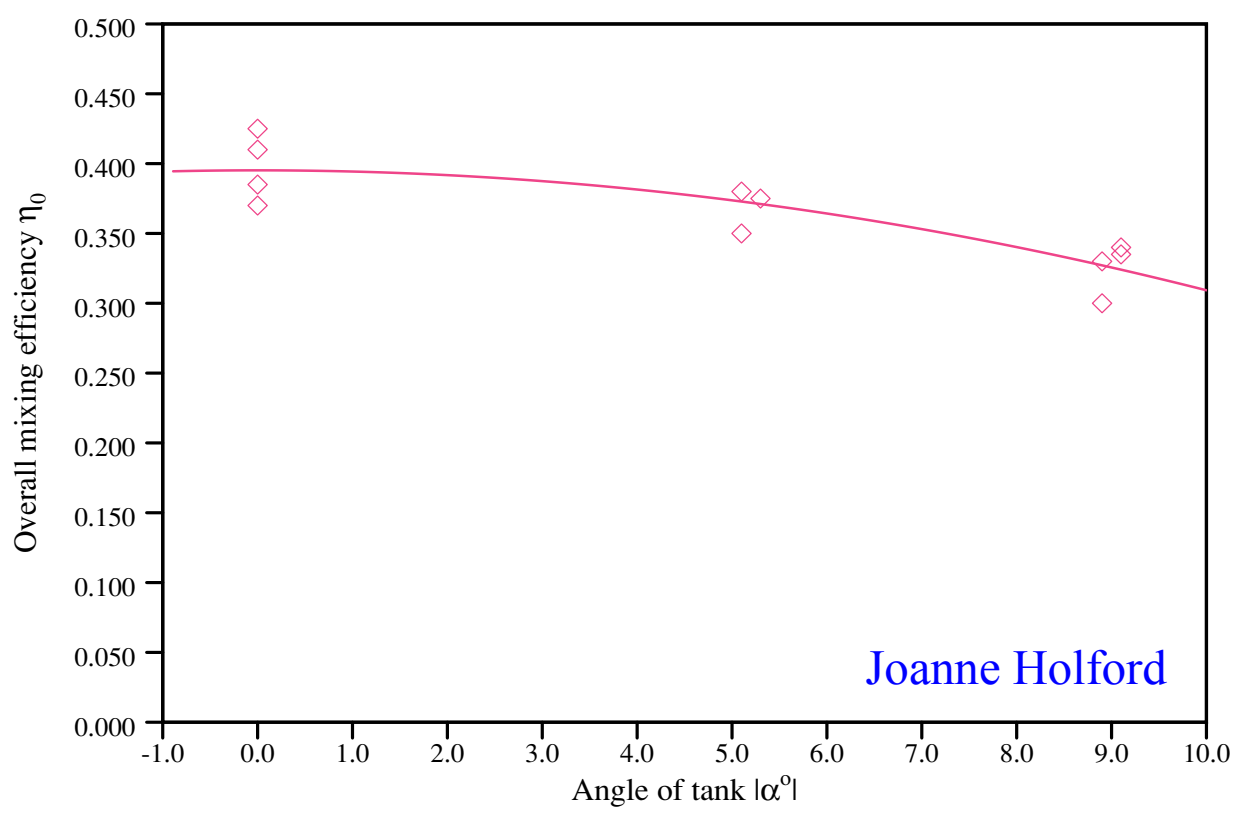

$\eta_{\text {instantaneous }}=\frac{\delta P E_{\text {Back }}}{-\delta E_{\text {Avail }}}=\frac{\delta P E_{\text {Back }}}{\delta K E+\delta P E_{\text {Avail }}}$

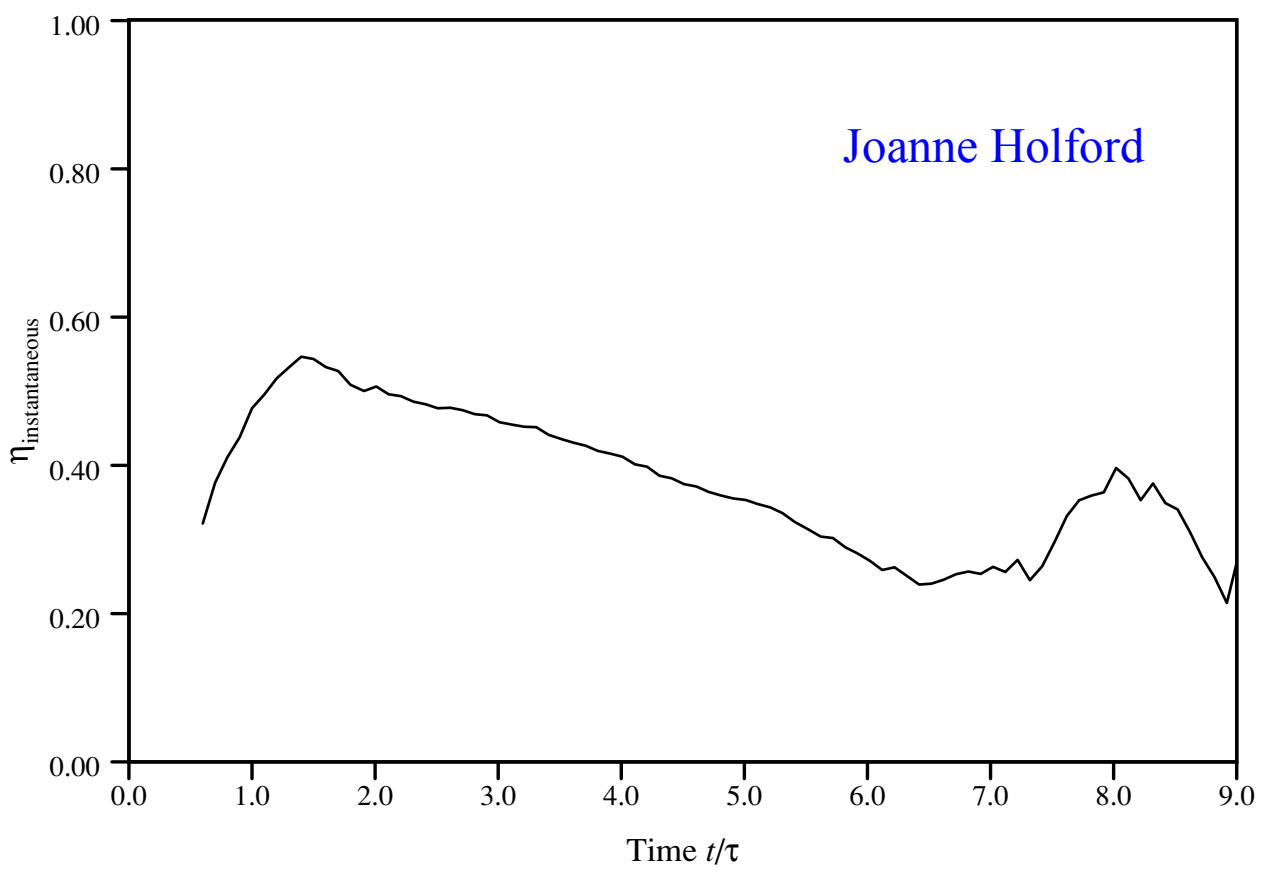




\section{Thermal}

\section{Entrainment into a thermal}

$$
\frac{d V}{d t}=\beta w A
$$

$$
\beta=0.18 .
$$

\section{Energetics of a thermal}

Mixing efficiency not well defined: depends on size of domain!

Rayleigh-Taylor
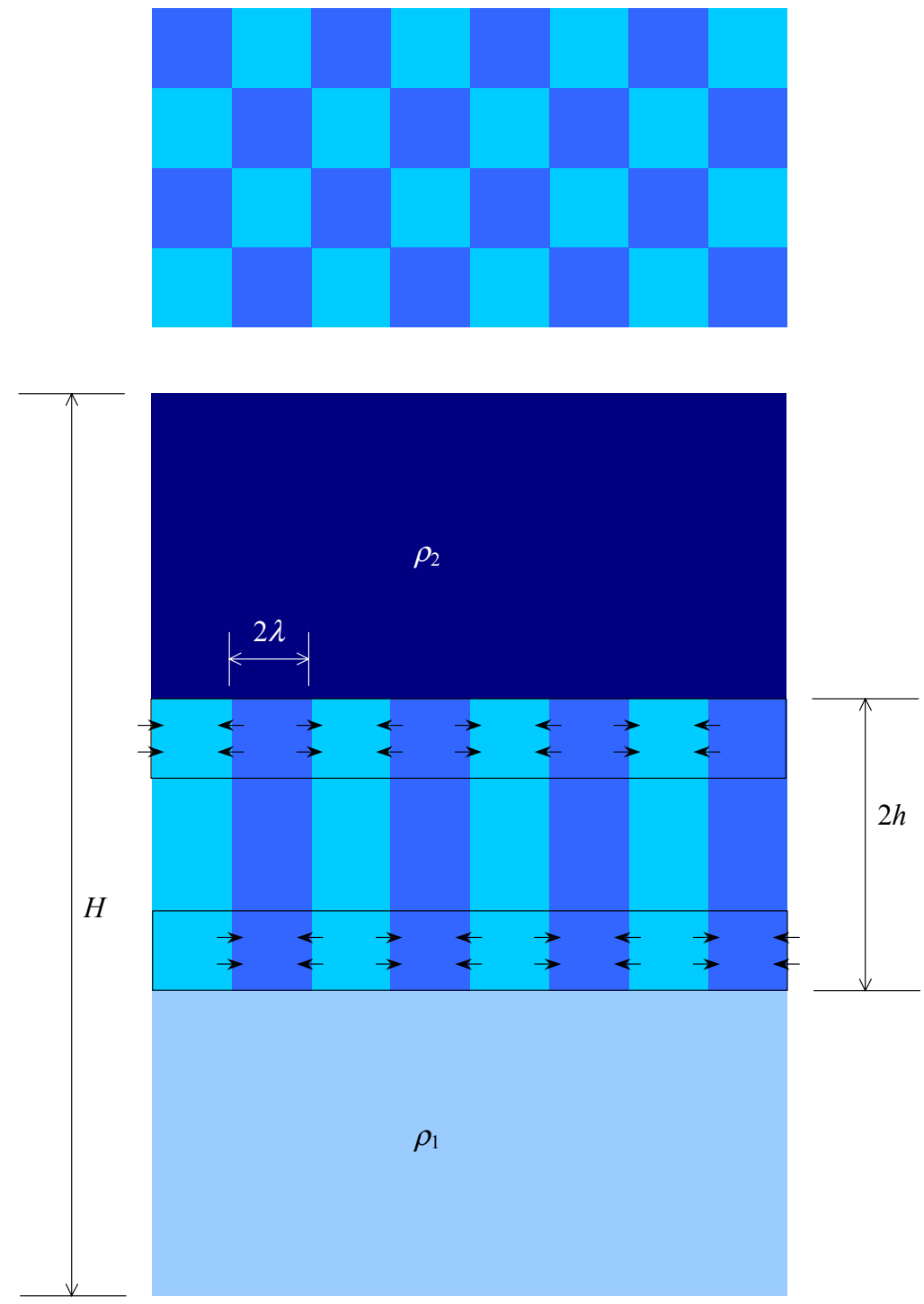


$$
\begin{array}{lr}
h=\alpha A g t^{2} & \delta=\alpha \tau^{2} \\
w=2 \alpha A g t & \omega=2 \alpha \tau \\
V=2 L^{2} h &
\end{array}
$$

\section{Total potential energy}

$$
P E_{\text {Total }}^{*}=\frac{P E_{\text {Total }}}{P E_{0}}=1-4 \alpha^{2} \tau^{4}
$$

\section{Background potential energy}
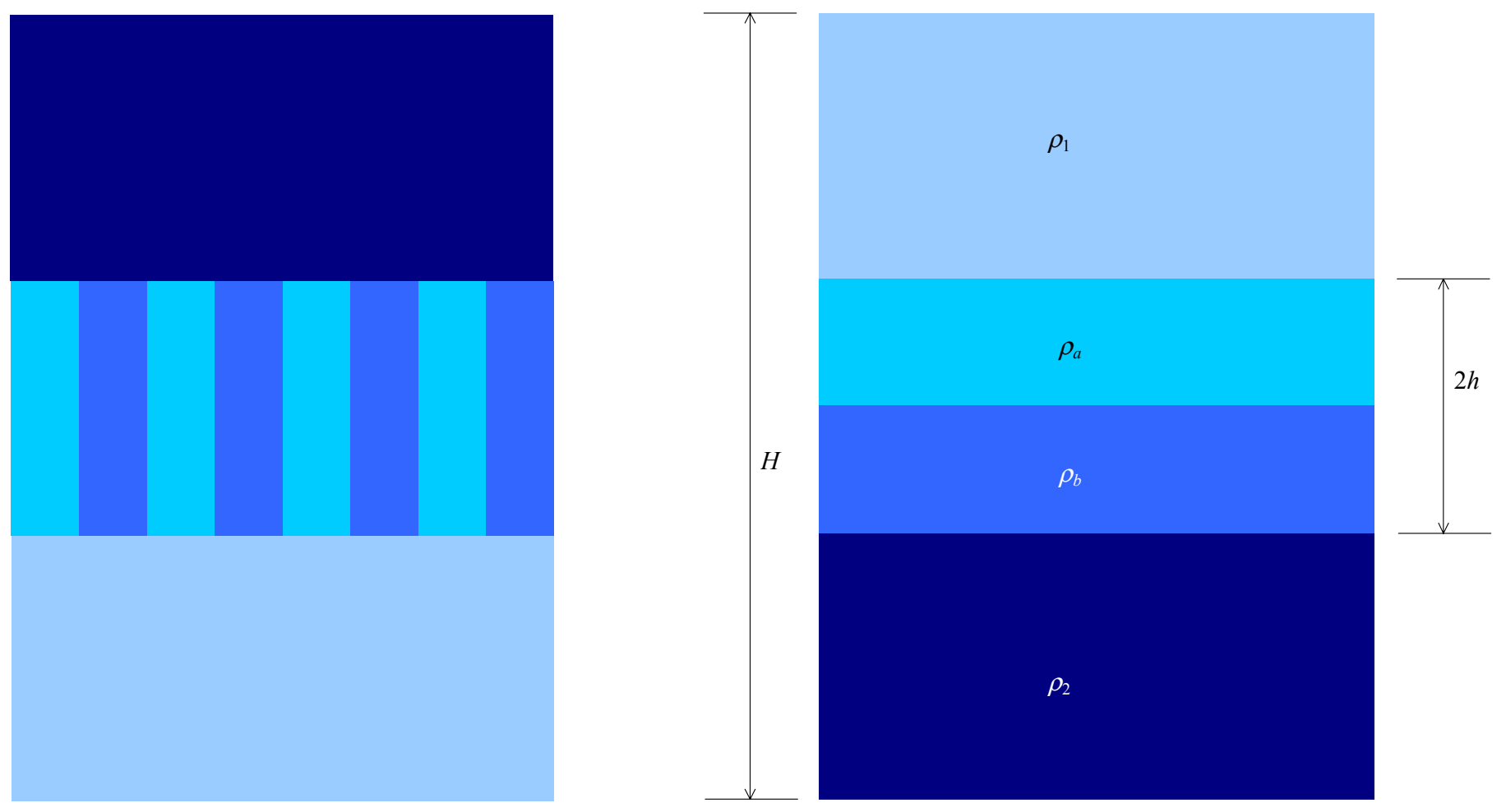

Changes due to entrainment between counter-flowing streams.

Invoke entrainment hypothesis: $u_{\mathrm{e}}=\beta w$

Area of entrainment independent of $h$

$\Leftrightarrow$ depth of entrainment comparable with $\lambda$

$\Rightarrow$ entraining area $=\varphi \times$ plan area.

$$
P E_{\text {Back }}^{*}=-\left(1-\varphi \beta \alpha^{2} \tau^{4}\right)
$$




\section{Available potential energy}

$$
P E_{\text {Avail }}^{*}=P E_{\text {Tot }}^{*}-P E_{\text {Back }}^{*}=2-(4+\varphi \beta) \alpha^{2} \tau^{4}
$$
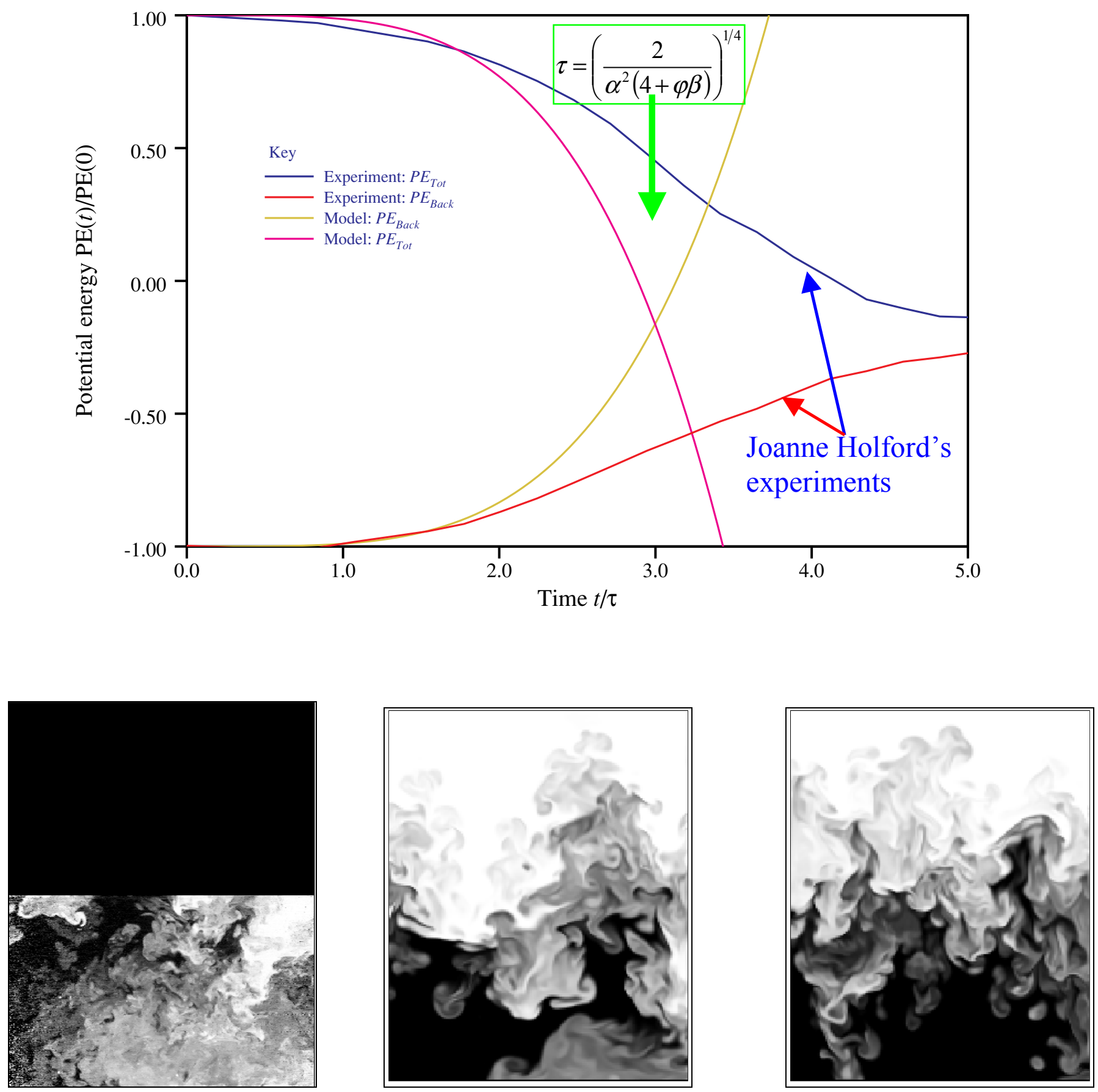


\section{Kinetic energy}

$$
K E^{*}=16 \sigma \alpha^{3} \tau^{4}
$$

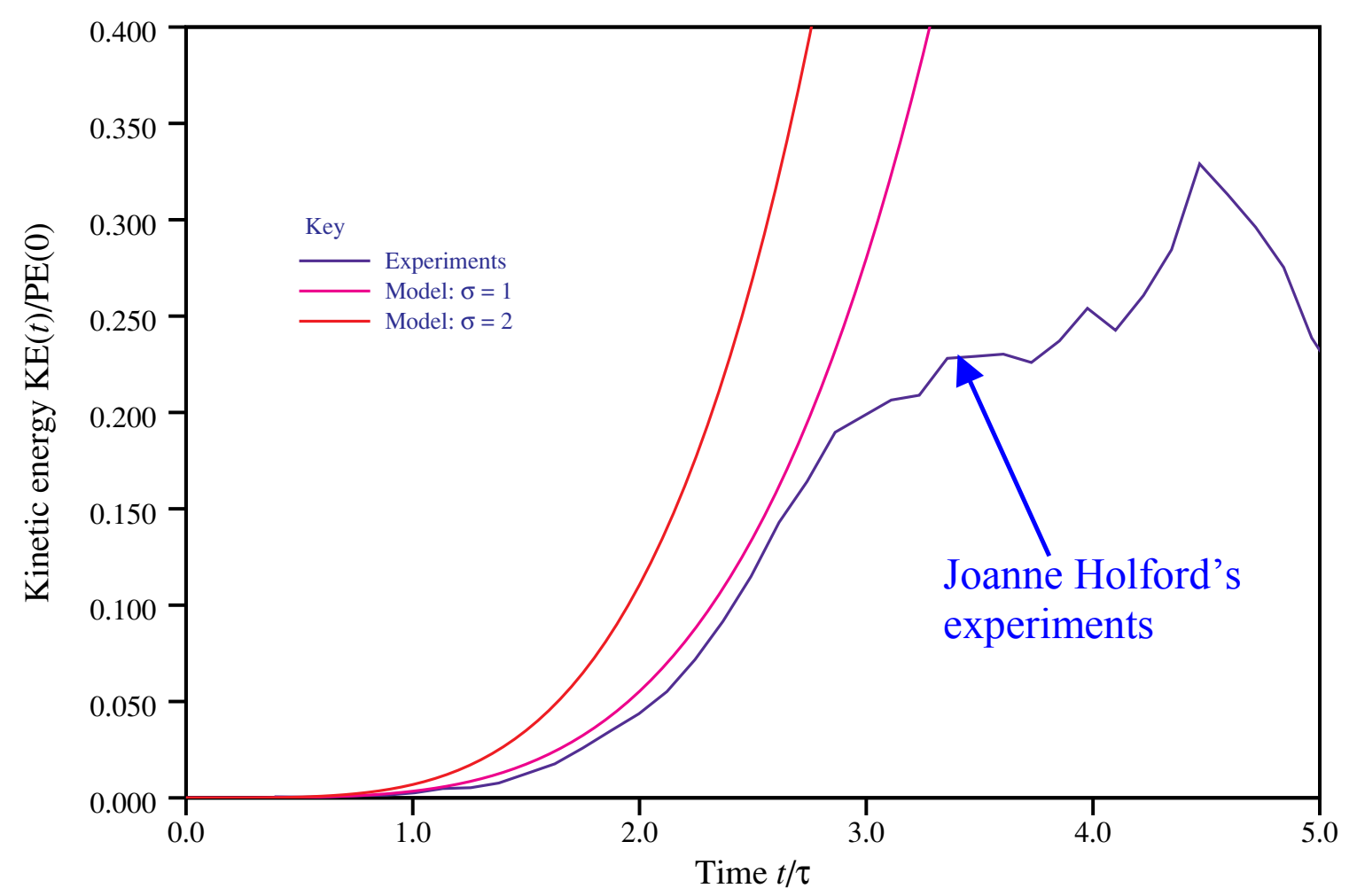

Available energy changing

$$
\begin{aligned}
& \frac{d E_{\text {Avail }}^{*}}{d \tau}=\frac{d K E^{*}}{d \tau}+\frac{d P E_{\text {Avail }}^{*}}{d \tau} \\
& =-4(4+\varphi \beta-16 \sigma \alpha) \alpha^{2} \tau^{3}
\end{aligned}
$$

Hence, energy is lost whenever $\alpha<1 / 4$ (for $\beta=0, \sigma=1)$. 
Instantaneous mixing efficiency

$$
\begin{aligned}
\eta_{\text {Inst }} & =-\frac{\frac{d P E_{\text {Back }}^{*}}{d \tau}}{\frac{d P E_{\text {Avail }}^{*}+\frac{d K E^{*}}{d \tau}}{d \tau}} \\
& =\frac{\varphi \beta}{4+\varphi \beta-16 \sigma \alpha}
\end{aligned}
$$

So for $\varphi=16, \beta=0.18, \sigma=1$, and $\alpha=0.06$, then $\eta_{\text {Inst }}=0.49$.

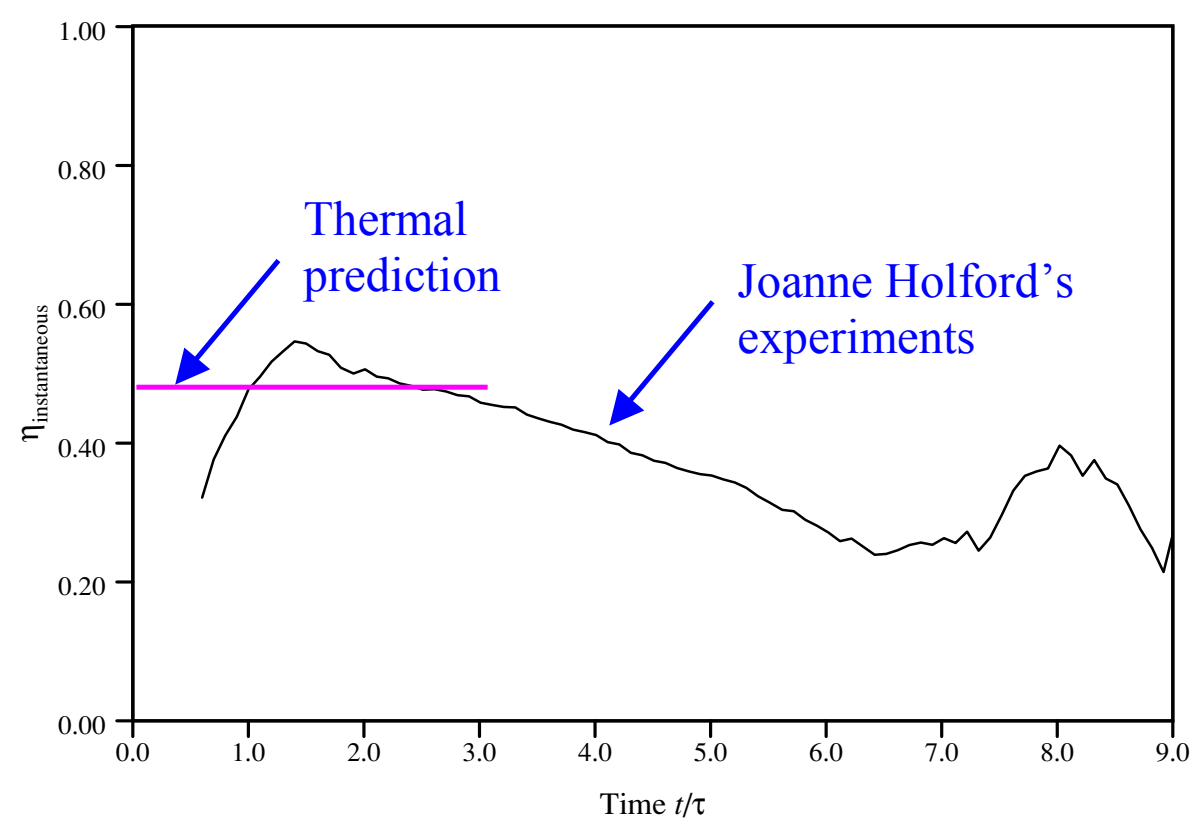




\section{Integral mixing efficiency}

\section{If there no mixing after reaching the bottom...}

$$
\eta_{\text {Integral }}=\frac{P E_{\text {Back }}^{(\text {bot })}-P E_{\text {Back }}^{(0)}}{P E_{\text {Avail }}^{(0)}}
$$
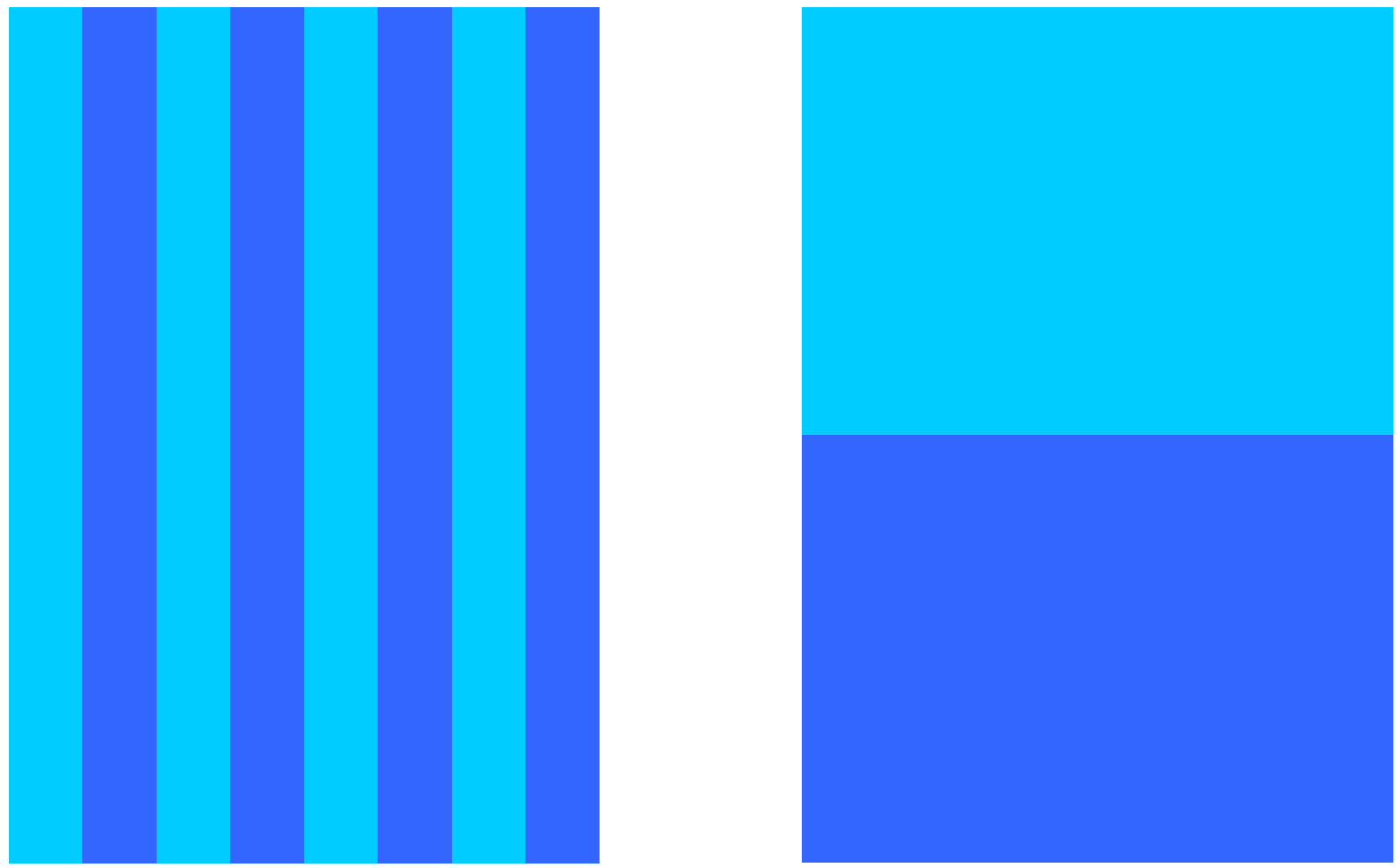

$$
\eta_{\text {Integral }}=\frac{1}{8} \varphi \beta
$$

For $\varphi=16$ and $\beta=0.18$, then $\eta_{\text {Integral }}=0.36$. 


\section{If there is mixing after reaching the bottom...}
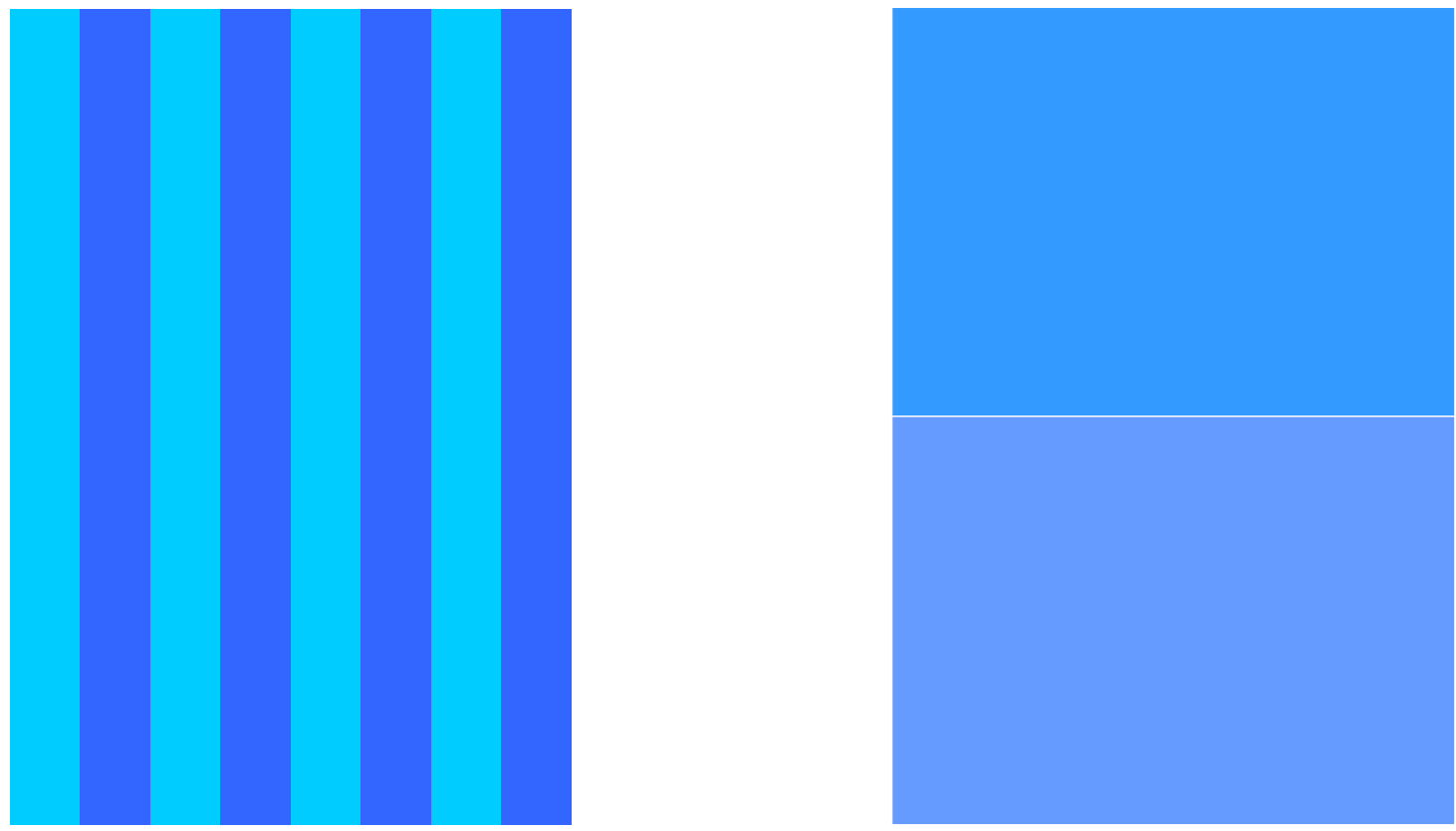

$$
E_{\text {Avail }}^{*(b o t)}=\left(1+4 \sigma \alpha-\frac{1}{4} \varphi \beta\right)
$$

If $\Delta E_{\text {Back }}^{(\text {Affer bot })}=\eta_{\text {stab }} E_{\text {Avail }}^{(\text {bot })}$, then

$$
\eta_{\text {Integral }}=\frac{1}{8} \varphi \beta+\frac{1}{2} \eta_{\text {stab }}\left(1+4 \sigma \alpha-\frac{1}{4} \varphi \beta\right)
$$

For $\eta_{\text {stab }}=0.2$, then $\eta_{\text {Integral }}=0.41$.

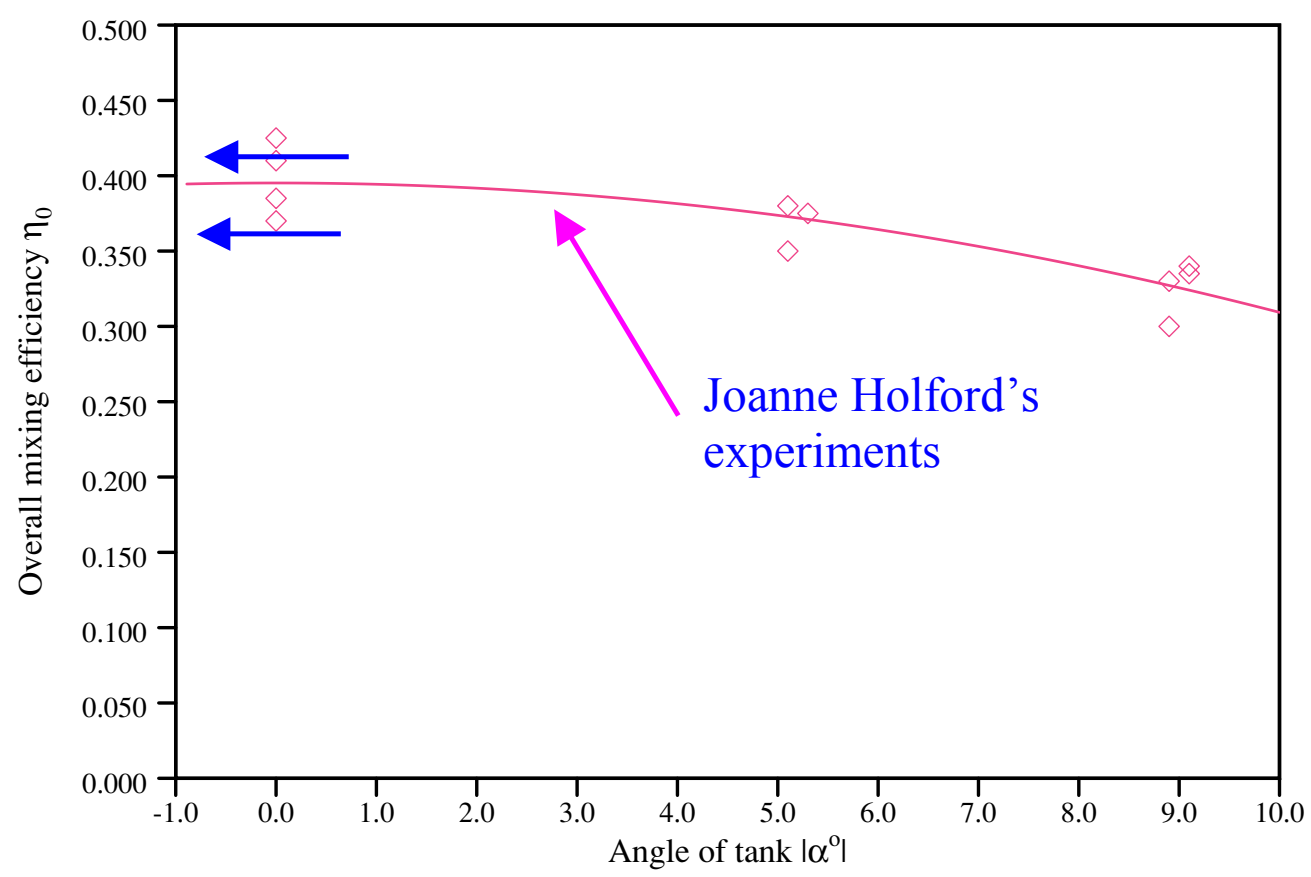




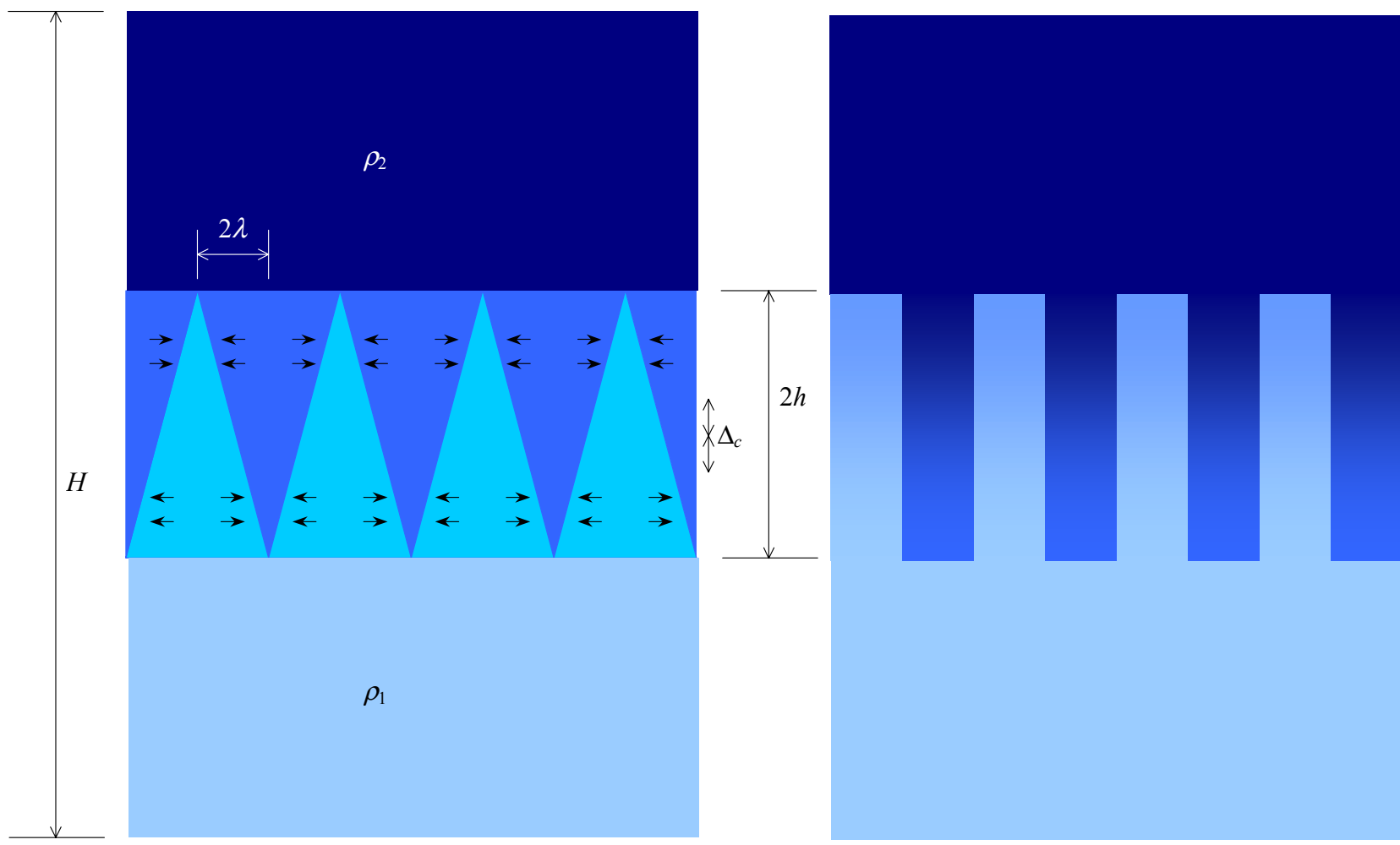

Let $\Delta_{\mathrm{c}}$ be the fractional displacement of the centroid of the bubble from $z=0$.

$\rightarrow$

$$
\begin{gathered}
\eta_{\text {Inst }}=-\frac{\delta P E_{\text {Back }}^{*}}{\delta P E_{\text {Avail }}^{*}+\delta K E^{*}} \\
=\frac{\varphi \beta}{4+\varphi \beta-4(4-\varphi \beta) \Delta_{c}-16 \sigma \alpha}
\end{gathered}
$$

Pyramid $\left(\Delta_{c}=1 / 4\right): \quad \eta_{\text {Inst }}=0.6$.

Parabolic $\left(\Delta_{c}=1 / 6\right): \quad \eta_{\text {Inst }}=0.56$.

(gives linear mean concentration) 
How can we avoid having to specify $C_{D}$ ?

\section{Shell model}

GOY model (Gledzer-Ohkitani-Yamada):

$$
\begin{gathered}
\frac{d U_{n}}{d t}=\left(a k_{n} U_{n+1}^{*} U_{n+2}^{*}+b k_{n-1} U_{n-1}^{*} U_{n+1}^{*}+c k_{n-2} U_{n-1}^{*} U_{n-2}^{*}\right) \\
-v k_{n}^{2} U_{n}+F_{n}
\end{gathered}
$$

with $k_{\mathrm{n}}=\beta^{\mathrm{n}} k_{0}, a=1, b=-\varepsilon$ and $c=-1+\varepsilon$.

In Rayleigh-Taylor instability, energy input at all scales.

$$
\begin{gathered}
\frac{d U_{n}}{d t}=\left(k_{n} U_{n+1} U_{n+2}-\varepsilon k_{n-1} U_{n-1} U_{n+1}-(1-\varepsilon) k_{n-2} U_{n-1} U_{n-2}\right) \\
-v k_{n}^{2} U_{n}+F_{n}
\end{gathered}
$$

Recall Layzer model: $(2+E) \frac{d w}{d t}=A g(1-E)-C_{D} \frac{w^{2}}{\lambda}$

Hence $F_{n}=A_{n} g \frac{1-E_{n}}{2+E_{n}}$, where

$$
E_{n}=\exp \left(-\frac{6 \pi h_{n}}{\lambda_{n}}\right) \text { and } A_{\mathrm{n}}=A h_{\mathrm{n}} / h \text {. }
$$

The mode penetrations $h_{\mathrm{n}}$ and total penetration $h$ are obtained from

$$
\frac{d h_{n}}{d t}=U_{n} \quad \text { and } \quad h=\max _{n} h_{n} .
$$




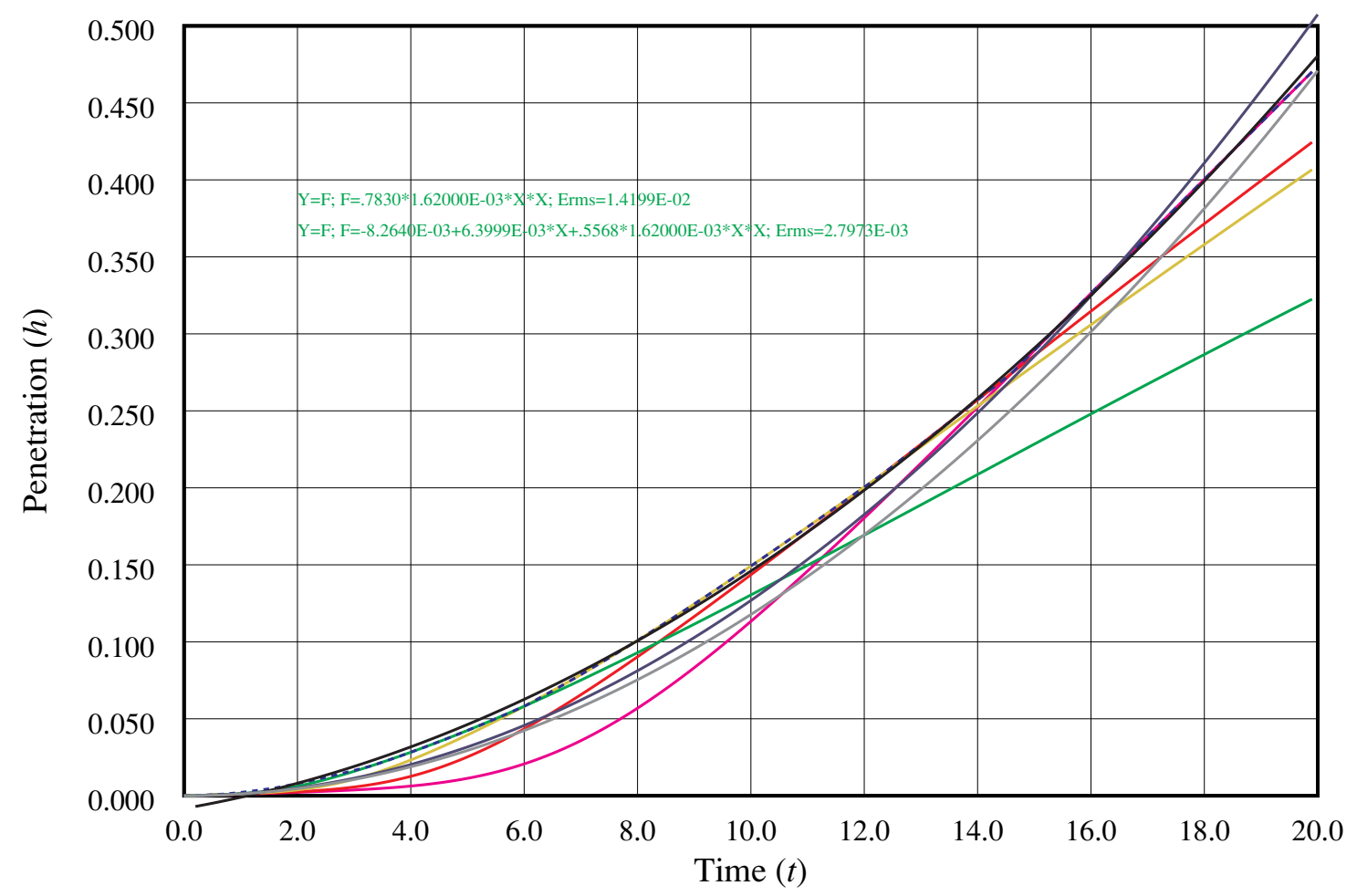

$\diamond$ Approximate quadratic growth

$\diamond$ Coefficient depends on initial spectrum

$\diamond$ Possible to replicate $\alpha \sim 0.06$ 


\section{Conclusions}

\section{General}

- Initial conditions are important for gross features

- Internal details relatively insensitive to initial conditions

- Appropriate modelling of initial conditions gives close agreement

\section{Thermals model}

- Single-mode growth rate consistent with isolated thermal

- Simple model for transfer between modes replicates $t^{2}$ growth

- Mixing efficiency consistent with thermal entrainment

\section{Shell model}

- Baroclinic input at all scales

- Very simple model replicates $t^{2}$ growth

- Growth rate sensitive to initial spectrum

\section{An explanation?}

No, but it helps. 
A General Buoyancy-Drag Model for the Evolution of the Rayleigh-Taylor and Richtmyer-Meshkov Instabilities

\section{Y. Elbaz, Y. Srebro, O. Sadot and D. Shvarts}

Nuclear Research Center - Negev, Israel.

Ben-Gurion Universiy, Beer-Sheva, Israel. 


\section{Abstract}

The growth of a single-mode perturbation is described by a buoyancydrag equation, which describes all instability stages (linear, non-linear and asymptotic) at time-dependant Atwood number and acceleration profile. The evolution of a multi-mode spectrum of perturbations from a short wavelength random noise is described using a single characteristic wavelength. The temporal evolution of this wavelength allows the description of both the linear stage and the late time selfsimilar behavior. The model includes additional effects, such as shock compression and spherical convergence.

Model results are compared to full 2D numerical simulations and shock-tube experiments of random perturbations, studying the various stages of the evolution. 


\section{Ideal Model Requirements}

- Calculate mix region for:

- general acceleration profile (RT and RM).

- all instability stages (linear, early nonlinear, asymptotic)

- general geometry (planar, cylindrical, spherical)

- compressibility and coupling to 1D flow.

- ablation.

- Describe internal structure of mixing zone:

- density, temperature and pressure of every material.

- degree of mixing.

- Feedback to 1D simulation:

- material flow. 


\section{Definitions}

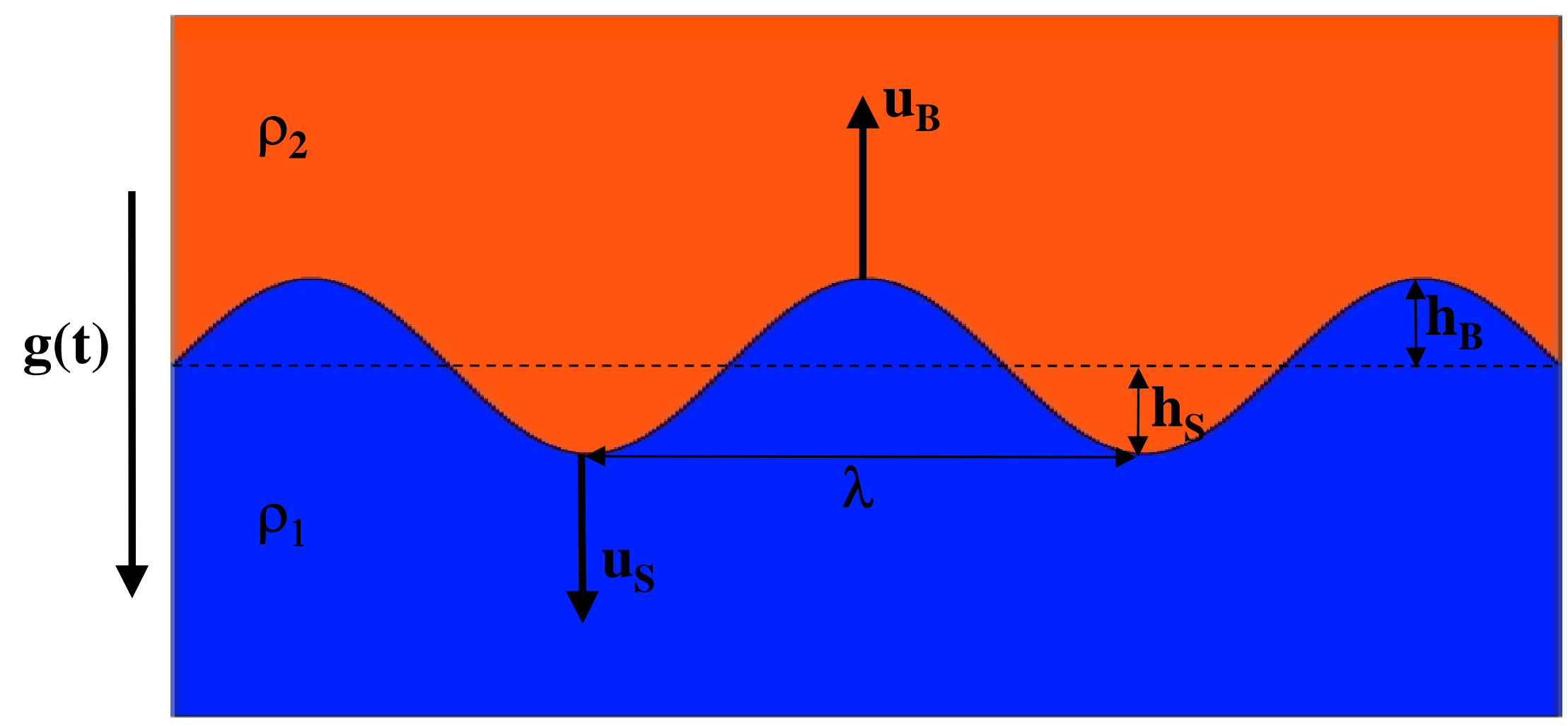

Atwood number

$$
A=\frac{\rho_{2}-\rho_{1}}{\rho_{2}+\rho_{1}} \quad \mathrm{k}=\frac{2 \pi}{\lambda}
$$




\section{Layzer model}

(2D) $\frac{d u_{B}}{d t}=\left(\frac{1-E}{2+E}\right) \cdot g(t)-\left(\frac{6 \pi}{2+E}\right) \cdot \frac{u_{B}^{2}}{\lambda} \quad, \quad E=e^{-3 k h_{B}}$
(3D) $\frac{d u_{B}}{d t}=\left(\frac{1-E}{1+E}\right) \cdot g(t)-\left(\frac{2 \pi}{1+E}\right) \cdot \frac{u_{B}^{2}}{\lambda} \quad, \quad E=e^{-2 k h_{B}}$

- Single mode (periodic array of bubbles and spikes).

- Describes all instability stages.

- Valid for a general acceleration profile.

- Limited to $A=1$. 


\section{Buoyancy-drag equations}

$$
\begin{aligned}
& \left(\rho_{1}+C_{a} \rho_{2}\right) \frac{d u_{B}}{d t}=\left(\rho_{2}-\rho_{1}\right) \cdot g(t)-\frac{C_{d}}{\lambda} \rho_{2} \cdot u_{B}^{2} \\
& \left(\rho_{2}+C_{a} \rho_{1}\right) \frac{d u_{S}}{d t}=\left(\rho_{2}-\rho_{1}\right) \cdot g(t)-\frac{C_{d}}{\lambda} \rho_{1} \cdot u_{S}^{2}
\end{aligned}
$$

- Single mode (periodic array of bubbles and spikes).

- Describes only asymptotic stage.

- Valid for a general acceleration profile.

- Valid for every A. 


\section{New model for single-mode}

\section{perturbation}

- We combine Layzer model with buoyancy-drag equations.

- $\mathrm{C}_{\mathrm{a}}, \mathrm{C}_{\mathrm{d}}, \mathrm{C}_{\mathrm{e}}$ are determined from Layzer model for $\mathrm{A}=1$, and assumed to be Atwood independent.

$$
\begin{gathered}
{\left[\left(C_{a} \cdot E(t)+1\right) \rho_{1}+\left(C_{a}+E(t)\right) \rho_{2}\right] \frac{d u_{B}}{d t}=} \\
(1-E(t)) \cdot\left(\rho_{2}-\rho_{1}\right) \cdot g(t)-\frac{C_{d}}{\lambda} \rho_{2} \cdot u_{B}^{2} \\
\left(E(t)=e^{-C_{e} \cdot k \cdot h_{B}}\right)
\end{gathered}
$$




\section{Multimode evolution}

Mixing fronts (bubbles and spikes) are described by one characteristic wavelength: $\langle\lambda\rangle=\left\langle\lambda_{\mathrm{BUB}}\right\rangle$.

- Linear stage: $\frac{d\langle\lambda\rangle}{d t}=0$

-Asymptotic self-similar behavior:

$$
\frac{h_{B}}{\langle\lambda\rangle}=b(A)
$$

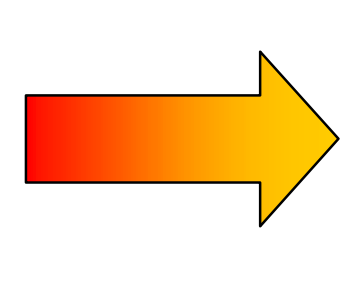

$\frac{d\langle\lambda\rangle}{d t}=\frac{u_{B}}{b(A)}$

-Transition from linear to asymptotic is at:

$$
h_{B}=\left\langle\lambda_{0}\right\rangle \cdot b(A)
$$




\section{Model properties}

- Linear stage:

reproduces theoretical result (first order):

$$
\ddot{h}(t)=\operatorname{Akgh}(t)
$$

- Early nonlinear stage:

for $A \rightarrow 1$, correct to second order (Layzer model)

- Asymptotic stage:

buoyancy-drag equation for all $\mathrm{A}$.

Limited to planar geometry and incompressible flow. 


\section{D Hydrodynamic coupling}

The dynamic front equation is solved coupled to the 1D lagrangian motion:

- Change in Atwood number:

$$
\rho_{i}=\left|\int_{h_{1 d}}^{h_{i}} \rho_{i} V d x\right| /\left|\int_{h_{1 d}}^{h_{i}} V d x\right| \quad i=1,2
$$

- 1D Lagrangian "drift" of the mixing zone boundaries:

$$
\begin{aligned}
& \mathrm{u}_{\mathrm{B}} \rightarrow \mathrm{u}_{\mathrm{B}}+\mathrm{U}_{1 \mathrm{~d}}\left(\mathrm{~h}_{\mathrm{B}}\right) \\
& \mathrm{u}_{\mathrm{S}} \rightarrow \mathrm{u}_{\mathrm{S}}+\mathrm{U}_{1 \mathrm{~d}}\left(\mathrm{~h}_{\mathrm{S}}\right)
\end{aligned}
$$




\section{Corrections required for non-planar geometry}

Non-planar geometry introduces two effects:

- change in amplitude due to 1D motion (Bell-Plesset)

- included in 1D coupling to lagrangian flow.

- Change in wavelength (conservation of wavenumber, $\ell=\lambda / \mathrm{R}$ ).

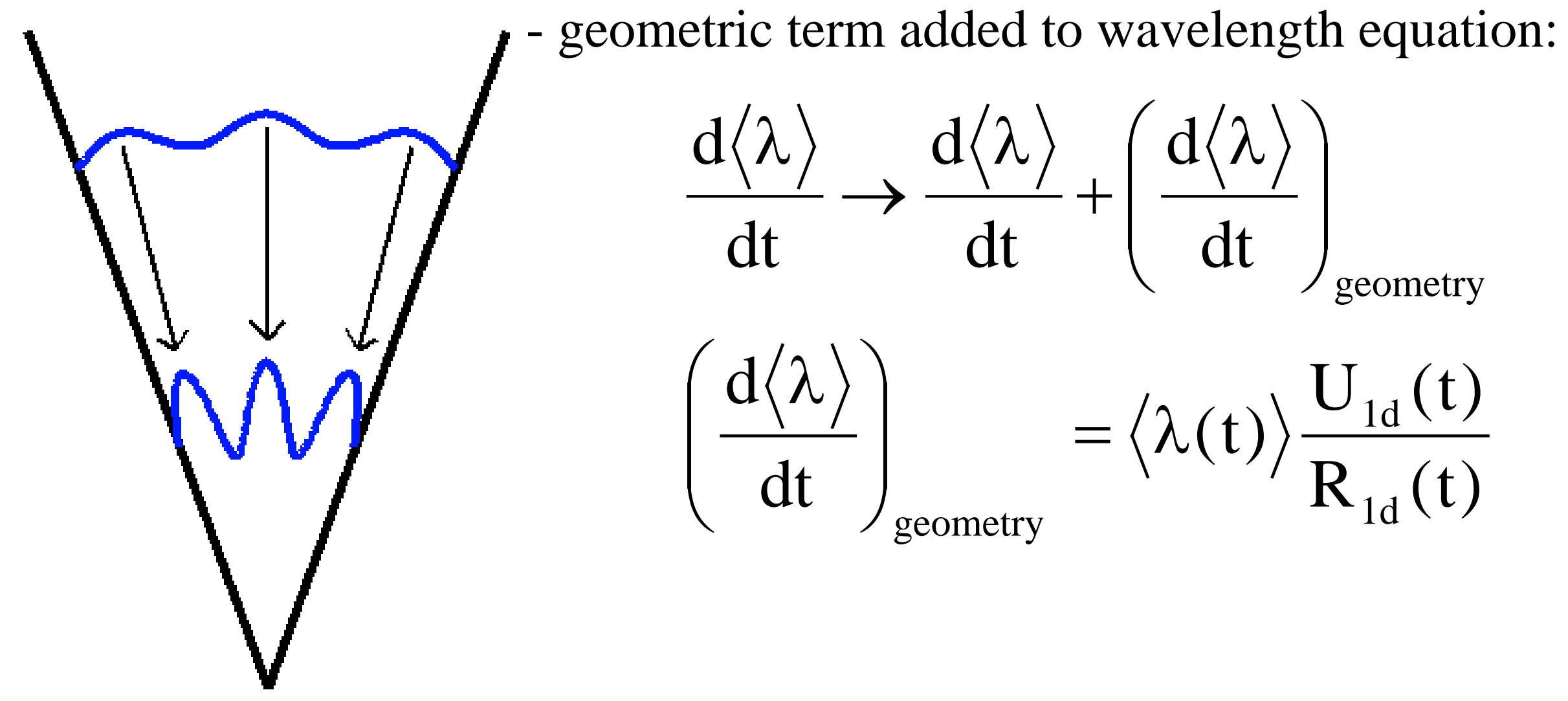




\section{Shock tube experiments}

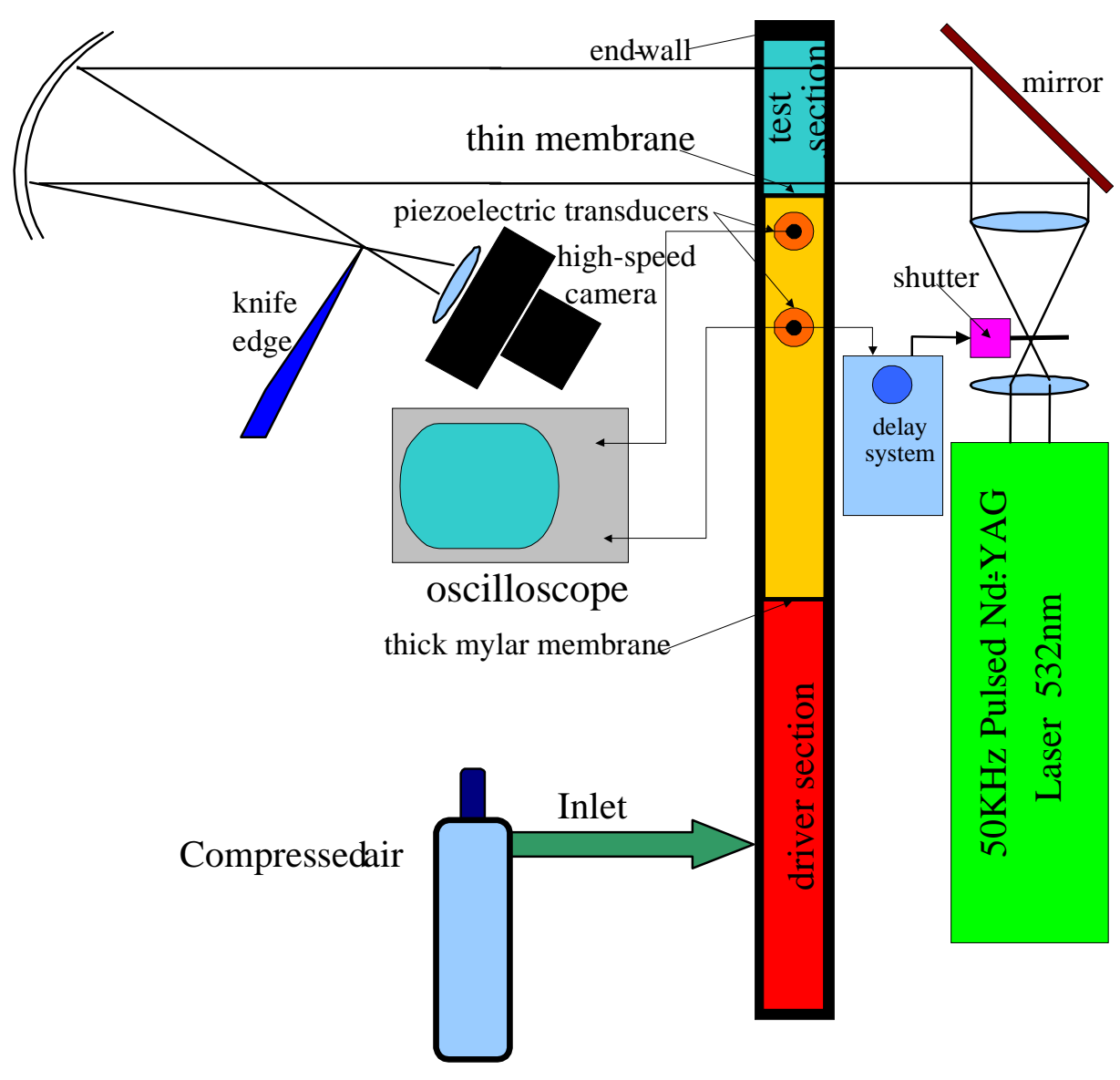

Mach=1.2

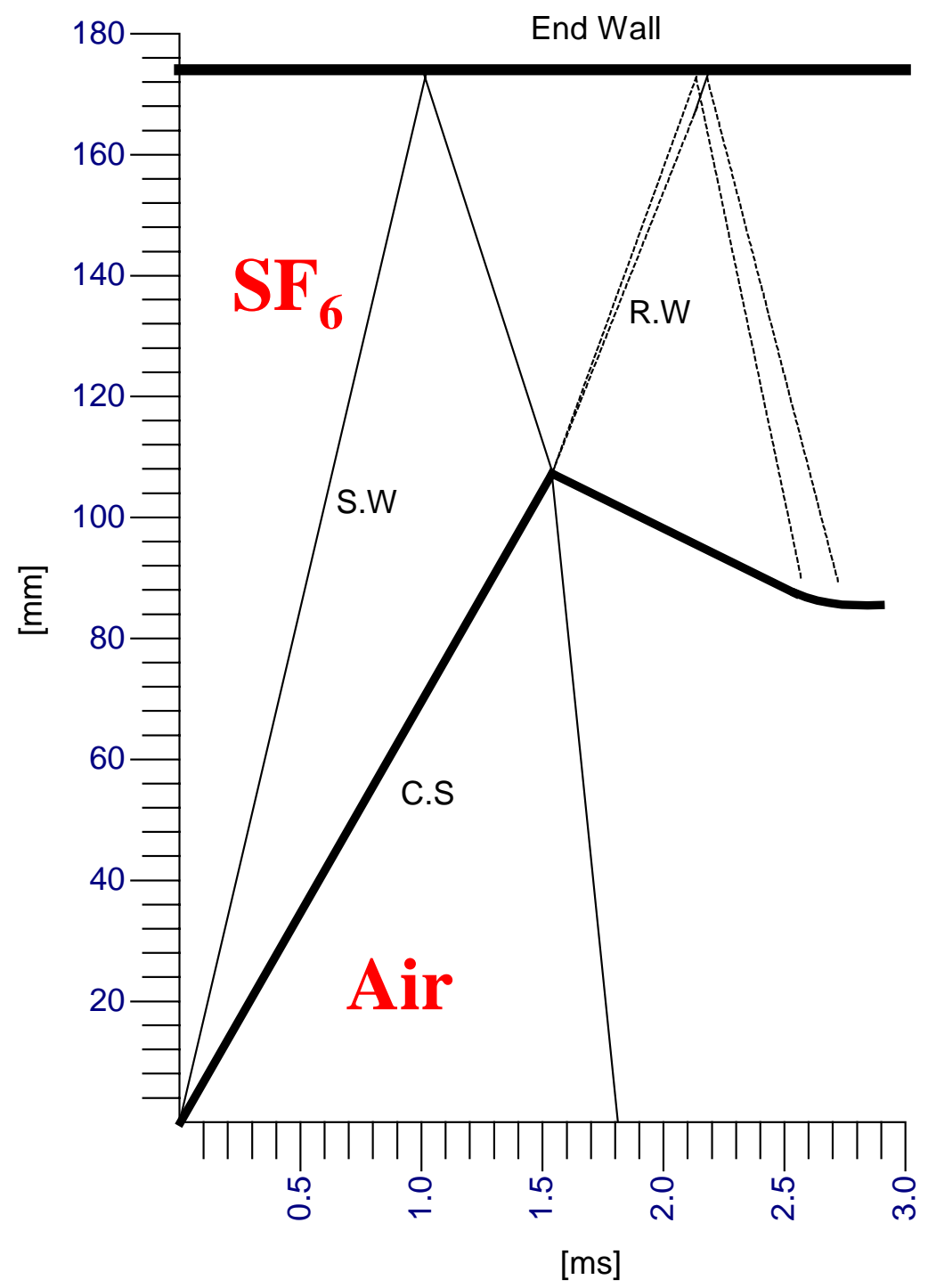




\section{Experimental results (random initial conditions)}

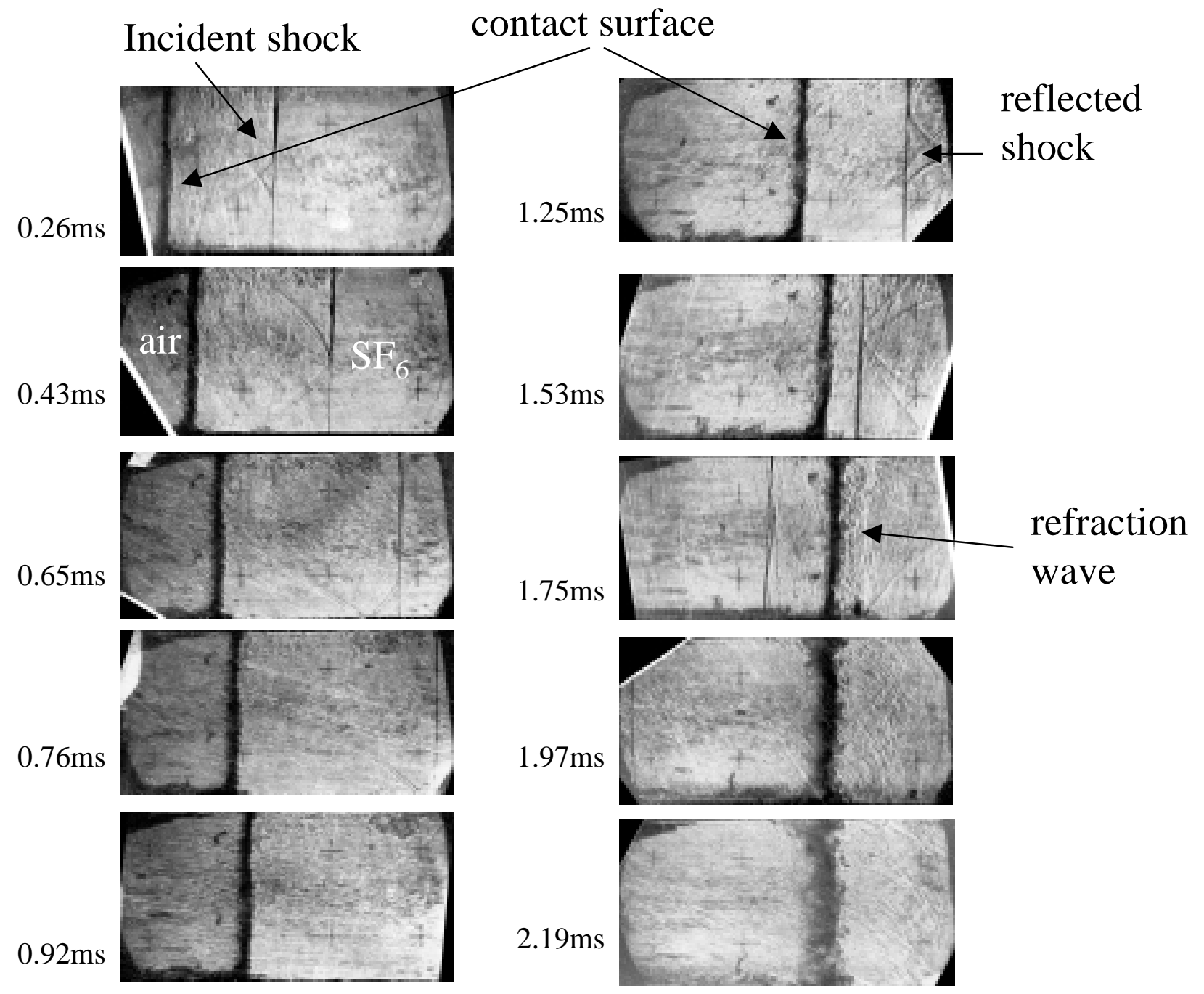


2D numerical simulations

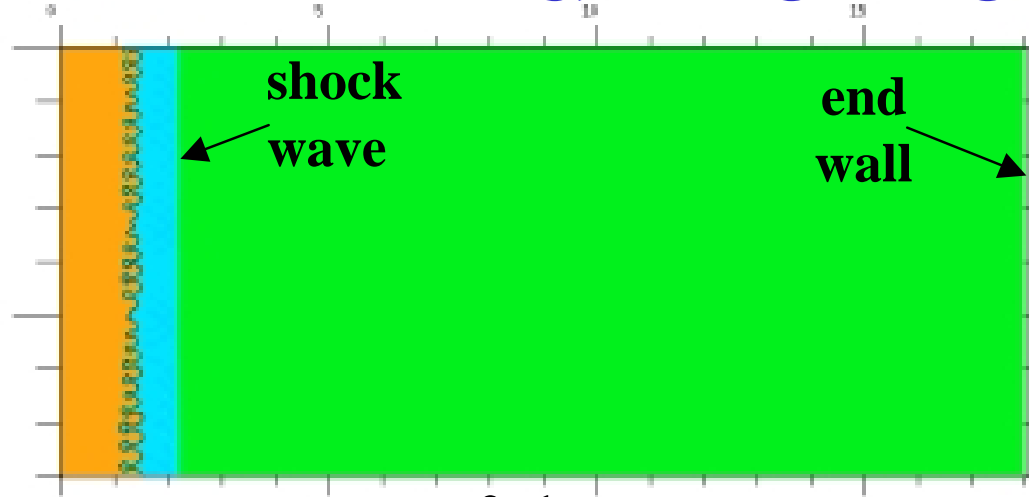

\section{$\mathrm{t}=\mathbf{0 . 1 \mathrm { ms }}$}
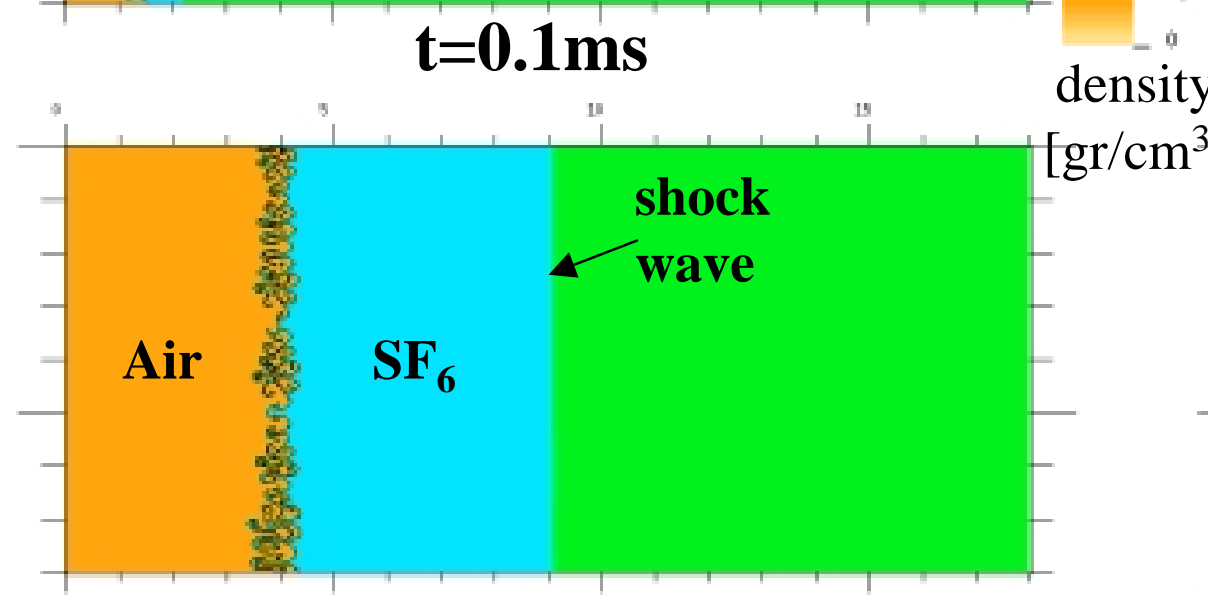

0.000

$\mathrm{t}=0.5 \mathrm{~ms}$

$\mathrm{t}=1.5 \mathrm{~ms}$ $\left[\mathrm{gr} / \mathrm{cm}^{3}\right]$

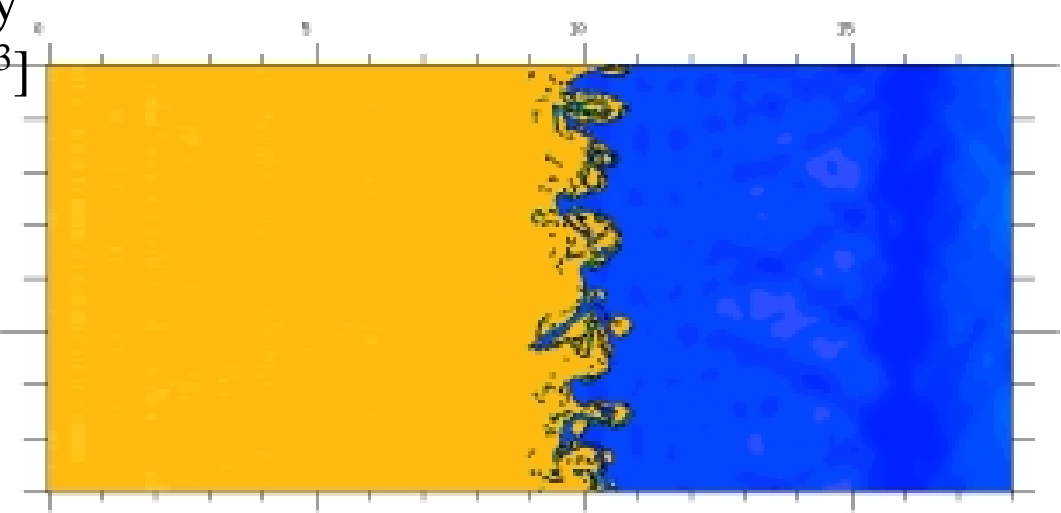

$\mathrm{t}=2.2 \mathrm{~ms}$

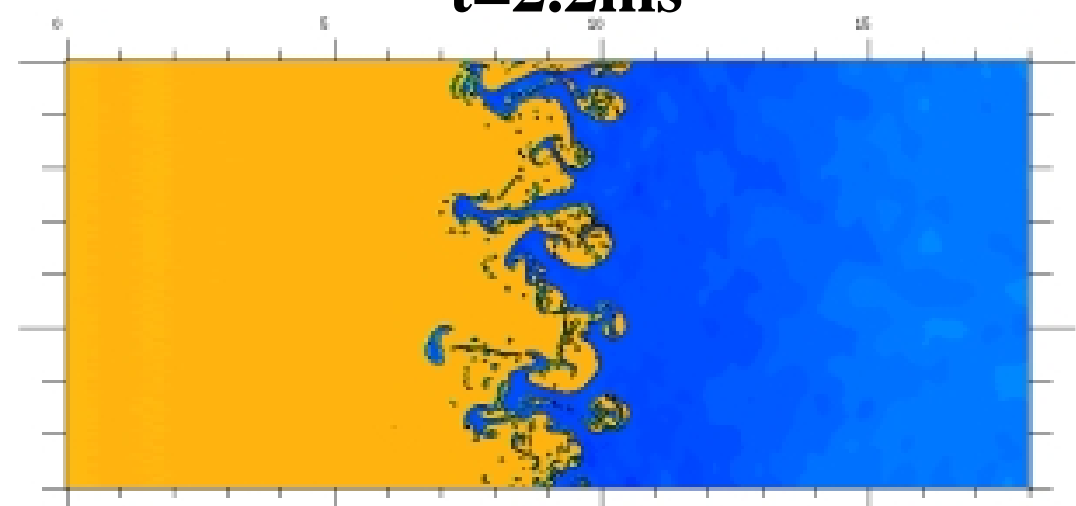

$\mathrm{t}=3.0 \mathrm{~ms}$ 
Good agreement between mix

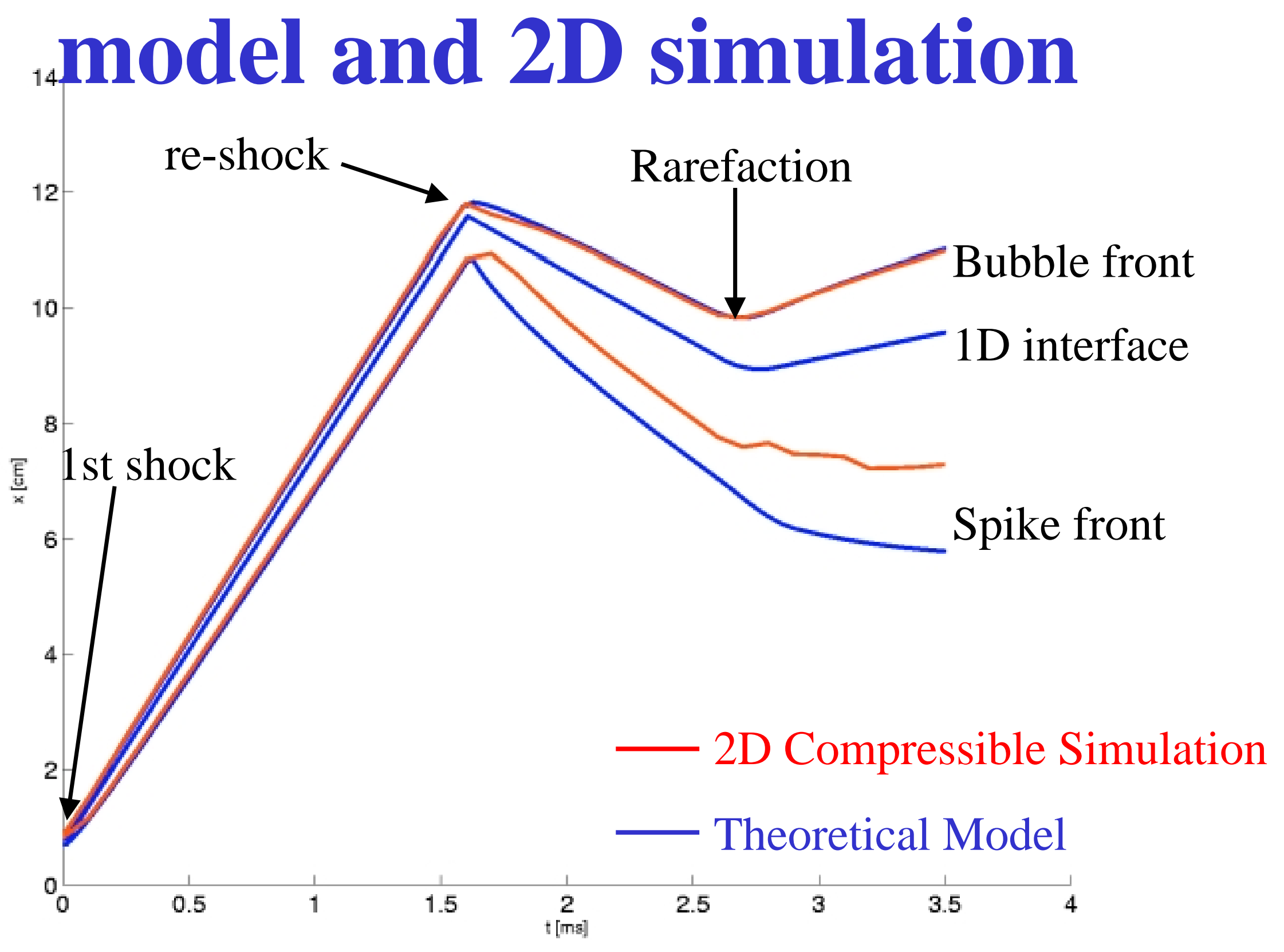




\section{Model agrees with}

\section{experimental results}

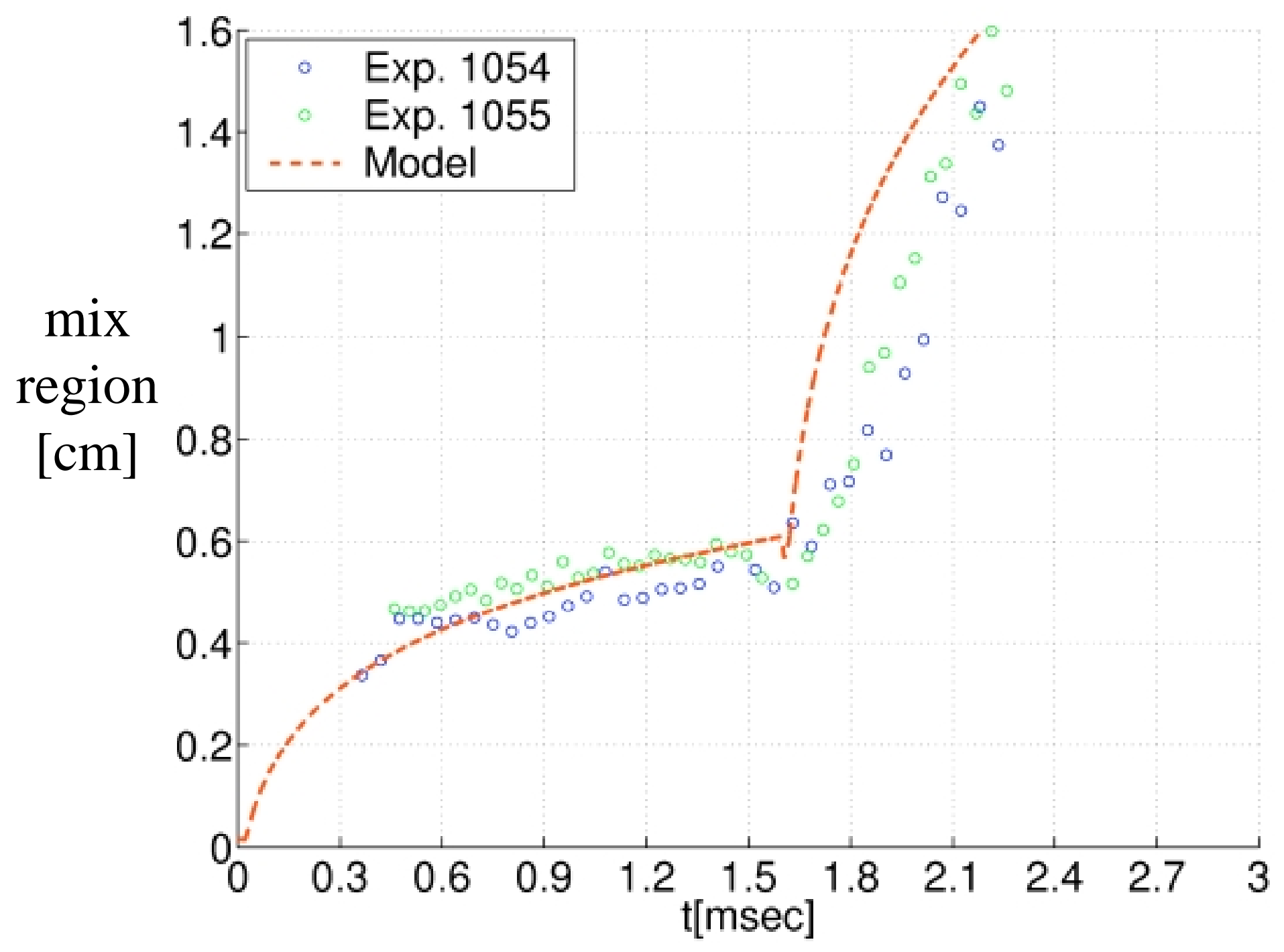




\section{Summary}

- Layzer model and buoyancy-drag equation have been combined to describe all instability stages for all Atwood numbers and a general acceleration profile.

- Multi-mode spectrum is described by one characteristic wavelength.

-1D compressibility and scale change effects are introduced through Lagrangian "drift" of the mixing zone boundaries and by time dependant Atwood number.

- Model results have been compared to experiments and to full 2D numerical simulations.

- Non-planar geometry may be introduced by modifying characteristic wavelength. 
NON LINEAR $R T$ and $R M$ SINGLE MODES (ANALYTIC)

- Nail INOGAMOV (MOSCOU)

- Matthien TRICOTTET (CEA-PAR IS)

- Alexer OPARIN (MOSCOU)

- Serge BOUQUET (CEA-PARIS)

Astro-ph 0104024<smiles>CCC1CCC1C</smiles> 
OUTLINE

- ASSUMPTIONS

$$
\begin{aligned}
& \text { INITIAL 2D/3D } \\
& \text { PERTURBATION }
\end{aligned}
$$

- AnALYTICAL MODEL

- STRUCTURE OF THE INTERFACE

- SHAPE factor

- COMP. OTHER WORKS. 
ASSUMPTIONS:

- $g=f(t)$ arbitrary

$$
\begin{cases}g \sim \text { cote } & R T I \\ g \sim \delta(t) & R M I\end{cases}
$$

- INCOMPRESSIBLE (compressible - Linear)

- potential flow

$$
\begin{aligned}
& \vec{V}=\vec{\nabla} \phi \quad \Rightarrow \nabla^{2} \phi=0 \\
& -A t=\frac{\rho_{H}-\rho_{L}}{\rho_{H}+\rho_{L}} \rightarrow 1 \\
& - \text { SINGLE MODE STUDY } \\
& 2 D \text { and } 3 D
\end{aligned}
$$

- Influence of the LATTICE (PERIODIC) $B 3(J 6), B 4(J 4), B 6(J 3)$ 


\section{Perturbations}

$\cdot 2 \mathrm{D}$

RIPPLES

-3D B4

3 D PERIODIC LATT.

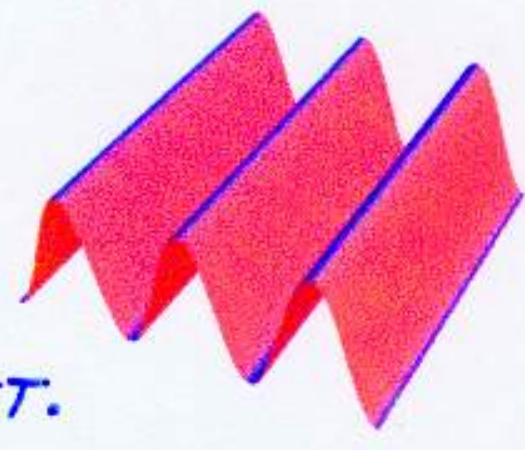

-3D B3/B6

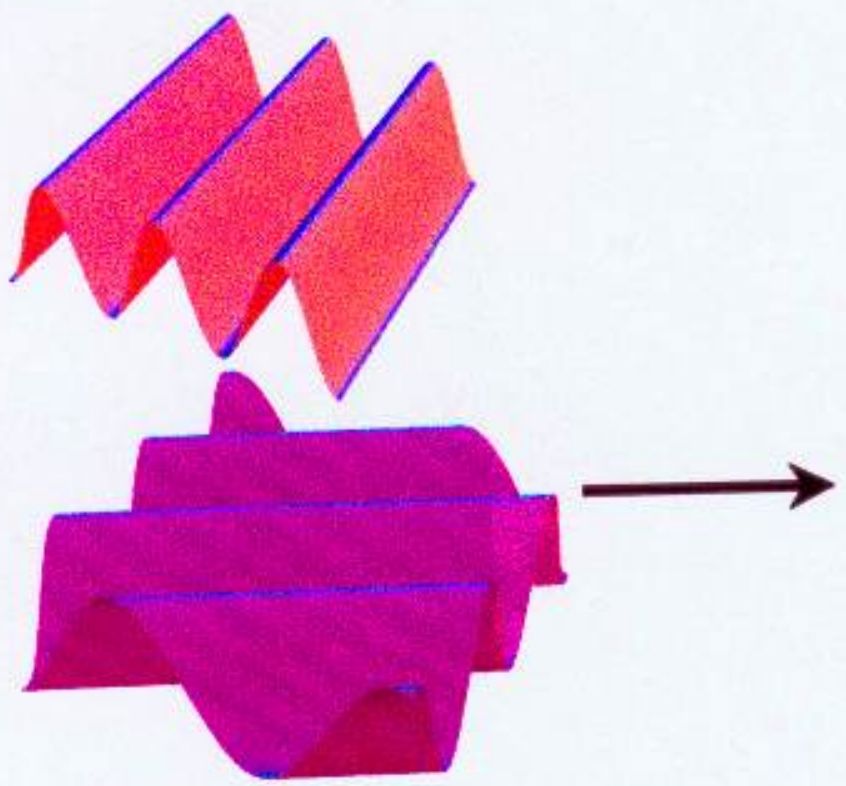

B4-Egg Box
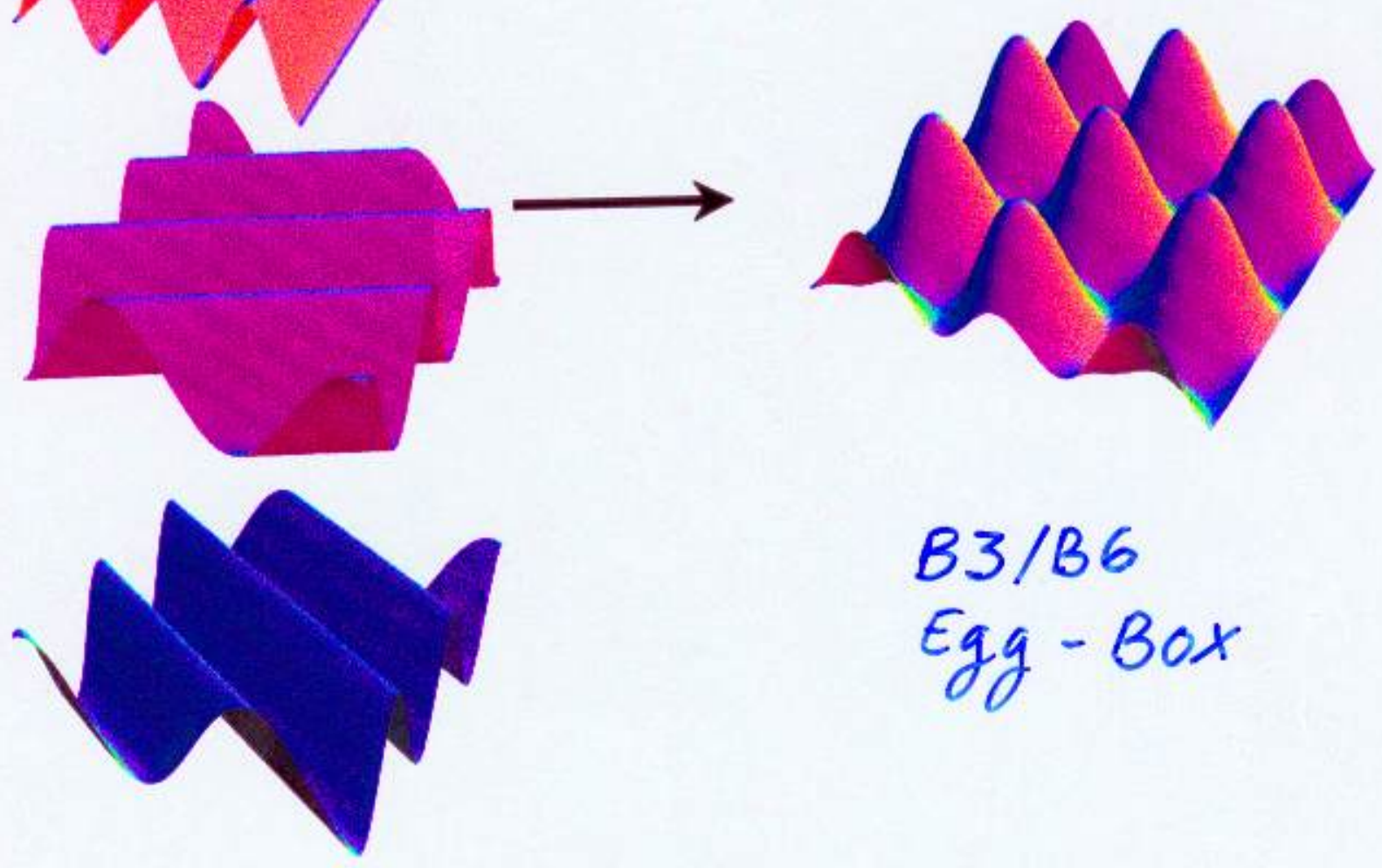

$B 3 / B 6$

Egg - BOX 


$$
\text { EQUATIONS }
$$

- INTERFACE $\begin{cases}2 D & z=\eta(x, t) \\ 3 D & z=\eta(x, y, t)\end{cases}$

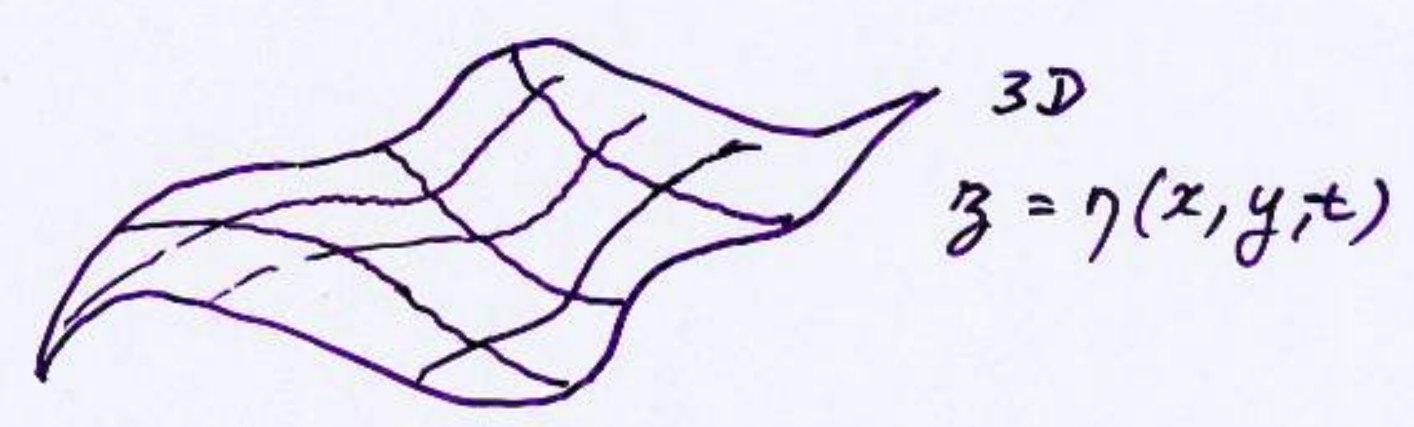

- PARTICLES Of FLYID ATTACHED TO THE INTERFACE

$$
\frac{\partial \eta}{\partial t}=v_{z}-v_{x} \frac{\partial \eta}{\partial x}-\underbrace{v_{y} \frac{\partial \eta}{\partial y}}_{3 D}
$$

- BERNOULLI EQUATION

$$
\frac{\partial \phi}{\partial t}=\frac{1}{2}(\vec{v})^{2}+g(t) \eta
$$


MODEL

Extension of LAYZER Approach

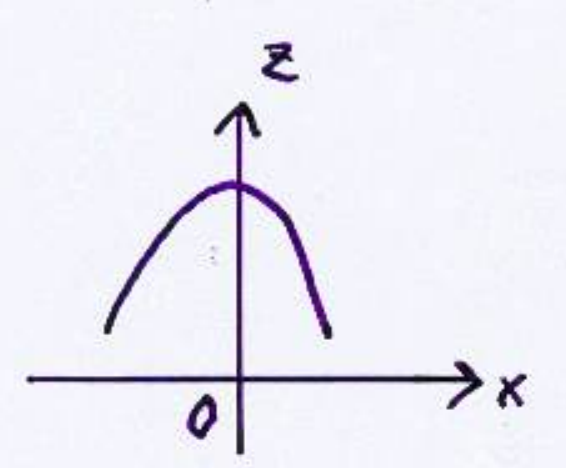
ADJ 122 1 (1955)

Top_ of a bubble:

1) $\eta(x, t)=\eta_{0}(t)+\frac{1}{2} \eta_{1}(t) x^{2}+\cdots$

2) monomode potential $\phi(x, 3, t) \sim a(t)(\cos k x) e^{-k z}$

OUR WORK:

LD:

$$
\begin{aligned}
& \phi(x, z, t)=\sum_{n=1}^{N} \phi_{n}(t) \cos (n x) e^{-n z} \\
& \eta=\eta_{0}+\sum_{n=1}^{N} k_{n}(t) \frac{x^{2 n}}{(2 n) !}
\end{aligned}
$$

$N$ - TRUNCATION NUMBER (up to 6 for $J$, for the first time)

$\underline{3 D}\left\{\begin{aligned} \phi & \sim \sum_{n m}^{n+m<N} \phi_{n m}(t) \cos x x \cos m y e^{-q_{n m} z} \\ \eta & \sim \sum_{n m} K_{n}(t) \frac{x^{2 n} y \cdot 2 m}{(2 n !)} \frac{(2 m !)}{(2 m}\end{aligned}\right.$ 
STAGNATION POINTS

- TOP OF BUBBLES

- TIP OF JETS

- SADDLE POINTS

for the first time 


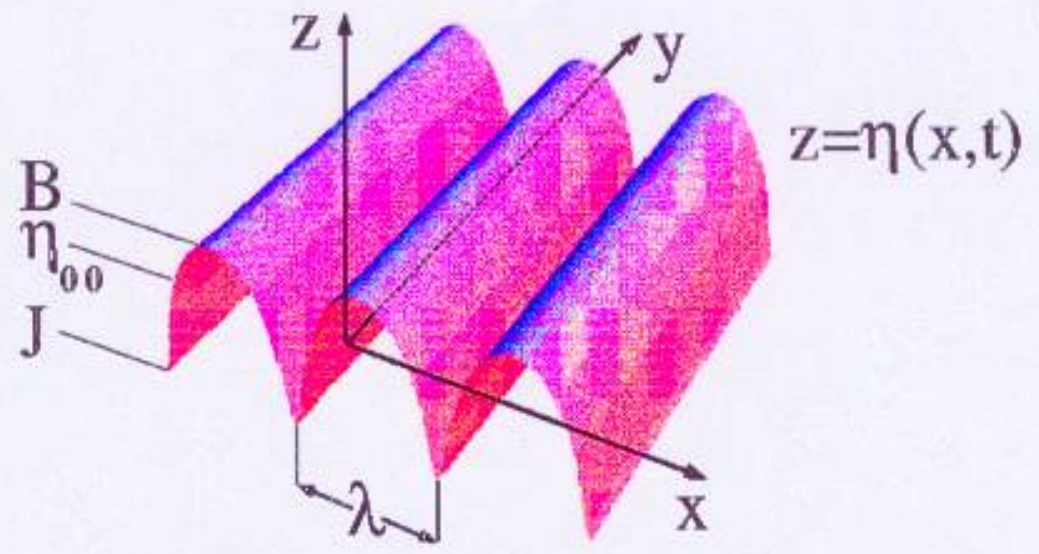

2D - Nonlinear Analytical Study (parrallel ripples)

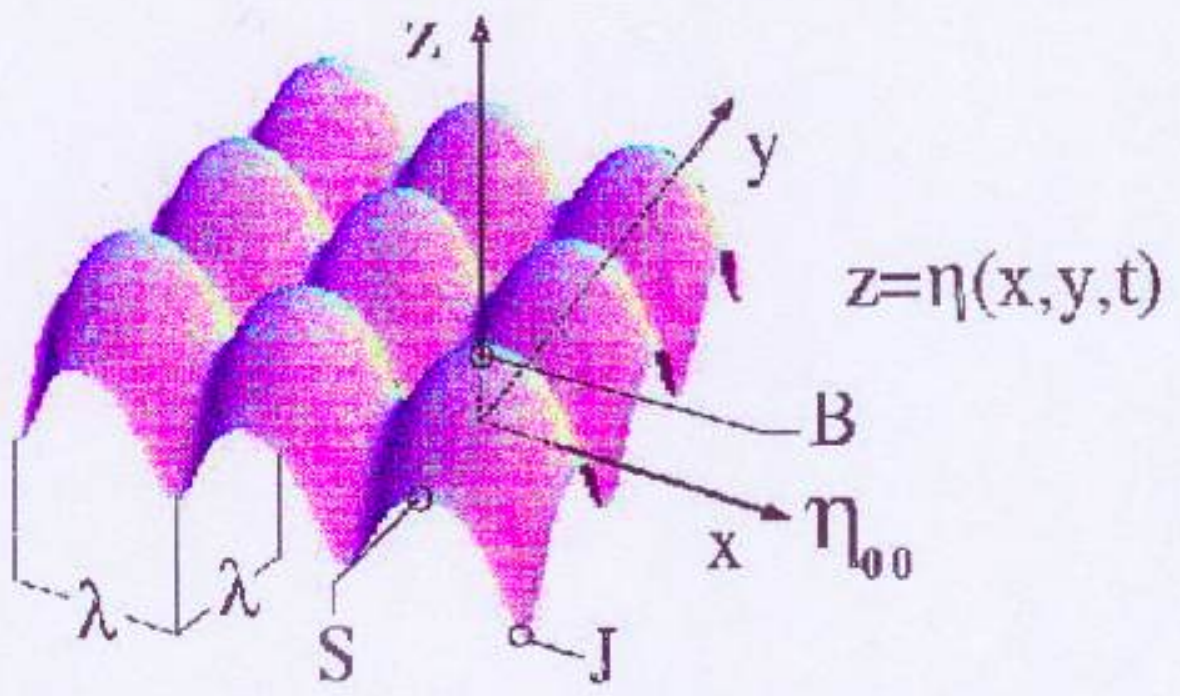

2D - Nonlinear Analytical Study (egg box type initial perturbation)

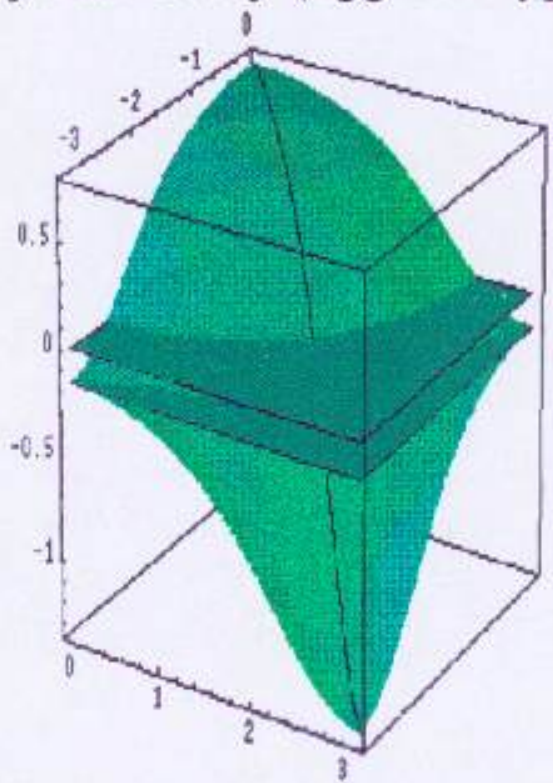

Detail of a Local System "Bubble+Jet+Saddle Point » 


\section{$B L O W-U P$ \\ $B+S+J$}

$\left.\begin{array}{l}h_{B} \simeq 0.7 \\ l_{J} \simeq 1.4\end{array}\right\} \ell_{J} \simeq 2 h_{B} \quad$ Highly $N L$

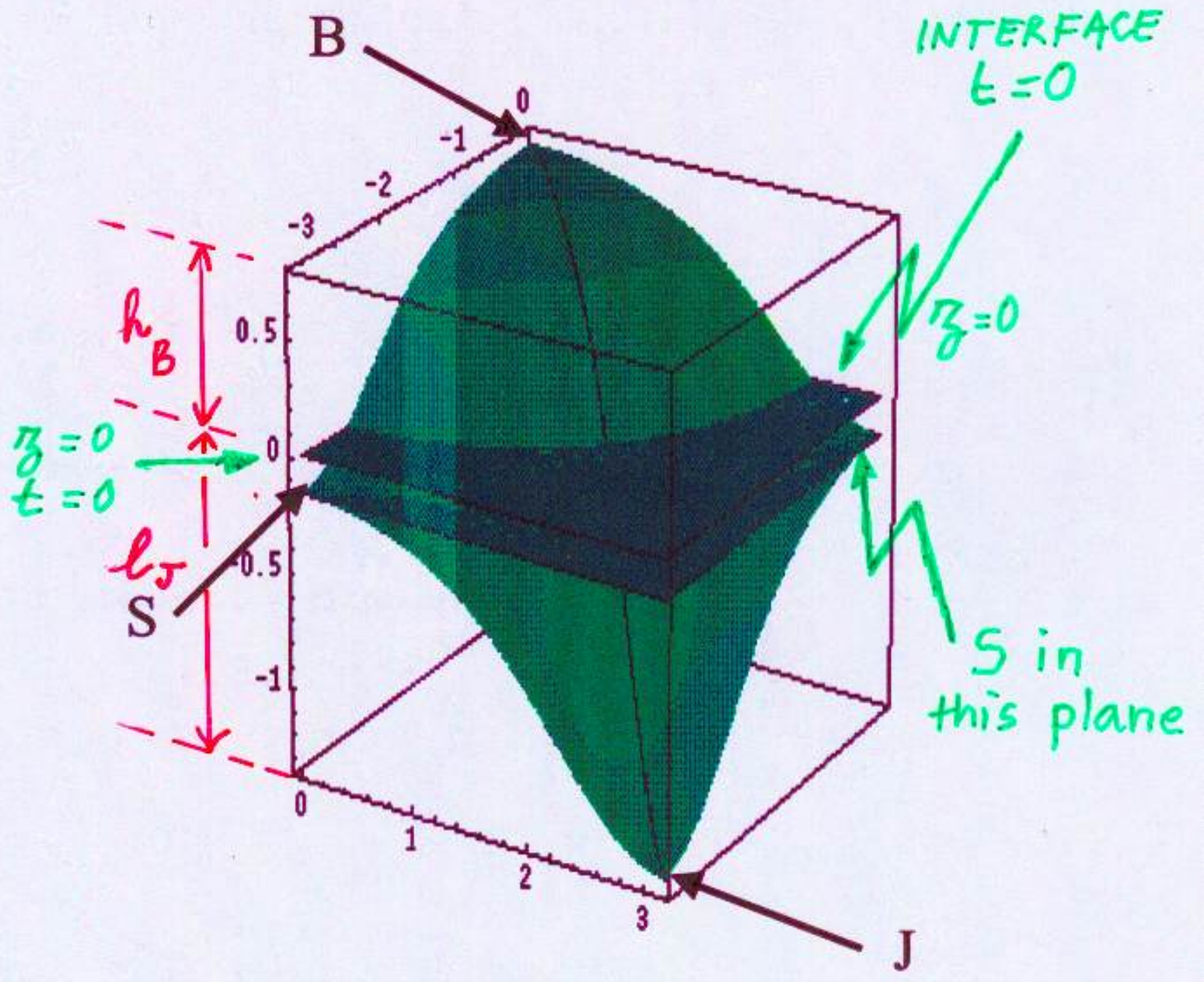



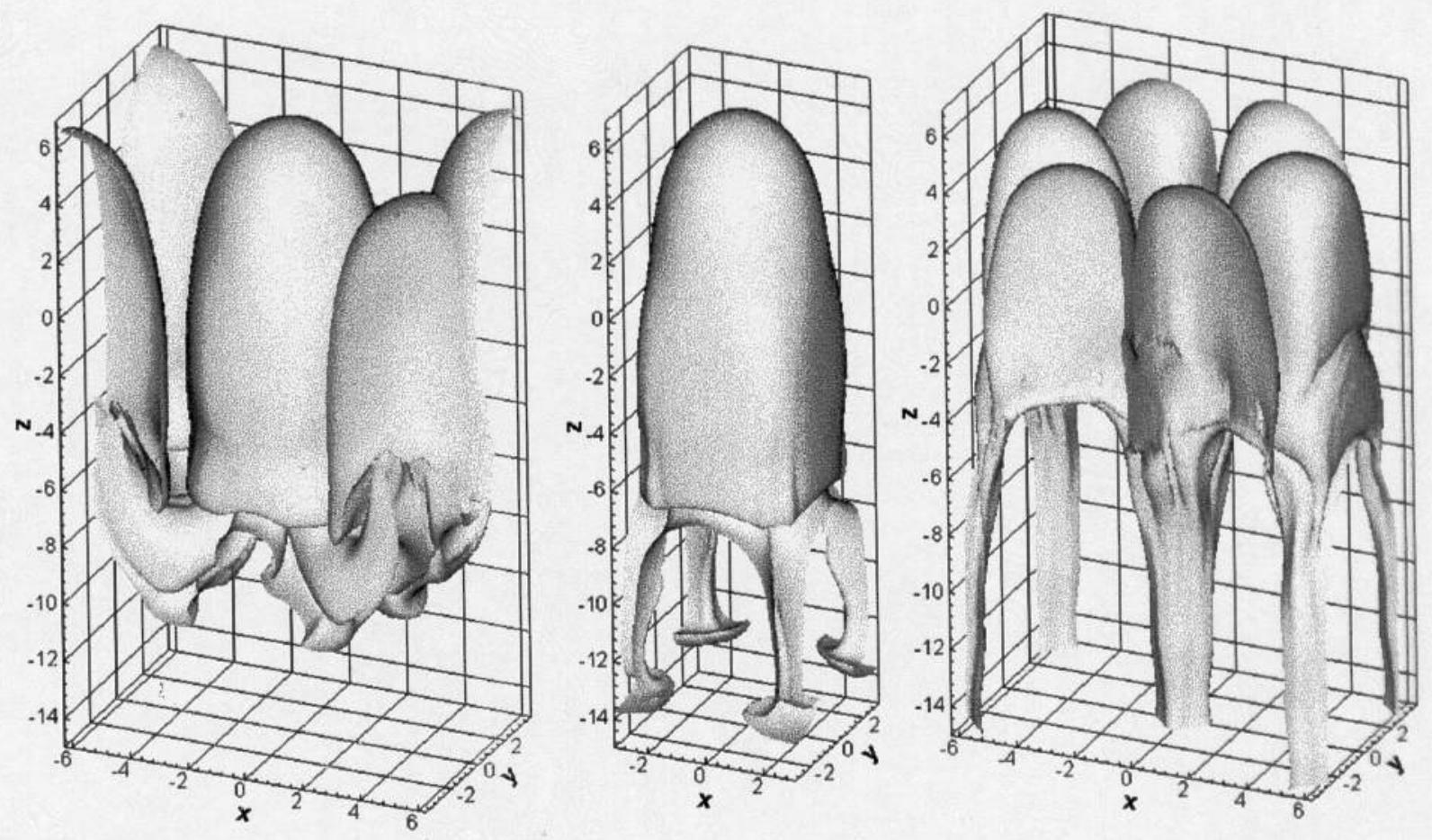

Numerical Simulation of the Rayleigh-Taylor Instability (B6, B4, B3)
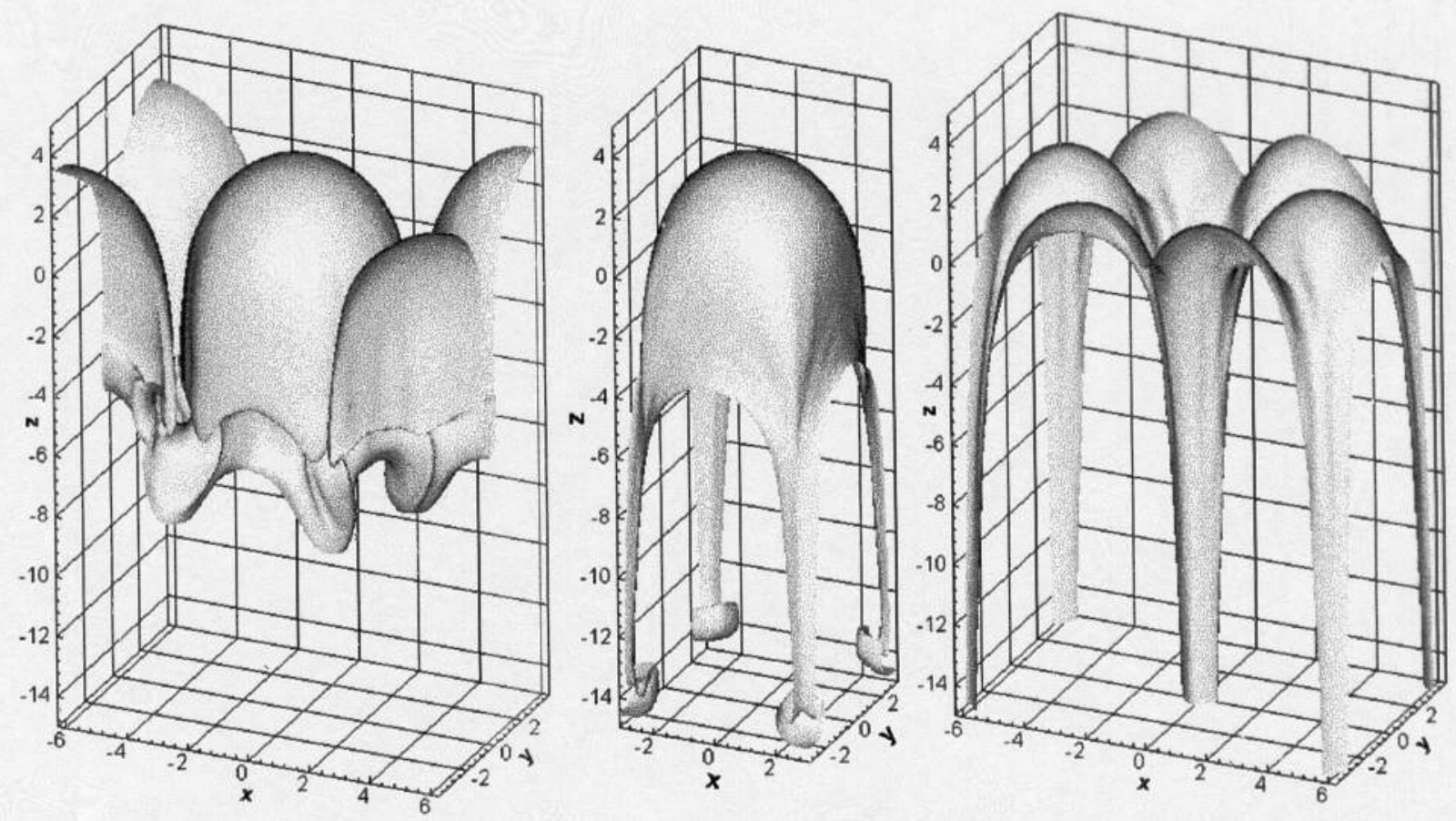

Numerical Simulation of the Richtmyer-Meshkov Instability (B6, B4, B3) 


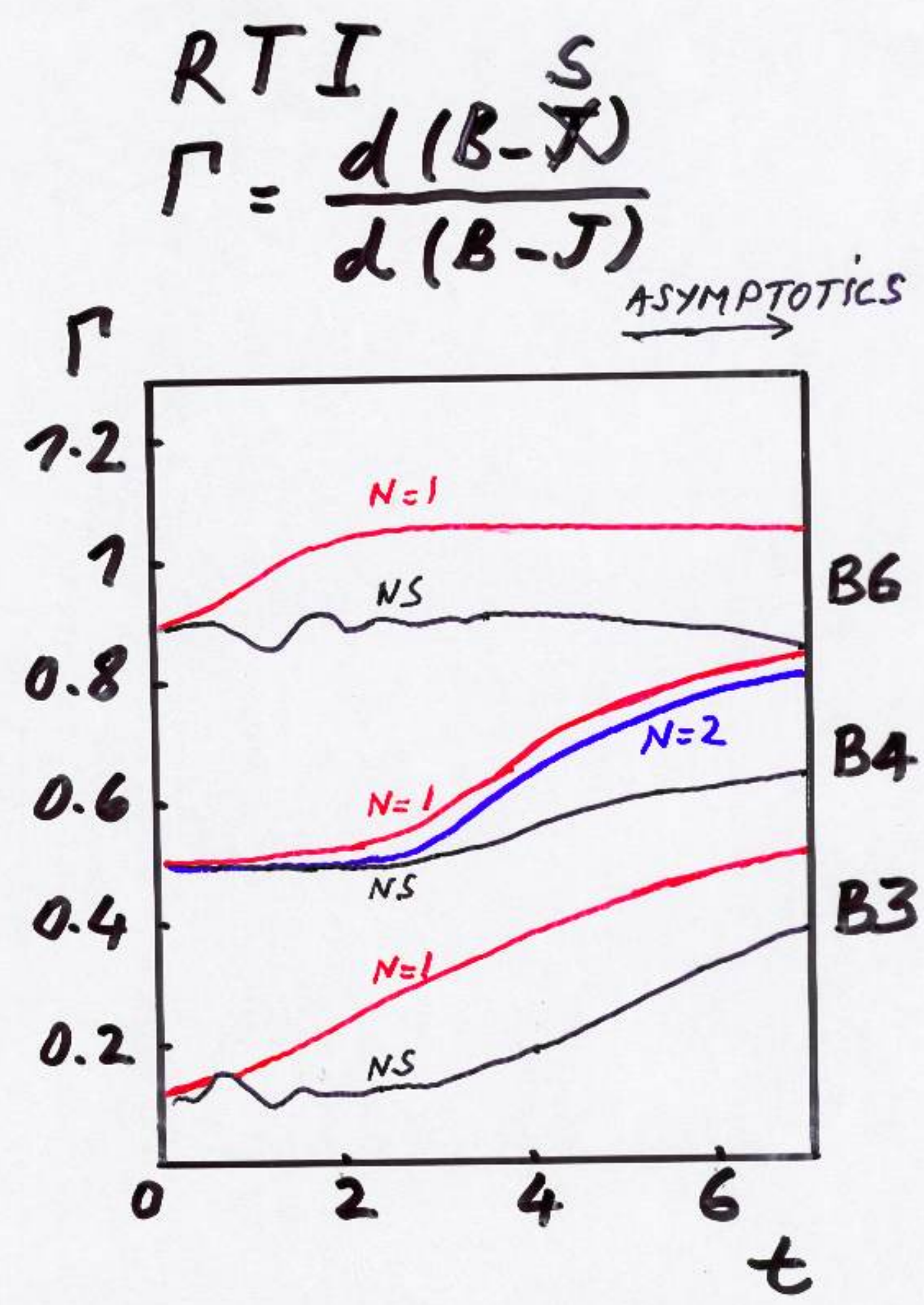

- Numb. Sim. (OPARIN)

- Theory $N=1$

- Theory $N=2$

GOOD TENDENCY ( $r$ is global) 
RM

$$
r=\frac{d(B-S)}{d(B-J)}
$$

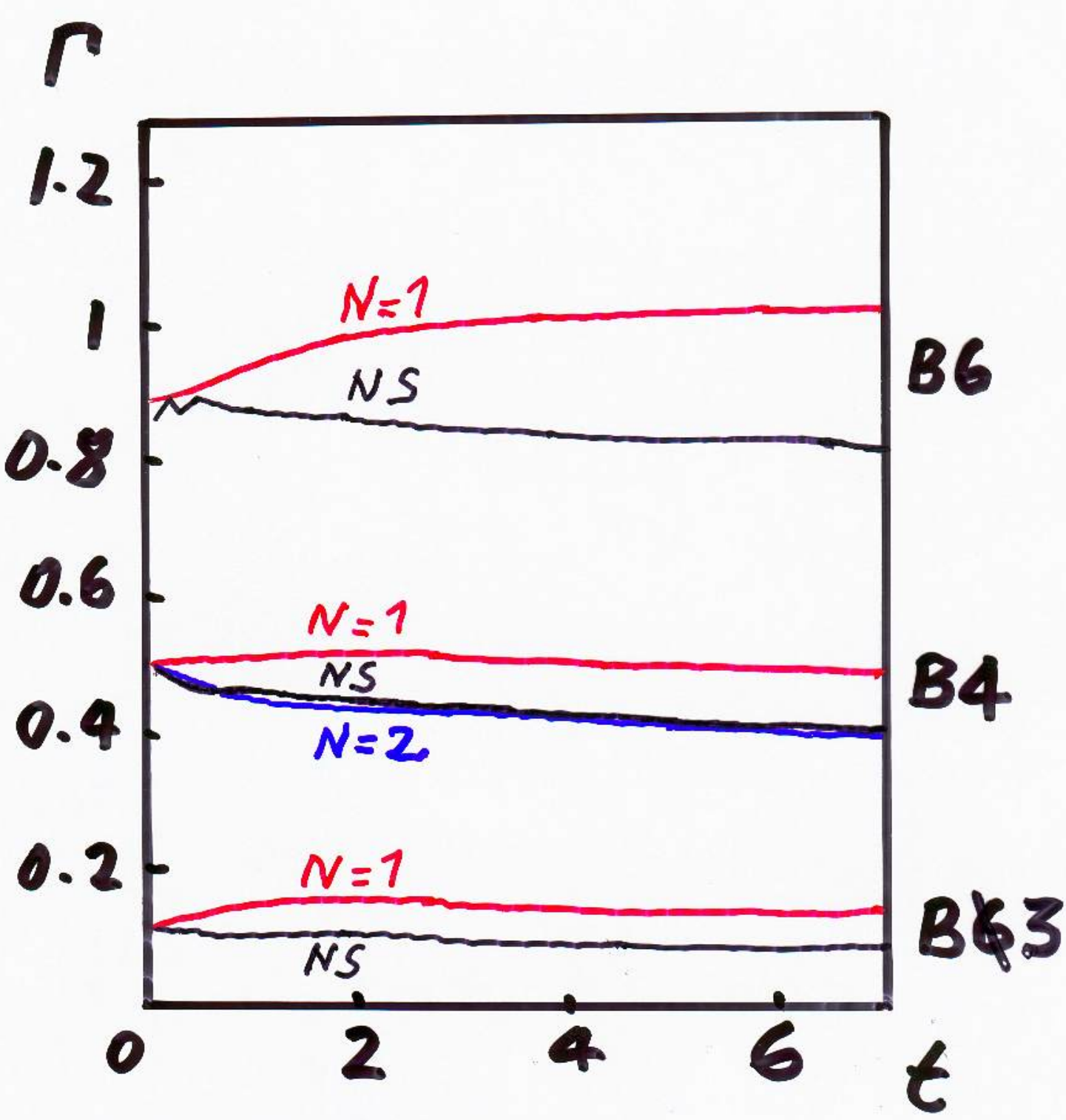

$N=2$ Good agreement.

Rather good " for asymptotic values. 


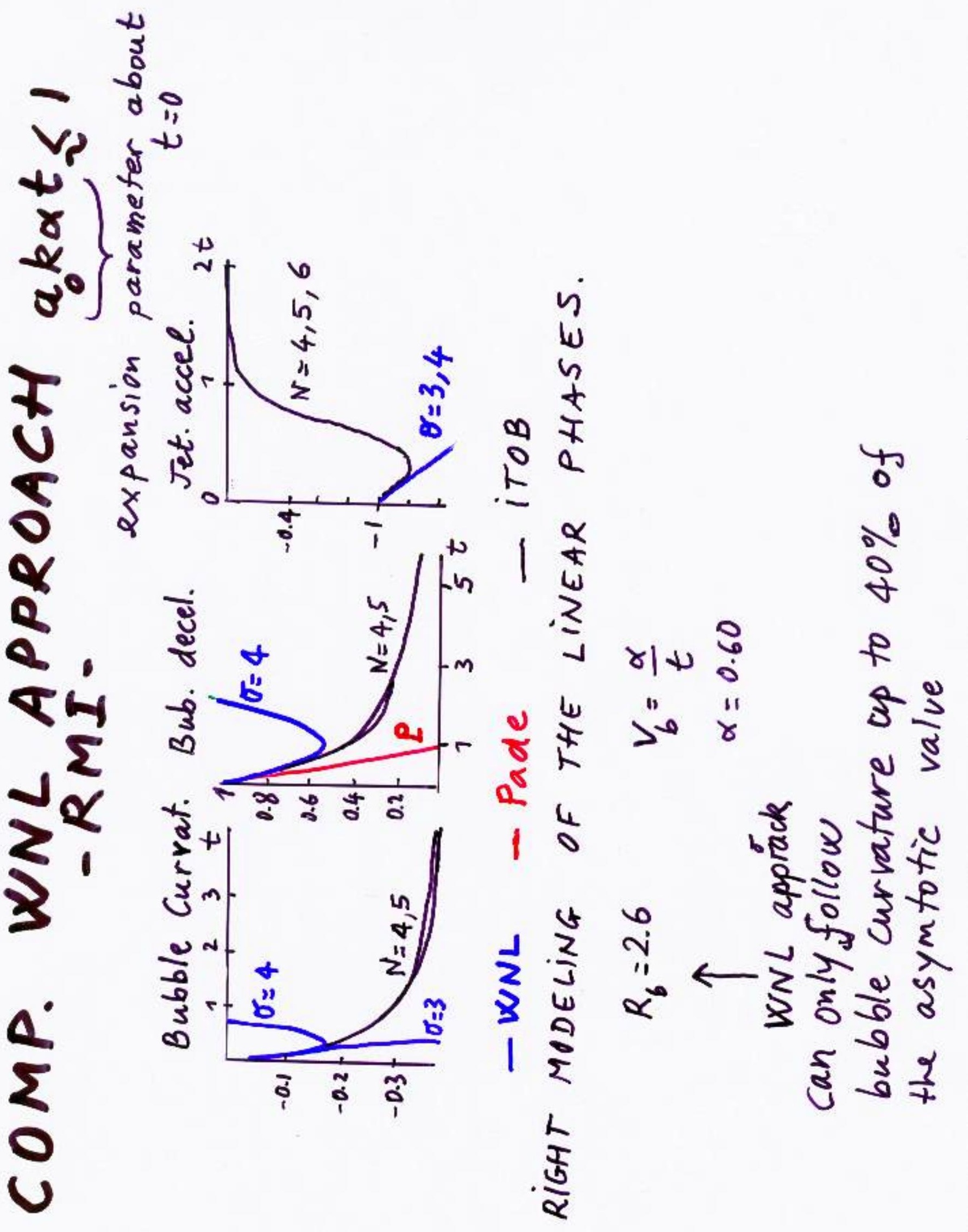


CONCLUSION

- Analytical NL asymptotic stages (t transients)

- comparisons with 2D/3D CODES which compute hydro in such NL regimes 


\section{Three Dimensional Multi-Mode Rayleigh-Taylor and Richtmyer-Meshkov Instabilities at All Density Ratios}

D. Kartoon ${ }^{1,2}$, D. Oron ${ }^{3}$, L. Arazi ${ }^{4}$, A. Rikanati ${ }^{1,2}$,

O. Sadot' ${ }^{1,5}$, A. Yosef-Hai ${ }^{1,5}$, U. Alon ${ }^{3}$, G. Ben-Dor ${ }^{5}$ and D. Shvarts $1,2,5$

1. Dept. of Physics, Nuclear Research Center Negev, Israel

2. Dept. of Physics, Ben-Gurion University, Beer-Sheva, Israel

3. Fac. of Physics, The Weizmann Institute of Science, Rehovot, Israel

4. School of Physics and Astronomy, Tel-Aviv University, Tel-Aviv, Israel

5. Dept. of Mech. Eng., Ben-Gurion University, Beer-Sheva, Israel 
Oron et al. PoP 2001.

Alon et al, PRL 1994, 1995.

Hecht et al. PoF 1994.

Oron et al. PoP 1998.

Rikanati et al. PRE 1998.

Sadot et al. PRL 1998.

Layzer APJ 1955.

Dimonte PoF, PoP 2000. 


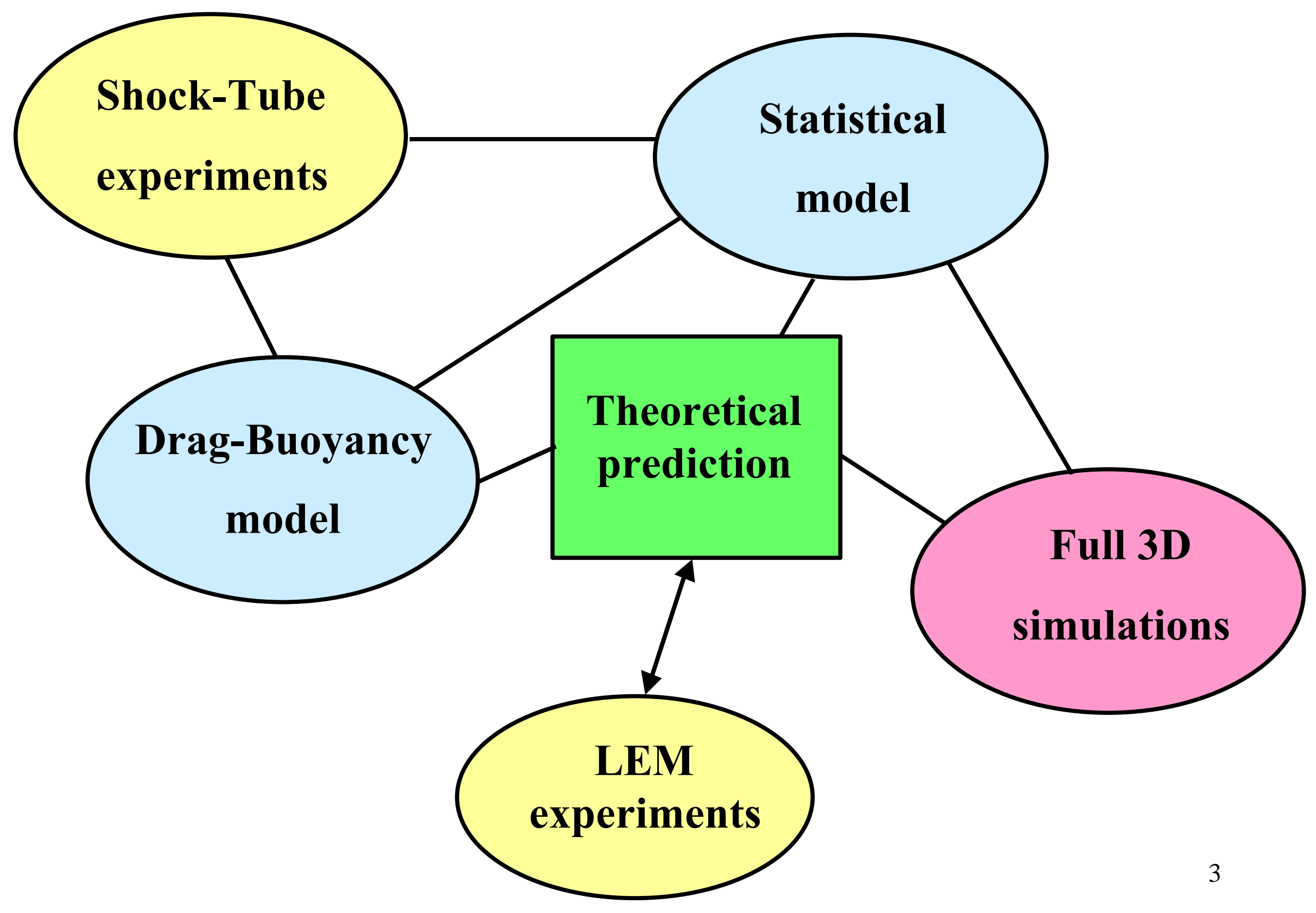


The Drag-Buoyancy Model for Bubbles

2D Statistical Model

Full 3D Numerical Simulations

3D Statistical Model

The Drag-Buoyancy Model for Spikes

Conclusion 


$$
\begin{aligned}
& \left(\rho_{l} \cdot V+C_{a} \cdot \rho_{h} \cdot V\right) \cdot \frac{d u}{d t}=\left(\rho_{h}-\rho_{l}\right) V \cdot g-\rho_{h} \cdot S \cdot u^{2} \\
& \text { (inertia) }+(\text { added mass })=(\text { buoyancy }) \quad \text { (kinematic drag) }
\end{aligned}
$$

$$
\text { Where } \mathrm{V} / \mathrm{S} \propto \lambda
$$
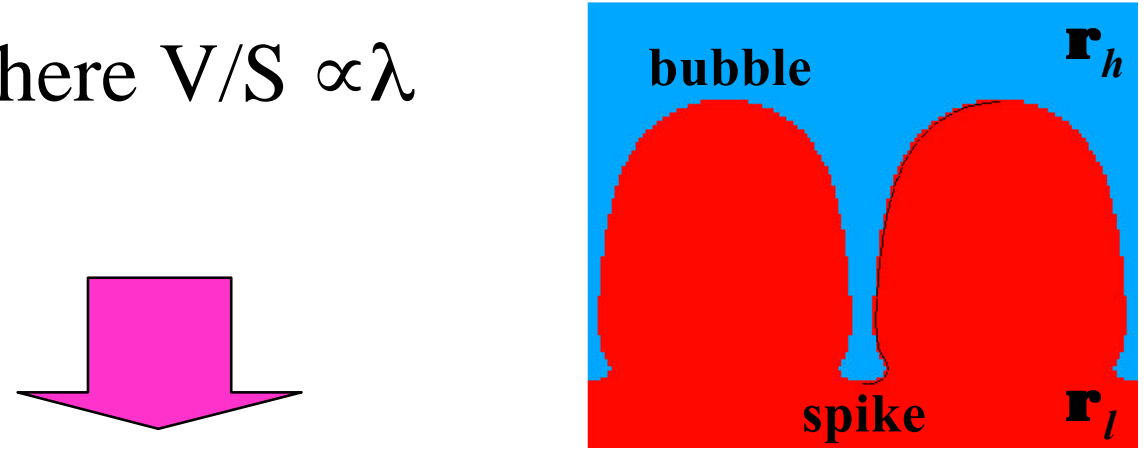

$$
\left(\rho_{l}+C_{a} \rho_{h}\right) \dot{u}=\left(\rho_{h}-\rho_{l}\right) \cdot g-\frac{C_{d}}{\lambda} \rho_{h} \cdot u^{2}
$$

Where $\mathrm{C}_{\mathrm{a}}$ and $\mathrm{C}_{\mathrm{d}}$ are geometric constants. 


\section{Single Mode Asymptotic Velocities}

$$
\left(\rho_{l}+C_{a} \rho_{h}\right) \dot{u}=\left(\rho_{h}-\rho_{l}\right) \cdot g-\frac{C_{d}}{\lambda} \rho_{h} \cdot u^{2}
$$

$\mathrm{RT}: \quad \mathrm{u}_{\mathrm{B}}=\sqrt{\frac{1}{\mathrm{C}_{\mathrm{d}}}\left(\frac{2 \mathrm{~A}}{1+\mathrm{A}}\right) \mathrm{g} \lambda}$

RM: $\quad \mathrm{u}_{\mathrm{B}}=\frac{1}{\mathrm{C}_{\mathrm{d}}} \cdot\left(\frac{1-\mathrm{A}}{1+\mathrm{A}}+\mathrm{C}_{\mathrm{a}}\right) \cdot \frac{\lambda}{\mathrm{t}}$

The geometric constants depend on the dimensionality:

$$
\begin{array}{ll}
\mathrm{C}_{\mathrm{a}}^{2 \mathrm{D}}=2, & \mathrm{C}_{\mathrm{d}}^{2 \mathrm{D}}=6 \pi \\
\mathrm{C}_{\mathrm{a}}^{3 \mathrm{D}}=1, & \mathrm{C}_{\mathrm{d}}^{3 \mathrm{D}}=2 \pi
\end{array}
$$


RM Single Mode Experimental Results

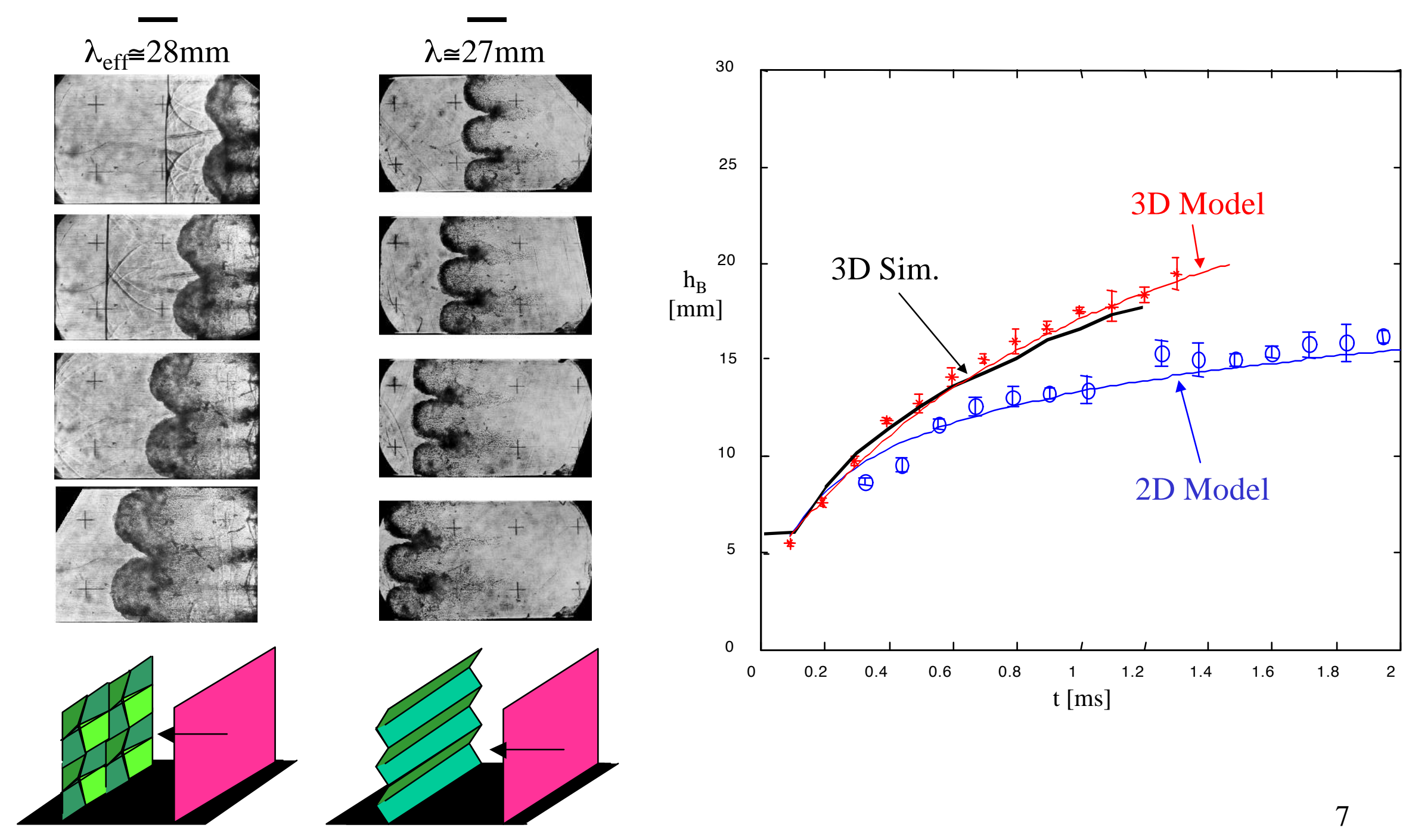




\section{Multi Mode Drag Buoyancy Model}

Generalization of the drag-buoyancy equation, using the self-similarity assumption:

$$
h_{B}^{(M M)}(t)=b(A) \cdot\langle\lambda\rangle(t)
$$

$\underline{\mathrm{RT}}: \quad u=\dot{h}=\sqrt{c_{1}(A) \cdot g \cdot h / b(A)}=\alpha \cdot A \cdot g t^{2}$

$\underline{\mathrm{RM}}: u=\dot{h}=c_{2}(A) \cdot h / b(A) \cdot \frac{1}{t}=c \cdot t^{\theta}$

$$
\alpha=\frac{1}{2(1+A)} \frac{1}{C_{d}} \frac{1}{b^{R T}(A)}
$$

$$
\theta=\left(\frac{1-A}{1+A}+C_{a}\right) \cdot \frac{1}{C_{d}} \cdot \frac{1}{b^{R M}(A)}
$$




\section{D Multi Mode the $\alpha \quad \theta \quad b$ Relations}

Using the 3D coefficients in the expressions relating $\alpha, \theta$ and $b$, and assuming that $b^{(\mathrm{RM})}=\mathrm{b}^{(\mathrm{RT})}$, the differences between 2D and 3D bubble front growth are obtained:

\begin{tabular}{|c|c|c|c|c|}
\hline & \multicolumn{2}{|c|}{$\begin{array}{c}\text { 2D } \\
\left(C_{a}=2, C_{d}=6 \pi\right)\end{array}$} & & $\begin{array}{l}3 \mathbf{D} \\
\left., \mathrm{C}_{\mathrm{d}}=2 \pi\right)\end{array}$ \\
\hline$\stackrel{\alpha}{a s s u m e d}$ & \multicolumn{2}{|c|}{0.05} & & 0.05 \\
\hline$\theta$ & $0.1(\mathrm{~A}+3)$ & $(=0.3-0.4)$ & & 0.2 \\
\hline b & $\frac{1}{2(A+1)}$ & $(=0.25-0.5)$ & $\frac{3}{2(A+1)}$ & $(=0.75-1.5)$ \\
\hline
\end{tabular}


The Drag-Buoyancy Model for Bubbles

2D Statistical Model

Full 3D Numerical Simulations

3D Statistical Model

The Drag-Buoyancy Model for Spikes 


\section{Simulation of 2D Multimode Perturbation Evolution}

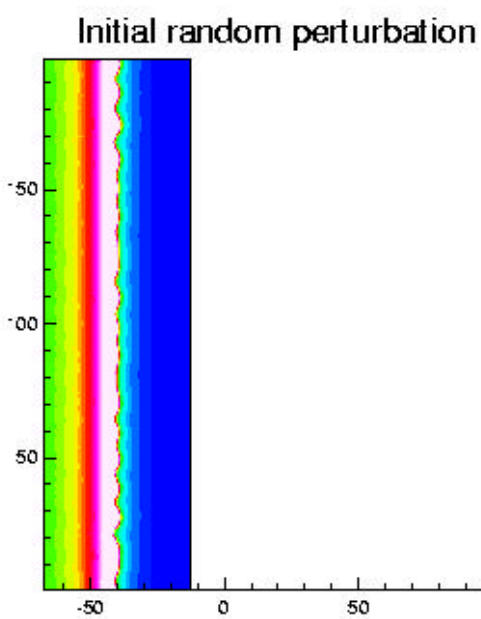

Bubble competition begins

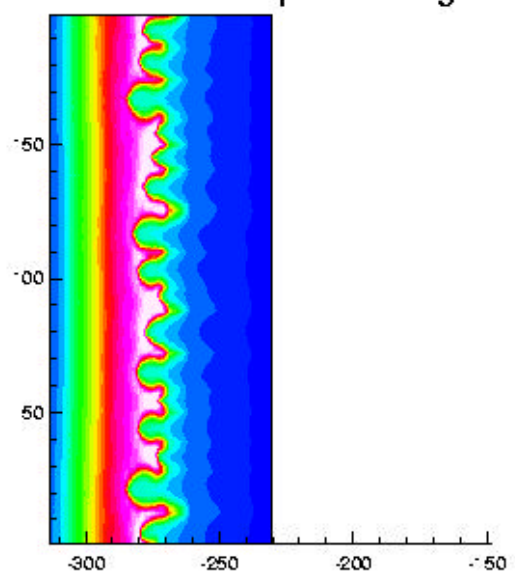

Perturbation growth

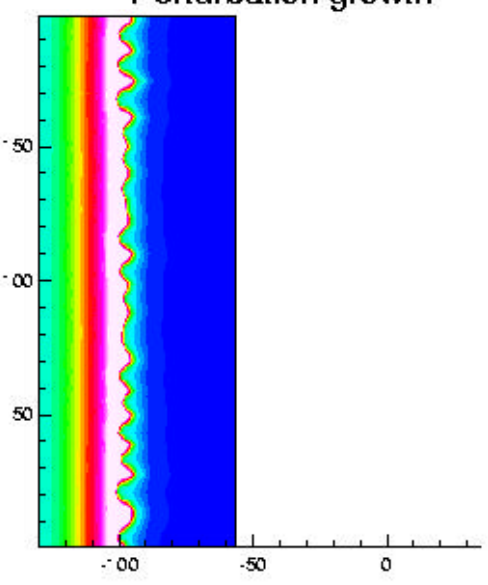

Small bubbles washed downstream

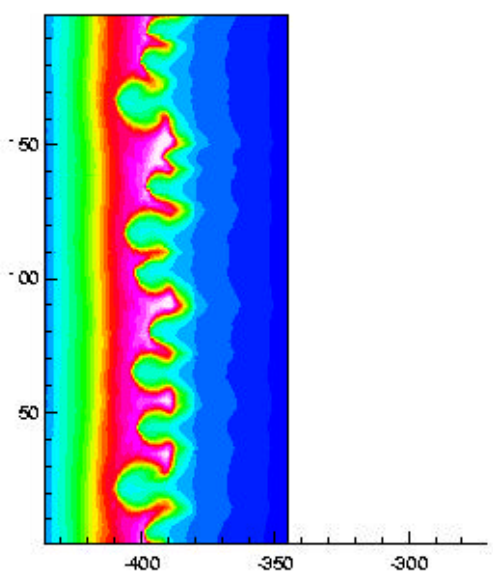

Coexistence

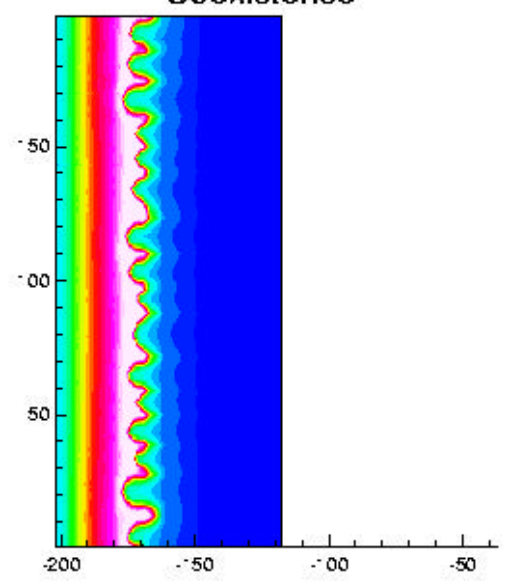

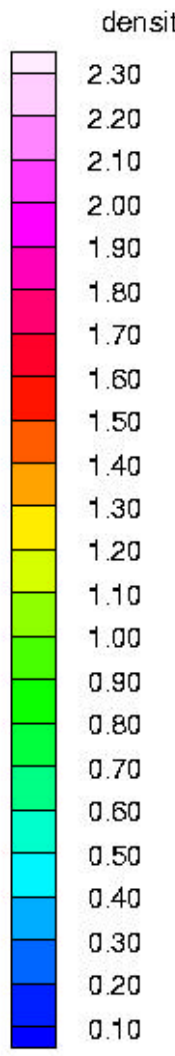

.10

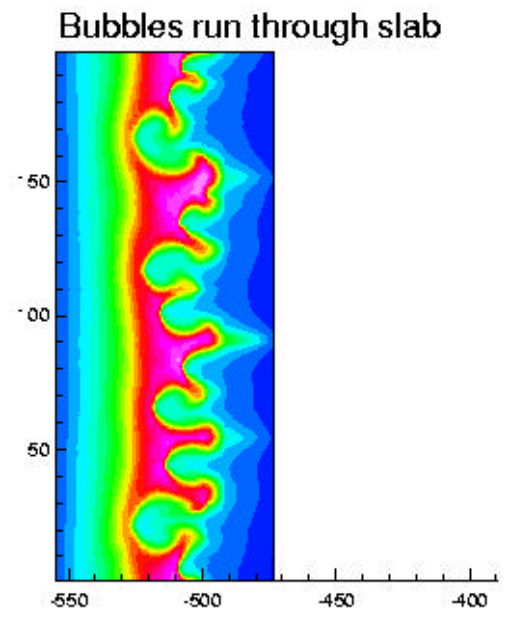




\section{Multi Mode 2D Statistical Model (Alon et. Al.)}

\section{Bubble Envelope (Simulation)}

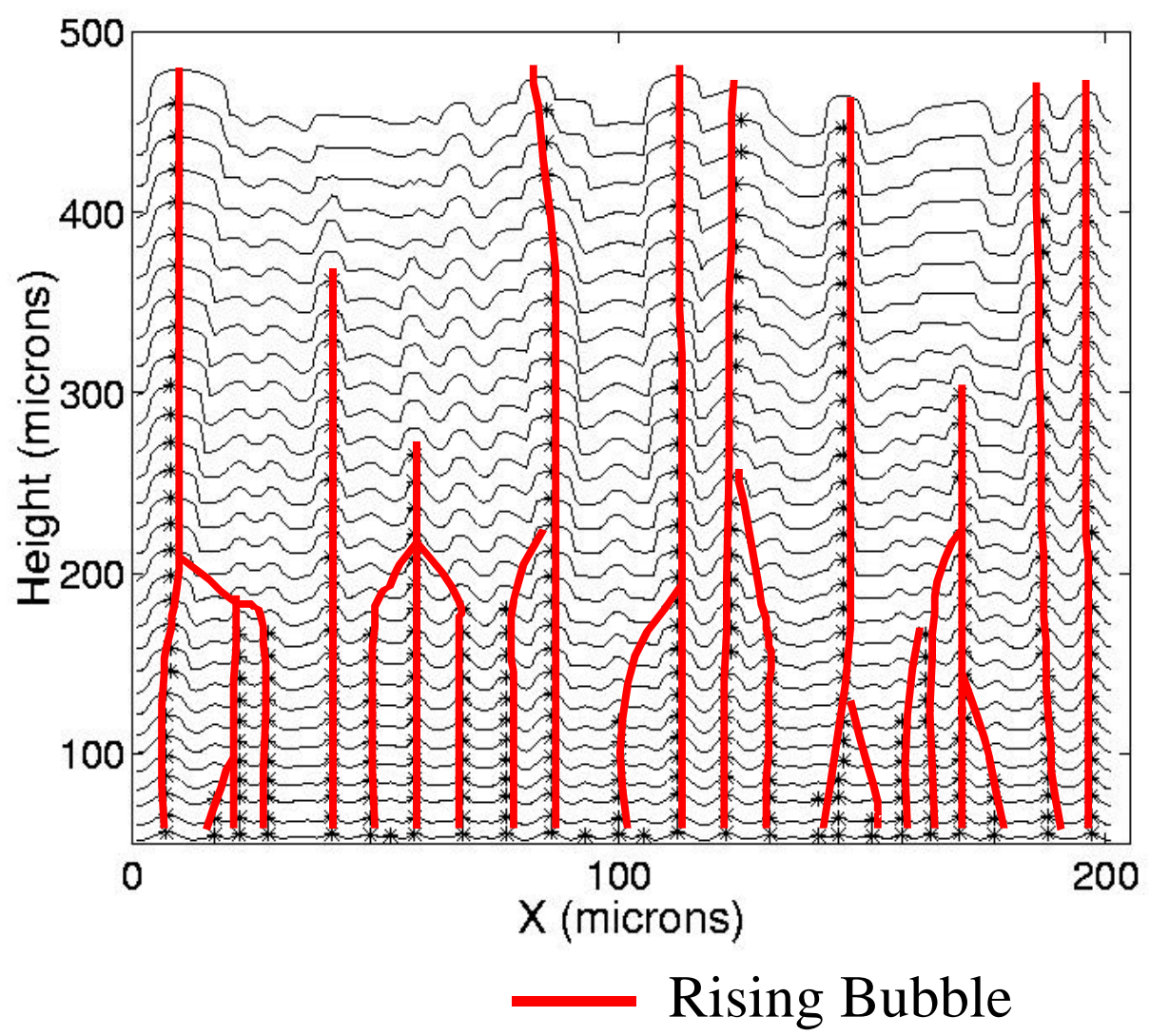

Each bubble grows with its asymptotic velocity, according to its wavelength:

$$
\mathrm{u}_{\mathrm{i}}=\mathrm{u}_{2 \mathrm{D}}^{\text {(asy) }}\left(\lambda_{i}, \mathrm{t}\right)
$$




\section{Multi Mode Bubble Merger}

Velocity evolution of two nonidentical adjacent bubbles:

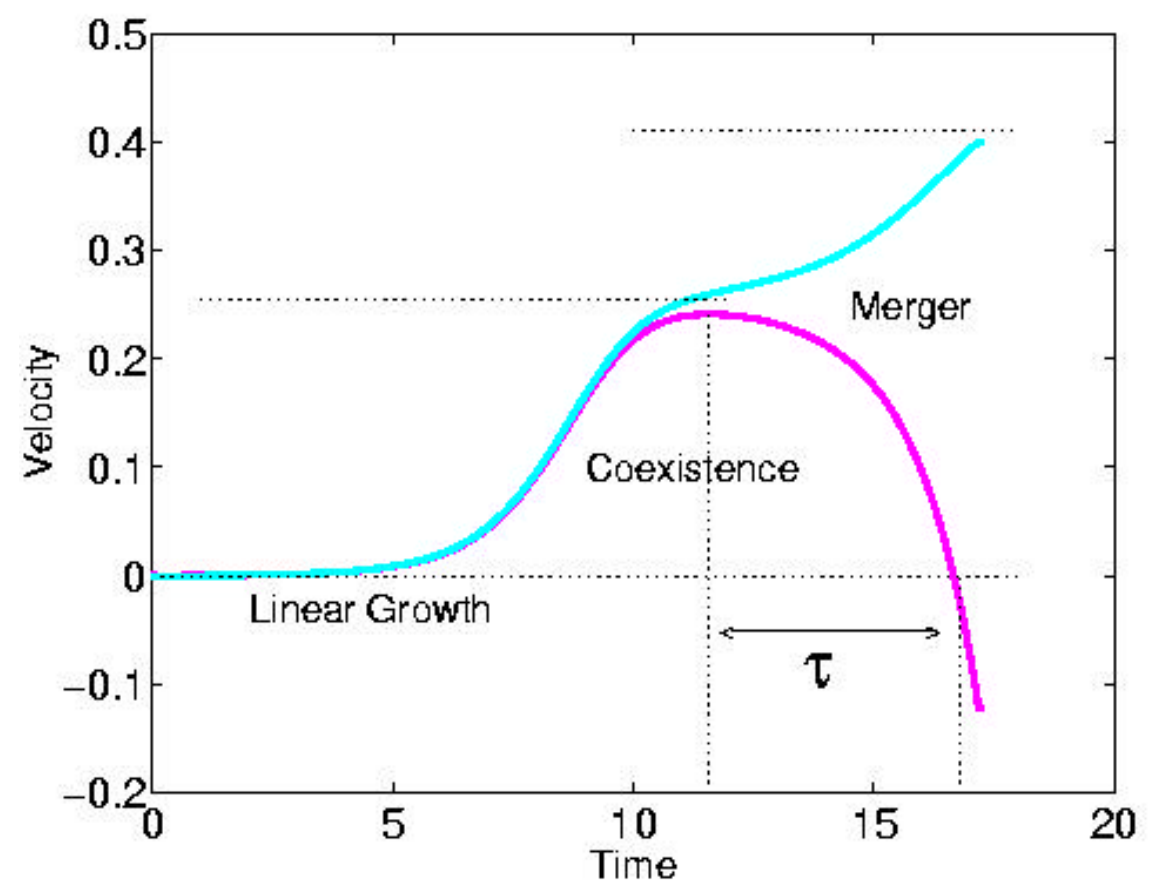

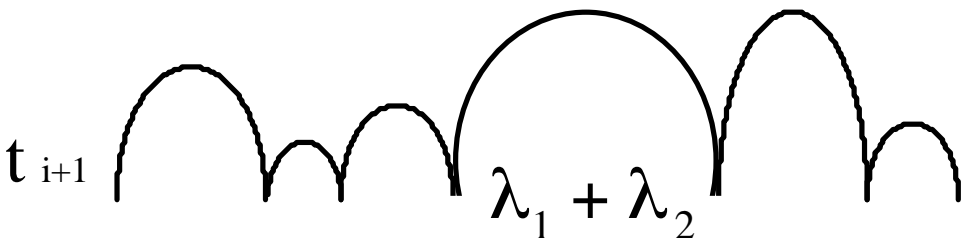

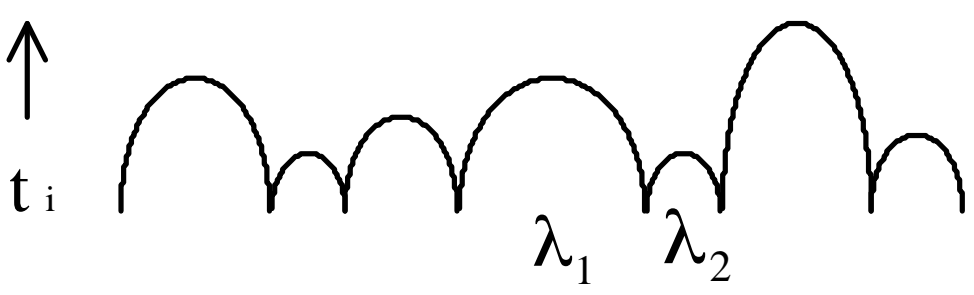

\section{Merger Rate:}

$\left(\lambda_{1}, \lambda_{1+1}\right)$

$\downarrow \omega_{\text {merge }}\left(\lambda_{1}, \lambda_{1+1}, t\right) \sim 1 / \tau$

$\left(\lambda_{1}+\lambda_{1+1}\right)$ 


\section{Multi Mode 2D Statistical Model}

$$
\begin{array}{rlr}
N(t) \cdot \frac{\partial g(\lambda, t)}{\partial t}= & -2 g(\lambda, t) \int_{0}^{\infty} g\left(\lambda^{\prime}, t\right) \omega\left(\lambda, \lambda^{\prime}\right) d \lambda^{\prime} & \text { death } \\
& +\int_{0}^{\infty} g\left(\lambda-\lambda^{\prime}, t\right) g\left(\lambda^{\prime}, t\right) \omega\left(\lambda-\lambda^{\prime}, \lambda^{\prime}\right) d \lambda^{\prime} & \text { birth }
\end{array}
$$

Where $\mathrm{g}(\lambda, \mathrm{t})$ is the number of bubbles with wavelength $\lambda$ within interval $\mathrm{d} \lambda$ at time $\mathrm{t}$, and $\mathrm{N}(\mathrm{t})$ is the total number of bubbles:

$$
\frac{\partial N(t)}{\partial t}=-\langle\omega\rangle_{g} \cdot N(t)
$$




\section{Multi Mode 2D Statistical Model Results}

1. The $\lambda$ distribution reaches an asymptotic function:

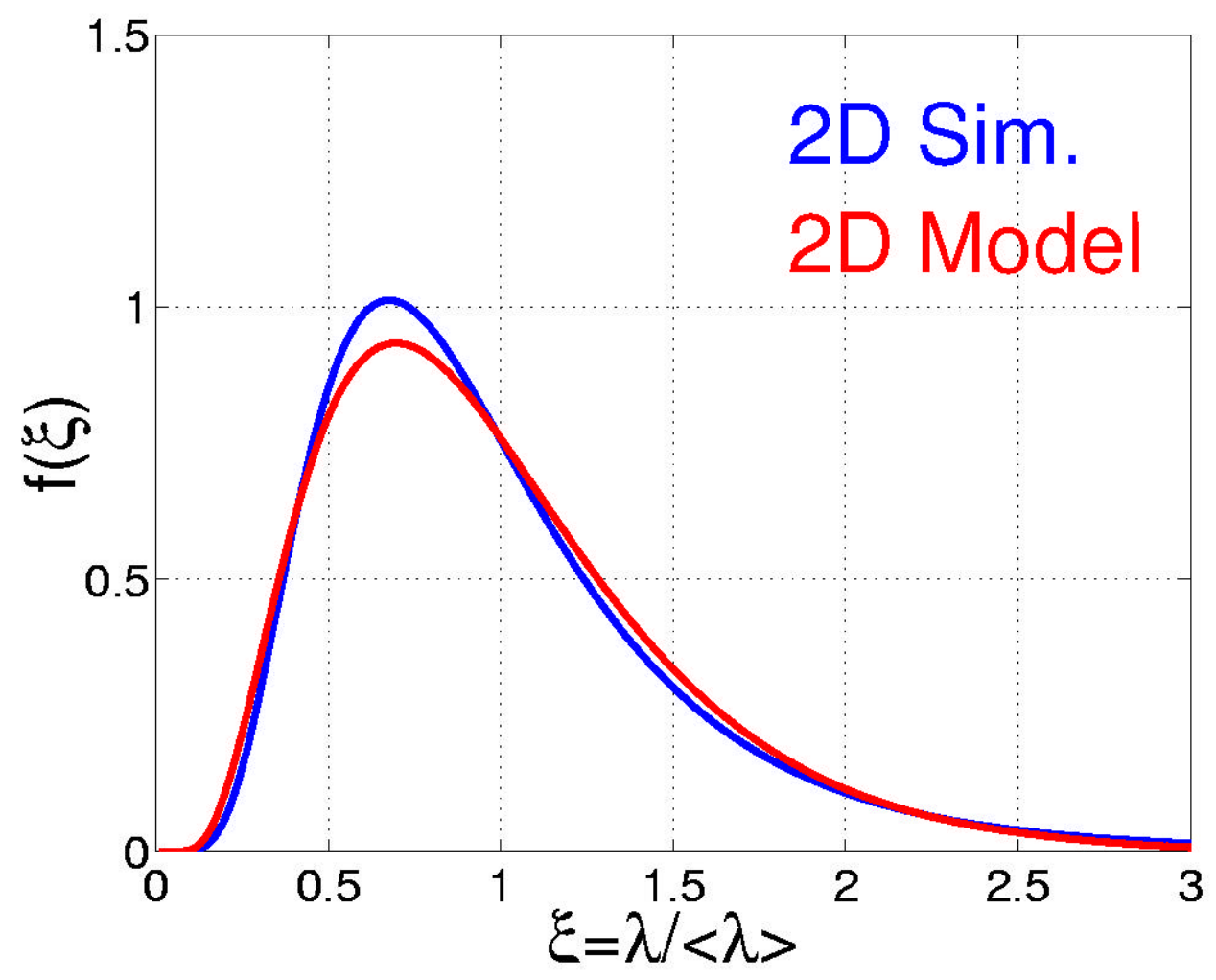

RT simulation, $\mathrm{A}=0.5$ 


\section{Multi Mode 2D Statistical Model Results}

2. The average bubble and spike heights are obtained for both the RT and the RM case:

$\mathrm{RT}: \mathrm{u}_{\mathrm{B}}^{\text {asy }} \propto \sqrt{\mathrm{g} \lambda} \stackrel{\text { self }- \text { similarity }}{\longrightarrow} \dot{\mathrm{h}}_{\mathrm{B}} \propto \sqrt{\mathrm{gh}} \rightarrow \mathrm{h}_{\mathrm{B}}=\alpha_{\mathrm{B}} \cdot \mathrm{gt}^{2}$

RM: $\quad u_{B}^{\text {asy }} \propto \frac{\lambda}{t} \stackrel{\text { self }- \text { similarity }}{\longrightarrow} \dot{h}_{B} \propto \frac{h_{B}}{t} \rightarrow h_{B}=c \cdot t^{\theta_{B}}$

Self-similarity parameter: $\quad b^{R T}=b^{R M}=\frac{h_{B}}{\langle\lambda\rangle}$

2D Statistical Model Results:

$$
\alpha_{\mathrm{B}} \cong 0.05 \quad \theta_{\mathrm{B}} \cong 0.4 \quad \mathrm{~b} \cong 0.2
$$




\section{LEM Experimental Results}

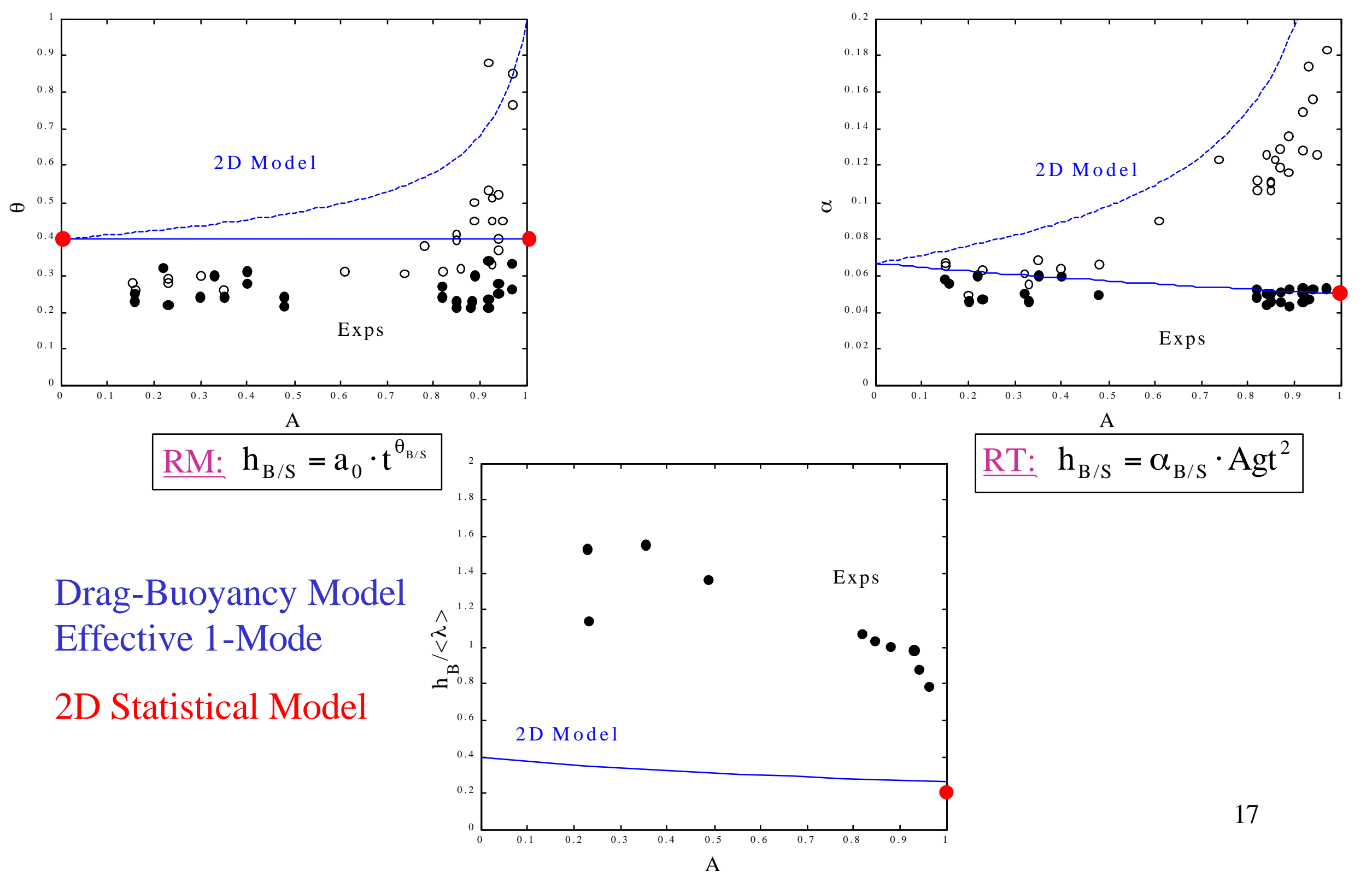


The Drag-Buoyancy Model for Bubbles

2D Statistical Model

Full 3D Numerical Simulations

3D Statistical Model

The Drag-Buoyancy Model for Spikes 
Full 3D Numerical Simulation of the RT $A=0.5$ Case
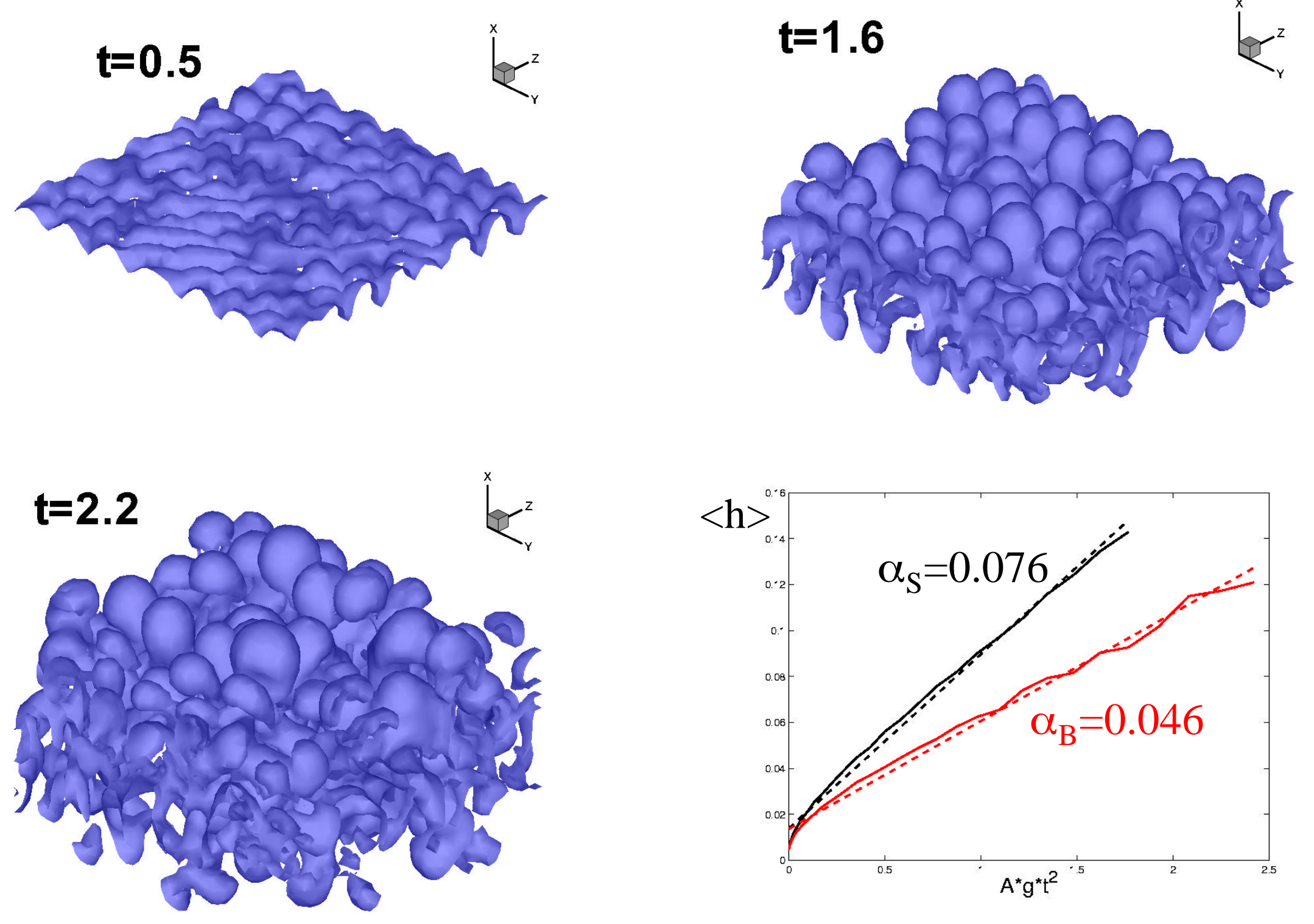
Voronoi Cell Structure of the Bubble Front

\section{Demonstrates the 3D Bubble Merger}
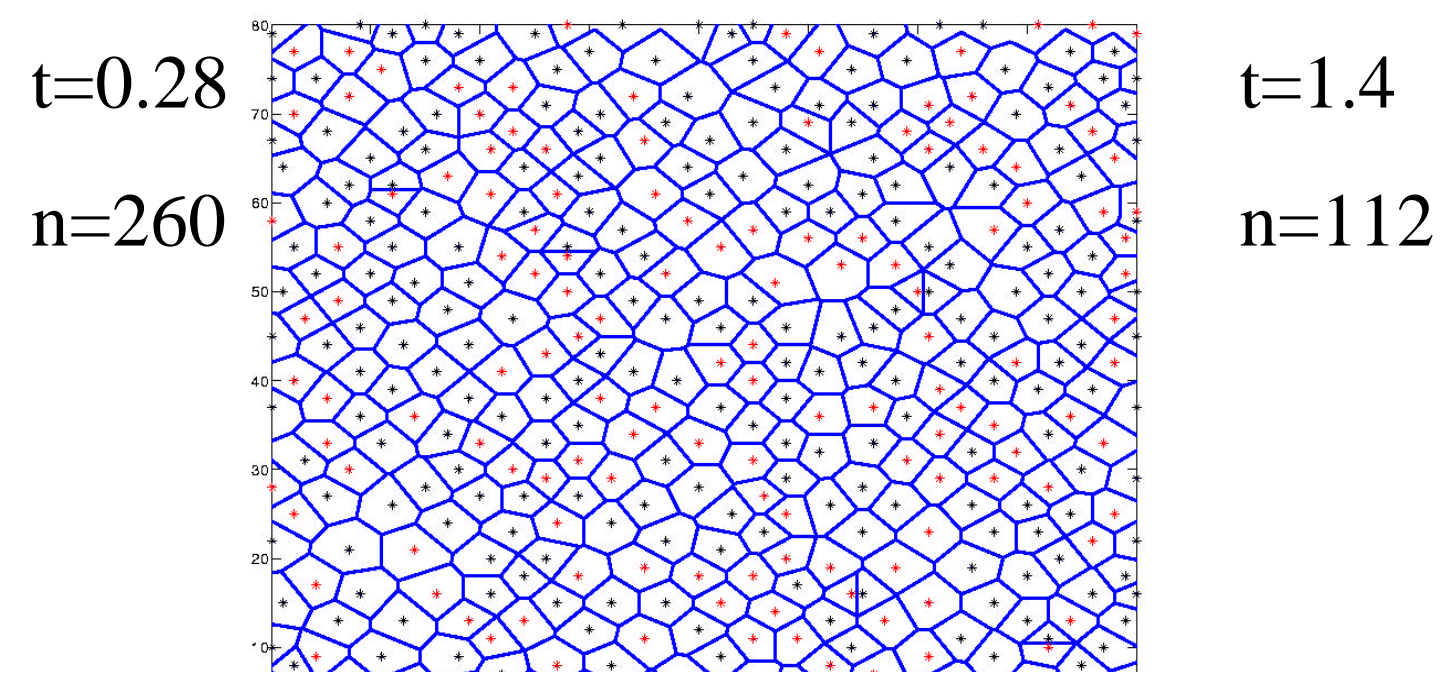

$\mathrm{t}=2.20$, No. of Rising Bubs: 30
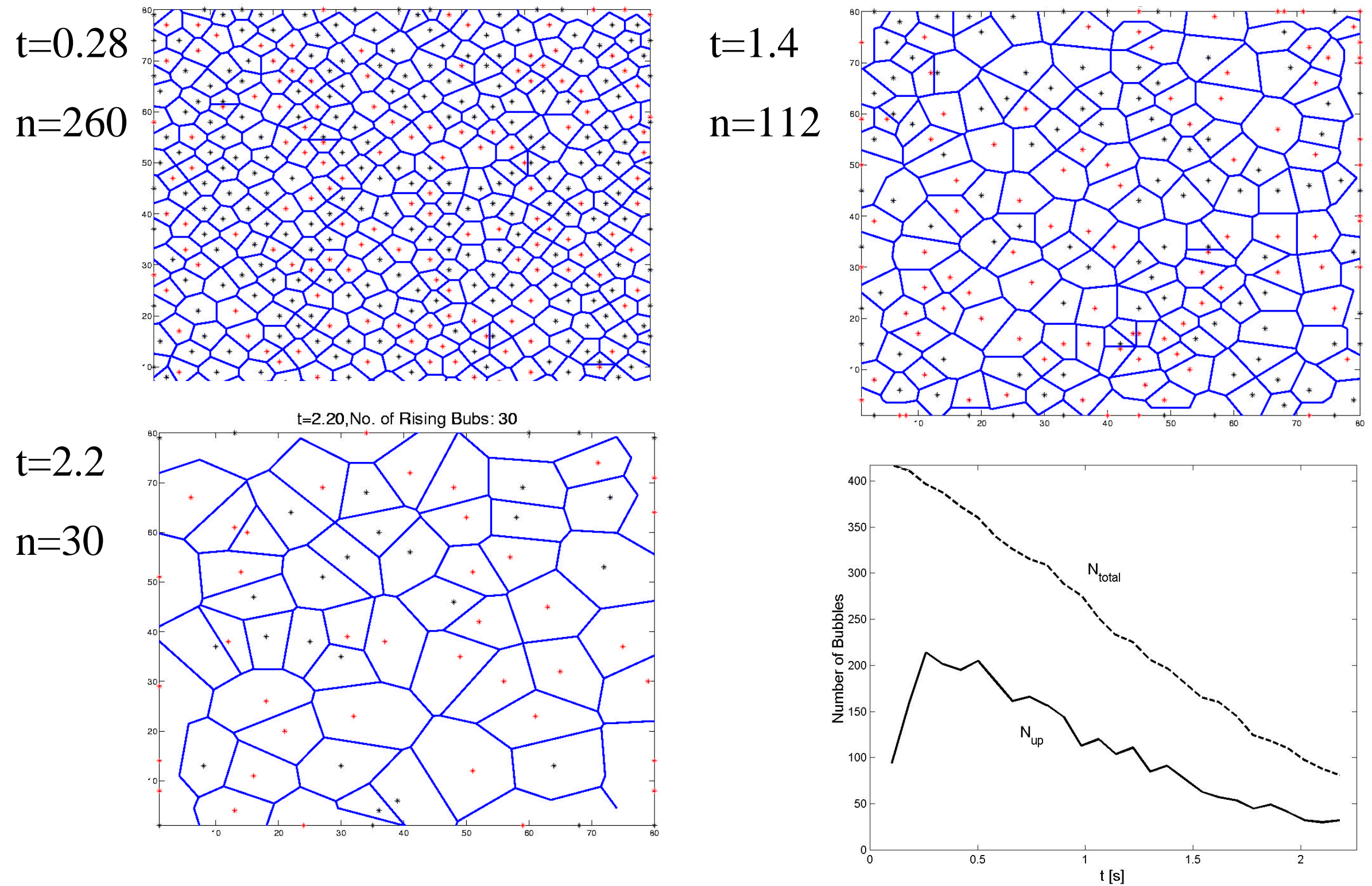

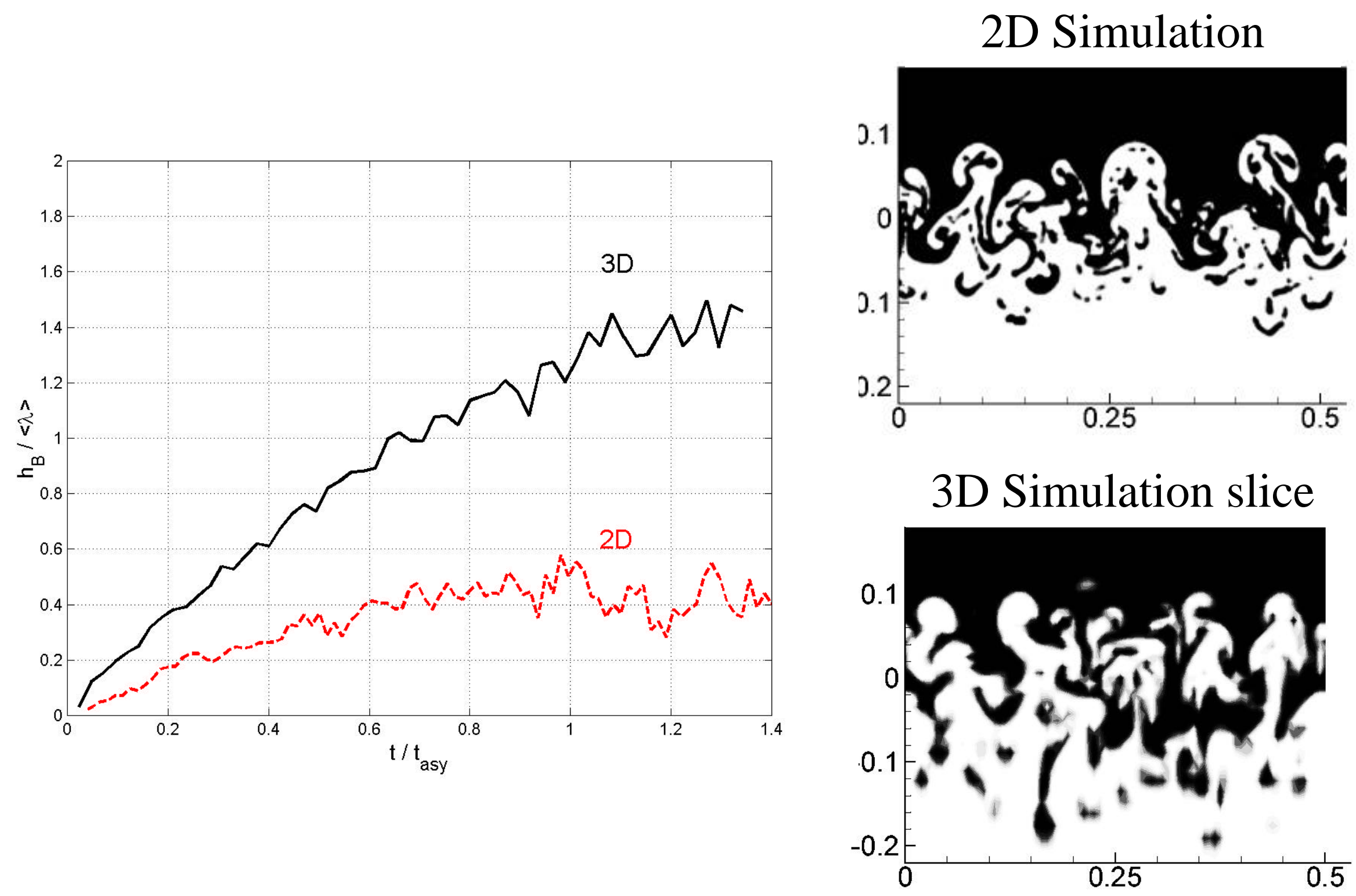
The Drag-Buoyancy Model for Bubbles

2D Statistical Model

Full 3D Numerical Simulations

\section{D Statistical Model}

The Drag-Buoyancy Model for Spikes 


\section{Multi Mode 3D Statistical Model}

Dimensionality effects on the statistical model:

Asymptotic velocity of each bubble 1.5-2 times higher than in the 2D case:

$$
\mathrm{u}_{3 \mathrm{D}}=1.5-2 \mathrm{u}_{2 \mathrm{D}}
$$

Average number of neighbors per bubbles $\approx 6$ in $3 \mathrm{D}$, rather than 2 in $2 \mathrm{D}$ :
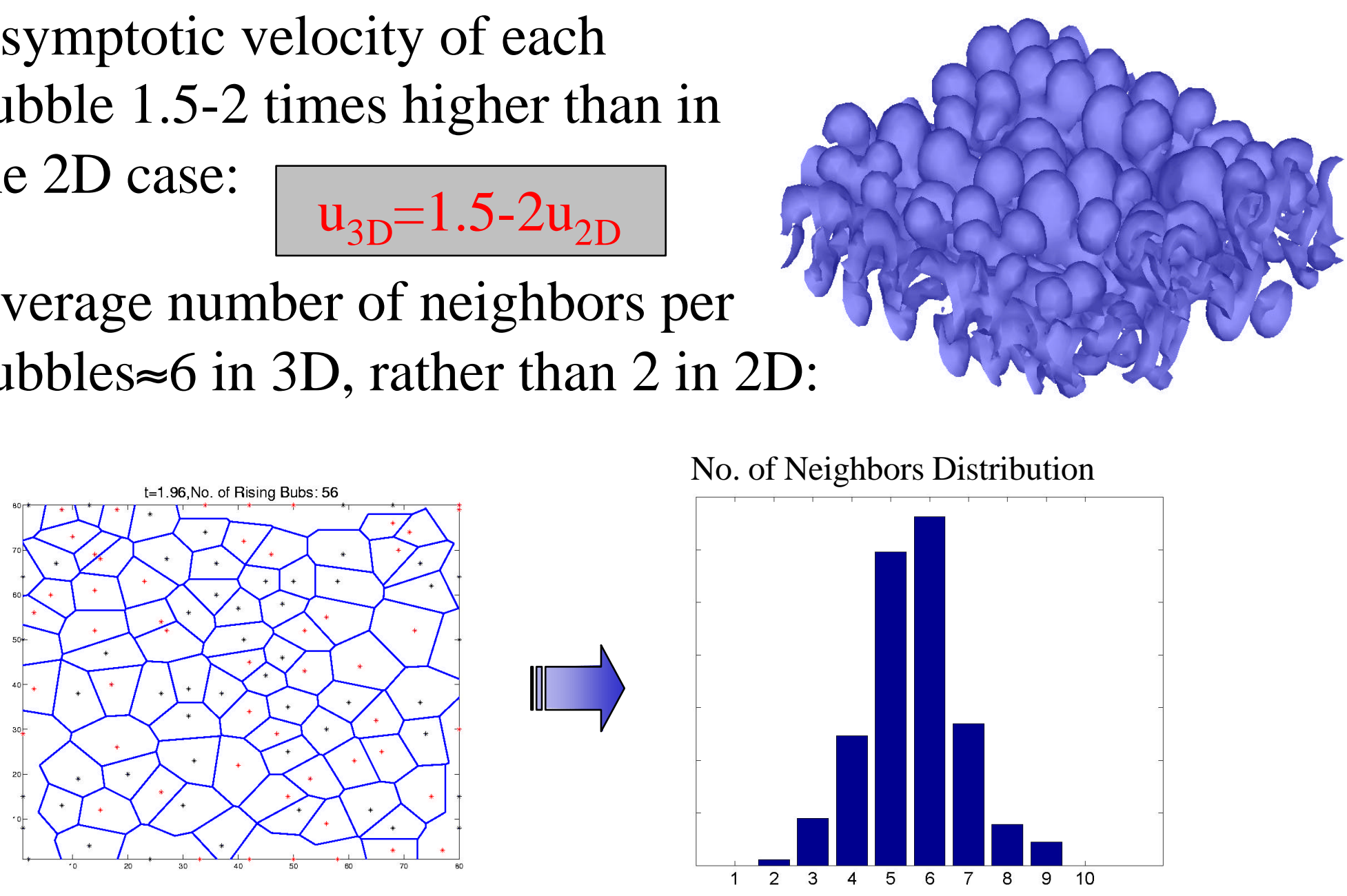


\section{Multi Mode 3D Statistical Model}

Dimensionality effects on the bubble merger:

Bubble merging in 3D conserves area, rather than length in 2D:

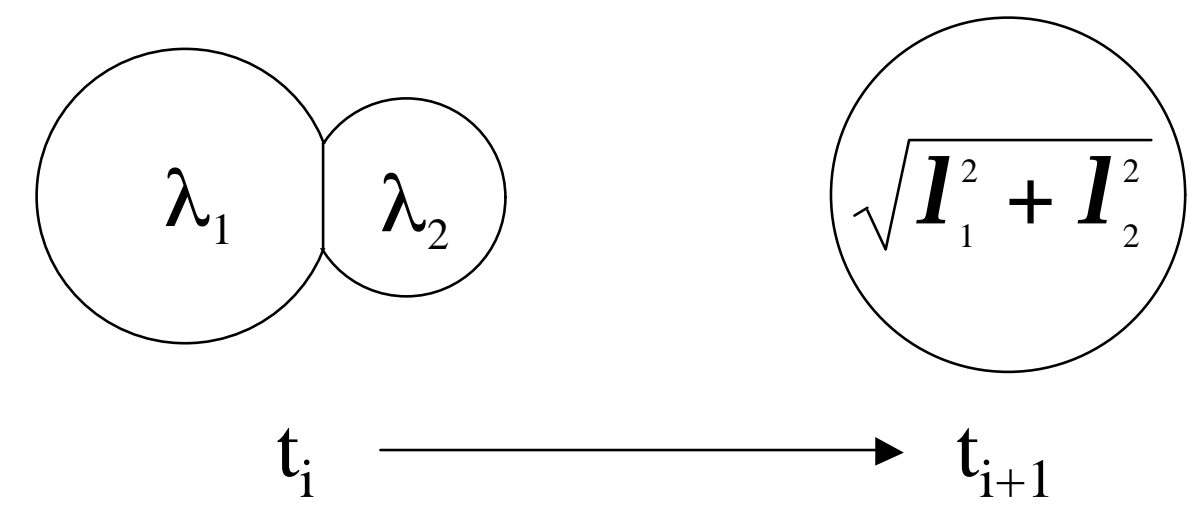

The merges occur with rate $\omega\left(\lambda_{1}, \lambda_{2}\right)$. At first step $\omega^{3 \mathrm{D}}$ was taken to be equal to $\left(\mathrm{u}_{3 \mathrm{D}}^{\text {asy }} / \mathrm{u}_{2 \mathrm{D}}\right.$ asy $) \cdot \omega^{2 \mathrm{D}}$

Because of the area conservation, a 3D bubble has to merge with more of its neighbors in order to reach the same $\lambda$. This effect reduces $d \lambda / d t$, which in turn reduces both $\alpha$ and $\theta$ (and increases $b$ ). 


\section{Multi Mode 3D Statistical Model Results}

The segment distribution $\mathrm{g}^{\prime}(\mathrm{d})$ is obtained from the simulation:

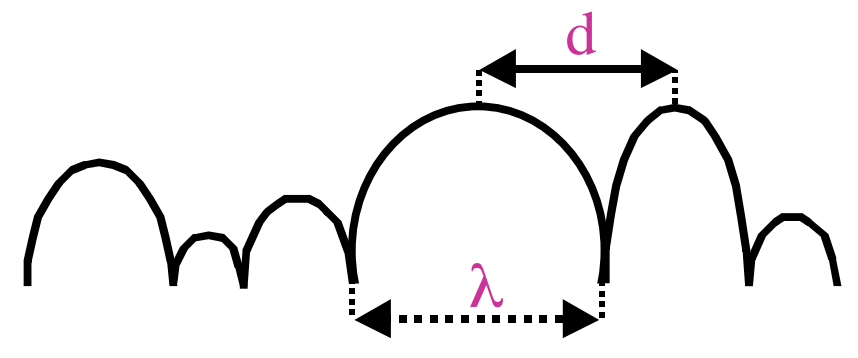

The relation between $\mathrm{g}^{`}(\mathrm{~d})$ and $\mathrm{g}(\lambda)$ is given by:

$$
g^{-}(d)=2 \int_{0}^{d} g(2 d-2 \lambda) g(2 \lambda) d \lambda
$$

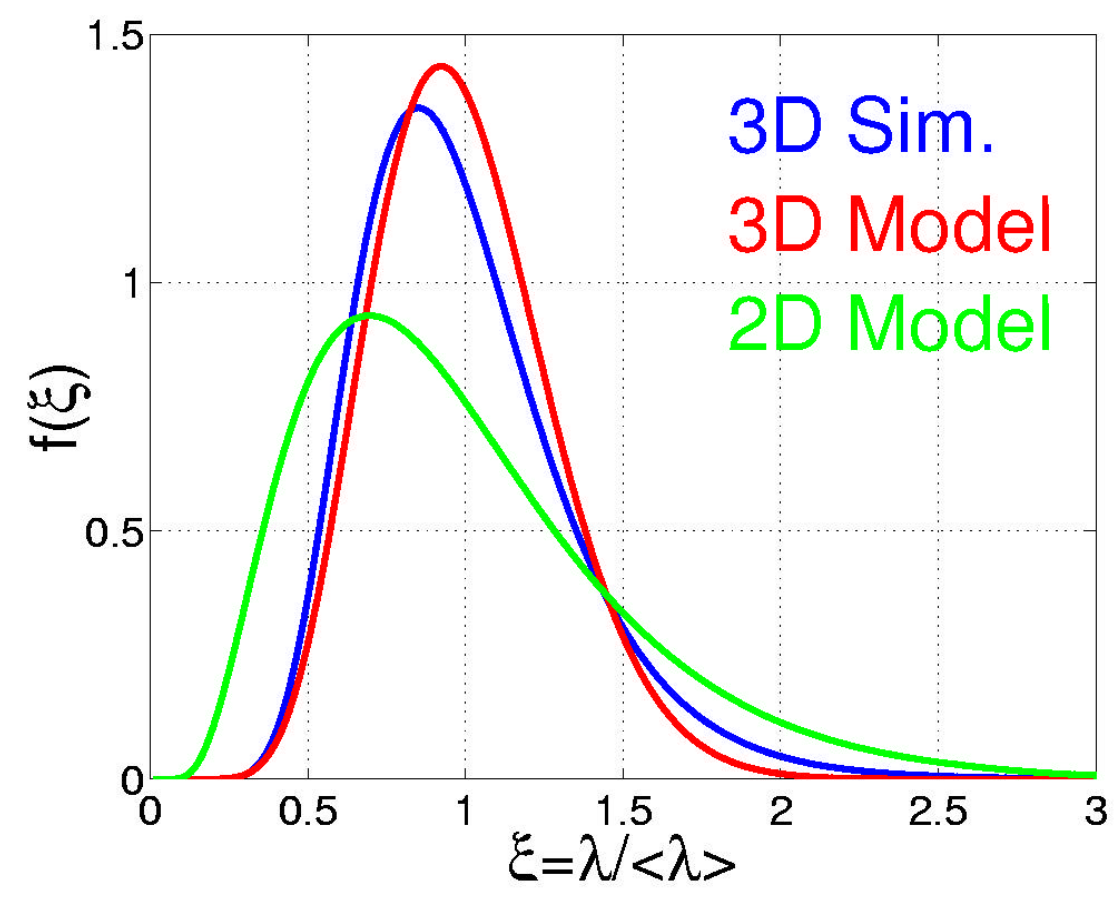




\section{Multi Mode 3D Statistical Model Results}

3D Statistical model results agree well with 3D Simulations.

$$
\alpha_{B}=0.055 \quad \theta_{B}=0.18 \quad b=0.67
$$

The 3D wavelength distribution is narrower than the 2D distribution. The narrowing of the $\lambda$ distribution is due to:

a) Reduction of $\mathrm{d} \lambda / \mathrm{dt}$.

b) Increased number of neighbors.

Simulations results indicate that the 3D statistical model may be applicable to a wide range of $\mathrm{A}$ :

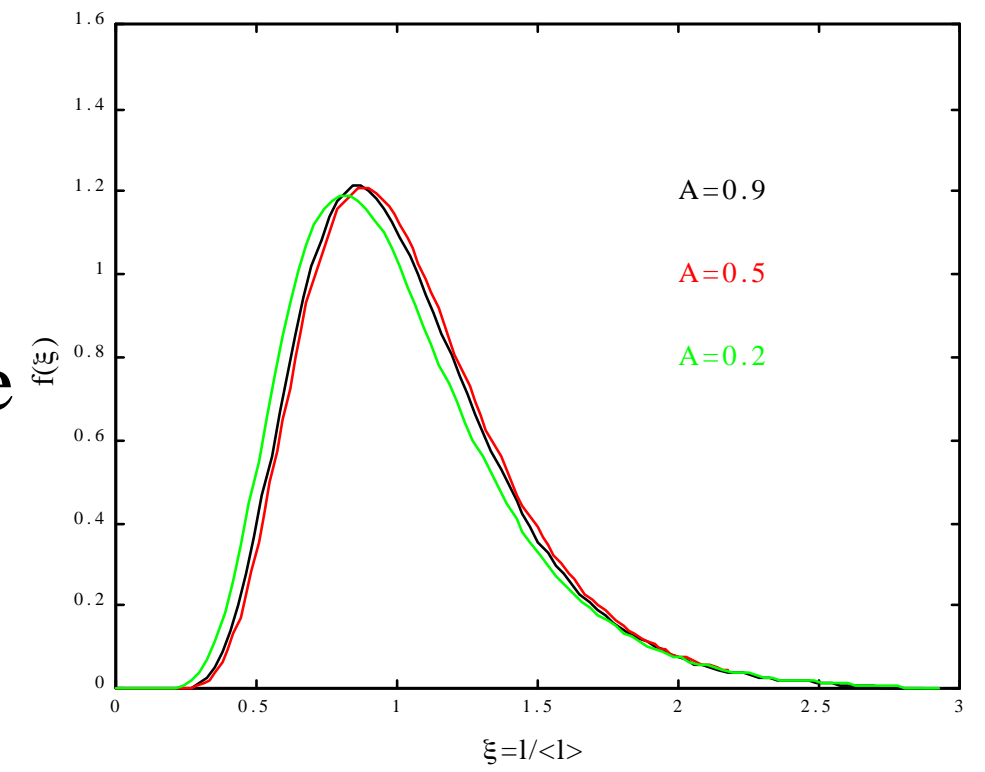




\section{D Statistical Model Dependence on Initial Distribution}

Using the initial wavelength distribution derived from the voronoi diagram in the statistical model gives the $\alpha$ dependence on the generation number:
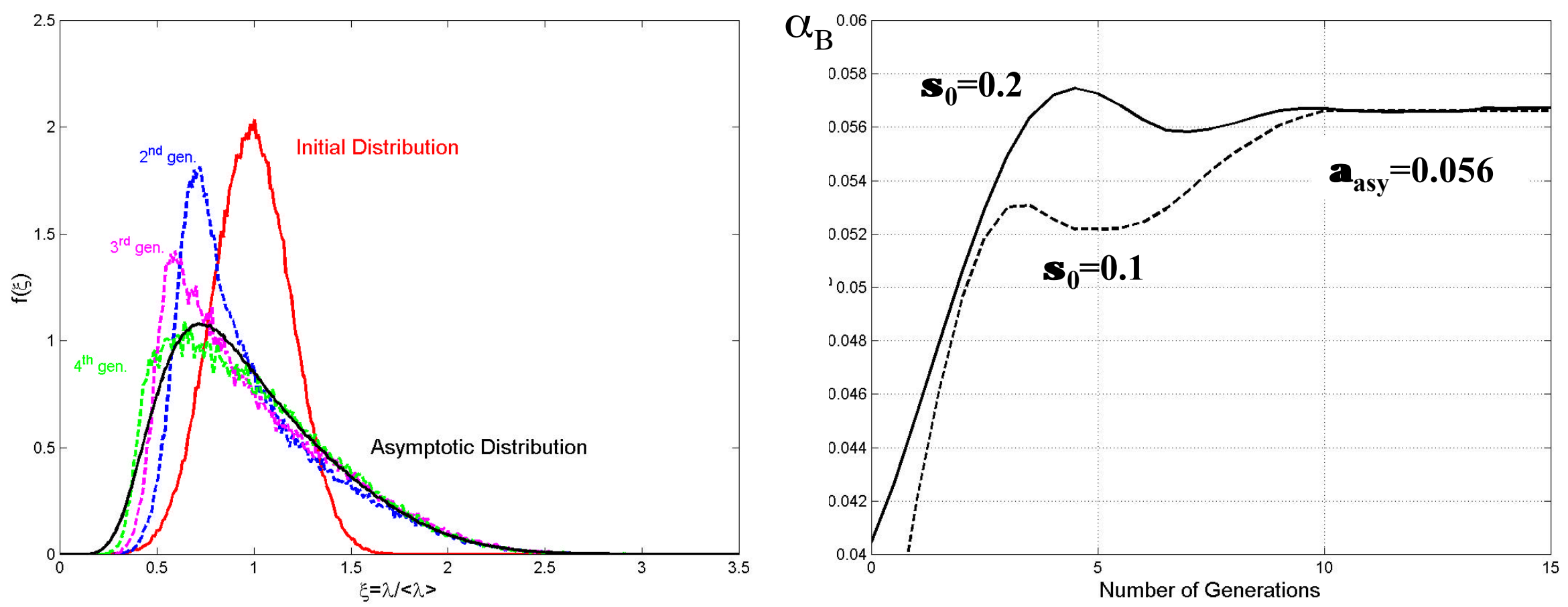


\section{LEM Experimental Results Vs. 3D Models and Simulations}
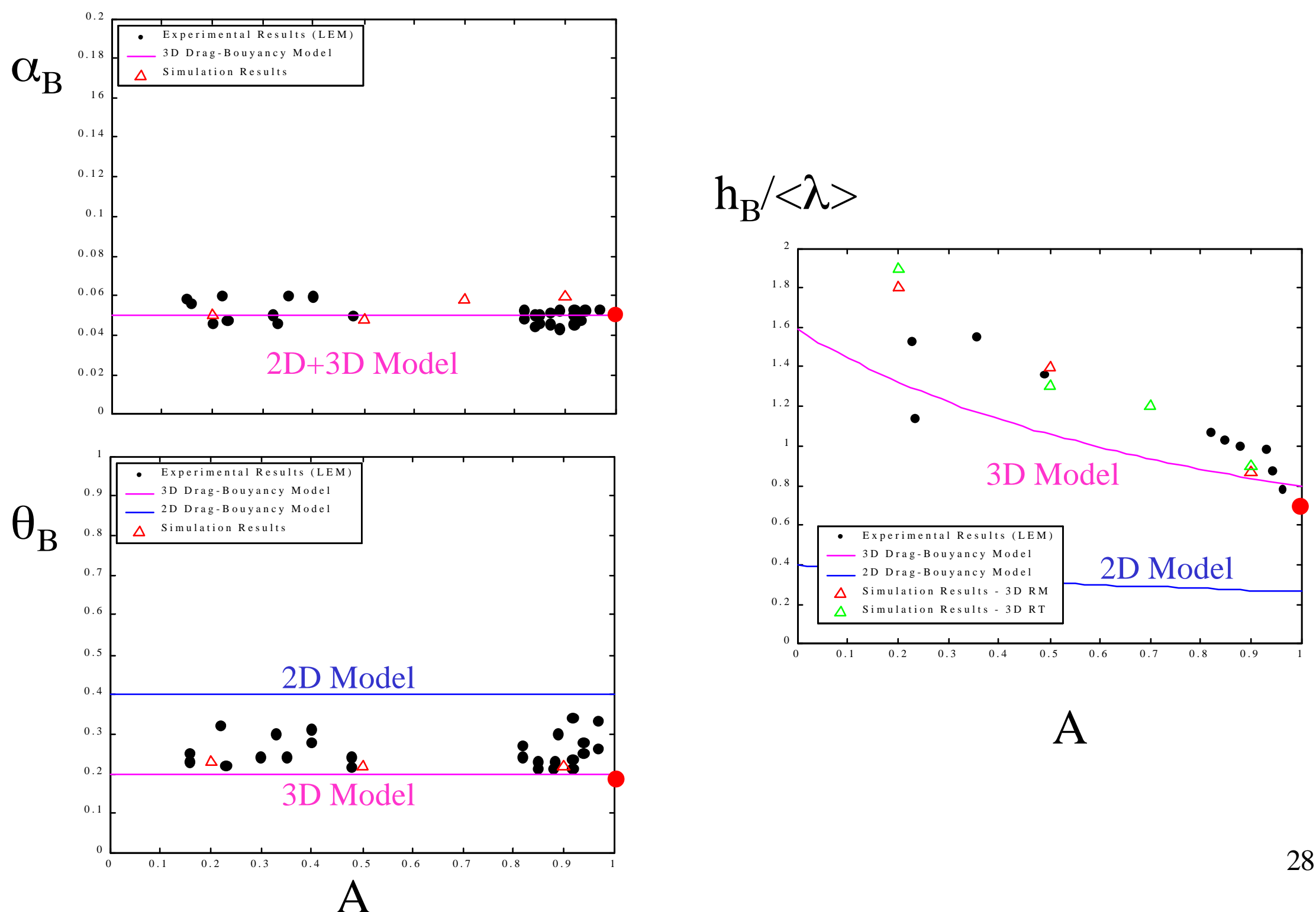

A 


\section{LEM Experimental Results Vs. 3D Simulations}
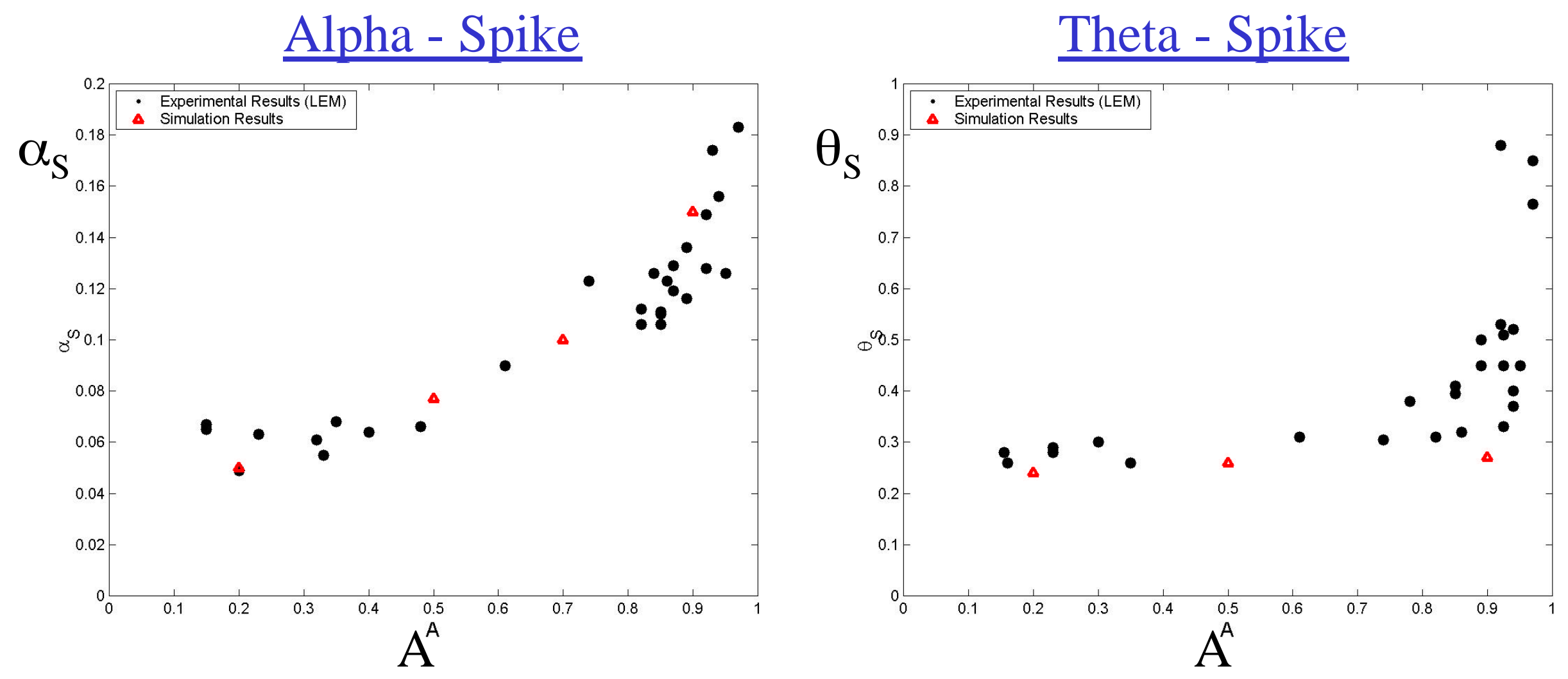
Sensitivity of $\theta_{S}$ to percentage criterion
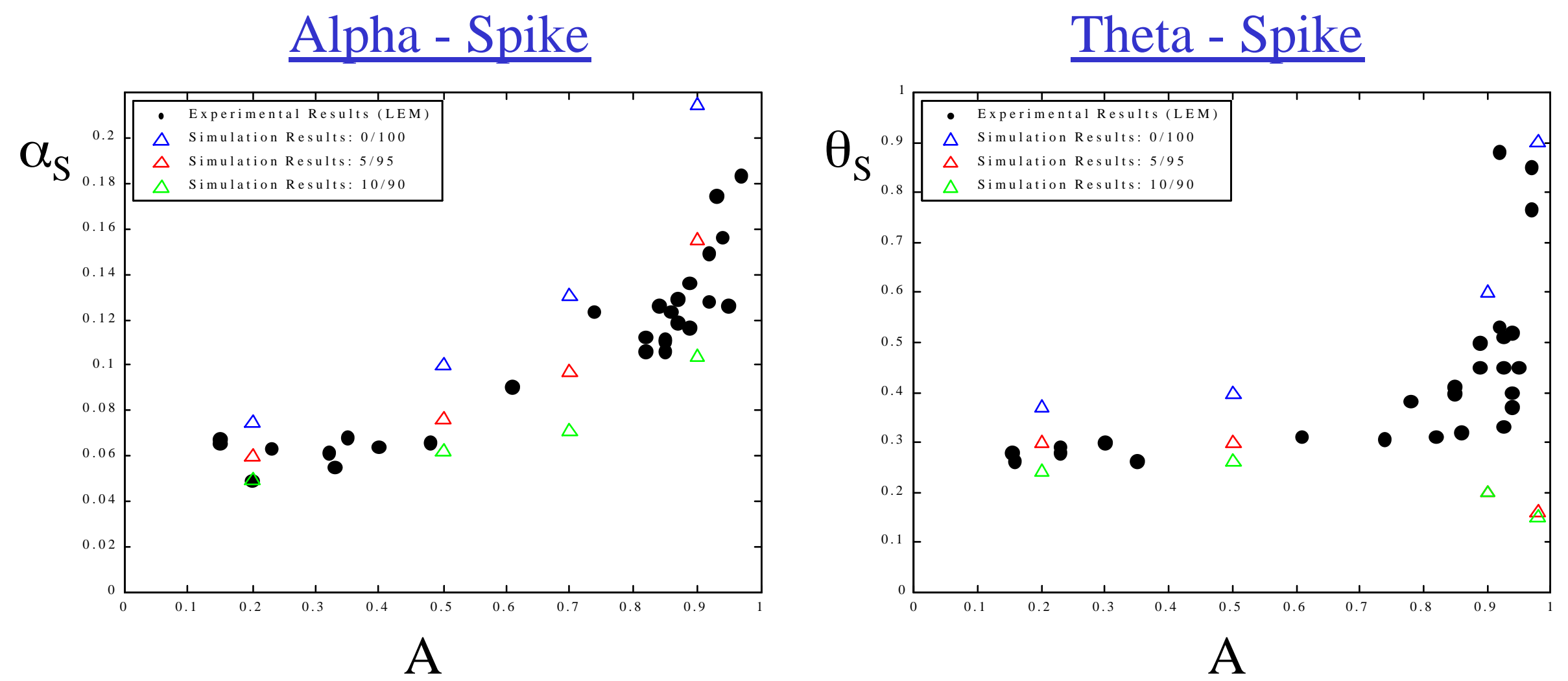
$\theta_{B}$ is not sensitive to percentage criterion

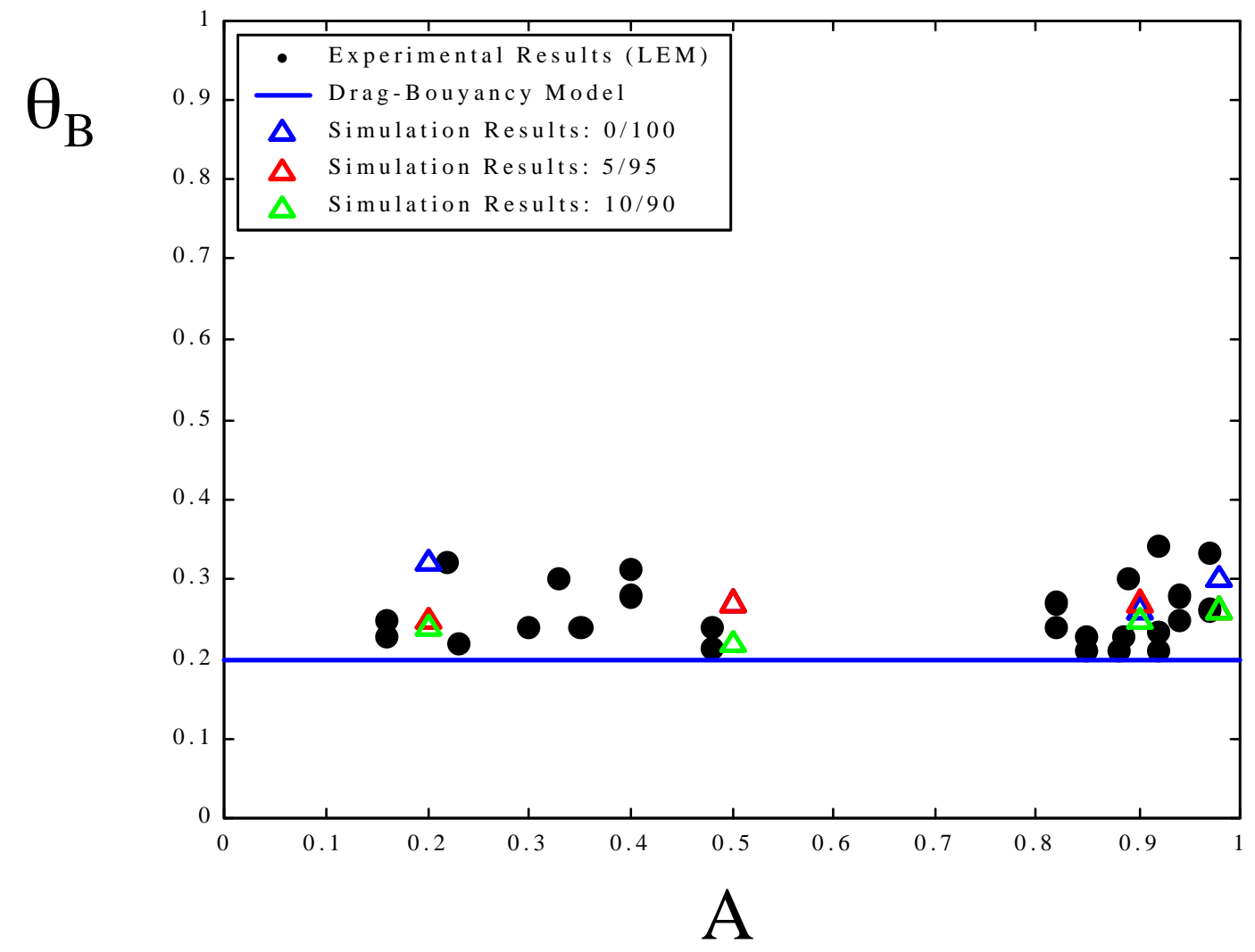


The Drag-Buoyancy Model for Bubbles

2D Statistical Model

Full 3D Numerical Simulations

3D Statistical Model

The Drag-Buoyancy Model for Spikes 


\section{Drag-Buoyancy Model for the Spike Front -}

\section{Single Mode}

Since the spikes develop a rounded tip, one can apply the drag-buoyancy equation to them:

$$
\left(\rho_{h}+C_{a} \rho_{l}\right) \dot{u}_{s}=\left(\rho_{h}-\rho_{l}\right) \cdot g-\frac{C_{d}}{\lambda} \rho_{l} \cdot u_{s}^{2}
$$

The spikes velocity is obtained using the assumptions:

$$
\begin{aligned}
& C_{d S}(A)=C_{d B}(A)= \begin{cases}6 \pi & 2 D \\
2 \pi & 3 D\end{cases} \\
& C_{a S}(A)=C_{a B}(A)= \begin{cases}2 & 2 D \\
1 & 3 D\end{cases}
\end{aligned}
$$


Drag-Buoyancy Model for the Spike Front I. Single Mode Shock Tube Experimental Results

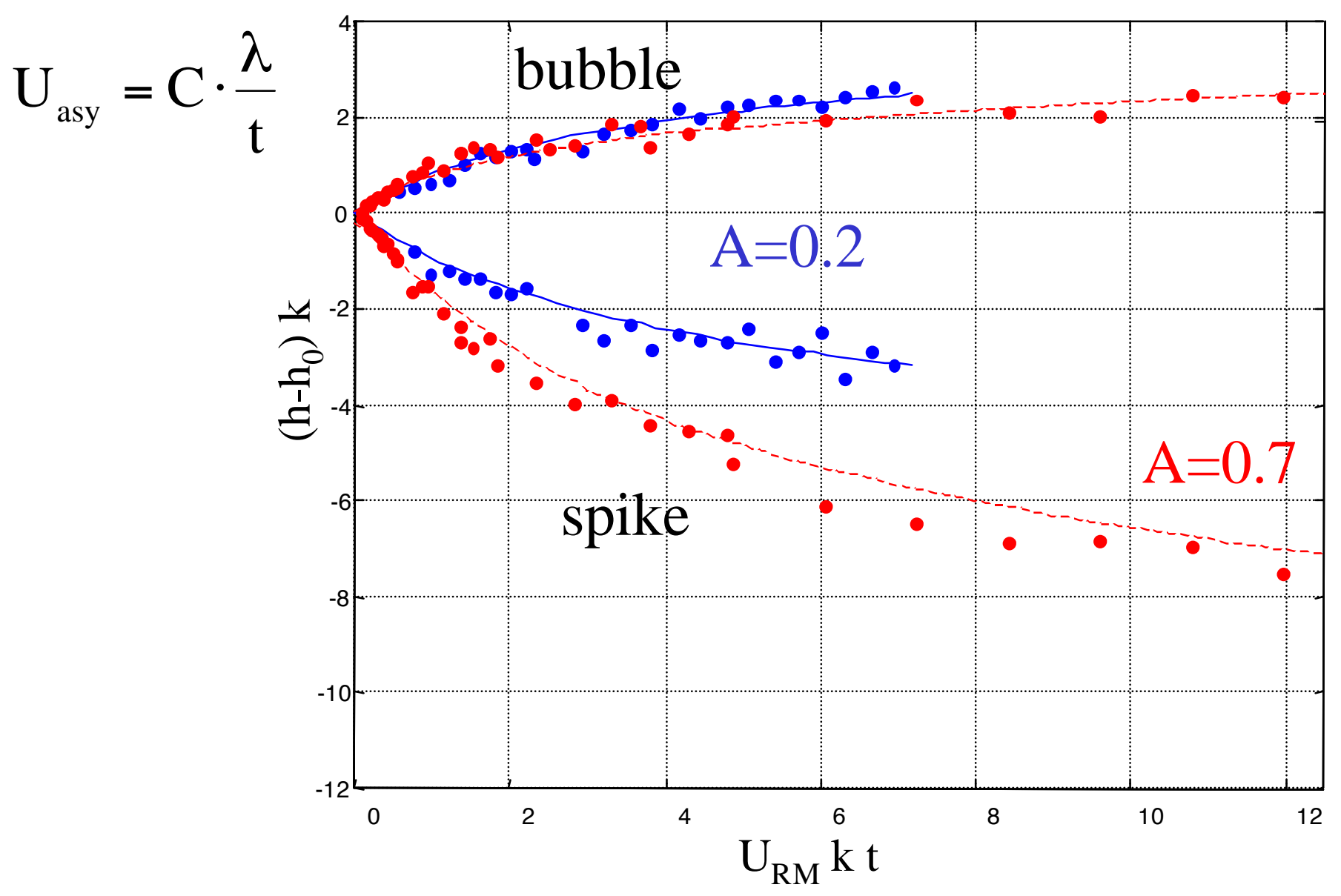




\section{Drag-Buoyancy Model for the Spike Front - II. Multi Mode}

$\underline{1^{\text {st }} \text { assumption: }}$

Periodicity of the spikes $\equiv$ Periodicity of the bubbles

$$
\lambda_{S}(t) \equiv \lambda_{B}(t)
$$
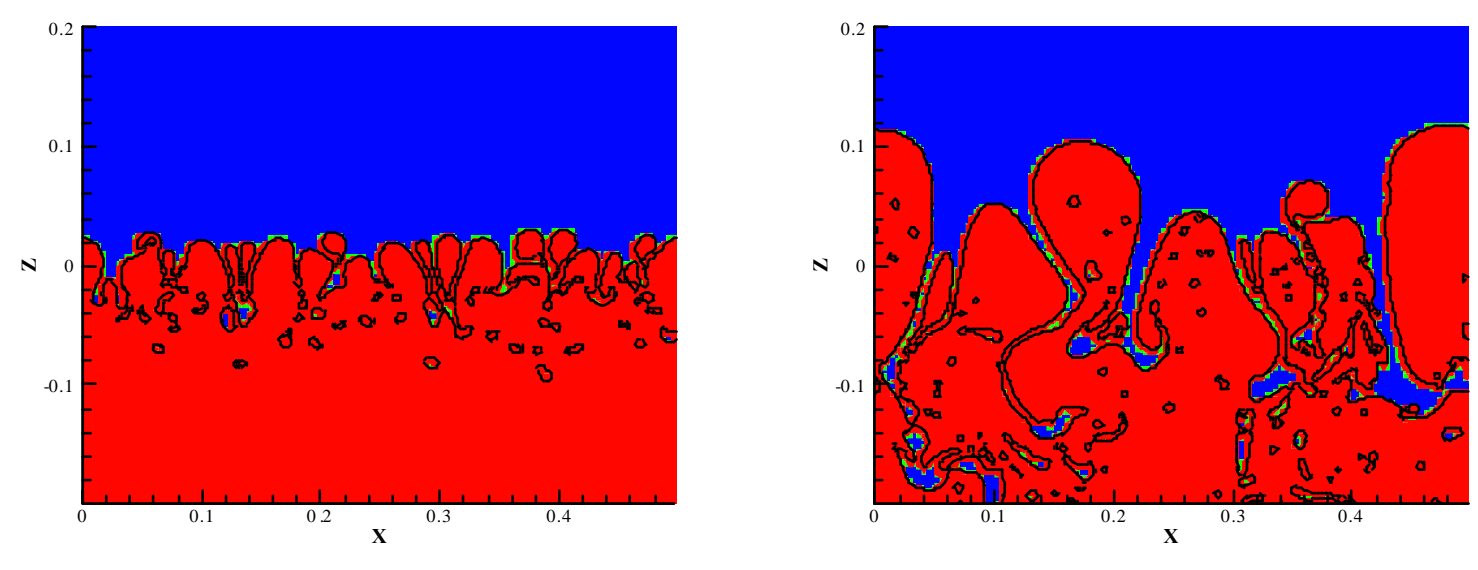

2D RT Simulation with $\mathrm{A}=0.9$ 


\section{Drag-Buoyancy Model for the Spike Front - II. Multi Mode - RT}

Na ve approach:

The ratio between the momentary velocities of the spikes and the bubbles equals the ratio between their asymptotic velocities:

$$
\frac{\mathrm{u}_{\mathrm{S}}(\mathrm{t})}{\mathrm{u}_{\mathrm{B}}(\mathrm{t})}=\frac{2 \alpha_{\mathrm{S}} \mathrm{Agt}}{2 \alpha_{\mathrm{B}} \mathrm{Agt}}=\frac{\alpha_{\mathrm{S}}}{\alpha_{\mathrm{B}}}=\frac{\mathrm{u}_{\mathrm{S}}^{\text {asy }}}{\mathrm{u}_{\mathrm{B}}^{\text {asy }}}=\sqrt{\frac{1+\mathrm{A}}{1-\mathrm{A}}}=\sqrt{\frac{\rho_{\mathrm{h}}}{\rho_{1}}}
$$

\section{$2^{\text {nd }}$ assumption:}

The ratio between the momentary velocities of the spikes and the bubbles equals the ratio between their velocities at the time $t_{b}$ in which the bubbles height reaches its self-similar value: $h_{B}\left(t_{b}\right)=b \lambda$

$$
\frac{\mathrm{u}_{\mathrm{S}}(\mathrm{t})}{\mathrm{u}_{\mathrm{B}}(\mathrm{t})}=\frac{2 \alpha_{\mathrm{S}} A g t}{2 \alpha_{\mathrm{B}} A g t}=\frac{\alpha_{\mathrm{S}}}{\alpha_{B}}=\frac{\mathrm{u}_{\mathrm{S}}\left(\mathrm{t}_{\mathrm{b}}\right)}{\mathrm{u}_{\mathrm{B}}\left(\mathrm{t}_{\mathrm{b}}\right)} \cong \frac{\mathrm{u}_{\mathrm{S}}\left(\mathrm{t}_{\mathrm{b}}\right)}{\mathrm{u}_{\mathrm{B}}^{\text {asy }}}
$$




\section{Drag-Buoyancy Model for the Spike Front - II. Multi Mode - RT Results}

$$
\text { 3D: } \quad \frac{\alpha_{S}}{\alpha_{B}}=\sqrt{\frac{1+A}{1-A}} \cdot \tanh \left[\sqrt{\frac{1-A}{1+A}} \cdot \cosh ^{-1} \cdot \exp (\pi b(1+A))\right]
$$

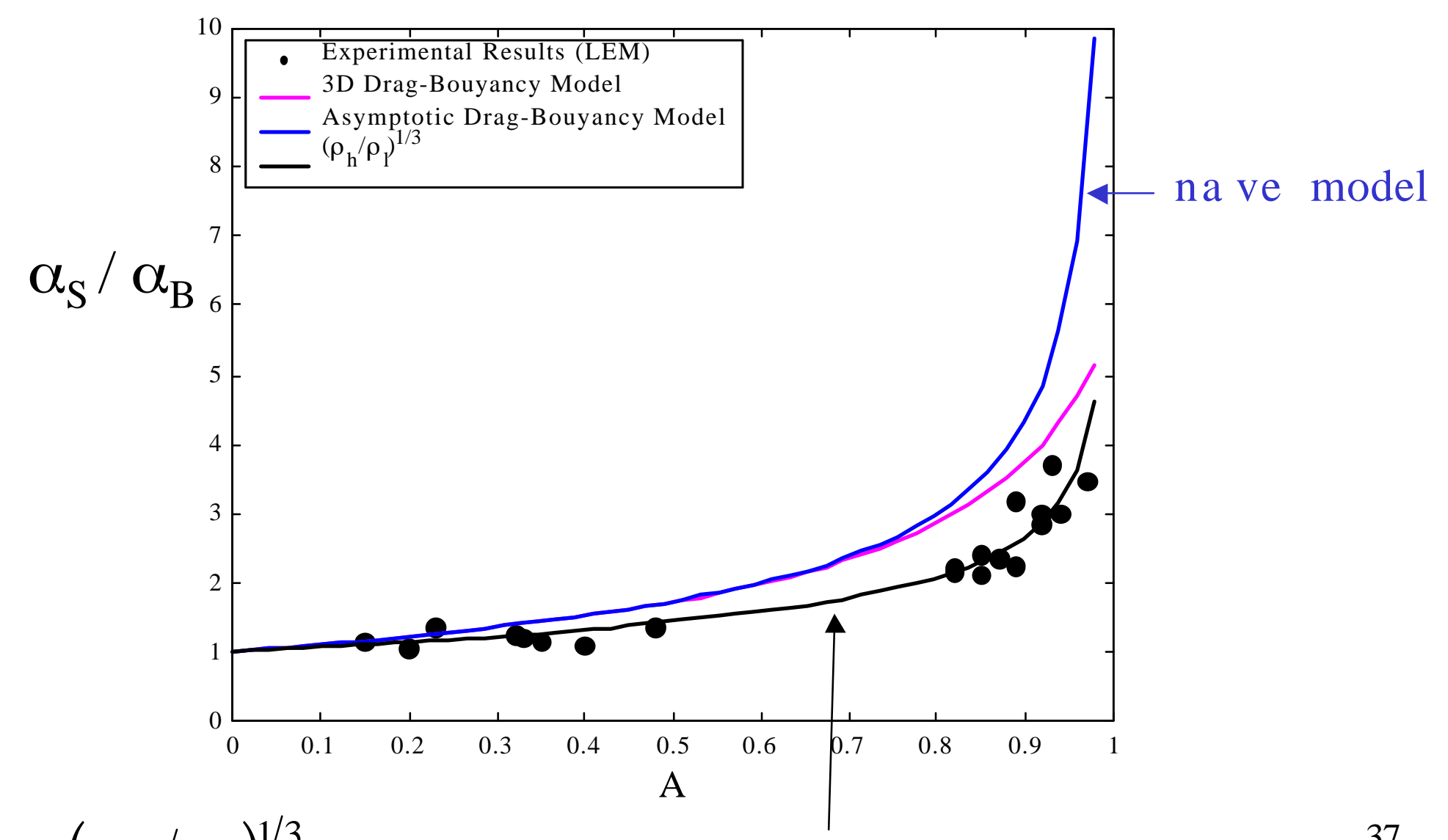

$\alpha_{\mathrm{S}} / \alpha_{\mathrm{B}} \sim\left(\rho_{\mathrm{h}} / \rho_{1}\right)^{1 / 3}:$ Best fit by Dimonte et. Al. 


\section{Drag-Buoyancy Model for the Spike Front - II. Single Mode - RM}

At late times, the bubble velocity in RM goes like $\lambda / \mathrm{t}$.

At finite time $t$ its velocity can be expressed by a time-dependent coefficient $\gamma(\mathrm{t})$ :

$$
\begin{aligned}
& \frac{\mathrm{u}_{\mathrm{B}}(\mathrm{t})}{\mathrm{u}_{0}} \propto\left(\frac{\mathrm{u}_{0} \mathrm{t}}{\lambda}\right)^{\gamma_{\mathrm{B}}(\mathrm{t})} \quad \gamma_{\mathrm{B}}(\mathrm{t} \rightarrow \infty)=-1 \quad \text { for all } A s \\
& \frac{\mathrm{u}_{\mathrm{S}}(\mathrm{t})}{\mathrm{u}_{0}} \propto\left(\frac{\mathrm{u}_{0} \mathrm{t}}{\lambda}\right)^{\gamma_{\mathrm{S}}(\mathrm{t})} \quad \gamma_{\mathrm{S}}(\mathrm{t} \rightarrow \infty)= \begin{cases}-1 & \mathrm{~A}<1 \\
0 & \mathrm{~A}=1\end{cases} \\
& \text { Where, from assumption 1: } \quad\langle\lambda(\mathrm{t})\rangle=\frac{\mathrm{h}_{\mathrm{B}}(\mathrm{t})}{\mathrm{b}} \propto \mathrm{t}^{\theta_{\mathrm{B}}}
\end{aligned}
$$




\section{Drag-Buoyancy Model for the Spike Front - II. Multi Mode - RM}

$$
\Rightarrow \mathrm{u}_{\mathrm{S}}^{\mathrm{MM}} \propto \mathrm{t}^{\gamma_{\mathrm{S}}\left(\mathrm{t}_{\mathrm{b}}\right) \cdot\left(1-\theta_{\mathrm{B}}\right)} \quad \text { also: } \quad \mathrm{u}_{\mathrm{S}}^{\mathrm{MM}}(\mathrm{t})=\frac{\mathrm{dh}_{\mathrm{S}}(\mathrm{t})}{\mathrm{dt}} \propto \mathrm{t}^{\theta_{\mathrm{S}}-1}
$$

Na ve approach:

$$
\gamma_{\mathrm{S}}^{\text {asy }}=\left\{\begin{array}{rlll}
0 & \mathrm{~A}=1 & \rightarrow & \theta_{\mathrm{S}}=1 \\
-1 & \mathrm{~A}<1 & \rightarrow & \theta_{\mathrm{S}}=\theta_{\mathrm{B}}
\end{array}\right.
$$




\section{Drag-Buoyancy Model for the Spike Front - II. Multi Mode - RM Results}

$$
\text { 3D: } \theta_{\mathrm{S}}=1+\gamma_{\mathrm{S}}\left(\mathrm{t}_{\mathrm{b}}\right) \cdot\left(1-\theta_{\mathrm{B}}\right)=1-\frac{\left(\frac{1-\mathrm{A}}{1+\mathrm{A}}\right) \cdot(\exp (\pi \mathrm{b}(1+\mathrm{A}))-1)}{1+\left(\frac{1-\mathrm{A}}{1+\mathrm{A}}\right) \cdot(\exp (\pi \mathrm{b}(1+\mathrm{A}))-1)} \cdot\left(1-\theta_{\mathrm{B}}\right)
$$

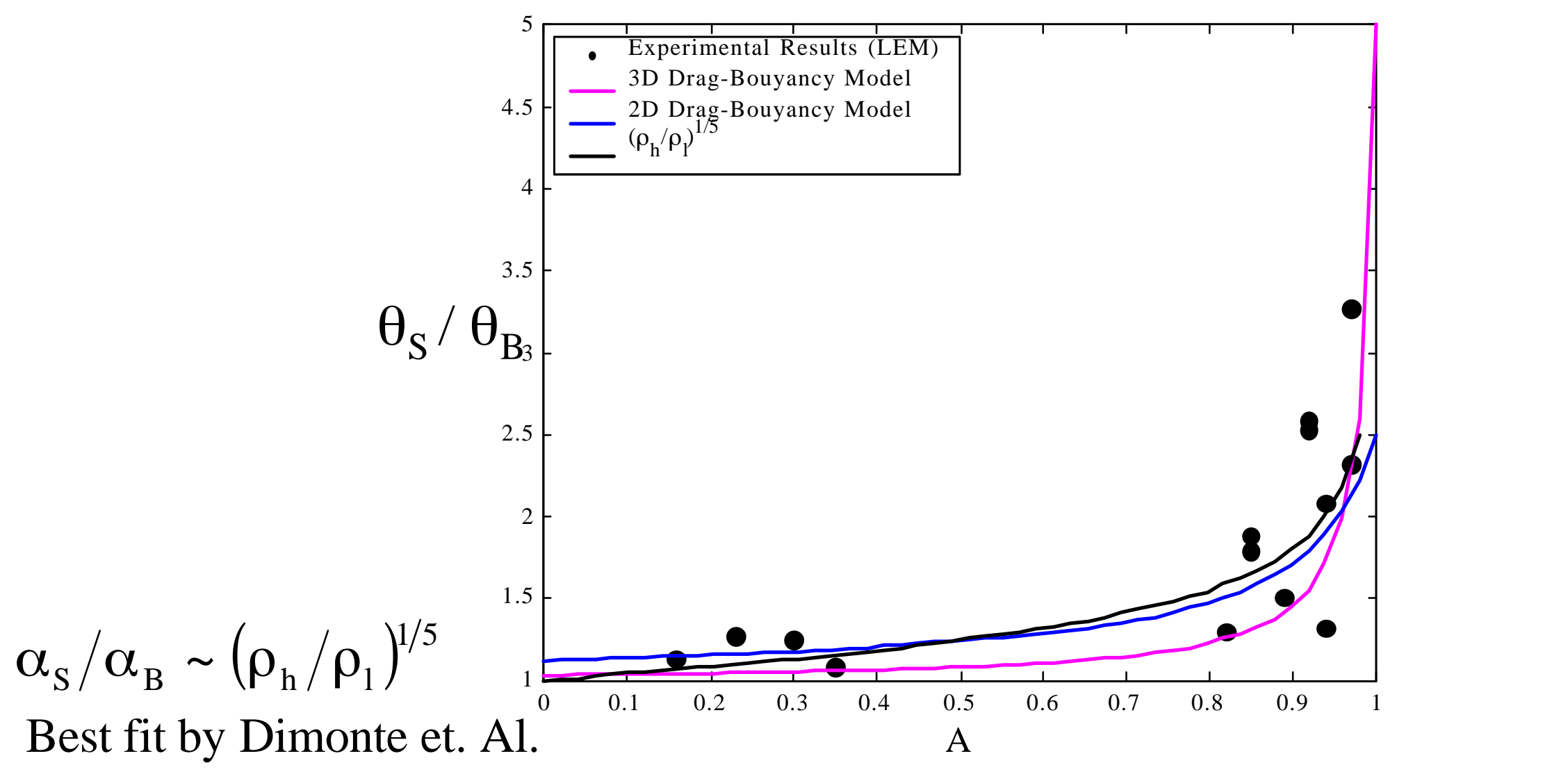




\section{Summary}

Good agreement between 3D drag-buoyancy model, statistical model, full 3D simulations and experimental results.

2D and 3D RT and RM scaling laws:

$$
\begin{aligned}
& \text { RT: } h_{B(S)}=\alpha_{B(S)} A t^{2} \\
& \text { RM: } h_{B(S)}=a_{B(S)} t^{\theta_{B(S)}}
\end{aligned}
$$

Geometrical effect results in different scaling parameters:

$$
\begin{aligned}
\alpha_{\mathrm{B}} \approx 0.05 & \text { in 2D and 3D } \\
\theta_{\mathrm{B}} \approx 0.2-0.3 & \text { in 3D } \\
\text { Vs. } 0.4 & \text { in 2D } \\
\mathrm{b} \approx 0.75-1.5 & \text { in 3D } \\
\text { Vs. } 0.25-0.5 & \text { in 2D }
\end{aligned}
$$

Spikes scaling laws are obtained from the drag-buoyancy modêl. 


\title{
STABILITY OF DIVERGING SHOCK WAVES
}

\author{
V.M.Ktitorov \\ Russian Federal Nuclear Center (VNIIEF), Sarov, $R U$
}

\section{Introduction}

The problem of a search for cases of unstable evolution of expanding shock waves with non-radial perturbations growing in amplitude was formulated long ago but there was no essential progress in this field until 1980-ths. The first proof of an existence of the unstable regime of perturbation evolution was published in the paper by E.Vishniac (Ref.1) where stability of point blast wave in an ideal gas with a specific heat ratio equal to unit $(\gamma=1)$ was considered. In this case the gas behind the shock front is compressed into thin dense layer moving under influence of pressure of hot gas in the center region. The calculation of this layer movement can be done simply by application of conservation laws. This calculation was made in Ref. 1 for small blast wave perturbations those being expanded in spherical harmonics. Results of these calculations were as follows:

- small perturbation evolution had oscillating pattern,

-perturbations with big enough harmonic numbers were growing in amplitude with power dependence of time the power exponent being complex number,

- the discovered instability was Raleigh-Taylor type.

Later investigations revealed that all these features are characteristic for the perturbations evolution in the general case of an arbitrary value of $\gamma$.

V.Ktitorov ${ }^{2}$ and E.Vishniac and D.Ryu ${ }^{3}$ first published the solution of the point blast wave stability problem in the general case of an arbitrary $\gamma>1$. There was considered the point blast wave in a uniform gas with constant density. There were found values determining the perturbation evolution: the increment of perturbation growth, and the period of oscillations. These values were calculated for a large number of sets: harmonic number $n$ and gas specific heat ratio $\gamma$.

The critical value of $\gamma$ determining the blast wave stability was found too (Ref.2): $\gamma_{c}=1.20$. If $\gamma$ were greater than this value the blast wave would be stable with respect to perturbations of all harmonic numbers; if $\gamma<1.20$, on the contrary, there would exist growing in amplitude harmonics.

Later there was found an experimental validation of these calculation results (Ref.4,5).

This solution was obtained owing to using in Refs. 2,3 a self-similar approach for calculation of perturbation structure. The physical sense of this approach was in considering an asymptotically regime of perturbation evolution. This regime takes place when time of perturbation evolution is large enough to make the influence of initial conditions become negligibly small. The analogy could be made between the role of this solution in describing the perturbation evolution and the role of the well-known Taylor-Sedov solution Ref.6 in describing an evolution of spherically symmetric blasts.

Later on the number of systems with blast waves that were studied using the self-similar approach was extended; and the following cases were considered:

- Cylindrical blast wave (Ref.7),

- Isothermal blast wave (Ref.8),

- Blast wave in gas which has initial density depending on radius as power function (Ref.9),

- Blast wave in a non-ideal gas, which specific heat ratio $\gamma$ is a function of gas density $\rho$ (Ref.10).

In this paper we use the self-similar approach in order to consider the stability problem of these and some other systems in the unified manner. 


\section{STABILITY 0F A POINT BLAST WAVE IN AN IDEAL GAS}

\subsection{Formulating the stability problem}

Let us consider a spherical $(s=3)$ or cylindrical $(s=2)$ blast wave in an ideal gas in the case when the initial gas density $\rho_{0}$ is a power function of radius $r: r_{0} \sim r^{k}$. According to ${ }^{2}$ we shall write the hydrodynamic system of equations in the Lagrangian form.

The following notation is used below:

$\bar{R}$ - the Eulerian co-ordinates

$\bar{r}$ - the Lagrangian co-ordinates (the initial co-ordinates before shock front are taken as the Lagrangian co-ordinates),

$\frac{d^{3}(\bar{R})}{d^{3}(\bar{r})} \equiv \frac{D\left(R^{3}, \cos \Theta, \Phi\right)}{D\left(r^{3}, \cos \theta, \varphi\right)}$ - the Jacobian of the transformation from the Lagrangian to the Eulerian co-ordinates,

$P$ - pressure, $r$ - density, $r_{0}$ - the initial density $\left(r_{0} \sim r^{k}\right)$,

We start considering the stability problem from writing 3D hydrodynamic equations in the Lagrangian form: (here time derivative is to be understood in the Lagrangian sense - it is the derivative along the flow line). The first two equations are obvious:

$$
\begin{aligned}
& \frac{\partial}{\partial t}\left(\frac{P}{\rho^{\gamma}}\right)=0, \\
& \frac{\rho_{0}}{\rho}=\frac{d^{3}(\bar{R})}{d^{3}(\bar{r})}
\end{aligned}
$$

The equation of motion (the third equation) can be deduced from its Eulerian form (for the sake of simplicity we use here Cartesian coordinates $\bar{R}=\left(X_{1}, X_{2}, X_{3}\right)$ and

$$
\begin{aligned}
& \left.\bar{r}=\left(x_{1}, x_{2}, x_{3}\right)\right): \\
& \frac{\partial^{2} X_{k}}{\partial t^{2}}=-\frac{\partial P}{\rho \partial X_{k}}
\end{aligned}
$$
formula:

We change the arguments in the equation of motion written in the Eulerian form using

$$
\frac{\partial}{\partial x_{i}}=\sum_{k} \frac{\partial X_{k}}{\partial x_{i}} \frac{\partial}{\partial X_{k}}
$$

And we get finally:

$$
\frac{\partial P}{\rho \partial x_{i}}=-\sum_{k} \frac{\partial X_{k}}{\partial x_{i}} \frac{\partial^{2} X_{k}}{\partial t^{2}}
$$

For the non-perturbed blast wave the system of equation (1),(2),(5) looks as follows:

$$
\begin{aligned}
& \frac{\partial}{\partial t}\left(\frac{P}{\rho^{\gamma}}\right)=0, \\
& \frac{\rho_{0}(r)}{\rho}=\frac{R^{s-1} \partial R}{r^{s-1} \partial r} \\
& \frac{\partial P}{\rho \partial r}=-\frac{\partial R}{\partial r} \frac{\partial^{2} R}{\partial t^{2}}
\end{aligned}
$$

The corresponding spherically (or cylindrically) symmetric self-similar solution of Eqs.

(6-8) is well known. It can be written using the shock wave radius value $S(t) \sim t^{2 /(k+s+2}$ and functions $x(z), p(z), r(z)$ of the self-similar argument $z=r / S$,: 


$$
\begin{aligned}
& R_{0}(t, r)=S x(z), \\
& P_{0}(t, r)=\frac{2}{\gamma+1} \rho^{0}(S) \dot{S}^{2} p(z), \\
& \rho_{0}(t, r)=\frac{\gamma+1}{\gamma-1} \rho^{0}(S) \rho(z), \\
& \Theta_{0}=\theta, \\
& \Phi_{0}=\varphi
\end{aligned}
$$

where $x, P, r$ should satisfy to ordinary differential equations:

$$
\begin{aligned}
& \rho=\frac{\gamma-1}{\gamma+1} \frac{z^{s+k}}{x^{s} w}, \\
& p=\frac{\rho^{\gamma}}{x^{s} z^{s}}, \\
& \frac{p^{\prime}}{p}=-\gamma \frac{a b}{w} ;
\end{aligned}
$$

With boundary conditions: $x(1)=p(1)=r(1)=1$,

Here we denote:

$$
\begin{aligned}
& w=\frac{z d x}{x d z}=\frac{x^{\prime} z}{z}, \\
& a=w^{\prime} z+(w-1)\left(w+\frac{s+k}{2}\right), \\
& b=\frac{\gamma+1}{2 \gamma} \frac{w z^{s+k}}{p x} ;
\end{aligned}
$$

We insert to the system of equations (1),(2),(5) hydrodynamic values corresponding to the perturbed blast wave $(\tilde{\bar{R}}, \widetilde{P}, \tilde{\rho})$ presented as sums of the non-perturbed blast wave values $(\bar{R}, P, \rho)$, and small perturbations $\left(\bar{R}_{1}, P_{1}, \rho_{1}\right)$ :

$$
\begin{aligned}
& \tilde{R}(t, \bar{r})=R(t, r)+R_{1}(t, \bar{r}), \\
& \widetilde{\Theta}(t, \bar{r})=\theta+\Theta_{1}(t, \bar{r}), \\
& \widetilde{\Phi}(t, \bar{r})=\varphi+\Phi_{1}(t, \bar{r}), \\
& \widetilde{P}(t, \bar{r})=P(t, r)+P_{1}(t, \bar{r}), \\
& \tilde{\rho}(t, \bar{r})=\rho(t, r)+\rho_{1}(t, \bar{r}) .
\end{aligned}
$$

Also we suppose that an angular motion is the potential one:

$$
\Theta_{1}=\frac{\partial F_{1}}{\partial \cos \theta}, \Phi_{1}=\frac{\partial F_{1}}{\partial \varphi},
$$

and that supposition will make the Jacobian being equal to:

$$
\frac{d^{3}\left(\bar{R}+\bar{R}_{1}\right)}{d^{3} \bar{r}}=\frac{R^{2} \partial R}{r^{2} \partial r}\left(1+\Delta_{\theta \varphi} F_{1}\right)+\frac{\partial R^{2} R_{1}}{r^{2} \partial r}
$$

We insert expressions $(12,13)$ to the system of equations Eqs.(1,2,5), and after finishing linearization procedure we get the following equations for perturbations:

Equation of entropy conservation:

$$
\frac{\partial}{\partial t}\left(\frac{P_{1}}{P}-\gamma \frac{\rho_{1}}{\rho}\right)=0
$$

Continuity equation: 
$\frac{\rho_{1}}{\rho}+\Delta_{\theta \varphi} F_{1}+\frac{\frac{\partial\left(R^{s-1} R_{1}\right)}{r^{s-1} \partial r}}{\frac{R^{s-1} \partial R}{r^{s-1} \partial r}}=0$

Angular component of the equation of motion (here we denote: $\dot{R} \equiv \frac{\partial R}{\partial t}$ ):

$$
\frac{P_{1}}{\rho}+R_{1} \ddot{R}+\left(R^{2} \dot{F}_{1} \dot{)}=0\right.
$$

Radial component of the equation of motion:

$$
\frac{\partial P_{1}}{\rho \partial r}+\ddot{R} \frac{\partial R_{1}}{\partial r}+\ddot{R}_{1} \frac{\partial R}{\partial r}-\frac{\rho_{1} \partial P}{\rho^{2} \partial r}=0
$$

Here and after $S$ stands for the non-perturbed shock front radius, $S(t) \sim t^{2 /(k+s+2)}$, and $S_{1}$ stands for the value of the shock front radius perturbation.

Boundary conditions for the system of equations Eqs.(15-18) should be put on the shock front $\left(r=S+S_{1}\right)$ using the following scheme:

$$
\begin{aligned}
& \left(R+R_{1}\right)\left(r=S+S_{1}, t\right)=S+S_{1} \\
& \left(R+R_{1}\right)\left(r=S+S_{1}, t\right)=R\left(r=S+S_{1}, t\right)+R_{1}\left(r=S+S_{1}, t\right) \\
& R\left(r=S+S_{1}, t\right)=R(r=S, t)+S_{1} \frac{\partial R}{\partial r}(r=S, t)=S+S_{1} \cdot \frac{w x}{z}(z=1) \\
& R_{1}\left(r=S+S_{1}, t\right)=R_{1}(r=S, t)
\end{aligned}
$$

From Eqs. $(10,11)$ we have:

$$
w(1)=\frac{\gamma-1}{\gamma+1}, x(1)=1
$$

Hence we get:

$$
R_{1}(r=S, t)=\frac{2}{\gamma+1} S_{1}
$$

The next front conditions are calculated in the same way. We get:

$$
\begin{aligned}
& F_{1}(r=S, t)=0 \\
& \dot{F}_{1}(r=S, t)=-\frac{2}{\gamma+1} \frac{\dot{S}}{S^{2}} S_{1} \\
& P_{1}(r=S, t)=\frac{2 \rho_{0}}{\gamma+1} \frac{\dot{S}^{2}}{S} S_{1}(2 \lambda+s+2+a(1)) \\
& \rho(r=S, t)=\frac{(\gamma+1) \rho_{0}}{\gamma+1} \frac{S_{1}}{S} a(1)
\end{aligned}
$$

According to Ref. 2 the perturbations are expanded in spherical harmonics $Y_{n m} \sim P_{n m}(\cos \theta) e^{i m \varphi}$ ( or in axial harmonics $e^{i n \varphi}$ in the cylindrical case), and the components of expansion are presented in the self-similar form.

Shock wave radius perturbations are expanded too: $\left(S_{1}=\sum S_{1 m n} Y_{m n}\right)$. We suppose that the components of expansion are power functions of time for each harmonic number:

$S_{1 m n} \sim S^{\lambda_{m n}+1} \sim t^{\frac{2\left(\lambda_{m n}+1\right)}{k+s+2}}$,

Here $\lambda_{m n}$ is a complex number.

So we write: 


$$
\begin{aligned}
& \tilde{R}(t, \bar{r})=R(t, r)+\frac{2}{\gamma+1} \sum_{n m} S_{1 n m}(t) x(z) x_{1 n m}(z) z^{\lambda_{n m}} Y_{n m}(\theta, \varphi), \\
& \widetilde{P}(t, \bar{r})=P(t, r)+\frac{2}{\gamma+1} \rho_{0}(r) \frac{\dot{S}^{2}(t)}{S} \sum_{n m} S_{1 n m}(t) p_{1 n m}(z) z^{\lambda_{n m}+S+k} Y_{n m}(\theta, \varphi), \\
& \tilde{\rho}(t, \bar{r})=\rho(t, r)+\frac{\gamma+1}{\gamma-1} \rho_{0}(r) \frac{1}{S} \sum_{n m} S_{1 n m}(t) \rho_{1 n m}(z) z^{\lambda_{n n}} Y_{n m}(\theta, \varphi), \\
& \Theta(t, \bar{r})=\theta+\frac{2}{\gamma+1} \frac{1}{S} \sum_{n m} S_{1 n m}(t) f_{1 n m}(z) z^{\lambda_{n m}} \frac{\partial Y(\theta, \varphi)}{\partial \cos \theta}, \\
& \Phi(t, \bar{r})=\varphi+\frac{2}{\gamma+1} \frac{1}{S} \sum_{n m} S_{1 n m}(t) f_{1 n m}(z) z^{\lambda_{n m}} \frac{\partial Y(\theta, \varphi)}{\partial \varphi}
\end{aligned}
$$

\subsection{Formulating an eigenvalue problem.}

At first, using boundary conditions we integrate adiabatic equation (15). We get (here and after we omit indices $n, m$ of $\left.p_{1}, r_{1}, x_{1}, f_{1}, l, S_{1}\right)$ :

$$
\frac{P_{1}}{P}-\gamma \frac{\rho_{1}}{\rho}=(2 \lambda+s+2) z^{\lambda} \frac{S_{1}}{S}
$$

After that using the assumption of the self-similarity (24) we convert the system of equations (16), (17), (18), (25) into the system of ordinary differential equations. We write it having excluded $\rho_{1}$ :

$$
\begin{aligned}
& b p_{1}+\left(\frac{z d}{d z}+\lambda+s w\right) x_{1}-n(n+s-1) w f_{1}=\frac{\gamma+1}{2 \gamma}(2 \lambda+s+k+2) w \\
& w p_{1}+a x_{1}-\left(\frac{z d}{d z}+2 w+\frac{s+k-2}{2}\right) \frac{z d}{d z} f_{1}=0 \\
& \left.\left[\frac{z d}{d z}+\lambda+s+k-(s+k-2) w\right] p_{1}+\left[\left(\frac{z d}{d z}+2 w+\frac{s+k-2}{2}\right) \frac{z d}{d z}-(s+k-2) a\right] x_{1}+28\right) \\
& +n(n+s-1) a f_{1}=0
\end{aligned}
$$

With boundary conditions as follows:

- On the right edge $z=1$ (shock front):

$f_{1}(1)=0$,

$f_{1}^{\prime}(1)=1$,

$x_{1}(1)=1$,

$p_{1}(1)=2 \lambda+\frac{6 \gamma+2-s(3 \gamma-1)+k(\gamma+1)}{(\gamma+1)^{2}}$;

- On the left edge $z=0$ (at the center):

$p_{1}(1)=0$;

The system of equations Eqs.(26-28) is of the forth order one, so four edge conditions Eqs.(29) construct the complete set of boundary conditions necessary for solution. The fifth edge condition Eq.(30) at $z=0$ converts the problem of solving the Eqs.(26-29) to an eigenvalue problem. Solving this problem includes calculation of the complex exponent $\lambda$ as eigenvalue. Coefficients in (26)-(30) are independent of index $m$, so $\lambda \equiv \lambda(n)$.

\subsection{Numerical solution results}

In general the case $\gamma>1$ the eigenvalue problem Eqs.(26-30) was solved numerically. The values of the complex exponents $\lambda_{n}$ were calculated in the wide region of $\gamma$, and $n$ for spherical 
$(s=3)$ and cylindrical $(s=2)$ blast waves. The values of $k$ were $k=0,-1,-2$. The results are presented on Figs.1,2.

The instability region on the plane $n-\gamma$ is shown on Fig.3. Blast wave is unstable in the right lower corner of the chart. If the function $\gamma_{0}(n)$ corresponds to the boundary line of the instability region then the critical value of specific ratio $\gamma_{c}$ can be defined:

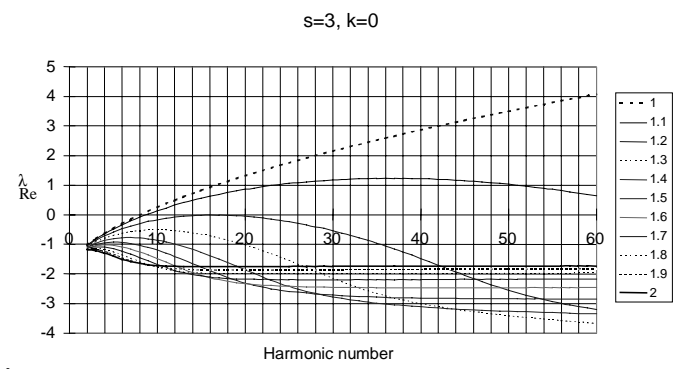

$\mathrm{s}=3, \mathrm{k}=-1$
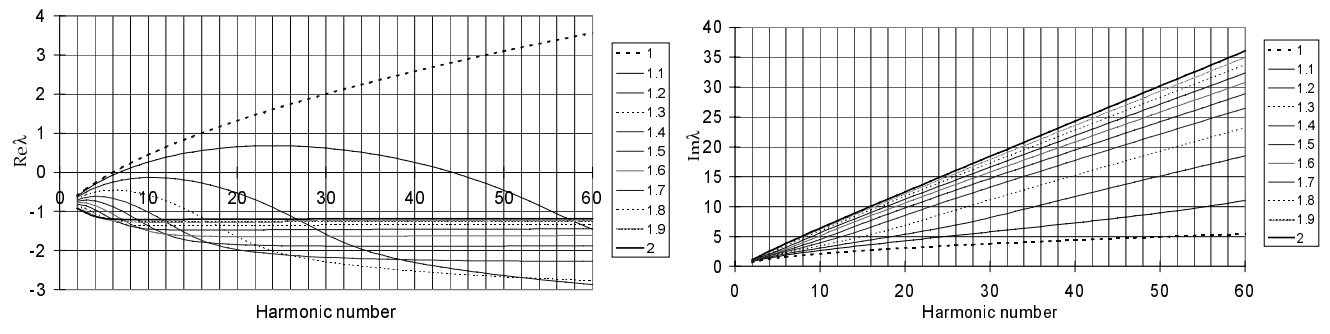

$s=3, k=-2$
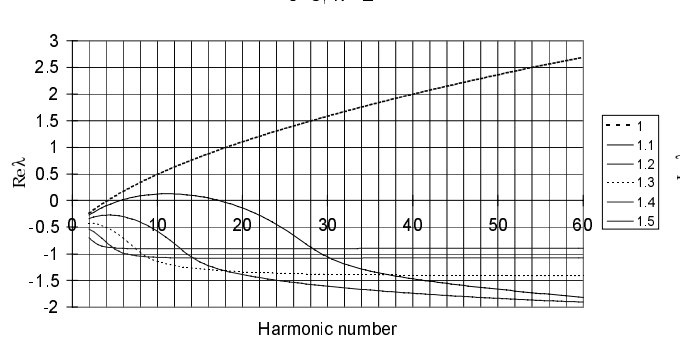

$s=2, k=0$
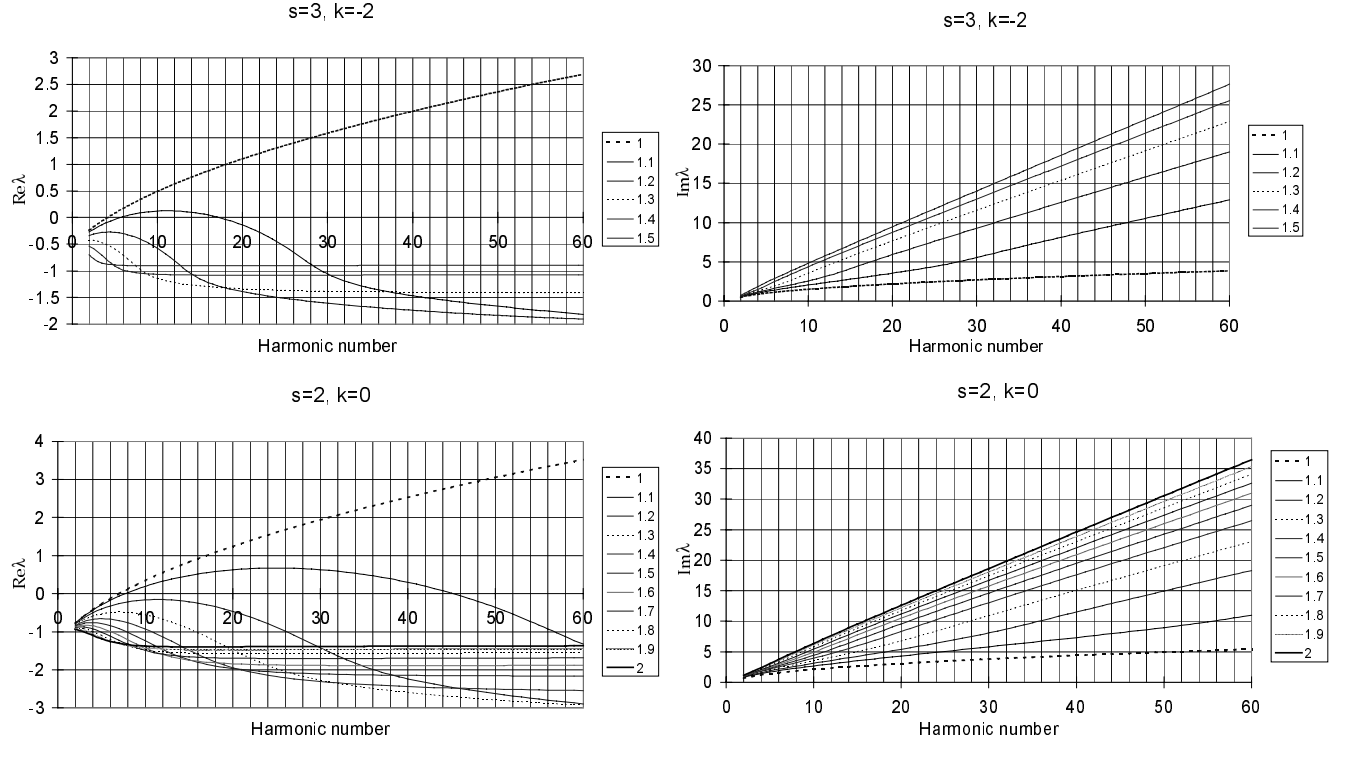

$s=2, k=-1$
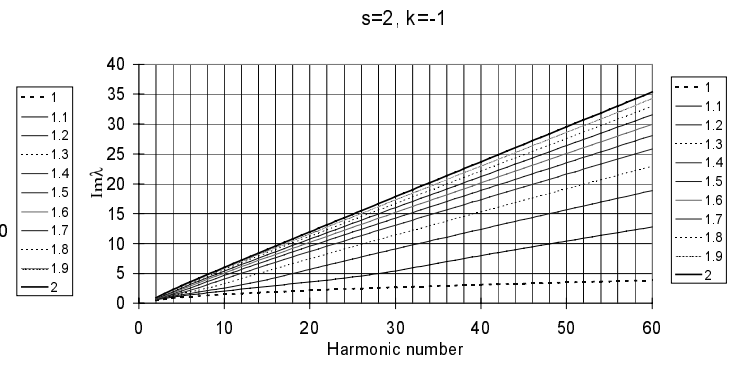

Fig.1 Values of complex exponent $\lambda$ in the cases of spherical $(s=3)$ and cylindrical $(s=2)$ blast wave in a gas which initial density $\rho_{0}$ is power function of radius: $\rho_{0} \sim r^{k}$ for a number of values of gas specific ratio $\gamma$. For the case $\gamma=1$ values are calculated using analytic expressions Eq.(36). 


\section{Instability region}

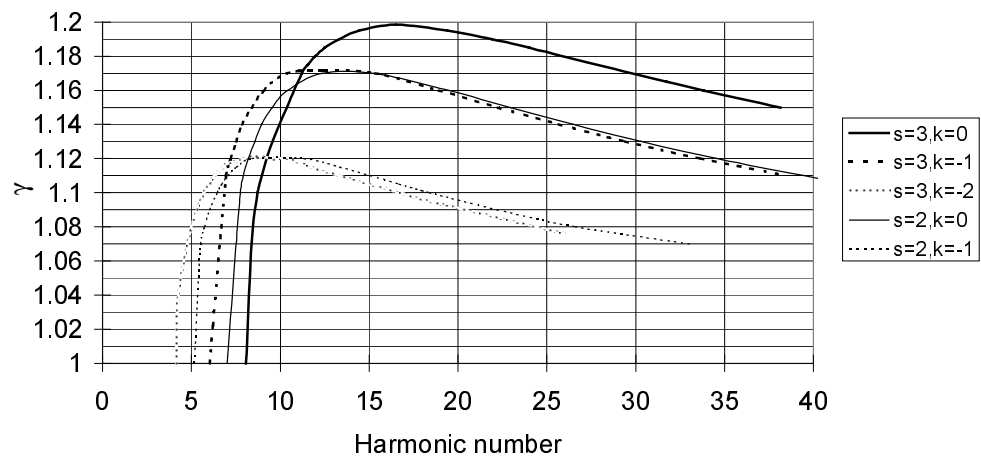

Fig. 2 Instability region on the plane $n-\gamma$

\subsection{Analytical solution results}

Let us consider some cases when eigenvalue problem has an analytical solution .

\subsubsection{Thin shell approximation: $\quad \gamma-1<1$}

The problem has an analytic solution in the case when the value of $g$ is close to unit. In this case we let in the equations (26)-(30): $\gamma=1, w=0, a=-(s+k) / 2, b=0$.

We get:

$$
\begin{aligned}
& \left(\frac{z d}{d z}+\lambda\right) x_{1}=0 \\
& -\frac{s+k}{2} x_{1}+\left(\frac{z d}{d z}+\frac{s+k-2}{2}\right) \frac{z d}{d z} f_{1}=0 \\
& \left(\frac{z d}{d z}+\lambda+s+k\right) p_{1}+\left(\left(\frac{z d}{d z}+\frac{s+k-2}{2}\right) \frac{z d}{d z}+(s+k-2) \frac{s+k}{2}\right) x_{1}- \\
& -n(n+s-1) \frac{s+k}{2} f_{1}=0
\end{aligned}
$$

with boundary conditions:

$$
\begin{aligned}
& f_{1}(1)=0, \\
& f_{1}^{\prime}(1)=1, \\
& x_{1}(1)=1, \\
& p_{1}(1)=2 \lambda+\frac{4-s+k}{2}, \\
& \left(p_{1} z^{s+k}\right)(z \rightarrow 0)=0
\end{aligned}
$$

Solution of (31)-(34) is as follows: 


$$
\begin{aligned}
& x_{1}=z^{-\lambda}, \\
& f_{1}=\frac{s+k}{2 \lambda\left(\lambda-\frac{s+k-2}{2}\right)} z^{-\lambda}-\frac{2}{s=k-2} \frac{\lambda+1}{\lambda-\frac{s+k-2}{2}} z^{-\frac{s+k-2}{2}}+\frac{2}{s=k-2} \frac{\lambda+\frac{s+k}{2}}{\lambda}, \\
& p_{1}=\left[\frac{\lambda\left(\lambda-\frac{s+k-2}{2}\right)-\frac{(s+k-2)(s+k)}{2}}{s+k}+\frac{n(n+s-1)(s+k)}{2 \lambda\left(\lambda-\frac{s+k-2}{2}\right)}\right] z^{-\lambda}- \\
& -\frac{(s+k)(\lambda+1) n(n+s-1)}{\left(\lambda-\frac{s+k-2}{2}\right)\left(\lambda+\frac{s+k+2}{2}\right)} z^{-\frac{s+k-2}{2}}+\frac{(s+k)\left(\lambda+\frac{s+k}{2}\right) n(n+s-1)}{\lambda(\lambda-+s+k)},
\end{aligned}
$$

-and after simple calculations we get the equation for $\lambda$ ( note that it is possible to get this equation in a simpler way applying conservation laws for calculating thin layer motion ${ }^{1}$ ):

$$
\begin{aligned}
& (\lambda+s+k)^{2}\left(\lambda+\frac{s+k+2}{2}\right)^{2}-\frac{s(s+k)}{2}(\lambda+s+k)\left(\lambda+\frac{s+k+2}{2}\right)+ \\
& +n(n+s-1) \frac{(s+k)^{2}}{4}=0 ;
\end{aligned}
$$

One of two pairs of the complexly conjugated roots of this equation with the greater real part is the solution of the problem in the case $n>1$.

In the case $n=1$ the equation (34) has four real negative roots. The two of them satisfying boundary condition at the center are solutions:

One of these solutions is determined with formula:

$$
\lambda=-\frac{s+k}{2},
$$

this case corresponds to explosion direct motion with momentum conservation. This value of 1 can be calculated from dimension consideration.

The other is the greater root of equation:

$$
\lambda^{2}+\lambda \frac{3 s+3 k+2}{2}+\frac{(s+k)(k+3)}{2}=0
$$

it is equal to - 1 if $k=0$; this case corresponds to the blast being displaced.

.We can continue analytical calculations if we take into account next terms of expansion of solution of eigenvalue problem (26)-(30) by powers of the small parameter $(g-1)$ :

$\lambda=\lambda^{(0)}+(\gamma-1) \lambda^{(1)}$

where $\lambda^{(0)}$ is determined by (36)

In this case we get for the first approximation terms the equation system with constant coefficients analogous to (31) but with non-uniform equations. The right parts of these equations are of the first order of smallness. This equation system is integrated quite similar to that was done for $(31,32)$, and (omitting cumbersome transformations) we arrive to the following formula ${ }^{2,7}$ for the first order correction of $l$ (to avoid bulky expressions here we present only the special case $s=3, k=0)$; here $b(a)$ stands for integral $\beta(\alpha)=\int_{0}^{1} \frac{t^{\alpha} d t}{1+t}$, and $l$ is from (36)): 


$$
\begin{aligned}
& \lambda^{(1)}=\frac{1}{3} \frac{(\lambda+1)\left(\lambda+\frac{3}{2}\right)\left(\lambda+\frac{5}{2}\right)(\lambda+3)(\lambda+4)}{\left(\lambda+\frac{11}{4}\right)\left(\lambda^{2}+\frac{11}{2} \lambda+\frac{21}{4}\right)}\left\{\left(1-2 \ln 2 \frac{\left(\lambda+\frac{3}{2}\right)\left(\lambda+\frac{5}{2}\right)}{\lambda\left(\lambda-\frac{1}{2}\right)}-\right.\right. \\
& \left.-\frac{4}{3}\left(\lambda+\frac{5}{2}\right)\left[\frac{\left(\lambda^{2}+6 \lambda+\frac{9}{2}\right)}{\lambda} \beta\left(\frac{\lambda+3}{3}\right)-\frac{\left(\lambda^{2}+\frac{11}{2} \lambda+3\right)}{\lambda-\frac{1}{2}} \beta\left(\frac{\lambda+\frac{5}{2}}{3}\right)\right]\right\}
\end{aligned}
$$

The results of (39),(40) are in a good agreement with the results of numerical calculation for the imaginary parts of 1 and in the worse one for the real parts. For example, in the case: $s=3, k=0, n=8, \gamma=1.2$, we have ${ }^{2}: \lambda_{\text {analyt }}=0.15+3.14 i$, and $\lambda_{\text {numer }}=-0.30+3.07 i$.

\subsubsection{The first harmonic: $\quad n=1$}

Note that both solutions (35,38): $\lambda=-1$, and $\lambda=-(s+k) / 2$, are independent of $\gamma$, and they are solutions of eigenvalue problem (26)-(30) in the general case of an arbitrary $\gamma>1$ :

1) The displacement solution. $\lambda=-1$

We can verify it by a direct substitution of the following expressions to the system of equations (26)-(28):

$$
\begin{aligned}
& n=1, k=0, \lambda=-1, \\
& f_{1}=\frac{\gamma+1}{2}\left(\frac{z}{x}-1\right) \\
& x_{1}=\frac{\gamma+1}{2}\left(\frac{z}{x}-w\right) \\
& p_{1}=\frac{\gamma+1}{2} a
\end{aligned}
$$

So $\lambda=-1$ is the correct solution for the case: $n=1, k=0$

2) $n=1, \lambda=-\frac{s+k}{2}$

We subtract equation (27) from equation (28) multiplied by $(s-1)$, and integrate the result. Then we get:

$$
p_{1}+\left(\frac{z d}{d z}+w+\frac{s+k}{2}\right) x_{1}+\left(\frac{z d}{d z}+w+\frac{s+k}{2}\right) f_{1}=0
$$

This equation together with Egs. $(25,27)$ forms equation system. It can easily be shown that all solutions of this system have the required convergence at the center independently of shock front boundary conditions. So $\lambda=-\frac{s+k}{2}$, is the correct solution of the eigenvalue problem for $n=1$.

\subsubsection{Investigation of the features of the solution in the limit $n \rightarrow \infty$.}

The next case of analytical approach to solving the eigenvalue problem is a short wavelength approximation: $n>>1$. In this case we seek for the solution, which looks like:

$$
\exp \left(\int \frac{q(z) d z}{z}\right), \text { where }|q| \sim|l| \sim n>>1 \text {. }
$$

We substitute so defined values to Eqs. (26-28), remove all terms of the lowest order, and get the following equation system: 


$$
\begin{aligned}
& b p_{1}+(q+\lambda) x_{1}-n^{2} w f_{1}=\frac{\gamma+1}{\gamma} \lambda w, \\
& w p_{1}+q^{2} f_{1}=0, \\
& (q+\lambda) p_{1}+q^{2} x_{1}=0, \\
& \text { and boundary conditions: } \\
& x_{1}(z=1)=1, \\
& f_{1}(z=1)=0, \\
& \frac{z d}{d z} f_{1}(z=1)=1, \\
& p_{1}(z=1)=2 \lambda, \\
& \operatorname{Re} a l(q)>0,
\end{aligned}
$$

Solution of (44),(45) is as follows:

$$
\begin{aligned}
& x_{1}(z)=\frac{\gamma^{2}-1}{2 \gamma^{2}}\left(\exp \left(\int_{1} \frac{q(z) d z}{z}\right)-1\right)-\frac{\lambda}{\gamma} \ln z+1, \\
& f_{1}(z)=-\frac{\gamma^{2}-1}{2 \gamma^{2} \lambda}\left(\exp \left(\int_{1} \frac{q(z) d z}{z}\right)-1\right)-\frac{1}{\gamma} \ln z, \\
& p_{1}(z)=2 \lambda \exp \left(\int_{1} \frac{q(z) d z}{z}\right)
\end{aligned}
$$

where

$$
\lambda=\operatorname{in} \sqrt{\frac{\gamma-1}{\gamma+1}}
$$

and $q(z)$ is the root with a positive real part of the following equation: $q^{2}(1-b)+2 \lambda q+\lambda^{2}-n^{2} w^{2}=0$,

Data of Fig.1 show that formula Eq.(47) is in a reasonable agreement with numerical results. We should note also that Eq.(47) has the right limit of $n \rightarrow \infty$, it is in an agreement with the corresponding formulas describing perturbation oscillations in plane shock waves. ${ }^{10}$

\section{Evolution of shock front radius small perturbations in the case when the gas density before shock front has small perturbation.}

We considered above free oscillations of blast wave. We can develop an analogy between the blast wave perturbations and other oscillating systems and consider the perturbations generated by perpetually operating external sources. One of these cases is when perturbations of density before shock front $r_{o 1} \sim r^{l+1}$ take place. This problem was considered earlier in Ref. 2. In this case hydrodynamic equation system for perturbations coincides with (16),(17),(18),(22) except for continuity equation which has a non-zero right part:

$$
\frac{\rho_{1}}{\rho}+\Delta_{\theta \varphi} F_{1}+\frac{\frac{\partial\left(R^{s-1} R_{1}\right)}{r^{s-1} \partial r}}{\frac{R^{s-1} \partial R}{r^{s-1} \partial r}}=\frac{\rho_{01}(r)}{\rho_{0}(r)}
$$

and for boundary conditions:

$$
\begin{aligned}
& P_{1}(r=S, t)=\frac{2 \rho_{0}}{\gamma+1} \frac{\dot{S}^{2}}{S} S_{1}(2 \lambda+s+2+a(1)) \\
& \rho(r=S, t)=\frac{(\gamma+1) \rho_{0}}{\gamma+1} \frac{S_{1}}{S} a(1)
\end{aligned}
$$


Using that we get the self-similar equation system and boundary conditions analogous to Eqs. (26)-(30). The difference between eigenvalue problem in this case and stability problem considered above is that the role of eigenvalues is played by the ratio of shock front radius perturbation to initial density perturbation $d$ :

$$
d=d(\lambda, n)=\frac{\frac{S_{1}}{S}(r)}{\frac{\rho_{10}}{\rho}(r)} ;
$$

The eigenvalue problem is linear of $1 / \mathrm{d}$. The system of equations is the following::

$$
\begin{aligned}
& b p_{1}+\left(\frac{z d}{d z}+\lambda+s w\right) x_{1}-n(n+s-1) w f_{1}=\frac{\gamma+1}{2 \gamma}\left(2 \lambda+s+k+2+\frac{1}{d}\right) w, \\
& w p_{1}+a x_{1}-\left(\frac{z d}{d z}+2 w+\frac{s+k-2}{2}\right) \frac{z d}{d z} f_{1}=0, \\
& \left(\frac{z d}{d z}+\lambda+s+k-(s+k-2) w\right) p_{1}+\left(\left(\frac{z d}{d z}+2 w+\frac{s+k-2}{2}\right) \frac{z d}{d z}-(s+k-1)\right) a x_{1}+ \\
& +n(n+s-1) a f_{1}=-\frac{\gamma+1}{2} \frac{a}{d} ;
\end{aligned}
$$

Boundary conditions are the same as in $(29,30)$, except:

$$
p_{1}(1)=2 \lambda+\frac{6 \gamma+2-s(3 \gamma-1)+k(\gamma+1)}{(\gamma+1)^{2}}+\frac{1}{d} \text {; }
$$

\subsection{The forced oscillations of the shock front radius.}

Earlier the case of $\lambda=0$ was considered in Ref. 2.

In this paper we consider pure imaginary values of $l: l=i w$. The problem is solved numerically. The calculated values of $|d(w, n)|$ are presented on Fig.3. The sharp resonance pattern (in the vicinity of $\omega=\sqrt{\frac{\gamma-1}{\gamma+1}} n$ ) is demonstrated for $\gamma=1.2$ and the smeared one is demonstrated for $\gamma=5 / 3$.

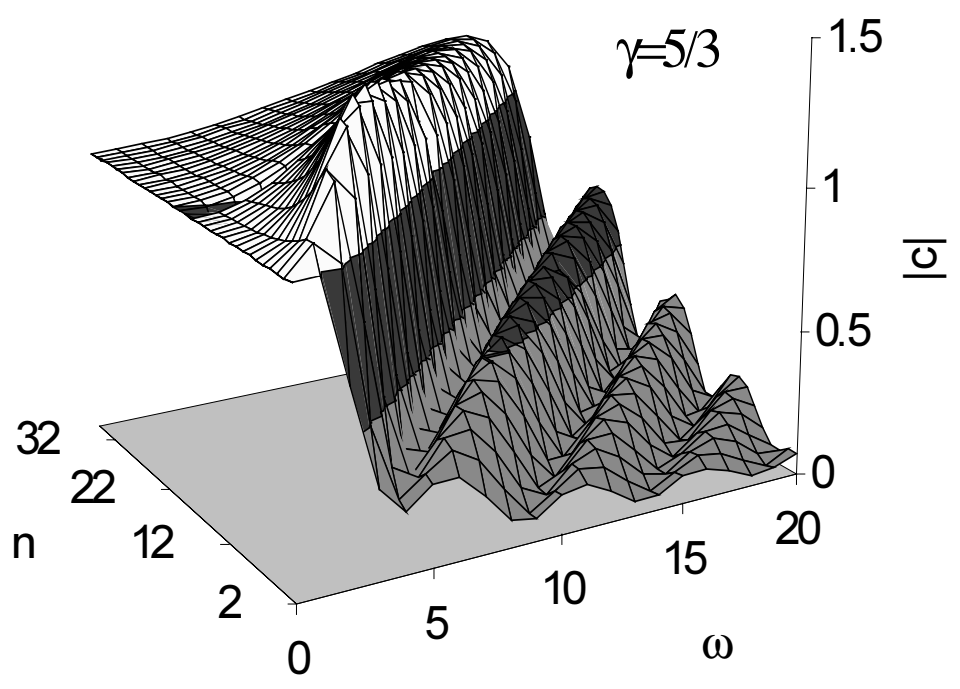

Fig.4a. Resonance patterns $|c(\omega, n)|$ for $\gamma=1.667$. Results are normalized to $q(0, n)$ : $c(\omega, n)=\left|\frac{q(\omega, n)}{q(0, n)}\right| .\left(\right.$ Note that $q(0, n) \cong \frac{1}{n} \sqrt{\frac{\gamma+1}{2 \gamma}}$ for $\left.n>>1\right)$ 


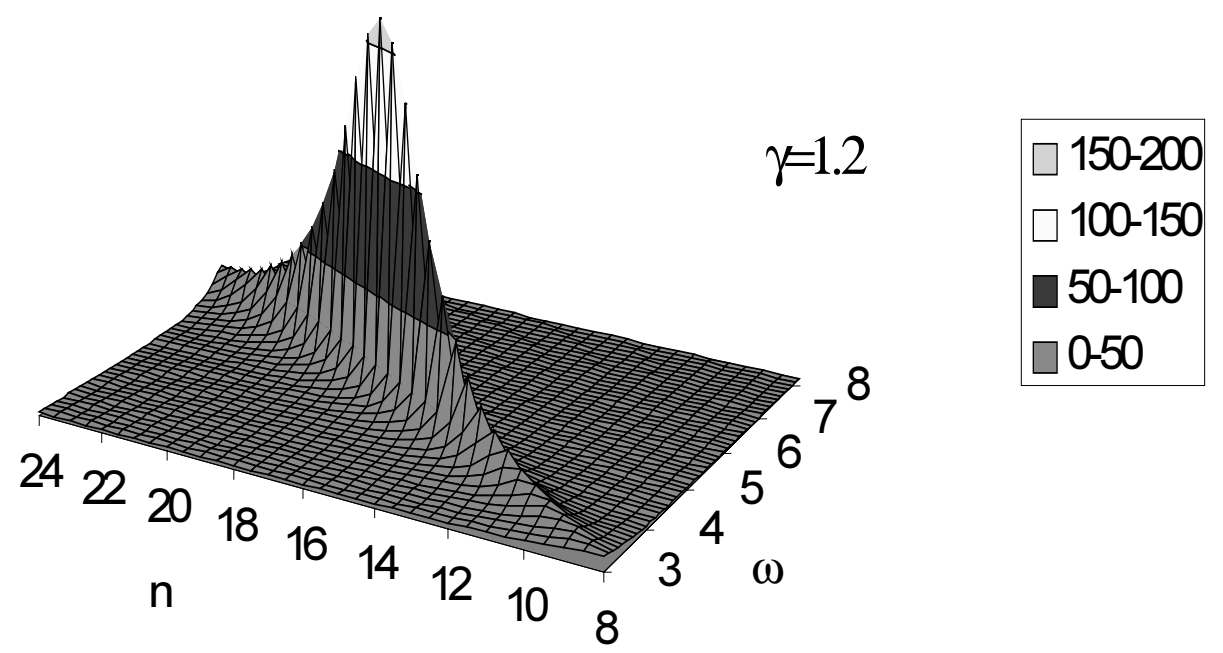

Fig.4b. Resonance patterns $|c(\omega, n)|$ for $\gamma=1$.2. Results are normalized to $q(0, n)$ :

$c(\omega, n)=\left|\frac{q(\omega, n)}{q(0, n)}\right|$.

Analytical solution can be obtained for $n>>1, k=0$.

We again seek solution of (43) type, reduce equation system (52), and get finally the following solution:

$$
\begin{aligned}
& q=n w \sqrt{\frac{1}{1-b}}, \\
& d=-\frac{1}{n} \sqrt{\frac{\gamma+1}{2 \gamma}}
\end{aligned}
$$

This result is in a good agreement with the well-known formulas for front perturbations in a stationary shock wave (Ref.11).

\section{Stability of the blast wave in a non-ideal gas.}

For the sake of convenience of comparison with an experiment we however need a solution of the stability problem for the case of non-ideal gas. In actual practice gas adiabatic exponent is a function of density and temperature: it's getting lower while degree of ionization is increasing, that is while density is lowing or temperature is rising. In Ref.12 the authors considered the stability problem for one of the cases of blast wave in non-ideal gas, namely, the only case when the blast wave in the non-ideal gas is self-similar: we supposed that the gas adiabatic exponent $\gamma$ was a function of the gas density (i.e. $\gamma$ was independent from the gas energy). In this case we can use the self-similar technique for solving the blast wave stability problem.

In Ref.13 the authors supposed that the gas equation of state (EOS) was as follows:

$\mathrm{P}=\left(\gamma^{*}(\rho)-1\right) \rho \mathrm{E}$

where coefficient $\gamma^{*}$ was a two-parameter function of $\rho$ :

$$
\gamma^{*}(\rho)-1=\frac{\gamma_{c}-1}{1-\frac{\gamma_{f}-\gamma_{c}}{\gamma_{f}+1} \rho}
$$

Here parameters: $\gamma_{\mathrm{c}}, \gamma_{\mathrm{f}}$ stand for values of the gas adiabatic exponent in the blast center and at the shock front. 
The eigenvalue problem in this case is the same (See Ref.13) as in Eqs.(26-31). Fig.4 presents results of numerical solution of the eigenvalue problem.
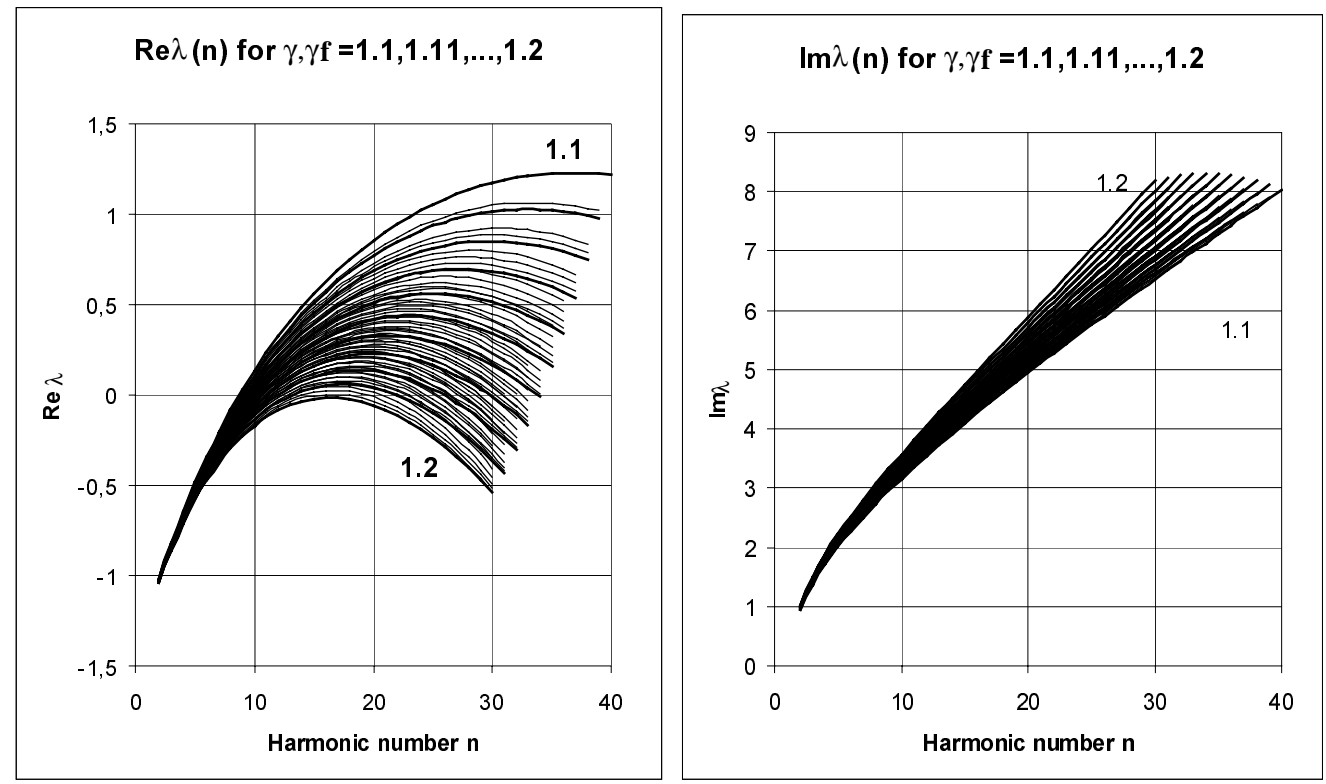

Fig.4 The components of complex eigenvalue $\lambda$ for some sets of values of parameters of gas EOS. Heavy curves correspond to the cases of ideal gas with $\gamma_{f}=\gamma_{c}=\gamma=1.10,1.11,1.12, \ldots 1.20$. the other lines correspond to the cases when $\gamma_{f}>\gamma_{c}$.

\section{Conclusion}

Blast wave perturbation evolution was considered. The solutions obtained have applications in laboratory physics and astrophysics; they also can serve the tests for elaboration of the 2D and 3D hydrodynamic codes.

Stability of some cases of spherical or cylindrical blast wave was considered using selfsimilar approach in the unified manner. Namely, stability of the cases in which gas density before shock front was a power function of radius (with power exponent being equal to $k$ ) was considered. Blast wave small perturbations were expanded in spherical harmonics. The perturbation value was supposed to be a power function of time (with complex exponent), stability problem was reduced to eigenvalue problem, and the increments of perturbation growth and periods of oscillation were calculated as the eigenvalues for each harmonic number. The calculations were made numerically both for spherical and cylindrical blasts for a large set of blast wave parameters: gas specific heat ratio $\gamma$, harmonic number $n$, value of $k$. The instability region on the plane $n-\gamma$ was determined. The critical value of specific heat ratio $\gamma_{c}$ was calculated for each case considered.

The evolution of perturbations generated by perpetually operating external sources was considered too. Namely, there were considered the forced oscillations of shock front radius perturbations caused by a presence of spatially oscillating perturbations of initial gas density. The eigenvalue problem in this case was formulated using the self-similar technique.

4. The short wavelength approximation was used to obtain an analytical solution of eigenvalue problem. It was shown that the obtained solutions agreed with the corresponding solutions describing plane shock wave perturbations.

5. The analytical solutions of stability problem were also obtained in the case of values of $g$ being close to unit. In this case expansion of the solution by powers of $\gamma-1$ was used. The terms of the zero order, and the first order of negligibility were calculated. It was shown that they were in a reasonable agreement with the numerical results.

6. The spherical (or cylindrical) blast wave stability was considered using the self-similar approach. Using this technique helped us to reduce rather complicated 3D hydrodynamic 
problem of small perturbation evolution to the simpler eigenvalue problem. Blast wave should satisfy two conditions to be studied using this approach: it should be self-similar and 1D solution for this blast wave should be smooth at the center. A sound speed should tend to infinity, and a particle velocity should be negligible compared with the sound speed at the center.

The main assumptions of the self-similar approach are:

- Pattern of the perturbation evolution is a self-similar one.

- The value of pressure small perturbations tends to zero at the center.

In the spherical case one more assumption was made. It is:

- Pattern of an angular motion is a potential one.

It seems to us that all three these assumptions were proved in experiments (see Ref. 4 where perturbation oscillation period was measured and the period value proved to be in a good agreement with the self-similar theory results). But for the better proof of the third assumption we need direct computer simulation of blast waves using 3D hydrodynamic codes.

\section{Acknowledgments}

This work was sponsored by the International Science and Technology Center (Project No222,No874).

\section{References}

1.E.T.Vishniac,"The dynamic and gravitational instabilities of spherical shocks," Astrophys.J. 274, p.152 (1983);

2.V.M.Ktitorov, "Asymptotical development of point blast wave small perturbations," Voprosy Atomnoi Nauki i Tekhniki, Ser.Teoreticheskaya i Prikladnaya.Fizika (Atomic Science and Technique Issues, Ser. Theoretical and Applied Physics), No2, p.28, (1984);

3.D.Ryu and E.T.Vishniac, "The growth of linear perturbations of adiabatic shock waves," Astrophys.J. 313, 820 (1987);

4.J.Grun, J.Stamper, C.Manca, et al., "Instabilities of Taylor-Sedov blast wave propagating through uniform gas," Phys.Rev.Let., V.66, No21, p.2738, (1991);

5.C.Manca, J.Grun, J.Stamper, et al., "Images of unstable Taylor-Sedov Blast Waves propagating through uniform gas," IEEE Transactions on Plasma Science vol.24,No1, p.35,(1995);

6.L.D.Landau, and E.M.Lifshits, Fluid Mechanics, 2-nd ed. (Pergamon, NY 1987);

7.V.M.Ktitorov, "Cylindrical blast wave stability and two-dimensional perturbation development," Voprosy Atomnoi Nauki i Tekhniki, Ser.Teoreticheskaya i Prikladnaya.Fizika (Atomic Science and Technique Issues, Ser. Theoretical and Applied Physics), No2, p.37, (1985);

8.E.T.Vishniac and D.Ryu, Astrophys.J "On the stability of decelerating shocks," 337, 917 (1989);

9.V.M.Ktitorov, "Stability of blast wave propagating through a gas with a power depending density", Khimicheskaya Fizika (Chemical Physics Issues) V.14, No 2-3, p.169, (1995);

10.R.M.Zeidel, "Perturbation evolution in plane shock waves," Zhurnal Prikladnoi Matematiki I Tekhnicheskoi Fiziki (Applied Mathematics and Physics Journal) v.4, p.30, (1968);

11.V.M.Ktitorov, "Perturbations of stationary shock wave moving along a channel", Khimicheskaya Fizika (Chemical Physics Issues) V.14, No 2-3, p.171, (1995);

12. V.M.Ktitorov, V.Yu.Meltsas, "Study of point blast wave instability in numerical experiment", Proceedings of the 6th IWPCTM, p.251

13. V.M.Ktitorov, "Stability of Blast wave in non-ideal gas", Report at the 7th IWPCTM, St-Peterbourg,, July,1999 


\section{STABILITY OF REFLECTED FROM THE CENTER SELF-SIMILAR \\ CONVERGING SHOCK WAVE}

V.M.Ktitorov

Russian Federal Nuclear Center (VNIIEF), Sarov, $R U$

In this paper the technique first used in Ref.1,2 for solving the point blast wave stability problem is applied for the one of the self-similar converging shock wave after focusing.

Equations for the case of converging self-similar shock wave

The obtained in Ref.1-4 system of equations can be applied for the case under consideration.

Let us consider the uniform equation for perturbations (we use the notification of Ref.4):

$$
\begin{aligned}
& \frac{P_{1}}{\rho}+{ }_{1}+\gamma \frac{\left(R^{2} R_{1}\right)^{\prime}}{R^{2} R^{\prime}}-n(n+1) \gamma F_{1}=0 \\
& \frac{P_{1}}{\rho}+R_{1} \ddot{R}+\left(R^{2} \dot{F}_{1} \dot{)}=0\right. \\
& \frac{P_{1}^{\prime}}{\rho}+\ddot{R} \frac{R_{1}}{R}+\ddot{R}_{1}+n(n+1) \ddot{R} F_{1}=0
\end{aligned}
$$

With boundary conditions:

$$
\begin{aligned}
& R_{(}(R=S, t)=R_{1}\left(1-R^{\prime}\right)+\frac{S_{1}}{3} R^{\prime}(S) \\
& F(S, t)=\frac{\lambda+3}{3 N} S_{1}(S) \\
& \dot{F}(S, t)=-\frac{\dot{R}(S)}{\dot{S}} \\
& P_{(}(S, t)=P_{1}(\text { Shock })-\frac{s_{1}}{3} P^{\prime}(S)
\end{aligned}
$$

According to the self-similar approach we look for the solution in the self-similar form:

$$
\left(F_{1}, R_{1}, P_{1}\right) \sim r^{\lambda} f(r / S) \text {, and: } s_{1} \sim r^{\lambda}
$$

After inserting of self-similar variables (functions of $z=r / S$ ) we get:

$$
\begin{aligned}
& P_{1} b-(D+\lambda+3 w) r_{1}-N w F_{1}=0 \\
& P_{1} w+r_{1} a+\left(D+2 w+\frac{1}{\alpha}-1\right) D F_{1}=0 \\
& (D+\lambda+3-w) P_{1}+\left(\left(D+2 w+\frac{1}{\alpha}-1\right) D-a\right) r_{1}+N a F_{1}=0
\end{aligned}
$$

(Here $D$ stands for differential operator: $D f \equiv \frac{d}{d \ln z} f$ ) 
Because of presence of non-zero initial velocity before shock front (we denote this velocity $\mathrm{u}_{0}$ ) the mass conservation equation is changing:

$$
\frac{1}{\rho}=\frac{1}{1+u_{0}} \frac{R^{s-1} \partial R}{r^{s-1} \partial r}
$$

So coefficients in (Eqs.27) look like as follows:

$$
\begin{aligned}
& w=\frac{D x}{x} \\
& a=D w+(w-1)\left(w+\frac{1}{\alpha}-1\right) \\
& b=\left(1+u_{0}\right) \frac{w z^{3}}{2 x P}
\end{aligned}
$$

(Here variables $x=R / S, P=P / S^{2}$ are functions of $\mathrm{z}$ ), the front value of coefficient $\mathrm{w}$ is $w(1)=\frac{1+u_{0}}{\delta}$, (here $\delta$ stands for front compression), the front value of coefficient a(1) is calculated from spherically symmetric solution.

Equation system (6) has the fourth order; there are four boundary conditions on the front edge $(\mathrm{z}=1)$ :

$$
\begin{aligned}
& x_{1}(1)=1-\frac{1+u_{0}}{\delta}+\frac{s_{1}}{3} \frac{1+u_{0}}{\delta} \\
& F_{1}(1)=\frac{\lambda+3}{3 N} s_{1} \\
& D F_{1}(1)=1-\frac{1+u_{0}}{\delta} \\
& P_{1}(1)=P_{1}(\text { Shock })+\frac{s_{1}}{3} a(1)
\end{aligned}
$$

These conditions construct full set of boundary conditions necessary to determine a solution. (To derive the conditions one should take into account the characteristics of gas flow before the shock front because values $P_{1}$ (shock) and $s_{1}$ in (31) depend on values of gradients of pressure and velocity before the shock front).

In order to construct the eigenvalue problem we add the fifth boundary condition (at the center):

$$
P_{1}(0)=0
$$

We solve the eigenvalue problem (Eqs.6,9,10) and we calculate the values of power exponent $\lambda$ (see Eq.5) as eigenvalues.

We used the simultaneous solving of spherically symmetric equations and equations for perturbations. The variables $P 1, F 1, x l$ are expressed as functions of $\mathrm{w}$ and coefficients $a, b$, in Eq. 6 are also expressed as functions of w: 


$$
\begin{aligned}
& a=\frac{b}{1-b}\left[(w-1)\left(w-1+\frac{1}{\alpha}\right)-3 w\left(w-1+\frac{2 / \alpha-2}{3 \gamma}\right)\right] \\
& D \equiv\left[a-(w-1)\left(w-1+\frac{1}{\alpha}\right)\right] \frac{d}{d w} \\
& \frac{d b}{b d w}=\frac{6-3 \gamma+w(3 \gamma-1)(b-1)}{3 w\left(w-1+\frac{2 / \alpha-2}{3 \gamma}\right)-b(w-1)\left(w-1+\frac{1}{\alpha}\right)}+\frac{\gamma+1}{w}
\end{aligned}
$$

Using this approach the eigenvalue problem was solved numerically. The values of $\lambda$ were calculated as eigenvalues. The dependence of $\lambda$ on $\gamma$ and harmonic number $n$ is shown on Fig.1, Fig2.

The case under consideration differs from other cases with diverging shock waves considered earlier. Curves on figures look like irregular ones. The reason of it is that self-similar solutions exist only in small region on the plane $n-\gamma$. For big harmonic numbers $(n \geq 15$ for $\gamma=1.2$, $\gamma=1.4$ and $n \geq 7$ for $\gamma=1.667$ ) self-similar solution supposedly does not exist at all.

Fig.3, Fig.4 present the computer validation of self-similar results shown on Fig.1, Fig.2. V.Yu Meltsas performed the computer modeling of the reflected from the center shock wave in the way he did it in Ref.5. The results of computer modeling agree the results of self-similar calculations: we see the exponential perturbation grow on Fig. 3 and oscillation regime of perturbation evolution for the case $n=2, \gamma=1.667$ on Fig. 4 .

\section{Acknowledgments}

The author wishes to acknowledge the financial support by ISTC (Project No 874).

\section{References:}

1. V.M.Ktitorov (Russian Atomic Science and Technique Issues, Ser. Theoretical and Applied Physics), No2, p.28, (1984);

2. D.Ryu and E.T.Vishniac, Astrophys.J. 313, 820 (1987);

3. V.M.Ktitorov, Khimicheskaya Fizika (Chemical Physics Issues) V.14, No 2-3, p.169, (1995)

4. V.M.Ktitorov Stability of Diverging Shock Waves, Report at the $8^{\text {th }}$ IWCTM, Pasadena, Dec, 9-14, 200112.

5. V.M.Ktitorov,V.Yu.Meltsas, "Study of point blast wave instability in numerical experiment", Proceedings of the 6th IWPCTM, p.251 


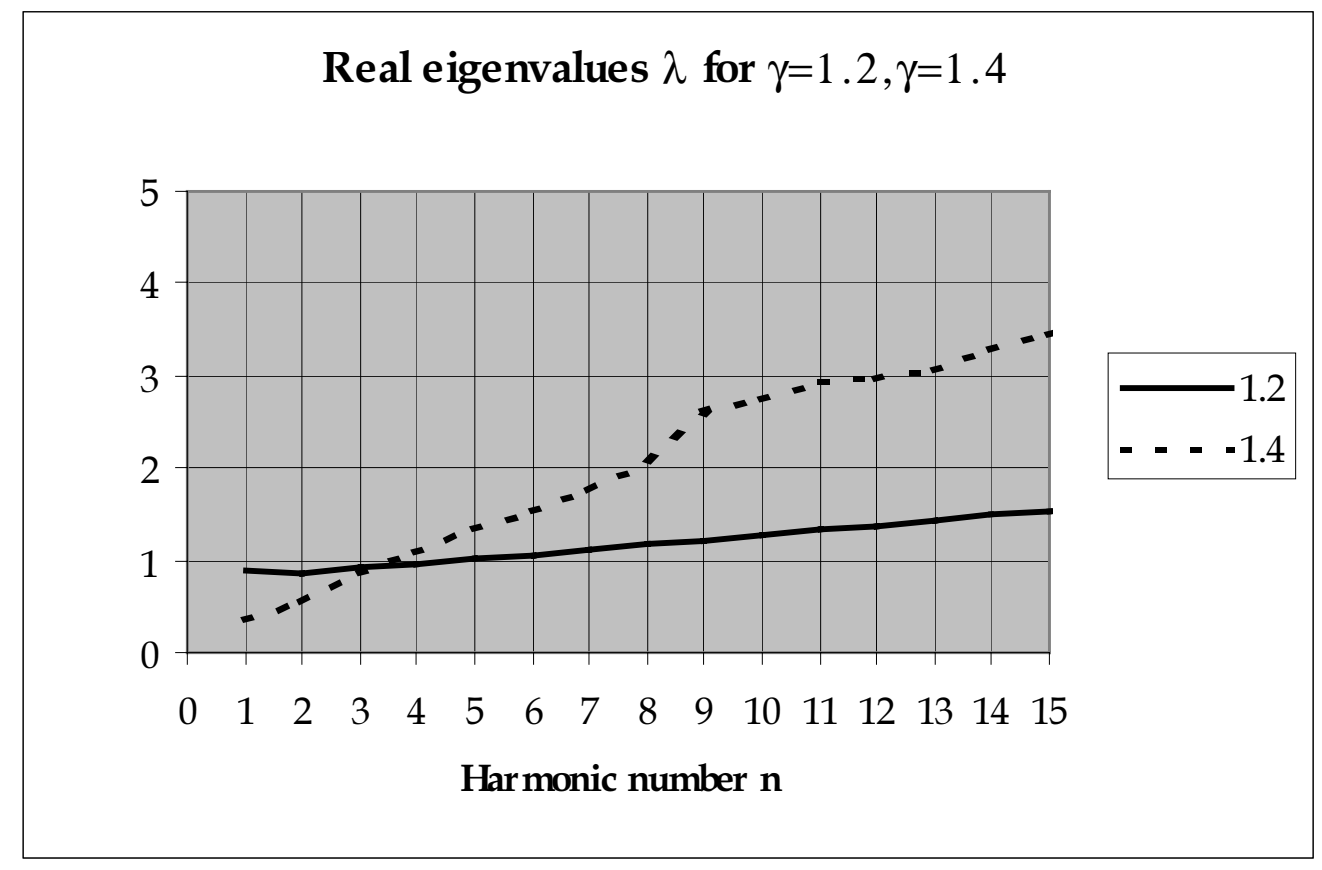

Fig. 1 Real eigenvalues $\lambda(\mathrm{n})$ for $\gamma=1.2, \gamma=1.4$. Shock wave is unstable with respect to perturbations of all harmonic numbers.

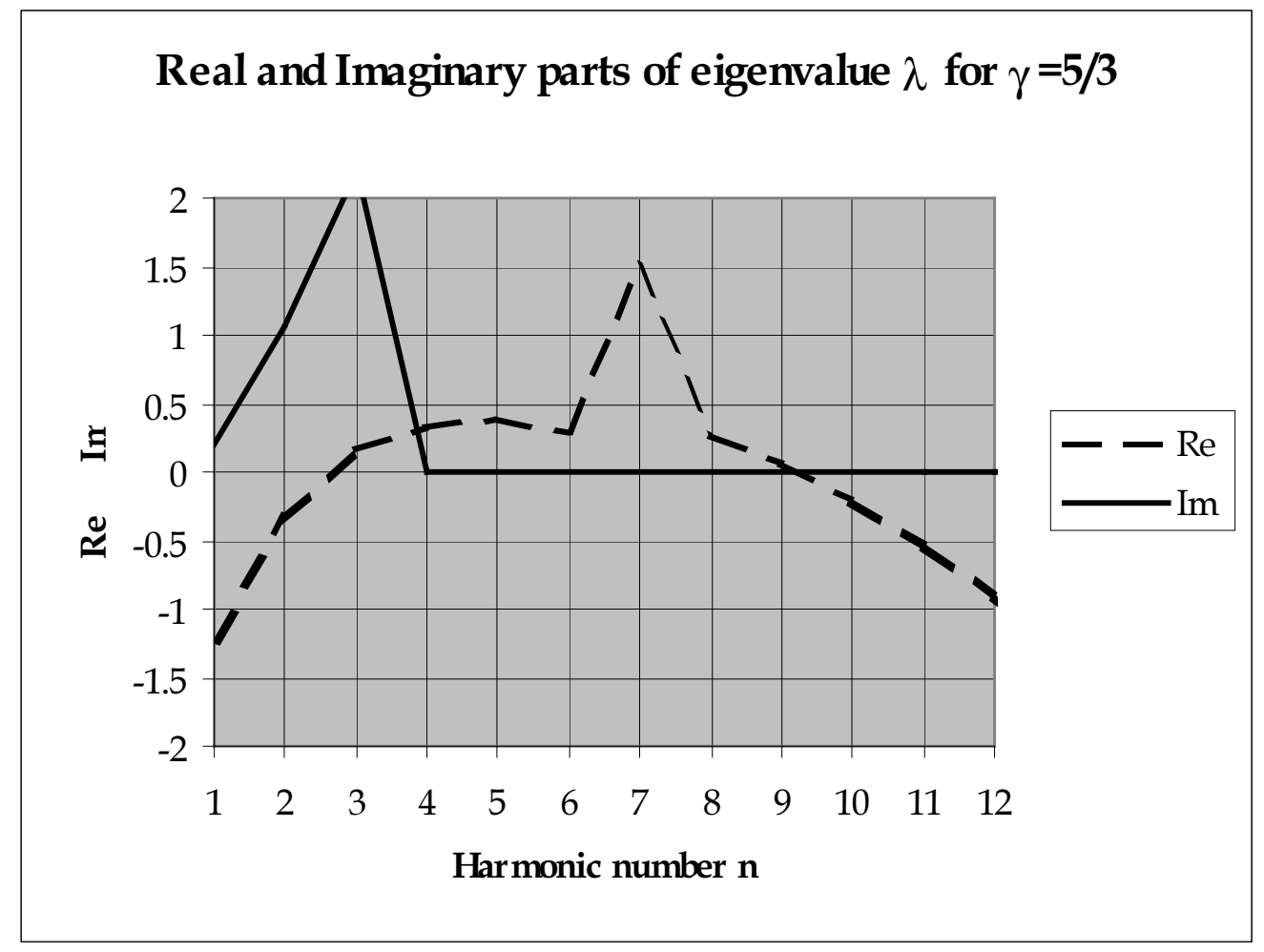

Fig.2 Complex (for $\mathrm{n}=1,2,3$ ) and real (for $\mathrm{n}>3$ ) power exponents (eigenvalues) $\lambda(n)$ for gases with $\gamma=1.667$. There appears to be no self-similar perturbations for $n>6$. 


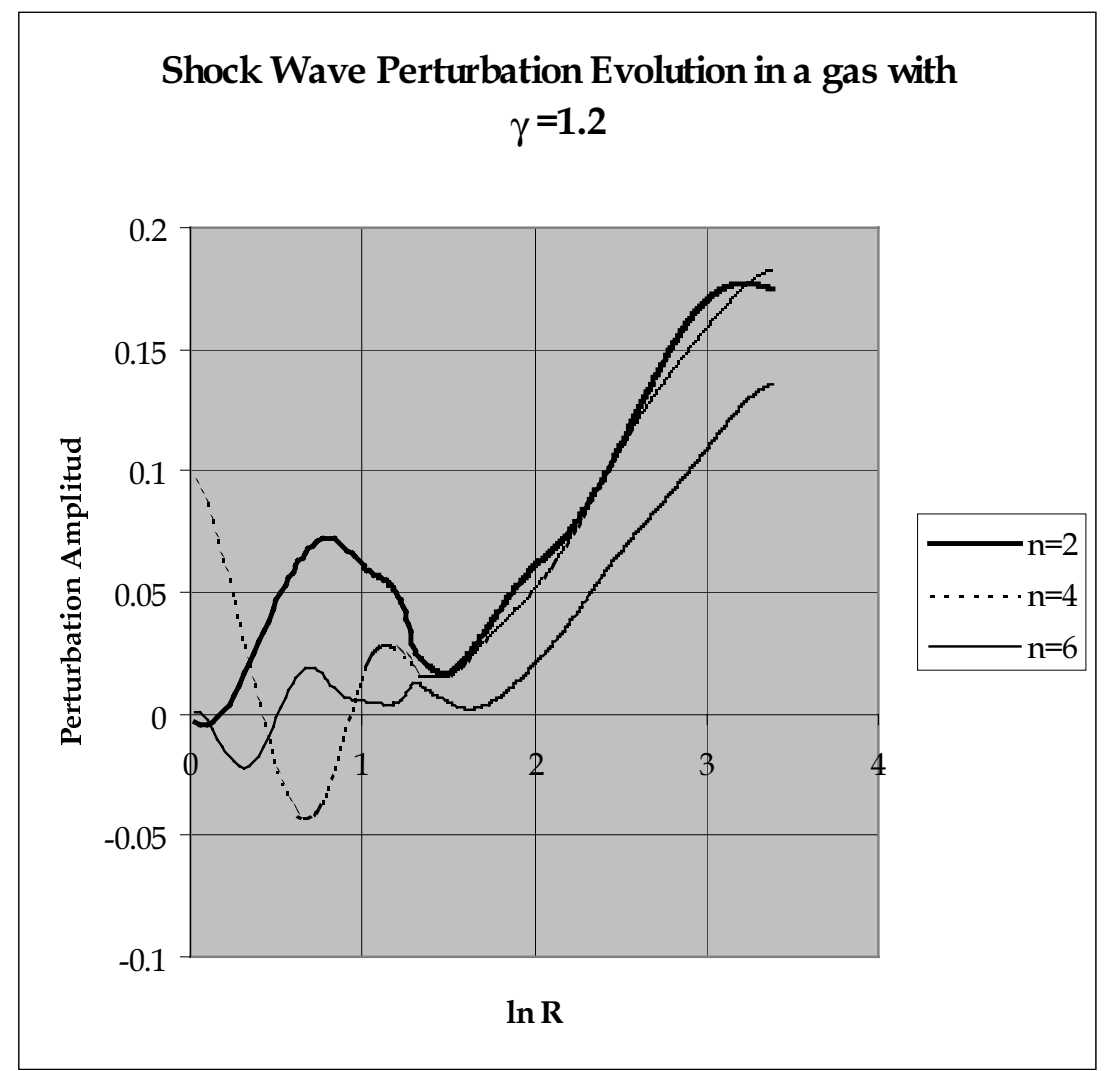

Fig.3 Perturbation evolution for shock wave in a gas with $\gamma=1.2$

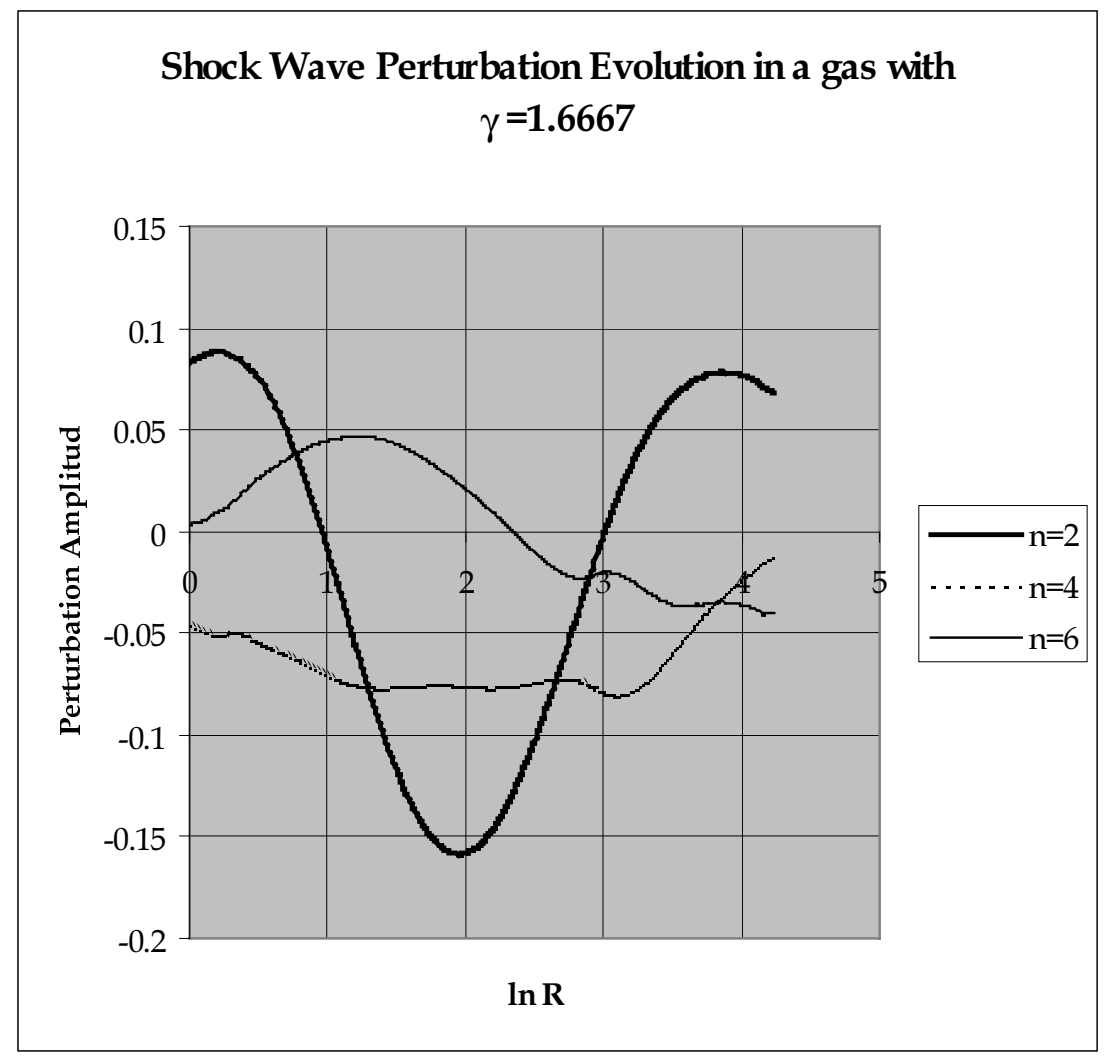

Fig.4 Perturbation evolution for shock wave in a gas with $\gamma=1.667$ 


\title{
Response of Turbulent RANS Models to Self-Similar Variable Acceleration RT-Mixing: an Analytical "0D" Analysis
}

\author{
Antoine LLOR \\ Commissariat à l'Energie Atomique \\ B.P.12 91680 Bruyères-le-Châtel Cedex
}

December 13th, 2001

\begin{abstract}
So far, the validation of RANS models applicable to variable acceleration RT mixing flows (as found in ICF) has mostly been carried out by fitting experimental or numerical data obtained for constant (RT) and impulsive (RM) accelerations. Further checks are also possible on the few available data for variable acceleration, such as in mixing-demixing flows induced by reverting the gravity field. Although this approach is widely applied and accepted, it is unsatisfactory because of the complex relationship between the model features and coefficients and the experimental measurements.

It is shown here that self-similar variable acceleration RT (SSVART) provides an appealing alternative since it extends the usual calibration techniques of turbulent RANS models based on simple self-similar flows. The general model equations in 1D (PDEs) are still too complex for full analytical calculations of SSVART flows, but using reasonable assumptions, simple 0D (ODEs) approximations can be derived and solved analytically.

This approach is applied to an extended $k-\varepsilon$ model derived from Andronov's (1979) and to Youngs' (1989) two-fluid model. The behaviour of the mixing layer growth rate and integral turbulent scales provides important informations on the accuracy of these models.

Finally, general qualitative arguments will be discussed showing the importance and the difficulty of capturing accurately a broad range of SSVARTs with a single simple model.
\end{abstract}




\section{Introduction}

Qualitative understanding of large scale turbulence in Rayleigh-Taylor (RT)

and incompressible Richtmyer-Meshkov (IRM) is not available as in other mixing layers (wakes, jets...).

Our aim is to provide such analysis for:

- ideal incompressible self-similar turbulent regime,

- vanishingly small density differences $(\mathcal{A} \rightarrow 0)$,

- and "OD" (global) energy balance.

"OD" approximation is averaging over TMZ width, $L(t)$.

Two-fluid analysis will be applied.

Kelvin-Helmoltz $(\mathrm{KH})$ will be used as reference.

Experimental, simulated and modeled results will be examined.

Extension to SSVARTs will be considered (Self-Similar Variable Acceleration Raleigh-Taylor mixing). 


\section{II "OD" averaging}

II.1 Fluid motion

In $\mathcal{A} \rightarrow 0$ limit

experiments and simulations show

linear profiles of fluid volume fractions:

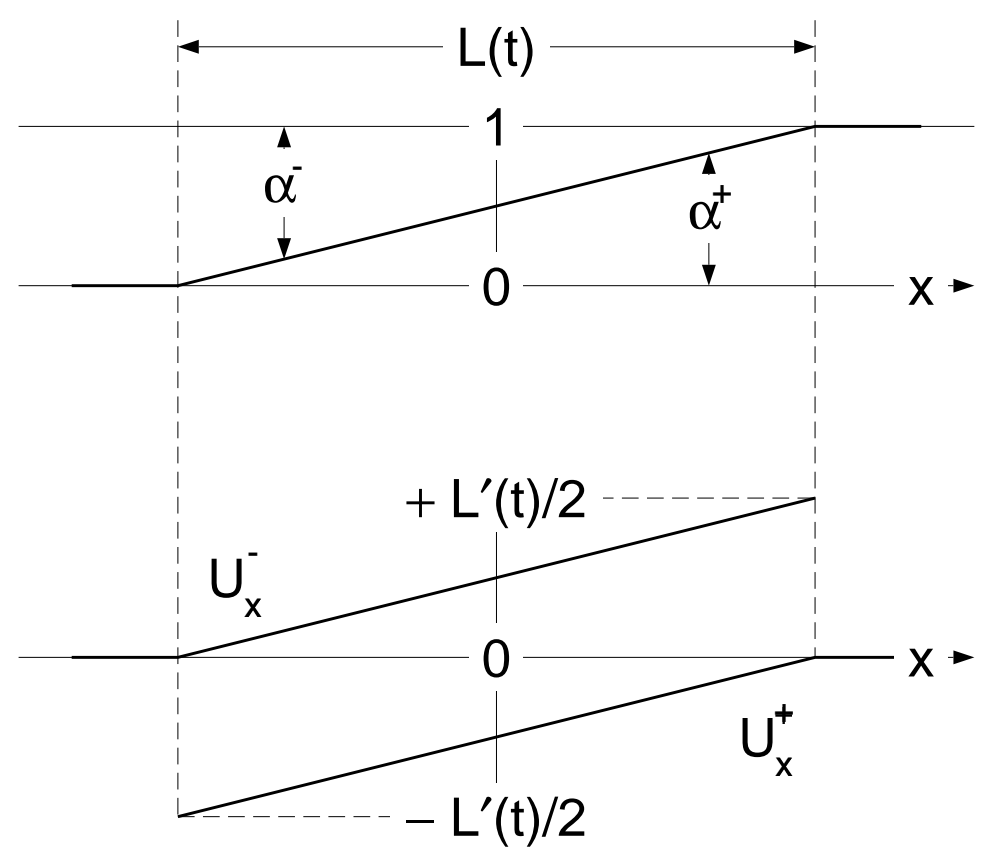

From mass conservation equations for fluids + and - , fluid velocities $U^{ \pm}$are also found linear.

Therefore:

$$
\delta U_{x}=U_{x}^{+}-U_{x}^{-}=\frac{\mathrm{d}}{\mathrm{d} t} L(t)
$$




\section{II.2 TMZ-growth related energies}

From growth law and fluid velocities, one can extract:

- Total input of energy:

$$
\begin{array}{r}
K_{I}=\frac{-2 g}{\left(\rho^{+}+\rho^{-}\right) L}\left(\int_{-L / 2}^{+L / 2}\left(\alpha^{+}(x) \rho^{+}+\alpha^{-}(x) \rho^{-}\right) x \mathrm{~d} x\right. \\
\left.-\int_{-L / 2}^{0} \rho^{-} x \mathrm{~d} x-\int_{0}^{+L / 2} \rho^{+} x \mathrm{~d} x\right) .
\end{array}
$$

- Mean kinetic energy:

$$
\begin{aligned}
K_{M} & =\int_{-L / 2}^{+L / 2} \bar{\rho}\left(U_{x}\right)^{2} / 2 \mathrm{~d} x / \int_{-L / 2}^{+L / 2} \bar{\rho} \mathrm{d} x \\
& \approx \frac{1}{15} \mathcal{A}^{2}\left(\delta U_{x}\right)^{2} \quad \text { for } \mathcal{A} \rightarrow 0 .
\end{aligned}
$$

- Directed kinetic energy:

$$
\begin{aligned}
& K_{D}= \int_{-L / 2}^{+L / 2}\left[\alpha^{+} \rho^{+}\left(U_{x}^{+}\right)^{2}+\alpha^{-} \rho^{-}\left(U_{x}^{-}\right)^{2}\right. \\
&\left.\quad-\bar{\rho}\left(U_{x}\right)^{2}\right]_{2} \mathrm{~d} x / \int_{-L / 2}^{+L / 2} \bar{\rho} \mathrm{d} x \\
& \approx \frac{1}{12}\left(\delta U_{x}\right)^{2}=\frac{1}{48}\left(L^{\prime}\right)^{2} \quad \text { for } \mathcal{A} \rightarrow 0 .
\end{aligned}
$$

$K_{M}$ will thus be ignored. 
II.3 Basic self-similar growth laws

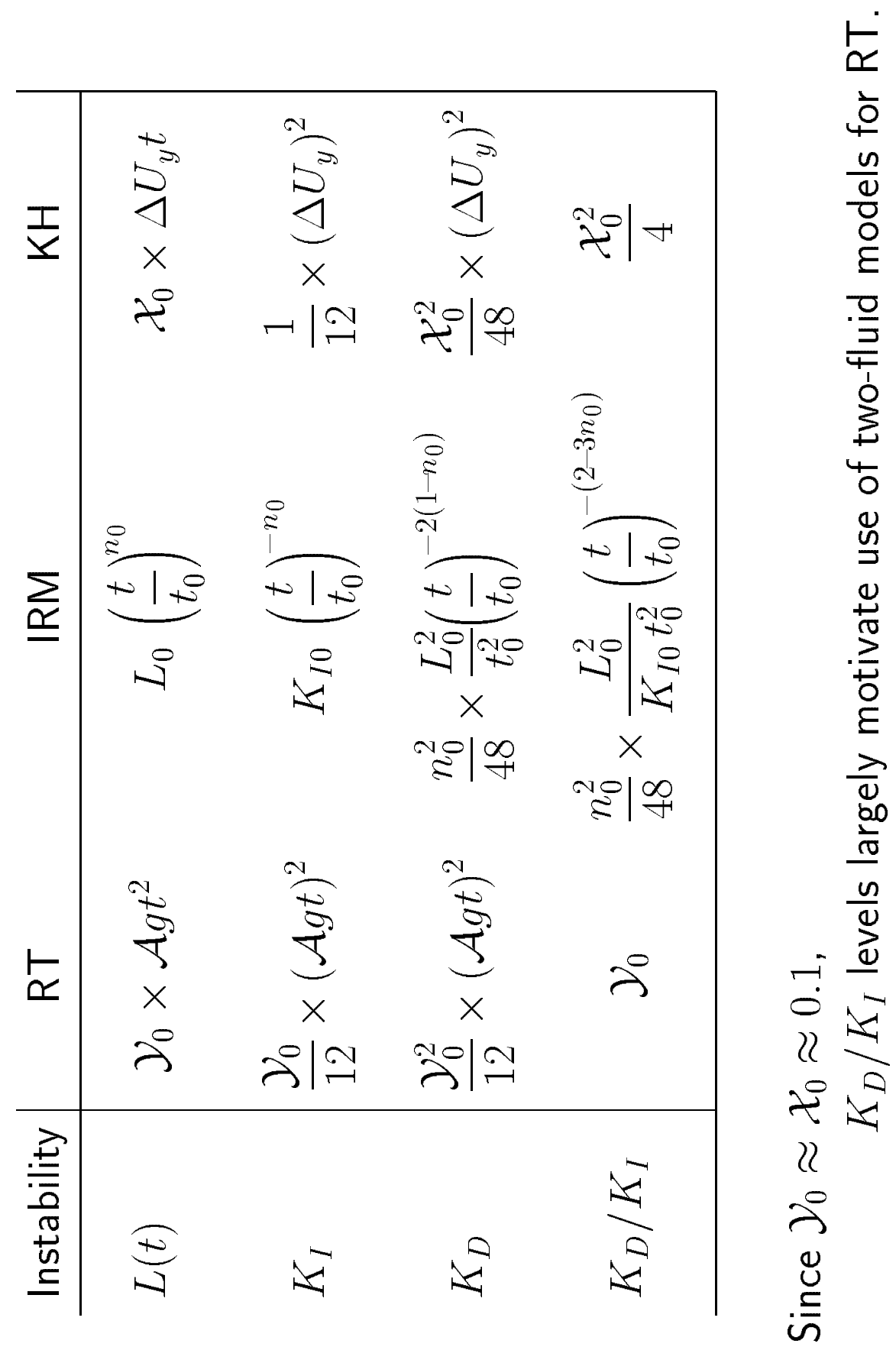


II.4 Energy balance

The knowledge of the total kinetic energy in TMZ, $K$, obtained from experiments or simulations, completes balance and provides dissipation, $E$.

The ratio of integral length scale to TMZ width:

$\kappa_{T}=\frac{K_{T}^{3 / 2}}{E L}$,

will be called Von Kármán number

by analogy with the Von Kármán Constant of turbulent boundary layers, with the two-fluid turbulence defined as:

$K_{T}=K-K_{D}$

\begin{tabular}{l|ccc|} 
Instability & $\mathrm{RT}$ & $\mathrm{IRM}$ & $\mathrm{KH}$ \\
\hline Growth coefficient & $\mathcal{Y}_{0}=0.12$ & $n_{0}=0.3$ & $\mathcal{X}_{0}=0.1$ \\
$K_{T} / K_{D}$ & 3 & 58 & 116 \\
$\kappa_{T}$ & 0.09 & 0.3 & 0.63 \\
\hline
\end{tabular}

see references in Llor 2001.

The Von Kármán number is 7 times smaller in RT than in $\mathrm{KH}$ : assuming there is one large eddy in $\mathrm{KH} \mathrm{TMZ \text {, }}$ there are seven large eddies in RT TMZ !

(confirmed in experiments or simulations, Dimonte 2000, Dalziel 1999, Inogamov 2001...) 


\section{Results from "OD" averaged models}

\section{III.1 "OD" averaging of model PDEs}

From equation for hydro quantity $a$ in model:

$\frac{\partial}{\partial t} a+\left(\phi_{x}^{a}\right)_{, x}=s^{a}$,

averaging over TMZ width, $A=\langle a\rangle$, yields:

$\frac{1}{L} \frac{\mathrm{d}(L A)}{\mathrm{d} t}=\frac{\mathrm{d} A}{\mathrm{~d} t}+\frac{\mathrm{d} L}{L \mathrm{~d} t} A=S^{a}$,

since (usually) $\phi_{x}^{a}=0$ at edges of TMZ.

Supplementary asumptions/approximations are needed to provide closed expression of $S^{a}$.

For instance, center of $\mathrm{TMZ}$ is often dominant, so:

$S^{a} \approx \frac{1}{\zeta} s^{a}(\zeta B)$,

if $s^{a}=s^{a}(b)$ and defining $\zeta \approx a(0) / A \approx b(0) / B \approx 3 / 2$.

Models are thus reduced from PDEs to ODEs.

Two models considered here:

- Andronov's modified single-fluid $k-\varepsilon$ model (1979),

- Youngs' two-fluid model (1989). 
III.2 PDEs of modified $k-\varepsilon$ and two-fluid models

Basic relevant PDEs for Andronov's modified $k-\varepsilon$ :

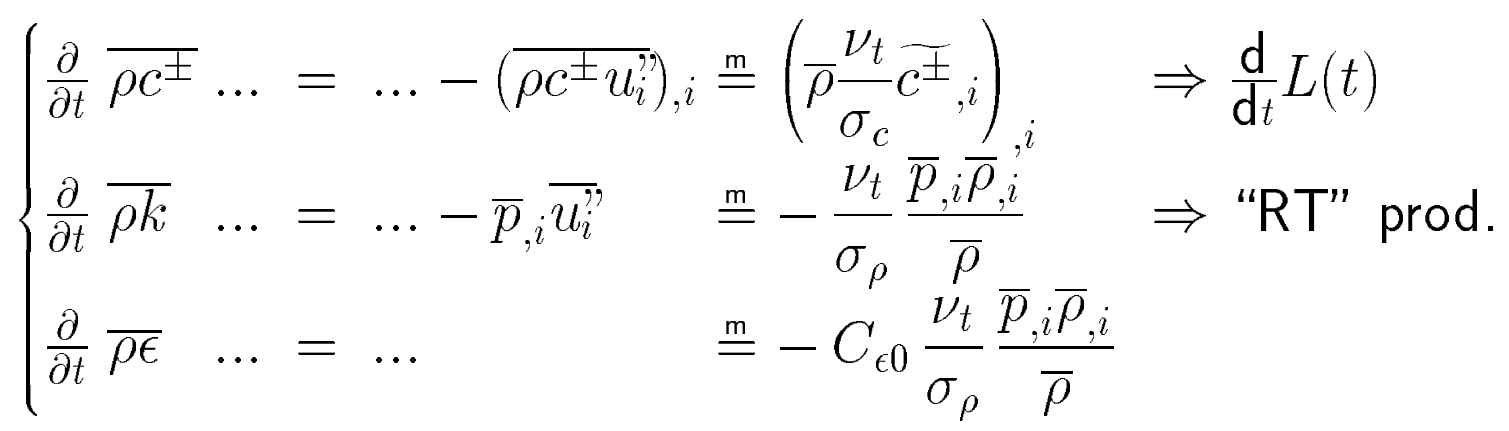

with $\sigma_{\rho} \approx 2$ and $C_{\epsilon 0} \approx 0.85$

(other constants as usual).

Basic relevant PDEs for Youngs' two-fluid:

$$
\begin{aligned}
& \left\{\begin{array}{l}
\frac{\partial}{\partial t}\left(\alpha^{ \pm} \rho^{ \pm}\right) \quad \ldots=\ldots \\
\frac{\partial}{\partial t}\left(\alpha^{ \pm} \rho^{ \pm} U_{i}^{ \pm}\right) \ldots=\ldots-\left(R_{i j}\right)_{j} \mp D_{i}\left(\lambda_{d}\right) \quad \Rightarrow \frac{\mathrm{d}}{\mathrm{d} t} L(t) \\
\frac{\partial}{\partial t}\left(\bar{\rho} k_{b}\right) \quad \ldots=\ldots \pi_{\mathrm{KH}}+D_{i} \delta U_{i} \\
\frac{\partial}{\partial t} \lambda_{d} \quad \ldots=\ldots \sqrt{\frac{2 \bar{\rho}}{\rho^{+}+\rho^{-}}} n_{i} \delta U_{i} \quad \Rightarrow \text { Length scale }
\end{array}\right. \\
& D_{i} \stackrel{\mathrm{m}}{=} C_{d} \bar{\rho} \frac{\alpha^{+} \alpha^{-}}{\lambda_{d}}\left\|U_{i}^{+}-U_{i}^{-}-\vec{W}\right\|\left(U_{i}^{+}-U_{i}^{-}-W_{i}\right)+a . m .+\ldots \\
& \nu_{t}=C_{\mu} \sqrt{k_{b}} \lambda_{i}, \\
& \text { with } C_{d} \approx 20 \text { and } C_{i} \approx 0.105 \\
& C_{\mu} \lambda_{d},
\end{aligned}
$$


III.3 ODEs of modified $k-\varepsilon$ and two-fluid models

"OD" ODEs for Andronov's modified $k-\varepsilon$ :

$$
\left\{\begin{array}{l}
\frac{\mathrm{d}}{\mathrm{d} t} L=\frac{8 \zeta C_{\mu}}{\sigma_{c}} \frac{K^{2}}{E L}, \\
\frac{\mathrm{d}}{\mathrm{d} t}=-\frac{\mathrm{d} L}{L \mathrm{~d} t} K+\Pi_{K}-E \\
\frac{\mathrm{d}}{\mathrm{d} t} E=-\frac{\mathrm{d} L}{L \mathrm{~d} t} E+C_{\epsilon \pi} \frac{E}{K} \Pi_{K}-C_{\epsilon 2} \frac{E^{2}}{K}
\end{array}\right.
$$

$$
\Pi_{K}^{\mathrm{RT}} \approx \frac{2 C_{\mu}}{\sigma_{\rho}} \frac{K^{2}}{E L} \mathcal{A} \Gamma, \quad \Pi_{K}^{\mathrm{KH}} \approx C_{\mu} \frac{K^{2}}{E}\left(\frac{\Delta U_{y}}{L}\right)^{2} .
$$

"OD" ODEs for Youngs' two-fluid:

$$
\left\{\begin{array}{l}
\frac{\mathrm{d}}{\mathrm{d} t} L=4 \sqrt{3 K_{D}} \\
\frac{\mathrm{d}}{\mathrm{d} t} K_{D}=-\frac{\mathrm{d} L}{L \mathrm{~d} t} K_{D}-\Pi_{D}+\frac{2}{3} \mathcal{A} \Gamma \sqrt{3 K_{D}} \\
\frac{\mathrm{d}}{\mathrm{d} t} K_{T}=-\frac{\mathrm{d} L}{L \mathrm{~d} t} K_{T}+\Pi_{D}-\frac{2 C_{\mu}}{C_{i}} \sqrt{\zeta} \frac{K_{T}^{3 / 2}}{L}
\end{array}\right.
$$

$\Pi_{D}=\left[\frac{C_{d}}{2 L}\left(2 \sqrt{3 K_{D}}-4 C_{i} \sqrt{\zeta K_{T}}\right)^{2}+\frac{\mathrm{d}}{\mathrm{d} t} \frac{\sqrt{3 K_{D}}}{4}+\frac{3 K_{D}}{2 L}\right] \frac{2 \sqrt{3 K_{D}}}{\zeta}$.

$\mathrm{KH}$ more complex but can be treated in similar way. 
III.4 Self-similar solutions of model ODEs

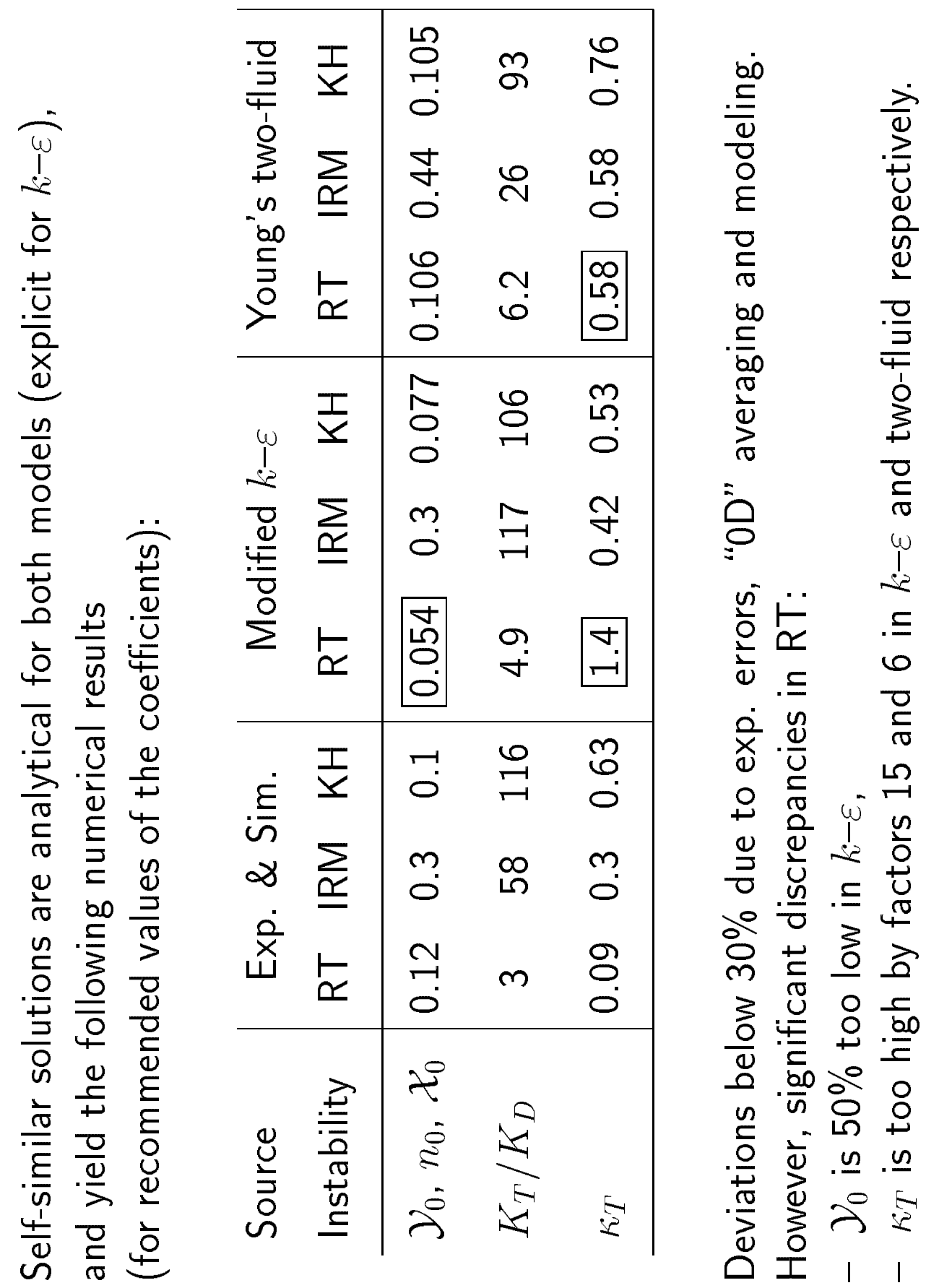




\section{III.5 Conclusion on models}

Inconsistencies for $k-\varepsilon$ due to purely diffusive TMZ growth.

In $k-\varepsilon$, directed transport in RT

is replaced by enhanced diffusion (sufficient for $\mathrm{KH}$ and IRM).

$L(t)$ equation in (10) for RT case can be rewritten as:

$1=\frac{2 \zeta C_{\mu}}{\sigma_{c}} \kappa \sqrt{\frac{K}{3 K_{D}}}$,

which yields unphysically small value $\sigma_{c} \approx 10^{-2}$, for known estimates of $\kappa$ and $\sqrt{K / K_{D}}$.

In two-fluid model, production term of $\lambda$ is $\propto \delta U$ ( $\approx$ uniform over TMZ). Therefore, $\kappa_{T}=\langle\lambda\rangle / L=C_{i} /\left(2 C_{\mu}\right) \approx 0.58$ is constant.

Variations in $\kappa$ cannot be reproduced; but it has little consequence since

diffusion is properly captured in IRM and $\mathrm{KH}$, whereas transport is dominated by directed energy in RT. 
IV Expanding horizons: SSVARTs

IV.1 Definition and usefulness of SSVARTs

Self-Similar Variable Acceleration Rayleigh-Taylor flows, or SSVARTs, defined by acceleration profiles:

$$
\begin{array}{ll}
\text { if } n>-2, g \propto t^{n} \quad \text { for } t>0, \\
\text { if } n<-2, g \propto(-t)^{n} \text { for } t<0 .
\end{array}
$$

Since $L(t) \propto t^{n+2}$, growth of $L(t)$ demands the $-t$ inversion for $n<-2$.

May be out of reach from experiments, but can be studied numerically (Youngs, this conference).

Will be assumed to exist as Reynolds (ensemble) averages.

Response will be:

$L(t)=\mathcal{Y}_{n} \mathcal{A} g(t) t^{2} \quad$ where $\quad \mathcal{Y}_{n}>0$.

Testing models in variable acceleration is difficult, but SSVARTs are helpful in testing robustness and accuracy, since they represent a "basis" set of all possible $g(t)$. 


\section{IV.2 Stability of SSVARTs}

Self-similar $g(t)$ can always be considered, but response of system is VART that may not be SSVART.

For stability (and existence),

a SSVART must be an attractor of motion and eventually forget its initial condition.

Behavior of input energy provides criterion:

- $g(t)$ energy input from $t_{0}$ to $t$ behaves as $K_{I} \propto( \pm t)^{2 n+2}$,

- initially present energy at $t_{0}$ is dilluted as $K_{0} \propto( \pm t)^{-(n+2)}$.

Therefore, initial condition is not forgotten if:

$K_{0} / K_{I} \rightarrow \infty$ for $\pm t \rightarrow \infty$ or 0 ,

$$
\text { which yields }-2<n<-4 / 3 \text {. }
$$

Since $K_{0}$ is both diluted and dissipated during growth, the forbiden interval is less restrictive :

$-2<n<-2+n_{0}$,

Within this range of "forbidden" values, SSVARTs eventually behave as Richtmyer-Meshkov, $L(t) \propto t^{n_{0}}$ (the evolution is dominated by the initial energy). 
IV.3 SSVARTs as seen by "OD" models

- For Andronov's modified $k-\varepsilon$ :

$$
\mathcal{Y}_{n}=\frac{2 C_{\mu}}{\sigma_{\rho}} \frac{\left(C_{\epsilon 2}-C_{\epsilon 0}\right)^{2}}{\left[\left(3 C_{\epsilon 2}-3\right) n+\left(4 C_{\epsilon 2}-3\right)\right]\left[\left(3 C_{\epsilon 0}-3\right) n+\left(4 C_{\epsilon 0}-3\right)\right]} .
$$

- For Youngs' two-fluid model:

$$
\mathcal{Y}_{n}=\frac{1 /(1+n / 2)}{C_{d}\left(1-\sqrt{2} C_{i} \mathcal{U}_{n}\right)^{2}(1+n / 2)+3(1+3 n / 4) / 2},
$$

where $\mathcal{U}_{n}=\sqrt{K_{T} / K_{D}}$ is largest real solution of:

$$
\begin{aligned}
C_{\mu} \mathcal{U}_{n}^{3} & -4 \sqrt{2} C_{i}\left[2 C_{i}^{2} C_{d}-\frac{4+3 n}{2(2+n)}\right] \mathcal{U}_{n}^{2} \\
& +16 C_{i}^{2} C_{d} \mathcal{U}_{n}-2 \sqrt{2} C_{i}\left[C_{d}+\frac{4+3 n}{2(2+n)}\right]=0
\end{aligned}
$$

- For scaled free fall (obtained by canceling $\Pi$ in two-fluid $K_{D}$ equation):

$$
\mathcal{Y}_{n}=\frac{\mathcal{Y}_{0}}{(1+n / 2)(1+3 n / 4)} \text {, }
$$

- For Read's formula $\left(L \propto \mathcal{A}\left(\int \sqrt{g}\right)^{2}\right)$ :

$$
\mathcal{Y}_{n}=\frac{\mathcal{Y}_{0}}{(1+n / 2)^{2}}
$$


All models generate two main poles for $\mathcal{Y}_{n}$.

$k-\varepsilon$ provides negative values outside pole interval $[-1.7,0.89]$ for recommended values of $\sigma_{\rho}$ and $C_{\epsilon 0}$.

Positive values outside interval $[-2,-1.7]$ requires $C_{\epsilon 0} \geq 3 / 2$, but then $\mathcal{Y}_{0} \approx 10^{-3}$ !

Again $k-\varepsilon$ cannot capture directed transport

which is enhanced as $n$ is increased.

Behavior of all other models is similar (common $n=-2$ pole) except near poles associated with IRM growth coefficients:

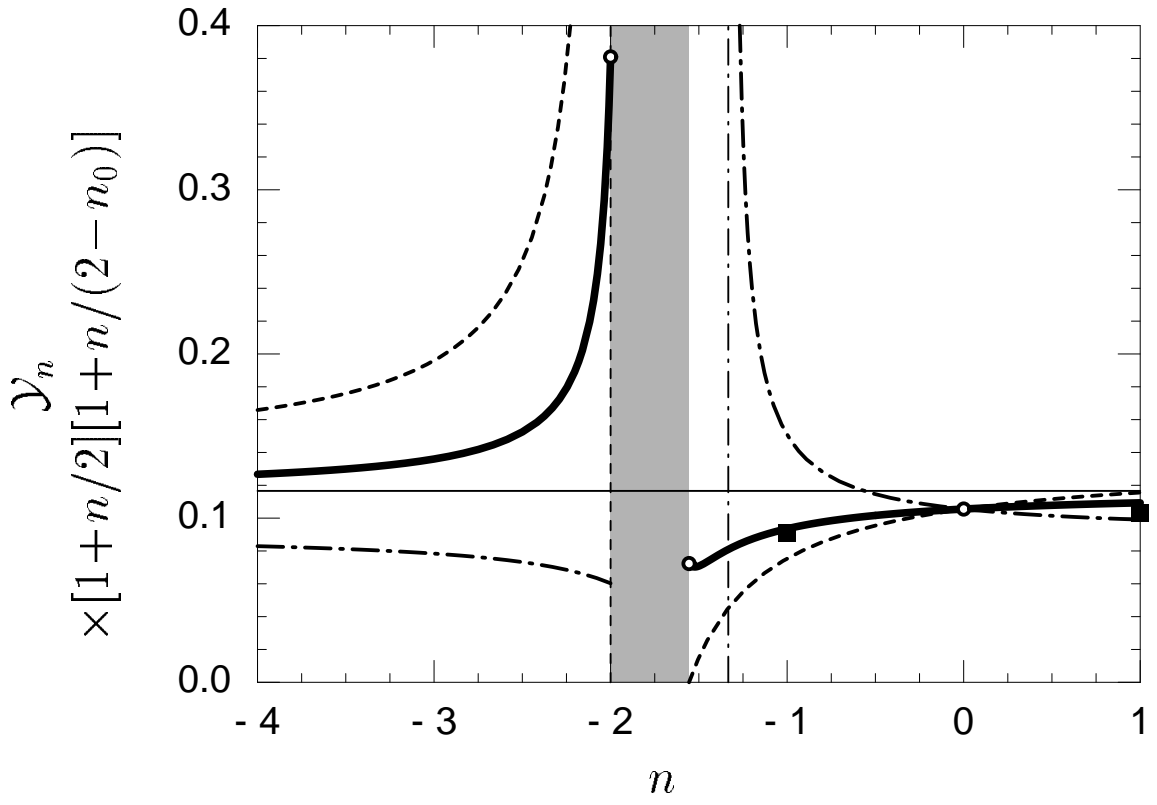

Normalized growth rates of various models:

Youngs' two-fluid (dot-dashed), free fall (dashed) Read's formula (dotted), and Youngs' preliminary simulations presented at this conference (filled squares). 
Free fall and Read's formula respectively provide

highest $(n=-4 / 3)$ and lowest $(n=-2)$ positions

of "IRM" pole.

Youngs' two-fluid model is intermediate

between free fall and Read's formula.

Free fall and Read's formula are good approximations around RT, but can diverge severly in other regimes.

Three continuously connected regimes are found:

$-n_{0}-2<n<-1$ :

directed transport is dominated by diffusion,

$--1<n$ and $n<-5 / 2$ :

directed transport is limited by turbulent viscosity,

$--5 / 2<n<-2$ :

directed transport is limited by free fall.

Single-fluid models seem limited to first regime, approximate models are accurate to $30 \%$ over second regime, but only two-fluid approaches can capture all three. 


\section{Conclusions}

RANS models can efficiently be analysed as "OD" averages, without heavy numerical developments.

Such analysis show importance, of energy balance and Von Kármán number in characterizing transport and turbulence structure.

Two-fluid approaches seem superior to single-fluid approaches in capturing directed transport with robust behaviour.

SSVARTs have been shown to provide a useful set of benchmark flows for models.

Appropriate capture of SSVARTs seems to require two-fluid model.

RT and IRM are limiting cases of SSVARTs.

SSVARTs are currently being investigated by DNS/LES.

Extention of SSVARTs to complex $n$ ? 


\title{
Acknowledgements
}

\section{Benoît Dejardins, Philippe Mignon, Eric Van Renterghem...}

\author{
(Commissariat à l'Energie Atomique, Bruyères-le-Châtel, France),
}

David L. Youngs (Atomic Weapons Establishment, United Kingdom),

\section{References}

V.A. Andronov, S.M. Bakhrakh, V.N. Mokhov, V.V. Nikiforov, and A.V. Pevnitskii, Pis'ma Zh. Eksp. Teor. Fiz. 1, 62 (1979) and Sov. Phys. JETP Lett. 29, 57 (1979); M. Bonnet, S. Gauthier, and P. Spitz, in Proceedings of the 1st International Workshop on the Physics of Compressible Turbulent Mixing, Princeton, New Jersey, October 1988, edited by W.P. Dannavik, A.C. Buckingham, and C.E. Leith, LLNL Report Conf-8810234, 1992; M. Bonnet, and S. Gauthier, Phys. Fluids A 2, 1685 (1990).

S.B. Dalziel, P.F. Linden, and D.L. Youngs, J. Fluid Mech. 399, 1 (1999).

G. Dimonte, and M. Schneider, Phys. Fluids 12, 304 (2000).

N.A. Inogamov, A.M. Oparin, A.Yu. Dem'yanov, L.N. Dembitskii, and V.A. Khokhlov, J. Exp. Theor. Phys. 92, 715 (2001).

A. Llor, CEA Report in print R-5983, France, 2001.

D.L. Youngs, Physica D37, 270 (1989).

D.L. Youngs, and A. Llor, This conference. 


\section{Nonlinear Evolution of an Interface in the Richtmyer-Meshkov Instability}

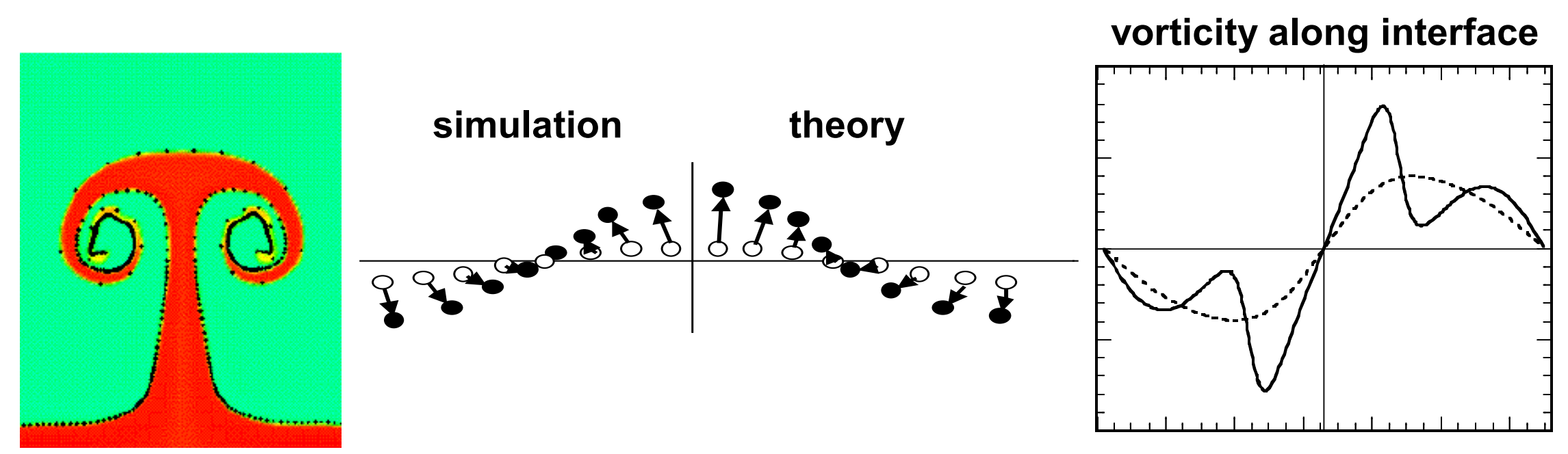

K. Nishihara, C. Matsuokaa, Y. Fukuda Institute of Laser Engineering, Osaka University

18 IWPCTM

a) Facluty of Science, Ehime University

Dec. 10-14, 2001

Pasadena, USA 


\section{Introduction}

Wouchuk-Nishihara formula(1997) of RMI growth rate indicates that RM instability is driven by velocity shear left by the shocks at the interface without the impulsive gravitational acceleration.
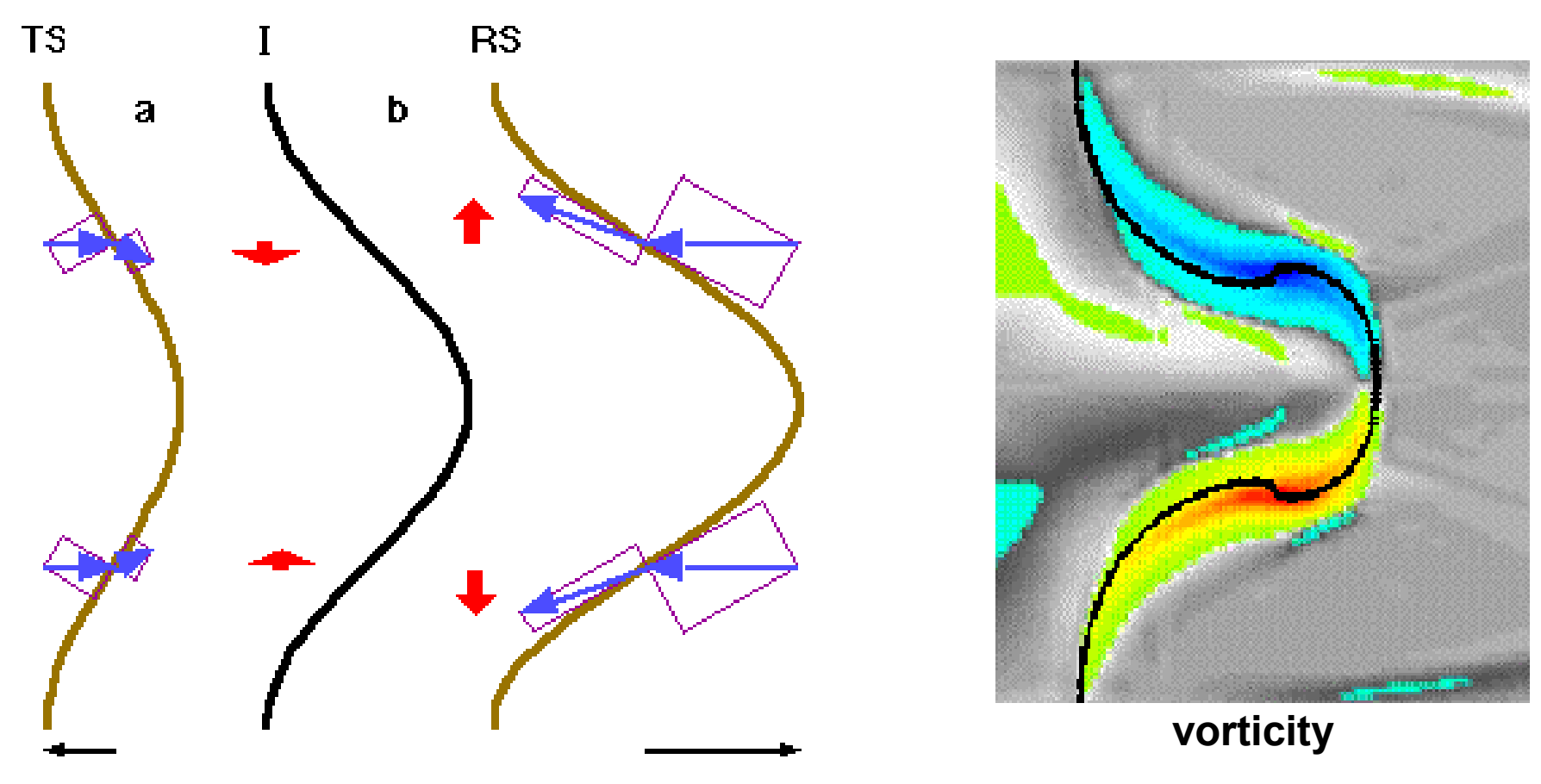

ILE-OSAKA

$$
\mathbf{v}_{\text {liu }}=\frac{\rho^{\mathbf{b}} \delta \mathbf{v}_{\mathbf{o}}^{\mathbf{b}}-\rho^{\mathbf{a}} \delta \mathbf{v}_{\mathbf{0}}^{\mathrm{a}}}{\rho^{\mathbf{a}}+\rho^{\mathbf{b}}}
$$

$$
\begin{aligned}
& \delta \mathbf{v}_{\mathbf{o}}^{\mathbf{a}}=\mathbf{k} \xi_{\mathbf{o}}\left(1-\frac{\mathbf{u}_{\mathbf{s t}}}{\mathbf{u}_{\mathbf{s i}}}\right) \mathbf{v}_{\mathbf{i}} \\
& \delta \mathbf{v}_{\mathbf{o}}^{\mathbf{b}}=\mathbf{k} \xi_{\mathbf{o}}\left(1+\frac{\mathbf{u}_{\mathbf{s r}}}{\mathbf{u}_{\mathbf{s i}}}\right)\left(\mathbf{v}_{\mathbf{l}}-\mathbf{v}_{\mathbf{i}}\right)
\end{aligned}
$$


- Nonlinear evolution of Richtmyer-Meshkov instability is described with nonlinear self-interaction of a vortex sheet with a density jump across the sheet.

- Lagrangian description of the vortex sheet reveals nonlinear dynamics, such as local stretching and shrinking of the sheet.

- Theory shows local increase and decrease of vorticity along the sheet. They results in spiral formation of spike and appearance of opposite vorticity sign at joint of mushroom umbrella, respectively.

-We have investigated dependence of nonlinear growth and nonlinear evolution of circulation on the sheet on the Atwood number and initial corrugation amplitude of the sheet. 
- Shocked interface vs vortex sheet

- Analytical model for nonlinear dynamics of vortex sheet

Lagrangian description, finite Atwood number

- Result and comparison with simulations

nonlinear growth of spike and bubble

local strethcing and shrinking of interface

local increase and decrease of vorticity along interface

dependence of nonlinear growth and circulation on interface on Atwood number and initial corrugation amplitude 
By treating the interface as a vortex sheet, fully nonlinear evolution of RM instability is described.

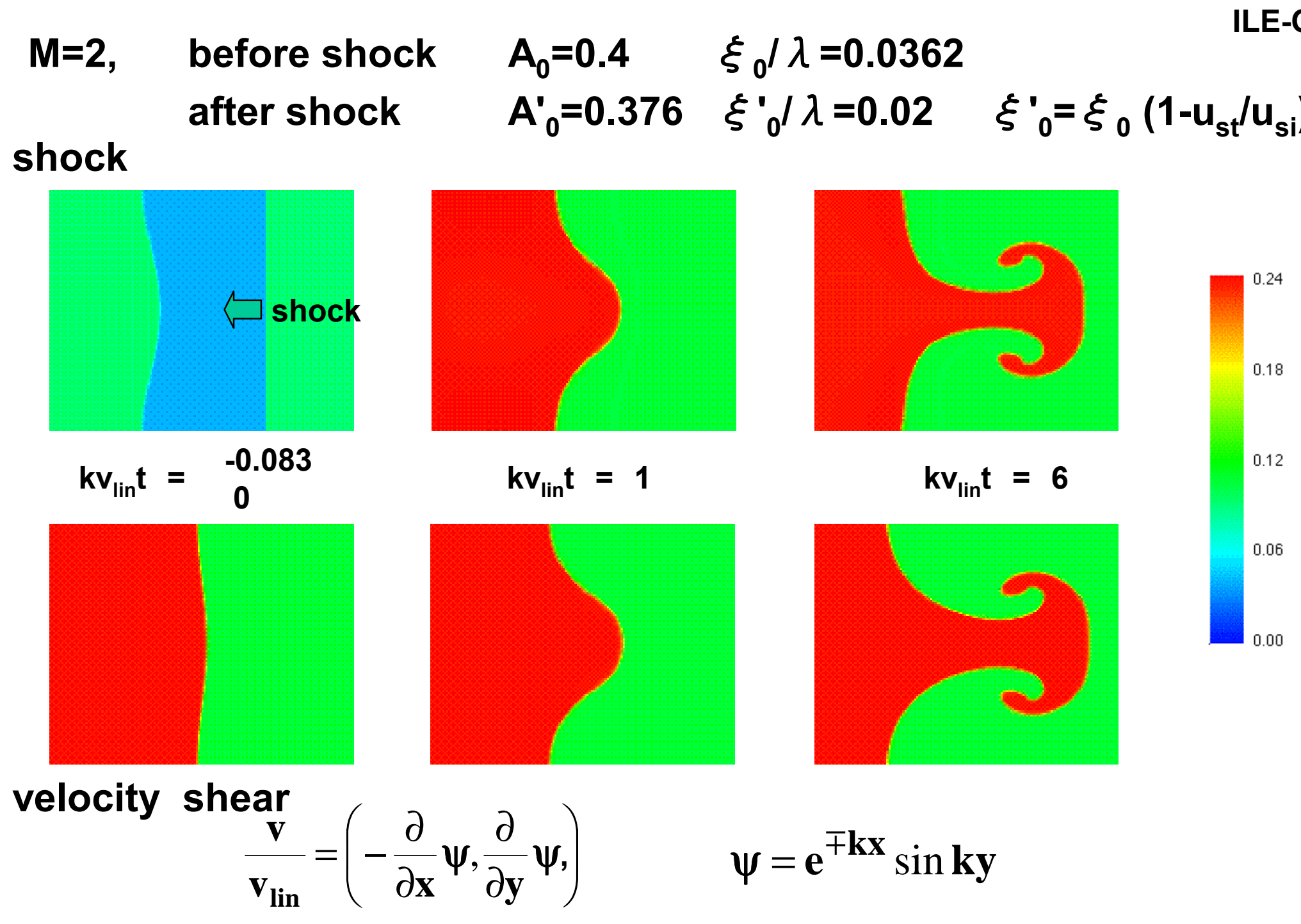


In case of finite Atwood number, baroclinic term induces vorticity on interface, and streching and shrinking of interface occurs locally in tangential direction

By introducing fluid velocity on the interface

$$
\mathbf{u}_{+} \equiv \frac{\rho_{1} \mathbf{u}_{1}+\rho_{2} \mathbf{u}_{2}}{\rho_{1}+\rho_{2}}
$$

we obtain from Euler equation

$$
\frac{\mathbf{D} \Gamma}{\mathbf{D} \mathbf{t}}=\left(\frac{\partial}{\partial \mathbf{t}}+\mathbf{u}_{+} \cdot \nabla\right) \Gamma=2 \mathbf{A}\left[\frac{\mathbf{D} \Phi}{\mathbf{D} \mathbf{t}}-\frac{1}{2} \mathbf{q} \cdot \mathbf{q}-\frac{1}{8} \boldsymbol{\kappa} \cdot \boldsymbol{\kappa}+\frac{\mathbf{A}}{2} \boldsymbol{\kappa} \cdot \mathbf{q}\right]
$$

where

$$
\begin{aligned}
& \mathbf{A}=\frac{\rho_{2}-\rho_{1}}{\rho_{2}+\rho_{1}} \quad \text { (Atwood number), } \Gamma=\phi_{1}-\phi_{2} \quad \text { (circulation), } \Phi=\phi_{1}+\phi_{2} \\
& \boldsymbol{\kappa}=\nabla \Gamma=\mathbf{u}_{1}-\mathbf{u}_{2} \text { (vorticity), } \mathbf{q}=\frac{1}{2}\left(\mathbf{u}_{1}+\mathbf{u}_{2}\right), \quad \mathbf{u}_{\mathbf{i}}=\nabla \phi_{\mathbf{i}}(\mathbf{i}=1,2)
\end{aligned}
$$

Modified Birkhoff-Rott equation becomes

$$
\frac{\partial}{\partial \mathbf{t}} \mathbf{Z}^{*}=\mathbf{u}_{+}=\mathbf{q}-\frac{\mathbf{A}}{2} \kappa=\frac{1}{2 \pi \mathbf{i}} \int \frac{\mathbf{K d s}^{\prime}}{\mathbf{Z}-\mathbf{Z}^{\prime}}-\frac{\mathbf{A}}{2} \kappa, \quad \mathbf{Z}(\mathbf{t})=\mathbf{x}+\mathbf{i} \mathbf{y} \quad \text { (position of the interface) }
$$


Nonlinear Theory of a Vortex Sheet(1): Basic Equations

Circulation on vortex sheet

$$
\frac{\mathbf{D}}{\mathbf{D t}} \Gamma=\left(\frac{\partial}{\partial \mathbf{t}}+\mathbf{u}_{+} \cdot \nabla\right) \Gamma=2 \mathbf{A}\left[\frac{\mathbf{D} \Phi}{\mathbf{D t}}-\frac{1}{2} \mathbf{q} \cdot \mathbf{q}-\frac{1}{8} \boldsymbol{\kappa} \cdot \boldsymbol{\kappa}+\frac{\mathbf{A}}{2} \boldsymbol{\kappa} \cdot \mathbf{q}\right]
$$

where

$$
\begin{aligned}
& \mathbf{A}=\frac{\rho_{2}-\rho_{1}}{\rho_{2}+\rho_{1}}, \Gamma=\phi_{1}-\phi_{2}, \Phi \equiv \phi_{1}-\phi_{2}, \\
& \mathbf{u}_{+}=\frac{\rho_{1} \mathbf{u}_{1}+\rho_{2} \mathbf{u}_{2}}{\rho_{1}+\rho_{2}}, \boldsymbol{\kappa}=\mathbf{u}_{1}-\mathbf{u}_{2}, \mathbf{q}=\frac{1}{2}\left(\mathbf{u}_{1}+\mathbf{u}_{2}\right)
\end{aligned}
$$

incompressible and irrotational fluid

$$
\nabla^{2} \phi_{\mathbf{i}}=0, \mathbf{u}_{\mathbf{i}}=\nabla \phi_{\mathbf{i}}(\mathbf{i}=1,2)
$$

kinematic boundary conditions

$$
\mathbf{r}_{\mathbf{t}} \cdot \mathbf{n}=\mathbf{u}_{+} \cdot \mathbf{n}, \quad \mathbf{r}_{\mathbf{t}} \cdot \mathbf{t}=\mathbf{u}_{+} \cdot \mathbf{t} \quad, \quad \mathbf{u}_{1} \cdot \mathbf{n}=\mathbf{u}_{2} \cdot \mathbf{n}
$$


Nonlinear Theory of a Vortex Sheet(2) : Expantion

Lagrangian marker of vortex sheet

$$
\begin{aligned}
& \mathbf{x}(\mathbf{t})=\theta+\mathbf{X}(\boldsymbol{\theta}, \mathbf{t}) \\
& \mathbf{y}(\mathbf{t})=\mathbf{Y}(\boldsymbol{\theta}, \mathbf{t})
\end{aligned}
$$

expansion

$$
\begin{aligned}
& \mathbf{X}=\sum_{\mathbf{n}} \varepsilon^{\mathbf{n}} \mathbf{X}^{(\mathbf{n})} \quad, \quad \mathbf{Y}=\sum_{\mathbf{n}} \varepsilon^{\mathbf{n}} \mathbf{Y}^{(\mathbf{n})} \\
& \phi_{1}=\sum_{\mathbf{n}} \boldsymbol{\varepsilon}^{\mathbf{n}} \phi_{\mathbf{i}}^{(\mathbf{n})} \mathbf{e}^{\mp \mathbf{n k y}} \cos \mathbf{n k x},
\end{aligned}
$$

By introducing normalization, $k x, k v_{\text {lin }} t$, and $k \phi_{i} / v_{\text {lin }}$, nonlinear evolution of a vortex sheet is determined from two parameters A (Atwood number) and $\mathrm{k} \xi_{0}$ (initial corrugation of sheet), where $\mathbf{v}_{\text {lin }}=\frac{\rho_{1} \delta \mathbf{v}_{10}-\rho_{2} \delta \mathbf{v}_{20}}{\rho_{1}+\rho_{2}}, \quad$ (Wouchuk - Nishihara formula $)$ 


$$
M=2 \quad A_{0}=0.4 \quad \xi{ }^{\prime} / \lambda=0.02
$$

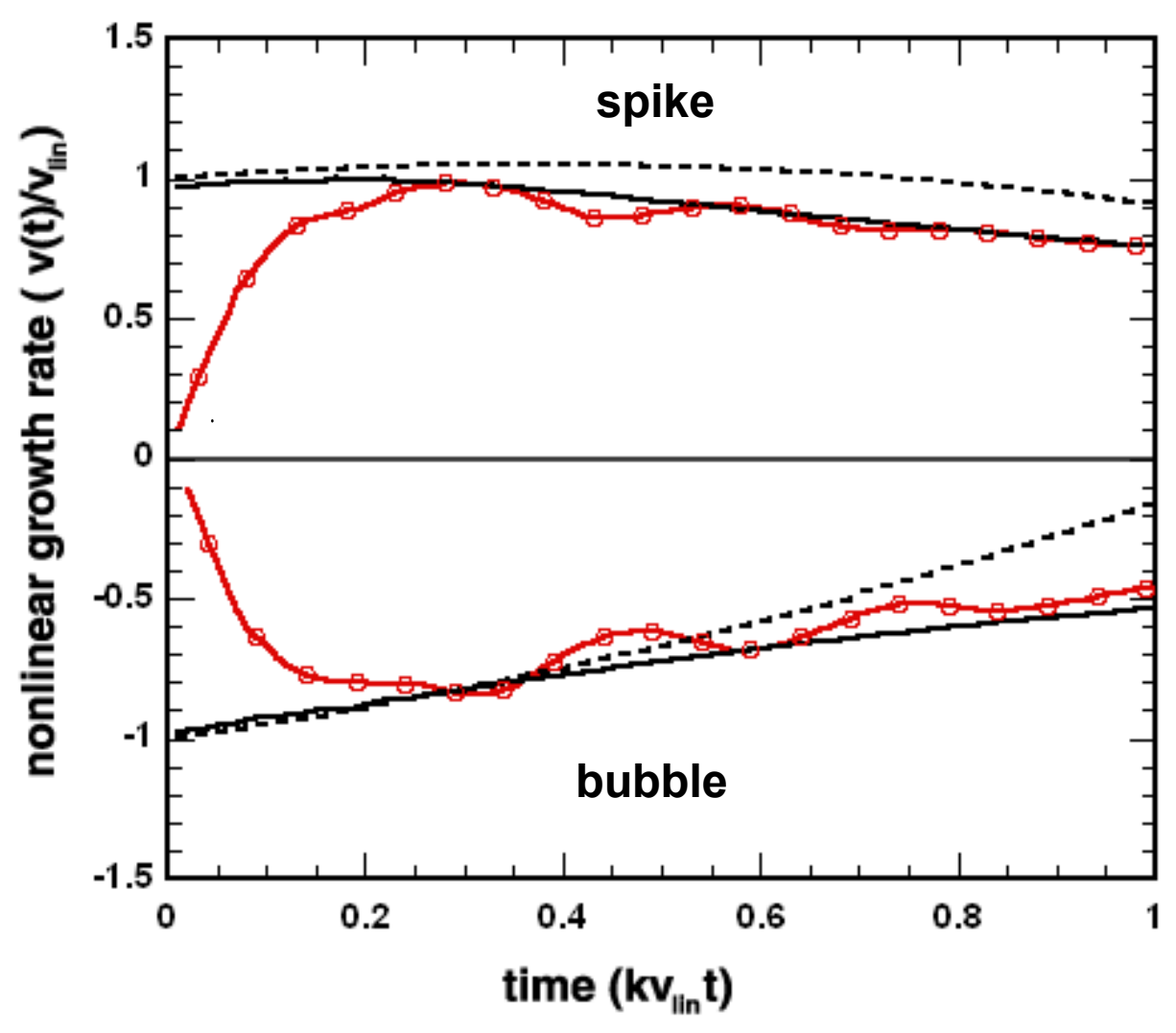

$\simeq$ Incident shock hits corrugated interface

Nonuniform velocity shear at corrugated interface

3rd order nonlinear theory of vortex sheet 

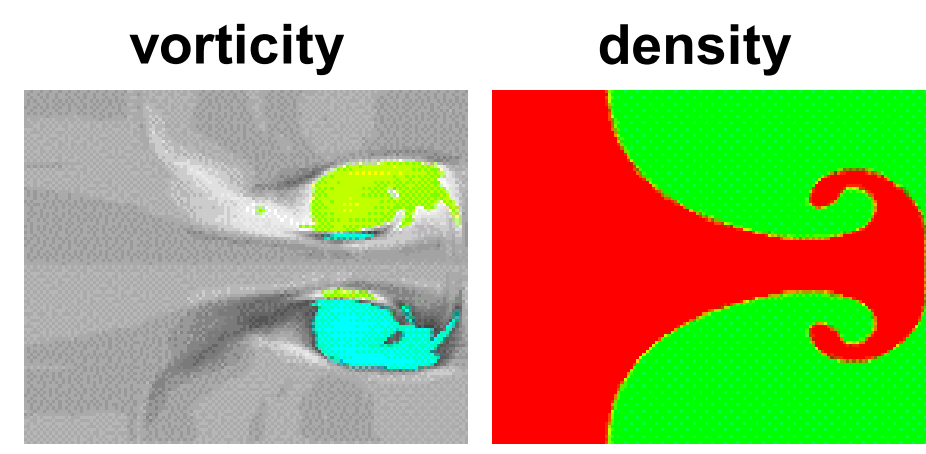

$\mathrm{A}_{0}=\mathbf{0 . 6}$

$\xi_{0}^{\prime} / \lambda=0.02$
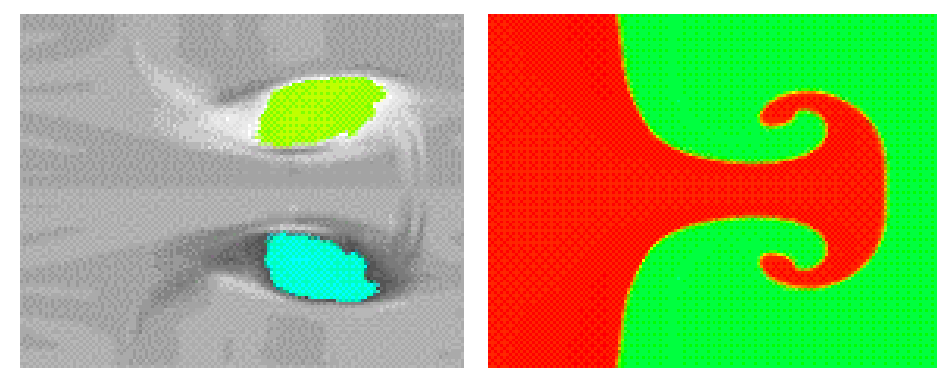

$A_{0}=0.4$

$\xi_{0}^{\prime} \prime \lambda=0.02$
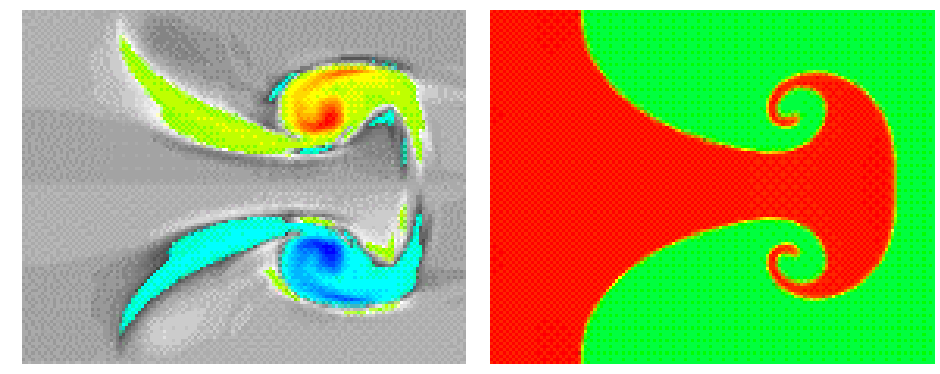

$\mathrm{A}_{0}=\mathbf{0 . 4}$

$\xi_{0}^{\prime} / \lambda=0.08$

$$
k v_{\text {lin }} t=6
$$

Larger Atwood number leads to larger nonlinear growth

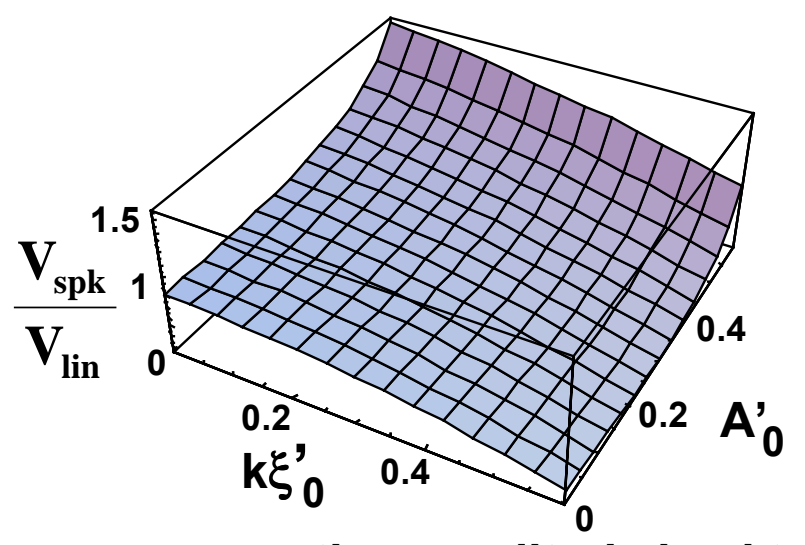

Larger corragation amplitude lead to rapid increase of circulation

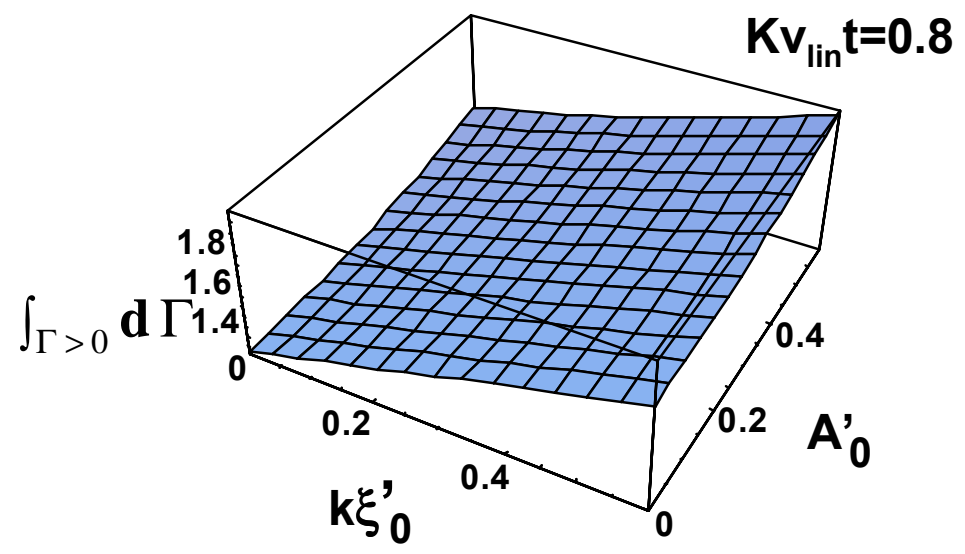


Lagrangian markers move both $\mathrm{x}$ and $\mathrm{y}$ directions. Stretch and shrink of vortex sheet occur locally.

Theoretical results agrees well with simulations

simulation

(shocked interface)

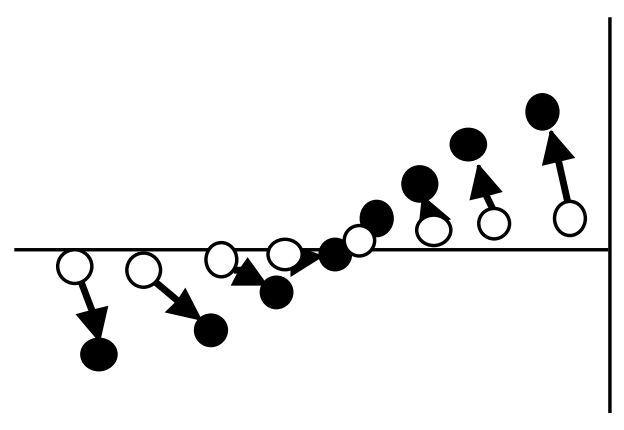

- $\mathrm{Kv}_{\text {lin }} \mathrm{t}=\mathbf{0 . 8 0}$

O $K \mathbf{v}_{\text {lin }} \mathbf{t}=\mathbf{0 . 0 5}$

$A_{0}{ }^{\prime}=0.376$

$\xi^{\prime}{ }_{0} / \lambda=0.02$

$\mathbf{M}=\mathbf{2}$ simulation

(vortex sheet)

theory

$(x(\theta, t), y(\theta, t))$
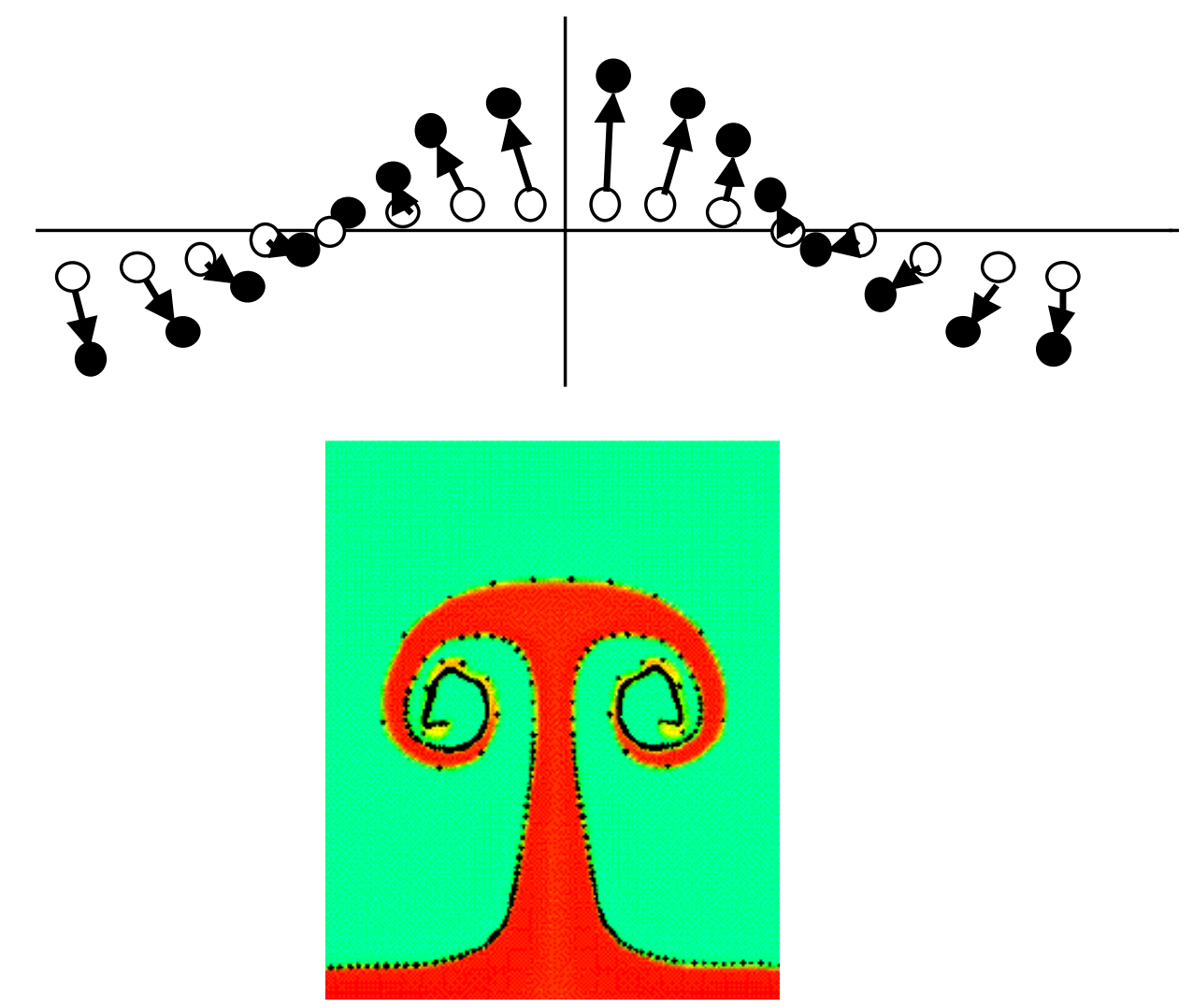
Theory shows local increase of vorticity (tip of spiral), and local decrease of vorticity ( joint of mushroom umbrella ).

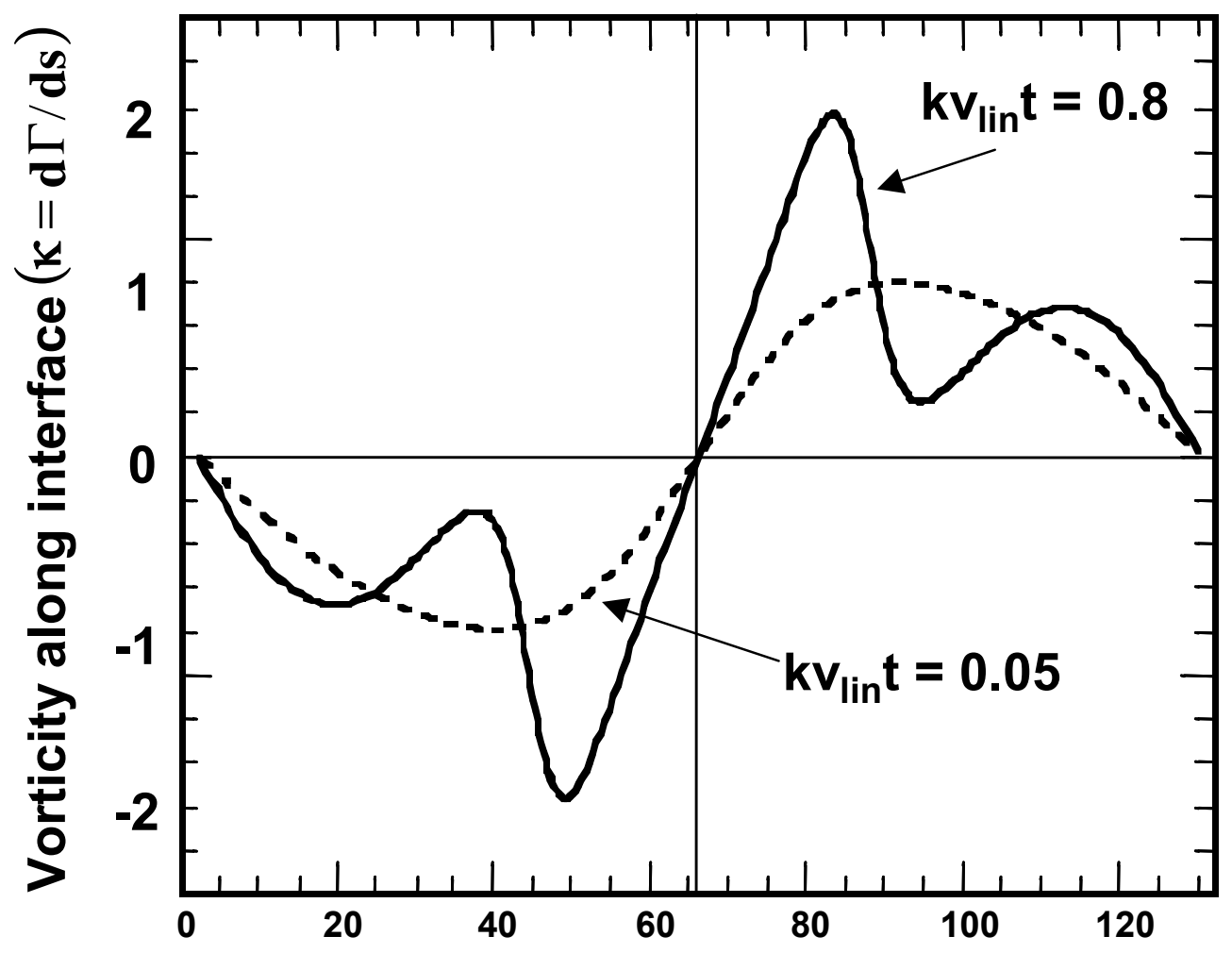

ILE OSAKA
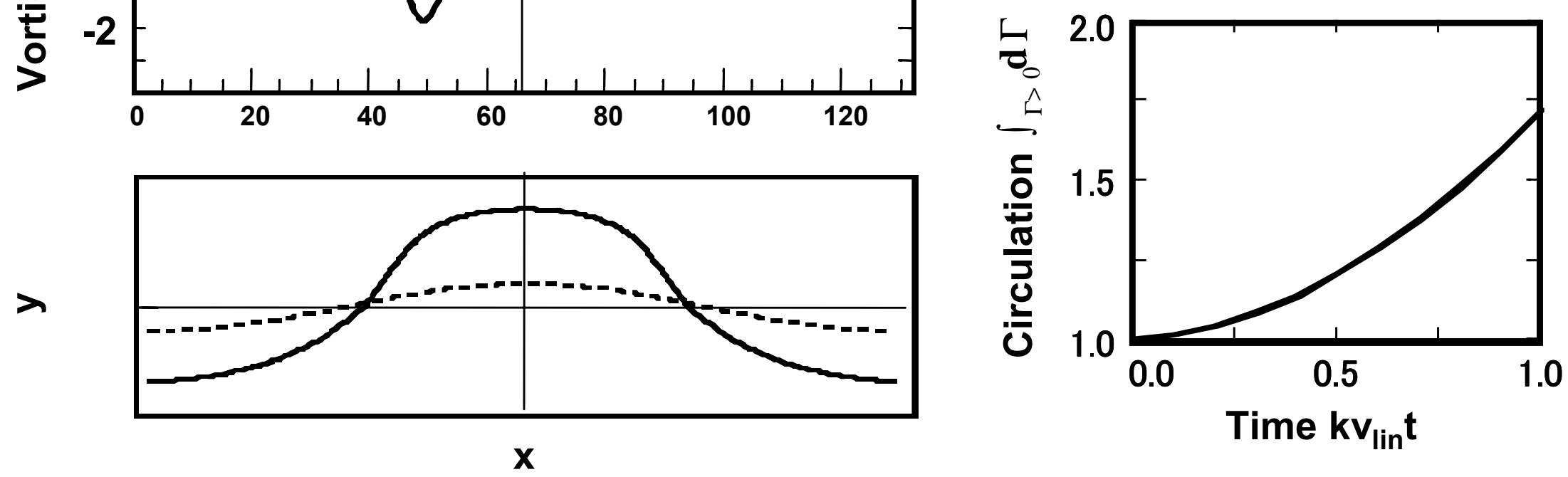
- Nonlinear evolution of Richtmyer-Meshkov instability is described with nonlinear self-interaction of a vortex sheet with a density jump across the sheet.

- Lagrangian description of the vortex sheet reveals nonlinear dynamics, such as local stretching and shrinking of the sheet.

- Theory shows local increase and decrease of vorticity along the sheet. They results in spiral formation of spike and appearance of opposite vorticity sign at joint of mushroom umbrella, respectively.

-We have investigated dependence of nonlinear growth and nonlinear evolution of circulation on the sheet on the Atwood number and initial corrugation amplitude of the sheet. 


\section{Vortex-Merger Statistical Model for the Late Time Self-Similar Evolution of the Kelvin-Helmholtz Instability}

\section{Avi Rikanati ${ }^{[1,2]}$, U. Alon ${ }^{[3]}$, Dov Shvarts ${ }^{[1,2]}$}

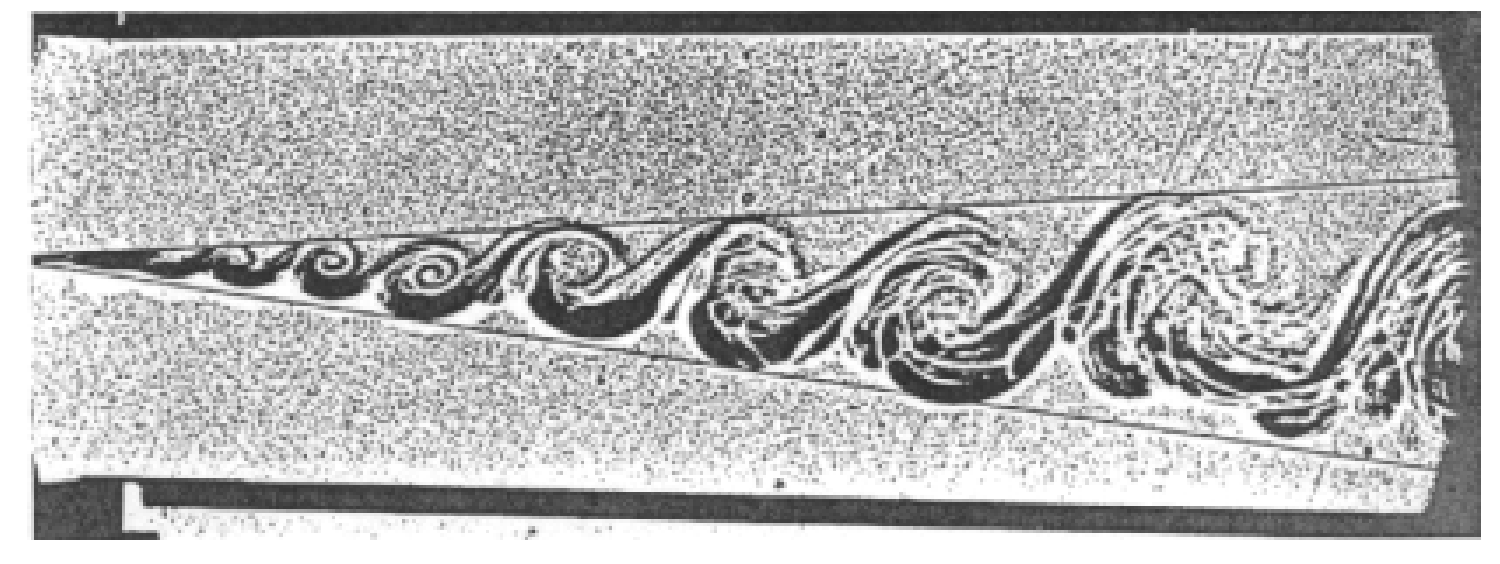

${ }^{1}$ Nuclear Research Center, Negev, ISRAEL

${ }^{2}$ Ben Gurion University, Beer-Sheva, ISRAEL

${ }^{3}$ Weizmann Institute of Science, Rehovot, ISRAEL 


\section{$\underline{\text { Abstract }}$}

The nonlinear growth of the multi-mode incompressible Kelvin-Helmholtz $(\mathrm{KH})$ shear flow instability at all density ratios is treated by a non empirical large scale statistical-mechanics eddy-pairing model, based on the single eddy behavior and the process of two eddy-pairing. From the model, a linear time growth of the mixing zone is obtained, resulting in the linear time growth coefficient for several density ratios as well as an asymptotic lognormal eddy size distribution and the average eddy life time probability. Very good agreement with previous works, full numerical simulations and experiments is achieved.

\section{Example of Shadowgraph Photography*}

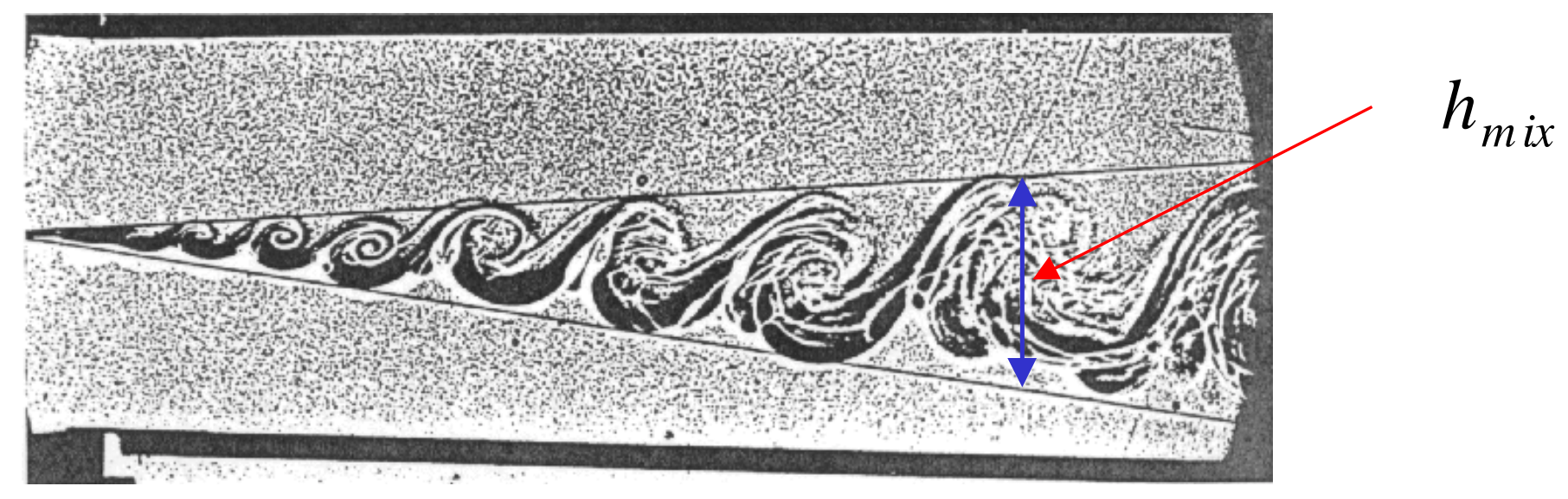

*Roshko \& Brown, J. Fluid Mech, 1974. 


\section{Numerical Simulations for the Single Mode case}

Material interface
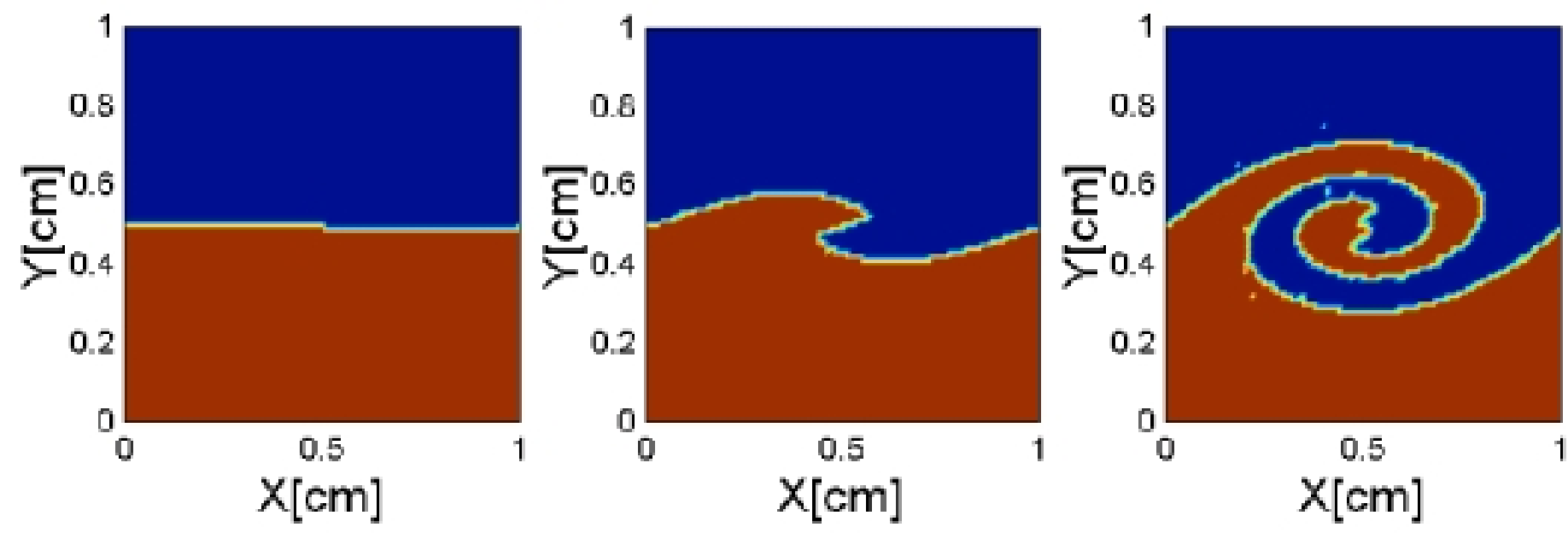

Vorticity Colormaps
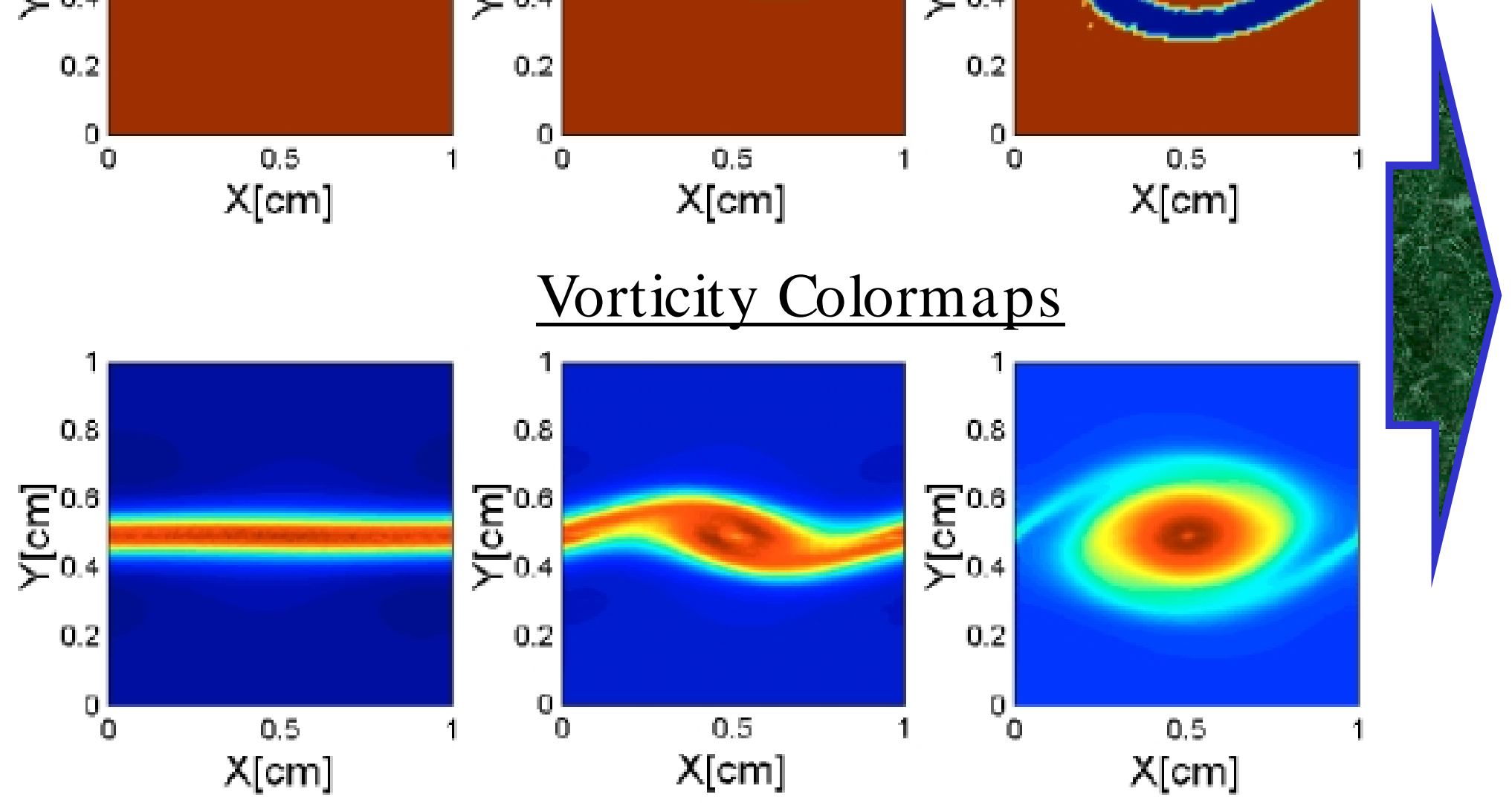

Fast

Vortex

Formation 


\section{Vortex Line - Analytical Solution}

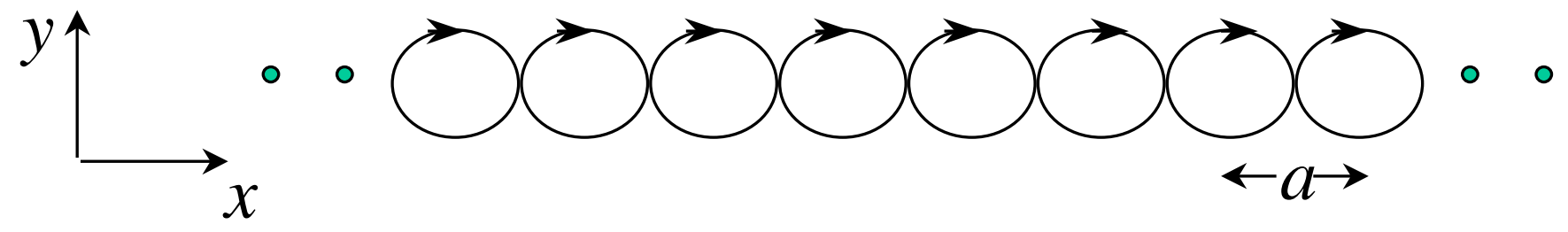

- Vorticity Definition: $\vec{\omega}=\nabla \times V$

- Vortex Strength set by Kelvin Theorem of Circulation:

$$
\Gamma=\int_{\text {Surface }} \vec{\omega} \cdot d s=\oint_{\text {Contour }} v \cdot d l=\text { Const }
$$

- Resulting Complex Potential of a Vortex Line :

$W(z)=\sum_{n=-\infty}^{\infty}\left(i \cdot \frac{\Gamma}{2 \pi}\right) \ln (z-n a)=\frac{i \cdot \Gamma}{2 \pi} \ln (\sin (\pi \cdot z / a))$

$$
(z=x+i \cdot y)
$$




\section{Vortex Model for a Single Mode Initial Perturbation}

Velocity field calculated as a derivative of the complex potential

$$
u(z)-i \cdot v(z)=d W(z) / d Z
$$

\section{$\underline{\text { Interface Evolution }}$}

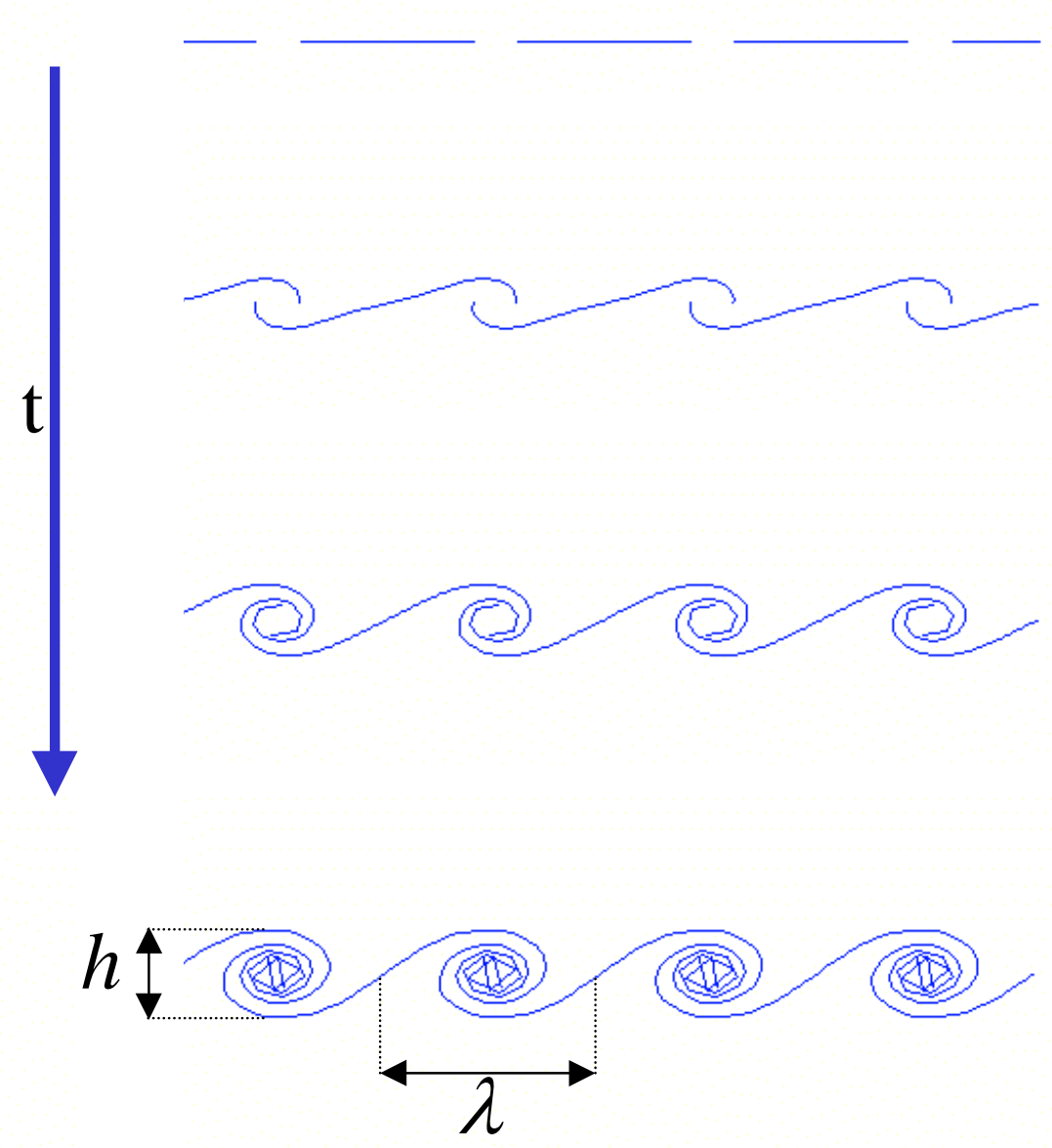

\section{Eddy Height Vs. Time*}

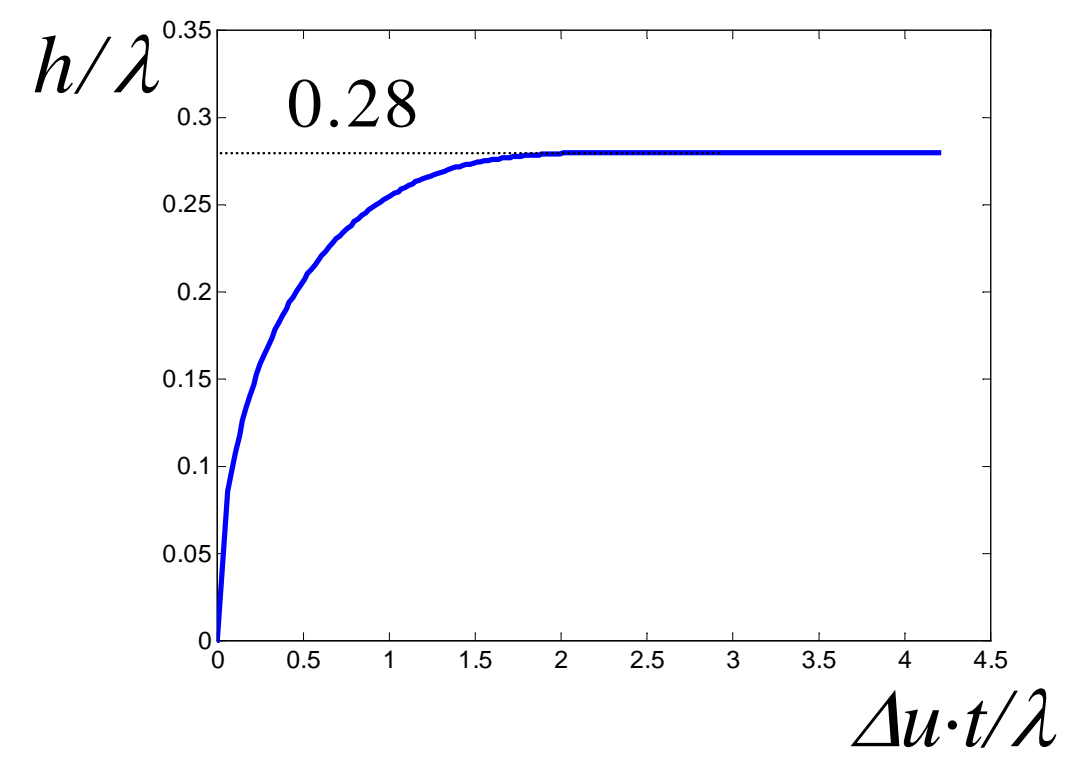

$$
h(\lambda, t \rightarrow \infty)=0.28 \lambda
$$




\section{Experimental eddies trajectories show the vortex merger dominance}

\section{$\underline{\text { Vortex Trajectories }}^{*}$}

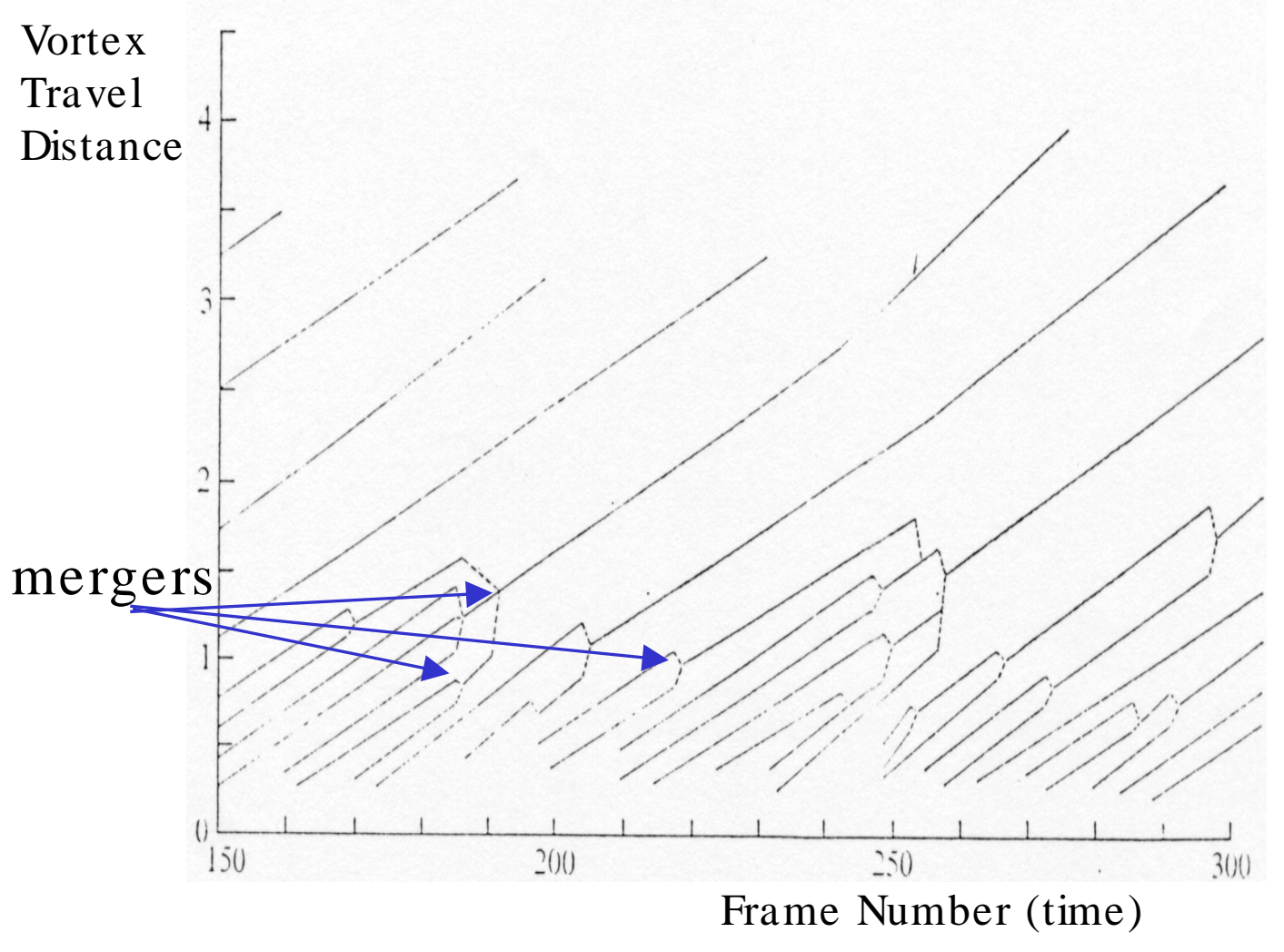

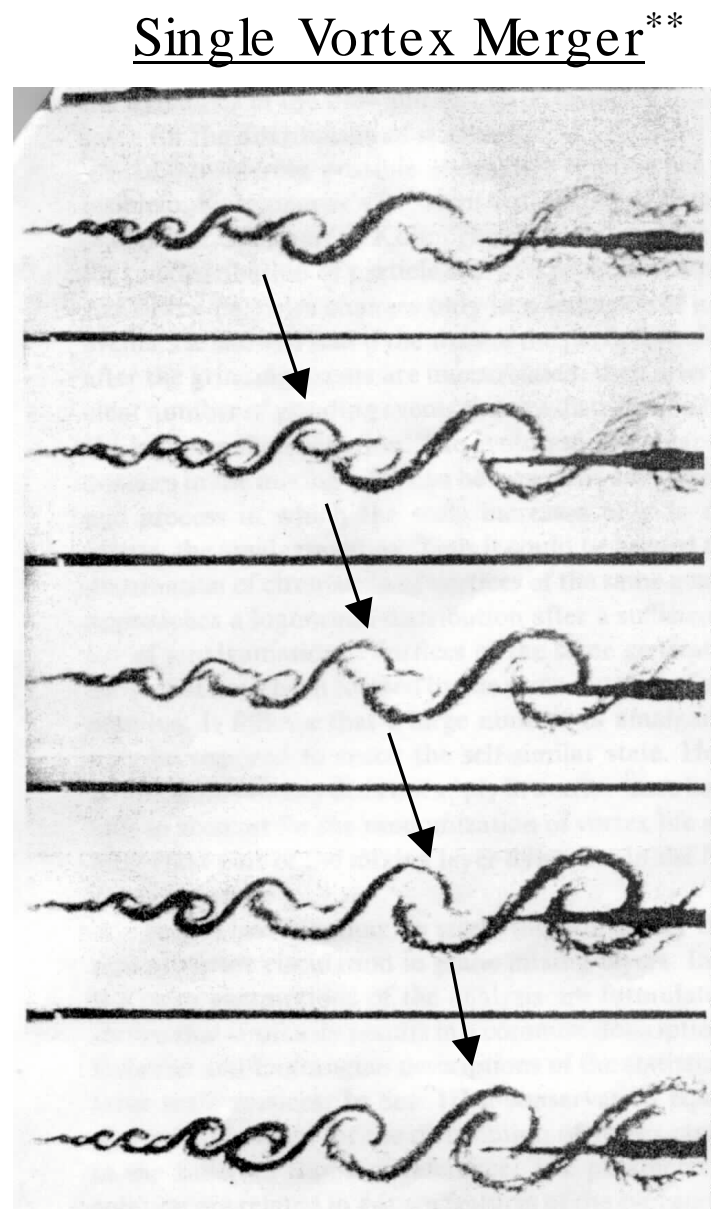

MPry

* Roshko \& Brown (1974)

${ }^{* *}$ Bernal (1988) 


\section{Two Vortex Pairing - Model Results}

\section{Two Vortex Setup}

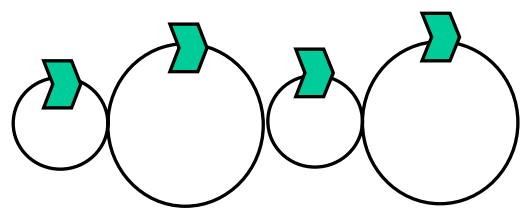

Eddies Interface
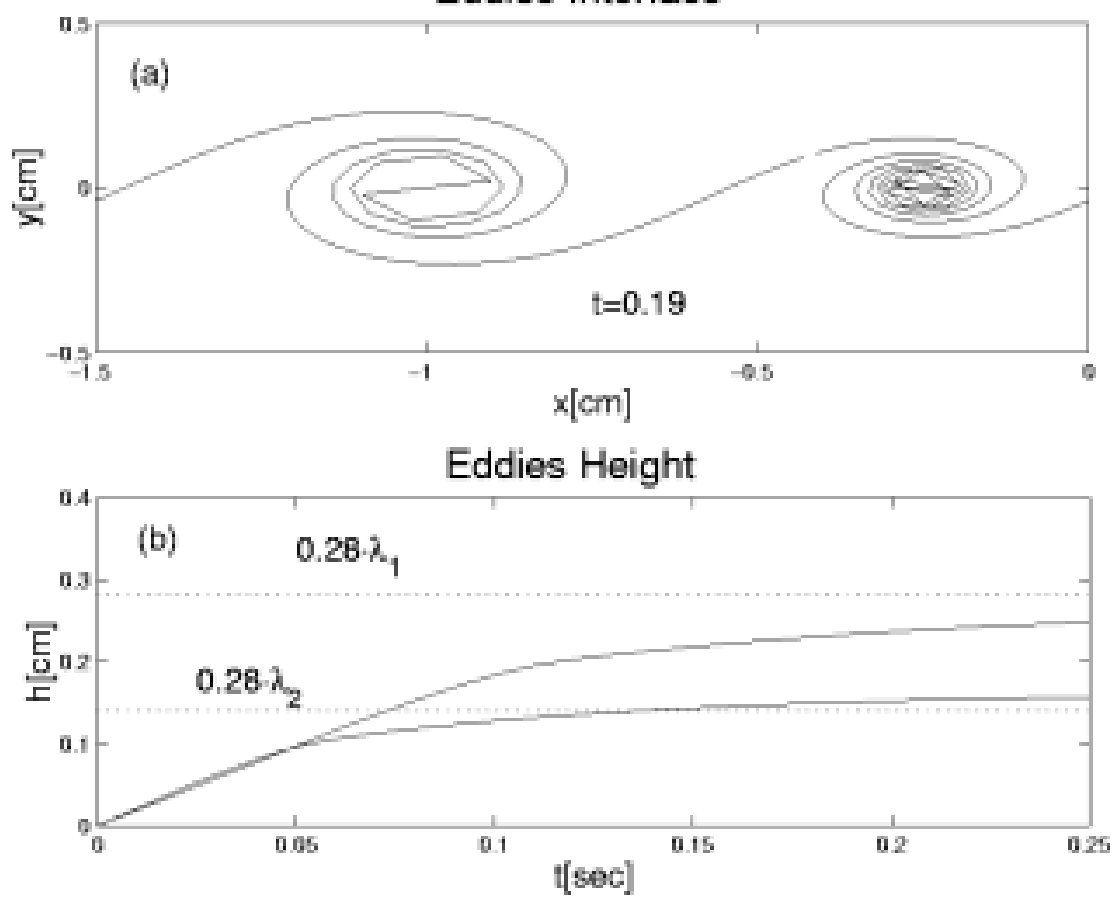

NO MERGER!

3 eddies must be introduced
Three Vortex Setup
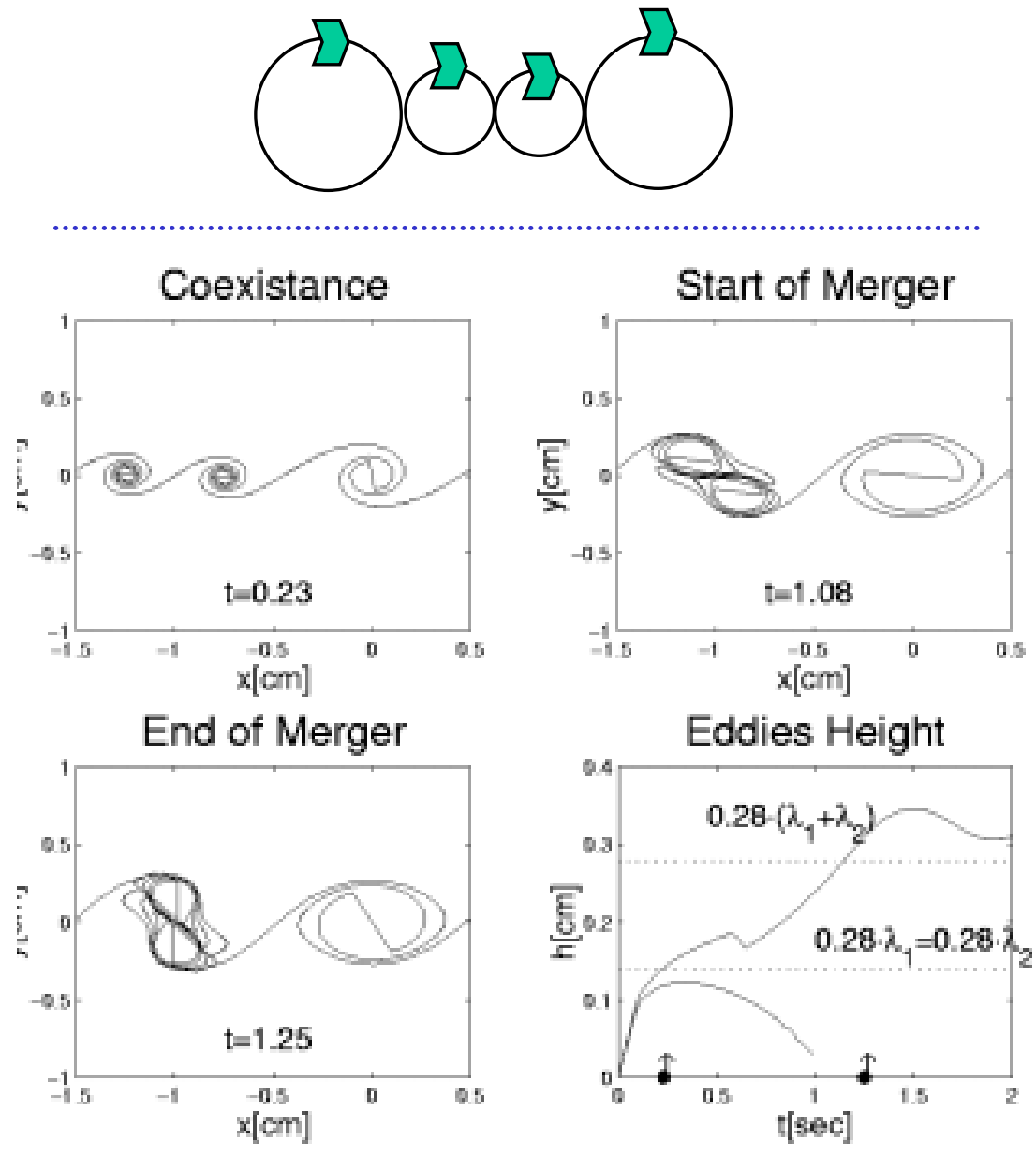

New vortex at predicted height of $\lambda_{1}+\lambda_{2}$ 


\section{Kelvin-Helmholtz Instability}

A large ensemble of eddies is set along a line.

Each eddy is assumed to possess its asymptotic height $\quad h(\lambda, t)=0.28 \lambda$ according to the vortex model.

Eddies increase in size through occasional mergers, according to the previously calculated merger rate.

$$
\lambda_{i}, \lambda_{i+1}
$$
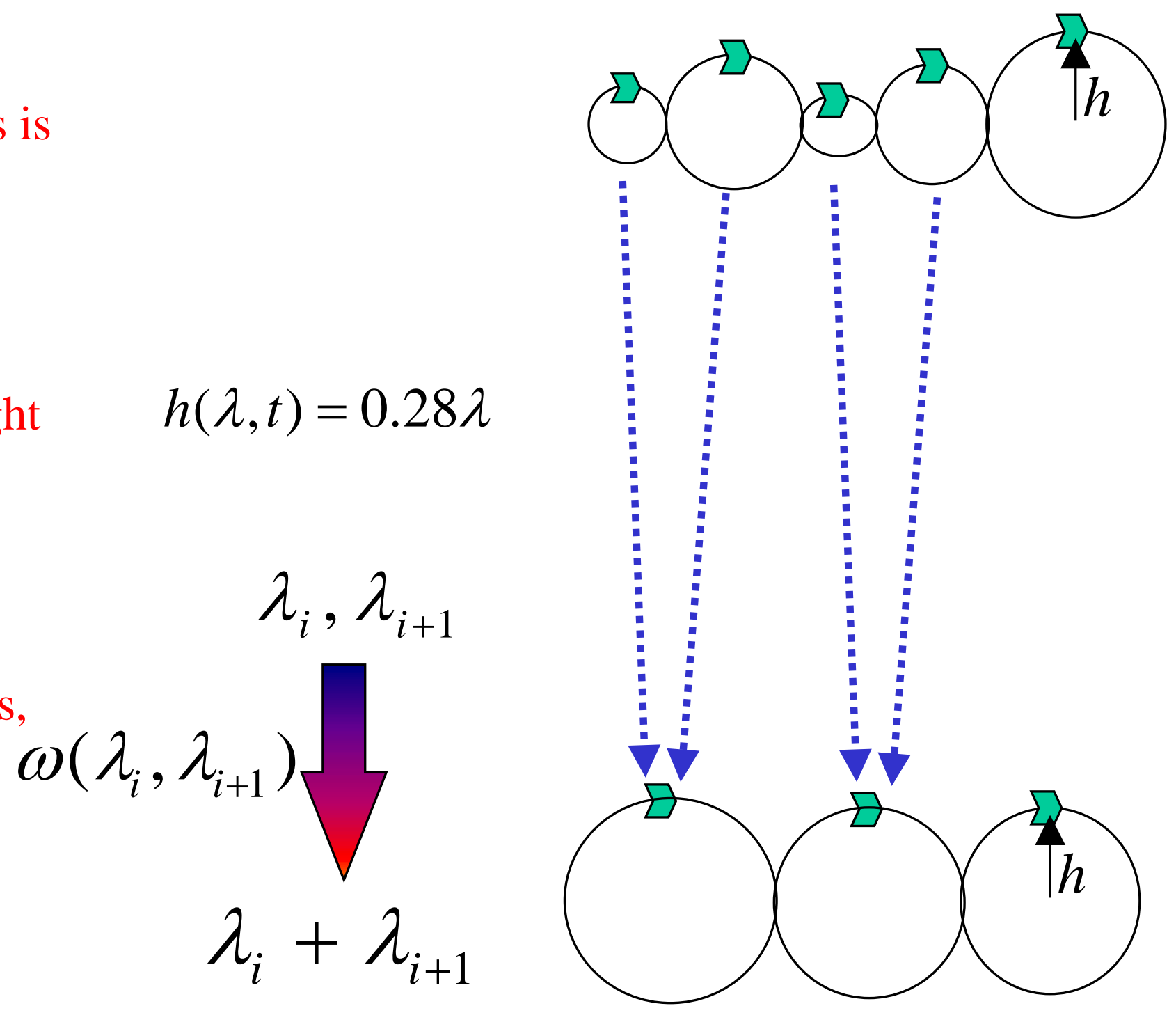


\section{Self-Similar Analyses of the Multi-Mode KH}

\section{Instability}

-Average width:

$\langle h(t)\rangle=0.56<\lambda>=0.56 L / N(t)$

-Number of eddies change through mergers:

$d(N(t)) / d t=-<\omega>N(t) / 2$

-Merger rate scales as:

$\langle\omega\rangle=\Delta u\left\langle\omega_{0}\right\rangle /\langle\lambda\rangle$

-Resulting number of eddies:

$$
N(t)=0.56 L /(\Delta u \cdot t) /\left\langle\omega_{0}\right\rangle
$$

Integrating the above, linearity is achieved through simple arguments.

$<\boldsymbol{h}(\boldsymbol{t})>=<\omega_{0}>\Delta u \cdot t$ 


\section{Results from the Large-Scale Statistical Model}

$\underline{\text { Statistical Model }}$

Experiment

$h_{\text {mix }}(t)=a_{0} \cdot t \quad ; \quad a_{0} \approx \underline{0.2}$

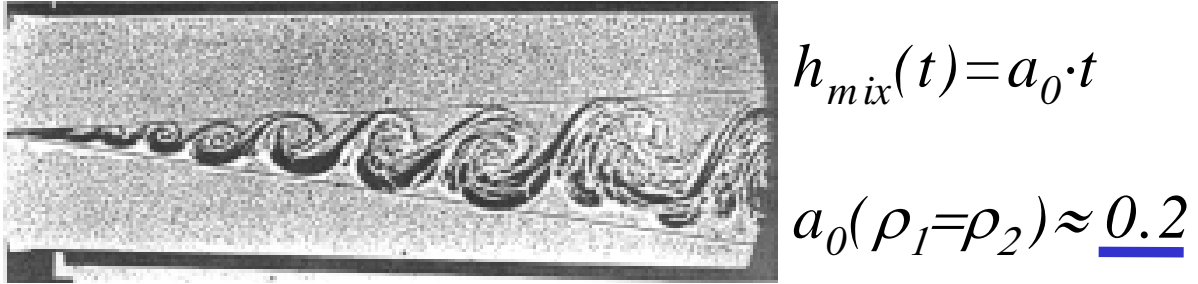

Eddy Size Distribution

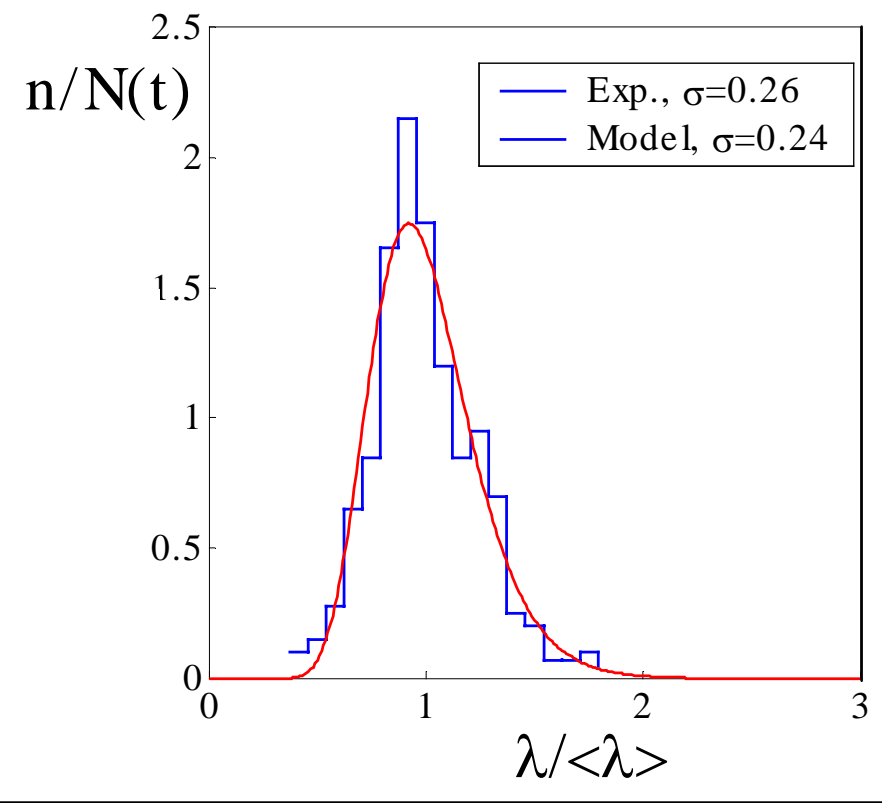

\section{Life-time Probability}

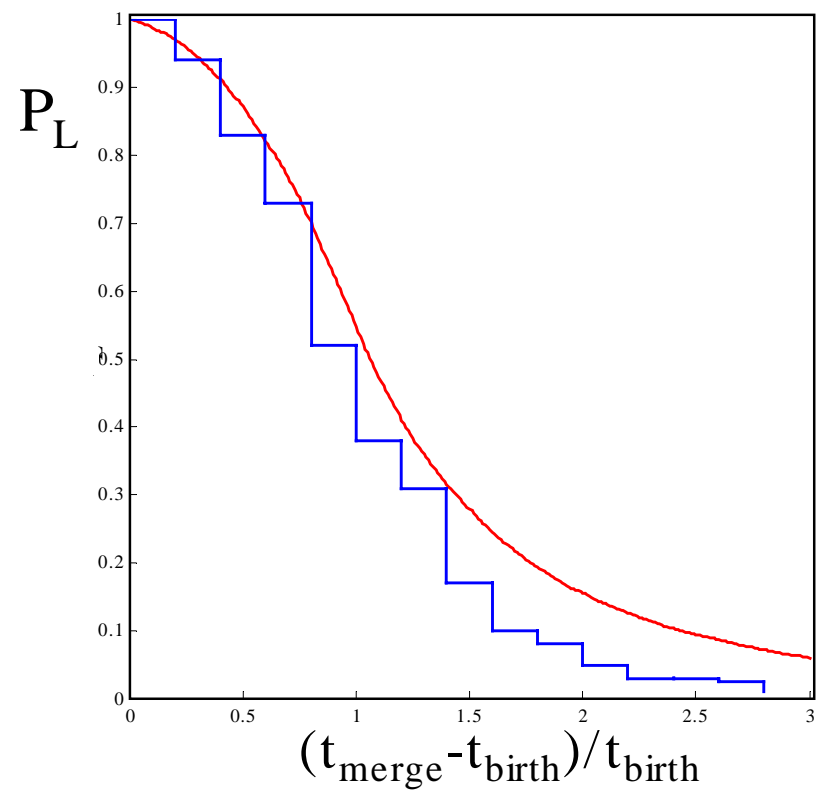

Experimental results taken from Roshko \& Brown, J. Fluid Mech, 1974 \& L.P. Bernal, Phys Fluids (1988). 


\section{Multi Mode Numerical Simulation}
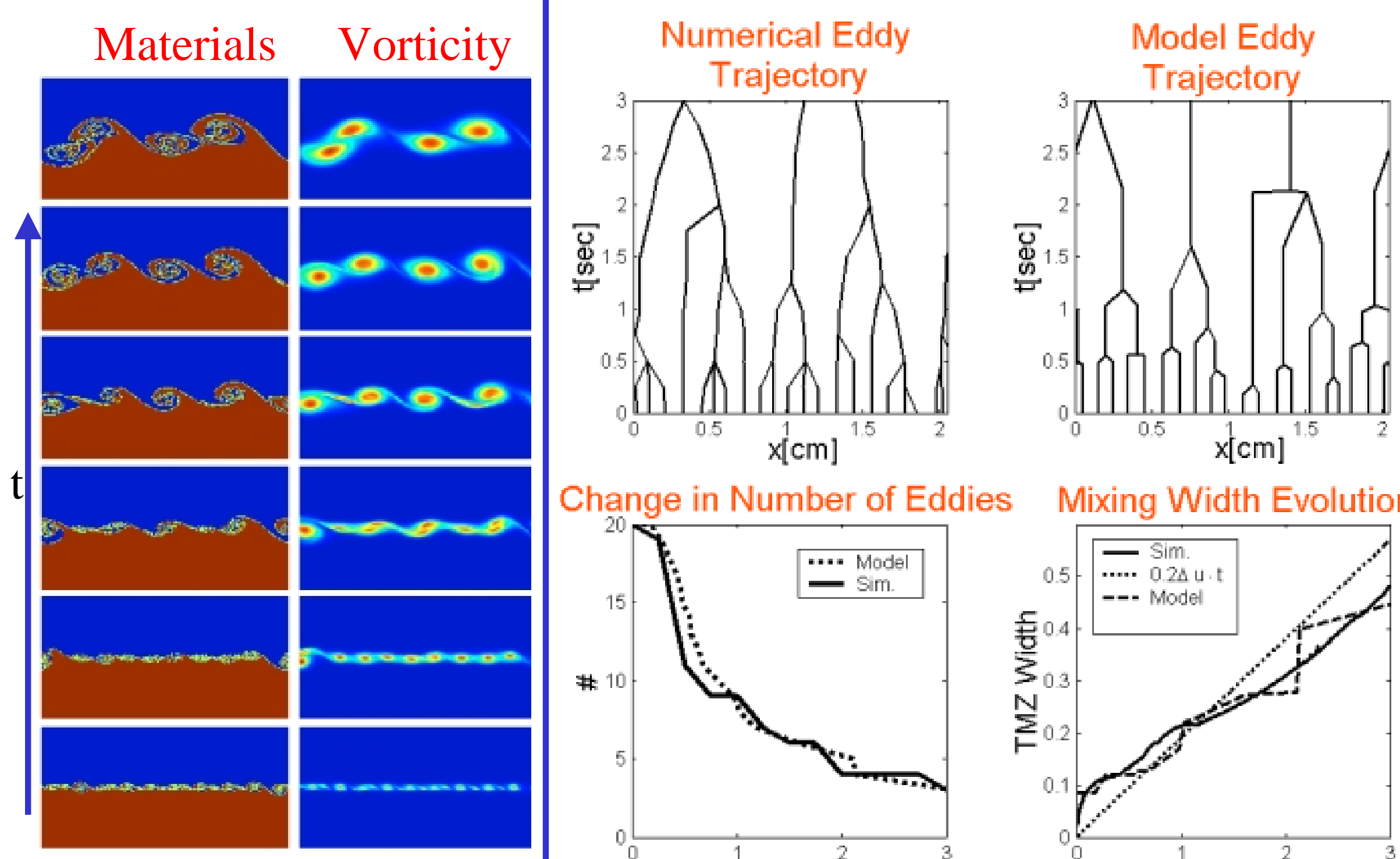

Change in Number of Eddies
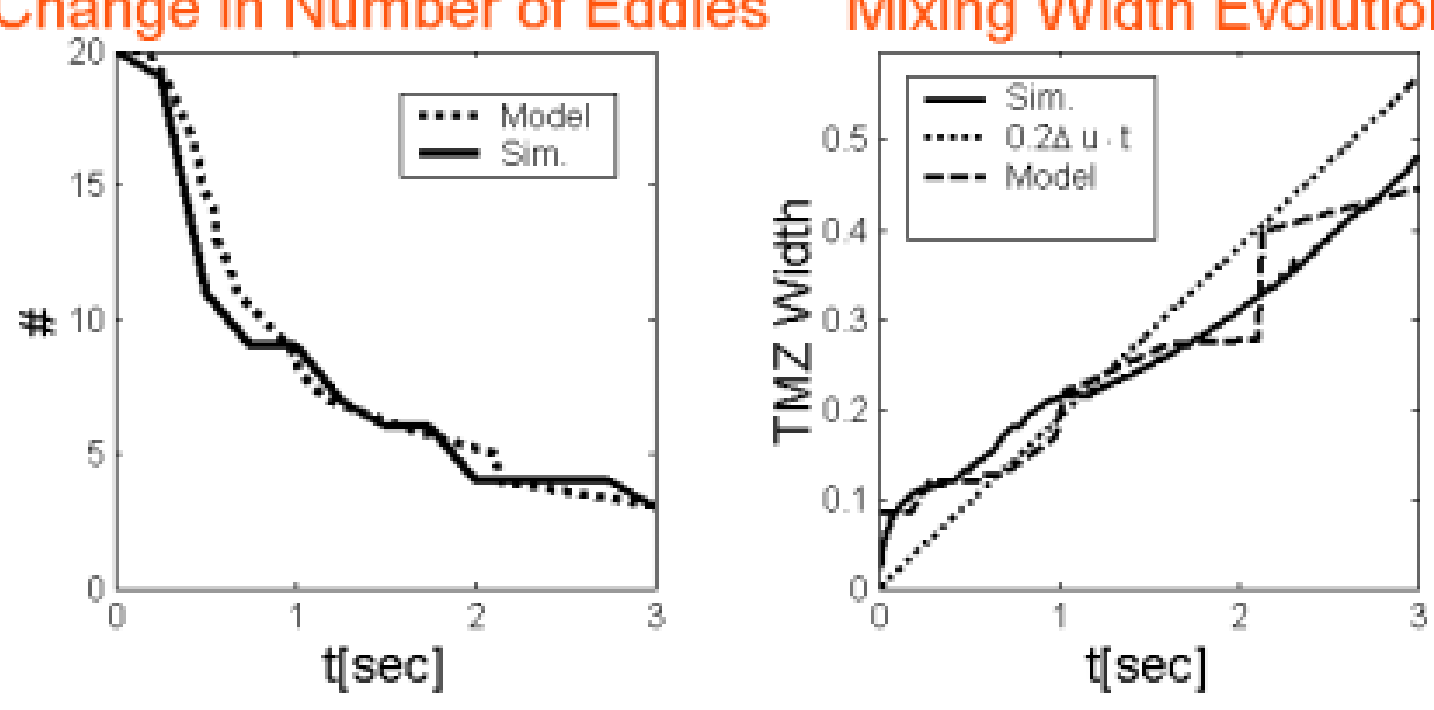


\section{Density Ratio Effects on the KH Instability:}

\section{Single Mode - Numerical Simulations}

$$
\rho_{1}=\rho_{2} ; v_{1}=v_{2}
$$
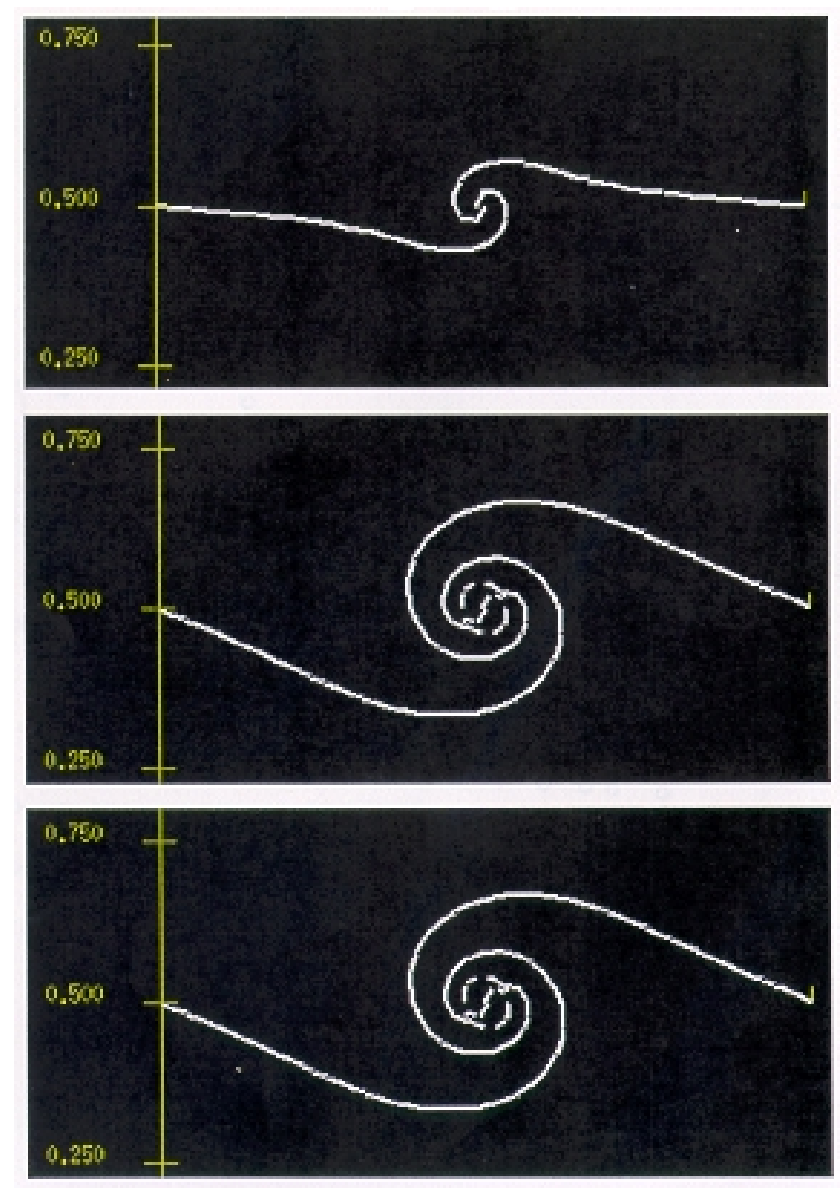

$$
\rho_{1}=7 \rho_{2} ; v_{1}=v_{2}
$$
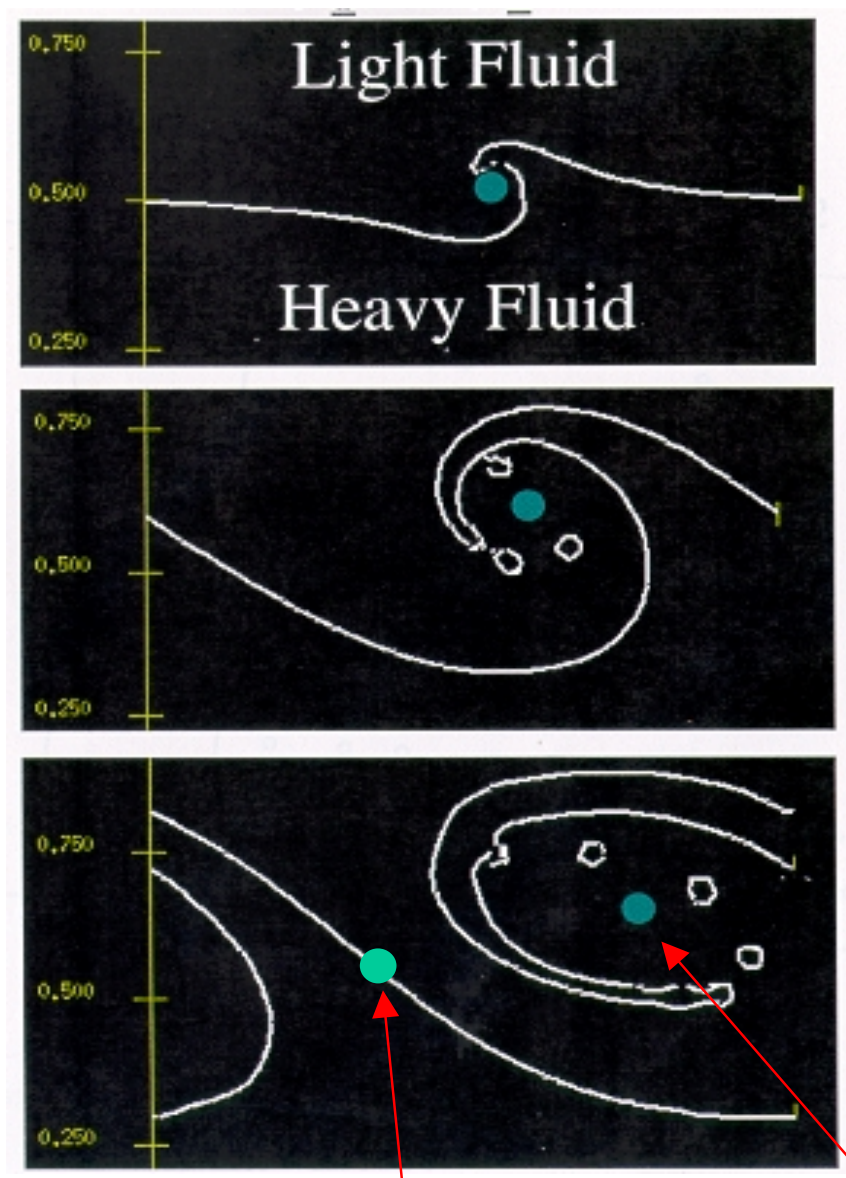

Stagnation point
Vortex drifts at

$$
v_{c} \neq \frac{v_{1}+v_{2}}{2}
$$

according to pressure equilibrium on stagnation points between neighboring vortices*:

$\rho_{1}\left(v_{1}-v_{c}\right)^{2}=\rho_{2}\left(v_{c}-v_{2}\right)^{2}$ 


\section{Multi-Mode Case: Numerical Simulation}
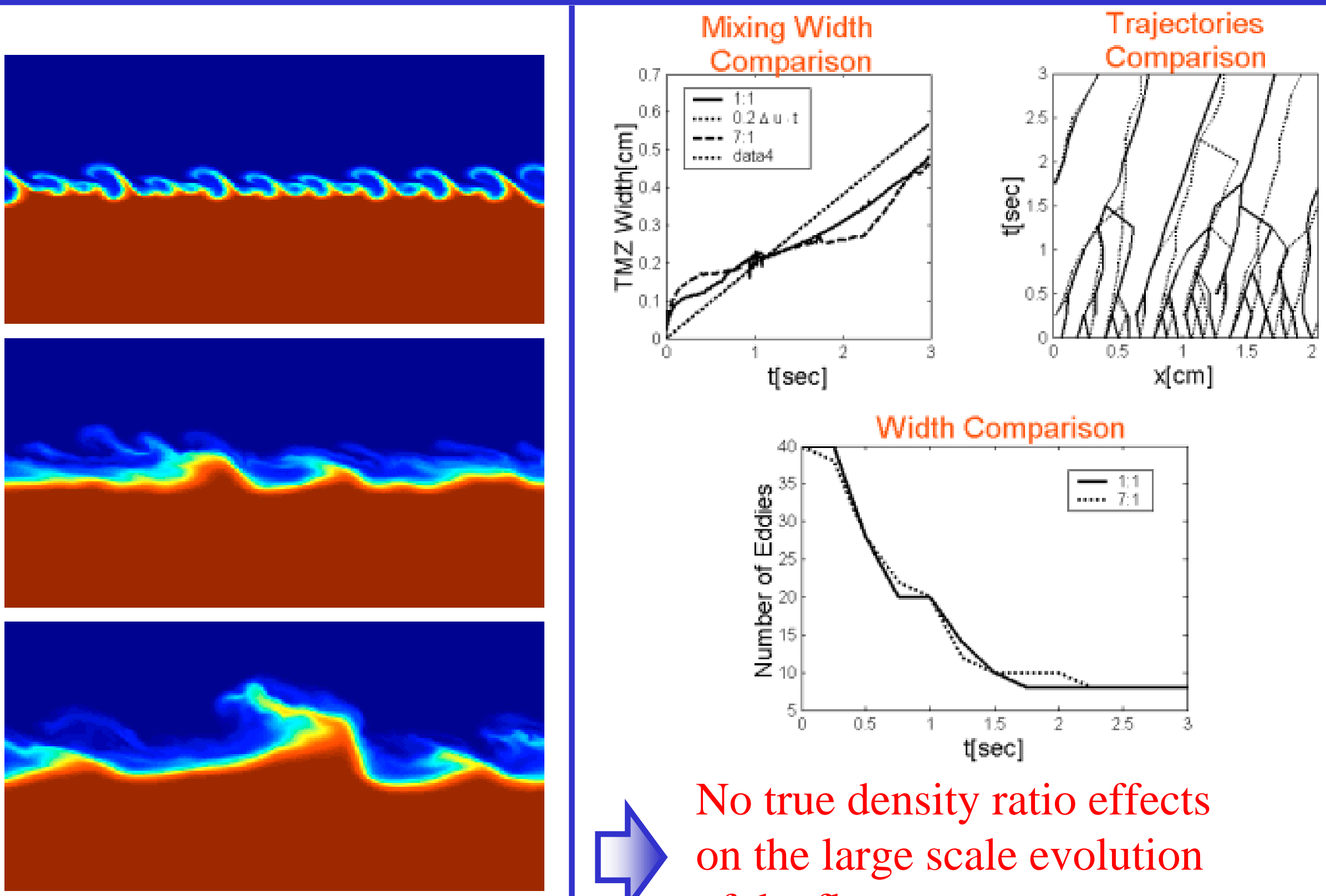

No true density ratio effects on the large scale evolution of the flow 
Drift Velocity Effect on the Linear Growth Coefficient

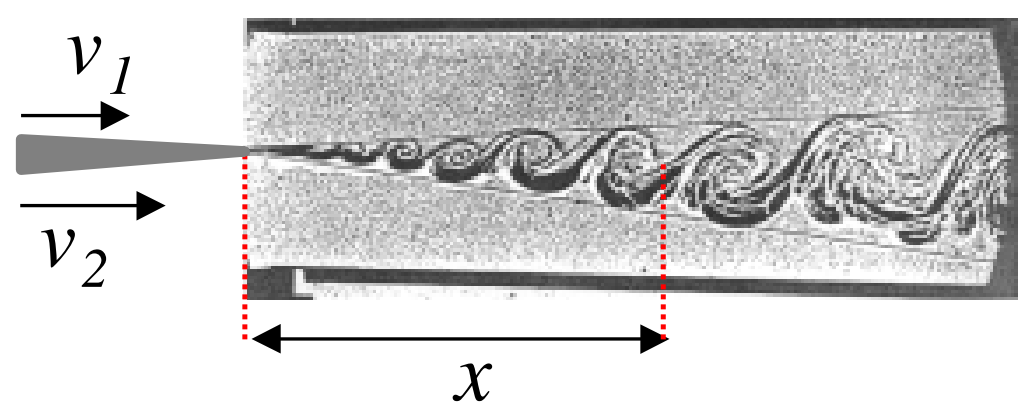

$$
\begin{aligned}
& \bar{v}=\left(v_{1}+v_{2}\right) / 2 \\
& v_{d}=\text { drift velocity }
\end{aligned}
$$

$$
\begin{gathered}
v_{d}=0\left(\rho_{I}=\rho_{2}\right) \\
t=\frac{x}{\bar{v}} \\
\downarrow \\
h_{\text {mix }}=0.2 \cdot \Delta u \cdot t=0.4 \frac{v_{1}-v_{2}}{v_{1}+v_{2}} x
\end{gathered}
$$

$$
\begin{gathered}
v_{d} \neq 0\left(\rho_{1} \neq \rho_{2}\right) \\
t=\frac{x}{v_{c}}=\frac{x}{\bar{v}+v_{d}} \\
\downarrow
\end{gathered}
$$$$
h_{\text {mix }}=0.2 \cdot \Delta u \cdot t=0.4 \frac{f}{1 \pm c \cdot f} x
$$$$
\text { with } f=\frac{v_{1}-v_{2}}{v_{1}+v_{2}}
$$ 


\section{Linear Growth Coefficient in Agreement with Experiments}

For mixing zone height of: $h(x)=\delta \cdot x$

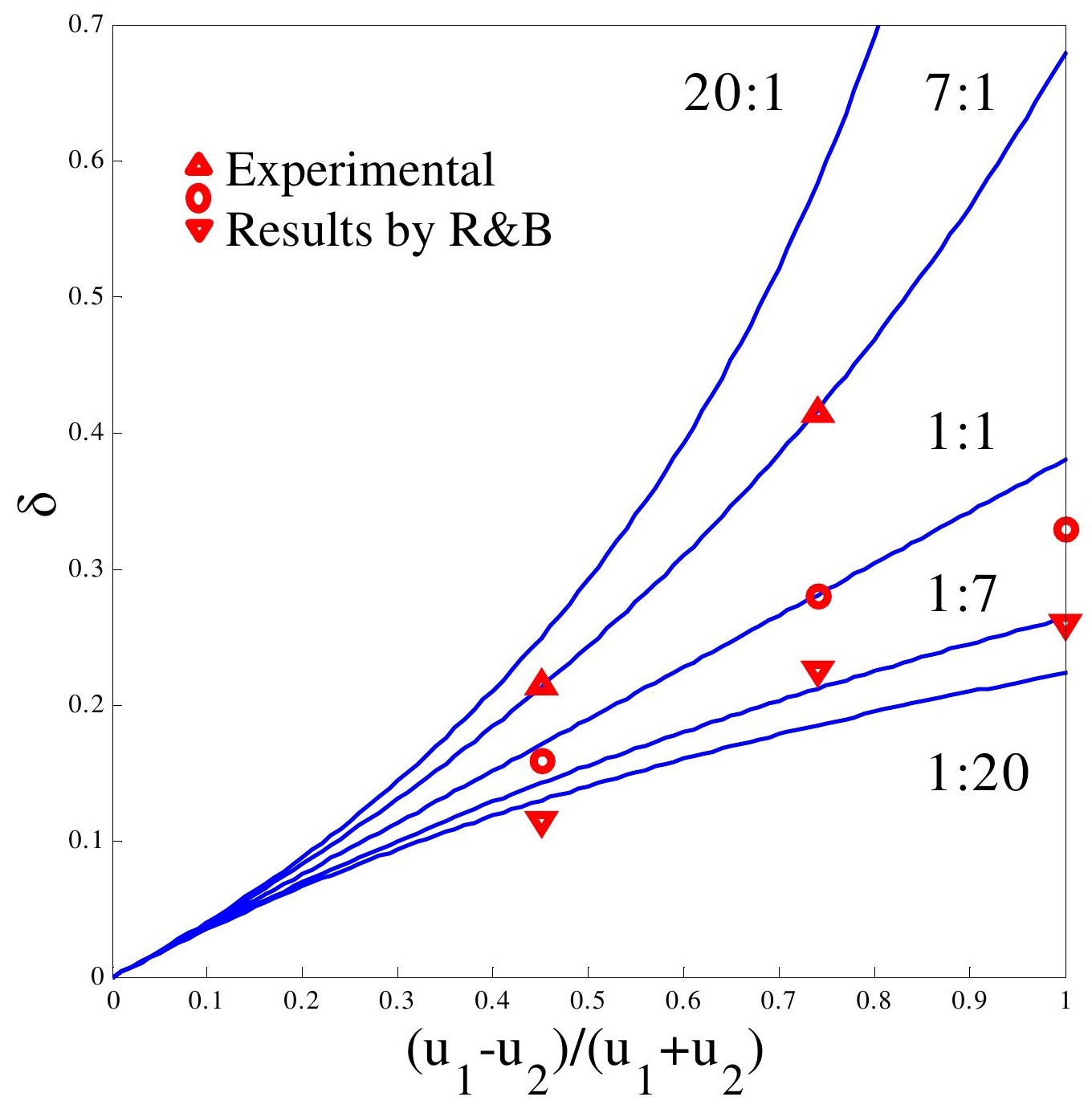

$h_{\text {mix }}(x)$ not linear with $\Delta \mathrm{u}$ for $v_{d} \neq 0$ 


\section{Summary}

- A large scale statistical model for the $\mathrm{KH}$ instability was developed resulting in:

- Linear growth rate coefficient for all density ratios.

- Full statistical characterization of the flow.

- The vortex merger is established as the growth mechanism of the mixing zone.

- Very good agreement with simulations and experiments is achieved. 
High Mach Number and High Initial Amplitude Effects on the Evolution of the Single-Mode Richtmyer-Meshkov

\author{
Instability - Theoretical Study
}

A. Rikanati, D. Oron, O. Sadot \& D. Shvarts 
Impulsive Models for the Small Amplitude Single-Mode RM Instability

Assuming low mach (SW effects as a delta function acceleration)

and small amplitudes $(\mathrm{ak}<<1)$ :

$\mathrm{k}$ - wavelength

$$
u_{\text {bubble }}=\Delta u_{1 d} \cdot k \cdot \frac{\rho_{1}-\rho_{2}}{\rho_{1}+\rho_{2}} a_{0}
$$

$\Delta \mathrm{u}_{1 \mathrm{~d}}$-velocity of unperturbed contact surface induced by shock wave $\rho_{1}, \rho_{2}$ - shocked densities before and after contact surface

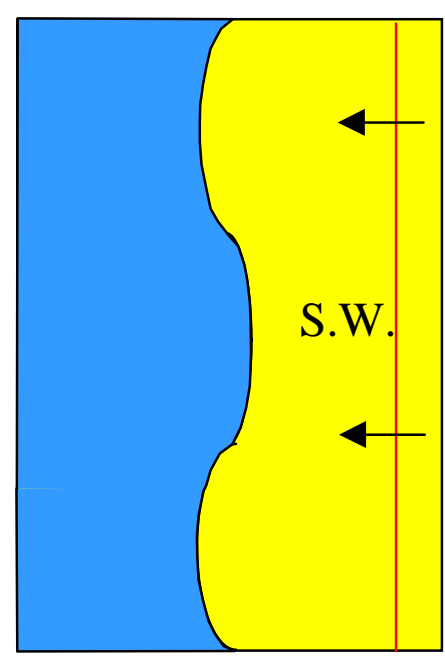

\section{Fast - Slow interaction}

Richtmyer Formula :

$$
a_{0}=a_{0}^{+}
$$

$a_{0}^{+}$- post shock amplitude
Slow - Fast interaction (phase inversion)

Meyer-Blewett correction :

$$
a_{0}=\frac{a_{0}^{+}+a_{0}^{-}}{2}
$$

$a_{0}^{+}$- post shock amplitude

$a_{0}^{-}$- pre shock amplitude 
Results from New Shock Tube Experimental by Sadot et. al. M=1.2 (E36)

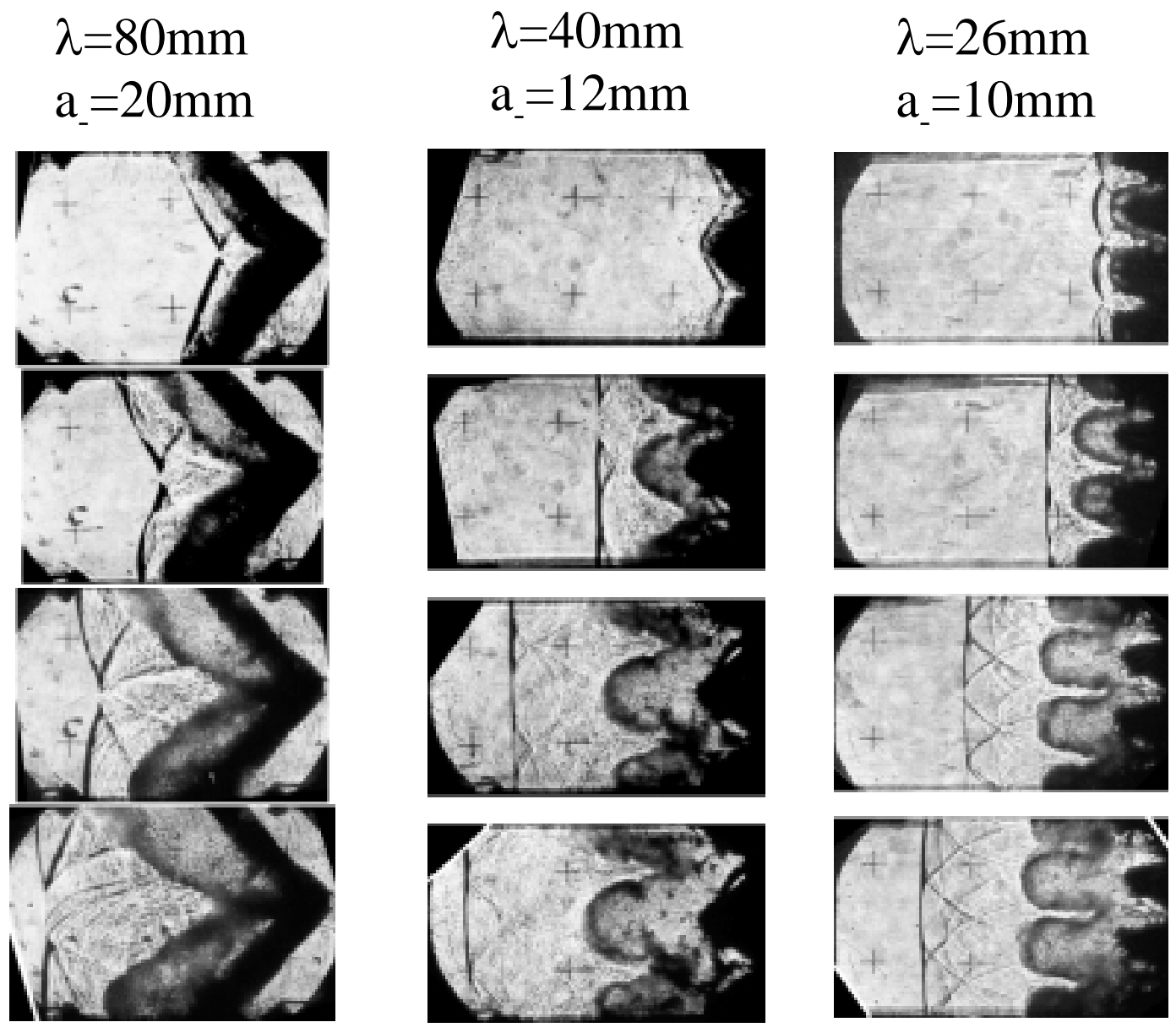




\section{Experimental Velocity Reduction}

Class A Experiments:

Similar reduction at a range of Mach numbers (1.2-15.3)

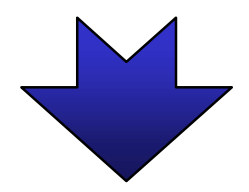

Apparent High Amplitude Effect

Class B Experiments:

Can be High Mach Effect

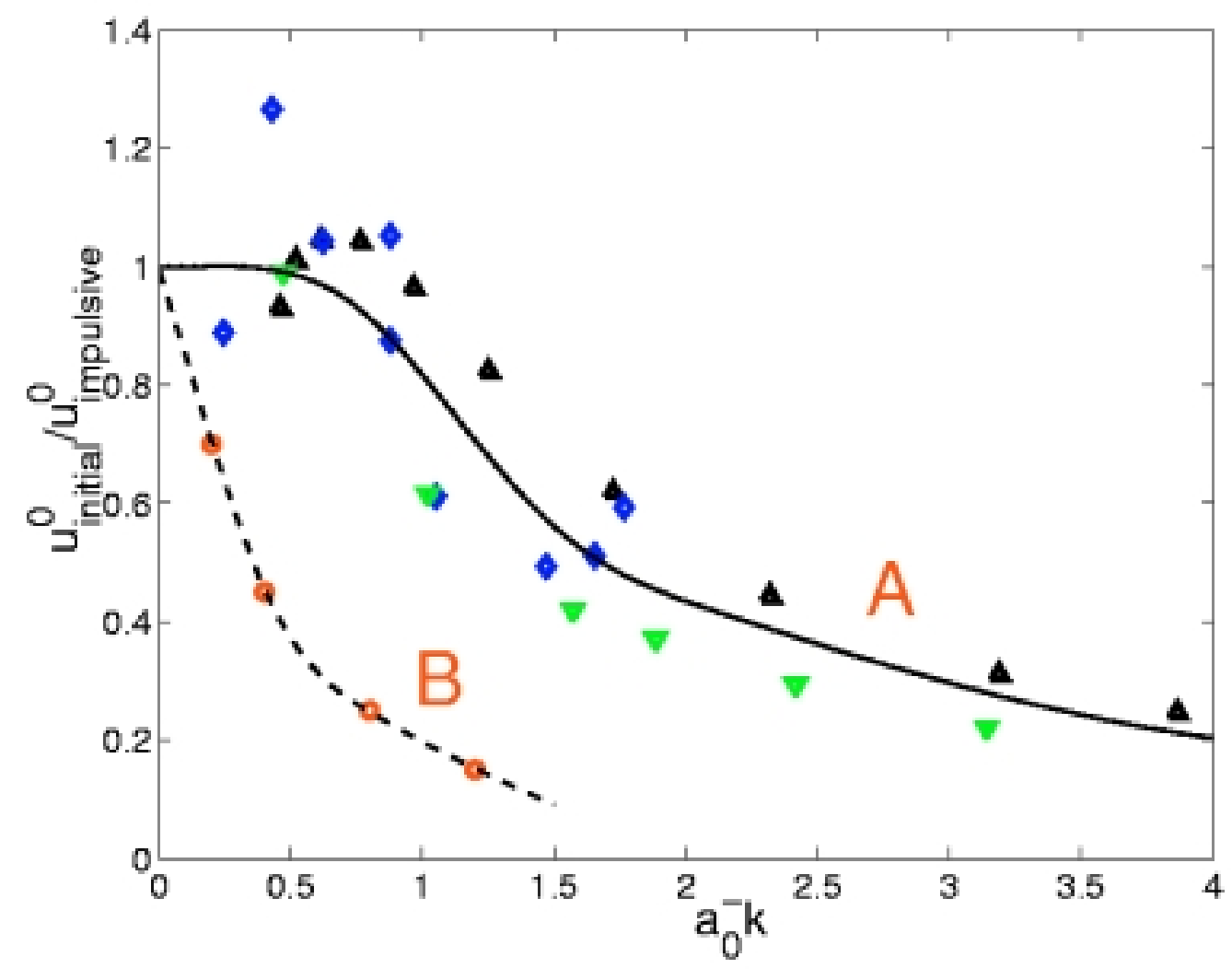

$\diamond \quad$ Dimonte $\mathrm{Be} \rightarrow$ Foam $(\mathrm{M}=15.3)$

$\Delta \quad$ Aleshin $\mathrm{Ar} \rightarrow \mathrm{Xe}(\mathrm{M}=2.5)$

$\nabla \quad$ Sadot Air $\rightarrow \mathrm{SF}_{6}(\mathrm{M}=1.2)$

- Aleshin $\mathrm{He} \rightarrow \mathrm{Xe}(\mathrm{M}=2.5)$ 

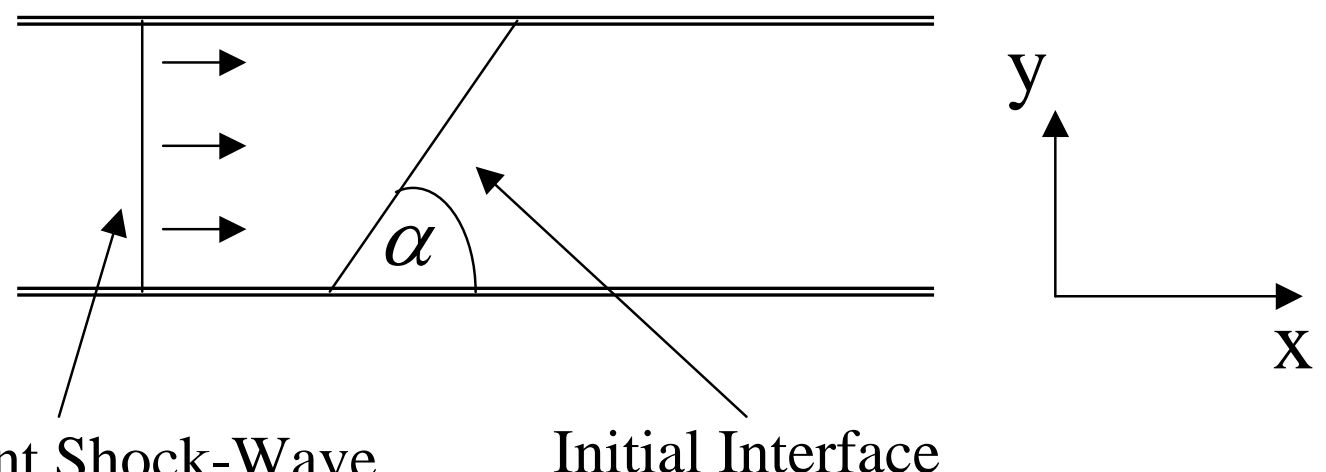

Incident Shock-Wave

Initial Interface

- Local vorticity deposition per unit length*:

$$
\Gamma d s=\Gamma_{0}\left(M, \gamma_{1}, \gamma_{2}, \rho_{1}, \rho_{2}\right) \sin (\alpha) \mathrm{ds}
$$

- Bubble tip velocity:

$$
u(z)-i \cdot v(z)=2 \pi \int_{\text {interface }} \Gamma(z) \cot \left(\left(z-z^{\prime}\right) \cdot \pi / d\right) d z^{\prime}
$$

$z=x+i \cdot y$

* R. Samtaney and N. J. Zabusky, Phys. Fluids A 5, 1285 (1993) 
Model Velocity Reduction Compared with class 'A' Experiments and Simulations

Experiments by Dimonte and Aleshin

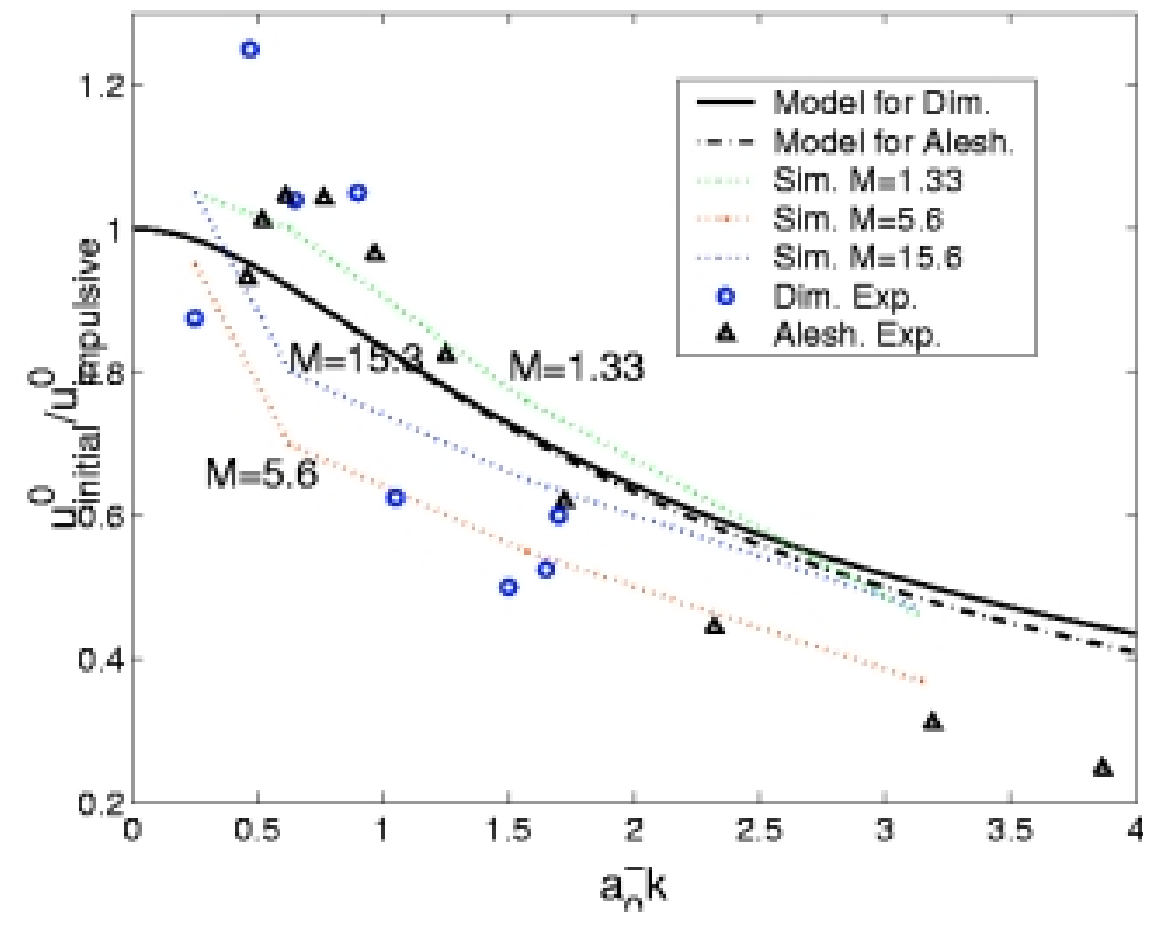

New shock Tube Experiments

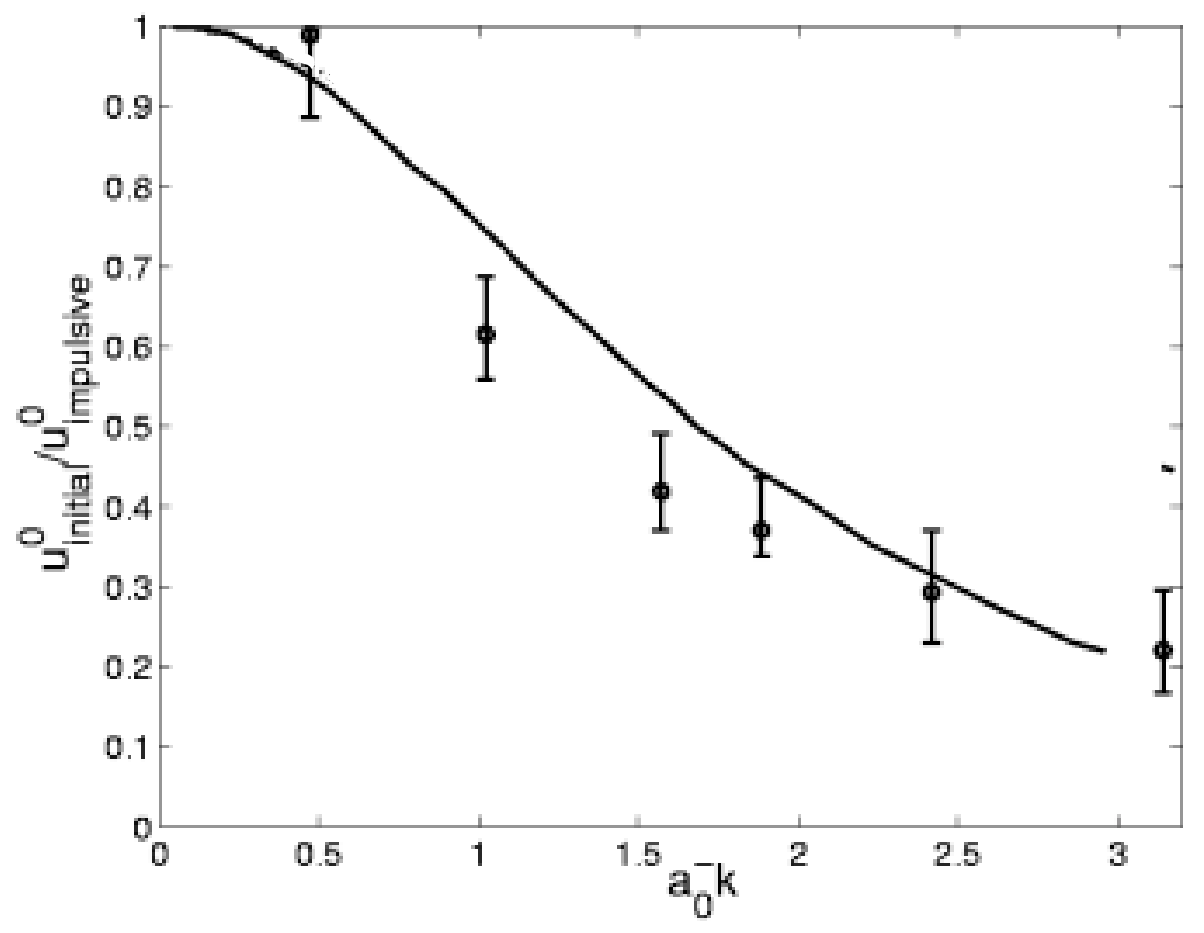

In class ' $A$ ' experiments the velocity reduction is mainly attributed to high amplitudes effects. 


\section{Compressibility dominated regimes}

Compressibility effects are expected to dominate the flow when the shock wave is in proximity with the interface.

Shock front

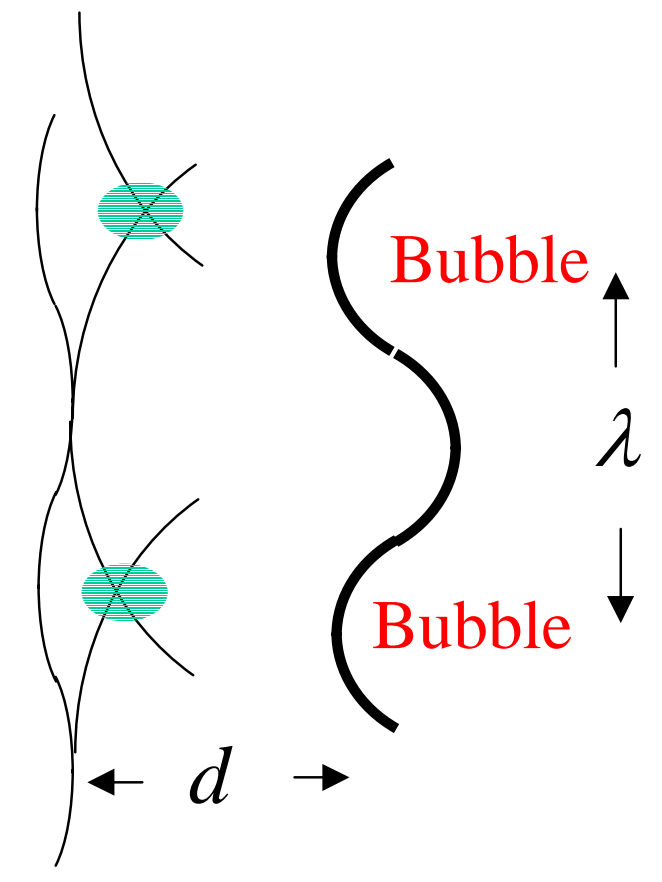

Proximity criterion: $f_{c}=\frac{u_{\text {bouble }}}{u_{\text {shock }}-u_{1 d}}$

Conjecture: $f_{c}$ characterizes the flow at moderate Mach numbers

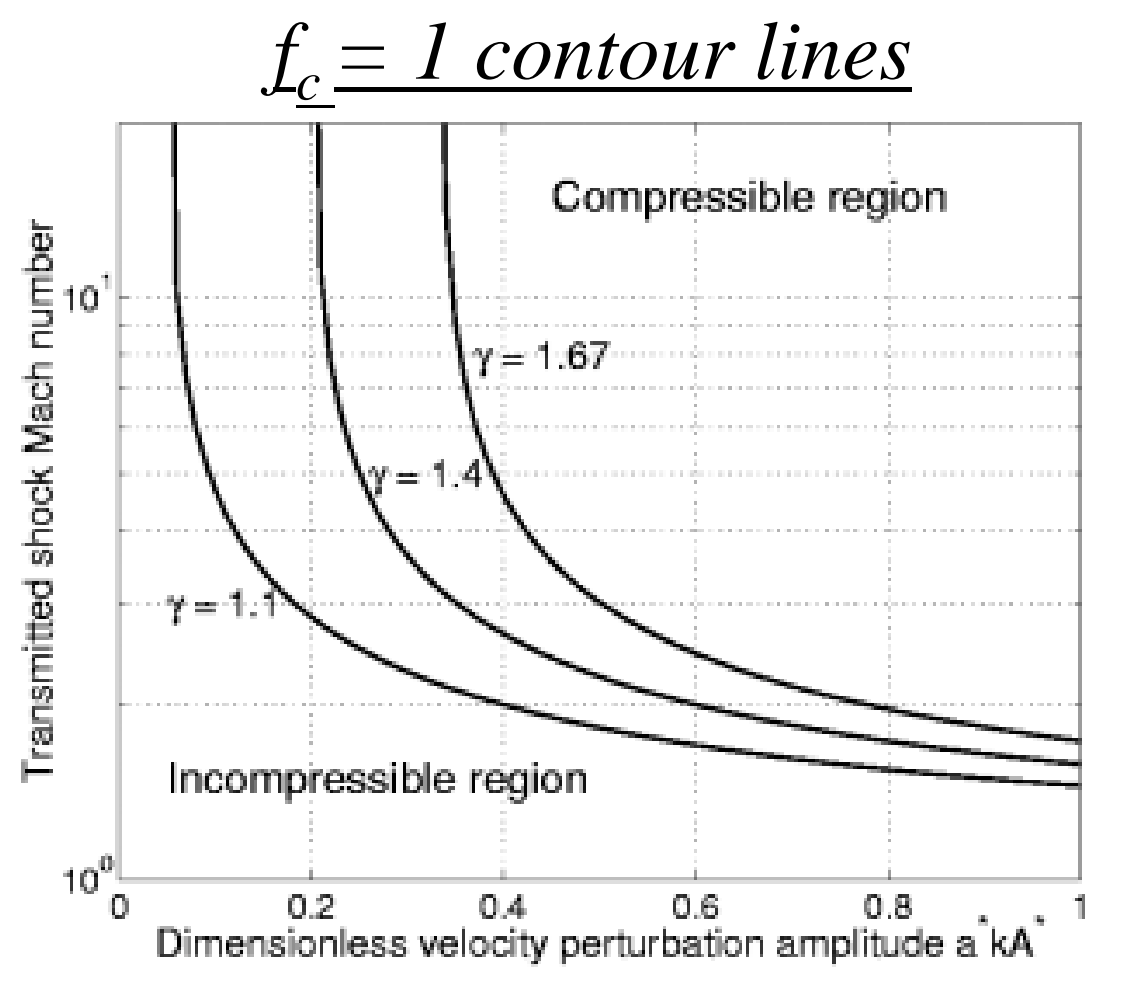




\section{"Wall" model for moderate Mach RM instability}

- Shock wave is treated as a rigid straight wall moving in the $1 d$ shock velocity.

- Secondary high pressure points are not considered.

- Model reduction depends only on $f_{c \text {. }}$.

- Model is solved by using

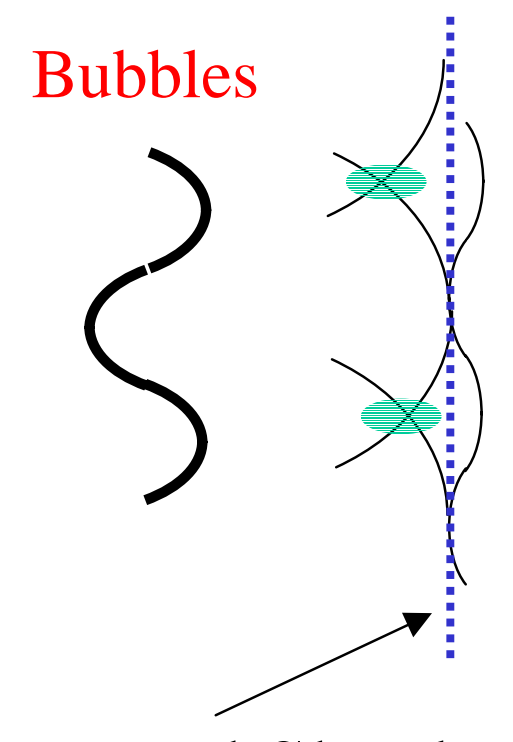
previous models* while inhibiting the shock as a moving boundary condition. 


\section{Example of results from the Wall model}

-As the shock velocity increases ( $f_{c}$ decreases) the velocity profile is closer to the incompressible case.

-The reduction factor is calculated by:

Reduced Velocity Richtmyer Velocity

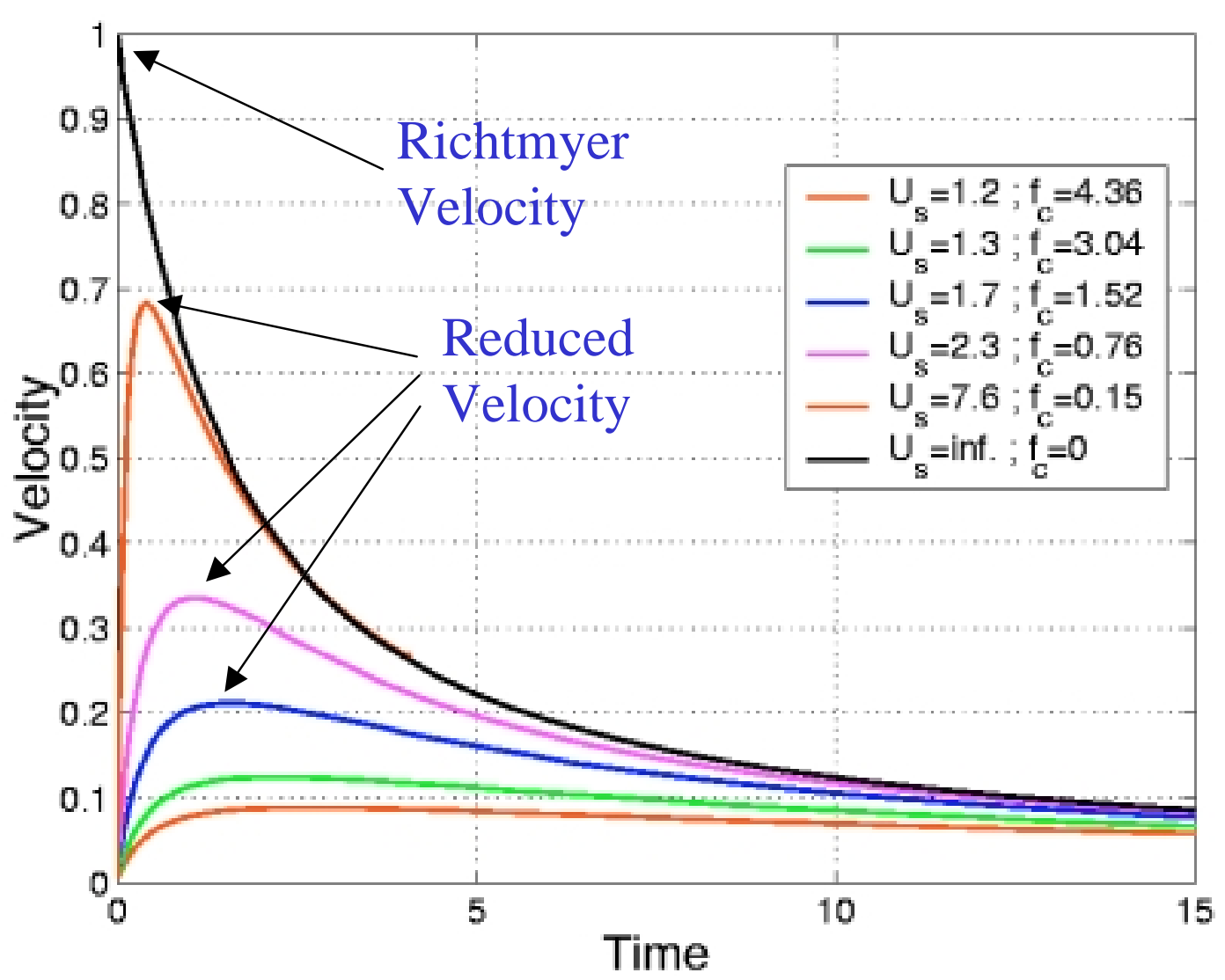




\section{Comparison with Aleshin $\mathrm{He} \rightarrow$ Xe experiments}

— Rictmyer Veloctiy

Classical Model

— Wall Model

— Sim./Exp.

Reverberation

$\uparrow$ arrivel time
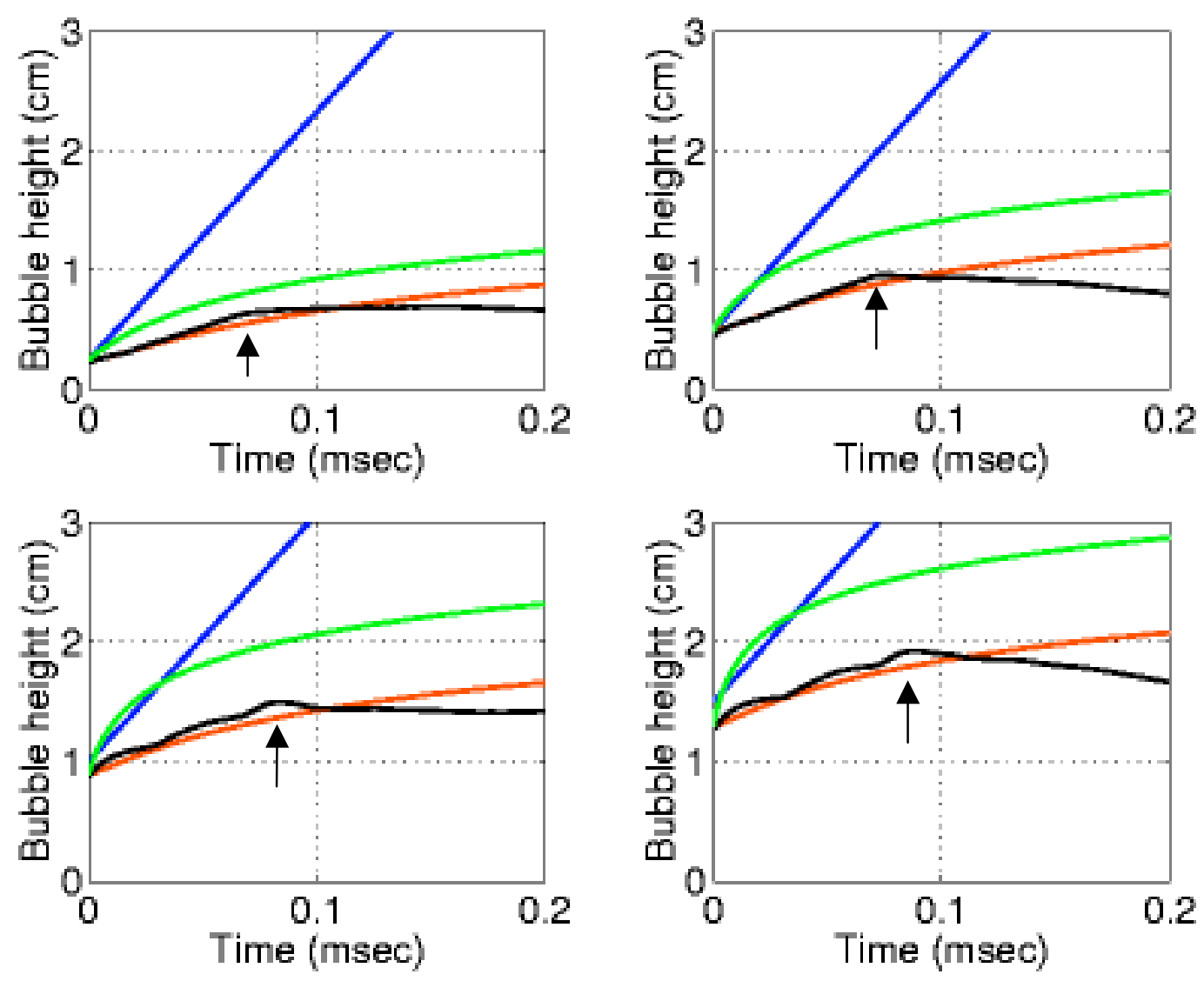

$\Rightarrow$ By Introducing $f_{c}$ to the potential model, good agreement is achieved with experiments. 


\section{Class B Reduction Factor - Theory Vs. Experiments}

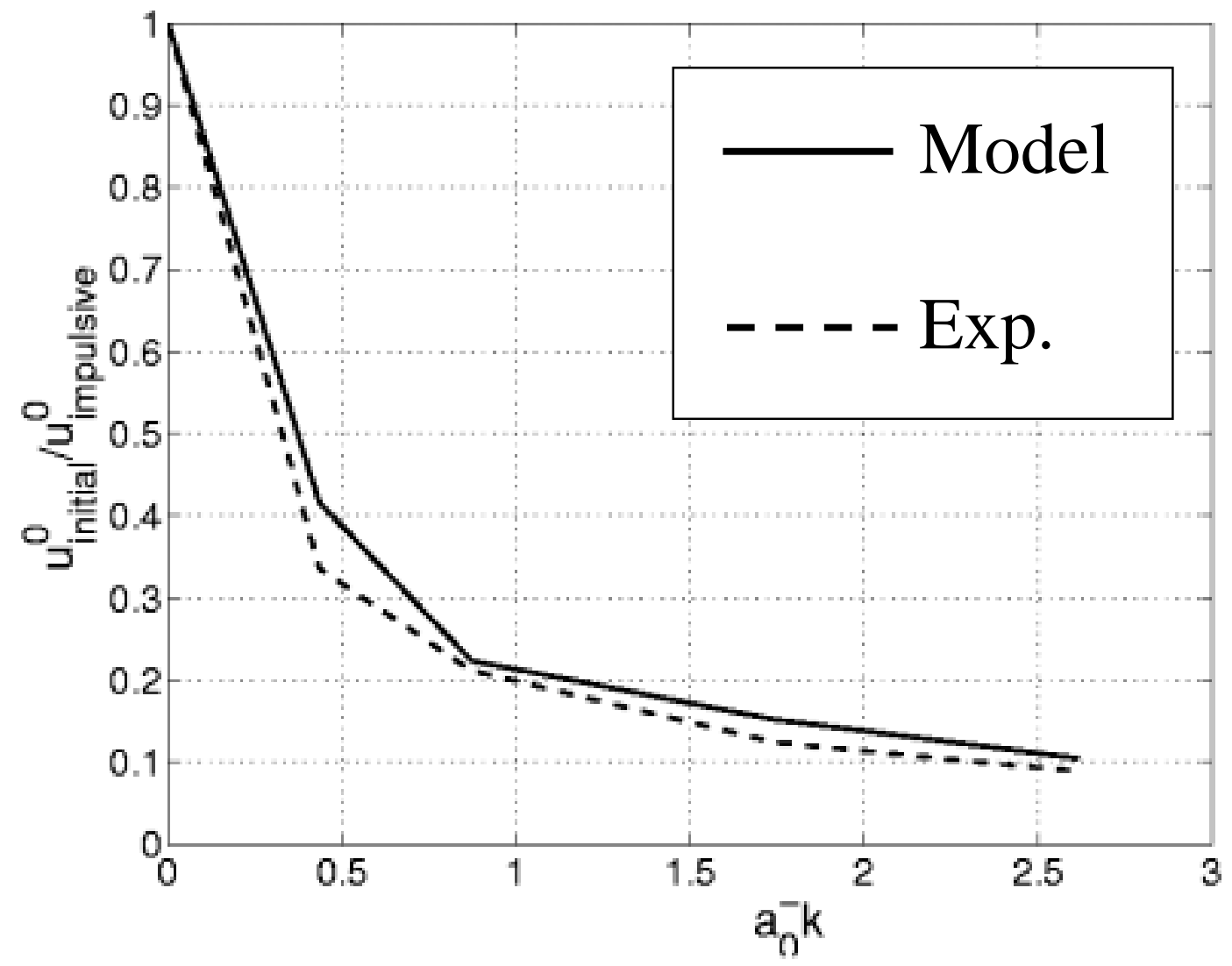

In class ' $\mathrm{B}$ ' experiments the velocity reduction is mainly attributed to high Mach effects. 
Late Nonlinear Stages of the Flow - Numerical Simulations at $f_{c}=0.05$ and $a k=0.175-1.75$.
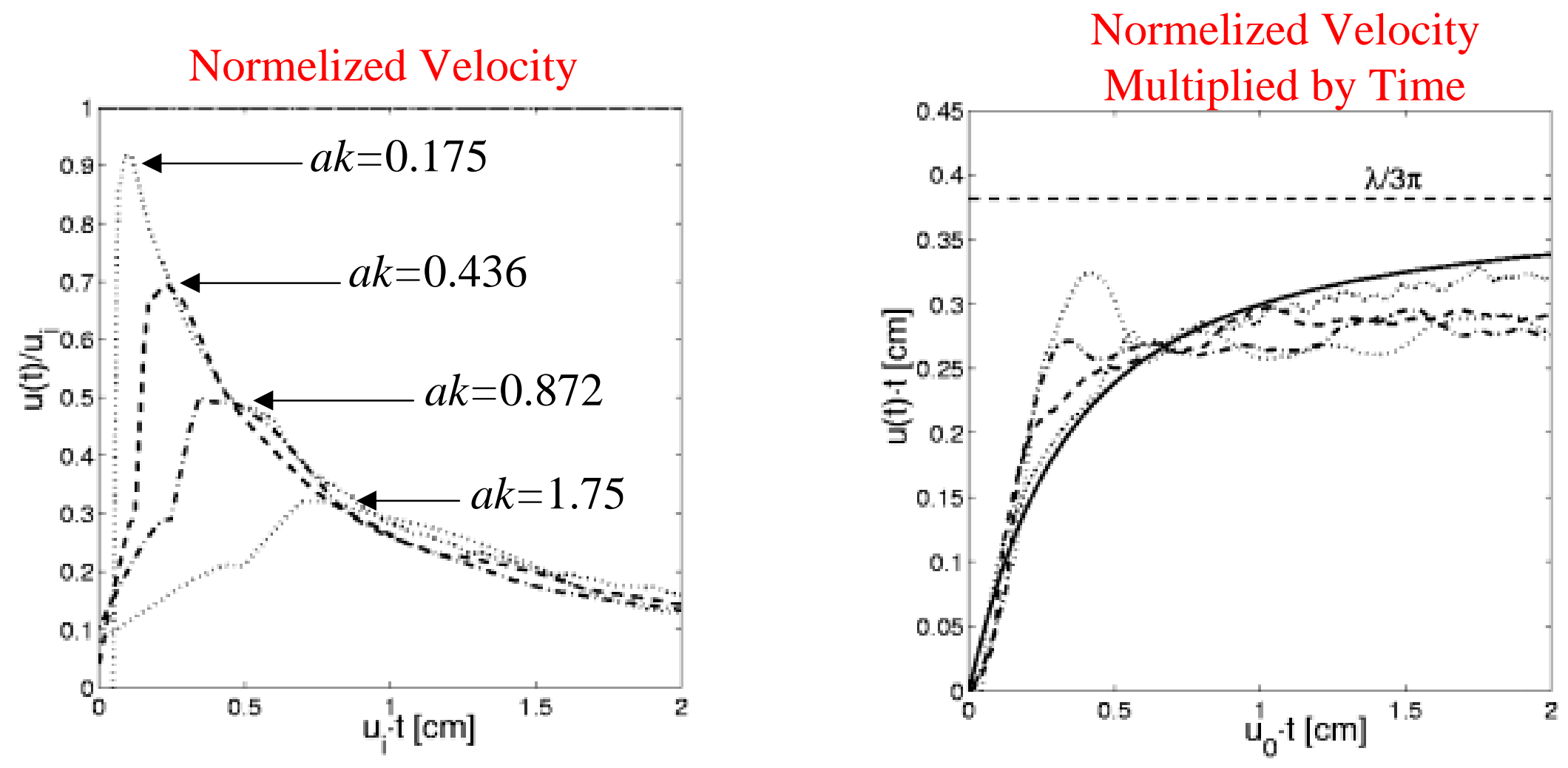

$\Rightarrow$ Normalizing the late stages of the flow by the initial velocity from the High Amplitudes Model, deduces high amplitudes effects. Hence the classic behavior is regained. 
Late Nonlinear Stages of the Flow - Numerical Simulations for $f_{c}=0.05-0.625$ and $a k=0.43$.

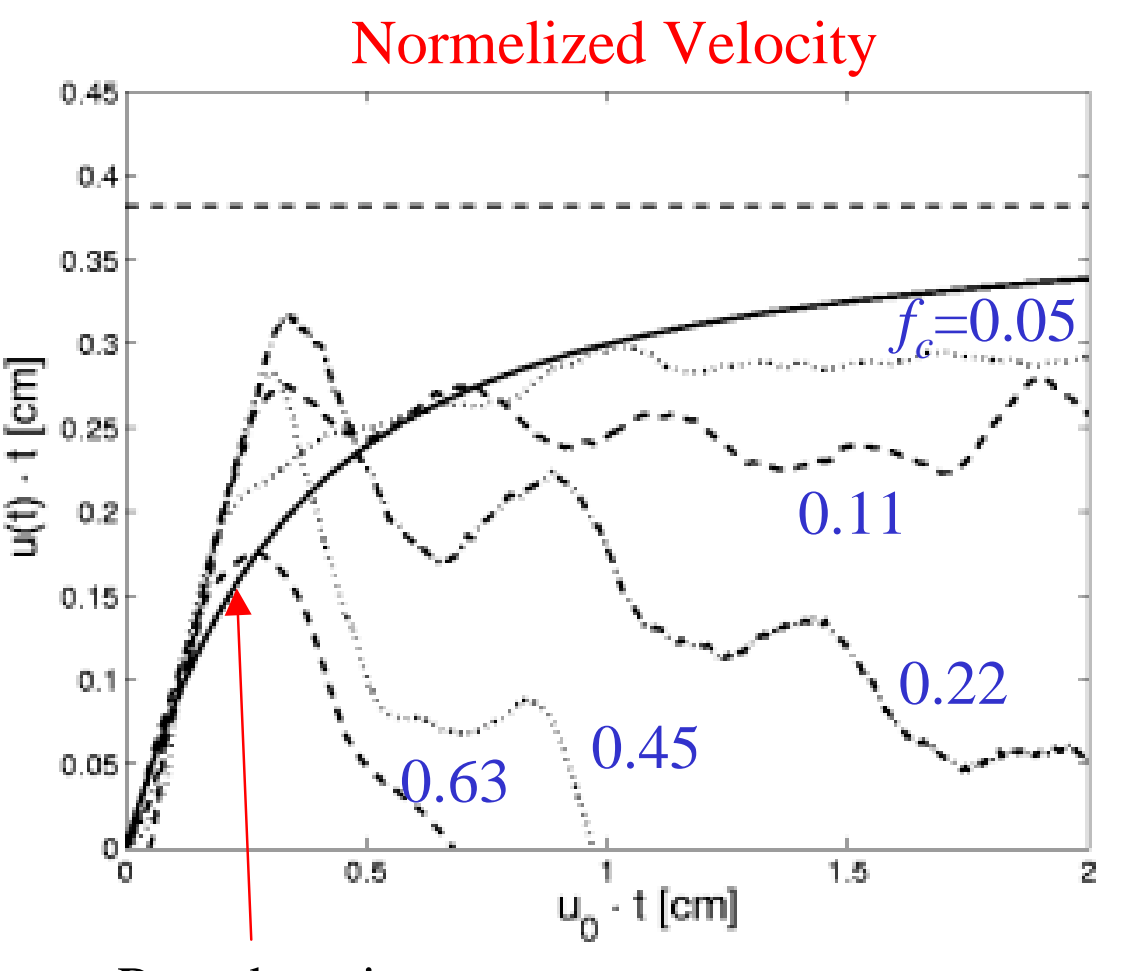

Reverberation
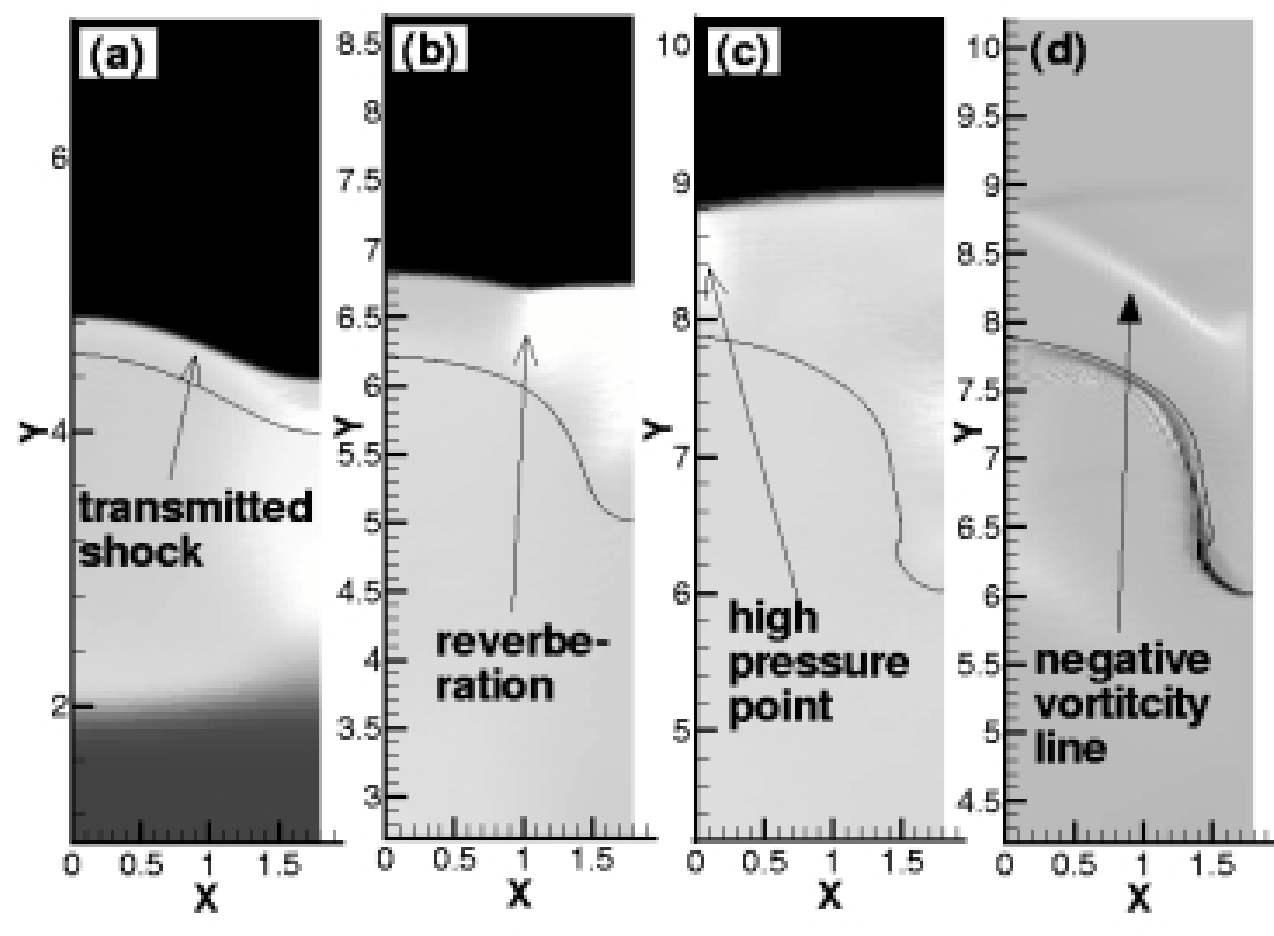

time

$\Rightarrow$ At High values of $f_{c}$ new phenomena arises due to secondary high pressure points, drastically affecting the flow. 


\section{Summary}

- Effects of high initial amplitudes and Mach numbers were quantified for the early linear stages of the flow.

- Classes 'A' and 'B' of experiments were recognized, distinguishing between the two effects.

- For the late nonlinear stages of the flow:

- No true effects were found for high initial amplitudes.

- New dominant effects were found for high Mach numbers. 
Poster T25

\title{
Compressible MHD Turbulence in Strongly Radiating Molecular Clouds in Astrophysics*
}

\author{
D.D. Ryutov
}

\author{
LLNL, Livermore, CA 94551, USA
}

Presented at $8^{\text {th }}$ International Workshop on the Physics of Compressible Turbulent Mixing, December 10-14, 2001, Pasadena CA

\footnotetext{
*Work performed for the U.S. DoE by UC LLNL under contract W-7405-Eng-48.
} 


\section{ABSTRACT}

Molecular clouds in astrophysics are often subjected to intense irradiation by nearby young stars. The ablation process ensues and a strong shock is driven into the cloud. In a number of cases, the radiative cooling time of the shocked matter is much shorter than the dynamical time of the cloud evolution. In such situations, possible pre-existing turbulent motions and turbulent magnetic fields can potentially contribute to the "stiffness" of the shocked material. We suggest simple models allowing quick evaluation of these effects. We conclude that the presence of a turbulent magnetic field can play a significant role, provided its amplitude is beyond some critical level, whereas the turbulent ram pressure of the unmagnetized medium can play only a relatively minor role. Implications for the dynamics of astrophysical molecular clouds are discussed.

Work performed under the auspices of the U.S. Department of Energy by University of California Lawrence Livermore National Laboratory under contract W-7405-Eng-48. 
Eagle Nebula

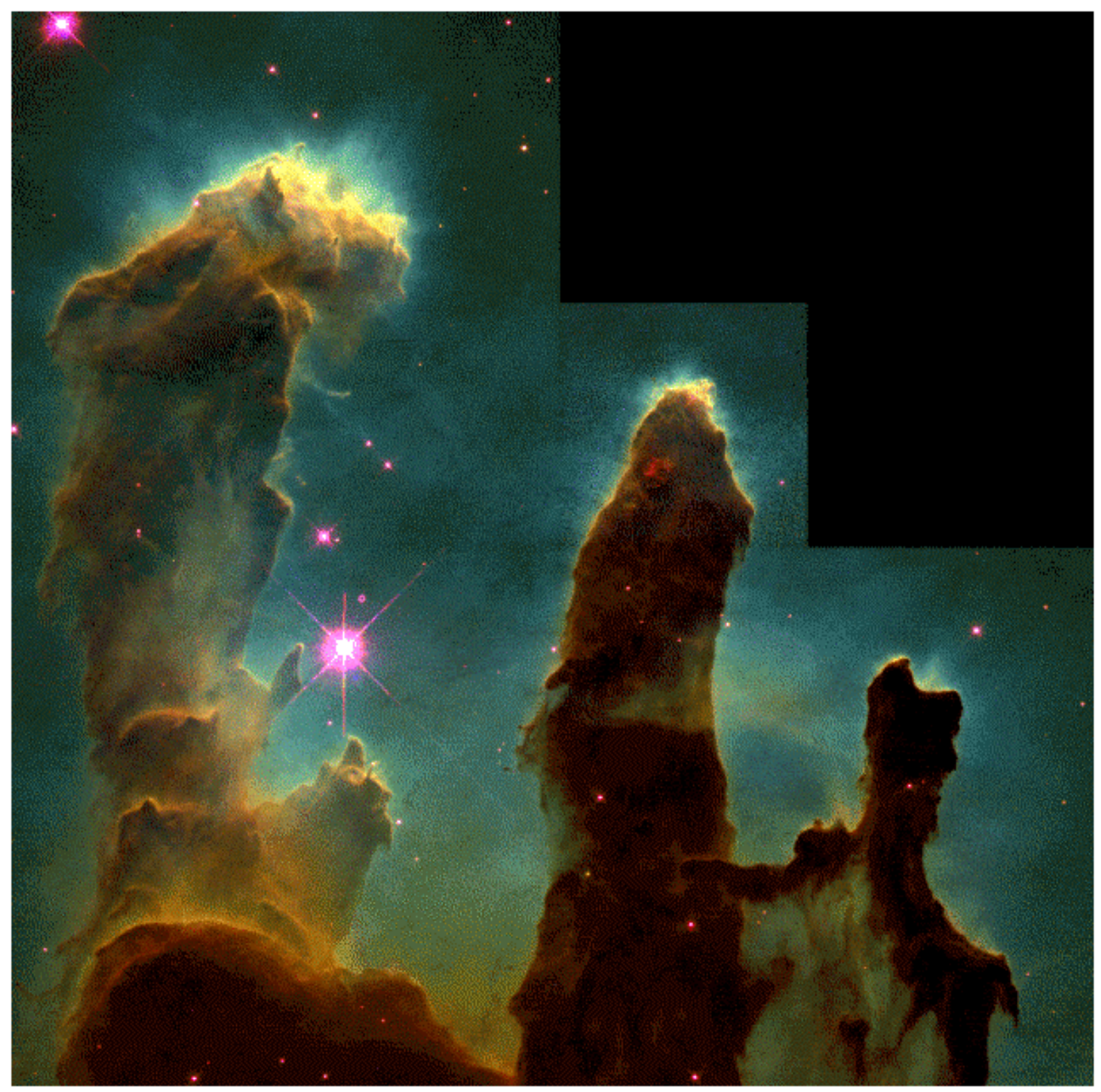




\section{OUTLINE}

Formulation of the problem (supersonic turbulence decays very quickly)

Possible experiment on the temporal evolution of the trans-sonic turbulence.

MHD turbulence: can it provide necessary stiffness? - Probably, not, if radiation is fast.

"Static" MHD turbulence (random, forcefree magnetic field) - Yes, it can provide necessary stiffness.

Acknowledgment: discussions with Bruce Remington (LLNL), Jave Kane (LLNL) and Marc Pound (U. of Maryland, College Park) are gratefully acknowledged 


\section{A PROBLEM:}

The molecular matter stays at a low temperature $\sim 30 \mathrm{~K}$ : at higher temperatures, extremely strong heat losses begin (radiation in $1 \mathrm{~mm}$ range)

The gas pressure inferred from simple hydrodynamic arguments is much (10 to 100 times) higher than the pressure found as a product of density and temperature.

A "canonical" solution: attribute the hydrodynamic pressure to the turbulent ram pressure of a small-scale hydrodynamic turbulence. This explanation to be valid, one has to assume that the turbulence is strongly supersonic. This, in turn, would mean extremely fast dissipation $(\sim 1$ turn-over time for characteristic vortex size), incompatible with any available energy sources.

A conclusion: hydrodynamic turbulence cannot provide required "stiffness" to molecular clouds. 


\section{A CONCEPT OF A LASER EXPERIMENT}

As it would be desirable to obtain a direct experimental information on the decay of a transonic turbulence, we suggest the following experiment:

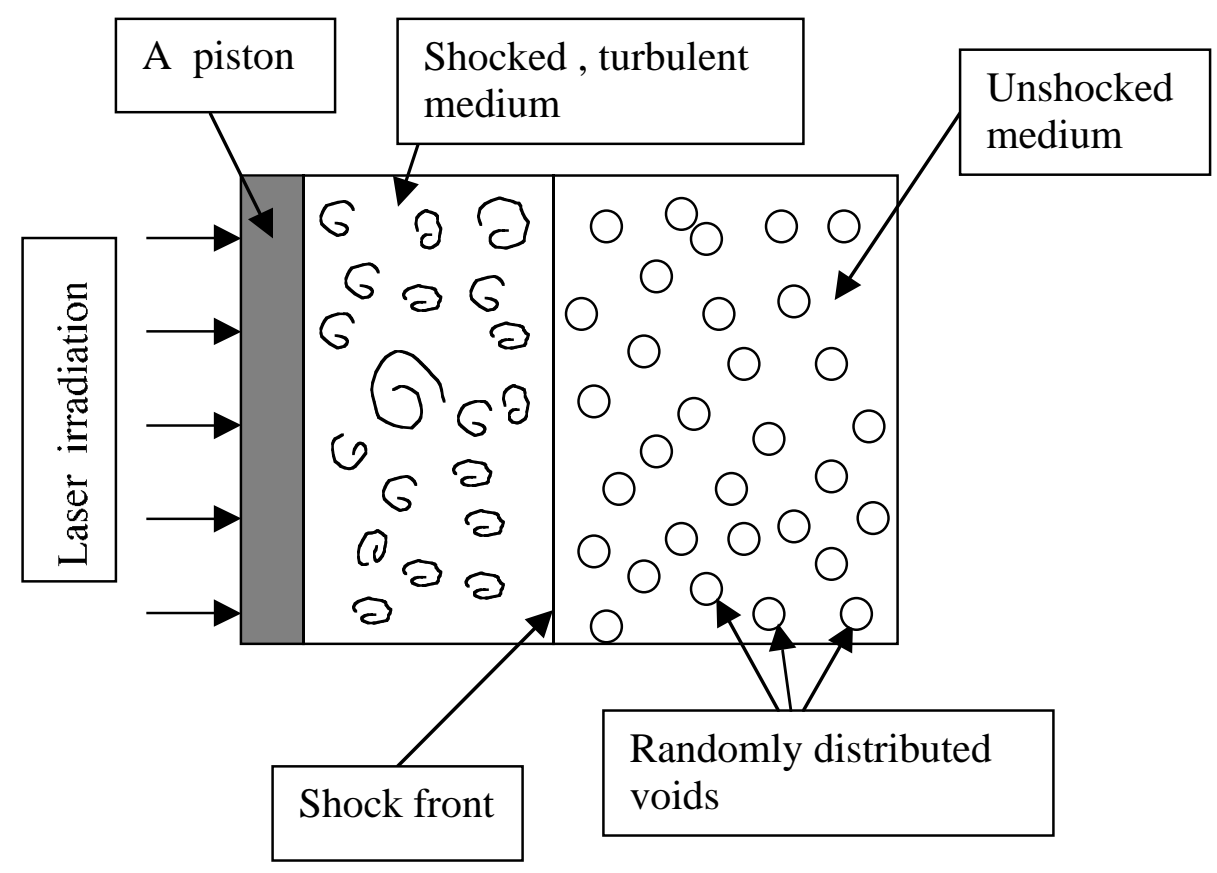

Using voids as a source of turbulent vortices is attractive because it allows one to eliminate complexities associated with mixing of different materials. Filling factor $\sim 1$. The shape of voids is not very important.

For a strong shock, the fluid behind the shock will be strongly turbulent, with a characteristic pulsation velocity of order of the sound velocity in the shocked material. 
How to observe: add a strip seeded with a tracer; its turbulent broadening will be a measure of the turbulent diffusion.

One can introduce a spherical marker (no alignment issues). Both face-on radiography, and a side-on radiography are possible. The side-on radiography can be used to study possible anisotropy of the turbulence.

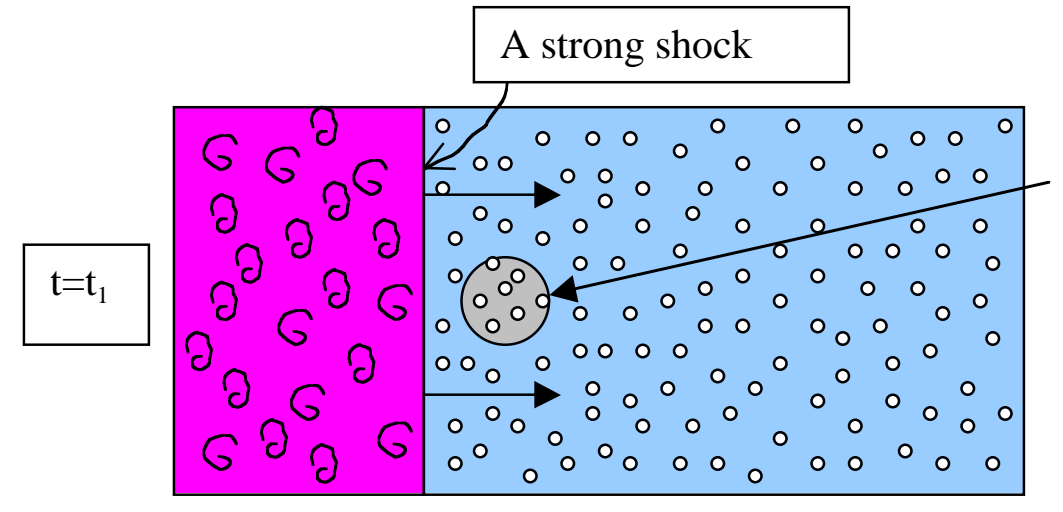

A tagged ball comprises a large number of voids

\section{$\mathrm{t}=\mathrm{t}_{2}$}

$\mathrm{t}=\mathrm{t}_{3}$
Just behind the shock, the ball is compressed in the normal direction

Reference experiment: compressing a "uniform" matter. 


\section{SUSTAINING SUPERSONIC TURBULENCE}

In the molecular cloud, the cooling time is typically orders of magnitude shorter than the sound transit time. Whence, even if initially the matter was hot and the turbulence was initially subsonic, very quickly the turbulent velocity becomes greater than the sound velocity. The resulting formation of shocks gives rise to a much faster decay of the turbulence than in the case of a subsonic (incompressible) turbulence.

One can try to study this process experimentally, by using the following techniques:

1) Creating conditions where the shock-heated plasma would be strongly radiating (rapidly cooling).

2) Letting the turbulent plasma to expand (e.g., in the rarefaction wave).

3) Compressing the turbulent plasma

The first approach would require reaching high temperatures of the shocked matter (may become feasible with the NIF facility)

The second (the third) approach works for the matter with a "stiff" ("soft") equation of state [effective adiabat index higher (lower) than 5/3] 


\section{MHD TURBULENCE ALSO CANNOT PROVIDE NECESSARY STIFFNESS}

By MHD turbulence we mean flows with a tangled magnetic field, with the average magnetic field much less than the turbulent field.

This

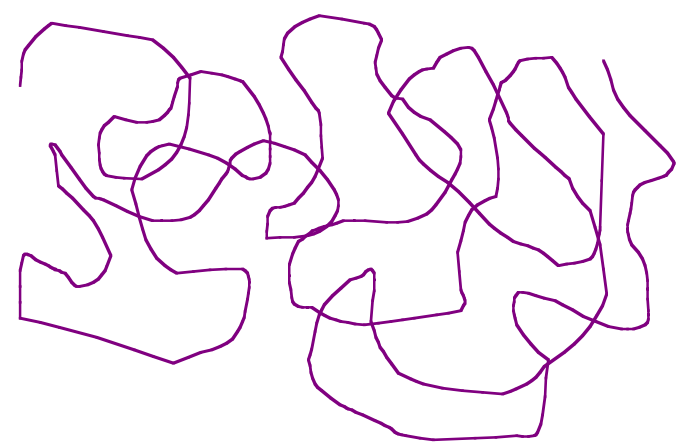

Not this

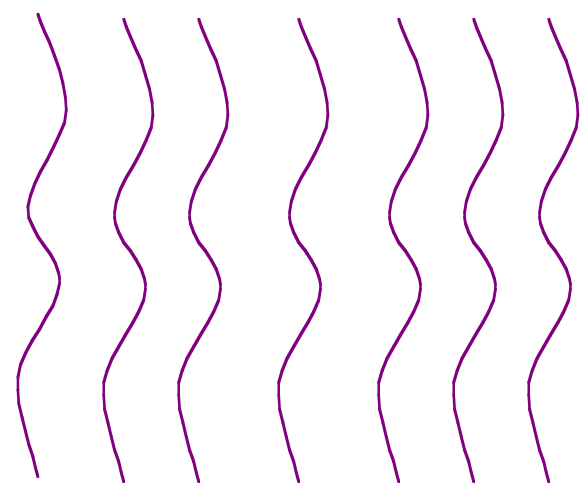

Mac Low et al (1998): turbulent energy decays as

$$
W_{\text {turb }} \sim t^{-\eta}, \quad 0.85<\eta<1.2
$$

Decay occurs within a few turn-over times for the largestscale vortices

(M.-M. Mac Low, R.S. Klessen, A. Burkert, M.D. Smith. "Decay Timescales of MHD Turbulence in Molecular Clouds." In: Interstellar turbulence, J. Franco, A Caraminiana, Editors, Cambridge University Press, 1999. p. 256; E.C. Ostriker, J.M. Stone, C.F. Gammie. "Density, velocity, and magnetic field structure in turbulent molecular cloud models. Astropysical Journal, 546, 980, 2001) 


\section{EXISTING MODELS OF THE STIFFNESS OF MOLECULAR CLOUDS}

\begin{tabular}{|l|l|l|}
\hline A Model & Main difficulty & Representative reference \\
\hline $\begin{array}{l}\text { Supersonic } \\
\text { turbulence }\end{array}$ & $\begin{array}{l}\text { Very high dissipation } \\
\text { rate related to shocks }\end{array}$ & $\begin{array}{l}\text { Mestel. MNRAS, 6, 161 } \\
\text { (1965) }\end{array}$ \\
\hline MHD turbulence & $\begin{array}{l}\text { Formation of shocks } \\
\text { parallel to the magnetic } \\
\text { field and very fast } \\
\text { dissipation of the } \\
\text { turbulence }\end{array}$ & $\begin{array}{l}\text { McLow et al., PRL, 80, } \\
\text { 2754 (1998) }\end{array}$ \\
\hline $\begin{array}{l}\text { A medium composed of } \\
\text { clumps and non- } \\
\text { interacting shells } \\
\text { moving at supersonic } \\
\text { velocities }\end{array}$ & $\begin{array}{l}\text { A relatively short time } \\
\text { for collisions between } \\
\text { dense structures (?) }\end{array}$ & $\begin{array}{l}\text { Melnick et al, In } \\
\text { "Interstellar turbulence." } \\
\text { J. Franco, A } \\
\text { Caraminiana, Editors, } \\
\text { Cambridge University } \\
\text { Press, 1999, p. 148 }\end{array}$ \\
\hline $\begin{array}{l}\text { A large-scale magnetic } \\
\text { field permeating a cloud }\end{array}$ & $\begin{array}{l}\text { In most cases, the } \\
\text { observed magnetic field } \\
\text { strength is insufficient } \\
\text { to provide a required } \\
\text { stiffness }\end{array}$ & $\begin{array}{l}\text { R.M. Crutcher, Ap.J. } \\
\text { 520, 706 (1991) }\end{array}$ \\
\hline
\end{tabular}




\section{POSSIBLE LONG-LASTING TURBULENT SUPPORT: "STATIC" MHD TURBLENCE}

Force-free magnetic field

$$
\nabla \times \mathbf{B}=\lambda \mathbf{B}(\mathbf{j}=4 \pi \lambda \mathbf{B} / c)
$$

Characteristic vortex size: $1 / \lambda$. The parameter $\lambda$ may vary in space and time.

A plausible scenario leading to a formation of a force-free random magnetic field:
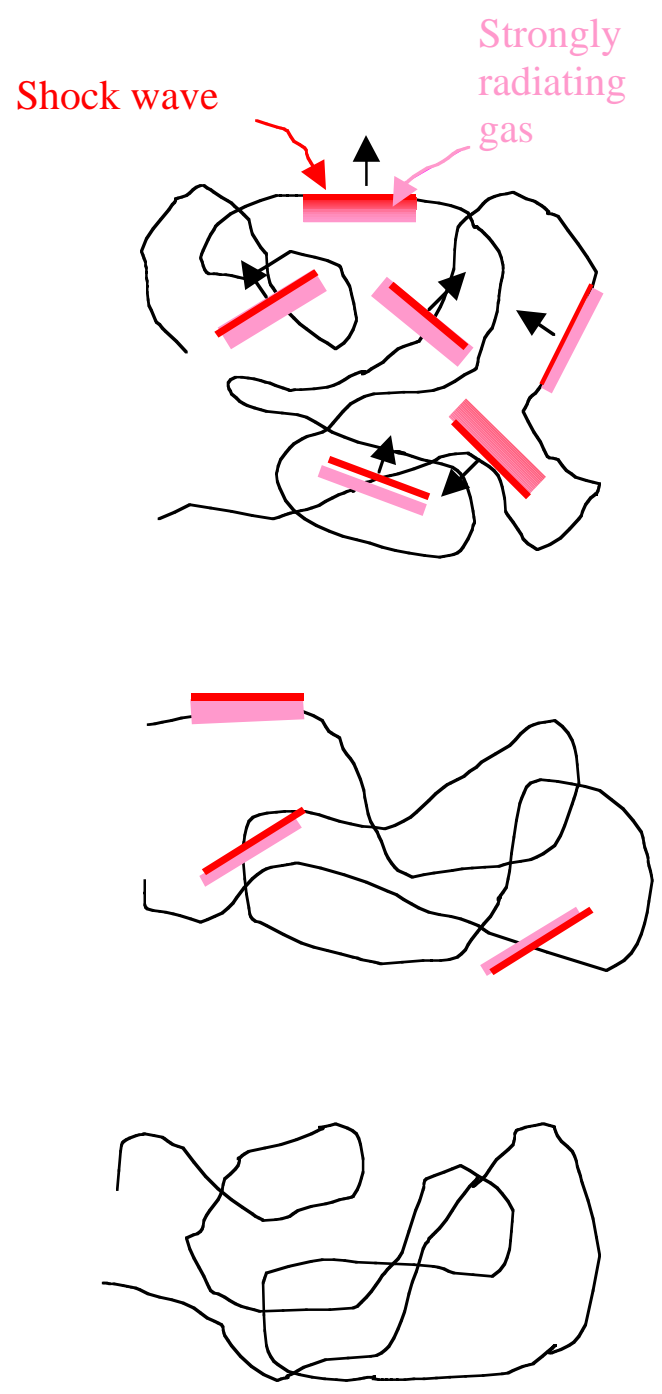

1) Initial (not a force-free) MHD turbulence stirs the gas, generates shocks, and transfers energy to the gas that quickly radiates it; the gas pressure remains low during this whole process;

2) The system evolves in the direction of a force-free state, leading to a gradual slowing down of a stirring (and dissipation);

3) A force-free state is reached whose lifetime is determined by a very slow resistive dissipation 


\section{REACTION OF A "STATIC" TURBULENCE TO COMPRESSION}

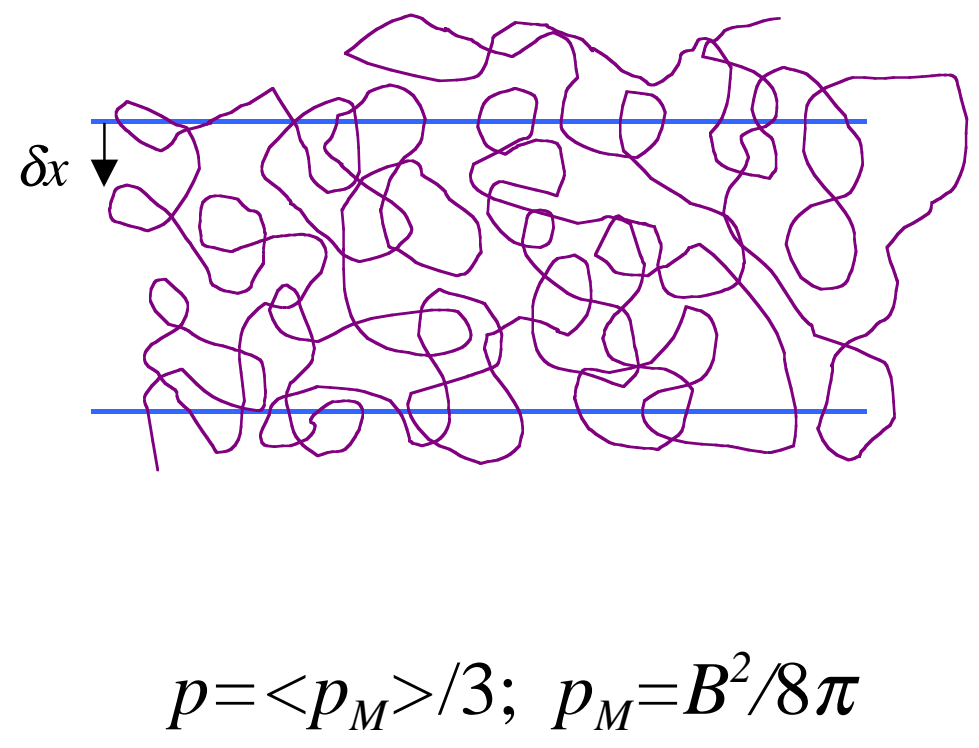

The energy density:

$$
W=\left\langle p_{M}\right\rangle
$$

The adiabat index

$$
\gamma=4 / 3
$$

(because $p=W / 3$ ) 


\section{A DERIVATION:}

Magnetic field stress tensor

$$
\pi_{\alpha \beta}=-p_{M} b_{\alpha} b_{\beta}+p_{M}\left(\delta_{\alpha \beta}-b_{\alpha} b_{\beta}\right), \boldsymbol{b}=\boldsymbol{B} /|\boldsymbol{B}|
$$

For a surface oriented perpendicularly to an axis $\mathrm{z}$, the $p_{z z}$ component (the "pressure" acting on this surface) is

$$
\pi_{z z}=\frac{B_{x}^{2}+B_{y}^{2}-B_{z}^{2}}{8 \pi}
$$

For isotropic turbulence,

$$
p=\left\langle p_{z z}\right\rangle=\left\langle p_{M}\right\rangle / 3
$$




\section{REACTION OF A STATIC TURBULENCE TO SHEAR DEFORMATION}

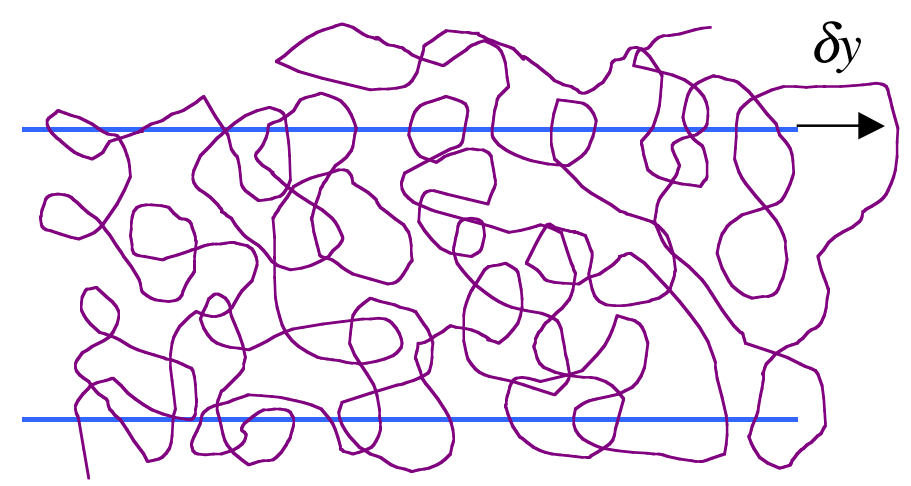

Shear stress:

$$
\sigma=-\left(p_{M} / 6\right)(d \delta y / d x)
$$

There is a rheological decay of the shear stress. 


\section{DISSIPATIVE PROCESSES}

Generally speaking, both compression and shear deformation trigger reconnection process that leads to some dissipation

This gives rise to appearance of the following terms in the momentum equation:

$$
\sigma_{\alpha \beta}=\eta\left(\frac{\partial v_{\alpha}}{\partial x_{\beta}}+\frac{\partial v_{\beta}}{\partial x_{\alpha}}-\frac{2}{3} \delta_{\alpha \beta} \frac{\partial v_{\gamma}}{\partial x_{\gamma}}\right)+\varsigma \delta_{\alpha \beta} \frac{\partial v_{\gamma}}{\partial x_{\gamma}}
$$

with

$$
\varsigma \approx 3 \eta=\frac{p_{M}}{\tau}
$$

where $\tau$ is a characteristic time of the reconnection over the scale $1 / \lambda$. 


\section{SUMMARY OF THE "STATIC TURBULENCE” EFFECTS}

"Static turbulence" has a very long decay time and is, therefore, an excellent candidate for a factor providing "stiffness" of molecular clouds

When a medium with initially present "static turbulence" is forced to move, the reaction is the following:

- For compressional waves, $\gamma=4 / 3$

- For shear waves, the shear stress is present, with a rheological decay

- Dissipative processes accompany both compressional and shear flow
A random magnetic field would not contribute a lot to a line-of sight average of polarization; small measured $\langle\mathbf{B}\rangle$ may correspond to a large $\left\langle\mathrm{B}^{2}\right\rangle^{1 / 2}$ 


\section{WHAT IS THE SOURCE OF THE}

STATIC TURBULENCE?

Initial large-scale weak magnetic field threading the cloud would be tangled in the course of fluid convection during the early stage of the cloud existence.

The magnetic pressure of this random field will be of the order of the gaseous pressure.

When the ablation pressure "turns on," the random magnetic field is compressed and its pressure provides the necessary stiffness.

Observed line broadening in molecular transitions can be explained by two effects: radiation of the just shocked matter; the presence of non-uniform large-scale motions integrated along the line of sight. 
Direct observations of the line shapes show that there exists considerable suprathermal broadening, How can this be compatible with the "static" turbulence?

An answer: locally static turbulence is compatible with supersonic velocity variation of the global flow .

\section{A relevant quotation:}

"A clear isolation of the effects of turbulent motions from observed line profiles, however, is not readily obtainable, because of the uncertainties introduced by the possible existence of large scale mass motions along the line of sight"

G. Munch. "Turbulence in the Interstellar Medium: a Retrospective Review," In: "Interstellar turbulence." J. Franco, A Caraminiana, Editors, Cambridge University Press, 1999. p.1.

\section{Another possibility: the line radiation comes predominantly from the just shocked hot regions}




\section{SUMMARY}

An experiment directed towards studies of decaying compressible turbulence has been proposed

"Static" (force-free) magnetic turbulence has been suggested as a slowly-decaying agent providing a necessary stiffness to molecular clouds

Basic equations describing effects of such turbulence on macroscopic motions of matter have been formulated 


\section{Single-Velocity, Multi-Component Turbulent Transport Models for Interfacial Instability-Driven Flows}

\section{Oleg Schilling}

University of California, Lawrence Livermore National Laboratory

P.O. Box 808, L-22, Livermore, CA 94551

(925) 423-6879, schilling1@IInl.gov

\section{Presented at the}

$8^{\text {th }}$ International Workshop on the Physics of Compressible Turbulent Mixing

California Institute of Technology, Pasadena, CA

9-14 December 2001 


\section{Outline of presentation}

- Motivation

- The need for turbulent transport and mixing models

- Single- vs. multiple-velocity, multi-component fluid formulations

- Derivation of the Favre-Reynolds averaged single-velocity equations

- Two-equation turbulence models

- The general $K-Z$ model

- The $K-\varepsilon$ model

- Derivation of consistent $K-I, K-\omega$, and $K-\tau$ models

- Work in progress: a priori model tests

- Determination of model coefficients from experimental data

- Determination of model coefficients from simulation data

- Conclusions 


\section{An averaged description of turbulent transport and mixing is needed due to the very wide range of spatio-temporal scales in turbulent mixing layers

- Direct numerical simulation (DNS) cannot attain parameter regimes of interest for astrophysical and inertial confinement fusion (ICF) applications

- Large-eddy simulation (LES) is not yet sufficiently developed

- Interim solution: turbulent transport and mixing models, which have similarities with LES

- Transport models are based on closing terms in the density-weighted averaged equations

- Reynolds stress tensor

- Density and energy flux

- These quantities are modeled using an eddy viscosity approximation
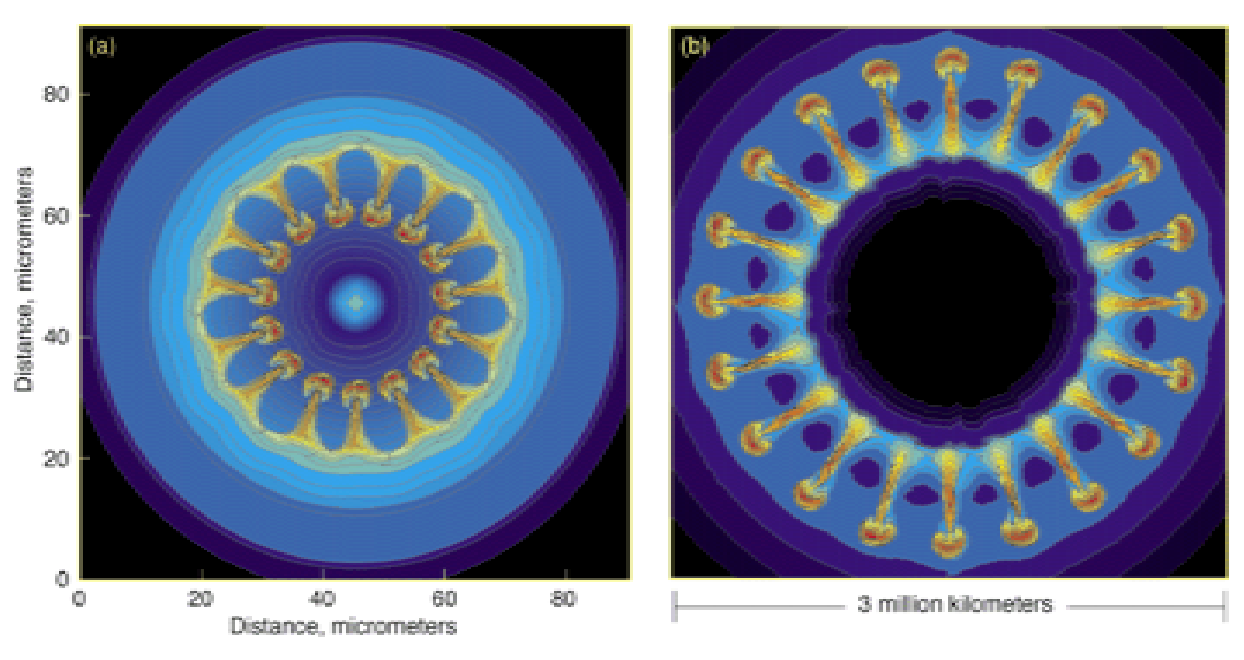

Striking similarites exist betwsen hydrodynamic instabilities in (a) inertal confinemant fusion capsule implosions and (b) core-collapse supenova explosions. [Image (a) is from Sakagami and Nishihara. Physics of Fuids B2. ICF

supernova 


\section{Single-velocity formulations of multi- component flow are significantly less complex than multiple-velocity formulations}

- Single-velocity, multi-component fluid formulations:

- Equations systematically derived from reacting flow theory

- Equations have nearly the same form as the single-fluid, compressible fluid dynamics equations

- Additional fluxes involving a diffusion velocity are present

- The diffusion velocity is obtained, and these fluxes are expressed in terms of a mass diffusion flux

- Multiple-velocity, multi-component fluid formulations:

- Require multiple advection terms equal to number of fluids

- Require fluid dynamic fields for every fluid, so the number of equations to model and solve is large

- Require phenomenological modeling of interfacial source terms arising from interfacial averaging: drag, added mass terms, etc. 


\section{The derivation of the single-velocity equations} begins with the full, $N$-fluid equations expressing

\section{mass, momentum, and energy conservation}

- In compact form, these equations are ( $r$ labels each fluid):

$$
\frac{\partial}{\partial t}\left(\rho^{r} \phi_{\alpha}^{r}\right)+\frac{\partial J_{\alpha \beta}^{r}\left[\phi^{r}\right]}{\partial x_{\beta}}=F_{\alpha}^{r}+S_{\alpha}^{r}
$$

- where the fields, fluxes, forces, and sources are

$$
\begin{aligned}
& \rho^{r} \phi_{\alpha}^{r}=\left(\begin{array}{c}
\hline \rho^{r} \\
\hline \rho^{r} v_{\alpha}^{r} \\
\hline \rho^{r} e^{r} \\
\hline \rho^{r} \phi^{r}
\end{array}\right), \quad J_{\alpha \beta}^{r}=\left(\begin{array}{c}
\rho^{r} v_{\beta}^{r} \\
\hline \begin{array}{c}
\rho^{r} v_{\alpha}^{r} v_{\beta}^{r}+p^{r} \delta_{\alpha \beta}-\sigma_{\alpha \beta}^{r} \\
\left(\rho^{r} e^{r}+p^{r}\right) v_{\beta}^{r}-v_{\alpha}^{r} \sigma_{\alpha \beta}^{r}+\Phi_{\beta}^{\mathrm{rad}, r}
\end{array} \\
\rho^{r} \phi^{r} v_{\beta}^{r}+\Phi_{\beta}^{\phi, r}
\end{array}\right) \\
& F_{\alpha}^{r}=\left(\begin{array}{c}
0 \\
\hline \rho^{r} g_{\alpha} \\
0 \\
0
\end{array}\right), \quad S_{\alpha}^{r}=\left(\begin{array}{c}
R^{r} \\
\hline v_{\alpha}^{r} R^{r}+\rho^{r} g_{\alpha} v_{\alpha}^{r} \\
\hline \phi^{r} R^{r}
\end{array}\right)
\end{aligned}
$$




\section{These fields are defined so that summing appropriate expressions over each fluids recovers the non-reacting, single-fluid equations}

- The quantities $\rho^{r}, v_{\alpha}^{r}, U^{r}, \varphi^{r}, \Phi_{\alpha}{ }^{r a d, r}, \Phi, g_{\alpha}, R^{r}$, and $H^{r}$ are the density, velocity, internal energy, scalar, radiative flux, scalar flux, acceleration, reaction rate, and heat of formation

- The pressure, viscous stress tensor, and total energy are

$$
\begin{gathered}
p^{r}=p^{r}\left(\rho^{r}, U^{r}\right) \\
\sigma_{\alpha \beta}^{r} \equiv \mu^{r}\left(\frac{\partial v_{\alpha}^{r}}{\partial x^{\beta}}+\frac{\partial v_{\beta}^{r}}{\partial x^{\alpha}}\right)+\left(\xi^{r}-\frac{2 \mu^{r}}{d}\right) \delta_{\alpha \beta} \frac{\partial v_{\gamma}^{r}}{\partial x_{\gamma}} \\
e^{r}=\frac{v_{r}^{2}}{2}+U^{r}+\rho m^{r} H^{r}-\mathbf{g} \cdot \mathbf{x}
\end{gathered}
$$

- Consistency with the single-fluid equations is obtained with the constraints

$$
\begin{gathered}
\sum_{r=1}^{N} \rho^{r} \phi_{\alpha}^{r}=\rho \phi_{\alpha} \quad, \quad \sum_{r=1}^{N} J_{\alpha \beta}^{r}=J_{\alpha \beta} \\
\sum_{r=1}^{N} F_{\alpha}^{r}=F_{\alpha} \quad, \quad \sum_{r=1}^{N} S_{\alpha}^{r}=S_{\alpha} \\
\sum_{r=1}^{N} R^{r}=0 \quad, \quad \sum_{r=1}^{N} \phi_{\alpha}^{r} R^{r}=0
\end{gathered}
$$




\section{The single-velocity equations are obtained by decomposing the velocity into a mean velocity plus a diffusion velocity}

- Introduce the local mass fraction of fluid $r$

$$
m^{r}(\mathbf{x}, t) \equiv \frac{\rho^{r}}{\rho} \quad, \quad \sum_{r=1}^{N} m^{r}(\mathbf{x}, t)=1
$$

- Write the velocity of fluid $r$ as

$$
\mathbf{v}^{r}=\mathbf{v}+\mathbf{V}^{r}, \quad \mathbf{v} \equiv \sum_{r=1}^{N} m^{r} \mathbf{v}^{r}
$$

where $V^{r}$ is the diffusion velocity, which expresses the molecular transport caused by the concentration gradient in fluid $r$

- The identity

$$
\sum_{r=1}^{N} m^{r} \mathbf{V}^{r}=0
$$

is central to the derivation of the single-velocity equations 


\section{The single-velocity equations are a consequence of the previous identities}

- Substituting the velocity decomposition into the multi-component equations, summing, and using the previous identities gives the single-velocity equations

$$
\frac{\partial}{\partial t}\left(\rho \phi_{\alpha}\right)+\frac{\partial J_{\alpha \beta}}{\partial x_{\beta}}=F_{\alpha}+S_{\alpha}
$$

- The fields and fluxes are

$$
\rho \phi_{\alpha}=\left(\begin{array}{c}
\rho \\
\hline \rho v_{\alpha} \\
\hline \rho e \\
\hline \rho \phi
\end{array}\right) \quad J_{\alpha \beta}=\left(\begin{array}{c}
\hline \rho v_{\beta} \\
\hline \frac{\rho v_{\alpha} v_{\beta}+p \delta_{\alpha \beta}-\sigma_{\alpha \beta}}{(\rho e+p) v_{\beta}-v_{\alpha} \sigma_{\alpha \beta}+\Phi_{\beta}^{\mathrm{rad}}} \\
\hline \rho \phi v_{\beta}+\Phi_{\beta}^{\phi}
\end{array}\right)+\left(\begin{array}{c}
0 \\
\hline-\sigma_{\alpha \beta}^{D} \\
\hline J_{\beta}^{e} \\
\hline J_{\beta}^{\phi}
\end{array}\right)
$$

where the last term in $J_{\alpha \beta}$ depends on the diffusion velocity and must be modeled 


\section{The forces, sources, and other quantities are defined as follows}

- The forces and sources are

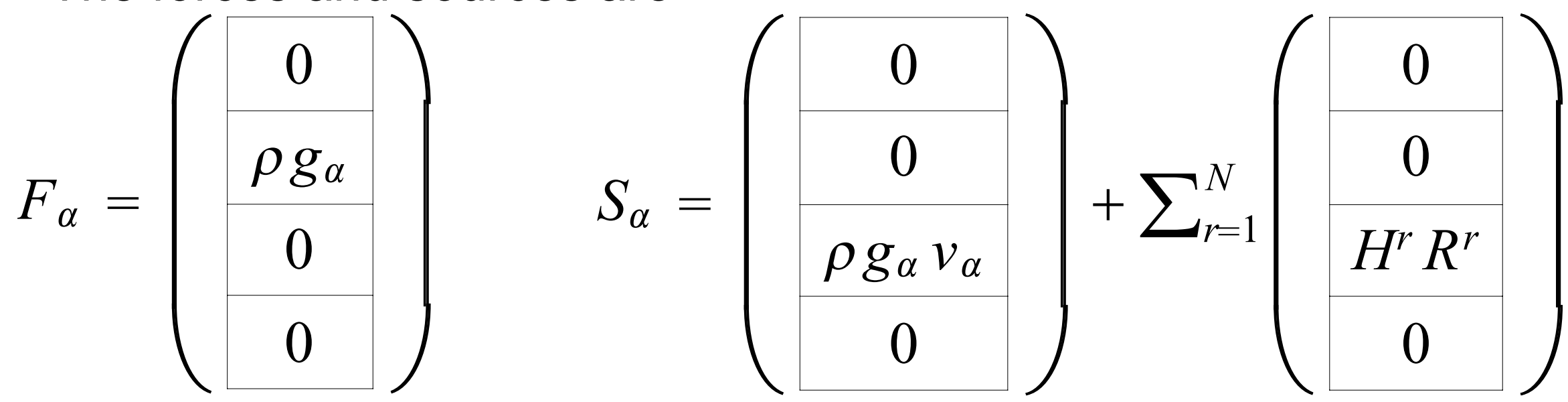

- The total density, pressure, radiative flux, viscous stress tensor, dynamic viscosity, and bulk viscosity are

$$
\begin{gathered}
\rho \equiv \sum_{r=1}^{N} \rho^{r} \quad p \equiv \sum_{r=1}^{N} p^{r}\left(\rho^{r}, U^{r}\right) \quad \Phi_{\alpha}^{\mathrm{rad}} \equiv \sum_{r=1}^{N} \Phi_{\alpha}^{\mathrm{rad}, r} \\
\sigma_{\alpha \beta} \equiv \mu\left(\frac{\partial v_{\alpha}}{\partial x^{\beta}}+\frac{\partial v_{\beta}}{\partial x^{\alpha}}\right)+\left(\xi-\frac{2 \mu}{d}\right) \delta_{\alpha \beta} \frac{\partial v_{\gamma}}{\partial x_{\gamma}} \\
+\sum_{r=1}^{N} \mu^{r}\left(\frac{\partial V_{\alpha}^{r}}{\partial x^{\beta}}+\frac{\partial V_{\beta}^{r}}{\partial x^{\alpha}}\right)+\left(\xi^{r}-\frac{2 \mu^{r}}{d}\right) \delta_{\alpha \beta} \frac{\partial V_{\gamma}^{r}}{\partial x_{\gamma}} \\
\mu \equiv \sum_{r=1}^{N} \mu^{r}, \xi \equiv \sum_{r=1}^{N} \xi^{r}
\end{gathered}
$$




\section{The diffusive fluxes are defined as follows}

- The multi-component viscous diffusion stress tensor is

$$
\sigma_{\alpha \beta}^{D} \equiv-\rho \sum_{r=1}^{N} m^{r} V_{\alpha}^{r} V
$$

- The diffusive energy flux is

$$
J_{\alpha}^{e} \equiv \rho \sum_{r=1}^{N} m^{r} e^{r} V_{\alpha}^{r}
$$

- The diffusive scalar flux is

$$
J_{\alpha}^{\phi} \equiv \rho \sum_{r=1}^{N} m^{r} \phi^{r} V_{\alpha}^{r}
$$




\section{The averaged equations are obtained by introducing the Favre-Reynolds decompositions and averaging}

- The Favre-Reynolds decompositions are

$$
\begin{gathered}
\phi_{\alpha}^{r}(\mathbf{x}, t)=\not_{\alpha}^{r}(\mathbf{x}, t)+\phi_{\alpha}^{r}(\mathbf{x}, t)^{\prime \prime} \\
\rho^{r}(\mathbf{x}, t)=\bar{\rho}^{r}(\mathbf{x}, t)+\rho^{r}(\mathbf{x}, t)^{\prime} \quad p^{r}(\mathbf{x}, t)=\bar{p}^{r}(\mathbf{x}, t)+p^{r}(\mathbf{x}, t)^{\prime}
\end{gathered}
$$

- The Favre average is

$$
\widetilde{\phi}_{\alpha} \equiv \frac{\overline{\rho \phi_{\alpha}}}{\bar{\rho}}
$$

- The Favre-averaged multi-component fluid dynamics equations are

$$
\frac{\partial}{\partial t}\left(\bar{\rho} \oiint_{\alpha}\right)+\frac{\partial \bar{J}_{\alpha \beta}}{\partial x_{\beta}}=\bar{F}_{\alpha}+\bar{S}_{\alpha}
$$




\section{The Favre-averaged fields and fluxes are defined as follows}

- The fields and fluxes are

$$
\bar{\rho}{\phi_{\alpha}}_{\alpha}\left(\begin{array}{c}
\hline \bar{\rho} \\
\hline \bar{\rho} \widetilde{v}_{\alpha} \\
\hline \bar{\rho} \widetilde{e} \\
\hline \bar{\rho} \widetilde{\phi}
\end{array}\right)
$$

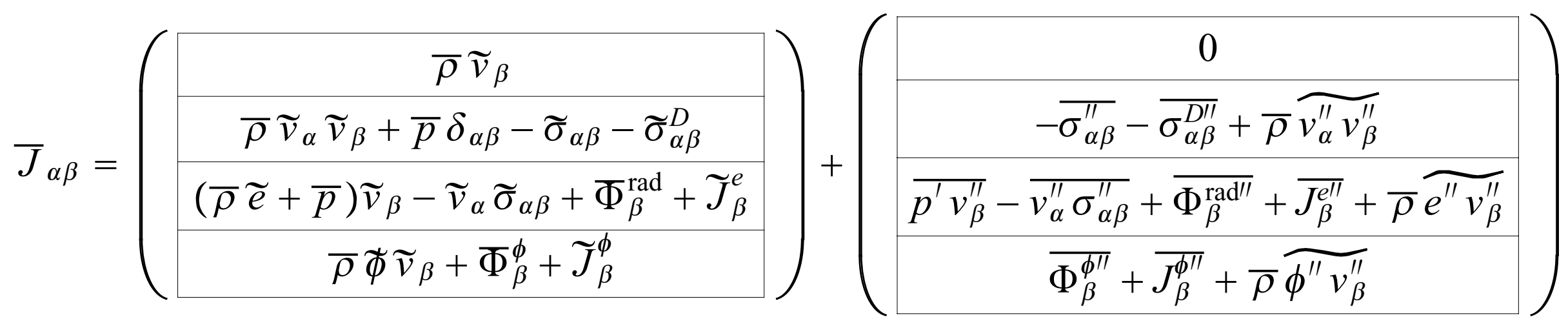




\section{The Favre-averaged forces and sources are defined as follows}

- The forces and sources are

$$
\begin{aligned}
& \bar{F}_{\alpha}=\left(\begin{array}{c}
\hline 0 \\
\hline \bar{\rho} g_{\alpha} \\
\hline 0 \\
\hline 0 \\
\hline
\end{array}\right) \\
& \bar{S}_{\alpha}=\left(\begin{array}{c}
\hline 0 \\
\hline 0 \\
\hline \rho g_{\alpha} \widetilde{v}_{\alpha} \\
\hline 0
\end{array}\right)+\left(\begin{array}{c}
0 \\
\frac{0}{\rho^{\prime} v_{\alpha}^{\prime \prime} \mathrm{g}_{\alpha}+\sum_{r}\left(\bar{H}^{r} \bar{R}^{r}+\overline{H^{r \prime \prime} R^{r / 1}}\right)} \\
0
\end{array}\right)
\end{aligned}
$$

- At large Reynolds numbers, the viscous stress terms and diffusive fluxes are assumed to be negligible compared to the Reynolds stress tensor and turbulent fluxes 


\section{A gradient diffusion approximation is usually used to model the turbulent stresses and fluxes}

- The gradient diffusion approximation is

$$
\begin{gathered}
\overline{\rho \phi_{\alpha}^{\prime \prime} v_{j}^{\prime \prime}}=\bar{\rho} \widetilde{\phi_{\alpha}^{\prime \prime} v_{\beta}^{\prime \prime}}=-\frac{\partial}{\partial x^{j}}\left(\frac{v_{t}}{\sigma_{\phi_{\alpha}}} \bar{\rho} \Phi_{\alpha}\right) \\
\tau_{i j} \equiv \overline{\rho v_{i}^{\prime \prime} v_{j}^{\prime \prime}}=\bar{\rho} \widetilde{v_{i}^{\prime \prime} v_{j}^{\prime \prime}} \\
=2 \bar{\rho} \widetilde{E^{\prime \prime}} \frac{\delta_{i j}}{3}-2 \bar{\rho} v_{t}\left(\widetilde{S}_{k j}-\frac{\delta_{k j}}{3} \frac{\partial \widetilde{v}_{l}}{\partial x_{l}}\right) \\
\overline{\rho^{\prime} v_{j}^{\prime \prime}}=-\frac{\partial}{\partial x_{j}}\left(\frac{v_{t}}{\sigma_{\rho}} \bar{\rho}\right)
\end{gathered}
$$

- The eddy viscosity

$$
v_{t}=C_{\mu}\left(\widetilde{E^{\prime \prime}}\right)^{\frac{m+2 n}{n}}\left(\widetilde{Z^{\prime \prime}}\right)^{-\frac{1}{n}}
$$

is determined by the solution of transport equations for two turbulence variables $K\left(=E^{\prime \prime}\right)$ and

$$
\widetilde{Z^{\prime \prime}} \equiv C_{Z}\left(\widetilde{E^{\prime \prime}}\right)^{m}\left(\widetilde{\epsilon^{\prime \prime}}\right)^{n}
$$




\section{The turbulent kinetic energy equation is closed as follows}

- The unclosed turbulent kinetic energy equation is

$$
\begin{gathered}
\frac{\partial}{\partial t}\left(\bar{\rho} \widetilde{E^{\prime \prime}}\right)+\frac{\partial}{\partial x_{j}}\left(\bar{\rho} \widetilde{E}^{\prime \prime} \widetilde{v}_{j}\right) \\
=\underbrace{\left(\bar{\rho} \overline{v_{i}^{\prime \prime}}+\overline{\rho^{\prime} v_{i}^{\prime \prime}}\right) g_{i}}_{\text {force production }}-\underbrace{\frac{\partial}{\partial x_{j}}\left(\frac{\overline{\rho v^{\prime \prime 2} v_{j}^{\prime \prime}}}{2}+\overline{p^{\prime} v_{j}^{\prime \prime}}-\overline{v_{i}^{\prime \prime} \sigma_{i j}^{\prime \prime}}-\overline{v_{i}^{\prime \prime} \sigma_{i j}^{D^{\prime \prime}}}\right)}_{\text {turbulentdiffusion }}
\end{gathered}
$$

$+\underbrace{\widetilde{v}_{i} \frac{\partial}{\partial x_{j}}\left(\overline{\rho v_{i}^{\prime \prime} v_{j}^{\prime \prime}}\right)}-\underbrace{\overline{v_{i}^{\prime \prime} \frac{\partial \bar{p}}{\partial x_{i}}}}+\underbrace{\overline{p^{\prime} \frac{\partial v_{j}^{\prime \prime}}{\partial x_{j}}}}-\underbrace{\overline{\sigma_{i j}^{\prime \prime} \frac{\partial v_{i}^{\prime \prime}}{\partial x_{j}}}-\overline{\sigma_{i j}^{D^{\prime \prime}} \frac{\partial v_{i}^{\prime \prime}}{\partial x_{j}}}}$ meanvelocityproduction pressurework pressure-dilatation kineticenergydissipationrate

- Use the gradient diffusion approximation to close the diffusion term and density flux, and $\left(M a_{t}\right.$ is the turbulent Mach number)

$$
\begin{gathered}
\overline{p^{\prime} \frac{\partial v_{i}^{\prime \prime}}{\partial x_{j}}}=\bar{\rho}\left(\alpha_{2} \tau_{i j} \frac{\partial \widetilde{v}_{i}}{\partial x_{j}}+\alpha_{3} M a_{t} \widetilde{\epsilon}^{\prime \prime}\right) M a_{t}^{2} \\
\overline{v_{i}^{\prime \prime}}=-\frac{\overline{\rho^{\prime} v_{i}^{\prime \prime}}}{\bar{\rho}}=\frac{v_{t}}{\bar{\rho} \sigma_{\rho}} \frac{\partial \bar{\rho}}{\partial x^{i}}
\end{gathered}
$$




\section{The modeled turbulent kinetic energy dissipation rate transport equation is obtained as follows}

- The turbulent kinetic energy dissipation rate equation is obtained by multiplying the turbulent kinetic energy equation by $\varepsilon / K$ and a dimensionless constant for each term:

$$
\begin{aligned}
& \frac{\partial}{\partial t}\left(\bar{\rho} \widetilde{\epsilon^{\prime \prime}}\right)+\frac{\partial}{\partial x_{j}}\left(\bar{\rho} \widetilde{\epsilon^{\prime \prime}} \widetilde{v}_{j}\right) \\
& =\underbrace{C_{\epsilon 0} \frac{\widetilde{\epsilon^{\prime \prime}}}{\widetilde{E^{\prime \prime}}}\left(\bar{\rho} \overline{v_{i}^{\prime \prime}}+\overline{\rho^{\prime} v_{i}^{\prime \prime}}\right) g_{i}}+\underbrace{C_{\epsilon 1} \frac{\widetilde{\epsilon^{\prime \prime}}}{{\widetilde{E^{\prime \prime}}}^{\prime \prime}} \widetilde{v}_{i} \frac{\partial}{\partial x_{j}}\left(\overline{\rho v_{i}^{\prime \prime} v_{j}^{\prime \prime}}\right)} \\
& \text { force production } \\
& -\underbrace{C_{\epsilon 2} \bar{\rho} \frac{\left(\widetilde{\epsilon^{\prime \prime}}\right)^{2}}{\widetilde{E^{\prime \prime}}}} \\
& \text { mean velocity production } \\
& -\underbrace{\frac{\partial}{\partial x_{j}}\left(\frac{\mu_{t}}{\sigma_{\epsilon}} \frac{\partial \epsilon^{\prime \prime}}{\partial x^{j}}\right)} \\
& -\underbrace{C_{\epsilon 3} \frac{\widetilde{\epsilon^{\prime \prime}}}{\widetilde{E^{\prime \prime}}} \overline{v_{i}^{\prime \prime}} \frac{\partial \bar{p}}{\partial x_{i}}}_{\text {pressure work }}+\underbrace{C_{\epsilon 4} \frac{\widetilde{\epsilon^{\prime \prime}}}{\widetilde{E^{\prime \prime}}} \overline{p^{\prime} \frac{\partial v_{i}^{\prime \prime}}{\partial x_{i}}}}_{\text {pressure-dilatation }}
\end{aligned}
$$




\section{The modeled $Z$ transport equation is obtained from the $K$ and $\varepsilon$ equations as follows}

- Using the $K$ and $\varepsilon$ equations,

$$
\begin{gathered}
\frac{\partial}{\partial t}\left(\bar{\rho} \widetilde{Z^{\prime \prime}}\right)+\frac{\partial}{\partial x_{j}}\left(\bar{\rho} \widetilde{Z^{\prime \prime}} \widetilde{v}_{j}\right)=\bar{\rho}\left(\frac{\partial}{\partial t}+\widetilde{v}_{j} \frac{\partial}{\partial x_{j}}\right) \widetilde{Z^{\prime \prime}} \\
=\widetilde{\rho} \widetilde{Z^{\prime \prime}}\left[\frac{m}{\widetilde{E^{\prime \prime}}}\left(\frac{\partial}{\partial t}+\widetilde{v}_{j} \frac{\partial}{\partial x_{j}}\right) \widetilde{E^{\prime \prime}}+\frac{n}{\widetilde{\epsilon^{\prime \prime}}}\left(\frac{\partial}{\partial t}+\widetilde{v}_{j} \frac{\partial}{\partial x_{j}}\right) \widetilde{\epsilon^{\prime \prime}}\right] \\
=m \frac{\widetilde{Z^{\prime \prime}}}{\widetilde{E^{\prime \prime}}}\left[\frac{\partial}{\partial t}\left(\bar{\rho} \widetilde{E^{\prime \prime}}\right)+\frac{\partial}{\partial x_{j}}\left(\bar{\rho} \widetilde{E^{\prime \prime}} \widetilde{v}_{j}\right)\right] \\
+n \frac{\widetilde{Z^{\prime \prime}}}{\widetilde{\epsilon^{\prime \prime}}}\left[\frac{\partial}{\partial t}\left(\bar{\rho} \widetilde{\epsilon^{\prime \prime}}\right)+\frac{\partial}{\partial x_{j}}\left(\widetilde{\rho} \widetilde{\epsilon^{\prime \prime}} \widetilde{v}_{j}\right)\right] \\
=\frac{\widetilde{Z^{\prime \prime}}}{\widetilde{E^{\prime \prime}}}\left(m+n C_{\epsilon_{K}}\right)\left[\frac{\partial}{\partial t}\left(\bar{\rho} \widetilde{E^{\prime \prime}}\right)+\frac{\partial}{\partial x_{j}}\left(\widetilde{\rho} \widetilde{E^{\prime \prime}} \widetilde{v}_{j}\right)\right]
\end{gathered}
$$




\section{The turbulent diffusion term is transformed as}

\section{follows}

- Substituting

$$
\widetilde{\epsilon^{\prime \prime}}=\left[\frac{\widetilde{Z^{\prime \prime}}}{C_{Z}\left(\widetilde{E^{\prime \prime}}\right)^{m}}\right]^{1 / n}
$$

it follows that

$$
\begin{gathered}
\widetilde{Z^{\prime \prime}}\left[\frac{m}{\widetilde{E^{\prime \prime}}} \frac{\partial}{\partial x_{j}}\left(\frac{\mu_{t}}{\sigma_{k}} \frac{\partial \widetilde{E}^{\prime \prime}}{\partial x^{j}}\right)+\frac{n}{\widetilde{\epsilon^{\prime \prime}}} \frac{\partial}{\partial x_{j}}\left(\frac{\mu_{t}}{\sigma_{\epsilon}} \frac{\partial \epsilon^{\prime \prime}}{\partial x^{j}}\right)\right] \\
=\frac{m \widetilde{Z^{\prime \prime}}}{\widetilde{E^{\prime \prime}}} \frac{\partial}{\partial x_{j}}\left(\frac{\mu_{t}}{\sigma_{k}} \frac{\partial \widetilde{E^{\prime \prime}}}{\partial x^{j}}\right)+\frac{\partial}{\partial x_{j}}\left[\frac{\mu_{t}}{\sigma_{\epsilon}}\left(\frac{\partial \widetilde{Z}^{\prime \prime}}{\partial x^{j}}-\frac{m \widetilde{Z^{\prime \prime}}}{\widetilde{E^{\prime \prime}}} \frac{\partial \widetilde{E}^{\prime \prime}}{\partial x^{j}}\right)\right] \\
+\frac{\mu_{t}}{n \sigma_{\epsilon}}\left(\frac{\partial \widetilde{Z}^{\prime \prime}}{\partial x^{j}}-\frac{m \widetilde{Z^{\prime \prime}}}{\widetilde{E^{\prime \prime}}} \frac{\partial \widetilde{E}^{\prime \prime}}{\partial x^{j}}\right)\left(\frac{1-n}{\widetilde{Z^{\prime \prime}}} \frac{\partial \widetilde{Z}^{\prime \prime}}{\partial x_{j}}-\frac{m}{\widetilde{E^{\prime \prime}}} \frac{\partial \widetilde{E}^{\prime \prime}}{\partial x_{j}}\right)
\end{gathered}
$$




\section{Finally, the modeled form of the $Z$ transport equation is as follows}

$$
\begin{aligned}
& \frac{\partial}{\partial t}\left(\bar{\rho} \widetilde{Z^{\prime \prime}}\right)+\frac{\partial}{\partial x_{j}}\left(\bar{\rho} \widetilde{Z^{\prime \prime}} \widetilde{v}_{j}\right)=\bar{\rho}\left(\frac{\partial}{\partial t}+\widetilde{v}_{j} \frac{\partial}{\partial x_{j}}\right) \widetilde{Z^{\prime \prime}} \\
& \begin{array}{c}
\underbrace{C_{Z 0} \frac{\widetilde{Z^{\prime \prime}}}{\widetilde{E^{\prime \prime}}}\left(\bar{\rho} \overline{v_{i}^{\prime \prime}}+\overline{\rho^{\prime} v_{i}^{\prime \prime}}\right) g_{i}}_{\text {force production }}+\underbrace{C_{Z 1} \frac{\widetilde{Z^{\prime \prime}}}{\widetilde{E}^{\prime \prime}} \widetilde{v}_{i} \frac{\partial}{\partial x_{j}}\left(\overline{\rho v_{i}^{\prime \prime} v_{j}^{\prime \prime}}\right)}_{\text {mean velocity production }}- \\
-\underbrace{\frac{\partial}{\partial x_{j}}\left[\left(\mu+\frac{\mu_{t}}{\sigma_{\epsilon}}\right) \frac{\partial \widetilde{Z^{\prime \prime}}}{\partial x^{j}}\right]-\frac{m \widetilde{Z^{\prime \prime}}}{\widetilde{E^{\prime \prime}}} \frac{\partial}{\partial x_{j}}\left(\frac{\mu_{t}}{\sigma_{k}} \frac{\partial \widetilde{E^{\prime \prime}}}{\partial x^{j}}\right)}
\end{array} \\
& \text { turbulent diffusion } \\
& +m \frac{\partial}{\partial x_{j}}\left(\frac{\mu_{t}}{\sigma_{\epsilon}} \frac{\widetilde{Z^{\prime \prime}}}{\widetilde{E^{\prime \prime}}} \frac{\partial E^{\prime \prime}}{\partial x^{j}}\right)-\frac{\mu_{t}}{n \sigma_{\epsilon}}\left(\frac{\partial \widetilde{Z^{\prime \prime}}}{\partial x^{j}}-\frac{m \widetilde{Z^{\prime \prime}}}{\widetilde{E^{\prime \prime}}} \frac{\partial{E^{\prime \prime}}^{\prime \prime}}{\partial x^{j}}\right)\left(\frac{1-n}{\widetilde{Z^{\prime \prime}}} \frac{\partial \widetilde{Z^{\prime \prime}}}{\partial x_{j}}-\frac{m}{\widetilde{E^{\prime \prime}}} \frac{\partial \widetilde{E_{k}^{\prime \prime}}}{\partial x_{j}}\right)
\end{aligned}
$$

kinetic energy dissipation rate

turbulent diffusion

$$
-\underbrace{C_{Z 3} \frac{\widetilde{Z^{\prime \prime}}}{\widetilde{E^{\prime \prime}}} \overline{v_{i}^{\prime \prime}} \frac{\partial \bar{p}}{\partial x_{i}}}_{\text {pressure work }}+\underbrace{C_{Z 4} \frac{\widetilde{Z^{\prime \prime}}}{\widetilde{E^{\prime \prime}}} \overline{p^{\prime} \frac{\partial v_{i}^{\prime \prime}}{\partial x_{i}}}}_{\text {pressure-dilatation }}
$$




\section{The coefficients in the modeled Z transport equation are obtained from those in the $\varepsilon$ equation}

- The coefficients in the standard $K-\varepsilon$ model are

$$
\begin{gathered}
\sigma_{k}=1.0, \sigma_{\epsilon}=1.3, C_{\epsilon 1}=1.44, C_{\epsilon 2}=1.92 \\
C_{\epsilon 0}=C_{\epsilon 4}=1.0
\end{gathered}
$$

- The coefficients in the $Z$ equation are

$$
C_{Z 0} \equiv m+n C_{\epsilon 0} \quad, \quad C_{Z 1} \equiv m+n C_{\epsilon 1} \quad, \quad C_{Z 2} \equiv m+n C_{\epsilon 2}
$$

$$
C_{Z 3} \equiv m+n C_{\epsilon 3} \quad, \quad C_{Z 4} \equiv m+n C_{\epsilon 4}
$$

- Different choices of $m$ and $n$ yield different 2-equation models:

- $\widetilde{E^{\prime \prime}}-\widetilde{\epsilon^{\prime \prime}}$ with $m=0$ and $n=1$ (turbulent energy dissipation)

- $\widetilde{E^{\prime \prime}}-\widetilde{\ell^{\prime \prime}}$ with $m=3 / 2$ and $n=-1$ (turbulent lengthscale)

- $\widetilde{E^{\prime \prime}}-\widetilde{\omega^{\prime \prime}}$ with $m=-1$ and $n=1$ (turbulent frequency)

- $\widetilde{E}^{\prime \prime}-\widetilde{\tau}^{\prime \prime}$ with $m=1$ and $n=-1$ (turbulent timescale) 


\section{The $K-Z Z$ model simplifies for several special types of turbulent flows, which can be used to tune the model coefficients}

- Isotropic turbulence: power-law decaying solutions

- Production terms proportional to $\tau_{i j}$, the turbulent diffusion terms, and the mean velocity vanish

- Free shear flows (plane wake; mixing layer; plane, round, and radial jet): far-field, self-similar, statistically-stationary solutions

- Solutions depend on the similarity variable $\eta=y / x$

- Turbulent boundary layers: power-law solutions in the logarithmic layer

- Sufficiently far from the boundary, the eddy viscosity dominates the molecular viscosity and the advection terms are negligible 


\section{The $K-Z$ model equations have power-law solutions for isotropic turbulence}

- The model equations reduce to coupled ordinary differential equations

$$
\frac{d K}{d t}=-\epsilon \quad \frac{d Z}{d t}=-C_{Z 2} \frac{Z}{K} \epsilon
$$

- The initial conditions are $\quad K(0)=K$ and

$$
Z(0)=Z_{0}=C_{Z} K_{0}^{m} \epsilon_{0}^{n}
$$

- The corresponding solutions are

$$
\begin{gathered}
\frac{K(t)}{K_{0}}=\left[1+\left(\frac{C_{Z 2}-m-n}{n}\right) \frac{\epsilon_{0}}{K_{0}} t\right]^{-n /\left(C_{Z 2}-m-n\right)} \\
=\left[1+\left(C_{\epsilon 2}-1\right) \frac{\epsilon_{0}}{K_{0}} t\right]^{-1 /\left(C_{\epsilon 2}-1\right)} \\
\frac{Z(t)}{Z_{0}}=\left[\frac{K(t)}{K_{0}}\right]^{C_{Z 2}}=\left[\frac{K(t)}{K_{0}}\right]^{m+n C_{\epsilon 2}}
\end{gathered}
$$

- Experimentally, $K(t) \propto t^{1.34}$, which determines $C_{\varepsilon 2}$ (or $C_{\mathrm{Z} 2}$ ) 


\section{The $K-Z$ model equations have similarity solutions for free shear flows}

- The model equations reduce to (and are solved by transforming to the similarity variable)

$$
\begin{gathered}
\left(\bar{v}_{x} \frac{\partial}{\partial x}+\bar{v}_{y} \frac{\partial}{\partial y}\right) K=\tau_{x y} \frac{\partial \bar{v}_{x}}{\partial y}-\epsilon+\frac{1}{y^{r}} \frac{\partial}{\partial y}\left(y^{r} \frac{v_{t}}{\sigma_{k}} \frac{\partial K}{\partial y}\right) \\
\left(\bar{v}_{x} \frac{\partial}{\partial x}+\bar{v}_{y} \frac{\partial}{\partial y}\right) Z=C_{Z 1} \frac{Z}{K} v_{t}\left(\frac{\partial \bar{v}_{i}}{\partial x_{j}}\right)^{2}-C_{Z 2} \frac{Z}{K} \epsilon \\
+\frac{1}{y^{r}} \frac{\partial}{\partial y}\left(y^{r} \frac{v_{t}}{\sigma_{\epsilon}} \frac{\partial Z}{\partial y}\right)+\frac{m Z}{K y^{r}} \frac{\partial}{\partial y}\left(y^{r} \frac{v_{t}}{\sigma_{k}} \frac{\partial K}{\partial y}\right)-\frac{m}{y^{r}} \frac{\partial}{\partial y}\left(y^{r} \frac{v_{t}}{\sigma_{\epsilon}} \frac{Z}{K} \frac{\partial K}{\partial y}\right) \\
+\frac{v_{t}}{n \sigma_{\epsilon}}\left(\frac{\partial Z}{\partial y}-\frac{m Z}{K} \frac{\partial K}{\partial y}\right)\left(\frac{1-n}{Z} \frac{\partial Z}{\partial y}-\frac{m}{K} \frac{\partial K}{\partial y}\right)
\end{gathered}
$$

where $r=1$ corresponds to a round jet and $r=0$ otherwise, and the shear stress is

$$
\tau_{x y}=v_{t} \frac{\partial \bar{v}_{x}}{\partial y}
$$




\section{The $K-Z$ model equations have similarity solutions for the mixing layer}

- The solutions have the form

$$
\begin{gathered}
\bar{v}_{x}(x, y)=\Delta v \bar{v}_{x}(\eta) \\
K(x, y)=(\Delta v)^{2} K(\eta) \\
Z(x, y)=C_{Z}(\Delta v)^{2 m} K(\eta)^{m}\left[\frac{(\Delta v)^{3}}{x}\right]^{n} \epsilon(\eta)^{n}
\end{gathered}
$$

where $\quad v=v_{1}-v_{2}$ is the velocity difference between the two streams 


\section{The $K-Z Z$ model equations have solutions consistent with the law-of-the-wall in bounded flows}

- The Reynolds-averaged and $K-Z$ equations reduce to

$$
\begin{gathered}
\frac{\partial}{\partial y}\left(\mu_{t} \frac{\partial \bar{v}_{x}}{\partial y}\right)=0 \\
\mu_{t}\left(\frac{\partial \bar{v}_{x}}{\partial y}\right)^{2}+\frac{\partial}{\partial y}\left(\frac{\mu_{t}}{\sigma_{K}} \frac{\partial K}{\partial y}\right)-\rho \epsilon=0 \\
C_{Z 1} \frac{Z}{K} \mu_{t}\left(\frac{\partial \bar{v}_{x}}{\partial y}\right)^{2}-C_{Z 2} \rho \frac{Z}{K} \epsilon+\frac{\partial}{\partial y}\left(\frac{\mu_{t}}{\sigma_{\epsilon}} \frac{\partial Z}{\partial y}\right) \\
+\frac{m Z}{K} \frac{\partial}{\partial y}\left(\frac{\mu_{t}}{\sigma_{k}} \frac{\partial K}{\partial y}\right)-m \frac{\partial}{\partial y}\left(\frac{\mu_{t}}{\sigma_{\epsilon}} \frac{Z}{K} \frac{\partial K}{\partial y}\right) \\
+\frac{\mu_{t}}{n \sigma_{\epsilon}}\left(\frac{\partial Z}{\partial y}-\frac{m Z}{K} \frac{\partial K}{\partial y}\right)\left(\frac{1-n}{Z} \frac{\partial Z}{\partial y}-\frac{m}{K} \frac{\partial K}{\partial y}\right)=0
\end{gathered}
$$

- The solutions have the form (where $v_{\tau}$ is the friction velocity, $\kappa$ is the von Kármán constant, and $C, D, C_{Z}$ are constants)

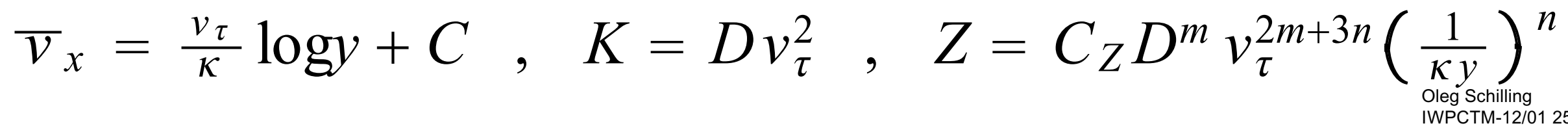




\section{Application to asymptotically self-similar Rayleigh-Taylor mixing}

- The turbulence production term is of the form

$$
P_{Z}=C_{Z 1} \frac{\widetilde{Z^{\prime \prime}}}{\widetilde{E^{\prime \prime}}} \tau_{i j} \frac{\partial \widetilde{v}_{i}}{\partial x_{j}}
$$

- Assume that at sufficiently late times, the scaling of the mixing layer width is

$$
h(t)=\alpha A t g t^{2}
$$

and that turbulence variables are proportional to this lengthscale and the corresponding velocity scale

- Then, $K, \varepsilon$, and $Z$ are

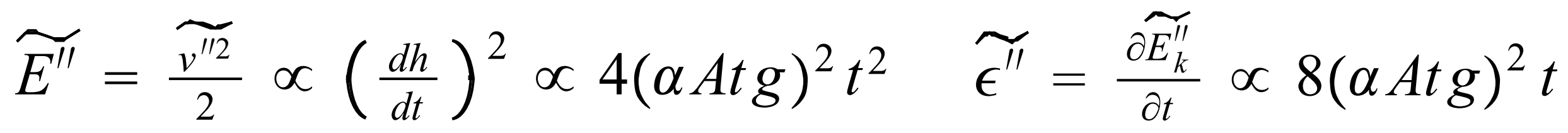

$$
\begin{aligned}
& \widetilde{Z^{\prime \prime}}=C_{Z}\left(\widetilde{E^{\prime \prime}}\right)^{m}\left(\widetilde{\epsilon^{\prime \prime}}\right)^{n} \propto(\alpha A t g)^{2 m+2 n} t^{2 m+n}
\end{aligned}
$$




\section{In Rayleigh-Taylor mixing the eddy viscosity and Reynolds stress tensor scale as follows}

- The eddy viscosity scales as

$$
v_{t}=\left(\widetilde{E^{\prime \prime}}\right)^{\frac{m+2 n}{n}}\left(\widetilde{Z^{\prime \prime}}\right)^{-\frac{1}{n}} \propto(\alpha A t g)^{2} t^{3}
$$

- The Reynolds stress scales as

$$
\begin{gathered}
\tau_{i j}=2 \bar{\rho} \widetilde{E}^{\prime \prime} \frac{\delta_{i j}}{d}-2 \bar{\rho} v_{t}\left(\widetilde{S}_{i j}-\frac{\delta_{i j}}{d} \frac{\partial \widetilde{v}_{k}}{\partial x_{k}}\right) \\
\propto 2 \bar{\rho}(\alpha A t g)^{2} t^{2}\left[\frac{4}{d} \delta_{i j}-\left(\widetilde{S}_{i j}-\frac{\delta_{i j}}{d} \frac{\partial \widetilde{v}_{k}}{\partial x_{k}}\right) t\right]
\end{gathered}
$$

- Therefore, if the Favre-averaged strain-rate tensor dimensionally scales as $\left(q_{\mathrm{ij}}\right.$ is dimensionless)

$$
\widetilde{S}_{i j}=\frac{1}{2}\left(\frac{\partial \widetilde{v}_{i}}{\partial x_{j}}+\frac{\partial \widetilde{v}_{j}}{\partial x_{i}}\right) \propto q_{i j} \frac{1}{h(t)} \frac{d h(t)}{d t} \propto \frac{1}{t}
$$

then $\tau_{i j} \propto 2 \bar{\rho}(\alpha A \operatorname{tg})^{2} t^{2}\left(\frac{5}{d} \delta_{i j}-q_{i j}\right)$ and

$$
P_{Z} \propto(\alpha A t g)^{2(m+n)} t^{2 m+n-1} \bar{\rho}\left(\frac{5}{d} \delta_{i j}-q_{i j}\right) q_{i j}
$$




\section{Conclusions}

- The methodology presented here provides a systematic and selfconsistent approach to the derivation of 2-equation turbulent transport models

- This provides an improved I transport equation

- Also provides a consistent expression for the diffusion and cross diffusion terms, which are important in many flow (e.g., near a boundary)

- Several canonical turbulent flows can be used to reduce the model equations and specify model parameters before application to interfacial-instability induced turbulence

- The general $Z$ equation is consistent with the $t^{2}$ scaling of the mixing layer width

- Both an $\omega$ and a $\tau$ equation were derived as alternatives to the $\varepsilon$ and I equation

- $\tau$ may be a better physical variable than $\varepsilon$ and I 


\section{Work in progress}

- Completion of solutions for canonical turbulent flows

- Completion of solutions for Rayleigh-Taylor instability-induced turbulence

- Commencement of examination of model parameters and forms of modeled terms using high-resolution DNS data

- Eventually, application to Richtmyer-Meshkov instability-induced turbulence 


\section{Large- and Small-Scale Dynamics of Variable-Density Rayleigh-Taylor Instability-Induced Turbulent Mixing}

Oleg Schilling and Andrew W. Cook

University of California, Lawrence Livermore National Laboratory

P.O. Box 808, L-22, Livermore, CA 94551 (925) 423-6879, schilling1@IInl.gov

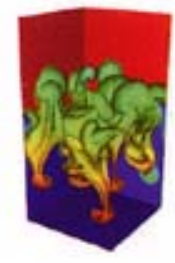

\section{Presented at the}

$8^{\text {th }}$ International Workshop on the Physics of Compressible Turbulent Mixing California Institute of Technology, Pasadena, CA

9-14 December 2001 
The dynamical effects of 'small' (unresolved) scales on 'large' (resolved) scales cannot be neglected in a turbulent mixing layer

- The dynamical effect of small scales on large scales is a bidirectional, competing process

- Energy transferred up and down scale through 'backscatter' and a cascade, respectively, over a wide range of eddies

- As $R e$ increases, contribution from the small scales increases

- Rate of scalar mixing is proportional to dissipation rate of the squared scalar $(\langle\cdot\rangle$ is an average and $D$ is the scalar diffusion coefficient)

$$
\left\langle\epsilon_{\phi}\right\rangle \equiv-\frac{\partial}{\partial t}\left\langle\frac{\phi^{2}}{2}\right\rangle=D\left\langle\phi \nabla^{2} \phi\right\rangle
$$

- Contribution to this quantity is dominated by the small scales, where gradients are large

- Turbulent quantities (e.g., the kinetic energy) are contributed by all scales, not only by large-scale, integral quantities

- Simulation of Rayleigh-Taylor mixing requires capability to simulate smaller scales as well as the largest scales 


\section{Schematic of direct numerical simulation (DNS) and large-eddy simulation (LES) for turbulent mixing}

- Turbulence enhances mass, momentum, energy transport

- Coherent bubble/spike structures associated with energy containing subrange

- Energy transferred among eddies at all scales

- Inertial subrange 'mediates' between largest and smallest scales

- Molecular (atomic) mixing associated with dissipation subrange, in which structures are too small to be resolved

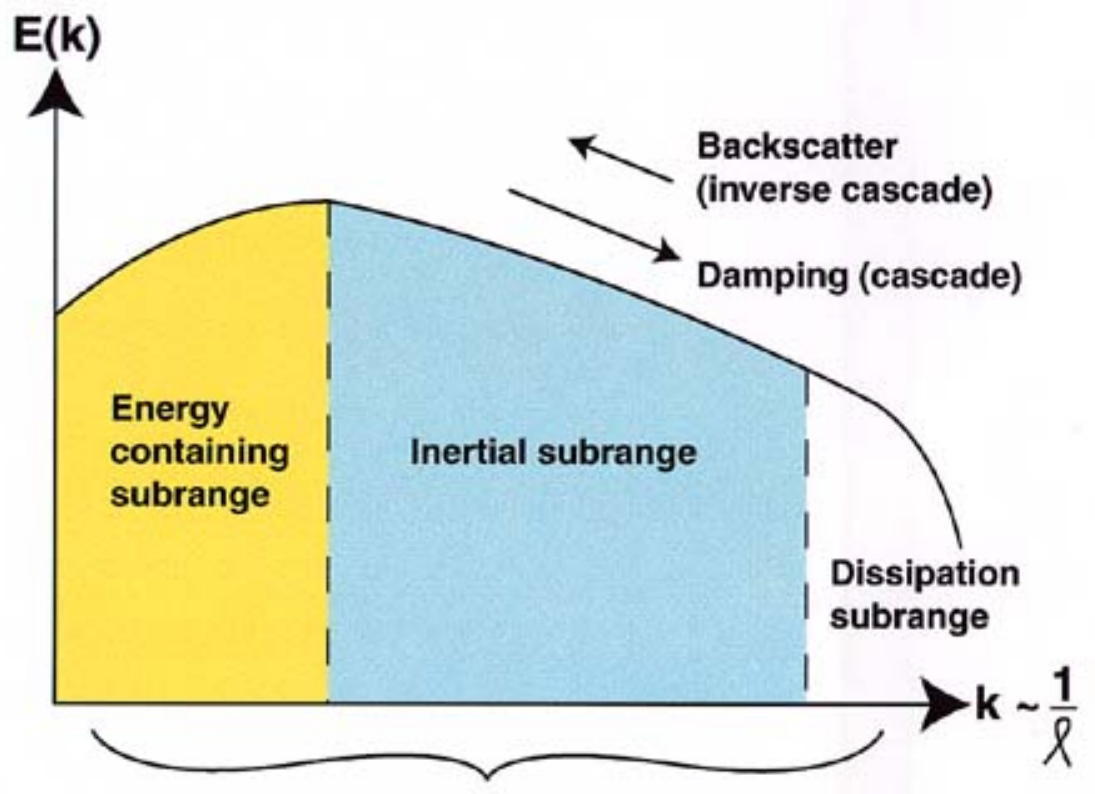

Resolve all scales (DNS)

Resolve largest scales
Model effects of small scales on large scales

(LES) 
The approaches used thus far to simulate

Rayleigh-Taylor mixing have important physical

and computational limitations

- Monotone integrated LES (MILES)

- Implicit numerical dissipation (finite-volume simulations of the non-dissipative equations)

- Advantage: modest computational requirements

- Disadvantage: small-scale structures and statistics not estimated well, while large-scale structures and lowest-order integral properties are

- DNS

- Physical dissipation (spectral and finite-difference simulations of the dissipative equations)

- Advantage: all structures and statistics estimated well

- Disadvantage: very high computational and data storage/processing requirements 
High-resolution DNS is too computationally expensive to simulate Rayleigh-Taylor mixing: an LES capability is needed

- DNS is too expensive to simulate a mixing layer that exceeds the mixing transition Reynolds number $R e \sim 10^{4}$

- Issue is more acute for miscible, reacting flows in which all scales are below any possible grid resolution

- Therefore, development of a true LES capability to predict mean hydrodynamic fields and lowestorder transport and mixing statistics is essential

$$
\operatorname{Re} \sim 2.5 \times 10^{3} \quad \operatorname{Re} \sim 1 \times 10^{4}
$$
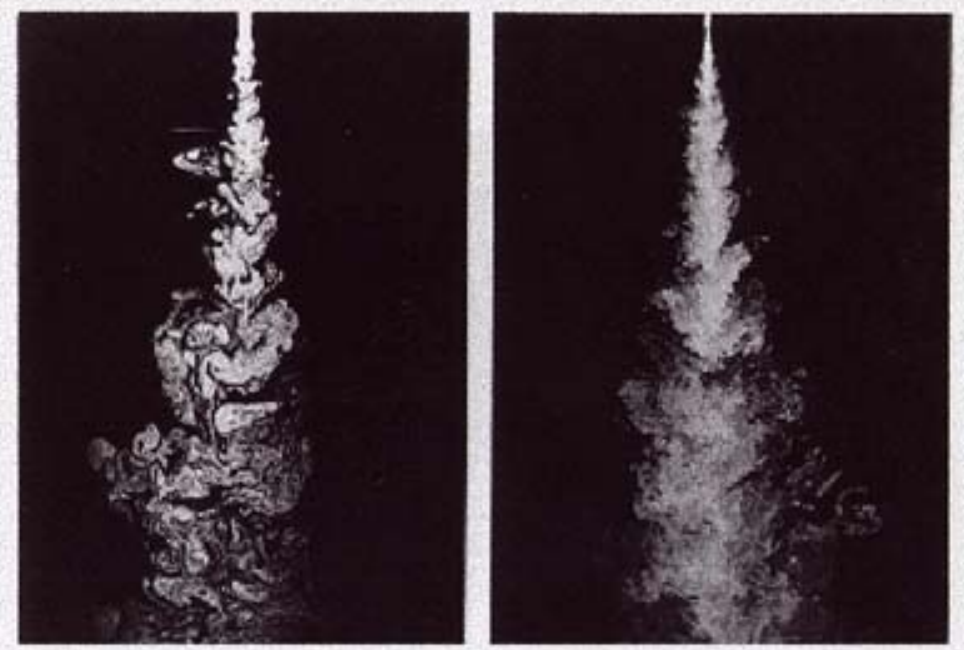

Liquid-jet concentration in a round turbulent jet (Dimotakis 1983) 
To develop an LES capability, an examination of interaction between unresolved and resolved scales in Rayleigh-Taylor mixing layers is needed

- Study transfer (exchange) of $\rho^{2} / 2$ and $v^{2} / 2$ among different wavenumber regions (corresponding to spatial scales or eddies)

- $\rho$ and $v_{\mathrm{i}}$ fields obtained from high-resolution DNS data

- An artificial cutoff scale is used to partition scales into computationally resolved and unresolved scales

- Fourier space representation determines magnitude and other properties of transfer process as a function of scale $k$ and height in the domain

- Wavelet transforms can also be used to add information as a function of position

- Data obtained from this study will be used to develop and assess subgrid-scale and backscatter models for LES

- Work will improve the classical Smagorinsky eddy viscosity and scale-similarity subgrid-scale models 
A high-resolution $\left(512^{2} \times 2040\right)$ DNS was performed for miscible, variable-density,

\section{Rayleigh-Taylor unstable fluids with $A t=1 / 2$}

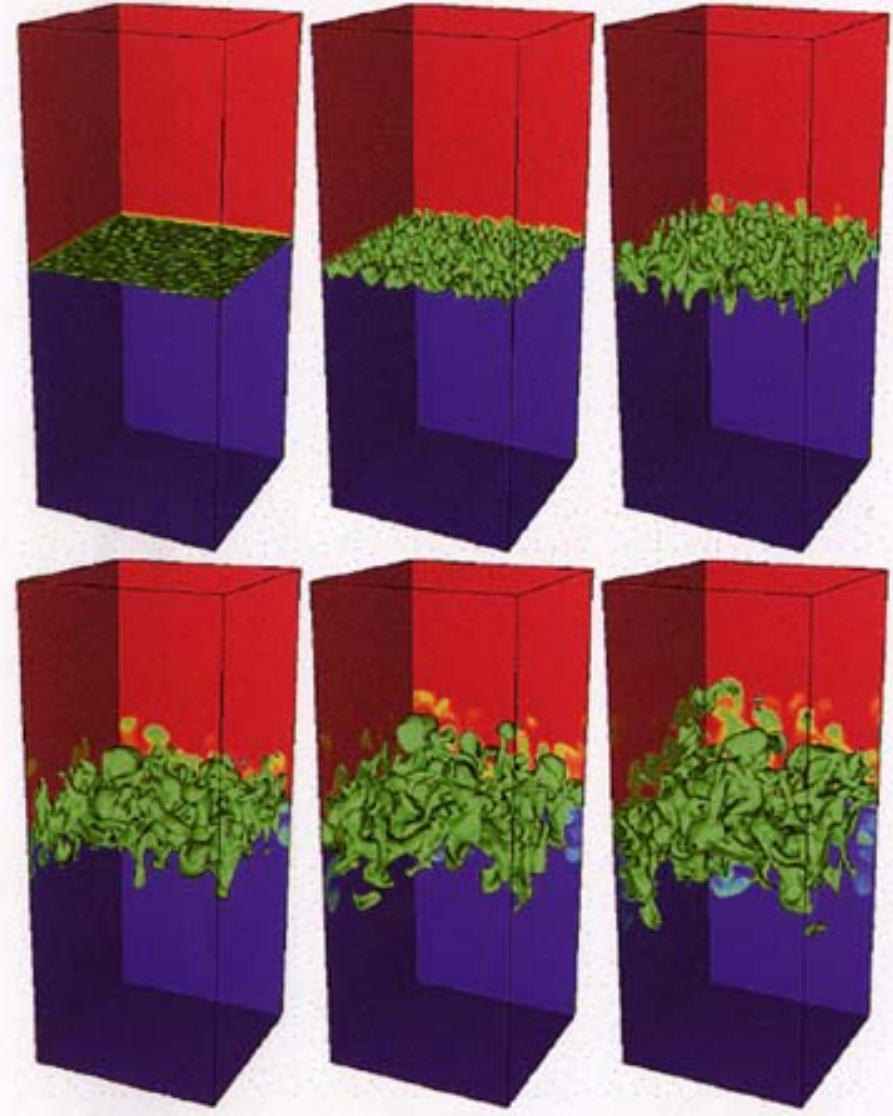

Density isosurface at times 1, 2, 3, 4, 5, 6 red: $\rho=3$; green: $\rho=2$; blue: $\rho=1$
- Spectral//8 ${ }^{\text {th }}$-order compact finite-difference method with $3^{\text {rd }}$-order, Adams-Bashforth time-evolution

- Small-scale initial perturbations on fluid interface

- Turbulence attains late-time Reynolds number based on mixing layer width

$$
\begin{aligned}
\operatorname{Re}_{h}(t) & =\frac{\left(\rho_{1}+\rho_{2}\right)}{2 \mu} h(t) \frac{d h(t)}{d t} \\
& \approx 5.5 \times 10^{3}
\end{aligned}
$$




\section{Similarly, the analysis of kinetic energy transfer dynamics begins with a 2-dimensional Fourier transform of the momentum equation}

- Applying the Fourier transform to the momentum equation in homogeneous directions gives ( $V=1 / \rho$ is the specific volume)

$$
\begin{gathered}
\frac{\partial v_{i}\left(\mathbf{k}_{\perp}, z, t\right)}{\partial t} \equiv \underbrace{F_{i}\left(\mathbf{k}_{\perp}, z, t\right)}_{\text {forcing term }}+\underbrace{N_{i}\left(\mathbf{k}_{\perp}, z, t\right)}_{\text {advection term }}+\underbrace{D_{i}\left(\mathbf{k}_{\perp}, z, t\right)}_{\text {dissipation terms }} \\
=-g(t) \delta_{i 3} \\
-\iint V\left(\mathbf{p}_{\perp}, z, t\right)\left(i q_{\perp}^{i}+\delta^{i 3} \frac{\partial}{\partial z}\right) p\left(\mathbf{q}_{\perp}, z, t\right) \delta^{2}\left(\mathbf{k}_{\perp}-\mathbf{p}_{\perp}-\mathbf{q}_{\perp}\right) d^{2} p_{\perp} d^{2} q_{\perp} \\
-\iint v_{j}\left(\mathbf{p}_{\perp}, z, t\right)\left(i q_{\perp}^{j}+\delta^{j 3} \frac{\partial}{\partial z}\right) v_{i}\left(\mathbf{q}_{\perp}, z, t\right) \delta^{2}\left(\mathbf{k}_{\perp}-\mathbf{p}_{\perp}-\mathbf{q}_{\perp}\right) d^{2} p_{\perp} d^{2} q_{\perp} \\
-v\left(k_{\perp}^{2}-\frac{\partial^{2}}{\partial z^{2}}\right) v_{i}\left(\mathbf{k}_{\perp}, z, t\right)-\frac{4}{3} v k_{\perp}^{i} k_{\perp}^{j} v_{j}\left(\mathbf{k}_{\perp}, z, t\right)
\end{gathered}
$$




\section{For a given $k_{\perp}, N_{\rho}$ and $N_{i}$ describe the} integrated nonlinear interactions of all other scales with $k_{\perp}$

- Decompose nonlinear terms into partial sum $N_{\rho}{ }^{A}$ and $N_{i}^{A}$ corresponding to different disjoint regions $A \ni \mathrm{k}_{\perp}$

- Each term represents nonlinear interactions contributed by any 2 such regions

- Define regions

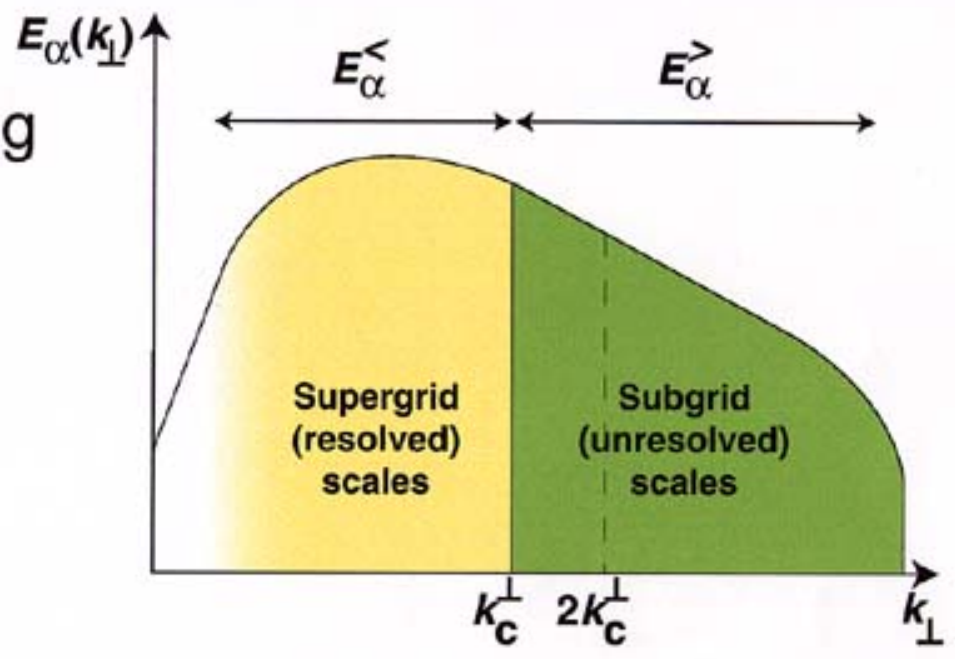

$$
k_{0}^{\perp}=0<k_{1}^{\perp}=k_{c}^{\perp}<k_{2}^{\perp}=2 k_{c}^{\perp}<k_{3}^{\perp}=k_{\text {max }}^{\perp}
$$

where $k_{c}{ }^{\perp}$ and $k_{\max }{ }^{\perp}$ are cutoff and maximum wavenumbers

- Partial nonlinear terms computed by truncating $v_{i}$ and $\rho$ to region $A$,

$$
\phi_{\alpha}^{\mathcal{A}}\left(\mathbf{k}_{\perp}, z, t\right) \equiv\left\{\begin{array}{l|l|}
\hline \phi_{\alpha}\left(\mathbf{k}_{\perp}, z, t\right) & \mathbf{k}_{\perp} \in \mathcal{A} \\
\hline 0 & \text { otherwise } \\
\hline
\end{array}\right.
$$


The kinetic energy spectrum at the midplane $(z=0)$ evolves to a state with a very short inertial subrange

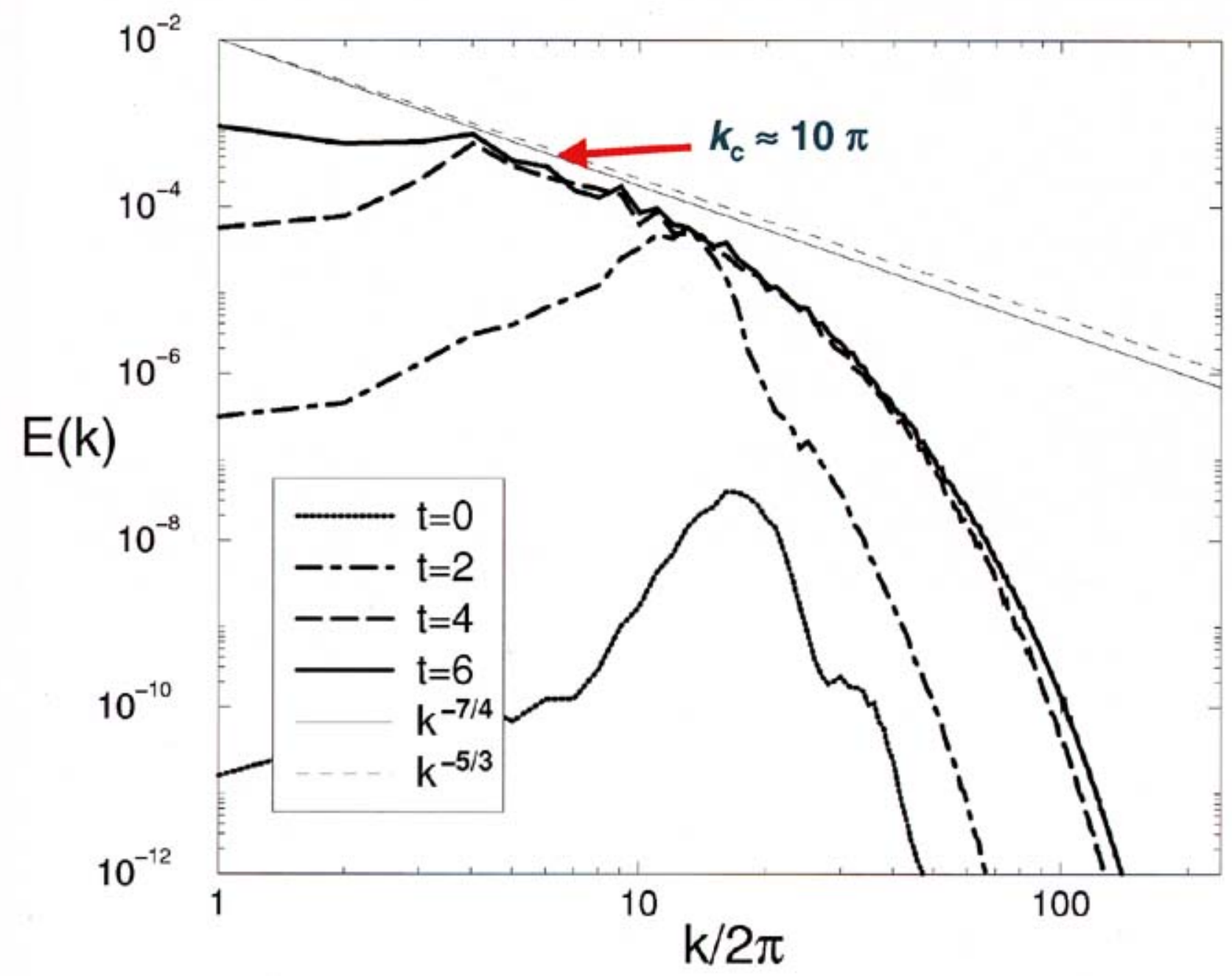




\section{Previous decomposition of nonlinear terms}

\section{can be used to derive expressions for}

\section{supergrid and subgrid nonlinear terms}

- The nonlinear term describing interactions of modes $k_{\perp} \in K$ with modes in regions $P$ and $Q$ can be expressed as a sum

$$
N_{\alpha}\left(\mathbf{k}_{\perp}, z, t\right)=\sum_{\mathcal{K}=1}^{3} \sum_{\mathcal{P}=1}^{3} \sum_{\mathcal{Q} \geq \mathcal{P}=1}^{3} N_{\alpha}^{\mathcal{N P \mathcal { Q }}}\left(\mathbf{k}_{\perp}, z, t\right)
$$

- Supergrid (resolved) nonlinear terms corresponding to modes $k_{\perp}<$ $k_{c}{ }^{\perp}$ and to modes $k_{\perp}<k_{2}{ }^{\perp}=2 k_{c}{ }^{\perp}$ :

$$
N_{\alpha}^{<}\left(\mathbf{k}_{\perp} \mid \mathbf{k}_{\perp}^{c}, z, t\right)=N_{\alpha}^{111}
$$

$$
N_{\alpha}^{<}\left(\mathbf{k}_{\perp} \mid 2 \mathbf{k}_{\perp}^{c}, z, t\right)=N_{\alpha}^{111}+N_{\alpha}^{121}+N_{\alpha}^{221}+N_{\alpha}^{112}+N_{\alpha}^{122}+N_{\alpha}^{222}
$$

- Subgrid (unresolved) nonlinear terms corresponding to modes $k_{\perp}>$ $k_{c}{ }^{\perp}$ and to modes $k_{c}{ }^{\perp}>k_{2}{ }^{\perp}=2 k_{c}{ }^{\perp}$ :

$$
\begin{gathered}
N_{\alpha}^{>}\left(\mathbf{k}_{\perp} \mid \mathbf{k}_{\perp}^{c}, z, t\right)=N_{\alpha}^{121}+N_{\alpha}^{131}+N_{\alpha}^{221}+N_{\alpha}^{231}+N_{\alpha}^{331} \\
N_{\alpha}^{>}\left(\mathbf{k}_{\perp} \mid 2 \mathbf{k}_{\perp}^{c}, z, t\right)=N_{\alpha}^{131}+N_{\alpha}^{231}+N_{\alpha}^{331}+N_{\alpha}^{132}+N_{\alpha}^{232}+N_{\alpha}^{332}
\end{gathered}
$$


The supergrid and subgrid energy density evolution equations can be formed in terms of

\section{the nonlinear terms}

- Partial transfer spectra describe rate of $\rho^{2} / 2$ and $v^{2} / 2$ change in modes $k_{\perp} \in K$ due to nonlinear interactions between modes $p_{\perp} \in P$ and $q_{\perp} \in Q$ :

$$
T_{\alpha}^{\mathcal{K P \mathcal { Q }}}\left(\mathbf{k}_{\perp}, z, t\right)=\operatorname{Re}\left[\phi_{\alpha}\left(\mathbf{k}_{\perp}, z, t\right)^{*} N_{\alpha}^{\mathcal{K} \mathcal{L Q}}\left(\mathbf{k}_{\perp}, z, t\right)\right]
$$

- Introducing large-scale density and velocity truncated at $k_{2}{ }^{\perp}=2 k_{c}{ }^{\perp}$,

$$
\rho^{<}=\rho^{1}+\rho^{2}, \quad v_{i}^{<}=v_{i}^{1}+v_{i}^{2}
$$

the supergrid (resolved) density squared and kinetic energy are

$$
E_{\rho}^{<}(\mathbf{x}, t)=\frac{\rho^{<}(\mathbf{x}, t)^{2}}{2} \quad, \quad E^{<}(\mathbf{x}, t)=\frac{v^{<}(\mathbf{x}, t)^{2}}{2}
$$

- The equations for these quantities contain the supergrid and subgrid transfers 
The subgrid-scale energy transfer spectra

$T_{\alpha}\left(k_{\perp} \mid k_{\perp}{ }^{c} ; z, t\right)$ provide information on magnitude

and direction of the energy transfer

- Computation of subgrid-scale energy transfers requires summation over all $k_{\perp}$ in a spherical shell centered around $\mathrm{k}_{\perp}$ :

$$
T_{\alpha}^{>}\left(k_{\perp} \mid k_{c}^{\perp} ; z, t\right)=\sum_{k_{\perp}-\frac{\Delta k_{\perp}}{2}<\left|\mathbf{k}_{\perp}\right|<k_{\perp}+\frac{\Delta k_{\perp}}{2}} T_{\alpha}^{>\mathcal{P Q}}\left(k_{\perp} \mid k_{c}^{\perp} ; z, t\right)
$$

where $P$ and $Q$ are spherical wavenumber bands centered around $\mathrm{p}_{\perp}$ and $\mathrm{q}_{\perp}$

- Computation involves interactions in which components of the sum can be either positive or negative

- Positive component (forward energy cascade):

$$
T_{\alpha}^{(+)>}\left(k_{\perp} \mid k_{c}^{\perp} ; z, t\right) \equiv \frac{T_{\alpha}^{>}\left(k_{\perp} \mid k_{c}^{\perp} ; z, t\right)+\left|T_{\alpha}^{>}\left(k_{\perp} \mid k_{c}^{\perp} ; z, t\right)\right|}{2}
$$

- Negative component (backward energy cascade):

$$
T_{\alpha}^{(-)>}\left(k_{\perp} \mid k_{c}^{\perp} ; z, t\right) \equiv \frac{T_{\alpha}^{>}\left(k_{\perp} \mid k_{c}^{\perp} ; z, t\right)-\left|T_{\alpha}^{>}\left(k_{\perp} \mid k_{c}^{\perp} ; z, t\right)\right|}{2}
$$




\section{Net eddy viscosities $v_{\alpha}{ }^{n}\left(k_{\perp} \mid k_{\perp}{ }^{c} ; z, t\right)$ can be defined for $\boldsymbol{k}_{\perp}<\boldsymbol{k}_{\perp} \mathrm{c}$ in terms of eddy and backscatter viscosities}

- Net eddy viscosities:

$$
v_{\alpha}^{n}\left(k_{\perp} \mid k_{c}^{\perp} ; z, t\right) \equiv-\frac{T_{\alpha}^{>}\left(k_{\perp} \mid k_{c}^{\perp} ; z, t\right)}{2 k_{\perp}^{2} E_{\alpha}^{<}\left(k_{\perp}, z, t\right)}
$$

- Eddy viscosities: (representing energy removal by small scales)

$v_{\alpha}\left(k_{\perp} \mid k_{c}^{\perp} ; z, t\right) \equiv-\frac{T_{\alpha}^{(+)>}\left(k_{\perp} \mid k_{c}^{\perp} ; z, t\right)}{2 k_{\perp}^{2} E_{\alpha}^{<}\left(k_{\perp}, z, t\right)}$

- Backscatter viscosities: (representing energy transfer from small to larger scales)

$v_{\alpha}^{b}\left(k_{\perp} \mid k_{c}^{\perp} ; z, t\right) \equiv \frac{T_{\alpha}^{(-)>}\left(k_{\perp} \mid k_{c}^{\perp} ; z, t\right)}{2 k_{\perp}^{2} E_{\alpha}^{<}\left(k_{\perp}, z, t\right)}$

- Thus, net viscosities are

$v_{\alpha}^{n}\left(k_{\perp} \mid k_{c}^{\perp} ; z, t\right)=v_{\alpha}\left(k_{\perp} \mid k_{c}^{\perp} ; z, t\right)-v_{\alpha}^{b}\left(k_{\perp} \mid k_{c}^{\perp} ; z, t\right)$

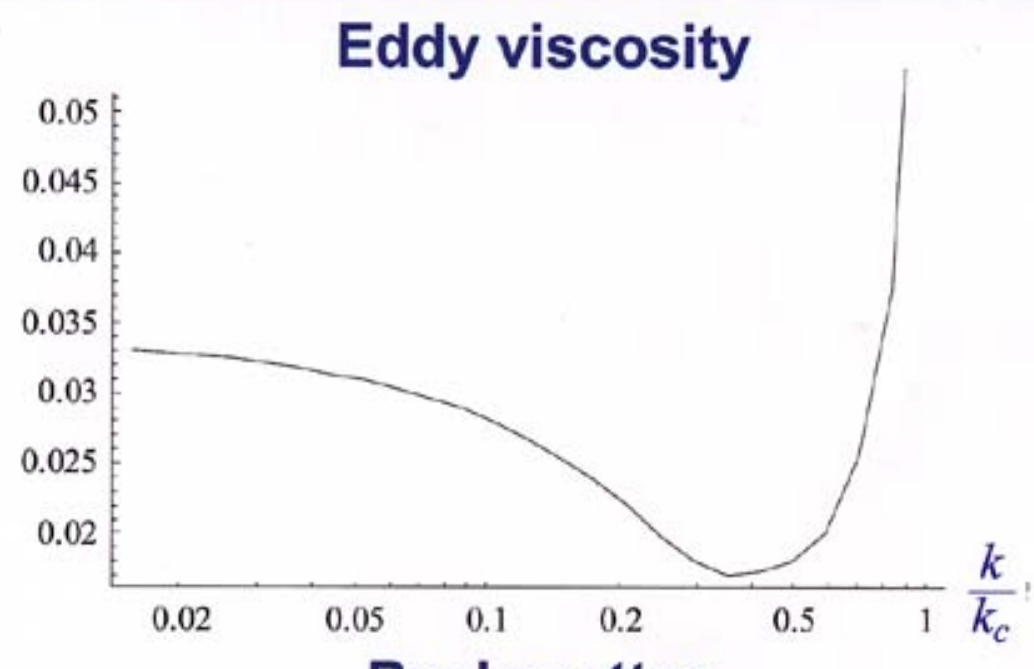

Backscatter

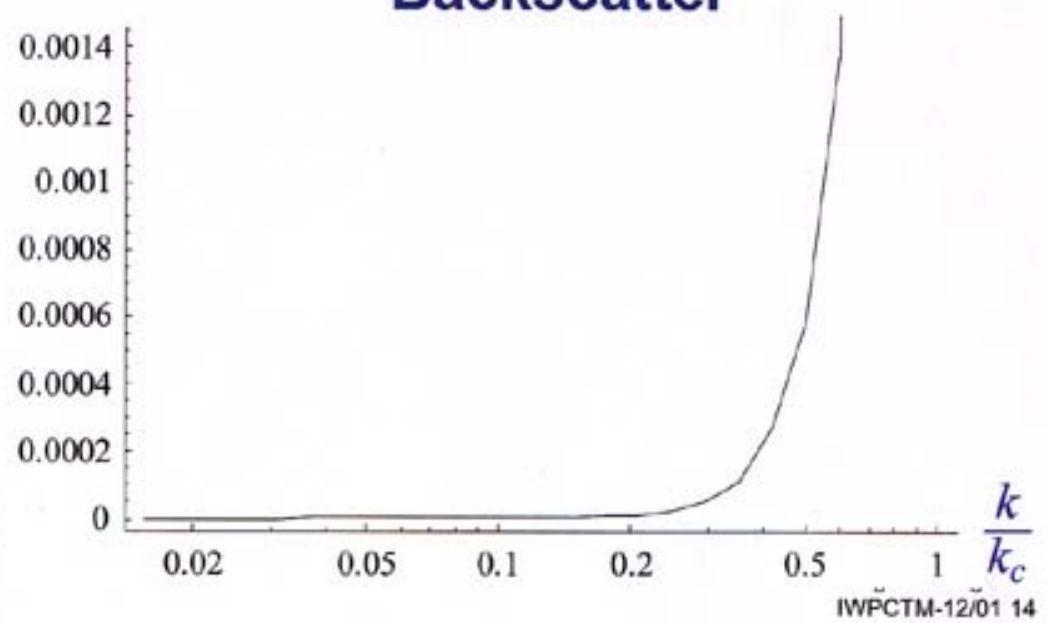




\section{Given the eddy and backscatter viscosities, a subgrid-scale model can be defined}

- Large-eddy equations have the form (only the Navier-Stokes equation is shown)

$$
\frac{\partial}{\partial t}\left(\rho^{<} v_{i}^{<}\right)+\frac{\partial}{\partial x_{j}}\left(\rho^{<} v_{i}^{<} v_{j}^{<}+p \delta_{i j}-\sigma_{i j}\right)-\rho^{<} g_{i}=-\frac{\partial \tau_{i j}}{\partial x_{j}}
$$

- Left side is computed explicitly Spectrum of turbulence

- Right side involves the Reynolds stress tensor $\tau_{i j}$

- The Smagorinsky model is

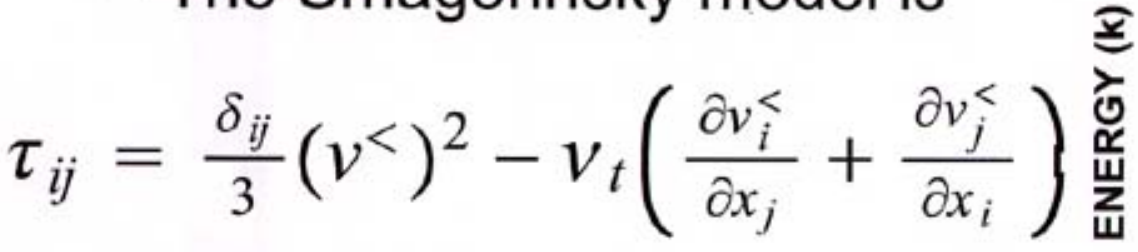

- A similar parameterization may be reasonable for resolved density equation

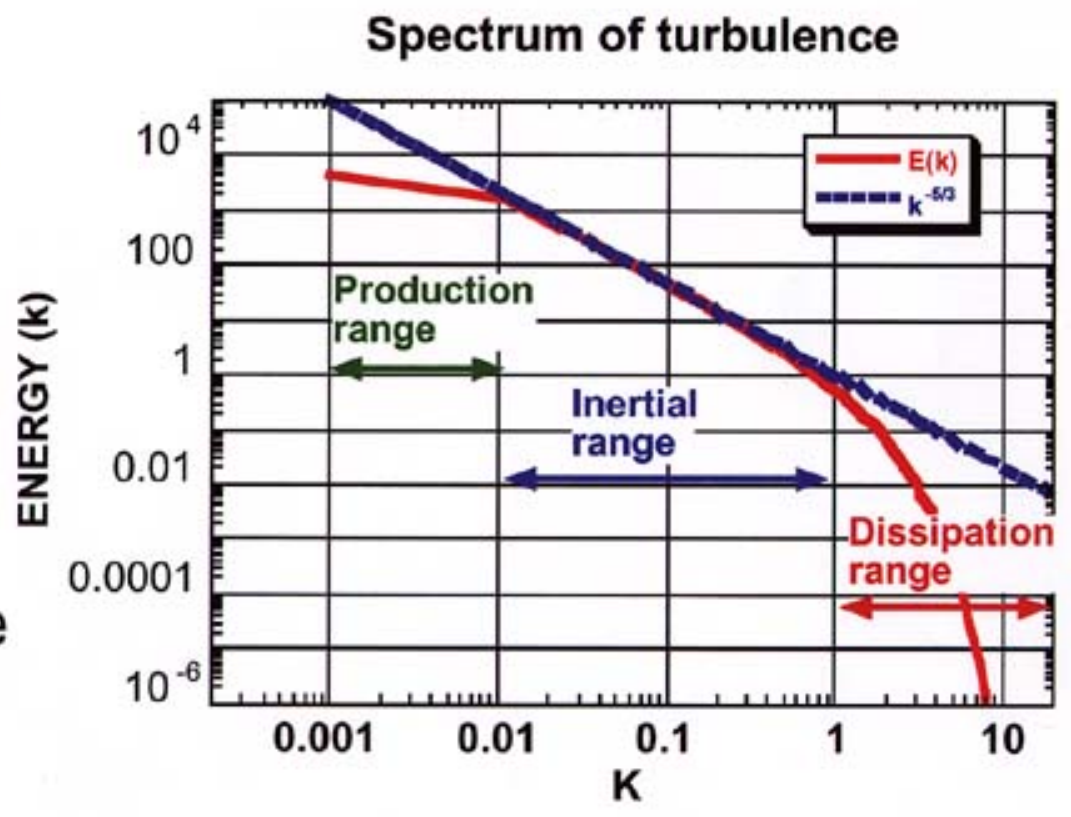




\section{Turbulence dynamics of enstrophy (vorticity squared) transfer is particularly relevant to interfacial instability-induced turbulent mixing}

- Dynamics of enstrophy $\rho \Omega=\rho \omega^{2} / 2$ will also be examined (dissipation terms are not written)

$$
\frac{\partial}{\partial t}(\rho \Omega)+\frac{\partial}{\partial x_{j}}\left(\rho \Omega v_{j}\right)=\underbrace{\epsilon_{i j k} \frac{\omega^{i}}{\rho} \frac{\partial \rho}{\partial x_{j}} \frac{\partial p}{\partial x_{k}}}_{\text {baroclinic production }}+\underbrace{\rho \omega_{i} S^{i j} \omega_{j}}_{\text {enstrophy stretching }}-\underbrace{2 \rho \Omega \frac{\partial v_{j}}{\partial x_{j}}}_{\text {dilatation }}
$$

- Baroclinic production term is an instability source term, existing due to misalignment of $\nabla \rho$ and $\nabla p$

- Stretching term is related to development of turbulence

- Dilatation term is small in a variable-density flow, but is important in a compressible (Richtmyer-Meshkov) flow

- Baroclinic production and stretching terms exist in 3D, but only the former exists in $2 \mathrm{D}$

- Nature of turbulent energy and enstrophy cascades is, therefore, very different in $2 \mathrm{D}$ and $3 \mathrm{D}$ 


\section{Conclusions and work in progress}

- A subgrid-scale transfer analysis can be applied to RayleighTaylor and Richtmyer-Meshkov mixing layers

- Provides time- and scale-dependent measure of energy transfer process

- Provides parameterizable eddy viscosities/backscatters for LES of Rayleigh-Taylor mixing at large $R e$

- Also provides information for scale-similarity and more recent deconvolution subgrid-scale models

- Data processing at LLNL requires 256 IBM SP nodes $(1,024$ CPUs) and sufficient computer time

- Purpose of this research is to acquire physical insight into coupling of the large and small scales, in order to develop and assess subgrid-scale models for LES 
ANALYTICAL STUDY of the RT in COMPRESSIBLE FLUIDS M.TRICOTTET (PhD.St.) Serge BOUQUET De COMMISSARIAT ENERGIE ATOMIQUE - EADept. App. Theor. Physics bouquet @bruyeres.cea.fr 
USE Of COMPRESSIBILITY for the RTI

Sound speed: $\quad c_{s}^{2}=(\Delta p / \Delta \rho)$ adiab.

incompressible fluids: $\Delta \rho$ finite $\rightarrow \Delta p=\infty$ ! $c_{s} \rightarrow \infty$

NOT NECESSARLY REPRESENTATIVE OF PHYSICS

- IVF

- ASTROPHYSICS

$H$-envelope Type II -SN progenitor:

$T \simeq 10^{6} \mathrm{~K}, \rho \simeq 10^{-3} \mathrm{~g} / C \mathrm{C}, D \simeq C / 10 \quad \mathrm{M} \approx 100 \mathrm{~s}$ :

INCOMPRESSIBILITY IS NO LONGER VALID

1980 's:

- Bernstein-Book (1983): $\alpha_{\text {comp }}>\alpha_{\text {incomp. }}$

- Baker (1983): compressibility either enhance or decrease $\alpha$

- Sharp (1984): $\alpha_{\text {comp }}<\alpha_{\text {income. }}$

Since... very few analytical work 
DIMENSIONAL ANALYSIS

INCOMPRESS. $\quad[g]=L T^{-2},[\lambda]=L \quad\left([\rho]=M L^{-3}\right)$

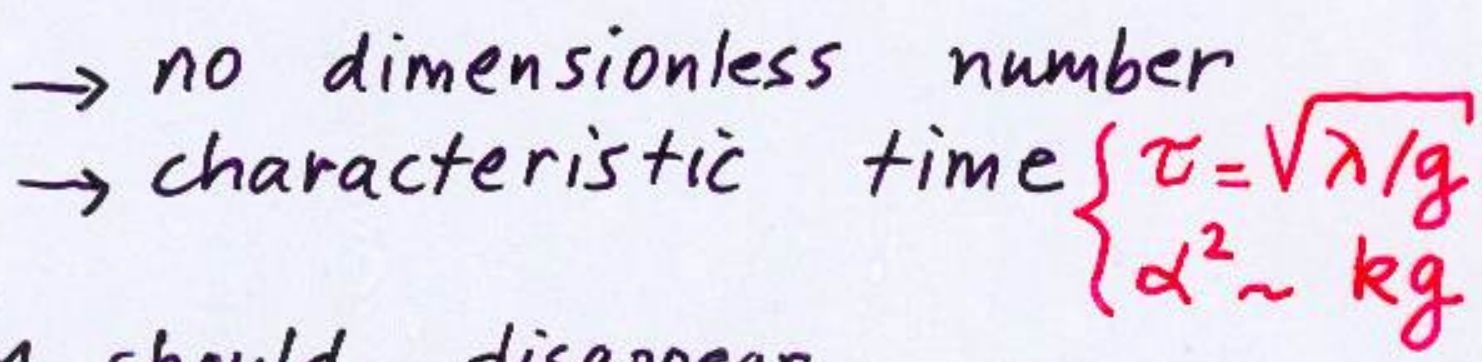

$[\rho]$ ? M should disappear

$$
\rho_{1} \rightarrow \rho_{2} \quad R \equiv \rho_{1} / \rho_{2} \rightarrow \text { At. }
$$

COMPRESSIBLE: $[g]=L T^{2},[\lambda]=L,\left[C_{S}\right]=L T^{-1}$

II -Theorem: $\quad \pi=c_{s}^{2} /(\lambda g)$

$$
\left\{\begin{array} { l } 
{ c _ { s } \text { finite } } \\
{ \lambda \rightarrow 0 }
\end{array} \approx \left\{\begin{array}{l}
c_{s} \rightarrow \infty \\
\lambda \text { finite }
\end{array} \quad(\pi \rightarrow \infty)\right.\right.
$$

small $\lambda$ 's $\left(\lambda \ll c_{s}^{2} / g\right)$ not affected by compress. (Sharp 1983)

3 QUANT. REPRESENT. Of. COMPRESS.

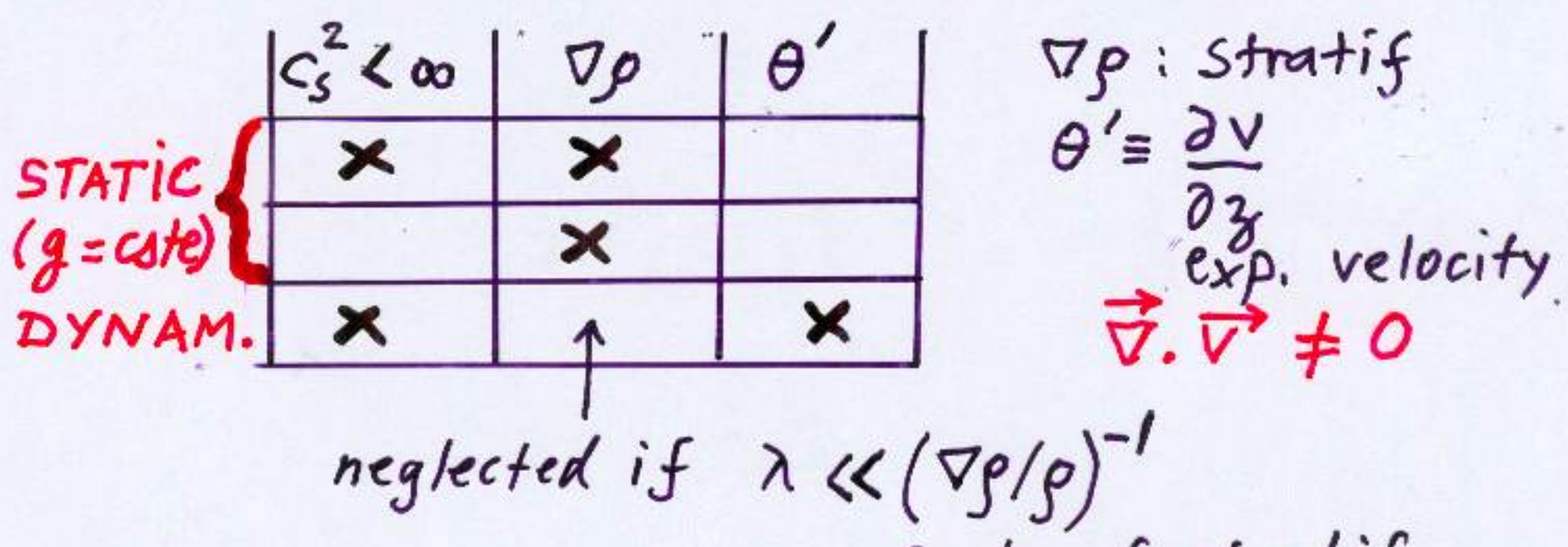

scale of stratify. 
ASSUMPTIONS

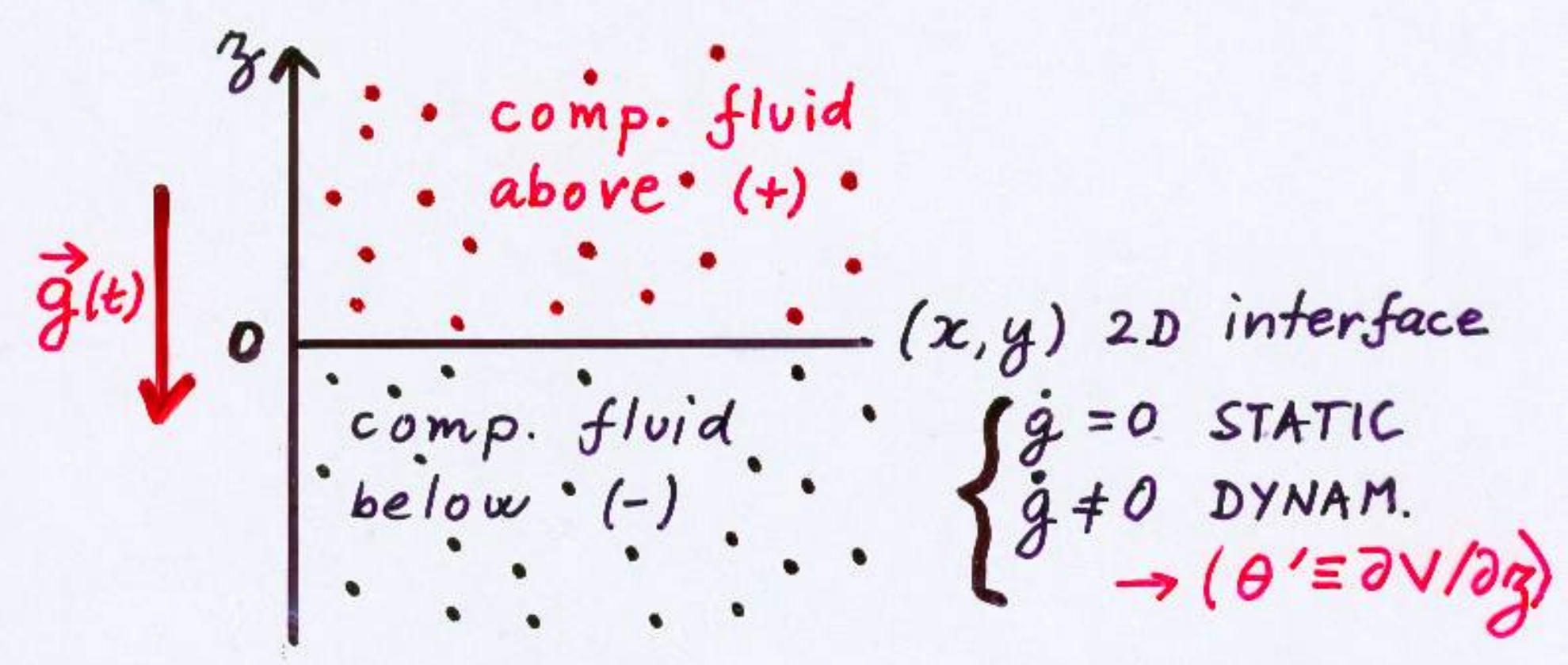

Assume: - initial state "o" $\left(\vec{v}_{0}=0\right.$ pr $\left.\vec{v}_{0} \neq 0\right)$

- no vorticity $\vec{v}=\vec{\nabla} \phi$

$\frac{d}{d t} \int \vec{v} \cdot d \vec{l}=c u t e=\frac{d}{d t} \int(\vec{\nabla} \wedge \vec{v}) d \vec{S}=\overrightarrow{0}$ if $\vec{v}_{0}=0$

$\Rightarrow \vec{\nabla} \wedge \vec{v}=0 \Rightarrow \vec{v}=\vec{\nabla} \phi$

Idem $V_{0} \neq 0$

- arbitrary EOS: $P_{\gamma}^{ \pm}=f^{ \pm}\left(\rho^{ \pm}\right)$

especially $p \sim \rho^{\gamma}, \gamma=\frac{1+n}{n}\left(\gamma^{+} \neq \gamma^{-}\right)$

For "t" and "-", hydro. Eggs. are:

$$
\begin{aligned}
& \left\{\begin{array}{l}
\rho \nabla^{2} \phi+\vec{\nabla} \phi \cdot \vec{\nabla} \rho+\partial \rho / \partial t=0 \\
\partial \phi / \partial t+(\vec{\nabla} \phi)^{2} / 2+g z+\underline{h}=0
\end{array}\right. \\
& \left\{\begin{array}{l}
\frac{h}{d h}: \text { enthalpy } \\
d \phi / \rho=f^{\prime}(\rho) d \rho / \rho \\
\text { Id. Gas: } \left.h=c_{s}^{2}\right)(\gamma-1)
\end{array}\right.
\end{aligned}
$$


THE PERTURBATION (STATIC)

Phys. quantity: $q(x, y, z ; t)=\underbrace{q_{0}(z)}_{\text {Eq. }}+\underbrace{q_{1}(x, y, z ; t)}_{\text {1rst o. pert. }}$

$3 D+t$ analytical stability analysis 4 dimensions!!!

Velocity_potential: $\phi(\vec{r} ; t)=\phi_{\text {dim }} S(x, y) Z(z) T(t)$

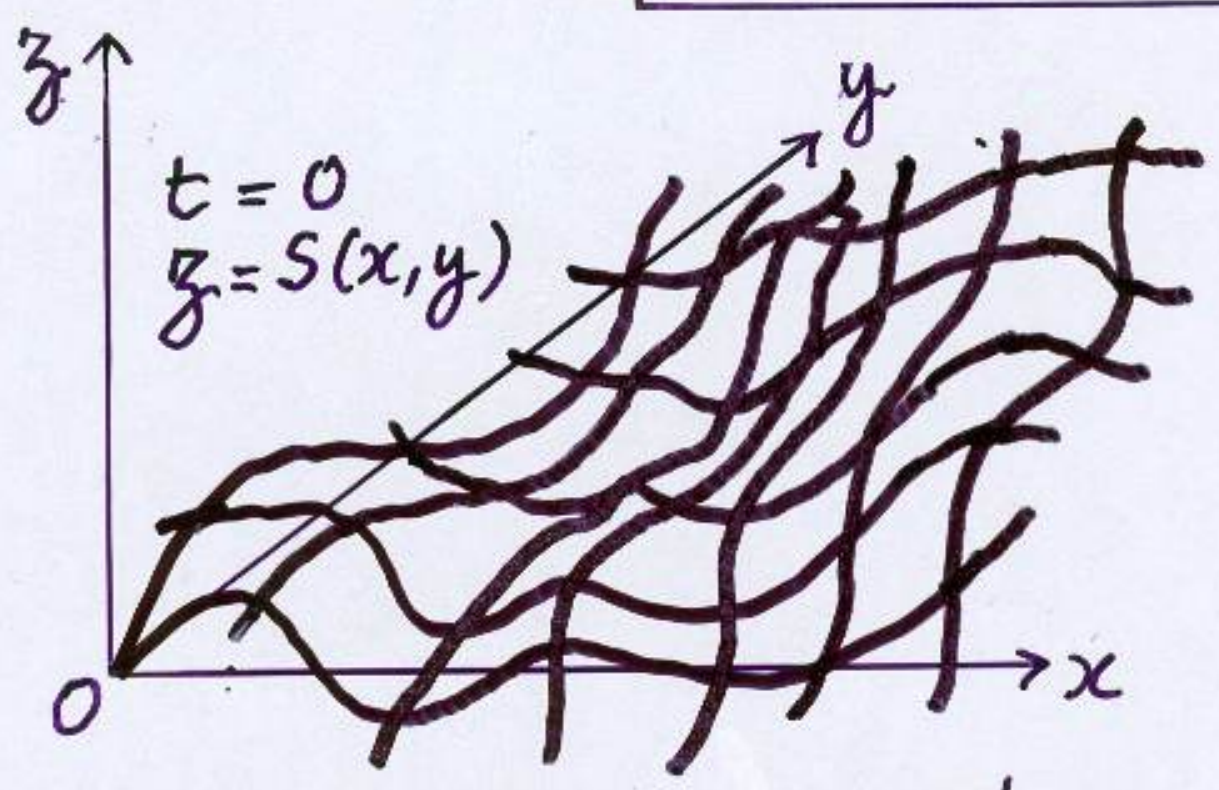

$$
z=S(x, y)
$$

3D-interface

LINEAR SYSTEM OF 3-ODE'S:

$$
\begin{aligned}
& \left\{\begin{array}{l}
-\ddot{T}(t)-\alpha^{2} T(t)=0 \Rightarrow T(t)=e^{\alpha t} ; \alpha=G \text {. Rate } \\
\partial_{x}^{2} S(x, y)+\partial_{y}^{2}(x, y)=-k^{2} S(x, y)
\end{array}\right. \\
& \text { - } \partial_{x}^{2} S(x, y)+\partial_{y}^{2}(x, y)=-k^{2} S(x, y) \\
& k^{2}: \text { eigen values of } 2 D-\nabla^{2} \\
& \text { - } c_{0}^{2}(z) Z^{\prime \prime}(z)-\underset{\substack{g \\
Z^{\prime}(z)}}{Z_{j}=0}\left[k^{2} c_{0}^{2}(z)+\alpha^{2}\right] z(z)=0 ; c_{0}=\frac{d p}{d \rho}=f^{\prime}(\rho)
\end{aligned}
$$

NO EXPONENTIAL SOLUTION !!!

$\rightarrow$ YET DISPERSION RELATION 
ISOTHERMAL CASE $p^{ \pm}=K^{ \pm} \rho^{ \pm}$

$$
\begin{aligned}
& \tilde{\alpha}^{2}=2\left(\frac{1}{R}-1\right) \frac{\left[1-\left(1+\tilde{\alpha}^{2}+\tilde{k}^{2}\right)^{1 / 2}\right]\left[1+\left(1+R \tilde{\alpha}^{2}+R^{2} \tilde{k}^{2}\right)^{1 / 2}\right]}{\left(1+\tilde{\alpha}^{2}+\tilde{k}^{2}\right)^{1 / 2}+\left(1+R \tilde{\alpha}^{2}+R^{2} \tilde{k}^{2}\right)^{1 / 2}} \\
& \left\{\begin{array}{l}
\tilde{\alpha}=\alpha /\left(g / 2 \sqrt{k^{+}}\right) \quad \text { implimensionless } \\
\tilde{k}=k /\left(g / 2 k^{+}\right)
\end{array}\right.
\end{aligned}
$$

UNIVERSAL 1-PARAMATER $R$ - CURVES

$R=\rho^{+} / \rho^{-}$instead of $A t=(R-1) /(R+1)$

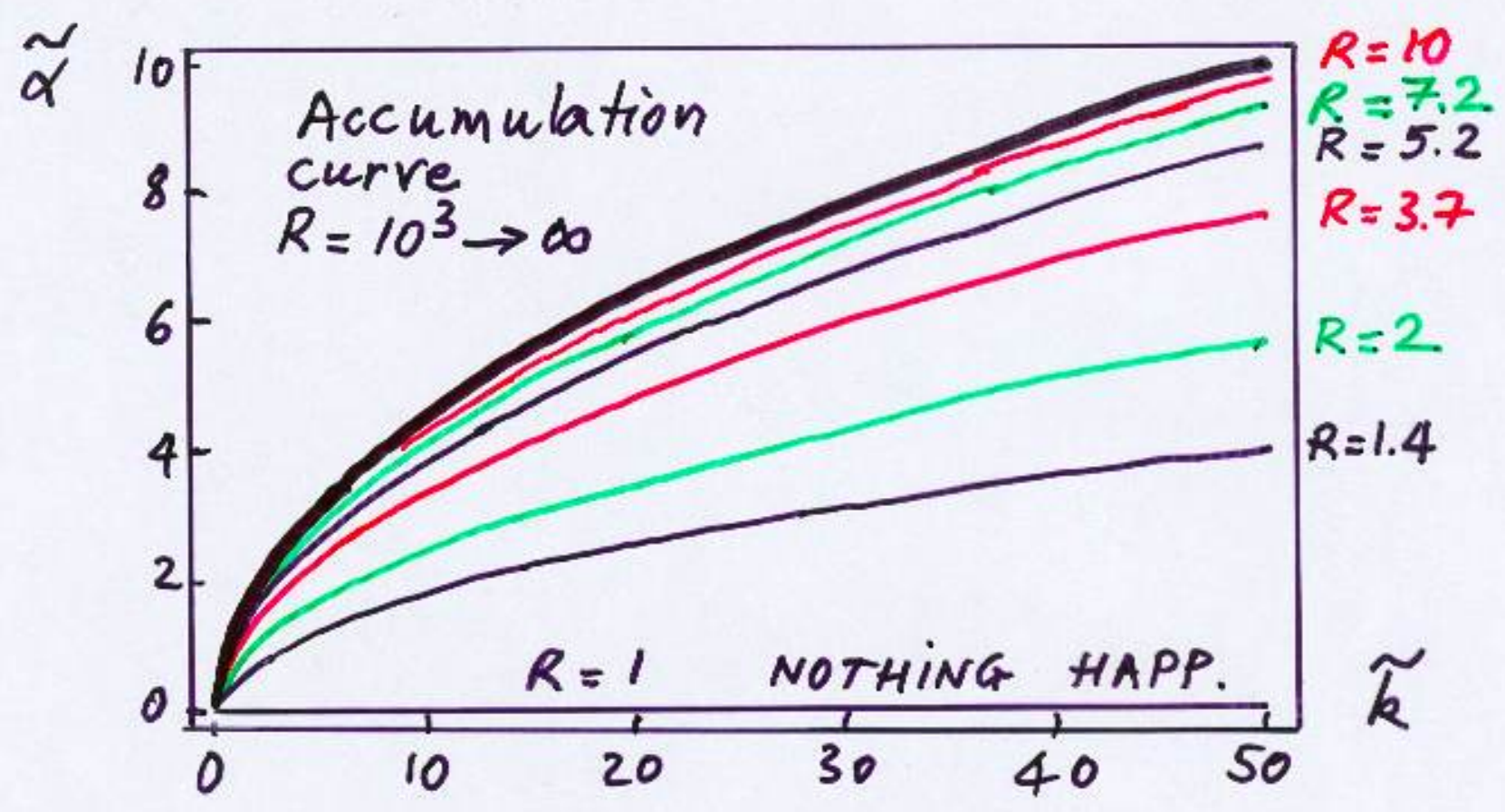

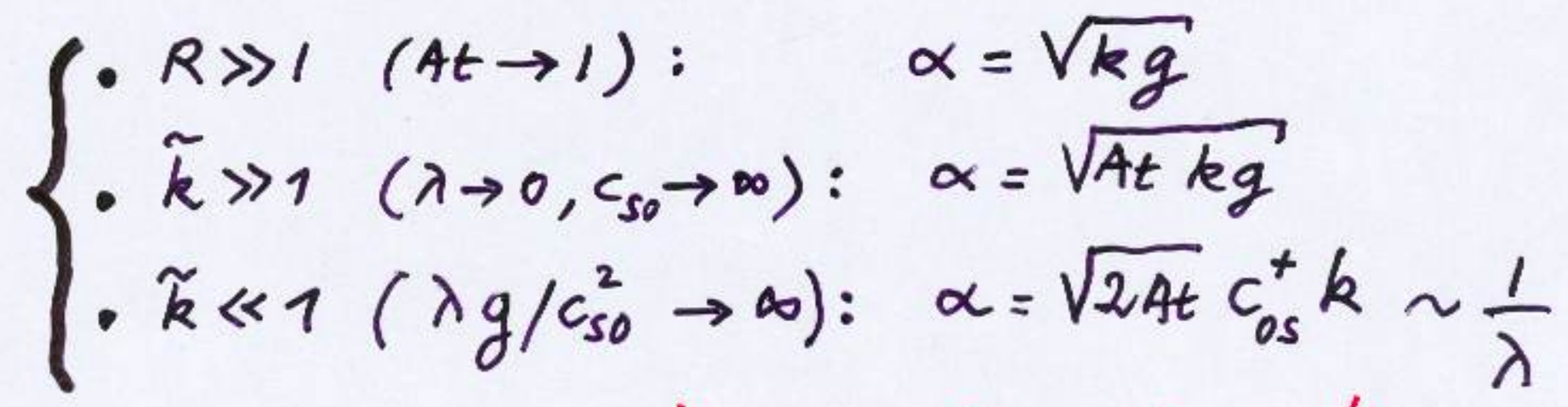

incompress. $\quad \alpha_{\text {inc }} \sim \frac{1}{\sqrt{\lambda}}$ $\alpha_{\text {comp }}<\alpha_{\text {incomp. }}$. 
POLYTROPIC CASE $p^{ \pm}=K^{ \pm}\left(\rho^{ \pm}\right)^{\Gamma^{ \pm}}$ 2 parameters $(R, \Gamma)$ universal family curves

$$
R=\rho^{+} / \rho^{-}=1.1 \quad \text { (At \# 0.05) }
$$

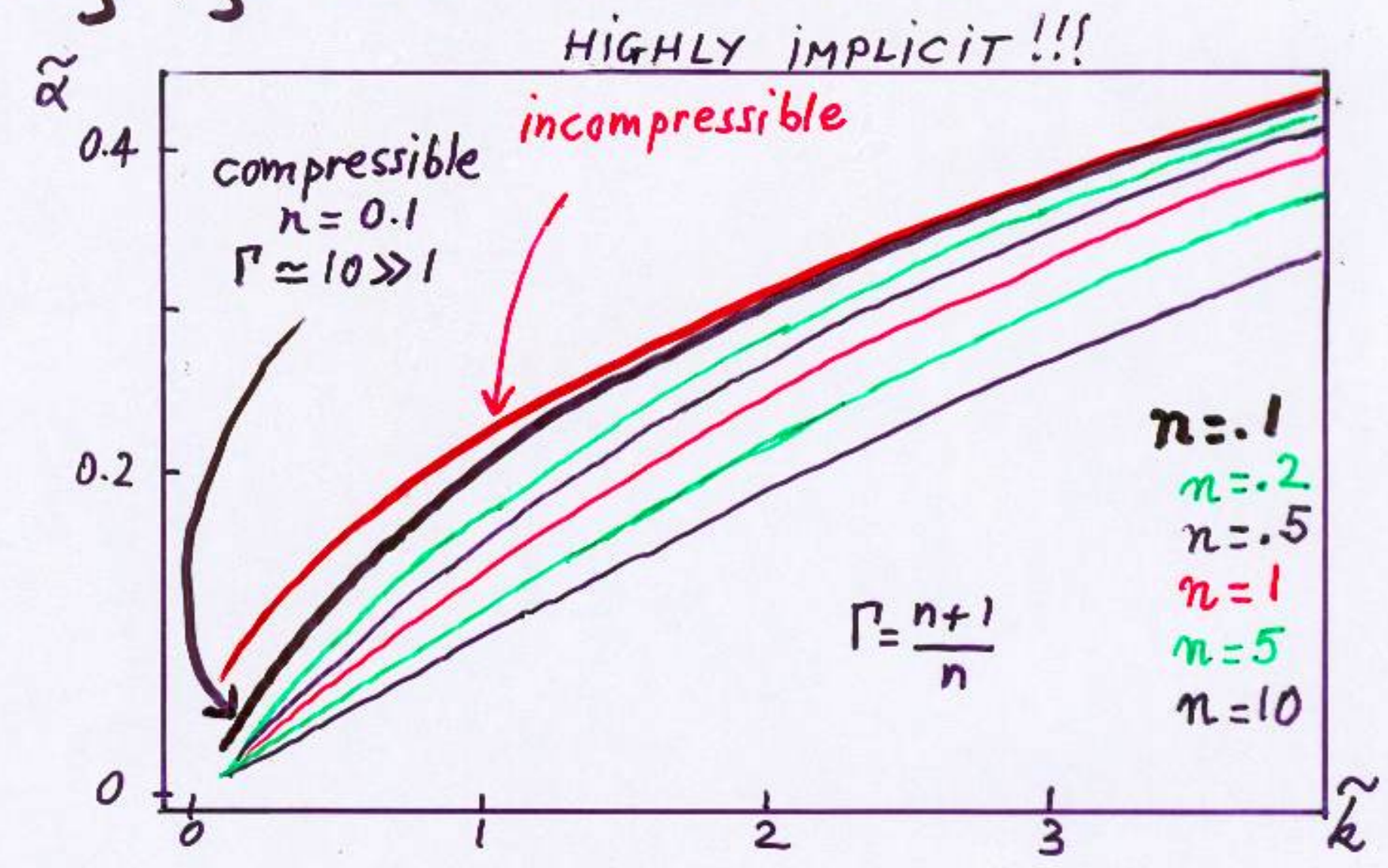

- $\tilde{\underline{k} \gg 1:} \quad \alpha \rightarrow \sqrt{A_{t} k g}$

. $n \rightarrow 0, \Gamma \rightarrow \infty:-\tilde{k}<2$ large $\lambda \quad \alpha_{\text {comp. }}<\alpha_{\text {income. }}(-$ below -$)$

- comparison: $\tilde{k}=2$

$\left\{\begin{array}{l}\tilde{\alpha}_{\text {comp }} \simeq .15 \\ \tilde{\alpha}_{\text {inc }} \simeq .3\end{array} \quad \sim 50 \%\right.$ deviation !!! (and into an exponential) 


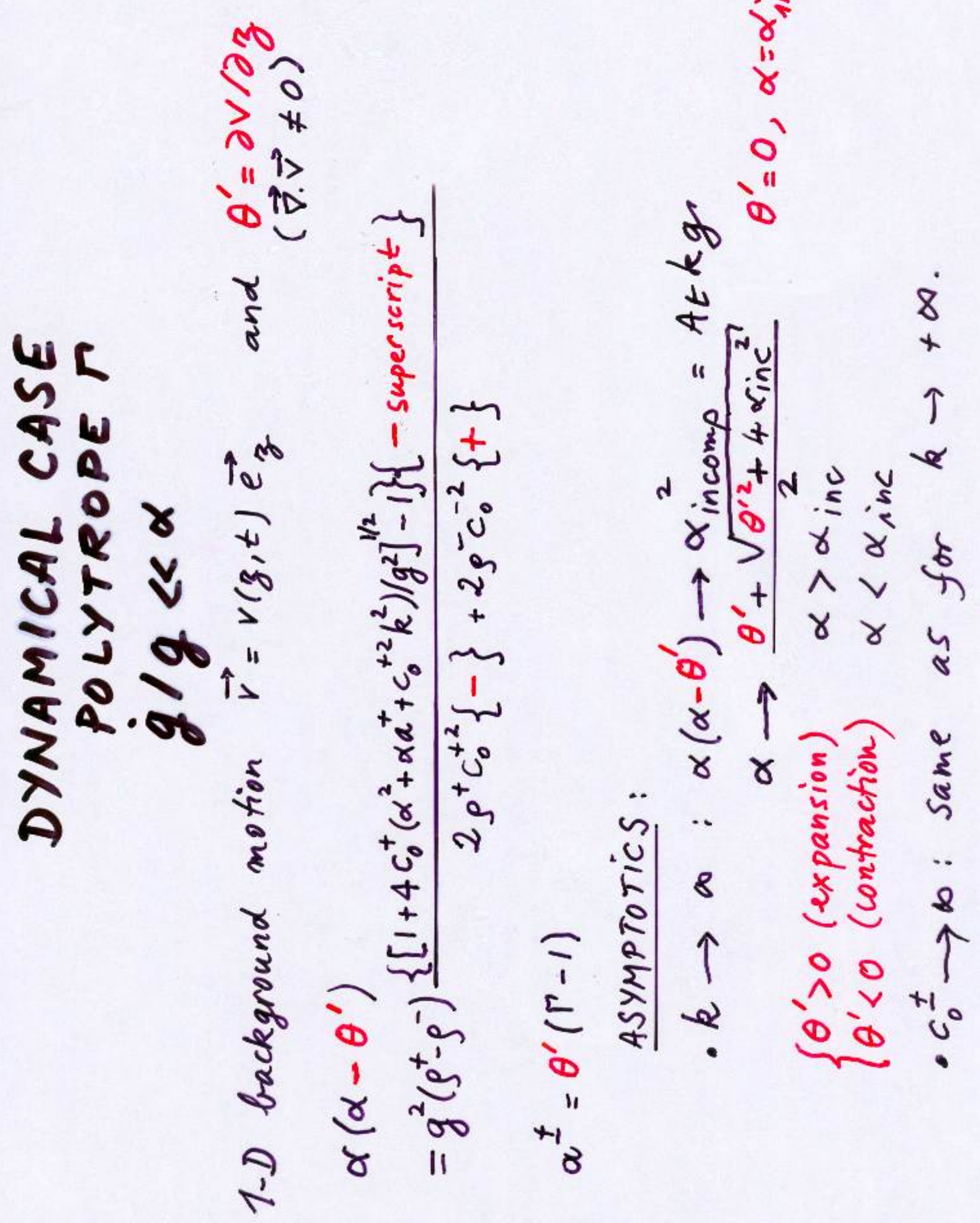


CONCLUSION

- Compressibility requires careful study (comparison with income. Should continued)

- Equilibrium initial State:

$$
\alpha_{\text {comp }}<\alpha_{\text {incomp }}=\sqrt{A_{t} \mathrm{~kg}}
$$

Similar to $\alpha_{\nabla} \simeq \sqrt{\frac{A_{t} k g}{1+k L_{\nabla}}}$

Stratification due to compressibility similar to the existence of a $\vec{\nabla} \rho$

- Incompressible:

$\vec{V}=\vec{\nabla} \Phi$ and $\vec{\nabla} \cdot \vec{v}=0 \Rightarrow \nabla^{2} \Phi=0$

compressible $\phi=\phi_{\text {dim }} S(x, y) z(z) T(t)$ with $\nabla^{2} S=-k^{2} S$

Similar to $\nabla^{2} \Phi=0$, but...

... additional equation for $Z(z), T(t)$ !

- To be used as a starting point for :

- Non Linear Study

- Comparison with Codes. 


\section{ANALYTICAL GROWTH RATE OF A NON-}

M. Vandenboomgaerde - Commissariat à l'Energie Atomique -

\section{ACCURATE APPROXIMATE PERTURBATION}

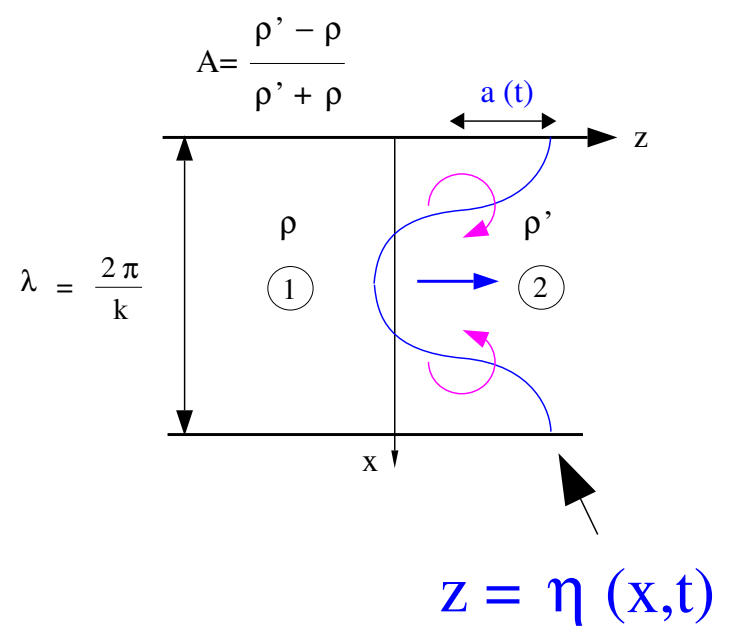

$$
\begin{aligned}
& k \eta=\sum_{n=1}^{\infty}\left(a_{0} k \sigma t\right)^{n} \sum_{j=1}^{n} a_{j}^{(n)} \cos (j k x) \quad \Rightarrow \\
& \mathbf{P} 1[\mathrm{~A}]=1 \\
& \mathrm{P3}[\mathrm{A}]=\frac{-1+2 * \mathrm{~A}^{2}}{6} \\
& \mathrm{P5}[\mathrm{A}]=\frac{19-125 * \mathrm{~A}^{2}+92 * \mathrm{~A}^{4}}{240} \\
& P 7[A]=\frac{-264+3686 * A^{2}-6997 * A^{4}+3234 * A^{6}}{5040}
\end{aligned}
$$

* See T32 Oral : Efficient perturbation method for the RM and the RT instabilities: Weakly nonlinear stage and beyond 


\section{LINEAR SINGLE-MODE R-M INSTABILIT}

BP 12 - 91680 Bruyeres-Le-Chatel- FRANCE

E-mail: marc.vandenboomgaerde@cea.fr

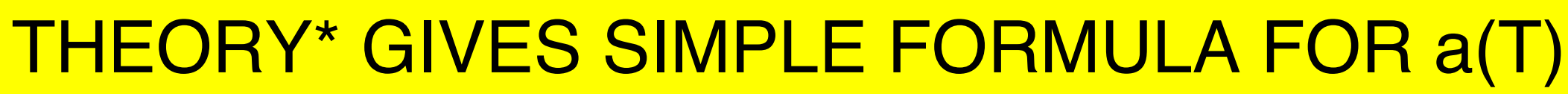

$k a(T)=\sum_{p=0}^{\infty} P_{2 p+1}[A] T^{2 p+1} \quad$ with $\quad T=a_{0} k \sigma t$

$\mathrm{P} 9[\mathrm{~A}]=\frac{117663-2855274 * \mathrm{~A}^{2}+10086083 * \mathrm{~A}^{4}-11093856 * \mathrm{~A}^{6}+3805728 * \mathrm{~A}^{8}}{2903040}$

$P 11[A]=\left(-5507319+206796915 * A^{2}-1168865775 * A^{4}+2250383605 * A^{6}\right.$

$\left.-1755444326 * A^{8}+483163144 * A^{10}\right) / 159667200$

$P 13[A]=\left(3117139337-167864265395 * A^{2}+1387729381613 * A^{4}-4085608349133 * A^{6}\right.$

$\left.+\mathbf{5 3 8 0 1 1 5 3 2 7 4 4 2} * \mathrm{~A}^{8}-\mathbf{3 2 7 2 2 6 9 5 5 4 9 6 8} * \mathrm{~A}^{10}+\mathbf{7 5 5 6 5 3 5 8 7 6 4 8} * \mathrm{~A}^{12}\right) / \mathbf{9 9 6 3 2 3 3 2 8 0 0}$ 


\section{$P_{2 \mathrm{P}+1}[A]$ POLYNOMIALS HAVE PECULIAR SHAPES}

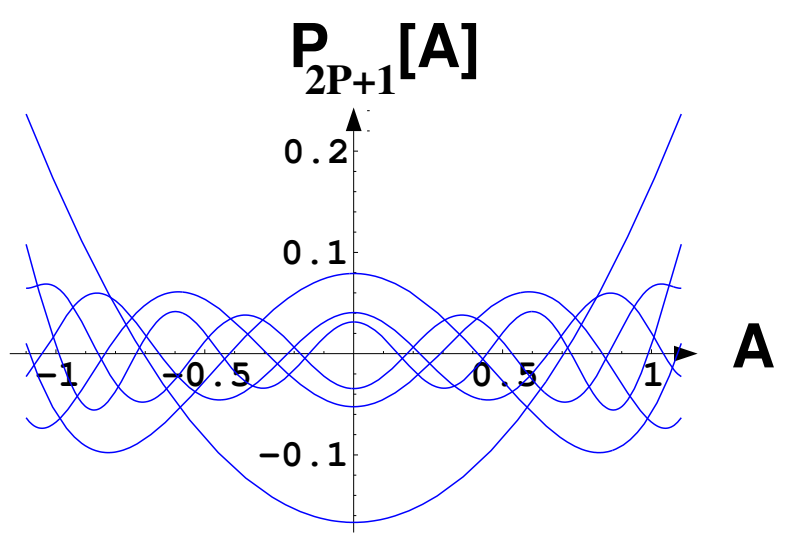

JACOBI'S POLYNOMIALS

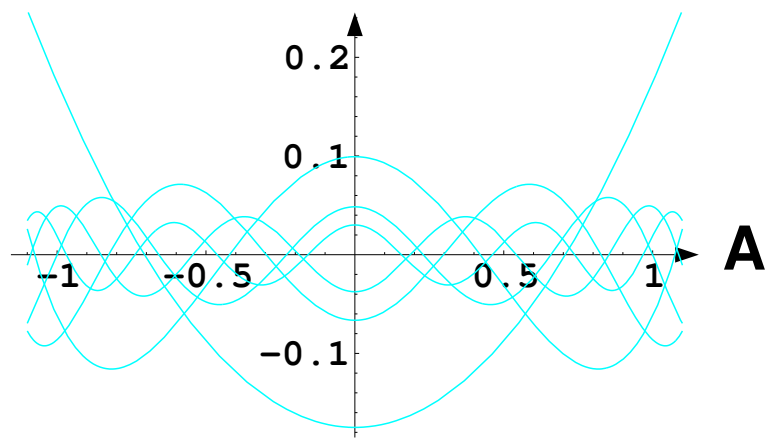

HYPOTHESIS $1: \mathrm{P}_{2 \mathrm{P}+1}[\mathrm{~A}]=\frac{1}{\mathrm{P}+2} \operatorname{JACOBIP}[2 \mathrm{P}, 0.1,0.1,0.79 \mathrm{~A}]$

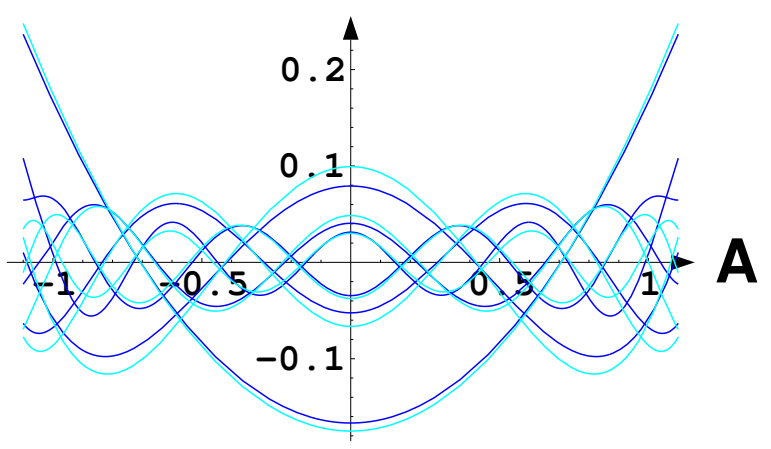




\section{A BASIS OF JACOBI'S POLYNOMIALS IS USED FOR a(T)}

$k\left(a(T)-a_{\mathbf{0}}\right)=\sum_{p=\mathbf{0}}^{\infty} P_{2 p+\mathbf{1}}[A] T^{\mathbf{2} p+\mathbf{1}}=\sum_{p=\mathbf{0}}^{\infty} \frac{\mathbf{1}}{p+\mathbf{2}} \mathbf{J a c o b i P}[2 p, \mathbf{0 . 1}, \mathbf{0 . 1}, \mathbf{0 . 7 9} A] T^{\mathbf{2} p+\mathbf{1}}+\operatorname{remainder}_{2 p+\mathbf{1}}[A] T^{\mathbf{2} p+\mathbf{1}}$ $=\frac{\mathbf{1}}{\mathbf{2}} T+\frac{\mathbf{1}}{T^{\mathbf{3}}} \int_{\mathbf{0}}^{T} \frac{\tau^{\mathbf{3}}}{\mathbf{2}}\{F(A, \tau)+F(A,-\tau)\} d \tau \quad+$ RemainderFunction $[A, T]$ $F(A, \tau)=$ Generating function of Jacobi's polynomials

HYPOTHESIS $2: \quad \mathrm{k}\left(\mathrm{a}(\mathrm{T})-\mathrm{a}_{0}\right) \approx \frac{1}{2} \mathrm{~T}+\frac{1}{\mathrm{~T}^{3}} \int_{0}^{\mathrm{T}} \frac{\tau^{3}}{2}\{\mathrm{~F}(\mathrm{~A}, \tau)+\mathrm{F}(\mathrm{A},-\tau)\} \mathrm{d} \tau$

EXTENDED GROWTH RATE BY GENERATING FUNCTION FITS WITH TAYLOR EXPANSION

$\mathrm{ka}(\mathrm{T}) \quad \mathrm{A}=0.1$

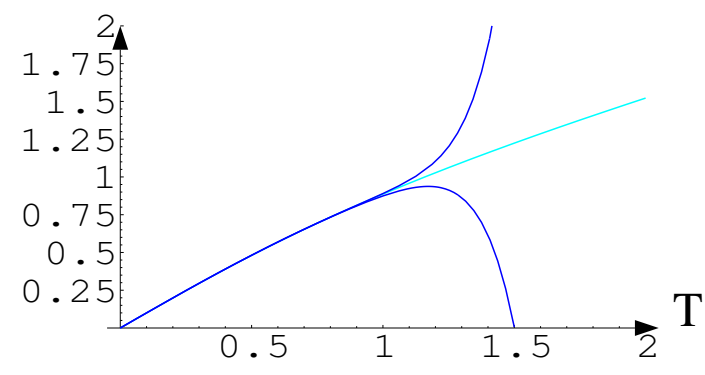

$\mathrm{k} \mathrm{a}(\mathrm{T}) \quad \mathrm{A}=0.5$

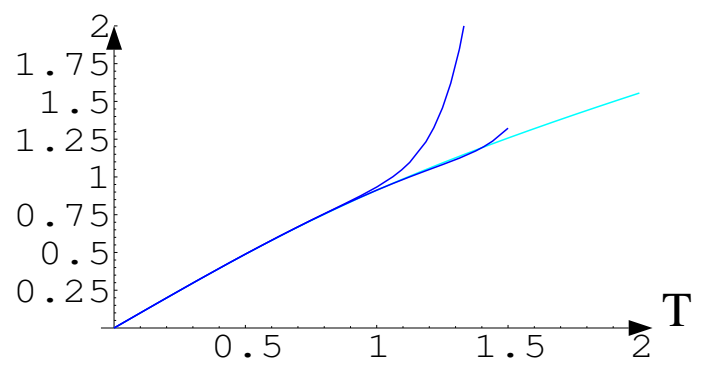

$\mathrm{k} \mathrm{a}(\mathrm{T}) \quad \mathrm{A}=0.9$

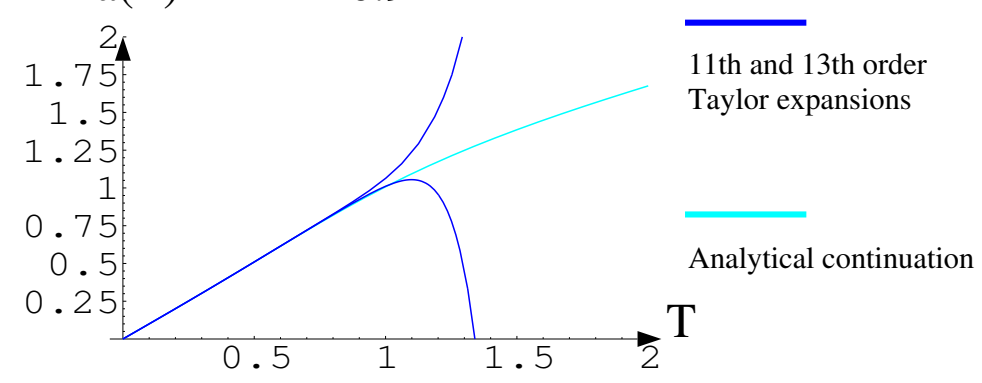




\section{EXTENTED GROWTH RATE BY GENERATING FUNCTION FITS NONLINEAR R-M INSTABILITIES}

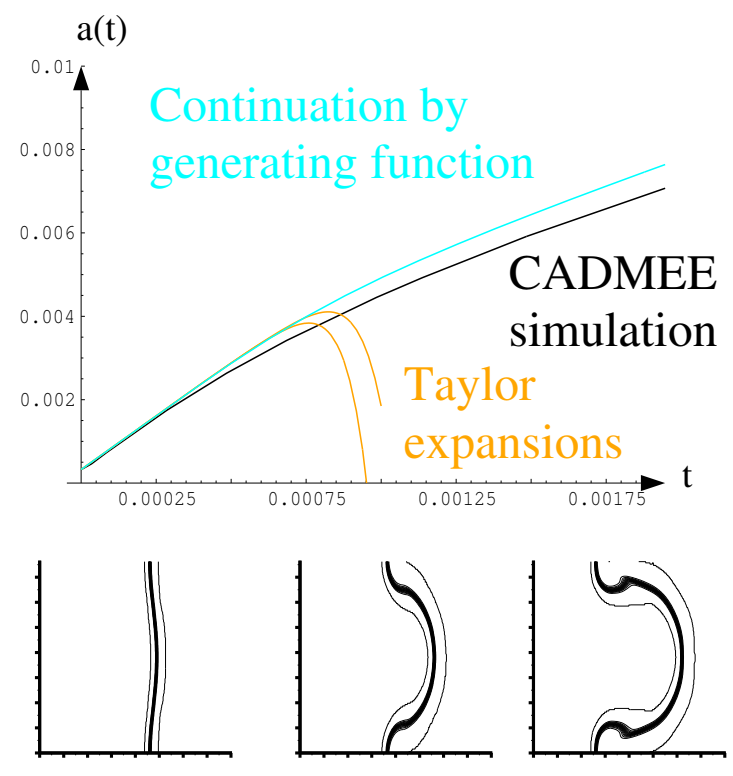

CADMEE simulation

$\mathrm{He} / \mathrm{Air} \mathrm{M} 0=1.09$

$k=224.855$

$A=0.764$

$\mathrm{a} 0=0.35 \mathrm{~mm}$

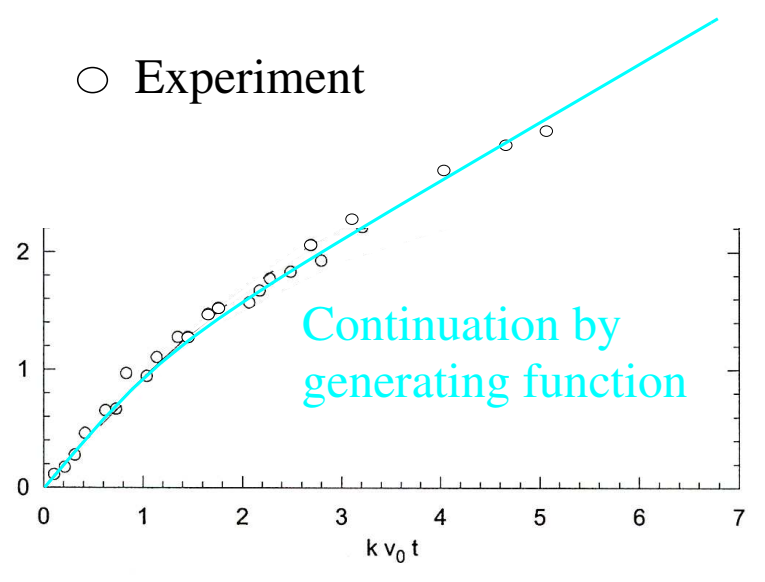

From Jacobs \& Krivets 2001 Proceedings of the 23rd ISSW

Air/SF6 $\mathrm{M} 0=1.3$

aok $=0.23$

$A=0.635$

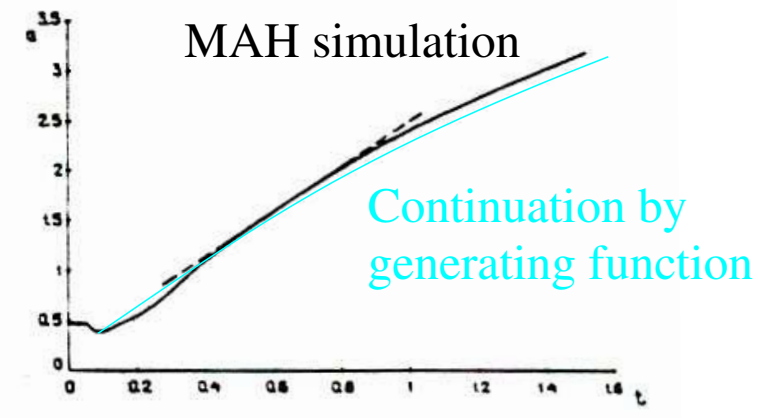

Fig. 6 Air-SF6.

Time dependence

$a(t)=x_{\max }(t)-x_{\min }(t)$.

From Anuchina \& Volkov

Proceedings IWPCTM 91

Air/SF6 $\mathrm{M} 0=1.32$

$\mathrm{k}=1.112$

$A=0.585$

$\mathrm{a} 0=0.183$ 


\section{ANALYTICAL CONTINUATION IS ACCURATE FOR $a(T) k<5.5$}
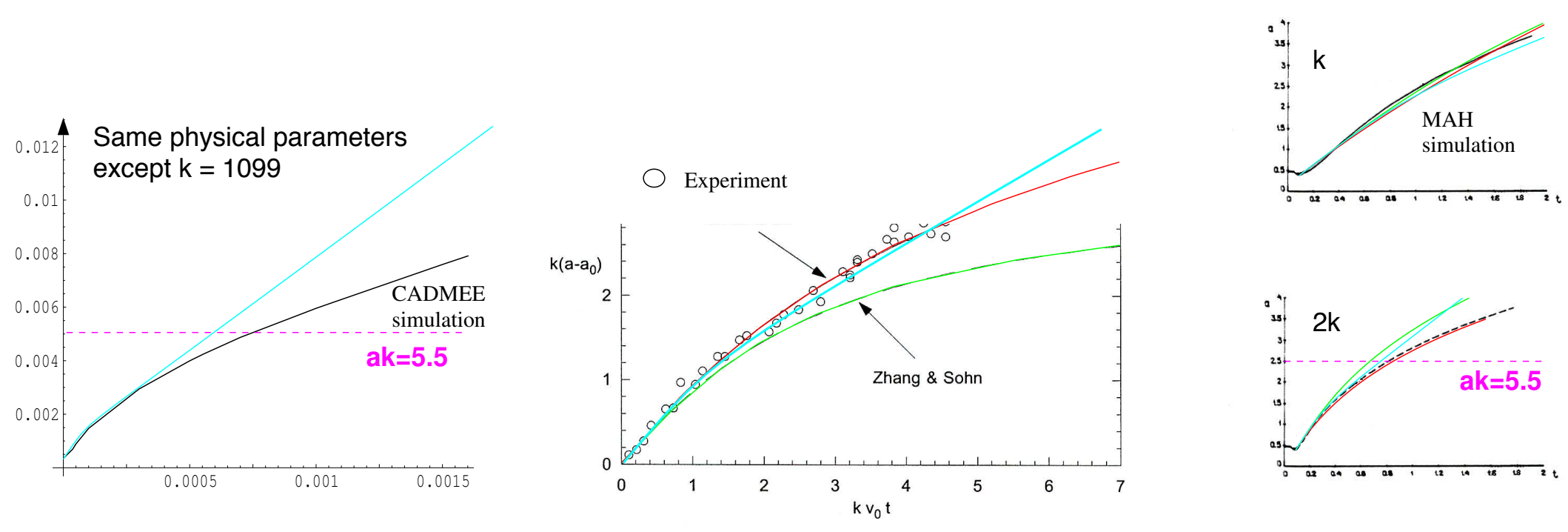

Analytical continuation

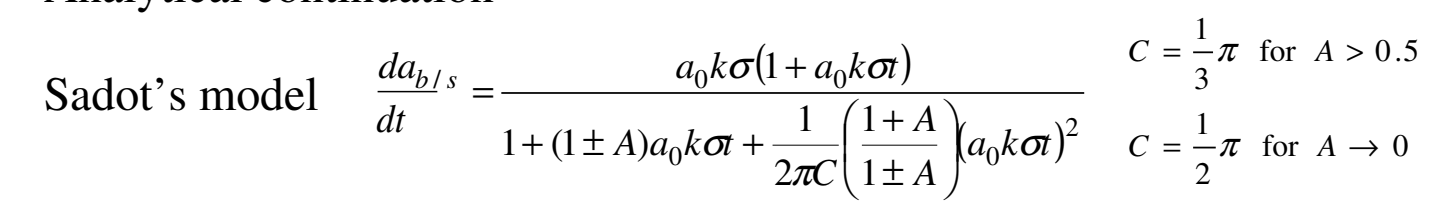

Zhang's model $\frac{d a}{d t}=\frac{a_{0} \sigma}{1+a_{0}^{2} k^{2} \sigma t+\operatorname{Max}\left[0, a_{0}^{2} k^{2}-A^{2}+1 / 2\right]\left(a_{0} k \sigma t\right)^{2}}$

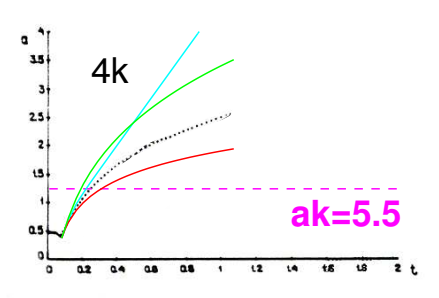




\section{CONCLUSIONS}

- Continuation of the growth rate by generating function has a reliable range of validity

* for ak $<5.5$ Relative error $<15 \%$

* for ak $>5.5$ non-physical linear behavior :

- remainder part no more negligeable

- other polynomial basis should be used

- single-evaluated interface model has reached its limit

- No adjustable parameter in the model

- The growth rate is solution of governing equations 


\section{Efficient perturbation methods for Richtmyer-Meshkov and Rayleigh-Taylor instabilities: Weakly nonlinear stage and Beyond.}

M. Vandenboomgaerde, S. Gauthier, D. Galmiche, C. Cherfils, P.A. Raviart CEA/DAM - Ile de France - DCSA

- I- Perturbation methods for the Richtmyer-Meshkov instability Summary, new approach and applications

- II- Beyond the weakly nonlinear stage

Inferences and numerical tests

- III- Accurate approximation for the Rayleigh-Taylor instability Some examples

- Concluding remarks 
I-Perturbation methods for the Richtmyer-Meshkov instability

$\underline{\text { Principle and hypothesis }}$
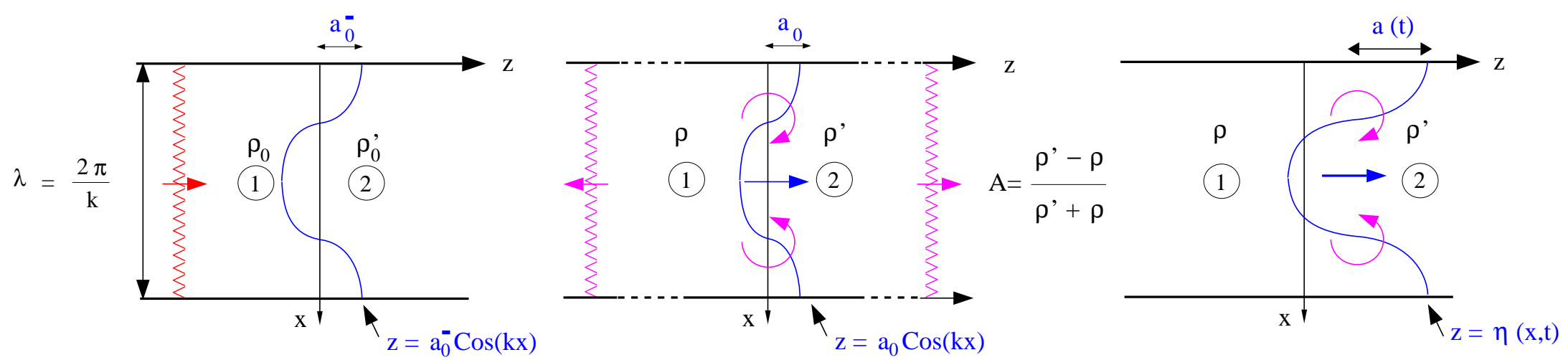

- Fluids are considered incompressible and irrotational

- No more effect of the reflected and transmitted waves

Equations

- Velocities derive from potentials: $\quad \vec{v}=-G \overrightarrow{r a d} \Phi \quad \overrightarrow{v^{\prime}}=-G \vec{r} a d \Phi^{\prime}$

- Motion of the interface: $-\frac{\partial \Phi}{\partial z}=\frac{\partial \eta}{\partial t}-\frac{\partial \Phi}{\partial x} \frac{\partial \eta}{\partial x} \quad$ and $\quad-\frac{\partial \Phi^{\prime}}{\partial z}=\frac{\partial \eta}{\partial t}-\frac{\partial \Phi^{\prime}}{\partial x} \frac{\partial \eta}{\partial x} \quad$ at $z=\eta$

- Bernoulli's equation: $-\rho^{\prime} \frac{\partial \Phi^{\prime}}{\partial t}+\rho \frac{\partial \Phi}{\partial t}+\frac{1}{2} \rho^{\prime}\left[\left(\frac{\partial \Phi^{\prime}}{\partial x}\right)^{2}+\left(\frac{\partial \Phi^{\prime}}{\partial z}\right)^{2}\right]-\frac{1}{2} \rho\left[\left(\frac{\partial \Phi}{\partial x}\right)^{2}+\left(\frac{\partial \Phi}{\partial z}\right)^{2}\right]=0$ at $z=\eta$ 
I-Perturbation methods for the Richtmyer-Meshkov instability

$\underline{\text { Summary of the full perturbation theory }}$

- Find a small parameter: $a_{0} k<<1$

- Expand unknowns $\eta, \Phi, \Phi^{\prime}$ as series: $\quad \eta=\sum \eta^{(n)} \quad$ with $\eta^{(n)} \#\left(a_{0} k\right)^{n}$

- Introduce expansions in the 3-equation system

- Collect terms of the same order

- Solve resulting systems $\Longrightarrow$ appearance of "secular terms"

Ex:

$$
X^{(n)}=\left(a_{0} k\right)^{n} \times\left(x_{1}^{(n)} t^{n}+x_{2}^{(n)} t^{n-1}+\ldots+x_{n}^{(n)}\right)
$$

- At the $n^{\text {th }}$ order, $3 n^{2}$ coefficients must be evaluated

$k \eta^{(3)}=-\frac{1}{24}\left(a_{0} k\right)^{3}\left[\left(4 A^{2}+1\right)(\sigma t)^{3}+3(\sigma t)^{2}+6 \sigma t\right] \cos k x+\frac{1}{8}\left(a_{0} k\right)^{3}\left[\left(4 A^{2}-1\right)(\sigma t)^{3}-3(\sigma t)^{2}\right] \cos 3 k x$ 
How to simplify the full perturbation theory

C.M. Bender, S.A. Orszag, "Advanced mathematical methods ...", 1978

\section{At each order, keep ONLY the MOST SECULAR TERMS}

- A new small parameter: $a_{0} k \sigma t<<1$

- Expand unknowns $\eta, \Phi, \Phi^{\prime}$ as series: $\quad \eta=\sum \eta^{(n)}$ with $\eta^{(n)} \#\left(a_{0} k \sigma t\right)^{n}$

- Introduce expansions in the 3-equation system

- Collect terms of the same order $\Longleftrightarrow$ at the $n^{t h}$ order, $\frac{\partial \eta}{\partial t} \Rightarrow t^{n-1}$ terms

- Solve resulting systems: results are written in a new form

$$
\begin{aligned}
\mathrm{Ex}: \quad X^{(n)}= & \left(a_{0} k\right)^{n} \times\left(x_{1}^{(n)} t^{n}+x_{2}^{(n)} t^{n-1}+\ldots+x_{n}^{(n)}\right) \\
\Downarrow & X^{(n)} \approx\left(a_{0} k\right)^{n} \times x_{1}^{(n)} t^{n}
\end{aligned}
$$

- At the $n^{\text {th }}$ order, only $3 n$ coefficients must be evaluated

$$
\begin{gathered}
k \eta^{(3)}=-\frac{1}{24}\left(a_{0} k\right)^{3}\left[\left(4 A^{2}+1\right)(\sigma t)^{3}+3(\sigma t)^{2}+6 \sigma t\right] \cos k x+\frac{1}{8}\left(a_{0} k\right)^{3}\left[\left(4 A^{2}-1\right)(\sigma t)^{3}-3(\sigma t)^{2}\right] \cos 3 k x \\
\Downarrow \\
k \eta^{(3)} \approx\left(a_{0} k \sigma t\right)^{3}\left[-\frac{1}{24}\left(4 A^{2}-1\right) \cos k x+\frac{1}{8}\left(4 A^{2}-1\right) \cos 3 k x\right]
\end{gathered}
$$

- Same algorithm for multimode configuration 
I-Perturbation methods for the Richtmyer-Meshkov instability

Some remarks about the approximate perturbation theory

- The approximate pertubation theory gives accurate results within its range of validity

- The range of validity is clearly defined: $a_{0} k \sigma t_{v}=1$

- The approximation makes solution easier and faster: $n^{2}$-algorithm $\rightarrow n$-algorithm

- The approximation makes multimode configurations tractable

- Sign of the initial amplitudes (or the phase between modes) have effects upon the mode-competition process

$\Downarrow$

Mode-competition models must take into account the phases of the initial perturbation spectrum 

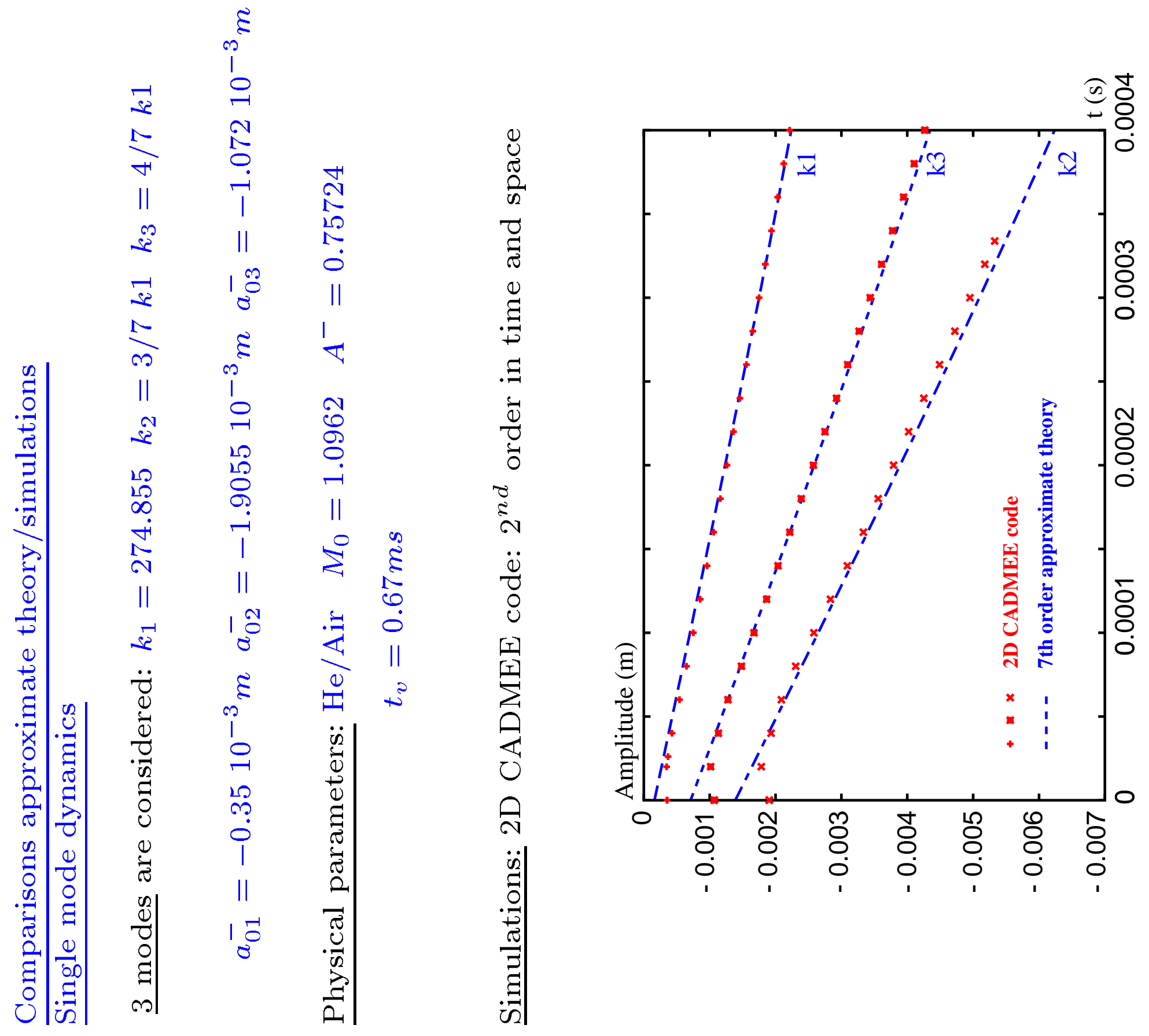

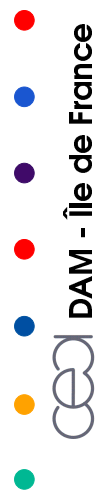


CEA / DAM - Île de France

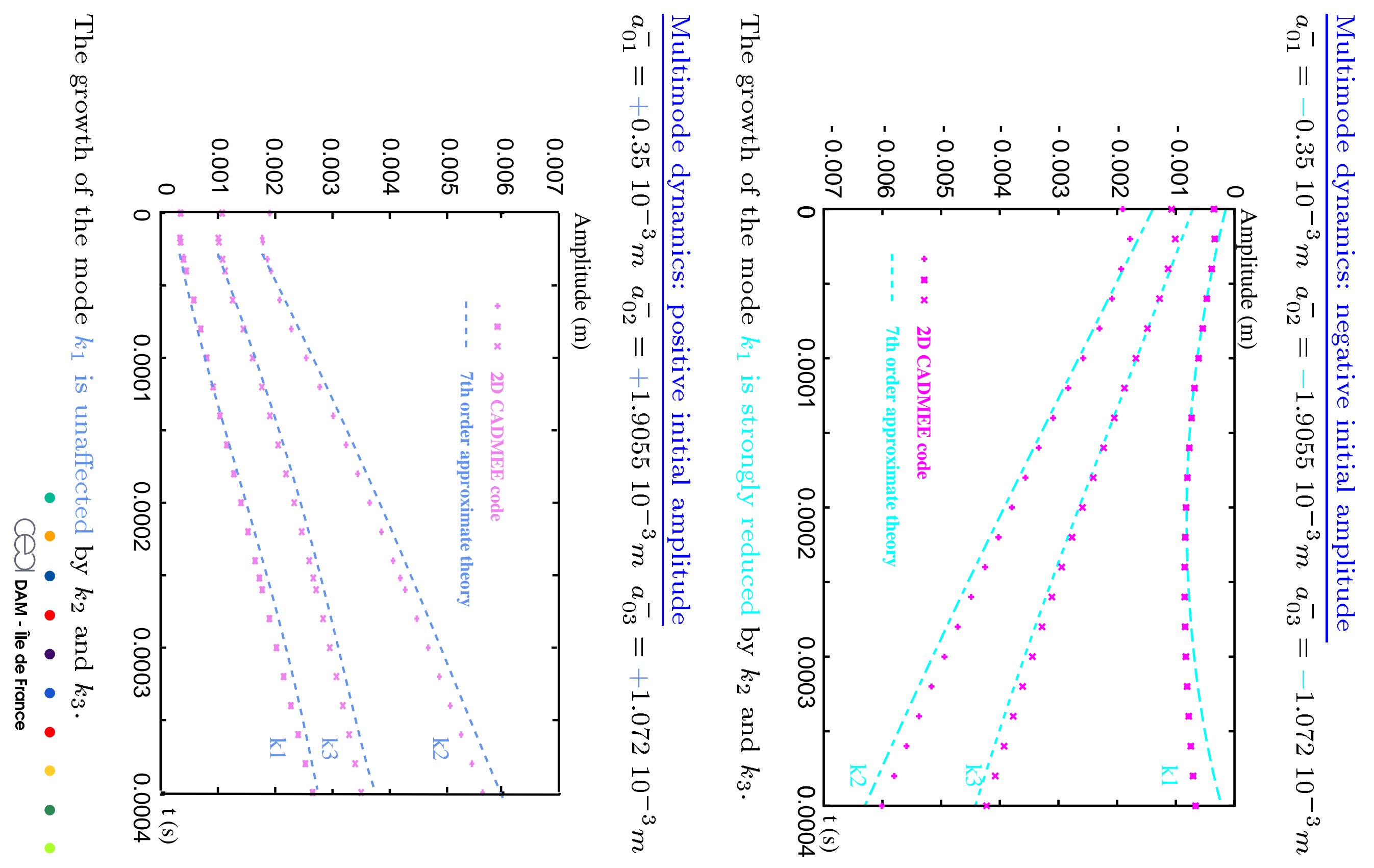


CEA / DAM - Île de France

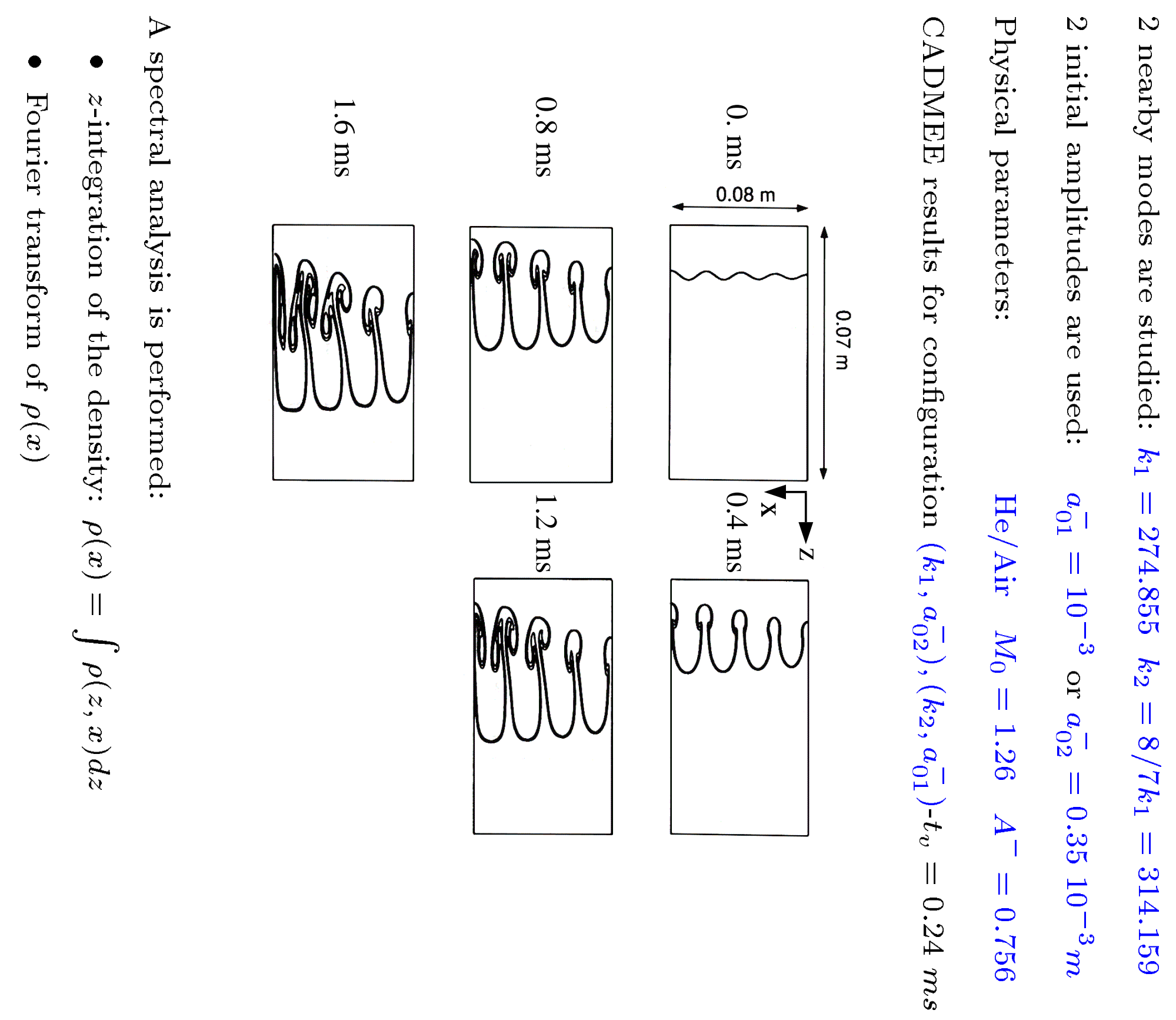


CEA / DAM - Île de France

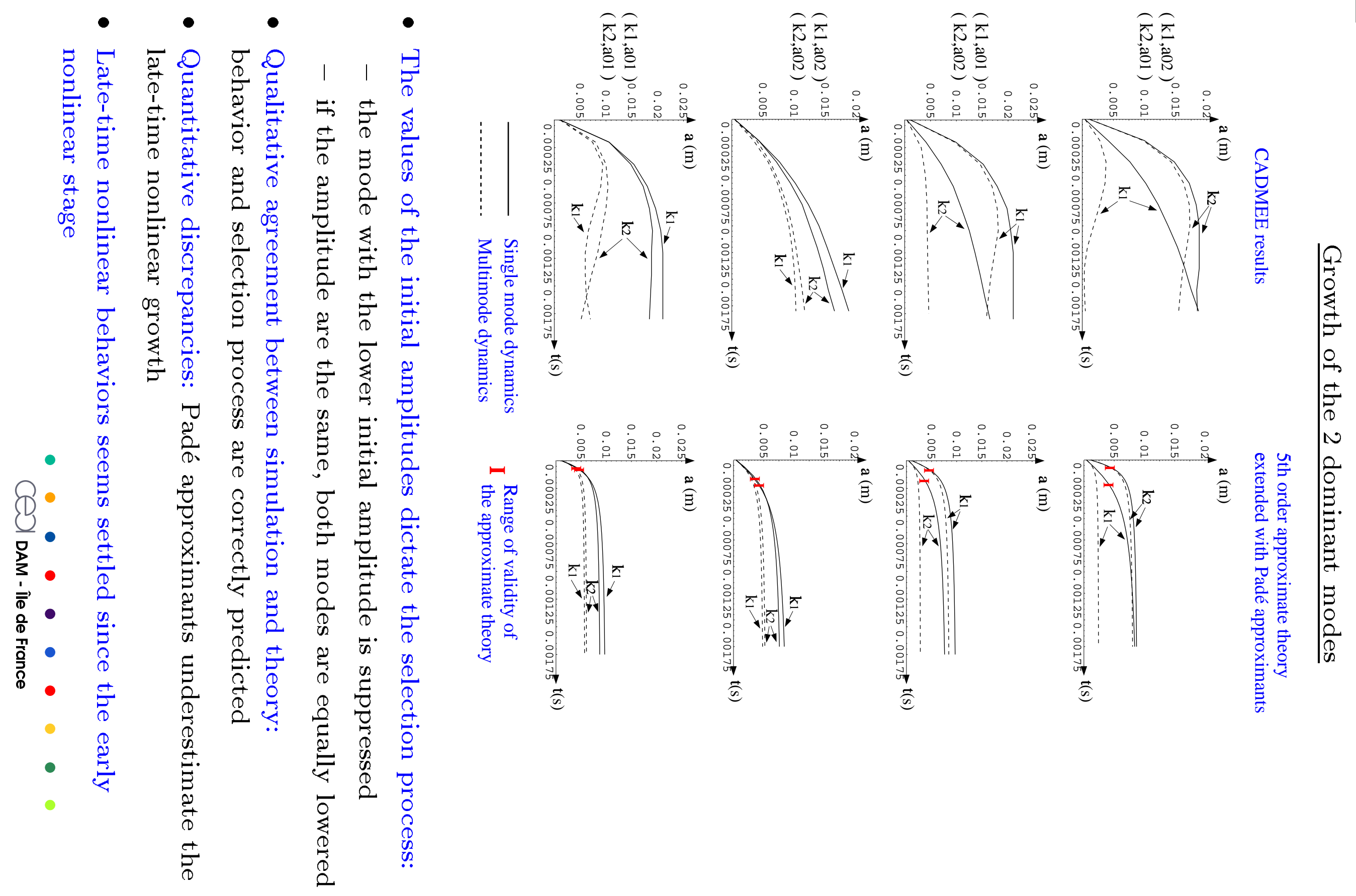


CEA / DAM - île de France

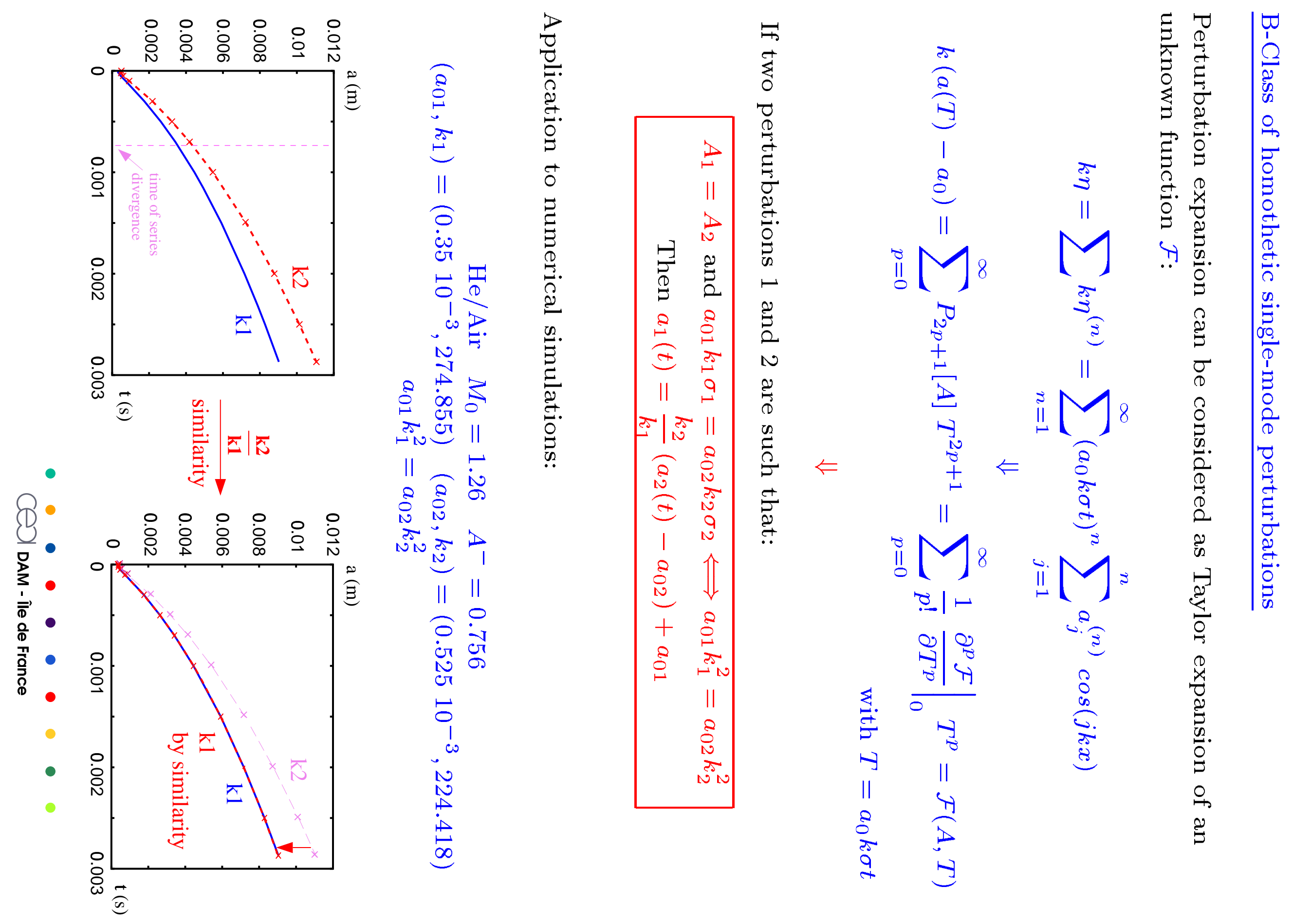


CEA / DAM - Île de France
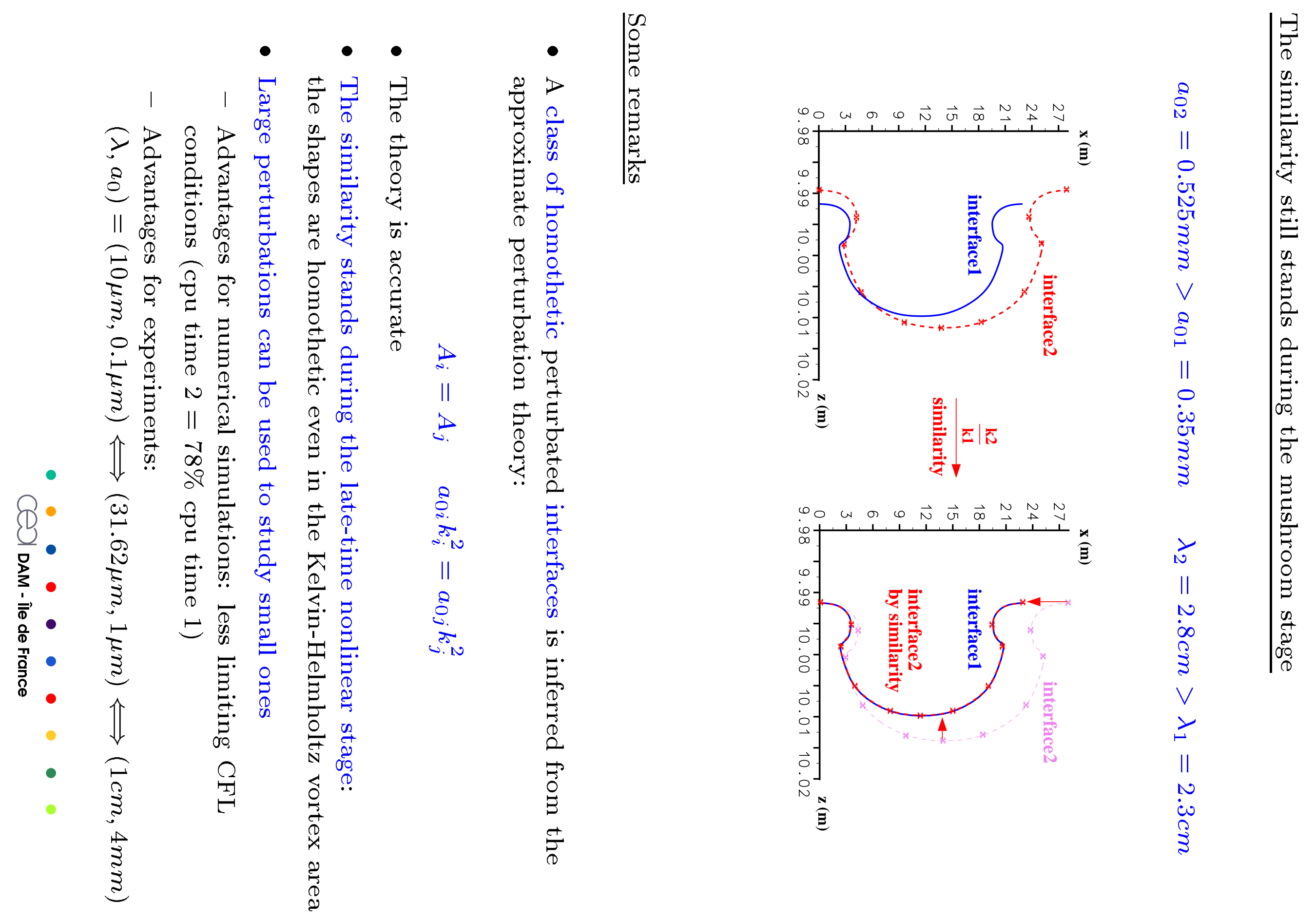
III- Accurate approximation for the Rayleigh-Taylor instability

- Same hypothesis for the fluids as for the RM instability

- Same 3-equation system as for the RM instability except for the additional

$$
\left(\rho^{\prime}-\rho\right) g \eta \text { term in Bernoulli's equation }
$$

- Same solution algorithm except that the "secular" terms write as

$$
\left(a_{0} k e^{\gamma t}\right)^{i} \text { with } \gamma=\sqrt{A g k}
$$

- Unknowns are written in a similar form:

$$
\eta=\sum \eta^{(n)}=\sum\left(a_{0} k e^{\gamma t}\right)^{n} \sum_{j=1}^{n} a_{j}^{(n)} \cos (j k x)
$$

- Range of validity: $a_{0} k e^{\gamma t v}=1$

- Same advantage: $n^{2}$-algorithm $\rightarrow n$-algorithm

- Example:

$$
\begin{gathered}
k \eta^{(2)}=-\frac{1}{4} A a_{0}^{2} k^{2}[2 \operatorname{Cosh}(\sqrt{2} \gamma t)-1-\operatorname{Cosh}(2 \gamma t)] \cos 2 k x \\
\downarrow \\
k \eta^{(2)}=-\frac{A}{8}\left(a_{0} k\right)^{2} e^{2 \gamma t} \cos 2 k x
\end{gathered}
$$


CEA / DAM - Île de France
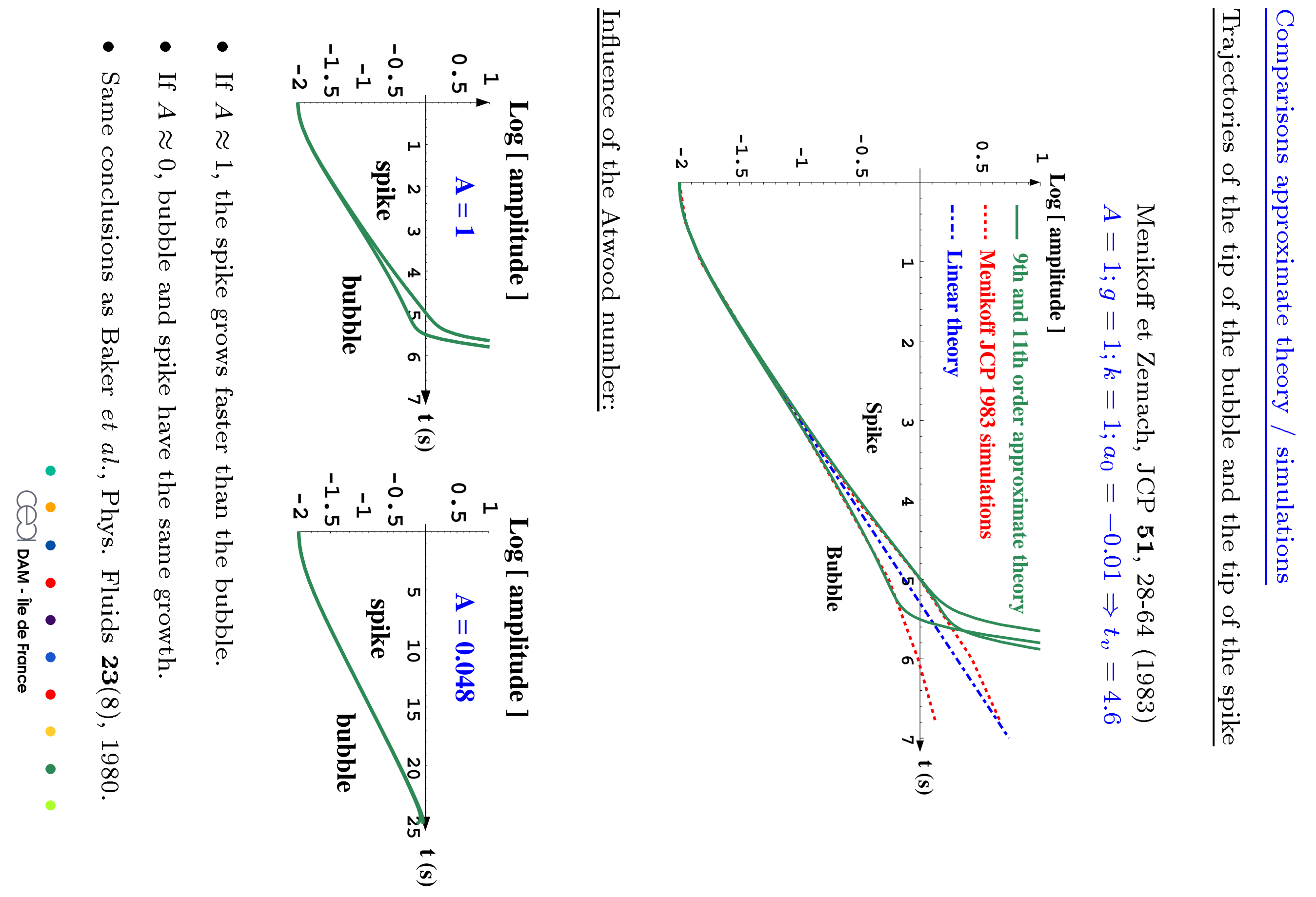
$\underline{\text { Concluding remarks }}$

- Approximate perturbation theory is accurate within its range of validity for RM and RT instabilities

- Solutions are easier and faster: $n^{2}$-algorithm $\rightarrow n$-algorithm

- For the Richtmyer-Meshkov instabilities:

- Approximate theory makes multimode configurations tractable

- Padé extended approximate theory allows us to predict late-time selection process: the origin of such process occurs in the weakly nonlinear stage

- Mode spectrum, amplitude values but also, the phase between modes must be known to determine the modes leading to the bubble stage.

- Existence of a class of homothetic perturbated interfaces:

$$
A_{i}=A_{j} \quad \text { and } \quad a_{0 i} k_{i}^{2}=a_{0 j} k_{j}^{2}
$$




\title{
Combined Shear and \\ Buoyancy Instabilities
}

Peter Wilson, Malcolm Andrews and Francis Harlow

$8^{\text {th }}$ International Workshop on the Physics of Compressible Turbulent Mixing

\author{
Pasadena, CA
}

December 9-14, 2001 
Spectral Turbulence Model for Variable Density

Turbulence

- Steinkamp, Clark and Harlow, Int. J. of Multiphase Flow

(1999)

- $\left.\hat{\mathrm{R}}_{\mathrm{ij}}(\overrightarrow{\mathrm{x}}, \overrightarrow{\mathrm{k}}, \mathrm{t})=\iiint_{-\infty} \mathrm{R}_{\mathrm{ij}} \overrightarrow{\mathrm{x}}_{1}, \overrightarrow{\mathrm{x}}_{2}, \mathrm{t}\right) \mathrm{e}^{-\mathrm{i} \mathrm{k} \overrightarrow{\mathrm{k}}} \mathrm{d} \overrightarrow{\mathrm{r}}$

where $\vec{x}=\frac{\vec{x}_{1}+\vec{x}_{2}}{2}, \vec{r}=\vec{x}_{2}-\vec{x}_{1}$

- $\mathrm{R}_{\mathrm{ij}}(\overrightarrow{\mathrm{x}}, \mathrm{k}, \mathrm{t})=\int_{\Omega_{\mathrm{k}}} \hat{\mathrm{R}}_{\mathrm{ij}}(\overrightarrow{\mathrm{x}}, \overrightarrow{\mathrm{k}}, \mathrm{t}) \frac{\mathrm{k}^{2} \mathrm{~d} \Omega_{\mathrm{k}}}{(2 \pi)^{3}}$

- $\mathrm{R}_{\mathrm{ij}}(\overrightarrow{\mathrm{x}}, \mathrm{t})=\int_{0}^{\infty} \mathrm{R}_{\mathrm{ij}}(\overrightarrow{\mathrm{x}}, \mathrm{k}, \mathrm{t}) \mathrm{dk}$ 
Advantages of Spectral Transport Models Over SinglePoint Models (i.e., k- $\varepsilon$ ):

- More generality, such as in the case of non-equilibrium transients

- Does not require a length scale or dissipation equation

- Greater flexibility with modeling, such as with non-local interactions in both physical and wavenumber space

Disadvantages:

- Greater Complexity

- More computationally expensive

- More Flexibility! 


\section{Governing Equations}

$$
\begin{aligned}
\frac{D \tilde{\mathrm{u}}_{\mathrm{i}}}{\mathrm{Dt}} & =-\frac{1}{\bar{\rho}}\left[\frac{\partial \mathrm{R}_{\mathrm{in}}}{\partial \mathrm{x}_{\mathrm{n}}}+\frac{\partial \overline{\mathrm{p}}}{\partial \mathrm{x}_{\mathrm{i}}}\right]+v_{m} \frac{\partial^{2} \overline{\mathrm{u}}_{\mathrm{i}}}{\partial \mathrm{x}_{\mathrm{n}}^{2}}+\mathrm{g}_{\mathrm{i}} \\
\tilde{\mathrm{u}}_{\mathrm{i}} & =\frac{\overline{\rho \mathrm{u}_{\mathrm{i}}}}{\bar{\rho}}, \mathrm{R}_{\mathrm{in}}=\overline{\rho \mathrm{u}_{\mathrm{i}}^{\prime \prime} \mathrm{u}^{\prime \prime}} \quad \text { where } \mathrm{u}_{\mathrm{i}}=\tilde{\mathrm{u}}_{\mathrm{i}}+\mathrm{u}_{\mathrm{i}}^{\prime \prime}
\end{aligned}
$$

$\frac{\partial \bar{\rho}}{\partial \mathrm{t}}+\frac{\partial \tilde{\rho}_{\mathrm{u}}}{\partial \mathrm{x}_{\mathrm{n}}}=0$

$\left.\frac{D R_{i j}(k)}{D t}=\iiint_{-\infty t 0 \infty}\left[a_{i}(k) \frac{\partial \bar{p}}{\partial x_{j}}+a_{j}(k) \frac{\partial \bar{p}}{\partial x_{i}}\right] \hat{\mathbf{f}}\right]_{\overline{x^{\prime}}}\left(\vec{x}, \overrightarrow{x^{\prime}}\right) d \overrightarrow{x^{\prime}}-R_{i n} \frac{\partial \tilde{u}_{j}}{\partial x_{n}}-R_{j n} \frac{\partial \widetilde{u}_{i}}{\partial x_{n}}$

$+c_{d} \frac{\partial}{\partial x_{n}}\left[v_{t} \frac{\partial R_{i j}}{\partial x_{n}}\right]+c_{m}\left(\frac{1}{3} \delta_{i j} R_{n n}-R_{i j}\right) \int_{0}^{\infty} \sqrt{\frac{k R_{n n}}{\bar{\rho}}} d k$

$+\frac{\partial}{\partial k}\left\{k^{2} \sqrt{\frac{k R_{n n}}{\bar{\rho}}}\left[-c_{1} R_{i j}+c_{2} k \frac{\partial R_{i j}}{\partial k}\right]\right\}$

+ Non - Local $(\mathrm{T}(\mathrm{k}))$

where $a_{i}\left(\vec{x}_{1}, \vec{x}_{2}, t\right)=-\overline{u_{i}^{\prime \prime}\left(\vec{x}_{1}\right) \rho\left(\vec{x}_{1}\right) v\left(\vec{x}_{2}\right)}$ and $v_{t}=\int_{0}^{\infty} \sqrt{\frac{k R_{n n}}{\bar{\rho}}} \frac{d k}{k^{2}}$ 
Governing Equations (cont.)

$\frac{D a_{i}(k)}{D t}=\frac{b(k)}{\bar{\rho}} \frac{\partial \bar{p}}{\partial x_{i}}-\frac{R_{i n}}{\bar{\rho}^{2}} \frac{\partial \bar{\rho}}{\partial x_{n}}+c_{d} \frac{\partial}{\partial x_{n}}\left[v_{t} \frac{\partial a_{i}}{\partial x_{n}}\right]$

$$
\begin{aligned}
& -\left[c_{r 1} k^{2} \sqrt{a_{n} a_{n}}+c_{r 2} k \sqrt{\frac{k R_{n n}}{\bar{\rho}}}\right] a_{i} \\
& +\frac{\partial}{\partial k}\left\{k^{2} \sqrt{\frac{k R_{n n}}{\bar{\rho}}}\left[-c_{1} a_{i}+c_{2} k \frac{\partial a_{i}}{\partial k}\right]\right\}
\end{aligned}
$$

where $b\left(\vec{x}_{1}, \vec{x}_{2}, t\right)=-\overline{\rho^{\prime}\left(\vec{x}_{1}\right) v^{\prime}\left(\vec{x}_{2}\right)}$

$$
\begin{aligned}
\frac{D b(k)}{D t}= & u_{n} \frac{\partial b}{\partial x_{n}}+\frac{2 \bar{\rho}-\rho_{1}-\rho_{2}}{\rho_{1} \rho_{2}} \frac{\partial \bar{\rho} a_{n}}{\partial x_{n}}+c_{d} \frac{\partial}{\partial x_{n}}\left[v_{t} \frac{\partial b}{\partial x_{n}}\right] \\
& -c_{f b}\left[\bar{v}^{2} \frac{\partial \bar{\rho} / \bar{v}}{\partial x_{n}}\right] \frac{\partial k a_{n}}{\partial k}+\frac{\partial}{\partial k}\left\{k^{2} \sqrt{\frac{k R_{n n}}{\bar{\rho}}}\left[-c_{1} b+c_{2} k \frac{\partial b}{\partial k}\right]\right\} \\
& -2 v_{t} k^{2} b
\end{aligned}
$$


Non-Local Behavior in Physical and Wavenumber Space

Physical Space:

$\frac{D R_{n n}}{D t}=2 \int_{-\infty}^{\infty} a_{2}\left(y^{\prime}\right) \frac{\partial \bar{p}}{\partial y^{\prime}} \hat{f}\left(y, y^{\prime}\right) d y^{\prime}+\cdots$

$$
\begin{aligned}
& \int_{-\infty}^{\infty} \hat{\mathrm{f}}\left(\mathrm{y}, \mathrm{y}^{\prime}\right) \mathrm{dy}=1 \\
& \hat{\mathrm{f}}\left(\mathrm{y}, \mathrm{y}^{\prime}\right)=\frac{\exp \left(-2 \mathrm{k}\left|\mathrm{y}-\mathrm{y}^{\prime}\right|\right)}{\int_{-\infty}^{\infty} \exp \left(-2 \mathrm{k}\left|\mathrm{y}-\mathrm{y}^{\prime \prime}\right|\right) \mathrm{dy^{ \prime \prime }}}
\end{aligned}
$$

Wavenumber Space: Kraichnan and Spiegal Model for Energy Transfer (1962)

$T(k)=\int\left[S_{a}(k / p)-S_{e}(k / p)\right] d p$

$S_{e}(p / k)=\eta \sqrt{p E(p)} E(p)\left(\frac{p}{k}\right)^{m} g\left(\frac{p}{k}\right)$

$\mathrm{S}_{\mathrm{a}}(\mathrm{k} / \mathrm{p})=\mathrm{S}_{\mathrm{e}}(\mathrm{p} / \mathrm{k})$

$\mathrm{T}(\mathrm{k})=-\Sigma(\mathrm{k})+\int \Sigma(\mathrm{p}) \breve{\mathrm{f}}(\mathrm{k}, \mathrm{p}) \mathrm{dp}$ where $\Sigma(\mathrm{k})=\eta[\mathrm{kE}(\mathrm{k})]^{\frac{3}{2}}$ and $\breve{f}(k, p)=p^{-1}\left(\frac{k}{p}\right)^{m} g\left(\frac{k}{p}\right)$ 


\section{Modeling Constants}

\section{$\underline{c}_{1}, c_{2}, c_{m}, c_{r 1}, c_{12}, c_{d}$ also $\eta$ and $g(x)$}

$c_{1} / c_{2}=2$ for equipartition of energy $\left(c_{1}=.5\right)$

$\mathrm{c}_{\mathrm{r} 1} \approx \mathrm{c}_{\mathrm{r} 2}=5.0$ to match buoyancy experiments

$\mathrm{c}_{\mathrm{m}}=1.0$ original $\mathrm{SCH}$ model constant

Given that $\int \mathrm{f}(\mathrm{k}, \mathrm{p}) \mathrm{dk}=1 \Rightarrow \int \mathrm{x}^{\mathrm{m}} \mathrm{g}(\mathrm{x}) \mathrm{dx}=1$ where $\mathrm{x}=\mathrm{k} / \mathrm{p}$

Let $g(x)=\exp \left\lfloor-\alpha\left(x+x^{-1}\right)\right] / N$

and for $2 k$ coupling $(m=-2, \alpha=2 / 3)$

Lastly,

$\varepsilon=\int_{\mathrm{k}}^{\infty} \int_{0}^{\mathrm{k}} \mathrm{S}(\mathrm{q} / \mathrm{p}) \mathrm{dpdq}$

$\eta=C_{k}-3 / 2\left[\int_{1}^{\infty} \ln (x)\left(x^{m}-x^{-m-2}\right) g(x) d x\right]^{-1} \Rightarrow \eta=1.36$ 


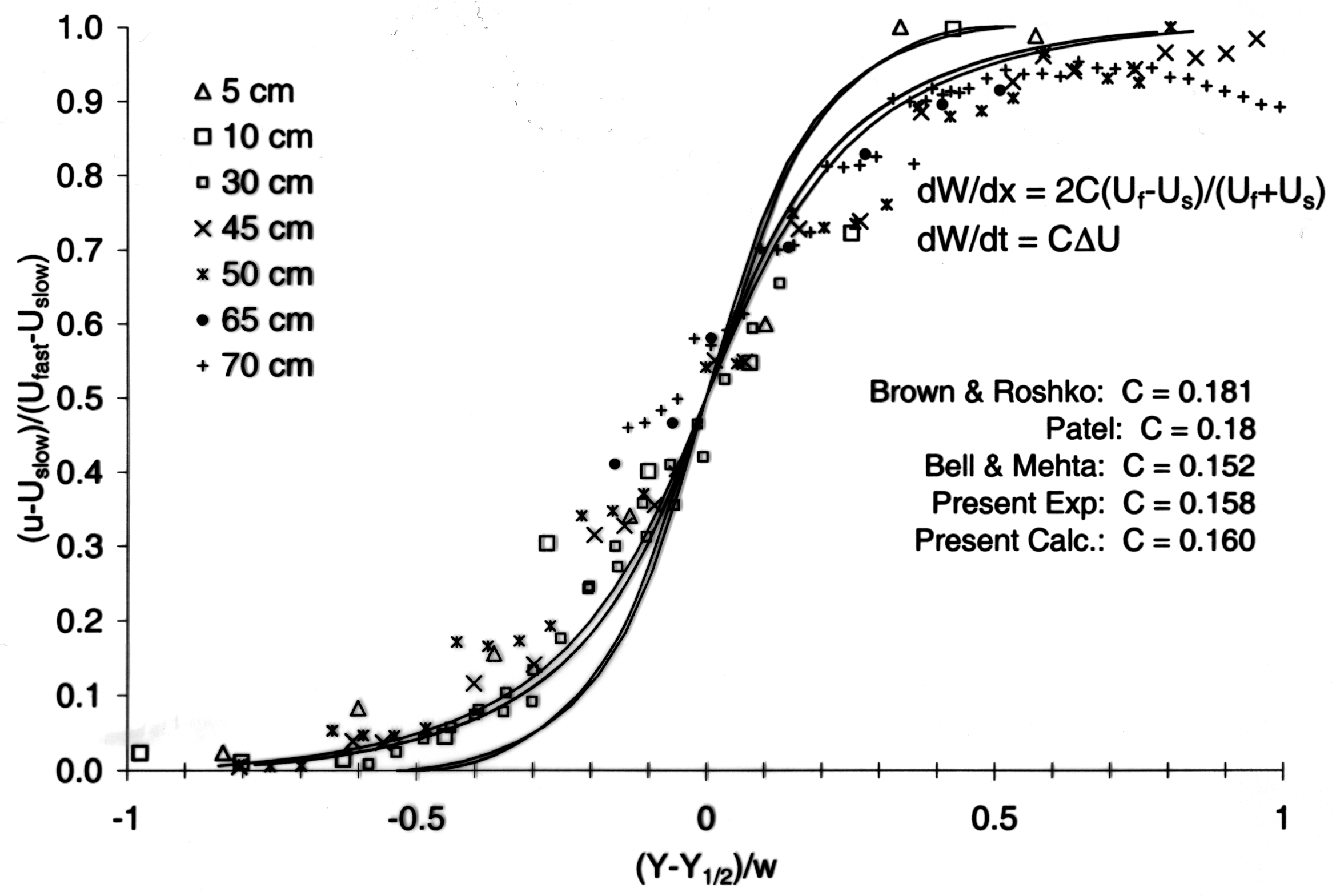




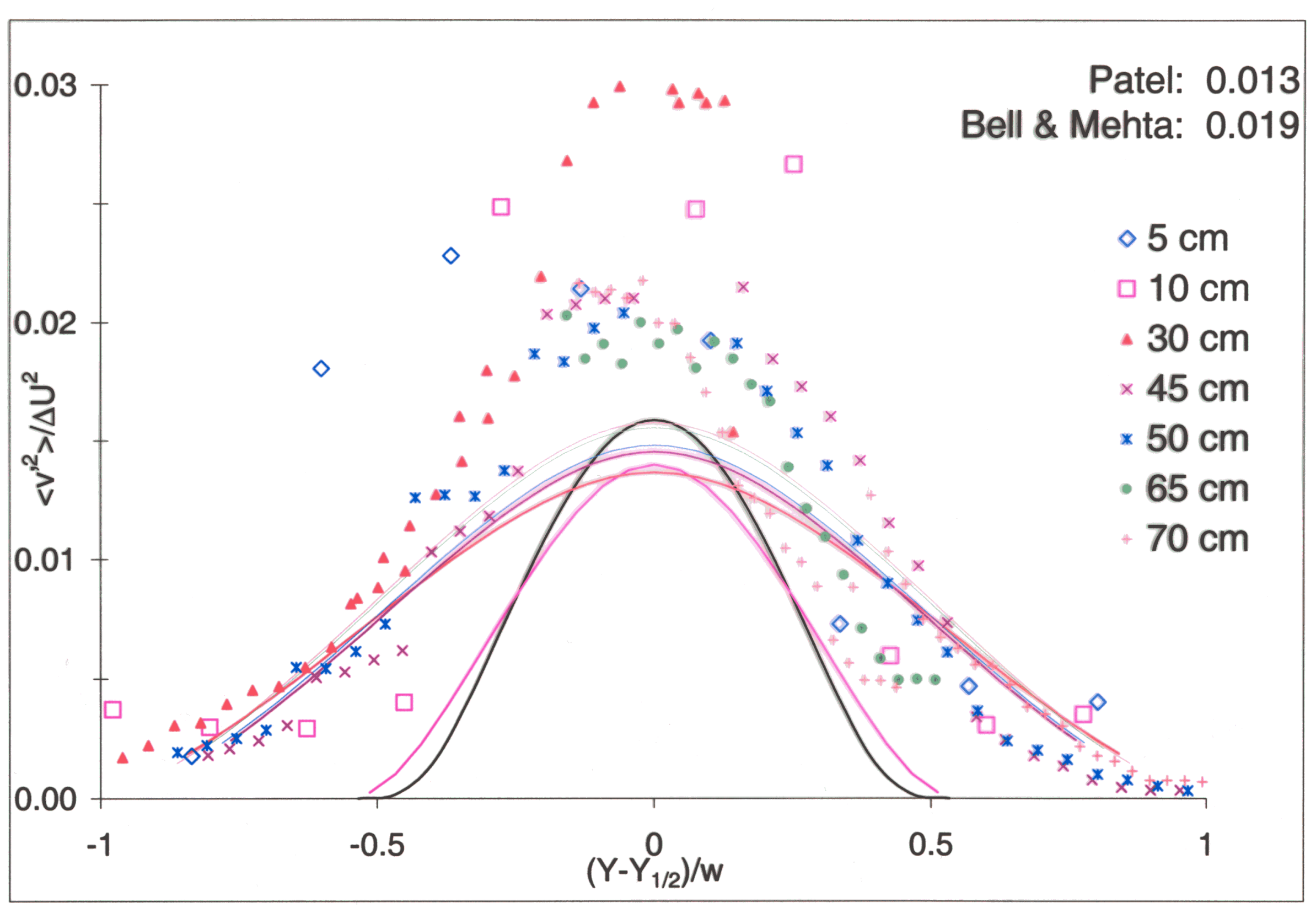


$\left(Y-Y_{1 / 2}\right) / w$

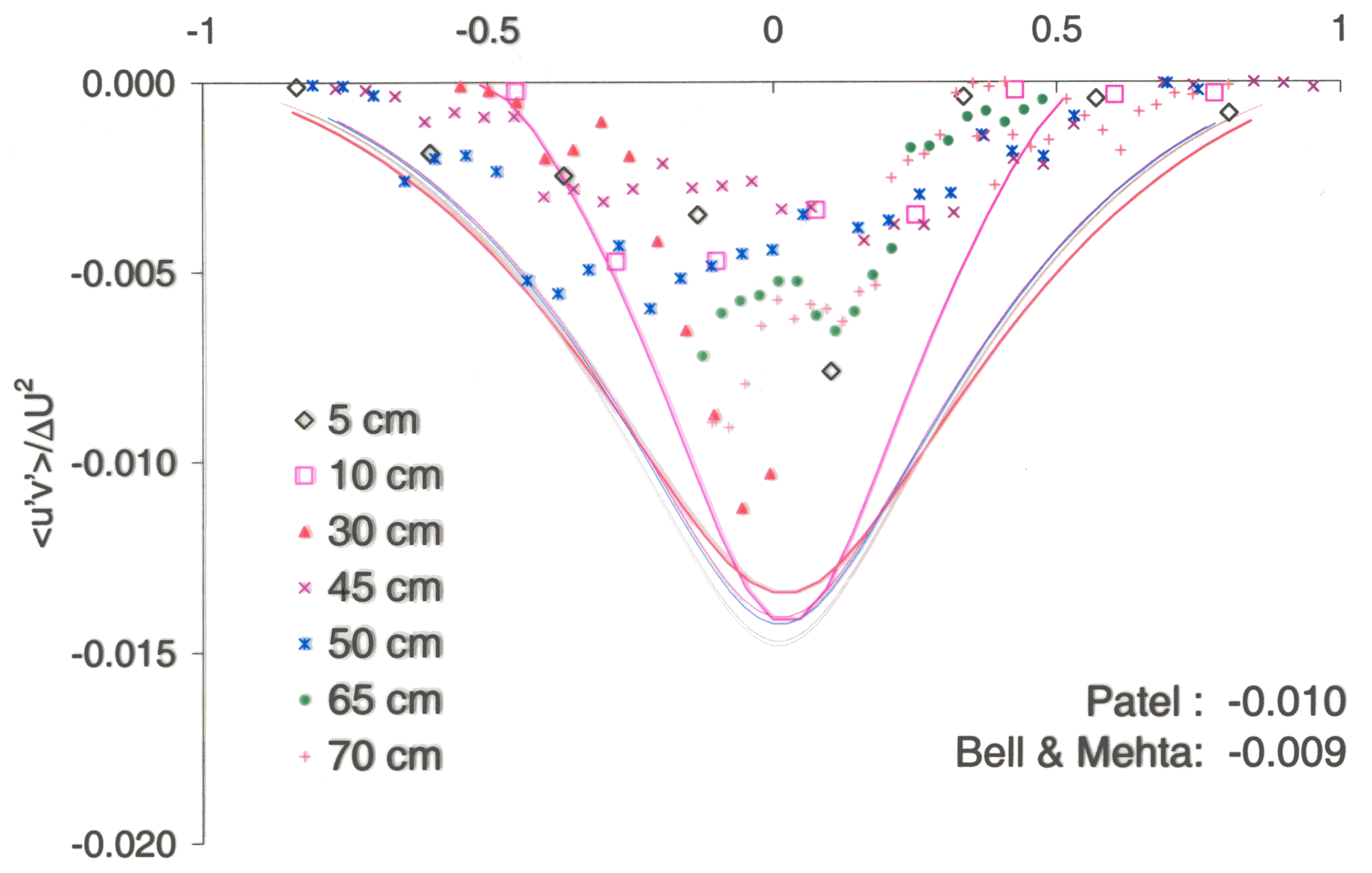


Shear Energy Spectra

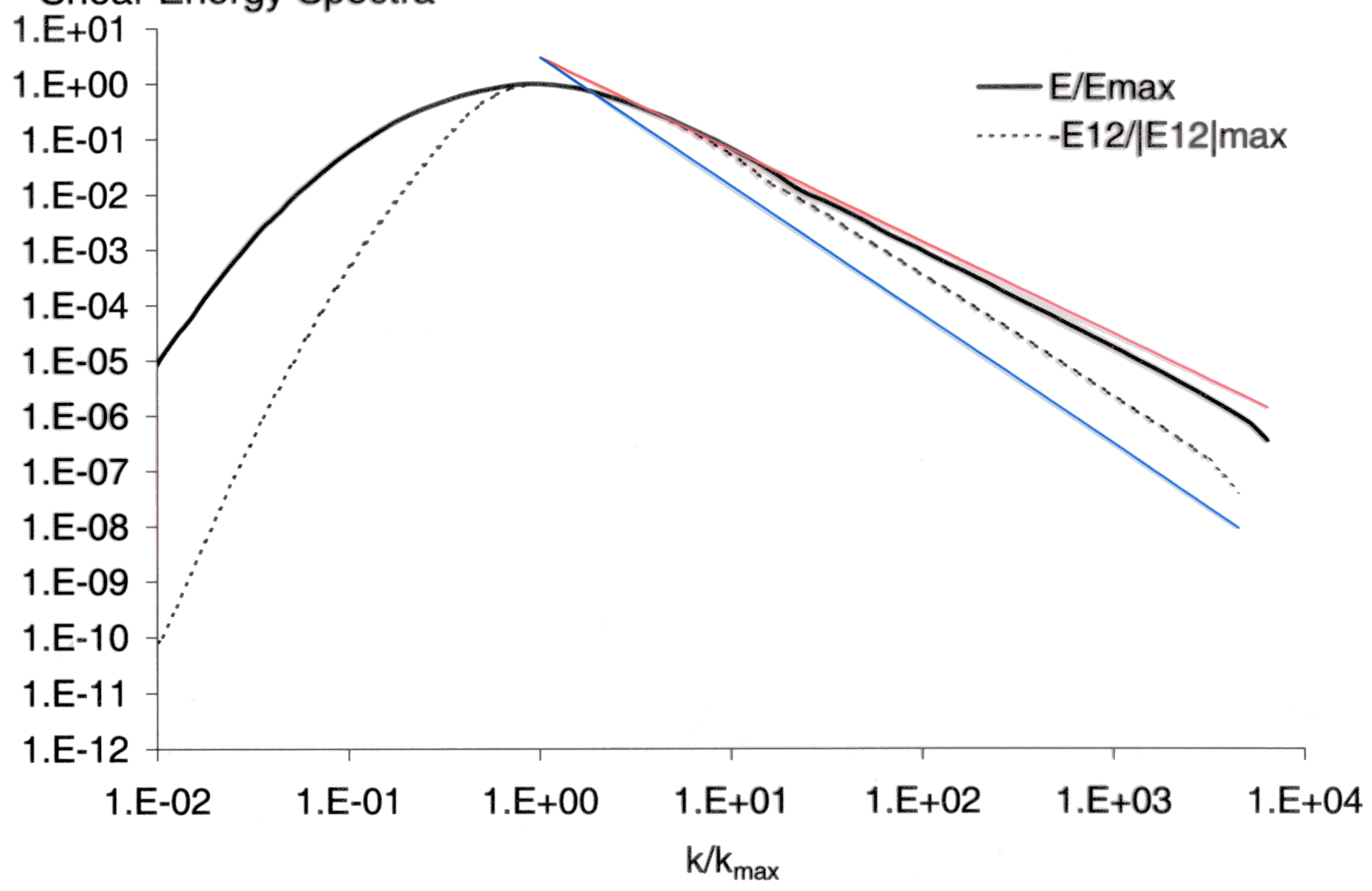




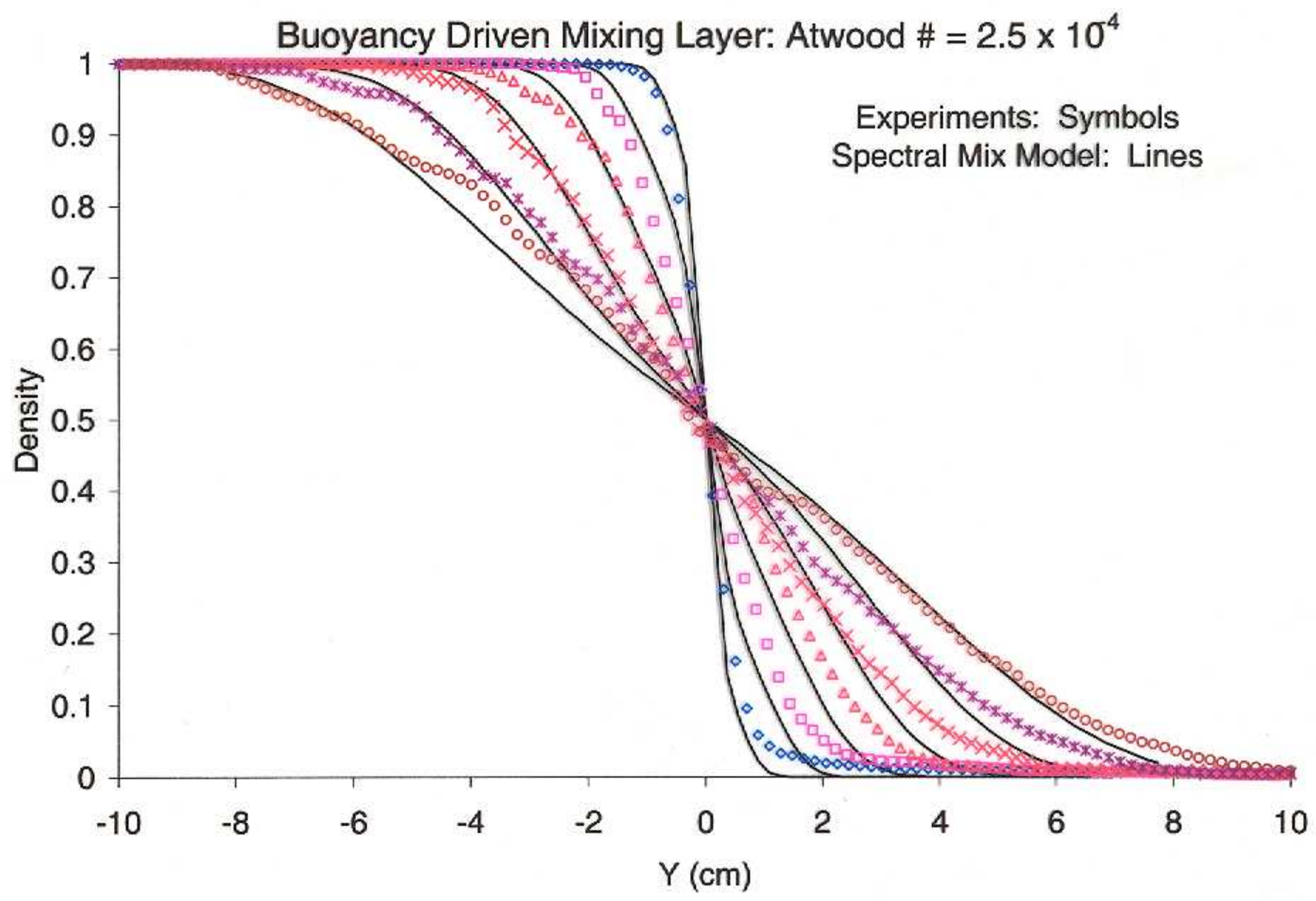




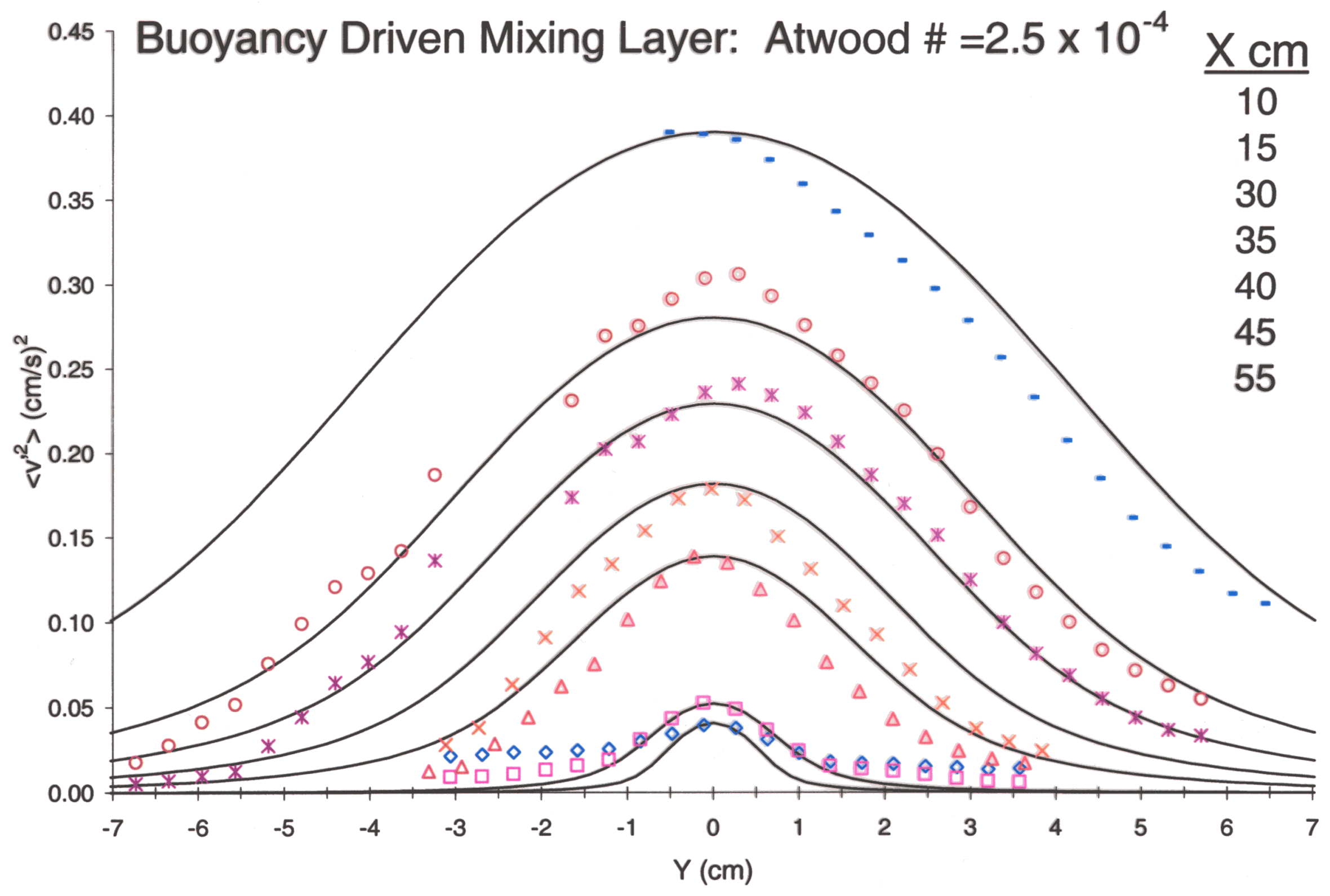




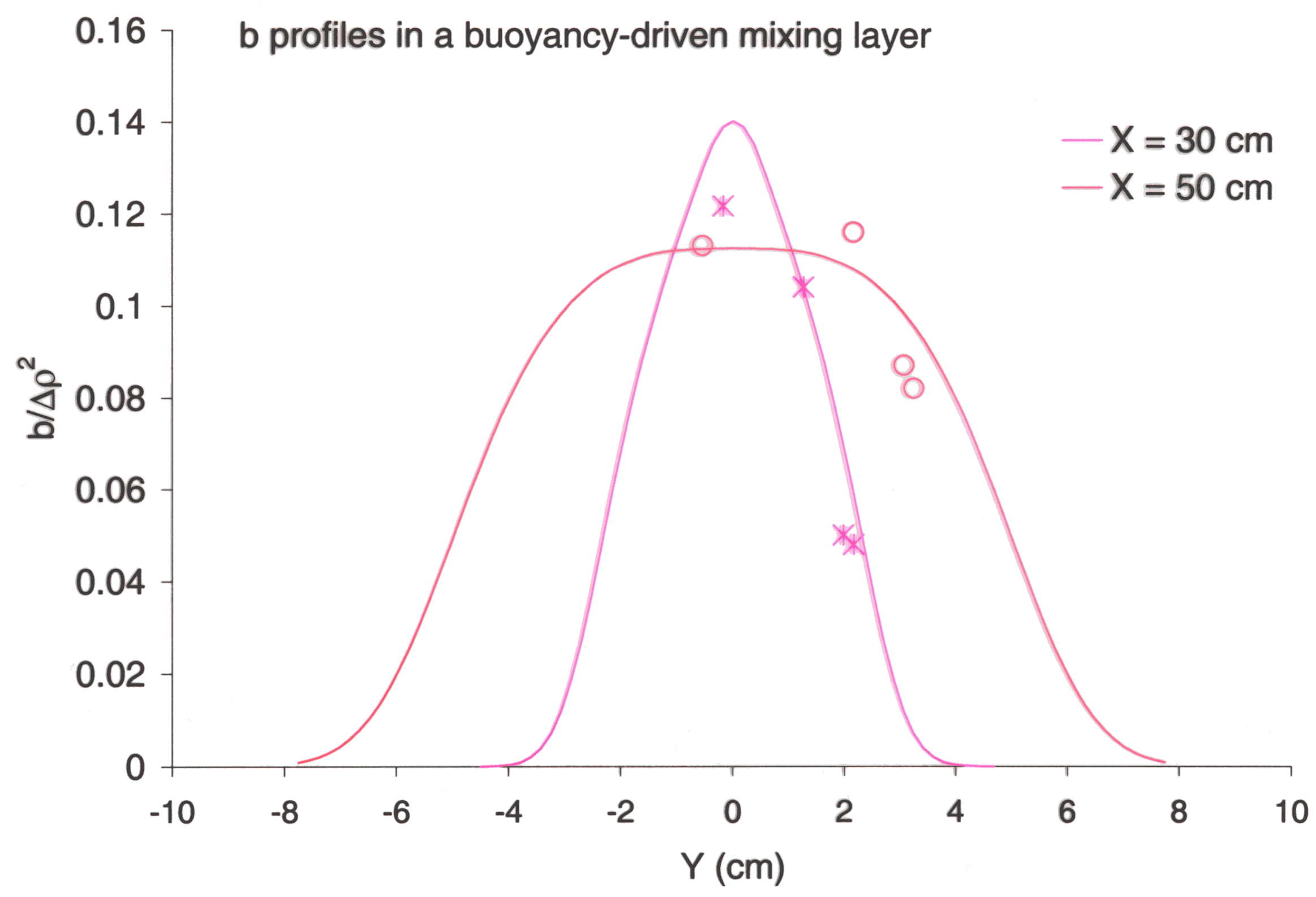



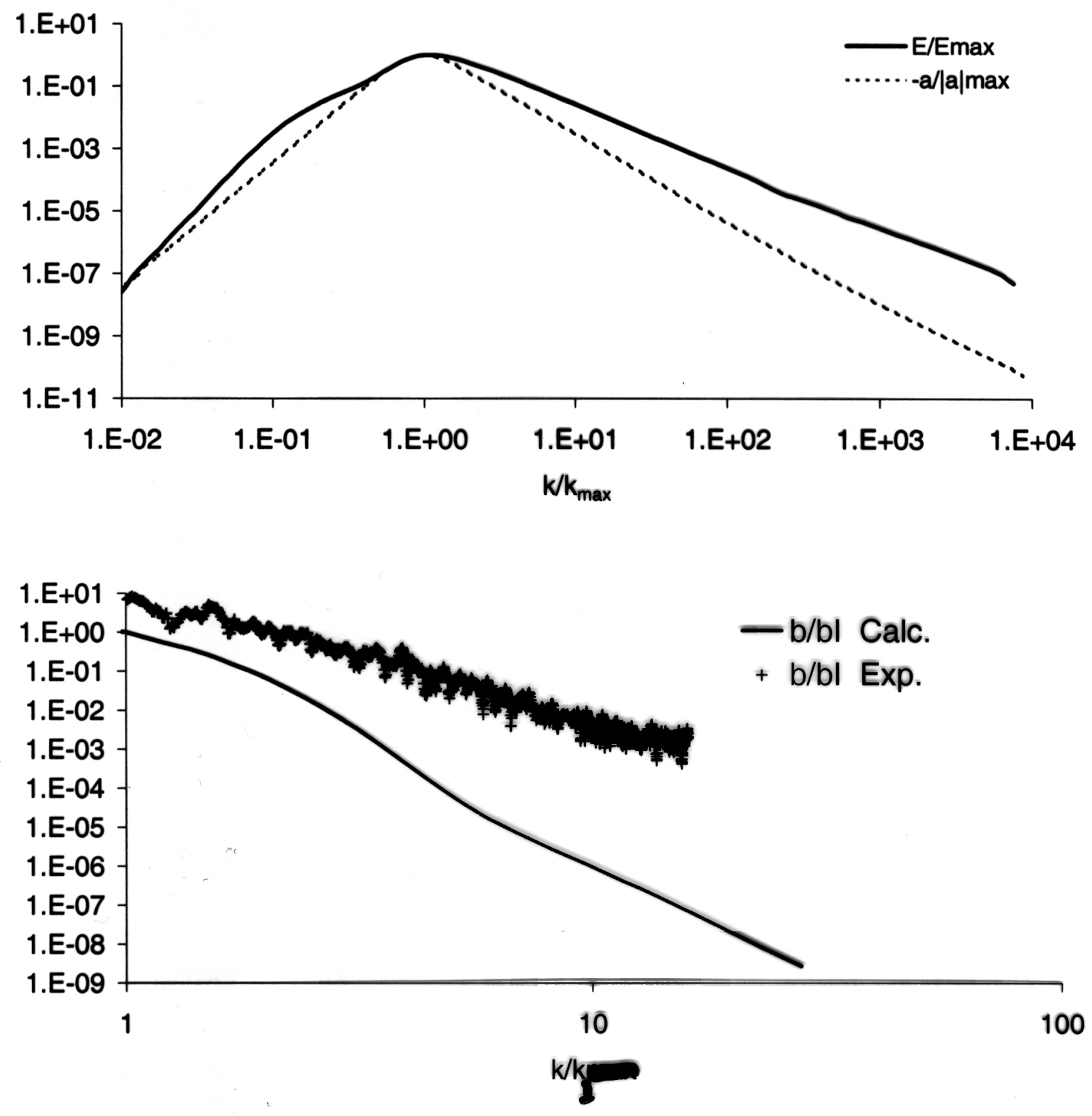


\title{
囚
}

Rate of growth of the linear Richtmyer-Meshkov instability

J. G. Wouchuk

\author{
E.T.S.I.Industriales \\ University of Castilla La Mancha \\ Ciudad Real \\ Spain
}

Presented at the $8^{\text {th }}$ IWPCTM California Institute of Technology

December 2001 
RM instability: perturbation growth due to the vorticity generation at both sides of a contact surface by means of corrugated shock fronts.

We distinguish two situations:

1) A shock is reflected back or,

2) a rarefaction is reflected instead 
Two possible approaches to get the growth rate are:

1-heuristic models based on an impulsive formulation (RM, VMG, MB),

2-rigorous linear theory:

-numerical solution as in Richtmyer, CPaAM 13, 297 (1960) or in Yang, Zhang and Sharp (PoF 6, 1856 (1994)),

- series expansions as in Velikovich, PoF 8,1666 (1996), Wouchuk and Nishihara, PoP $\underline{3}, 3761$ (1996).

-closed analytical formulas deduced from linear theory [Fraley, PoF 29 , 376 (1986) Wouchuk and Nishihara, PoP 3,3761 (1996);4, 1028 (1997)] 
It is clear that an estimate by means of a closed formula would be useful be it for numerical or experimental studies.

It is always possible to get asymptotic expressions of the rate of growth truncating the temporal expansions. However, the validity of such expressions is limited to very weak incident shocks.

The same restriction applies to any heuristic approach like the ones based on an impulsive formulation of the instability.

Before discussing an exact formulation that leads to a very accurate determination of the growth rate, we discuss some of the hypothesis underlying the heuristic models. 
The impulsive formulations make the assumptions:

a) Incompressible fluid flow after shock transit, and

b) Irrotational perturbations left by the shocks. 
The general form of the vorticity profile left by a rippled shock is:

$$
\begin{aligned}
& \delta \omega(x, y)=\Omega \delta p\left(x, t=\frac{x}{U_{S}}\right) \sin (k y) \\
& \delta \omega(x, y)=\Omega \delta p_{S}\left(k x / \sinh \left(\theta_{S}\right)\right) \sin (k y) \\
& \delta \omega(x, y)=\sum_{n \geq 0} A_{2 n+1} J_{2 n+1}\left(k x / \sinh \left(\theta_{S}\right)\right) \sin (k y)
\end{aligned}
$$


We see that vorticity production could be substantial, and this fact depends on fluids properties as well as on the incident shock intensity.

The vorticity left by the shock fronts is the memory of the effect of the "rippled compression" of the fluid elements.

For a strictly planar shock the flow behind it will be trivially irrotational.

For a corrugated front, the flow behind it will be rotational.

The vorticity deposited in the interior of the fluids will have a definite effect on the interface growth as we are going to see. It can not be neglected for strong shocks or highly compressible fluids. 


\section{Tangential velocity:}

$$
\frac{\partial v_{y}}{\partial t}=\delta p_{i}(t) \stackrel{\int_{0+}^{\infty} \ldots d t}{\longrightarrow} \rho_{a f}\left(\delta v_{y a}^{\infty}-\delta v_{y a}^{0}\right)=\rho_{b f}\left(\delta v_{y b}^{\infty}-\delta v_{y b}^{0}\right)
$$

Normal velocity:

$$
\frac{\partial \delta v_{x}}{\partial t}=-\frac{1}{\rho_{m f}} \frac{\partial \delta p_{i}}{\partial x} \stackrel{\int_{0+}^{\infty} \ldots d t}{\longrightarrow} \delta v_{i}^{\infty}
$$




$$
\delta v_{i}^{\infty}, \quad \delta v_{y a}^{\infty}, \text { and } \delta v_{y b}^{\infty}
$$

are not equal in the general case.

In fact, the parameters:

$$
\begin{aligned}
& F_{a}=\delta v_{y a}^{\infty}+\delta v_{i}^{\infty} \\
& F_{b}=\delta v_{y b}^{\infty}-\delta v_{i}^{\infty}
\end{aligned}
$$

are different from zero and become important for very strong shocks and highly compressible fluids. 


\section{How do we relate the parameters $F_{a}$ and $F_{b}$ with the shock compression history?}

Asymptotic velocity fields:

-Incompressibility:

$$
\frac{d \delta v_{x}}{d x}+\delta v_{y}=0
$$

-Vorticity:

$$
\frac{d \delta v_{y}}{d x}+\delta v_{x}=\Omega \delta p\left(x / \sinh \theta_{s}\right)
$$


We get, for example:

$$
\frac{d^{2} \delta v_{x}}{d x^{2}}-\delta v_{x}=-\Omega \delta p\left(x / \sinh \theta_{s}\right)
$$

The right hand side can not be made zero for moderate to strong shocks. 
In fact, not only the qualitative form of the velocity profile is modified because of the bulk vorticity: the asymptotic value of the interface velocity could be seriously inferior to any value deduced from an irrotational assumption. 
An exact expression of the linear asymptotic growth rate is obtained:

$$
\delta v_{i}^{\infty}=\frac{\rho_{b f} \delta v_{y b}^{0}-\rho_{a f} \delta v_{y a}^{0}}{\rho_{b f}+\rho_{a f}}+\frac{-\rho_{b f} F_{b}+\rho_{a f} F_{a}}{\rho_{b f}+\rho_{a f}}
$$

$F_{a}$ and $F_{b}$ must be related with the compressible evolution of the instability. 
For very weak shocks we have the scaling laws:

$$
\left\{\begin{array} { l } 
{ F _ { a } \propto ( M _ { t } ^ { 2 } - 1 ) ^ { 7 / 2 } } \\
{ F _ { b } \propto ( M _ { r } ^ { 2 } - 1 ) ^ { 7 / 2 } }
\end{array} \quad \left\{\begin{array}{lll}
\delta v_{y a}^{0} & \propto & \left(M_{t}^{2}-1\right) \\
\delta v_{y a}^{0} & \propto & M_{r}^{2}-1
\end{array}\right.\right.
$$

And then, an irrotational estimation for the asymptotic velocity is justified:

$$
\delta v_{i r r o t}^{\infty}=\frac{\rho_{b f} \delta v_{y b}^{0}-\rho_{a f} \delta v_{y a}^{0}}{\rho_{b f}+\rho_{a f}}
$$


We make a Laplace transform of the equation for $\delta v_{x}$ and get:

$$
\delta V_{x b}(\sigma)=\frac{\sigma \delta v_{i}^{\infty}-\delta v_{y b}^{\infty}-\Omega_{b} \sinh \theta_{r} \delta P_{r}\left(\sigma \sinh \theta_{r}\right)}{\sigma^{2}-1}
$$

A similar equation holds in fluid " $a$ ".

The function $\delta P$ is the time Laplace transform of the shock front pressure perturbations.

To get bounded perturbations, we see that it must be: 


$$
F_{b}=-\delta v_{i}^{\infty}+\delta v_{y b}^{\infty}=\Omega_{b} \sinh \theta_{r} \delta P_{r}\left(\sinh \theta_{r}\right)
$$

And an analogous relationship holds in fluid " $a$ ".

If a rarefaction were reflected, then: $F_{b}=0$.

Thus, to get the values of $F_{a}$ and $F_{b}$, we need temporal averages of the shock pressure functions.

How do we calculate the parameters $F_{a}$ and $F_{b}$ ? 
We change to the coordinate system:

$$
\left\{\begin{array}{l}
k x=r \sinh \theta \\
k c_{f} t=r \cosh \theta
\end{array}\right.
$$

The shock-fronts coordinates are defined by:

$$
\tanh \theta_{t}=-\frac{U_{t}}{c_{a f}} \quad \tanh \theta_{r}=\frac{U_{r}}{c_{b f}}
$$


It can also be seen that the Laplace transform of the shock pressure in each fluid can be written as:

$$
\delta P_{m}(\theta, q)=\frac{F_{m 1}(q-\theta)+F_{m 2}(q+\theta)}{\cosh q}
$$

Where " $q$ " is related to the Laplace variable " $s$ " through:

$$
s=\sinh q
$$


In principle we have four unknown functions:

$$
\left\{\begin{array}{l}
F_{a 1}\left(q_{a}\right), F_{a 2}\left(q_{a}\right) \\
F_{b 1}\left(q_{b}\right), F_{b 2}\left(q_{b}\right)
\end{array}\right.
$$

After some algebra, at the shock fronts and at the interface, we can relate Fa1 and Fb2 in the following way:

$$
\left\{\begin{array}{l}
F_{a 1}\left(q_{a}\right)+\phi_{b 3} F_{b 2}\left(q_{b}\right)=\phi_{b 1}+\phi_{b 2} F_{b 2}\left(q_{b}+2 \theta_{r}\right) \\
F_{b 2}\left(q_{b}\right)+\phi_{a 3} F_{a 1}\left(q_{a}\right)=\phi_{a 1}+\phi_{a 2} F_{a 1}\left(q_{a}-2 \theta_{t}\right)
\end{array}\right.
$$


Besides, it can be seen that the desired parameters $F_{a}$ and $F_{b}$ are easily related to specific values of $F_{a 1}$ and $F_{b 2}$ :

$$
\left\{\begin{array}{l}
F_{a}=\varepsilon_{a 1} F_{a 1}\left(-2 \theta_{t}\right)+\varepsilon_{a 0} \\
F_{b}=\varepsilon_{b 1} F_{b 2}\left(2 \theta_{r}\right)+\varepsilon_{b 0}
\end{array}\right.
$$

$A$ very fast and accurate algorithm can be implemented to get $F_{a}$ and $F_{b}$. 
We define an iteration sequence:

$$
F_{a}^{[n]} \quad \text { and } \quad F_{b}^{[n]}
$$

with which we get the parameters $F_{a}$ and $F_{b}$.

With just the starting values $(n=0)$ we can get up to 3 digits in the asymptotic velocities even for very strong shocks and highly compressible fluids. For details see Phys. Plasmas8, 2890 (2001), Phys. Rev. E 63, 056303 (2001). 


$$
\begin{gathered}
\gamma_{\text {light }}=1.3, \gamma_{\text {heavy }}=1.8, \\
\text { initial Atwood }=3 \\
\text { incident Mach }=2.84
\end{gathered}
$$

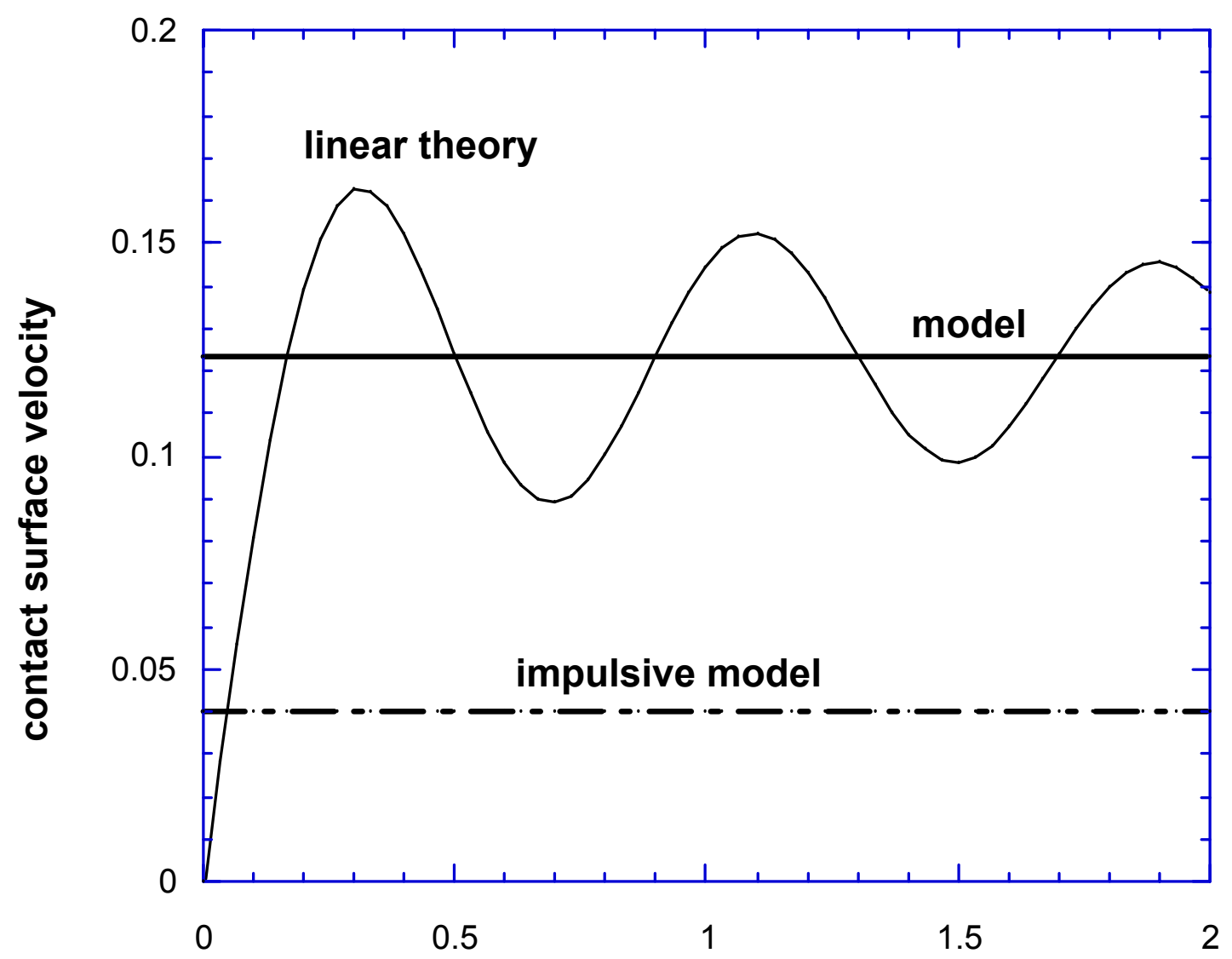




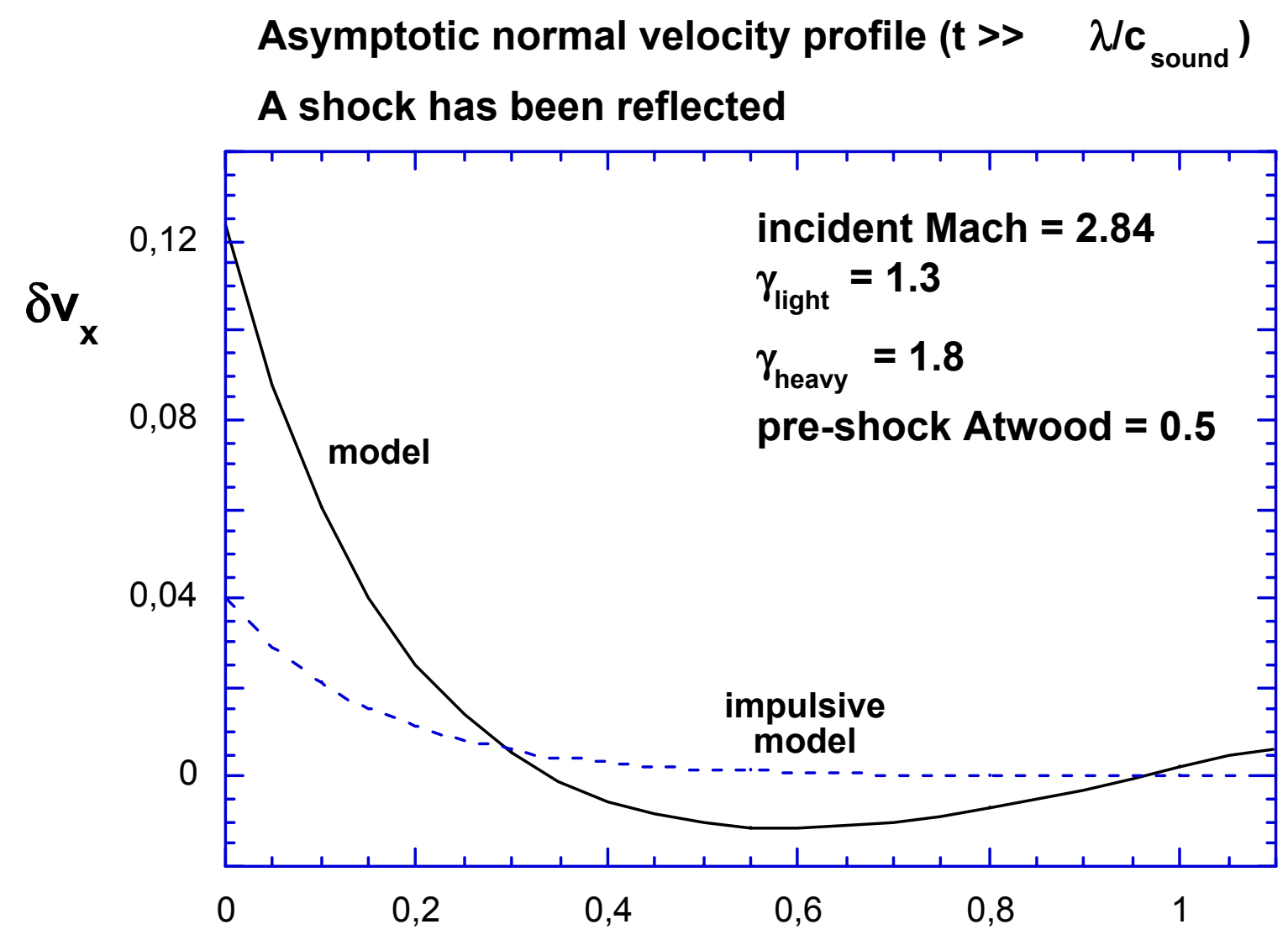


Comparison between the model and simulations together with two impulsive prescriptions.

A shock has been reflected.

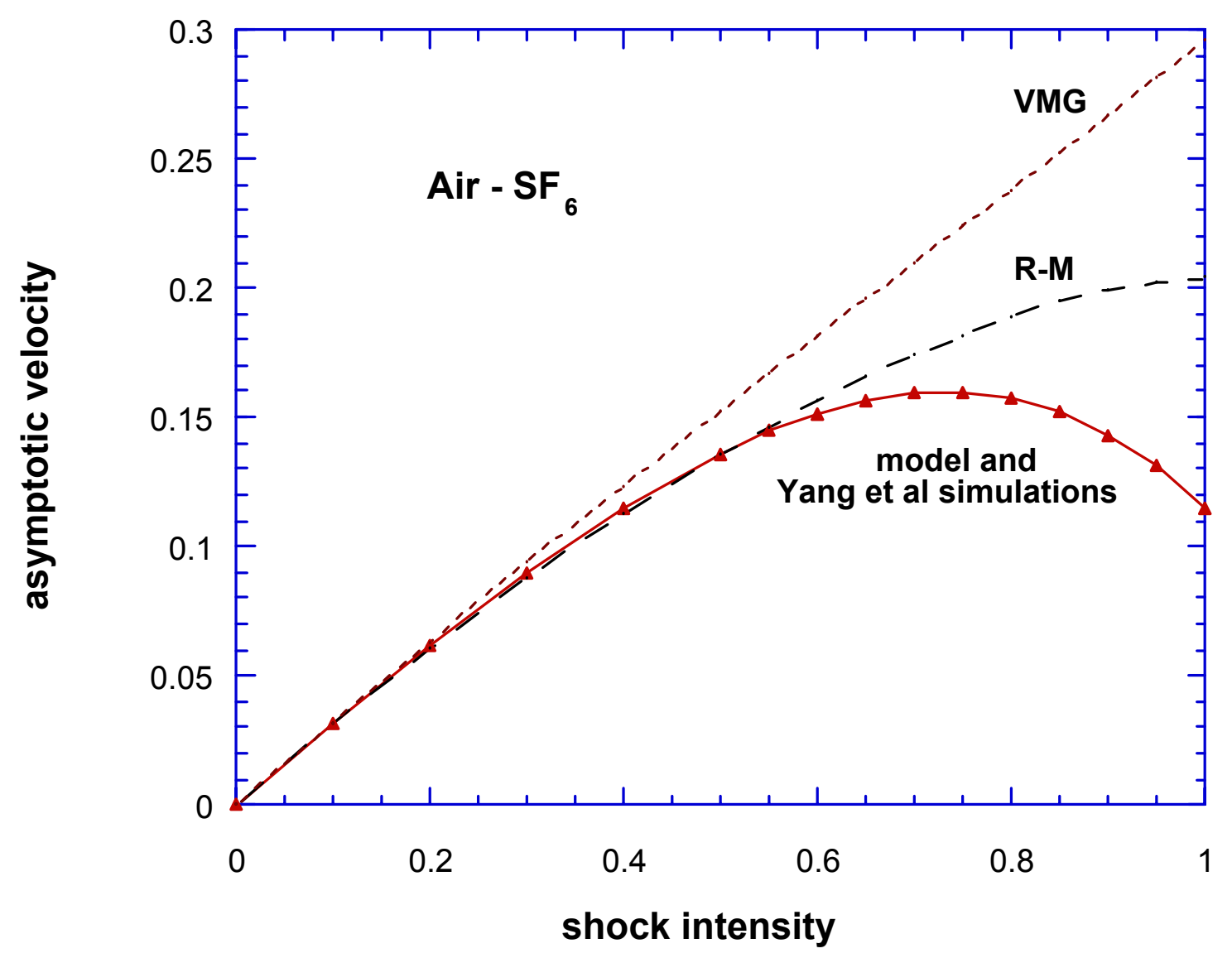


Comparison between the model and the irrotational approx. which does not include the vorticity corrections.

A shock impinged upon a Air-He interface

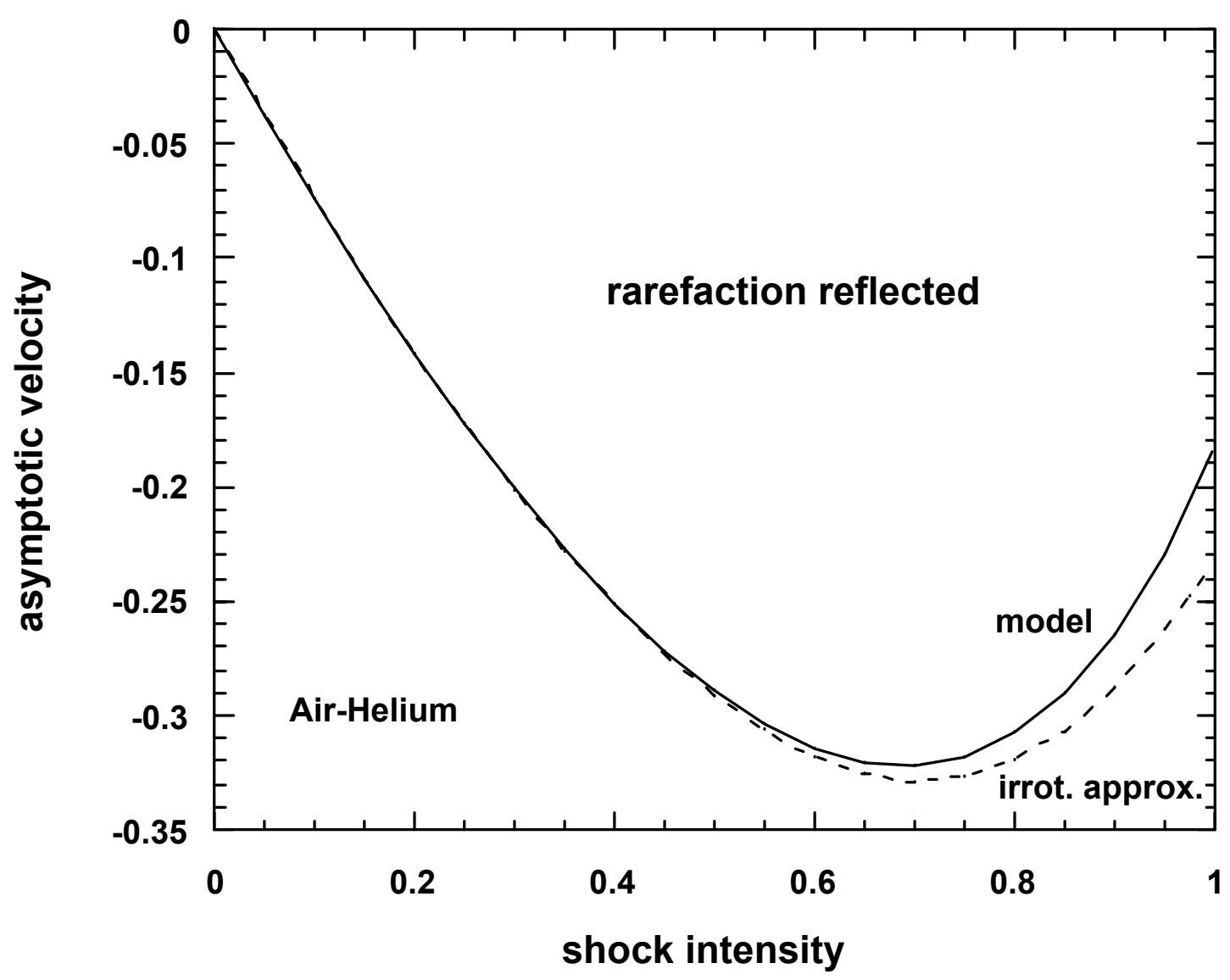


The same as the former picture but with a comparison to different impulsive prescriptions.

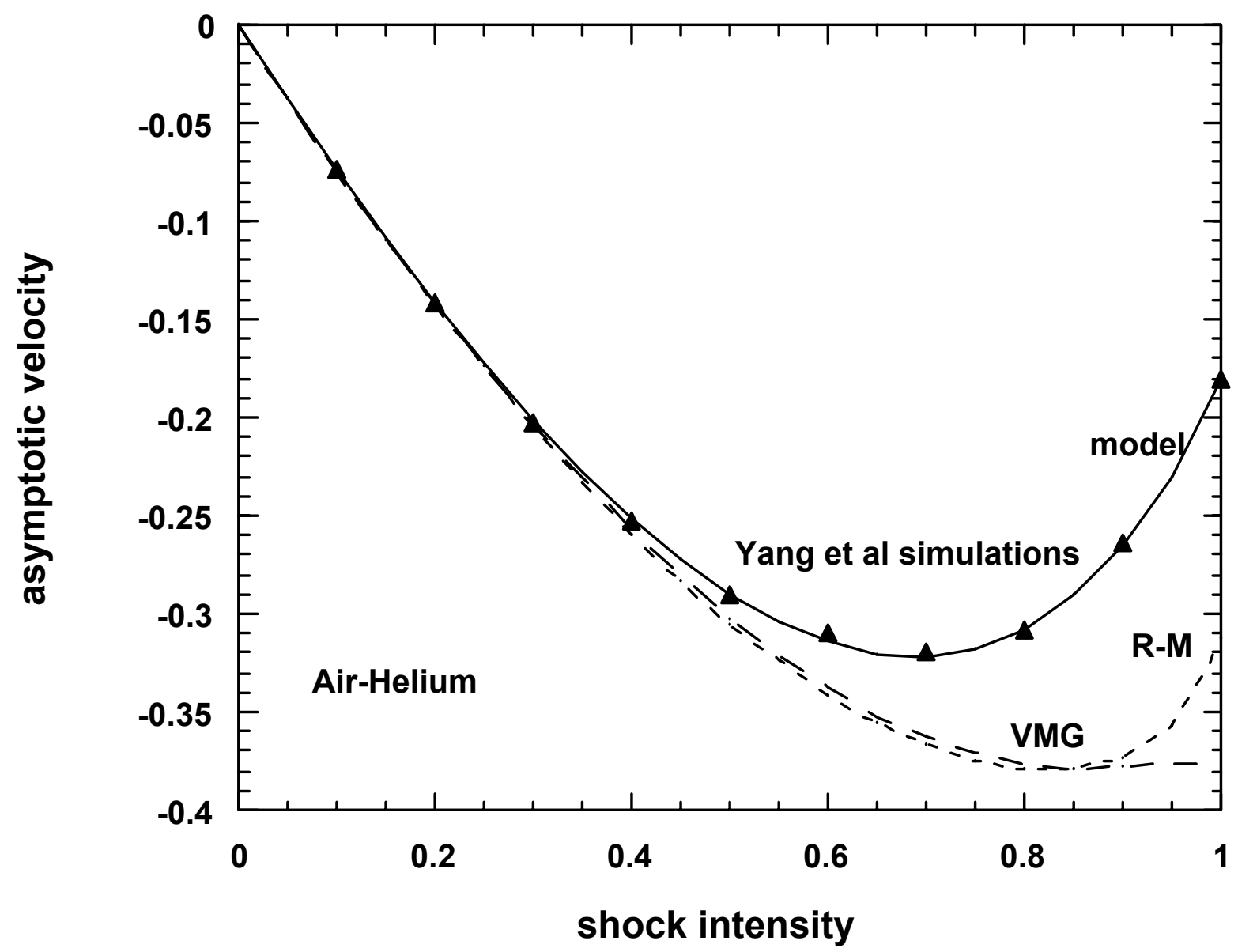


asymptotic velocity (model and simulations)

a rarefaction is reflected back

different pairs of gases has been considered

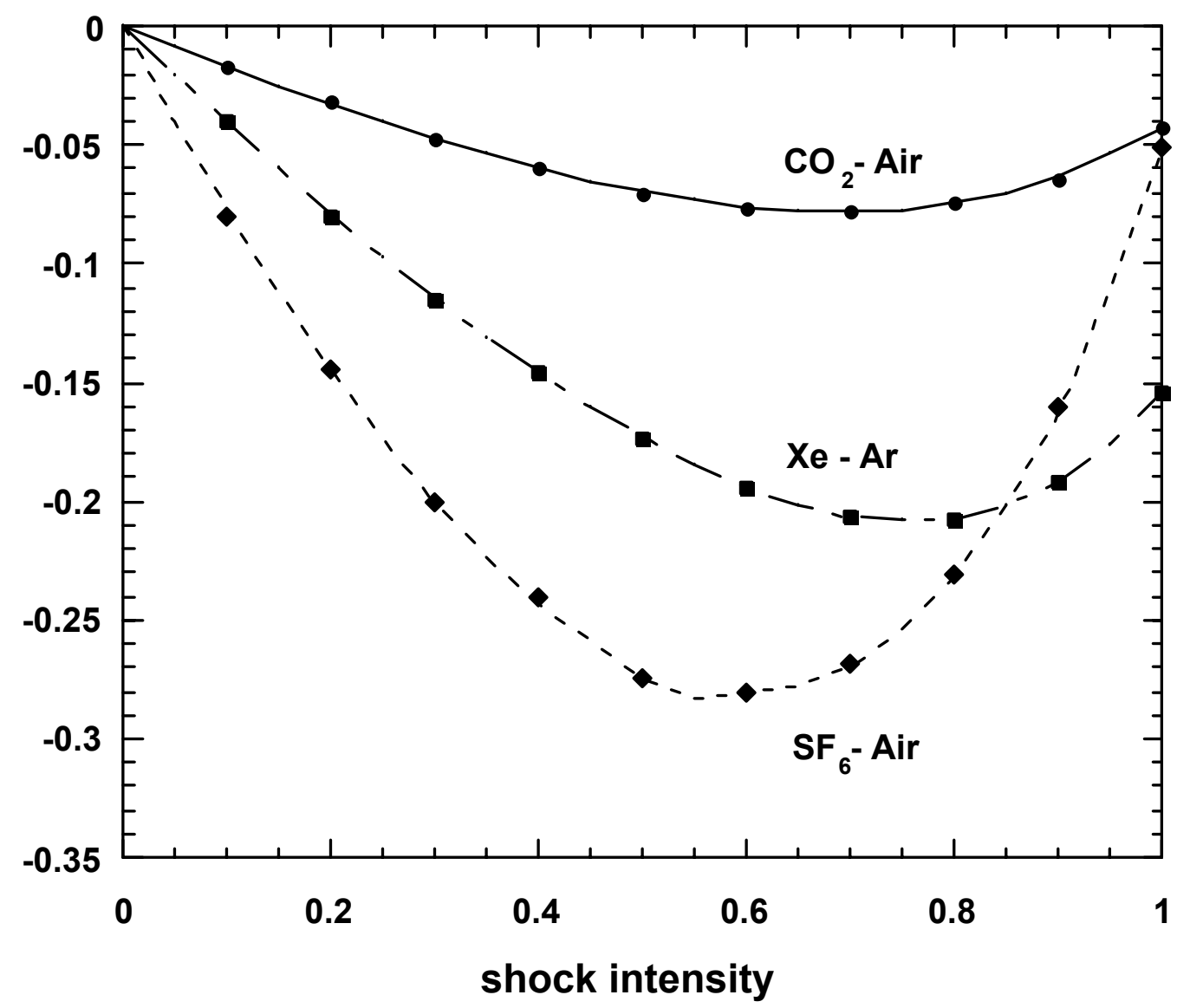




\title{
Spherical Combustion Layer in a TNT Explosion
}

\author{
A. L. Kuhl ${ }^{\dagger} \&$ R. E. Ferguson ${ }^{*}$ \\ $\nmid$ Lawrence Livermore National Laboratory, Livermore, CA \\ *Krispin Technologies, Inc, Rockville, MD
}

$8^{\text {th }}$ Int. Workshop on the Physics of Compressible Turbulent Mixing

California Institue of Technology

Pasadena, California

December 9-14, 2001

UCRL-JC-14658 


\section{Background: mixing at HE-air interface}

1977: S. I. Anisimov \& Ya. B. Zel'dovich, "Rayleigh-Taylor instabilitiy of the boundary between deotnation products and gas in a spherical explosion", Pis'ma Zh. Eksp. Teor. Fiz, 3, pp. 1081-1084.

1983: S. I. Anisimov, Ya. B. Zel'dovich, M. A. Inogamov \& M. F. Ivanov, “ "Taylor instability of contact boundary between expanding detonation products and a surrounding gas", Shock Waves, Explosions \& Detonations" Prog. Astronautics \& Astronautics Series, 87, AIAA, Wash., DC, pp 218-227.

1996: A. L. Kuhl, "Spherical Mixing Layers in Explosions", Dynamics of

Exothermicity, Ed. J. R. Bowen, Gordon \& Breach, Amsterdam, pp. 291-320. 


\section{LLNL Bomb Calorimeter ( $\mathrm{V}=5.28$ I)}

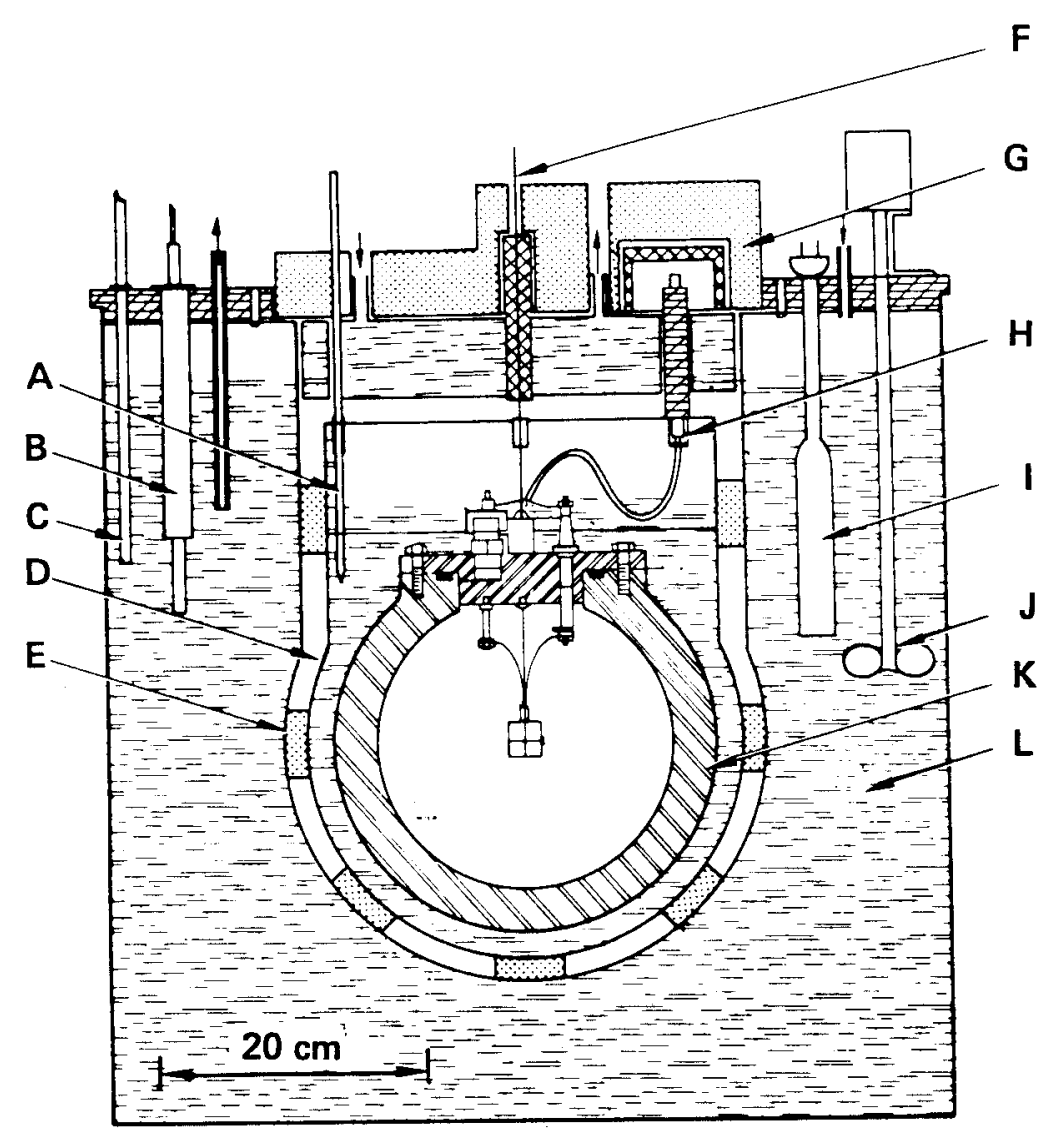




\section{Composition: TNT products in $\mathrm{O}_{2}$ \& vacuum}

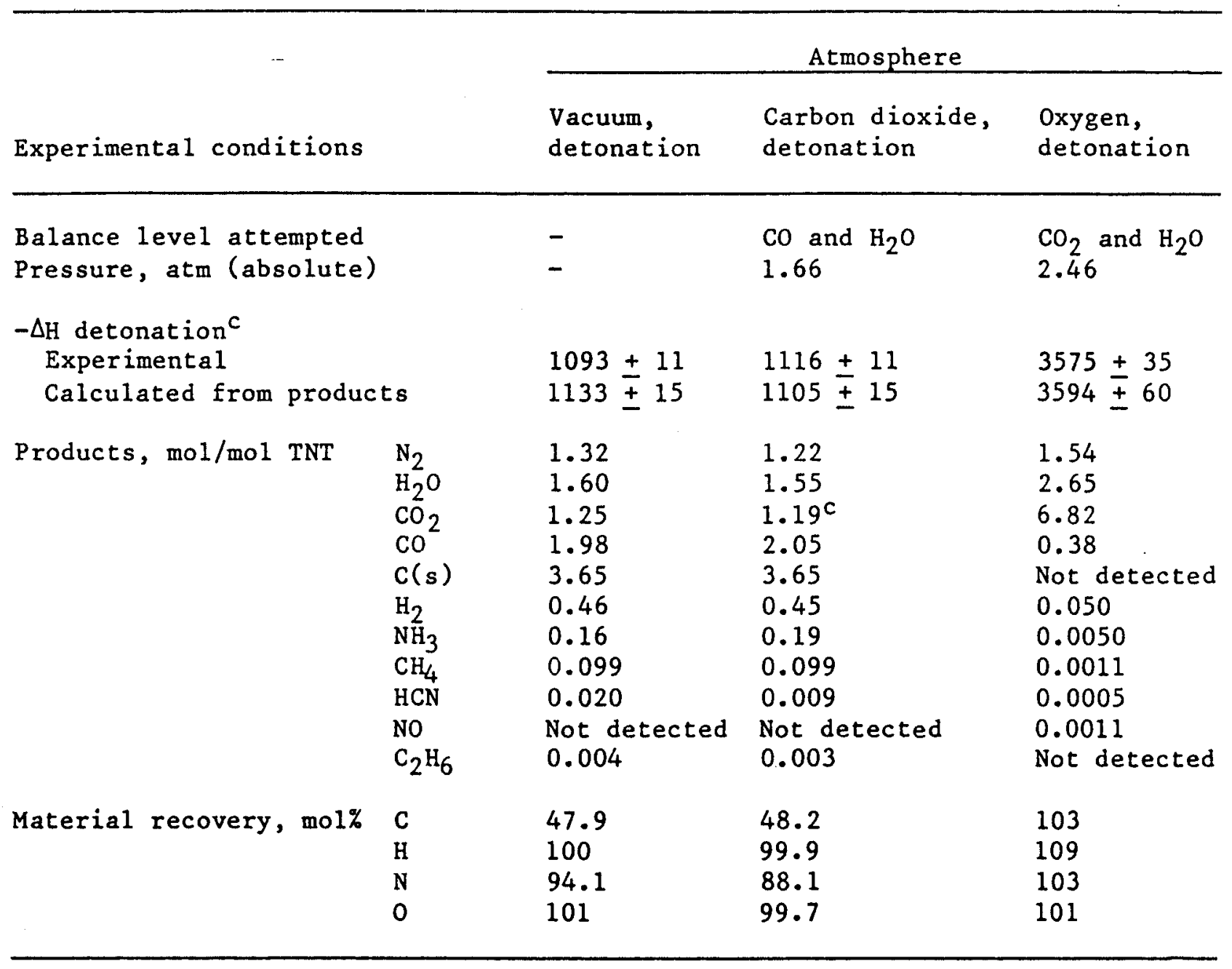




\section{Multi-Fluid Model}

FORMULATION: turbulent combustion in un-mixed gases

- Three Fluids: Fuel- $F$, Air- $A \&$ Products- $P$ defined by: $\left\{\rho_{K}, u_{K}\right\}$ where $K=F, A, P$

- Asymptotic Limit: $R e=P e=D a \rightarrow \infty$

- Compressible Flow: $M>0$

\section{CONSERVATION EQUATIONS: mixture}

- Mass: $\partial_{t} \rho_{m}+\nabla \cdot \rho_{m} \mathbf{u}=0$

- Momentum: $\partial_{t} \rho_{m} \mathbf{u}+\nabla \cdot \rho_{m} \mathbf{u u}=-\nabla p_{m}$

- Energy: $\partial_{t} \rho_{m} U_{T}+\nabla \cdot \rho_{m} U_{T} \mathbf{u}=-\nabla \cdot\left(p_{m} \mathbf{u}\right) \quad$ where $\quad U_{T}=u_{m}+\mathbf{u} \cdot \mathbf{u} / 2$

THERMODYANMIC FIELDS: fluids

- Fuel: $\quad \partial_{t} \rho_{F}+\nabla \cdot \rho_{F} \mathbf{u}=-\rho_{s} \quad \& \quad \partial_{t} \rho_{F} u_{F}+\nabla \cdot \rho_{F} u_{F} \mathbf{u}=-p_{F} \nabla \cdot \mathbf{u}-\rho_{s} u_{F}$

- Air: $\quad \partial_{t} \rho_{A}+\nabla \cdot \rho_{A} \mathbf{u}=-\sigma \rho_{s} \quad \& \quad \partial_{t} \rho_{A} u_{A}+\nabla \cdot \rho_{A} u_{A} \mathbf{u}=-p_{A} \nabla \cdot \mathbf{u}-\sigma \rho_{s} u_{A}$

- Products: $\partial_{t} \rho_{P}+\nabla \cdot \rho_{P} \mathbf{u}=(1+\sigma) \partial_{s} \quad \& \quad \partial_{t} \rho_{P} u_{P}+\nabla \cdot \rho_{P} u_{P} \mathbf{u}=-p_{P} \nabla \cdot \mathbf{u}+(1+\sigma) \partial_{s} u_{P}$

- Stoichiometric Source: $\dot{p}_{s}=\left\{\begin{array}{l}\rho_{F}\left(\mathbf{x}_{s}, t_{s}\right) \delta\left(t-t_{s}\right) \text { for } \lambda_{e} \geq 1 \\ \rho_{A}\left(\mathbf{x}_{s}, t_{s}\right) \delta\left(t-t_{s}\right) / \sigma \text { for } \lambda_{e}<1\end{array}\right.$

- Adiabatic Constraint: $\sum_{K} \dot{\rho}_{K} u_{K}=0$

SOLUTION: high-order Godunov scheme \& AMR to follow turbulent mixing 


\section{Thermodynamic Model}

Equations of State: fluid $K(=F, A \& P)$

- Perfect Gas Equation: $p_{K} v_{K} \equiv w_{K} \equiv R_{K} T_{K}$

- Caloric Equation: $u_{K}=F_{K}\left(w_{K}\right) \cong-\left|\mathrm{q}_{K}\right|+\mathrm{C}_{K} w_{K} \Leftrightarrow w_{K}=F_{K}^{-1}\left(u_{K}\right) \cong\left[u_{K}+\left|\mathrm{q}_{K}\right|\right] / \mathrm{C}_{K}$

\section{Pressures \& Temperatures}

- fluid $K: \quad p_{K} \equiv \rho_{K} w_{K}=\rho_{K}\left[u_{K}+\left|\mathrm{q}_{K}\right|\right] / \mathrm{C}_{K} \quad \& \quad T_{K}=w_{K} / R_{K}$

- mixture m: $\quad p_{m} \equiv \rho_{m} w_{m}=\rho_{m}\left[u_{m}+\left|\mathrm{q}_{m}\right|\right] / \mathrm{C}_{m} \quad \& \quad T_{m}=w_{m} / R_{m}$ where $\rho_{m} \equiv \sum_{K} \rho_{K} ; Y_{K} \equiv \rho_{K} / \rho_{m} ; u_{m} \equiv \sum_{K} Y_{K} u_{K} ; w_{m} \equiv \sum_{K} Y_{K} w_{K} ; \mathrm{q}_{m} \equiv \sum_{K} Y_{K} \mathrm{q}_{K} ; \mathrm{C}_{m}=\sum_{K} Y_{K} \mathrm{C}_{K} w_{K} / w_{m}$ 
Le Chatelier Diagram: combustion of TNT in air

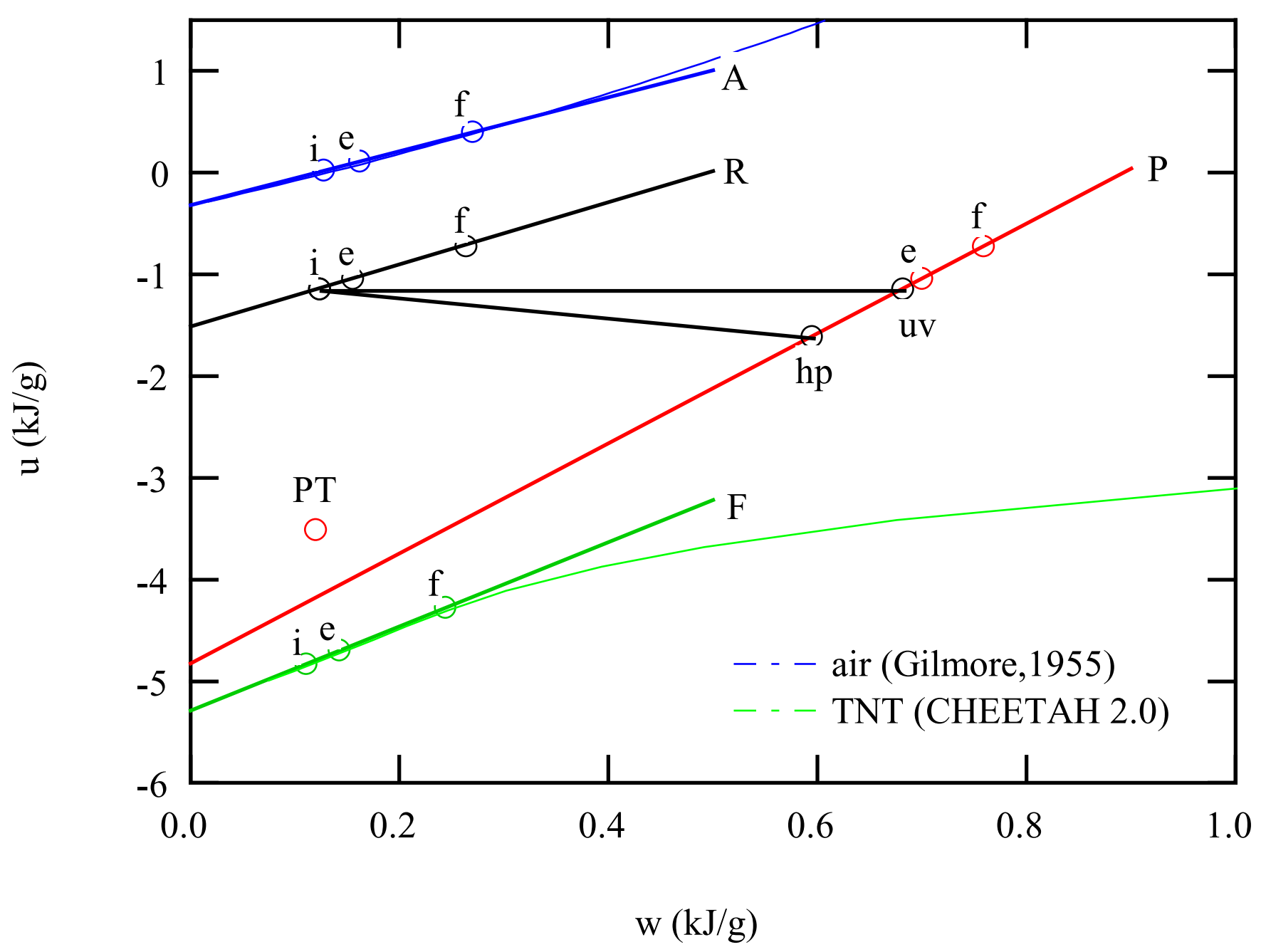




\section{Combustion Model}

1. Reactants Formation: stoichiometric sub-grid mass mixing:

$$
u_{R}=\left(u_{F}+\sigma u_{A}\right) /(1+\sigma) \quad \& \quad w_{R}=\left(w_{F}+\sigma w_{A}\right) /(1+\sigma)
$$

2. Combustion $\equiv$ material transformations in the Le Chatelier plane

- $\quad$ at $u v=$ constant $\left(\right.$ closed systems): $u_{P}=u_{R}$

- at $h p=$ constant (deflagrations): $\quad u_{P}=u_{R}-\frac{\Delta \mathrm{Q}-\left(\mathrm{C}_{P}-\mathrm{C}_{R}\right) w_{R}}{\mathrm{C}_{P}+1}$

3. Thermal Equilibration $\Leftrightarrow$ sub-grid energy mixing: $T_{K}^{e}=T_{m}$

$$
\begin{aligned}
w_{K}^{e} & =R_{K} T_{m} \\
u_{K}^{e} & =-\left|\mathrm{q}_{K}\right|+\mathrm{C}_{K} w_{K}^{e}
\end{aligned}
$$




\section{Initial Conditions: Self-Similar CJ Detonation}

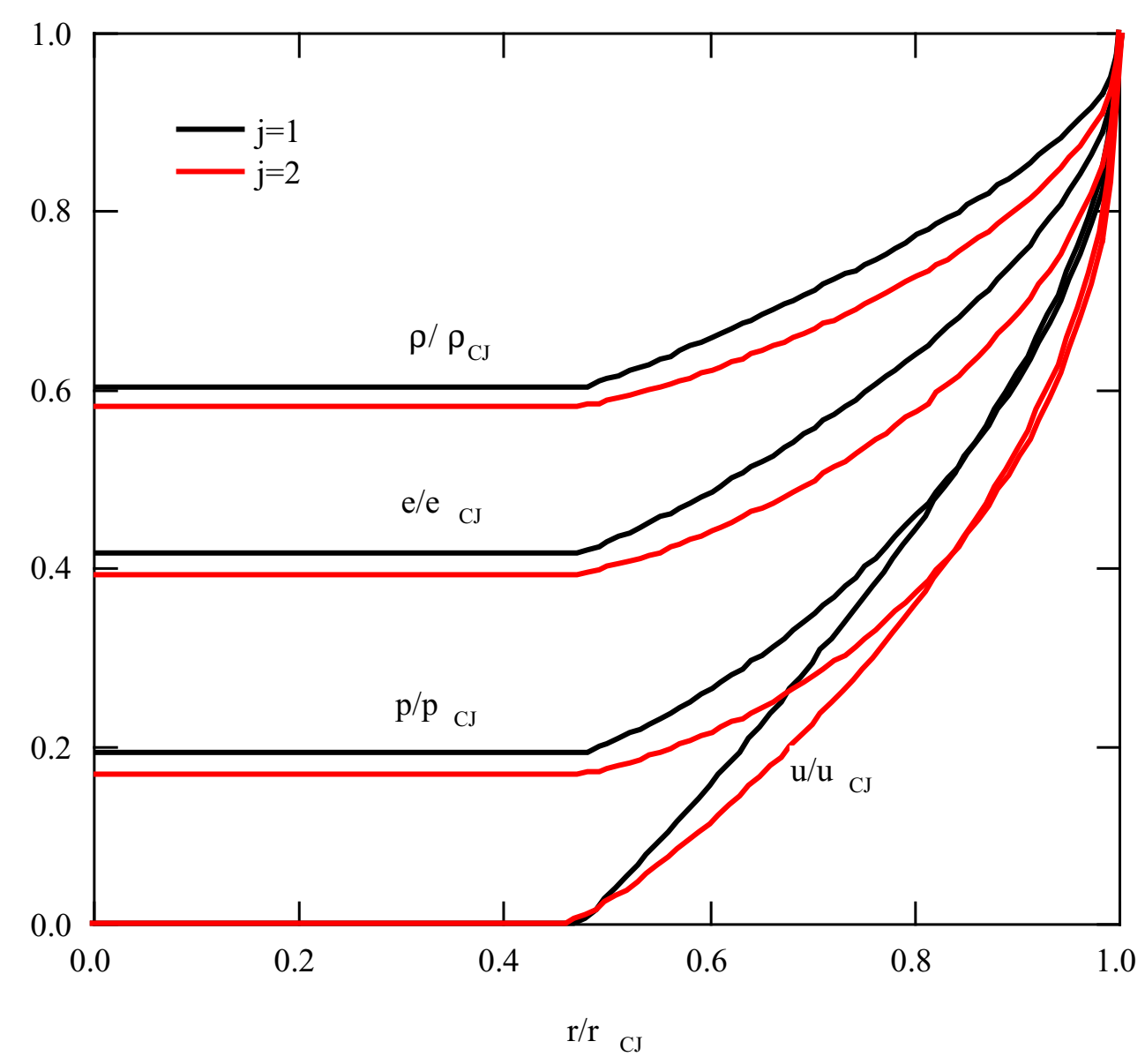

8th IWPCTM 


\section{Evolution: Material \& Vorticity Fields}

QuickTime ${ }^{\mathrm{TM}}$ and a

Qeiderinimests and a

are needed todscentisesseture. are needed to see this picture. 

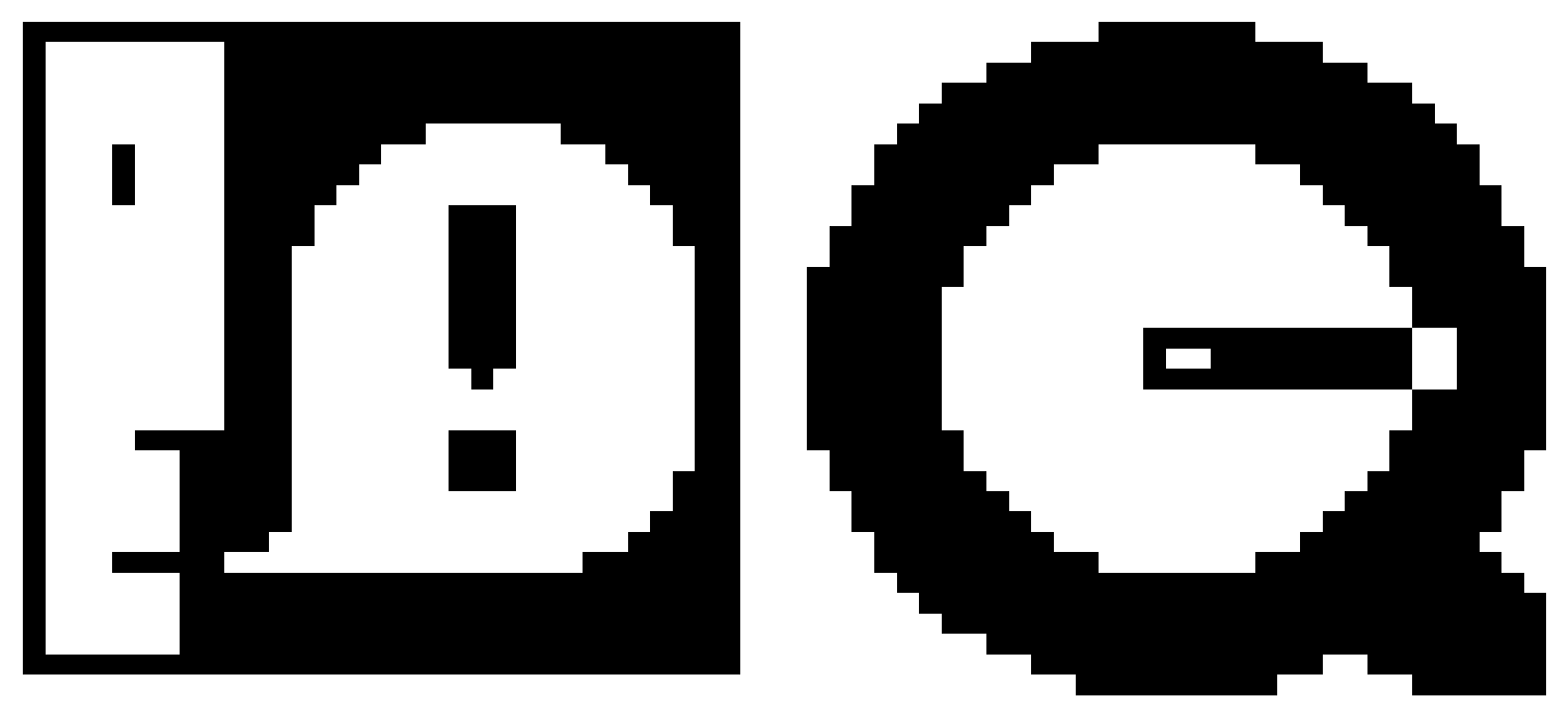

QuidrTirne FICT

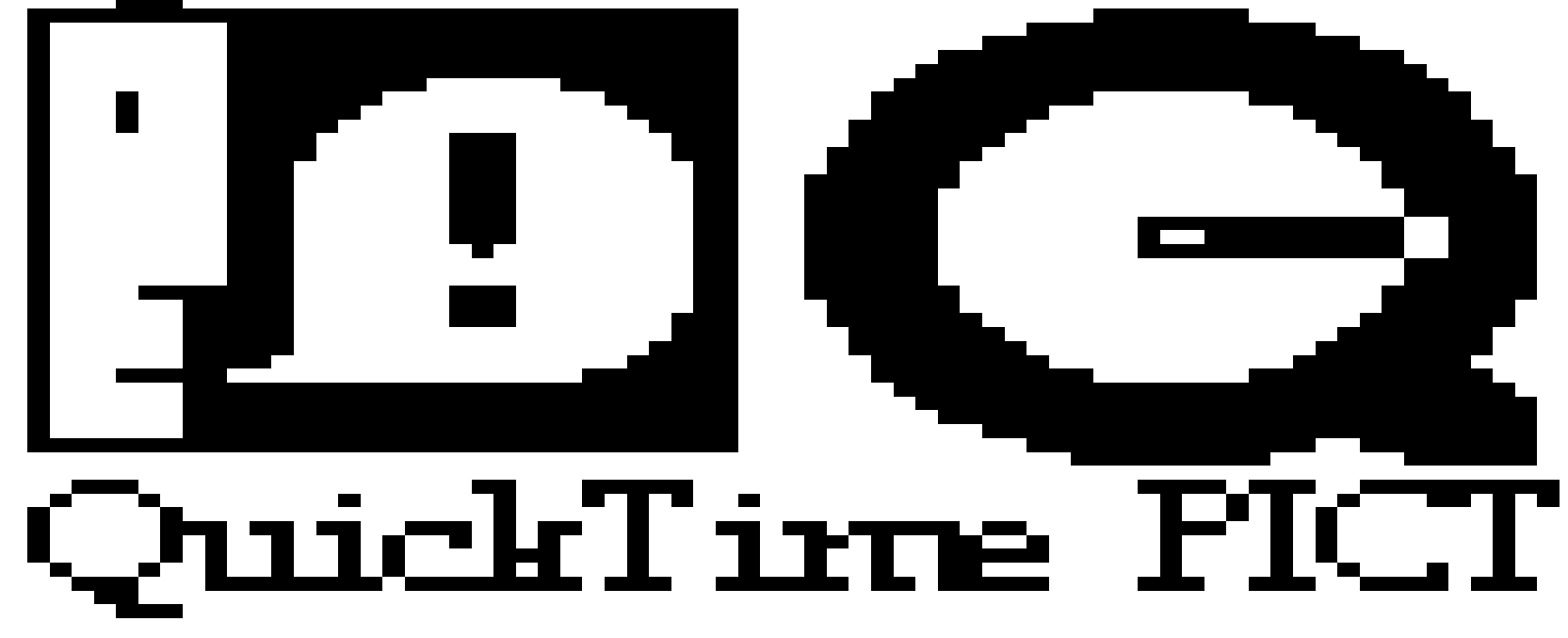




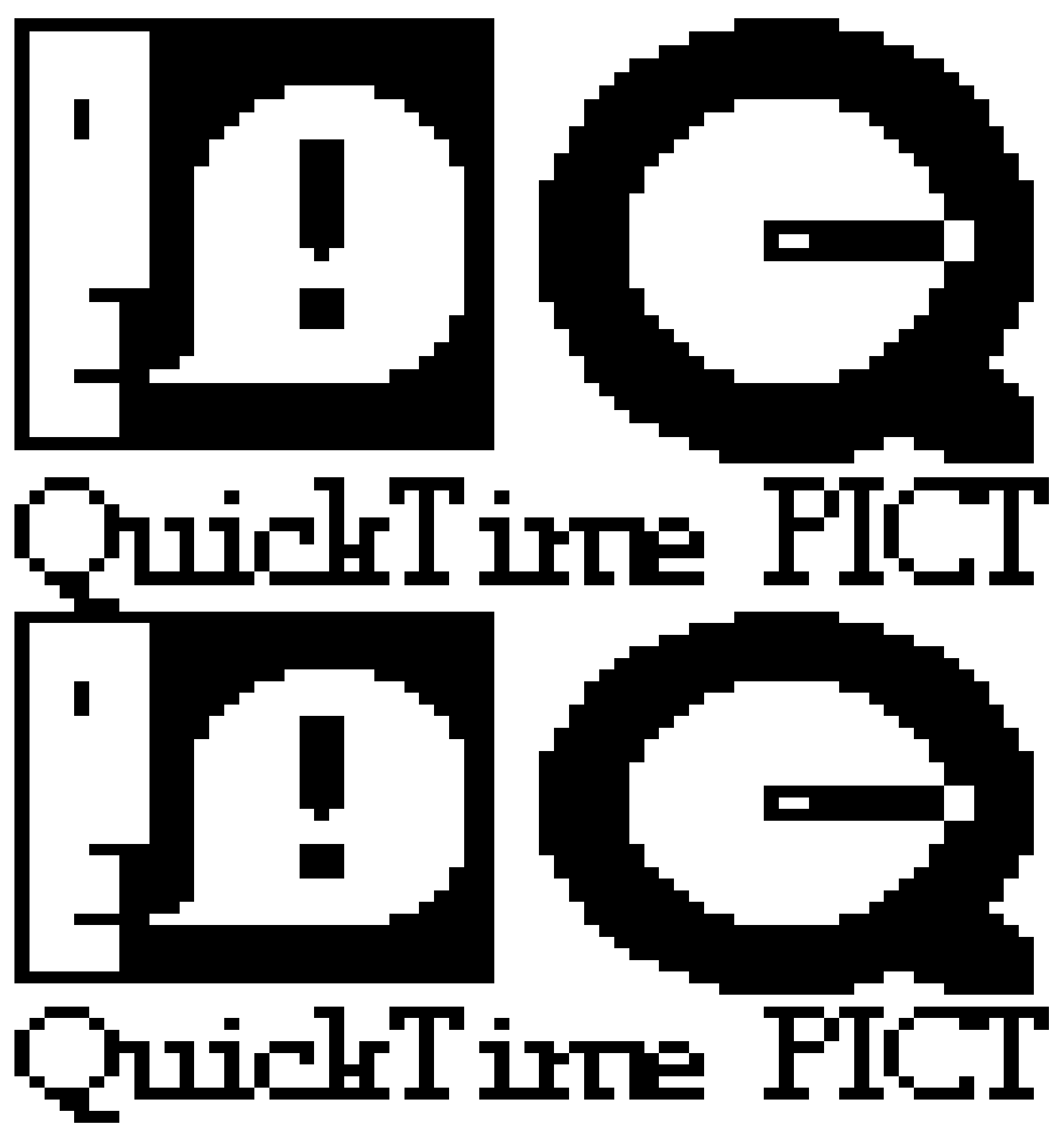




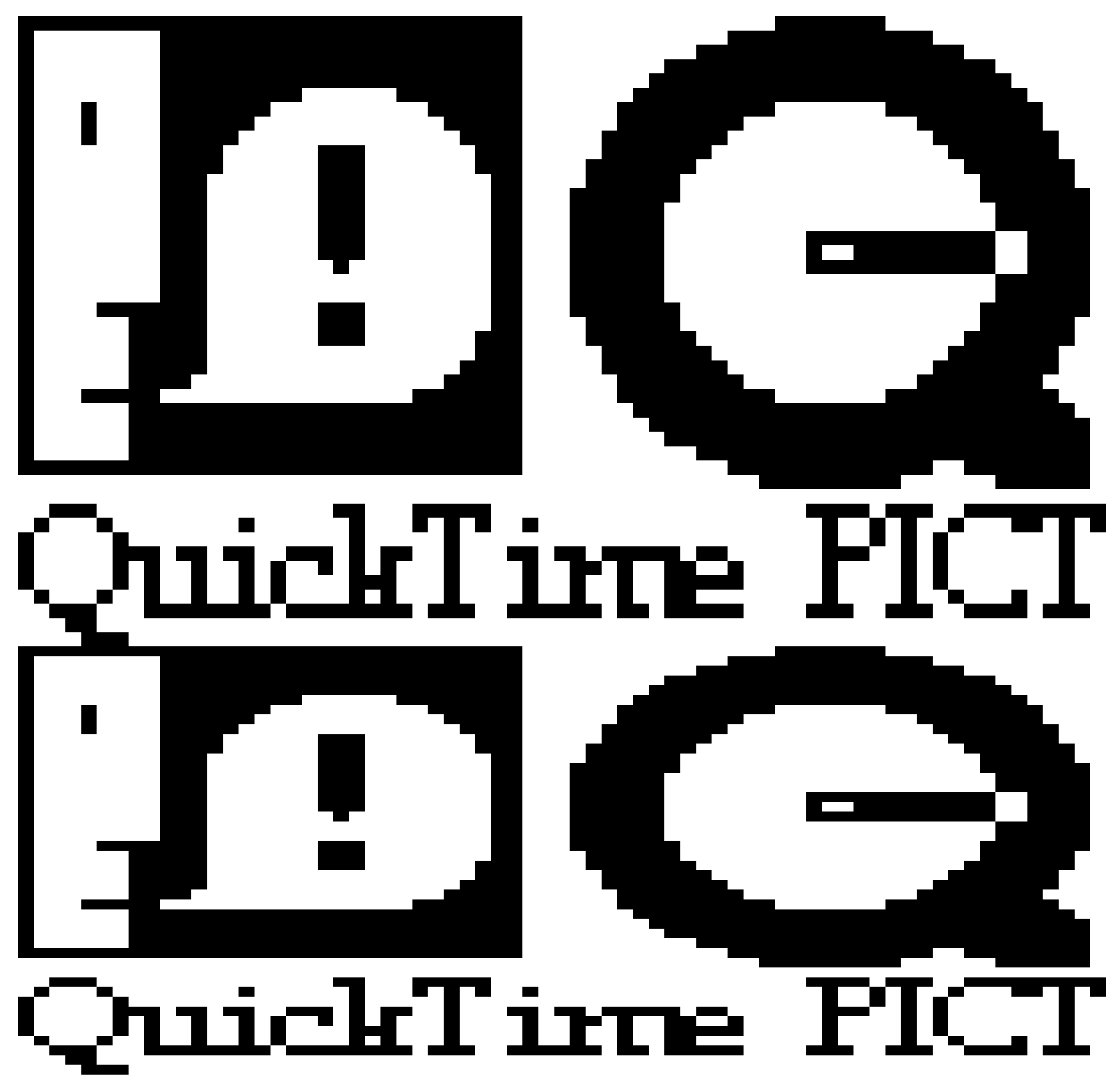




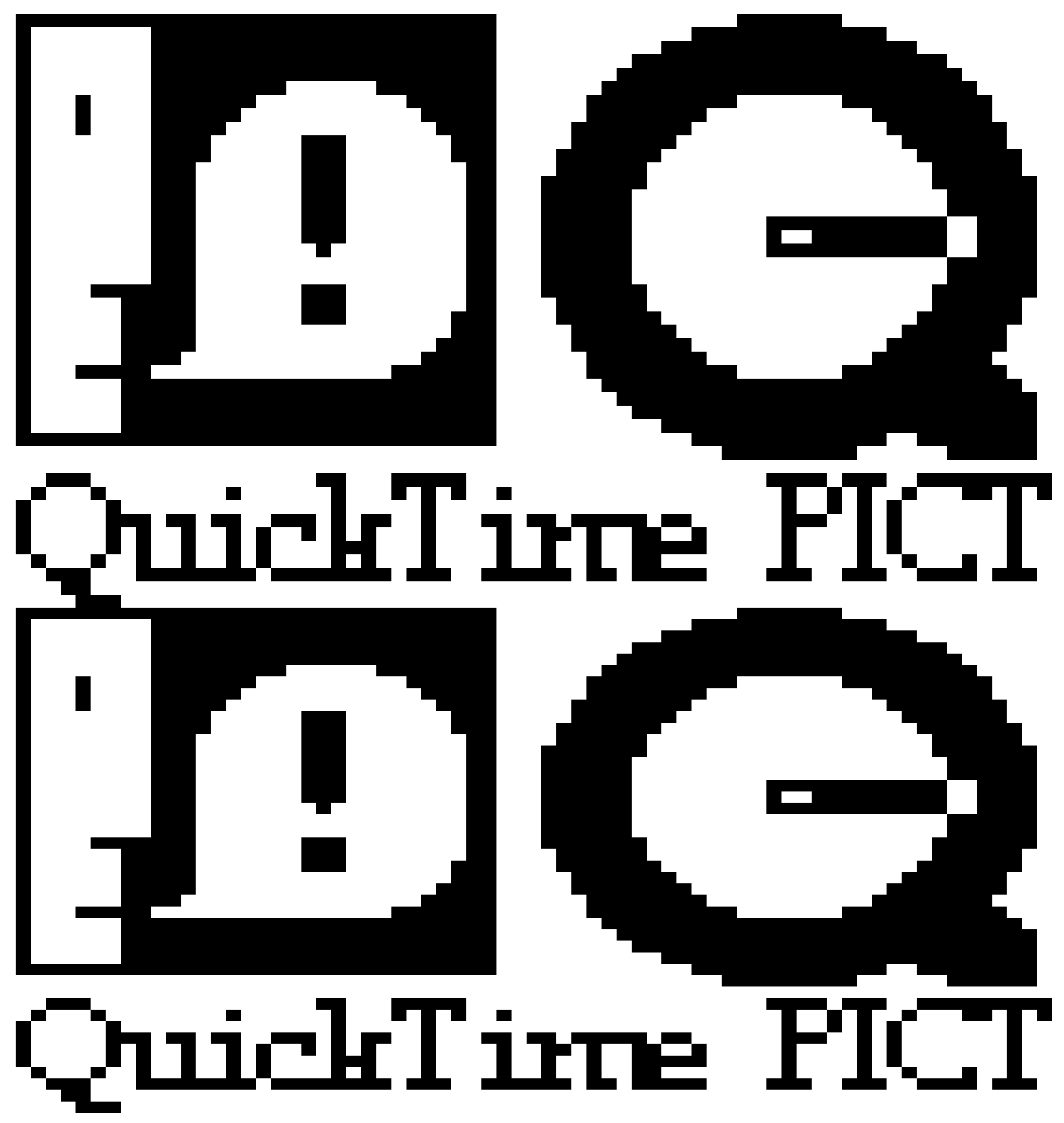




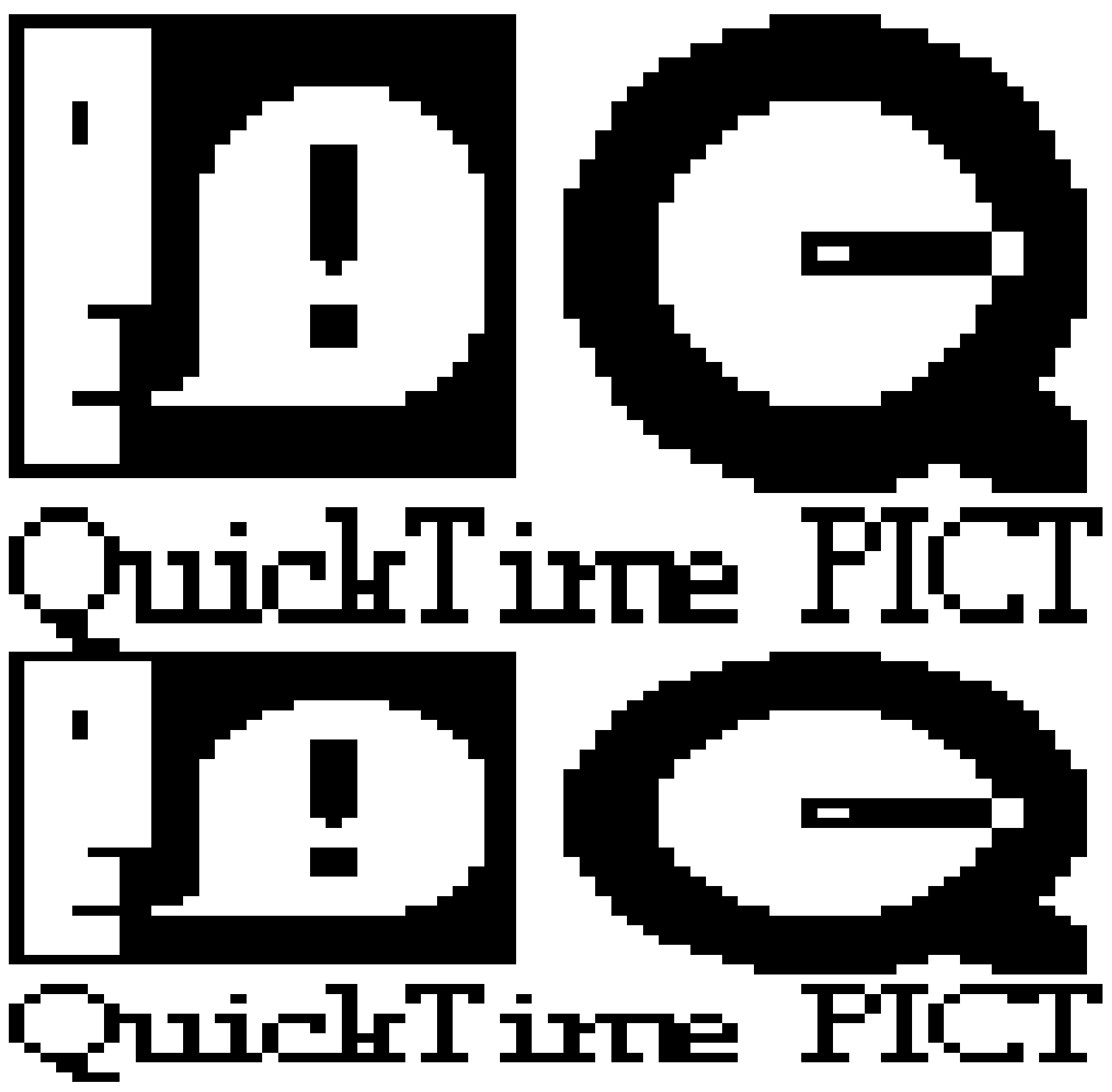



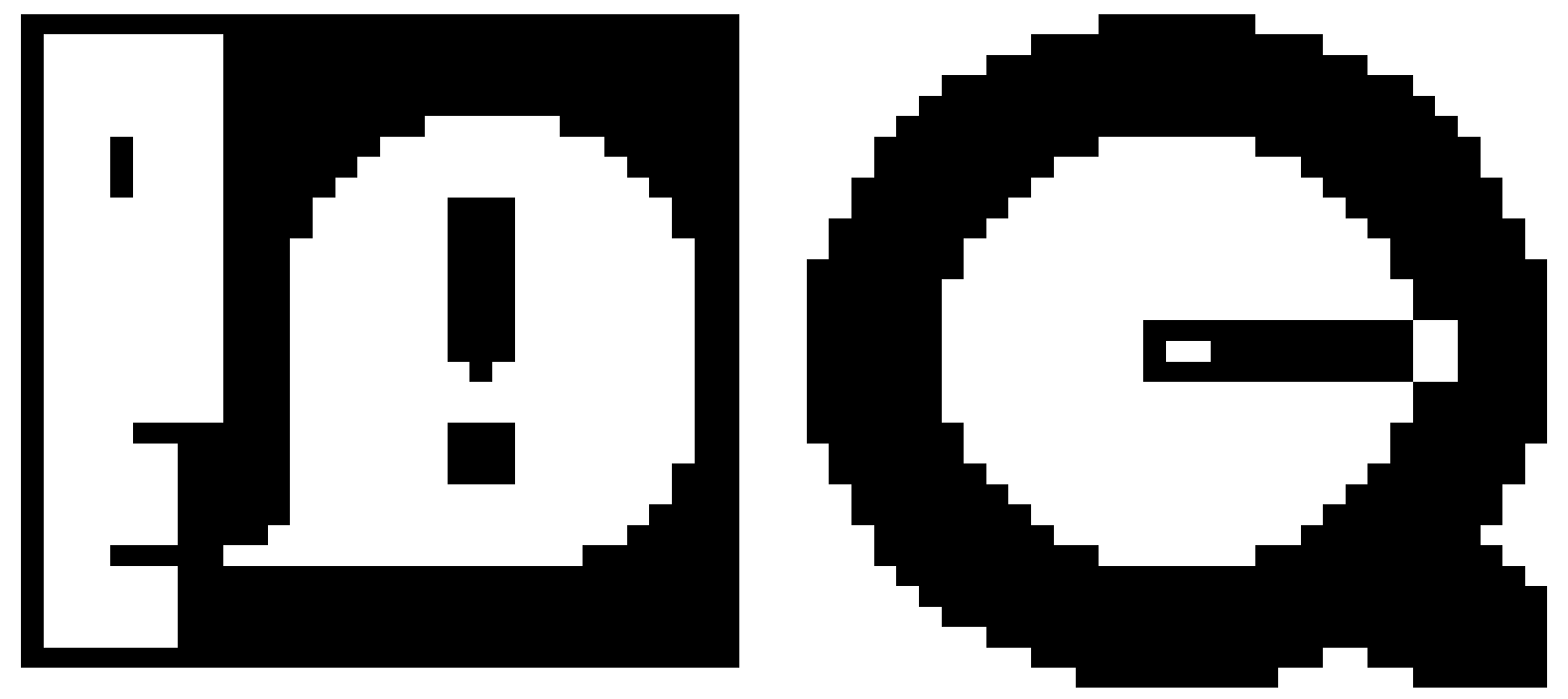

QuichTirne PICT

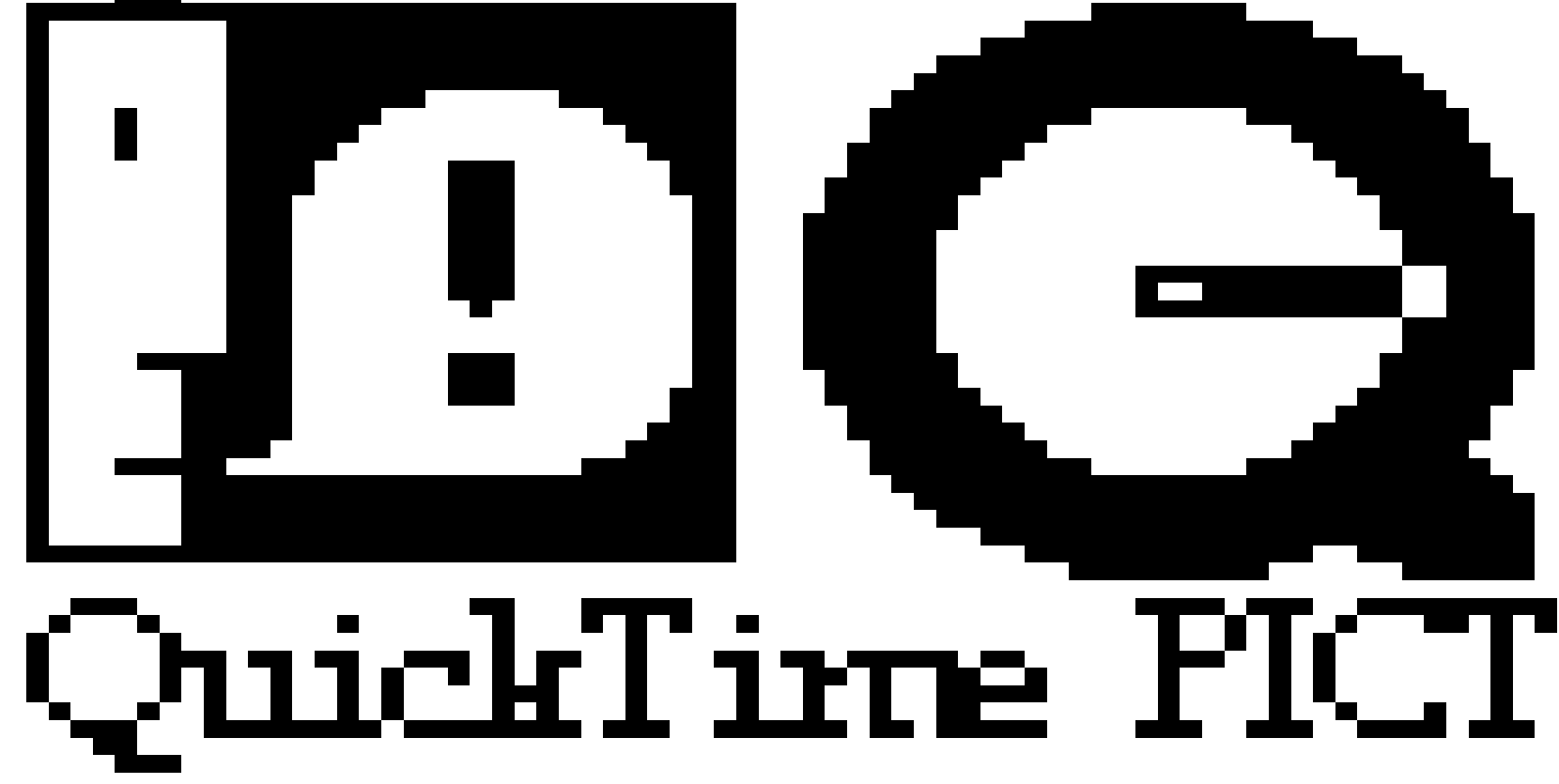




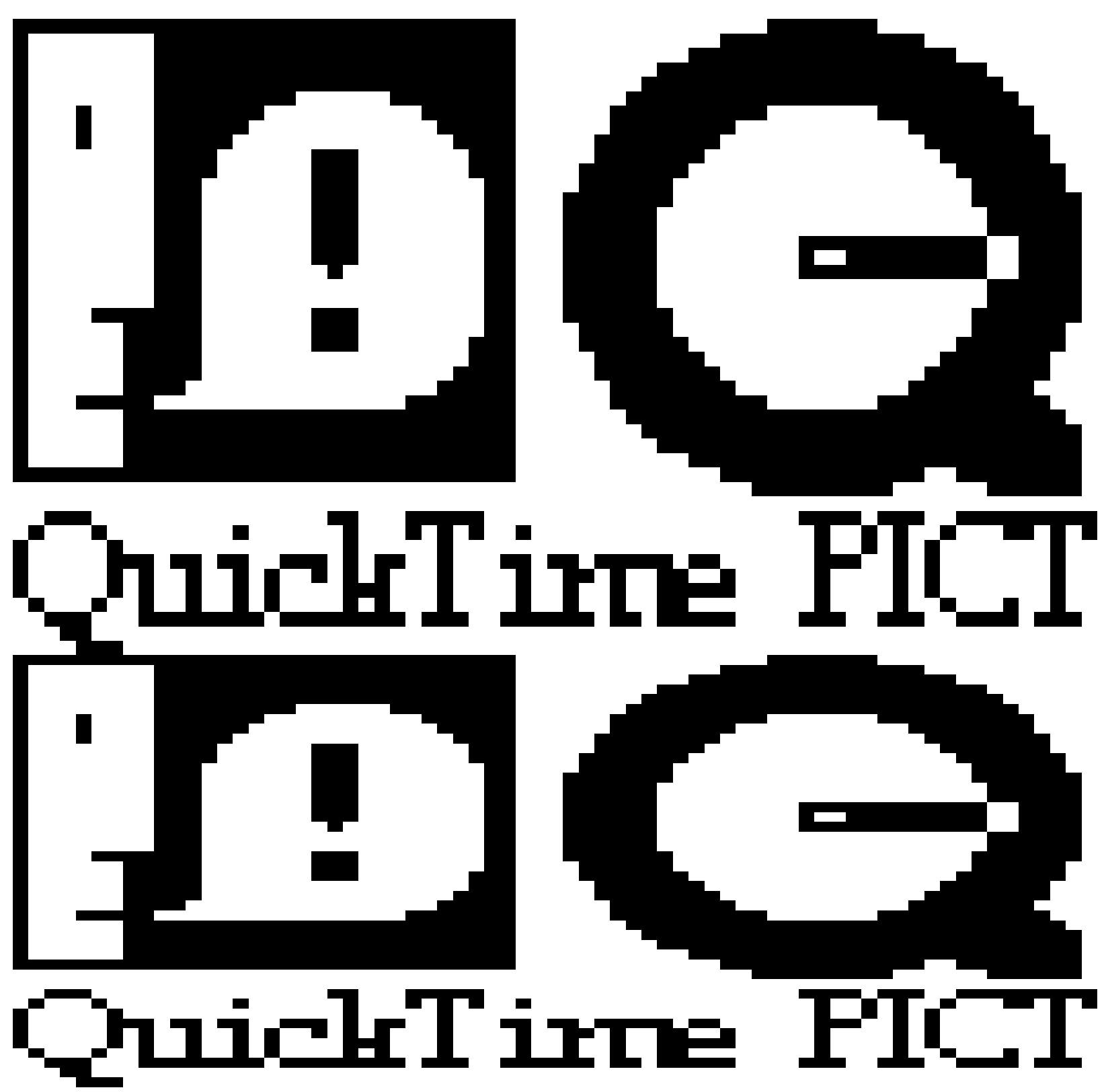



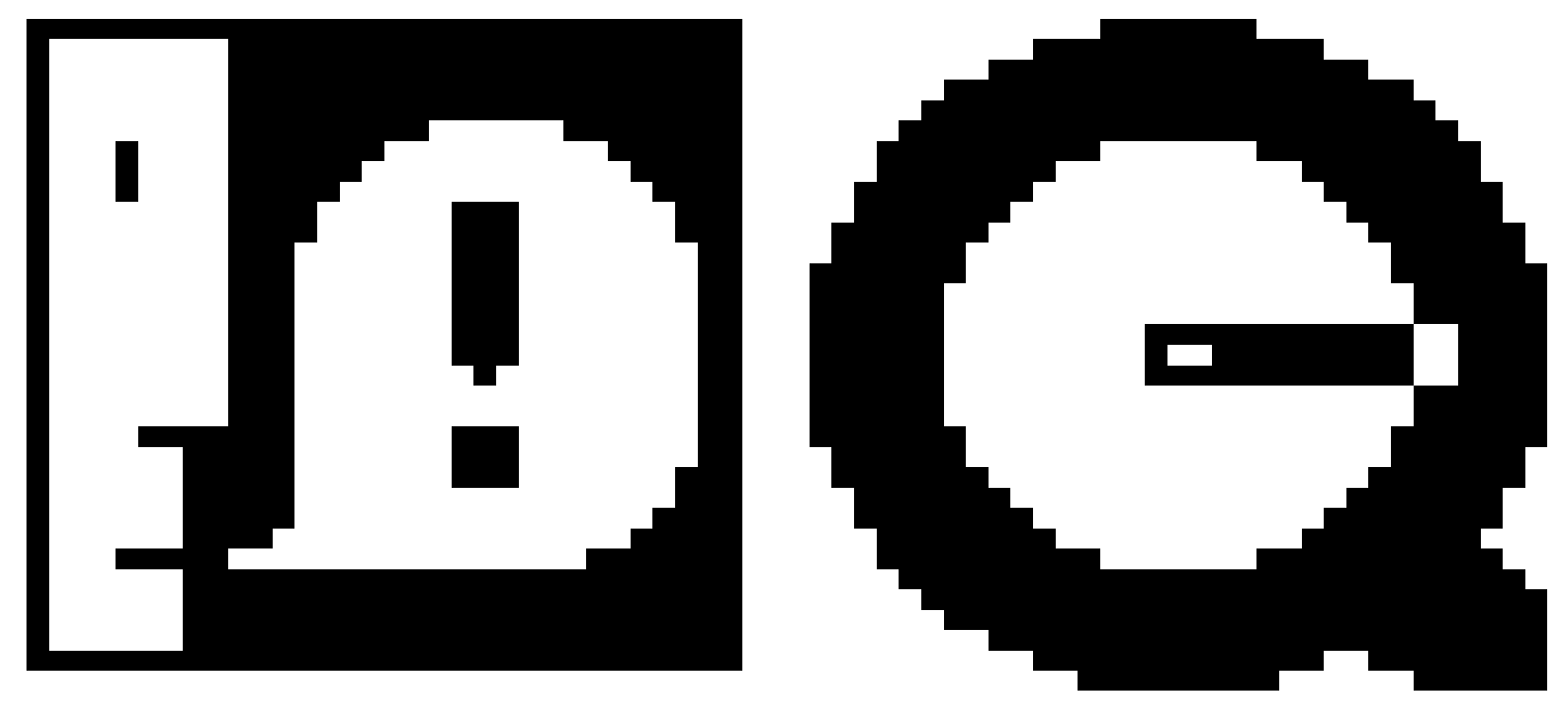

QuichTirne FICT

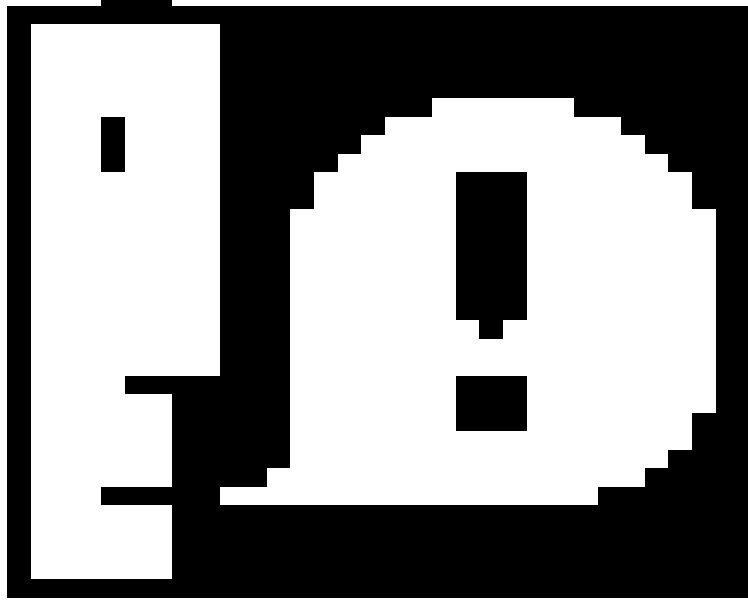

QuighTirne FICT 


\section{Visualization of Exothermic Fields}
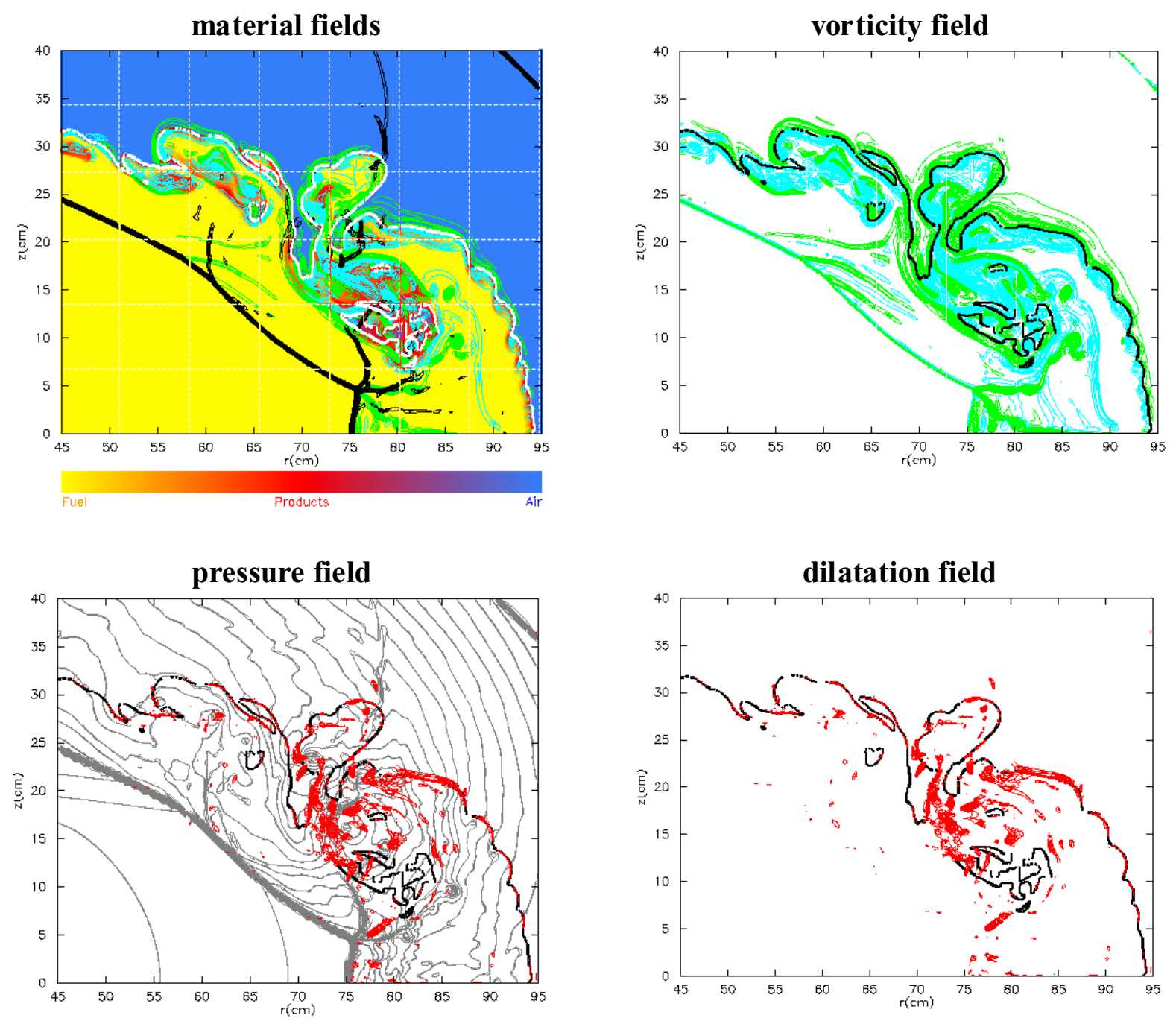

8th IWPCTM 


\section{Post-Explosion Combustion of HE in Chamber}

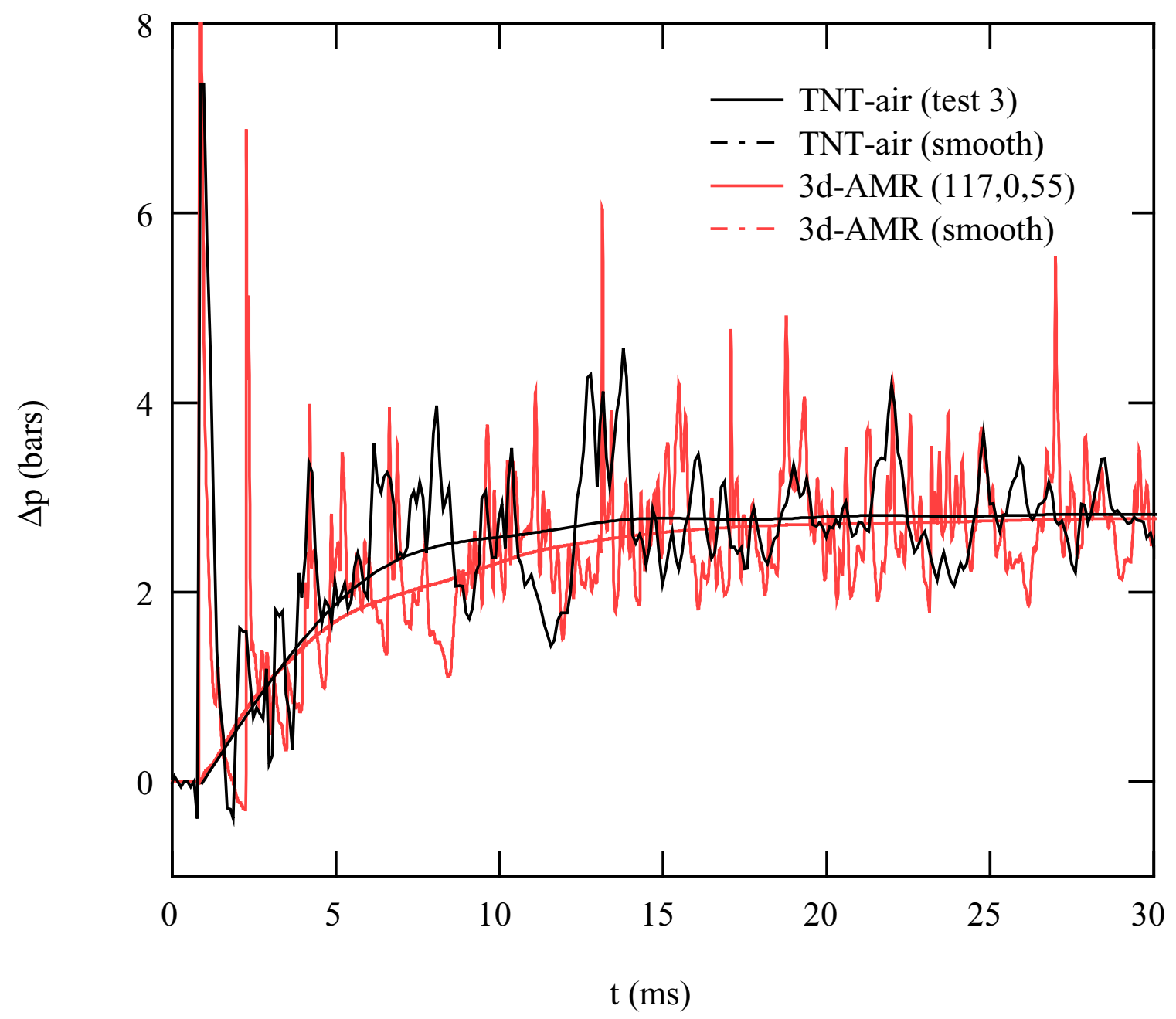




\section{Fuel Consumption}

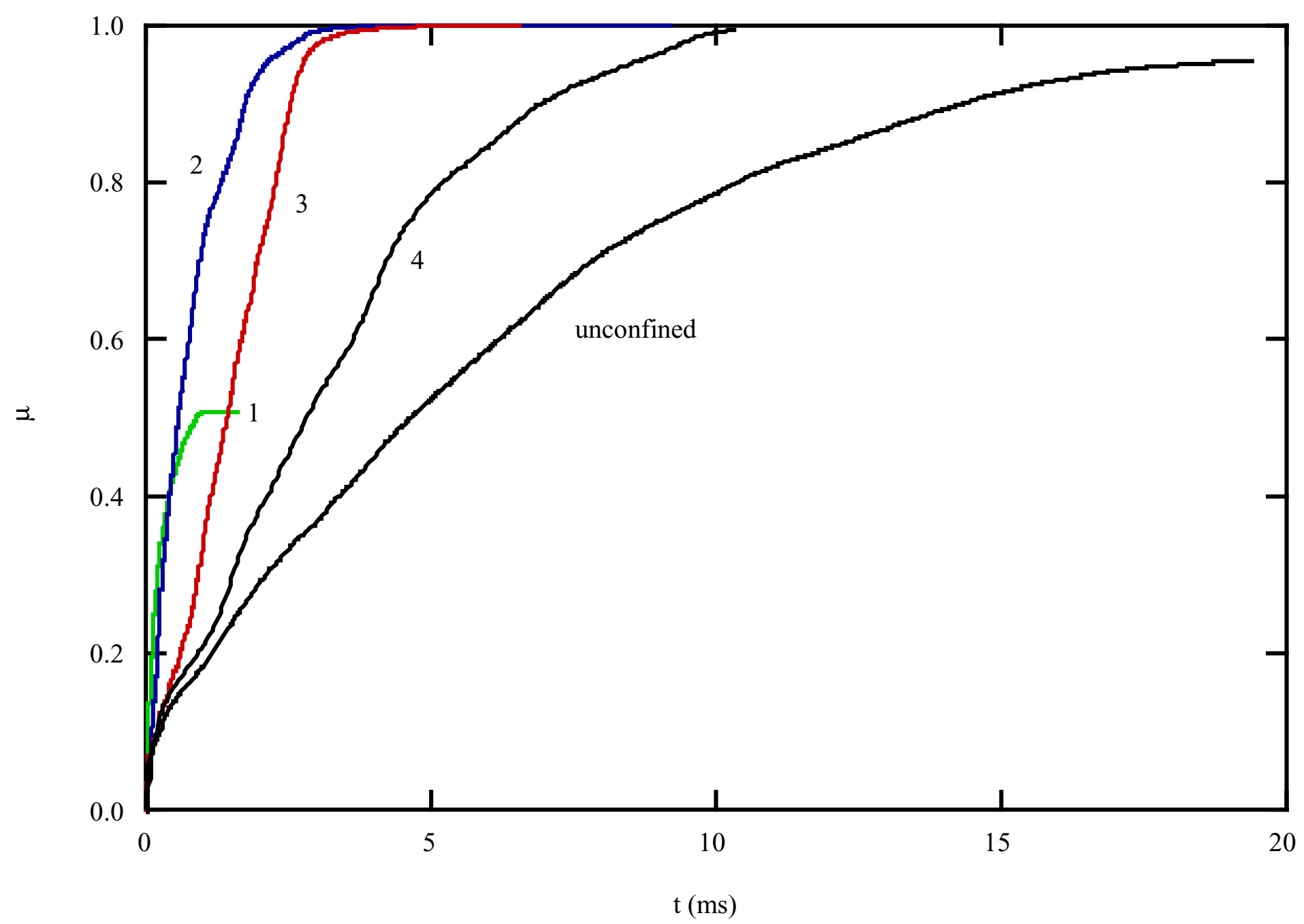

8th IWPCTM 


\section{Résumé}

\section{Multi-fluid Model}

- Gas-dynamic Conservation Equations for the mixture

- Mass \& Energy Conservation Equations for each fluid, with source/sink terms

\section{Thermodynamic Model}

- Equations of State: for each fluid

- Thermodynamic-Equilibrium Relations: for mixed cells

Combustion Model

- combustion occurs at thin exothermic sheets: $\mathbf{x}_{s}\left(t_{s}\right) \quad$ (stoichiometric surface)

- $\quad$ sink for Fuel \& Air mass and energy

- source for Products mass and energy

- Combustion $\equiv$ material transformations in the Le Chatelier plane:

$$
u_{R}\left(w_{R}\right) \Rightarrow u_{P}\left(w_{P}\right)
$$




\section{Conclusions}

- This Model elucidates the link between turbulence (三 vorticity) and exothermicity (三 dilatation) in the limit of fast chemistry.

- It thus illustrates the dynamics of turbulent combustion where exothermic effects are controlled by mixing — rather than by the reaction-diffusion mechanism of Zel'dovich \& Frank-Kamenetzkii (1938) 


\section{Discrete Wavelet Transform}

Techniques for Denoising, Pattern

Detection and Compression of

Turbulent Rayleigh-Taylor Mix

\section{Data}

Bedros Afeyan, Polymath Research Inc. Praveen Ramaprabhu \& Malcolm J. Andrews, Texas A\&M University

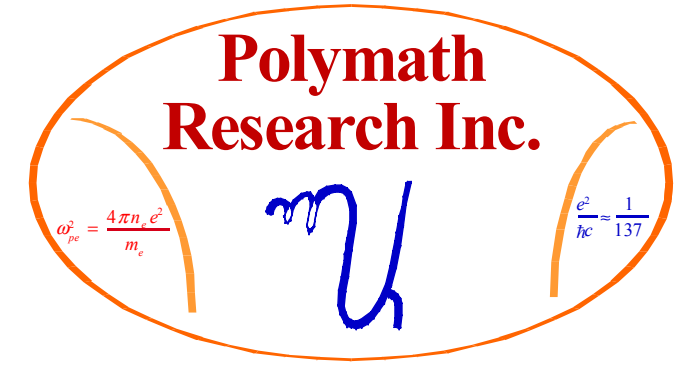

International Workshop on the Physics of Compressible Turbulent Mixing Cal Tech

Pasadena, CA December 9-14, 2001 
What Are Wavelets?

\section{Start@(www.wavelets.org) surf (Mathsoft, amara, ...)}

Polymath Research Inc.

Mallat, Meyer, Daubechies, Beylkin, Coifman, Strang, Sweldens, Jawerth...

- Wavelets are localized kernels or atoms in PHASE SPACE.

- You may think of them as basis functions with prescribed dilation and translation properties.

- They may or may not be orthonormal or have compact support or be differentiable everywhere, or be fractal, or have many zero momemts.

- Wavelets are like breathing wave packets which can home in on structures in phase space better than FT or WFT ever could.

$$
\begin{aligned}
& \psi_{j, k}(x)=2^{j / 2} \Psi\left[2^{j}\left(x-\frac{k}{2^{j}}\right)\right] ; j, k \in Z \\
& \Psi_{n}(x)=(-1)^{n} \frac{d^{n}}{d x^{n}}\left[\exp \left(-\kappa\left(x-x_{c}\right)^{2} / 2\right)\right]
\end{aligned}
$$

When the scale is decreased translation steps between wavelets should likewise be decreased 


\section{What is MRD or Multi-resolution Decomposition?}

- Multiresolution: Zoom in and out on a number of successively finer scales in a sequence of nested approximation subspaces $\left\{\mathrm{V}_{\mathrm{j}}\right\}_{\mathrm{j} \text { in } \mathrm{Z}}$.

- In general, get an overcomplete basis set in $\mathrm{L}_{2}(\mathrm{R})$.

Approximate (or truncate) by bounding the scales of interest.

\section{Scaling functions and the scaling equation:}

\section{Low pass filter}

$$
\begin{aligned}
& \varphi(x)=2 \sum_{k=0}^{2 N-1} h_{k} \varphi(2 x-k) \\
& \sum_{k} h_{k}=1 \quad \int_{-\infty}^{\infty} \varphi(x) \mathrm{d} x=1
\end{aligned}
$$

\section{The Wavelets:}

$$
\begin{aligned}
& \psi(x)=2 \sum_{k=0}^{2 N-1} g_{k} \varphi(2 x-k) \\
& g_{k}=(-1)^{k} h_{2 N-1-k}
\end{aligned}
$$

These filters decompose a sampled signal into 2 sub-sampled channels: the coarse approximation of the signal and the missing details at finer scales. The original signal can be reconstructed from these channels by interpolation. 


\section{What Are Discrete Wavelet Decompositions Good for?}

- Wavelet decompositions are very useful for the analysis of intermittent or bursty data.

- Spatial and scale localized information is efficiently represented.

- Because the trends you want are captured efficiently (get large coefficients in the expansion)very high quality denoising is possible.

- Similarly, pattern recognition and detection capability is enhanced.

- Compression is achieved where a few coefficients can represent what is needed in the data.

- All this depends on nonlinear (or largest coefficient)thresholding and not scale or level thresholding. The latter is rather reminiscent of what is done with Fourier expansions. 


\section{The Scaling Function and Wavelet for Haar or Daubechies 1 in X-Space}
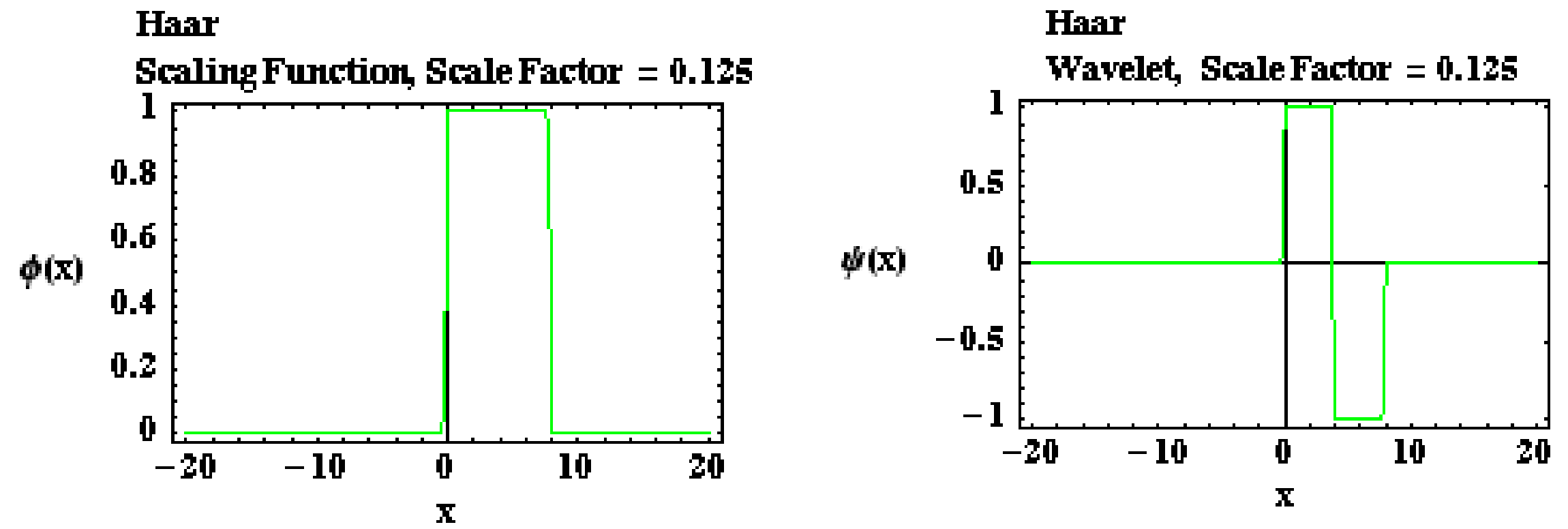


\section{The Scaling Function and Wavelet for Haar or Daubechies 1 in K-Space}

K space: Hair

Sealing Furction Scale Factor = 0.125

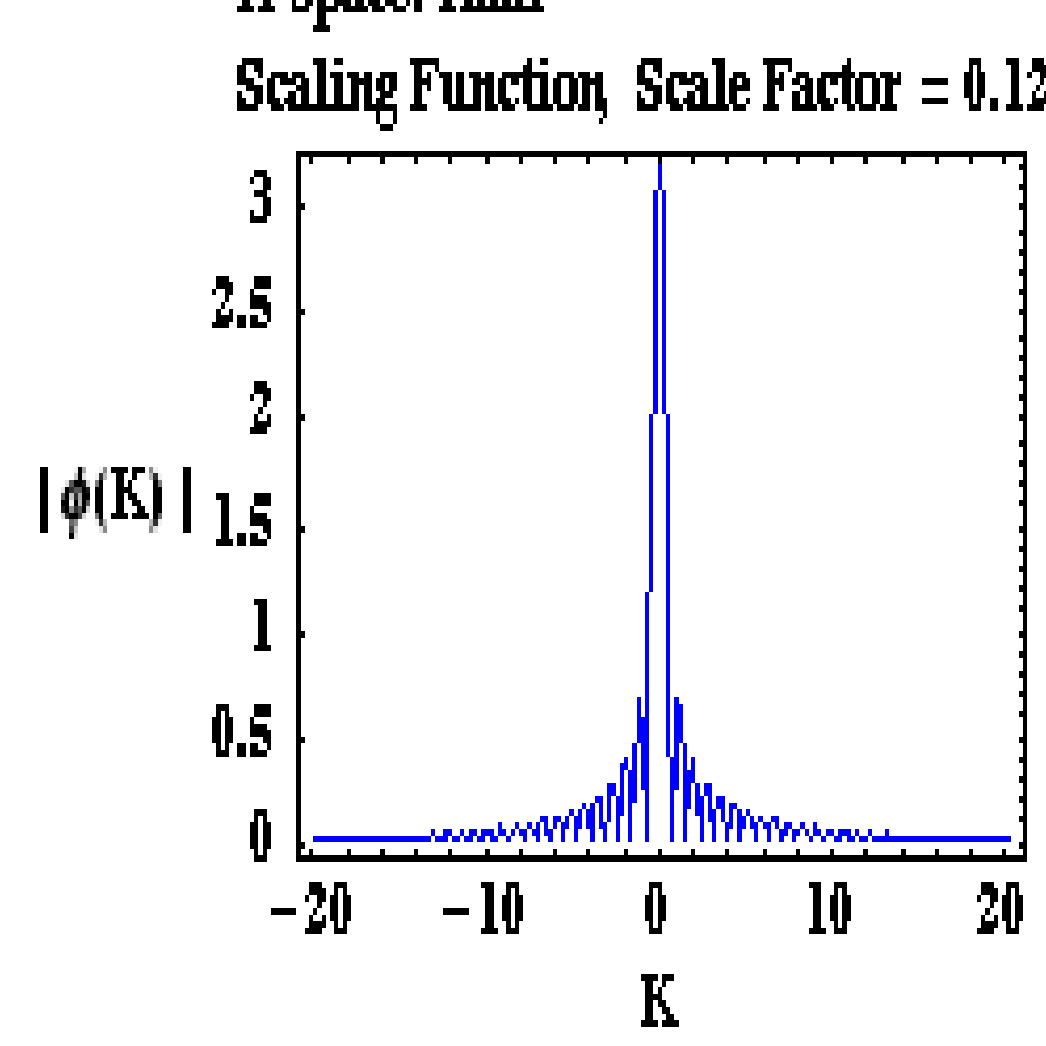

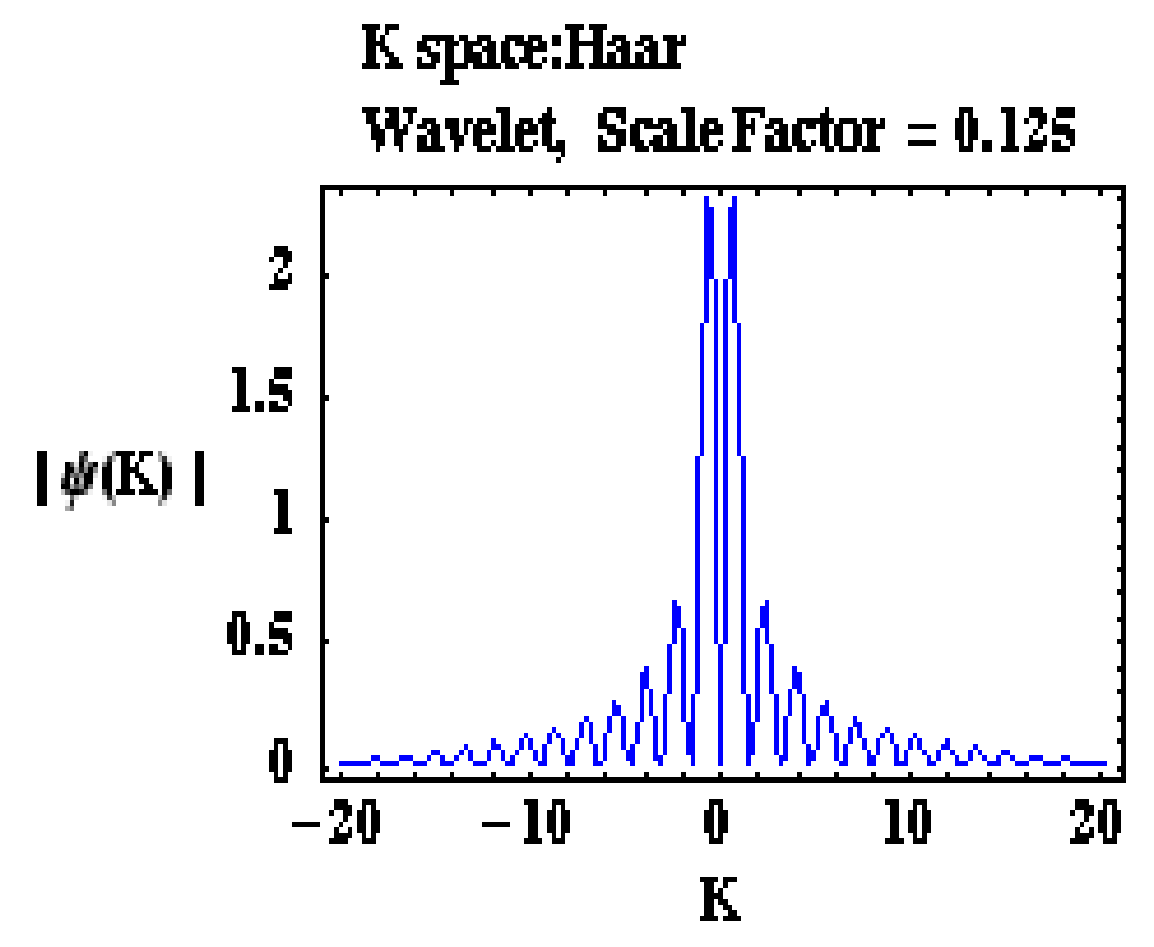




\section{The Scaling Functions and Wavelets for Daubechies 2-6 in X-Space}

Polymath

Research Inc.

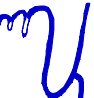

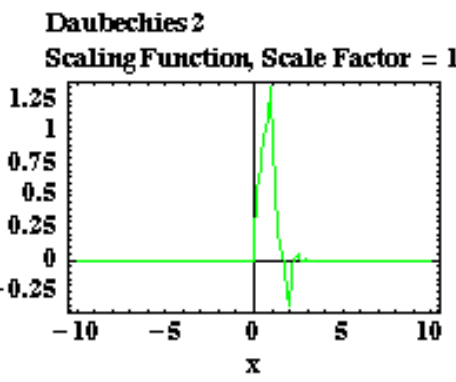

\section{Daubechies 4}

Sealing Function, Scale Factor = 1

$\phi(\mathrm{x})$

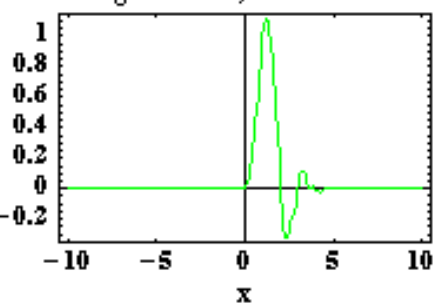

Daubechies 6

Scaling Function Scale Factor =

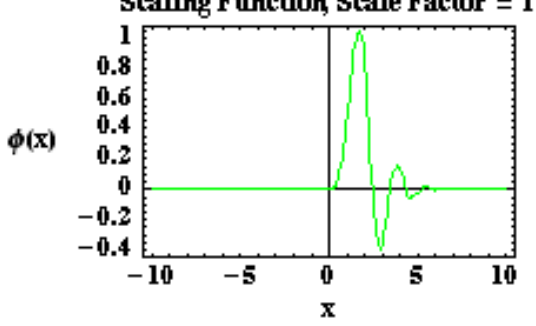

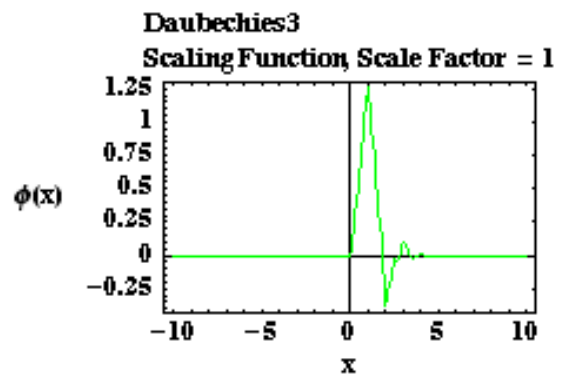

Daubechiess

SealingFunction, Scale Factor $=1$

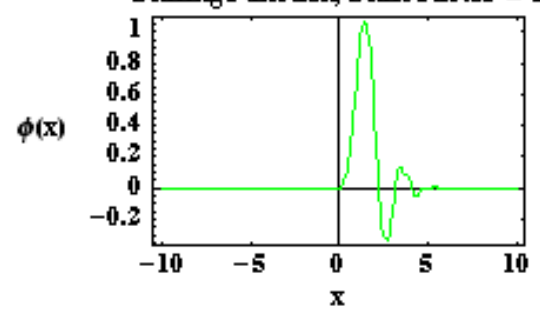

$\psi(x)$

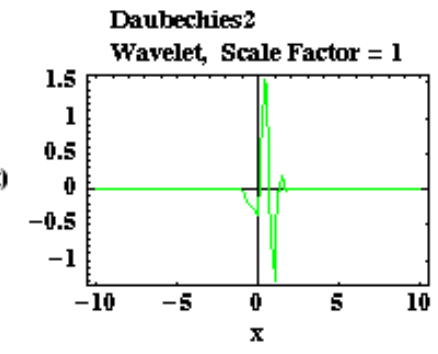

Daubechies4

Wavelet, Scale Factor $=1$

$\psi(x)$

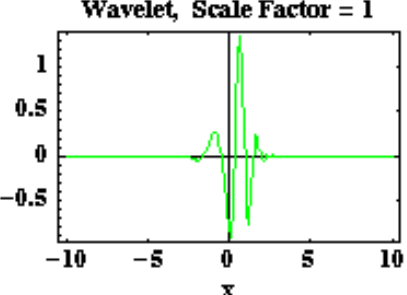

Daubechies6

Wavelet, Scale Factor $=1$

$\psi(x)$

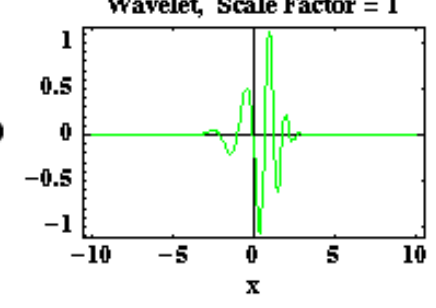




\section{The Scaling Functions and Wavelets for Daubechies 2-6 in k-Space}

K space: Daubechies2

Sealing Function, Seale Factor = 1

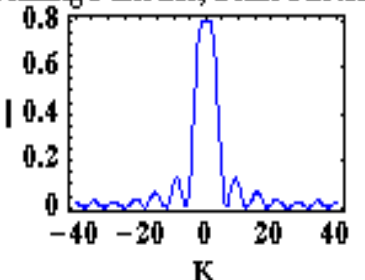

K space: Daubechies4

Scaling Function Seale Factor = 1

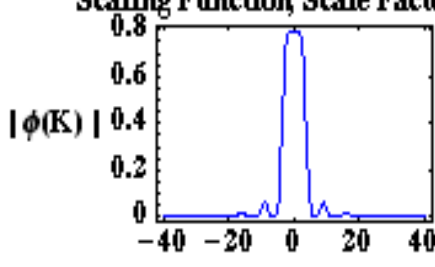

$\mathbf{K}$

K space: Daubechies6

Saling Function, Sale Factor = 1

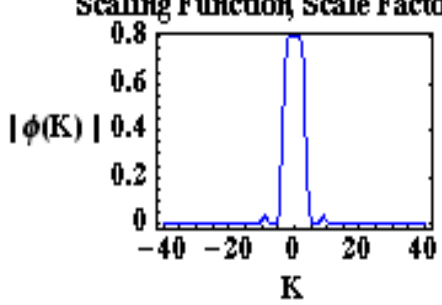

K space: Daubechies3

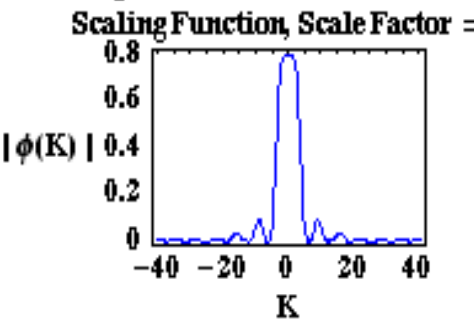

K space: Daubechies5

SalingFunction, Sale Factor =

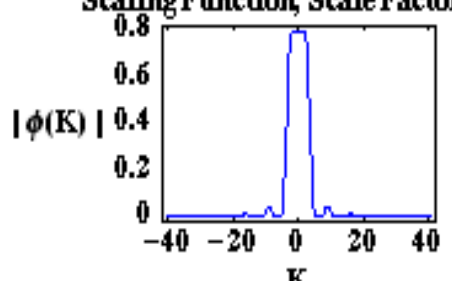

K

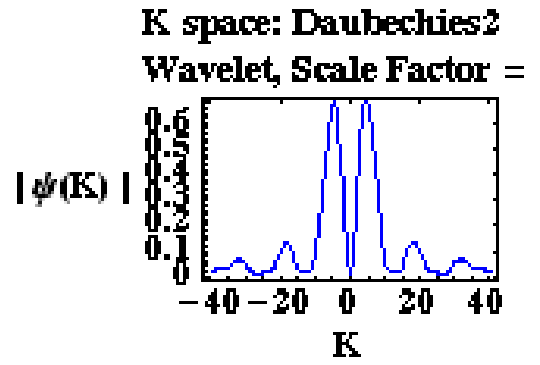

K space: Daubechies4

Wavelet, Scale Factor $=1$

| $\psi(\mathbf{K}) \mid$

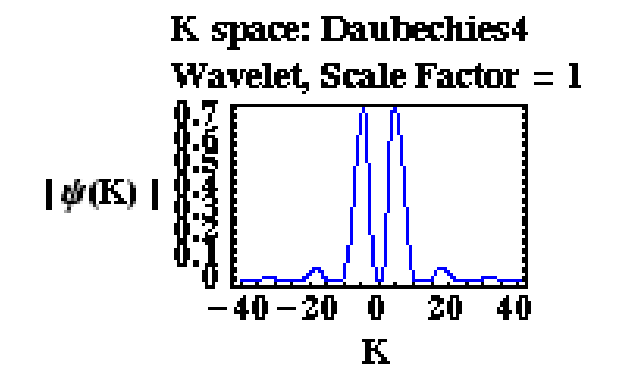

$\mathbf{K}$

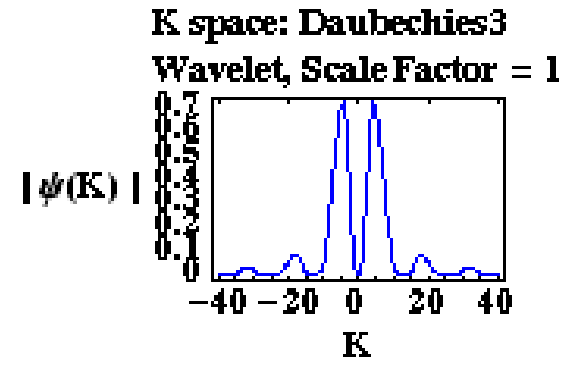

K space: Daubechies5

Wavelet, Sale Factor $=1$

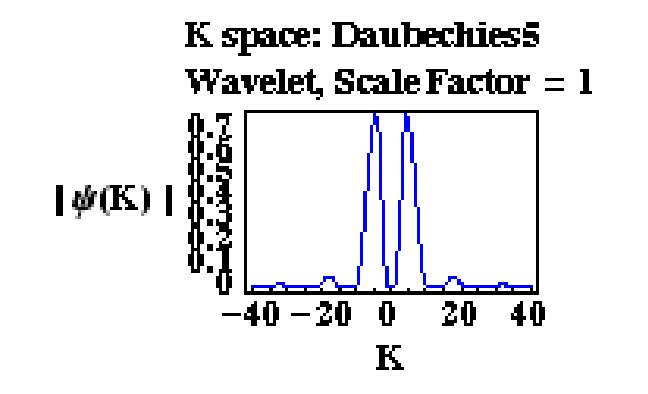

K space: Daubechies6

Wavelet, Scale Factor = 1

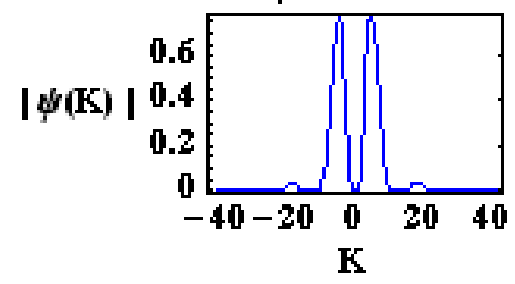

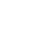




\section{Raw Thermocouple RT Strong Mix}

\section{Data $(30 \mathrm{~cm}$ Downstream,}

theta $\sim 0.71)$ from Texas A\&M

Polymath

Research Inc.

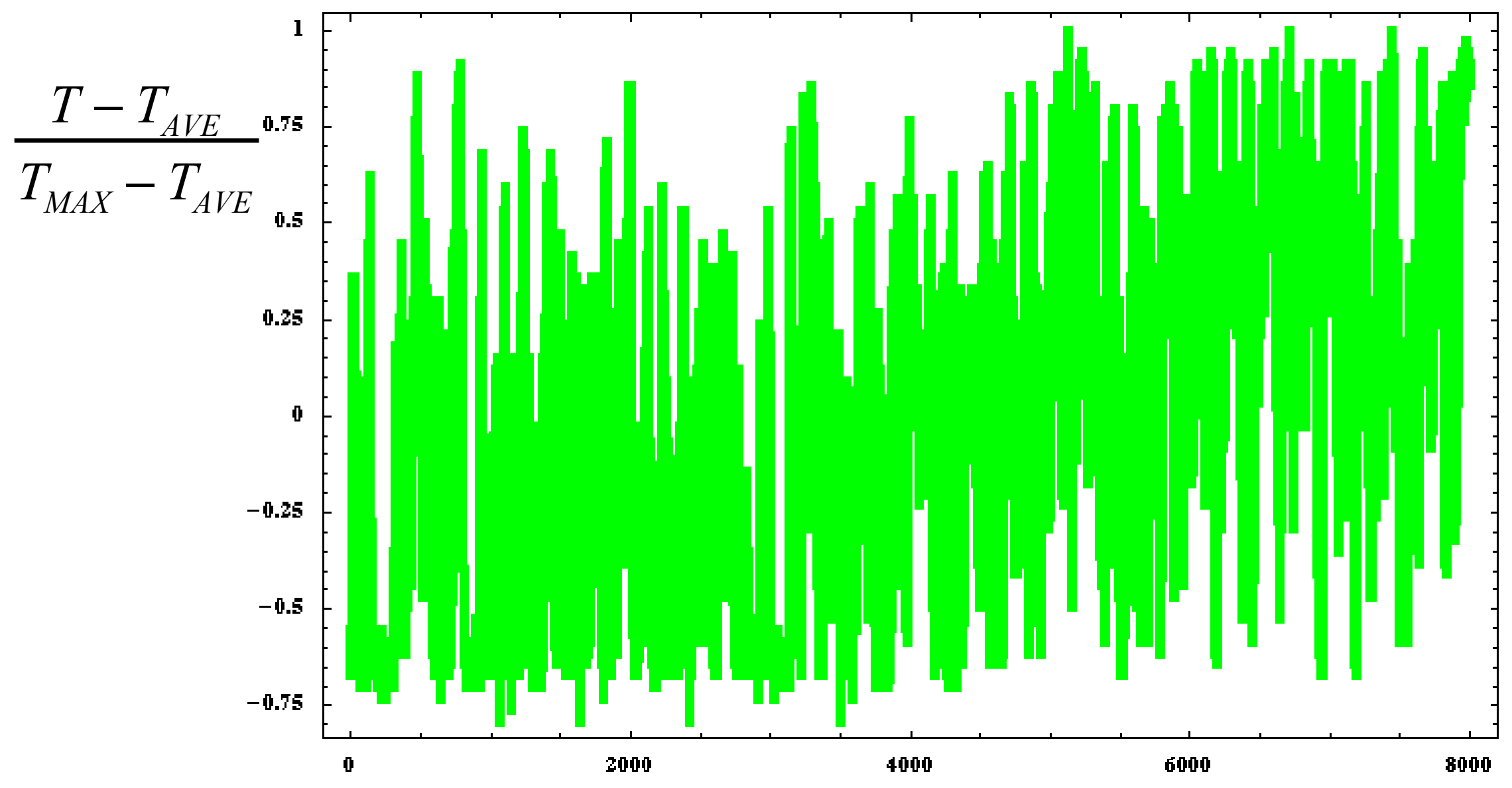

Time, arb. units (Delta $\mathrm{t}=0.012 \mathrm{sec}$, Sampling Rate $=85 \mathrm{~Hz}$ ) $\begin{gathered}\text { Bal Tech Pasadena CA } \\ \text { 8th IW PCTM 12-11-01 }\end{gathered}$ 


\section{The Faded and Padded Version of the Data 8192 Points Long}

$2^{13}$ data points faded and padded

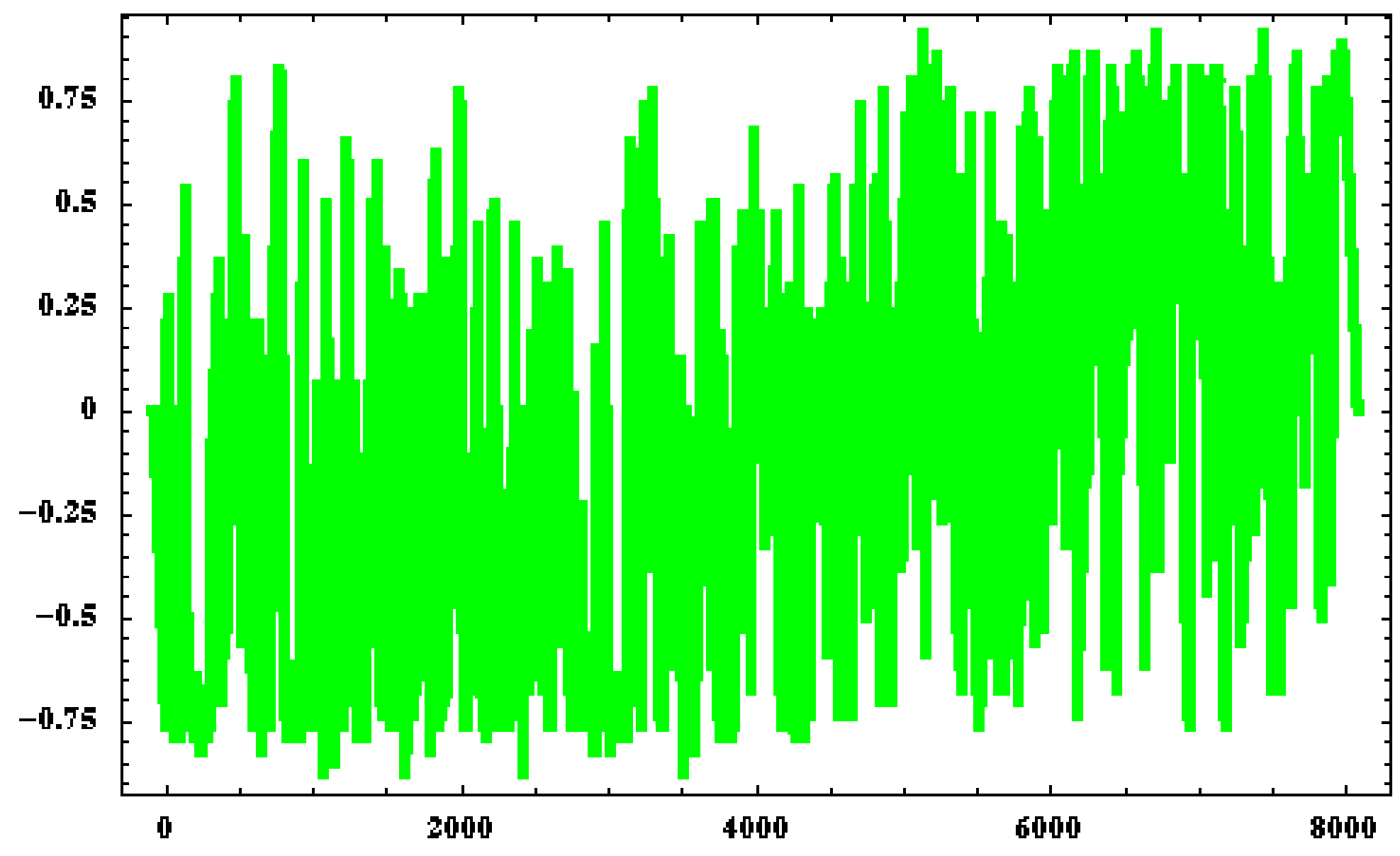

80 pts to fade 16 pts to pad per side 


\section{The Fourier Transform of the RT Mix Data}

FFT of Tapered Padded RT

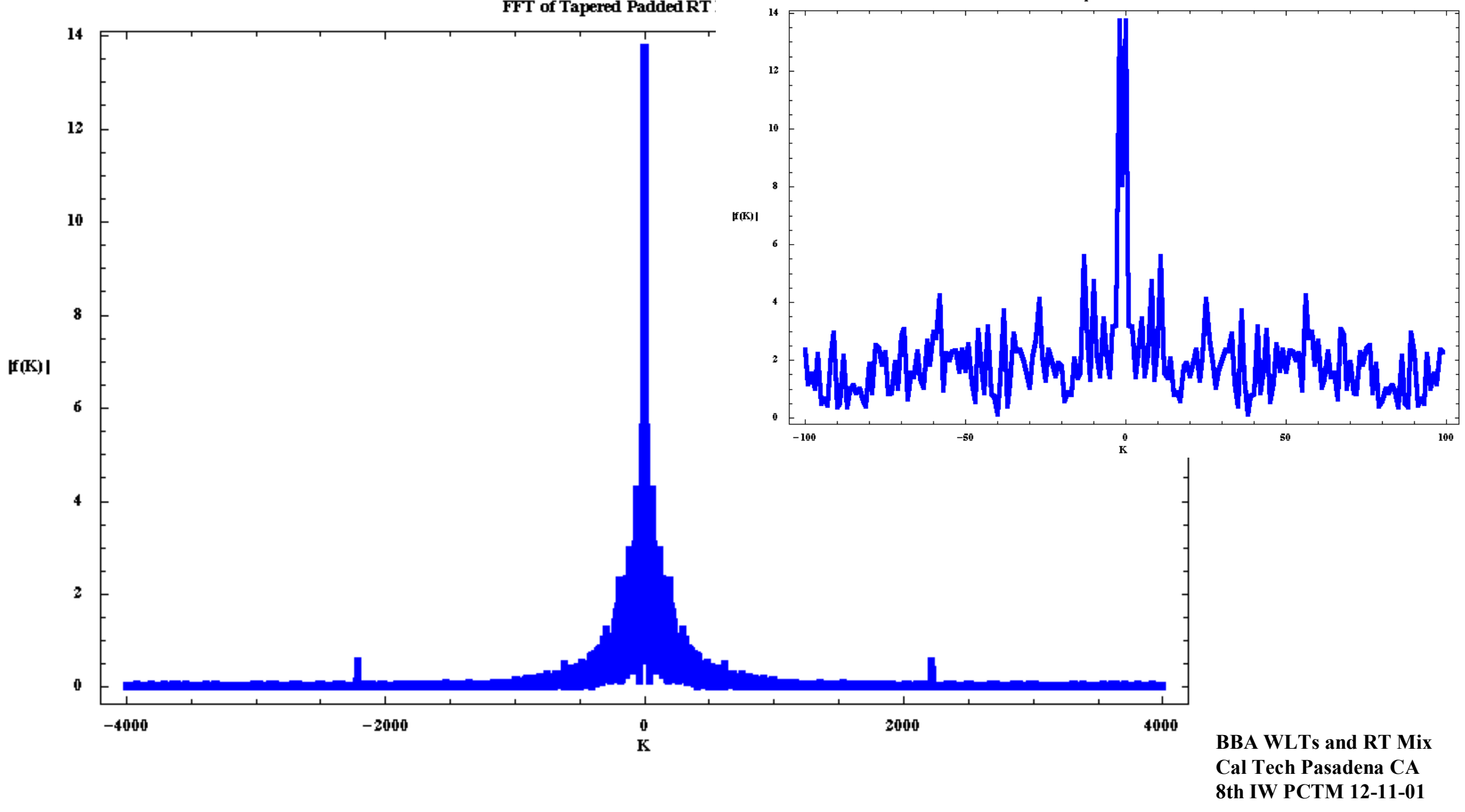




\section{MRDs of the RT Mix Data in 6 Different Daubechies WLT Bases}

Polymath Research Inc.

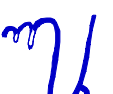

\section{Haax
MIRD}

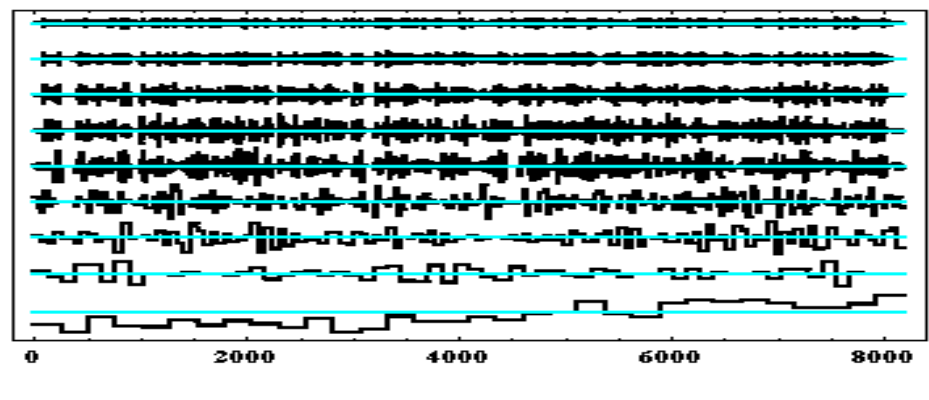

Daubechies 3

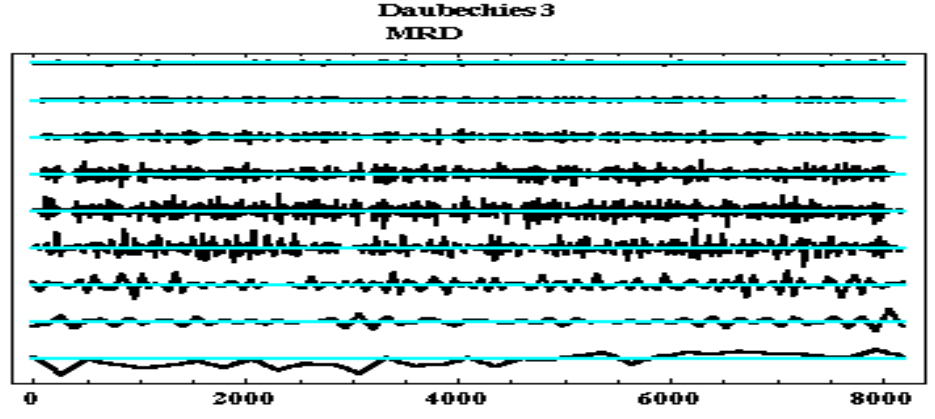

Daubechies:

\section{MIRD}

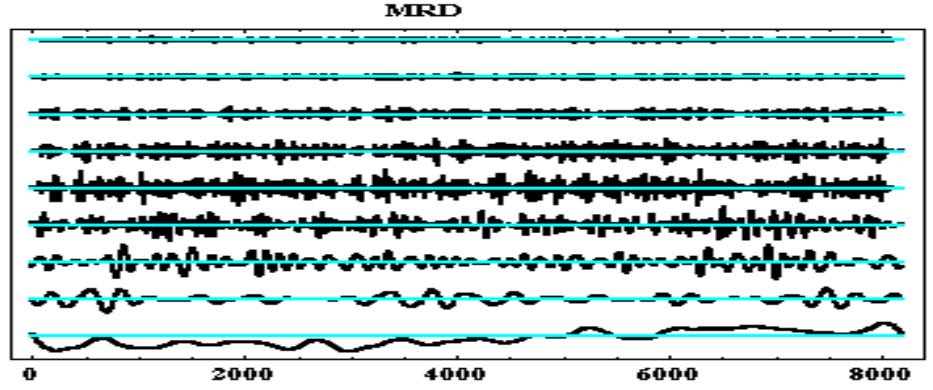

Daubechies :

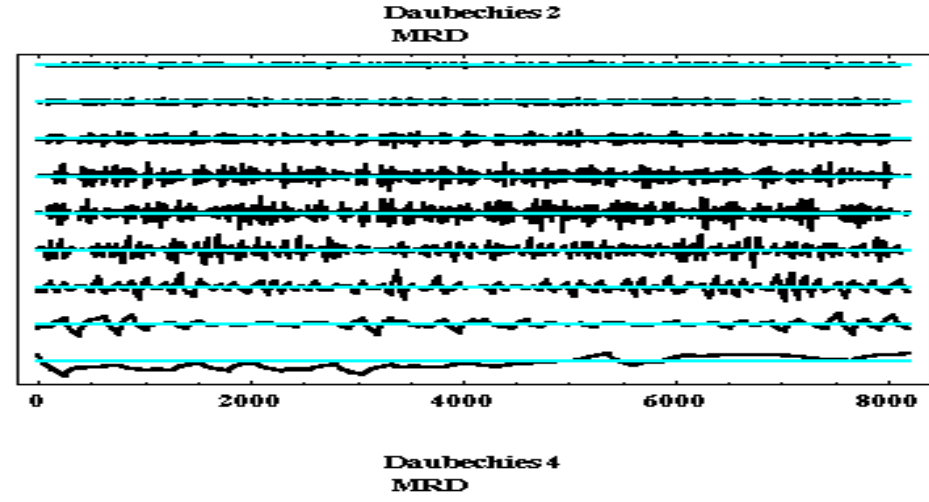

Maube

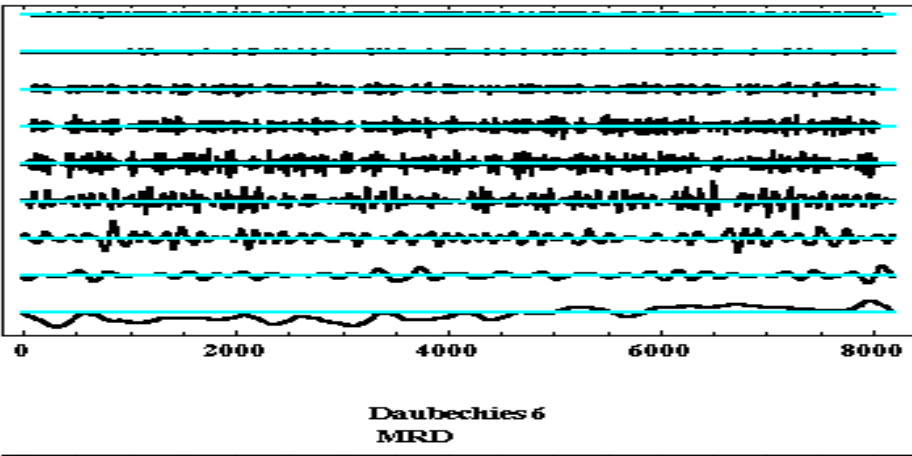
MIRD

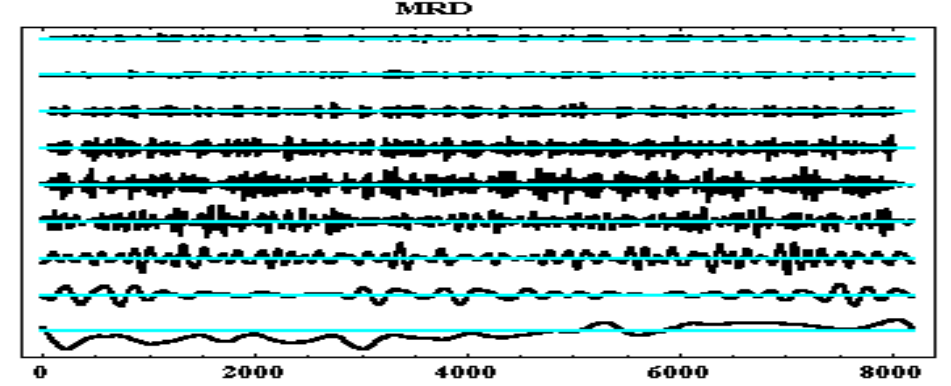




\section{Decay Rate of Largest Coefficient vs Number of Coefficients Kept in 6 Different Daub WLT Decomps}
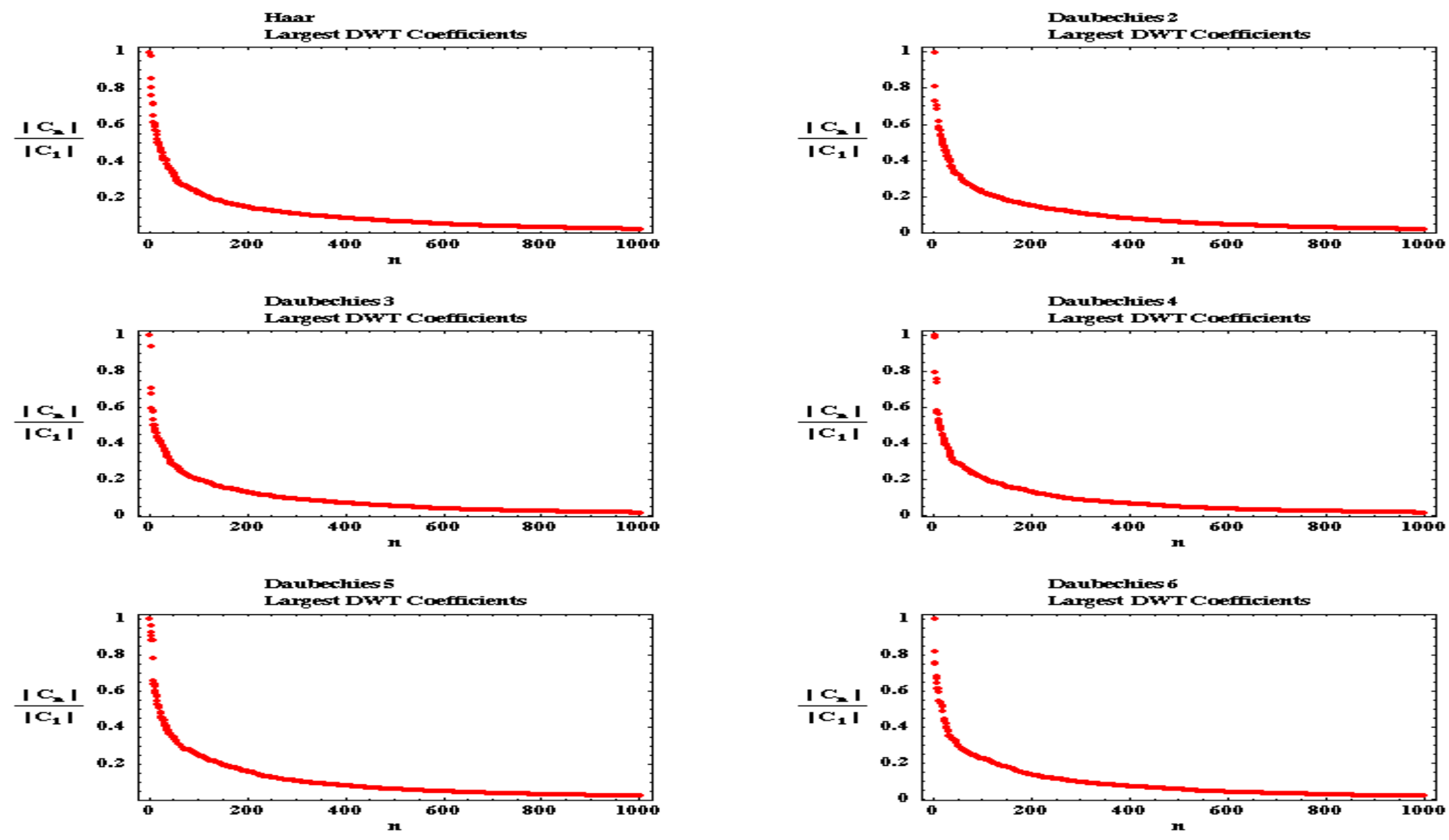


\section{Energy Accumulation Rate in}

\section{Coefficient Space vs \# of WLTs}

Kept in 6 Different Daub Decomps

Polymath Research Inc.
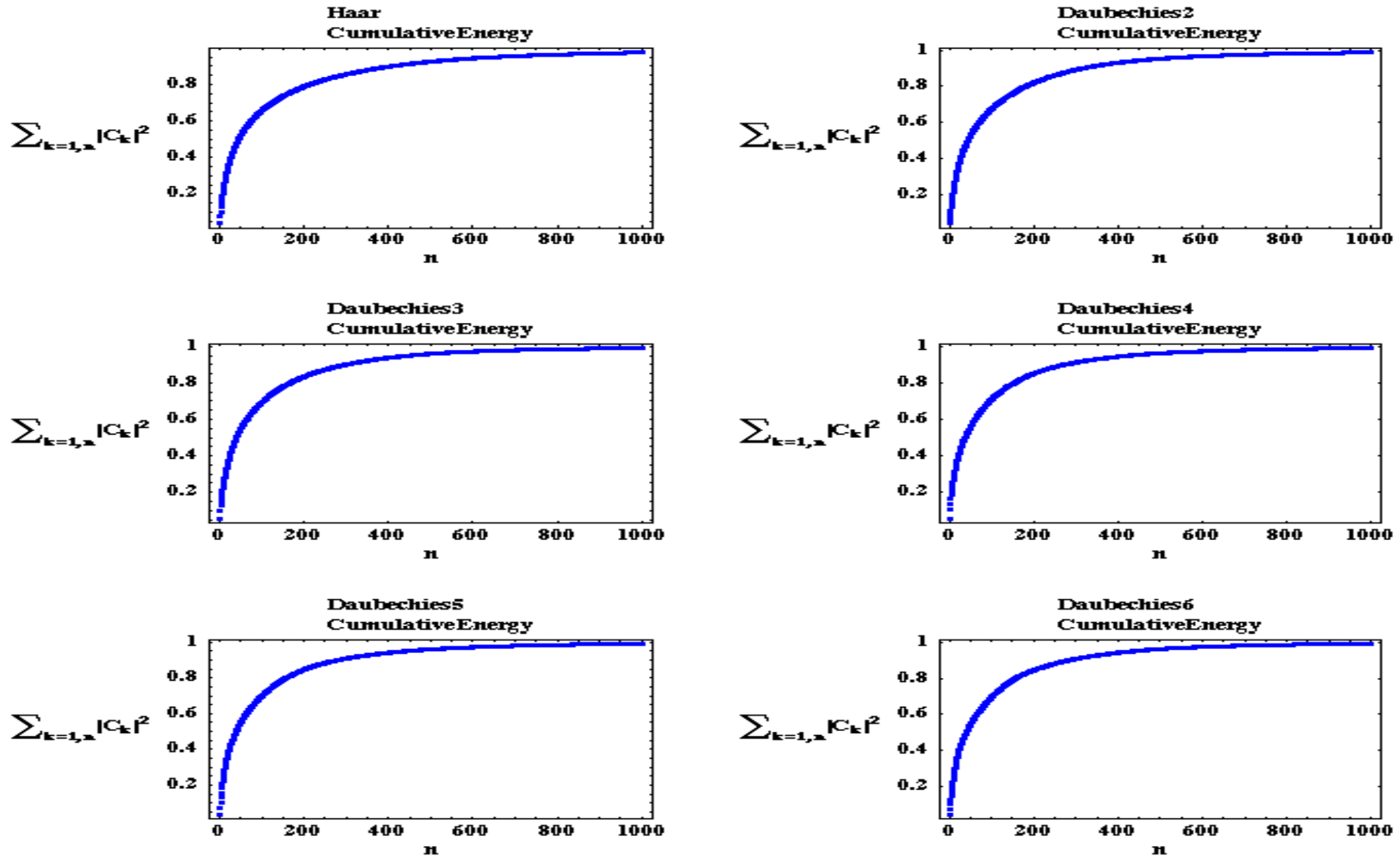


\section{Scaleograms: Waveleters \\ Preferred Way of Judging Tiling in Scale-Translation Space}
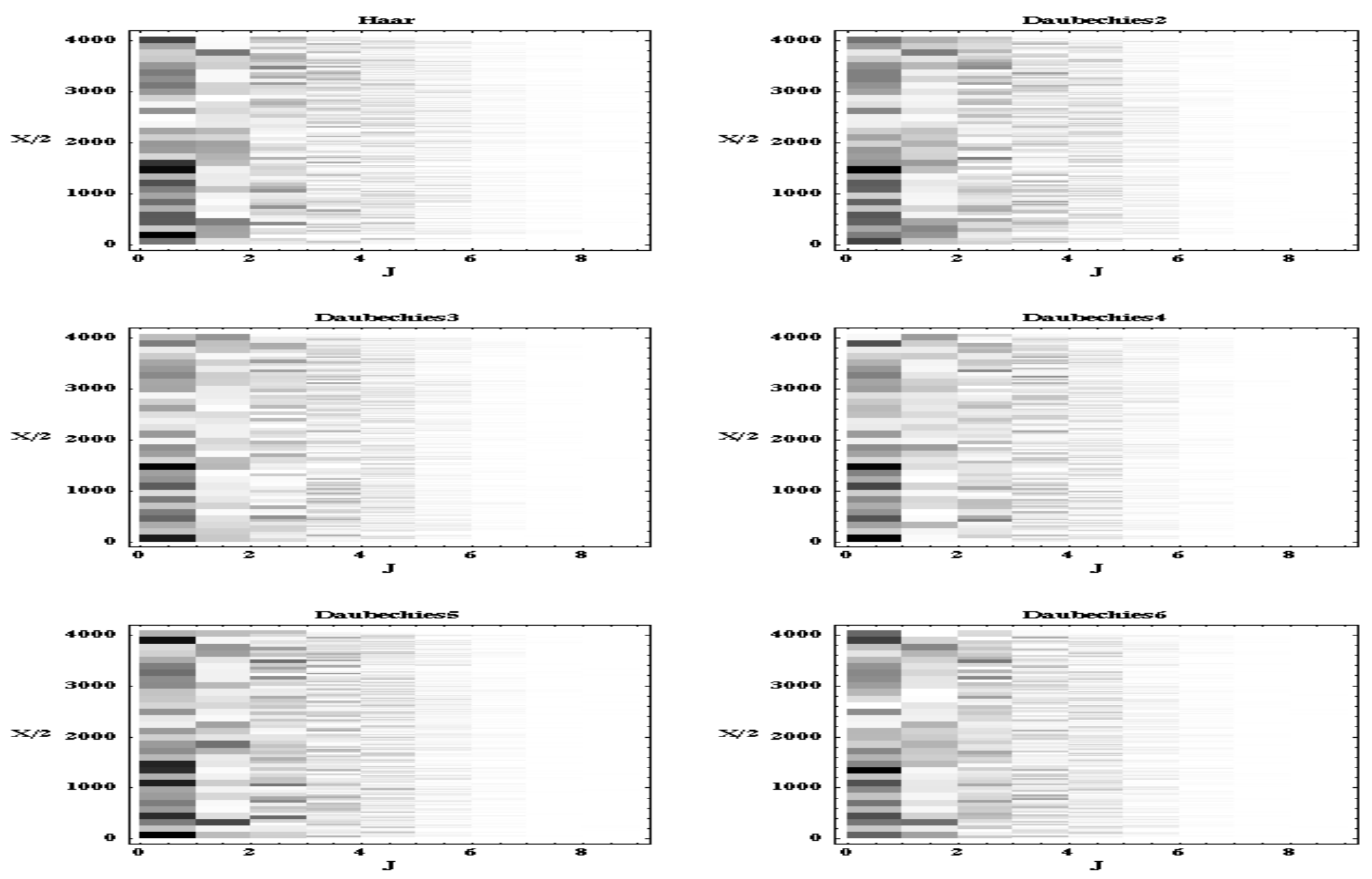

Polymath Research Inc. 


\section{Least Square Error Incurred}

\section{By Truncating the WLT Series at $\mathrm{N}$ of its Largest Coefficients}

Polymath Research Inc.
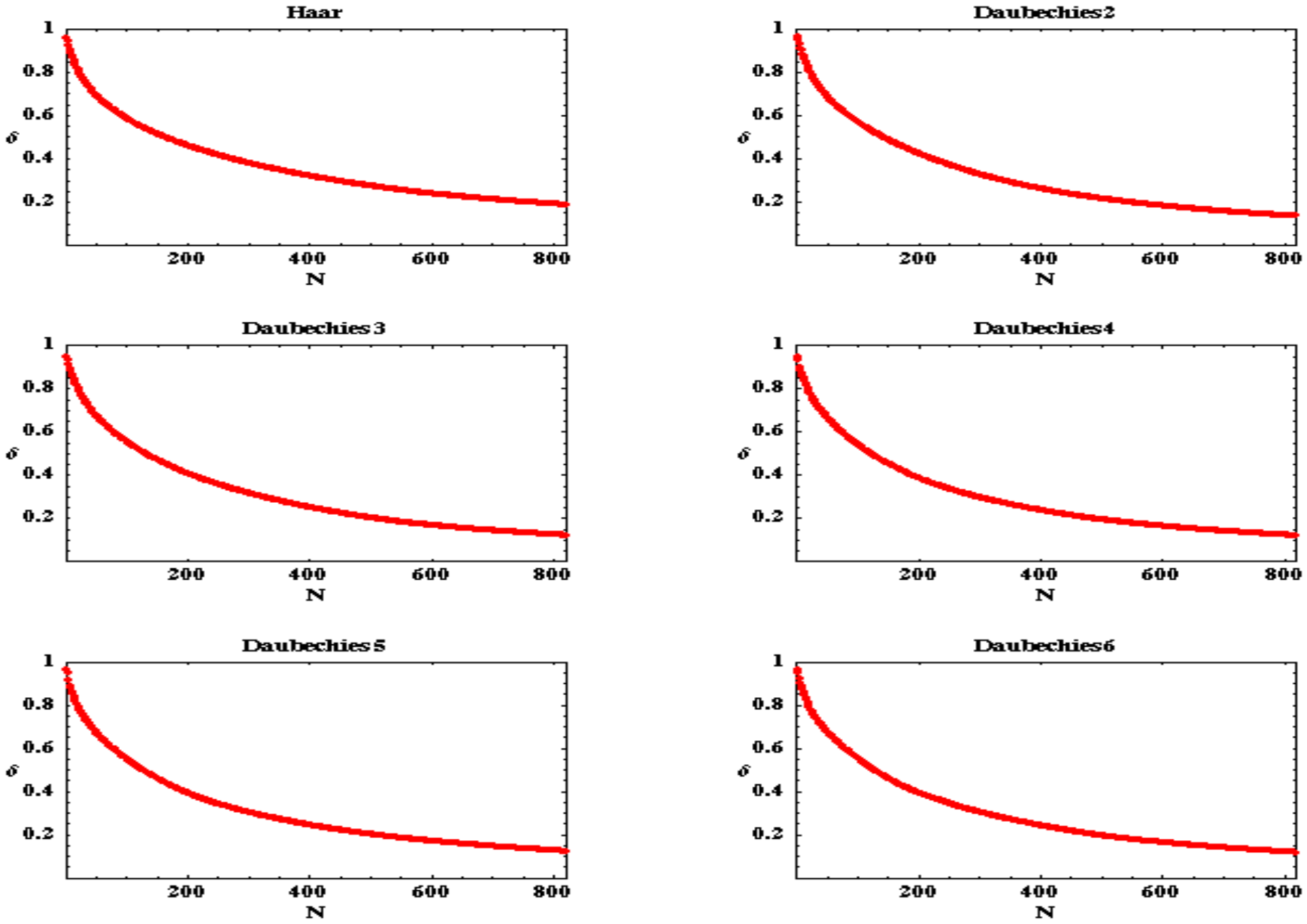


\section{Least Square Error Incurred by Level Thresholding the DWT}

Polymath Research Inc.

m
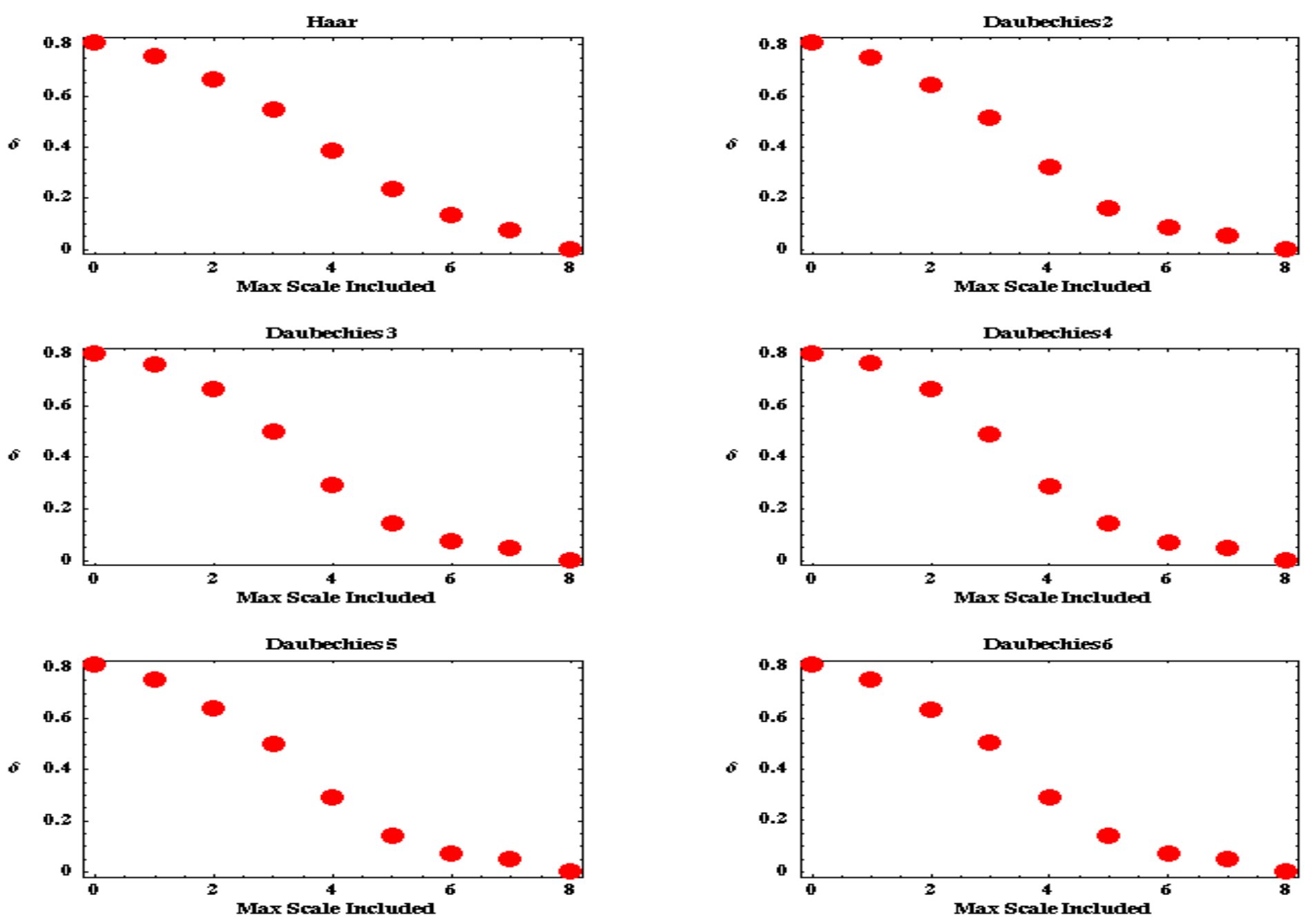


\section{Daubechies 5 Does Much Better than Haar: 5 Quantitative Measures}

Polymath Research Inc.
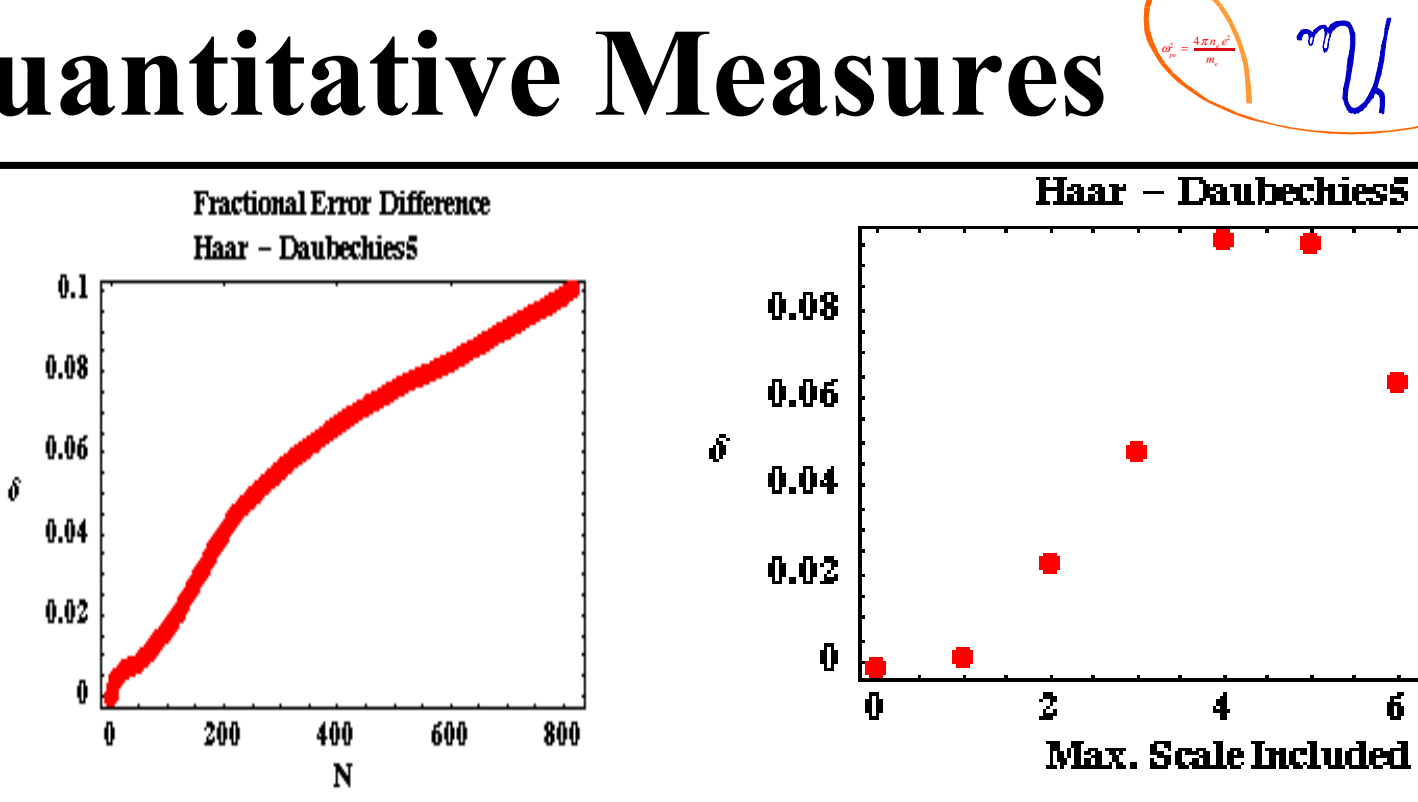

Haar - Daubechies

CunulativeEnergy

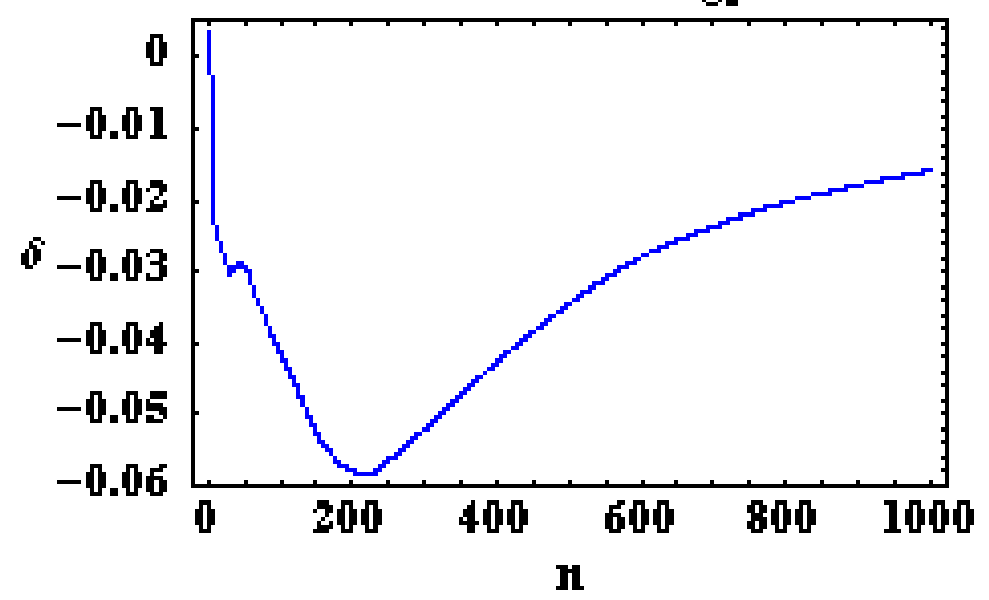

Haar - Daubechies5

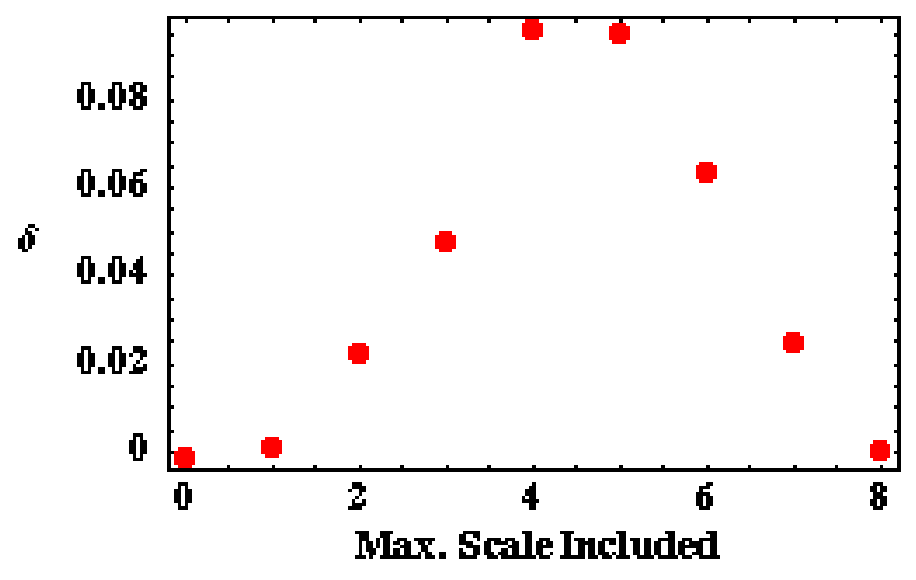

Hatar - Daubechies 5

Largest Coeficients

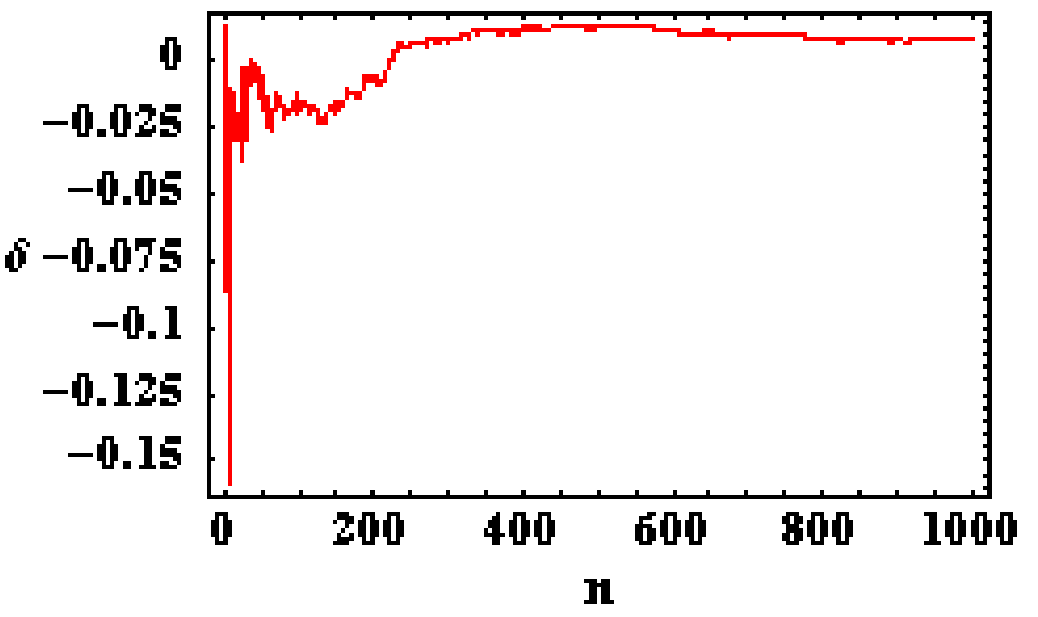




\section{Level by Level Decomposition of the RT Mix Data Using Daub5 WLTs}

Daubechiess
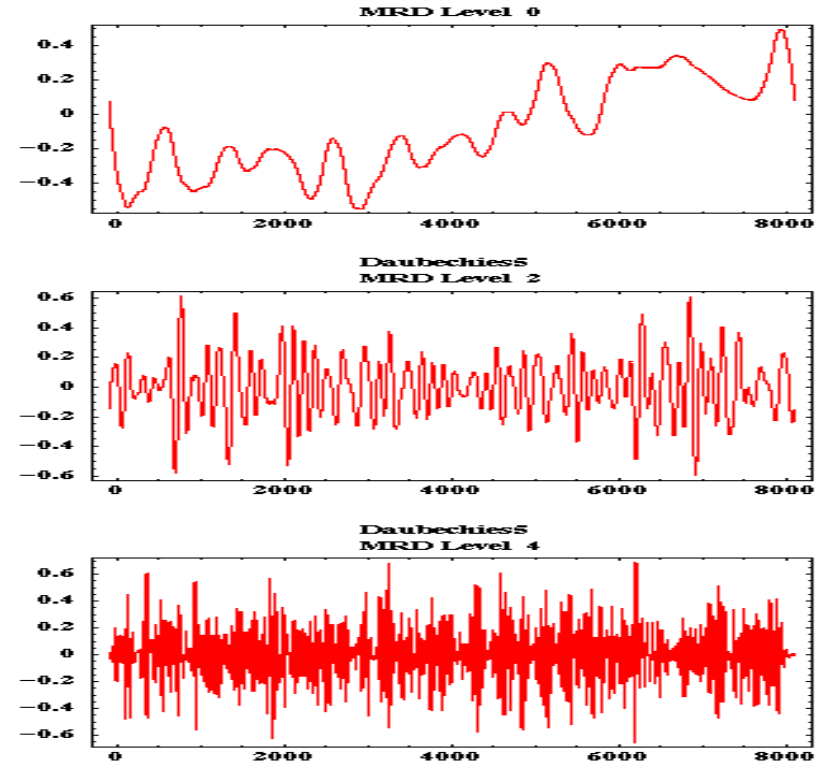

Daubechiess

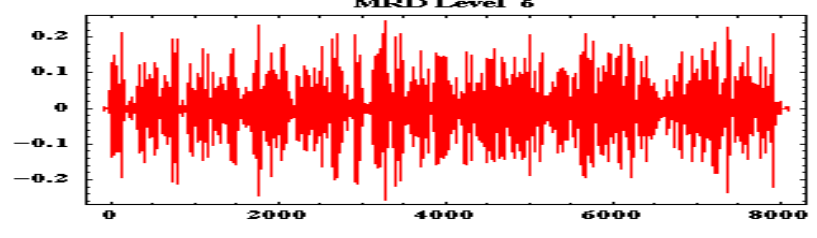

Daubechiess:

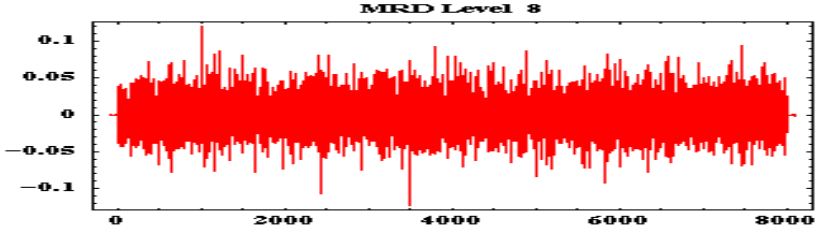

Daubechiess,
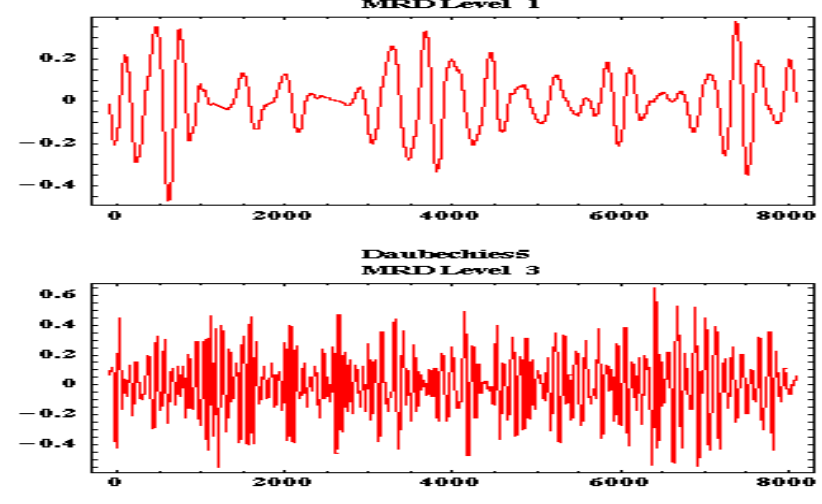

Daubechiess

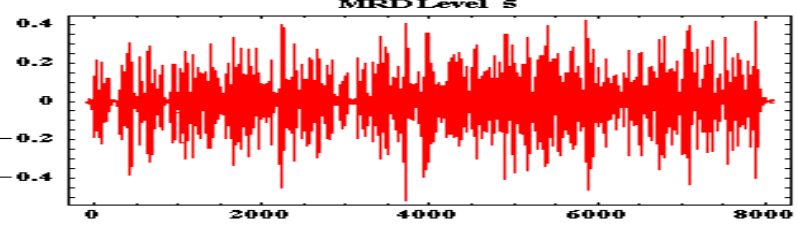

Daubechiess,
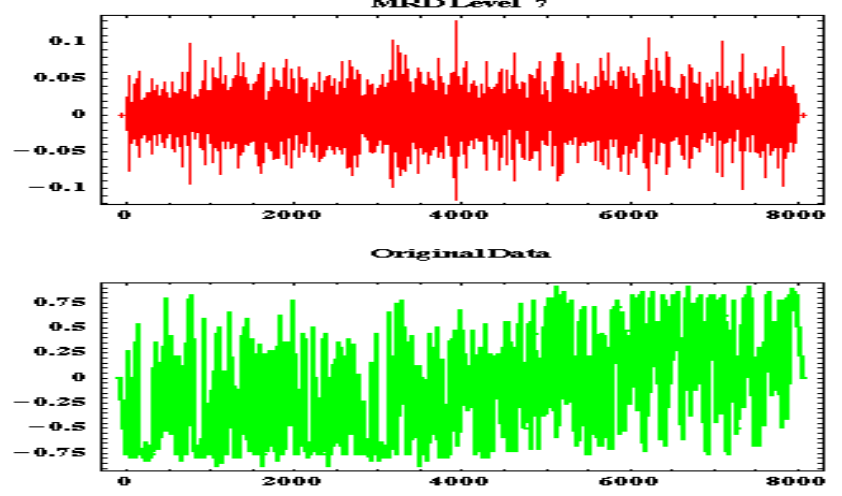

BBA WLTs and RT Mix Cal Tech Pasadena CA 8th IW PCTM 12-11-01 


\section{Reconstruction of the Data Using} the 5 Largest WLT Coefficients

Polymath Research Inc.
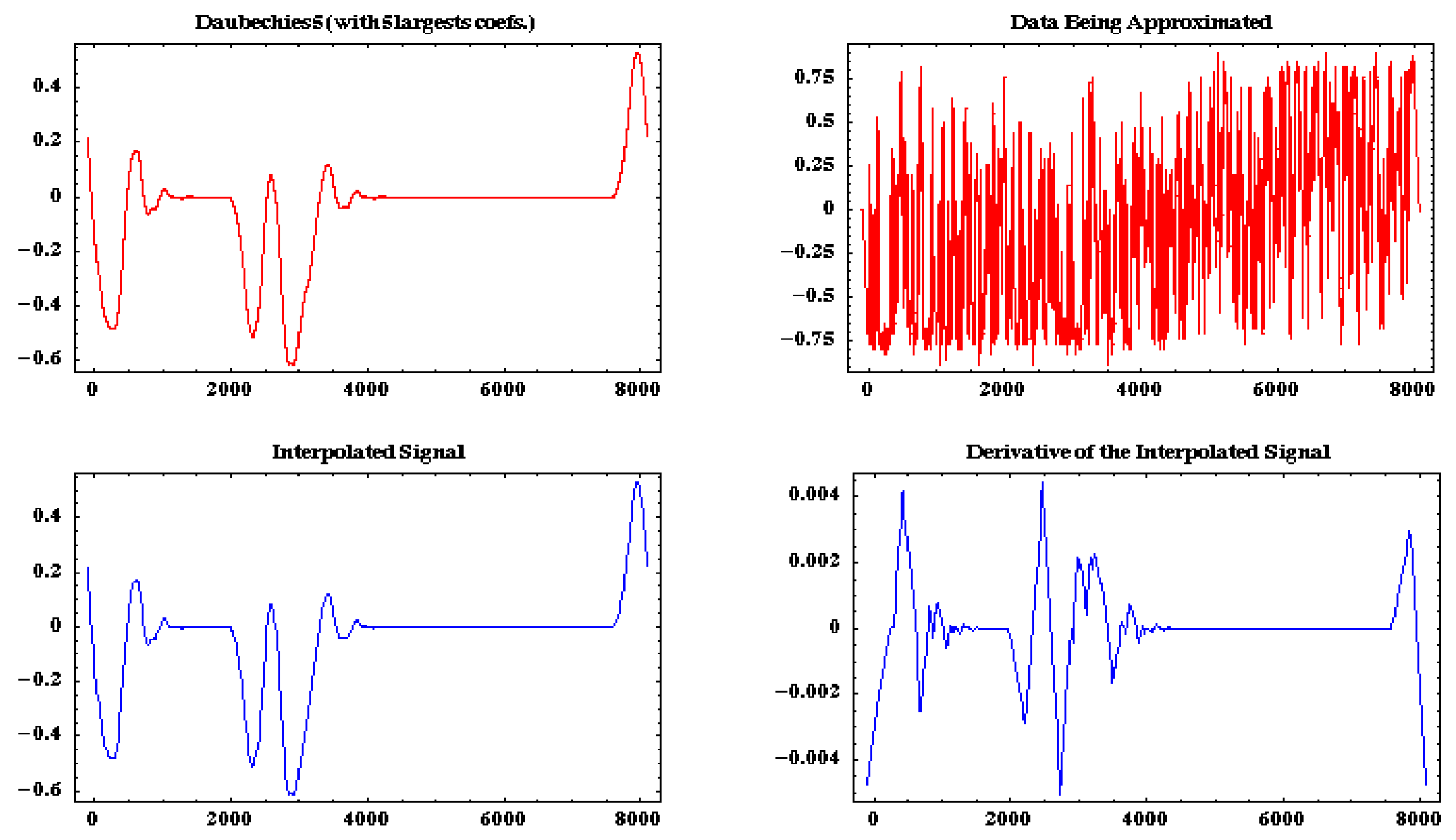


\section{Reconstruction of the Data Using} the 10 Largest WLT Coefficients

Polymath Research Inc.
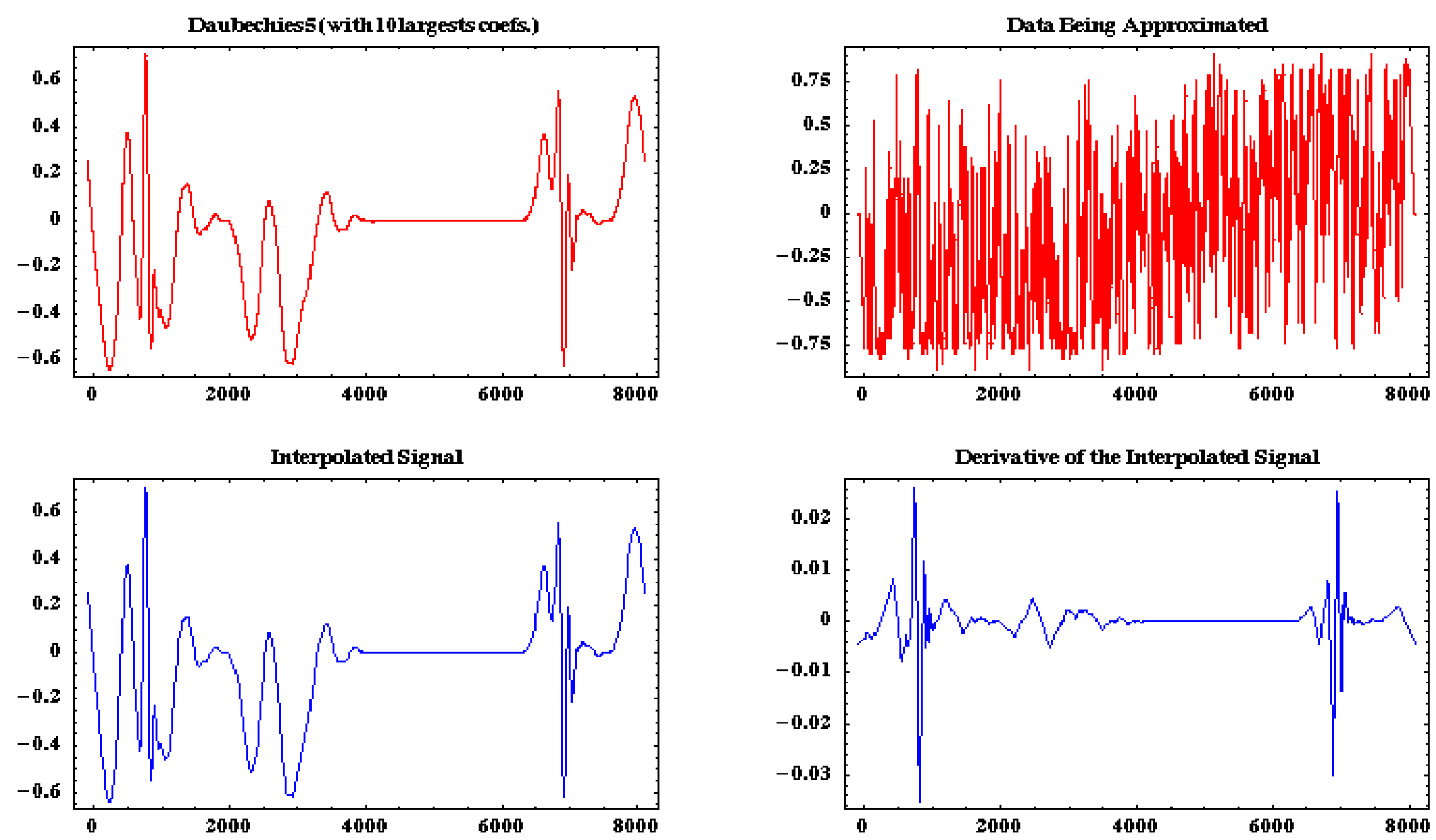


\section{Reconstruction of the Data Using}

\section{the 15 Largest WLT Coefficients}

Polymath

Research Inc.
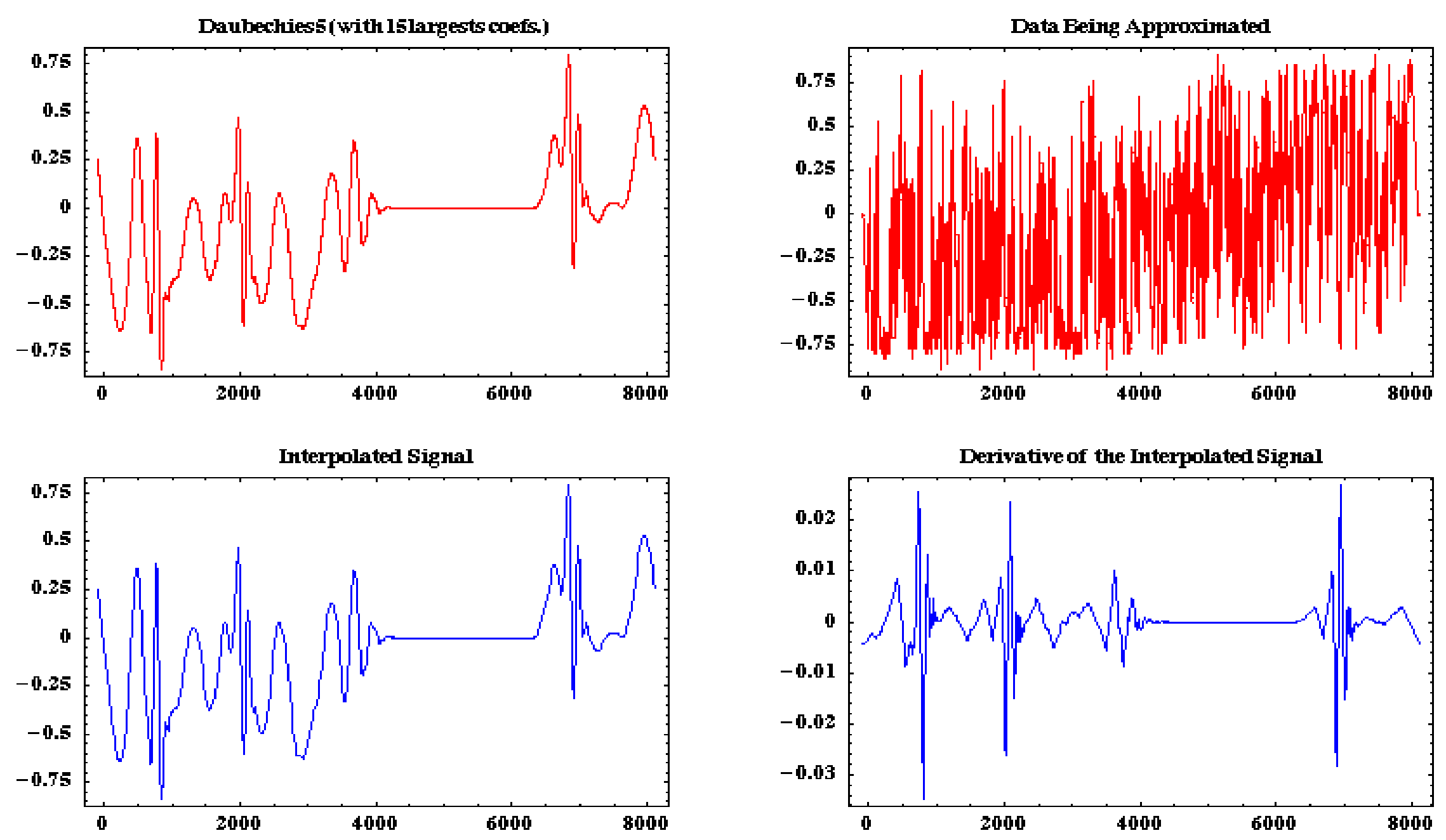


\section{Reconstruction of the Data Using the 20 Largest WLT Coefficients}
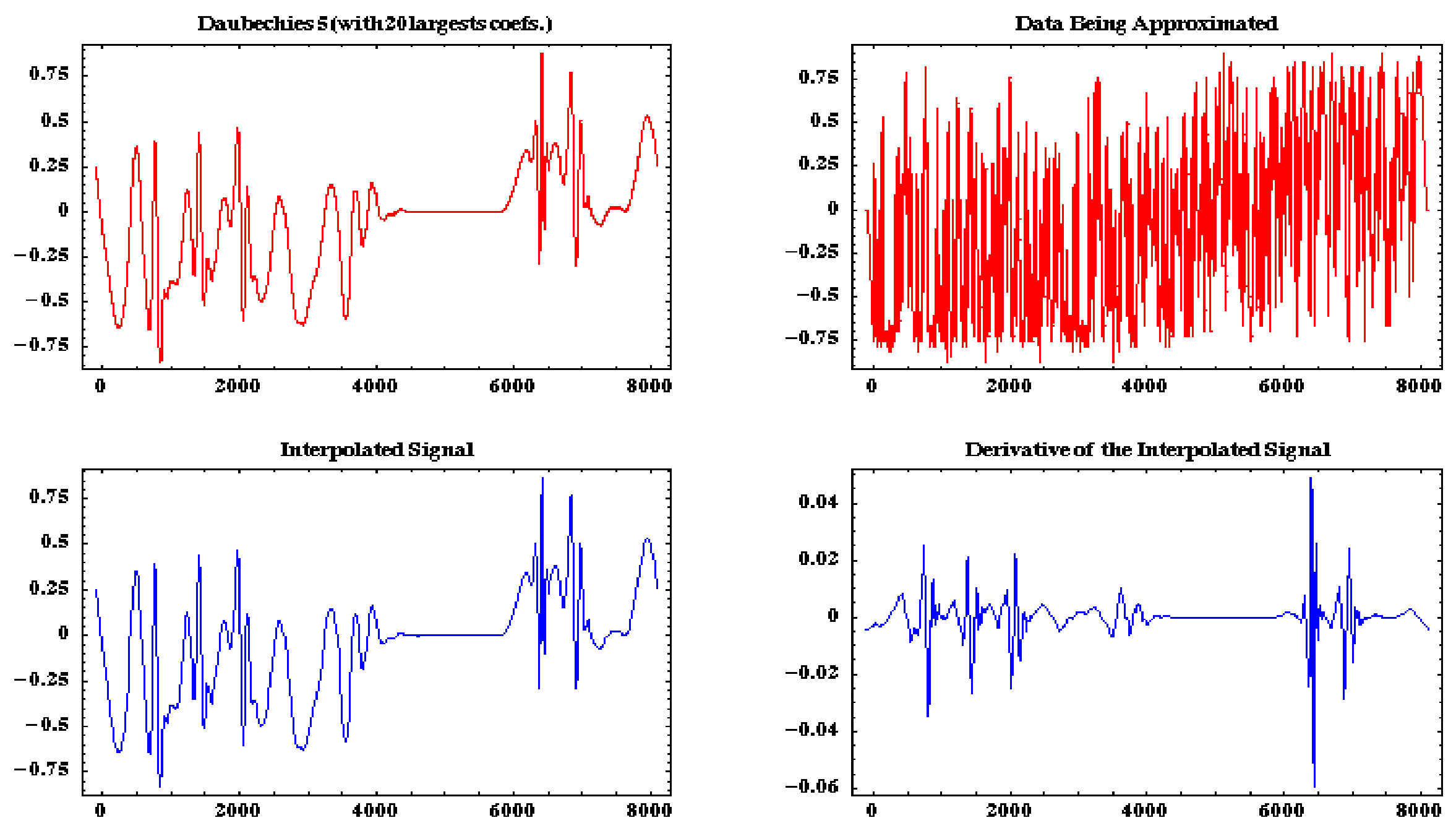


\section{Reconstruction of the Data Using the 30 Largest WLT Coefficients}

Polymath

Research Inc.
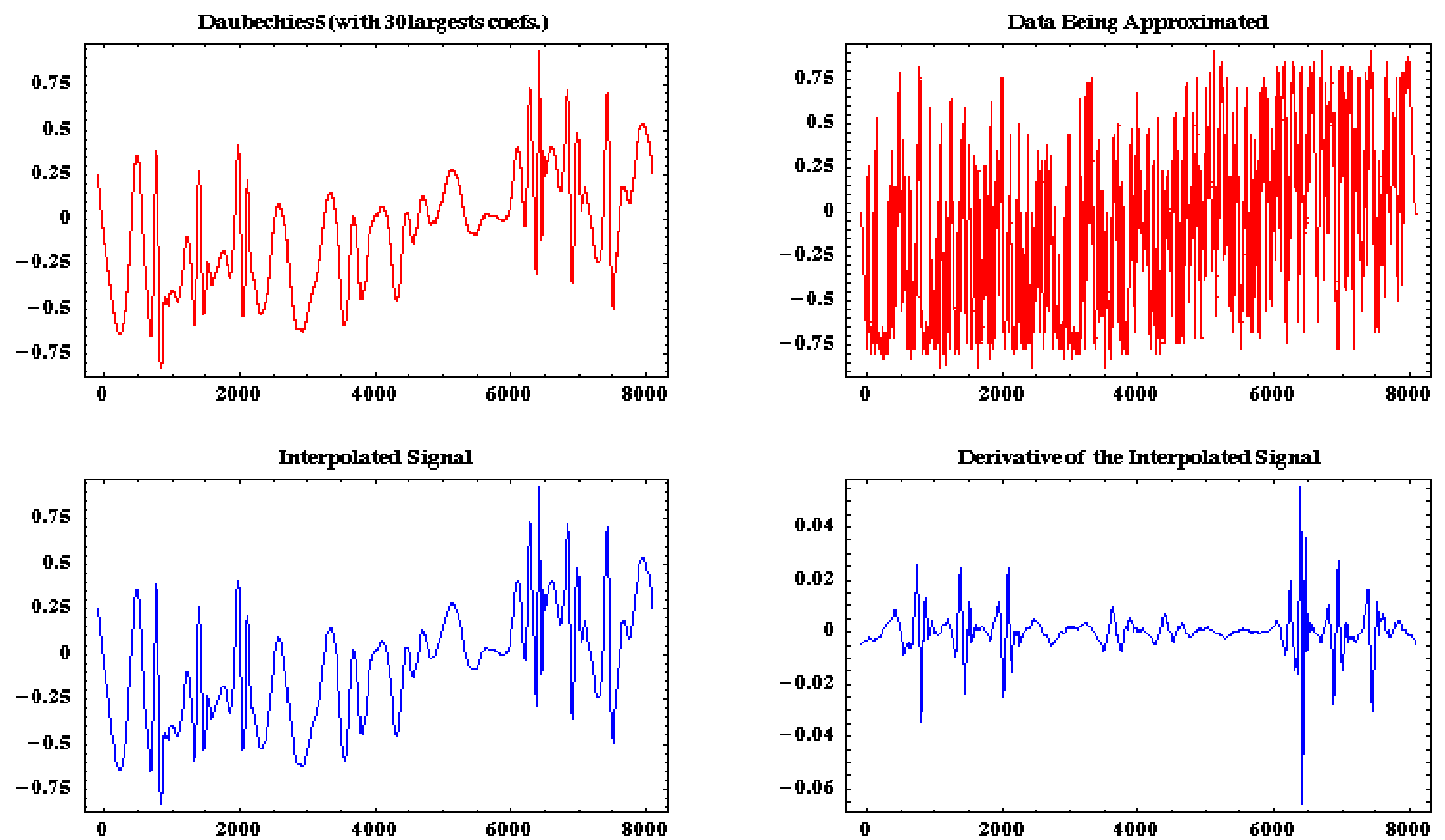


\section{Reconstruction of the Data Using} the 50 Largest WLT Coefficients
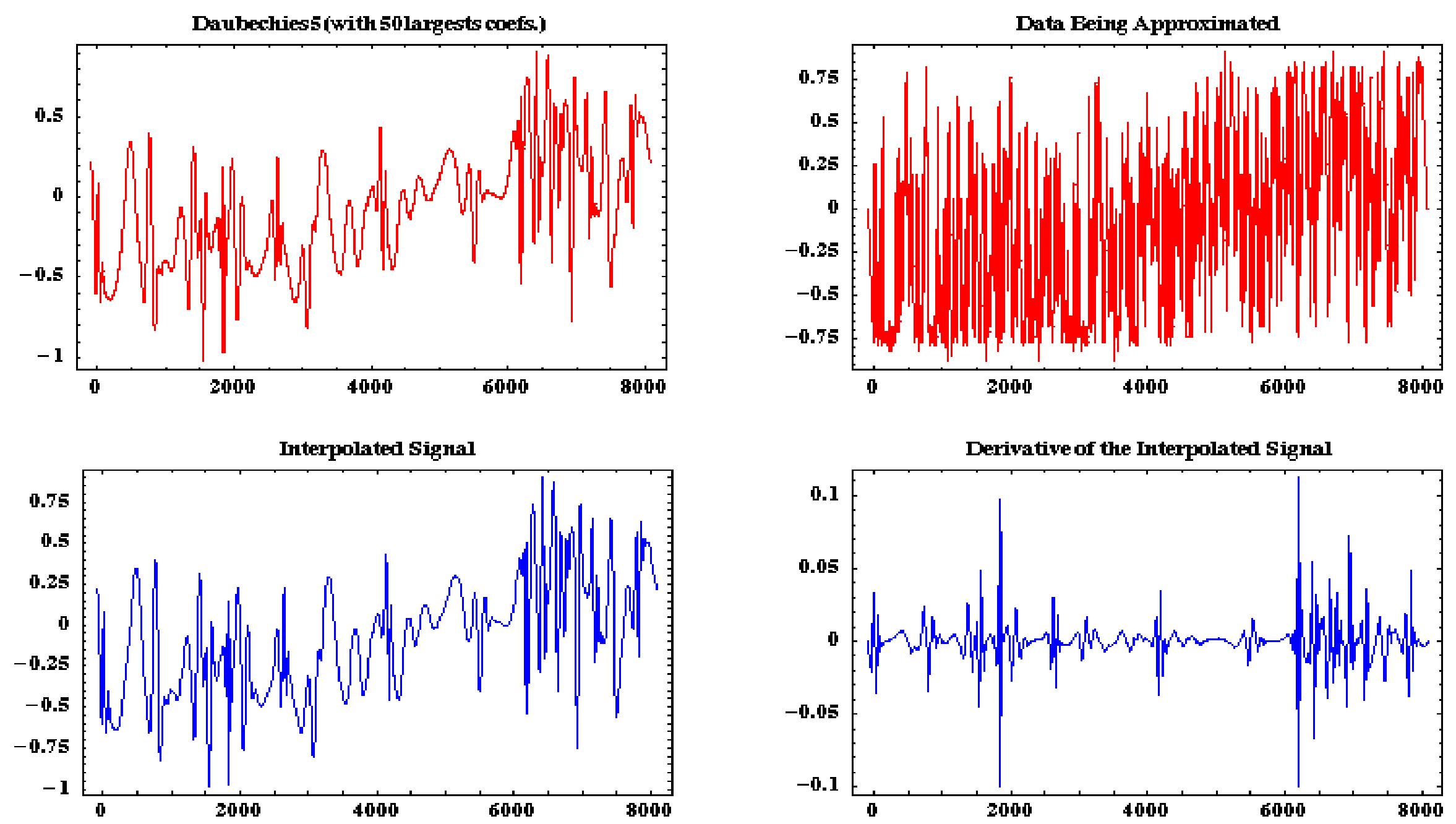


\section{Reconstruction of the Data Using Polymath 100 Largest WLT Coefficients Research Inc.}
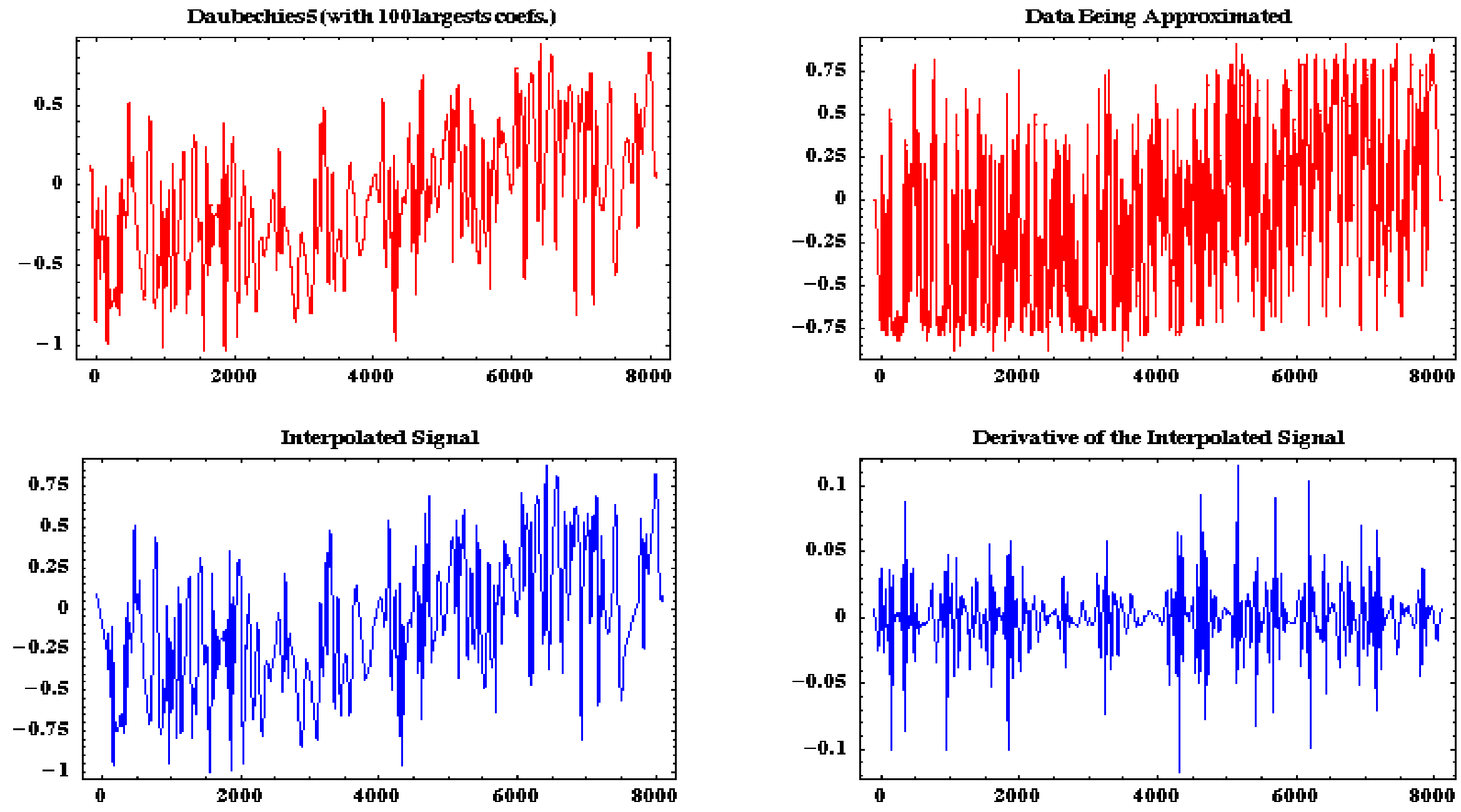


\section{Reconstruction of the Data Using Polymath 200 Largest WLT Coefficients
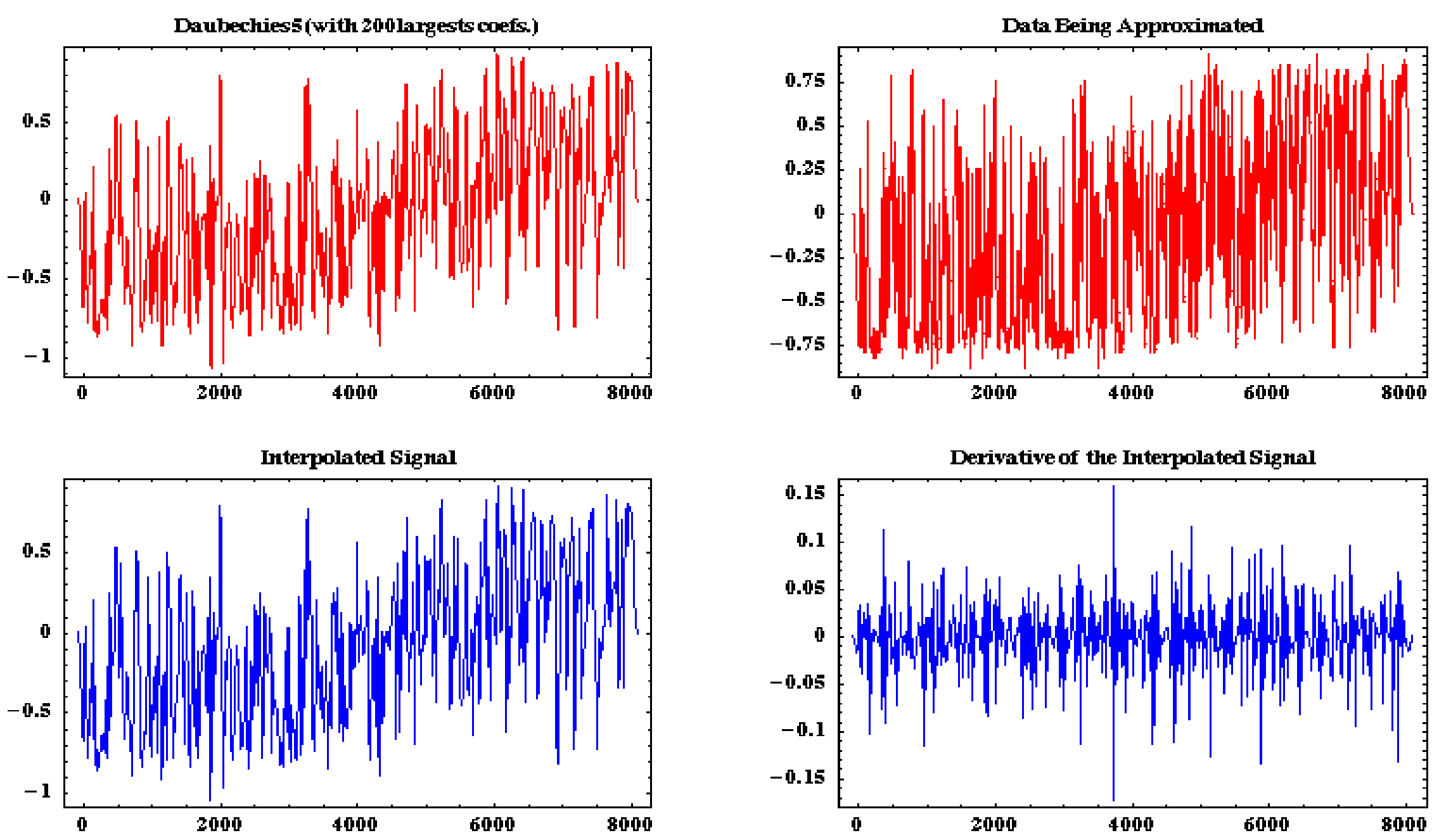


\section{Reconstruction of the Data Using Polymath 400 Largest WLT Coefficients Research Inc.}
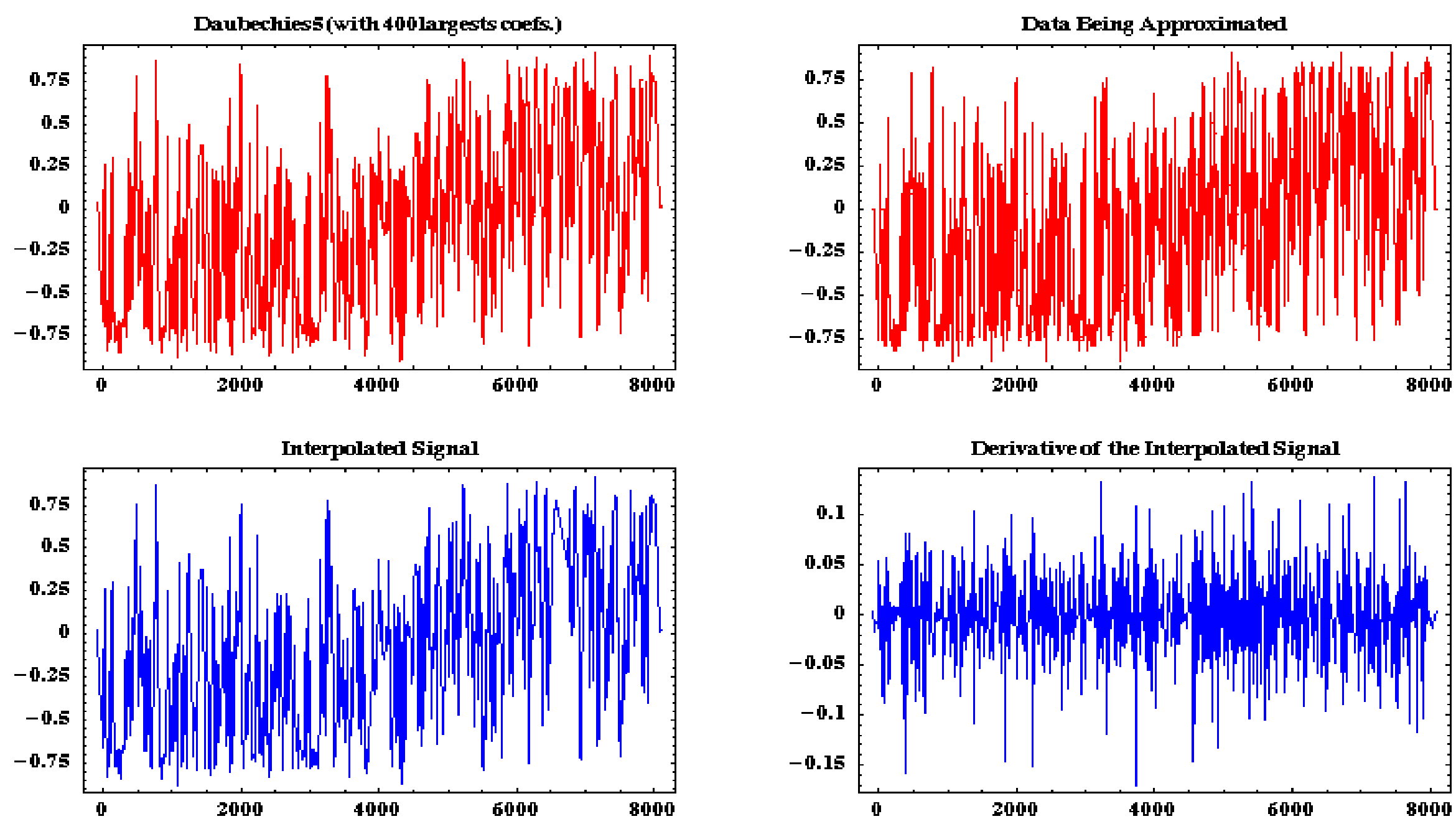


\section{Reconstruction of the Data Using}

\section{Up to 0.75 times the Largest WLT Coefficient}
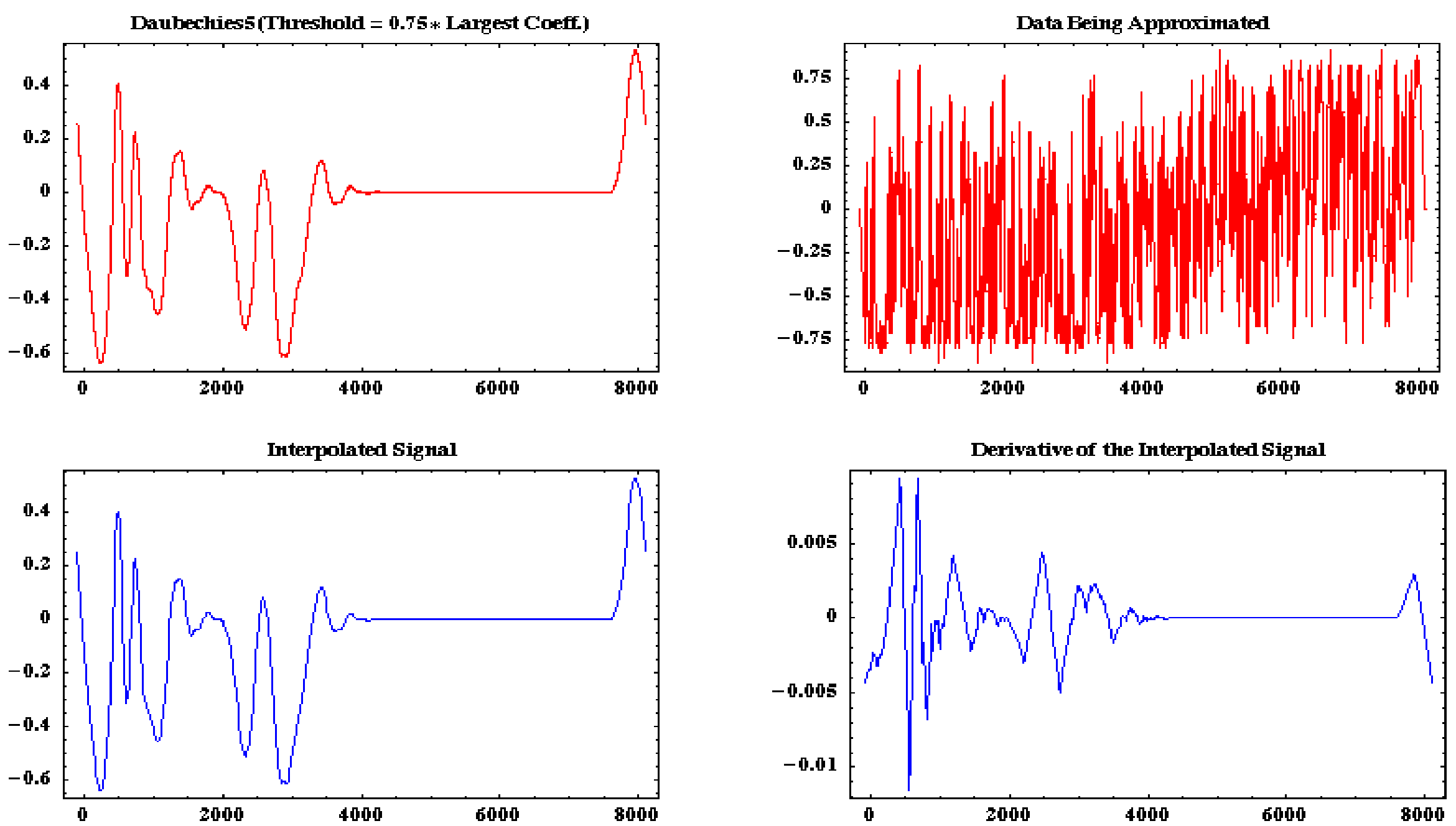


\section{Reconstruction of the Data Using}

Up to 0.5 times the Largest

WLT Coefficient
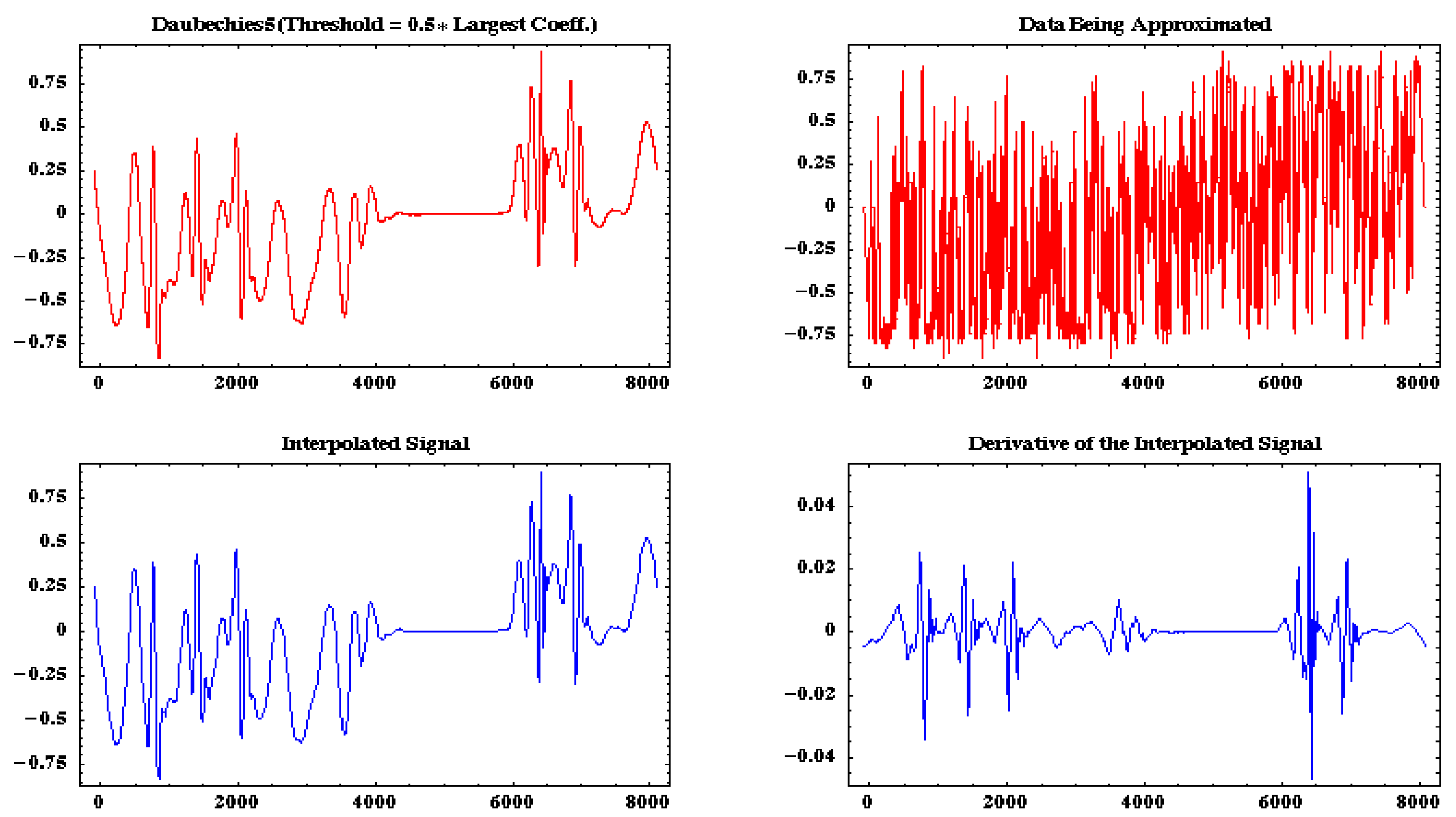


\section{Reconstruction of the Data Using}

\section{Up to 0.25 times the Largest WLT Coefficient}
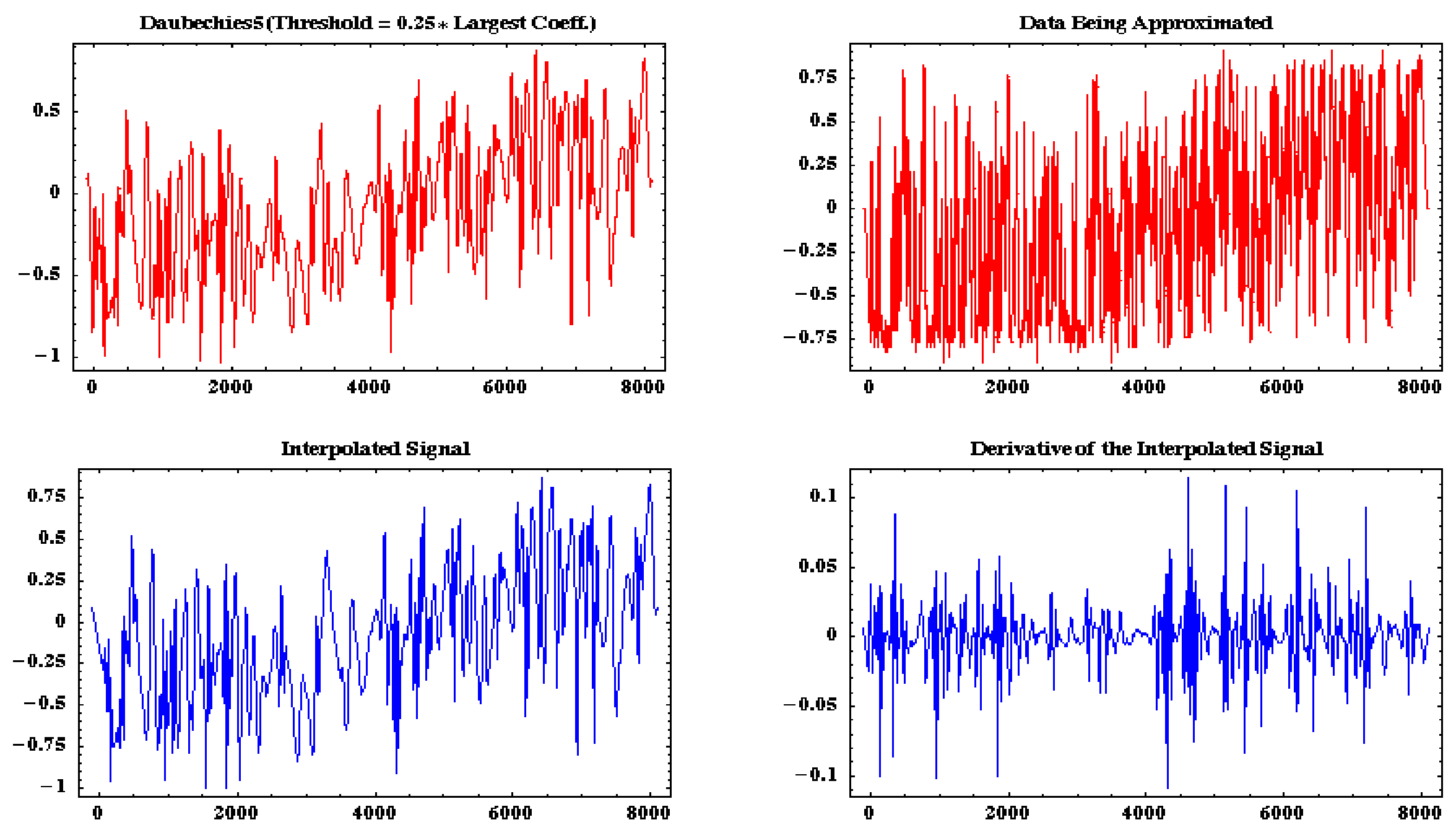


\section{Reconstruction of the Data Using}

\section{Up to 0.1 times the Largest}

WLT Coefficient
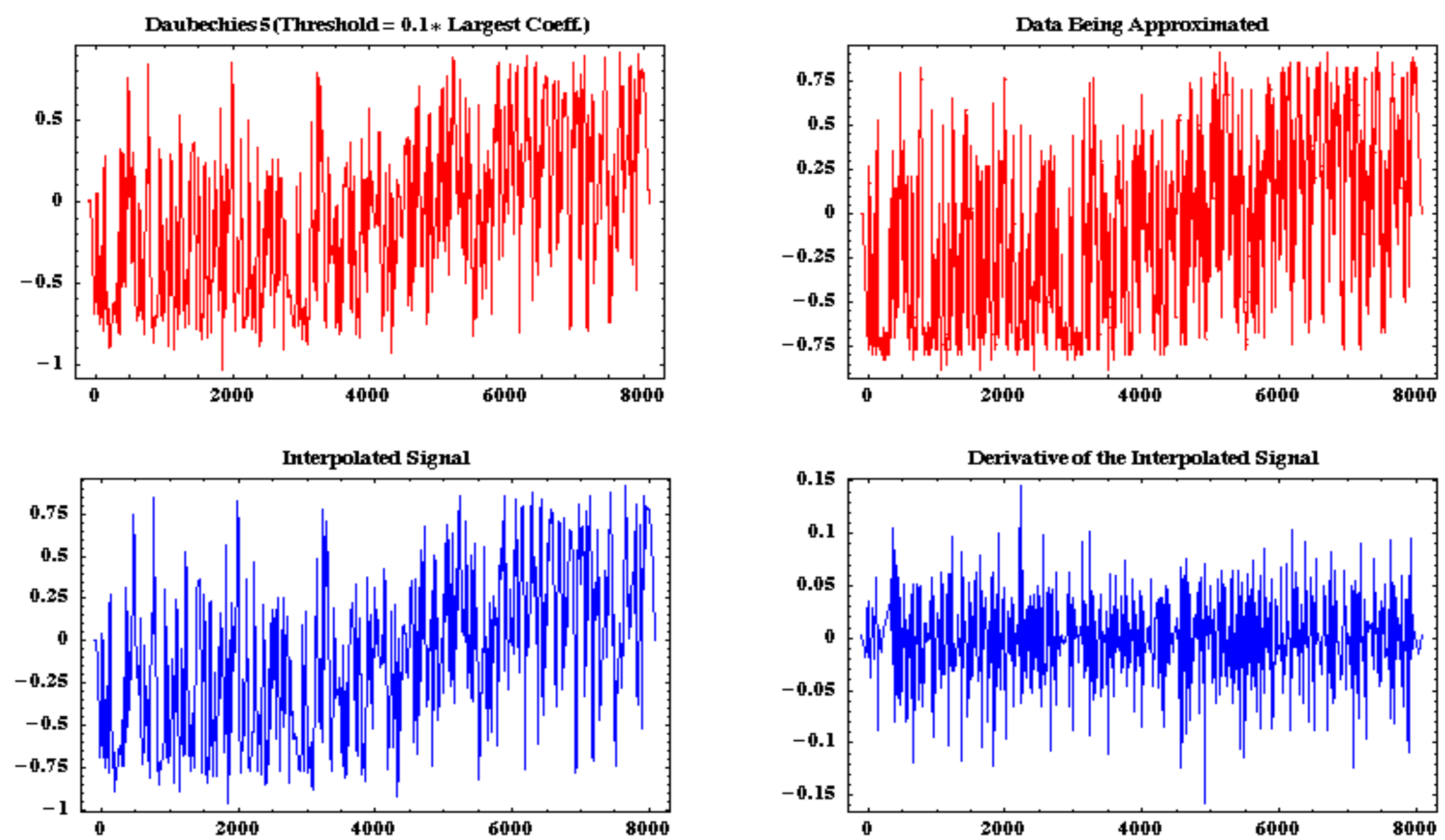


\section{Reconstruction of the Data Using \\ Up to 0.05 times the Largest WLT Coefficient}

Polymath Research Inc.
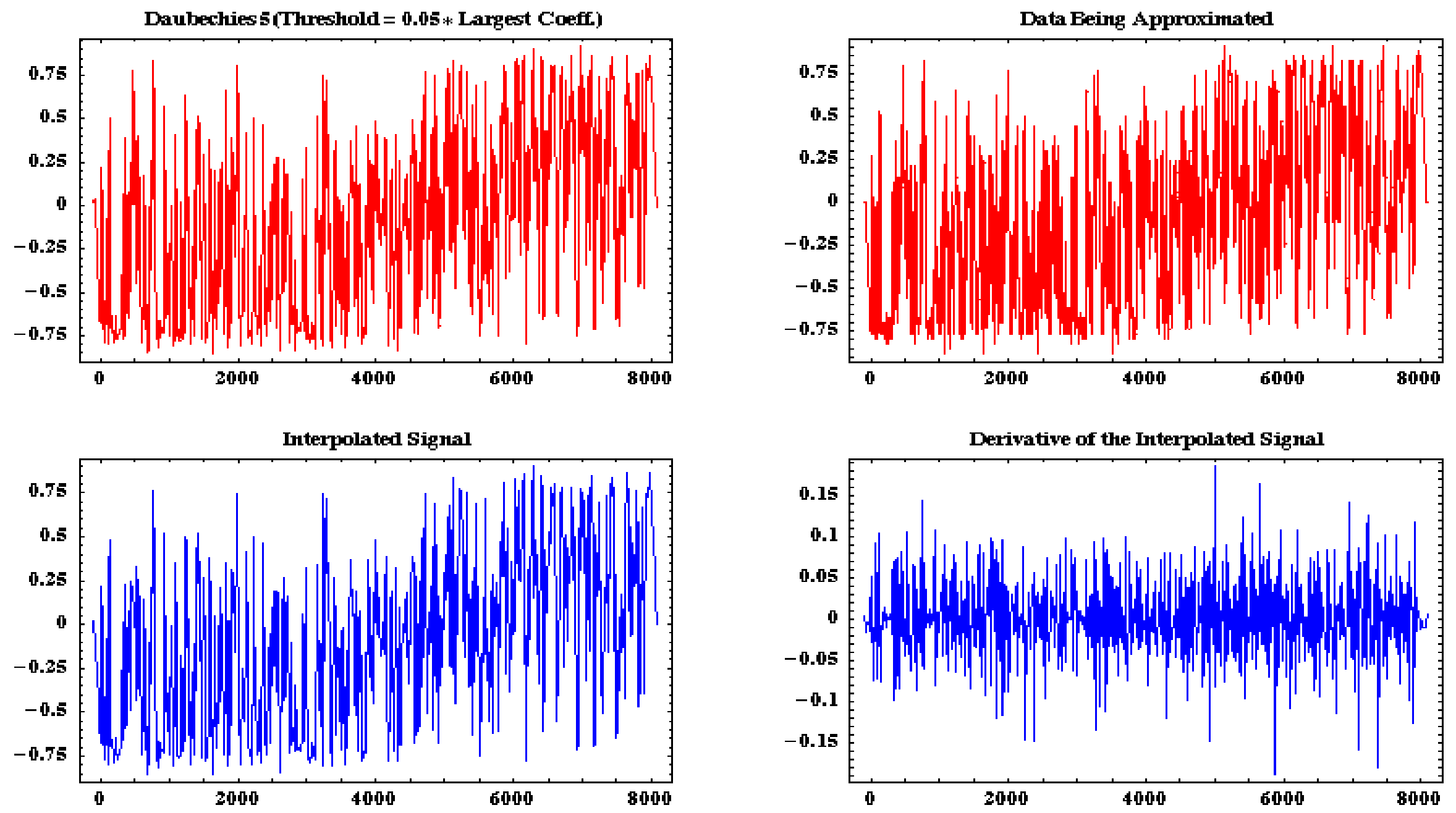


\section{Reconstruction of the Data}

\section{Using the First (of 10) Level of the MRD}
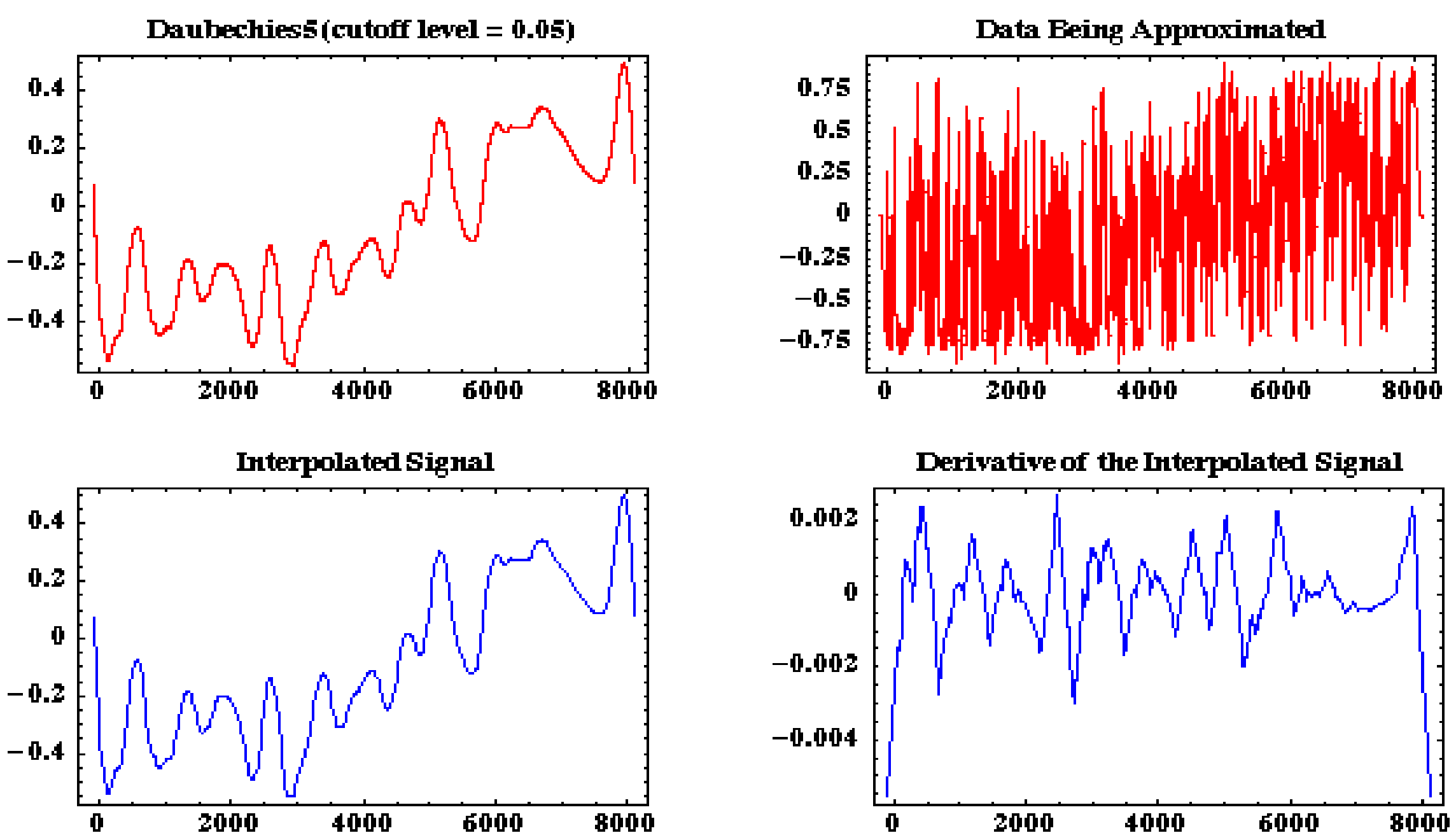


\section{Reconstruction of the Data}

\section{Using the First Two (of 10) Levels of the MRD}

Polymath Research Inc.
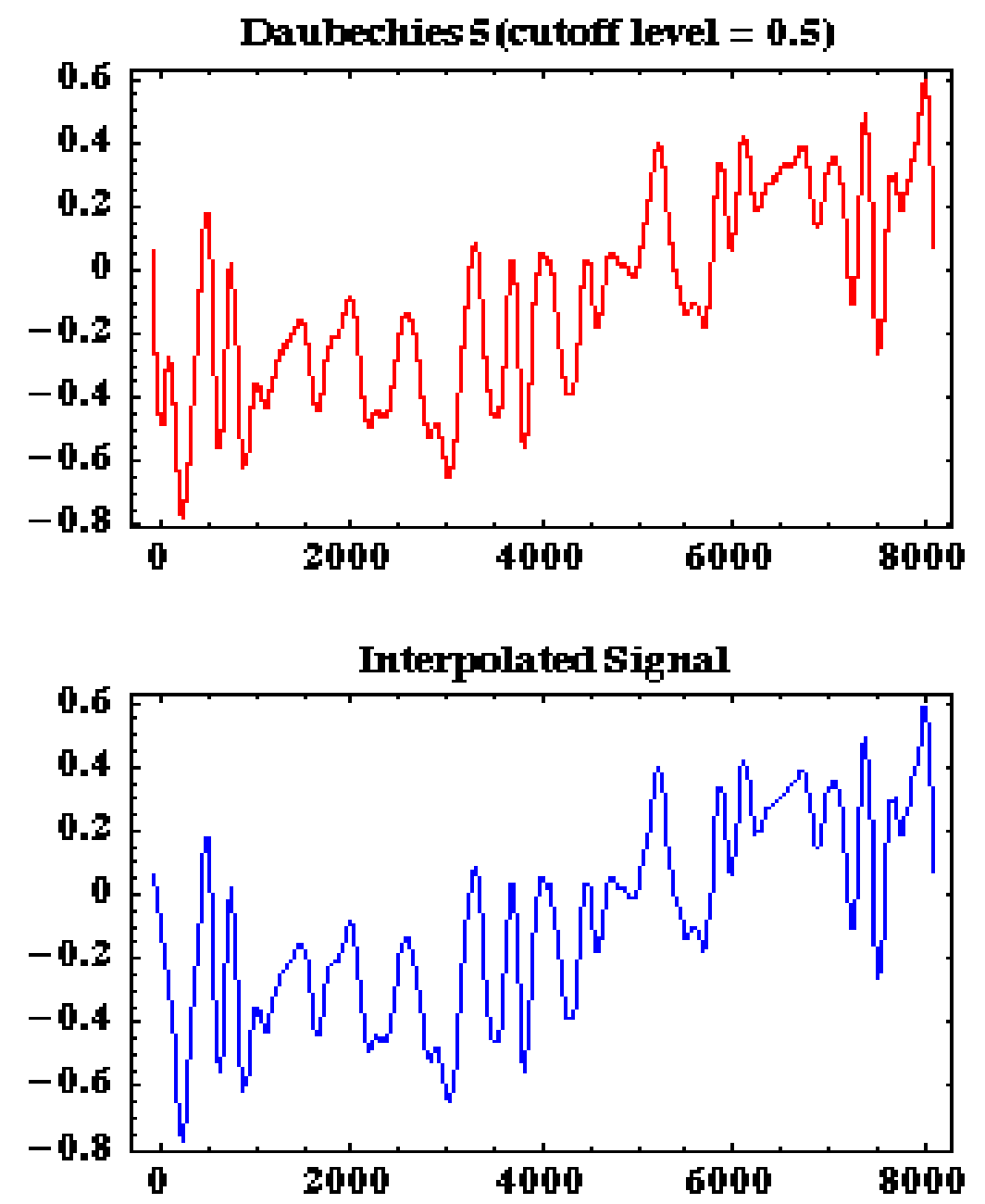

Data Eeing Approximated
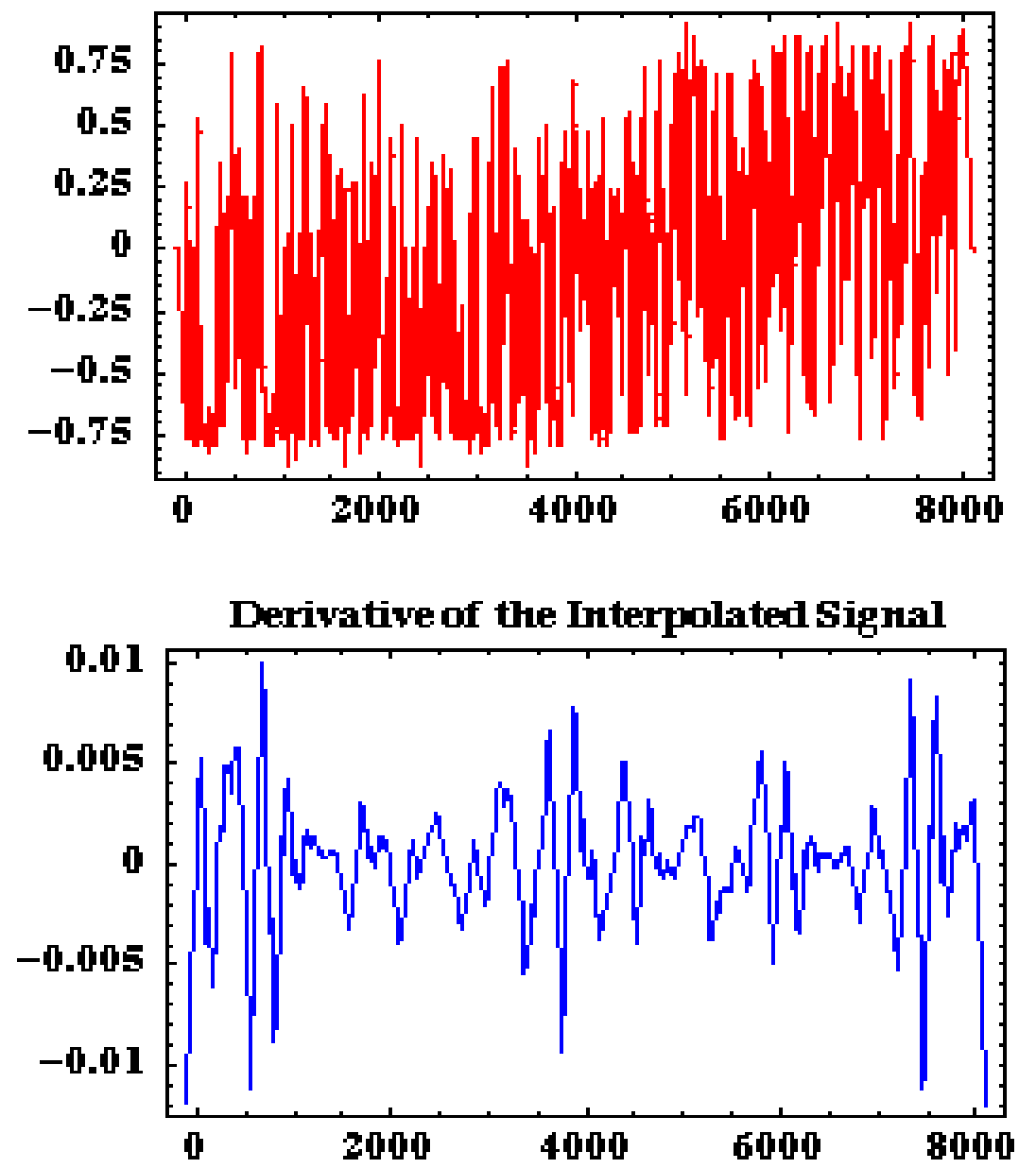


\section{Reconstruction of the Data Using the First Three (of 10) Levels of}

the MRD
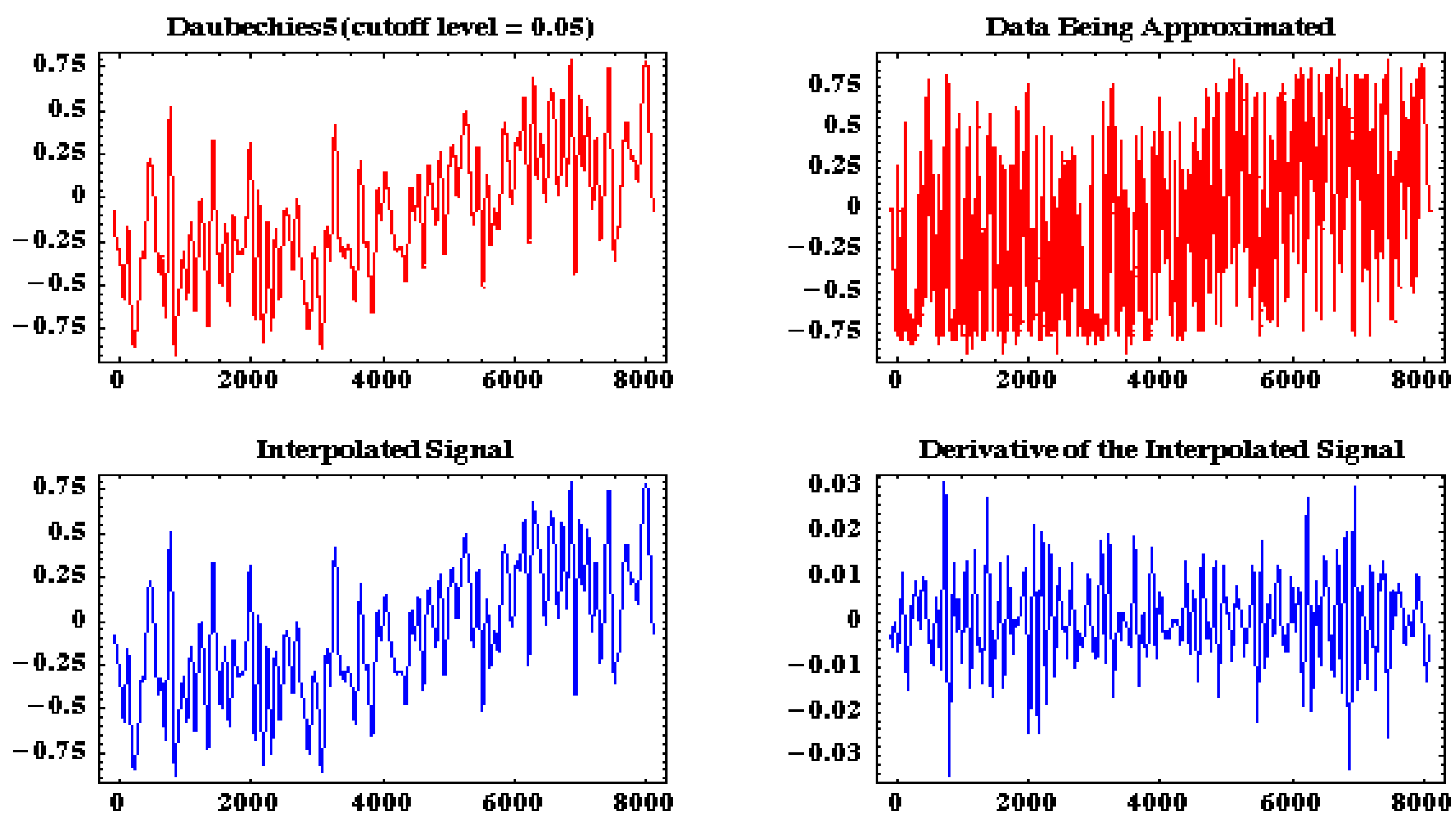


\section{Reconstruction of the Data}

\section{Using the First Four (of 10) Levels of the MRD}
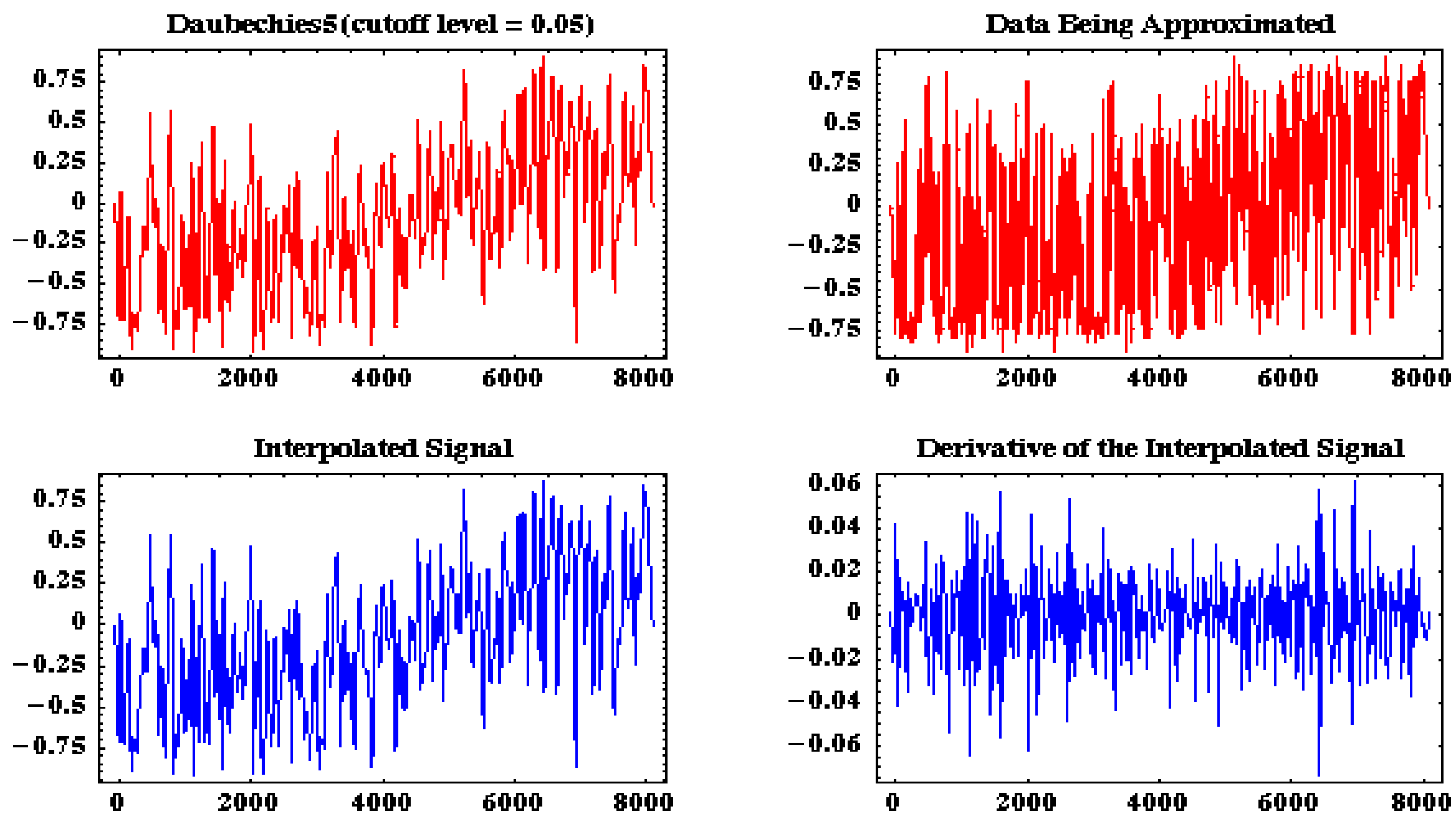


\section{Reconstruction of the Data}

\section{Using the First Five (of 10) Levels of the MRD}
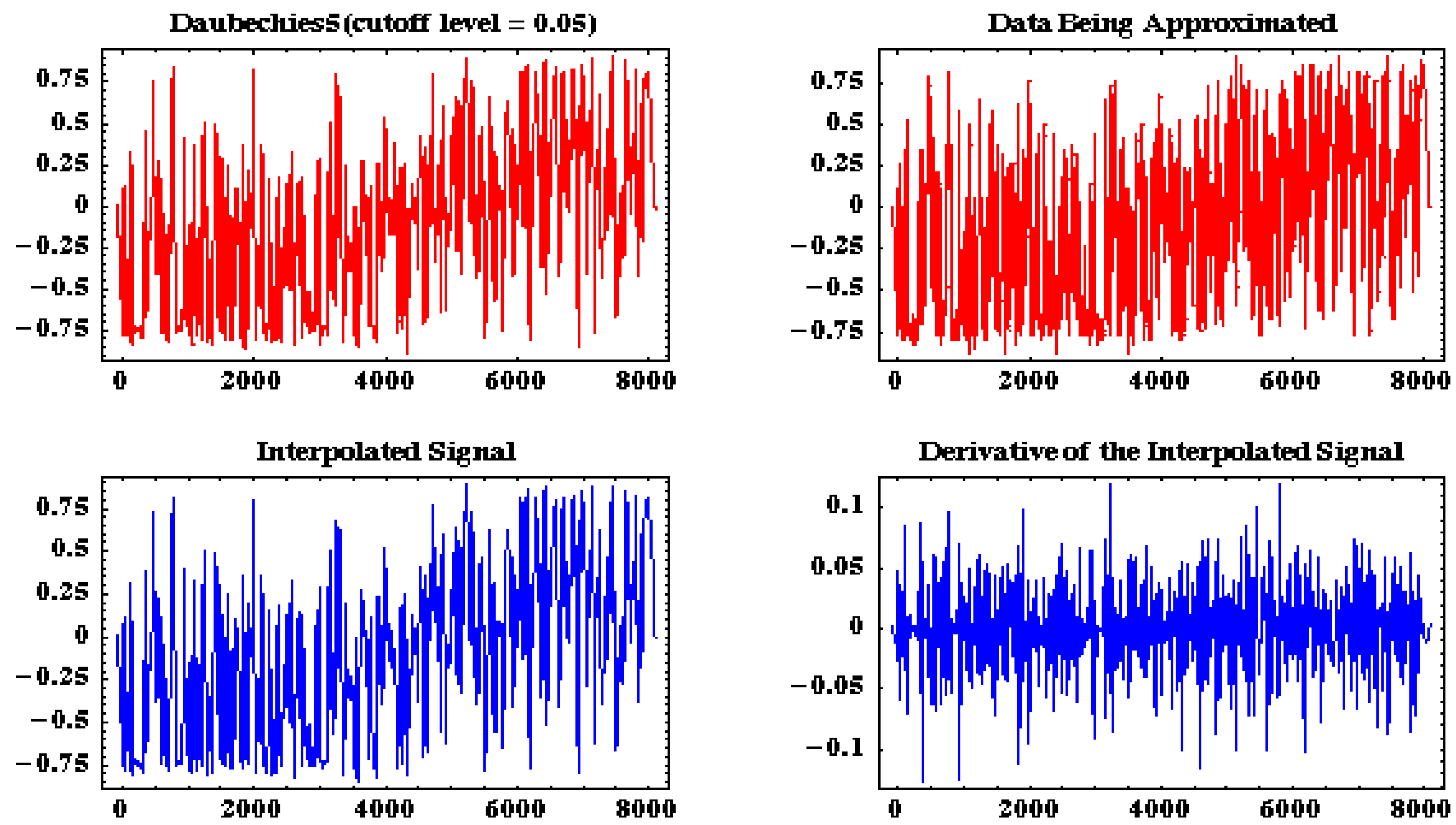


\section{Reconstruction of the Data}

\section{Using the First Six (of 10) \\ Levels of the MRD}
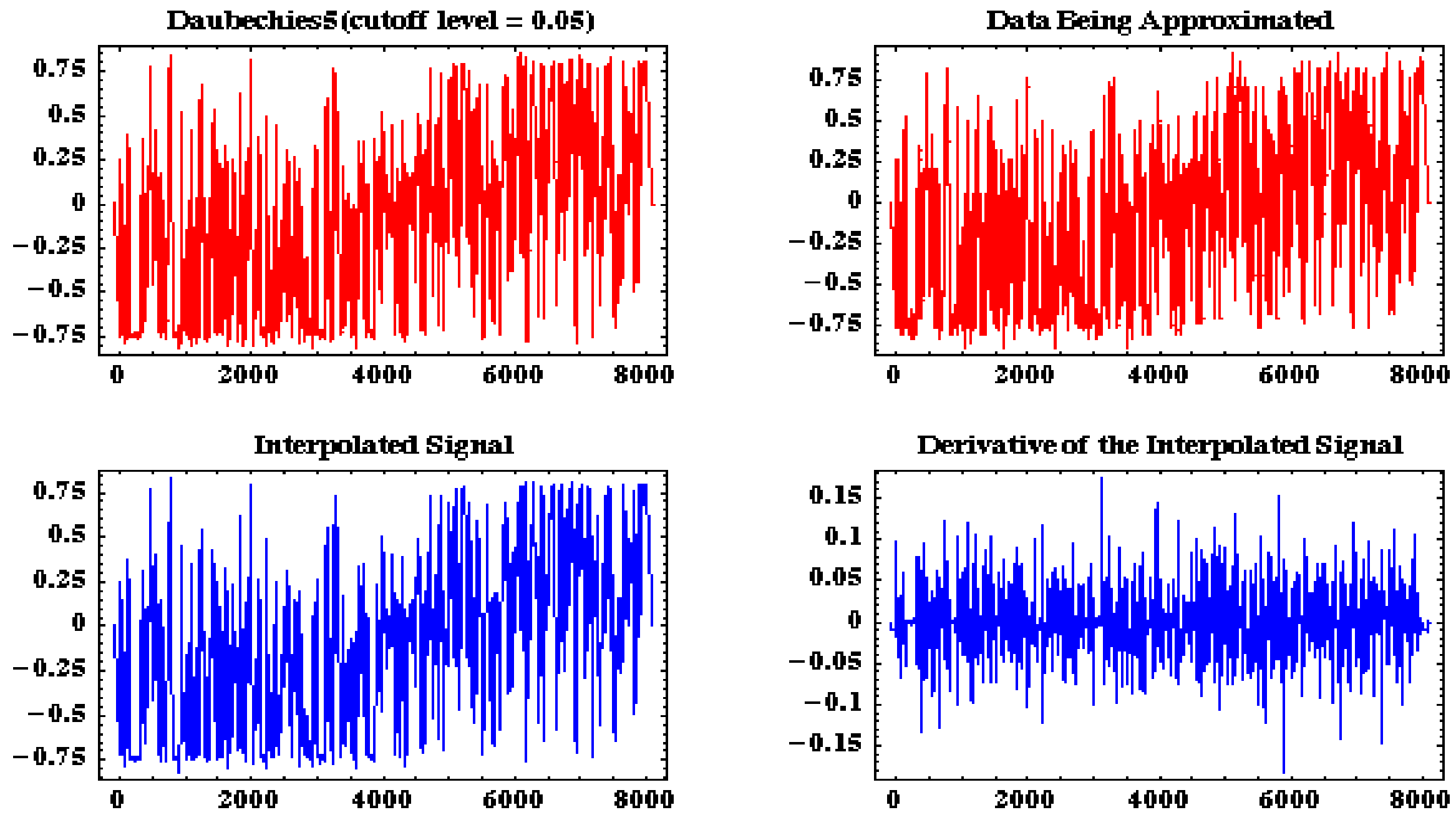


\section{Reconstruction of the Data}

\section{Using the First Seven (of 10) Levels of the MRD}
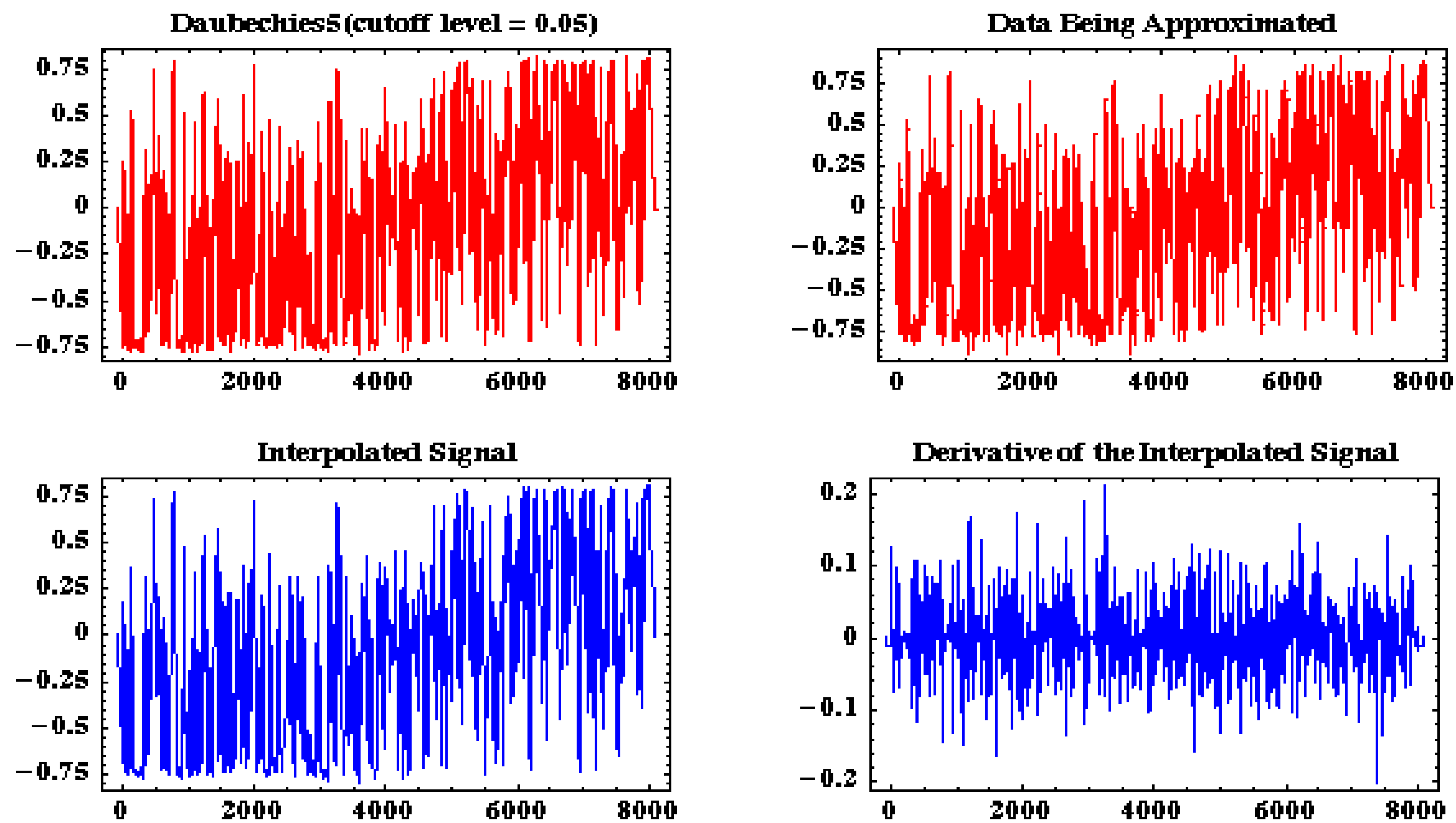


\section{Conclusions on Raw RT Mix Data Analysis Using DWT}

- Compression of around a factor of 20 seems likely with full data set.

- Will see what low pass filtering will do to initial data and its subsequent WLT analysis.

- Looks like $25 \%$ of the largest coefficients are enough to reconstruct the clean parts of the data.

- We should compare different stages of evolution of RT Mix in terms of their optimum WLT representations.

- Significant dynamical degrees of freedom vs insignificant ones which vary more slowly or not at all or randomly might be obtainable if we keep at it! 


\section{Low Pass Filtered RT Mix Data}

Polymath

Research Inc.

n

Filtered Data

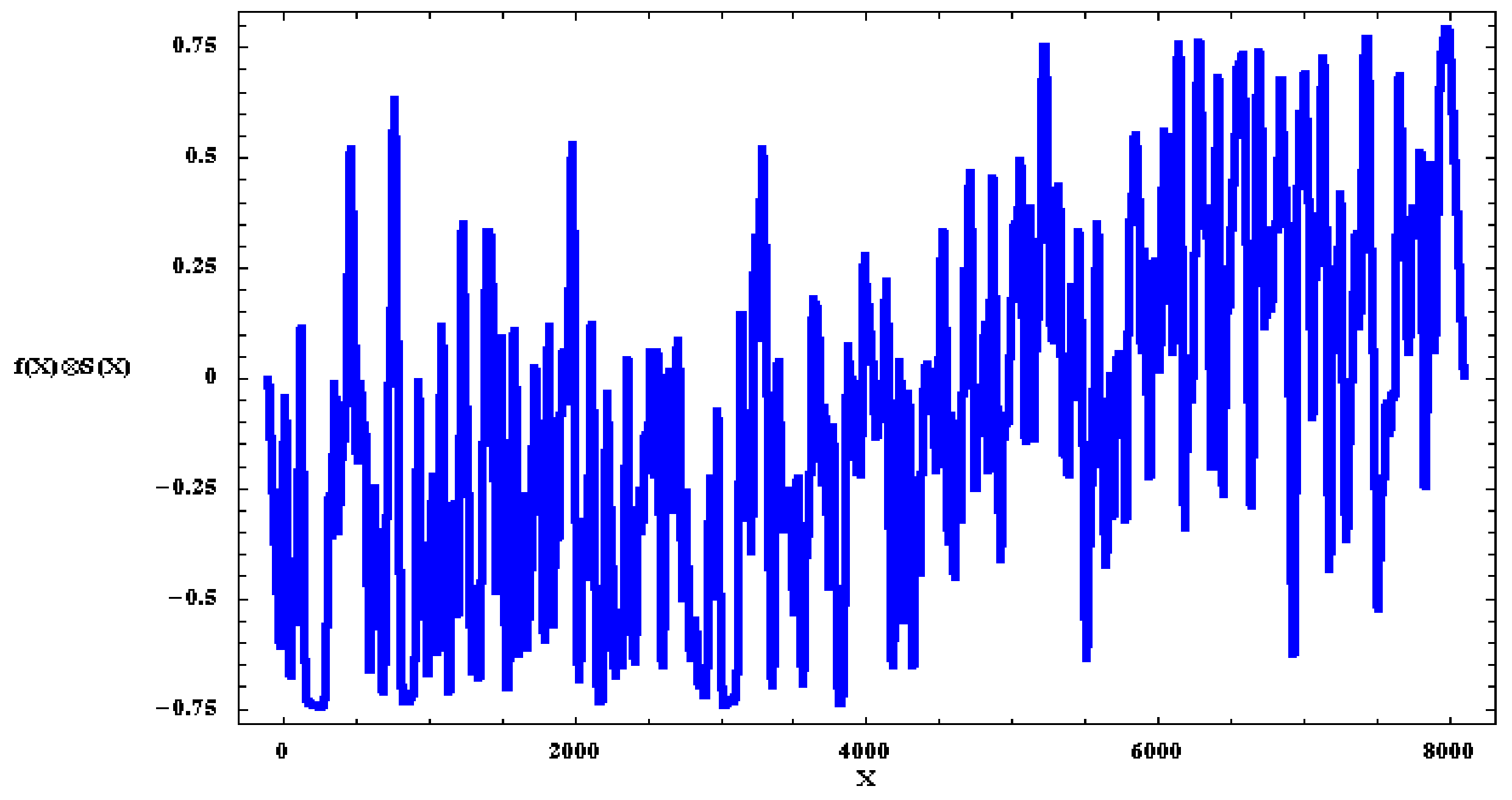




\section{The Filtering Has This Form and Effect in k-Space}
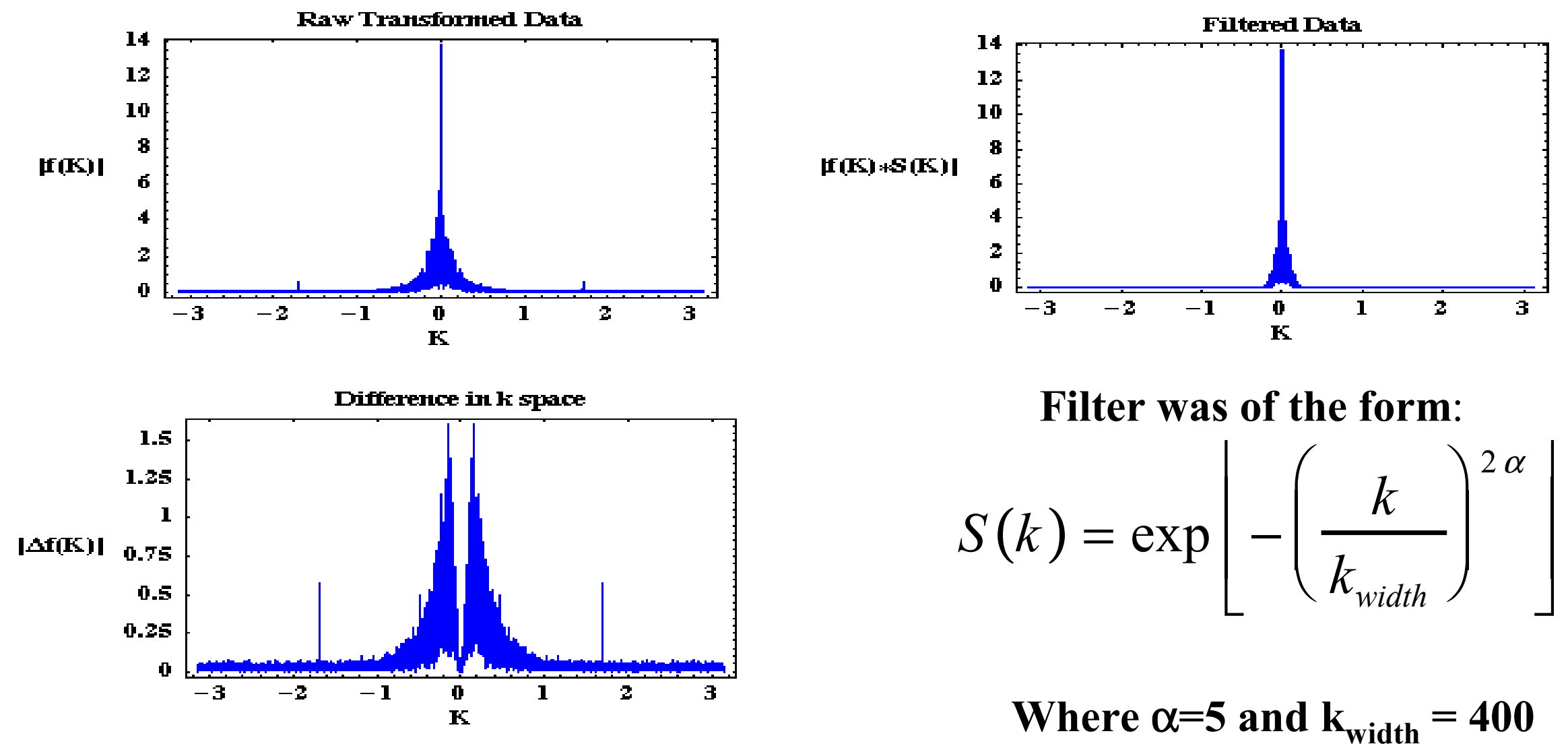

Filter was of the form:

$$
\begin{gathered}
S(k)=\exp \left\lfloor-\left(\frac{k}{k_{\text {width }}}\right)^{2 \alpha}\right\rfloor \\
\text { Where } \alpha=5 \text { and } \mathbf{k}_{\text {width }}=\mathbf{4 0 0}
\end{gathered}
$$




\section{MRDs of the LP Filtered RT Mix}

Data in 6 Different Daubechies

WLT Bases
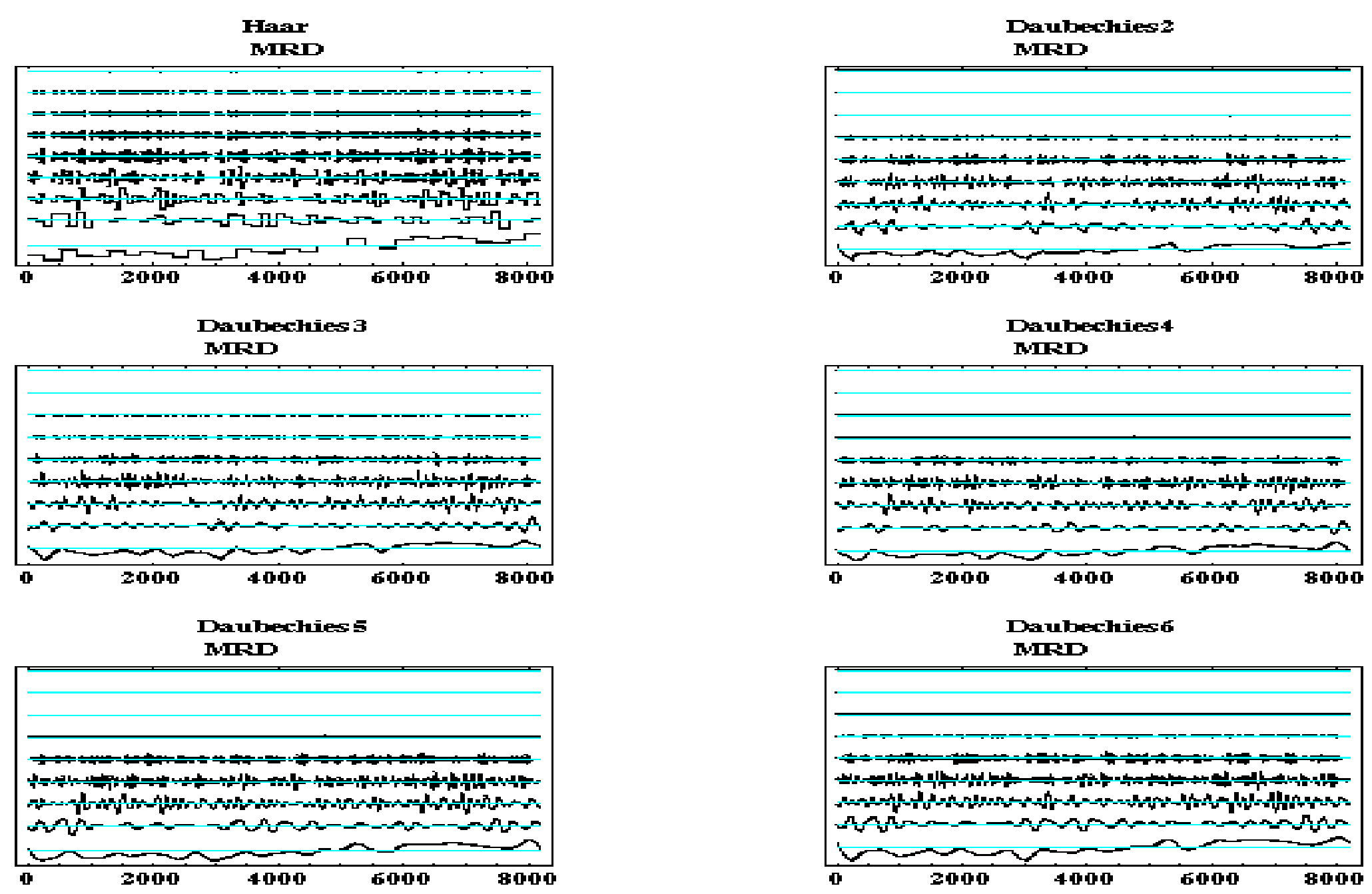


\section{Decay Rate of Largest Coefficient vs Number of Coeffs Kept in LPF RT Mix Data}
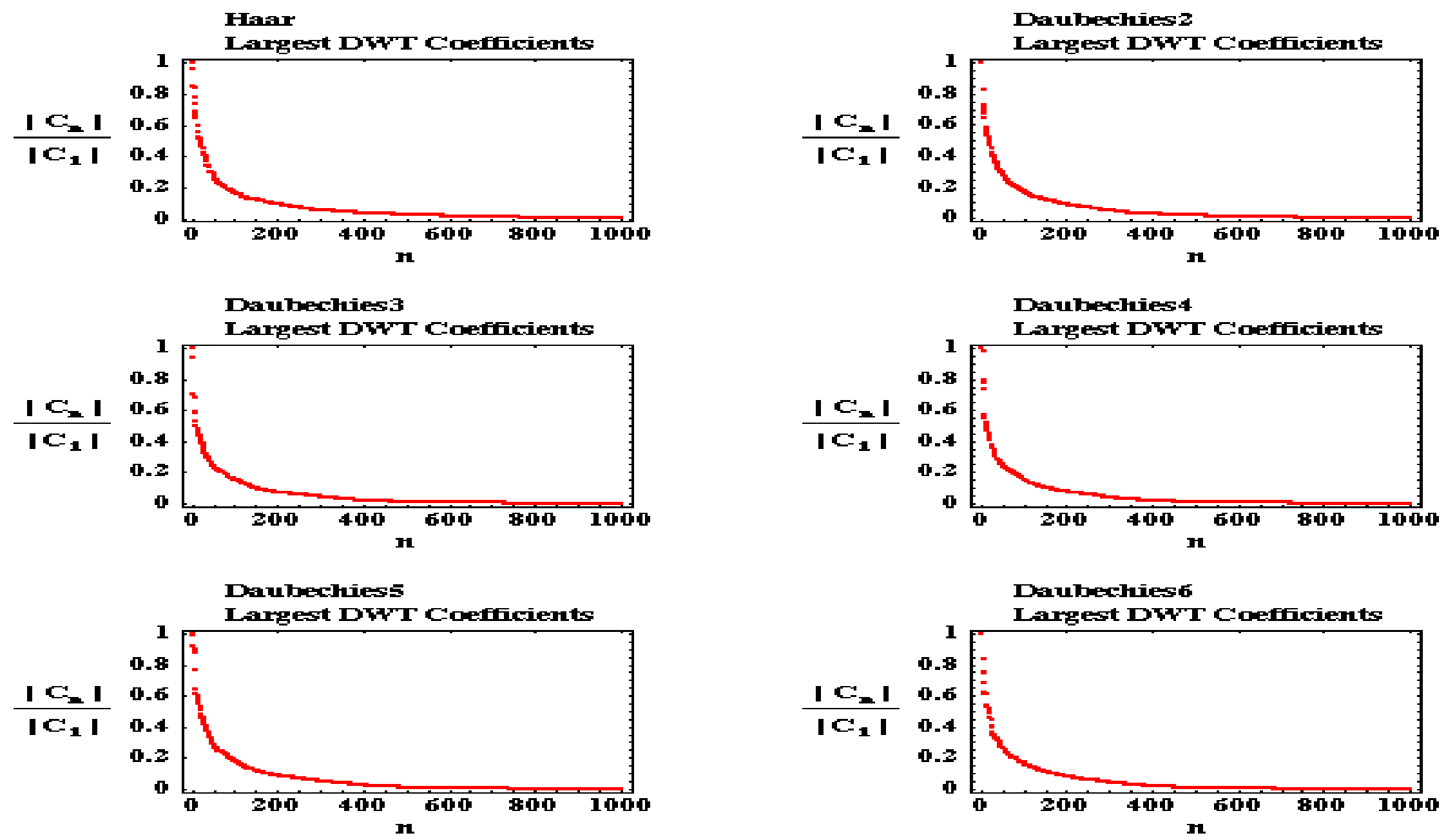


\section{Energy Accumulation Rate in Coefficient Space vs \# of WLTs Kept for LPF RT Mix Data}
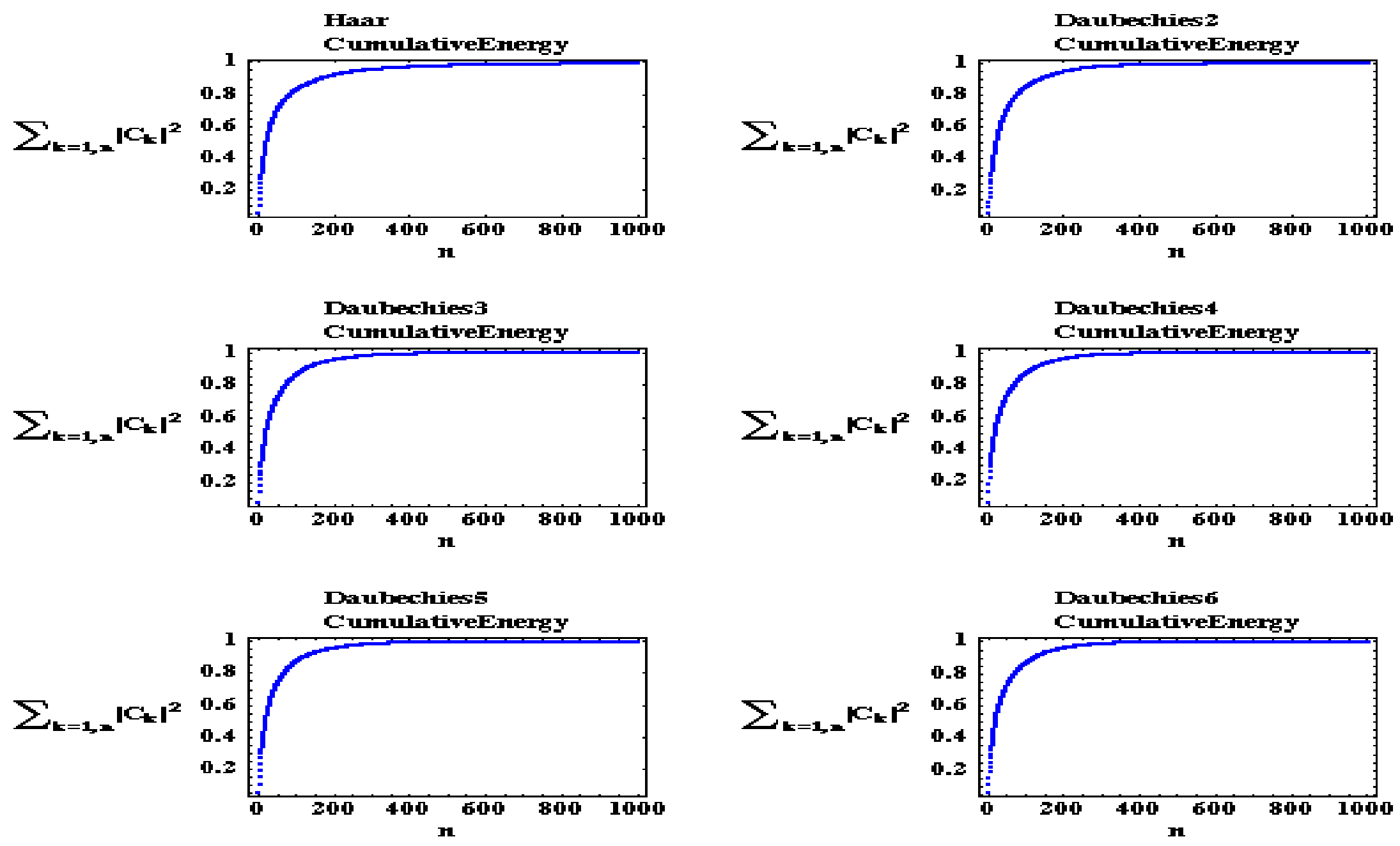


\section{Scaleograms: Waveleters Preferred}

Way of Judging Tiling in ScaleTranslation Space for LPF RT Mix
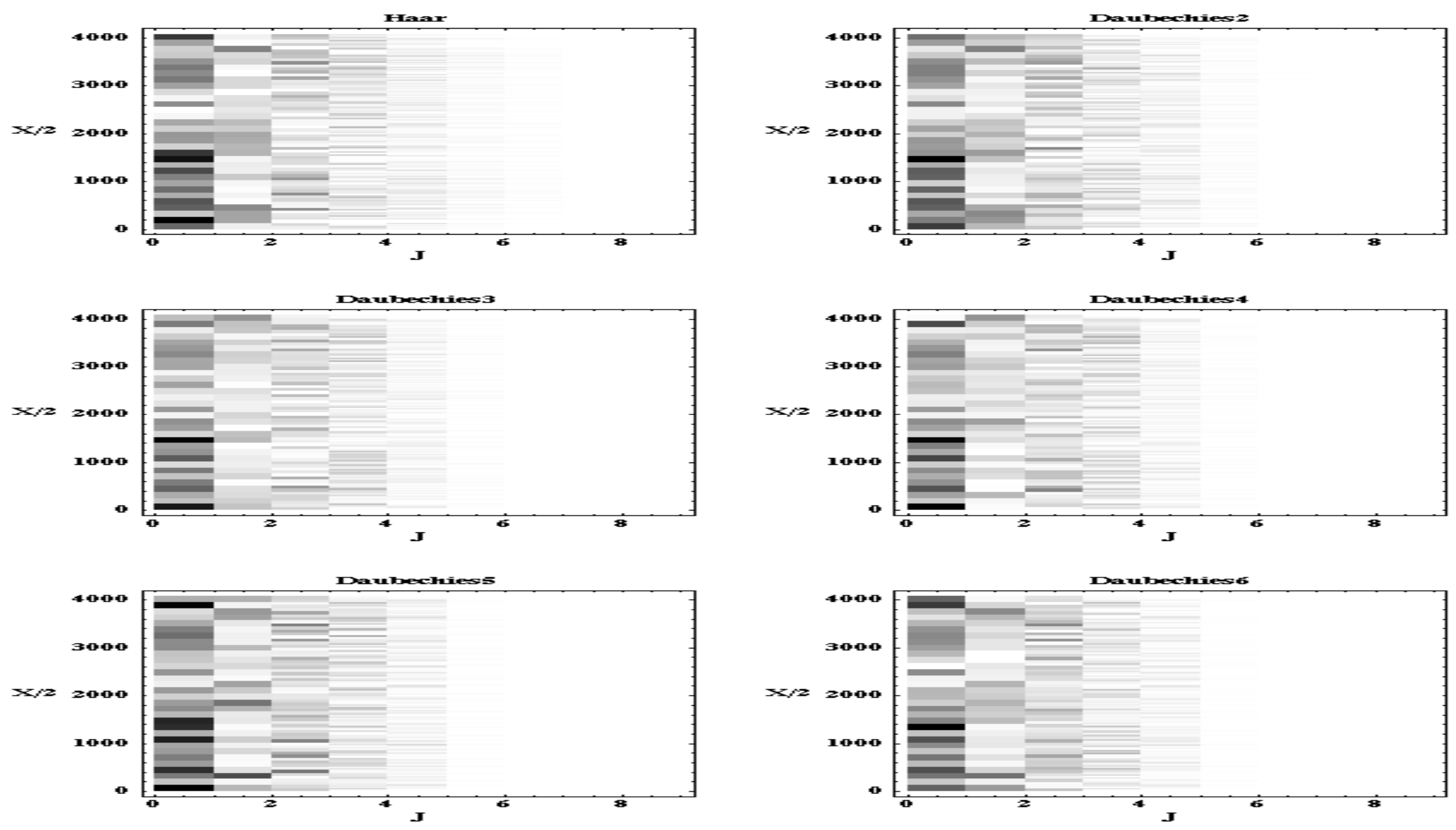


\section{Least Square Error Incurred By}

\section{Truncating the WLT Series at N of its Largest Coeffs LPF RT Mix Data}
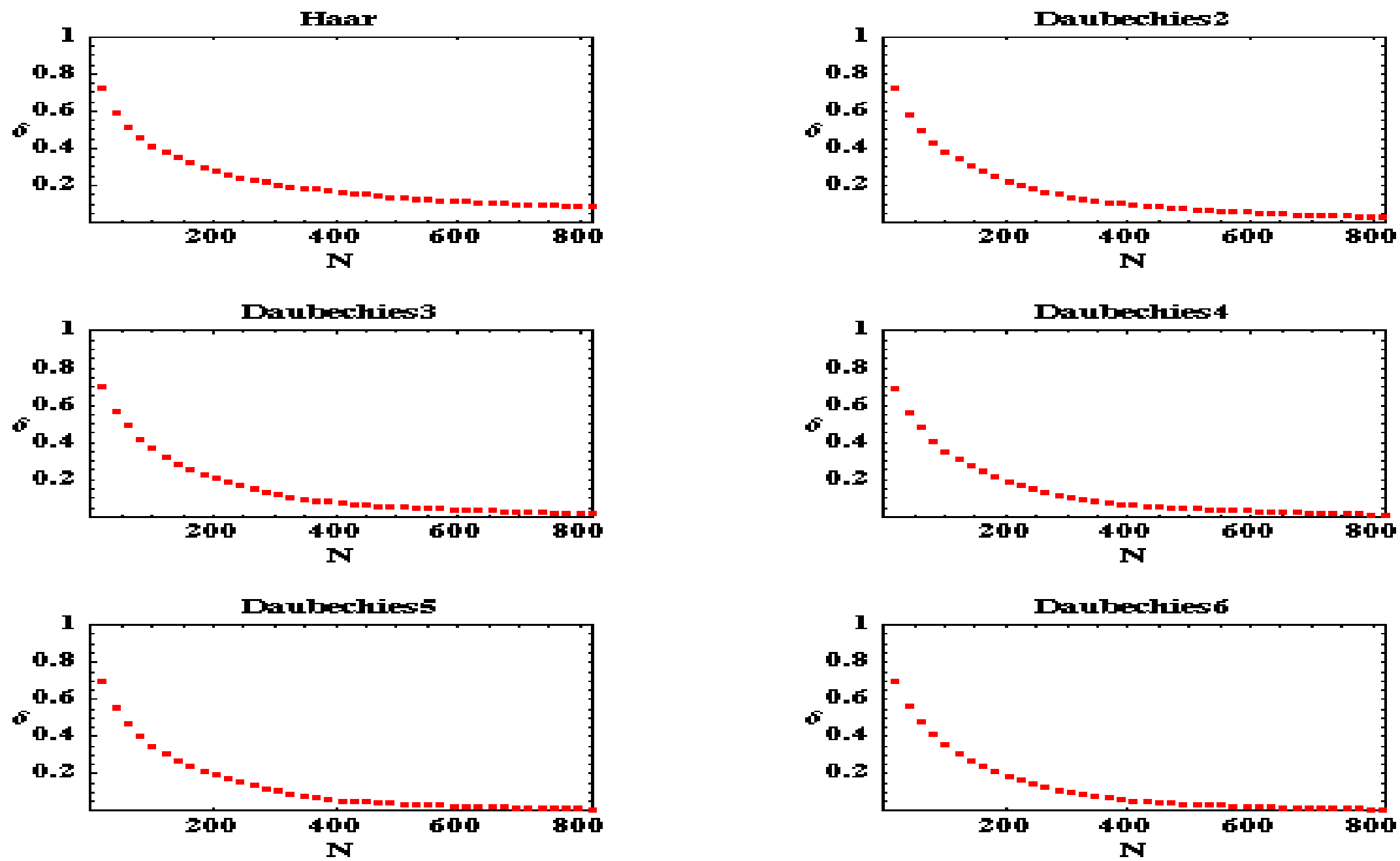


\section{Least Square Error Incurred by Level Thresholding the DWT of LPF RT Mix Data}
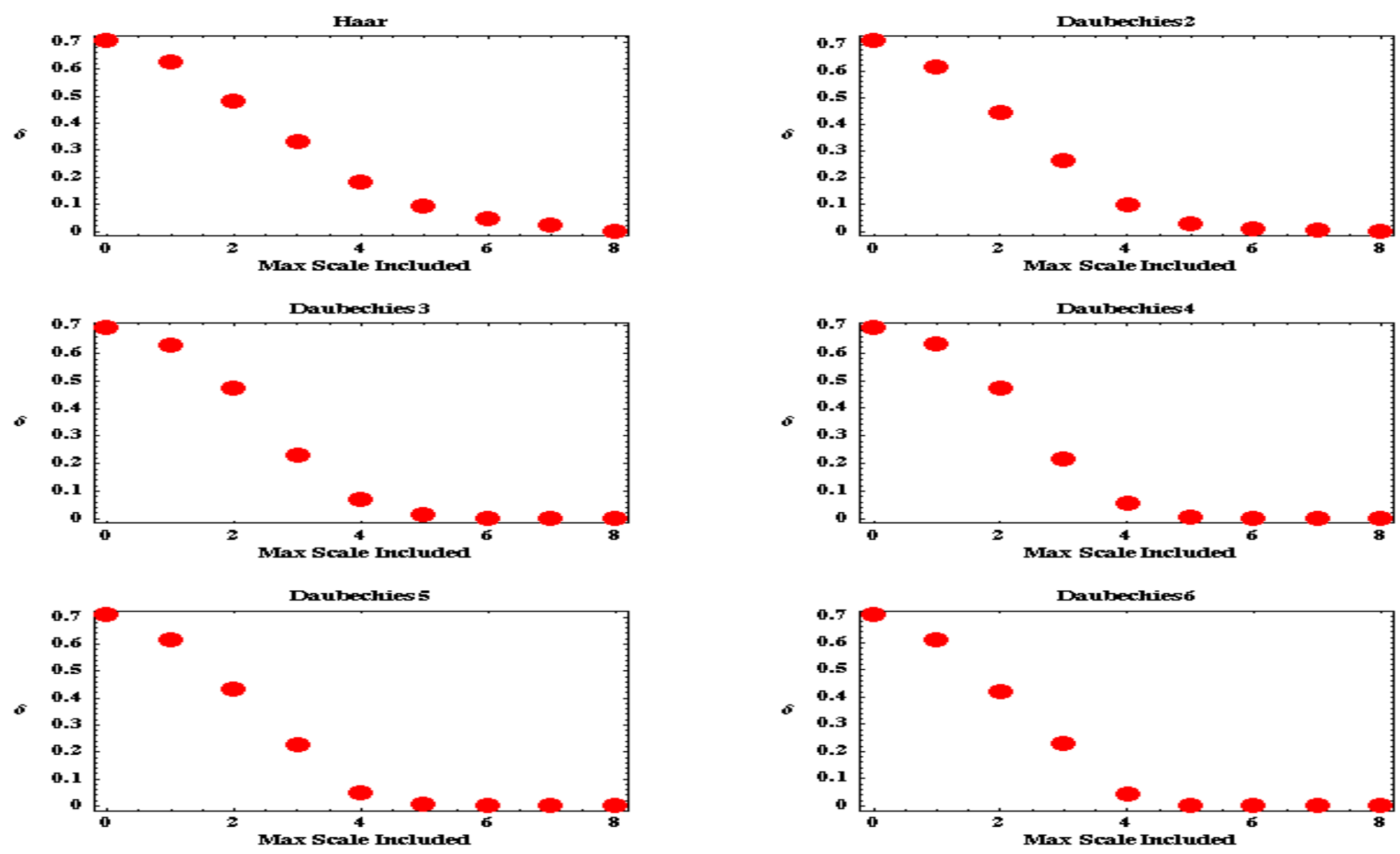


\section{Daubechies 5 Does Much Better than}

\section{Haar: 5 Quantitative Measures for LPF RT Mix Data}

Polymath Research Inc.

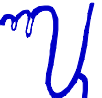

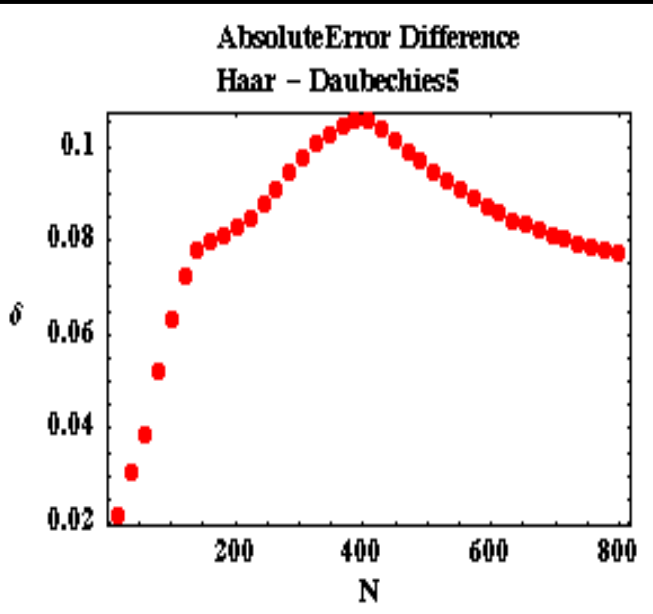
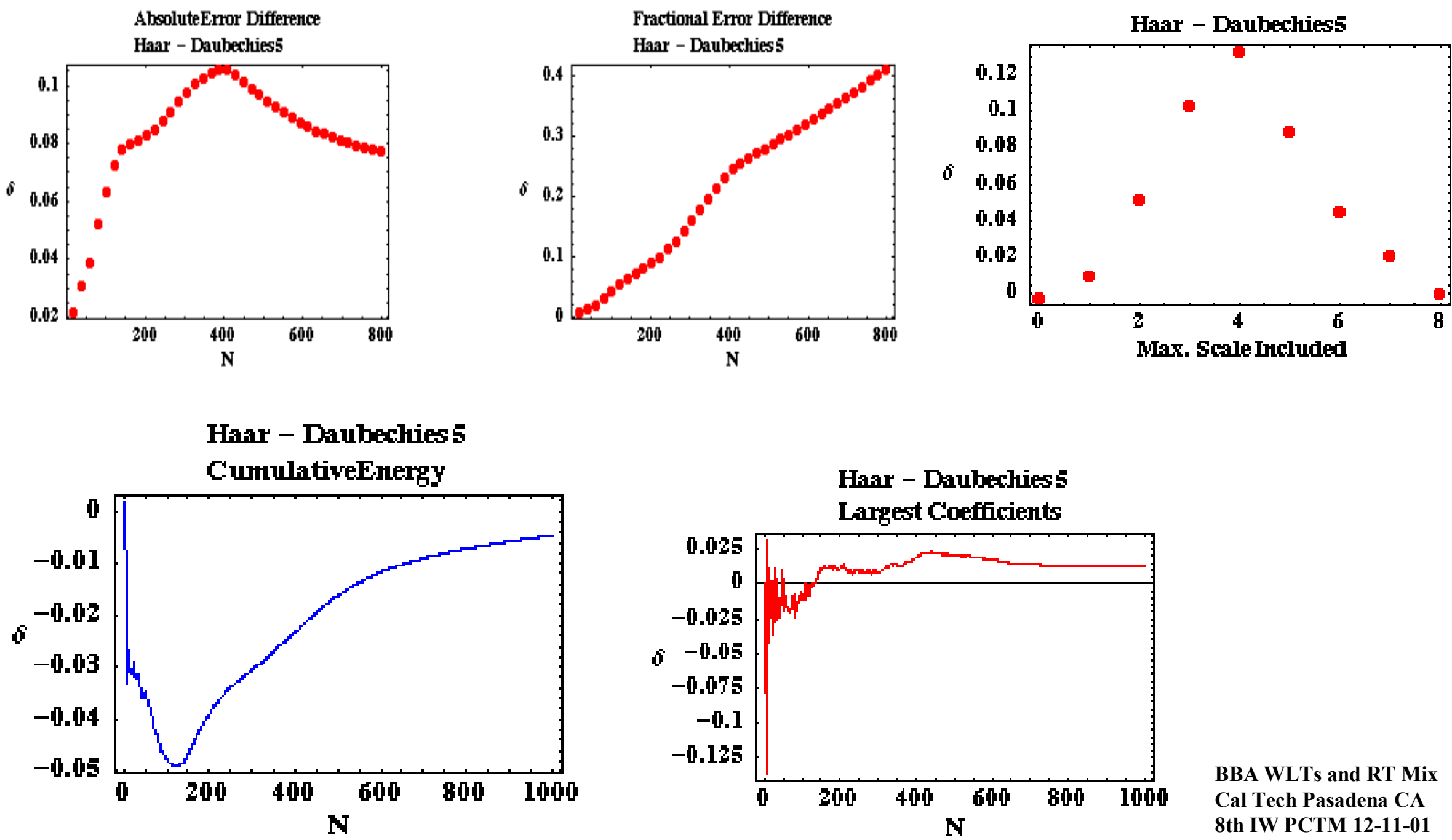


\section{Level by Level Decomposition of the LPF RT Mix Data Using Daub5 WLTs}

Polymath Research Inc.

Daubechess.
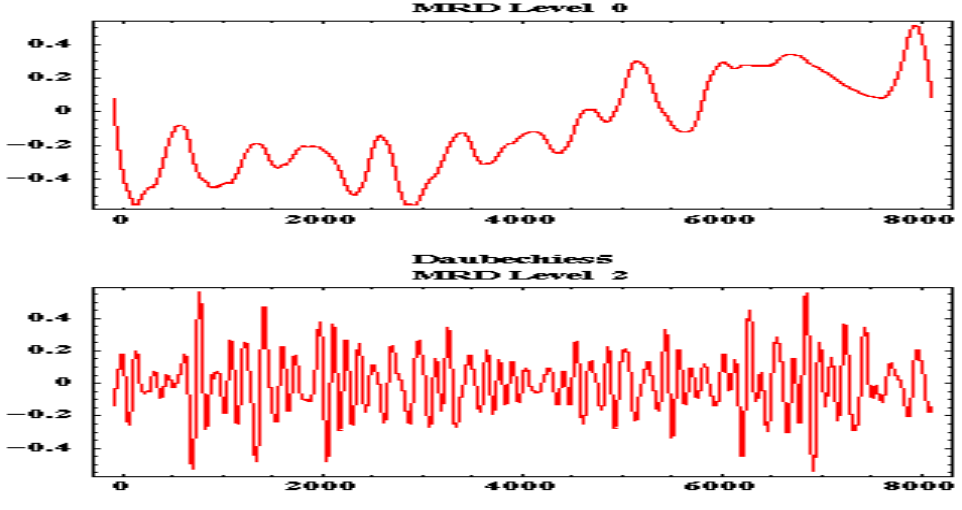

Dawberiess

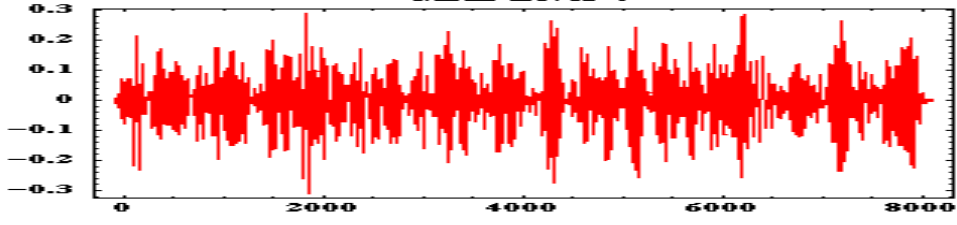

nautocises:

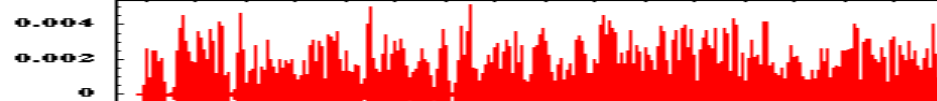

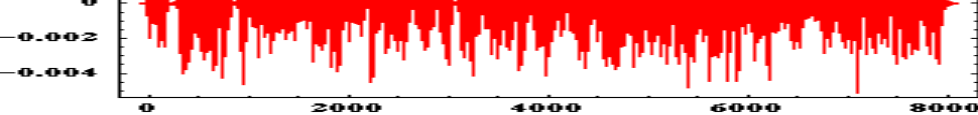

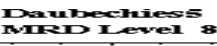

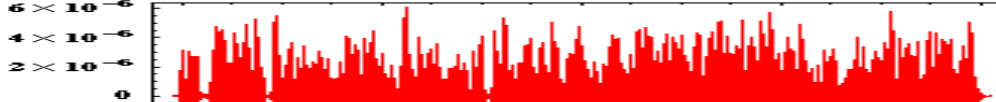

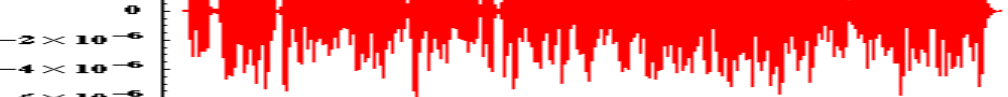

Maubechess.
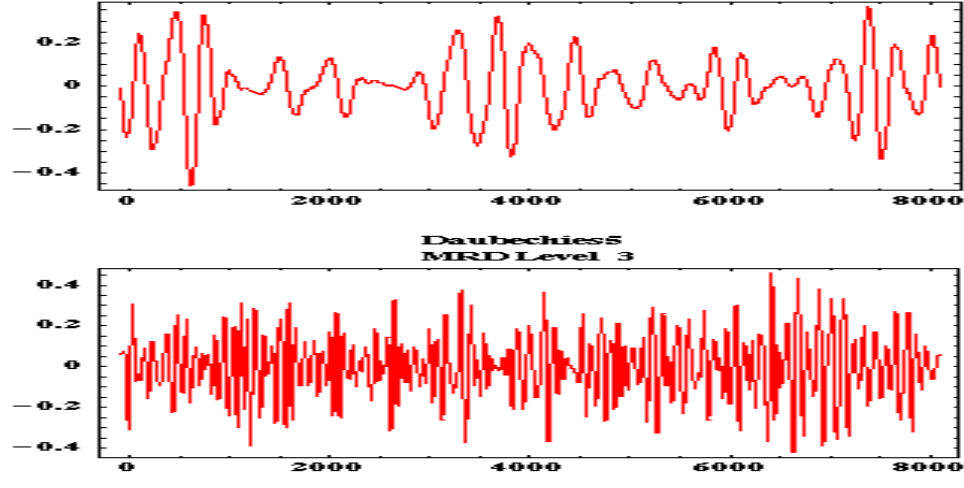

Davbrevers

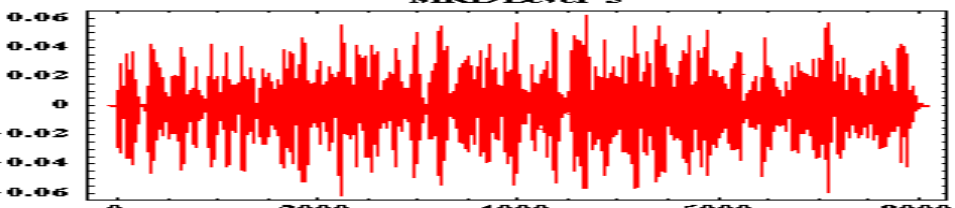

Maubsenes:

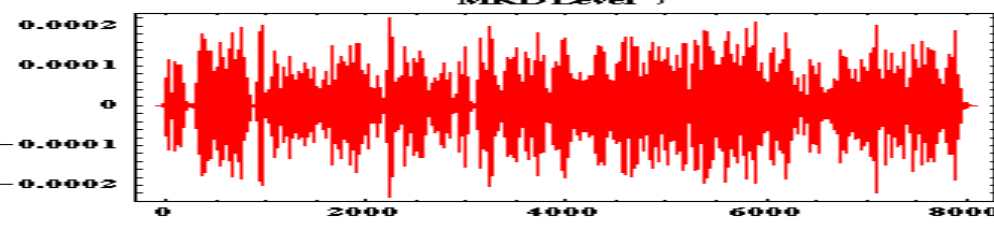

Origimandate

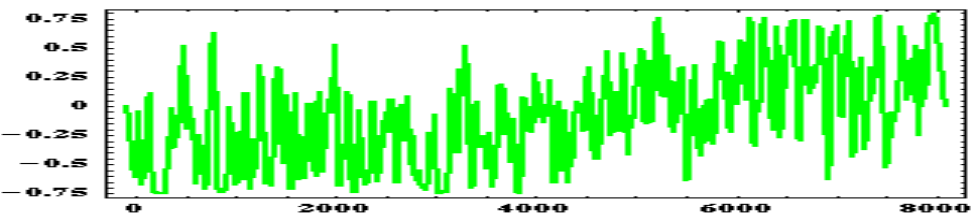




\section{Reconstruction of the LPF Data}

\section{with 5 Largest WLT Coeffs}

Polymath

Research Inc.
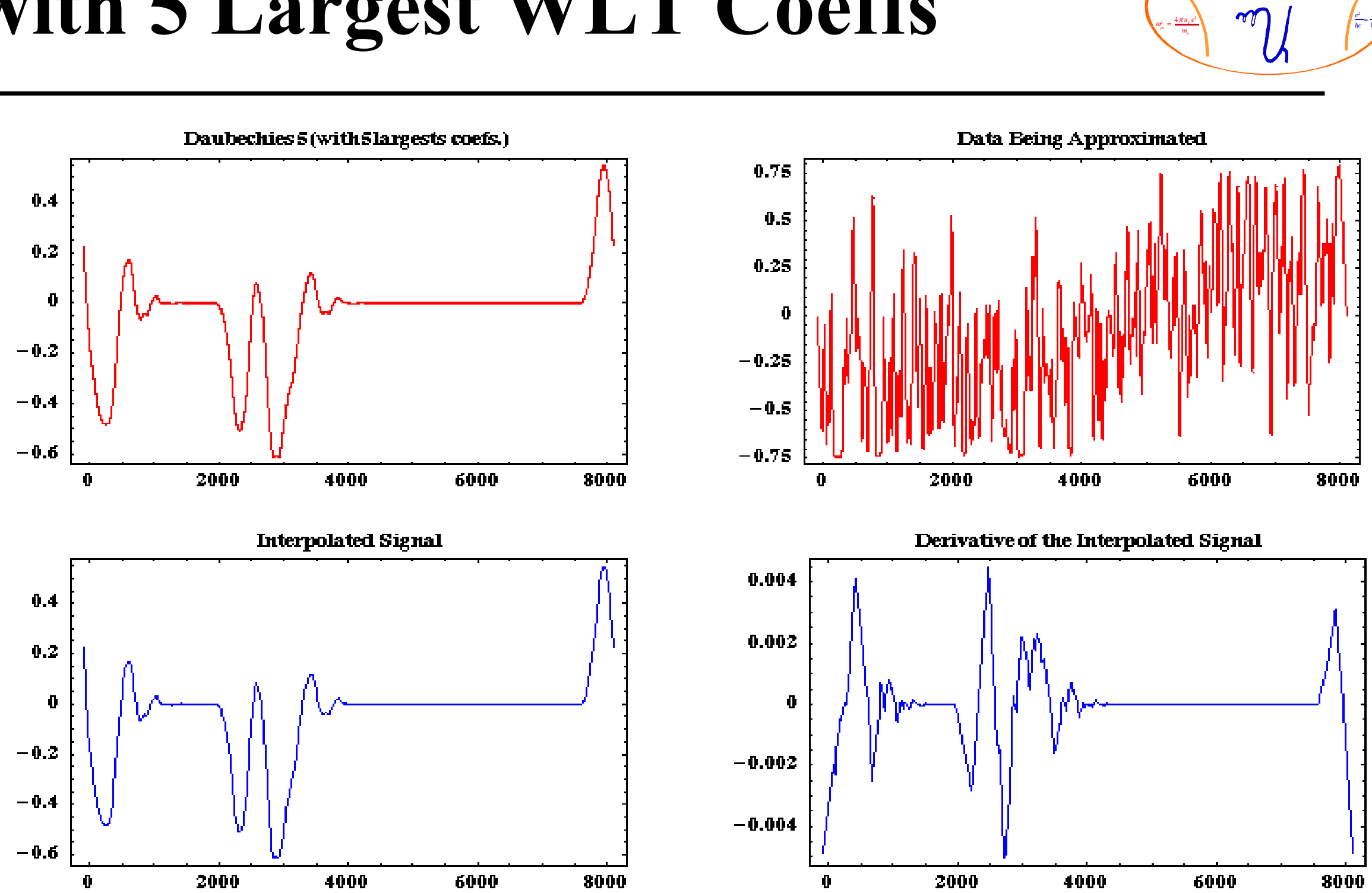


\section{Reconstruction of the LPF Data with 10 Largest WLT Coeffs}
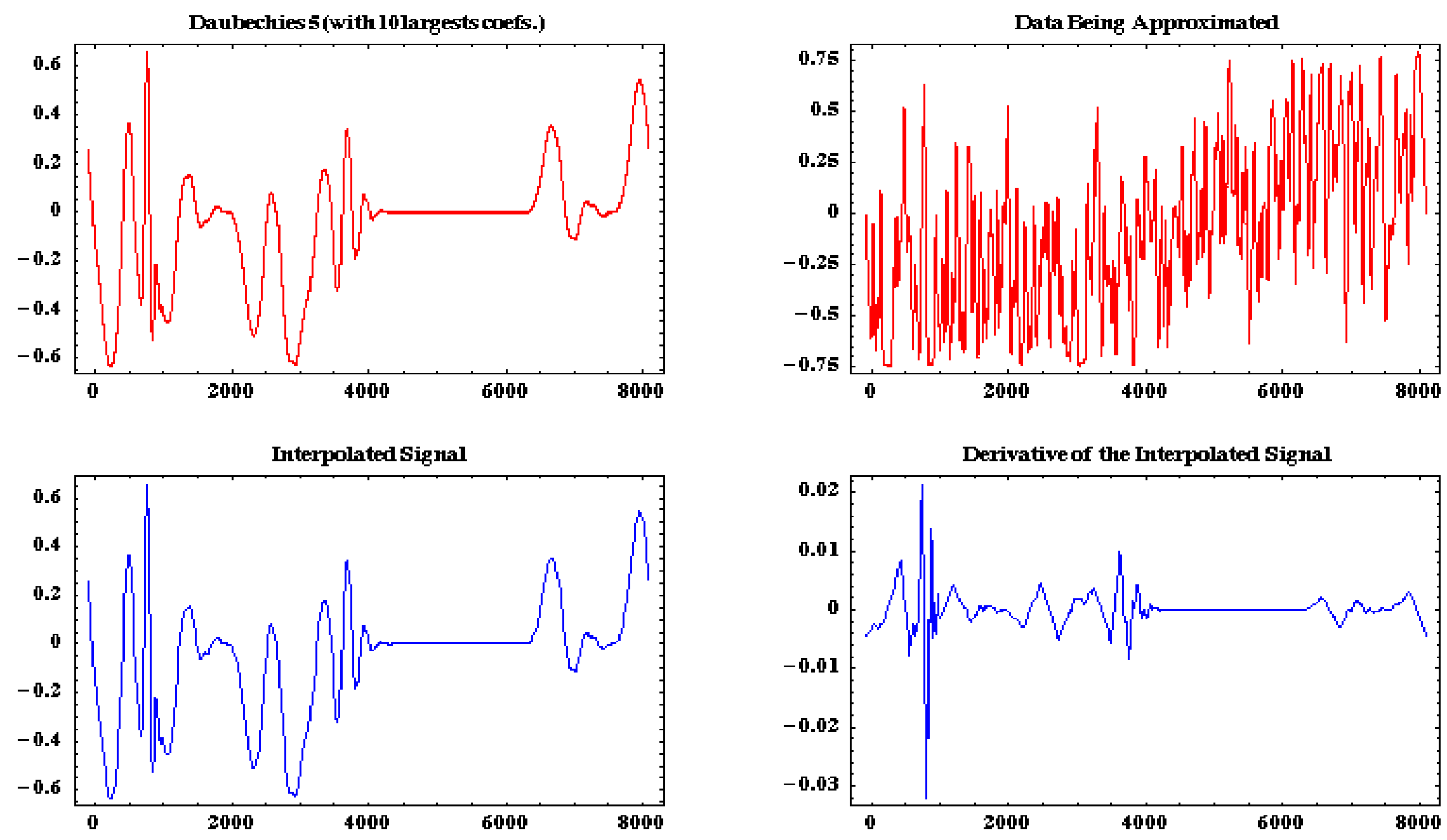


\section{Reconstruction of the LPF Data with 15 Largest WLT Coeffs}
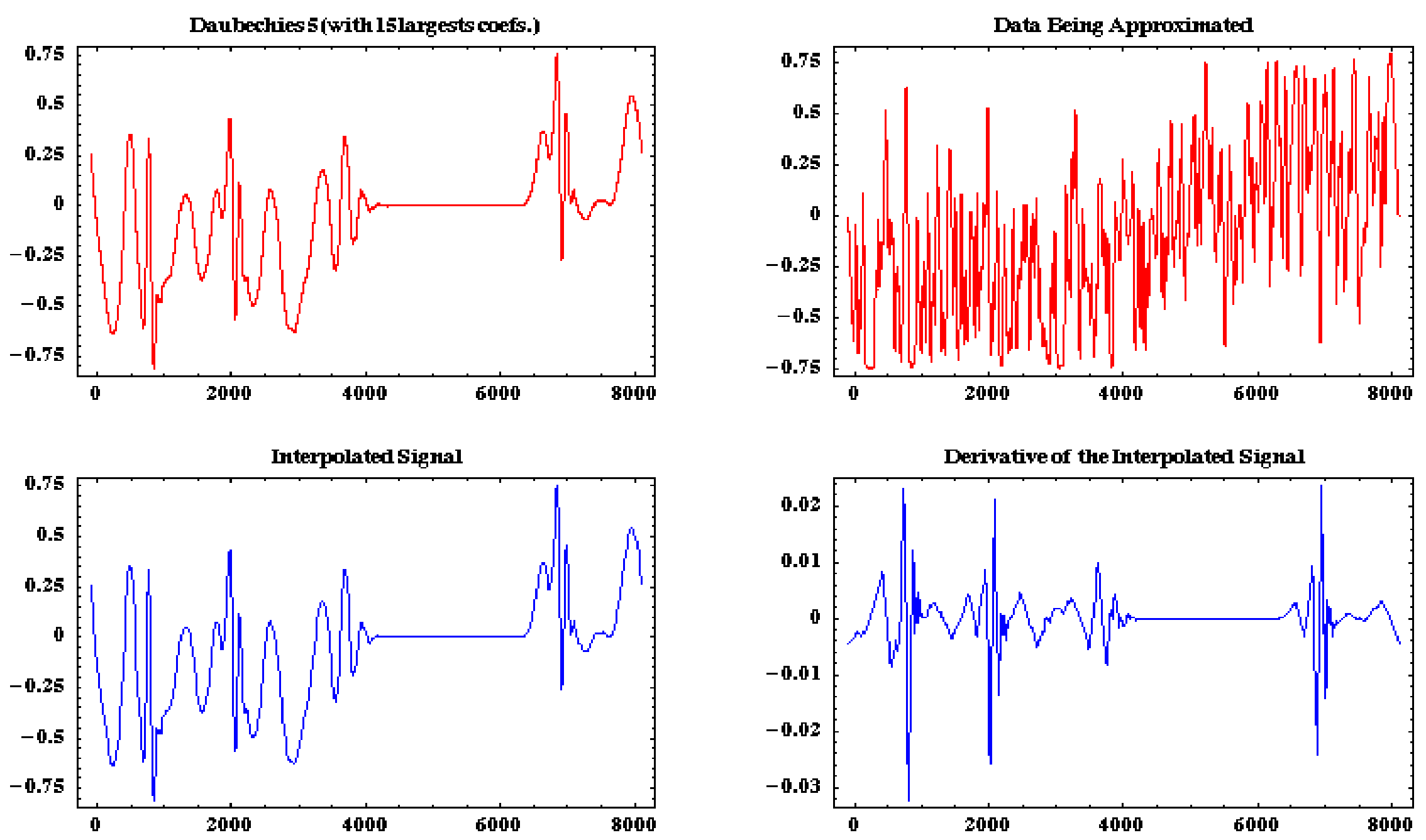


\section{Reconstruction of the LPF Data with 20 Largest WLT Coeffs}
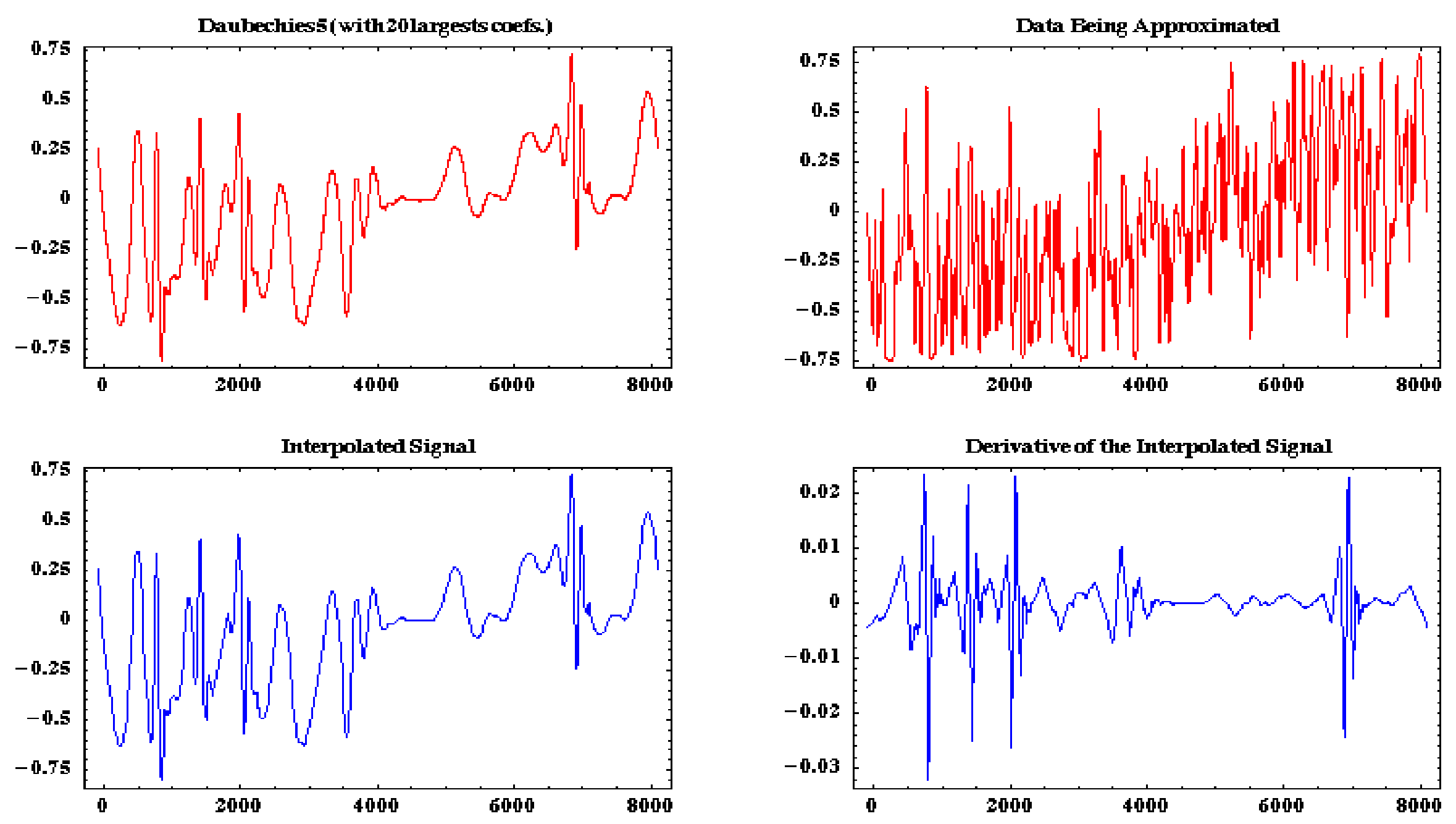


\section{Reconstruction of the LPF Data with 30 Largest WLT Coeffs}
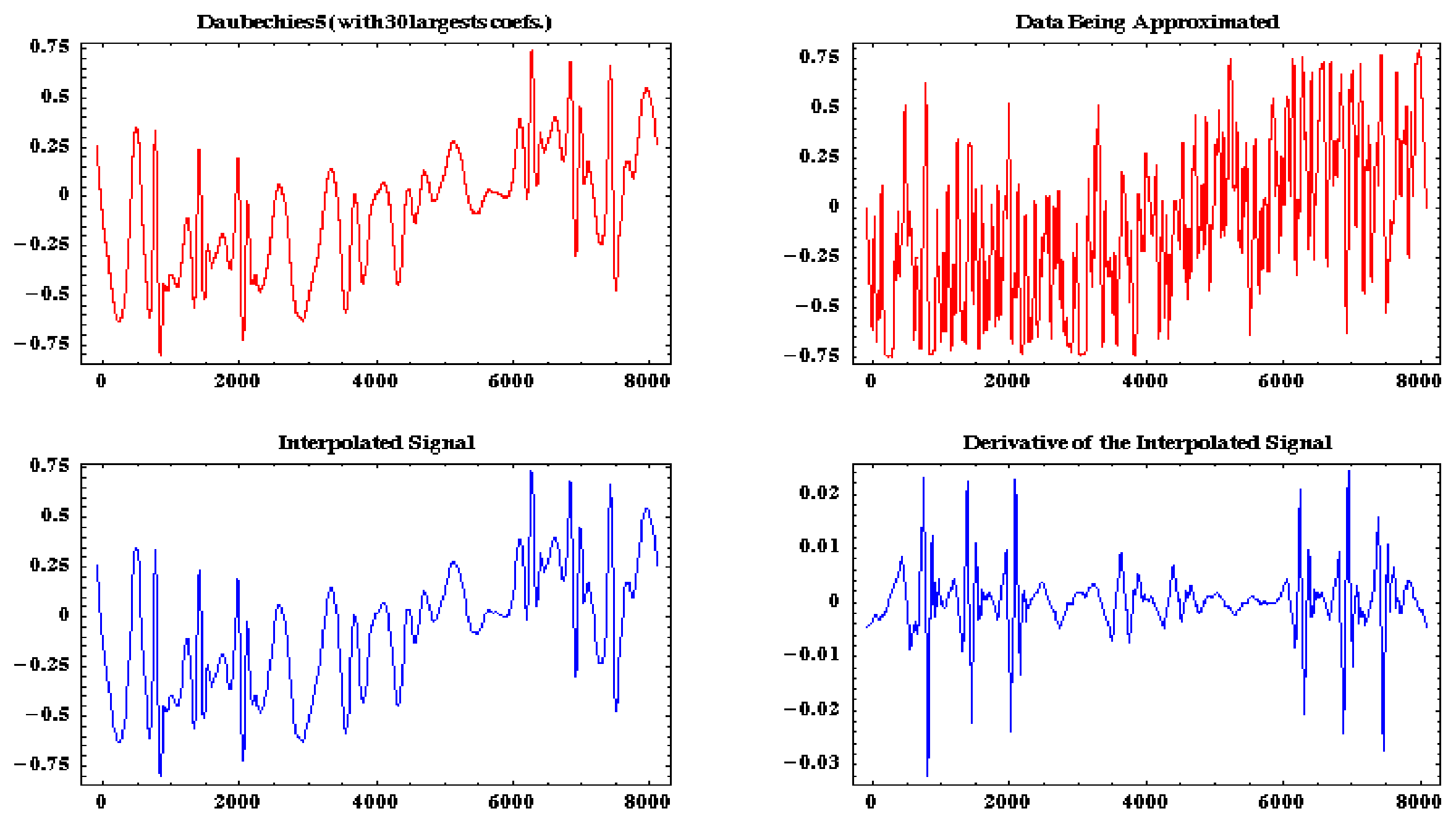


\section{Reconstruction of the LPF Data with 50 Largest WLT Coeffs}
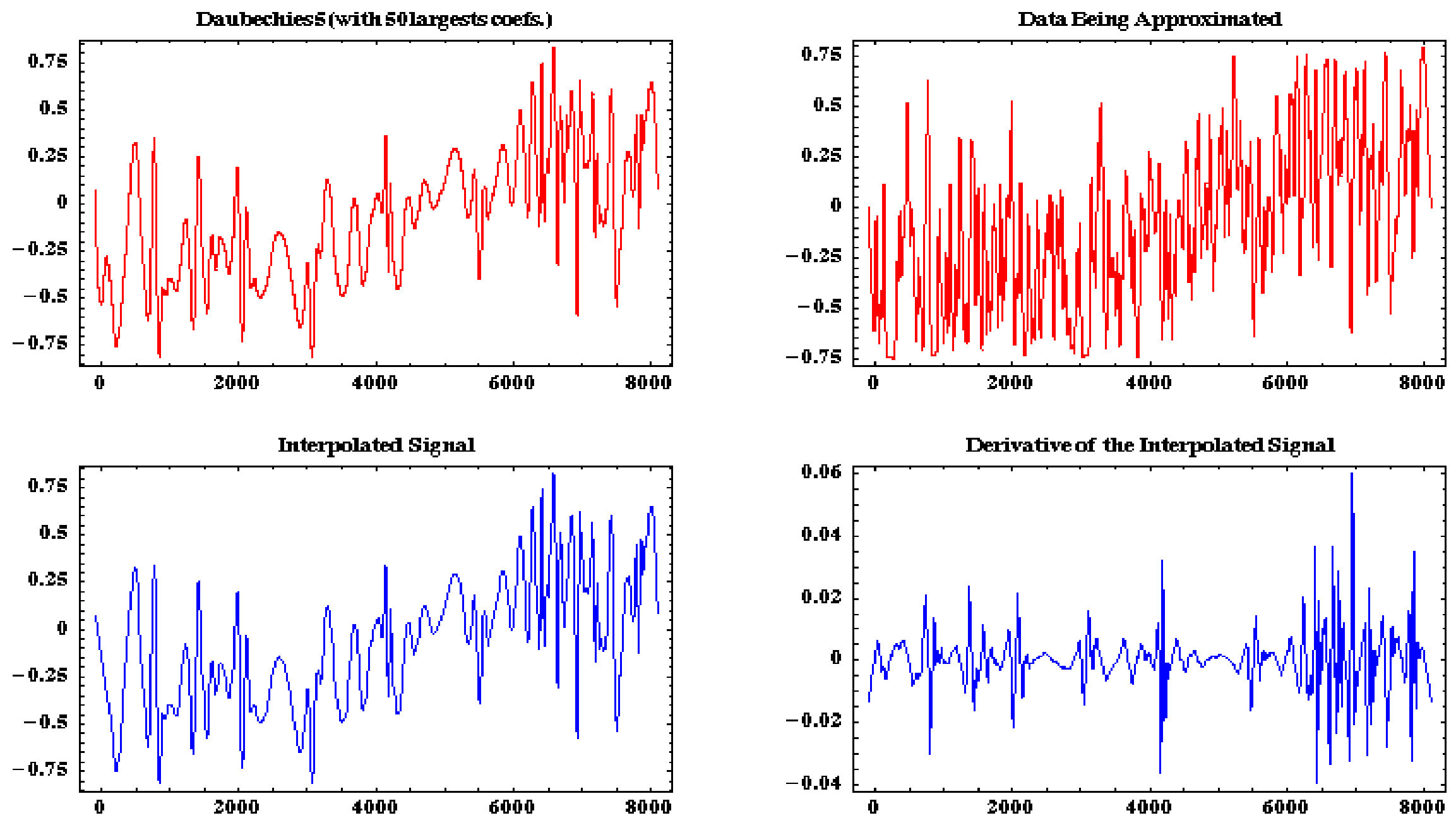


\section{Reconstruction of the LPF Data with 100 Largest WLT Coeffs}

Polymath Research Inc.
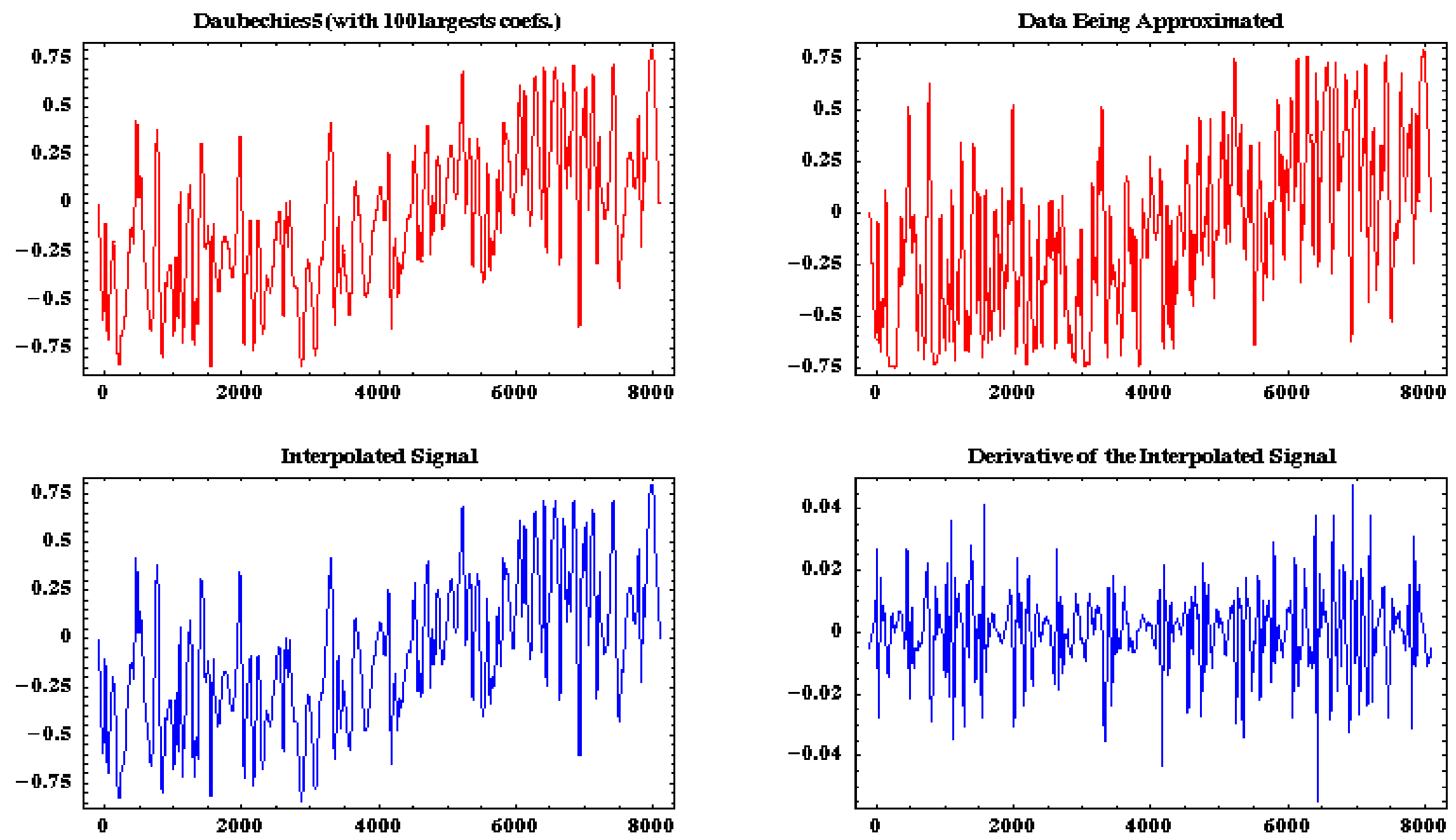


\section{Reconstruction of the LPF Data with 200 Largest WLT Coeffs}
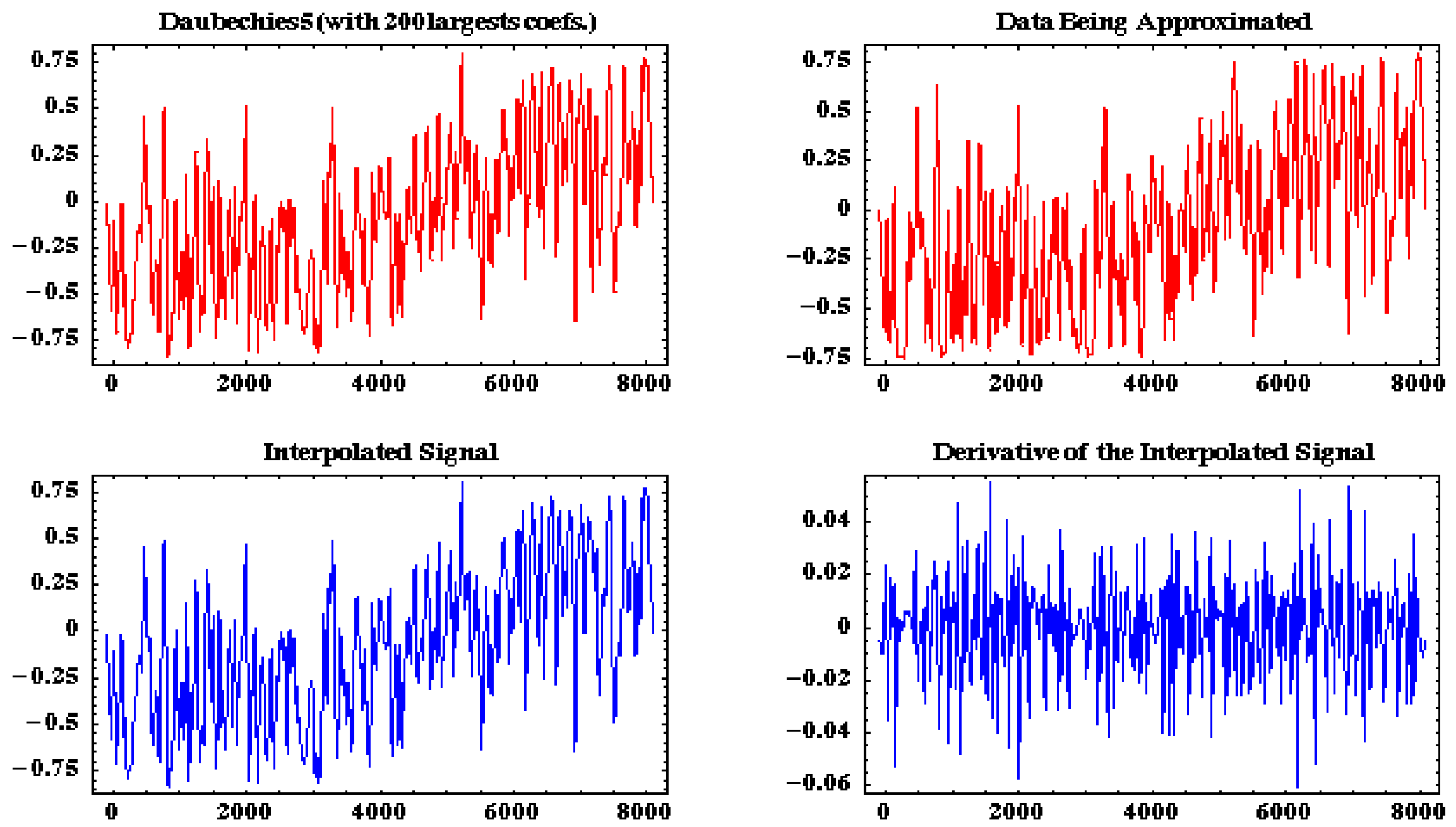


\section{Reconstruction of the LPF Data with 400 Largest WLT Coeffs}
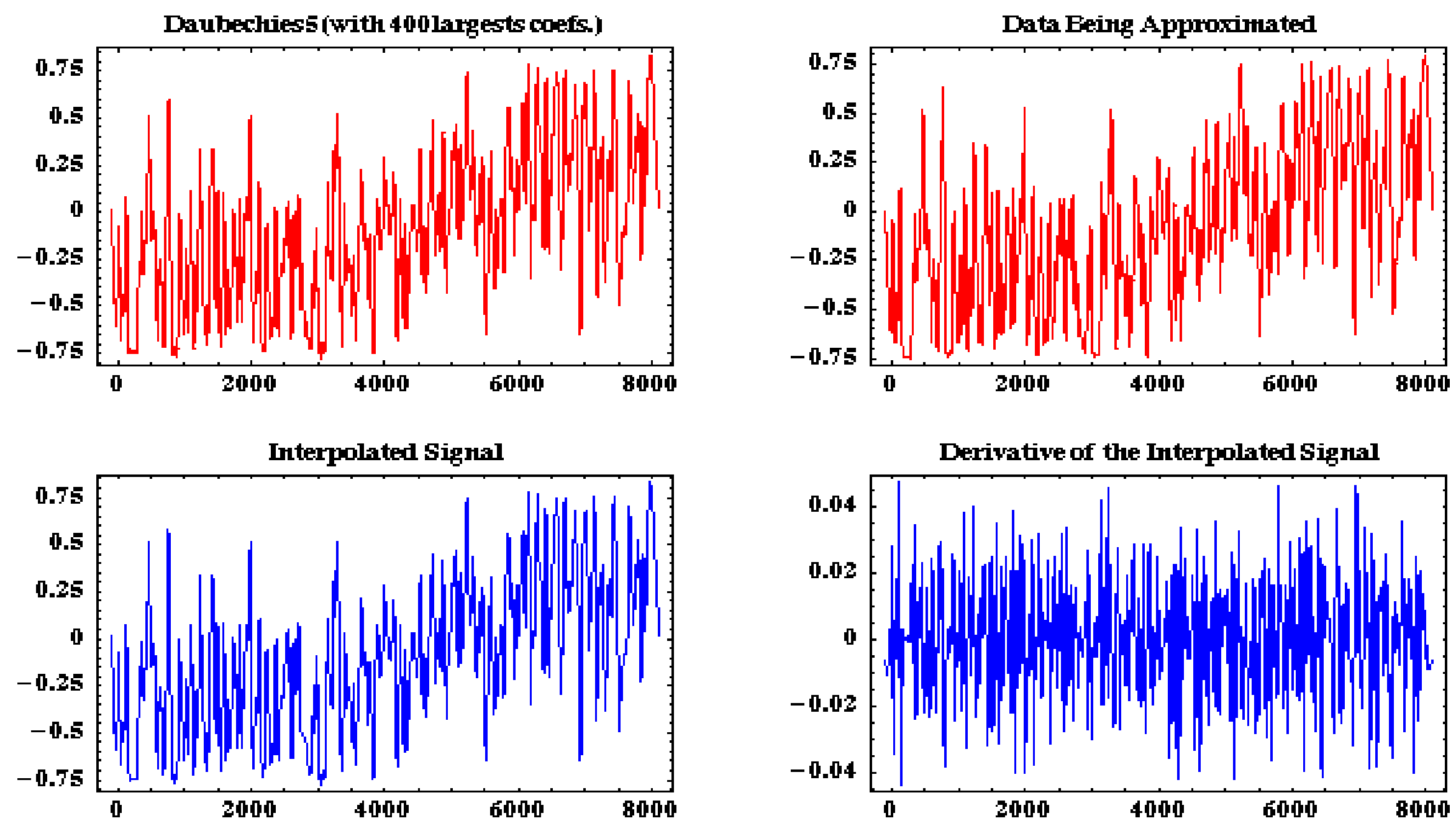


\section{Recons. of the LPF Data Using Up to $0.75 x$ the Largest WLTs}
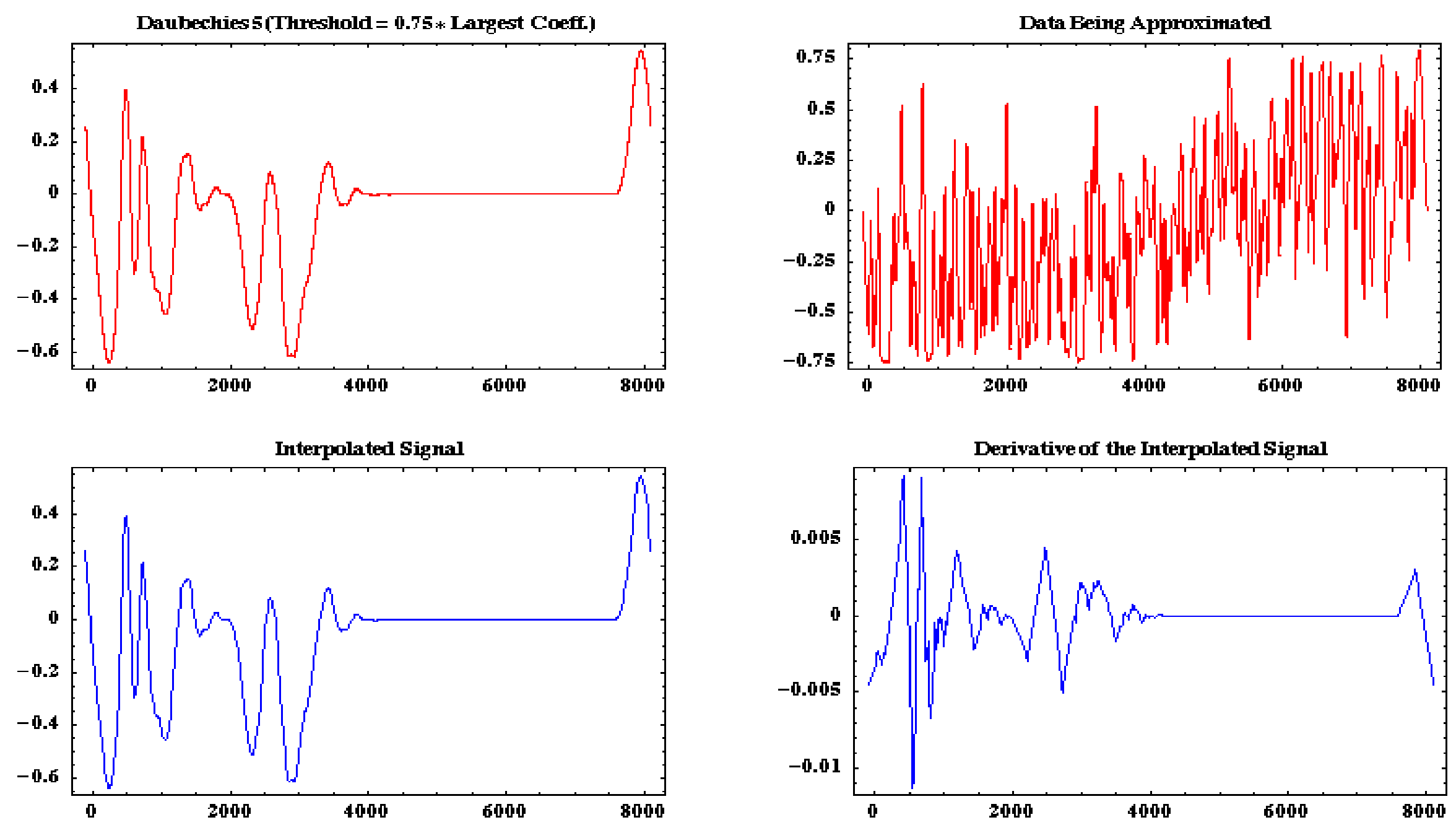


\section{Recons. of the LPF Data Using} Up to $0.5 x$ the Largest WLTs
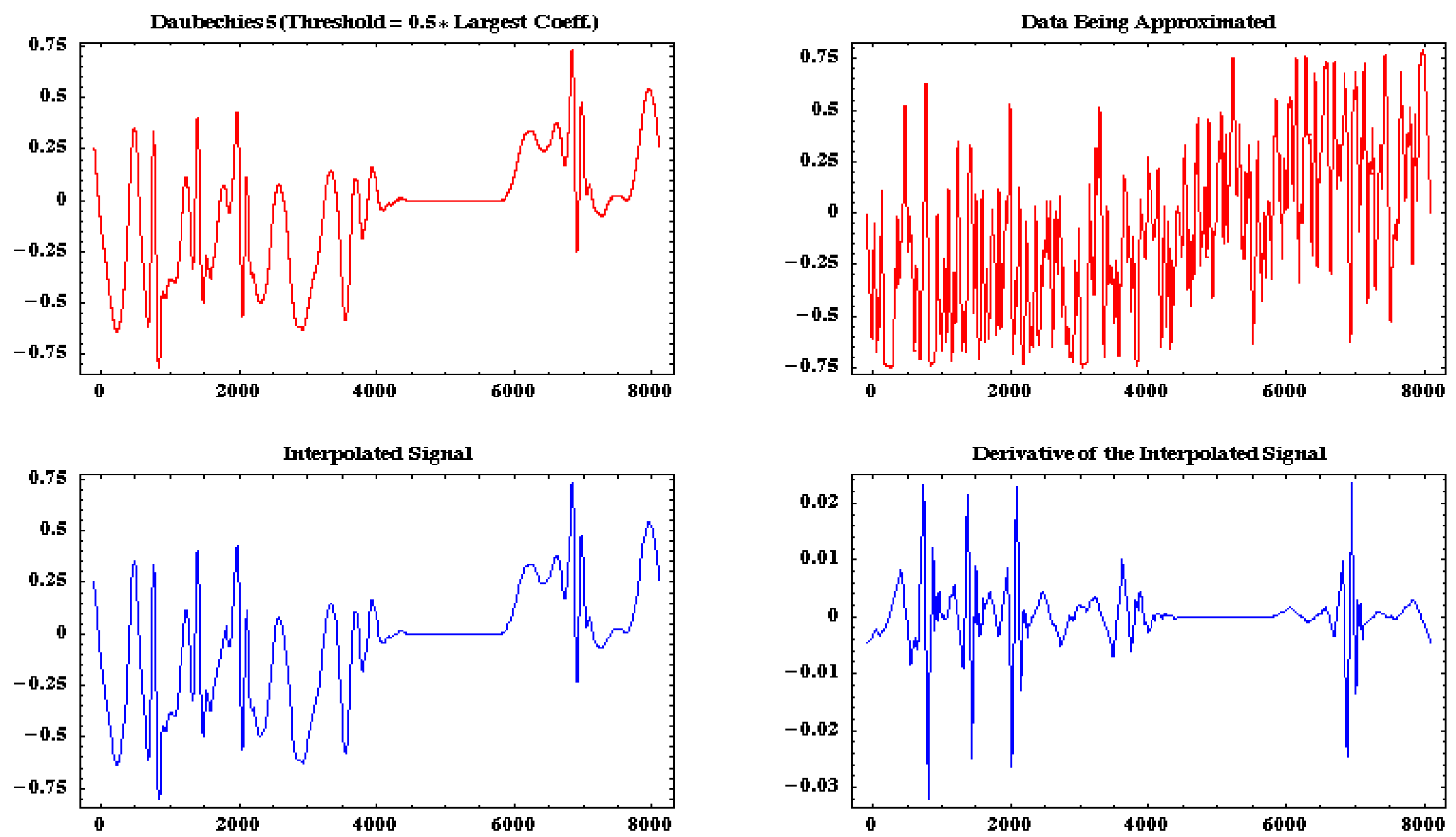


\section{Recons. of the LPF Data Using Up to $0.25 \mathrm{x}$ the Largest WLTs}
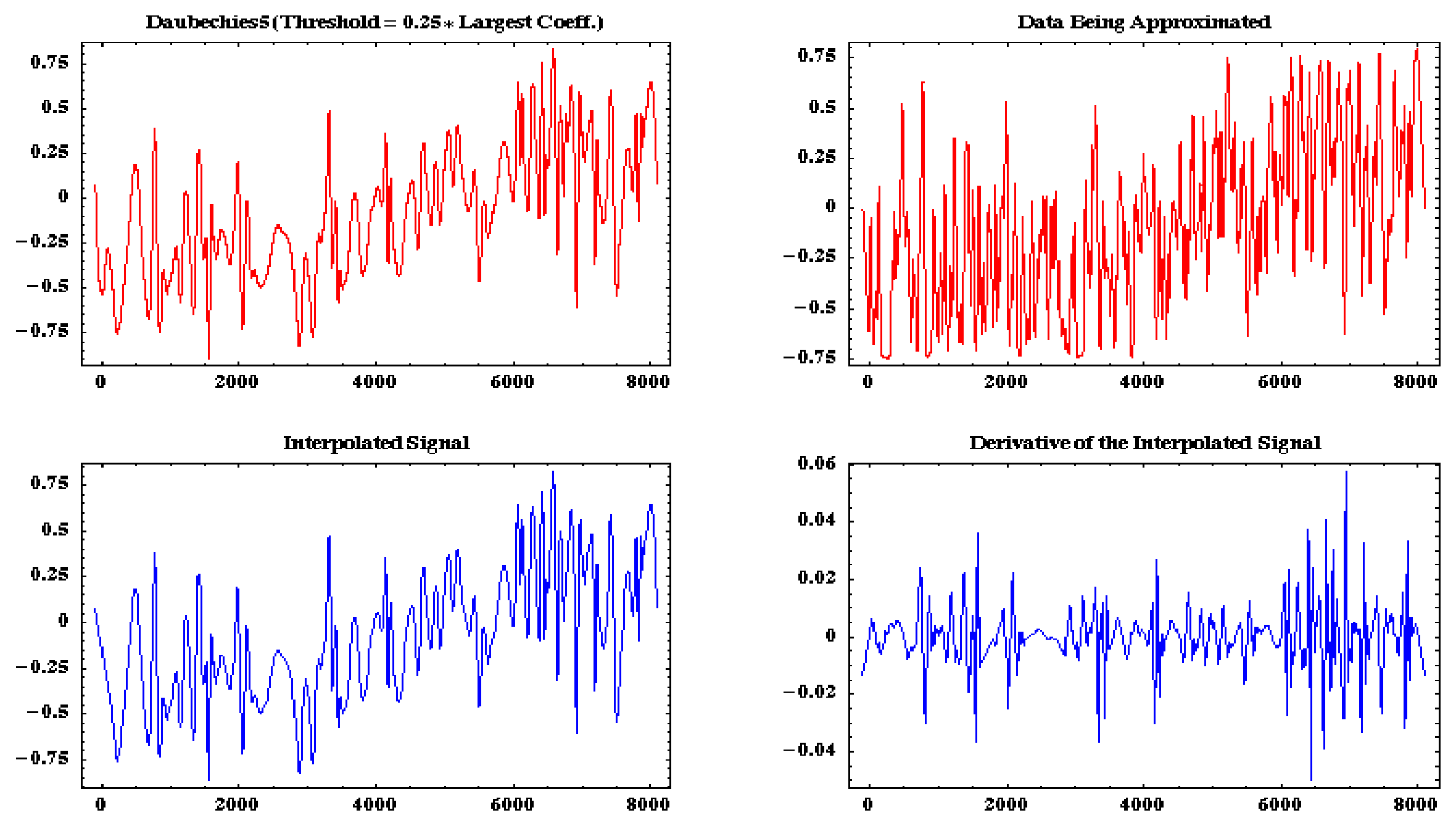


\section{Recons. of the LPF Data Using} Up to $0.1 \times$ the Largest WLTs

Polymath Research Inc.
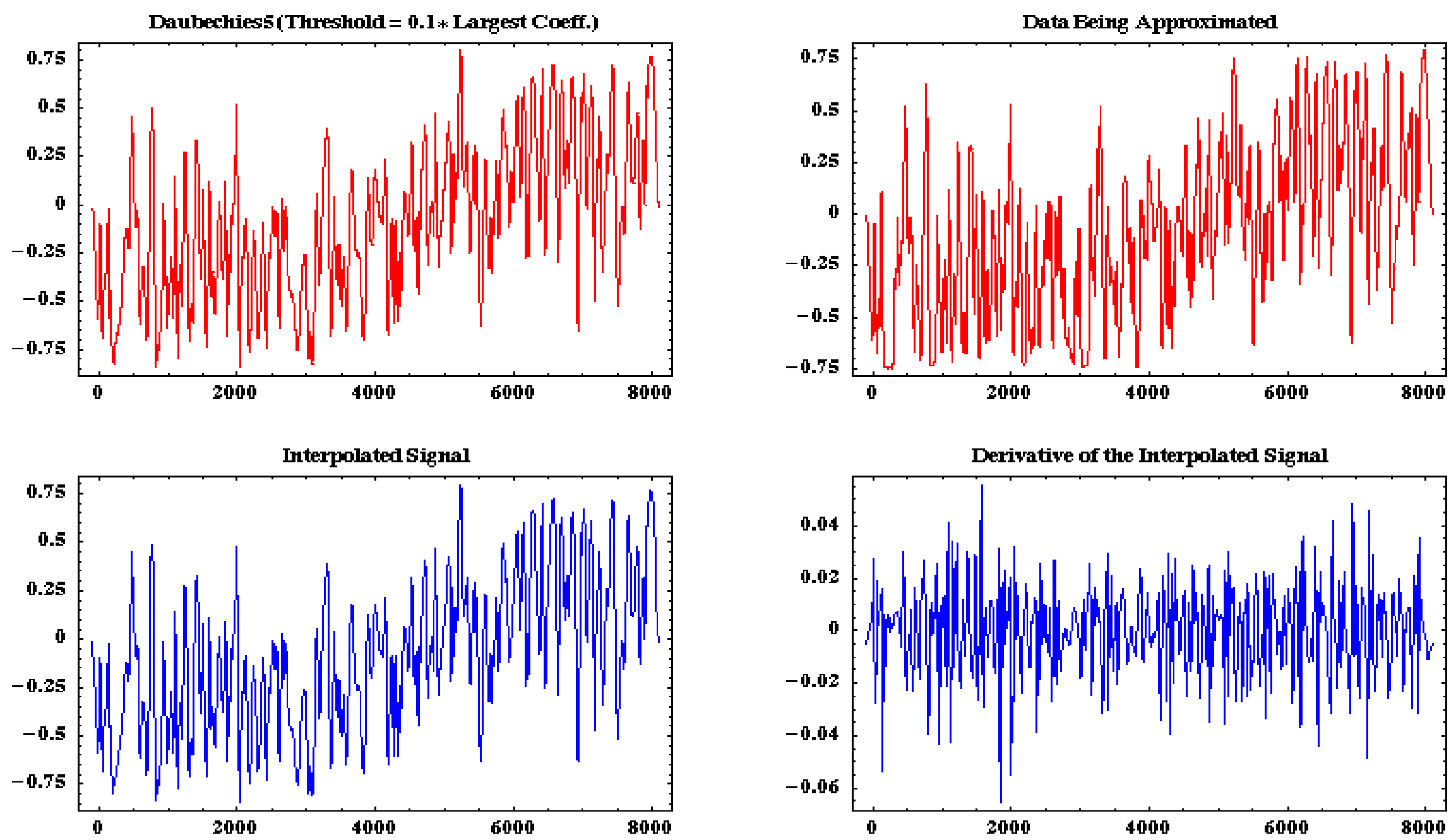


\section{Recons. of the LPF Data Using Up to $0.05 x$ the Largest WLTs}

Daubechies5 (Thueshold $=0.05 *$ Largest Coeff.)

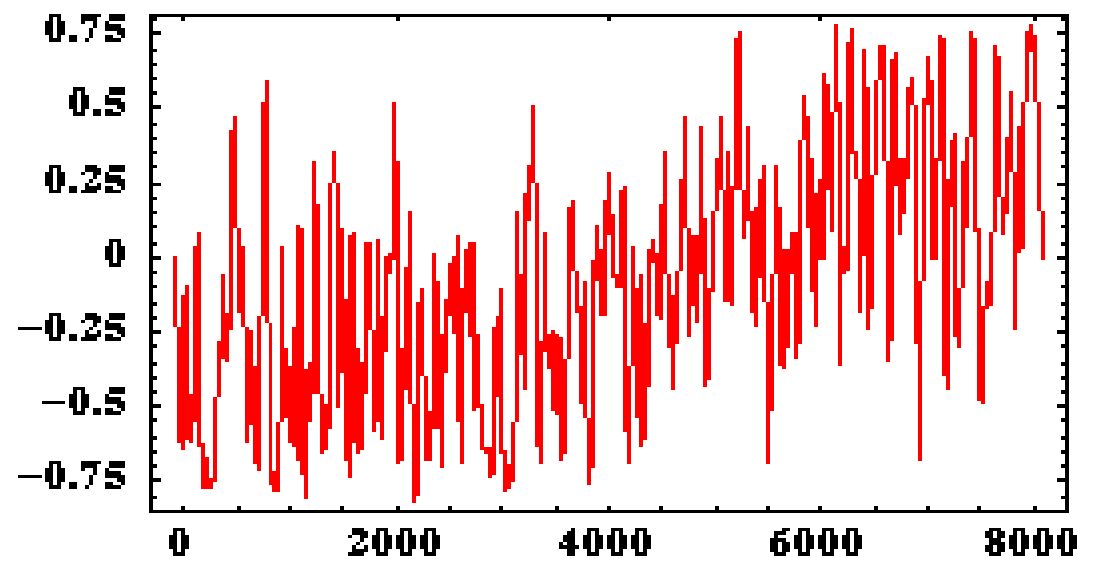

Interpolated Signal

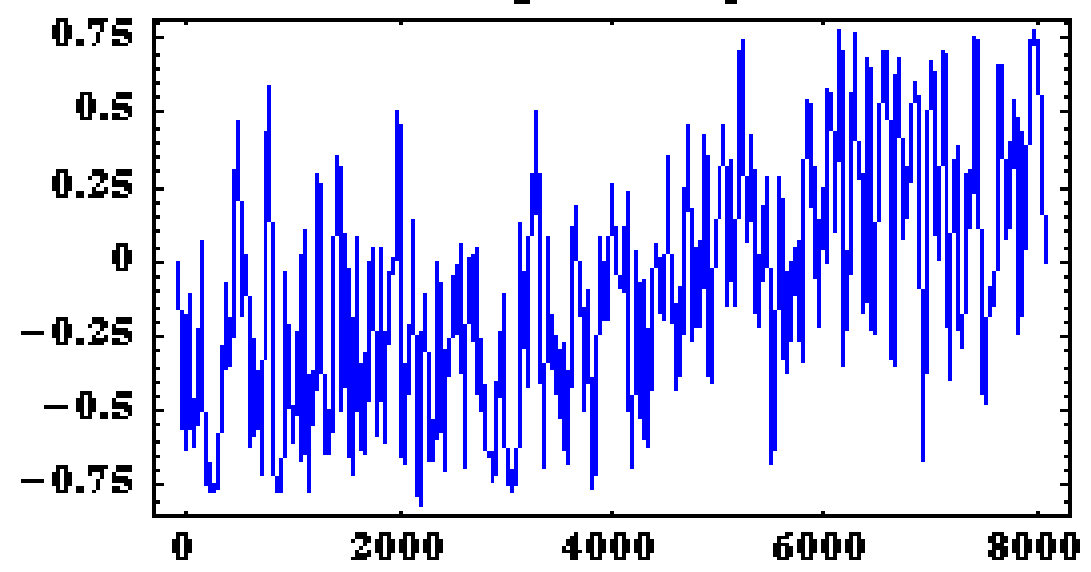

Data Being Approximated

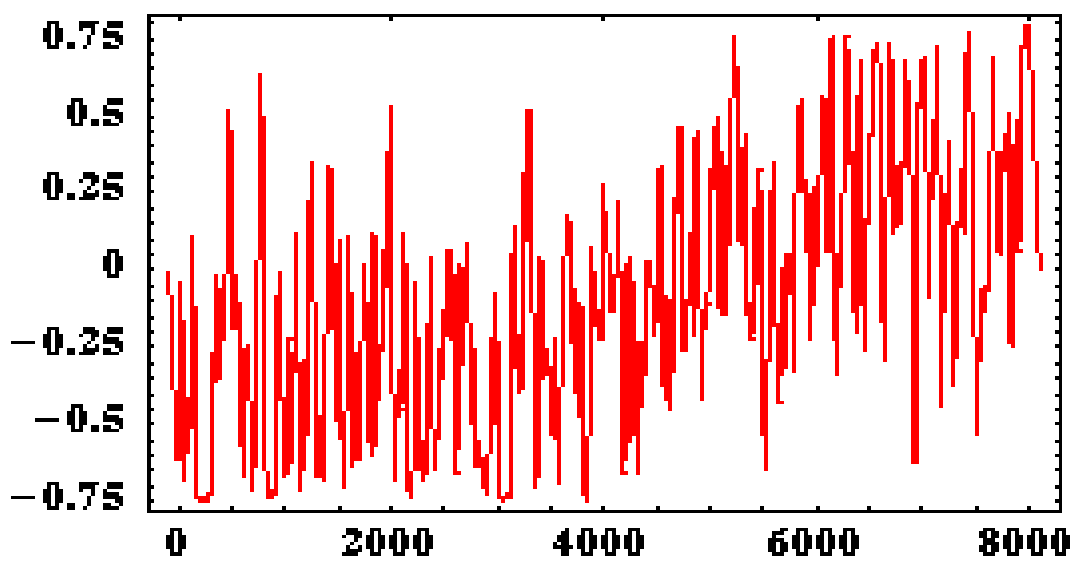

Derivative of the Interpolated Signal

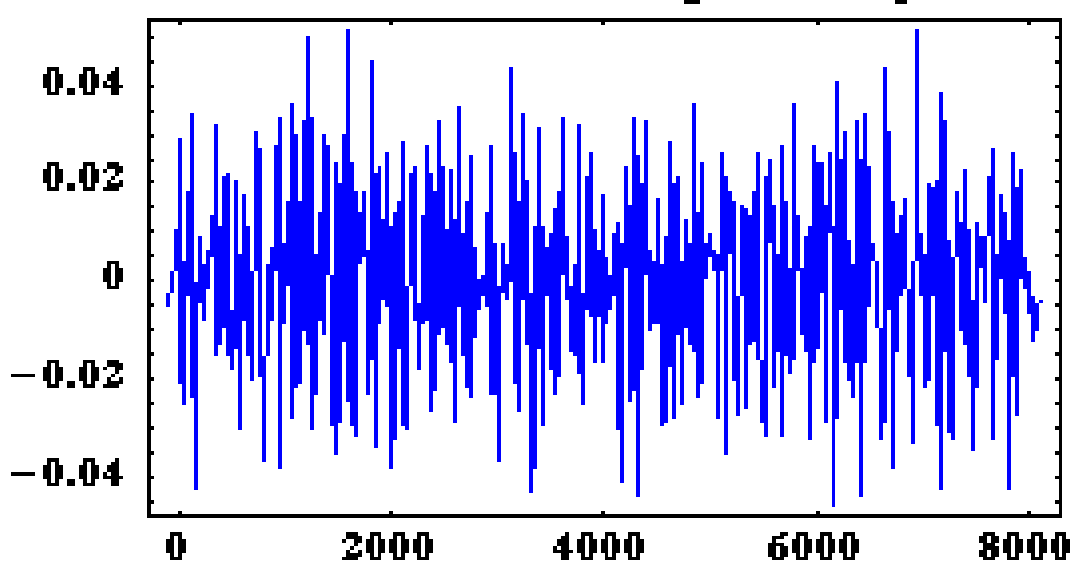




\section{Reconstruction of the LPF Data}

\section{Using the First MRD Level}

Polymath

Research Inc.
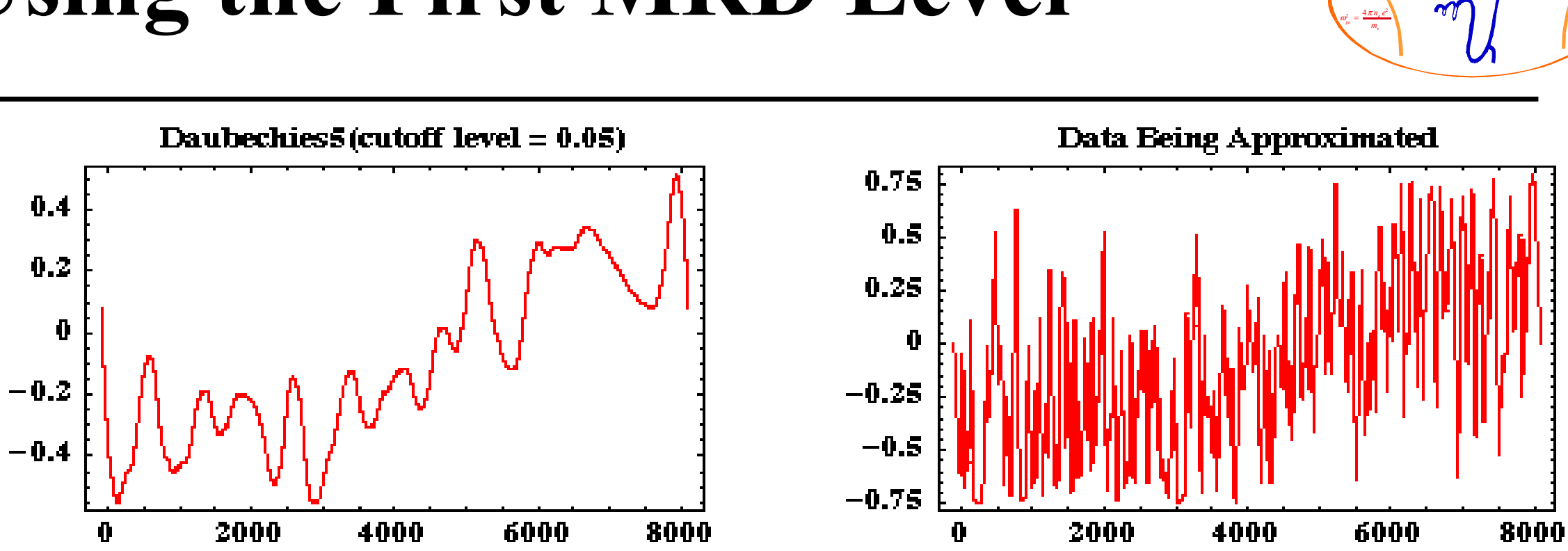

Interpolated Sigial
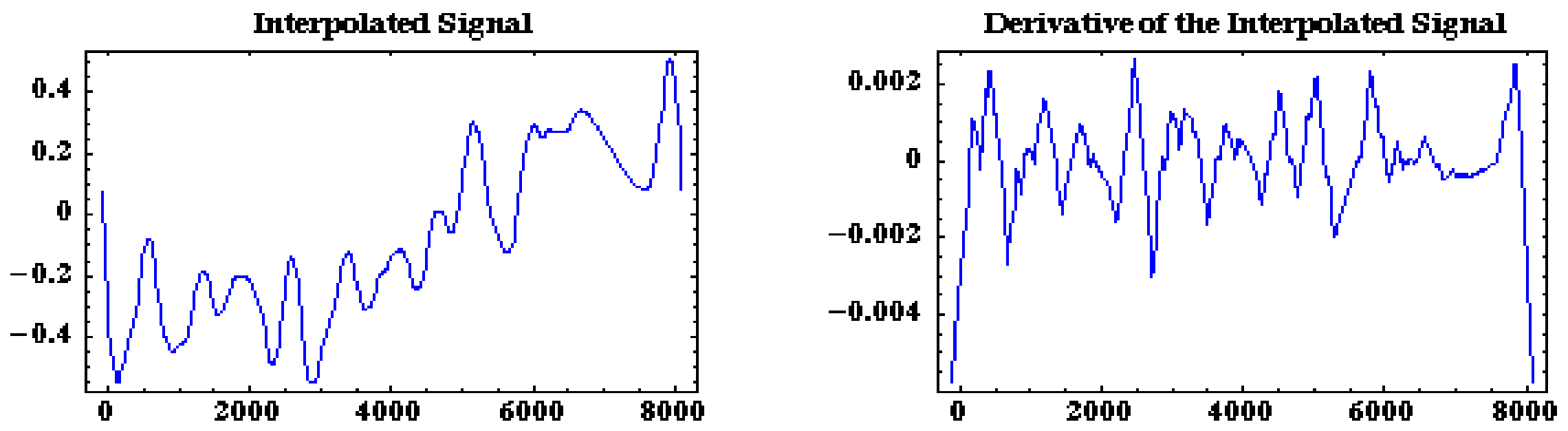


\section{Reconstruction of the LPF Data} Using the First 2 MRD Levels
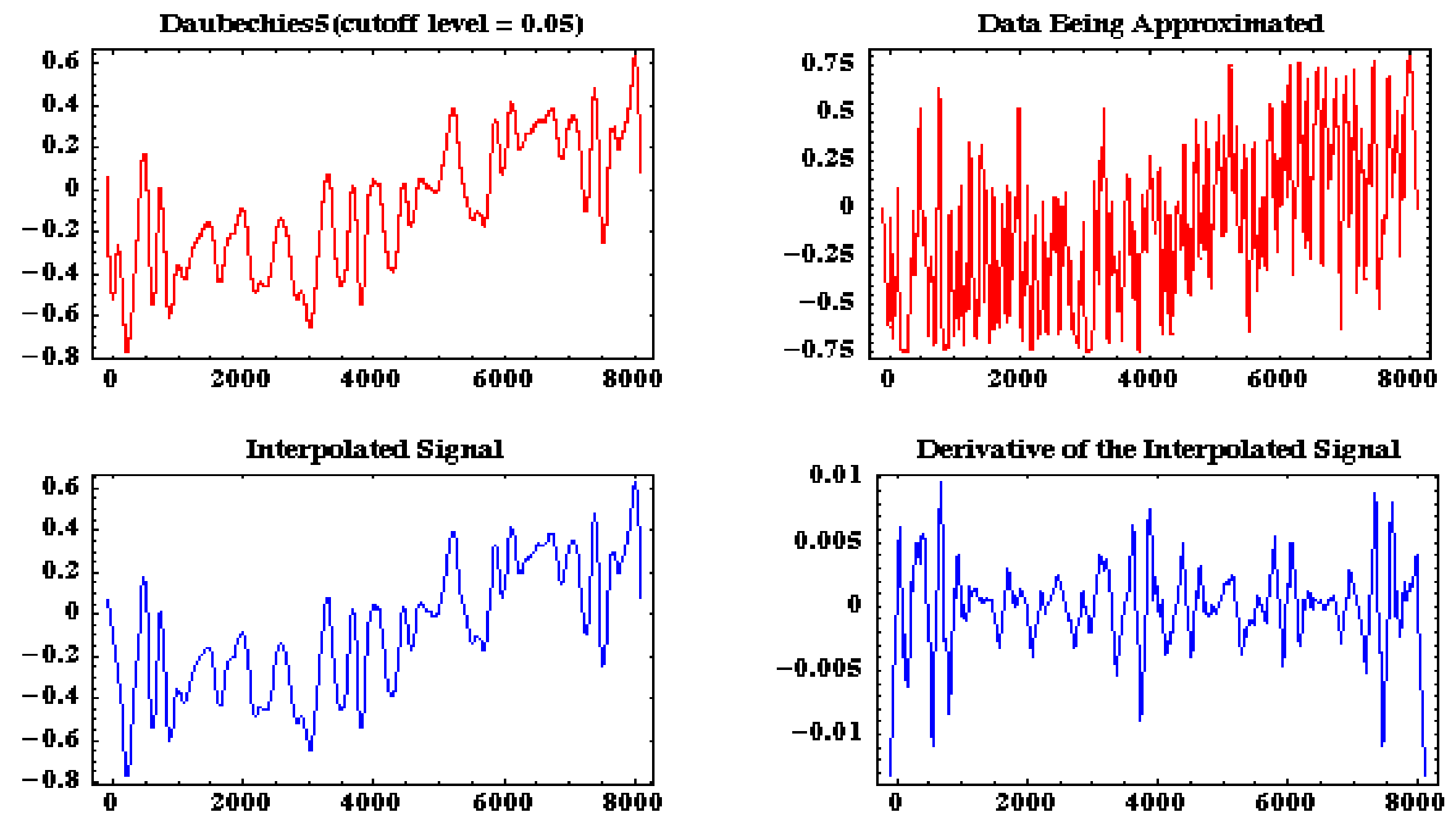


\section{Reconstruction of the LPF Data} Using the First 3 MRD Levels
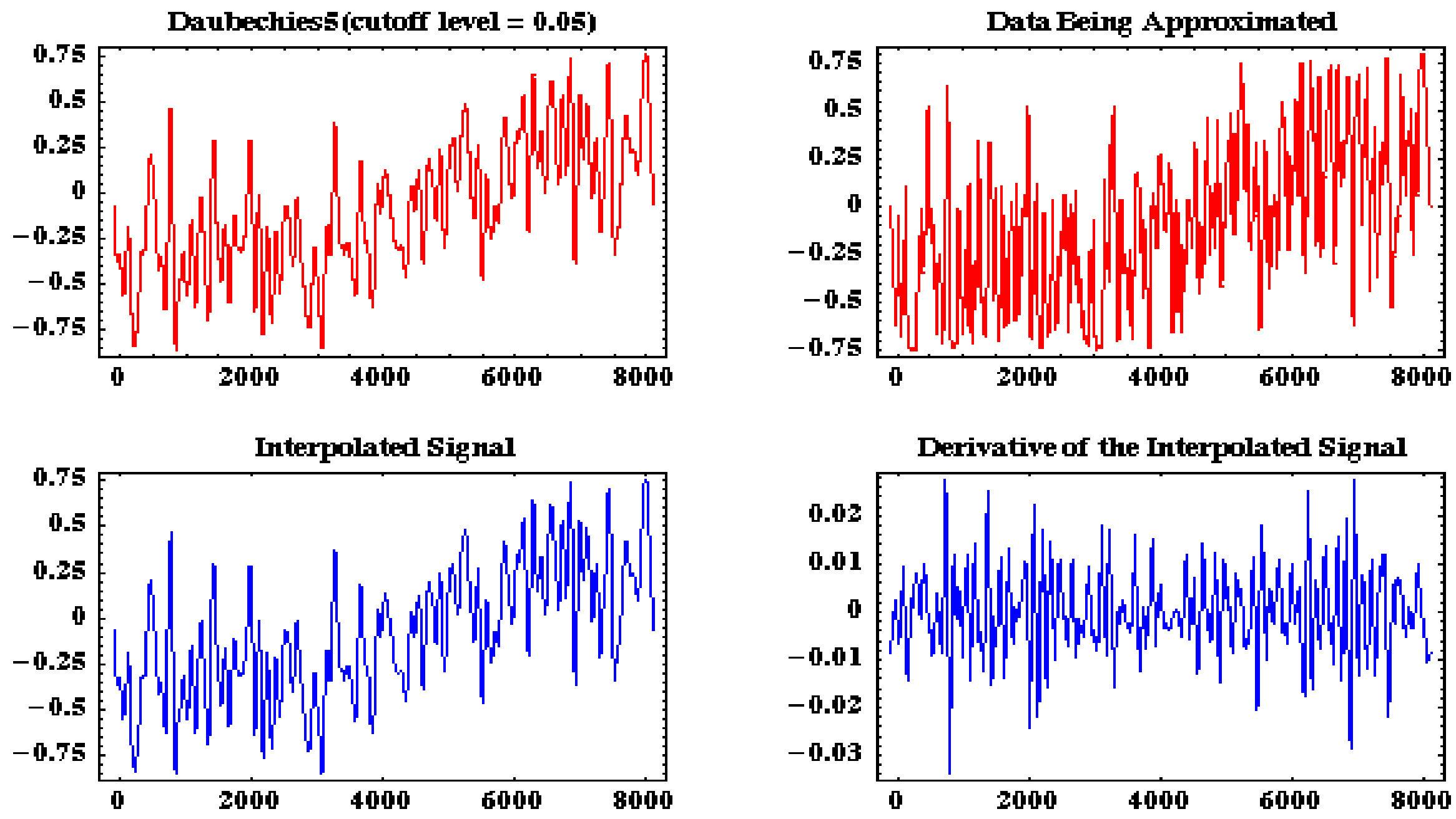


\section{Reconstruction of the LPF Data} Using the First 4 MRD Levels
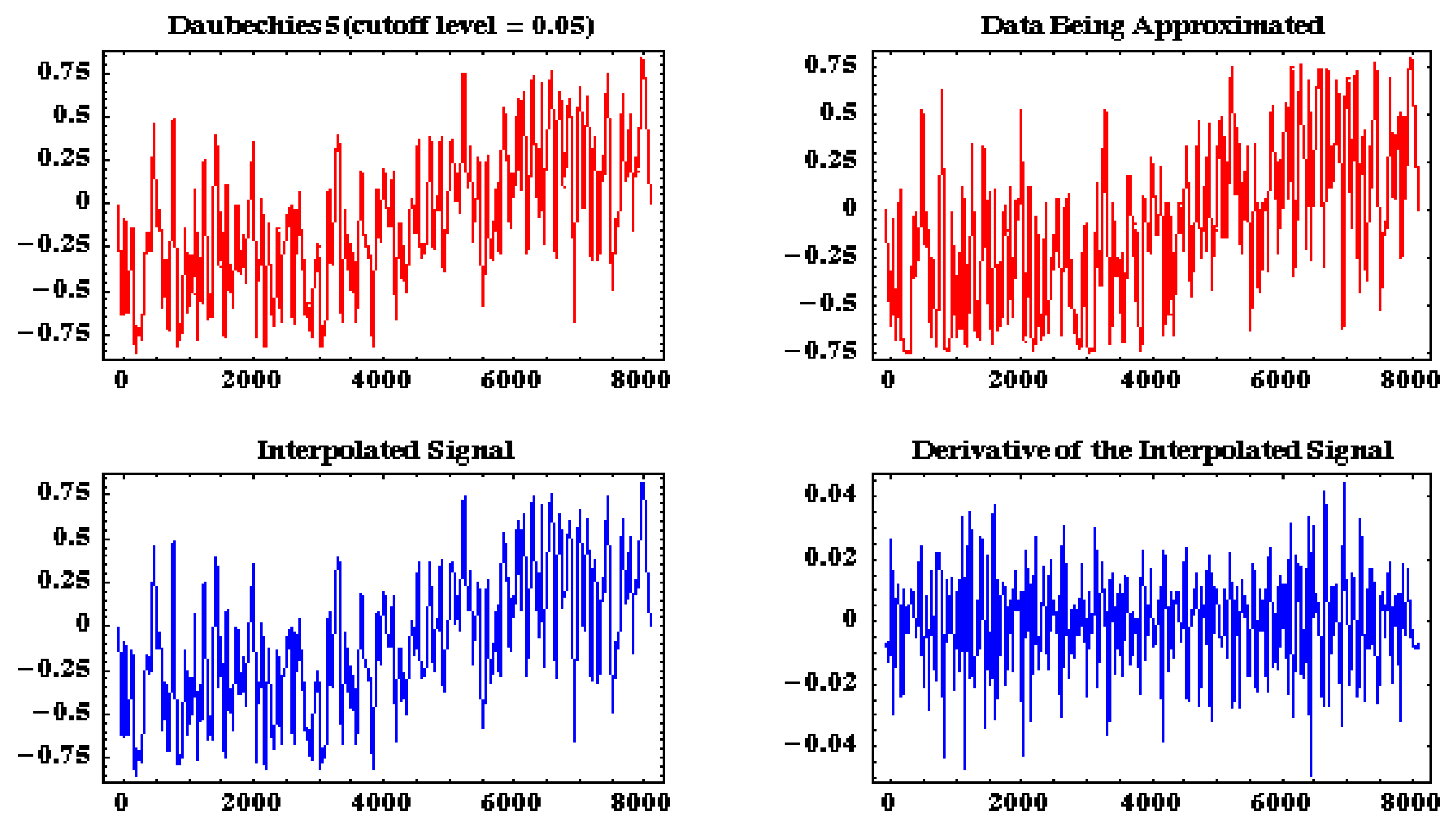


\section{Reconstruction of the LPF Data} Using the First 5 MRD Levels
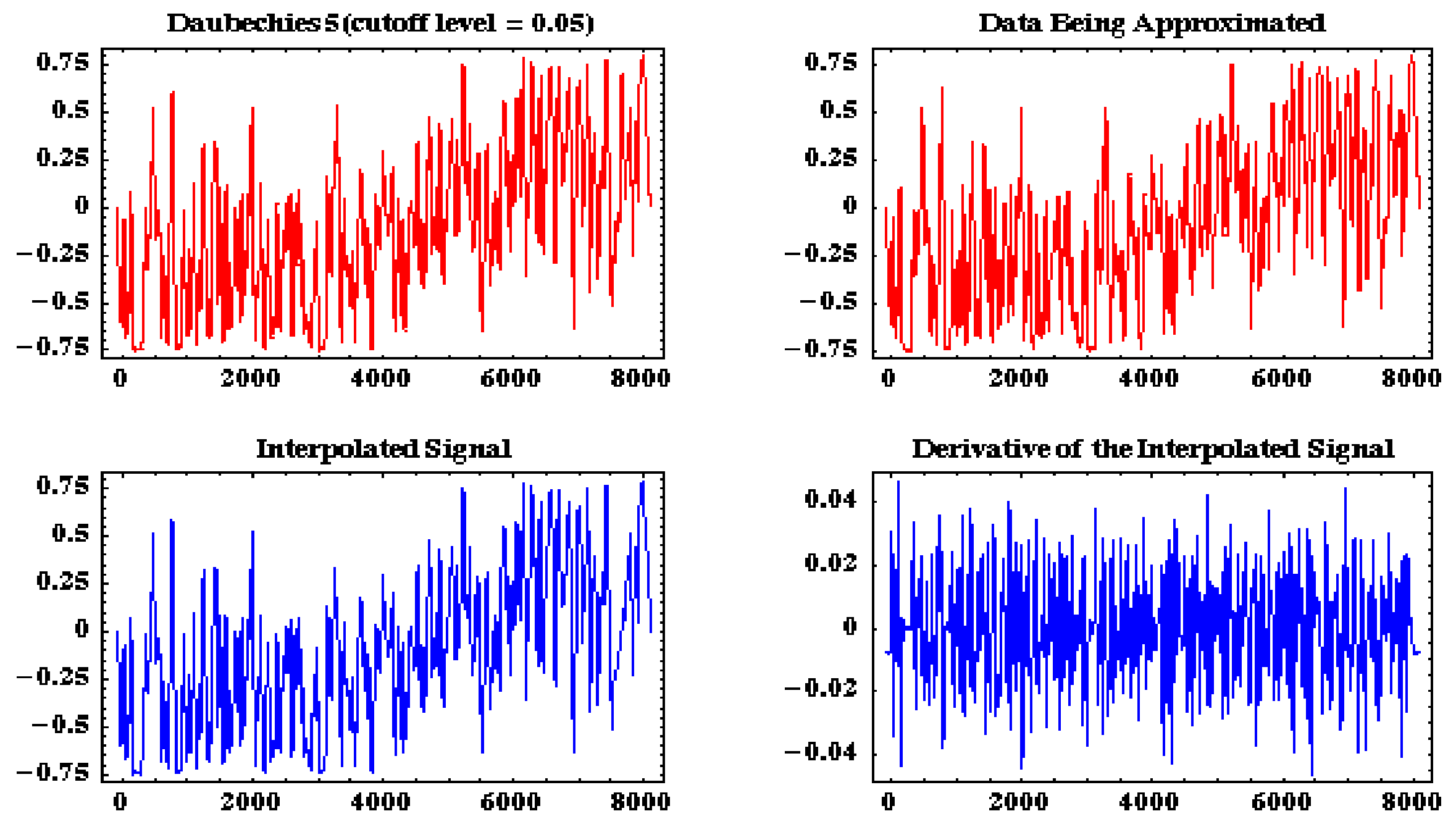


\section{Conclusions Regarding the WLT Analysis of the LPF RT Mix Data}

- Far better compression and denoising is achieved once a modest amount of initial low pass filtering is done on the data.

- Note the extremely small contributions levels 5 and above make to the MRD while with the unfiltered data that contribution was of order 1 or 0.1

- Far cleaner structures are observable in levels 1,2 and 3, periodic correlations in time, or so it seems to the eye!

- The reconstruction with largest wavelets kept shows long patches of flatness surrounded by localized structures which could be indicative of the correlation properties of the data.

- More to come! 
Raw RT Weak Mix Data $(2 \mathrm{~cm}$

\section{Downstream, Theta $=0.7$ )}

from Texas A\&M

Raw Weak RT Mix Data

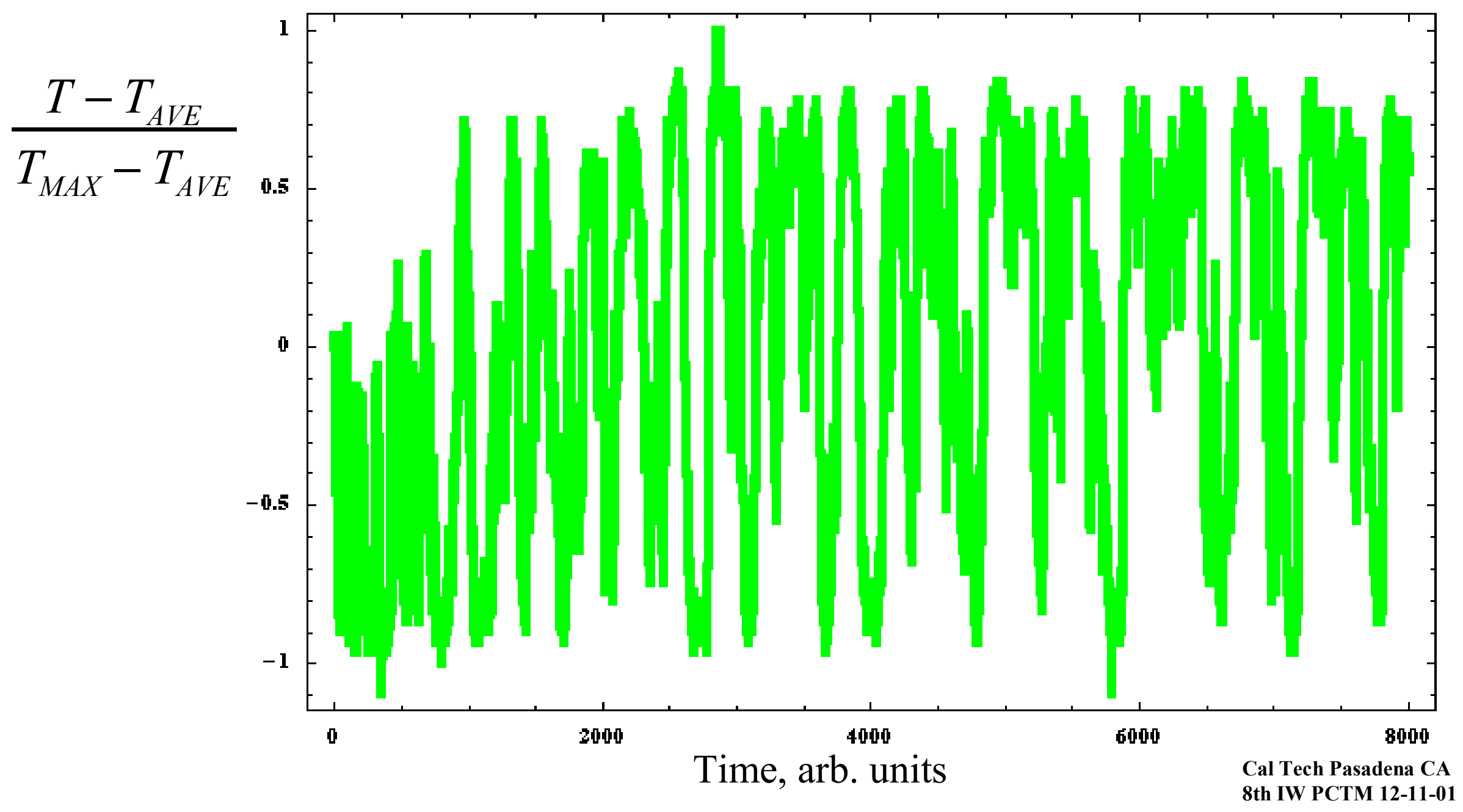




\section{The Faded and Padded Version of the RT Weak Mix Data: 8192 Points}

$2^{13}$ data points, faded and padded

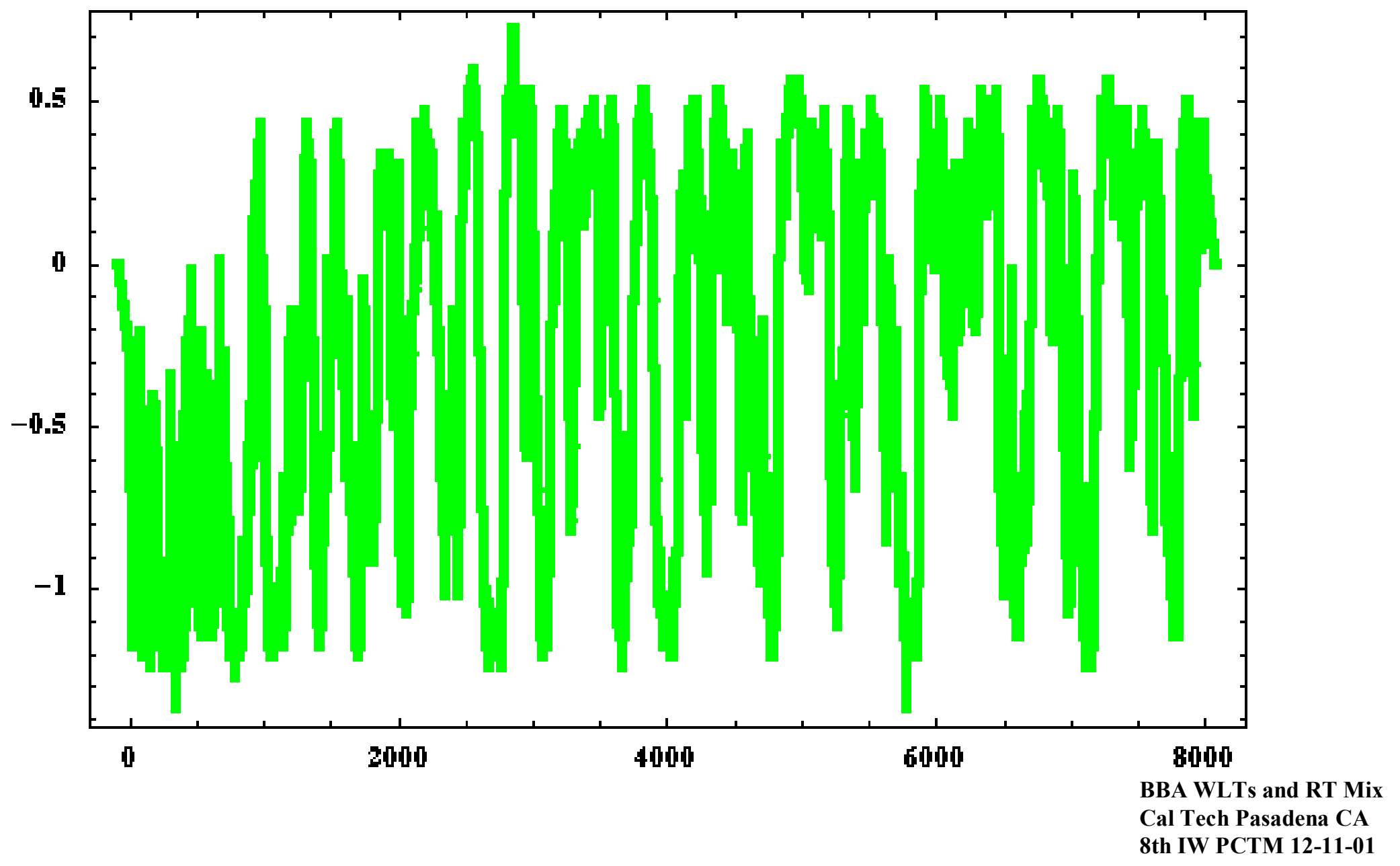




\section{The Fourier Transform of the RT Weak Mix Data}

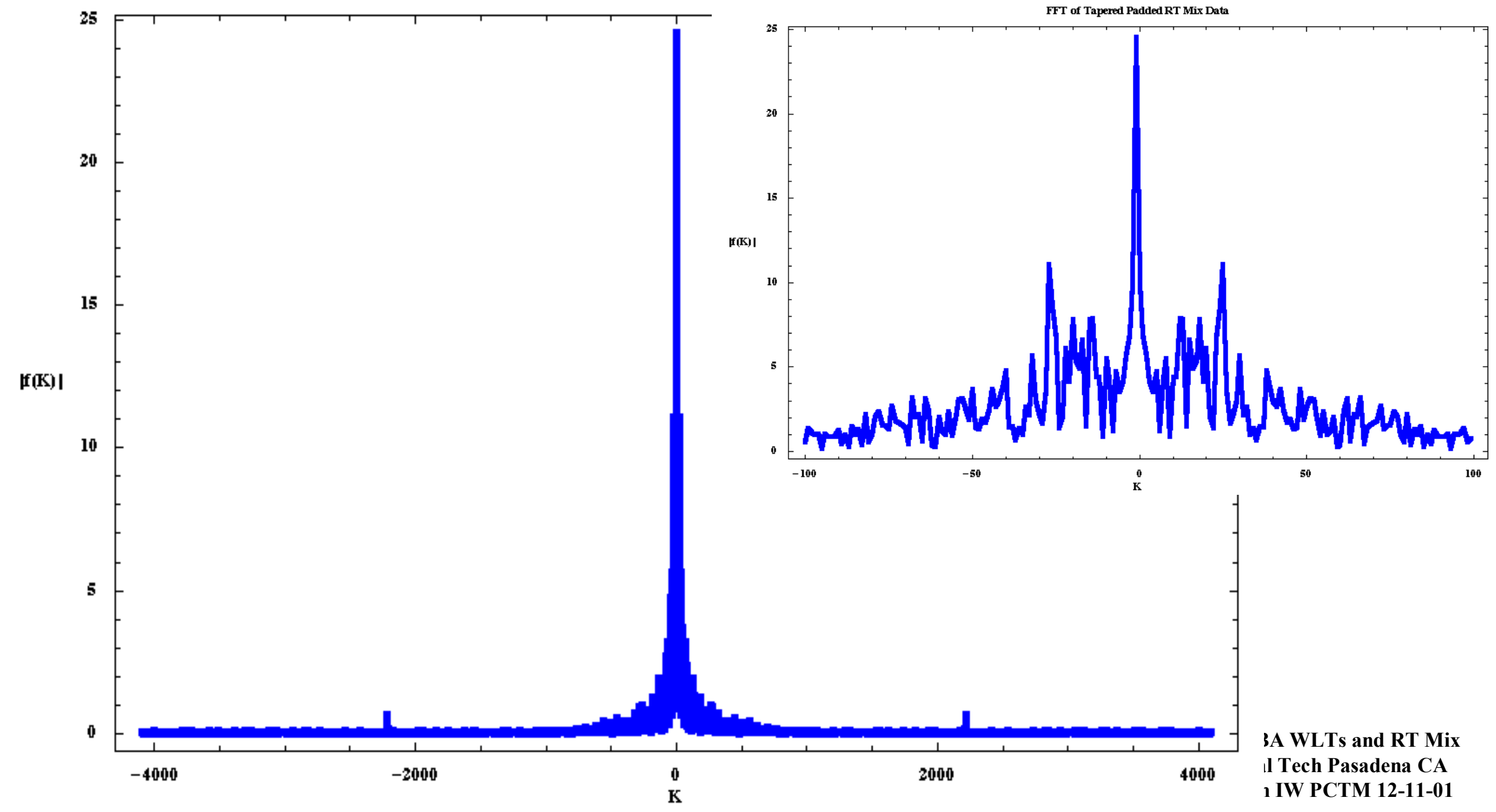




\section{MRD Coefficients of the RT Weak Mix Data in 6 Different Daubechies WLT Bases}
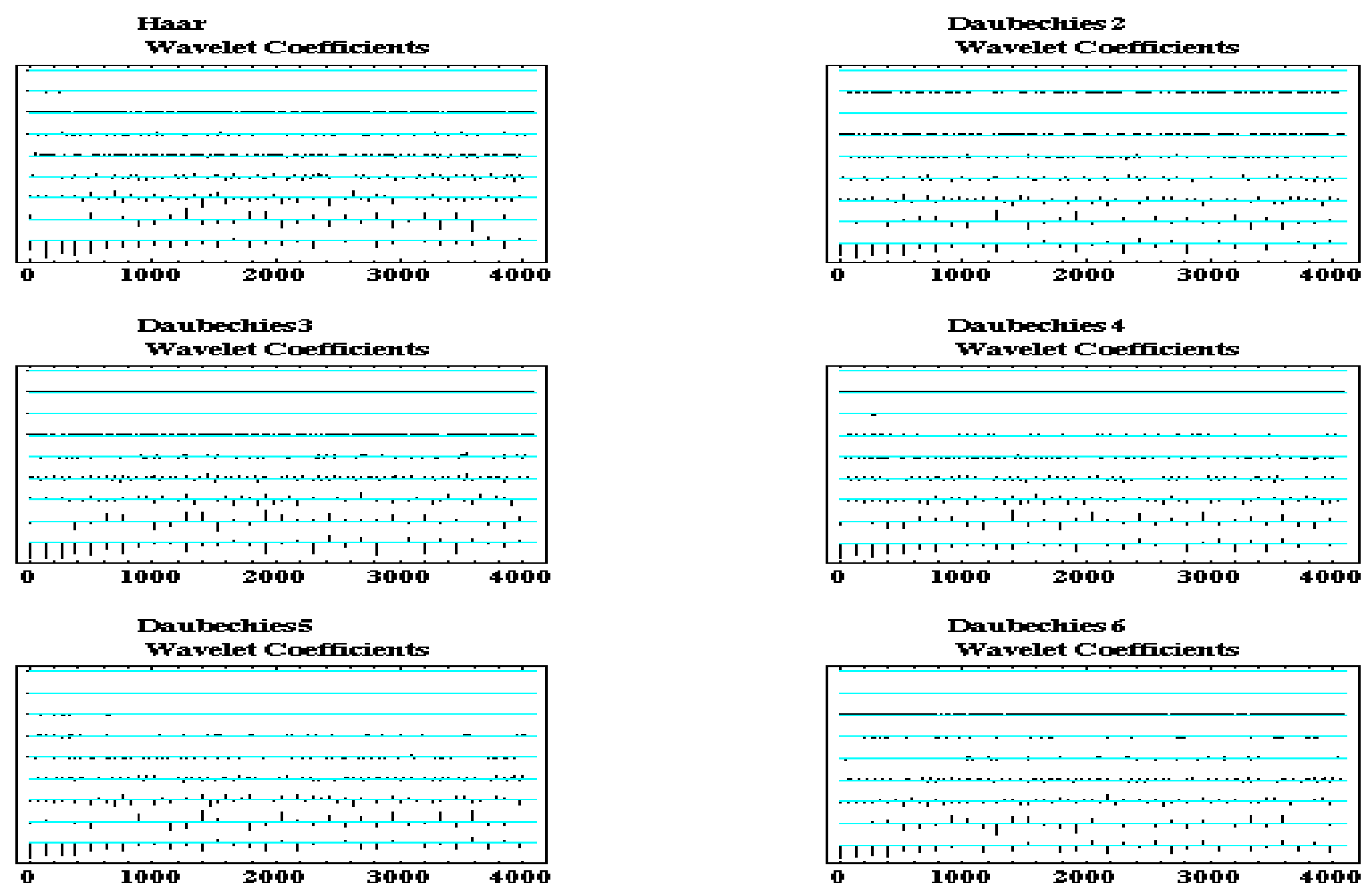


\section{Actual MRDs of the RT Weak Mix Data in 6 Different Daubechies WLT Bases}

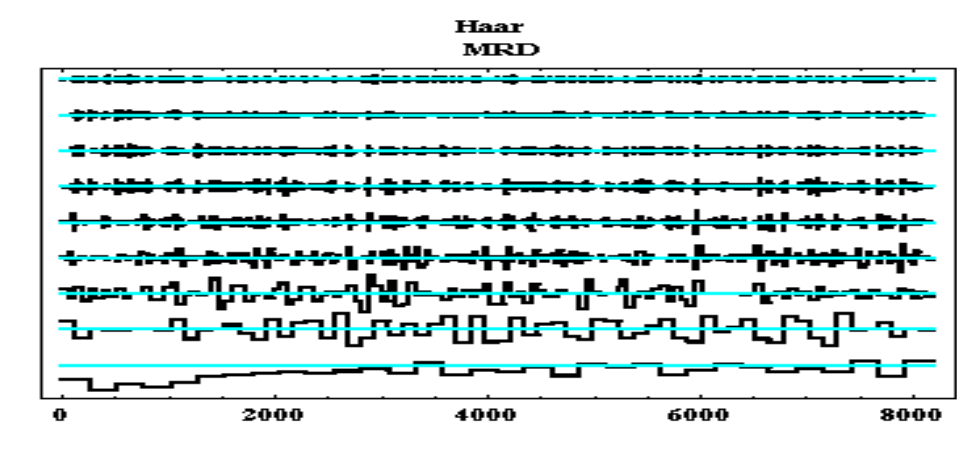

Daubechies 3

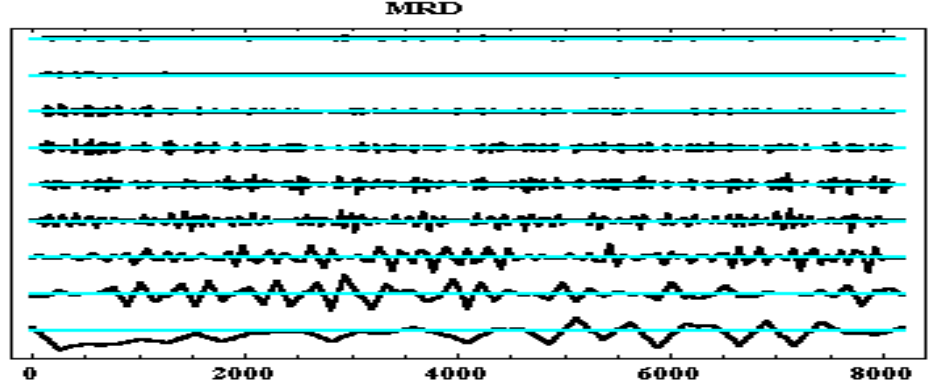

Daubechies

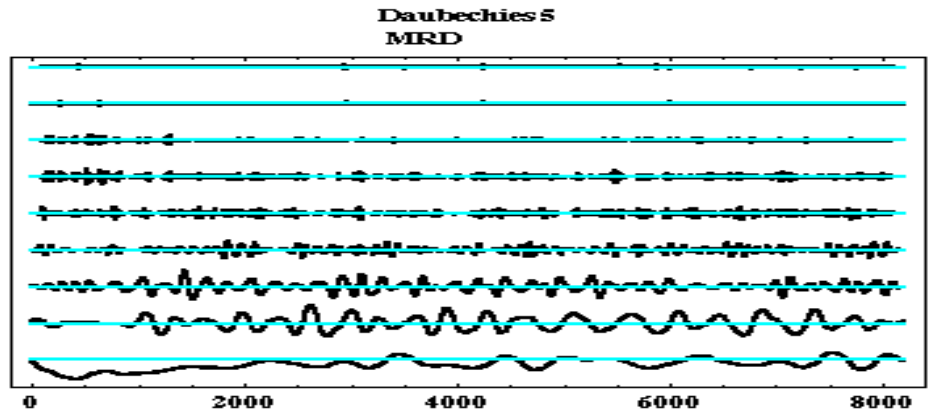

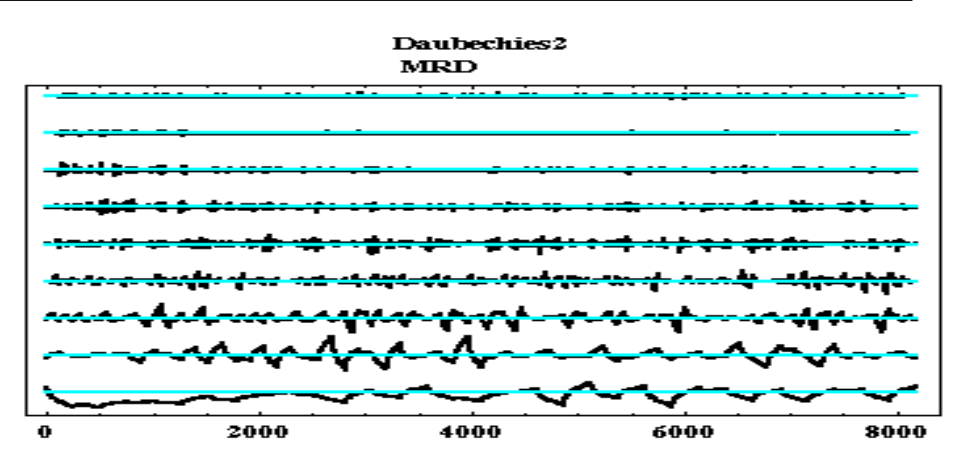

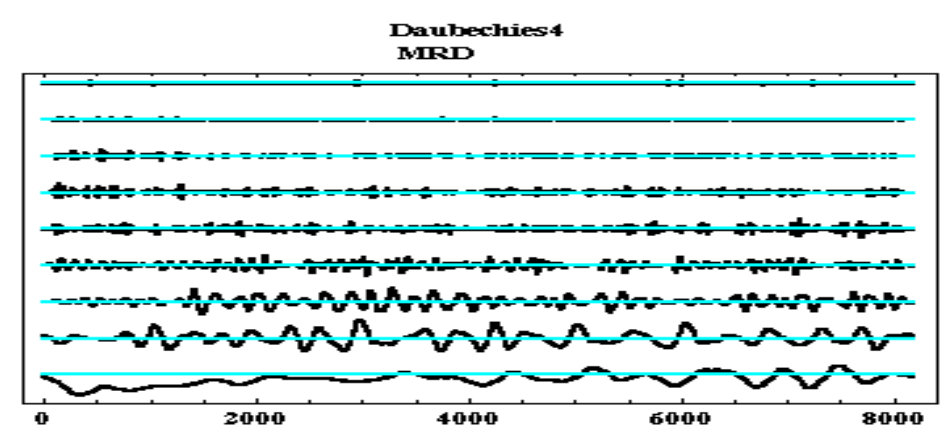

Daubechies6

MIRD

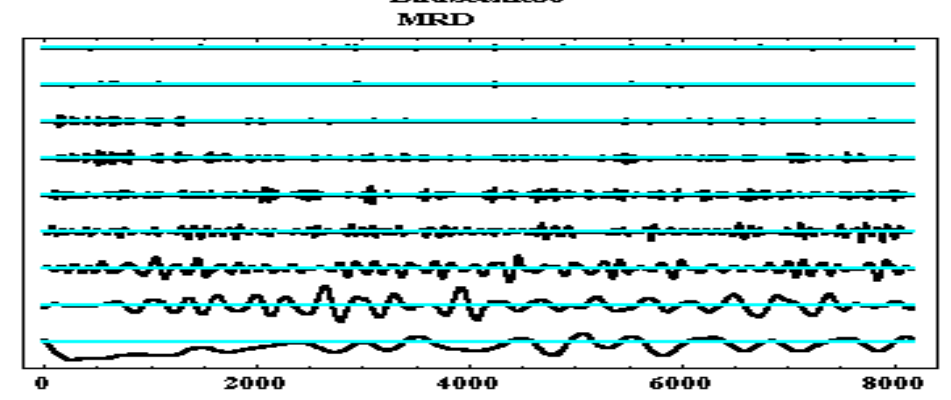




\section{Decay Rate of Largest Coefficient vs Number of Coefficients Kept in 6 Different Daub WLT Decomps}
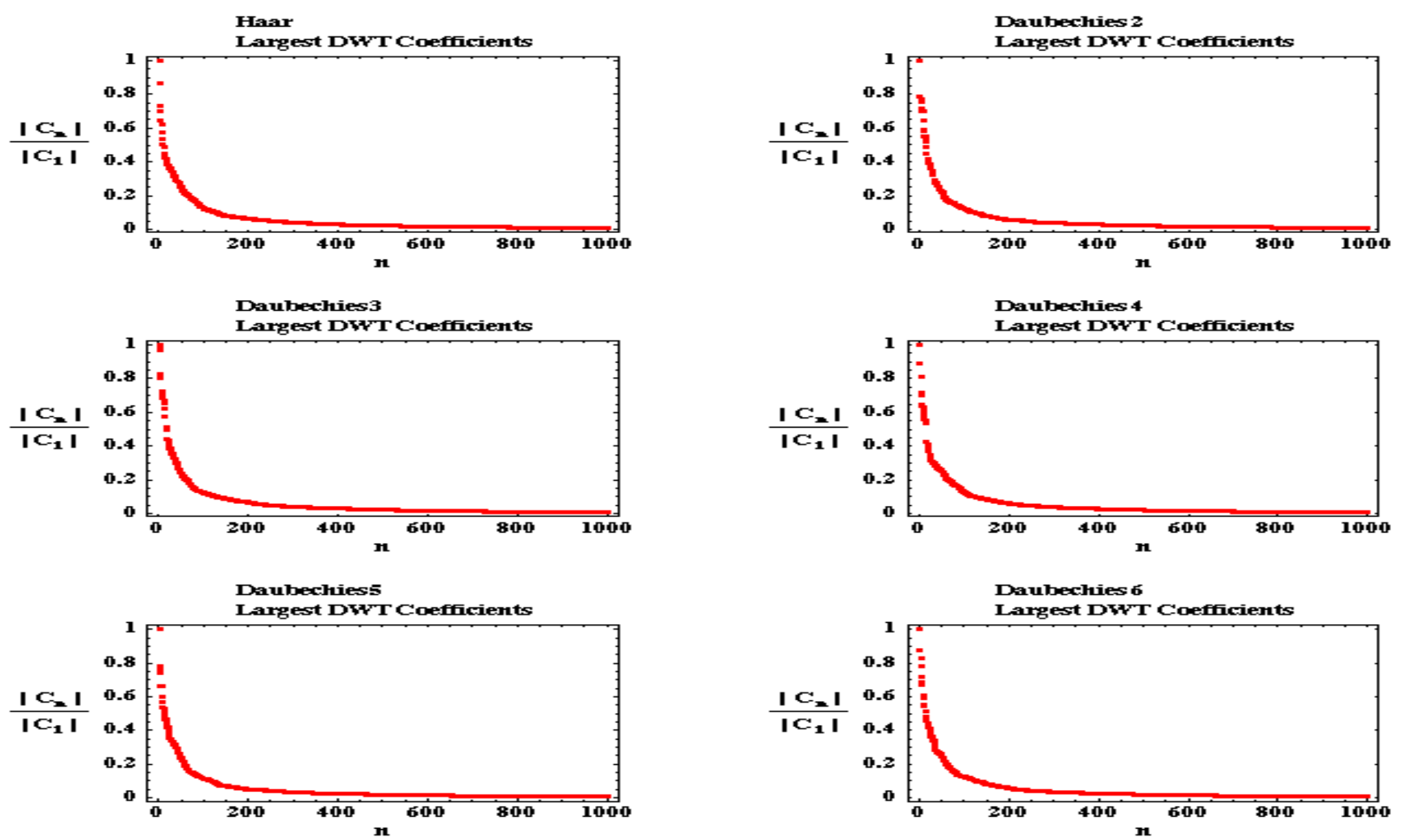


\section{Energy Accumulation Rate in Coefficient Space vs \# of WLTs Kept in 6 Different Daub Decomps}
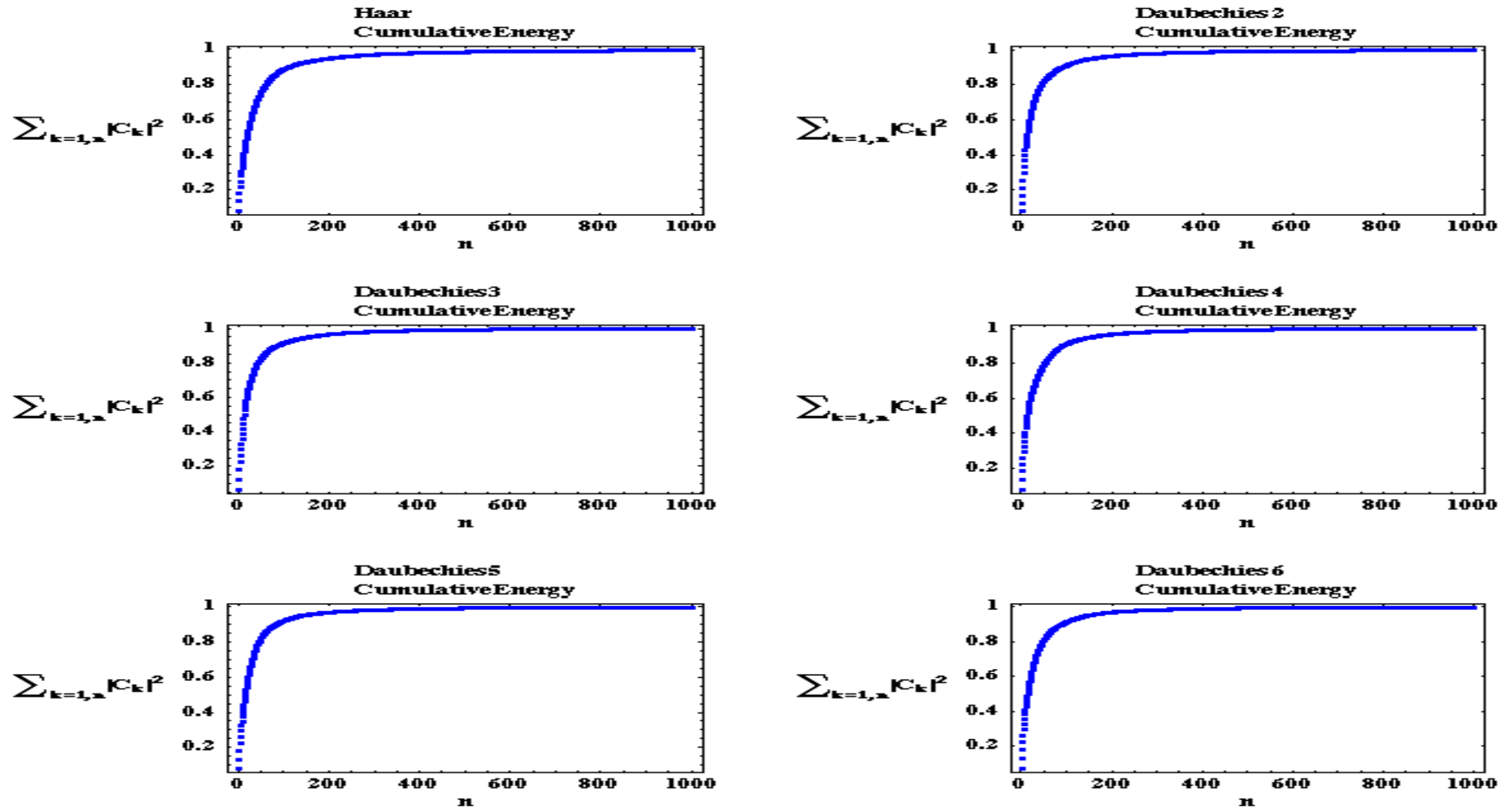


\section{Scaleograms: Waveleters}

\section{Preferred Way of Judging Tiling}

\section{in Scale-Translation Space}
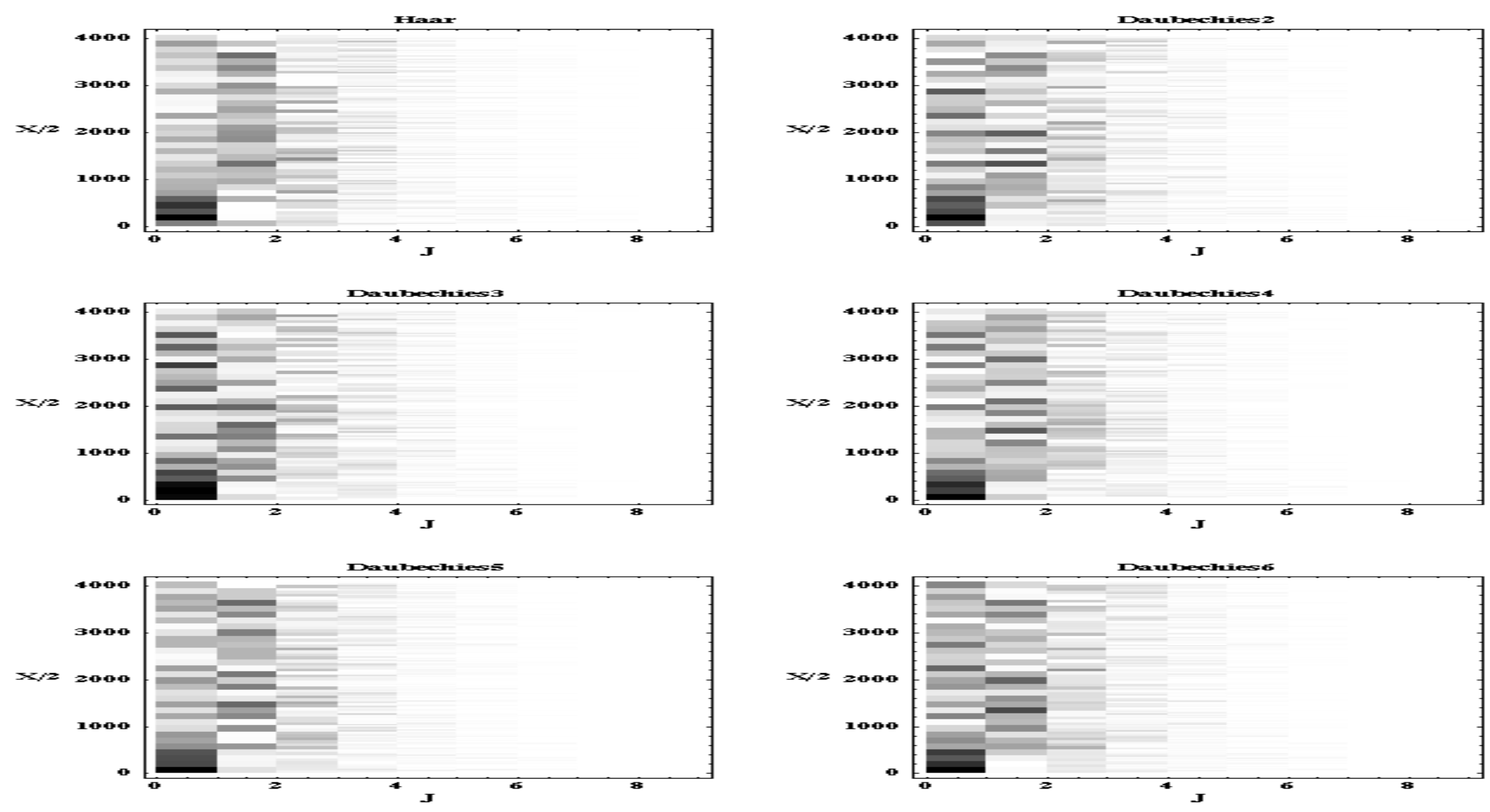


\section{Least Square Error Incurred By Truncating the WLT Series at $N$ of its Largest Coefficients}
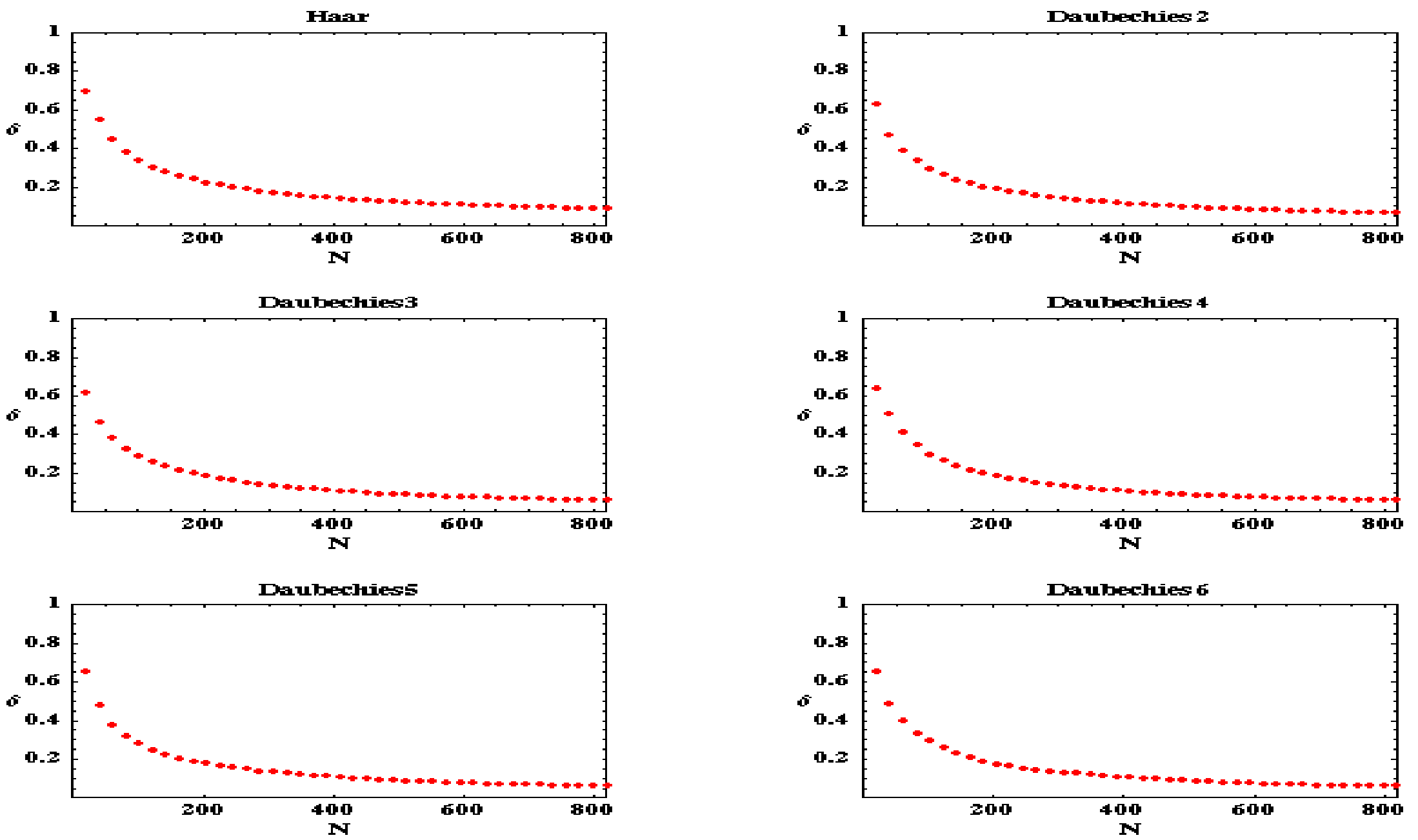


\section{Least Square Error Incurred by Level Thresholding the DWT}

Polymath Research Inc.

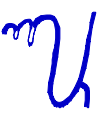

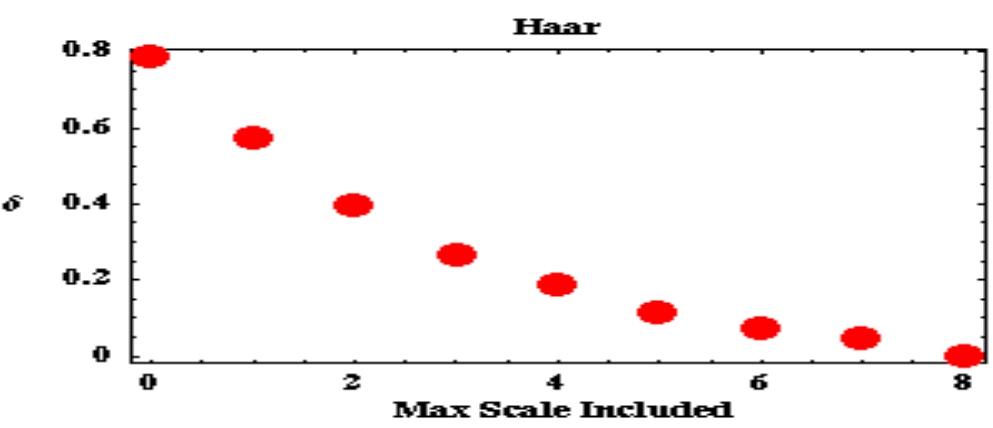
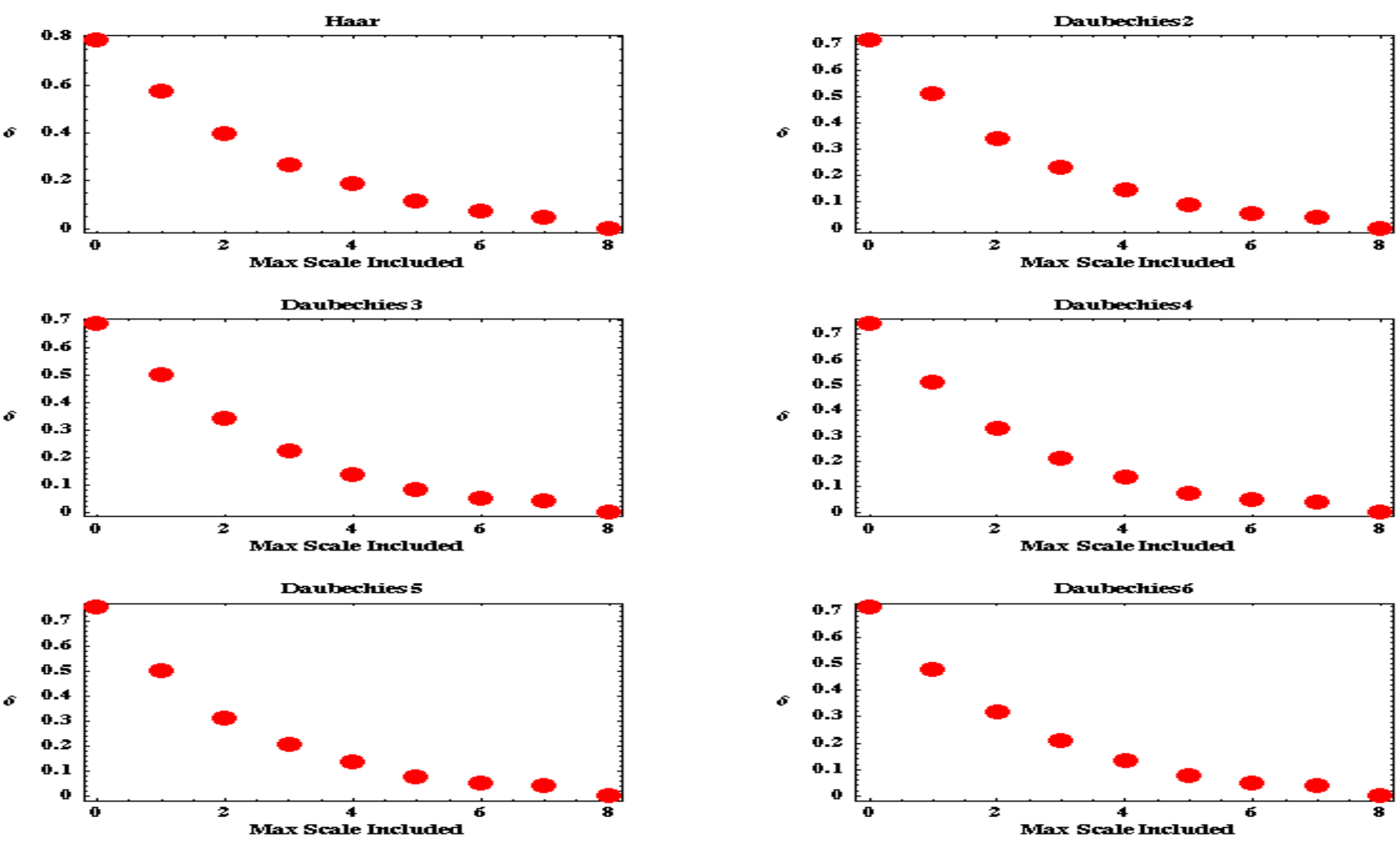


\section{Daubechies 5 Does Much Better than Haar: 5 Quantitative Measures}
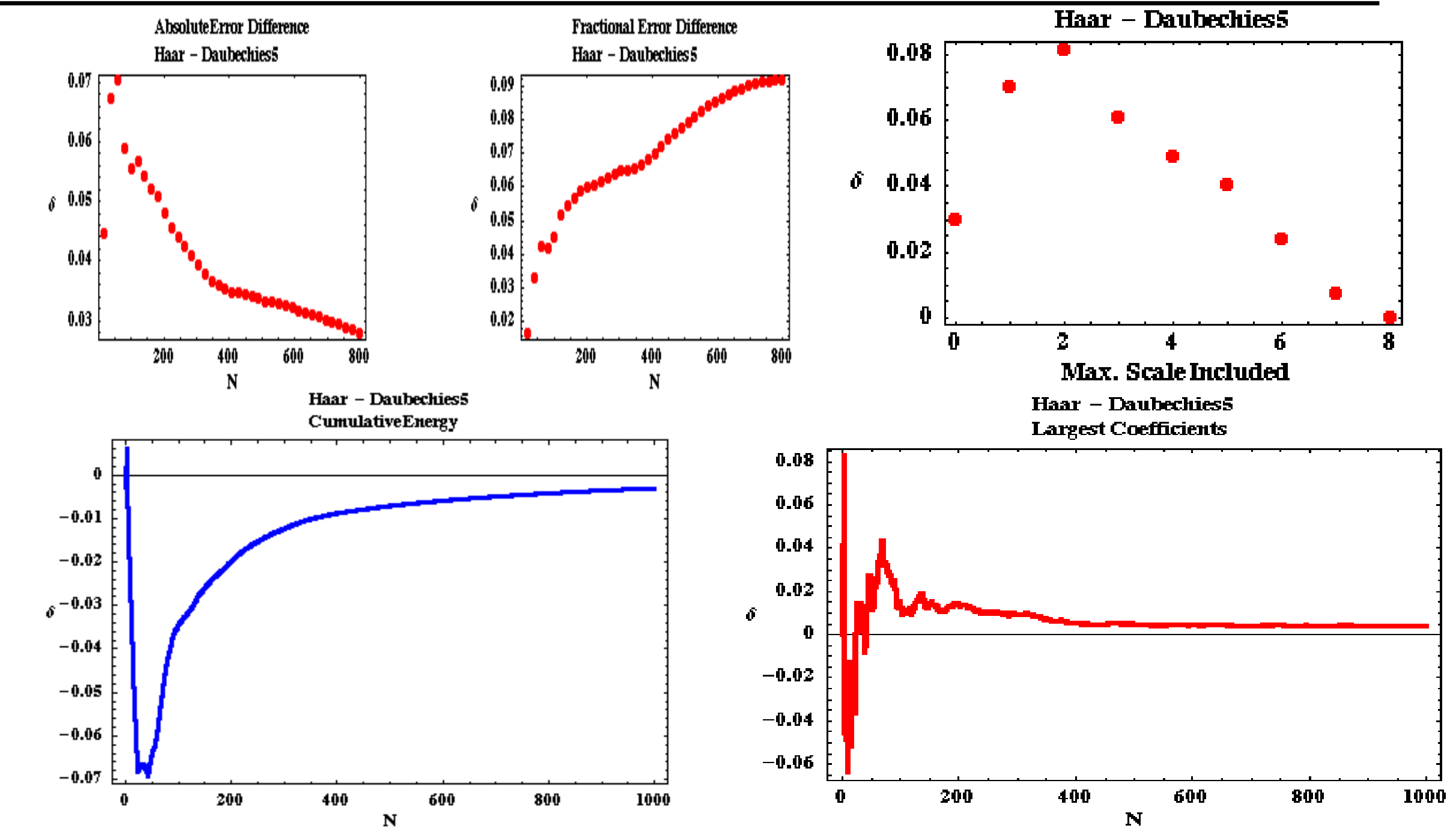


\section{Level by Level Decomposition of the $\mathrm{RT}_{\text {olymath }}$ Weak Mix Data Using Daub5 WLTs V}
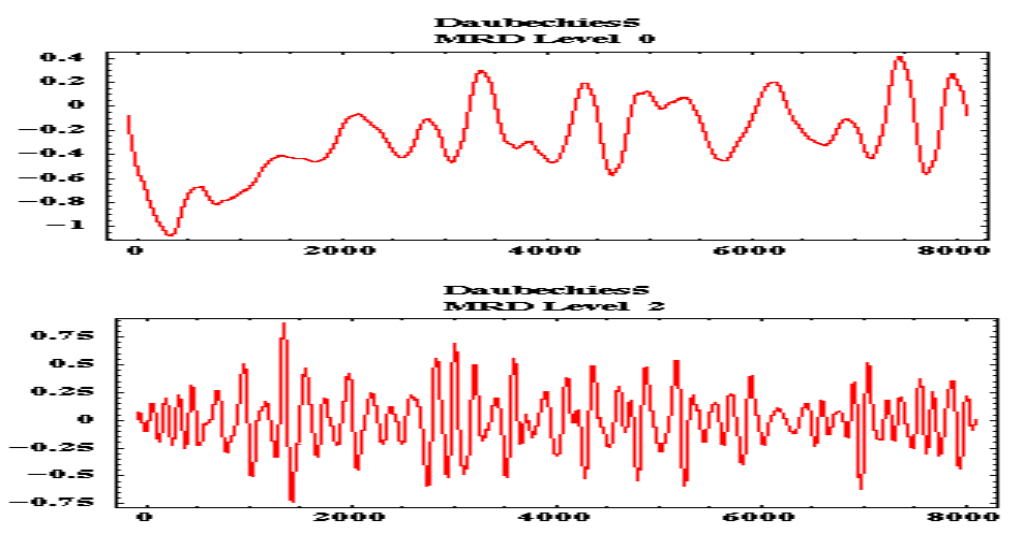
natbrenes:
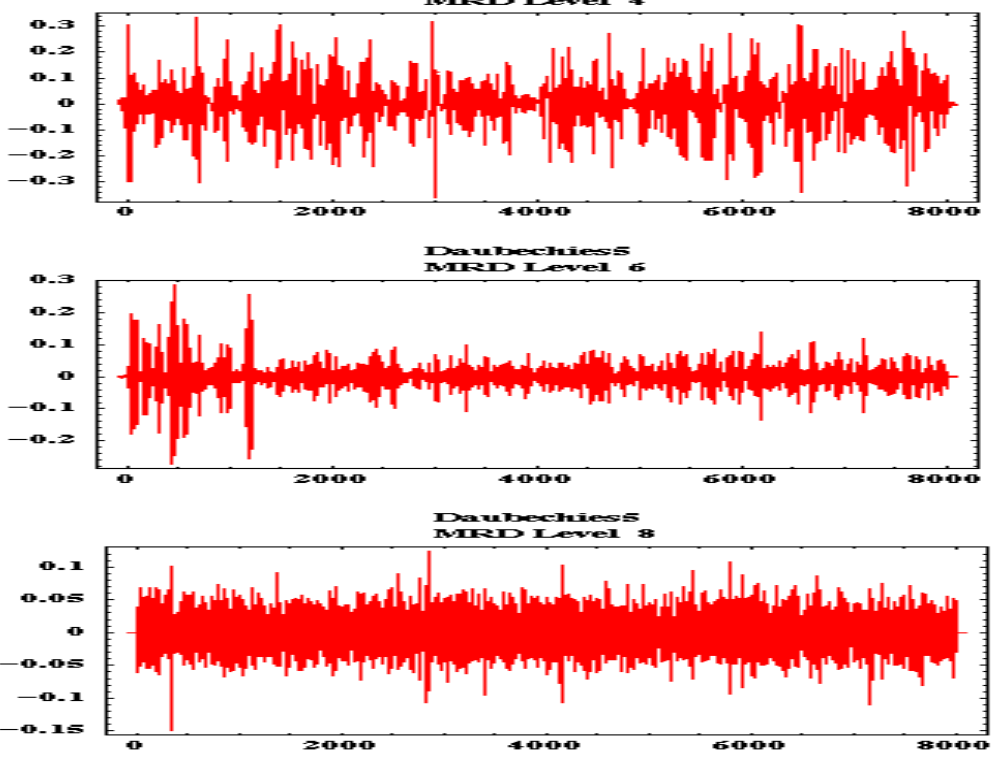

Dinsocheses.

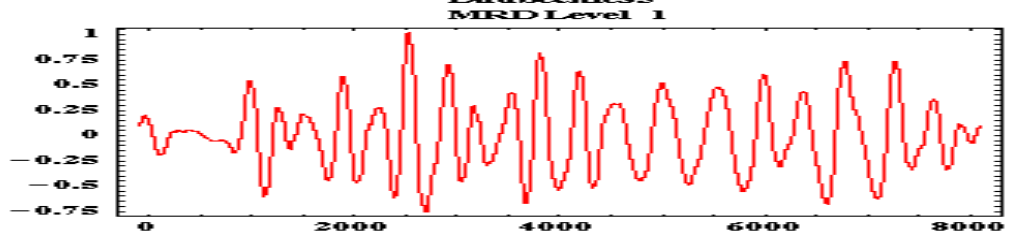

Dawberies:

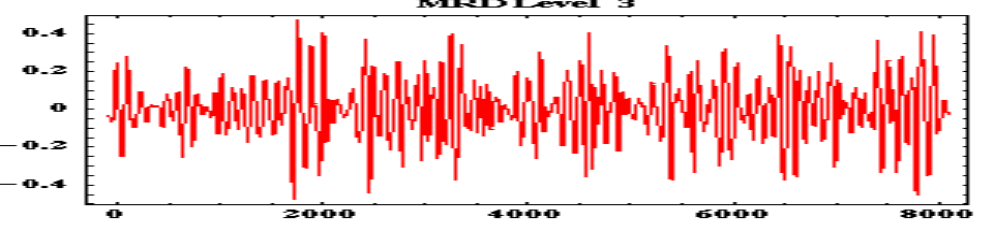

Davbrevers

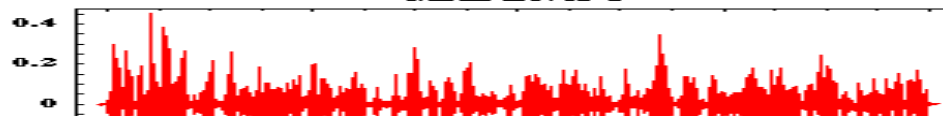

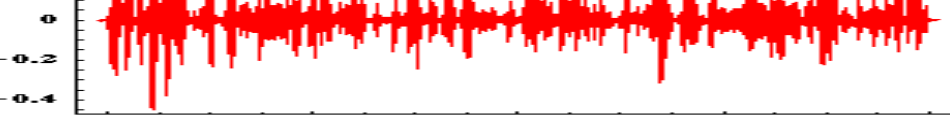

-0.4 .

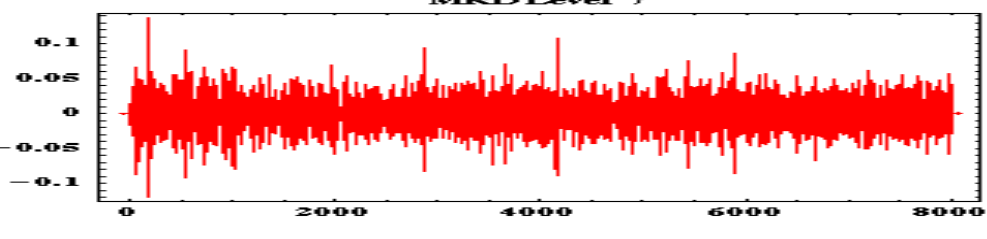

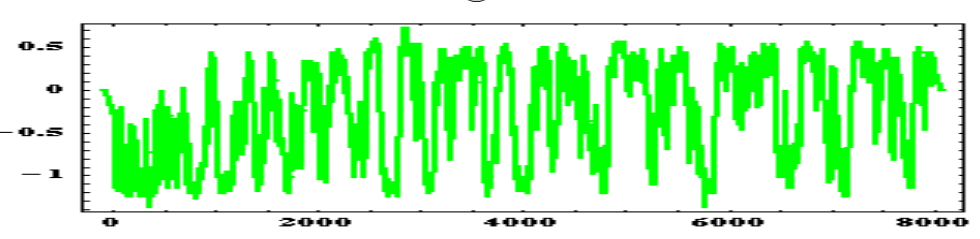




\section{Reconstruction of the Data Using} the 5 Largest WLT Coefficients

Polymath

Research Inc.
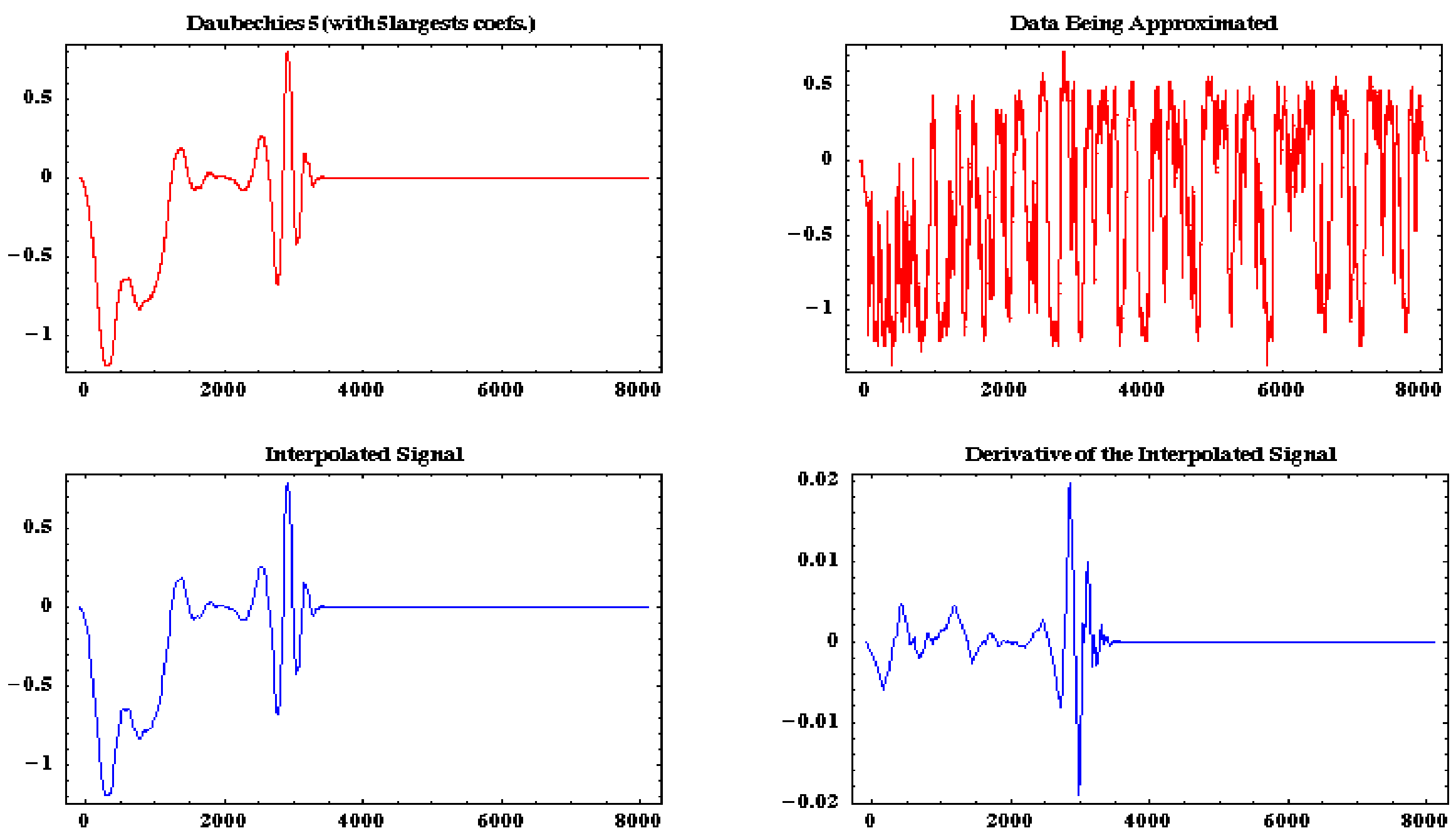


\section{Reconstruction of the Data Using the 10 Largest WLT Coefficients}

Polymath

Research Inc.
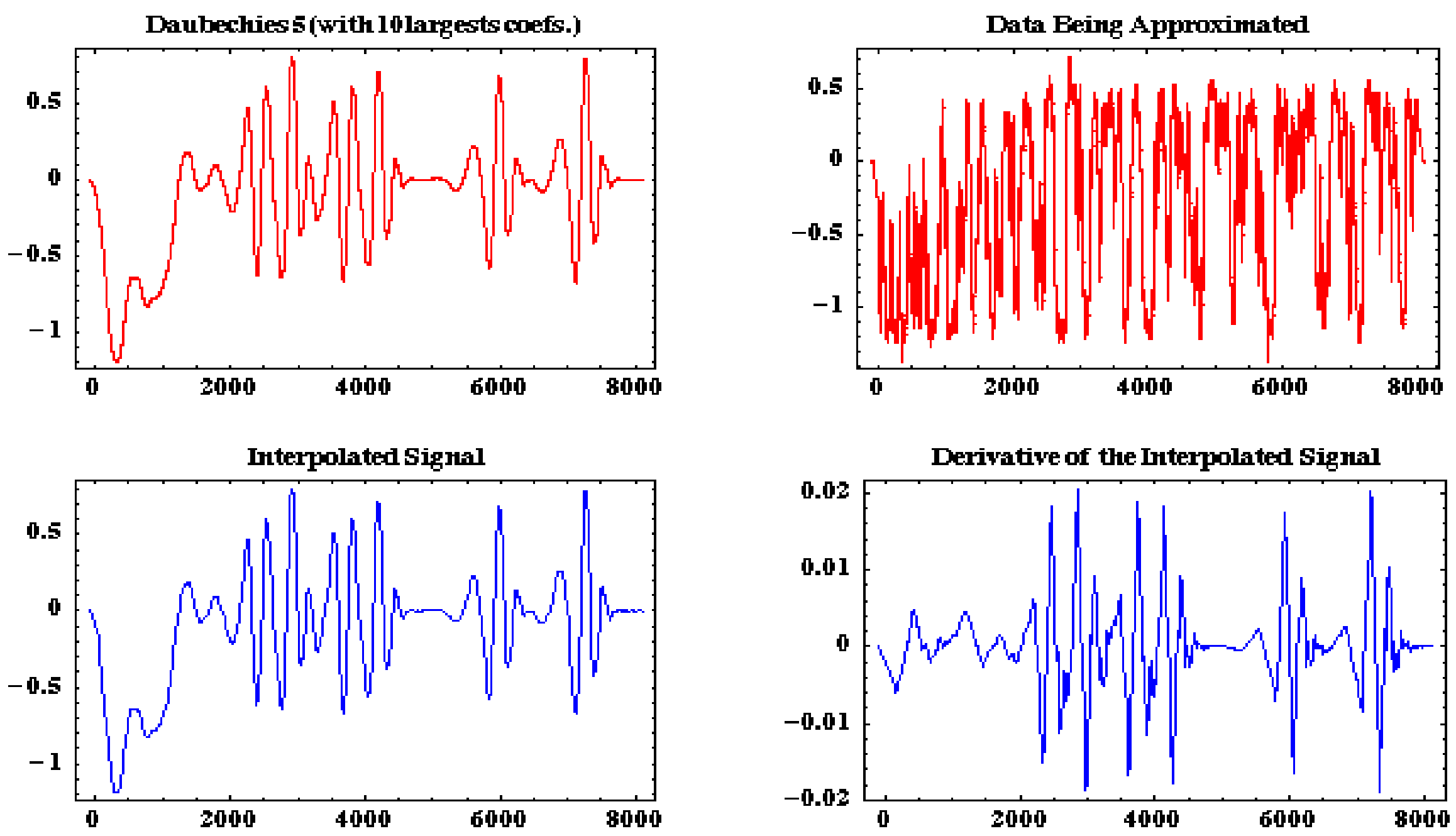


\section{Reconstruction of the Data Using the 15 Largest WLT Coefficients}

Polymath

Research Inc.
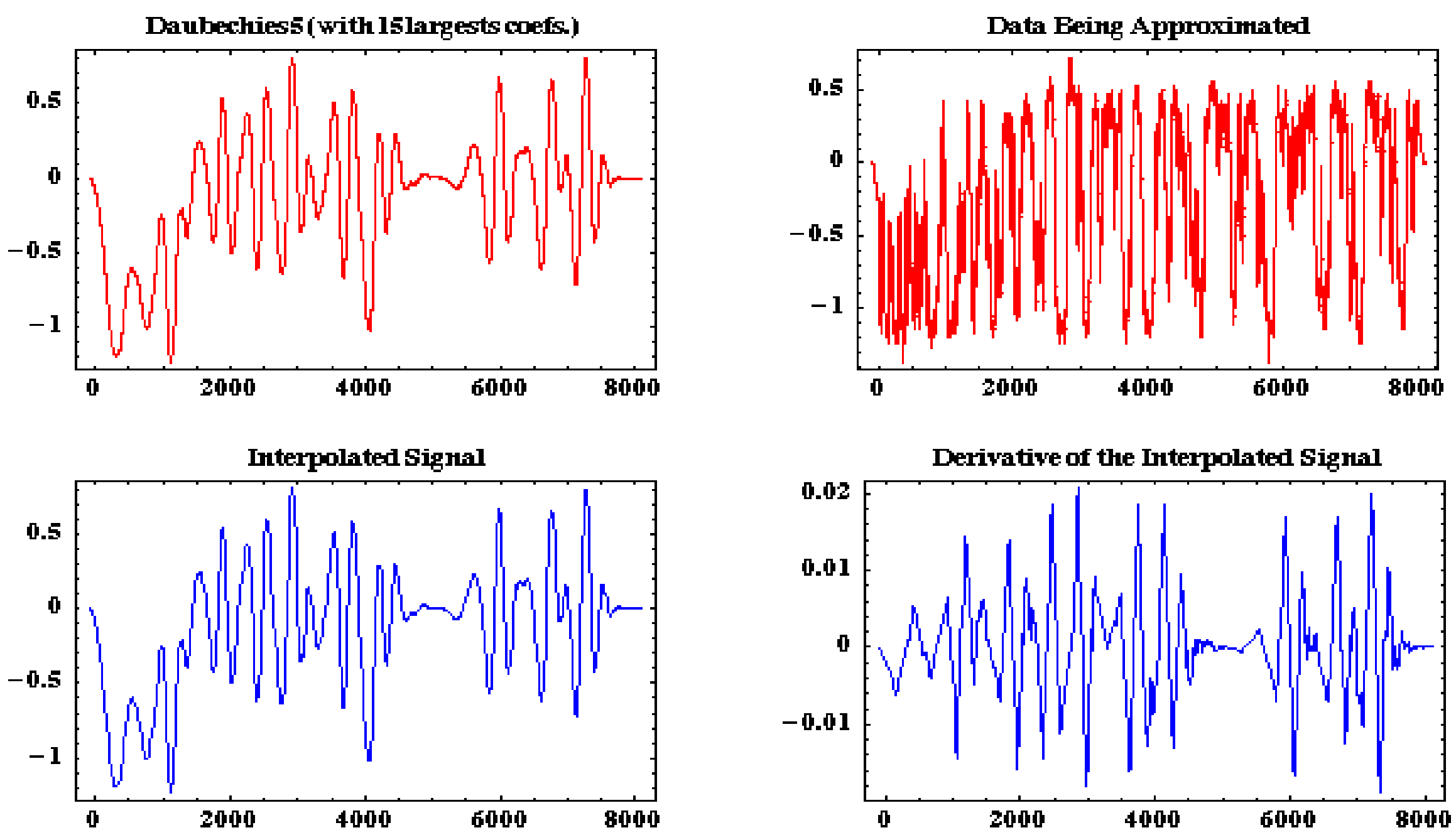


\section{Reconstruction of the Data Using the 20 Largest WLT Coefficients}
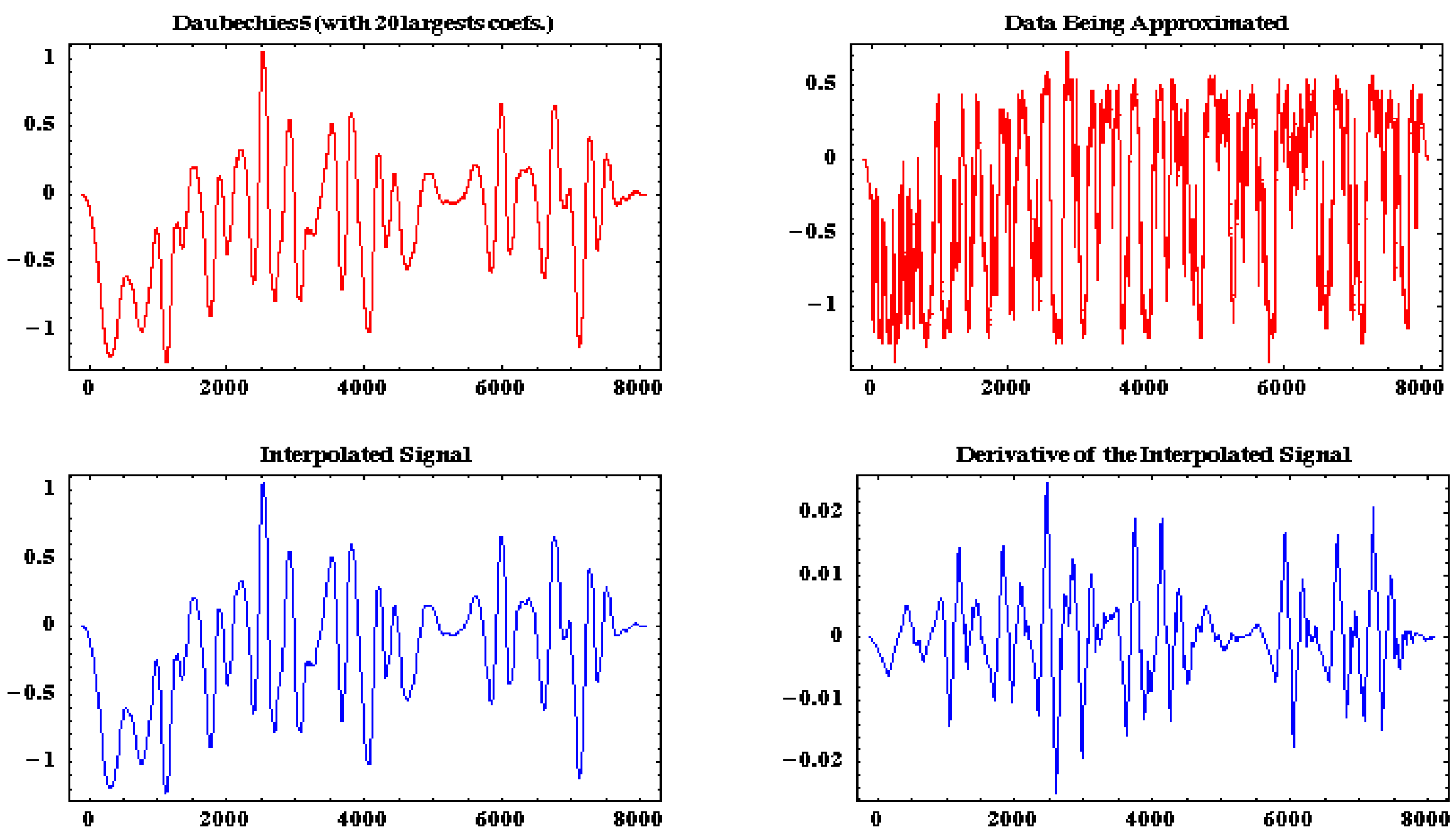


\section{Reconstruction of the Data Using} the 25 Largest WLT Coefficients
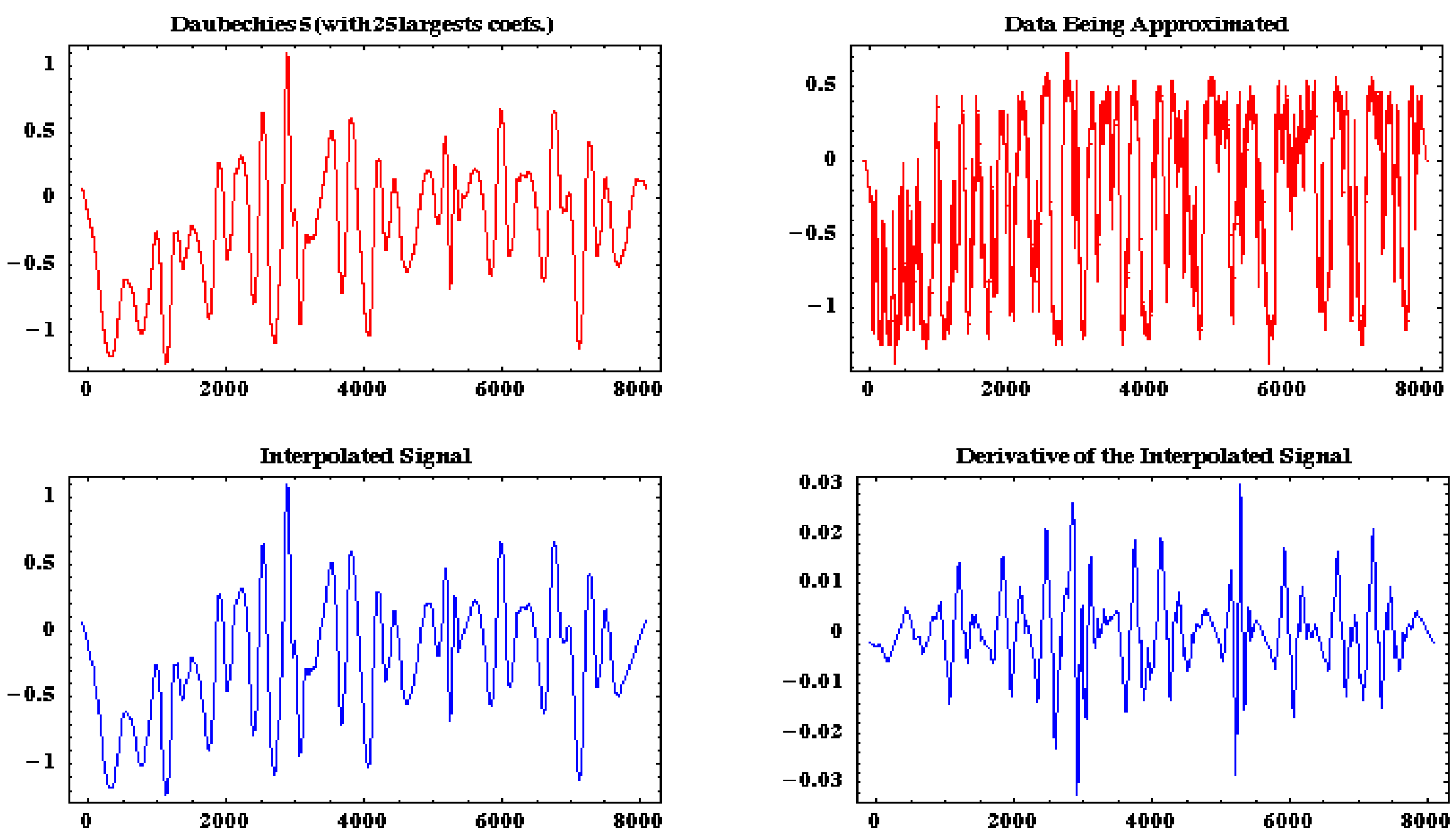


\section{Reconstruction of the Data Using}

\section{the 30 Largest WLT Coefficients}

Polymath

Research Inc.
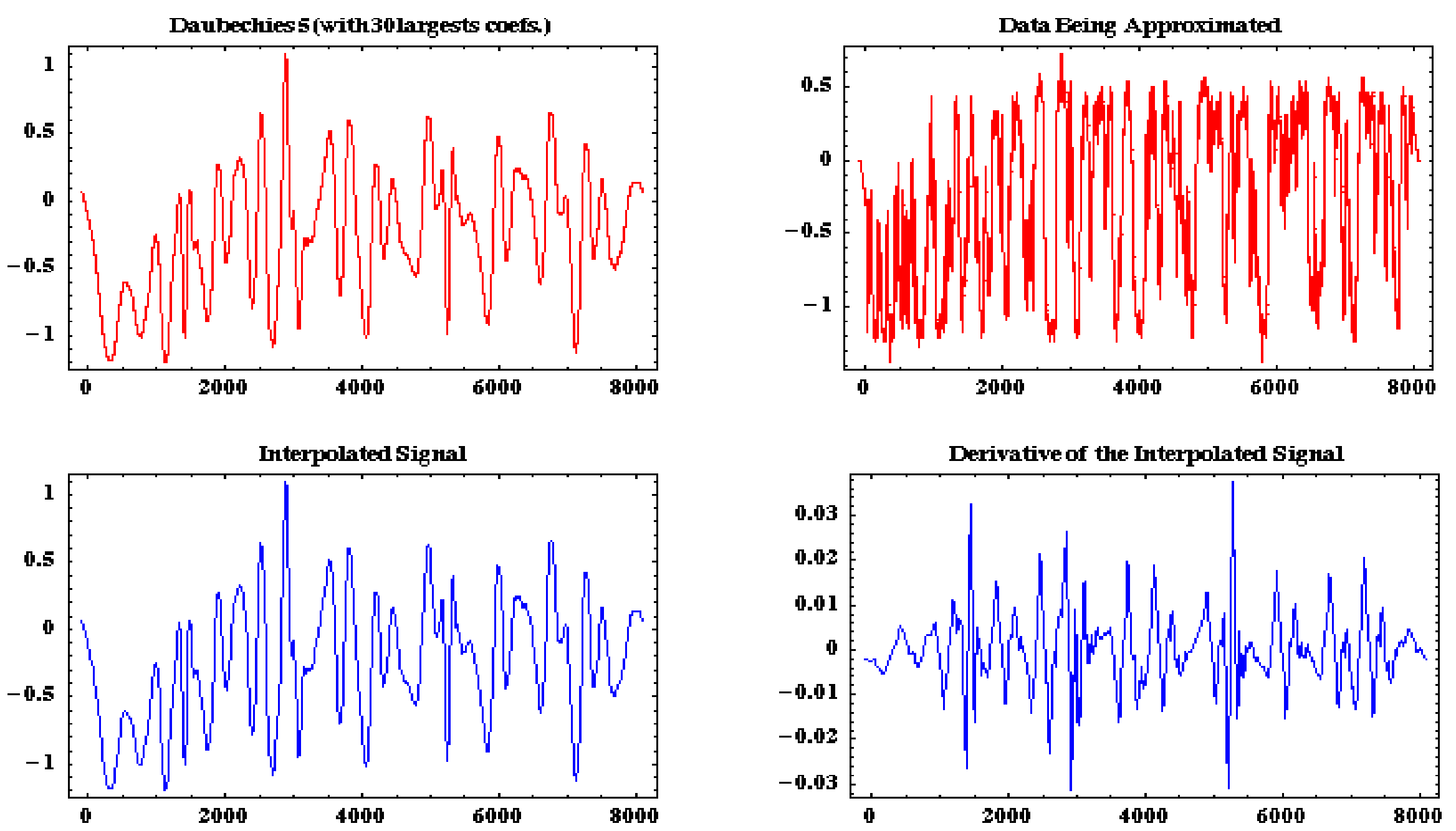


\section{Reconstruction of the Data Using} Polymath

\section{the 35 Largest WLT Coefficients}
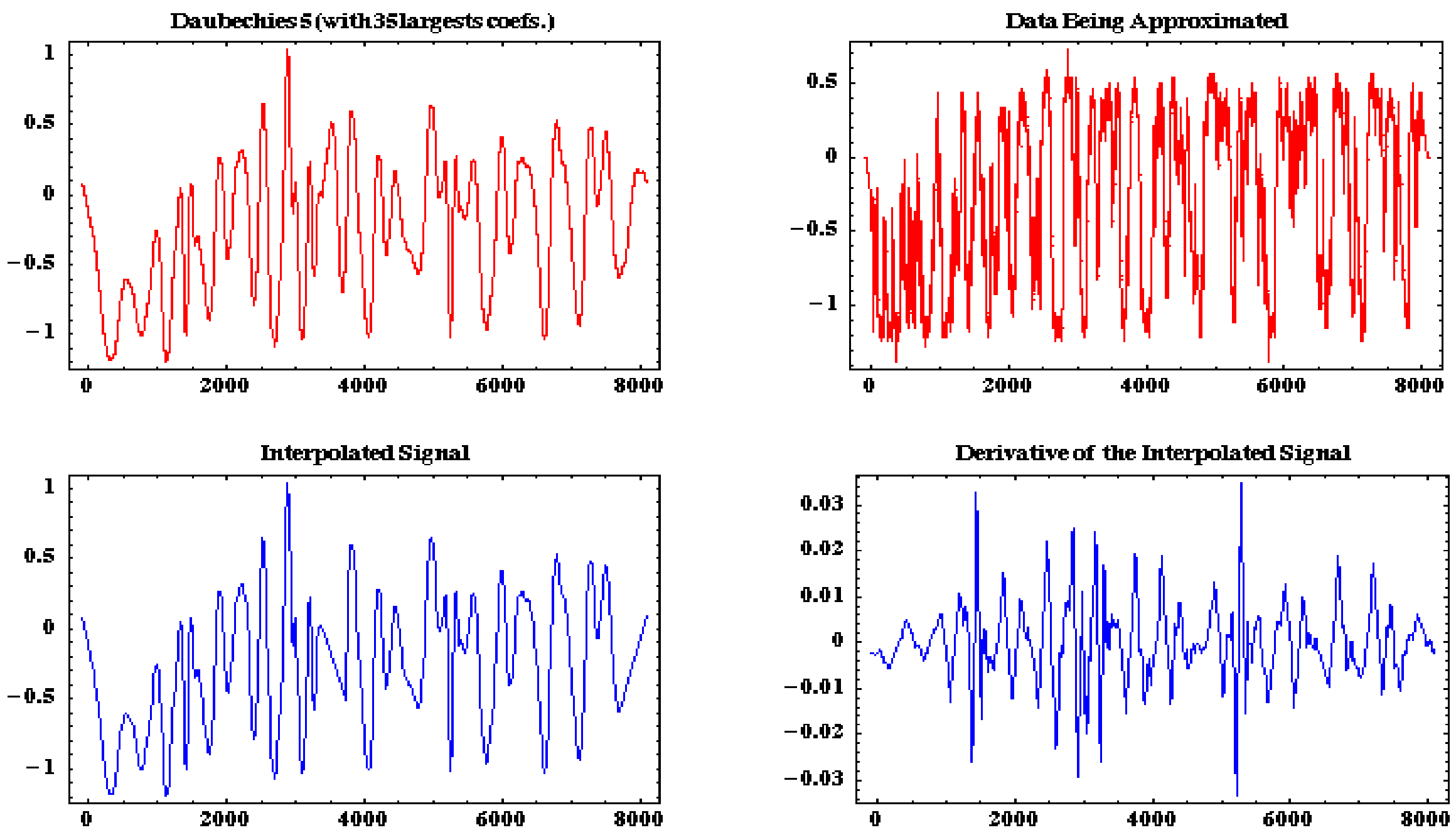


\section{Reconstruction of the Data Using}

\section{the 40 Largest WLT Coefficients}

Polymath

Research Inc.
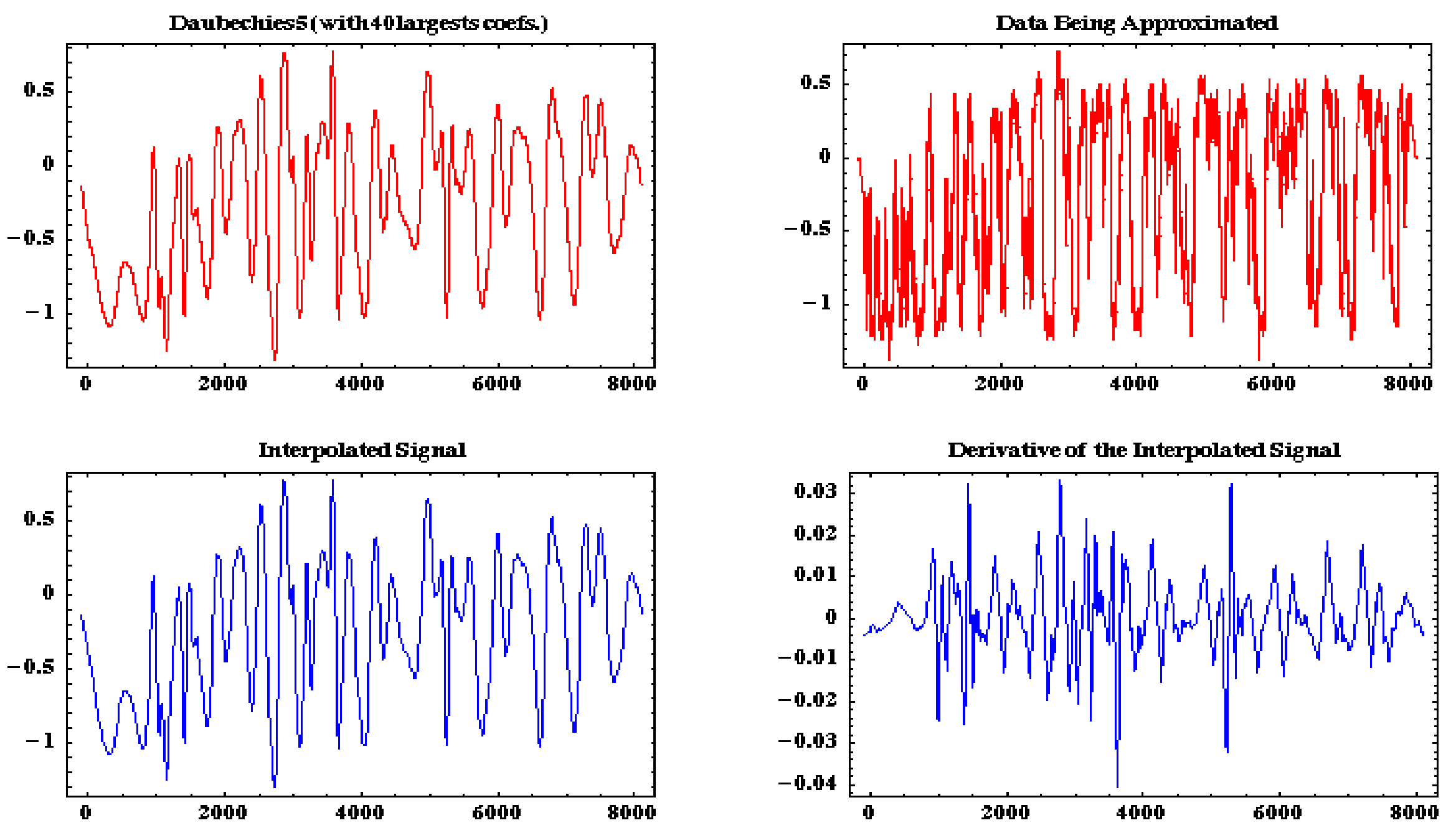


\section{Reconstruction of the Data Using} the 45 Largest WLT Coefficients
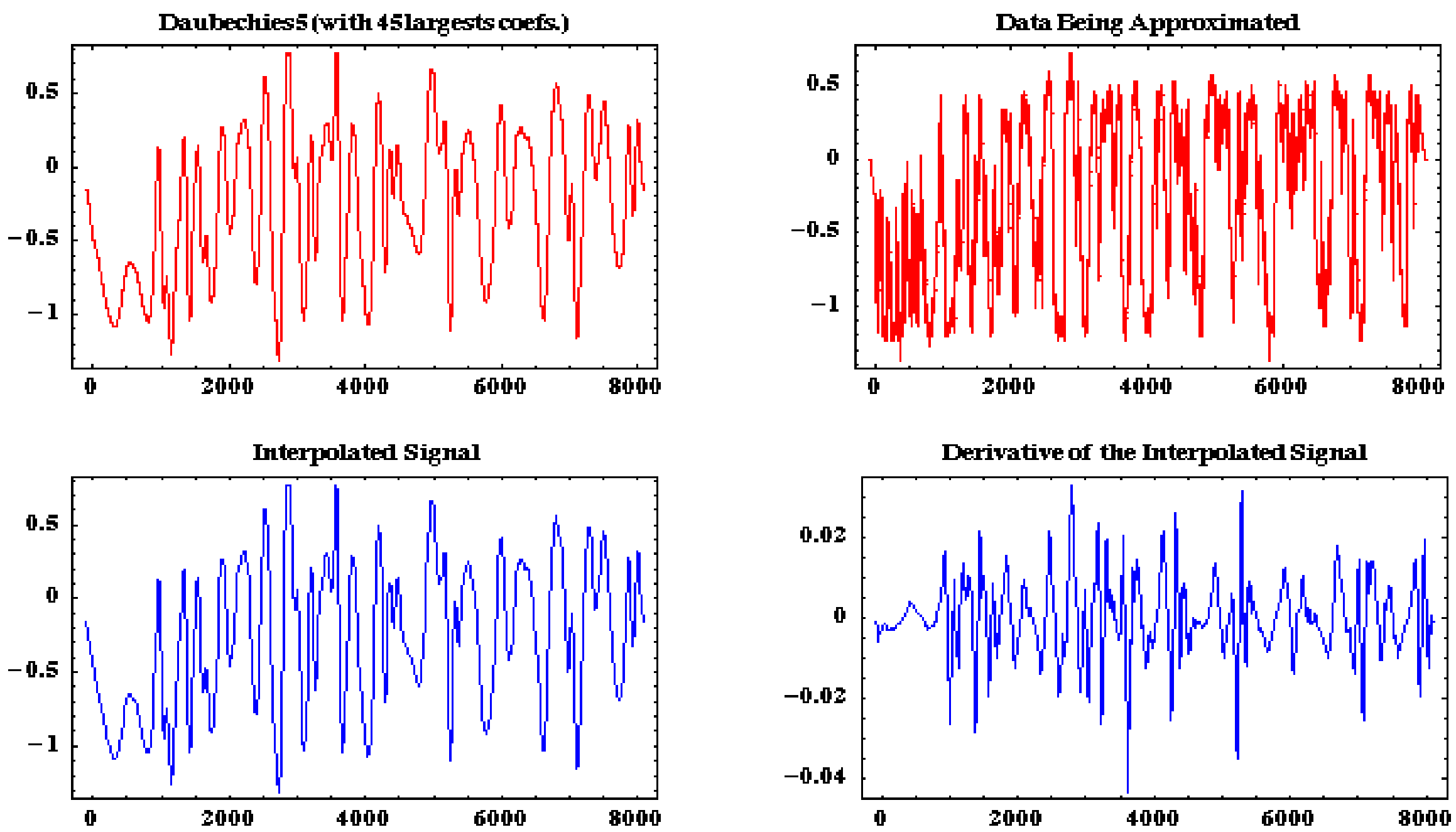


\section{Reconstruction of the Data Using} the 50 Largest WLT Coefficients
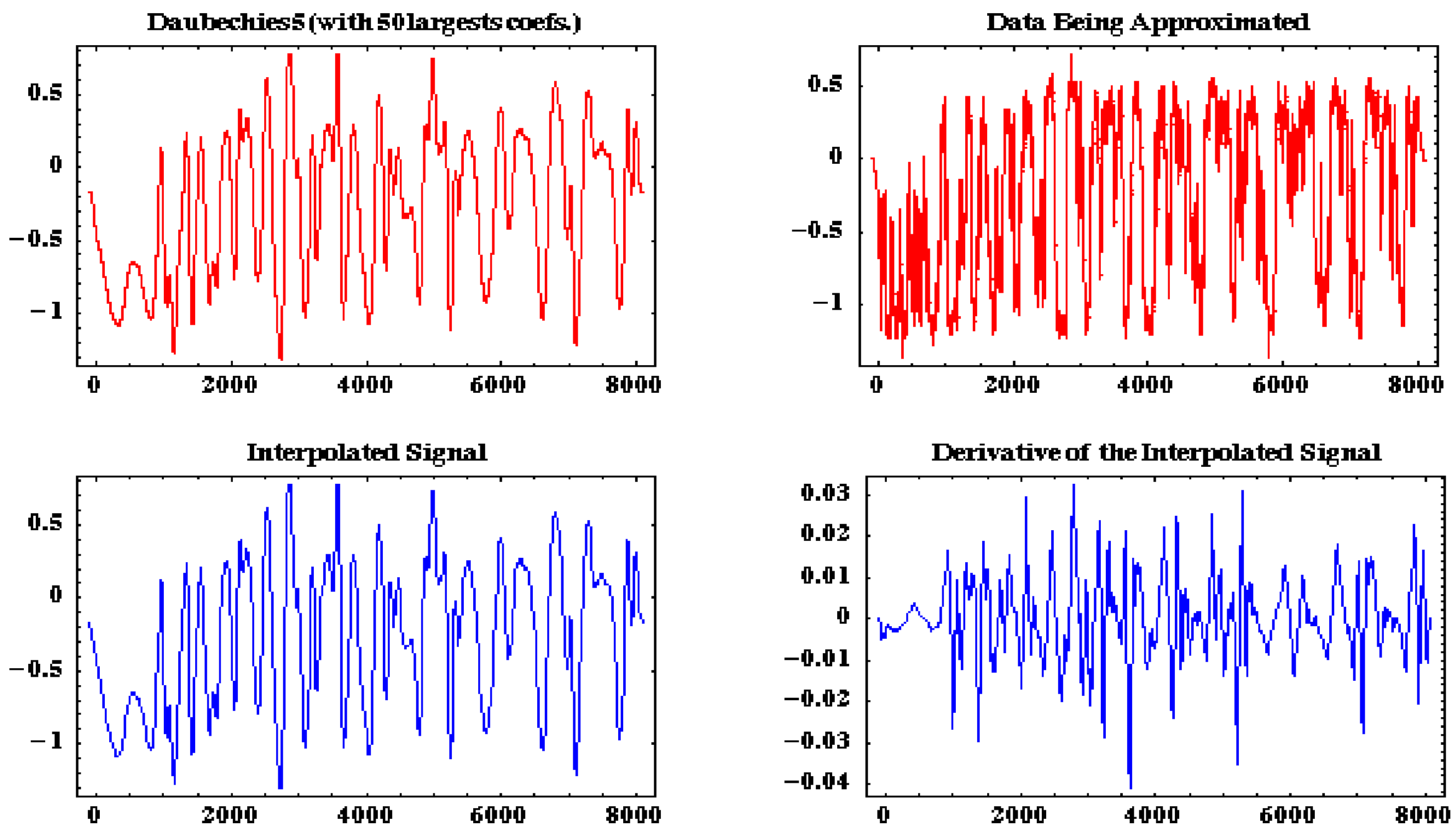


\section{Reconstruction of the Data Using}

Polymath

the 100 Largest WLT Coefficients $U$
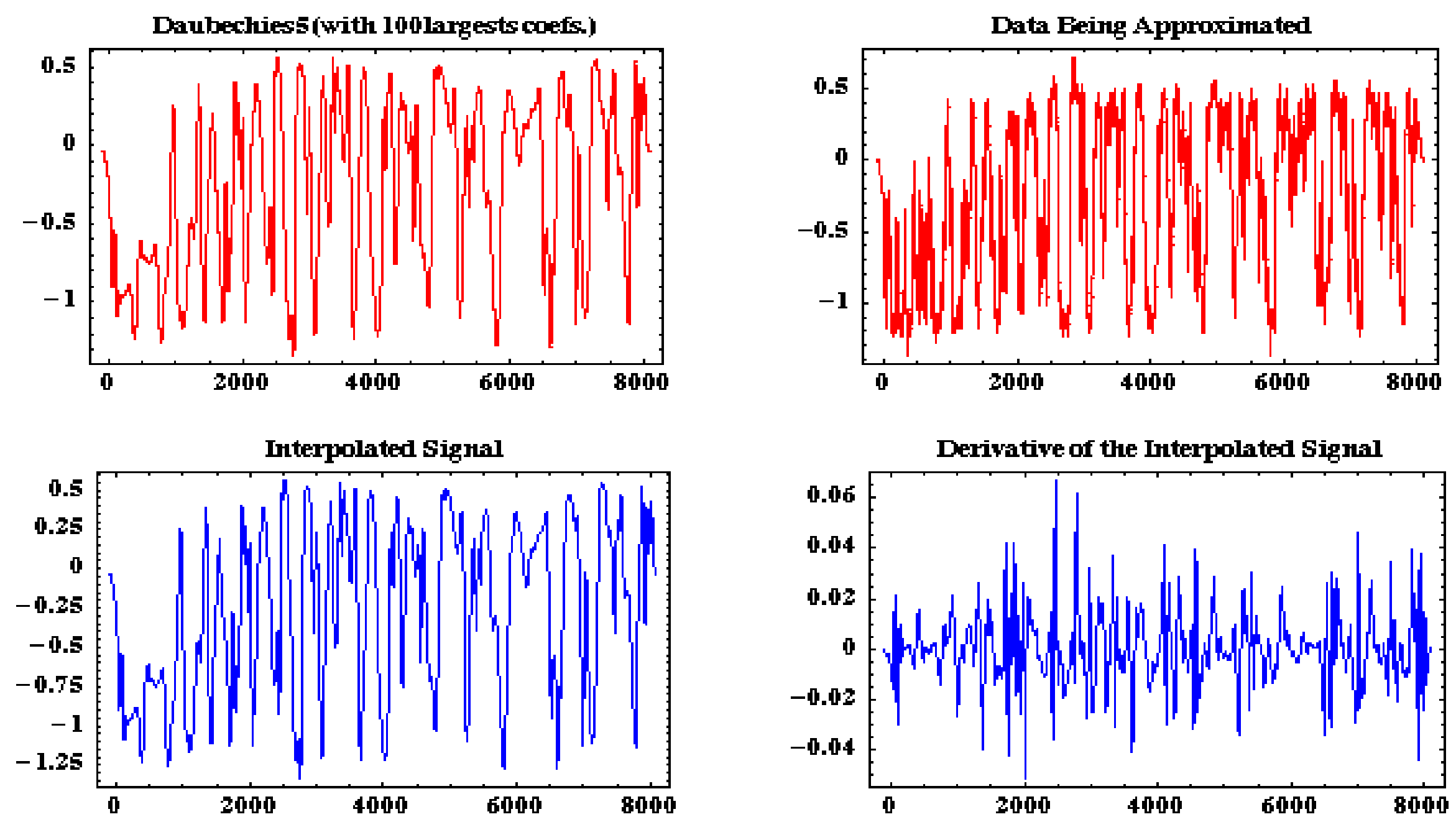


\section{Reconstruction of the Data Using}

Polymath

the 200 Largest WLT Coefficients U
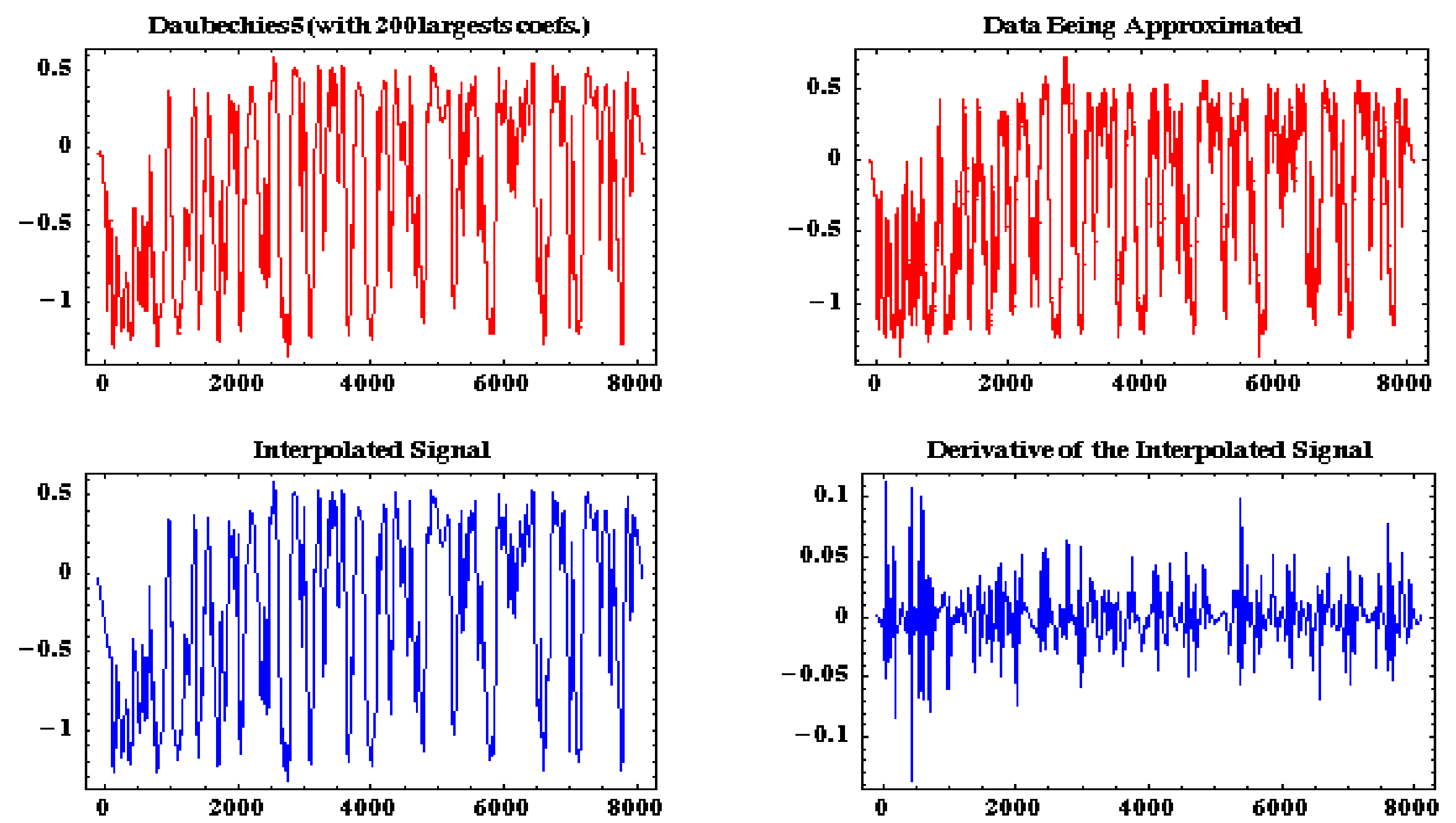


\section{Reconstruction of the LPF Data Using the First MRD Level}
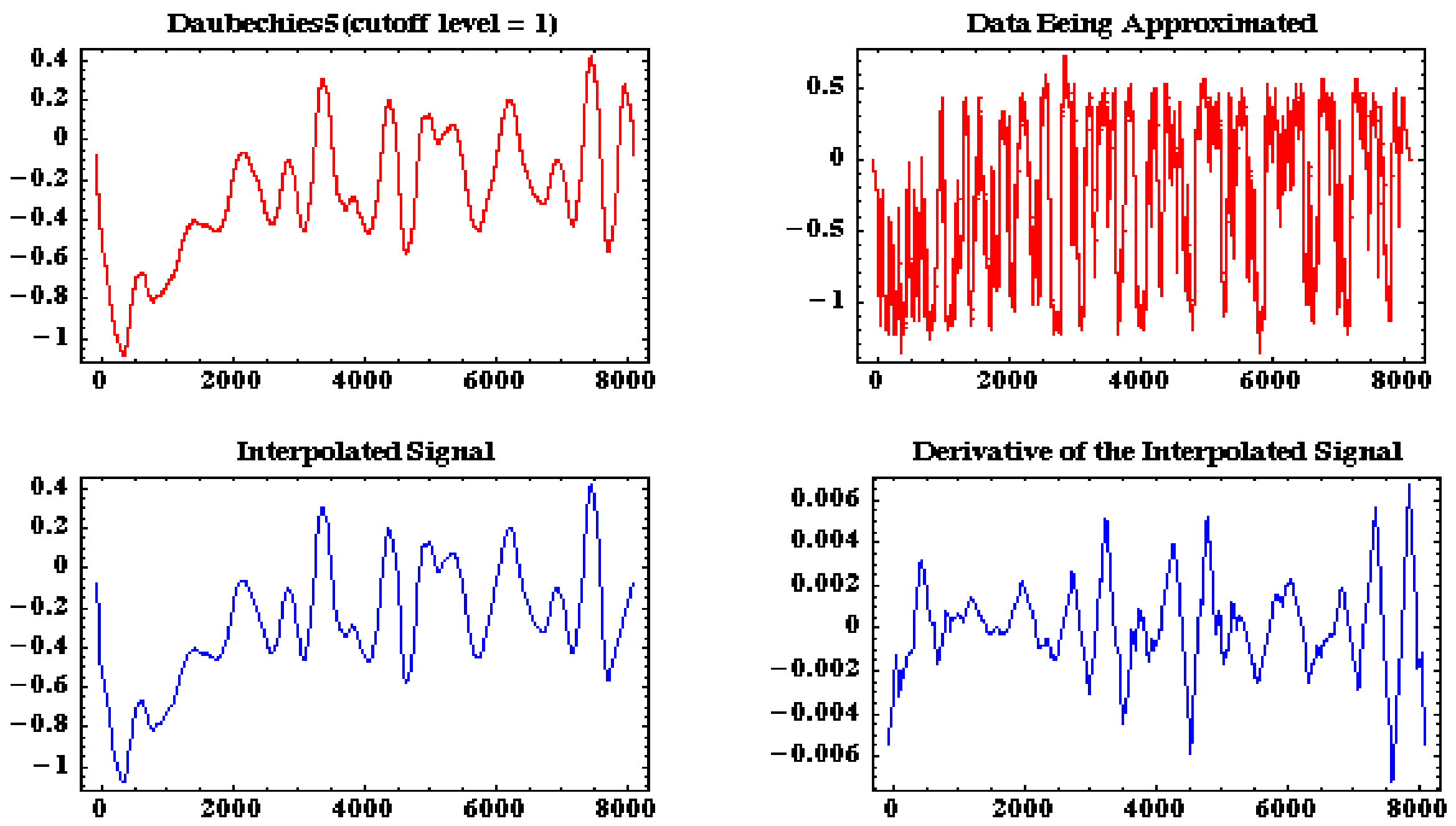


\section{Reconstruction of the Weak Mix}

Data Using First 2 MRD Levels

Polymath

Research Inc.
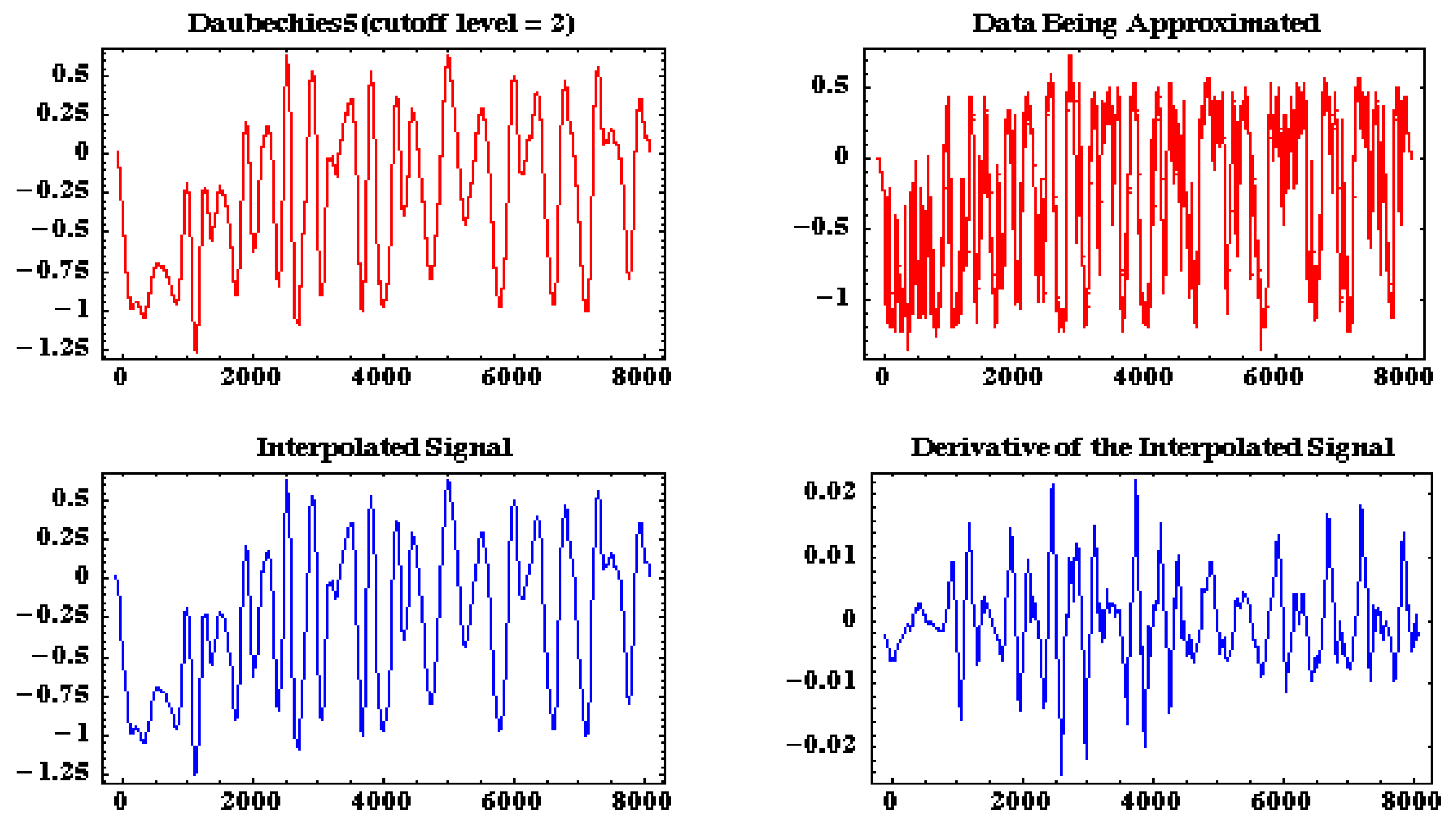


\section{Reconstruction of the Weak Mix}

\section{Data Using First 3 MRD Levels}

Polymath

Research Inc.
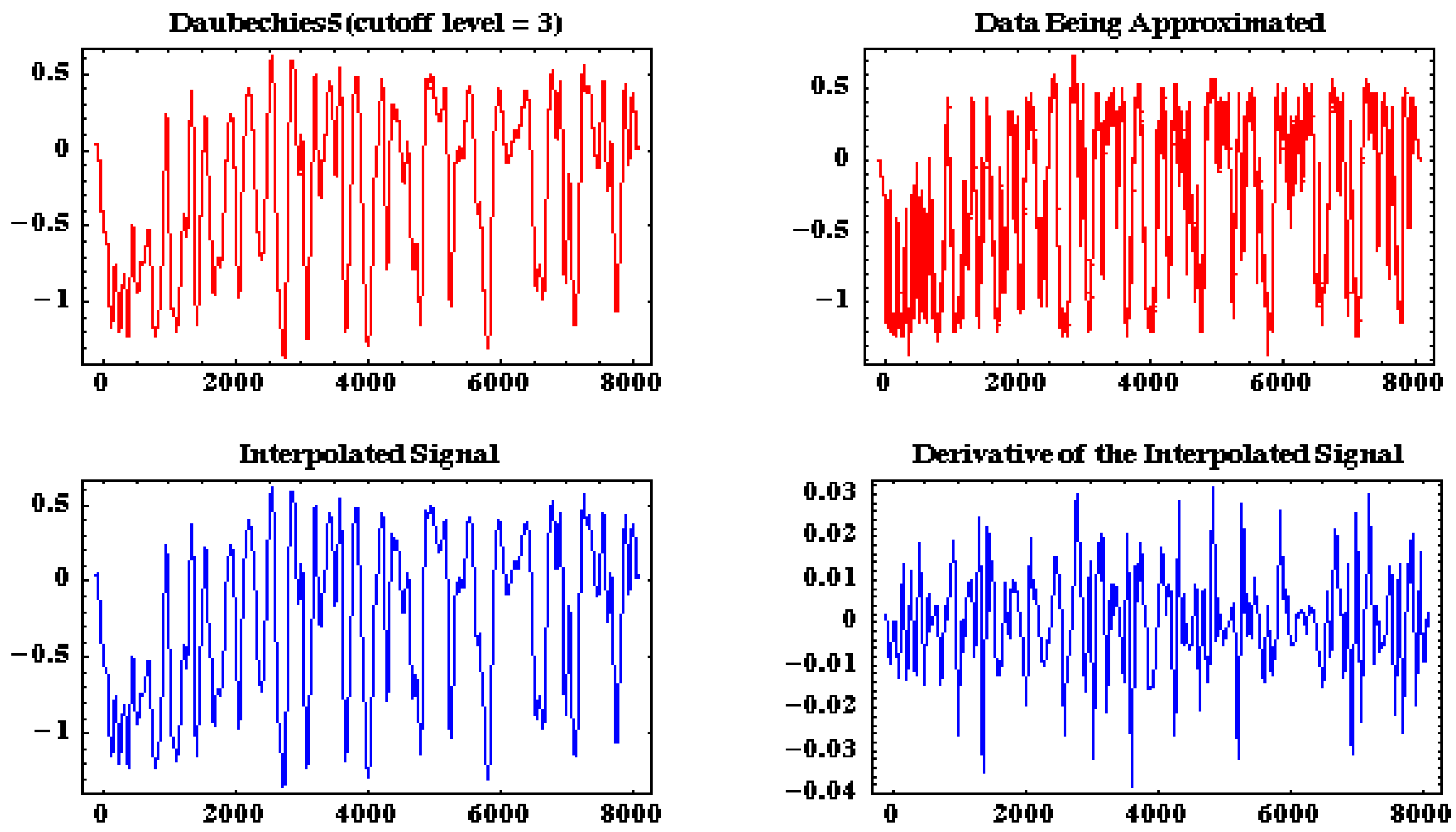


\section{Reconstruction of the Weak Mix} Data Using First 4 MRD Levels
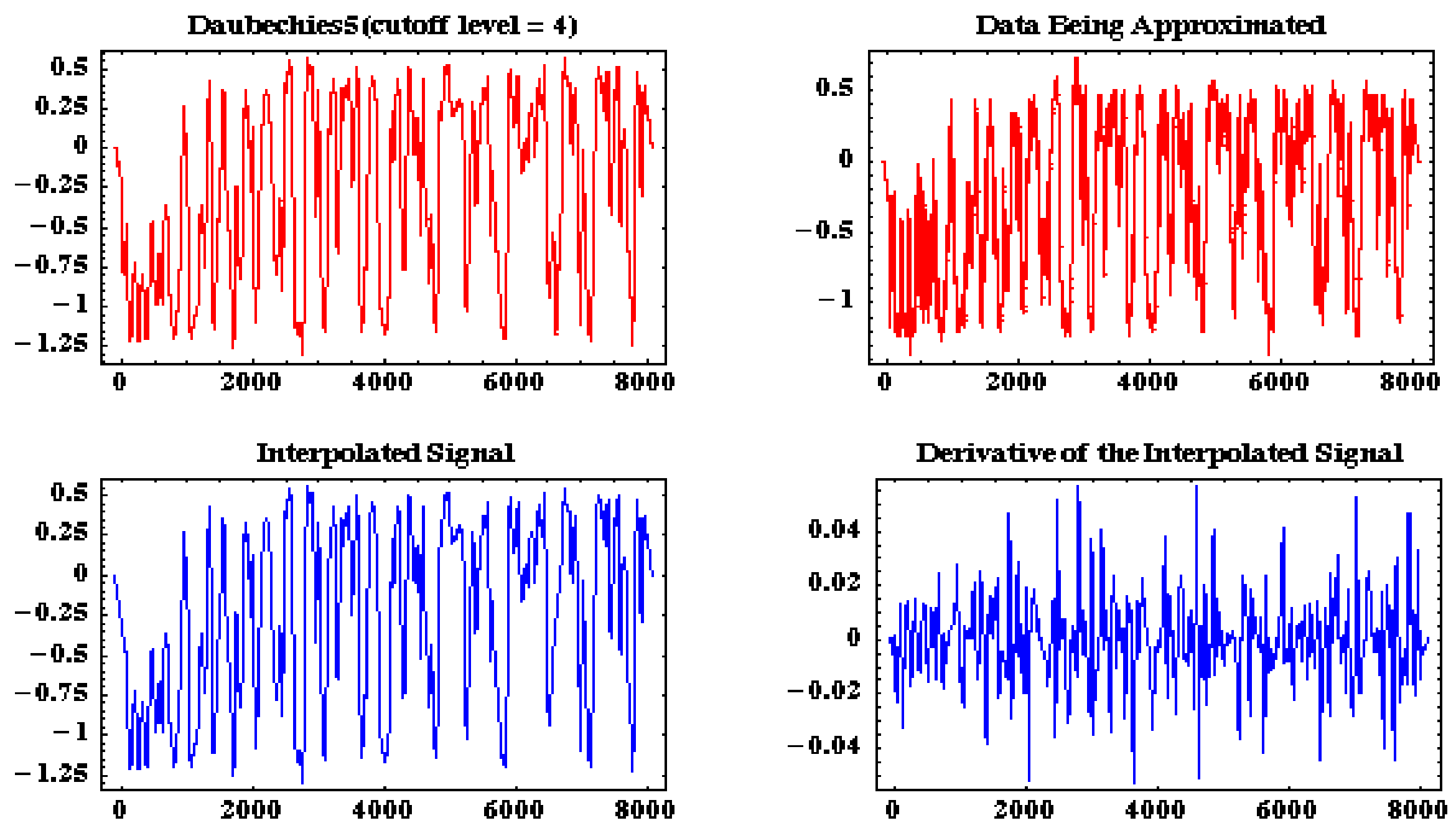


\section{Reconstruction of the Weak Mix Data Using First 5 MRD Levels
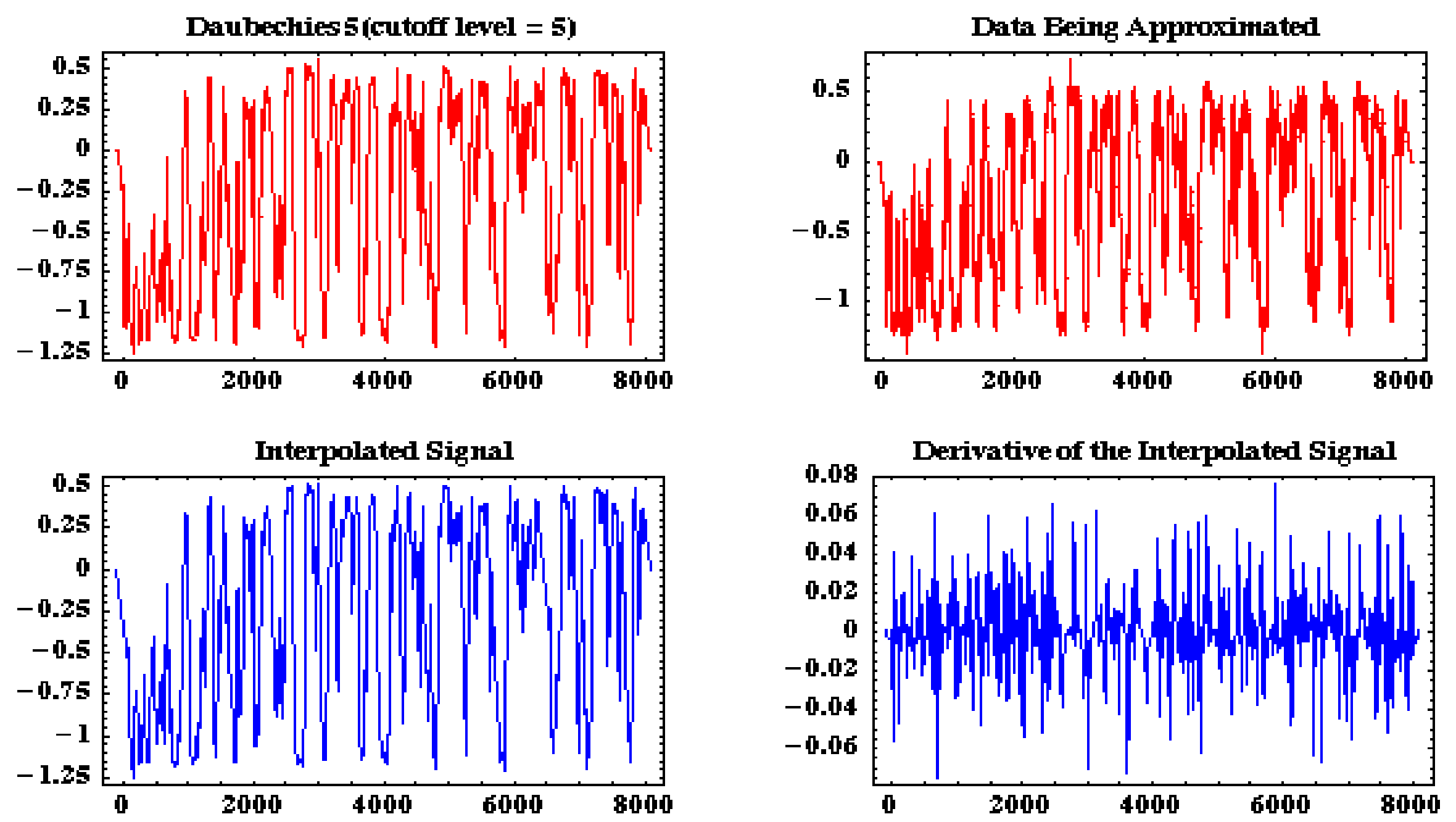


\section{Conclusions Regarding the WLT Analysis of the RT Weak Mix Data}




\section{Low Pass Filtered (LPF) Padded and} Faded RT Weak Mix Data

Filtered Data

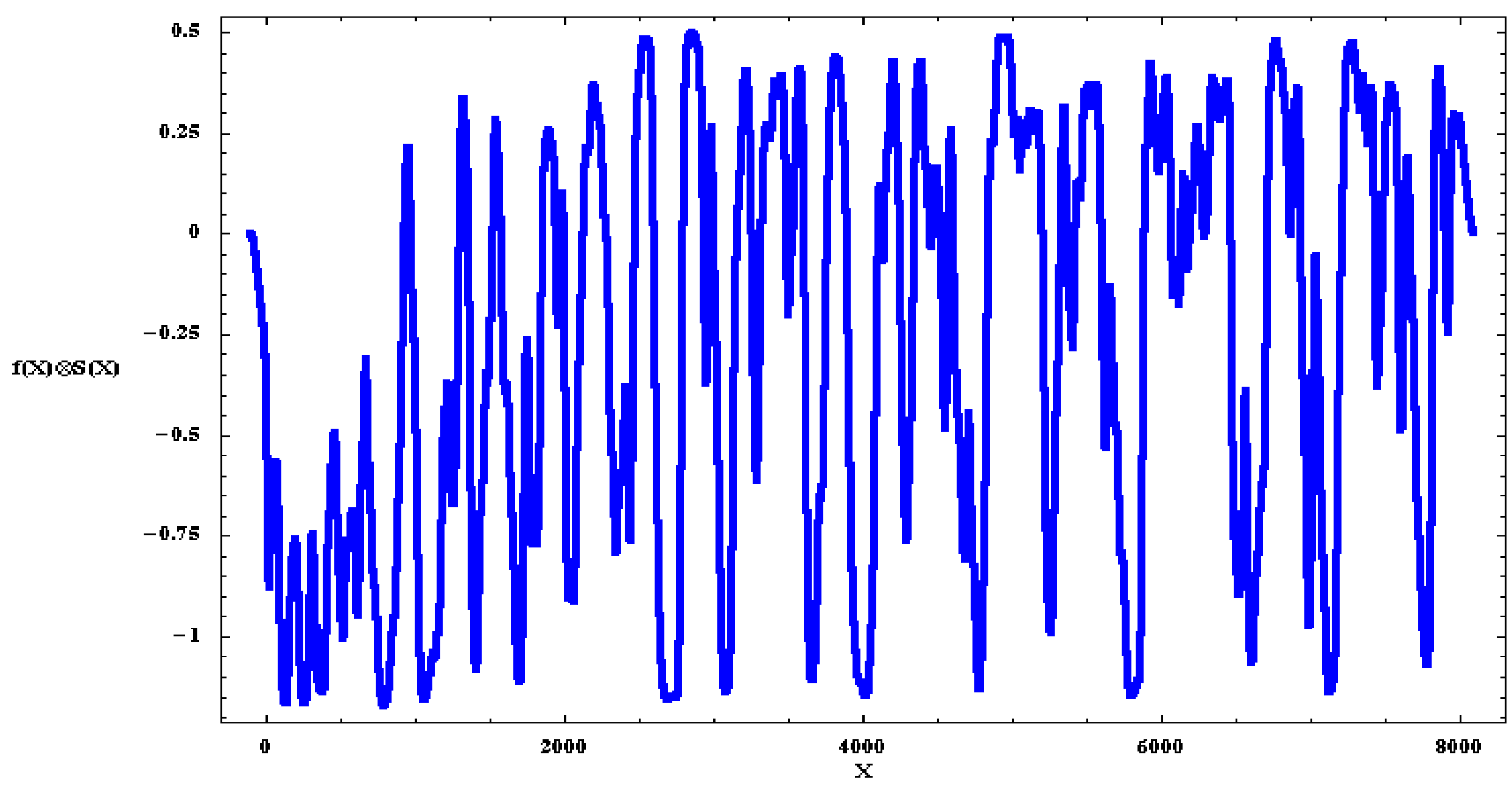




\section{The Filtering Has This Form and Effect on the Data in k-Space}

Polymath

Research Inc.

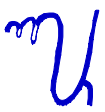

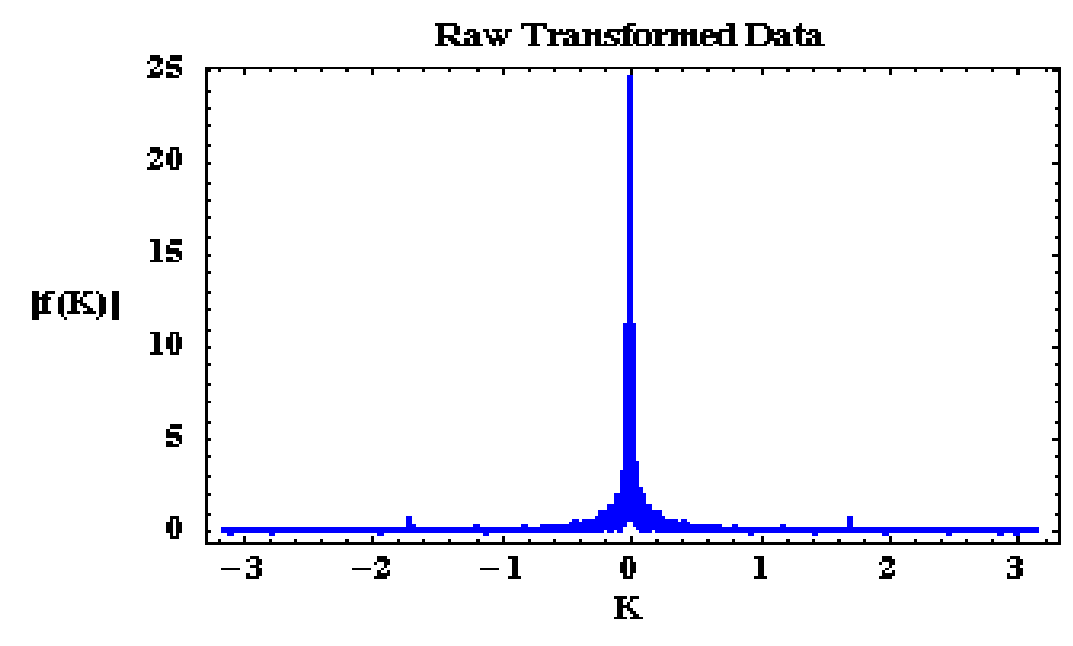

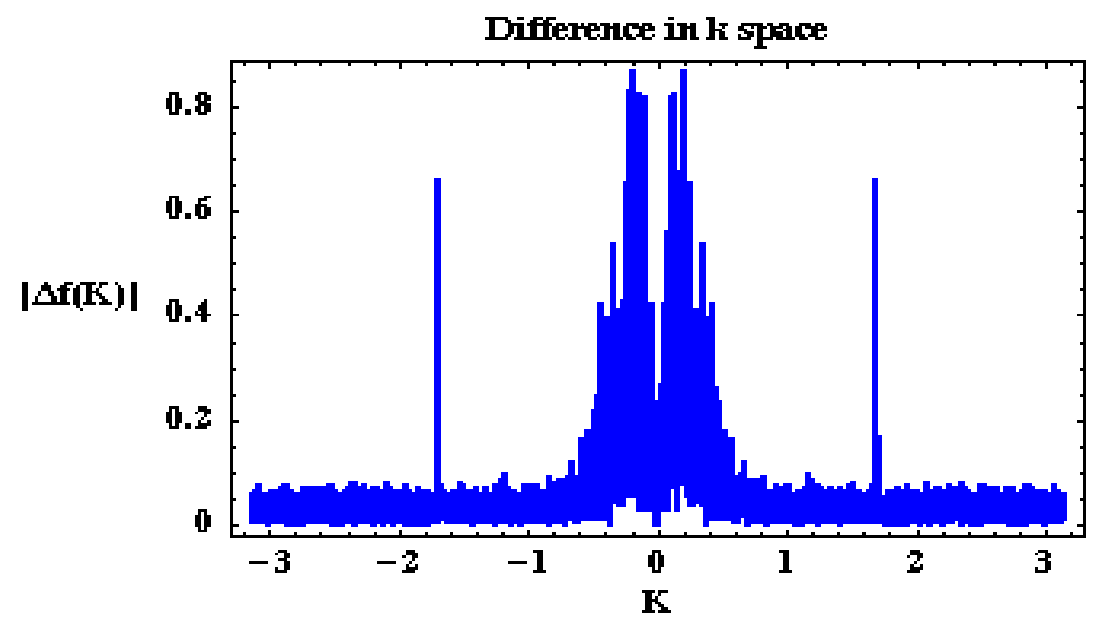

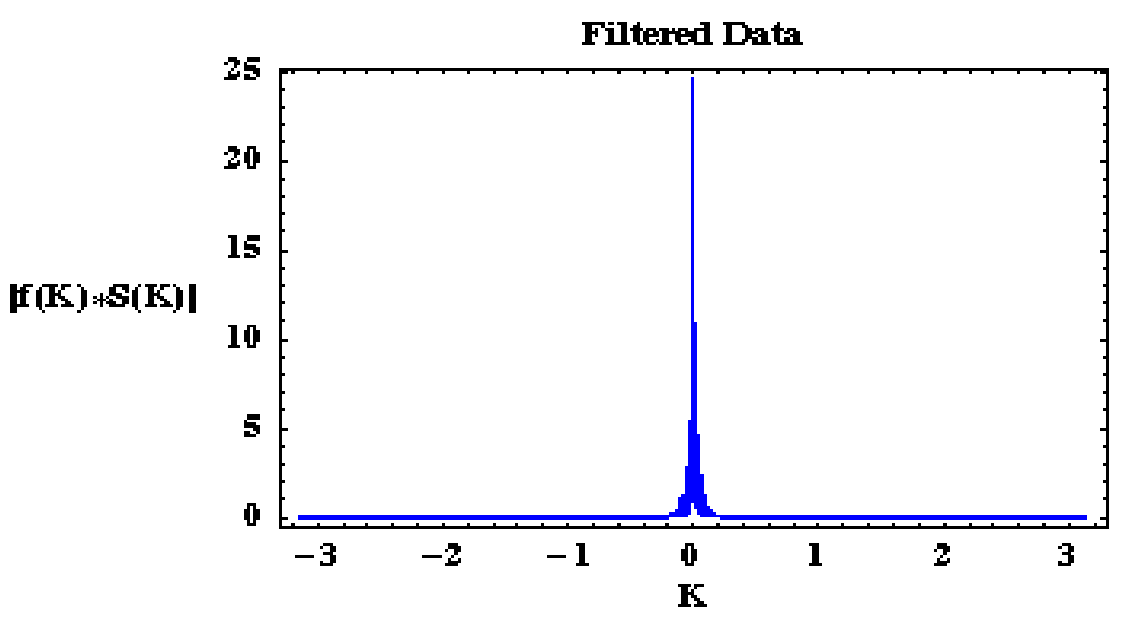

Filter was of the form:

$S(k)=\exp \left\lfloor-\left(\frac{k}{k_{\text {width }}}\right)^{2 \alpha}\right\rfloor$

Where $\alpha=5$ and $k_{\text {width }}=400$ 


\section{MRD Coefficients of the LPF RT Weak Mix Data in 6 Different Daubechies WLT Bases}
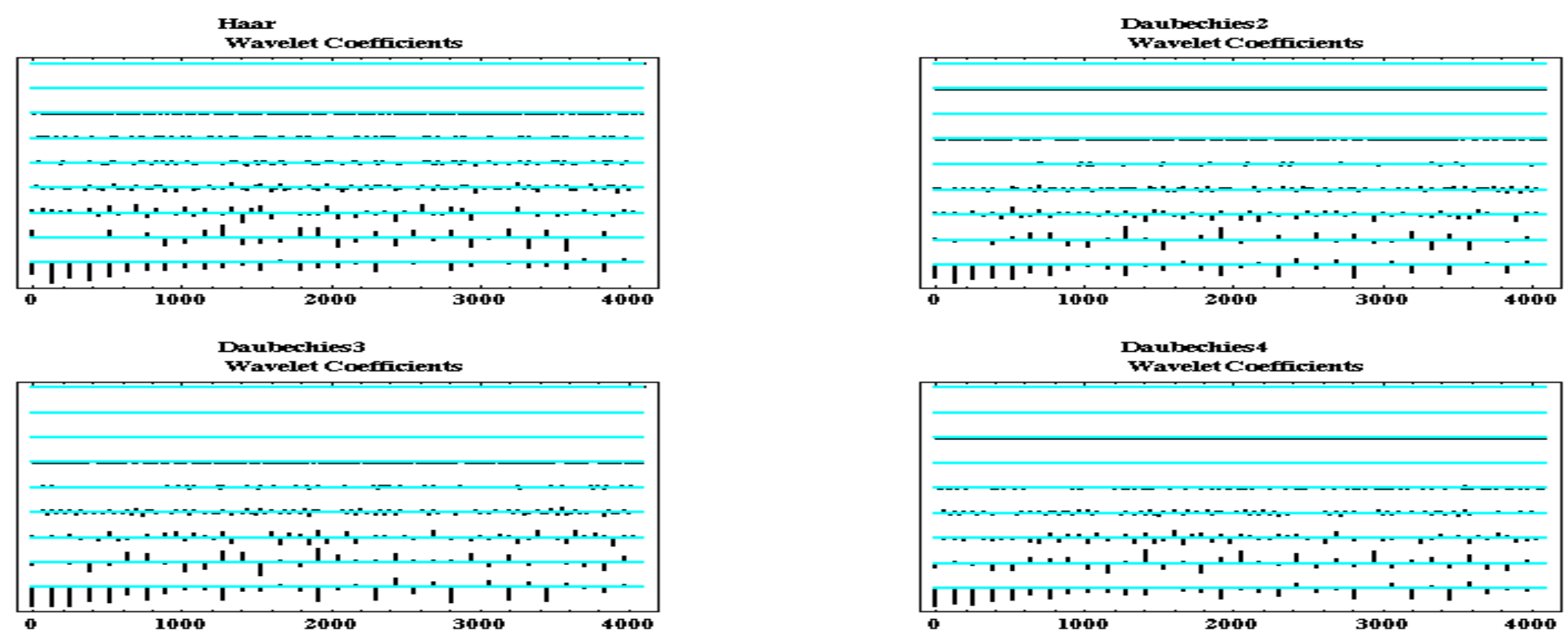

Daubechies4

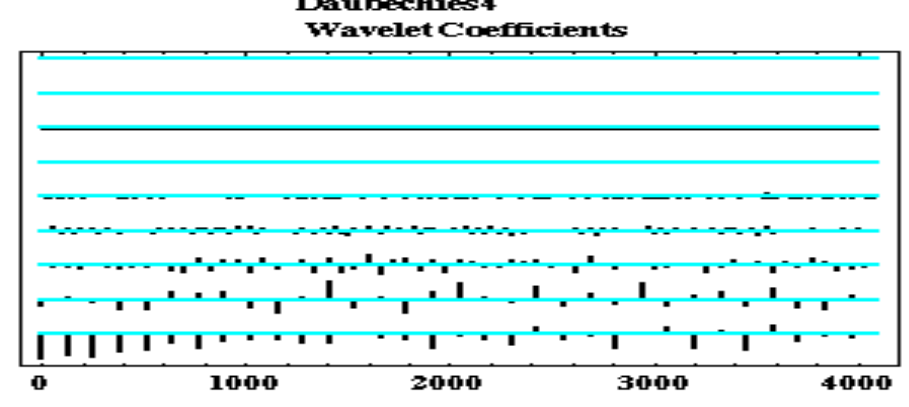

Daubechiess

wavelet Coefficients

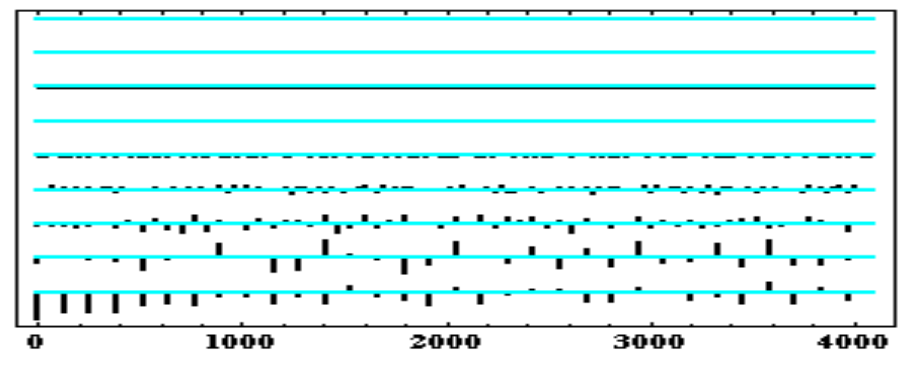

Daubechies6

Wavelet Coefficients

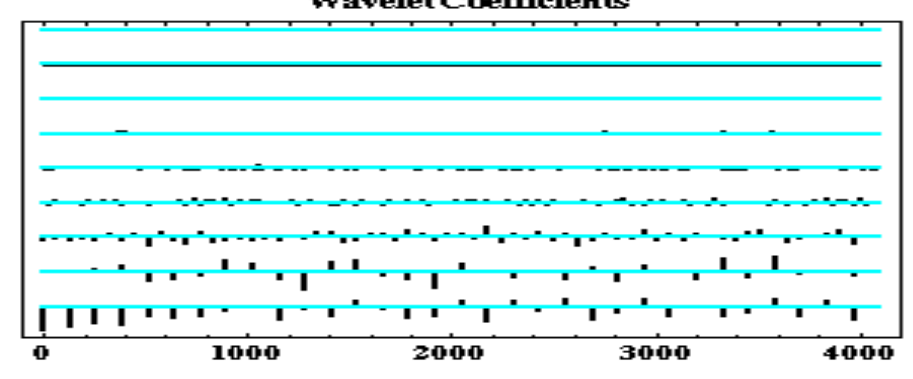




\section{Actual MRDs of the LPF RT Weak Mix Data in 6 Different Daubechies WLT Bases}
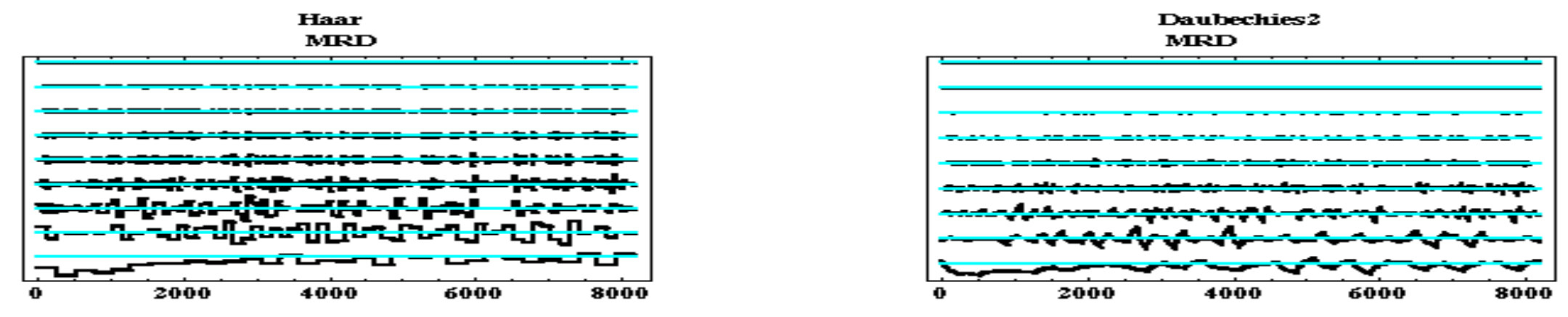

Daubechies 3

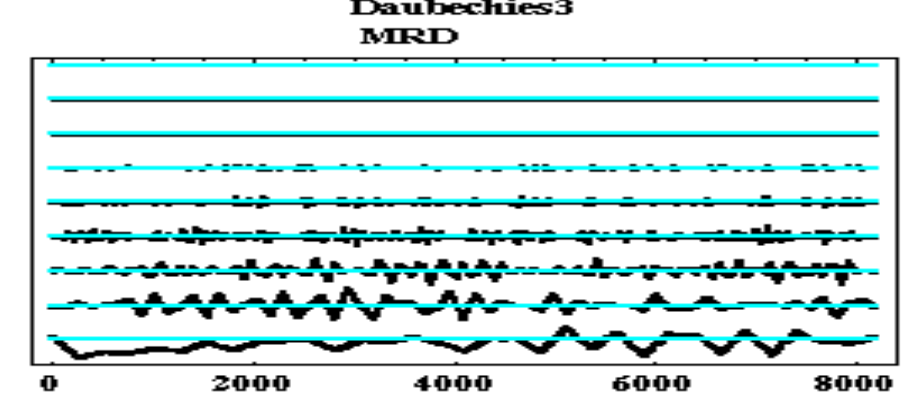

Daubechies 4

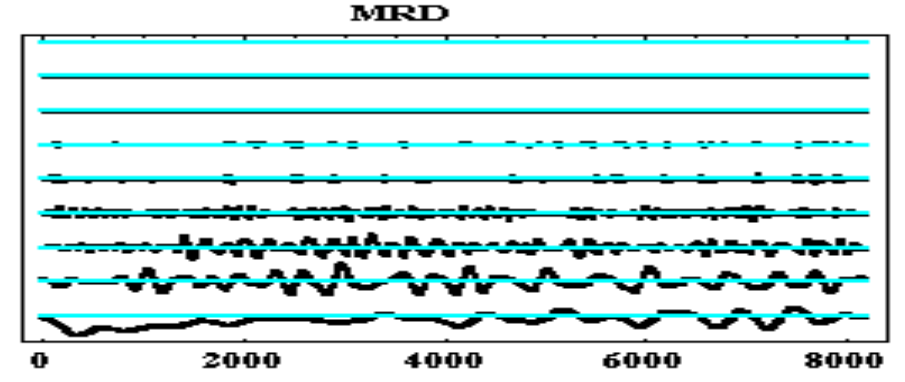

Daubechiess

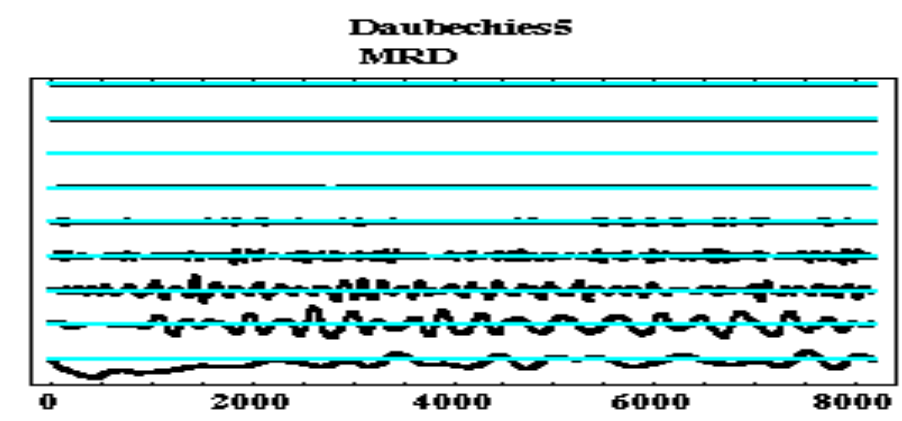

Daubechies6

NIRD

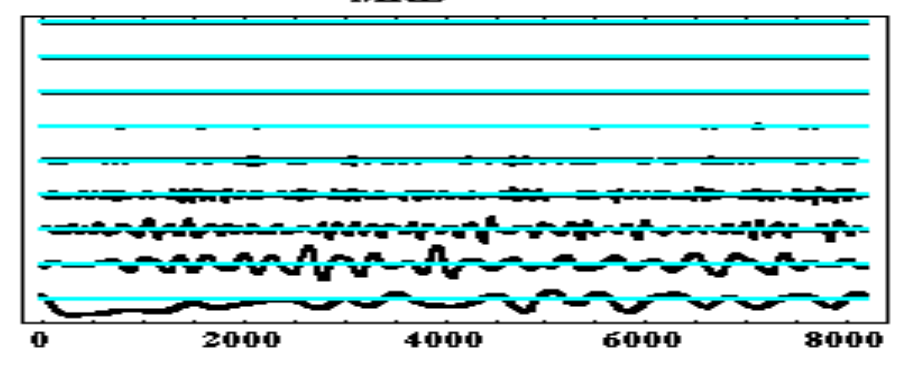

Polymath Research Inc. 


\section{Decay Rate of Largest Coefficient vs Number of Coefficients Kept in 6 Different Daub WLT Decomps}
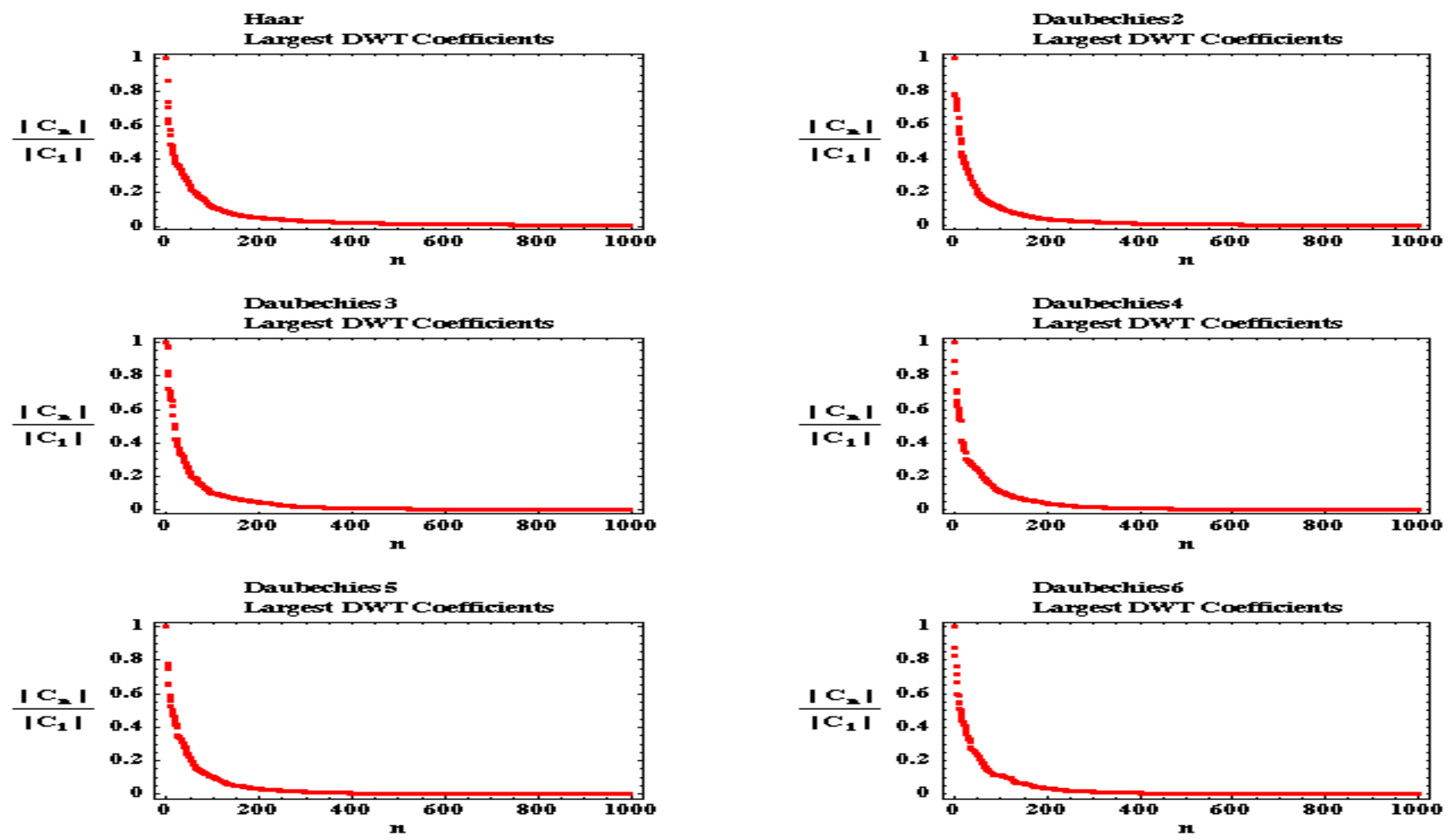


\section{Energy Accumulation Rate in Coefficient Space vs \# of WLTs Kept in 6 Different Daub Decomps.}
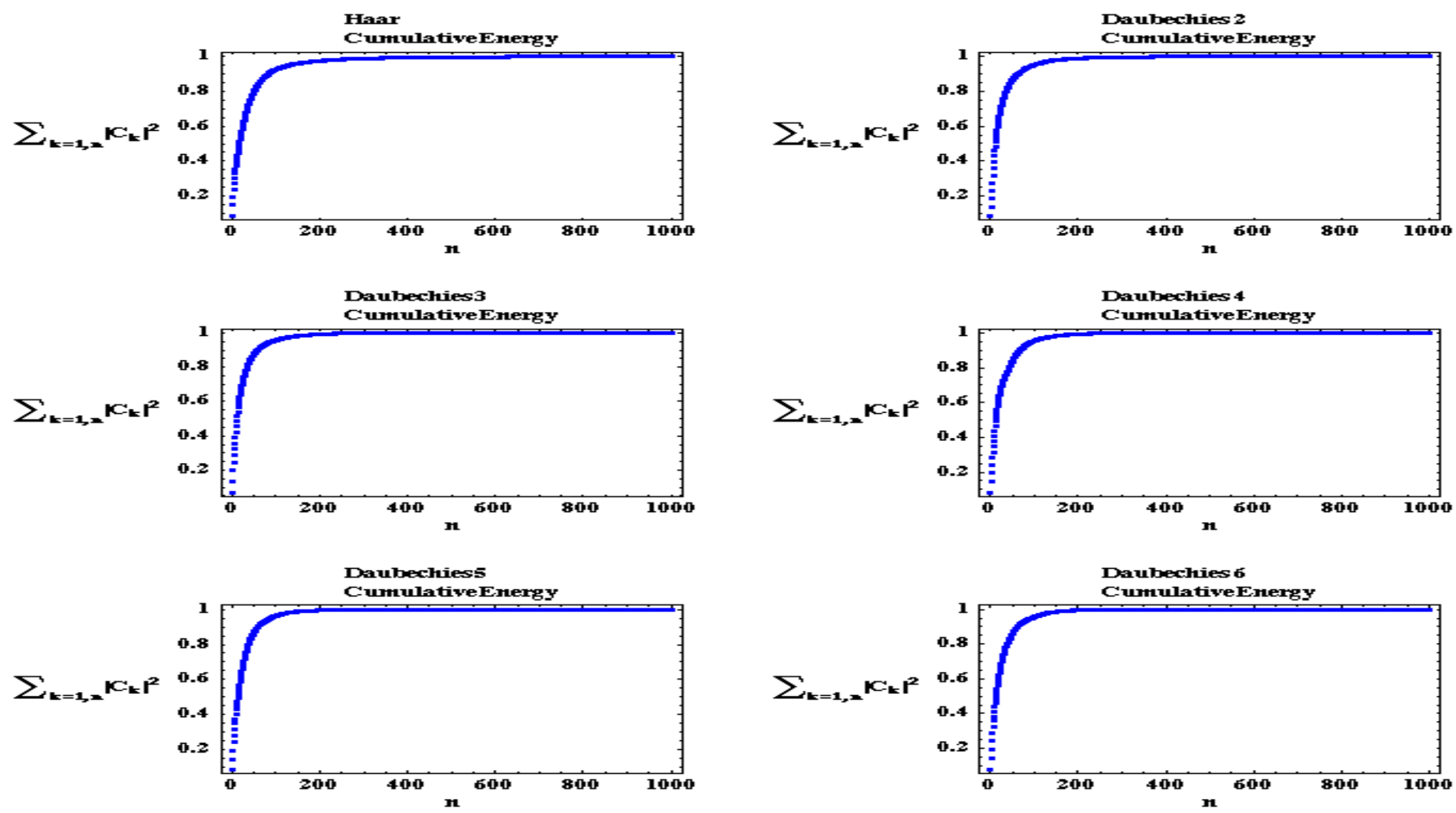


\section{Scaleograms: Waveleters Preferred Way of Judging Tiling in Scale- Translation Space}
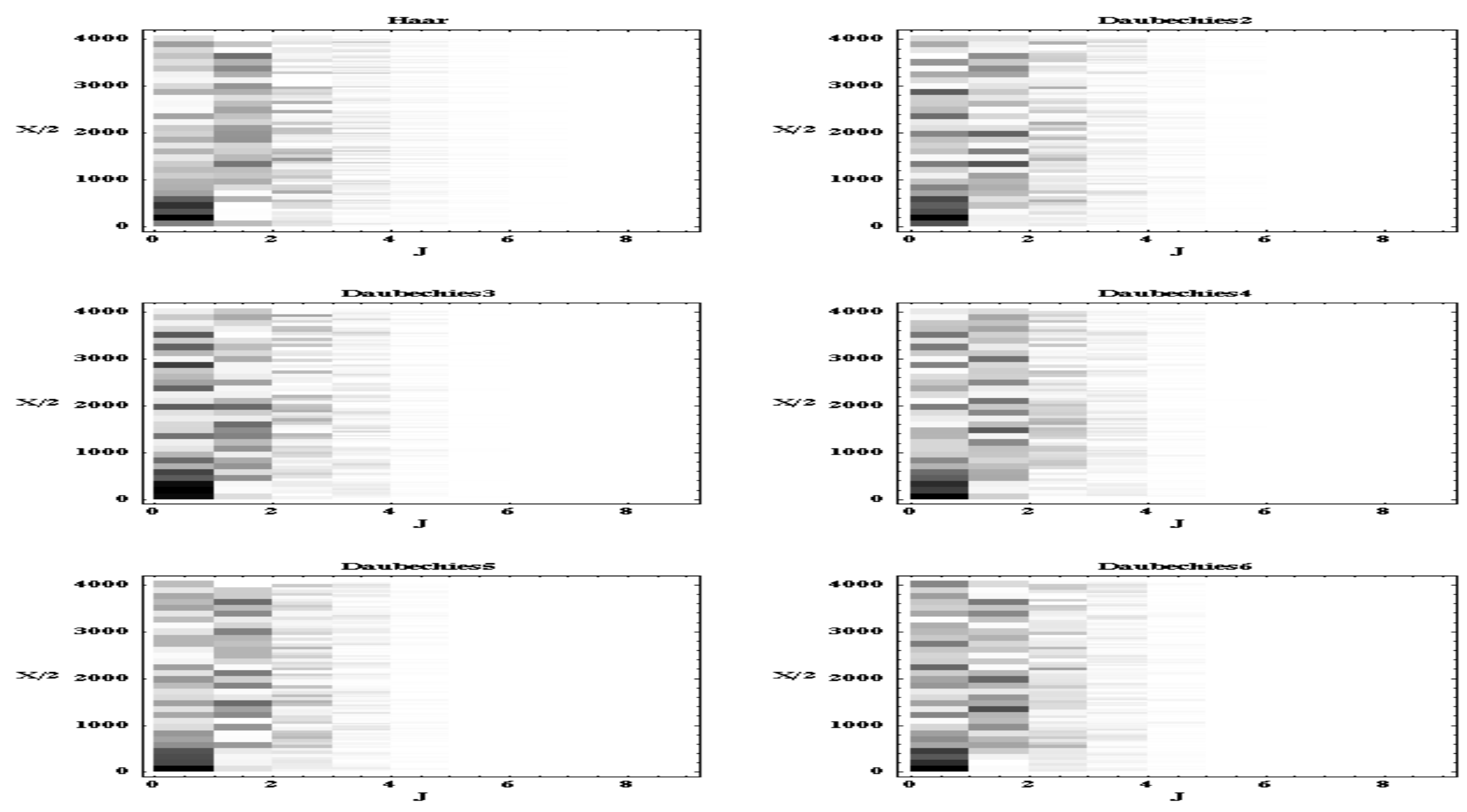


\section{Least Square Error Incurred By Truncating the WLT Series at N of its Largest Coefficients}
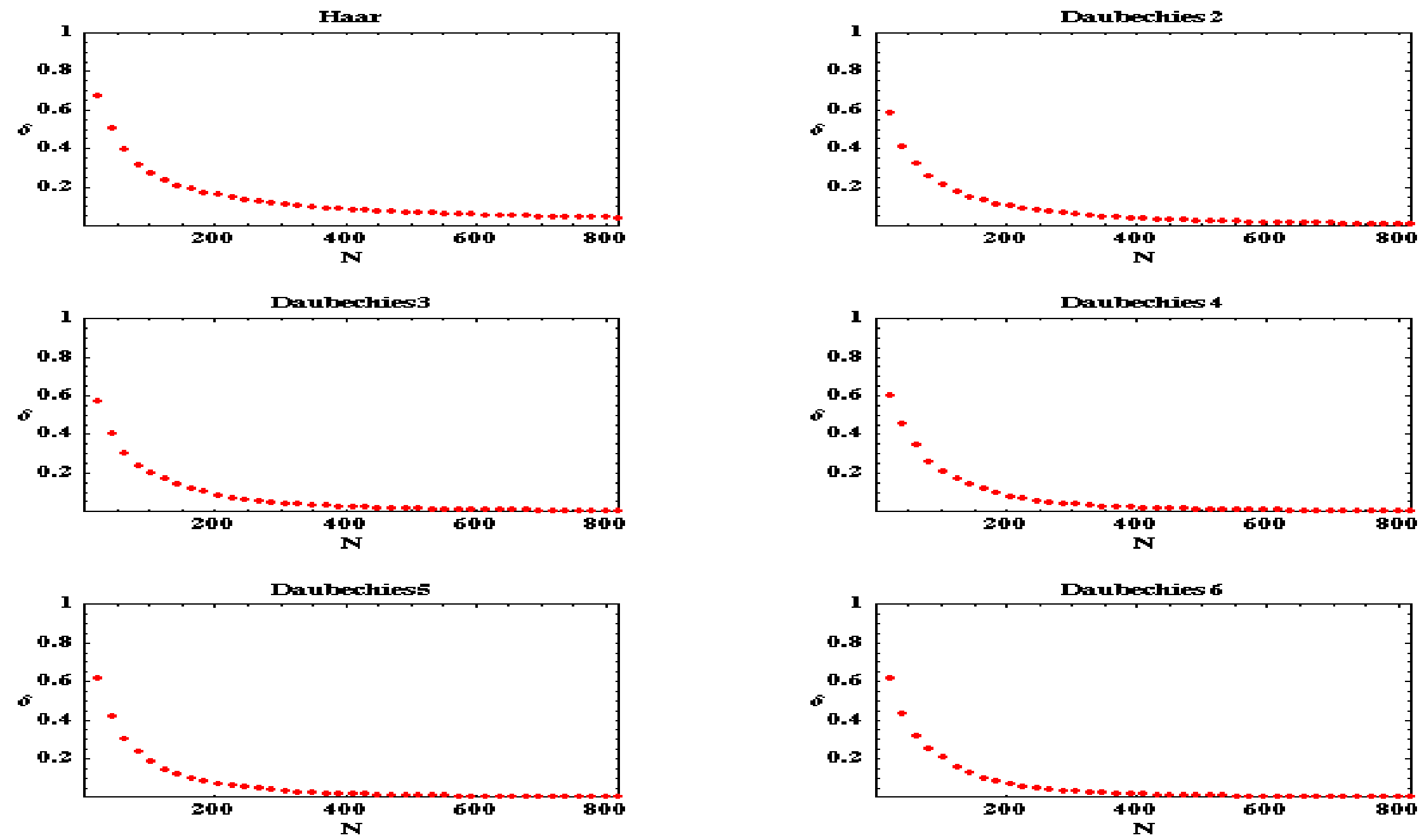


\section{Least Square Error Incurred by Level Thresholding the DWT}

Polymath Research Inc.

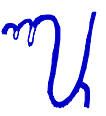

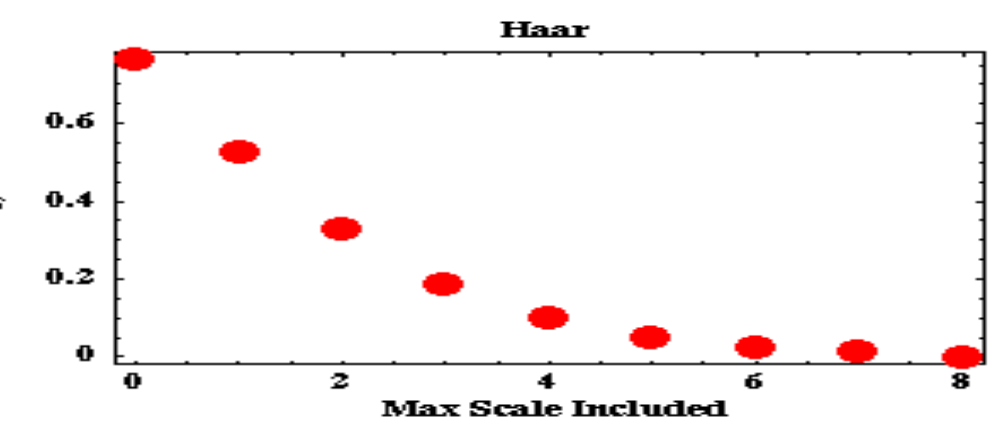
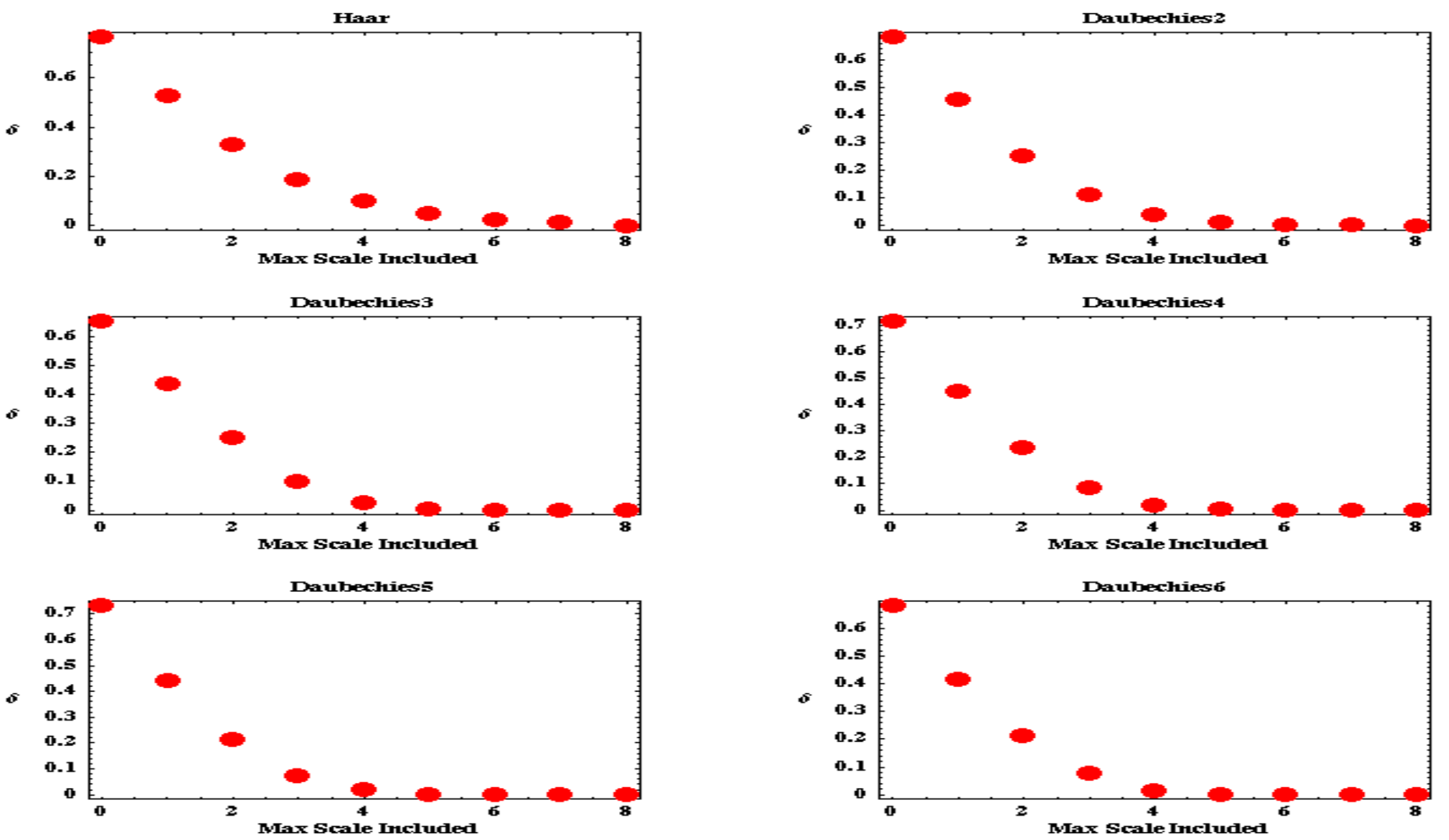


\section{Daubechies 5 Does Much Better than}

\section{Haar: 5 Quantitative Measures}
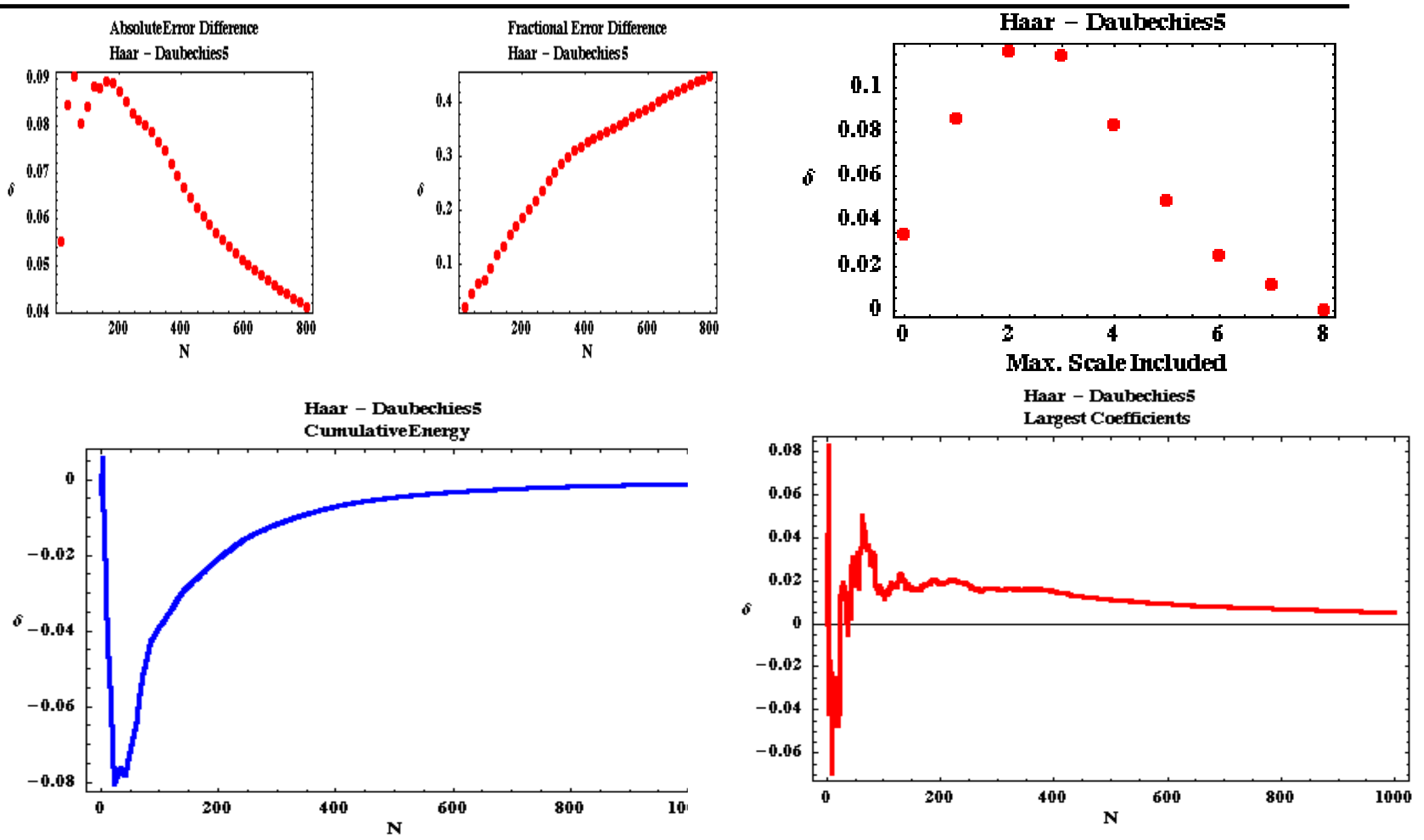


\section{Level by Level Decomposition of the LPF RT Weak Mix Data Using Daub5 WLTs}
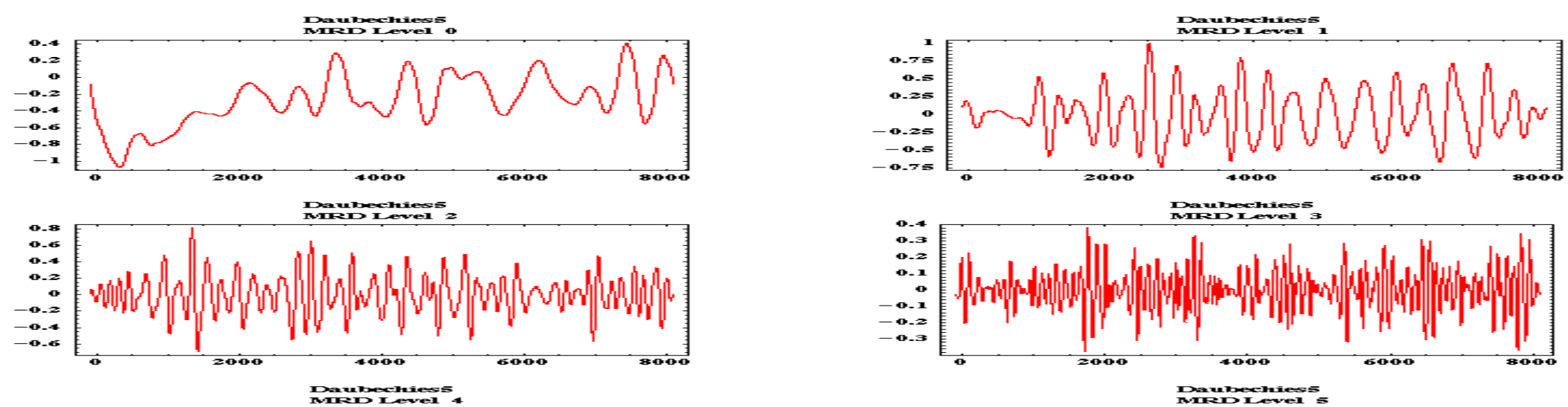

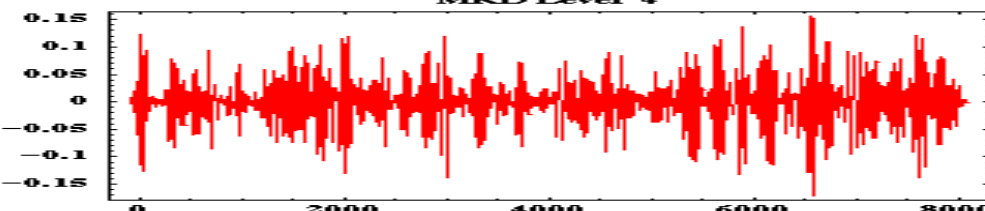

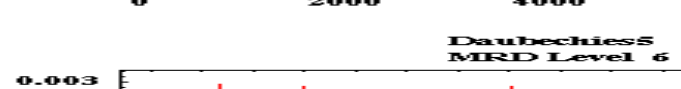

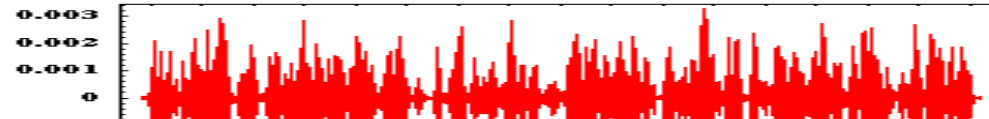

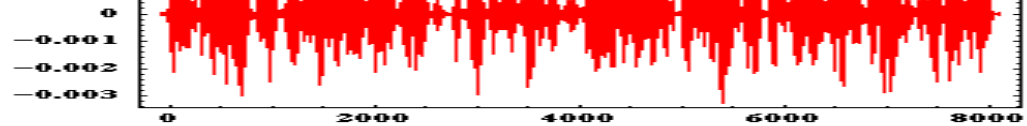

Davbeniess

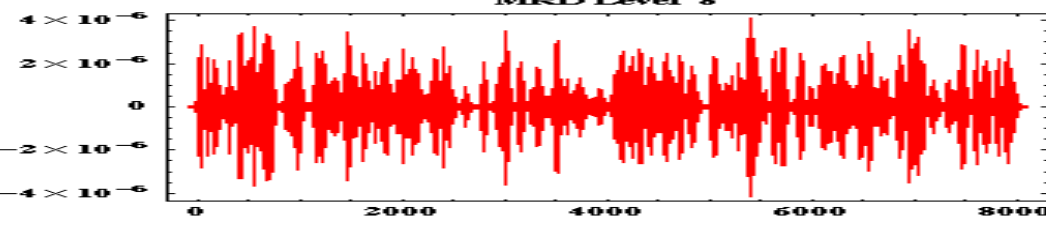

Polymath Research Inc.
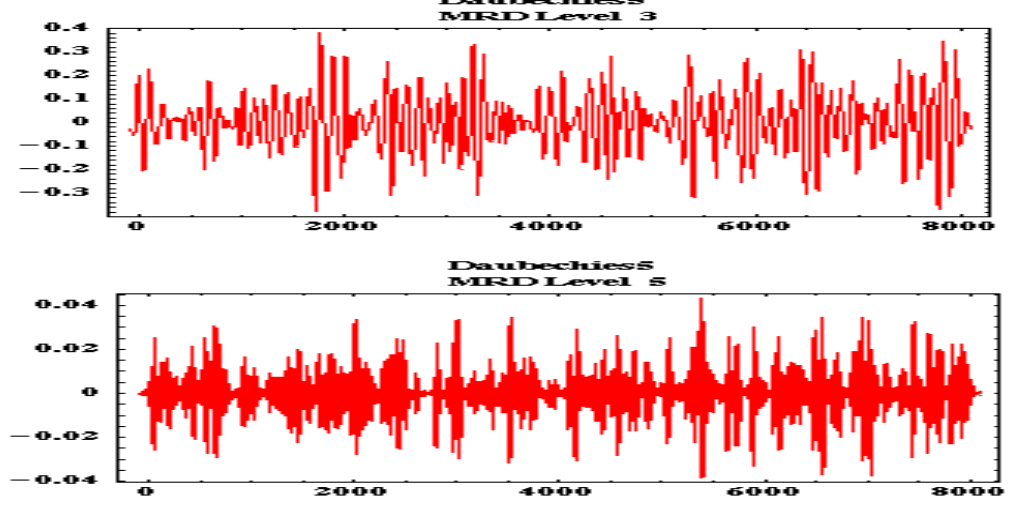

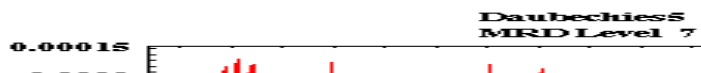

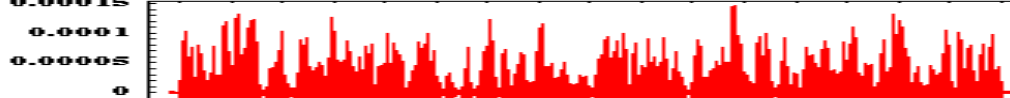

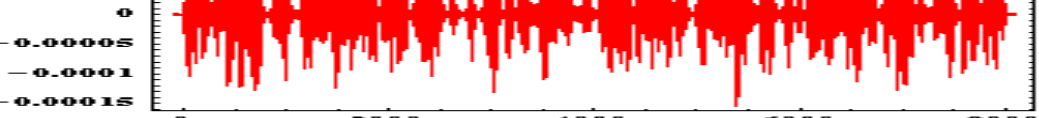

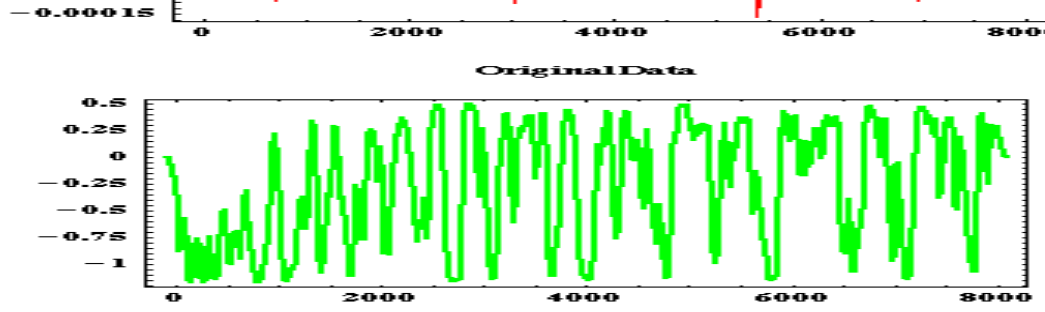




\section{Reconstruction of the LPF RT}

Weak Mix Data Using the 5

\section{Largest WLT Coefficients}
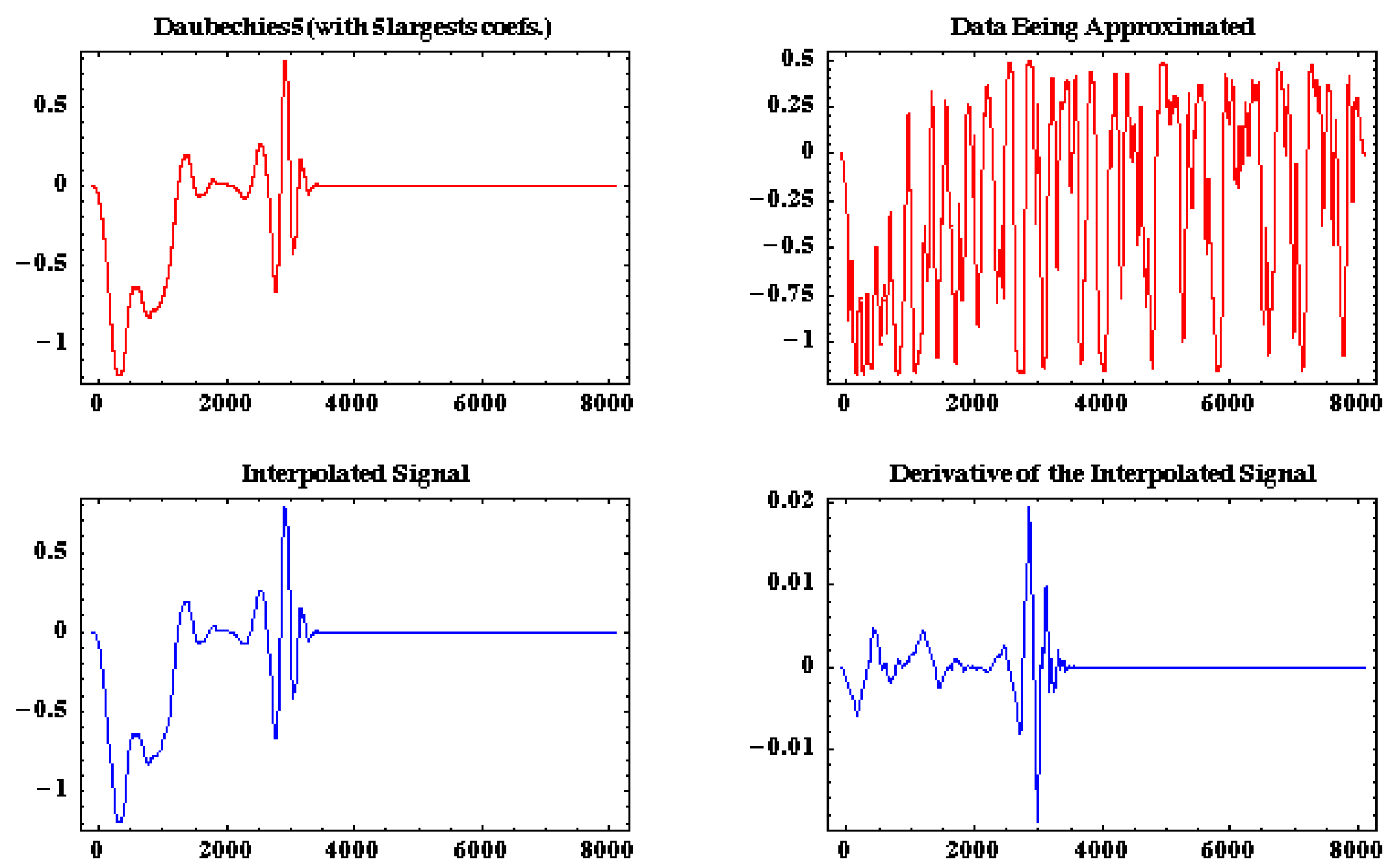


\section{Reconstruction of the LPF RT}

Weak Mix Data Using the 10 Largest WLT Coefficients
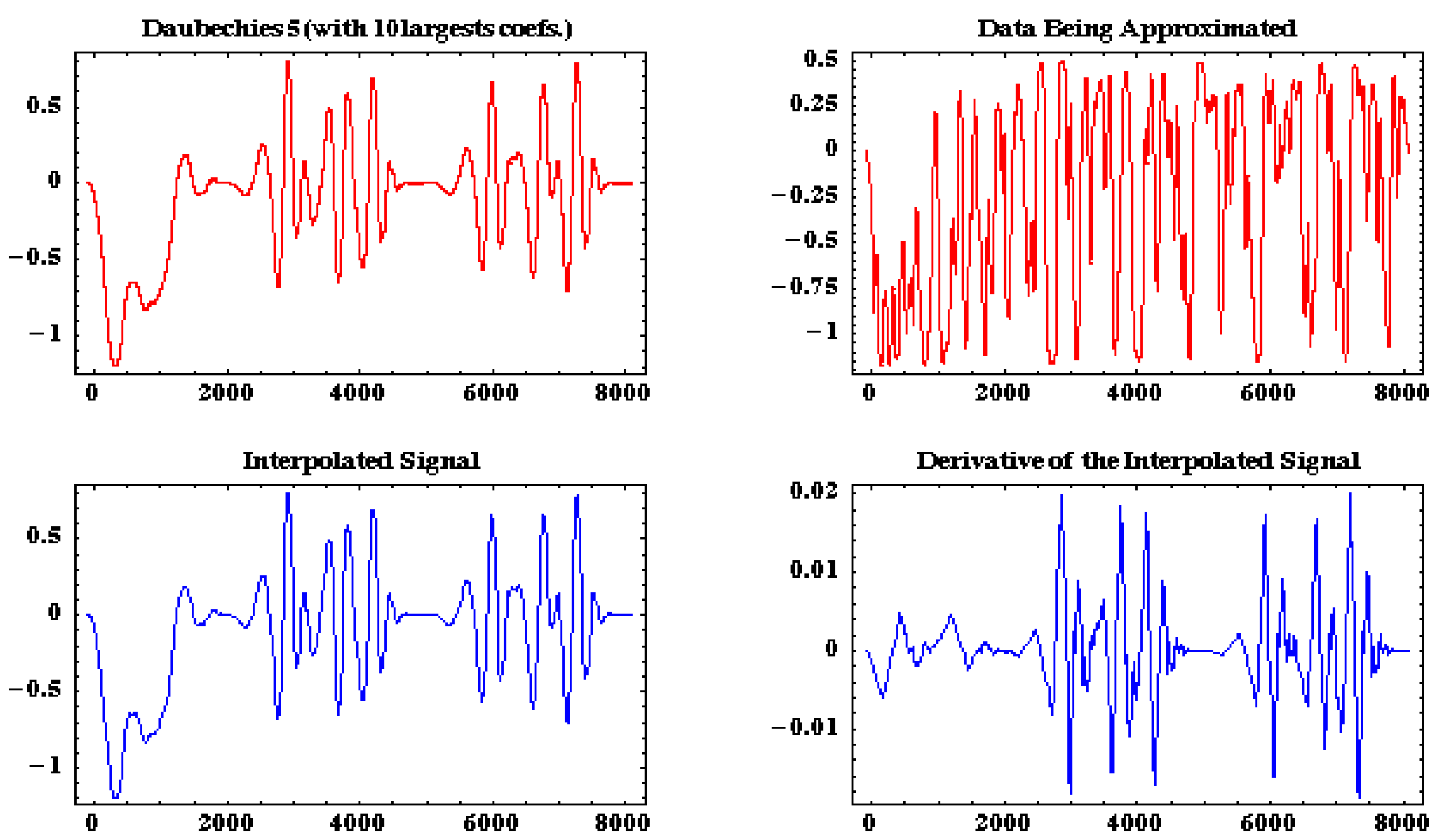


\section{Reconstruction of the LPF RT}

Weak Mix Data Using the 15

Largest WLT Coefficients
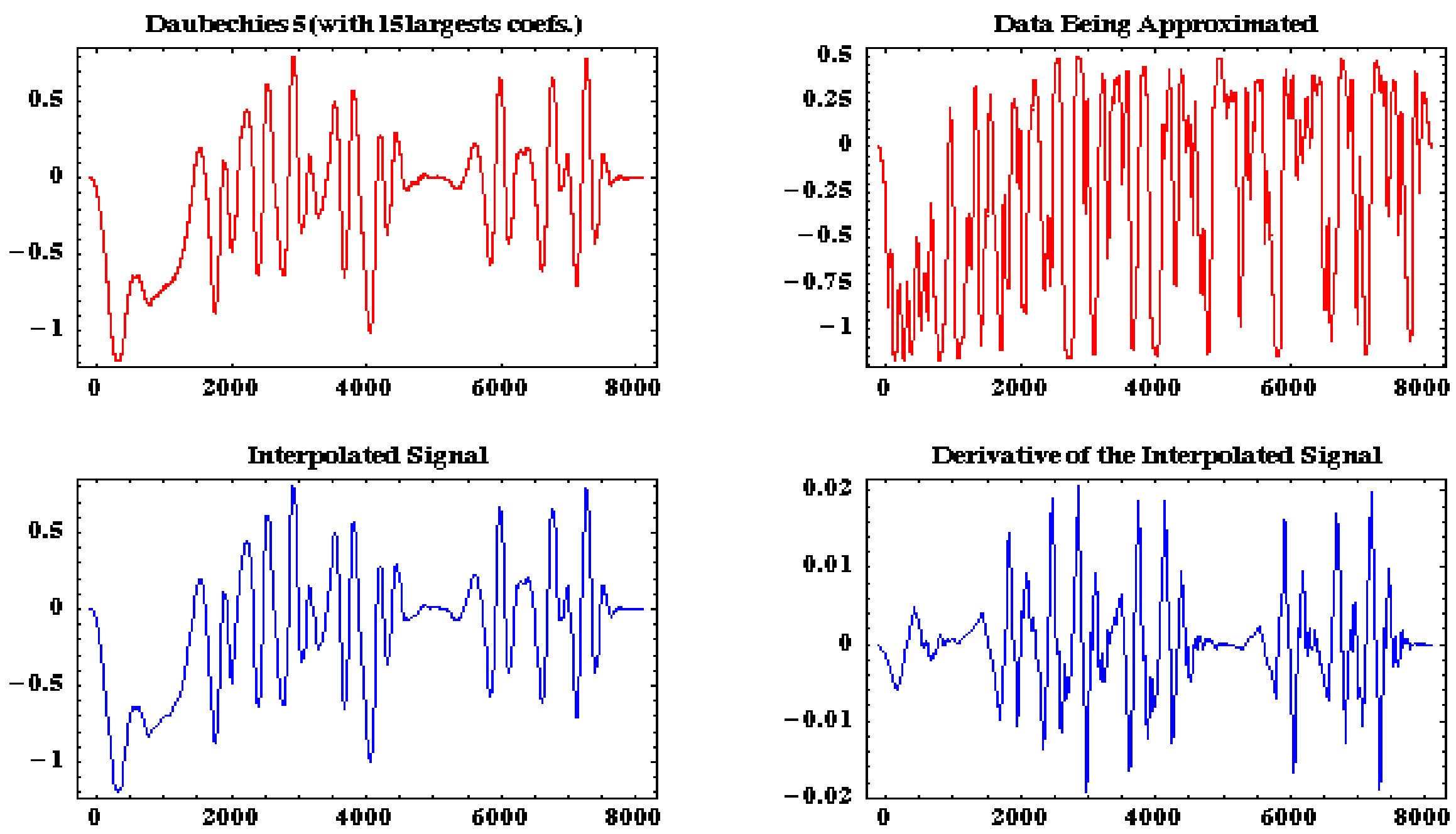


\section{Reconstruction of the LPF RT}

\section{Weak Mix Data Using the 20 Largest WLT Coefficients}

Polymath Research Inc.

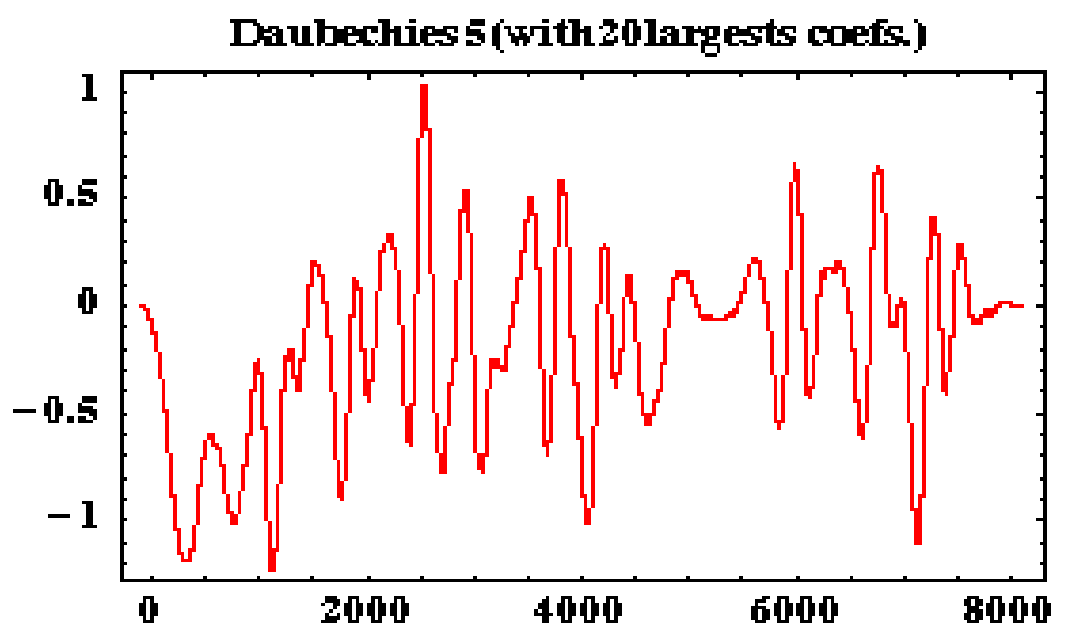

Interpolated Signal

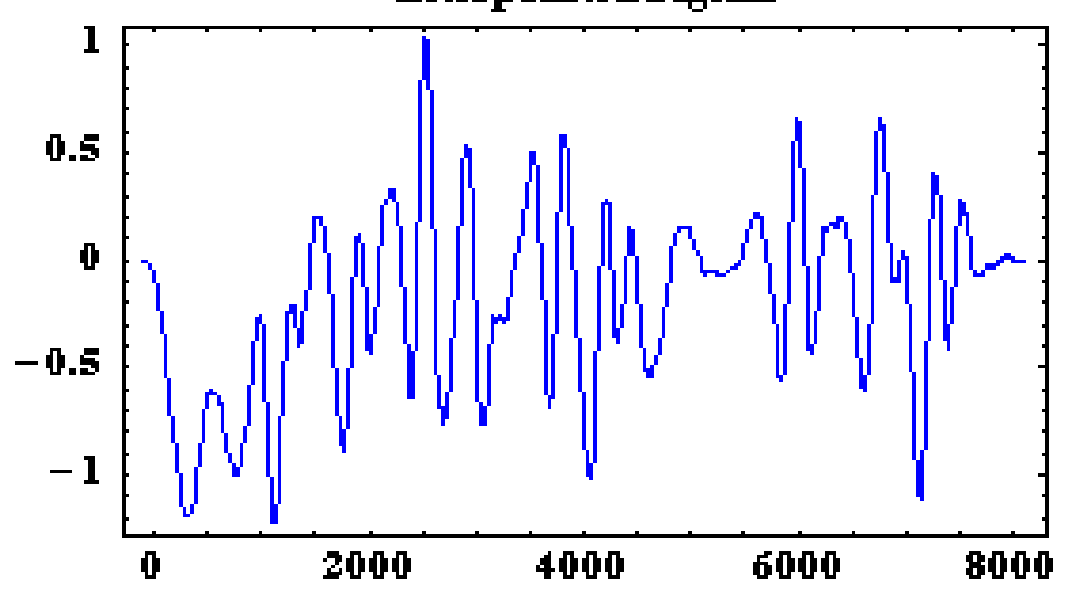

Data Being Approximated
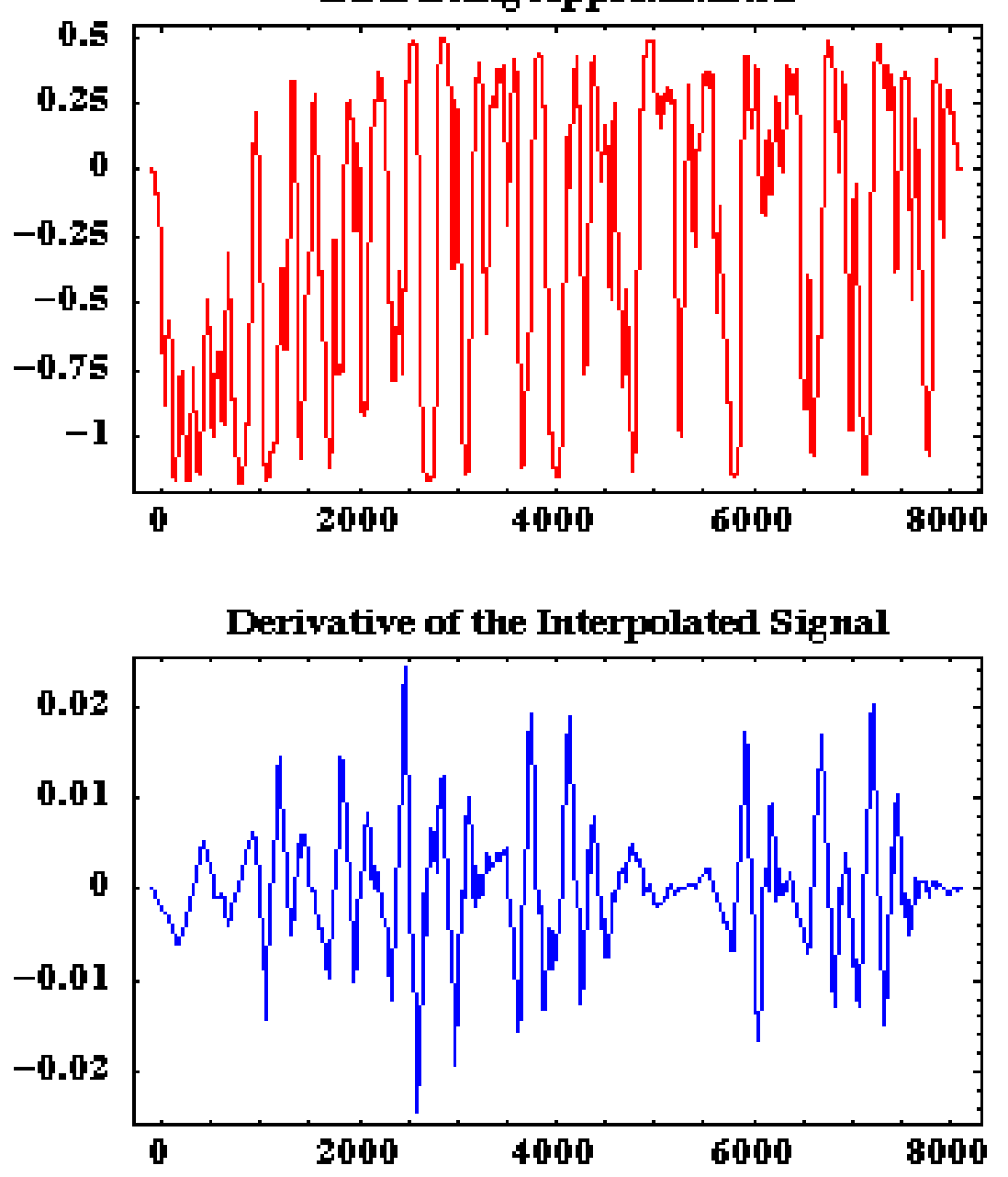


\section{Reconstruction of the LPF RT}

Weak Mix Data Using the 25

Largest WLT Coefficients
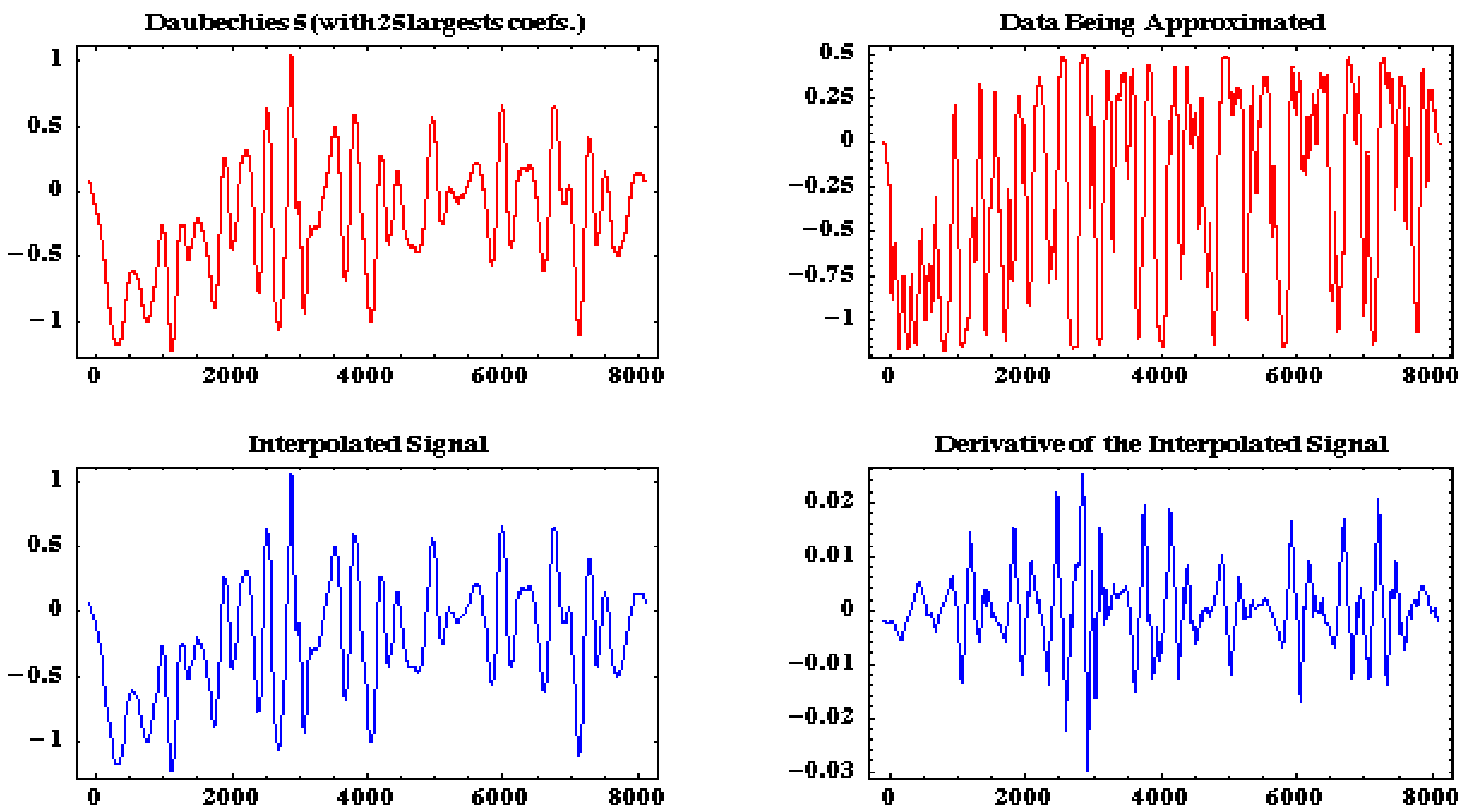


\section{Reconstruction of the LPF RT}

Weak Mix Data Using the 30

\section{Largest WLT Coefficients}
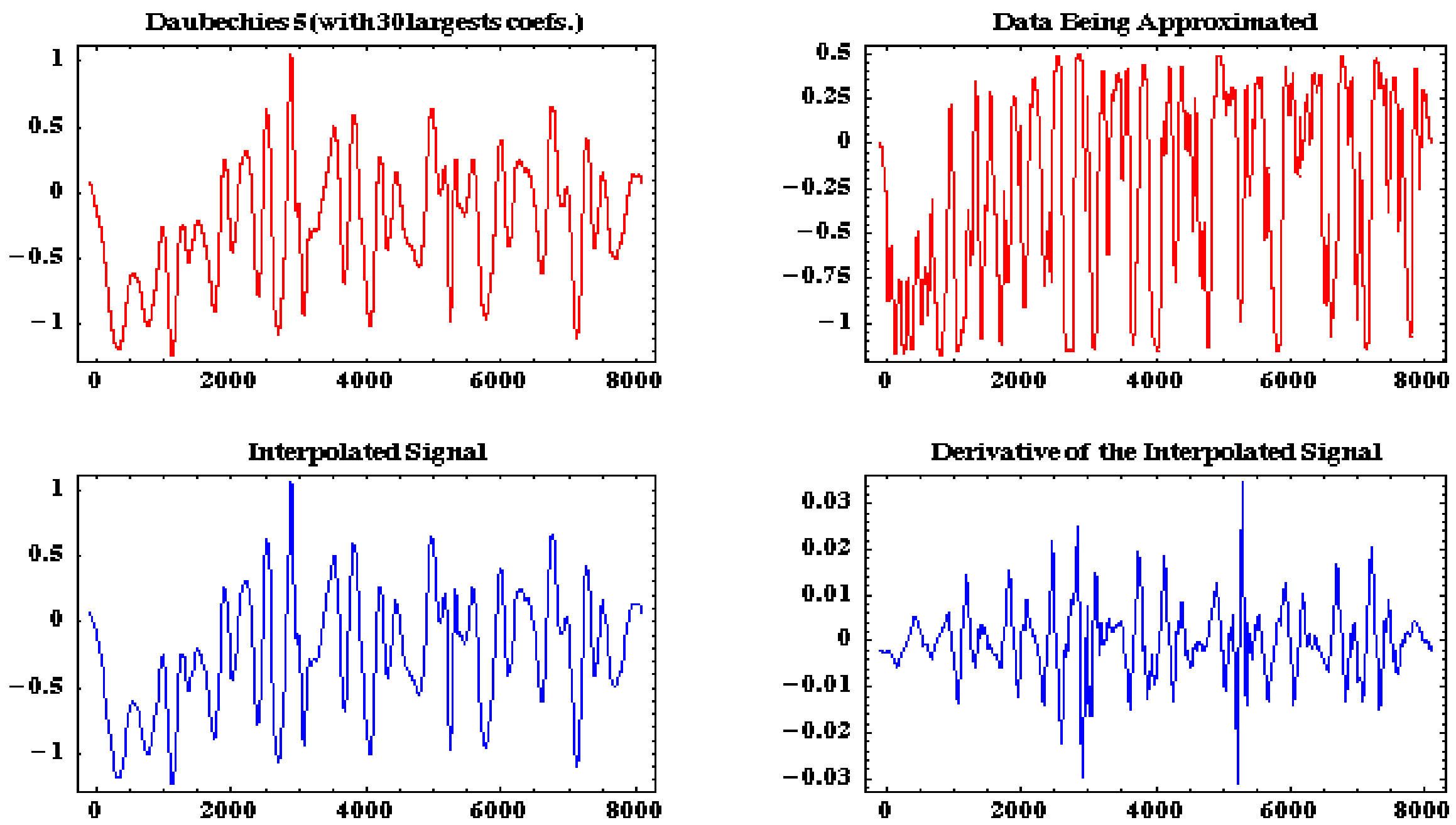


\section{Reconstruction of the LPF RT}

\section{Weak Mix Data Using the 35 \\ Largest WLT Coefficients}

Polymath

Research Inc.
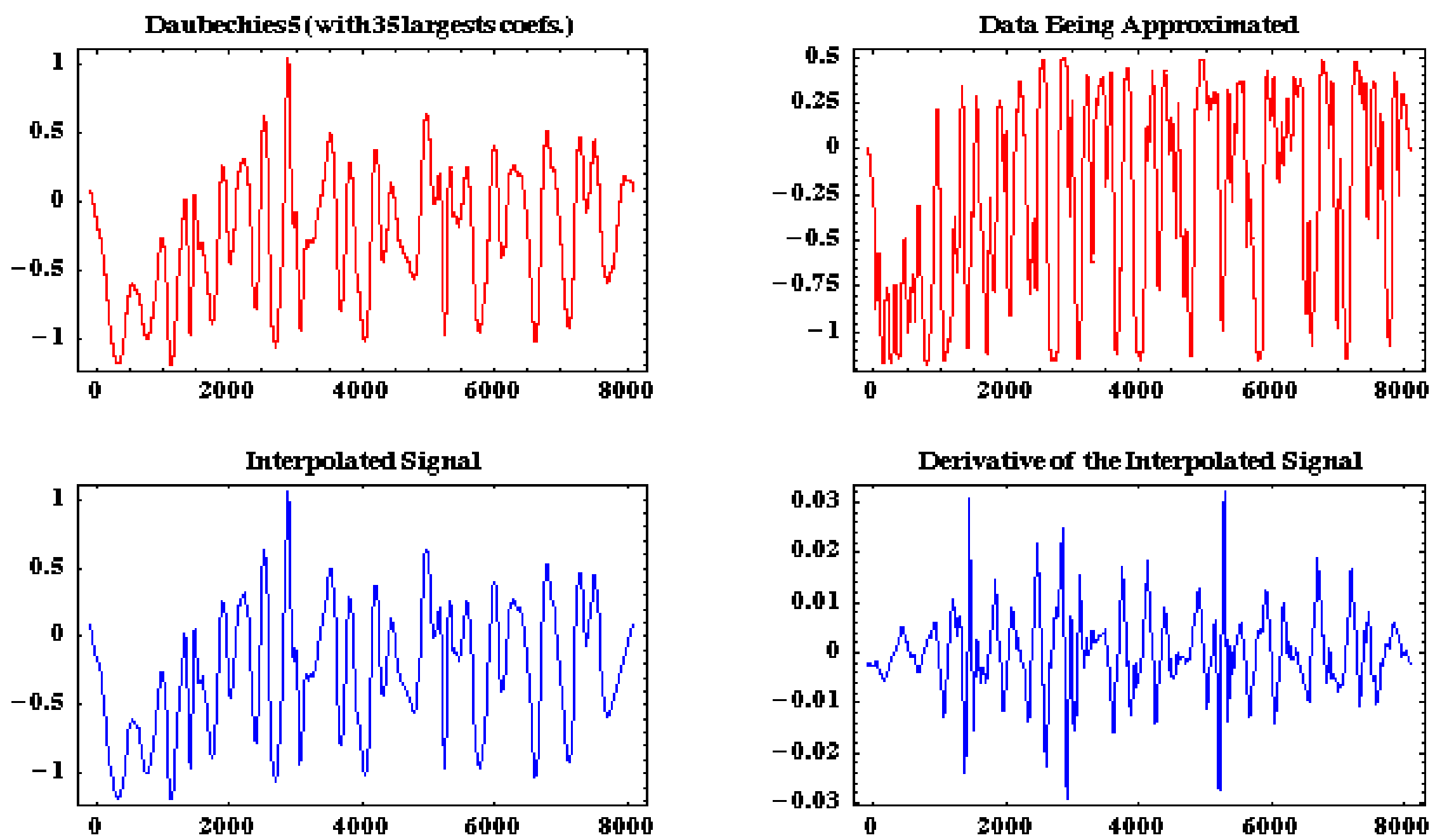


\section{Reconstruction of the LPF RT}

Weak Mix Data Using the 40

\section{Largest WLT Coefficients}

Polymath

Research Inc.
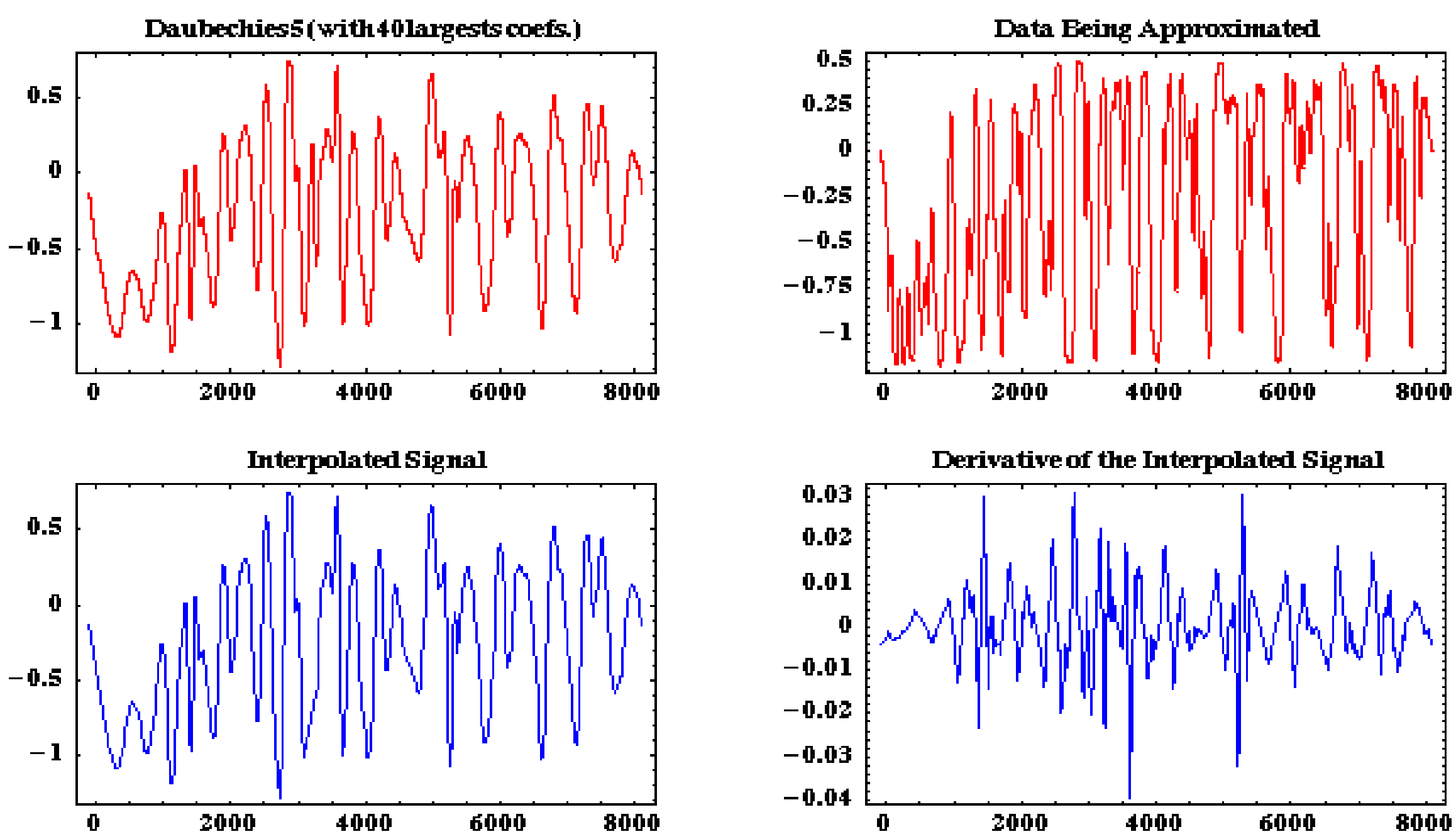


\section{Reconstruction of the LPF RT}

Weak Mix Data Using the 45

Largest WLT Coefficients

Polymath

Research Inc.
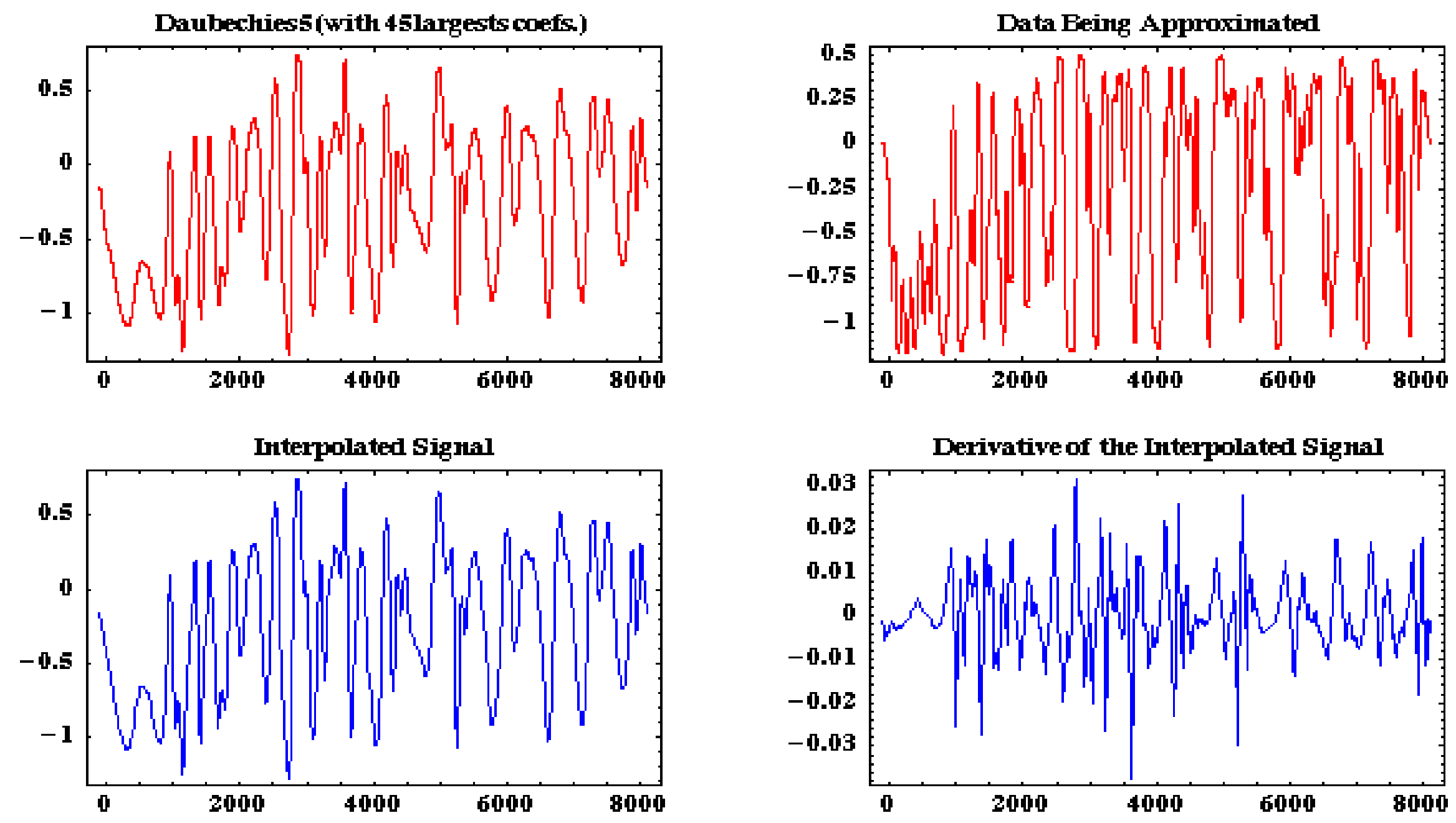


\section{Reconstruction of the LPF RT}

Weak Mix Data Using the 50

Largest WLT Coefficients

Polymath

Research Inc.
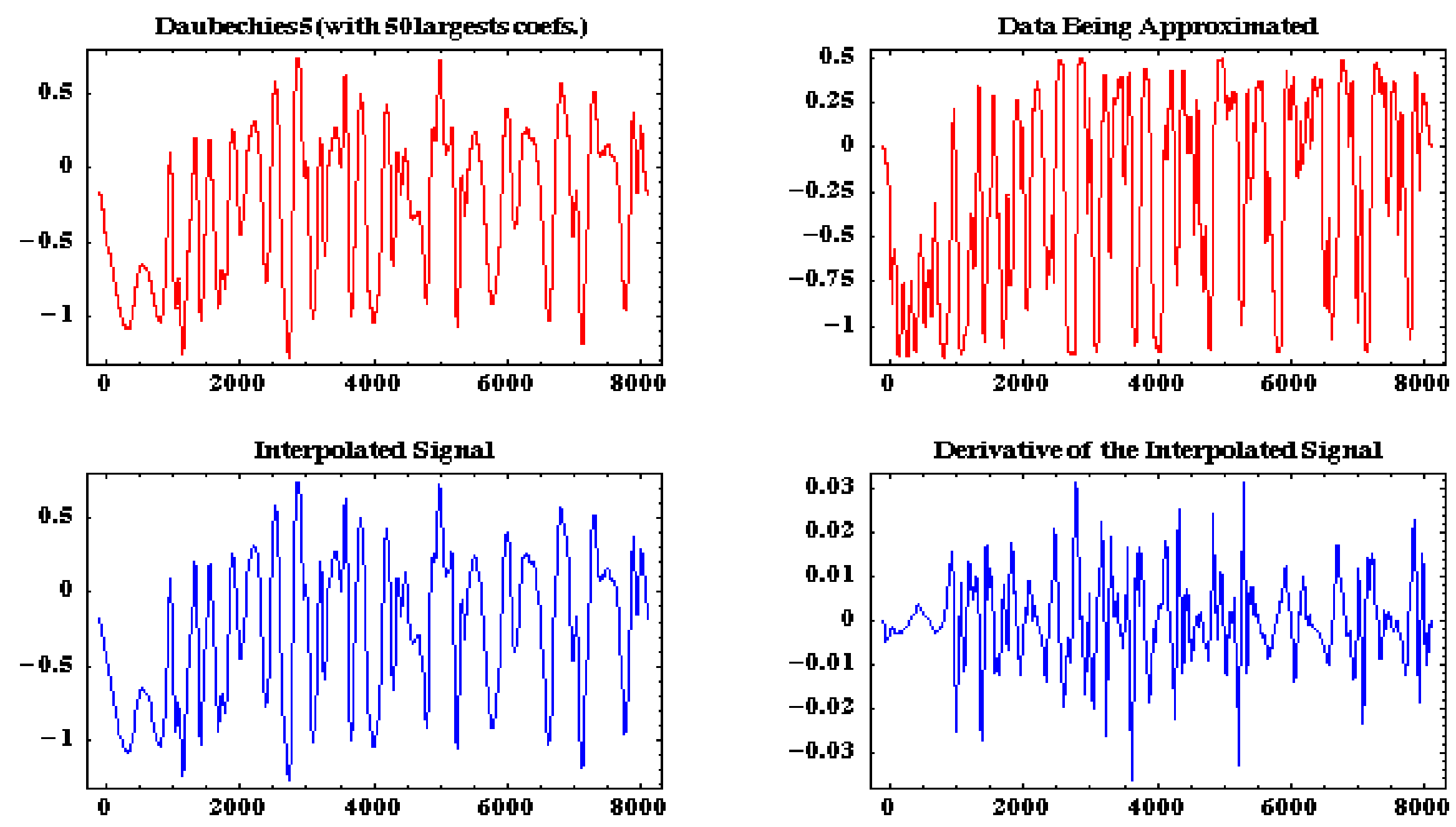


\section{Reconstruction of the LPF RT}

\section{Weak Mix Data Using the 100 Largest WLT Coefficients}
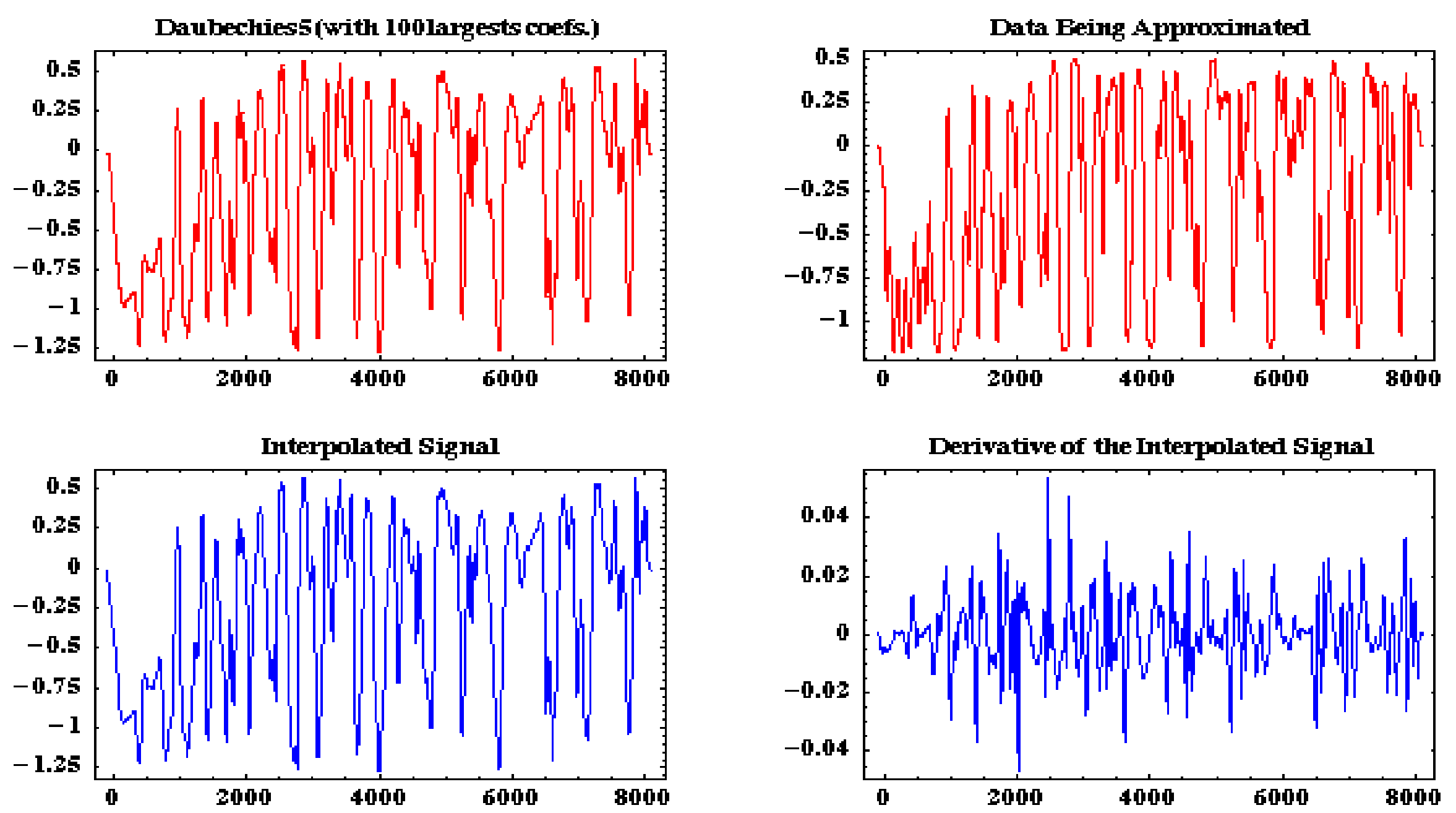

Research Inc.

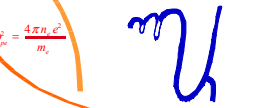




\section{Reconstruction of the LPF RT}

\section{Weak Mix Data Using the 200 Largest WLT Coefficients}
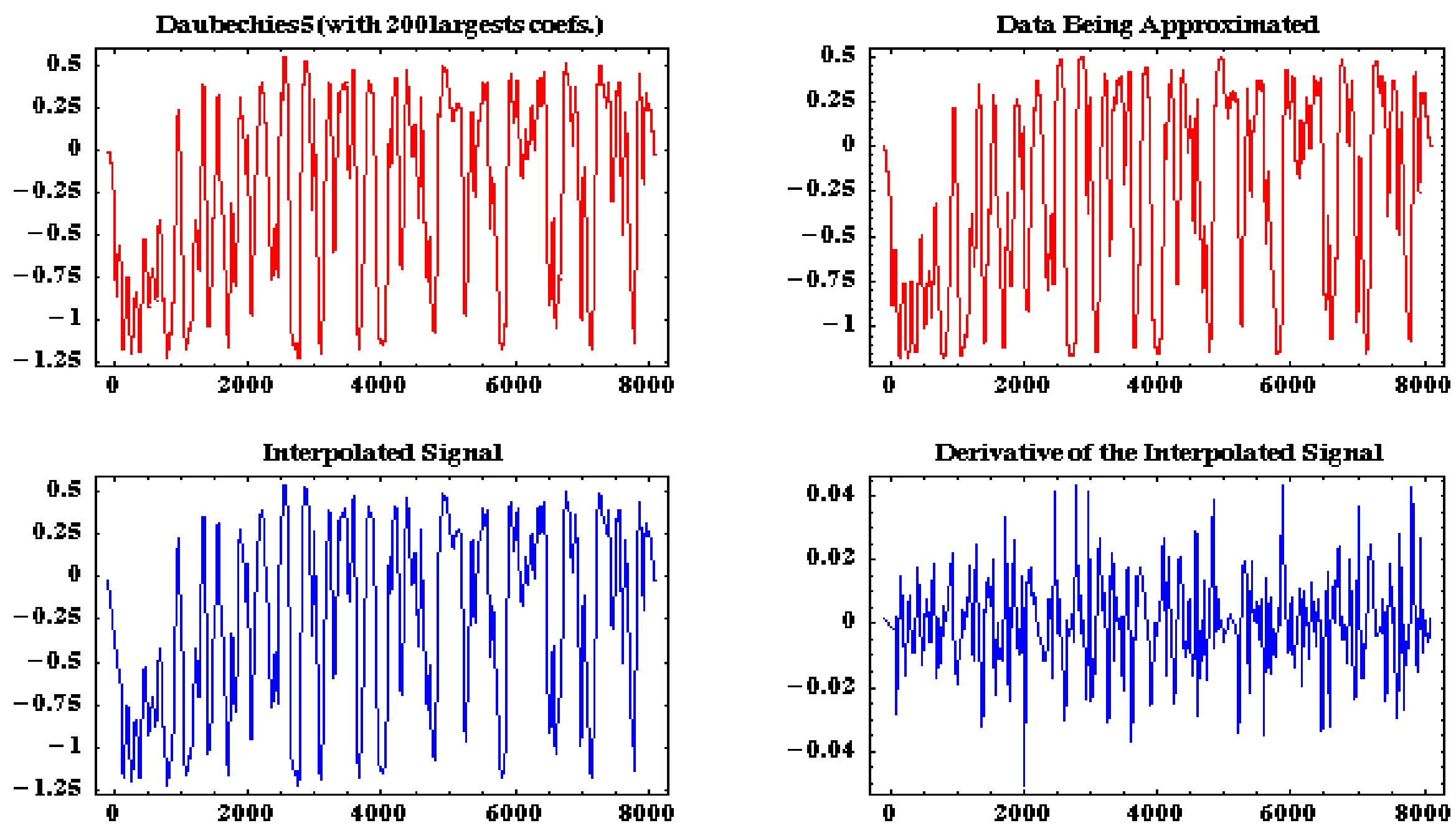


\section{Reconstruction of the LPF RT}

\section{Weak Mix Data Using the First MRD Level}
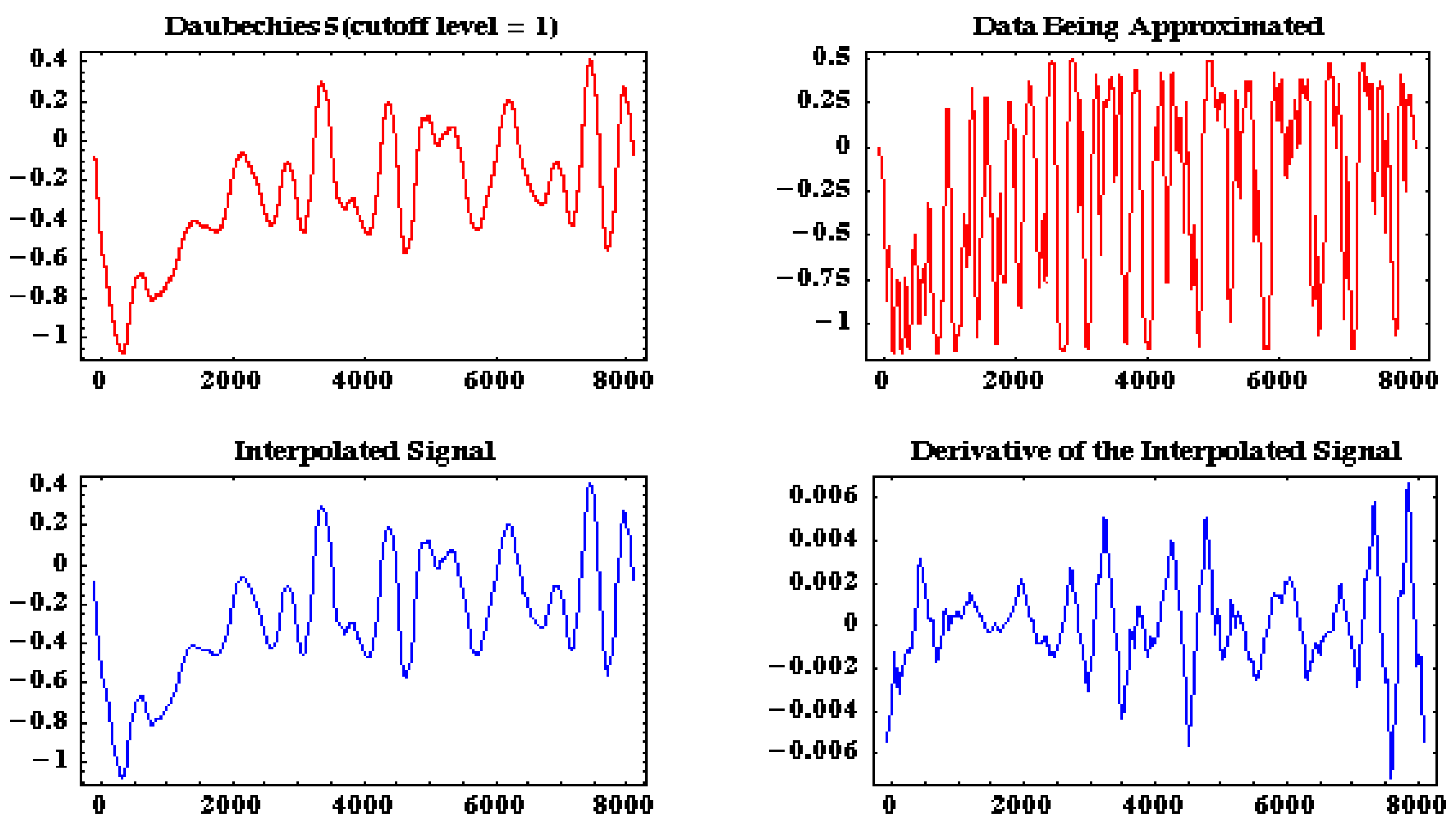


\section{Reconstruction of the LPF RT}

\section{Weak Mix Data Using the First 2 MRD Levels}
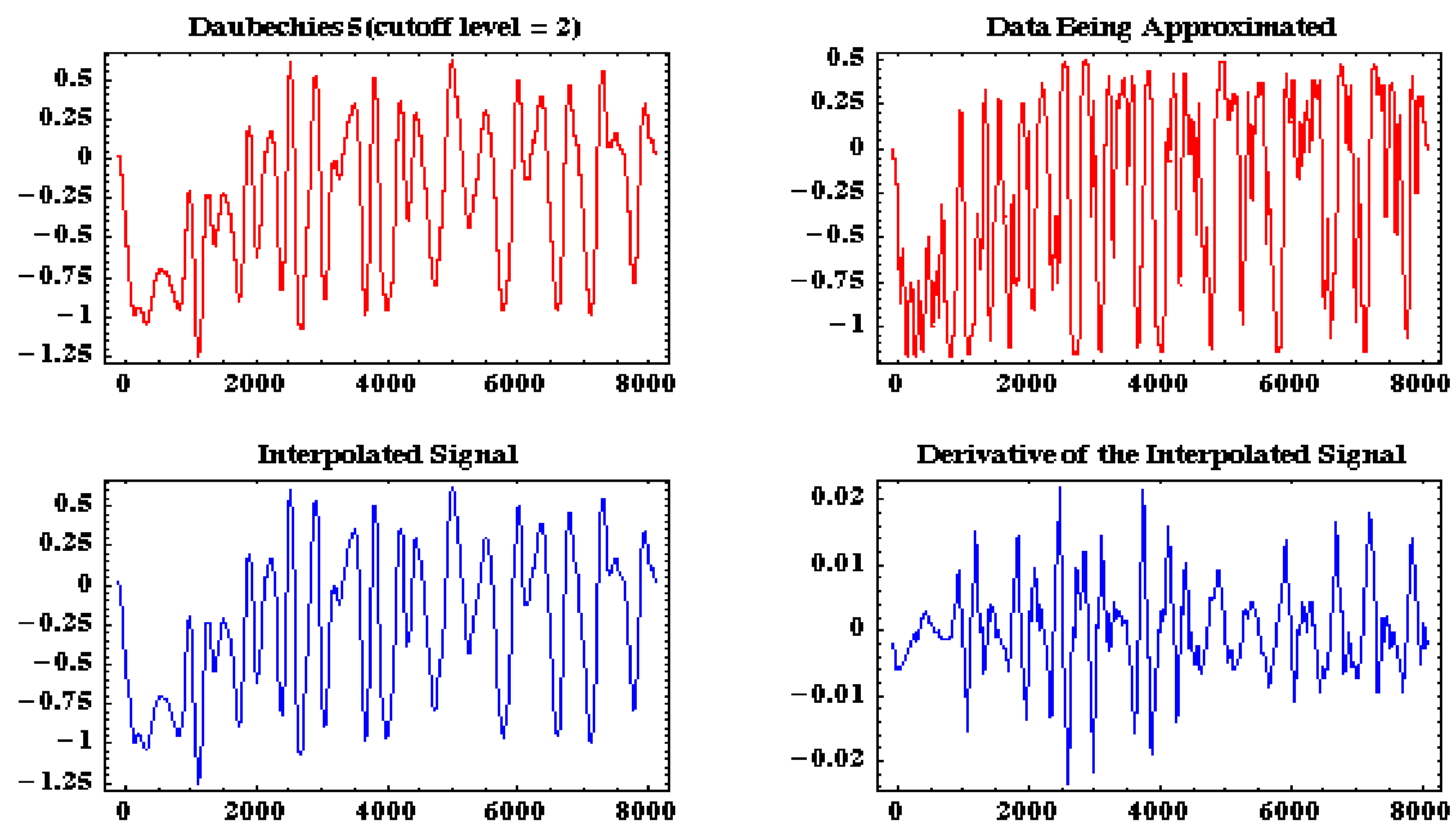


\section{Reconstruction of the LPF}

\section{RT Weak Mix Data Using the First 3 MRD Levels}

Polymath Research Inc.
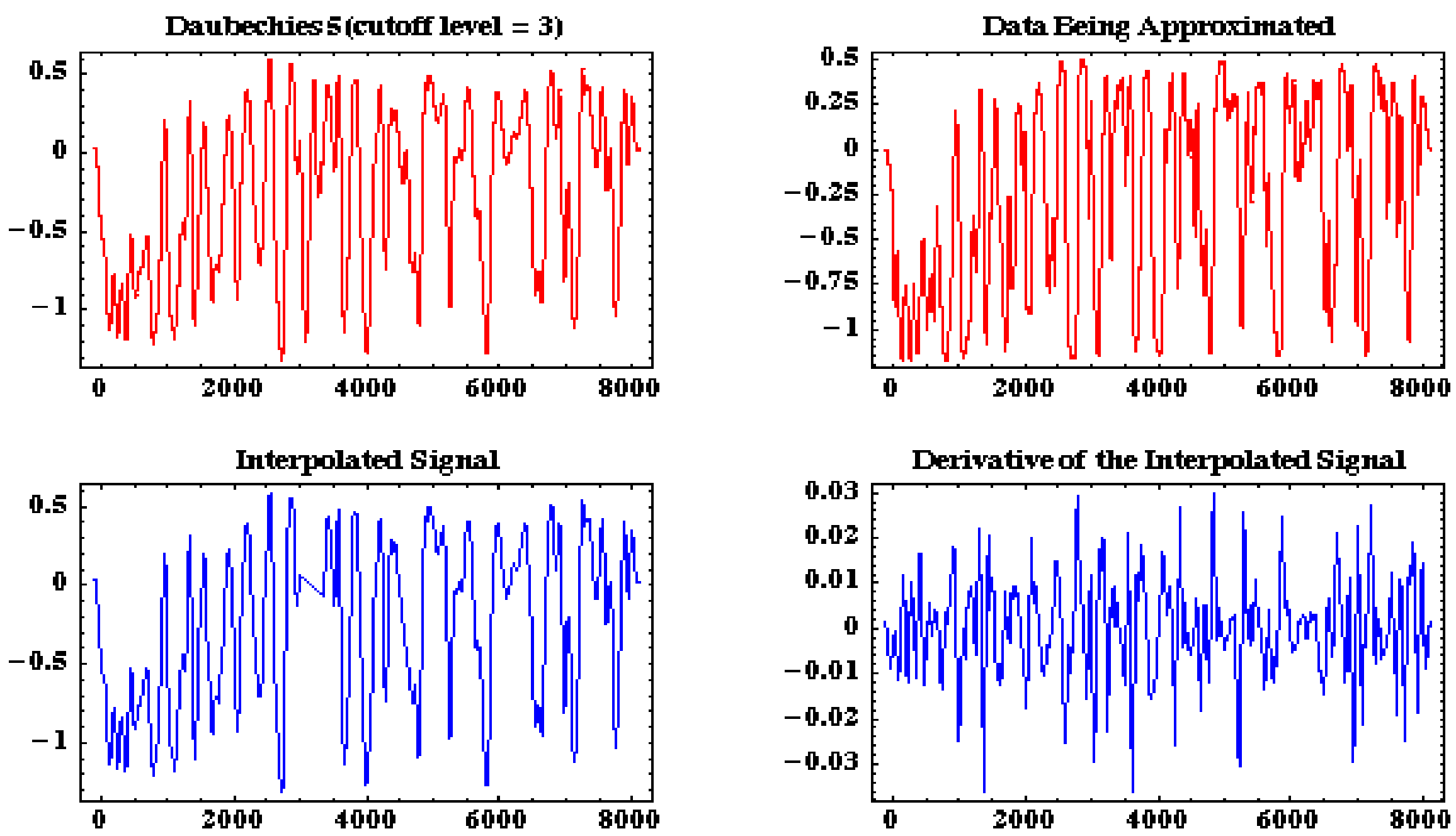


\section{Reconstruction of the LPF RT}

\section{Weak Mix Data Using the First 4 MRD Levels}
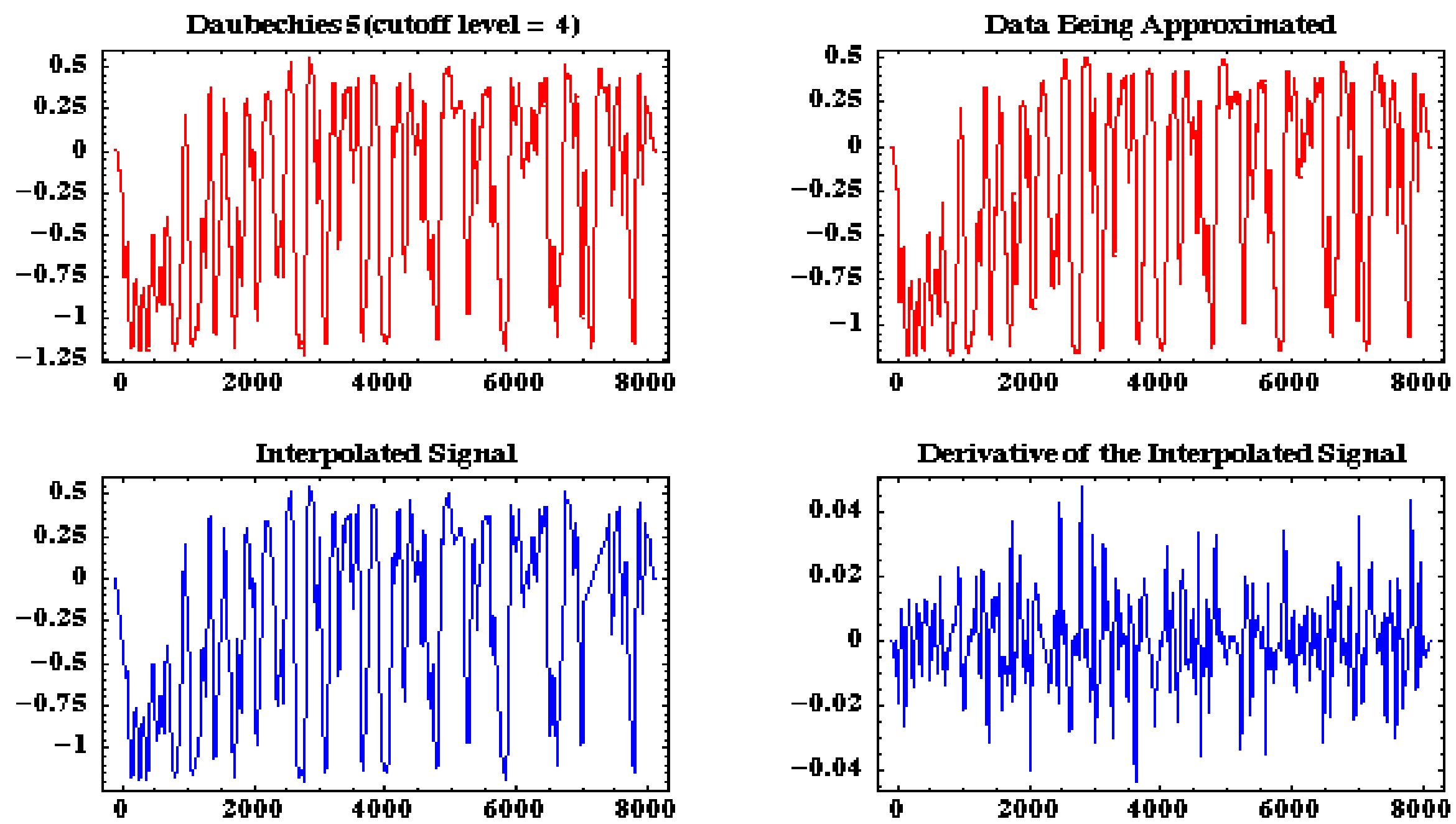


\section{Reconstruction of the LPF}

\section{RT Weak Mix Data Using the First 5 MRD Levels}

Polymath

Research Inc.
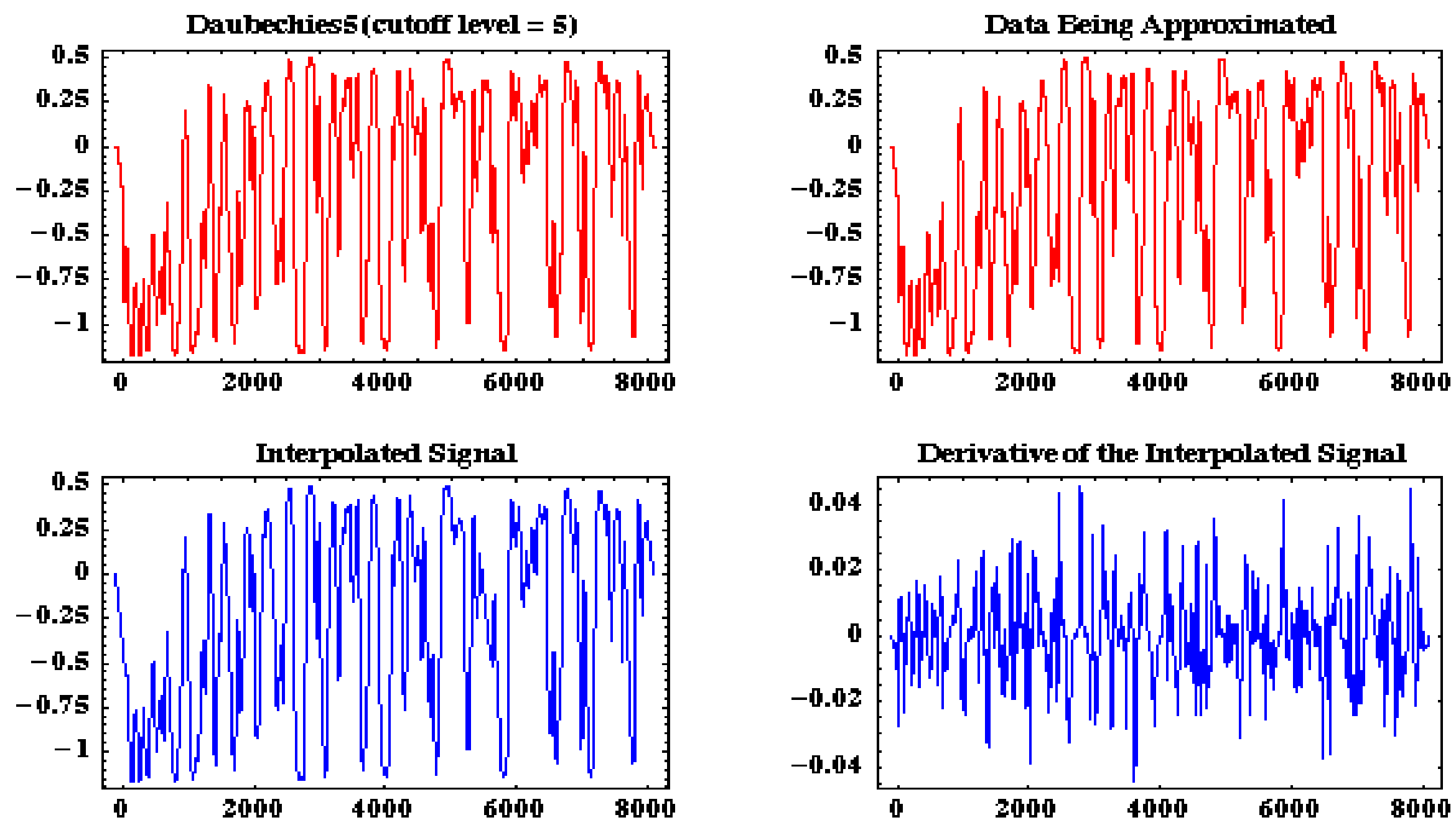


\section{Conclusions Based on the LPF RT Weak Mix Data's WLT Analyses}




\section{REVIEW OF NUMERICAL SIMULATION OF MIXING DUE TO \\ RAYLEIGH-TAYLOR AND \\ RICHTMYER-MESHKOV \\ INSTABILITIES}

David Youngs

AWE

Aldermaston

UK 
Scope: 2D and 3D numerical simulation (DNS or LES) of the non-linear growth of Rayleigh-Taylor and RichtmyerMeshkov instabilities.

Reasons for numerical simulation

(a) gain understanding of the mixing processes which is not available from experiment

(b) explain experimental results

(c) design experiments

(d) provide results for the calibration of engineering models (eg RANS models)

(e) full simulation of engineering applications 
(a) 2D single mode

(b) 2D multimode

(c) Additional physics

(d) 3D single mode/few modes

(e) 3D turbulence modelling of the unresolved scales

(f) Future role of numerical simulation AWE examples

\begin{abstract}
Aim to illustrate the progress made with examples - not a complete review of all the work done.
\end{abstract}


Turbulent mixing is a 3D process.

However, the dynamics of the large scale structures within the mixing layer is the key aspect of mixing and much has been/can still be learnt about this from single-mode or 2D multimode simulations.

The fine-scale structure (dissipation at high wave numbers) is essentially a 3D process and for this $3 \mathrm{D}$ simulation is essential.

The possibilities of 3D simulation on present-day super computers should be fully exploited - however, simpler 2D simulation still has an essential role especially for complex problems with additional physics. 


\section{D SINGLE MODE}

The first 2D simulations of RT were carried out in Frank Harlow's group at LANL in the late 1960s.

e.g. B J Daly, Phys Fluids Vol 10, p297 (1967)

MAC code: incompressible

'Marker and Cell'

$$
\frac{\rho_{\mathbf{1}}}{\rho_{\mathbf{2}}}=1.1,2,10
$$

Roll-up of the spike due to Kelvin-Helmholtz instability seen for

$$
\frac{\rho_{1}}{\rho_{2}}=1.1, \text { and } 2, \quad \text { but not for } \quad \frac{\rho_{1}}{\rho_{2}}=10 .
$$

not observed experimentally until Ratafia, Phys Fluids Vol 16 p1207 (1973).

results explained by drag force on bubble and spike - as in buoyancy - drag models which are widely used today. 

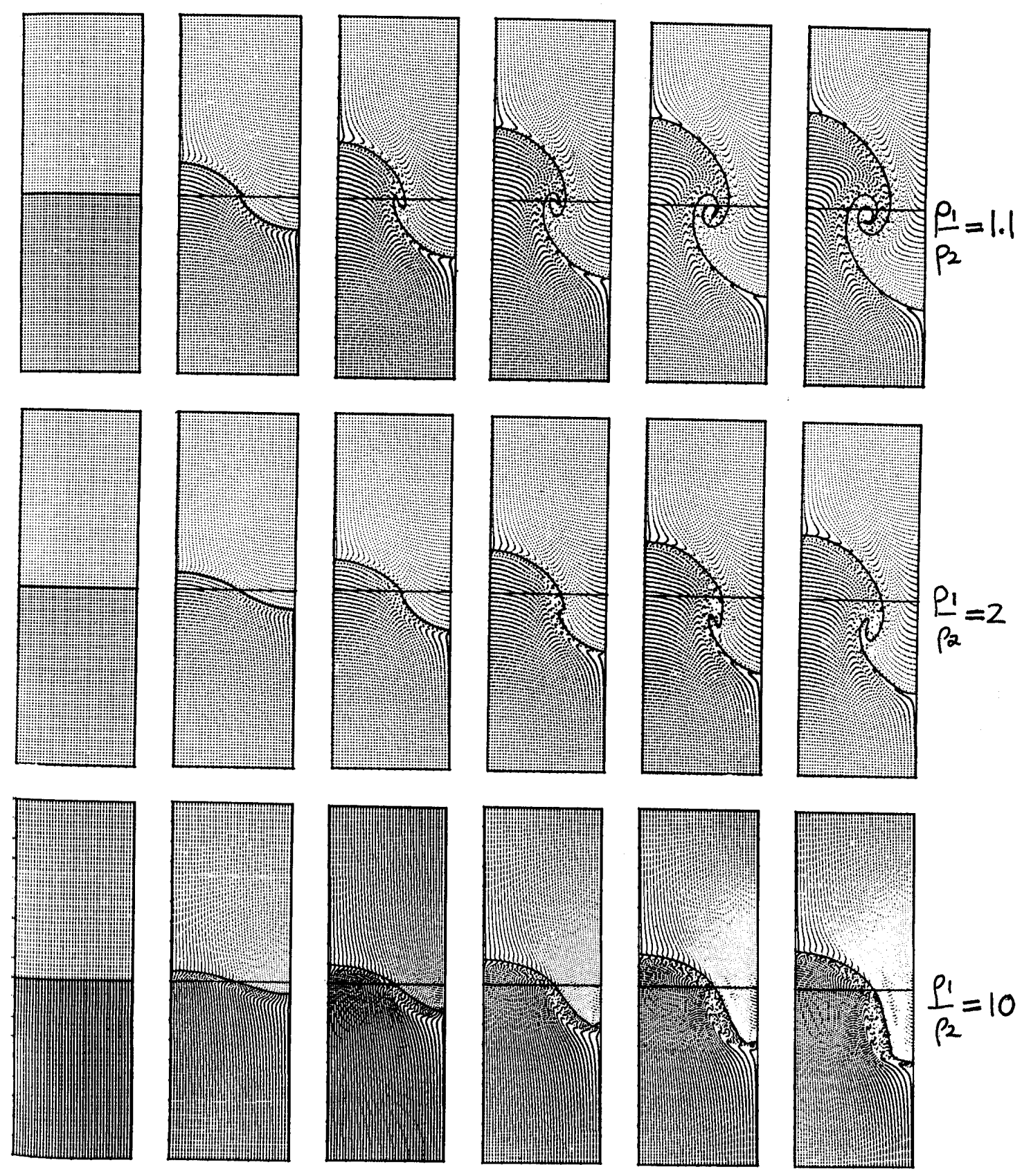

Physics of Fluids, vol 10, p297 (1967) 
Youngs, Physica 12D, p32 (1984)

2D Eulerian code

- Interface tracking (VOF technique)

- Monotonic advection method of Van Leer for all fluid variables

$\Rightarrow \quad$ improved numerical stability over the earlier hydrocodes

Shows KH instability on the spike at high density ratio, $\frac{\rho_{1}}{\rho_{2}}=20$
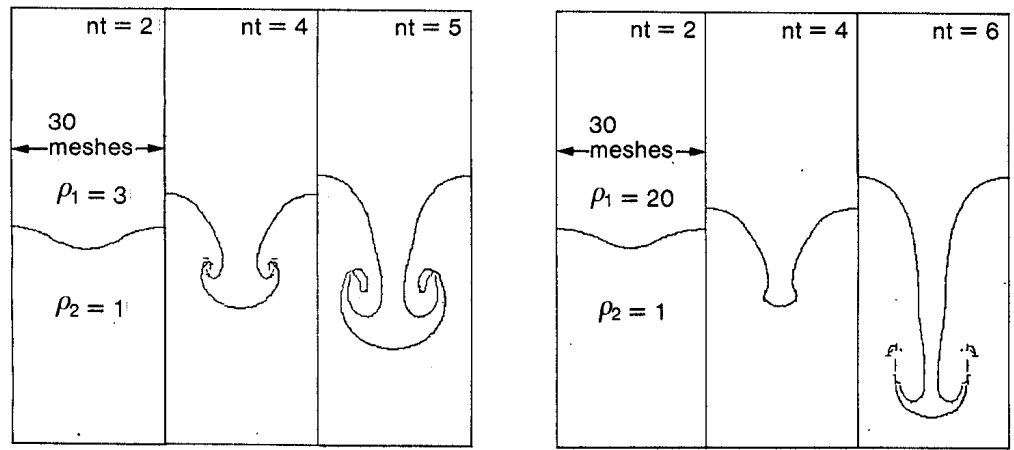

Fig. 1. Rayleigh-Taylor instability, single wavelength initial perturbation 
Youngs, Physica 12D, p19 (1984)

2D Multimode Rayleigh-Taylor triggered by short wavelength random perturbations. Incompressible hydrocode similar to MAC code but interface tracking was abandoned as fine-scale mixing expected.

Solved equation for fluid 1 volume fraction

$$
\frac{\partial \mathrm{f}_{1}}{\partial \mathrm{t}}+\operatorname{div}\left(\mathrm{f}_{1} \underline{\mathrm{u}}\right)=0
$$

Showed self-similar growth with bubble penetration $\left(h_{1}\right)$ given by

$$
\mathbf{h}_{1}=\alpha \frac{\rho_{1}-\rho_{2}}{\rho_{1}+\rho_{2}} \mathbf{g t}^{2}
$$

$\alpha \approx 0.04$ to 0.05 independent of density ratio, for growth by mode coupling.

Subsequent experiments, Read, Physica 12D, p45 (1984) gave $\alpha \sim 0.06$ to 0.07 .

At the time difference between $\alpha$ in calculation and experiment was attributed to 2D vs 3D effects - but this has not turned out to be so simple. 
I-L Chern, J Glimm et al

J Comp Phys, vol 62, p64 (1986)

Fron Tier: Accurate front tracking technique (more accurate than the VOF techniques)

Applied to multimode 2D RT in Glimm et al Phys Fluids A2 p2046 (1990)

Note that most of RT experiments use immiscible liquids - so need to eliminate mass diffusion.

Results used to develop bubble merger models.

For two generations of bubble merger simulations give $\alpha \sim 0.055$ to 0.065 in agreement with experiment.

For more than two generations $\alpha$ drops to $\sim 0.04$ (fine-scale structure develops).

Glimm et al (1990)

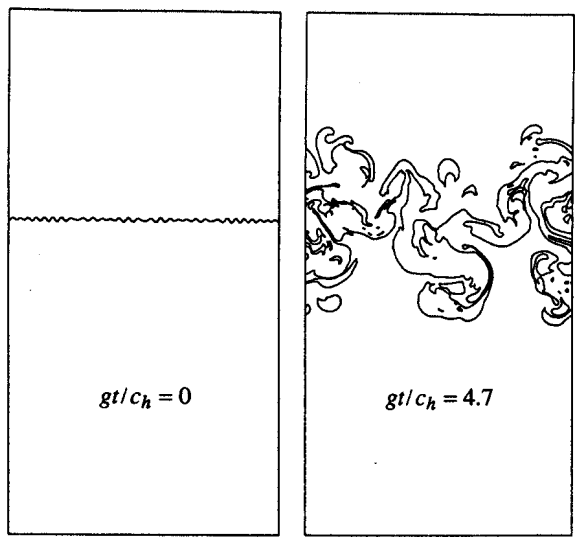

FIG. 10. Plots of interfaces in the random disturbance simulation of Rayleigh-Taylor instability. The Atwood number is $A=\{$ and the compressibility is $M^{2}=0.1$. The acceleration rate of the bubble envelope has good

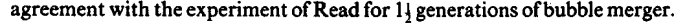
The acceleration rate decreases after this time because of the multiphase connectivity, which is different in the exactly two-dimensional computation and in the approximately two-dimensional experiments. 
Inogamov ( $3^{\text {rd }}$ IWPCTM) argued that self similar gt $^{2}$ growth should be obtained if a multimode perturbation with

$$
\frac{\text { amplitude }}{\text { wavelength }}=\boldsymbol{\varepsilon} \text { (a constant) }
$$

is used. In this case $\alpha$ depends weakly on the initial conditions.

2D multimode calculations described by Atzeni and Guerrieri, Europhys Lett, Vol 22, p603 (1993).

$\alpha \sim 0.04$ to 0.05 lower bound for mix evolution.

Demonstrates the very useful role which numerical simulation can play in understanding the effect of initial conditions on turbulent mixing.

What happens in real problems?

Growth via mode coupling or growth directly from initial perturbations. 


\section{RICHTMYER-MESHKOV: 2D SINGLE MODE}

Impulsive linear model (Richtmyer)

$$
\frac{d a}{d t}=\frac{2 \pi}{\lambda} A^{+} a_{0}^{+} \Delta U
$$

$A^{+}, a_{0}^{+}:$post shock Atwood Number and amplitude

2D numerical simulation very useful in understanding the correct effect of compressibility on the linear theory and also the non-linear behaviour.

Highlight recent paper:-

Holmes, Dimonte, Fryxell, Gittings, Grove, Schneider, Sharp, Velikovich, Weaver, Zhang. J Fluid Mech, Vol 389, p55 (1999)

Compare three different hydrocodes (RAGE, PROMETHEUS and Fron Tier) with non-linear theory, and with a NOVA experiment.

Fron Tier: $\quad$ interface tracking

RAGE: no interface tracking, AMR

PROMETHEUS: no interface tracking (MUSCL) 

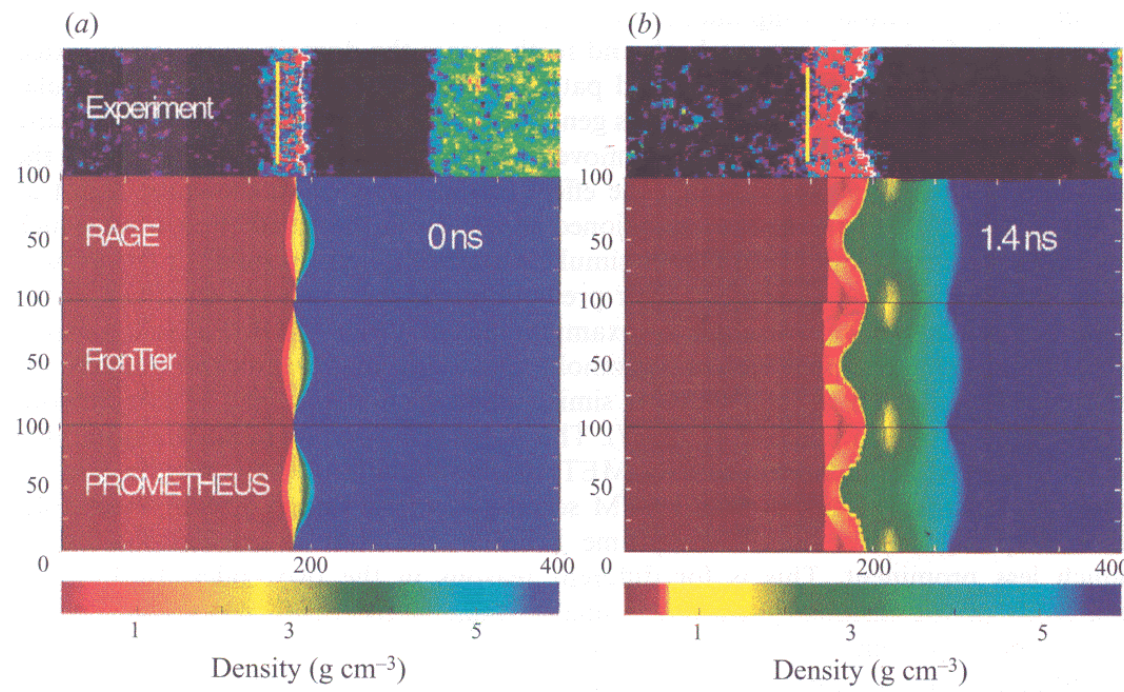

(c)
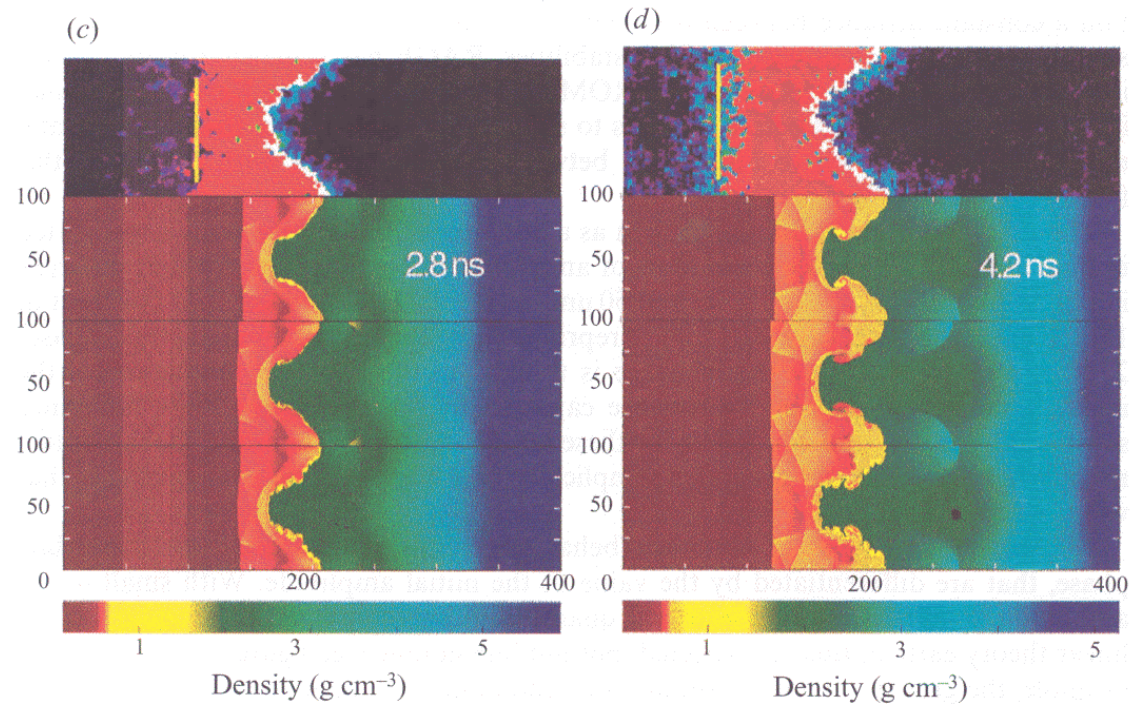

FIGURE 3. Simulation density plots and experimental radiographs for case $10 / 15.3$. (a) $t=0.0 \mathrm{~ns}$, (b) $t=1.4 \mathrm{~ns},(c) t=2.8 \mathrm{~ns},(d) t=4.2 \mathrm{~ns}$. Each figure shows the results obtained with the three codes (RAGE, FronTier and PROMETHEUS, top-to-bottom) along with an experimental image. The incident shock moves from right to left. The experimental radiograph is an averaged composite over four wavelengths. Note that at later times the experimental wavelength increases due to expansion of the experimental target.

J Fluid Mech, vol 389, p55 (1999) 


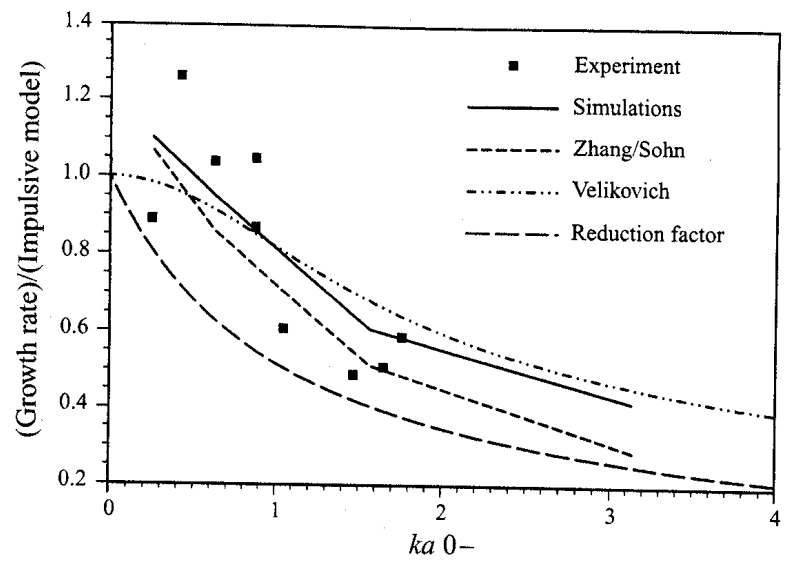

FIGURE 7. Comparison of experimental, simulation and theoretical peak growth rates as a function of initial amplitude with incident Mach number 15.3. Growth rates are scaled to the Meyer-Blewett formulation of the Impulsive Model. For the simulations we averaged the peak growth rates of
each of the three codes.

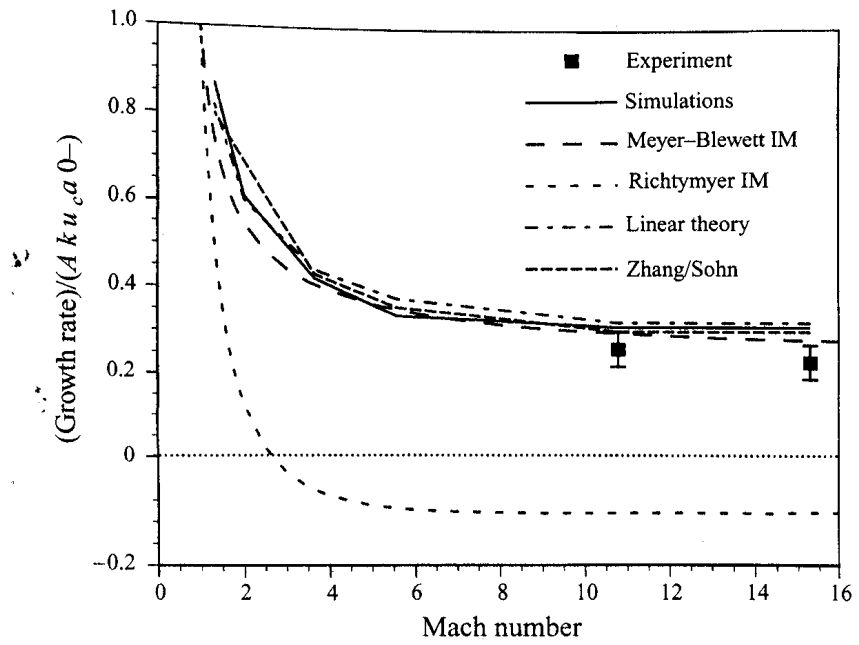

FIGURE 10. Comparison of experimental, simulation and theoretical peak growth rates as a function of incident shock Mach number. The growth rate is scaled by $A k u_{c} a_{0-}$.

J Fluid Mech, vol 389, p55 (1999) 
Richtmyer-Meshkov: Late-time behaviour (Multimode)

Theory suggests $h \sim t^{\theta}$ for single shock $R M$

Nova experiment (Dimonte, Frerking, Schneider, PRL, vol 74, p4855 (1995)

Be/foam interface, random perturbations experiment gives

$\theta \sim 0.6$.

2D CALE simulation with representation of the measured surface finish.

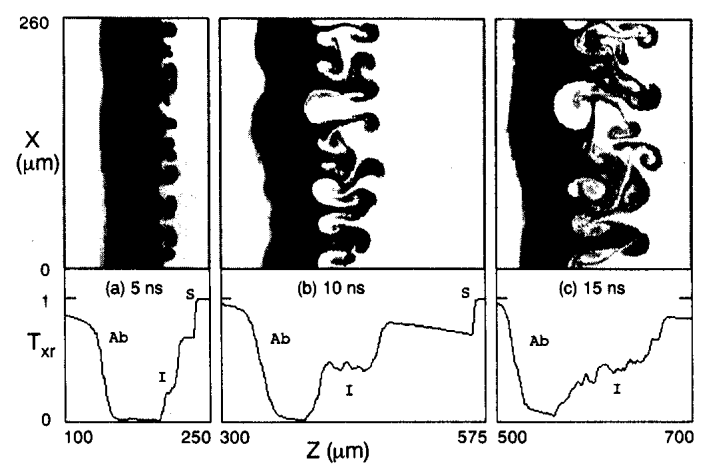

FIG. 4. Simulation side-on radiographs and spatial $x$-ray transmission profiles at $t=0,5$, and $10 \mathrm{~ns}$.

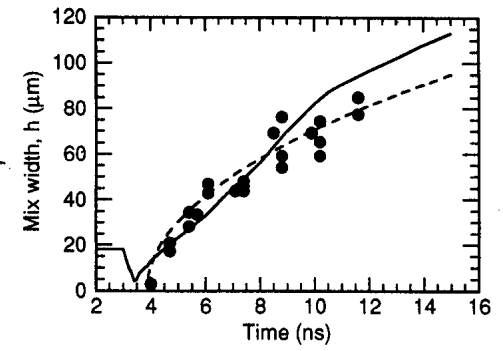

FIG. 5. The turbulent mix width at the interface vs time for the experiment (points), CALE (solid line), and Eq. (3) (dashed line) with $\eta_{\mathrm{rms}} \sim 4.6 \mu \mathrm{m}, h_{0 e}^{*}=5.6 \mu \mathrm{m}$, and $t_{i}=3.9 \mathrm{~ns}$. 
Mügler and Gauthier. Physics of Fluids, vol 12, p1783 (2000)

\section{D multimode $R M$ mixing \\ compare with shock tube experimental data.}

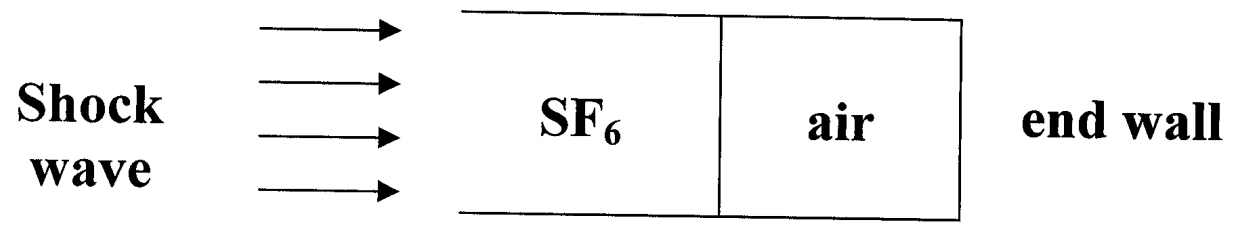

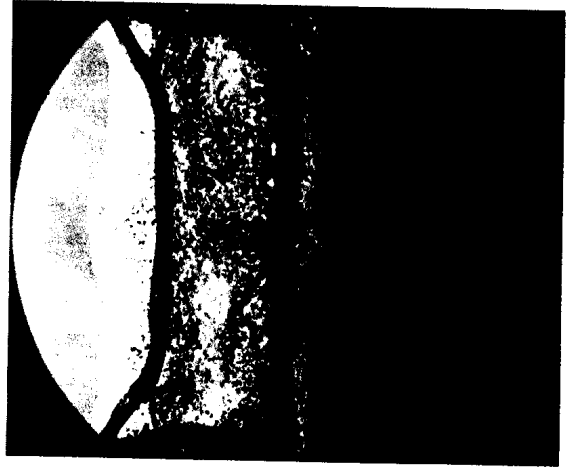

(a)

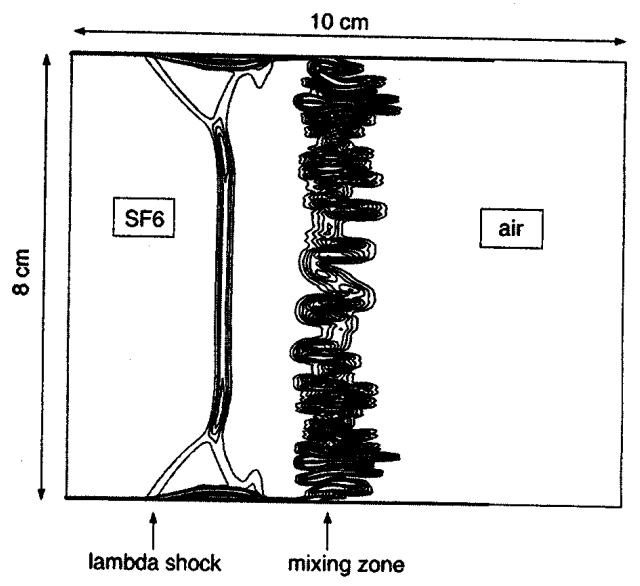

(b)

FIG. 7. (Color) (a) Experimental, from Galametz (Ref. 5), and (b) numerical schlieren pictures at a time $t$ just after the first reflected shock wave-mixing zone interaction. Because of its interaction with the boundary layer, the transmitted shock in the $\mathrm{SF}_{6}$ bifurcates.

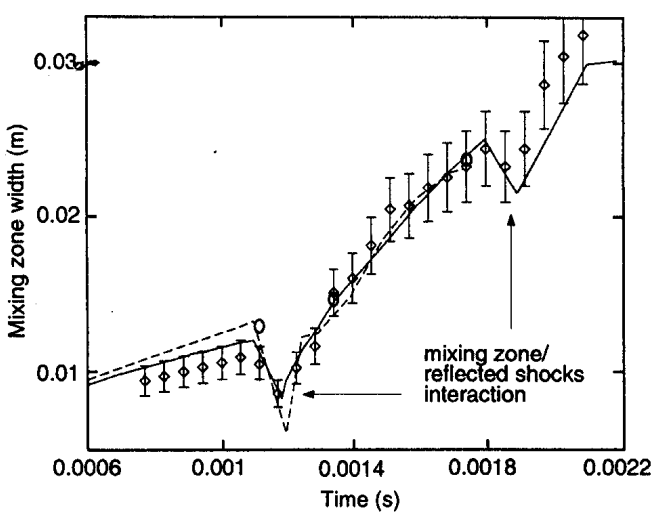

FIG. 2. Evolution of the mixing-zone width vs time. Diamonds correspond to the experimental width measured from schlieren pictures. The errorbars of this visual measurement are equal to $\pm 10 \%$. The three small circles correspond to microdensitometry measurements of some schlieren pictures. Full and dotted lines correspond to numerical widths obtained from the coarse and fine grids, respectively. 


\section{ADDITIONAL PHYSICS}

Numerical simulation has played a major role in the understanding of additional physics on RT/RM instability.

(a) Material strength (solids)

(b) Density gradient stabilisation

(c) Ablation front stabilisation 


\section{Effect of Material Strength}

Linear theory not straightforward.

Growth rate depends on

amplitude

wavelength

shear modulus

yield strength

Simplest model : amplitude threshold (Drucker)

Initial paper : J F Barnes et al J Appl Phys, vol 45, p727 (1974) (LANL)

Also : Swegle and Robinson. J Appl Phys, vol 66, p2838 (1989) (SNLA)

2D Lagrangian numerical simulation.

Swegle and Robinson

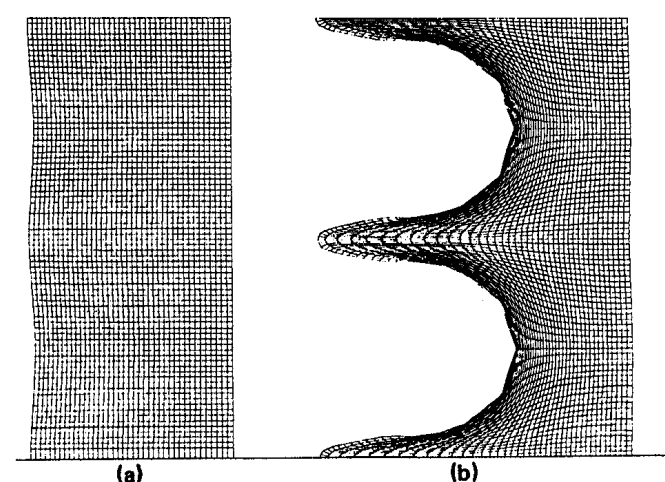

FIG. 2. Typical mesh plot for an unstable calculation. The small initial perturbation in (a) grows to that in (b) after a few microseconds of application of the driving pressure. There are 40 zones through the plate thickness of 2 $\mathrm{mm}$, with 80 zones in the other direction. 
Hydrodynamic Instability in Strong Media UCRL - CR - 126710 (LLNL)

- Review of open VNIIEF publications

Theory, experiment, numerical simulation

2D (planar) vs 3D (axisymmetric)

2D grows faster than 3D (opposite to the fluid case)

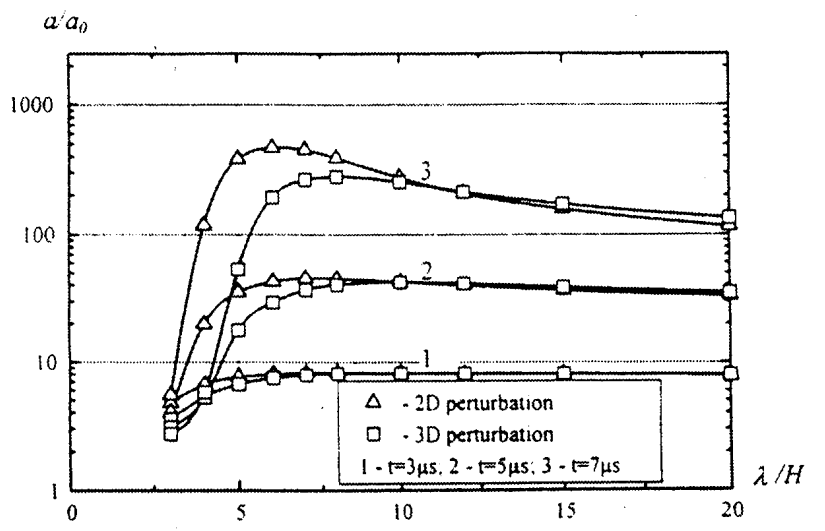

Fig. 4.3-14. Perturbation amplitude vs wavelength at various times for $2 D$ and $3 D$ perturbations. $a_{0}=3.3 \mu \mathrm{m}, \sigma_{\mathrm{T}}=1.5 \mathrm{GPa}, \Delta \tau=2 \mu \mathrm{s}$. 
UCRL - CR - 126710

IV. Results of Experimental Study

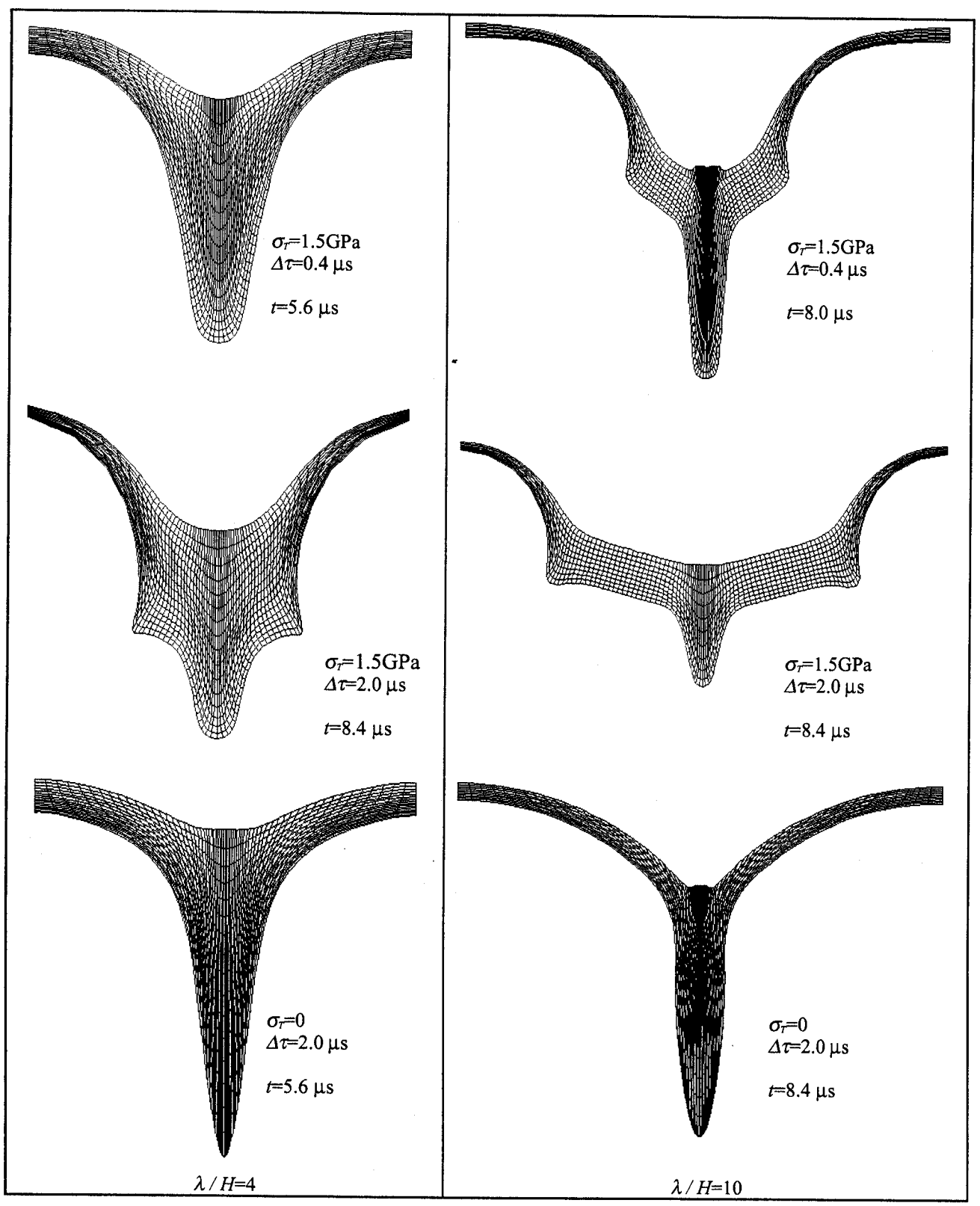

Fig. 4.3-13. Characteristic layer shapes. 


\section{Density gradient stabilisation}

Pham and Meiron, Physics of Fluids, vol A5, p344 (1993)

\section{RM + continuously stratified fluids} 2D single mode and multimode.

\section{Implusive acceleration}

$$
\rho(\mathbf{x}, \mathbf{y})=\frac{\mathbf{1}}{\mathbf{2}}\left[\mathbf{1}+\mathbf{A} \tanh \left\{\frac{\mathbf{y}-\zeta(\mathbf{x})}{\mathbf{L}}\right\}\right]
$$
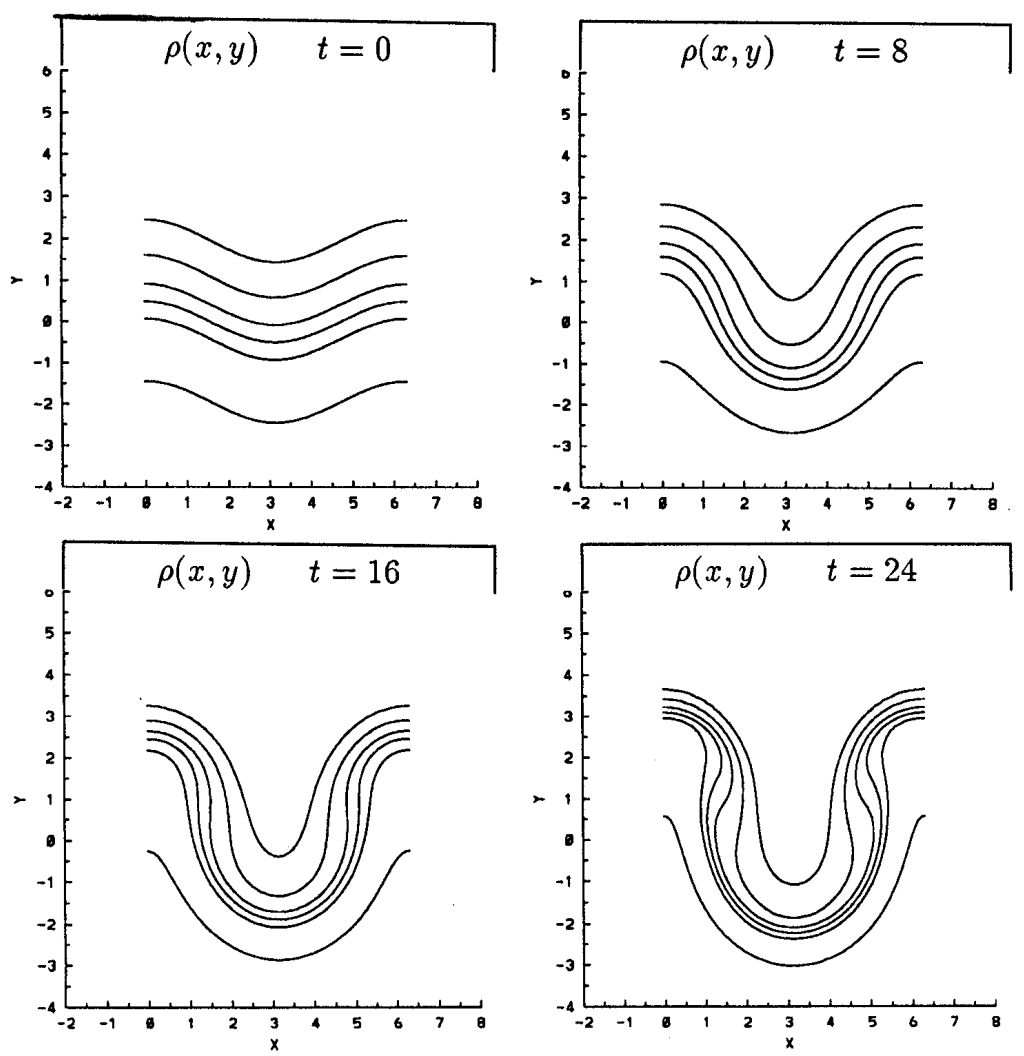

FIG. 1. Time evolution of the density contours for the single-scale profile $L=1.0, A=-0.5, \epsilon=0.5, t=0,8,16,24$. The contours are at $\rho=0.26,0.3,0.4,0.5,0.6,0.74$ in that order from top to bottom of each figure. 


\section{Ablative Rayleigh-Taylor instability}

Ablation stabilisation is a key issue for direct-drive ICF as this reduces the growth of Rayleigh-Taylor instability.

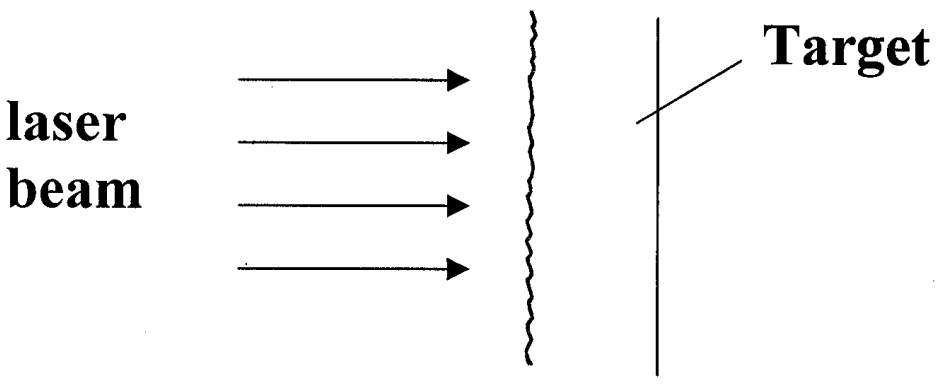

2D and 3D numerical simulations of ablative RT have been performed by the NRL group - also some of the first 3D RT calculations - Dahlburg and Gardner, Phys Rev A, vol 41, p5695 (1990) - mixing rate higher in 3D that 2D

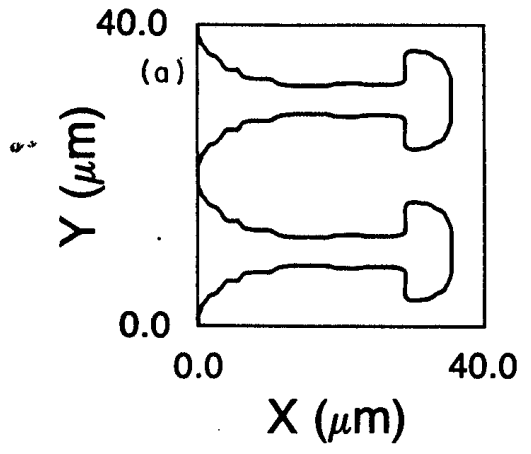

2D

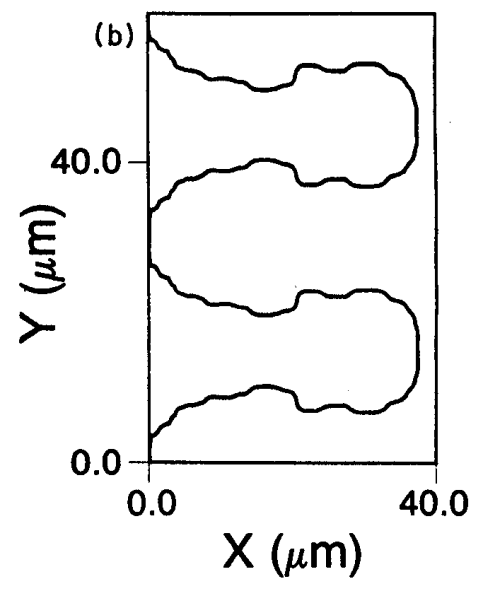

3D 


\section{T Multimode ablative RT instability}

Dahlburg et al, Phys Plasmas, vol 2, p2453 (1995)

FAST3D code - FCT method

$220 \times 128 \times 128$ cells

Random perturbation $\mathrm{k}^{-2}$ spectrum

In the case considered, ablative RT did not give fully developed turbulence.
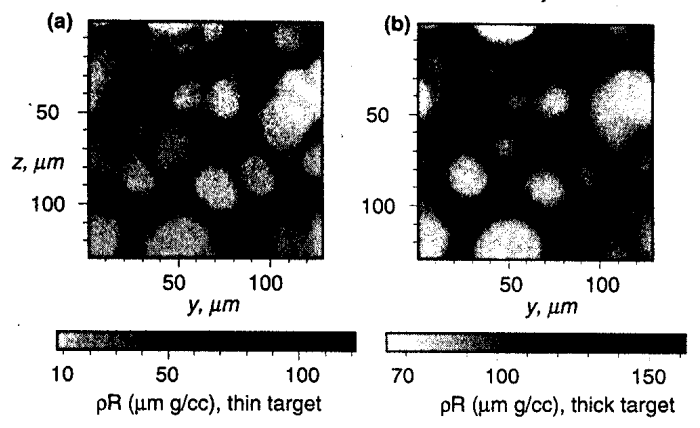

FIG. 4. Comparison of $\rho R$ between the thinner (a) and thicker (b) targets, at $4 \mathrm{~ns}$ when the thinner target has burned through. In these plots, the darkest shades of gray scale indicate the regions of greatest $\rho R$. 


\section{D SIMULATION}

(a) Single mode/few modes

Difference between the behaviour of large scale structure in 2D and 3D.

3D growth rate is higher than $2 \mathrm{D}$ growth rate. (Layzer theory).

Use of interface tracking is an advantage.

(b) Turbulent Mixing

Formation of a Kolmogorov-like $\left(k^{-\frac{5}{3}}\right)$ inertial
range.

Formation of a Kolmogorov-like $\left(k^{-\frac{5}{3}}\right)$ inertial
range.

Dissipation in 3D much higher than in 2D. Counteracts the higher growth rate of the large scale structures in 3D. 
Tryggvason and Unverdi Phys Fluids, vol A2, p656 (1990)

Single mode RT (2D vs 3D)

Boussinesq limit + viscosity

Accurate interface tracking method. Interface represented by triangular elements.

3D growth greater than 2D growth.

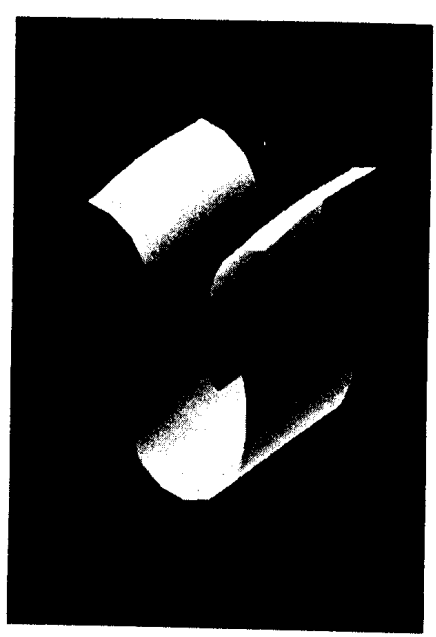

FIG. 2. The large-amplitude stage for a single initial mode. Here $t=2.75$.
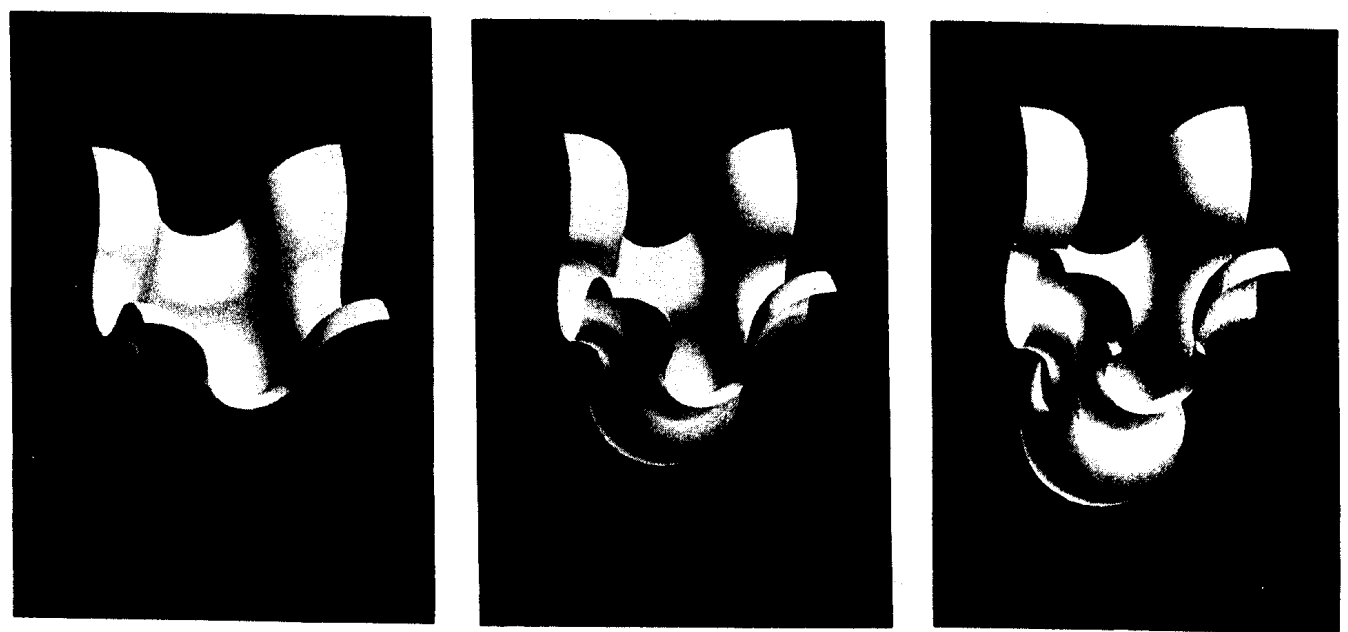

FIG. 1. The evolution of an interface disturbed by two modes. The nondimensional times are 2.0, 2.5, and 3.0. 


\section{RT Single Mode at Higher Atwood No.}

Oparin and Abarzhi. Phys Fluids, vol 11, p3306 (1999) quasi-monotonic grid - characteristic method.
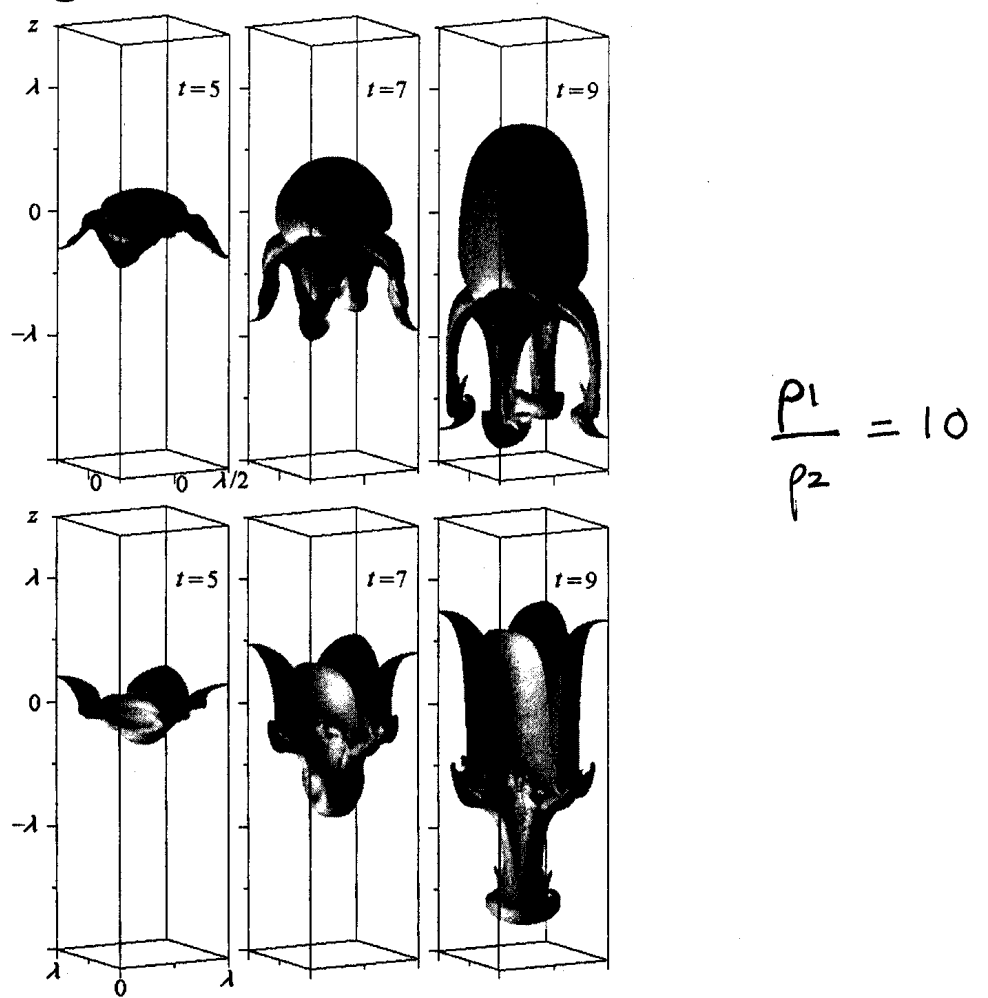

FIG. 1. Bubble (above) and jet (below) evolution. Grid $30 \times 30 \times 210$.

He, Zhang, Chen and Doolen. Physics of Fluids Vol 11, p1143 (1999) - Lattice Boltzmann scheme

Mixing of immiscible fluids. $\frac{p_{1}}{p_{2}}=3$

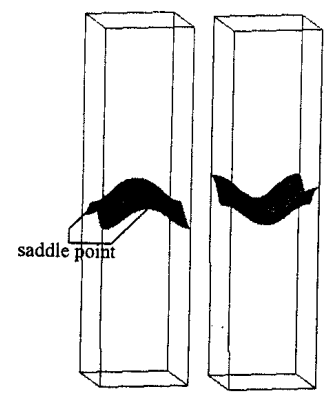

(a)

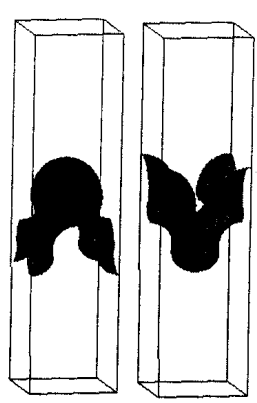

(b)

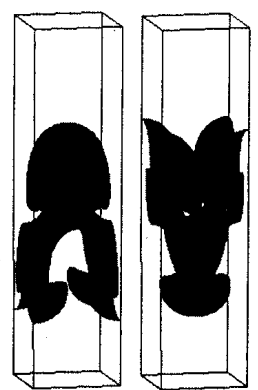

(c)

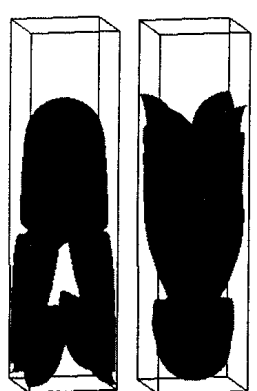

(d) 
V.V.Nikishin et al. $6^{\text {th }}$ IWPCTM (Marseille, 1997)

Institute for Mathematical Modelling

Lebedev Institute

3D single single mode/ few mode RM
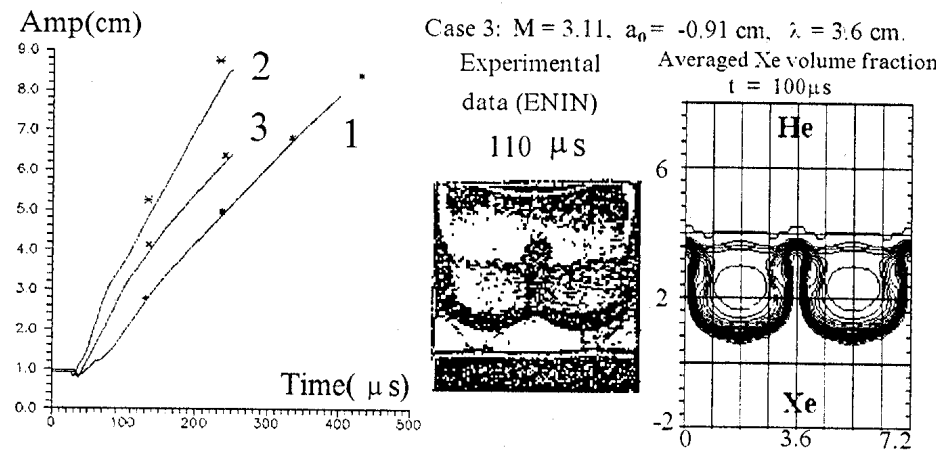

Figure 5. Comparison $\mathrm{He}-X e 3 \mathrm{D}$ results (ENIN,IMM,AWE).

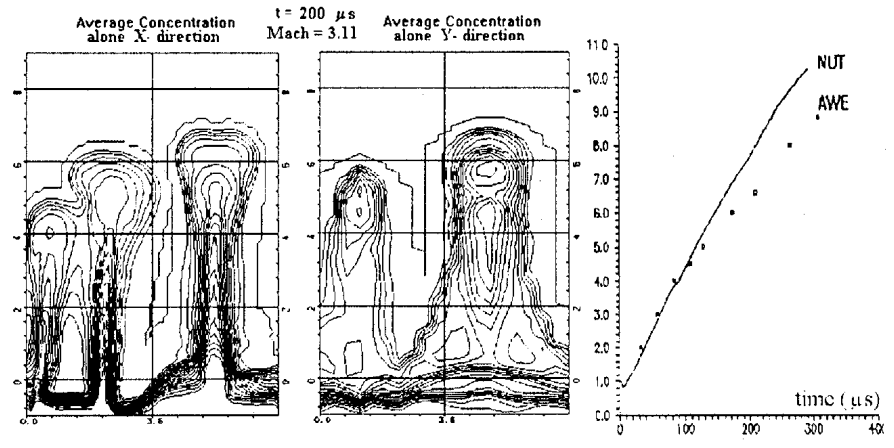

Figure 6. Multimode $H \epsilon-X e 3 \mathrm{D}$ results. 
Li and Zhang. Phys Fluids, vol 9, p3069 (1997)

2D and 3D single mode

TVD method + Artificial Compression

Single shock : good agreement with non-linear theory of Zhang and Sohn.

$3 D$ growth $>2 D$ growth.

Also single shock + reflected shock.

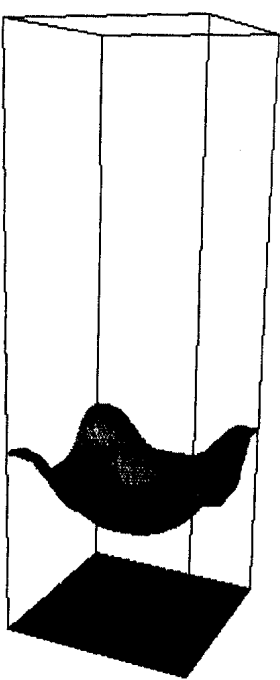

$t=22.1$

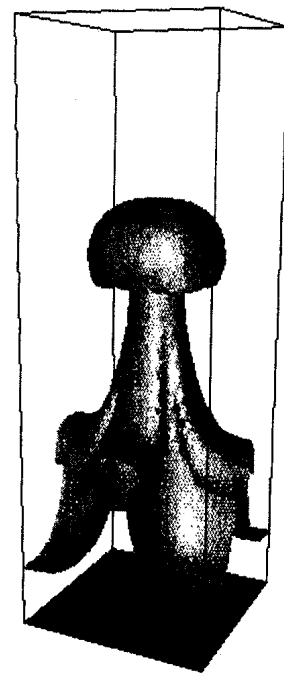

$t=58.6$

FIG. 10. Evolution of fluid interface in the Richtmyer-Meshkov re-shock experiment. The fluid interface first moves downward after transition of the incident shock. It is then hit by the reflected shock Since the reflected thock travels from heavy fluid to light fluid it is the rected reck which reverses the phase of the interace is which reverses the phase of the interface motion. A mushroom-like spike is
formed at $t=58.6$. 
SPHERICAL IMPLOSIONS (ICF)

Sakagami and Nishihara - PRL, vol 65, p432 (1990)

Town and Bell - PRL, vol 67, p1863 (1991)

$(\mathbf{r}, \theta, \phi)$ grid $\quad: \frac{1}{\mathbf{1 2 0}}$ of the sphere.

RT at inner fuel/shell interface.

Single mode spherical harmonic perturbation.

3D growth $>2 D$ growth.

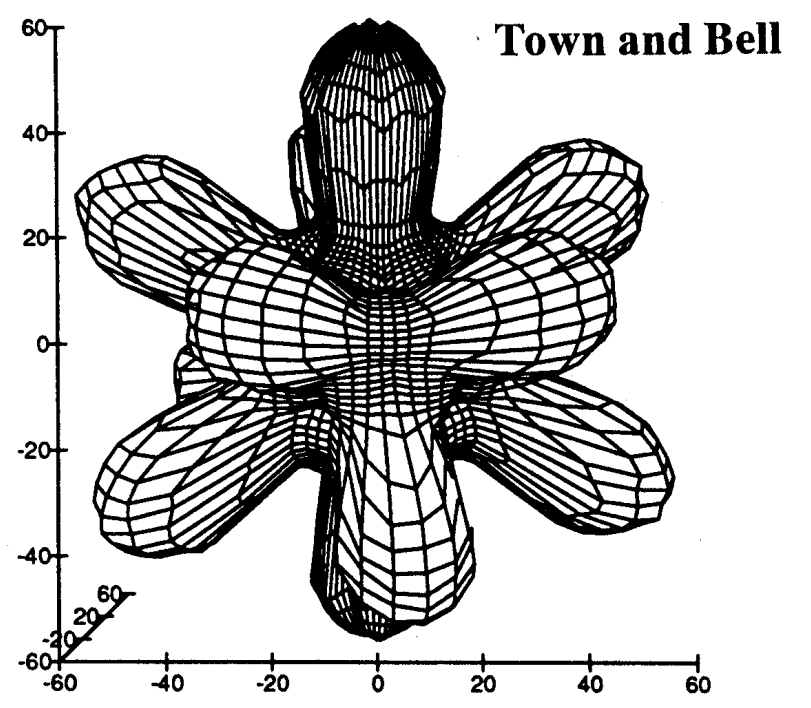

FIG. 1. The inner surface of the shell (defined as the surface of constant density equal to $1 / e$ of the maximum density) at $1.226 \mathrm{~ns}$. This shows the bubble-ridge arrangement. 
Marinak et al, Physics of Plasmas, vol 5, p1125 (1998)

3D multimode RT calculations for NIF capsules.

$(\mathbf{r}, \theta, \phi)$ mesh

$170 \times 64 \times 64$ zones

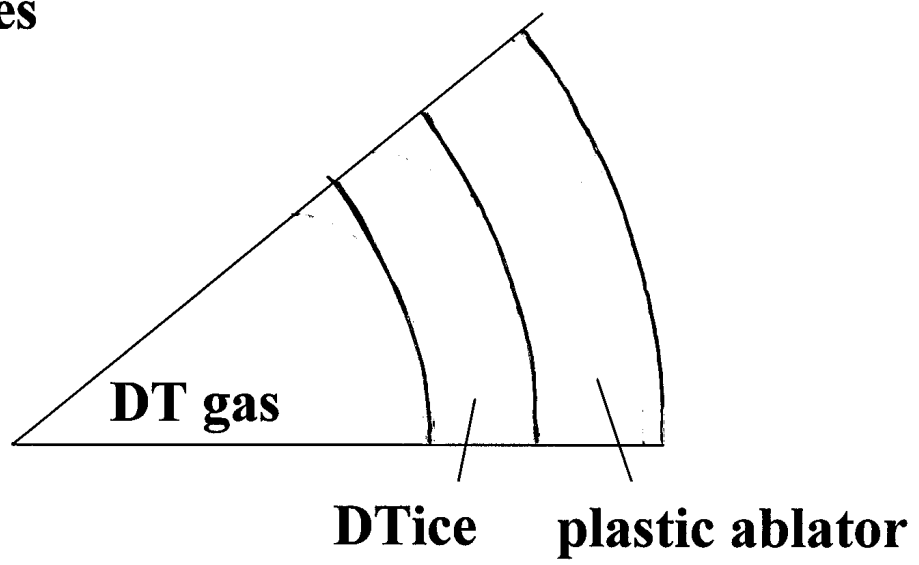

Realistic surface finish

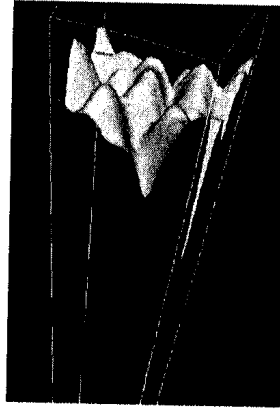

(a)

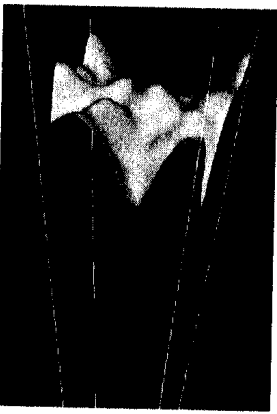

(b)

FIG. 9. Density isosurfaces close to the ablator-fuel interface $20 \mathrm{ps}$ before the respective ignition time for each capsule. (a) For the PT capsule and (b)
for the $\mathrm{BeCu}$ capsule. 


\section{D TURBULENCE SIMULATION}

Most detailed analysis for RT mixing - the simple case $\rho_{1}, \rho_{2}$, g constant - for which bubble growth is given by

$$
\mathbf{h}_{1}=\alpha \frac{\rho_{1}-\rho_{2}}{\rho_{1}+\rho_{2}} \mathbf{g t}^{2}
$$

Major area of controversy is the treatment of the small scales

Techniques used

- Direct Numerical Simulation (DNS) - viscosity and diffusivity included in the calculation - all scales present are resolved

- Interface tracking - for immiscible mixing

- Large Eddy Simulation (LES) - only large scales resolved - dissipation at small scales modelled 


\section{The high-Reynolds number limit}

In high-Reynolds number turbulent mixing, turbulence KE and density fluctuations are dissipated by a cascade to high wave numbers.

Power spectrum: $\quad \sigma^{2}=\int_{0}^{\infty} \mathrm{P}(\mathrm{k}) \mathrm{dk}$

$$
\text { where } \sigma^{2}=\left\langle\left(\mathbf{u}_{i}-\overline{\mathbf{u}}_{i}\right)^{2}>\text { or }\left\langle(\rho-\bar{\rho})^{2}\right\rangle\right.
$$

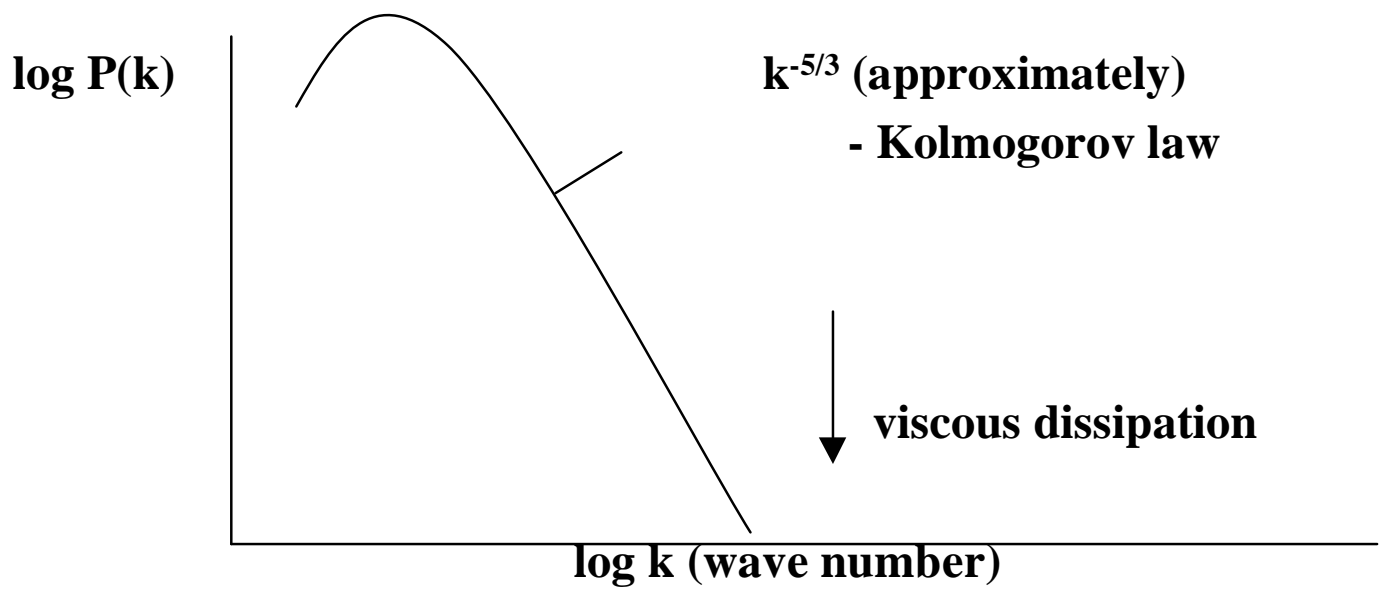

Viscosity/diffusivity determines the scale at which dissipation occurs, not the rate.

LES works if some of the $\mathrm{k}^{-5 / 3}$ spectrum can be resolved - dissipation occurs at an artificially large scale determined by the mesh resolution.

(Conclusions given here are not necessarily applicable to simulation of turbulent boundary layers. 
J Fluid Mech, vol 399, p1 (1999)

S. B. Dalziel, P. F. Linden and D. L. Youngs

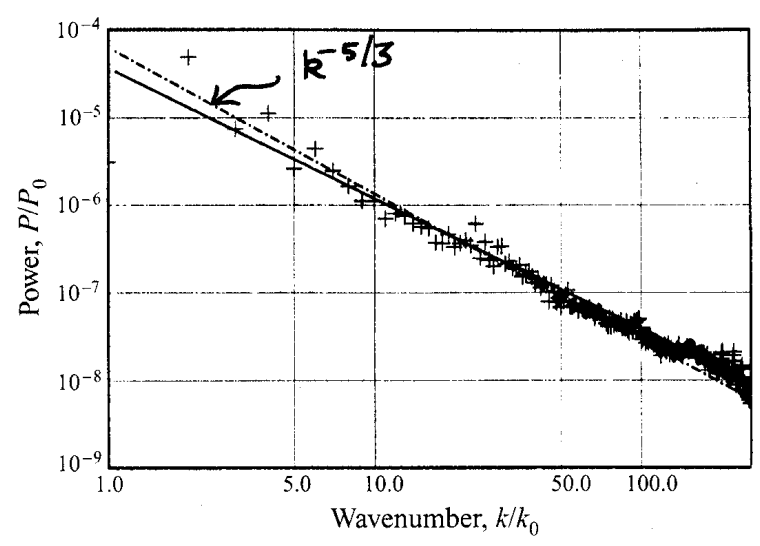

$\mathrm{Sc}=1000$

Wilson and Andrews, Phys. Fluids, vol 14, p938 (2002)

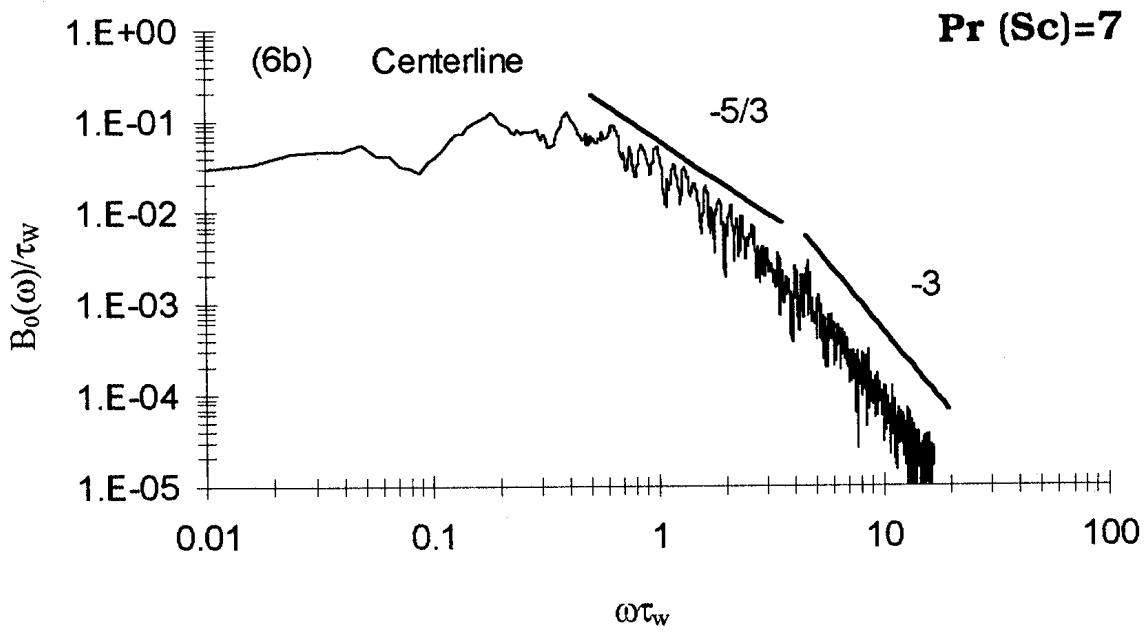


Two approaches to LES

See recent text books:-

Turbulent Flows: Stephen Pope, Cambridge University Press (2000)

Numerical Simulation of Reactive Flow (second edition) : Elaine Oran and Jay Boris, Cambridge University Press (2000)

(a) The numerical method should have negligible dissipation.

$>80 \%$ of the turbulence KE should be resolved

A sub-grid scale model should be used to represent the effect of the unresolved scales.

(b) Many numerical schemes (FCT, van Leer, TVD) have implicit dissipation at high-wave numbers. No additional sub-grid model should be used.

- MILES, Monotone Integrated Large-Eddy Simulation

(a) is most popular within the turbulence community - a controversial issue but not given much attention so far at the IWPCTMs.

Limited application of sub-grid models to RT/RM mixing. 


\section{$\underline{\text { LES + Smagorinsky model }}$}

Simplest and most well-known sub-grid model.

For incompressible uniform density flow

$$
\frac{\partial \mathbf{u}_{\mathbf{i}}}{\partial \mathbf{t}}+\frac{\partial}{\partial \mathbf{x}_{\mathbf{j}}}\left(\mathbf{u}_{\mathbf{i}} \mathbf{u}_{\mathbf{j}}\right)=-\frac{\mathbf{1}}{\boldsymbol{\rho}} \frac{\partial \mathbf{p}}{\partial \mathbf{x}_{\mathbf{i}}}
$$

$\overline{\mathbf{u}}_{\mathbf{i}}=$ filtered value of $\mathbf{u}_{\mathbf{i}}$ ie averaged over a small region of space just sufficient for $\overline{\mathbf{u}} \mathbf{i}$ to be resolved by the numerical mesh

$$
\begin{aligned}
& \frac{\partial \overline{\mathbf{u}}_{\mathbf{i}}}{\partial \mathbf{t}}+\frac{\partial}{\partial \mathbf{x}_{\mathbf{j}}}\left(\overline{\mathbf{u}}_{\mathbf{i}} \overline{\mathbf{u}}_{\mathbf{j}}\right)=-\frac{\mathbf{1}}{\boldsymbol{\rho}} \frac{\partial \overline{\mathbf{p}}_{\mathbf{i}}}{\partial \mathbf{x}_{\mathbf{i}}}+\boldsymbol{\tau}_{\mathbf{j}} \\
& \tau_{i j}=\overline{\mathbf{u}}_{\mathbf{i}} \overline{\mathbf{u}}_{\mathbf{j}}-\overline{\mathbf{u}_{\mathbf{i}} \mathbf{u}_{\mathbf{j}}} \\
& =2 v_{t} \bar{S}_{i j}+\frac{1}{3} \tau_{k k} \delta_{i j} \\
& \overline{\mathbf{S}}_{\mathbf{i j}}=\frac{\mathbf{1}}{\mathbf{2}}\left(\frac{\partial \overline{\mathbf{u}}_{\mathbf{i}}}{\partial \mathbf{x}_{\mathbf{j}}}+\frac{\partial \overline{\mathbf{u}}_{\mathbf{j}}}{\partial \mathbf{x}_{\mathbf{i}}}\right) \\
& v_{t}=C_{d} \Delta x^{2}|\bar{S}|_{\text {where }} \bar{S}^{2}=\bar{S}_{i j} \bar{S}_{i j} \\
& \frac{1}{3} \tau_{k k} \text { add to } \bar{p}
\end{aligned}
$$


Vremen et al J Fluid Mech, Vol 399, p357, (1997)

Six subgrid scale models applied to the free shear layer ( $32^{3}$ grid).

Smagorinsky model with constant coefficient did not perform well - too dissipative in laminar regions.

Best results with dynamic eddy-viscosity model, Germano, J Fluid Mech, Vol 238, p325 (1992).

$$
\tau_{\mathrm{ij}}=\mathbf{c}_{\mathrm{d}} \Delta \mathbf{x}^{2}|\overline{\mathbf{S}}| \overline{\mathbf{S}}_{\mathrm{ij}}
$$

$c_{d}$ : a variable coefficient estimated from the velocity field filtered at two different levels $\Delta x$ and $2 \Delta x$. 
Subgrid Scale Models

Theoretical analysis available.

eg for Smagorinsky model (Fureby et al, Physics of Fluids, Vol 9, p1416, 1997) - assuming spectrum

$$
\begin{aligned}
v_{\mathrm{t}} & =\mathbf{c}_{\mathrm{D}} \Delta^{2}|\mathrm{~S}| \\
\mathrm{C}_{\mathrm{D}} & =\frac{4}{\pi^{2}} \frac{1}{\left(3 \mathrm{C}_{\mathrm{K}}\right)^{3 / 2}}=0.042 \\
\Delta & =\text { filter width } \\
\mathrm{C}_{\mathrm{K}} & =\text { Kolmogorov constant }
\end{aligned}
$$

\section{MILES technique}

Theoretical analysis lacking, except for recent work by Margolin and Rider, ECCOMAS, Swansea, UK (2001).

Analysis of truncation terms in nonoscillatory finite volume schemes - relate to SGS models.

"It appears that the reluctance of the community in general to accept implicit turbulence modelling is more due to lack of justification of the approach rather than any failure of application." 
DNS - low to moderate Reynolds no.

needed to understand the transition to Turbulence.

Understand the behaviour of the high wave number end of the spectra.

Effect of Schmidt no.

\section{LES or MILES}

- used to model the high-Reynolds number limit - relevant to many applications eg shock tube experiments.

\section{INTERFACE TRACKING}

- useful to understand the effect of surface tension for mixing of immiscible fluids (a number of RT experiments have used immiscible fluids).

Immiscible fluids with negligible surface tension should give fine-scale mixing which behaves like miscible mixing, at high Reynolds number. 


\section{TURMOIL3D experience}

Numerical method used based on the 2D Eulerian technique for multimaterial flow developed $\sim 1980$.

- $\quad$ Lagrangian phase + rezone (advection) phase

- $\quad$ Interface tracking

- Monotonic advection method of van Leer used in rezone phase for all fluid variables.

Van Leer method very successful at giving a robust method with low numerical diffusion.

Applied to a wide range of compressible flows with shocks and density discontinuities.

- TURMOIL3D - same basic numerical method as the $2 D$ code

- $\quad$ As simple as possible eg perfect gas EoS

- Interface tracking not used, as dissipation of density fluctuations at small scales expected

- Use of the van Leer method implied non- linear numerical dissipation at scales of order the mesh size 


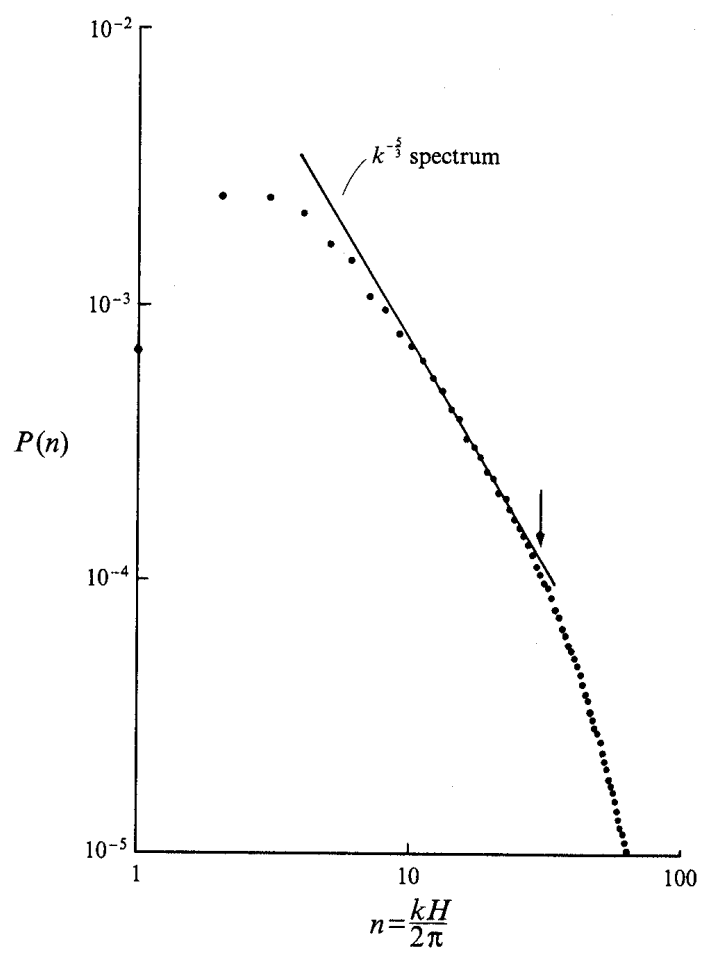

Figure 14. Power spectrum $P(n)$ for concentration fluctuations at $\tau=3$ from the numerical calculation without the long-wave perturbation. The line drawn corresponds to a $k^{-\frac{5}{5}}$ spectrum, where $k=\left(k_{x}^{2}+k_{y}^{2}\right)^{\frac{1}{2}}$. The arrow indicates the point where the wavelength $2 \pi / k$ equals 6 mesh widths.

\section{TURMOIL3D density fluctuation spectrum}

Linden, Redondo, Youngs J Fluid Mech, vol 265, p97 (1994)

No need for additional sub-grid dissipation model

Could be argued that there is more high-wavenumber dissipation than desirable 


\section{MILES vs LES with explicit sub-grid model}

TVD schemes (such as the van Leer advection method used in TURMOIL3D) have become very popular for compressible flow with shocks and contact discontinuities. It seems appropriate to continue using them for compressible turbulent flow. The dissipation implicit in the numerical scheme should be sufficient to make sub-grid models unnecessary $\rightarrow$ MILES approach.

The rationale for LES + explicit sub-grid model requires the use of a basic numerical technique with negligible dissipation $\Rightarrow$ TVD schemes cannot be used.

Does this mean that this approach is most useful for uniform density incompressible flow? 
Many disagreements about best method to use for 3D RT turbulent mixing.

Need to compare results for agreed test problems.

Guy Dimonte (alpha group comparison).

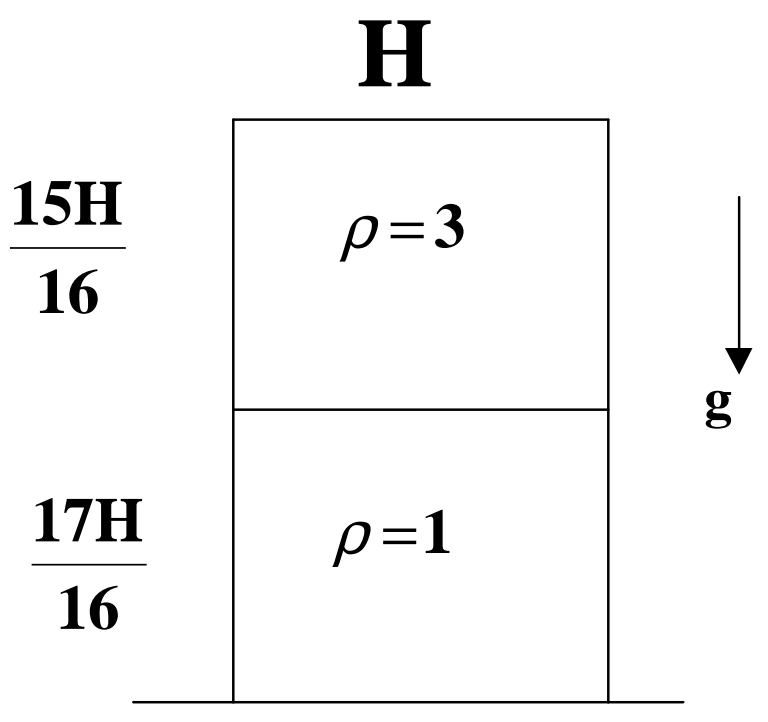

$256 \times 256$ x 512 zones

Initial perturbation : wavelengths in the range $4 \Delta x$ to $8 \Delta \mathbf{x}$.

Growth by mode coupling $\Rightarrow$ loss of memory of the initial conditions. 


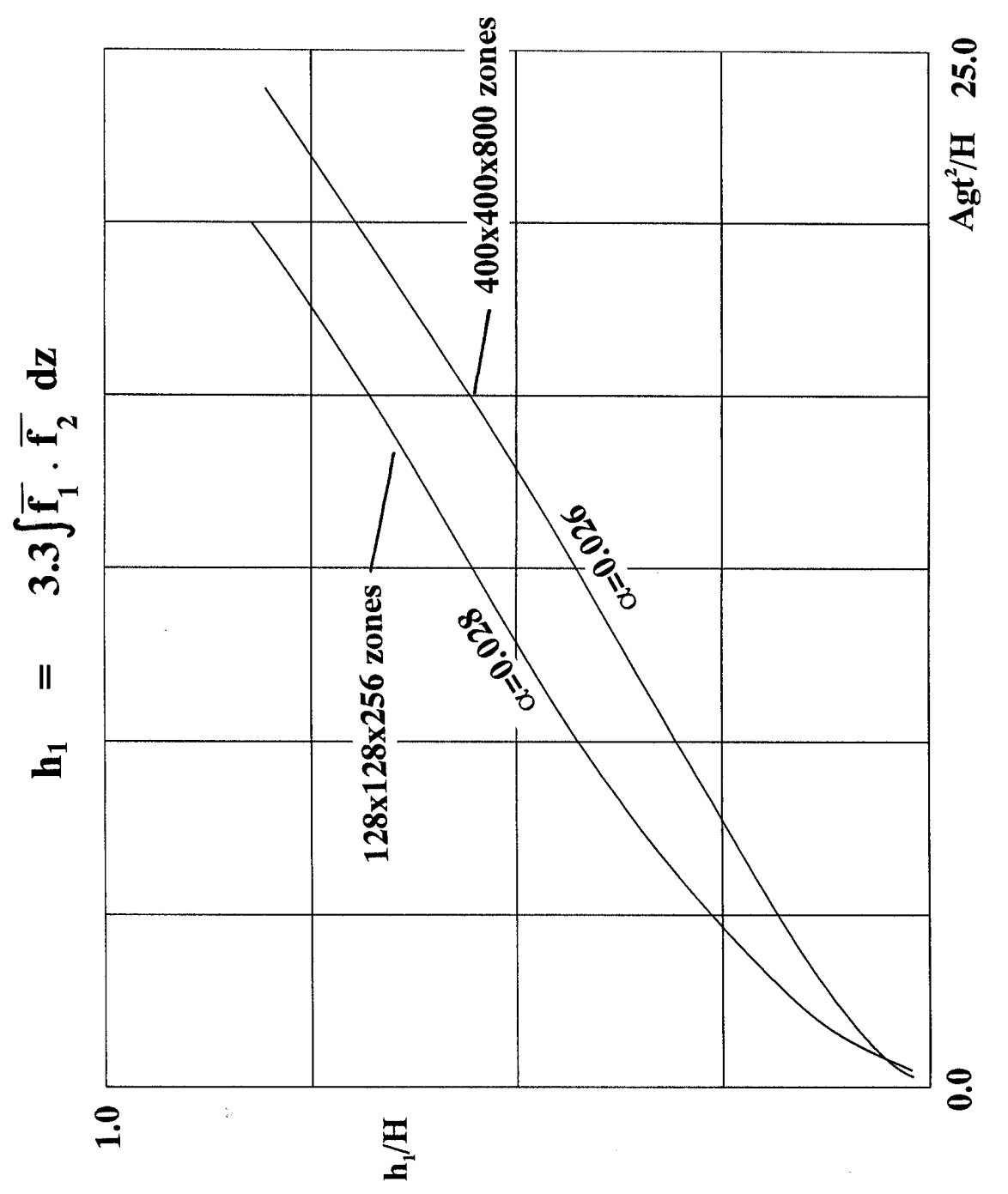




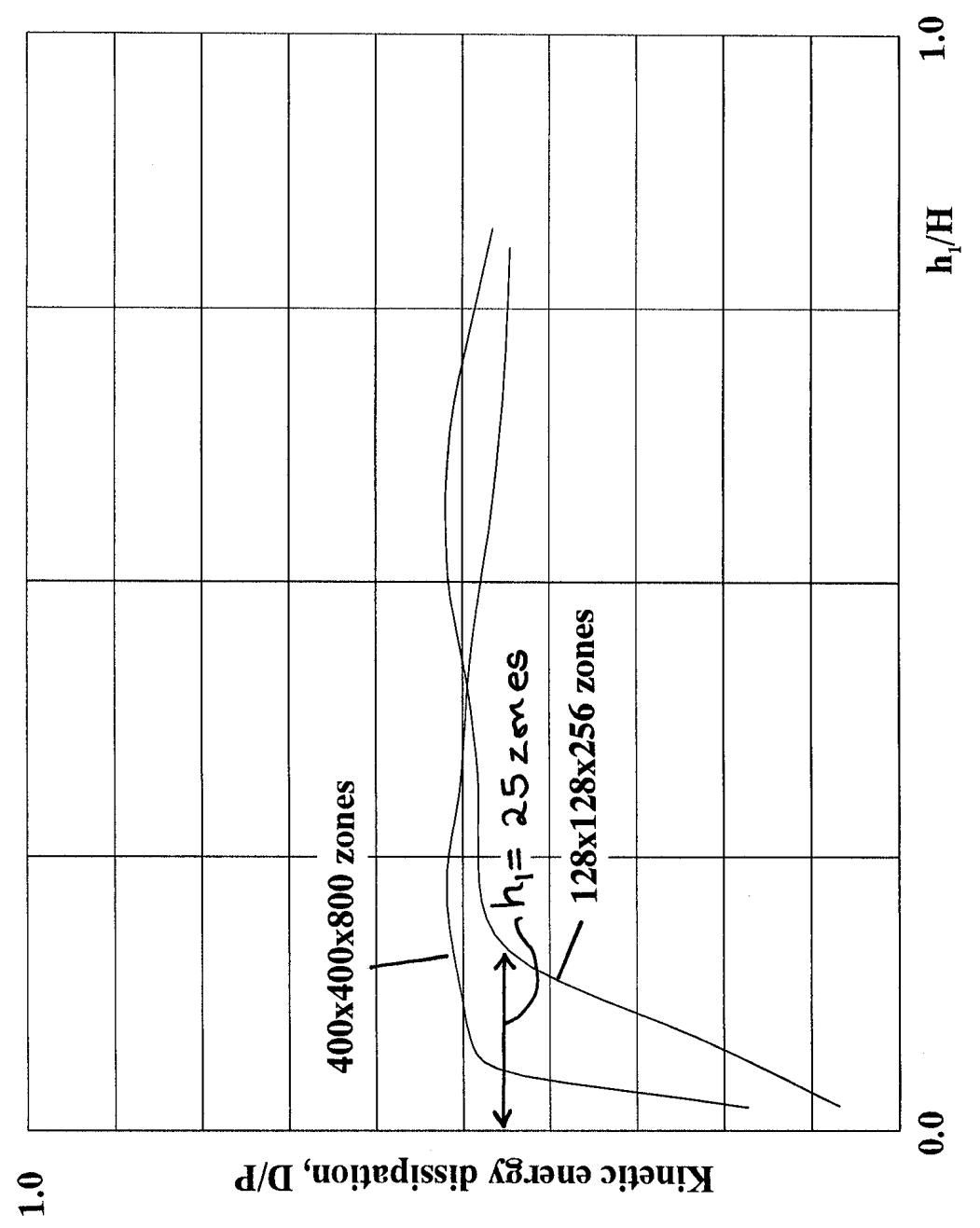




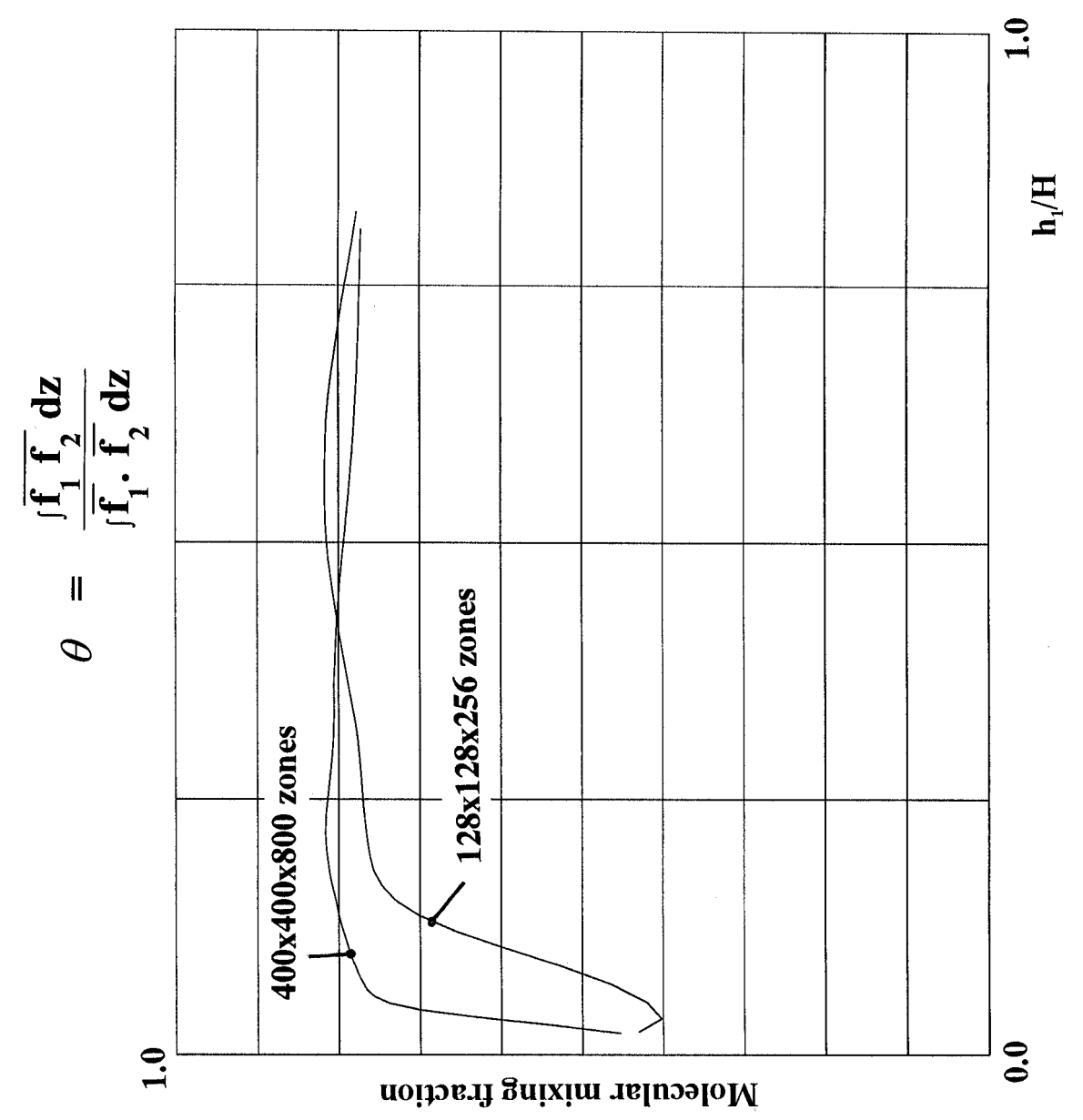




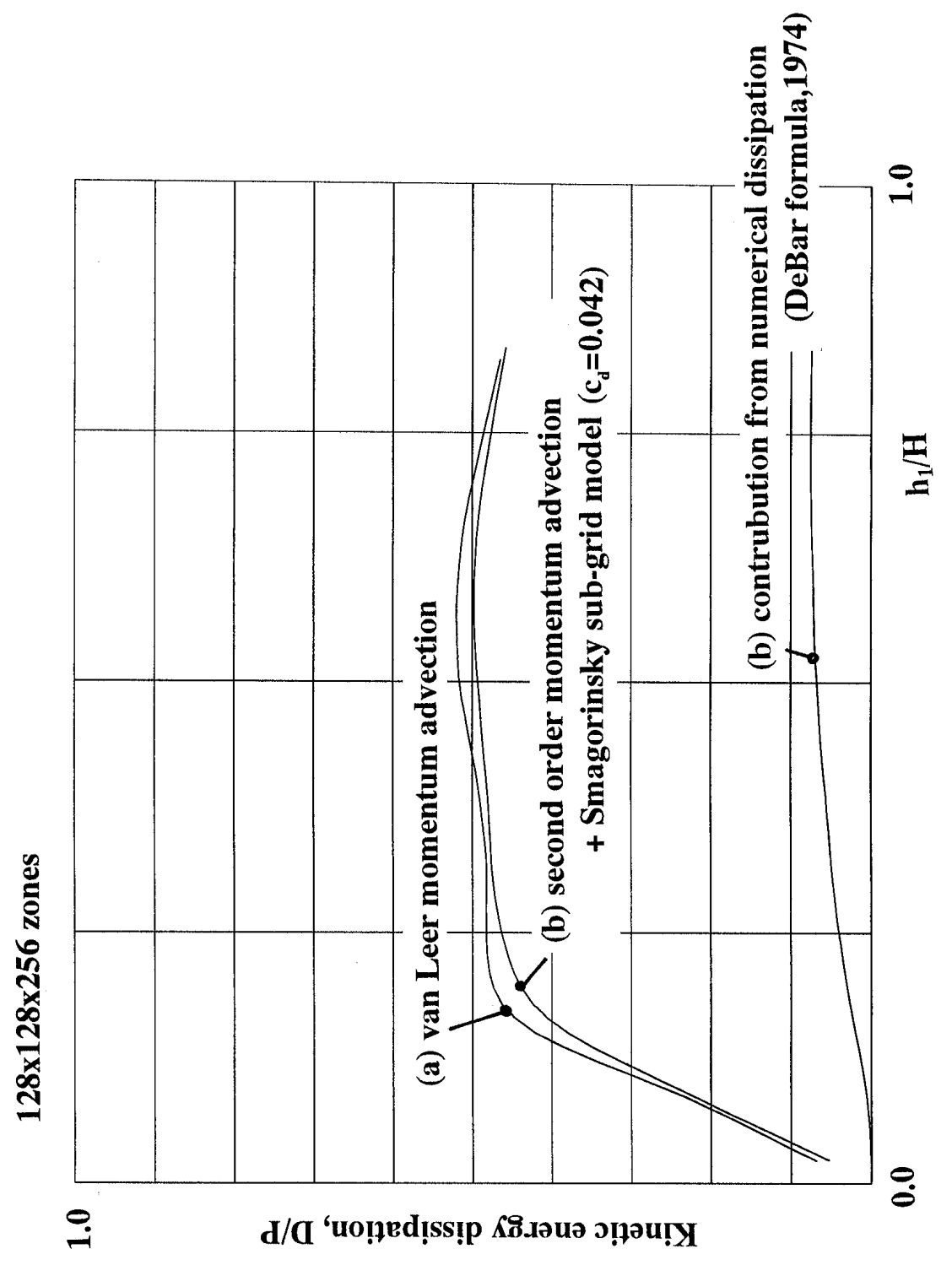




\section{THE INITIAL PERTURBATION}

RT experiments with constant g give bubble penetration

$$
\mathbf{h}_{1}=\alpha \frac{\rho_{1}-\rho_{2}}{\rho_{1}+\rho_{2}} \mathbf{g t}^{2} \quad, \text { with } \alpha \sim 0.05 \text { to } 0.06
$$

TURMOIL3D calculations with short wavelength initial perturbations (growth purely by mode coupling) give

$$
\alpha \sim \mathbf{0 . 0 3} \text {. }
$$

Need to assume long wavelength initial perturbations with amplitude $\propto$ wavelength (as proposed by Inogamov) to give self-similar growth with $\alpha \sim \mathbf{0 . 0 5}$.

Perturbation used $\zeta(\mathbf{x}, \mathbf{y})=\zeta_{\mathrm{S}}+\zeta_{\mathrm{L}}$

$\zeta_{\mathrm{S}}$ : wavelengths $4 \Delta \mathrm{x}$ to $8 \Delta \mathrm{x}$

$$
\text { s.d }=0.005 \Delta x
$$

$\zeta_{\mathrm{L}}$ : power spectrum $\mathbf{P}(\mathbf{k})$

$$
\begin{aligned}
\sigma_{\lambda}=\left\{\int_{2 \pi / \lambda}^{\infty} \mathbf{P}(\mathbf{k}) \mathbf{d} \mathbf{k}\right\}^{\frac{1}{2}}=\varepsilon \lambda \\
\Rightarrow \mathbf{P}(\mathbf{k}) \propto \mathbf{1} / \mathbf{k}^{3} \text { (ocean surface spectrum) } \\
\varepsilon=\mathbf{0 . 0 0 0 5}
\end{aligned}
$$

wavelengths in the range $4 \Delta x$ to $\frac{H}{2}$

$\left(\right.$ ICF surface finish $\left.P(k) \propto 1 / k^{2}\right)$ 


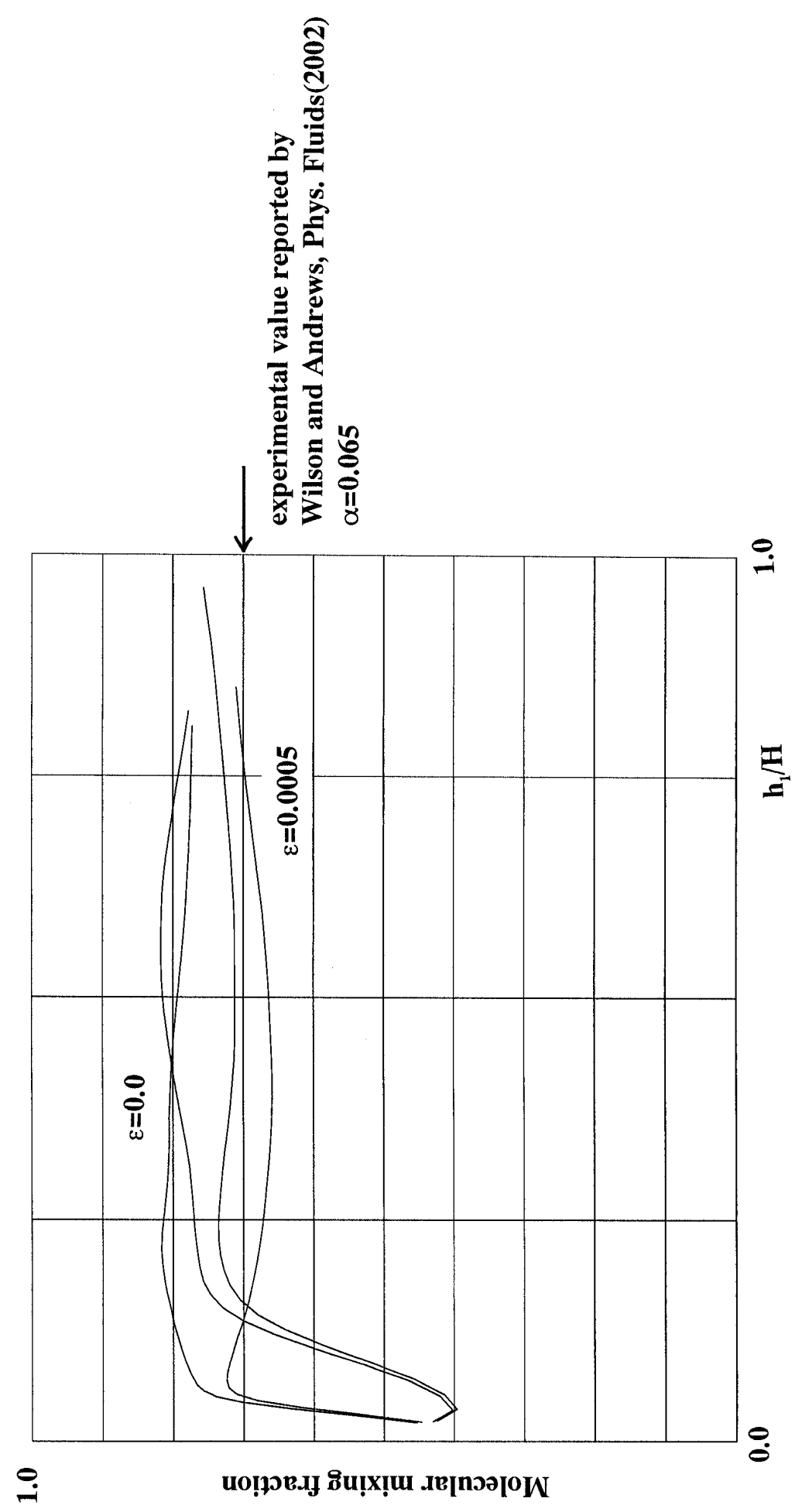




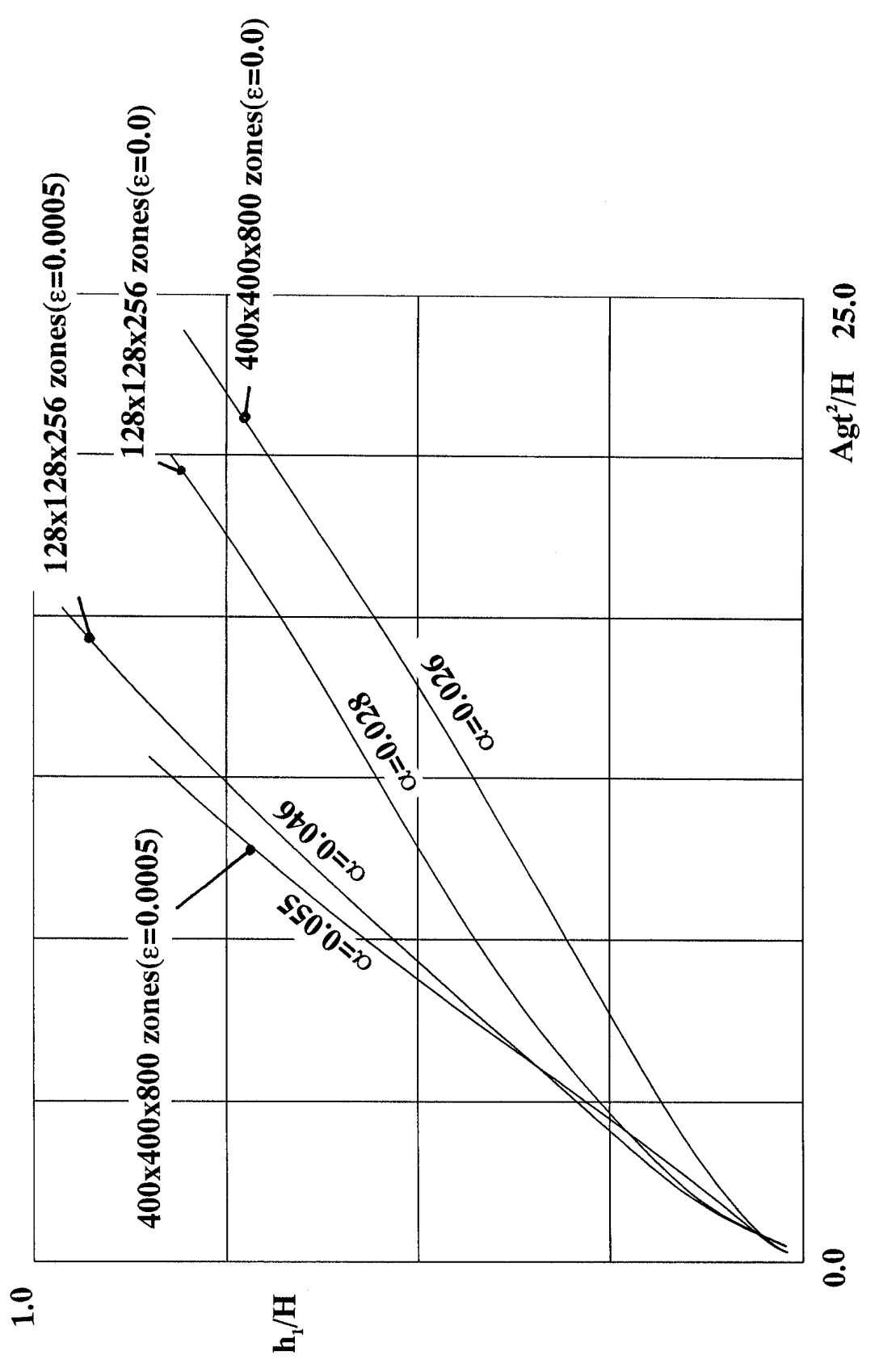


Figure7 : Probability distributions for $f_{1}$ within a plane $z=$ constant

Wilson and Andrews At $=0.001$
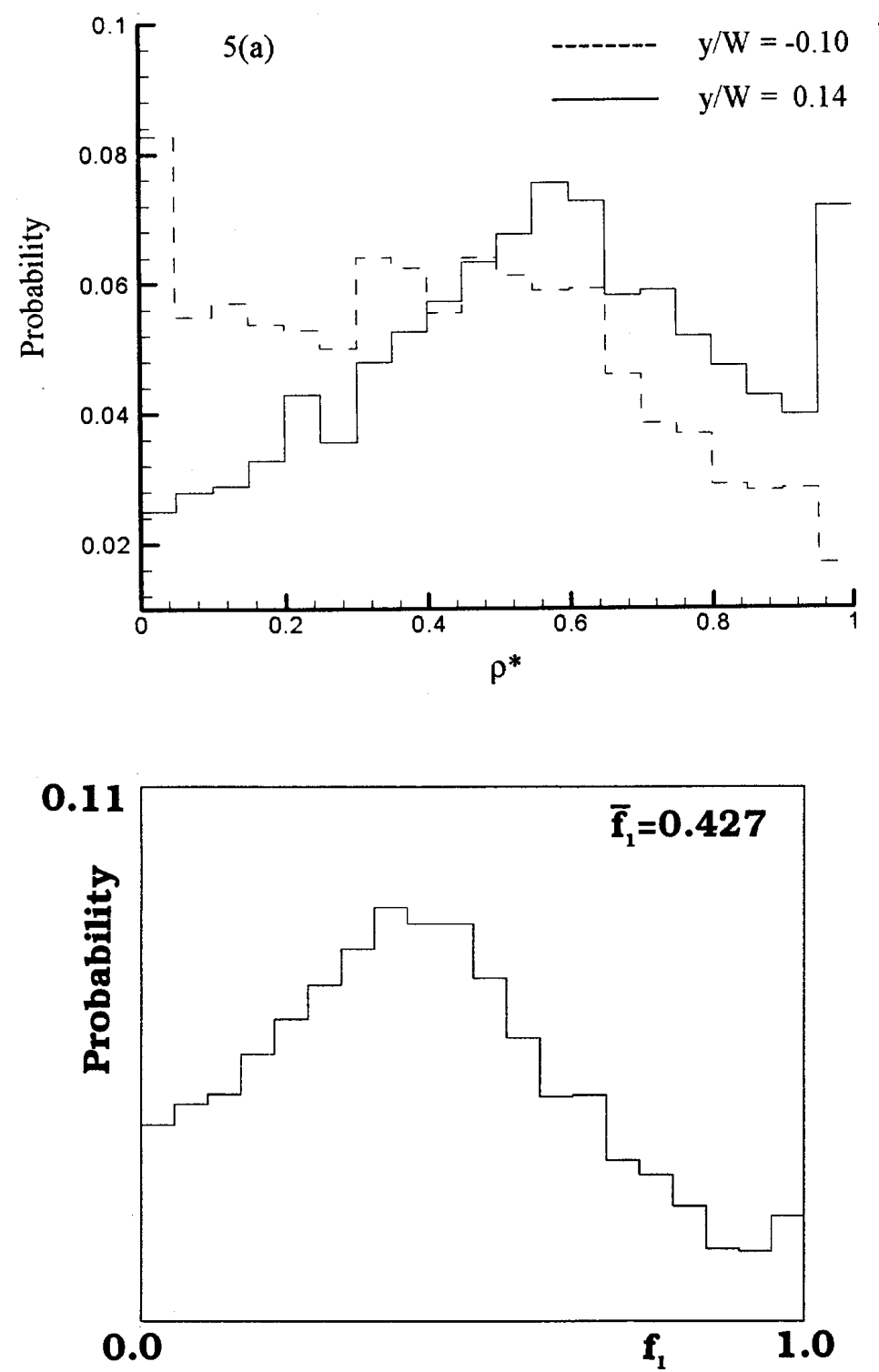

TURMOIL3D calculation

At $=0.5$

$128 \times 128 \times 256$ zones

$g=$ constant

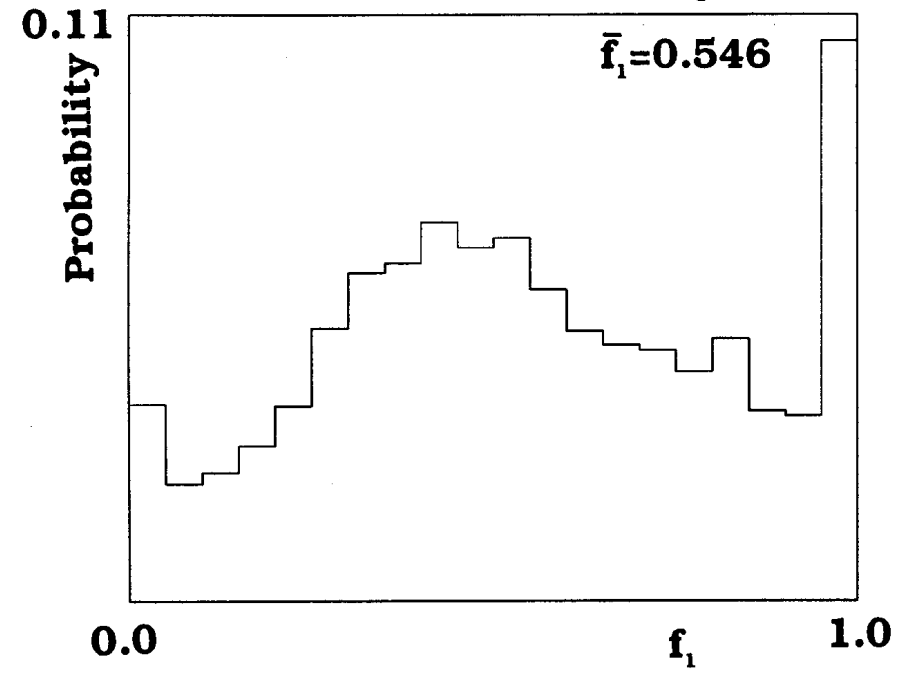


Cook and Dimotakis, J Fluid Mech, vol 443, p69 (2001)

DNS : $256 \times 256 \times 1024$ zones

$\mathrm{Sc}=1, \quad \operatorname{Re} \sim 140, \quad \rho_{1} / \rho_{2}=3$

Diffuse initial interface

Eighth-order compact scheme (Lele 1992) - spectrallike resolution.

Calculated transition to turbulence, which was found to be very dependent on the initial conditions.

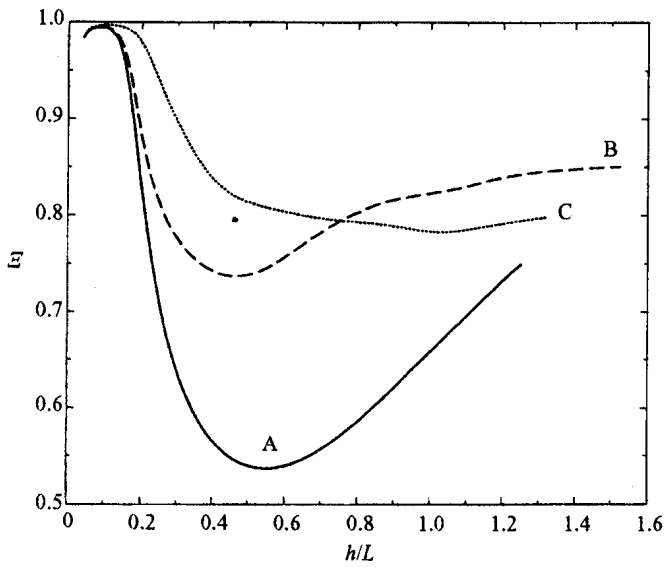

FIGURE 16. Mixing parameter, $\Xi, v s . h / L$, for the three cases. 


\section{Young, Tufo, Dubey, Rosner (Chicago U) - J Fluid}

Mech, vol 447, p377 (2001)

\section{Miscible RT}

\section{Spectral method or spectral-element method}

Low Atwood no. (Boussinesq approximation)

256 × 256 × 512 zones.

Initial modes with wavelengths around $8 \Delta x$.

2D

$$
\alpha \sim 0.017 ?
$$

Y.-N. Young, H. Tufo, A. Dubey and R. Rosner

3D

$$
\alpha \sim \mathbf{0 . 0 3}
$$

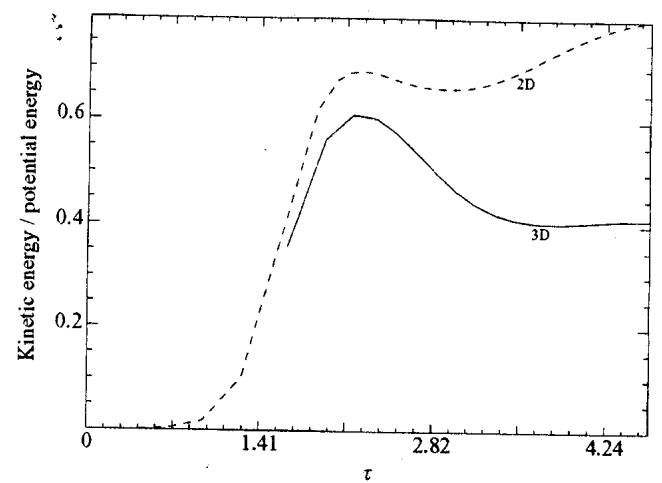

FIGURE 8. Ratio of kinetic energy to gravitational potential energy: two-dimensional versus three-dimensional simulations. The kinetic energy is the volume integral of the kinetic energy density, and the potential energy is the volume integral of the potential energy available in the system. For $\tau \leqslant 1.4$, the growth is exponential and is similar for both two and three dimensions, after this period, two-dimensional motions are much more efficient in extracting potential energy
than three-dimensional motions.

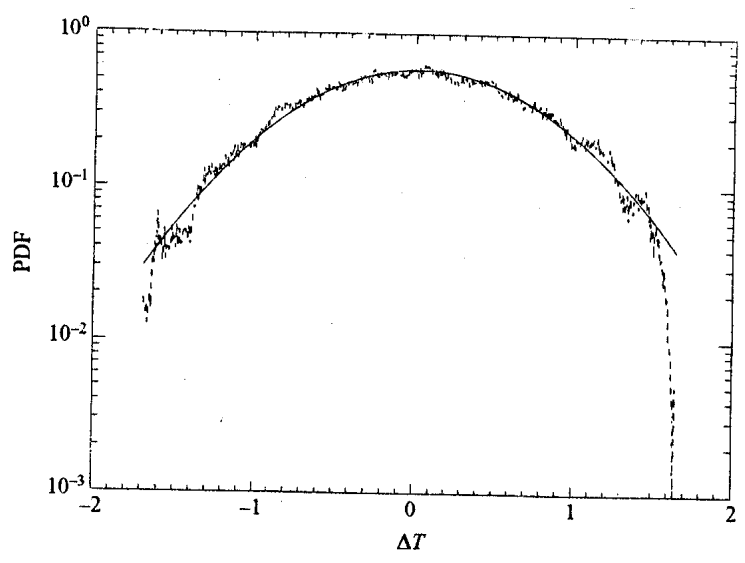

FIGURE 13. PDF of the temperature fluctuation $\left(\delta T=T-T_{0}\right)$ within the mixing zone $(\Delta z=0.025)$ at the position of the original interface $(z=0.06)$. 


\section{Calculations with Interface Tracking}

A number of researchers have considered turbulent mixing of immiscible fluids using interface tracking techniques.

J Glimm et al J Comp Phys, Vol 162, p652 (2001)

Frontier method - represents both the velocity and density discontinuity at the interface.

$$
\begin{aligned}
& 112 \times 112 \times 224 \text { zones } \\
& \alpha \sim 0.07
\end{aligned}
$$

Oron, Arazi, Kartoon, Rikanati, Alon, Shvarts Physics of Plasmas, Vol 8, p2883 (2001). See also Shvarts et al ShockInduced instability of interfaces, in Handbook of Shock Waves, Vol 2, Academic Press (2001).

$$
\begin{aligned}
& 80 \times 80 \times 80 \text { zones } \\
& \alpha \sim 0.05
\end{aligned}
$$

Anuchina et al - Proceeding of 5th Zababakhin Scientific Talks (1999).

$$
\begin{array}{ll}
60 \times 60 \times 60 \text { zones } & \alpha=0.064 \\
120 \times 120 \times 120 \text { zones } & \alpha=0.074
\end{array}
$$

Evidence for $\mathbf{k}^{-5 / 3}$ energy spectrum.

(Also, Yu. V. Yanilkin, VNIIEF, $1^{3}{ }^{3}$ mesh, $\alpha=0.06$ ) 


\section{$\underline{\text { Rayleigh-Taylor Summary }}$}

- Many 3D calculations with significant differences between results

$\alpha \sim 0.03$ to 0.07

- Effect of initial conditions important - very good reason for pursuing the numerical simulation.

- Controversy over the numerical techniques which should be used.

Use of sub-grid scale models is recommended by many but has not been widely used here.

Interface tracking calculations have given higher values of $\alpha$ but have not used the highest resolution.

- Need some test problems to resolve the disagreements (see talk by Guy Dimonte). 


\section{$\underline{\text { RM Turbulent Mixing }}$}

Fewer 3D simulations available.

Scaling laws for single shock RM :

$\begin{array}{lll}\text { Bubbles } & : & \mathbf{h}_{\mathrm{B}} \sim \mathbf{t}^{\mathrm{\theta B}} \\ \text { Spikes } & : & \mathbf{h}_{\mathrm{S}} \sim \mathbf{t}^{\mathrm{AS}}\end{array}$

Youngs, Laser and Particle Beams, Vol 12, p725, (1994)

$160 \times 160 \times 270$ zones, assumed $\theta_{\mathbf{B}}=\theta_{\mathrm{S}}$

then $\theta \simeq 0.30$ (based on growth of integral mix width)

for a flat spectrum

$\mathbf{P}(\mathbf{k})=$ const for $0<\mathbf{k}<\mathbf{k}_{\max }$

$k_{\text {max }}=\frac{2 \pi}{\lambda_{\text {min }}}, \lambda_{\text {min }}=16 \Delta x$ 
Cohen et al. IWPCTM6 (Marseille)

High resolution RM calculations up to $512^{3}$

\section{PPM method}

Single-shock and double shock calculations

Single - shock random perturbations, but with longer wavelengths present $\theta=\mathbf{0 . 7 5}$
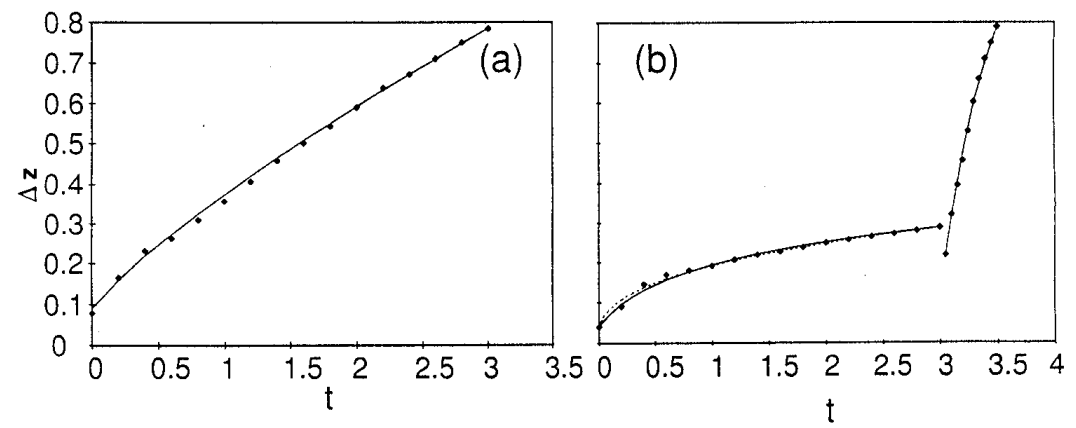

Figure 1. Mixing layer growth vs. time for (a) random-phase sum initial condition; (b) single mode initial condition with two shocks 
Oron, Arazi, Kartoon, Rikanati, Alon, Shvarts Physics of Plasmas, vol 8, p2883 (2001)

\section{LEEOR-3D (interface tracking)}

$80 \times 80$ x 80 zones

$$
\begin{array}{ll}
\theta_{\mathbf{B}} \simeq 0.35(2 \mathrm{D}) & , \quad \sim 0.22(3 \mathrm{D}) \\
\theta_{\mathrm{S}} \simeq 0.45(2 \mathrm{D}) & , \quad \sim 0.3(3 \mathrm{D})
\end{array}
$$

Results used to construct a simple buoyancy - drag model.

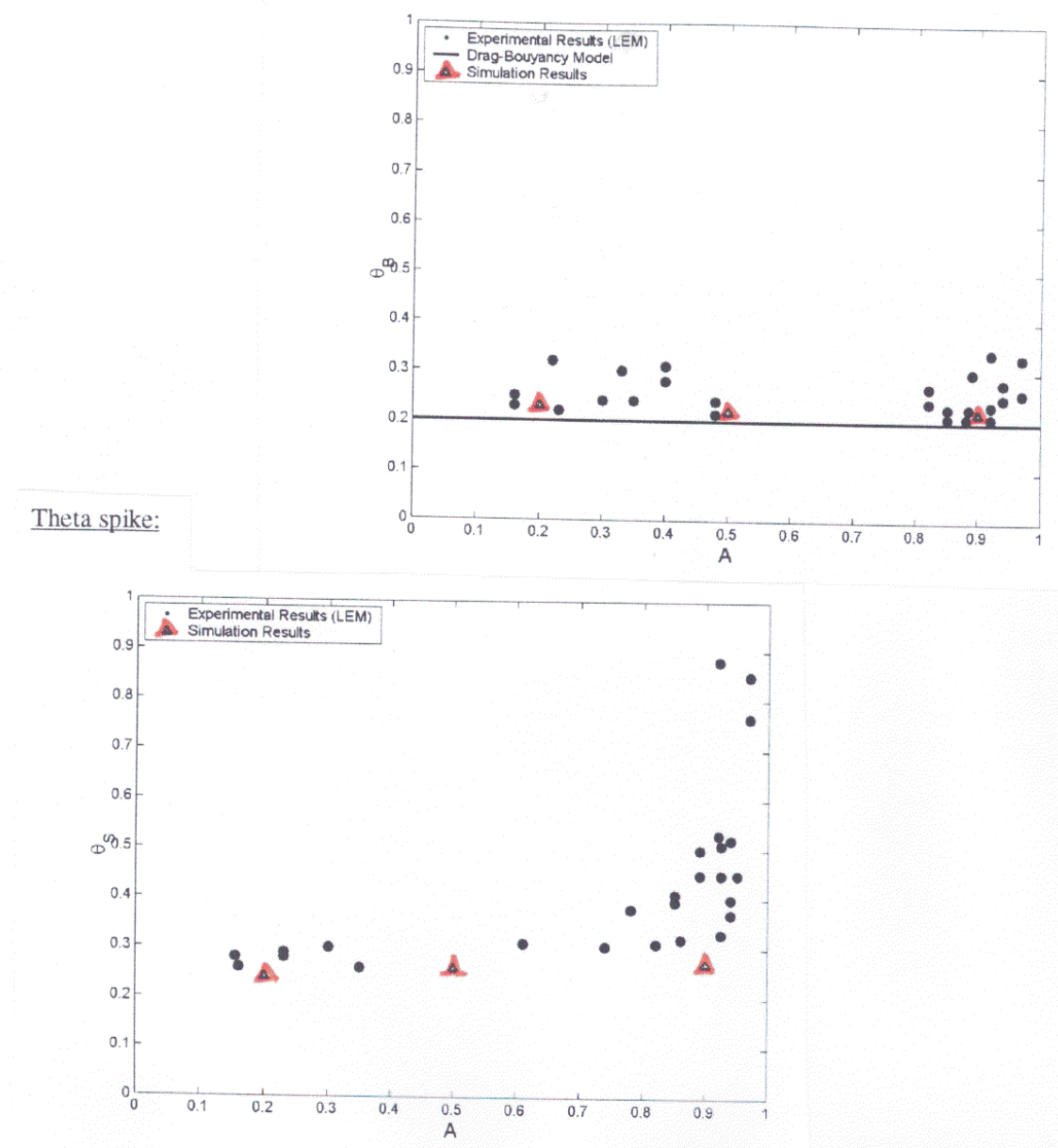




\section{Single Shock RM}

3D simulation should be used to investigate the effect of initial conditions in more detail.

An initial amplitude spectrum

$$
\mathbf{P}(\mathbf{k}) \sim \mathbf{k}^{-2}
$$

may be more appropriate to real applications $\Rightarrow$ higher values of $\theta_{\mathrm{S}}, \theta_{\mathrm{B}}$ ?

\section{Double Shock RM}

3D simulation has been applied to experiments where several shocks are present.

However, no detailed 3D studies (development of scaling laws) for double shock RM.

Second shock : shock-turbulence interaction and shock-density fluctuation interaction. 


\title{
FUTURE ROLE OF NUMERICAL SIMULATION
}

\author{
$\underline{\text { 2D Simulation }}$
}

- Will continue to be essential for complex problems with additional physics

3D Simulation

- Fundamental understanding of turbulent mixing in simple flows (DNS and LES)

- More complex flows - LES now feasible

- LES results should be used to validate engineering models (Bouyancy - drag models, RANS models)

- Not yet feasible for complex real applications 


\section{AWE SHOCK TUBE EXPERIMENT}

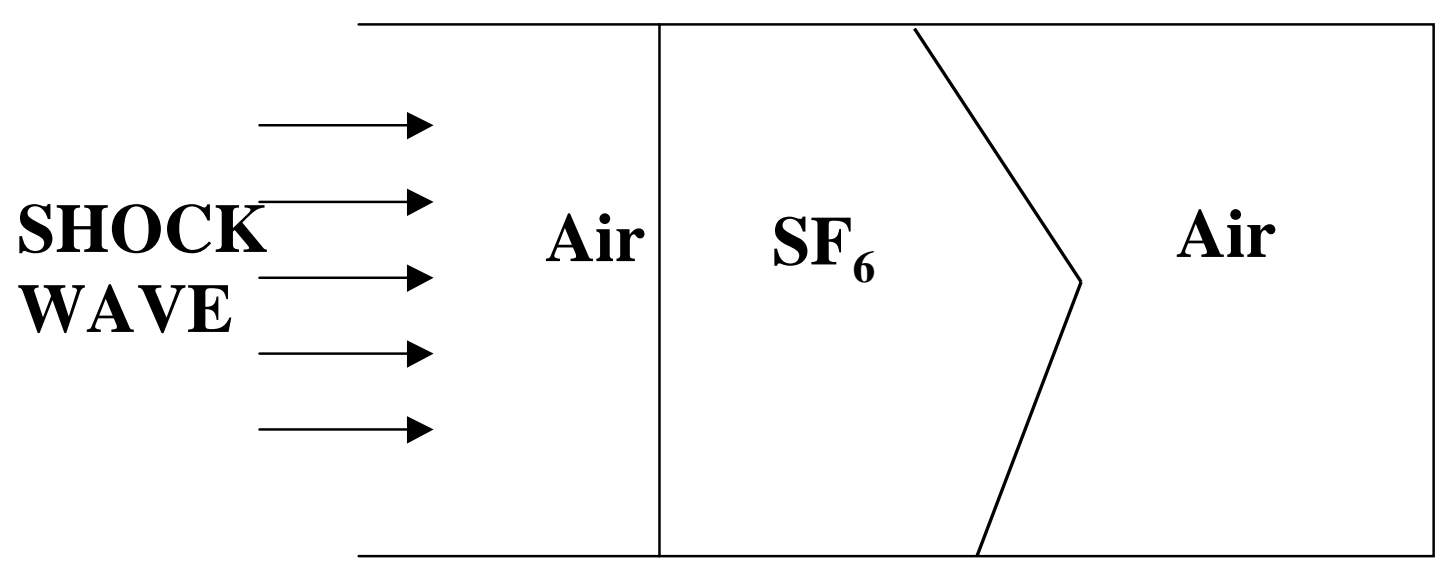

The purpose of the shock tube experiment is validation of a 2D RANS model

Experiment is 2D on average

3D Simulation (TURMOIL3D) : $400 \times 320 \times 160$ zones interfaces randomly perturbed

2D turbulence model (RANS model) calculation : $\quad 200 \times 160$ zones

Compare average behaviour extracted from 3D simulation with 2D RANS model 


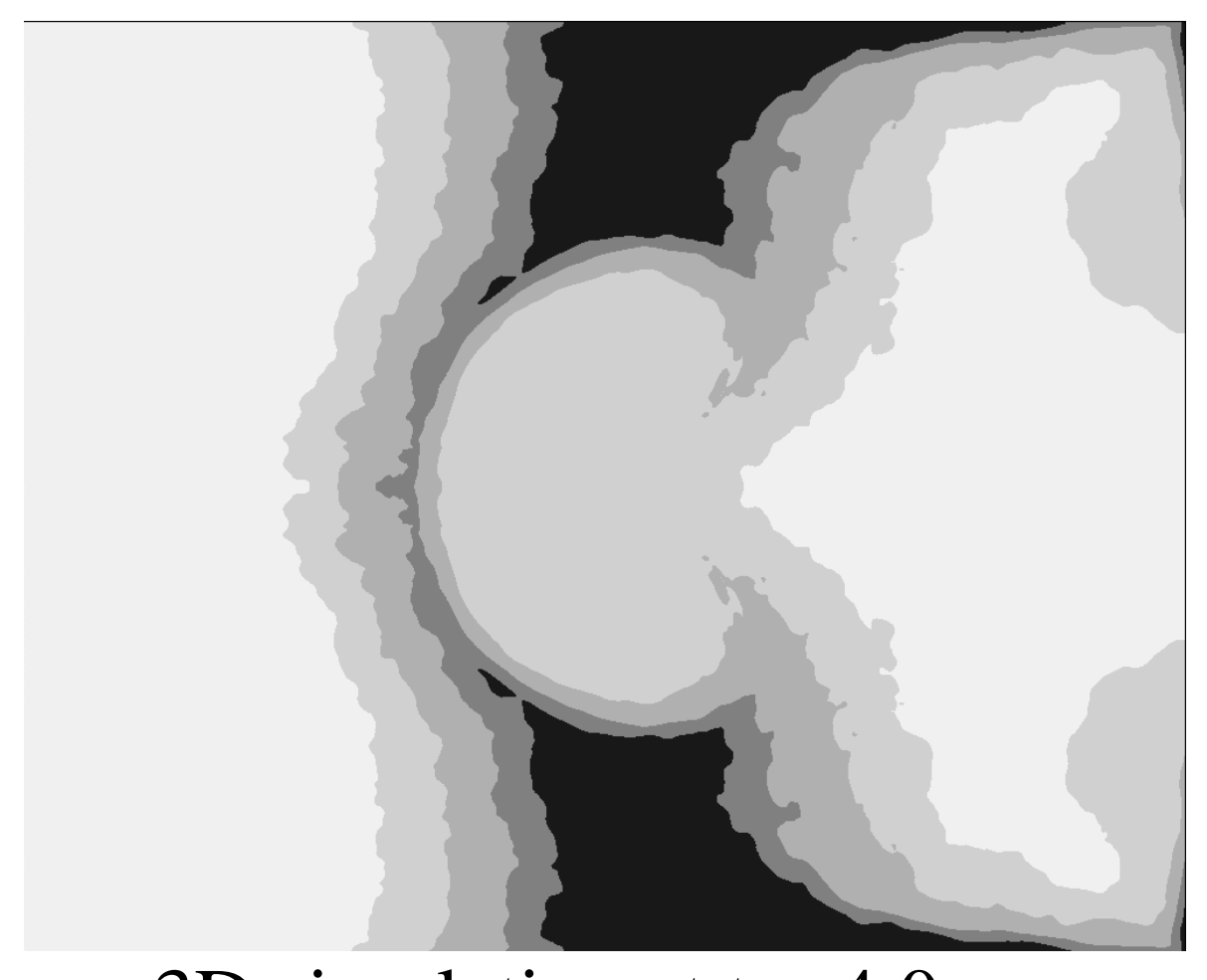

$3 \mathrm{D}$ simulation at $\mathrm{t}=4.0 \mathrm{~ms}$

2D RANS model at $\mathrm{t}=4.0 \mathrm{~ms}$

Mean volume fraction levels -

$0.0,0.05,0.3,0.7,0.95,1.0$ 


\section{FINAL REMARKS}

- Numerical simulation has made a major contribution to the understanding of $R T$ and RM instability over the last 40 years.

- Need to focus more now on 3D turbulence simulation.

- Reasonably good 3D LES can be performed with mesh sizes $\sim 256^{3}$, well within the capability of present-day supercomputers.

- 3D simulation not yet practical for complex real applications but can have a major impact on engineering models. 
$8^{\text {th }}$ International Workshop on the Physics of Compressible Turbulent Mixing,

Pasadena, CA (2001)

\title{
Authors Contact Information
}

\author{
Abarzhi, Snejana I. \\ Department of Applied Mathematics \\ State University of New York at Stony Brook \\ Stony Brook, NY 11794-3600 \\ Phone: 631-632-9360 \\ Fax: 631-632-8490 \\ E-mail: snezha@ams.sunysb.edu
}

Abe, $M$.

Institute of Laser Engineering

Osaka University

Suita, Osaka 565 Japan

Afeyan, Bedros

Polymath Research Incorporated

827 Bonde Court

Pleasanton, CA 94566

Phone: (925) 417-0609

Fax: (925) 417-0684

E-mail: bedros@polymath-usa.com

Allen, A. M.

University of Oxford

University Offices

Wellington Square

Oxford OX1 2JD. UK

Phone: +44 1865270000

Fax: $\quad+441865270708$

Alon, U.

Weizmann Institute of Science

PO Box 26

Rehovot 76100 Israel

Phone: + 972-8-934-2111

Fax: + 972-8-934-4107

E-mail: uri.alon@weizmann.ac.il

Anderson, M. H.

University of Wisconsin at Madison

1500 Engineering Drive

Madison, WI 53706

Phone: (608) 263-2802

Fax: (608) 263-7541

E-mail: manderson@engr.wisc.edu 
Andrews, Malcolm

Department of Mechanical Engineering

Texas A \& M University

MS 3123

College Station, TX 77843-3123

Phone: (409) 847-8843

Fax: (409) 8453081

E-mail: malcolm@chagal.tamu.edu

Anisimov, V. I.

Russian Federal Nuclear Center-VNIITF

P.O. Box 245

456770 Snezhinsk, Russia

E-mail: v.i.anisimov@vniitf.ru

Anuchin, M. G.

Russian Federal Nuclear Center-VNIITF

P.O. Box 245

456770 Snezhinsk, Russia

Arazi, L.

Tel Aviv University

P.O. Box 39040

Tel Aviv 69978

Israel

Ardashova, R. I.

Russian Federal Nuclear Center-VNIITF

P.O. Box 245

456770 Snezhinsk, Russia

Arnett, D.

The University of Arizona

P.O. Box: 210096

Tucson, AZ 85721

Phone: (520) 621-9587

E-mail:: darnett@as.arizona.edu

Ashurst, William T.

Sandia National Laboratory

P.O. Box 969, MS 9051

Livermore, CA 94551-0969

Phone: (925) 294-2274

Fax: (925) 294-2595

E-mail:: ASHURS@sandia.gov

Azechi, Hiroshi

Institute of Laser Engineering

Osaka University

Suita, Osaka 565 Japan

Phone: 81-879-8770

Fax: 81-877-4799

E-mail: azechi@ile.osaka-u.ac.jp 
$8^{\text {th }}$ International Workshop on the Physics of Compressible Turbulent Mixing,

Pasadena, CA (2001)

Bailly, P.

Commissariat à l'Energie Atomique

BP 12 - 91680 Bruyères-le-Châtel

Baishev, A. I.

Russian Federal Nuclear Center-VNIITF

P.O. Box 245

456770 Snezhinsk, Russia

Balabin, Serguei

Russian Federal Nuclear Center-VNIITF

P.O. Box 245

456770 Snezhinsk, Russia

Fax: (351-72) 32077

E-mail: kucherenko@five.ch70.chel.su

Barnes, Christopher W.

Los Alamos National Laboratory

P.O. Box 1663

Los Alamos, NM 87545

Phone: (505) 665-5687

E-mail:: cbarnes@lanl.gov

Barton, C.

Atomic Weapons Establishment

Aldermaston

Reading, Berkshire

RG7 4PR UK

Phone: +44 (0)1734814111

Fax: $\quad+44(0) 1734815320$

Batha, Steven

Los Alamos National Laboratory

P.O. Box 1663, MS E526

Los Alamos, NM 87545

Phone: (505) 665-5898

Fax: (505) 665-4409

E-mail: sbatha@lanl.gov

Belak, James F.

Lawrence Livermore National Laboratory

P.O. Box 808, L-045

Livermore, CA 94551

Phone: (925) 422-6061

Fax: (925) 422-2851

E-mail: belak1@1lnlg.gov

Belomestnih, A. V.

Russian Federal Nuclear Center-VNIITF

P.O. Box 245

456770 Snezhinsk, Russia 
Ben-Dor, Gabi

Ben-Gurion University of the Negev

Faculty of Engineering Sciences

Beer Sheva, 84105, Israel

Phone: 972-7-6461212

Fax: 972-7-6472936

E-mail: bendor@menix.bgu.ac.il

Benjamin, Robert F.

Los Alamos National Laboratory

P.O. Box 1663, MS P940

Los Alamos, NM 87545

Phone: (505) 667-8116

Fax: (505) 665-3359

E-mail: rfb@lanl.gov

Biello, J.

University of Chicago

933 East $56^{\text {th }}$ Street

Chicago, IL 60637

Phone: (773) 702-1234

Bliznetsov, M. V.

Russian Federal Nuclear Center-VNIIEF

Mir Avenue, 37, Sarov

Nizhnii Novgorod Region, 607190, Russia

Fax: (831-30) 459-58

E-mail: root@gdd.vniief.ru

Boehly, T. R.

Laboratory for Laser Energetics

University of Rochester

250 East River Road

Rochester, New York 14623-1299

Phone: (716) 275-0254

E-mail: trb@1le.rochester.edu

Bonazza, Riccardo

Department of Engineering. Physics

University of Wisconsin at Madison

1500 Engineering Drive

Madison, WI 53706

Phone: (608) 265-2337

Fax: (608) 262-6707

E-mail: bonazza@engr.wisc.edu

Bouquet, Serge

Commissariat à l'Energie Atomique

PDPTA-BP 12

Bruyeres-le-Chatel, France 91680

Phone: +33-1-69-26-51-83

Fax: +33-1-69-26-71-06

E-mail: bouquet@bruyeres.cea.fr 
$8^{\text {th }}$ International Workshop on the Physics of Compressible Turbulent Mixing,

Pasadena, CA (2001)

Braun, David G.

Lawrence Livermore National Laboratory

P.O. Box 808, L-031

Livermore, CA 94551

Phone: (925) 423-6275

Fax: $\quad$ (925) 423-9969

E-mail: braun1@1lnl.gov

Breidenthal, Robert

Department of Aeronautics and Astronautics

University of Washington

Box 352400

Seattle, WA 98195-2400

Phone: (206) 685-1098

Fax: (206) 685-0217

E-mail: breidenthal@aa.washington.edu

Brouillette, Martin

Department of Mechanical Engineering

Université de Sherbrooke

Sherbrooke, Quebec J1K 2R1 Canada

Phone: (819) 821-7144

Fax: (819) 821-7163

E-mail: martin.brouillette@gme.usherb.ca

Buckingham, Alfred C.

Lawrence Livermore National Laboratory

P.O. Box 808, L-23

Livermore, CA 94551

Phone: (925) 423-4828

Fax: (925) 422-2644

E-mail: alfredcb@1lnl.gov

Budil, Kimberly S.

Lawrence Livermore National Laboratory

P. O. Box 808, L-97

Livermore, CA 94551

Phone: (925) 423-8098

Fax: $\quad$ (925) 422-0779

E-mail: budil1@1lnl.gov

Cabot, William H.

Lawrence Livermore National Laboratory

P.O. Box 808, L-22

Livermore, CA 94551

Phone: (925) 423-9272

Fax: (925) 423-0925

E-mail: cabot1@1lnl.gov 
Calder, A. C.

Department of Astronomy and Astrophysics

University of Chicago

5640 South Ellis Ave.

Chicago, IL 60637

Phone: (773) 834-3904

Fax: (773) 834-3230

E-mail:: calder@flash.uchicago.edu

Chebotareva, E. I.

Krzhizhanovsky Power Engineering Institute

ENIN, Leninsky pr. 19, GSP-1

Moscow, 117927 Russia

Phone: (7-095) 955-31-07

Fax: (7-095) 954-42-50

E-mail: S.G.Zaytsev@mtu-net.ru

Cheng, Baolian

Los Alamos National Laboratory

P.O. Box 1663, MS D413

Los Alamos, NM 87545

Phone: (505) 667-4701

Fax: (505) 667-3726

E-mail: bcheng@lanl.gov

Clark, Timothy T.

Los Alamos National Laboratory

P.O. Box 1663, MS B213

Los Alamos, NM 87545

Phone: (505) 665-4858

Fax: (505) 665-3003

E-mail: ttc@1lnl.gov

Clerouin-Cherfils, Catherine

Commissariat à l'Energie Atomique

CEA/DRIF

BP 12, 91680, Bruyeres-le-Chatel, France

Phone: 33169265738

Fax: $\quad 33169267094$

E-mail: cherfils@bruyeres.cea.fr

Colvin, Jeffrey. D.

Lawrence Livermore National Laboratory

P.O. Box 808, L-021

Livermore, CA 94551

Phone: (925) 422-3273

Fax: (925) 423-8945

E-mail: colvin5@1lnl.gov 
$8^{\text {th }}$ International Workshop on the Physics of Compressible Turbulent Mixing,

Pasadena, CA (2001)

Cook, Andrew W.

Lawrence Livermore National Laboratory

P.O. Box 808, L-22

Livermore, CA 94551

Phone: (925) 423-2856

Fax: (925) 423-0925

E-mail: awcook@1lnl.gov

Dalziel, Stuart

DAMTP, University of Cambridge

Silver Street, Cambridge CB3 9EW, England

Phone: (44) (1223) 337911

Fax: (44) (1223) 337918

E-mail: s.dalziel@damtp.cam.ac.uk

Dannenberg, K.

University of Michigan

Space Research Lab, Rm 1216

2245 Hayward

Ann Arbor, MI 48104

Darlington, Rebecca M.

Lawrence Livermore National Laboratory

P.O. Box 808, L-95

Livermore, CA 94551

Phone: (925) 424-3422

E-mail: darlington1@1lnl.gov

Delettrez, J. A.

Laboratory for Laser Energetics

University of Rochester

Rochester, NY 14623-1299

Phone: (716) 275-5374

E-mail: jdel@1le.rochester.edu

Demyanov, A. Yu.

Landau Institute for Theoretical Physics

Kosygnina St. 2, Moscow

117940, GSP-1

V-334 RUSSIA

Dimits, Andris M.

Lawrence Livermore National Laboratory

P.O. Box 808, L-630

Livermore, CA 94551

Phone: (925) 422-0211

Fax: (925) 423-3484

E-mail: dimits1@1lnl.gov 
Dimonte, Guy

Lawrence Livermore National Laboratory

P.O. Box 808 , L-43

Livermore, CA 94551

Phone: (925) 423-0596

Fax: (925) 423-5998

E-mail: dimonte1@1lnl.gov

Dittrich, Thomas R.

Lawrence Livermore National Laboratory

P.O. Box 808, L-023

Livermore, CA 94551

Phone: (925) 422-4706

Fax: (925) 423-8945

E-mail: dittrich1@1lnl.gov

Don, Wai-Sun

Division of Applied Mathematics

Brown University

Box F

Providence, RI 02912

Phone: (401) 863-2250

E-mail:: Wai-Sun_Don@brown.edu

Drake, R. Paul

University of Michigan

2245 Hayward

Ann Arbor, MI 48104

Phone: (734) 763-4072

Fax: (734) 647-3083

E-mail: rpdrake@umich.edu

Drennov, O. B.

Russian Federal Nuclear Center-VNIIEF

Mir Avenue, 37, Sarov

Nizhnii Novgorod Region, 607190, Russia

Fax: (831-30) 459-58

E-mail: root@gdd.vniief.ru

Dudin, V. D.

Russian Federal Nuclear Center-VNIIEF

Mir Avenue, 37, Sarov

Nizhnii Novgorod Region, 607190, Russia

Fax: (831-30) 459-58

E-mail: root@gdd.vniief.ru

Dulov, A. V.

Russian Federal Nuclear Center-VNIITF

P.O. Box 245

456770 Snezhinsk, Russia 
$8^{\text {th }}$ International Workshop on the Physics of Compressible Turbulent Mixing,

Pasadena, CA (2001)

Dunne, A. M.

Atomic Weapons Establishment

Aldermaston

Reading, Berkshire

RG7 4PR UK

Dursi, L.

Department of Astronomy and Astrophysics

University of Chicago

5640 South Ellis Ave.

Chicago, IL 60637

Phone: (773) 834-1059

Fax: (773) 834-3230

Dutta, S.

State University of New York at Stony Brook

P-138A Math Tower

Stony Brook, NY 11794-3600

Edwards, Michael J.

Lawrence Livermore National Laboratory

P.O. Box 808, L-21

Livermore, CA 94551

Phone: (925) 422-1189

Fax: (925) 423-8945

E-mail: edwards39@1lnl.gov

Elbaz, Y.

Ben-Gurion University

Beer-Sheva

Israel 84015

Phone: 972-8-6568836

Fax: 972-8-6567878

E-mail: elbazyo@bgumail.bgu.ac.il

Eliason, Donald E.

Lawrence Livermore National Laboratory

P.O. Box 808, L-103

Livermore, CA 94551

Phone: (925) 423-5625

Fax: (925) 422-6388

E-mail: eliason@merlin.llnl.gov

Epstein, Reuben

Laboratory for Laser Energetics

University of Rochester

Rochester, NY 14623-1299

Phone: (716) 275-5405

E-mail: reps@lle.rochester.edu 
Farley, David

Institute of Laser Engineering

Osaka University

Suita, Osaka 565, Japan

E-mail: dfarley@epri.com

Fryxell, Bruce

Enrico Fermi Institute and Laboratory for Astrophysics and

Space Research

University of Chicago

933 East $56^{\text {th }}$ Street

Chicago, IL 60637

Phone: (773) 704-3226

Fax: (773) 704-3230

E-mail:: fryxell@uffda.asci.uchicago.edu

Fujioka, S.

Institute of Laser Engineering,

2-6, Yamada-oka, Suita,

Osaka 565-0871 Japan

Fukuda, Y.

Institute of Laser Engineering,

2-6, Yamada-oka, Suita,

Osaka 565-0871 Japan

Galmiche, Didier Jean Marie

Commissariat à l'Energie Atomique

BP12 91680 Bruyeres-le-Chatel, France

Phone: 33169264005

Fax: $\quad 33169267094$

E-mail: galmiche@bruyeres.cea.fr

Garasi, C.

Sandia National Laboratory

P.O. Box 969, 0819

Livermore, CA 94551-0969

Phone: (505) 284-2691

Fax: (505) 844-0918

E-mail: CJGARAS@sandia.gov

Gauthier, Serge

Centre d'Etudes de Limeil-Valenton

BP 27 Villeneuve St. Georges cedex

94195, FRANCE

E-mail: gauthier@limeil.cea.fr

Gavrilova, E. S.

Russian Federal Nuclear Center-VNIIEF

Mir Avenue, 37, Sarov

Nizhnii Novgorod Region, 607190, Russia

Fax: (831-30) 459-58

E-mail: root@gdd.vniief.ru 
$8^{\text {th }}$ International Workshop on the Physics of Compressible Turbulent Mixing,

Pasadena, CA (2001)

George, E.

State University of New York at Stony Brook

P-138A Math Tower

Stony Brook, NY 11794-3600

Gerasimov, Serguei

Russian Federal Nuclear Center, VNIIEF

Mir Avenue, 37, Sarov

Nizhnii Novgorod Region, 607190, Russia

Phone: (7-83130) 45009

Fax: $\quad(7-83130) 45958$

E-mail: root@gdd.vniief.ru

Glebov, V. Yu.

Laboratory for Laser Energetics

University of Rochester

250 East River Road

Rochester, New York 14623-1299

Phone: (716) 275-7454

E-mail:vgle@lle.rochester.edu

Glendinning, Gail

Lawrence Livermore National Laboratory

P.O. Box 808, L-21

Livermore, CA 94551

Phone: (925) 423-3065

Fax: (925) 423-8945

E-mail: glendinning1@1lnl.gov

Glimm, James

Department of Applied Mathematics and Statistics

State University of New York at Stony Brook

P-138A Math Tower

Stony Brook, NY 11794-3600

Phone: (631) 632-8355

FAX: (631) 632-8490

E-mail: glimm@ams.sunysb.edu

Goncharov, V. N.

Laboratory for Laser Energetics

University of Rochester

250 East River Road

Rochester, New York 14623-1299

Phone: (716) 275-1017

E-mail:vgon@lle.rochester.edu

Goodwin, Bruce T.

Lawrence Livermore National Laboratory

P.O. Box 808, L-160

Livermore, CA 94551

Phone: (925) 423-7736

Fax: (925) 424-2723

E-mail: goodwin2@1lnl.gov 
Gottlieb, David I.

Division of Applied Mathematics

Brown University

Providence, RI 02912

Phone: (401) 863-2266

E-mail:: David.Gottlieb@brown.edu

Graham, Mary J.

Lawrence Livermore National Laboratory

P.O. Box 808, L-312

Livermore, CA 94551

Phone: (925) 424-4222

Fax: (925) 422-8920

E-mail: mjgraham@1lnl.gov

Greenough, Jeffrey A.

Lawrence Livermore National Laboratory

P.O. Box 808, L-30

Livermore, CA 94551

Phone: (925) 423-3211

Fax: (925) 424-6764

E-mail: greenough1@1lnl.gov

Grieves, Brian

Atomic Weapons Establishment

Aldermaston

Reading, Berkshire

RG7 4PR UK

Phone: +44 (0)1734814111

Fax: $\quad+44(0) 1734815320$

E-mail:: brian.grieves@awe.co.uk

Grove, John W.

Los Alamos National Laboratory

P.O. Box 1663, MS D413

Los Alamos, New Mexico, 87545

Phone: (505) 667-0723

Fax: (505) 665-4972

E-mail: jgrove@lanl.gov

Gubkov, E. V.

Russian Federal Nuclear Center-VNIIEF

Mir Avenue, 37, Sarov

Nizhnii Novgorod Region, 607190, Russia

Phone: (831-30) 45009

Fax: (831-30) 45958

E-mail: root@gdd.vniief.ru 
$8^{\text {th }}$ International Workshop on the Physics of Compressible Turbulent Mixing,

Pasadena, CA (2001)

Gulak, Y.

Department of Mechanical and Aerospace Engineering

Rutgers University

98 Brett Rd.

Piscataway, NJ 08854

Phone: (732) 445-5627

Fax: (732) 445-3124

E-mail: YGULAK@RCI.RUTGERS.EDU

Gupta, S.

Department of Mechanical and Aerospace Engineering

Rutgers University

98 Brett Rd.

Piscataway, NJ 08854

Phone: (732) 445-5627

Fax: (732) 445-3124

Haan, Steven W.

Lawrence Livermore National Laboratory

P.O. Box 808, L-023

Livermore, CA 94551

Phone: (925) 422-4715

Fax: (925) 423-8945

E-mail: haan1@1lnl.gov

Haas, Jean Francois

Commissariat à l'Energie Atomique

CEA/DIF/DCRE/SCSE BP 12

Bruyeres le Chatel, 91680

France

Phone: 33-1-69-26-52-94

Fax: 33-1-69-26-70-62

E-mail: jean-francois.haas@cea.fr

Harlow, Francis H.

Los Alamos National Laboratory

P.O. Box 1663, MS B216

Los Alamos, NM 87545

Phone: (505) 667-9090

E-mail: fhharlow@lanl.gov

Hatchett, Stephen P.

Lawrence Livermore National Laboratory

P.O. Box 808, L-016

Livermore, CA 94551

Phone: (925) 422-5916

Fax: (925) 423-5112

E-mail: hatchett1@1lnl.gov 
Hauer, A.

Los Alamos National Laboratory

P.O. Box 1663

Los Alamos, NM 87545

Phone: (505) 667-5167

Fax: (505) 667-0405

E-mail: hauer@lanl.gov

Hébert, C. H.

Department of Mechanical Engineering

Université de Sherbrooke

Sherbrooke, Quebec J1K 2R1 Canada

Hinkel, Denise

Lawrence Livermore National Laboratory

P.O. Box 808, L-038

Livermore, CA 94551

Phone: (925) 423-2626

Fax: (925) 423-2157

E-mail: hinkel1@1lnl.gov

Holder, David

Atomic Weapons Establishment

Building H27, Aldermaston

Reading, Berkshire, RG7 4PR, United Kingdom

Phone: 01189825592

Fax: 01189824816

E-mail:: david.holder@awe.co.uk

Holford, Joanne M.

University of Cambridge

Silver Street

Cambridge, UK CB3 9EW

Phone: +44 1223337858

Fax: $\quad+441223337918$

E-mail:: J.M.Holford@DAMTP.CAM.AC.UK

Holmes, Richard L.

Los Alamos National Laboratory

P.O. Box 1663, MS B220

Los Alamos, NM 87545

Phone: (505) 667-3598

Fax: (505) 665-2227

E-mail: holmes@lanl.gov

Hosseini, S. H. R.

Shock Wave Research Center, Institute of Fluid Science

Tohoku University

2-1-1 Katahira, Aoba-ku

Sendai 980-77, Japan

E-mail:: hosseini@rainbow.ifs.tohoku.ac.jp 
$8^{\text {th }}$ International Workshop on the Physics of Compressible Turbulent Mixing,

Pasadena, CA (2001)

Houas, Lazhar

I.U.S.T.I. / Umr CNRS 6595

Technopole de Chateau Gombert

5 rue Enrico Fermi

13453 Marseille Cedex 13 France

Phone: $33(0) 491106930$

Fax: $\quad 33(0) 491106969$

E-mail: houas@iusti.univ-mrs.fr

Hsing, Warren W.

Lawrence Livermore National Laboratory

P.O. Box 808, L-21

Livermore, CA 94551

Phone: (925) 423-2849

Fax: (925) 423-8945

E-mail:: hsing@1lnl.gov

Hurricane, Omar

Lawrence Livermore National Laboratory

P.O. Box 808, L-22

Livermore, CA 94551

Phone: (925) 424-2701

Fax: (925) 423-0925

E-mail: hurricane1@1lnl.gov

Inogamov, Nail

Landau Inst. for Theor. Phys.

Kosygnina St. 2, Moscow

117940, GSP-1

V-334 RUSSIA

Phone: 0954258767

FAX: 0959382077

E-mail: itf@ips.ac.msk.su

Jacobs, Jeffrey W.

Department of Aerospace and Mechanical Engineering

University of Arizona

Bldg. 16, Room 301

Tucson, AZ 85721

Phone: (602) 621-8459

Fax: (602) 621-8191

E-mail: jacobs@ame.arizona.edu

Jameson, Leland M.

Lawrence Livermore National Laboratory

P.O. Box 808, L-312

Livermore, CA 94551

Phone: (925) 424-6160

E-mail: jameson3@1lnl.gov 
Jones, Oggden

Lawrence Livermore National Laboratory

P.O. Box 808, L-030

Livermore, CA 94551

Phone: (925) 423-1872

Fax: (925) 423-9969

E-mail:: jones96@llnl.gov

Jourdan, Georges

Universite de Provence

IUSTI-CNRS Umr 139

Centre Saint Jerome -- Case 321

13397 Marseille Cedex 20 France

Phone: 33 (0) 491106930

Fax: $\quad 33$ (0) 491106969

E-mail: jourdan@iusti-dipha.univ-mrs.fr

Kalantar, Daniel

Lawrence Livermore National Laboratory

P.O. Box 808, L- 472

Livermore, CA 94551

Phone: (925) 422-6147

Fax: (925) 422-8395

E-mail: kalantar1@1lnl.gov

Kamm, R. J.

Los Alamos National Laboratory

P.O. Box 1663

Los Alamos NM, 87545

Phone: (505) 667-1918

E-mail:: kammj@lanl.gov

Kane, Jave O.

Lawrence Livermore National Laboratory

P.O. Box 808, L-411

Livermore, CA 94551

Phone: (925) 424-5805

Fax: (925) 423-2463

E-mail:: jave@1lnl.gov

Kang, Young-Gwang

Kwangju Institute of Science and Technology

1 Oryong-dong

Kwangju, 500-712, South Korea

Phone: 82-62-970-2310

Fax: 82-62-970-2304

E-mail: ygkang@kjist.ac.kr 
$8^{\text {th }}$ International Workshop on the Physics of Compressible Turbulent Mixing,

Pasadena, CA (2001)

Kartoon, Daniela

Department of Physics

Nuclear Research Center Negev

P.O. Box 9001

Beer-Sheva, 84190, Israel

Phone: 972-8-6568845

Fax: $\quad 972-8-6567878$

E-mail:: danyk@bgumail.bgu.ac.il

Keiter, P.

University of Michigan

Space Research Lab, Rm 1216

2245 Hayward

Ann Arbor, MI 48104

Kerstein, Alan R.

Sandia National Laboratory

P.O. Box 969, MS 9051

Livermore, CA 94551-0969

Phone: (925) 294-2390

Fax: (925) 294-1004

E-mail: kerstein@ca.sandia.gov

Kim, H.

Department of Mechanical and Aerospace Engineering

Rutgers University

98 Brett Rd.

Piscataway, NJ 08854

Klein, Richard I.

Lawrence Livermore National Laboratory

P.O. Box 808, L-023

Livermore, CA 94551

Phone: (925) 422-3548

Fax: (925) 423-0925

E-mail: rklein@1lnl.gov

Knauer, J.

Department of Mechanical and Aerospace Engineering

Rutgers University

98 Brett Rd.

Piscataway, NJ 08854

Kobayashi, K.

Osaka University

2-6, Yamada-oka, Suita,

Osaka 565-0871 Japan

Korreck, K. E.

University of Michigan

Space Research Lab, Rm 1216

2245 Hayward

Ann Arbor, MI 48104 
Kozelkov, O. E.

Russian Federal Nuclear Center-VNIITF

P.O. Box 245

456770 Snezhinsk, Russia

Kozlov, V. I.

Russian Federal Nuclear Center-VNIIEF

Mir Avenue, 37, Sarov

Nizhnii Novgorod Region, 607190 Russia

Fax: (831-30) 4-57-72

E-mail: otd1_0903@spd.vniief.ru

Ktitorov, Vladimir M.

Russian Federal Nuclear Center-VNIIEF

Mir Avenue, 37, Sarov

Nizhnii Novgorod Region, 607190 Russia

Phone: 831-30 1-36-65

Fax: $\quad 831-30$ 5-45-65

E-mail: mvn_072e@rfnc.nnov.su

Kucherenko, Yuri A.

Russian Federal Nuclear Center-VNIITF

P.O. Box 245

456770 Snezhinsk, Russia

Fax: (351-72) 32077

E-mail: kucherenko@five.ch70.chel.su

Kuhl, Allen L.

Lawrence Livermore National Laboratory

P.O. Box 808, L-30

Livermore, CA 94551

Phone: (925) 422-4777

Fax: (925) 424-6764

E-mail: kuhl2@1lnl.gov

Kumar, M.

Department of Physics

Nuclear Research Center Negev

P.O. Box 9001

Beer-Sheva, 84190, Israel

Kushnir, D.

Department of Physics

Nuclear Research Center Negev

P.O. Box 9001

Beer-Sheva, 84190, Israel

Lanier, N. E.

Los Alamos National Laboratory

P.O. Box 1663, MS

Los Alamos NM, 87545

Phone: (505) 665-0236

Fax: (505) 665-3686

E-mail:: nlanier@lanl.gov 
$8^{\text {th }}$ International Workshop on the Physics of Compressible Turbulent Mixing,

Pasadena, CA (2001)

Lasinski, Barbara F.

Lawrence Livermore National Laboratory

P.O. Box 808, L-038

Livermore, CA 94551

Phone: (925) 422-5443

Fax: (925) 423-9208

E-mail:: blasinski@1lnl.gov

Lassis, A.

Commissariat à l'Energie Atomique

BP 12

Bruyeres le Chatel, 91680, France

Layes, G.

IUSTI

Technopole Chateau-Gombert - 5,

Rue Enrico Fermi

Marseille, 13013 France

Lebedev, Alexander

Russian Federal Nuclear Center-VNIIEF

Mir Avenue, 37, Sarov

Nizhnii Novgorod Region, 607190, Russia

Phone: (831-30) 45009

Fax: $\quad$ (831-30) 45958

E-mail: root@gdd.vniief.ru

Lebo, Ivan

Laser Fusion Department

PN Lebedev Physics Institute

Leninsky prospect 53

117294 Russia, Moscow

Phone: 095-132-68-47

Fax: 095-132-11-96

E-mail: lebo@sci.fian.msk.su

Legrand, Michel

Commissariat à l'Energie Atomique

DAM Ile de France

BP12, Bruyeres le Chatel, 91680, France

Phone: 33-1-69-26-57-09

Fax: 33-1-69-26-70-94

E-mail: michel.legrand@cea.fr

Levy, Kedem

Physics Department

Ben Gurion University of the Negev

P.O. B 653, Beer-Sheva 84105, Israel

Phone: 972-8-6568844

Fax: $\quad 972-8-6567878$

E-Mail: klevy@bgumail.bgu.ac.il 


\section{Li, Xiaolin}

Department of Applied Mathematics and Statistics

State University of New York at Stony Brook

1-119 Math Tower

Stony Brook, NY 11794-3600

Phone: (631) 632-8353

Fax: (631) 632-8490

E-mail: linli@ams.sunysb.edu

Linn, Rodman R.

Los Alamos National Laboratory

P.O. Box 1663

Los Alamos NM, 87545

Phone: (505) 665-6254

E-mail:: rrl@lanl.gov

Llor, Antoine

Commissariat à l'Energie Atomique

BP 12

Bruyeres le Chatel, 91680, France

Phone: 33169264938

Fax: $\quad 33169267097$

E-mail: antoine.llor@cea.fr

Logvinov, A.

Russian Federal Nuclear Center, VNIIEF

Mir Avenue, 37, Sarov

Nizhnii Novgorod Region, 607190, Russia

E-mail: root@gdd.vniief.ru

Lorenz, Karl T.

Lawrence Livermore National Laboratory

P.O. Box 808, L-472

Livermore, CA 94551

Phone: (925) 424-4200

E-mail: lorenz3@1lnl.gov

Louis, Hedley

Lawrence Livermore National Laboratory

P.O. Box 808, L-472

Livermore, CA 94551

Phone: (925) 4242-9937

E-mail: louis1@1lnl.gov

Loveridge -Smith, A.

Oxford University

University Offices

Wellington, Oxford, OX1 2JD UK

Phone: +44 1865270000

Fax: $\quad+441865270708$ 
$8^{\text {th }}$ International Workshop on the Physics of Compressible Turbulent Mixing,

Pasadena, CA (2001)

MacNeice, $P$.

NASA Goddard Space Flight Center

Greenbelt, MD

Magelssen, Glenn R.

Los Alamos National Laboratory

P.O. Box 1663

Los Alamos NM, 87545

Phone: (505) 667-6519

E-mail:: grm@lanl.gov

Magnaudet, J.

Institut de Mécanique des Fluides de Toulouse

31400 Toulouse

Toulouse, France

Marchese, A.

State University of New York at Stony Brook

P-138A Math Tower

Stony Brook, NY 11794-3600

Marinak, Michael M.

Lawrence Livermore National Laboratory

P.O. Box 808, L-023

Livermore, CA 94551

Phone: (925) 423-8458

Fax: (925) 423-9969

E-mail:: marinak1@1lnl.gov

Matsuoka, C.

10-13,Dogo-Himata,Matsuyama 790-8577

Japan

Mazilin, I. M.

Krzhizhanovsky Power Engineering Institute ENIN, Leninsky pr. 19, GSP-1

Moscow, 117927 Russia

McAbee, Thomas L.

Lawrence Livermore National Laboratory

P.O. Box 808, L-170

Livermore, CA 94551

Phone: (925) 422-3398

Fax: (925) 424-2723

E-mail: mcabee@viper.llnl.gov

McCray, R.

University of Colorado, Boulder

Colorado 80309

Phone: (303) 492-1411 
McKenty, P. W.

Laboratory for Laser Energetics

University of Rochester

250 East River Road

Rochester, New York 14623-1299

Medvedev, V. M.

Russian Federal Nuclear Center-VNIITF

P.O. Box 245

456770 Snezhinsk, Russia

Meiron, Daniel I.

Department of Applied and Computational Mathematics

California Institute of Technology

MC 217-50

Pasadena, CA 91125

Phone: (626) 395-4563

Fax: (626) 578-0124

E-mail:dim@acm.caltech.edu

Meshkov, Evgeni E.

Russian Federal Nuclear Center-VNIIEF

Mir Avenue, 37, Sarov

Nizhnii Novgorod Region, 607190, Russia

Fax: 831-305-4565

E-mail: root@gdd.vniief.ru

Meyers, M. A.

University of California at San Diego

9500 Gilman Drive

La Jolla, CA 92093-0411

Mikaelian, Karnig O.

Lawrence Livermore National Laboratory

P.O. Box 808, L-97

Livermore, CA 94551

Phone: (925) 422-5449

Fax: (925) 423-7228

Mikhaylov, A. L.

Russian Federal Nuclear Center, VNIIEF

Mir Avenue, 37, Sarov

Nizhnii Novgorod Region, 607190, Russia

E-mail: root@gdd.vniief.ru

Miles, Aaron

Lawrence Livermore National Laboratory

P.O. Box 808, L-12

Livermore, CA 94551

Phone: (925) 423-8131

Fax: (925) 422-8920

E-Mail: miles15@1lnl.gov 
$8^{\text {th }}$ International Workshop on the Physics of Compressible Turbulent Mixing,

Pasadena, CA (2001)

Miller, Paul L.

Lawrence Livermore National Laboratory

P.O. Box 808, L-22

Livermore, CA 94551

Phone: (925) 423-6455

Fax: (925) 423-0925

E-mail: pmiller@1lnl.gov

Minich, Roger

Lawrence Livermore National Laboratory

P. O. Box 808, L-097

Livermore, CA 94551

Phone: (925) 422-4552

Fax: (925) 422-0779

E-mail:: minich1@1lnl.gov

Miyanaga, N.

Osaka University

2-6, Yamada-oka, Suita,

Osaka 565-0871 Japan

Mizuta, A.

Osaka University

2-6, Yamada-oka, Suita,

Osaka 565-0871 Japan

Montlaurent, P.

Commissariat à l'Energie Atomique

BP 12, 91680

Bruyeres-le-Chatel, France

Moreno, Juan

Lawrence Livermore National Laboratory

P. O. Box 808, L-031

Livermore, CA 94551

Phone: (925) 423-4170

Fax: (925) 424-6764

E-mail:: jcmoreno@1lnl.gov

Morris, A. P.

Atomic Weapons Establishment

Aldermaston

Reading

Berkshire RG7 4PR England

Munro, David H.

Lawrence Livermore National Laboratory

P. O. Box 808, L-023

Livermore, CA 94551

Phone: (925) 422-5428

Fax: (925) 423-9969

E-mail:: dmunro@llnl.gov 
Murphy, T. J.

Los Alamos National Laboratory

P.O. Box 1663

Los Alamos NM, 87545

Phone: (505) 665-5697

Fax: (505) 996-4366

E-mail:: tjmurphy@lanl.gov

Murzakov, V. D.

Russian Federal Nuclear Center-VNIITF

P.O. Box 245

456770 Snezhinsk, Russia

Nagatomo, H.

Osaka University

2-6, Yamada-oka, Suita,

Osaka 565-0871 Japan

Nakai, M.

2-6, Yamada-oka, Suita,

Osaka 565-0871 Japan

Nash, Jeffrey K.

Lawrence Livermore National Laboratory

P. O. Box 808, L-15

Livermore, CA 94551

Phone: (925) 422-7255

E-mail:: jknash@1lnl.gov

Neuvazhayev, Vladimir

Russian Federal Nuclear Center-VNIITF

P.O. Box 245

456770 Snezhinsk, Russia

Phone: 351-72-55675

Fax: 351-72-32077

E-mail: nio3@ch70.chel.su

Nevmerzhitsky, Nikolay

Russian Federal Nuclear Center, VNIIEF

Mir Avenue, 37, Sarov

Nizhnii Novgorod Region, 607190, Russia

Phone: (831-30) 45009

Fax: (831-30) 45958

E-mail: root@gdd.vniief.ru

Niederhaus, Charles E.

NASA Glenn Research Center

21000 Brookpark Rd., MS-77-5

Cleveland, $\mathrm{OH} 44135$

Phone: (216) 433-5461

Fax: (216) 433-8050

E-mail:: charles.niederhaus@grc.nasa.gov 
$8^{\text {th }}$ International Workshop on the Physics of Compressible Turbulent Mixing,

Pasadena, CA (2001)

Nikishin, Vladislav

Institute for Mathematical Modeling (IMM)

Russian Academy of Science

Miusskaya 4a, 125047, Moscow, Russia

Phone: 007(095) 250-79-35

Fax: 007(095) 972-07-23

E-mail: nikishin\\#4@imamod.msk.su

Nikulin, A.

Russian Federal Nuclear Center-VNIIEF

Mir Avenue, 37, Sarov

Nizhnii Novgorod Region, 607190 Russia

E-mail:: root@gdd.vniief.ru

Nishihara, Katsunobu

Institute of Laser Engineering

Osaka University

2-6, Yamada-oka, Suita,

Osaka 565-0871 Japan

Phone: 81-6-6879-8725

Fax: 81-6-6877-4799

E-mail: nishihara@ile.osaka-u.ac.jp

Nishikino, M.

2-6, Yamada-oka, Suita,

Osaka 565-0871 Japan

Nizovtsev, P. N.

Russian Federal Nuclear Center-VNIIEF

Mir Avenue, 37, Sarov

Nizhnii Novgorod Region, 607190 Russia

E-mail:: root@gdd.vniief.ru

Nomoto, K.

University of Tokyo

7-3-1 Hongo, Bunkyo-ku, Tokyo 113-8654, Japan

Phone: +81-3-3812-2111

Oakley, J. G.

Department of Engineering. Physics

University of Wisconsin at Madison

1500 Engineering Drive

Madison, WI 53706

Olson, K.

NASA Goddard Space Flight Center

Greenbelt, MD

Oparin, A. M.

Institute for Computer Aided Design,

Vtoraya Brestskaya ul., 19/18, 123056

Moscow, Russia

Phone: (095) 250-96-30

Fax: (095) 250-89-28

E-mail: oparin@cpd.landau.ac.ru 
Oron, Dan

Department of Physics

Nuclear Research Center - Negev

P.O. Box 9001

Beer-Sheva, 84190, Israel

Phone: 972-7-6568736

Fax: $\quad 972-7-6567878$

E-mail: danor@bgumail.bgu.ac.il

Paisley, Dennis

Los Alamos National Laboratory

P.O. Box 1663

Los Alamos NM, 87545

Phone: (505) 667-7837

E-mail:: dxp@lanl.gov

Pantano, C.

University of California at San Diego

9500 Gilman Drive

La Jolla, CA 92093-0411

Parker, Kenneth W.

Atomic Weapons Establishment

Aldermaston

Reading

Berkshire RG7 4PR England

Phone: +44 1189827578

Fax: $\quad+441189824844$

E-mail:: Kenneth.W.Parker@awe.co.uk

Parshukov, I. E.

Russian Federal Nuclear Center-VNIITF

P.O. Box 245

456770 Snezhinsk, Russia

Peng, O.

Rutgers University

98 Brett Rd.

Piscataway, NJ 08854

Perry, Theodore S.

Lawrence Livermore National Laboratory

P.O. Box 808, L-23

Livermore, CA 94551

Phone: (925) 423-2065

Fax: (925) 424-3294

E-mail: tedperry@1lnl.gov 
$8^{\text {th }}$ International Workshop on the Physics of Compressible Turbulent Mixing,

Pasadena, CA (2001)

Peyser, Thomas A.

Lawrence Livermore National Laboratory

P.O. Box 808, L-23

Livermore, CA 94551

Phone: (925) 423-6454

Fax: (925) 423-0925

E-mail: tpeyser@1lnl.gov

Piskunov, Yu. A.

Russian Federal Nuclear Center-VNIITF

P.O. Box 245

456770 Snezhinsk, Russia

Polionov, Arkadi

Russian Federal Nuclear Center

Institute of Technical Physics

Chelyabinsk 70

P.O. Box 245, Russia

Phone: 351-722-3977

Fax: 351-723-2077

E-mail: nio3@ch70.chel.su

Pollaine, Steven

Lawrence Livermore National Laboratory

P.O. Box 808, L-30

Livermore, CA 94551

Phone: (925) 422-5950

Fax: (925) 423-9208

E-mail: pollaine@llnl.gov

Popov, V. N.

Russian Federal Nuclear Center-VNIITF

P.O. Box 245

456770 Snezhinsk, Russia

Pound, $\mathrm{M}$.

University of Maryland

College Park, MD 20742

Phone (301) 405-1000

Prestridge, Katherine P.

Los Alamos National Laboratory

P.O. Box 1663, MS P940

Los Alamos NM, 87545

Phone: (505) 667-8861

Fax: (505) 665-3359

E-mail: kpp@lanl.gov 
Pullin, Dale I.

Department of Aeronautics

California Institute of Technology

1200 East California Boulevard, MS 105-50

Pasadena, CA 91125

Phone: (626) 395-6081

Fax: (626) 441-2222

E-mail:: dale@galcit.caltech.edu

Pylaev, Anatoly

Russian Federal Nuclear Center-VNIITF

P.O. Box 245

456770 Snezhinsk, Chelyabinsk region, Russia

Fax: (351-72) 32077

E-mail: kucherenko@five.ch70.chel.su

Radha, P. B.

Laboratory for Laser Energetics

University of Rochester

Rochester, NY 14623-1299

Raevskii, Viktor

All Russian Sci \& Research Institute of Experimental Physics

607200, Arzamas - 16

Nizhny Novgorod Region, Russia

Fax: 831-305-4565

E-mail: otd3_2305@rfnc.nnov.su

Rampaprabhu, P.

Department of Mechanical Engineering

Texas A \& M University

MS 3123

College Station, TX 77843-3123

Raviard, P. A.

Commissariat à l'Energie Atomique

BP 12, 91680

Bruyeres-le-Chatel, France

Rayer, C.

Commissariat à l'Energie Atomique

BP 12, 91680

Bruyeres-le-Chatel, France

Razin, A. N.

Russian Federal Nuclear Center-VNIIEF

Mir Avenue, 37, Sarov

Nizhnii Novgorod Region, 607190

E-mail:: root@gdd.vniief.ru 
$8^{\text {th }}$ International Workshop on the Physics of Compressible Turbulent Mixing,

Pasadena, CA (2001)

Rebrov, S. V.

Russian Federal Nuclear Center-VNIIEF

Mir Avenue, 37, Sarov

Nizhnii Novgorod Region, 607190

E-mail:: root@gdd.vniief.ru

Remington, Bruce A.

Lawrence Livermore National Laboratory

P.O. Box 808, L-21

Livermore, CA 94551

Phone: (925) 423-2712

Fax: (925) 422-8395

E-mail: remington2@1lnl.gov

Renaud, Francois

Commissariat à l'Energie Atomique

CEA/DRIF/DCSA/SET

BP 12, 91680

Bruyeres-le-Chatel, France

Phone: 33169264081

Fax: $\quad 33169267102$

Ricker, P.

University of Chicago

933 East $56^{\text {th }}$ Street

Chicago, IL 60637

Rider, William J.

Los Alamos National Laboratory

P.O. Box 1663, MS D413

Los Alamos NM, 87545

Phone: (505) 665-4162

Fax: (505) 667-3726

E-mail: wjr@lanl.gov

Rightley, Paul M.

Los Alamos National Laboratory

P.O. Box 1663, MS P940

Los Alamos NM, 87545

Phone: (505) 667-0460

Fax: (505) 665-3359

E-mail: pright@lanl.gov

Rikanati, Avi

Physics Department

Nuclear Research Center - Negev

P.O. Box 9001

Beer-Sheva, 84190, Israel

Phone: 972-7-6568736

Fax: 972-7-6567878

E-mail: rkavi@bgumail.bgu.ac.il 
Robey, Harry F.

Lawrence Livermore National Laboratory

P.O. Box 808, L-21

Livermore, CA 94551

Phone: (925) 423-5669

FAX: (925) 422-3358

E-mail: robey1@1lnl.gov

Robinson, A.

Sandia National Laboratory

P.O. Box 969, MS 9051

Livermore, CA 94551-0969

Rosner, Robert L.

Enrico Fermi Institute and Laboratory for Astrophysics and Space Research

University of Chicago

933 East $56^{\text {th }}$ Street

Chicago, IL 60637

Phone: (773) 702-0560

Fax: (773) 704-3230

E-mail:: rosner@uchicago.edu

Rothman, S.

Atomic Weapons Establishment

Aldermaston

Reading

Berkshire RG7 4PR England

Roualdes, P.

Centre d'Etudes de Gramat

Gramat. France

Rozanov, I. A.

Lebedev Physical Institute

Leninsky Pr. 53

117924, Moscow, Russia

E-mail:: Rozanov@sci.lebedev.ru

Rozanov, Vladislav

Lebedev Physical Institute

Leninsky Pr. 53

117924, Moscow, Russia

Phone: (095)-132-68-47

Fax: (095)-132 -11-96

E-mail: lebo@neur.lpi.msk.su

Ryutov, Dimitri D.

Lawrence Livermore National Laboratory

P.O. Box 808, L-630

Livermore, CA 94551

Phone: (925) 422-9832

E-mail: ryutov1@1lnl.gov 
$8^{\text {th }}$ International Workshop on the Physics of Compressible Turbulent Mixing,

Pasadena, CA (2001)

Sadot, Oren

Department of Physics

Nuclear Research Center - Negev

P.O.B. 9001, Beer-Sheva 84109, Israel

Phone: 972-7-6567278

Fax: 972-7-6567878

E-mail: sorens@bgumail.bgu.ac.il

Sakaiya, T.

Institute of Laser Engineering

Osaka University

2-6 Yamadaoka Suita

Osaka, 565 Japan

Samtaney, Ravi

Department of Aeronautics

California Institute of Technology

MS 205-45

Pasadena, CA 91125

Phone: (626) 395-8030

Fax: (626) 449-2677

E-mail: ravi@ama.caltech.edu

Sapozhnikov, I. V.

Russian Federal Nuclear Center-VNIIEF

Mir Avenue, 37, Sarov

Nizhnii Novgorod Region, 607190, Russia

Sarid, Eli

Physics Department

Nuclear Research Center - Negev

P.O. Box 9001

Beer-Sheva, 84190, Israel

Phone: 972-7-6568736

Fax: $\quad$ 972-7-6567878

E-mail: fnsarid@weizmann.weizmann.ac.il

Sarkar, Sutanu

Department of Mechanical and Aerospace Engineering

University of California at San Diego

9500 Gilman Drive

La Jolla, CA 92093-0411

Phone: (858) 534-8243

Fax: (858) 534-7599

E-mail:: sarkar@mae.ucsd.edu 
Schilling, Oleg

Lawrence Livermore National Laboratory

P.O. Box 808, L-22

Livermore, CA 94551

Phone: (925) 423-6879

Fax: (925) 423-0925

E-mail: schilling1@1lnl.gov

Schurtz, G.

Commissariat à l'Energie Atomique

CEA/DRIF/DCSA/SET

BP12, 91680 Bruyeres-le-Chatel, France

Schwaederlè, Laurent

IUSTI

Technopole Chateau-Gombert - 5,

Rue Enrico Fermi

Marseille, 13013 France

Phone: 0491106935

Fax: $\quad 0491106969$

E-Mail: schwaed@iusti.univ-mrs.fr

Sharp, David H.

Los Alamos National Laboratory

P.O. Box 1663, MS B285

Los Alamos, NM 87545

Phone: (505) 667-5266

Fax: (505) 665-3003

E-mail: dhs@lanl.gov

Shestachenko, Oleg

Russian Federal Nuclear Center-VNIITF

P.O. Box 245

456770 Snezhinsk, Chelyabinsk region, Russia

Fax: (351-72) 32077

E-mail: kucherenko@five.ch70.chel.su

Shestakov, Alex

Lawrence Livermore National Laboratory

P.O. Box 808, L-38

Livermore, CA 94551

Phone: (925) 422-4213

Fax: (925) 423-9208

E-mail: shestakov1@1lnl.gov

Shigemori, K.

Institute of Laser Engineering

Osaka University

2-6 Yamadaoka Suita

Osaka, 565 Japan 
$8^{\text {th }}$ International Workshop on the Physics of Compressible Turbulent Mixing,

Pasadena, CA (2001)

Shiraga, $\mathrm{H}$.

Institute of Laser Engineering

Osaka University

2-6 Yamadaoka Suita

Osaka, 565 Japan

Shu, Chi-Wang

Division of Applied Mathematics

Brown University

Providence, RI 02912

Phone: (401) 863-2549

E-mail:: Chi-Wang_Shu@brown.edu

Shvarts, Dov

Department of Physics

Nuclear Research Center - Negev

P.O. Box 9001

Beer-Sheva, 84190, Israel

Phone: 972-7-6567500

Fax: 972-7-6567665

E-mail: shvarts@bgumail.bgu.ac.il

Silvani, X.

Institut de Mécanique des Fluides de Toulouse

31400 Toulouse

Toulouse, France

Sinitsina, S. N.

Russian Federal Nuclear Center-VNIIEF

Mir Avenue, 37, Sarov

Nizhnii Novgorod Region, 607190, Russia

E-mail:: root@gdd.vniief.ru

Sinkova, O. G.

Russian Federal Nuclear Center-VNIIEF

Mir Avenue, 37, Sarov

Nizhnii Novgorod Region, 607190, Russia

E-mail:: root@gdd.vniief.ru

Skupsky, S.

Laboratory for Laser Energetics

University of Rochester

Rochester, NY 14623-1299

Smalyuk, V. A.

Laboratory for Laser Energetics

University of Rochester

Rochester, NY 14623-1299 
Smith, Alan V.

Building H27

Atomic Weapons Establishment

Aldermaston

Reading

Berkshire RG7 4PR England

Phone: 01189-825592

Fax: 01734-815320

E-mail: alan.smith@awe.co.uk

Sotskov, E.A.

Russian Federal Nuclear Center-VNIIEF

Mir Avenue, 37, Sarov

Nizhnii Novgorod Region, 607190, Russia

Fax: (831-30) 45958

E-mail: root@gdd.vniief.ru

Souffland, Denis

Commissariat à l'Energie Atomique

CEA/DRIF/DCSA/SET

BP12, 91680 Bruyeres-le-Chatel, France

Phone: 33169265977

Fax: $\quad 33169267094$

E-mail: soufflan@bruyeres.cea.fr

Srebro, Yair

Department of Physics

Ben-Gurion University of the Negev

Beer Sheva, 84105, Israel

Phone: 972-8-6568416

Fax: $\quad 972-8-656-7878$

E-mail:: sibo@bgumail.bgu.ac.il

Stadnik, A. L.

Russian Federal Nuclear Center-VNIIEF

Mir Avenue, 37, Sarov

Nizhnii Novgorod Region, 607190, Russia

E-mail: root@gdd.vniief.ru

Statsenko, V. P.

Russian Federal Nuclear Center-VNIIEF

Mir Avenue, 37, Sarov

Nizhnii Novgorod Region, 607190, Russia

E-mail: root@gdd.vniief.ru

Steinkamp, Michael J.

Los Alamos National Laboratory

P.O. Box 1663, MS D413

Los Alamos, NM 87545

Phone: (505) 667-5837

Fax: (505) 667-3726

E-mail: steinmj@lanl.gov 
$8^{\text {th }}$ International Workshop on the Physics of Compressible Turbulent Mixing,

Pasadena, CA (2001)

Stoeckl, C.

Laboratory for Laser Energetics

University of Rochester

Rochester, NY 14623-1299

Stone, J.

University of Maryland

College Park, MD 20742

Phone (301) 405-1000

Sunahara, A.

Institute of Laser Engineering

Osaka University

2-6 Yamadaoka Suita

Osaka, 565 Japan

Phone: 81-6-879-8742

Fax: $\quad 81-6-877-4799$

E-mail: suna@ile.osaka-u.ac.jp

Suter, Laurance

Lawrence Livermore National Laboratory

P. O. Box 808, L-031

Livermore, CA 94551

Phone: (925) 422-5423

Fax: (925) 423-9969

E-mail:: suter1@1lnl.gov

Sviridov, E. V.

Russian Federal Nuclear Center-VNIITF

P.O. Box 245

456770 Snezhinsk, Russia

Takabe, Hideaki

Institute of Laser Engineering

Osaka University

Yamada-oka 2-6, Suita

Osaka 565, Japan

Phone: 81-6-877-5111 Ext. 6553

Fax: $\quad 81-6-877-4779$

E-mail: takabe@ile.osaka-u.ac.jp

Takayama, Kazuyoshi

Shock Wave Research Center

Institute of Fluid Science

Tohoku University

2-1-1 Katahira, Aoba-ku

Sendai 980-77, Japan

Phone: 81-22-263-0895

Fax: 81-22-227-7390

E-mail: paul@bellanca.ifs.tohoku.ac.jp 
Tamari, Y.

Institute of Laser Engineering (ILE)

Osaka University

2-6 Yamadoaka

Suita, Osaka 565-0871, Japan

Teyssier, R.

Commissariat a 'Energie Atomique

BP 12, 91680

Bruyeres-le-Chatel, France

Timmes, F.

University of Chicago

933 East $56^{\text {th }}$ Street

Chicago, IL 60637

Tishkin, Vladimir

Institute for Mathematical Modeling

Russian Academy of Science

Miusskaya 4a

125047, Moscow, Russia

Phone: 007(095) 250-79-35

Fax: $\quad$ 007(095) 972-07-23

E-mail: tishkin@imamod.msk.su

Titov, S. N.

Krzhizhanovsky Power Engineering Institute

ENIN, Leninsky pr. 19, GSP-1

Moscow, 117927 Russia

Tomkins, Christopher

Los Alamos National Laboratory

P.O. Box 1663, MS 0454

Los Alamos, NM 87545

Phone: (505) 665-6228

Fax: (505) 665-4507

E-mail:: ctomkins@lanl.gov

Toqué, Nathalie

Universite de Montreal

\#1, 6685 Saint Denis

Montreal, Canada H-85-851

Phone: 514-276-9014

E-mail:: Toque@astro.umontreal.ca

Tricottet, Matthieu

Commissariat à l'Energie Atomique

BP 12, 91680

Bruyeres-le-Chatel, France

E-mail:: Matthieu.Tricottet@cea.fr 
$8^{\text {th }}$ International Workshop on the Physics of Compressible Turbulent Mixing,

Pasadena, CA (2001)

Tufo, $\mathrm{H}$.

University of Chicago

933 East $56^{\text {th }}$ Street

Chicago, IL 60637

Turano, Edward J.

Lawrence Livermore National Laboratory

P.O. Box 808 , L-15

Livermore, CA 94551

Phone: (925) 424-3508

E-mail:: turano1@1lnl.gov

Turner, N.

University of Maryland

College Park, MD 20742

Phone (301) 405-1000

Tyaktev, A. A.

Russian Federal Nuclear Center-VNIITF

P.O. Box 245

456770 Snezhinsk, Russia

Uchayev, A. Ya.

Russian Federal Nuclear Center, VNIIEF

Mir Avenue, 37, Sarov

Nizhnii Novgorod Region, 607190, Russia

Vandenboomgaerde, Marc

Commissariat à l'Energie Atomique

BP 12, 91680

Bruyeres-le-Chatel, France

Phone: 330169266668

Fax: 330169267094

E-mail: marc.vandenboomgaerde@bruyeres.cea.fr

Verdon, Charles P.

Lawrence Livermore National Laboratory

P.O. Box 808, L-30

Livermore, CA 94551

Phone: (925) 423-4449

Fax: (925) 423-9969

E-mail:: verdon1@1lnl.gov

Vlasov, Yu.

Russian Federal Nuclear Center, VNIIEF

Mir Avenue, 37, Sarov

Nizhnii Novgorod Region, 607190, Russia 
Voelkl, Tobias

Department of Applied and Computational Mathematics

California Institute of Technology

$\mathrm{M} / \mathrm{C}$ 217-50

Pasadena, CA 91125

Phone: (626) 395-8534

E-mail: tobias@its.caltech.edu

Vold, Erik L.

Los Alamos National Laboratory

P.O. Box 1663, MS D413

Los Alamos, NM 87545

Phone: (505) 665-5646

Fax: (505) 667-3726

E-mail: elv@lanl.gov

Volkov, V. I.

Russian Federal Nuclear Center-VNIITF

P.O. Box 245456770

Snezhinsk, Chelyabinsk Region, Russia

E-mail: v.a.gordeychuk@vniitf.ru

Vorobieff, Peter V.

Department of Mechanical Engineering

University of New Mexico

ME Bldg. Rm. 424

Albuquerque, NM 87131

Phone: (505) 277-8347

Fax: (505) 277-1571

E-mail:kalmoth@me.unm.edu

Ward, David J.

Lawrence Livermore National Laboratory

P.O. Box 808, L-30

Livermore, CA 94551

Phone: (925) 423-3211

Fax: (925) 424-6764

E-mail: djw@1lnl.gov

Ward, Richard A.

Lawrence Livermore National Laboratory

P.O. Box 808, L-13

Livermore, CA 94551

Phone: (925) 423-2679

Fax: (925) 422-4643

E-mail: raward@llnl.gov 
$8^{\text {th }}$ International Workshop on the Physics of Compressible Turbulent Mixing,

Pasadena, CA (2001)

Wark, J. S.

Oxford University

University Offices

Wellington, Oxford, OX1 2JD UK

Phone: +44 1865270000

Fax: $\quad+441865270708$

Weber, Stephen V.

Lawrence Livermore National Laboratory

P.O. Box 808, L-16

Livermore, CA 94551

Phone: (925) 422-5433

Fax: (925) 423-9208

E-mail: svweber@1lnl.gov

Wiley, Larry G.

Lawrence Livermore National Laboratory

P.O. Box 808, L-97

Livermore, CA 94551

Phone: (925) 423-2461

Fax: (925) 422-0779

E-mail: wiley1@1lnl.gov

Wilson, P. N.

Department of Mechanical Engineering

Texas A \& M University

MS 3123

College Station, TX 77843-3123

Wolfer, Wilhelm G.

Lawrence Livermore National Laboratory

P.O. Box 808, L-97

Livermore, CA 94551

Phone: (925) 423-1501

Fax: (925) 423-7040

E-mail:: wolfer1@1lnl.gov

Wouchuk, Juan G.

E.T.S.I. Industriales

Universidad Castilla-La Mancha

Campus Universitario $\mathrm{s} / \mathrm{n}$

13071 Ciudad Real, Spain

Phone: (34) 926-29-53-00 x3826

Fax: (34) 926-29-53-61

E-mail: gwouchuk@ind-cr.uclm.es

Wunsch, Scott E.

Sandia National Laboratory

P.O. Box 969, MS 9051

Livermore, CA 94551-0969

Phone: (925) 294-4621

Fax: (925) 294-2595

E-mail: sewunsch@sandia.gov 
$\mathrm{Xu}, \mathrm{Z}$.

State University of New York at Stony Brook

P-138A Math Tower

Stony Brook, NY 11794-3600

Yamada, Shoichi

Institute of Laser Engineering (ILE)

Osaka University

2-6 Yamadoaka

Suita, Osaka 565-0871, Japan

Phone: 81-6-6879-8747

Fax: 81-6-6879-8747

E-mail: syamada@ile.osaka-u.ac.jp

Yanilkin, Yury

Russian Federal Nuclear Center-VNIIEF

Mir Avenue, 37, Sarov

Nizhnii Novgorod Region, 607190, Russia

Phone: (831-30) 11190

Fax: $\quad$ (831-30) 54665

E-mail: yan@md08.vniief.ru

Yosef-Hai, Arnon

Department of Physics

Nuclear Research Center-Negev

P.O.B. 9001 Beer-Sheva, 84190, Israel

Phone: 972-8-6568913

Fax: $\quad 972-8-6567878$

E-mail: ajoseph@bgumail.bgu.ac.il

Young, Y.-N.

University of Chicago

933 East $56^{\text {th }}$ Street

Chicago, IL 60637

Youngs, David L.

Atomic Weapons Establishment

Building E3, AWE(A)

Reading, Berkshire

RG7 4PR, England

Phone: 01189826766

Fax: 01189815320

E-mail: dyoungs@awe.co.uk

Zabusky, Norman J.

Department of Mechanical and Aerospace Engineering

Rutgers University

98 Brett Rd.

Piscataway, NJ 08854

Phone: (732) 445-5869

Fax: (732) 445-3124

E-mail: nzabusky@caip.rutgers.edu 
$8^{\text {th }}$ International Workshop on the Physics of Compressible Turbulent Mixing,

Pasadena, CA (2001)

Zaytsev, Sergey G.

Krzhizhanovsky Power Engineering Institute

ENIN, Leninsky pr. 19, GSP-1

Moscow, 117927 Russia

Phone: (7-095) 955-31-07

Fax: (7-095) 954-42-50

E-mail: S.G.Zaytsev@mtu-net.ru

Zhakhovskii, V.

Institute of Laser Engineering

Osaka University

Suita, Osaka 565 Japan

Zhang, S.

Department of Mechanical and Aerospace Engineering

Rutgers University

98 Brett Rd.

Piscataway, NJ 08854

Zhang, Y.

State University of New York at Stony Brook

P-138A Math Tower

Stony Brook, NY 11794-3600

Zhmailo, Vadim A.

Russian Federal Nuclear Center-VNIIEF

Mir Avenue, 37, Sarov

Nizhnii Novgorod Region, 607190, Russia

Fax: 8313054565

E-mail: rvg_092C@rfnc.nnov.su

Zhou, Ye K.

Lawrence Livermore National Laboratory

P.O. Box 808, L-23

Livermore, CA 94551

Phone: (925) 424-3624

E-mail:: zhou3@1lnl.gov

Zingdale, N. V.

University of Chicago

933 East $56^{\text {th }}$ Street

Chicago, IL 60637

Zmitrenko, Nikolay

Institute for Mathematical Modeling

Russian Academy of Science

Miusskaya 4a, 125047, Moscow, Russia

Phone: 007(095) 250-79-16

Fax: $\quad$ 007(095) 972-07-23

E-mail: nickzmit/\#9@imamod.msk.su 
Zoldi, Cindy A.

Los Alamos National Laboratory

P.O. Box 1663, MS B220

Los Alamos, NM 87544

Phone: (505) 667-6455

Fax: (505) 665-2227

E-mail: czoldi@lanl.gov

Zvorykin, V. D.

PN Lebedev Physics Institute

Leninsky prospect 53

117294 Russia, Moscow 
$8^{\text {th }}$ International Workshop on the Physics of Compressible Turbulent Mixing,

Pasadena, CA (2001)

\section{Author Index}

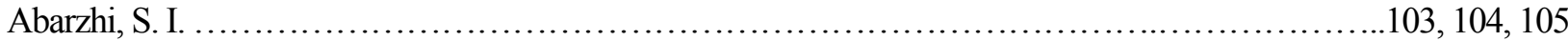

Abe $\mathrm{M}$

Afeyan, B. B.

...138

Allen, A. M.

Alon, U.

Anderson, M. H

33,87

Andrews, M. J. $38,59,131,138$

Anisimov, V. I. . .54

Anuchin, M. G. . .55

Arazi, L.

Ardashova, R. I. 7,8

Arnett, D. ... 15,71

Ashurst Wm. T. . .56

Azechi, H. .51

Bailly, P. . .106

Baishev, A. I

$.34,44$

Balabin, S. I.

$.7,8,26$

Barnes, C. W $9,10,76$

Barton, C. 18,45

Batha, S. H.

$9,10,76$

Belak, J. 25,35

Belomestnih, A. V .37

Ben-Dor, G. $30,41,133$

Benjamin, R. F. $36,46,94$

Biello, J. ....59

Bliznetsov, M. $.11,12$

Boehly, T.R. 35,39

Bonazza, R. ... .33, 87

Bouquet, $\mathrm{S}$. 4,128

Braun, D. G. . .17

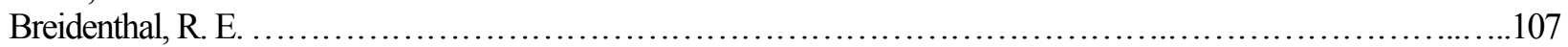

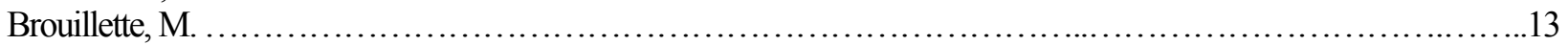

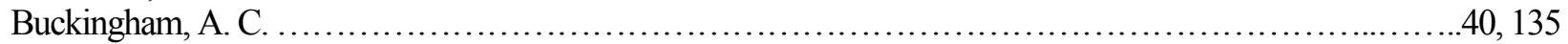

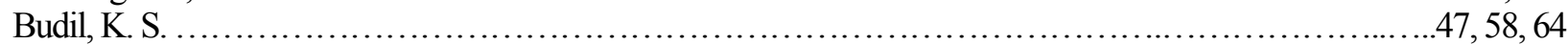

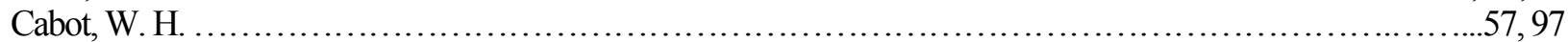

Calder, A. $\quad$.

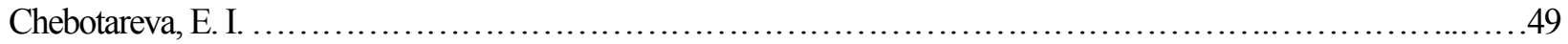

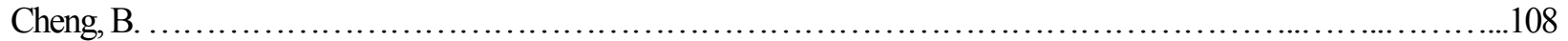

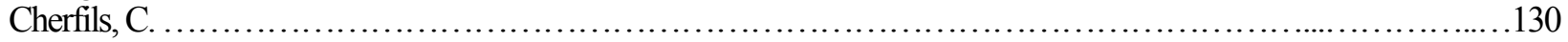

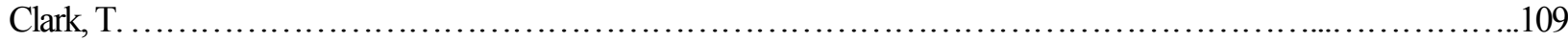

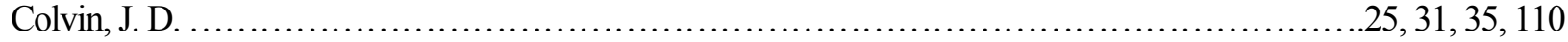

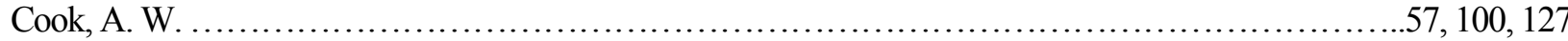

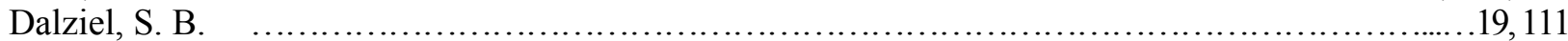

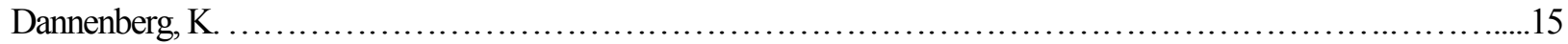

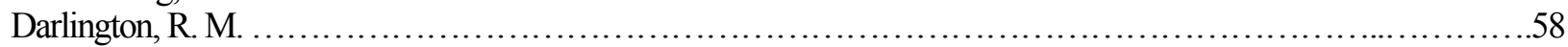

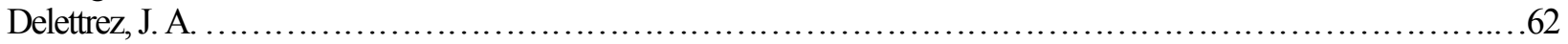

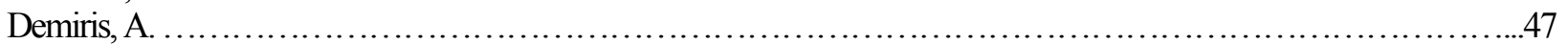

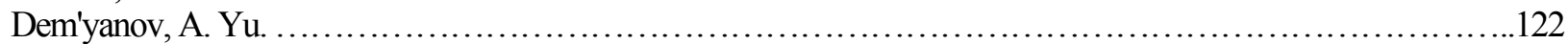


Dimits, A 59,99

Dimonte, G $.59,88$

Dimotakis, P. E. . 100

Dittrich, T. . .70

Don, W.-S. .98

Drake, R. P. $14,15,40$

Drennov, O. B. ..16

Dudin, V. D. ..11

Dulov, A. V. .8

Dunne, A. M. $9,10,76$

Dursi, L. . . . .59

Dutta, S.

Edwards, M. J. . .60

Elbaz, Y. $.17,31,35,34,101$

Eliason, D. E. $30,61,83,112$

Epstein, J. A. .. .97

Ferguson, R. E ...62

Fryxell, B. .136

Fujioka, S. 15,59

Fukuda, Y. ...51

Galmiche, D. 120

Garasi, C. . 130

Gauthier, S. ..... .59

Gavrilova, E. S. 130

George, E.

Gerasimov, S. 60

Glebov, V. Yu.

Glendinning, S. G. 62

Glimm, J. $17,52,71,101$

Goncharov, V.N. $15,60,108$

Gottlieb, D. .

Graham, M. J. .62

Greenough, J. A. ... .. .98

Grieves, B. ....64

Grove, J.

Gubkov, E. V.

Gulak, Y.

Gupta, S.

Haan, S. W.

Haas, J.-F.

Harlow, F.

Hatchett, S.

$39,48,57,65$

Hauer, A.

Hérbert, C. H.

Hinkel, D. ...

Holder, D. A.

Holford, J. M. .

Hosseini, S. H. R.

Houas, L.

Hsing, W. W.

$15,60,64,67$ ..63, 68 
$8^{\text {th }}$ International Workshop on the Physics of Compressible Turbulent Mixing,

Pasadena, CA (2001)

Hurricane, O. A.

Inogamov, N. A.

$113,114,122$

Jacobs, J. W. ...

$.24,32$

Jameson, L. ...

.. .98

Jones, $\mathrm{O}$.

. .70

Jourdan, G.

$11,22,29,42$

Kalantar, D. H.

$25,31,35$

Kamm, R. J. 65,79

Kane, J. O.

$15,39,71$

Kang, Y.-G. ...93

Kartoon, D.

115,133

Keiter, P.

15,40

Kerstein, A. R

. .56

$\mathrm{Kim}, \mathrm{H}$.

Klein, R.

39,48

Knauer, J.

. .15

Kobayashi, K . .84

Korreck, K. E. . .15

Kozelkov, O. E. $.7,8$

Kozlov, V. I. 95,96

Krivets, V. V.

24,49

Ktitorov, V. M

116,117

Kucherenko, Yu. A.

Kuhl, A. L.

$7,8,26,34,37,44$

Kumar, M.

136

Kushnir, D.

Lanier, N. E.

. .83

Lasinski, B. F.

$9,10,76$

Lassis, A .17

Layes, $\mathrm{G}$ . .27

Lebedev, A. .29

Lebo, I. G. . . .50

LeGrand, M. .72

Levy, K.

$.81,110$

$\mathrm{Li}, \mathrm{X}$. . .30

Linn, R. R. ..60

Llor, A. .118

Logvinov, A. $1,106,119$

Lorenz, K. T.

$25,31,35$

Louis, $\mathrm{H}$. 17,47

Loveridge-Smith, A.

$.25,35$

MacNeice, P. . .59

Magelssen, G. R.

$9,10,76$

Magnaudet, J. ... .91

Marchese, A. .60

Marinak, M. M. 70,88

Matsuoka, C. .120

Mazilin, I. M. ...49

McCray, R ...15

McKenty, P. W. .62 
Medvedev, V. M.

34,44

Meshkov, E.

$11,12,22$

Meyers, M. A. 25,35

Mikaelian, K. O. $25,35,121$

Mikhaylov, A. L. 16

Miles, A 101

Miller, P. L. ... . .47

Minich, R. .35

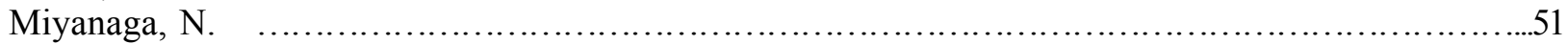

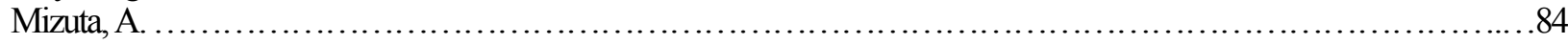

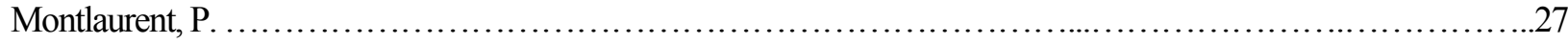

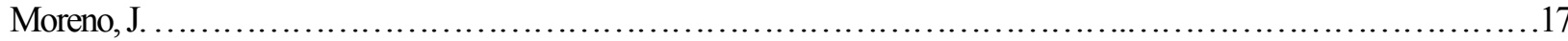

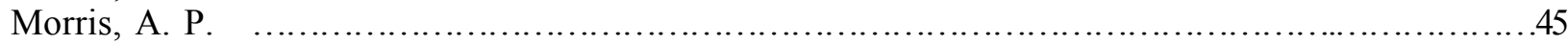

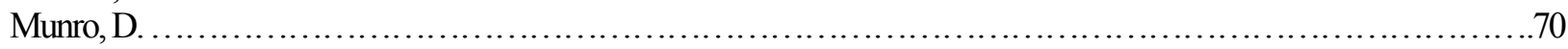

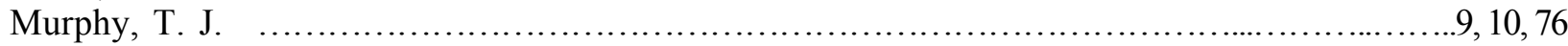

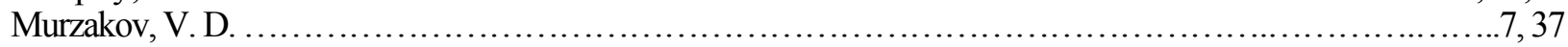

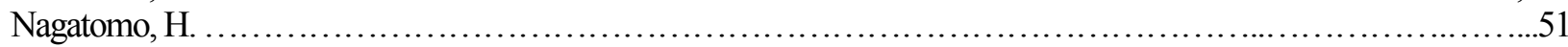

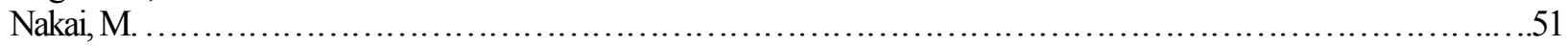

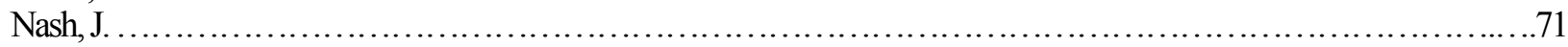

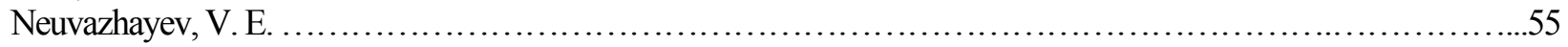

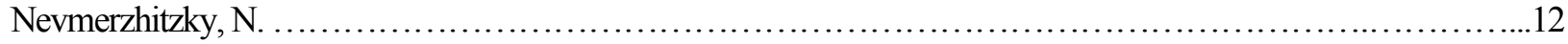

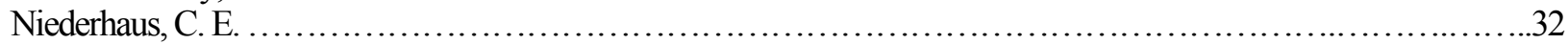

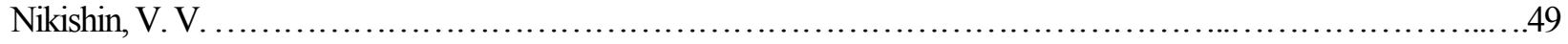

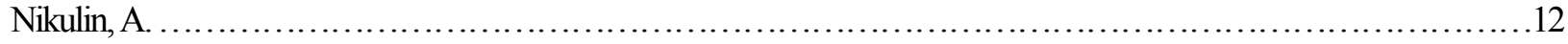

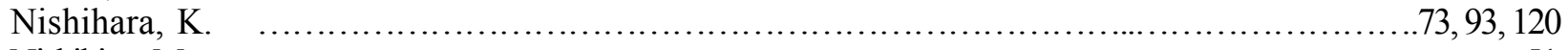

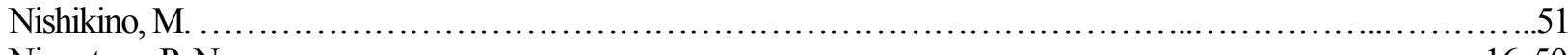

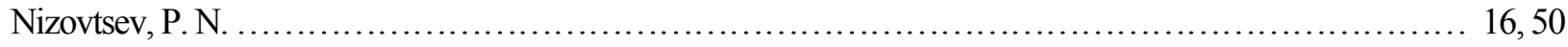

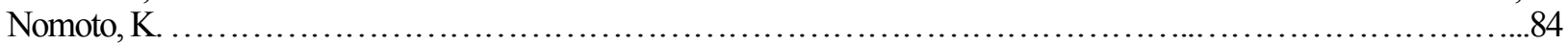

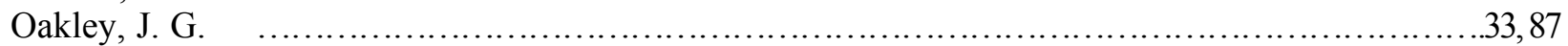

Olson, K . . . . .

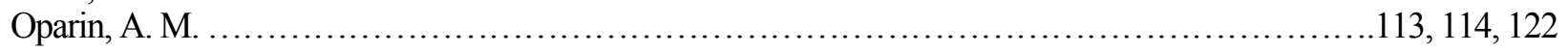

Oron, D. ................................................................... 41, 61, 115, 124, 133

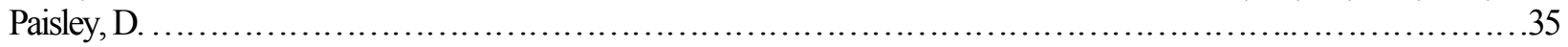

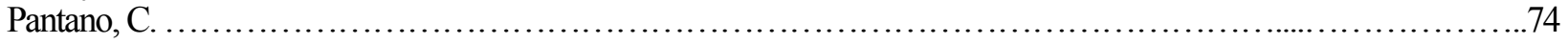

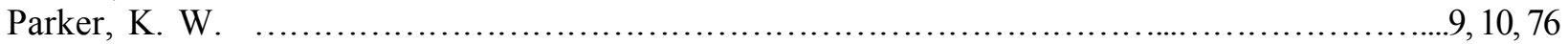

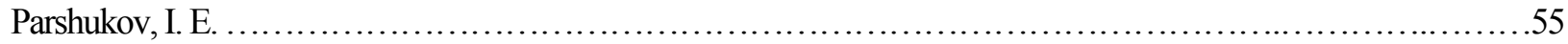

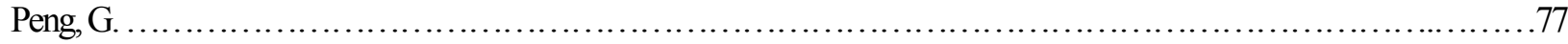

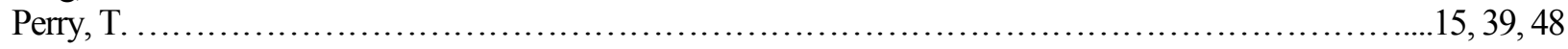

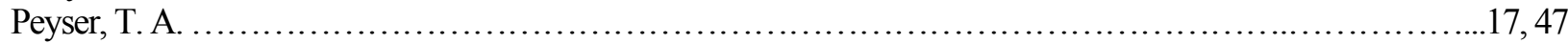

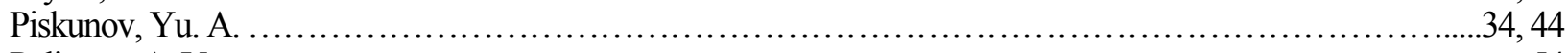

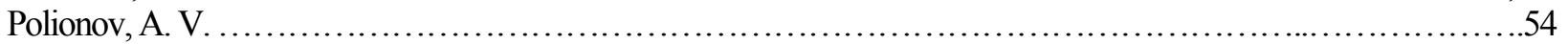

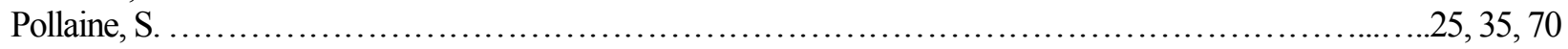

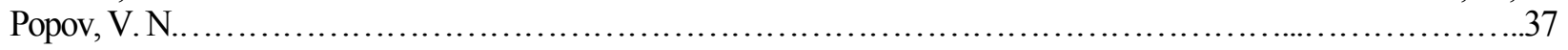

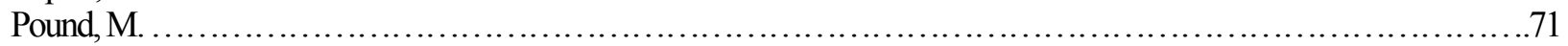

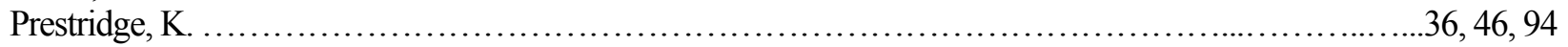

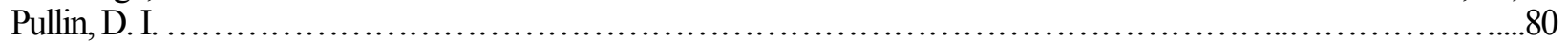

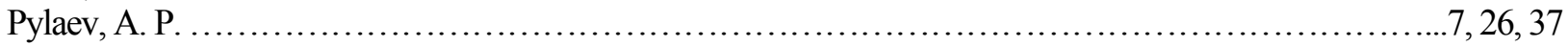

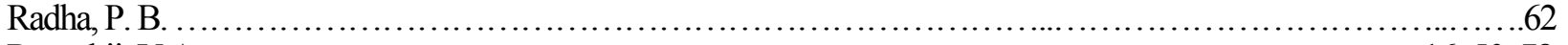

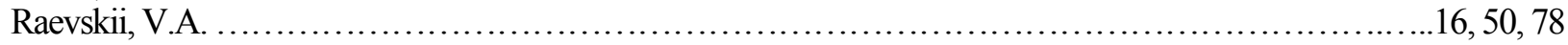

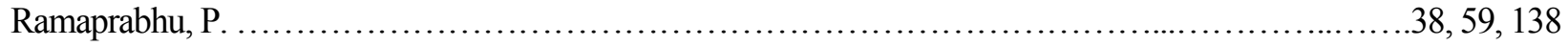


$8^{\text {th }}$ International Workshop on the Physics of Compressible Turbulent Mixing,

Pasadena, CA (2001)

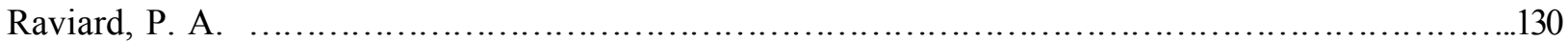

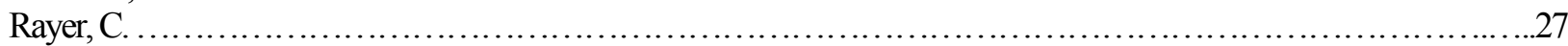

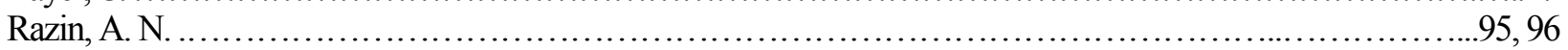

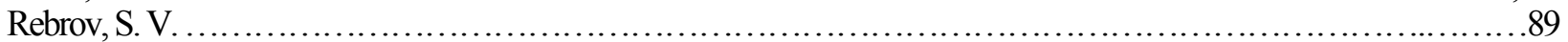

Remington, B. A. ..........................................15, 17, 25, 31, 35, 40, 47, 64, 71, 110, 125

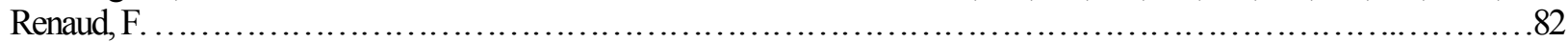

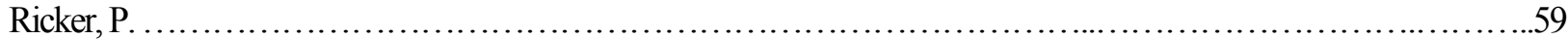

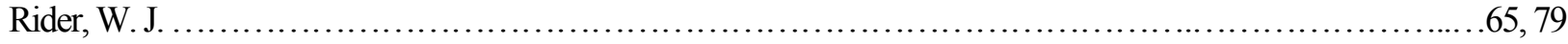

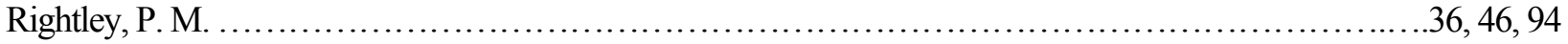

Rikanati, A...................................................................... $41,61,115,123,124$

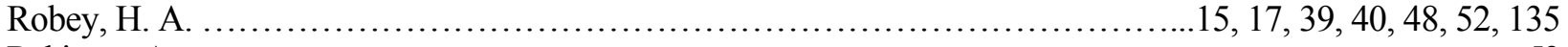

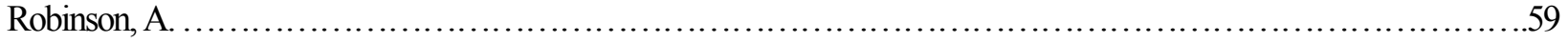

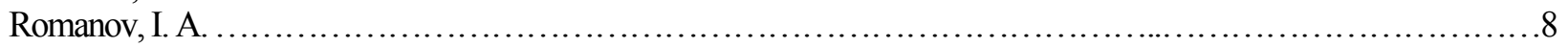

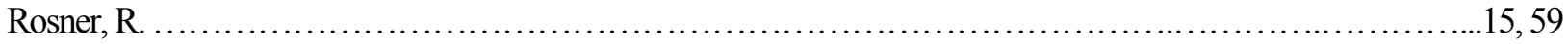

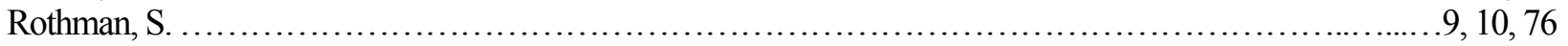

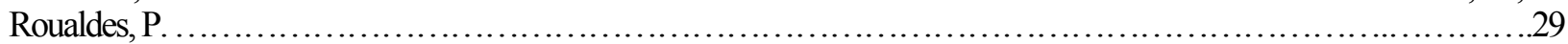

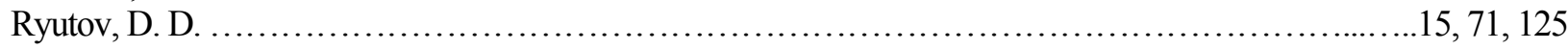

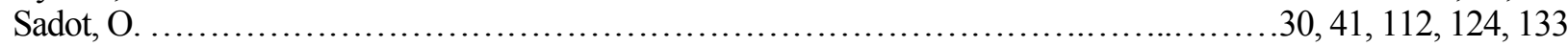

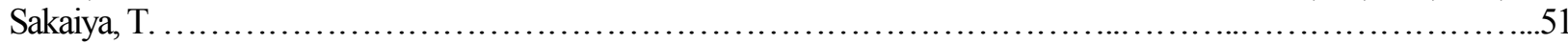

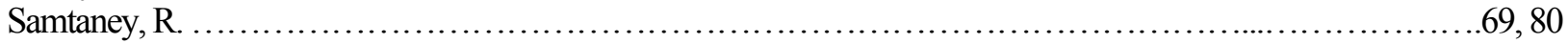

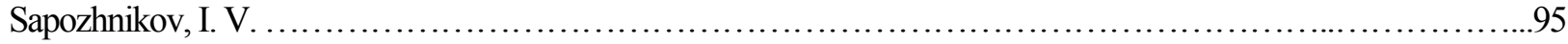

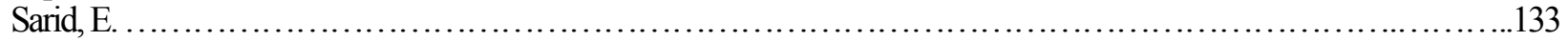

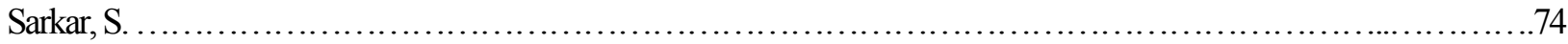

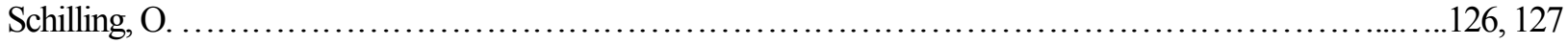

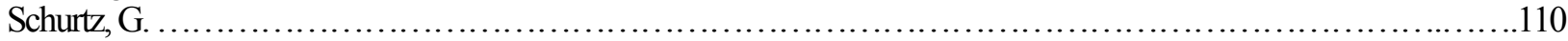

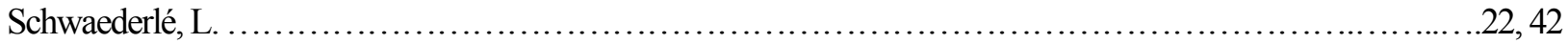

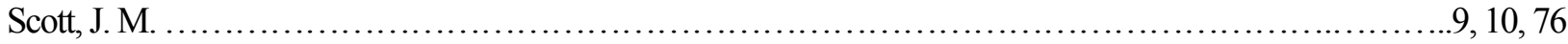

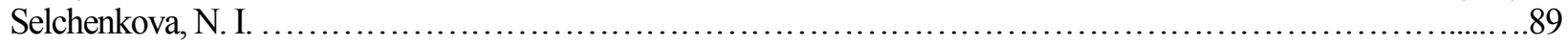

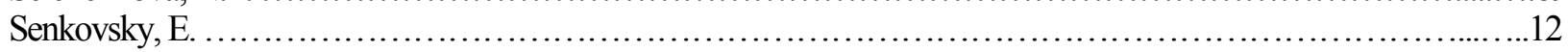

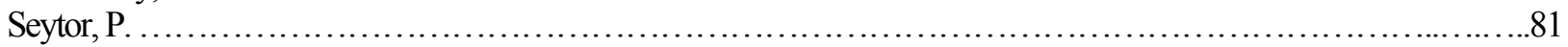

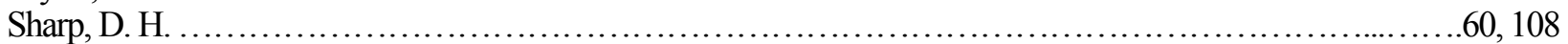

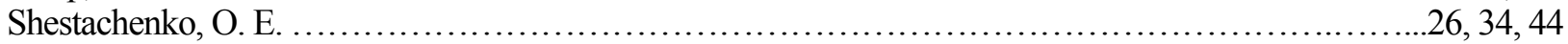

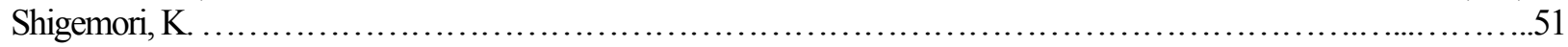

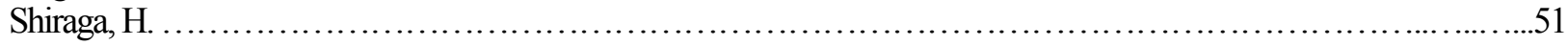

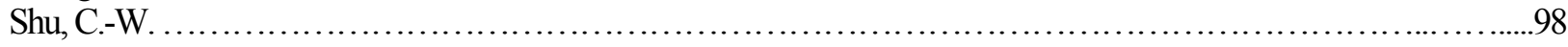

Shvarts, D. ................................................ 30, 41, 61, 83, 112, 115, 123, 124, 133

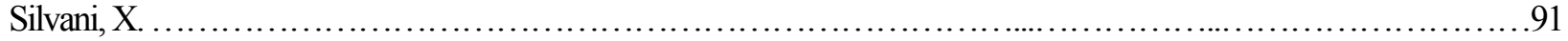

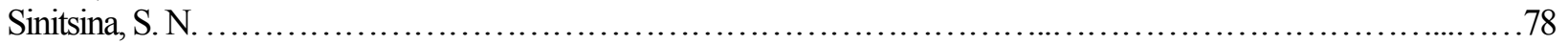

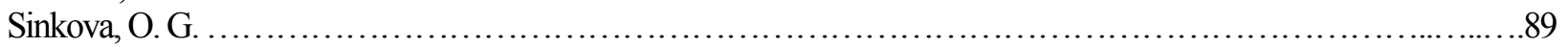

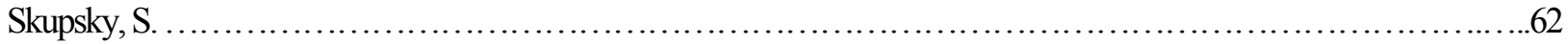

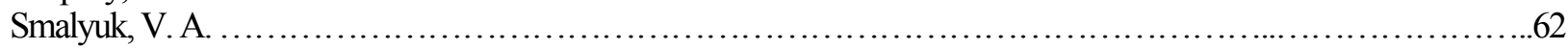

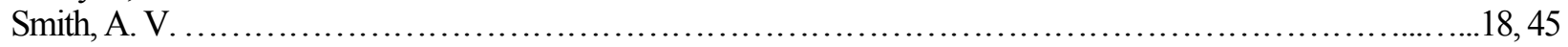

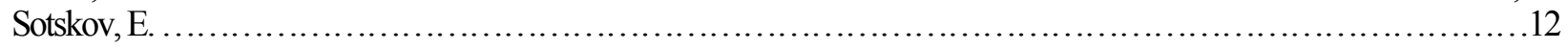

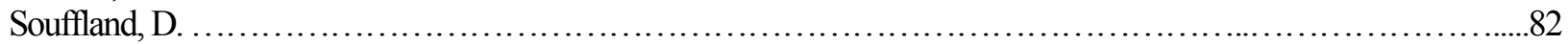

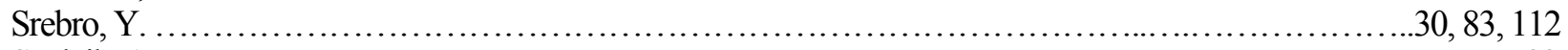

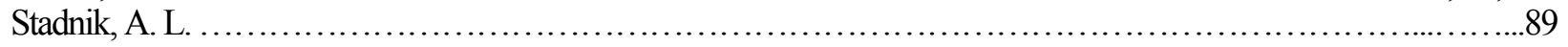

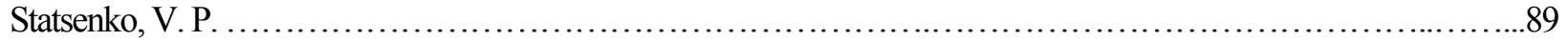

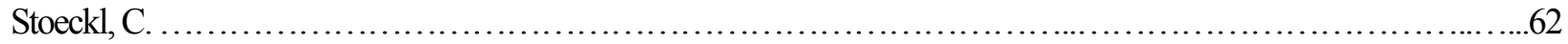

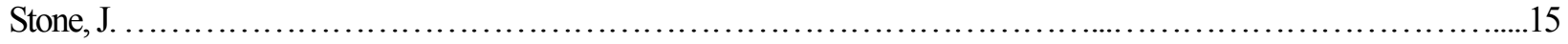

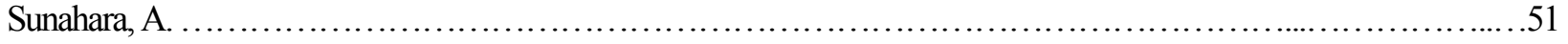




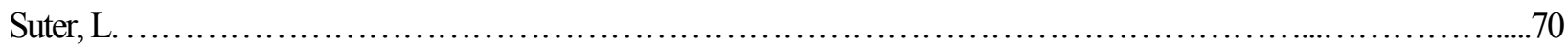

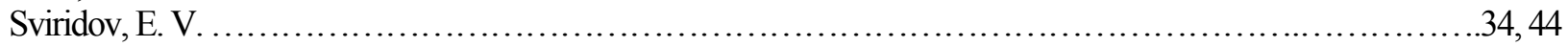

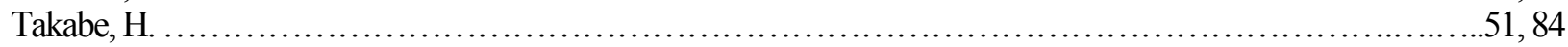

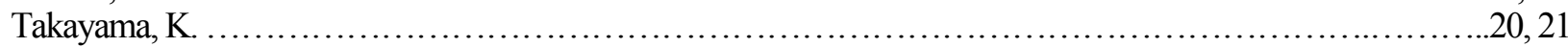

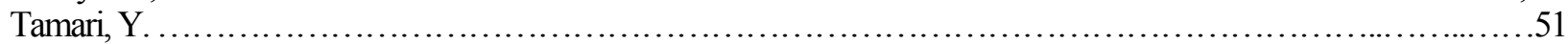

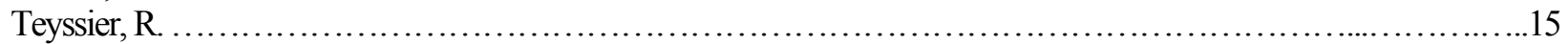

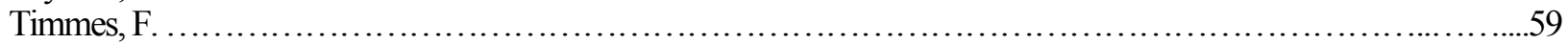

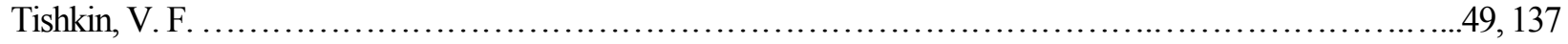

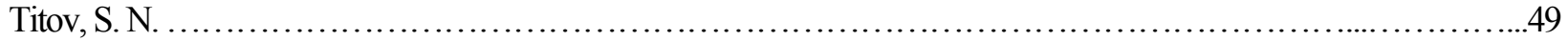

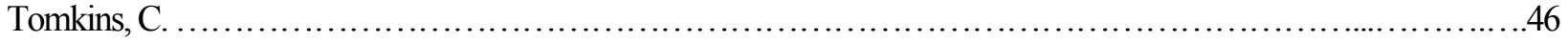

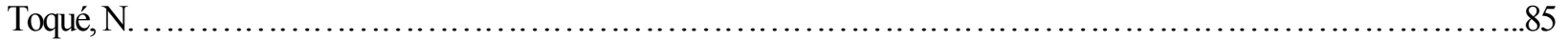

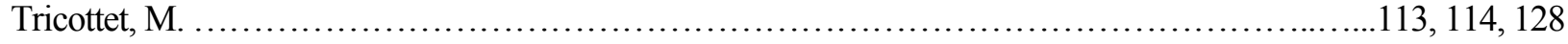

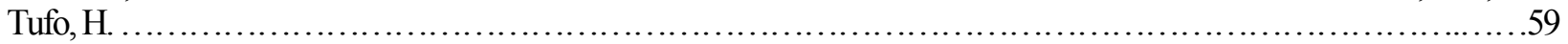

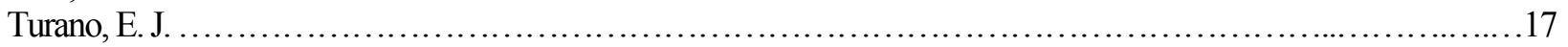

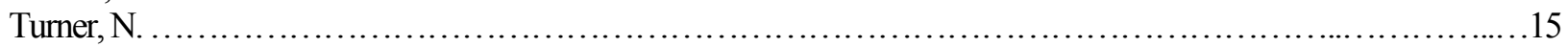

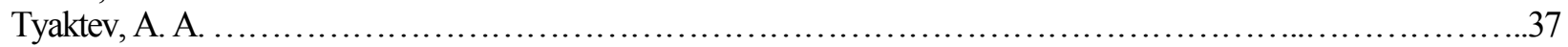

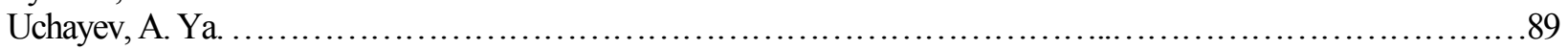

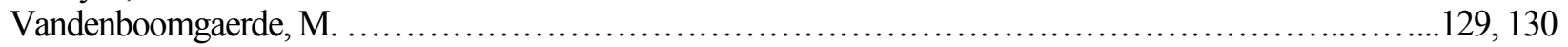

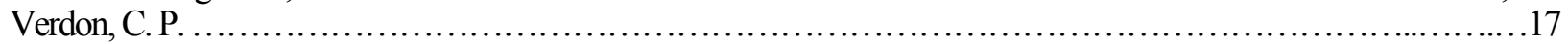

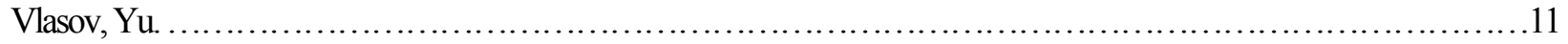

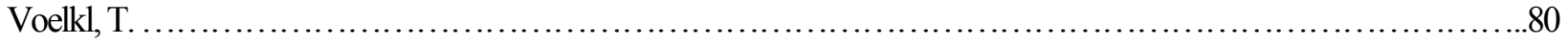

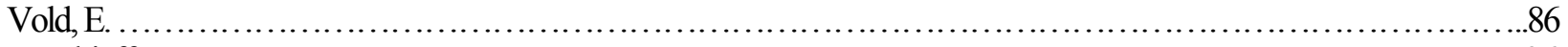

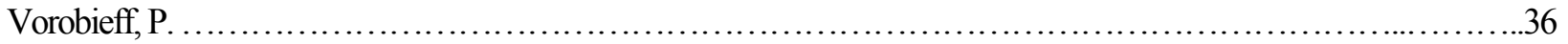

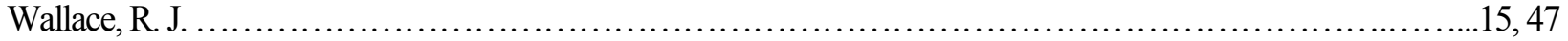

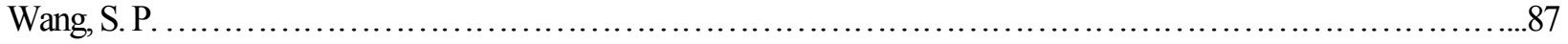

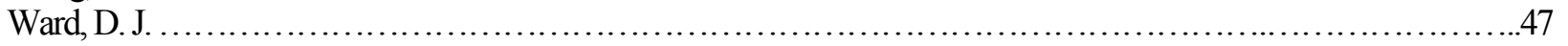

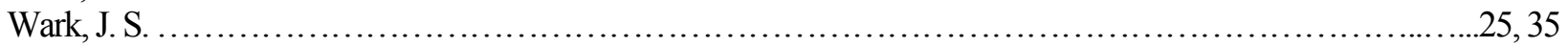

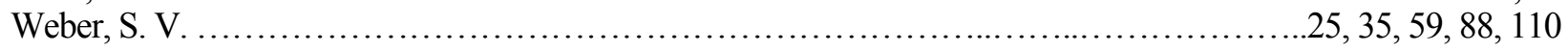

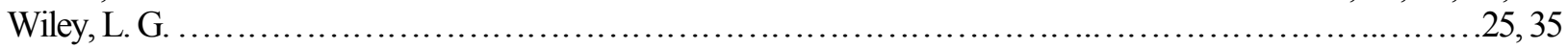

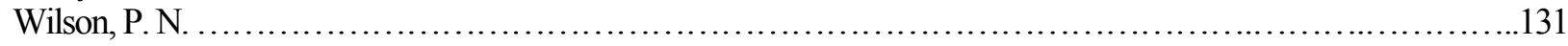

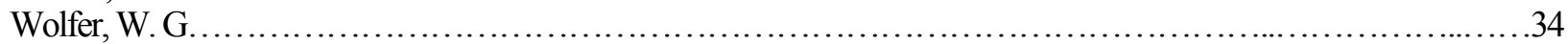

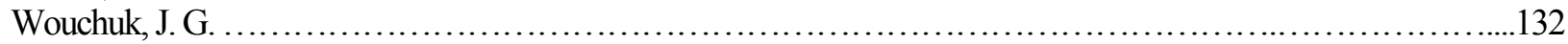

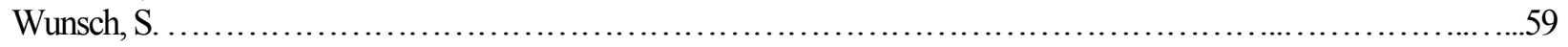

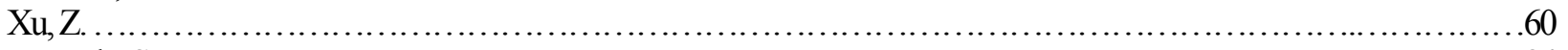

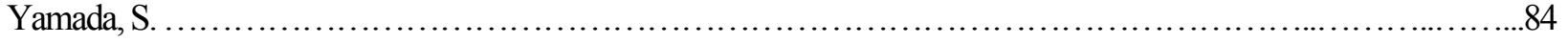

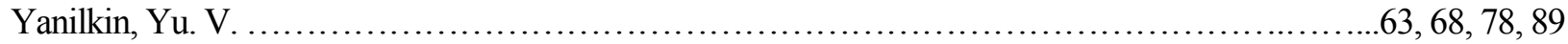

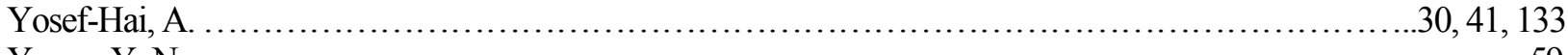

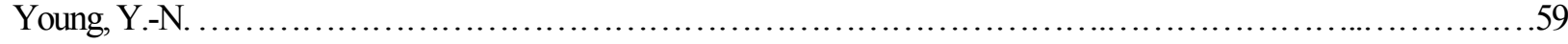

Youngs, D. L. .......................................................... 9, 10, 19, 45, 59, 76, 90, 91

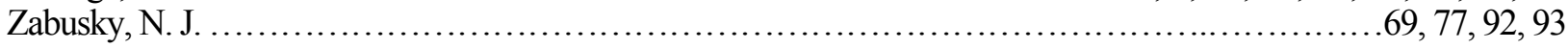

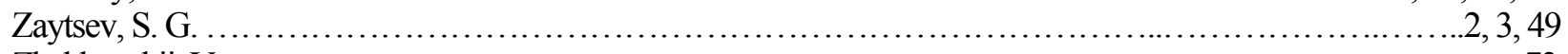

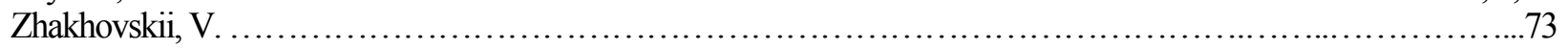

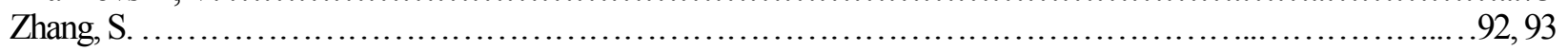

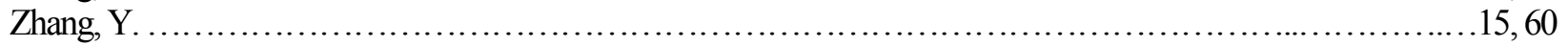

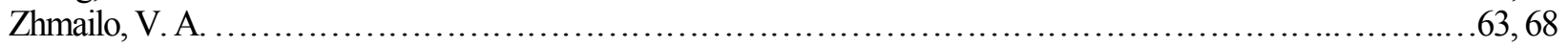

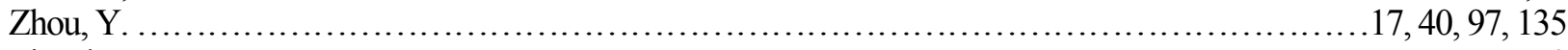

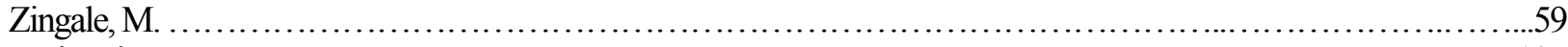

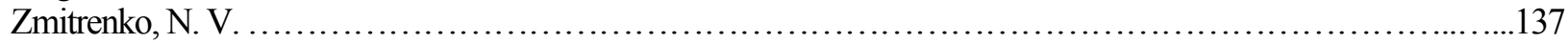

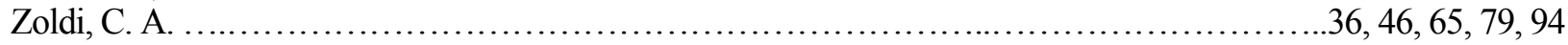

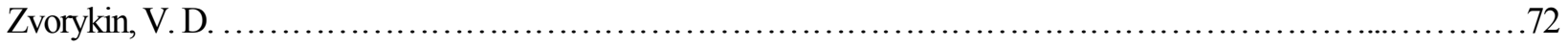


$8^{\text {th }}$ International Workshop on the Physics of Compressible Turbulent Mixing,

Pasadena, CA (2001)

\section{Authors Contact Information}

Abarzhi, Snejana I.

Department of Applied Mathematics

State University of New York at Stony Brook

Stony Brook, NY 11794-3600

Phone: (631) 632-9360

Fax: (631) 632-8490

E-mail: snezha@ams.sunysb.edu

Abe, M.

Institute of Laser Engineering

Osaka University

Suita, Osaka 565 Japan

Afeyan, Bedros

Polymath Research Incorporated

827 Bonde Court

Pleasanton, CA 94566

Phone: (925) 417-0609

Fax: (925) 417-0684

E-mail: bedros@polymath-usa.com

Allen, A. M.

University of Oxford

University Offices

Wellington Square

Oxford OX1 2JD United Kingdom

Phone: +44 1865270000

Fax: $\quad$ +44 1865270708

Alon, Uri

Weizmann Institute of Science

PO Box 26

Rehovot 76100 Israel

Phone: + 972-8-934-2111

Fax: + 972-8-934-4107

E-mail: uri.alon@weizmann.ac.il

Anderson, M. H.

University of Wisconsin at Madison

1500 Engineering Drive

Madison, WI 53706

Phone: (608) 263-2802

Fax: (608) 263-7541

E-mail: manderson@engr.wisc.edu 
Andrews, Malcolm

Department of Mechanical Engineering

Texas A \& M University

MS 3123

College Station, TX 77843-3123

Phone: (409) 847-8843

Fax: (409) 8453081

E-mail: malcolm@chagal.tamu.edu

Anisimov, V. I.

Russian Federal Nuclear Center-VNIITF

P.O. Box 245

456770 Snezhinsk, Russia

E-mail: v.i.anisimov@vniitf.ru

Anuchin, M. G.

Russian Federal Nuclear Center-VNIITF

P.O. Box 245

456770 Snezhinsk, Russia

Arazi, L.

Tel Aviv University

P.O. Box 39040

Tel Aviv 69978 Israel

Ardashova, Rufina I.

Russian Federal Nuclear Center-VNIITF

P.O. Box 245

456770 Snezhinsk, Russia

Arnett, David

University of Arizona

P.O. Box 210096

Tucson, AZ 85721

Phone: (520) 621-9587

E-mail: darnett@as.arizona.edu

Ashurst, William T.

Sandia National Laboratory

P.O. Box 969, MS 9051

Livermore, CA 94551-0969

Phone: (925) 294-2274

Fax: (925) 294-2595

E-mail: ashurs@sandia.gov

Azechi, Hiroshi

Institute of Laser Engineering

Osaka University

Suita, Osaka 565 Japan

Phone: 81-879-8770

Fax: 81-877-4799

E-mail: azechi@ile.osaka-u.ac.jp 
$8^{\text {th }}$ International Workshop on the Physics of Compressible Turbulent Mixing,

Pasadena, CA (2001)

Bailly, P.

Commissariat à l'Energie Atomique

BP 12 - 91680 Bruyères-le-Châtel France

Baishev, A. I.

Russian Federal Nuclear Center-VNIITF

P.O. Box 245

456770 Snezhinsk, Russia

Balabin, Serguei

Russian Federal Nuclear Center-VNIITF

P.O. Box 245

456770 Snezhinsk, Russia

Fax: (351-72) 32077

E-mail: kucherenko@ five.ch70.chel.su

Barnes, Christopher W.

Los Alamos National Laboratory

P.O. Box 1663

Los Alamos, NM 87545

Phone: (505) 665-5687

E-mail: cbarnes@lanl.gov

Barton, C.

Atomic Weapons Establishment

Aldermaston

Reading, Berkshire

RG7 4PR United Kingdom

Phone: +44 (0)1734814111

Fax: $\quad+44(0) 1734815320$

Batha, Steven

Los Alamos National Laboratory

P.O. Box 1663, MS E526

Los Alamos, NM 87545

Phone: (505) 665-5898

Fax: (505) 665-4409

E-mail: sbatha@lanl.gov

Belak, James F.

Lawrence Livermore National Laboratory

P.O. Box 808, L-45

Livermore, CA 94551

Phone: (925) 422-6061

Fax: (925) 422-2851

E-mail: belak1@1lnlg.gov

Belomestnih, A. V.

Russian Federal Nuclear Center-VNIITF

P.O. Box 245

456770 Snezhinsk, Russia 
Ben-Dor, Gabi

Faculty of Engineering Sciences

Ben-Gurion University of the Negev

Beer Sheva, 84105, Israel

Phone: 972-7-6461212

Fax: 972-7-6472936

E-mail: bendor@menix.bgu.ac.il

Benjamin, Robert F.

Los Alamos National Laboratory

P.O. Box 1663, MS P940

Los Alamos, NM 87545

Phone: (505) 667-8116

Fax: (505) 665-3359

E-mail: rfb@lanl.gov

Biello, J.

University of Chicago

933 East $56^{\text {th }}$ Street

Chicago, IL 60637

Phone: (773) 702-1234

Bliznetsov, M. V.

Russian Federal Nuclear Center-VNIIEF

Mir Avenue, 37, Sarov

Nizhnii Novgorod Region, 607190, Russia

Fax: (831-30) 459-58

E-mail: root@gdd.vniief.ru

Boehly, T. R.

Laboratory for Laser Energetics

University of Rochester

250 East River Road

Rochester, New York 14623-1299

Phone: (716) 275-0254

E-mail: trb@1le.rochester.edu

Bonazza, Riccardo

Department of Engineering Physics

University of Wisconsin at Madison

1500 Engineering Drive

Madison, WI 53706

Phone: (608) 265-2337

Fax: (608) 262-6707

E-mail: bonazza@engr.wisc.edu 
$8^{\text {th }}$ International Workshop on the Physics of Compressible Turbulent Mixing,

Pasadena, CA (2001)

Bouquet, Serge

Commissariat à l'Energie Atomique

PDPTA-BP 12

Bruyeres-le-Chatel, France 91680

Phone: +33-1-69-26-51-83

Fax: +33-1-69-26-71-06

E-mail: bouquet@bruyeres.cea.fr

Braun, David G.

Lawrence Livermore National Laboratory

P.O. Box 808, L-31

Livermore, CA 94551

Phone: (925) 423-6275

Fax: (925) 423-9969

E-mail: braun1@1lnl.gov

Breidenthal, Robert

Department of Aeronautics and Astronautics

University of Washington

Box 352400

Seattle, WA 98195-2400

Phone: (206) 685-1098

Fax: (206) 685-0217

E-mail: breidenthal@aa.washington.edu

Brouillette, Martin

Department of Mechanical Engineering

Université de Sherbrooke

Sherbrooke, Quebec J1K 2R1 Canada

Phone: (819) 821-7144

Fax: (819) 821-7163

E-mail: martin.brouillette@gme.usherb.ca

Buckingham, Alfred C.

Lawrence Livermore National Laboratory

P.O. Box 808, L-23

Livermore, CA 94551

Phone: (925) 423-4828

Fax: (925) 422-2644

E-mail: alfredcb@1lnl.gov

Budil, Kimberly S.

Lawrence Livermore National Laboratory

P. O. Box 808, L-97

Livermore, CA 94551

Phone: (925) 423-8098

Fax: (925) 422-0779

E-mail: budil1 @1lnl.gov 
Cabot, William H.

Lawrence Livermore National Laboratory

P.O. Box 808, L-22

Livermore, CA 94551

Phone: (925) 423-9272

Fax: (925) 423-0925

E-mail: cabot1@1lnl.gov

Calder, A. C.

Department of Astronomy and Astrophysics

University of Chicago

5640 South Ellis Ave.

Chicago, IL 60637

Phone: (773) 834-3904

Fax: (773) 834-3230

E-mail: calder@flash.uchicago.edu

Chebotareva, E. I.

Krzhizhanovsky Power Engineering Institute ENIN, Leninsky pr. 19, GSP-1

Moscow, 117927 Russia

Phone: (7-095) 955-31-07

Fax: (7-095) 954-42-50

E-mail: S.G.Zaytsev@mtu-net.ru

Cheng, Baolian

Los Alamos National Laboratory

P.O. Box 1663, MS D413

Los Alamos, NM 87545

Phone: (505) 667-4701

Fax: (505) 667-3726

E-mail: bcheng@lanl.gov

Clark, Timothy $\mathrm{T}$.

Los Alamos National Laboratory

P.O. Box 1663, MS B213

Los Alamos, NM 87545

Phone: (505) 665-4858

Fax: (505) 665-3003

E-mail: ttc@1lnl.gov

Clerouin-Cherfils, Catherine

Commissariat à l'Energie Atomique

CEA/DRIF

BP 12, 91680, Bruyeres-le-Chatel, France

Phone: 33169265738

Fax: $\quad 33169267094$

E-mail: cherfils@bruyeres.cea.fr 
$8^{\text {th }}$ International Workshop on the Physics of Compressible Turbulent Mixing,

Pasadena, CA (2001)

Colvin, Jeffrey. D.

Lawrence Livermore National Laboratory

P.O. Box 808, L-21

Livermore, CA 94551

Phone: (925) 422-3273

Fax: (925) 423-8945

E-mail: colvin5@1lnl.gov

Cook, Andrew W.

Lawrence Livermore National Laboratory

P.O. Box 808, L-22

Livermore, CA 94551

Phone: (925) 423-2856

Fax: (925) 423-0925

E-mail: awcook@1lnl.gov

Dalziel, Stuart

DAMTP, University of Cambridge

Silver Street, Cambridge CB3 9EW, United Kingdom

Phone: (44) (1223) 337911

Fax: (44) (1223) 337918

E-mail: s.dalziel@damtp.cam.ac.uk

Dannenberg, K.

University of Michigan

Space Research Lab, Rm 1216

2245 Hayward

Ann Arbor, MI 48104

Darlington, Rebecca M.

Lawrence Livermore National Laboratory

P.O. Box 808, L-95

Livermore, CA 94551

Phone: (925) 424-3422

E-mail: darlington1@1lnl.gov

Delettrez, J. A.

Laboratory for Laser Energetics

University of Rochester

Rochester, NY 14623-1299

Phone: (716) 275-5374

E-mail: jdel@lle.rochester.edu

Demyanov, A. Yu.

Landau Institute for Theoretical Physics

Kosygnina St. 2, Moscow

117940, GSP-1

V-334 Russia 
Dimits, Andris M.

Lawrence Livermore National Laboratory

P.O. Box 808 , L-630

Livermore, CA 94551

Phone: (925) 422-0211

Fax: (925) 423-3484

E-mail: dimits1@1lnl.gov

Dimonte, Guy

Lawrence Livermore National Laboratory

P.O. Box 808, L-43

Livermore, CA 94551

Phone: (925) 423-0596

Fax: (925) 423-5998

E-mail: dimonte1@1lnl.gov

Dittrich, Thomas R.

Lawrence Livermore National Laboratory

P.O. Box 808, L-23

Livermore, CA 94551

Phone: (925) 422-4706

Fax: (925) 423-8945

E-mail: dittrich1@1lnl.gov

Don, Wai-Sun

Division of Applied Mathematics

Brown University

Box F

Providence, RI 02912

Phone: (401) 863-2250

E-mail: Wai-Sun_Don@brown.edu

Drake, R. Paul

University of Michigan

2245 Hayward

Ann Arbor, MI 48104

Phone: (734) 763-4072

Fax: (734) 647-3083

E-mail: rpdrake@umich.edu

Drennov, O. B.

Russian Federal Nuclear Center-VNIIEF

Mir Avenue, 37, Sarov

Nizhnii Novgorod Region, 607190, Russia

Fax: (831-30) 459-58

E-mail: root@gdd.vniief.ru 
$8^{\text {th }}$ International Workshop on the Physics of Compressible Turbulent Mixing,

Pasadena, CA (2001)

Dudin, V. D.

Russian Federal Nuclear Center-VNIIEF

Mir Avenue, 37, Sarov

Nizhnii Novgorod Region, 607190, Russia

Fax: (831-30) 459-58

E-mail: root@gdd.vniief.ru

Dulov, A. V.

Russian Federal Nuclear Center-VNIITF

P.O. Box 245

456770 Snezhinsk, Russia

Dunne, A. M.

Atomic Weapons Establishment

Aldermaston

Reading, Berkshire

RG7 4PR United Kingdom

Dursi, J. L.

Department of Astronomy and Astrophysics

University of Chicago

5640 South Ellis Ave.

Chicago, IL 60637

Phone: (773) 834-1059

Fax: (773) 834-3230

Dutta, S.

State University of New York at Stony Brook

P-138A Math Tower

Stony Brook, NY 11794-3600

Edwards, Michael J.

Lawrence Livermore National Laboratory

P.O. Box 808, L-21

Livermore, CA 94551

Phone: (925) 422-1189

E-mail: edwards39@1ln1.gov

Elbaz, Y.

Ben-Gurion University

Beer-Sheva Israel 84015

Phone: 972-8-6568836

Fax: $\quad 972-8-6567878$

E-mail: elbazyo@bgumail.bgu.ac.il

Eliason, Donald E.

Lawrence Livermore National Laboratory

P.O. Box 808, L-103

Livermore, CA 94551

Phone: (925) 423-5625

Fax: (925) 422-6388

E-mail: eliason@merlin.llnl.gov 
Epstein, Reuben

Laboratory for Laser Energetics

University of Rochester

Rochester, NY 14623-1299

Phone: (716) 275-5405

E-mail: reps@1le.rochester.edu

Farley, David

Institute of Laser Engineering

Osaka University

Suita, Osaka 565, Japan

E-mail: dfarley@epri.com

Fryxell, Bruce

Enrico Fermi Institute and Laboratory for Astrophysics and

Space Research

University of Chicago

933 East $56^{\text {th }}$ Street

Chicago, IL 60637

Phone: (773) 704-3226

Fax: (773) 704-3230

E-mail: fryxell@uffda.asci.uchicago.edu

Fujioka, S.

Institute of Laser Engineering,

2-6, Yamada-oka, Suita,

Osaka 565-0871 Japan

Fukuda, Y.

Institute of Laser Engineering, 2-6, Yamada-oka, Suita,

Osaka 565-0871 Japan

Galmiche, Didier Jean Marie

Commissariat à l'Energie Atomique

BP12 91680 Bruyeres-le-Chatel, France

Phone: 33169264005

Fax: $\quad 33169267094$

E-mail: galmiche@bruyeres.cea.fr

Garasi, C.

Sandia National Laboratory

P.O. Box 969, 0819

Livermore, CA 94551

Phone: (505) 284-2691

Fax: (505) 844-0918

E-mail: cjgaras@sandia.gov

Gauthier, Serge

Centre d'Etudes de Limeil-Valenton

BP 27 Villeneuve St. Georges

94195, France

E-mail: gauthier@limeil.cea.fr 
$8^{\text {th }}$ International Workshop on the Physics of Compressible Turbulent Mixing,

Pasadena, CA (2001)

Gavrilova, E. S.

Russian Federal Nuclear Center-VNIIEF

Mir Avenue, 37, Sarov

Nizhnii Novgorod Region, 607190, Russia

Fax: (831-30) 459-58

E-mail: root@gdd.vniief.ru

George, E.

State University of New York at Stony Brook

P-138A Math Tower

Stony Brook, NY 11794-3600

Gerasimov, Serguei

Russian Federal Nuclear Center-VNIIEF

Mir Avenue, 37, Sarov

Nizhnii Novgorod Region, 607190, Russia

Phone: (7-83130) 45009

Fax: (7-83130) 45958

E-mail: root@gdd.vniief.ru

Glebov, V. Yu.

Laboratory for Laser Energetics

University of Rochester

250 East River Road

Rochester, NY 14623-1299

Phone: (716) 275-7454

E-mail:vgle@1le.rochester.edu

Glendinning, Sharon G.

Lawrence Livermore National Laboratory

P.O. Box 808, L-21

Livermore, CA 94551

Phone: (925) 423-3065

Fax: (925) 423-8945

E-mail: glendinning1@1lnl.gov

Glimm, James

Department of Applied Mathematics and Statistics

State University of New York at Stony Brook

P-138A Math Tower

Stony Brook, NY 11794-3600

Phone: (631) 632-8355

Fax: (631) 632-8490

E-mail: glimm@ams.sunysb.edu

Goncharov, V. N.

Laboratory for Laser Energetics

University of Rochester

250 East River Road

Rochester, NY 14623-1299

Phone: (716) 275-1017

E-mail: vgon@1le.rochester.edu 
Goodwin, Bruce T.

Lawrence Livermore National Laboratory

P.O. Box 808 , L-160

Livermore, CA 94551

Phone: (925) 423-7736

Fax: (925) 424-2723

E-mail: goodwin2@1lnl.gov

Gottlieb, David I.

Division of Applied Mathematics

Brown University

Providence, RI 02912

Phone: (401) 863-2266

E-mail: David.Gottlieb@brown.edu

Graham, Mary J.

Lawrence Livermore National Laboratory

P.O. Box 808 , L-312

Livermore, CA 94551

Phone: (925) 424-4222

Fax: (925) 422-8920

E-mail: mjgraham@1lnl.gov

Greenough, Jeffrey A.

Lawrence Livermore National Laboratory

P.O. Box 808, L-30

Livermore, CA 94551

Phone: (925) 423-3211

Fax: (925) 424-6764

E-mail: greenough1@1lnl.gov

Grieves, Brian

Atomic Weapons Establishment

Aldermaston

Reading, Berkshire

RG7 4PR United Kingdom

Phone: +44 (0)1734 814111

Fax: $\quad+44(0) 1734815320$

E-mail: brian.grieves@awe.co.uk

Grove, John W.

Los Alamos National Laboratory

P.O. Box 1663, MS D413

Los Alamos, NM 87545

Phone: (505) 667-0723

Fax: (505) 665-4972

E-mail: jgrove@lanl.gov 
$8^{\text {th }}$ International Workshop on the Physics of Compressible Turbulent Mixing,

Pasadena, CA (2001)

Gubkov, E. V.

Russian Federal Nuclear Center-VNIIEF

Mir Avenue, 37, Sarov

Nizhnii Novgorod Region, 607190, Russia

Phone: (831-30) 45009

Fax: (831-30) 45958

E-mail: root@gdd.vniief.ru

Gulak, Y.

Department of Mechanical and Aerospace Engineering

Rutgers University

98 Brett Road

Piscataway, NJ 08854

Phone: (732) 445-5627

Fax: (732) 445-3124

E-mail:ygulak@rci.rutgers.edu

Gupta, S.

Department of Mechanical and Aerospace Engineering

Rutgers University

98 Brett Road

Piscataway, NJ 08854

Phone: (732) 445-5627

Fax: (732) 445-3124

Haan, Steven W.

Lawrence Livermore National Laboratory

P.O. Box 808, L-23

Livermore, CA 94551

Phone: (925) 422-4715

Fax: (925) 423-8945

E-mail: haan1@1lnl.gov

Haas, Jean Francois

Commissariat à l'Energie Atomique

CEA/DIF/DCRE/SCSE BP 12

Bruyeres le Chatel, 91680, France

Phone: 33-1-69-26-52-94

Fax: 33-1-69-26-70-62

E-mail: jean-francois.haas@cea.fr

Harlow, Francis H.

Los Alamos National Laboratory

P.O. Box 1663, MS B216

Los Alamos, NM 87545

Phone: (505) 667-9090

E-mail: fhharlow@lanl.gov 
Hatchett, Stephen P.

Lawrence Livermore National Laboratory

P.O. Box 808, L-16

Livermore, CA 94551

Phone: (925) 422-5916

Fax: (925) 423-5112

E-mail: hatchett1@1lnl.gov

Hauer, A.

Los Alamos National Laboratory

P.O. Box 1663

Los Alamos, NM 87545

Phone: (505) 667-5167

Fax: (505) 667-0405

E-mail: hauer@lanl.gov

Hebert, C. H.

Department of Mechanical Engineering

Universite de Sherbrooke

Sherbrooke, Quebec J1K 2R1 Canada

Hinkel, Denise

Lawrence Livermore National Laboratory

P.O. Box 808, L-38

Livermore, CA 94551

Phone: (925) 423-2626

Fax: (925) 423-2157

E-mail: hinkel1@1lnl.gov

Holder, David

AWE

Building H27, Aldermaston

Reading, Berkshire, RG7 4PR, United Kingdom

Phone: 01189825592

Fax: 01189824816

E-mail: david.holder@awe.co.uk

Holford, Joanne M.

University of Cambridge

Silver Street

Cambridge, United Kingdom CB3 9EW

Phone: +44 1223337858

Fax: $\quad$ +44 1223337918

E-mail: J.M.Holford@damtp.cam.ac.uk

Holmes, Richard L.

Los Alamos National Laboratory

P.O. Box 1663, MS B220

Los Alamos, NM 87545

Phone: (505) 667-3598

Fax: (505) 665-2227

E-mail: holmes@lanl.gov 
$8^{\text {th }}$ International Workshop on the Physics of Compressible Turbulent Mixing,

Pasadena, CA (2001)

Hosseini, S. H. R.

Shock Wave Research Center, Institute of Fluid Science

Tohoku University

2-1-1 Katahira, Aoba-ku

Sendai 980-77, Japan

E-mail: hosseini@ rainbow.ifs.tohoku.ac.jp

Houas, Lazhar

I.U.S.T.I. / Umr CNRS 6595

Technopole de Chateau Gombert

5 rue Enrico Fermi

13453 Marseille 13 France

Phone: $33(0) 491106930$

Fax: $\quad 33(0) 491106969$

E-mail: houas@iusti.univ-mrs.fr

Hsing, Warren W.

Lawrence Livermore National Laboratory

P.O. Box 808, L-21

Livermore, CA 94551

Phone: (925) 423-2849

Fax: (925) 423-8945

E-mail: hsing@1lnl.gov

Hurricane, Omar

Lawrence Livermore National Laboratory

P.O. Box 808, L-22

Livermore, CA 94551

Phone: (925) 424-2701

Fax: (925) 423-0925

E-mail: hurricane1@1lnl.gov

Inogamov, Nail

Landau Institute for Theoretical Physics

Kosygnina St. 2, Moscow

117940, GSP-1

V-334 Russia

Phone: 0954258767

Fax: 0959382077

E-mail: itf@ips.ac.msk.su

Jacobs, Jeffrey W.

Department of Aerospace and Mechanical Engineering

University of Arizona

Bldg. 16, Room 301

Tucson, AZ 85721

Phone: (602) 621-8459

Fax: (602) 621-8191

E-mail: jacobs@ame.arizona.edu 
Jameson, Leland M.

Lawrence Livermore National Laboratory

P.O. Box 808, L-39

Livermore, CA 94551

Phone: (925) 424-6160

E-mail: jameson3@1lnl.gov

Jones, Oggden

Lawrence Livermore National Laboratory

P.O. Box 808, L-30

Livermore, CA 94551

Phone: (925) 423-1872

Fax: (925) 423-9969

E-mail: jones96@1lnl.gov

Jourdan, Georges

Universite de Provence

IUSTI-CNRS Umr 139

Centre Saint Jerome - Case 321

13397 Marseille Cedex 20 France

Phone: 33 (0) 491106930

Fax: 33 (0) 491106969

E-mail: jourdan@iusti-dipha.univ-mrs.fr

Kalantar, Daniel

Lawrence Livermore National Laboratory

P.O. Box 808, L-472

Livermore, CA 94551

Phone: (925) 422-6147

Fax: (925) 422-8395

E-mail: kalantar1@1lnl.gov

Kamm, R. J.

Los Alamos National Laboratory

P.O. Box 1663, MS

Los Alamos, NM 87545

Phone: (505) 667-1918

E-mail:kammj@lanl.gov

Kane, Jave O.

Lawrence Livermore National Laboratory

P.O. Box 808, L-411

Livermore, CA 94551

Phone: (925) 424-5805

Fax: (925) 423-2463

E-mail: jave@1lnl.gov 
$8^{\text {th }}$ International Workshop on the Physics of Compressible Turbulent Mixing,

Pasadena, CA (2001)

Kang, Young-Gwang

Kwangju Institute of Science and Technology

1 Oryong-dong

Kwangju, 500-712, South Korea

Phone: 82-62-970-2310

Fax: 82-62-970-2304

E-mail: ygkang@kjist.ac.kr

Kartoon, Daniela

Department of Physics

Nuclear Research Center Negev

P.O. Box 9001

Beer-Sheva, 84190, Israel

Phone: 972-8-6568845

Fax: $\quad 972-8-6567878$

E-mail:danyk@bgumail.bgu.ac.il

Keiter, P.

University of Michigan

Space Research Lab, Rm 1216

2245 Hayward

Ann Arbor, MI 48104

Kerstein, Alan R.

Sandia National Laboratory

P.O. Box 969, MS 9051

Livermore, CA 94551-0969

Phone: (925) 294-2390

Fax: (925) 294-1004

E-mail: kerstein@ca.sandia.gov

Kim, H.

Department of Mechanical and Aerospace Engineering

Rutgers University

98 Brett Road

Piscataway, NJ 08854

Klein, Richard I.

Lawrence Livermore National Laboratory

P.O. Box 808, L-23

Livermore, CA 94551

Phone: (925) 422-3548

Fax: (925) 423-0925

E-mail: rklein@1lnl.gov

Knauer, J.

Department of Mechanical and Aerospace Engineering

Rutgers University

98 Brett Road

Piscataway, NJ 08854 
Kobayashi, K.

Osaka University

2-6, Yamada-oka, Suita,

Osaka 565-0871 Japan

Korreck, K. E.

University of Michigan

Space Research Lab, Rm 1216

2245 Hayward

Ann Arbor, MI 48104

Kozelkov, O. E.

Russian Federal Nuclear Center-VNIITF

P.O. Box 245

456770 Snezhinsk, Russia

Kozlov, V. I.

Russian Federal Nuclear Center-VNIIEF

Mir Avenue, 37, Sarov

Nizhnii Novgorod Region, 607190, Russia

Fax: $\quad$ (831-30) 4-57-72

E-mail: otd1_0903@spd.vniief.ru

Ktitorov, Vladimir M.

Russian Federal Nuclear Center-VNIIEF

Mir Avenue, 37, Sarov

Nizhnii Novgorod Region, 607190 Russia

Phone: 831-30 1-36-65

Fax: 831-30 5-45-65

E-mail: mvn_072e@rfnc.nnov.su

Kucherenko, Yuri A.

Russian Federal Nuclear Center-VNIITF

P.O. Box 245

456770 Snezhinsk, Russia

Fax: (351-72) 32077

E-mail: kucherenko@five.ch70.chel.su

Kuhl, Allen L.

Lawrence Livermore National Laboratory

P.O. Box 808, L-30

Livermore, CA 94551

Phone: (925) 422-4777

Fax: (925) 424-6764

E-mail:kuhl2@1lnl.gov

Kumar, M.

Department of Physics

Nuclear Research Center Negev

P.O. Box 9001

Beer-Sheva, 84190, Israel 
$8^{\text {th }}$ International Workshop on the Physics of Compressible Turbulent Mixing,

Pasadena, CA (2001)

Kushnir, D.

Department of Physics

Nuclear Research Center Negev

P.O. Box 9001

Beer-Sheva, 84190, Israel

Lanier, N. E.

Los Alamos National Laboratory

P.O. Box 1663, MS

Los Alamos NM, 87545

Phone: (505) 665-0236

Fax: (505) 665-3686

E-mail: nlanier@lanl.gov

Lasinski, Barbara F.

Lawrence Livermore National Laboratory

P.O. Box 808, L-38

Livermore, CA 94551

Phone: (925) 422-5443

Fax: (925) 423-9208

E-mail: blasinski@1lnl.gov

Lassis, A.

Commissariat à l'Energie Atomique

BP 12

Bruyeres le Chatel, 91680, France

Layes, G.

IUSTI

Technopole Chateau-Gombert

5 Rue Enrico Fermi

Marseille, 13013 France

Lebedev, Alexander

Russian Federal Nuclear Center-VNIIEF

Mir Avenue, 37, Sarov

Nizhnii Novgorod Region, 607190, Russia

Phone: (831-30) 45009

Fax: $\quad(831-30) 45958$

E-mail: root@gdd.vniief.ru

Lebo, Ivan

Laser Fusion Department

PN Lebedev Physics Institute

Leninsky prospect 53

117294 Russia, Moscow

Phone: 095-132-68-47

Fax: 095-132-11-96

E-mail: lebo@sci.fian.msk.su 
Legrand, Michel

Commissariat à l'Energie Atomique

DAM Ile de France

BP12, Bruyeres le Chatel, 91680, France

Phone: 33-1-69-26-57-09

Fax: 33-1-69-26-70-94

E-mail: michel.legrand@cea.fr

Levy, Kedem

Physics Department

Ben Gurion University of the Negev

P.O. B 653, Beer-Sheva 84105, Israel

Phone: 972-8-6568844

Fax: $\quad 972-8-6567878$

E-Mail: klevy@bgumail.bgu.ac.il

Li, Xiaolin

Department of Applied Mathematics and Statistics

State University of New York at Stony Brook

1-119 Math Tower

Stony Brook, NY 11794-3600

Phone: (631) 632-8353

Fax: (631) 632-8490

E-mail:1inli@ams.sunysb.edu

Linn, Rodman R.

Los Alamos National Laboratory

P.O. Box 1663, MS

Los Alamos, NM 87545

Phone: (505) 665-6254

E-mail:rrl@lanl.gov

Llor, Antoine

Commissariat à l'Energie Atomique

BP 12

Bruyeres le Chatel, 91680, France

Phone: 33169264938

Fax: $\quad 33169267097$

E-mail: antoine.llor@cea.fr

Logvinov, A.

Russian Federal Nuclear Center-VNIIEF

Mir Avenue, 37, Sarov

Nizhnii Novgorod Region, 607190, Russia

E-mail: root@gdd.vniief.ru

Lorenz, Karl T.

Lawrence Livermore National Laboratory

P.O. Box 808, L-472

Livermore, CA 94551

Phone: (925) 424-4200

E-mail: lorenz3@1lnl.gov 
$8^{\text {th }}$ International Workshop on the Physics of Compressible Turbulent Mixing,

Pasadena, CA (2001)

Louis, Hedley

Lawrence Livermore National Laboratory

P.O. Box 808 , L-472

Livermore, CA 94551

Phone: (925) 4242-9937

E-mail: louis1@1lnl.gov

Loveridge-Smith, A.

Oxford University

University Offices

Wellington, Oxford, OX1 2JD United Kingdom

Phone: +44 1865270000

Fax: +44 1865270708

MacNeice, $P$.

NASA Goddard Space Flight Center

Greenbelt, MD

Magelssen, Glenn R.

Los Alamos National Laboratory

P.O. Box 1663, MS

Los Alamos, NM 87545

Phone: (505) 667-6519

E-mail: grm@lanl.gov

Magnaudet, J.

Institut de Mécanique des Fluides de Toulouse 31400 Toulouse

Toulouse, France

Marchese, A.

State University of New York at Stony Brook

P-138A Math Tower

Stony Brook, NY 11794-3600

Marinak, Michael M.

Lawrence Livermore National Laboratory

P.O. Box 808, L-23

Livermore, CA 94551

Phone: (925) 423-8458

Fax: (925) 423-9969

E-mail: marinak1@1lnl.gov

Matsuoka, C.

10-13,Dogo-Himata, Matsuyama 790-8577

Japan

Mazilin, I. M.

Krzhizhanovsky Power Engineering Institute ENIN, Leninsky pr. 19, GSP-1

Moscow, 117927 Russia 
McAbee, Thomas L.

Lawrence Livermore National Laboratory

P.O. Box 808 , L-170

Livermore, CA 94551

Phone: (925) 422-3398

Fax: (925) 424-2723

E-mail: mcabee@viper.llnl.gov

McCray, R.

University of Colorado at Boulder

Boulder, CO 80309

Phone: (303) 492-1411

McKenty, P. W.

Laboratory for Laser Energetics

University of Rochester

250 East River Road

Rochester, NY 14623-1299

Medvedev, V. M.

Russian Federal Nuclear Center-VNIITF

P.O. Box 245

456770 Snezhinsk, Russia

Meiron, Daniel I.

Department of Applied and Computational Mathematics

California Institute of Technology

MC 217-50

Pasadena, CA 91125

Phone: (626) 395-4563

Fax: (626) 578-0124

E-mail:dim@acm.caltech.edu

Meshkov, Evgeni E.

Russian Federal Nuclear Center-VNIIEF

Mir Avenue, 37, Sarov

Nizhny Novgorod Region, 607190, Russia

Fax: 831-305-4565

E-mail: root@gdd.vniief.ru

Meyers, M. A.

University of California at San Diego

9500 Gilman Drive

La Jolla, CA 92093-0411

Mikaelian, Karnig O.

Lawrence Livermore National Laboratory

P.O. Box 808, L-97

Livermore, CA 94551

Phone: (925) 422-5449

Fax: (925) 423-7228 
$8^{\text {th }}$ International Workshop on the Physics of Compressible Turbulent Mixing,

Pasadena, CA (2001)

Mikhaylov, A. L.

Russian Federal Nuclear Center-VNIIEF

Mir Avenue, 37, Sarov

Nizhnii Novgorod Region, 607190, Russia

E-mail: root@gdd.vniief.ru

Miles, Aaron

Lawrence Livermore National Laboratory

P.O. Box 808, L-12

Livermore, CA 94551

Phone: (925) 423-8131

Fax: (925) 422-8920

E-Mail: miles15@1lnl.gov

Miller, Paul L.

Lawrence Livermore National Laboratory

P.O. Box 808, L-22

Livermore, CA 94551

Phone: (925) 423-6455

Fax: (925) 423-0925

E-mail: pmiller@1lnl.gov

Minich, Roger

Lawrence Livermore National Laboratory

P. O. Box 808 , L-97

Livermore, CA 94551

Phone: (925) 422-4552

Fax: (925) 422-0779

E-mail: minich1@1lnl.gov

Miyanaga, N.

Osaka University

2-6, Yamada-oka, Suita,

Osaka 565-0871 Japan

Mizuta, A.

Osaka University

2-6, Yamada-oka, Suita,

Osaka 565-0871 Japan

Montlaurent, P.

Commissariat à l'Energie Atomique

BP 12, 91680

Bruyeres-le-Chatel, France

Moreno, Juan

Lawrence Livermore National Laboratory

P. O. Box 808 , L-31

Livermore, CA 94551

Phone: (925) 423-4170

Fax: (925) 424-6764

E-mail: jcmoreno@1lnl.gov 
Morris, A. P.

AWE Aldermaston

Reading

Berkshire RG7 4PR United Kingdom

Munro, David H.

Lawrence Livermore National Laboratory

P. O. Box 808 , L-23

Livermore, CA 94551

Phone: (925) 422-5428

Fax: (925) 423-9969

E-mail:dmunro@llnl.gov

Murphy, T. J.

Los Alamos National Laboratory

P.O. Box 1663, MS

Los Alamos, NM 87545

Phone: (505) 665-5697

Fax: (505) 996-4366

E-mail: tjmurphy@lanl.gov

Murzakov, V. D.

Russian Federal Nuclear Center-VNIITF

P.O. Box 245

456770 Snezhinsk, Russia

Nagatomo, $\mathrm{H}$.

Osaka University

2-6, Yamada-oka, Suita,

Osaka 565-0871 Japan

Nakai, M.

2-6, Yamada-oka, Suita,

Osaka 565-0871 Japan

Nash, Jeffrey K.

Lawrence Livermore National Laboratory

P. O. Box 808, L-15

Livermore, CA 94551

Phone: (925) 422-7255

E-mail: jknash@1lnl.gov

Neuvazhayev, Vladimir

Russian Federal Nuclear Center -VNIITF

P.O. Box 245

456770 Snezhinsk, Russia

Phone: 351-72-55675

Fax: $351-72-32077$

E-mail:nio3@ch70.chel.su 
$8^{\text {th }}$ International Workshop on the Physics of Compressible Turbulent Mixing,

Pasadena, CA (2001)

Nevmerzhitsky, Nikolay

Russian Federal Nuclear Center-VNIIEF

Mir Avenue, 37, Sarov

Nizhnii Novgorod Region, 607190, Russia

Phone: (831-30) 45009

Fax: $\quad(831-30) 45958$

E-mail: root@gdd.vniief.ru

Niederhaus, Charles E.

NASA Glenn Research Center

21000 Brookpark Road

MS-77-5

Cleveland, $\mathrm{OH} 44135$

Phone: (216) 433-5461

Fax: (216) 433-8050

E-mail: charles.niederhaus@grc.nasa.gov

Nikishin, Vladislav

Institute for Mathematical Modeling

Russian Academy of Science

Miusskaya 4a, 125047, Moscow, Russia

Phone: 007(095) 250-79-35

Fax: 007(095) 972-07-23

E-mail: nikishin\\#4@imamod.msk.su

Nikulin, A.

Russian Federal Nuclear Center-VNIIEF

Mir Avenue, 37, Sarov

Nizhnii Novgorod Region, 607190, Russia

E-mail: root@gdd.vniief.ru

Nishihara, Katsunobu

Osaka University, Institute of Laser Engineering,

2-6, Yamada-oka, Suita,

Osaka 565-0871 Japan

Phone: 81-6-6879-8725

Fax: 81-6-6877-4799

E-mail: nishihara@ile.osaka-u.ac.jp

Nishikino, M.

2-6, Yamada-oka, Suita,

Osaka 565-0871 Japan

Nizovtsev, P. N.

Russian Federal Nuclear Center-VNIIEF

Mir Avenue, 37, Sarov

Nizhnii Novgorod Region, 607190, Russia

E-mail: root@gdd.vniief.ru

Nomoto, K.

University of Tokyo

7-3-1 Hongo, Bunkyo-ku, Tokyo 113-8654, Japan

Phone: +81-3-3812-2111 
Oakley, J. G.

Department of Engineering Physics

University of Wisconsin at Madison 1500 Engineering Drive

Madison, WI 53706

Olson, K.

NASA Goddard Space Flight Center

Greenbelt, MD

Oparin, A. M.

Institute for Computer Aided Design

Vtoraya Brestskaya ul., 19/18, 123056

Moscow, Russia

Phone: (095) 250-96-30

Fax: (095) 250-89-28

E-mail: oparin@cpd.landau.ac.ru

Oron, Dan

Department of Physics

Nuclear Research Center - Negev

P.O. Box 9001

Beer-Sheva, 84190, Israel

Phone: 972-7-6568736

Fax: $\quad 972-7-6567878$

E-mail: danor@bgumail.bgu.ac.il

Paisley, Dennis

Los Alamos National Laboratory

P.O. Box 1663, MS

Los Alamos, NM 87545

Phone: (505) 667-7837

E-mail: dxp@lanl.gov

Pantano, C.

University of California at San Diego

9500 Gilman Drive

La Jolla, CA 92093-0411

Parker, Kenneth W.

AWE Aldermaston

Reading

Berkshire RG7 4PR United Kingdom

Phone: +44 1189827578

Fax: $\quad$ +44 1189824844

E-mail: Kenneth.W.Parker@awe.co.uk

Parshukov, I. E.

Russian Federal Nuclear Center-VNIITF

P.O. Box 245

456770 Snezhinsk, Russia 
$8^{\text {th }}$ International Workshop on the Physics of Compressible Turbulent Mixing,

Pasadena, CA (2001)

Peng, O.

Rutgers University

98 Brett Road

Piscataway, NJ 08854

Perry, Theodore S.

Lawrence Livermore National Laboratory

P.O. Box 808, L-23

Livermore, CA 94551

Phone: (925) 423-2065

Fax: (925) 424-3294

E-mail: tedperry@1lnl.gov

Peyser, Thomas A.

Lawrence Livermore National Laboratory

P.O. Box 808, L-23

Livermore, CA 94551

Phone: (925) 423-6454

Fax: (925) 423-0925

E-mail: tpeyser@1lnl.gov

Piskunov, Yu. A.

Russian Federal Nuclear Center-VNIITF

P.O. Box 245

456770 Snezhinsk, Russia

Polionov, Arkadi

Russian Federal Nuclear Center-VNIITF

P.O. Box 245

456770 Snezhinsk, Russia

Phone: 351-722-3977

Fax: 351-723-2077

E-mail:nio3@ch70.chel.su

Pollaine, Steven

Lawrence Livermore National Laboratory

P.O. Box 808, L-30

Livermore, CA 94551

Phone: (925) 422-5950

Fax: (925) 423-9208

E-mail: pollaine@1lnl.gov

Popov, V. N.

Russian Federal Nuclear Center-VNIITF

P.O. Box 245

456770 Snezhinsk, Russia

Pound, $\mathrm{M}$.

University of Maryland

College Park, MD 20742

Phone: (301) 405-1000 
Prestridge, Katherine P.

Los Alamos National Laboratory

P.O. Box 1663, MS P940

Los Alamos, NM 87545

Phone: (505) 667-8861

Fax: (505) 665-3359

E-mail: kpp@lanl.gov

Pullin, Dale I.

Department of Aeronautics

California Institute of Technology

1200 East California Boulevard, MS 105-50

Pasadena, CA 91125

Phone: (626) 395-6081

Fax: (626) 441-2222

E-mail: dale@galcit.caltech.edu

Pylaev, Anatoly

Russian Federal Nuclear Center-VNIITF

P.O. Box 245

456770 Snezhinsk, Russia

Fax: (351-72) 32077

E-mail: kucherenko@five.ch70.chel.su

Radha, P. B.

Laboratory for Laser Energetics

University of Rochester

Rochester, NY 14623-1299

Raevskii, Viktor

Russian Federal Nuclear Center-VNIIEF

Mir Avenue, 37, Sarov

Nizhny Novgorod Region, 607190, Russia

Fax: 831-305-4565

E-mail: otd3_2305@rfnc.nnov.su

Rampaprabhu, P.

Department of Mechanical Engineering

Texas A \& M University

MS 3123

College Station, TX 77843-3123

Raviard, P. A.

Commissariat à l'Energie Atomique

BP 12,91680

Bruyeres-le-Chatel, France

Rayer, C.

Commissariat à l'Energie Atomique

BP 12, 91680

Bruyeres-le-Chatel, France 
$8^{\text {th }}$ International Workshop on the Physics of Compressible Turbulent Mixing,

Pasadena, CA (2001)

Razin, A. N.

Russian Federal Nuclear Center-VNIIEF

Mir Avenue, 37, Sarov

Nizhnii Novgorod Region, 607190, Russia

E-mail: root@gdd.vniief.ru

Rebrov, S. V.

Mir Avenue, 37, Sarov

Nizhnii Novgorod Region, 607190, Russia

E-mail: root@gdd.vniief.ru

Remington, Bruce A.

Lawrence Livermore National Laboratory

P.O. Box 808, L-21

Livermore, CA 94551

Phone: (925) 423-2712

Fax: (925) 422-8395

E-mail: remington2@1lnl.gov

Renaud, Francois

Commissariat à l'Energie Atomique

CEA/DRIF/DCSA/SET

BP 12, 91680

Bruyeres-le-Chatel, France

Phone: 33169264081

Fax: 33169267102

Ricker, P.

University of Chicago

933 East $56^{\text {th }}$ Street

Chicago, IL 60637

Rider, William J.

Los Alamos National Laboratory

P.O. Box 1663, MS D413

Los Alamos, NM 87545

Phone: (505) 665-4162

Fax: (505) 667-3726

E-mail: wjr@lanl.gov

Rightley, Paul M.

Los Alamos National Laboratory

P.O. Box 1663, MS P940

Los Alamos, NM 87545

Phone: (505) 667-0460

Fax: (505) 665-3359

E-mail:pright@lanl.gov 
Rikanati, Avi

Physics Department

Nuclear Research Center - Negev

P.O. Box 9001

Beer-Sheva, 84190, Israel

Phone: 972-7-6568736

Fax: 972-7-6567878

E-mail: rkavi@bgumail.bgu.ac.il

Robey, Harry F.

Lawrence Livermore National Laboratory

P.O. Box 808, L-21

Livermore, CA 94551

Phone: (925) 423-5669

Fax: (925) 422-3358

E-mail: robey1@llnl.gov

Robinson, A.

Sandia National Laboratory

P.O. Box 969, MS 9051

Livermore, CA 94551-0969

Rozanov, I. A.

Lebedev Physical Institute

Leninsky Pr. 53

117924, Moscow, Russia

E-mail: Rozanov@sci.lebedev.ru

Rosner, Robert L.

Enrico Fermi Institute and Laboratory for Astrophysics and Space Research

University of Chicago

933 East $56^{\text {th }}$ Street

Chicago, IL 60637

Phone: (773) 702-0560

Fax: (773) 704-3230

E-mail: rosner@uchicago.edu

Rothman, S.

AWE Aldermaston

Reading

Berkshire RG7 4PR United Kingdom

Roualdes, P.

Centre d'Etudes de Gramat

Gramat, France

Rozanov, Vladislav

Lebedev Physical Institute

Leninsky Pr. 53

117924, Moscow, Russia

Phone: (095)-132-68-47

Fax: (095)-132 -11-96

E-mail: lebo@neur.lpi.msk.su 
$8^{\text {th }}$ International Workshop on the Physics of Compressible Turbulent Mixing,

Pasadena, CA (2001)

Ryutov, Dimitri D.

Lawrence Livermore National Laboratory

P.O. Box 808 , L-630

Livermore, CA 94551

Phone: (925) 422-9832

E-mail: ryutov1@1lnl.gov

Sadot, Oren

Department of Physics

Nuclear Research Center - Negev

P.O. Box 9001

Beer-Sheva 84109, Israel

Phone: 972-7-6567278

Fax: 972-7-6567878

E-mail: sorens@bgumail.bgu.ac.il

Sakaiya, T.

Institute of Laser Engineering

Osaka University

2-6 Yamadaoka Suita

Osaka, 565 Japan

Samtaney, Ravi

Department of Aeronautics

California Institute of Technology

MS 205-45

Pasadena, CA 91125

Phone: (626) 395-8030

Fax: (626) 449-2677

E-mail: ravi@ama.caltech.edu

Sapozhnikov, I. V.

Russian Federal Nuclear Center-VNIIEF

Mir Avenue, 37, Sarov

Nizhnii Novgorod Region, 607190, Russia

Sarid, Eli

Department of Physics

Nuclear Research Center - Negev

P.O. Box 9001

Beer-Sheva, 84190, Israel

Phone: 972-7-6568736

Fax: $\quad 972-7-6567878$

E-mail: fnsarid@weizmann.weizmann.ac.il 
Sarkar, Sutanu

Department of Mechanical and Aerospace Engineering

University of California at San Diego

9500 Gilman Drive

La Jolla, CA 92093-0411

Phone: (858) 534-8243

Fax: (858) 534-7599

E-mail: sarkar@mae.ucsd.edu

Schilling, Oleg

Lawrence Livermore National Laboratory

P.O. Box 808, L-22

Livermore, CA 94551

Phone: (925) 423-6879

Fax: (925) 423-0925

E-mail: schilling1@1lnl.gov

Schurtz, G.

Commissariat à l'Energie Atomique

CEA/DRIF/DCSA/SET

BP12, 91680 Bruyeres-le-Chatel, France

Schwaederlè, Laurent

IUSTI

Technopole Chateau-Gombert

5 Rue Enrico Fermi

Marseille, 13013 France

Phone: 0491106935

Fax: 0491106969

E-Mail: schwaed@iusti.univ-mrs.fr

Sharp, David H.

Los Alamos National Laboratory

P.O. Box 1663, MS B285

Los Alamos, NM 87545

Phone: (505) 667-5266

Fax: (505) 665-3003

E-mail: dhs@lanl.gov

Shestachenko, Oleg

Russian Federal Nuclear Center-VNIITF

P.O. Box 245

456770 Snezhinsk, Russia

Fax: (351-72) 32077

E-mail: kucherenko@five.ch70.chel.su 
$8^{\text {th }}$ International Workshop on the Physics of Compressible Turbulent Mixing,

Pasadena, CA (2001)

Shestakov, Alex

Lawrence Livermore National Laboratory

P.O. Box 808, L-38

Livermore, CA 94551

Phone: (925) 422-4213

Fax: (925) 423-9208

E-mail: shestakov1@1lnl.gov

Shigemori, K.

Institute of Laser Engineering

Osaka University

2-6 Yamadaoka Suita

Osaka, 565 Japan

Shiraga, $\mathrm{H}$.

Institute of Laser Engineering

Osaka University

2-6 Yamadaoka Suita

Osaka, 565 Japan

Shu, Chi-Wang

Division of Applied Mathematics

Brown University

Providence, RI 02912

Phone: (401) 863-2549

E-mail: Chi-Wang_Shu@brown.edu

Shvarts, Dov

Nuclear Research Center - Negev

Department of Physics

P.O. Box 9001

Beer-Sheva, 84190, Israel

Phone: 972-7-6567500

Fax: 972-7-6567665

E-mail: shvarts@bgumail.bgu.ac.il

Silvani, X.

Institut de Mécanique des Fluides de Toulouse

31400 Toulouse

Toulouse, France

Sinitsina, S. N.

Russian Federal Nuclear Center-VNIIEF

Mir Avenue, 37, Sarov

Nizhnii Novgorod Region, 607190, Russia

E-mail: root@gdd.vniief.ru

Sinkova, O. G.

Russian Federal Nuclear Center-VNIIEF

Mir Avenue, 37, Sarov

Nizhnii Novgorod Region, 607190, Russia

E-mail: root@gdd.vniief.ru 
Skupsky, S.

Laboratory for Laser Energetics

University of Rochester

Rochester, NY 14623-1299

Smalyuk, V. A.

Laboratory for Laser Energetics

University of Rochester

Rochester, NY 14623-1299

Smith, Alan V.

Building H27

AWE Aldermaston

Reading

Berkshire RG7 4PR United Kingdom

Phone: 01189-825592

Fax: 01734-815320

E-mail: alan.smith@awe.co.uk

Sotskov, E.A.

Russian Federal Nuclear Center-VNIIEF

Mir Avenue, 37, Sarov

Nizhnii Novgorod Region, 607190, Russia

Fax: (831-30) 45958

E-mail: root@gdd.vniief.ru

Souffland, Denis

Commissariat à l'Energie Atomique

CEA/DRIF/DCSA/SET

BP12, 91680 Bruyeres-le-Chatel, France

Phone: 33169265977

Fax: $\quad 33169267094$

E-mail: soufflan@bruyeres.cea.fr

Srebro, Yair

Department of Physics

Ben-Gurion University of the Negev

Beer Sheva, 84105, Israel

Phone: 972-8-6568416

Fax: 972-8-656-7878

E-mail: sibo@bgumail.bgu.ac.il

Stadnik, A. L.

Russian Federal Nuclear Center-VNIIEF

Mir Avenue, 37, Sarov

Nizhnii Novgorod Region, 607190, Russia

E-mail: root@gdd.vniief.ru 
$8^{\text {th }}$ International Workshop on the Physics of Compressible Turbulent Mixing,

Pasadena, CA (2001)

Statsenko, V. P.

Russian Federal Nuclear Center-VNIIEF

Mir Avenue, 37, Sarov

Nizhnii Novgorod Region, 607190, Russia

E-mail: root@gdd.vniief.ru

Steinkamp, Michael J.

Los Alamos National Laboratory

P.O. Box 1663, MS D413

Los Alamos, NM 87545

Phone: (505) 667-5837

Fax: (505) 667-3726

E-mail: steinmj@lanl.gov

Stoeckl, C.

Laboratory for Laser Energetics

University of Rochester

Rochester, NY 14623-1299

Stone, J.

University of Maryland

College Park, MD 20742

Phone: (301) 405-1000

Sunahara, A.

Institute of Laser Engineering

Osaka University

2-6 Yamadaoka Suita

Osaka, 565 Japan

Phone: 81-6-879-8742

Fax: 81-6-877-4799

E-mail: suna@ile.osaka-u.ac.jp

Suter, Laurance

Lawrence Livermore National Laboratory

P.O. Box 808, L-31

Livermore, CA 94551

Phone: (925) 422-5423

Fax: (925) 423-9969

E-mail: suter1@1lnl.gov

Sviridov, E. V.

Russian Federal Nuclear Center-VNIITF

P.O. Box 245

456770 Snezhinsk, Russia 
Takabe, Hideaki

Institute of Laser Engineering

Osaka University

Yamada-oka 2-6, Suita

Osaka 565, Japan

E-mail: takabe@ile.osaka-u.ac.jp

Phone: 81-6-877-5111 Ext. 6553

Fax: $81-6-877-4779$

Takayama, Kazuyoshi

Shock Wave Research Center

Institute of Fluid Science

Tohoku University

2-1-1 Katahira, Aoba-ku

Sendai 980-77, Japan

Phone: 81-22-263-0895

Fax: $\quad 81-22-227-7390$

E-mail:paul@bellanca.ifs.tohoku.ac.jp

Tamari, Y.

Institute of Laser Engineering (ILE)

Osaka University

2-6 Yamadoaka

Suita, Osaka 565-0871, Japan

Teyssier, R.

Commissariat à l'Energie Atomique

BP 12, 91680

Bruyeres-le-Chatel, France

Timmes, F.

University of Chicago

933 East $56^{\text {th }}$ Street

Chicago, IL 60637

Tishkin, Vladimir

Institute for Mathematical Modeling

Russian Academy of Science

Miusskaya 4a

125047, Moscow, Russia

Phone: 007(095) 250-79-35

Fax: 007(095) 972-07-23

E-mail: tishkin@imamod.msk.su

Titov, S. N.

Krzhizhanovsky Power Engineering Institute ENIN, Leninsky pr. 19, GSP-1

Moscow, 117927 Russia 
$8^{\text {th }}$ International Workshop on the Physics of Compressible Turbulent Mixing,

Pasadena, CA (2001)

Tomkins, Christopher

Los Alamos National Laboratory

P.O. Box 1663, MS 0454

Los Alamos, NM 87545

Phone: (505) 665-6228

Fax: (505) 665-4507

E-mail: ctomkins@lanl.gov

Toqué, Nathalie

Universite de Montreal

\#1, 6685 Saint Denis

Montreal, Canada H-85-851

Phone: 514-276-9014

E-mail:Toque@astro.umontreal.ca

Tricottet, Matthieu

Commissariat à l'Energie Atomique

BP 12, 91680

Bruyeres-le-Chatel, France

E-mail: Matthieu.Tricottet@cea.fr

Tufo, $\mathrm{H}$.

University of Chicago

933 East $56^{\text {th }}$ Street

Chicago, IL 60637

Turano, Edward J.

Lawrence Livermore National Laboratory

P.O. Box 808, L-15

Livermore, CA 94551

Phone: (925) 424-3508

E-mail: turano1@1lnl.gov

Turner, N.

University of Maryland

College Park, MD 20742

Phone (301) 405-1000

Tyaktev, A. A.

Russian Federal Nuclear Center-VNIITF

P.O. Box 245

456770 Snezhinsk, Russia

Uchayev, A. Ya.

Russian Federal Nuclear Center-VNIIEF

Mir Avenue, 37, Sarov

Nizhnii Novgorod Region, 607190, Russia 
Vandenboomgaerde, Marc

Commissariat à l'Energie Atomique

BP 12, 91680

Bruyeres-le-Chatel, France

Phone: 330169266668

Fax: $\quad 330169267094$

E-mail: marc.vandenboomgaerde@ bruyeres.cea.fr

Verdon, Charles P.

Lawrence Livermore National Laboratory

P.O. Box 808, L-30

Livermore, CA 94551

Phone: (925) 423-4449

Fax: (925) 423-9969

E-mail: verdon1@1lnl.gov

Vlasov, Yu.

Russian Federal Nuclear Center-VNIIEF

Mir Avenue, 37, Sarov

Nizhnii Novgorod Region, 607190, Russia

Voelkl, Tobias

Department of Applied and Computational Mathematics

California Institute of Technology

$\mathrm{M} / \mathrm{C} 217-50$

Pasadena, CA 91125

Phone: (626) 395-8534

E-mail: tobias@its.caltech.edu

Vold, Erik L.

Los Alamos National Laboratory

P.O. Box 1663, MS D413

Los Alamos, NM 87545

Phone: (505) 665-5646

Fax: (505) 667-3726

E-mail: elv@lanl.gov

Volkov, V. I.

Russian Federal Nuclear Center-VNIITF

P.O. Box 245

Snezhinsk, 456770 Russia

E-mail: v.a.gordeychuk@vniitf.ru

Vorobieff, Peter V.

Department of Mechanical Engineering

University of New Mexico

ME Bldg. Rm. 424

Albuquerque, NM 87131

Phone: (505) 277-8347

Fax: (505) 277-1571

E-mail: kalmoth@me.unm.edu 
$8^{\text {th }}$ International Workshop on the Physics of Compressible Turbulent Mixing,

Pasadena, CA (2001)

Ward, David J.

Lawrence Livermore National Laboratory

P.O. Box 808, L-30

Livermore, CA 94551

Phone: (925) 423-3211

Fax: (925) 424-6764

E-mail: djw@1lnl.gov

Ward, Richard A.

Lawrence Livermore National Laboratory

P.O. Box 808, L-13

Livermore, CA 94551

Phone: (925) 423-2679

Fax: (925) 422-4643

E-mail: raward@1lnl.gov

Wark, J. S.

Oxford University

University Offices

Wellington, Oxford, OX1 2JD United Kingdom

Phone: +44 1865270000

Fax: $\quad+441865270708$

Weber, Stephen V.

Lawrence Livermore National Laboratory

P.O. Box 808, L-16

Livermore, CA 94551

Phone: (925) 422-5433

Fax: (925) 423-9208

E-mail: svweber@1lnl.gov

Wiley, Larry G.

Lawrence Livermore National Laboratory

P.O. Box 808, L-97

Livermore, CA 94551

Phone: (925) 423-2461

Fax: (925) 422-0779

E-mail: wiley1@1lnl.gov

Wilson, P. N.

Department of Mechanical Engineering

Texas A \& M University

MS 3123

College Station, TX 77843-3123

Wolfer, Wilhelm G.

Lawrence Livermore National Laboratory

P.O. Box 808, L-97

Livermore, CA 94551

Phone: (925) 423-1501

Fax: (925) 423-7040

E-mail: wolfer1@1lnl.gov 
Wouchuk, Juan G.

E.T.S.I. Industriales

Universidad Castilla-La Mancha

Campus Universitario s/n

13071 Ciudad Real, Spain

Phone: (34) 926-29-53-00 x3826

Fax: (34) 926-29-53-61

E-mail: gwouchuk@ind-cr.uclm.es

Wunsch, Scott E.

Sandia National Laboratory

P.O. Box 969, MS 9051

Livermore, CA 94551-0969

Phone: (925) 294-4621

Fax: (925) 294-2595

E-mail: sewunsch@sandia.gov

$\mathrm{Xu}, \mathrm{Z}$.

State University of New York at Stony Brook

P-138A Math Tower

Stony Brook, NY 11794-3600

Yamada, Shoichi

Institute of Laser Engineering (ILE)

Osaka University

2-6 Yamadoaka

Suita, Osaka 565-0871, Japan

Phone: 81-6-6879-8747

Fax: $\quad 81-6-6879-8747$

E-mail: syamada@ile.osaka-u.ac.jp

Yanilkin, Yury

Russian Federal Nuclear Center-VNIIEF

Mir Avenue, 37, Sarov

Nizhnii Novgorod Region, 607190, Russia

Phone: (831-30) 11190

Fax: (831-30) 54665

E-mail: yan@md08.vniief.ru

Yosef-Hai, Arnon

Department of Physics

Nuclear Research Center-Negev

P.O. Box 9001

Beer-Sheva, 84190, Israel

Phone: 972-8-6568913

Fax: 972-8-6567878

E-mail: ajoseph@bgumail.bgu.ac.il

Young, Y.-N.

University of Chicago

933 East $56^{\text {th }}$ Street

Chicago, IL 60637 
$8^{\text {th }}$ International Workshop on the Physics of Compressible Turbulent Mixing,

Pasadena, CA (2001)

Youngs, David L.

AWE

Building E3, AWE(A)

Reading, Berkshire

RG7 4PR, United Kingdom

Phone: 01189826766

Fax: 01189815320

E-mail: dyoungs@awe.co.uk

Zabusky, Norman J.

Department of Mechanical and Aerospace Engineering

Rutgers University

98 Brett Road

Piscataway, NJ 08854

Phone: (732) 445-5869

Fax: (732) 445-3124

E-mail: nzabusky@ caip.rutgers.edu

Zaytsev, Sergey G.

Krzhizhanovsky Power Engineering Institute

ENIN, Leninsky pr. 19, GSP-1

Moscow, 117927 Russia

Phone: (7-095) 955-31-07

Fax: (7-095) 954-42-50

E-mail: S.G.Zaytsev@mtu-net.ru

Zhakhovskii, V.

Institute of Laser Engineering

Osaka University

Suita, Osaka 565 Japan

Zhang, S.

Department of Mechanical and Aerospace Engineering

Rutgers University

98 Brett Road

Piscataway, NJ 08854

Zhang, Y.

State University of New York at Stony Brook

P-138A Math Tower

Stony Brook, NY 11794-3600

Zhmailo, Vadim A.

Russian Federal Nuclear Center-VNIIEF

Mir Avenue, 37, Sarov

Nizhnii Novgorod Region, 607190, Russia

Fax: 8313054565

E-mail:rvg_092C@rfnc.nnov.su 
Zhou, Ye K.

Lawrence Livermore National Laboratory

P.O. Box 808, L-23

Livermore, CA 94551

Phone: (925) 424-3624

E-mail: zhou3@1lnl.gov

Zingdale, N. V.

University of Chicago

933 East $56^{\text {th }}$ Street

Chicago, IL 60637

Zmitrenko, Nikolay

Institute for Mathematical Modeling

Russian Academy of Science

Miusskaya 4a, 125047, Moscow, Russia

Phone: 007(095) 250-79-16

Fax: 007(095) 972-07-23

E-mail: nickzmitł\#9@imamod.msk.su

Zoldi, Cindy A.

Los Alamos National Laboratory

P.O. Box 1663, MS B220

Los Alamos, NM 87544

Phone: (505) 667-6455

Fax: (505) 665-2227

E-mail: czoldi@lanl.gov

Zvorykin, V. D.

PN Lebedev Physics Institute

Leninsky prospect 53

117294 Russia, Moscow 


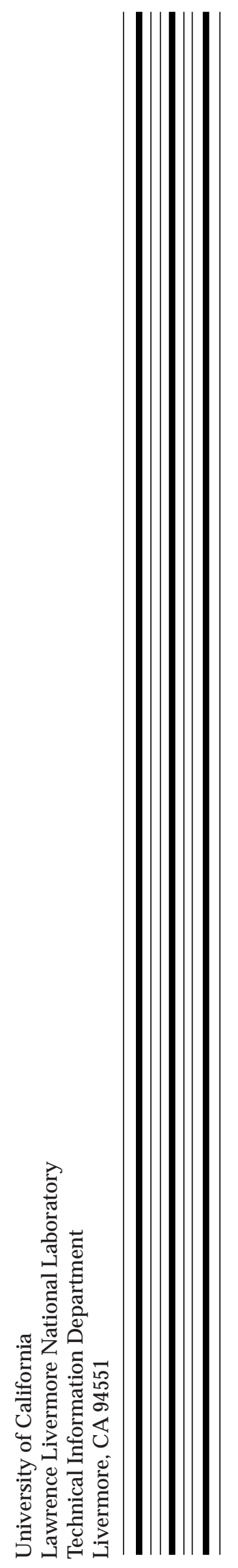

